\title{
Beyond DP4: an improved probability for the stereochemical assignment of isomeric compounds using quantum chemical calculations of NMR shifts
}

\author{
Nicolás Grimblat, ${ }^{1}$ María M. Zanardi ${ }^{1,2}$ and Ariel M. Sarotti ${ }^{1, *}$
}

sarotti@iquir-conicet.gov.ar

1) Instituto de Química Rosario (CONICET), Facultad de Ciencias Bioquímicas y Farmacéuticas, Universidad Nacional de Rosario, Suipacha 531, Rosario (2000), Argentina.

2) Facultad de Química e Ingeniería "Fray Rogelio Bacón", Pontificia Universidad Católica Argentina, Av. Pellegrini 3314, Rosario (2000), Argentina.

\section{Supporting Information}

\section{Table of contents}

- Instructions for using the Excel file to compute the DP4+ probability (with tutorial). Pages S2-S5.

- Complete set of compounds 1-72 used to compute the $[\mu, \sigma, v]$ statistical parameters. Page S6.

- Experimental chemical shifts, and Boltzmann averaged GIAO isotropic magnetic shielding values calculated for B3LYP/6-31G* geometries of compounds 1-72 at the 24 levels of theory under study. Pages S7-S147.

- Values of the $[\mu, \sigma, v]$ statistical parameters at the 24 levels of theory. Page S148.

- Experimental chemical shifts, and Boltzmann averaged GIAO isotropic magnetic shielding values calculated for B3LYP/6-31G* geometries of compounds 73-90 at the 24 levels of theory under study. Pages S149-S233.

- sDP4+, uDP4+ and DP4+ probabilities (\%) for compounds 73-90 at the 24 levels of theory. Pages S234-S473.

- Performance evaluation of all DP4+ probabilities. Pages S474-S478. 


\section{Instructions for using the Excel file to compute the DP4+ probability}

- Despite the DP4+ calculations can be made "by hand", an Excel file is provided from the authors as part of the Supporting Information of this paper that considerably simplifies the procedure.

- The file contains 2 sheets: "Main" and "Detailed Results" (Figure S1).

- The "Main" sheet is divided in 3 different zones: A, B and C.

Zone A

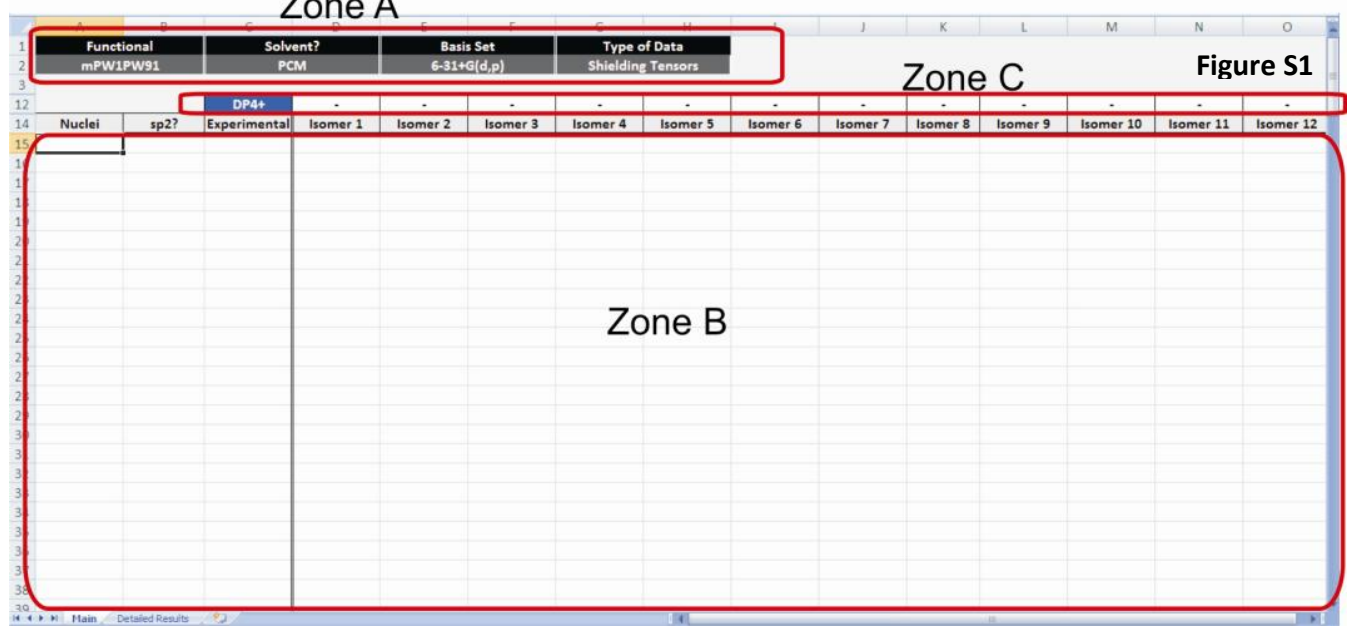

Zone A: user selection (drop down lists):

- Functional: functional used to perform the GIAO NMR calculations (B3LYP or mPW1PW91).

- Solvent?: inclusion of solvent effect in the GIAO NMR calculations (PCM or Gas Phase).

- Basis Set: basis set used in the GIAO NMR calculations (6-31G*, 6-31G**, 6-31+G**, 6-311G*, 6-311G** or 6-311+G**)

- Type of Data: indicates the type of data entered in Zone B (Shielding Tensors, Unscaled Shifts or Scaled Shifts).

Zone B: enabled cells to enter the data:

- Nuclei: enter the letter "C" (without quotes) for ${ }^{13} \mathrm{C}$ data or the letter "H" (without quotes) for ${ }^{1} \mathrm{H}$ data.

- sp2?: enter the letter " $\mathrm{x}$ " (without quotes) if the carbon is $\mathrm{sp}$-sp ${ }^{2}$ hybridized (or if the proton is attached to an $\mathrm{sp}$-sp ${ }^{2}$ hybridized carbon).

- Experimental: enter the experimental ${ }^{13} \mathrm{C}$ and/or ${ }^{1} \mathrm{H}$ chemical shifts.

- Isomer 1, Isomer 2, etc: enter the computed data for the candidate structures.

Zone C: displays the DP4+ probabilities once the data is entered in Zone B.

Tutorial: compounds 78a-d (four candidates). NMR data from: 78b (correct isomer).<smiles>CCC[C@H]1O[C@@H](c2ccccc2)C[C@H]1O</smiles><smiles>CCC[C@H]1O[C@@H](c2ccccc2)C[C@H]1O</smiles><smiles>CCC[C@H]1O[C@@H](c2ccccc2)CC1O</smiles><smiles>CCC[C@H]1O[C@@H](c2ccccc2)C[C@H]1O</smiles>

Step 1: perform a conformational search using a suitable package (Hyperchem, Spartan, MacroModel, etc).

Step 2: optimize the most significant conformations at the B3LYP/6-31G* level (gas phase).

Step 3: perform the NMR GIAO calculations at the selected level of theory (in this case, at the PCM/mPW1PW91/6-31G**) for all representative conformations.

Step 4: compute all the Boltzmann-averaged shielding tensors values for each compound.

Step 5: open the Excel file.

Step 6: select the correct level of theory that was used to perform the NMR shift calculation (Figure S2). In this particular case, $\mathrm{PCM} / \mathrm{mPW} 1 \mathrm{PW} 91 / 6-31 \mathrm{G} * *$. 


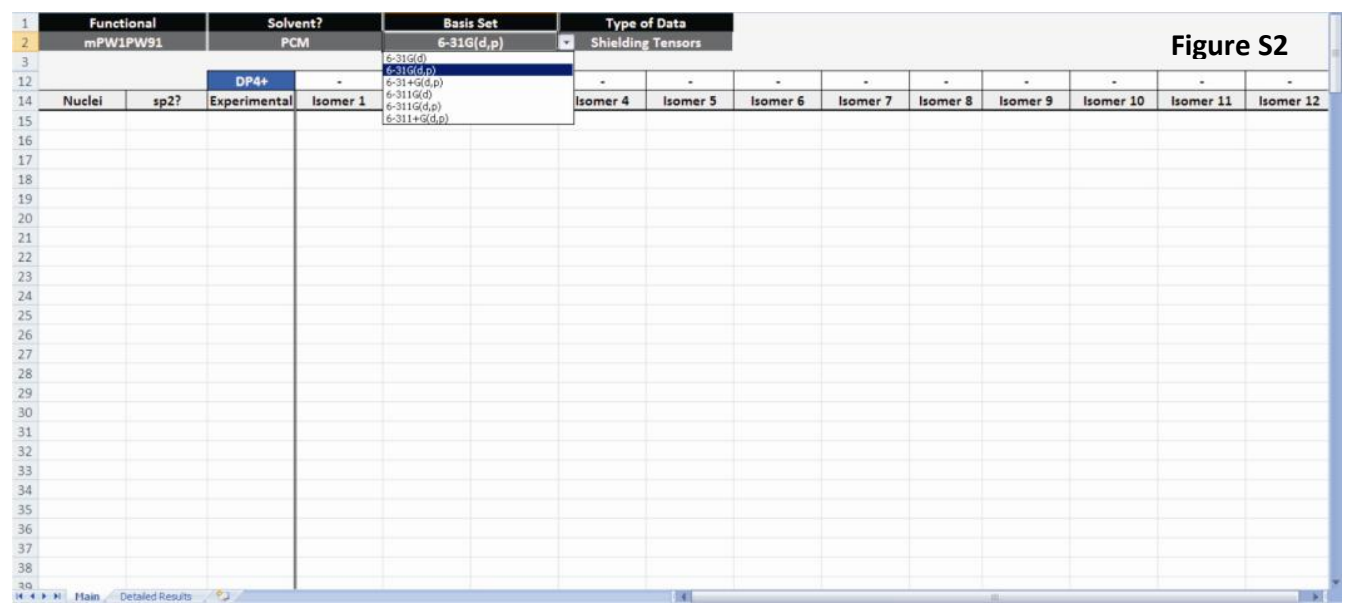

Step 7: Introduce the experimental data (Figure S3). Important: mark the sp ${ }^{2}$ nuclei.

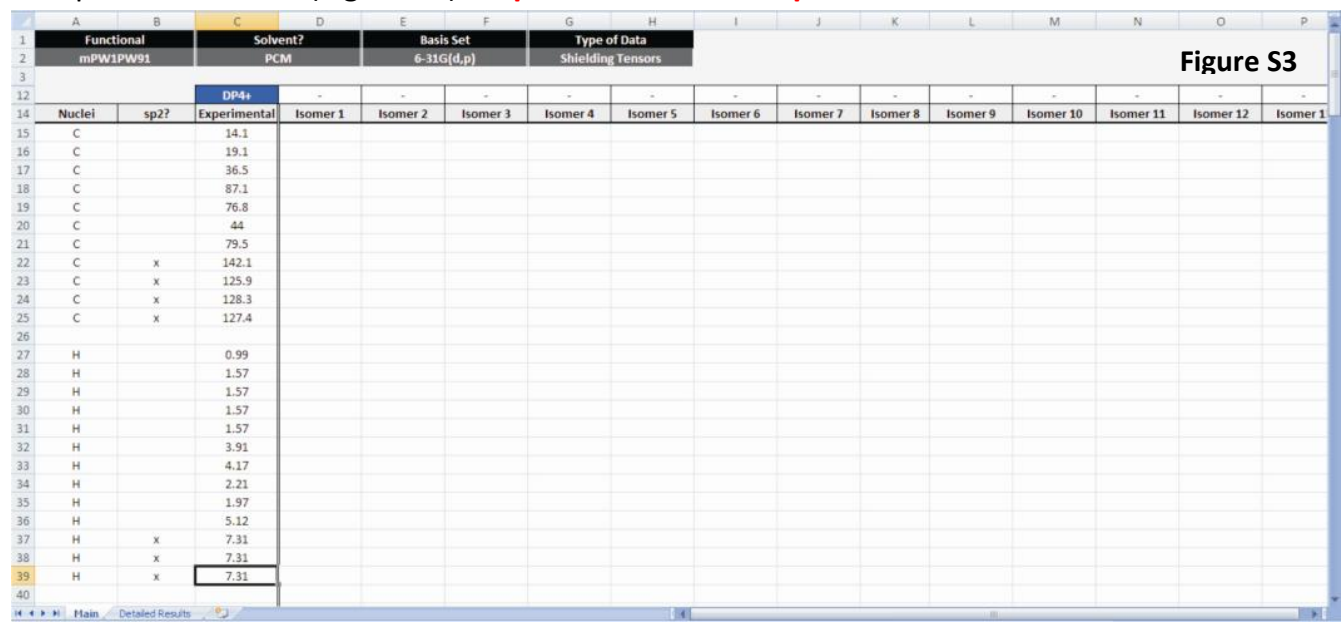

Step 8: Introduce the calculated shielding tensors for each possible candidate structure (4 in this case) (Figure S4). The DP4+ values are automatically calculated and displayed for each isomer above the number of isomer. Important: the data must be assigned (know which shift corresponds to which nuclei).

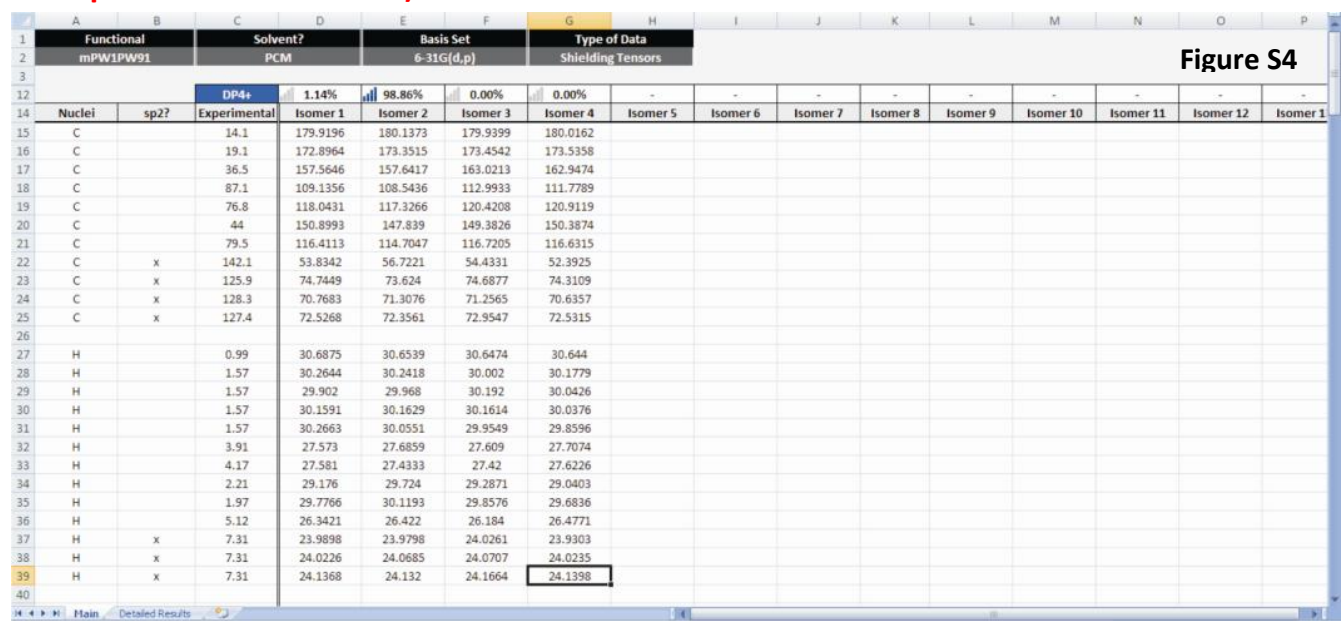


Step 9: The probabilities computed using unscaled ( $u \mathrm{DP} 4+)$ and scaled (sDP4+), as well as using carbon and/or proton data, is shown in the sheet "Detailed Results" (Figure S5)

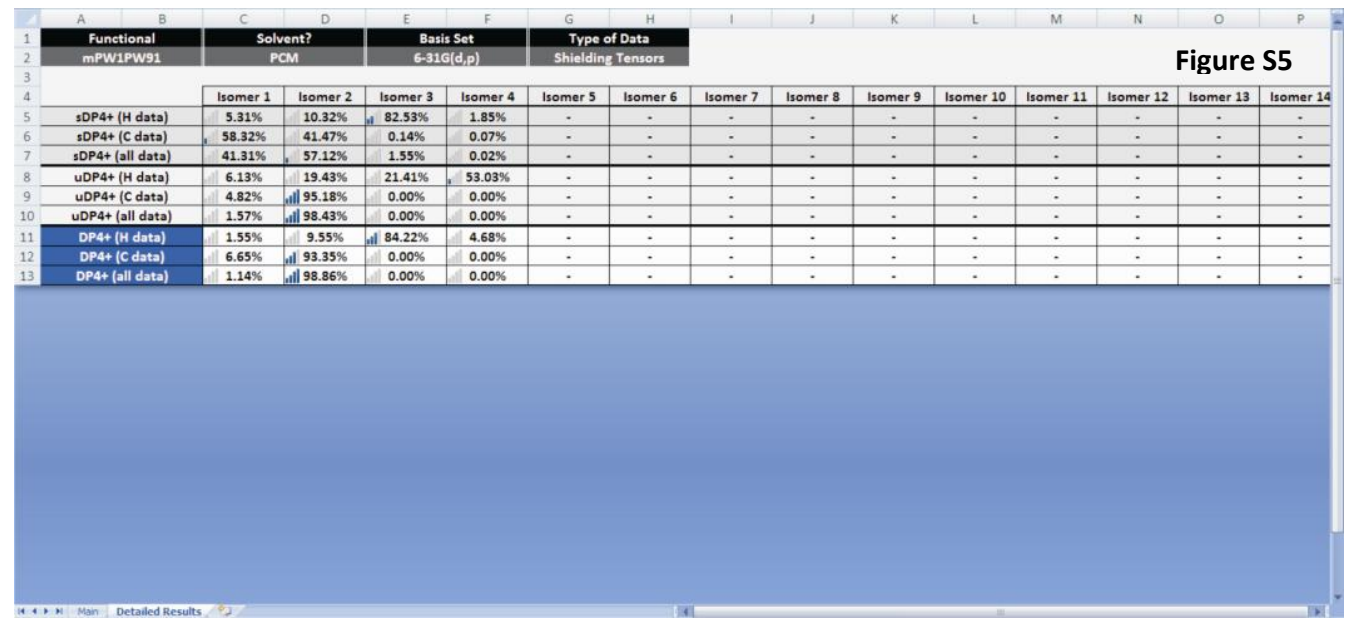

Note 1: the same results are obtained using unscaled shifts (Figure S6).

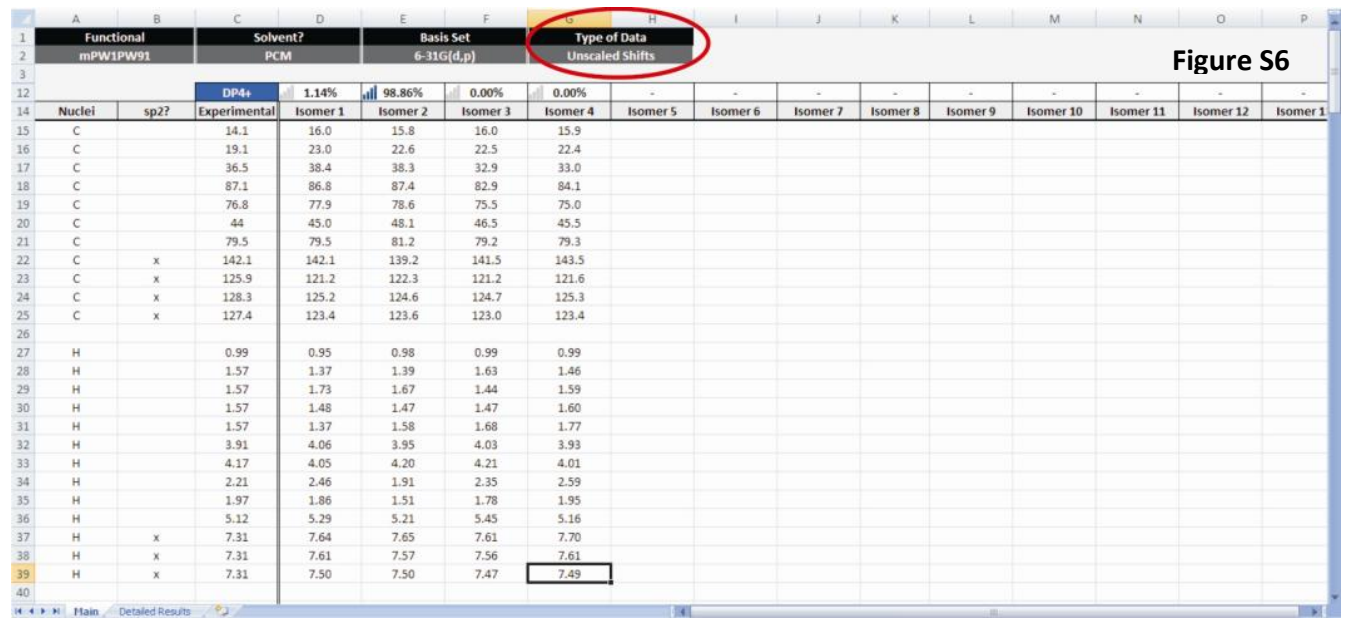

Note 2: if scaled shifts are introduced, the Excel file cannot compute the DP4+ probability (Figure S7), but instead the sDP4+ values can be seen in the "Detailed Results" sheet (Figure S8).

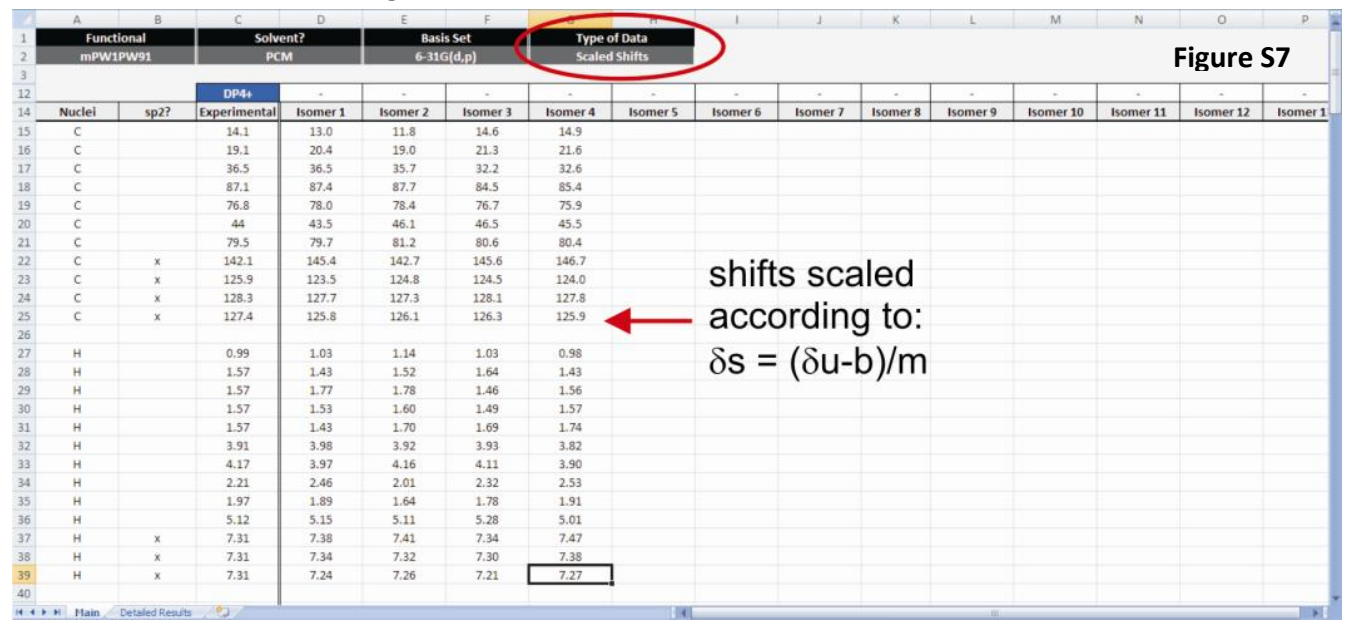




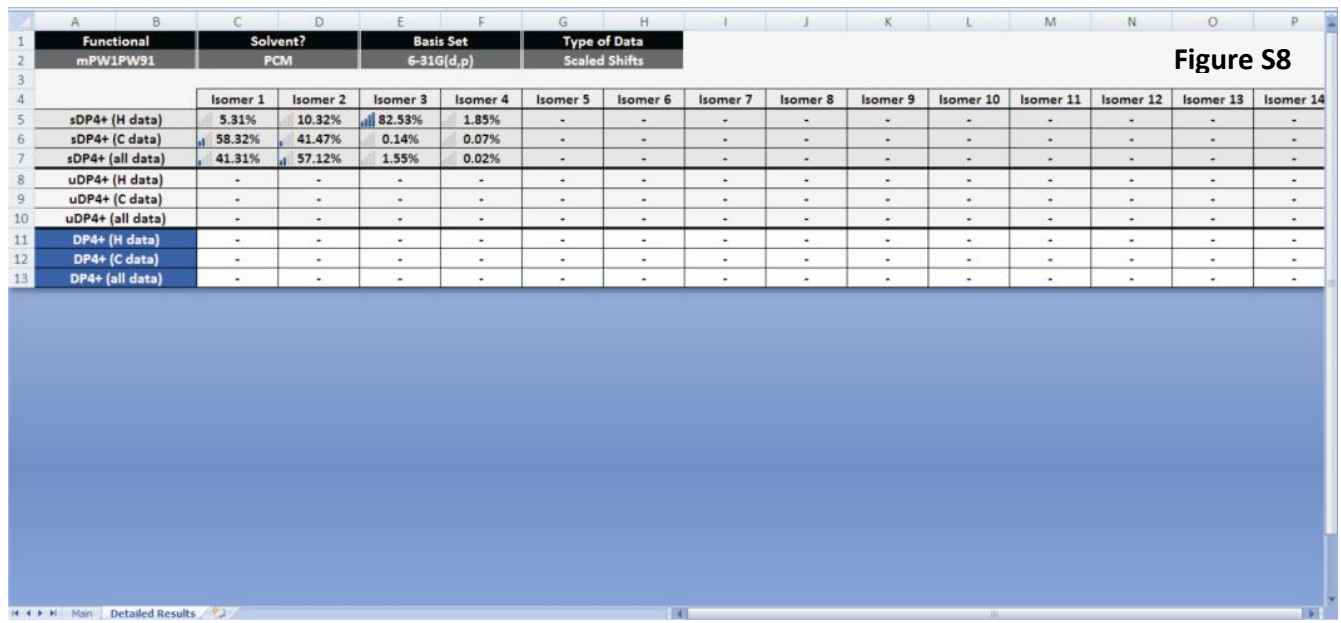




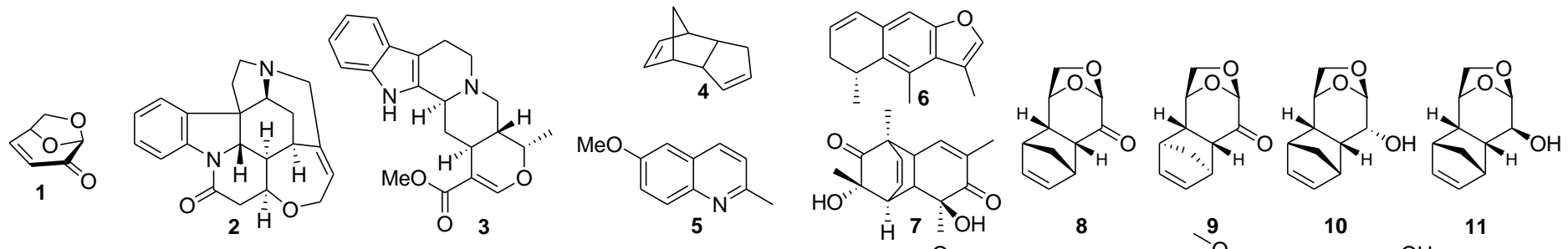

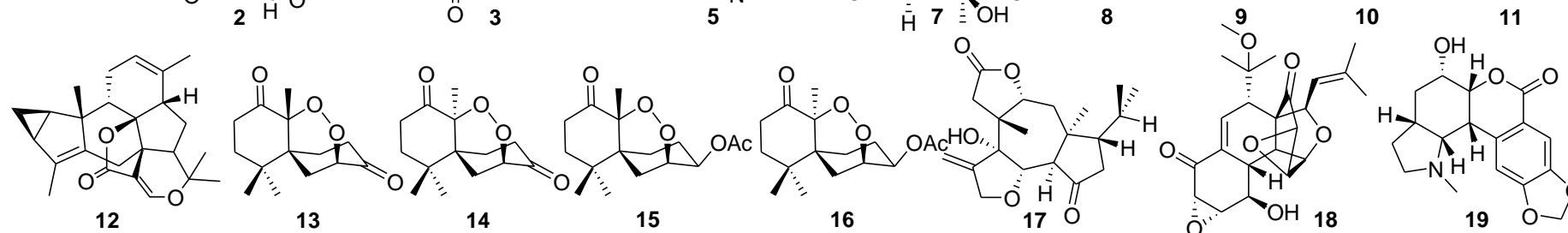

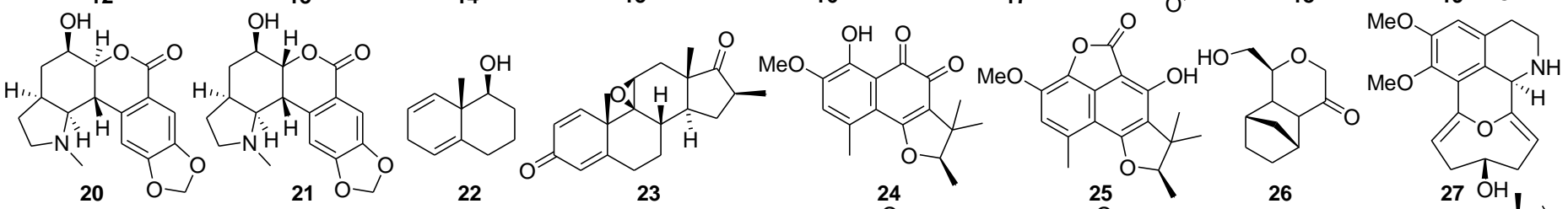
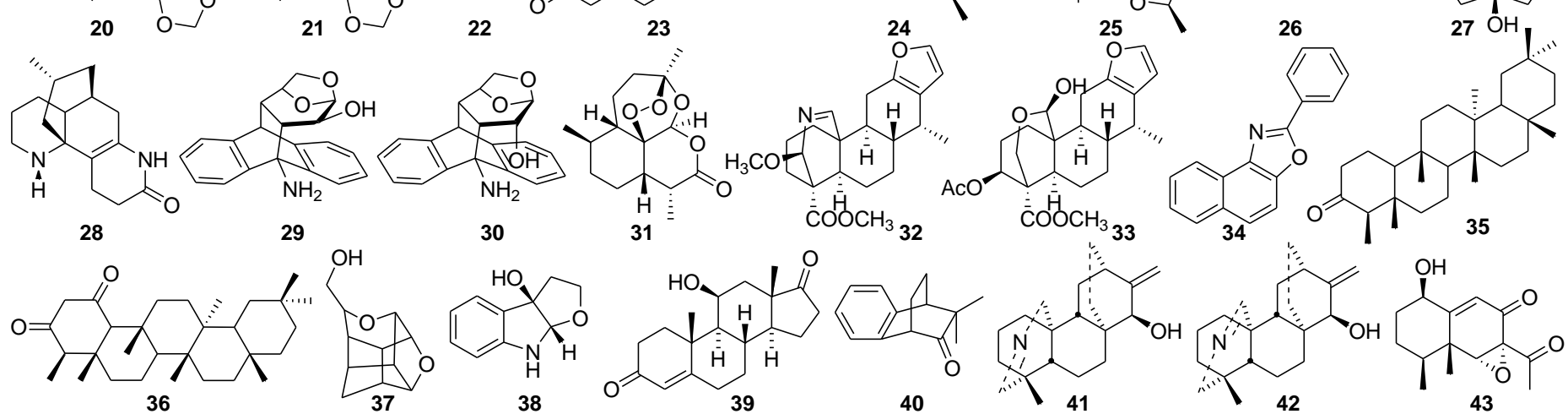

36

38

39

40

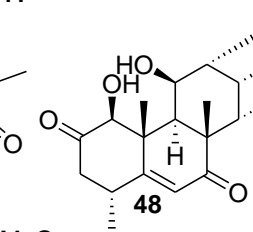

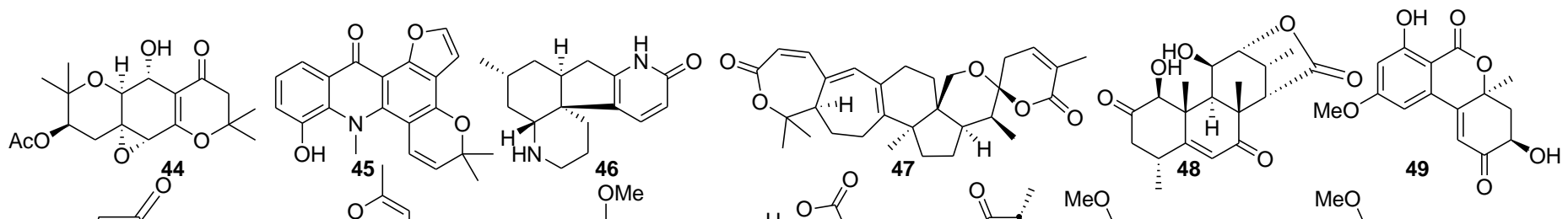

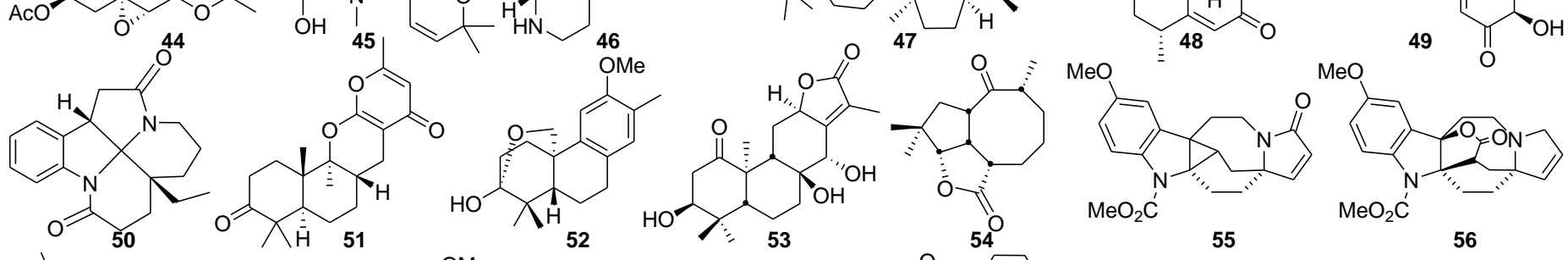

(1)

(1)

56

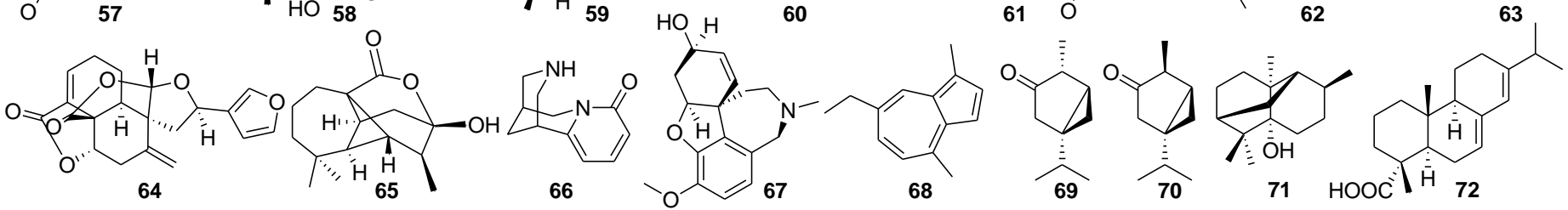

Figure S9. Complete set of compounds (1-72) used to compute the $[\mu, \sigma, v]$ statistical parameters. 


\section{Compound 1}

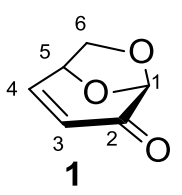

\begin{tabular}{|c|c|c|c|c|c|c|c|c|c|c|c|c|c|}
\hline & \multirow[b]{3}{*}{ Exp } & \multicolumn{12}{|c|}{ B3LYP } \\
\hline & & \multicolumn{6}{|c|}{ Gas phase } & \multicolumn{6}{|c|}{ PCM } \\
\hline & & 6-31G* & $6-31 G * *$ & $6-31+G * *$ & 6-311G* & $6-311 G * *$ & $6-311+G * *$ & 6-31G* & $6-31 G * *$ & $6-31+G * *$ & 6-311G* & $6-311 G * *$ & $6-311+G * *$ \\
\hline \multicolumn{14}{|c|}{${ }^{13} \mathrm{C}$} \\
\hline 2 & 188.6 & 9.8818 & 10.0285 & 8.3177 & -8.3684 & -8.3790 & -12.3137 & 8.0507 & 8.1918 & 5.9922 & -10.5490 & -10.5607 & -15.0056 \\
\hline 4 & 148.1 & 48.8330 & 49.4415 & 48.2215 & 30.2434 & 29.3234 & 27.7745 & 44.9689 & 45.5763 & 43.6916 & 25.7660 & 24.8621 & 22.8675 \\
\hline 3 & 126.5 & 66.3646 & 66.7892 & 66.2640 & 48.7397 & 47.8730 & 47.1995 & 67.3532 & 67.7784 & 67.4183 & 49.9138 & 49.0698 & 48.3769 \\
\hline 1 & 101.4 & 88.5099 & 89.2210 & 87.7433 & 75.2300 & 74.5432 & 74.7918 & 88.7542 & 89.4616 & 87.8959 & 75.4004 & 74.7162 & 74.9114 \\
\hline 5 & 71.5 & 116.8430 & 117.6813 & 116.8268 & 106.1462 & 105.5951 & 105.4601 & 116.7127 & 117.5463 & 116.5987 & 105.9181 & 105.3525 & 105.1632 \\
\hline 6 & 66.3 & 123.5105 & 124.8472 & 124.6554 & 113.8143 & 113.1225 & 112.6648 & 124.1203 & 125.4436 & 125.1352 & 114.3101 & 113.5959 & 113.1623 \\
\hline \multicolumn{14}{|c|}{${ }^{1} \mathrm{H}$} \\
\hline 1 & 5.36 & 27.1190 & 26.5477 & 26.4234 & 27.0631 & 26.7499 & 26.7241 & 27.1071 & 26.5306 & 26.3933 & 27.0307 & 26.7170 & 26.6860 \\
\hline 3 & 6.12 & 26.3026 & 25.5955 & 25.4801 & 26.2467 & 25.7809 & 25.7014 & 26.2505 & 25.5364 & 25.4118 & 26.1937 & 25.7272 & 25.6378 \\
\hline 4 & 7.27 & 25.2230 & 24.5273 & 24.3196 & 25.1193 & 24.6296 & 24.5869 & 24.8704 & 24.1771 & 23.9348 & 24.7641 & 24.2839 & 24.2087 \\
\hline 5 & 5.00 & 27.7494 & 27.1704 & 26.9875 & 27.6718 & 27.3029 & 27.2715 & 27.5052 & 26.9169 & 26.7079 & 27.4223 & 27.0464 & 26.9951 \\
\hline 6 & 3.77 & 28.6379 & 28.1031 & 27.9762 & 28.6299 & 28.3120 & 28.2252 & 28.5320 & 27.9921 & 27.8520 & 28.5252 & 28.2012 & 28.1024 \\
\hline 6 & 3.90 & 28.4424 & 27.9020 & 27.6977 & 28.3510 & 28.0463 & 27.9599 & 28.3369 & 27.7974 & 27.5715 & 28.2397 & 27.9387 & 27.8355 \\
\hline
\end{tabular}

\begin{tabular}{|c|c|c|c|c|c|c|c|c|c|c|c|c|c|}
\hline & & \multicolumn{12}{|c|}{ mPW1PW91 } \\
\hline & \multirow[b]{2}{*}{ Exp } & \multicolumn{6}{|c|}{ Gas phase } & \multicolumn{6}{|c|}{ PCM } \\
\hline & & 6-31G* & 6-31G** & $6-31+G * *$ & 6-311G* & $6-311 G * *$ & $6-311+G * *$ & 6-31G* & $6-31 G * *$ & $6-31+G * *$ & 6-311G* & $6-311 G * *$ & $6-311+G * *$ \\
\hline \multicolumn{14}{|c|}{${ }^{13} \mathrm{C}$} \\
\hline 2 & 188.6 & 11.7650 & 11.8990 & 10.8844 & -3.6925 & -3.7103 & -7.2668 & 9.8538 & 9.9809 & 8.5184 & -5.9117 & -5.9319 & -9.9444 \\
\hline 4 & 148.1 & 51.3370 & 51.8558 & 50.7585 & 34.8948 & 33.9338 & 32.5415 & 47.3149 & 47.8313 & 46.1456 & 30.3601 & 29.4147 & 27.6347 \\
\hline 3 & 126.5 & 68.8678 & 69.2305 & 68.7595 & 53.4219 & 52.5248 & 51.8998 & 69.9168 & 70.2828 & 69.9655 & 54.6090 & 53.7395 & 53.1060 \\
\hline 1 & 101.4 & 92.9187 & 93.6070 & 92.4936 & 81.9174 & 81.2512 & 81.3817 & 93.1661 & 93.8509 & 92.6583 & 82.0807 & 81.4175 & 81.4990 \\
\hline 5 & 71.5 & 121.2405 & 122.0256 & 121.3879 & 112.0163 & 111.4700 & 111.2987 & 121.0702 & 121.8516 & 121.1246 & 111.7454 & 111.1871 & 110.9716 \\
\hline 6 & 66.3 & 127.5267 & 128.7905 & 128.8792 & 119.2124 & 118.5485 & 118.0821 & 128.1067 & 129.3592 & 129.3195 & 119.6601 & 118.9761 & 118.5280 \\
\hline \multicolumn{14}{|c|}{ ( } \\
\hline 1 & 5.36 & 27.0041 & 26.4385 & 26.3414 & 27.0023 & 26.6549 & 26.6457 & 26.9856 & 26.4145 & 26.3059 & 26.9648 & 26.6174 & 26.6038 \\
\hline 3 & 6.12 & 26.0798 & 25.3740 & 25.2858 & 26.0720 & 25.5726 & 25.5069 & 26.0225 & 25.3098 & 25.2139 & 26.0161 & 25.5165 & 25.4411 \\
\hline 4 & 7.27 & 25.0322 & 24.3332 & 24.1557 & 24.9587 & 24.4365 & 24.4005 & 24.6611 & 23.9641 & 23.7576 & 24.5901 & 24.0769 & 24.0126 \\
\hline 5 & 5.00 & 27.6477 & 27.0674 & 26.9100 & 27.5769 & 27.1885 & 27.1702 & 27.3905 & 26.7998 & 26.6213 & 27.3185 & 26.9226 & 26.8870 \\
\hline 6 & 3.77 & 28.5744 & 28.0396 & 27.9312 & 28.5574 & 28.2261 & 28.1494 & 28.4608 & 27.9205 & 27.8022 & 28.4483 & 28.1105 & 28.0235 \\
\hline 6 & 3.90 & 28.3816 & 27.8439 & 27.6626 & 28.2922 & 27.9767 & 27.9031 & 28.2634 & 27.7262 & 27.5260 & 28.1716 & 27.8596 & 27.7706 \\
\hline
\end{tabular}




\section{Compound 2}

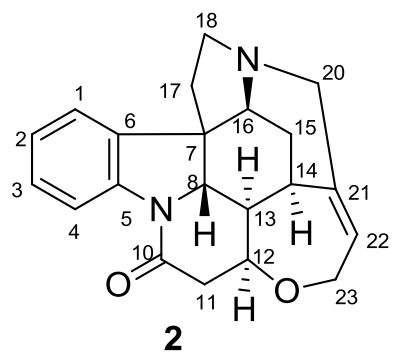

\begin{tabular}{|c|c|c|c|c|c|c|c|c|c|c|c|c|c|}
\hline & \multirow[b]{3}{*}{ Exp } & \multicolumn{12}{|c|}{ B3LYP } \\
\hline & & \multicolumn{6}{|c|}{ Gas phase } & \multicolumn{6}{|c|}{ PCM } \\
\hline & & 6-31G* & 6-31G** & $6-31+G * *$ & 6-311G* & 6-311G** & $6-311+G * *$ & 6-31G* & $6-31 G * *$ & $6-31+\mathrm{G} * *$ & 6-311G* & 6-311G** & $6-311+G^{* *}$ \\
\hline \multicolumn{14}{|l|}{${ }^{13} \mathrm{C}$} \\
\hline 1 & 122.2 & 74.2906 & 74.9682 & 74.0708 & 57.9054 & 57.1145 & 56.4324 & 73.3809 & 74.0452 & 72.9871 & 56.6999 & 55.9066 & 55.1208 \\
\hline 2 & 124.2 & 73.1252 & 73.7344 & 73.4344 & 56.2475 & 55.3579 & 54.5740 & 72.8770 & 73.4878 & 73.0806 & 55.9140 & 55.0532 & 54.2451 \\
\hline 3 & 128.5 & 67.4046 & 68.0092 & 67.6181 & 50.4523 & 49.5492 & 48.7949 & 67.9267 & 68.5328 & 68.1591 & 50.9272 & 50.0547 & 49.4012 \\
\hline 4 & 116.2 & 78.5650 & 79.1821 & 78.6554 & 62.4959 & 61.6607 & 59.9007 & 79.6824 & 80.3081 & 79.8394 & 63.7867 & 62.9775 & 61.2112 \\
\hline 5 & 142.2 & 52.5504 & 52.4744 & 51.1477 & 33.3506 & 33.2401 & 32.9697 & 52.6598 & 52.5785 & 51.2020 & 33.3954 & 33.2853 & 32.9776 \\
\hline 6 & 132.8 & 64.0494 & 63.8720 & 62.0848 & 44.9034 & 44.6940 & 43.6520 & 62.5946 & 62.4165 & 60.4733 & 43.2763 & 43.0804 & 41.9381 \\
\hline 7 & 51.9 & 135.2377 & 135.4622 & 135.3071 & 124.2562 & 124.2788 & 124.0568 & 135.2214 & 135.4434 & 135.3096 & 124.2401 & 124.2615 & 124.0050 \\
\hline 8 & 60.1 & 128.3966 & 129.1052 & 128.2554 & 117.9789 & 117.5965 & 117.1819 & 128.2136 & 128.9178 & 128.0977 & 117.8017 & 117.4142 & 116.9707 \\
\hline 10 & 169.3 & 31.1468 & 31.3289 & 29.5688 & 11.4076 & 11.5444 & 8.9658 & 29.3195 & 29.4983 & 27.4111 & 9.2280 & 9.3613 & 6.5030 \\
\hline 11 & 42.5 & 146.4474 & 147.6738 & 146.6091 & 136.2320 & 135.5995 & 135.1926 & 146.2620 & 147.4798 & 146.2380 & 136.0061 & 135.3631 & 134.7827 \\
\hline 12 & 77.6 & 111.7248 & 112.4905 & 111.2194 & 99.4292 & 99.0366 & 98.6798 & 112.2750 & 113.0390 & 111.8569 & 100.1161 & 99.7190 & 99.3744 \\
\hline 13 & 48.2 & 140.4402 & 141.2135 & 141.1294 & 130.1719 & 129.9006 & 129.1930 & 140.5425 & 141.3115 & 141.3160 & 130.3038 & 130.0323 & 129.3367 \\
\hline 14 & 31.6 & 154.9767 & 155.7426 & 154.3037 & 145.1402 & 144.8788 & 144.8230 & 155.2256 & 155.9893 & 154.6448 & 145.4754 & 145.2095 & 145.1624 \\
\hline 15 & 26.9 & 160.5885 & 161.9693 & 160.9409 & 152.5734 & 152.2914 & 152.0593 & 160.8433 & 162.2171 & 161.2057 & 152.8500 & 152.5582 & 152.3615 \\
\hline 16 & 60.2 & 128.4861 & 129.1674 & 128.0247 & 116.9473 & 116.6498 & 116.4968 & 128.4460 & 129.1237 & 127.9571 & 116.8675 & 116.5675 & 116.4219 \\
\hline 17 & 42.7 & 145.2537 & 146.5716 & 146.2417 & 136.1480 & 135.7304 & 135.8414 & 145.3358 & 146.6438 & 146.2834 & 136.1840 & 135.7590 & 135.8933 \\
\hline 18 & 50.3 & 139.8140 & 141.1624 & 140.3822 & 130.5507 & 130.0355 & 129.8518 & 139.8216 & 141.1687 & 140.3233 & 130.5289 & 130.0025 & 129.7995 \\
\hline 20 & 52.9 & 137.2386 & 138.4727 & 136.9727 & 127.2395 & 126.5816 & 125.9090 & 137.7162 & 138.9396 & 137.4701 & 127.7505 & 127.0852 & 126.4130 \\
\hline 21 & 140.6 & 52.4880 & 52.3516 & 50.0693 & 31.3877 & 31.0126 & 28.2832 & 52.1941 & 52.0616 & 49.6659 & 31.0352 & 30.6753 & 27.8489 \\
\hline 22 & 127.2 & 66.9073 & 67.7594 & 67.6401 & 49.9815 & 49.5684 & 48.3575 & 66.8566 & 67.7035 & 67.6095 & 49.8855 & 49.4750 & 48.2807 \\
\hline $\begin{array}{l}23 \\
{ }^{\mathbf{1}} \mathbf{H}\end{array}$ & 64.6 & 125.5978 & 126.9259 & 125.8356 & 115.2492 & 114.5057 & 114.2207 & 125.8385 & 127.1553 & 125.9774 & 115.4325 & 114.6759 & 114.3691 \\
\hline 1 & 7.16 & 25.2635 & 24.5926 & 24.3715 & 25.2219 & 24.7756 & 24.6298 & 25.1046 & 24.4329 & 24.1891 & 25.0452 & 24.6030 & 24.4461 \\
\hline 2 & 7.10 & 25.3462 & 24.7175 & 24.4473 & 25.2163 & 24.8124 & 24.7311 & 25.2339 & 24.6056 & 24.3304 & 25.0937 & 24.6973 & 24.6099 \\
\hline 3 & 7.25 & 25.1173 & 24.5138 & 24.3763 & 24.9621 & 24.6038 & 24.5106 & 25.0583 & 24.4553 & 24.3120 & 24.8951 & 24.5451 & 24.4452 \\
\hline 4 & 8.09 & 24.1327 & 23.3423 & 23.0988 & 23.9807 & 23.4656 & 23.2959 & 24.2687 & 23.4799 & 23.2348 & 24.1074 & 23.5963 & 23.4244 \\
\hline 8 & 3.85 & 28.2795 & 27.8386 & 27.7639 & 28.4479 & 28.2089 & 28.0938 & 28.2419 & 27.7982 & 27.7130 & 28.4054 & 28.1635 & 28.0414 \\
\hline 12 & 4.28 & 28.1239 & 27.7030 & 27.6284 & 28.0671 & 27.8413 & 27.8190 & 27.9969 & 27.5722 & 27.4797 & 27.9317 & 27.7054 & 27.6725 \\
\hline 13 & 1.27 & 31.1231 & 30.8279 & 30.7697 & 31.1977 & 31.0851 & 30.9986 & 31.0448 & 30.7476 & 30.6807 & 31.1191 & 31.0064 & 30.9113 \\
\hline 14 & 3.15 & 29.3707 & 28.8921 & 28.8132 & 29.3393 & 29.0530 & 29.0213 & 29.2360 & 28.7529 & 28.6710 & 29.2077 & 28.9176 & 28.8820 \\
\hline 16 & 3.93 & 28.3374 & 27.8697 & 27.6281 & 28.3003 & 28.0621 & 27.9230 & 28.3095 & 27.8405 & 27.5744 & 28.2561 & 28.0171 & 27.8653 \\
\hline 22 & 5.90 & 26.4717 & 25.8105 & 25.4155 & 26.3946 & 25.9054 & 25.7481 & 26.3933 & 25.7343 & 25.3338 & 26.3087 & 25.8297 & 25.6698 \\
\hline $11 a$ & 3.11 & 29.4483 & 28.9397 & 28.6827 & 29.2649 & 28.9609 & 28.8576 & 29.4569 & 28.9409 & 28.6821 & 29.2666 & 28.9580 & 28.8559 \\
\hline
\end{tabular}




\begin{tabular}{|c|c|c|c|c|c|c|c|c|c|c|c|c|c|}
\hline $11 b$ & 2.67 & 29.5501 & 29.0997 & 28.9659 & 29.5110 & 29.2518 & 29.2021 & 29.5478 & 29.0947 & 28.9589 & 29.5110 & 29.2497 & 29.1956 \\
\hline $15 a$ & 2.35 & 30.0228 & 29.6037 & 29.4398 & 29.9619 & 29.7632 & 29.7282 & 29.9405 & 29.5182 & 29.3478 & 29.8771 & 29.6774 & 29.6387 \\
\hline $15 b$ & 1.45 & 30.7970 & 30.4000 & 30.3547 & 30.7982 & 30.5999 & 30.5838 & 30.7723 & 30.3736 & 30.3288 & 30.7812 & 30.5809 & 30.5599 \\
\hline & 1.88 & 30.3927 & 29.9611 & 29.8594 & 30.4158 & 30.1761 & 30.0968 & 30.3439 & 29.9084 & 29.7926 & 30.3658 & 30.1224 & 30.0334 \\
\hline $17 b$ & 1.89 & 30.2846 & 29.8923 & 29.8457 & 30.2527 & 30.0823 & 30.0146 & 30.2540 & 29.8600 & 29.7937 & 30.2165 & 30.0453 & 29.9665 \\
\hline $18 a$ & 3.19 & 29.1965 & 28.6843 & 28.4743 & 29.1219 & 28.8154 & 28.7774 & 29.1753 & 28.6626 & 28.4351 & 29.0920 & 28.7858 & 28.7398 \\
\hline $18 b$ & 2.87 & 29.2487 & 28.7553 & 28.6778 & 29.3726 & 29.0802 & 29.0083 & 29.2108 & 28.7178 & 28.6282 & 29.3332 & 29.0424 & 28.9635 \\
\hline $20 a$ & 3.70 & 28.5212 & 28.0567 & 27.8355 & 28.4574 & 28.2026 & 28.0550 & 28.4981 & 28.0318 & 27.8016 & 28.4293 & 28.1735 & 28.0200 \\
\hline $20 b$ & 2.72 & 29.6892 & 29.2942 & 29.1358 & 29.6186 & 29.4393 & 29.3630 & 29.6221 & 29.2233 & 29.0480 & 29.5425 & 29.3618 & 29.2776 \\
\hline $23 a$ & 4.07 & 28.2894 & 27.8113 & 27.4979 & 28.1658 & 27.8858 & 27.7502 & 28.1794 & 27.6981 & 27.3737 & 28.0560 & 27.7743 & 27.6271 \\
\hline $23 b$ & 4.14 & 28.2643 & 27.7397 & 27.3981 & 28.1068 & 27.7952 & 27.7021 & 28.2255 & 27.7010 & 27.3593 & 28.0652 & 27.7548 & 27.6628 \\
\hline \multicolumn{14}{|c|}{ mPW1PW91 } \\
\hline & & \multicolumn{6}{|c|}{ Gas phase } & \multicolumn{6}{|c|}{ PCM } \\
\hline & Exp & $6-31 G^{*}$ & $6-31 G * *$ & $6-31+G * *$ & 6-311G* & $6-311 G * *$ & $6-311+G * *$ & $6-31 G *$ & $6-31 G * *$ & $6-31+G * *$ & 6-311G* & $6-311 G * *$ & $6-311+G * *$ \\
\hline \multicolumn{14}{|c|}{${ }^{13} \mathrm{C}$} \\
\hline 1 & 122.2 & 76.6114 & 77.2378 & 76.4172 & 62.2170 & 61.4026 & 60.7022 & 75.6526 & 76.2650 & 75.3080 & 60.9816 & 60.1655 & 59.3774 \\
\hline 2 & 124.2 & 75.6697 & 76.1931 & 76.0255 & 60.8672 & 59.9260 & 59.1911 & 75.3738 & 75.8988 & 75.6341 & 60.4750 & 59.5649 & 58.8209 \\
\hline 3 & 128.5 & 69.8512 & 70.3685 & 69.9891 & 54.9648 & 54.0135 & 53.2143 & 70.3355 & 70.8539 & 70.4877 & 55.3749 & 54.4562 & 53.7609 \\
\hline 4 & 116.2 & 81.1271 & 81.6845 & 81.3769 & 67.0350 & 66.1709 & 64.4074 & 82.2888 & 82.8545 & 82.5785 & 68.3189 & 67.4829 & 65.7178 \\
\hline 5 & 142.2 & 55.4567 & 55.3702 & 54.3592 & 38.6568 & 38.5418 & 38.2429 & 55.6182 & 55.5262 & 54.4641 & 38.7564 & 38.6418 & 38.2988 \\
\hline 6 & 132.8 & 67.3503 & 67.1622 & 65.6814 & 50.5403 & 50.3183 & 49.3284 & 65.8984 & 65.7104 & 64.0967 & 48.9544 & 48.7476 & 47.6705 \\
\hline 7 & 51.9 & 139.9720 & 140.1871 & 140.0024 & 130.1538 & 130.1818 & 129.9573 & 139.9583 & 140.1706 & 140.0034 & 130.1413 & 130.1677 & 129.9109 \\
\hline 8 & 60.1 & 133.1325 & 133.7861 & 133.2228 & 123.9546 & 123.5802 & 123.1726 & 132.9372 & 133.5869 & 133.0529 & 123.7582 & 123.3788 & 122.9422 \\
\hline 10 & 169.3 & 33.5190 & 33.6847 & 32.5210 & 16.3342 & 16.4601 & 14.1385 & 31.6387 & 31.8008 & 30.3403 & 14.1513 & 14.2737 & 11.6984 \\
\hline 11 & 42.5 & 150.8332 & 151.9779 & 151.1684 & 141.7638 & 141.1356 & 140.6797 & 150.6543 & 151.7897 & 150.8171 & 141.5427 & 140.9034 & 140.2994 \\
\hline 12 & 77.6 & 116.5293 & 117.2275 & 116.2562 & 105.8976 & 105.4985 & 105.0848 & 117.0774 & 117.7754 & 116.8775 & 106.5498 & 106.1469 & 105.7428 \\
\hline 13 & 48.2 & 145.8390 & 146.5357 & 146.4878 & 136.5822 & 136.3247 & 135.6651 & 145.9159 & 146.6097 & 146.6446 & 136.6794 & 136.4218 & 135.7746 \\
\hline 14 & 31.6 & 159.9016 & 160.5918 & 159.2890 & 151.0375 & 150.7597 & 150.6291 & 160.1485 & 160.8375 & 159.6208 & 151.3545 & 151.0732 & 150.9485 \\
\hline 15 & 26.9 & 165.5681 & 166.8129 & 165.1524 & 158.1049 & 157.8142 & 157.5973 & 165.8294 & 167.0679 & 165.4202 & 158.3735 & 158.0738 & 157.8911 \\
\hline 16 & 60.2 & 133.3873 & 133.9988 & 132.8300 & 123.0618 & 122.7603 & 122.6463 & 133.3638 & 133.9720 & 132.7787 & 122.9875 & 122.6843 & 122.5790 \\
\hline 17 & 42.7 & 149.9833 & 151.1965 & 150.5563 & 141.6975 & 141.2944 & 141.4674 & 150.0701 & 151.2742 & 150.6102 & 141.7279 & 141.3197 & 141.5194 \\
\hline 18 & 50.3 & 143.9473 & 145.2108 & 144.7758 & 135.8125 & 135.3023 & 135.0895 & 143.9738 & 145.2366 & 144.7436 & 135.8028 & 135.2825 & 135.0550 \\
\hline 20 & 52.9 & 141.5716 & 142.7155 & 141.3081 & 132.7576 & 132.1230 & 131.4962 & 142.0847 & 143.2179 & 141.8307 & 133.2706 & 132.6301 & 132.0065 \\
\hline 21 & 140.6 & 55.6280 & 55.4646 & 53.7492 & 36.8836 & 36.4980 & 34.0361 & 55.3393 & 55.1808 & 53.3584 & 36.5480 & 36.1805 & 33.6289 \\
\hline 22 & 127.2 & 69.4653 & 70.2583 & 70.2506 & 54.3479 & 53.9182 & 52.7734 & 69.3731 & 70.1606 & 70.1753 & 54.2002 & 53.7734 & 52.6450 \\
\hline $\begin{array}{l}23 \\
{ }^{1} \mathbf{H}\end{array}$ & 64.6 & 129.6751 & 130.9249 & 130.1295 & 120.8609 & 120.1361 & 119.7789 & 129.9304 & 131.1697 & 130.2858 & 121.0343 & 120.2979 & 119.9236 \\
\hline 1 & 7.16 & 25.0599 & 24.3902 & 24.1898 & 25.0310 & 24.5578 & 24.4275 & 24.8856 & 24.2149 & 23.9953 & 24.8434 & 24.3740 & 24.2342 \\
\hline 2 & 7.10 & 25.1439 & 24.5119 & 24.2587 & 25.0397 & 24.6011 & 24.5320 & 25.0151 & 24.3832 & 24.1289 & 24.9039 & 24.4730 & 24.3992 \\
\hline 3 & 7.25 & 24.9108 & 24.3037 & 24.1630 & 24.7800 & 24.3849 & 24.2864 & 24.8363 & 24.2295 & 24.0864 & 24.7004 & 24.3140 & 24.2102 \\
\hline 4 & 8.09 & 23.9493 & 23.1588 & 22.9227 & 23.8181 & 23.2713 & 23.1199 & 24.0776 & 23.2888 & 23.0491 & 23.9376 & 23.3957 & 23.2399 \\
\hline 8 & 3.85 & 28.2021 & 27.7621 & 27.6797 & 28.3066 & 28.0769 & 27.9635 & 28.1649 & 27.7219 & 27.6287 & 28.2653 & 28.0322 & 27.9114 \\
\hline 12 & 4.28 & 28.0513 & 27.6298 & 27.5723 & 27.9646 & 27.7433 & 27.7276 & 27.9145 & 27.4886 & 27.4156 & 27.8228 & 27.6006 & 27.5749 \\
\hline 13 & 1.27 & 31.0841 & 30.7919 & 30.7085 & 31.0829 & 30.9752 & 30.8843 & 31.0083 & 30.7141 & 30.6232 & 31.0088 & 30.9010 & 30.8011 \\
\hline 14 & 3.15 & 29.3026 & 28.8188 & 28.7506 & 29.2246 & 28.9340 & 28.9074 & 29.1611 & 28.6723 & 28.6029 & 29.0889 & 28.7941 & 28.7637 \\
\hline
\end{tabular}




\begin{tabular}{llllllllllllll}
\hline 16 & 3.93 & 28.2684 & 27.8034 & 27.5702 & 28.2066 & 27.9652 & 27.8265 & 28.2357 & 27.7694 & 27.5134 & 28.1589 & 27.9167 & 27.7661 \\
22 & 5.90 & 26.3159 & 25.6466 & 25.2708 & 26.2437 & 25.7310 & 25.5826 & 26.2261 & 25.5591 & 25.1794 & 26.1488 & 25.6467 & 25.4958 \\
11a 3.11 & 29.3197 & 28.8061 & 28.5867 & 29.1424 & 28.8233 & 28.7339 & 29.3183 & 28.7968 & 28.5759 & 29.1349 & 28.8110 & 28.7230 \\
11b 2.67 & 29.4895 & 29.0252 & 28.9106 & 29.4133 & 29.1538 & 29.1121 & 29.4838 & 29.0163 & 28.8989 & 29.4083 & 29.1467 & 29.1005 \\
15a 2.35 & 29.9743 & 29.5630 & 29.4341 & 29.8877 & 29.6990 & 29.6801 & 29.8841 & 29.4693 & 29.3343 & 29.7957 & 29.6060 & 29.5835 \\
15b 1.45 & 30.7333 & 30.3379 & 30.3164 & 30.6852 & 30.4860 & 30.4763 & 30.7091 & 30.3121 & 30.2913 & 30.6691 & 30.4678 & 30.4537 \\
17a 1.88 & 30.3037 & 29.8693 & 29.7818 & 30.2947 & 30.0500 & 29.9691 & 30.2550 & 29.8167 & 29.7149 & 30.2452 & 29.9967 & 29.9066 \\
17b 1.89 & 30.1963 & 29.8044 & 29.7665 & 30.1378 & 29.9711 & 29.9037 & 30.1631 & 29.7693 & 29.7132 & 30.0990 & 29.9313 & 29.8542 \\
18a 3.19 & 29.1292 & 28.6175 & 28.4360 & 29.0536 & 28.7333 & 28.7176 & 29.0996 & 28.5873 & 28.3900 & 29.0163 & 28.6965 & 28.6741 \\
18b 2.87 & 29.2138 & 28.7236 & 28.6298 & 29.2675 & 28.9864 & 28.9152 & 29.1739 & 28.6843 & 28.5808 & 29.2279 & 28.9483 & 28.8717 \\
20a 3.70 & 28.5060 & 28.0443 & 27.8461 & 28.4074 & 28.1616 & 28.0197 & 28.4798 & 28.0161 & 27.8091 & 28.3761 & 28.1294 & 27.9818 \\
20b 2.72 & 29.5669 & 29.1776 & 29.0427 & 29.4950 & 29.3100 & 29.2406 & 29.4914 & 29.0978 & 28.9490 & 29.4124 & 29.2256 & 29.1501 \\
23a 4.07 & 28.2335 & 27.7574 & 27.4374 & 28.0766 & 27.7981 & 27.6551 & 28.1159 & 27.6363 & 27.3081 & 27.9619 & 27.6816 & 27.5282 \\
23b 4.14 & 28.1766 & 27.6554 & 27.3399 & 28.0460 & 27.7138 & 27.6225 & 28.1284 & 27.6071 & 27.2925 & 27.9957 & 27.6648 & 27.5749 \\
\hline
\end{tabular}

\section{Compound 3}

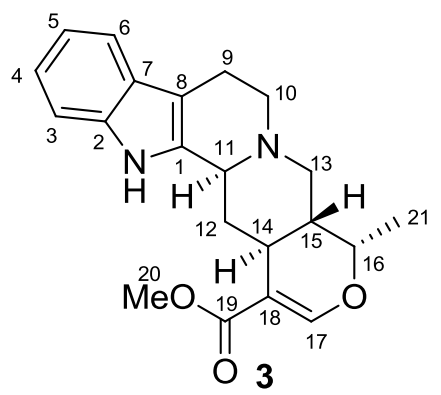

\begin{tabular}{|c|c|c|c|c|c|c|c|c|c|c|c|c|c|}
\hline & \multirow[b]{3}{*}{ Exp } & \multicolumn{12}{|c|}{ B3LYP } \\
\hline & & \multicolumn{6}{|c|}{ Gas phase } & \multicolumn{6}{|c|}{ PCM } \\
\hline & & 6-31G* & $6-31 G * *$ & $6-31+G * *$ & 6-311G* & $6-311 G * *$ & $6-311+G * *$ & 6-31G* & $6-31 G * *$ & $6-31+G * *$ & 6-311G* & $6-311 G * *$ & $6-311+G * *$ \\
\hline \multicolumn{14}{|c|}{${ }^{13} \mathrm{C}$} \\
\hline 1 & 134.5 & 61.8630 & 61.9154 & 60.3999 & 43.3656 & 43.3145 & 42.1092 & 60.4938 & 60.5526 & 58.9427 & 41.7369 & 41.7152 & 40.4677 \\
\hline 2 & 136.0 & 62.4035 & 62.2407 & 60.6649 & 42.6094 & 42.4598 & 40.6270 & 62.4545 & 62.2966 & 60.6315 & 42.6389 & 42.5009 & 40.6693 \\
\hline 3 & 110.8 & 86.2718 & 86.8936 & 86.4241 & 70.8202 & 69.8935 & 69.1486 & 85.8031 & 86.4170 & 85.7707 & 70.0876 & 69.1954 & 68.5334 \\
\hline 4 & 121.4 & 74.5862 & 75.2375 & 76.1842 & 57.9915 & 57.1227 & 56.4542 & 75.1736 & 75.8221 & 76.6843 & 58.5801 & 57.7412 & 57.1117 \\
\hline 5 & 119.4 & 76.3008 & 76.9201 & 77.8011 & 60.0122 & 59.0758 & 58.2005 & 76.8925 & 77.5094 & 78.3052 & 60.5757 & 59.6663 & 58.8425 \\
\hline 6 & 118.0 & 77.4464 & 78.1280 & 78.4322 & 61.6480 & 60.8262 & 61.2608 & 77.8542 & 78.5215 & 78.7078 & 61.9155 & 61.0953 & 61.5615 \\
\hline 7 & 127.3 & 69.0919 & 68.8705 & 67.4149 & 51.0235 & 50.7932 & 48.6359 & 69.3954 & 69.1757 & 67.6182 & 51.2831 & 51.0612 & 48.8218 \\
\hline 8 & 106.8 & 86.3357 & 86.3619 & 85.8488 & 69.8960 & 69.7884 & 68.0494 & 86.6171 & 86.6448 & 86.0599 & 70.1226 & 70.0198 & 68.2669 \\
\hline 9 & 21.9 & 165.5289 & 166.8151 & 165.7526 & 157.4455 & 157.1310 & 156.3709 & 165.8087 & 167.0911 & 165.9497 & 157.6630 & 157.3392 & 156.5584 \\
\hline 10 & 53.2 & 136.4015 & 137.5638 & 136.4356 & 126.5955 & 126.1883 & 126.0108 & 136.8880 & 138.0447 & 136.9379 & 127.1130 & 126.6948 & 126.5185 \\
\hline 11 & 60.1 & 131.3015 & 131.7730 & 130.6398 & 119.8094 & 119.3714 & 118.6815 & 131.6089 & 132.0803 & 131.0728 & 120.1980 & 119.7581 & 119.0727 \\
\hline 12 & 33.0 & 155.8141 & 156.9865 & 155.8813 & 146.6254 & 146.0673 & 145.6103 & 155.6797 & 156.8562 & 155.6101 & 146.4256 & 145.8687 & 145.3980 \\
\hline 13 & 56.9 & 133.0557 & 134.1742 & 132.9209 & 123.1022 & 122.3869 & 122.0986 & 133.5851 & 134.6986 & 133.6003 & 123.7284 & 123.0047 & 122.7407 \\
\hline 14 & 30.7 & 157.0747 & 157.6928 & 156.7024 & 147.4204 & 147.2069 & 146.7069 & 157.4026 & 158.0168 & 157.1143 & 147.7729 & 147.5526 & 147.0155 \\
\hline 15 & 41.0 & 148.4340 & 149.0380 & 148.2445 & 137.6605 & 137.5216 & 136.9991 & 148.3466 & 148.9466 & 147.9628 & 137.5422 & 137.4035 & 136.7772 \\
\hline 16 & 73.8 & 115.7575 & 116.5090 & 115.1202 & 103.4765 & 103.1917 & 102.9558 & 115.6186 & 116.3675 & 114.8157 & 103.2878 & 102.9903 & 102.7153 \\
\hline
\end{tabular}




\begin{tabular}{|c|c|c|c|c|c|c|c|c|c|c|c|c|c|}
\hline 17 & 154.6 & 39.6939 & 40.5146 & 39.0157 & 20.9494 & 20.1298 & 19.0443 & 38.9394 & 39.7619 & 38.2310 & 20.1103 & 19.2894 & 18.2050 \\
\hline 18 & 107.9 & 87.0866 & 86.9308 & 86.4338 & 71.4816 & 71.3157 & 70.1933 & 86.9587 & 86.8036 & 86.1549 & 71.3394 & 71.1762 & 69.9346 \\
\hline 19 & 167.5 & 32.6254 & 32.5414 & 27.8153 & 10.3249 & 10.3663 & 9.8531 & 31.7053 & 31.6317 & 26.8820 & 9.3072 & 9.3570 & 8.5832 \\
\hline 20 & 50.9 & 139.9306 & 141.6767 & 141.6103 & 131.2355 & 130.6845 & 130.3684 & 139.8064 & 141.5377 & 141.4189 & 131.0619 & 130.4888 & 130.1652 \\
\hline $\begin{array}{l}21 \\
{ }^{\mathbf{1}} \mathbf{H}\end{array}$ & 15.0 & 173.5072 & 175.3790 & 176.2489 & 167.1515 & 167.0407 & 166.6095 & 173.6504 & 175.5062 & 176.3242 & 167.2461 & 167.1152 & 166.6758 \\
\hline 3 & 7.27 & 25.3129 & 24.6617 & 24.2994 & 25.1057 & 24.6713 & 24.5959 & 25.1504 & 24.4971 & 24.1266 & 24.9257 & 24.4984 & 24.4117 \\
\hline 4 & 7.08 & 25.2229 & 24.6158 & 24.3394 & 25.0476 & 24.6815 & 24.6000 & 25.1684 & 24.5605 & 24.2732 & 24.9811 & 24.6220 & 24.5332 \\
\hline 5 & 7.12 & 25.2489 & 24.6398 & 24.4176 & 25.0870 & 24.7174 & 24.6602 & 25.2049 & 24.5949 & 24.3672 & 25.0296 & 24.6667 & 24.6071 \\
\hline 6 & 7.46 & 24.9591 & 24.3296 & 24.1477 & 24.7778 & 24.3621 & 24.4068 & 24.8913 & 24.2594 & 24.0486 & 24.6901 & 24.2785 & 24.3083 \\
\hline $9 a x$ & 3.00 & 29.2064 & 28.7249 & 28.5137 & 29.1246 & 28.8504 & 28.7813 & 29.2507 & 28.7724 & 28.5611 & 29.1688 & 28.8999 & 28.8306 \\
\hline 9eq & 2.73 & 29.7041 & 29.2165 & 29.0253 & 29.5409 & 29.2591 & 29.1786 & 29.6562 & 29.1656 & 28.9602 & 29.4860 & 29.2024 & 29.1220 \\
\hline $10 \mathrm{~b}$ & 3.08 & 29.3868 & 28.9961 & 28.9135 & 29.3591 & 29.2010 & 29.1144 & 29.3684 & 28.9766 & 28.9041 & 29.3478 & 29.1878 & 29.1054 \\
\hline 10a & 2.66 & 29.5219 & 29.0880 & 28.8520 & 29.3553 & 29.1058 & 29.0349 & 29.4257 & 28.9876 & 28.7453 & 29.2494 & 28.9971 & 28.9253 \\
\hline 11 & 3.37 & 28.7141 & 28.3359 & 28.2937 & 28.7489 & 28.5429 & 28.4822 & 28.6383 & 28.2596 & 28.2082 & 28.6739 & 28.4681 & 28.3989 \\
\hline $12 a$ & 3.21 & 28.9462 & 28.3558 & 27.9878 & 28.8488 & 28.4594 & 28.3054 & 29.0001 & 28.4180 & 28.0484 & 28.8918 & 28.5095 & 28.3561 \\
\hline $12 b$ & 1.31 & 31.0216 & 30.6464 & 30.4693 & 31.0575 & 30.9180 & 30.7701 & 31.0582 & 30.6815 & 30.5003 & 31.0913 & 30.9510 & 30.7846 \\
\hline $13 a$ & 2.95 & 29.6007 & 29.1582 & 28.9930 & 29.5063 & 29.2251 & 29.1657 & 29.4839 & 29.0373 & 28.8594 & 29.3823 & 29.0982 & 29.0346 \\
\hline $13 b$ & 2.21 & 29.8486 & 29.4591 & 29.4818 & 29.8680 & 29.6975 & 29.6887 & 29.7709 & 29.3790 & 29.3864 & 29.7885 & 29.6166 & 29.5920 \\
\hline 14 & 2.41 & 29.7711 & 29.3689 & 29.3431 & 29.8668 & 29.6400 & 29.5844 & 29.7167 & 29.3109 & 29.2653 & 29.8067 & 29.5777 & 29.5111 \\
\hline 15 & 2.13 & 30.0223 & 29.6845 & 29.5190 & 30.0535 & 29.9202 & 29.8301 & 30.0487 & 29.7128 & 29.5386 & 30.0746 & 29.9456 & 29.8426 \\
\hline 16 & 4.41 & 27.8760 & 27.3840 & 27.3299 & 27.8108 & 27.5583 & 27.5994 & 27.7519 & 27.2554 & 27.2012 & 27.6833 & 27.4255 & 27.4726 \\
\hline 17 & 7.54 & 24.7442 & 24.0756 & 23.8269 & 24.5885 & 24.1835 & 23.9928 & 24.6904 & 24.0231 & 23.7583 & 24.5282 & 24.1251 & 23.9453 \\
\hline 20 & 3.73 & 28.6216 & 28.1082 & 27.9293 & 28.5630 & 28.2309 & 28.2172 & 28.5580 & 28.0445 & 27.8643 & 28.4999 & 28.1685 & 28.1491 \\
\hline 21 & 1.16 & 31.0553 & 30.5857 & 30.4903 & 31.0389 & 30.7693 & 30.7108 & 31.0113 & 30.5394 & 30.4506 & 30.9968 & 30.7263 & 30.6685 \\
\hline
\end{tabular}

\begin{tabular}{|c|c|c|c|c|c|c|c|c|c|c|c|c|c|}
\hline \multirow{3}{*}{\multicolumn{2}{|c|}{$\operatorname{Exp}$}} & \multicolumn{12}{|c|}{ mPW1PW91 } \\
\hline & & \multicolumn{6}{|c|}{ Gas phase } & \multicolumn{6}{|c|}{ PCM } \\
\hline & & 6-31G* & $6-31 G^{* *}$ & $6-31+G^{* *}$ & 6-311G* & 6-311G** & $6-311+G^{* *}$ & 6-31G* & $6-31 G^{* *}$ & $6-31+G^{* *}$ & 6-311G* & $6-311 G * *$ & $6-311+G^{* *}$ \\
\hline \multicolumn{14}{|c|}{ e } \\
\hline 1 & 134.5 & 64.8463 & 64.8690 & 63.8826 & 48.6786 & 48.5996 & 47.4760 & 63.4628 & 63.4917 & 62.4002 & 47.0594 & 47.0096 & 45.8448 \\
\hline 2 & 136.0 & 65.4592 & 65.2696 & 63.9910 & 48.2108 & 48.0280 & 46.3859 & 65.5534 & 65.3702 & 64.0048 & 48.2637 & 48.0953 & 46.4407 \\
\hline 3 & 110.8 & 88.6869 & 89.2459 & 89.1024 & 75.0840 & 74.1303 & 73.3213 & 88.1789 & 88.7295 & 88.3815 & 74.3105 & 73.3941 & 72.6680 \\
\hline 4 & 121.4 & 77.1104 & 77.6766 & 78.7356 & 62.5337 & 61.6159 & 60.9875 & 77.6300 & 78.1938 & 79.1557 & 63.0235 & 62.1389 & 61.5438 \\
\hline 5 & 119.4 & 78.9611 & 79.4950 & 80.6326 & 64.6769 & 63.6900 & 62.8706 & 79.4877 & 80.0199 & 81.0500 & 65.1488 & 64.1921 & 63.4284 \\
\hline 6 & 118.0 & 79.7064 & 80.3222 & 80.3459 & 65.8519 & 65.0009 & 65.3779 & 80.0993 & 80.7005 & 80.6271 & 66.0866 & 65.2386 & 65.6638 \\
\hline 7 & 127.3 & 72.3376 & 72.0980 & 70.6929 & 56.6122 & 56.3679 & 54.3167 & 72.7163 & 72.4792 & 70.9726 & 56.9264 & 56.6919 & 54.5411 \\
\hline 8 & 106.8 & 89.8121 & 89.8176 & 89.6205 & 75.2956 & 75.1772 & 73.4311 & 90.1052 & 90.1127 & 89.8381 & 75.5298 & 75.4188 & 73.6528 \\
\hline 9 & 21.9 & 169.6734 & 170.8742 & 170.1254 & 162.6403 & 162.3091 & 161.5487 & 169.9985 & 171.1949 & 170.3694 & 162.8824 & 162.5415 & 161.7616 \\
\hline 10 & 53.2 & 141.3621 & 142.4342 & 141.3167 & 132.6217 & 132.2259 & 132.0279 & 141.8856 & 142.9530 & 141.8496 & 133.1456 & 132.7391 & 132.5378 \\
\hline 11 & 60.1 & 136.0696 & 136.4824 & 135.5513 & 126.1221 & 125.6848 & 125.0097 & 136.3950 & 136.8083 & 135.9892 & 126.5066 & 126.0676 & 125.3887 \\
\hline 12 & 33.0 & 160.8425 & 161.9059 & 160.9820 & 152.6374 & 152.0913 & 151.6369 & 160.7211 & 161.7880 & 160.7237 & 152.4416 & 151.8964 & 151.4287 \\
\hline 13 & 56.9 & 138.0194 & 139.0370 & 137.9782 & 129.3226 & 128.6133 & 128.3532 & 138.5769 & 139.5912 & 138.6791 & 129.9368 & 129.2209 & 128.9786 \\
\hline 14 & 30.7 & 162.0106 & 162.5727 & 161.6820 & 153.4758 & 153.2737 & 152.7147 & 162.3587 & 162.9173 & 162.1201 & 153.8388 & 153.6299 & 153.0280 \\
\hline 15 & 41.0 & 153.9596 & 154.5048 & 153.7822 & 144.1344 & 143.9980 & 143.4762 & 153.8523 & 154.3943 & 153.4911 & 143.9984 & 143.8624 & 143.2473 \\
\hline
\end{tabular}




\begin{tabular}{|c|c|c|c|c|c|c|c|c|c|c|c|c|c|}
\hline 16 & 73.8 & 120.6233 & 121.3216 & 120.2085 & 109.7869 & 109.5095 & 109.2384 & 120.4651 & 121.1614 & 119.9106 & 109.5656 & 109.2768 & 108.9695 \\
\hline 17 & 154.6 & 41.7897 & 42.5834 & 42.0381 & 25.4105 & 24.5884 & 23.3524 & 40.9921 & 41.7867 & 41.1913 & 24.5208 & 23.6976 & 22.4882 \\
\hline 18 & 107.9 & 90.6775 & 90.4896 & 90.0941 & 77.2213 & 77.0256 & 75.8288 & 90.5963 & 90.4092 & 89.8903 & 77.1408 & 76.9477 & 75.6499 \\
\hline 19 & 167.5 & 34.9684 & 34.8842 & 31.1100 & 15.4840 & 15.5276 & 14.9382 & 34.0359 & 33.9617 & 30.1665 & 14.4698 & 14.5210 & 13.6945 \\
\hline 20 & 50.9 & 143.3831 & 145.0557 & 145.0385 & 135.7235 & 135.2130 & 134.9593 & 143.2618 & 144.9196 & 144.8579 & 135.5460 & 135.0151 & 134.7558 \\
\hline $\begin{array}{l}21 \\
{ }^{1} \mathbf{H}\end{array}$ & 15.0 & 177.5488 & 179.2987 & 179.8244 & 171.6225 & 171.5364 & 171.1830 & 177.7151 & 179.4494 & 179.8948 & 171.7243 & 171.6198 & 171.2686 \\
\hline 3 & 7.27 & 25.0954 & 24.4399 & 24.1188 & 24.9224 & 24.4546 & 24.3901 & 24.9144 & 24.2563 & 23.9289 & 24.7276 & 24.2669 & 24.1921 \\
\hline 4 & 7.08 & 25.0083 & 24.3996 & 24.1657 & 24.8636 & 24.4628 & 24.3923 & 24.9342 & 24.3246 & 24.0818 & 24.7816 & 24.3882 & 24.3106 \\
\hline 5 & 7.12 & 25.0426 & 24.4302 & 24.2379 & 24.9101 & 24.5050 & 24.4531 & 24.9795 & 24.3660 & 24.1718 & 24.8376 & 24.4397 & 24.3865 \\
\hline 6 & 7.46 & 24.7380 & 24.1040 & 23.9762 & 24.5889 & 24.1383 & 24.1991 & 24.6537 & 24.0170 & 23.8641 & 24.4884 & 24.0421 & 24.0912 \\
\hline 9ax & 3.00 & 29.1371 & 28.6458 & 28.4480 & 29.0320 & 28.7551 & 28.6985 & 29.1792 & 28.6916 & 28.4910 & 29.0732 & 28.8021 & 28.7438 \\
\hline 9eq & 2.73 & 29.6277 & 29.1380 & 28.9580 & 29.4535 & 29.1649 & 29.0835 & 29.5677 & 29.0744 & 28.8821 & 29.3887 & 29.0983 & 29.0174 \\
\hline $10 b$ & 3.08 & 29.3331 & 28.9374 & 28.8419 & 29.2439 & 29.0889 & 28.9998 & 29.3104 & 28.9137 & 28.8289 & 29.2284 & 29.0718 & 28.9881 \\
\hline $10 a$ & 2.66 & 29.4340 & 29.0070 & 28.8144 & 29.2839 & 29.0197 & 28.9651 & 29.3250 & 28.8930 & 28.6949 & 29.1684 & 28.9010 & 28.8455 \\
\hline 11 & 3.37 & 28.6481 & 28.2629 & 28.1991 & 28.5936 & 28.4030 & 28.3369 & 28.5641 & 28.1779 & 28.1055 & 28.5116 & 28.3208 & 28.2470 \\
\hline $12 a$ & 3.21 & 28.9009 & 28.3100 & 27.9688 & 28.7780 & 28.3947 & 28.2514 & 28.9495 & 28.3665 & 28.0199 & 28.8153 & 28.4388 & 28.2927 \\
\hline $12 b$ & 1.31 & 30.9094 & 30.5338 & 30.3742 & 30.9120 & 30.7667 & 30.6416 & 30.9547 & 30.5776 & 30.4102 & 30.9525 & 30.8065 & 30.6610 \\
\hline $13 a$ & 2.95 & 29.5243 & 29.0841 & 28.9460 & 29.4206 & 29.1318 & 29.0778 & 29.3969 & 28.9522 & 28.8026 & 29.2895 & 28.9974 & 28.9392 \\
\hline $13 b$ & 2.21 & 29.8164 & 29.4248 & 29.4298 & 29.7526 & 29.5910 & 29.5739 & 29.7299 & 29.3357 & 29.3271 & 29.6662 & 29.5029 & 29.4729 \\
\hline 14 & 2.41 & 29.7299 & 29.3234 & 29.2727 & 29.7481 & 29.5402 & 29.4782 & 29.6717 & 29.2612 & 29.1927 & 29.6853 & 29.4748 & 29.4013 \\
\hline 15 & 2.13 & 29.9474 & 29.6142 & 29.4509 & 29.9314 & 29.8079 & 29.7277 & 29.9804 & 29.6491 & 29.4764 & 29.9576 & 29.8390 & 29.7437 \\
\hline 16 & 4.41 & 27.7947 & 27.3038 & 27.2636 & 27.7200 & 27.4558 & 27.4979 & 27.6623 & 27.1663 & 27.1261 & 27.5872 & 27.3174 & 27.3652 \\
\hline 17 & 7.54 & 24.5837 & 23.9223 & 23.7143 & 24.4820 & 24.0398 & 23.8609 & 24.5211 & 23.8613 & 23.6411 & 24.4153 & 23.9752 & 23.8071 \\
\hline 20 & 3.73 & 28.5492 & 28.0359 & 27.8839 & 28.4963 & 28.1506 & 28.1503 & 28.4788 & 27.9651 & 27.8126 & 28.4276 & 28.0826 & 28.0770 \\
\hline 21 & 1.16 & 31.0018 & 30.5293 & 30.4398 & 30.9554 & 30.6783 & 30.6207 & 30.9540 & 30.4791 & 30.3968 & 30.9097 & 30.6315 & 30.5752 \\
\hline
\end{tabular}

\section{Compound 4}

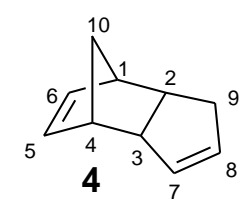

\begin{tabular}{|c|c|c|c|c|c|c|c|c|c|c|c|c|c|}
\hline & \multirow[b]{3}{*}{ Exp } & \multicolumn{12}{|c|}{ B3LYP } \\
\hline & & \multicolumn{6}{|c|}{ Gas phase } & \multicolumn{6}{|c|}{ PCM } \\
\hline & & 6-31G* & $6-31 G * *$ & $6-31+G * *$ & 6-311G* & $6-311 G * *$ & $6-311+G * *$ & 6-31G* & $6-31 G * *$ & $6-31+G * *$ & 6-311G* & $6-311 G * *$ & $6-311+G * *$ \\
\hline \multicolumn{14}{|c|}{${ }^{13} \mathrm{C}$} \\
\hline 1 & 46.4 & 141.4963 & 142.3841 & 141.4714 & 130.8436 & 130.5327 & 130.3544 & 141.5263 & 142.4118 & 141.4723 & 130.8696 & 130.5534 & 130.3567 \\
\hline 2 & 41.5 & 144.7163 & 145.6311 & 145.7046 & 135.2085 & 135.0443 & 134.9905 & 144.8767 & 145.7838 & 145.8992 & 135.3950 & 135.2168 & 135.1713 \\
\hline 3 & 55.1 & 132.0635 & 132.7773 & 132.7550 & 120.9931 & 120.6384 & 120.6668 & 132.1481 & 132.8590 & 132.8736 & 121.1038 & 120.7437 & 120.7801 \\
\hline 4 & 45.5 & 141.7283 & 142.6108 & 142.0648 & 131.3989 & 131.0586 & 130.8228 & 141.7814 & 142.6611 & 142.1226 & 131.4660 & 131.1190 & 130.8736 \\
\hline 5 & 136.0 & 57.8098 & 58.2907 & 58.3059 & 38.9337 & 38.0633 & 37.5234 & 57.8257 & 58.3064 & 58.3133 & 38.8924 & 38.0434 & 37.4938 \\
\hline 6 & 132.0 & 62.0601 & 62.5642 & 62.6236 & 43.6485 & 42.7444 & 41.9717 & 61.6525 & 62.1531 & 62.0129 & 43.0067 & 42.1308 & 41.2498 \\
\hline 7 & 132.0 & 62.9052 & 63.5095 & 63.0439 & 44.7365 & 43.9406 & 42.8426 & 62.6554 & 63.2655 & 62.7339 & 44.3866 & 43.6321 & 42.5106 \\
\hline
\end{tabular}




\begin{tabular}{cccccccccccccc}
\hline 8 & 131.0 & 62.0535 & 62.6679 & 62.3809 & 43.8209 & 42.9501 & 42.2588 & 62.0642 & 62.6704 & 62.3562 & 43.7548 & 42.8942 & 42.2269 \\
9 & 34.9 & 153.4767 & 154.7519 & 154.6059 & 144.3111 & 143.8874 & 143.6412 & 153.6194 & 154.8861 & 154.7099 & 144.4381 & 143.9997 & 143.7527 \\
10 & 50.5 & 138.0649 & 139.3758 & 139.0536 & 128.2229 & 127.8342 & 127.5150 & 138.1611 & 139.4655 & 139.1193 & 128.3345 & 127.9321 & 127.5805 \\
${ }^{1} \mathbf{H}$ & & & & & & & & & & & & & \\
1 & 2.87 & 29.4947 & 28.9682 & 28.8362 & 29.4132 & 29.1224 & 29.0596 & 29.4517 & 28.9222 & 28.7789 & 29.3636 & 29.0702 & 29.0017 \\
\hline 2 & 2.73 & 29.4543 & 29.0113 & 28.8750 & 29.4178 & 29.1643 & 29.0995 & 29.4092 & 28.9645 & 28.8160 & 29.3667 & 29.1128 & 29.0415 \\
3 & 3.21 & 28.8426 & 28.3804 & 28.2057 & 28.8153 & 28.5373 & 28.4403 & 28.8085 & 28.3451 & 28.1617 & 28.7776 & 28.4987 & 28.3962 \\
4 & 2.78 & 29.5679 & 29.0378 & 28.9259 & 29.5016 & 29.2086 & 29.1445 & 29.5185 & 28.9856 & 28.8626 & 29.4450 & 29.1500 & 29.0803 \\
5 & 5.93 & 26.1104 & 25.4288 & 25.2721 & 26.1174 & 25.6640 & 25.6089 & 26.0642 & 25.3827 & 25.2246 & 26.0613 & 25.6142 & 25.5586 \\
6 & 5.98 & 26.1400 & 25.4432 & 25.2517 & 26.1159 & 25.6504 & 25.5832 & 26.0671 & 25.3702 & 25.1685 & 26.0285 & 25.5690 & 25.4969 \\
7 & 5.49 & 26.5411 & 25.9097 & 25.7817 & 26.5708 & 26.1574 & 26.1147 & 26.4806 & 25.8488 & 25.7138 & 26.4988 & 26.0903 & 26.0436 \\
8 & 5.49 & 26.7081 & 26.0819 & 25.8658 & 26.6165 & 26.1978 & 26.1486 & 26.6616 & 26.0345 & 25.8105 & 26.5564 & 26.1411 & 26.0893 \\
9 & 1.62 & 30.4050 & 29.9265 & 29.8917 & 30.4141 & 30.1546 & 30.1162 & 30.3843 & 29.9046 & 29.8680 & 30.3932 & 30.1326 & 30.0935 \\
9 & 2.18 & 30.0214 & 29.5271 & 29.3410 & 29.9103 & 29.6328 & 29.5512 & 29.9984 & 29.5027 & 29.3139 & 29.8860 & 29.6078 & 29.5229 \\
$10 \mathrm{a}$ & 1.48 & 30.6104 & 30.2056 & 30.0692 & 30.5487 & 30.3364 & 30.2782 & 30.6554 & 30.2501 & 30.1233 & 30.5997 & 30.3862 & 30.3266 \\
$10 \mathrm{~b}$ & 1.30 & 30.8856 & 30.4657 & 30.3768 & 30.8176 & 30.6158 & 30.5612 & 30.8478 & 30.4261 & 30.3263 & 30.7756 & 30.5728 & 30.5128 \\
\hline
\end{tabular}

\begin{tabular}{|c|c|c|c|c|c|c|c|c|c|c|c|c|c|}
\hline & \multirow[b]{3}{*}{ Exp } & \multicolumn{12}{|c|}{ mPW1PW91 } \\
\hline & & \multicolumn{6}{|c|}{ Gas phase } & \multicolumn{6}{|c|}{ PCM } \\
\hline & & 6-31G* & 6-31G** & $6-31+G^{* *}$ & 6-311G* & 6-311G** & $6-311+G^{* *}$ & 6-31G* & 6-31G** & $6-31+G * *$ & 6-311G* & 6-311G** & $6-311+G * *$ \\
\hline${ }^{3} \mathrm{C}$ & & & & & & & & & & & & & \\
\hline 1 & 46.4 & 146.1364 & 146.9406 & 146.0178 & 136.4325 & 136.1005 & 135.8962 & 146.1740 & 146.9755 & 146.0297 & 136.4572 & 136.1201 & 135.8993 \\
\hline 2 & 41.5 & 149.6978 & 150.5216 & 150.4744 & 140.8975 & 140.7060 & 140.5922 & 149.8841 & 150.6999 & 150.6923 & 141.0957 & 140.8902 & 140.7848 \\
\hline 3 & 55.1 & 136.8521 & 137.4807 & 137.3509 & 126.6916 & 126.3173 & 126.2721 & 136.9503 & 137.5758 & 137.4811 & 126.8050 & 126.4257 & 126.3872 \\
\hline 4 & 45.5 & 146.3184 & 147.1209 & 146.4912 & 136.8850 & 136.5244 & 136.2508 & 146.3831 & 147.1823 & 146.5623 & 136.9523 & 136.5855 & 136.3044 \\
\hline 5 & 136.0 & 60.3622 & 60.7493 & 60.6986 & 43.3906 & 42.4769 & 41.9852 & 60.3799 & 60.7670 & 60.7166 & 43.3388 & 42.4488 & 41.9570 \\
\hline 6 & 132.0 & 64.5699 & 64.9919 & 65.0324 & 48.0105 & 47.0681 & 46.4145 & 64.1068 & 64.5250 & 64.3912 & 47.3228 & 46.4098 & 45.6640 \\
\hline 7 & 132.0 & 65.4638 & 65.9904 & 65.6183 & 49.3083 & 48.4761 & 47.4446 & 65.1968 & 65.7296 & 65.2869 & 48.9466 & 48.1577 & 47.0997 \\
\hline 8 & 131.0 & 64.2322 & 64.7685 & 64.5274 & 48.0184 & 47.1088 & 46.4133 & 64.2172 & 64.7455 & 64.4755 & 47.9075 & 47.0114 & 46.3448 \\
\hline 9 & 34.9 & 157.5057 & 158.6739 & 158.5810 & 149.3616 & 148.9034 & 148.5900 & 157.6812 & 158.8403 & 158.7144 & 149.5023 & 149.0296 & 148.7158 \\
\hline $\begin{array}{l}10 \\
{ }^{1} \mathrm{H}\end{array}$ & 50.5 & 142.4504 & 143.6471 & 143.1687 & 133.4419 & 133.0401 & 132.7288 & 142.5679 & 143.7582 & 143.2607 & 133.5581 & 133.1429 & 132.8059 \\
\hline 1 & 2.87 & 29.3952 & 28.8675 & 28.7420 & 29.2988 & 28.9918 & 28.9322 & 29.3448 & 28.8137 & 28.6781 & 29.2438 & 28.9339 & 28.8690 \\
\hline 2 & 2.73 & 29.3860 & 28.9319 & 28.7898 & 29.3005 & 29.0457 & 28.9798 & 29.3349 & 28.8786 & 28.7256 & 29.2457 & 28.9904 & 28.9180 \\
\hline 3 & 3.21 & 28.7772 & 28.3044 & 28.1259 & 28.6942 & 28.4156 & 28.3202 & 28.7379 & 28.2635 & 28.0766 & 28.6528 & 28.3732 & 28.2718 \\
\hline 4 & 2.78 & 29.4608 & 28.9301 & 28.8168 & 29.3817 & 29.0706 & 29.0055 & 29.4040 & 28.8700 & 28.7469 & 29.3197 & 29.0063 & 28.9361 \\
\hline 5 & 5.93 & 25.9604 & 25.2756 & 25.1352 & 25.9896 & 25.5025 & 25.4426 & 25.9070 & 25.2223 & 25.0819 & 25.9282 & 25.4480 & 25.3877 \\
\hline 6 & 5.98 & 25.9878 & 25.2900 & 25.1183 & 25.9896 & 25.4911 & 25.4204 & 25.9042 & 25.2063 & 25.0264 & 25.8945 & 25.4024 & 25.3271 \\
\hline 7 & 5.49 & 26.4144 & 25.7785 & 25.6576 & 26.4443 & 26.0023 & 25.9592 & 26.3455 & 25.7092 & 25.5827 & 26.3666 & 25.9297 & 25.8830 \\
\hline 8 & 5.49 & 26.5744 & 25.9462 & 25.7545 & 26.4925 & 26.0436 & 25.9971 & 26.5184 & 25.8892 & 25.6915 & 26.4249 & 25.9797 & 25.9313 \\
\hline 9 & 1.62 & 30.3605 & 29.8784 & 29.8217 & 30.3104 & 30.0511 & 30.0067 & 30.3357 & 29.8525 & 29.7957 & 30.2864 & 30.0263 & 29.9823 \\
\hline 9 & 2.18 & 29.9670 & 29.4701 & 29.2957 & 29.8229 & 29.5415 & 29.4667 & 29.9374 & 29.4390 & 29.2620 & 29.7923 & 29.5106 & 29.4324 \\
\hline 10a & 1.48 & 30.5399 & 30.1361 & 30.0077 & 30.4542 & 30.2365 & 30.1785 & 30.5850 & 30.1809 & 30.0594 & 30.5040 & 30.2854 & 30.2245 \\
\hline $10 \mathrm{~b}$ & 1.30 & 30.8241 & 30.4112 & 30.3247 & 30.7166 & 30.5197 & 30.4650 & 30.7801 & 30.3652 & 30.2688 & 30.6700 & 30.4722 & 30.4122 \\
\hline
\end{tabular}




\section{Compound 5}

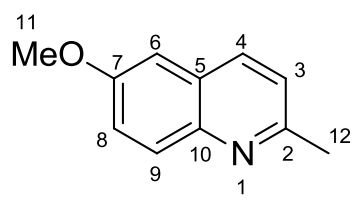

5

\begin{tabular}{|c|c|c|c|c|c|c|c|c|c|c|c|c|c|}
\hline & \multirow[b]{3}{*}{ Exp } & \multicolumn{12}{|c|}{ B3LYP } \\
\hline & & \multicolumn{6}{|c|}{ Gas phase } & \multicolumn{6}{|c|}{ PCM } \\
\hline & & 6-31G* & $6-31 G * *$ & $6-31+G * *$ & 6-311G* & $6-311 G * *$ & $6-311+G * *$ & 6-31G* & $6-31 G * *$ & $6-31+G * *$ & 6-311G* & $6-311 G * *$ & $6-311+G * *$ \\
\hline \multicolumn{14}{|c|}{${ }^{13} \mathrm{C}$} \\
\hline 2 & 157.2 & 40.5644 & 40.6598 & 39.9812 & 22.2516 & 22.1611 & 20.4110 & 39.8238 & 39.9234 & 39.0512 & 21.2806 & 21.2112 & 19.3056 \\
\hline 3 & 122.2 & 74.5182 & 75.1579 & 75.5905 & 58.6625 & 57.9175 & 57.3101 & 73.8744 & 74.5105 & 74.8499 & 57.8297 & 57.0987 & 56.4546 \\
\hline 4 & 135.0 & 62.4159 & 63.0565 & 62.4972 & 45.5561 & 44.7477 & 43.8326 & 61.4357 & 62.0693 & 61.2898 & 44.3931 & 43.5848 & 42.5718 \\
\hline 5 & 127.3 & 68.5141 & 68.3437 & 68.3934 & 51.0118 & 50.8836 & 49.3576 & 68.2803 & 68.1157 & 68.1063 & 50.7995 & 50.6806 & 49.1078 \\
\hline 6 & 105.3 & 89.9542 & 90.6733 & 91.6887 & 76.9588 & 76.3746 & 75.6872 & 89.1534 & 89.8621 & 90.6430 & 75.8579 & 75.2655 & 74.5115 \\
\hline 7 & 156.3 & 40.5804 & 40.2633 & 38.4363 & 18.7887 & 18.6231 & 17.7751 & 40.3987 & 40.0845 & 38.3739 & 18.6225 & 18.4687 & 17.6393 \\
\hline 8 & 121.8 & 74.8964 & 75.5360 & 75.0747 & 57.9623 & 57.2355 & 56.3223 & 74.7996 & 75.4348 & 74.8966 & 57.7650 & 57.0406 & 56.1445 \\
\hline 9 & 130.1 & 63.9198 & 64.5050 & 63.4587 & 46.3958 & 45.4680 & 44.7448 & 64.8608 & 65.4457 & 64.3844 & 47.3669 & 46.4589 & 45.7409 \\
\hline 10 & 144.0 & 52.0753 & 51.9880 & 50.7177 & 33.4331 & 33.3128 & 31.5805 & 52.7313 & 52.6375 & 51.2469 & 34.0389 & 33.9182 & 32.1450 \\
\hline 11 & 55.4 & 137.1858 & 138.9946 & 138.3703 & 128.5260 & 127.8427 & 127.5736 & 137.0283 & 138.8115 & 137.8944 & 128.1563 & 127.4374 & 127.1392 \\
\hline $\begin{array}{l}12 \\
{ }^{1} \mathbf{H}\end{array}$ & 25.0 & 164.6283 & 166.3289 & 166.1226 & 156.4636 & 156.1717 & 155.7035 & 164.6634 & 166.3455 & 165.9313 & 156.3716 & 156.0476 & 155.5029 \\
\hline 3 & 7.21 & 25.1093 & 24.4640 & 24.3706 & 24.9974 & 24.5917 & 24.5508 & 24.9387 & 24.2912 & 24.1817 & 24.8132 & 24.4120 & 24.3637 \\
\hline 4 & 7.91 & 24.5992 & 23.9798 & 23.7308 & 24.4016 & 23.9781 & 23.9103 & 24.3921 & 23.7721 & 23.5014 & 24.1807 & 23.7643 & 23.6832 \\
\hline 6 & 7.01 & 25.6782 & 24.9676 & 24.7725 & 25.5436 & 25.0655 & 24.9172 & 25.4606 & 24.7466 & 24.5274 & 25.3086 & 24.8352 & 24.6743 \\
\hline 8 & 7.33 & 25.0364 & 24.3729 & 24.1274 & 24.8329 & 24.4233 & 24.3709 & 24.9697 & 24.3049 & 24.0498 & 24.7560 & 24.3507 & 24.2942 \\
\hline 9 & 7.92 & 24.3857 & 23.7362 & 23.5841 & 24.1738 & 23.7551 & 23.7557 & 24.4226 & 23.7709 & 23.6119 & 24.1970 & 23.7819 & 23.7804 \\
\hline 11 & 3.88 & 28.4092 & 27.9097 & 27.7745 & 28.3769 & 28.0829 & 28.0003 & 28.3117 & 27.8124 & 27.6654 & 28.2757 & 27.9835 & 27.8942 \\
\hline 12 & 2.69 & 29.6404 & 29.1502 & 28.9981 & 29.4812 & 29.2029 & 29.1384 & 29.6089 & 29.1178 & 28.9626 & 29.4496 & 29.1729 & 29.1044 \\
\hline
\end{tabular}

\begin{tabular}{|c|c|c|c|c|c|c|c|c|c|c|c|c|c|}
\hline & \multirow[b]{3}{*}{ Exp } & \multicolumn{12}{|c|}{ mPW1PW91 } \\
\hline & & \multicolumn{6}{|c|}{ Gas phase } & \multicolumn{6}{|c|}{ PCM } \\
\hline & & 6-31G* & $6-31 G * *$ & $6-31+G * *$ & 6-311G* & $6-311 G * *$ & $6-311+G * *$ & 6-31G* & $6-31 G * *$ & $6-31+G * *$ & 6-311G* & $6-311 G * *$ & $6-311+G * *$ \\
\hline \multicolumn{14}{|c|}{${ }^{13} \mathrm{C}$} \\
\hline 2 & 157.2 & 43.1441 & 43.2206 & 42.8282 & 27.2693 & 27.1690 & 25.5663 & 42.2754 & 42.3570 & 41.7866 & 26.1801 & 26.1034 & 24.3697 \\
\hline 3 & 122.2 & 76.9340 & 77.5086 & 78.1029 & 63.0921 & 62.3236 & 61.6744 & 76.2306 & 76.8022 & 77.3050 & 62.2069 & 61.4543 & 60.7861 \\
\hline 4 & 135.0 & 64.8415 & 65.4055 & 64.9339 & 50.0252 & 49.1793 & 48.2379 & 63.8070 & 64.3643 & 63.6868 & 48.8230 & 47.9794 & 46.9627 \\
\hline 5 & 127.3 & 71.7233 & 71.5345 & 71.8803 & 56.6214 & 56.4685 & 54.8377 & 71.5256 & 71.3443 & 71.6327 & 56.4405 & 56.2988 & 54.6315 \\
\hline 6 & 105.3 & 92.6592 & 93.3532 & 94.3742 & 81.3882 & 80.8044 & 79.9082 & 91.8505 & 92.5320 & 93.3208 & 80.2936 & 79.7006 & 78.7527 \\
\hline 7 & 156.3 & 43.5720 & 43.2217 & 42.0158 & 24.7987 & 24.6045 & 23.7468 & 43.3832 & 43.0375 & 41.9495 & 24.6253 & 24.4444 & 23.6042 \\
\hline 8 & 121.8 & 77.1733 & 77.7520 & 77.7060 & 62.3369 & 61.5892 & 60.7165 & 77.0572 & 77.6314 & 77.4982 & 62.1106 & 61.3666 & 60.5134 \\
\hline 9 & 130.1 & 66.4191 & 66.9377 & 66.2396 & 51.1006 & 50.1394 & 49.4075 & 67.3613 & 67.8799 & 67.1492 & 52.0365 & 51.0978 & 50.3762 \\
\hline
\end{tabular}




\begin{tabular}{llllllllllllll}
\hline 10 & 144.0 & 55.0080 & 54.9083 & 54.2129 & 38.9329 & 38.8058 & 37.1856 & 55.7225 & 55.6168 & 54.7916 & 39.5825 & 39.4557 & 37.7833 \\
11 & 55.4 & 140.7359 & 142.4784 & 142.0906 & 133.3287 & 132.6905 & 132.4331 & 140.5636 & 142.2815 & 141.6244 & 132.9491 & 132.2773 & 132.0001 \\
12 & 25.0 & 168.1851 & 169.7871 & 169.7943 & 160.8517 & 160.5692 & 160.1649 & 168.2376 & 169.8202 & 169.6311 & 160.7626 & 160.4500 & 159.9801 \\
${ }^{1} \mathbf{H}$ & & & & & & & & & & & & & \\
3 & 7.21 & 24.9124 & 24.2669 & 24.1921 & 24.8250 & 24.3856 & 24.3547 & 24.7235 & 24.0754 & 23.9856 & 24.6266 & 24.1918 & 24.1545 \\
4 & 7.91 & 24.3694 & 23.7476 & 23.5121 & 24.2158 & 23.7562 & 23.6884 & 24.1438 & 23.5210 & 23.2675 & 23.9816 & 23.5287 & 23.4490 \\
6 & 7.01 & 25.4649 & 24.7563 & 24.5773 & 25.3606 & 24.8620 & 24.7243 & 25.2299 & 24.5172 & 24.3192 & 25.1139 & 24.6196 & 24.4704 \\
8 & 7.33 & 24.8161 & 24.1534 & 23.9678 & 24.6419 & 24.2018 & 24.1809 & 24.7369 & 24.0725 & 23.8778 & 24.5553 & 24.1196 & 24.0939 \\
9 & 7.92 & 24.1456 & 23.4964 & 23.3884 & 23.9671 & 23.5184 & 23.5377 & 24.1689 & 23.5171 & 23.4019 & 23.9792 & 23.5348 & 23.5511 \\
11 & 3.88 & 28.3381 & 27.8418 & 27.7136 & 28.3068 & 28.0025 & 27.9268 & 28.2308 & 27.7344 & 27.5964 & 28.1983 & 27.8956 & 27.8143 \\
12 & 2.69 & 29.5311 & 29.0355 & 28.9072 & 29.3625 & 29.0733 & 29.0299 & 29.4905 & 28.9935 & 28.8622 & 29.3230 & 29.0355 & 28.9882 \\
\hline
\end{tabular}

\section{Compound 6}

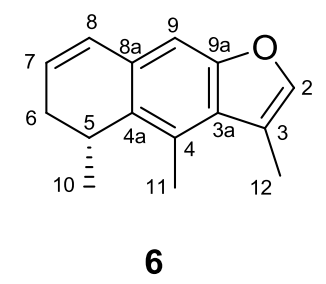

\begin{tabular}{|c|c|c|c|c|c|c|c|c|c|c|c|c|c|}
\hline \multirow{3}{*}{\multicolumn{2}{|c|}{ Exp }} & \multicolumn{12}{|c|}{ B3LYP } \\
\hline & & \multicolumn{6}{|c|}{ Gas phase } & \multicolumn{6}{|c|}{ PCM } \\
\hline & & 6-31G* & $6-31 G^{* *}$ & $6-31+G * *$ & 6-311G* & 6-311G** & $6-311+\mathrm{G} * *$ & 6-31G* & $6-31 G^{* *}$ & $6-31+G * *$ & 6-311G* & 6-311G** & $6-311+G * *$ \\
\hline \\
\hline $4 a$ & 133.2 & 61.7103 & 61.7482 & 60.1214 & 42.4758 & 42.6608 & 41.9330 & 61.2612 & 61.2994 & 59.6107 & 41.9328 & 42.1184 & 41.3190 \\
\hline 4 & 127.9 & 66.4568 & 66.6347 & 66.6124 & 49.1571 & 49.1542 & 48.7671 & 65.5967 & 65.7652 & 65.5258 & 48.0426 & 48.0281 & 47.5538 \\
\hline $3 a$ & 126.6 & 68.5484 & 68.5593 & 67.6999 & 51.3804 & 51.4635 & 50.5657 & 68.6648 & 68.6747 & 67.7974 & 51.4682 & 51.5515 & 50.6739 \\
\hline 3 & 116.5 & 78.2675 & 78.1501 & 77.4027 & 61.1616 & 60.8941 & 59.3928 & 77.4587 & 77.3341 & 76.4521 & 60.2300 & 59.9558 & 58.3149 \\
\hline 2 & 141.7 & 53.9689 & 54.8036 & 54.3772 & 36.3321 & 35.6369 & 34.6090 & 53.8621 & 54.6958 & 54.2218 & 36.0231 & 35.3530 & 34.4718 \\
\hline & 154.4 & 42.6995 & 42.5858 & 40.1437 & 22.0862 & 21.8853 & 20.3055 & 43.1499 & 43.0375 & 40.5517 & 22.5008 & 22.3103 & 20.7677 \\
\hline 9 & 107.5 & 86.6221 & 87.3696 & 87.9686 & 71.8452 & 71.2649 & 70.4550 & 87.3439 & 88.0870 & 88.7334 & 72.5500 & 71.9788 & 71.2459 \\
\hline & 130.0 & 65.3262 & 65.2483 & 64.4207 & 47.1861 & 47.1507 & 45.9427 & 65.5014 & 65.4289 & 64.5605 & 47.3727 & 47.3424 & 46.1141 \\
\hline 8 & 128.2 & 65.3475 & 65.8539 & 66.4420 & 48.0540 & 47.1705 & 45.8390 & 66.0409 & 66.5401 & 67.1719 & 48.7953 & 47.9245 & 46.6463 \\
\hline 7 & 125.3 & 69.0149 & 69.8007 & 69.5644 & 51.9058 & 51.1783 & 50.6063 & 67.8692 & 68.6528 & 68.2395 & 50.4619 & 49.7460 & 49.0910 \\
\hline 6 & 31.1 & 156.8129 & 158.1059 & 157.4381 & 148.0618 & 147.7990 & 147.6145 & 157.0746 & 158.3561 & 157.6955 & 148.3307 & 148.0506 & 147.8552 \\
\hline 5 & 27.5 & 157.9055 & 158.8946 & 158.8384 & 149.5976 & 149.5218 & 148.9635 & 158.1298 & 159.1147 & 159.1085 & 149.8885 & 149.8051 & 149.2381 \\
\hline 10 & 19.6 & 169.4735 & 171.2177 & 172.3943 & 163.3000 & 163.3012 & 163.3150 & 169.7217 & 171.4599 & 172.6960 & 163.6280 & 163.6064 & 163.6523 \\
\hline 11 & 14.1 & 173.9309 & 175.7443 & 175.6051 & 167.8899 & 167.6505 & 167.6567 & 174.1615 & 175.9537 & 175.7403 & 168.0155 & 167.7525 & 167.7649 \\
\hline $\begin{array}{l}12 \\
{ }^{1} \mathbf{H}\end{array}$ & 11.4 & 176.3552 & 178.1741 & 178.1788 & 169.9320 & 169.5760 & 169.3504 & 176.8621 & 178.6597 & 178.6527 & 170.4085 & 170.0277 & 169.8321 \\
\hline 2 & 7.33 & 25.2211 & 24.5058 & 24.2817 & 25.1154 & 24.6448 & 24.5415 & 25.0941 & 24.3808 & 24.1584 & 24.9796 & 24.5175 & 24.4238 \\
\hline 9 & 7.05 & 25.6038 & 24.9311 & 24.5782 & 25.3595 & 24.9044 & 24.8030 & 25.5494 & 24.8751 & 24.5117 & 25.2846 & 24.8361 & 24.7333 \\
\hline 8 & 6.54 & 25.8580 & 25.2357 & 24.9929 & 25.7095 & 25.2916 & 25.2307 & 25.8043 & 25.1811 & 24.9307 & 25.6382 & 25.2278 & 25.1659 \\
\hline 7 & 5.94 & 26.4011 & 25.7948 & 25.6126 & 26.2921 & 25.8882 & 25.7869 & 26.2777 & 25.6709 & 25.4829 & 26.1525 & 25.7544 & 25.6549 \\
\hline $6 a$ & 2.63 & 29.4838 & 29.0008 & 28.7927 & 29.3803 & 29.1331 & 29.0561 & 29.4798 & 28.9970 & 28.7883 & 29.3803 & 29.1339 & 29.0527 \\
\hline
\end{tabular}




\begin{tabular}{cccccccccccccc}
\hline 6b & 2.30 & 30.0787 & 29.6462 & 29.4691 & 29.9199 & 29.6909 & 29.6449 & 29.9942 & 29.5584 & 29.3742 & 29.8281 & 29.5981 & 29.5501 \\
5 & 3.36 & 29.1341 & 28.6829 & 28.5772 & 29.0589 & 28.8437 & 28.8218 & 29.0548 & 28.5984 & 28.4772 & 28.9725 & 28.7513 & 28.7221 \\
10 & 1.16 & 30.9909 & 30.5555 & 30.4811 & 31.0060 & 30.7606 & 30.7304 & 30.9927 & 30.5555 & 30.4845 & 31.0097 & 30.7624 & 30.7331 \\
11 & 2.63 & 29.7925 & 29.2832 & 29.1289 & 29.6362 & 29.3260 & 29.3154 & 29.7397 & 29.2283 & 29.0712 & 29.5841 & 29.2734 & 29.2602 \\
12 & 2.44 & 29.9502 & 29.4534 & 29.2520 & 29.7867 & 29.4973 & 29.4696 & 29.8952 & 29.3958 & 29.1936 & 29.7330 & 29.4426 & 29.4117 \\
\hline
\end{tabular}

\begin{tabular}{|c|c|c|c|c|c|c|c|c|c|c|c|c|c|}
\hline \multirow{3}{*}{\multicolumn{2}{|c|}{ Exp }} & \multicolumn{12}{|c|}{ mPW1PW91 } \\
\hline & & \multicolumn{6}{|c|}{ Gas phase } & \multicolumn{6}{|c|}{ PCM } \\
\hline & & 6-31G* & $6-31 G * *$ & $6-31+G * *$ & 6-311G* & $6-311 G * *$ & $6-311+G * *$ & 6-31G* & $6-31 G * *$ & $6-31+G * *$ & $6-311 G^{*}$ & $6-311 G * *$ & $6-311+G * *$ \\
\hline \multicolumn{14}{|c|}{${ }^{13} \mathrm{C}$} \\
\hline $4 a$ & 133.2 & 65.2597 & 65.2953 & 63.8550 & 48.3356 & 48.5140 & 47.7827 & 64.7780 & 64.8135 & 63.3306 & 47.7707 & 47.9498 & 47.1620 \\
\hline 4 & 127.9 & 69.5477 & 69.7150 & 69.8004 & 54.4139 & 54.4038 & 53.8561 & 68.6571 & 68.8153 & 68.7059 & 53.2758 & 53.2560 & 52.6438 \\
\hline $3 a$ & 126.6 & 71.5423 & 71.5568 & 71.0107 & 56.6435 & 56.7366 & 55.6361 & 71.7190 & 71.7326 & 71.1378 & 56.7741 & 56.8670 & 55.7805 \\
\hline 3 & 116.5 & 81.7624 & 81.6124 & 80.9790 & 66.7877 & 66.4854 & 64.7936 & 80.9693 & 80.8136 & 80.0730 & 65.8849 & 65.5794 & 63.7776 \\
\hline 2 & 141.7 & 56.1981 & 57.0125 & 57.0160 & 40.8936 & 40.2003 & 39.1804 & 56.0136 & 56.8268 & 56.7536 & 40.4958 & 39.8277 & 38.9512 \\
\hline $9 a$ & 154.4 & 45.8562 & 45.7260 & 44.0785 & 27.9715 & 27.7556 & 26.0644 & 46.3374 & 46.2097 & 44.5167 & 28.3967 & 28.1924 & 26.5391 \\
\hline 9 & 107.5 & 89.1793 & 89.8942 & 90.5515 & 76.2219 & 75.6369 & 74.8336 & 89.9187 & 90.6282 & 91.2991 & 76.8982 & 76.3225 & 75.5889 \\
\hline $8 a$ & 130.0 & 68.4883 & 68.3917 & 67.7253 & 52.7977 & 52.7342 & 51.5238 & 68.6746 & 68.5841 & 67.8832 & 52.9916 & 52.9336 & 51.7076 \\
\hline 8 & 128.2 & 68.1635 & 68.6029 & 69.5090 & 52.9057 & 52.0002 & 50.6446 & 68.9414 & 69.3741 & 70.3069 & 53.7039 & 52.8137 & 51.5035 \\
\hline 7 & 125.3 & 71.5170 & 72.1988 & 72.0088 & 56.3532 & 55.5662 & 54.9860 & 70.1853 & 70.8645 & 70.5222 & 54.7429 & 53.9695 & 53.3358 \\
\hline 6 & 31.1 & 161.6397 & 162.8071 & 162.1818 & 153.5766 & 153.3067 & 153.0827 & 161.9294 & 163.0853 & 162.4628 & 153.8462 & 153.5598 & 153.3242 \\
\hline 5 & 27.5 & 162.9124 & 163.8347 & 163.7312 & 155.3596 & 155.2848 & 154.7684 & 163.1510 & 164.0690 & 164.0076 & 155.6453 & 155.5630 & 155.0393 \\
\hline 10 & 19.6 & 173.7680 & 175.3722 & 176.4526 & 167.9681 & 167.9743 & 167.9494 & 174.0491 & 175.6473 & 176.7808 & 168.2973 & 168.2826 & 168.2870 \\
\hline 11 & 14.1 & 177.4680 & 179.1697 & 179.1330 & 172.0614 & 171.8257 & 171.8435 & 177.7273 & 179.4069 & 179.2870 & 172.1879 & 171.9304 & 171.9571 \\
\hline $\begin{array}{l}12 \\
\mathbf{1}_{\mathbf{H}}\end{array}$ & 11.4 & 180.0393 & 181.7672 & 181.8491 & 174.3496 & 174.0027 & 173.8310 & 180.5933 & 182.2994 & 182.3493 & 174.8374 & 174.4680 & 174.3215 \\
\hline 2 & 7.33 & 25.0478 & 24.3361 & 24.1651 & 24.9864 & 24.4776 & 24.3995 & 24.9046 & 24.1943 & 24.0253 & 24.8371 & 24.3369 & 24.2679 \\
\hline 9 & 7.05 & 25.3924 & 24.7146 & 24.4143 & 25.1722 & 24.6890 & 24.6270 & 25.3219 & 24.6421 & 24.3341 & 25.0841 & 24.6077 & 24.5442 \\
\hline 8 & 6.54 & 25.6464 & 25.0263 & 24.8145 & 25.5380 & 25.0882 & 25.0546 & 25.5816 & 24.9602 & 24.7439 & 25.4590 & 25.0170 & 24.9822 \\
\hline 7 & 5.94 & 26.2241 & 25.6132 & 25.4401 & 26.1329 & 25.7001 & 25.5961 & 26.0795 & 25.4680 & 25.2916 & 25.9765 & 25.5495 & 25.4487 \\
\hline $6 a$ & 2.63 & 29.4408 & 28.9534 & 28.7530 & 29.3001 & 29.0504 & 28.9768 & 29.4347 & 28.9476 & 28.7452 & 29.2977 & 29.0492 & 28.9705 \\
\hline $6 b$ & 2.30 & 30.0017 & 29.5705 & 29.4139 & 29.8260 & 29.5912 & 29.5496 & 29.9011 & 29.4658 & 29.3041 & 29.7213 & 29.4850 & 29.4423 \\
\hline 5 & 3.36 & 29.0469 & 28.5989 & 28.4969 & 28.9225 & 28.7124 & 28.6907 & 28.9595 & 28.5057 & 28.3892 & 28.8310 & 28.6144 & 28.5857 \\
\hline 10 & 1.16 & 30.9488 & 30.5108 & 30.4442 & 30.9303 & 30.6795 & 30.6550 & 30.9480 & 30.5081 & 30.4446 & 30.9310 & 30.6782 & 30.6547 \\
\hline 11 & 2.63 & 29.7037 & 29.1896 & 29.0404 & 29.5367 & 29.2197 & 29.2048 & 29.6419 & 29.1254 & 28.9733 & 29.4768 & 29.1591 & 29.1416 \\
\hline 12 & 2.44 & 29.8660 & 29.3667 & 29.1850 & 29.6965 & 29.3996 & 29.3775 & 29.8021 & 29.2999 & 29.1168 & 29.6351 & 29.3371 & 29.3111 \\
\hline
\end{tabular}




\section{Compound 7}

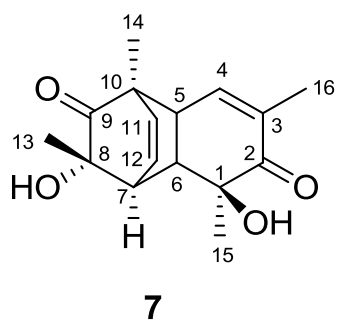

\begin{tabular}{|c|c|c|c|c|c|c|c|c|c|c|c|c|c|}
\hline \multirow{3}{*}{\multicolumn{2}{|c|}{ Exp }} & \multicolumn{12}{|c|}{ B3LYP } \\
\hline & & \multicolumn{6}{|c|}{ Gas phase } & \multicolumn{6}{|c|}{ PCM } \\
\hline & & $6-31 G *$ & $6-31 G * *$ & $6-31+G * *$ & 6-311G* & $6-311 G * *$ & $6-311+G^{* *}$ & $6-31 G *$ & $6-31 G * *$ & $6-31+G * *$ & 6-311G* & $6-311 G * *$ & $6-311+G * *$ \\
\hline \multicolumn{14}{|c|}{${ }^{13} \mathrm{C}$} \\
\hline 1 & 73.7 & 117.1325 & 117.4004 & 116.5036 & 105.9388 & 106.0204 & 104.6866 & 117.0518 & 117.3176 & 116.3023 & 105.8150 & 105.8853 & 104.4707 \\
\hline 2 & 203.1 & -6.3273 & -6.3171 & -7.2675 & -27.3808 & -27.2634 & -29.6565 & -7.0034 & -7.0094 & -8.1769 & -28.2992 & -28.2410 & -30.8033 \\
\hline 3 & 135.6 & 57.7505 & 57.7470 & 57.3887 & 38.9548 & 38.6983 & 37.1981 & 57.9228 & 57.9187 & 57.6444 & 39.2250 & 38.9776 & 37.4584 \\
\hline 4 & 139.4 & 52.9639 & 53.8424 & 53.9191 & 34.6313 & 34.1897 & 32.9222 & 51.3151 & 52.1973 & 51.9129 & 32.6821 & 32.2230 & 30.7106 \\
\hline 5 & 43.4 & 142.3684 & 143.0502 & 142.1551 & 131.5892 & 131.4683 & 130.9871 & 142.5725 & 143.2469 & 142.3452 & 131.8205 & 131.6853 & 131.1496 \\
\hline 6 & 42.7 & 144.8148 & 145.5765 & 145.3561 & 135.3572 & 134.9774 & 135.1297 & 144.9335 & 145.6938 & 145.5603 & 135.4794 & 135.1099 & 135.3181 \\
\hline 7 & 44.5 & 144.0030 & 144.6038 & 144.4063 & 133.5588 & 133.2271 & 133.0447 & 144.0314 & 144.6337 & 144.4469 & 133.5698 & 133.2483 & 133.0793 \\
\hline 8 & 72.6 & 116.4782 & 116.6874 & 116.7222 & 105.5752 & 105.7147 & 104.5811 & 116.2648 & 116.4724 & 116.4153 & 105.3247 & 105.4448 & 104.2088 \\
\hline 9 & 214.6 & -22.7296 & -22.8647 & -23.9754 & -44.3380 & -44.2013 & -46.7533 & -24.1197 & -24.2769 & -25.8103 & -46.0192 & -45.9565 & -48.8453 \\
\hline 10 & 53.7 & 132.8507 & 133.1529 & 132.6371 & 122.0384 & 122.1416 & 121.4378 & 132.4603 & 132.7644 & 132.1487 & 121.5438 & 121.6556 & 120.8589 \\
\hline 11 & 133.1 & 62.8164 & 63.1076 & 62.6092 & 44.3694 & 43.2269 & 42.7252 & 62.2273 & 62.5208 & 61.9482 & 43.6528 & 42.5269 & 41.9777 \\
\hline 12 & 135.4 & 56.9856 & 57.5618 & 55.7061 & 37.3419 & 36.6235 & 35.6542 & 57.6591 & 58.2335 & 56.4212 & 38.0659 & 37.3398 & 36.4514 \\
\hline 13 & 26.1 & 162.6983 & 164.4144 & 165.0077 & 155.4111 & 155.1581 & 155.0233 & 162.8478 & 164.5489 & 165.0563 & 155.5099 & 155.2376 & 155.0426 \\
\hline 14 & 15.7 & 171.8720 & 173.6714 & 174.6137 & 165.6860 & 165.5490 & 165.2411 & 172.2361 & 174.0226 & 175.0394 & 166.1025 & 165.9481 & 165.6673 \\
\hline 15 & 31.7 & 157.2894 & 158.9424 & 158.4340 & 148.7897 & 148.5977 & 148.0957 & 157.3130 & 158.9493 & 158.2857 & 148.7665 & 148.5483 & 147.9232 \\
\hline $\begin{array}{l}16 \\
\mathbf{1}_{\mathbf{H}}\end{array}$ & 16.3 & 170.6771 & 172.4108 & 173.2669 & 163.9136 & 163.6686 & 163.6650 & 171.1030 & 172.8203 & 173.7590 & 164.4049 & 164.1438 & 164.1662 \\
\hline 4 & 6.28 & 25.8170 & 25.2085 & 25.1235 & 25.8260 & 25.4100 & 25.3529 & 25.6195 & 25.0141 & 24.9119 & 25.6271 & 25.2185 & 25.1503 \\
\hline 5 & 2.85 & 29.4532 & 29.0208 & 28.9782 & 29.4748 & 29.2341 & 29.1978 & 29.3100 & 28.8735 & 28.8104 & 29.3235 & 29.0824 & 29.0345 \\
\hline 6 & 3.22 & 28.7431 & 28.3014 & 28.1570 & 28.7641 & 28.5602 & 28.4695 & 28.8084 & 28.3660 & 28.2153 & 28.8259 & 28.6134 & 28.5201 \\
\hline 7 & 3.33 & 28.4426 & 27.9525 & 27.7778 & 28.4129 & 28.2437 & 28.1128 & 28.5527 & 28.0654 & 27.8790 & 28.5165 & 28.3412 & 28.2044 \\
\hline 11 & 5.44 & 26.5434 & 25.9303 & 25.8607 & 26.6443 & 26.2596 & 26.1859 & 26.4273 & 25.8153 & 25.7375 & 26.5245 & 26.1459 & 26.0634 \\
\hline 12 & 6.22 & 25.7348 & 25.1213 & 24.9694 & 25.7345 & 25.3573 & 25.3071 & 25.6960 & 25.0829 & 24.9253 & 25.6877 & 25.3151 & 25.2631 \\
\hline 13 & 1.17 & 31.0818 & 30.6111 & 30.5369 & 30.9894 & 30.7222 & 30.7301 & 31.0537 & 30.5809 & 30.4978 & 30.9584 & 30.6893 & 30.6907 \\
\hline 14 & 1.28 & 30.9427 & 30.4833 & 30.4251 & 30.9496 & 30.6755 & 30.6548 & 30.9038 & 30.4429 & 30.3791 & 30.9095 & 30.6355 & 30.6087 \\
\hline 15 & 1.26 & 30.9700 & 30.5433 & 30.3889 & 30.9205 & 30.7052 & 30.6343 & 30.9380 & 30.5085 & 30.3450 & 30.8861 & 30.6673 & 30.5894 \\
\hline 16 & 1.79 & 30.4337 & 29.9450 & 29.8421 & 30.3621 & 30.0635 & 30.0282 & 30.3994 & 29.9085 & 29.8068 & 30.3279 & 30.0288 & 29.9938 \\
\hline
\end{tabular}

\section{mPW1PW91}

\section{Gas phase}

PCM

Exp 6-31G* 6-31G** 6-31+G** 6-311G* 6-311G** 6-311+G** 6-31G* 6-31G** 6-31+G** 6-311G* 6-311G** 6-311+G** ${ }^{13} \mathrm{C}$ 


\begin{tabular}{|c|c|c|c|c|c|c|c|c|c|c|c|c|c|}
\hline 2 & 203.1 & -3.7362 & -3.7332 & -4.4834 & -21.9243 & -21.8073 & -23.8503 & -4.4819 & -4.4946 & -5.4460 & -22.8924 & -22.8358 & -25.0306 \\
\hline 3 & 135.6 & 60.7699 & 60.7456 & 60.5826 & 44.1793 & 43.9098 & 42.4081 & 60.9807 & 60.9569 & 60.8642 & 44.4628 & 44.2057 & 42.6882 \\
\hline 4 & 139.4 & 55.7944 & 56.6145 & 56.8387 & 39.5640 & 39.1104 & 37.9331 & 54.0740 & 54.8979 & 54.8125 & 37.5949 & 37.1222 & 35.7376 \\
\hline 5 & 43.4 & 147.5013 & 148.1103 & 147.3049 & 137.6830 & 137.5615 & 137.0239 & 147.6813 & 148.2839 & 147.4727 & 137.8791 & 137.7443 & 137.1608 \\
\hline 6 & 42.7 & 149.9675 & 150.6396 & 150.5613 & 141.3342 & 140.9759 & 141.0380 & 150.0900 & 150.7601 & 150.7595 & 141.4537 & 141.1046 & 141.2134 \\
\hline 7 & 44.5 & 148.9924 & 149.5244 & 149.1534 & 139.5046 & 139.1943 & 139.0431 & 149.0345 & 149.5674 & 149.2034 & 139.5258 & 139.2248 & 139.0824 \\
\hline 8 & 72.6 & 120.8205 & 121.0282 & 121.2975 & 111.4565 & 111.5905 & 110.5464 & 120.5995 & 120.8057 & 120.9939 & 111.1946 & 111.3101 & 110.1771 \\
\hline 9 & 214.6 & -19.6305 & -19.7792 & -20.4862 & -38.5025 & -38.3690 & -40.5618 & -21.0779 & -21.2490 & -22.3335 & -40.2108 & -40.1530 & -42.6436 \\
\hline 10 & 53.7 & 137.6306 & 137.9217 & 137.5052 & 127.9079 & 128.0171 & 127.3918 & 137.2360 & 137.5288 & 137.0237 & 127.4145 & 127.5323 & 126.8248 \\
\hline 11 & 133.1 & 65.6887 & 65.9069 & 65.3632 & 49.0137 & 47.8574 & 47.4158 & 65.0982 & 65.3187 & 64.7060 & 48.3036 & 47.1639 & 46.6822 \\
\hline 12 & 135.4 & 59.9890 & 60.4670 & 58.8108 & 42.2461 & 41.4896 & 40.6632 & 60.6258 & 61.1030 & 59.4783 & 42.9205 & 42.1587 & 41.4085 \\
\hline 13 & 26.1 & 166.9851 & 168.5720 & 168.8670 & 160.2855 & 160.0709 & 159.9358 & 167.1653 & 168.7379 & 168.9605 & 160.4020 & 160.1706 & 159.9831 \\
\hline 14 & 15.7 & 176.3786 & 178.0615 & 178.8749 & 170.5082 & 170.3960 & 170.1289 & 176.7622 & 178.4329 & 179.3128 & 170.9171 & 170.7902 & 170.5387 \\
\hline 15 & 31.7 & 161.8195 & 163.3396 & 162.6545 & 153.9531 & 153.8031 & 153.3526 & 161.8631 & 163.3671 & 162.5499 & 153.9351 & 153.7617 & 153.2037 \\
\hline 16 & 16.3 & 174.3887 & 176.0147 & 176.8146 & 168.2103 & 167.9750 & 167.9591 & 174.8606 & 176.4697 & 177.3373 & 168.7078 & 168.4587 & 168.4667 \\
\hline 4 & 6.28 & 25.6894 & 25.0843 & 25.0049 & 25.6895 & 25.2582 & 25.2108 & 25.4811 & 24.8787 & 24.7866 & 25.4834 & 25.0588 & 25.0027 \\
\hline 5 & 2.85 & 29.3896 & 28.9531 & 28.8913 & 29.3415 & 29.1039 & 29.0601 & 29.2419 & 28.8007 & 28.7221 & 29.1885 & 28.9501 & 28.8967 \\
\hline 6 & 3.22 & 28.6890 & 28.2422 & 28.0925 & 28.6498 & 28.4506 & 28.3690 & 28.7530 & 28.3052 & 28.1502 & 28.7109 & 28.5025 & 28.4187 \\
\hline 7 & 3.33 & 28.3562 & 27.8722 & 27.6994 & 28.2804 & 28.1120 & 27.9923 & 28.4634 & 27.9821 & 27.7985 & 28.3821 & 28.2071 & 28.0817 \\
\hline 11 & 5.44 & 26.3915 & 25.7781 & 25.7117 & 26.5023 & 26.0887 & 26.0108 & 26.2693 & 25.6568 & 25.5838 & 26.3783 & 25.9707 & 25.8848 \\
\hline 12 & 6.22 & 25.5894 & 24.9681 & 24.8277 & 25.6009 & 25.1934 & 25.1484 & 25.5427 & 24.9217 & 24.7766 & 25.5480 & 25.1454 & 25.0979 \\
\hline 13 & 1.17 & 31.0189 & 30.5448 & 30.4804 & 30.9027 & 30.6283 & 30.6360 & 30.9877 & 30.5112 & 30.4399 & 30.8688 & 30.5925 & 30.5950 \\
\hline 14 & 1.28 & 30.8684 & 30.4097 & 30.3552 & 30.8474 & 30.5687 & 30.5508 & 30.8250 & 30.3645 & 30.3055 & 30.8032 & 30.5246 & 30.5015 \\
\hline 15 & 1.26 & 30.9106 & 30.4809 & 30.3372 & 30.8302 & 30.6099 & 30.5465 & 30.8750 & 30.4424 & 30.2914 & 30.7926 & 30.5686 & 30.4999 \\
\hline 16 & 1.79 & 30.3546 & 29.8609 & 29.7621 & 30.2675 & 29.9576 & 29.9217 & 30.3150 & 29.8189 & 29.7216 & 30.2284 & 29.9181 & 29.8829 \\
\hline
\end{tabular}

\section{Compound 8}

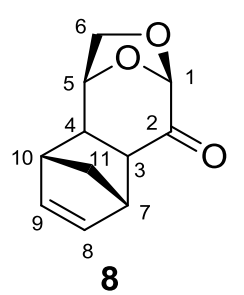

\begin{tabular}{|c|c|c|c|c|c|c|c|c|c|c|c|c|c|}
\hline & \multirow[b]{3}{*}{ Exp } & \multicolumn{12}{|c|}{ B3LYP } \\
\hline & & \multicolumn{6}{|c|}{ Gas phase } & \multicolumn{6}{|c|}{ PCM } \\
\hline & & 6-31G* & 6-31G** & $6-31+\mathrm{G} * *$ & 6-311G* & $6-311 G * *$ & $6-311+\mathrm{G} * *$ & 6-31G* & 6-31G** & $6-31+G * *$ & 6-311G* & $6-311 G * *$ & $6-311+G * *$ \\
\hline \multicolumn{14}{|c|}{${ }^{13} \mathrm{C}$} \\
\hline 1 & 99.3 & 91.4941 & 92.1997 & 90.3272 & 77.9826 & 77.3532 & 77.7059 & 91.6633 & 92.3662 & 90.3873 & 78.0771 & 77.4488 & 77.7262 \\
\hline 2 & 200.2 & -2.3964 & -2.1850 & -4.6473 & -22.4884 & -22.3081 & -25.8472 & -5.4397 & -5.2360 & -8.3298 & -25.9477 & -25.7739 & -29.9710 \\
\hline 3 & 46.9 & 140.9016 & 141.6198 & 141.3370 & 131.1145 & 130.8462 & 130.2028 & 140.5196 & 141.2294 & 140.8188 & 130.6093 & 130.3371 & 129.5082 \\
\hline 4 & 42.3 & 143.7348 & 144.4543 & 144.0924 & 133.8086 & 133.5722 & 133.5203 & 144.0092 & 144.7242 & 144.5718 & 134.1832 & 133.9371 & 133.9434 \\
\hline 5 & 74.8 & 114.4650 & 115.2709 & 114.4175 & 103.1233 & 102.6477 & 102.3729 & 114.3759 & 115.1802 & 114.2703 & 102.9911 & 102.5079 & 102.1875 \\
\hline 6 & 70.2 & 120.5227 & 121.8447 & 121.4405 & 110.1425 & 109.3700 & 108.9160 & 120.6666 & 121.9780 & 121.3788 & 110.1771 & 109.3775 & 108.8678 \\
\hline
\end{tabular}




\begin{tabular}{ccccccccccccccc}
\hline 7 & 46.9 & 140.0102 & 140.8993 & 140.5271 & 129.7626 & 129.4258 & 129.2026 & 139.9638 & 140.8508 & 140.4374 & 129.6965 & 129.3619 & 129.0854 \\
8 & 135.5 & 57.6353 & 58.1091 & 57.2456 & 38.6669 & 37.7644 & 36.9751 & 58.2730 & 58.7452 & 57.9168 & 39.3117 & 38.4349 & 37.6523 \\
9 & 134.1 & 60.9358 & 61.4713 & 60.7252 & 42.2190 & 41.3559 & 40.4501 & 59.9630 & 60.4989 & 59.4597 & 40.9924 & 40.1469 & 39.0826 \\
10 & 46.3 & 140.2645 & 141.1351 & 140.2234 & 129.4569 & 129.1327 & 128.9407 & 140.3660 & 141.2307 & 140.3767 & 129.5954 & 129.2659 & 129.0897 \\
11 & 49.4 & 139.3786 & 140.7025 & 140.2474 & 129.2998 & 128.8591 & 128.6809 & 139.4041 & 140.7210 & 140.2486 & 129.3631 & 128.9039 & 128.6935 \\
${ }^{1} \mathbf{H}$ & & & & & & & & & & & & & & \\
1 & 4.80 & 27.5748 & 27.0139 & 26.9454 & 27.5300 & 27.2366 & 27.2384 & 27.5823 & 27.0157 & 26.9414 & 27.5171 & 27.2214 & 27.2247 \\
3 & 3.00 & 29.2327 & 28.7787 & 28.6624 & 29.2626 & 28.9746 & 28.9223 & 29.1284 & 28.6692 & 28.5304 & 29.1530 & 28.8617 & 28.7931 \\
4 & 2.38 & 29.9854 & 29.5800 & 29.4968 & 30.0245 & 29.7833 & 29.7136 & 29.7838 & 29.3730 & 29.2659 & 29.8217 & 29.5784 & 29.4901 \\
5 & 4.62 & 27.8950 & 27.3568 & 27.2348 & 27.9108 & 27.5898 & 27.5512 & 27.7190 & 27.1744 & 27.0315 & 27.7311 & 27.4055 & 27.3510 \\
$6 e n d o$ & 3.84 & 28.5289 & 28.0183 & 27.9028 & 28.5131 & 28.1871 & 28.1373 & 28.3832 & 27.8690 & 27.7356 & 28.3688 & 28.0383 & 27.9728 \\
6 exo & 3.78 & 28.5267 & 27.9911 & 27.7894 & 28.4080 & 28.1048 & 28.0486 & 28.4623 & 27.9285 & 27.7162 & 28.3411 & 28.0421 & 27.9754 \\
7 & 3.38 & 28.9332 & 28.3930 & 28.2510 & 28.8853 & 28.5762 & 28.4919 & 28.9488 & 28.4089 & 28.2602 & 28.8945 & 28.5875 & 28.4980 \\
8 & 6.01 & 26.0446 & 25.3596 & 25.1981 & 26.0490 & 25.6051 & 25.5540 & 26.0594 & 25.3738 & 25.2039 & 26.0489 & 25.6097 & 25.5558 \\
9 & 6.23 & 25.9524 & 25.2766 & 25.1004 & 25.9317 & 25.4998 & 25.4325 & 25.8691 & 25.1932 & 25.0019 & 25.8345 & 25.4085 & 25.3311 \\
10 & 3.03 & 29.4549 & 28.9372 & 28.7916 & 29.3885 & 29.1081 & 29.0518 & 29.3243 & 28.8010 & 28.6418 & 29.2566 & 28.9718 & 28.9055 \\
$11 \mathrm{a}$ & 1.46 & 30.7175 & 30.3051 & 30.2009 & 30.6837 & 30.4753 & 30.4073 & 30.7296 & 30.3161 & 30.2222 & 30.7072 & 30.4975 & 30.4260 \\
$11 \mathrm{~b}$ & 1.30 & 30.9932 & 30.5583 & 30.4906 & 30.9199 & 30.7070 & 30.6814 & 30.8989 & 30.4603 & 30.3769 & 30.8241 & 30.6083 & 30.5722 \\
\hline
\end{tabular}

\begin{tabular}{|c|c|c|c|c|c|c|c|c|c|c|c|c|c|}
\hline & \multirow[b]{3}{*}{ Exp } & \multicolumn{12}{|c|}{ mPW1PW91 } \\
\hline & & \multicolumn{6}{|c|}{ Gas phase } & \multicolumn{6}{|c|}{ PCM } \\
\hline & & 6-31G* & 6-31G** & $6-31+G * *$ & 6-311G* & $6-311 G * *$ & $6-311+G * *$ & 6-31G* & 6-31G** & $6-31+G * *$ & 6-311G* & $6-311 G^{* *}$ & $6-311+G * *$ \\
\hline \multicolumn{14}{|l|}{${ }^{13} \mathrm{C}$} \\
\hline 1 & 99.3 & 95.8414 & 96.5271 & 95.0263 & 84.5881 & 83.9766 & 84.2287 & 96.0242 & 96.7071 & 95.1123 & 84.6896 & 84.0788 & 84.2622 \\
\hline 2 & 200.2 & -0.2721 & -0.0720 & -1.8389 & -17.5040 & -17.3306 & -20.5051 & -3.4063 & -3.2152 & -5.5438 & -20.9913 & -20.8254 & -24.5834 \\
\hline 3 & 46.9 & 145.6057 & 146.2546 & 146.0043 & 136.7101 & 136.4398 & 135.8021 & 145.1977 & 145.8386 & 145.4775 & 136.1776 & 135.9034 & 135.1064 \\
\hline 4 & 42.3 & 149.0529 & 149.6830 & 149.2327 & 139.8720 & 139.6160 & 139.4695 & 149.3169 & 149.9440 & 149.6802 & 140.2076 & 139.9432 & 139.8461 \\
\hline 5 & 74.8 & 119.0849 & 119.8395 & 119.0898 & 109.3287 & 108.8573 & 108.5765 & 118.9642 & 119.7183 & 118.9130 & 109.1561 & 108.6787 & 108.3577 \\
\hline 6 & 70.2 & 124.5923 & 125.8426 & 125.6047 & 115.6269 & 114.8753 & 114.4623 & 124.7212 & 125.9628 & 125.5355 & 115.6297 & 114.8530 & 114.3916 \\
\hline 7 & 46.9 & 144.6179 & 145.4269 & 144.9843 & 135.2619 & 134.9142 & 134.6833 & 144.5873 & 145.3937 & 144.9183 & 135.2039 & 134.8579 & 134.5822 \\
\hline 8 & 135.5 & 60.1754 & 60.5639 & 59.7837 & 43.1713 & 42.2343 & 41.5518 & 60.8220 & 61.2090 & 60.4650 & 43.8011 & 42.8918 & 42.2138 \\
\hline 9 & 134.1 & 63.3349 & 63.7856 & 63.2184 & 46.5320 & 45.6296 & 44.8315 & 62.3117 & 62.7619 & 61.9304 & 45.2706 & 44.3861 & 43.4471 \\
\hline 10 & 46.3 & 144.9403 & 145.7335 & 144.7419 & 135.0997 & 134.7560 & 134.5291 & 145.0310 & 145.8186 & 144.8751 & 135.2105 & 134.8622 & 134.6478 \\
\hline $\begin{array}{l}11 \\
{ }^{1} \mathrm{H}\end{array}$ & 49.4 & 143.8023 & 145.0111 & 144.3180 & 134.5935 & 134.1439 & 133.9543 & 143.8400 & 145.0431 & 144.3348 & 134.6505 & 134.1838 & 133.9684 \\
\hline 1 & 4.80 & 27.4769 & 26.9234 & 26.8754 & 27.4799 & 27.1516 & 27.1698 & 27.4798 & 26.9204 & 26.8674 & 27.4632 & 27.1328 & 27.1526 \\
\hline 3 & 3.00 & 29.1753 & 28.7107 & 28.5960 & 29.1562 & 28.8674 & 28.8185 & 29.0671 & 28.5968 & 28.4629 & 29.0445 & 28.7523 & 28.6882 \\
\hline 4 & 2.38 & 29.9403 & 29.5318 & 29.4222 & 29.9095 & 29.6715 & 29.5963 & 29.7267 & 29.3124 & 29.1836 & 29.6995 & 29.4590 & 29.3677 \\
\hline 5 & 4.62 & 27.8222 & 27.2805 & 27.1608 & 27.8170 & 27.4810 & 27.4474 & 27.6371 & 27.0883 & 26.9517 & 27.6316 & 27.2907 & 27.2433 \\
\hline 6endo & 3.84 & 28.4664 & 27.9554 & 27.8589 & 28.4393 & 28.0998 & 28.0590 & 28.3096 & 27.7946 & 27.6836 & 28.2879 & 27.9436 & 27.8891 \\
\hline $6 e x o$ & 3.78 & 28.4699 & 27.9355 & 27.7575 & 28.3559 & 28.0399 & 27.9926 & 28.3959 & 27.8633 & 27.6757 & 28.2811 & 27.9694 & 27.9126 \\
\hline 7 & 3.38 & 28.8209 & 28.2780 & 28.1590 & 28.7675 & 28.4419 & 28.3691 & 28.8315 & 28.2887 & 28.1635 & 28.7726 & 28.4490 & 28.3709 \\
\hline 8 & 6.01 & 25.8819 & 25.1935 & 25.0609 & 25.9110 & 25.4323 & 25.3847 & 25.8913 & 25.2023 & 25.0628 & 25.9069 & 25.4334 & 25.3829 \\
\hline 9 & 6.23 & 25.7869 & 25.1089 & 24.9479 & 25.7945 & 25.3297 & 25.2659 & 25.6948 & 25.0165 & 24.8426 & 25.6907 & 25.2320 & 25.1589 \\
\hline 10 & 3.03 & 29.3411 & 28.8236 & 28.6876 & 29.2612 & 28.9642 & 28.9157 & 29.2011 & 28.6774 & 28.5302 & 29.1227 & 28.8208 & 28.7636 \\
\hline
\end{tabular}




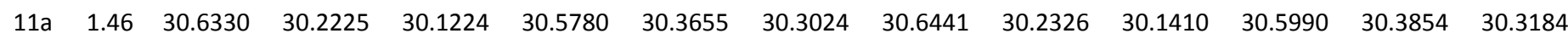

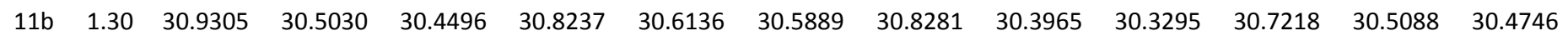

\section{Compound 9}

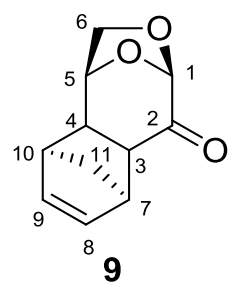

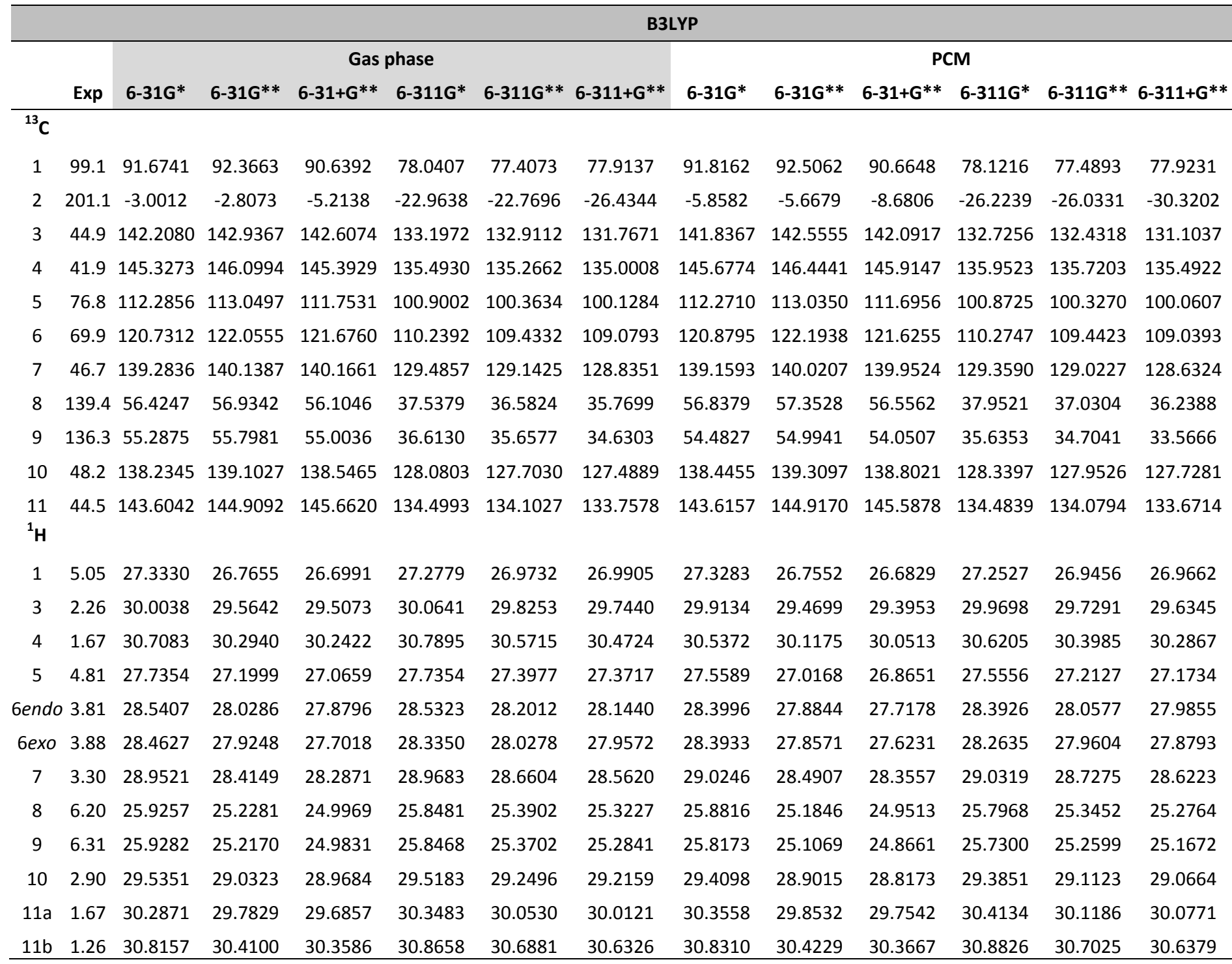

\begin{tabular}{|c|c|c|c|c|c|c|c|c|c|c|c|c|c|}
\hline & \multirow[b]{3}{*}{ Exp } & \multicolumn{12}{|c|}{ mPW1PW91 } \\
\hline & & \multicolumn{6}{|c|}{ Gas phase } & \multicolumn{6}{|c|}{ PCM } \\
\hline & & 6-31G* & $6-31 G * *$ & $6-31+\mathrm{G} * *$ & 6-311G* & 6-311G** & $6-311+G * *$ & 6-31G* & $6-31 G * *$ & $6-31+\mathrm{G} * *$ & 6-311G* & $6-311 G * *$ & $6-311+G * *$ \\
\hline & & & & & & & & & & & & & \\
\hline 1 & 99.1 & 96.0334 & 96.7068 & 95.3131 & 84.6650 & 84.0493 & 84.4191 & 96.1817 & 96.8530 & 95.3580 & 84.7465 & 84.1319 & 84.4381 \\
\hline
\end{tabular}




\begin{tabular}{|c|c|c|c|c|c|c|c|c|c|c|c|c|c|}
\hline 2 & 201.1 & -0.9446 & -0.7617 & -2.5303 & -18.0410 & -17.8530 & -21.1490 & -3.8866 & -3.7083 & -6.0223 & -21.3240 & -21.1407 & -24.9880 \\
\hline 3 & 44.9 & 146.9027 & 147.5622 & 147.3313 & 138.6936 & 138.4049 & 137.3237 & 146.5264 & 147.1762 & 146.8305 & 138.2166 & 137.9198 & 136.6790 \\
\hline 4 & 41.9 & 150.5156 & 151.2021 & 150.4733 & 141.4768 & 141.2303 & 140.8909 & 150.8494 & 151.5318 & 150.9610 & 141.8899 & 141.6397 & 141.3336 \\
\hline 5 & 76.8 & 116.9603 & 117.6714 & 116.6163 & 107.1351 & 106.6018 & 106.3421 & 116.9230 & 117.6350 & 116.5387 & 107.0745 & 106.5339 & 106.2487 \\
\hline 6 & 69.9 & 124.7813 & 126.0340 & 125.9062 & 115.7516 & 114.9682 & 114.6537 & 124.9090 & 126.1536 & 125.8374 & 115.7511 & 114.9435 & 114.5874 \\
\hline 7 & 46.7 & 143.9343 & 144.7142 & 144.6823 & 135.0215 & 134.6676 & 134.3462 & 143.8408 & 144.6266 & 144.5153 & 134.9194 & 134.5718 & 134.1788 \\
\hline 8 & 139.4 & 58.9489 & 59.3674 & 58.5244 & 42.0661 & 41.0560 & 40.3273 & 59.3577 & 59.7825 & 58.9716 & 42.4600 & 41.4860 & 40.7763 \\
\hline 9 & 136.3 & 57.8861 & 58.3057 & 57.5016 & 41.1471 & 40.1448 & 39.2589 & 57.0307 & 57.4519 & 56.5223 & 40.1304 & 39.1542 & 38.1742 \\
\hline 10 & 48.2 & 142.9872 & 143.7782 & 143.2074 & 133.7534 & 133.3564 & 133.1224 & 143.2034 & 143.9909 & 143.4609 & 133.9967 & 133.5911 & 133.3475 \\
\hline $\begin{array}{l}11 \\
{ }^{1} \mathbf{H}\end{array}$ & 44.5 & 147.7168 & 148.9037 & 149.4267 & 139.3635 & 138.9608 & 138.6090 & 147.7562 & 148.9390 & 149.3853 & 139.3653 & 138.9543 & 138.5481 \\
\hline 1 & 5.05 & 27.2297 & 26.6685 & 26.6237 & 27.2253 & 26.8856 & 26.9155 & 27.2194 & 26.6523 & 26.6022 & 27.1956 & 26.8538 & 26.8870 \\
\hline 3 & 2.26 & 29.9467 & 29.4987 & 29.4433 & 29.9531 & 29.7137 & 29.6382 & 29.8557 & 29.4034 & 29.3333 & 29.8599 & 29.6185 & 29.5308 \\
\hline 4 & 1.67 & 30.6799 & 30.2650 & 30.1921 & 30.6788 & 30.4694 & 30.3679 & 30.5022 & 30.0815 & 29.9977 & 30.5072 & 30.2934 & 30.1809 \\
\hline 5 & 4.81 & 27.6539 & 27.1151 & 26.9886 & 27.6395 & 27.2886 & 27.2731 & 27.4668 & 26.9207 & 26.7804 & 27.4529 & 27.0967 & 27.0697 \\
\hline 6endo & 3.81 & 28.4827 & 27.9698 & 27.8485 & 28.4600 & 28.1146 & 28.0719 & 28.3316 & 27.8152 & 27.6797 & 28.3141 & 27.9647 & 27.9087 \\
\hline 6exo & 3.88 & 28.4019 & 27.8655 & 27.6641 & 28.2805 & 27.9606 & 27.9027 & 28.3224 & 27.7876 & 27.5763 & 28.2006 & 27.8849 & 27.8175 \\
\hline 7 & 3.30 & 28.8346 & 28.2973 & 28.1835 & 28.8387 & 28.5179 & 28.4300 & 28.9034 & 28.3693 & 28.2487 & 28.8992 & 28.5820 & 28.4876 \\
\hline 8 & 6.20 & 25.7491 & 25.0468 & 24.8531 & 25.7093 & 25.2151 & 25.1568 & 25.6962 & 24.9946 & 24.8001 & 25.6514 & 25.1640 & 25.1042 \\
\hline 9 & 6.31 & 25.7536 & 25.0390 & 24.8407 & 25.7055 & 25.1952 & 25.1159 & 25.6309 & 24.9169 & 24.7138 & 25.5796 & 25.0758 & 24.9906 \\
\hline 10 & 2.90 & 29.4206 & 28.9191 & 28.8587 & 29.3925 & 29.1071 & 29.0807 & 29.2858 & 28.7781 & 28.7008 & 29.2531 & 28.9632 & 28.9263 \\
\hline $11 a$ & 1.67 & 30.2437 & 29.7431 & 29.6512 & 30.2580 & 29.9663 & 29.9218 & 30.3144 & 29.8158 & 29.7218 & 30.3239 & 30.0330 & 29.9877 \\
\hline $11 \mathrm{~b}$ & 1.26 & 30.7344 & 30.3312 & 30.2724 & 30.7571 & 30.5796 & 30.5264 & 30.7496 & 30.3439 & 30.2800 & 30.7729 & 30.5931 & 30.5311 \\
\hline
\end{tabular}

\section{Compound 10}

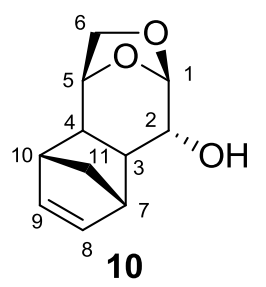

\begin{tabular}{|c|c|c|c|c|c|c|c|c|c|c|c|c|c|}
\hline & \multirow[b]{3}{*}{ Exp } & \multicolumn{12}{|c|}{ B3LYP } \\
\hline & & \multicolumn{6}{|c|}{ Gas phase } & \multicolumn{6}{|c|}{ PCM } \\
\hline & & 6-31G* & $6-31 G * *$ & $6-31+\mathrm{G} * *$ & 6-311G* & $6-311 G * *$ & $6-311+G * *$ & 6-31G* & $6-31 G * *$ & $6-31+\mathrm{G} * *$ & 6-311G* & $6-311 G * *$ & $6-311+\mathrm{G} * *$ \\
\hline \multicolumn{14}{|c|}{${ }^{13} \mathrm{C}$} \\
\hline 1 & 101.9 & 88.3226 & 89.0406 & 87.8734 & 75.4822 & 74.9326 & 75.0026 & 88.4405 & 89.1602 & 87.8828 & 75.4929 & 74.9465 & 74.9851 \\
\hline 2 & 67.9 & 122.1943 & 122.9135 & 120.8208 & 110.4657 & 110.1002 & 109.6150 & 122.1894 & 122.9094 & 120.6948 & 110.3569 & 110.0002 & 109.4439 \\
\hline 3 & 39.4 & 145.0634 & 145.8922 & 145.7343 & 134.4425 & 134.4576 & 134.7437 & 145.5334 & 146.3542 & 146.2662 & 134.9417 & 134.9393 & 135.2565 \\
\hline 4 & 42.2 & 143.2190 & 143.9336 & 143.0772 & 132.9596 & 132.7673 & 132.5355 & 143.5193 & 144.2295 & 143.5157 & 133.3525 & 133.1484 & 132.9580 \\
\hline 5 & 74.4 & 114.9357 & 115.7678 & 114.8132 & 103.5842 & 103.1043 & 102.7496 & 114.8881 & 115.7192 & 114.7011 & 103.5020 & 103.0163 & 102.6239 \\
\hline 6 & 71.2 & 118.3888 & 119.6850 & 119.1423 & 107.8007 & 106.9966 & 106.8336 & 118.5352 & 119.8239 & 119.1198 & 107.8606 & 107.0352 & 106.8231 \\
\hline 7 & 46.4 & 140.4390 & 141.3481 & 140.5577 & 129.8435 & 129.6034 & 129.4347 & 140.5375 & 141.4424 & 140.6240 & 129.9402 & 129.6956 & 129.5063 \\
\hline 8 & 137.7 & 55.2350 & 55.7115 & 55.0859 & 35.7393 & 34.8204 & 33.9342 & 55.5472 & 56.0117 & 55.3464 & 35.9922 & 35.0655 & 34.1619 \\
\hline 9 & 132.9 & 62.4504 & 62.9595 & 62.6283 & 43.6580 & 42.9493 & 42.0431 & 61.5968 & 62.1138 & 61.5555 & 42.5670 & 41.9125 & 40.8761 \\
\hline
\end{tabular}




\begin{tabular}{cccccccccccccccc}
\hline 10 & 47.6 & 140.2906 & 141.1509 & 139.8834 & 129.2828 & 128.9301 & 128.7474 & 140.3535 & 141.2099 & 139.9787 & 129.3696 & 129.0151 & 128.8391 \\
11 & 50.0 & 137.8533 & 139.1873 & 138.6134 & 127.7667 & 127.3722 & 126.9847 & 137.8703 & 139.2002 & 138.5811 & 127.8049 & 127.3971 & 126.9704 \\
${ }^{1} \mathbf{H}$ & & & & & & & & & & & & & & \\
1 & 5.07 & 27.2379 & 26.7120 & 26.5195 & 27.1683 & 26.8921 & 26.8301 & 27.2459 & 26.7172 & 26.5146 & 27.1586 & 26.8797 & 26.8165 \\
1 & 3.82 & 28.5500 & 28.1003 & 27.9693 & 28.5397 & 28.3034 & 28.2305 & 28.5417 & 28.0883 & 27.9352 & 28.5114 & 28.2689 & 28.1877 \\
3 & 2.76 & 29.4451 & 29.0195 & 28.9385 & 29.4705 & 29.2195 & 29.2045 & 29.3844 & 28.9564 & 28.8576 & 29.4012 & 29.1480 & 29.1219 \\
4 & 2.11 & 30.1415 & 29.7443 & 29.6918 & 30.1993 & 29.9474 & 29.8832 & 30.0058 & 29.6062 & 29.5360 & 30.0580 & 29.8076 & 29.7319 \\
5 & 4.32 & 28.1371 & 27.6067 & 27.4857 & 28.1607 & 27.8306 & 27.7952 & 27.9924 & 27.4566 & 27.3200 & 28.0098 & 27.6768 & 27.6313 \\
6endo & 3.73 & 28.6257 & 28.1149 & 27.9804 & 28.5825 & 28.2391 & 28.2063 & 28.5003 & 27.9869 & 27.8356 & 28.4555 & 28.1098 & 28.0648 \\
6 6xo & 3.61 & 28.5959 & 28.0735 & 27.8822 & 28.4603 & 28.1767 & 28.1580 & 28.5576 & 28.0377 & 27.8357 & 28.4169 & 28.1383 & 28.1109 \\
7 & 3.08 & 29.2054 & 28.6855 & 28.5888 & 29.1488 & 28.8788 & 28.8263 & 29.2124 & 28.6908 & 28.5846 & 29.1494 & 28.8764 & 28.8184 \\
8 & 6.32 & 25.7253 & 25.0296 & 24.8029 & 25.7266 & 25.2692 & 25.1408 & 25.7163 & 25.0201 & 24.7877 & 25.7058 & 25.2520 & 25.1246 \\
9 & 6.23 & 26.0553 & 25.3512 & 25.1201 & 25.9966 & 25.5225 & 25.4487 & 25.9599 & 25.2566 & 25.0138 & 25.8887 & 25.4237 & 25.3429 \\
10 & 2.91 & 29.5978 & 29.0803 & 28.9513 & 29.5248 & 29.2284 & 29.1777 & 29.4893 & 28.9675 & 28.8269 & 29.4122 & 29.1135 & 29.0560 \\
$11 \mathrm{a}$ & 1.38 & 30.7141 & 30.3168 & 30.2082 & 30.6731 & 30.4762 & 30.4130 & 30.7396 & 30.3418 & 30.2426 & 30.7070 & 30.5099 & 30.4441 \\
$11 \mathrm{~b}$ & 1.25 & 30.9325 & 30.5078 & 30.4692 & 30.8860 & 30.6717 & 30.6707 & 30.8646 & 30.4378 & 30.3860 & 30.8140 & 30.5987 & 30.5906 \\
\hline
\end{tabular}

\begin{tabular}{|c|c|c|c|c|c|c|c|c|c|c|c|c|c|}
\hline & \multirow[b]{3}{*}{ Exp } & \multicolumn{12}{|c|}{ mPW1PW91 } \\
\hline & & \multicolumn{6}{|c|}{ Gas phase } & \multicolumn{6}{|c|}{ PCM } \\
\hline & & 6-31G* & $6-31 G^{* *}$ & $6-31+\mathrm{G} * *$ & 6-311G* & 6-311G** & $6-311+G^{* *}$ & 6-31G* & $6-31 G^{* *}$ & $6-31+G * *$ & 6-311G* & 6-311G** & $6-311+G^{* *}$ \\
\hline \multicolumn{14}{|c|}{ ( } \\
\hline 1 & 101.9 & 92.9400 & 93.6192 & 92.7160 & 82.0954 & 81.5631 & 81.5619 & 93.0694 & 93.7499 & 92.7472 & 82.1172 & 81.5865 & 81.5567 \\
\hline 2 & 67.9 & 126.8238 & 127.4913 & 125.7285 & 116.8573 & 116.4696 & 115.8631 & 126.8264 & 127.4948 & 125.6239 & 116.7542 & 116.3747 & 115.7044 \\
\hline 3 & 39.4 & 150.4717 & 151.2139 & 151.0483 & 140.7085 & 140.6988 & 140.8267 & 150.9446 & 151.6798 & 151.5713 & 141.1899 & 141.1638 & 141.3188 \\
\hline 4 & 42.2 & 148.6452 & 149.2706 & 148.4311 & 139.1591 & 138.9430 & 138.6236 & 148.9439 & 149.5663 & 148.8541 & 139.5245 & 139.2979 & 139.0172 \\
\hline 5 & 74.4 & 119.5042 & 120.2853 & 119.5215 & 109.7412 & 109.2613 & 108.9054 & 119.4376 & 120.2183 & 119.3940 & 109.6293 & 109.1448 & 108.7567 \\
\hline 6 & 71.2 & 122.5587 & 123.7781 & 123.3724 & 113.4020 & 112.6162 & 112.4589 & 122.6999 & 123.9133 & 123.3534 & 113.4385 & 112.6335 & 112.4351 \\
\hline 7 & 46.4 & 145.1572 & 145.9803 & 145.1590 & 135.4772 & 135.2199 & 135.0183 & 145.2771 & 146.0954 & 145.2489 & 135.5856 & 135.3228 & 135.1021 \\
\hline 8 & 137.7 & 58.0421 & 58.4302 & 57.9001 & 40.4797 & 39.5173 & 38.7614 & 58.3467 & 58.7232 & 58.1574 & 40.7139 & 39.7443 & 38.9718 \\
\hline 9 & 132.9 & 64.7121 & 65.1325 & 64.8846 & 47.7862 & 47.0334 & 46.2254 & 63.8289 & 64.2570 & 63.8120 & 46.6798 & 45.9823 & 45.0632 \\
\hline 10 & 47.6 & 144.9631 & 145.7409 & 144.4246 & 134.9547 & 134.5823 & 134.3723 & 145.0231 & 145.7972 & 144.5108 & 135.0229 & 134.6498 & 134.4455 \\
\hline $\begin{array}{l}11 \\
{ }^{1} \mathbf{H}\end{array}$ & 50.0 & 142.2920 & 143.5093 & 142.7599 & 133.0584 & 132.6500 & 132.2776 & 142.3229 & 143.5371 & 142.7442 & 133.0925 & 132.6719 & 132.2656 \\
\hline 1 & 5.07 & 27.1739 & 26.6514 & 26.4871 & 27.1321 & 26.8317 & 26.7811 & 27.1776 & 26.6523 & 26.4792 & 27.1189 & 26.8162 & 26.7650 \\
\hline 2 & 3.82 & 28.4867 & 28.0334 & 27.9088 & 28.4571 & 28.2111 & 28.1455 & 28.4746 & 28.0173 & 27.8729 & 28.4262 & 28.1741 & 28.1010 \\
\hline 3 & 2.76 & 29.4017 & 28.9711 & 28.8799 & 29.3670 & 29.1228 & 29.1030 & 29.3369 & 28.9035 & 28.7973 & 29.2957 & 29.0490 & 29.0191 \\
\hline 4 & 2.11 & 30.1253 & 29.7239 & 29.6417 & 30.1083 & 29.8605 & 29.7919 & 29.9811 & 29.5770 & 29.4806 & 29.9622 & 29.7156 & 29.6370 \\
\hline 5 & 4.32 & 28.0659 & 27.5310 & 27.4121 & 28.0708 & 27.7242 & 27.6944 & 27.9139 & 27.3731 & 27.2420 & 27.9156 & 27.5661 & 27.5276 \\
\hline Gendo & 3.73 & 28.5697 & 28.0597 & 27.9523 & 28.5217 & 28.1654 & 28.1428 & 28.4339 & 27.9210 & 27.8000 & 28.3878 & 28.0291 & 27.9961 \\
\hline 6exo & 3.61 & 28.5521 & 28.0325 & 27.8733 & 28.4203 & 28.1260 & 28.1180 & 28.5059 & 27.9888 & 27.8200 & 28.3703 & 28.0813 & 28.0654 \\
\hline 7 & 3.08 & 29.1096 & 28.5882 & 28.4989 & 29.0394 & 28.7525 & 28.7026 & 29.1136 & 28.5903 & 28.4921 & 29.0377 & 28.7475 & 28.6925 \\
\hline 8 & 6.32 & 25.5565 & 24.8629 & 24.6639 & 25.5802 & 25.0972 & 24.9820 & 25.5407 & 24.8466 & 24.6428 & 25.5539 & 25.0749 & 24.9604 \\
\hline 9 & 6.23 & 25.8767 & 25.1689 & 24.9633 & 25.8536 & 25.3422 & 25.2676 & 25.7726 & 25.0657 & 24.8508 & 25.7395 & 25.2376 & 25.1568 \\
\hline 10 & 2.91 & 29.4888 & 28.9701 & 28.8502 & 29.4033 & 29.0883 & 29.0450 & 29.3721 & 28.8487 & 28.7193 & 29.2851 & 28.9675 & 28.9181 \\
\hline $11 a$ & 1.38 & 30.6319 & 30.2359 & 30.1339 & 30.5700 & 30.3683 & 30.3077 & 30.6576 & 30.2614 & 30.1662 & 30.6022 & 30.4005 & 30.3366 \\
\hline
\end{tabular}




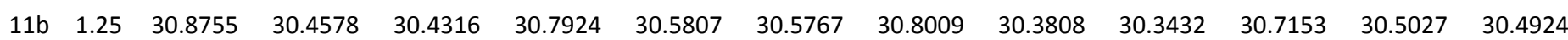

\section{Compound 11}

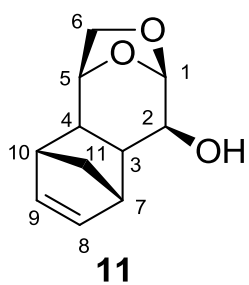

\begin{tabular}{|c|c|c|c|c|c|c|c|c|c|c|c|c|c|}
\hline & \multirow[b]{3}{*}{ Exp } & \multicolumn{12}{|c|}{ B3LYP } \\
\hline & & \multicolumn{6}{|c|}{ Gas phase } & \multicolumn{6}{|c|}{ PCM } \\
\hline & & 6-31G* & $6-31 G * *$ & $6-31+G * *$ & 6-311G* & $6-311 G * *$ & $6-311+G * *$ & $6-31 G *$ & $6-31 G * *$ & $6-31+G^{* *}$ & 6-311G* & $6-311 G * *$ & $6-311+G * *$ \\
\hline \multicolumn{14}{|l|}{${ }^{13} \mathrm{C}$} \\
\hline 1 & 99.9 & 89.9687 & 90.7286 & 89.6052 & 77.4514 & 76.9117 & 77.1715 & 90.0845 & 90.8429 & 89.6330 & 77.4183 & 76.8895 & 77.0968 \\
\hline 2 & 69.8 & 117.8326 & 118.7216 & 117.8398 & 106.3264 & 106.2860 & 106.0626 & 117.9132 & 118.7977 & 117.8700 & 106.3504 & 106.2996 & 106.0592 \\
\hline 3 & 46.9 & 141.7298 & 142.4570 & 142.1253 & 131.4820 & 131.2348 & 131.1575 & 141.9609 & 142.6847 & 142.4475 & 131.6867 & 131.4455 & 131.4257 \\
\hline 4 & 44.2 & 139.6448 & 140.3662 & 139.5582 & 129.1097 & 128.9252 & 128.4328 & 139.8892 & 140.6051 & 139.9197 & 129.4011 & 129.2064 & 128.7358 \\
\hline 5 & 74.4 & 114.7964 & 115.6276 & 114.8261 & 103.6137 & 103.1391 & 102.9061 & 114.7529 & 115.5835 & 114.7430 & 103.5314 & 103.0519 & 102.7889 \\
\hline 6 & 71.1 & 118.9815 & 120.3303 & 120.0099 & 108.6509 & 107.9182 & 107.7780 & 119.1851 & 120.5256 & 119.9992 & 108.7460 & 107.9880 & 107.7952 \\
\hline 7 & 46.3 & 142.1994 & 143.0917 & 142.8475 & 131.9256 & 131.6205 & 131.6117 & 142.1266 & 143.0164 & 142.7351 & 131.8424 & 131.5374 & 131.4775 \\
\hline 8 & 135.7 & 59.1311 & 59.6330 & 59.3320 & 40.6690 & 39.7280 & 38.9636 & 58.6100 & 59.1096 & 58.6683 & 39.9268 & 39.0306 & 38.1707 \\
\hline 9 & 135.7 & 58.2120 & 58.6739 & 58.0208 & 38.9191 & 38.0017 & 37.2089 & 58.0455 & 58.5071 & 57.7195 & 38.6750 & 37.7781 & 36.8881 \\
\hline 10 & 47.8 & 140.0113 & 140.8574 & 139.9080 & 129.0574 & 128.6939 & 128.4039 & 140.0298 & 140.8736 & 139.9456 & 129.0999 & 128.7361 & 128.4524 \\
\hline $\begin{array}{l}11 \\
{ }^{\mathbf{1}_{\mathbf{H}}}\end{array}$ & 50.3 & 138.0538 & 139.3805 & 138.8548 & 128.0816 & 127.6229 & 127.4584 & 138.1941 & 139.5120 & 138.9895 & 128.2577 & 127.7803 & 127.5783 \\
\hline 1 & 5.14 & 27.0162 & 26.4796 & 26.3245 & 26.9291 & 26.6428 & 26.6230 & 27.0429 & 26.5035 & 26.3376 & 26.9356 & 26.6459 & 26.6259 \\
\hline 2 & 3.16 & 28.9692 & 28.5069 & 28.4477 & 29.0132 & 28.7315 & 28.7299 & 28.9178 & 28.4504 & 28.3701 & 28.9528 & 28.6663 & 28.6519 \\
\hline 3 & 2.23 & 29.7484 & 29.3432 & 29.3155 & 29.8254 & 29.6208 & 29.5691 & 29.7421 & 29.3339 & 29.3004 & 29.8117 & 29.6041 & 29.5507 \\
\hline 4 & 2.23 & 29.9345 & 29.5430 & 29.5010 & 30.0109 & 29.7783 & 29.7044 & 29.8094 & 29.4148 & 29.3498 & 29.8811 & 29.6466 & 29.5578 \\
\hline 5 & 4.41 & 28.0939 & 27.5676 & 27.4486 & 28.1166 & 27.7997 & 27.7737 & 27.9508 & 27.4184 & 27.2806 & 27.9666 & 27.6454 & 27.6070 \\
\hline Gendo & 3.73 & 28.5364 & 28.0352 & 27.9115 & 28.5182 & 28.1992 & 28.1760 & 28.4290 & 27.9248 & 27.7847 & 28.4091 & 28.0856 & 28.0500 \\
\hline $6 e x o$ & 3.73 & 28.5177 & 27.9990 & 27.8119 & 28.3932 & 28.1272 & 28.0911 & 28.4891 & 27.9723 & 27.7748 & 28.3599 & 28.0965 & 28.0516 \\
\hline 7 & 3.16 & 29.4750 & 28.9310 & 28.8132 & 29.4469 & 29.1141 & 29.0644 & 29.3412 & 28.7934 & 28.6672 & 29.3074 & 28.9772 & 28.9232 \\
\hline 8 & 6.18 & 26.0701 & 25.3634 & 25.1741 & 26.0752 & 25.6001 & 25.5144 & 25.9856 & 25.2785 & 25.0784 & 25.9758 & 25.5071 & 25.4186 \\
\hline 9 & 6.28 & 25.8566 & 25.1701 & 24.9543 & 25.8239 & 25.3808 & 25.2831 & 25.8132 & 25.1259 & 24.9006 & 25.7670 & 25.3284 & 25.2266 \\
\hline 10 & 2.89 & 29.5739 & 29.0585 & 28.9362 & 29.5097 & 29.2224 & 29.1768 & 29.4766 & 28.9569 & 28.8217 & 29.4097 & 29.1188 & 29.0648 \\
\hline $11 a$ & 1.44 & 30.6791 & 30.2693 & 30.1577 & 30.6391 & 30.4278 & 30.3443 & 30.7121 & 30.3020 & 30.2022 & 30.6818 & 30.4701 & 30.3849 \\
\hline $11 b$ & 1.28 & 30.9184 & 30.4865 & 30.3906 & 30.8662 & 30.6392 & 30.5888 & 30.8633 & 30.4296 & 30.3201 & 30.8070 & 30.5796 & 30.5208 \\
\hline
\end{tabular}

\begin{tabular}{|c|c|c|c|c|c|c|c|c|c|c|c|}
\hline \multirow[b]{3}{*}{ Exp } & \multicolumn{11}{|c|}{ mPW1PW91 } \\
\hline & \multicolumn{6}{|c|}{ Gas phase } & \multicolumn{5}{|c|}{ PCM } \\
\hline & 6-31G* & $6-31 G * *$ & $6-31+G * *$ & 6-311G* & $6-311 G * *$ & $6-311+G * *$ & 6-31G* & $6-31 \mathrm{G} * *$ & $6-31+G * *$ & 6-311G* & $6-311 G * * 6-311+G * *$ \\
\hline
\end{tabular}




\begin{tabular}{|c|c|c|c|c|c|c|c|c|c|c|c|c|c|}
\hline 1 & 99.9 & 94.4136 & 95.1392 & 94.3500 & 83.9302 & 83.4094 & 83.5741 & 94.5434 & 95.2664 & 94.3987 & 83.9017 & 83.3903 & 83.5059 \\
\hline 2 & 69.8 & 122.5333 & 123.3650 & 122.7513 & 112.7972 & 112.7446 & 112.4365 & 122.5901 & 123.4197 & 122.7591 & 112.7932 & 112.7324 & 112.4100 \\
\hline 3 & 46.9 & 147.2845 & 147.9290 & 147.5560 & 137.9530 & 137.6940 & 137.5387 & 147.5179 & 148.1599 & 147.8687 & 138.1462 & 137.8941 & 137.7874 \\
\hline 4 & 44.2 & 145.0756 & 145.7067 & 144.8239 & 135.3536 & 135.1487 & 134.6103 & 145.3182 & 145.9454 & 145.1717 & 135.6187 & 135.4047 & 134.8862 \\
\hline 5 & 74.4 & 119.3827 & 120.1625 & 119.4746 & 109.7815 & 109.3049 & 109.0722 & 119.3183 & 120.0981 & 119.3727 & 109.6675 & 109.1871 & 108.9279 \\
\hline 6 & 71.1 & 123.1624 & 124.4377 & 124.2420 & 114.2505 & 113.5354 & 113.4171 & 123.3539 & 124.6225 & 124.2256 & 114.3122 & 113.5740 & 113.4076 \\
\hline 7 & 46.3 & 146.8048 & 147.6164 & 147.2800 & 137.4104 & 137.0855 & 137.0437 & 146.7284 & 147.5379 & 147.1688 & 137.3172 & 136.9943 & 136.9072 \\
\hline 8 & 135.7 & 61.8049 & 62.2272 & 61.9762 & 45.1937 & 44.2203 & 43.5666 & 61.2438 & 61.6640 & 61.2955 & 44.4214 & 43.4952 & 42.7585 \\
\hline 9 & 135.7 & 60.5104 & 60.8781 & 60.3522 & 43.1948 & 42.2294 & 41.4889 & 60.3226 & 60.6901 & 60.0386 & 42.9187 & 41.9752 & 41.1455 \\
\hline 10 & 47.8 & 144.6845 & 145.4477 & 144.4361 & 134.7131 & 134.3288 & 134.0370 & 144.7012 & 145.4620 & 144.4667 & 134.7412 & 134.3572 & 134.0694 \\
\hline $\begin{array}{l}11 \\
{ }^{1} \mathbf{H}\end{array}$ & 50.3 & 142.5014 & 143.7107 & 142.9677 & 133.3956 & 132.9327 & 132.7398 & 142.6642 & 143.8653 & 143.1219 & 133.5718 & 133.0906 & 132.8622 \\
\hline 1 & 5.14 & 26.9496 & 26.4146 & 26.2821 & 26.8942 & 26.5828 & 26.5683 & 26.9724 & 26.4345 & 26.2921 & 26.8975 & 26.5828 & 26.5685 \\
\hline 2 & 3.16 & 28.9797 & 28.5208 & 28.4448 & 28.9721 & 28.6947 & 28.6825 & 28.9226 & 28.4585 & 28.3645 & 28.9080 & 28.6260 & 28.6028 \\
\hline 3 & 2.23 & 29.6856 & 29.2734 & 29.2349 & 29.7097 & 29.5035 & 29.4556 & 29.6770 & 29.2617 & 29.2192 & 29.6947 & 29.4855 & 29.4362 \\
\hline 4 & 2.23 & 29.9067 & 29.5129 & 29.4420 & 29.9113 & 29.6842 & 29.6080 & 29.7731 & 29.3758 & 29.2856 & 29.7767 & 29.5473 & 29.4578 \\
\hline 5 & 4.41 & 28.0231 & 27.4930 & 27.3732 & 28.0258 & 27.6929 & 27.6727 & 27.8718 & 27.3350 & 27.1998 & 27.8706 & 27.5332 & 27.5022 \\
\hline Gendo & 3.73 & 28.4801 & 27.9793 & 27.8853 & 28.4566 & 28.1237 & 28.1106 & 28.3625 & 27.8585 & 27.7505 & 28.3404 & 28.0029 & 27.9787 \\
\hline 6exo & 3.73 & 28.4727 & 27.9563 & 27.8012 & 28.3516 & 28.0746 & 28.0520 & 28.4356 & 27.9211 & 27.7563 & 28.3109 & 28.0368 & 28.0059 \\
\hline 7 & 3.16 & 29.3819 & 28.8376 & 28.7276 & 29.3414 & 28.9897 & 28.9487 & 29.2396 & 28.6909 & 28.5744 & 29.1957 & 28.8464 & 28.8017 \\
\hline 8 & 6.18 & 25.9043 & 25.1982 & 25.0284 & 25.9353 & 25.4306 & 25.3457 & 25.8102 & 25.1038 & 24.9261 & 25.8292 & 25.3313 & 25.2444 \\
\hline 9 & 6.28 & 25.6854 & 24.9955 & 24.7968 & 25.6834 & 25.2047 & 25.1106 & 25.6340 & 24.9433 & 24.7369 & 25.6206 & 25.1468 & 25.0486 \\
\hline 10 & 2.89 & 29.4613 & 28.9455 & 28.8269 & 29.3845 & 29.0800 & 29.0410 & 29.3560 & 28.8354 & 28.7057 & 29.2789 & 28.9705 & 28.9238 \\
\hline $11 a$ & 1.44 & 30.5995 & 30.1915 & 30.0837 & 30.5365 & 30.3223 & 30.2459 & 30.6327 & 30.2246 & 30.1260 & 30.5775 & 30.3632 & 30.2842 \\
\hline $11 \mathrm{~b}$ & 1.28 & 30.8550 & 30.4296 & 30.3504 & 30.7696 & 30.5453 & 30.4977 & 30.7937 & 30.3664 & 30.2748 & 30.7052 & 30.4805 & 30.4253 \\
\hline
\end{tabular}

\section{Compound 12}

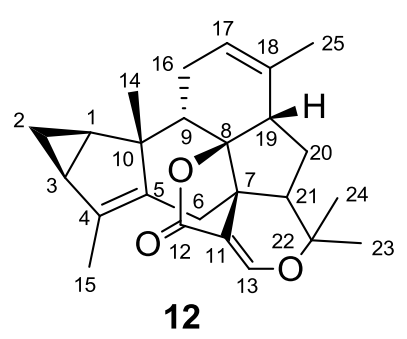

\begin{tabular}{|c|c|c|c|c|c|c|c|c|c|c|c|c|c|}
\hline & \multirow[b]{3}{*}{ Exp } & \multicolumn{12}{|c|}{ B3LYP } \\
\hline & & \multicolumn{6}{|c|}{ Gas phase } & \multicolumn{6}{|c|}{ PCM } \\
\hline & & 6-31G* & $6-31 G * *$ & $6-31+G * *$ & 6-311G* & $6-311 \mathrm{G} * *$ & $6-311+G * *$ & 6-31G* & $6-31 G * *$ & $6-31+G * *$ & 6-311G* & $6-311 G * *$ & $6-311+G * *$ \\
\hline \multicolumn{14}{|c|}{${ }^{13} \mathrm{C}$} \\
\hline 1 & 27.1 & 160.6048 & 161.4754 & 161.9820 & 151.9937 & 151.5984 & 150.8130 & 160.8251 & 161.6917 & 162.1914 & 152.2292 & 151.8254 & 151.0571 \\
\hline 2 & 15.1 & 172.2262 & 173.8077 & 174.5607 & 166.3145 & 165.8093 & 165.8042 & 172.5399 & 174.1043 & 174.8939 & 166.6668 & 166.1421 & 166.1279 \\
\hline 3 & 26.7 & 161.4014 & 162.2713 & 162.7433 & 152.4794 & 152.1073 & 151.8918 & 161.5072 & 162.3708 & 162.8402 & 152.6056 & 152.2182 & 151.9816 \\
\hline 4 & 136.2 & 56.9289 & 57.2141 & 56.6305 & 37.7578 & 37.9431 & 37.4089 & 55.7795 & 56.0557 & 55.3634 & 36.4214 & 36.5926 & 35.9649 \\
\hline 5 & 129.8 & 59.9804 & 60.1632 & 60.0853 & 41.0677 & 41.2148 & 40.0647 & 60.8862 & 61.0756 & 61.1020 & 42.0866 & 42.2402 & 41.1828 \\
\hline 6 & 27.2 & 159.4777 & 160.8437 & 160.8732 & 151.8611 & 151.4330 & 151.2804 & 159.6317 & 160.9899 & 161.0176 & 151.9976 & 151.5630 & 151.4130 \\
\hline 7 & 54.4 & 132.8203 & 133.0315 & 131.9894 & 121.5368 & 121.6123 & 121.1623 & 132.5905 & 132.7967 & 131.6961 & 121.3190 & 121.3886 & 120.8487 \\
\hline
\end{tabular}




\begin{tabular}{|c|c|c|c|c|c|c|c|c|c|c|c|c|c|}
\hline 8 & 97.8 & 94.5116 & 94.7198 & 93.7666 & 81.0016 & 81.1555 & 80.3912 & 93.7108 & 93.9191 & 92.7639 & 80.0873 & 80.2434 & 79.3282 \\
\hline 9 & 48.5 & 140.4053 & 141.1075 & 140.5639 & 129.4033 & 129.0121 & 128.3221 & 140.2357 & 140.9350 & 140.3412 & 129.1734 & 128.7820 & 128.0908 \\
\hline 10 & 48.6 & 137.6989 & 138.0860 & 139.0736 & 126.8275 & 126.9898 & 126.1519 & 137.8868 & 138.2755 & 139.2910 & 127.0763 & 127.2327 & 126.3961 \\
\hline 11 & 110.1 & 82.9115 & 82.6792 & 83.7530 & 66.6435 & 66.4178 & 64.2104 & 83.0516 & 82.8155 & 83.8706 & 66.8431 & 66.6199 & 64.3191 \\
\hline 12 & 170.5 & 31.3065 & 31.3579 & 29.5252 & 11.0737 & 11.0968 & 8.4708 & 29.0572 & 29.1066 & 26.8314 & 8.4398 & 8.4583 & 5.4576 \\
\hline 13 & 150.0 & 45.8793 & 46.6886 & 44.8510 & 27.3028 & 26.4810 & 25.8688 & 44.6579 & 45.4665 & 43.5242 & 25.9372 & 25.1153 & 24.3283 \\
\hline 14 & 24.5 & 164.5758 & 166.3416 & 165.4315 & 157.6525 & 157.4598 & 157.4041 & 164.6791 & 166.4493 & 165.6056 & 157.8543 & 157.6513 & 157.6083 \\
\hline 15 & 13.7 & 174.6961 & 176.4414 & 177.6518 & 167.9750 & 167.7718 & 167.3067 & 174.9096 & 176.6392 & 177.8550 & 168.1294 & 167.9071 & 167.4542 \\
\hline 16 & 23.5 & 163.5889 & 164.9033 & 164.3665 & 155.4353 & 155.1891 & 154.7314 & 163.8620 & 165.1696 & 164.6359 & 155.7319 & 155.4773 & 155.0286 \\
\hline 17 & 121.1 & 70.9340 & 71.8227 & 71.7890 & 54.4485 & 54.0839 & 53.5042 & 70.9912 & 71.8918 & 71.7934 & 54.4615 & 54.1232 & 53.5076 \\
\hline 18 & 139.8 & 52.8471 & 52.8433 & 50.3255 & 31.5548 & 31.3277 & 29.4160 & 52.6943 & 52.6752 & 50.1615 & 31.3347 & 31.1003 & 29.2295 \\
\hline 19 & 50.9 & 137.3534 & 138.1088 & 139.7430 & 126.3229 & 126.0616 & 124.0166 & 137.3450 & 138.0970 & 139.6447 & 126.2368 & 125.9738 & 123.8741 \\
\hline 20 & 36.5 & 150.5852 & 152.0556 & 151.3057 & 141.7994 & 141.4608 & 140.7425 & 150.5881 & 152.0516 & 151.2626 & 141.7633 & 141.4174 & 140.7065 \\
\hline 21 & 45.2 & 144.0463 & 144.7586 & 144.5470 & 133.6927 & 133.3769 & 133.0164 & 144.2931 & 144.9998 & 144.8804 & 133.9791 & 133.6598 & 133.3451 \\
\hline 22 & 79.4 & 110.9314 & 111.4230 & 111.0120 & 98.2767 & 98.3868 & 97.5846 & 110.2213 & 110.7119 & 110.2029 & 97.4488 & 97.5620 & 96.6978 \\
\hline 23 & 27.7 & 161.8711 & 163.5446 & 163.8746 & 154.8123 & 154.5177 & 154.5634 & 162.2428 & 163.8966 & 164.1822 & 155.1895 & 154.8688 & 154.8825 \\
\hline 24 & 27.7 & 161.7285 & 163.4226 & 164.0347 & 154.8457 & 154.4270 & 154.4301 & 161.9431 & 163.6185 & 164.1770 & 155.0167 & 154.5760 & 154.5461 \\
\hline $\begin{array}{l}25 \\
{ }^{1} \mathrm{H}\end{array}$ & 22.5 & 165.9328 & 167.6549 & 166.9670 & 158.3425 & 158.0597 & 157.9121 & 166.2618 & 167.9671 & 167.2648 & 158.6634 & 158.3521 & 158.2058 \\
\hline 1 & 1.52 & 30.7490 & 30.3318 & 30.1899 & 30.7925 & 30.5922 & 30.5467 & 30.6757 & 30.2561 & 30.1047 & 30.7083 & 30.5080 & 30.4592 \\
\hline $2 a$ & 0.02 & 32.0117 & 31.6240 & 31.5068 & 32.0683 & 31.9063 & 31.8962 & 32.0324 & 31.6439 & 31.5296 & 32.0891 & 31.9286 & 31.9161 \\
\hline $2 b$ & 0.72 & 31.4581 & 31.0531 & 31.1514 & 31.4517 & 31.2840 & 31.2395 & 31.4219 & 31.0132 & 31.1124 & 31.4114 & 31.2416 & 31.2008 \\
\hline 3 & 1.65 & 30.5502 & 30.1173 & 30.1069 & 30.5611 & 30.3603 & 30.3669 & 30.4711 & 30.0346 & 30.0248 & 30.4779 & 30.2766 & 30.2848 \\
\hline $6 a$ & 1.97 & 30.1800 & 29.7372 & 29.6652 & 30.2505 & 30.0132 & 29.9522 & 30.2177 & 29.7758 & 29.6963 & 30.2865 & 30.0505 & 29.9814 \\
\hline $6 b$ & 2.89 & 29.5987 & 29.0368 & 28.9768 & 29.5105 & 29.1732 & 29.1993 & 29.4738 & 28.9063 & 28.8369 & 29.3846 & 29.0434 & 29.0614 \\
\hline 9 & 1.97 & 30.1173 & 29.7421 & 29.5705 & 30.1847 & 29.9987 & 29.8565 & 30.1876 & 29.8154 & 29.6387 & 30.2508 & 30.0687 & 29.9185 \\
\hline 13 & 7.40 & 24.9766 & 24.3270 & 24.1971 & 24.9150 & 24.5190 & 24.4161 & 24.8988 & 24.2473 & 24.1063 & 24.8232 & 24.4284 & 24.3252 \\
\hline 16 & 1.51 & 30.3934 & 29.9509 & 29.8309 & 30.5225 & 30.2832 & 30.2101 & 30.3639 & 29.9189 & 29.8013 & 30.4952 & 30.2533 & 30.1799 \\
\hline 16 & 2.37 & 30.0672 & 29.5740 & 29.4092 & 29.9841 & 29.6976 & 29.6551 & 29.9951 & 29.5011 & 29.3309 & 29.9084 & 29.6224 & 29.5785 \\
\hline 17 & 5.57 & 26.5656 & 25.9355 & 25.8034 & 26.4584 & 26.0096 & 26.0409 & 26.5134 & 25.8845 & 25.7458 & 26.3921 & 25.9505 & 25.9809 \\
\hline 19 & 2.51 & 29.6444 & 29.2135 & 28.9931 & 29.7296 & 29.4879 & 29.2180 & 29.6596 & 29.2251 & 28.9937 & 29.7351 & 29.4896 & 29.2151 \\
\hline 20 & 1.37 & 30.8477 & 30.4280 & 30.3557 & 30.9488 & 30.7366 & 30.7155 & 30.7718 & 30.3508 & 30.2762 & 30.8789 & 30.6665 & 30.6400 \\
\hline 20 & 2.26 & 29.7653 & 29.2960 & 29.2467 & 29.9408 & 29.6633 & 29.5612 & 29.6998 & 29.2296 & 29.1723 & 29.8724 & 29.5956 & 29.4882 \\
\hline 21 & 2.15 & 29.9323 & 29.5330 & 29.6617 & 30.1112 & 29.9002 & 29.9842 & 29.7954 & 29.3931 & 29.5065 & 29.9680 & 29.7561 & 29.8304 \\
\hline 23 & 1.34 & 30.9610 & 30.5072 & 30.4469 & 30.9371 & 30.6654 & 30.6413 & 30.9015 & 30.4455 & 30.3838 & 30.8800 & 30.6072 & 30.5817 \\
\hline 24 & 1.56 & 30.7527 & 30.2719 & 30.1727 & 30.7254 & 30.4343 & 30.4052 & 30.6987 & 30.2165 & 30.1158 & 30.6728 & 30.3816 & 30.3511 \\
\hline 25 & 1.68 & 30.5178 & 30.0585 & 29.8820 & 30.4362 & 30.1744 & 30.1313 & 30.4937 & 30.0322 & 29.8527 & 30.4096 & 30.1474 & 30.1016 \\
\hline 14 & 1.26 & 30.8968 & 30.4428 & 30.3249 & 30.8736 & 30.6305 & 30.5280 & 30.9424 & 30.4881 & 30.3690 & 30.9159 & 30.6715 & 30.5677 \\
\hline 15 & 1.76 & 30.5260 & 30.0296 & 29.9520 & 30.4238 & 30.1212 & 30.1258 & 30.4779 & 29.9799 & 29.9090 & 30.3803 & 30.0773 & 30.0854 \\
\hline
\end{tabular}

mPW1PW91

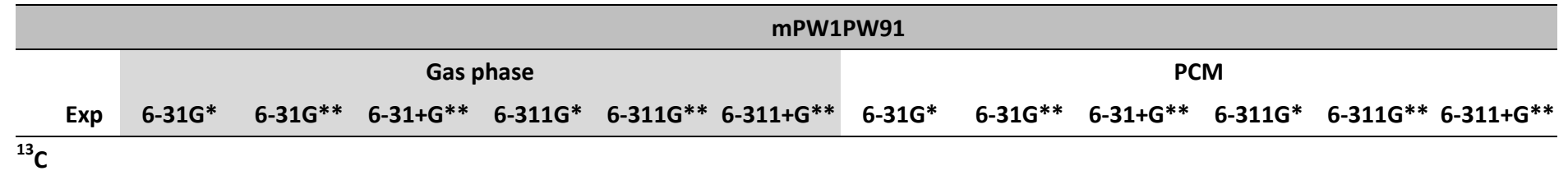

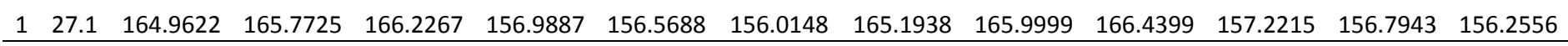




\begin{tabular}{|c|c|c|c|c|c|c|c|c|c|c|c|c|c|}
\hline 2 & 15.1 & 176.2935 & 177.7837 & 178.0851 & 170.8339 & 170.3057 & 170.3200 & 176.6266 & 178.0990 & 178.4274 & 171.1734 & 170.6270 & 170.6325 \\
\hline 3 & 26.7 & 65.2741 & 166.0925 & 66.7024 & 157.1387 & 156.7395 & 156.4500 & & 166.2159 & 166.8174 & 157.2736 & 156.8600 & \\
\hline 4 & 136.2 & 60.3483 & 60.6252 & 60.0330 & 43.0730 & 43.2481 & 42.5502 & 59.1425 & 59.4092 & 58.7273 & 41.6802 & 41.8422 & 41.0678 \\
\hline 5 & 129.8 & 63.4321 & 63.6160 & 63.6687 & 46.5438 & 46.6946 & 45.5551 & 64.3693 & 64.5608 & 64.6960 & 47.5853 & 47.7430 & 46.6752 \\
\hline 6 & 27.2 & 163.6375 & 164.9133 & 164.9623 & 156.9305 & 156.5248 & 156.3880 & 163.8129 & 165.0804 & 165.1234 & 157.0813 & 156.6689 & 156.5347 \\
\hline 7 & 54.4 & 137.8066 & 138.0079 & 136.9856 & 127.6801 & 127.7654 & 127.2487 & 137.5730 & 137.7695 & 136.7001 & 127.4601 & 127.5395 & 126.9451 \\
\hline 8 & 97.8 & 99.2401 & 99.4364 & 98.8498 & 87.4447 & 87.5992 & 86.8927 & 98.4522 & 98.6481 & 97.8801 & 86.5510 & 86.7067 & 85.8707 \\
\hline 9 & 48.5 & 145.6717 & 146.3164 & 145.7909 & 135.8318 & 135.4684 & 134.7394 & 145.5018 & 146.1435 & 145.5705 & 135.6039 & 135.2406 & 134.5127 \\
\hline 10 & 48.6 & 142.5464 & 142.9358 & 143.7646 & 132.6426 & 132.8093 & 132.0787 & 142.7424 & 143.1331 & 143.9876 & 132.8948 & 133.0556 & 132.3224 \\
\hline 1. & 110.1 & 86.9503 & 86.6960 & 87.9227 & 72.5661 & 72.3115 & 70.2512 & 87.1255 & 86.8691 & 88.0684 & 72.8083 & 72.5571 & 70.3929 \\
\hline & 170.5 & 33.7677 & 33.8214 & 32.4966 & 16.0976 & 16.1236 & 13.8106 & 31.5119 & 31.5633 & 29.8392 & 13.5070 & 13.5277 & 10.8919 \\
\hline & 150.0 & 47.9263 & 48.7084 & 47.4117 & 31.6293 & 30.8118 & 30.2627 & 46.6569 & 47.4362 & 46.0548 & 30.2273 & 29.4085 & 28.7222 \\
\hline 14 & 24.5 & 168.8633 & 170.4854 & 169.0775 & 162.4357 & 162.2676 & 162.3367 & 168.9927 & 170.6179 & 169.2819 & 162.6361 & 162.4578 & 162.5526 \\
\hline 15 & 13.7 & 178.1158 & 179.7512 & 181.1308 & 172.0636 & 171.8704 & 171.3945 & 178.3650 & 179.9837 & 181.3629 & 172.2229 & 172.0125 & 171.5511 \\
\hline 16 & 23.5 & 168.0276 & 169.2330 & 168.7320 & 160.6265 & 160.3829 & 159.9363 & 168.3124 & 169.5119 & 169.0063 & 160.9186 & 160.6677 & 160.2268 \\
\hline & 121.1 & 73.3822 & 74.2107 & 74.3806 & 58.6454 & 58.2490 & 57.5318 & 73.4438 & 74.2843 & 74.3922 & 58.6559 & 58.2867 & 57.5376 \\
\hline $1 \varepsilon$ & 139.8 & 56.3570 & 56.3190 & 54.0173 & 37.4386 & 37.1943 & 35.4539 & 56.1616 & 56.1089 & 53.8124 & 37.1675 & 36.9194 & 35.2172 \\
\hline 19 & 50.9 & 142.1975 & 142.8921 & 144.8460 & 132.4764 & 132.2241 & 130.2860 & 142.1916 & 142.8828 & 144.7502 & 132.3884 & 132.1345 & 130.1420 \\
\hline 20 & 36.5 & 155.5164 & 156.8825 & 156.1072 & 147.5833 & 147.2422 & 146.5541 & 155.5001 & 156.8611 & 156.0483 & 147.5226 & 147.1756 & 146.4954 \\
\hline 21 & 45.2 & 149.0334 & 149.6727 & 149.4703 & 139.5849 & 139.2978 & 138.9833 & 149.2599 & 149.8957 & 149.7725 & 139.8339 & 139.5452 & 139.2749 \\
\hline 22 & 79.4 & 115.7313 & 116.2144 & 116.0963 & 104.4902 & 104.6018 & 103.8584 & 115.0176 & 115.4994 & 115.3019 & 103.6611 & 103.7759 & 102.9821 \\
\hline 23 & 27.7 & 166.0498 & 167.5861 & 167.7084 & 159.5067 & 159.2384 & 159.2941 & 166.4504 & 167.9682 & 168.0407 & 159.8818 & 159.5907 & 159.6167 \\
\hline 24 & 27.7 & 165.9938 & 167.5580 & 167.9637 & 159.6793 & 159.2983 & 159.3946 & 166.2309 & 167.7780 & 168.1325 & 159.8515 & 159.4520 & 159.5210 \\
\hline & 22.5 & 169.7486 & 171.3544 & 170.6576 & 162.8188 & 162.5429 & 162.3834 & 170.1227 & 171.7103 & 170.9946 & 163.1473 & 162.8451 & 162.6886 \\
\hline 1 & 1.52 & 30.6552 & 30.2368 & 30.0952 & 30.6548 & 30.4473 & 30.4116 & 30.5754 & 30.1540 & 30.0047 & 30.5670 & 30.3591 & 30.3205 \\
\hline $2 a$ & 0.02 & 31.9190 & 31.5359 & 31.4341 & 31.9367 & 31.7754 & 31.7669 & 31.9384 & 31.5546 & 31.4544 & 31.9558 & 31.7964 & 31.7850 \\
\hline $2 b$ & 0.72 & 31.3711 & 30.9663 & 31.0515 & 31.3519 & 31.1749 & 31.1386 & 31.3246 & 30.9155 & 31.0017 & 31.3019 & 31.1228 & 31.0900 \\
\hline 3 & 1.65 & 30.4589 & 30.0195 & 29.9809 & 30.4468 & 30.2263 & 30.2214 & 30.3726 & 29.9289 & 29.8919 & 30.3583 & 30.1367 & 30.1336 \\
\hline $6 a$ & 1.97 & 30.1213 & 29.6752 & 29.5985 & 30.1266 & 29.8989 & 29.8473 & 30.1601 & 29.7149 & 29.6292 & 30.1638 & 29.9373 & 29.8768 \\
\hline $6 b$ & 2.89 & 29.5093 & 28.9436 & 28.8808 & 29.3840 & 29.0500 & 29.0695 & 29.3812 & 28.8098 & 28.7385 & 29.2555 & 28.9174 & 28.9286 \\
\hline 9 & 1.97 & 30.0394 & 29.6683 & 29.4927 & 30.0403 & 29.8606 & 29.7127 & 30.1135 & 29.7454 & 29.5621 & 30.1099 & 29.9340 & 29.7763 \\
\hline 13 & 7.40 & 24.8031 & 24.1599 & 24.0697 & 24.7922 & 24.3634 & 24.2839 & 24.7178 & 24.0725 & 23.9749 & 24.6947 & 24.2673 & 24.1891 \\
\hline 16 & 1.51 & 30.3660 & 29.9154 & 29.7431 & 30.4069 & 30.1723 & 30.0816 & 30.3410 & 29.8879 & 29.7184 & 30.3840 & 30.1465 & 30.0558 \\
\hline 16 & 2.37 & 30.0001 & 29.5101 & 29.3498 & 29.8794 & 29.5930 & 29.5498 & 29.9205 & 29.4293 & 29.2650 & 29.7979 & 29.5119 & 29.4677 \\
\hline 17 & 5.57 & 26.4037 & 25.7690 & 25.6375 & 26.3099 & 25.8376 & 25.8536 & 26.3407 & 25.7071 & 25.5715 & 26.2349 & 25.7702 & 25.7868 \\
\hline 19 & 2.51 & 29.6011 & 29.1674 & 28.9108 & 29.6171 & 29.3778 & 29.0833 & 29.6173 & 29.1797 & 28.9134 & 29.6241 & 29.3807 & 29.0819 \\
\hline 20 & 1.37 & 30.8069 & 30.3831 & 30.2925 & 30.8584 & 30.6389 & 30.6215 & 30.7305 & 30.3053 & 30.2144 & 30.7891 & 30.5692 & 30.5468 \\
\hline 20 & 2.26 & 29.7641 & 29.2980 & 29.2232 & 29.8643 & 29.5987 & 29.4993 & 29.6934 & 29.2261 & 29.1453 & 29.7914 & 29.5263 & 29.4225 \\
\hline 21 & 2.15 & 29.8759 & 29.4762 & 29.5673 & 29.9764 & 29.7636 & 29.8393 & 29.7392 & 29.3364 & 29.4157 & 29.8371 & 29.6229 & 29.6895 \\
\hline 23 & 1.34 & 30.8918 & 30.4361 & 30.3738 & 30.8406 & 30.5623 & 30.5329 & 30.8276 & 30.3694 & 30.3072 & 30.7794 & 30.4998 & 30.4700 \\
\hline 24 & 1.56 & 30.6855 & 30.2063 & 30.0980 & 30.6312 & 30.3374 & 30.3042 & 30.6282 & 30.1473 & 30.0380 & 30.5757 & 30.2817 & 30.2472 \\
\hline 25 & 1.68 & 30.4476 & 29.9832 & 29.8318 & 30.3450 & 30.0736 & 30.0315 & 30.4174 & 29.9507 & 29.7974 & 30.3129 & 30.0411 & 29.9970 \\
\hline 14 & 1.26 & 30.8360 & 30.3823 & 30.2790 & 30.7695 & 30.5264 & 30.4257 & 30.8803 & 30.4264 & 30.3209 & 30.8097 & 30.5655 & 30.4629 \\
\hline 15 & 1.76 & 30.4596 & 29.9587 & 29.8811 & 30.3321 & 30.0200 & 30.0259 & 30.4051 & 29.9023 & 29.8313 & 30.2826 & 29.9700 & 29.9791 \\
\hline
\end{tabular}




\section{Compound 13}

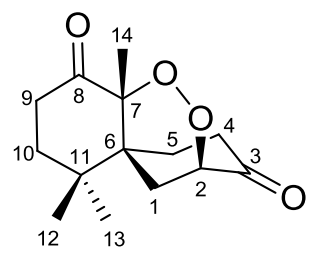

13

\begin{tabular}{|c|c|c|c|c|c|c|c|c|c|c|c|c|c|}
\hline & \multirow[b]{3}{*}{ Exp } & \multicolumn{12}{|c|}{ B3LYP } \\
\hline & & \multicolumn{6}{|c|}{ Gas phase } & \multicolumn{6}{|c|}{ PCM } \\
\hline & & 6-31G* & 6-31G** & $6-31+\mathrm{G} * *$ & 6-311G* & $6-311 G * *$ & $6-311+G * *$ & 6-31G* & 6-31G** & $6-31+\mathrm{G} * *$ & 6-311G* & $6-311 G * *$ & $6-311+G * *$ \\
\hline \multicolumn{14}{|l|}{${ }^{13} \mathrm{C}$} \\
\hline 1 & 25.7 & 159.5486 & 160.8208 & 160.8839 & 151.4387 & 151.1089 & 151.1239 & 159.6620 & 160.9135 & 160.8773 & 151.4862 & 151.1362 & 151.0780 \\
\hline 2 & 80.7 & 108.2793 & 108.9799 & 106.8536 & 95.4527 & 94.9241 & 94.9352 & 107.7660 & 108.4605 & 106.1427 & 94.7872 & 94.2595 & 94.0861 \\
\hline 3 & 207.7 & -8.2132 & -8.0220 & -10.8475 & -28.2742 & -28.0610 & -33.3973 & -11.7015 & -11.5225 & -15.1221 & -32.2937 & -32.0880 & -38.2343 \\
\hline 4 & 38.6 & 148.7615 & 149.9940 & 148.6873 & 138.7556 & 138.2648 & 137.6951 & 148.0912 & 149.3163 & 147.7466 & 137.9584 & 137.4577 & 136.6603 \\
\hline 5 & 27.2 & 157.6269 & 158.9208 & 158.7158 & 148.7818 & 148.5582 & 147.8198 & 157.6097 & 158.8875 & 158.5961 & 148.7190 & 148.4779 & 147.6612 \\
\hline 6 & 43.7 & 141.5530 & 141.7916 & 141.2765 & 130.5297 & 130.7183 & 130.4682 & 141.1458 & 141.3822 & 140.7494 & 129.9835 & 130.1793 & 129.8533 \\
\hline 7 & 90.6 & 99.0057 & 99.1722 & 98.8851 & 87.0219 & 87.1286 & 85.7340 & 98.1299 & 98.2917 & 97.8483 & 85.9358 & 86.0392 & 84.5948 \\
\hline 8 & 207.4 & -9.2618 & -9.1239 & -10.7557 & -29.8188 & -29.6816 & -33.1713 & -13.0334 & -12.9083 & -15.2962 & -34.3057 & -34.1846 & -38.3420 \\
\hline 9 & 35.4 & 154.0282 & 155.3001 & 154.3067 & 143.9752 & 143.5006 & 142.8672 & 153.3390 & 154.5955 & 153.3918 & 143.0987 & 142.6035 & 141.7408 \\
\hline 10 & 36.5 & 150.7513 & 151.9679 & 152.1361 & 141.5001 & 141.1802 & 140.6338 & 150.7802 & 151.9889 & 152.1120 & 141.5506 & 141.2175 & 140.6089 \\
\hline 11 & 36.4 & 150.5302 & 150.9274 & 151.5295 & 140.3676 & 140.3736 & 139.5346 & 150.3582 & 150.7606 & 151.3026 & 140.1385 & 140.1513 & 139.2591 \\
\hline 12 & 24.7 & 164.3415 & 166.0639 & 167.2267 & 157.4602 & 157.2032 & 156.7058 & 164.6622 & 166.3665 & 167.5478 & 157.8145 & 157.5338 & 157.0405 \\
\hline 13 & 26.6 & 161.8962 & 163.5940 & 163.7250 & 155.1828 & 154.8750 & 154.7856 & 162.2896 & 163.9730 & 164.1455 & 155.6288 & 155.2954 & 155.2351 \\
\hline $\begin{array}{l}14 \\
{ }^{1} \mathbf{H}\end{array}$ & 23.0 & 163.8569 & 165.6681 & 166.0389 & 156.5283 & 156.2330 & 156.1088 & 163.9087 & 165.6936 & 165.9266 & 156.4733 & 156.1412 & 155.9587 \\
\hline 1eq & 2.69 & 29.5603 & 29.0641 & 29.0007 & 29.5914 & 29.2945 & 29.2966 & 29.4138 & 28.9133 & 28.8307 & 29.4391 & 29.1407 & 29.1284 \\
\hline $1 a x$ & 1.81 & 30.6086 & 30.1446 & 30.1159 & 30.7238 & 30.4202 & 30.4146 & 30.4585 & 29.9876 & 29.9422 & 30.5656 & 30.2577 & 30.2390 \\
\hline 2 & 4.21 & 28.4157 & 27.8996 & 27.9049 & 28.3346 & 28.0852 & 28.1373 & 28.3482 & 27.8220 & 27.8154 & 28.2532 & 27.9938 & 28.0420 \\
\hline 4 eq & 2.88 & 28.6578 & 28.1650 & 28.1176 & 28.6782 & 28.4024 & 28.3811 & 28.7477 & 28.2560 & 28.1851 & 28.7593 & 28.4838 & 28.4409 \\
\hline $4 a x$ & 2.47 & 29.9411 & 29.4521 & 29.3685 & 29.8705 & 29.6048 & 29.5411 & 29.9002 & 29.4041 & 29.3037 & 29.8202 & 29.5483 & 29.4726 \\
\hline 5 eq & 2.11 & 30.1752 & 29.6963 & 29.6284 & 30.3178 & 30.0192 & 29.9794 & 30.1524 & 29.6735 & 29.5957 & 30.2883 & 29.9883 & 29.9409 \\
\hline $5 a x$ & 1.84 & 30.2539 & 29.7580 & 29.7077 & 30.3628 & 30.0566 & 30.0262 & 30.0816 & 29.5812 & 29.5116 & 30.1858 & 29.8767 & 29.8331 \\
\hline $9 e q$ & 2.85 & 29.3418 & 28.8026 & 28.7098 & 30.1065 & 29.8550 & 29.7410 & 29.1843 & 28.6420 & 28.5256 & 30.0824 & 29.8229 & 29.7027 \\
\hline $9 a x$ & 2.33 & 30.1961 & 29.6998 & 29.5747 & 29.4021 & 29.0372 & 28.9886 & 30.1688 & 29.6658 & 29.5348 & 29.2407 & 28.8768 & 28.8113 \\
\hline 10 eq & 2.06 & 30.0781 & 29.6151 & 29.5485 & 30.6449 & 30.4003 & 30.3468 & 30.0208 & 29.5591 & 29.4860 & 30.5172 & 30.2703 & 30.2041 \\
\hline $10 a x$ & 1.67 & 30.5916 & 30.1605 & 30.1151 & 30.1539 & 29.9040 & 29.8297 & 30.4637 & 30.0300 & 29.9704 & 30.0959 & 29.8487 & 29.7688 \\
\hline 12 & 1.30 & 30.9810 & 30.5055 & 30.4677 & 31.0160 & 30.7304 & 30.6867 & 30.9312 & 30.4564 & 30.4140 & 30.9688 & 30.6847 & 30.6379 \\
\hline 13 & 1.05 & 31.2918 & 30.8368 & 30.8541 & 31.3317 & 31.0602 & 31.0611 & 31.2474 & 30.7916 & 30.8048 & 31.2873 & 31.0158 & 31.0152 \\
\hline 14 & 1.94 & 30.2994 & 29.7943 & 29.6828 & 30.3490 & 30.0328 & 29.9552 & 30.2445 & 29.7370 & 29.6089 & 30.2873 & 29.9697 & 29.8798 \\
\hline
\end{tabular}




\begin{tabular}{|c|c|c|c|c|c|c|c|c|c|c|c|c|c|}
\hline & & \multicolumn{12}{|c|}{ mPW1PW91 } \\
\hline & \multirow[b]{2}{*}{ Exp } & \multicolumn{6}{|c|}{ Gas phase } & \multicolumn{6}{|c|}{ PCM } \\
\hline & & 6-31G* & 6-31G** & $6-31+\mathrm{G} * *$ & 6-311G* & $6-311 G * *$ & $6-311+G * *$ & 6-31G* & $6-31 \mathrm{G} * *$ & $6-31+G * *$ & 6-311G* & $6-311 G * *$ & $6-311+G * *$ \\
\hline \multicolumn{14}{|l|}{${ }^{13} \mathrm{C}$} \\
\hline 1 & 25.7 & 164.4969 & 165.6624 & 165.4319 & 156.9499 & 156.6411 & 156.6536 & 164.5871 & 165.7344 & 165.4113 & 156.9642 & 156.6383 & 156.5842 \\
\hline 2 & 80.7 & 113.3327 & 113.9804 & 112.2680 & 102.3725 & 101.8552 & 101.7514 & 112.7912 & 113.4335 & 111.5564 & 101.6792 & 101.1633 & 100.9027 \\
\hline 3 & 207.7 & -6.0352 & -5.8606 & -7.9597 & -23.1136 & -22.9081 & -27.6215 & -9.6325 & -9.4706 & -12.2666 & -27.1710 & -26.9726 & -32.4074 \\
\hline 4 & 38.6 & 152.9110 & 154.0536 & 152.9997 & 144.0241 & 143.5273 & 142.9695 & 152.2388 & 153.3738 & 152.0827 & 143.2334 & 142.7260 & 141.9683 \\
\hline 5 & 27.2 & 162.7521 & 163.9212 & 163.6737 & 154.4637 & 154.2643 & 153.5531 & 162.7236 & 163.8781 & 163.5437 & 154.3803 & 154.1649 & 153.3845 \\
\hline 6 & 43.7 & 146.9011 & 147.1426 & 146.5970 & 136.5993 & 136.7898 & 136.4960 & 146.4990 & 146.7378 & 146.0898 & 136.0692 & 136.2662 & 135.9106 \\
\hline 7 & 90.6 & 103.9067 & 104.0690 & 104.1434 & 93.2912 & 93.4013 & 92.1250 & 103.0138 & 103.1716 & 103.1093 & 92.1951 & 92.3028 & 90.9896 \\
\hline 8 & 207.4 & -6.4563 & -6.3275 & -7.4936 & -24.2613 & -24.1270 & -27.2029 & -10.3369 & -10.2198 & -12.0525 & -28.7849 & -28.6649 & -32.3180 \\
\hline 9 & 35.4 & 158.3573 & 159.5454 & 158.7325 & 149.3115 & 148.8439 & 148.2081 & 157.6527 & 158.8265 & 157.8341 & 148.4266 & 147.9395 & 147.1093 \\
\hline 10 & 36.5 & 155.8022 & 156.9029 & 156.9499 & 147.2284 & 146.9270 & 146.4306 & 155.8418 & 156.9364 & 156.9427 & 147.2746 & 146.9624 & 146.4163 \\
\hline 11 & 36.4 & 155.8324 & 156.2446 & 156.7931 & 146.2400 & 146.2527 & 145.5149 & 155.6626 & 156.0798 & 156.5743 & 146.0193 & 146.0388 & 145.2549 \\
\hline 12 & 24.7 & 168.6032 & 170.1695 & 170.9627 & 162.2380 & 161.9863 & 161.5755 & 168.9585 & 170.5082 & 171.3114 & 162.5949 & 162.3234 & 161.9191 \\
\hline 13 & 26.6 & 166.4007 & 167.9548 & 167.8205 & 160.0837 & 159.7980 & 159.7574 & 166.8266 & 168.3678 & 168.2651 & 160.5304 & 160.2230 & 160.2111 \\
\hline $\begin{array}{l}14 \\
{ }^{1} \mathbf{H}\end{array}$ & 23.0 & 168.1436 & 169.8192 & 169.8473 & 161.4233 & 161.1426 & 160.9896 & 168.2170 & 169.8688 & 169.7710 & 161.3779 & 161.0645 & 160.8602 \\
\hline 1 eq & 2.69 & 29.5217 & 29.0312 & 28.9634 & 29.5055 & 29.2200 & 29.2187 & 29.3671 & 28.8721 & 28.7890 & 29.3472 & 29.0599 & 29.0463 \\
\hline $1 \mathrm{ax}$ & 1.81 & 30.5747 & 30.1146 & 30.0781 & 30.6316 & 30.3335 & 30.3277 & 30.4191 & 29.9514 & 29.9014 & 30.4705 & 30.1675 & 30.1503 \\
\hline 2 & 4.21 & 28.3035 & 27.7955 & 27.8164 & 28.2425 & 27.9718 & 28.0363 & 28.2316 & 27.7129 & 27.7238 & 28.1582 & 27.8774 & 27.9391 \\
\hline $4 e q$ & 2.88 & 28.6166 & 28.1164 & 28.0712 & 28.5764 & 28.3075 & 28.2947 & 28.7081 & 28.2092 & 28.1403 & 28.6581 & 28.3893 & 28.3550 \\
\hline $4 a x$ & 2.47 & 29.8167 & 29.3304 & 29.2606 & 29.7448 & 29.4715 & 29.4264 & 29.7701 & 29.2764 & 29.1923 & 29.6898 & 29.4103 & 29.3547 \\
\hline $5 e q$ & 2.11 & 30.1491 & 29.6724 & 29.5828 & 30.2257 & 29.9377 & 29.8951 & 30.1251 & 29.6483 & 29.5500 & 30.1960 & 29.9063 & 29.8567 \\
\hline $5 a x$ & 1.84 & 30.2165 & 29.7261 & 29.6704 & 30.2740 & 29.9747 & 29.9508 & 30.0393 & 29.5440 & 29.4729 & 30.0944 & 29.7919 & 29.7570 \\
\hline $9 e q$ & 2.85 & 29.3237 & 28.7798 & 28.6660 & 29.9652 & 29.7061 & 29.6072 & 29.1617 & 28.6145 & 28.4808 & 29.9375 & 29.6700 & 29.5658 \\
\hline $9 a x$ & 2.33 & 30.0583 & 29.5664 & 29.4539 & 29.3120 & 28.9547 & 28.9050 & 30.0268 & 29.5276 & 29.4110 & 29.1463 & 28.7898 & 28.7264 \\
\hline 10 eq & 2.06 & 30.0307 & 29.5749 & 29.4993 & 30.5384 & 30.2942 & 30.2469 & 29.9717 & 29.5173 & 29.4365 & 30.4058 & 30.1585 & 30.1002 \\
\hline 10ax & 1.67 & 30.5328 & 30.1079 & 30.0681 & 30.0555 & 29.8152 & 29.7448 & 30.3977 & 29.9696 & 29.9185 & 29.9968 & 29.7591 & 29.6837 \\
\hline 12 & 1.30 & 30.9167 & 30.4423 & 30.3929 & 30.9106 & 30.6244 & 30.5793 & 30.8640 & 30.3903 & 30.3373 & 30.8609 & 30.5762 & 30.5285 \\
\hline 13 & 1.05 & 31.2352 & 30.7837 & 30.7931 & 31.2363 & 30.9641 & 30.9615 & 31.1871 & 30.7348 & 30.7412 & 31.1891 & 30.9168 & 30.9133 \\
\hline 14 & 1.94 & 30.2471 & 29.7419 & 29.6318 & 30.2608 & 29.9438 & 29.8604 & 30.1890 & 29.6812 & 29.5566 & 30.1961 & 29.8776 & 29.7834 \\
\hline
\end{tabular}




\section{Compound 14}

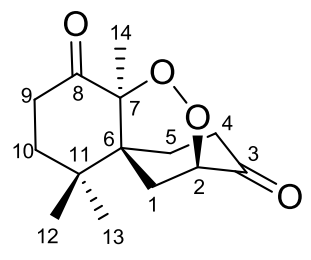

14

\begin{tabular}{|c|c|c|c|c|c|c|c|c|c|c|c|c|c|}
\hline & \multirow[b]{3}{*}{ Exp } & \multicolumn{12}{|c|}{ B3LYP } \\
\hline & & \multicolumn{6}{|c|}{ Gas phase } & \multicolumn{6}{|c|}{ PCM } \\
\hline & & $6-31 G *$ & $6-31 G * *$ & $6-31+G * *$ & 6-311G* & $6-311 G * *$ & $6-311+G * *$ & 6-31G* & 6-31G** & $6-31+G * *$ & 6-311G* & $6-311 G * *$ & $6-311+G * *$ \\
\hline \multicolumn{14}{|l|}{${ }^{13} \mathrm{C}$} \\
\hline 1 & 32.3 & 155.6087 & 156.8757 & 156.2893 & 146.8772 & 146.5604 & 146.4028 & 155.7386 & 156.9844 & 156.3370 & 146.9394 & 146.6035 & 146.4182 \\
\hline 2 & 81.7 & 108.5311 & 109.2479 & 107.7020 & 96.1664 & 95.6648 & 95.1802 & 108.1218 & 108.8327 & 106.9879 & 95.5582 & 95.0550 & 94.3572 \\
\hline 3 & 206.7 & -8.4519 & -8.2562 & -11.6370 & -28.4518 & -28.2317 & -33.2195 & -12.0983 & -11.9147 & -16.0920 & -32.6624 & -32.4497 & -38.2719 \\
\hline 4 & 39.4 & 149.4113 & 150.6609 & 149.2781 & 139.3025 & 138.8119 & 138.3395 & 148.6711 & 149.9108 & 148.2521 & 138.3905 & 137.8896 & 137.1637 \\
\hline 5 & 26.3 & 159.9756 & 161.2689 & 161.1630 & 152.1196 & 151.8170 & 151.1833 & 160.0832 & 161.3596 & 161.1758 & 152.2017 & 151.8815 & 151.1738 \\
\hline 6 & 42.1 & 144.2948 & 144.5050 & 144.0994 & 133.2378 & 133.4394 & 132.8095 & 143.8788 & 144.0859 & 143.5522 & 132.6708 & 132.8797 & 132.1702 \\
\hline 7 & 91.1 & 98.9134 & 99.1142 & 99.1033 & 86.8219 & 86.9642 & 85.2115 & 97.8032 & 97.9965 & 97.7417 & 85.4934 & 85.6317 & 83.7201 \\
\hline 8 & 207.0 & -8.0807 & -7.9461 & -10.2306 & -28.2665 & -28.1167 & -31.4304 & -12.4616 & -12.3415 & -15.4274 & -33.3893 & -33.2545 & -37.3205 \\
\hline 9 & 35.8 & 153.8489 & 155.1412 & 154.0859 & 144.0715 & 143.5705 & 143.1120 & 153.2118 & 154.4873 & 153.2065 & 143.2426 & 142.7219 & 142.0191 \\
\hline 10 & 35.9 & 152.2206 & 153.4003 & 153.2300 & 143.3514 & 143.0216 & 142.9375 & 152.4425 & 153.6149 & 153.4454 & 143.6408 & 143.2965 & 143.1806 \\
\hline 11 & 37.8 & 149.4084 & 149.7859 & 149.0699 & 138.8201 & 138.8083 & 138.6891 & 149.1845 & 149.5668 & 148.7690 & 138.5313 & 138.5255 & 138.3441 \\
\hline 12 & 26.5 & 162.7986 & 164.5253 & 164.4000 & 156.3046 & 155.9933 & 156.1725 & 163.1568 & 164.8681 & 164.7883 & 156.7099 & 156.3722 & 156.5793 \\
\hline 13 & 24.9 & 164.2862 & 166.0148 & 166.3243 & 157.4227 & 157.1485 & 156.9768 & 164.5734 & 166.2815 & 166.5972 & 157.7229 & 157.4235 & 157.2480 \\
\hline $\begin{array}{l}14 \\
{ }^{1} \mathbf{H}\end{array}$ & 22.1 & 166.1651 & 167.9148 & 168.2393 & 159.1397 & 158.8807 & 158.5685 & 166.2721 & 167.9884 & 168.1362 & 159.1278 & 158.8356 & 158.4304 \\
\hline 1 eq & 2.27 & 30.0163 & 29.5441 & 29.4849 & 30.1299 & 29.8469 & 29.7602 & 29.9532 & 29.4794 & 29.4058 & 30.0553 & 29.7733 & 29.6754 \\
\hline $1 a x$ & 1.90 & 30.4597 & 30.0172 & 30.0390 & 30.6029 & 30.3358 & 30.3178 & 30.2862 & 29.8356 & 29.8409 & 30.4242 & 30.1509 & 30.1184 \\
\hline 2 & 4.16 & 28.4456 & 27.9350 & 27.9361 & 28.3871 & 28.1330 & 28.1647 & 28.4112 & 27.8923 & 27.8807 & 28.3356 & 28.0733 & 28.0968 \\
\hline $4 e q$ & 2.93 & 29.0903 & 28.6116 & 28.5292 & 29.7814 & 29.5099 & 29.4689 & 29.0836 & 28.6043 & 28.5011 & 29.7320 & 29.4534 & 29.4015 \\
\hline $4 a x$ & 2.59 & 29.8394 & 29.3438 & 29.2945 & 29.1410 & 28.8646 & 28.8111 & 29.7955 & 29.2929 & 29.2287 & 29.1221 & 28.8466 & 28.7805 \\
\hline $5 e q$ & 2.33 & 29.9726 & 29.4823 & 29.3600 & 30.0360 & 29.7397 & 29.6376 & 29.8571 & 29.3631 & 29.2262 & 29.9208 & 29.6202 & 29.5053 \\
\hline $5 a x$ & 1.93 & 30.3602 & 29.8885 & 29.8521 & 30.4766 & 30.1933 & 30.1658 & 30.2059 & 29.7304 & 29.6754 & 30.3150 & 30.0299 & 29.9894 \\
\hline $9 e q$ & 2.73 & 29.5065 & 29.0026 & 28.9255 & 29.9757 & 29.7147 & 29.5732 & 29.3321 & 28.8234 & 28.7218 & 29.9702 & 29.7014 & 29.5538 \\
\hline $9 a x$ & 2.48 & 30.0620 & 29.5597 & 29.4107 & 29.5547 & 29.2358 & 29.1922 & 30.0555 & 29.5468 & 29.3912 & 29.3748 & 29.0557 & 28.9951 \\
\hline 10 eq & 2.04 & 30.1150 & 29.6517 & 29.5987 & 30.7261 & 30.5068 & 30.4724 & 30.0551 & 29.5924 & 29.5343 & 30.6015 & 30.3792 & 30.3313 \\
\hline $10 a x$ & 1.64 & 30.6863 & 30.2775 & 30.2456 & 30.1898 & 29.9371 & 29.8962 & 30.5620 & 30.1503 & 30.1040 & 30.1259 & 29.8761 & 29.8305 \\
\hline 12 & 1.02 & 31.2845 & 30.8309 & 30.8433 & 31.3285 & 31.0542 & 31.0142 & 31.2380 & 30.7835 & 30.7928 & 31.2823 & 31.0077 & 30.9665 \\
\hline 13 & 1.30 & 30.9646 & 30.4888 & 30.4661 & 30.9931 & 30.7071 & 30.6334 & 30.9115 & 30.4361 & 30.4082 & 30.9433 & 30.6585 & 30.5804 \\
\hline 14 & 1.50 & 30.8309 & 30.3254 & 30.2396 & 30.8603 & 30.5384 & 30.4682 & 30.7551 & 30.2455 & 30.1455 & 30.7798 & 30.4548 & 30.3740 \\
\hline
\end{tabular}




\begin{tabular}{|c|c|c|c|c|c|c|c|c|c|c|c|c|c|}
\hline & \multirow[b]{3}{*}{$\operatorname{Exp}$} & \multicolumn{12}{|c|}{ mPW1PW91 } \\
\hline & & \multicolumn{6}{|c|}{ Gas phase } & \multicolumn{6}{|c|}{ PCM } \\
\hline & & 6-31G* & $6-31 G * *$ & $6-31+G * *$ & 6-311G* & $6-311 G * *$ & $6-311+G * *$ & 6-31G* & $6-31 G * *$ & $6-31+G * *$ & 6-311G* & $6-311 G * *$ & $6-311+\mathrm{G} * *$ \\
\hline \multicolumn{14}{|l|}{${ }^{13} \mathrm{C}$} \\
\hline 1 & 32.3 & 160.8383 & 161.9875 & 161.2124 & 152.6000 & 152.3098 & 152.0938 & 160.9404 & 162.0711 & 161.2401 & 152.6197 & 152.3130 & 152.0716 \\
\hline 2 & 81.7 & 113.5211 & 114.1846 & 113.0946 & 103.0367 & 102.5455 & 101.9650 & 113.0939 & 113.7515 & 112.3992 & 102.4162 & 101.9234 & 101.1593 \\
\hline 3 & 206.7 & -6.2236 & -6.0423 & -8.5897 & -23.2225 & -23.0075 & -27.4752 & -9.9812 & -9.8125 & -13.0709 & -27.4686 & -27.2605 & -32.4709 \\
\hline 4 & 39.4 & 153.6381 & 154.7971 & 153.5722 & 144.6516 & 144.1637 & 143.6391 & 152.8732 & 154.0232 & 152.5535 & 143.7236 & 143.2258 & 142.4841 \\
\hline 5 & 26.3 & 164.9180 & 166.0950 & 166.0420 & 157.6119 & 157.3337 & 156.7208 & 165.0126 & 166.1748 & 166.0472 & 157.6698 & 157.3763 & 156.6963 \\
\hline 6 & 42.1 & 149.6210 & 149.8343 & 149.3406 & 139.2567 & 139.4594 & 138.8164 & 149.2094 & 149.4194 & 148.8122 & 138.7054 & 138.9149 & 138.2077 \\
\hline 7 & 91.1 & 103.9025 & 104.0995 & 104.4800 & 93.2796 & 93.4268 & 91.7733 & 102.7689 & 102.9588 & 103.1192 & 91.9385 & 92.0830 & 90.3015 \\
\hline 8 & 207.0 & -5.3496 & -5.2217 & -7.0422 & -22.7759 & -22.6280 & -25.6079 & -9.8638 & -9.7495 & -12.2621 & -27.9467 & -27.8118 & -31.4386 \\
\hline 9 & 35.8 & 158.1295 & 159.3366 & 158.4251 & 149.3544 & 148.8603 & 148.3608 & 157.4698 & 158.6613 & 157.5625 & 148.5087 & 147.9959 & 147.2867 \\
\hline 10 & 35.9 & 157.3195 & 158.3828 & 158.0766 & 149.0788 & 148.7689 & 148.6810 & 157.5560 & 158.6140 & 158.3076 & 149.3616 & 149.0398 & 148.9248 \\
\hline 11 & 37.8 & 154.6640 & 155.0555 & 154.3781 & 144.6886 & 144.6858 & 144.5869 & 154.4417 & 154.8378 & 154.0887 & 144.4093 & 144.4125 & 144.2593 \\
\hline 12 & 26.5 & 167.2922 & 168.8754 & 168.4176 & 161.1794 & 160.8956 & 161.1056 & 167.6820 & 169.2511 & 168.8318 & 161.5853 & 161.2792 & 161.5178 \\
\hline 13 & 24.9 & 168.5439 & 170.1159 & 170.0325 & 162.1691 & 161.8983 & 161.7631 & 168.8671 & 170.4202 & 170.3357 & 162.4740 & 162.1823 & 162.0491 \\
\hline $\begin{array}{l}14 \\
{ }^{1} \mathbf{H}\end{array}$ & 22.1 & 170.4856 & 172.0952 & 172.1821 & 164.0045 & 163.7616 & 163.4110 & 170.6183 & 172.1973 & 172.1284 & 164.0064 & 163.7344 & 163.3000 \\
\hline $1 e q$ & 2.27 & 29.9743 & 29.5055 & 29.4528 & 30.0362 & 29.7610 & 29.6764 & 29.9077 & 29.4372 & 29.3727 & 29.9598 & 29.6853 & 29.5907 \\
\hline $1 a x$ & 1.90 & 30.4355 & 29.9960 & 30.0128 & 30.5169 & 30.2534 & 30.2399 & 30.2566 & 29.8084 & 29.8122 & 30.3353 & 30.0651 & 30.0391 \\
\hline 2 & 4.16 & 28.3356 & 27.8316 & 27.8486 & 28.2933 & 28.0187 & 28.0631 & 28.2969 & 27.7842 & 27.7905 & 28.2387 & 27.9561 & 27.9936 \\
\hline 4 eq & 2.93 & 29.0525 & 28.5663 & 28.4687 & 29.6590 & 29.3809 & 29.3452 & 29.0453 & 28.5584 & 28.4415 & 29.6041 & 29.3187 & 29.2733 \\
\hline $4 a x$ & 2.59 & 29.7195 & 29.2271 & 29.1870 & 29.0429 & 28.7716 & 28.7042 & 29.6688 & 29.1691 & 29.1164 & 29.0230 & 28.7525 & 28.6737 \\
\hline $5 e q$ & 2.33 & 29.9401 & 29.4555 & 29.3113 & 29.9467 & 29.6596 & 29.5513 & 29.8180 & 29.3293 & 29.1727 & 29.8261 & 29.5340 & 29.4145 \\
\hline $5 a x$ & 1.93 & 30.3161 & 29.8507 & 29.8050 & 30.3754 & 30.1004 & 30.0774 & 30.1568 & 29.6872 & 29.6266 & 30.2116 & 29.9342 & 29.9002 \\
\hline $9 e q$ & 2.73 & 29.4867 & 28.9772 & 28.8925 & 29.8398 & 29.5711 & 29.4352 & 29.3077 & 28.7931 & 28.6877 & 29.8306 & 29.5536 & 29.4131 \\
\hline $9 a x$ & 2.48 & 29.9264 & 29.4285 & 29.2906 & 29.4641 & 29.1513 & 29.1095 & 29.9159 & 29.4111 & 29.2686 & 29.2800 & 28.9668 & 28.9111 \\
\hline $10 e q$ & 2.04 & 30.0669 & 29.6108 & 29.5531 & 30.6210 & 30.4024 & 30.3674 & 30.0055 & 29.5500 & 29.4883 & 30.4909 & 30.2685 & 30.2217 \\
\hline $10 a x$ & 1.64 & 30.6275 & 30.2257 & 30.1903 & 30.0895 & 29.8465 & 29.8060 & 30.4955 & 30.0901 & 30.0428 & 30.0249 & 29.7848 & 29.7402 \\
\hline 12 & 1.02 & 31.2266 & 30.7761 & 30.7780 & 31.2308 & 30.9554 & 30.9190 & 31.1765 & 30.7250 & 30.7251 & 31.1819 & 30.9060 & 30.8693 \\
\hline 13 & 1.30 & 30.9007 & 30.4261 & 30.3947 & 30.8873 & 30.6009 & 30.5281 & 30.8445 & 30.3705 & 30.3352 & 30.8350 & 30.5498 & 30.4736 \\
\hline 14 & 1.50 & 30.7678 & 30.2603 & 30.1730 & 30.7609 & 30.4376 & 30.3705 & 30.6879 & 30.1760 & 30.0770 & 30.6768 & 30.3503 & 30.2742 \\
\hline
\end{tabular}




\section{Compound 15}

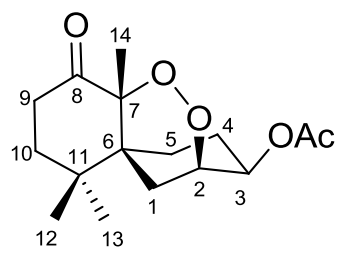

15

\begin{tabular}{|c|c|c|c|c|c|c|c|c|c|c|c|c|c|}
\hline & \multirow[b]{3}{*}{ Exp } & \multicolumn{12}{|c|}{ B3LYP } \\
\hline & & \multicolumn{6}{|c|}{ Gas phase } & \multicolumn{6}{|c|}{ PCM } \\
\hline & & 6-31G* & 6-31G** & $6-31+\mathrm{G} * *$ & 6-311G* & 6-311G** & $6-311+G * *$ & 6-31G* & 6-31G** & $6-31+\mathrm{G} * *$ & 6-311G* & $6-311 G * *$ & $6-311+G * *$ \\
\hline \multicolumn{14}{|l|}{${ }^{13} \mathrm{C}$} \\
\hline 1 & 26.3 & 161.1337 & 162.4342 & 162.8238 & 153.6849 & 153.2864 & 153.2597 & 161.3541 & 162.6364 & 163.0135 & 153.8576 & 153.4433 & 153.3732 \\
\hline 2 & 77.3 & 113.5097 & 114.2798 & 113.2809 & 102.1144 & 101.6678 & 101.3789 & 112.7278 & 113.4945 & 112.1847 & 101.0676 & 100.6182 & 100.2210 \\
\hline 3 & 72.8 & 116.8035 & 117.6315 & 116.9708 & 105.4437 & 105.3522 & 104.7753 & 116.7295 & 117.5534 & 116.8809 & 105.3226 & 105.2273 & 104.6425 \\
\hline 4 & 28.3 & 159.9648 & 161.3383 & 160.5368 & 151.2406 & 151.0192 & 150.7753 & 159.6749 & 161.0538 & 160.2864 & 150.9587 & 150.7351 & 150.4559 \\
\hline 5 & 28.1 & 159.5152 & 160.8299 & 160.9589 & 150.5776 & 150.3655 & 149.7760 & 159.7763 & 161.0784 & 161.2109 & 150.8451 & 150.6171 & 150.0795 \\
\hline 6 & 43.3 & 141.9853 & 142.2402 & 141.8837 & 131.2688 & 131.4754 & 131.3520 & 141.5579 & 141.8117 & 141.3888 & 130.7326 & 130.9474 & 130.7635 \\
\hline 7 & 89.8 & 99.7835 & 99.9518 & 99.6907 & 87.7721 & 87.8631 & 86.5229 & 98.9229 & 99.0869 & 98.5983 & 86.7291 & 86.8188 & 85.5142 \\
\hline 8 & 208.1 & -9.3554 & -9.2121 & -10.7719 & -29.8682 & -29.7410 & -33.4736 & -13.2343 & -13.1037 & -15.4748 & -34.4847 & -34.3735 & -38.7734 \\
\hline 9 & 35.5 & 154.0088 & 155.2869 & 154.1676 & 143.9911 & 143.5147 & 142.8631 & 153.3391 & 154.6018 & 153.2430 & 143.1422 & 142.6469 & 141.7719 \\
\hline 10 & 37.0 & 150.8936 & 152.1179 & 152.2880 & 141.7039 & 141.3889 & 140.8840 & 150.9242 & 152.1411 & 152.2386 & 141.7551 & 141.4270 & 140.8659 \\
\hline 11 & 36.1 & 150.6708 & 151.0686 & 151.4115 & 140.4730 & 140.4831 & 139.9125 & 150.5043 & 150.9074 & 151.2273 & 140.2463 & 140.2638 & 139.6519 \\
\hline 12 & 25.2 & 164.4427 & 166.1722 & 167.1024 & 157.5880 & 157.3414 & 156.8591 & 164.7885 & 166.5024 & 167.5854 & 157.9815 & 157.7098 & 157.2164 \\
\hline 13 & 27.5 & 161.7540 & 163.4658 & 163.6123 & 155.0791 & 154.7600 & 154.8683 & 162.1502 & 163.8482 & 163.9920 & 155.5251 & 155.1826 & 155.3179 \\
\hline 14 & 24.2 & 163.5386 & 165.3574 & 165.5377 & 156.2381 & 155.9448 & 155.8641 & 163.6390 & 165.4317 & 165.4981 & 156.2364 & 155.9086 & 155.7561 \\
\hline 15 & 171.3 & 28.0441 & 28.2008 & 25.7456 & 6.5757 & 6.7131 & 4.4930 & 26.6384 & 26.7882 & 23.9800 & 4.8749 & 5.0036 & 2.4706 \\
\hline $\begin{array}{l}16 \\
{ }^{1} \mathbf{H}\end{array}$ & 21.4 & 169.1509 & 170.8684 & 170.6859 & 161.2132 & 160.9101 & 160.4771 & 169.0477 & 170.7384 & 170.2726 & 160.9092 & 160.5713 & 159.9731 \\
\hline 1 eq & 2.52 & 29.8304 & 29.3566 & 29.2899 & 29.8467 & 29.5678 & 29.5447 & 29.7148 & 29.2360 & 29.1665 & 29.7238 & 29.4420 & 29.4081 \\
\hline $1 \mathrm{ax}$ & 1.40 & 30.9131 & 30.4638 & 30.4463 & 31.0225 & 30.7223 & 30.7126 & 30.7745 & 30.3200 & 30.2857 & 30.8719 & 30.5697 & 30.5457 \\
\hline 2 & 4.30 & 28.0229 & 27.4775 & 27.4662 & 28.0017 & 27.6713 & 27.7294 & 28.0330 & 27.4898 & 27.4685 & 27.9877 & 27.6622 & 27.7139 \\
\hline 3 & 4.88 & 27.4849 & 26.9630 & 26.8602 & 27.5557 & 27.2071 & 27.1449 & 27.4098 & 26.8839 & 26.7623 & 27.4703 & 27.1173 & 27.0446 \\
\hline 4 eq & 2.61 & 29.1374 & 28.6411 & 28.6140 & 30.3493 & 30.1063 & 30.0702 & 29.3846 & 28.8927 & 28.8728 & 30.2443 & 29.9937 & 29.9468 \\
\hline $4 a x$ & 2.00 & 30.3936 & 29.9620 & 29.9108 & 29.1539 & 28.8968 & 28.8623 & 30.2918 & 29.8541 & 29.7799 & 29.4032 & 29.1470 & 29.1178 \\
\hline $5 e q$ & 1.85 & 30.3325 & 29.8546 & 29.8044 & 30.4710 & 30.1719 & 30.1120 & 30.3400 & 29.8633 & 29.8207 & 30.4747 & 30.1752 & 30.1283 \\
\hline $5 a x$ & 1.77 & 30.4601 & 29.9908 & 29.8651 & 30.5191 & 30.2335 & 30.1827 & 30.3084 & 29.8340 & 29.7023 & 30.3672 & 30.0771 & 30.0185 \\
\hline $9 e q$ & 2.81 & 29.3930 & 28.8557 & 28.7570 & 30.1651 & 29.9208 & 29.7867 & 29.2422 & 28.7027 & 28.5858 & 30.1454 & 29.8925 & 29.7530 \\
\hline $9 a x$ & 2.30 & 30.2536 & 29.7646 & 29.6354 & 29.4513 & 29.0854 & 29.0136 & 30.2302 & 29.7342 & 29.6002 & 29.2974 & 28.9343 & 28.8499 \\
\hline 10eq & 2.03 & 30.0784 & 29.6151 & 29.5263 & 30.6831 & 30.4416 & 30.3918 & 30.0216 & 29.5596 & 29.4599 & 30.5640 & 30.3190 & 30.2575 \\
\hline $10 a x$ & 1.67 & 30.6316 & 30.2046 & 30.1659 & 30.1499 & 29.8976 & 29.8337 & 30.5117 & 30.0815 & 30.0294 & 30.0927 & 29.8433 & 29.7708 \\
\hline 12 & 1.25 & 31.0123 & 30.5390 & 30.4972 & 31.0394 & 30.7572 & 30.6832 & 30.9699 & 30.4971 & 30.4539 & 30.9995 & 30.7187 & 30.6447 \\
\hline 13 & 0.99 & 31.3149 & 30.8620 & 30.8555 & 31.3363 & 31.0690 & 31.0773 & 31.2719 & 30.8181 & 30.8057 & 31.2944 & 31.0267 & 31.0281 \\
\hline 14 & 1.90 & 30.3805 & 29.8790 & 29.8020 & 30.4330 & 30.1196 & 30.0418 & 30.3324 & 29.8285 & 29.7289 & 30.3782 & 30.0629 & 29.9715 \\
\hline 16 & 2.08 & 30.2924 & 29.7761 & 29.6604 & 30.2187 & 29.9006 & 29.8906 & 30.2199 & 29.6985 & 29.5699 & 30.1434 & 29.8230 & 29.8005 \\
\hline
\end{tabular}




\begin{tabular}{|c|c|c|c|c|c|c|c|c|c|c|c|c|c|}
\hline & & \multicolumn{12}{|c|}{ mPW1PW91 } \\
\hline & \multirow[b]{2}{*}{ Exp } & \multicolumn{6}{|c|}{ Gas phase } & \multicolumn{6}{|c|}{ PCM } \\
\hline & & 6-31G* & $6-31 G * *$ & $6-31+G * *$ & 6-311G* & $6-311 G * *$ & $6-311+G * *$ & 6-31G* & $6-31 G * *$ & $6-31+G * *$ & 6-311G* & $6-311 G * *$ & $6-311+G * *$ \\
\hline \multicolumn{14}{|l|}{${ }^{13} \mathrm{C}$} \\
\hline 1 & 26.3 & 166.0117 & 167.2046 & 167.2205 & 159.1372 & 158.7547 & 158.7580 & 166.2292 & 167.4061 & 167.4399 & 159.2893 & 158.8934 & 158.8506 \\
\hline 2 & 77.3 & 118.4475 & 119.1539 & 118.3721 & 108.6345 & 108.1994 & 107.9118 & 117.6688 & 118.3722 & 117.3249 & 107.6179 & 107.1792 & 106.8102 \\
\hline 3 & 72.8 & 121.5239 & 122.3013 & 121.8154 & 111.5206 & 111.4413 & 110.8759 & 121.4390 & 122.2129 & 121.7297 & 111.3806 & 111.2983 & 110.7257 \\
\hline 4 & 28.3 & 164.8014 & 166.0743 & 165.3179 & 156.9349 & 156.7070 & 156.4457 & 164.5259 & 165.8032 & 165.0728 & 156.6655 & 156.4348 & 156.1465 \\
\hline 5 & 28.1 & 164.6554 & 165.8462 & 165.7559 & 156.2972 & 156.1036 & 155.5501 & 164.9103 & 166.0900 & 166.0012 & 156.5416 & 156.3336 & 155.8198 \\
\hline 6 & 43.3 & 147.3731 & 147.6292 & 147.1665 & 137.3590 & 137.5616 & 137.3724 & 146.9483 & 147.2029 & 146.6935 & 136.8342 & 137.0446 & 136.8083 \\
\hline 7 & 89.8 & 104.6777 & 104.8416 & 104.9991 & 94.0375 & 94.1320 & 92.8621 & 103.7963 & 103.9563 & 103.9009 & 92.9786 & 93.0729 & 91.8424 \\
\hline 8 & 208.1 & -6.5572 & -6.4226 & -7.4784 & -24.3153 & -24.1912 & -27.5359 & -10.5454 & -10.4225 & -12.1794 & -28.9647 & -28.8549 & -32.7808 \\
\hline 9 & 35.5 & 158.3582 & 159.5523 & 158.6158 & 149.3491 & 148.8790 & 148.2363 & 157.6727 & 158.8526 & 157.7065 & 148.4902 & 148.0020 & 147.1672 \\
\hline 10 & 37.0 & 155.9423 & 157.0497 & 157.0874 & 147.4389 & 147.1418 & 146.6979 & 155.9853 & 157.0871 & 157.0695 & 147.4873 & 147.1793 & 146.6893 \\
\hline 11 & 36.1 & 155.9629 & 156.3751 & 156.5813 & 146.3474 & 146.3632 & 145.8642 & 155.7987 & 156.2162 & 156.3920 & 146.1288 & 146.1516 & 145.6161 \\
\hline 12 & 25.2 & 168.6964 & 170.2673 & 170.9080 & 162.3478 & 162.1049 & 161.7115 & 169.0768 & 170.6334 & 171.3994 & 162.7421 & 162.4780 & 162.0673 \\
\hline 13 & 27.5 & 166.2704 & 167.8375 & 167.6860 & 159.9968 & 159.6995 & 159.8293 & 166.6982 & 168.2528 & 168.0981 & 160.4434 & 160.1266 & 160.2873 \\
\hline 14 & 24.2 & 167.8454 & 169.5272 & 169.2988 & 161.1518 & 160.8726 & 160.7583 & 167.9706 & 169.6286 & 169.3051 & 161.1626 & 160.8530 & 160.6819 \\
\hline 15 & 171.3 & 30.1804 & 30.3357 & 28.4538 & 11.5210 & 11.6587 & 9.6711 & 28.7415 & 28.8891 & 26.6919 & 9.8098 & 9.9394 & 7.6742 \\
\hline $\begin{array}{l}16 \\
{ }^{1} \mathbf{H}\end{array}$ & 21.4 & 172.4753 & 174.0947 & 174.2202 & 165.3287 & 165.0445 & 164.6143 & 172.3899 & 173.9824 & 173.8528 & 165.0344 & 164.7181 & 164.1432 \\
\hline $1 e q$ & 2.52 & 29.7874 & 29.3196 & 29.2431 & 29.7562 & 29.4883 & 29.4576 & 29.6659 & 29.1928 & 29.1154 & 29.6287 & 29.3579 & 29.3179 \\
\hline $1 a x$ & 1.40 & 30.8934 & 30.4490 & 30.4097 & 30.9340 & 30.6420 & 30.6251 & 30.7479 & 30.2977 & 30.2457 & 30.7803 & 30.4855 & 30.4552 \\
\hline 2 & 4.30 & 27.9405 & 27.3987 & 27.3799 & 27.9190 & 27.5742 & 27.6264 & 27.9475 & 27.4076 & 27.3808 & 27.9031 & 27.5623 & 27.6091 \\
\hline 3 & 4.88 & 27.4485 & 26.9323 & 26.8331 & 27.4639 & 27.1228 & 27.0722 & 27.3705 & 26.8498 & 26.7328 & 27.3782 & 27.0323 & 26.9709 \\
\hline 4 eq & 2.61 & 29.0721 & 28.5768 & 28.5455 & 30.2752 & 30.0287 & 29.9894 & 29.3252 & 28.8348 & 28.8045 & 30.1665 & 29.9125 & 29.8646 \\
\hline $4 a x$ & 2.00 & 30.3390 & 29.9071 & 29.8493 & 29.0336 & 28.7871 & 28.7570 & 30.2323 & 29.7940 & 29.7188 & 29.2848 & 29.0392 & 29.0103 \\
\hline $5 e q$ & 1.85 & 30.3053 & 29.8299 & 29.7439 & 30.3817 & 30.0933 & 30.0183 & 30.3130 & 29.8387 & 29.7610 & 30.3855 & 30.0964 & 30.0332 \\
\hline $5 a x$ & 1.77 & 30.4383 & 29.9742 & 29.8394 & 30.4384 & 30.1628 & 30.1132 & 30.2816 & 29.8119 & 29.6738 & 30.2836 & 30.0032 & 29.9468 \\
\hline $9 e q$ & 2.81 & 29.3773 & 28.8353 & 28.7124 & 30.0268 & 29.7739 & 29.6462 & 29.2225 & 28.6780 & 28.5399 & 30.0032 & 29.7415 & 29.6098 \\
\hline $9 a x$ & 2.30 & 30.1189 & 29.6338 & 29.5112 & 29.3622 & 29.0049 & 28.9283 & 30.0911 & 29.5984 & 29.4723 & 29.2045 & 28.8493 & 28.7626 \\
\hline 10 eq & 2.03 & 30.0353 & 29.5791 & 29.4829 & 30.5767 & 30.3372 & 30.2894 & 29.9767 & 29.5218 & 29.4146 & 30.4525 & 30.2087 & 30.1502 \\
\hline $10 a x$ & 1.67 & 30.5741 & 30.1537 & 30.1168 & 30.0550 & 29.8121 & 29.7486 & 30.4469 & 30.0228 & 29.9738 & 29.9968 & 29.7568 & 29.6842 \\
\hline 12 & 1.25 & 30.9497 & 30.4771 & 30.4215 & 30.9337 & 30.6510 & 30.5730 & 30.9047 & 30.4326 & 30.3756 & 30.8915 & 30.6101 & 30.5321 \\
\hline 13 & 0.99 & 31.2612 & 30.8118 & 30.7934 & 31.2434 & 30.9758 & 30.9745 & 31.2146 & 30.7642 & 30.7411 & 31.1987 & 30.9305 & 30.9238 \\
\hline 14 & 1.90 & 30.3331 & 29.8318 & 29.7473 & 30.3477 & 30.0340 & 29.9459 & 30.2821 & 29.7782 & 29.6741 & 30.2902 & 29.9745 & 29.8762 \\
\hline 16 & 2.08 & 30.2067 & 29.6832 & 29.5847 & 30.1218 & 29.7881 & 29.7875 & 30.1277 & 29.5984 & 29.4891 & 30.0406 & 29.7043 & 29.6932 \\
\hline
\end{tabular}




\section{Compound 16}

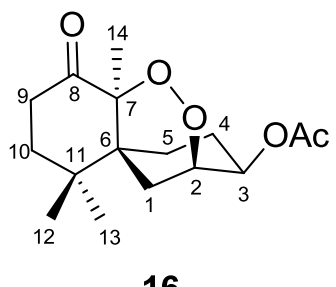

16

\begin{tabular}{|c|c|c|c|c|c|c|c|c|c|c|c|c|c|}
\hline & \multirow[b]{3}{*}{ Exp } & \multicolumn{12}{|c|}{ B3LYP } \\
\hline & & \multicolumn{6}{|c|}{ Gas phase } & \multicolumn{6}{|c|}{ PCM } \\
\hline & & 6-31G* & 6-31G** & $6-31+\mathrm{G} * *$ & $6-311 G *$ & $6-311 G * *$ & $6-311+G * *$ & 6-31G* & 6-31G** & $6-31+G * *$ & 6-311G* & $6-311 G * *$ & $6-311+G * *$ \\
\hline \multicolumn{14}{|l|}{${ }^{13} \mathrm{C}$} \\
\hline 1 & 27.5 & 157.4103 & 158.7046 & 158.3473 & 149.2843 & 148.9321 & 148.5154 & 157.5984 & 158.8742 & 158.3958 & 149.4110 & 149.0440 & 148.6409 \\
\hline 2 & 76.0 & 114.3355 & 115.1318 & 114.5073 & 103.4288 & 103.0342 & 102.3378 & 113.5851 & 114.3781 & 113.4006 & 102.3839 & 101.9866 & 101.1204 \\
\hline 3 & 71.7 & 117.8632 & 118.6825 & 117.5079 & 106.4912 & 106.3978 & 105.9754 & 117.6918 & 118.5061 & 117.3419 & 106.2354 & 106.1417 & 105.7449 \\
\hline 4 & 30.5 & 160.5651 & 161.9314 & 161.1701 & 151.5794 & 151.3490 & 151.2233 & 160.4896 & 161.8613 & 161.0813 & 151.5152 & 151.2844 & 151.1160 \\
\hline 5 & 25.4 & 161.7722 & 163.1037 & 162.9457 & 153.7887 & 153.5139 & 153.1055 & 162.1154 & 163.4325 & 163.2644 & 154.1724 & 153.8777 & 153.5243 \\
\hline 6 & 41.4 & 144.6259 & 144.8541 & 144.7048 & 133.7648 & 133.9799 & 133.1635 & 144.1826 & 144.4084 & 144.1229 & 133.1974 & 133.4205 & 132.5522 \\
\hline 7 & 90.0 & 99.7553 & 99.9629 & 100.1037 & 87.6697 & 87.7929 & 86.0445 & 98.6938 & 98.8946 & 98.8449 & 86.3952 & 86.5161 & 84.6231 \\
\hline 8 & 207.9 & -8.5966 & -8.4541 & -10.9842 & -28.7869 & -28.6367 & -32.0360 & -13.0686 & -12.9391 & -16.3174 & -34.0086 & -33.8716 & -38.0401 \\
\hline 9 & 35.7 & 153.8101 & 155.1084 & 153.5627 & 144.0745 & 143.5635 & 143.1722 & 153.1879 & 154.4695 & 152.7370 & 143.2545 & 142.7259 & 142.0925 \\
\hline 10 & 35.8 & 152.4146 & 153.6041 & 153.3810 & 143.5823 & 143.2526 & 143.0921 & 152.6113 & 153.7940 & 153.6159 & 143.8477 & 143.5044 & 143.3189 \\
\hline 11 & 37.4 & 149.4835 & 149.8548 & 149.1328 & 138.9661 & 138.9586 & 138.7666 & 149.2948 & 149.6709 & 148.8355 & 138.7145 & 138.7131 & 138.4814 \\
\hline 12 & 26.3 & 162.9122 & 164.6545 & 164.6015 & 156.4948 & 156.1733 & 156.3625 & 163.2604 & 164.9882 & 164.9361 & 156.8888 & 156.5416 & 156.7848 \\
\hline 13 & 24.7 & 164.4174 & 166.1526 & 166.9419 & 157.5896 & 157.3223 & 156.9727 & 164.7056 & 166.4221 & 167.2724 & 157.9004 & 157.6087 & 157.2758 \\
\hline 14 & 21.5 & 166.5060 & 168.2581 & 168.8196 & 159.5846 & 159.3507 & 158.9966 & 166.5870 & 168.3087 & 168.7168 & 159.5505 & 159.2818 & 158.8016 \\
\hline 15 & 171.0 & 28.1525 & 28.3143 & 25.7507 & 6.8058 & 6.9462 & 4.7408 & 26.6589 & 26.8120 & 23.9111 & 5.0048 & 5.1336 & 2.6220 \\
\hline $\begin{array}{l}16 \\
{ }^{1} \mathbf{H}\end{array}$ & 21.3 & 169.2271 & 170.9469 & 170.6691 & 161.3478 & 161.0373 & 160.5747 & 169.1194 & 170.8114 & 170.2660 & 161.0297 & 160.6843 & 160.0519 \\
\hline $1 e q$ & 2.41 & 30.2740 & 29.8233 & 29.7263 & 30.3746 & 30.1052 & 30.0264 & 30.2425 & 29.7908 & 29.6876 & 30.3302 & 30.0621 & 29.9775 \\
\hline $1 a x$ & 2.02 & 30.7746 & 30.3426 & 30.3529 & 30.8989 & 30.6345 & 30.5979 & 30.6158 & 30.1776 & 30.1720 & 30.7302 & 30.4629 & 30.4166 \\
\hline 2 & 4.23 & 28.0880 & 27.5458 & 27.5149 & 28.0768 & 27.7395 & 27.7872 & 28.1061 & 27.5664 & 27.5285 & 28.0699 & 27.7371 & 27.7847 \\
\hline 3 & 4.87 & 27.4479 & 26.9238 & 26.8620 & 27.5263 & 27.1751 & 27.1434 & 27.3877 & 26.8596 & 26.7822 & 27.4575 & 27.1001 & 27.0574 \\
\hline $4 e q$ & 1.99 & 29.4951 & 29.0046 & 28.9375 & 29.5358 & 29.2706 & 29.2083 & 29.6339 & 29.1472 & 29.0976 & 29.6745 & 29.4128 & 29.3640 \\
\hline $4 a x$ & 1.57 & 30.2898 & 29.8500 & 29.7939 & 30.2574 & 30.0053 & 29.9734 & 30.2091 & 29.7644 & 29.6903 & 30.1750 & 29.9160 & 29.8747 \\
\hline $5 e q$ & 2.14 & 30.1271 & 29.6429 & 29.5551 & 30.1902 & 29.8971 & 29.8090 & 30.0415 & 29.5544 & 29.4638 & 30.1065 & 29.8099 & 29.7171 \\
\hline $5 a x$ & 1.61 & 30.5880 & 30.1364 & 30.0929 & 30.6502 & 30.3851 & 30.3590 & 30.4611 & 30.0060 & 29.9502 & 30.5193 & 30.2520 & 30.2168 \\
\hline $9 e q$ & 2.65 & 29.5590 & 29.0578 & 28.9887 & 29.6114 & 29.2916 & 29.2396 & 29.3897 & 28.8838 & 28.7880 & 29.4368 & 29.1171 & 29.0486 \\
\hline $9 a x$ & 2.39 & 30.1141 & 29.6168 & 29.4692 & 30.0324 & 29.7739 & 29.6270 & 30.1105 & 29.6064 & 29.4553 & 30.0297 & 29.7627 & 29.6103 \\
\hline 10eq & 1.97 & 30.1440 & 29.6816 & 29.5833 & 30.2130 & 29.9615 & 29.9071 & 30.0916 & 29.6296 & 29.5273 & 30.1567 & 29.9075 & 29.8468 \\
\hline $10 a x$ & 1.55 & 30.7433 & 30.3375 & 30.3027 & 30.7800 & 30.5635 & 30.5329 & 30.6237 & 30.2152 & 30.1673 & 30.6603 & 30.4413 & 30.3995 \\
\hline 12 & 0.95 & 31.3121 & 30.8587 & 30.8473 & 31.3390 & 31.0662 & 31.0275 & 31.2702 & 30.8160 & 30.8040 & 31.2982 & 31.0252 & 30.9845 \\
\hline 13 & 1.23 & 30.9950 & 30.5203 & 30.4771 & 31.0233 & 30.7354 & 30.6417 & 30.9472 & 30.4732 & 30.4264 & 30.9794 & 30.6930 & 30.5960 \\
\hline 14 & 1.38 & 30.9243 & 30.4255 & 30.3567 & 30.9632 & 30.6483 & 30.5654 & 30.8563 & 30.3535 & 30.2729 & 30.8903 & 30.5723 & 30.4784 \\
\hline 16 & 2.06 & 30.2817 & 29.7670 & 29.6364 & 30.2113 & 29.8943 & 29.8575 & 30.2095 & 29.6894 & 29.5476 & 30.1362 & 29.8167 & 29.7693 \\
\hline
\end{tabular}




\begin{tabular}{|c|c|c|c|c|c|c|c|c|c|c|c|c|c|}
\hline & \multirow[b]{3}{*}{ Exp } & \multicolumn{12}{|c|}{ mPW1PW91 } \\
\hline & & \multicolumn{6}{|c|}{ Gas phase } & \multicolumn{6}{|c|}{ PCM } \\
\hline & & 6-31G* & $6-31 G * *$ & $6-31+G * *$ & 6-311G* & $6-311 G * *$ & $6-311+G * *$ & 6-31G* & $6-31 G * *$ & $6-31+G * *$ & 6-311G* & $6-311 G * *$ & $6-311+G * *$ \\
\hline \multicolumn{14}{|l|}{${ }^{13} \mathrm{C}$} \\
\hline 1 & 27.5 & 162.5817 & 163.7580 & 163.2516 & 154.9314 & 154.5979 & 154.1361 & 162.7600 & 163.9200 & 163.2972 & 155.0305 & 154.6846 & 154.2359 \\
\hline 2 & 76.0 & 119.2241 & 119.9559 & 119.5841 & 109.8948 & 109.5086 & 108.8238 & 118.4762 & 119.2047 & 118.5412 & 108.8799 & 108.4902 & 107.6626 \\
\hline 3 & 71.7 & 122.5898 & 123.3609 & 122.4354 & 112.5899 & 112.5113 & 112.0309 & 122.4102 & 123.1768 & 122.2733 & 112.3206 & 112.2425 & 111.7844 \\
\hline 4 & 30.5 & 165.4457 & 166.7113 & 165.9976 & 157.3252 & 157.0979 & 156.9054 & 165.3731 & 166.6442 & 165.8980 & 157.2581 & 157.0306 & 156.8102 \\
\hline 5 & 25.4 & 166.7333 & 167.9471 & 167.8075 & 159.3110 & 159.0542 & 158.6422 & 167.0678 & 168.2694 & 168.1111 & 159.6672 & 159.3925 & 159.0174 \\
\hline 6 & 41.4 & 149.9851 & 150.2157 & 149.9335 & 139.8098 & 140.0202 & 139.1967 & 149.5444 & 149.7723 & 149.3790 & 139.2547 & 139.4727 & 138.6075 \\
\hline 7 & 90.0 & 104.7589 & 104.9630 & 105.4473 & 94.1185 & 94.2460 & 92.5908 & 103.6713 & 103.8690 & 104.1873 & 92.8279 & 92.9545 & 91.1822 \\
\hline 8 & 207.9 & -5.8752 & -5.7397 & -7.7657 & -23.3037 & -23.1555 & -26.1993 & -10.4806 & -10.3571 & -13.1208 & -28.5708 & -28.4343 & -32.1441 \\
\hline 9 & 35.7 & 158.1086 & 159.3203 & 157.9142 & 149.3700 & 148.8669 & 148.4078 & 157.4633 & 158.6597 & 157.1018 & 148.5342 & 148.0142 & 147.3548 \\
\hline 10 & 35.8 & 157.5188 & 158.5908 & 158.2419 & 149.3128 & 149.0032 & 148.8532 & 157.7297 & 158.7970 & 158.4891 & 149.5728 & 149.2517 & 149.0817 \\
\hline 11 & 37.4 & 154.7375 & 155.1230 & 154.4052 & 144.8245 & 144.8259 & 144.6447 & 154.5482 & 154.9383 & 154.1120 & 144.5792 & 144.5863 & 144.3691 \\
\hline 12 & 26.3 & 167.3973 & 168.9945 & 168.5278 & 161.3566 & 161.0630 & 161.2973 & 167.7780 & 169.3622 & 168.8933 & 161.7546 & 161.4392 & 161.7255 \\
\hline 13 & 24.7 & 168.6751 & 170.2521 & 170.6891 & 162.3410 & 162.0739 & 161.7664 & 169.0010 & 170.5605 & 171.0517 & 162.6569 & 162.3695 & 162.0797 \\
\hline 14 & 21.5 & 170.8242 & 172.4361 & 172.6133 & 164.4439 & 164.2235 & 163.8651 & 170.9323 & 172.5163 & 172.5605 & 164.4266 & 164.1758 & 163.6993 \\
\hline 15 & 171.0 & 30.3077 & 30.4676 & 28.4808 & 11.7531 & 11.8932 & 9.9045 & 28.7796 & 28.9301 & 26.6391 & 9.9447 & 10.0738 & 7.8148 \\
\hline $\begin{array}{l}16 \\
{ }^{1} \mathbf{H}\end{array}$ & 21.3 & 172.5494 & 174.1719 & 174.2013 & 165.4521 & 165.1642 & 164.7067 & 172.4605 & 174.0553 & 173.8413 & 165.1482 & 164.8277 & 164.2168 \\
\hline $1 e q$ & 2.41 & 30.2296 & 29.7815 & 29.6911 & 30.2783 & 30.0161 & 29.9323 & 30.1969 & 29.7478 & 29.6517 & 30.2338 & 29.9727 & 29.8840 \\
\hline $1 a x$ & 2.02 & 30.7610 & 30.3329 & 30.3215 & 30.8140 & 30.5566 & 30.5108 & 30.5952 & 30.1605 & 30.1382 & 30.6418 & 30.3813 & 30.3282 \\
\hline 2 & 4.23 & 28.0079 & 27.4685 & 27.4353 & 27.9928 & 27.6426 & 27.6838 & 28.0223 & 27.4850 & 27.4445 & 27.9834 & 27.6367 & 27.6770 \\
\hline 3 & 4.87 & 27.4115 & 26.8931 & 26.8127 & 27.4314 & 27.0888 & 27.0513 & 27.3480 & 26.8254 & 26.7315 & 27.3617 & 27.0126 & 26.9656 \\
\hline 4 eq & 1.99 & 29.4373 & 28.9485 & 28.8543 & 29.4227 & 29.1677 & 29.0856 & 29.5791 & 29.0941 & 29.0139 & 29.5610 & 29.3093 & 29.2390 \\
\hline $4 a x$ & 1.57 & 30.2373 & 29.7970 & 29.7425 & 30.1854 & 29.9298 & 29.8917 & 30.1512 & 29.7059 & 29.6369 & 30.0988 & 29.8365 & 29.7907 \\
\hline $5 e q$ & 2.14 & 30.0971 & 29.6180 & 29.5008 & 30.1059 & 29.8206 & 29.7195 & 30.0060 & 29.5239 & 29.4051 & 30.0171 & 29.7280 & 29.6235 \\
\hline $5 a x$ & 1.61 & 30.5581 & 30.1120 & 30.0507 & 30.5561 & 30.3011 & 30.2736 & 30.4262 & 29.9762 & 29.9043 & 30.4225 & 30.1648 & 30.1302 \\
\hline $9 e q$ & 2.65 & 29.5451 & 29.0378 & 28.9551 & 29.5253 & 29.2110 & 29.1552 & 29.3711 & 28.8588 & 28.7531 & 29.3463 & 29.0318 & 28.9635 \\
\hline $9 a x$ & 2.39 & 29.9822 & 29.4896 & 29.3558 & 29.8991 & 29.6331 & 29.4915 & 29.9744 & 29.4743 & 29.3382 & 29.8923 & 29.6175 & 29.4713 \\
\hline 10 eq & 1.97 & 30.0988 & 29.6431 & 29.5384 & 30.1142 & 29.8720 & 29.8173 & 30.0451 & 29.5897 & 29.4824 & 30.0573 & 29.8173 & 29.7572 \\
\hline $10 a x$ & 1.55 & 30.6866 & 30.2881 & 30.2465 & 30.6759 & 30.4610 & 30.4260 & 30.5591 & 30.1572 & 30.1054 & 30.5505 & 30.3321 & 30.2884 \\
\hline 12 & 0.95 & 31.2563 & 30.8060 & 30.7853 & 31.2428 & 30.9693 & 30.9313 & 31.2109 & 30.7595 & 30.7393 & 31.1992 & 30.9254 & 30.8863 \\
\hline 13 & 1.23 & 30.9350 & 30.4611 & 30.4069 & 30.9198 & 30.6312 & 30.5338 & 30.8842 & 30.4111 & 30.3544 & 30.8733 & 30.5862 & 30.4868 \\
\hline 14 & 1.38 & 30.8652 & 30.3644 & 30.2897 & 30.8681 & 30.5516 & 30.4690 & 30.7934 & 30.2884 & 30.2039 & 30.7918 & 30.4721 & 30.3808 \\
\hline 16 & 2.06 & 30.1968 & 29.6747 & 29.5616 & 30.1144 & 29.7818 & 29.7567 & 30.1181 & 29.5900 & 29.4670 & 30.0335 & 29.6982 & 29.6634 \\
\hline
\end{tabular}




\section{Compound 17}

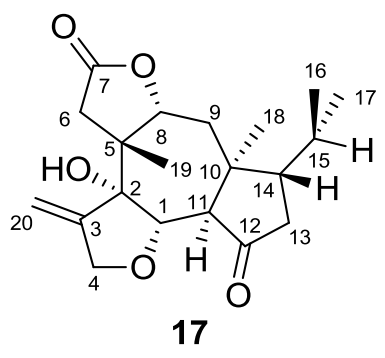

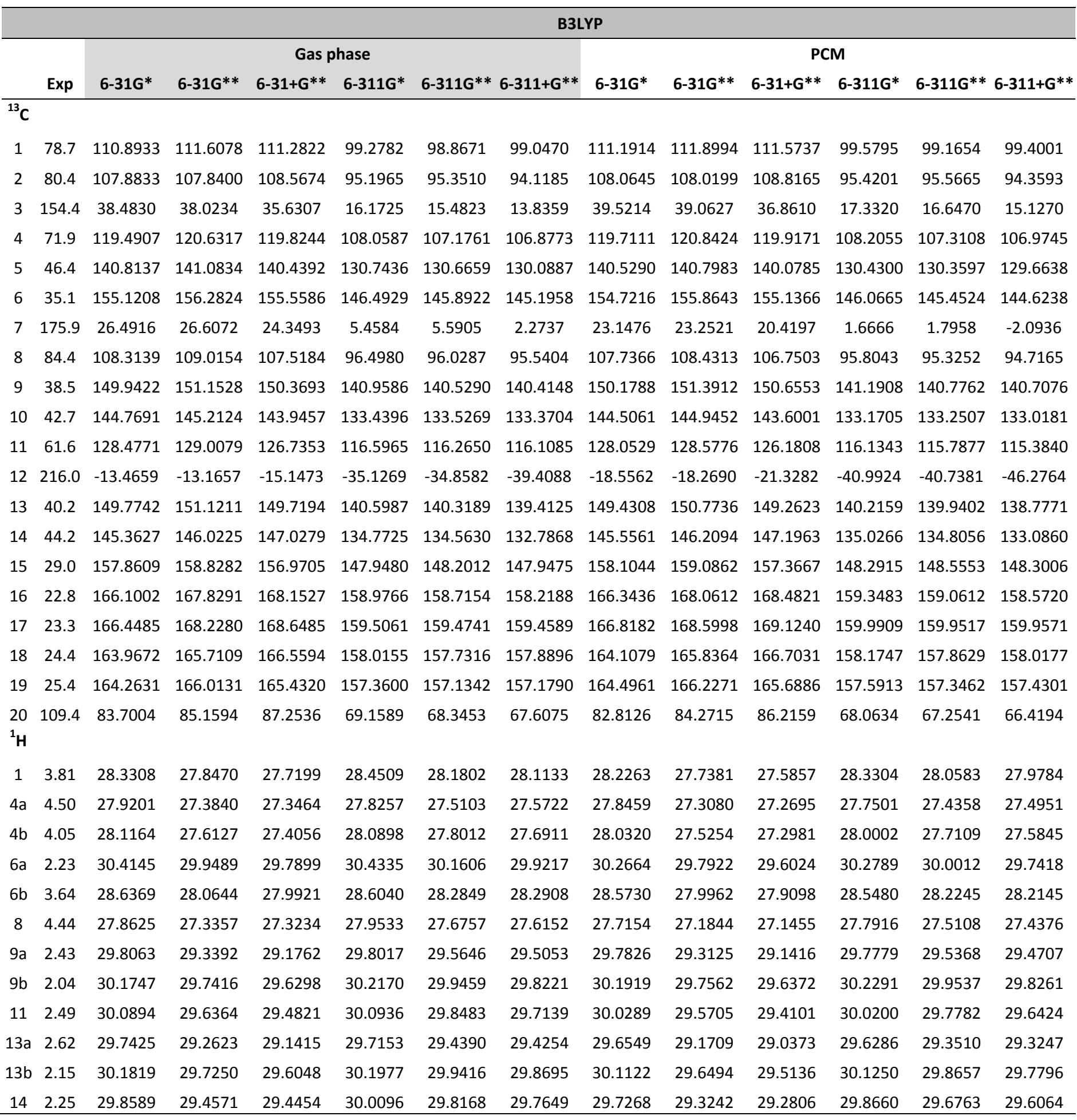




\begin{tabular}{cccccccccccccc}
\hline 15 & 1.80 & 30.2208 & 29.8028 & 29.8182 & 30.3624 & 30.1752 & 30.0688 & 30.1669 & 29.7470 & 29.7468 & 30.3039 & 30.1140 & 30.0002 \\
$20 \mathrm{a}$ & 5.23 & 26.9031 & 26.2670 & 26.1913 & 26.8975 & 26.4931 & 26.5747 & 26.8372 & 26.2018 & 26.1165 & 26.8306 & 26.4289 & 26.5019 \\
$20 \mathrm{~b}$ & 5.15 & 26.9031 & 26.2670 & 26.1913 & 26.9595 & 26.5201 & 26.4963 & 26.8372 & 26.2018 & 26.1165 & 26.8181 & 26.3869 & 26.3566 \\
16 & 0.96 & 31.0014 & 30.5721 & 30.5190 & 31.0001 & 30.7692 & 30.7010 & 31.0067 & 30.5768 & 30.5228 & 31.0056 & 30.7742 & 30.7066 \\
17 & 1.10 & 31.2024 & 30.7726 & 30.7270 & 31.1845 & 30.9566 & 30.9510 & 31.1910 & 30.7603 & 30.7167 & 31.1771 & 30.9489 & 30.9437 \\
18 & 1.01 & 31.2199 & 30.8006 & 30.7370 & 31.2661 & 31.0279 & 31.0048 & 31.1908 & 30.7704 & 30.7012 & 31.2368 & 30.9976 & 30.9711 \\
19 & 1.31 & 30.9969 & 30.5470 & 30.5103 & 31.0601 & 30.8006 & 30.6941 & 30.9345 & 30.4833 & 30.4363 & 30.9977 & 30.7374 & 30.6235 \\
\hline
\end{tabular}

\begin{tabular}{|c|c|c|c|c|c|c|c|c|c|c|c|c|c|}
\hline & & \multicolumn{12}{|c|}{ mPW1PW91 } \\
\hline & \multirow[b]{2}{*}{ Exp } & \multicolumn{6}{|c|}{ Gas phase } & \multicolumn{6}{|c|}{ PCM } \\
\hline & & 6-31G* & 6-31G** & $6-31+G^{* *}$ & 6-311G* & 6-311G** & $6-311+G^{* *}$ & 6-31G* & $6-31 G * *$ & $6-31+G^{* *}$ & 6-311G* & $6-311 G * *$ & $6-311+G * *$ \\
\hline \multicolumn{14}{|c|}{ ( } \\
\hline 1 & 78.7 & 115.4443 & 116.1100 & 116.0966 & 105.5455 & 105.1580 & 105.2222 & 115.7217 & 116.3820 & 116.3683 & 105.8124 & 105.4223 & 105.5325 \\
\hline 2 & 80.4 & 112.1845 & 112.1371 & 112.8345 & 101.3787 & 101.5233 & 100.3551 & 112.3624 & 112.3147 & 113.0801 & 101.6020 & 101.7387 & 100.5974 \\
\hline 3 & 154.4 & 42.1832 & 41.6796 & 39.7380 & 22.5510 & 21.8378 & 20.2625 & 43.2538 & 42.7526 & 40.9695 & 23.7392 & 23.0336 & 21.5580 \\
\hline 4 & 71.9 & 123.3313 & 124.4186 & 123.8996 & 113.6535 & 112.7959 & 112.4574 & 123.5420 & 124.6202 & 123.9814 & 113.7700 & 112.9016 & 112.5324 \\
\hline 5 & 46.4 & 145.8058 & 146.0755 & 145.5041 & 136.6651 & 136.5936 & 136.0795 & 145.5190 & 145.7882 & 145.1569 & 136.3556 & 136.2919 & 135.6773 \\
\hline 6 & 35.1 & 159.1361 & 160.2231 & 159.5517 & 151.4476 & 150.8757 & 150.1332 & 158.7223 & 159.7909 & 159.1101 & 150.9984 & 150.4124 & 149.5481 \\
\hline 7 & 175.9 & 28.6921 & 28.8050 & 27.2432 & 10.3489 & 10.4809 & 7.5374 & 25.3120 & 25.4126 & 23.3529 & 6.6018 & 6.7300 & 3.2877 \\
\hline 8 & 84.4 & 112.8258 & 113.4779 & 112.3679 & 102.6508 & 102.2047 & 101.6810 & 112.2378 & 112.8838 & 111.6044 & 101.9451 & 101.4900 & 100.8644 \\
\hline 9 & 38.5 & 154.6751 & 155.7731 & 155.3066 & 146.6180 & 146.2269 & 145.9888 & 154.9164 & 156.0167 & 155.5843 & 146.8513 & 146.4754 & 146.2803 \\
\hline 10 & 42.7 & 149.9281 & 150.3639 & 149.1464 & 139.4199 & 139.5104 & 139.2713 & 149.6553 & 150.0867 & 148.8062 & 139.1506 & 139.2339 & 138.9333 \\
\hline 11 & 61.6 & 133.2487 & 133.7287 & 131.7067 & 122.6948 & 122.3959 & 122.1520 & 132.8049 & 133.2797 & 131.1480 & 122.2107 & 121.8983 & 121.4288 \\
\hline 12 & 216.0 & -10.7555 & -10.4577 & -11.8383 & -29.5446 & -29.2782 & -33.2120 & -15.9542 & -15.6701 & -17.9880 & -35.4053 & -35.1552 & -39.9449 \\
\hline 13 & 40.2 & 153.8101 & 155.0530 & 153.6899 & 145.5773 & 145.3253 & 144.5656 & 153.4253 & 154.6640 & 153.2157 & 145.1489 & 144.9000 & 143.9073 \\
\hline 14 & 44.2 & 150.5483 & 151.1282 & 152.4155 & 140.9166 & 140.7225 & 138.9610 & 150.7280 & 151.3026 & 152.5629 & 141.1367 & 140.9328 & 139.2212 \\
\hline 15 & 29.0 & 162.9991 & 163.8977 & 162.3233 & 153.9005 & 154.1695 & 153.9278 & 163.2422 & 164.1553 & 162.6997 & 154.2315 & 154.5125 & 154.2716 \\
\hline 16 & 22.8 & 170.4589 & 172.0552 & 172.3302 & 163.9349 & 163.6543 & 163.1219 & 170.7373 & 172.3224 & 172.6976 & 164.3068 & 164.0016 & 163.4763 \\
\hline 17 & 23.3 & 170.6833 & 172.3186 & 172.6809 & 164.1969 & 164.1939 & 164.2150 & 171.0890 & 172.7261 & 173.1744 & 164.6858 & 164.6784 & 164.7176 \\
\hline 18 & 24.4 & 168.5111 & 170.1135 & 170.2403 & 162.8948 & 162.6387 & 162.8853 & 168.6707 & 170.2594 & 170.3961 & 163.0523 & 162.7717 & 163.0191 \\
\hline 19 & 25.4 & 168.7434 & 170.3479 & 169.4876 & 162.2656 & 162.0646 & 162.1468 & 168.9892 & 170.5770 & 169.7557 & 162.4893 & 162.2732 & 162.3912 \\
\hline $\begin{array}{l}20 \\
{ }^{1} \mathbf{H}\end{array}$ & 109.4 & 85.2537 & 86.6489 & 88.6678 & 72.2581 & 71.4285 & 70.7317 & 84.2974 & 85.6936 & 87.5920 & 71.1096 & 70.2854 & 69.5156 \\
\hline 1 & 3.81 & 28.2924 & 27.8166 & 27.6663 & 28.3416 & 28.0921 & 28.0282 & 28.1864 & 27.7061 & 27.5334 & 28.2213 & 27.9701 & 27.8950 \\
\hline $4 a$ & 4.50 & 27.8167 & 27.2822 & 27.2626 & 27.7472 & 27.4146 & 27.4814 & 27.7351 & 27.1983 & 27.1790 & 27.6656 & 27.3340 & 27.3991 \\
\hline $4 b$ & 4.05 & 28.0929 & 27.5893 & 27.3850 & 28.0460 & 27.7570 & 27.6553 & 28.0014 & 27.4949 & 27.2727 & 27.9511 & 27.6617 & 27.5452 \\
\hline $6 a$ & 2.23 & 30.2962 & 29.8302 & 29.6842 & 30.2996 & 30.0234 & 29.8052 & 30.1403 & 29.6651 & 29.4950 & 30.1403 & 29.8592 & 29.6237 \\
\hline $6 b$ & 3.64 & 28.5716 & 27.9957 & 27.9375 & 28.5157 & 28.1998 & 28.1964 & 28.5030 & 27.9226 & 27.8537 & 28.4559 & 28.1354 & 28.1177 \\
\hline 8 & 4.44 & 27.7984 & 27.2775 & 27.2495 & 27.8292 & 27.5596 & 27.4948 & 27.6473 & 27.1219 & 27.0711 & 27.6658 & 27.3928 & 27.3161 \\
\hline $9 a$ & 2.43 & 29.7345 & 29.2679 & 29.0942 & 29.6734 & 29.4420 & 29.3606 & 29.7114 & 29.2416 & 29.0615 & 29.6510 & 29.4154 & 29.3285 \\
\hline $9 b$ & 2.04 & 30.1141 & 29.6815 & 29.5704 & 30.0974 & 29.8358 & 29.7172 & 30.1301 & 29.6951 & 29.5768 & 30.1076 & 29.8415 & 29.7198 \\
\hline 11 & 2.49 & 29.9661 & 29.5206 & 29.3624 & 29.9229 & 29.6748 & 29.5529 & 29.9052 & 29.4542 & 29.2910 & 29.8503 & 29.6059 & 29.4822 \\
\hline $13 a$ & 2.62 & 29.6537 & 29.1654 & 29.0576 & 29.5940 & 29.3138 & 29.3129 & 29.5614 & 29.0686 & 28.9501 & 29.5034 & 29.2218 & 29.2090 \\
\hline $13 b$ & 2.15 & 30.1063 & 29.6470 & 29.5235 & 30.0765 & 29.8186 & 29.7368 & 30.0314 & 29.5656 & 29.4282 & 29.9998 & 29.7383 & 29.6442 \\
\hline 14 & 2.25 & 29.8015 & 29.4078 & 29.3436 & 29.8629 & 29.6848 & 29.6138 & 29.6661 & 29.2717 & 29.1807 & 29.7199 & 29.5446 & 29.4584 \\
\hline
\end{tabular}




\begin{tabular}{cccccccccccccc}
\hline 15 & 1.80 & 30.1871 & 29.7761 & 29.7722 & 30.2436 & 30.0680 & 29.9524 & 30.1330 & 29.7198 & 29.7022 & 30.1863 & 30.0077 & 29.8857 \\
$20 \mathrm{a}$ & 5.23 & 26.7187 & 26.0785 & 26.0188 & 26.7073 & 26.2866 & 26.3593 & 26.6437 & 26.0044 & 25.9386 & 26.6336 & 26.2155 & 26.2809 \\
\hline 20b & 5.15 & 26.7187 & 26.0785 & 26.0188 & 26.7762 & 26.3175 & 26.2749 & 26.6437 & 26.0044 & 25.9386 & 26.6252 & 26.1749 & 26.1268 \\
16 & 0.96 & 30.9512 & 30.5224 & 30.4762 & 30.9076 & 30.6737 & 30.5997 & 30.9548 & 30.5255 & 30.4779 & 30.9114 & 30.6770 & 30.6032 \\
17 & 1.10 & 31.1484 & 30.7195 & 30.6722 & 31.0949 & 30.8637 & 30.8564 & 31.1344 & 30.7045 & 30.6592 & 31.0850 & 30.8534 & 30.8466 \\
18 & 1.01 & 31.1625 & 30.7455 & 30.6861 & 31.1659 & 30.9274 & 30.9209 & 31.1309 & 30.7128 & 30.6485 & 31.1344 & 30.8950 & 30.8852 \\
19 & 1.31 & 30.9466 & 30.4976 & 30.4516 & 30.9755 & 30.7161 & 30.6128 & 30.8817 & 30.4313 & 30.3769 & 30.9115 & 30.6512 & 30.5417 \\
\hline
\end{tabular}

\section{Compound 18}

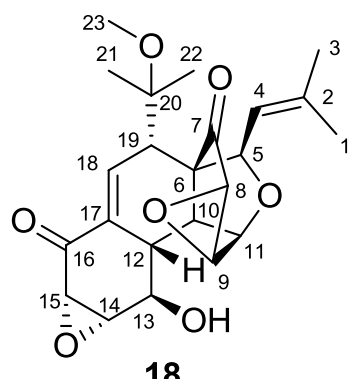

18

\begin{tabular}{|c|c|c|c|c|c|c|c|c|c|c|c|c|c|}
\hline & \multirow[b]{3}{*}{ Exp } & \multicolumn{12}{|c|}{ B3LYP } \\
\hline & & \multicolumn{6}{|c|}{ Gas phase } & \multicolumn{6}{|c|}{ PCM } \\
\hline & & 6-31G* & $6-31 G * *$ & $6-31+G * *$ & 6-311G* & $6-311 G * *$ & $6-311+G * *$ & 6-31G* & $6-31 G * *$ & $6-31+G * *$ & 6-311G* & $6-311 G * *$ & $6-311+G * *$ \\
\hline \multicolumn{14}{|l|}{${ }^{13} \mathrm{C}$} \\
\hline 1 & 18.6 & 170.3026 & 171.9477 & 171.3551 & 162.6891 & 162.2876 & 161.8664 & 170.4781 & 172.1216 & 171.5960 & 162.8920 & 162.4822 & 162.1371 \\
\hline 2 & 142.2 & 54.6361 & 54.5201 & 49.2220 & 33.2533 & 32.6804 & 29.8683 & 53.4426 & 53.3387 & 47.7430 & 32.0067 & 31.4493 & 28.6917 \\
\hline 3 & 26.1 & 163.1022 & 164.7672 & 165.5353 & 155.1352 & 154.8274 & 154.7816 & 163.5493 & 165.1924 & 165.9123 & 155.6252 & 155.2683 & 155.1742 \\
\hline 4 & 120.7 & 70.6270 & 71.4777 & 70.9393 & 54.1899 & 53.8729 & 54.5795 & 71.3709 & 72.2302 & 72.1165 & 55.0547 & 54.7422 & 55.6285 \\
\hline 5 & 75.8 & 112.2394 & 112.8483 & 113.1221 & 100.3262 & 99.9942 & 100.9096 & 112.5714 & 113.1623 & 113.1570 & 100.7076 & 100.3135 & 101.1608 \\
\hline 6 & 60.5 & 126.4073 & 126.5838 & 126.2738 & 114.2227 & 114.2627 & 114.0833 & 126.4853 & 126.6573 & 126.7635 & 114.3805 & 114.3920 & 114.1090 \\
\hline 7 & 202.9 & -9.7637 & -9.6719 & -13.7538 & -31.4815 & -31.5684 & -33.7467 & -12.1034 & -11.9972 & -16.2284 & -34.2681 & -34.3063 & -36.6238 \\
\hline 8 & 53.1 & 136.7290 & 137.5909 & 136.6617 & 126.6020 & 125.9042 & 125.0809 & 136.8425 & 137.7022 & 136.9484 & 126.6778 & 125.9678 & 124.9783 \\
\hline 9 & 54.5 & 134.5385 & 135.4859 & 134.4839 & 124.9715 & 124.2701 & 122.9193 & 134.6721 & 135.6241 & 134.5860 & 125.1179 & 124.4126 & 122.9570 \\
\hline 10 & 47.8 & 140.4484 & 141.1397 & 140.3411 & 130.3751 & 130.2403 & 130.2408 & 140.7100 & 141.4125 & 140.3601 & 130.6549 & 130.5393 & 130.6700 \\
\hline 11 & 71.5 & 117.2099 & 118.0816 & 118.0505 & 105.7594 & 105.3666 & 105.2155 & 117.2736 & 118.1475 & 118.0911 & 105.7695 & 105.4010 & 105.2171 \\
\hline 12 & 40.4 & 145.8993 & 146.4087 & 146.7674 & 136.5387 & 136.1211 & 136.8525 & 146.9124 & 147.4173 & 148.1039 & 137.7794 & 137.2619 & 137.9223 \\
\hline 13 & 72.7 & 117.6956 & 118.2773 & 116.7136 & 105.2378 & 104.9633 & 104.0772 & 117.0667 & 117.6772 & 116.0429 & 104.4221 & 104.2952 & 103.5110 \\
\hline 14 & 61.0 & 129.7559 & 130.6094 & 128.3730 & 119.3978 & 118.7834 & 117.4153 & 129.2354 & 130.0772 & 127.9507 & 118.8064 & 118.1622 & 116.7560 \\
\hline 15 & 53.2 & 137.0491 & 137.9018 & 137.3439 & 127.1636 & 126.4624 & 125.9930 & 137.2068 & 138.0531 & 137.4344 & 127.3482 & 126.6015 & 125.9270 \\
\hline 16 & 192.8 & 5.8503 & 5.9332 & 4.2926 & -13.1869 & -13.1147 & -16.4789 & 3.7499 & 3.8337 & 1.7352 & -15.7321 & -15.6194 & -19.3401 \\
\hline 17 & 132.5 & 61.4409 & 61.2002 & 61.9935 & 43.4083 & 43.2181 & 41.9510 & 60.8756 & 60.6390 & 61.2201 & 42.6808 & 42.5578 & 41.3906 \\
\hline 18 & 139.6 & 51.6944 & 52.5633 & 52.4071 & 33.4186 & 32.9849 & 30.7679 & 50.0335 & 50.9010 & 50.0769 & 31.6089 & 31.1831 & 28.7299 \\
\hline 19 & 40.9 & 145.2008 & 145.7315 & 147.0693 & 135.8145 & 135.6574 & 135.8276 & 145.2215 & 145.7318 & 147.2732 & 135.7623 & 135.5623 & 135.8431 \\
\hline 20 & 77.3 & 111.1387 & 111.4787 & 111.0339 & 98.3704 & 98.4158 & 97.7843 & 110.9182 & 111.2617 & 110.7395 & 98.1130 & 98.1588 & 97.5800 \\
\hline 21 & 26.6 & 164.9658 & 166.6334 & 167.0409 & 158.1097 & 157.7362 & 157.2270 & 165.1009 & 166.7605 & 166.9730 & 158.2878 & 157.9027 & 157.1117 \\
\hline 22 & 24.7 & 165.6163 & 167.3041 & 166.1769 & 158.7320 & 158.2375 & 156.7068 & 165.3917 & 167.0845 & 165.9075 & 158.4544 & 157.9831 & 156.6360 \\
\hline $\begin{array}{l}23 \\
{ }^{1} \mathbf{H}\end{array}$ & 49.1 & 141.7199 & 143.5998 & 143.5111 & 134.1520 & 133.5313 & 133.0445 & 141.6617 & 143.5347 & 143.2551 & 133.9386 & 133.3170 & 132.8063 \\
\hline
\end{tabular}




\begin{tabular}{rrrlllllllllll}
\hline 4 & 4.82 & 27.0953 & 26.4788 & 26.4607 & 27.1074 & 26.6534 & 26.7283 & 27.1295 & 26.5088 & 26.4738 & 27.1249 & 26.6688 & 26.7547 \\
5 & 5.46 & 26.5225 & 25.8571 & 25.7617 & 26.6347 & 26.1768 & 26.0935 & 26.5221 & 25.8615 & 25.7707 & 26.6337 & 26.1775 & 26.1042 \\
8 & 3.23 & 29.3350 & 28.8209 & 28.7984 & 29.2416 & 28.9638 & 29.1222 & 29.2494 & 28.7315 & 28.7219 & 29.1399 & 28.8639 & 29.0295 \\
9 & 3.64 & 28.8432 & 28.3581 & 28.2534 & 28.7627 & 28.5077 & 28.5540 & 28.6963 & 28.2060 & 28.0891 & 28.5985 & 28.3469 & 28.3746 \\
10 & 2.74 & 29.5046 & 29.1140 & 28.9361 & 29.5208 & 29.3338 & 29.2003 & 29.3867 & 28.9894 & 28.8040 & 29.3831 & 29.1919 & 29.0706 \\
11 & 4.99 & 27.2035 & 26.6656 & 26.4769 & 27.1562 & 26.8613 & 26.7887 & 27.1835 & 26.6399 & 26.3934 & 27.1220 & 26.8160 & 26.7073 \\
12 & 3.55 & 28.8345 & 28.2258 & 27.9116 & 28.9647 & 28.5756 & 28.3349 & 28.6324 & 28.0330 & 27.6639 & 28.7029 & 28.3659 & 28.1383 \\
13 & 3.81 & 28.2888 & 27.8514 & 27.8460 & 28.4078 & 28.2007 & 28.1671 & 28.2442 & 27.8041 & 27.7632 & 28.3434 & 28.1192 & 28.0683 \\
14 & 3.51 & 28.9253 & 28.4701 & 28.3330 & 28.7917 & 28.5841 & 28.5902 & 28.8032 & 28.3362 & 28.1802 & 28.6813 & 28.4530 & 28.4452 \\
15 & 3.29 & 29.3771 & 28.8773 & 28.6443 & 29.1581 & 28.8968 & 28.8670 & 29.2809 & 28.7786 & 28.5164 & 29.0575 & 28.8007 & 28.7619 \\
18 & 6.73 & 25.1370 & 24.5075 & 24.2611 & 25.1179 & 24.7127 & 24.5620 & 25.0972 & 24.4654 & 24.2074 & 25.0649 & 24.6543 & 24.4935 \\
19 & 3.59 & 28.5200 & 27.9528 & 27.9027 & 28.5906 & 28.1895 & 28.1915 & 28.5391 & 27.9756 & 27.8582 & 28.5943 & 28.1993 & 28.1255 \\
21 & 1.26 & 30.9659 & 30.4938 & 30.4242 & 30.9653 & 30.6873 & 30.6827 & 30.9438 & 30.4702 & 30.3821 & 30.9457 & 30.6656 & 30.6496 \\
22 & 1.15 & 31.0418 & 30.5709 & 30.5642 & 31.0764 & 30.8040 & 30.7861 & 31.0084 & 30.5390 & 30.5478 & 31.0417 & 30.7719 & 30.7633 \\
23 & 3.02 & 29.0351 & 28.5348 & 28.4891 & 29.0865 & 28.7800 & 28.7688 & 28.9912 & 28.4922 & 28.4375 & 29.0420 & 28.7376 & 28.7284 \\
1 & 1.77 & 30.3392 & 29.8456 & 29.7340 & 30.2388 & 29.9575 & 29.9213 & 30.3380 & 29.8467 & 29.7309 & 30.2383 & 29.9588 & 29.9142 \\
3 & 1.72 & 30.5681 & 30.1064 & 29.9649 & 30.4587 & 30.1917 & 30.0779 & 30.5306 & 30.0673 & 29.9326 & 30.4191 & 30.1528 & 30.0448 \\
\hline
\end{tabular}

\begin{tabular}{|c|c|c|c|c|c|c|c|c|c|c|c|c|c|}
\hline \multirow{3}{*}{\multicolumn{2}{|c|}{ Exp }} & \multicolumn{12}{|c|}{ mPW1PW91 } \\
\hline & & \multicolumn{6}{|c|}{ Gas phase } & \multicolumn{6}{|c|}{ PCM } \\
\hline & & 6-31G* & $6-31 G * *$ & $6-31+G^{* *}$ & $6-311 G^{*}$ & 6-311G** & $6-311+G^{* *}$ & 6-31G* & 6-31G** & $6-31+G^{* *}$ & 6-311G* & $6-311 \mathrm{G} * *$ & $6-311+G^{* *}$ \\
\hline \\
\hline 1 & 18.6 & 173.8251 & 175.3504 & 175.0539 & 166.9616 & 166.5642 & 166.1016 & 174.0142 & 175.5368 & 175.2707 & 167.1415 & 166.7363 & 166.3463 \\
\hline 2 & 142.2 & 58.3265 & 58.1929 & 53.9063 & 39.1863 & 38.5943 & 35.9084 & 56.9718 & 56.8424 & 52.1036 & 37.7448 & 37.1672 & 34.5370 \\
\hline 3 & 26.1 & 166.7761 & 168.3152 & 169.2155 & 159.5338 & 159.2253 & 159.1530 & 167.2733 & 168.7916 & 169.6853 & 160.0337 & 159.6783 & 159.5715 \\
\hline 4 & 120.7 & 73.4584 & 74.2659 & 73.6472 & 58.9132 & 58.6028 & 59.1421 & 74.2426 & 75.0602 & 74.9349 & 59.8087 & 59.5049 & 60.2461 \\
\hline 5 & 75.8 & 116.5374 & 117.0914 & 117.2831 & 106.1296 & 105.8257 & 106.6676 & 116.9436 & 117.4836 & 117.4357 & 106.5824 & 106.2147 & 107.0714 \\
\hline 6 & 60.5 & 131.2991 & 131.4601 & 130.8329 & 120.3026 & 120.3481 & 120.1336 & 131.3781 & 131.5358 & 131.4176 & 120.4683 & 120.4814 & 120.2149 \\
\hline 7 & 202.9 & -6.5927 & -6.5082 & -9.7515 & -25.5171 & -25.6127 & -27.5730 & -9.0691 & -8.9739 & -12.3827 & -28.3914 & -28.4374 & -30.5442 \\
\hline 8 & 53.1 & 140.6459 & 141.4764 & 140.6274 & 131.8695 & 131.1822 & 130.5205 & 140.7572 & 141.5864 & 140.9443 & 131.9333 & 131.2372 & 130.4077 \\
\hline 9 & 54.5 & 138.7273 & 139.6249 & 138.7812 & 130.3039 & 129.6074 & 128.3872 & 138.8334 & 139.7361 & 138.8650 & 130.4041 & 129.7067 & 128.3905 \\
\hline 10 & 47.8 & 146.1940 & 146.7925 & 145.9404 & 136.7673 & 136.6390 & 136.5183 & 146.4456 & 147.0550 & 145.7965 & 137.0339 & 136.9289 & 136.9528 \\
\hline 11 & 71.5 & 121.6449 & 122.4618 & 122.7287 & 111.7046 & 111.3124 & 111.1548 & 121.7141 & 122.5338 & 122.7815 & 111.7211 & 111.3537 & 111.1647 \\
\hline 12 & 40.4 & 150.3856 & 150.8332 & 150.9406 & 142.2302 & 141.8614 & 142.3222 & 151.3505 & 151.7992 & 152.3941 & 143.3786 & 142.9146 & 143.4768 \\
\hline 13 & 72.7 & 122.8203 & 123.3630 & 122.3192 & 112.3599 & 112.0162 & 111.2180 & 122.1933 & 122.7601 & 121.5245 & 111.5445 & 111.3565 & 110.5057 \\
\hline 14 & 61.0 & 133.7648 & 134.5822 & 132.5586 & 124.9800 & 124.3607 & 123.0770 & 133.2523 & 134.0568 & 132.1423 & 124.3909 & 123.7428 & 122.3882 \\
\hline 15 & 53.2 & 141.0435 & 141.8624 & 141.4270 & 132.5021 & 131.8157 & 131.2728 & 141.1921 & 142.0059 & 141.5420 & 132.6568 & 131.9268 & 131.2443 \\
\hline & 192.8 & 7.9452 & 8.0200 & 6.8950 & -8.0776 & -8.0274 & -11.0370 & 5.8033 & 5.8756 & 4.3175 & -10.6003 & -10.5158 & -13.8903 \\
\hline & 132.5 & 64.7035 & 64.4335 & 65.4838 & 48.9980 & 48.7737 & 47.4955 & 64.1930 & 63.9269 & 64.7351 & 48.3380 & 48.1799 & 46.9834 \\
\hline & 139.6 & 54.5527 & 55.3816 & 55.7551 & 38.2697 & 37.8244 & 35.8741 & 52.8485 & 53.6729 & 53.3962 & 36.4478 & 36.0110 & 33.8079 \\
\hline 19 & 40.9 & 149.8145 & 150.2842 & 151.5141 & 141.6296 & 141.4540 & 141.5324 & 149.8716 & 150.3190 & 151.7906 & 141.6194 & 141.3941 & 141.4978 \\
\hline 20 & 77.3 & 115.9565 & 116.2902 & 116.0266 & 104.6290 & 104.6725 & 104.0555 & 115.7308 & 116.0650 & 115.7341 & 104.3692 & 104.4106 & 103.8319 \\
\hline 21 & 26.6 & 168.9411 & 170.4894 & 170.7348 & 162.4479 & 162.1305 & 161.6624 & 169.0817 & 170.6237 & 170.6873 & 162.6149 & 162.2940 & 161.6625 \\
\hline 22 & 24.7 & 170.0577 & 171.6325 & 170.4772 & 163.7235 & 163.2582 & 161.9233 & 169.7927 & 171.3690 & 170.1114 & 163.3724 & 162.9355 & 161.7483 \\
\hline 23 & 49.1 & 145.3495 & 147.1561 & 147.1655 & 138.8031 & 138.2326 & 137.8218 & 145.2975 & 147.0974 & 146.9243 & 138.5840 & 138.0176 & 137.5930 \\
\hline
\end{tabular}




\begin{tabular}{cccccccccccccc}
\hline${ }^{1} \mathbf{H}$ & & & & & & & & & & & & & \\
4 & 4.82 & 26.9630 & 26.3500 & 26.3315 & 26.9493 & 26.4901 & 26.5455 & 26.9951 & 26.3768 & 26.3403 & 26.9671 & 26.5039 & 26.5723 \\
5 & 5.46 & 26.4899 & 25.8272 & 25.7035 & 26.5154 & 26.0775 & 25.9803 & 26.4872 & 25.8296 & 25.7203 & 26.5115 & 26.0753 & 26.0016 \\
8 & 3.23 & 29.2416 & 28.7249 & 28.7276 & 29.1633 & 28.8598 & 29.0220 & 29.1504 & 28.6292 & 28.6458 & 29.0579 & 28.7555 & 28.9247 \\
9 & 3.64 & 28.7627 & 28.2722 & 28.2022 & 28.6965 & 28.4169 & 28.4687 & 28.6063 & 28.1102 & 28.0264 & 28.5263 & 28.2493 & 28.2849 \\
10 & 2.74 & 29.4576 & 29.0726 & 28.9109 & 29.4513 & 29.2716 & 29.1512 & 29.3303 & 28.9381 & 28.7613 & 29.3054 & 29.1224 & 29.0010 \\
11 & 4.99 & 27.1107 & 26.5708 & 26.3913 & 27.0603 & 26.7553 & 26.7005 & 27.0855 & 26.5393 & 26.3123 & 27.0251 & 26.7066 & 26.6194 \\
12 & 3.55 & 28.8321 & 28.2207 & 27.9207 & 28.9195 & 28.5225 & 28.3140 & 28.6207 & 28.0163 & 27.6448 & 28.6499 & 28.3055 & 28.0824 \\
13 & 3.81 & 28.2085 & 27.7683 & 27.7422 & 28.3041 & 28.0960 & 28.0544 & 28.1656 & 27.7237 & 27.6686 & 28.2416 & 28.0179 & 27.9654 \\
14 & 3.51 & 28.8263 & 28.3658 & 28.2275 & 28.7004 & 28.4744 & 28.4603 & 28.6971 & 28.2239 & 28.0806 & 28.5865 & 28.3375 & 28.3242 \\
15 & 3.29 & 29.2550 & 28.7525 & 28.5410 & 29.0745 & 28.7826 & 28.7643 & 29.1494 & 28.6432 & 28.4072 & 28.9662 & 28.6781 & 28.6526 \\
18 & 6.73 & 24.9831 & 24.3556 & 24.1263 & 24.9824 & 24.5487 & 24.4047 & 24.9368 & 24.3072 & 24.0720 & 24.9242 & 24.4864 & 24.3342 \\
19 & 3.59 & 28.4246 & 27.8606 & 27.8091 & 28.4012 & 28.0178 & 28.0285 & 28.4453 & 27.8853 & 27.7839 & 28.4088 & 28.0319 & 27.9798 \\
21 & 1.26 & 30.8927 & 30.4225 & 30.3574 & 30.8466 & 30.5710 & 30.5685 & 30.8692 & 30.3975 & 30.3194 & 30.8256 & 30.5478 & 30.5382 \\
22 & 1.15 & 30.9945 & 30.5239 & 30.4988 & 30.9908 & 30.7146 & 30.6927 & 30.9582 & 30.4891 & 30.4823 & 30.9536 & 30.6798 & 30.6680 \\
23 & 3.02 & 29.0042 & 28.5062 & 28.4871 & 29.0350 & 28.7209 & 28.7158 & 28.9563 & 28.4595 & 28.4280 & 28.9876 & 28.6757 & 28.6728 \\
1 & 1.77 & 30.2687 & 29.7682 & 29.6618 & 30.1371 & 29.8452 & 29.8075 & 30.2640 & 29.7661 & 29.6591 & 30.1339 & 29.8438 & 29.7996 \\
3 & 1.72 & 30.4991 & 30.0345 & 29.8950 & 30.3682 & 30.0916 & 29.9816 & 30.4548 & 29.9884 & 29.8559 & 30.3221 & 30.0463 & 29.9420 \\
\hline
\end{tabular}

\section{Compound 19}

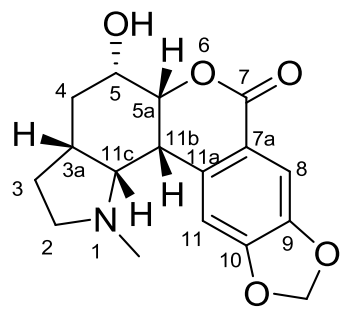

19

\begin{tabular}{|c|c|c|c|c|c|c|c|c|c|c|c|}
\hline & \multicolumn{11}{|c|}{ B3LYP } \\
\hline \multirow[b]{2}{*}{ Exp } & \multicolumn{6}{|c|}{ Gas phase } & \multicolumn{5}{|c|}{ PCM } \\
\hline & 6-31G* & $6-31 G * *$ & $6-31+G * *$ & 6-311G* & $6-311 G * *$ & $6-311+G * *$ & 6-31G* & $6-31 G * *$ & $6-31+G * *$ & 6-311G* & $6-311 \mathrm{G} * * 6-311+\mathrm{G} * *$ \\
\hline
\end{tabular}

\begin{tabular}{|c|c|c|c|c|c|c|c|c|c|c|c|c|}
\hline${ }^{13} \mathrm{C}$ & & & & & & & & & & & & \\
\hline 2 & 55.0135 .8884 & 137.1949 & 136.7560 & 125.9230 & 125.3497 & 125.3177 & 136.0113 & 137.3184 & 136.8848 & 126.0516 & 125.4718 & 125.4365 \\
\hline 3 & 29.8157 .8466 & 159.2340 & 159.0951 & 149.9526 & 149.6976 & 148.9108 & 157.9833 & 159.3652 & 159.2047 & 150.1014 & 149.8328 & 149.0439 \\
\hline $3 a$ & 39.4148 .3032 & 149.1475 & 146.1588 & 137.6706 & 137.4694 & 137.5323 & 148.5413 & 149.3826 & 146.4451 & 137.9838 & 137.7792 & 137.8564 \\
\hline 4 & 30.9157 .1203 & 158.3852 & 157.8583 & 148.6052 & 148.2605 & 148.1775 & 157.2398 & 158.5033 & 158.0285 & 148.7549 & 148.4169 & 148.3391 \\
\hline 5 & 69.7121 .1396 & 121.8394 & 119.7443 & 109.7602 & 109.2590 & 109.1097 & 121.3828 & 122.0854 & 119.9199 & 109.8906 & 109.4182 & 109.2267 \\
\hline $5 a$ & 78.0111 .9994 & 112.6741 & 113.0257 & 100.7556 & 100.4475 & 100.1097 & 111.5196 & 112.1916 & 112.4466 & 100.1949 & 99.8842 & 99.5041 \\
\hline 7 & 164.735 .8202 & 35.8065 & 33.0422 & 16.3261 & 16.3897 & 14.4194 & 34.0158 & 34.0008 & 30.9290 & 14.3184 & 14.3719 & 12.0196 \\
\hline $7 a$ & 121.071 .7854 & 71.6598 & 71.3713 & 55.2213 & 55.1044 & 53.9288 & 72.8771 & 72.7512 & 72.5915 & 56.4445 & 56.3379 & 55.1845 \\
\hline 8 & 109.883 .3614 & 84.0999 & 83.5941 & 68.1665 & 67.5713 & 66.4028 & 84.4281 & 85.1681 & 84.7749 & 69.4611 & 68.8721 & 67.6800 \\
\hline 9 & 147.649 .7527 & 49.5700 & 47.9310 & 29.9401 & 29.8229 & 29.0883 & 49.5750 & 49.3912 & 47.7649 & 29.7225 & 29.6074 & 28.8864 \\
\hline 10 & 151.946 .3067 & 46.1416 & 44.8252 & 26.1834 & 26.0414 & 24.4898 & 45.2275 & 45.0652 & 43.7411 & 25.0678 & 24.9294 & 23.3176 \\
\hline 11 & 106.688 .7552 & 89.5143 & 89.5129 & 73.5968 & 73.0758 & 72.1794 & 87.9276 & 88.6823 & 88.4706 & 72.5756 & 72.0437 & 71.0201 \\
\hline $11 a$ & 137.857 .9776 & 57.9364 & 55.2389 & 38.6073 & 38.5630 & 37.8293 & 56.5717 & 56.5334 & 53.7053 & 37.0615 & 37.0188 & 36.1640 \\
\hline $11 b$ & 41.0145 .8356 & 146.5248 & 145.2524 & 135.4810 & 135.2956 & 134.5937 & 146.4142 & 147.0964 & 146.0102 & 136.2243 & 136.0259 & 135.3477 \\
\hline
\end{tabular}




\begin{tabular}{|c|c|c|c|c|c|c|c|c|c|c|c|c|c|}
\hline $11 \mathrm{c}$ & 67.7 & 122.3185 & 122.9120 & 121.9549 & 110.0674 & 109.8293 & 109.3234 & 122.6268 & 123.2148 & 122.3641 & 110.4748 & 110.2294 & 109.7799 \\
\hline $\mathrm{NCH}_{3}$ & 45.2 & 146.5492 & 148.1883 & 148.0840 & 138.1691 & 137.7425 & 137.5020 & 146.8279 & 148.4600 & 148.3592 & 138.4935 & 138.0524 & 137.8084 \\
\hline $\begin{array}{c}\mathrm{OCH}_{2} \mathrm{O} \\
{ }^{1} \mathrm{H}\end{array}$ & 101.9 & 89.2451 & 90.2767 & 89.5376 & 76.4061 & 75.4558 & 75.4915 & 89.1399 & 90.1616 & 89.3001 & 76.1273 & 75.1537 & 75.2066 \\
\hline 2 & 3.31 & 28.7202 & 28.1874 & 28.0673 & 28.7240 & 28.4302 & 28.4354 & 28.7766 & 28.2454 & 28.1120 & 28.7667 & 28.4761 & 28.4765 \\
\hline 2 & 2.24 & 29.9183 & 29.4874 & 29.4783 & 29.9299 & 29.7033 & 29.7034 & 29.8517 & 29.4176 & 29.3973 & 29.8586 & 29.6294 & 29.6258 \\
\hline 3 & 1.93 & 30.3326 & 29.8666 & 29.7836 & 30.2706 & 30.0066 & 29.9671 & 30.2553 & 29.7891 & 29.7060 & 30.2003 & 29.9382 & 29.8954 \\
\hline 3 & 1.45 & 30.8897 & 30.4576 & 30.3172 & 30.8326 & 30.6060 & 30.5730 & 30.8497 & 30.4158 & 30.2722 & 30.7957 & 30.5674 & 30.5331 \\
\hline $3 a$ & 2.34 & 30.0095 & 29.5509 & 29.3747 & 29.9888 & 29.7060 & 29.6874 & 29.8859 & 29.4244 & 29.2400 & 29.8681 & 29.5820 & 29.5580 \\
\hline 4 & 1.88 & 30.2172 & 29.7831 & 29.7846 & 30.2331 & 30.0006 & 30.1081 & 30.2638 & 29.8321 & 29.8238 & 30.2671 & 30.0407 & 30.1440 \\
\hline 4 & 1.78 & 30.5448 & 30.1526 & 30.0017 & 30.4193 & 30.2302 & 30.2000 & 30.5262 & 30.1315 & 29.9742 & 30.4005 & 30.2081 & 30.1733 \\
\hline 5 & 3.74 & 28.6211 & 28.1954 & 28.0464 & 28.5834 & 28.3947 & 28.3507 & 28.5049 & 28.0718 & 27.9015 & 28.4590 & 28.2549 & 28.2005 \\
\hline $5 a$ & 4.61 & 27.8074 & 27.3595 & 27.0948 & 27.7537 & 27.5407 & 27.4862 & 27.7241 & 27.2715 & 26.9968 & 27.6691 & 27.4509 & 27.3866 \\
\hline 8 & 7.54 & 24.8462 & 24.1191 & 23.8071 & 24.7421 & 24.2439 & 24.1048 & 24.9572 & 24.2274 & 23.9064 & 24.8391 & 24.3401 & 24.1963 \\
\hline 11 & 6.70 & 25.9263 & 25.2457 & 24.9782 & 25.7550 & 25.2994 & 25.1918 & 25.7449 & 25.0628 & 24.7794 & 25.5679 & 25.1155 & 24.9956 \\
\hline $11 b$ & 2.87 & 29.7854 & 29.3517 & 29.2236 & 29.7258 & 29.4917 & 29.4360 & 29.5665 & 29.1228 & 28.9740 & 29.5066 & 29.2634 & 29.1928 \\
\hline $11 \mathrm{c}$ & 2.60 & 29.4666 & 29.0314 & 28.9799 & 29.5358 & 29.3343 & 29.2554 & 29.3682 & 28.9309 & 28.8692 & 29.4352 & 29.2314 & 29.1468 \\
\hline $\mathrm{OCH}_{2} \mathrm{O}$ & 6.05 & 26.0423 & 25.4571 & 25.3375 & 26.1322 & 25.8107 & 25.7512 & 25.9397 & 25.3562 & 25.2376 & 26.0370 & 25.7214 & 25.6618 \\
\hline $\mathrm{OCH}_{2} \mathrm{O}$ & 6.05 & 26.1343 & 25.5597 & 25.4940 & 25.9904 & 25.6479 & 25.5862 & 26.0423 & 25.4713 & 25.4011 & 25.8857 & 25.5473 & 25.4905 \\
\hline $\mathrm{NCH}_{3}$ & 1.45 & 30.7408 & 30.2877 & 30.2855 & 30.8102 & 30.5685 & 30.4896 & 30.7326 & 30.2795 & 30.2712 & 30.8014 & 30.5600 & 30.4755 \\
\hline
\end{tabular}

\begin{tabular}{|c|c|c|c|c|c|c|c|c|c|c|c|c|c|}
\hline & \multicolumn{13}{|c|}{ mPW1PW91 } \\
\hline & \multirow[b]{2}{*}{ Exp } & \multicolumn{6}{|c|}{ Gas phase } & \multicolumn{6}{|c|}{ PCM } \\
\hline & & 6-31G* & 6-31G** & $6-31+G^{* *}$ & 6-311G* & $6-311 G^{* *}$ & $6-311+G * *$ & 6-31G* & $6-31 G^{* *}$ & $6-31+G * *$ & 6-311G* & 6-311G** & $6-311+G * *$ \\
\hline${ }^{13} \mathrm{C}$ & & & & & & & & & & & & & \\
\hline 2 & 55.0 & 140.2618 & 141.4787 & 141.2970 & 131.4879 & 130.9248 & 130.8747 & 140.4142 & 141.6319 & 141.4566 & 131.6296 & 131.0602 & 131.0100 \\
\hline 3 & 29.8 & 162.3185 & 163.6042 & 163.1910 & 155.0204 & 154.7548 & 154.1056 & 162.4733 & 163.7540 & 163.3273 & 155.1786 & 154.9001 & 154.2526 \\
\hline $3 a$ & 39.4 & 153.3842 & 154.1532 & 151.3470 & 143.7003 & 143.4961 & 143.4717 & 153.6135 & 154.3805 & 151.6175 & 143.9890 & 143.7818 & 143.7698 \\
\hline 4 & 30.9 & 162.4454 & 163.6005 & 162.9907 & 154.6844 & 154.3595 & 154.2490 & 162.5803 & 163.7341 & 163.1734 & 154.8393 & 154.5206 & 154.4190 \\
\hline 5 & 69.7 & 125.7095 & 126.3577 & 124.6708 & 116.1651 & 115.6601 & 115.3986 & 125.9438 & 126.5961 & 124.8347 & 116.2616 & 115.7851 & 115.4862 \\
\hline $5 a$ & 78.0 & 117.1206 & 117.7377 & 118.2883 & 107.2433 & 106.9503 & 106.5910 & 116.5988 & 117.2134 & 117.6816 & 106.6468 & 106.3495 & 105.9591 \\
\hline 7 & 164.7 & 38.4303 & 38.4180 & 36.1091 & 21.3893 & 21.4493 & 19.7340 & 36.6014 & 36.5877 & 34.0016 & 19.3985 & 19.4479 & 17.3964 \\
\hline $7 a$ & 121.0 & 74.9438 & 74.8127 & 75.2553 & 60.6206 & 60.4967 & 59.2156 & 76.0956 & 75.9641 & 76.4943 & 61.8849 & 61.7706 & 60.4976 \\
\hline 8 & 109.8 & 85.4918 & 86.1982 & 85.8271 & 72.1388 & 71.5331 & 70.4427 & 86.5901 & 87.2967 & 87.0227 & 73.4107 & 72.8119 & 71.7031 \\
\hline 9 & 147.6 & 52.4888 & 52.2937 & 51.2472 & 35.4499 & 35.3236 & 34.5479 & 52.3304 & 52.1346 & 51.0979 & 35.2504 & 35.1269 & 34.3711 \\
\hline 10 & 151.9 & 48.9260 & 48.7491 & 48.2363 & 31.5974 & 31.4465 & 29.9235 & 47.8354 & 47.6622 & 47.1490 & 30.4847 & 30.3387 & 28.7676 \\
\hline 11 & 106.6 & 91.2013 & 91.9288 & 91.9964 & 77.7870 & 77.2593 & 76.5625 & 90.3504 & 91.0729 & 90.9557 & 76.7578 & 76.2191 & 75.4228 \\
\hline $11 a$ & 137.8 & 61.4509 & 61.3990 & 59.2040 & 44.3870 & 44.3270 & 43.5923 & 59.9798 & 59.9311 & 57.6413 & 42.8207 & 42.7619 & 41.9326 \\
\hline $11 \mathrm{~b}$ & 41.0 & 150.8624 & 151.4871 & 150.2002 & 141.6727 & 141.4936 & 140.7912 & 151.4240 & 152.0436 & 150.9215 & 142.3571 & 142.1658 & 141.4856 \\
\hline $11 \mathrm{c}$ & 67.7 & 127.4474 & 127.9710 & 127.2544 & 116.6512 & 116.4297 & 115.9331 & 127.7435 & 128.2631 & 127.6406 & 117.0247 & 116.7971 & 116.3482 \\
\hline $\mathrm{NCH}_{3}$ & 45.2 & 150.5528 & 152.0646 & 151.9293 & 143.0967 & 142.6925 & 142.4964 & 150.8650 & 152.3708 & 152.2377 & 143.4330 & 143.0166 & 142.8101 \\
\hline $\begin{array}{c}\mathrm{OCH}_{2} \mathrm{O} \\
{ }^{1} \mathrm{H}\end{array}$ & 101.9 & 92.9103 & 93.9200 & 93.6799 & 82.3074 & 81.3874 & 81.2792 & 92.7618 & 93.7637 & 93.3740 & 81.9851 & 81.0430 & 80.9616 \\
\hline 2 & 3.31 & 28.6834 & 28.1529 & 28.0489 & 28.6604 & 28.3678 & 28.3890 & 28.7404 & 28.2117 & 28.0939 & 28.7036 & 28.4143 & 28.4301 \\
\hline 2 & 2.24 & 29.8593 & 29.4316 & 29.4192 & 29.8419 & 29.6049 & 29.6156 & 29.7881 & 29.3567 & 29.3354 & 29.7672 & 29.5276 & 29.5357 \\
\hline
\end{tabular}




\begin{tabular}{|c|c|c|c|c|c|c|c|c|c|c|c|c|c|}
\hline 3 & 1.93 & 30.2768 & 29.8112 & 29.7306 & 30.1757 & 29.9156 & 29.8864 & 30.1939 & 29.7279 & 29.6486 & 30.1004 & 29.8423 & 29.8110 \\
\hline 3 & 1.45 & 30.8159 & 30.3843 & 30.2577 & 30.7283 & 30.4971 & 30.4769 & 30.7695 & 30.3359 & 30.2073 & 30.6859 & 30.4530 & 30.4316 \\
\hline $3 a$ & 2.34 & 29.9601 & 29.5015 & 29.3431 & 29.8877 & 29.6069 & 29.6052 & 29.8287 & 29.3667 & 29.2026 & 29.7625 & 29.4779 & 29.4717 \\
\hline 4 & 1.88 & 30.1558 & 29.7215 & 29.7237 & 30.1339 & 29.9027 & 30.0102 & 30.2061 & 29.7744 & 29.7660 & 30.1705 & 29.9455 & 30.0480 \\
\hline 4 & 1.78 & 30.4536 & 30.0618 & 29.9406 & 30.3323 & 30.1339 & 30.1131 & 30.4285 & 30.0341 & 29.9077 & 30.3079 & 30.1059 & 30.0808 \\
\hline 5 & 3.74 & 28.5489 & 28.1196 & 27.9862 & 28.4893 & 28.2919 & 28.2580 & 28.4249 & 27.9876 & 27.8370 & 28.3605 & 28.1474 & 28.1046 \\
\hline $5 a$ & 4.61 & 27.7283 & 27.2820 & 27.0562 & 27.6678 & 27.4495 & 27.4106 & 27.6432 & 27.1924 & 26.9573 & 27.5830 & 27.3595 & 27.3098 \\
\hline 8 & 7.54 & 24.6397 & 23.9093 & 23.6279 & 24.5704 & 24.0352 & 23.9135 & 24.7408 & 24.0075 & 23.7192 & 24.6584 & 24.1227 & 23.9974 \\
\hline 11 & 6.70 & 25.7258 & 25.0414 & 24.8264 & 25.5808 & 25.0957 & 25.0120 & 25.5291 & 24.8426 & 24.6148 & 25.3821 & 24.8997 & 24.8061 \\
\hline $11 b$ & 2.87 & 29.7201 & 29.2880 & 29.1878 & 29.6051 & 29.3702 & 29.3322 & 29.4884 & 29.0452 & 28.9284 & 29.3779 & 29.1331 & 29.0817 \\
\hline $11 \mathrm{c}$ & 2.60 & 29.4159 & 28.9857 & 28.9241 & 29.4098 & 29.2158 & 29.1411 & 29.3116 & 28.8790 & 28.8103 & 29.3064 & 29.1096 & 29.0305 \\
\hline $\mathrm{OCH}_{2} \mathrm{O}$ & 6.05 & 25.9889 & 25.4062 & 25.3072 & 26.1165 & 25.7823 & 25.7158 & 25.8747 & 25.2934 & 25.1961 & 26.0117 & 25.6835 & 25.6168 \\
\hline $\mathrm{OCH}_{2} \mathrm{O}$ & 6.05 & 26.1042 & 25.5326 & 25.4589 & 25.9615 & 25.6024 & 25.5419 & 25.9999 & 25.4320 & 25.3550 & 25.8477 & 25.4927 & 25.4363 \\
\hline $\mathrm{NCH}_{3}$ & 1.45 & 30.7037 & 30.2500 & 30.2417 & 30.7403 & 30.4944 & 30.4160 & 30.6951 & 30.2415 & 30.2281 & 30.7310 & 30.4855 & 30.4017 \\
\hline
\end{tabular}

\section{Compound 20}

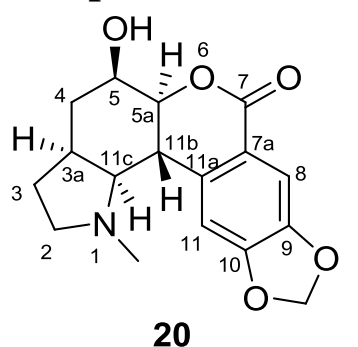

\begin{tabular}{|c|c|c|c|c|c|c|c|c|c|c|c|c|c|}
\hline & \multirow[b]{3}{*}{ Exp } & \multicolumn{12}{|c|}{ B3LYP } \\
\hline & & \multicolumn{6}{|c|}{ Gas phase } & \multicolumn{6}{|c|}{ PCM } \\
\hline & & 6-31G* & $6-31 G * *$ & $6-31+\mathrm{G} * *$ & 6-311G* & $6-311 \mathrm{G} * *$ & $6-311+\mathrm{G} * *$ & 6-31G* & 6-31G** & $6-31+\mathrm{G} * *$ & 6-311G* & 6-311G** & $6-311+G * *$ \\
\hline \multicolumn{14}{|l|}{${ }^{13} \mathrm{C}$} \\
\hline 2 & 53.0 & 138.3791 & 139.7221 & 138.5846 & 128.9671 & 128.4928 & 128.5091 & 138.6036 & 139.9418 & 138.8155 & 129.2027 & 128.7181 & 128.7417 \\
\hline 3 & 30.8 & 157.5915 & 159.0330 & 158.4196 & 149.2383 & 149.0167 & 148.4892 & 157.4693 & 158.9153 & 158.2843 & 149.1185 & 148.9004 & 148.3538 \\
\hline $3 a$ & 33.4 & 153.7304 & 154.6679 & 154.4480 & 144.6739 & 144.5975 & 144.5562 & 153.7627 & 154.6961 & 154.4880 & 144.7070 & 144.6221 & 144.5918 \\
\hline 4 & 28.7 & 160.8740 & 162.1924 & 160.8737 & 152.1257 & 151.8207 & 151.8850 & 161.0659 & 162.3787 & 161.0725 & 152.3369 & 152.0322 & 152.0809 \\
\hline 5 & 67.4 & 121.5280 & 122.2143 & 120.3226 & 109.9832 & 109.7452 & 109.7000 & 121.4332 & 122.1187 & 120.0642 & 109.8151 & 109.5681 & 109.4356 \\
\hline $5 a$ & 81.8 & 109.0023 & 109.6322 & 108.6850 & 97.4734 & 97.1511 & 96.5439 & 108.6878 & 109.3090 & 108.3403 & 97.1414 & 96.8043 & 96.1483 \\
\hline 7 & 164.7 & 35.5911 & 35.5774 & 34.0924 & 16.1429 & 16.2259 & 14.0137 & 33.8715 & 33.8581 & 32.0527 & 14.2130 & 14.2898 & 11.7373 \\
\hline $7 a$ & 118.7 & 74.8106 & 74.7229 & 74.8219 & 58.5819 & 58.5152 & 57.0119 & 75.5506 & 75.4644 & 75.6479 & 59.4142 & 59.3653 & 57.8749 \\
\hline 8 & 109.3 & 84.0518 & 84.8136 & 84.6966 & 69.0269 & 68.4302 & 67.2924 & 84.9382 & 85.7024 & 85.6375 & 70.0974 & 69.5090 & 68.3552 \\
\hline 9 & 146.7 & 50.7380 & 50.5753 & 50.2810 & 30.9258 & 30.8300 & 29.3053 & 50.6399 & 50.4784 & 50.1821 & 30.7903 & 30.6961 & 29.1895 \\
\hline 10 & 152.7 & 45.2180 & 45.0633 & 45.1554 & 25.2668 & 25.2090 & 23.2129 & 44.3782 & 44.2265 & 44.2638 & 24.3806 & 24.3202 & 22.2487 \\
\hline 11 & 107.2 & 87.2413 & 87.9851 & 88.4676 & 71.9540 & 71.4400 & 71.0006 & 86.9624 & 87.7026 & 88.0040 & 71.5993 & 71.0673 & 70.4935 \\
\hline $11 a$ & 140.8 & 54.8446 & 54.8178 & 51.9020 & 35.5560 & 35.5472 & 34.6210 & 53.3124 & 53.2781 & 50.2046 & 33.9017 & 33.8855 & 32.8208 \\
\hline $11 b$ & 33.1 & 156.4880 & 157.1105 & 157.0994 & 147.8469 & 147.7079 & 146.9325 & 156.5907 & 157.2098 & 157.2510 & 147.9695 & 147.8316 & 147.0197 \\
\hline $11 c$ & 69.5 & 119.8785 & 120.3846 & 119.7167 & 107.8584 & 107.5732 & 107.2486 & 120.1262 & 120.6268 & 120.0357 & 108.1488 & 107.8634 & 107.5740 \\
\hline $\mathrm{N} \mathrm{CH}_{3}$ & 45.2 & 145.5782 & 147.1691 & 147.4012 & 137.4748 & 136.9200 & 136.6086 & 145.8504 & 147.4290 & 147.6616 & 137.7507 & 137.1768 & 136.8772 \\
\hline $\begin{array}{c}\mathrm{OCH}_{2} \mathrm{O} \\
{ }^{1} \mathbf{H}\end{array}$ & 101.8 & 89.4464 & 90.4885 & 88.2805 & 76.3875 & 75.4407 & 75.9983 & 89.3718 & 90.4043 & 88.0766 & 76.1317 & 75.1600 & 75.7372 \\
\hline
\end{tabular}




\begin{tabular}{|c|c|c|c|c|c|c|c|c|c|c|c|c|c|}
\hline 2 & 3.29 & 28.8492 & 28.3413 & 28.1310 & 28.8431 & 28.5729 & 28.4683 & 28.8736 & 28.3652 & 28.1503 & 28.8631 & 28.5916 & 28.4833 \\
\hline 2 & 2.27 & 29.7098 & 29.2526 & 29.1203 & 29.6713 & 29.4209 & 29.3232 & 29.6296 & 29.1693 & 29.0343 & 29.5899 & 29.3383 & 29.2403 \\
\hline 3 & 2.53 & 29.5577 & 29.0709 & 28.9075 & 29.5393 & 29.3416 & 29.2590 & 29.6944 & 29.2093 & 29.0614 & 29.6820 & 29.4797 & 29.4035 \\
\hline 3 & 2.27 & 30.2291 & 29.7476 & 29.6161 & 30.1882 & 29.8751 & 29.8463 & 30.1679 & 29.6841 & 29.5521 & 30.1295 & 29.8159 & 29.7857 \\
\hline $3 a$ & 2.53 & 29.7557 & 29.2329 & 29.1738 & 29.7213 & 29.4070 & 29.4203 & 29.6622 & 29.1387 & 29.0841 & 29.6317 & 29.3179 & 29.3315 \\
\hline 4 & 2.10 & 29.8336 & 29.3961 & 29.2322 & 29.7293 & 29.5365 & 29.4664 & 29.8696 & 29.4310 & 29.2730 & 29.7716 & 29.5727 & 29.5024 \\
\hline 4 & 1.80 & 30.6879 & 30.2412 & 29.9961 & 30.5565 & 30.3227 & 30.2439 & 30.5497 & 30.0990 & 29.8424 & 30.4188 & 30.1833 & 30.0969 \\
\hline 5 & 4.24 & 28.0653 & 27.5860 & 27.4645 & 27.9060 & 27.6766 & 27.7272 & 28.0205 & 27.5390 & 27.4141 & 27.8506 & 27.6193 & 27.6708 \\
\hline $5 a$ & 4.09 & 28.3137 & 27.8844 & 27.6844 & 28.3527 & 28.1487 & 28.0491 & 28.1407 & 27.7047 & 27.4785 & 28.1697 & 27.9613 & 27.8436 \\
\hline 8 & 7.46 & 24.9579 & 24.2136 & 23.9350 & 24.8255 & 24.3019 & 24.2350 & 25.0560 & 24.3100 & 24.0192 & 24.9099 & 24.3867 & 24.3124 \\
\hline 11 & 7.74 & 24.2557 & 23.4232 & 23.0989 & 24.1758 & 23.6139 & 23.5004 & 24.1895 & 23.3527 & 23.0094 & 24.0993 & 23.5321 & 23.4058 \\
\hline $11 \mathrm{~b}$ & 3.22 & 28.8815 & 28.4438 & 28.2654 & 29.0830 & 28.7820 & 28.6282 & 28.8521 & 28.4136 & 28.2272 & 29.0452 & 28.7464 & 28.5874 \\
\hline $11 \mathrm{c}$ & 2.89 & 29.3487 & 28.9397 & 28.8558 & 29.4430 & 29.2480 & 29.2013 & 29.2244 & 28.8104 & 28.7166 & 29.3155 & 29.1164 & 29.0653 \\
\hline $\mathrm{N} \mathrm{CH}_{3}$ & 2.53 & 29.7150 & 29.2457 & 29.1269 & 29.6689 & 29.3967 & 29.3378 & 29.6818 & 29.2127 & 29.0943 & 29.6366 & 29.3652 & 29.3080 \\
\hline $\mathrm{OCH}_{2} \mathrm{O}$ & 6.02 & 26.0644 & 25.4810 & 25.3874 & 26.0055 & 25.6691 & 25.5188 & 25.9552 & 25.3736 & 25.2814 & 25.8946 & 25.5625 & 25.4180 \\
\hline $\mathrm{OCH}_{2} \mathrm{O}$ & 6.02 & 26.1410 & 25.5666 & 25.4906 & 26.1423 & 25.8229 & 25.6532 & 26.0542 & 25.4839 & 25.4005 & 26.0514 & 25.7384 & 25.5685 \\
\hline
\end{tabular}

mPW1PW91

Gas phase

PCM

$\operatorname{Exp} 6-31 G * 6-31 G * * 6-31+G * * 6-311 G * 6-311 G * * 6-311+G * * 6-31 G * 6-31 G * * 6-31+G * * 6-311 G * 6-311 G * * 6-311+G * *$

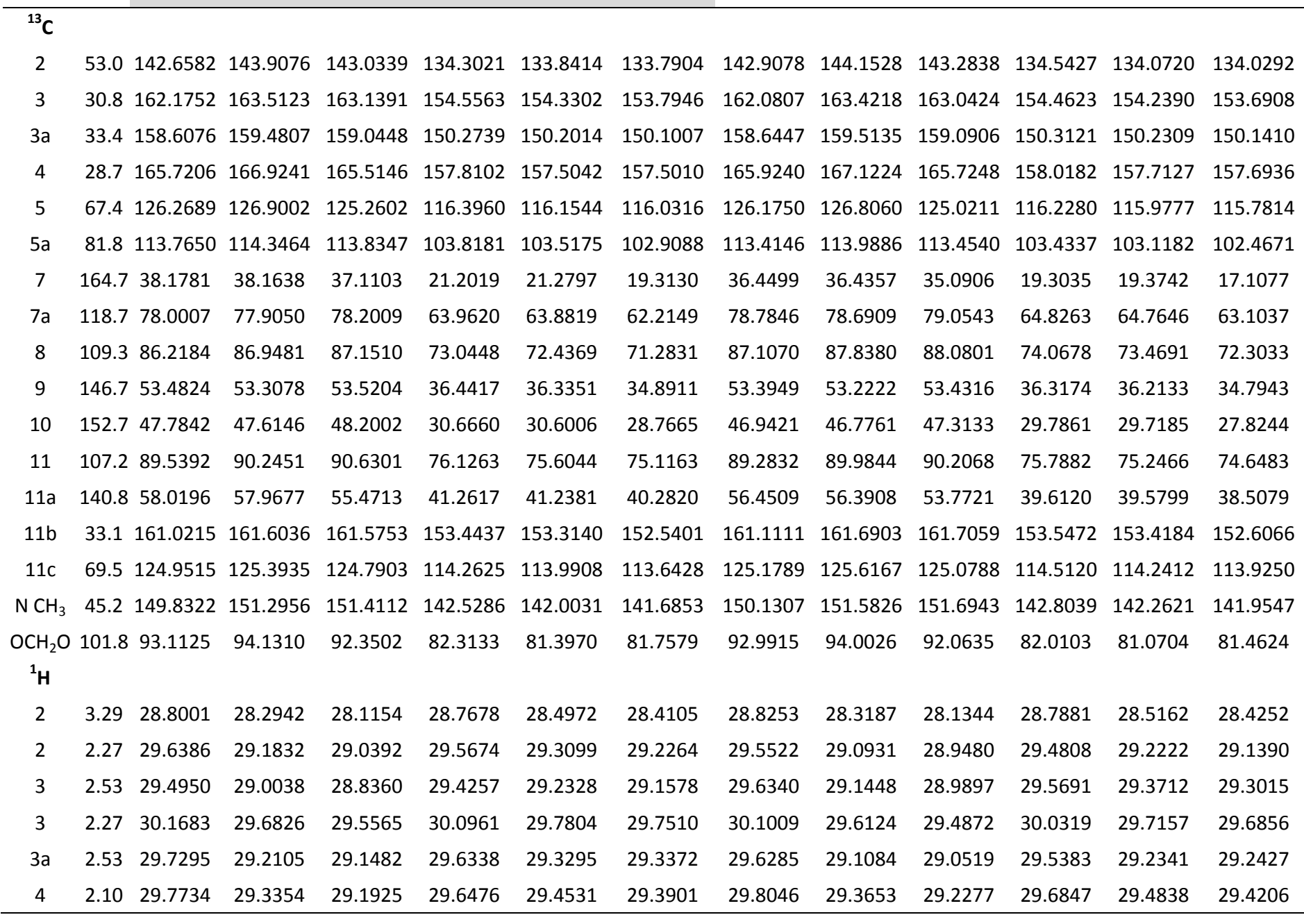




\begin{tabular}{cccccccccccccc}
\hline 4 & 1.80 & 30.6440 & 30.1998 & 29.9645 & 30.4781 & 30.2452 & 30.1665 & 30.4979 & 30.0494 & 29.8054 & 30.3356 & 30.1008 & 30.0159 \\
5 & 4.24 & 28.0029 & 27.5255 & 27.4374 & 27.8426 & 27.6087 & 27.6628 & 27.9508 & 27.4710 & 27.3809 & 27.7823 & 27.5461 & 27.6018 \\
$5 \mathrm{a}$ & 4.09 & 28.2373 & 27.8095 & 27.6160 & 28.2386 & 28.0317 & 27.9288 & 28.0592 & 27.6244 & 27.4092 & 28.0547 & 27.8432 & 27.7239 \\
8 & 7.46 & 24.7582 & 24.0105 & 23.7863 & 24.6611 & 24.0994 & 24.0437 & 24.8441 & 24.0943 & 23.8614 & 24.7347 & 24.1736 & 24.1126 \\
11 & 7.74 & 24.0688 & 23.2367 & 22.9566 & 23.9965 & 23.4175 & 23.3350 & 23.9976 & 23.1606 & 22.8626 & 23.9161 & 23.3313 & 23.2362 \\
$11 \mathrm{~b}$ & 3.22 & 28.8105 & 28.3633 & 28.1803 & 28.9526 & 28.6641 & 28.5199 & 28.7826 & 28.3343 & 28.1428 & 28.9165 & 28.6300 & 28.4801 \\
$11 \mathrm{C}$ & 2.89 & 29.2608 & 28.8541 & 28.7589 & 29.2965 & 29.0996 & 29.0396 & 29.1350 & 28.7230 & 28.6211 & 29.1709 & 28.9696 & 28.9061 \\
$\mathrm{~N} \mathrm{CH}_{3}$ & 2.53 & 29.6638 & 29.1980 & 29.0898 & 29.6000 & 29.3233 & 29.2613 & 29.6258 & 29.1599 & 29.0532 & 29.5635 & 29.2877 & 29.2276 \\
$\mathrm{OCH}_{2} \mathrm{O}$ & 6.02 & 26.0118 & 25.4314 & 25.3643 & 25.9777 & 25.6243 & 25.4879 & 25.8900 & 25.3110 & 25.2459 & 25.8569 & 25.5077 & 25.3757 \\
$\mathrm{OCH}_{2} \mathrm{O}$ & 6.02 & 26.1096 & 25.5387 & 25.4662 & 26.1251 & 25.7935 & 25.6399 & 26.0106 & 25.4437 & 25.3639 & 26.0245 & 25.6994 & 25.5448 \\
\hline
\end{tabular}

\section{Compound 21}

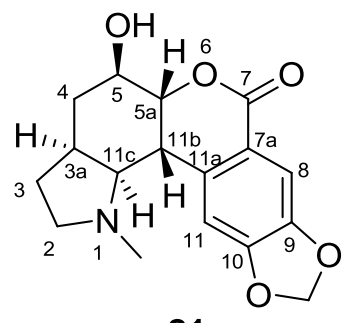

21

\begin{tabular}{|c|c|c|c|c|c|c|c|c|c|c|c|c|c|}
\hline & \multicolumn{13}{|c|}{ B3LYP } \\
\hline & \multirow[b]{2}{*}{ Exp } & \multicolumn{6}{|c|}{ Gas phase } & \multicolumn{6}{|c|}{ PCM } \\
\hline & & 6-31G* & $6-31 \mathrm{G} * *$ & $6-31+\mathrm{G} * *$ & 6-311G* & $6-311 G * *$ & $6-311+G * *$ & 6-31G* & 6-31G** & $6-31+G * *$ & 6-311G* & 6-311G** & $6-311+G * *$ \\
\hline \multicolumn{14}{|l|}{${ }^{13} \mathrm{C}$} \\
\hline 2 & 54.9 & 135.6425 & 136.8041 & 136.1916 & 125.2112 & 124.6853 & 125.0235 & 135.8106 & 136.9708 & 136.5185 & 125.4290 & 124.9189 & 125.4950 \\
\hline 3 & 30.1 & 158.4610 & 159.8119 & 160.3534 & 149.4100 & 149.0694 & 149.8761 & 158.5695 & 159.9205 & 160.9083 & 149.5899 & 149.2993 & 150.6190 \\
\hline $3 a$ & 34.7 & 152.2903 & 153.1906 & 151.6721 & 142.5230 & 142.4561 & 142.7893 & 152.4595 & 153.3559 & 151.8782 & 142.7540 & 142.6870 & 143.1278 \\
\hline 4 & 33.7 & 156.9255 & 158.3157 & 156.0035 & 148.9375 & 148.7056 & 147.1867 & 156.9844 & 158.3660 & 155.3246 & 148.8736 & 148.5571 & 146.2725 \\
\hline 5 & 68.6 & 121.8797 & 122.6542 & 121.1982 & 110.0617 & 109.8540 & 110.1293 & 122.0682 & 122.8439 & 121.3496 & 110.2176 & 110.0233 & 110.2262 \\
\hline $5 a$ & 81.4 & 109.5347 & 110.2100 & 109.3091 & 98.0254 & 97.6323 & 97.4119 & 109.2854 & 109.9604 & 108.6990 & 97.7912 & 97.3728 & 96.8583 \\
\hline 7 & 164.0 & 36.0111 & 35.9869 & 33.5504 & 16.4447 & 16.5086 & 14.9636 & 34.3309 & 34.3045 & 31.5738 & 14.5374 & 14.6093 & 12.9655 \\
\hline $7 a$ & 118.6 & 674.5457 & 74.4461 & 73.4341 & 57.9212 & 57.8336 & 56.7466 & 75.4671 & 75.3665 & 74.4388 & 58.9265 & 58.8354 & 57.6435 \\
\hline 8 & 109.7 & 783.3989 & 84.1485 & 83.1129 & 68.3149 & 67.7018 & 66.5738 & 84.3613 & 85.1132 & 84.0597 & 69.4790 & 68.8684 & 67.6821 \\
\hline 9 & 147.2 & 250.3443 & 50.1537 & 48.7336 & 30.7073 & 30.5898 & 29.9895 & 50.1422 & 49.9537 & 48.5463 & 30.4788 & 30.3739 & 29.9024 \\
\hline 10 & 152.6 & 645.5802 & 45.4236 & 43.6802 & 25.7777 & 25.6710 & 24.3921 & 44.5563 & 44.4046 & 42.5278 & 24.7141 & 24.5999 & 23.1517 \\
\hline 11 & 106.5 & 89.4264 & 90.1140 & 90.6228 & 73.8637 & 73.2846 & 73.7552 & 88.7594 & 89.4516 & 90.0011 & 73.1412 & 72.6049 & 73.1944 \\
\hline $11 a$ & 137.3 & 57.2763 & 57.1384 & 56.6345 & 37.6767 & 37.5764 & 37.4503 & 55.9411 & 55.7996 & 55.5716 & 36.2463 & 36.1924 & 36.3362 \\
\hline $11 b$ & 36.6 & 150.9920 & 151.6253 & 151.9904 & 141.4101 & 141.1193 & 141.7556 & 151.0605 & 151.6919 & 152.5395 & 141.5461 & 141.3057 & 142.4170 \\
\hline $11 c$ & 66.6 & 123.6576 & 124.2279 & 123.3576 & 112.0122 & 111.6756 & 111.5238 & 123.8470 & 124.4122 & 123.7503 & 112.2430 & 111.9068 & 111.9481 \\
\hline $\mathrm{NCH}_{3}$ & 41.8 & 149.4384 & 151.0574 & 151.7289 & 141.1037 & 140.5870 & 141.4876 & 149.6766 & 151.2873 & 152.3740 & 141.3904 & 140.9105 & 142.3058 \\
\hline $\begin{array}{c}\mathrm{OCH}_{2} \mathrm{O} \\
{ }^{1} \mathbf{H}\end{array}$ & 102.0 & 89.2811 & 90.3148 & 89.4451 & 76.4580 & 75.5130 & 75.4570 & 89.1721 & 90.1966 & 89.2433 & 76.1748 & 75.2042 & 75.1308 \\
\hline 2 & 3.24 & 29.0661 & 28.5630 & 28.3263 & 28.9828 & 28.6936 & 28.6119 & 29.0627 & 28.5593 & 28.3061 & 28.9725 & 28.6834 & 28.5939 \\
\hline 2 & 2.30 & 29.8967 & 29.4744 & 29.4423 & 29.8801 & 29.6875 & 29.6298 & 29.8366 & 29.4137 & 29.3741 & 29.8261 & 29.6329 & 29.5658 \\
\hline 3 & 2.00 & 30.1861 & 29.7361 & 29.7960 & 30.0713 & 29.8785 & 30.0039 & 30.2062 & 29.7560 & 29.8995 & 30.1144 & 29.9280 & 30.1309 \\
\hline 3 & 1.62 & 30.5126 & 30.0498 & 29.8616 & 30.4384 & 30.1784 & 30.0418 & 30.4445 & 29.9799 & 29.7810 & 30.3761 & 30.1131 & 29.9664 \\
\hline
\end{tabular}




\begin{tabular}{|c|c|c|c|c|c|c|c|c|c|c|c|c|c|}
\hline $3 a$ & 2.27 & 30.0696 & 29.6389 & 29.5626 & 30.0856 & 29.8575 & 29.8394 & 29.9991 & 29.5673 & 29.4834 & 30.0170 & 29.7879 & 29.7700 \\
\hline 4 & 2.02 & 30.3011 & 29.8631 & 29.7514 & 30.1761 & 29.9389 & 29.9456 & 30.3166 & 29.8795 & 29.7891 & 30.1974 & 29.9652 & 29.9935 \\
\hline 4 & 1.62 & 30.2663 & 29.8636 & 29.7844 & 30.2068 & 30.0377 & 30.0579 & 30.2955 & 29.8893 & 29.8495 & 30.2329 & 30.0624 & 30.1276 \\
\hline 5 & 3.96 & 28.2787 & 27.7988 & 27.8168 & 28.1747 & 27.9353 & 28.0428 & 28.2278 & 27.7437 & 27.7649 & 28.1225 & 27.8829 & 28.0043 \\
\hline $5 a$ & 4.65 & 27.9244 & 27.4684 & 27.2960 & 27.9231 & 27.6967 & 27.6153 & 27.8167 & 27.3532 & 27.1712 & 27.7897 & 27.5545 & 27.4733 \\
\hline 8 & 7.53 & 24.8709 & 24.1315 & 23.7999 & 24.7383 & 24.2309 & 24.0937 & 24.9739 & 24.2325 & 23.8885 & 24.8280 & 24.3200 & 24.1775 \\
\hline 11 & 7.05 & 25.5866 & 24.8487 & 24.6570 & 25.4485 & 24.9572 & 24.9337 & 25.4749 & 24.7383 & 24.5339 & 25.3385 & 24.8502 & 24.8146 \\
\hline $11 b$ & 3.34 & 28.9954 & 28.5663 & 28.2480 & 29.0805 & 28.8372 & 28.6125 & 28.9056 & 28.4719 & 28.0614 & 28.9842 & 28.7295 & 28.4180 \\
\hline $11 \mathrm{c}$ & 2.67 & 29.4197 & 28.9915 & 28.8741 & 29.5596 & 29.3475 & 29.2038 & 29.3504 & 28.9228 & 28.7307 & 29.4875 & 29.2718 & 29.0558 \\
\hline $\mathrm{NCH}_{3}$ & 2.24 & 30.0427 & 29.5854 & 29.4104 & 30.0084 & 29.7627 & 29.6729 & 30.0168 & 29.5595 & 29.3601 & 29.9824 & 29.7350 & 29.6243 \\
\hline $\mathrm{OCH}_{2} \mathrm{O}$ & 6.05 & 26.0953 & 25.5154 & 25.3560 & 26.0698 & 25.7433 & 25.6507 & 25.9943 & 25.4174 & 25.2531 & 25.9664 & 25.6438 & 25.5526 \\
\hline $\mathrm{OCH}_{2} \mathrm{O}$ & 6.05 & 26.1118 & 25.5299 & 25.3849 & 26.0759 & 25.7411 & 25.6258 & 26.0110 & 25.4320 & 25.3072 & 25.9736 & 25.6459 & 25.5483 \\
\hline
\end{tabular}

\section{mPW1PW91}

\begin{tabular}{|c|c|c|c|c|c|c|c|c|c|c|c|c|c|}
\hline & \multirow[b]{2}{*}{ Exp } & \multicolumn{6}{|c|}{ Gas phase } & \multicolumn{6}{|c|}{ PCM } \\
\hline & & 6-31G* & $6-31 G * *$ & $6-31+G * *$ & 6-311G* & $6-311 G^{* *}$ & $6-311+G * *$ & 6-31G* & 6-31G** & $6-31+G * *$ & $6-311 G^{*}$ & $6-311 G * *$ & $6-311+G * *$ \\
\hline \multicolumn{14}{|l|}{${ }^{13} \mathrm{C}$} \\
\hline 2 & 54.9 & 140.5545 & 141.6547 & 140.9863 & 131.5098 & 131.0095 & 131.1523 & 140.7491 & 141.8475 & 141.2842 & 131.7233 & 131.2387 & 131.5322 \\
\hline 3 & 30.1 & 163.6261 & 164.9523 & 165.2021 & 155.7511 & 155.4237 & 155.7984 & 163.7667 & 165.0905 & 165.6188 & 155.9264 & 155.6433 & 156.3344 \\
\hline $3 a$ & 34.7 & 157.5441 & 158.3729 & 156.8419 & 148.7105 & 148.6180 & 148.7893 & 157.7070 & 158.5327 & 157.0281 & 148.9278 & 148.8354 & 149.0625 \\
\hline 4 & 33.7 & 160.6803 & 161.8361 & 159.9793 & 153.0938 & 152.7725 & 151.7844 & 160.8047 & 161.9574 & 159.6160 & 153.1324 & 152.7386 & 151.2546 \\
\hline 5 & 68.6 & 126.7310 & 127.4613 & 126.4452 & 116.8427 & 116.6183 & 116.5232 & 126.8407 & 127.5699 & 126.4881 & 116.8491 & 116.6364 & 116.4728 \\
\hline $5 a$ & 81.4 & 114.5589 & 115.1793 & 114.3037 & 104.4174 & 104.0410 & 103.8876 & 114.1593 & 114.7734 & 113.6175 & 103.9644 & 103.5617 & 103.1977 \\
\hline 7 & 164.0 & 38.7251 & 38.7151 & 36.6524 & 21.7308 & 21.7908 & 20.3638 & 37.0619 & 37.0506 & 34.7440 & 19.8776 & 19.9434 & 18.3702 \\
\hline $7 a$ & 118.6 & 77.5554 & 77.4303 & 76.6142 & 62.9909 & 62.8757 & 61.9741 & 78.5530 & 78.4301 & 77.6768 & 64.0775 & 63.9637 & 62.9906 \\
\hline 8 & 109.7 & 85.4345 & 86.1458 & 85.5305 & 72.1951 & 71.5660 & 70.3804 & 86.4292 & 87.1430 & 86.5396 & 73.3436 & 72.7203 & 71.4892 \\
\hline 9 & 147.2 & 53.1985 & 53.0054 & 52.1640 & 36.3753 & 36.2583 & 35.6223 & 53.0002 & 52.8088 & 51.9826 & 36.1446 & 36.0401 & 35.4938 \\
\hline 10 & 152.6 & 48.0403 & 47.8620 & 46.9186 & 30.9824 & 30.8641 & 29.5366 & 46.9912 & 46.8176 & 45.7840 & 29.9108 & 29.7852 & 28.3292 \\
\hline 11 & 106.5 & 92.4996 & 93.2173 & 93.4103 & 79.0817 & 78.5281 & 78.6715 & 91.7411 & 92.4541 & 92.6079 & 78.2196 & 77.6990 & 77.8858 \\
\hline $11 a$ & 137.3 & 61.3307 & 61.2258 & 60.8632 & 44.3179 & 44.2332 & 43.6719 & 59.9677 & 59.8576 & 59.6444 & 42.8980 & 42.8523 & 42.4399 \\
\hline $11 \mathrm{~b}$ & 36.6 & 156.7840 & 157.3968 & 157.3721 & 148.3861 & 148.0888 & 148.2953 & 156.8209 & 157.4301 & 157.7251 & 148.4534 & 148.2049 & 148.6993 \\
\hline $11 \mathrm{c}$ & 66.6 & 128.9524 & 129.4199 & 128.5369 & 118.5503 & 118.2232 & 117.9820 & 129.1720 & 129.6378 & 128.9199 & 118.8223 & 118.4972 & 118.3876 \\
\hline $\mathrm{NCH}_{3}$ & 41.8 & 154.2616 & 155.8418 & 156.1341 & 147.0652 & 146.6009 & 147.1738 & 154.5401 & 156.1098 & 156.6794 & 147.3516 & 146.9234 & 147.8153 \\
\hline $\begin{array}{c}\mathrm{OCH}_{2} \mathrm{O} \\
{ }^{1} \mathrm{H}\end{array}$ & 102.0 & 92.9213 & 93.9317 & 93.7516 & 82.3266 & 81.4121 & 81.2268 & 92.7632 & 93.7663 & 93.4668 & 81.9946 & 81.0558 & 80.8696 \\
\hline 2 & 3.24 & 28.9970 & 28.4922 & 28.2805 & 28.8990 & 28.6012 & 28.5261 & 28.9908 & 28.4856 & 28.2605 & 28.8866 & 28.5891 & 28.5074 \\
\hline 2 & 2.30 & 29.8564 & 29.4361 & 29.3727 & 29.7803 & 29.5860 & 29.5166 & 29.7847 & 29.3634 & 29.2949 & 29.7150 & 29.5201 & 29.4426 \\
\hline 3 & 2.00 & 30.3080 & 29.8756 & 29.8849 & 30.2089 & 30.0150 & 30.0616 & 30.3058 & 29.8714 & 29.9344 & 30.2217 & 30.0341 & 30.1296 \\
\hline 3 & 1.62 & 30.4309 & 29.9629 & 29.7828 & 30.3250 & 30.0572 & 29.9295 & 30.3517 & 29.8818 & 29.6964 & 30.2531 & 29.9831 & 29.8499 \\
\hline $3 a$ & 2.27 & 30.0316 & 29.5950 & 29.5032 & 29.9968 & 29.7616 & 29.7368 & 29.9606 & 29.5231 & 29.4265 & 29.9284 & 29.6931 & 29.6666 \\
\hline 4 & 2.02 & 30.2990 & 29.8726 & 29.7582 & 30.1716 & 29.9505 & 29.9285 & 30.3016 & 29.8744 & 29.7705 & 30.1778 & 29.9586 & 29.9480 \\
\hline 4 & 1.62 & 30.2256 & 29.8344 & 29.7580 & 30.1738 & 30.0169 & 30.0134 & 30.2646 & 29.8702 & 29.8191 & 30.2076 & 30.0484 & 30.0735 \\
\hline 5 & 3.96 & 28.2832 & 27.8010 & 27.8006 & 28.2036 & 27.9600 & 28.0118 & 28.2270 & 27.7401 & 27.7377 & 28.1446 & 27.8996 & 27.9560 \\
\hline $5 a$ & 4.65 & 27.7383 & 27.2792 & 27.1520 & 27.7204 & 27.4819 & 27.4368 & 27.6539 & 27.1889 & 27.0475 & 27.6163 & 27.3680 & 27.3175 \\
\hline 8 & 7.53 & 24.6622 & 23.9160 & 23.6384 & 24.5674 & 24.0145 & 23.9134 & 24.7553 & 24.0072 & 23.7207 & 24.6487 & 24.0960 & 23.9919 \\
\hline
\end{tabular}




\begin{tabular}{|c|c|c|c|c|c|c|c|c|c|c|c|c|c|}
\hline 11 & 7.05 & 25.4589 & 24.7083 & 24.5212 & 25.3430 & 24.8085 & 24.7816 & 25.3213 & 24.5712 & 24.3752 & 25.2069 & 24.6760 & 24.6401 \\
\hline $11 b$ & 3.34 & 28.8160 & 28.3601 & 28.1008 & 28.8304 & 28.5667 & 28.4054 & 28.7147 & 28.2537 & 27.9287 & 28.7231 & 28.4483 & 28.2274 \\
\hline $11 \mathrm{c}$ & 2.67 & 29.2966 & 28.8653 & 28.7224 & 29.3441 & 29.1318 & 28.9820 & 29.2075 & 28.7757 & 28.5804 & 29.2524 & 29.0358 & 28.8377 \\
\hline $\mathrm{NCH}_{3}$ & 2.24 & 29.9602 & 29.5009 & 29.3412 & 29.8924 & 29.6355 & 29.5669 & 29.9281 & 29.4687 & 29.2914 & 29.8609 & 29.6026 & 29.5188 \\
\hline $\mathrm{OCH}_{2} \mathrm{O}$ & 6.05 & 26.0388 & 25.4584 & 25.3283 & 26.0219 & 25.6744 & 25.6123 & 25.9232 & 25.3452 & 25.2129 & 25.9066 & 25.5627 & 25.5024 \\
\hline $\mathrm{OCH}_{2} \mathrm{O}$ & 6.05 & 26.0883 & 25.5122 & 25.3835 & 26.0825 & 25.7384 & 25.6232 & 25.9771 & 25.4042 & 25.2897 & 25.9720 & 25.6356 & 25.5350 \\
\hline
\end{tabular}

\section{Compound 22}

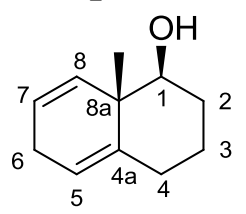

22

\begin{tabular}{|c|c|c|c|c|c|c|c|c|c|c|c|c|c|}
\hline & & \multicolumn{12}{|c|}{ B3LYP } \\
\hline & \multirow[b]{2}{*}{ Exp } & \multicolumn{6}{|c|}{ Gas phase } & \multicolumn{6}{|c|}{ PCM } \\
\hline & & 6-31G* & $6-31 G * *$ & $6-31+G * *$ & 6-311G* & 6-311G** & $6-311+G * *$ & 6-31G* & $6-31 G * *$ & $6-31+\mathrm{G} * *$ & 6-311G* & $6-311 G * *$ & $6-311+G * *$ \\
\hline \multicolumn{14}{|l|}{${ }^{13} \mathrm{C}$} \\
\hline 1 & 76.7 & 114.4685 & 115.1583 & 113.5112 & 101.3411 & 101.2485 & 101.4967 & 114.4542 & 115.1417 & 113.4018 & 101.2827 & 101.1859 & 101.3666 \\
\hline 2 & 30.8 & 156.1924 & 157.4687 & 155.9680 & 146.7858 & 146.4300 & 146.0342 & 156.7033 & 157.9686 & 156.6040 & 147.4023 & 147.0173 & 146.6578 \\
\hline 3 & 24.9 & 163.3856 & 164.7625 & 163.4651 & 154.1374 & 154.0092 & 153.3665 & 163.5478 & 164.9226 & 163.6274 & 154.3313 & 154.1939 & 153.5473 \\
\hline 4 & 26.7 & 156.1501 & 157.4597 & 157.0598 & 146.9649 & 146.5951 & 145.8522 & 156.3518 & 157.6534 & 157.2964 & 147.2100 & 146.8289 & 146.0977 \\
\hline $4 a$ & 140.4 & 54.0674 & 53.9220 & 51.6396 & 33.1397 & 33.0060 & 31.0082 & 53.6778 & 53.5323 & 51.2192 & 32.7088 & 32.5942 & 30.5561 \\
\hline 5 & 118.3 & 72.8462 & 73.7847 & 74.2137 & 56.8729 & 56.4555 & 56.0251 & 73.1677 & 74.1024 & 74.5042 & 57.1611 & 56.7429 & 56.3096 \\
\hline 6 & 31.4 & 161.0179 & 162.1529 & 160.5088 & 151.2913 & 150.9466 & 150.6072 & 161.2746 & 162.4023 & 160.6878 & 151.4927 & 151.1358 & 150.7991 \\
\hline 7 & 122.1 & 73.1923 & 73.8756 & 73.6249 & 56.2930 & 55.4411 & 54.6625 & 72.7416 & 73.4249 & 73.0058 & 55.6446 & 54.8178 & 53.9478 \\
\hline 8 & 132.2 & 60.5086 & 61.0731 & 60.1126 & 42.2718 & 41.6204 & 39.9630 & 60.6786 & 61.2357 & 60.3566 & 42.5237 & 41.8789 & 40.2152 \\
\hline $8 a$ & 41.0 & 145.5306 & 145.7935 & 144.6027 & 135.5050 & 135.6123 & 135.4153 & 145.7178 & 145.9721 & 144.8439 & 135.7039 & 135.7947 & 135.6077 \\
\hline $\begin{array}{c}\text { 8a-Me } \\
{ }^{1} \mathbf{H}\end{array}$ & 18.2 & 169.9984 & 171.8062 & 172.3346 & 163.7511 & 163.6682 & 163.4606 & 169.9675 & 171.7718 & 172.2532 & 163.6990 & 163.6074 & 163.3925 \\
\hline 1 & 3.51 & 28.4218 & 28.0098 & 27.9417 & 28.4064 & 28.2454 & 28.2389 & 28.4066 & 27.9936 & 27.9186 & 28.3855 & 28.2220 & 28.2132 \\
\hline $2 * a x$ & 2.18 & 30.3671 & 29.9494 & 29.7876 & 30.3571 & 30.1476 & 30.0855 & 30.4070 & 29.9891 & 29.8335 & 30.4022 & 30.1897 & 30.1274 \\
\hline $2 * *$ & 1.95 & 30.8443 & 30.3954 & 30.2757 & 30.7252 & 30.4528 & 30.4363 & 30.6849 & 30.2319 & 30.1046 & 30.5641 & 30.2946 & 30.2760 \\
\hline $3^{*}$ & 1.61 & 30.5696 & 30.1212 & 30.0149 & 30.4574 & 30.2054 & 30.1798 & 30.5267 & 30.0756 & 29.9604 & 30.4128 & 30.1578 & 30.1272 \\
\hline $3 * *$ & 1.30 & 30.7497 & 30.3445 & 30.2534 & 30.7359 & 30.5549 & 30.5023 & 30.7383 & 30.3335 & 30.2481 & 30.7272 & 30.5484 & 30.4965 \\
\hline 4 & 1.77 & 30.0865 & 29.6425 & 29.4937 & 30.0076 & 29.7726 & 29.7088 & 30.0600 & 29.6148 & 29.4603 & 29.9781 & 29.7428 & 29.6759 \\
\hline 5 & 5.41 & 26.8913 & 26.3201 & 26.0755 & 26.6814 & 26.2774 & 26.2474 & 26.8402 & 26.2680 & 26.0062 & 26.6140 & 26.2138 & 26.1770 \\
\hline 6 & 2.63 & 29.6416 & 29.1446 & 28.8399 & 29.4348 & 29.1455 & 29.0232 & 29.5989 & 29.1007 & 28.7961 & 29.3915 & 29.1017 & 28.9789 \\
\hline 7 & 5.66 & 26.6863 & 26.1222 & 25.7845 & 26.4991 & 26.1353 & 26.0176 & 26.6143 & 26.0494 & 25.6953 & 26.4110 & 26.0517 & 25.9277 \\
\hline 8 & 5.84 & 25.8726 & 25.3419 & 25.1474 & 25.8425 & 25.5142 & 25.4125 & 25.9255 & 25.3916 & 25.1955 & 25.8867 & 25.5524 & 25.4516 \\
\hline $8 \mathrm{a}-\mathrm{Me}$ & 1.11 & 30.9415 & 30.5036 & 30.4152 & 30.9608 & 30.7478 & 30.6804 & 30.9614 & 30.5222 & 30.4343 & 30.9826 & 30.7655 & 30.6956 \\
\hline
\end{tabular}

\section{mPW1PW91}

\section{Gas phase}

PCM

Exp 6-31G* 6-31G** 6-31+G** 6-311G* 6-311G** 6-311+G** 6-31G* 6-31G** 6-31+G** 6-311G* 6-311G** 6-311+G** 


\begin{tabular}{|c|c|c|c|c|c|c|c|c|c|c|c|c|c|}
\hline${ }^{13} \mathrm{C}$ & & & & & & & & & & & & & \\
\hline 1 & 76.7 & 119.7964 & 120.4305 & 118.9424 & 108.1615 & 108.0799 & 108.2548 & 119.7343 & 120.3687 & 118.7942 & 108.0443 & 107.9625 & 108.0804 \\
\hline 2 & 30.8 & 161.3976 & 162.5611 & 161.1833 & 152.8972 & 152.5377 & 152.0742 & 161.9096 & 163.0638 & 161.8055 & 153.4857 & 153.0999 & 152.6652 \\
\hline 3 & 24.9 & 168.4181 & 169.6891 & 168.4056 & 159.8989 & 159.7595 & 159.1079 & 168.5959 & 169.8651 & 168.5871 & 160.0942 & 159.9457 & 159.2886 \\
\hline 4 & 26.7 & 160.9555 & 162.1552 & 161.8650 & 152.6116 & 152.2356 & 151.5069 & 161.1982 & 162.3893 & 162.1332 & 152.8681 & 152.4822 & 151.7662 \\
\hline $4 a$ & 140.4 & 57.8867 & 57.7176 & 55.9055 & 39.3160 & 39.1542 & 37.2659 & 57.4596 & 57.2922 & 55.4423 & 38.8505 & 38.7115 & 36.7816 \\
\hline 5 & 118.3 & 75.7273 & 76.6061 & 76.9392 & 61.4492 & 61.0106 & 60.5262 & 76.0493 & 76.9227 & 77.2236 & 61.7131 & 61.2744 & 60.7851 \\
\hline 6 & 31.4 & 165.2004 & 166.2504 & 164.9805 & 156.5498 & 156.1766 & 155.6930 & 165.4946 & 166.5367 & 165.1841 & 156.7623 & 156.3770 & 155.8943 \\
\hline 7 & 122.1 & 76.0978 & 76.6921 & 76.3669 & 61.0259 & 60.1338 & 59.3217 & 75.6065 & 76.2001 & 75.7206 & 60.3289 & 59.4644 & 58.5716 \\
\hline 8 & 132.2 & 63.6804 & 64.1749 & 63.4363 & 47.4162 & 46.7398 & 45.2702 & 63.8650 & 64.3518 & 63.6795 & 47.6696 & 47.0010 & 45.5197 \\
\hline $8 a$ & 41.0 & 150.4421 & 150.6974 & 149.4781 & 141.4062 & 141.5040 & 141.3387 & 150.6421 & 150.8886 & 149.7219 & 141.6008 & 141.6827 & 141.5288 \\
\hline $\begin{array}{c}\text { 8a-Me } \\
{ }^{1} \mathrm{H}\end{array}$ & 18.2 & 174.2910 & 175.9601 & 176.0445 & 168.3921 & 168.3416 & 168.1842 & 174.2787 & 175.9436 & 175.9854 & 168.3402 & 168.2815 & 168.1178 \\
\hline 1 & 3.51 & 28.3816 & 27.9766 & 27.8949 & 28.3256 & 28.1705 & 28.1572 & 28.3678 & 27.9617 & 27.8740 & 28.3075 & 28.1500 & 28.1346 \\
\hline $2 * a x$ & 2.18 & 30.3198 & 29.9023 & 29.7372 & 30.2640 & 30.0575 & 30.0018 & 30.3582 & 29.9407 & 29.7812 & 30.3070 & 30.0973 & 30.0416 \\
\hline $2^{* *}$ & 1.95 & 30.7840 & 30.3375 & 30.2326 & 30.6531 & 30.3750 & 30.3575 & 30.6150 & 30.1637 & 30.0528 & 30.4846 & 30.2092 & 30.1901 \\
\hline $3^{*}$ & 1.61 & 30.5300 & 30.0848 & 29.9870 & 30.4040 & 30.1455 & 30.1169 & 30.4776 & 30.0294 & 29.9237 & 30.3510 & 30.0892 & 30.0563 \\
\hline $3 * *$ & 1.30 & 30.7080 & 30.3035 & 30.2033 & 30.6494 & 30.4675 & 30.4117 & 30.6973 & 30.2933 & 30.1985 & 30.6412 & 30.4617 & 30.4066 \\
\hline 4 & 1.77 & 30.0200 & 29.5771 & 29.4369 & 29.9122 & 29.6774 & 29.6125 & 29.9882 & 29.5440 & 29.3985 & 29.8785 & 29.6431 & 29.5752 \\
\hline 5 & 5.41 & 26.7321 & 26.1570 & 25.9463 & 26.5324 & 26.1104 & 26.0855 & 26.6691 & 26.0929 & 25.8664 & 26.4554 & 26.0372 & 26.0059 \\
\hline 6 & 2.63 & 29.6139 & 29.1099 & 28.8159 & 29.3704 & 29.0753 & 28.9569 & 29.5625 & 29.0572 & 28.7636 & 29.3191 & 29.0236 & 28.9046 \\
\hline 7 & 5.66 & 26.5255 & 25.9541 & 25.6458 & 26.3505 & 25.9612 & 25.8486 & 26.4417 & 25.8694 & 25.5470 & 26.2530 & 25.8685 & 25.7500 \\
\hline 8 & 5.84 & 25.7236 & 25.1944 & 25.0006 & 25.6982 & 25.3530 & 25.2496 & 25.7714 & 25.2388 & 25.0434 & 25.7384 & 25.3874 & 25.2840 \\
\hline 8a-Me & 1.11 & 30.8883 & 30.4520 & 30.3655 & 30.8673 & 30.6535 & 30.5933 & 30.9066 & 30.4690 & 30.3826 & 30.8870 & 30.6692 & 30.6062 \\
\hline
\end{tabular}

\section{Compound 23}

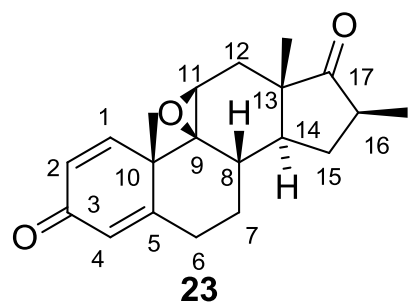

\begin{tabular}{|c|c|c|c|c|c|c|c|c|c|c|c|c|c|}
\hline & \multirow[b]{3}{*}{ Exp } & \multicolumn{12}{|c|}{ B3LYP } \\
\hline & & \multicolumn{6}{|c|}{ Gas phase } & \multicolumn{6}{|c|}{ PCM } \\
\hline & & 6-31G* & $6-31 G * *$ & $6-31+\mathrm{G} * *$ & 6-311G* & $6-311 G * *$ & $6-311+G * *$ & 6-31G* & 6-31G** & $6-31+\mathrm{G} * *$ & 6-311G* & $6-311 G * *$ & $6-311+G * *$ \\
\hline \multicolumn{14}{|l|}{${ }^{13} \mathrm{C}$} \\
\hline 1 & 151.9 & 45.9548 & 46.4809 & 44.7682 & 27.1546 & 26.3339 & 24.6457 & 42.4964 & 43.0123 & 40.6064 & 23.1506 & 22.3146 & 20.1190 \\
\hline 2 & 128.1 & 65.3381 & 65.7950 & 65.3445 & 47.9896 & 47.1643 & 46.6179 & 66.3714 & 66.8305 & 66.5062 & 49.1759 & 48.3659 & 47.8234 \\
\hline 3 & 185.9 & 16.5114 & 16.5754 & 14.6065 & -2.8894 & -3.0065 & -6.5474 & 15.2935 & 15.3479 & 12.9630 & -4.4680 & -4.5875 & -8.5156 \\
\hline 4 & 125.1 & 67.5790 & 68.2672 & 68.4583 & 51.2490 & 50.7080 & 50.1630 & 68.6844 & 69.3686 & 69.6637 & 52.4776 & 51.9387 & 51.3928 \\
\hline 5 & 164.3 & 35.2797 & 35.0856 & 32.9206 & 13.7056 & 13.5248 & 11.0586 & 30.7312 & 30.5344 & 27.5446 & 8.5672 & 8.3874 & 5.3052 \\
\hline 6 & 29.4 & 157.5996 & 158.9111 & 158.1167 & 148.5188 & 148.1068 & 147.7567 & 157.4880 & 158.7890 & 157.9899 & 148.4005 & 147.9686 & 147.5527 \\
\hline 7 & 30.0 & 154.3054 & 155.6415 & 155.0920 & 145.1119 & 144.9218 & 144.1984 & 154.5768 & 155.9056 & 155.4062 & 145.4235 & 145.2265 & 144.4981 \\
\hline 8 & 33.4 & 153.2710 & 154.0047 & 153.8833 & 143.2436 & 143.1235 & 142.4074 & 153.4500 & 154.1762 & 154.1074 & 143.4622 & 143.3377 & 142.6266 \\
\hline 9 & 66.8 & 121.7277 & 121.8085 & 121.7476 & 110.3121 & 110.3165 & 108.5400 & 121.1443 & 121.2241 & 121.0885 & 109.6744 & 109.6811 & 107.8002 \\
\hline
\end{tabular}




\begin{tabular}{|c|c|c|c|c|c|c|c|c|c|c|c|c|c|}
\hline 10 & 44.1 & 143.3235 & 143.5225 & 143.3662 & 132.7190 & 132.7491 & 131.8343 & 142.6742 & 142.8677 & 142.5127 & 131.9277 & 131.9532 & 130.8939 \\
\hline 11 & 62.3 & 126.7605 & 127.5693 & 127.2513 & 115.7081 & 115.2678 & 114.5918 & 126.5023 & 127.3121 & 126.9186 & 115.4465 & 114.9972 & 114.2658 \\
\hline 12 & 32.5 & 155.4588 & 156.6569 & 156.6677 & 146.3756 & 145.9856 & 145.7177 & 155.8067 & 156.9975 & 157.0811 & 146.7702 & 146.3787 & 146.1221 \\
\hline 13 & 46.1 & 141.2529 & 141.6020 & 141.1827 & 130.3877 & 130.5833 & 129.5767 & 140.8531 & 141.2030 & 140.6668 & 129.8986 & 130.1017 & 128.9746 \\
\hline 14 & 48.5 & 138.2073 & 138.8334 & 137.5006 & 127.6583 & 127.5437 & 127.7100 & 138.8672 & 139.4832 & 138.2896 & 128.4608 & 128.3375 & 128.5543 \\
\hline 15 & 31.6 & 156.9578 & 158.2613 & 157.2936 & 147.8489 & 147.5369 & 147.5371 & 157.1911 & 158.4874 & 157.5657 & 148.1196 & 147.7956 & 147.7946 \\
\hline 16 & 42.9 & 145.1007 & 145.9021 & 144.3009 & 134.4484 & 134.3304 & 133.1947 & 144.7227 & 145.5157 & 143.8118 & 134.0283 & 133.8988 & 132.5706 \\
\hline 17 & 221.0 & $0-20.7163$ & -20.6350 & -21.9330 & -44.0951 & -43.7869 & -47.7171 & -24.3725 & -24.2947 & -26.4649 & -48.3304 & -48.0268 & -52.7201 \\
\hline 18 & 16.7 & 171.7244 & 173.3859 & 175.3280 & 165.1636 & 165.0239 & 164.8840 & 171.8761 & 173.5289 & 175.4527 & 165.3256 & 165.1681 & 165.0393 \\
\hline 19 & 23.7 & 164.5068 & 166.2185 & 167.6460 & 157.5097 & 157.3489 & 156.6254 & 164.7638 & 166.4583 & 167.9026 & 157.7380 & 157.5580 & 156.8848 \\
\hline $\begin{array}{c}16 \mathrm{CH}_{3} \\
{ }^{1} \mathbf{H}\end{array}$ & 17.1 & 171.3044 & 173.1374 & 173.6170 & 164.6829 & 164.6117 & 164.4405 & 171.5886 & 173.4151 & 173.9518 & 165.0446 & 164.9522 & 164.7869 \\
\hline 1 & 6.59 & 25.6866 & 25.0946 & 25.0806 & 25.6888 & 25.2933 & 25.3155 & 25.4555 & 24.8633 & 24.8123 & 25.4431 & 25.0517 & 25.0457 \\
\hline 2 & 6.19 & 26.2630 & 25.6052 & 25.3853 & 26.1184 & 25.6876 & 25.5901 & 26.2532 & 25.5901 & 25.3630 & 26.1014 & 25.6719 & 25.5670 \\
\hline 4 & 6.14 & 26.3534 & 25.6871 & 25.5436 & 26.2152 & 25.7228 & 25.7625 & 26.3332 & 25.6591 & 25.4972 & 26.1845 & 25.6893 & 25.7134 \\
\hline $6^{*}$ & 2.71 & 29.4850 & 28.9939 & 28.9027 & 29.4777 & 29.1925 & 29.1737 & 29.3862 & 28.8933 & 28.7968 & 29.3823 & 29.0968 & 29.0714 \\
\hline $6^{* *}$ & 2.49 & 30.0399 & 29.5932 & 29.5007 & 29.8940 & 29.6637 & 29.6711 & 29.9318 & 29.4792 & 29.3741 & 29.7858 & 29.5505 & 29.5489 \\
\hline $7^{*}$ & 2.48 & 29.8792 & 29.4176 & 29.2975 & 29.8025 & 29.5458 & 29.4968 & 29.7762 & 29.3106 & 29.1824 & 29.7024 & 29.4415 & 29.3887 \\
\hline $7^{* *}$ & 1.24 & 30.7968 & 30.4083 & 30.2962 & 30.8413 & 30.6575 & 30.5961 & 30.7371 & 30.3459 & 30.2346 & 30.7846 & 30.6003 & 30.5367 \\
\hline 8 & 2.38 & 29.7477 & 29.3262 & 29.3702 & 29.9207 & 29.6735 & 29.6968 & 29.6337 & 29.2099 & 29.2340 & 29.8012 & 29.5542 & 29.5631 \\
\hline 11 & 3.23 & 28.9691 & 28.4574 & 28.3514 & 28.9174 & 28.6233 & 28.6657 & 28.9163 & 28.4053 & 28.2997 & 28.8628 & 28.5715 & 28.6138 \\
\hline $12^{*}$ & 2.21 & 29.9929 & 29.5461 & 29.5211 & 30.0198 & 29.7649 & 29.7512 & 30.0311 & 29.5831 & 29.5556 & 30.0492 & 29.7941 & 29.7803 \\
\hline $12^{* *}$ & 1.63 & 30.7772 & 30.3176 & 30.2528 & 30.7382 & 30.4969 & 30.4676 & 30.7223 & 30.2574 & 30.1925 & 30.6816 & 30.4355 & 30.4053 \\
\hline 14 & 1.25 & 30.9241 & 30.6485 & 30.6209 & 31.0098 & 30.9249 & 30.8965 & 30.8103 & 30.5310 & 30.4954 & 30.8935 & 30.8075 & 30.7754 \\
\hline $15^{*}$ & 2.25 & 30.1644 & 29.6926 & 29.5646 & 30.0891 & 29.8233 & 29.8326 & 30.0670 & 29.5923 & 29.4611 & 29.9988 & 29.7308 & 29.7334 \\
\hline $15^{* *}$ & 1.23 & 30.8511 & 30.4225 & 30.4141 & 30.9263 & 30.7037 & 30.7227 & 30.7649 & 30.3346 & 30.3133 & 30.8374 & 30.6161 & 30.6240 \\
\hline 16 & 2.08 & 30.1944 & 29.7629 & 29.7549 & 30.1809 & 29.9534 & 29.9705 & 30.0943 & 29.6599 & 29.6350 & 30.0802 & 29.8512 & 29.8567 \\
\hline 18 & 1.01 & 31.0151 & 30.5225 & 30.4961 & 31.1014 & 30.8037 & 30.7875 & 31.0155 & 30.5216 & 30.4887 & 31.0988 & 30.7996 & 30.7776 \\
\hline 19 & 1.45 & 30.7467 & 30.2877 & 30.2488 & 30.7817 & 30.5375 & 30.4644 & 30.7082 & 30.2462 & 30.1989 & 30.7427 & 30.4949 & 30.4157 \\
\hline $16 \mathrm{CH}_{3}$ & 1.19 & 31.0152 & 30.5630 & 30.4742 & 30.9633 & 30.7082 & 30.6810 & 31.0197 & 30.5660 & 30.4768 & 30.9674 & 30.7111 & 30.6820 \\
\hline
\end{tabular}

\begin{tabular}{|c|c|c|c|c|c|c|c|c|c|c|c|c|c|}
\hline & \multirow[b]{3}{*}{ Exp } & \multicolumn{12}{|c|}{ mPW1PW91 } \\
\hline & & \multicolumn{6}{|c|}{ Gas phase } & \multicolumn{6}{|c|}{ PCM } \\
\hline & & $6-31 G^{*}$ & 6-31G** & $6-31+G * *$ & 6-311G* & $6-311 G * *$ & $6-311+G * *$ & 6-31G* & $6-31 G^{* *}$ & $6-31+G * *$ & 6-311G* & 6-311G** & $6-311+G * *$ \\
\hline \multicolumn{14}{|l|}{${ }^{13} \mathrm{C}$} \\
\hline 1 & 151.9 & 48.9660 & 49.4231 & 47.8494 & 32.3660 & 31.5157 & 30.0874 & 45.4044 & 45.8499 & 43.6579 & 28.3303 & 27.4639 & 25.5835 \\
\hline 2 & 128.1 & 67.9426 & 68.3357 & 68.0207 & 52.6525 & 51.7903 & 51.2227 & 69.0197 & 69.4166 & 69.2157 & 53.8396 & 52.9958 & 52.4424 \\
\hline 3 & 185.9 & 18.5301 & 18.5828 & 17.5187 & 2.1704 & 2.0432 & -1.2502 & 17.2759 & 17.3183 & 15.8687 & 0.5930 & 0.4622 & -3.1788 \\
\hline 4 & 125.1 & 70.1754 & 70.8256 & 71.0303 & 55.7979 & 55.2468 & 54.6660 & 71.3498 & 71.9954 & 72.2932 & 57.0537 & 56.5060 & 55.9226 \\
\hline 5 & 164.3 & 38.6568 & 38.4320 & 36.5906 & 19.6192 & 19.4063 & 17.2301 & 33.9768 & 33.7496 & 31.1699 & 14.4307 & 14.2207 & 11.4932 \\
\hline 6 & 29.4 & 162.1644 & 163.3717 & 162.5891 & 153.9781 & 153.5601 & 153.2571 & 162.0528 & 163.2497 & 162.4638 & 153.8423 & 153.4056 & 153.0436 \\
\hline 7 & 30.0 & 159.5108 & 160.7437 & 160.4916 & 151.0953 & 150.9140 & 150.2185 & 159.7682 & 160.9950 & 160.7842 & 151.3753 & 151.1876 & 150.4861 \\
\hline 8 & 33.4 & 158.3076 & 158.9895 & 159.0231 & 149.5129 & 149.3972 & 148.7363 & 158.4710 & 159.1464 & 159.2275 & 149.7030 & 149.5831 & 148.9259 \\
\hline 9 & 66.8 & 126.3778 & 126.4564 & 126.5726 & 116.3544 & 116.3545 & 114.7729 & 125.8048 & 125.8821 & 125.9209 & 115.7333 & 115.7355 & 114.0559 \\
\hline 10 & 44.1 & 147.9663 & 148.1638 & 147.9966 & 138.5505 & 138.5846 & 137.7327 & 147.3080 & 147.4992 & 147.1529 & 137.7534 & 137.7830 & 136.8022 \\
\hline
\end{tabular}




\begin{tabular}{|c|c|c|c|c|c|c|c|c|c|c|c|c|c|}
\hline 11 & 62.3 & 130.8325 & 131.5927 & 131.3981 & 121.3805 & 120.9339 & 120.4122 & 130.5673 & 131.3284 & 131.0615 & 121.1167 & 120.6616 & 120.0865 \\
\hline 12 & 32.5 & 160.2401 & 161.3492 & 161.3541 & 152.0289 & 151.6508 & 151.3001 & 160.5943 & 161.6964 & 161.7706 & 152.4082 & 152.0292 & 151.6893 \\
\hline 13 & 46.1 & 146.0415 & 146.3827 & 146.0181 & 136.2361 & 136.4274 & 135.5238 & 145.6406 & 145.9825 & 145.5114 & 135.7471 & 135.9462 & 134.9340 \\
\hline 14 & 48.5 & 143.9168 & 144.4732 & 143.2545 & 134.3105 & 134.2015 & 134.3862 & 144.5414 & 145.0904 & 143.9895 & 135.0418 & 134.9259 & 135.1589 \\
\hline 15 & 31.6 & 161.6816 & 162.8885 & 162.2897 & 153.4918 & 153.1701 & 153.0947 & 161.9221 & 163.1229 & 162.5708 & 153.7555 & 153.4236 & 153.3430 \\
\hline 16 & 42.9 & 149.8359 & 150.5934 & 149.1552 & 140.3249 & 140.2042 & 139.1090 & 149.4222 & 150.1724 & 148.6546 & 139.8670 & 139.7362 & 138.4740 \\
\hline 17 & 221.0 & -17.6897 & -17.6080 & -18.2647 & -38.1106 & -37.8068 & -41.2719 & -21.4346 & -21.3568 & -22.7879 & -42.3619 & -42.0630 & -46.2074 \\
\hline 18 & 16.7 & 176.0534 & 177.5768 & 179.3143 & 169.9761 & 169.8498 & 169.6919 & 176.2297 & 177.7444 & 179.4674 & 170.1476 & 170.0048 & 169.8536 \\
\hline 19 & 23.7 & 169.0735 & 170.6450 & 171.3144 & 162.4153 & 162.2891 & 161.7633 & 169.3393 & 170.8949 & 171.5667 & 162.6327 & 162.4901 & 162.0065 \\
\hline $\begin{array}{c}16 \mathrm{CH}_{3} \\
{ }^{\mathbf{1}} \mathbf{H}\end{array}$ & 17.1 & 175.3690 & 177.0733 & 177.1256 & 169.2707 & 169.2052 & 168.9716 & 175.6850 & 177.3822 & 177.4919 & 169.6372 & 169.5519 & 169.3239 \\
\hline 1 & 6.59 & 25.5110 & 24.9264 & 24.9360 & 25.5018 & 25.1059 & 25.1433 & 25.2674 & 24.6823 & 24.6596 & 25.2475 & 24.8553 & 24.8674 \\
\hline 2 & 6.19 & 26.0366 & 25.3791 & 25.1926 & 25.9302 & 25.4635 & 25.3873 & 26.0183 & 25.3554 & 25.1635 & 25.9063 & 25.4415 & 25.3584 \\
\hline 4 & 6.14 & 26.1233 & 25.4607 & 25.3491 & 26.0203 & 25.5016 & 25.5358 & 26.0942 & 25.4236 & 25.2963 & 25.9825 & 25.4615 & 25.4816 \\
\hline 6* & 2.71 & 29.4505 & 28.9559 & 28.8549 & 29.3812 & 29.1037 & 29.0786 & 29.3452 & 28.8485 & 28.7428 & 29.2795 & 29.0017 & 28.9707 \\
\hline $6 * *$ & 2.49 & 29.9184 & 29.4761 & 29.4105 & 29.7733 & 29.5352 & 29.5507 & 29.7980 & 29.3490 & 29.2730 & 29.6554 & 29.4116 & 29.4196 \\
\hline 7* & 2.48 & 29.8430 & 29.3828 & 29.2531 & 29.7386 & 29.4774 & 29.4191 & 29.7271 & 29.2624 & 29.1271 & 29.6277 & 29.3617 & 29.3011 \\
\hline $7 * *$ & 1.24 & 30.7312 & 30.3440 & 30.2197 & 30.7249 & 30.5396 & 30.4827 & 30.6743 & 30.2845 & 30.1607 & 30.6712 & 30.4856 & 30.4258 \\
\hline 8 & 2.38 & 29.6908 & 29.2619 & 29.2684 & 29.7872 & 29.5461 & 29.5527 & 29.5742 & 29.1428 & 29.1317 & 29.6669 & 29.4258 & 29.4195 \\
\hline 11 & 3.23 & 28.9172 & 28.4075 & 28.3189 & 28.8540 & 28.5429 & 28.5967 & 28.8608 & 28.3515 & 28.2651 & 28.7972 & 28.4887 & 28.5427 \\
\hline $12^{*}$ & 2.21 & 29.9142 & 29.4648 & 29.4471 & 29.9061 & 29.6480 & 29.6423 & 29.9479 & 29.4972 & 29.4771 & 29.9311 & 29.6727 & 29.6670 \\
\hline $12^{* *}$ & 1.63 & 30.7091 & 30.2497 & 30.1806 & 30.6223 & 30.3803 & 30.3520 & 30.6531 & 30.1879 & 30.1201 & 30.5659 & 30.3188 & 30.2899 \\
\hline 14 & 1.25 & 30.8493 & 30.5821 & 30.5330 & 30.8591 & 30.7921 & 30.7630 & 30.7389 & 30.4680 & 30.4131 & 30.7485 & 30.6803 & 30.6476 \\
\hline $15^{*}$ & 2.25 & 30.1036 & 29.6283 & 29.5110 & 29.9993 & 29.7272 & 29.7388 & 29.9976 & 29.5187 & 29.3999 & 29.9023 & 29.6275 & 29.6333 \\
\hline $15^{* *}$ & 1.23 & 30.7849 & 30.3581 & 30.3401 & 30.8033 & 30.5830 & 30.6041 & 30.6947 & 30.2661 & 30.2362 & 30.7113 & 30.4922 & 30.5034 \\
\hline 16 & 2.08 & 30.1324 & 29.6974 & 29.6920 & 30.0584 & 29.8365 & 29.8639 & 30.0294 & 29.5909 & 29.5707 & 29.9561 & 29.7326 & 29.7492 \\
\hline 18 & 1.01 & 30.9624 & 30.4693 & 30.4307 & 31.0104 & 30.7105 & 30.6940 & 30.9609 & 30.4665 & 30.4221 & 31.0056 & 30.7043 & 30.6826 \\
\hline 19 & 1.45 & 30.6929 & 30.2353 & 30.1901 & 30.6889 & 30.4429 & 30.3748 & 30.6501 & 30.1891 & 30.1369 & 30.6461 & 30.3963 & 30.3226 \\
\hline $16 \mathrm{CH}_{3}$ & 1.19 & 30.9471 & 30.4933 & 30.4054 & 30.8759 & 30.6129 & 30.5826 & 30.9483 & 30.4930 & 30.4047 & 30.8764 & 30.6124 & 30.5806 \\
\hline
\end{tabular}

\section{Compound 24}

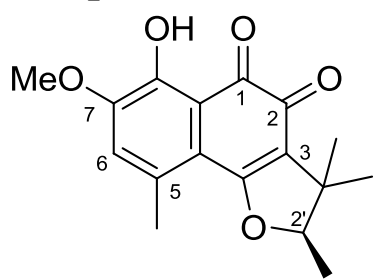

24

\begin{tabular}{|c|c|c|c|c|c|c|c|c|c|c|c|c|c|}
\hline & \multirow[b]{3}{*}{ Exp } & \multicolumn{12}{|c|}{ B3LYP } \\
\hline & & \multicolumn{6}{|c|}{ Gas phase } & \multicolumn{6}{|c|}{ PCM } \\
\hline & & 6-31G* & $6-31 \mathrm{G} * *$ & $6-31+G * *$ & $6-311 G *$ & $6-311 G * *$ & $6-311+G * *$ & 6-31G* & $6-31 G * *$ & $6-31+G * *$ & 6-311G* & $6-311 G * *$ & $6-311+G * *$ \\
\hline \multicolumn{14}{|l|}{${ }^{13} \mathrm{C}$} \\
\hline 1 & 186.9 & 10.7567 & 10.7977 & 11.3474 & -7.6870 & -7.7104 & -9.7100 & 9.4763 & 9.5242 & 9.9439 & -8.9977 & -9.0213 & -11.2349 \\
\hline 2 & 174.4 & 22.7108 & 22.7464 & 22.2230 & 4.6238 & 4.5984 & 3.7173 & 22.6245 & 22.6622 & 22.1639 & 4.3783 & 4.3458 & 3.4773 \\
\hline 3 & 122.8 & 72.1657 & 72.1093 & 71.5312 & 56.2657 & 56.3119 & 55.6813 & 71.4664 & 71.4100 & 70.7186 & 55.4553 & 55.5148 & 54.6947 \\
\hline 4 & 170.6 & 28.5534 & 28.5762 & 27.5111 & 9.0054 & 9.0387 & 8.1121 & 26.5520 & 26.5838 & 25.1579 & 6.8917 & 6.9315 & 5.6821 \\
\hline
\end{tabular}




\begin{tabular}{|c|c|c|c|c|c|c|c|c|c|c|c|c|c|}
\hline 5 & 132.6 & 63.7533 & 63.8668 & 63.1490 & 46.7194 & 46.6095 & 45.2473 & 60.9013 & 61.0172 & 59.7922 & 43.7007 & 43.5797 & 41.7536 \\
\hline 6 & 119.8 & 76.6614 & 77.4653 & 78.9015 & 60.9294 & 60.5478 & 60.3229 & 74.9459 & 75.7408 & 76.7448 & 60.5441 & 60.0240 & 59.1503 \\
\hline 7 & 152.5 & 43.9540 & 43.6437 & 41.6563 & 22.9582 & 22.8570 & 22.2699 & 43.6086 & 43.2942 & 41.4197 & 22.6729 & 22.5451 & 22.1379 \\
\hline 8 & 156.0 & 37.8479 & 37.6825 & 38.0505 & 17.5818 & 17.7412 & 17.3442 & 38.2806 & 38.1061 & 38.4533 & 18.0722 & 18.1838 & 17.8714 \\
\hline 9 & 114.1 & 78.0110 & 77.9393 & 79.0375 & 64.3208 & 64.0533 & 62.3592 & 77.9512 & 77.8857 & 78.9523 & 64.4079 & 64.1534 & 62.2684 \\
\hline 10 & 115.6 & 77.4340 & 77.4242 & 77.5370 & 61.6804 & 61.6869 & 61.0901 & 77.8503 & 77.8524 & 77.9439 & 62.4702 & 62.4915 & 61.7656 \\
\hline $1^{\prime}$ & 14.7 & 174.6411 & 176.4841 & 176.5161 & 168.0727 & 167.9994 & 168.2951 & 174.9607 & 176.7848 & 176.7671 & 168.3283 & 168.2327 & 168.4985 \\
\hline $2^{\prime}$ & 92.4 & 100.3108 & 100.9313 & 100.8054 & 86.8076 & 86.4824 & 85.1737 & 100.0819 & 100.6992 & 100.5727 & 86.5702 & 86.2351 & 84.9464 \\
\hline $3^{\prime}$ & 43.1 & 143.2387 & 143.6178 & 143.1147 & 133.0162 & 133.1158 & 132.5124 & 143.2015 & 143.5823 & 143.0406 & 132.9842 & 133.0838 & 132.4412 \\
\hline $4^{\prime}$ & 20.2 & 168.4823 & 170.1688 & 171.6629 & 162.2718 & 162.0949 & 161.7775 & 168.5986 & 170.2755 & 171.8050 & 162.4327 & 162.2356 & 161.944 \\
\hline $5^{\prime}$ & 25.7 & 164.0388 & 165.7423 & 166.3083 & 156.8269 & 156.6425 & 156.2742 & 164.3827 & 166.0790 & 166.7064 & 157.2394 & 157.0356 & 156.7251 \\
\hline $5-\mathrm{Me}$ & 22.2 & 165.1811 & 166.8743 & 167.6695 & 158.1675 & 157.7992 & 157.7547 & 165.3399 & 167.0085 & 167.7251 & 158.2237 & 157.8293 & 157.7354 \\
\hline $\begin{array}{c}\text { 7-OMe } \\
{ }^{1} \mathbf{H}\end{array}$ & 56.2 & 136.7388 & 138.5251 & 138.7817 & 127.3603 & 126.7507 & 126.4021 & 136.4427 & 138.2005 & 138.1560 & 127.5399 & 126.8388 & 126.2955 \\
\hline 6 & 6.71 & 26.1319 & 25.4715 & & & & 25.3525 & & & & 25.7319 & 25.2845 & 25.0818 \\
\hline $1^{\prime}$ & 1.45 & 30.8152 & 30.3430 & 30.2238 & 30.7765 & 30.5007 & 30.4775 & 30.7542 & 30.2792 & 30.1578 & 30.7187 & 30.4415 & 30.4163 \\
\hline $2^{\prime}$ & 4.61 & 27.6872 & 27.2125 & 27.0612 & 27.7169 & 27.4829 & 27.4848 & 27.5616 & 27.0850 & 26.9177 & 27.5864 & 27.3530 & 27.3482 \\
\hline $4^{\prime}$ & 1.25 & 30.9498 & 30.5026 & 30.4242 & 30.9897 & 30.7433 & 30.7010 & 30.9443 & 30.4962 & 30.4067 & 30.9790 & 30.7315 & 30.6839 \\
\hline $5^{\prime}$ & 1.43 & 30.6981 & 30.2428 & 30.1537 & 30.7046 & 30.4408 & 30.4128 & 30.7082 & 30.2522 & 30.1550 & 30.7101 & 30.4455 & 30.4109 \\
\hline $5-\mathrm{Me}$ & 2.54 & 29.9236 & 29.4317 & 29.2584 & 29.7840 & 29.4887 & 29.4696 & 29.8698 & 29.3751 & 29.2030 & 29.7277 & 29.4310 & 29.4115 \\
\hline 7-OMe & 3.95 & 28.4715 & 27.9688 & 27.8410 & 28.3922 & 28.1029 & 28.0182 & 28.3456 & 27.8428 & 27.7048 & 28.3029 & 28.0117 & 27.9138 \\
\hline
\end{tabular}

\begin{tabular}{|c|c|c|c|c|c|c|c|c|c|c|c|c|c|}
\hline & \multicolumn{13}{|c|}{ mPW1PW91 } \\
\hline & \multirow[b]{2}{*}{ Exp } & \multicolumn{6}{|c|}{ Gas phase } & \multicolumn{6}{|c|}{ PCM } \\
\hline & & 6-31G* & 6-31G** & $6-31+G * *$ & 6-311G* & 6-311G** & $6-311+G * *$ & 6-31G* & 6-31G** & $6-31+G * *$ & 6-311G* & 6-311G** & $6-311+G * *$ \\
\hline \multicolumn{14}{|l|}{${ }^{13} \mathrm{C}$} \\
\hline 1 & 186.9 & 12.7600 & 12.7963 & 13.9701 & -2.7620 & -2.7803 & -4.6822 & 11.4915 & 11.5349 & 12.5766 & -4.0503 & -4.0691 & -6.1444 \\
\hline 2 & 174.4 & 24.8910 & 24.9219 & 25.0233 & 9.5939 & 9.5650 & 8.6523 & 24.7791 & 24.8126 & 24.9125 & 9.3321 & 9.2971 & 8.4000 \\
\hline 3 & 122.8 & 75.6706 & 75.6216 & 75.2617 & 61.7248 & 61.7692 & 61.0906 & 74.9872 & 74.9372 & 74.4848 & 60.9547 & 61.0120 & 60.1734 \\
\hline 4 & 170.6 & 31.2680 & 31.2956 & 30.6484 & 14.4330 & 14.4743 & 13.5773 & 29.2579 & 29.2942 & 28.3254 & 12.3221 & 12.3689 & 11.1845 \\
\hline 5 & 132.6 & 67.0789 & 67.1611 & 66.6951 & 52.3542 & 52.2215 & 50.8622 & 64.1714 & 64.2559 & 63.3590 & 49.2485 & 49.1093 & 47.3855 \\
\hline 6 & 119.8 & 79.1308 & 79.9025 & 81.2415 & 66.0263 & 65.6044 & 65.1086 & 77.3850 & 78.1464 & 79.0993 & 64.9240 & 64.3953 & 63.5136 \\
\hline 7 & 152.5 & 46.8140 & 46.4743 & 45.1256 & 28.6633 & 28.5575 & 27.9718 & 46.4625 & 46.1192 & 44.8795 & 28.3677 & 28.2325 & 27.7854 \\
\hline 8 & 156.0 & 40.8744 & 40.6895 & 41.3894 & 23.4983 & 23.6675 & 23.3793 & 41.3499 & 41.1550 & 41.8305 & 24.0060 & 24.1264 & 23.9376 \\
\hline 9 & 114.1 & 81.2601 & 81.1851 & 82.4657 & 69.6843 & 69.4070 & 67.3626 & 81.2872 & 81.2186 & 82.4531 & 69.8026 & 69.5412 & 67.3728 \\
\hline 10 & 115.6 & 80.6237 & 80.6180 & 80.7070 & 67.1845 & 67.1916 & 66.5110 & 81.1119 & 81.1193 & 81.1839 & 67.9309 & 67.9592 & 67.1905 \\
\hline $1^{\prime}$ & 14.7 & 178.5659 & 180.2950 & 180.4412 & 172.4893 & 172.4376 & 172.6626 & 178.9118 & 180.6224 & 180.7184 & 172.7493 & 172.6779 & 172.8863 \\
\hline $2^{\prime}$ & 92.4 & 104.9598 & 105.5441 & 105.8246 & 93.1571 & 92.8399 & 91.7795 & 104.6818 & 105.2643 & 105.5473 & 92.8457 & 92.5213 & 91.4724 \\
\hline $3^{\prime}$ & 43.1 & 148.0551 & 148.4366 & 148.0231 & 138.8181 & 138.9143 & 138.3356 & 148.0074 & 148.3903 & 147.9471 & 138.7778 & 138.8738 & 138.2540 \\
\hline $4^{\prime}$ & 20.2 & 172.5347 & 174.0886 & 175.2403 & 166.7275 & 166.5693 & 166.3699 & 172.6732 & 174.2173 & 175.3943 & 166.8809 & 166.7035 & 166.5353 \\
\hline $5^{\prime}$ & 25.7 & 168.4532 & 170.0141 & 170.4207 & 161.7213 & 161.5574 & 161.2858 & 168.8202 & 170.3741 & 170.8295 & 162.1298 & 161.9481 & 161.7332 \\
\hline 5-Me & 22.2 & 169.0688 & 170.6465 & 171.5665 & 162.6144 & 162.2481 & 162.1823 & 169.2521 & 170.8047 & 171.6399 & 162.6871 & 162.2990 & 162.1951 \\
\hline $\begin{array}{c}\text { 7-OMe } \\
{ }^{1} \mathrm{H}\end{array}$ & 56.2 & 140.2947 & 142.0154 & 142.1814 & 132.5313 & 131.9561 & 131.6493 & 139.9693 & 141.6627 & 141.5482 & 132.3935 & 131.7430 & 131.3458 \\
\hline 6 & 6.71 & 25.9330 & 25.2709 & 25.1827 & 25.8144 & 25.3371 & 25.1945 & 25.6249 & 24.9596 & 24.8549 & 25.5376 & 25.0633 & 24.8982 \\
\hline
\end{tabular}




\begin{tabular}{cccccccccccccc}
\hline $1^{\prime}$ & 1.45 & 30.7439 & 30.2686 & 30.1705 & 30.6787 & 30.3946 & 30.3803 & 30.6764 & 30.1979 & 30.0985 & 30.6150 & 30.3291 & 30.3129 \\
$2^{\prime}$ & 4.61 & 27.6221 & 27.1440 & 26.9998 & 27.6052 & 27.3696 & 27.3782 & 27.4912 & 27.0109 & 26.8526 & 27.4714 & 27.2366 & 27.2381 \\
$4^{\prime}$ & 1.25 & 30.8855 & 30.4401 & 30.3613 & 30.8928 & 30.6444 & 30.5976 & 30.8772 & 30.4308 & 30.3427 & 30.8800 & 30.6305 & 30.5779 \\
$5^{\prime}$ & 1.43 & 30.6316 & 30.1770 & 30.0972 & 30.6073 & 30.3407 & 30.3172 & 30.6378 & 30.1825 & 30.0952 & 30.6098 & 30.3425 & 30.3128 \\
5-Me & 2.54 & 29.8257 & 29.3296 & 29.1695 & 29.6690 & 29.3667 & 29.3568 & 29.7614 & 29.2622 & 29.1037 & 29.6053 & 29.3013 & 29.2899 \\
7-OMe & 3.95 & 28.4019 & 27.9029 & 27.7957 & 28.3436 & 28.0439 & 27.9768 & 28.2649 & 27.7654 & 27.6500 & 28.2293 & 27.9285 & 27.8556 \\
\hline
\end{tabular}

\section{Compound 25}

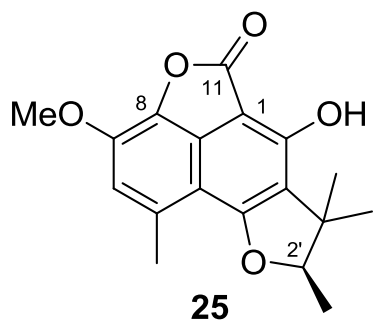

\begin{tabular}{|c|c|c|c|c|c|c|c|c|c|c|c|c|c|}
\hline & \multirow[b]{3}{*}{ Exp } & \multicolumn{12}{|c|}{ B3LYP } \\
\hline & & \multicolumn{6}{|c|}{ Gas phase } & \multicolumn{6}{|c|}{ PCM } \\
\hline & & 6-31G* & $6-31 G * *$ & $6-31+G * *$ & 6-311G* & $6-311 \mathrm{G} * *$ & $6-311+G * *$ & 6-31G* & $6-31 G * *$ & $6-31+\mathrm{G} * *$ & 6-311G* & $6-311 G * *$ & $6-311+G^{* *}$ \\
\hline \multicolumn{14}{|l|}{${ }^{13} \mathrm{C}$} \\
\hline 1 & 95.0 & 95.3584 & 95.3008 & 95.9364 & 82.6828 & 82.6435 & 81.7490 & 96.7970 & 96.7380 & 97.4112 & 84.3115 & 84.2467 & 83.3055 \\
\hline 2 & 155.9 & 42.5161 & 42.4741 & 39.5156 & 21.7738 & 21.8832 & 22.4448 & 41.5903 & 41.5346 & 38.5656 & 20.7918 & 20.8798 & 21.3388 \\
\hline 3 & 121.0 & 78.3398 & 78.1812 & 79.9646 & 62.4034 & 62.0732 & 60.8180 & 75.9785 & 75.8222 & 77.5688 & 59.8119 & 59.5143 & 58.1040 \\
\hline 4 & 164.6 & 33.9093 & 33.9524 & 32.7852 & 14.5906 & 14.5992 & 14.1729 & 32.2090 & 32.2585 & 30.9475 & 12.7673 & 12.8078 & 12.2363 \\
\hline 5 & 132.4 & 64.1636 & 64.2075 & 64.3100 & 46.2338 & 46.0266 & 45.6284 & 62.3935 & 62.4372 & 62.3537 & 44.2484 & 44.0572 & 43.4830 \\
\hline 6 & 118.5 & 74.3970 & 75.1669 & 76.1292 & 58.3827 & 57.8700 & 56.7643 & 75.2090 & 75.9786 & 76.8728 & 59.2497 & 58.7320 & 57.6295 \\
\hline 7 & 140.7 & 55.6138 & 55.5278 & 54.5033 & 36.3514 & 36.2946 & 35.5212 & 55.2327 & 55.1463 & 54.0880 & 35.9117 & 35.8649 & 35.0624 \\
\hline 8 & 129.5 & 64.3453 & 64.3867 & 64.4392 & 47.6613 & 47.7134 & 47.6052 & 65.3511 & 65.3901 & 65.3974 & 48.6758 & 48.7203 & 48.6044 \\
\hline 9 & 134.7 & 58.9713 & 59.0079 & 57.4200 & 41.6150 & 41.6633 & 40.3688 & 59.6007 & 59.6323 & 57.9478 & 42.2595 & 42.2956 & 40.9704 \\
\hline 10 & 107.8 & 88.0781 & 88.0400 & 86.9004 & 72.2602 & 72.2709 & 71.6214 & 87.9128 & 87.8800 & 86.6173 & 72.0870 & 72.1104 & 71.3788 \\
\hline 11 & 167.5 & 36.3074 & 36.3429 & 33.6812 & 17.0458 & 17.0037 & 14.4455 & 34.5880 & 34.6251 & 31.6057 & 15.0579 & 15.0361 & 12.0863 \\
\hline $1^{\prime}$ & 14.5 & 175.3436 & 177.1920 & 177.2402 & 168.6594 & 168.5992 & 168.6897 & 175.6572 & 177.4870 & 177.4763 & 168.9092 & 168.8277 & 168.8964 \\
\hline $2^{\prime}$ & 92.0 & 102.0284 & 102.6560 & 103.1347 & 89.1207 & 88.8402 & 87.2977 & 101.5609 & 102.1838 & 102.6623 & 88.5633 & 88.2773 & 86.7785 \\
\hline $3^{\prime}$ & 43.4 & 144.3936 & 144.7196 & 143.1451 & 134.1750 & 134.2107 & 133.7272 & 143.9407 & 144.2693 & 142.6537 & 133.6599 & 133.7106 & 133.1662 \\
\hline $4^{\prime}$ & 21.0 & 166.7011 & 168.4366 & 169.6606 & 160.5541 & 160.3750 & 160.2610 & 166.9548 & 168.6703 & 169.8774 & 160.7589 & 160.5493 & 160.4430 \\
\hline $5^{\prime}$ & 25.7 & 162.7994 & 164.6044 & 163.3530 & 155.7200 & 155.4320 & 155.5337 & 163.5829 & 165.3572 & 164.1185 & 156.5143 & 156.1975 & 156.3334 \\
\hline 5-Me & 20.1 & 167.2962 & 169.0094 & 169.4201 & 160.1800 & 159.9303 & 160.0279 & 167.5566 & 169.2473 & 169.6103 & 160.4014 & 160.1226 & 160.2136 \\
\hline $\begin{array}{c}\text { 7-OMe } \\
{ }^{1} \mathbf{H}\end{array}$ & 58.9 & 132.6529 & 134.4402 & 133.2347 & 123.6658 & 122.9390 & 122.3752 & 132.5644 & 134.3374 & 132.9398 & 123.4539 & 122.7064 & 122.1236 \\
\hline 6 & 6.70 & 25.8071 & 25.1349 & 25.0182 & 25.6241 & 25.1295 & 25.0408 & 25.7557 & 25.0807 & 24.9641 & 25.5622 & 25.0694 & 24.9802 \\
\hline $1^{\prime}$ & 1.47 & 30.7638 & 30.2845 & 30.2156 & 30.7293 & 30.4417 & 30.4350 & 30.7031 & 30.2203 & 30.1498 & 30.6678 & 30.3783 & 30.3711 \\
\hline $2^{\prime}$ & 4.68 & 27.5904 & 27.1219 & 26.9801 & 27.6385 & 27.4105 & 27.3953 & 27.4477 & 26.9770 & 26.8226 & 27.4812 & 27.2565 & 27.2389 \\
\hline $4^{\prime}$ & 1.28 & 30.9542 & 30.5055 & 30.3806 & 31.0136 & 30.7487 & 30.6399 & 30.9055 & 30.4546 & 30.3187 & 30.9584 & 30.6939 & 30.5808 \\
\hline $5^{\prime}$ & 1.52 & 30.7734 & 30.3048 & 30.3124 & 30.7963 & 30.4886 & 30.4759 & 30.6842 & 30.2146 & 30.2165 & 30.7057 & 30.4013 & 30.3867 \\
\hline 5-Me & 2.66 & 29.7139 & 29.2311 & 29.0170 & 29.6041 & 29.3259 & 29.2582 & 29.6625 & 29.1767 & 28.9652 & 29.5520 & 29.2722 & 29.2056 \\
\hline
\end{tabular}




\begin{tabular}{|c|c|c|c|c|c|c|c|c|c|c|c|c|c|}
\hline \multicolumn{2}{|c|}{ 7-OMe 4.19} & 28.0828 & 27.5778 & 27.3902 & 28.0072 & 27.7056 & 27.5842 & 28.0391 & 27.5347 & 27.3473 & 27.9600 & 27.6606 & 27.5411 \\
\hline & \multirow[b]{3}{*}{ Exp } & \multicolumn{12}{|c|}{ mPW1PW91 } \\
\hline & & \multicolumn{6}{|c|}{ Gas phase } & \multicolumn{6}{|c|}{ PCM } \\
\hline & & 6-31G* & $6-31 G * *$ & $6-31+\mathrm{G} * *$ & 6-311G* & 6-311G** & $6-311+G * *$ & 6-31G* & $6-31 G * *$ & $6-31+G * *$ & 6-311G* & 6-311G** & $6-311+\mathrm{G} * *$ \\
\hline \multicolumn{14}{|l|}{${ }^{13} \mathrm{C}$} \\
\hline 1 & 95.0 & 98.6395 & 98.5745 & 99.5527 & 87.7705 & 87.7343 & 86.8000 & 100.1623 & 100.0970 & 101.0767 & 89.4468 & 89.3849 & 88.3935 \\
\hline 2 & 155.9 & 45.1830 & 45.1191 & 42.6151 & 27.2952 & 27.3868 & 27.9959 & 44.2169 & 44.1391 & 41.6545 & 26.2839 & 26.3549 & 26.8574 \\
\hline 3 & 121.0 & 81.8724 & 81.7187 & 83.8730 & 67.9808 & 67.6492 & 66.3780 & 79.4796 & 79.3271 & 81.4585 & 65.4022 & 65.1034 & 63.7075 \\
\hline 4 & 164.6 & 36.6078 & 36.6597 & 36.0319 & 19.9365 & 19.9551 & 19.4413 & 34.8828 & 34.9401 & 34.1932 & 18.1171 & 18.1672 & 17.5323 \\
\hline 5 & 132.4 & 67.1841 & 67.1975 & 67.2542 & 51.6130 & 51.3870 & 50.8480 & 65.3415 & 65.3542 & 65.2669 & 49.5740 & 49.3663 & 48.6939 \\
\hline 6 & 118.5 & 77.1406 & 77.8713 & 79.0334 & 63.1391 & 62.6120 & 61.5332 & 77.9607 & 78.6903 & 79.7880 & 63.9755 & 63.4435 & 62.3870 \\
\hline 7 & 140.7 & 58.4948 & 58.3947 & 57.8850 & 41.9975 & 41.9305 & 41.1636 & 58.0879 & 57.9873 & 57.4576 & 41.5343 & 41.4772 & 40.6920 \\
\hline 8 & 129.5 & 67.7787 & 67.8255 & 68.2203 & 53.3875 & 53.4474 & 52.9144 & 68.8195 & 68.8644 & 69.2025 & 54.4134 & 54.4657 & 53.9383 \\
\hline 9 & 134.7 & 61.9407 & 61.9746 & 60.8171 & 46.8921 & 46.9480 & 45.8374 & 62.6556 & 62.6849 & 61.4132 & 47.6041 & 47.6477 & 46.4990 \\
\hline 10 & 107.8 & 91.1374 & 91.0994 & 90.0839 & 77.5231 & 77.5403 & 76.8226 & 91.0324 & 90.9996 & 89.8652 & 77.4044 & 77.4341 & 76.6533 \\
\hline 11 & 167.5 & 38.8966 & 38.9293 & 36.8973 & 22.1313 & 22.0900 & 19.7333 & 37.1850 & 37.2192 & 34.8697 & 20.1887 & 20.1677 & 17.4669 \\
\hline $1^{\prime}$ & 14.5 & 179.2617 & 180.9944 & 181.1771 & 173.0733 & 173.0340 & 173.0428 & 179.6002 & 181.3146 & 181.4404 & 173.3265 & 173.2681 & 173.2564 \\
\hline $2^{\prime}$ & 92.0 & 106.6130 & 107.2102 & 107.9387 & 95.3600 & 95.0949 & 93.8516 & 106.0962 & 106.6897 & 107.4089 & 94.7414 & 94.4726 & 93.2721 \\
\hline $3^{\prime}$ & 43.4 & 149.2640 & 149.5897 & 148.0566 & 139.9973 & 140.0211 & 139.5650 & 148.7809 & 149.1093 & 147.5399 & 139.4594 & 139.4994 & 138.9885 \\
\hline $4^{\prime}$ & 21.0 & 170.7887 & 172.3919 & 173.3809 & 165.0505 & 164.8940 & 164.8410 & 171.0686 & 172.6528 & 173.6235 & 165.2588 & 165.0752 & 165.0332 \\
\hline $5^{\prime}$ & 25.7 & 167.3485 & 169.0052 & 167.6665 & 160.7962 & 160.5246 & 160.5545 & 168.1612 & 169.7899 & 168.4497 & 161.5729 & 161.2779 & 161.3430 \\
\hline 5-Me & 20.1 & 171.0723 & 172.6723 & 173.5451 & 164.5872 & 164.3333 & 164.3745 & 171.3624 & 172.9389 & 173.7436 & 164.8021 & 164.5222 & 164.5562 \\
\hline $\begin{array}{c}\text { 7-OMe } \\
{ }^{{ }^{1}} \mathbf{H}\end{array}$ & 58.9 & 136.2591 & 137.9831 & 137.1012 & 128.5855 & 127.9016 & 127.4796 & 136.1622 & 137.8723 & 136.8023 & 128.3631 & 127.6599 & 127.2320 \\
\hline 6 & 6.70 & 25.5754 & 24.9163 & 24.8125 & 25.4167 & 24.9137 & 24.8614 & 25.5082 & 24.8456 & 24.7441 & 25.3417 & 24.8405 & 24.7880 \\
\hline $1^{\prime}$ & 1.47 & 30.6882 & 30.2061 & 30.1585 & 30.6281 & 30.3322 & 30.3368 & 30.6210 & 30.1349 & 30.0859 & 30.5611 & 30.2630 & 30.2672 \\
\hline $2^{\prime}$ & 4.68 & 27.5148 & 27.0445 & 26.9269 & 27.5186 & 27.2904 & 27.2886 & 27.3663 & 26.8935 & 26.7649 & 27.3585 & 27.1339 & 27.1302 \\
\hline $4^{\prime}$ & 1.28 & 30.8899 & 30.4426 & 30.3235 & 30.9168 & 30.6489 & 30.5410 & 30.8374 & 30.3876 & 30.2587 & 30.8585 & 30.5908 & 30.4788 \\
\hline $5^{\prime}$ & 1.52 & 30.7083 & 30.2422 & 30.2458 & 30.7003 & 30.3897 & 30.3692 & 30.6128 & 30.1454 & 30.1443 & 30.6054 & 30.2979 & 30.2759 \\
\hline 5-Me & 2.66 & 29.6180 & 29.1303 & 28.9518 & 29.4890 & 29.2020 & 29.1705 & 29.5571 & 29.0660 & 28.8896 & 29.4286 & 29.1398 & 29.1087 \\
\hline 7-OMe & 4.19 & 28.0090 & 27.5065 & 27.3357 & 27.9343 & 27.6219 & 27.5089 & 27.9574 & 27.4554 & 27.2859 & 27.8811 & 27.5708 & 27.4601 \\
\hline
\end{tabular}




\section{Compound 26}

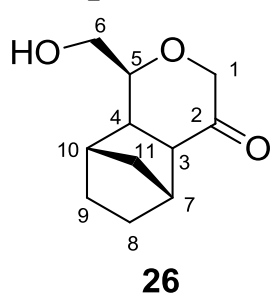

\begin{tabular}{|c|c|c|c|c|c|c|c|c|c|c|c|c|c|}
\hline & & \multicolumn{12}{|c|}{ B3LYP } \\
\hline & \multirow[b]{2}{*}{ Exp } & \multicolumn{6}{|c|}{ Gas phase } & \multicolumn{6}{|c|}{ PCM } \\
\hline & & 6-31G* & $6-31 G * *$ & $6-31+G * *$ & 6-311G* & $6-311 G * *$ & $6-311+\mathrm{G} * *$ & $6-31 G *$ & $6-31 G * *$ & $6-31+G * *$ & 6-311G* & $6-311 G * *$ & $6-311+G * *$ \\
\hline \multicolumn{14}{|l|}{${ }^{13} \mathrm{C}$} \\
\hline 1 & 74.8 & 117.2048 & 118.3681 & 115.8910 & 105.4648 & 104.4894 & 104.0078 & 117.0959 & 118.2448 & 115.7207 & 105.2832 & 104.3089 & 103.7162 \\
\hline 2 & 213.4 & -15.0557 & -14.7413 & -17.1883 & -37.0711 & -36.8111 & -39.9649 & -18.2106 & -17.9038 & -21.0726 & -40.7844 & -40.5351 & -44.3614 \\
\hline 3 & 50.2 & 139.0827 & 139.7863 & 138.4873 & 128.1464 & 127.8440 & 126.7978 & 138.7109 & 139.4045 & 137.9707 & 127.6748 & 127.3633 & 126.1434 \\
\hline 4 & 39.9 & 148.0425 & 148.8052 & 147.9599 & 138.5736 & 138.3311 & 137.3904 & 147.7763 & 148.5313 & 147.7805 & 138.2801 & 138.0601 & 137.1926 \\
\hline 5 & 79.3 & 112.0837 & 112.8089 & 111.2883 & 100.5757 & 100.1358 & 99.2570 & 112.0551 & 112.7742 & 111.2784 & 100.5498 & 100.1069 & 99.2175 \\
\hline 6 & 65.0 & 125.8641 & 127.1190 & 125.2327 & 115.1716 & 114.7212 & 114.0811 & 125.9389 & 127.1855 & 125.1151 & 115.0669 & 114.6078 & 113.9399 \\
\hline 7 & 39.2 & 148.2356 & 149.1840 & 148.3743 & 138.4832 & 138.1567 & 138.0465 & 148.3636 & 149.3169 & 148.5143 & 138.6395 & 138.3196 & 138.1836 \\
\hline 8 & 24.5 & 163.1591 & 164.5965 & 164.4715 & 155.2946 & 155.0615 & 154.7472 & 163.1826 & 164.6186 & 164.5084 & 155.3651 & 155.1309 & 154.7571 \\
\hline 9 & 22.8 & 165.2237 & 166.6882 & 166.6927 & 157.7744 & 157.5746 & 157.1877 & 165.3600 & 166.8199 & 166.8217 & 157.9410 & 157.7275 & 157.3205 \\
\hline 10 & 38.7 & 148.8254 & 149.7783 & 148.4663 & 139.1392 & 138.8350 & 138.4690 & 148.8777 & 149.8322 & 148.5657 & 139.2006 & 138.9000 & 138.5454 \\
\hline 11 & 39.3 & 149.9099 & 151.1981 & 150.7890 & 141.1877 & 140.7909 & 140.4724 & 150.1318 & 151.4091 & 151.0454 & 141.4729 & 141.0536 & 140.7733 \\
\hline \multicolumn{14}{|l|}{${ }^{1} \mathrm{H}$} \\
\hline 1 & 4.25 & 28.1409 & 27.6214 & 27.3835 & 27.9954 & 27.6634 & 27.5970 & 28.0676 & 27.5445 & 27.3040 & 27.9188 & 27.5876 & 27.5192 \\
\hline 1 & 3.80 & 28.4487 & 27.9735 & 27.8314 & 28.2983 & 28.0613 & 28.0313 & 28.3793 & 27.8990 & 27.7430 & 28.2188 & 27.9796 & 27.9421 \\
\hline 3 & 2.65 & 29.6034 & 29.1948 & 29.0396 & 29.6583 & 29.3985 & 29.2911 & 29.4791 & 29.0670 & 28.8905 & 29.5298 & 29.2707 & 29.1443 \\
\hline 4 & 2.37 & 29.6742 & 29.2567 & 29.3057 & 29.6961 & 29.4744 & 29.5111 & 29.7026 & 29.2879 & 29.2940 & 29.7266 & 29.5010 & 29.5029 \\
\hline 5 & 3.43 & 28.9366 & 28.5162 & 28.3279 & 28.9965 & 28.7872 & 28.6479 & 28.8192 & 28.3987 & 28.1976 & 28.8700 & 28.6641 & 28.5188 \\
\hline 6endo & 3.61 & 28.7007 & 28.2454 & 28.1026 & 28.6059 & 28.3807 & 28.3544 & 28.6565 & 28.2001 & 28.0481 & 28.5507 & 28.3240 & 28.2917 \\
\hline $6 e x o$ & 3.80 & 28.4404 & 27.9672 & 27.8561 & 28.3251 & 28.0878 & 28.1311 & 28.4097 & 27.9368 & 27.7970 & 28.3020 & 28.0569 & 28.0767 \\
\hline 7 & 2.75 & 29.5576 & 29.0476 & 28.9321 & 29.5810 & 29.2595 & 29.1920 & 29.6001 & 29.0917 & 28.9742 & 29.6191 & 29.3002 & 29.2297 \\
\hline 8 & 1.25 & 30.7307 & 30.3028 & 30.2401 & 30.7562 & 30.5347 & 30.4805 & 30.7100 & 30.2786 & 30.2069 & 30.7332 & 30.5068 & 30.4473 \\
\hline 8 & 1.46 & 30.6816 & 30.1983 & 30.1539 & 30.7340 & 30.4492 & 30.4270 & 30.7692 & 30.2900 & 30.2499 & 30.8199 & 30.5382 & 30.5180 \\
\hline 9 & 1.25 & 30.8218 & 30.3972 & 30.3239 & 30.8451 & 30.6249 & 30.5486 & 30.7758 & 30.3513 & 30.2754 & 30.8023 & 30.5833 & 30.5044 \\
\hline 9 & 1.46 & 30.7619 & 30.2749 & 30.1918 & 30.8394 & 30.5623 & 30.4675 & 30.7078 & 30.2198 & 30.1449 & 30.7851 & 30.5083 & 30.4186 \\
\hline 10 & 2.29 & 30.0589 & 29.5682 & 29.4740 & 30.0928 & 29.8085 & 29.7483 & 30.0050 & 29.5122 & 29.4026 & 30.0359 & 29.7489 & 29.6784 \\
\hline $11 a$ & 1.42 & 30.7621 & 30.3490 & 30.2478 & 30.7922 & 30.5821 & 30.5397 & 30.7225 & 30.3077 & 30.2023 & 30.7519 & 30.5395 & 30.4938 \\
\hline $11 b$ & 1.42 & 30.8604 & 30.4325 & 30.3530 & 30.8201 & 30.6021 & 30.5795 & 30.8086 & 30.3776 & 30.2848 & 30.7706 & 30.5486 & 30.5147 \\
\hline
\end{tabular}

\begin{tabular}{|c|c|c|c|c|c|c|c|c|c|c|c|c|c|}
\hline & \multirow[b]{3}{*}{ Exp } & \multicolumn{12}{|c|}{ mPW1PW91 } \\
\hline & & \multicolumn{6}{|c|}{ Gas phase } & \multicolumn{6}{|c|}{ PCM } \\
\hline & & 6-31G* & $6-31 \mathrm{G} * *$ & $6-31+G * *$ & 6-311G* & $6-311 G * *$ & $6-311+G^{* *}$ & $6-31 G *$ & $6-31 G * *$ & $6-31+G * *$ & $6-311 G *$ & $6-311 G * *$ & $6-311+G * *$ \\
\hline \multicolumn{14}{|c|}{${ }^{13} \mathrm{C}$} \\
\hline 1 & 74.8 & 121.0528 & 122.1499 & 120.0525 & 110.9869 & 110.0485 & 109.6114 & 120.9269 & 122.0098 & 119.8684 & 110.7711 & 109.8341 & 109.3030 \\
\hline 2 & 213.4 & -12.7617 & -12.4526 & -14.2472 & -31.5780 & -31.3077 & -34.1266 & -15.9917 & -15.6917 & -18.1312 & -35.2948 & -35.0360 & -38.4482 \\
\hline
\end{tabular}




\begin{tabular}{|c|c|c|c|c|c|c|c|c|c|c|c|c|c|}
\hline 3 & 50.2 & 143.7570 & 144.3915 & 143.2994 & 133.8035 & 133.4900 & 132.5375 & 143.3662 & 143.9911 & 142.7749 & 133.3161 & 132.9941 & 131.8871 \\
\hline 4 & 39.9 & 153.1844 & 153.8681 & 153.1039 & 144.5165 & 144.2641 & 143.4480 & 152.9265 & 153.6021 & 152.9121 & 144.2363 & 144.0050 & 143.2362 \\
\hline 5 & 79.3 & 116.8661 & 117.5403 & 116.3699 & 107.0704 & 106.6461 & 105.8010 & 116.8241 & 117.4931 & 116.3450 & 107.0259 & 106.5977 & 105.7388 \\
\hline 6 & 65.0 & 30.1815 & 131.3691 & 130.0182 & 121.1059 & 120.6593 & 120.0783 & 130.2478 & 131.4286 & 129.9151 & 120.9934 & 120.5395 & 119.9230 \\
\hline 7 & 39.2 & 53.0280 & 53.8952 & 153.0341 & 144.0896 & 143.7472 & 143.6029 & 153.1772 & 154.0488 & 153.2023 & 144.2626 & 143.9259 & 143.7587 \\
\hline 8 & 24.5 & 67.7807 & 69.0908 & 168.7833 & 160.4138 & 160.1639 & 159.8408 & 167.8357 & 169.1436 & 168.8556 & 160.4993 & 160.2474 & 159.8757 \\
\hline 9 & 22.8 & 169.8581 & 171.1959 & 171.0226 & 162.8827 & 162.6606 & 162.2735 & 170.0185 & 171.3519 & 171.1783 & 163.0569 & 162.8222 & 162.4177 \\
\hline 10 & 38.7 & 153.6247 & 154.4978 & 153.2485 & 144.7445 & 144.4229 & 144.0260 & 153.6888 & 154.5635 & 153.3542 & 144.8119 & 144.4934 & 144.1046 \\
\hline $\begin{array}{l}11 \\
{ }^{1} \mathbf{H}\end{array}$ & 39.3 & 154.4281 & 155.5902 & 154.9368 & 146.4273 & 146.0119 & 145.7016 & 154.6676 & 155.8196 & 155.2204 & 146.7083 & 146.2723 & 145.9928 \\
\hline 1 & 4.25 & 28.0578 & 27.5335 & 27.3316 & 27.9409 & 27.5902 & 27.5370 & 27.9785 & 27.4501 & 27.2463 & 27.8587 & 27.5087 & 27.4540 \\
\hline 1 & 3.80 & 28.3838 & 27.9102 & 27.7777 & 28.2268 & 27.9849 & 27.9610 & 28.3082 & 27.8290 & 27.6845 & 28.1423 & 27.8981 & 27.8680 \\
\hline 3 & 2.65 & 29.5726 & 29.1509 & 28.9931 & 29.5632 & 29.3019 & 29.2022 & 29.4468 & 29.0210 & 28.8455 & 29.4346 & 29.1737 & 29.0567 \\
\hline 4 & 2.37 & 29.6193 & 29.1986 & 29.2075 & 29.5808 & 29.3600 & 29.3655 & 29.6456 & 29.2280 & 29.2004 & 29.6095 & 29.3842 & 29.3621 \\
\hline 5 & 3.43 & 28.8737 & 28.4586 & 28.2598 & 28.8785 & 28.6779 & 28.5454 & 28.7534 & 28.3379 & 28.1289 & 28.7520 & 28.5551 & 28.4166 \\
\hline 6endo & 3.61 & 28.6468 & 28.1928 & 28.0631 & 28.5508 & 28.3153 & 28.2940 & 28.5948 & 28.1397 & 28.0015 & 28.4894 & 28.2531 & 28.2257 \\
\hline 6exo & 3.80 & 28.3884 & 27.9153 & 27.8062 & 28.2814 & 28.0314 & 28.0658 & 28.3510 & 27.8779 & 27.7445 & 28.2532 & 27.9942 & 28.0096 \\
\hline 7 & 2.75 & 29.4710 & 28.9570 & 28.8578 & 29.4721 & 29.1430 & 29.0807 & 29.5108 & 28.9984 & 28.8975 & 29.5082 & 29.1819 & 29.1162 \\
\hline 8 & 1.25 & 30.6770 & 30.2493 & 30.1917 & 30.6686 & 30.4452 & 30.3880 & 30.6527 & 30.2211 & 30.1555 & 30.6423 & 30.4139 & 30.3520 \\
\hline 8 & 1.46 & 30.6453 & 30.1605 & 30.1082 & 30.6548 & 30.3652 & 30.3409 & 30.7368 & 30.2565 & 30.2073 & 30.7432 & 30.4573 & 30.4343 \\
\hline 9 & 1.25 & 30.7715 & 30.3466 & 30.2726 & 30.7583 & 30.5353 & 30.4539 & 30.7200 & 30.2949 & 30.2196 & 30.7110 & 30.4888 & 30.4053 \\
\hline 9 & 1.46 & 30.7383 & 30.2528 & 30.1586 & 30.7640 & 30.4839 & 30.3902 & 30.6818 & 30.1954 & 30.1093 & 30.7084 & 30.4285 & 30.3389 \\
\hline 10 & 2.29 & 29.9972 & 29.5046 & 29.4067 & 29.9986 & 29.7051 & 29.6398 & 29.9374 & 29.4422 & 29.3318 & 29.9380 & 29.6413 & 29.5677 \\
\hline $11 a$ & 1.42 & 30.6942 & 30.2874 & 30.1948 & 30.6945 & 30.4847 & 30.4465 & 30.6502 & 30.2411 & 30.1457 & 30.6505 & 30.4381 & 30.3973 \\
\hline $11 \mathrm{~b}$ & 1.42 & 30.7979 & 30.3750 & 30.3141 & 30.7233 & 30.5096 & 30.4905 & 30.7418 & 30.3156 & 30.2429 & 30.6704 & 30.4527 & 30.4235 \\
\hline
\end{tabular}

\section{Compound 27}

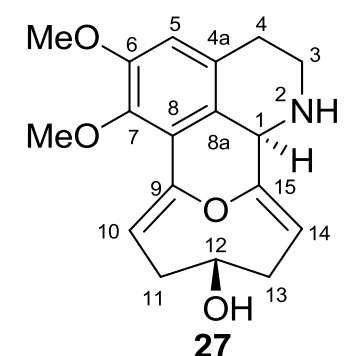

\begin{tabular}{|c|c|c|c|c|c|c|c|c|c|c|c|c|c|}
\hline & \multirow[b]{3}{*}{ Exp } & \multicolumn{12}{|c|}{ B3LYP } \\
\hline & & \multicolumn{6}{|c|}{ Gas phase } & \multicolumn{6}{|c|}{ PCM } \\
\hline & & 6-31G* & $6-31 G * *$ & $6-31+\mathrm{G} * *$ & 6-311G* & $6-311 G * *$ & $6-311+G * *$ & 6-31G* & $6-31 G * *$ & $6-31+G * *$ & 6-311G* & $6-311 G * *$ & $6-311+G * *$ \\
\hline \multicolumn{14}{|l|}{${ }^{13} \mathrm{C}$} \\
\hline 1 & 55.2 & 133.2456 & 133.6259 & 132.7200 & 122.4085 & 122.1342 & 122.0953 & 133.4463 & 133.8228 & 132.8813 & 122.6222 & 122.3453 & 122.2837 \\
\hline $3^{*}$ & 43.3 & 144.8846 & 146.0580 & 144.7869 & 134.8374 & 134.5363 & 133.9261 & 145.2168 & 146.3854 & 145.2121 & 135.2046 & 134.8972 & 134.1902 \\
\hline $4 *$ & 27.4 & 161.3436 & 162.4395 & 162.1135 & 152.6639 & 152.3271 & 151.1372 & 161.5697 & 162.6569 & 162.4262 & 152.9614 & 152.5977 & 151.4484 \\
\hline $4 a$ & 129.6 & 65.2873 & 65.3610 & 64.2154 & 47.3898 & 47.3570 & 46.8636 & 64.0223 & 64.1087 & 62.8255 & 46.0247 & 46.0292 & 45.3649 \\
\hline 5 & 112.2 & 82.8191 & 83.5690 & 85.0262 & 67.6649 & 67.0788 & 68.1406 & 82.8584 & 83.6127 & 84.4850 & 68.1108 & 67.6226 & 67.6864 \\
\hline 6 & 152.0 & 43.5706 & 43.4081 & 41.9745 & 22.7378 & 22.6858 & 22.4968 & 43.6017 & 43.4291 & 42.0469 & 22.7852 & 22.7467 & 22.5323 \\
\hline 7 & 143.1 & 52.3745 & 52.3542 & 51.5801 & 32.3273 & 32.3163 & 33.2296 & 53.3567 & 53.3384 & 52.3903 & 33.4692 & 33.4882 & 34.2428 \\
\hline
\end{tabular}




\begin{tabular}{|c|c|c|c|c|c|c|c|c|c|c|c|c|c|}
\hline 8 & 123.6 & 68.7253 & 68.7636 & 69.2962 & 51.7922 & 51.7645 & 51.0564 & 69.6136 & 69.6529 & 70.2193 & 52.7072 & 52.6826 & 52.0039 \\
\hline $8 a$ & 128.6 & 64.5602 & 64.5180 & 63.2966 & 46.1190 & 46.0932 & 44.8347 & 65.3217 & 65.2892 & 64.0707 & 47.1414 & 47.1444 & 45.7589 \\
\hline 9 & 155.5 & 37.5163 & 37.3490 & 37.1954 & 17.4873 & 17.4256 & 16.0323 & 37.0750 & 36.9073 & 36.7582 & 16.9717 & 16.9298 & 15.5673 \\
\hline 10 & 112.6 & 76.8208 & 77.7232 & 77.2610 & 60.5495 & 59.8972 & 59.6902 & 77.6899 & 78.5917 & 78.0483 & 61.4035 & 60.7129 & 60.4815 \\
\hline $11^{*}$ & 34.4 & 153.2161 & 154.5544 & 154.8319 & 144.7124 & 144.3423 & 144.1061 & 153.5016 & 154.8340 & 155.0985 & 144.9968 & 144.6122 & 144.3802 \\
\hline 12 & 69.4 & 119.1401 & 119.8483 & 117.6638 & 106.6049 & 106.3079 & 106.3856 & 119.3414 & 120.0554 & 117.8155 & 106.7274 & 106.4455 & 106.5427 \\
\hline $13^{*}$ & 34.6 & 152.6191 & 153.9415 & 153.3764 & 144.0997 & 143.8192 & 143.5452 & 152.9269 & 154.2429 & 153.6435 & 144.4004 & 144.1073 & 143.8458 \\
\hline 14 & 103.8 & 87.1791 & 88.1760 & 88.5028 & 72.4812 & 72.1119 & 71.7475 & 87.5636 & 88.5571 & 88.8193 & 72.8090 & 72.4174 & 72.0062 \\
\hline 15 & 160.5 & 33.8530 & 33.6690 & 31.9880 & 12.3022 & 12.2089 & 10.2622 & 33.2854 & 33.0983 & 31.4143 & 11.6656 & 11.5882 & 9.6346 \\
\hline C6-OMe & 56.0 & 136.4733 & 138.2599 & 139.1612 & 127.5910 & 126.8453 & 126.5797 & 136.7916 & 138.5646 & 139.1749 & 127.9636 & 127.2283 & 126.6256 \\
\hline $\begin{array}{c}\text { C7-OMe } \\
{ }^{1} \mathbf{H}\end{array}$ & 60.6 & & 134.8031 & 133.6990 & 124.2467 & 123.5922 & & & & & & & \\
\hline 1 & 4.57 & 27.7520 & 27.3450 & 27.0995 & 27.6488 & 27.3913 & 27.2968 & 27.7298 & 27.3179 & 27.0381 & 27.6056 & 27.3437 & 27.2340 \\
\hline $3^{*}$ & 3.23 & 29.1488 & 28.7437 & 28.4269 & 28.9728 & 28.7821 & 28.6513 & 29.1273 & 28.7195 & 28.3778 & 28.9417 & 28.7501 & 28.6078 \\
\hline $3 * *$ & 3.38 & 29.0334 & 28.5738 & 28.2426 & 28.7934 & 28.5572 & 28.4964 & 28.9956 & 28.5315 & 28.1926 & 28.7537 & 28.5099 & 28.4513 \\
\hline $4^{*}$ & 2.77 & 29.7371 & 29.2697 & 29.0526 & 29.5778 & 29.3034 & 29.2921 & 29.6437 & 29.1732 & 28.9475 & 29.4883 & 29.2123 & 29.1882 \\
\hline $4 * *$ & 2.85 & 29.6529 & 29.1733 & 28.9893 & 29.5173 & 29.2428 & 29.2274 & 29.5437 & 29.0651 & 28.8839 & 29.4166 & 29.1478 & 29.1312 \\
\hline 5 & 6.60 & 26.1497 & 25.4426 & 25.2020 & 25.9887 & 25.5271 & 25.5276 & 25.9694 & 25.2576 & 24.9786 & 25.8206 & 25.3676 & 25.3191 \\
\hline 10 & 5.80 & 26.3143 & 25.5796 & 25.3252 & 26.2242 & 25.7281 & 25.6091 & 26.3582 & 25.6241 & 25.3524 & 26.2514 & 25.7563 & 25.6374 \\
\hline $11^{*}$ & 2.51 & 29.8301 & 29.3756 & 29.2200 & 29.6932 & 29.4601 & 29.3636 & 29.7969 & 29.3414 & 29.1744 & 29.6494 & 29.4151 & 29.3144 \\
\hline $11^{* *}$ & 3.10 & 29.1621 & 28.6799 & 28.5412 & 29.0571 & 28.7761 & 28.7295 & 29.1490 & 28.6672 & 28.5142 & 29.0385 & 28.7601 & 28.7030 \\
\hline 12 & 4.36 & 28.2613 & 27.7946 & 27.6297 & 28.2041 & 27.9650 & 27.8509 & 28.2205 & 27.7513 & 27.5645 & 28.1434 & 27.8972 & 27.7749 \\
\hline $13^{*}$ & 2.47 & 29.8974 & 29.4508 & 29.2581 & 29.7307 & 29.5124 & 29.3866 & 29.8691 & 29.4214 & 29.2135 & 29.6911 & 29.4725 & 29.3359 \\
\hline $13^{* *}$ & 2.87 & 29.3771 & 28.9046 & 28.6823 & 29.2638 & 28.9902 & 28.8827 & 29.3586 & 28.8849 & 28.6397 & 29.2387 & 28.9648 & 28.8426 \\
\hline 14 & 5.03 & 27.2456 & 26.5635 & 26.4501 & 27.2012 & 26.7519 & 26.7442 & 27.2289 & 26.5455 & 26.4181 & 27.1722 & 26.7234 & 26.7084 \\
\hline C6-OM e & 3.85 & 28.5185 & 28.0104 & 27.7886 & 28.4770 & 28.1664 & 28.1448 & 28.4376 & 27.9313 & 27.6911 & 28.3990 & 28.0934 & 28.0557 \\
\hline C7-OMe & 3.82 & 28.5361 & 28.0351 & 27.9162 & 28.5129 & 28.2079 & 28.1536 & 28.4946 & 27.9948 & 27.8677 & 28.4659 & 28.1641 & 28.1038 \\
\hline
\end{tabular}

\begin{tabular}{|c|c|c|c|c|c|c|c|c|c|c|c|c|c|}
\hline & \multirow[b]{3}{*}{ Exp } & \multicolumn{12}{|c|}{ mPW1PW91 } \\
\hline & & \multicolumn{6}{|c|}{ Gas phase } & \multicolumn{6}{|c|}{ PCM } \\
\hline & & 6-31G* & 6-31G** & $6-31+G * *$ & 6-311G* & $6-311 \mathrm{G} * *$ & $6-311+G * *$ & 6-31G* & 6-31G** & $6-31+G^{* *}$ & 6-311G* & 6-311G** & $6-311+G * *$ \\
\hline \multicolumn{14}{|l|}{${ }^{13} \mathrm{C}$} \\
\hline 1 & 55.2 & 137.7487 & 138.0996 & 137.4877 & 128.4077 & 128.1147 & 127.9116 & 137.9543 & 138.3015 & 137.6670 & 128.6102 & 128.3153 & 128.0887 \\
\hline $3^{*}$ & 43.3 & 149.7244 & 150.8121 & 149.9388 & 140.7988 & 140.4730 & 139.8164 & 150.0752 & 151.1588 & 150.3818 & 141.1622 & 140.8311 & 140.1075 \\
\hline $4^{*}$ & 27.4 & 165.9653 & 166.9698 & 166.7183 & 158.2166 & 157.8576 & 156.7428 & 166.1904 & 167.1872 & 167.0045 & 158.5043 & 158.1197 & 157.0348 \\
\hline $4 a$ & 129.6 & 68.6274 & 68.6724 & 67.4506 & 53.0657 & 53.0046 & 52.3846 & 67.2617 & 67.3193 & 65.9847 & 51.5943 & 51.5727 & 50.8372 \\
\hline 5 & 112.2 & 85.8029 & 86.5260 & 87.7544 & 72.8152 & 72.2370 & 72.7529 & 85.5706 & 86.3026 & 87.0177 & 72.7988 & 72.3281 & 72.0798 \\
\hline 6 & 152.0 & 46.7179 & 46.5312 & 45.6053 & 28.7206 & 28.6639 & 28.5147 & 46.7089 & 46.5146 & 45.6567 & 28.7252 & 28.6801 & 28.5311 \\
\hline 7 & 143.1 & 55.7179 & 55.6931 & 55.0813 & 38.3742 & 38.3719 & 39.1707 & 56.6542 & 56.6340 & 55.8680 & 39.4173 & 39.4502 & 40.1266 \\
\hline 8 & 123.6 & 71.6082 & 71.6478 & 72.2106 & 57.0047 & 56.9809 & 56.2380 & 72.5511 & 72.5924 & 73.1686 & 57.9687 & 57.9490 & 57.2345 \\
\hline $8 a$ & 128.6 & 68.2286 & 68.1765 & 67.4053 & 52.1264 & 52.0912 & 50.6867 & 68.9593 & 68.9193 & 68.1628 & 53.0757 & 53.0752 & 51.5496 \\
\hline 9 & 155.5 & 41.1285 & 40.9464 & 41.3876 & 23.7004 & 23.6455 & 22.2662 & 40.7615 & 40.5782 & 41.0150 & 23.2653 & 23.2293 & 21.8717 \\
\hline 10 & 112.6 & 79.5046 & 80.3502 & 79.8938 & 65.0972 & 64.4322 & 64.2056 & 80.2443 & 81.0890 & 80.5507 & 65.8034 & 65.1005 & 64.8597 \\
\hline $11^{*}$ & 34.4 & 157.7642 & 158.9910 & 159.1034 & 150.0952 & 149.7315 & 149.4311 & 158.0775 & 159.2984 & 159.3935 & 150.3919 & 150.0142 & 149.7171 \\
\hline 12 & 69.4 & 123.9153 & 124.5760 & 122.8151 & 113.2196 & 112.9140 & 112.8100 & 124.1057 & 124.7722 & 122.9626 & 113.3143 & 113.0250 & 112.9421 \\
\hline
\end{tabular}




\begin{tabular}{|c|c|c|c|c|c|c|c|c|c|c|c|c|c|}
\hline $13^{*}$ & 34.6 & 157.2587 & 158.4714 & 157.7953 & 149.5788 & 149.2990 & 148.9736 & 157.5932 & 158.7996 & 158.0985 & 149.8884 & 149.5967 & 149.2794 \\
\hline 14 & 103.8 & 89.8455 & 90.7974 & 91.5018 & 76.8072 & 76.4268 & 76.0783 & 90.1546 & 91.1026 & 91.7709 & 77.0495 & 76.6455 & 76.2791 \\
\hline 15 & 160.5 & 37.4017 & 37.1890 & & 18.6031 & & & & 36.6712 & 35.3531 & 18.0230 & 17.9444 & 16.1014 \\
\hline C6-OMe & 56.0 & 140.2696 & 141.9840 & 142.6011 & 132.7471 & 132.0494 & 131.7544 & 140.4789 & 142.1828 & 142.5163 & 132.9455 & 132.2642 & 131.7126 \\
\hline $\begin{array}{c}\text { C7-OMe } \\
{ }^{1} \mathbf{H}\end{array}$ & 60.6 & 136.5057 & 138.1843 & 137.6789 & 128.9436 & 128.3337 & 127.6025 & 136.5090 & 138.1723 & 137.3997 & 128.7377 & 128.1191 & 127.3677 \\
\hline 1 & 4.57 & 27.7141 & 27.2892 & 27.0537 & 27.5576 & 27.3083 & 27.2174 & 27.6888 & 27.2589 & 26.9906 & 27.5119 & 27.2589 & 27.1518 \\
\hline $3^{*}$ & 3.23 & 29.0783 & 28.6753 & 28.3737 & 28.8880 & 28.7030 & 28.5747 & 29.0517 & 28.6460 & 28.3208 & 28.8521 & 28.6663 & 28.5273 \\
\hline $3^{* *}$ & 3.38 & 28.9561 & 28.4996 & 28.2315 & 28.7369 & 28.4852 & 28.4420 & 28.9053 & 28.4439 & 28.1704 & 28.6864 & 28.4265 & 28.3857 \\
\hline $4^{*}$ & 2.77 & 29.6850 & 29.2144 & 29.0093 & 29.5034 & 29.2271 & 29.2118 & 29.5765 & 29.1021 & 28.8905 & 29.4002 & 29.1223 & 29.0961 \\
\hline $4^{* *}$ & 2.85 & 29.5969 & 29.1143 & 28.9400 & 29.4369 & 29.1620 & 29.1495 & 29.4783 & 28.9964 & 28.8273 & 29.3282 & 29.0593 & 29.0466 \\
\hline 5 & 6.60 & 25.9616 & 25.2578 & 24.9547 & 25.8322 & 25.3514 & 25.3147 & 25.7543 & 25.0455 & 24.7103 & 25.6337 & 25.1616 & 25.0859 \\
\hline 10 & 5.80 & 26.1734 & 25.4348 & 25.1960 & 26.0942 & 25.5749 & 25.4657 & 26.2079 & 25.4698 & 25.2169 & 26.1137 & 25.5961 & 25.4864 \\
\hline $11^{*}$ & 2.51 & 29.7314 & 29.2794 & 29.1266 & 29.5929 & 29.3500 & 29.2648 & 29.6861 & 29.2327 & 29.0722 & 29.5389 & 29.2949 & 29.2069 \\
\hline $11^{* *}$ & 3.10 & 29.1098 & 28.6300 & 28.5061 & 28.9561 & 28.6816 & 28.6457 & 29.0909 & 28.6112 & 28.4742 & 28.9329 & 28.6609 & 28.6145 \\
\hline 12 & 4.36 & 28.1892 & 27.7227 & 27.5333 & 28.1253 & 27.8778 & 27.7640 & 28.1375 & 27.6679 & 27.4602 & 28.0571 & 27.8019 & 27.6816 \\
\hline $13^{*}$ & 2.47 & 29.8001 & 29.3552 & 29.1746 & 29.6346 & 29.4062 & 29.2906 & 29.7608 & 29.3143 & 29.1218 & 29.5860 & 29.3566 & 29.2337 \\
\hline $13^{* *}$ & 2.87 & 29.3368 & 28.8635 & 28.6592 & 29.1727 & 28.9027 & 28.7959 & 29.3120 & 28.8374 & 28.6119 & 29.1425 & 28.8726 & 28.7508 \\
\hline 14 & 5.03 & 27.0794 & 26.3942 & 26.2780 & 27.0593 & 26.5886 & 26.5796 & 27.0500 & 26.3632 & 26.2357 & 27.0188 & 26.5493 & 26.5349 \\
\hline $\mathrm{C} 6-\mathrm{OM} \mathrm{e}$ & e 3.85 & 28.4618 & 27.9578 & 27.7562 & 28.4248 & 28.1051 & 28.0903 & 28.3656 & 27.8631 & 27.6487 & 28.3321 & 28.0171 & 27.9915 \\
\hline C7-OMe & 3.82 & 28.4822 & 27.9811 & 27.8691 & 28.4488 & 28.1332 & 28.0934 & 28.4326 & 27.9325 & 27.8138 & 28.3953 & 28.0828 & 28.0386 \\
\hline
\end{tabular}

\section{Compound 28}

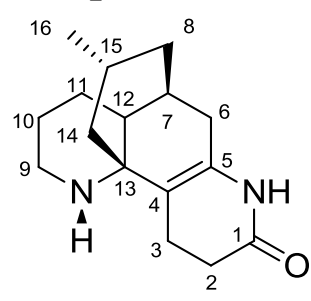

28

\begin{tabular}{|c|c|c|c|c|c|c|c|c|c|c|c|c|c|}
\hline & \multirow[b]{3}{*}{ Exp } & \multicolumn{12}{|c|}{ B3LYP } \\
\hline & & \multicolumn{6}{|c|}{ Gas phase } & \multicolumn{6}{|c|}{ PCM } \\
\hline & & 6-31G* & 6-31G** & $6-31+\mathrm{G} * *$ & 6-311G* & $6-311 G * *$ & $6-311+G^{* *}$ & 6-31G* & $6-31 G * *$ & $6-31+G * *$ & 6-311G* & $6-311 G * *$ & $6-311+G * *$ \\
\hline \multicolumn{14}{|l|}{${ }^{13} \mathrm{C}$} \\
\hline 1 & 170.8 & 34.2651 & 34.1702 & 30.7297 & 14.6177 & 14.6075 & 10.8467 & 32.2382 & 32.1314 & 28.2151 & 12.2596 & 12.2327 & 7.9867 \\
\hline $2^{*}$ & 30.5 & 158.5231 & 159.8089 & 158.7786 & 149.3723 & 148.9573 & 148.2665 & 158.4024 & 159.6781 & 158.5776 & 149.2300 & 148.8080 & 147.9763 \\
\hline $3^{*}$ & 19.7 & 167.4876 & 168.8282 & 167.4861 & 159.5840 & 159.2489 & 158.9557 & 167.8311 & 169.1667 & 167.7742 & 159.9534 & 159.6078 & 159.3120 \\
\hline 4 & 110.6 & 76.7478 & 76.8579 & 75.8857 & 60.0727 & 60.1574 & 59.2758 & 76.0878 & 76.2034 & 75.0048 & 59.3569 & 59.4357 & 58.2842 \\
\hline 5 & 133.5 & 63.6993 & 63.9498 & 62.8485 & 44.9311 & 45.1795 & 44.8787 & 63.2728 & 63.5299 & 62.4680 & 44.4606 & 44.7312 & 44.5141 \\
\hline $6^{*}$ & 34.4 & 153.2877 & 154.4803 & 153.9905 & 144.1091 & 143.6696 & 143.5524 & 153.5844 & 154.7677 & 154.3099 & 144.4317 & 143.9805 & 143.8643 \\
\hline 7 & 32.7 & 153.3451 & 154.2733 & 152.7264 & 143.4248 & 143.1930 & 142.9349 & 153.4865 & 154.4175 & 152.9053 & 143.6248 & 143.3943 & 143.1406 \\
\hline $8^{*}$ & 35.5 & 152.8701 & 154.1368 & 152.6239 & 143.2767 & 143.0361 & 142.8531 & 153.0250 & 154.2863 & 152.8079 & 143.4978 & 143.2453 & 143.0731 \\
\hline $9 *$ & 39.9 & 147.8272 & 149.1500 & 148.3074 & 138.4739 & 138.2841 & 137.9124 & 148.0327 & 149.3515 & 148.4217 & 138.6419 & 138.4410 & 138.0537 \\
\hline $10^{*}$ & 22.4 & 161.9419 & 163.3051 & 162.1245 & 152.6552 & 152.4506 & 151.8881 & 161.9143 & 163.2778 & 162.0038 & 152.5758 & 152.3705 & 151.7536 \\
\hline 11 & 22.8 & 162.9772 & 164.3574 & 163.0243 & 153.7562 & 153.5009 & 153.1618 & 163.1849 & 164.5601 & 163.2532 & 154.0153 & 153.7491 & 153.4241 \\
\hline
\end{tabular}




\begin{tabular}{|c|c|c|c|c|c|c|c|c|c|c|c|c|c|}
\hline 12 & 40.1 & 146.3985 & 147.1914 & 145.8362 & 136.2388 & 136.1148 & 136.0603 & 146.2027 & 146.9934 & 145.5904 & 135.9592 & 135.8391 & 135.7688 \\
\hline 13 & 59.5 & 133.9495 & 134.2770 & 133.7031 & 123.8384 & 123.9076 & 123.5796 & 133.8860 & 134.2143 & 133.6032 & 123.7291 & 123.8004 & 123.4303 \\
\hline 14 & 32.2 & 154.5142 & 155.5966 & 154.9709 & 145.3007 & 144.9778 & 144.3287 & 154.7697 & 155.8431 & 155.3324 & 145.6187 & 145.2867 & 144.7222 \\
\hline 15 & 26.2 & 161.2705 & 162.2633 & 160.0231 & 150.9076 & 150.8845 & 150.8299 & 161.2900 & 162.2837 & 160.0442 & 150.9579 & 150.9330 & 150.8885 \\
\hline $\begin{array}{l}16 \\
{ }^{1} \mathrm{H}\end{array}$ & 21.7 & 166.6895 & 168.4759 & 169.0027 & 159.8791 & 159.8333 & 159.3262 & 166.9549 & 168.7349 & 169.3494 & 160.2572 & 160.1875 & 159.6917 \\
\hline $2^{*}$ & 2.42 & 30.1239 & 29.6464 & 29.4202 & 30.0303 & 29.7749 & 29.5955 & 30.1702 & 29.6865 & 29.4502 & 30.0770 & 29.8124 & 29.6231 \\
\hline $2^{* *}$ & 2.68 & 29.9651 & 29.5343 & 29.3700 & 29.9432 & 29.7279 & 29.6467 & 29.8530 & 29.4183 & 29.2284 & 29.8290 & 29.6136 & 29.5105 \\
\hline $3^{*}$ & 2.56 & 30.4160 & 29.9195 & 29.7792 & 30.3696 & 30.0463 & 29.9908 & 30.2627 & 29.7631 & 29.6051 & 30.2124 & 29.8893 & 29.8200 \\
\hline $3^{* *}$ & 2.75 & 29.7295 & 29.2533 & 28.9514 & 29.7244 & 29.4729 & 29.3323 & 29.7751 & 29.2977 & 28.9812 & 29.7694 & 29.5154 & 29.3615 \\
\hline $6^{*}$ & 1.84 & 30.3721 & 29.9605 & 29.8724 & 30.3171 & 30.0962 & 30.0877 & 30.2941 & 29.8803 & 29.7896 & 30.2381 & 30.0178 & 30.0086 \\
\hline $6 * *$ & 2.60 & 29.8401 & 29.3866 & 29.1894 & 29.7218 & 29.4753 & 29.4380 & 29.7606 & 29.3070 & 29.1076 & 29.6465 & 29.4033 & 29.3618 \\
\hline 7 & 2.09 & 30.3390 & 29.9303 & 29.8730 & 30.2913 & 30.1158 & 30.0990 & 30.2894 & 29.8779 & 29.8101 & 30.2382 & 30.0596 & 30.0373 \\
\hline $8^{*}$ & 1.34 & 30.7899 & 30.4214 & 30.3592 & 30.7566 & 30.6028 & 30.5919 & 30.7587 & 30.3891 & 30.3239 & 30.7243 & 30.5701 & 30.5585 \\
\hline $8^{* *}$ & 1.42 & 30.7508 & 30.3564 & 30.3273 & 30.7773 & 30.5944 & 30.6144 & 30.7328 & 30.3382 & 30.3069 & 30.7599 & 30.5772 & 30.5959 \\
\hline 9* & 3.02 & 29.5864 & 29.1351 & 28.9278 & 29.3997 & 29.1648 & 29.1375 & 29.5898 & 29.1374 & 28.9291 & 29.3990 & 29.1637 & 29.1358 \\
\hline $9^{* *}$ & 3.19 & 29.0951 & 28.6233 & 28.4704 & 29.0010 & 28.7761 & 28.7811 & 29.0664 & 28.5957 & 28.4569 & 28.9797 & 28.7549 & 28.7656 \\
\hline $10^{*}$ & 1.54 & 30.7112 & 30.2742 & 30.1154 & 30.6162 & 30.3713 & 30.3104 & 30.6710 & 30.2319 & 30.0652 & 30.5719 & 30.3253 & 30.2609 \\
\hline $10^{* *}$ & 1.82 & 30.4965 & 30.1305 & 30.0741 & 30.4637 & 30.3229 & 30.3550 & 30.5688 & 30.2038 & 30.1490 & 30.5325 & 30.3921 & 30.4233 \\
\hline 11 & 1.95 & 30.6661 & 30.2412 & 30.1695 & 30.6637 & 30.4448 & 30.4240 & 30.6282 & 30.2019 & 30.1257 & 30.6239 & 30.4046 & 30.3804 \\
\hline 12 & 2.06 & 30.2367 & 29.9312 & 29.8004 & 30.3460 & 30.2570 & 30.2081 & 30.3072 & 30.0043 & 29.8657 & 30.4121 & 30.3249 & 30.2663 \\
\hline 14 & 1.69 & 30.6761 & 30.2534 & 30.2062 & 30.7380 & 30.5086 & 30.5048 & 30.6211 & 30.1988 & 30.1515 & 30.6818 & 30.4546 & 30.4524 \\
\hline 15 & 1.72 & 30.1071 & 29.6818 & 29.7695 & 30.3107 & 30.1047 & 30.0980 & 30.1167 & 29.6935 & 29.7792 & 30.3205 & 30.1157 & 30.1094 \\
\hline 16 & 0.95 & 31.2381 & 30.8255 & 30.7541 & 31.2057 & 30.9926 & 30.9642 & 31.2377 & 30.8239 & 30.7552 & 31.2073 & 30.9937 & 30.9662 \\
\hline
\end{tabular}

\begin{tabular}{|c|c|c|c|c|c|c|c|c|c|c|c|c|c|}
\hline & \multirow[b]{3}{*}{ Exp } & \multicolumn{12}{|c|}{ mPW1PW91 } \\
\hline & & \multicolumn{6}{|c|}{ Gas phase } & \multicolumn{6}{|c|}{ PCM } \\
\hline & & 6-31G* & $6-31 G * *$ & $6-31+G^{* *}$ & 6-311G* & $6-311 G * *$ & $6-311+G^{* *}$ & 6-31G* & $6-31 G * *$ & $6-31+G * *$ & 6-311G* & $6-311 G * *$ & $6-311+G^{* *}$ \\
\hline \multicolumn{14}{|l|}{${ }^{13} \mathrm{C}$} \\
\hline 1 & 170.8 & 36.3563 & 36.2379 & 33.6289 & 19.3479 & 19.3193 & 15.9383 & 34.2810 & 34.1496 & 31.1156 & 16.9884 & 16.9423 & 13.1293 \\
\hline $2^{*}$ & 30.5 & 162.9973 & 164.1928 & 163.1892 & 154.8481 & 154.4351 & 153.6956 & 162.8777 & 164.0632 & 162.9942 & 154.6966 & 154.2761 & 153.4092 \\
\hline $3^{*}$ & 19.7 & 171.8697 & 173.1200 & 171.8588 & 164.8137 & 164.4761 & 164.1538 & 172.2377 & 173.4830 & 172.1641 & 165.1905 & 164.8432 & 164.5165 \\
\hline 4 & 110.6 & 80.8056 & 80.9091 & 80.2587 & 65.9003 & 65.9751 & 65.1921 & 80.1488 & 80.2587 & 79.4128 & 65.2067 & 65.2760 & 64.2482 \\
\hline 5 & 133.5 & 66.9353 & 67.1639 & 66.4774 & 50.2718 & 50.5031 & 50.0422 & 66.4980 & 66.7333 & 66.0740 & 49.7855 & 50.0402 & 49.6612 \\
\hline $6 *$ & 34.4 & 158.0996 & 159.1818 & 158.8611 & 149.8915 & 149.4436 & 149.2843 & 158.3905 & 159.4650 & 159.1721 & 150.1868 & 149.7294 & 149.5685 \\
\hline 7 & 32.7 & 158.7602 & 159.6039 & 158.2258 & 149.7389 & 149.4971 & 149.2019 & 158.9186 & 159.7652 & 158.4209 & 149.9445 & 149.7038 & 149.4110 \\
\hline $8^{*}$ & 35.5 & 158.2647 & 159.4007 & 157.7660 & 149.4336 & 149.1998 & 149.0143 & 158.4443 & 159.5748 & 157.9702 & 149.6619 & 149.4167 & 149.2417 \\
\hline $9 *$ & 39.9 & 152.5492 & 153.7676 & 153.1078 & 144.2271 & 144.0161 & 143.6759 & 152.7818 & 153.9963 & 153.2515 & 144.4079 & 144.1857 & 143.8328 \\
\hline $10 *$ & 22.4 & 166.9915 & 168.2492 & 167.1375 & 158.5254 & 158.3037 & 157.6905 & 167.0001 & 168.2575 & 167.0620 & 158.4773 & 158.2542 & 157.5875 \\
\hline 11 & 22.8 & 167.9701 & 169.2400 & 168.0907 & 159.5925 & 159.3233 & 158.9532 & 168.2051 & 169.4703 & 168.3421 & 159.8605 & 159.5812 & 159.2217 \\
\hline 12 & 40.1 & 152.2781 & 152.9915 & 151.6238 & 142.8921 & 142.7745 & 142.7011 & 152.0646 & 152.7763 & 151.3643 & 142.5965 & 142.4839 & 142.3931 \\
\hline 13 & 59.5 & 138.8741 & 139.1864 & 138.6739 & 129.9418 & 129.9974 & 129.6237 & 138.8127 & 139.1260 & 138.5802 & 129.8344 & 129.8928 & 129.4789 \\
\hline 14 & 32.2 & 159.5403 & 160.5136 & 159.8993 & 151.1067 & 150.8095 & 150.1684 & 159.8134 & 160.7786 & 160.2715 & 151.4203 & 151.1159 & 150.5586 \\
\hline 15 & 26.2 & 166.5565 & 167.4875 & 165.5595 & 157.0312 & 157.0127 & 156.8631 & 166.5681 & 167.5008 & 165.5716 & 157.0681 & 157.0489 & 156.9071 \\
\hline 16 & 21.7 & 171.0290 & 172.6908 & 172.9524 & 164.7300 & 164.6767 & 164.2834 & 171.3387 & 172.9934 & 173.3364 & 165.1192 & 165.0440 & 164.6616 \\
\hline
\end{tabular}




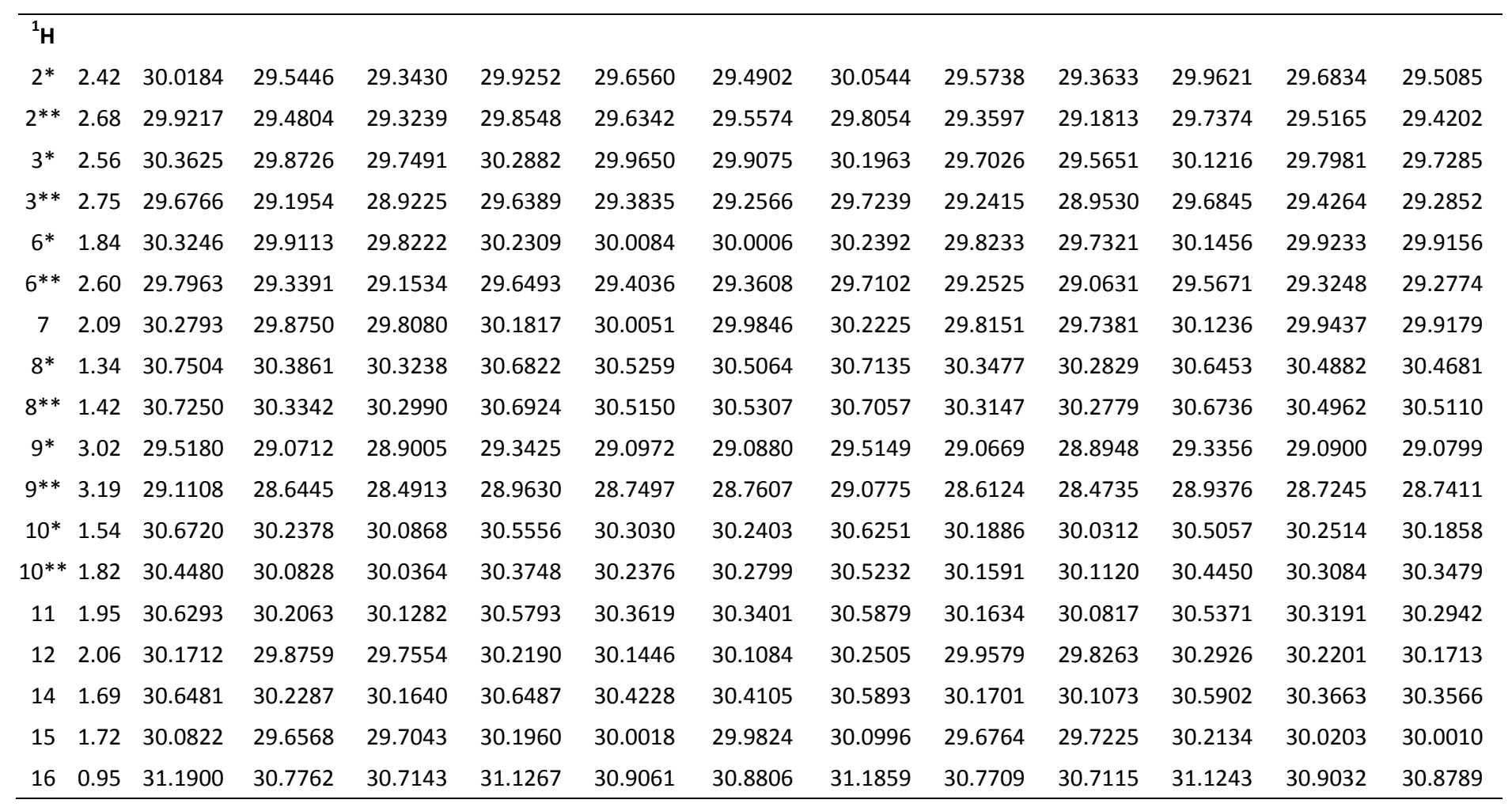

\section{Compound 29}

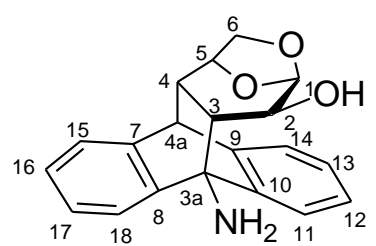

29

\begin{tabular}{|c|c|c|c|c|c|c|c|c|c|c|c|c|c|}
\hline & & \multicolumn{12}{|c|}{ B3LYP } \\
\hline & \multirow[b]{2}{*}{ Exp } & \multicolumn{6}{|c|}{ Gas phase } & \multicolumn{6}{|c|}{ PCM } \\
\hline & & 6-31G* & $6-31 G * *$ & $6-31+\mathrm{G} * *$ & 6-311G* & $6-311 G * *$ & $6-311+G * *$ & $6-31 G *$ & $6-31 G * *$ & $6-31+G * *$ & 6-311G* & $6-311 G * *$ & $6-311+G * *$ \\
\hline \multicolumn{14}{|l|}{${ }^{13} \mathrm{C}$} \\
\hline 1 & 100.0 & 88.7829 & 89.5546 & 88.2946 & 76.1258 & 75.5773 & 75.9193 & 89.0030 & 89.7703 & 88.4009 & 76.2052 & 75.6614 & 75.9522 \\
\hline 2 & 69.4 & 118.4402 & 119.2618 & 117.9474 & 106.9462 & 106.9037 & 106.5877 & 118.3817 & 119.2037 & 117.8356 & 106.7825 & 106.7373 & 106.4623 \\
\hline 3 & 46.6 & 140.5073 & 141.1846 & 140.9170 & 130.0259 & 129.8902 & 129.7975 & 140.8245 & 141.4899 & 141.2393 & 130.2890 & 130.1476 & 130.0328 \\
\hline $3 a$ & 62.4 & 125.7421 & 125.9217 & 124.9966 & 113.1664 & 113.3908 & 113.2818 & 125.6861 & 125.8642 & 124.9359 & 113.1538 & 113.3757 & 113.2583 \\
\hline 4 & 47.7 & 139.3095 & 140.1305 & 138.2909 & 128.6052 & 128.4136 & 127.8246 & 139.5045 & 140.3221 & 138.6358 & 128.8413 & 128.6468 & 128.0894 \\
\hline $4 a$ & 49.6 & 138.4041 & 139.0156 & 137.1689 & 125.9637 & 125.6707 & 125.5831 & 138.9916 & 139.5967 & 137.9058 & 126.8004 & 126.4876 & 126.4289 \\
\hline 5 & 76.6 & 112.2263 & 113.0186 & 112.1048 & 101.0723 & 100.5318 & 100.0237 & 112.3345 & 113.1243 & 112.1935 & 101.1803 & 100.6299 & 100.1024 \\
\hline $6 * *$ & 70.6 & 118.9737 & 120.3421 & 119.8720 & 108.6434 & 107.9143 & 107.8382 & 119.1875 & 120.5470 & 119.8804 & 108.7348 & 107.9797 & 107.8531 \\
\hline 7 & 144.6 & 49.6872 & 49.6688 & 49.8474 & 29.2200 & 29.0997 & 27.0029 & 49.6074 & 49.5861 & 49.7038 & 29.0863 & 28.9701 & 26.7393 \\
\hline 8 & 143.6 & 51.3213 & 51.0291 & 49.9726 & 30.9143 & 30.6457 & 28.1298 & 51.2782 & 50.9874 & 49.9741 & 30.8867 & 30.6342 & 28.1140 \\
\hline 9 & 143.1 & 53.1816 & 52.9989 & 51.0048 & 32.8631 & 32.6063 & 31.1933 & 52.6914 & 52.5133 & 50.4476 & 32.2859 & 32.0516 & 30.6115 \\
\hline 10 & 140.0 & 55.7088 & 55.6241 & 53.5335 & 35.4998 & 35.3679 & 33.4942 & 55.2264 & 55.1481 & 52.8933 & 34.9117 & 34.7961 & 32.7956 \\
\hline 11 & 126.4 & 70.1570 & 70.7592 & 70.2547 & 52.9339 & 52.0429 & 51.2293 & 70.2779 & 70.8786 & 70.1792 & 52.9487 & 52.0886 & 51.2504 \\
\hline 12 & 126.1 & 70.4169 & 71.0279 & 70.2603 & 53.1131 & 52.2237 & 51.2751 & 70.3894 & 71.0012 & 70.3341 & 52.9910 & 52.1319 & 51.3507 \\
\hline
\end{tabular}




\begin{tabular}{|c|c|c|c|c|c|c|c|c|c|c|c|c|c|}
\hline 13 & 126.0 & 70.4885 & 71.1572 & 70.5255 & 53.5010 & 52.5914 & 51.6564 & 70.5675 & 71.1819 & 70.4845 & 53.3503 & 52.4780 & 51.6426 \\
\hline 14 & 125.8 & 70.6494 & 71.2611 & 70.7252 & 53.7265 & 52.8261 & 51.9480 & 70.7267 & 71.3855 & 70.7864 & 53.7168 & 52.8428 & 52.0356 \\
\hline 15 & 124.7 & 70.7702 & 71.3988 & 70.8355 & 53.8042 & 52.9789 & 52.5396 & 70.8451 & 71.4697 & 71.0741 & 53.9164 & 53.0985 & 52.6581 \\
\hline 16 & 122.1 & 73.5113 & 74.1971 & 73.5031 & 57.2452 & 56.4331 & 56.2327 & 73.5296 & 74.2042 & 73.4597 & 57.1094 & 56.2983 & 55.8848 \\
\hline 17 & 121.5 & 74.1767 & 74.8840 & 75.3023 & 58.0345 & 57.2731 & 56.4443 & 73.8059 & 74.5016 & 74.8790 & 57.4228 & 56.6822 & 56.0981 \\
\hline $\begin{array}{l}18 \\
{ }^{1} \mathrm{H}\end{array}$ & 120.5 & 76.8366 & 77.4891 & 77.3622 & 61.0130 & 60.1048 & 59.2700 & 76.0893 & 76.7429 & 76.6152 & 59.9793 & 59.1193 & 58.2969 \\
\hline 1 & 5.05 & 27.1015 & 26.5799 & 26.5053 & 27.0257 & 26.7621 & 26.7760 & 27.1271 & 26.6021 & 26.5151 & 27.0285 & 26.7624 & 26.7761 \\
\hline 2 & 3.16 & 29.0466 & 28.5628 & 28.6615 & 29.1443 & 28.8694 & 28.9059 & 29.0672 & 28.5769 & 28.6473 & 29.1524 & 28.8668 & 28.8845 \\
\hline 3 & 2.02 & 30.1325 & 29.7839 & 29.5957 & 30.2095 & 30.0835 & 30.0052 & 30.0998 & 29.7473 & 29.5377 & 30.1683 & 30.0361 & 29.9462 \\
\hline 4 & 2.18 & 30.3673 & 29.9643 & 29.6610 & 30.3595 & 30.1526 & 29.9245 & 30.2489 & 29.8428 & 29.5098 & 30.2444 & 30.0350 & 29.7796 \\
\hline $4 a$ & 4.22 & 28.3980 & 27.9618 & 27.7805 & 28.2579 & 28.0548 & 27.8994 & 28.2111 & 27.7658 & 27.5478 & 28.0505 & 27.8410 & 27.6708 \\
\hline 5 & 4.68 & 27.9233 & 27.4100 & 27.2507 & 27.9394 & 27.6265 & 27.5581 & 27.7495 & 27.2288 & 27.0463 & 27.7557 & 27.4373 & 27.3542 \\
\hline 6exo & 3.80 & 28.4846 & 27.9652 & 27.7916 & 28.3702 & 28.1118 & 28.0368 & 28.4394 & 27.9222 & 27.7364 & 28.3207 & 28.0660 & 27.9817 \\
\hline Gendo & 3.71 & 28.6413 & 28.1453 & 27.9667 & 28.6472 & 28.3338 & 28.2045 & 28.5183 & 28.0195 & 27.8201 & 28.5240 & 28.2060 & 28.0602 \\
\hline 11 & 7.52 & 24.9830 & 24.3178 & 24.0917 & 24.8675 & 24.4468 & 24.3594 & 24.8725 & 24.2050 & 23.9606 & 24.7483 & 24.3226 & 24.2396 \\
\hline 12 & 7.47 & 25.0124 & 24.3849 & 24.1978 & 24.9124 & 24.4544 & 24.4683 & 24.9112 & 24.2825 & 24.0075 & 24.7838 & 24.3421 & 24.3392 \\
\hline 13 & 7.40 & 25.1019 & 24.4940 & 24.2825 & 24.9606 & 24.5840 & 24.4779 & 25.0209 & 24.3585 & 24.1661 & 24.8654 & 24.4266 & 24.3792 \\
\hline 14 & 7.26 & 25.1077 & 24.5001 & 24.2834 & 24.9682 & 24.5853 & 24.5165 & 25.0236 & 24.4093 & 24.1904 & 24.8711 & 24.4852 & 24.4036 \\
\hline 15 & 7.24 & 25.1335 & 24.5112 & 24.3059 & 24.9816 & 24.6059 & 24.5366 & 25.0357 & 24.4139 & 24.2168 & 24.8735 & 24.4939 & 24.4306 \\
\hline 16 & 7.24 & 25.1835 & 24.5547 & 24.3177 & 25.0349 & 24.6180 & 24.5874 & 25.0609 & 24.4188 & 24.2362 & 24.9086 & 24.4979 & 24.4436 \\
\hline 17 & 7.18 & 25.2105 & 24.5565 & 24.3799 & 25.0615 & 24.6198 & 24.6005 & 25.0717 & 24.4438 & 24.2868 & 24.9307 & 24.5182 & 24.5026 \\
\hline 18 & 7.17 & 25.2604 & 24.5935 & 24.5359 & 25.1378 & 24.6717 & 24.6416 & 25.1201 & 24.5033 & 24.4148 & 24.9586 & 24.5762 & 24.5196 \\
\hline \multicolumn{14}{|c|}{ mPW1PW91 } \\
\hline & & \multicolumn{6}{|c|}{ Gas phase } & \multicolumn{6}{|c|}{ PCM } \\
\hline & Exp & 6-31G* & 6-31G** & $6-31+G * *$ & 6-311G* & 6-311G** & $6-311+G * *$ & 6-31G* & $6-31 G * *$ & $6-31+G * *$ & 6-311G* & 6-311G** & $6-311+G * *$ \\
\hline \multicolumn{14}{|l|}{${ }^{13} \mathrm{C}$} \\
\hline 1 & 100.0 & 93.1741 & 93.9095 & 92.9968 & 82.6069 & 82.0821 & 82.2763 & 93.4120 & 94.1424 & 93.1300 & 82.6953 & 82.1741 & 82.3222 \\
\hline 2 & 69.4 & 123.0813 & 123.8476 & 122.7514 & 113.3055 & 113.2683 & 112.9339 & 123.0058 & 123.7725 & 122.6278 & 113.1278 & 113.0877 & 112.7872 \\
\hline 3 & 46.6 & 146.3236 & 146.9215 & 146.2433 & 136.6933 & 136.5710 & 136.4284 & 146.6363 & 147.2235 & 146.5582 & 136.9334 & 136.8062 & 136.6400 \\
\hline $3 a$ & 62.4 & 130.1150 & 130.2854 & 129.3539 & 118.9268 & 119.1391 & 118.9350 & 130.0443 & 130.2137 & 129.2829 & 118.9096 & 119.1200 & 118.9076 \\
\hline 4 & 47.7 & 144.9263 & 145.6580 & 143.9254 & 135.0840 & 134.8923 & 134.3061 & 145.1051 & 145.8342 & 144.2426 & 135.2760 & 135.0822 & 134.5218 \\
\hline $4 a$ & 49.6 & 143.3430 & 143.8857 & 141.8764 & 132.2314 & 131.9444 & 131.9422 & 143.9564 & 144.4938 & 142.6081 & 133.0472 & 132.7427 & 132.7608 \\
\hline 5 & 76.6 & 116.9078 & 117.6461 & 117.0085 & 107.3279 & 106.7860 & 106.2699 & 116.9989 & 117.7358 & 117.0763 & 107.3988 & 106.8487 & 106.3144 \\
\hline $6^{* *}$ & 70.6 & 123.2175 & 124.5080 & 124.1712 & 114.3150 & 113.6080 & 113.5478 & 123.4107 & 124.6946 & 124.1644 & 114.3650 & 113.6347 & 113.5348 \\
\hline 7 & 144.6 & 53.4037 & 53.3725 & 53.5635 & 35.2133 & 35.0831 & 33.3140 & 53.3202 & 53.2871 & 53.4178 & 35.0821 & 34.9568 & 33.0534 \\
\hline 8 & 143.6 & 54.7164 & 54.4134 & 53.6105 & 36.6210 & 36.3362 & 34.1824 & 54.6712 & 54.3702 & 53.6031 & 36.5996 & 36.3318 & 34.1667 \\
\hline 9 & 143.1 & 56.7275 & 56.5305 & 54.7008 & 38.5735 & 38.3151 & 37.0945 & 56.2229 & 56.0305 & 54.1457 & 37.9962 & 37.7612 & 36.5232 \\
\hline 10 & 140.0 & 59.1180 & 59.0155 & 57.4062 & 41.1597 & 41.0119 & 39.2845 & 58.6259 & 58.5309 & 56.7621 & 40.5767 & 40.4469 & 38.5909 \\
\hline 11 & 126.4 & 72.6349 & 73.1498 & 72.8617 & 57.5019 & 56.5619 & 55.7068 & 72.7194 & 73.2332 & 72.7441 & 57.4582 & 56.5517 & 55.7044 \\
\hline 12 & 126.1 & 72.9233 & 73.4481 & 72.8720 & 57.7228 & 56.7859 & 55.7778 & 72.8472 & 73.3733 & 72.9002 & 57.5346 & 56.6310 & 55.7735 \\
\hline 13 & 126.0 & 72.9543 & 73.5555 & 73.0373 & 58.1031 & 57.1414 & 56.1515 & 73.0253 & 73.5530 & 72.9494 & 57.8870 & 56.9662 & 56.0863 \\
\hline 14 & 125.8 & 73.1511 & 73.6754 & 73.3659 & 58.2909 & 57.3575 & 56.4350 & 73.1916 & 73.7829 & 73.3861 & 58.2401 & 57.3208 & 56.4695 \\
\hline 15 & 124.7 & 73.3072 & 73.8491 & 73.3913 & 58.3058 & 57.4440 & 56.9345 & 73.3493 & 73.8874 & 73.6249 & 58.3802 & 57.5426 & 57.0429 \\
\hline 16 & 122.1 & 75.9911 & 76.6101 & 76.1872 & 61.6662 & 60.8346 & 60.4041 & 75.9932 & 76.6009 & 76.1355 & 61.5017 & 60.6735 & 60.2486 \\
\hline 17 & 121.5 & 76.6848 & 77.3392 & 77.7034 & 62.4589 & 61.6719 & 60.8473 & 76.2863 & 76.9283 & 77.2507 & 61.8255 & 61.0612 & 60.2693 \\
\hline
\end{tabular}




\begin{tabular}{ccccccccccccccc}
\hline 18 & 120.5 & 79.2163 & 79.8102 & 79.7241 & 65.3106 & 64.3755 & 63.5231 & 78.4418 & 79.0356 & 78.9572 & 64.2602 & 63.3750 & 62.5334 \\
${ }^{1} \mathbf{H}$ & & & & & & & & & & & & & \\
1 & 5.05 & 27.0314 & 26.5103 & 26.4522 & 26.9902 & 26.6980 & 26.7160 & 27.0518 & 26.5272 & 26.4580 & 26.9889 & 26.6944 & 26.7125 \\
2 & 3.16 & 29.0398 & 28.5573 & 28.6071 & 29.0723 & 28.8067 & 28.8294 & 29.0631 & 28.5744 & 28.5985 & 29.0839 & 28.8080 & 28.8129 \\
3 & 2.02 & 30.0423 & 29.6887 & 29.5353 & 30.0766 & 29.9444 & 29.8862 & 30.0080 & 29.6504 & 29.4784 & 30.0360 & 29.8974 & 29.8285 \\
4 & 2.18 & 30.3380 & 29.9367 & 29.6602 & 30.2653 & 30.0645 & 29.8729 & 30.2150 & 29.8105 & 29.5080 & 30.1484 & 29.9450 & 29.7277 \\
$4 a$ & 4.22 & 28.2144 & 27.7827 & 27.6247 & 28.0583 & 27.8443 & 27.7211 & 28.0090 & 27.5672 & 27.3773 & 27.8391 & 27.6180 & 27.4813 \\
5 & 4.68 & 27.8431 & 27.3261 & 27.1742 & 27.8507 & 27.5199 & 27.4594 & 27.6561 & 27.1311 & 26.9598 & 27.6582 & 27.3216 & 27.2481 \\
6 exo & 3.80 & 28.4399 & 27.9222 & 27.7867 & 28.3307 & 28.0610 & 28.0068 & 28.3834 & 27.8680 & 27.7216 & 28.2715 & 28.0058 & 27.9435 \\
6 endo & 3.71 & 28.5939 & 28.0976 & 27.9605 & 28.5901 & 28.2639 & 28.1495 & 28.4596 & 27.9601 & 27.8062 & 28.4592 & 28.1284 & 27.9993 \\
11 & 7.52 & 24.7739 & 24.1085 & 23.9047 & 24.6811 & 24.2292 & 24.1605 & 24.6507 & 23.9826 & 23.7623 & 24.5501 & 24.0954 & 24.0299 \\
12 & 7.47 & 24.7995 & 24.1695 & 24.0151 & 24.7200 & 24.2371 & 24.2279 & 24.6829 & 24.0515 & 23.8099 & 24.5818 & 24.1133 & 24.0897 \\
13 & 7.40 & 24.9040 & 24.2888 & 24.0751 & 24.7877 & 24.3716 & 24.2630 & 24.8071 & 24.1321 & 23.9481 & 24.6800 & 24.1979 & 24.1517 \\
14 & 7.26 & 24.9065 & 24.2966 & 24.0772 & 24.7902 & 24.3725 & 24.3126 & 24.8074 & 24.1883 & 23.9684 & 24.6807 & 24.2592 & 24.1738 \\
15 & 7.24 & 24.9297 & 24.3023 & 24.0939 & 24.8053 & 24.3948 & 24.3275 & 24.8154 & 24.1887 & 23.9923 & 24.6837 & 24.2654 & 24.2157 \\
16 & 7.24 & 24.9657 & 24.3349 & 24.1092 & 24.8455 & 24.3977 & 24.3698 & 24.8346 & 24.1995 & 24.0158 & 24.7068 & 24.2694 & 24.2226 \\
17 & 7.18 & 25.0056 & 24.3450 & 24.1617 & 24.8843 & 24.4034 & 24.3812 & 24.8375 & 24.2056 & 24.0550 & 24.7310 & 24.2949 & 24.2710 \\
18 & 7.17 & 25.0506 & 24.3840 & 24.2896 & 24.9504 & 24.4587 & 24.4141 & 24.8986 & 24.2770 & 24.1524 & 24.7681 & 24.3502 & 24.2794 \\
\hline
\end{tabular}

\section{Compound 30}

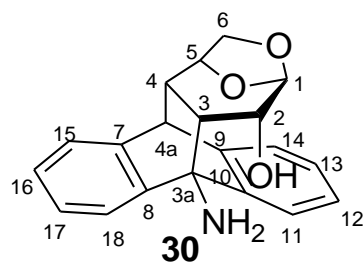

\begin{tabular}{|c|c|c|c|c|c|c|c|c|c|c|c|c|c|}
\hline \multirow{3}{*}{\multicolumn{2}{|c|}{$\operatorname{Exp}$}} & \multicolumn{12}{|c|}{ B3LYP } \\
\hline & & \multicolumn{6}{|c|}{ Gas phase } & \multicolumn{6}{|c|}{ PCM } \\
\hline & & 6-31G* & 6-31G** & $6-31+G * *$ & 6-311G* & 6-311G** & $6-311+G * *$ & $6-31 G^{*}$ & $6-31 G * *$ & $6-31+G^{* *}$ & 6-311G* & $6-311 \mathrm{G} * *$ & $6-311+G * *$ \\
\hline \multicolumn{14}{|c|}{${ }^{13} \mathrm{C}$} \\
\hline 1 & 102.3 & 87.1868 & 87.9005 & 86.4669 & 74.3353 & 73.7814 & 73.9149 & 87.3469 & 88.0613 & 86.4974 & 74.3856 & 73.8340 & 73.9185 \\
\hline 2 & 68.6 & 122.0410 & 122.7665 & 121.3835 & 110.7294 & 110.4773 & 110.0166 & 122.1410 & 122.8670 & 121.3772 & 110.7336 & 110.4890 & 109.9908 \\
\hline 3 & 40.5 & 145.6010 & 146.3962 & 146.2267 & 135.4755 & 135.5635 & 134.8743 & 146.0927 & 146.8735 & 146.7790 & 135.9856 & 136.0543 & 135.3934 \\
\hline $3 a$ & 61.8 & 126.6762 & 126.8825 & 126.3555 & 113.9970 & 114.3036 & 114.0565 & 126.6265 & 126.8267 & 126.2872 & 113.9651 & 114.2567 & 114.0144 \\
\hline 4 & 44.2 & 140.7767 & 141.5948 & 139.9845 & 130.2094 & 130.0518 & 129.4284 & 141.0519 & 141.8643 & 140.3967 & 130.5510 & 130.3858 & 129.8122 \\
\hline $4 a$ & 50.6 & 138.1317 & 138.7429 & 136.4823 & 125.6927 & 125.3617 & 125.4008 & 138.7067 & 139.3107 & 137.2348 & 126.5235 & 126.1737 & 126.2453 \\
\hline 5 & 76.0 & 113.2957 & 114.0863 & 112.8925 & 102.0116 & 101.4799 & 101.2889 & 113.4170 & 114.2073 & 112.9869 & 102.1484 & 101.6096 & 101.3991 \\
\hline 6* & 71.3 & 117.9112 & 119.2103 & 118.9620 & 107.3331 & 106.5428 & 106.1764 & 118.0659 & 119.3579 & 118.9875 & 107.3996 & 106.5884 & 106.1933 \\
\hline 7 & 144.7 & 48.9596 & 48.6887 & 46.5930 & 28.1288 & 27.9200 & 25.9387 & 48.9091 & 48.7455 & 46.7281 & 28.2304 & 28.0158 & 26.1095 \\
\hline 8 & 144.6 & 49.0862 & 49.0662 & 47.7069 & 28.6237 & 28.4854 & 26.7434 & 49.0229 & 48.8841 & 47.1231 & 28.3869 & 28.2564 & 26.4092 \\
\hline 9 & 143.9 & 50.4758 & 50.2961 & 49.0659 & 30.5073 & 30.2943 & 30.1508 & 49.9746 & 49.7913 & 48.8314 & 29.8334 & 29.6185 & 29.4703 \\
\hline 10 & 140.1 & 56.9337 & 56.8364 & 54.3670 & 36.5774 & 36.3819 & 35.0851 & 56.1189 & 56.0304 & 53.4349 & 35.6236 & 35.4516 & 34.0612 \\
\hline 11 & 126.1 & 70.7119 & 71.3208 & 70.3802 & 53.4692 & 52.5666 & 51.5314 & 70.6664 & 71.2779 & 70.3084 & 53.3192 & 52.4526 & 51.5038 \\
\hline 12 & 126.1 & 70.8433 & 71.4599 & 70.7911 & 53.6706 & 52.7809 & 51.8124 & 70.8698 & 71.4892 & 70.8418 & 53.6342 & 52.7777 & 51.9204 \\
\hline 13 & 126.0 & 70.9611 & 71.5911 & 70.7911 & 53.9355 & 53.0683 & 52.1450 & 71.2157 & 71.8427 & 71.0274 & 53.9772 & 53.1403 & 52.3154 \\
\hline
\end{tabular}




\begin{tabular}{|c|c|c|c|c|c|c|c|c|c|c|c|c|}
\hline 14126.0 & 71.1855 & 71.8421 & 71.0209 & 54.0329 & 53.1518 & 52.2752 & 71.2910 & 71.8983 & 71.0328 & 54.1217 & 53.2818 & 52.4082 \\
\hline 15124.5 & 71.2379 & 71.8987 & 71.1720 & 54.3385 & 53.6749 & 53.0897 & 71.4458 & 72.0939 & 71.7779 & 54.6718 & 53.8498 & 53.3524 \\
\hline 16122.4 & 71.6837 & 72.3348 & 72.4270 & 55.0958 & 54.2451 & 53.8037 & 71.6718 & 72.3704 & 72.1307 & 54.8136 & 54.1307 & 53.6639 \\
\hline 17121.1 & 74.1415 & 74.8316 & 74.4616 & 57.9264 & 57.1079 & 56.9740 & 74.0726 & 74.7520 & 74.2930 & 57.6821 & 56.8707 & 56.6768 \\
\hline $\begin{array}{l}18119.7 \\
{ }^{1} \mathbf{H}\end{array}$ & 76.2377 & 76.8922 & 76.9257 & 60.2615 & 59.3922 & 58.7180 & 75.7570 & 76.4150 & 76.4587 & 59.5531 & 58.7202 & 58.0665 \\
\hline 15.03 & 27.2470 & 26.7406 & 26.5821 & 27.1753 & 26.9249 & 26.8973 & 27.2542 & 26.7446 & 26.5763 & 27.1661 & 26.9124 & 26.8849 \\
\hline 24.01 & 28.2706 & 27.8307 & 27.6485 & 28.3173 & 28.0824 & 27.9533 & 28.2689 & 27.8235 & 27.6146 & 28.2955 & 28.0501 & 27.9138 \\
\hline $\begin{array}{ll}3 & 2.42\end{array}$ & 30.1135 & 29.7402 & 29.4685 & 30.1406 & 29.9420 & 29.7688 & 30.0001 & 29.6238 & 29.3243 & 30.0149 & 29.8165 & 29.6264 \\
\hline $\begin{array}{ll}4 & 2.07\end{array}$ & 30.4708 & 30.0689 & 29.7789 & 30.4432 & 30.2275 & 29.9902 & 30.3601 & 29.9557 & 29.6465 & 30.3348 & 30.1179 & 29.8623 \\
\hline $\begin{array}{ll}\text { 4a } & 4.18\end{array}$ & 28.4895 & 28.0570 & 27.8723 & 28.3369 & 28.1235 & 28.0011 & 28.2881 & 27.8457 & 27.6283 & 28.1148 & 27.8950 & 27.7602 \\
\hline $\begin{array}{ll}5 & 4.58\end{array}$ & 27.9725 & 27.4514 & 27.3515 & 27.9812 & 27.6493 & 27.6336 & 27.8025 & 27.2746 & 27.1543 & 27.8032 & 27.4665 & 27.4392 \\
\hline $6^{*} 3.64$ & 28.5934 & 28.0695 & 27.8798 & 28.4664 & 28.1845 & 28.1331 & 28.5407 & 28.0195 & 27.8169 & 28.4097 & 28.1334 & 28.0713 \\
\hline $6 * * 3.68$ & 28.7243 & 28.2182 & 28.0201 & 28.7040 & 28.3730 & 28.2933 & 28.5895 & 28.0810 & 27.8644 & 28.5695 & 28.2363 & 28.1437 \\
\hline 117.46 & 24.5400 & 23.9158 & 23.7853 & 24.4935 & 24.1056 & 24.1699 & 24.5681 & 23.9393 & 23.7763 & 24.5047 & 24.1123 & 24.1556 \\
\hline 127.42 & 25.0532 & 24.4127 & 24.2628 & 24.9061 & 24.4709 & 24.4652 & 24.9244 & 24.2838 & 24.1137 & 24.7590 & 24.3324 & 24.3165 \\
\hline $\begin{array}{ll}13 & 7.39\end{array}$ & 25.1188 & 24.5030 & 24.2932 & 24.9842 & 24.5625 & 24.4753 & 25.0415 & 24.3411 & 24.1599 & 24.9024 & 24.4002 & 24.3823 \\
\hline $14 \quad 7.23$ & 25.1662 & 24.5217 & 24.3036 & 25.0059 & 24.6172 & 24.5456 & 25.0563 & 24.4571 & 24.2040 & 24.9027 & 24.5218 & 24.4733 \\
\hline $\begin{array}{ll}15 & 7.18\end{array}$ & 25.1905 & 24.5485 & 24.3159 & 25.0608 & 24.6274 & 24.5828 & 25.0743 & 24.4589 & 24.2365 & 24.9076 & 24.5410 & 24.4801 \\
\hline 167.18 & 25.2077 & 24.5758 & 24.3737 & 25.0784 & 24.6699 & 24.6290 & 25.1050 & 24.4906 & 24.2771 & 24.9604 & 24.5581 & 24.5013 \\
\hline $17 \quad 7.18$ & 25.2481 & 24.6176 & 24.3980 & 25.0969 & 24.6780 & 24.6493 & 25.1283 & 24.4969 & 24.3087 & 24.9610 & 24.5863 & 24.5360 \\
\hline $\begin{array}{lll}18 & 7.18\end{array}$ & 25.2613 & 24.6456 & 24.4635 & 25.1052 & 24.7157 & 24.6867 & 25.1679 & 24.5525 & 24.3290 & 24.9980 & 24.6165 & 24.5531 \\
\hline
\end{tabular}

\begin{tabular}{|c|c|c|c|c|c|c|c|c|c|c|c|c|c|}
\hline \multirow{3}{*}{\multicolumn{2}{|c|}{ Exp }} & \multicolumn{12}{|c|}{ mPW1PW91 } \\
\hline & & \multicolumn{6}{|c|}{ Gas phase } & \multicolumn{6}{|c|}{ PCM } \\
\hline & & 6-31G* & $6-31 G^{* *}$ & $6-31+G^{* *}$ & 6-311G* & 6-311G** & $6-311+G^{* *}$ & 6-31G* & 6-31G** & $6-31+G^{* *}$ & 6-311G* & $6-311 \mathrm{G} * *$ & $6-311+G^{* *}$ \\
\hline \multicolumn{14}{|c|}{ 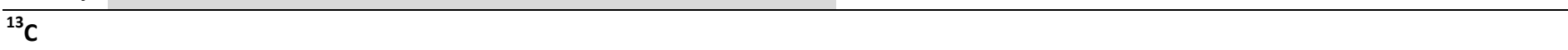 } \\
\hline 1 & 102.3 & 91.7813 & 92.4552 & 91.3113 & 80.9556 & 80.4268 & 80.4839 & 91.9536 & 92.6278 & 91.3666 & 81.0173 & 80.4892 & 80.5050 \\
\hline 2 & 68.6 & 126.7043 & 127.3780 & 126.3838 & 117.0648 & 116.8027 & 116.1710 & 126.8048 & 127.4790 & 126.3882 & 117.0646 & 116.8089 & 116.1457 \\
\hline 3 & 40.5 & 151.3287 & 152.0344 & 151.6591 & 141.9433 & 142.0219 & 141.3325 & 151.8138 & 152.5063 & 152.2071 & 142.4178 & 142.4793 & 141.8159 \\
\hline 3a & 61.8 & 131.0536 & 131.2479 & 130.7341 & 119.8259 & 120.1171 & 119.7692 & 130.9879 & 131.1763 & 130.6552 & 119.7859 & 120.0628 & 119.7156 \\
\hline 4 & 44.2 & 146.5222 & 147.2493 & 145.6909 & 136.7860 & 136.6259 & 135.9747 & 146.7830 & 147.5052 & 146.0793 & 137.0839 & 136.9170 & 136.3149 \\
\hline $4 a$ & 50.6 & 143.1253 & 143.6645 & 141.2867 & 132.0280 & 131.7055 & 131.8045 & 143.7230 & 144.2562 & 142.0251 & 132.8302 & 132.4913 & 132.6108 \\
\hline 5 & 76.0 & 117.9104 & 118.6496 & 117.7235 & 108.1980 & 107.6658 & 107.4653 & 118.0184 & 118.7584 & 117.8036 & 108.3011 & 107.7633 & 107.5451 \\
\hline 6* & 71.3 & 122.1408 & 123.3612 & 123.1662 & 112.9915 & 112.2210 & 111.8937 & 122.2825 & 123.4978 & 123.1848 & 113.0272 & 112.2382 & 111.8886 \\
\hline 7 & 144.7 & 52.4161 & 52.1328 & 50.3878 & 33.9116 & 33.6902 & 32.0109 & 52.4778 & 52.1880 & 50.5038 & 34.0188 & 33.7912 & 32.1712 \\
\hline 8 & 144.6 & 52.7653 & 52.7319 & 51.6306 & 34.5876 & 34.4399 & 32.9535 & 52.5869 & 52.5491 & 51.0508 & 34.3523 & 34.2130 & 32.6222 \\
\hline 9 & 143.9 & 54.0685 & 53.8707 & 52.8142 & 36.1798 & 35.9653 & 35.7699 & 53.5512 & 53.3492 & 52.5774 & 35.5117 & 35.2945 & 35.1017 \\
\hline 10 & 140.1 & 60.3061 & 60.1954 & 58.1988 & 42.2252 & 42.0197 & 40.8572 & 59.4780 & 59.3771 & 57.2639 & 41.2807 & 41.1006 & 39.8485 \\
\hline 11 & 126.1 & 73.2205 & 73.7431 & 72.9873 & 58.0630 & 57.1132 & 56.0147 & 73.1266 & 73.6525 & 72.8633 & 57.8472 & 56.9366 & 55.9324 \\
\hline 12 & 126.1 & 73.3548 & 73.8832 & 73.3474 & 58.2713 & 57.3298 & 56.3093 & 73.3375 & 73.8696 & 73.3462 & 58.1692 & 57.2646 & 56.3588 \\
\hline 13 & 126.0 & 73.4914 & 74.0372 & 73.5051 & 58.5008 & 57.5891 & 56.6655 & 73.7188 & 74.2615 & 73.6524 & 58.5143 & 57.6342 & 56.8043 \\
\hline 14 & 126.0 & 73.7209 & 74.2700 & 73.6753 & 58.6275 & 57.7002 & 56.8088 & 73.7676 & 74.2914 & 73.7074 & 58.6308 & 57.7489 & 56.8745 \\
\hline 15 & 124.5 & 73.7498 & 74.3759 & 73.7215 & 58.8950 & 58.2061 & 57.6448 & 73.9275 & 74.5091 & 74.2922 & 59.1205 & 58.2792 & 57.7445 \\
\hline 16 & 122.4 & 74.1780 & 74.7626 & 74.7732 & 59.5652 & 58.6928 & 58.2027 & 74.2072 & 74.8458 & 74.4709 & 59.3459 & 58.6380 & 58.1633 \\
\hline
\end{tabular}




\begin{tabular}{llllllllllllll}
\hline 17 & 121.1 & 76.6069 & 77.2307 & 77.0885 & 62.3233 & 61.4862 & 61.0468 & 76.5247 & 77.1377 & 76.9136 & 62.0546 & 61.2268 & 60.7369 \\
18 & 119.7 & 78.6465 & 79.2399 & 79.3516 & 64.5876 & 63.6898 & 62.9662 & 78.1362 & 78.7326 & 78.8658 & 63.8555 & 62.9962 & 62.2924 \\
${ }^{1} \boldsymbol{H}$ & & & & & & & & & & & & & \\
1 & 5.03 & 27.1853 & 26.6821 & 26.5552 & 27.1434 & 26.8681 & 26.8568 & 27.1875 & 26.6809 & 26.5456 & 27.1300 & 26.8517 & 26.8411 \\
2 & 4.01 & 28.2106 & 27.7654 & 27.5848 & 28.2340 & 27.9893 & 27.8696 & 28.2036 & 27.7526 & 27.5475 & 28.2087 & 27.9533 & 27.8269 \\
3 & 2.42 & 30.0572 & 29.6799 & 29.4302 & 30.0269 & 29.8270 & 29.6735 & 29.9394 & 29.5590 & 29.2850 & 29.9006 & 29.7007 & 29.5313 \\
4 & 2.07 & 30.4573 & 30.0556 & 29.7856 & 30.3603 & 30.1499 & 29.9473 & 30.3428 & 29.9386 & 29.6520 & 30.2505 & 30.0389 & 29.8193 \\
4 4a & 4.18 & 28.3025 & 27.8746 & 27.7195 & 28.1357 & 27.9110 & 27.8134 & 28.0830 & 27.6441 & 27.4605 & 27.9021 & 27.6703 & 27.5616 \\
5 & 4.58 & 27.8876 & 27.3628 & 27.2708 & 27.8887 & 27.5380 & 27.5305 & 27.7058 & 27.1735 & 27.0649 & 27.7031 & 27.3474 & 27.3298 \\
$6^{*}$ & 3.64 & 28.5465 & 28.0255 & 27.8768 & 28.4229 & 28.1317 & 28.0941 & 28.4838 & 27.9655 & 27.8052 & 28.3578 & 28.0724 & 28.0253 \\
$6^{* *}$ & 3.68 & 28.6743 & 28.1678 & 28.0045 & 28.6446 & 28.3005 & 28.2327 & 28.5285 & 28.0193 & 27.8413 & 28.5029 & 28.1564 & 28.0779 \\
11 & 7.46 & 24.3306 & 23.7053 & 23.5914 & 24.2982 & 23.8857 & 23.9319 & 24.3491 & 23.7190 & 23.5744 & 24.3016 & 23.8846 & 23.9112 \\
12 & 7.42 & 24.8372 & 24.1945 & 24.0783 & 24.7192 & 24.2524 & 24.2606 & 24.6927 & 24.0497 & 23.9164 & 24.5603 & 24.1022 & 24.1047 \\
13 & 7.39 & 24.9185 & 24.2980 & 24.0901 & 24.8084 & 24.3477 & 24.2646 & 24.8199 & 24.1191 & 23.9301 & 24.7041 & 24.1730 & 24.1557 \\
14 & 7.23 & 24.9625 & 24.3166 & 24.0925 & 24.8288 & 24.4043 & 24.3394 & 24.8416 & 24.2316 & 23.9881 & 24.7118 & 24.2959 & 24.2390 \\
15 & 7.18 & 24.9912 & 24.3397 & 24.0996 & 24.8868 & 24.4155 & 24.3711 & 24.8539 & 24.2392 & 24.0140 & 24.7200 & 24.3083 & 24.2562 \\
16 & 7.18 & 25.0030 & 24.3724 & 24.1686 & 24.8921 & 24.4494 & 24.3981 & 24.8904 & 24.2583 & 24.0590 & 24.7588 & 24.3348 & 24.2565 \\
17 & 7.18 & 25.0299 & 24.3954 & 24.2021 & 24.9071 & 24.4683 & 24.4231 & 24.8938 & 24.2718 & 24.0953 & 24.7740 & 24.3645 & 24.3190 \\
18 & 7.18 & 25.0562 & 24.4357 & 24.2457 & 24.9286 & 24.5030 & 24.4657 & 24.9462 & 24.3257 & 24.1015 & 24.8082 & 24.3907 & 24.3194 \\
\hline
\end{tabular}

\section{Compound 31}

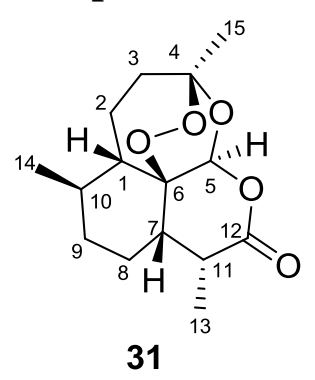

\begin{tabular}{|c|c|c|c|c|c|c|c|c|c|c|c|c|c|}
\hline & \multirow[b]{3}{*}{ Exp } & \multicolumn{12}{|c|}{ B3LYP } \\
\hline & & \multicolumn{6}{|c|}{ Gas phase } & \multicolumn{6}{|c|}{ PCM } \\
\hline & & 6-31G* & $6-31 G * *$ & $6-31+G * *$ & 6-311G* & $6-311 G * *$ & $6-311+G * *$ & 6-31G* & $6-31 G * *$ & $6-31+\mathrm{G} * *$ & 6-311G* & 6-311G** & $6-311+G * *$ \\
\hline \multicolumn{14}{|l|}{${ }^{13} \mathrm{C}$} \\
\hline 1 & 50.1 & 138.6524 & 139.2464 & 137.8699 & 127.4356 & 127.2099 & 126.9743 & 138.8432 & 139.4293 & 138.1063 & 127.6538 & 127.4181 & 127.1962 \\
\hline $2^{*}$ & 24.9 & 162.4825 & 163.8924 & 163.1894 & 153.6978 & 153.5560 & 153.3169 & 162.7235 & 164.1249 & 163.4327 & 153.9133 & 153.7653 & 153.5386 \\
\hline $3^{*}$ & 35.9 & 153.1665 & 154.4528 & 153.7802 & 143.7672 & 143.3288 & 143.0400 & 153.2877 & 154.5652 & 153.8934 & 143.8411 & 143.3901 & 143.1315 \\
\hline 4 & 105.4 & 85.7188 & 86.0959 & 85.3356 & 72.6467 & 72.8054 & 71.8803 & 85.3731 & 85.7461 & 84.8677 & 72.1330 & 72.2930 & 71.3040 \\
\hline 5 & 93.7 & 96.9749 & 97.6675 & 97.7578 & 85.6417 & 85.2260 & 85.2354 & 96.7013 & 97.3885 & 97.3299 & 85.2394 & 84.8066 & 84.7284 \\
\hline 6 & 79.5 & 110.1892 & 110.3944 & 109.6771 & 98.2248 & 98.3540 & 97.2380 & 109.4923 & 109.6956 & 108.8590 & 97.4201 & 97.5530 & 96.3205 \\
\hline 7 & 45.0 & 142.7833 & 143.4021 & 143.3391 & 132.4399 & 132.1993 & 131.6970 & 143.1045 & 143.7174 & 143.7507 & 132.8217 & 132.5726 & 132.0887 \\
\hline $8^{*}$ & 23.4 & 164.4793 & 165.8375 & 164.9312 & 156.0669 & 155.8383 & 155.2070 & 164.5046 & 165.8542 & 164.9468 & 156.0708 & 155.8349 & 155.1829 \\
\hline $9 *$ & 33.6 & 155.1806 & 156.3770 & 154.7711 & 145.5616 & 145.2203 & 144.7838 & 155.5042 & 156.6969 & 155.1867 & 146.0163 & 145.6600 & 145.2360 \\
\hline 10 & 37.5 & 150.8051 & 151.5503 & 150.5098 & 140.2852 & 140.2668 & 139.5420 & 150.8982 & 151.6431 & 150.6351 & 140.4651 & 140.4391 & 139.7035 \\
\hline 11 & 32.9 & 155.3590 & 156.1176 & 155.3936 & 146.1654 & 146.0254 & 145.1777 & 154.7668 & 155.5228 & 154.7205 & 145.4785 & 145.3400 & 144.3467 \\
\hline 12 & 172.1 & 29.3288 & 29.3682 & 26.9939 & 8.8294 & 9.0002 & 6.4362 & 26.5021 & 26.5400 & 23.7276 & 5.6343 & 5.8035 & 2.7361 \\
\hline
\end{tabular}




\begin{tabular}{|c|c|c|c|c|c|c|c|c|c|c|c|c|c|}
\hline 13 & .6 & 4.9639 & 6.8357 & 7.5439 & 168.8764 & 8.8454 & 168.7580 & 175.2756 & 177.1402 & 177.8781 & 169.2471 & 169.2088 & 1 \\
\hline 14 & .8 & & & & & & & & & & & & \\
\hline 15 & & & & & & & & & & & & & \\
\hline 1 & 39 & 832 & & & & & & & & & & & \\
\hline $2^{*}$ & 2.00 & 0.3701 & 85 & .8484 & & & & & & & 380 & & 656 \\
\hline * & 1.47 & 0.5068 & & & & & & & & & & & \\
\hline 5 & 43 & & & & & & & & & & & & \\
\hline & 2.06 & & & & & & & & & & & & \\
\hline 5 & 5.86 & & & & & & & & & & & & \\
\hline 7 & 1.76 & 1.5624 & & & & & & & & & & & \\
\hline $8^{*}$ & 1.88 & .4504 & 2 & 9783 & & & & & & & & & 038 \\
\hline ** & 1.08 & & & & & & & & & & & & \\
\hline $9^{*}$ & & & & & & & & & & & & & \\
\hline $9^{* *}$ & 1.08 & & & & & & & & & & & & \\
\hline 10 & 1.42 & 30.5745 & & & & & & & & & & 30.5435 & 30.4999 \\
\hline 11 & 3.40 & 8935 & & & & & 270 & & 4002 & & 439 & 5575 & 28.5795 \\
\hline 13 & 1.21 & 1214 & & 5665 & & 8606 & 7934 & .004 & 475 & 5372 & 31.0946 & 30.8358 & 30.7613 \\
\hline 14 & 1.00 & 31.2304 & 30.7935 & 30.7099 & 31.1892 & 30.9560 & 30.9144 & 2057 & 30.7671 & 30.6874 & 31.1691 & 30.9342 & 30.8946 \\
\hline 15 & & Ju.J1<0 & 30.4483 & 30.3139 & 30.8451 & 30.5652 & 30.4971 & & 30.4122 & 30.2745 & 30.8093 & 30.5278 & 30.40 \\
\hline
\end{tabular}

\begin{tabular}{|c|c|c|c|c|c|c|c|c|c|c|c|c|c|}
\hline & \multirow[b]{3}{*}{ Exp } & \multicolumn{12}{|c|}{ mPW1PW91 } \\
\hline & & \multicolumn{6}{|c|}{ Gas phase } & \multicolumn{6}{|c|}{ PCM } \\
\hline & & 6-31G* & $6-31 \mathrm{G} * *$ & $6-31+G * *$ & 6-311G* & $6-311 \mathrm{G} * *$ & $6-311+G^{* *}$ & 6-31G* & 6-31G** & $6-31+G^{* *}$ & 6-311G* & $6-311 G * *$ & $6-311+G * *$ \\
\hline \multicolumn{14}{|l|}{${ }^{13} \mathrm{C}$} \\
\hline 1 & 50.1 & 144.3248 & 144.8471 & 143.5314 & 134.1547 & 133.9428 & 133.6800 & 144.5008 & 145.0173 & 143.7477 & 134.3369 & 134.1168 & 133.8693 \\
\hline $2^{*}$ & 24.9 & 167.4352 & 168.7438 & 168.2510 & 159.4600 & 159.3162 & 159.0637 & 167.6920 & 168.9929 & 168.5005 & 159.6773 & 159.5281 & 159.2857 \\
\hline $3^{*}$ & 35.9 & 157.7566 & 158.9507 & 158.2424 & 149.3366 & 148.9011 & 148.6336 & 157.8862 & 159.0727 & 158.3688 & 149.4103 & 148.9635 & 148.7283 \\
\hline 4 & 105.4 & 90.2606 & 90.6360 & 90.2158 & 79.1600 & 79.3212 & 78.5062 & 89.9071 & 90.2782 & 89.7476 & 78.6315 & 78.7944 & 77.9277 \\
\hline 5 & 93.7 & 101.4453 & 102.1203 & 102.4618 & 91.8992 & 91.5237 & 91.4698 & 101.1408 & 101.8111 & 102.0170 & 91.4663 & 91.0741 & 90.9444 \\
\hline 6 & 79.5 & 115.1683 & 115.3637 & 114.9167 & 104.7192 & 104.8418 & 103.7685 & 114.4671 & 114.6602 & 114.1254 & 103.9265 & 104.0524 & 102.8850 \\
\hline 7 & 45.0 & 148.3730 & 148.9168 & 148.7937 & 138.8870 & 138.6614 & 138.1709 & 148.6866 & 149.2263 & 149.1823 & 139.2343 & 139.0018 & 138.5280 \\
\hline $8^{*}$ & 23.4 & 169.2736 & 170.5302 & 169.8643 & 161.5696 & 161.3411 & 160.7367 & 169.3089 & 170.5574 & 169.9003 & 161.5737 & 161.3388 & 160.7236 \\
\hline $9^{*}$ & 33.6 & 160.3319 & 161.4191 & 159.8053 & 151.6258 & 151.2739 & 150.7808 & 160.6739 & 161.7587 & 160.2330 & 152.0759 & 151.7102 & 151.2273 \\
\hline 10 & 37.5 & 156.3087 & 156.9898 & 156.2005 & 146.7264 & 146.7141 & 145.9726 & 156.4027 & 157.0846 & 156.3283 & 146.8889 & 146.8704 & 146.1233 \\
\hline 11 & 32.9 & 159.8987 & 160.6073 & 159.9812 & 151.5738 & 151.4285 & 150.6170 & 159.2921 & 159.9982 & 159.3128 & 150.8824 & 150.7389 & 149.8041 \\
\hline 12 & 172.1 & 31.7531 & 31.7903 & 30.0003 & 13.8975 & 14.0641 & 11.7740 & 28.8722 & 28.9071 & 26.7386 & 10.7148 & 10.8794 & 8.1418 \\
\hline 13 & 12.6 & 179.2842 & 181.0377 & 181.5961 & 173.6398 & 173.6170 & 173.5244 & 179.6224 & 181.3688 & 181.9400 & 174.0080 & 173.9791 & 173.8905 \\
\hline 14 & 19.8 & 173.0262 & 174.7048 & 175.1795 & 166.8075 & 166.6961 & 166.6107 & 173.3953 & 175.0674 & 175.6119 & 167.2415 & 167.1108 & 167.0412 \\
\hline $\begin{array}{l}15 \\
{ }^{1} \mathbf{H}\end{array}$ & 25.2 & 168.7308 & 170.3459 & 170.5073 & 161.6279 & 161.5006 & 161.2222 & 168.9946 & 170.5911 & 170.6041 & 161.7835 & 161.6308 & 161.2532 \\
\hline 1 & 1.39 & 30.7048 & 30.3752 & 30.3638 & 30.7245 & 30.5774 & 30.5584 & 30.6042 & 30.2734 & 30.2489 & 30.6192 & 30.4738 & 30.4446 \\
\hline $2^{*}$ & 2.00 & 30.3260 & 29.8662 & 29.7943 & 30.2702 & 30.0236 & 30.0045 & 30.2104 & 29.7463 & 29.6701 & 30.1570 & 29.9063 & 29.8839 \\
\hline $2^{* *}$ & 1.47 & 30.4809 & 30.0718 & 29.9273 & 30.5242 & 30.3407 & 30.2964 & 30.4699 & 30.0625 & 29.9231 & 30.5057 & 30.3251 & 30.2849 \\
\hline $3 *$ & 2.43 & 29.6935 & 29.2215 & 29.0930 & 29.6570 & 29.4073 & 29.3654 & 29.7044 & 29.2323 & 29.1017 & 29.6665 & 29.4175 & 29.3730 \\
\hline
\end{tabular}




\begin{tabular}{cccccccccccccc}
\hline $3^{* *}$ & 2.06 & 30.2804 & 29.8176 & 29.7105 & 30.2441 & 29.9833 & 29.9350 & 30.1363 & 29.6683 & 29.5528 & 30.0959 & 29.8325 & 29.7811 \\
5 & 5.86 & 26.3363 & 25.7535 & 25.8572 & 26.4306 & 26.1213 & 26.2174 & 26.2217 & 25.6341 & 25.7236 & 26.3003 & 25.9862 & 26.0777 \\
7 & 1.76 & 30.4829 & 30.1274 & 30.1113 & 30.4854 & 30.3174 & 30.2859 & 30.3411 & 29.9812 & 29.9451 & 30.3439 & 30.1713 & 30.1235 \\
$8^{*}$ & 1.88 & 30.4004 & 29.9238 & 29.9238 & 30.3992 & 30.1343 & 30.0873 & 30.3292 & 29.8503 & 29.8467 & 30.3329 & 30.0659 & 30.0138 \\
$8^{* *}$ & 1.08 & 30.8935 & 30.4966 & 30.4594 & 31.0211 & 30.8277 & 30.7737 & 30.8662 & 30.4683 & 30.4250 & 30.9858 & 30.7931 & 30.7372 \\
$9^{*}$ & 1.78 & 30.5145 & 30.0855 & 30.0825 & 30.4436 & 30.2029 & 30.1888 & 30.4582 & 30.0268 & 30.0235 & 30.3905 & 30.1468 & 30.1327 \\
$9^{* *}$ & 1.08 & 31.0147 & 30.6462 & 30.6479 & 30.9661 & 30.8137 & 30.8277 & 30.9380 & 30.5682 & 30.5691 & 30.8939 & 30.7418 & 30.7529 \\
10 & 1.42 & 30.5321 & 30.1723 & 30.1475 & 30.6431 & 30.5143 & 30.4624 & 30.4649 & 30.1047 & 30.0743 & 30.5645 & 30.4377 & 30.3874 \\
11 & 3.40 & 28.8574 & 28.3790 & 28.2411 & 28.8706 & 28.5892 & 28.5433 & 28.8386 & 28.3598 & 28.1946 & 28.8469 & 28.5671 & 28.4971 \\
13 & 1.21 & 31.0529 & 30.6020 & 30.5120 & 31.0257 & 30.7622 & 30.7043 & 31.0272 & 30.5736 & 30.4790 & 30.9992 & 30.7330 & 30.6689 \\
14 & 1.00 & 31.1706 & 30.7333 & 30.6541 & 31.1063 & 30.8666 & 30.8310 & 31.1424 & 30.7033 & 30.6278 & 31.0824 & 30.8411 & 30.8077 \\
15 & 1.45 & 30.8251 & 30.3552 & 30.2417 & 30.7418 & 30.4515 & 30.3935 & 30.7888 & 30.3154 & 30.1988 & 30.7026 & 30.4106 & 30.3499 \\
\hline
\end{tabular}

\section{Compound 32}

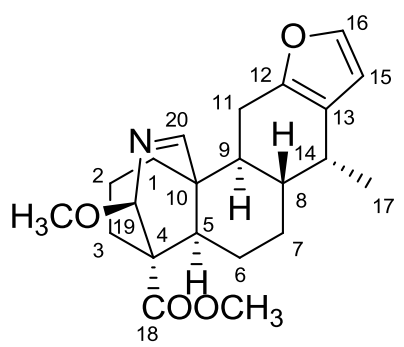

32

\begin{tabular}{|c|c|c|c|c|c|c|c|c|c|c|c|}
\hline & \multicolumn{11}{|c|}{ B3LYP } \\
\hline \multirow[b]{2}{*}{ Exp } & \multicolumn{6}{|c|}{ Gas phase } & \multicolumn{5}{|c|}{ PCM } \\
\hline & 6-31G* & $6-31 G * *$ & $6-31+G * *$ & $6-311 G *$ & $6-311 G * *$ & $6-311+G * *$ & 6-31G* & $6-31 G * *$ & $6-31+G * *$ & 6-311G* & $6-311 \mathrm{G} * * 6-311+\mathrm{G} * *$ \\
\hline
\end{tabular}

${ }^{13} \mathrm{C}$

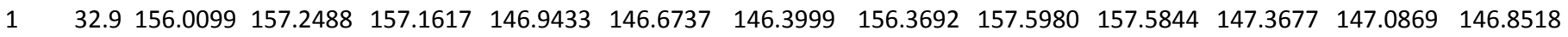

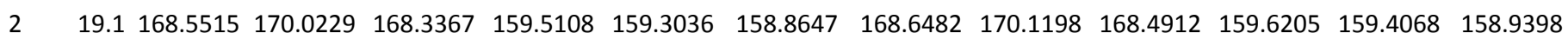

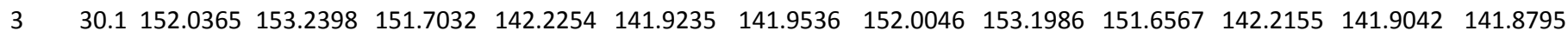

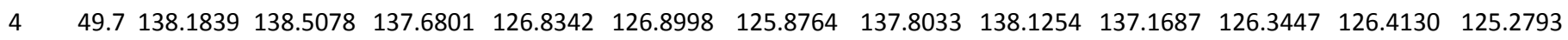

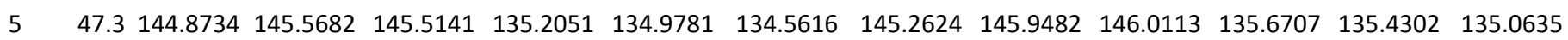

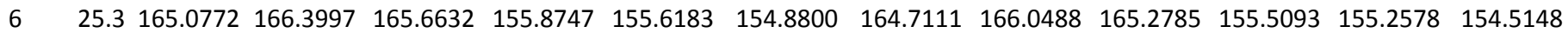

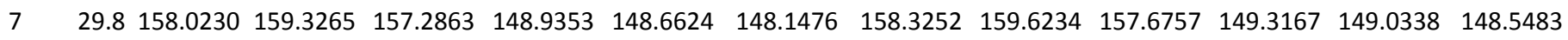

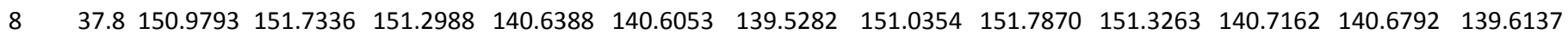

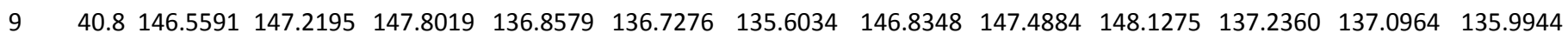

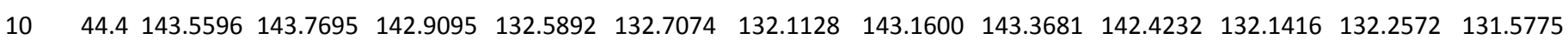

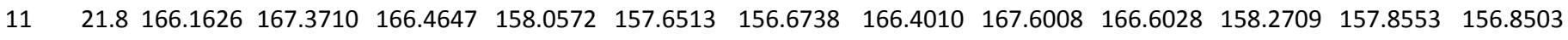

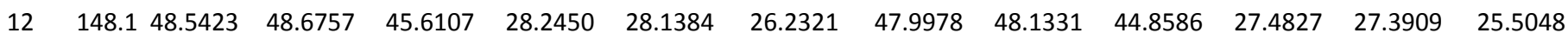

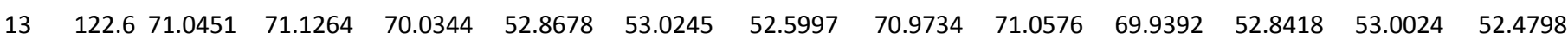

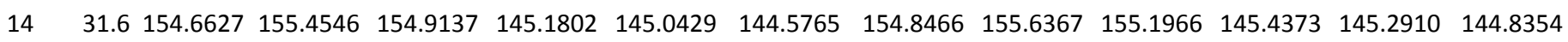

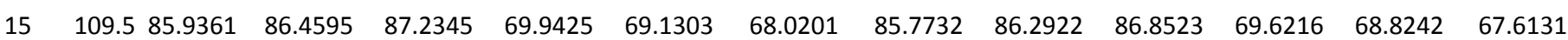

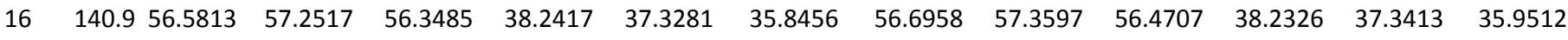

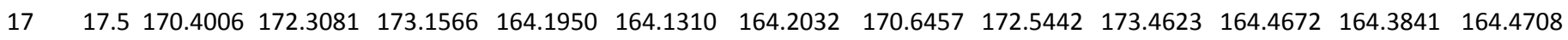

$\begin{array}{llllllllllllll}18 & 175.2 & 23.4599 & 23.3426 & 20.8991 & 0.1600 & 0.2080 & -0.4581 & 22.1979 & 22.0820 & 19.3746 & -1.3760 & -1.3229 & -2.1517\end{array}$

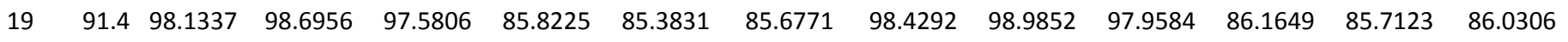




\begin{tabular}{|c|c|c|c|c|c|c|c|c|c|c|c|c|c|}
\hline 20 & 164.6 & 29.6789 & 30.3306 & 28.7319 & 9.9924 & 8.9224 & 7.8834 & 28.0592 & 28.7219 & 26.7633 & 8.0847 & 7.0176 & 5.7227 \\
\hline 18-OMe & 52.0 & 139.3176 & 141.0769 & 140.6871 & 130.7372 & 130.1677 & 130.1942 & 139.1335 & 140.8742 & 140.4342 & 130.4693 & 129.8732 & 129.8950 \\
\hline 19-OMe & 57.2 & 134.8909 & 136.6549 & 136.3118 & 126.4079 & 125.8016 & 125.4282 & 135.0415 & 136.7905 & 136.2994 & 126.4345 & 125.8131 & 125.4836 \\
\hline \multicolumn{14}{|l|}{${ }^{1} \mathrm{H}$} \\
\hline $1^{*}$ & 1.33 & 31.0821 & 30.7040 & 30.6267 & 30.4431 & 30.1955 & 30.1145 & 30.9790 & 30.5985 & 30.5080 & 30.3905 & 30.1392 & 30.0578 \\
\hline $1^{* *}$ & 1.88 & 30.5190 & 30.0519 & 29.9875 & 31.0601 & 30.8854 & 30.8145 & 30.4592 & 29.9892 & 29.9263 & 30.9561 & 30.7796 & 30.6998 \\
\hline $2^{*}$ & 1.62 & 30.8207 & 30.3668 & 30.2880 & 30.7362 & 30.5014 & 30.4513 & 30.7320 & 30.2734 & 30.1894 & 30.6511 & 30.4115 & 30.3588 \\
\hline $2^{* *}$ & 1.62 & 30.8048 & 30.3645 & 30.2876 & 30.8449 & 30.6312 & 30.5692 & 30.8200 & 30.3810 & 30.3091 & 30.8616 & 30.6489 & 30.5864 \\
\hline $3^{*}$ & 1.69 & 30.8177 & 30.4238 & 30.3269 & 30.7580 & 30.5793 & 30.5413 & 30.7465 & 30.3494 & 30.2385 & 30.6840 & 30.5032 & 30.4572 \\
\hline $3^{* *}$ & 2.17 & 30.2458 & 29.8228 & 29.7087 & 30.1946 & 29.9739 & 29.8822 & 30.2427 & 29.8177 & 29.7044 & 30.1875 & 29.9647 & 29.8797 \\
\hline 5 & 1.76 & 30.4772 & 30.1557 & 30.1106 & 30.5448 & 30.4112 & 30.3502 & 30.4421 & 30.1192 & 30.0563 & 30.4979 & 30.3625 & 30.2921 \\
\hline 6* & 1.40 & 29.9139 & 29.3891 & 29.3289 & 29.8985 & 29.5896 & 29.5190 & 30.0624 & 29.5421 & 29.4794 & 30.0370 & 29.7314 & 29.6595 \\
\hline $6^{* *}$ & 1.40 & 30.5847 & 30.1451 & 30.1230 & 30.6703 & 30.4554 & 30.3726 & 30.6414 & 30.2025 & 30.1665 & 30.7211 & 30.5039 & 30.4110 \\
\hline $7^{*}$ & 1.39 & 30.7210 & 30.3517 & 30.2545 & 30.7401 & 30.5869 & 30.5172 & 30.6975 & 30.3260 & 30.2254 & 30.7165 & 30.5600 & 30.4897 \\
\hline $7^{* *}$ & 1.67 & 30.6187 & 30.2157 & 30.1961 & 30.5980 & 30.3797 & 30.3802 & 30.5944 & 30.1891 & 30.1607 & 30.5695 & 30.3490 & 30.3451 \\
\hline 8 & 1.65 & 30.3559 & 30.0144 & 30.0432 & 30.4589 & 30.3162 & 30.3011 & 30.3543 & 30.0131 & 30.0488 & 30.4553 & 30.3141 & 30.3002 \\
\hline 9 & 1.78 & 30.2582 & 29.9117 & 29.9229 & 30.3527 & 30.1762 & 30.1336 & 30.1614 & 29.8132 & 29.8086 & 30.2498 & 30.0744 & 30.0238 \\
\hline $11^{*}$ & 2.81 & 29.4499 & 28.8866 & 28.7293 & 29.4365 & 29.0555 & 28.9513 & 29.4252 & 28.8616 & 28.7014 & 29.4106 & 29.0314 & 28.9222 \\
\hline $11^{* *}$ & 2.81 & 29.5629 & 29.0723 & 28.9606 & 29.4596 & 29.1673 & 29.0602 & 29.5013 & 29.0077 & 28.8943 & 29.4019 & 29.1083 & 28.9944 \\
\hline 14 & 2.66 & 29.5162 & 29.0891 & 29.0879 & 29.5071 & 29.2909 & 29.2774 & 29.4652 & 29.0365 & 29.0152 & 29.4498 & 29.2331 & 29.2049 \\
\hline 15 & 6.19 & 26.2626 & 25.5149 & 25.1191 & 26.1516 & 25.6537 & 25.5409 & 26.1441 & 25.3941 & 24.9885 & 26.0152 & 25.5231 & 25.3998 \\
\hline 16 & 7.25 & 25.1891 & 24.4598 & 24.2475 & 25.0933 & 24.6437 & 24.5920 & 25.0975 & 24.3696 & 24.1643 & 24.9938 & 24.5528 & 24.5106 \\
\hline 19 & 5.11 & 27.4379 & 27.0442 & 26.9731 & 27.4922 & 27.3549 & 27.2628 & 27.4150 & 27.0166 & 26.9377 & 27.4545 & 27.3136 & 27.2209 \\
\hline 20 & 7.78 & 24.1405 & 23.5396 & 23.5338 & 24.1764 & 23.7997 & 23.7337 & 24.0928 & 23.4916 & 23.4825 & 24.1258 & 23.7510 & 23.6846 \\
\hline 17 & 0.97 & 31.1665 & 30.7288 & 30.6807 & 31.1668 & 30.9393 & 30.8850 & 31.1538 & 30.7146 & 30.6654 & 31.1551 & 30.9267 & 30.8728 \\
\hline 18-OMe & 3.72 & 28.6015 & 28.0956 & 27.9809 & 28.5656 & 28.2377 & 28.2656 & 28.5348 & 28.0285 & 27.9171 & 28.4987 & 28.1715 & 28.2021 \\
\hline 19-OMe & 3.73 & 28.6436 & 28.1379 & 27.9477 & 28.6136 & 28.2939 & 28.2344 & 28.6124 & 28.1077 & 27.9200 & 28.5815 & 28.2636 & 28.2063 \\
\hline
\end{tabular}

\begin{tabular}{|c|c|c|c|c|c|c|c|c|c|c|c|c|c|}
\hline & \multicolumn{13}{|c|}{ mPW1PW91 } \\
\hline & \multirow[b]{2}{*}{ Exp } & \multicolumn{6}{|c|}{ Gas phase } & \multicolumn{6}{|c|}{ PCM } \\
\hline & & 6-31G* & $6-31 \mathrm{G} * *$ & $6-31+G * *$ & $6-311 G *$ & 6-311G** & $6-311+\mathrm{G} * *$ & 6-31G* & 6-31G** & $6-31+G * *$ & 6-311G* & 6-311G** & $6-311+G * *$ \\
\hline \multicolumn{14}{|l|}{${ }^{13} \mathrm{C}$} \\
\hline 1 & 32.9 & 161.2401 & 162.3655 & 162.0719 & 152.8255 & 152.5660 & 152.3322 & 161.6088 & 162.7256 & 162.4823 & 153.2316 & 152.9626 & 152.7660 \\
\hline 2 & 19.1 & 173.3077 & 174.6728 & 172.9117 & 165.0373 & 164.8136 & 164.4680 & 173.4197 & 174.7854 & 173.0748 & 165.1547 & 164.9248 & 164.5614 \\
\hline 3 & 30.1 & 157.6012 & 158.6857 & 157.1634 & 148.4878 & 148.1984 & 148.1985 & 157.5783 & 158.6550 & 157.1279 & 148.4740 & 148.1770 & 148.1352 \\
\hline 4 & 49.7 & 143.2742 & 143.5825 & 142.9439 & 133.0004 & 133.0682 & 132.0951 & 142.8858 & 143.1923 & 142.4571 & 132.5091 & 132.5798 & 131.5120 \\
\hline 5 & 47.3 & 150.8556 & 151.4599 & 151.2697 & 141.8919 & 141.6741 & 141.3710 & 151.2602 & 151.8561 & 151.7424 & 142.3511 & 142.1215 & 141.8651 \\
\hline 6 & 25.3 & 169.8653 & 171.0761 & 170.5877 & 161.4801 & 161.2227 & 160.5402 & 169.5163 & 170.7411 & 170.2352 & 161.1378 & 160.8842 & 160.1943 \\
\hline 7 & 29.8 & 163.0109 & 164.2072 & 162.0277 & 154.8448 & 154.5634 & 154.1469 & 163.3412 & 164.5322 & 162.4353 & 155.2311 & 154.9406 & 154.5479 \\
\hline 8 & 37.8 & 156.4599 & 157.1364 & 156.8010 & 147.0994 & 147.0738 & 146.0703 & 156.5026 & 157.1770 & 156.8205 & 147.1575 & 147.1293 & 146.1324 \\
\hline 9 & 40.8 & 152.0657 & 152.6495 & 153.3218 & 143.2419 & 143.1239 & 141.9800 & 152.3432 & 152.9217 & 153.6460 & 143.5999 & 143.4739 & 142.3514 \\
\hline 10 & 44.4 & 148.7421 & 148.9313 & 148.1871 & 138.6846 & 138.7907 & 138.1785 & 148.3271 & 148.5144 & 147.7001 & 138.2324 & 138.3364 & 137.6515 \\
\hline 11 & 21.8 & 170.3117 & 171.4458 & 170.9135 & 163.1848 & 162.7803 & 161.8705 & 170.5683 & 171.6935 & 171.0657 & 163.3967 & 162.9823 & 162.0450 \\
\hline 12 & 148.1 & 51.5579 & 51.6693 & 49.3657 & 33.8060 & 33.6903 & 31.8522 & 51.0138 & 51.1269 & 48.6381 & 33.0625 & 32.9614 & 31.1201 \\
\hline 13 & 122.6 & 74.6559 & 74.7259 & 73.7861 & 58.5944 & 58.7302 & 58.2030 & 74.5940 & 74.6676 & 73.7076 & 58.5784 & 58.7184 & 58.1093 \\
\hline
\end{tabular}




\begin{tabular}{|c|c|c|c|c|c|c|c|c|c|c|c|c|c|}
\hline 14 & 31.6 & 159.6125 & 160.3499 & 159.8717 & 151.1151 & 150.9703 & 150.4892 & 159.8146 & 160.5505 & 160.1611 & 151.3721 & 151.2191 & 150.7457 \\
\hline 15 & 109.5 & 88.4945 & 88.9520 & 89.7517 & 74.3814 & 73.5319 & 72.4394 & 88.3467 & 88.7994 & 89.4192 & 74.0804 & 73.2468 & 72.0695 \\
\hline 16 & 140.9 & 58.7368 & 59.3602 & 59.0073 & 42.7546 & 41.8265 & 40.3840 & 58.7947 & 59.4121 & 59.0331 & 42.6651 & 41.7613 & 40.4182 \\
\hline 17 & 17.5 & 174.6764 & 176.4549 & 176.9752 & 168.8665 & 168.8296 & 168.9553 & 174.9473 & 176.7164 & 177.3019 & 169.1364 & 169.0820 & 169.2217 \\
\hline 18 & 175.2 & 26.4187 & 26.3058 & 24.2938 & 5.7229 & 5.7684 & 5.2289 & 25.1337 & 25.0224 & 22.7992 & 4.1938 & 4.2443 & 3.5662 \\
\hline 19 & 91.4 & 103.2638 & 103.7839 & 102.9661 & 92.6693 & 92.2588 & 92.4696 & 103.5656 & 104.0806 & 103.3315 & 92.9916 & 92.5687 & 92.7931 \\
\hline 20 & 164.6 & 32.1303 & 32.7294 & 31.3499 & 14.5628 & 13.4932 & 12.6903 & 30.4605 & 31.0708 & 29.3929 & 12.6479 & 11.5813 & 10.5517 \\
\hline 18-OMe & 52.0 & 142.7459 & 144.4327 & 144.0909 & 135.2028 & 134.6773 & 134.7020 & 142.5781 & 144.2468 & 143.8532 & 134.9467 & 134.3968 & 134.4274 \\
\hline 19-OMe & 57.2 & 138.5854 & 140.2630 & 140.1706 & 131.2693 & 130.6999 & 130.2975 & 138.7642 & 140.4273 & 140.1696 & 131.3018 & 130.7193 & 130.3653 \\
\hline \multicolumn{14}{|l|}{${ }^{1} \mathrm{H}$} \\
\hline $1^{*}$ & 1.33 & 31.0279 & 30.6510 & 30.5556 & 30.3698 & 30.1202 & 30.0403 & 30.9189 & 30.5391 & 30.4329 & 30.3130 & 30.0596 & 29.9792 \\
\hline $1 * *$ & 1.88 & 30.4847 & 30.0191 & 29.9517 & 30.9526 & 30.7817 & 30.7109 & 30.4204 & 29.9516 & 29.8867 & 30.8456 & 30.6727 & 30.5936 \\
\hline $2^{*}$ & 1.62 & 30.7733 & 30.3195 & 30.2485 & 30.6607 & 30.4235 & 30.3704 & 30.6745 & 30.2156 & 30.1407 & 30.5666 & 30.3246 & 30.2692 \\
\hline $2^{* *}$ & 1.62 & 30.7935 & 30.3518 & 30.2583 & 30.7718 & 30.5640 & 30.5052 & 30.8114 & 30.3712 & 30.2815 & 30.7902 & 30.5834 & 30.5243 \\
\hline $3^{*}$ & 1.69 & 30.7542 & 30.3631 & 30.2711 & 30.6520 & 30.4797 & 30.4490 & 30.6816 & 30.2871 & 30.1827 & 30.5780 & 30.4032 & 30.3655 \\
\hline $3^{* *}$ & 2.17 & 30.2049 & 29.7847 & 29.6802 & 30.1106 & 29.8925 & 29.8061 & 30.1972 & 29.7749 & 29.6705 & 30.1001 & 29.8796 & 29.7977 \\
\hline 5 & 1.76 & 30.3820 & 30.0647 & 29.9993 & 30.3954 & 30.2692 & 30.2129 & 30.3457 & 30.0267 & 29.9478 & 30.3499 & 30.2217 & 30.1566 \\
\hline $6^{*}$ & 1.40 & 29.8610 & 29.3371 & 29.2752 & 29.8121 & 29.5014 & 29.4305 & 30.0109 & 29.4918 & 29.4242 & 29.9503 & 29.6430 & 29.5698 \\
\hline $6 * *$ & 1.40 & 30.5606 & 30.1180 & 30.0675 & 30.5667 & 30.3606 & 30.2855 & 30.6252 & 30.1833 & 30.1179 & 30.6234 & 30.4151 & 30.3294 \\
\hline $7^{*}$ & 1.39 & 30.6829 & 30.3134 & 30.2001 & 30.6370 & 30.4904 & 30.4228 & 30.6573 & 30.2853 & 30.1696 & 30.6122 & 30.4620 & 30.3940 \\
\hline $7^{* *}$ & 1.67 & 30.5773 & 30.1744 & 30.1723 & 30.5203 & 30.2962 & 30.3055 & 30.5463 & 30.1408 & 30.1308 & 30.4865 & 30.2600 & 30.2648 \\
\hline 8 & 1.65 & 30.3042 & 29.9619 & 29.9600 & 30.3404 & 30.1999 & 30.1829 & 30.3084 & 29.9665 & 29.9665 & 30.3425 & 30.2034 & 30.1844 \\
\hline 9 & 1.78 & 30.1878 & 29.8450 & 29.8349 & 30.2053 & 30.0398 & 29.9916 & 30.0877 & 29.7431 & 29.7192 & 30.1022 & 29.9377 & 29.8825 \\
\hline $11^{*}$ & 2.81 & 29.4043 & 28.8370 & 28.6721 & 29.3429 & 28.9606 & 28.8661 & 29.3741 & 28.8062 & 28.6387 & 29.3124 & 28.9318 & 28.8314 \\
\hline $11^{* *}$ & 2.81 & 29.4945 & 28.9994 & 28.8940 & 29.3627 & 29.0743 & 28.9724 & 29.4249 & 28.9265 & 28.8197 & 29.2971 & 29.0072 & 28.8997 \\
\hline 14 & 2.66 & 29.4542 & 29.0266 & 29.0232 & 29.3946 & 29.1815 & 29.1623 & 29.3978 & 28.9682 & 28.9472 & 29.3339 & 29.1199 & 29.0876 \\
\hline 15 & 6.19 & 26.0919 & 25.3394 & 25.0106 & 26.0103 & 25.4721 & 25.3778 & 25.9604 & 25.2050 & 24.8688 & 25.8645 & 25.3323 & 25.2306 \\
\hline 16 & 7.25 & 25.0194 & 24.2916 & 24.1094 & 24.9646 & 24.4754 & 24.4251 & 24.9146 & 24.1883 & 24.0157 & 24.8543 & 24.3740 & 24.3327 \\
\hline 19 & 5.11 & 27.3865 & 26.9993 & 26.9225 & 27.3874 & 27.2530 & 27.1814 & 27.3607 & 26.9684 & 26.8828 & 27.3488 & 27.2104 & 27.1356 \\
\hline 20 & 7.78 & 23.9974 & 23.4014 & 23.4061 & 24.0364 & 23.6451 & 23.5961 & 23.9460 & 23.3501 & 23.3521 & 23.9840 & 23.5947 & 23.5435 \\
\hline 17 & 0.97 & 31.1062 & 30.6692 & 30.6208 & 31.0793 & 30.8473 & 30.7968 & 31.0902 & 30.6515 & 30.6023 & 31.0642 & 30.8313 & 30.7818 \\
\hline 18-OMe & 3.72 & 28.5391 & 28.0328 & 27.9476 & 28.5005 & 28.1592 & 28.1960 & 28.4666 & 27.9598 & 27.8782 & 28.4291 & 28.0885 & 28.1289 \\
\hline 19-OMe & 3.73 & 28.6014 & 28.0968 & 27.9344 & 28.5618 & 28.2310 & 28.1829 & 28.5653 & 28.0617 & 27.9022 & 28.5258 & 28.1970 & 28.1506 \\
\hline
\end{tabular}




\section{Compound 33}

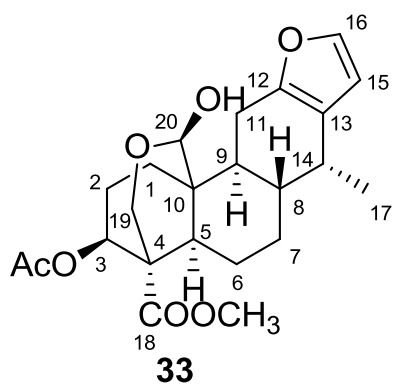

\begin{tabular}{|c|c|c|c|c|c|c|c|c|c|c|c|c|c|}
\hline & \multicolumn{13}{|c|}{ B3LYP } \\
\hline & \multirow[b]{2}{*}{ Exp } & \multicolumn{6}{|c|}{ Gas phase } & \multicolumn{6}{|c|}{ PCM } \\
\hline & & 6-31G* & 6-31G** & $6-31+\mathrm{G} * *$ & 6-311G* & $6-311 G * *$ & $6-311+G * *$ & 6-31G* & $6-31 \mathrm{G} * *$ & $6-31+G * *$ & 6-311G* & 6-311G** & $6-311+G * *$ \\
\hline \multicolumn{14}{|l|}{${ }^{13} \mathrm{C}$} \\
\hline 1 & 36.0 & 151.4965 & 152.7899 & 152.6933 & 142.8425 & 142.5674 & 142.3181 & 151.9701 & 153.2550 & 153.2316 & 143.3977 & 143.1151 & 142.8877 \\
\hline 2 & 26.3 & 162.5365 & 163.9093 & 163.5594 & 153.8964 & 153.5797 & 153.7084 & 162.2857 & 163.6621 & 163.3490 & 153.6203 & 153.2991 & 153.4605 \\
\hline 3 & 76.0 & 112.7049 & 113.3836 & 110.5929 & 100.8663 & 100.7472 & 100.8654 & 112.3504 & 113.0311 & 110.1906 & 100.4963 & 100.3827 & 100.4871 \\
\hline 4 & 49.4 & 138.5163 & 138.7330 & 138.0535 & 128.1891 & 128.1592 & 127.3593 & 138.3666 & 138.5805 & 137.7571 & 127.9818 & 127.9507 & 127.1502 \\
\hline 5 & 44.4 & 142.2895 & 142.9342 & 143.4790 & 132.4355 & 132.3220 & 131.5702 & 142.3599 & 142.9977 & 143.5981 & 132.5660 & 132.4452 & 131.7262 \\
\hline 6 & 23.2 & 164.4458 & 165.8393 & 164.4818 & 155.7329 & 155.5205 & 155.1292 & 164.5342 & 165.9301 & 164.5562 & 155.8449 & 155.6316 & 155.2241 \\
\hline 7 & 29.4 & 158.4700 & 159.8006 & 158.4746 & 149.5580 & 149.3318 & 149.0559 & 158.7535 & 160.0791 & 158.7900 & 149.9020 & 149.6720 & 149.4363 \\
\hline 8 & 36.3 & 151.6237 & 152.3784 & 151.0397 & 141.2657 & 141.1911 & 140.6859 & 151.4878 & 152.2465 & 150.8635 & 141.0879 & 141.0274 & 140.5252 \\
\hline 9 & 42.0 & 145.7213 & 146.3972 & 145.6379 & 135.9314 & 135.8183 & 135.3722 & 145.9861 & 146.6577 & 145.9388 & 136.2764 & 136.1590 & 135.7605 \\
\hline 10 & 38.0 & 148.8555 & 149.1448 & 149.1175 & 138.3088 & 138.4788 & 138.0923 & 148.9059 & 149.1936 & 149.1593 & 138.3694 & 138.5331 & 138.1335 \\
\hline 11 & 22.7 & 164.7187 & 165.9732 & 164.8773 & 156.5655 & 156.2317 & 155.9418 & 164.9267 & 166.1716 & 164.9744 & 156.7367 & 156.3898 & 156.0795 \\
\hline 12 & 149.0 & 47.5605 & 47.6838 & 44.5682 & 27.0559 & 27.0281 & 25.1704 & 47.0989 & 47.2231 & 43.9360 & 26.3742 & 26.3676 & 24.4768 \\
\hline 13 & 122.5 & 71.6642 & 71.7492 & 70.6571 & 53.6584 & 53.8089 & 53.3007 & 71.4516 & 71.5399 & 70.4187 & 53.4575 & 53.6050 & 53.0110 \\
\hline 14 & 31.3 & 155.1757 & 155.9584 & 155.5763 & 145.7996 & 145.6384 & 145.2046 & 155.3393 & 156.1211 & 155.7790 & 146.0076 & 145.8419 & 145.3990 \\
\hline 15 & 109.7 & 785.5854 & 86.1310 & 85.1629 & 69.7493 & 68.9710 & 68.6402 & 85.3851 & 85.9249 & 84.8674 & 69.3844 & 68.6187 & 68.1419 \\
\hline 16 & 140.4 & 456.8983 & 57.5764 & 56.5759 & 38.5793 & 37.6435 & 36.3664 & 57.0034 & 57.6768 & 56.6882 & 38.5549 & 37.6462 & 36.4699 \\
\hline 17 & 16.8 & 170.6480 & 172.5774 & 173.2556 & 164.5915 & 164.5068 & 164.4911 & 170.8981 & 172.8172 & 173.5531 & 164.8536 & 164.7517 & 164.7463 \\
\hline 18 & 173.1 & 123.9827 & 23.9208 & 21.6114 & 0.9664 & 1.0042 & 0.1426 & 22.7805 & 22.7192 & 20.1722 & -0.5317 & -0.4833 & -1.4856 \\
\hline 19 & 57.0 & 131.3506 & 132.6393 & 132.9915 & 122.1248 & 121.6353 & 121.5765 & 131.6195 & 132.9081 & 133.2090 & 122.4062 & 121.9118 & 121.8715 \\
\hline 20 & 97.2 & 92.6286 & 93.2412 & 91.4545 & 79.6373 & 79.3438 & 79.2882 & 92.8704 & 93.4829 & 91.6118 & 79.8782 & 79.5709 & 79.4799 \\
\hline 18-OMe & 51.8 & 139.0789 & 140.8143 & 141.3440 & 130.4125 & 129.8130 & 129.3487 & 138.7570 & 140.4747 & 140.8852 & 129.9873 & 129.3581 & 128.8896 \\
\hline $20-\mathrm{OAc}-\mathrm{Me}$ & 21.0 & 169.2706 & 170.9812 & 170.4204 & 161.3591 & 161.0491 & 160.5437 & 169.1422 & 170.8237 & 170.0131 & 161.0269 & 160.6817 & 160.0279 \\
\hline $20-\mathrm{OAC}-\mathrm{CO}$ & 169.9 & 29.6581 & 29.8414 & 27.3300 & 8.2226 & 8.3417 & 6.1025 & 27.7957 & 27.9688 & 25.0463 & 5.9975 & 6.1097 & 3.5464 \\
\hline \multicolumn{14}{|l|}{${ }^{1} \mathrm{H}$} \\
\hline $1^{*}$ & 1.33 & 30.9051 & 30.5274 & 30.3426 & 30.2246 & 29.9875 & 29.8219 & 30.8386 & 30.4599 & 30.2607 & 30.1621 & 29.9207 & 29.7522 \\
\hline $1 * *$ & 2.07 & 30.2680 & 29.8214 & 29.6633 & 30.8437 & 30.6598 & 30.6190 & 30.2045 & 29.7548 & 29.5878 & 30.7777 & 30.5920 & 30.5372 \\
\hline $2^{*}$ & 2.05 & 30.1771 & 29.7100 & 29.5681 & 30.1129 & 29.8155 & 29.7568 & 30.2029 & 29.7368 & 29.5907 & 30.1353 & 29.8388 & 29.7786 \\
\hline $2^{* *}$ & 2.26 & 29.9455 & 29.4317 & 29.3834 & 29.9359 & 29.6523 & 29.6072 & 29.9631 & 29.4497 & 29.3956 & 29.9529 & 29.6706 & 29.6183 \\
\hline $3^{*}$ & 5.31 & 26.8133 & 26.2986 & 26.1881 & 26.8374 & 26.5497 & 26.4828 & 26.8789 & 26.3665 & 26.2446 & 26.8992 & 26.6084 & 26.5318 \\
\hline 5 & 1.62 & 30.4059 & 30.0993 & 30.0319 & 30.4733 & 30.3611 & 30.3339 & 30.3571 & 30.0499 & 29.9704 & 30.4183 & 30.3041 & 30.2721 \\
\hline $6 *$ & 1.77 & 31.0636 & 30.6613 & 30.6091 & 31.0060 & 30.7925 & 30.8345 & 31.0554 & 30.6504 & 30.5930 & 30.9913 & 30.7736 & 30.8163 \\
\hline $6 * *$ & 2.33 & 29.6033 & 29.1183 & 29.0222 & 29.5696 & 29.3392 & 29.3743 & 29.6318 & 29.1440 & 29.0519 & 29.6003 & 29.3656 & 29.4007 \\
\hline $7^{*}$ & 1.38 & 30.6819 & 30.3084 & 30.2227 & 30.6731 & 30.5132 & 30.5177 & 30.6406 & 30.2661 & 30.1758 & 30.6352 & 30.4740 & 30.4743 \\
\hline
\end{tabular}




\begin{tabular}{|c|c|c|c|c|c|c|c|c|c|c|c|c|c|}
\hline $7^{* *}$ & 1.73 & 30.5038 & 30.1104 & 30.0265 & 30.4416 & 30.2441 & 30.2275 & 30.4735 & 30.0777 & 29.9880 & 30.4102 & 30.2088 & 30.1914 \\
\hline 8 & 2.40 & 29.4959 & 29.1283 & 29.0305 & 29.5107 & 29.3842 & 29.3262 & 29.5609 & 29.1927 & 29.0891 & 29.5816 & 29.4506 & 29.3869 \\
\hline 9 & 1.49 & 30.5485 & 30.2028 & 30.1806 & 30.6651 & 30.4981 & 30.4490 & 30.4831 & 30.1353 & 30.0962 & 30.5957 & 30.4267 & 30.3656 \\
\hline $11 *$ & 2.64 & 29.6753 & 29.1638 & 29.0107 & 29.5852 & 29.2668 & 29.1464 & 29.6256 & 29.1113 & 28.9562 & 29.5315 & 29.2115 & 29.0900 \\
\hline $11^{* *}$ & 2.73 & 29.4195 & 28.9315 & 28.8126 & 29.4240 & 29.1495 & 29.0737 & 29.4081 & 28.9187 & 28.8082 & 29.4122 & 29.1393 & 29.0686 \\
\hline 14 & 2.63 & 29.5123 & 29.0943 & 29.0048 & 29.4955 & 29.2975 & 29.1989 & 29.4698 & 29.0507 & 28.9462 & 29.4483 & 29.2494 & 29.1440 \\
\hline 15 & 6.18 & 26.3128 & 25.5582 & 25.2066 & 26.2000 & 25.6872 & 25.6415 & 26.1913 & 25.4343 & 25.0551 & 26.0603 & 25.5530 & 25.5040 \\
\hline 16 & 7.21 & 25.2412 & 24.5119 & 24.2695 & 25.1477 & 24.6965 & 24.5465 & 25.1498 & 24.4220 & 24.1894 & 25.0472 & 24.6051 & \\
\hline $19^{*}$ & 4.03 & 28.1538 & 27.6703 & 27.5718 & 28.1118 & 27.8401 & 27.7973 & 28.2046 & 27.7208 & 27.6259 & 28.1557 & 27.8847 & 27.8435 \\
\hline $19 * *$ & 4.33 & 27.7782 & 27.2952 & 27.0053 & 27.7234 & 27.4948 & 27.4160 & 27.7682 & 27.2814 & 26.9844 & 27.7052 & 27.4742 & 27.3927 \\
\hline 20 & 5.06 & 27.1190 & 26.6523 & 26.6842 & 27.1196 & 26.9442 & 26.8474 & 27.0536 & 26.5842 & 26.6168 & 27.0479 & 26.8717 & 26.7801 \\
\hline 17 & 0.97 & 31.1842 & 30.7485 & 30.6919 & 31.1643 & 30.9383 & 30.9111 & 31.1678 & 30.7304 & 30.6715 & 31.1491 & 30.9218 & 30.8940 \\
\hline 18-OMe & 3.67 & 28.6490 & 28.1419 & 28.0404 & 28.6310 & 28.2980 & 28.3182 & 28.5660 & 28.0584 & 27.9530 & 28.5470 & 28.2150 & 28.2320 \\
\hline 20-OAc-Me & 2.02 & 30.4233 & 29.9038 & 29.7896 & 30.3396 & 30.0162 & 29.9823 & 30.3309 & 29.8058 & 29.6810 & 30.2447 & 29.9188 & 29.8793 \\
\hline
\end{tabular}

\begin{tabular}{|c|c|c|c|c|c|c|c|c|c|}
\hline & \multicolumn{9}{|c|}{ mPW1PW91 } \\
\hline \multirow[b]{2}{*}{ Exp } & \multicolumn{5}{|c|}{ Gas phase } & \multicolumn{4}{|c|}{ PCM } \\
\hline & $6-31 G^{*}$ & 6-31G** & $6-31+G^{* *}$ & 6-311G* & $6-311 G * * 6-311+G * *$ & 6-31G* & $6-31 G * * 6-31+G * *$ & 6-311G* & $6-311 G * * 6-311+G * *$ \\
\hline
\end{tabular}

${ }^{13} \mathrm{C}$

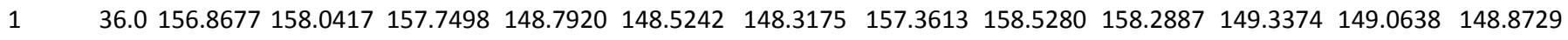

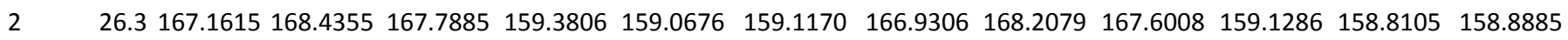

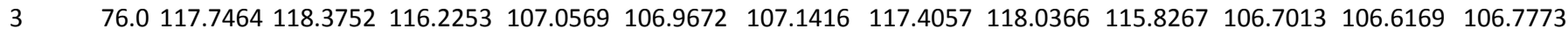

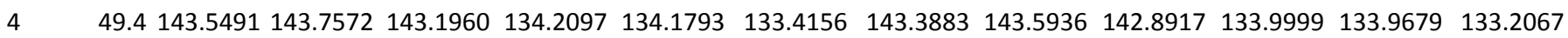

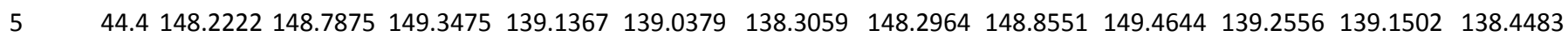

$\begin{array}{llllllllllllll}6 & 23.2 & 169.1978 & 170.4773 & 169.6946 & 161.2888 & 161.0631 & 160.6568 & 169.2951 & 170.5770 & 169.7771 & 161.4033 & 161.1768 & 160.7550\end{array}$

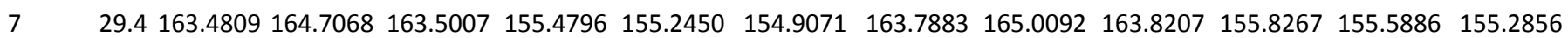

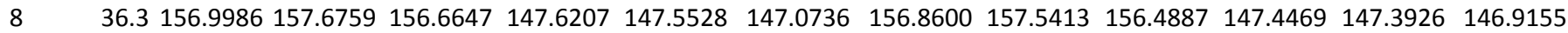

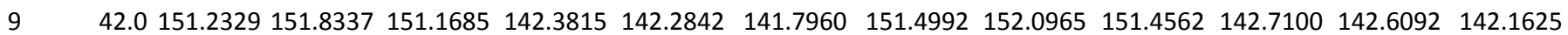

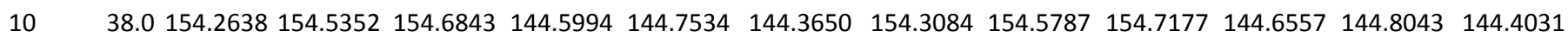

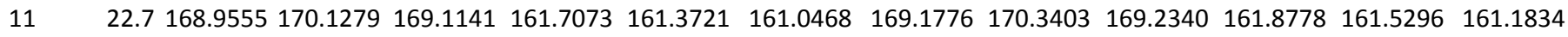

$12 \quad \begin{array}{lllllllllllll}149.0 & 50.6102 & 50.7118 & 48.4314 & 32.6627 & 32.6268 & 30.8031 & 50.1565 & 50.2590 & 47.8220 & 32.0014 & 31.9871 & 30.1216\end{array}$

$\begin{array}{llllllllllllll}13 & 122.5 & 75.2948 & 75.3662 & 74.6683 & 59.3917 & 59.5189 & 58.9090 & 75.0864 & 75.1614 & 74.4276 & 59.1973 & 59.3218 & 58.6374\end{array}$

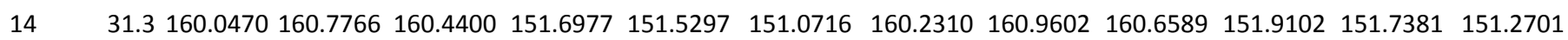

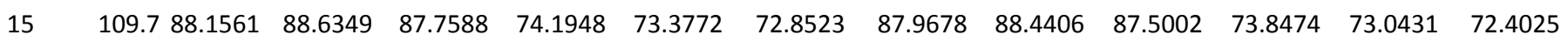

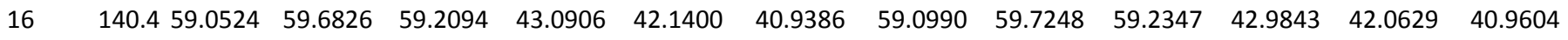

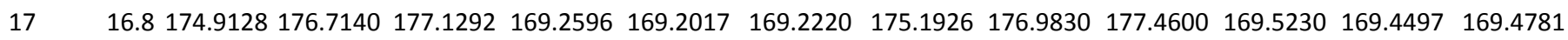

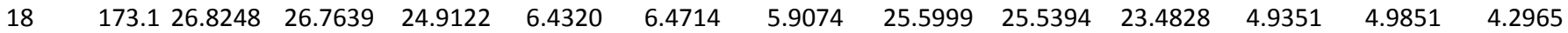

$\begin{array}{llllllllllllll}19 & 57.0 & 135.9909 & 137.2089 & 137.2464 & 127.8091 & 127.3709 & 127.3858 & 136.2766 & 137.4941 & 137.4871 & 128.0959 & 127.6530 & 127.6854\end{array}$

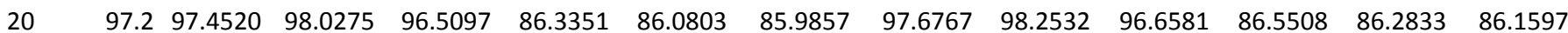

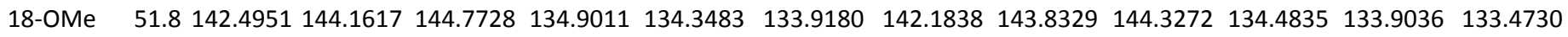
20-OAc-Me $21.0172 .5972174 .2117 \quad 174.1080165 .4810165 .1910 \quad 164.6681 \quad 172.4815174 .0672173 .7314165 .1537 \quad 164.8315164 .1810$

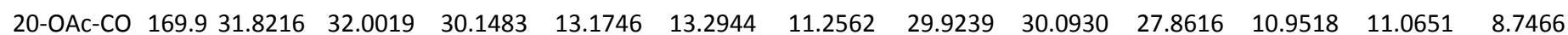
${ }^{1} \mathrm{H}$

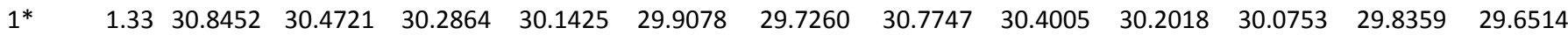

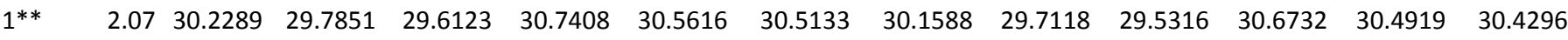




\begin{tabular}{|c|c|c|c|c|c|c|c|c|c|c|c|c|c|}
\hline $2^{*}$ & 2.05 & 30.1203 & 29.6521 & 29.5146 & 30.0277 & 29.7335 & 29.6796 & 30.1422 & 29.6748 & 29.5342 & 30.0466 & 29.7532 & 29.6986 \\
\hline $2^{* *}$ & 2.26 & 29.8951 & 29.3814 & 29.2877 & 29.8306 & 29.5549 & 29.4773 & 29.9151 & 29.4018 & 29.3014 & 29.8484 & 29.5741 & 29.4886 \\
\hline $3^{*}$ & 5.31 & 26.7434 & 26.2383 & 26.1128 & 26.7117 & 26.4408 & 26.3782 & 26.8091 & 26.3060 & 26.1715 & 26.7755 & 26.5012 & 26.4288 \\
\hline 5 & 1.62 & 30.3315 & 30.0302 & 29.9593 & 30.3341 & 30.2315 & 30.2010 & 30.2814 & 29.9793 & 29.8989 & 30.2812 & 30.1760 & 30.1414 \\
\hline $6^{*}$ & 1.77 & 31.0159 & 30.6164 & 30.5348 & 30.9278 & 30.7115 & 30.7335 & 31.0065 & 30.6044 & 30.5178 & 30.9128 & 30.6922 & 30.7140 \\
\hline $6^{* *}$ & 2.33 & 29.5707 & 29.0830 & 28.9512 & 29.4694 & 29.2525 & 29.2560 & 29.6005 & 29.1100 & 28.9821 & 29.5002 & 29.2792 & 29.2816 \\
\hline 7* & 1.38 & 30.6490 & 30.2753 & 30.1650 & 30.5788 & 30.4238 & 30.4175 & 30.6054 & 30.2309 & 30.1165 & 30.5401 & 30.3833 & 30.3724 \\
\hline $7^{* *}$ & 1.73 & 30.4604 & 30.0668 & 29.9805 & 30.3662 & 30.1611 & 30.1392 & 30.4221 & 30.0257 & 29.9356 & 30.3289 & 30.1196 & 30.0972 \\
\hline 8 & 2.40 & 29.4386 & 29.0708 & 28.9561 & 29.3873 & 29.2713 & 29.2023 & 29.5094 & 29.1410 & 29.0185 & 29.4622 & 29.3419 & 29.2659 \\
\hline 9 & 1.49 & 30.4895 & 30.1476 & 30.1289 & 30.5358 & 30.3733 & 30.3212 & 30.4239 & 30.0798 & 30.0464 & 30.4688 & 30.3039 & 30.2398 \\
\hline $11^{*}$ & 2.64 & 29.6102 & 29.0944 & 28.9613 & 29.4910 & 29.1725 & 29.0531 & 29.5514 & 29.0325 & 28.8981 & 29.4296 & 29.1093 & 28.9890 \\
\hline $11^{* *}$ & 2.73 & 29.3836 & 28.8890 & 28.7624 & 29.3315 & 29.0616 & 28.9832 & 29.3704 & 28.8741 & 28.7545 & 29.3172 & 29.0489 & 28.9743 \\
\hline 14 & 2.63 & 29.4551 & 29.0352 & 28.9512 & 29.3850 & 29.1893 & 29.0931 & 29.4074 & 28.9861 & 28.8891 & 29.3347 & 29.1378 & 29.0352 \\
\hline 15 & 6.18 & 26.1404 & 25.3826 & 25.0841 & 26.0568 & 25.5069 & 25.4555 & 26.0055 & 25.2447 & 24.9228 & 25.9075 & 25.3632 & 25.3124 \\
\hline 16 & 7.21 & 25.0747 & 24.3466 & 24.1335 & 25.0220 & 24.5310 & 24.3881 & 24.9700 & 24.2434 & 24.0409 & 24.9106 & 24.4290 & 24.2930 \\
\hline $19^{*}$ & 4.03 & 28.0576 & 27.5783 & 27.5084 & 28.0152 & 27.7377 & 27.7105 & 28.1076 & 27.6278 & 27.5604 & 28.0580 & 27.7812 & 27.7556 \\
\hline $19 * *$ & 4.33 & 27.7334 & 27.2616 & 26.9749 & 27.6512 & 27.4358 & 27.3461 & 27.7227 & 27.2470 & 26.9550 & 27.6324 & 27.4146 & 27.3237 \\
\hline 20 & 5.06 & 27.0793 & 26.6257 & 26.6544 & 27.0732 & 26.9008 & 26.8025 & 27.0095 & 26.5528 & 26.5836 & 26.9981 & 26.8248 & 26.7315 \\
\hline 17 & 0.97 & 31.1242 & 30.6893 & 30.6326 & 31.0799 & 30.8485 & 30.8187 & 31.1039 & 30.6673 & 30.6079 & 31.0611 & 30.8285 & 30.7978 \\
\hline 18-OMe & 3.67 & 28.5886 & 28.0807 & 27.9972 & 28.5683 & 28.2214 & 28.2501 & 28.4994 & 27.9907 & 27.9046 & 28.4798 & 28.1337 & 28.1596 \\
\hline 20-OAc-Me & 2.02 & 30.3386 & 29.8118 & 29.7149 & 30.2428 & 29.9042 & 29.8809 & 30.2393 & 29.7060 & 29.6006 & 30.1420 & 29.8004 & 29.7728 \\
\hline
\end{tabular}

\section{Compound 34}

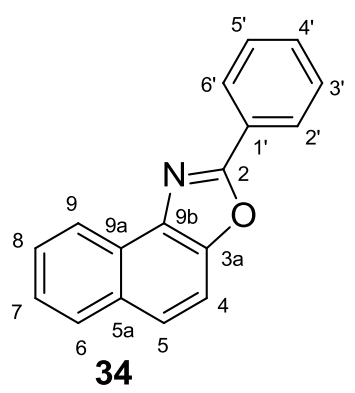

\begin{tabular}{|c|c|c|c|c|c|c|c|c|c|c|c|c|c|}
\hline \multirow{3}{*}{\multicolumn{2}{|c|}{ Exp }} & \multicolumn{12}{|c|}{ B3LYP } \\
\hline & & \multicolumn{6}{|c|}{ Gas phase } & \multicolumn{6}{|c|}{ PCM } \\
\hline & & $6-31 G^{*}$ & $6-31 G * *$ & $6-31+G * *$ & 6-311G* & $6-311 G * *$ & $6-311+G^{* *}$ & $6-31 G *$ & $6-31 G * *$ & $6-31+G^{* *}$ & 6-311G* & $6-311 G * *$ & $6-311+\mathrm{G} * *$ \\
\hline \multicolumn{14}{|c|}{${ }^{13} \mathrm{C}$} \\
\hline 2 & 162.3 & 34.6487 & 34.6431 & 32.8135 & 14.9205 & 14.9674 & 13.4043 & 34.2098 & 34.2049 & 32.2885 & 14.3372 & 14.3857 & 12.7481 \\
\hline $3 a$ & a 148.0 & 48.0682 & 47.9684 & 46.6405 & 29.4672 & 29.3772 & 28.1355 & 47.8070 & 47.7100 & 46.4099 & 29.1776 & 29.0994 & 27.8471 \\
\hline 4 & 110.8 & 85.2665 & 85.8996 & 85.4927 & 69.8695 & 69.0758 & 69.0425 & 84.7498 & 85.3751 & 84.8544 & 69.1186 & 68.3419 & 68.3544 \\
\hline 5 & 126.0 & 69.4808 & 70.0870 & 70.3612 & 53.5192 & 52.6568 & 51.4961 & 68.9301 & 69.5299 & 69.7512 & 52.8193 & 51.9658 & 50.8434 \\
\hline $5 a$ & a 131.2 & 64.9691 & 64.7381 & 64.3332 & 46.8422 & 46.6555 & 44.7316 & 65.0658 & 64.8386 & 64.3765 & 46.9707 & 46.7937 & 44.8275 \\
\hline 6 & 128.6 & 67.1930 & 67.7705 & 67.4505 & 50.7682 & 49.8234 & 48.9237 & 66.7659 & 67.3377 & 66.8969 & 50.1653 & 49.2357 & 48.3724 \\
\hline 7 & 125.4 & 70.9096 & 71.5255 & 71.4925 & 54.0081 & 53.0909 & 52.3738 & 70.5624 & 71.1820 & 71.1061 & 53.5075 & 52.6272 & 51.9949 \\
\hline 8 & 127.0 & 69.3545 & 69.9619 & 70.2649 & 52.6006 & 51.6643 & 50.9298 & 69.0099 & 69.6200 & 69.8643 & 52.0800 & 51.1735 & 50.5240 \\
\hline 9 & 122.3 & 71.6600 & 72.3172 & 71.7187 & 55.7857 & 54.9004 & 54.3292 & 72.3619 & 73.0161 & 72.4069 & 56.4881 & 55.6206 & 55.0737 \\
\hline & 126.6 & 68.9698 & 68.8129 & 67.8647 & 51.3252 & 51.1888 & 49.9476 & 69.5333 & 69.3712 & 68.3589 & 51.8908 & 51.7544 & 50.4770 \\
\hline
\end{tabular}




\begin{tabular}{|c|c|c|c|c|c|c|c|c|c|c|c|c|}
\hline $9 \mathrm{~b} 137.6$ & 56.2615 & 56.2394 & 56.9403 & 39.1975 & 39.1447 & 37.1435 & 56.9173 & 56.8920 & 57.5186 & 39.8064 & 39.7566 & 37.7523 \\
\hline 1' 127.6 & 67.9752 & 67.6888 & 65.7133 & 49.9566 & 49.6783 & 48.2333 & 68.5699 & 68.2788 & 66.2367 & 50.5527 & 50.2809 & 48.8192 \\
\hline $4^{\prime} 131.0$ & 66.3284 & 66.9464 & 66.6500 & 48.5430 & 47.6416 & 46.9133 & 65.3926 & 66.0173 & 65.6244 & 47.3977 & 46.5344 & 45.8359 \\
\hline 2' 127.3 & 68.2106 & 68.8745 & 68.2295 & 52.7568 & 51.9454 & 50.7898 & 68.3421 & 69.0022 & 68.3123 & 52.5108 & 51.7078 & 50.6011 \\
\hline $\begin{array}{l}6^{\prime} 127.3 \\
\end{array}$ & 68.2106 & 68.8745 & 68.2295 & 50.3387 & 49.4970 & 48.1627 & 68.3421 & 69.0022 & 68.3123 & 50.7797 & 49.9534 & 48.6426 \\
\hline 3' 128.9 & 67.8082 & 68.3873 & 68.1883 & 50.7969 & 49.8345 & 48.9298 & 67.3343 & 67.9128 & 67.6095 & 50.0402 & 49.1043 & 48.2492 \\
\hline $\begin{array}{l}5 ' 128.9 \\
{ }^{1} \mathbf{H}\end{array}$ & 67.8082 & 68.3873 & 68.1883 & 50.5029 & 49.5373 & 48.5650 & 67.3343 & 67.9128 & 67.6095 & 49.9500 & 49.0108 & 48.0810 \\
\hline $\begin{array}{ll}4 & 7.71\end{array}$ & 24.7609 & 24.0870 & 23.8129 & 24.5267 & 24.1017 & 24.0797 & 24.5956 & 23.9198 & 23.6418 & 24.3480 & 23.9287 & 23.9087 \\
\hline $\begin{array}{ll}5 & 7.8\end{array}$ & 24.6672 & 24.0529 & 23.8245 & 24.4338 & 24.0419 & 23.9125 & 24.4919 & 23.8758 & 23.6351 & 24.2467 & 23.8615 & 23.7269 \\
\hline $\begin{array}{ll}6 & 7.98\end{array}$ & 24.4949 & 23.8611 & 23.7228 & 24.2725 & 23.8361 & 23.7453 & 24.3367 & 23.7021 & 23.5488 & 24.1013 & 23.6726 & 23.5749 \\
\hline $\begin{array}{ll}7 & 7.58\end{array}$ & 24.7052 & 24.0990 & 23.8870 & 24.5573 & 24.1938 & 24.1751 & 24.5710 & 23.9649 & 23.7475 & 24.4108 & 24.0548 & 24.0343 \\
\hline 87.68 & 24.5829 & 23.9775 & 23.7954 & 24.4190 & 24.0509 & 24.0626 & 24.4592 & 23.8539 & 23.6656 & 24.2840 & 23.9236 & 23.9312 \\
\hline $\begin{array}{ll}9 & 8.61\end{array}$ & 23.5169 & 22.8612 & 22.6684 & 23.3382 & 22.8991 & 22.8887 & 23.5427 & 22.8865 & 22.6761 & 23.3488 & 22.9138 & 22.8935 \\
\hline $\begin{array}{ll}4^{\prime} \quad 7.69\end{array}$ & 24.8840 & 24.2548 & 24.0241 & 24.7286 & 24.3382 & 24.2700 & 24.7137 & 24.0851 & 23.8457 & 24.5476 & 24.1651 & 24.0905 \\
\hline $2^{\prime} \quad 8.36$ & 23.8538 & 23.1819 & 22.9949 & 23.9252 & 23.4854 & 23.3135 & 23.8324 & 23.1606 & 22.9624 & 23.8454 & 23.4099 & 23.2355 \\
\hline $\begin{array}{ll}6^{\prime} & 8.36\end{array}$ & 23.8538 & 23.1819 & 22.9949 & 23.4663 & 23.0244 & 22.8424 & 23.8324 & 23.1606 & 22.9624 & 23.4824 & 23.0450 & 22.8537 \\
\hline $\begin{array}{ll}3^{\prime} & 7.69\end{array}$ & 24.8240 & 24.1951 & 23.9498 & 24.6944 & 24.2949 & 24.2286 & 24.6803 & 24.0513 & 23.7981 & 24.5326 & 24.1406 & 24.0695 \\
\hline $\begin{array}{ll}5^{\prime} & 7.69\end{array}$ & 24.8240 & 24.1951 & 23.9498 & 24.6505 & 24.2531 & 24.1940 & 24.6803 & 24.0513 & 23.7981 & 24.5048 & 24.1154 & 24.0498 \\
\hline
\end{tabular}

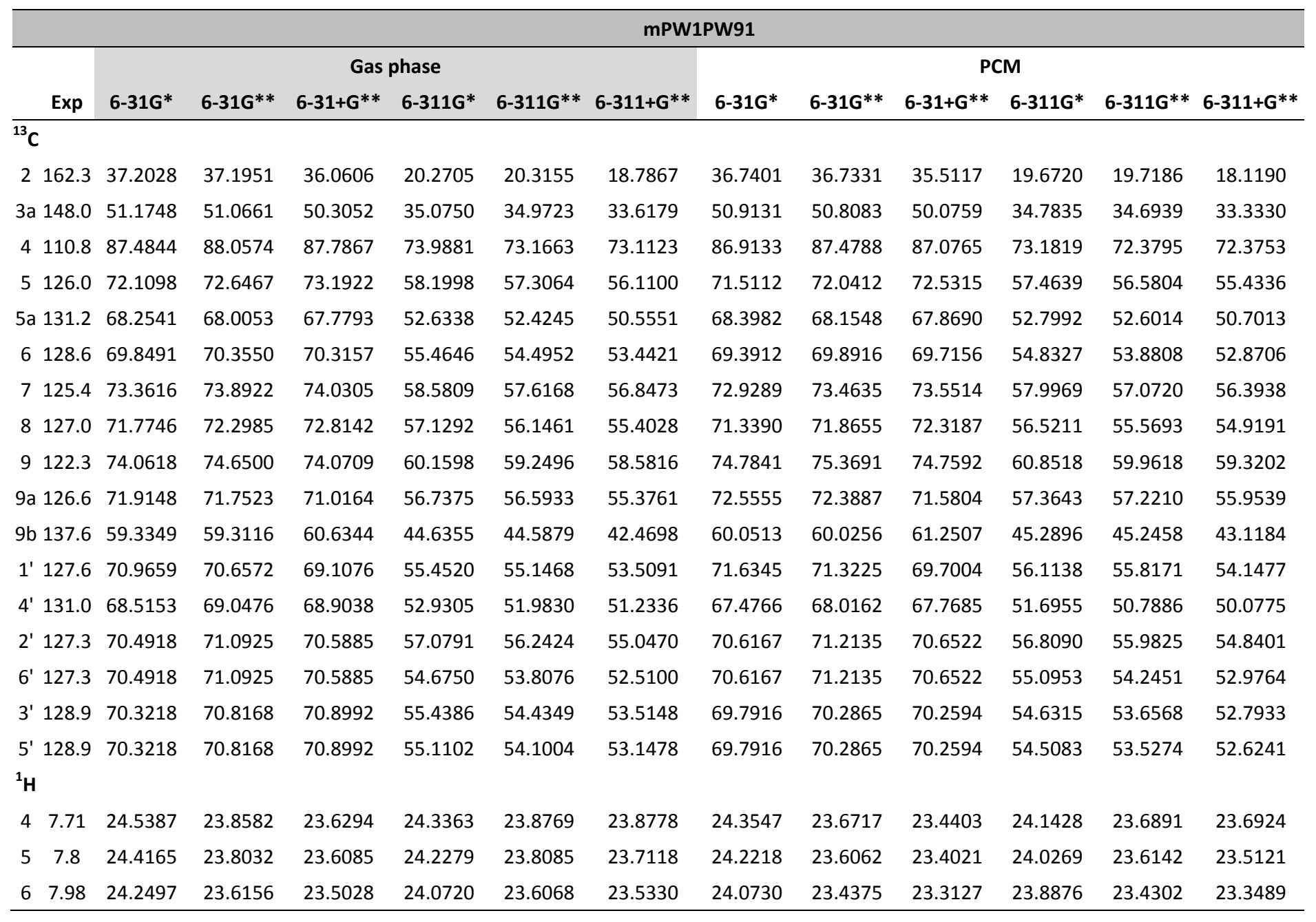




\begin{tabular}{llllllllllllll}
\hline 7 & 7.58 & 24.4897 & 23.8794 & 23.7164 & 24.3711 & 23.9713 & 23.9707 & 24.3362 & 23.7255 & 23.5595 & 24.2098 & 23.8177 & 23.8158 \\
8 & 7.68 & 24.3652 & 23.7558 & 23.6130 & 24.2340 & 23.8294 & 23.8558 & 24.2224 & 23.6127 & 23.4665 & 24.0843 & 23.6875 & 23.7106 \\
9 & 8.61 & 23.2819 & 22.6243 & 22.4672 & 23.1485 & 22.6751 & 22.6748 & 23.2952 & 22.6369 & 22.4631 & 23.1497 & 22.6810 & 22.6702 \\
$4^{\prime}$ & 7.69 & 24.6547 & 24.0235 & 23.8451 & 24.5298 & 24.1034 & 24.0506 & 24.4656 & 23.8348 & 23.6493 & 24.3346 & 23.9160 & 23.8570 \\
$2^{\prime}$ & 8.36 & 23.6209 & 22.9478 & 22.7931 & 23.7291 & 23.2516 & 23.0978 & 23.5877 & 22.9147 & 22.7497 & 23.6399 & 23.1669 & 23.0098 \\
$6^{\prime}$ & 8.36 & 23.6209 & 22.9478 & 22.7931 & 23.2730 & 22.7945 & 22.6243 & 23.5877 & 22.9147 & 22.7497 & 23.2810 & 22.8076 & 22.6279 \\
$3^{\prime}$ & 7.69 & 24.6027 & 23.9706 & 23.7623 & 24.5031 & 24.0690 & 24.0106 & 24.4418 & 23.8095 & 23.5948 & 24.3286 & 23.9019 & 23.8390 \\
$5^{\prime}$ & 7.69 & 24.6027 & 23.9706 & 23.7623 & 24.4585 & 24.0257 & 23.9704 & 24.4418 & 23.8095 & 23.5948 & 24.3003 & 23.8755 & 23.8142 \\
\hline
\end{tabular}

\section{Compound 35}

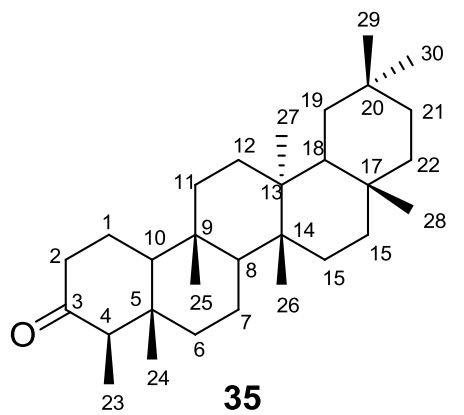

\begin{tabular}{|c|c|c|c|c|c|c|c|c|c|c|c|c|c|}
\hline & \multirow[b]{3}{*}{ Exp } & \multicolumn{12}{|c|}{ B3LYP } \\
\hline & & \multicolumn{6}{|c|}{ Gas phase } & \multicolumn{6}{|c|}{ PCM } \\
\hline & & 6-31G* & $6-31 G * *$ & $6-31+G * *$ & 6-311G* & 6-311G** & $6-311+G * *$ & 6-31G* & 6-31G** & $6-31+\mathrm{G} * *$ & 6-311G* & 6-311G** & $6-311+\mathrm{G} * *$ \\
\hline \multicolumn{14}{|l|}{${ }^{13} \mathrm{C}$} \\
\hline 1 & 22.3 & 164.6279 & 166.0033 & 165.9707 & 156.5332 & 156.2557 & 156.3945 & 164.4775 & 165.8501 & 165.7356 & 156.3835 & 156.0977 & 156.1242 \\
\hline 2 & 41.5 & 148.8889 & 150.0712 & 148.0703 & 138.0015 & 137.4668 & 137.4711 & 148.5265 & 149.7000 & 147.5354 & 137.5625 & 137.0167 & 136.7971 \\
\hline 3 & 213.2 & -11.6806 & -11.4531 & -15.0640 & -34.0440 & -33.7011 & -37.6120 & -15.5539 & -15.3263 & -19.8598 & -38.5701 & -38.2278 & -43.0090 \\
\hline 4 & 58.2 & 131.1681 & 131.6851 & 128.7654 & 119.9892 & 119.7105 & 119.6945 & 131.1844 & 131.6965 & 128.8129 & 120.0640 & 119.7728 & 119.6822 \\
\hline 5 & 42.1 & 144.4471 & 144.8300 & 145.7114 & 133.7697 & 133.9067 & 133.4795 & 144.0508 & 144.4344 & 145.2195 & 133.3839 & 133.5230 & 132.9787 \\
\hline 6 & 41.3 & 147.4753 & 148.6972 & 147.1857 & 138.1233 & 137.7025 & 136.8748 & 147.7012 & 148.9192 & 147.4757 & 138.4385 & 138.0125 & 137.2118 \\
\hline 7 & 18.2 & 168.2190 & 169.6469 & 170.4326 & 160.8990 & 160.6657 & 160.7662 & 168.2838 & 169.7125 & 170.5085 & 160.9793 & 160.7454 & 160.8367 \\
\hline 8 & 53.1 & 132.9351 & 133.5621 & 132.4353 & 122.7900 & 122.4324 & 122.0948 & 133.1160 & 133.7396 & 132.6720 & 123.0611 & 122.6979 & 122.3830 \\
\hline 9 & 37.4 & 148.8507 & 149.3006 & 149.0214 & 138.9063 & 138.9262 & 138.1212 & 148.7878 & 149.2387 & 148.9591 & 138.8680 & 138.8884 & 138.0656 \\
\hline 10 & 59.5 & 129.3108 & 129.8798 & 127.6535 & 117.8097 & 117.4568 & 117.3054 & 129.7436 & 130.3038 & 128.2213 & 118.4258 & 118.0566 & 117.9389 \\
\hline 11 & 35.6 & 152.7154 & 154.0597 & 153.2109 & 144.0485 & 143.6172 & 142.9126 & 152.8674 & 154.2095 & 153.3718 & 144.2299 & 143.7971 & 143.1041 \\
\hline 12 & 30.5 & 158.6573 & 160.0067 & 159.9169 & 150.7253 & 150.4426 & 150.2483 & 158.7473 & 160.0946 & 160.0249 & 150.8522 & 150.5632 & 150.3758 \\
\hline 13 & 39.7 & 145.7190 & 146.1229 & 145.2768 & 135.7537 & 135.8244 & 136.2861 & 145.6766 & 146.0839 & 145.2236 & 135.7064 & 135.7819 & 136.2369 \\
\hline 14 & 38.3 & 148.6372 & 149.0795 & 151.1098 & 138.5471 & 138.6360 & 137.8132 & 148.5982 & 149.0426 & 151.0680 & 138.5254 & 138.6163 & 137.7783 \\
\hline 15 & 32.8 & 152.3801 & 153.8222 & 154.8460 & 144.1243 & 143.7927 & 142.8075 & 152.5378 & 153.9763 & 155.0258 & 144.3455 & 144.0024 & 143.0286 \\
\hline 16 & 36.0 & 154.1970 & 155.5623 & 154.0364 & 145.3290 & 145.0491 & 144.1207 & 154.2903 & 155.6537 & 154.1403 & 145.4594 & 145.1716 & 144.2537 \\
\hline 17 & 30.0 & 152.4982 & 152.9582 & 151.1064 & 142.5935 & 142.7306 & 143.0790 & 152.4782 & 152.9412 & 151.0887 & 142.6063 & 142.7437 & 143.0906 \\
\hline 18 & 42.8 & 139.4292 & 140.1135 & 137.4603 & 129.5532 & 129.1221 & 129.1086 & 139.4728 & 140.1567 & 137.5190 & 129.6339 & 129.1995 & 129.1928 \\
\hline 19 & 35.3 & 151.4081 & 152.6196 & 152.6106 & 143.0288 & 142.5095 & 141.1252 & 151.5227 & 152.7332 & 152.7574 & 143.1983 & 142.6719 & 141.2958 \\
\hline 20 & 28.2 & 155.2045 & 155.8144 & 156.5775 & 144.9845 & 144.9359 & 144.0087 & 155.1645 & 155.7803 & 156.5300 & 144.9921 & 144.9443 & 144.0174 \\
\hline 21 & 32.4 & 154.5878 & 155.8233 & 155.6373 & 145.2539 & 144.8554 & 144.3916 & 154.6616 & 155.8974 & 155.7208 & 145.3622 & 144.9591 & 144.4964 \\
\hline 22 & 39.2 & 142.8366 & 144.1266 & 143.9565 & 133.2350 & 132.7393 & 132.4363 & 142.9771 & 144.2649 & 144.1081 & 133.4505 & 132.9427 & 132.6282 \\
\hline
\end{tabular}




\begin{tabular}{|c|c|c|c|c|c|c|c|c|c|c|c|c|c|}
\hline 23 & .8 & 9.9108 & 1.8315 & 2.6613 & 4.2652 & 74.2818 & & & 181.9969 & & 74.5293 & 174.5409 & \\
\hline 24 & & & & & & 67.2732 & & & & & & & \\
\hline 25 & 7.9 & & 4188 & & & & & & & & & & \\
\hline 26 & 0.3 & 53.3581 & 5.2593 & 6.5552 & 6.8436 & 008 & 56.0665 & & 191 & 1570 & 814 & 3256 & 156.302 \\
\hline 27 & 8.7 & 5.8469 & 7.7655 & 8.9596 & 9.6022 & 907 & 69 & & 7.9280 & 1724 & & 172 & \\
\hline 28 & 2.1 & 9.8020 & 1.6220 & 480 & 3.0421 & 52.7280 & 393 & & 1.7880 & 162.9572 & & & \\
\hline 29 & 1.8 & & & & & & & & & & & & \\
\hline $\begin{array}{l}30 \\
{ }^{1} \mathrm{H}\end{array}$ & 5.0 & & & & & & & & & & & & \\
\hline $1^{*}$ & 1.68 & 558 & .8683 & 374 & 10 & & & & & & & & 1597 \\
\hline $1^{* *}$ & 96 & 30.4003 & 29.9141 & 29.94 & 30.38 & & & & & & & & \\
\hline $2^{*}$ & .28 & 0.0492 & & & & & & & & & & & \\
\hline $2^{* *}$ & .39 & 0.0124 & 29.5716 & 29.3 & & & & & & & & & \\
\hline 4 & 2.25 & 29.9963 & 29.6474 & 22 & & & & & & & & & $20 s$ \\
\hline $6^{*}$ & 1.28 & 19 & & & & & & & & & & & \\
\hline ** & & & & & & & & & & & & & \\
\hline 7* & 37 & & & & & & & & & & & & \\
\hline ** & 49 & 0.6535 & & & & & & & & & & & \\
\hline 8 & 1.39 & 0.4777 & & & & & & & & & & & \\
\hline 10 & 1.53 & & & & & & & & & & & & \\
\hline 1* & & & & & & & & & & & & & \\
\hline & 46 & & & & & & & & & & & & \\
\hline $12 *$ & .34 & 0.4310 & 30.0200 & 9.9879 & 5495 & & & & & 812 & & & 2990 \\
\hline$* *$ & 34 & 9716 & & & & & & & & & & & \\
\hline $15 *$ & .27 & 5661 & & & & & & & & & & & \\
\hline & 46 & & & & & & & & & & & & \\
\hline $16^{*}$ & .36 & 30.5509 & 30.1768 & 0.2518 & 30.6079 & 30.4126 & 806 & & 1886 & 30.2647 & & & 4945 \\
\hline $6^{* *}$ & 1.57 & .4828 & & & & & & & & & & & \\
\hline 18 & 1.56 & & & & & & & & & & & & \\
\hline & & & & & & & & & & & & & \\
\hline$* *$ & 1.38 & 30.4819 & & & & & & & & & & & \\
\hline $21^{*}$ & .30 & 30.9384 & 30.5322 & 30.5162 & 30.9838 & 30.7738 & 0.7584 & 410 & 0.5354 & 30.5189 & 9834 & & 7607 \\
\hline I** & 1.51 & 30.5242 & .1008 & .0684 & 806 & & & & & 640 & & & 3334 \\
\hline $22 *$ & 0.94 & 0.8693 & & & & & & & & & & & \\
\hline $22^{* *}$ & 1.50 & & & & & & & & & & & & \\
\hline 23 & 0.87 & & & & & & & & & & & & \\
\hline 24 & 0.71 & & & & & & & & & & & & 2269 \\
\hline 25 & 0.86 & 31.0956 & 30.6534 & 30.6151 & 31.2196 & 30.9604 & & & 30.6410 & 30.6044 & 31.2097 & 3514 & 30.9106 \\
\hline 26 & & & & & & & & & & & & & \\
\hline 27 & & & & & & & & & & & & & \\
\hline 28 & & & & & & & & & & & & & \\
\hline 29 & 1. & & & & & & & & & & 087 & & 30.9380 \\
\hline 30 & & & & & & & & & & & & 30.9244 & ו \\
\hline
\end{tabular}




\begin{tabular}{|c|c|c|c|c|c|c|c|c|c|c|c|c|c|}
\hline & \multirow[b]{3}{*}{ Exp } & \multicolumn{12}{|c|}{ mPW1PW91 } \\
\hline & & \multicolumn{6}{|c|}{ Gas phase } & \multicolumn{6}{|c|}{ PCM } \\
\hline & & $6-31 G *$ & $6-31 G * *$ & $6-31+G * *$ & 6-311G* & $6-311 G * *$ & $6-311+G * *$ & $6-31 G *$ & $6-31 G * *$ & $6-31+G * *$ & 6-311G* & $6-311 G * *$ & $6-311+G * *$ \\
\hline \multicolumn{14}{|l|}{${ }^{13} \mathrm{C}$} \\
\hline 1 & 22.3 & 169.3756 & 170.6435 & 170.5694 & 162.0067 & 161.7372 & 161.7711 & 169.2331 & 170.4988 & 170.3560 & 161.8616 & 161.5848 & 161.5212 \\
\hline 2 & 41.5 & 153.2483 & 154.3413 & 152.4402 & 143.5521 & 143.0178 & 142.9141 & 152.8843 & 153.9691 & 151.9241 & 143.1088 & 142.5635 & 142.2673 \\
\hline 3 & 213.2 & -9.1229 & -8.9079 & -11.8100 & -28.4776 & -28.1472 & -31.5052 & -13.1093 & -12.8937 & -16.6145 & -33.0340 & -32.7033 & -36.8226 \\
\hline 4 & 58.2 & 136.0656 & 136.5309 & 134.2487 & 125.7971 & 125.5277 & 125.4562 & 136.0674 & 136.5302 & 134.2787 & 125.8327 & 125.5529 & 125.4219 \\
\hline 5 & 42.1 & 149.7242 & 150.0962 & 150.7895 & 139.8177 & 139.9554 & 139.5741 & 149.3276 & 149.7005 & 150.3138 & 139.4349 & 139.5749 & 139.0954 \\
\hline 6 & 41.3 & 152.6365 & 153.7412 & 152.3645 & 144.1523 & 143.7420 & 142.9875 & 152.8784 & 153.9802 & 152.6607 & 144.4629 & 144.0486 & 143.3218 \\
\hline 7 & 18.2 & 172.8653 & 174.1908 & 175.0764 & 166.2612 & 166.0240 & 165.9603 & 172.9475 & 174.2737 & 175.1723 & 166.3524 & 166.1146 & 166.0435 \\
\hline 8 & 53.1 & 138.5741 & 139.1010 & 138.0528 & 129.3180 & 128.9889 & 128.7364 & 138.7531 & 139.2775 & 138.2799 & 129.5721 & 129.2383 & 129.0095 \\
\hline 9 & 37.4 & 154.2055 & 154.6338 & 154.5583 & 145.1691 & 145.1826 & 144.3402 & 154.1444 & 154.5738 & 154.5002 & 145.1324 & 145.1466 & 144.2909 \\
\hline 10 & 59.5 & 135.1039 & 135.5648 & 133.4804 & 124.6195 & 124.2789 & 124.0717 & 135.5424 & 135.9970 & 134.0343 & 125.2076 & 124.8532 & 124.6791 \\
\hline 11 & 35.6 & 157.7589 & 158.9941 & 157.8904 & 149.9158 & 149.5079 & 148.8774 & 157.9208 & 159.1545 & 158.0589 & 150.0973 & 149.6885 & 149.0701 \\
\hline 12 & 30.5 & 163.7285 & 164.9670 & 164.8626 & 156.4707 & 156.1923 & 156.0479 & 163.8301 & 165.0671 & 164.9834 & 156.5987 & 156.3152 & 156.1782 \\
\hline 13 & 39.7 & 150.9922 & 151.3864 & 150.1948 & 141.7822 & 141.8500 & 142.3186 & 150.9523 & 151.3494 & 150.1469 & 141.7399 & 141.8122 & 142.2742 \\
\hline 14 & 38.3 & 153.9527 & 154.3856 & 156.4959 & 144.6140 & 144.6953 & 143.8493 & 153.9128 & 154.3477 & 156.4547 & 144.5949 & 144.6778 & 143.8192 \\
\hline 15 & 32.8 & 157.6711 & 158.9891 & 159.9613 & 150.1200 & 149.7911 & 148.8104 & 157.8449 & 159.1602 & 160.1554 & 150.3418 & 150.0028 & 149.0343 \\
\hline 16 & 36.0 & 159.1679 & 160.4133 & 158.8873 & 151.1749 & 150.8971 & 150.0993 & 159.2870 & 160.5304 & 159.0196 & 151.3211 & 151.0358 & 150.2495 \\
\hline 17 & 30.0 & 157.9696 & 158.4164 & 156.8634 & 148.7782 & 148.9015 & 149.2257 & 157.9484 & 158.3981 & 156.8455 & 148.7930 & 148.9167 & 149.2395 \\
\hline 18 & 42.8 & 144.5806 & 145.1867 & 142.7778 & 135.7166 & 135.2930 & 135.3613 & 144.6244 & 145.2306 & 142.8343 & 135.7914 & 135.3648 & 135.4420 \\
\hline 19 & 35.3 & 156.2566 & 157.3407 & 157.4078 & 148.7213 & 148.2173 & 146.9300 & 156.3834 & 157.4665 & 157.5639 & 148.8902 & 148.3798 & 147.1032 \\
\hline 20 & 28.2 & 160.4789 & 161.0798 & 162.0463 & 151.0032 & 150.9661 & 150.0477 & 160.4405 & 161.0469 & 162.0035 & 151.0170 & 150.9810 & 150.0618 \\
\hline 21 & 32.4 & 159.5649 & 160.6788 & 160.3294 & 151.0023 & 150.6104 & 150.1473 & 159.6593 & 160.7735 & 160.4375 & 151.1234 & 150.7271 & 150.2684 \\
\hline 22 & 39.2 & 148.0660 & 149.2080 & 148.8927 & 139.2552 & 138.7582 & 138.5317 & 148.2395 & 149.3796 & 149.0758 & 139.4868 & 138.9786 & 138.7425 \\
\hline 23 & 6.8 & 184.0921 & 185.8861 & 186.4842 & 178.7889 & 178.8140 & 178.9477 & 184.2857 & 186.0796 & 186.7338 & 179.0631 & 179.0828 & 179.2265 \\
\hline 24 & 14.7 & 177.3511 & 179.0918 & 179.7821 & 171.9393 & 171.7989 & 171.5278 & 177.5042 & 179.2353 & 179.9476 & 172.0993 & 171.9440 & 171.6640 \\
\hline 25 & 17.9 & 171.6902 & 173.5206 & 173.7098 & 166.3214 & 165.9752 & 165.7673 & 171.9336 & 173.7552 & 173.9712 & 166.6051 & 166.2438 & 166.0409 \\
\hline 26 & 20.3 & 167.5421 & 169.2745 & 170.1108 & 161.5616 & 161.3269 & 160.8005 & 167.7273 & 169.4576 & 170.3343 & 161.8060 & 161.5598 & 161.0484 \\
\hline 27 & 18.7 & 170.0391 & 171.8161 & 172.8403 & 164.3609 & 164.0524 & 164.1499 & 170.2326 & 172.0024 & 173.0712 & 164.6075 & 164.2828 & 164.3855 \\
\hline 28 & 32.1 & 164.2534 & 165.9030 & 166.6409 & 157.9435 & 157.6375 & 157.6842 & 164.4495 & 166.0918 & 166.8676 & 158.1889 & 157.8658 & 157.9238 \\
\hline 29 & 31.8 & 161.1329 & 162.6103 & 163.3220 & 154.2083 & 154.0078 & 153.0482 & 161.4299 & 162.8970 & 163.6699 & 154.5961 & 154.3673 & 153.4089 \\
\hline 30 & 35.0 & 166.6288 & 168.2403 & 168.7830 & 160.2945 & 159.9917 & 159.8656 & 166.9032 & 168.5061 & 169.1069 & 160.6457 & 160.3194 & 160.1979 \\
\hline \multicolumn{14}{|l|}{${ }^{1} \mathrm{H}$} \\
\hline $1^{*}$ & 1.68 & 30.3320 & 29.8389 & 29.8756 & 30.3996 & 30.1035 & 30.1271 & 30.2820 & 29.7875 & 29.8210 & 30.3450 & 30.0497 & 30.0702 \\
\hline $1 * *$ & 1.96 & 30.3507 & 29.8641 & 29.8567 & 30.3084 & 30.0087 & 30.0644 & 30.2732 & 29.7843 & 29.7723 & 30.2347 & 29.9316 & 29.9811 \\
\hline $2^{*}$ & 2.28 & 29.9196 & 29.4267 & 29.4591 & 29.7952 & 29.5122 & 29.5332 & 29.9732 & 29.4752 & 29.5146 & 29.8495 & 29.5594 & 29.5869 \\
\hline $2 * *$ & 2.39 & 29.9736 & 29.5289 & 29.2976 & 29.8457 & 29.6132 & 29.5681 & 29.8134 & 29.3640 & 29.1145 & 29.6842 & 29.4506 & 29.3911 \\
\hline 4 & 2.25 & 29.9421 & 29.5969 & 29.6385 & 29.9253 & 29.7731 & 29.6819 & 29.7809 & 29.4314 & 29.4495 & 29.7602 & 29.6055 & 29.4953 \\
\hline $6^{*}$ & 1.28 & 30.7691 & 30.4041 & 30.3001 & 30.7159 & 30.5388 & 30.4676 & 30.7149 & 30.3493 & 30.2385 & 30.6610 & 30.4845 & 30.4075 \\
\hline $6 * *$ & 1.75 & 30.3551 & 29.9513 & 29.9281 & 30.3614 & 30.1389 & 30.0823 & 30.3557 & 29.9523 & 29.9318 & 30.3650 & 30.1429 & 30.0891 \\
\hline $7^{*}$ & 1.37 & 30.5399 & 30.0926 & 30.1669 & 30.6287 & 30.3741 & 30.3608 & 30.5158 & 30.0688 & 30.1415 & 30.6055 & 30.3523 & 30.3385 \\
\hline $7 * *$ & 1.49 & 30.6078 & 30.1651 & 30.2605 & 30.6126 & 30.3465 & 30.3373 & 30.5918 & 30.1487 & 30.2431 & 30.5983 & 30.3320 & 30.3228 \\
\hline 8 & 1.39 & 30.4391 & 30.1224 & 30.2334 & 30.5150 & 30.3629 & 30.3449 & 30.3955 & 30.0798 & 30.1875 & 30.4672 & 30.3177 & 30.2996 \\
\hline 10 & 1.53 & 30.3414 & 30.0588 & 30.0303 & 30.3483 & 30.2355 & 30.2154 & 30.2385 & 29.9545 & 29.9173 & 30.2399 & 30.1272 & 30.1041 \\
\hline
\end{tabular}




\begin{tabular}{|c|c|c|c|c|c|c|c|c|c|c|c|c|c|}
\hline $11^{*}$ & 26 & 90 & 34 & 33 & 04 & 14 & 86 & 55 & 30.2142 & 30.31 & 46 & 30.4244 & \\
\hline & & 5060 & .0933 & 43 & & & & & & & & & \\
\hline & & & & & & & & & & & & & \\
\hline & & & & & & & & & & & & & \\
\hline & & & & & & & & & & & & & \\
\hline & 46 & .3375 & .9492 & 3 & & 1152 & & & & & & & \\
\hline $16^{*}$ & 36 & .4970 & .1247 & 95 & & .3081 & & & & & & & \\
\hline ;** & ${ }^{k} 1$ & & & & & & & & & & & & \\
\hline 18 & 56 & & & & & & & & & & & & \\
\hline $19 *$ & & & & & & & & & & & & & \\
\hline & ${ }^{k} 1.38$ & .4603 & & & & & & & & & & & \\
\hline $21 *$ & .30 & .8968 & 0.4955 & 30.46 & .8829 & & & & & & & & \\
\hline $1^{* *}$ & ${ }^{k} 1.51$ & .4891 & .0673 & 9.99 & .4793 & 52 & & & & & & & \\
\hline $22^{*}$ & 34 & & & & & & & & & & & & \\
\hline & & & & & & & & & & & & & \\
\hline 23 & & & & & & & & & & & & & \\
\hline 24 & .71 & & & & & & & & & & & & \\
\hline 25 & 86 & & & & & & & & & & & & 3182 \\
\hline 26 & D0 & & & & & & & & & & & & 0.1846 \\
\hline 27 & 1.05 & 18927 & & 30.4463 & & & 30.0004 & & 207 & 30.4343 & 30.9109 & 30.6227 & 30.6602 \\
\hline 28 & 1.11 & & & & & & & & & & & & \\
\hline 29 & & & & & & & & & & & & & \\
\hline 30 & 0.95 & .0991 & .6633 & 6513 & . 0807 & 8272 & .8752 & & 30.6565 & 30.6453 & 31.0746 & 30.8220 & 308708 \\
\hline
\end{tabular}

\section{Compound 36}

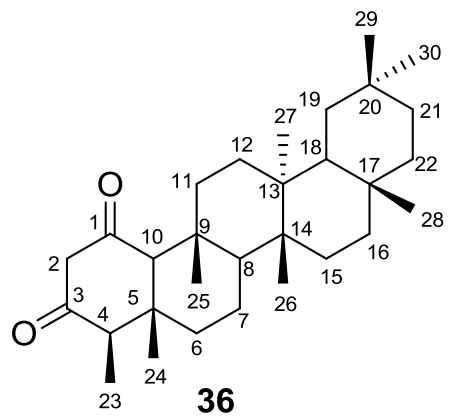

\begin{tabular}{|c|c|c|c|c|c|c|c|c|c|c|c|c|c|}
\hline & \multirow[b]{3}{*}{ Exp } & \multicolumn{12}{|c|}{ B3LYP } \\
\hline & & \multicolumn{6}{|c|}{ Gas phase } & \multicolumn{6}{|c|}{ PCM } \\
\hline & & 6-31G* & $6-31 G * *$ & $6-31+\mathrm{G} * *$ & 6-311G* & $6-311 G * *$ & $6-311+\mathrm{G} * *$ & 6-31G* & $6-31 G * *$ & $6-31+\mathrm{G} * *$ & 6-311G* & $6-311 G * *$ & $6-311+G * *$ \\
\hline \multicolumn{14}{|c|}{${ }^{13} \mathrm{C}$} \\
\hline 1 & 202.8 & -6.1471 & -5.9851 & -8.5329 & -28.3171 & -28.0664 & -30.1999 & -8.4297 & -8.2603 & -11.2822 & -31.0246 & -30.7697 & -33.2967 \\
\hline 2 & 60.7 & 129.9753 & 130.8991 & 130.4798 & 117.7673 & 116.8741 & 116.3481 & 129.4759 & 130.3882 & 129.7622 & 117.1188 & 116.2089 & 115.4916 \\
\hline 3 & 204.2 & -4.8382 & -4.6249 & -7.4223 & -26.5523 & -26.2308 & -29.5720 & -7.7969 & -7.5856 & -11.0162 & -30.0186 & -29.7006 & -33.6778 \\
\hline 4 & 59.1 & 130.1767 & 130.6568 & 129.7020 & 118.4215 & 118.1332 & 118.1435 & 130.3516 & 130.8266 & 129.9869 & 118.6999 & 118.3992 & 118.3616 \\
\hline 5 & 37.9 & 148.2963 & 148.6692 & 150.9524 & 138.3599 & 138.5032 & 137.6039 & 148.0148 & 148.3850 & 150.6321 & 138.1203 & 138.2687 & 137.2959 \\
\hline 6 & 40.7 & 147.8284 & 149.0189 & 148.0939 & 138.2717 & 137.8130 & 136.9093 & 148.2108 & 149.3953 & 148.5751 & 138.7813 & 138.3159 & 137.4383 \\
\hline 7 & 18.1 & 168.4155 & 169.8260 & 169.4227 & 161.0908 & 160.8523 & 160.9606 & 168.5221 & 169.9326 & 169.5492 & 161.2143 & 160.9748 & 161.0864 \\
\hline
\end{tabular}




\begin{tabular}{|c|c|c|c|c|c|c|c|c|c|c|c|c|c|}
\hline 8 & 52.2 & 134.0785 & 134.6765 & 133.3380 & 124.1589 & 123.7983 & 123.7030 & 134.4396 & 135.0332 & 133.8002 & 124.6424 & 124.2780 & 124.2248 \\
\hline 9 & 37.2 & 148.7164 & 149.1856 & 148.0181 & 138.7705 & 138.8291 & 138.7284 & 148.7725 & 149.2414 & 148.0874 & 138.8627 & 138.9209 & 138.8149 \\
\hline 10 & 71.9 & 118.5250 & 118.8816 & 116.6840 & 106.1230 & 105.6675 & 104.9428 & 118.8157 & 119.1671 & 117.0217 & 106.5487 & 106.0813 & 105.2808 \\
\hline 11 & 34.6 & 154.3934 & 155.7283 & 155.0823 & 145.7688 & 145.3732 & 145.1426 & 154.3382 & 155.6759 & 155.0215 & 145.7324 & 145.3394 & 145.1016 \\
\hline 12 & 30.2 & 159.1489 & 160.4852 & 160.2549 & 151.2043 & 150.9182 & 150.9501 & 159.2757 & 160.6099 & 160.4226 & 151.3846 & 151.0919 & 151.1354 \\
\hline 13 & 39.5 & 145.9341 & 146.3367 & 145.3220 & 135.9127 & 135.9957 & 136.4420 & 145.8931 & 146.2991 & 145.2714 & 135.8668 & 135.9539 & 136.3993 \\
\hline 14 & 38.3 & 148.7847 & 149.2268 & 151.3484 & 138.6085 & 138.6954 & 137.7969 & 148.7560 & 149.2001 & 151.3169 & 138.5975 & 138.6870 & 137.7704 \\
\hline 15 & 32.4 & 152.0720 & 153.5099 & 154.1923 & 143.7810 & 143.4367 & 142.6437 & 152.3100 & 153.7434 & 154.4694 & 144.0941 & 143.7370 & 142.9644 \\
\hline 16 & 35.9 & 154.1651 & 155.5269 & 153.7692 & 145.2952 & 145.0214 & 143.8495 & 154.2735 & 155.6328 & 153.8911 & 145.4413 & 145.1601 & 144.0014 \\
\hline 17 & 30.0 & 152.5183 & 152.9781 & 150.4307 & 142.5833 & 142.7277 & 143.1946 & 152.4873 & 152.9501 & 150.4055 & 142.5835 & 142.7286 & 143.1955 \\
\hline 18 & 42.7 & 139.5098 & 140.1980 & 137.2700 & 129.6279 & 129.1963 & 129.2877 & 139.5522 & 140.2397 & 137.3300 & 129.7122 & 129.2769 & 129.3756 \\
\hline 19 & 35.3 & 151.4620 & 152.6693 & 152.6053 & 143.0309 & 142.5114 & 141.3284 & 151.5691 & 152.7755 & 152.7462 & 143.1934 & 142.6669 & 141.4907 \\
\hline 20 & 28.2 & 55.1930 & 155.8069 & 156.7375 & 144.9586 & 144.9176 & 144.0154 & 155.1528 & 155.7726 & 156.6919 & 144.9676 & 144.9270 & 144.0290 \\
\hline 21 & 32.8 & 154.5728 & 155.8072 & 155.2406 & 145.2723 & 144.8846 & 144.1472 & 154.6544 & 155.8891 & 155.3357 & 145.3902 & 144.9979 & 144.2660 \\
\hline 22 & 39.3 & 143.0215 & 144.3110 & 143.6055 & 133.4767 & 132.9802 & 132.8381 & 143.1739 & 144.4609 & 143.7733 & 133.7080 & 133.1988 & 133.0488 \\
\hline 23 & 7.3 & 179.5488 & 181.4446 & 182.1418 & 173.7158 & 173.6957 & 173.7261 & 179.7110 & 181.6043 & 182.3332 & 173.9434 & 173.9160 & 173.9296 \\
\hline 24 & 16.0 & 172.6385 & 74.4465 & 176.4535 & 166.3359 & 166.1221 & 165.6080 & 172.6441 & 174.4414 & 176.4003 & 166.3300 & 166.0978 & 165.5409 \\
\hline 25 & 18.0 & 167.5830 & 169.5439 & 169.8165 & 161.7804 & 161.3792 & 161.2551 & 167.6765 & 169.6322 & 169.9318 & 161.9219 & 161.5082 & 161.3806 \\
\hline 26 & 20.3 & 163.3434 & 165.2295 & 165.8556 & 156.7322 & 156.4781 & 156.0104 & 163.5052 & 165.3893 & 166.0629 & 156.9771 & 156.7109 & 156.2516 \\
\hline 27 & 18.8 & 165.9205 & 167.8380 & 168.8972 & 159.6994 & 159.3874 & 159.5396 & 166.0996 & 168.0095 & 169.1201 & 159.9533 & 159.6233 & 159.7798 \\
\hline 28 & 32.1 & 159.8479 & 1.6669 & 162.6632 & 153.1070 & 152.7949 & 152.8905 & 160.0484 & 161.8569 & 162.8802 & 153.3626 & 153.0298 & 153.1308 \\
\hline 29 & 31.8 & 156.5301 & 158.1555 & 159.4279 & 149.1392 & 148.9079 & 147.7000 & 156.7927 & 158.4073 & 159.7458 & 149.5252 & 149.2620 & 148.0529 \\
\hline $\begin{array}{l}30 \\
{ }^{1} \mathbf{H}\end{array}$ & 35.0 & 162.2033 & 163.9775 & 165.4485 & 155.4384 & 155.1221 & 154.7146 & 162.4296 & 164.1947 & 165.7195 & 155.7679 & 155.4249 & 155.0196 \\
\hline $2^{*}$ & 3.24 & 29.2490 & 28.6932 & 28.6241 & 29.0508 & 28.7276 & 28.6804 & 29.3485 & 28.7867 & 273 & 29.1526 & 220 & 28.7792 \\
\hline $2^{* *}$ & 3.46 & 28.8322 & 28.3286 & 28.3321 & 28.7866 & 28.4664 & 28.5421 & 28.5763 & 28.0682 & 28.0501 & 28.5234 & 28.2056 & 28.2691 \\
\hline 4 & 2.58 & 29.7148 & 29.3479 & 29.3071 & 29.7577 & 29.5972 & 29.4408 & 29.4936 & 29.1213 & 29.0614 & 29.5321 & 29.3687 & 29.1976 \\
\hline $6^{*}$ & 1.39 & 30.7562 & 30.3760 & 30.3754 & 30.7719 & 30.5821 & 30.5909 & 30.6635 & 30.2823 & 30.2713 & 30.6775 & 30.4889 & 30.4901 \\
\hline $6^{* *}$ & 1.90 & 30.2756 & 29.8645 & 29.8327 & 30.3433 & 30.1260 & 30.0703 & 30.2430 & 29.8313 & 29.7974 & 30.3166 & 30.0980 & 30.0397 \\
\hline $7^{*}$ & 1.43 & 30.6582 & 30.2078 & 30.3369 & 30.7096 & 30.4350 & 30.4444 & 30.6272 & 30.1767 & 30.3046 & 30.6815 & 30.4070 & 30.4154 \\
\hline $7^{* *}$ & 1.54 & 30.5224 & 30.0613 & 30.1897 & 30.6874 & 30.4074 & 30.4720 & 30.5009 & 30.0422 & 30.1724 & 30.6680 & 30.3926 & 30.4577 \\
\hline 8 & 1.25 & 30.6913 & 30.3584 & 30.4670 & 30.8774 & 30.7014 & 30.6780 & 30.6175 & 30.2844 & 30.3840 & 30.7967 & 30.6228 & 30.5967 \\
\hline 10 & 2.38 & 29.7060 & 29.3845 & 29.2693 & 29.7727 & 29.6147 & 29.5474 & 29.4961 & 29.1695 & 29.0307 & 29.5541 & 29.3932 & 29.3132 \\
\hline $11^{*}$ & 1.14 & 30.7929 & 30.3915 & 30.3579 & 30.9189 & 30.6985 & 30.6436 & 30.7074 & 30.3039 & 30.2580 & 30.8280 & 30.6074 & 30.5469 \\
\hline $11^{* *}$ & 2.15 & 29.8211 & 29.3877 & 29.4861 & 29.8739 & 29.6504 & 29.6456 & 29.9649 & 29.5364 & 29.6429 & 30.0160 & 29.7956 & 29.7947 \\
\hline $12^{*}$ & 1.41 & 30.4092 & 29.9950 & 29.9589 & 30.5304 & 30.3236 & 30.3017 & 30.4074 & 29.9935 & 29.9527 & 30.5262 & 30.3197 & 30.2957 \\
\hline $12 * *$ & 1.41 & 31.0392 & 30.6691 & 30.6116 & 31.1276 & 30.9350 & 30.8901 & 31.0270 & 30.6569 & 30.5923 & 31.1087 & 30.9174 & 30.8699 \\
\hline $15 *$ & 1.27 & 30.6170 & 30.2011 & 30.1663 & 30.7194 & 30.4871 & 30.4264 & 30.5873 & 30.1713 & 30.1347 & 30.6913 & 30.4593 & 30.3980 \\
\hline $15^{* *}$ & 1.52 & 30.3648 & 29.9713 & 30.1210 & 30.4458 & 30.2043 & 30.2369 & 30.3355 & 29.9420 & 30.0908 & 30.4154 & 30.1755 & 30.2089 \\
\hline $16 *$ & 1.35 & 30.5742 & 30.1987 & 30.3251 & 30.6329 & 30.4338 & 30.5020 & 30.5812 & 30.2058 & 30.3336 & 30.6412 & 30.4429 & 30.5117 \\
\hline $16^{* *}$ & 1.56 & 30.4815 & 30.0464 & 30.0880 & 30.5355 & 30.2543 & 30.2143 & 30.4641 & 30.0290 & 30.0714 & 30.5174 & 30.2379 & 30.1981 \\
\hline 18 & 1.57 & 30.3352 & 29.9725 & 30.1315 & 30.5683 & 30.3982 & 30.4794 & 30.3089 & 29.9481 & 30.1017 & 30.5374 & 30.3706 & 30.4523 \\
\hline $19 *$ & 1.36 & 30.6160 & 30.2158 & 30.2412 & 30.7139 & 30.4874 & 30.5363 & 30.6139 & 30.2151 & 30.2358 & 30.7075 & 30.4834 & 30.5316 \\
\hline $19 * *$ & 1.41 & 30.4765 & 30.0517 & 30.1923 & 30.6670 & 30.4051 & 30.5026 & 30.4582 & 30.0340 & 30.1714 & 30.6465 & 30.3863 & 30.4829 \\
\hline $21^{*}$ & 1.27 & 30.9337 & 30.5300 & 30.5092 & 30.9761 & 30.7687 & 30.7154 & 30.9366 & 30.5336 & 30.5124 & 30.9761 & 30.7705 & 30.7181 \\
\hline $21 * *$ & 1.46 & 30.5189 & 30.0961 & 29.9916 & 30.5757 & 30.3406 & 30.3328 & 30.5136 & 30.0911 & 29.9858 & 30.5706 & 30.3356 & 30.3277 \\
\hline
\end{tabular}




\begin{tabular}{rrllllllllllll}
\hline $22^{*}$ & 0.94 & 30.8809 & 30.5104 & 30.4443 & 30.9500 & 30.7511 & 30.6943 & 30.8854 & 30.5149 & 30.4493 & 30.9523 & 30.7543 & 30.6986 \\
$22^{* *}$ & 1.49 & 30.6969 & 30.3165 & 30.1702 & 30.7715 & 30.5656 & 30.5207 & 30.6838 & 30.3040 & 30.1566 & 30.7559 & 30.5518 & 30.5066 \\
23 & 1.05 & 31.2458 & 30.7691 & 30.6997 & 31.2716 & 30.9808 & 30.9581 & 31.2481 & 30.7708 & 30.6984 & 31.2738 & 30.9836 & 30.9549 \\
24 & 0.69 & 31.4402 & 30.9961 & 31.1221 & 31.5702 & 31.3158 & 31.3070 & 31.4326 & 30.9889 & 31.1128 & 31.5602 & 31.3070 & 31.2963 \\
25 & 1.20 & 30.7629 & 30.2915 & 30.1524 & 30.8692 & 30.5878 & 30.5260 & 30.7766 & 30.3050 & 30.1625 & 30.8805 & 30.5983 & 30.5361 \\
26 & 1.03 & 31.0301 & 30.5766 & 30.6029 & 31.1705 & 30.9026 & 30.8777 & 31.0312 & 30.5786 & 30.6049 & 31.1733 & 30.9065 & 30.8826 \\
27 & 1.02 & 30.9880 & 30.5214 & 30.5710 & 31.0819 & 30.7858 & 30.8327 & 30.9692 & 30.5035 & 30.5530 & 31.0654 & 30.7714 & 30.8183 \\
28 & 1.18 & 31.0456 & 30.6161 & 30.5640 & 31.1532 & 30.9107 & 30.8053 & 31.0403 & 30.6109 & 30.5581 & 31.1481 & 30.9065 & 30.8016 \\
29 & 1.00 & 31.1935 & 30.7836 & 30.6959 & 31.2003 & 30.9814 & 30.9315 & 31.1989 & 30.7884 & 30.7034 & 31.2067 & 30.9878 & 30.9388 \\
30 & 0.94 & 31.1548 & 30.7168 & 30.7120 & 31.1859 & 30.9345 & 30.9742 & 31.1511 & 30.7131 & 30.7086 & 31.1825 & 30.9319 & 30.9720 \\
\hline
\end{tabular}

\begin{tabular}{|c|c|c|c|c|c|c|c|c|c|c|c|c|c|}
\hline & & \multicolumn{12}{|c|}{ mPW1PW91 } \\
\hline & \multirow[b]{2}{*}{ Exp } & \multicolumn{6}{|c|}{ Gas phase } & \multicolumn{6}{|c|}{ PCM } \\
\hline & & 6-31G* & $6-31 G * *$ & $6-31+\mathrm{G} * *$ & $6-311 G *$ & $6-311 G * *$ & $6-311+G * *$ & $6-31 G *$ & $6-31 \mathrm{G} * *$ & $6-31+\mathrm{G} * *$ & 6-311G* & $6-311 G * *$ & $6-311+G * *$ \\
\hline \multicolumn{14}{|l|}{${ }^{13} \mathrm{C}$} \\
\hline 1 & 202.8 & -3.5169 & -3.3681 & -5.3169 & -22.7744 & -22.5443 & -24.4485 & -5.8596 & -5.7029 & -8.0571 & -25.4846 & -25.2498 & -27.5024 \\
\hline 2 & 60.7 & 133.7454 & 134.6004 & 134.2192 & 123.0098 & 122.1414 & 121.6136 & 133.2541 & 134.0977 & 133.5277 & 122.3840 & 121.4994 & 120.8089 \\
\hline 3 & 204.2 & -2.2697 & -2.0622 & -4.0277 & -21.1129 & -20.7953 & -23.7689 & -5.3235 & -5.1179 & -7.6470 & -24.6025 & -24.2882 & -27.8082 \\
\hline 4 & 59.1 & 135.0965 & 135.5294 & 134.9511 & 124.3350 & 124.0591 & 124.0697 & 135.2500 & 135.6807 & 135.1984 & 124.5601 & 124.2742 & 124.2427 \\
\hline 5 & 37.9 & 153.6017 & 153.9659 & 155.7699 & 144.3272 & 144.4693 & 143.7550 & 153.3296 & 153.6913 & 155.4691 & 144.0960 & 144.2433 & 143.4647 \\
\hline 6 & 40.7 & 153.0017 & 154.0812 & 153.1899 & 144.3864 & 143.9426 & 143.0669 & 153.3974 & 154.4730 & 153.6727 & 144.8814 & 144.4326 & 143.5854 \\
\hline 7 & 18.1 & 173.0536 & 174.3615 & 174.0637 & 166.4569 & 166.2132 & 166.2503 & 173.1766 & 174.4847 & 174.2104 & 166.5888 & 166.3443 & 166.3840 \\
\hline 8 & 52.2 & 139.7548 & 140.2575 & 138.9960 & 130.7058 & 130.3748 & 130.3639 & 140.1169 & 140.6168 & 139.4462 & 131.1695 & 130.8353 & 130.8625 \\
\hline 9 & 37.2 & 154.1720 & 154.6156 & 153.5859 & 145.1396 & 145.1887 & 145.0156 & 154.2306 & 154.6743 & 153.6603 & 145.2331 & 145.2822 & 145.1040 \\
\hline 10 & 71.9 & 123.6093 & 123.8825 & 121.7660 & 112.4847 & 112.0376 & 111.3998 & 123.8960 & 124.1675 & 122.0986 & 112.8715 & 112.4150 & 111.7099 \\
\hline 11 & 34.6 & 159.3589 & 160.5758 & 159.8605 & 151.4632 & 151.0900 & 150.7255 & 159.3196 & 160.5395 & 159.8158 & 151.4391 & 151.0680 & 150.6939 \\
\hline 12 & 30.2 & 164.2048 & 165.4318 & 165.1363 & 156.9129 & 156.6333 & 156.6780 & 164.3466 & 165.5718 & 165.3165 & 157.0960 & 156.8108 & 156.8687 \\
\hline 13 & 39.5 & 151.2172 & 151.6099 & 150.3039 & 141.9602 & 142.0400 & 142.4411 & 151.1778 & 151.5734 & 150.2582 & 141.9190 & 142.0023 & 142.4032 \\
\hline 14 & 38.3 & 154.1039 & 154.5361 & 156.6890 & 144.6927 & 144.7709 & 143.8467 & 154.0744 & 154.5085 & 156.6583 & 144.6837 & 144.7642 & 143.8243 \\
\hline 15 & 32.4 & 157.3699 & 158.6821 & 159.3093 & 149.7853 & 149.4418 & 148.6395 & 157.6236 & 158.9325 & 159.5957 & 150.0954 & 149.7408 & 148.9578 \\
\hline 16 & 35.9 & 159.1443 & 160.3860 & 158.6365 & 151.1517 & 150.8779 & 149.8877 & 159.2777 & 160.5170 & 158.7855 & 151.3120 & 151.0311 & 150.0539 \\
\hline 17 & 30.0 & 157.9895 & 158.4358 & 156.2464 & 148.7717 & 148.9020 & 149.3340 & 157.9572 & 158.4062 & 156.2211 & 148.7734 & 148.9045 & 149.3367 \\
\hline 18 & 42.7 & 144.6665 & 145.2758 & 142.6060 & 135.7867 & 135.3662 & 135.4818 & 144.7082 & 145.3174 & 142.6622 & 135.8639 & 135.4401 & 135.5643 \\
\hline 19 & 35.3 & 156.3060 & 157.3867 & 157.4547 & 148.7239 & 148.2195 & 147.0954 & 156.4251 & 157.5049 & 157.6048 & 148.8858 & 148.3750 & 147.2597 \\
\hline 20 & 28.2 & 160.4697 & 161.0743 & 162.2362 & 150.9853 & 150.9554 & 150.0410 & 160.4309 & 161.0409 & 162.1947 & 151.0001 & 150.9709 & 150.0590 \\
\hline 21 & 32.8 & 159.5441 & 160.6570 & 159.9309 & 151.0135 & 150.6306 & 149.9394 & 159.6459 & 160.7590 & 160.0497 & 151.1434 & 150.7561 & 150.0737 \\
\hline 22 & 39.3 & 148.2374 & 149.3794 & 148.5874 & 139.4729 & 138.9773 & 138.8816 & 148.4225 & 149.5623 & 148.7858 & 139.7191 & 139.2118 & 139.1099 \\
\hline 23 & 7.3 & 183.7549 & 185.5254 & 185.9798 & 178.2912 & 178.2836 & 178.2932 & 183.9445 & 185.7118 & 186.1976 & 178.5292 & 178.5146 & 178.5132 \\
\hline 24 & 16.0 & 176.6829 & 178.3453 & 179.4691 & 170.7684 & 170.5658 & 170.0993 & 176.7028 & 178.3548 & 179.4522 & 170.7620 & 170.5427 & 170.0434 \\
\hline 25 & 18.0 & 171.7796 & 173.5994 & 173.8606 & 166.5458 & 166.1584 & 166.0377 & 171.8843 & 173.6991 & 173.9785 & 166.6829 & 166.2837 & 166.1544 \\
\hline 26 & 20.3 & 167.5355 & 169.2523 & 169.4364 & 161.4466 & 161.2011 & 160.7303 & 167.7195 & 169.4348 & 169.6616 & 161.6957 & 161.4396 & 160.9808 \\
\hline 27 & 18.8 & 170.1180 & 171.8931 & 172.7629 & 164.4604 & 164.1502 & 164.3265 & 170.3205 & 172.0887 & 173.0039 & 164.7159 & 164.3899 & 164.5691 \\
\hline 28 & 32.1 & 164.3028 & 165.9511 & 166.5021 & 158.0079 & 157.7044 & 157.8631 & 164.5256 & 166.1642 & 166.7361 & 158.2613 & 157.9402 & 158.1044 \\
\hline 29 & 31.8 & 161.0663 & 162.5470 & 163.2636 & 154.1913 & 153.9774 & 153.0547 & 161.3621 & 162.8325 & 163.6146 & 154.5780 & 154.3354 & 153.4162 \\
\hline 30 & 35.0 & 166.6462 & 168.2598 & 169.6409 & 160.3455 & 160.0392 & 159.6757 & 166.9112 & 168.5162 & 169.9474 & 160.6839 & 160.3539 & 159.9927 \\
\hline
\end{tabular}




\begin{tabular}{|c|c|c|c|c|c|c|c|c|c|c|c|c|c|}
\hline \multicolumn{14}{|l|}{${ }^{1} \mathrm{H}$} \\
\hline $2^{*}$ & 3.24 & 29.0396 & 28.4898 & 28.4441 & 28.8698 & 28.5321 & 28.5153 & 29.1350 & 28.5786 & 28.5404 & 28.9658 & 28.6201 & 28.6071 \\
\hline $2^{* *}$ & 3.46 & 28.8013 & 28.2896 & 28.2784 & 28.6914 & 28.3753 & 28.4495 & 28.5394 & 28.0220 & 27.9935 & 28.4247 & 28.1106 & 28.1743 \\
\hline 4 & 2.58 & 29.6450 & 29.2822 & 29.2019 & 29.6109 & 29.4622 & 29.2967 & 29.4189 & 29.0501 & 28.9551 & 29.3850 & 29.2327 & 29.0547 \\
\hline $6^{*}$ & 1.39 & 30.7194 & 30.3436 & 30.3375 & 30.6698 & 30.4858 & 30.4960 & 30.6230 & 30.2459 & 30.2315 & 30.5746 & 30.3914 & 30.3941 \\
\hline $6 * *$ & 1.90 & 30.2430 & 29.8357 & 29.8084 & 30.2581 & 30.0456 & 29.9858 & 30.2073 & 29.7992 & 29.7704 & 30.2282 & 30.0141 & 29.9523 \\
\hline $7^{*}$ & 1.43 & 30.6089 & 30.1576 & 30.2844 & 30.6162 & 30.3381 & 30.3549 & 30.5739 & 30.1223 & 30.2484 & 30.5851 & 30.3069 & 30.3229 \\
\hline $7^{* *}$ & 1.54 & 30.4965 & 30.0360 & 30.1670 & 30.5840 & 30.3169 & 30.3790 & 30.4755 & 30.0175 & 30.1491 & 30.5645 & 30.3020 & 30.3644 \\
\hline 8 & 1.25 & 30.6580 & 30.3355 & 30.3967 & 30.7353 & 30.5806 & 30.5416 & 30.5837 & 30.2610 & 30.3157 & 30.6570 & 30.5041 & 30.4626 \\
\hline 10 & 2.38 & 29.6385 & 29.3269 & 29.1609 & 29.6120 & 29.4688 & 29.3947 & 29.4236 & 29.1068 & 28.9223 & 29.3940 & 29.2474 & 29.1616 \\
\hline $11^{*}$ & 1.14 & 30.7596 & 30.3611 & 30.3085 & 30.8135 & 30.6016 & 30.5437 & 30.6747 & 30.2739 & 30.2112 & 30.7241 & 30.5121 & 30.4493 \\
\hline $11 * *$ & 2.15 & 29.7694 & 29.3387 & 29.4557 & 29.7709 & 29.5500 & 29.5328 & 29.9152 & 29.4894 & 29.6123 & 29.9133 & 29.6952 & 29.6817 \\
\hline $12^{*}$ & 1.41 & 30.3751 & 29.9621 & 29.8902 & 30.4275 & 30.2296 & 30.2028 & 30.3747 & 29.9623 & 29.8856 & 30.4244 & 30.2269 & 30.1983 \\
\hline $12 * *$ & 1.41 & 30.9974 & 30.6326 & 30.5714 & 31.0231 & 30.8351 & 30.8059 & 30.9826 & 30.6174 & 30.5499 & 31.0030 & 30.8159 & 30.7841 \\
\hline $15^{*}$ & 1.27 & 30.5936 & 30.1795 & 30.1123 & 30.6261 & 30.3984 & 30.3166 & 30.5646 & 30.1504 & 30.0822 & 30.5991 & 30.3716 & 30.2893 \\
\hline $15^{* *}$ & 1.52 & 30.3421 & 29.9495 & 30.0614 & 30.3513 & 30.1192 & 30.1333 & 30.3082 & 29.9154 & 30.0273 & 30.3180 & 30.0872 & 30.1024 \\
\hline $16^{*}$ & 1.35 & 30.5190 & 30.1455 & 30.2721 & 30.5234 & 30.3298 & 30.3779 & 30.5231 & 30.1496 & 30.2771 & 30.5293 & 30.3363 & 30.3849 \\
\hline $16^{* *}$ & 1.56 & 30.4529 & 30.0203 & 30.0692 & 30.4359 & 30.1635 & 30.1128 & 30.4346 & 30.0022 & 30.0520 & 30.4172 & 30.1466 & 30.0961 \\
\hline 18 & 1.57 & 30.3072 & 29.9489 & 30.0605 & 30.4098 & 30.2572 & 30.3284 & 30.2815 & 29.9250 & 30.0327 & 30.3813 & 30.2317 & 30.3033 \\
\hline $19 *$ & 1.36 & 30.5710 & 30.1720 & 30.1738 & 30.5999 & 30.3746 & 30.4167 & 30.5681 & 30.1706 & 30.1681 & 30.5941 & 30.3709 & 30.4122 \\
\hline $19 * *$ & 1.41 & 30.4561 & 30.0326 & 30.1500 & 30.5565 & 30.3037 & 30.3852 & 30.4384 & 30.0156 & 30.1303 & 30.5370 & 30.2859 & 30.3666 \\
\hline $21^{*}$ & 1.27 & 30.8918 & 30.4929 & 30.4583 & 30.8755 & 30.6684 & 30.6068 & 30.8918 & 30.4933 & 30.4585 & 30.8733 & 30.6677 & 30.6071 \\
\hline $21^{* *}$ & 1.46 & 30.4842 & 30.0630 & 29.9272 & 30.4753 & 30.2419 & 30.2321 & 30.4798 & 30.0589 & 29.9225 & 30.4711 & 30.2378 & 30.2282 \\
\hline $22^{*}$ & 0.94 & 30.8347 & 30.4698 & 30.3739 & 30.8462 & 30.6529 & 30.5837 & 30.8349 & 30.4697 & 30.3743 & 30.8453 & 30.6526 & 30.5844 \\
\hline $22^{* *}$ & 1.49 & 30.6624 & 30.2875 & 30.1449 & 30.6645 & 30.4678 & 30.4365 & 30.6496 & 30.2753 & 30.1319 & 30.6495 & 30.4546 & 30.4231 \\
\hline 23 & 1.05 & 31.1774 & 30.6993 & 30.6259 & 31.1766 & 30.8790 & 30.8502 & 31.1770 & 30.6982 & 30.6225 & 31.1757 & 30.8787 & 30.8454 \\
\hline 24 & 0.69 & 31.3799 & 30.9343 & 31.0588 & 31.4654 & 31.2092 & 31.2028 & 31.3725 & 30.9275 & 31.0496 & 31.4550 & 31.2003 & 31.1911 \\
\hline 25 & 1.20 & 30.7024 & 30.2347 & 30.0897 & 30.7634 & 30.4864 & 30.4220 & 30.7162 & 30.2484 & 30.0996 & 30.7740 & 30.4963 & 30.4315 \\
\hline 26 & 1.03 & 30.9782 & 30.5209 & 30.5489 & 31.0601 & 30.7924 & 30.7695 & 30.9797 & 30.5233 & 30.5513 & 31.0628 & 30.7963 & 30.7746 \\
\hline 27 & 1.02 & 30.9434 & 30.4804 & 30.5161 & 30.9756 & 30.6853 & 30.7261 & 30.9233 & 30.4612 & 30.4972 & 30.9580 & 30.6699 & 30.7108 \\
\hline 28 & 1.18 & 30.9964 & 30.5683 & 30.5011 & 31.0510 & 30.8089 & 30.6911 & 30.9903 & 30.5623 & 30.4946 & 31.0452 & 30.8040 & 30.6869 \\
\hline 29 & 1.00 & 31.1321 & 30.7231 & 30.6318 & 31.0992 & 30.8774 & 30.8309 & 31.1354 & 30.7257 & 30.6369 & 31.1030 & 30.8813 & 30.8360 \\
\hline 30 & 0.94 & 31.1061 & 30.6707 & 30.6565 & 31.0870 & 30.8336 & 30.8729 & 31.1006 & 30.6652 & 30.6513 & 31.0818 & 30.8293 & 30.8690 \\
\hline
\end{tabular}

\section{Compound 37}

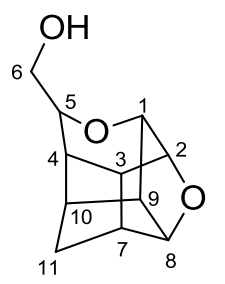

37

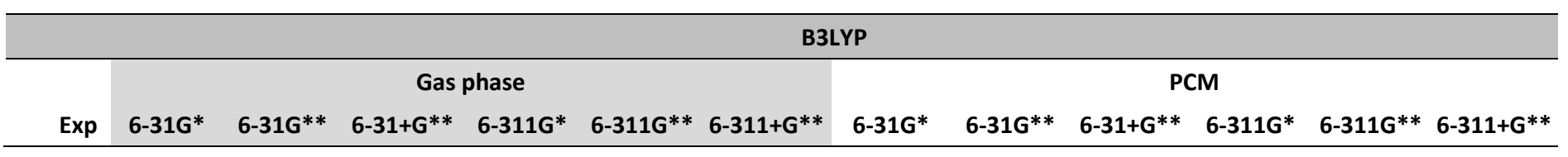




\begin{tabular}{|c|c|c|c|c|c|c|c|c|c|c|c|c|c|}
\hline \multicolumn{14}{|l|}{${ }^{13} \mathrm{C}$} \\
\hline 1 & 74.4 & 114.8454 & 115.7024 & 113.7315 & 102.1614 & 101.7392 & 102.0587 & 115.1425 & 116.0012 & 113.9352 & 102.3938 & 101.9745 & 102.232 \\
\hline 2 & 6.6 & 112.8145 & 113.7619 & 112.4287 & 101.2604 & 100.7870 & 100.5714 & 113.0131 & 113.9552 & 112.4183 & 101.3386 & 100.8605 & 100.551 \\
\hline 3 & 36.1 & 149.8415 & 150.8532 & 150.9326 & 139.5975 & 139.3451 & 139.5658 & 150.1042 & 151.1072 & 151.2042 & 139.8374 & 139.5719 & 139.783 \\
\hline 4 & 39.7 & 147.6988 & 148.4485 & 47.7313 & 137.1486 & 136.9304 & 137.1879 & 148.2372 & 148.9824 & 148.2889 & 137.8011 & & \\
\hline 5 & 72.0 & 117.1694 & 17.9242 & 6.9509 & 105.4362 & 104.9602 & 105.0931 & 117.0742 & 117.8323 & 116.7568 & 105.2807 & 104.8010 & 104.875 \\
\hline 6 & 65.9 & 125.1337 & 126.4040 & 2.6883 & 114.3381 & 113.7511 & 113.4002 & 125.4587 & 126.7239 & 124.8959 & 114.5511 & 113.9524 & 113.605 \\
\hline 7 & 49.1 & 138.8844 & 139.8369 & 139.0133 & 128.2432 & 127.8587 & 127.6698 & 138.8889 & 139.8410 & 138.9697 & 128.2039 & 127.8200 & 127.557 \\
\hline 8 & 87.1 & 103.7678 & 104.6263 & 3.3045 & 91.2000 & 90.7075 & 90.48 & 103.7 & 104.6269 & 103.0627 & 91.0732 & 90.5801 & 0.2386 \\
\hline 9 & 36.7 & 149.5926 & 150.5727 & 0.1562 & 139.6666 & 139.3620 & 138.9810 & 149.7273 & & & 139.7 & & \\
\hline 10 & 39.4 & & & & 136.9 & & & & & & & & \\
\hline $\begin{array}{l}11 \\
{ }^{1} \mathbf{H}\end{array}$ & 31.9 & 156.3245 & 157.7258 & & 148.1538 & & 792 & 156.5408 & & & & & 858 \\
\hline 1 & 3.75 & 28.5447 & 8.0674 & 27.9 & & & & & & & & & 28.2378 \\
\hline 2 & 4.34 & 8.1685 & & & & & & & & & & & אובכס \\
\hline 3 & 2.32 & & & & & & & & & & & & \\
\hline 4 & 1.73 & 30.6475 & 30.2399 & 30.1674 & 30.7426 & 30.5098 & 30.4615 & 30.5140 & 30.0999 & 30.0095 & 30.6095 & 30.3688 & 30.3073 \\
\hline 5 & 4.00 & 28.3200 & 27.8579 & 27.6913 & 28.2621 & 28.0116 & 27.9668 & 28.2743 & 27.8119 & 27.6474 & 28.2131 & 27.9605 & 27.9226 \\
\hline $6 a$ & 3.59 & 3.8082 & 3.3443 & 28.2773 & 3.6981 & 28.4596 & 28.5294 & 28.7868 & 225 & 088 & 28.6777 & 1329 & 28.4605 \\
\hline $6 b$ & 3.51 & 28.7208 & 28.2692 & 28.0623 & 28.6283 & 28.4146 & & 28.6481 & 28.1948 & 27.9853 & & 28.3341 & 28.2582 \\
\hline 7 & 2.57 & 29.7935 & 29.2790 & 29.1766 & 29.8057 & 29.4860 & & & 29.2508 & 29.1436 & 29.7755 & 29.4561 & 29.4105 \\
\hline 8 & 4.63 & 27.8395 & 27.2928 & 27.0939 & 27.7804 & 27.4688 & 27.4284 & 27.7716 & 27.2213 & 27.0051 & 27.7035 & 27.3874 & 27.3386 \\
\hline 9 & 2.17 & 30.2302 & 9.7431 & 29.6340 & 30.2402 & 29.9596 & 29.8901 & 30.1478 & 29.6582 & 29.5364 & 30.1516 & 29.8721 & 29.7936 \\
\hline 10 & 2.22 & 30.1506 & 29.6801 & 29.5850 & 30.1360 & 29.8785 & 29.8334 & 30.0837 & 29.6104 & 29.5082 & 30.0652 & 29.8062 & 29.7559 \\
\hline $11 a$ & 1.56 & 30.7028 & 30.2531 & 30.1409 & 30.6850 & 30.4609 & 30.3876 & 30.6377 & 30.1847 & 30.0652 & 30.6214 & 30.3944 & 30.3151 \\
\hline & 1.36 & 30.8801 & 30.4498 & 30.3624 & 30.8800 & 30.6459 & 30.6132 & 30.8106 & 30.3769 & 30.2794 & 30.8141 & 30.5759 & 30.5345 \\
\hline
\end{tabular}

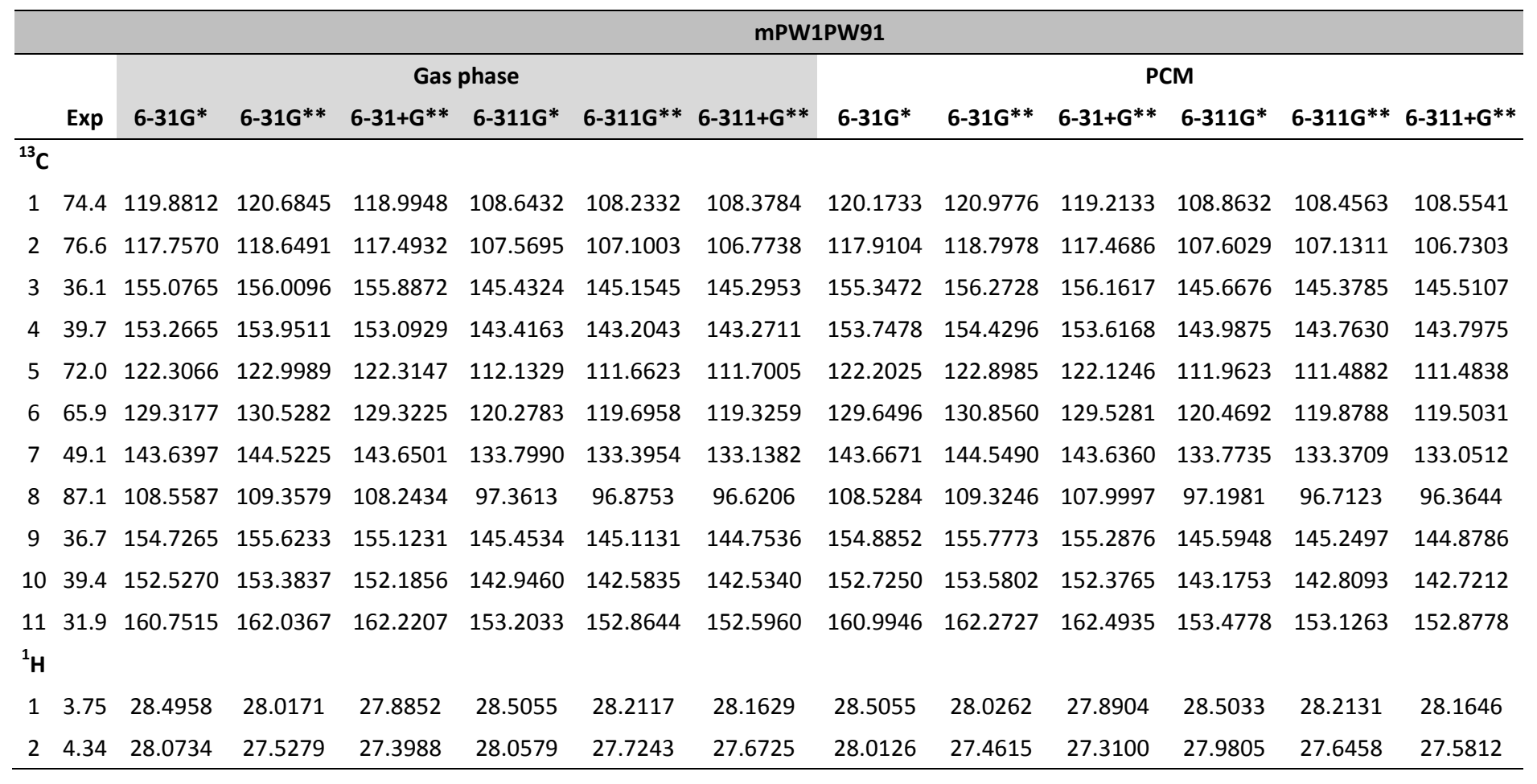




\begin{tabular}{cccccccccccccc}
\hline 3 & 2.32 & 29.8958 & 29.4049 & 29.3800 & 29.9281 & 29.6608 & 29.6418 & 29.8363 & 29.3427 & 29.2868 & 29.8672 & 29.5970 & 29.5524 \\
4 & 1.73 & 30.6154 & 30.2080 & 30.1270 & 30.6528 & 30.4166 & 30.3684 & 30.4734 & 30.0597 & 29.9642 & 30.5147 & 30.2713 & 30.2114 \\
5 & 4.00 & 28.2518 & 27.7927 & 27.6386 & 28.1830 & 27.9283 & 27.8910 & 28.2052 & 27.7455 & 27.5919 & 28.1344 & 27.8776 & 27.8448 \\
$6 \mathrm{a}$ & 3.59 & 28.7819 & 28.3209 & 28.2497 & 28.6765 & 28.4286 & 28.4736 & 28.7419 & 28.2798 & 28.1729 & 28.6388 & 28.3834 & 28.3979 \\
$6 \mathrm{~b}$ & 3.51 & 28.6712 & 28.2213 & 28.0458 & 28.5695 & 28.3462 & 28.2918 & 28.5981 & 28.1468 & 27.9650 & 28.4933 & 28.2666 & 28.2081 \\
7 & 2.57 & 29.7109 & 29.1916 & 29.0968 & 29.7093 & 29.3778 & 29.3328 & 29.6787 & 29.1580 & 29.0597 & 29.6744 & 29.3436 & 29.2973 \\
8 & 4.63 & 27.7593 & 27.2095 & 27.0382 & 27.6979 & 27.3667 & 27.3362 & 27.6839 & 27.1302 & 26.9441 & 27.6156 & 27.2799 & 27.2424 \\
9 & 2.17 & 30.1467 & 29.6510 & 29.5499 & 30.1406 & 29.8475 & 29.7840 & 30.0614 & 29.5630 & 29.4508 & 30.0504 & 29.7583 & 29.6868 \\
10 & 2.22 & 30.0676 & 29.5963 & 29.5088 & 30.0369 & 29.7698 & 29.7263 & 29.9973 & 29.5230 & 29.4292 & 29.9647 & 29.6962 & 29.6470 \\
$11 \mathrm{a}$ & 1.56 & 30.6329 & 30.1867 & 30.0703 & 30.5783 & 30.3526 & 30.2754 & 30.5627 & 30.1130 & 29.9902 & 30.5105 & 30.2821 & 30.1995 \\
$11 \mathrm{~b}$ & 1.36 & 30.8183 & 30.3924 & 30.3013 & 30.7885 & 30.5506 & 30.5087 & 30.7413 & 30.3119 & 30.2123 & 30.7166 & 30.4746 & 30.4253 \\
\hline
\end{tabular}

\section{Compound 38}<smiles>OC12CCO[C@H]1Nc1ccccc12</smiles>

38

\begin{tabular}{|c|c|c|c|c|c|c|c|c|c|c|c|c|c|}
\hline & \multirow[b]{3}{*}{ Exp } & \multicolumn{12}{|c|}{ B3LYP } \\
\hline & & \multicolumn{6}{|c|}{ Gas phase } & \multicolumn{6}{|c|}{ PCM } \\
\hline & & 6-31G* & 6-31G** & $6-31+G * *$ & 6-311G* & 6-311G** & $6-311+G^{* *}$ & 6-31G* & $6-31 G^{* *}$ & $6-31+G^{* *}$ & 6-311G* & 6-311G** & $6-311+G * *$ \\
\hline \multicolumn{14}{|c|}{ 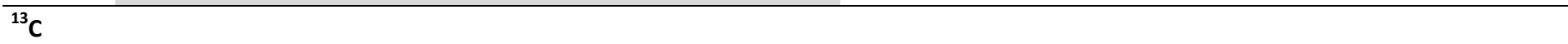 } \\
\hline 2 & 67.3 & 122.4263 & 123.7363 & 122.3929 & 111.4548 & 110.8646 & 110.1942 & 122.4456 & 123.7509 & 122.1674 & 111.3577 & 110.7479 & 109.9975 \\
\hline 3 & 40.8 & 148.2787 & 149.5768 & 149.1284 & 139.0162 & 138.5516 & 138.3815 & 148.3688 & 149.6596 & 149.1681 & 139.0769 & 138.6046 & 138.3561 \\
\hline $3 a$ & 89.3 & 99.7521 & 99.8580 & 98.4164 & 85.6531 & 85.8709 & 85.7002 & 99.8807 & 99.9839 & 98.5167 & 85.7413 & 85.9516 & 85.7609 \\
\hline $3 b$ & 130.1 & 66.6562 & 66.3200 & 65.8487 & 48.4443 & 47.9330 & 46.4827 & 66.4710 & 66.1303 & 65.7347 & 48.2132 & 47.7006 & 46.3399 \\
\hline 4 & 109.5 & 87.3321 & 87.9674 & 87.1342 & 71.6372 & 70.7636 & 69.6042 & 87.2432 & 87.8749 & 86.9320 & 71.3992 & 70.5525 & 69.3690 \\
\hline 5 & 130.3 & 65.4624 & 66.1076 & 65.7204 & 47.9309 & 47.0598 & 46.0708 & 65.4708 & 66.1188 & 65.6189 & 47.8287 & 46.9972 & 46.0029 \\
\hline 6 & 119.8 & 77.3568 & 77.9514 & 77.4918 & 60.8105 & 59.9062 & 58.9245 & 77.6222 & 78.2209 & 77.6897 & 61.0674 & 60.1922 & 59.2175 \\
\hline 7 & 124.1 & 71.2322 & 71.9234 & 70.0173 & 54.0850 & 53.3511 & 52.7299 & 71.1351 & 71.8212 & 69.8216 & 53.8416 & 53.1170 & 52.4410 \\
\hline $7 a$ & 149.5 & 46.4337 & 46.3444 & 42.9940 & 24.9778 & 24.9739 & 24.5381 & 46.2189 & 46.1303 & 42.7503 & 24.7044 & 24.7135 & 24.2347 \\
\hline $\begin{array}{l}8 \mathrm{a} \\
{ }^{1} \mathrm{H}\end{array}$ & 99.4 & 93.5573 & 94.1739 & 92.4276 & 79.5622 & 79.0097 & 79.0547 & 93.6232 & 94.2429 & 92.4136 & 79.6045 & 79.0529 & 79.0555 \\
\hline 2 & 4.01 & 28.2626 & 27.7381 & 27.5863 & 28.1766 & 27.8741 & 27.8381 & 28.1934 & 27.6676 & 27.5017 & 28.1047 & 27.8005 & 27.7554 \\
\hline 2 & 3.63 & 28.5792 & 28.0886 & 27.9601 & 28.5515 & 28.3287 & 28.2703 & 28.5492 & 28.0596 & 27.9197 & 28.5224 & 28.3007 & 28.2297 \\
\hline $3^{*}$ & 2.42 & 29.6363 & 29.1950 & 28.9778 & 29.4924 & 29.3049 & 29.2862 & 29.6723 & 29.2311 & 29.0029 & 29.5280 & 29.3361 & 29.3088 \\
\hline $3^{* *}$ & 2.32 & 30.2152 & 29.7352 & 29.5433 & 30.1304 & 29.8422 & 29.7542 & 30.1058 & 29.6219 & 29.4008 & 30.0167 & 29.7259 & 29.6175 \\
\hline 4 & 6.59 & 25.9567 & 25.2984 & 25.0037 & 25.7715 & 25.3360 & 25.2057 & 25.8250 & 25.1659 & 24.8539 & 25.6252 & 25.1981 & 25.0547 \\
\hline 5 & 7.12 & 25.1798 & 24.5764 & 24.3651 & 25.0295 & 24.6620 & 24.5950 & 25.0877 & 24.4849 & 24.2585 & 24.9245 & 24.5655 & 24.4889 \\
\hline 6 & 6.80 & 25.6165 & 24.9771 & 24.7379 & 25.4872 & 25.0758 & 24.9885 & 25.5456 & 24.9068 & 24.6593 & 25.4061 & 25.0030 & 24.9110 \\
\hline 7 & 7.29 & 25.0593 & 24.4129 & 24.2218 & 24.9326 & 24.5196 & 24.4126 & 24.9814 & 24.3335 & 24.1261 & 24.8427 & 24.4319 & 24.3147 \\
\hline $8 a$ & 5.34 & 27.1619 & 26.6330 & 26.3572 & 27.0086 & 26.7188 & 26.6707 & 27.1557 & 26.6214 & 26.3296 & 26.9844 & 26.6873 & 26.6337 \\
\hline
\end{tabular}




\begin{tabular}{|c|c|c|c|c|c|c|c|c|c|c|c|c|c|}
\hline \multicolumn{14}{|c|}{ mPW1PW91 } \\
\hline & \multirow[b]{2}{*}{ Exp } & \multicolumn{6}{|c|}{ Gas phase } & \multicolumn{6}{|c|}{ PCM } \\
\hline & & 6-31G* & $6-31 G * *$ & $6-31+\mathrm{G} * *$ & 6-311G* & $6-311 G * *$ & $6-311+G * *$ & 6-31G* & $6-31 G * *$ & $6-31+\mathrm{G} * *$ & 6-311G* & $6-311 G * *$ & $6-311+G * *$ \\
\hline \multicolumn{14}{|l|}{${ }^{13} \mathrm{C}$} \\
\hline 2 & 67.3 & 126.5185 & 127.7600 & 126.9361 & 117.0764 & 116.5029 & 115.8049 & 126.5232 & 127.7611 & 126.7186 & 116.9595 & 116.3675 & 115.6037 \\
\hline 3 & 40.8 & 152.7261 & 153.9306 & 153.4696 & 144.4040 & 143.9678 & 143.7913 & 152.8350 & 154.0329 & 153.5347 & 144.4715 & 144.0282 & 143.7790 \\
\hline $3 a$ & 89.3 & 104.0912 & 104.1817 & 103.1833 & 92.1689 & 92.3799 & 92.0323 & 104.2120 & 104.3004 & 103.2830 & 92.2472 & 92.4511 & 92.0888 \\
\hline $3 b$ & 130.1 & 70.0769 & 69.7161 & 69.5802 & 54.0976 & 53.5726 & 52.2855 & 69.8753 & 69.5108 & 69.4344 & 53.8659 & 53.3412 & 52.1297 \\
\hline 4 & 109.5 & 89.7785 & 90.3537 & 89.7363 & 75.9414 & 75.0479 & 73.8843 & 89.6841 & 90.2558 & 89.5242 & 75.6852 & 74.8215 & 73.6413 \\
\hline 5 & 130.3 & 67.8611 & 68.4202 & 68.1205 & 52.4503 & 51.5334 & 50.5676 & 67.8308 & 68.3934 & 67.9822 & 52.2933 & 51.4190 & 50.4585 \\
\hline 6 & 119.8 & 79.9541 & 80.4671 & 80.2470 & 65.4266 & 64.4775 & 63.4782 & 80.1870 & 80.7052 & 80.4079 & 65.6337 & 64.7166 & 63.7356 \\
\hline 7 & 124.1 & 73.3679 & 73.9998 & 72.2408 & 58.2588 & 57.5003 & 56.9150 & 73.2468 & 73.8734 & 72.0267 & 57.9913 & 57.2434 & 56.6159 \\
\hline $7 a$ & 149.5 & 49.2053 & 49.0949 & 46.2529 & 30.5316 & 30.5081 & 29.9414 & 49.0120 & 48.9027 & 46.0311 & 30.2802 & 30.2708 & 29.6651 \\
\hline $8 a$ & 99.4 & 97.8964 & 98.4821 & 97.2783 & 86.1489 & 85.5998 & 85.5306 & 97.9697 & 98.5581 & 97.2792 & 86.1896 & 85.6413 & 85.5330 \\
\hline \multicolumn{14}{|l|}{${ }^{1} \mathrm{H}$} \\
\hline 2 & 4.01 & 28.2007 & 27.6776 & 27.5422 & 28.1263 & 27.8091 & 27.7735 & 28.1204 & 27.5958 & 27.4488 & 28.0458 & 27.7269 & 27.6836 \\
\hline 2 & 3.63 & 28.5668 & 28.0772 & 27.9426 & 28.5076 & 28.2801 & 28.2144 & 28.5361 & 28.0477 & 27.9027 & 28.4788 & 28.2527 & 28.1751 \\
\hline $3^{*}$ & 2.42 & 29.5398 & 29.0986 & 28.9120 & 29.3921 & 29.2026 & 29.1815 & 29.5698 & 29.1288 & 28.9323 & 29.4226 & 29.2284 & 29.1994 \\
\hline $3 * *$ & 2.32 & 30.1227 & 29.6381 & 29.4517 & 30.0165 & 29.7214 & 29.6299 & 30.0047 & 29.5158 & 29.3045 & 29.8974 & 29.5996 & 29.4901 \\
\hline 4 & 6.59 & 25.7491 & 25.0882 & 24.8283 & 25.5990 & 25.1308 & 25.0176 & 25.6025 & 24.9404 & 24.6657 & 25.4408 & 24.9813 & 24.8558 \\
\hline 5 & 7.12 & 24.9673 & 24.3605 & 24.1841 & 24.8465 & 24.4429 & 24.3863 & 24.8593 & 24.2530 & 24.0655 & 24.7292 & 24.3344 & 24.2694 \\
\hline 6 & 6.80 & 25.4120 & 24.7685 & 24.5504 & 25.3104 & 24.8646 & 24.7864 & 25.3255 & 24.6825 & 24.4598 & 25.2174 & 24.7802 & 24.6989 \\
\hline 7 & 7.29 & 24.8378 & 24.1893 & 24.0362 & 24.7363 & 24.2912 & 24.2017 & 24.7460 & 24.0959 & 23.9292 & 24.6367 & 24.1939 & 24.0956 \\
\hline $8 a$ & 5.34 & 27.0690 & 26.5382 & 26.3035 & 26.9309 & 26.6227 & 26.5832 & 27.0568 & 26.5202 & 26.2705 & 26.9024 & 26.5870 & 26.5427 \\
\hline
\end{tabular}

\section{Compound 39}

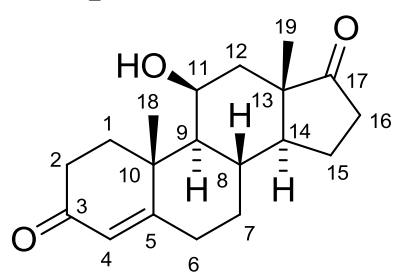

39

\begin{tabular}{|c|c|c|c|c|c|c|c|c|c|c|c|c|c|}
\hline & \multirow[b]{3}{*}{ Exp } & \multicolumn{12}{|c|}{ B3LYP } \\
\hline & & \multicolumn{6}{|c|}{ Gas phase } & \multicolumn{6}{|c|}{ PCM } \\
\hline & & 6-31G* & $6-31 \mathrm{G} * *$ & $6-31+G * *$ & 6-311G* & $6-311 G * *$ & $6-311+G * *$ & 6-31G* & 6-31G** & $6-31+G^{*} *$ & 6-311G* & 6-311G** & $6-311+\mathrm{G} * *$ \\
\hline \multicolumn{14}{|c|}{${ }^{13} \mathrm{C}$} \\
\hline 1 & 34.9 & 156.4716 & 157.7997 & 157.3584 & 148.0850 & 147.7988 & 147.2516 & 156.6900 & 158.0184 & 157.5599 & 148.3513 & 148.0576 & 147.4864 \\
\hline 2 & 33.7 & 156.3044 & 157.6219 & 156.3173 & 146.7859 & 146.3243 & 145.3250 & 155.9899 & 157.2954 & 155.7949 & 146.3685 & 145.8963 & 144.6852 \\
\hline 3 & 199.4 & 4.3365 & 4.4960 & 1.4880 & -15.7148 & -15.6435 & -20.1128 & 1.5657 & 1.7076 & -2.0198 & -19.0360 & -18.9774 & -24.1870 \\
\hline 4 & 122.5 & 71.5004 & 72.2057 & 72.5969 & 55.2519 & 54.7254 & 54.2057 & 72.5464 & 73.2514 & 73.6900 & 56.4583 & 55.9336 & 55.3377 \\
\hline 5 & 171.6 & 25.9462 & 25.8667 & 22.1642 & 2.9391 & 2.8431 & 0.9460 & 21.2940 & 21.2131 & 16.7010 & -2.3278 & -2.4115 & -4.9839 \\
\hline 6 & 31.8 & 155.6789 & 157.0306 & 156.1714 & 146.6025 & 146.1298 & 145.9904 & 155.4634 & 156.8094 & 155.9295 & 146.3640 & 145.8803 & 145.6777 \\
\hline 7 & 31.4 & 153.7794 & 155.0789 & 153.6847 & 144.2805 & 144.0932 & 143.7251 & 153.7504 & 155.0443 & 153.6590 & 144.2576 & 144.0675 & 143.6839 \\
\hline 8 & 30.9 & 156.2252 & 156.9628 & 156.0995 & 146.4909 & 146.4216 & 146.4583 & 156.3098 & 157.0445 & 156.2232 & 146.6296 & 146.5597 & 146.5947 \\
\hline
\end{tabular}




\begin{tabular}{|c|c|c|c|c|c|c|c|c|c|c|c|c|c|}
\hline 9 & 56.6 & 136.6833 & 137.2676 & 136.3685 & 125.7087 & 125.5395 & 124.8984 & 136.9416 & 137.5159 & 136.7241 & 125.9897 & 125.8236 & 125.23 \\
\hline 10 & 9.2 & 46.9842 & 7.3560 & 6.9621 & 6.6570 & 136.8329 & 36.3550 & 46.5644 & 146.9347 & 146.4405 & 136.1579 & 136.3308 & 135.763 \\
\hline 11 & 7.8 & 18.7547 & 19.5587 & 8.0639 & 6.5799 & 106.4235 & 06.0192 & 18.6664 & 119.4711 & & 106.5411 & & \\
\hline 12 & 40.8 & 6.3692 & 7.4919 & & & & & & & & & & \\
\hline 13 & 46.7 & 140.4365 & 140.7973 & 141.1289 & 130.6689 & 130.8212 & 129.6373 & 139.8395 & 140.2017 & 140.3781 & 129.9504 & 130.1150 & 128.790 \\
\hline 14 & 52.3 & 137.0539 & 137.6311 & 136.7138 & 125.9029 & 125.7596 & 125.2226 & 137.4798 & 138.0441 & 137.2120 & 126.4467 & 126.2893 & 125.784 \\
\hline 15 & 21.6 & 165.4678 & 166.9101 & 7.0770 & 57.8058 & 157.6176 & 157.0845 & 165.5733 & 167.0097 & 167.1887 & 157.9348 & 157.7309 & 157.183 \\
\hline 16 & 35.2 & 153.9743 & 155.3160 & 53.9362 & 14.8533 & 144.4200 & 143.4302 & 153.4665 & 154.7925 & 153.1571 & 144.2521 & 143.8014 & 142.4614 \\
\hline 17 & 219.2 & -18.2263 & -18.0067 & -20.5707 & -40.7414 & -40.4877 & -44.2619 & -22.3042 & -22.0904 & -25.6637 & -45.4585 & -45.2125 & סברם \\
\hline 18 & 15.8 & 172.9883 & 174.6675 & 175.1840 & 166.0463 & 165.8649 & 165.8324 & 173.1356 & 174.8043 & 175.2705 & 166.1989 & 165.9999 & 165.959 \\
\hline $\begin{array}{l}19 \\
{ }^{1} \mathbf{H}\end{array}$ & 21.0 & 163.4762 & 165.2733 & 165.7516 & 156.3413 & 156.1090 & 155.9908 & 163.5986 & 165.3847 & 165.8311 & 156.4783 & 156.2250 & 156.098 \\
\hline $1^{*}$ & 2.17 & & & & & & & & & & & & 29.5889 \\
\hline $1^{* *}$ & .82 & 30.3274 & 29.9638 & 29.8840 & 30.3407 & & & & & & & & \\
\hline $2^{*}$ & 2.33 & 30.2574 & 29.7866 & 29.6255 & 30.1023 & 29.8647 & 29.7673 & 30.3075 & 29.8331 & 29.6653 & 30.1508 & 29.9096 & 29.8047 \\
\hline $2^{* *}$ & 2.42 & 29.9344 & 29.4785 & 29.4374 & 29.9354 & 29.6819 & 29.6310 & 29.7929 & 29.3318 & 29.2663 & 29.7868 & 29.5314 & 29.4622 \\
\hline 4 & 5.67 & 5.6884 & 5.0130 & 25.8815 & 571 & & 327 & & & 25.8826 & & 783 & 5.0321 \\
\hline $6^{*}$ & 2.24 & 0.1848 & 9.7451 & & & & & & & & & & \\
\hline $6^{* *}$ & & 9.4240 & & & & & & & & & & & \\
\hline $7^{*}$ & 1.10 & 30.9082 & 30.5416 & 30.5392 & 30.9462 & 30.8063 & 30.8156 & 30.8515 & 30.4830 & 30.4751 & 30.8889 & 30.7480 & 30.7521 \\
\hline $7^{* *}$ & 2.06 & 0.0505 & 29.6246 & 29.4904 & 30.0111 & 29.7995 & 29.7182 & 29.9660 & 29.5367 & 29.3951 & 29.9297 & 29.7142 & 29.6279 \\
\hline 8 & 2.14 & .5683 & .1594 & 9.2263 & 29.7636 & כוע & 9.0313 & $9.3<44$ & 29.1148 & 29.1717 & 29.7184 & 29.5237 & $29.3 / 02$ \\
\hline 9 & & 1.5177 & & & & & & & & & & & \\
\hline 11 & 4.44 & 27.6753 & 27.1681 & 27.1670 & 27.6778 & 27.4034 & 27.4138 & 27.6158 & 27.1085 & 27.1039 & 27.6183 & 27.3435 & 27.3542 \\
\hline $12^{*}$ & 1.94 & 30.5157 & 30.0793 & 30.0906 & 30.5217 & 30.2072 & 30.1929 & 30.4566 & 30.0197 & 30.0316 & 30.4592 & 30.1524 & 30.1395 \\
\hline $2^{* *}$ & 1.45 & 30.7133 & 30.2661 & 30.1954 & 30.6976 & 30.4661 & 30.4306 & 30.7135 & 30.2644 & 30.1892 & 30.6957 & 30.4601 & 30.4231 \\
\hline 14 & 1.23 & 30.7860 & 30.4962 & 30.4219 & 30.8841 & (3.198 & 30.6734 & 30.6831 & 30.3919 & 30.2997 & 30.7746 & 30.6886 & 30.5530 \\
\hline $15^{*}$ & 1.64 & 30.4733 & 30.0313 & 29.9745 & 30.5302 & 30.3074 & 30.2601 & 30.3833 & 29.9398 & 29.8681 & 30.4391 & 30.2182 & 30.1598 \\
\hline $15^{* *}$ & 1.99 & 30.3380 & 29.8864 & 29.7031 & 30.2650 & 30.0318 & 29.9720 & 30.2774 & 29.8236 & 29.6356 & 30.2120 & 29.9767 & 29.9105 \\
\hline $16^{*}$ & 2.04 & 30.2643 & 29.7867 & 29.6729 & 30.1641 & 29.9009 & 29.8665 & 30.2157 & 29.7336 & 29.6063 & 30.1159 & 29.8491 & 29.8024 \\
\hline $16 * *$ & 2.48 & 29.8909 & 29.3909 & 29.2735 & 29.8105 & 29.5011 & 29.4826 & 29.8433 & 29.3386 & 29.2081 & 29.7572 & 29.4446 & 29.4152 \\
\hline 18 & 1.14 & 30.9357 & 30.4644 & 30.3998 & 31.0022 & 30.7426 & 30.6841 & 30.9094 & 30.4352 & 30.3619 & 30.9750 & 30.7119 & 30.6468 \\
\hline 19 & 1.45 & 30.7371 & 30.3020 & 30.2237 & 30.7442 & 30.5342 & 30.5141 & & & & & 30.5275 & 30.5015 \\
\hline
\end{tabular}

\begin{tabular}{|c|c|c|c|c|c|c|c|c|c|c|c|c|c|}
\hline & \multirow[b]{3}{*}{ Exp } & \multicolumn{12}{|c|}{ mPW1PW91 } \\
\hline & & \multicolumn{6}{|c|}{ Gas phase } & \multicolumn{6}{|c|}{ PCM } \\
\hline & & 6-31G* & $6-31 G * *$ & $6-31+\mathrm{G} * *$ & 6-311G* & $6-311 G * *$ & $6-311+G^{* *}$ & 6-31G* & $6-31 G * *$ & $6-31+G * *$ & 6-311G* & $6-311 G * *$ & $6-311+\mathrm{G} * *$ \\
\hline \multicolumn{14}{|c|}{${ }^{13} \mathrm{C}$} \\
\hline 1 & 34.9 & 161.4881 & 162.7061 & 162.2531 & 153.6871 & 153.4243 & 152.9214 & 161.7034 & 162.9229 & 162.4546 & 153.9420 & 153.6727 & 153.1480 \\
\hline 2 & 33.7 & 160.5967 & 161.8229 & 160.6194 & 152.1360 & 151.6775 & 150.6002 & 160.2695 & 161.4838 & 160.1116 & 151.7048 & 151.2360 & 149.9834 \\
\hline 3 & 199.4 & 6.3696 & 6.5133 & 4.5753 & -10.6509 & -10.5831 & -14.7379 & 3.5015 & 3.6267 & 1.0309 & -14.0034 & -13.9492 & -18.7571 \\
\hline 4 & 122.5 & 74.1404 & 74.8120 & 75.1990 & 59.7185 & 59.1826 & 58.6439 & 75.2745 & 75.9452 & 76.3787 & 60.9775 & 60.4440 & 59.8425 \\
\hline 5 & 171.6 & 29.5886 & 29.4737 & 26.3504 & 9.2254 & 9.1076 & 7.4273 & 24.7969 & 24.6813 & 20.8408 & 3.8919 & 3.7883 & 1.5161 \\
\hline 6 & 31.8 & 160.2227 & 161.4715 & 160.5218 & 152.0338 & 151.5629 & 151.3550 & 160.0121 & 161.2550 & 160.2857 & 151.7845 & 151.3037 & 151.0429 \\
\hline 7 & 31.4 & 158.9460 & 160.1452 & 158.6605 & 150.2771 & 150.0980 & 149.7505 & 158.9055 & 160.1000 & 158.6222 & 150.2316 & 150.0509 & 149.6904 \\
\hline
\end{tabular}




\begin{tabular}{|c|c|c|c|c|c|c|c|c|c|c|c|c|c|}
\hline 8 & 30.9 & 161.3167 & 161.9941 & 161.5447 & 152.5516 & 152.4886 & 152.2817 & 161.3956 & 162.0706 & 161.6605 & 152.6774 & 152.6137 & 152.4092 \\
\hline 9 & 56.6 & 142.1733 & 142.6818 & 141.9411 & 132.2694 & 132.1213 & 131.4750 & 142.4072 & 142.9072 & 142.2639 & 132.5048 & 132.3617 & 131.7634 \\
\hline 10 & 39.2 & 152.0729 & 152.4342 & 152.1612 & 142.6456 & 142.8128 & 142.3569 & 151.6496 & 152.0088 & 151.6528 & 142.1493 & 142.3136 & 141.7803 \\
\hline 11 & 67.8 & 123.5574 & 124.2931 & 123.0605 & 113.1265 & 112.9616 & 112.5147 & 123.4512 & 124.1898 & 122.9058 & 113.0753 & 112.9063 & 112.4101 \\
\hline 12 & 40.8 & 151.5796 & 152.5866 & 153.2263 & 142.8542 & 142.3821 & 142.1100 & 152.3885 & 153.3856 & 154.2405 & 143.8078 & 143.3103 & 143.1604 \\
\hline 13 & 46.7 & 145.2422 & 145.5955 & 145.9375 & 136.3814 & 136.5232 & 135.4593 & 144.6386 & 144.9932 & 145.1989 & 135.6638 & 135.8184 & 134.6260 \\
\hline 14 & 52.3 & 142.8173 & 143.3232 & 142.5950 & 132.6157 & 132.4850 & 132.0053 & 143.2199 & 143.7150 & 143.0587 & 133.1071 & 132.9645 & 132.5148 \\
\hline 15 & 21.6 & 169.9470 & 171.2982 & 171.5709 & 162.9561 & 162.7487 & 162.2459 & 170.0595 & 171.4053 & 171.6929 & 163.0824 & 162.8604 & 162.3431 \\
\hline 16 & 35.2 & 158.0246 & 159.2755 & 158.0458 & 149.8753 & 149.4344 & 148.5286 & 157.4904 & 158.7256 & 157.2832 & 149.2505 & 148.7925 & 147.5851 \\
\hline 17 & 219.2 & -15.3364 & -15.1145 & -16.8584 & -34.9095 & -34.6512 & -38.0326 & -19.5215 & -19.3063 & -21.9462 & -39.6475 & -39.3977 & -43.6027 \\
\hline 18 & 15.8 & 177.3734 & 178.9125 & 179.2503 & 170.9058 & 170.7400 & 170.6682 & 177.5449 & 179.0741 & 179.3636 & 171.0672 & 170.8859 & 170.8051 \\
\hline 19 & 21.0 & 168.0065 & 169.6621 & 169.7353 & 161.3633 & 161.1611 & 161.0543 & 168.1398 & 169.7850 & 169.8236 & 161.4921 & 161.2711 & 161.1584 \\
\hline${ }^{1} \mathrm{H}$ & & & & & & & & & & & & & \\
\hline $1^{*}$ & 2.17 & 29.8657 & 29.4453 & 29.4044 & 29.7736 & 29.5603 & 29.5610 & 29.8032 & 29.3786 & 29.3372 & 29.7131 & 29.4950 & 29.4917 \\
\hline $1 * *$ & 1.82 & 30.2513 & 29.8915 & 29.8155 & 30.2191 & 30.1007 & 30.0675 & 30.2371 & 29.8774 & 29.7954 & 30.2088 & 30.0901 & 30.0474 \\
\hline $2^{*}$ & 2.33 & 30.1317 & 29.6626 & 29.5214 & 29.9737 & 29.7272 & 29.6386 & 30.1741 & 29.7010 & 29.5536 & 30.0146 & 29.7645 & 29.6688 \\
\hline $2^{* *}$ & 2.42 & 29.9084 & 29.4458 & 29.4080 & 29.8474 & 29.5966 & 29.5486 & 29.7626 & 29.2943 & 29.2363 & 29.6968 & 29.4437 & 29.3794 \\
\hline 4 & 5.67 & 26.4783 & 25.8065 & 25.6799 & 26.3811 & 25.8681 & 25.8410 & 26.4925 & 25.8124 & 25.6788 & 26.3862 & 25.8697 & 25.8388 \\
\hline $6^{*}$ & 2.24 & 30.0754 & 29.6396 & 29.5917 & 29.9453 & 29.7054 & 29.6878 & 29.9987 & 29.5577 & 29.5005 & 29.8675 & 29.6234 & 29.6004 \\
\hline $6 * *$ & 2.48 & 29.3782 & 28.9035 & 28.8174 & 29.2901 & 29.0619 & 29.0154 & 29.2986 & 28.8223 & 28.7272 & 29.2155 & 28.9854 & 28.9283 \\
\hline $7^{*}$ & 1.10 & 30.8455 & 30.4799 & 30.4593 & 30.8259 & 30.6893 & 30.7063 & 30.7897 & 30.4219 & 30.3977 & 30.7709 & 30.6331 & 30.6457 \\
\hline $7 * *$ & 2.06 & 30.0100 & 29.5851 & 29.4616 & 29.9449 & 29.7311 & 29.6578 & 29.9170 & 29.4883 & 29.3588 & 29.8560 & 29.6381 & 29.5603 \\
\hline 8 & 2.14 & 29.5246 & 29.1152 & 29.1470 & 29.6379 & 29.4526 & 29.4820 & 29.4820 & 29.0719 & 29.0950 & 29.5947 & 29.4066 & 29.4290 \\
\hline 9 & 0.98 & 30.4746 & 30.1127 & 30.0575 & 30.4999 & 30.3474 & 30.2782 & 30.4137 & 30.0506 & 29.9911 & 30.4381 & 30.2836 & 30.2112 \\
\hline 11 & 4.44 & 27.6618 & 27.1627 & 27.1486 & 27.6219 & 27.3569 & 27.3576 & 27.5973 & 27.0979 & 27.0818 & 27.5593 & 27.2937 & 27.2948 \\
\hline $12^{*}$ & 1.94 & 30.4286 & 29.9911 & 30.0120 & 30.4101 & 30.0896 & 30.0752 & 30.3644 & 29.9261 & 29.9478 & 30.3435 & 30.0307 & 30.0174 \\
\hline $12 * *$ & 1.45 & 30.6687 & 30.2253 & 30.1455 & 30.6018 & 30.3726 & 30.3390 & 30.6678 & 30.2225 & 30.1395 & 30.6003 & 30.3668 & 30.3318 \\
\hline 14 & 1.23 & 30.7240 & 30.4407 & 30.3294 & 30.7435 & 30.6674 & 30.5424 & 30.6219 & 30.3372 & 30.2118 & 30.6381 & 30.5621 & 30.4274 \\
\hline $15^{*}$ & 1.64 & 30.4100 & 29.9675 & 29.8989 & 30.4160 & 30.1975 & 30.1513 & 30.3163 & 29.8722 & 29.7902 & 30.3222 & 30.1056 & 30.0496 \\
\hline $15^{* *}$ & 1.99 & 30.2633 & 29.8093 & 29.6415 & 30.1684 & 29.9308 & 29.8842 & 30.1952 & 29.7387 & 29.5674 & 30.1092 & 29.8691 & 29.8168 \\
\hline $16^{*}$ & 2.04 & 30.1700 & 29.6875 & 29.5805 & 30.0488 & 29.7812 & 29.7604 & 30.1169 & 29.6295 & 29.5102 & 29.9959 & 29.7246 & 29.6930 \\
\hline $16^{* *}$ & 2.48 & 29.8135 & 29.3081 & 29.2093 & 29.7088 & 29.3912 & 29.3900 & 29.7598 & 29.2492 & 29.1386 & 29.6498 & 29.3288 & 29.3185 \\
\hline 18 & 1.14 & 30.8814 & 30.4102 & 30.3408 & 30.9109 & 30.6496 & 30.5863 & 30.8539 & 30.3797 & 30.3028 & 30.8825 & 30.6176 & 30.5484 \\
\hline 19 & 1.45 & 30.6834 & 30.2499 & 30.1829 & 30.6464 & 30.4355 & 30.4143 & 30.6768 & 30.2403 & 30.1719 & 30.6435 & 30.4269 & 30.3998 \\
\hline
\end{tabular}




\section{Compound 40}

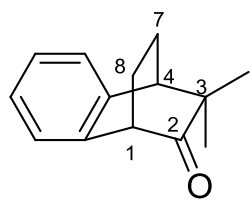

40

\begin{tabular}{|c|c|c|c|c|c|c|c|c|c|c|c|c|c|}
\hline & \multirow[b]{3}{*}{ Exp } & \multicolumn{12}{|c|}{ B3LYP } \\
\hline & & \multicolumn{6}{|c|}{ Gas phase } & \multicolumn{6}{|c|}{ PCM } \\
\hline & & 6-31G* & $6-31 G * *$ & $6-31+\mathrm{G} * *$ & 6-311G* & $6-311 G * *$ & $6-311+G * *$ & $6-31 G *$ & 6-31G** & $6-31+G * *$ & 6-311G* & $6-311 G * *$ & $6-311+G * *$ \\
\hline \multicolumn{14}{|l|}{${ }^{13} \mathrm{C}$} \\
\hline 1 & 52.6 & 136.3585 & 137.0880 & 134.2985 & 123.7259 & 123.4710 & 123.2712 & 136.0267 & 136.7491 & 133.7680 & 123.3799 & 123.1196 & 122.7057 \\
\hline 2 & 216.7 & -16.5275 & -16.5422 & -18.7052 & -38.2963 & -38.1975 & -42.1086 & -20.1854 & -20.2066 & -23.2919 & -42.5677 & -42.4636 & -47.1770 \\
\hline 3 & 44.3 & 143.6848 & 144.0771 & 143.1473 & 133.6962 & 133.7344 & 132.3624 & 143.1119 & 143.5054 & 142.4298 & 133.0369 & 133.0773 & 131.4944 \\
\hline 4 & 48.1 & 140.8238 & 141.4282 & 139.8494 & 128.8983 & 128.6820 & 128.2887 & 141.1065 & 141.7027 & 140.2177 & 129.3039 & 129.0710 & 128.6658 \\
\hline Me exo & 23.8 & 164.3039 & 165.9653 & 166.6365 & 157.4529 & 157.1989 & 156.9027 & 164.6398 & 166.2811 & 166.9770 & 157.8343 & 157.5537 & 157.2604 \\
\hline Me endo & 26.0 & 162.5325 & 164.2181 & 164.9016 & 155.4167 & 155.1930 & 154.9252 & 162.6792 & 164.3545 & 165.0614 & 155.6229 & 155.3713 & 155.1143 \\
\hline 7 & 23.1 & 164.3945 & 165.9017 & 166.9946 & 157.2217 & 157.0768 & 156.2727 & 164.6340 & 166.1298 & 167.2518 & 157.4447 & 157.2819 & 156.4714 \\
\hline 8 & 21.4 & 163.4467 & 164.8852 & 165.5614 & 155.4091 & 155.3333 & 154.5050 & 163.5983 & 165.0260 & 165.6369 & 155.5447 & 155.4547 & 154.5765 \\
\hline Car & 135.2 & 59.9223 & 59.7873 & 57.9024 & 40.1859 & 40.0068 & 38.9735 & 60.0391 & 59.9066 & 57.9835 & 40.2583 & 40.0943 & 39.0730 \\
\hline Car & 143.6 & 52.2763 & 52.1934 & 49.7950 & 31.8824 & 31.6995 & 30.5300 & 51.6809 & 51.6020 & 49.1141 & 31.1737 & 31.0095 & 29.7684 \\
\hline Car & 124.8 & 70.7048 & 71.3675 & 70.8788 & 54.0802 & 53.2990 & 52.8932 & 70.5556 & 71.2131 & 70.6797 & 53.7794 & 53.0106 & 52.5766 \\
\hline Car & 125.3 & 70.4319 & 71.0797 & 70.9669 & 53.8089 & 52.9692 & 52.2425 & 70.4837 & 71.1248 & 71.0036 & 53.7457 & 52.9187 & 52.1868 \\
\hline Car & 126.8 & 69.3896 & 69.9868 & 69.5394 & 52.0240 & 51.1214 & 50.3619 & 69.5015 & 70.0968 & 69.6487 & 52.0682 & 51.1964 & 50.5206 \\
\hline $\begin{array}{c}\mathrm{C} \text { ar } \\
{ }^{1} \mathbf{H}\end{array}$ & 127.1 & 69.4333 & 70.0410 & 69.6485 & 52.1341 & 51.2201 & 50.3634 & 69.3643 & 69.9705 & 69.5321 & 51.9779 & 51.0928 & 50.2628 \\
\hline 1 & 3.55 & 28.8976 & 28.4034 & 28.3392 & 28.7938 & 28.5481 & 28.5179 & 28.8588 & 28.3572 & 28.2787 & 28.7403 & 28.4891 & 28.4538 \\
\hline 4 & 2.93 & 29.2781 & 28.8574 & 28.8899 & 29.3242 & 29.1232 & 29.1367 & 29.1473 & 28.7220 & 28.7256 & 29.1791 & 28.9750 & 28.9742 \\
\hline 7 syn & 1.71 & 30.7283 & 30.3227 & 30.1942 & 30.7128 & 30.5414 & 30.4514 & 30.6979 & 30.2911 & 30.1596 & 30.6897 & 30.5176 & 30.4202 \\
\hline 7 anti & 1.99 & 29.9488 & 29.4703 & 29.3294 & 29.9831 & 29.7045 & 29.6256 & 29.8869 & 29.4073 & 29.2588 & 29.9210 & 29.6435 & 29.5602 \\
\hline 8 syn & 1.47 & 30.5584 & 30.1279 & 29.9825 & 30.5638 & 30.3531 & 30.3097 & 30.4905 & 30.0569 & 29.8996 & 30.5005 & 30.2871 & 30.2308 \\
\hline 8 anti & 2.20 & 30.2018 & 29.7491 & 29.5605 & 30.2164 & 29.9721 & 29.8829 & 30.1435 & 29.6871 & 29.4921 & 30.1547 & 29.9083 & 29.8153 \\
\hline Car & 7.19 & 25.1161 & 24.4973 & 24.4155 & 24.9890 & 24.5740 & 24.5610 & 25.0043 & 24.3852 & 24.2914 & 24.8642 & 24.4551 & 24.4356 \\
\hline Car & 7.19 & 25.0457 & 24.4324 & 24.2915 & 24.8986 & 24.5187 & 24.4957 & 24.9537 & 24.3403 & 24.1975 & 24.7955 & 24.4228 & 24.3996 \\
\hline Car & 7.19 & 25.0434 & 24.4310 & 24.2390 & 24.8968 & 24.5212 & 24.4695 & 24.9619 & 24.3491 & 24.1555 & 24.8043 & 24.4359 & 24.3832 \\
\hline Car & 7.19 & 25.1468 & 24.5179 & 24.2589 & 25.0047 & 24.5888 & 24.4546 & 25.0651 & 24.4351 & 24.1629 & 24.9090 & 24.4990 & 24.3576 \\
\hline Me exo & 1.23 & 30.9670 & 30.5161 & 30.4664 & 30.9831 & 30.7225 & 30.6777 & 30.9403 & 30.4875 & 30.4309 & 30.9553 & 30.6934 & 30.6428 \\
\hline Me endo & 0.73 & 31.3818 & 30.9255 & 30.9321 & 31.4147 & 31.1564 & 31.1379 & 31.3872 & 30.9298 & 30.9306 & 31.4186 & 31.1592 & 31.1352 \\
\hline
\end{tabular}

\begin{tabular}{|c|c|c|c|c|c|c|c|c|c|c|c|c|c|}
\hline & \multirow[b]{3}{*}{ Exp } & \multicolumn{12}{|c|}{ mPW1PW91 } \\
\hline & & \multicolumn{6}{|c|}{ Gas phase } & \multicolumn{6}{|c|}{ PCM } \\
\hline & & 6-31G* & 6-31G** & $6-31+\mathrm{G} * *$ & 6-311G* & 6-311G** & $6-311+G * *$ & 6-31G* & 6-31G** & $6-31+\mathrm{G} * *$ & 6-311G* & $6-311 G * *$ & $6-311+G * *$ \\
\hline \multicolumn{14}{|l|}{${ }^{13} \mathrm{C}$} \\
\hline 1 & 52.6 & 141.2408 & 141.9151 & 139.2465 & 129.9675 & 129.7122 & 129.5148 & 140.9130 & 141.5800 & 138.7484 & 129.6164 & 129.3565 & 128.9738 \\
\hline
\end{tabular}




\begin{tabular}{|c|c|c|c|c|c|c|c|c|c|c|c|c|c|}
\hline 2 & 216.7 & -13.4492 & -13.4666 & -15.0592 & -32.5635 & -32.4737 & -35.8786 & -17.2184 & -17.2425 & -19.6578 & -36.8698 & -36.7749 & -40.8915 \\
\hline 3 & 44.3 & 148.5648 & 148.9543 & 148.1961 & 139.3581 & 139.4021 & 138.1852 & 147.9793 & 148.3703 & 147.4897 & 138.6883 & 138.7361 & 137.3374 \\
\hline 4 & 48.1 & 146.0120 & 146.5516 & 145.0023 & 135.1237 & 134.9201 & 134.5805 & 146.2951 & 146.8276 & 145.3558 & 135.4982 & 135.2807 & 134.9300 \\
\hline Me exo & 23.8 & 168.7752 & 170.3013 & 170.7145 & 162.4138 & 162.1829 & 161.8760 & 169.1436 & 170.6510 & 171.0854 & 162.7956 & 162.5421 & 162.2376 \\
\hline Me endo & 26.0 & 166.9726 & 168.5167 & 169.0230 & 160.2927 & 160.0914 & 159.8539 & 167.1517 & 168.6864 & 169.2136 & 160.5081 & 160.2813 & 160.0519 \\
\hline 7 & 23.1 & 169.3500 & 170.7273 & 171.8540 & 162.5405 & 162.3904 & 161.5027 & 169.6048 & 170.9712 & 172.1265 & 162.7645 & 162.5967 & 161.7020 \\
\hline 8 & 21.4 & 168.5279 & 169.8393 & 170.3761 & 160.8593 & 160.7833 & 159.9490 & 168.6845 & 169.9856 & 170.4648 & 160.9886 & 160.8992 & 160.0203 \\
\hline C ar & 135.2 & 63.2713 & 63.1267 & 61.4203 & 45.6973 & 45.5056 & 44.5276 & 63.3771 & 63.2362 & 61.4900 & 45.7694 & 45.5945 & 44.6261 \\
\hline Car & 143.6 & 55.8560 & 55.7656 & 53.5441 & 37.7061 & 37.5111 & 36.4463 & 55.2114 & 55.1263 & 52.8314 & 36.9685 & 36.7944 & 35.6691 \\
\hline C ar & 124.8 & 73.2195 & 73.8159 & 73.3670 & 58.5494 & 57.7437 & 57.3670 & 73.0578 & 73.6489 & 73.1628 & 58.2312 & 57.4397 & 57.0445 \\
\hline C ar & 125.3 & 72.8796 & 73.4602 & 73.3681 & 58.2413 & 57.3804 & 56.6388 & 72.9395 & 73.5125 & 73.4123 & 58.1707 & 57.3241 & 56.5764 \\
\hline C ar & 126.8 & 71.8864 & 72.3973 & 72.0951 & 56.6268 & 55.6770 & 54.8565 & 71.9838 & 72.4926 & 72.1753 & 56.6340 & 55.7172 & 54.9787 \\
\hline $\begin{array}{c}\mathrm{C} \text { ar } \\
{ }^{{ }^{1}} \mathbf{H}\end{array}$ & 127.1 & 71.9823 & 72.5045 & 72.3130 & 56.7777 & 55.8159 & 54.9629 & 71.8888 & 72.4094 & 72.1620 & 56.5786 & 55.6482 & 54.8257 \\
\hline 1 & 3.55 & 28.7327 & 28.2482 & 28.2046 & 28.6096 & 28.3539 & 28.3500 & 28.6857 & 28.1929 & 28.1363 & 28.5502 & 28.2887 & 28.2800 \\
\hline 4 & 2.93 & 29.1737 & 28.7598 & 28.7865 & 29.1606 & 28.9597 & 28.9761 & 29.0324 & 28.6129 & 28.6140 & 29.0102 & 28.8056 & 28.8088 \\
\hline 7 syn & 1.71 & 30.6779 & 30.2713 & 30.1506 & 30.6220 & 30.4501 & 30.3615 & 30.6474 & 30.2394 & 30.1145 & 30.5983 & 30.4256 & 30.3291 \\
\hline 7 anti & 1.99 & 29.9284 & 29.4488 & 29.2908 & 29.9072 & 29.6317 & 29.5367 & 29.8571 & 29.3763 & 29.2120 & 29.8372 & 29.5628 & 29.4642 \\
\hline 8 syn & 1.47 & 30.5086 & 30.0738 & 29.9405 & 30.4821 & 30.2642 & 30.2244 & 30.4375 & 29.9991 & 29.8546 & 30.4158 & 30.1949 & 30.1426 \\
\hline 8 anti & 2.20 & 30.1627 & 29.7068 & 29.5258 & 30.1322 & 29.8844 & 29.8015 & 30.0968 & 29.6370 & 29.4509 & 30.0647 & 29.8146 & 29.7287 \\
\hline C ar & 7.19 & 24.9091 & 24.2865 & 24.2185 & 24.8001 & 24.3546 & 24.3493 & 24.7836 & 24.1605 & 24.0822 & 24.6652 & 24.2257 & 24.2145 \\
\hline C ar & 7.19 & 24.8480 & 24.2295 & 24.0929 & 24.7239 & 24.3085 & 24.2814 & 24.7420 & 24.1233 & 23.9874 & 24.6095 & 24.2016 & 24.1751 \\
\hline C ar & 7.19 & 24.8441 & 24.2265 & 24.0451 & 24.7202 & 24.3094 & 24.2567 & 24.7490 & 24.1308 & 23.9503 & 24.6169 & 24.2134 & 24.1600 \\
\hline C ar & 7.19 & 24.9378 & 24.3059 & 24.0772 & 24.8190 & 24.3729 & 24.2534 & 24.8435 & 24.2103 & 23.9701 & 24.7136 & 24.2737 & 24.1472 \\
\hline Me exo & 1.23 & 30.9078 & 30.4576 & 30.4035 & 30.8900 & 30.6267 & 30.5727 & 30.8767 & 30.4245 & 30.3646 & 30.8586 & 30.5940 & 30.5349 \\
\hline Me endo & 0.73 & 31.3341 & 30.8774 & 30.8758 & 31.3262 & 31.0647 & 31.0448 & 31.3393 & 30.8816 & 30.8750 & 31.3298 & 31.0672 & 31.0423 \\
\hline
\end{tabular}

\section{Compound 41}

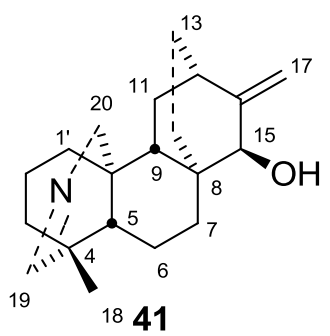

\begin{tabular}{|c|c|c|c|c|c|c|c|c|c|c|c|c|c|}
\hline \multirow{3}{*}{\multicolumn{2}{|c|}{ Exp }} & \multicolumn{12}{|c|}{ B3LYP } \\
\hline & & \multicolumn{6}{|c|}{ Gas phase } & \multicolumn{6}{|c|}{ PCM } \\
\hline & & 6-31G* & $6-31 \mathrm{G} * *$ & $6-31+G * *$ & 6-311G* & $6-311 G * *$ & $6-311+G * *$ & 6-31G* & $6-31 G * *$ & $6-31+\mathrm{G} * *$ & $6-311 G *$ & $6-311 G * *$ & $6-311+\mathrm{G} * *$ \\
\hline \multicolumn{14}{|c|}{${ }^{13} \mathrm{C}$} \\
\hline 1 & 41.4 & 146.7544 & 147.9650 & 147.3103 & 137.1139 & 136.7851 & 136.9501 & 147.0023 & 148.2056 & 147.6045 & 137.4421 & 137.0989 & 137.2872 \\
\hline 2 & 20.2 & 167.7888 & 169.2563 & 168.0099 & 158.6487 & 158.4201 & 158.5272 & 167.7677 & 169.2385 & 167.9847 & 158.6335 & 158.4030 & 158.5052 \\
\hline 3 & 37.6 & 151.2277 & 152.3978 & 150.9617 & 141.7078 & 141.3703 & 140.7706 & 151.4956 & 152.6562 & 151.2617 & 142.0476 & 141.6948 & 141.1140 \\
\hline 4 & 38.9 & 149.3355 & 149.7680 & 149.4360 & 139.3875 & 139.4648 & 138.7878 & 148.9773 & 149.4108 & 148.9724 & 138.9880 & 139.0603 & 138.2649 \\
\hline 5 & 47.6 & 140.8379 & 141.5055 & 140.7798 & 130.5982 & 130.3456 & 130.0277 & 141.0588 & 141.7171 & 141.0809 & 130.8901 & 130.6228 & 130.3383 \\
\hline
\end{tabular}




\begin{tabular}{|c|c|c|c|c|c|c|c|c|c|c|c|c|c|}
\hline 6 & 9.6 & 7.6528 & 9.1495 & 8.7160 & 59.5788 & 59.3973 & 59.0140 & 67.6792 & 169.1756 & 58.7295 & 159.6210 & 159.4364 & 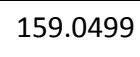 \\
\hline & 31.0 & & 8.5895 & 8.2925 & & 48.9355 & & & 58.5115 & & & & \\
\hline & 6.4 & .9609 & & 48.0783 & & & & 48.9712 & 49.2933 & & & & \\
\hline y & 38.4 & 8.2343 & 8.9816 & 48.8111 & 38.9618 & 38.9104 & 38.2687 & 48.4764 & 49.2142 & 0897 & 139.2555 & & 138.548 \\
\hline & 37.6 & 0.7352 & 1.1138 & 50.5842 & 0.6949 & 0.7001 & 10.0221 & 50.6270 & 51.0032 & 0.4276 & 5949 & 004 & 139.846 \\
\hline & 28.4 & 7.9673 & 9.3805 & 58.1752 & 9.5413 & 49.2248 & 48.3527 & 58.1238 & 159.5297 & 58.3534 & 49.7228 & & 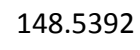 \\
\hline & 36.3 & 0.7627 & & & & & & & & & & & \\
\hline & 26.3 & 1.8411 & 3.2831 & 62.3137 & 3.4031 & 3.1870 & 52.6483 & 52.0183 & 163.4548 & 62.4736 & & & 152827 \\
\hline & 27.0 & 9.7745 & 1.2377 & 159.9230 & 2.1619 & 51.9414 & 52.2499 & 59.9761 & 161.4364 & 60.1661 & 4400 & 2099 & 152524 \\
\hline & 77.0 & 3.0656 & 3.5694 & 112.4636 & 00.8588 & 00.6627 & 00.4878 & 1430 & 113.6488 & 12.4936 & 3955 & 055 & 100.524 \\
\hline & 156.6 & 6.5254 & 36.1349 & 31.1544 & 12.5656 & 2.0464 & 096 & 569 & 35.8675 & & 12.1797 & & 5621 \\
\hline & 109.1 & 83.5804 & 84.9212 & 86.3563 & & & & & & & & & 6197 \\
\hline & 3.7 & & 166.4101 & 166.6106 & 8.1484 & & & & 166.7782 & & & & \\
\hline & 169.0 & 29.2126 & 29.8781 & 29.1277 & .7772 & 8088 & & & 494 & 3624 & & & 3635 \\
\hline 0 & 55.5 & 32.6989 & 3.8647 & $131 . \varepsilon$ & & & & & & & & & \\
\hline 1 & 70 & & & & & & & & & & & & \\
\hline 1 & 1.00 & 526 & 0.8028 & 437 & & & & & & & & & \\
\hline 2 & 1.50 & 0.6418 & 84 & 0.21 & 30.7 & & & & & & & & \\
\hline 2 & 27 & .7990 & & & & & & & & & & & \\
\hline 3 & ד. & & & & & & & & & & & & \\
\hline 3 & & & & & & & & & & & & & \\
\hline 5 & 0.98 & & & 44 & & & & & & & & & \\
\hline 6 & 1.56 & 6696 & & & & & & & & & & & \\
\hline 6 & 99 & & & & & & & & & & & & \\
\hline 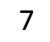 & & & & & & & & & & & & & \\
\hline 7 & 1.12 & & & & & & & & & & & & \\
\hline 9 & 1.58 & .7986 & & & & & & & & & & & \\
\hline & 72 & & & & & & & & & & & & \\
\hline & & & & & & & & & & & & & \\
\hline & & & & & & & & & & & & & \\
\hline & 1.57 & & & & & & & & & & & & \\
\hline & 1.57 & & & & & & & & & & & & \\
\hline & 2.14 & & & & & & & & & & & & 1292 \\
\hline & 0.02 & & & & & & & & & & & & 30.8806 \\
\hline & & & & & & & & & & & & & \\
\hline & 5.10 & & & & & & & & & & & & \\
\hline & 5.04 & & & & & 0021 & & & 4667 & 4011 & 0007 & & 26.6048 \\
\hline 18 & 1.07 & 31.1897 & 30.7648 & 30.6959 & 31.1802 & 30.9521 & 30.9005 & & 7312 & 6578 & 1490 & 30.9184 & 30.8629 \\
\hline - & 7.43 & & & & & & & & & & & & \\
\hline & & & & & & & & & & & & & \\
\hline 0 & & & & & & & & & .0860 & 0008 & 28.5755 & 28.3444 & 28.1277 \\
\hline
\end{tabular}




\begin{tabular}{|c|c|c|c|c|c|c|c|c|c|c|c|c|c|}
\hline & & \multicolumn{12}{|c|}{ mPW1PW91 } \\
\hline & \multirow[b]{2}{*}{ Exp } & \multicolumn{6}{|c|}{ Gas phase } & \multicolumn{6}{|c|}{ PCM } \\
\hline & & 6-31G* & $6-31 G * *$ & $6-31+G * *$ & 6-311G* & 6-311G** & $6-311+G^{* *}$ & 6-31G* & $6-31 G * *$ & $6-31+G * *$ & 6-311G* & $6-311 G * *$ & $6-311+\mathrm{G} * *$ \\
\hline \multicolumn{14}{|l|}{${ }^{13} \mathrm{C}$} \\
\hline 1 & 41.4 & 152.1823 & 153.2541 & 152.6982 & 143.2156 & 142.8944 & 142.9116 & 152.4460 & 153.5114 & 153.0020 & 143.5336 & 143.1999 & 143.2376 \\
\hline 2 & 20.2 & 172.5380 & 173.8946 & 172.7407 & 164.1645 & 163.9239 & 163.9118 & 172.5403 & 173.9000 & 172.7419 & 164.1662 & 163.9228 & 163.9102 \\
\hline 3 & 37.6 & 156.4448 & 157.4983 & 155.8017 & 147.6619 & 147.3322 & 146.8333 & 156.7322 & 157.7773 & 156.1189 & 147.9959 & 147.6527 & 147.1734 \\
\hline 4 & 38.9 & 154.4124 & 154.8266 & 154.4883 & 145.2175 & 145.2916 & 144.6669 & 154.0442 & 154.4594 & 154.0291 & 144.8141 & 144.8842 & 144.1549 \\
\hline 5 & 47.6 & 146.7941 & 147.3624 & 146.6204 & 137.3365 & 137.0880 & 136.7801 & 147.0192 & 147.5797 & 146.9162 & 137.6101 & 137.3488 & 137.0723 \\
\hline 6 & 19.6 & 172.4083 & 173.7960 & 173.2602 & 165.1158 & 164.9305 & 164.5842 & 172.4468 & 173.8341 & 173.2886 & 165.1644 & 164.9759 & 164.6284 \\
\hline 7 & 31.0 & 162.3907 & 163.5395 & 163.0646 & 154.9858 & 154.7192 & 154.5626 & 162.3371 & 163.4918 & 163.0675 & 155.0017 & 154.7366 & 154.5901 \\
\hline 8 & 36.4 & 154.2877 & 154.5965 & 153.5889 & 144.7062 & 144.7677 & 144.4939 & 154.2944 & 154.6032 & 153.5679 & 144.7098 & 144.7690 & 144.4892 \\
\hline 9 & 38.4 & 153.7764 & 154.4405 & 154.4154 & 145.2023 & 145.1519 & 144.5178 & 154.0164 & 154.6722 & 154.6799 & 145.4755 & 145.4025 & 144.7748 \\
\hline 10 & 37.6 & 156.0173 & 156.3781 & 155.7579 & 146.7785 & 146.7757 & 146.0897 & 155.9045 & 156.2629 & 155.6043 & 146.6782 & 146.6759 & 145.9256 \\
\hline 11 & 28.4 & 163.1073 & 164.3954 & 163.1169 & 155.2691 & 154.9580 & 154.2176 & 163.2668 & 164.5484 & 163.2972 & 155.4409 & 155.1186 & 154.3963 \\
\hline 12 & 36.3 & 155.9828 & 156.8678 & 154.9308 & 145.8258 & 145.5566 & 144.8804 & 156.1497 & 157.0326 & 155.1202 & 146.0324 & 145.7519 & 145.0652 \\
\hline 13 & 26.3 & 166.9649 & 168.2768 & 167.3111 & 159.0554 & 158.8290 & 158.3089 & 167.1682 & 168.4745 & 167.4996 & 159.2622 & 159.0237 & 158.5008 \\
\hline 14 & 27.0 & 164.9876 & 166.3157 & 164.9906 & 157.8997 & 157.6781 & 157.9458 & 165.2190 & 166.5445 & 165.2609 & 158.1877 & 157.9571 & 158.2325 \\
\hline 15 & 77.0 & 117.9587 & 118.4190 & 117.4026 & 107.2582 & 107.0645 & 106.7813 & 118.0406 & 118.5037 & 117.4494 & 107.2953 & 107.1073 & 106.8178 \\
\hline 16 & 156.6 & 40.4170 & 39.9676 & 35.6181 & 19.1323 & 18.5783 & 16.6911 & 40.1301 & 39.6833 & 35.2425 & 18.7615 & 18.2018 & 16.2600 \\
\hline 17 & 109.1 & 85.2823 & 86.5562 & 87.6763 & 72.9819 & 72.1024 & 72.2170 & 85.5729 & 86.8397 & 88.0625 & 73.2303 & 72.3832 & 72.5437 \\
\hline 18 & 23.7 & 169.2049 & 170.8868 & 170.5873 & 163.1380 & 162.9423 & 162.7540 & 169.6131 & 171.2821 & 171.0355 & 163.5876 & 163.3720 & 163.2091 \\
\hline 19 & 169.0 & 31.7382 & 32.3483 & 32.1085 & 14.4981 & 13.5307 & 11.8856 & 29.9365 & 30.5522 & 29.8489 & 12.4574 & 11.4809 & 9.4585 \\
\hline $\begin{array}{l}20 \\
{ }^{1} \mathbf{H}\end{array}$ & 55.5 & 137.4894 & 138.5491 & 136.8155 & 128.6273 & 127.8604 & 127.6607 & 137.7568 & 138.8023 & 136.8993 & 128.7596 & 127.9837 & 127.7447 \\
\hline 1 & 1.70 & 30.4394 & 30.0297 & 29.9672 & 30.3932 & 30.1820 & 30.0840 & 30.4005 & 29.9895 & 29.9269 & 30.3577 & 30.1453 & 30.0482 \\
\hline 1 & 1.00 & 31.1071 & 30.7615 & 30.6840 & 31.0685 & 30.9051 & 30.8637 & 31.0485 & 30.7022 & 30.6213 & 31.0094 & 30.8469 & 30.8029 \\
\hline 2 & 1.50 & 30.6233 & 30.1902 & 30.1713 & 30.6492 & 30.4450 & 30.4093 & 30.6890 & 30.2596 & 30.2400 & 30.7090 & 30.5073 & 30.4721 \\
\hline 2 & 1.27 & 30.7637 & 30.3101 & 30.2402 & 30.6740 & 30.4303 & 30.3063 & 30.7137 & 30.2571 & 30.1803 & 30.6238 & 30.3778 & 30.2495 \\
\hline 3 & 1.49 & 30.6857 & 30.2647 & 30.1655 & 30.6441 & 30.4127 & 30.3169 & 30.6374 & 30.2141 & 30.1091 & 30.5937 & 30.3608 & 30.2610 \\
\hline 3 & 1.28 & 30.8057 & 30.4371 & 30.3586 & 30.7437 & 30.5845 & 30.5525 & 30.7381 & 30.3679 & 30.2789 & 30.6764 & 30.5156 & 30.4749 \\
\hline 5 & 0.98 & 30.9557 & 30.6795 & 30.5612 & 30.9953 & 30.8905 & 30.8233 & 30.8957 & 30.6200 & 30.4917 & 30.9301 & 30.8266 & 30.7534 \\
\hline 6 & 1.56 & 30.6321 & 30.2040 & 30.1295 & 30.6081 & 30.4072 & 30.3537 & 30.5870 & 30.1560 & 30.0766 & 30.5602 & 30.3563 & 30.3005 \\
\hline 6 & 0.99 & 30.8516 & 30.4446 & 30.4613 & 30.9507 & 30.7494 & 30.7599 & 30.8787 & 30.4725 & 30.4873 & 30.9723 & 30.7706 & 30.7781 \\
\hline 7 & 1.68 & 30.0124 & 29.5995 & 29.4618 & 29.9359 & 29.7760 & 29.7245 & 30.1286 & 29.7178 & 29.5859 & 30.0556 & 29.8900 & 29.8393 \\
\hline 7 & 1.12 & 31.0554 & 30.6834 & 30.5703 & 31.0248 & 30.8468 & 30.7914 & 31.0188 & 30.6449 & 30.5213 & 30.9811 & 30.8015 & 30.7418 \\
\hline 9 & 1.58 & 30.7705 & 30.4109 & 30.3248 & 30.8473 & 30.6228 & 30.5388 & 30.7108 & 30.3513 & 30.2681 & 30.7804 & 30.5626 & 30.4845 \\
\hline 11 & 1.72 & 30.5745 & 30.1358 & 30.0885 & 30.5143 & 30.2708 & 30.2145 & 30.5239 & 30.0850 & 30.0450 & 30.4739 & 30.2323 & 30.1785 \\
\hline 11 & 1.36 & 30.6561 & 30.2403 & 30.1072 & 30.6560 & 30.4161 & 30.3231 & 30.6282 & 30.2132 & 30.0765 & 30.6261 & 30.3884 & 30.2961 \\
\hline 12 & 2.34 & 29.9086 & 29.4710 & 29.3599 & 29.8468 & 29.6010 & 29.5815 & 29.8414 & 29.4008 & 29.2785 & 29.7727 & 29.5268 & 29.5035 \\
\hline 13 & 1.57 & 30.5248 & 30.1257 & 30.0140 & 30.4273 & 30.2322 & 30.2169 & 30.4883 & 30.0881 & 29.9703 & 30.3877 & 30.1926 & 30.1762 \\
\hline 13 & 1.57 & 30.5483 & 30.1244 & 30.0067 & 30.4505 & 30.2487 & 30.2356 & 30.5478 & 30.1229 & 30.0041 & 30.4536 & 30.2512 & 30.2347 \\
\hline 14 & 2.14 & 29.8649 & 29.3799 & 29.3731 & 29.9021 & 29.6124 & 29.6291 & 29.8734 & 29.3896 & 29.3806 & 29.9061 & 29.6173 & 29.6327 \\
\hline 14 & 0.92 & 31.1681 & 30.8136 & 30.6551 & 31.1337 & 30.9828 & 30.8412 & 31.1293 & 30.7724 & 30.6032 & 31.0917 & 30.9384 & 30.7892 \\
\hline 15 & 3.61 & 28.4110 & 28.0176 & 27.8976 & 28.3453 & 28.1895 & 28.1540 & 28.4087 & 28.0138 & 27.8720 & 28.3293 & 28.1674 & 28.1210 \\
\hline 17 & 5.10 & 26.7887 & 26.1382 & 25.9626 & 26.7122 & 26.2619 & 26.1871 & 26.7995 & 26.1488 & 25.9779 & 26.7160 & 26.2685 & 26.1982 \\
\hline
\end{tabular}




\begin{tabular}{llllllllllllll}
\hline 17 & 5.04 & 26.9820 & 26.3287 & 26.2943 & 26.8871 & 26.4272 & 26.4835 & 26.9085 & 26.2563 & 26.2170 & 26.8060 & 26.3555 & 26.4085 \\
18 & 1.07 & 31.1173 & 30.6933 & 30.6220 & 31.0731 & 30.8415 & 30.7973 & 31.0818 & 30.6553 & 30.5804 & 31.0380 & 30.8039 & 30.7565 \\
19 & 7.43 & 24.3116 & 23.7966 & 23.6991 & 24.3564 & 24.0528 & 23.9648 & 24.2839 & 23.7687 & 23.6733 & 24.3246 & 24.0207 & 23.9351 \\
20 & 3.92 & 28.0212 & 27.4992 & 27.4246 & 28.0069 & 27.6943 & 27.6829 & 28.0405 & 27.5165 & 27.4357 & 28.0179 & 27.7041 & 27.6945 \\
20 & 3.42 & 28.4574 & 28.0315 & 27.9585 & 28.4870 & 28.2595 & 28.0650 & 28.4618 & 28.0337 & 27.9574 & 28.4830 & 28.2550 & 28.0657 \\
\hline
\end{tabular}

\section{Compound 42}

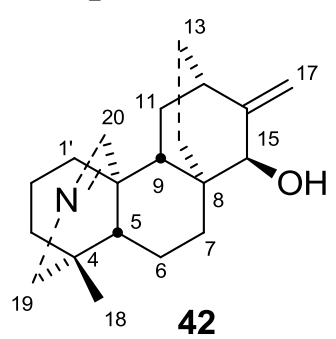

\begin{tabular}{|c|c|c|c|c|c|c|c|c|c|c|c|c|c|}
\hline & & \multicolumn{12}{|c|}{ B3LYP } \\
\hline & \multirow[b]{2}{*}{ Exp } & \multicolumn{6}{|c|}{ Gas phase } & \multicolumn{6}{|c|}{ PCM } \\
\hline & & 6-31G* & $6-31 G * *$ & $6-31+G * *$ & 6-311G* & $6-311 G * *$ & $6-311+G * *$ & 6-31G* & $6-31 G * *$ & $6-31+G * *$ & 6-311G* & $6-311 G * *$ & $6-311+\mathrm{G} * *$ \\
\hline \multicolumn{14}{|l|}{${ }^{13} \mathrm{C}$} \\
\hline 1 & 34.2 & 153.3321 & 154.5775 & 154.4135 & 144.3721 & 144.1301 & 144.1968 & 153.6353 & 154.8708 & 154.7451 & 144.7364 & 144.4803 & 144.5732 \\
\hline 2 & 20.0 & 167.9593 & 169.4240 & 167.9907 & 158.8192 & 158.6017 & 158.7008 & 167.9237 & 169.3922 & 167.9518 & 158.7901 & 158.5704 & 158.6656 \\
\hline 3 & 42.4 & 147.0708 & 148.2238 & 146.7275 & 136.9571 & 136.5609 & 136.0860 & 147.2838 & 148.4303 & 146.9961 & 137.2630 & 136.8511 & 136.3995 \\
\hline 4 & 32.9 & 154.6917 & 155.1994 & 154.5085 & 144.5589 & 144.5845 & 144.0526 & 154.5807 & 155.0904 & 154.3618 & 144.4774 & 144.5028 & 143.9121 \\
\hline 5 & 46.9 & 141.3474 & 142.0059 & 140.4182 & 131.1901 & 130.9490 & 130.9115 & 141.6004 & 142.2490 & 140.7671 & 131.5281 & 131.2711 & 131.2762 \\
\hline 6 & 19.5 & 167.8519 & 169.3415 & 169.1395 & 159.6603 & 159.4779 & 159.0167 & 167.8793 & 169.3692 & 169.1587 & 159.7061 & 159.5206 & 159.0526 \\
\hline 7 & 30.9 & 157.4139 & 158.6423 & 158.6340 & 149.1992 & 148.9397 & 148.8316 & 157.3568 & 158.5914 & 158.6433 & 149.2184 & 148.9617 & 148.8663 \\
\hline 8 & 37.3 & 149.4368 & 149.7650 & 148.1368 & 139.2682 & 139.3459 & 139.2949 & 149.4405 & 149.7688 & 148.0989 & 139.2621 & 139.3380 & 139.2724 \\
\hline 9 & 38.0 & 148.5839 & 149.3200 & 149.4256 & 139.4830 & 139.3468 & 138.4452 & 148.7689 & 149.4945 & 149.6261 & 139.7049 & 139.5469 & 138.6383 \\
\hline 10 & 42.5 & 145.8468 & 146.1412 & 145.4137 & 135.4915 & 135.5116 & 134.7177 & 145.4733 & 145.7644 & 144.9449 & 135.0870 & 135.1058 & 134.1896 \\
\hline 11 & 28.0 & 158.4340 & 159.8134 & 158.4563 & 149.6258 & 149.3404 & 148.5659 & 158.6400 & 160.0086 & 158.6882 & 149.8759 & 149.5729 & 148.8099 \\
\hline 12 & 35.9 & 151.0159 & 151.9728 & 149.6429 & 139.7549 & 139.5079 & 139.1811 & 151.1900 & 152.1443 & 149.8471 & 139.9937 & 139.7336 & 139.3909 \\
\hline 13 & 26.0 & 162.1135 & 163.5646 & 163.1245 & 153.5484 & 153.3928 & 152.7034 & 162.2940 & 163.7393 & 163.2828 & 153.7565 & 153.5874 & 152.8915 \\
\hline 14 & 25.1 & 161.7158 & 163.1263 & 162.1693 & 154.1043 & 153.8971 & 154.1938 & 161.7901 & 163.1996 & 162.2805 & 154.2471 & 154.0316 & 154.3279 \\
\hline 15 & 75.8 & 114.1474 & 114.6564 & 113.4895 & 101.8539 & 101.6802 & 101.3731 & 114.2260 & 114.7374 & 113.5265 & 101.8921 & 101.7258 & 101.4086 \\
\hline 16 & 156.6 & 36.3707 & 35.9847 & 30.9201 & 12.5499 & 12.0257 & 9.9761 & 36.1773 & 35.7925 & 30.6277 & 12.2501 & 11.7205 & 9.6279 \\
\hline 171 & 109.1 & 83.6876 & 85.0477 & 86.4213 & 70.0521 & 69.2044 & 69.6376 & 83.9845 & 85.3372 & 86.8127 & 70.3462 & 69.5245 & 69.9847 \\
\hline 18 & 25.9 & 162.7851 & 164.5824 & 165.2947 & 156.2861 & 156.0370 & 155.9210 & 163.1204 & 164.9066 & 165.7015 & 156.7177 & 156.4440 & 156.3574 \\
\hline 19 & 60.7 & 128.5460 & 129.6750 & 127.9829 & 118.0101 & 117.1695 & 116.6476 & 128.9029 & 130.0208 & 128.2332 & 118.3169 & 117.4618 & 116.9051 \\
\hline 201 & 165.8 & 31.6756 & 32.3943 & 30.8196 & 12.3138 & 11.3108 & 9.8467 & 29.7849 & 30.5142 & 28.4118 & 10.0603 & 9.0625 & 7.1817 \\
\hline \multicolumn{14}{|l|}{${ }^{1} \mathrm{H}$} \\
\hline 1 & 1.69 & 30.5500 & 30.1057 & 30.0359 & 30.5419 & 30.3078 & 30.2154 & 30.5077 & 30.0609 & 29.9866 & 30.5027 & 30.2661 & 30.1696 \\
\hline 1 & 1.09 & 31.1002 & 30.7394 & 30.6554 & 31.1206 & 30.9494 & 30.9229 & 31.0188 & 30.6568 & 30.5609 & 31.0368 & 30.8656 & 30.8301 \\
\hline 2 & 1.49 & 30.6013 & 30.1659 & 30.1101 & 30.6720 & 30.4606 & 30.4209 & 30.6667 & 30.2350 & 30.1786 & 30.7340 & 30.5251 & 30.4839 \\
\hline 2 & 1.32 & 30.8183 & 30.3666 & 30.2728 & 30.7559 & 30.5169 & 30.4036 & 30.7736 & 30.3192 & 30.2158 & 30.7106 & 30.4692 & 30.3492 \\
\hline 3 & 1.45 & 30.7564 & 30.3623 & 30.2442 & 30.7536 & 30.5435 & 30.4133 & 30.7265 & 30.3317 & 30.2099 & 30.7214 & 30.5117 & 30.3795 \\
\hline 3 & 1.22 & 30.8725 & 30.5086 & 30.4744 & 30.8644 & 30.7065 & 30.6655 & 30.8284 & 30.4640 & 30.4228 & 30.8204 & 30.6620 & 30.6150 \\
\hline
\end{tabular}




\begin{tabular}{|c|c|c|c|c|c|c|c|c|c|c|c|c|c|}
\hline 5 & 1.01 & 31.0119 & 30.7246 & 30.7178 & 31.1467 & 31.0286 & 30.9833 & 30.9476 & 30.6610 & 30.6400 & 31.0734 & 30.9573 & 30.9042 \\
\hline 6 & 1.60 & 30.6440 & 30.2054 & 30.1637 & 30.6703 & 30.4512 & 30.4065 & 30.6054 & 30.1648 & 30.1170 & 30.6297 & 30.4087 & 30.3601 \\
\hline 6 & 1.07 & 30.7975 & 30.3850 & 30.3975 & 30.9958 & 30.7867 & 30.7917 & 30.8100 & 30.3981 & 30.4074 & 31.0015 & 30.7925 & 30.7951 \\
\hline 7 & 1.80 & 29.9925 & 29.5744 & 29.4484 & 29.9726 & 29.8048 & 29.7378 & 30.1022 & 29.6859 & 29.5664 & 30.0871 & 29.9136 & 29.8476 \\
\hline 7 & 1.13 & 31.1092 & 30.7323 & 30.6461 & 31.1237 & 30.9491 & 30.8782 & 31.0816 & 30.7031 & 30.6053 & 31.0868 & 30.9108 & 30.8352 \\
\hline 9 & 1.81 & 30.5527 & 30.1924 & 30.1249 & 30.6992 & 30.4857 & 30.4195 & 30.4816 & 30.1208 & 30.0502 & 30.6195 & 30.4113 & 30.3454 \\
\hline 11 & 1.75 & 30.3030 & 29.8589 & 29.7620 & 30.3956 & 30.1222 & 30.0171 & 30.2863 & 29.8442 & 29.7448 & 30.3743 & 30.1041 & 29.9986 \\
\hline 11 & 1.75 & 30.6335 & 30.1972 & 30.1462 & 30.5753 & 30.3453 & 30.2899 & 30.5619 & 30.1244 & 30.0790 & 30.5156 & 30.2871 & 30.2320 \\
\hline 12 & 2.39 & 29.9253 & 29.4917 & 29.4786 & 29.8991 & 29.6796 & 29.7415 & 29.8593 & 29.4231 & 29.3962 & 29.8252 & 29.6054 & 29.6604 \\
\hline 13 & 1.60 & 30.4947 & 30.1050 & 29.9797 & 30.4832 & 30.2847 & 30.2668 & 30.4714 & 30.0810 & 29.9476 & 30.4525 & 30.2539 & 30.2341 \\
\hline 13 & 1.60 & 30.5939 & 30.1750 & 30.0418 & 30.5454 & 30.3533 & 30.2727 & 30.5912 & 30.1715 & 30.0387 & 30.5487 & 30.3559 & 30.2718 \\
\hline 14 & 1.93 & 30.0380 & 29.5786 & 29.4923 & 30.1108 & 29.8637 & 29.8555 & 30.1154 & 29.6583 & 29.5774 & 30.1857 & 29.9382 & 29.9332 \\
\hline 14 & 0.88 & 31.2509 & 30.8901 & 30.7980 & 31.3063 & 31.1397 & 31.0199 & 31.2072 & 30.8439 & 30.7390 & 31.2562 & 31.0873 & 30.9590 \\
\hline 15 & 3.70 & 28.3797 & 27.9940 & 27.9505 & 28.3641 & 28.2131 & 28.2456 & 28.3793 & 27.9922 & 27.9245 & 28.3483 & 28.1912 & 28.2106 \\
\hline 17 & 5.10 & 26.9557 & 26.3152 & 26.2062 & 26.8804 & 26.4551 & 26.3654 & 26.9755 & 26.3345 & 26.2302 & 26.8929 & 26.4694 & 26.3846 \\
\hline 17 & 5.04 & 27.1457 & 26.5000 & 26.4117 & 27.0419 & 26.6078 & 26.5696 & 27.0828 & 26.4380 & 26.3411 & 26.9705 & 26.5449 & 26.5007 \\
\hline 18 & 0.84 & 31.3961 & 30.9712 & 30.9569 & 31.4034 & 31.1659 & 31.1206 & 31.3768 & 30.9503 & 30.9355 & 31.3859 & 31.1468 & 31.1010 \\
\hline 19 & 3.82 & 28.5559 & 28.1014 & 27.9310 & 28.5223 & 28.3118 & 28.2416 & 28.5786 & 28.1225 & 27.9457 & 28.5392 & 28.3270 & 28.2556 \\
\hline 19 & 3.82 & 28.5314 & 28.1016 & 27.9820 & 28.5467 & 28.3313 & 28.2347 & 28.5637 & 28.1343 & 28.0109 & 28.5720 & 28.3587 & 28.2632 \\
\hline 20 & 7.88 & 24.0924 & 23.5110 & 23.5101 & 24.1195 & 23.7638 & 23.6678 & 24.0646 & 23.4833 & 23.4759 & 24.0842 & 23.7297 & 23.6329 \\
\hline
\end{tabular}

\begin{tabular}{|c|c|c|c|c|c|c|c|c|c|c|c|c|c|}
\hline \multicolumn{14}{|c|}{ mPW1PW91 } \\
\hline \multirow{2}{*}{\multicolumn{2}{|c|}{ Exp }} & \multicolumn{6}{|c|}{ Gas phase } & \multicolumn{6}{|c|}{ PCM } \\
\hline & & 6-31G* & 6-31G** & $6-31+G^{* *}$ & 6-311G* & $6-311 G^{* *}$ & $6-311+G * *$ & 6-31G* & $6-31 \mathrm{G} * *$ & $6-31+G * *$ & 6-311G* & $6-311 \mathrm{G} * *$ & $6-311+G * *$ \\
\hline \multicolumn{14}{|l|}{${ }^{33} \mathrm{C}$} \\
\hline 1 & 34.2 & 158.5683 & 159.6909 & 159.3823 & 150.2454 & 150.0125 & 150.0906 & 158.8850 & 159.9986 & 159.7185 & 150.5990 & 150.3536 & 150.4541 \\
\hline 2 & 20.0 & 172.7106 & 174.0652 & 172.7301 & 164.3226 & 164.0935 & 164.0906 & 172.6980 & 174.0560 & 172.7175 & 164.3107 & 164.0786 & 164.0763 \\
\hline 3 & 42.4 & 152.4281 & 153.4519 & 151.7785 & 143.0948 & 142.6996 & 142.3174 & 152.6652 & 153.6837 & 152.0670 & 143.3981 & 142.9894 & 142.6296 \\
\hline 4 & 32.9 & 159.9916 & 160.4862 & 159.8863 & 150.5363 & 150.5607 & 150.0104 & 159.8757 & 160.3723 & 159.7412 & 150.4530 & 150.4780 & 149.8744 \\
\hline 5 & 46.9 & 147.3329 & 147.8919 & 146.2752 & 137.9204 & 137.6860 & 137.7023 & 147.5897 & 148.1404 & 146.6152 & 138.2382 & 137.9901 & 138.0477 \\
\hline 6 & 19.5 & 172.6269 & 174.0067 & 173.7342 & 165.2063 & 165.0190 & 164.6022 & 172.6648 & 174.0447 & 173.7629 & 165.2559 & 165.0654 & 164.6413 \\
\hline 7 & 30.9 & 162.5008 & 163.6272 & 163.3739 & 155.0102 & 154.7576 & 154.6042 & 162.4764 & 163.6081 & 163.4074 & 155.0515 & 154.8008 & 154.6569 \\
\hline 8 & 37.3 & 154.7390 & 155.0560 & 153.6413 & 145.4265 & 145.4971 & 145.4364 & 154.7390 & 155.0560 & 153.6053 & 145.4185 & 145.4870 & 145.4166 \\
\hline 9 & 38.0 & 154.1458 & 154.7960 & 155.0092 & 145.7594 & 145.6297 & 144.7457 & 154.3265 & 154.9676 & 155.1980 & 145.9591 & 145.8092 & 144.9195 \\
\hline 10 & 42.5 & 150.9741 & 151.2461 & 150.5176 & 141.5019 & 141.5151 & 140.6697 & 150.5904 & 150.8591 & 150.0541 & 141.0953 & 141.1072 & 140.1567 \\
\hline 11 & 28.0 & 163.5851 & 164.8378 & 163.3892 & 155.4281 & 155.1396 & 154.4388 & 163.7927 & 165.0356 & 163.6216 & 155.6633 & 155.3587 & 154.6706 \\
\hline 12 & 35.9 & 156.2472 & 157.1207 & 154.9146 & 146.0086 & 145.7548 & 145.3348 & 156.4418 & 157.3131 & 155.1367 & 146.2537 & 145.9877 & 145.5524 \\
\hline 13 & 26.0 & 167.2113 & 168.5260 & 168.1024 & 159.1739 & 159.0048 & 158.3232 & 167.4261 & 168.7349 & 168.2981 & 159.3981 & 159.2152 & 158.5276 \\
\hline 14 & 25.1 & 166.9493 & 168.2268 & 167.1134 & 159.8058 & 159.5979 & 159.8855 & 167.0501 & 168.3268 & 167.2482 & 159.9590 & 159.7436 & 160.0340 \\
\hline 15 & 75.8 & 119.0303 & 119.4982 & 118.4315 & 108.2731 & 108.0961 & 107.7217 & 119.1126 & 119.5839 & 118.4826 & 108.3114 & 108.1417 & 107.7572 \\
\hline 161 & 156.6 & 40.2978 & 39.8544 & 35.4397 & 19.1495 & 18.5933 & 16.6759 & 40.0859 & 39.6451 & 35.1465 & 18.8645 & 18.3024 & 16.3424 \\
\hline 171 & 109.1 & 85.3982 & 86.6911 & 87.7545 & 73.1518 & 72.2914 & 72.6126 & 85.6580 & 86.9437 & 88.0974 & 73.3672 & 72.5365 & 72.8877 \\
\hline 18 & 25.9 & 167.3127 & 168.9790 & 169.2735 & 161.2382 & 161.0109 & 160.9006 & 167.6788 & 169.3348 & 169.7035 & 161.6661 & 161.4178 & 161.3355 \\
\hline 19 & 60.7 & 133.4535 & 134.4815 & 132.9839 & 124.1838 & 123.3803 & 122.8610 & 133.8217 & 134.8389 & 133.2568 & 124.4800 & 123.6623 & 123.1204 \\
\hline 201 & 165.8 & 34.2913 & 34.9631 & 33.6742 & 17.0593 & 16.0665 & 14.7695 & 32.3526 & 33.0354 & 31.3032 & 14.8068 & 13.8193 & 12.1638 \\
\hline
\end{tabular}




\begin{tabular}{|c|c|c|c|c|c|c|c|c|c|c|c|c|c|}
\hline \multicolumn{14}{|l|}{${ }^{1} \mathrm{H}$} \\
\hline 1 & 1.69 & 30.5158 & 30.0698 & 29.9943 & 30.4673 & 30.2289 & 30.1387 & 30.4719 & 30.0234 & 29.9441 & 30.4262 & 30.1853 & 30.0920 \\
\hline 1 & 1.09 & 31.0548 & 30.6970 & 30.5968 & 31.0160 & 30.8505 & 30.8157 & 30.9694 & 30.6102 & 30.5003 & 30.9305 & 30.7647 & 30.7219 \\
\hline 2 & 1.49 & 30.5831 & 30.1468 & 30.0606 & 30.5917 & 30.3881 & 30.3397 & 30.6541 & 30.2217 & 30.1336 & 30.6576 & 30.4564 & 30.4061 \\
\hline 2 & 1.32 & 30.7782 & 30.3279 & 30.2294 & 30.6858 & 30.4446 & 30.3276 & 30.7273 & 30.2741 & 30.1675 & 30.6347 & 30.3912 & 30.2688 \\
\hline 3 & 1.45 & 30.7082 & 30.3183 & 30.2141 & 30.6602 & 30.4458 & 30.3338 & 30.6731 & 30.2822 & 30.1746 & 30.6241 & 30.4099 & 30.2959 \\
\hline 3 & 1.22 & 30.8209 & 30.4616 & 30.4162 & 30.7533 & 30.6021 & 30.5683 & 30.7755 & 30.4155 & 30.3643 & 30.7089 & 30.5571 & 30.5179 \\
\hline 5 & 1.01 & 30.9390 & 30.6572 & 30.6326 & 30.9987 & 30.8858 & 30.8462 & 30.8735 & 30.5923 & 30.5566 & 30.9279 & 30.8166 & 30.7702 \\
\hline 6 & 1.60 & 30.6059 & 30.1706 & 30.1170 & 30.5924 & 30.3722 & 30.3299 & 30.5624 & 30.1248 & 30.0665 & 30.5474 & 30.3253 & 30.2798 \\
\hline 6 & 1.07 & 30.7921 & 30.3780 & 30.3378 & 30.9060 & 30.7048 & 30.7036 & 30.8074 & 30.3941 & 30.3510 & 30.9146 & 30.7136 & 30.7099 \\
\hline 7 & 1.80 & 29.9533 & 29.5363 & 29.4274 & 29.8764 & 29.7168 & 29.6647 & 30.0652 & 29.6503 & 29.5472 & 29.9921 & 29.8265 & 29.7751 \\
\hline 7 & 1.13 & 31.0474 & 30.6725 & 30.5670 & 31.0193 & 30.8418 & 30.7670 & 31.0148 & 30.6381 & 30.5225 & 30.9790 & 30.8000 & 30.7211 \\
\hline 9 & 1.81 & 30.5199 & 30.1652 & 30.0700 & 30.5761 & 30.3714 & 30.2972 & 30.4473 & 30.0920 & 29.9955 & 30.4973 & 30.2977 & 30.2242 \\
\hline 11 & 1.75 & 30.2733 & 29.8337 & 29.7161 & 30.3031 & 30.0348 & 29.9211 & 30.2515 & 29.8140 & 29.6940 & 30.2780 & 30.0128 & 29.8987 \\
\hline 11 & 1.75 & 30.5704 & 30.1329 & 30.0819 & 30.4757 & 30.2423 & 30.1895 & 30.4976 & 30.0589 & 30.0141 & 30.4156 & 30.1835 & 30.1315 \\
\hline 12 & 2.39 & 29.8362 & 29.4058 & 29.3568 & 29.7781 & 29.5476 & 29.5902 & 29.7640 & 29.3305 & 29.2694 & 29.6998 & 29.4687 & 29.5057 \\
\hline 13 & 1.60 & 30.4612 & 30.0710 & 29.9278 & 30.4072 & 30.2089 & 30.1812 & 30.4322 & 30.0412 & 29.8911 & 30.3720 & 30.1736 & 30.1446 \\
\hline 13 & 1.60 & 30.5549 & 30.1336 & 29.9938 & 30.4668 & 30.2694 & 30.1832 & 30.5537 & 30.1316 & 29.9905 & 30.4702 & 30.2723 & 30.1816 \\
\hline 14 & 1.93 & 30.0109 & 29.5483 & 29.4468 & 30.0227 & 29.7785 & 29.7551 & 30.0930 & 29.6331 & 29.5362 & 30.1009 & 29.8562 & 29.8358 \\
\hline 14 & 0.88 & 31.2150 & 30.8569 & 30.7501 & 31.2202 & 31.0550 & 30.9353 & 31.1672 & 30.8062 & 30.6873 & 31.1677 & 30.9998 & 30.8719 \\
\hline 15 & 3.70 & 28.3226 & 27.9347 & 27.8735 & 28.2671 & 28.1186 & 28.1383 & 28.3203 & 27.9308 & 27.8466 & 28.2508 & 28.0961 & 28.1031 \\
\hline 17 & 5.10 & 26.7671 & 26.1223 & 26.0125 & 26.6903 & 26.2485 & 26.1535 & 26.7783 & 26.1332 & 26.0281 & 26.6945 & 26.2552 & 26.1651 \\
\hline 17 & 5.04 & 26.9532 & 26.3030 & 26.2361 & 26.8597 & 26.4047 & 26.3812 & 26.8785 & 26.2293 & 26.1556 & 26.7779 & 26.3320 & 26.3035 \\
\hline 18 & 0.84 & 31.3296 & 30.9061 & 30.8842 & 31.2987 & 31.0579 & 31.0166 & 31.3068 & 30.8815 & 30.8596 & 31.2779 & 31.0355 & 30.9941 \\
\hline 19 & 3.82 & 28.5062 & 28.0579 & 27.8853 & 28.4432 & 28.2354 & 28.1640 & 28.5283 & 28.0783 & 27.8993 & 28.4589 & 28.2494 & 28.1772 \\
\hline 19 & 3.82 & 28.4560 & 28.0306 & 27.9087 & 28.4463 & 28.2278 & 28.1245 & 28.4858 & 28.0606 & 27.9345 & 28.4694 & 28.2529 & 28.1505 \\
\hline 20 & 7.88 & 23.9469 & 23.3711 & 23.3769 & 23.9736 & 23.6104 & 23.5211 & 23.9165 & 23.3413 & 23.3427 & 23.9370 & 23.5754 & 23.4862 \\
\hline
\end{tabular}

\section{Compound 43}

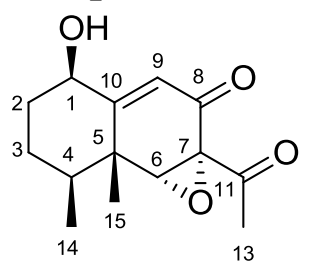

43

\begin{tabular}{|c|c|c|c|c|c|c|c|c|c|c|c|c|c|}
\hline \multirow{3}{*}{\multicolumn{2}{|c|}{ Exp }} & \multicolumn{12}{|c|}{ B3LYP } \\
\hline & & \multicolumn{6}{|c|}{ Gas phase } & \multicolumn{6}{|c|}{ PCM } \\
\hline & & 6-31G* & 6-31G** & $6-31+G * *$ & 6-311G* & $6-311 G * *$ & $6-311+G * *$ & 6-31G* & $6-31 G * *$ & $6-31+G * *$ & 6-311G* & $6-311 G * *$ & $6-311+G * *$ \\
\hline \multicolumn{14}{|c|}{${ }^{13} \mathrm{C}$} \\
\hline 1 & 73.6 & 113.9399 & 114.6834 & 114.3028 & 101.9034 & 101.6449 & 101.3160 & 114.0753 & 114.8215 & 114.4317 & 102.1037 & 101.8423 & 101.4493 \\
\hline 2 & 33.0 & 151.9431 & 153.1981 & 152.2407 & 142.4699 & 142.1009 & 141.4259 & 152.4609 & 153.7011 & 152.8872 & 143.0958 & 142.6848 & 142.0700 \\
\hline 3 & 24.5 & 163.3169 & 164.6182 & 163.8688 & 154.9076 & 154.6882 & 154.4186 & 163.4646 & 164.7622 & 164.0464 & 155.0827 & 154.8561 & 154.5894 \\
\hline 4 & 37.8 & 148.9866 & 149.7295 & 148.8332 & 139.0524 & 139.0741 & 139.1062 & 148.4607 & 149.2015 & 148.2042 & 138.4697 & 138.4898 & 138.4740 \\
\hline 5 & 40.9 & 144.9290 & 145.1911 & 143.7048 & 134.0913 & 134.2153 & 133.8818 & 144.5563 & 144.8157 & 143.2678 & 133.6554 & 133.7673 & 133.3557 \\
\hline 6 & 66.7 & 123.6592 & 124.4419 & 122.9700 & 113.2971 & 112.8025 & 112.1717 & 123.1004 & 123.8776 & 122.3520 & 112.6816 & 112.1718 & 111.4678 \\
\hline
\end{tabular}




\begin{tabular}{|c|c|c|c|c|c|c|c|c|c|c|c|c|c|}
\hline 7 & 63.6 & 127.3984 & 127.2953 & 127.2238 & 116.8992 & 116.7552 & 115.6141 & 127.3012 & 127.2012 & 127.0621 & 116.7525 & 116.6156 & 115.4254 \\
\hline 8 & 191.3 & 3.3085 & 3.3215 & 2.1230 & -16.7700 & -16.8676 & -19.9213 & 2.6092 & 2.6153 & 1.1955 & -17.7307 & -17.8349 & -21.0946 \\
\hline 9 & 123.2 & 68.2429 & 69.0309 & 69.2064 & 52.0035 & 51.5071 & 51.0746 & 69.0482 & 69.8289 & 69.9661 & 52.8528 & 52.3351 & 51.8216 \\
\hline 10 & 163.5 & 31.6723 & 31.4166 & 30.4837 & 10.0836 & 9.7868 & 8.0325 & 28.2824 & 28.0268 & 26.6446 & 6.3284 & 6.0699 & 3.9564 \\
\hline 11 & 200.6 & -5.4933 & -5.2976 & -7.2741 & -25.9893 & -25.7754 & -29.1938 & -7.9702 & -7.7872 & -10.2537 & -28.8557 & -28.6509 & -32.5861 \\
\hline 13 & 28.0 & 160.8768 & 162.5328 & 162.6500 & 152.7931 & 152.4076 & 151.8446 & 160.4085 & 162.0435 & 161.8131 & 152.1269 & 151.7044 & 150.9501 \\
\hline 14 & 15.7 & 172.4651 & 174.3027 & 174.5090 & 165.7739 & 165.7204 & 165.6910 & 172.6902 & 174.5160 & 174.7454 & 166.0171 & 165.9430 & 165.9138 \\
\hline 15 & 19.3 & 169.1395 & 170.8850 & 171.5910 & 162.5167 & 162.3965 & 162.3383 & 169.2647 & 171.0046 & 171.6863 & 162.6625 & 162.5284 & 162.4659 \\
\hline 1 & 4.40 & 27.8101 & 27.3364 & 27.2303 & 27.6799 & 27.4374 & 27.4655 & 27.7181 & 27.2399 & 27.1254 & 27.5802 & 27.3344 & 27.3592 \\
\hline 2 & 1.94 & 30.4278 & 29.9962 & 29.8468 & 30.3574 & 30.1432 & 30.0674 & 30.4203 & 29.9872 & 29.8364 & 30.3557 & 30.1395 & 30.0587 \\
\hline 2 & 1.70 & 30.6568 & 30.2005 & 30.1630 & 30.5572 & 30.2693 & 30.2847 & 30.4648 & 30.0032 & 29.9569 & 30.3645 & 30.0791 & 30.0909 \\
\hline 3 & 1.76 & 29.9879 & 29.5640 & 29.5082 & 30.0608 & 29.8375 & 29.8165 & 29.9674 & 29.5430 & 29.4963 & 30.0435 & 29.8221 & 29.8063 \\
\hline 3 & 1.52 & 30.8433 & 30.4199 & 30.3758 & 30.8118 & 30.5877 & 30.5676 & 30.7719 & 30.3466 & 30.2933 & 30.7404 & 30.5147 & 30.4896 \\
\hline 4 & 1.98 & 30.0542 & 29.6619 & 29.5945 & 30.1918 & 30.0134 & 29.9564 & 30.0857 & 29.6962 & 29.6234 & 30.2187 & 30.0431 & 29.9816 \\
\hline 6 & 3.49 & 28.5642 & 28.0771 & 28.0146 & 28.6018 & 28.3284 & 28.3125 & 28.4632 & 27.9696 & 27.8777 & 28.4869 & 28.2080 & 28.1716 \\
\hline 9 & 5.90 & 26.5640 & 25.8951 & 25.6892 & 26.4025 & 25.9149 & 25.9369 & 26.4911 & 25.8150 & 25.5999 & 26.3170 & 25.8264 & 25.8471 \\
\hline 13 & 2.34 & 29.7315 & 29.1944 & 29.0251 & 29.6624 & 29.3367 & 29.2510 & 29.6812 & 29.1409 & 28.9533 & 29.6070 & 29.2792 & 29.1773 \\
\hline 14 & 1.11 & 31.0213 & 30.5749 & 30.5543 & 31.0388 & 30.7914 & 30.7679 & 30.9926 & 30.5452 & 30.5217 & 31.0111 & 30.7628 & 30.7372 \\
\hline 15 & 1.39 & 30.7175 & 30.2633 & 30.1840 & 30.7819 & 30.5453 & 30.4659 & 30.7076 & 30.2496 & 30.1686 & 30.7725 & 30.5300 & 30.4479 \\
\hline
\end{tabular}

\begin{tabular}{|c|c|c|c|c|c|c|c|c|c|c|c|c|c|}
\hline \multirow{3}{*}{\multicolumn{2}{|c|}{ Exp }} & \multicolumn{12}{|c|}{ mPW1PW91 } \\
\hline & & \multicolumn{6}{|c|}{ Gas phase } & \multicolumn{6}{|c|}{ PCM } \\
\hline & & 6-31G* & 6-31G** & $6-31+G * *$ & 6-311G* & 6-311G** & $6-311+G * *$ & 6-31G* & $6-31 G^{* *}$ & $6-31+\mathrm{G} * *$ & 6-311G* & $6-311 G^{* *}$ & $6-311+G^{* *}$ \\
\hline \multicolumn{14}{|c|}{ 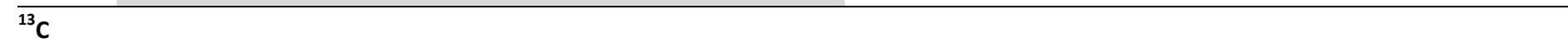 } \\
\hline 1 & 73.6 & 118.8709 & 119.5509 & 119.3253 & 108.5384 & 108.2738 & 107.9196 & 118.9804 & 119.6657 & 119.4388 & 108.6993 & 108.4354 & 108.0285 \\
\hline 2 & 33.0 & 157.1726 & 158.3113 & 157.4175 & 148.5492 & 148.1691 & 147.5133 & 157.6698 & 158.7968 & 158.0364 & 149.1269 & 148.7093 & 148.1094 \\
\hline 3 & 24.5 & 168.3404 & 169.5347 & 169.0489 & 160.6338 & 160.4084 & 160.1175 & 168.5052 & 169.6967 & 169.2446 & 160.8126 & 160.5816 & 160.2937 \\
\hline 4 & 37.8 & 154.5490 & 155.2370 & 154.5320 & 145.3074 & 145.3374 & 145.3478 & 154.0189 & 154.7052 & 153.9150 & 144.7256 & 144.7552 & 144.7341 \\
\hline 5 & 40.9 & 149.8927 & 150.1522 & 148.7976 & 140.1180 & 140.2389 & 139.9102 & 149.5140 & 149.7705 & 148.3600 & 139.6781 & 139.7868 & 139.3925 \\
\hline 6 & 66.7 & 127.9938 & 128.7467 & 127.2657 & 118.9851 & 118.5094 & 118.0906 & 127.4222 & 128.1693 & 126.6569 & 118.3608 & 117.8701 & 117.3839 \\
\hline 7 & 63.6 & 131.2123 & 131.1094 & 131.2180 & 122.3906 & 122.2534 & 121.2100 & 131.1161 & 131.0162 & 131.0613 & 122.2467 & 122.1158 & 121.0266 \\
\hline 8 & 191.3 & 5.4175 & 5.4215 & 4.8291 & -11.5359 & -11.6375 & -14.3949 & 4.6758 & 4.6727 & 3.8708 & -12.5107 & -12.6198 & -15.5651 \\
\hline 9 & 123.2 & 71.1187 & 71.8724 & 72.0757 & 56.7971 & 56.2912 & 55.8086 & 71.9785 & 72.7250 & 72.8960 & 57.6731 & 57.1472 & 56.6007 \\
\hline 10 & 163.5 & 35.3110 & 35.0197 & 34.5019 & 16.3518 & 16.0314 & 14.3976 & 31.8308 & 31.5382 & 30.6458 & 12.5514 & 12.2712 & 10.3262 \\
\hline 11 & 200.6 & -3.2682 & -3.0776 & -4.5135 & -20.7354 & -20.5226 & -23.5435 & -5.8160 & -5.6391 & -7.5018 & -23.6291 & -23.4246 & -26.9002 \\
\hline 13 & 28.0 & 164.1166 & 165.6644 & 165.7979 & 156.7022 & 156.3319 & 155.8634 & 163.6486 & 165.1751 & 164.9905 & 156.0364 & 155.6316 & 154.9994 \\
\hline 14 & 15.7 & 176.6679 & 178.3782 & 178.7826 & 170.4307 & 170.3777 & 170.3039 & 176.9258 & 178.6242 & 179.0437 & 170.6790 & 170.6074 & 170.5372 \\
\hline $\begin{array}{l}15 \\
{ }^{1} \mathbf{H}\end{array}$ & 19.3 & 173.5242 & 175.1377 & 175.5402 & 167.2974 & 167.2088 & 167.1380 & 173.6599 & 175.2678 & 175.6391 & 167.4350 & 167.3335 & 167.2676 \\
\hline 1 & 4.40 & 27.7465 & 27.2799 & 27.1813 & 27.6165 & 27.3668 & 27.4035 & 27.6448 & 27.1732 & 27.0691 & 27.5102 & 27.2571 & 27.2911 \\
\hline 2 & 1.94 & 30.4009 & 29.9703 & 29.8303 & 30.2779 & 30.0643 & 29.9929 & 30.3917 & 29.9596 & 29.8177 & 30.2751 & 30.0595 & 29.9826 \\
\hline 2 & 1.70 & 30.5954 & 30.1420 & 30.1114 & 30.4804 & 30.1867 & 30.2017 & 30.3902 & 29.9309 & 29.8947 & 30.2777 & 29.9861 & 29.9992 \\
\hline 3 & 1.76 & 29.9606 & 29.5400 & 29.4629 & 29.9703 & 29.7604 & 29.7365 & 29.9395 & 29.5185 & 29.4507 & 29.9518 & 29.7436 & 29.7257 \\
\hline 3 & 1.52 & 30.7840 & 30.3645 & 30.3203 & 30.7282 & 30.4997 & 30.4753 & 30.7047 & 30.2829 & 30.2312 & 30.6507 & 30.4201 & 30.3914 \\
\hline
\end{tabular}




\begin{tabular}{rrrlllllllllll}
\hline 4 & 1.98 & 29.9996 & 29.6124 & 29.5474 & 30.0693 & 29.8999 & 29.8465 & 30.0344 & 29.6499 & 29.5784 & 30.1002 & 29.9334 & 29.8741 \\
6 & 3.49 & 28.4952 & 28.0123 & 27.9595 & 28.5104 & 28.2347 & 28.2359 & 28.3869 & 27.8969 & 27.8188 & 28.3911 & 28.1095 & 28.0925 \\
9 & 5.90 & 26.3546 & 25.6915 & 25.5242 & 26.2335 & 25.7208 & 25.7557 & 26.2742 & 25.6034 & 25.4306 & 26.1432 & 25.6275 & 25.6620 \\
13 & 2.34 & 29.6210 & 29.0757 & 28.9347 & 29.5378 & 29.1996 & 29.1249 & 29.5645 & 29.0156 & 28.8587 & 29.4771 & 29.1366 & 29.0474 \\
14 & 1.11 & 30.9729 & 30.5257 & 30.5026 & 30.9528 & 30.7003 & 30.6807 & 30.9401 & 30.4918 & 30.4667 & 30.9214 & 30.6681 & 30.6471 \\
15 & 1.39 & 30.6662 & 30.2120 & 30.1372 & 30.6823 & 30.4440 & 30.3656 & 30.6551 & 30.1970 & 30.1217 & 30.6718 & 30.4275 & 30.3474 \\
\hline
\end{tabular}

\section{Compound 44}

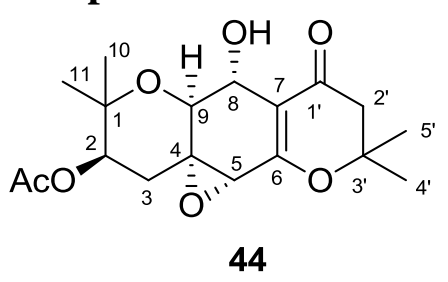

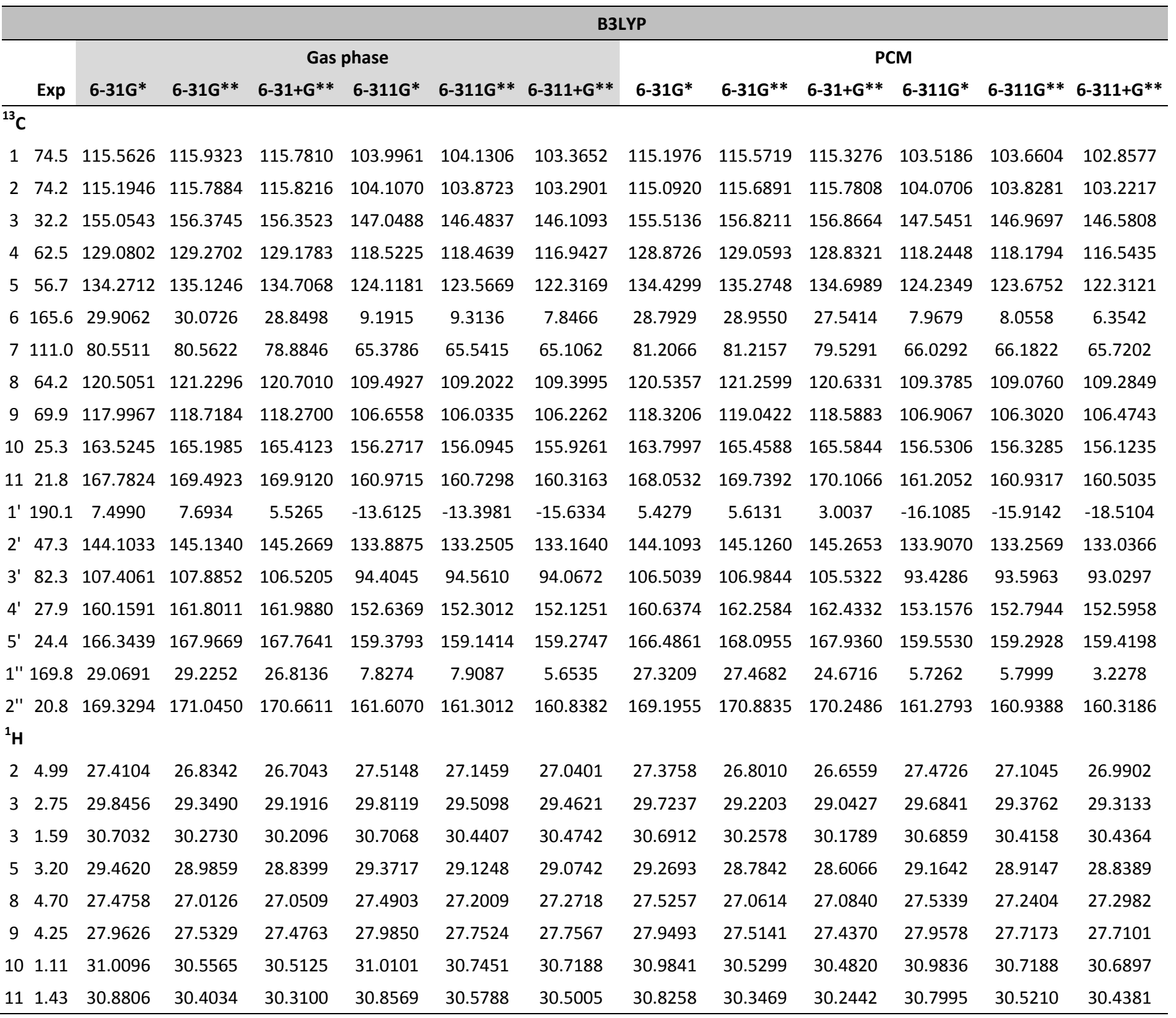




\begin{tabular}{llllllllllllll}
\hline $2^{\prime}$ & 2.70 & 29.5412 & 29.0988 & 28.9080 & 29.5127 & 29.2577 & 29.1663 & 29.3815 & 28.9349 & 28.7236 & 29.3510 & 29.0962 & 28.9876 \\
2' $^{\prime}$ & 2.51 & 30.1873 & 29.6931 & 29.5345 & 30.0991 & 29.8068 & 29.7415 & 30.0957 & 29.5956 & 29.4312 & 30.0018 & 29.7056 & 29.6391 \\
4' $^{\prime}$ & 1.53 & 30.7587 & 30.3047 & 30.2097 & 30.7010 & 30.4263 & 30.4162 & 30.6827 & 30.2250 & 30.1232 & 30.6254 & 30.3483 & 30.3343 \\
$5^{\prime}$ & 1.42 & 30.8027 & 30.3334 & 30.3018 & 30.8356 & 30.5599 & 30.5617 & 30.7739 & 30.3030 & 30.2655 & 30.8035 & 30.5271 & 30.5248 \\
2" $^{\prime 2}$ & 2.08 & 30.3122 & 29.7926 & 29.6579 & 30.2709 & 29.9421 & 29.8892 & 30.2198 & 29.6950 & 29.5510 & 30.1764 & 29.8457 & 29.7858 \\
\hline
\end{tabular}

\begin{tabular}{|c|c|c|c|c|c|c|c|c|c|c|c|c|c|}
\hline \multirow{3}{*}{\multicolumn{2}{|c|}{ Exp }} & \multicolumn{12}{|c|}{ mPW1PW91 } \\
\hline & & \multicolumn{6}{|c|}{ Gas phase } & \multicolumn{6}{|c|}{ PCM } \\
\hline & & 6-31G* & $6-31 G * *$ & $6-31+G * *$ & 6-311G* & $6-311 G * *$ & $6-311+G * *$ & 6-31G* & $6-31 G * *$ & $6-31+G * *$ & 6-311G* & $6-311 G * *$ & $6-311+G * *$ \\
\hline \multicolumn{14}{|l|}{${ }^{3} \mathrm{C}$} \\
\hline 1 & 74.5 & 120.1844 & 120.5563 & 120.6048 & 110.0178 & 110.1488 & 109.4463 & 119.8034 & 120.1796 & 120.1395 & 109.5222 & 109.6607 & 108.9268 \\
\hline 2 & 74.2 & 119.8454 & 120.3941 & 120.5130 & 109.9988 & 109.7824 & 109.2081 & 119.7446 & 120.2971 & 120.4713 & 109.9603 & 109.7361 & 109.1374 \\
\hline 3 & 32.2 & 159.7241 & 160.9426 & 160.6390 & 152.6560 & 152.1196 & 151.7364 & 160.1944 & 161.4008 & 161.1573 & 153.1388 & 152.5930 & 152.1946 \\
\hline 4 & 62.5 & 133.3528 & 133.5306 & 133.6720 & 124.2039 & 124.1429 & 122.6974 & 133.1157 & 133.2908 & 133.3109 & 123.9031 & 123.8357 & 122.2875 \\
\hline 5 & 56.7 & 138.4470 & 139.2753 & 139.0704 & 129.8831 & 129.3406 & 128.1071 & 138.6188 & 139.4390 & 139.0667 & 129.9954 & 129.4459 & 128.1144 \\
\hline 6 & 165.6 & 32.4565 & 32.6228 & 31.9467 & 14.4667 & 14.5977 & 13.2781 & 31.3375 & 31.4988 & 30.6414 & 13.2471 & 13.3412 & 11.8099 \\
\hline 7 & 111.0 & 84.0004 & 84.0104 & 82.3863 & 70.7731 & 70.9314 & 70.5281 & 84.7072 & 84.7144 & 83.0856 & 71.4762 & 71.6241 & 71.1954 \\
\hline 8 & 64.2 & 125.0456 & 125.7309 & 125.4613 & 115.8895 & 115.6045 & 115.7174 & 125.1156 & 125.8004 & 125.4293 & 115.8082 & 115.5117 & 115.6339 \\
\hline 9 & 69.9 & 122.6220 & 123.3028 & 123.1088 & 113.0399 & 112.4454 & 112.5940 & 122.9659 & 123.6465 & 123.4423 & 113.3007 & 112.7224 & 112.8440 \\
\hline 10 & 25.3 & 167.9241 & 169.4666 & 169.3928 & 161.1341 & 160.9820 & 160.9282 & 168.2294 & 169.7581 & 169.5935 & 161.3966 & 161.2225 & 161.1316 \\
\hline 11 & 21.8 & 171.9433 & 173.5304 & 173.4993 & 165.5936 & 165.3720 & 164.9887 & 172.2393 & 173.8039 & 173.7147 & 165.8254 & 165.5770 & 165.1820 \\
\hline $1^{\prime}$ & 190.1 & 9.5885 & 9.7723 & 8.3186 & -8.4906 & -8.2789 & -10.3321 & 7.4672 & 7.6410 & 5.7887 & -10.9901 & -10.7999 & -13.1718 \\
\hline $2^{\prime}$ & 47.3 & 148.5516 & 149.4898 & 149.6185 & 139.3695 & 138.7545 & 138.6033 & 148.5328 & 149.4590 & 149.5969 & 139.3391 & 138.7125 & 138.4500 \\
\hline $3^{\prime}$ & 82.3 & 111.9854 & 112.4644 & 111.6095 & 100.4137 & 100.5767 & 100.0251 & 111.0549 & 111.5352 & 110.6114 & 99.4152 & 99.5899 & 98.9792 \\
\hline $4^{\prime}$ & 27.9 & 164.4354 & 165.9481 & 165.8875 & 157.4718 & 157.1670 & 157.0254 & 164.9373 & 166.4307 & 166.3492 & 157.9762 & 157.6480 & 157.4878 \\
\hline $5^{\prime}$ & 24.4 & 170.3510 & 171.8325 & 171.6925 & 163.7991 & 163.5851 & 163.6559 & 170.5186 & 171.9875 & 171.8885 & 163.9716 & 163.7385 & 163.8111 \\
\hline 1" & 169.8 & 31.2069 & 31.3614 & 29.5864 & 12.7577 & 12.8417 & 10.8024 & 29.4277 & 29.5730 & 27.4453 & 10.6594 & 10.7363 & 8.4206 \\
\hline $\begin{array}{l}2^{\prime \prime} \\
{ }^{1} \mathrm{H}\end{array}$ & 20.8 & 172.6392 & 174.2616 & 174.2621 & 165.7074 & 165.4241 & 164.9496 & 172.5184 & 174.1134 & 173.8836 & 165.3851 & 165.0697 & 164.4617 \\
\hline 2 & 4.99 & 27.3746 & 26.8155 & 26.6798 & 27.4172 & 27.0580 & 26.9579 & 27.3358 & 26.7780 & 26.6293 & 27.3737 & 27.0147 & 26.9068 \\
\hline 3 & 2.75 & 29.7928 & 29.2967 & 29.1474 & 29.7082 & 29.4163 & 29.3621 & 29.6638 & 29.1604 & 28.9935 & 29.5746 & 29.2764 & 29.2081 \\
\hline 3 & 1.59 & 30.6077 & 30.1774 & 30.1202 & 30.5985 & 30.3285 & 30.3725 & 30.5913 & 30.1574 & 30.0854 & 30.5746 & 30.3001 & 30.3315 \\
\hline 5 & 3.20 & 29.3719 & 28.8935 & 28.7644 & 29.2957 & 29.0213 & 28.9906 & 29.1698 & 28.6812 & 28.5246 & 29.0817 & 28.8043 & 28.7510 \\
\hline 8 & 4.70 & 27.4367 & 26.9698 & 27.0162 & 27.4211 & 27.1364 & 27.2151 & 27.4829 & 27.0148 & 27.0477 & 27.4624 & 27.1741 & 27.2407 \\
\hline 9 & 4.25 & 27.9018 & 27.4701 & 27.4185 & 27.8759 & 27.6519 & 27.6725 & 27.8836 & 27.4460 & 27.3764 & 27.8452 & 27.6127 & 27.6230 \\
\hline 10 & 1.11 & 30.9443 & 30.4897 & 30.4526 & 30.9116 & 30.6410 & 30.6172 & 30.9157 & 30.4598 & 30.4193 & 30.8821 & 30.6116 & 30.5852 \\
\hline 11 & 1.43 & 30.8234 & 30.3476 & 30.2592 & 30.7686 & 30.4874 & 30.4051 & 30.7634 & 30.2856 & 30.1894 & 30.7069 & 30.4251 & 30.3393 \\
\hline $2^{\prime}$ & 2.70 & 29.4704 & 29.0196 & 28.8252 & 29.4061 & 29.1459 & 29.0604 & 29.3061 & 28.8507 & 28.6392 & 29.2414 & 28.9814 & 28.8805 \\
\hline $2^{\prime}$ & 2.51 & 30.0711 & 29.5800 & 29.4358 & 29.9614 & 29.6627 & 29.6079 & 29.9694 & 29.4717 & 29.3250 & 29.8568 & 29.5537 & 29.4993 \\
\hline $4^{\prime}$ & 1.53 & 30.6742 & 30.2190 & 30.1401 & 30.5956 & 30.3143 & 30.3073 & 30.5924 & 30.1330 & 30.0493 & 30.5152 & 30.2312 & 30.2213 \\
\hline $5^{\prime}$ & 1.42 & 30.7394 & 30.2689 & 30.2450 & 30.7402 & 30.4598 & 30.4672 & 30.7086 & 30.2363 & 30.2074 & 30.7064 & 30.4252 & 30.4288 \\
\hline $2^{\prime \prime}$ & 2.08 & 30.2279 & 29.7008 & 29.5835 & 30.1709 & 29.8272 & 29.7896 & 30.1288 & 29.5960 & 29.4706 & 30.0707 & 29.7248 & 29.6809 \\
\hline
\end{tabular}




\section{Compound 45}

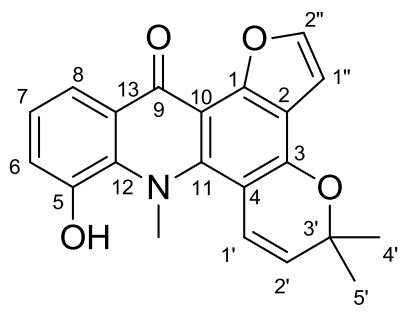

45

\begin{tabular}{|c|c|c|c|c|c|c|c|c|c|c|c|c|c|}
\hline & \multirow[b]{3}{*}{ Exp } & \multicolumn{12}{|c|}{ B3LYP } \\
\hline & & \multicolumn{6}{|c|}{ Gas phase } & \multicolumn{6}{|c|}{ PCM } \\
\hline & & 6-31G* & $6-31 G * *$ & $6-31+\mathrm{G} * *$ & 6-311G* & $6-311 G * *$ & $6-311+G * *$ & 6-31G* & $6-31 G * *$ & $6-31+G * *$ & 6-311G* & $6-311 G * *$ & $6-311+G * *$ \\
\hline \multicolumn{14}{|l|}{${ }^{13} \mathrm{C}$} \\
\hline 1 & 154.2 & 41.3762 & 41.3037 & 39.5778 & 21.7846 & 21.6169 & 20.5630 & 42.1606 & 42.0906 & 40.1887 & 22.5304 & 22.3801 & 21.3359 \\
\hline 2 & 113.7 & 79.0899 & 79.1448 & 79.2411 & 62.9509 & 62.9563 & 62.1028 & 79.7748 & 79.8490 & 79.8281 & 63.8155 & 63.7580 & 62.8232 \\
\hline 3 & 152.8 & 44.4841 & 44.5271 & 43.2020 & 25.3035 & 25.3029 & 24.6190 & 43.6798 & 43.7275 & 42.3189 & 24.4268 & 24.4420 & 23.8502 \\
\hline 4 & 106.5 & 84.8658 & 84.8978 & 84.7195 & 70.2774 & 70.2150 & 70.3011 & 84.3805 & 84.4475 & 84.2641 & 69.9496 & 69.8610 & 69.8446 \\
\hline 5 & 148.4 & 48.2250 & 48.0237 & 46.5972 & 27.1584 & 27.0349 & 27.5768 & 48.1870 & 47.9790 & 46.5739 & 27.1659 & 26.9833 & 27.4196 \\
\hline 6 & 119.0 & 77.8385 & 78.4756 & 78.8891 & 61.5372 & 60.7214 & 60.0880 & 77.6436 & 78.2501 & 78.1112 & 61.7044 & 60.6797 & 59.8236 \\
\hline 7 & 123.4 & 70.8481 & 71.5393 & 72.0725 & 54.1785 & 53.2975 & 53.4622 & 71.6808 & 72.3907 & 72.7448 & 55.2062 & 54.2160 & 54.2306 \\
\hline 8 & 117.5 & 75.2705 & 75.9092 & 75.4301 & 59.4470 & 58.6470 & 57.3531 & 76.3411 & 77.0015 & 76.7088 & 60.7720 & 59.9900 & 59.0938 \\
\hline 9 & 177.5 & 21.8386 & 21.8947 & 21.4579 & 4.1150 & 4.1578 & 2.4934 & 21.7366 & 21.8206 & 21.0685 & 3.9215 & 3.8980 & 1.9773 \\
\hline 10 & 108.2 & 81.6002 & 81.6586 & 82.0921 & 67.7675 & 67.7370 & 67.8473 & 82.6608 & 82.7393 & 83.2010 & 69.1045 & 69.0284 & 69.1520 \\
\hline 11 & 147.2 & 49.6354 & 49.5047 & 49.0274 & 31.3729 & 31.2510 & 30.3643 & 48.8585 & 48.7459 & 48.0192 & 30.5895 & 30.5107 & 29.5517 \\
\hline 12 & 137.2 & 59.0794 & 58.9177 & 58.4781 & 41.4460 & 41.0769 & 40.4284 & 59.0803 & 58.9731 & 58.6704 & 41.3916 & 41.0464 & 40.3222 \\
\hline 13 & 127.7 & 64.6637 & 64.5070 & 63.9210 & 48.3215 & 48.0978 & 47.1853 & 65.4483 & 65.3118 & 64.8771 & 49.2303 & 48.9985 & 48.1753 \\
\hline $1^{\prime}$ & 121.8 & 72.4415 & 72.9845 & 71.9167 & 55.9364 & 55.0796 & 53.6958 & 72.4640 & 72.9765 & 71.9165 & 55.7142 & 54.9391 & 53.6202 \\
\hline $2^{\prime}$ & 124.1 & 71.2138 & 71.7643 & 71.7653 & 54.7933 & 53.8454 & 53.6375 & 70.6101 & 71.2217 & 71.1601 & 54.2176 & 53.1812 & 52.8270 \\
\hline $3^{\prime}$ & 77.3 & 112.5732 & 113.0848 & 112.7267 & 100.5703 & 100.6124 & 99.9351 & 112.0746 & 112.5950 & 112.2209 & 99.9928 & 100.0263 & 99.2777 \\
\hline $4^{\prime}$ & 27.2 & 162.7685 & 164.4786 & 165.8135 & 156.3160 & 156.0766 & 156.1035 & 163.1887 & 164.8944 & 166.1914 & 156.7929 & 156.5086 & 156.5591 \\
\hline $5^{\prime}$ & 27.2 & 160.8918 & 162.5218 & 162.7550 & 153.2434 & 152.9490 & 152.3135 & 161.2319 & 162.8465 & 162.9057 & 153.5835 & 153.2743 & 152.5833 \\
\hline $1 "$ & 103.6 & 90.9349 & 91.4734 & 93.3350 & 76.4031 & 75.6395 & 74.8046 & 90.6520 & 91.1900 & 92.9874 & 75.9789 & 75.2272 & 74.3048 \\
\hline $2 "$ & 144.5 & 51.9761 & 52.6651 & 52.0324 & 33.8051 & 32.7984 & 31.5431 & 52.1605 & 52.8593 & 52.1277 & 33.8390 & 32.8181 & 31.6020 \\
\hline $\begin{array}{c}\mathrm{N}-\mathrm{CH}_{3} \\
{ }^{\mathbf{1}} \mathbf{H}\end{array}$ & 3 48.4 & 143.9487 & 145.4974 & 144.5542 & 135.9710 & 135.4351 & 134.0789 & 143.1952 & 144.7517 & 143.1823 & 134.7789 & 134.3041 & 133.1230 \\
\hline 6 & 7.07 & 25.3091 & 24.6400 & 24.5252 & 25.1112 & 24.6702 & 24.7957 & 25.2996 & 24.6192 & 24.4445 & 25.1261 & 24.6397 & 24.6974 \\
\hline 7 & 7.09 & 24.9691 & 24.3673 & 24.2626 & 24.8487 & 24.4945 & 24.5604 & 24.9121 & 24.3079 & 24.1904 & 24.7959 & 24.4347 & 24.4897 \\
\hline 8 & 7.89 & 24.3346 & 23.6513 & 23.3015 & 24.1880 & 23.7508 & 23.4922 & 24.3897 & 23.6983 & 23.3682 & 24.2261 & 23.7856 & 23.5457 \\
\hline $1^{\prime}$ & 6.77 & 25.5255 & 24.8468 & 24.6445 & 25.4335 & 24.9662 & 24.8334 & 25.3940 & 24.7364 & 24.5369 & 25.2864 & 24.8600 & 24.7475 \\
\hline $2^{\prime}$ & 5.54 & 26.6964 & 26.0866 & 25.9122 & 26.6804 & 26.2599 & 26.1955 & 26.5159 & 25.9157 & 25.7302 & 26.4905 & 26.0844 & 26.0113 \\
\hline $4^{\prime}$ & 1.53 & 30.7099 & 30.2449 & 30.1667 & 30.6605 & 30.3910 & 30.3551 & 30.6452 & 30.1788 & 30.0938 & 30.5869 & 30.3212 & 30.2805 \\
\hline $5^{\prime}$ & 1.53 & 30.6322 & 30.1747 & 30.0598 & 30.5386 & 30.2734 & 30.2416 & 30.5862 & 30.1291 & 30.0026 & 30.4857 & 30.2246 & 30.1859 \\
\hline $1 "$ & 6.83 & 25.6175 & 24.8623 & 24.5595 & 25.4917 & 24.9931 & 24.9238 & 25.4826 & 24.7256 & 24.3902 & 25.3406 & 24.8492 & 24.7614 \\
\hline $2^{\prime \prime}$ & 7.68 & 24.7724 & 24.0614 & 23.8357 & 24.6684 & 24.2356 & 24.1026 & 24.6712 & 23.9623 & 23.7254 & 24.5565 & 24.1305 & 23.9952 \\
\hline $\mathrm{N}-\mathrm{CH}_{3}$ & 3.73 & 29.2028 & 28.6873 & 28.3917 & 29.1749 & 28.8644 & 28.5477 & 28.9075 & 28.3881 & 28.0779 & 28.8135 & 28.5463 & 28.2254 \\
\hline
\end{tabular}




\begin{tabular}{|c|c|c|c|c|c|c|c|c|c|c|c|c|c|}
\hline & \multirow[b]{3}{*}{ Exp } & \multicolumn{12}{|c|}{ mPW1PW91 } \\
\hline & & \multicolumn{6}{|c|}{ Gas phase } & \multicolumn{6}{|c|}{ PCM } \\
\hline & & $6-31 G^{*}$ & $6-31 G * *$ & $6-31+G * *$ & 6-311G* & $6-311 G * *$ & $6-311+G * *$ & $6-31 G *$ & $6-31 G * *$ & $6-31+G * *$ & 6-311G* & $6-311 G * *$ & $6-311+G^{* *}$ \\
\hline \multicolumn{14}{|l|}{${ }^{13} \mathrm{C}$} \\
\hline 1 & 154.2 & 44.3759 & 44.2908 & 43.0074 & 27.3980 & 27.2337 & 26.2585 & 45.2122 & 45.1299 & 43.6802 & 28.1669 & 28.0224 & 27.0617 \\
\hline 2 & 113.7 & 82.3539 & 82.3892 & 82.6560 & 68.4106 & 68.3914 & 67.5364 & 83.1869 & 83.2294 & 83.3557 & 69.3741 & 69.2800 & 68.3345 \\
\hline 3 & 152.8 & 47.1488 & 47.1945 & 46.2834 & 30.5648 & 30.5759 & 29.9273 & 46.3241 & 46.3739 & 45.3753 & 29.6717 & 29.6997 & 29.1300 \\
\hline 4 & 106.5 & 88.4096 & 88.4343 & 88.6778 & 75.9401 & 75.8626 & 75.5943 & 88.0754 & 88.1248 & 88.3638 & 75.7617 & 75.6463 & 75.2854 \\
\hline 5 & 148.4 & 51.2795 & 51.0437 & 50.1262 & 33.1610 & 32.9971 & 33.2715 & 51.3313 & 51.0834 & 50.1841 & 33.2579 & 33.0337 & 33.2371 \\
\hline 6 & 119.0 & 80.1048 & 80.6683 & 81.2281 & 65.8264 & 64.9521 & 64.1823 & 79.9779 & 80.5049 & 80.5506 & 66.0573 & 64.9429 & 64.0007 \\
\hline 7 & 123.4 & 73.3587 & 73.9501 & 74.6380 & 58.7654 & 57.8081 & 57.8390 & 74.2614 & 74.8567 & 75.3531 & 59.8222 & 58.7392 & 58.6676 \\
\hline 8 & 117.5 & 77.7268 & 78.3166 & 77.4698 & 63.8485 & 63.0272 & 61.8697 & 78.8126 & 79.4205 & 78.7059 & 65.1364 & 64.3226 & 63.5011 \\
\hline 9 & 177.5 & 24.1495 & 24.1918 & 24.1200 & 9.1071 & 9.1331 & 7.4822 & 24.1654 & 24.2208 & 23.7874 & 8.9992 & 8.9480 & 7.0313 \\
\hline 10 & 108.2 & 84.9310 & 84.9814 & 85.4859 & 73.0787 & 73.0379 & 72.9186 & 86.1980 & 86.2567 & 86.7574 & 74.5839 & 74.4861 & 74.4032 \\
\hline 11 & 147.2 & 52.5994 & 52.4717 & 52.3207 & 36.8072 & 36.6957 & 35.8039 & 51.7848 & 51.6773 & 51.4003 & 36.0158 & 35.9545 & 34.9458 \\
\hline 12 & 137.2 & 62.1931 & 62.0163 & 61.6821 & 46.8601 & 46.4806 & 45.7856 & 62.3064 & 62.1587 & 61.9248 & 46.8975 & 46.5437 & 45.7347 \\
\hline 13 & 127.7 & 67.7116 & 67.5416 & 67.1192 & 53.6518 & 53.4164 & 52.4630 & 68.6779 & 68.5188 & 68.2777 & 54.7242 & 54.4704 & 53.6021 \\
\hline $1^{\prime}$ & 121.8 & 75.4294 & 75.9283 & 74.9028 & 60.8300 & 59.9742 & 58.7199 & 75.4037 & 75.8853 & 74.8981 & 60.5836 & 59.8218 & 58.6292 \\
\hline $2^{\prime}$ & 124.1 & 73.8871 & 74.3331 & 74.3925 & 59.2123 & 58.1969 & 57.9248 & 73.3463 & 73.8321 & 73.8211 & 58.6497 & 57.5333 & 57.1252 \\
\hline $3^{\prime}$ & 77.3 & 117.0481 & 117.5507 & 117.4466 & 106.4834 & 106.5217 & 105.8566 & 116.5462 & 117.0538 & 116.9524 & 105.8961 & 105.9239 & 105.1918 \\
\hline $4^{\prime}$ & 27.2 & 166.7418 & 168.3179 & 169.4322 & 160.7865 & 160.5721 & 160.5808 & 167.2215 & 168.7880 & 169.8380 & 161.2866 & 161.0289 & 161.0543 \\
\hline $5^{\prime}$ & 27.2 & 165.2566 & 166.7548 & 166.8426 & 158.1325 & 157.8671 & 157.3454 & 165.6264 & 167.1107 & 167.0058 & 158.4730 & 158.1963 & 157.6242 \\
\hline 1" & 103.6 & 93.4752 & 93.9444 & 95.6566 & 80.6463 & 79.8417 & 79.0512 & 93.2239 & 93.6928 & 95.3598 & 80.2560 & 79.4651 & 78.5900 \\
\hline $2 "$ & 144.5 & 54.0980 & 54.7334 & 54.5423 & 38.4053 & 37.3727 & 36.1665 & 54.2197 & 54.8586 & 54.5464 & 38.3511 & 37.2994 & 36.1300 \\
\hline $\begin{array}{c}\mathrm{N}-\mathrm{CH}_{3} \\
{ }^{{ }^{\mathbf{H}}}\end{array}$ & 48.4 & 147.7605 & 149.2133 & 148.5943 & 140.6269 & 140.1577 & 139.0673 & 146.8940 & 148.3761 & 147.1639 & 139.3918 & 138.9827 & 138.0422 \\
\hline 6 & 7.07 & 25.0842 & 24.4149 & 24.3164 & 24.9288 & 24.4530 & 24.5670 & 25.0747 & 24.3922 & 24.2441 & 24.9454 & 24.4226 & 24.4828 \\
\hline 7 & 7.09 & 24.7646 & 24.1576 & 24.0604 & 24.6729 & 24.2795 & 24.3402 & 24.6985 & 24.0868 & 23.9793 & 24.6130 & 24.2102 & 24.2654 \\
\hline 8 & 7.89 & 24.1126 & 23.4295 & 23.1369 & 24.0038 & 23.5288 & 23.3042 & 24.1507 & 23.4593 & 23.1859 & 24.0280 & 23.5498 & 23.3388 \\
\hline $1^{\prime}$ & 6.77 & 25.3428 & 24.6683 & 24.4980 & 25.2723 & 24.7824 & 24.6659 & 25.2029 & 24.5513 & 24.3876 & 25.1183 & 24.6712 & 24.5800 \\
\hline $2^{\prime}$ & 5.54 & 26.5301 & 25.9215 & 25.7723 & 26.5194 & 26.0740 & 26.0235 & 26.3381 & 25.7387 & 25.5810 & 26.3194 & 25.8888 & 25.8342 \\
\hline $4^{\prime}$ & 1.53 & 30.6389 & 30.1728 & 30.0945 & 30.5627 & 30.2882 & 30.2488 & 30.5680 & 30.1005 & 30.0173 & 30.4833 & 30.2134 & 30.1689 \\
\hline $5^{\prime}$ & 1.53 & 30.5402 & 30.0814 & 29.9877 & 30.4279 & 30.1562 & 30.1291 & 30.4884 & 30.0299 & 29.9247 & 30.3692 & 30.1021 & 30.0687 \\
\hline 1" & 6.83 & 25.4311 & 24.6674 & 24.4280 & 25.3412 & 24.7988 & 24.7653 & 25.2829 & 24.5167 & 24.2445 & 25.1790 & 24.6439 & 24.5909 \\
\hline 2" & 7.68 & 24.5942 & 23.8855 & 23.6962 & 24.5328 & 24.0633 & 23.9496 & 24.4775 & 23.7706 & 23.5698 & 24.4080 & 23.9454 & 23.8294 \\
\hline $\mathrm{N}-\mathrm{CH}_{3}$ & 3.73 & 29.1184 & 28.6061 & 28.3308 & 29.0736 & 28.7652 & 28.4872 & 28.7784 & 28.2674 & 27.9911 & 28.6799 & 28.4195 & 28.1411 \\
\hline
\end{tabular}




\section{Compound 46}

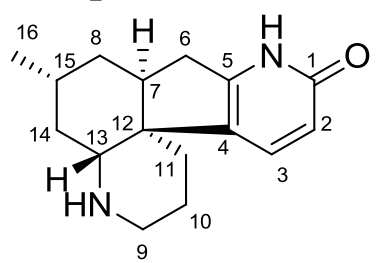

46

\begin{tabular}{|c|c|c|c|c|c|c|c|c|c|c|c|c|c|}
\hline \multirow{3}{*}{\multicolumn{2}{|c|}{ Exp }} & \multicolumn{12}{|c|}{ B3LYP } \\
\hline & & \multicolumn{6}{|c|}{ Gas phase } & \multicolumn{6}{|c|}{ PCM } \\
\hline & & 6-31G* & $6-31 \mathrm{G} * *$ & $6-31+G * *$ & 6-311G* & 6-311G** & $6-311+G * *$ & 6-31G* & $6-31 G * *$ & $6-31+\mathrm{G} * *$ & 6-311G* & $6-311 G * *$ & $6-311+\mathrm{G} * *$ \\
\hline \multicolumn{14}{|c|}{${ }^{13} \mathrm{C}$} \\
\hline 1 & 165.7 & 41.0185 & 40.8891 & 37.7480 & 24.8300 & 24.4999 & 19.2670 & 39.7955 & 39.6515 & 36.2101 & 20.8952 & 20.7874 & 16.5548 \\
\hline 2 & 115.9 & 76.0878 & 76.7247 & 76.7102 & 60.4995 & 59.8061 & 58.9793 & 78.3173 & 78.9593 & 79.0369 & 63.0800 & 62.4002 & 61.4944 \\
\hline 3 & 139.8 & 59.6614 & 60.2974 & 58.8005 & 42.4583 & 41.5065 & 40.2998 & 58.0623 & 58.7012 & 56.8842 & 40.3855 & 39.4398 & 38.1241 \\
\hline 4 & 129.3 & 68.6358 & 68.4928 & 67.3576 & 57.5707 & 57.0620 & 52.1252 & 67.3935 & 67.2560 & 65.7741 & 51.7724 & 51.7238 & 48.7996 \\
\hline 5 & 148.8 & 48.6650 & 48.7259 & 47.1192 & 37.9487 & 37.4354 & 30.8517 & 46.6217 & 46.6831 & 44.9387 & 29.4057 & 29.5507 & 26.2045 \\
\hline 6 & 34.0 & 154.3953 & 155.5829 & 154.6162 & 136.7457 & 136.8640 & 141.5648 & 154.4497 & 155.6307 & 154.6679 & 142.4724 & 141.9946 & 143.6629 \\
\hline 7 & 46.0 & 141.1355 & 141.9542 & 141.7357 & 131.2208 & 130.9848 & 129.3369 & 141.2676 & 142.0816 & 141.8635 & 130.6629 & 130.4966 & 129.1877 \\
\hline 8 & 33.4 & 156.2244 & 157.5389 & 155.6880 & 145.2734 & 145.0255 & 145.5590 & 156.5392 & 157.8506 & 156.0774 & 146.8965 & 146.5080 & 146.4131 \\
\hline 9 & 39.7 & 149.8758 & 151.2311 & 149.8816 & 139.3682 & 139.2951 & 139.5750 & 150.1553 & 151.5059 & 150.1278 & 140.0323 & 139.9250 & 139.9560 \\
\hline 10 & 23.8 & 163.6029 & 165.0052 & 163.9141 & 153.7443 & 153.6532 & 153.3328 & 163.9182 & 165.3168 & 164.3019 & 154.7008 & 154.5444 & 153.9553 \\
\hline 11 & 30.0 & 158.1763 & 159.5725 & 158.7324 & 149.8192 & 149.6497 & 149.0440 & 158.3998 & 159.7871 & 159.0100 & 149.8011 & 149.6544 & 149.2410 \\
\hline 12 & 45.3 & 142.3889 & 142.6244 & 141.5588 & 132.6229 & 132.8114 & 131.6968 & 142.2017 & 142.4362 & 141.3391 & 131.5344 & 131.8185 & 131.1265 \\
\hline 13 & 57.8 & 129.9422 & 130.7625 & 131.5576 & 120.5382 & 120.3239 & 118.3398 & 130.3820 & 131.1940 & 131.9804 & 119.7859 & 119.6878 & 118.3969 \\
\hline 14 & 35.3 & 155.4441 & 156.6963 & 154.0115 & 145.5589 & 145.2228 & 145.3664 & 155.3473 & 156.5990 & 153.7705 & 145.4712 & 145.1158 & 145.1417 \\
\hline 15 & 27.3 & 159.3909 & 160.2791 & 160.6493 & 147.9916 & 148.1983 & 149.0292 & 159.5645 & 160.4530 & 159.0405 & 149.3034 & 149.3927 & 149.6901 \\
\hline 16 & 22.4 & 166.5682 & 168.3372 & 169.1669 & 152.1550 & 152.5048 & 156.6506 & 166.8221 & 168.5857 & 169.4991 & 157.6001 & 157.4191 & 158.8470 \\
\hline \multicolumn{14}{|c|}{${ }^{1} \mathbf{H}$} \\
\hline 2 & 6.36 & 26.2917 & 25.6109 & 25.4052 & 26.2253 & 25.7554 & 25.6730 & 26.3206 & 25.6356 & 25.4005 & 26.2456 & 25.7771 & 25.6693 \\
\hline 3 & 7.90 & 24.7845 & 24.0728 & 23.8813 & 24.7913 & 24.2621 & 24.1370 & 24.5458 & 23.8359 & 23.6205 & 24.5189 & 23.9932 & 23.8668 \\
\hline 6 & 2.81 & 29.9949 & 29.5247 & 29.3333 & 29.8368 & 29.5809 & 29.5414 & 29.7903 & 29.3151 & 29.1113 & 29.6513 & 29.3935 & 29.3318 \\
\hline 6 & 2.81 & 29.5048 & 28.9939 & 28.8238 & 29.6406 & 29.3398 & 29.1388 & 29.3884 & 28.8772 & 28.7005 & 29.4421 & 29.1473 & 28.9866 \\
\hline 7 & 2.18 & 30.0582 & 29.7118 & 29.6325 & 30.0873 & 29.9478 & 29.8843 & 29.9957 & 29.6466 & 29.5549 & 30.0207 & 29.8831 & 29.8053 \\
\hline 8 & 1.34 & 30.8277 & 30.4280 & 30.3827 & 30.8371 & 30.6345 & 30.5972 & 30.7886 & 30.3893 & 30.3414 & 30.7943 & 30.5937 & 30.5562 \\
\hline 8 & 1.56 & 30.6374 & 30.2460 & 30.1744 & 30.5755 & 30.4084 & 30.4190 & 30.5832 & 30.1886 & 30.1126 & 30.5324 & 30.3622 & 30.3654 \\
\hline 9 & 2.83 & 29.5490 & 29.1181 & 28.9248 & 29.3698 & 29.1608 & 29.1452 & 29.5368 & 29.1036 & 28.9002 & 29.3501 & 29.1356 & 29.1197 \\
\hline 9 & 2.95 & 29.2854 & 28.8706 & 28.7115 & 29.2014 & 29.0136 & 29.0046 & 29.2827 & 28.8659 & 28.6768 & 29.1877 & 29.0015 & 28.9734 \\
\hline 10 & 1.62 & 30.8174 & 30.3649 & 30.2611 & 30.6788 & 30.4431 & 30.4511 & 30.7891 & 30.3353 & 30.2261 & 30.6641 & 30.4224 & 30.4200 \\
\hline 10 & 1.99 & 30.2900 & 29.8633 & 29.8131 & 30.2995 & 30.0584 & 29.9901 & 30.2311 & 29.8063 & 29.7506 & 30.2051 & 29.9664 & 29.9181 \\
\hline 11 & 1.49 & 30.6693 & 30.2812 & 30.2670 & 30.6377 & 30.4556 & 30.5011 & 30.6559 & 30.2670 & 30.2540 & 30.6228 & 30.4432 & 30.4879 \\
\hline 11 & 1.92 & 30.1167 & 29.7049 & 29.6768 & 30.1710 & 29.9710 & 29.9325 & 30.0453 & 29.6335 & 29.5942 & 30.0836 & 29.8852 & 29.8484 \\
\hline 13 & 2.59 & 29.5752 & 29.2221 & 29.1069 & 29.7163 & 29.5879 & 29.5314 & 29.5728 & 29.2169 & 29.0941 & 29.6635 & 29.5367 & 29.4995 \\
\hline 14 & 1.34 & 30.5918 & 30.2089 & 30.1936 & 30.4760 & 30.3135 & 30.4240 & 30.6960 & 30.3136 & 30.3029 & 30.6234 & 30.4568 & 30.5454 \\
\hline 14 & 1.56 & 30.5312 & 30.1046 & 30.1587 & 30.4955 & 30.2741 & 30.3580 & 30.4607 & 30.0339 & 30.0657 & 30.4973 & 30.2675 & 30.2975 \\
\hline
\end{tabular}




\begin{tabular}{|c|c|c|c|c|c|c|c|c|c|c|c|c|}
\hline 151.64 & 30.3310 & 29.9248 & 29.8861 & 30.4685 & 30.2816 & 30.2235 & 30.2632 & 29.8557 & 29.8073 & 30.3887 & 30.2020 & 30.1424 \\
\hline 0.95 & 31.1569 & 30.7396 & 30.6648 & 31.0371 & 30.8265 & 30.8013 & 31.1592 & 30.7406 & 30.6688 & 31.0812 & 30.8658 & 30.8209 \\
\hline
\end{tabular}

\begin{tabular}{|c|c|c|c|c|c|c|c|c|c|c|c|c|c|}
\hline \multicolumn{14}{|c|}{ mPW1PW91 } \\
\hline & \multirow[b]{2}{*}{ Exp } & \multicolumn{6}{|c|}{ Gas phase } & \multicolumn{6}{|c|}{ PCM } \\
\hline & & 6-31G* & $6-31 G * *$ & $6-31+\mathrm{G} * *$ & 6-311G* & $6-311 G * *$ & $6-311+G * *$ & 6-31G* & $6-31 G * *$ & $6-31+G * *$ & 6-311G* & $6-311 G * *$ & $6-311+G * *$ \\
\hline \multicolumn{14}{|c|}{${ }^{13} \mathrm{C}$} \\
\hline 1 & 165.7 & 43.1637 & 43.0085 & 40.6802 & 31.3903 & 31.1016 & 25.0559 & 41.9611 & 41.7900 & 39.2059 & 26.2591 & 26.2129 & 21.8766 \\
\hline 2 & 115.9 & 78.8870 & 79.4653 & 79.6231 & 65.2767 & 64.5605 & 63.5734 & 81.1677 & 81.7531 & 81.9752 & 67.8460 & 67.1471 & 66.0850 \\
\hline 3 & 139.8 & 61.7564 & 62.3224 & 61.1427 & 46.8172 & 45.8327 & 44.7109 & 60.0989 & 60.6681 & 59.1962 & 44.6769 & 43.6961 & 42.5098 \\
\hline 4 & 129.3 & 72.5954 & 72.4395 & 71.5841 & 66.6683 & 66.2360 & 59.4417 & 71.3436 & 71.1949 & 70.0371 & 58.7444 & 58.8232 & 55.2265 \\
\hline 5 & 148.8 & 51.3408 & 51.3719 & 50.3274 & 47.5099 & 47.1162 & 37.7229 & 49.2328 & 49.2641 & 48.0934 & 35.7861 & 36.1233 & 31.6711 \\
\hline 6 & 34.0 & 158.5086 & 159.6169 & 158.8969 & 137.8282 & 137.8200 & 144.9986 & 158.5340 & 159.6373 & 158.9191 & 146.3602 & 145.6900 & 148.3214 \\
\hline 7 & 46.0 & 146.4815 & 147.2377 & 147.2721 & 138.0496 & 137.8389 & 135.9713 & 146.6088 & 147.3608 & 147.3935 & 137.1572 & 137.0237 & 135.6566 \\
\hline 8 & 33.4 & 161.3069 & 162.4972 & 160.8499 & 150.4335 & 150.1620 & 151.2141 & 161.6492 & 162.8370 & 161.2616 & 152.6195 & 152.1946 & 152.3199 \\
\hline 9 & 39.7 & 154.7570 & 156.0179 & 155.1098 & 145.0005 & 144.9014 & 145.2731 & 155.0531 & 156.3095 & 155.3725 & 145.8481 & 145.7167 & 145.7422 \\
\hline 10 & 23.8 & 168.5675 & 169.8473 & 168.9713 & 159.1558 & 159.0265 & 158.8695 & 168.9128 & 170.1897 & 169.3749 & 160.3670 & 160.1680 & 159.5899 \\
\hline 11 & 30.0 & 163.5274 & 164.8012 & 163.9397 & 155.9937 & 155.8334 & 155.1355 & 163.7569 & 165.0224 & 164.2165 & 155.8548 & 155.7239 & 155.2716 \\
\hline 12 & 45.3 & 147.4932 & 147.7194 & 146.3589 & 139.3559 & 139.5346 & 137.9492 & 147.3017 & 147.5270 & 146.1424 & 137.7996 & 138.0905 & 137.1852 \\
\hline 13 & 57.8 & 135.3714 & 136.1130 & 137.1597 & 127.9256 & 127.7363 & 125.3058 & 135.7966 & 136.5305 & 137.5636 & 126.5832 & 126.5238 & 125.0774 \\
\hline 14 & 35.3 & 160.5158 & 161.6577 & 159.3322 & 151.4969 & 151.1830 & 151.3165 & 160.4461 & 161.5874 & 159.1421 & 151.4995 & 151.1563 & 151.1602 \\
\hline 15 & 27.3 & 164.6567 & 165.4893 & 164.1088 & 153.4039 & 153.5813 & 154.7805 & 164.8343 & 165.6681 & 164.3283 & 155.2275 & 155.2772 & 155.6655 \\
\hline 16 & 22.4 & 170.9277 & 172.5738 & 173.2453 & 153.4588 & 153.6852 & 160.1076 & 171.2267 & 172.8667 & 173.6211 & 161.3976 & 161.0464 & 163.4241 \\
\hline \multicolumn{14}{|c|}{${ }^{1} \mathrm{H}$} \\
\hline 2 & 6.36 & 26.0955 & 25.4177 & 25.2105 & 26.0592 & 25.5573 & 25.4727 & 26.1148 & 25.4329 & 25.2008 & 26.0729 & 25.5731 & 25.4651 \\
\hline 3 & 7.90 & 24.6118 & 23.9018 & 23.7063 & 24.6390 & 24.0863 & 23.9576 & 24.3618 & 23.6540 & 23.4384 & 24.3475 & 23.7965 & 23.6744 \\
\hline 6 & 2.81 & 29.8994 & 29.4256 & 29.2723 & 29.7211 & 29.4582 & 29.4354 & 29.6819 & 29.2020 & 29.0368 & 29.5313 & 29.2664 & 29.2181 \\
\hline 6 & 2.81 & 29.4391 & 28.9232 & 28.7669 & 29.5902 & 29.2944 & 29.0569 & 29.3140 & 28.7976 & 28.6364 & 29.3416 & 29.0510 & 28.8778 \\
\hline 7 & 2.18 & 29.9826 & 29.6351 & 29.5569 & 29.9597 & 29.8173 & 29.7548 & 29.9183 & 29.5681 & 29.4783 & 29.8862 & 29.7476 & 29.6733 \\
\hline 8 & 1.34 & 30.7896 & 30.3952 & 30.3399 & 30.7414 & 30.5470 & 30.5108 & 30.7465 & 30.3525 & 30.2950 & 30.6917 & 30.4995 & 30.4653 \\
\hline 8 & 1.56 & 30.5935 & 30.2042 & 30.1551 & 30.4833 & 30.3120 & 30.3320 & 30.5330 & 30.1400 & 30.0875 & 30.4409 & 30.2664 & 30.2760 \\
\hline 9 & 2.83 & 29.4766 & 29.0511 & 28.8807 & 29.3130 & 29.0968 & 29.0815 & 29.4582 & 29.0301 & 28.8505 & 29.2873 & 29.0632 & 29.0503 \\
\hline 9 & 2.95 & 29.2557 & 28.8446 & 28.6916 & 29.1419 & 28.9530 & 28.9496 & 29.2522 & 28.8390 & 28.6576 & 29.1269 & 28.9427 & 28.9199 \\
\hline 10 & 1.62 & 30.7715 & 30.3247 & 30.2243 & 30.5975 & 30.3621 & 30.3687 & 30.7358 & 30.2875 & 30.1826 & 30.5832 & 30.3398 & 30.3336 \\
\hline 10 & 1.99 & 30.2684 & 29.8429 & 29.7835 & 30.2538 & 30.0215 & 29.9249 & 30.2067 & 29.7833 & 29.7201 & 30.1438 & 29.9109 & 29.8435 \\
\hline 11 & 1.49 & 30.6133 & 30.2258 & 30.2216 & 30.5497 & 30.3617 & 30.4045 & 30.5999 & 30.2116 & 30.2079 & 30.5293 & 30.3453 & 30.3908 \\
\hline 11 & 1.92 & 30.0994 & 29.6894 & 29.6448 & 30.0952 & 29.9034 & 29.8479 & 30.0230 & 29.6128 & 29.5591 & 29.9999 & 29.8094 & 29.7583 \\
\hline 13 & 2.59 & 29.5136 & 29.1639 & 29.0424 & 29.6401 & 29.5103 & 29.4249 & 29.5148 & 29.1623 & 29.0343 & 29.5678 & 29.4408 & 29.3872 \\
\hline 14 & 1.34 & 30.5302 & 30.1495 & 30.1636 & 30.3534 & 30.1832 & 30.3217 & 30.6355 & 30.2553 & 30.2722 & 30.5260 & 30.3522 & 30.4536 \\
\hline 14 & 1.56 & 30.5079 & 30.0834 & 30.1091 & 30.3437 & 30.1314 & 30.2440 & 30.4350 & 30.0100 & 30.0159 & 30.3872 & 30.1631 & 30.2026 \\
\hline 15 & 1.64 & 30.2925 & 29.8910 & 29.8527 & 30.3665 & 30.1900 & 30.1224 & 30.2240 & 29.8210 & 29.7746 & 30.2826 & 30.1066 & 30.0398 \\
\hline 16 & 0.95 & 31.0957 & 30.6772 & 30.6218 & 30.9198 & 30.7010 & 30.7030 & 31.0947 & 30.6749 & 30.6223 & 30.9814 & 30.7572 & 30.7288 \\
\hline
\end{tabular}




\section{Compound 47}

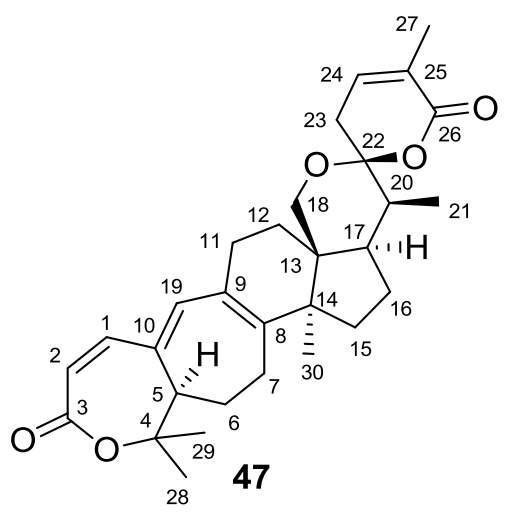

\begin{tabular}{|c|c|c|c|c|c|c|c|c|c|c|c|c|c|}
\hline \multicolumn{14}{|c|}{ B3LYP } \\
\hline & \multirow[b]{2}{*}{ Exp } & \multicolumn{6}{|c|}{ Gas phase } & \multicolumn{6}{|c|}{ PCM } \\
\hline & & 6-31G* & $6-31 G * *$ & $6-31+G * *$ & 6-311G* & $6-311 G * *$ & $6-311+G * *$ & 6-31G* & $6-31 G * *$ & $6-31+G * *$ & 6-311G* & $6-311 G * *$ & $6-311+G * *$ \\
\hline \multicolumn{14}{|c|}{${ }^{13} \mathrm{C}$} \\
\hline 1 & 143.5 & 54.7034 & 55.3009 & 54.8039 & 36.9318 & 35.9160 & 34.7368 & 51.8001 & 52.3959 & 51.3786 & 33.5877 & 32.5647 & 31.0114 \\
\hline 2 & 117.9 & 74.1079 & 74.6488 & 74.2332 & 57.9451 & 57.0935 & 57.0458 & 76.7305 & 77.2739 & 77.4210 & 60.9349 & 60.1117 & 60.3614 \\
\hline 3 & 166.9 & 35.2635 & 35.3051 & 32.8124 & 15.2545 & 15.2830 & 13.1585 & 33.1428 & 33.1808 & 30.2642 & 12.8301 & 12.8518 & 10.2555 \\
\hline 4 & 80.2 & 112.6174 & 113.0058 & 109.5843 & 100.7486 & 100.9218 & 100.7242 & 111.6771 & 112.0636 & 108.4977 & 99.6547 & 99.8330 & 99.5080 \\
\hline 5 & 49.0 & 140.9403 & 141.4987 & 138.6947 & 129.5247 & 129.1566 & 129.7352 & 141.2159 & 141.7677 & 139.1211 & 129.8289 & 129.4524 & 130.1542 \\
\hline 6 & 39.2 & 144.2576 & 145.5936 & 146.2662 & 135.2450 & 135.0536 & 133.9135 & 144.4639 & 145.7942 & 146.5121 & 135.4631 & 135.2669 & 134.1666 \\
\hline 7 & 27.6 & 159.8189 & 161.2523 & 163.0452 & 150.9424 & 150.6446 & 149.1293 & 159.8934 & 161.3261 & 163.1300 & 151.0241 & 150.7216 & 149.1795 \\
\hline 8 & 149.3 & 39.6352 & 39.8597 & 37.3722 & 18.5083 & 18.7238 & 17.8013 & 38.5335 & 38.7636 & 36.0715 & 17.3214 & 17.5364 & 16.4513 \\
\hline 9 & 129.8 & 60.9460 & 61.1813 & 62.8586 & 42.9313 & 43.0473 & 41.6707 & 60.6618 & 60.8954 & 62.5427 & 42.6091 & 42.7340 & 41.3182 \\
\hline 10 & 139.6 & 52.5828 & 52.3467 & 50.3789 & 32.4273 & 32.1792 & 31.7414 & 53.4181 & 53.1748 & 51.3216 & 33.4274 & 33.1777 & 32.8022 \\
\hline 11 & 25.8 & 161.1668 & 162.4588 & 162.0316 & 152.9077 & 152.6115 & 152.0689 & 161.3700 & 162.6545 & 162.2786 & 153.1146 & 152.8110 & 152.2739 \\
\hline 12 & 22.1 & 164.3269 & 165.7375 & 165.1143 & 156.9057 & 156.7008 & 156.2536 & 164.4872 & 165.8921 & 165.2918 & 157.0806 & 156.8655 & 156.4321 \\
\hline 13 & 44.5 & 142.3423 & 142.5329 & 143.2402 & 132.0805 & 132.1393 & 131.6506 & 142.2776 & 142.4691 & 143.1608 & 131.9779 & 132.0387 & 131.5463 \\
\hline 14 & 48.9 & 137.5012 & 137.7521 & 138.9499 & 127.1299 & 127.0722 & 126.4905 & 137.3078 & 137.5638 & 138.7377 & 126.9108 & 126.8590 & 126.2456 \\
\hline 15 & 31.0 & 156.8063 & 158.2695 & 157.2783 & 149.0259 & 148.7115 & 148.9944 & 156.9955 & 158.4545 & 157.4938 & 149.2577 & 148.9328 & 149.2430 \\
\hline 16 & 22.4 & 164.5888 & 166.1061 & 167.8905 & 156.7972 & 156.7283 & 154.1342 & 164.4912 & 166.0089 & 167.8719 & 156.7258 & 156.6517 & 154.0844 \\
\hline 17 & 42.9 & 144.0113 & 144.7859 & 142.0050 & 133.8735 & 133.6473 & 134.1662 & 144.3176 & 145.0867 & 142.3830 & 134.2474 & 134.0109 & 134.5635 \\
\hline 18 & 61.0 & 129.0564 & 130.4031 & 130.7985 & 119.9328 & 119.4080 & 118.5903 & 129.0354 & 130.3772 & 130.6884 & 119.8572 & 119.3281 & 118.4716 \\
\hline 19 & 142.8 & 51.8832 & 52.3697 & 52.1318 & 34.6574 & 33.9107 & 32.0723 & 48.9686 & 49.4593 & 48.7358 & 31.3160 & 30.5762 & 28.4042 \\
\hline 20 & 35.3 & 152.0449 & 152.7814 & 151.5827 & 142.4041 & 142.2506 & 142.0082 & 152.2892 & 153.0236 & 151.8906 & 142.7024 & 142.5455 & 142.3205 \\
\hline 21 & 14.8 & 172.5568 & 174.4030 & 174.9286 & 166.5327 & 166.5061 & 166.1041 & 172.8094 & 174.6470 & 175.2050 & 166.8485 & 166.7981 & 166.3966 \\
\hline & 104.6 & 86.2498 & 86.3787 & 86.2095 & 73.3821 & 73.5734 & 73.0878 & 85.6552 & 85.7819 & 85.5132 & 72.6902 & 72.8821 & 72.3429 \\
\hline 23 & 32.9 & 155.5565 & 156.7440 & 156.9734 & 146.8916 & 146.4520 & 145.8244 & 155.5292 & 156.7022 & 156.8777 & 146.7589 & 146.2984 & 145.6085 \\
\hline & 136.9 & 57.7255 & 58.6813 & 58.9948 & 39.9101 & 39.3625 & 38.0686 & 54.4349 & 55.3844 & 55.1396 & 36.0401 & 35.4859 & 33.8112 \\
\hline & 126.8 & 65.8117 & 65.7820 & 65.0883 & 47.5114 & 47.3169 & 46.6007 & 66.9836 & 66.9583 & 66.4770 & 48.9045 & 48.7264 & 48.1608 \\
\hline 26 & 164.3 & 35.6189 & 35.6969 & 34.4310 & 15.5724 & 15.6680 & 12.8575 & 33.9161 & 33.9959 & 32.3429 & 13.5872 & 13.6849 & 10.5025 \\
\hline
\end{tabular}




\begin{tabular}{|c|c|c|c|c|c|c|c|c|c|c|c|c|c|}
\hline 27 & 17.0 & 9.8705 & 1.6066 & 171.9058 & 52.9505 & 162.7110 & 162.7618 & 170.3855 & 172.1068 & 172.5202 & 163.5455 & 163.2906 & 163.3906 \\
\hline 28 & 6.2 & 53.7003 & 5.3766 & 165.8201 & 56.9316 & 156.7430 & 156.5527 & 164.0203 & 165.6767 & 166.1597 & 157.2342 & 157.0230 & 156.8706 \\
\hline 29 & 29.0 & 9.6284 & 51.2940 & 158.0784 & 52.2008 & 151.8761 & 52.0969 & 159.9613 & 161.6086 & 158.1837 & 152.5225 & 152.1749 & 52.2843 \\
\hline 30 & 26.8 & 61.0849 & 162.8265 & 162.9817 & 154.3585 & 154.0933 & 153.9072 & 161.3948 & 163.1164 & 163.2826 & 154.6888 & 154.4014 & 154.2359 \\
\hline 1 & 6.63 & 25.9668 & 25.3778 & 25.0951 & 25.7617 & 25.3457 & 25.3053 & 25.7151 & 25.1256 & 24.8171 & 25.4986 & 25.0905 & 25.0274 \\
\hline 2 & 5.78 & 26.7019 & 26.0548 & 25.8831 & 26.5976 & 26.1519 & 26.0490 & 26.7816 & 26.1241 & 25.9557 & 26.6690 & & 26.1197 \\
\hline 5 & 2.45 & 29.6204 & 29.2188 & 29.4229 & 29.7602 & 29.5990 & 29.6500 & 29.5283 & 29.1227 & 29.3132 & 29.6663 & 29.5021 & 29.5435 \\
\hline 6 & 2.20 & 29.6326 & 29.1627 & 29.1197 & 29.6778 & 29.4040 & 29.3244 & 29.5521 & 29.0807 & 29.0258 & 29.5957 & 29.3223 & 29.2382 \\
\hline 6 & 2.40 & 29.5509 & 29.0851 & 29.0932 & 29.6078 & 29.3599 & 29.3752 & 29.6552 & 29.1913 & 29.2014 & 29.7088 & 29.4626 & 29.4783 \\
\hline 7 & 1.83 & 30.1671 & 29.6919 & 29.4185 & 30.1412 & 29.8718 & 29.8197 & 30.1076 & 29.6305 & 29.3494 & 30.0770 & 29.8063 & 29.7502 \\
\hline 7 & 2.50 & 30.1179 & 29.6866 & 29.4296 & 30.1954 & & & & & 29.4590 & & & 29.6819 \\
\hline 11 & 2.10 & 30.0689 & 29.5983 & 29.5818 & 30.0079 & 29.7463 & 29.7591 & 30.0111 & 29.5375 & 29.5240 & 29.9578 & 29.6935 & 29.7042 \\
\hline 11 & 2.30 & 29.9000 & 29.4381 & 29.2938 & 29.8633 & 29.6051 & 29.5435 & 29.8376 & 29.3747 & 29.2289 & 29.7971 & 29.5404 & 29.4771 \\
\hline 12 & 1.59 & 30.5935 & 30.1498 & 30.1025 & 30.6214 & 30.3534 & 30.2964 & 30.5161 & 30.0710 & 30.0208 & 30.5462 & 30.2786 & 30.2177 \\
\hline 12 & 2.07 & 30.0954 & 29.6253 & 29.5148 & 30.1152 & 29.8304 & 29.7693 & 30.0980 & 29.6283 & 29.5208 & 30.1137 & 29.8302 & 29.7711 \\
\hline 15 & 1.50 & 30.6998 & 30.2802 & 30.4269 & 30.6936 & 30.4680 & 30.6118 & & & 30.3663 & 6379 & & .5516 \\
\hline 15 & 1.88 & 30.1969 & 29.7320 & 29.8632 & 30.2746 & 30.0184 & 29.9985 & 30.2103 & 29.7453 & 29.8729 & 30.2828 & 270 & 0039 \\
\hline 16 & 1.87 & 30.3944 & 29.9362 & 29.8087 & 30.4200 & 30.1454 & 30.1561 & 30.3386 & 29.8780 & 29.7440 & 30.3649 & 30.0895 & 30.0959 \\
\hline 16 & 2.09 & 29.3539 & 28.8245 & 28.6345 & 29.4359 & 29.1321 & 29.0428 & 29.5145 & 28.9887 & 28.7983 & 29.5924 & 29.2888 & 29.1977 \\
\hline 17 & 2.01 & 0.1103 & 29.7030 & 29.7305 & 30.2213 & 29.9965 & 29.9965 & 30.0159 & 29.6082 & 29.6246 & 30.1237 & 29.9002 & 9.8929 \\
\hline 18 & 3.17 & 28.9302 & 28.4773 & 28.2984 & & 28.8239 & & & & & & & 6161 \\
\hline 18 & 3.61 & 28.2739 & 27.7923 & 27.7204 & 28.4193 & 28.1668 & 28.0676 & 28.3120 & 27.8312 & 27.7456 & 28.4451 & 28.1928 & 28.0843 \\
\hline 19 & 6.13 & 26.1611 & 25.5336 & 25.2880 & 26.0251 & 25.5711 & 25.4472 & 25.9376 & 25.3120 & 25.0561 & 25.7932 & 25.3478 & 25.2075 \\
\hline 20 & 1.81 & 30.5013 & 30.1040 & 29.9557 & 30.5117 & 30.3219 & 30.3144 & 30.3783 & 29.9758 & 29.8157 & 30.3843 & 30.1898 & 30.1755 \\
\hline 23 & 2.20 & 30.2982 & 29.8184 & 29.6245 & 30.1826 & 29.9009 & 29.7820 & 30.1617 & 29.6760 & 29.4765 & 30.0399 & 29.7542 & 29.6325 \\
\hline 23 & 2.83 & 29.6096 & 29.0651 & 28.8979 & & & & & 28.9224 & 28.7556 & 29.4508 & & 28.9512 \\
\hline 24 & 6.44 & 25.9823 & 25.3504 & 25.0650 & 25.8501 & 25.3772 & 25.3419 & 25.7045 & 25.0725 & 24.7649 & 25.5620 & 25.0950 & 25.0434 \\
\hline 27 & 1.87 & 30.4051 & 29.9230 & 29.8355 & 30.3507 & 30.0693 & 30.0783 & 30.4009 & 29.9165 & 29.8291 & 30.3416 & 30.0590 & 30.0686 \\
\hline 28 & 1.50 & 30.7444 & 30.2761 & 30.2671 & 30.7518 & 30.4805 & 30.4705 & 30.7163 & 30.2458 & 30.2372 & 30.7235 & 30.4507 & 30.4417 \\
\hline 29 & 1.38 & 30.8887 & 30.4418 & 30.4068 & 30.8598 & 30.6029 & 30.5249 & 30.8531 & 30.4037 & 30.3651 & 30.8221 & 30.5637 & 30.4843 \\
\hline 30 & 1.06 & 30.9991 & 30.5647 & 30.6538 & & & & & & & & & 30.7815 \\
\hline 21 & 1.09 & 31.1099 & 30.6621 & 30.5896 & 31.0975 & 30.8382 & 30.7949 & 31.1030 & 30.6540 & 30.5842 & 31.0930 & 30.8329 & 30.7900 \\
\hline
\end{tabular}

\section{mPW1PW91}

\section{Gas phase}

\section{PCM}

Exp 6-31G* 6-31G** 6-31+G** 6-311G* 6-311G** 6-311+G** 6-31G* 6-31G** 6-31+G** 6-311G* 6-311G** 6-311+G** ${ }^{13} \mathrm{C}$

\begin{tabular}{|c|c|c|c|c|c|c|c|c|c|c|c|c|c|}
\hline & 143.5 & 57.2958 & 57.8218 & 57.2422 & 41.6884 & 40.6464 & 39.6231 & 54.3260 & 54.8492 & 53.8053 & 38.3222 & 37.2724 & 35.9341 \\
\hline & 117.9 & 76.7315 & 77.2038 & 76.8297 & 62.5926 & 61.7045 & 61.5367 & 79.4134 & 79.8912 & 80.0157 & 65.5612 & 64.7060 & 64.7991 \\
\hline & 166.9 & 37.4386 & 37.4755 & 35.5843 & 20.0595 & 20.0833 & 18.1315 & 35.2721 & 35.3050 & 33.0364 & 17.6407 & 17.6571 & 15.2882 \\
\hline & 80.2 & 117.1194 & 117.5091 & 114.5922 & 106.6095 & 106.7794 & 106.5358 & 116.1609 & 116.5484 & 113.5003 & 105.5022 & 105.6773 & 105.3210 \\
\hline & 49.0 & 145.6181 & 146.1142 & 143.3002 & 135.4255 & 135.0724 & 135.6547 & 145.8759 & 146.3668 & 143.7011 & 135.6922 & 135.3316 & 136.0331 \\
\hline & 39.2 & 148.7146 & 149.9547 & 150.6878 & 140.5219 & 140.3533 & 139.2439 & 148.9271 & 150.1616 & 150.9303 & 140.7350 & 140.5613 & 139.4873 \\
\hline & 27.6 & 164.0548 & 165.3944 & 167.2136 & 156.0521 & 155.7647 & 154.3468 & 164.1399 & 165.4790 & 167.3017 & 156.1363 & 155.8443 & 154.4035 \\
\hline
\end{tabular}




\begin{tabular}{|c|c|c|c|c|c|c|c|c|c|c|c|c|}
\hline 8149.3 & 43.1839 & 43.3934 & 41.1137 & 24.2851 & 24.4978 & 23.5316 & 42.0824 & 42.2999 & 39.8417 & 23.1127 & 23.3271 & 22.2250 \\
\hline 9129.8 & 64.7572 & 64.9938 & 66.6393 & 48.6900 & 48.8126 & 47.3398 & 64.4706 & 64.7037 & 66.3207 & 48.3741 & 48.5053 & 47.0042 \\
\hline 10139.6 & 55.9713 & 55.6996 & 53.8671 & 38.2553 & 37.9662 & 37.5581 & 56.8544 & 56.5765 & 54.8413 & 39.2838 & 38.9957 & 38.6545 \\
\hline 1125.8 & 165.6409 & 166.8418 & 166.5854 & 158.2424 & 157.9453 & 157.4404 & 165.8528 & 167.0469 & 166.8359 & 158.4423 & 158.1382 & 157.6357 \\
\hline $12 \quad 22.1$ & 169.1609 & 170.4605 & 169.9048 & 162.2974 & 162.0974 & 161.6456 & 169.3401 & 170.6346 & 170.1003 & 162.4784 & 162.2690 & 161.8294 \\
\hline 1344.5 & 147.5202 & 147.7074 & 148.0809 & 137.9730 & 138.0277 & 137.5755 & 147.4532 & 147.6407 & 148.0041 & 137.8706 & 137.9265 & 137.4731 \\
\hline 1448.9 & 142.6216 & 142.8792 & 144.1814 & 133.0289 & 132.9795 & 132.5013 & 142.4265 & 142.6892 & 143.9720 & 132.8104 & 132.7676 & 132.2606 \\
\hline 1531.0 & 161.5345 & 162.8977 & 161.7340 & 154.4036 & 154.1037 & 154.2826 & 161.7467 & 163.1055 & 161.9653 & 154.6421 & 154.3321 & 154.5360 \\
\hline 1622.4 & 169.1549 & 170.5669 & 172.7170 & 162.0053 & 161.9146 & 159.5865 & 169.0827 & 170.4946 & 172.7264 & 161.9522 & 161.8558 & 159.5528 \\
\hline $17 \quad 42.9$ & 149.4525 & 150.1595 & 147.5844 & 140.2249 & 140.0113 & 140.4048 & 149.7637 & 150.4664 & 147.9589 & 140.5823 & 140.3593 & 140.7811 \\
\hline 1861.0 & 133.5850 & 134.8428 & 135.0862 & 125.5039 & 125.0182 & 124.3052 & 133.5639 & 134.8171 & 134.9790 & 125.4230 & 124.9334 & 124.1880 \\
\hline 19142.8 & 54.4775 & 54.9237 & 54.8800 & 39.2216 & 38.4638 & 36.7430 & 51.4984 & 51.9478 & 51.4732 & 35.8730 & 35.1213 & 33.1154 \\
\hline 2035.3 & 157.0848 & 157.7635 & 156.2657 & 148.3917 & 148.2435 & 148.0600 & 157.3207 & 157.9984 & 156.5550 & 148.6629 & 148.5120 & 148.3437 \\
\hline 2114.8 & 176.8551 & 178.5726 & 178.8923 & 171.2318 & 171.2122 & 170.9559 & 177.1456 & 178.8546 & 179.1968 & 171.5558 & 171.5144 & 171.2608 \\
\hline 22104.6 & 90.7567 & 90.8786 & 91.0875 & 79.8547 & 80.0442 & 79.4819 & 90.1498 & 90.2692 & 90.3917 & 79.1535 & 79.3431 & 78.7361 \\
\hline 2332.9 & 160.0387 & 161.1365 & 161.4102 & 152.2653 & 151.8358 & 151.1885 & 160.0024 & 161.0874 & 161.3032 & 152.1099 & 151.6619 & 150.9569 \\
\hline 24136.9 & 60.1132 & 61.0057 & 61.4767 & 44.4432 & 43.8726 & 42.7717 & 56.7004 & 57.5856 & 57.5683 & 40.5241 & 39.9466 & 38.5269 \\
\hline 25126.8 & 68.8857 & 68.8374 & 68.4864 & 52.8664 & 52.6637 & 51.9194 & 70.1217 & 70.0803 & 69.9002 & 54.2871 & 54.1039 & 53.4888 \\
\hline 26164.3 & 38.0615 & 38.1451 & 37.5747 & 20.6254 & 20.7266 & 18.1170 & 36.3317 & 36.4169 & 35.5031 & 18.6525 & 18.7552 & 15.8157 \\
\hline 2717.0 & 173.6967 & 175.3227 & 175.6946 & 167.3942 & 167.1674 & 167.3239 & 174.2706 & 175.8810 & 176.3212 & 168.0085 & 167.7685 & 167.9607 \\
\hline $28 \quad 26.2$ & 167.8180 & 169.3570 & 169.2197 & 161.5228 & 161.3539 & 161.1089 & 168.1604 & 169.6810 & 169.5820 & 161.8244 & 161.6366 & 161.4296 \\
\hline 2929.0 & 164.1112 & 165.6460 & 162.1385 & 157.2187 & 156.9277 & 157.3049 & 164.4677 & 165.9851 & 162.2657 & 157.5325 & 157.2216 & 157.5003 \\
\hline $\begin{array}{l}3026.8 \\
{ }^{1} \mathbf{H}\end{array}$ & 165.5866 & 167.1780 & 166.2850 & 159.2929 & 159.0581 & 158.9944 & 165.9065 & 167.4794 & 166.5963 & & 159.3546 & \\
\hline 16.63 & 25.7573 & 25.1665 & 24.9032 & 25.5912 & 25.1407 & 25.0969 & 25.4900 & 24.8985 & 24.6139 & 25.3166 & 24.8735 & 24.8094 \\
\hline 25.78 & 26.5149 & 25.8753 & 25.6911 & 26.4299 & 25.9614 & 25.8604 & 26.5855 & 25.9355 & 25.7555 & 26.4930 & 26.0199 & 25.9232 \\
\hline $\begin{array}{ll}5 & 2.45\end{array}$ & 29.5693 & 29.1741 & 29.3414 & 29.6186 & 29.4713 & 29.5042 & 29.4794 & 29.0800 & 29.2355 & 29.5289 & 29.3783 & 29.4018 \\
\hline $\begin{array}{ll}6 & 2.20\end{array}$ & 29.5604 & 29.0898 & 29.0209 & 29.5705 & 29.2949 & 29.2137 & 29.4732 & 29.0009 & 28.9223 & 29.4837 & 29.2085 & 29.1236 \\
\hline $\begin{array}{ll}6 & 2.40\end{array}$ & 29.4634 & 28.9955 & 28.9901 & 29.4809 & 29.2297 & 29.2439 & 29.5693 & 29.1034 & 29.0983 & 29.5824 & 29.3329 & 29.3467 \\
\hline $\begin{array}{ll}7 & 1.83\end{array}$ & 30.1213 & 29.6440 & 29.3915 & 30.0526 & 29.7759 & 29.7341 & 30.0550 & 29.5757 & 29.3174 & 29.9833 & 29.7051 & 29.6598 \\
\hline $\begin{array}{ll}7 \quad 2.50\end{array}$ & 30.0579 & 29.6242 & 29.3843 & 30.0647 & 29.8545 & 29.5695 & 30.0928 & 29.6585 & 29.4181 & 30.1032 & 29.8901 & 29.6000 \\
\hline 112.10 & 30.0191 & 29.5471 & 29.5339 & 29.9218 & 29.6590 & 29.6775 & 29.9574 & 29.4822 & 29.4723 & 29.8678 & 29.6024 & 29.6187 \\
\hline 112.30 & 29.8648 & 29.4039 & 29.2535 & 29.7790 & 29.5280 & 29.4691 & 29.7954 & 29.3332 & 29.1815 & 29.7070 & 29.4575 & 29.3964 \\
\hline 121.59 & 30.5416 & 30.0981 & 30.0365 & 30.5111 & 30.2496 & 30.2062 & 30.4598 & 30.0149 & 29.9520 & 30.4328 & 30.1717 & 30.1252 \\
\hline $12 \quad 2.07$ & 30.0504 & 29.5831 & 29.4696 & 30.0157 & 29.7353 & 29.6732 & 30.0494 & 29.5823 & 29.4720 & 30.0114 & 29.7320 & 29.6716 \\
\hline 151.50 & 30.6302 & 30.2132 & 30.3358 & 30.5802 & 30.3556 & 30.4815 & 30.5711 & 30.1528 & 30.2705 & 30.5206 & 30.2962 & 30.4177 \\
\hline 151.88 & 30.1602 & 29.6992 & 29.8118 & 30.1776 & 29.9296 & 29.9097 & 30.1739 & 29.7131 & 29.8218 & 30.1866 & 29.9392 & 29.9152 \\
\hline $\begin{array}{ll}16 & 1.87\end{array}$ & 30.3420 & 29.8802 & 29.7707 & 30.3206 & 30.0446 & 30.0478 & 30.2815 & 29.8168 & 29.7017 & 30.2614 & 29.9846 & 29.9844 \\
\hline $16 \quad 2.09$ & 29.3164 & 28.7861 & 28.5806 & 29.3241 & 29.0277 & 28.9395 & 29.4808 & 28.9544 & 28.7462 & 29.4826 & 29.1864 & 29.0949 \\
\hline $17 \quad 2.01$ & 30.0829 & 29.6762 & 29.6559 & 30.1066 & 29.8853 & 29.8760 & 29.9832 & 29.5758 & 29.5472 & 30.0069 & 29.7866 & 29.7708 \\
\hline $18 \quad 3.17$ & 28.8647 & 28.4146 & 28.2362 & 28.9459 & 28.7219 & 28.5667 & 28.8369 & 28.3860 & 28.2031 & 28.9147 & 28.6910 & 28.5296 \\
\hline 183.61 & 28.2605 & 27.7881 & 27.6976 & 28.3450 & 28.1050 & 27.9897 & 28.3005 & 27.8290 & 27.7238 & 28.3728 & 28.1331 & 28.0077 \\
\hline 196.13 & 26.0047 & 25.3784 & 25.1441 & 25.8780 & 25.4078 & 25.2968 & 25.7672 & 25.1427 & 24.9008 & 25.6358 & 25.1741 & 25.0482 \\
\hline 201.81 & 30.4439 & 30.0521 & 29.9012 & 30.3842 & 30.2069 & 30.2049 & 30.3160 & 29.9187 & 29.7589 & 30.2548 & 30.0724 & 30.0647 \\
\hline $23 \quad 2.20$ & 30.2344 & 29.7509 & 29.5598 & 30.0778 & 29.7930 & 29.6733 & 30.0856 & 29.5953 & 29.4004 & 29.9257 & 29.6366 & 29.5147 \\
\hline $\begin{array}{ll}23 & 2.83\end{array}$ & 29.5742 & 29.0212 & 28.8451 & 29.5071 & 29.1290 & 29.0082 & 29.4259 & 28.8701 & 28.6957 & 29.3650 & 28.9872 & 28.8646 \\
\hline
\end{tabular}




\begin{tabular}{llllllllllllll}
\hline 24 & 6.44 & 25.8203 & 25.1870 & 24.9435 & 25.7082 & 25.2123 & 25.1894 & 25.5260 & 24.8921 & 24.6309 & 25.4077 & 24.9172 & 24.8802 \\
27 & 1.87 & 30.3206 & 29.8352 & 29.7660 & 30.2542 & 29.9629 & 29.9872 & 30.3115 & 29.8237 & 29.7552 & 30.2407 & 29.9485 & 29.9737 \\
28 & 1.50 & 30.6934 & 30.2227 & 30.2137 & 30.6623 & 30.3871 & 30.3536 & 30.6625 & 30.1896 & 30.1818 & 30.6314 & 30.3547 & 30.3229 \\
29 & 1.38 & 30.8158 & 30.3685 & 30.3432 & 30.7591 & 30.4966 & 30.4288 & 30.7752 & 30.3252 & 30.2977 & 30.7172 & 30.4531 & 30.3847 \\
30 & 1.06 & 30.9551 & 30.5221 & 30.6014 & 30.9823 & 30.7297 & 30.7243 & 30.9147 & 30.4810 & 30.5606 & 30.9459 & 30.6934 & 30.6880 \\
21 & 1.09 & 31.0571 & 30.6068 & 30.5502 & 31.0109 & 30.7448 & 30.7128 & 31.0471 & 30.5956 & 30.5420 & 31.0029 & 30.7361 & 30.7048 \\
\hline
\end{tabular}

\section{Compound 48}

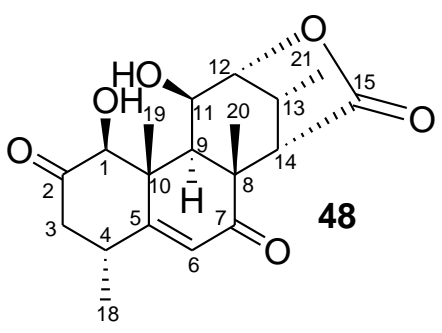

\begin{tabular}{|c|c|c|c|c|c|c|c|c|c|c|c|c|c|}
\hline \multirow{3}{*}{\multicolumn{2}{|c|}{ Exp }} & \multicolumn{12}{|c|}{ B3LYP } \\
\hline & & \multicolumn{6}{|c|}{ Gas phase } & \multicolumn{6}{|c|}{ PCM } \\
\hline & & 6-31G* & $6-31 G * *$ & $6-31+G * *$ & 6-311G* & $6-311 G * *$ & $6-311+G * *$ & 6-31G* & $6-31 G * *$ & $6-31+G * *$ & 6-311G* & $6-311 G * *$ & $6-311+G * *$ \\
\hline \multicolumn{14}{|c|}{${ }^{13} \mathrm{C}$} \\
\hline 1 & 81.4 & 109.7156 & 110.1000 & 109.0181 & 98.1805 & 97.6548 & 97.1824 & 109.9968 & 110.3805 & 109.2825 & 98.5000 & 97.9714 & 97.4732 \\
\hline 2 & 206.9 & -13.4625 & -13.2965 & -16.0206 & -34.6240 & -34.3512 & -36.2701 & -14.8373 & -14.6893 & -17.8905 & -36.2746 & -36.0784 & -38.4378 \\
\hline 3 & 45.0 & 145.5209 & 146.5530 & 145.1693 & 134.7831 & 134.2554 & 133.7073 & 145.6505 & 146.6722 & 145.2304 & 134.9144 & 134.3709 & 133.7105 \\
\hline 4 & 34.2 & 152.1038 & 152.8500 & 152.7274 & 141.8313 & 141.8121 & 141.0816 & 151.7687 & 152.5086 & 152.3474 & 141.4464 & 141.4173 & 140.5974 \\
\hline 5 & 165.5 & 30.8497 & 30.5604 & 30.2016 & 8.3473 & 8.0900 & 6.2463 & 26.5149 & 26.2340 & 25.4629 & 3.5757 & 3.3723 & 1.3067 \\
\hline 6 & 122.7 & 70.1983 & 70.9546 & 70.7751 & 53.4936 & 53.0670 & 52.4713 & 70.9519 & 71.7124 & 71.3720 & 54.2664 & 53.8362 & 53.0474 \\
\hline 7 & 198.6 & 0.7619 & 0.8260 & -0.5966 & -20.7520 & -20.6144 & -23.1652 & -1.2232 & -1.1761 & -3.0871 & -23.1685 & -23.0545 & -25.9942 \\
\hline 8 & 48.1 & 139.3700 & 139.6975 & 137.5652 & 129.2091 & 129.2128 & 128.6502 & 139.1291 & 139.4509 & 137.2368 & 128.8767 & 128.8706 & 128.1918 \\
\hline 9 & 47.6 & 140.5235 & 141.1792 & 140.5321 & 130.1534 & 130.0096 & 129.6347 & 140.8122 & 141.4621 & 140.8503 & 130.4713 & 130.3182 & 129.9687 \\
\hline 10 & 49.4 & 136.4235 & 136.7527 & 135.3893 & 124.8861 & 125.1205 & 124.8077 & 136.2275 & 136.5508 & 135.1689 & 124.6861 & 124.9026 & 124.5503 \\
\hline 11 & 69.3 & 119.2947 & 120.0523 & 118.1309 & 107.3363 & 107.1891 & 107.0684 & 119.7107 & 120.4663 & 118.5828 & 107.7406 & 107.5941 & 107.4979 \\
\hline 12 & 83.4 & 107.9815 & 108.6909 & 108.2162 & 96.2074 & 95.7715 & 95.1165 & 107.5887 & 108.2907 & 107.7083 & 95.7239 & 95.2801 & 94.4964 \\
\hline 13 & 31.7 & 154.5015 & 155.4292 & 155.4182 & 145.6773 & 145.6824 & 145.0163 & 154.1271 & 155.0580 & 154.9778 & 145.2541 & 145.2559 & 144.5055 \\
\hline 14 & 52.2 & 137.1047 & 137.6981 & 136.7261 & 126.4280 & 126.0805 & 126.0263 & 136.7004 & 137.2836 & 136.2809 & 125.9752 & 125.6147 & 125.4677 \\
\hline 15 & 176.4 & 23.7296 & 23.8266 & 22.1840 & 2.6683 & 2.8312 & 0.2023 & 20.7772 & 20.8658 & 18.7377 & -0.7296 & -0.5851 & -3.6565 \\
\hline 18 & 18.2 & 170.4512 & 172.2585 & 172.5203 & 163.6388 & 163.4999 & 162.9560 & 170.8196 & 172.6153 & 172.9187 & 164.0706 & 163.9086 & 163.3681 \\
\hline 19 & 18.2 & 167.9864 & 169.7769 & 170.7585 & 161.8515 & 161.6844 & 161.6321 & 168.0925 & 169.8652 & 170.8404 & 161.9363 & 161.7464 & 161.6973 \\
\hline 20 & 22.7 & 165.7397 & 167.5127 & 167.5698 & 159.1768 & 158.8709 & 158.7975 & 165.8943 & 167.6490 & 167.6437 & 159.2830 & 158.9578 & 158.8567 \\
\hline $\begin{array}{l}21 \\
{ }^{1} \mathbf{H}\end{array}$ & 16.5 & 172.1955 & 173.9787 & 174.3352 & 165.4721 & 165.4468 & 165.2810 & 172.4134 & 174.1848 & 174.6017 & 165.7246 & 165.6798 & 165.5224 \\
\hline 1 & 4.08 & 28.1562 & 27.7328 & 27.5517 & 28.1369 & 27.9417 & 27.7970 & 28.0282 & 27.6014 & 27.3967 & 27.9958 & 27.7986 & 27.6393 \\
\hline 3 & 2.72 & 29.7736 & 29.2704 & 29.1712 & 29.6633 & 29.3807 & 29.2631 & 29.6983 & 29.1904 & 29.0899 & 29.5889 & 29.3018 & 29.1857 \\
\hline 3 & 2.37 & 29.8347 & 29.3844 & 29.2718 & 29.8414 & 29.6013 & 29.4512 & 29.6417 & 29.1850 & 29.0484 & 29.6469 & 29.4047 & 29.2373 \\
\hline
\end{tabular}




\begin{tabular}{rrrlllllllllll}
\hline 4 & 2.82 & 29.3502 & 28.8716 & 28.8728 & 29.5007 & 29.2124 & 29.1918 & 29.2203 & 28.7400 & 28.7273 & 29.3667 & 29.0784 & 29.0477 \\
6 & 5.90 & 26.5935 & 25.9011 & 25.7919 & 26.4490 & 25.9903 & 25.9875 & 26.5640 & 25.8597 & 25.7360 & 26.4074 & 25.9384 & 25.9321 \\
9 & 2.23 & 29.9687 & 29.6306 & 29.7177 & 30.0533 & 29.9432 & 29.9114 & 29.9818 & 29.6454 & 29.7171 & 30.0551 & 29.9460 & 29.8998 \\
11 & 4.79 & 27.6027 & 27.1452 & 27.0876 & 27.5881 & 27.3600 & 27.2988 & 27.5630 & 27.1006 & 27.0338 & 27.5342 & 27.3029 & 27.2367 \\
12 & 4.36 & 27.9874 & 27.5105 & 27.3861 & 27.9664 & 27.7188 & 27.6389 & 27.8773 & 27.3936 & 27.2556 & 27.8480 & 27.5920 & 27.5012 \\
13 & 2.92 & 29.4226 & 28.9486 & 28.8308 & 29.4776 & 29.2363 & 29.1568 & 29.3404 & 28.8613 & 28.7266 & 29.3945 & 29.1446 & 29.0500 \\
14 & 2.98 & 29.4166 & 28.9250 & 28.8281 & 29.4253 & 29.1348 & 29.0627 & 29.4341 & 28.9422 & 28.8152 & 29.4271 & 29.1399 & 29.0438 \\
18 & 1.26 & 30.9816 & 30.5285 & 30.4625 & 30.9503 & 30.6957 & 30.7172 & 30.9481 & 30.4927 & 30.4244 & 30.9171 & 30.6609 & 30.6800 \\
19 & 1.38 & 30.8372 & 30.3454 & 30.2851 & 30.9505 & 30.6399 & 30.6521 & 30.8017 & 30.3110 & 30.2465 & 30.9141 & 30.6062 & 30.6146 \\
20 & 1.44 & 30.8158 & 30.3653 & 30.3376 & 30.8356 & 30.6051 & 30.6433 & 30.7582 & 30.3043 & 30.2613 & 30.7758 & 30.5413 & 30.5662 \\
21 & 1.18 & 31.0583 & 30.6304 & 30.5365 & 31.0001 & 30.7839 & 30.7380 & 31.0301 & 30.6002 & 30.5021 & 30.9723 & 30.7542 & 30.7031 \\
\hline
\end{tabular}

\section{mPW1PW91}

\section{Gas phase}

$\operatorname{Exp} 6-31 G * 6-31 G * * 6-31+G * * 6-311 G * 6-311 G * * 6-311+G * * \quad 6-31 G *$ ${ }^{13} \mathrm{C}$ 


\begin{tabular}{llllllllllllll}
\hline 14 & 2.98 & 29.2974 & 28.8092 & 28.7257 & 29.2836 & 28.9816 & 28.9120 & 29.3107 & 28.8219 & 28.7118 & 29.2838 & 28.9850 & 28.8925 \\
18 & 1.26 & 30.9055 & 30.4546 & 30.3888 & 30.8568 & 30.5981 & 30.6148 & 30.8667 & 30.4133 & 30.3462 & 30.8184 & 30.5580 & 30.5730 \\
19 & 1.38 & 30.7771 & 30.2834 & 30.2131 & 30.8553 & 30.5450 & 30.5480 & 30.7391 & 30.2464 & 30.1739 & 30.8169 & 30.5091 & 30.5092 \\
20 & 1.44 & 30.7505 & 30.3006 & 30.2856 & 30.7414 & 30.5115 & 30.5487 & 30.6896 & 30.2362 & 30.2093 & 30.6793 & 30.4455 & 30.4714 \\
21 & 1.18 & 30.9942 & 30.5637 & 30.4758 & 30.9169 & 30.6927 & 30.6483 & 30.9619 & 30.5292 & 30.4380 & 30.8854 & 30.6589 & 30.6101 \\
\hline
\end{tabular}

\section{Compound 49}<smiles>COc1cc(O)c2c(c1)C1=CC(=O)[C@@H](O)C[C@]1(C)OC2=O</smiles>

49

\begin{tabular}{|c|c|c|c|c|c|c|c|c|c|c|c|c|c|}
\hline & \multirow[b]{3}{*}{ Exp } & \multicolumn{12}{|c|}{ B3LYP } \\
\hline & & \multicolumn{6}{|c|}{ Gas phase } & \multicolumn{6}{|c|}{ PCM } \\
\hline & & 6-31G* & $6-31 G * *$ & $6-31+G * *$ & 6-311G* & $6-311 G * *$ & $6-311+G * *$ & 6-31G* & $6-31 G * *$ & $6-31+G * *$ & 6-311G* & $6-311 G * *$ & $6-311+G * *$ \\
\hline \multicolumn{14}{|l|}{${ }^{13} \mathrm{C}$} \\
\hline 1 & 120.8 & 74.0999 & 74.8234 & 75.7765 & 58.0351 & 57.3813 & 56.3262 & 73.8493 & 74.5864 & 75.4448 & 57.7015 & 57.0678 & 55.8775 \\
\hline 2 & 197.4 & -1.2977 & -1.2491 & -2.8211 & -21.2100 & -21.1886 & -24.2060 & -2.1073 & -2.0873 & -3.9047 & -22.2510 & -22.3022 & -25.5486 \\
\hline 3 & 70.6 & 119.9389 & 120.6010 & 119.2744 & 108.5937 & 108.0760 & 107.0727 & 120.2429 & 120.8955 & 119.4836 & 108.8426 & 108.3052 & 107.2629 \\
\hline 4 & 44.1 & 144.2738 & 145.3940 & 144.0919 & 134.5429 & 134.0839 & 133.8276 & 144.6165 & 145.7290 & 144.4288 & 134.9462 & 134.4881 & 134.1727 \\
\hline $4 a$ & 80.7 & 109.3706 & 109.6855 & 109.6162 & 97.2138 & 97.3784 & 96.3925 & 108.5892 & 108.9008 & 108.6950 & 96.3546 & 96.5100 & 95.4220 \\
\hline 4a-Me & 25.8 & 163.4534 & 165.1625 & 167.1484 & 156.5008 & 156.2762 & 156.1965 & 163.7301 & 165.4108 & 167.3835 & 156.6737 & 156.4269 & 156.3366 \\
\hline 6 & 166.7 & 30.7170 & 30.7473 & 29.8407 & 11.5634 & 11.6102 & 9.7888 & 29.9700 & 30.0010 & 28.9709 & 10.7237 & 10.7530 & 8.7603 \\
\hline $6 a$ & 100.1 & 93.0042 & 92.8922 & 92.7214 & 79.7274 & 79.3956 & 77.5996 & 93.0745 & 92.9656 & 92.7850 & 79.8142 & 79.5082 & 77.6397 \\
\hline 7 & 164.7 & 31.2714 & 31.0110 & 30.0724 & 10.0200 & 9.9552 & 9.1240 & 31.8741 & 31.6056 & 30.7373 & 10.6494 & 10.5604 & 9.8531 \\
\hline 8 & 104.0 & 91.6811 & 92.3538 & 92.3224 & 77.5922 & 77.1946 & 75.8937 & 91.0041 & 91.6746 & 91.2760 & 76.6471 & 76.2249 & 74.6295 \\
\hline 9 & 166.2 & 33.2160 & 32.9192 & 30.9391 & 11.1433 & 11.0530 & 10.0271 & 32.4281 & 32.1359 & 30.1437 & 10.3605 & 10.2754 & 9.1804 \\
\hline 10 & 105.0 & 90.4735 & 91.2782 & 90.3820 & 75.3865 & 74.9683 & 74.0560 & 89.6265 & 90.4241 & 89.3290 & 74.4698 & 74.0152 & 72.8866 \\
\hline $10 a$ & 134.3 & 59.9509 & 59.7739 & 59.0136 & 41.9027 & 41.6093 & 41.1348 & 59.7462 & 59.5743 & 58.9064 & 41.7788 & 41.5077 & 41.0292 \\
\hline $10 b$ & 153.3 & 38.3875 & 38.1196 & 36.0788 & 18.5632 & 18.4961 & 17.3294 & 37.4740 & 37.1894 & 34.9479 & 17.6365 & 17.5188 & 16.1512 \\
\hline $\begin{array}{c}\text { 9-OMe } \\
{ }^{1} \mathbf{H}\end{array}$ & 56.0 & 136.6826 & 138.4710 & 138.6389 & 127.8913 & 127.1951 & 126.7906 & 136.4107 & 138.1747 & 138.1310 & 127.4290 & 126.6936 & 126.2860 \\
\hline 1 & 6.53 & 26.0946 & 25.3583 & 25.1909 & 25.9212 & 25.3822 & 25.3779 & 25.9892 & 25.2501 & 25.0731 & 25.8123 & 25.2734 & 25.2629 \\
\hline 3 & 4.29 & 27.9439 & 27.4948 & 27.3699 & 27.8849 & 27.6858 & 27.6493 & 27.8176 & 27.3593 & 27.2040 & 27.7497 & 27.5396 & 27.4784 \\
\hline $4^{*}$ & 2.82 & 29.6387 & 29.2076 & 29.0151 & 29.4721 & 29.2350 & 29.1550 & 29.6140 & 29.1789 & 28.9747 & 29.4414 & 29.1973 & 29.1127 \\
\hline $4 * *$ & 2.47 & 29.8619 & 29.4359 & 29.2801 & 29.7799 & 29.6123 & 29.5165 & 29.8682 & 29.4384 & 29.2802 & 29.7852 & 29.6145 & 29.5113 \\
\hline 4a-Me & 1.74 & 30.6377 & 30.1587 & 30.0229 & 30.6418 & 30.3615 & 30.2919 & 30.5579 & 30.0750 & 29.9346 & 30.5601 & 30.2774 & 30.2082 \\
\hline 8 & 6.64 & 26.1631 & 25.3929 & 25.1026 & 25.9536 & 25.4813 & 25.3259 & 26.0329 & 25.2609 & 24.9416 & 25.8004 & 25.3268 & 25.1616 \\
\hline 10 & 6.70 & 25.9628 & 25.2272 & 24.9773 & 25.7683 & 25.2638 & 25.2383 & 25.8063 & 25.0699 & 24.8107 & 25.6128 & 25.1120 & 25.0720 \\
\hline 9-OMe & 3.89 & 28.4520 & 27.9469 & 27.7664 & 28.4164 & 28.1203 & 28.0556 & 28.3432 & 27.8380 & 27.6523 & 28.3048 & 28.0101 & 27.9438 \\
\hline
\end{tabular}




\begin{tabular}{|c|c|c|c|c|c|c|c|c|c|c|c|c|c|}
\hline & & \multicolumn{12}{|c|}{ mPW1PW91 } \\
\hline & \multirow[b]{2}{*}{ Exp } & \multicolumn{6}{|c|}{ Gas phase } & \multicolumn{6}{|c|}{ PCM } \\
\hline & & 6-31G* & $6-31 G * *$ & $6-31+G * *$ & 6-311G* & $6-311 G * *$ & $6-311+G * *$ & 6-31G* & $6-31 G * *$ & $6-31+G * *$ & 6-311G* & $6-311 G * *$ & $6-311+G^{* *}$ \\
\hline \multicolumn{14}{|l|}{${ }^{13} \mathrm{C}$} \\
\hline 1 & 120.8 & 76.4482 & 77.1454 & 78.2360 & 62.3449 & 61.6750 & 60.6284 & 76.1801 & 76.8907 & 77.9119 & 61.9968 & 61.3480 & 60.1966 \\
\hline 2 & 197.4 & 0.6264 & 0.6539 & -0.2101 & -16.1473 & -16.1319 & -18.9562 & -0.2672 & -0.2692 & -1.3600 & -17.2321 & -17.2925 & -20.3255 \\
\hline 3 & 70.6 & 123.8178 & 124.4355 & 123.6410 & 114.3611 & 113.8464 & 112.8104 & 124.0646 & 124.6743 & 123.7938 & 114.5390 & 114.0056 & 112.9339 \\
\hline 4 & 44.1 & 149.5014 & 150.5186 & 149.2985 & 140.5520 & 140.1445 & 139.8807 & 149.8491 & 150.8582 & 149.6299 & 140.9367 & 140.5290 & 140.2086 \\
\hline $4 a$ & 80.7 & 113.7880 & 114.0902 & 114.2453 & 103.1902 & 103.3522 & 102.4539 & 112.9812 & 113.2797 & 113.3205 & 102.3142 & 102.4673 & 101.4848 \\
\hline 4a-Me & 25.8 & 167.6499 & 169.2268 & 171.0999 & 161.1341 & 160.9448 & 160.8281 & 167.9362 & 169.4865 & 171.3523 & 161.2988 & 161.0916 & 160.9720 \\
\hline 6 & 166.7 & 33.2689 & 33.2953 & 32.8294 & 16.5602 & 16.6072 & 14.9808 & 32.5208 & 32.5478 & 31.9744 & 15.7420 & 15.7706 & 13.9964 \\
\hline $6 a$ & 100.1 & 96.2939 & 96.1808 & 96.0620 & 84.8545 & 84.5160 & 82.6536 & 96.4247 & 96.3148 & 96.1872 & 85.0044 & 84.6930 & 82.7606 \\
\hline 7 & 164.7 & 34.0131 & 33.7152 & 33.3639 & 15.7770 & 15.6948 & 14.9231 & 34.6324 & 34.3273 & 34.0357 & 16.4190 & 16.3125 & 15.6505 \\
\hline 8 & 104.0 & 94.3298 & 94.9704 & 94.8842 & 81.9536 & 81.5630 & 80.2260 & 93.6597 & 94.2959 & 93.8972 & 81.0271 & 80.6090 & 79.0462 \\
\hline 9 & 166.2 & 35.8535 & 35.5215 & 34.2287 & 16.8543 & 16.7371 & 15.7713 & 35.0352 & 34.7088 & 33.4118 & 16.0521 & 15.9406 & 14.9161 \\
\hline 10 & 105.0 & 93.0354 & 93.8222 & 93.0369 & 79.8301 & 79.4132 & 78.5672 & 92.2035 & 92.9819 & 92.0010 & 78.9306 & 78.4749 & 77.4492 \\
\hline $10 a$ & 134.3 & 63.0019 & 62.8056 & 62.3199 & 47.4322 & 47.1218 & 46.5586 & 62.8248 & 62.6338 & 62.2290 & 47.3345 & 47.0487 & 46.4833 \\
\hline $10 \mathrm{~b}$ & 153.3 & 41.7243 & 41.4323 & 39.8125 & 24.4605 & 24.3849 & 23.1926 & 40.8864 & 40.5773 & 38.7708 & 23.6262 & 23.4981 & 22.1166 \\
\hline $\begin{array}{c}\text { 9-OMe } \\
{ }^{{ }^{H}} \mathbf{H}\end{array}$ & 56.0 & 140.1772 & 141.9018 & 141.9918 & 132.6579 & 132.0119 & 131.7126 & 139.8852 & 141.5864 & 141.4775 & 132.1813 & 131.4979 & 131.1998 \\
\hline 1 & 6.53 & 25.8666 & 25.1408 & 25.0078 & 25.7456 & 25.1801 & 25.1963 & 25.7526 & 25.0236 & 24.8848 & 25.6305 & 25.0649 & 25.0762 \\
\hline 3 & 4.29 & 27.9072 & 27.4510 & 27.3181 & 27.8160 & 27.6151 & 27.5643 & 27.7693 & 27.3034 & 27.1448 & 27.6717 & 27.4595 & 27.3876 \\
\hline $4^{*}$ & 2.82 & 29.5234 & 29.0903 & 28.9305 & 29.3653 & 29.1191 & 29.0404 & 29.4886 & 29.0510 & 28.8806 & 29.3264 & 29.0724 & 28.9894 \\
\hline $4 * *$ & 2.47 & 29.7161 & 29.2891 & 29.1712 & 29.6230 & 29.4510 & 29.3688 & 29.7239 & 29.2930 & 29.1721 & 29.6294 & 29.4541 & 29.3641 \\
\hline 4a-Me & 1.74 & 30.5658 & 30.0837 & 29.9526 & 30.5479 & 30.2616 & 30.1889 & 30.4806 & 29.9941 & 29.8611 & 30.4624 & 30.1734 & 30.1020 \\
\hline 8 & 6.64 & 25.9476 & 25.1803 & 24.9138 & 25.7800 & 25.2785 & 25.1441 & 25.8018 & 25.0323 & 24.7440 & 25.6167 & 25.1135 & 24.9722 \\
\hline 10 & 6.70 & 25.7443 & 25.0173 & 24.8124 & 25.5920 & 25.0674 & 25.0682 & 25.5780 & 24.8494 & 24.6371 & 25.4283 & 24.9069 & 24.8942 \\
\hline 9-OMe & 3.89 & 28.3740 & 27.8722 & 27.7211 & 28.3460 & 28.0397 & 27.9974 & 28.2557 & 27.7534 & 27.5991 & 28.2273 & 27.9222 & 27.8791 \\
\hline
\end{tabular}

\section{Compound 50}

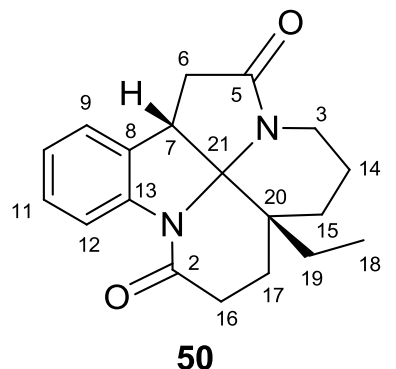

\begin{tabular}{|c|c|c|c|c|c|c|c|c|c|c|c|c|c|}
\hline \multirow{3}{*}{\multicolumn{2}{|c|}{ Exp }} & \multicolumn{12}{|c|}{ B3LYP } \\
\hline & & \multicolumn{6}{|c|}{ Gas phase } & \multicolumn{6}{|c|}{ PCM } \\
\hline & & 6-31G* & $6-31 G * *$ & $6-31+G * *$ & 6-311G* & $6-311 G * *$ & $6-311+G * *$ & 6-31G* & 6-31G** & $6-31+G * *$ & 6-311G* & $6-311 G * *$ & $6-311+G * *$ \\
\hline \multicolumn{14}{|c|}{${ }^{13} \mathrm{C}$} \\
\hline 2 & 172.9 & 27.7018 & 27.8853 & 25.6445 & 7.6511 & 7.8141 & 5.3653 & 25.3009 & 25.4796 & 22.7898 & 4.8327 & 4.9907 & 2.1505 \\
\hline 3 & 36.8 & 152.3292 & 153.6808 & 152.9526 & 144.7757 & 144.3773 & 144.0746 & 152.0134 & 153.3696 & 152.6395 & 144.4521 & 144.0499 & 143.7034 \\
\hline
\end{tabular}




\begin{tabular}{|c|c|c|c|c|c|c|c|c|c|c|c|c|}
\hline $\begin{array}{ll}5 & 170.8\end{array}$ & 30.6106 & 30.7172 & 28.4874 & 10.0628 & 10.1556 & 7.6150 & 28.6697 & 28.7703 & 26.0980 & 7.7660 & 7.8541 & 4.9275 \\
\hline $\begin{array}{ll}6 & 37.6\end{array}$ & 152.3448 & 153.6690 & 152.5143 & 143.1928 & 142.5788 & 141.6420 & 152.1688 & 153.4785 & 152.1314 & 142.9212 & 142.2940 & 141.1470 \\
\hline $\begin{array}{ll}7 & 41.9\end{array}$ & 145.4990 & 146.2122 & 146.9813 & 135.7089 & 135.3677 & 134.4874 & 145.4421 & 146.1486 & 146.9157 & 135.6439 & 135.2890 & 134.3944 \\
\hline 8135.4 & 61.1206 & 60.9774 & 60.2895 & 42.1905 & 41.9097 & 40.5455 & 60.1114 & 59.9707 & 59.2021 & 41.0229 & 40.7597 & 39.3386 \\
\hline 9123.8 & 73.1318 & 73.8522 & 73.2987 & 56.4516 & 55.7126 & 55.3517 & 72.3163 & 73.0237 & 72.3611 & 55.3790 & 54.6417 & 54.2391 \\
\hline 10125.5 & 71.5533 & 72.1640 & 72.0441 & 54.5154 & 53.6210 & 52.8527 & 71.4258 & 72.0374 & 71.8346 & 54.3122 & 53.4493 & 52.6885 \\
\hline 11128.0 & 67.9695 & 68.5941 & 69.4923 & 50.9803 & 50.1285 & 49.2904 & 68.3643 & 68.9896 & 69.8557 & 51.3058 & 50.4838 & 49.6623 \\
\hline 12120.1 & 75.2455 & 75.8960 & 74.6255 & 58.5801 & 57.7408 & 57.2653 & 76.2883 & 76.9418 & 75.7029 & 59.7105 & 58.8964 & 58.4459 \\
\hline 13142.1 & 52.4155 & 52.3830 & 52.0381 & 32.3188 & 32.2318 & 30.5771 & 52.5512 & 52.5162 & 52.1911 & 32.3979 & 32.3125 & 30.6746 \\
\hline 1420.1 & 167.1578 & 168.6028 & 169.0631 & 159.3632 & 159.1804 & 158.8418 & 167.1991 & 168.6423 & 169.1500 & 159.4391 & 159.2475 & 158.9109 \\
\hline 1526.2 & 161.9246 & 163.2426 & 165.1957 & 153.4157 & 153.1691 & 152.3830 & 162.1719 & 163.4814 & 165.4691 & 153.6998 & 153.4405 & 152.6597 \\
\hline 1629.4 & 159.3437 & 160.7151 & 159.7941 & 151.0334 & 150.5153 & 149.7329 & 158.9793 & 160.3412 & 159.2864 & 150.5763 & 150.0537 & 149.1075 \\
\hline 1726.6 & 160.7092 & 162.0037 & 162.4939 & 153.0664 & 152.8749 & 152.4145 & 161.1082 & 162.3938 & 162.9264 & 153.5488 & 153.3427 & 152.8687 \\
\hline 7.3 & 180.3494 & 182.2649 & 182.4327 & 174.3030 & 174.3447 & 174.0735 & 180.5515 & 182.4632 & 182.6632 & 174.5521 & 174.5814 & 174.3093 \\
\hline 1926.9 & 161.5857 & 162.8432 & 161.8023 & 153.1731 & 153.0628 & 152.9095 & 161.7808 & 163.0358 & 162.0003 & 153.4182 & 153.3021 & 153.1692 \\
\hline 2038.1 & 149.7603 & 150.0142 & 149.2141 & 139.1075 & 139.1570 & 138.5325 & 149.4512 & 149.7028 & 148.8250 & 138.7233 & 138.7768 & 138.0871 \\
\hline $\begin{array}{l}2192.5 \\
{ }^{1} \mathbf{H}\end{array}$ & 96.8551 & 96.9668 & 97.8466 & 85.1159 & 85.1432 & 83.6799 & 96.3200 & 96.4285 & 97.1869 & 84.5123 & 84.5377 & 82.9548 \\
\hline $\begin{array}{ll}3 & 2.80\end{array}$ & 29.4870 & 29.0398 & 28.9074 & 29.4847 & 29.2663 & 29.1163 & 29.4369 & 28.9888 & 28.8364 & 29.4280 & 29.2099 & 29.0425 \\
\hline 33.95 & 28.2769 & 27.6903 & 27.5305 & 28.2803 & 27.8760 & 27.7170 & 28.4057 & 27.8230 & 27.6625 & 28.4066 & 28.0037 & 27.8387 \\
\hline $\begin{array}{ll}6 & 2.68\end{array}$ & 29.8052 & 29.3394 & 29.2046 & 29.7279 & 29.4675 & 29.4083 & 29.7737 & 29.3051 & 29.1552 & 29.6915 & 29.4324 & 29.3607 \\
\hline $\begin{array}{ll}6 & 2.87\end{array}$ & 29.7598 & 29.2815 & 29.1226 & 29.6615 & 29.3929 & 29.3101 & 29.6001 & 29.1159 & 28.9304 & 29.4950 & 29.2247 & 29.1248 \\
\hline $\begin{array}{ll}7 & 3.82\end{array}$ & 28.6544 & 28.1110 & 27.8566 & 28.6358 & 28.2671 & 28.1705 & 28.4631 & 27.9136 & 27.6452 & 28.4453 & 28.0747 & 27.9653 \\
\hline $\begin{array}{ll}9 & 7.17\end{array}$ & 25.2410 & 24.5838 & 24.4374 & 25.1730 & 24.7336 & 24.7576 & 25.0726 & 24.4142 & 24.2509 & 24.9893 & 24.5558 & 24.5697 \\
\hline 107.14 & 25.1875 & 24.5690 & 24.4051 & 25.0599 & 24.6767 & 24.6637 & 25.0849 & 24.4664 & 24.3010 & 24.9461 & 24.5701 & 24.5563 \\
\hline 117.25 & 25.0864 & 24.4745 & 24.2043 & 24.9365 & 24.5594 & 24.4438 & 25.0239 & 24.4119 & 24.1315 & 24.8635 & 24.4941 & 24.3706 \\
\hline 127.77 & 24.2958 & 23.5814 & 23.3333 & 24.1812 & 23.7234 & 23.5982 & 24.3978 & 23.6843 & 23.4332 & 24.2672 & 23.8150 & 23.6889 \\
\hline 141.60 & 30.8921 & 30.4435 & 30.4136 & 30.8774 & 30.6382 & 30.5796 & 30.7970 & 30.3438 & 30.3043 & 30.7862 & 30.5427 & 30.4762 \\
\hline 141.60 & 30.4571 & 30.0425 & 29.9748 & 30.5495 & 30.3371 & 30.2691 & 30.4285 & 30.0121 & 29.9418 & 30.5212 & 30.3079 & 30.2355 \\
\hline $\begin{array}{ll}15 & 1.60\end{array}$ & 30.1541 & 29.7170 & 29.5054 & 30.1960 & 29.9751 & 29.8685 & 30.1275 & 29.6919 & 29.4849 & 30.1703 & 29.9535 & 29.8489 \\
\hline $\begin{array}{ll}15 & 1.60\end{array}$ & 30.5501 & 30.1107 & 30.1312 & 30.6356 & 30.3925 & 30.3615 & 30.4336 & 29.9913 & 30.0096 & 30.5228 & 30.2774 & 30.2422 \\
\hline $16 \quad 2.49$ & 29.9880 & 29.5125 & 29.3940 & 29.8996 & 29.6151 & 29.5506 & 29.9677 & 29.4890 & 29.3736 & 29.8796 & 29.5938 & 29.5310 \\
\hline $\begin{array}{ll}16 & 2.78\end{array}$ & 29.6148 & 29.1249 & 29.0299 & 29.5733 & 29.2975 & 29.2513 & 29.5035 & 29.0094 & 28.9052 & 29.4612 & 29.1854 & 29.1310 \\
\hline 171.86 & 30.6426 & 30.2323 & 30.0950 & 30.6717 & 30.4512 & 30.3849 & 30.5321 & 30.1197 & 29.9725 & 30.5636 & 30.3442 & 30.2698 \\
\hline 171.97 & 30.5584 & 30.0958 & 30.0200 & 30.5710 & 30.2967 & 30.2232 & 30.4414 & 29.9751 & 29.8949 & 30.4576 & 30.1793 & 30.1000 \\
\hline $\begin{array}{ll}18 & 0.93\end{array}$ & 31.2928 & 30.8376 & 30.7803 & 31.2934 & 31.0234 & 30.9810 & 31.2599 & 30.8034 & 30.7485 & 31.2658 & 30.9951 & 30.9534 \\
\hline 191.37 & 30.9943 & 30.5758 & 30.4616 & 30.9947 & 30.7672 & 30.7175 & 30.8931 & 30.4722 & 30.3486 & 30.9009 & 30.6722 & 30.6185 \\
\hline 191.78 & 30.2415 & 29.7726 & 29.7697 & 30.4177 & 30.1601 & 30.1341 & 30.2782 & 29.8114 & 29.8134 & 30.4442 & 30.1881 & 30.1674 \\
\hline
\end{tabular}

\section{mPW1PW91}

\section{Gas phase}

PCM

Exp 6-31G* 6-31G** 6-31+G** 6-311G* 6-311G** 6-311+G** 6-31G* 6-31G** 6-31+G** 6-311G* 6-311G** 6-311+G** ${ }^{13} \mathrm{C}$

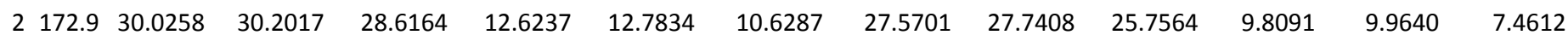

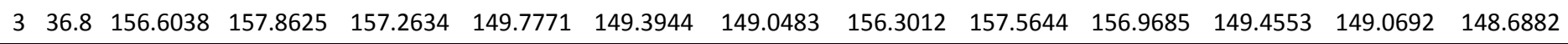




\begin{tabular}{|c|c|c|c|c|c|c|c|c|c|c|c|c|c|}
\hline 5 & 170.8 & 32.9883 & 33.0931 & 31.4479 & 14.9908 & 15.0894 & 12.8265 & 31.0040 & 31.1025 & 29.0757 & 12.7005 & 12.7938 & 10.1896 \\
\hline 6 & 37.6 & 156.5791 & 157.8138 & 156.6936 & 148.4316 & 147.8392 & 146.9288 & 156.3876 & 157.6092 & 156.3129 & 148.1391 & 147.5345 & 146.4419 \\
\hline 7 & 41.9 & 149.9343 & 150.5962 & 151.2101 & 141.1173 & 140.7723 & 140.0047 & 149.8581 & 150.5146 & 151.1217 & 141.0272 & 140.6698 & 139.8856 \\
\hline 8 & 135.4 & 64.4731 & 64.3146 & 63.9969 & 47.8360 & 47.5468 & 46.1545 & 63.4545 & 63.3002 & 62.9108 & 46.6840 & 46.4147 & 44.9778 \\
\hline 9 & 123.8 & 75.4855 & 76.1514 & 75.5579 & 60.8199 & 60.0558 & 59.6682 & 74.6202 & 75.2722 & 74.5879 & 59.7076 & 58.9461 & 58.5383 \\
\hline 10 & 125.5 & 74.0907 & 74.6159 & 74.7580 & 59.1366 & 58.1927 & 57.4598 & 73.9181 & 74.4440 & 74.4988 & 58.8731 & 57.9629 & 57.2409 \\
\hline 11 & 128.0 & 70.3991 & 70.9399 & 71.8250 & 55.4649 & 54.5664 & 53.8340 & 70.7595 & 71.3006 & 72.1478 & 55.7312 & 54.8643 & 54.1458 \\
\hline 12 & 120.1 & 77.7885 & 78.3830 & 77.2588 & 63.1902 & 62.3247 & 61.9596 & 78.8675 & 79.4647 & 78.3571 & 64.3142 & 63.4761 & 63.1373 \\
\hline 13 & 142.1 & 55.5072 & 55.4626 & 55.5923 & 37.8802 & 37.7918 & 36.1931 & 55.6903 & 55.6436 & 55.7822 & 38.0050 & 37.9183 & 36.3304 \\
\hline 14 & 20.1 & 171.9075 & 173.2452 & 173.6638 & 164.7626 & 164.5676 & 164.1419 & 171.9611 & 173.2975 & 173.7635 & 164.8440 & 164.6404 & 164.2142 \\
\hline 15 & 26.2 & 166.8713 & 168.0754 & 169.7454 & 158.8952 & 158.6638 & 157.9243 & 167.1215 & 168.3184 & 170.0279 & 159.1696 & 158.9273 & 158.1932 \\
\hline 16 & 29.4 & 163.5334 & 164.8193 & 164.1707 & 156.1700 & 155.6648 & 154.8633 & 163.1523 & 164.4289 & 163.6726 & 155.6968 & 155.1870 & 154.2521 \\
\hline 17 & 26.6 & 165.6698 & 166.8542 & 166.9661 & 158.4736 & 158.2956 & 157.9167 & 166.0765 & 167.2542 & 167.3988 & 158.9401 & 158.7499 & 158.3574 \\
\hline 18 & 7.3 & 184.3545 & 186.1563 & 186.3673 & 178.7563 & 178.7965 & 178.5259 & 184.5937 & 186.3911 & 186.6318 & 179.0200 & 179.0489 & 178.7790 \\
\hline 19 & 26.9 & 166.6386 & 167.7965 & 166.5826 & 158.8086 & 158.7134 & 158.5929 & 166.8423 & 167.9991 & 166.7818 & 159.0453 & 158.9461 & 158.8444 \\
\hline 20 & 38.1 & 154.8632 & 155.1103 & 154.4467 & 145.1023 & 145.1474 & 144.5138 & 154.5599 & 154.8044 & 154.0666 & 144.7271 & 144.7764 & 144.0857 \\
\hline $\begin{array}{l}21 \\
{ }^{1} H\end{array}$ & 92.5 & 101.6605 & 101.7755 & 102.7563 & 91.4050 & 91.4415 & 89.9543 & 101.1249 & 101.2364 & 102.1084 & 90.8071 & 90.8412 & 89.2477 \\
\hline 3 & 2.80 & 29.4147 & 28.9708 & 28.8491 & 29.3687 & 29.1561 & 29.0161 & 29.3670 & 28.9221 & 28.7801 & 29.3142 & 29.1018 & 28.9442 \\
\hline 3 & 3.95 & 28.2150 & 27.6303 & 27.4821 & 28.1979 & 27.7833 & 27.6201 & 28.3412 & 27.7608 & 27.6117 & 28.3211 & 27.9079 & 27.7385 \\
\hline 6 & 2.68 & 29.6797 & 29.2117 & 29.1043 & 29.5973 & 29.3348 & 29.2780 & 29.6402 & 29.1692 & 29.0484 & 29.5539 & 29.2925 & 29.2247 \\
\hline 6 & 2.87 & 29.6791 & 29.1983 & 29.0563 & 29.5661 & 29.2937 & 29.2124 & 29.5066 & 29.0196 & 28.8550 & 29.3900 & 29.1157 & 29.0195 \\
\hline 7 & 3.82 & 28.5673 & 28.0187 & 27.7674 & 28.5011 & 28.1387 & 28.0477 & 28.3670 & 27.8115 & 27.5483 & 28.3044 & 27.9397 & 27.8365 \\
\hline 9 & 7.17 & 25.0378 & 24.3793 & 24.2571 & 24.9860 & 24.5164 & 24.5450 & 24.8525 & 24.1926 & 24.0571 & 24.7896 & 24.3257 & 24.3465 \\
\hline 10 & 7.14 & 24.9879 & 24.3662 & 24.2204 & 24.8865 & 24.4669 & 24.4644 & 24.8690 & 24.2471 & 24.1034 & 24.7594 & 24.3474 & 24.3454 \\
\hline 11 & 7.25 & 24.8806 & 24.2662 & 24.0089 & 24.7526 & 24.3416 & 24.2372 & 24.8030 & 24.1884 & 23.9241 & 24.6673 & 24.2644 & 24.1527 \\
\hline 12 & 7.77 & 24.1178 & 23.4034 & 23.1633 & 24.0204 & 23.5271 & 23.4291 & 24.2110 & 23.4978 & 23.2551 & 24.0986 & 23.6118 & 23.5123 \\
\hline 14 & 1.60 & 30.8406 & 30.3952 & 30.3609 & 30.7972 & 30.5524 & 30.4960 & 30.7369 & 30.2865 & 30.2448 & 30.6994 & 30.4499 & 30.3865 \\
\hline 14 & 1.60 & 30.4278 & 30.0122 & 29.9040 & 30.4545 & 30.2434 & 30.1620 & 30.3991 & 29.9817 & 29.8720 & 30.4265 & 30.2144 & 30.1291 \\
\hline 15 & 1.60 & 30.0965 & 29.6665 & 29.4290 & 30.0785 & 29.8705 & 29.7539 & 30.0705 & 29.6419 & 29.4087 & 30.0539 & 29.8497 & 29.7346 \\
\hline 15 & 1.60 & 30.5143 & 30.0794 & 30.0889 & 30.5492 & 30.3069 & 30.2733 & 30.3901 & 29.9518 & 29.9604 & 30.4306 & 30.1857 & 30.1484 \\
\hline 16 & 2.49 & 29.8778 & 29.4035 & 29.3003 & 29.7868 & 29.4953 & 29.4357 & 29.8491 & 29.3711 & 29.2716 & 29.7593 & 29.4661 & 29.4081 \\
\hline 16 & 2.78 & 29.5569 & 29.0621 & 28.9562 & 29.4814 & 29.2083 & 29.1626 & 29.4377 & 28.9384 & 28.8248 & 29.3625 & 29.0893 & 29.0362 \\
\hline 17 & 1.86 & 30.5792 & 30.1701 & 30.0336 & 30.5529 & 30.3344 & 30.2752 & 30.4664 & 30.0548 & 29.9097 & 30.4443 & 30.2268 & 30.1595 \\
\hline 17 & 1.97 & 30.5067 & 30.0499 & 29.9870 & 30.4778 & 30.2052 & 30.1375 & 30.3799 & 29.9187 & 29.8531 & 30.3564 & 30.0790 & 30.0064 \\
\hline 18 & 0.93 & 31.2451 & 30.7890 & 30.7260 & 31.2135 & 30.9359 & 30.8973 & 31.2080 & 30.7504 & 30.6898 & 31.1820 & 30.9037 & 30.8660 \\
\hline 19 & 1.37 & 30.9643 & 30.5500 & 30.4410 & 30.8977 & 30.6743 & 30.6302 & 30.8581 & 30.4412 & 30.3247 & 30.8027 & 30.5778 & 30.5298 \\
\hline 19 & 1.78 & 30.2200 & 29.7526 & 29.7218 & 30.3153 & 30.0644 & 30.0305 & 30.2590 & 29.7938 & 29.7688 & 30.3440 & 30.0946 & 30.0656 \\
\hline
\end{tabular}




\section{Compound 51}

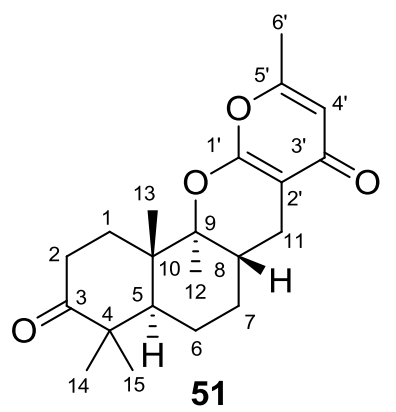

\begin{tabular}{|c|c|c|c|c|c|c|c|c|c|c|c|c|c|}
\hline \multirow{3}{*}{\multicolumn{2}{|c|}{ Exp }} & \multicolumn{12}{|c|}{ B3LYP } \\
\hline & & \multicolumn{6}{|c|}{ Gas phase } & \multicolumn{6}{|c|}{ PCM } \\
\hline & & 6-31G* & $6-31 \mathrm{G} * *$ & $6-31+\mathrm{G} * *$ & 6-311G* & $6-311 G * *$ & $6-311+G * *$ & 6-31G* & $6-31 G * *$ & $6-31+\mathrm{G} * *$ & 6-311G* & $6-311 G * *$ & $6-311+G * *$ \\
\hline \multicolumn{14}{|c|}{${ }^{13} \mathrm{C}$} \\
\hline 1 & 30.8 & 157.4614 & 158.6520 & 158.8787 & 149.0531 & 148.7519 & 148.2399 & 157.4439 & 158.6285 & 158.7939 & 149.0419 & 148.7305 & 148.1769 \\
\hline 2 & 34.5 & 154.3829 & 155.7011 & 153.9850 & 144.8661 & 144.3866 & 144.1260 & 153.9205 & 155.2286 & 153.2259 & 144.2593 & 143.7675 & 143.2365 \\
\hline 3 & 215.5 & -14.8435 & -14.7539 & -18.1376 & -36.4430 & -36.2041 & -40.4135 & -18.3339 & -18.2489 & -22.5192 & -40.5403 & -40.3046 & -45.3386 \\
\hline 4 & 47.8 & 139.2791 & 139.6763 & 138.7590 & 128.7342 & 128.8132 & 127.4687 & 138.8082 & 139.2070 & 138.1736 & 128.2183 & 128.3006 & 126.8088 \\
\hline 5 & 48.8 & 139.3606 & 139.9269 & 139.7455 & 129.0941 & 128.8432 & 127.2156 & 139.6203 & 140.1778 & 140.0663 & 129.4309 & 129.1727 & 127.5449 \\
\hline 6 & 22.1 & 165.2738 & 166.6157 & 166.8884 & 157.3808 & 157.1321 & 156.4040 & 165.4722 & 166.8092 & 167.0877 & 157.5995 & 157.3422 & 156.6221 \\
\hline 7 & 28.7 & 159.2789 & 160.5814 & 160.0441 & 150.3546 & 150.0856 & 149.7784 & 159.5767 & 160.8759 & 160.4246 & 150.7352 & 150.4598 & 150.1594 \\
\hline 8 & 28.7 & 154.4481 & 155.1876 & 155.2823 & 144.6431 & 144.6781 & 144.5147 & 154.6494 & 155.3842 & 155.5673 & 144.9104 & 144.9417 & 144.7973 \\
\hline 9 & 88.8 & 101.3894 & 101.5694 & 102.0829 & 89.1872 & 89.2595 & 88.6626 & 100.7201 & 100.9026 & 101.3195 & 88.3882 & 88.4654 & 87.8159 \\
\hline 10 & 41.5 & 144.7303 & 144.9882 & 144.6847 & 134.5406 & 134.5355 & 134.4322 & 144.6901 & 144.9490 & 144.6199 & 134.4670 & 134.4684 & 134.3699 \\
\hline 11 & 22.0 & 164.8628 & 166.1057 & 166.2599 & 157.5234 & 157.0993 & 156.8495 & 165.0557 & 166.2986 & 166.5720 & 157.8289 & 157.4097 & 157.1874 \\
\hline 12 & 13.2 & 175.9967 & 177.9539 & 178.1628 & 170.5169 & 170.3619 & 170.2420 & 175.9690 & 177.9099 & 178.0544 & 170.3900 & 170.2203 & 170.0746 \\
\hline 13 & 14.1 & 175.0409 & 176.9698 & 177.0666 & 169.5524 & 169.3693 & 169.3829 & 175.1745 & 177.0883 & 177.1570 & 169.6696 & 169.4660 & 169.4697 \\
\hline 14 & 26.2 & 163.4887 & 165.2566 & 165.9751 & 156.9704 & 156.7898 & 156.6121 & 163.8076 & 165.5688 & 166.4372 & 157.4280 & 157.2261 & 157.0724 \\
\hline 15 & 21.8 & 167.1744 & 168.8755 & 170.5999 & 160.2058 & 160.0486 & 159.3446 & 167.3146 & 168.9962 & 170.5813 & 160.3064 & 160.1237 & 159.3601 \\
\hline $1^{\prime}$ & 163.0 & 33.5332 & 33.6733 & 33.1128 & 14.0801 & 14.1770 & 13.0036 & 32.6190 & 32.7607 & 32.0983 & 13.0469 & 13.1427 & 11.8722 \\
\hline $2^{\prime}$ & 98.1 & 92.9658 & 93.1477 & 93.8222 & 79.3033 & 79.5061 & 77.4887 & 92.6388 & 92.8225 & 93.3029 & 78.8769 & 79.0902 & 76.8763 \\
\hline $3^{\prime}$ & 180.1 & 24.1984 & 24.2257 & 22.1385 & 4.5925 & 4.5664 & 1.5637 & 22.9424 & 22.9630 & 20.5405 & 2.9961 & 2.9691 & -0.3330 \\
\hline $4^{\prime}$ & 111.9 & 79.1999 & 79.9951 & 81.0455 & 64.8135 & 64.3722 & 63.7848 & 80.2648 & 81.0586 & 82.0131 & 65.9859 & 65.5436 & 64.7914 \\
\hline $5^{\prime}$ & 160.7 & 37.1452 & 37.1805 & 36.0232 & 17.5106 & 17.3743 & 15.9774 & 34.6083 & 34.6361 & 33.2297 & 14.6272 & 14.4859 & 12.9179 \\
\hline $6^{\prime}$ & 19.3 & 170.0158 & 171.7192 & 171.6089 & 162.4980 & 162.2024 & 161.9471 & 170.1589 & 171.8321 & 171.6270 & 162.5347 & 162.2061 & 161.9140 \\
\hline \multicolumn{14}{|c|}{${ }^{1} \mathrm{H}$} \\
\hline 1 & 2.02 & 30.2269 & 29.7810 & 29.8688 & 30.3140 & 30.0833 & 30.1290 & 30.1257 & 29.6774 & 29.7581 & 30.2090 & 29.9784 & 30.0204 \\
\hline 1 & 2.03 & 30.0897 & 29.6514 & 29.5682 & 30.1241 & 29.8674 & 29.8383 & 30.1045 & 29.6661 & 29.5830 & 30.1404 & 29.8819 & 29.8508 \\
\hline 2 & 2.42 & 30.0537 & 29.5725 & 29.4910 & 29.9343 & 29.6799 & 29.5707 & 30.0879 & 29.6023 & 29.5255 & 29.9683 & 29.7093 & 29.6042 \\
\hline 2 & 2.70 & 29.4477 & 28.9353 & 28.8301 & 29.4878 & 29.1455 & 29.0632 & 29.3137 & 28.7979 & 28.6692 & 29.3480 & 29.0059 & 28.9068 \\
\hline 5 & 1.70 & 30.3152 & 29.9257 & 29.9635 & 30.5660 & 30.3457 & 30.2960 & 30.2167 & 29.8255 & 29.8511 & 30.4601 & 30.2404 & 30.1849 \\
\hline 6 & 1.62 & 30.6429 & 30.1601 & 30.0921 & 30.6875 & 30.3774 & 30.4054 & 30.6145 & 30.1326 & 30.0654 & 30.6630 & 30.3538 & 30.3836 \\
\hline 6 & 1.61 & 30.3861 & 29.9356 & 30.0501 & 30.5275 & 30.2655 & 30.2970 & 30.3127 & 29.8618 & 29.9724 & 30.4546 & 30.1950 & 30.2236 \\
\hline 7 & 1.21 & 30.8668 & 30.4703 & 30.4975 & 30.9370 & 30.7418 & 30.7152 & 30.7990 & 30.3998 & 30.4221 & 30.8683 & 30.6725 & 30.6427 \\
\hline 7 & 1.94 & 30.3557 & 29.9133 & 29.8601 & 30.3380 & 30.0892 & 30.0732 & 30.3208 & 29.8770 & 29.8220 & 30.3050 & 30.0544 & 30.0374 \\
\hline
\end{tabular}




\begin{tabular}{rrrlllllllllll}
\hline 8 & 2.10 & 30.0178 & 29.6293 & 29.6009 & 30.1643 & 29.9939 & 30.0070 & 29.9337 & 29.5430 & 29.5075 & 30.0777 & 29.9063 & 29.9122 \\
11 & 1.95 & 30.2827 & 29.8342 & 29.7878 & 30.3624 & 30.1187 & 30.0727 & 30.2576 & 29.8054 & 29.7478 & 30.3309 & 30.0856 & 30.0270 \\
11 & 2.58 & 29.7503 & 29.2612 & 29.1131 & 29.7253 & 29.4319 & 29.3276 & 29.8469 & 29.3614 & 29.2116 & 29.8149 & 29.5254 & 29.4150 \\
12 & 1.27 & 31.0079 & 30.4982 & 30.4666 & 31.1218 & 30.7937 & 30.7355 & 30.9623 & 30.4520 & 30.4169 & 31.0739 & 30.7468 & 30.6864 \\
13 & 1.29 & 30.7795 & 30.2768 & 30.2599 & 30.8821 & 30.5735 & 30.5097 & 30.7519 & 30.2502 & 30.2327 & 30.8559 & 30.5489 & 30.4843 \\
14 & 1.11 & 31.1011 & 30.6437 & 30.5962 & 31.1232 & 30.8380 & 30.8016 & 31.1155 & 30.6578 & 30.6081 & 31.1353 & 30.8507 & 30.8098 \\
15 & 1.09 & 31.1039 & 30.6477 & 30.6890 & 31.1523 & 30.9009 & 30.8841 & 31.0608 & 30.6030 & 30.6350 & 31.1100 & 30.8570 & 30.8331 \\
$4^{\prime}$ & 5.98 & 26.7007 & 25.9743 & 25.6988 & 26.5899 & 26.0538 & 25.9553 & 26.6564 & 25.9236 & 25.6343 & 26.5301 & 25.9958 & 25.8894 \\
$6^{\prime}$ & 2.21 & 30.2153 & 29.7168 & 29.5698 & 30.0871 & 29.7812 & 29.8013 & 30.1074 & 29.6031 & 29.4516 & 29.9816 & 29.6717 & 29.6873 \\
\hline
\end{tabular}

\begin{tabular}{|c|c|c|c|c|c|c|c|c|c|c|c|c|c|}
\hline \multicolumn{14}{|c|}{ mPW1PW91 } \\
\hline \multirow{2}{*}{\multicolumn{2}{|c|}{ Exp }} & \multicolumn{6}{|c|}{ Gas phase } & \multicolumn{6}{|c|}{ PCM } \\
\hline & & 6-31G* & 6-31G** & $6-31+G * *$ & 6-311G* & $6-311 G * *$ & $6-311+G * *$ & 6-31G* & $6-31 G^{* *}$ & $6-31+G * *$ & $6-311 G^{*}$ & $6-311 \mathrm{G} * *$ & $6-311+G * *$ \\
\hline \\
\hline 1 & 30.8 & 162.4246 & 163.5098 & 163.5451 & 154.5633 & 154.2921 & 153.7972 & 162.4059 & 163.4857 & 163.4580 & 154.5449 & 154.2645 & 153.7347 \\
\hline 2 & 34.5 & 158.6773 & 159.9127 & 158.4599 & 150.2118 & 149.7327 & 149.3475 & 158.2218 & 159.4473 & 157.7443 & 149.6181 & 149.1270 & 148.5097 \\
\hline 3 & 215.5 & -12.0496 & -11.9658 & -14.5776 & -30.7875 & -30.5578 & -34.3538 & -15.6404 & -15.5600 & -18.9650 & -34.9162 & -34.6878 & -39.2137 \\
\hline 4 & 47.8 & 144.1048 & 144.4974 & 143.7130 & 134.5400 & 134.6129 & 133.4234 & 143.6227 & 144.0172 & 143.1376 & 134.0173 & 134.0942 & 132.7766 \\
\hline 5 & 48.8 & 144.7974 & 145.2838 & 145.2258 & 135.3905 & 135.1628 & 133.5862 & 145.0389 & 145.5180 & 145.5166 & 135.6881 & 135.4539 & 133.8791 \\
\hline 6 & 22.1 & 170.0622 & 171.2961 & 171.4315 & 162.9049 & 162.6555 & 161.9680 & 170.2720 & 171.5016 & 171.6407 & 163.1254 & 162.8679 & 162.1892 \\
\hline 7 & 28.7 & 164.2409 & 165.4427 & 164.9762 & 156.1559 & 155.8802 & 155.5122 & 164.5549 & 165.7542 & 165.3713 & 156.5358 & 156.2543 & 155.8922 \\
\hline 8 & 28.7 & 159.7501 & 160.4243 & 160.3431 & 150.6848 & 150.7261 & 150.5698 & 159.9233 & 160.5940 & 160.5901 & 150.9167 & 150.9549 & 150.8128 \\
\hline 9 & 88.8 & 106.3539 & 106.5381 & 107.1608 & 95.3545 & 95.4296 & 94.9264 & 105.6728 & 105.8595 & 106.4000 & 94.5547 & 94.6347 & 94.0889 \\
\hline 10 & 41.5 & 149.9638 & 150.2192 & 149.8558 & 140.5406 & 140.5435 & 140.3641 & 149.9280 & 150.1839 & 149.7952 & 140.4704 & 140.4791 & 140.3052 \\
\hline 11 & 22.0 & 169.4799 & 170.6544 & 170.7175 & 162.9922 & 162.5859 & 162.3709 & 169.7071 & 170.8806 & 171.0433 & 163.3181 & 162.9152 & 162.7202 \\
\hline 12 & 13.2 & 180.0277 & 181.8616 & 181.6867 & 174.8730 & 174.7481 & 174.7269 & 180.0074 & 181.8259 & 181.5912 & 174.7447 & 174.6079 & 174.5672 \\
\hline 13 & 14.1 & 179.2080 & 180.9961 & 180.7670 & 174.1107 & 173.9419 & 173.9221 & 179.3663 & 181.1399 & 180.8797 & 174.2362 & 174.0492 & 174.0213 \\
\hline 14 & 26.2 & 168.1186 & 169.7311 & 169.9305 & 161.8796 & 161.7179 & 161.5880 & 168.4661 & 170.0719 & 170.4132 & 162.3312 & 162.1502 & 162.0419 \\
\hline 15 & 21.8 & 171.4896 & 173.0412 & 174.2305 & 165.0002 & 164.8540 & 164.2535 & 171.6566 & 173.1896 & 174.2460 & 165.1113 & 164.9428 & 164.2925 \\
\hline $1^{\prime}$ & 163.0 & 36.6525 & 36.7975 & 36.8378 & 19.6728 & 19.7772 & 18.6534 & 35.7222 & 35.8684 & 35.8101 & 18.6389 & 18.7419 & 17.5248 \\
\hline $2^{\prime}$ & 98.1 & 96.6601 & 96.8412 & 97.7928 & 84.7649 & 84.9636 & 82.9378 & 96.3503 & 96.5329 & 97.3137 & 84.3723 & 84.5812 & 82.3796 \\
\hline $3^{\prime}$ & 180.1 & 26.3284 & 26.3481 & 25.0945 & 9.5948 & 9.5655 & 6.7303 & 25.0581 & 25.0706 & 23.5085 & 8.0124 & 7.9814 & 4.8783 \\
\hline $4^{\prime}$ & 111.9 & 82.0220 & 82.7829 & 83.8717 & 69.5195 & 69.0804 & 68.3726 & 83.1543 & 83.9133 & 84.9166 & 70.7284 & 70.2893 & 69.4446 \\
\hline $5^{\prime}$ & 160.7 & 39.8751 & 39.8906 & 39.0325 & 22.9031 & 22.7533 & 21.4260 & 37.2394 & 37.2466 & 36.1607 & 19.9519 & 19.7986 & 18.3333 \\
\hline $\begin{array}{c}6^{\prime} \\
{ }^{1} \mathrm{H}\end{array}$ & 19.3 & 173.5302 & 175.1321 & 175.2985 & 166.7216 & 166.4478 & 166.1743 & 173.6777 & 175.2490 & 175.3157 & 166.7336 & 166.4297 & 166.1249 \\
\hline 1 & 2.02 & 30.1714 & 29.7296 & 29.8066 & 30.2018 & 29.9782 & 30.0265 & 30.0709 & 29.6267 & 29.6986 & 30.0988 & 29.8752 & 29.9203 \\
\hline 1 & 2.03 & 30.0391 & 29.6053 & 29.5216 & 30.0292 & 29.7726 & 29.7503 & 30.0512 & 29.6170 & 29.5321 & 30.0428 & 29.7839 & 29.7584 \\
\hline 2 & 2.42 & 29.9098 & 29.4350 & 29.3780 & 29.7886 & 29.5289 & 29.4500 & 29.9388 & 29.4592 & 29.4067 & 29.8176 & 29.5529 & 29.4775 \\
\hline 2 & 2.70 & 29.4342 & 28.9162 & 28.7780 & 29.3970 & 29.0642 & 28.9597 & 29.2970 & 28.7753 & 28.6163 & 29.2542 & 28.9217 & 28.8026 \\
\hline 5 & 1.70 & 30.2752 & 29.8968 & 29.8951 & 30.4237 & 30.2211 & 30.1670 & 30.1777 & 29.7975 & 29.7857 & 30.3213 & 30.1190 & 30.0592 \\
\hline 6 & 1.62 & 30.6233 & 30.1406 & 30.0914 & 30.6187 & 30.3027 & 30.3460 & 30.5893 & 30.1073 & 30.0597 & 30.5897 & 30.2743 & 30.3191 \\
\hline 6 & 1.61 & 30.3725 & 29.9230 & 30.0112 & 30.4289 & 30.1801 & 30.2029 & 30.2966 & 29.8466 & 29.9316 & 30.3544 & 30.1079 & 30.1277 \\
\hline 7 & 1.21 & 30.8374 & 30.4402 & 30.4317 & 30.8395 & 30.6483 & 30.6199 & 30.7671 & 30.3671 & 30.3543 & 30.7690 & 30.5768 & 30.5460 \\
\hline 7 & 1.94 & 30.3040 & 29.8636 & 29.8124 & 30.2590 & 30.0046 & 29.9879 & 30.2612 & 29.8190 & 29.7671 & 30.2195 & 29.9629 & 29.9455 \\
\hline
\end{tabular}




\begin{tabular}{rrrlllllllllll}
\hline 8 & 2.10 & 29.9618 & 29.5764 & 29.5305 & 30.0423 & 29.8801 & 29.8852 & 29.8806 & 29.4930 & 29.4401 & 29.9592 & 29.7958 & 29.7937 \\
11 & 1.95 & 30.2288 & 29.7784 & 29.7263 & 30.2580 & 30.0190 & 29.9953 & 30.1999 & 29.7458 & 29.6833 & 30.2231 & 29.9827 & 29.9472 \\
11 & 2.58 & 29.6555 & 29.1679 & 29.0317 & 29.6187 & 29.3248 & 29.2308 & 29.7470 & 29.2629 & 29.1249 & 29.7035 & 29.4136 & 29.3138 \\
12 & 1.27 & 30.9525 & 30.4437 & 30.4061 & 31.0259 & 30.6965 & 30.6442 & 30.9046 & 30.3952 & 30.3551 & 30.9762 & 30.6476 & 30.5937 \\
13 & 1.29 & 30.7284 & 30.2285 & 30.2056 & 30.7773 & 30.4716 & 30.4107 & 30.6992 & 30.2004 & 30.1768 & 30.7496 & 30.4455 & 30.3836 \\
14 & 1.11 & 31.0381 & 30.5813 & 30.5464 & 31.0236 & 30.7341 & 30.6990 & 31.0499 & 30.5928 & 30.5559 & 31.0331 & 30.7443 & 30.7053 \\
15 & 1.09 & 31.0561 & 30.5996 & 30.6379 & 31.0591 & 30.8057 & 30.7901 & 31.0106 & 30.5524 & 30.5831 & 31.0147 & 30.7598 & 30.7381 \\
$4^{\prime}$ & 5.98 & 26.4953 & 25.7659 & 25.5336 & 26.4146 & 25.8441 & 25.7845 & 26.4409 & 25.7047 & 25.4607 & 26.3474 & 25.7792 & 25.7114 \\
$6^{\prime}$ & 2.21 & 30.1170 & 29.6113 & 29.4904 & 29.9826 & 29.6633 & 29.6916 & 29.9985 & 29.4863 & 29.3614 & 29.8675 & 29.5439 & 29.5687 \\
\hline
\end{tabular}

\section{Compound 52}

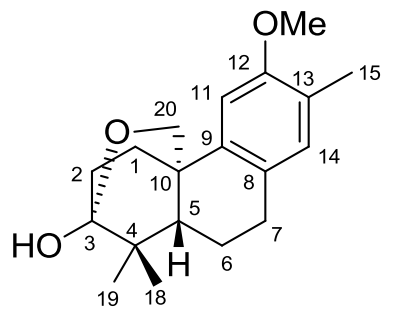

52

\begin{tabular}{|c|c|c|c|c|c|c|c|c|c|c|c|c|c|}
\hline & \multirow[b]{3}{*}{ Exp } & \multicolumn{12}{|c|}{ B3LYP } \\
\hline & & \multicolumn{6}{|c|}{ Gas phase } & \multicolumn{6}{|c|}{ PCM } \\
\hline & & 6-31G* & $6-31 G * *$ & $6-31+\mathrm{G} * *$ & 6-311G* & $6-311 G * *$ & $6-311+G * *$ & 6-31G* & $6-31 \mathrm{G} * *$ & $6-31+G * *$ & 6-311G* & 6-311G** & $6-311+\mathrm{G} * *$ \\
\hline \multicolumn{14}{|l|}{${ }^{13} \mathrm{C}$} \\
\hline 1 & 34.9 & 151.7819 & 153.0498 & 152.7669 & 143.2553 & 142.8962 & 142.8323 & 152.1873 & 153.4421 & 153.2283 & 143.7144 & 143.3393 & 143.3043 \\
\hline 2 & 29.4 & 156.3709 & 157.6949 & 156.9032 & 147.8937 & 147.4670 & 146.8778 & 156.8519 & 158.1607 & 157.4422 & 148.4711 & 148.0105 & 147.4069 \\
\hline 3 & 99.1 & 91.8725 & 91.9949 & 92.3971 & 78.9316 & 79.1654 & 78.5606 & 91.7285 & 91.8503 & 92.1952 & 78.7070 & 78.9358 & 78.2957 \\
\hline 4 & 40.3 & 147.1239 & 147.4822 & 147.6231 & 137.0202 & 137.0098 & 136.1472 & 147.1477 & 147.5064 & 147.6654 & 137.0445 & 137.0255 & 136.1510 \\
\hline 5 & 48.4 & 140.7168 & 141.3482 & 140.2147 & 129.6100 & 129.2753 & 128.5069 & 141.0304 & 141.6520 & 140.6193 & 130.0206 & 129.6705 & 128.9553 \\
\hline 6 & 20.6 & 166.8907 & 168.2687 & 167.5947 & 159.0675 & 158.9235 & 158.8961 & 167.0894 & 168.4614 & 167.8097 & 159.3076 & 159.1529 & 159.1258 \\
\hline 7 & 30.0 & 157.2629 & 158.4597 & 157.3760 & 148.7720 & 148.4509 & 148.3828 & 157.5270 & 158.7180 & 157.6621 & 149.0837 & 148.7509 & 148.6874 \\
\hline 8 & 129.2 & 66.7574 & 66.7524 & 65.1259 & 48.0349 & 47.9856 & 47.0901 & 66.4934 & 66.5010 & 64.7881 & 47.7081 & 47.6761 & 46.7081 \\
\hline 9 & 136.6 & 58.0178 & 57.8984 & 57.7600 & 38.5231 & 38.5141 & 38.2035 & 57.7015 & 57.5950 & 57.4821 & 38.1724 & 38.1937 & 37.8978 \\
\hline 10 & 36.2 & 151.1265 & 151.3851 & 150.0357 & 140.9739 & 140.9600 & 140.2003 & 151.0913 & 151.3498 & 149.9795 & 140.9465 & 140.9357 & 140.1778 \\
\hline 11 & 108.0 & 87.8434 & 88.6533 & 90.8326 & 74.3580 & 73.8374 & 72.8975 & 87.4396 & 88.2308 & 90.2559 & 73.6788 & 73.1438 & 72.1360 \\
\hline 12 & 156.0 & 41.6783 & 41.4217 & 40.4604 & 20.1093 & 20.0953 & 19.2644 & 41.7635 & 41.5062 & 40.5651 & 20.1804 & 20.1692 & 19.4026 \\
\hline 13 & 125.1 & 70.2977 & 70.3914 & 69.9064 & 51.8213 & 51.7477 & 50.1851 & 70.5605 & 70.6471 & 70.1800 & 52.0714 & 51.9919 & 50.4723 \\
\hline 14 & 131.1 & 64.1159 & 64.8808 & 64.8223 & 47.8819 & 47.2556 & 46.7273 & 64.5420 & 65.2980 & 65.2173 & 48.2810 & 47.6425 & 47.1823 \\
\hline 15 & 15.7 & 170.9977 & 172.7453 & 174.3241 & 164.3906 & 164.2110 & 163.7247 & 171.3561 & 173.0879 & 174.6422 & 164.7722 & 164.5724 & 164.0825 \\
\hline 18 & 26.9 & 162.8103 & 164.5851 & 165.4453 & 156.0806 & 155.8754 & 155.4412 & 163.1334 & 164.8936 & 165.8116 & 156.4897 & 156.2591 & 155.8439 \\
\hline 19 & 18.0 & 169.2882 & 171.0658 & 171.6304 & 163.6511 & 163.5596 & 163.6380 & 169.4349 & 171.2119 & 171.8797 & 163.8955 & 163.7936 & 163.9113 \\
\hline 20 & 72.4 & 117.5329 & 118.7623 & 118.0265 & 106.9458 & 106.1534 & 106.0521 & 117.8455 & 119.0645 & 118.2069 & 107.2255 & 106.4231 & 106.2774 \\
\hline $\begin{array}{l}\text { OMe } \\
{ }^{{ }^{1}} \mathbf{H}\end{array}$ & 55.4 & 137.4271 & 139.2581 & 139.8902 & 129.0169 & 128.3320 & 127.6443 & 137.3573 & 139.1664 & 139.5984 & 128.7485 & 128.0323 & 127.3967 \\
\hline 1 & 2.44 & 29.8883 & 29.4573 & 29.3546 & 29.8469 & 29.6002 & 29.5939 & 29.8055 & 29.3734 & 29.2555 & 29.7561 & 29.5115 & 29.4996 \\
\hline 1 & 1.67 & 30.5538 & 30.1556 & 30.0912 & 30.5083 & 30.3367 & 30.3472 & 30.5218 & 30.1219 & 30.0466 & 30.4803 & 30.3066 & 30.3059 \\
\hline
\end{tabular}




\begin{tabular}{rrrlllllllllll}
\hline 2 & 2.28 & 29.9753 & 29.4740 & 29.3534 & 29.9231 & 29.5995 & 29.5871 & 29.9691 & 29.4651 & 29.3369 & 29.9152 & 29.5875 & 29.5686 \\
2 & 1.93 & 30.7992 & 30.3623 & 30.3320 & 30.7092 & 30.4275 & 30.4360 & 30.6848 & 30.2440 & 30.2087 & 30.5824 & 30.3055 & 30.3174 \\
5 & 1.64 & 30.4271 & 30.1111 & 30.1022 & 30.5155 & 30.3940 & 30.3733 & 30.3497 & 30.0326 & 30.0027 & 30.4314 & 30.3087 & 30.2764 \\
6 & 1.83 & 30.4088 & 30.0008 & 29.9007 & 30.4574 & 30.2755 & 30.2166 & 30.4138 & 30.0070 & 29.9059 & 30.4649 & 30.2854 & 30.2231 \\
6 & 1.65 & 30.4942 & 30.0613 & 29.8668 & 30.4521 & 30.2127 & 30.0710 & 30.4463 & 30.0129 & 29.8092 & 30.4008 & 30.1614 & 30.0149 \\
7 & 2.63 & 29.6571 & 29.2154 & 29.0923 & 29.4780 & 29.2452 & 29.2204 & 29.6104 & 29.1667 & 29.0365 & 29.4272 & 29.1935 & 29.1662 \\
7 & 2.74 & 29.5636 & 29.1104 & 28.9208 & 29.4880 & 29.2318 & 29.1709 & 29.5313 & 29.0783 & 28.8889 & 29.4606 & 29.2059 & 29.1423 \\
11 & 6.68 & 25.9897 & 25.2274 & 25.1625 & 26.0533 & 25.5308 & 25.4695 & 25.8406 & 25.0763 & 24.9904 & 25.8850 & 25.3661 & 25.2955 \\
14 & 6.85 & 25.5384 & 24.9405 & 24.7772 & 25.3947 & 24.9615 & 24.8679 & 25.4705 & 24.8715 & 24.6900 & 25.3120 & 24.8835 & 24.7786 \\
15 & 2.15 & 30.1685 & 29.6917 & 29.4760 & 30.0388 & 29.7638 & 29.6373 & 30.1610 & 29.6822 & 29.4632 & 30.0286 & 29.7524 & 29.6244 \\
18 & 1.12 & 31.0520 & 30.6327 & 30.5161 & 31.0243 & 30.8014 & 30.7532 & 31.0477 & 30.6269 & 30.5074 & 31.0205 & 30.7945 & 30.7441 \\
19 & 1.05 & 31.0504 & 30.6304 & 30.5893 & 31.0301 & 30.8200 & 30.8008 & 31.0778 & 30.6568 & 30.6119 & 31.0574 & 30.8437 & 30.8205 \\
20 & 4.00 & 28.2051 & 27.7251 & 27.6014 & 28.1685 & 27.9206 & 27.8078 & 28.1503 & 27.6691 & 27.5284 & 28.1055 & 27.8582 & 27.7371 \\
20 & 4.10 & 27.9232 & 27.4078 & 27.3736 & 27.9918 & 27.7018 & 27.6654 & 27.9181 & 27.4022 & 27.3457 & 27.9790 & 27.6896 & 27.6376 \\
OMe 3.79 & 28.5212 & 28.0232 & 27.8850 & 28.5092 & 28.2082 & 28.1456 & 28.4378 & 27.9407 & 27.7933 & 28.4207 & 28.1226 & 28.0552 \\
\hline
\end{tabular}

\begin{tabular}{|c|c|c|c|c|c|c|c|c|c|c|c|c|c|}
\hline & & \multicolumn{12}{|c|}{ mPW1PW91 } \\
\hline & \multirow[b]{2}{*}{ Exp } & \multicolumn{6}{|c|}{ Gas phase } & \multicolumn{6}{|c|}{ PCM } \\
\hline & & 6-31G* & $6-31 G^{* *}$ & $6-31+G * *$ & 6-311G* & 6-311G** & $6-311+G^{* *}$ & $6-31 G^{*}$ & 6-31G** & $6-31+G^{* *}$ & 6-311G* & 6-311G** & $6-311+G^{* *}$ \\
\hline \multicolumn{14}{|c|}{ 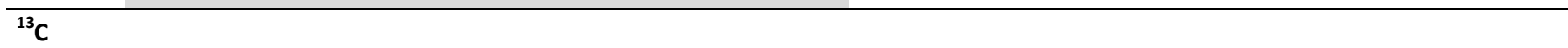 } \\
\hline 1 & 34.9 & 157.0768 & 158.2191 & 157.7289 & 149.1216 & 148.7696 & 148.7134 & 157.4951 & 158.6261 & 158.1919 & 149.5586 & 149.1939 & 149.1601 \\
\hline 2 & 29.4 & 161.3349 & 162.5531 & 161.8607 & 153.6765 & 153.2594 & 152.6060 & 161.8282 & 163.0331 & 162.4042 & 154.2381 & 153.7890 & 153.1218 \\
\hline 3 & 99.1 & 96.3252 & 96.4444 & 97.3633 & 85.2897 & 85.5206 & 84.7252 & 96.1675 & 96.2864 & 97.1586 & 85.0536 & 85.2794 & 84.4506 \\
\hline 4 & 40.3 & 152.2491 & 152.6028 & 152.6780 & 142.8949 & 142.8851 & 142.2404 & 152.2666 & 152.6205 & 152.7128 & 142.9080 & 142.8902 & 142.2378 \\
\hline 5 & 48.4 & 146.2787 & 146.8359 & 146.0746 & 136.1600 & 135.8460 & 135.0994 & 146.5793 & 147.1286 & 146.4546 & 136.5323 & 136.2048 & 135.5057 \\
\hline 6 & 20.6 & 171.6954 & 172.9714 & 172.1248 & 164.6214 & 164.4728 & 164.4279 & 171.9170 & 173.1872 & 172.3612 & 164.8678 & 164.7091 & 164.6631 \\
\hline 7 & 30.0 & 161.9904 & 163.0903 & 162.2680 & 154.3793 & 154.0440 & 153.8148 & 162.2865 & 163.3807 & 162.5759 & 154.6991 & 154.3528 & 154.1257 \\
\hline 8 & 129.2 & 70.0312 & 69.9982 & 68.7908 & 53.6386 & 53.5730 & 52.5441 & 69.7345 & 69.7147 & 68.4347 & 53.2856 & 53.2385 & 52.1546 \\
\hline 9 & 136.6 & 61.2529 & 61.1272 & 61.1043 & 44.0906 & 44.0687 & 43.6804 & 60.9050 & 60.7933 & 60.7959 & 43.7243 & 43.7338 & 43.3595 \\
\hline 10 & 36.2 & 156.2619 & 156.5086 & 155.2886 & 146.9952 & 146.9749 & 146.2063 & 156.2261 & 156.4728 & 155.2345 & 146.9673 & 146.9508 & 146.1822 \\
\hline 11 & 108.0 & 90.3728 & 91.1695 & 93.3868 & 78.6991 & 78.1766 & 77.2664 & 89.9681 & 90.7445 & 92.8008 & 78.0290 & 77.4908 & 76.5219 \\
\hline 12 & 156.0 & 44.7165 & 44.4405 & 43.9620 & 25.9859 & 25.9673 & 25.1060 & 44.8113 & 44.5351 & 44.0699 & 26.0523 & 26.0372 & 25.2361 \\
\hline 13 & 125.1 & 73.5096 & 73.5810 & 73.0982 & 57.3132 & 57.2223 & 55.7137 & 73.7433 & 73.8083 & 73.3336 & 57.4979 & 57.4042 & 55.9284 \\
\hline 14 & 131.1 & 66.6585 & 67.3717 & 67.3054 & 52.4274 & 51.7879 & 51.3412 & 67.0978 & 67.8010 & 67.7008 & 52.8024 & 52.1515 & 51.7704 \\
\hline 15 & 15.7 & 174.8213 & 176.4542 & 178.2802 & 168.7380 & 168.5585 & 168.2202 & 175.2378 & 176.8534 & 178.6295 & 169.1411 & 168.9432 & 168.5968 \\
\hline 18 & 26.9 & 167.2482 & 168.8724 & 169.2221 & 160.9873 & 160.8060 & 160.4387 & 167.6028 & 169.2132 & 169.6141 & 161.3964 & 161.1927 & 160.8451 \\
\hline 19 & 18.0 & 173.7654 & 175.3853 & 175.3769 & 168.4129 & 168.3335 & 168.4355 & 173.9475 & 175.5664 & 175.6468 & 168.6694 & 168.5805 & 168.7148 \\
\hline 20 & 72.4 & 122.2276 & 123.3609 & 122.7969 & 112.9452 & 112.2023 & 112.0264 & 122.5519 & 123.6753 & 122.9896 & 113.2100 & 112.4587 & 112.2433 \\
\hline $\begin{array}{c}\text { OMe } \\
{ }^{1} \mathbf{H}\end{array}$ & 55.4 & 141.0102 & 142.7721 & 143.3368 & 133.8166 & 133.1782 & 132.6200 & 140.9355 & 142.6762 & 143.0487 & 133.5423 & 132.8743 & 132.3670 \\
\hline 1 & 2.44 & 29.8442 & 29.4141 & 29.3122 & 29.7668 & 29.5258 & 29.5046 & 29.7549 & 29.3236 & 29.2088 & 29.6708 & 29.4320 & 29.4063 \\
\hline 1 & 1.67 & 30.4841 & 30.0856 & 30.0200 & 30.4018 & 30.2303 & 30.2302 & 30.4525 & 30.0521 & 29.9760 & 30.3750 & 30.2009 & 30.1897 \\
\hline 2 & 2.28 & 29.9653 & 29.4614 & 29.3339 & 29.8575 & 29.5386 & 29.5225 & 29.9535 & 29.4468 & 29.3134 & 29.8447 & 29.5215 & 29.5000 \\
\hline 2 & 1.93 & 30.7005 & 30.2617 & 30.2247 & 30.5915 & 30.3058 & 30.3098 & 30.5794 & 30.1362 & 30.0953 & 30.4589 & 30.1784 & 30.1862 \\
\hline 5 & 1.64 & 30.3550 & 30.0408 & 29.9947 & 30.3685 & 30.2512 & 30.2187 & 30.2778 & 29.9623 & 29.8977 & 30.2870 & 30.1683 & 30.1246 \\
\hline
\end{tabular}




\begin{tabular}{rlllllllllllll}
\hline 6 & 1.83 & 30.3692 & 29.9615 & 29.8716 & 30.3579 & 30.1825 & 30.1235 & 30.3765 & 29.9701 & 29.8786 & 30.3669 & 30.1941 & 30.1317 \\
6 & 1.65 & 30.4457 & 30.0174 & 29.8381 & 30.3647 & 30.1247 & 29.9887 & 30.3874 & 29.9581 & 29.7708 & 30.3055 & 30.0650 & 29.9242 \\
7 & 2.63 & 29.5664 & 29.1267 & 29.0143 & 29.3884 & 29.1481 & 29.1183 & 29.5084 & 29.0661 & 28.9481 & 29.3280 & 29.0865 & 29.0550 \\
7 & 2.74 & 29.5181 & 29.0578 & 28.8706 & 29.3955 & 29.1437 & 29.0828 & 29.4812 & 29.0211 & 28.8339 & 29.3640 & 29.1140 & 29.0499 \\
11 & 6.68 & 25.8062 & 25.0525 & 24.9724 & 25.8584 & 25.3233 & 25.2408 & 25.6450 & 24.8890 & 24.7909 & 25.6826 & 25.1508 & 25.0602 \\
14 & 6.85 & 25.3390 & 24.7428 & 24.5915 & 25.2205 & 24.7602 & 24.6808 & 25.2557 & 24.6580 & 24.4912 & 25.1249 & 24.6693 & 24.5789 \\
15 & 2.15 & 30.0832 & 29.6021 & 29.3935 & 29.9344 & 29.6517 & 29.5419 & 30.0690 & 29.5856 & 29.3741 & 29.9178 & 29.6340 & 29.5229 \\
18 & 1.12 & 31.0012 & 30.5838 & 30.4675 & 30.9351 & 30.7112 & 30.6680 & 30.9934 & 30.5744 & 30.4558 & 30.9282 & 30.7011 & 30.6561 \\
19 & 1.05 & 30.9952 & 30.5765 & 30.5298 & 30.9366 & 30.7267 & 30.7033 & 31.0213 & 30.6015 & 30.5518 & 30.9622 & 30.7488 & 30.7221 \\
20 & 4.00 & 28.1541 & 27.6823 & 27.5696 & 28.1123 & 27.8643 & 27.7514 & 28.0943 & 27.6212 & 27.4934 & 28.0454 & 27.7979 & 27.6779 \\
20 & 4.10 & 27.9023 & 27.3945 & 27.3765 & 27.9318 & 27.6466 & 27.6082 & 27.8955 & 27.3873 & 27.3485 & 27.9180 & 27.6331 & 27.5803 \\
OMe & 3.79 & 28.4562 & 27.9623 & 27.8418 & 28.4453 & 28.1347 & 28.0844 & 28.3637 & 27.8704 & 27.7428 & 28.3499 & 28.0421 & 27.9881 \\
\hline
\end{tabular}

\section{Compound 53}

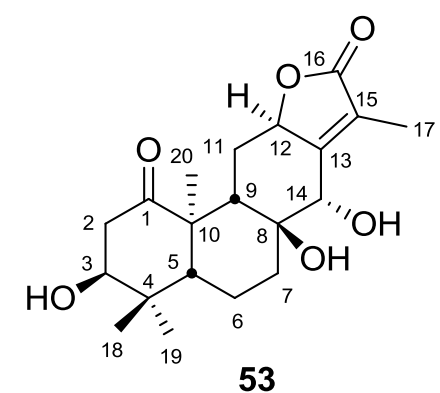

\begin{tabular}{|c|c|c|c|c|c|c|c|c|c|c|c|c|c|}
\hline \multirow{3}{*}{\multicolumn{2}{|c|}{ Exp }} & \multicolumn{12}{|c|}{ B3LYP } \\
\hline & & \multicolumn{6}{|c|}{ Gas phase } & \multicolumn{6}{|c|}{ PCM } \\
\hline & & 6-31G* & $6-31 G * *$ & $6-31+G * *$ & 6-311G* & $6-311 G * *$ & $6-311+\mathrm{G} * *$ & 6-31G* & $6-31 G * *$ & $6-31+G * *$ & $6-311 G *$ & $6-311 G * *$ & $6-311+G * *$ \\
\hline \multicolumn{14}{|c|}{${ }^{13} \mathrm{C}$} \\
\hline 1 & 216.0 & -15.1814 & -15.1454 & -16.5250 & -37.0875 & -37.0532 & -39.5046 & -18.8581 & -18.8179 & -20.9602 & -41.4071 & -41.3456 & -44.4140 \\
\hline 2 & 43.9 & 148.6468 & 149.8846 & 148.5918 & 139.1451 & 138.4651 & 138.8071 & 147.7539 & 148.9804 & 147.2995 & 137.9680 & 137.2723 & 137.3399 \\
\hline 3 & 80.1 & 108.8488 & 109.5279 & 107.3088 & 96.5813 & 96.4217 & 96.5360 & 108.8046 & 109.4804 & 107.2634 & 96.5585 & 96.3831 & 96.4610 \\
\hline 4 & 39.0 & 149.1688 & 149.5505 & 148.2854 & 139.5077 & 139.4502 & 138.3975 & 149.0842 & 149.4699 & 148.0828 & 139.3638 & 139.3124 & 138.1885 \\
\hline 5 & 51.1 & 139.5789 & 140.1911 & 139.3620 & 129.4537 & 129.3290 & 128.5563 & 139.2720 & 139.8825 & 138.9741 & 129.1177 & 128.9999 & 128.2070 \\
\hline 6 & 21.5 & 166.7029 & 168.1285 & 168.9835 & 159.2588 & 159.0219 & 158.7199 & 166.7988 & 168.2226 & 169.0909 & 159.3869 & 159.1399 & 158.8416 \\
\hline 7 & 42.3 & 149.5191 & 150.7555 & 149.8483 & 140.5745 & 140.0931 & 140.2823 & 149.5244 & 150.7585 & 149.8560 & 140.5841 & 140.1194 & 140.2775 \\
\hline 8 & 75.9 & 114.4699 & 114.7594 & 113.9143 & 102.5587 & 102.8308 & 101.8951 & 114.3638 & 114.6489 & 113.7203 & 102.3360 & 102.5959 & 101.6039 \\
\hline 9 & 48.7 & 139.7964 & 140.4191 & 140.1167 & 129.4025 & 129.1136 & 129.0740 & 139.6743 & 140.2953 & 139.9898 & 129.2368 & 128.9588 & 128.9622 \\
\hline 10 & 54.4 & 135.1906 & 135.4886 & 133.7013 & 123.8867 & 124.0367 & 123.4724 & 134.6234 & 134.9227 & 132.9876 & 123.2075 & 123.3661 & 122.6504 \\
\hline 11 & 32.7 & 155.6795 & 157.0063 & 156.9188 & 146.7583 & 146.3982 & 146.1098 & 156.1395 & 157.4594 & 157.4120 & 147.2877 & 146.9155 & 146.5956 \\
\hline 12 & 78.9 & 115.1183 & 115.8821 & 115.3165 & 102.5626 & 102.2468 & 101.8125 & 114.5872 & 115.3428 & 114.6431 & 101.9728 & 101.6323 & 101.0799 \\
\hline 13 & 164.8 & 35.8148 & 35.9178 & 34.0453 & 16.6313 & 16.6739 & 14.5655 & 34.0864 & 34.1743 & 32.0766 & 14.8226 & 14.8042 & 12.4731 \\
\hline 14 & 73.5 & 113.9402 & 114.5656 & 114.1281 & 102.3312 & 102.1378 & 102.5275 & 114.3238 & 114.9447 & 114.5112 & 102.8094 & 102.6093 & 102.9264 \\
\hline 15 & 122.8 & 65.1406 & 65.3945 & 65.3611 & 47.5596 & 47.9092 & 47.2436 & 65.4319 & 65.6921 & 65.7222 & 47.8797 & 48.2547 & 47.5495 \\
\hline 16 & 177.2 & 28.0749 & 28.0847 & 25.2602 & 7.1958 & 7.2008 & 4.7617 & 25.9402 & 25.9482 & 22.7978 & 4.8037 & 4.8086 & 1.9875 \\
\hline 17 & 8.3 & 178.7328 & 180.4607 & 181.3567 & 172.6715 & 172.4956 & 172.2211 & 178.9679 & 180.6824 & 181.6702 & 172.9076 & 172.7249 & 172.4211 \\
\hline 18 & 28.5 & 161.2062 & 162.9474 & 163.1141 & 154.8674 & 154.4520 & 154.0604 & 161.6324 & 163.3538 & 163.5645 & 155.3282 & 154.8834 & 154.5467 \\
\hline
\end{tabular}




\begin{tabular}{|c|c|c|c|c|c|c|c|c|c|c|c|c|c|}
\hline & & 7.1671 & 8.9841 & 168.6444 & 0.7206 & 160.4953 & 60.1241 & 167.4714 & 169.2739 & 168.9591 & 161.0680 & 160.8201 & 100.7 \\
\hline & & & & & & & & & & & & & \\
\hline 2 & 2.23 & 8511 & 4005 & 2366 & .6883 & 9,1887 & 281 & (2981 & 9.5324 & 9.3758 & 29.8240 & 29.6195 & 29.510 \\
\hline 2 & 3.26 & 9.2124 & 28.6669 & 8.5354 & .2021 & 88319 & & & & & & & 5916 \\
\hline 3 & 82 & 3.3222 & 8893 & & & & & & & 891 & & 526 & 421 \\
\hline 5 & 76 & & & & & & & & & & & & 9.9250 \\
\hline 6 & בנ כנ & & & & & & & & & & & & \\
\hline 6 & & & & & & & & & & & & & \\
\hline 7 & 62 & & & & & & & & & & & & \\
\hline 7 & 2.26 & 9.9611 & .5656 & .5078 & & & & & & & & & 851 \\
\hline 9 & 2.34 & .5948 & 2240 & 267 & 2 & 5099 & 3 & & & & 58 & & 5674 \\
\hline & 81 & & & & & & & & & & & & \\
\hline & & & & & & & & & & & & & \\
\hline & & & & & & & & & & & & & \\
\hline & 4.38 & 27.5895 & 27.1425 & 27.0863 & & 27.3158 & 273901 & 27.4860 & & & & & 27.2743 \\
\hline 17 & 1.84 & 0.4621 & & & & & 0907 & 108 & 9.8955 & & 538 & 30.0307 & 30.0345 \\
\hline $1 \varepsilon_{2}$ & 1.01 & 31.2580 & & & & 104 & 9000 & 933 & 530 & 093 & 173 & 30.9466 & 0.8979 \\
\hline 19 & 1.14 & 31.0883 & 30.6437 & 30.5961 & & 30.8619 & & 31.0494 & 30.6044 & 30.5507 & 31.0768 & 30.8232 & 30.6958 \\
\hline & & 30.6136 & 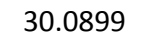 & 29.9592 & 30.6599 & 30.3695 & t5. & 30.5622 & 30.0353 & 29.9000 & 30.6073 & 30.3127 & 30.2410 \\
\hline
\end{tabular}

\begin{tabular}{|c|c|c|c|c|c|c|c|c|c|c|c|c|c|}
\hline \multicolumn{14}{|c|}{ mPW1PW91 } \\
\hline \multirow{2}{*}{\multicolumn{2}{|c|}{ Exp }} & \multicolumn{6}{|c|}{ Gas phase } & \multicolumn{6}{|c|}{ PCM } \\
\hline & & $6-31 G *$ & $6-31 G * *$ & $6-31+G * *$ & $6-311 G *$ & $6-311 G * *$ & $6-311+G^{* *}$ & $6-31 G *$ & $6-31 G * *$ & $6-31+G * *$ & 6-311G* & $6-311 G * *$ & $6-311+\mathrm{G} * *$ \\
\hline \multicolumn{14}{|c|}{${ }^{13} \mathrm{C}$} \\
\hline 1 & 216.0 & -12.2707 & -12.2486 & -13.0072 & -31.3612 & -31.3387 & -33.5074 & -16.0387 & -16.0111 & -17.4400 & -35.7079 & -35.6554 & -38.3613 \\
\hline 2 & 43.9 & 153.0507 & 154.1992 & 152.6580 & 144.5510 & 143.8805 & 144.1444 & 152.1557 & 153.2935 & 151.4101 & 143.3952 & 142.7102 & 142.7437 \\
\hline 3 & 80.1 & 113.9030 & 114.5212 & 112.3261 & 103.1327 & 102.9871 & 103.1083 & 113.8356 & 114.4533 & 112.2642 & 103.0713 & 102.9126 & 103.0117 \\
\hline 4 & 39.0 & 154.2448 & 154.6204 & 153.7869 & 145.3696 & 145.3216 & 144.4688 & 154.1654 & 154.5445 & 153.6015 & 145.2322 & 145.1898 & 144.2779 \\
\hline 5 & 51.1 & 145.1499 & 145.6839 & 144.9836 & 135.8834 & 135.7794 & 135.0317 & 144.8365 & 145.3687 & 144.5948 & 135.5417 & 135.4454 & 134.6870 \\
\hline 6 & 21.5 & 171.4319 & 172.7467 & 173.6366 & 164.7136 & 164.4814 & 164.1369 & 171.5338 & 172.8474 & 173.7489 & 164.8417 & 164.5999 & 164.2599 \\
\hline 7 & 42.3 & 154.6307 & 155.7618 & 154.7491 & 146.5770 & 146.1164 & 146.2452 & 154.6628 & 155.7912 & 154.7852 & 146.5987 & 146.1544 & 146.2561 \\
\hline 8 & 75.9 & 119.2052 & 119.4713 & 118.8963 & 109.0029 & 109.2479 & 108.3438 & 119.0827 & 119.3448 & 118.7039 & 108.7655 & 108.9990 & 108.0493 \\
\hline 9 & 48.7 & 145.3857 & 145.9329 & 145.8221 & 135.9011 & 135.6349 & 135.5595 & 145.2746 & 145.8196 & 145.7071 & 135.7538 & 135.4978 & 135.4612 \\
\hline 10 & 54.4 & 140.0792 & 140.3670 & 138.5014 & 129.8824 & 130.0277 & 129.4935 & 139.5111 & 139.8002 & 137.8117 & 129.2089 & 129.3629 & 128.7009 \\
\hline 11 & 32.7 & 160.7335 & 161.9364 & 161.7646 & 152.6268 & 152.2851 & 151.9757 & 161.1818 & 162.3776 & 162.2378 & 153.1213 & 152.7665 & 152.4268 \\
\hline 12 & 78.9 & 119.2015 & 119.9244 & 119.7255 & 108.3295 & 108.0223 & 107.5668 & 118.6367 & 119.3523 & 119.0257 & 107.7102 & 107.3779 & 106.8157 \\
\hline 13 & 164.8 & 39.0155 & 39.1143 & 37.5812 & 22.2158 & 22.2508 & 20.3306 & 37.2283 & 37.3118 & 35.5863 & 20.3924 & 20.3638 & 18.2434 \\
\hline 14 & 73.5 & 118.7261 & 119.2990 & 118.6984 & 108.7880 & 108.6074 & 108.9299 & 119.0750 & 119.6461 & 119.0523 & 109.2201 & 109.0352 & 109.2954 \\
\hline 15 & 122.8 & 68.0867 & 68.3371 & 68.2969 & 52.5139 & 52.8713 & 52.0930 & 68.3941 & 68.6513 & 68.6684 & 52.8359 & 53.2205 & 52.4150 \\
\hline 16 & 177.2 & 30.4425 & 30.4499 & 28.3644 & 12.2917 & 12.2978 & 10.0456 & 28.2842 & 28.2896 & 25.9112 & 9.9244 & 9.9297 & 7.3430 \\
\hline 17 & 8.3 & 182.1754 & 183.8029 & 184.8257 & 176.6494 & 176.4927 & 176.1975 & 182.4441 & 184.0575 & 185.1624 & 176.8934 & 176.7324 & 176.4142 \\
\hline 18 & 28.5 & 165.7638 & 167.3705 & 167.1505 & 159.9289 & 159.5436 & 159.1993 & 166.2153 & 167.8042 & 167.6244 & 160.3817 & 159.9716 & 159.6749 \\
\hline 19 & 22.7 & 171.4158 & 173.0851 & 172.4801 & 165.4862 & 165.2673 & 164.8938 & 171.7524 & 173.4080 & 172.8180 & 165.8369 & 165.5986 & 165.2329 \\
\hline 20 & 16.9 & 175.7568 & 177.4025 & 177.9654 & 169.9879 & 169.7870 & 169.1476 & 175.9670 & 177.5961 & 178.1180 & 170.1552 & 169.9330 & 169.2696 \\
\hline
\end{tabular}




\begin{tabular}{lllllllllllllll}
\hline${ }^{1} \mathbf{H}$ & & & & & & & & & & & & & \\
2 & 2.23 & 29.6951 & 29.2476 & 29.1156 & 29.5351 & 29.3321 & 29.2330 & 29.8246 & 29.3759 & 29.2491 & 29.6664 & 29.4587 & 29.3573 \\
2 & 3.26 & 29.1788 & 28.6302 & 28.5135 & 29.1143 & 28.7518 & 28.7267 & 28.9809 & 28.4297 & 28.2936 & 28.9080 & 28.5495 & 28.5094 \\
3 & 3.82 & 28.2747 & 27.8514 & 27.8803 & 28.2111 & 28.0135 & 28.1115 & 28.1534 & 27.7268 & 27.7388 & 28.0838 & 27.8817 & 27.9657 \\
5 & 1.76 & 29.9361 & 29.6140 & 29.6204 & 29.9764 & 29.8775 & 29.7897 & 29.9659 & 29.6436 & 29.6410 & 29.9964 & 29.8947 & 29.8012 \\
6 & 1.53 & 30.6951 & 30.2523 & 30.1764 & 30.6951 & 30.4469 & 30.4327 & 30.5855 & 30.1376 & 30.0519 & 30.5820 & 30.3296 & 30.3112 \\
6 & 2.08 & 29.7953 & 29.3667 & 29.3271 & 29.8239 & 29.6524 & 29.6343 & 29.8031 & 29.3711 & 29.3303 & 29.8338 & 29.6548 & 29.6322 \\
7 & 1.62 & 30.2844 & 29.9194 & 29.8103 & 30.1721 & 30.0329 & 29.9915 & 30.3476 & 29.9826 & 29.8723 & 30.2392 & 30.0924 & 30.0485 \\
7 & 2.26 & 29.8969 & 29.5001 & 29.4461 & 29.8256 & 29.6144 & 29.6355 & 29.8519 & 29.4505 & 29.3862 & 29.7743 & 29.5566 & 29.5739 \\
9 & 2.34 & 29.5375 & 29.1731 & 29.1801 & 29.5479 & 29.3993 & 29.4934 & 29.6369 & 29.2721 & 29.2696 & 29.6380 & 29.4830 & 29.5702 \\
11 & 1.81 & 30.7612 & 30.3085 & 30.2469 & 30.7399 & 30.4705 & 30.3924 & 30.7129 & 30.2551 & 30.1749 & 30.6757 & 30.4075 & 30.3215 \\
11 & 2.62 & 29.1120 & 28.5703 & 28.5339 & 29.0673 & 28.7486 & 28.7458 & 29.2173 & 28.6783 & 28.6375 & 29.1655 & 28.8432 & 28.8400 \\
12 & 5.13 & 27.1985 & 26.6339 & 26.5148 & 27.1779 & 26.8391 & 26.8088 & 27.0622 & 26.4870 & 26.3556 & 27.0379 & 26.6880 & 26.6479 \\
14 & 4.38 & 27.5254 & 27.0872 & 27.0286 & 27.4511 & 27.2399 & 27.3131 & 27.4135 & 26.9673 & 26.9094 & 27.3320 & 27.1127 & 27.1932 \\
17 & 1.84 & 30.3763 & 29.8584 & 29.7649 & 30.3091 & 29.9749 & 29.9904 & 30.3185 & 29.7968 & 29.7029 & 30.2512 & 29.9152 & 29.9289 \\
18 & 1.01 & 31.1997 & 30.7639 & 30.7212 & 31.1919 & 30.9180 & 30.8682 & 31.1304 & 30.6940 & 30.6424 & 31.1218 & 30.8506 & 30.7973 \\
19 & 1.14 & 31.0238 & 30.5798 & 30.5233 & 31.0125 & 30.7555 & 30.6220 & 30.9823 & 30.5379 & 30.4766 & 30.9713 & 30.7145 & 30.5779 \\
20 & 1.61 & 30.5653 & 30.0393 & 29.8885 & 30.5646 & 30.2775 & 30.1951 & 30.5122 & 29.9830 & 29.8295 & 30.5108 & 30.2194 & 30.1350 \\
\hline
\end{tabular}

\section{Compound 54}

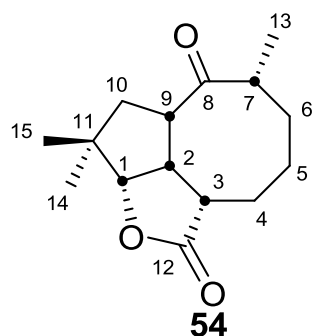

\begin{tabular}{|c|c|c|c|c|c|c|c|c|c|c|c|c|c|}
\hline & & \multicolumn{12}{|c|}{ B3LYP } \\
\hline & \multirow[b]{2}{*}{ Exp } & \multicolumn{6}{|c|}{ Gas phase } & \multicolumn{6}{|c|}{ PCM } \\
\hline & & 6-31G* & $6-31 G * *$ & $6-31+G * *$ & 6-311G* & $6-311 G * *$ & $6-311+G * *$ & 6-31G* & $6-31 G * *$ & $6-31+G * *$ & 6-311G* & $6-311 G * *$ & $6-311+\mathrm{G} * *$ \\
\hline \\
\hline 1 & 90.9 & 101.5040 & 102.0796 & 102.2154 & 88.9032 & 88.4696 & 88.1958 & 100.9954 & 101.5685 & 101.6284 & 88.3253 & 87.8879 & 87.5265 \\
\hline 2 & 45.7 & 142.1142 & 142.8443 & 142.6771 & 131.5427 & 131.3950 & 130.8235 & 141.9148 & 142.6445 & 142.3374 & 131.3136 & 131.1528 & 130.4773 \\
\hline 3 & 43.1 & 144.9221 & 145.6703 & 144.9182 & 134.7453 & 134.5460 & 133.6192 & 144.9945 & 145.7258 & 145.0779 & 134.7988 & 134.5951 & 133.6220 \\
\hline 4 & 22.5 & 164.1379 & 165.5640 & 164.9487 & 155.8758 & 155.7265 & 155.2591 & 164.0064 & 165.4355 & 164.8302 & 155.7574 & 155.6220 & 155.1374 \\
\hline 5 & 22.9 & 163.1909 & 164.7508 & 162.9035 & 154.3735 & 154.3463 & 153.7495 & 163.6702 & 165.2143 & 163.5150 & 154.9513 & 154.9162 & 154.3559 \\
\hline 6 & 27.9 & 157.4100 & 159.0009 & 156.8370 & 148.0893 & 148.0962 & 147.1011 & 157.9519 & 159.5295 & 157.4091 & 148.6524 & 148.6465 & 147.6272 \\
\hline 7 & 45.7 & 142.1116 & 142.8790 & 141.8087 & 131.2761 & 131.1031 & 130.4076 & 142.0246 & 142.7798 & 141.7683 & 131.1693 & 130.9808 & 130.2457 \\
\hline 8 & 213.8 & -16.7266 & -16.4955 & -19.6970 & -39.9644 & -39.5463 & -41.9385 & -19.7296 & -19.4982 & -23.3493 & -43.5732 & -43.1608 & -46.0676 \\
\hline 9 & 50.1 & 137.0870 & 137.9714 & 137.0842 & 126.5872 & 126.4825 & 125.3808 & 137.2534 & 138.1196 & 137.2660 & 126.7066 & 126.5821 & 125.4244 \\
\hline 10 & 38.5 & 150.6862 & 151.7587 & 151.7274 & 141.7338 & 141.3185 & 141.2786 & 150.7314 & 151.8062 & 151.8240 & 141.8428 & 141.4277 & 141.4038 \\
\hline 11 & 40.8 & 146.4720 & 146.9522 & 146.5912 & 135.8458 & 135.8387 & 135.4205 & 146.4731 & 146.9573 & 146.6492 & 135.9064 & 135.9015 & 135.4532 \\
\hline 12 & 177.9 & 23.8263 & 23.8983 & 21.4024 & 2.3967 & 2.5593 & 0.0776 & 20.8170 & 20.8872 & 17.8817 & -0.9869 & -0.8252 & -3.8583 \\
\hline 13 & 13.2 & 172.8098 & 174.8238 & 174.9186 & 166.5257 & 166.6325 & 165.9548 & 173.2241 & 175.2208 & 175.4346 & 167.0442 & 167.1287 & 166.4715 \\
\hline
\end{tabular}




\begin{tabular}{llllllllllllllll}
\hline 14 & 24.5 & 165.8681 & 167.5852 & 168.3903 & 158.7557 & 158.5718 & 158.1184 & 166.0792 & 167.7903 & 168.6928 & 159.0965 & 158.8873 & 158.4509 \\
15 & 23.0 & 164.0338 & 165.7222 & 166.2038 & 157.1544 & 156.9063 & 156.7593 & 164.5102 & 166.1795 & 166.7130 & 157.6917 & 157.4166 & 157.2995 \\
${ }^{1}$ H & & & & & & & & & & & & & & \\
1 & 4.29 & 28.0430 & 27.5450 & 27.4848 & 28.0820 & 27.8110 & 27.7763 & 27.8872 & 27.3830 & 27.3118 & 27.9214 & 27.6446 & 27.6017 \\
2 & 3.79 & 28.7858 & 28.2757 & 28.1552 & 28.7904 & 28.4512 & 28.3718 & 28.5507 & 28.0365 & 27.8971 & 28.5567 & 28.2190 & 28.1236 \\
3 & 2.77 & 29.7241 & 29.3334 & 29.2215 & 29.7721 & 29.5729 & 29.4971 & 29.5182 & 29.1208 & 28.9862 & 29.5673 & 29.3659 & 29.2697 \\
4 & 1.96 & 30.2554 & 29.8147 & 29.6290 & 30.2622 & 29.9916 & 29.9069 & 30.3470 & 29.9071 & 29.7276 & 30.3474 & 30.0796 & 29.9964 \\
4 & 1.45 & 30.7090 & 30.2292 & 30.2675 & 30.8491 & 30.5813 & 30.5994 & 30.8073 & 30.3305 & 30.3593 & 30.9390 & 30.6718 & 30.6851 \\
5 & 1.58 & 30.7540 & 30.3380 & 30.3022 & 30.7007 & 30.5089 & 30.5072 & 30.6502 & 30.2307 & 30.1845 & 30.6004 & 30.4048 & 30.3947 \\
5 & 1.93 & 30.2409 & 29.7846 & 29.7152 & 30.2072 & 29.9628 & 29.9418 & 30.2243 & 29.7677 & 29.7009 & 30.1972 & 29.9531 & 29.9316 \\
6 & 1.83 & 30.3986 & 29.9850 & 29.8303 & 30.3450 & 30.1465 & 30.1382 & 30.3863 & 29.9647 & 29.8260 & 30.3312 & 30.1270 & 30.1283 \\
6 & 2.43 & 30.0825 & 29.6087 & 29.6303 & 30.0204 & 29.7631 & 29.8371 & 29.9415 & 29.4672 & 29.4821 & 29.8880 & 29.6317 & 29.7045 \\
7 & 2.53 & 29.7117 & 29.3143 & 29.1951 & 29.7028 & 29.5294 & 29.4797 & 29.5507 & 29.1495 & 29.0179 & 29.5438 & 29.3672 & 29.3060 \\
9 & 3.28 & 29.1017 & 28.6394 & 28.5563 & 29.1633 & 28.8747 & 28.8176 & 28.9055 & 28.4374 & 28.3404 & 28.9662 & 28.6764 & 28.6067 \\
10 & 2.16 & 29.7286 & 29.2473 & 29.2856 & 29.8253 & 29.5719 & 29.5867 & 29.8680 & 29.3925 & 29.4170 & 29.9601 & 29.7101 & 29.7156 \\
10 & 1.38 & 30.9003 & 30.4904 & 30.4395 & 31.0004 & 30.7634 & 30.7219 & 30.8449 & 30.4321 & 30.3794 & 30.9428 & 30.7055 & 30.6593 \\
13 & 1.11 & 31.0715 & 30.6213 & 30.5507 & 31.0522 & 30.7925 & 30.7812 & 31.0868 & 30.6368 & 30.5639 & 31.0684 & 30.8088 & 30.7950 \\
14 & 1.19 & 30.9363 & 30.4914 & 30.4490 & 30.9297 & 30.6786 & 30.6431 & 30.9610 & 30.5163 & 30.4757 & 30.9545 & 30.7040 & 30.6677 \\
15 & 1.02 & 31.2270 & 30.7875 & 30.7169 & 31.2398 & 30.9953 & 30.9298 & 31.1678 & 30.7263 & 30.6528 & 31.1843 & 30.9384 & 30.8721 \\
\hline
\end{tabular}

\begin{tabular}{|c|c|c|c|c|c|c|c|c|c|c|c|c|c|}
\hline \multicolumn{14}{|c|}{ mPW1PW91 } \\
\hline & \multirow[b]{2}{*}{ Exp } & \multicolumn{6}{|c|}{ Gas phase } & \multicolumn{6}{|c|}{ PCM } \\
\hline & & $6-31 G *$ & $6-31 G * *$ & $6-31+G * *$ & 6-311G* & $6-311 G * *$ & $6-311+G^{* *}$ & $6-31 G *$ & $6-31 G * *$ & $6-31+\mathrm{G} * *$ & $6-311 G *$ & $6-311 G * *$ & $6-311+\mathrm{G} * *$ \\
\hline \multicolumn{14}{|c|}{${ }^{13} \mathrm{C}$} \\
\hline 1 & 90.9 & 105.8151 & 106.3539 & 106.6930 & 94.7424 & 94.3467 & 93.9471 & 105.2931 & 105.8318 & 106.0947 & 94.1483 & 93.7490 & 93.2746 \\
\hline 2 & 45.7 & 146.3512 & 147.0644 & 146.7560 & 136.8920 & 136.7901 & 136.1180 & 146.1744 & 146.8907 & 146.4525 & 136.6912 & 136.5753 & 135.8024 \\
\hline 3 & 43.1 & 149.7976 & 150.4627 & 149.9896 & 140.8110 & 140.5938 & 139.6918 & 149.8072 & 150.4564 & 150.0805 & 140.7841 & 140.5636 & 139.6250 \\
\hline 4 & 22.5 & 169.3237 & 170.6220 & 170.0133 & 161.8416 & 161.6972 & 161.2129 & 169.1852 & 170.4842 & 169.8960 & 161.7155 & 161.5833 & 161.0944 \\
\hline 5 & 22.9 & 168.6370 & 170.0434 & 168.6096 & 160.6609 & 160.5939 & 160.0741 & 169.0825 & 170.4728 & 169.1733 & 161.1630 & 161.0909 & 160.6099 \\
\hline 6 & 27.9 & 163.6994 & 165.0755 & 163.3540 & 155.2455 & 155.1481 & 154.4139 & 164.2368 & 165.5880 & 163.9465 & 155.7717 & 155.6579 & 154.9385 \\
\hline 7 & 45.7 & 146.9243 & 147.6250 & 146.9602 & 137.0129 & 136.8517 & 136.2481 & 146.7732 & 147.4657 & 146.8520 & 136.8296 & 136.6567 & 136.0128 \\
\hline 8 & 213.8 & -13.0861 & -12.9048 & -15.3566 & -33.2321 & -32.8938 & -34.9245 & -16.2107 & -16.0330 & -19.0505 & -36.9103 & -36.5760 & -39.0408 \\
\hline 9 & 50.1 & 142.2050 & 142.9985 & 142.6297 & 133.0482 & 132.8892 & 131.8245 & 142.3316 & 143.1054 & 142.7675 & 133.1068 & 132.9290 & 131.8277 \\
\hline 10 & 38.5 & 155.1689 & 156.1565 & 156.0433 & 146.9617 & 146.5813 & 146.4620 & 155.2343 & 156.2255 & 156.1446 & 147.0813 & 146.7016 & 146.5891 \\
\hline 11 & 40.8 & 151.5889 & 152.0688 & 151.9059 & 141.7812 & 141.7863 & 141.3730 & 151.5801 & 152.0633 & 151.9395 & 141.8271 & 141.8345 & 141.3937 \\
\hline 12 & 177.9 & 26.2201 & 26.2962 & 24.4155 & 7.4735 & 7.6338 & 5.3840 & 23.1393 & 23.2144 & 20.8794 & 4.0907 & 4.2516 & 1.5109 \\
\hline 13 & 13.2 & 178.0070 & 179.8269 & 180.1148 & 172.2133 & 172.2434 & 171.8386 & 178.3978 & 180.1982 & 180.6062 & 172.6815 & 172.6929 & 172.3256 \\
\hline 14 & 24.5 & 170.2495 & 171.8354 & 172.3988 & 163.6212 & 163.4486 & 163.0498 & 170.4854 & 172.0652 & 172.7074 & 163.9566 & 163.7606 & 163.3841 \\
\hline 15 & 23.0 & 168.5931 & 170.1322 & 170.3547 & 162.1147 & 161.8776 & 161.7969 & 169.1024 & 170.6241 & 170.8927 & 162.6483 & 162.3888 & 162.3304 \\
\hline \multicolumn{14}{|c|}{${ }^{1} \mathrm{H}$} \\
\hline 1 & 4.29 & 28.0025 & 27.5011 & 27.4638 & 27.9995 & 27.7171 & 27.6925 & 27.8387 & 27.3307 & 27.2840 & 27.8332 & 27.5452 & 27.5133 \\
\hline 2 & 3.79 & 28.7528 & 28.2478 & 28.0995 & 28.6925 & 28.3647 & 28.2757 & 28.5087 & 27.9988 & 27.8379 & 28.4535 & 28.1267 & 28.0249 \\
\hline 3 & 2.77 & 29.6882 & 29.2866 & 29.1593 & 29.6807 & 29.4808 & 29.4057 & 29.4732 & 29.0646 & 28.9188 & 29.4699 & 29.2678 & 29.1748 \\
\hline 4 & 1.96 & 30.2279 & 29.7898 & 29.6434 & 30.2133 & 29.9434 & 29.8758 & 30.3088 & 29.8718 & 29.7302 & 30.2867 & 30.0206 & 29.9545 \\
\hline 4 & 1.45 & 30.6379 & 30.1589 & 30.1674 & 30.7349 & 30.4638 & 30.4637 & 30.7525 & 30.2766 & 30.2765 & 30.8389 & 30.5681 & 30.5636 \\
\hline
\end{tabular}




\begin{tabular}{rrrlllllllllll}
\hline 5 & 1.58 & 30.6880 & 30.2722 & 30.2125 & 30.5888 & 30.3980 & 30.3821 & 30.5847 & 30.1651 & 30.0985 & 30.4925 & 30.2973 & 30.2745 \\
5 & 1.93 & 30.2188 & 29.7638 & 29.6958 & 30.1561 & 29.9120 & 29.8897 & 30.1991 & 29.7438 & 29.6774 & 30.1413 & 29.8975 & 29.8749 \\
6 & 1.83 & 30.3963 & 29.9775 & 29.8218 & 30.2997 & 30.0923 & 30.0944 & 30.3526 & 29.9270 & 29.7802 & 30.2561 & 30.0438 & 30.0523 \\
6 & 2.43 & 29.9046 & 29.4362 & 29.3931 & 29.7913 & 29.5455 & 29.5897 & 29.7655 & 29.2980 & 29.2491 & 29.6645 & 29.4205 & 29.4624 \\
7 & 2.53 & 29.6875 & 29.2922 & 29.1852 & 29.6306 & 29.4598 & 29.4200 & 29.5242 & 29.1241 & 29.0090 & 29.4725 & 29.2973 & 29.2486 \\
9 & 3.28 & 29.0817 & 28.6130 & 28.4906 & 29.0566 & 28.7738 & 28.7023 & 28.8781 & 28.4034 & 28.2710 & 28.8553 & 28.5711 & 28.4882 \\
10 & 2.16 & 29.5523 & 29.0785 & 29.0778 & 29.6069 & 29.3603 & 29.3476 & 29.7125 & 29.2450 & 29.2328 & 29.7617 & 29.5178 & 29.4964 \\
10 & 1.38 & 30.8400 & 30.4245 & 30.3825 & 30.8986 & 30.6511 & 30.6121 & 30.7767 & 30.3581 & 30.3135 & 30.8335 & 30.5862 & 30.5422 \\
13 & 1.11 & 30.9996 & 30.5484 & 30.4739 & 30.9612 & 30.6929 & 30.6811 & 31.0182 & 30.5673 & 30.4919 & 30.9792 & 30.7112 & 30.6981 \\
14 & 1.19 & 30.8610 & 30.4173 & 30.3807 & 30.8193 & 30.5645 & 30.5289 & 30.8847 & 30.4411 & 30.4057 & 30.8425 & 30.5881 & 30.5517 \\
15 & 1.02 & 31.1747 & 30.7375 & 30.6629 & 31.1445 & 30.8977 & 30.8374 & 31.1113 & 30.6719 & 30.5957 & 31.0858 & 30.8373 & 30.7766 \\
\hline
\end{tabular}

\section{Compound 55}

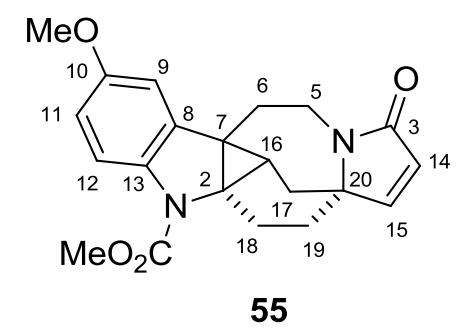

\begin{tabular}{|c|c|c|c|c|c|c|c|c|c|c|c|c|c|}
\hline & \multicolumn{13}{|c|}{ B3LYP } \\
\hline & \multirow[b]{2}{*}{ Exp } & \multicolumn{6}{|c|}{ Gas phase } & \multicolumn{6}{|c|}{ PCM } \\
\hline & & 6-31G* & 6-31G** & $6-31+G * *$ & 6-311G* & $6-311 G * *$ & $6-311+G * *$ & 6-31G* & $6-31 G * *$ & $6-31+G * *$ & 6-311G* & $6-311 G * *$ & $6-311+G * *$ \\
\hline \multicolumn{14}{|l|}{${ }^{13} \mathrm{C}$} \\
\hline 2 & 50.1 & 136.4842 & 136.8167 & 137.0084 & 126.8065 & 126.9929 & 126.0061 & 136.6990 & 137.0278 & 137.3068 & 126.9061 & 127.0974 & 126.0956 \\
\hline 3 & 169.8 & 32.7022 & 32.7234 & 29.9924 & 11.7843 & 11.7974 & 9.1986 & 31.3011 & 31.3222 & 28.2860 & 10.1286 & 10.1416 & 7.2021 \\
\hline 5 & 36.1 & 153.4122 & 154.7487 & 153.8652 & 145.4211 & 145.1269 & 144.6786 & 153.2595 & 154.6021 & 153.7706 & 145.3328 & 145.0330 & 144.5625 \\
\hline 6 & 27.9 & 159.0326 & 160.3680 & 159.3883 & 149.7368 & 149.4590 & 149.2451 & 159.4396 & 160.7701 & 159.7920 & 150.1848 & 149.9049 & 149.7923 \\
\hline 7 & 33.6 & 153.4401 & 153.5856 & 153.2315 & 142.5325 & 142.6134 & 142.2754 & 153.2623 & 153.4080 & 152.9193 & 142.3416 & 142.4242 & 142.0253 \\
\hline 8 & 139.4 & 56.2974 & 56.1544 & 53.6101 & 36.8768 & 36.6590 & 35.8481 & 55.7444 & 55.6066 & 52.9661 & 36.2531 & 36.0640 & 35.2852 \\
\hline 9 & 108.9 & 86.9217 & 87.7278 & 87.7723 & 71.7619 & 71.1548 & 70.3071 & 86.8111 & 87.6106 & 87.6194 & 71.5509 & 70.9664 & 70.1173 \\
\hline 10 & 156.1 & 42.2047 & 41.8769 & 39.4439 & 20.1420 & 19.9513 & 18.8421 & 42.1990 & 41.8727 & 39.4634 & 20.1573 & 19.9741 & 18.8657 \\
\hline 11 & 111.2 & 84.5694 & 85.3210 & 86.6104 & 70.1257 & 69.6877 & 68.9254 & 84.6785 & 85.4128 & 86.5091 & 70.0809 & 69.6054 & 68.7394 \\
\hline 12 & 116.3 & 78.2491 & 78.9323 & 78.4024 & 62.3312 & 61.5384 & 60.8834 & 78.7838 & 79.4672 & 78.9100 & 62.9367 & 62.1492 & 61.4972 \\
\hline 13 & 135.0 & 60.7297 & 60.6628 & 59.4003 & 41.4771 & 41.4221 & 40.6992 & 60.5846 & 60.5181 & 59.1326 & 41.3188 & 41.2672 & 40.5282 \\
\hline 14 & 125.1 & 68.3825 & 68.8345 & 68.8876 & 50.8626 & 49.9518 & 48.8954 & 69.7706 & 70.2274 & 70.4339 & 52.4217 & 51.5433 & 50.5492 \\
\hline 15 & 154.1 & 42.1829 & 42.6117 & 41.8664 & 22.7273 & 21.8881 & 20.5010 & 39.7560 & 40.1826 & 39.0225 & 19.8764 & 19.0433 & 17.4064 \\
\hline 16 & 26.0 & 160.6468 & 161.5396 & 163.0335 & 151.3722 & 151.0704 & 149.6145 & 160.7317 & 161.6212 & 163.1418 & 151.4497 & 151.1471 & 149.7109 \\
\hline 17 & 30.6 & 156.4313 & 157.7432 & 155.6940 & 147.2748 & 146.9351 & 147.2099 & 157.1013 & 158.4014 & 156.5047 & 148.0084 & 147.6588 & 147.9374 \\
\hline 18 & 20.5 & 166.1970 & 167.6806 & 168.2696 & 159.2726 & 158.9652 & 158.7064 & 166.3812 & 167.8600 & 168.5480 & 159.4492 & 159.1315 & 158.9012 \\
\hline 19 & 28.9 & 158.6426 & 159.9669 & 159.4408 & 149.5794 & 149.4928 & 149.5407 & 159.1324 & 160.4486 & 159.9445 & 150.1131 & 150.0138 & 150.1530 \\
\hline 20 & 65.6 & 125.4683 & 125.7472 & 125.0859 & 112.9279 & 112.9818 & 111.7475 & 124.8975 & 125.1720 & 124.4414 & 112.2391 & 112.2931 & 110.8929 \\
\hline 10-OMe & 55.7 & 137.3989 & 139.2039 & 139.2776 & 128.7784 & 128.0975 & 127.6717 & 137.3305 & 139.1121 & 138.9235 & 128.5080 & 127.7951 & 127.3514 \\
\hline $\mathrm{NCO}_{2} \mathrm{Me}$ & 154.8 & 44.4024 & 44.4020 & 42.2520 & 22.6559 & 22.6426 & 21.9350 & 43.8082 & 43.8046 & 41.6240 & 21.9206 & 21.9146 & 21.1178 \\
\hline
\end{tabular}




\begin{tabular}{|c|c|c|c|c|c|c|c|c|c|c|c|c|c|}
\hline${ }^{1} \mathbf{H}$ & & & & 1 & 129.85 & 04 & 53 & 138.2 & 139.9 & 139.7 & 129.5 & & \\
\hline 5 & 3.53 & 28.8699 & 28.3308 & 28.0898 & 28.8502 & 28.5461 & & & & & 28.7533 & & 29207 \\
\hline 5 & 4.30 & 0619 & & & & & & & & & & & \\
\hline 6 & 2.64 & .8495 & 3943 & 1328 & 29.8030 & 5 & & .7948 & 53 & & & & $333 \varepsilon^{3}$ \\
\hline 6 & 2. & & & & & & & & & & & & \\
\hline 9 & & & & & & & & & & & & & \\
\hline 11 & & & & & & & & & & & & & \\
\hline & & & & & & & & & & & & & \\
\hline 14 & 6.09 & & & & & & & & & & & & \\
\hline 15 & 6.88 & & & & & & & & & & & & \\
\hline 16 & 1.13 & & 0 & & & & & & & & & & \\
\hline 17 & & & & & & & & & & & & & \\
\hline 17 & & & & & & & & & & & & & \\
\hline 18 & & & & & & & & & & & & & \\
\hline 18 & 2.91 & 29.2224 & 28.6795 & 28.5430 & 29.2254 & 28.8944 & 28.8544 & 29.2830 & 28.7442 & 28.6198 & 29.2836 & 28.9573 & 28.9186 \\
\hline 19 & 1.5 & 602 & & & & & & 165 & & & 199 & 3958 & 30.3325 \\
\hline 19 & 2.13 & .0441 & 6332 & & D.0206 & & & 9.9894 & & 29.4658 & & 575 & 29.6869 \\
\hline $\mathrm{OMe}$ & 3.79 & 28.5722 & & & 28.5371 & 28.2407 & & 28.4854 & & 27.7730 & 28.4470 & 28.1529 & 28.0470 \\
\hline & & & & & & & & & & & וזכנים & & \\
\hline
\end{tabular}

\begin{tabular}{|c|c|c|c|c|c|c|c|c|c|c|c|c|c|}
\hline & \multirow[b]{3}{*}{ Exp } & \multicolumn{12}{|c|}{ mPW1PW91 } \\
\hline & & \multicolumn{6}{|c|}{ Gas phase } & \multicolumn{6}{|c|}{ PCM } \\
\hline & & 6-31G* & 6-31G** & $6-31+G * *$ & 6-311G* & 6-311G** & $6-311+G^{* *}$ & 6-31G* & 6-31G** & $6-31+G * *$ & 6-311G* & 6-311G** & $6-311+G^{* *}$ \\
\hline${ }^{13} \mathrm{C}$ & & & & & & & & & & & & & \\
\hline 2 & 50.1 & 141.0789 & 141.3971 & 141.9001 & 132.2949 & 132.4687 & 131.5530 & 141.2835 & 141.5989 & 142.1625 & 132.3890 & 132.5678 & 131.6360 \\
\hline 3 & 169.8 & 34.9685 & 34.9793 & 32.9049 & 16.7461 & 16.7569 & 14.3766 & 33.5547 & 33.5652 & 31.2388 & 15.1092 & 15.1196 & 12.4343 \\
\hline 5 & 36.1 & 157.4733 & 158.7223 & 157.9424 & 150.3974 & 150.1165 & 149.6863 & 157.3345 & 158.5895 & 157.8796 & 150.3111 & 150.0247 & 149.5687 \\
\hline 6 & 27.9 & 163.6634 & 164.9092 & 164.1719 & 155.4285 & 155.1626 & 154.8398 & 164.0766 & 165.3186 & 164.5762 & 155.8621 & 155.5945 & 155.3497 \\
\hline 7 & 33.6 & 157.7359 & 157.8783 & 157.7684 & 147.8498 & 147.9288 & 147.5505 & 157.5687 & 157.7110 & 157.4832 & 147.6637 & 147.7449 & 147.3103 \\
\hline 8 & 139.4 & 59.5871 & 59.4219 & 57.3690 & 42.5620 & 42.3218 & 41.5165 & 59.0468 & 58.8880 & 56.7557 & 41.9659 & 41.7572 & 40.9776 \\
\hline 9 & 108.9 & 89.2595 & 90.0365 & 90.2120 & 76.0367 & 75.4159 & 74.5852 & 89.1255 & 89.8946 & 90.0356 & 75.7821 & 75.1859 & 74.3488 \\
\hline 10 & 156.1 & 45.1914 & 44.8300 & 43.0958 & 26.1261 & 25.9051 & 24.7800 & 45.1697 & 44.8109 & 43.1188 & 26.1214 & 25.9089 & 24.7902 \\
\hline 11 & 111.2 & 87.0486 & 87.7530 & 88.9181 & 74.3580 & 73.9181 & 73.1234 & 87.1394 & 87.8250 & 88.8042 & 74.2943 & 73.8134 & 72.9463 \\
\hline 12 & 116.3 & 80.7976 & 81.4190 & 81.1157 & 66.8566 & 66.0351 & 65.3128 & 81.3306 & 81.9517 & 81.6217 & 67.4187 & 66.6036 & 65.8827 \\
\hline 13 & 135.0 & 63.8491 & 63.7693 & 62.9200 & 47.0029 & 46.9364 & 46.1245 & 63.7513 & 63.6717 & 62.6951 & 46.8857 & 46.8234 & 45.9957 \\
\hline 14 & 125.1 & 70.7336 & 71.1297 & 71.2317 & 55.2214 & 54.2834 & 53.1605 & 72.1647 & 72.5675 & 72.8162 & 56.7691 & 55.8678 & 54.7947 \\
\hline 15 & 154.1 & 44.8867 & 45.2494 & 44.4733 & 27.6313 & 26.7445 & 25.5886 & 42.3614 & 42.7226 & 41.5781 & 24.7357 & 23.8549 & 22.4836 \\
\hline 16 & 26.0 & 165.5875 & 166.4230 & 167.4897 & 157.0829 & 156.7712 & 155.6399 & 165.6715 & 166.5040 & 167.5876 & 157.1464 & 156.8344 & 155.7167 \\
\hline 17 & 30.6 & 161.3328 & 162.5356 & 160.4207 & 152.9535 & 152.6230 & 152.9179 & 161.9940 & 163.1871 & 161.1958 & 153.6512 & 153.3128 & 153.6008 \\
\hline 18 & 20.5 & 170.6716 & 172.0509 & 172.6739 & 164.5797 & 164.2753 & 164.0068 & 170.8881 & 172.2628 & 172.9609 & 164.7675 & 164.4527 & 164.2095 \\
\hline 19 & 28.9 & 163.7065 & 164.9196 & 164.2623 & 155.2953 & 155.2219 & 155.2592 & 164.2009 & 165.4075 & 164.7492 & 155.8100 & 155.7250 & 155.8395 \\
\hline 20 & 65.6 & 130.0034 & 130.2676 & 129.6655 & 118.8213 & 118.8719 & 117.7176 & 129.4297 & 129.6891 & 129.0359 & 118.1378 & 118.1886 & 116.8829 \\
\hline 10-OMe & 55.7 & 140.9539 & 142.6918 & 142.7239 & 133.5962 & 132.9609 & 132.6151 & 140.8750 & 142.5900 & 142.3667 & 133.3155 & 132.6498 & 132.2972 \\
\hline $\mathrm{NCO}_{2} \mathrm{Me}$ & 154.8 & 47.1022 & 47.0995 & 45.4141 & 27.8010 & 27.7920 & 27.2042 & 46.4849 & 46.4792 & 44.7780 & 27.0656 & 27.0635 & 26.3876 \\
\hline
\end{tabular}




\begin{tabular}{|c|c|c|c|c|c|c|c|c|c|c|c|c|c|}
\hline $\begin{array}{c}\mathrm{CO}_{2} \mathrm{M} \\
{ }^{1} \mathbf{H}\end{array}$ & & & & & 134.3 & 96 & 01 & 141.6 & & 143.2 & 134.0 & 3 & \\
\hline 5 & 3.53 & 28.8279 & 28.2848 & 28.0309 & 28.7539 & 28.4579 & 28.3063 & 28.7290 & 28.1851 & 27.9231 & 28.6548 & 28.3620 & 0,202 \\
\hline 5 & 4.30 & 9662 & 3610 & & & & & .0833 & 27.4839 & & & & 4566 \\
\hline 6 & 2.64 & .8001 & .3433 & 9.0953 & 297134 & 94410 & & .7393 & 29.2790 & 29.0249 & & & 2399 \\
\hline 6 & 2.64 & .5305 & .0621 & 88 & 14 & & & 666 & 949 & 614 & & & 516 \\
\hline 9 & 6.72 & & & & & & & & & & & & \\
\hline 11 & & & & & & & & & & & & & \\
\hline 12 & & & & & & & & & & & & & \\
\hline 14 & 6.09 & & & & & & & & & & & & \\
\hline 15 & 6.88 & .3623 & .6930 & & & & & & & & & & \\
\hline 16 & 1.13 & 1815 & 3006 & .4997 & & & & & & & & 250 & 905 \\
\hline 17 & 1.8 & & & & & & & & & & & & \\
\hline 17 & & & & & & & & & & & & & \\
\hline 18 & & & & & & & & & & & & & \\
\hline 18 & 2.91 & & & & & & & & & & & & \\
\hline 19 & 1.5 & & & & & & & & & & & & 2405 \\
\hline 19 & & & & & & & & 509 & 368 & 296 & & 656 & 29.6005 \\
\hline $\mathrm{Me}$ & 3.79 & 28.5039 & & & 28.4696 & & & 28.4071 & 118 & 27.7227 & 28.3721 & 28.0681 & 27.9821 \\
\hline$C_{2} \mathrm{w}$ & & 28.4304 & 27.9169 & 27.7940 & 28.4033 & 28.0541 & 28.0434 & 28.3518 & 27.8381 & 27.7244 & 28.3270 & 27.9793 & ביוניר \\
\hline
\end{tabular}

\section{Compound 56}

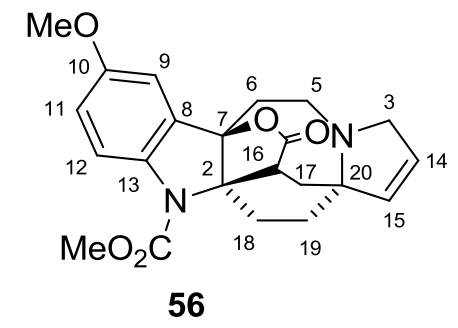

\begin{tabular}{|c|c|c|c|c|c|c|c|c|c|c|c|c|c|}
\hline & \multirow[b]{3}{*}{ Exp } & \multicolumn{12}{|c|}{ B3LYP } \\
\hline & & \multicolumn{6}{|c|}{ Gas phase } & \multicolumn{6}{|c|}{ PCM } \\
\hline & & $6-31 G *$ & $6-31 G * *$ & $6-31+G * *$ & 6-311G* & $6-311 G * *$ & $6-311+G^{* *}$ & 6-31G* & 6-31G** & $6-31+\mathrm{G} * *$ & $6-311 G *$ & $6-311 G * *$ & $6-311+G * *$ \\
\hline \multicolumn{14}{|l|}{${ }^{13} \mathrm{C}$} \\
\hline 2 & 73.9 & 112.8143 & 113.1432 & 113.0550 & 101.5514 & 101.6694 & 100.8578 & 112.7012 & 113.0274 & 112.8967 & 101.2988 & 101.4219 & 100.6056 \\
\hline 3 & 61.7 & 129.2733 & 130.3513 & 129.6345 & 118.2926 & 117.5923 & 117.2279 & 129.3450 & 130.4281 & 129.6656 & 118.3960 & 117.6911 & 117.3343 \\
\hline 5 & 47.1 & 141.4531 & 142.7105 & 142.5059 & 132.1374 & 131.7977 & 131.4641 & 141.7165 & 142.9689 & 142.7204 & 132.4140 & 132.0651 & 131.7794 \\
\hline 6 & 29.8 & 157.1978 & 158.5166 & 157.7925 & 148.6909 & 148.4916 & 148.0052 & 157.7383 & 159.0376 & 158.4340 & 149.1938 & 148.9779 & 148.5506 \\
\hline 7 & 90.8 & 100.5691 & 100.6859 & 101.4089 & 88.5059 & 88.5532 & 87.5314 & 99.6757 & 99.7881 & 100.3313 & 87.4945 & 87.5393 & 86.3686 \\
\hline 8 & 130.1 & 64.3288 & 64.1748 & 62.7271 & 46.2620 & 46.0362 & 44.7276 & 64.6284 & 64.4795 & 62.9976 & 46.4783 & 46.2781 & 45.0111 \\
\hline 9 & 108.7 & 85.4944 & 86.2727 & 85.7112 & 70.4091 & 69.7862 & 69.0668 & 85.2576 & 86.0280 & 85.3368 & 69.9860 & 69.3811 & 68.7094 \\
\hline 10 & 156.0 & 42.3936 & 42.0595 & 39.1956 & 20.3911 & 20.1971 & 19.1994 & 42.2313 & 41.9008 & 39.0584 & 20.2556 & 20.0710 & 19.0940 \\
\hline 11 & 116.4 & 80.0822 & 80.8193 & 81.3670 & 65.0180 & 64.5135 & 63.3801 & 79.7868 & 80.5084 & 80.8122 & 64.5722 & 64.0339 & 62.7653 \\
\hline 12 & 116.6 & 78.3582 & 79.0296 & 78.0079 & 62.5541 & 61.7510 & 60.8569 & 78.5997 & 79.2708 & 78.1237 & 62.8743 & 62.0748 & 61.1380 \\
\hline 13 & 134.5 & 60.6590 & 60.5617 & 58.9145 & 41.5107 & 41.4684 & 41.1741 & 60.4907 & 60.3905 & 58.5524 & 41.2879 & 41.2458 & 40.9319 \\
\hline
\end{tabular}




\begin{tabular}{|c|c|c|c|c|c|c|c|c|c|c|c|c|c|}
\hline 14 & 126.7 & 67.5797 & 68.1861 & 67.6344 & 49.8551 & 48.9522 & 47.8973 & 67.3898 & 68.0012 & 67.3661 & 49.5691 & 48.6942 & 47.6628 \\
\hline 15 & 135.8 & 57.9567 & 58.4383 & 58.2673 & 39.9032 & 39.1318 & 38.0839 & 57.8319 & 58.3088 & 58.1573 & 39.6674 & 38.9143 & 37.8534 \\
\hline 16 & 45.2 & 144.0558 & 144.8167 & 144.6083 & 133.8419 & 133.4372 & 132.4042 & 143.6690 & 144.4221 & 144.2134 & 133.3818 & 132.9700 & 131.8180 \\
\hline 17 & 38.5 & 148.2997 & 149.6151 & 149.4712 & 139.0286 & 138.7688 & 138.3586 & 147.9701 & 149.2839 & 149.2059 & 138.6371 & 138.3724 & 137.8673 \\
\hline 18 & 22.4 & 165.1819 & 166.5994 & 167.3076 & 157.6687 & 157.5039 & 156.8525 & 165.1349 & 166.5443 & 167.4359 & 157.5851 & 157.4074 & 156.6029 \\
\hline 19 & 25.1 & 162.5107 & 163.9551 & 162.4991 & 154.4610 & 154.2196 & 154.2916 & 162.9618 & 164.3930 & 162.9979 & 154.9550 & 154.6938 & 154.7302 \\
\hline 20 & 67.4 & 121.4080 & 121.6900 & 121.1312 & 109.6244 & 109.6447 & 109.7747 & 121.3644 & 121.6480 & 121.1084 & 109.5638 & 109.5851 & 109.6239 \\
\hline 10-OMe & 55.7 & 137.2868 & 139.0882 & 139.4075 & 128.6309 & 127.9453 & 127.2743 & 137.1716 & 138.9483 & 139.0222 & 128.3121 & 127.5935 & 126.9219 \\
\hline $\mathrm{NCO}_{2} \mathrm{Me}$ & 52.7 & 138.7053 & 140.4192 & 140.3684 & 130.1132 & 129.5307 & 129.1956 & 138.4098 & 140.1091 & 140.0526 & 129.7896 & 129.1850 & 128.8515 \\
\hline $\mathrm{NCO}_{2} \mathrm{Me}$ & 152.4 & 46.0252 & 46.0100 & 43.9369 & 24.4401 & 24.4293 & 23.7994 & 45.4981 & 45.4822 & 43.4279 & 23.7768 & 23.7740 & 23.0672 \\
\hline $\begin{array}{c}\mathrm{CO} \text { lac } \\
{ }^{1} \mathbf{H}\end{array}$ & 177.3 & 22.8830 & 22.9621 & 21.1873 & 1.6609 & 1.7624 & -0.5715 & 20.5998 & 20.6705 & 18.5142 & -0.9713 & -0.8785 & -3.4892 \\
\hline 3 & 3.18 & 29.2080 & 28.7157 & 28.5159 & 29.1317 & 28.8419 & 28.7542 & 29.0988 & 28.6017 & 28.3905 & 29.0198 & 28.7268 & 28.6258 \\
\hline 3 & 3.80 & 28.2370 & 27.6625 & 27.5107 & 28.2040 & 27.8470 & 27.8167 & 28.3587 & 27.7863 & 27.6221 & 28.3146 & 27.9579 & 27.9199 \\
\hline 5 & 2.91 & 29.2347 & 28.8255 & 28.6884 & 29.0427 & 28.7432 & 28.6517 & 29.2316 & 28.8243 & 28.6915 & 28.9668 & 28.6654 & 28.5827 \\
\hline 5 & 3.24 & 29.0350 & 28.5373 & 28.4326 & 29.2425 & 29.0185 & 28.9087 & 28.9556 & 28.4560 & 28.3695 & 29.2324 & 29.0131 & 28.9117 \\
\hline 6 & 2.15 & 30.2748 & 29.8104 & 29.5568 & 30.2547 & 29.9854 & 29.8032 & 30.1572 & 29.6879 & 29.4434 & 30.1420 & 29.8710 & 29.6969 \\
\hline 6 & 2.70 & 29.8422 & 29.3581 & 29.1838 & 29.7647 & 29.4683 & 29.3504 & 29.7408 & 29.2533 & 29.0669 & 29.6617 & 29.3636 & 29.2462 \\
\hline 9 & 6.94 & 25.6635 & 24.9262 & 24.7107 & 25.5366 & 25.0535 & 24.9491 & 25.5430 & 24.8014 & 24.5594 & 25.4024 & 24.9203 & 24.7998 \\
\hline 11 & 6.90 & 25.6781 & 25.0020 & 24.7236 & 25.5018 & 25.0940 & 24.9432 & 25.5541 & 24.8782 & 24.5680 & 25.3653 & 24.9643 & 24.8040 \\
\hline 12 & 7.71 & 24.6499 & 23.8838 & 23.6679 & 24.4442 & 23.9435 & 23.8503 & 24.5721 & 23.7993 & 23.5438 & 24.3648 & 23.8630 & 23.7487 \\
\hline 14 & 5.73 & 26.6551 & 25.9952 & 25.7841 & 26.5504 & 26.0904 & 26.0104 & 26.5628 & 25.9023 & 25.6892 & 26.4482 & 25.9933 & 25.9145 \\
\hline 15 & 5.53 & 26.6126 & 25.9933 & 25.9642 & 26.6367 & 26.2324 & 26.1949 & 26.4984 & 25.8783 & 25.8411 & 26.5120 & 26.1121 & 26.0729 \\
\hline 16 & 3.36 & 29.0524 & 28.5319 & 28.4445 & 29.0947 & 28.7618 & 28.7194 & 29.0402 & 28.5161 & 28.4245 & 29.0696 & 28.7371 & 28.6863 \\
\hline 17 & 1.99 & 30.0066 & 29.5898 & 29.3945 & 30.0356 & 29.8400 & 29.7454 & 29.9219 & 29.5015 & 29.2993 & 29.9385 & 29.7410 & 29.6390 \\
\hline 17 & 2.25 & 30.1101 & 29.6970 & 29.6047 & 30.1230 & 29.9017 & 29.8834 & 30.2101 & 29.7983 & 29.6952 & 30.2201 & 29.9977 & 29.9622 \\
\hline 18 & 1.71 & 30.7344 & 30.2911 & 30.0809 & 30.7523 & 30.4863 & 30.3366 & 30.6217 & 30.1719 & 29.9521 & 30.6445 & 30.3736 & 30.2084 \\
\hline 18 & 2.52 & 29.5530 & 29.0325 & 28.7630 & 29.6080 & 29.2596 & 29.1352 & 29.5836 & 29.0656 & 28.8063 & 29.6315 & 29.2880 & 29.1665 \\
\hline 19 & 1.99 & 30.3737 & 29.9424 & 29.9367 & 30.1060 & 29.8681 & 29.7170 & 30.2491 & 29.8150 & 29.8118 & 30.0436 & 29.8037 & 29.6463 \\
\hline 19 & 2.05 & 30.1075 & 29.6636 & 29.5642 & 30.3358 & 30.1144 & 30.0388 & 30.0365 & 29.5902 & 29.4894 & 30.2123 & 29.9904 & 29.9144 \\
\hline 10-OMe & 3.80 & 28.5388 & 28.0392 & 27.8586 & 28.4988 & 28.2036 & 28.1326 & 28.4460 & 27.9468 & 27.7554 & 28.4027 & 28.1095 & 28.0325 \\
\hline $\mathrm{NCO}_{2} \mathrm{Me}$ & 3.88 & 28.4901 & 27.9729 & 27.8107 & 28.4690 & 28.1270 & 28.1158 & 28.4219 & 27.9044 & 27.7507 & 28.4037 & 28.0629 & 28.0510 \\
\hline
\end{tabular}

\begin{tabular}{|c|c|c|c|c|c|c|c|c|c|c|c|c|c|}
\hline & \multicolumn{13}{|c|}{ mPW1PW91 } \\
\hline & \multirow[b]{2}{*}{ Exp } & \multicolumn{6}{|c|}{ Gas phase } & \multicolumn{6}{|c|}{ PCM } \\
\hline & & $6-31 G^{*}$ & $6-31 G^{* *}$ & $6-31+\mathrm{G} * *$ & 6-311G* & 6-311G** & $6-311+G * *$ & 6-31G* & 6-31G** & $6-31+G * *$ & 6-311G* & $6-311 G * *$ & $6-311+G * *$ \\
\hline \multicolumn{14}{|l|}{${ }^{13} \mathrm{C}$} \\
\hline 2 & 73.9 & 117.7064 & 118.0221 & 117.7949 & 107.6074 & 107.7189 & 106.9756 & 117.5892 & 117.9022 & 117.6455 & 107.3642 & 107.4811 & 106.7211 \\
\hline 3 & 61.7 & 133.1183 & 134.1309 & 133.3927 & 123.5621 & 122.8655 & 122.5478 & 133.2234 & 134.2406 & 133.4398 & 123.6823 & 122.9809 & 122.6700 \\
\hline 5 & 47.1 & 146.1092 & 147.2627 & 146.9990 & 137.9020 & 137.5641 & 137.2623 & 146.3828 & 147.5330 & 147.2283 & 138.1733 & 137.8271 & 137.5744 \\
\hline 6 & 29.8 & 161.7671 & 162.9783 & 162.3053 & 154.0925 & 153.9033 & 153.5086 & 162.3186 & 163.5119 & 162.9354 & 154.5751 & 154.3711 & 154.0307 \\
\hline 7 & 90.8 & 105.0551 & 105.1656 & 106.2783 & 94.6485 & 94.6955 & 93.6762 & 104.1625 & 104.2682 & 105.2059 & 93.6443 & 93.6890 & 92.5478 \\
\hline 8 & 130.1 & 67.5556 & 67.3928 & 66.3892 & 51.7635 & 51.5269 & 50.2615 & 67.8681 & 67.7119 & 66.6617 & 52.0024 & 51.7937 & 50.5660 \\
\hline 9 & 108.7 & 87.8380 & 88.5925 & 88.0963 & 74.6933 & 74.0565 & 73.3650 & 87.5706 & 88.3155 & 87.6956 & 74.2246 & 73.6071 & 72.9614 \\
\hline 10 & 156.0 & 45.3890 & 45.0189 & 43.0401 & 26.4001 & 26.1775 & 25.1012 & 45.2081 & 44.8427 & 42.9085 & 26.2421 & 26.0300 & 24.9820 \\
\hline
\end{tabular}




\begin{tabular}{|c|c|c|c|c|c|c|c|c|c|c|c|c|c|}
\hline 11 & 116.4 & 82.4788 & 83.1605 & 83.8038 & 69.2459 & 68.7355 & 67.6077 & 82.1694 & 82.8351 & 83.2508 & 68.7882 & 68.2419 & 67.0181 \\
\hline 12 & 116.6 & 80.9469 & 81.5547 & 80.9360 & 67.0977 & 66.2640 & 65.2445 & 81.1869 & 81.7940 & 81.0835 & 67.3763 & 66.5473 & 65.4820 \\
\hline 13 & 134.5 & 63.6664 & 63.5558 & 62.4346 & 46.9041 & 46.8504 & 46.3356 & 63.5448 & 63.4312 & 62.1537 & 46.7288 & 46.6763 & 46.1364 \\
\hline 14 & 126.7 & 69.8851 & 70.4126 & 69.8563 & 54.0722 & 53.1298 & 52.0153 & 69.6488 & 70.1810 & 69.5689 & 53.7301 & 52.8182 & 51.7300 \\
\hline 15 & 135.8 & 60.6856 & 61.1058 & 60.8934 & 44.6121 & 43.8066 & 42.8626 & 60.5641 & 60.9810 & 60.8057 & 44.3912 & 43.6062 & 42.6548 \\
\hline 16 & 45.2 & 148.6176 & 149.3271 & 149.1743 & 139.5105 & 139.1125 & 138.1759 & 148.2307 & 148.9319 & 148.7984 & 139.0494 & 138.6442 & 137.6189 \\
\hline 17 & 38.5 & 153.0208 & 154.2196 & 153.6887 & 144.5350 & 144.2918 & 143.8558 & 152.6996 & 153.8965 & 153.4352 & 144.1490 & 143.9009 & 143.3901 \\
\hline 18 & 22.4 & 169.8641 & 171.1669 & 171.9024 & 163.0301 & 162.8751 & 162.1652 & 169.8139 & 171.1100 & 172.0278 & 162.9377 & 162.7718 & 161.9454 \\
\hline 19 & 25.1 & 167.2015 & 168.5410 & 166.8249 & 159.8528 & 159.6128 & 159.6423 & 167.6764 & 169.0042 & 167.3271 & 160.3477 & 160.0902 & 160.0917 \\
\hline 20 & 67.4 & 126.0159 & 126.2794 & 125.7349 & 115.5439 & 115.5471 & 115.6859 & 125.9757 & 126.2409 & 125.7157 & 115.4879 & 115.4927 & 115.5614 \\
\hline 10-OMe & 55.7 & 140.8605 & 142.5948 & 142.7235 & 133.4642 & 132.8261 & 132.2424 & 140.7304 & 142.4409 & 142.3381 & 133.1323 & 132.4628 & 131.8798 \\
\hline $\mathrm{NCO}_{2} \mathrm{Me}$ & 52.7 & 142.1231 & 143.7645 & 143.8790 & 134.5660 & 134.0271 & 133.7474 & 141.8275 & 143.4544 & 143.5742 & 134.2420 & 133.6822 & 133.3959 \\
\hline $\mathrm{NCO}_{2} \mathrm{Me}$ & 152.4 & 48.7917 & 48.7743 & 47.1836 & 29.5261 & 29.5204 & 29.0290 & 48.2507 & 48.2327 & 46.6492 & 28.8725 & 28.8739 & 28.3137 \\
\hline $\begin{array}{c}\text { CO lac } \\
{ }^{1} \mathbf{H}\end{array}$ & 177.3 & 25.6004 & 25.6718 & 24.4297 & 6.8750 & 6.9758 & 4.9156 & 23.2934 & 23.3567 & 21.7714 & 4.2665 & 4.3587 & 2.0515 \\
\hline 3 & 3.18 & 29.1235 & 28.6321 & 28.4332 & 29.0231 & 28.7229 & 28.6356 & 29.0064 & 28.5097 & 28.3008 & 28.9049 & 28.6016 & 28.5011 \\
\hline 3 & 3.80 & 28.1869 & 27.6062 & 27.4635 & 28.1156 & 27.7515 & 27.7412 & 28.3067 & 27.7284 & 27.5716 & 28.2246 & 27.8613 & 27.8425 \\
\hline 5 & 2.91 & 29.1628 & 28.7560 & 28.6204 & 28.9690 & 28.6704 & 28.5652 & 29.1551 & 28.7501 & 28.6163 & 28.8874 & 28.5869 & 28.4877 \\
\hline 5 & 3.24 & 29.0194 & 28.5212 & 28.3986 & 29.1420 & 28.9173 & 28.8098 & 28.9328 & 28.4326 & 28.3263 & 29.1278 & 28.9079 & 28.8052 \\
\hline 6 & 2.15 & 30.2277 & 29.7584 & 29.5079 & 30.1512 & 29.8826 & 29.7193 & 30.1064 & 29.6316 & 29.3895 & 30.0362 & 29.7652 & 29.6089 \\
\hline 6 & 2.70 & 29.7603 & 29.2755 & 29.1140 & 29.6566 & 29.3596 & 29.2538 & 29.6488 & 29.1603 & 28.9893 & 29.5455 & 29.2468 & 29.1407 \\
\hline 9 & 6.94 & 25.4493 & 24.7173 & 24.5154 & 25.3421 & 24.8394 & 24.7482 & 25.3141 & 24.5778 & 24.3547 & 25.1961 & 24.6947 & 24.5896 \\
\hline 11 & 6.90 & 25.4657 & 24.7911 & 24.5259 & 25.3139 & 24.8764 & 24.7484 & 25.3267 & 24.6515 & 24.3631 & 25.1666 & 24.7350 & 24.5996 \\
\hline 12 & 7.71 & 24.4753 & 23.7121 & 23.4985 & 24.3005 & 23.7728 & 23.6812 & 24.3857 & 23.6154 & 23.3696 & 24.2091 & 23.6802 & 23.5708 \\
\hline 14 & 5.73 & 26.4890 & 25.8233 & 25.6216 & 26.4075 & 25.9122 & 25.8354 & 26.3846 & 25.7180 & 25.5169 & 26.2953 & 25.8054 & 25.7320 \\
\hline 15 & 5.53 & 26.4540 & 25.8345 & 25.8027 & 26.4960 & 26.0582 & 26.0132 & 26.3307 & 25.7101 & 25.6738 & 26.3661 & 25.9326 & 25.8867 \\
\hline 16 & 3.36 & 28.9726 & 28.4441 & 28.3414 & 28.9686 & 28.6316 & 28.5947 & 28.9612 & 28.4290 & 28.3212 & 28.9449 & 28.6085 & 28.5624 \\
\hline 17 & 1.99 & 29.9545 & 29.5411 & 29.3413 & 29.9350 & 29.7399 & 29.6495 & 29.8652 & 29.4479 & 29.2406 & 29.8370 & 29.6398 & 29.5415 \\
\hline 17 & 2.25 & 30.0139 & 29.6022 & 29.4985 & 29.9886 & 29.7762 & 29.7680 & 30.1149 & 29.7044 & 29.5899 & 30.0855 & 29.8719 & 29.8473 \\
\hline 18 & 1.71 & 30.6755 & 30.2330 & 30.0359 & 30.6387 & 30.3802 & 30.2366 & 30.5619 & 30.1124 & 29.9062 & 30.5297 & 30.2660 & 30.1083 \\
\hline 18 & 2.52 & 29.5175 & 28.9958 & 28.7414 & 29.5183 & 29.1780 & 29.0683 & 29.5471 & 29.0279 & 28.7810 & 29.5421 & 29.2063 & 29.0989 \\
\hline 19 & 1.99 & 30.2949 & 29.8667 & 29.8697 & 30.0052 & 29.7680 & 29.6239 & 30.1614 & 29.7298 & 29.7387 & 29.9390 & 29.6998 & 29.5484 \\
\hline 19 & 2.05 & 30.0608 & 29.6141 & 29.5101 & 30.2190 & 29.9988 & 29.9344 & 29.9864 & 29.5373 & 29.4319 & 30.0891 & 29.8680 & 29.8053 \\
\hline 10-OMe & 3.80 & 28.4700 & 27.9739 & 27.8163 & 28.4305 & 28.1259 & 28.0704 & 28.3671 & 27.8712 & 27.7062 & 28.3267 & 28.0239 & 27.9646 \\
\hline $\mathrm{NCO}_{2} \mathrm{Me}$ & 3.88 & 28.4182 & 27.9008 & 27.7673 & 28.4024 & 28.0471 & 28.0536 & 28.3428 & 27.8248 & 27.6984 & 28.3312 & 27.9769 & 27.9820 \\
\hline
\end{tabular}




\section{Compound 57}

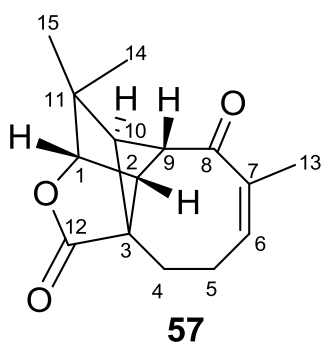

\begin{tabular}{|c|c|c|c|c|c|c|c|c|c|c|c|c|c|}
\hline & \multirow[b]{3}{*}{ Exp } & \multicolumn{12}{|c|}{ B3LYP } \\
\hline & & \multicolumn{6}{|c|}{ Gas phase } & \multicolumn{6}{|c|}{ PCM } \\
\hline & & 6-31G* & 6-31G** & $6-31+\mathrm{G} * *$ & 6-311G* & 6-311G** & $6-311+G^{* *}$ & 6-31G* & 6-31G** & $6-31+\mathrm{G} * *$ & 6-311G* & 6-311G** & $6-311+G * *$ \\
\hline \multicolumn{14}{|l|}{${ }^{13} \mathrm{C}$} \\
\hline 1 & 83.3 & 107.4247 & 108.2349 & 107.1518 & 94.9638 & 94.5316 & 94.3200 & 107.0167 & 107.8240 & 106.6362 & 94.4708 & 94.0371 & 93.7254 \\
\hline 2 & 54.8 & 135.3381 & 136.2572 & 135.2626 & 124.9367 & 124.7437 & 124.6632 & 134.7845 & 135.7001 & 134.5521 & 124.2794 & 124.0783 & 123.8381 \\
\hline 3 & 62.8 & 127.3638 & 127.5633 & 125.9283 & 115.4352 & 115.3550 & 114.4777 & 127.2713 & 127.4726 & 125.8608 & 115.3184 & 115.2393 & 114.3342 \\
\hline 4 & 22.5 & 165.3278 & 166.7739 & 166.2830 & 157.5018 & 157.0858 & 156.3869 & 165.1161 & 166.5634 & 166.0378 & 157.2616 & 156.8363 & 156.1411 \\
\hline 5 & 30.5 & 159.4664 & 160.7148 & 159.8610 & 150.0998 & 149.8380 & 149.2315 & 159.5744 & 160.8175 & 159.9644 & 150.1831 & 149.9173 & 149.3006 \\
\hline 6 & 131.1 & 61.7592 & 62.5845 & 61.2992 & 43.6485 & 43.2278 & 42.1408 & 60.4072 & 61.2277 & 59.6386 & 42.0142 & 41.5809 & 40.2646 \\
\hline 7 & 135.1 & 57.0621 & 57.0169 & 55.8995 & 38.1020 & 37.8560 & 36.4539 & 57.4735 & 57.4277 & 56.3922 & 38.5877 & 38.3496 & 36.9460 \\
\hline 8 & 211.9 & -13.1506 & -12.9789 & -15.4493 & -34.5414 & -34.3322 & -36.7047 & -15.7334 & -15.5668 & -18.6157 & -37.6195 & -37.4144 & -40.3136 \\
\hline 9 & 54.5 & 134.7892 & 135.6202 & 135.3880 & 125.3127 & 124.8872 & 123.8770 & 134.5133 & 135.3414 & 135.0470 & 124.9299 & 124.5007 & 123.3894 \\
\hline 10 & 62.6 & 125.7083 & 126.4046 & 124.6960 & 115.0057 & 114.6463 & 114.2791 & 125.2386 & 125.9308 & 124.0703 & 114.5615 & 114.1993 & 113.7211 \\
\hline 11 & 41.9 & 145.7330 & 146.1811 & 145.9772 & 134.8826 & 134.8014 & 134.4208 & 145.5319 & 145.9825 & 145.7229 & 134.6404 & 134.5599 & 134.1252 \\
\hline 12 & 177.5 & 22.5496 & 22.5919 & 19.9167 & 0.8531 & 0.8787 & -1.3737 & 20.1158 & 20.1587 & 17.0360 & -1.9856 & -1.9611 & -4.5870 \\
\hline 13 & 22.2 & 165.0605 & 166.8036 & 166.3993 & 157.5866 & 157.2727 & 157.3383 & 165.4358 & 167.1633 & 166.8050 & 158.0184 & 157.6835 & 157.7717 \\
\hline 14 & 22.6 & 167.1444 & 168.8917 & 168.8301 & 160.1931 & 159.8670 & 159.7149 & 167.5844 & 169.3143 & 169.2660 & 160.6677 & 160.3181 & 160.1767 \\
\hline 15 & 22.8 & 166.9984 & 168.6675 & 168.8756 & 159.7999 & 159.5913 & 159.3867 & 167.1718 & 168.8384 & 169.0953 & 160.0844 & 159.8553 & 159.6611 \\
\hline \multicolumn{14}{|l|}{${ }^{1} \mathbf{H}$} \\
\hline 1 & 4.48 & 28.0321 & 27.5019 & 27.4326 & 28.0781 & 27.7841 & 27.7415 & 27.8562 & 27.3192 & 27.2343 & 27.8931 & 27.5936 & 27.5432 \\
\hline 2 & 3.26 & 29.1294 & 28.6214 & 28.5827 & 29.2361 & 28.9505 & 28.9355 & 28.9841 & 28.4710 & 28.4130 & 29.0992 & 28.8120 & 28.7796 \\
\hline 4 & 2.52 & 29.7592 & 29.2282 & 29.0014 & 29.7856 & 29.4515 & 29.3423 & 29.8359 & 29.3082 & 29.0841 & 29.8561 & 29.5272 & 29.4196 \\
\hline 4 & 1.96 & 30.4277 & 30.0055 & 29.8490 & 30.3392 & 30.1282 & 30.0610 & 30.4037 & 29.9819 & 29.8183 & 30.3137 & 30.1025 & 30.0263 \\
\hline 5 & 2.35 & 30.0262 & 29.5432 & 29.4104 & 29.9233 & 29.6470 & 29.6584 & 29.9536 & 29.4671 & 29.3262 & 29.8478 & 29.5691 & 29.5727 \\
\hline 5 & 2.03 & 30.1303 & 29.6685 & 29.5124 & 30.0633 & 29.8217 & 29.8116 & 30.0869 & 29.6228 & 29.4621 & 30.0128 & 29.7686 & 29.7525 \\
\hline 6 & 5.85 & 26.4098 & 25.8122 & 25.6015 & 26.2332 & 25.7969 & 25.8227 & 26.2572 & 25.6602 & 25.4315 & 26.0700 & 25.6392 & 25.6533 \\
\hline 9 & 2.92 & 29.4367 & 28.9440 & 28.8370 & 29.4991 & 29.1673 & 29.0805 & 29.3306 & 28.8336 & 28.7148 & 29.3869 & 29.0533 & 28.9609 \\
\hline 10 & 2.64 & 29.7015 & 29.2612 & 29.2756 & 29.8222 & 29.5710 & 29.5659 & 29.6050 & 29.1625 & 29.1564 & 29.7080 & 29.4578 & 29.4347 \\
\hline 13 & 1.87 & 30.3570 & 29.8700 & 29.7826 & 30.2604 & 29.9694 & 29.9560 & 30.3311 & 29.8426 & 29.7516 & 30.2322 & 29.9413 & 29.9256 \\
\hline 14 & 1.05 & 31.1126 & 30.6619 & 30.6335 & 31.1303 & 30.8730 & 30.8308 & 31.0583 & 30.6052 & 30.5730 & 31.0779 & 30.8183 & 30.7732 \\
\hline 15 & 1.19 & 31.1803 & 30.7423 & 30.6580 & 31.1936 & 30.9517 & 30.8834 & 31.1963 & 30.7584 & 30.6725 & 31.2074 & 30.9655 & 30.8954 \\
\hline
\end{tabular}




\begin{tabular}{|c|c|c|c|c|c|c|c|c|c|c|c|c|c|}
\hline & \multirow[b]{2}{*}{ Exp } & \multicolumn{6}{|c|}{ Gas phase } & \multicolumn{6}{|c|}{ PCM } \\
\hline & & 6-31G* & 6-31G** & $6-31+G * *$ & 6-311G* & 6-311G** & $6-311+\mathrm{G} * *$ & 6-31G* & 6-31G** & $6-31+G * *$ & 6-311G* & 6-311G** & $6-311+G * *$ \\
\hline \multicolumn{14}{|l|}{${ }^{13} \mathrm{C}$} \\
\hline 1 & 83.3 & 112.1648 & 112.9267 & 111.9853 & 101.0797 & 100.6562 & 100.4386 & 111.7364 & 112.4957 & 111.4588 & 100.5651 & 100.1409 & 99.8375 \\
\hline 2 & 54.8 & 140.2101 & 141.0601 & 139.9876 & 130.7538 & 130.5462 & 130.4568 & 139.6500 & 140.4975 & 139.2942 & 130.1082 & 129.8931 & 129.6683 \\
\hline 3 & 62.8 & 132.4397 & 132.6325 & 131.2976 & 121.5783 & 121.5198 & 120.6381 & 132.3450 & 132.5397 & 131.2233 & 121.4520 & 121.3937 & 120.4883 \\
\hline 4 & 22.5 & 170.0415 & 171.3874 & 170.9558 & 163.0079 & 162.6008 & 161.8856 & 169.8522 & 171.1988 & 170.7357 & 162.7885 & 162.3719 & 161.6569 \\
\hline 5 & 30.5 & 163.8096 & 164.9565 & 164.1429 & 155.3768 & 155.1070 & 154.5131 & 163.9211 & 165.0626 & 164.2437 & 155.4516 & 155.1780 & 154.5750 \\
\hline 6 & 131.1 & 64.4530 & 65.2176 & 64.1059 & 48.3443 & 47.9046 & 46.8789 & 63.0330 & 63.7926 & 62.4232 & 46.6758 & 46.2238 & 45.0081 \\
\hline 7 & 135.1 & 60.2072 & 60.1438 & 59.2722 & 43.5198 & 43.2638 & 42.0434 & 60.6516 & 60.5889 & 59.7814 & 44.0191 & 43.7735 & 42.5456 \\
\hline 8 & 211.9 & -10.5743 & -10.4126 & -12.4200 & -28.9466 & -28.7410 & -30.7464 & -13.2118 & -13.0558 & -15.5782 & -32.0268 & -31.8264 & -34.2916 \\
\hline 9 & 54.5 & 139.3009 & 140.0735 & 140.0293 & 130.6697 & 130.2554 & 129.3881 & 139.0335 & 139.8033 & 139.7015 & 130.2951 & 129.8768 & 128.9216 \\
\hline 10 & 62.6 & 130.6676 & 131.2972 & 129.6443 & 120.9428 & 120.5957 & 120.2396 & 130.2005 & 130.8257 & 129.0356 & 120.4842 & 120.1353 & 119.6786 \\
\hline 11 & 41.9 & 151.0735 & 151.5250 & 151.4496 & 140.9566 & 140.8834 & 140.5008 & 150.8689 & 151.3229 & 151.2006 & 140.7122 & 140.6406 & 140.2129 \\
\hline 12 & 177.5 & 25.2706 & 25.3071 & 23.2327 & 6.2220 & 6.2416 & 4.3234 & 22.8158 & 22.8523 & 20.3879 & 3.4221 & 3.4391 & 1.1975 \\
\hline 13 & 22.2 & 168.8568 & 170.4842 & 169.8825 & 162.0605 & 161.7584 & 161.7873 & 169.2822 & 170.8935 & 170.3314 & 162.4995 & 162.1790 & 162.2325 \\
\hline 14 & 22.6 & 171.3537 & 172.9791 & 172.7110 & 165.0096 & 164.7067 & 164.5250 & 171.8187 & 173.4281 & 173.1682 & 165.4780 & 165.1556 & 164.9845 \\
\hline 15 & 22.8 & 171.2754 & 172.8226 & 172.8464 & 164.5602 & 164.3745 & 164.1964 & 171.4740 & 173.0189 & 173.0852 & 164.8426 & 164.6385 & 164.4663 \\
\hline \multicolumn{14}{|l|}{${ }^{1} \mathrm{H}$} \\
\hline 1 & 4.48 & 27.9245 & 27.3948 & 27.3419 & 27.9699 & 27.6524 & 27.6265 & 27.7413 & 27.2040 & 27.1372 & 27.7805 & 27.4571 & 27.4234 \\
\hline 2 & 3.26 & 29.0577 & 28.5460 & 28.5087 & 29.1282 & 28.8322 & 28.8205 & 28.9128 & 28.3957 & 28.3435 & 28.9925 & 28.6945 & 28.6683 \\
\hline 4 & 2.52 & 29.6757 & 29.1467 & 28.9486 & 29.6648 & 29.3273 & 29.2223 & 29.7479 & 29.2221 & 29.0270 & 29.7312 & 29.3988 & 29.2950 \\
\hline 4 & 1.96 & 30.3528 & 29.9341 & 29.7857 & 30.2290 & 30.0228 & 29.9766 & 30.3318 & 29.9135 & 29.7587 & 30.2062 & 30.0000 & 29.9453 \\
\hline 5 & 2.35 & 29.9726 & 29.4884 & 29.3761 & 29.8447 & 29.5625 & 29.5783 & 29.8908 & 29.4027 & 29.2835 & 29.7615 & 29.4767 & 29.4848 \\
\hline 5 & 2.03 & 30.0804 & 29.6143 & 29.4605 & 29.9680 & 29.7239 & 29.7095 & 30.0335 & 29.5649 & 29.4075 & 29.9153 & 29.6685 & 29.6487 \\
\hline 6 & 5.85 & 26.2609 & 25.6598 & 25.4881 & 26.1013 & 25.6421 & 25.6719 & 26.0954 & 25.4946 & 25.3089 & 25.9282 & 25.4744 & 25.4951 \\
\hline 9 & 2.92 & 29.3884 & 28.8918 & 28.7908 & 29.3993 & 29.0645 & 28.9962 & 29.2773 & 28.7759 & 28.6651 & 29.2840 & 28.9471 & 28.8734 \\
\hline 10 & 2.64 & 29.6012 & 29.1540 & 29.1788 & 29.7025 & 29.4319 & 29.4316 & 29.5018 & 29.0522 & 29.0591 & 29.5896 & 29.3197 & 29.3030 \\
\hline 13 & 1.87 & 30.2736 & 29.7836 & 29.7134 & 30.1665 & 29.8655 & 29.8553 & 30.2424 & 29.7507 & 29.6774 & 30.1336 & 29.8328 & 29.8206 \\
\hline 14 & 1.05 & 31.0514 & 30.6023 & 30.5745 & 31.0318 & 30.7699 & 30.7303 & 30.9926 & 30.5408 & 30.5103 & 30.9756 & 30.7114 & 30.6691 \\
\hline 15 & 1.19 & 31.1202 & 30.6817 & 30.5987 & 31.0962 & 30.8502 & 30.7907 & 31.1346 & 30.6961 & 30.6121 & 31.1081 & 30.8623 & 30.8011 \\
\hline
\end{tabular}




\section{Compound 58}

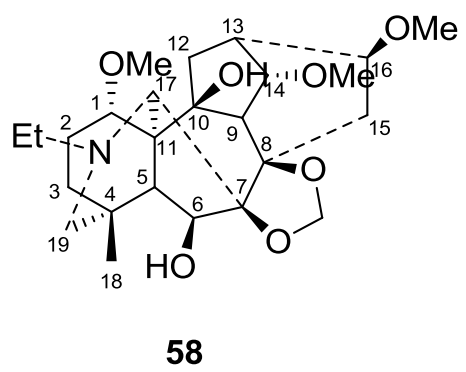

\begin{tabular}{|c|c|c|c|c|c|c|c|c|c|c|c|c|c|}
\hline & \multicolumn{13}{|c|}{ B3LYP } \\
\hline & \multirow[b]{2}{*}{ Exp } & \multicolumn{6}{|c|}{ Gas phase } & \multicolumn{6}{|c|}{ PCM } \\
\hline & & 6-31G* & 6-31G** & $6-31+\mathrm{G} * *$ & 6-311G* & $6-311 G * *$ & $6-311+G * *$ & 6-31G* & $6-31 G * *$ & $6-31+\mathrm{G} * *$ & 6-311G* & $6-311 G * *$ & $6-311+\mathrm{G} * *$ \\
\hline \multicolumn{14}{|l|}{${ }^{13} \mathrm{C}$} \\
\hline 1 & 77.3 & 113.4157 & 114.1402 & 113.8783 & 99.9329 & 101.2031 & 101.4193 & 113.3451 & 114.0725 & 113.7460 & 100.0377 & 101.1245 & 101.3336 \\
\hline 2 & 27.0 & 161.3141 & 162.7020 & 161.1537 & 152.9007 & 152.4513 & 152.7505 & 161.2195 & 162.6051 & 161.0249 & 152.7459 & 152.3162 & 152.6223 \\
\hline 3 & 36.8 & 151.9750 & 153.2124 & 152.1216 & 142.6516 & 142.2943 & 142.6189 & 152.3774 & 153.6069 & 152.5825 & 143.2928 & 142.7914 & 143.1426 \\
\hline 4 & 33.6 & 154.0011 & 154.5118 & 153.7649 & 143.5696 & 144.0974 & 144.1129 & 153.9565 & 154.4692 & 153.8974 & 143.4280 & 144.0797 & 144.0954 \\
\hline 5 & 51.3 & 136.5098 & 137.1837 & 138.7372 & 124.8612 & 125.1568 & 125.6023 & 136.7444 & 137.4103 & 139.2159 & 124.8989 & 125.3878 & 125.8365 \\
\hline 6 & 80.1 & 110.6986 & 111.4305 & 110.0804 & 96.7605 & 97.2945 & 97.8369 & 110.7424 & 111.4719 & 109.7236 & 96.8156 & 97.2786 & 97.8251 \\
\hline 7 & 92.4 & 98.7320 & 98.8113 & 97.8033 & 85.0722 & 85.5717 & 85.5040 & 98.7237 & 98.8028 & 97.7469 & 84.9633 & 85.5489 & 85.4921 \\
\hline 8 & 82.4 & 105.4194 & 105.6465 & 105.5213 & 92.5322 & 93.0615 & 92.9186 & 105.5948 & 105.8186 & 105.6279 & 92.6470 & 93.1771 & 93.0426 \\
\hline 9 & 50.8 & 134.9354 & 135.5538 & 135.5161 & 123.9890 & 123.9301 & 124.3141 & 135.3645 & 135.9750 & 135.9060 & 124.5257 & 124.3682 & 124.7608 \\
\hline 10 & 83.5 & 105.1672 & 105.4334 & 104.3516 & 92.5338 & 92.5137 & 92.3196 & 105.0779 & 105.3437 & 104.3825 & 92.3779 & 92.3556 & 92.1642 \\
\hline 11 & 56.0 & 130.6462 & 130.7835 & 133.2957 & 117.6409 & 119.1965 & 119.3448 & 130.6332 & 130.7694 & 133.3303 & 117.5018 & 119.0954 & 119.2388 \\
\hline 12 & 38.7 & 151.7734 & 152.9964 & 152.4996 & 141.8005 & 141.5911 & 142.1491 & 151.6487 & 152.8684 & 152.2690 & 141.6236 & 141.4601 & 142.0192 \\
\hline 13 & 37.4 & 149.9995 & 150.8531 & 150.4453 & 139.4595 & 139.7609 & 139.9559 & 150.3981 & 151.2385 & 150.9801 & 139.9147 & 140.2335 & 140.4472 \\
\hline 14 & 81.6 & 109.5903 & 110.3512 & 108.8135 & 96.3313 & 96.5250 & 96.9374 & 109.5721 & 110.3309 & 108.8149 & 96.2564 & 96.4659 & 96.8752 \\
\hline 15 & 34.3 & 154.7987 & 156.1649 & 155.4216 & 145.9421 & 145.7820 & 146.2439 & 154.5240 & 155.8928 & 155.0649 & 145.5423 & 145.4011 & 145.8627 \\
\hline 16 & 81.6 & 108.7998 & 109.6360 & 107.3343 & 96.1677 & 96.1938 & 96.6599 & 109.0558 & 109.8902 & 107.6425 & 96.4094 & 96.4621 & 96.9391 \\
\hline 17 & 63.2 & 124.0609 & 124.8165 & 123.9915 & 112.4192 & 112.8589 & 113.3840 & 123.7342 & 124.4794 & 123.6469 & 111.9663 & 112.4092 & 112.8922 \\
\hline 18 & 25.6 & 163.2204 & 164.9158 & 164.8893 & 155.8845 & 156.3149 & 156.4277 & 163.5964 & 165.2807 & 165.2504 & 156.3023 & 156.7599 & 156.8960 \\
\hline 19 & 57.2 & 134.4146 & 135.5459 & 134.8597 & 123.6674 & 124.0849 & 124.5089 & 134.7640 & 135.8930 & 135.1979 & 124.0952 & 124.4763 & 124.9434 \\
\hline $\mathrm{NCH}_{2} \mathrm{Me}$ & 50.4 & 138.1720 & 139.3386 & 138.7375 & 127.6953 & 128.3479 & 128.7731 & 138.0141 & 139.1782 & 138.5542 & 127.4878 & 128.1083 & 128.5269 \\
\hline $\mathrm{NCH}_{2} \mathrm{Me}$ & 13.9 & 174.5174 & 176.4002 & 176.5427 & 167.4415 & 167.9696 & 168.0313 & 174.6184 & 176.4932 & 176.6097 & 167.4935 & 168.0446 & 168.1245 \\
\hline $\mathrm{OCH}_{2} \mathrm{O}$ & 93.3 & 98.0876 & 99.2716 & 99.0864 & 85.9890 & 86.0039 & 87.0086 & 98.2931 & 99.4645 & 99.1959 & 86.1151 & 86.0895 & 87.1012 \\
\hline 1-OMe & 55.5 & 136.0097 & 137.8367 & 137.9986 & 127.6448 & 127.4376 & 128.0396 & 136.0979 & 137.9104 & 137.9236 & 127.5849 & 127.3734 & 127.9936 \\
\hline 14-OMe & 57.8 & 134.2325 & 136.0077 & 135.8069 & 124.9155 & 125.1357 & 125.7958 & 134.3624 & 136.1223 & 135.7217 & 124.8392 & 125.1009 & 125.7734 \\
\hline $\begin{array}{c}\text { 16-OMe } \\
{ }^{\mathbf{1}_{\mathbf{H}}}\end{array}$ & 56.2 & 135.2984 & 137.1176 & 136.9717 & 126.0949 & 126.1755 & 126.7567 & 135.5524 & 137.3476 & 137.0193 & 126.1610 & 126.2568 & 126.8553 \\
\hline 1 & 3.55 & 28.3606 & 27.9627 & 27.9990 & 28.1694 & 28.2678 & 28.4307 & 28.3937 & 27.9943 & 28.0382 & 28.2010 & 28.2908 & 28.4602 \\
\hline 2 & 2.03 & 30.1046 & 29.6305 & 29.5888 & 29.7108 & 29.7641 & 30.0269 & 30.0271 & 29.5506 & 29.5035 & 29.6168 & 29.6839 & 29.9484 \\
\hline 2 & 2.13 & 29.8199 & 29.3618 & 29.3526 & 29.6791 & 29.6675 & 29.9106 & 29.8919 & 29.4351 & 29.4164 & 29.7278 & 29.7330 & 29.9756 \\
\hline 3 & 1.21 & 30.8664 & 30.5000 & 30.3584 & 30.6139 & 30.6684 & 30.8457 & 30.8270 & 30.4592 & 30.3151 & 30.5762 & 30.6263 & 30.8046 \\
\hline 3 & 1.57 & 30.6313 & 30.2374 & 30.1585 & 30.3345 & 30.4037 & 30.5938 & 30.5854 & 30.1900 & 30.1240 & 30.3017 & 30.3553 & 30.5471 \\
\hline 5 & 1.48 & 30.8645 & 30.4598 & 30.4651 & 30.6622 & 30.7234 & 30.9528 & 30.8047 & 30.3973 & 30.3907 & 30.5932 & 30.6518 & 30.8817 \\
\hline 6 & 4.24 & 27.7834 & 27.2913 & 27.3060 & 27.6798 & 27.6347 & 27.9206 & 27.7667 & 27.2706 & 27.2604 & 27.6362 & 27.5985 & 27.8886 \\
\hline
\end{tabular}




\begin{tabular}{|c|c|c|c|c|c|c|c|c|c|c|c|c|c|}
\hline 9 & 3.45 & 28.9580 & 28.3430 & 28.4151 & 28.4062 & 28.5438 & 28.9831 & 28.9812 & 28.3653 & 28.4297 & 28.4255 & 28.5610 & 28.9995 \\
\hline 12 & 1.76 & 30.5051 & 30.0760 & 30.0323 & 30.2440 & 30.2754 & 30.4559 & 30.5320 & 30.1017 & 30.0533 & 30.2573 & 30.2973 & 30.4848 \\
\hline 12 & 3.08 & 28.8227 & 28.1260 & 28.0650 & 28.3874 & 28.4613 & 28.9033 & 28.8024 & 28.1030 & 28.0301 & 28.3537 & 28.4309 & 28.8761 \\
\hline 13 & 2.53 & 29.8168 & 29.3332 & 29.2462 & 29.5033 & 29.5645 & 29.8486 & 29.7640 & 29.2742 & 29.1746 & 29.4379 & 29.5029 & 29.7980 \\
\hline 14 & 4.13 & 28.0910 & 27.6964 & 27.5393 & 27.8269 & 27.8786 & 28.0304 & 28.0866 & 27.6885 & 27.5163 & 27.8006 & 27.8575 & 28.0153 \\
\hline 15 & 1.83 & 30.0339 & 29.5693 & 29.4470 & 29.7569 & 29.8767 & 30.1145 & 30.1709 & 29.7099 & 29.5937 & 29.8933 & 30.0056 & 30.2406 \\
\hline 15 & 2.50 & 29.7935 & 29.2753 & 29.1261 & 29.4459 & 29.5417 & 29.8649 & 29.7265 & 29.2090 & 29.0527 & 29.3800 & 29.4695 & 29.7894 \\
\hline 16 & 3.18 & 28.9814 & 28.6247 & 28.5674 & 28.8578 & 28.9152 & 29.0756 & 28.9294 & 28.5707 & 28.4990 & 28.7923 & 28.8468 & 29.0117 \\
\hline 17 & 3.01 & 29.1575 & 28.7016 & 28.6633 & 29.0083 & 29.0256 & 29.2871 & 29.1618 & 28.7079 & 28.6447 & 28.9873 & 29.0121 & 29.2741 \\
\hline 18 & 0.94 & 31.2472 & 30.8186 & 30.8364 & 30.9524 & 31.0012 & 31.2436 & 31.2336 & 30.8034 & 30.8215 & 30.9342 & 30.9864 & 31.2309 \\
\hline 19 & 2.25 & 29.7690 & 29.3579 & 29.3744 & 29.6423 & 29.6780 & 29.8708 & 29.8099 & 29.4012 & 29.4367 & 29.6922 & 29.7208 & 29.9150 \\
\hline 19 & 2.66 & 29.4349 & 29.0026 & 28.9626 & 29.2626 & 29.3044 & 29.5396 & 29.3423 & 28.9070 & 28.8632 & 29.1405 & 29.2050 & 29.4397 \\
\hline $\mathrm{NCH}_{2} \mathrm{Me}$ & 2.64 & 29.3456 & 28.9110 & 28.7913 & 29.0386 & 29.1409 & 29.3701 & 29.3556 & 28.9205 & 28.8162 & 29.0554 & 29.1550 & 29.3775 \\
\hline $\mathrm{NCH}_{2} \mathrm{Me}$ & 2.73 & 29.5240 & 29.0762 & 28.9969 & 29.2260 & 29.2882 & 29.5364 & 29.5222 & 29.0761 & 28.9724 & 29.2110 & 29.2748 & 29.5282 \\
\hline $\mathrm{NCH}_{2} \mathrm{Me}$ & 1.04 & 31.1290 & 30.6753 & 30.5678 & 30.8156 & 30.8472 & 31.0996 & 31.1183 & 30.6636 & 30.5558 & 30.8011 & 30.8364 & 31.0904 \\
\hline $\mathrm{OCH}_{2} \mathrm{O}$ & 5.05 & 27.0871 & 26.5208 & 26.5824 & 26.8918 & 26.7601 & 27.0965 & 27.0328 & 26.4679 & 26.5278 & 26.8407 & 26.7073 & 27.0380 \\
\hline $\mathrm{OCH}_{2} \mathrm{O}$ & 5.13 & 27.1262 & 26.5560 & 26.5610 & 26.8931 & 26.7936 & 27.1482 & 27.0390 & 26.4686 & 26.4894 & 26.8143 & 26.7065 & 27.0562 \\
\hline 1-OMe & 3.25 & 28.9278 & 28.4448 & 28.3211 & 28.6473 & 28.6311 & 28.9129 & 28.8801 & 28.3974 & 28.2711 & 28.5964 & 28.5848 & 28.8662 \\
\hline 14-OMe & 3.43 & 28.8342 & 28.3498 & 28.1553 & 28.4391 & 28.5117 & 28.8026 & 28.7989 & 28.3151 & 28.1085 & 28.3991 & 28.4762 & 28.7670 \\
\hline 16-OMe & 3.33 & 28.9333 & 28.4439 & 28.2999 & 28.5700 & 28.5961 & 28.8867 & 28.8768 & 28.3882 & 28.2291 & 28.5042 & 28.5423 & 28.8319 \\
\hline
\end{tabular}

\begin{tabular}{|c|c|c|c|c|c|c|c|c|c|c|c|c|c|}
\hline & \multicolumn{13}{|c|}{ mPW1PW91 } \\
\hline & \multirow[b]{2}{*}{ Exp } & \multicolumn{6}{|c|}{ Gas phase } & \multicolumn{6}{|c|}{ PCM } \\
\hline & & 6-31G* & 6-31G** & $6-31+G * *$ & 6-311G* & 6-311G** & $6-311+G^{* *}$ & 6-31G* & 6-31G** & $6-31+G * *$ & 6-311G* & 6-311G** & $6-311+G^{* *}$ \\
\hline \multicolumn{14}{|l|}{${ }^{13} \mathrm{C}$} \\
\hline 1 & 77.3 & 118.4286 & 119.0966 & 119.3657 & 106.5718 & 107.7146 & 107.9118 & 118.3640 & 119.0349 & 119.2525 & 106.6778 & 107.6364 & 107.8271 \\
\hline 2 & 27.0 & 166.0180 & 167.3071 & 165.5803 & 158.3630 & 157.9828 & 158.2751 & 165.9287 & 167.2152 & 165.4682 & 158.2191 & 157.8515 & 158.1499 \\
\hline 3 & 36.8 & 157.1119 & 158.2320 & 156.8787 & 148.5391 & 148.1485 & 148.4738 & 157.5364 & 158.6500 & 157.3429 & 149.1515 & 148.6419 & 148.9918 \\
\hline 4 & 33.6 & 159.2136 & 159.7088 & 159.1304 & 149.5737 & 149.9790 & 149.9848 & 159.1691 & 159.6663 & 159.2506 & 149.4383 & 149.9622 & 149.9681 \\
\hline 5 & 51.3 & 142.1002 & 142.6855 & 143.9110 & 131.5050 & 131.6491 & 132.0747 & 142.3419 & 142.9204 & 144.3910 & 131.5555 & 131.8713 & 132.2998 \\
\hline 6 & 80.1 & 115.6303 & 116.2964 & 115.6407 & 103.3864 & 103.9178 & 104.4481 & 115.6706 & 116.3346 & 115.2994 & 103.4251 & 103.8924 & 104.4267 \\
\hline 7 & 92.4 & 103.3098 & 103.3805 & 102.7578 & 91.3361 & 91.8178 & 91.7402 & 103.3023 & 103.3727 & 102.7087 & 91.2505 & 91.8050 & 91.7397 \\
\hline 8 & 82.4 & 110.2367 & 110.4480 & 110.4743 & 98.7784 & 99.3428 & 99.2017 & 110.4251 & 110.6332 & 110.5950 & 98.9058 & 99.4670 & 99.3353 \\
\hline 9 & 50.8 & 140.0390 & 140.5851 & 140.6474 & 130.0323 & 130.1144 & 130.4860 & 140.4887 & 141.0267 & 141.0349 & 130.5541 & 130.5624 & 130.9410 \\
\hline 10 & 83.5 & 110.1777 & 110.4329 & 109.6474 & 99.1183 & 99.0974 & 98.9104 & 110.0766 & 110.3314 & 109.7073 & 98.9745 & 98.9374 & 98.7534 \\
\hline 11 & 56.0 & 135.9092 & 136.0384 & 138.6597 & 124.0705 & 125.5010 & 125.6325 & 135.8925 & 136.0205 & 138.6804 & 123.9242 & 125.3973 & 125.5240 \\
\hline 12 & 38.7 & 156.4722 & 157.5785 & 157.1520 & 147.2688 & 147.1270 & 147.6819 & 156.3665 & 157.4698 & 156.9463 & 147.1079 & 147.0101 & 147.5659 \\
\hline 13 & 37.4 & 154.6939 & 155.4849 & 154.8518 & 144.9688 & 145.3651 & 145.5396 & 155.1195 & 155.8972 & 155.3910 & 145.4326 & 145.8385 & 146.0308 \\
\hline 14 & 81.6 & 114.6892 & 115.3697 & 113.8172 & 102.9960 & 103.0596 & 103.4550 & 114.6737 & 115.3521 & 113.8302 & 102.9256 & 102.9925 & 103.3855 \\
\hline 15 & 34.3 & 159.9713 & 161.2440 & 160.1055 & 151.7167 & 151.7350 & 152.1663 & 159.6949 & 160.9711 & 159.7550 & 151.3144 & 151.3626 & 151.7928 \\
\hline 16 & 81.6 & 113.8675 & 114.6187 & 112.7986 & 102.8239 & 102.9436 & 103.4128 & 114.1090 & 114.8602 & 113.0829 & 103.0259 & 103.1715 & 103.6503 \\
\hline 17 & 63.2 & 129.1420 & 129.8256 & 128.8563 & 118.8913 & 119.0856 & 119.6287 & 128.7586 & 129.4297 & 128.4608 & 118.3323 & 118.5669 & 119.0569 \\
\hline 18 & 25.6 & 167.7198 & 169.2794 & 168.7916 & 160.9363 & 161.2542 & 161.3475 & 168.1315 & 169.6804 & 169.1865 & 161.3703 & 161.7027 & 161.8162 \\
\hline 19 & 57.2 & 139.2402 & 140.2620 & 139.6204 & 129.6292 & 130.0135 & 130.3893 & 139.6320 & 140.6528 & 139.9918 & 130.1021 & 130.4362 & 130.8602 \\
\hline $\mathrm{NCH}_{2} \mathrm{Me}$ & 50.4 & 142.9987 & 144.0871 & 143.6384 & 133.5278 & 134.2050 & 134.6394 & 142.8367 & 143.9218 & 143.4416 & 133.3074 & 133.9453 & 134.3675 \\
\hline
\end{tabular}




\begin{tabular}{|c|c|c|c|c|c|c|c|c|c|c|c|c|c|}
\hline $\mathrm{CH}_{2} \mathrm{Me}$ & 3.9 & 78.6035 & 80.3720 & 80.6406 & 72.0421 & 72.5842 & 72.6464 & 178.7335 & 180.4931 & 180.7463 & 172.1038 & 172.6699 & 172.7484 \\
\hline $\mathrm{OCH}_{2} \mathrm{O}$ & 93.3 & 02.1534 & 03.2876 & 103.2671 & 91.8442 & 91.8540 & 92.8200 & & 103.4626 & 103.3764 & 1.9306 & & 92.8815 \\
\hline 1-OMe & 55.5 & 139.7875 & 141.5280 & 141.6823 & 132.5331 & 132.3562 & 32.9292 & 39.8971 & 141.6233 & 141.6360 & 132.4780 & 132.2990 & 132.8882 \\
\hline -OMe & 57.8 & 7.9349 & & 139.6674 & & & & & & & & & 7180 \\
\hline $\begin{array}{l}\text { 6-OMe } \\
{ }^{1} \mathbf{H}\end{array}$ & 6.2 & & & & & & & & & & & & \\
\hline 1 & 3.55 & 28.3123 & 27.9178 & 27.9312 & & & & & & & & & 28.3300 \\
\hline 2 & 2.03 & & & & & & & & & & & & \\
\hline 2 & .13 & 29.7825 & & 29.2818 & & 29.5688 & & 29.8596 & & 29.3458 & 29.6235 & & 29.8698 \\
\hline 3 & .21 & & & & & & & & & & & & \\
\hline 3 & 1.57 & 30.5821 & & & & & & & & & & & \\
\hline 5 & .48 & & & & & & & & & & & & 514 \\
\hline 6 & 4.24 & & & & & & & & & & & & \\
\hline 9 & 3.45 & 28.9030 & 28.2869 & & & & & & & & 28.3432 & & .8895 \\
\hline 12 & .76 & & & & & & & & & & & & \\
\hline 12 & 3.08 & & & & & & & & & & & & \\
\hline 13 & 53 & 29.7570 & & & & & & & & & & & \\
\hline 14 & .13 & & & & & & & & & & & & \\
\hline 15 & 1.83 & 090 & & & & & & & & & & & \\
\hline 15 & 2.50 & 29.7490 & & 29.0759 & & & & & & & & & \\
\hline 16 & 18 & & & & & & & & & & & & \\
\hline 17 & 3.01 & & & & & & & & & & & & \\
\hline 18 & 0.94 & & & & & & & & & & & & \\
\hline 19 & 2.25 & & & & & & & & & & & & \\
\hline 19 & 2.66 & & & & & & & & & & & & \\
\hline $\mathrm{NCH}_{2} \mathrm{Me}$ & .64 & & & & & & & & & & & & \\
\hline $\mathrm{NCH}_{2} \mathrm{Me}$ & .73 & 29.4632 & 73 & 28.9481 & 208 & & & 29.4649 & & 360 & & & 342 \\
\hline $\mathrm{NCH}_{2} \mathrm{Me}$ & 1.04 & 31.0783 & 30.6235 & 30.5142 & 30.7242 & 30.7589 & 31.0181 & 31.0654 & 30.6095 & 30.5022 & 30.7096 & 30.7455 & 31.0066 \\
\hline $\mathrm{OCH}_{2} \mathrm{O}$ & .05 & 27.0600 & & & 26.8503 & & 27.0744 & & & & & & 27.0098 \\
\hline $\mathrm{OCH}_{2} \mathrm{O}$ & .13 & 27.0934 & & & 26.8344 & & & 26.9980 & & & & & 27.0333 \\
\hline 1-OMe & 3.25 & 28.8923 & & & & & & & & & & & \\
\hline 14-OMe & 3.43 & & & & & & & 7563 & & & & & \\
\hline 16-OMe & 3.33 & 28.8959 & 28.4081 & 28.2854 & 28.5289 & 28.5455 & 28.8480 & & & & & 28.4864 & 28.7880 \\
\hline
\end{tabular}




\section{Compound 59}

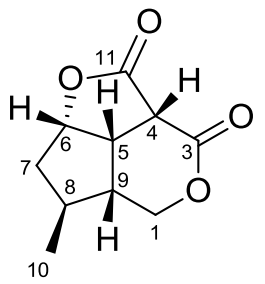

59

\section{B3LYP}

\begin{tabular}{|c|c|c|c|c|c|c|c|c|c|c|c|c|c|}
\hline \multirow{3}{*}{\multicolumn{2}{|c|}{ Exp }} & \multicolumn{12}{|c|}{ B3LYP } \\
\hline & & \multicolumn{6}{|c|}{ Gas phase } & \multicolumn{6}{|c|}{ PCM } \\
\hline & & 6-31G* & $6-31 G * *$ & $6-31+G * *$ & 6-311G* & $6-311 G * *$ & $6-311+G * *$ & 6-31G* & $6-31 G * *$ & $6-31+G * *$ & 6-311G* & $6-311 G * *$ & $6-311+G * *$ \\
\hline \\
\hline 1 & 68.8 & 123.0295 & 124.3462 & 123.2656 & 112.3332 & 111.6360 & 111.3982 & 122.4804 & 123.7833 & 122.4488 & 111.5935 & 110.8747 & 110.4525 \\
\hline 3 & 164.7 & 36.3067 & 36.3304 & 34.5599 & 16.3644 & 16.4943 & 13.9053 & 32.9735 & 32.9951 & 30.7128 & 12.6254 & 12.7611 & 9.6457 \\
\hline 4 & 45.7 & 142.9068 & 143.6140 & 143.0064 & 132.9753 & 132.5169 & 131.9247 & 142.1111 & 142.8045 & 142.0365 & 132.0577 & 131.5924 & 130.7485 \\
\hline 5 & 42.3 & 145.9770 & 146.8186 & 145.9881 & 135.4784 & 135.3053 & 134.7547 & 145.8391 & 146.6678 & 145.7290 & 135.2664 & 135.0818 & 134.4476 \\
\hline 6 & 85.1 & 107.3409 & 108.1622 & 107.5763 & 95.4411 & 94.9687 & 94.4604 & 106.3233 & 107.1323 & 106.3331 & 94.2308 & 93.7463 & 93.0825 \\
\hline 7 & 41.2 & 149.1005 & 150.4223 & 149.7230 & 139.4850 & 138.9319 & 138.6261 & 149.3786 & 150.6879 & 150.0095 & 139.7697 & 139.2055 & 138.8545 \\
\hline 8 & 34.2 & 151.0825 & 151.9558 & 151.5167 & 141.3547 & 141.1496 & 140.9566 & 151.1786 & 152.0552 & 151.6778 & 141.5393 & 141.3327 & 141.1361 \\
\hline 9 & 43.1 & 143.3548 & 144.0907 & 143.2731 & 132.6466 & 132.3073 & 131.9592 & 143.4893 & 144.2162 & 143.4046 & 132.7295 & 132.3920 & 131.9913 \\
\hline 10 & 19.4 & 166.5929 & 168.3538 & 168.5480 & 159.4504 & 159.2717 & 158.8633 & 166.9362 & 168.6903 & 168.9772 & 159.8944 & 159.7008 & 159.3092 \\
\hline 11 & 170.2 & 29.8213 & 29.9195 & 27.6351 & 9.5907 & 9.6987 & 7.3060 & 27.1592 & 27.2563 & 24.5655 & 6.5570 & 6.6616 & 3.8483 \\
\hline \\
\hline 1 & 4.47 & 28.1259 & 27.6387 & 27.5462 & 28.1202 & 27.8570 & 27.8173 & 27.9496 & 27.4585 & 27.3521 & 27.9478 & 27.6808 & 27.6265 \\
\hline 1 & 4.05 & 28.2054 & 27.7261 & 27.6670 & 28.3070 & 28.0752 & 28.0322 & 28.1531 & 27.6750 & 27.6009 & 28.2502 & 28.0190 & 27.9629 \\
\hline 4 & 3.80 & 28.8693 & 28.3777 & 28.1718 & 28.7928 & 28.5003 & 28.3649 & 28.7299 & 28.2290 & 27.9987 & 28.6468 & 28.3513 & 28.1969 \\
\hline 5 & 3.35 & 29.1478 & 28.6350 & 28.4726 & 29.1909 & 28.8541 & 28.7628 & 28.9066 & 28.3869 & 28.2050 & 28.9511 & 28.6136 & 28.5048 \\
\hline 6 & 5.04 & 27.3981 & 26.8330 & 26.6900 & 27.3891 & 27.0386 & 26.9661 & 27.2030 & 26.6320 & 26.4730 & 27.1927 & 26.8384 & 26.7517 \\
\hline 7 & 2.36 & 29.9838 & 29.4866 & 29.3978 & 30.0161 & 29.7394 & 29.6781 & 29.9865 & 29.4881 & 29.4023 & 30.0196 & 29.7440 & 29.6815 \\
\hline 7 & 1.68 & 30.1451 & 29.6997 & 29.6535 & 30.1500 & 29.9219 & 29.8883 & 30.0202 & 29.5705 & 29.5115 & 30.0252 & 29.7953 & 29.7525 \\
\hline 8 & 2.04 & 30.1108 & 29.6293 & 29.5878 & 30.2017 & 29.9402 & 29.8824 & 29.9677 & 29.4823 & 29.4292 & 30.0588 & 29.7944 & 29.7299 \\
\hline 9 & 2.10 & 30.0798 & 29.6767 & 29.5900 & 30.1701 & 29.9726 & 29.8812 & 29.9492 & 29.5430 & 29.4449 & 30.0359 & 29.8391 & 29.7380 \\
\hline 10 & 1.12 & 31.1706 & 30.7448 & 30.6496 & 31.1562 & 30.9255 & 30.8683 & 31.1327 & 30.7051 & 30.6114 & 31.1243 & 30.8921 & 30.8351 \\
\hline
\end{tabular}

\begin{tabular}{|c|c|c|c|c|c|c|c|c|c|c|c|c|c|}
\hline \multirow{3}{*}{\multicolumn{2}{|c|}{ Exp }} & \multicolumn{12}{|c|}{ mPW1PW91 } \\
\hline & & \multicolumn{6}{|c|}{ Gas phase } & \multicolumn{6}{|c|}{ PCM } \\
\hline & & 6-31G* & $6-31 G * *$ & $6-31+\mathrm{G} * *$ & 6-311G* & $6-311 G * *$ & $6-311+G * *$ & 6-31G* & 6-31G** & $6-31+\mathrm{G} * *$ & 6-311G* & $6-311 G * *$ & $6-311+G * *$ \\
\hline \\
\hline 1 & 68.8 & 127.4238 & 128.6640 & 127.8881 & 118.0851 & 117.4178 & 117.1778 & 126.8322 & 128.0598 & 127.0463 & 117.3023 & 116.6147 & 116.2147 \\
\hline 3 & 164.7 & 38.7454 & 38.7649 & 37.4955 & 21.3037 & 21.4254 & 19.1465 & 35.3626 & 35.3789 & 33.6597 & 17.5910 & 17.7174 & 14.9785 \\
\hline 4 & 45.7 & 147.0590 & 147.7186 & 147.1719 & 138.3904 & 137.9258 & 137.3364 & 146.2406 & 146.8874 & 146.2046 & 137.4647 & 136.9943 & 136.1874 \\
\hline 5 & 42.3 & 150.5961 & 151.3624 & 150.5114 & 141.0523 & 140.8635 & 140.3042 & 150.4373 & 151.1918 & 150.2415 & 140.8251 & 140.6263 & 139.9956 \\
\hline 6 & 85.1 & 111.5160 & 112.2849 & 112.0640 & 101.2469 & 100.7829 & 100.2153 & 110.4674 & 111.2247 & 110.8124 & 100.0246 & 99.5499 & 98.8522 \\
\hline 7 & 41.2 & 153.5688 & 154.7877 & 154.0562 & 144.9619 & 144.4120 & 144.0438 & 153.8445 & 155.0516 & 154.3369 & 145.2279 & 144.6679 & 144.2573 \\
\hline
\end{tabular}




\begin{tabular}{ccccccccccccccc}
\hline 8 & 34.2 & 156.0787 & 156.9001 & 156.5109 & 147.1849 & 146.9682 & 146.7235 & 156.1563 & 156.9825 & 156.6493 & 147.3416 & 147.1254 & 146.8753 \\
9 & 43.1 & 148.4779 & 149.1487 & 148.3422 & 138.7771 & 138.4379 & 138.0621 & 148.5921 & 149.2550 & 148.4560 & 138.8330 & 138.4966 & 138.0746 \\
10 & 19.4 & 171.0208 & 172.6496 & 172.6566 & 164.3521 & 164.1803 & 163.8032 & 171.3925 & 173.0149 & 173.1035 & 164.7891 & 164.6046 & 164.2431 \\
11 & 170.2 & 32.4658 & 32.5604 & 30.9107 & 14.7347 & 14.8421 & 12.7161 & 29.7521 & 29.8448 & 27.8379 & 11.7157 & 11.8187 & 9.3241 \\
${ }^{1} \mathbf{H}$ & & & & & & & & & & & & & & \\
1 & 4.47 & 28.0321 & 27.5492 & 27.4873 & 28.0490 & 27.7709 & 27.7424 & 27.8458 & 27.3582 & 27.2850 & 27.8690 & 27.5866 & 27.5447 \\
1 & 4.05 & 28.1655 & 27.6911 & 27.6249 & 28.2345 & 28.0069 & 27.9596 & 28.1089 & 27.6359 & 27.5569 & 28.1750 & 27.9479 & 27.8890 \\
4 & 3.80 & 28.7627 & 28.2686 & 28.0916 & 28.6712 & 28.3692 & 28.2540 & 28.6166 & 28.1125 & 27.9146 & 28.5205 & 28.2154 & 28.0831 \\
5 & 3.35 & 29.0895 & 28.5711 & 28.4196 & 29.0938 & 28.7568 & 28.6703 & 28.8382 & 28.3122 & 28.1455 & 28.8473 & 28.5089 & 28.4074 \\
6 & 5.04 & 27.3399 & 26.7674 & 26.6384 & 27.3070 & 26.9468 & 26.8771 & 27.1376 & 26.5586 & 26.4158 & 27.1056 & 26.7416 & 26.6589 \\
7 & 2.36 & 29.8968 & 29.3988 & 29.3221 & 29.8947 & 29.6175 & 29.5602 & 29.8975 & 29.3982 & 29.3241 & 29.8958 & 29.6196 & 29.5608 \\
7 & 1.68 & 30.0745 & 29.6289 & 29.5893 & 30.0487 & 29.8139 & 29.7800 & 29.9424 & 29.4921 & 29.4417 & 29.9182 & 29.6814 & 29.6394 \\
8 & 2.04 & 30.0481 & 29.5688 & 29.5215 & 30.0834 & 29.8245 & 29.7653 & 29.8994 & 29.4157 & 29.3596 & 29.9380 & 29.6758 & 29.6106 \\
9 & 2.10 & 30.0125 & 29.6064 & 29.5211 & 30.0534 & 29.8564 & 29.7682 & 29.8797 & 29.4704 & 29.3760 & 29.9192 & 29.7227 & 29.6258 \\
10 & 1.12 & 31.1158 & 30.6890 & 30.5960 & 31.0734 & 30.8350 & 30.7786 & 31.0744 & 30.6457 & 30.5546 & 31.0383 & 30.7983 & 30.7420 \\
\hline
\end{tabular}

\section{Compound 60}

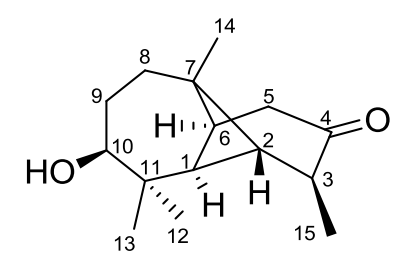

60

\begin{tabular}{|c|c|c|c|c|c|c|c|c|c|c|c|c|c|}
\hline & \multirow[b]{3}{*}{ Exp } & \multicolumn{12}{|c|}{ B3LYP } \\
\hline & & \multicolumn{6}{|c|}{ Gas phase } & \multicolumn{6}{|c|}{ PCM } \\
\hline & & 6-31G* & $6-31 G * *$ & $6-31+\mathrm{G} * *$ & 6-311G* & $6-311 G * *$ & $6-311+G * *$ & 6-31G* & $6-31 G * *$ & $6-31+\mathrm{G} * *$ & 6-311G* & $6-311 G * *$ & $6-311+G * *$ \\
\hline \multicolumn{14}{|c|}{${ }^{13} \mathrm{C}$} \\
\hline 1 & 61.2 & 129.1260 & 129.8861 & 127.8355 & 118.2508 & 117.8918 & 117.6917 & 129.4989 & 130.2489 & 128.3358 & 118.7801 & 118.4034 & 118.3172 \\
\hline 2 & 42.1 & 145.5807 & 146.4011 & 147.8282 & 136.1558 & 135.9252 & 135.6244 & 145.7999 & 146.6161 & 148.0777 & 136.4667 & 136.2189 & 135.8692 \\
\hline 3 & 53.8 & 134.9709 & 135.7101 & 134.2711 & 123.7514 & 123.4317 & 122.5185 & 134.7702 & 135.5071 & 134.0090 & 123.5383 & 123.2075 & 122.1570 \\
\hline 4 & 215.8 & -14.0210 & -13.6979 & -17.1162 & -36.8837 & -36.5226 & -40.2730 & -17.9572 & -17.6389 & -22.0380 & -41.4803 & -41.1304 & -45.7885 \\
\hline 5 & 47.4 & 143.1445 & 144.3550 & 142.7938 & 133.0059 & 132.4393 & 131.5872 & 142.7298 & 143.9294 & 142.2236 & 132.5262 & 131.9546 & 130.8906 \\
\hline 6 & 38.2 & 149.6273 & 150.5975 & 149.0061 & 140.3344 & 140.2273 & 139.5670 & 149.5197 & 150.4912 & 148.9727 & 140.2751 & 140.1736 & 139.5592 \\
\hline 7 & 40.8 & 147.1638 & 147.6831 & 147.2691 & 136.3837 & 136.5497 & 135.9832 & 147.1653 & 147.6864 & 147.2490 & 136.3894 & 136.5569 & 135.9745 \\
\hline 8 & 37.7 & 151.9870 & 153.2274 & 151.4960 & 142.2495 & 141.8393 & 141.1194 & 152.1544 & 153.3874 & 151.8247 & 142.4854 & 142.0663 & 141.4834 \\
\hline 9 & 30.5 & 156.4366 & 157.8428 & 156.0135 & 146.9796 & 146.6615 & 145.9661 & 156.8926 & 158.2861 & 156.6627 & 147.5062 & 147.1636 & 146.5608 \\
\hline 10 & 76.9 & 113.9356 & 114.6376 & 113.5907 & 101.4034 & 101.1901 & 101.5601 & 114.1479 & 114.8496 & 113.7895 & 101.6887 & 101.4564 & 101.7769 \\
\hline 11 & 37.1 & 150.4052 & 150.8939 & 150.5912 & 140.2468 & 140.1794 & 139.5166 & 150.3995 & 150.8866 & 150.6392 & 140.3293 & 140.2510 & 139.5463 \\
\hline 12 & 27.2 & 161.9791 & 163.7275 & 164.6532 & 155.3883 & 155.2653 & 154.9481 & 162.1287 & 163.8723 & 164.8959 & 155.6513 & 155.5093 & 155.1922 \\
\hline 13 & 17.2 & 168.3531 & 170.0293 & 172.6273 & 162.5348 & 162.3624 & 163.5994 & 168.9704 & 170.6454 & 173.2314 & 163.3603 & 163.1465 & 164.2139 \\
\hline 14 & 23.2 & 166.2435 & 168.0610 & 167.7292 & 159.1705 & 158.8353 & 158.6857 & 166.3792 & 168.1896 & 167.8830 & 159.3826 & 159.0289 & 158.8915 \\
\hline 15 & 17.2 & 171.3639 & 173.1906 & 173.3652 & 165.0003 & 164.9118 & 164.6955 & 171.5814 & 173.4048 & 173.6434 & 165.2953 & 165.1913 & 164.9927 \\
\hline
\end{tabular}




\begin{tabular}{llllllllllllll}
\hline 1 & 1.37 & 30.6949 & 30.4207 & 30.4331 & 30.8200 & 30.7082 & 30.6807 & 30.6406 & 30.3657 & 30.3681 & 30.7599 & 30.6483 & 30.6155 \\
2 & 2.25 & 29.5558 & 29.0960 & 29.1647 & 29.7503 & 29.5094 & 29.5557 & 29.5570 & 29.0970 & 29.1328 & 29.7525 & 29.5058 & 29.5203 \\
3 & 2.44 & 29.7062 & 29.3501 & 29.3649 & 29.7623 & 29.6030 & 29.5652 & 29.6260 & 29.2672 & 29.2577 & 29.6775 & 29.5166 & 29.4617 \\
5 & 2.50 & 29.7653 & 29.3376 & 29.2522 & 29.6843 & 29.4669 & 29.4592 & 29.6984 & 29.2678 & 29.1702 & 29.6162 & 29.3980 & 29.3817 \\
5 & 2.71 & 29.5571 & 29.0973 & 29.0232 & 29.5259 & 29.2729 & 29.2314 & 29.5386 & 29.0741 & 28.9900 & 29.5048 & 29.2478 & 29.2014 \\
6 & 2.12 & 29.9941 & 29.5229 & 29.5965 & 30.1683 & 29.8767 & 29.9286 & 29.9304 & 29.4591 & 29.5174 & 30.0995 & 29.8088 & 29.8516 \\
8 & 1.75 & 30.6468 & 30.2652 & 30.1527 & 30.6156 & 30.4204 & 30.4061 & 30.5691 & 30.1900 & 30.0684 & 30.5368 & 30.3465 & 30.3295 \\
8 & 1.64 & 30.3944 & 29.9723 & 29.9342 & 30.3982 & 30.1578 & 30.2006 & 30.3930 & 29.9691 & 29.9184 & 30.3978 & 30.1556 & 30.1844 \\
9 & 2.00 & 30.1684 & 29.7221 & 29.5062 & 30.1492 & 29.8974 & 29.7701 & 30.1292 & 29.6792 & 29.5016 & 30.1243 & 29.8710 & 29.7762 \\
9 & 1.70 & 30.7167 & 30.2646 & 30.3461 & 30.7081 & 30.4241 & 30.4942 & 30.6629 & 30.2122 & 30.2560 & 30.6515 & 30.3685 & 30.4058 \\
10 & 3.56 & 28.4075 & 27.9934 & 27.9927 & 28.4102 & 28.2383 & 28.3130 & 28.3566 & 27.9426 & 27.9227 & 28.3515 & 28.1795 & 28.2459 \\
12 & 1.02 & 31.0888 & 30.6774 & 30.5758 & 31.0740 & 30.8544 & 30.7915 & 31.0872 & 30.6753 & 30.5816 & 31.0736 & 30.8528 & 30.7972 \\
13 & 0.94 & 31.0859 & 30.6611 & 30.6301 & 31.1041 & 30.8793 & 30.8646 & 31.1218 & 30.6949 & 30.6608 & 31.1456 & 30.9153 & 30.8936 \\
14 & 0.86 & 31.2647 & 30.8347 & 30.8145 & 31.3267 & 31.0797 & 31.0487 & 31.2697 & 30.8391 & 30.8182 & 31.3313 & 31.0835 & 31.0511 \\
15 & 1.23 & 30.9680 & 30.5140 & 30.4225 & 30.9525 & 30.6909 & 30.6751 & 30.9775 & 30.5225 & 30.4269 & 30.9613 & 30.6982 & 30.6785 \\
\hline
\end{tabular}

\begin{tabular}{|c|c|c|c|c|c|c|c|c|c|c|c|c|c|}
\hline \multirow{3}{*}{\multicolumn{2}{|c|}{ Exp }} & \multicolumn{12}{|c|}{ mPW1PW91 } \\
\hline & & \multicolumn{6}{|c|}{ Gas phase } & \multicolumn{6}{|c|}{ PCM } \\
\hline & & 6-31G* & 6-31G** & $6-31+G^{* *}$ & 6-311G* & 6-311G** & $6-311+G^{* *}$ & 6-31G* & 6-31G** & $6-31+G^{* *}$ & $6-311 \mathrm{G}^{*}$ & 6-311G** & $6-311+G^{* *}$ \\
\hline \\
\hline 1 & 61.2 & 134.8145 & 135.5007 & 133.9018 & 124.8772 & 124.5353 & 124.3716 & 135.2048 & 135.8827 & 134.4018 & 125.3919 & 125.0342 & 124.9706 \\
\hline 2 & 42.1 & 150.7552 & 151.5066 & 152.7343 & 142.1549 & 141.9351 & 141.6965 & 150.9791 & 151.7266 & 152.9797 & 142.4556 & 142.2178 & 141.9343 \\
\hline 3 & 53.8 & 139.8112 & 140.4907 & 139.2591 & 129.6300 & 129.3141 & 128.4850 & 139.5982 & 140.2762 & 138.9946 & 129.3950 & 129.0689 & 128.1208 \\
\hline 4 & 215.8 & -11.2992 & -10.9836 & -13.6000 & -31.1731 & -30.8061 & -34.0924 & -15.3232 & -15.0131 & -18.5039 & -35.7745 & -35.4191 & -39.5072 \\
\hline 5 & 47.4 & 147.4024 & 148.5062 & 147.0541 & 138.2213 & 137.6713 & 136.8906 & 146.9741 & 148.0673 & 146.4961 & 137.7230 & 137.1685 & 136.2029 \\
\hline 6 & 38.2 & 154.5736 & 155.4581 & 154.0048 & 146.0746 & 145.9560 & 145.4238 & 154.4887 & 155.3747 & 153.9776 & 146.0275 & 145.9156 & 145.4153 \\
\hline 7 & 40.8 & 152.3869 & 152.9006 & 152.6433 & 142.4499 & 142.6185 & 141.9712 & 152.3989 & 152.9143 & 152.6336 & 142.4605 & 142.6308 & 141.9684 \\
\hline 8 & 37.7 & 157.0082 & 158.1318 & 156.5026 & 148.2050 & 147.7878 & 147.1804 & 157.2015 & 158.3198 & 156.8406 & 148.4517 & 148.0286 & 147.5311 \\
\hline 9 & 30.5 & 161.2513 & 162.5348 & 161.0443 & 152.8239 & 152.4928 & 151.8446 & 161.7248 & 162.9980 & 161.6908 & 153.3476 & 152.9956 & 152.4144 \\
\hline 10 & 76.9 & 118.9582 & 119.5975 & 118.7081 & 107.9469 & 107.7461 & 108.0415 & 119.1490 & 119.7908 & 118.8857 & 108.1931 & 107.9766 & 108.2306 \\
\hline 11 & 37.1 & 155.2767 & 155.7560 & 155.5437 & 145.9961 & 145.9434 & 145.4047 & 155.2690 & 155.7465 & 155.5845 & 146.0704 & 146.0072 & 145.4374 \\
\hline 12 & 27.2 & 166.4947 & 168.0857 & 168.4855 & 160.2874 & 160.1781 & 159.9668 & 166.6776 & 168.2635 & 168.7488 & 160.5518 & 160.4261 & 160.2159 \\
\hline 13 & 17.2 & 172.9045 & 174.4536 & 176.3711 & 167.3068 & 167.1740 & 168.1202 & 173.5411 & 175.0874 & 177.0155 & 168.1109 & 167.9349 & 168.7509 \\
\hline 14 & 23.2 & 170.4203 & 172.1187 & 171.8870 & 164.0311 & 163.7152 & 163.5721 & 170.5771 & 172.2684 & 172.0590 & 164.2390 & 163.9063 & 163.7755 \\
\hline $\begin{array}{l}15 \\
{ }^{1} \mathbf{H}\end{array}$ & 17.2 & 175.4666 & 177.1659 & 177.1541 & 169.6169 & 169.5395 & 169.3229 & 175.7163 & 177.4117 & 177.4558 & 169.9189 & 169.8266 & 169.6250 \\
\hline 1 & 1.37 & 30.6365 & 30.3612 & 30.3449 & 30.6780 & 30.5662 & 30.5418 & 30.5804 & 30.3044 & 30.2800 & 30.6190 & 30.5072 & 30.4781 \\
\hline 2 & 2.25 & 29.5427 & 29.0822 & 29.1047 & 29.6634 & 29.4152 & 29.4390 & 29.5396 & 29.0786 & 29.0727 & 29.6635 & 29.4088 & 29.4057 \\
\hline 3 & 2.44 & 29.6425 & 29.2834 & 29.2883 & 29.6323 & 29.4812 & 29.4449 & 29.5613 & 29.1992 & 29.1822 & 29.5484 & 29.3955 & 29.3433 \\
\hline 5 & 2.50 & 29.6742 & 29.2430 & 29.1752 & 29.5682 & 29.3511 & 29.3420 & 29.6038 & 29.1694 & 29.0905 & 29.4975 & 29.2795 & 29.2619 \\
\hline 5 & 2.71 & 29.4641 & 29.0053 & 28.9421 & 29.4158 & 29.1622 & 29.1209 & 29.4429 & 28.9795 & 28.9065 & 29.3918 & 29.1342 & 29.0885 \\
\hline 6 & 2.12 & 29.9570 & 29.4846 & 29.5203 & 30.0684 & 29.7700 & 29.8008 & 29.8877 & 29.4149 & 29.4367 & 29.9965 & 29.6983 & 29.7213 \\
\hline 8 & 1.75 & 30.5864 & 30.2058 & 30.1071 & 30.5076 & 30.3145 & 30.3066 & 30.5047 & 30.1263 & 30.0196 & 30.4255 & 30.2378 & 30.2272 \\
\hline 8 & 1.64 & 30.3605 & 29.9414 & 29.8928 & 30.3134 & 30.0727 & 30.1093 & 30.3554 & 29.9345 & 29.8741 & 30.3100 & 30.0664 & 30.0908 \\
\hline 9 & 2.00 & 30.1064 & 29.6574 & 29.4415 & 30.0457 & 29.7943 & 29.6734 & 30.0733 & 29.6209 & 29.4347 & 30.0226 & 29.7701 & 29.6756 \\
\hline
\end{tabular}




\begin{tabular}{rrrlllllllllll}
\hline 9 & 1.70 & 30.6903 & 30.2446 & 30.2885 & 30.6319 & 30.3496 & 30.3941 & 30.6233 & 30.1781 & 30.1914 & 30.5662 & 30.2841 & 30.3020 \\
10 & 3.56 & 28.3731 & 27.9699 & 27.9484 & 28.3207 & 28.1605 & 28.2331 & 28.3160 & 27.9129 & 27.8748 & 28.2578 & 28.0982 & 28.1638 \\
12 & 1.02 & 31.0301 & 30.6204 & 30.5237 & 30.9761 & 30.7562 & 30.7039 & 31.0276 & 30.6175 & 30.5276 & 30.9746 & 30.7537 & 30.7073 \\
13 & 0.94 & 31.0359 & 30.6122 & 30.5736 & 31.0074 & 30.7815 & 30.7669 & 31.0705 & 30.6446 & 30.6032 & 31.0469 & 30.8155 & 30.7949 \\
14 & 0.86 & 31.2213 & 30.7922 & 30.7660 & 31.2357 & 30.9860 & 30.9502 & 31.2249 & 30.7951 & 30.7683 & 31.2385 & 30.9881 & 30.9509 \\
15 & 1.23 & 30.8994 & 30.4423 & 30.3619 & 30.8636 & 30.5925 & 30.5789 & 30.9065 & 30.4483 & 30.3647 & 30.8695 & 30.5972 & 30.5803 \\
\hline
\end{tabular}

\section{Compound 61}

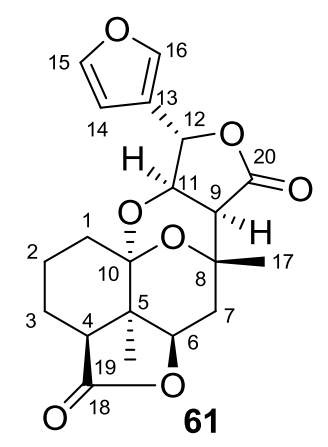

\begin{tabular}{|c|c|c|c|c|c|c|c|c|c|c|c|c|c|}
\hline & \multirow[b]{3}{*}{ Exp } & \multicolumn{12}{|c|}{ B3LYP } \\
\hline & & \multicolumn{6}{|c|}{ Gas phase } & \multicolumn{6}{|c|}{ PCM } \\
\hline & & 6-31G* & 6-31G** & $6-31+\mathrm{G} * *$ & 6-311G* & $6-311 G * *$ & $6-311+\mathrm{G} * *$ & 6-31G* & $6-31 G * *$ & $6-31+G * *$ & 6-311G* & 6-311G** & $6-311+G * *$ \\
\hline \multicolumn{14}{|l|}{${ }^{13} \mathrm{C}$} \\
\hline 1 & 32.4 & 150.7750 & 152.0264 & 151.9869 & 141.6134 & 141.2999 & 140.6444 & 150.8905 & 152.1391 & 151.9864 & 141.7270 & 141.3991 & 140.6956 \\
\hline 2 & 22.1 & 168.4265 & 169.8751 & 168.6197 & 160.9021 & 160.7753 & 160.1956 & 168.3118 & 169.7653 & 168.5468 & 160.8384 & 160.7088 & 160.1196 \\
\hline 3 & 18.1 & 165.5825 & 166.9936 & 167.5818 & 157.7898 & 157.6103 & 157.2543 & 165.8123 & 167.2205 & 167.8121 & 158.0459 & 157.8542 & 157.5244 \\
\hline 4 & 45.4 & 139.8357 & 140.4310 & 140.7520 & 129.4744 & 129.0261 & 128.3078 & 139.8766 & 140.4632 & 140.8015 & 129.5338 & 129.0753 & 128.2783 \\
\hline 5 & 43.3 & 143.3377 & 143.6295 & 145.1954 & 132.1961 & 132.3258 & 131.5801 & 142.8327 & 143.1241 & 144.5578 & 131.5828 & 131.7157 & 130.8788 \\
\hline 6 & 79.4 & 111.3414 & 111.9900 & 110.8979 & 99.0768 & 98.6301 & 97.7004 & 111.0023 & 111.6453 & 110.5452 & 98.7437 & 98.2868 & 97.3220 \\
\hline 7 & 40.8 & 155.2028 & 156.4789 & 155.6787 & 146.3246 & 145.7822 & 145.4782 & 155.5962 & 156.8610 & 156.0918 & 146.7135 & 146.1545 & 145.8722 \\
\hline 8 & 70.5 & 119.9260 & 120.3245 & 119.0929 & 108.6590 & 108.7276 & 108.7144 & 119.7717 & 120.1715 & 118.8930 & 108.4750 & 108.5434 & 108.4897 \\
\hline 9 & 42.8 & 145.0605 & 145.6789 & 145.5160 & 135.8686 & 135.5881 & 134.5328 & 144.8752 & 145.4848 & 145.2924 & 135.6178 & 135.3287 & 134.2028 \\
\hline 10 & 100.0 & 90.3714 & 90.5935 & 90.2926 & 77.7110 & 77.8704 & 76.7321 & 90.4350 & 90.6554 & 90.3000 & 77.7063 & 77.8664 & 76.7088 \\
\hline 11 & 75.2 & 113.7236 & 114.5635 & 113.7549 & 102.4915 & 102.0013 & 101.5334 & 113.9693 & 114.8020 & 114.0276 & 102.7509 & 102.2531 & 101.7749 \\
\hline 12 & 77.7 & 110.1384 & 110.7510 & 110.3599 & 98.6110 & 97.9908 & 97.9737 & 109.5464 & 110.1585 & 109.6305 & 97.9482 & 97.3222 & 97.1909 \\
\hline 13 & 121.2 & 72.4005 & 72.1002 & 70.6353 & 53.7837 & 53.5800 & 52.6601 & 72.3286 & 72.0334 & 70.5441 & 53.7207 & 53.5328 & 52.5276 \\
\hline 14 & 107.9 & 88.1489 & 88.6454 & 88.6273 & 72.2846 & 71.5476 & 71.1952 & 87.5317 & 88.0222 & 87.8406 & 71.4505 & 70.7242 & 70.2641 \\
\hline 15 & 144.8 & 53.6186 & 54.1430 & 51.9905 & 34.6539 & 33.4790 & 31.9644 & 53.2117 & 53.7330 & 51.5320 & 34.0701 & 32.9146 & 31.4674 \\
\hline 16 & 139.7 & 56.6592 & 57.3326 & 55.4983 & 38.0914 & 37.1205 & 35.3182 & 57.1570 & 57.8310 & 56.0592 & 38.5535 & 37.6047 & 35.8491 \\
\hline 17 & 26.2 & 163.5127 & 165.1677 & 165.0221 & 155.9164 & 155.7413 & 155.3988 & 163.5657 & 165.2237 & 165.0870 & 156.0773 & 155.8781 & 155.5251 \\
\hline 18 & 174.5 & 25.2447 & 25.3313 & 22.9277 & 3.5522 & 3.7442 & 1.5047 & 22.3077 & 22.3925 & 19.4853 & 0.2358 & 0.4200 & -2.3195 \\
\hline 19 & 16.7 & 166.2127 & 168.0704 & 169.3469 & 159.8807 & 159.7050 & 159.0398 & 166.8320 & 168.6705 & 169.9662 & 160.5440 & 160.3520 & 159.6942 \\
\hline 20 & 171.8 & 27.2726 & 27.3128 & 25.1216 & 6.0494 & 6.2003 & 4.4798 & 24.7736 & 24.8126 & 22.2348 & 3.2071 & 3.3524 & 1.2508 \\
\hline \multicolumn{14}{|c|}{${ }^{1} \mathrm{H}$} \\
\hline 1 & 1.69 & 30.5494 & 30.1453 & 29.9555 & 30.5318 & 30.3176 & 30.2543 & 30.4554 & 30.0471 & 29.8454 & 30.4328 & 30.2168 & 30.1454 \\
\hline 1 & 1.79 & 30.3606 & 29.9340 & 29.7456 & 30.2475 & 30.0396 & 29.9720 & 30.4366 & 30.0106 & 29.8190 & 30.3232 & 30.1157 & 30.0408 \\
\hline
\end{tabular}




\begin{tabular}{llllllllllllll}
\hline 2 & 1.55 & 30.8963 & 30.4523 & 30.4167 & 30.8170 & 30.5988 & 30.5441 & 30.8220 & 30.3732 & 30.3240 & 30.7388 & 30.5158 & 30.4530 \\
2 & 1.80 & 30.3756 & 29.9522 & 29.8413 & 30.4369 & 30.2299 & 30.0779 & 30.5352 & 30.1176 & 30.0020 & 30.5928 & 30.3888 & 30.2306 \\
3 & 1.46 & 30.8171 & 30.3997 & 30.2346 & 30.7447 & 30.5428 & 30.4967 & 30.6542 & 30.2312 & 30.0504 & 30.5814 & 30.3766 & 30.3195 \\
3 & 1.92 & 30.0346 & 29.5574 & 29.4027 & 29.9916 & 29.7263 & 29.6768 & 30.1574 & 29.6849 & 29.5378 & 30.1133 & 29.8537 & 29.8052 \\
4 & 2.81 & 29.8808 & 29.4651 & 29.5596 & 29.9049 & 29.6955 & 29.7251 & 29.6585 & 29.2375 & 29.3007 & 29.6776 & 29.4670 & 29.4733 \\
6 & 4.56 & 28.0982 & 27.6020 & 27.3842 & 28.1330 & 27.8618 & 27.7133 & 27.8975 & 27.3951 & 27.1590 & 27.9281 & 27.6495 & 27.4888 \\
7 & 2.27 & 30.0112 & 29.5790 & 29.4046 & 30.0062 & 29.7936 & 29.6847 & 29.9490 & 29.5154 & 29.3396 & 29.9475 & 29.7336 & 29.6237 \\
7 & 1.90 & 30.6140 & 30.1294 & 29.9806 & 30.6796 & 30.3816 & 30.2869 & 30.4039 & 29.9137 & 29.7573 & 30.4677 & 30.1686 & 30.0708 \\
9 & 2.36 & 30.2594 & 29.8386 & 29.5976 & 30.3458 & 30.0978 & 29.8669 & 30.0133 & 29.5830 & 29.3250 & 30.1021 & 29.8497 & 29.6018 \\
11 & 4.36 & 27.6735 & 27.1224 & 27.0543 & 27.6905 & 27.3775 & 27.2945 & 27.4834 & 26.9261 & 26.8519 & 27.4916 & 27.1768 & 27.0905 \\
12 & 5.36 & 26.9805 & 26.4520 & 26.2485 & 26.9032 & 26.5952 & 26.5884 & 26.8811 & 26.3444 & 26.1285 & 26.7988 & 26.4817 & 26.4637 \\
14 & 6.33 & 26.2265 & 25.4403 & 25.2468 & 26.1110 & 25.5818 & 25.5762 & 26.0337 & 25.2450 & 25.0377 & 25.9096 & 25.3855 & 25.3680 \\
15 & 7.48 & 24.9933 & 24.2562 & 24.0433 & 24.8889 & 24.4162 & 24.2920 & 24.8722 & 24.1386 & 23.9282 & 24.7616 & 24.2983 & 24.1793 \\
16 & 7.44 & 25.0154 & 24.2572 & 23.8975 & 24.9500 & 24.4445 & 24.1939 & 25.0042 & 24.2460 & 23.8854 & 24.9290 & 24.4288 & 24.1812 \\
17 & 1.74 & 30.4686 & 29.9988 & 29.8749 & 30.4490 & 30.1711 & 30.1174 & 30.5171 & 30.0474 & 29.9224 & 30.4942 & 30.2159 & 30.1605 \\
19 & 1.19 & 31.0462 & 30.6009 & 30.3790 & 31.0559 & 30.7927 & 30.7093 & 30.9605 & 30.5121 & 30.2863 & 30.9710 & 30.7062 & 30.6215 \\
\hline
\end{tabular}

\section{mPW1PW91}

\section{Gas phase PCM}

Exp 6-31G* 6-31G** 6-31+G** 6-311G* 6-311G**6-311+G** 6-31G* 6-31G** 6-31+G** 6-311G* 6-311G** 6-311+G**

\section{${ }^{13} \mathrm{C}$}

$\begin{array}{lllllll}2 & 22.1 & 173.1954 & 174.5376 & 173.0314 & 166.2394 & 166.1037\end{array}$

$\begin{array}{lllllll}3 & 18.1 & 170.5808 & 171.8685 & 172.2341 & 163.3989 & 163.2087\end{array}$

$\begin{array}{lllllll}4 & 45.4 & 144.5849 & 145.1214 & 145.3894 & 135.2576 & 134.8118\end{array}$

$\begin{array}{llllllll}5 & 43.3 & 148.5731 & 148.8606 & 150.3214 & 138.4807 & 138.5982\end{array}$

$\begin{array}{lllllll}10 & 100.0 & 94.9807 & 95.1994 & 95.1039 & 84.1825 & 84.3394\end{array}$

$\begin{array}{llllllll}11 & 75.2 & 118.3954 & 119.1828 & 118.5213 & 108.7198 & 108.2477 & 107\end{array}$

$\begin{array}{llllllll}12 & 77.7 & 114.3081 & 114.8917 & 114.8261 & 104.5563 & 103.9487\end{array}$

$\begin{array}{lllllll}13 & 121.2 & 75.9948 & 75.6504 & 74.3657 & 59.7389 & 59.4800\end{array}$

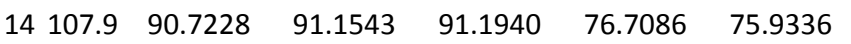

$15144.8 \quad 55.8073 \quad 56.2741$

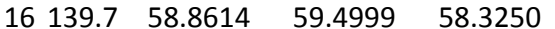

$\begin{array}{lllll}17 & 26.2 & 167.6799 & 169.2063 & 168.9546\end{array}$

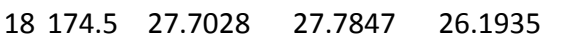

$\begin{array}{lllll}19 & 16.7 & 170.7675 & 172.4802 & 173.2260\end{array}$

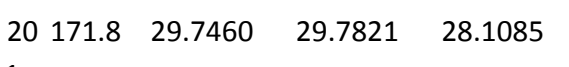
${ }^{1} \mathrm{H}$

\begin{tabular}{|c|c|c|c|c|c|c|c|c|c|c|c|c|c|}
\hline & 1.69 & 30.5102 & 30.1067 & 29.9287 & 30.4475 & 30.2358 & 30.1785 & 30.4114 & 30.0032 & 29.8149 & 30.3454 & 30.1316 & 30.0666 \\
\hline & 1.79 & 30.2673 & 29.8407 & 29.6851 & 30.1413 & 29.9301 & 29.8796 & 30.3456 & 29.9196 & 29.7599 & 30.2177 & 30.0070 & 29.9487 \\
\hline & 1.55 & 30.8225 & 30.3848 & 30.3533 & 30.7337 & 30.5069 & 30.4551 & 30.7423 & 30.2993 & 30.2562 & 30.6504 & 30.4188 & 30.3595 \\
\hline
\end{tabular}

46.7985 $\begin{array}{llllllll}65.5695 & 173.1071 & 174.4534 & 172.9837 & 166.1919 & 166.0527 & 165.5115\end{array}$ $\begin{array}{lllllll}62.7676 & 170.8308 & 172.1166 & 172.4756 & 163.6619 & 163.4598 & 163.0396\end{array}$ $\begin{array}{lllllll}34.1877 & 144.6061 & 145.1353 & 145.4164 & 135.2789 & 134.8238 & 134.1275\end{array}$ $\begin{array}{llllllll}37.9060 & 148.0583 & 148.3457 & 149.7040 & 137.8673 & 137.9889 & 137.2189\end{array}$ $\begin{array}{llllllll}04.0464 & 115.6709 & 116.2701 & 115.4253 & 105.0068 & 104.5710 & 103.6384\end{array}$ $\begin{array}{llllllll} & 16170 & 160.1973 & 161.3707 & 160.5363 & 152.1805 & 151.6463 & 151.2915\end{array}$ $\begin{array}{lllllll}4.8400 & 124.5985 & 124.9925 & 123.9224 & 114.5996 & 114.6701 & 114.6038\end{array}$ $\begin{array}{llllllll}39.9143 & 149.2213 & 149.7751 & 149.5810 & 140.8868 & 140.6150 & 139.5655\end{array}$ $\begin{array}{lllllll}8.2081 & 95.0339 & 95.2511 & 95.1141 & 84.1645 & 84.3222 & 83.1671\end{array}$ $\begin{array}{lllllll}07.8229 & 118.6213 & 119.4035 & 118.7672 & 108.9421 & 108.4634 & 108.0279\end{array}$ $\begin{array}{lllllll}03.8682 & 113.7132 & 114.2966 & 114.1090 & 103.8987 & 103.2855 & 103.1052\end{array}$ $\begin{array}{llllllll}58.5155 & 75.9228 & 75.5845 & 74.2809 & 59.6939 & 59.4513 & 58.3987\end{array}$ $\begin{array}{llllllll}75.5249 & 90.1163 & 90.5424 & 90.4290 & 75.9176 & 75.1548 & 74.6508\end{array}$ $\begin{array}{lllllll}36.6935 & 55.3539 & 55.8183 & 54.0490 & 38.6762 & 37.4996 & 36.1593\end{array}$ $\begin{array}{cccccccccc}42.6431 & 41.6768 & 39.9590 & 59.3521 & 59.9912 & 58.8710 & 43.0763 & 42.1327 & 40.4602 \\ 160.6858 & 160.5430 & 160.2242 & 167.7550 & 169.2837 & 169.0459 & 160.8475 & 160.6810 & 160.3507\end{array}$ $\begin{array}{lllllllll}8.6681 & 8.8581 & 6.8110 & 24.7152 & 24.7953 & 22.7683 & 5.3709 & 5.5527 & 3.0687\end{array}$ $\begin{array}{lllllllll}164.8345 & 164.6717 & 164.0765 & 171.4211 & 173.1171 & 173.8724 & 165.4893 & 165.3154 & 164.7219\end{array}$ $\begin{array}{lllllllll}11.1111 & 11.2573 & 9.7718 & 27.2184 & 27.2526 & 25.2523 & 8.3026 & 8.4427 & 6.6283\end{array}$ 


\begin{tabular}{llllllllllllll}
\hline 2 & 1.80 & 30.3292 & 29.9057 & 29.8072 & 30.3372 & 30.1355 & 29.9960 & 30.4953 & 30.0781 & 29.9713 & 30.4975 & 30.2990 & 30.1507 \\
3 & 1.46 & 30.7585 & 30.3458 & 30.1678 & 30.6556 & 30.4546 & 30.4073 & 30.5899 & 30.1711 & 29.9792 & 30.4885 & 30.2841 & 30.2272 \\
3 & 1.92 & 29.9721 & 29.4978 & 29.3518 & 29.9029 & 29.6396 & 29.5911 & 30.0921 & 29.6228 & 29.4817 & 30.0203 & 29.7632 & 29.7151 \\
4 & 2.81 & 29.8240 & 29.4047 & 29.4885 & 29.7970 & 29.5863 & 29.6212 & 29.5946 & 29.1696 & 29.2272 & 29.5666 & 29.3543 & 29.3690 \\
6 & 4.56 & 28.0313 & 27.5381 & 27.3268 & 28.0340 & 27.7627 & 27.6284 & 27.8230 & 27.3231 & 27.0975 & 27.8246 & 27.5456 & 27.4015 \\
7 & 2.27 & 29.9241 & 29.4910 & 29.3138 & 29.8922 & 29.6788 & 29.5681 & 29.8560 & 29.4212 & 29.2436 & 29.8283 & 29.6132 & 29.5022 \\
7 & 1.90 & 30.5835 & 30.1026 & 29.9512 & 30.5962 & 30.3042 & 30.2141 & 30.3656 & 29.8786 & 29.7232 & 30.3801 & 30.0864 & 29.9939 \\
9 & 2.36 & 30.2189 & 29.7935 & 29.5477 & 30.2482 & 30.0003 & 29.7667 & 29.9702 & 29.5347 & 29.2775 & 30.0051 & 29.7524 & 29.5050 \\
11 & 4.36 & 27.6202 & 27.0758 & 27.0073 & 27.6082 & 27.3000 & 27.2301 & 27.4204 & 26.8692 & 26.7982 & 27.4037 & 27.0931 & 27.0212 \\
12 & 5.36 & 26.8902 & 26.3510 & 26.1990 & 26.7977 & 26.4819 & 26.5032 & 26.7865 & 26.2385 & 26.0738 & 26.6895 & 26.3645 & 26.3741 \\
14 & 6.33 & 26.0538 & 25.2663 & 25.1089 & 25.9760 & 25.4087 & 25.4200 & 25.8490 & 25.0583 & 24.8908 & 25.7673 & 25.2049 & 25.2063 \\
15 & 7.48 & 24.8244 & 24.0884 & 23.9138 & 24.7645 & 24.2530 & 24.1447 & 24.6905 & 23.9578 & 23.7868 & 24.6271 & 24.1254 & 24.0224 \\
16 & 7.44 & 24.8430 & 24.0902 & 23.7849 & 24.8172 & 24.2783 & 24.0579 & 24.8241 & 24.0712 & 23.7656 & 24.7900 & 24.2569 & 24.0391 \\
17 & 1.74 & 30.3776 & 29.9033 & 29.7883 & 30.3357 & 30.0522 & 29.9985 & 30.4245 & 29.9504 & 29.8343 & 30.3789 & 30.0951 & 30.0397 \\
19 & 1.19 & 30.9958 & 30.5503 & 30.3435 & 30.9701 & 30.7036 & 30.6224 & 30.9049 & 30.4562 & 30.2480 & 30.8814 & 30.6131 & 30.5311 \\
\hline
\end{tabular}

\section{Compound 62}

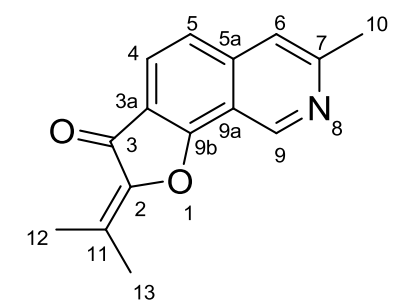

\section{2}

\begin{tabular}{|c|c|c|c|c|c|c|c|c|c|c|c|c|c|}
\hline \multirow{3}{*}{\multicolumn{2}{|c|}{ Exp }} & \multicolumn{12}{|c|}{ B3LYP } \\
\hline & & \multicolumn{6}{|c|}{ Gas phase } & \multicolumn{6}{|c|}{ PCM } \\
\hline & & 6-31G* & $6-31 G * *$ & $6-31+G * *$ & 6-311G* & $6-311 G * *$ & $6-311+G * *$ & 6-31G* & $6-31 G * *$ & $6-31+\mathrm{G} * *$ & 6-311G* & $6-311 G * *$ & $6-311+G * *$ \\
\hline \multicolumn{14}{|c|}{${ }^{13} \mathrm{C}$} \\
\hline 2 & 145.6 & 48.6767 & 48.7141 & 47.6083 & 30.7866 & 30.9148 & 30.1693 & 48.8602 & 48.8976 & 47.8267 & 31.0349 & 31.1590 & 30.3809 \\
\hline 3 & 182.1 & 17.1209 & 17.1523 & 17.6165 & -1.5942 & -1.5293 & -4.4493 & 16.5511 & 16.5856 & 16.8226 & -2.3842 & -2.3198 & -5.4469 \\
\hline $3 a$ & 119.3 & 74.2462 & 74.2049 & 73.7502 & 59.0097 & 58.9307 & 57.5648 & 74.3757 & 74.3375 & 73.8654 & 59.1872 & 59.1176 & 57.6827 \\
\hline 4 & 124.1 & 69.9942 & 70.6221 & 70.5727 & 53.9063 & 53.0533 & 52.5990 & 70.0451 & 70.6775 & 70.6697 & 53.9586 & 53.1300 & 52.7008 \\
\hline 5 & 120.5 & 75.3878 & 76.0154 & 75.6866 & 59.7629 & 58.9461 & 58.1651 & 74.8384 & 75.4596 & 75.0170 & 59.0633 & 58.2460 & 57.4578 \\
\hline $5 a$ & 141.3 & 56.0110 & 55.8332 & 54.9585 & 37.0466 & 36.9119 & 35.3029 & 55.6379 & 55.4670 & 54.5171 & 36.7099 & 36.5856 & 34.8669 \\
\hline 6 & 119.5 & 75.7441 & 76.5008 & 77.5803 & 61.2876 & 60.6408 & 59.9773 & 75.0845 & 75.8340 & 76.7278 & 60.4720 & 59.8132 & 59.0739 \\
\hline 7 & 156.7 & 37.8751 & 37.8794 & 35.4563 & 18.5159 & 18.2571 & 17.2814 & 37.5474 & 37.5471 & 35.1983 & 18.0313 & 17.7778 & 16.8615 \\
\hline 9 & 146.2 & 48.2455 & 48.9865 & 48.6802 & 32.3079 & 31.3922 & 29.9381 & 48.2537 & 48.9899 & 48.5417 & 32.1337 & 31.2223 & 29.6790 \\
\hline $9 a$ & 114.6 & 80.9657 & 80.7960 & 80.6901 & 65.3579 & 65.2611 & 64.0013 & 80.8504 & 80.6813 & 80.5309 & 65.2999 & 65.2050 & 63.8886 \\
\hline $9 b$ & 164.0 & 32.6005 & 32.6150 & 32.1267 & 13.4391 & 13.4120 & 11.8694 & 32.4469 & 32.4610 & 31.9038 & 13.2223 & 13.1997 & 11.6135 \\
\hline 11 & 133.7 & 59.8661 & 60.1214 & 57.7628 & 40.2807 & 40.2838 & 38.7878 & 56.1109 & 56.3578 & 53.2868 & 36.0048 & 35.9885 & 34.0119 \\
\hline 12 & 17.5 & 173.9357 & 175.5101 & 175.4575 & 166.4511 & 166.1371 & 165.5697 & 173.8877 & 175.4567 & 175.3131 & 166.4335 & 166.0886 & 165.4462 \\
\hline 13 & 20.4 & 169.6832 & 171.3277 & 171.6983 & 162.2476 & 161.9632 & 161.6370 & 169.6634 & 171.2864 & 171.6021 & 162.1585 & 161.8365 & 161.4757 \\
\hline 10 & 24.7 & 164.2321 & 165.9554 & 164.8111 & 156.6751 & 156.2597 & 156.2082 & 164.5066 & 166.2071 & 164.9438 & 156.8713 & 156.4327 & 156.3374 \\
\hline
\end{tabular}




\begin{tabular}{rlllllllllllll}
\hline 4 & 7.80 & 24.3788 & 23.7431 & 23.6390 & 24.2507 & 23.8489 & 23.8722 & 24.3343 & 23.6975 & 23.5916 & 24.1928 & 23.7939 & 23.8203 \\
5 & 7.35 & 25.1023 & 24.4728 & 24.3290 & 24.9222 & 24.5058 & 24.4838 & 24.9241 & 24.2934 & 24.1399 & 24.7304 & 24.3213 & 24.2944 \\
9 & 9.52 & 22.6137 & 21.9944 & 21.7827 & 22.4536 & 22.0476 & 21.9601 & 22.6050 & 21.9859 & 21.7709 & 22.4381 & 22.0348 & 21.9477 \\
6 & 7.52 & 25.0383 & 24.3838 & 24.1342 & 24.8784 & 24.3970 & 24.2482 & 24.8374 & 24.1807 & 23.9115 & 24.6610 & 24.1863 & 24.0270 \\
10 & 2.74 & 29.5889 & 29.1074 & 28.9813 & 29.4330 & 29.1485 & 29.1445 & 29.5525 & 29.0682 & 28.9418 & 29.3940 & 29.1082 & 29.1042 \\
12 & 2.43 & 29.8290 & 29.2669 & 28.9605 & 29.6834 & 29.3328 & 29.2092 & 29.7970 & 29.2330 & 28.9225 & 29.6501 & 29.2978 & 29.1687 \\
13 & 2.25 & 30.1210 & 29.6175 & 29.3440 & 29.9869 & 29.6857 & 29.5793 & 30.0321 & 29.5255 & 29.2509 & 29.8986 & 29.5961 & 29.4878 \\
\hline
\end{tabular}

\begin{tabular}{|c|c|c|c|c|c|c|c|c|c|c|c|c|c|}
\hline \multirow{3}{*}{\multicolumn{2}{|c|}{ Exp }} & \multicolumn{12}{|c|}{ mPW1PW91 } \\
\hline & & \multicolumn{6}{|c|}{ Gas phase } & \multicolumn{6}{|c|}{ PCM } \\
\hline & & 6-31G* & 6-31G** & $6-31+G * *$ & 6-311G* & 6-311G** & $6-311+G^{* *}$ & 6-31G* & $6-31 G^{* *}$ & $6-31+G * *$ & $6-311 G^{*}$ & $6-311 \mathrm{G} * *$ & $6-311+G^{* *}$ \\
\hline \multicolumn{14}{|c|}{${ }^{3} \mathrm{C}$} \\
\hline 2 & 145.6 & 51.9033 & 51.9488 & 51.2703 & 36.6220 & 36.7578 & 35.8670 & 52.1703 & 52.2159 & 51.5683 & 36.9514 & 37.0824 & 36.1676 \\
\hline 3 & 182.1 & 19.3404 & 19.3696 & 20.2201 & 3.3963 & 3.4640 & 0.8237 & 18.7859 & 18.8186 & 19.4354 & 2.6356 & 2.7027 & -0.1253 \\
\hline $3 a$ & 119.3 & 77.4890 & 77.4432 & 77.4468 & 64.3519 & 64.2656 & 62.6656 & 77.6679 & 77.6261 & 77.6113 & 64.5710 & 64.4951 & 62.8360 \\
\hline 4 & 124.1 & 71.8770 & 72.4404 & 72.5908 & 57.8923 & 57.0087 & 56.4413 & 71.8911 & 72.4591 & 72.6284 & 57.8909 & 57.0334 & 56.4969 \\
\hline 5 & 120.5 & 78.2510 & 78.8151 & 78.5864 & 64.6242 & 63.7823 & 62.9403 & 77.6666 & 78.2233 & 77.8826 & 63.8927 & 63.0510 & 62.2234 \\
\hline $5 a$ & 141.3 & 58.9008 & 58.7078 & 58.0026 & 42.4784 & 42.3171 & 40.7565 & 58.5628 & 58.3780 & 57.6025 & 42.1708 & 42.0217 & 40.3634 \\
\hline 6 & 119.5 & 78.5476 & 79.2583 & 80.4985 & 65.9548 & 65.3091 & 64.5266 & 77.9206 & 78.6235 & 79.6914 & 65.1817 & 64.5249 & 63.6903 \\
\hline 7 & 156.7 & 40.5586 & 40.5360 & 38.6995 & 23.8185 & 23.5454 & 22.5794 & 40.1239 & 40.0973 & 38.3277 & 23.2260 & 22.9605 & 22.0533 \\
\hline 9 & 146.2 & 50.2441 & 50.9369 & 50.7538 & 36.3443 & 35.4196 & 34.2305 & 50.2090 & 50.8961 & 50.5867 & 36.1202 & 35.2005 & 33.9369 \\
\hline $9 a$ & 114.6 & 84.1696 & 83.9918 & 84.2440 & 70.7115 & 70.6013 & 69.2658 & 84.1221 & 83.9460 & 84.1519 & 70.7175 & 70.6104 & 69.2250 \\
\hline $9 b$ & 164.0 & 35.3217 & 35.3382 & 35.3597 & 18.8990 & 18.8780 & 17.2921 & 35.2036 & 35.2200 & 35.1722 & 18.7144 & 18.6984 & 17.0723 \\
\hline 11 & 133.7 & 63.0174 & 63.2444 & 61.3443 & 45.7677 & 45.7460 & 44.2818 & 58.9900 & 59.2058 & 56.6957 & 41.2828 & 41.2424 & 39.3814 \\
\hline 12 & 17.5 & 177.3511 & 178.8105 & 178.7970 & 170.5362 & 170.2320 & 169.7360 & 177.2969 & 178.7497 & 178.6484 & 170.4789 & 170.1457 & 169.5899 \\
\hline 13 & 20.4 & 173.2557 & 174.7848 & 175.2719 & 166.4276 & 166.1533 & 165.8794 & 173.2286 & 174.7354 & 175.1602 & 166.3039 & 165.9943 & 165.6926 \\
\hline $\begin{array}{l}10 \\
{ }^{1} \mathrm{H}\end{array}$ & 24.7 & 167.8279 & 169.4443 & 168.6156 & 161.0424 & 160.6476 & 160.5713 & 168.1308 & 169.7234 & 168.7771 & 161.2372 & 160.8215 & 160.7108 \\
\hline 4 & 7.80 & 24.1472 & 23.5072 & 23.4106 & 24.0580 & 23.6142 & 23.6327 & 24.0873 & 23.4459 & 23.3490 & 23.9880 & 23.5472 & 23.5697 \\
\hline 5 & 7.35 & 24.8783 & 24.2461 & 24.1139 & 24.7399 & 24.2916 & 24.2781 & 24.6815 & 24.0475 & 23.9097 & 24.5345 & 24.0932 & 24.0761 \\
\hline 9 & 9.52 & 22.3937 & 21.7735 & 21.6226 & 22.2725 & 21.8345 & 21.7842 & 22.3713 & 21.7514 & 21.5969 & 22.2460 & 21.8111 & 21.7604 \\
\hline 6 & 7.52 & 24.8232 & 24.1682 & 23.9325 & 24.6998 & 24.1901 & 24.0901 & 24.6047 & 23.9468 & 23.6954 & 24.4701 & 23.9671 & 23.8571 \\
\hline 10 & 2.74 & 29.4794 & 28.9927 & 28.8957 & 29.3163 & 29.0205 & 29.0397 & 29.4334 & 28.9435 & 28.8458 & 29.2691 & 28.9720 & 28.9907 \\
\hline 12 & 2.43 & 29.7382 & 29.1709 & 28.9022 & 29.5801 & 29.2210 & 29.1231 & 29.6975 & 29.1278 & 28.8545 & 29.5390 & 29.1781 & 29.0748 \\
\hline 13 & 2.25 & 30.0305 & 29.5236 & 29.3058 & 29.8816 & 29.5704 & 29.4966 & 29.9309 & 29.4202 & 29.2011 & 29.7842 & 29.4715 & 29.3953 \\
\hline
\end{tabular}




\section{Compound 63}

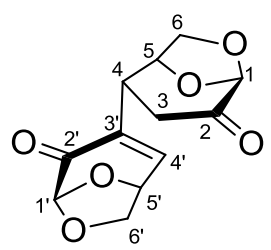

63

\begin{tabular}{|c|c|c|c|c|c|c|c|c|c|c|c|c|c|}
\hline & & \multicolumn{12}{|c|}{ B3LYP } \\
\hline & \multirow[b]{2}{*}{ Exp } & \multicolumn{6}{|c|}{ Gas phase } & \multicolumn{6}{|c|}{ PCM } \\
\hline & & 6-31G* & $6-31 G * *$ & $6-31+\mathrm{G} * *$ & 6-311G* & $6-311 G * *$ & $6-311+G * *$ & 6-31G* & $6-31 G * *$ & $6-31+G * *$ & 6-311G* & $6-311 G * *$ & $6-311+G * *$ \\
\hline \multicolumn{14}{|l|}{${ }^{13} \mathrm{C}$} \\
\hline 1 & 101.5 & 89.5614 & 90.2350 & 88.5247 & 76.0916 & 75.4199 & 75.5085 & 89.6888 & 90.3598 & 88.5879 & 76.1903 & 75.5206 & 75.5510 \\
\hline 2 & 200.5 & -2.0290 & -1.8401 & -4.5539 & -21.4694 & -21.3548 & -25.7917 & -4.9643 & -4.7854 & -8.1398 & -24.8371 & -24.7285 & -29.8513 \\
\hline 3 & 34.8 & 152.9811 & 154.1907 & 152.7995 & 143.7864 & 143.3946 & 142.6788 & 152.5561 & 153.7499 & 152.2077 & 143.2104 & 142.8052 & 141.9377 \\
\hline 4 & 38.6 & 150.3181 & 151.0455 & 150.5139 & 140.8986 & 140.6422 & 140.2197 & 150.0781 & 150.8048 & 150.3133 & 140.7240 & 140.4658 & 140.0121 \\
\hline 5 & 75.5 & 112.9375 & 113.6773 & 112.4694 & 101.4119 & 100.9408 & 100.6859 & 112.9283 & 113.6643 & 112.4431 & 101.3752 & 100.8976 & 100.6385 \\
\hline 6 & 66.7 & 122.1208 & 123.4509 & 123.1487 & 112.1446 & 111.2936 & 111.0018 & 122.2016 & 123.5228 & 123.0248 & 112.1261 & 111.2498 & 110.9125 \\
\hline $1^{\prime}$ & 102.0 & 89.0797 & 89.8274 & 88.0990 & 76.2204 & 75.5361 & 75.7229 & 89.2947 & 90.0391 & 88.2146 & 76.3446 & 75.6619 & 75.7980 \\
\hline $2^{\prime}$ & 188.9 & 9.0382 & 9.1301 & 7.7057 & -9.9109 & -9.8789 & -12.7331 & 7.8851 & 7.9820 & 6.2958 & -11.3309 & -11.2920 & -14.4143 \\
\hline $3^{\prime}$ & 137.1 & 53.7530 & 53.5571 & 51.3909 & 34.9565 & 34.6781 & 34.4905 & 54.1030 & 53.9065 & 51.9004 & 35.4346 & 35.1651 & 35.0681 \\
\hline $4^{\prime}$ & 145.4 & 51.6903 & 52.5157 & 50.6033 & 33.5085 & 32.8451 & 31.2657 & 49.2793 & 50.1097 & 47.7841 & 30.7326 & 30.0808 & 28.2099 \\
\hline $5^{\prime}$ & 72.9 & 116.6750 & 117.5057 & 116.8201 & 105.9527 & 105.3955 & 105.0964 & 116.5977 & 117.4266 & 116.6656 & 105.7992 & 105.2291 & 104.8854 \\
\hline $6^{\prime}$ & 68.1 & 124.0234 & 125.3507 & 125.1710 & 114.3175 & 113.6364 & 113.1551 & 124.4112 & 125.7264 & 125.3797 & 114.5744 & 113.8703 & 113.3680 \\
\hline \multicolumn{14}{|l|}{${ }^{1} \mathrm{H}$} \\
\hline 1 & 5.11 & 27.3237 & 26.7522 & 26.6710 & 27.2859 & 26.9719 & 26.9816 & 27.2883 & 26.7106 & 26.6229 & 27.2342 & 26.9180 & 26.9287 \\
\hline $3 e q$ & 2.23 & 30.2918 & 29.7995 & 29.8024 & 30.3257 & 30.0678 & 29.9882 & 30.3409 & 29.8450 & 29.8334 & 30.3723 & 30.1095 & 30.0167 \\
\hline $3 a x$ & 2.97 & 29.3405 & 28.8895 & 28.7968 & 29.4054 & 29.1341 & 29.0316 & 29.2085 & 28.7516 & 28.6330 & 29.2635 & 28.9909 & 28.8691 \\
\hline 4 & 3.43 & 29.0985 & 28.5372 & 28.3505 & 29.0701 & 28.7193 & 28.5505 & 29.0239 & 28.4639 & 28.2548 & 28.9937 & 28.6451 & 28.4600 \\
\hline 5 & 4.53 & 27.8492 & 27.3111 & 27.2405 & 27.8440 & 27.5436 & 27.5087 & 27.6686 & 27.1240 & 27.0369 & 27.6661 & 27.3620 & 27.3124 \\
\hline Gendo & 4.15 & 28.2088 & 27.6768 & 27.5885 & 28.2579 & 27.9102 & 27.8393 & 28.0645 & 27.5304 & 27.4215 & 28.1121 & 27.7633 & 27.6756 \\
\hline 6exo & 4.03 & 28.2861 & 27.7309 & 27.5554 & 28.1752 & 27.8560 & 27.8332 & 28.1944 & 27.6406 & 27.4554 & 28.0849 & 27.7693 & 27.7358 \\
\hline $1^{\prime}$ & 5.40 & 27.0840 & 26.5048 & 26.4272 & 26.9812 & 26.6632 & 26.6854 & 27.0638 & 26.4804 & 26.3973 & 26.9418 & 26.6235 & 26.6478 \\
\hline $4^{\prime}$ & 7.08 & 24.5331 & 23.7574 & 23.5007 & 24.4546 & 23.9156 & 23.8290 & 24.3734 & 23.6019 & 23.3253 & 24.2943 & 23.7597 & 23.6569 \\
\hline $5^{\prime}$ & 5.06 & 27.7079 & 27.1317 & 26.9410 & 27.6263 & 27.2571 & 27.2126 & 27.4895 & 26.9048 & 26.6914 & 27.4032 & 27.0279 & 26.9674 \\
\hline 6'endo & 3.71 & 28.6689 & 28.1359 & 28.0256 & 28.6924 & 28.3755 & 28.2853 & 28.5980 & 28.0619 & 27.9394 & 28.6200 & 28.2986 & 28.1968 \\
\hline 6'exo & 3.89 & 28.4639 & 27.9268 & 27.7569 & 28.3814 & 28.0826 & 27.9898 & 28.3645 & 27.8276 & 27.6358 & 28.2735 & 27.9780 & 27.8718 \\
\hline
\end{tabular}

\begin{tabular}{|c|c|c|c|c|c|c|c|c|c|c|c|c|c|}
\hline & \multirow[b]{3}{*}{ Exp } & \multicolumn{12}{|c|}{ mPW1PW91 } \\
\hline & & \multicolumn{6}{|c|}{ Gas phase } & \multicolumn{6}{|c|}{ PCM } \\
\hline & & 6-31G* & $6-31 G * *$ & $6-31+\mathrm{G} * *$ & 6-311G* & $6-311 G * *$ & $6-311+G * *$ & 6-31G* & $6-31 \mathrm{G} * *$ & $6-31+\mathrm{G} * *$ & 6-311G* & $6-311 G * *$ & $6-311+G * *$ \\
\hline \multicolumn{14}{|l|}{${ }^{13} \mathrm{C}$} \\
\hline 1 & 101.5 & 93.9433 & 94.5996 & 93.2832 & 82.6919 & 82.0423 & 82.0421 & 94.0742 & 94.7283 & 93.3562 & 82.7866 & 82.1394 & 82.0874 \\
\hline 2 & 200.5 & -0.1095 & 0.0685 & -1.7783 & -16.6398 & -16.5282 & -20.5540 & -3.1540 & -2.9875 & -5.4097 & -20.0500 & -19.9450 & -24.5843 \\
\hline
\end{tabular}




\begin{tabular}{|c|c|c|c|c|c|c|c|c|c|c|c|c|c|}
\hline 3 & 34.8 & 157.3783 & 158.4886 & 157.1045 & 149.0792 & 148.7075 & 148.0224 & 156.9308 & 158.0252 & 156.5022 & 148.4815 & 148.0964 & 147.2782 \\
\hline 4 & 38.6 & 155.1438 & 155.8106 & 155.4034 & 146.6734 & 146.4230 & 145.9914 & 154.9181 & 155.5841 & 155.2198 & 146.5120 & 146.2597 & 145.8028 \\
\hline 5 & 75.5 & 117.6734 & 118.3580 & 117.2314 & 107.6118 & 107.1509 & 106.9139 & 117.6268 & 118.3088 & 117.1658 & 107.5357 & 107.0702 & 106.8292 \\
\hline 6 & 66.7 & 126.0267 & 127.2874 & 127.1596 & 117.5063 & 116.6762 & 116.3792 & 126.0894 & 127.3428 & 127.0239 & 117.4602 & 116.6071 & 116.2701 \\
\hline $1^{\prime}$ & 102.0 & 93.4324 & 94.1564 & 92.7670 & 82.8184 & 82.1518 & 82.2341 & 93.6522 & 94.3729 & 92.8949 & 82.9394 & 82.2748 & 82.3133 \\
\hline $2^{\prime}$ & 188.9 & 10.9849 & 11.0703 & 10.3788 & -5.1559 & -5.1243 & -7.7492 & 9.7914 & 9.8813 & 8.9494 & -6.5911 & -6.5532 & -9.4159 \\
\hline $3^{\prime}$ & 137.1 & 57.1269 & 56.9126 & 54.8808 & 40.7370 & 40.4589 & 40.2396 & 57.5071 & 57.2928 & 55.4046 & 41.2362 & 40.9679 & 40.8334 \\
\hline $4^{\prime}$ & 145.4 & 54.0978 & 54.8613 & 53.2881 & 37.8852 & 37.1946 & 35.7415 & 51.6102 & 52.3779 & 50.4392 & 35.0810 & 34.4006 & 32.6990 \\
\hline $5^{\prime}$ & 72.9 & 121.0088 & 121.7865 & 121.3265 & 111.7626 & 111.2070 & 110.9213 & 120.9015 & 121.6784 & 121.1550 & 111.5776 & 111.0110 & 110.6884 \\
\hline $\begin{array}{l}6^{\prime} \\
{ }^{1} \mathrm{H}\end{array}$ & 68.1 & 128.0273 & 129.2816 & 129.2760 & 119.6651 & 119.0112 & 118.5510 & 128.3887 & 129.6325 & 129.4574 & 119.8830 & 119.2079 & 118.7260 \\
\hline 1 & 5.11 & 27.2044 & 26.6411 & 26.5991 & 27.2254 & 26.8782 & 26.9046 & 27.1631 & 26.5933 & 26.5460 & 27.1695 & 26.8203 & 26.8480 \\
\hline $3 e q$ & 2.23 & 30.1428 & 29.6506 & 29.6630 & 30.1836 & 29.9148 & 29.8451 & 30.1923 & 29.6965 & 29.6956 & 30.2300 & 29.9562 & 29.8744 \\
\hline $3 a x$ & 2.97 & 29.2995 & 28.8427 & 28.7432 & 29.3303 & 29.0601 & 28.9555 & 29.1604 & 28.6971 & 28.5754 & 29.1826 & 28.9111 & 28.7893 \\
\hline 4 & 3.43 & 29.0293 & 28.4740 & 28.2943 & 28.9546 & 28.6051 & 28.4459 & 28.9475 & 28.3931 & 28.1942 & 28.8736 & 28.5260 & 28.3517 \\
\hline 5 & 4.53 & 27.7557 & 27.2161 & 27.1620 & 27.7437 & 27.4300 & 27.4056 & 27.5697 & 27.0230 & 26.9550 & 27.5629 & 27.2453 & 27.2075 \\
\hline 6endo & 4.15 & 28.1396 & 27.6099 & 27.5390 & 28.1685 & 27.8135 & 27.7493 & 27.9843 & 27.4520 & 27.3645 & 28.0156 & 27.6592 & 27.5801 \\
\hline 6exo & 4.03 & 28.2062 & 27.6532 & 27.5079 & 28.1088 & 27.7772 & 27.7612 & 28.1048 & 27.5531 & 27.3992 & 28.0106 & 27.6826 & 27.6568 \\
\hline $1^{\prime}$ & 5.40 & 26.9646 & 26.3919 & 26.3303 & 26.9171 & 26.5661 & 26.5956 & 26.9365 & 26.3592 & 26.2927 & 26.8715 & 26.5203 & 26.5517 \\
\hline $4^{\prime}$ & 7.08 & 24.3585 & 23.5849 & 23.3715 & 24.2932 & 23.7330 & 23.6650 & 24.1894 & 23.4199 & 23.1894 & 24.1253 & 23.5692 & 23.4876 \\
\hline $5^{\prime}$ & 5.06 & 27.6032 & 27.0262 & 26.8570 & 27.5310 & 27.1419 & 27.1090 & 27.3719 & 26.7854 & 26.5977 & 27.2985 & 26.9028 & 26.8566 \\
\hline 6'endo & 3.71 & 28.5976 & 28.0639 & 27.9826 & 28.6136 & 28.2836 & 28.2008 & 28.5211 & 27.9840 & 27.8930 & 28.5382 & 28.2035 & 28.1100 \\
\hline 6'exo & 3.89 & 28.4005 & 27.8652 & 27.7220 & 28.3224 & 28.0117 & 27.9325 & 28.2887 & 27.7534 & 27.5914 & 28.2050 & 27.8976 & 27.8061 \\
\hline
\end{tabular}

\section{Compound 64}

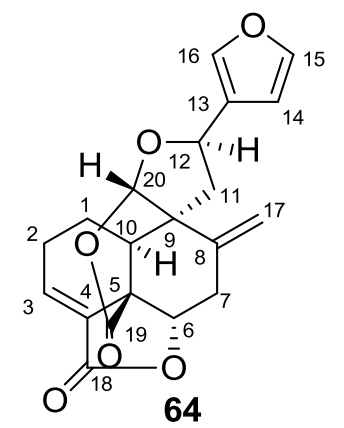

\begin{tabular}{|c|c|c|c|c|c|c|c|c|c|c|c|c|c|}
\hline & \multirow[b]{3}{*}{ Exp } & \multicolumn{12}{|c|}{ B3LYP } \\
\hline & & \multicolumn{6}{|c|}{ Gas phase } & \multicolumn{6}{|c|}{ PCM } \\
\hline & & 6-31G* & $6-31 G * *$ & $6-31+\mathrm{G} * *$ & 6-311G* & $6-311 \mathrm{G} * *$ & $6-311+G * *$ & 6-31G* & $6-31 G * *$ & $6-31+G * *$ & $6-311 G *$ & $6-311 G * *$ & $6-311+\mathrm{G} * *$ \\
\hline \multicolumn{14}{|c|}{${ }^{13} \mathrm{C}$} \\
\hline 1 & 20.7 & 166.6857 & 168.0838 & 167.0231 & 158.4300 & 158.2918 & 158.1553 & 166.7749 & 168.1615 & 167.0732 & 158.4855 & 158.3327 & 158.2083 \\
\hline 2 & 26.1 & 161.9606 & 163.2000 & 162.5484 & 152.7119 & 152.4334 & 151.8605 & 161.6720 & 162.8951 & 162.1796 & 152.3558 & 152.0536 & 151.3825 \\
\hline 3 & 139.8 & 56.3196 & 57.1928 & 55.8770 & 38.0349 & 37.4948 & 35.7102 & 52.1259 & 52.9950 & 51.0058 & 33.3365 & 32.7801 & 30.4243 \\
\hline 4 & 128.2 & 62.7399 & 62.5106 & 62.8142 & 44.9685 & 44.7826 & 44.1739 & 64.3950 & 64.1635 & 64.9010 & 46.9695 & 46.7902 & 46.4297 \\
\hline 5 & 48.2 & 140.5287 & 140.6338 & 139.9565 & 130.0248 & 130.1063 & 128.7122 & 140.0091 & 140.1093 & 139.2968 & 129.4441 & 129.5239 & 127.9710 \\
\hline 6 & 75.8 & 113.5504 & 114.2990 & 112.6729 & 101.0832 & 100.7564 & 100.3782 & 113.0466 & 113.7867 & 111.9973 & 100.4621 & 100.1289 & 99.6720 \\
\hline 7 & 36.5 & 151.3411 & 152.5265 & 151.8104 & 141.7689 & 141.1625 & 140.5723 & 151.9639 & 153.1363 & 152.4417 & 142.4451 & 141.8333 & 141.2351 \\
\hline
\end{tabular}




\begin{tabular}{|c|c|c|c|c|c|c|c|c|c|c|c|c|c|}
\hline 8 & 44.4 & .0803 & 47.5302 & 4.5883 & 25.9596 & 25.3041 & 4.4926 & 48.8561 & 48.3260 & 45.5320 & 26.8700 & 26.2834 & 25.558 \\
\hline 9 & 50.5 & 39.0765 & 39.2839 & 37.6068 & 28.0680 & 127.9952 & 128.2013 & 139.1627 & 139.3704 & 37.7685 & 128.1808 & 128.1173 & 8.323 \\
\hline 10 & 38.8 & & & & & & & & & & & & \\
\hline 11 & 1.6 & 3.5928 & 4.9783 & 145.5830 & 33.7883 & 133.1792 & 132.9013 & 43.9276 & 145.3068 & 145.9863 & 1413 & 133.5219 & 133.275 \\
\hline 12 & 5.5 & 117.9953 & 18.6890 & 117.9236 & 106.5942 & 106.1837 & 105.9786 & 117.8025 & 118.4956 & 117.6175 & 106.3267 & 105.9111 & 105.643 \\
\hline 13 & 124.6 & 66.1583 & 65.8424 & 64.3659 & 47.1660 & 46.9024 & 46.0831 & 65.6678 & 65.3542 & 63.7821 & 46.6019 & 46.3543 & 45.4297 \\
\hline & 108.5 & 87.3589 & 87.8886 & 87.9171 & 71.5257 & 70.7901 & 69.9903 & 87.0484 & 87.5687 & 87.4488 & 71.0192 & 70.2958 & 69.3787 \\
\hline & 144.2 & 53.5894 & 54.1105 & 52.6617 & 34.6282 & & 31.8801 & 53.5340 & 54.0508 & 52.5322 & & & 31.7508 \\
\hline & 140.0 & 58.8456 & 59.5305 & 57.4785 & 40.3583 & 39.4317 & 37.7633 & 59.0527 & 59.7423 & 577308 & 447 & 396326 & 37.9876 \\
\hline 17 & 115.3 & 77.7704 & 79.1973 & 80.2379 & 63.9052 & 62.8547 & 62.2815 & 76.8892 & 78.2986 & 79.2513 & 62.8081 & 61.7254 & 61.0813 \\
\hline 18 & 167.2 & 33.0857 & 33.1139 & 31.4088 & 12.8864 & 12.9692 & 10.4739 & 30.9369 & 30.9632 & 28.8407 & 10.4487 & 10.5282 & 7.6310 \\
\hline & 168.9 & 0.1024 & 30.1943 & 27.8333 & & & & & & & & .0912 & 4.8814 \\
\hline & 112.5 & & & & & & & & & & & & \\
\hline 1 & 1.96 & 30.5138 & 30.0438 & 29.9474 & 30.5073 & 30.2284 & 30.1 & 30.2981 & 212 & & 924 & 30.0092 & 29.9526 \\
\hline 1 & 2.16 & 29.9356 & 29.4676 & 29.3425 & 30.0196 & 29.7661 & 29.7021 & 30.0565 & 29.5929 & 29.4749 & 389 & 29.8883 & 29.8274 \\
\hline 2 & 2.73 & 9.6335 & 29.1346 & 8.9441 & & & & & & & & & 25.002 \\
\hline 2 & 2.54 & & & & & & & & & & & & \\
\hline 3 & 7.01 & 25.4569 & 24.8005 & 24.4975 & & 4.8931 & 24.7622 & & 24.6061 & & & & - \\
\hline 6 & 4.68 & 27.9445 & 27.4265 & 27.4364 & 27.8792 & 27.6265 & 27.6643 & 27.8620 & 27.3361 & 27.3268 & 27.7860 & 27.5248 & 27.5492 \\
\hline 7 & 2.60 & 29.8369 & 29.3523 & 29.2568 & 9.8110 & 29.5454 & 9.5719 & 29.7317 & 29.2396 & 391 & 9.7055 & 9.4350 & 29.452 \\
\hline 7 & 3.01 & . $.3 / 91$ & .1113 & 9.0594 & 29.4723 & 29.1895 & 29.1835 & 29.4785 & 29.0059 & 28.9449 & 3682 & .0820 & 29.071 \\
\hline & 2.14 & & & & & & & & & & & & 30.00 \\
\hline 11 & 2.51 & 29.7957 & 29.3145 & 29.1545 & 29.7953 & 29.5308 & 29.4627 & 29.6539 & 29.1702 & 28.9989 & 29.6546 & 29.3905 & 29.3165 \\
\hline 12 & 5.30 & 26.9764 & 26.4280 & 26.2184 & 26.9218 & 26.5985 & 26.5323 & 26.8710 & 26.3193 & 26.0942 & 26.8077 & 26.4843 & 26.406 \\
\hline 14 & 6.46 & 26.1386 & 25.3738 & 25.1214 & 26.0614 & 25.5489 & 25.4801 & 26.0275 & 25.2590 & 24.9844 & 25.9315 & 25.4223 & 25.3428 \\
\hline 15 & 7.46 & 24.9902 & 24.2579 & 24.0076 & 24.8881 & 24.4230 & 24.2730 & 24.8973 & 24.1673 & 23.9191 & 24.7857 & 24.3293 & 24.1866 \\
\hline 16 & 7.49 & & & & & & & & & & & & 24.1600 \\
\hline 17 & 5.33 & 27.1405 & 26.4554 & & & & & & & & & & 26.411 \\
\hline 17 & 5.35 & & & & & & & & & & & 2751 & 26.1749 \\
\hline 20 & 5.55 & 26.6697 & 26.1643 & 26.0707 & 26.6796 & 26.4596 & 26.3947 & 26.6077 & 26.0994 & 25.9928 & 26.6082 & 26.3848 & 26.3137 \\
\hline
\end{tabular}

\section{mPW1PW91}

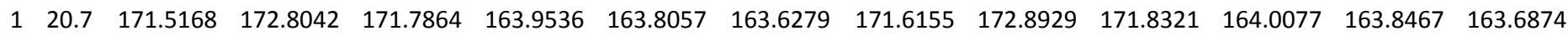

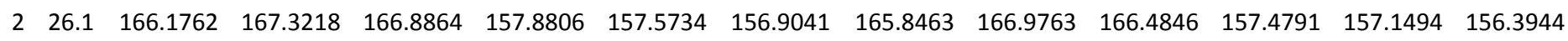

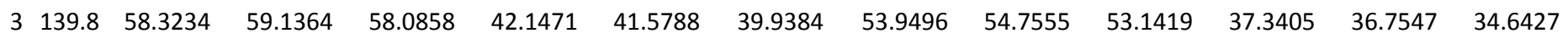

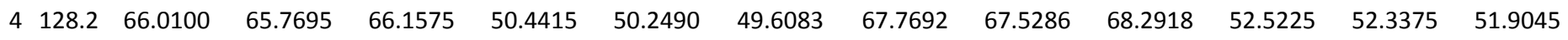

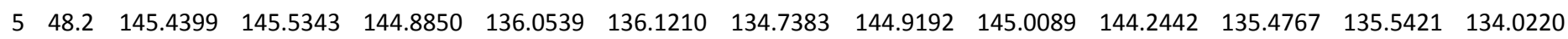

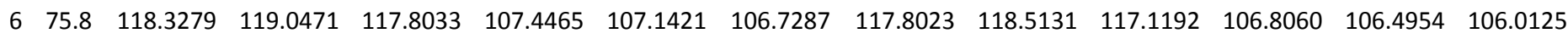

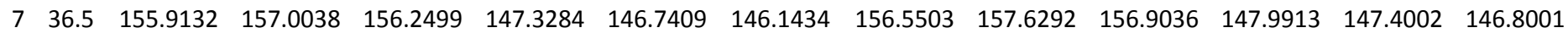

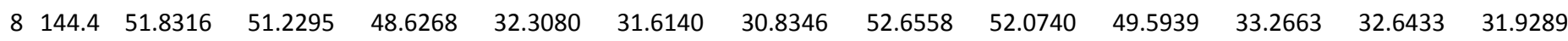

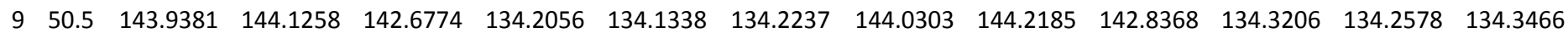




\begin{tabular}{|c|c|c|c|c|c|c|c|c|c|c|c|c|c|}
\hline 10 & 38.8 & 154.0994 & 154.7621 & 153.8073 & 145.4529 & 145.2266 & 145.4723 & 154.6087 & 155.2645 & 154.4601 & 146.0213 & 145.7937 & 146.0842 \\
\hline 11 & 41.6 & 148.2094 & 149.4987 & 149.9511 & 139.5192 & 138.9292 & 138.7196 & 148.5538 & 149.8388 & 150.3555 & 139.8655 & 139.2668 & 139.0820 \\
\hline 12 & 75.5 & 122.1905 & 122.8510 & 122.3535 & 112.5265 & 112.1305 & 111.9649 & 121.9760 & 122.6362 & 122.0379 & 112.2372 & 111.8362 & 111.6169 \\
\hline 13 & 124.6 & 69.7316 & 69.3634 & 68.1331 & 53.1324 & 52.8144 & 51.9856 & 69.2576 & 68.8957 & 67.5883 & 52.6250 & 52.3257 & 51.3977 \\
\hline 14 & 108.5 & 89.8636 & 90.3096 & 90.5082 & 75.8374 & 75.0559 & 74.2512 & 89.5773 & 90.0191 & 90.0831 & 75.3951 & 74.6301 & 73.7286 \\
\hline 15 & 144.2 & 55.7823 & 56.2449 & 55.1919 & 39.2901 & 38.0996 & 36.6252 & 55.6839 & 56.1435 & 55.0229 & 39.0171 & 37.8515 & 36.4507 \\
\hline 16 & 140.0 & 61.1382 & 61.7966 & 60.3595 & 45.0173 & 44.0964 & 42.5007 & 61.3235 & 61.9809 & 60.5752 & 45.1361 & 44.2269 & 42.6588 \\
\hline 17 & 115.3 & 79.3471 & 80.7106 & 81.7728 & 66.9819 & 65.9162 & 65.3427 & 78.3717 & 79.7191 & 80.7164 & 65.8021 & 64.7059 & 64.0828 \\
\hline 18 & 167.2 & 35.4917 & 35.5188 & 34.3574 & 17.9185 & 17.9979 & 15.7394 & 33.3386 & 33.3632 & 31.8239 & 15.5250 & 15.6003 & 12.9893 \\
\hline 19 & 168.9 & 33.1664 & 33.2552 & 31.1967 & 15.4193 & 15.4772 & 13.9528 & 30.5531 & 30.6346 & 28.2391 & 12.4861 & 12.5399 & 10.7028 \\
\hline 20 & 112.5 & 82.9917 & 83.6459 & 83.5038 & 72.1810 & 71.6505 & 71.0771 & 82.8342 & 83.4903 & 83.2231 & 71.9128 & 71.3858 & 70.7750 \\
\hline \multicolumn{14}{|c|}{${ }^{1} \mathbf{H}$} \\
\hline 1 & 1.96 & 30.4492 & 29.9800 & 29.9169 & 30.4160 & 30.1357 & 30.1061 & 30.2204 & 29.7435 & 29.6728 & 30.1924 & 29.9071 & 29.8706 \\
\hline 1 & 2.16 & 29.8956 & 29.4243 & 29.3088 & 29.9276 & 29.6779 & 29.6261 & 30.0209 & 29.5545 & 29.4445 & 30.0493 & 29.8026 & 29.7530 \\
\hline 2 & 2.73 & 29.5727 & 29.0665 & 28.8843 & 29.4510 & 29.1569 & 29.0956 & 29.4649 & 28.9538 & 28.7640 & 29.3440 & 29.0456 & 28.9775 \\
\hline 2 & 2.54 & 29.8966 & 29.3949 & 29.2290 & 29.7637 & 29.4720 & 29.3852 & 29.6891 & 29.1825 & 29.0217 & 29.5703 & 29.2765 & 29.1876 \\
\hline 3 & 7.01 & 25.2529 & 24.5984 & 24.3130 & 25.1747 & 24.6984 & 24.5815 & 25.0479 & 24.3897 & 24.0781 & 24.9599 & 24.4815 & 24.3466 \\
\hline 6 & 4.68 & 27.8676 & 27.3451 & 27.3623 & 27.7839 & 27.5216 & 27.5567 & 27.7828 & 27.2521 & 27.2528 & 27.6896 & 27.4189 & 27.4420 \\
\hline 7 & 2.60 & 29.7723 & 29.2878 & 29.2357 & 29.7034 & 29.4490 & 29.5106 & 29.6664 & 29.1745 & 29.1170 & 29.5980 & 29.3386 & 29.3906 \\
\hline 7 & 3.01 & 29.4503 & 28.9813 & 28.9500 & 29.3499 & 29.0541 & 29.0536 & 29.3392 & 28.8645 & 28.8264 & 29.2373 & 28.9377 & 28.9369 \\
\hline 10 & 2.14 & 30.2971 & 29.9452 & 29.7488 & 30.3458 & 30.2185 & 30.1283 & 30.1044 & 29.7460 & 29.5429 & 30.1578 & 30.0247 & 29.9243 \\
\hline 11 & 2.51 & 29.7082 & 29.2268 & 29.0593 & 29.6793 & 29.4157 & 29.3426 & 29.5587 & 29.0745 & 28.8992 & 29.5334 & 29.2701 & 29.1928 \\
\hline 12 & 5.30 & 26.9309 & 26.3705 & 26.1713 & 26.8471 & 26.5205 & 26.4640 & 26.8171 & 26.2529 & 26.0401 & 26.7260 & 26.3993 & 26.3334 \\
\hline 14 & 6.46 & 25.9612 & 25.1912 & 24.9710 & 25.9071 & 25.3540 & 25.2956 & 25.8405 & 25.0674 & 24.8278 & 25.7751 & 25.2257 & 25.1570 \\
\hline 15 & 7.46 & 24.8165 & 24.0853 & 23.8670 & 24.7590 & 24.2556 & 24.1171 & 24.7114 & 23.9825 & 23.7677 & 24.6472 & 24.1530 & 24.0218 \\
\hline 16 & 7.49 & 24.7836 & 24.0265 & 23.8339 & 24.7423 & 24.2123 & 24.0727 & 24.7154 & 23.9598 & 23.7688 & 24.6739 & 24.1507 & 24.0067 \\
\hline 17 & 5.33 & 26.9411 & 26.2545 & 26.1155 & 26.9151 & 26.4264 & 26.3124 & 26.8275 & 26.1417 & 25.9954 & 26.8006 & 26.3152 & 26.1945 \\
\hline 17 & 5.35 & 26.7377 & 26.0809 & 25.8534 & 26.6608 & 26.1876 & 26.0730 & 26.6057 & 25.9499 & 25.7219 & 26.5259 & 26.0601 & 25.9474 \\
\hline 20 & 5.55 & 26.6331 & 26.1322 & 26.0485 & 26.6364 & 26.4166 & 26.3474 & 26.5689 & 26.0646 & 25.9702 & 26.5645 & 26.3410 & 26.2664 \\
\hline
\end{tabular}

\section{Compound 65}

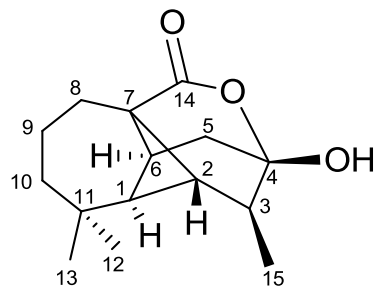

65

\begin{tabular}{|c|c|c|c|c|c|c|c|c|c|c|c|c|c|}
\hline \multirow{3}{*}{\multicolumn{2}{|c|}{ Exp }} & \multicolumn{12}{|c|}{ B3LYP } \\
\hline & & \multicolumn{6}{|c|}{ Gas phase } & \multicolumn{6}{|c|}{ PCM } \\
\hline & & 6-31G* & $6-31 \mathrm{G} * *$ & $6-31+\mathrm{G} * *$ & 6-311G* & $6-311 G * *$ & $6-311+G * *$ & 6-31G* & $6-31 G * *$ & $6-31+\mathrm{G} * *$ & 6-311G* & $6-311 G * *$ & $6-311+G * *$ \\
\hline \multicolumn{14}{|c|}{ 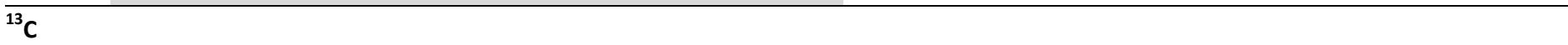 } \\
\hline 1 & 63.3 & 126.4882 & 127.1762 & 126.6491 & 116.0197 & 115.5941 & 115.1183 & 126.8542 & 127.5418 & 127.1773 & 116.5523 & 116.1241 & 115.7025 \\
\hline 2 & 43.8 & 144.3940 & 145.1841 & 144.6230 & 134.5207 & 134.2699 & 133.7443 & 144.4460 & 145.2317 & 144.6416 & 134.6079 & 134.3466 & 133.7797 \\
\hline
\end{tabular}




\begin{tabular}{|c|c|c|c|c|c|c|c|c|c|c|c|c|c|}
\hline 3 & 5.8 & 13.3492 & 4.0530 & 12.9695 & 2.4890 & 132.2027 & $131.8 \mathrm{C}$ & 143.5717 & 144.2713 & 143.2327 & 132.7591 & 132.4698 & 1 \\
\hline & 02.2 & 90.1354 & 0.2945 & 9.6524 & & & & & & & & & \\
\hline 5 & 3.1 & & & & & & & & & & & & \\
\hline 6 & 36.5 & 509331 & 1.8673 & 50.3831 & 1.8539 & 1.6961 & & 8943 & 151.8231 & 150.3128 & 8190 & & 53 \\
\hline 7 & 44.5 & 43.7781 & 4.1622 & 143.9324 & 33.2438 & 133.4554 & 31.8475 & 143.3110 & 143.6989 & 143.3237 & 132.6832 & 132.8990 & 131.184 \\
\hline 8 & 30.3 & 57.5141 & 8.7191 & 158.0344 & 18.6009 & 148.2247 & 47.8796 & 157.5798 & 158.7906 & 158.2559 & 148.8010 & 148.4338 & 148.121 \\
\hline 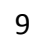 & 20.3 & 167.33 & 58.8180 & 166.9 & & 158.6 & & & & 167.1831 & & & 159.129 \\
\hline & 36.8 & 1500277 & 52.1531 & & & & & & & & & & \\
\hline & 32.8 & 54.9433 & 5.5276 & 155.0637 & & 629 & 43.7211 & 154 & 4486 & 9590 & & & 653 \\
\hline & 27.5 & 161.8488 & 53.5579 & 164.1482 & 55.0083 & 154.8336 & 163 & 162.1853 & 3809 & 164.5385 & 4538 & 2524 & .050 \\
\hline & 27.7 & 161.7051 & 63.3 & 164.2688 & 286 & 154.7081 & & 0348 & & 164.6599 & 3599 & 155.1133 & 154.956 \\
\hline & 174.7 & 26.7853 & 26.8399 & & & & & & & & & & \\
\hline & & & & & & & & & & & & & \\
\hline 1 & 1.32 & 30.7 & & & & & & & & & & & 30.6455 \\
\hline 2 & 1.99 & 30.0260 & 29.6045 & 29.6298 & 30.2 & 30.0792 & & & & & & & 8838 \\
\hline 3 & 2.23 & & & & & & & & & & & & \\
\hline 5 & & & & & & & & & & & & & \\
\hline 5 & 2.18 & 30.28 & & & & & & & & & & & \\
\hline 6 & 2.34 & 29.8346 & 29.3744 & 29.4060 & 30.0681 & 29.7927 & 29.7953 & 29.7224 & 29.2585 & 29.2707 & & 29.6706 & 29.6609 \\
\hline 8 & 1.99 & & & & & & & & & & & & 9323 \\
\hline 8 & 1.99 & & & & & & & & & & & & 30.0225 \\
\hline 9 & 1.67 & & & & & & & & & & & & \\
\hline 9 & 1.67 & & & & & & & & & & & & \\
\hline & 1.44 & & & & & & & & & & & & 4463 \\
\hline & 1.44 & & & & & & & & & 423 & & 1535 & 30.4148 \\
\hline 12 & 0.92 & 31.2305 & & 30. & & 0051 & & 2107 & 30.7897 & 30.7621 & 31.2244 & 30.9884 & 30.9634 \\
\hline 13 & 0.92 & & & & & & & & & & & & \\
\hline 15 & 0.96 & & & & & & & & & & & & \\
\hline
\end{tabular}

\begin{tabular}{|c|c|c|c|c|c|c|c|c|c|c|c|c|c|}
\hline & \multirow[b]{3}{*}{ Exp } & \multicolumn{12}{|c|}{ mPW1PW91 } \\
\hline & & \multicolumn{6}{|c|}{ Gas phase } & \multicolumn{6}{|c|}{ PCM } \\
\hline & & 6-31G* & $6-31 G * *$ & $6-31+\mathrm{G} * *$ & 6-311G* & $6-311 G * *$ & $6-311+G * *$ & 6-31G* & $6-31 G * *$ & $6-31+G * *$ & 6-311G* & $6-311 G * *$ & $6-311+\mathrm{G} * *$ \\
\hline \multicolumn{14}{|c|}{${ }^{13} \mathrm{C}$} \\
\hline 1 & 63.3 & 132.2865 & 132.8968 & 132.7050 & 122.6995 & 122.2904 & 121.7934 & 132.6738 & 133.2859 & 133.2380 & 123.2162 & 122.8064 & 122.3568 \\
\hline 2 & 43.8 & 149.6443 & 150.3586 & 149.8284 & 140.6469 & 140.3931 & 139.9287 & 149.6957 & 150.4070 & 149.8491 & 140.7224 & 140.4593 & 139.9580 \\
\hline 3 & 45.8 & 148.5615 & 149.2064 & 148.2423 & 138.6235 & 138.3464 & 137.9529 & 148.7921 & 149.4335 & 148.5101 & 138.8784 & 138.5988 & 138.1763 \\
\hline 4 & 102.2 & 94.4719 & 94.6154 & 94.5314 & 83.0100 & 83.2992 & 82.4224 & 93.7573 & 93.8983 & 93.7527 & 82.2059 & 82.4855 & 81.5293 \\
\hline 5 & 43.1 & 149.6939 & 150.7772 & 150.0909 & 140.6412 & 140.0344 & 139.6800 & 150.1143 & 151.1868 & 150.5805 & 141.0649 & 140.4479 & 140.1252 \\
\hline 6 & 36.5 & 156.0341 & 156.8954 & 155.3650 & 147.6564 & 147.4930 & 147.4151 & 155.9990 & 156.8557 & 155.3008 & 147.6147 & 147.4515 & 147.3558 \\
\hline 7 & 44.5 & 148.7796 & 149.1630 & 149.1945 & 139.1921 & 139.4097 & 137.7668 & 148.3179 & 148.7044 & 148.6062 & 138.6453 & 138.8664 & 137.1340 \\
\hline 8 & 30.3 & 162.5845 & 163.7011 & 163.1924 & 154.4758 & 154.1100 & 153.7298 & 162.6670 & 163.7885 & 163.4181 & 154.6806 & 154.3227 & 153.9720 \\
\hline 9 & 20.3 & 172.0011 & 173.3722 & 171.5450 & 164.2643 & 164.0825 & 164.1999 & 172.2333 & 173.6062 & 171.8046 & 164.5509 & 164.3646 & 164.4862 \\
\hline 10 & 36.8 & 155.9510 & 157.0425 & 155.8901 & 147.3007 & 146.9028 & 146.8729 & 156.2561 & 157.3454 & 156.2443 & 147.6645 & 147.2587 & 147.2506 \\
\hline 11 & 32.8 & 159.8348 & 160.4110 & 160.1242 & 150.2322 & 150.1132 & 149.5507 & 159.7491 & 160.3307 & 160.0225 & 150.1767 & 150.0615 & 149.4871 \\
\hline
\end{tabular}




\begin{tabular}{|c|c|c|c|c|c|c|c|c|c|c|c|c|c|}
\hline 12 & 27.5 & 166.3150 & 167.8768 & 168.0017 & 159.9225 & 159.7674 & 159.6427 & 166.6899 & 168.2387 & 168.4228 & 160.3703 & 160.1915 & 160.0827 \\
\hline 13 & 27.7 & 166.0924 & 167.6296 & 168.1000 & 159.7607 & 159.5526 & 159.4000 & 166.4593 & 167.9863 & 168.5239 & 160.1943 & 159.9637 & 159.8344 \\
\hline 14 & 174.7 & 29.3677 & 29.4238 & 27.8847 & 10.8767 & 10.8884 & 9.0816 & 26.7741 & 26.8319 & 24.8818 & 7.9560 & 7.9682 & 5.8298 \\
\hline $\begin{array}{l}15 \\
{ }^{1} \mathbf{H}\end{array}$ & 12.9 & 179.1928 & 180.8817 & 181.5619 & 173.5259 & 173.5795 & 173.3652 & 179.4765 & 181.1656 & 181.9535 & 173.8944 & 173.9317 & 173.7666 \\
\hline 1 & 1.32 & 30.7395 & 30.4548 & 30.3966 & 30.7878 & 30.6514 & 30.6235 & 30.6388 & 30.3513 & 30.2802 & 30.6840 & 30.5455 & 30.5133 \\
\hline 2 & 1.99 & 29.9913 & 29.5655 & 29.5590 & 30.1919 & 29.9715 & 29.8929 & 29.8810 & 29.4518 & 29.4263 & 30.0770 & 29.8540 & 29.7636 \\
\hline 3 & 2.23 & 29.9314 & 29.5553 & 29.4620 & 29.9288 & 29.7584 & 29.6886 & 29.8435 & 29.4626 & 29.3552 & 29.8400 & 29.6630 & 29.5823 \\
\hline 5 & 2.10 & 30.3985 & 29.9576 & 29.8567 & 30.3408 & 30.0821 & 30.0093 & 30.2637 & 29.8195 & 29.7155 & 30.2009 & 29.9483 & 29.8755 \\
\hline 5 & 2.18 & 30.2202 & 29.8218 & 29.7176 & 30.1602 & 29.9614 & 29.9191 & 30.1142 & 29.7094 & 29.5980 & 30.0555 & 29.8504 & 29.8035 \\
\hline 6 & 2.34 & 29.8027 & 29.3336 & 29.3421 & 29.9799 & 29.6931 & 29.6830 & 29.6861 & 29.2130 & 29.2053 & 29.8573 & 29.5690 & 29.5486 \\
\hline 8 & 1.99 & 30.0629 & 29.6255 & 29.4825 & 30.0280 & 29.7984 & 29.7768 & 30.1306 & 29.6960 & 29.5481 & 30.0877 & 29.8605 & 29.8368 \\
\hline 8 & 1.99 & 30.1593 & 29.7362 & 29.6080 & 30.1208 & 29.9074 & 29.8948 & 30.2122 & 29.7897 & 29.6559 & 30.1671 & 29.9542 & 29.9377 \\
\hline 9 & 1.67 & 30.4815 & 30.0494 & 30.0243 & 30.4019 & 30.1764 & 30.1369 & 30.4619 & 30.0282 & 29.9998 & 30.3842 & 30.1562 & 30.1138 \\
\hline 9 & 1.67 & 30.4068 & 29.9456 & 29.8937 & 30.4188 & 30.1685 & 30.1177 & 30.3408 & 29.8777 & 29.8213 & 30.3535 & 30.1018 & 30.0482 \\
\hline 10 & 1.44 & 30.6012 & 30.2153 & 30.1446 & 30.5810 & 30.3986 & 30.3821 & 30.5589 & 30.1731 & 30.0990 & 30.5390 & 30.3577 & 30.3405 \\
\hline 10 & 1.44 & 30.5848 & 30.1973 & 30.1171 & 30.5671 & 30.3808 & 30.3460 & 30.5494 & 30.1623 & 30.0787 & 30.5320 & 30.3467 & 30.3111 \\
\hline 12 & 0.92 & 31.1729 & 30.7542 & 30.7187 & 31.1430 & 30.9048 & 30.8811 & 31.1503 & 30.7309 & 30.6962 & 31.1235 & 30.8854 & 30.8623 \\
\hline 13 & 0.92 & 31.1620 & 30.7445 & 30.7192 & 31.1321 & 30.8949 & 30.8801 & 31.1428 & 30.7249 & 30.7000 & 31.1161 & 30.8791 & 30.8648 \\
\hline 15 & 0.96 & 31.1095 & 30.6792 & 30.5918 & 31.0589 & 30.8346 & 30.8002 & 31.1454 & 30.7147 & 30.6293 & 31.0958 & 30.8693 & 30.8346 \\
\hline
\end{tabular}

\section{Compound 66}

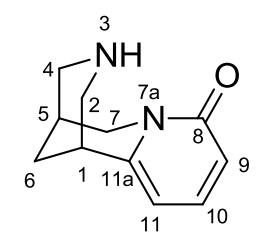

66

\begin{tabular}{|c|c|c|c|c|c|c|c|c|c|c|c|c|c|}
\hline & \multirow[b]{3}{*}{ Exp } & \multicolumn{12}{|c|}{ B3LYP } \\
\hline & & \multicolumn{6}{|c|}{ Gas phase } & \multicolumn{6}{|c|}{ PCM } \\
\hline & & 6-31G* & $6-31 G * *$ & $6-31+G * *$ & 6-311G* & $6-311 G * *$ & $6-311+G * *$ & 6-31G* & $6-31 G * *$ & $6-31+G * *$ & 6-311G* & $6-311 G * *$ & $6-311+G * *$ \\
\hline \multicolumn{14}{|l|}{${ }^{13} \mathrm{C}$} \\
\hline 8 & 163.7 & 38.4456 & 38.4089 & 35.8775 & 17.5535 & 17.5229 & 15.7698 & 37.6665 & 37.6257 & 34.8187 & 16.4888 & 16.4583 & 14.4934 \\
\hline $11 \mathrm{a}$ & 150.8 & 44.5578 & 44.4472 & 42.2324 & 23.8072 & 23.6930 & 22.6234 & 42.4025 & 42.3045 & 40.0592 & 21.4969 & 21.4160 & 20.2931 \\
\hline 10 & 138.8 & 59.9348 & 60.5903 & 59.6927 & 42.2923 & 41.3724 & 40.3852 & 58.5664 & 59.2218 & 58.0512 & 40.6128 & 39.7105 & 38.5803 \\
\hline 9 & 116.8 & 78.4354 & 79.0481 & 78.0381 & 62.1299 & 61.4077 & 60.8950 & 80.4103 & 81.0240 & 80.1330 & 64.3836 & 63.6823 & 63.1001 \\
\hline 11 & 105.1 & 92.7312 & 93.5091 & 93.3277 & 78.2707 & 77.6577 & 76.9104 & 91.6894 & 92.4489 & 91.9070 & 76.9760 & 76.3511 & 75.2953 \\
\hline 7 & 53.7 & 139.5812 & 140.7258 & 140.0677 & 130.2859 & 129.7290 & 129.7596 & 139.1184 & 140.2629 & 139.6863 & 129.8970 & 129.3433 & 129.3275 \\
\hline 4 & 52.7 & 136.1089 & 137.2523 & 135.6288 & 125.0143 & 124.6570 & 124.7425 & 136.3586 & 137.4979 & 135.8838 & 125.3019 & 124.9289 & 125.0301 \\
\hline 2 & 49.7 & 135.4264 & 136.5841 & 135.3002 & 124.3702 & 124.0327 & 124.1541 & 135.6809 & 136.8292 & 135.5683 & 124.6402 & 124.2836 & 124.4243 \\
\hline 1 & 35.4 & 151.7390 & 152.6317 & 150.9718 & 141.8135 & 141.6114 & 141.1508 & 151.7741 & 152.6608 & 151.0679 & 141.8582 & 141.6524 & 141.1779 \\
\hline 5 & 27.6 & 158.9179 & 159.9048 & 158.2971 & 148.8240 & 148.7224 & 148.4727 & 159.1952 & 160.1802 & 158.6664 & 149.1992 & 149.0916 & 148.8499 \\
\hline 6 & 26.2 & 160.9820 & 162.3698 & 161.8941 & 152.3978 & 152.1210 & 151.9607 & 161.4148 & 162.7909 & 162.4110 & 152.9317 & 152.6388 & 152.5103 \\
\hline
\end{tabular}




\begin{tabular}{cccccccccccccc}
\hline${ }^{1} \mathbf{H}$ & & & & & & & & & & & & & \\
10 & 7.30 & 25.3456 & 24.7322 & 24.4066 & 25.1724 & 24.7756 & 24.6675 & 25.1257 & 24.5126 & 24.1674 & 24.9410 & 24.5517 & 24.4293 \\
9 & 6.45 & 26.2143 & 25.5566 & 25.3477 & 26.0427 & 25.6429 & 25.5621 & 26.2501 & 25.5873 & 25.3636 & 26.0658 & 25.6689 & 25.5788 \\
11 & 6.00 & 26.7846 & 26.1176 & 25.8624 & 26.6791 & 26.2376 & 26.1349 & 26.5143 & 25.8463 & 25.5657 & 26.3982 & 25.9642 & 25.8430 \\
$7 \mathrm{a}$ & 4.13 & 28.2571 & 27.7800 & 27.5471 & 28.2710 & 27.9982 & 27.8900 & 28.3361 & 27.8630 & 27.6205 & 28.3371 & 28.0669 & 27.9485 \\
$7 \mathrm{~b}$ & 3.89 & 28.6005 & 28.1251 & 27.8250 & 28.5561 & 28.2688 & 28.1788 & 28.5939 & 28.1193 & 27.8172 & 28.5435 & 28.2603 & 28.1603 \\
$4 \mathrm{a}$ & 3.13 & 29.1975 & 28.7684 & 28.5856 & 29.0644 & 28.8367 & 28.7700 & 29.1763 & 28.7479 & 28.5671 & 29.0430 & 28.8174 & 28.7518 \\
$4 \mathrm{~b}$ & 3.02 & 29.2584 & 28.8340 & 28.6436 & 29.1135 & 28.9162 & 28.8171 & 29.1904 & 28.7656 & 28.5841 & 29.0555 & 28.8579 & 28.7593 \\
$2 \mathrm{a}$ & 3.08 & 29.2039 & 28.7837 & 28.6102 & 29.0871 & 28.9021 & 28.8097 & 29.1123 & 28.6907 & 28.5208 & 29.0008 & 28.8156 & 28.7201 \\
$2 \mathrm{~b}$ & 3.02 & 29.2415 & 28.7977 & 28.6435 & 29.0992 & 28.8883 & 28.8671 & 29.2260 & 28.7814 & 28.6250 & 29.0873 & 28.8763 & 28.8516 \\
1 & 2.92 & 29.7252 & 29.2736 & 29.1625 & 29.6420 & 29.4174 & 29.3729 & 29.5569 & 29.0988 & 28.9679 & 29.4727 & 29.2427 & 29.1845 \\
5 & 2.35 & 30.2297 & 29.7590 & 29.6200 & 30.1684 & 29.9089 & 29.8337 & 30.1090 & 29.6335 & 29.4868 & 30.0519 & 29.7853 & 29.7035 \\
6 & 1.96 & 30.4268 & 30.0317 & 29.8815 & 30.3507 & 30.1684 & 30.0916 & 30.3508 & 29.9533 & 29.8019 & 30.2765 & 30.0930 & 30.0130 \\
\hline
\end{tabular}

\begin{tabular}{|c|c|c|c|c|c|c|c|c|c|c|c|c|c|}
\hline & & \multicolumn{12}{|c|}{ mPW1PW91 } \\
\hline & \multirow[b]{2}{*}{ Exp } & \multicolumn{6}{|c|}{ Gas phase } & \multicolumn{6}{|c|}{ PCM } \\
\hline & & 6-31G* & 6-31G** & $6-31+G^{* *}$ & 6-311G* & 6-311G** & $6-311+\mathrm{G} * *$ & 6-31G* & 6-31G** & $6-31+\mathrm{G}^{* *}$ & 6-311G* & 6-311G** & $6-311+G^{* *}$ \\
\hline \\
\hline 8 & 163.7 & 40.8576 & 40.8185 & 38.9928 & 22.5779 & 22.5451 & 20.9374 & 40.0956 & 40.0519 & 37.9789 & 21.5509 & 21.5177 & 19.7206 \\
\hline $11 \mathrm{a}$ & 150.8 & 47.5658 & 47.4314 & 45.7531 & 29.2790 & 29.1400 & 28.0895 & 45.3474 & 45.2272 & 43.5353 & 26.9504 & 26.8471 & 25.7565 \\
\hline 10 & 138.8 & 62.0693 & 62.6340 & 62.0321 & 46.6029 & 45.6281 & 44.6539 & 60.6295 & 61.1940 & 60.3304 & 44.8668 & 43.9109 & 42.8184 \\
\hline 9 & 116.8 & 81.2095 & 81.7612 & 80.9359 & 66.9632 & 66.2146 & 65.4532 & 83.2414 & 83.7952 & 83.0637 & 69.2084 & 68.4840 & 67.6582 \\
\hline 11 & 105.1 & 95.8528 & 96.5789 & 96.6131 & 83.1031 & 82.4766 & 81.6387 & 94.8027 & 95.5090 & 95.2073 & 81.8117 & 81.1734 & 80.0681 \\
\hline 7 & 53.7 & 144.2983 & 145.3594 & 144.7233 & 135.9363 & 135.3949 & 135.4464 & 143.8442 & 144.9040 & 144.3455 & 135.5515 & 135.0124 & 135.0243 \\
\hline 4 & 52.7 & 141.1917 & 142.2185 & 140.8086 & 131.1870 & 130.8175 & 130.8674 & 141.4619 & 142.4862 & 141.0811 & 131.4745 & 131.0910 & 131.1543 \\
\hline 2 & 49.7 & 140.4986 & 141.5448 & 140.3871 & 130.5475 & 130.2087 & 130.2697 & 140.7537 & 141.7929 & 140.6623 & 130.8016 & 130.4460 & 130.5245 \\
\hline 1 & 35.4 & 156.9657 & 157.7862 & 156.2926 & 147.8707 & 147.6577 & 147.1912 & 156.9909 & 157.8058 & 156.3711 & 147.8855 & 147.6698 & 147.1919 \\
\hline 5 & 27.6 & 164.3091 & 165.2215 & 163.6771 & 155.0630 & 154.9444 & 154.6521 & 164.5856 & 165.4964 & 164.0391 & 155.4223 & 155.2976 & 155.0111 \\
\hline 6 & 26.2 & 166.2819 & 167.5385 & 167.0828 & 158.2049 & 157.9225 & 157.7159 & 166.7351 & 167.9814 & 167.6159 & 158.7362 & 158.4390 & 158.2574 \\
\hline \multicolumn{14}{|l|}{${ }^{1} H$} \\
\hline 10 & 7.30 & 25.1338 & 24.5181 & 24.2290 & 24.9958 & 24.5610 & 24.4634 & 24.8984 & 24.2827 & 23.9770 & 24.7520 & 24.3247 & 24.2137 \\
\hline 9 & 6.45 & 26.0149 & 25.3571 & 25.1705 & 25.8721 & 25.4398 & 25.3619 & 26.0415 & 25.3786 & 25.1794 & 25.8880 & 25.4593 & 25.3723 \\
\hline 11 & 6.00 & 26.6103 & 25.9403 & 25.7139 & 26.5219 & 26.0488 & 25.9560 & 26.3240 & 25.6525 & 25.4052 & 26.2293 & 25.7635 & 25.6551 \\
\hline $7 a$ & 4.13 & 28.1886 & 27.7122 & 27.4994 & 28.1785 & 27.9019 & 27.8116 & 28.2669 & 27.7944 & 27.5722 & 28.2437 & 27.9697 & 27.8690 \\
\hline $7 \mathrm{~b}$ & 3.89 & 28.5348 & 28.0608 & 27.7893 & 28.4655 & 28.1815 & 28.1087 & 28.5250 & 28.0516 & 27.7785 & 28.4506 & 28.1707 & 28.0884 \\
\hline $4 a$ & 3.13 & 29.1419 & 28.7164 & 28.5546 & 29.0063 & 28.7714 & 28.7167 & 29.1154 & 28.6907 & 28.5299 & 28.9798 & 28.7471 & 28.6928 \\
\hline $4 b$ & 3.02 & 29.2472 & 28.8266 & 28.6393 & 29.0627 & 28.8700 & 28.7736 & 29.1690 & 28.7478 & 28.5703 & 28.9969 & 28.8036 & 28.7083 \\
\hline $2 a$ & 3.08 & 29.2004 & 28.7838 & 28.6129 & 29.0394 & 28.8594 & 28.7620 & 29.0947 & 28.6765 & 28.5107 & 28.9424 & 28.7619 & 28.6626 \\
\hline $2 b$ & 3.02 & 29.1762 & 28.7344 & 28.5951 & 29.0297 & 28.8104 & 28.7899 & 29.1592 & 28.7167 & 28.5753 & 29.0156 & 28.7963 & 28.7728 \\
\hline 1 & 2.92 & 29.6276 & 29.1814 & 29.0699 & 29.5124 & 29.2868 & 29.2463 & 29.4495 & 28.9961 & 28.8681 & 29.3367 & 29.1050 & 29.0529 \\
\hline 5 & 2.35 & 30.1612 & 29.6938 & 29.5619 & 30.0620 & 29.7999 & 29.7382 & 30.0305 & 29.5579 & 29.4205 & 29.9391 & 29.6694 & 29.6024 \\
\hline 6 & 1.96 & 30.3718 & 29.9804 & 29.8373 & 30.2661 & 30.0823 & 30.0023 & 30.2906 & 29.8967 & 29.7529 & 30.1877 & 30.0023 & 29.9202 \\
\hline
\end{tabular}




\section{Compound 67}

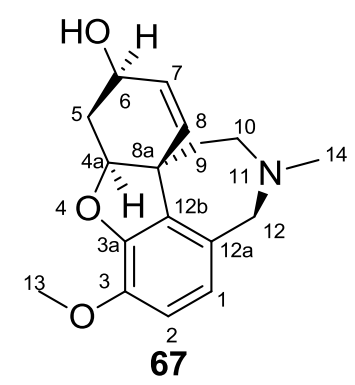

\begin{tabular}{|c|c|c|c|c|c|c|c|c|c|c|c|c|c|}
\hline & & \multicolumn{12}{|c|}{ B3LYP } \\
\hline & \multirow[b]{2}{*}{ Exp } & \multicolumn{6}{|c|}{ Gas phase } & \multicolumn{6}{|c|}{ PCM } \\
\hline & & 6-31G* & 6-31G** & $6-31+\mathrm{G} * *$ & 6-311G* & $6-311 G * *$ & $6-311+G * *$ & 6-31G* & $6-31 G * *$ & $6-31+\mathrm{G} * *$ & 6-311G* & $6-311 G * *$ & $6-311+G * *$ \\
\hline \multicolumn{14}{|l|}{${ }^{13} \mathrm{C}$} \\
\hline $3 a$ & 146.0 & 47.8311 & 47.8376 & 46.7378 & 29.0346 & 28.9589 & 28.8651 & 47.7212 & 47.7250 & 46.5687 & 28.8239 & 28.7616 & 28.7801 \\
\hline 3 & 144.3 & 53.3823 & 53.2289 & 50.6048 & 32.4293 & 32.2948 & 32.2769 & 53.8316 & 53.6784 & 51.0459 & 32.8622 & 32.7360 & 32.7846 \\
\hline $12 b$ & 133.2 & 64.1330 & 64.1660 & 62.2760 & 45.9764 & 45.7781 & 44.0829 & 63.2686 & 63.2973 & 61.3463 & 44.9346 & 44.7602 & 42.9159 \\
\hline $12 a$ & 129.6 & 60.8240 & 60.7483 & 61.0170 & 42.2361 & 42.2723 & 41.7644 & 60.3128 & 60.2342 & 60.3589 & 41.6597 & 41.6711 & 41.0697 \\
\hline 7 & 127.8 & 65.8451 & 66.3990 & 65.6306 & 48.1814 & 47.3911 & 45.7146 & 66.5081 & 67.0549 & 66.4436 & 48.9002 & 48.0755 & 46.4722 \\
\hline 8 & 127.0 & 66.9551 & 67.4409 & 65.3727 & 48.6007 & 47.8218 & 47.7748 & 66.1953 & 66.6956 & 64.3086 & 47.7651 & 47.0163 & 46.7065 \\
\hline 1 & 122.2 & 73.3255 & 74.0651 & 74.0325 & 57.6160 & 56.8497 & 56.3004 & 73.2985 & 74.0217 & 73.9993 & 57.4128 & 56.6633 & 56.2474 \\
\hline 2 & 111.4 & 74.5749 & 75.2644 & 75.0306 & 58.1669 & 57.4347 & 56.3919 & 75.1262 & 75.8100 & 75.6833 & 58.7670 & 58.0562 & 57.0584 \\
\hline $4 a$ & 88.9 & 101.1494 & 101.8161 & 100.6968 & 88.2097 & 87.8588 & 87.4865 & 101.2676 & 101.9282 & 100.8590 & 88.3483 & 87.9838 & 87.6404 \\
\hline 6 & 62.3 & 126.8994 & 127.7288 & 126.2281 & 115.5538 & 115.3438 & 115.2956 & 127.0092 & 127.8379 & 126.1808 & 115.5008 & 115.3090 & 115.2035 \\
\hline 12 & 60.9 & 128.3669 & 129.4061 & 128.6700 & 118.7996 & 117.8102 & 116.7174 & 128.5049 & 129.5109 & 129.0006 & 118.8355 & 117.8611 & 116.9861 \\
\hline 13 & 56.1 & 133.8831 & 135.6530 & 134.2257 & 124.6246 & 123.9997 & 123.5311 & 133.9012 & 135.6587 & 133.9434 & 124.4755 & 123.8322 & 123.2944 \\
\hline 10 & 54.0 & 135.0181 & 136.1287 & 134.3467 & 124.8131 & 124.1497 & 123.8789 & 135.2012 & 136.3036 & 134.5332 & 124.9470 & 124.2696 & 124.0964 \\
\hline $8 a$ & 48.4 & 140.0094 & 140.2610 & 139.7090 & 129.3585 & 129.4746 & 128.8537 & 139.8280 & 140.0753 & 139.4074 & 129.1107 & 129.2342 & 128.6347 \\
\hline 14 & 42.4 & 142.1621 & 143.7259 & 142.9704 & 133.9120 & 133.1974 & 132.4138 & 142.4110 & 143.9506 & 143.1507 & 134.0296 & 133.3128 & 132.5994 \\
\hline 9 & 34.0 & 150.8059 & 152.1197 & 149.8194 & 141.3602 & 140.9087 & 139.8313 & 150.8166 & 152.1080 & 149.6134 & 141.1743 & 140.7390 & 139.7593 \\
\hline 5 & 30.1 & 157.9578 & 159.3056 & 158.9091 & 148.8076 & 148.5639 & 148.6064 & 158.2115 & 159.5489 & 159.1023 & 149.0237 & 148.7703 & 148.7836 \\
\hline \multicolumn{14}{|l|}{${ }^{1} \mathbf{H}$} \\
\hline 7 & 6.00 & 26.1594 & 25.5782 & 25.1524 & 26.0668 & 25.6855 & 25.5592 & 26.1378 & 25.5566 & 25.1149 & 26.0329 & 25.6541 & 25.5253 \\
\hline 8 & 6.07 & 25.9483 & 25.3191 & 25.2354 & 25.9667 & 25.5731 & 25.5625 & 25.8728 & 25.2476 & 25.1294 & 25.8906 & 25.4999 & 25.4611 \\
\hline 1 & 6.62 & 25.8080 & 25.1947 & 25.1481 & 25.7193 & 25.3009 & 25.2737 & 25.6988 & 25.0845 & 25.0305 & 25.5924 & 25.1807 & 25.1482 \\
\hline 2 & 6.66 & 25.6588 & 24.9968 & 24.7392 & 25.4790 & 25.0584 & 24.8962 & 25.6452 & 24.9808 & 24.7177 & 25.4532 & 25.0349 & 24.8735 \\
\hline $4 a$ & 4.61 & 27.8139 & 27.3371 & 27.2076 & 27.7726 & 27.5279 & 27.4776 & 27.6992 & 27.2188 & 27.0819 & 27.6498 & 27.4043 & 27.3514 \\
\hline 6 & 4.14 & 28.1711 & 27.7075 & 27.6221 & 28.1353 & 27.8747 & 27.9131 & 28.1263 & 27.6605 & 27.5600 & 28.0758 & 27.8077 & 27.8428 \\
\hline $12 a$ & 4.08 & 28.6044 & 28.1564 & 28.1957 & 28.6361 & 28.3605 & 28.3894 & 28.5162 & 28.0668 & 28.1260 & 28.5505 & 28.2779 & 28.3233 \\
\hline $12 b$ & 3.68 & 28.9169 & 28.4551 & 28.4036 & 28.8189 & 28.5447 & 28.5571 & 28.8679 & 28.4092 & 28.3330 & 28.7633 & 28.4899 & 28.4834 \\
\hline $10 a$ & 3.26 & 29.3637 & 28.9322 & 28.9214 & 29.3562 & 29.1325 & 29.1190 & 29.3251 & 28.8953 & 28.8800 & 29.3285 & 29.1063 & 29.0810 \\
\hline $10 \mathrm{~b}$ & 3.05 & 29.6413 & 29.1990 & 29.0787 & 29.5799 & 29.3283 & 29.2017 & 29.5582 & 29.1110 & 28.9723 & 29.4851 & 29.2272 & 29.0987 \\
\hline $9 a$ & 1.57 & 30.5810 & 30.1387 & 30.0253 & 30.5921 & 30.3353 & 30.2468 & 30.4728 & 30.0273 & 29.9074 & 30.4779 & 30.2172 & 30.1307 \\
\hline $9 b$ & 2.08 & 30.3104 & 29.9112 & 29.7625 & 30.3095 & 30.0904 & 30.0108 & 30.3020 & 29.9028 & 29.7606 & 30.2930 & 30.0804 & 30.0051 \\
\hline $5 a$ & 2.01 & 30.4220 & 29.9644 & 29.7763 & 30.3463 & 30.0717 & 30.0124 & 30.2790 & 29.8156 & 29.6110 & 30.2020 & 29.9236 & 29.8499 \\
\hline $5 b$ & 2.69 & 29.7812 & 29.3505 & 29.1168 & 29.6418 & 29.4273 & 29.3467 & 29.7833 & 29.3500 & 29.1115 & 29.6417 & 29.4236 & 29.3393 \\
\hline
\end{tabular}




\begin{tabular}{|c|c|c|c|c|c|c|c|c|c|c|c|c|c|}
\hline 13 & 3.83 & 28.3363 & 27.8123 & 27.6289 & 28.3025 & 27.9806 & 27.8880 & 28.2844 & 27.7614 & 27.5712 & 28.2452 & 27.9261 & 27.8286 \\
\hline 14 & 2.40 & 29.8737 & 29.4178 & 29.1890 & 29.7895 & 29.5352 & 29.3856 & 29.8364 & 29.3803 & 29.1317 & 29.7460 & 29.4925 & 29.3272 \\
\hline
\end{tabular}

\begin{tabular}{|c|c|c|c|c|c|c|c|c|c|c|c|c|c|}
\hline & & \multicolumn{12}{|c|}{ mPW1PW91 } \\
\hline & \multirow[b]{2}{*}{ Exp } & \multicolumn{6}{|c|}{ Gas phase } & \multicolumn{6}{|c|}{ PCM } \\
\hline & & 6-31G* & $6-31 G * *$ & $6-31+G * *$ & $6-311 G *$ & $6-311 G * *$ & $6-311+G * *$ & 6-31G* & $6-31 G * *$ & $6-31+\mathrm{G} * *$ & 6-311G* & $6-311 G * *$ & $6-311+G * *$ \\
\hline \multicolumn{14}{|l|}{${ }^{13} \mathrm{C}$} \\
\hline $3 a$ & 146.0 & 51.0264 & 51.0364 & 50.4561 & 34.8035 & 34.7272 & 34.2776 & 50.9185 & 50.9269 & 50.2222 & 34.5928 & 34.5287 & 34.2137 \\
\hline 3 & 144.3 & 56.4220 & 56.2492 & 54.4258 & 38.2992 & 38.1562 & 37.9543 & 56.8819 & 56.7112 & 54.8537 & 38.7236 & 38.5875 & 38.4348 \\
\hline $12 b$ & b 133.2 & 67.2799 & 67.2902 & 65.6436 & 51.4509 & 51.2390 & 49.5793 & 66.2981 & 66.3026 & 64.6857 & 50.3214 & 50.1347 & 48.3253 \\
\hline $12 a$ & a 129.6 & 63.9043 & 63.8320 & 64.1979 & 47.4348 & 47.4725 & 46.9390 & 63.4361 & 63.3590 & 63.6101 & 46.9181 & 46.9345 & 46.3506 \\
\hline 7 & 127.8 & 68.7029 & 69.1654 & 68.5435 & 52.9232 & 52.0896 & 50.4696 & 69.3081 & 69.7634 & 69.3127 & 53.5633 & 52.6946 & 51.1463 \\
\hline 8 & 127.0 & 69.2152 & 69.6221 & 68.1325 & 52.7346 & 51.9618 & 52.1171 & 68.6425 & 69.0642 & 67.2257 & 52.1049 & 51.3752 & 51.3364 \\
\hline 1 & 122.2 & 76.3330 & 77.0099 & 76.7642 & 62.5340 & 61.7338 & 61.0443 & 76.1660 & 76.8250 & 76.5597 & 62.1824 & 61.3946 & 60.8424 \\
\hline 2 & 111.4 & 77.2763 & 77.9116 & 77.6396 & 62.8295 & 62.0676 & 61.0880 & 77.7823 & 78.4114 & 78.2393 & 63.3501 & 62.6089 & 61.6528 \\
\hline $4 a$ & 88.9 & 106.3592 & 106.9654 & 106.0313 & 94.9578 & 94.6139 & 94.2716 & 106.4389 & 107.0398 & 106.1374 & 95.0401 & 94.6825 & 94.3716 \\
\hline 6 & 62.3 & 131.7235 & 132.4889 & 131.2482 & 122.0772 & 121.8498 & 121.6226 & 131.8419 & 132.6072 & 131.2184 & 122.0231 & 121.8146 & 121.5388 \\
\hline 12 & 60.9 & 133.5479 & 134.5130 & 133.7774 & 125.3085 & 124.3120 & 123.0492 & 133.4875 & 134.4162 & 133.9200 & 125.1295 & 124.1413 & 123.0495 \\
\hline 13 & 56.1 & 137.3178 & 139.0074 & 137.9918 & 129.3987 & 128.8035 & 128.3726 & 137.3631 & 139.0406 & 137.7530 & 129.2630 & 128.6574 & 128.1644 \\
\hline 10 & 54.0 & 139.9593 & 140.9648 & 139.5458 & 130.9595 & 130.3074 & 129.9152 & 140.0615 & 141.0615 & 139.6457 & 131.0124 & 130.3406 & 130.0254 \\
\hline $8 a$ & 48.4 & 144.6721 & 144.9160 & 144.4668 & 135.2064 & 135.3056 & 134.5136 & 144.4508 & 144.6886 & 144.0791 & 134.9249 & 135.0318 & 134.3002 \\
\hline 14 & 42.4 & 146.7160 & 148.1752 & 147.4273 & 139.3937 & 138.6979 & 137.9012 & 146.9050 & 148.3389 & 147.5273 & 139.4143 & 138.7133 & 137.9563 \\
\hline 9 & 34.0 & 156.3988 & 157.6229 & 155.4611 & 147.9204 & 147.4764 & 146.2888 & 156.2659 & 157.4667 & 154.9133 & 147.5798 & 147.1441 & 146.0293 \\
\hline 5 & 30.1 & 162.9573 & 164.1898 & 163.7973 & 154.5427 & 154.3033 & 154.2826 & 163.2225 & 164.4452 & 164.0177 & 154.7601 & 154.5125 & 154.4461 \\
\hline \multicolumn{14}{|l|}{${ }^{1} \mathbf{H}$} \\
\hline 7 & 6.00 & 25.9792 & 25.3921 & 25.0264 & 25.9086 & 25.4984 & 25.3994 & 25.9493 & 25.3620 & 24.9743 & 25.8678 & 25.4599 & 25.3548 \\
\hline 8 & 6.07 & 25.7155 & 25.0844 & 25.0380 & 25.7312 & 25.3211 & 25.3350 & 25.6658 & 25.0399 & 24.9615 & 25.6803 & 25.2737 & 25.2641 \\
\hline 1 & 6.62 & 25.6276 & 25.0114 & 24.9688 & 25.5604 & 25.1137 & 25.0938 & 25.4978 & 24.8808 & 24.8351 & 25.4160 & 24.9767 & 24.9472 \\
\hline 2 & 6.66 & 25.4584 & 24.7997 & 24.5659 & 25.3038 & 24.8538 & 24.7146 & 25.4308 & 24.7689 & 24.5296 & 25.2663 & 24.8181 & 24.6791 \\
\hline $4 a$ & 4.61 & 27.7316 & 27.2547 & 27.1442 & 27.6710 & 27.4209 & 27.3710 & 27.6085 & 27.1275 & 27.0142 & 27.5430 & 27.2910 & 27.2397 \\
\hline 6 & 4.14 & 28.1089 & 27.6444 & 27.5633 & 28.0703 & 27.7973 & 27.8272 & 28.0569 & 27.5898 & 27.5024 & 28.0055 & 27.7247 & 27.7548 \\
\hline $12 a$ & 4.08 & 28.5752 & 28.1239 & 28.0852 & 28.5298 & 28.2546 & 28.2384 & 28.4673 & 28.0134 & 28.0073 & 28.4232 & 28.1530 & 28.1627 \\
\hline $12 b$ & 3.68 & 28.7500 & 28.2804 & 28.2222 & 28.6394 & 28.3586 & 28.3738 & 28.7120 & 28.2491 & 28.1742 & 28.5998 & 28.3189 & 28.3205 \\
\hline $10 a$ & 3.26 & 29.2943 & 28.8620 & 28.8030 & 29.2180 & 28.9945 & 28.9674 & 29.2533 & 28.8230 & 28.7655 & 29.1855 & 28.9661 & 28.9317 \\
\hline $10 b$ & 3.05 & 29.5818 & 29.1484 & 29.0388 & 29.4902 & 29.2471 & 29.1259 & 29.4871 & 29.0477 & 28.9228 & 29.3906 & 29.1364 & 29.0153 \\
\hline $9 a$ & 1.57 & 30.5461 & 30.0990 & 29.9804 & 30.5051 & 30.2442 & 30.1513 & 30.4330 & 29.9828 & 29.8686 & 30.3875 & 30.1226 & 30.0371 \\
\hline $9 b$ & 2.08 & 30.2566 & 29.8518 & 29.7268 & 30.2069 & 29.9822 & 29.9109 & 30.2341 & 29.8296 & 29.7143 & 30.1795 & 29.9623 & 29.8939 \\
\hline $5 a$ & 2.01 & 30.3959 & 29.9385 & 29.7486 & 30.2700 & 29.9961 & 29.9278 & 30.2444 & 29.7807 & 29.5802 & 30.1204 & 29.8424 & 29.7608 \\
\hline $5 b$ & 2.69 & 29.6826 & 29.2500 & 29.0438 & 29.5342 & 29.3136 & 29.2420 & 29.6759 & 29.2404 & 29.0275 & 29.5259 & 29.3014 & 29.2225 \\
\hline 13 & 3.83 & 28.2814 & 27.7576 & 27.5902 & 28.2447 & 27.9096 & 27.8219 & 28.2229 & 27.7001 & 27.5265 & 28.1815 & 27.8499 & 27.7569 \\
\hline 14 & 2.40 & 29.8018 & 29.3476 & 29.1498 & 29.7043 & 29.4428 & 29.3211 & 29.7591 & 29.3049 & 29.0846 & 29.6561 & 29.3956 & 29.2566 \\
\hline
\end{tabular}




\section{Compound 68}

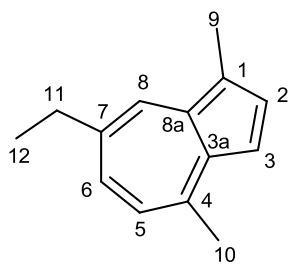

68

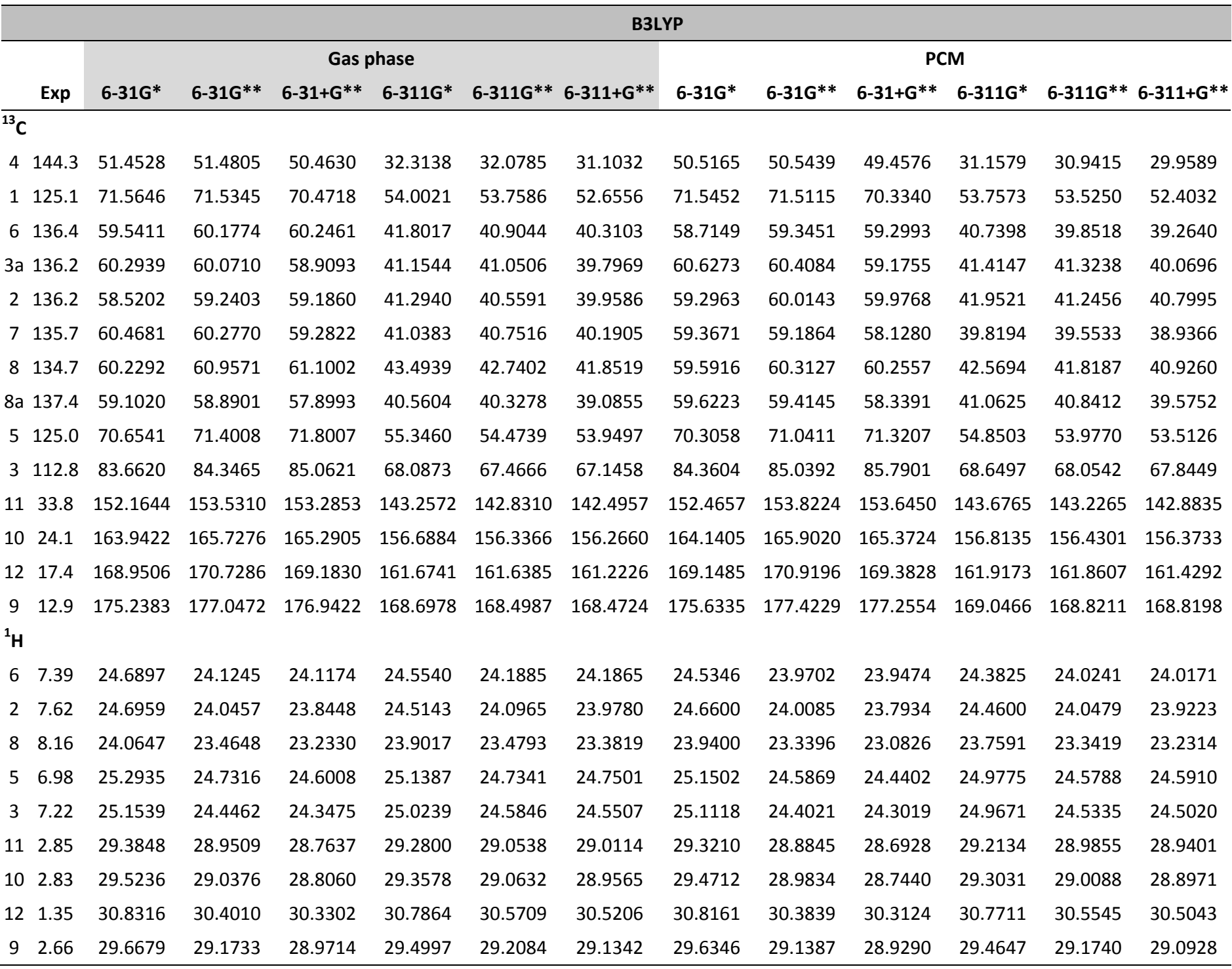

mPW1PW91

\begin{tabular}{|c|c|c|c|c|c|c|c|c|c|c|c|c|}
\hline \multirow[b]{3}{*}{ Exp } & \multicolumn{12}{|c|}{ mPW1PW91 } \\
\hline & \multicolumn{6}{|c|}{ Gas phase } & \multicolumn{6}{|c|}{ PCM } \\
\hline & 6-31G* & 6-31G** & $6-31+G^{* *}$ & 6-311G* & $6-311 \mathrm{G} * *$ & $6-311+G * *$ & 6-31G* & $6-31 \mathrm{G} * *$ & $6-31+G^{* *}$ & 6-311G* & $6-311 \mathrm{G} * *$ & $6-311+G * *$ \\
\hline${ }^{13} \mathrm{C}$ & & & & & & & & & & & & \\
\hline $\begin{array}{ll}4 & 144.3\end{array}$ & 53.9095 & 53.9172 & 53.0831 & 37.2657 & 37.0151 & 35.9595 & 52.8787 & 52.8870 & 51.9836 & 36.0188 & 35.7906 & 34.7371 \\
\hline $\begin{array}{ll}1 & 125.1\end{array}$ & 74.9547 & 74.9016 & 73.9632 & 59.4856 & 59.2209 & 58.0336 & 74.8860 & 74.8298 & 73.7796 & 59.1781 & 58.9278 & 57.7330 \\
\hline $\begin{array}{ll}6 & 136.4\end{array}$ & 61.5294 & 62.1045 & 62.2186 & 45.9985 & 45.0717 & 44.4479 & 60.6069 & 61.1755 & 61.1754 & 44.8461 & 43.9298 & 43.3254 \\
\hline
\end{tabular}




\begin{tabular}{|c|c|c|c|c|c|c|c|c|c|c|c|c|c|}
\hline $3 a$ & 136.2 & 63.2114 & 62.9747 & 61.9813 & 46.5596 & 46.4481 & 45.1457 & 63.6304 & 63.3979 & 62.3252 & 46.8845 & 46.7868 & 45.4793 \\
\hline 2 & 136.2 & 60.9900 & 61.6455 & 61.8015 & 45.8553 & 45.0821 & 44.4353 & 61.7531 & 62.4067 & 62.5543 & 46.4613 & 45.7190 & 45.2161 \\
\hline 7 & 135.7 & 63.6656 & 63.4349 & 62.4867 & 46.7428 & 46.4206 & 45.8235 & 62.4917 & 62.2739 & 61.2798 & 45.4623 & 45.1641 & 44.5250 \\
\hline 8 & 134.7 & 61.9914 & 62.6828 & 63.0551 & 47.4947 & 46.7321 & 45.7675 & 61.3025 & 61.9851 & 62.1588 & 46.5211 & 45.7600 & 44.8004 \\
\hline $8 a$ & 137.4 & 62.1311 & 61.8986 & 61.0154 & 46.0792 & 45.8351 & 44.6101 & 62.7481 & 62.5206 & 61.5588 & 46.6558 & 46.4241 & 45.1763 \\
\hline 5 & 125.0 & 73.2031 & 73.8755 & 74.4554 & 59.8802 & 58.9805 & 58.3806 & 72.8387 & 73.4999 & 73.9377 & 59.3538 & 58.4561 & 57.9201 \\
\hline 3 & 112.8 & 86.1615 & 86.8029 & 87.4880 & 72.4192 & 71.7800 & 71.3366 & 86.8274 & 87.4621 & 88.1661 & 72.9223 & 72.3090 & 71.9781 \\
\hline 11 & 33.8 & 157.0250 & 158.2855 & 157.8428 & 148.9140 & 148.4814 & 148.1699 & 157.3503 & 158.6017 & 158.2141 & 149.3217 & 148.8682 & 148.5534 \\
\hline 10 & 24.1 & 167.6524 & 169.3123 & 169.2701 & 161.0974 & 160.7345 & 160.6953 & 167.8710 & 169.5065 & 169.3517 & 161.2116 & 160.8201 & 160.7954 \\
\hline 12 & 17.4 & 173.2216 & 174.8850 & 173.5952 & 166.4760 & 166.4554 & 166.0431 & 173.4440 & 175.1007 & 173.8208 & 166.7191 & 166.6796 & 166.2552 \\
\hline $\begin{array}{c}9 \\
{ }^{1} \mathrm{H}\end{array}$ & 12.9 & 178.8181 & 180.5237 & 180.3389 & 172.9435 & 172.7469 & 172.7876 & 179.2674 & 180.9522 & 180.6885 & 173.3072 & 173.0867 & 173.1473 \\
\hline 6 & 7.39 & 24.4880 & 23.9206 & 23.9148 & 24.3637 & 23.9674 & 23.9805 & 24.3154 & 23.7485 & 23.7288 & 24.1789 & 23.7895 & 23.7976 \\
\hline 2 & 7.62 & 24.5002 & 23.8485 & 23.6724 & 24.3455 & 23.8906 & 23.7917 & 24.4490 & 23.7956 & 23.6071 & 24.2792 & 23.8303 & 23.7246 \\
\hline 8 & 8.16 & 23.8480 & 23.2548 & 23.0641 & 23.6837 & 23.2587 & 23.1604 & 23.7101 & 23.1161 & 22.9033 & 23.5318 & 23.1116 & 23.0020 \\
\hline 5 & 6.98 & 25.0932 & 24.5323 & 24.4280 & 24.9564 & 24.5286 & 24.5571 & 24.9333 & 24.3705 & 24.2522 & 24.7821 & 24.3600 & 24.3845 \\
\hline 3 & 7.22 & 24.9561 & 24.2508 & 24.1710 & 24.8491 & 24.3841 & 24.3556 & 24.8985 & 24.1909 & 24.1105 & 24.7805 & 24.3214 & 24.2943 \\
\hline 11 & 2.85 & 29.3041 & 28.8753 & 28.7220 & 29.1673 & 28.9438 & 28.9096 & 29.2326 & 28.8006 & 28.6430 & 29.0947 & 28.8690 & 28.8314 \\
\hline 10 & 2.83 & 29.4208 & 28.9320 & 28.7282 & 29.2461 & 28.9452 & 28.8587 & 29.3587 & 28.8678 & 28.6561 & 29.1828 & 28.8822 & 28.7905 \\
\hline 12 & 1.35 & 30.7747 & 30.3424 & 30.2608 & 30.7038 & 30.4804 & 30.4302 & 30.7553 & 30.3213 & 30.2391 & 30.6844 & 30.4601 & 30.4101 \\
\hline 9 & 2.66 & 29.5800 & 29.0828 & 28.9205 & 29.4005 & 29.1015 & 29.0457 & 29.5383 & 29.0396 & 28.8695 & 29.3582 & 29.0599 & 28.9971 \\
\hline
\end{tabular}

\section{Compound 69}

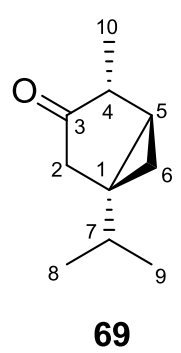

\begin{tabular}{|c|c|c|c|c|c|c|c|c|c|c|c|c|c|}
\hline & \multirow[b]{3}{*}{ Exp } & \multicolumn{12}{|c|}{ B3LYP } \\
\hline & & \multicolumn{6}{|c|}{ Gas phase } & \multicolumn{6}{|c|}{ PCM } \\
\hline & & 6-31G* & $6-31 G * *$ & $6-31+G * *$ & 6-311G* & $6-311 G * *$ & $6-311+G * *$ & 6-31G* & $6-31 G * *$ & $6-31+G * *$ & 6-311G* & $6-311 G * *$ & $6-311+\mathrm{G} * *$ \\
\hline \multicolumn{14}{|c|}{${ }^{13} \mathrm{C}$} \\
\hline 3 & 221.4 & -20.0242 & -19.7309 & -23.5825 & -42.5164 & -42.2037 & -46.7168 & -24.3531 & -24.0732 & -29.0024 & -47.5148 & -47.2169 & -52.7671 \\
\hline 4 & 47.4 & 141.7567 & 142.5172 & 140.8948 & 130.8612 & 130.4989 & 129.4141 & 141.1537 & 141.9090 & 140.0651 & 130.1861 & 129.8151 & 128.4749 \\
\hline 2 & 39.7 & 150.3379 & 151.5309 & 150.5692 & 141.0552 & 140.4939 & 139.5648 & 149.6908 & 150.8663 & 149.6854 & 140.3044 & 139.7315 & 138.4939 \\
\hline 7 & 32.9 & 153.2169 & 154.1037 & 153.1977 & 142.6393 & 142.4968 & 142.2013 & 153.4759 & 154.3619 & 153.5150 & 143.0014 & 142.8462 & 142.5676 \\
\hline 1 & 29.7 & 158.4686 & 158.5116 & 158.1608 & 148.3053 & 148.1093 & 147.8406 & 158.3911 & 158.4332 & 158.1183 & 148.2918 & 148.0911 & 147.7954 \\
\hline 5 & 25.5 & 161.8139 & 162.6658 & 161.8286 & 152.6368 & 152.1808 & 151.7184 & 162.1018 & 162.9506 & 162.1473 & 152.9711 & 152.5076 & 152.0395 \\
\hline 8 & 20.0 & 169.3213 & 171.0805 & 171.4195 & 162.7019 & 162.5886 & 162.2385 & 169.5655 & 171.3165 & 171.7142 & 163.0189 & 162.8852 & 162.5380 \\
\hline 9 & 19.7 & 168.8294 & 170.6094 & 171.1351 & 162.1999 & 162.1242 & 161.7560 & 169.0718 & 170.8478 & 171.4307 & 162.5243 & 162.4297 & 162.0624 \\
\hline 6 & 18.7 & 168.9302 & 170.4086 & 170.9826 & 162.1553 & 161.4629 & 161.0312 & 169.2127 & 170.6757 & 171.2487 & 162.4662 & 161.7564 & 161.2949 \\
\hline 10 & 18.2 & 169.8979 & 171.6930 & 172.1696 & 163.2457 & 163.1550 & 162.7903 & 170.1574 & 171.9392 & 172.3909 & 163.5121 & 163.3991 & 163.0082 \\
\hline
\end{tabular}




\begin{tabular}{cccccccccccccc}
\hline${ }^{1} \boldsymbol{H}$ & & & & & & & & & & & & & \\
4 & 2.22 & 30.0446 & 29.6129 & 29.5845 & 30.0568 & 29.8530 & 29.7731 & 30.0127 & 29.5768 & 29.5270 & 30.0196 & 29.8132 & 29.7159 \\
$2 \mathrm{a}$ & 2.55 & 29.7133 & 29.2073 & 29.1270 & 29.7713 & 29.4443 & 29.4024 & 29.6136 & 29.1038 & 28.9985 & 29.6632 & 29.3356 & 29.2742 \\
$2 \mathrm{~b}$ & 2.07 & 30.2604 & 29.7983 & 29.6439 & 30.1973 & 29.9694 & 29.8799 & 30.2163 & 29.7486 & 29.5825 & 30.1506 & 29.9188 & 29.8169 \\
7 & 1.36 & 30.8944 & 30.4981 & 30.4256 & 30.9891 & 30.7978 & 30.7678 & 30.8195 & 30.4215 & 30.3355 & 30.9081 & 30.7155 & 30.6779 \\
5 & 1.09 & 31.0830 & 30.6898 & 30.6140 & 31.1491 & 30.9753 & 30.9615 & 30.9814 & 30.5841 & 30.4894 & 31.0386 & 30.8634 & 30.8363 \\
6a & 0.76 & 31.4555 & 31.0315 & 30.9794 & 31.4746 & 31.2898 & 31.2680 & 31.3852 & 30.9579 & 30.8956 & 31.4008 & 31.2159 & 31.1856 \\
$6 \mathrm{~b}$ & 0.12 & 31.9274 & 31.5682 & 31.5010 & 31.9817 & 31.8640 & 31.8265 & 31.9111 & 31.5515 & 31.4794 & 31.9621 & 31.8464 & 31.8048 \\
8 & 0.96 & 31.1389 & 30.7143 & 30.6540 & 31.1267 & 30.9008 & 30.8791 & 31.1378 & 30.7123 & 30.6531 & 31.1282 & 30.9019 & 30.8793 \\
9 & 1.01 & 31.0930 & 30.6569 & 30.5714 & 31.0826 & 30.8370 & 30.8022 & 31.0802 & 30.6437 & 30.5584 & 31.0722 & 30.8269 & 30.7907 \\
10 & 1.16 & 31.0342 & 30.5988 & 30.5081 & 31.0066 & 30.7679 & 30.7373 & 31.0098 & 30.5723 & 30.4734 & 30.9813 & 30.7408 & 30.7024 \\
\hline
\end{tabular}

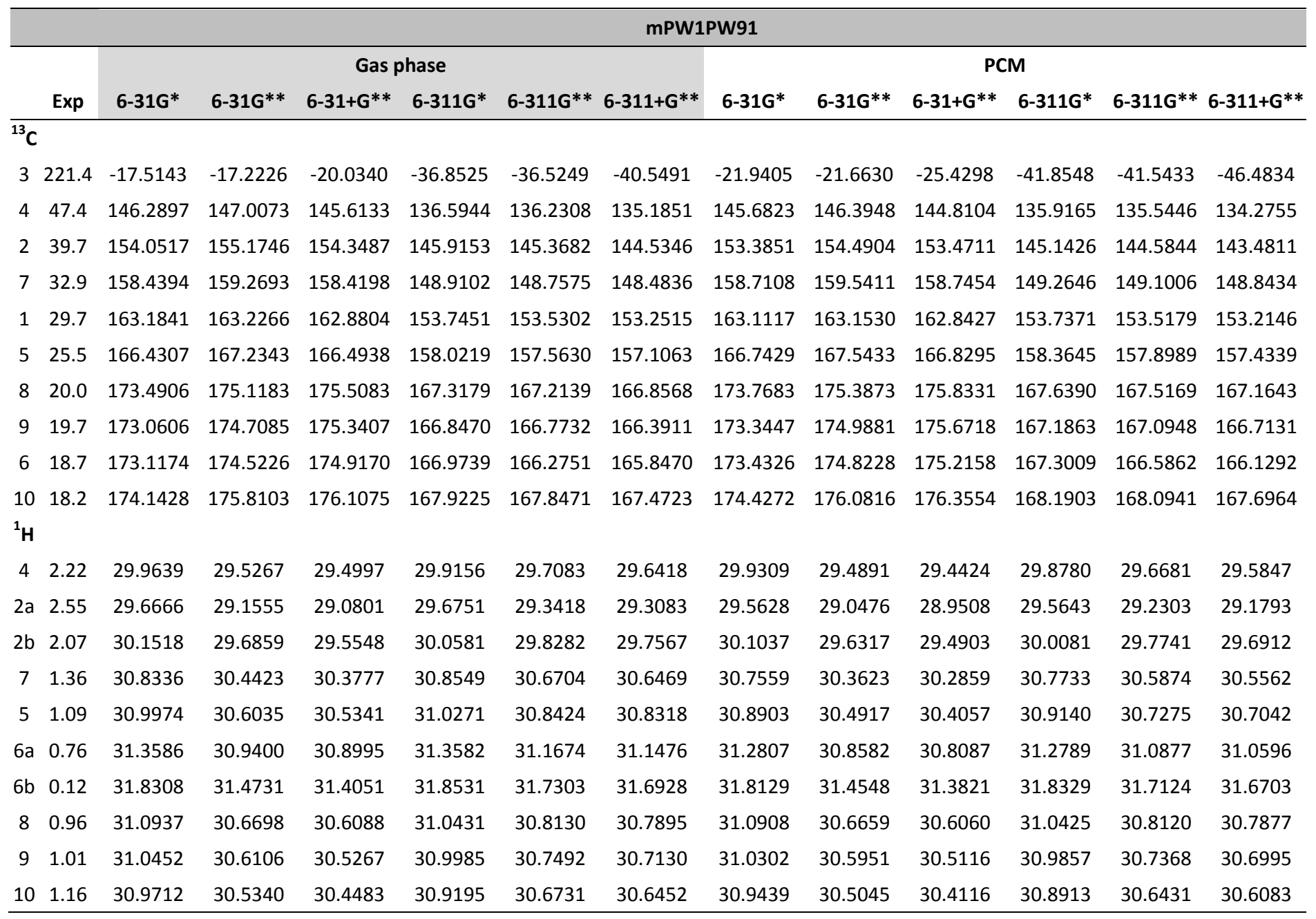




\section{Compound 70}

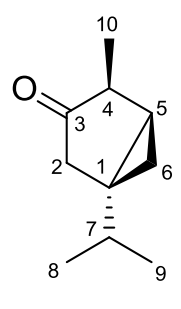

70

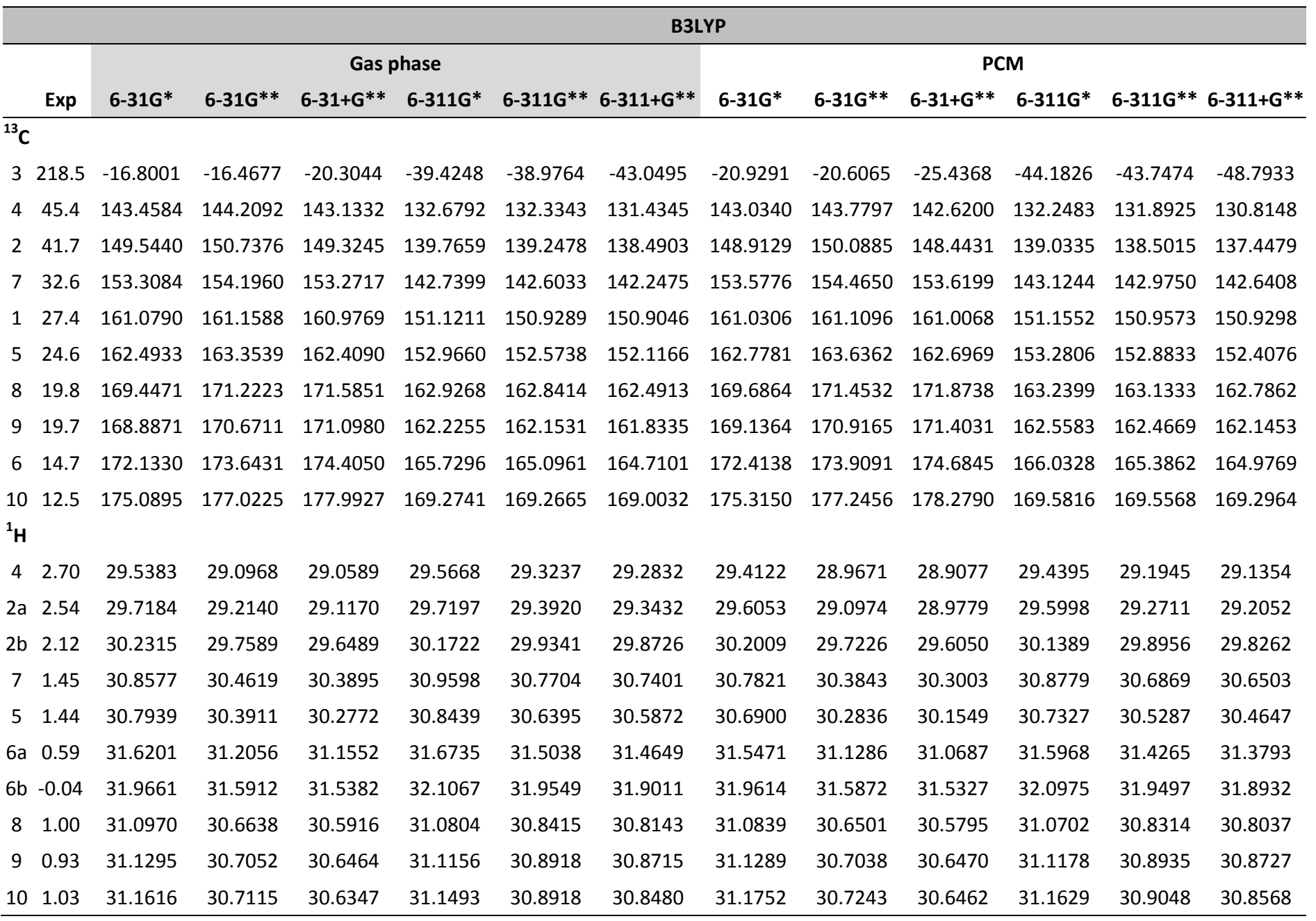

\begin{tabular}{|c|c|c|c|c|c|c|c|c|c|c|c|c|c|}
\hline \multirow{3}{*}{\multicolumn{2}{|c|}{ Exp }} & \multicolumn{12}{|c|}{ mPW1PW91 } \\
\hline & & \multicolumn{6}{|c|}{ Gas phase } & \multicolumn{6}{|c|}{ PCM } \\
\hline & & 6-31G* & $6-31 \mathrm{G} * *$ & $6-31+\mathrm{G} * *$ & 6-311G* & $6-311 G * *$ & $6-311+G * *$ & 6-31G* & $6-31 G * *$ & $6-31+G * *$ & 6-311G* & $6-311 G * *$ & $6-311+G * *$ \\
\hline \multicolumn{14}{|c|}{$13 \mathrm{c}$} \\
\hline 3 & 218.5 & -14.3033 & -13.9762 & -16.8284 & -33.8024 & -33.3425 & -36.9682 & -18.5316 & -18.2151 & -21.9425 & -38.5665 & -38.1211 & -42.6071 \\
\hline 4 & 45.4 & 147.6579 & 148.3617 & 147.4104 & 137.9854 & 137.6405 & 136.8182 & 147.2126 & 147.9116 & 146.8865 & 137.5289 & 137.1741 & 136.1933 \\
\hline 2 & 41.7 & 153.2845 & 154.4073 & 153.1644 & 144.6945 & 144.1927 & 143.5199 & 152.6363 & 153.7411 & 152.2944 & 143.9445 & 143.4290 & 142.4956 \\
\hline 7 & 32.6 & 158.5427 & 159.3750 & 158.5641 & 149.0380 & 148.8882 & 148.5481 & 158.8259 & 159.6593 & 158.9202 & 149.4142 & 149.2530 & 148.9340 \\
\hline 1 & 27.4 & 165.5713 & 165.6511 & 165.4629 & 156.3758 & 156.1686 & 156.1076 & 165.5293 & 165.6077 & 165.4911 & 156.4129 & 156.2002 & 156.1349 \\
\hline 5 & 24.6 & 167.0433 & 167.8524 & 167.0270 & 158.3349 & 157.9370 & 157.5046 & 167.3482 & 168.1549 & 167.3312 & 158.6559 & 158.2539 & 157.8011 \\
\hline
\end{tabular}




\begin{tabular}{|c|c|c|c|c|c|c|c|c|c|c|c|c|c|}
\hline 8 & 9.8 & 73.6236 & 75.2680 & 175.7173 & 167.5323 & 167.4570 & 167.1085 & 173.8976 & 175.5332 & 176.0366 & 167.8509 & 167.7567 & 167.4127 \\
\hline 9 & 19.7 & 173.1398 & 74.7932 & 175.3233 & 166.9129 & 166.8402 & 166.4917 & 173.4314 & 175.0804 & 175.6634 & 167.2608 & 167.1701 & 166.8199 \\
\hline 6 & 14.7 & 176.1782 & 177.6152 & 178.2397 & 170.4065 & 169.7656 & 169.3792 & 176.4917 & 177.9143 & 178.5492 & 170.7232 & 170.0706 & 169.6610 \\
\hline 10 & 12.5 & 179.0645 & 180.8727 & 181.6570 & 173.6577 & 173.6679 & 173.3647 & 179.3243 & 181.1292 & 181.9703 & 173.9736 & 173.9674 & 173.6676 \\
\hline 4 & 2.70 & 29.4821 & 29.0349 & 28.9917 & 29.4499 & 29.2071 & 29.1737 & 29.3542 & 28.9028 & 28.8409 & 29.3221 & 29.0772 & 29.0268 \\
\hline $2 a$ & 2.54 & 29.6727 & 29.1631 & 29.0678 & 29.6272 & 29.2953 & 29.2523 & 29.5557 & 29.0421 & 28.9275 & 29.5047 & 29.1717 & 29.1135 \\
\hline $2 b$ & 2.12 & 30.1181 & 29.6426 & 29.5465 & 30.0310 & 29.7900 & 29.7433 & 30.0832 & 29.6017 & 29.4987 & 29.9940 & 29.7478 & 29.6941 \\
\hline 7 & 1.45 & 30.7935 & 30.4023 & 30.3330 & 30.8172 & 30.6356 & 30.6122 & 30.7150 & 30.3212 & 30.2422 & 30.7349 & 30.5514 & 30.5217 \\
\hline 5 & 1.44 & 30.7124 & 30.3069 & 30.2033 & 30.7284 & 30.5119 & 30.4667 & 30.6017 & 30.1918 & 30.0755 & 30.6135 & 30.3967 & 30.3403 \\
\hline $6 a$ & 0.59 & 31.5185 & 31.1088 & 31.0689 & 31.5541 & 31.3754 & 31.3444 & 31.4379 & 31.0239 & 30.9753 & 31.4719 & 31.2923 & 31.2531 \\
\hline $6 b$ & -0.04 & 31.8827 & 31.5106 & 31.4539 & 31.9875 & 31.8307 & 31.7756 & 31.8766 & 31.5051 & 31.4472 & 31.9777 & 31.8248 & 31.7670 \\
\hline 8 & 1.00 & 31.0493 & 30.6176 & 30.5482 & 30.9953 & 30.7531 & 30.7259 & 31.0340 & 30.6017 & 30.5339 & 30.9829 & 30.7408 & 30.7132 \\
\hline 9 & 0.93 & 31.0856 & 30.6620 & 30.6023 & 31.0354 & 30.8069 & 30.7839 & 31.0831 & 30.6586 & 30.6008 & 31.0355 & 30.8064 & 30.7829 \\
\hline 10 & 1.03 & 31.0937 & 30.6426 & 30.5712 & 31.0559 & 30.7897 & 30.7509 & 31.1045 & 30.6524 & 30.5796 & 31.0662 & 30.7996 & 30.7566 \\
\hline
\end{tabular}

\section{Compound 71}

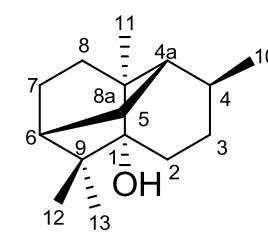

71

\begin{tabular}{|c|c|c|c|c|c|c|c|c|c|c|c|c|c|}
\hline & \multirow[b]{3}{*}{ Exp } & \multicolumn{12}{|c|}{ B3LYP } \\
\hline & & \multicolumn{6}{|c|}{ Gas phase } & \multicolumn{6}{|c|}{ PCM } \\
\hline & & 6-31G* & $6-31 G * *$ & $6-31+\mathrm{G} * *$ & 6-311G* & $6-311 G * *$ & $6-311+G * *$ & 6-31G* & $6-31 \mathrm{G} * *$ & $6-31+G * *$ & 6-311G* & $6-311 G * *$ & $6-311+G * *$ \\
\hline \multicolumn{14}{|c|}{${ }^{13} \mathrm{C}$} \\
\hline 1 & 75.7 & 113.4900 & 113.7278 & 113.3844 & 101.5317 & 101.8130 & 101.0850 & 113.3665 & 113.6083 & 113.1668 & 101.3387 & 101.6248 & 100.8553 \\
\hline 2 & 32.7 & 153.5136 & 154.8300 & 153.6411 & 144.8609 & 144.5059 & 143.9939 & 153.9967 & 155.3020 & 154.1573 & 145.3816 & 145.0069 & 144.4925 \\
\hline 3 & 28.6 & 159.6544 & 160.9822 & 158.9727 & 150.6160 & 150.4045 & 149.3575 & 159.8263 & 161.1539 & 159.1718 & 150.8549 & 150.6367 & 149.5944 \\
\hline 4 & 28.2 & 158.7409 & 159.6652 & 158.2657 & 149.1335 & 149.0991 & 148.9386 & 158.8329 & 159.7570 & 158.3731 & 149.2750 & 149.2329 & 149.0793 \\
\hline 5 & 24.4 & 162.2264 & 163.7013 & 163.6230 & 154.4905 & 154.2051 & 153.8340 & 162.3936 & 163.8632 & 163.8184 & 154.7014 & 154.4043 & 154.0491 \\
\hline 6 & 39.2 & 149.2358 & 150.0850 & 148.2245 & 138.2314 & 138.0953 & 137.8886 & 149.3274 & 150.1761 & 148.3441 & 138.3743 & 138.2305 & 138.0420 \\
\hline 7 & 24.6 & 162.7498 & 164.2794 & 164.2320 & 155.0943 & 154.7951 & 154.0871 & 162.8770 & 164.4031 & 164.3902 & 155.2715 & 154.9640 & 154.2652 \\
\hline 8 & 28.9 & 158.6568 & 160.0449 & 159.9349 & 150.9440 & 150.7077 & 150.4182 & 158.6791 & 160.0674 & 159.9929 & 151.0437 & 150.7939 & 150.5200 \\
\hline 9 & 40.2 & 146.9414 & 147.3777 & 146.5474 & 136.7172 & 136.7382 & 136.1349 & 146.9502 & 147.3897 & 146.5822 & 136.7463 & 136.7658 & 136.1607 \\
\hline 10 & 18.6 & 169.6469 & 171.4641 & 172.3292 & 163.0746 & 163.0993 & 162.6426 & 169.9012 & 171.7124 & 172.6519 & 163.4263 & 163.4297 & 162.9914 \\
\hline 11 & 20.7 & 168.6442 & 170.4608 & 170.5608 & 162.9025 & 162.5916 & 161.8720 & 168.8234 & 170.6246 & 170.7288 & 163.0912 & 162.7565 & 162.0589 \\
\hline 12 & 24.3 & 164.5109 & 166.2319 & 166.9685 & 158.0553 & 157.8006 & 157.4539 & 164.7020 & 166.4118 & 167.1643 & 158.3016 & 158.0231 & 157.6874 \\
\hline 13 & 26.9 & 162.3064 & 163.9959 & 164.7540 & 155.4234 & 155.1498 & 155.1073 & 162.3884 & 164.0785 & 164.9109 & 155.6543 & 155.3668 & 155.3622 \\
\hline $4 a$ & 43.8 & 144.4824 & 145.2903 & 144.6790 & 133.9529 & 133.7598 & 133.0629 & 144.6304 & 145.4296 & 144.8772 & 134.1851 & 133.9718 & 133.2837 \\
\hline $8 a$ & 37.7 & 148.4168 & 148.7731 & 148.0398 & 139.2671 & 139.2719 & 138.7521 & 148.4047 & 148.7646 & 147.9753 & 139.2205 & 139.2336 & 138.6943 \\
\hline \multicolumn{14}{|c|}{${ }^{1} \mathrm{H}$} \\
\hline 4 & 1.97 & 30.1413 & 29.7059 & 29.7290 & 30.2234 & 29.9843 & 30.0518 & 30.1022 & 29.6672 & 29.6833 & 30.1817 & 29.9438 & 30.0092 \\
\hline
\end{tabular}




\begin{tabular}{cccccccccccccc}
\hline 6 & 1.20 & 30.8417 & 30.4777 & 30.5274 & 31.0009 & 30.8390 & 30.8695 & 30.8272 & 30.4640 & 30.5035 & 30.9795 & 30.8187 & 30.8446 \\
2a & 1.72 & 30.2225 & 29.7985 & 29.7493 & 30.2240 & 29.9933 & 29.9903 & 30.2721 & 29.8475 & 29.7993 & 30.2760 & 30.0413 & 30.0393 \\
2b & 1.49 & 30.9950 & 30.5704 & 30.5982 & 31.0616 & 30.7523 & 30.7917 & 30.8701 & 30.4439 & 30.4652 & 30.9330 & 30.6295 & 30.6674 \\
3a & 1.48 & 30.5831 & 30.1251 & 30.0647 & 30.6632 & 30.4093 & 30.3772 & 30.5775 & 30.1210 & 30.0568 & 30.6566 & 30.4043 & 30.3710 \\
3b & 1.38 & 30.7513 & 30.3358 & 30.2441 & 30.7282 & 30.5006 & 30.4274 & 30.7182 & 30.3022 & 30.2052 & 30.6945 & 30.4672 & 30.3910 \\
4a & 1.45 & 30.4993 & 30.1651 & 30.1652 & 30.6235 & 30.4702 & 30.4356 & 30.4684 & 30.1356 & 30.1210 & 30.5827 & 30.4328 & 30.3918 \\
5a & 1.87 & 30.4562 & 29.9867 & 29.9699 & 30.6819 & 30.3645 & 30.3695 & 30.4362 & 29.9665 & 29.9481 & 30.6601 & 30.3437 & 30.3481 \\
5b & 1.26 & 30.8038 & 30.4240 & 30.3997 & 30.8230 & 30.6412 & 30.6182 & 30.7919 & 30.4121 & 30.3829 & 30.8106 & 30.6292 & 30.6030 \\
7a & 1.50 & 30.1663 & 29.7247 & 29.6445 & 30.1577 & 29.9396 & 29.9278 & 30.2025 & 29.7615 & 29.6830 & 30.1966 & 29.9773 & 29.9650 \\
7b & 1.29 & 30.8581 & 30.4867 & 30.3860 & 30.8168 & 30.6636 & 30.5980 & 30.8362 & 30.4646 & 30.3567 & 30.7920 & 30.6395 & 30.5698 \\
8a & 1.85 & 29.9061 & 29.4883 & 29.4973 & 29.9641 & 29.7472 & 29.7793 & 29.9807 & 29.5634 & 29.5801 & 30.0357 & 29.8172 & 29.8557 \\
8b & 1.06 & 31.1051 & 30.7431 & 30.7723 & 31.1456 & 30.9761 & 30.9511 & 31.0691 & 30.7070 & 30.7262 & 31.1045 & 30.9362 & 30.9056 \\
10 & 0.80 & 31.3514 & 30.9266 & 30.8484 & 31.3065 & 31.0704 & 31.0533 & 31.3516 & 30.9258 & 30.8500 & 31.3092 & 31.0725 & 31.0561 \\
11 & 0.85 & 31.3622 & 30.8966 & 30.8902 & 31.4434 & 31.1449 & 31.1483 & 31.3281 & 30.8627 & 30.8537 & 31.4081 & 31.1120 & 31.1159 \\
12 & 1.09 & 31.0426 & 30.6014 & 30.5659 & 31.0799 & 30.8262 & 30.8146 & 31.0331 & 30.5909 & 30.5524 & 31.0695 & 30.8144 & 30.8014 \\
13 & 1.07 & 30.9542 & 30.5392 & 30.4708 & 31.0012 & 30.7913 & 30.7342 & 30.9977 & 30.5820 & 30.5166 & 31.0447 & 30.8322 & 30.7747 \\
\hline
\end{tabular}

\section{mPW1PW91}

\section{Gas phase}

Exp 6-31G* 6-31G** 6-31+G** 6-311G* 6-311G** 6-311+G** 6-31G* 6-31G** 6-31+G** 6-311G* 6-311G** 6-311+G**

\section{${ }^{13} \mathrm{C}$}

$\begin{array}{llllllll}1 & 75.7 & 118.6615 & 118.8917 & 118.8239 & 108.0816 & 108.3341 & 107.5534\end{array}$

$\begin{array}{llllllll}2 & 32.7 & 158.6790 & 159.8624 & 158.7111 & 150.8405 & 150.4815 & 149.9755\end{array}$

$\begin{array}{llllllll}3 & 28.6 & 164.5559 & 165.7624 & 163.9188 & 156.3645 & 156.1420 & 155.1969\end{array}$

$\begin{array}{llllllll}4 & 28.2 & 164.0374 & 164.8912 & 163.6960 & 155.1375 & 155.1127 & 154.8974\end{array}$

$\begin{array}{lllllll}5 & 24.4 & 167.1772 & 168.5315 & 168.4151 & 160.0372 & 159.7345\end{array}$

$\begin{array}{lllllll}6 & 39.2 & 154.8420 & 155.6144 & 153.6957 & 144.6887 & 144.5496\end{array}$

$\begin{array}{lllllll}7 & 24.6 & 167.7239 & 169.1227 & 169.1749 & 160.6261 & 160.3107\end{array}$

$\begin{array}{lllllll}8 & 28.9 & 163.9474 & 165.2161 & 164.7644 & 156.7848 & 156.5513\end{array}$

$\begin{array}{lllllll}9 & 40.2 & 152.2020 & 152.6373 & 151.9668 & 142.7227 & 142.7463\end{array}$

$\begin{array}{lllllll}10 & 18.6 & 173.9550 & 175.6445 & 176.3232 & 167.8478 & 167.8676\end{array}$

$\begin{array}{lllllll}11 & 20.7 & 173.1410 & 174.8352 & 174.5384 & 167.8054 & 167.5156\end{array}$

$\begin{array}{lllllll}12 & 24.3 & 168.8776 & 170.4519 & 170.8843 & 162.8993 & 162.6472\end{array}$

$\begin{array}{lllllll}13 & 26.9 & 166.8653 & 168.4029 & 168.6846 & 160.4159 & 160.1568\end{array}$

$\begin{array}{lllllll}4 a & 43.8 & 150.2563 & 150.9750 & 150.4187 & 140.4834 & 140.2946\end{array}$

$\begin{array}{lllllll}8 \mathrm{a} & 37.7 & 153.8137 & 154.1685 & 153.7271 & 145.2136 & 145.2156\end{array}$ ${ }^{1} \mathbf{H}$

\begin{tabular}{cccccccccccccc} 
4 & 1.97 & 30.1097 & 29.6789 & 29.6804 & 30.1202 & 29.8911 & 29.9520 & 30.0711 & 29.6406 & 29.6361 & 30.0798 & 29.8518 & 29.9105 \\
6 & 1.20 & 30.8111 & 30.4513 & 30.4811 & 30.8843 & 30.7249 & 30.7543 & 30.7948 & 30.4355 & 30.4565 & 30.8631 & 30.7047 & 30.7297 \\
2a & 1.72 & 30.1909 & 29.7668 & 29.7146 & 30.1400 & 29.9133 & 29.9040 & 30.2413 & 29.8167 & 29.7652 & 30.1922 & 29.9612 & 29.9527 \\
2b & 1.49 & 30.9735 & 30.5532 & 30.5721 & 30.9764 & 30.6747 & 30.7073 & 30.8447 & 30.4225 & 30.4363 & 30.8453 & 30.5495 & 30.5809 \\
3a & 1.48 & 30.5782 & 30.1243 & 30.0420 & 30.5822 & 30.3396 & 30.3066 & 30.5729 & 30.1209 & 30.0350 & 30.5754 & 30.3344 & 30.3006 \\
3b & 1.38 & 30.7104 & 30.2964 & 30.2059 & 30.6542 & 30.4239 & 30.3441 & 30.6734 & 30.2586 & 30.1633 & 30.6173 & 30.3871 & 30.3047 \\
4a & 1.45 & 30.4702 & 30.1391 & 30.1134 & 30.5203 & 30.3639 & 30.3247 & 30.4380 & 30.1078 & 30.0693 & 30.4812 & 30.3278 & 30.2824 \\
5a & 1.87 & 30.4687 & 30.0032 & 29.9564 & 30.6037 & 30.2958 & 30.2914 & 30.4494 & 29.9840 & 29.9356 & 30.5818 & 30.2751 & 30.2704 \\
\hline
\end{tabular}

$\begin{array}{llllll}118.5293 & 118.7645 & 118.6065 & 107.8845 & 108.1427 & 107.3227\end{array}$ $\begin{array}{llllll}159.1709 & 160.3449 & 159.2344 & 151.3489 & 150.9719 & 150.4651\end{array}$ $\begin{array}{llllll}164.7520 & 165.9581 & 164.1422 & 156.6151 & 156.3859 & 155.4472\end{array}$ $\begin{array}{llllll}164.1326 & 164.9868 & 163.8060 & 155.2762 & 155.2446 & 155.0368\end{array}$ $\begin{array}{llllll}167.3716 & 168.7209 & 168.6341 & 160.2603 & 159.9466 & 159.5866\end{array}$ $\begin{array}{lllllll}144.3741 & 154.9474 & 155.7195 & 153.8283 & 144.8335 & 144.6876 & 144.5292\end{array}$ $\begin{array}{lllllll}159.5880 & 167.8839 & 169.2792 & 169.3618 & 160.8194 & 160.4957 & 159.7838\end{array}$ $\begin{array}{lllllll}156.2900 & 164.0019 & 165.2710 & 164.8542 & 156.9032 & 156.6570 & 156.4119\end{array}$ $\begin{array}{lllllll}142.1818 & 152.2120 & 152.6500 & 152.0009 & 142.7539 & 142.7759 & 142.2088\end{array}$ $\begin{array}{lllllll}167.4360 & 174.2482 & 175.9311 & 176.6785 & 168.2084 & 168.2086 & 167.7958\end{array}$ $\begin{array}{lllllll}166.9329 & 173.3451 & 175.0252 & 174.7307 & 167.9971 & 167.6876 & 167.1266\end{array}$ $\begin{array}{lllllll}162.3397 & 169.1003 & 170.6641 & 171.1155 & 163.1549 & 162.8820 & 162.5863\end{array}$ $\begin{array}{lllllll}160.0981 & 166.9790 & 168.5168 & 168.8667 & 160.6519 & 160.3802 & 160.3574\end{array}$ $\begin{array}{lllllll}139.5945 & 150.4096 & 151.1213 & 150.6173 & 140.6994 & 140.4933 & 139.8026\end{array}$ $\begin{array}{lllllll}144.8156 & 153.8061 & 154.1639 & 153.6751 & 145.1743 & 145.1842 & 144.7693\end{array}$ 


\begin{tabular}{llllllllllllll}
\hline 5b & 1.26 & 30.7696 & 30.3909 & 30.3545 & 30.7358 & 30.5575 & 30.5350 & 30.7554 & 30.3765 & 30.3356 & 30.7221 & 30.5441 & 30.5183 \\
7a & 1.50 & 30.1505 & 29.7093 & 29.6132 & 30.0917 & 29.8761 & 29.8621 & 30.1862 & 29.7459 & 29.6512 & 30.1295 & 29.9128 & 29.8984 \\
7b & 1.29 & 30.8205 & 30.4519 & 30.3462 & 30.7317 & 30.5810 & 30.5220 & 30.7966 & 30.4275 & 30.3154 & 30.7058 & 30.5556 & 30.4926 \\
8a & 1.85 & 29.9031 & 29.4879 & 29.4857 & 29.8838 & 29.6780 & 29.7074 & 29.9784 & 29.5638 & 29.5684 & 29.9555 & 29.7480 & 29.7834 \\
8b & 1.06 & 31.0622 & 30.7030 & 30.7158 & 31.0460 & 30.8795 & 30.8462 & 31.0237 & 30.6640 & 30.6678 & 31.0038 & 30.8382 & 30.7997 \\
10 & 0.80 & 31.3066 & 30.8798 & 30.8053 & 31.2334 & 30.9905 & 30.9706 & 31.3045 & 30.8768 & 30.8043 & 31.2333 & 30.9898 & 30.9707 \\
11 & 0.85 & 31.3169 & 30.8551 & 30.8300 & 31.3503 & 31.0503 & 31.0491 & 31.2799 & 30.8182 & 30.7911 & 31.3125 & 31.0150 & 31.0146 \\
12 & 1.09 & 30.9971 & 30.5584 & 30.5078 & 30.9858 & 30.7329 & 30.7146 & 30.9864 & 30.5467 & 30.4937 & 30.9744 & 30.7201 & 30.7006 \\
13 & 1.07 & 30.9141 & 30.5034 & 30.4207 & 30.9088 & 30.7017 & 30.6411 & 30.9574 & 30.5461 & 30.4656 & 30.9518 & 30.7420 & 30.6806 \\
\hline
\end{tabular}

\section{Compound 72}

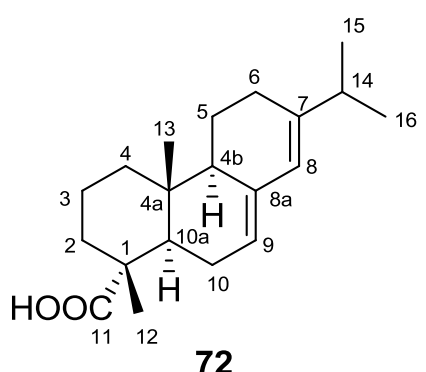

\begin{tabular}{|c|c|c|c|c|c|c|c|c|c|c|c|c|c|}
\hline & \multirow[b]{3}{*}{ Exp } & \multicolumn{12}{|c|}{ B3LYP } \\
\hline & & \multicolumn{6}{|c|}{ Gas phase } & \multicolumn{6}{|c|}{ PCM } \\
\hline & & 6-31G* & 6-31G** & $6-31+\mathrm{G} * *$ & 6-311G* & $6-311 G * *$ & $6-311+G * *$ & 6-31G* & 6-31G** & $6-31+G * *$ & 6-311G* & $6-311 G * *$ & $6-311+\mathrm{G} * *$ \\
\hline \multicolumn{14}{|l|}{${ }^{13} \mathrm{C}$} \\
\hline 11 & 184.8 & 20.7194 & 20.4682 & 17.3772 & -1.5140 & -1.4953 & -3.8355 & 19.1910 & 18.9322 & 15.5063 & -3.3078 & -3.3321 & -6.0150 \\
\hline 7 & 145.3 & 50.9645 & 50.8278 & 48.3718 & 30.0646 & 29.8991 & 29.2043 & 49.4428 & 49.3022 & 46.6082 & 28.2360 & 28.0810 & 27.2781 \\
\hline $8 a$ & 135.6 & 59.5127 & 59.3727 & 57.0046 & 39.4499 & 39.3688 & 37.8273 & 59.1959 & 59.0553 & 56.5504 & 39.0689 & 38.9890 & 37.3184 \\
\hline 8 & 122.4 & 68.3343 & 69.1207 & 69.4922 & 51.9768 & 51.3751 & 50.9667 & 69.3005 & 70.0820 & 70.5465 & 53.0405 & 52.4430 & 52.1199 \\
\hline 9 & 120.5 & 70.8094 & 71.7263 & 71.8575 & 54.6877 & 54.2828 & 54.1351 & 71.2281 & 72.1430 & 72.3512 & 55.0421 & 54.6517 & 54.5992 \\
\hline $4 b$ & 50.9 & 138.0437 & 138.5906 & 137.7595 & 127.1995 & 126.9539 & 126.4331 & 138.1722 & 138.7147 & 137.9029 & 127.3602 & 127.1056 & 126.5962 \\
\hline 1 & 46.3 & 141.8347 & 142.1306 & 141.4914 & 131.3614 & 131.3509 & 129.1441 & 141.5944 & 141.8922 & 141.1828 & 131.0651 & 131.0439 & 128.7436 \\
\hline $10 a$ & 44.9 & 143.6015 & 144.2537 & 144.4579 & 133.1528 & 133.0088 & 132.1538 & 143.3991 & 144.0542 & 144.1803 & 132.9028 & 132.7702 & 131.9063 \\
\hline 4 & 38.3 & 150.2793 & 151.4897 & 151.1479 & 141.0897 & 140.7406 & 140.5378 & 150.5132 & 151.7178 & 151.4226 & 141.3988 & 141.0396 & 140.8459 \\
\hline 2 & 37.2 & 151.8892 & 153.0776 & 151.6618 & 142.1479 & 141.9389 & 141.4986 & 151.9177 & 153.0974 & 151.6347 & 142.2004 & 141.9791 & 141.5214 \\
\hline 14 & 34.9 & 151.0987 & 152.0187 & 151.0400 & 140.4994 & 140.3596 & 139.8303 & 151.1818 & 152.0997 & 151.1332 & 140.6343 & 140.4781 & 139.9639 \\
\hline $4 a$ & 34.4 & 152.0049 & 152.4437 & 152.3284 & 141.9562 & 142.1384 & 141.8327 & 151.9743 & 152.4151 & 152.2779 & 141.9382 & 142.1189 & 141.7971 \\
\hline 6 & 27.5 & 162.8370 & 164.0320 & 163.1540 & 154.4919 & 154.1331 & 154.0508 & 162.9221 & 164.1119 & 163.1877 & 154.5428 & 154.1755 & 154.0736 \\
\hline 10 & 25.6 & 161.9974 & 163.2632 & 163.0683 & 153.4790 & 153.1834 & 153.0847 & 162.0645 & 163.3291 & 163.1183 & 153.5349 & 153.2293 & 153.1150 \\
\hline 5 & 22.5 & 164.8485 & 166.2734 & 165.3237 & 156.6233 & 156.4371 & 156.5197 & 164.9252 & 166.3478 & 165.3475 & 156.6719 & 156.4833 & 156.5388 \\
\hline 15 & 21.4 & 168.0651 & 169.8416 & 169.9496 & 160.9358 & 160.8538 & 160.5077 & 168.2884 & 170.0561 & 170.1801 & 161.1984 & 161.0931 & 160.7421 \\
\hline 16 & 20.9 & 168.2068 & 169.9329 & 170.3902 & 160.8287 & 160.7718 & 160.0588 & 168.4439 & 170.1608 & 170.6204 & 161.1075 & 161.0259 & 160.3066 \\
\hline 3 & 18.0 & 168.7142 & 170.1986 & 169.7908 & 160.6396 & 160.4765 & 159.9173 & 168.8906 & 170.3748 & 169.9795 & 160.8519 & 160.6845 & 160.1098 \\
\hline 12 & 16.7 & 170.6532 & 172.5153 & 173.2640 & 164.5394 & 164.4132 & 164.2775 & 170.8467 & 172.7023 & 173.4896 & 164.8001 & 164.6559 & 164.5142 \\
\hline 13 & 14.0 & 173.4123 & 175.3490 & 176.1674 & 167.9738 & 167.8527 & 168.4661 & 173.6080 & 175.5353 & 176.3986 & 168.2262 & 168.0867 & 168.7243 \\
\hline \multicolumn{14}{|l|}{${ }^{1} \mathrm{H}$} \\
\hline 8 & 5.77 & 26.5223 & 25.8940 & 25.5790 & 26.3591 & 25.9008 & 25.8066 & 26.5129 & 25.8843 & 25.5551 & 26.3292 & 25.8773 & 25.7763 \\
\hline
\end{tabular}




\begin{tabular}{cccccccccccccc}
\hline 9 & 5.38 & 26.9176 & 26.3208 & 26.1328 & 26.7494 & 26.3338 & 26.3822 & 26.8933 & 26.2963 & 26.0973 & 26.7024 & 26.2918 & 26.3398 \\
4b & 1.94 & 30.0549 & 29.7444 & 29.5749 & 30.1190 & 29.9643 & 29.9096 & 30.0333 & 29.7236 & 29.5365 & 30.0903 & 29.9380 & 29.8704 \\
10a & 2.07 & 29.7033 & 29.3592 & 29.2297 & 29.7597 & 29.6443 & 29.5887 & 29.7564 & 29.4138 & 29.2752 & 29.8082 & 29.6895 & 29.6261 \\
4a & 1.88 & 30.2981 & 29.8747 & 29.8978 & 30.2934 & 30.0660 & 30.0702 & 30.2606 & 29.8363 & 29.8524 & 30.2548 & 30.0264 & 30.0273 \\
4b & 1.15 & 30.8960 & 30.5397 & 30.4627 & 30.8771 & 30.7156 & 30.7113 & 30.8623 & 30.5066 & 30.4205 & 30.8410 & 30.6807 & 30.6713 \\
2a & 1.80 & 30.5252 & 30.1170 & 30.0894 & 30.5136 & 30.3059 & 30.2798 & 30.4892 & 30.0778 & 30.0430 & 30.4751 & 30.2634 & 30.2335 \\
2b & 1.67 & 30.2267 & 29.8359 & 29.6220 & 30.1733 & 30.0133 & 29.9322 & 30.2301 & 29.8404 & 29.6231 & 30.1789 & 30.0177 & 29.9330 \\
14 & 2.22 & 29.8657 & 29.4389 & 29.3866 & 29.8619 & 29.6546 & 29.5905 & 29.8352 & 29.4069 & 29.3497 & 29.8215 & 29.6141 & 29.5495 \\
$10 \mathrm{a}$ & 2.07 & 29.9300 & 29.4347 & 29.4801 & 30.0083 & 29.7184 & 29.7797 & 29.8910 & 29.3939 & 29.4335 & 29.9676 & 29.6763 & 29.7336 \\
$10 \mathrm{~b}$ & 1.88 & 30.3682 & 29.9212 & 29.8554 & 30.3105 & 30.0485 & 30.1038 & 30.4108 & 29.9642 & 29.8967 & 30.3419 & 30.0804 & 30.1385 \\
$5 \mathrm{a}$ & 1.80 & 30.5783 & 30.1264 & 30.0513 & 30.5032 & 30.2315 & 30.1660 & 30.5207 & 30.0664 & 29.9792 & 30.4401 & 30.1666 & 30.0967 \\
$5 \mathrm{~b}$ & 1.22 & 30.8359 & 30.4414 & 30.3254 & 30.9211 & 30.7417 & 30.6283 & 30.8517 & 30.4573 & 30.3414 & 30.9410 & 30.7623 & 30.6441 \\
15 & 1.00 & 31.1292 & 30.6936 & 30.5970 & 31.1029 & 30.8700 & 30.8135 & 31.1189 & 30.6822 & 30.5878 & 31.0935 & 30.8601 & 30.8043 \\
16 & 1.01 & 31.1371 & 30.7112 & 30.6158 & 31.1103 & 30.8946 & 30.8372 & 31.1267 & 30.6992 & 30.6059 & 31.1015 & 30.8848 & 30.8272 \\
3 & 1.58 & 30.5774 & 30.1401 & 30.0849 & 30.5654 & 30.3347 & 30.3087 & 30.5416 & 30.1034 & 30.0457 & 30.5324 & 30.3007 & 30.2725 \\
12 & 1.25 & 30.9198 & 30.4475 & 30.4473 & 30.9492 & 30.6759 & 30.6431 & 30.9226 & 30.4496 & 30.4502 & 30.9511 & 30.6764 & 30.6431 \\
13 & 0.83 & 31.1870 & 30.7297 & 30.7106 & 31.2919 & 31.0423 & 30.9983 & 31.1827 & 30.7252 & 30.7076 & 31.2909 & 31.0411 & 30.9973 \\
6 & 2.07 & 30.1400 & 29.6831 & 29.5326 & 30.1035 & 29.8484 & 29.7697 & 30.0941 & 29.6359 & 29.4832 & 30.0568 & 29.8020 & 29.7216 \\
\hline
\end{tabular}

\begin{tabular}{|c|c|c|c|c|c|c|c|c|c|c|c|c|c|}
\hline & \multirow[b]{3}{*}{ Exp } & \multicolumn{12}{|c|}{ mPW1PW91 } \\
\hline & & \multicolumn{6}{|c|}{ Gas phase } & \multicolumn{6}{|c|}{ PCM } \\
\hline & & 6-31G* & 6-31G** & $6-31+G * *$ & 6-311G* & $6-311 \mathrm{G} * *$ & $6-311+G^{* *}$ & $6-31 G *$ & $6-31 G^{* *}$ & $6-31+G^{* *}$ & 6-311G* & $6-311 G * *$ & $6-311+\mathrm{G}^{* *}$ \\
\hline \multicolumn{14}{|l|}{${ }^{13} \mathrm{C}$} \\
\hline 11 & 184.8 & 23.2468 & 22.9894 & 20.4936 & 3.7429 & 3.7673 & 1.7269 & 21.7048 & 21.4395 & 18.6506 & 1.9632 & 1.9433 & -0.3941 \\
\hline 7 & 145.3 & 54.5338 & 54.3490 & 52.3118 & 36.0360 & 35.8276 & 35.1765 & 52.8858 & 52.6973 & 50.4416 & 34.0939 & 33.8997 & 33.1627 \\
\hline $8 a$ & 135.6 & 62.9596 & 62.7874 & 60.6856 & 45.4109 & 45.2953 & 43.9015 & 62.6681 & 62.4953 & 60.2759 & 45.0639 & 44.9496 & 43.4338 \\
\hline 8 & 122.4 & 71.2186 & 71.9647 & 72.5218 & 56.7602 & 56.1508 & 55.6100 & 72.2692 & 73.0100 & 73.6324 & 57.8708 & 57.2664 & 56.8023 \\
\hline 9 & 120.5 & 73.7438 & 74.6020 & 74.7295 & 59.3534 & 58.9140 & 58.7362 & 74.1069 & 74.9639 & 75.1612 & 59.6240 & 59.2019 & 59.1171 \\
\hline $4 b$ & 50.9 & 143.6540 & 144.1256 & 143.3512 & 133.8545 & 133.6071 & 133.1481 & 143.7902 & 144.2577 & 143.5045 & 134.0069 & 133.7510 & 133.3048 \\
\hline 1 & 46.3 & 146.8570 & 147.1438 & 146.6498 & 137.4025 & 137.3875 & 135.3749 & 146.6119 & 146.9006 & 146.3514 & 137.1051 & 137.0804 & 134.9949 \\
\hline 10a & 44.9 & 149.2328 & 149.8195 & 150.1286 & 139.6273 & 139.5053 & 138.6557 & 149.0405 & 149.6291 & 149.8720 & 139.3898 & 139.2792 & 138.4228 \\
\hline 4 & 38.3 & 155.6852 & 156.7770 & 156.3708 & 147.2479 & 146.9049 & 146.5864 & 155.9438 & 157.0308 & 156.6670 & 147.5595 & 147.2082 & 146.8994 \\
\hline 2 & 37.2 & 157.3755 & 158.4430 & 156.8616 & 148.3659 & 148.1629 & 147.7383 & 157.4093 & 158.4697 & 156.8539 & 148.4107 & 148.1980 & 147.7625 \\
\hline 14 & 34.9 & 156.2682 & 157.1359 & 156.2287 & 146.6598 & 146.5060 & 146.0293 & 156.3675 & 157.2333 & 156.3361 & 146.7880 & 146.6197 & 146.1627 \\
\hline $4 a$ & 34.4 & 157.2195 & 157.6469 & 157.3918 & 147.9876 & 148.1584 & 147.8280 & 157.1909 & 157.6201 & 157.3475 & 147.9712 & 148.1407 & 147.7974 \\
\hline 6 & 27.5 & 167.1997 & 168.3090 & 167.6618 & 159.7344 & 159.3755 & 159.1541 & 167.3025 & 168.4058 & 167.7072 & 159.7856 & 159.4182 & 159.1768 \\
\hline 10 & 25.6 & 166.5785 & 167.7527 & 167.6543 & 158.8800 & 158.5828 & 158.4460 & 166.6640 & 167.8362 & 167.7248 & 158.9427 & 158.6355 & 158.4853 \\
\hline 5 & 22.5 & 169.7910 & 171.1076 & 170.2785 & 162.3013 & 162.1184 & 162.1412 & 169.8776 & 171.1916 & 170.3051 & 162.3491 & 162.1638 & 162.1615 \\
\hline 15 & 21.4 & 172.3843 & 174.0290 & 174.0673 & 165.7743 & 165.6972 & 165.3220 & 172.6466 & 174.2821 & 174.3365 & 166.0481 & 165.9494 & 165.5727 \\
\hline 16 & 20.9 & 172.5313 & 174.1244 & 174.3513 & 165.6462 & 165.5966 & 164.9519 & 172.8037 & 174.3870 & 174.6147 & 165.9315 & 165.8591 & 165.2098 \\
\hline 3 & 18.0 & 173.4980 & 174.8757 & 174.4809 & 166.1964 & 166.0106 & 165.4198 & 173.7046 & 175.0816 & 174.6979 & 166.4281 & 166.2374 & 165.6333 \\
\hline 12 & 16.7 & 175.0379 & 176.7578 & 177.4944 & 169.3053 & 169.1953 & 168.9774 & 175.2626 & 176.9756 & 177.7458 & 169.5780 & 169.4514 & 169.2211 \\
\hline $\begin{array}{l}13 \\
{ }^{1} \mathbf{H}\end{array}$ & 14.0 & 177.5923 & 179.3752 & 179.6768 & 172.5060 & 172.3916 & 172.9772 & 177.8159 & 179.5895 & 179.9328 & 172.7673 & 172.6360 & 173.2424 \\
\hline 8 & 5.77 & 26.3204 & 25.7003 & 25.3889 & 26.1860 & 25.7042 & 25.6277 & 26.3024 & 25.6818 & 25.3592 & 26.1494 & 25.6745 & 25.5911 \\
\hline
\end{tabular}




\begin{tabular}{|c|c|c|c|c|c|c|c|c|c|c|c|c|c|}
\hline 9 & 5.38 & 26.7535 & 26.1556 & 25.9908 & 26.6109 & 26.1684 & 26.2302 & 26.7156 & 26.1173 & 25.9445 & 26.5525 & 26.1153 & 26.1776 \\
\hline $4 b$ & 1.94 & 30.0007 & 29.6886 & 29.5276 & 29.9842 & 29.8419 & 29.8019 & 29.9801 & 29.6689 & 29.4903 & 29.9580 & 29.8180 & 29.7647 \\
\hline $10 a$ & 2.07 & 29.6495 & 29.3122 & 29.1588 & 29.6230 & 29.5234 & 29.4447 & 29.7068 & 29.3709 & 29.2089 & 29.6764 & 29.5728 & 29.4860 \\
\hline $4 a$ & 1.88 & 30.2666 & 29.8478 & 29.8553 & 30.2158 & 29.9912 & 29.9790 & 30.2242 & 29.8043 & 29.8061 & 30.1735 & 29.9476 & 29.9327 \\
\hline $4 b$ & 1.15 & 30.8501 & 30.4967 & 30.4141 & 30.7746 & 30.6181 & 30.6097 & 30.8146 & 30.4615 & 30.3716 & 30.7389 & 30.5832 & 30.5696 \\
\hline $2 a$ & 1.80 & 30.4804 & 30.0767 & 30.0380 & 30.4357 & 30.2269 & 30.1863 & 30.4391 & 30.0322 & 29.9874 & 30.3925 & 30.1797 & 30.1365 \\
\hline $2 b$ & 1.67 & 30.1833 & 29.7972 & 29.5828 & 30.0762 & 29.9268 & 29.8426 & 30.1878 & 29.8022 & 29.5865 & 30.0840 & 29.9327 & 29.8451 \\
\hline 14 & 2.22 & 29.7892 & 29.3704 & 29.3005 & 29.7295 & 29.5294 & 29.4670 & 29.7541 & 29.3333 & 29.2590 & 29.6861 & 29.4856 & 29.4220 \\
\hline $10 a$ & 2.07 & 29.9180 & 29.4198 & 29.4169 & 29.9230 & 29.6401 & 29.6916 & 29.8757 & 29.3755 & 29.3684 & 29.8794 & 29.5951 & 29.6436 \\
\hline $10 b$ & 1.88 & 30.3148 & 29.8653 & 29.8021 & 30.2249 & 29.9602 & 30.0075 & 30.3539 & 29.9048 & 29.8401 & 30.2528 & 29.9887 & 30.0387 \\
\hline $5 a$ & 1.80 & 30.5346 & 30.0848 & 30.0158 & 30.4330 & 30.1517 & 30.0920 & 30.4674 & 30.0150 & 29.9357 & 30.3626 & 30.0792 & 30.0157 \\
\hline $5 b$ & 1.22 & 30.7981 & 30.4014 & 30.2582 & 30.8229 & 30.6436 & 30.5179 & 30.8185 & 30.4220 & 30.2780 & 30.8461 & 30.6676 & 30.5369 \\
\hline 15 & 1.00 & 31.0752 & 30.6407 & 30.5363 & 31.0158 & 30.7787 & 30.7206 & 31.0618 & 30.6262 & 30.5240 & 31.0032 & 30.7655 & 30.7082 \\
\hline 16 & 1.01 & 31.0901 & 30.6642 & 30.5662 & 31.0293 & 30.8085 & 30.7493 & 31.0765 & 30.6490 & 30.5526 & 31.0171 & 30.7952 & 30.7358 \\
\hline 3 & 1.58 & 30.5397 & 30.1037 & 30.0420 & 30.4837 & 30.2540 & 30.2177 & 30.4998 & 30.0628 & 29.9993 & 30.4467 & 30.2160 & 30.1781 \\
\hline 12 & 1.25 & 30.8616 & 30.3898 & 30.3877 & 30.8565 & 30.5792 & 30.5485 & 30.8616 & 30.3891 & 30.3878 & 30.8554 & 30.5769 & 30.5457 \\
\hline 13 & 0.83 & 31.1418 & 30.6840 & 30.6557 & 31.1986 & 30.9483 & 30.9100 & 31.1370 & 30.6791 & 30.6525 & 31.1967 & 30.9462 & 30.9082 \\
\hline 6 & 2.07 & 30.1055 & 29.6483 & 29.5005 & 30.0239 & 29.7709 & 29.6873 & 30.0529 & 29.5941 & 29.4439 & 29.9710 & 29.7182 & 29.6329 \\
\hline
\end{tabular}


Values of the $[\mu, \sigma, v]$ statistical parameters of DP4+

$$
\begin{aligned}
& \mathrm{P}(I)=\frac{\prod_{k=1}^{N}\left[1-T_{s}^{v}\left(\mathrm{e}_{s, k}^{i} / \sigma_{s}\right)\right]\left[1-T_{u-s p x}\left(\left(\mathrm{e}_{u, k}^{i} \mu_{u-s p x}\right) / \sigma_{u-s p x}\right)\right]}{\sum_{j=1}^{m} \prod_{k=1}^{N}\left[1-T_{s}^{v}{ }_{s}\left(\mathrm{e}_{s, k}^{j} / \sigma_{s}\right)\right]\left[1-T_{u-s p x}^{v}\left(\left(\mathrm{e}_{u, k^{-}} \mu_{u-s p x}\right) / \sigma_{u-s p x}\right)\right]} \\
& T^{v}, \sigma, \mu \text {, and } e^{i} \text { computed from B3LYP/6-31G* geometries }
\end{aligned}
$$

\begin{tabular}{|c|c|c|c|c|c|c|c|c|}
\hline Level of theory & $\sigma_{s}$ & $v_{s}$ & $\mu_{u-s p 2}$ & $\sigma_{u-s p 2}$ & $v_{u-s p 2}$ & $\mu_{u-s p 3}$ & $\sigma_{u-s p 3}$ & $v_{u-s p 3}$ \\
\hline \multicolumn{9}{|c|}{${ }^{13} \mathrm{C}$ series } \\
\hline B3LYP/6-31G* & 1.581 & 5.970 & -6.157 & 2.488 & 6.532 & 1.302 & 1.648 & 6.289 \\
\hline B3LYP/6-31G** & 1.852 & 8.736 & -4.616 & 2.455 & 6.714 & 2.047 & 1.765 & 7.316 \\
\hline B3LYP/6-31+G** & 2.087 & 10.386 & -2.682 & 2.294 & 8.049 & 3.584 & 1.899 & 7.184 \\
\hline B3LYP/6-311G* & 1.921 & 7.164 & 5.918 & 2.349 & 5.859 & 4.733 & 2.285 & 15.728 \\
\hline B3LYP/6-311G** & 1.837 & 6.341 & 6.538 & 2.241 & 5.026 & 5.265 & 2.212 & 14.147 \\
\hline B3LYP/6-311+G** & 1.833 & 7.386 & 7.371 & 2.252 & 6.189 & 5.157 & 2.294 & 18.584 \\
\hline B3LYP/6-31G* (PCM) & 1.525 & 6.087 & -5.226 & 1.838 & 4.625 & 1.596 & 1.594 & 6.065 \\
\hline B3LYP/6-31G** (PCM) & 1.784 & 8.777 & -3.696 & 1.837 & 4.860 & 2.336 & 1.752 & 7.415 \\
\hline B3LYP/6-31+G** (PCM) & 2.034 & 10.172 & -1.417 & 1.994 & 6.562 & 4.044 & 1.895 & 7.359 \\
\hline B3LYP/6-311G* (PCM) & 1.841 & 7.315 & 7.279 & 2.647 & 14.742 & 5.165 & 2.355 & 20.697 \\
\hline B3LYP/6-311G** (PCM) & 1.738 & 6.366 & 7.809 & 2.409 & 8.781 & 5.678 & 2.271 & 17.407 \\
\hline B3LYP/6-311+G** (PCM) & 1.803 & 7.943 & 8.919 & 3.064 & 77.024 & 5.599 & 2.395 & 23.235 \\
\hline mPW1PW91/6-31G* & 1.267 & 4.192 & -4.787 & 2.410 & 7.026 & 0.745 & 1.484 & 5.691 \\
\hline mPW1PW91/6-31G** & 1.446 & 5.325 & -3.346 & 2.304 & 6.531 & 1.410 & 1.516 & 6.070 \\
\hline mPW1PW91/6-31+G** & 1.598 & 6.113 & -2.185 & 2.105 & 6.551 & 2.431 & 1.605 & 6.039 \\
\hline mPW1PW91/6-311G* & 1.603 & 4.905 & 6.084 & 2.040 & 4.276 & 4.284 & 1.740 & 6.303 \\
\hline mPW1PW91/6-311G** & 1.547 & 4.484 & 6.781 & 2.048 & 4.122 & 4.851 & 1.689 & 6.178 \\
\hline mPW1PW91/6-311+G** & 1.582 & 5.761 & 7.515 & 1.965 & 4.839 & 4.748 & 1.814 & 8.744 \\
\hline mPW1PW91/6-31G* (PCM) & 1.227 & 4.431 & -3.787 & 1.724 & 4.484 & 1.087 & 1.433 & 5.558 \\
\hline mPW1PW91/6-31G** (PCM) & 1.391 & 5.576 & -2.365 & 1.675 & 4.673 & 1.742 & 1.508 & 6.216 \\
\hline mPW1PW91/6-31+G** (PCM) & 1.557 & 6.227 & -0.920 & 1.748 & 5.364 & 2.909 & 1.600 & 6.269 \\
\hline mPW1PW91/6-311G* (PCM) & 1.560 & 5.798 & 6.321 & 2.310 & 9.605 & 3.641 & 1.858 & 9.397 \\
\hline mPW1PW91/6-311G** (PCM) & 1.491 & 5.226 & 6.885 & 2.144 & 6.663 & 4.154 & 1.787 & 8.592 \\
\hline mPW1PW91/6-311+G** (PCM) & 1.575 & 6.671 & 7.911 & 2.702 & 46.563 & 4.072 & 1.935 & 11.156 \\
\hline \multicolumn{9}{|c|}{${ }^{1} \mathrm{H}$ series } \\
\hline B3LYP/6-31G* & 0.140 & 5.942 & -0.172 & 0.194 & 13.808 & -0.056 & 0.169 & 6.950 \\
\hline B3LYP/6-31G** & 0.128 & 4.811 & 0.016 & 0.177 & 9.198 & -0.072 & 0.149 & 4.828 \\
\hline B3LYP/6-31+G** & 0.127 & 4.703 & 0.139 & 0.163 & 6.839 & -0.060 & 0.134 & 4.047 \\
\hline B3LYP/6-311G* & 0.116 & 3.295 & -0.035 & 0.169 & 10.935 & -0.029 & 0.149 & 3.925 \\
\hline B3LYP/6-311G** & 0.113 & 3.197 & 0.112 & 0.149 & 7.274 & -0.072 & 0.130 & 3.342 \\
\hline B3LYP/6-311+G** & 0.119 & 3.409 & 0.163 & 0.145 & 5.785 & -0.051 & 0.135 & 3.531 \\
\hline B3LYP/6-31G* (PCM) & 0.129 & 6.408 & -0.091 & 0.172 & 10.337 & -0.013 & 0.151 & 7.441 \\
\hline B3LYP/6-31G** (PCM) & 0.115 & 4.897 & 0.097 & 0.149 & 7.268 & -0.027 & 0.130 & 4.715 \\
\hline B3LYP/6-31+G** (PCM) & 0.110 & 4.273 & 0.238 & 0.132 & 5.347 & -0.003 & 0.119 & 3.972 \\
\hline B3LYP/6-311G* (PCM) & 0.103 & 3.228 & 0.063 & 0.148 & 8.854 & 0.019 & 0.126 & 3.466 \\
\hline B3LYP/6-311G** (PCM) & 0.095 & 2.850 & 0.206 & 0.124 & 5.987 & -0.022 & 0.105 & 2.756 \\
\hline B3LYP/6-311+G** (PCM) & 0.099 & 2.929 & 0.267 & 0.119 & 5.196 & 0.008 & 0.115 & 3.033 \\
\hline mPW1PW91/6-31G* & 0.128 & 5.030 & -0.044 & 0.185 & 13.042 & -0.065 & 0.156 & 6.129 \\
\hline mPW1PW91/6-31G** & 0.120 & 4.303 & 0.140 & 0.167 & 8.595 & -0.085 & 0.136 & 4.223 \\
\hline mPW1PW91/6-31+G** & 0.122 & 4.318 & 0.239 & 0.149 & 5.840 & -0.075 & 0.127 & 3.684 \\
\hline mPW1PW91/6-311G* & 0.113 & 3.160 & 0.305 & 0.168 & 10.205 & 0.239 & 0.148 & 3.878 \\
\hline mPW1PW91/6-311G** & 0.110 & 3.061 & 0.478 & 0.142 & 6.250 & 0.193 & 0.123 & 3.124 \\
\hline mPW1PW91/6-311+G** & 0.117 & 3.394 & 0.516 & 0.138 & 5.394 & 0.209 & 0.131 & 3.388 \\
\hline mPW1PW91/6-31G* (PCM) & 0.117 & 5.543 & 0.047 & 0.162 & 10.576 & -0.021 & 0.136 & 6.259 \\
\hline mPW1PW91/6-31G** (PCM) & 0.105 & 4.323 & 0.233 & 0.136 & 6.954 & -0.038 & 0.116 & 4.026 \\
\hline mPW1PW91/6-31+G** (PCM) & 0.104 & 3.893 & 0.347 & 0.118 & 4.911 & -0.018 & 0.112 & 3.651 \\
\hline mPW1PW91/6-311G* (PCM) & 0.101 & 3.157 & 0.133 & 0.147 & 8.764 & 0.009 & 0.125 & 3.536 \\
\hline mPW1PW91/6-311G** (PCM) & 0.093 & 2.784 & 0.297 & 0.117 & 5.315 & -0.039 & 0.098 & 2.599 \\
\hline mPW1PW91/6-311+G** (PCM) & 0.098 & 2.891 & 0.344 & 0.112 & 4.880 & -0.014 & 0.111 & 2.891 \\
\hline
\end{tabular}




\section{Compound 73a}<smiles>CCOC(=O)[C@H](C)[C@@H](O)c1ccccc1</smiles>

\begin{tabular}{|c|c|c|c|c|c|c|c|c|c|c|c|c|c|}
\hline \multirow{3}{*}{\multicolumn{2}{|c|}{ Exp }} & \multicolumn{12}{|c|}{ B3LYP } \\
\hline & & \multicolumn{6}{|c|}{ Gas phase } & \multicolumn{6}{|c|}{ PCM } \\
\hline & & 6-31G* & 6-31G** & $6-31+G * *$ & 6-311G* & $6-311 \mathrm{G} * *$ & $6-311+G * *$ & 6-31G* & 6-31G** & $6-31+G * *$ & 6-311G* & $6-311 \mathrm{G} * *$ & $6-311+G * *$ \\
\hline \multicolumn{14}{|c|}{ 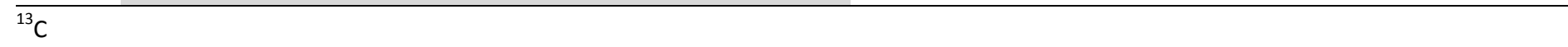 } \\
\hline 1 & 73.7 & 117.3852 & 117.9285 & 117.0934 & 106.1139 & 105.7994 & 105.3981 & 117.6212 & 118.1621 & 117.3211 & 106.3300 & 106.0059 & 105.6126 \\
\hline 2 & 46.5 & 142.3070 & 143.0319 & 141.7407 & 132.2790 & 132.0507 & 131.1076 & 142.4573 & 143.1766 & 141.9060 & 132.4294 & 132.1785 & 131.2214 \\
\hline 3 & 175.5 & 17.2455 & 17.1699 & 14.9769 & -5.8263 & -5.6437 & -7.0157 & 16.7222 & 16.6373 & 14.2557 & -6.4676 & -6.3283 & -7.8485 \\
\hline 4 & 11.0 & 177.4361 & 179.2199 & 180.0859 & 171.3702 & 171.5025 & 171.2463 & 177.6052 & 179.3780 & 180.2359 & 171.5430 & 171.6474 & 171.4079 \\
\hline 5 & 141.5 & 55.0216 & 54.7043 & 53.1603 & 35.2646 & 34.8075 & 33.5943 & 54.8403 & 54.5240 & 53.0048 & 34.9758 & 34.5552 & 33.3567 \\
\hline 6 & 125.9 & 70.1071 & 70.7544 & 69.5784 & 52.8133 & 52.1027 & 51.3155 & 70.2908 & 70.9304 & 69.7449 & 52.9274 & 52.2169 & 51.4430 \\
\hline 7 & 128.1 & 68.6502 & 69.2419 & 69.0637 & 51.3973 & 50.4245 & 49.4349 & 68.4977 & 69.0895 & 68.8188 & 51.1149 & 50.1740 & 49.2120 \\
\hline 8 & 127.3 & 69.9080 & 70.5170 & 69.9988 & 52.3751 & 51.5075 & 50.9547 & 69.7463 & 70.3585 & 69.7253 & 52.1026 & 51.2707 & 50.7405 \\
\hline 9 & 60.5 & 129.3816 & 130.6682 & 129.6931 & 118.6569 & 118.2592 & 117.8359 & 128.7953 & 130.0786 & 129.0613 & 118.0105 & 117.6072 & 117.1683 \\
\hline $\begin{array}{l}10 \\
{ }^{1} \mathrm{H}\end{array}$ & 13.9 & 174.8747 & 176.6831 & 176.9228 & 167.9320 & 167.8920 & 167.4887 & 175.1052 & 176.9052 & 177.1366 & 168.1779 & 168.1114 & 167.7194 \\
\hline 1 & 5.09 & 26.9691 & 26.5157 & 26.3921 & 26.8546 & 26.6796 & 26.6260 & 26.9008 & 26.4439 & 26.2956 & 26.7699 & 26.5918 & 26.5243 \\
\hline 2 & 2.77 & 29.7730 & 29.2951 & 29.0969 & 29.7107 & 29.4765 & 29.3194 & 29.6618 & 29.1800 & 28.9569 & 29.5904 & 29.3542 & 29.1864 \\
\hline 4 & 1.12 & 31.1834 & 30.7241 & 30.6458 & 31.2144 & 30.9679 & 30.8939 & 31.1777 & 30.7174 & 30.6424 & 31.2094 & 30.9618 & 30.8901 \\
\hline 6 & 7.29 & 24.8755 & 24.2163 & 24.0576 & 24.7638 & 24.3627 & 24.2574 & 24.8366 & 24.1763 & 24.0041 & 24.7131 & 24.3131 & 24.2011 \\
\hline 7 & 7.29 & 24.9778 & 24.3659 & 24.1104 & 24.8264 & 24.4490 & 24.3884 & 24.8715 & 24.2597 & 23.9919 & 24.7080 & 24.3385 & 24.2707 \\
\hline 8 & 7.29 & 25.0603 & 24.4494 & 24.2907 & 24.9034 & 24.5385 & 24.4997 & 24.9493 & 24.3385 & 24.1758 & 24.7818 & 24.4247 & 24.3832 \\
\hline 9 & 4.12 & 28.0013 & 27.4755 & 27.4058 & 27.9939 & 27.6564 & 27.6866 & 27.9477 & 27.4241 & 27.3622 & 27.9463 & 27.6105 & 27.6435 \\
\hline 10 & 1.21 & 30.9494 & 30.4768 & 30.3556 & 30.8888 & 30.6058 & 30.5649 & 30.9123 & 30.4386 & 30.3135 & 30.8503 & 30.5683 & 30.5233 \\
\hline
\end{tabular}

\begin{tabular}{|c|c|c|c|c|c|c|c|c|c|c|c|c|c|}
\hline & \multirow[b]{3}{*}{ Exp } & \multicolumn{12}{|c|}{ mPW1PW91 } \\
\hline & & \multicolumn{6}{|c|}{ Gas phase } & \multicolumn{6}{|c|}{ PCM } \\
\hline & & 6-31G* & $6-31 G * *$ & $6-31+G * *$ & 6-311G* & $6-311 G * *$ & $6-311+\mathrm{G} * *$ & 6-31G* & $6-31 G * *$ & $6-31+G * *$ & 6-311G* & 6-311G** & $6-311+G * *$ \\
\hline \multicolumn{14}{|c|}{${ }^{13} \mathrm{C}$} \\
\hline 1 & 73.7 & 122.0063 & 122.5092 & 121.8306 & 112.3134 & 112.0131 & 111.5619 & 122.2311 & 122.7322 & 122.0482 & 112.4980 & 112.1901 & 111.7481 \\
\hline 2 & 46.5 & 147.0215 & 147.6956 & 146.6317 & 138.1488 & 137.9357 & 137.0377 & 147.1732 & 147.8430 & 146.8083 & 138.2903 & 138.0554 & 137.1521 \\
\hline 3 & 175.5 & 19.8212 & 19.7363 & 18.1353 & -0.4002 & -0.2244 & -1.4642 & 19.2871 & 19.1938 & 17.4246 & -1.0388 & -0.9065 & -2.2754 \\
\hline 4 & 11.0 & 181.6821 & 183.3368 & 184.0796 & 175.8971 & 176.0349 & 175.8117 & 181.8760 & 183.5200 & 184.2439 & 176.0785 & 176.1899 & 175.9850 \\
\hline 5 & 141.5 & 58.2427 & 57.8891 & 56.6957 & 40.9956 & 40.5136 & 39.3473 & 58.0566 & 57.7050 & 56.5234 & 40.7131 & 40.2701 & 39.1189 \\
\hline 6 & 125.9 & 72.5715 & 73.1605 & 72.0269 & 57.3863 & 56.6569 & 55.9017 & 72.7536 & 73.3347 & 72.1833 & 57.4903 & 56.7622 & 56.0199 \\
\hline
\end{tabular}




\begin{tabular}{cccccccccccccc}
\hline 7 & 128.1 & 71.1145 & 71.6170 & 71.7898 & 55.9698 & 54.9487 & 53.9564 & 70.9303 & 71.4333 & 71.5051 & 55.6444 & 54.6588 & 53.7004 \\
8 & 127.3 & 72.3155 & 72.8395 & 72.2887 & 56.9033 & 55.9901 & 55.3923 & 72.1180 & 72.6460 & 71.9763 & 56.5823 & 55.7078 & 55.1420 \\
9 & 60.5 & 133.4593 & 134.6973 & 133.9286 & 124.0160 & 123.6368 & 123.2497 & 132.8705 & 134.1064 & 133.2937 & 123.3710 & 122.9866 & 122.5906 \\
10 & 13.9 & 178.8275 & 180.5265 & 180.7591 & 172.4131 & 172.3807 & 171.9573 & 179.0862 & 180.7761 & 181.0102 & 172.6676 & 172.6085 & 172.1951 \\
${ }^{1} \mathrm{H}$ & & & & & & & & & & & & & \\
\\
1 & 5.09 & 26.9024 & 26.4496 & 26.3248 & 26.7720 & 26.5974 & 26.5323 & 26.8273 & 26.3707 & 26.2226 & 26.6831 & 26.5052 & 26.4272 \\
2 & 2.77 & 29.6972 & 29.2259 & 29.0316 & 29.5878 & 29.3624 & 29.2031 & 29.5803 & 29.1049 & 28.8883 & 29.4648 & 29.2369 & 29.0673 \\
4 & 1.12 & 31.1290 & 30.6661 & 30.5780 & 31.1335 & 30.8818 & 30.7997 & 31.1219 & 30.6579 & 30.5736 & 31.1268 & 30.8741 & 30.7946 \\
6 & 7.29 & 24.6704 & 24.0132 & 23.8844 & 24.5815 & 24.1526 & 24.0592 & 24.6211 & 23.9626 & 23.8218 & 24.5239 & 24.0962 & 23.9959 \\
7 & 7.29 & 24.7710 & 24.1550 & 23.9229 & 24.6477 & 24.2348 & 24.1813 & 24.6501 & 24.0341 & 23.7919 & 24.5180 & 24.1133 & 24.0529 \\
8 & 7.29 & 24.8521 & 24.2376 & 24.1198 & 24.7247 & 24.3238 & 24.2937 & 24.7263 & 24.1118 & 23.9922 & 24.5914 & 24.1987 & 24.1663 \\
9 & 4.12 & 27.9457 & 27.4209 & 27.3656 & 27.9174 & 27.5725 & 27.6075 & 27.8881 & 27.3651 & 27.3174 & 27.8666 & 27.5231 & 27.5608 \\
10 & 1.21 & 30.8914 & 30.4162 & 30.3022 & 30.8113 & 30.5185 & 30.4786 & 30.8508 & 30.3745 & 30.2568 & 30.7700 & 30.4782 & 30.4340 \\
\hline
\end{tabular}

\section{Compound 73b}<smiles>CCOC(=O)[C@H](C)[C@H](O)c1ccccc1</smiles>

\begin{tabular}{|c|c|c|c|c|c|c|c|c|c|c|c|c|c|}
\hline \multirow{3}{*}{\multicolumn{2}{|c|}{ Exp }} & \multicolumn{12}{|c|}{ B3LYP } \\
\hline & & \multicolumn{6}{|c|}{ Gas phase } & \multicolumn{6}{|c|}{ PCM } \\
\hline & & 6-31G* & $6-31 G * *$ & $6-31+G * *$ & 6-311G* & $6-311 G^{* *}$ & $6-311+G^{* *}$ & 6-31G* & $6-31 G * *$ & $6-31+G^{* *}$ & 6-311G* & $6-311 G^{* *}$ & $6-311+G * *$ \\
\hline${ }^{13} \mathrm{C}$ & & & & & & & & & & & & & \\
\hline 1 & 76.1 & 114.6727 & 115.1729 & 114.0883 & 102.7317 & 102.3819 & 101.8053 & 114.9274 & 115.4278 & 114.2732 & 102.9378 & 102.6031 & 102.0163 \\
\hline 2 & 47.1 & 142.3634 & 143.0928 & 142.2462 & 131.9749 & 131.7543 & 131.0534 & 142.3120 & 143.0366 & 142.1556 & 131.8875 & 131.6506 & 130.9058 \\
\hline 3 & 175.7 & 19.1922 & 19.1467 & 17.2037 & -3.8587 & -3.6162 & -4.9663 & 18.4541 & 18.4003 & 16.2584 & -4.7807 & -4.5728 & -6.0219 \\
\hline 4 & 14.3 & 171.1104 & 172.8492 & 173.2258 & 164.3297 & 164.3399 & 164.0273 & 171.3542 & 173.0788 & 173.4681 & 164.5991 & 164.5853 & 164.2705 \\
\hline 5 & 141.6 & 52.4598 & 52.1204 & 49.0136 & 32.2004 & 31.8169 & 31.2551 & 52.4746 & 52.1345 & 49.0397 & 32.1458 & 31.7635 & 31.2764 \\
\hline 6 & 126.5 & 70.7229 & 71.3691 & 70.9115 & 53.6243 & 52.8226 & 51.9618 & 70.9408 & 71.5807 & 71.1205 & 53.7653 & 52.9731 & 52.1389 \\
\hline 7 & 128.2 & 68.6004 & 69.1996 & 68.5638 & 51.3456 & 50.3940 & 49.7493 & 68.4012 & 68.9989 & 68.2611 & 51.0026 & 50.0811 & 49.4418 \\
\hline 8 & 127.8 & 70.1060 & 70.7233 & 70.2812 & 52.6032 & 51.7371 & 51.1633 & 69.8494 & 70.4697 & 69.9212 & 52.2159 & 51.3932 & 50.8169 \\
\hline 9 & 60.6 & 130.1395 & 131.4728 & 130.4692 & 119.6235 & 119.2455 & 118.8289 & 129.6960 & 131.0263 & 129.9252 & 119.1083 & 118.7152 & 118.2672 \\
\hline $\begin{array}{l}10 \\
{ }^{1} \mathrm{H}\end{array}$ & 14.0 & 174.8246 & 176.6485 & 177.0610 & 168.0698 & 168.0555 & 167.6007 & 174.9226 & 176.7429 & 177.1545 & 168.1877 & 168.1559 & 167.6980 \\
\hline 1 & 4.75 & 27.4569 & 27.0159 & 26.8522 & 27.4085 & 27.2185 & 27.1694 & 27.3910 & 26.9461 & 26.7536 & 27.3268 & 27.1297 & 27.0663 \\
\hline 2 & 2.80 & 29.5395 & 29.0456 & 28.8782 & 29.4708 & 29.2063 & 29.0614 & 29.4338 & 28.9358 & 28.7488 & 29.3592 & 29.0916 & 28.9375 \\
\hline 4 & 1.02 & 30.7156 & 30.2639 & 30.1260 & 30.6574 & 30.4124 & 30.3496 & 30.6825 & 30.2288 & 30.0885 & 30.6241 & 30.3780 & 30.3124 \\
\hline 6 & 7.31 & 24.9946 & 24.3163 & 24.1579 & 24.9198 & 24.4538 & 24.3928 & 24.9376 & 24.2593 & 24.0829 & 24.8469 & 24.3870 & 24.3168 \\
\hline 7 & 7.31 & 25.0049 & 24.3892 & 24.2136 & 24.8603 & 24.4740 & 24.4402 & 24.8953 & 24.2796 & 24.0943 & 24.7378 & 24.3591 & 24.3211 \\
\hline 8 & 7.31 & 25.1171 & 24.5010 & 24.2918 & 24.9606 & 24.5836 & 24.4981 & 25.0006 & 24.3844 & 24.1701 & 24.8329 & 24.4637 & 24.3760 \\
\hline 9 & 4.18 & 28.2442 & 27.7104 & 27.6265 & 28.2456 & 27.9098 & 27.8943 & 28.1765 & 27.6432 & 27.5606 & 28.1807 & 27.8465 & 27.8306 \\
\hline 10 & 1.25 & 31.3273 & 30.8612 & 30.7476 & 31.3069 & 31.0275 & 30.9691 & 31.2945 & 30.8269 & 30.7126 & 31.2714 & 30.9919 & 30.9336 \\
\hline
\end{tabular}




\begin{tabular}{|c|c|c|c|c|c|c|c|c|c|c|c|c|c|}
\hline \multirow{3}{*}{\multicolumn{2}{|c|}{$\operatorname{Exp}$}} & \multicolumn{12}{|c|}{ mPW1PW91 } \\
\hline & & \multicolumn{6}{|c|}{ Gas phase } & \multicolumn{6}{|c|}{ PCM } \\
\hline & & 6-31G* & $6-31 G^{* *}$ & $6-31+G * *$ & 6-311G* & $6-311 G * *$ & $6-311+G * *$ & 6-31G* & $6-31 G^{* *}$ & $6-31+G * *$ & 6-311G* & $6-311 \mathrm{G} * *$ & $6-311+G * *$ \\
\hline \multicolumn{14}{|c|}{${ }^{13} \mathrm{C}$} \\
\hline 1 & 76.1 & 119.3585 & 119.8117 & 119.0463 & 109.0428 & 108.6924 & 108.1211 & 119.6060 & 120.0603 & 119.2141 & 109.2233 & 108.8888 & 108.3073 \\
\hline 2 & 47.1 & 147.1923 & 147.8711 & 147.1059 & 137.9125 & 137.7100 & 137.0457 & 147.1228 & 147.7980 & 146.9588 & 137.7985 & 137.5816 & 136.8850 \\
\hline 3 & 175.7 & 21.7673 & 21.7083 & 20.2974 & 1.5482 & 1.7796 & 0.5453 & 21.0025 & 20.9354 & 19.3177 & 0.6208 & 0.8171 & -0.5025 \\
\hline 4 & 14.3 & 175.5153 & 177.1263 & 177.4260 & 169.1537 & 169.1709 & 168.8638 & 175.7836 & 177.3813 & 177.6827 & 169.4210 & 169.4172 & 169.1101 \\
\hline 5 & 141.6 & 55.6822 & 55.3095 & 52.4625 & 37.9675 & 37.5634 & 36.9001 & 55.6780 & 55.3056 & 52.5972 & 37.9071 & 37.5045 & 36.9088 \\
\hline 6 & 126.5 & 73.1814 & 73.7708 & 73.5169 & 58.1805 & 57.3565 & 56.5308 & 73.4020 & 73.9849 & 73.6547 & 58.3144 & 57.5018 & 56.6974 \\
\hline 7 & 128.2 & 71.0850 & 71.5958 & 71.3585 & 55.9318 & 54.9313 & 54.2927 & 70.8507 & 71.3605 & 70.9867 & 55.5438 & 54.5769 & 53.9496 \\
\hline 8 & 127.8 & 72.5277 & 73.0604 & 72.7060 & 57.1350 & 56.2214 & 55.5618 & 72.2364 & 72.7726 & 72.2809 & 56.7025 & 55.8358 & 55.1806 \\
\hline 9 & 60.6 & 134.1658 & 135.4349 & 134.5932 & 124.8662 & 124.5072 & 124.1280 & 133.7357 & 135.0024 & 134.0055 & 124.3662 & 123.9939 & 123.5926 \\
\hline $\begin{array}{l}10 \\
{ }^{1} \mathrm{H}\end{array}$ & 14.0 & 178.7954 & 180.5145 & 180.7567 & 172.5159 & 172.5171 & 172.0685 & 178.9198 & 180.6353 & 180.8291 & 172.6419 & 172.6267 & 172.1760 \\
\hline 1 & 4.75 & 27.3667 & 26.9252 & 26.7666 & 27.3076 & 27.1133 & 27.0646 & 27.2934 & 26.8478 & 26.6580 & 27.2213 & 27.0197 & 26.9576 \\
\hline 2 & 2.80 & 29.4627 & 28.9783 & 28.8161 & 29.3519 & 29.0971 & 28.9597 & 29.3503 & 28.8613 & 28.6843 & 29.2366 & 28.9782 & 28.8318 \\
\hline 4 & 1.02 & 30.6501 & 30.1955 & 30.0712 & 30.5774 & 30.3253 & 30.2609 & 30.6116 & 30.1548 & 30.0355 & 30.5389 & 30.2857 & 30.2186 \\
\hline 6 & 7.31 & 24.7988 & 24.1221 & 23.9897 & 24.7436 & 24.2482 & 24.1963 & 24.7309 & 24.0542 & 23.9119 & 24.6634 & 24.1743 & 24.1136 \\
\hline 7 & 7.31 & 24.8028 & 24.1828 & 24.0229 & 24.6842 & 24.2623 & 24.2325 & 24.6788 & 24.0586 & 23.8846 & 24.5504 & 24.1365 & 24.1032 \\
\hline 8 & 7.31 & 24.9121 & 24.2929 & 24.1132 & 24.7815 & 24.3691 & 24.2862 & 24.7812 & 24.1617 & 23.9789 & 24.6424 & 24.2382 & 24.1542 \\
\hline 9 & 4.18 & 28.1970 & 27.6667 & 27.5910 & 28.1760 & 27.8343 & 27.8196 & 28.1252 & 27.5952 & 27.4987 & 28.1086 & 27.7682 & 27.7533 \\
\hline 10 & 1.25 & 31.2947 & 30.8257 & 30.7139 & 31.2478 & 30.9587 & 30.9044 & 31.2584 & 30.7876 & 30.3818 & 31.2086 & 30.9196 & 30.8657 \\
\hline
\end{tabular}

\section{Compound 74a}<smiles>C[C@H](O)[C@H](C)C(=O)c1ccccc1</smiles>

\begin{tabular}{|c|c|c|c|c|c|c|c|c|c|c|c|c|c|}
\hline \multirow{3}{*}{\multicolumn{2}{|c|}{ Exp }} & \multicolumn{12}{|c|}{ B3LYP } \\
\hline & & \multicolumn{6}{|c|}{ Gas phase } & \multicolumn{6}{|c|}{ PCM } \\
\hline & & 6-31G* & 6-31G** & $6-31+G * *$ & 6-311G* & 6-311G** & $6-311+G^{* *}$ & 6-31G* & 6-31G** & $6-31+G * *$ & 6-311G* & $6-311 G * *$ & $6-311+G * *$ \\
\hline \\
\hline 1 & 205.8 & -10.4618 & -10.4749 & -11.8585 & -31.9094 & -31.5716 & -34.2246 & -11.6831 & -11.7059 & -13.4246 & -33.3692 & -33.0812 & -36.0449 \\
\hline 2 & 45.8 & 144.6609 & 145.3597 & 144.1441 & 134.8438 & 134.7033 & 133.8356 & 144.7653 & 145.4532 & 144.2742 & 134.9299 & 134.7580 & 133.8519 \\
\hline 3 & 67.5 & 122.5744 & 123.3025 & 122.6882 & 111.2612 & 111.3045 & 110.9199 & 122.5425 & 123.2700 & 122.5412 & 111.1199 & 111.1506 & 110.7427 \\
\hline 4 & 20.3 & 169.2456 & 171.0557 & 171.2742 & 162.5019 & 162.3268 & 162.2364 & 169.5521 & 171.3484 & 171.4888 & 162.7852 & 162.5958 & 162.4411 \\
\hline 5 & 11.2 & 176.8192 & 178.6731 & 179.7351 & 171.2551 & 171.3286 & 170.8680 & 176.9455 & 178.7847 & 179.8509 & 171.3606 & 171.4059 & 170.9262 \\
\hline 6 & 135.9 & 62.0812 & 61.7711 & 60.2310 & 43.9142 & 43.6241 & 42.4736 & 62.3097 & 62.0012 & 60.4506 & 44.1647 & 43.8847 & 42.7339 \\
\hline 7 & 128.7 & 66.5274 & 67.1482 & 66.1383 & 49.4657 & 48.6105 & 47.4640 & 66.4950 & 67.1119 & 66.0243 & 49.3908 & 48.5444 & 47.3756 \\
\hline 8 & 128.4 & 67.9100 & 68.4857 & 67.9555 & 50.7804 & 49.8205 & 49.0634 & 67.5040 & 68.0789 & 67.4696 & 50.2249 & 49.2894 & 48.5791 \\
\hline
\end{tabular}




\begin{tabular}{|c|c|c|c|c|c|c|c|c|c|c|c|c|c|}
\hline 9 & 133.4 & 63.0034 & 63.6272 & 62.9666 & 45.0085 & 44.1353 & 43.3266 & 61.9542 & 62.5825 & 61.7491 & 43.7479 & 42.9064 & 42.0768 \\
\hline \multicolumn{14}{|c|}{${ }^{1} \mathrm{H}$} \\
\hline 2 & 3.42 & 29.2027 & 28.6857 & 28.5371 & 29.1856 & 28.8816 & 28.7175 & 29.0126 & 28.4908 & 28.3086 & 28.9840 & 28.6779 & 28.4929 \\
\hline 3 & 4.24 & 27.8392 & 27.3792 & 27.3032 & 27.8048 & 27.5863 & 27.5922 & 27.8149 & 27.3543 & 27.2572 & 27.7677 & 27.5472 & 27.5381 \\
\hline 4 & 1.26 & 31.0608 & 30.6171 & 30.4807 & 30.9891 & 30.7484 & 30.6952 & 31.0411 & 30.5948 & 30.4505 & 30.9665 & 30.7231 & 30.6650 \\
\hline 5 & 1.18 & 30.8784 & 30.4115 & 30.3176 & 30.9120 & 30.6511 & 30.5714 & 30.8620 & 30.3940 & 30.2960 & 30.8954 & 30.6335 & 30.5495 \\
\hline 7 & 7.94 & 24.2193 & 23.5211 & 23.3767 & 24.1188 & 23.6367 & 23.5255 & 24.1727 & 23.4760 & 23.3199 & 24.0648 & 23.5882 & 23.4676 \\
\hline 8 & 7.53 & 24.8434 & 24.2099 & 24.0050 & 24.7132 & 24.3023 & 24.2666 & 24.7095 & 24.0764 & 23.8632 & 24.5696 & 24.1668 & 24.1254 \\
\hline 9 & 7.53 & 24.7655 & 24.1418 & 23.9519 & 24.6149 & 24.2257 & 24.1613 & 24.5979 & 23.9751 & 23.7737 & 24.4366 & 24.0553 & 23.9822 \\
\hline
\end{tabular}

\begin{tabular}{|c|c|c|c|c|c|c|c|c|c|c|c|c|c|}
\hline \multirow{3}{*}{\multicolumn{2}{|c|}{ Exp }} & \multicolumn{12}{|c|}{ mPW1PW91 } \\
\hline & & \multicolumn{6}{|c|}{ Gas phase } & \multicolumn{6}{|c|}{ PCM } \\
\hline & & 6-31G* & $6-31 G * *$ & $6-31+G * *$ & 6-311G* & $6-311 G * *$ & $6-311+G * *$ & 6-31G* & $6-31 G * *$ & $6-31+G * *$ & 6-311G* & $6-311 G * *$ & $6-311+G^{* *}$ \\
\hline \multicolumn{14}{|c|}{${ }^{13} \mathrm{C}$} \\
\hline 1 & 205.8 & -8.1173 & -8.1344 & -8.8403 & -26.5482 & -26.2129 & -28.6356 & -9.4064 & -9.4333 & -10.4377 & -28.0408 & -27.7568 & -30.4550 \\
\hline 2 & 45.8 & 149.0742 & 149.7268 & 148.6548 & 140.4213 & 140.2940 & 139.4321 & 149.1457 & 149.7893 & 148.7543 & 140.4598 & 140.3025 & 139.4086 \\
\hline 3 & 67.5 & 127.4014 & 128.0850 & 127.8543 & 117.5804 & 117.6210 & 117.1527 & 127.3582 & 128.0418 & 127.6977 & 117.4155 & 117.4453 & 116.9545 \\
\hline 4 & 20.3 & 173.4172 & 175.1008 & 175.1797 & 167.2494 & 167.0896 & 167.0222 & 173.7586 & 175.4287 & 175.4341 & 167.5369 & 167.3645 & 167.2381 \\
\hline 5 & 11.2 & 181.0559 & 182.7797 & 183.7242 & 175.8109 & 175.8944 & 175.5167 & 181.2017 & 182.9113 & 183.8542 & 175.9227 & 175.9797 & 175.5858 \\
\hline 6 & 135.9 & 65.0542 & 64.7215 & 63.5941 & 49.3995 & 49.0870 & 47.7781 & 65.3321 & 65.0024 & 63.8587 & 49.6966 & 49.3953 & 48.0835 \\
\hline 7 & 128.7 & 68.9274 & 69.4904 & 68.4623 & 54.0219 & 53.1411 & 52.0251 & 68.8971 & 69.4561 & 68.3467 & 53.9411 & 53.0707 & 51.9373 \\
\hline 8 & 128.4 & 70.4395 & 70.9295 & 70.6436 & 55.4251 & 54.4195 & 53.6157 & 69.9994 & 70.4889 & 70.1168 & 54.8350 & 53.8568 & 53.1074 \\
\hline 9 & 133.4 & 65.3185 & 65.8532 & 65.2621 & 49.5561 & 48.6354 & 47.8269 & 64.2193 & 64.7585 & 63.9978 & 48.2553 & 47.3688 & 46.5552 \\
\hline \multicolumn{14}{|c|}{${ }^{1} \mathrm{H}$} \\
\hline 2 & 3.42 & 29.1366 & 28.6320 & 28.4889 & 29.0546 & 28.7638 & 28.6195 & 28.9388 & 28.4289 & 28.2565 & 28.8497 & 28.5565 & 28.3926 \\
\hline 3 & 4.24 & 27.7890 & 27.3331 & 27.2516 & 27.7343 & 27.5169 & 27.5116 & 27.7604 & 27.3039 & 27.2027 & 27.6950 & 27.4757 & 27.4560 \\
\hline 4 & 1.26 & 30.9929 & 30.5467 & 30.4264 & 30.9026 & 30.6536 & 30.6082 & 30.9683 & 30.5194 & 30.3922 & 30.8756 & 30.6236 & 30.5742 \\
\hline 5 & 1.18 & 30.8181 & 30.3494 & 30.2556 & 30.8241 & 30.5592 & 30.4848 & 30.7991 & 30.3291 & 30.2320 & 30.8045 & 30.5387 & 30.4607 \\
\hline 7 & 7.94 & 24.0190 & 23.3236 & 23.1996 & 23.9400 & 23.4274 & 23.3376 & 23.9626 & 23.2687 & 23.1343 & 23.8788 & 23.3721 & 23.2729 \\
\hline 8 & 7.53 & 24.6356 & 23.9986 & 23.8208 & 24.5345 & 24.0882 & 24.0535 & 24.4868 & 23.8501 & 23.6654 & 24.3797 & 23.9416 & 23.9018 \\
\hline 9 & 7.53 & 24.5497 & 23.9235 & 23.7835 & 24.4303 & 24.0031 & 23.9508 & 24.3672 & 23.7416 & 23.5920 & 24.2408 & 23.8215 & 23.7613 \\
\hline
\end{tabular}

\section{Compound 74b}<smiles>C[C@@H](O)[C@@H](C)C(=O)c1ccccc1</smiles>

74b

\begin{tabular}{|c|c|c|c|c|c|c|c|c|c|c|c|c|}
\hline \multirow[b]{3}{*}{ Exp } & \multicolumn{12}{|c|}{ B3LYP } \\
\hline & \multicolumn{6}{|c|}{ Gas phase } & \multicolumn{6}{|c|}{ PCM } \\
\hline & 6-31G* & $6-31 G * *$ & $6-31+G * *$ & 6-311G* & $6-311 G * *$ & $6-311+G * *$ & 6-31G* & $6-31 G * *$ & $6-31+G * *$ & 6-311G* & 6-311G** & $6-311+G * *$ \\
\hline \multicolumn{13}{|l|}{${ }^{13} \mathrm{C}$} \\
\hline 1205.2 & -10.2475 & -10.2718 & -10.7088 & -31.6684 & -31.5085 & -32.5746 & -11.4734 & -11.5022 & -12.2746 & -33.2035 & -33.0678 & -34.3816 \\
\hline
\end{tabular}




\begin{tabular}{lccccccccccccc}
\hline 2 & 47.6 & 144.2505 & 144.9567 & 141.9160 & 133.3577 & 133.7665 & 130.8628 & 144.3433 & 145.0345 & 141.9111 & 133.4602 & 133.8235 & 130.7181 \\
3 & 69.7 & 118.4742 & 119.1445 & 119.3908 & 106.7944 & 106.3927 & 107.0397 & 118.5066 & 119.1812 & 119.3158 & 106.6822 & 106.3162 & 106.9359 \\
4 & 20.7 & 167.2087 & 168.9802 & 170.2330 & 160.4108 & 160.0020 & 160.7328 & 167.3972 & 169.1610 & 170.3558 & 160.5523 & 160.1473 & 160.8395 \\
5 & 15.2 & 170.4217 & 172.1738 & 172.7802 & 163.8938 & 163.7365 & 163.9482 & 170.5453 & 172.2877 & 172.8340 & 164.0010 & 163.8431 & 163.9792 \\
6 & 136.4 & 61.5263 & 61.2223 & 59.9943 & 43.0796 & 42.7948 & 41.3718 & 61.7390 & 61.4363 & 60.2012 & 43.3041 & 43.0290 & 41.5856 \\
7 & 128.6 & 66.6517 & 67.2764 & 66.5856 & 49.5131 & 48.6954 & 47.4045 & 66.6128 & 67.2332 & 66.4947 & 49.4341 & 48.6215 & 47.3066 \\
8 & 128.3 & 67.9002 & 68.4792 & 67.9666 & 50.7575 & 49.7912 & 49.1078 & 67.4978 & 68.0760 & 67.4650 & 50.2062 & 49.2640 & 48.6217 \\
9 & 133.2 & 62.9589 & 63.5830 & 63.3469 & 44.9374 & 44.0434 & 43.3397 & 61.9069 & 62.5359 & 62.1189 & 43.6732 & 42.8111 & 42.0709 \\
${ }^{1} \mathbf{H}$ & & & & & & & & & & & & & \\
2 & 3.51 & 29.0960 & 28.5753 & 28.5311 & 29.0687 & 28.7562 & 28.7220 & 28.9172 & 28.3912 & 28.3372 & 28.8842 & 28.5645 & 28.5275 \\
3 & 4.14 & 28.3340 & 27.8925 & 27.7047 & 28.3010 & 28.1214 & 28.0318 & 28.2998 & 27.8576 & 27.6553 & 28.2565 & 28.0686 & 27.9714 \\
4 & 1.31 & 31.0323 & 30.5822 & 30.4647 & 30.9554 & 30.7017 & 30.6645 & 31.0122 & 30.5602 & 30.4335 & 30.9332 & 30.6779 & 30.6322 \\
5 & 1.26 & 30.7653 & 30.3110 & 30.3273 & 30.7975 & 30.5206 & 30.5398 & 30.7450 & 30.2898 & 30.2850 & 30.7681 & 30.4961 & 30.5002 \\
7 & 7.99 & 24.2147 & 23.5163 & 23.3099 & 24.1110 & 23.6341 & 23.5340 & 24.1667 & 23.4697 & 23.2534 & 24.0554 & 23.5836 & 23.4770 \\
8 & 7.55 & 24.8374 & 24.2064 & 24.0250 & 24.7053 & 24.3033 & 24.2679 & 24.7038 & 24.0731 & 23.8839 & 24.5622 & 24.1680 & 24.1279 \\
9 & 7.55 & 24.7687 & 24.1445 & 23.9072 & 24.6247 & 24.2325 & 24.1737 & 24.6013 & 23.9779 & 23.7281 & 24.4469 & 24.0622 & 23.9958 \\
\hline
\end{tabular}

\begin{tabular}{|c|c|c|c|c|c|c|c|c|c|c|c|c|c|}
\hline \multirow{3}{*}{\multicolumn{2}{|c|}{ Exp }} & \multicolumn{12}{|c|}{ mPW1PW91 } \\
\hline & & \multicolumn{6}{|c|}{ Gas phase } & \multicolumn{6}{|c|}{ PCM } \\
\hline & & 6-31G* & $6-31 G^{* *}$ & $6-31+G^{* *}$ & $6-311 G^{*}$ & $6-311 \mathrm{G} * *$ & $6-311+G^{* *}$ & 6-31G* & $6-31 G^{* *}$ & $6-31+G^{* *}$ & 6-311G* & $6-311 \mathrm{G} * *$ & $6-311+G * *$ \\
\hline \multicolumn{14}{|c|}{ 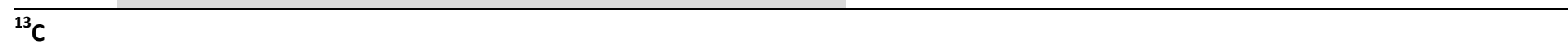 } \\
\hline 1 & 205.2 & -7.8113 & -7.8494 & -7.7749 & -26.2228 & -26.0963 & -27.0984 & -9.0929 & -9.1351 & -9.3542 & -27.7660 & -27.6685 & -28.8743 \\
\hline 2 & 47.6 & 148.5909 & 149.2627 & 146.6900 & 138.8635 & 139.3063 & 136.8578 & 148.6191 & 149.2779 & 146.6323 & 138.8631 & 139.2757 & 136.6373 \\
\hline 3 & 69.7 & 123.5650 & 124.1755 & 124.5829 & 113.5007 & 113.0780 & 113.4572 & 123.5976 & 124.2141 & 124.5253 & 113.3859 & 112.9939 & 113.3612 \\
\hline 4 & 20.7 & 171.4460 & 173.0817 & 174.1335 & 165.2574 & 164.8358 & 165.3971 & 171.6767 & 173.3053 & 174.3095 & 165.4327 & 165.0113 & 165.5520 \\
\hline 5 & 15.2 & 174.9248 & 176.5409 & 176.9922 & 168.8357 & 168.6730 & 168.8177 & 175.0726 & 176.6805 & 177.0818 & 168.9534 & 168.7893 & 168.8801 \\
\hline 6 & 136.4 & 64.5037 & 64.1770 & 63.3352 & 48.5799 & 48.2724 & 46.7573 & 64.7689 & 64.4448 & 63.5939 & 48.8547 & 48.5577 & 47.0196 \\
\hline 7 & 128.6 & 69.0433 & 69.6109 & 69.0106 & 54.0631 & 53.2237 & 51.9735 & 69.0067 & 69.5698 & 68.9169 & 53.9765 & 53.1444 & 51.8713 \\
\hline 8 & 128.3 & 70.4309 & 70.9240 & 70.6264 & 55.4056 & 54.3936 & 53.6376 & 69.9955 & 70.4881 & 70.0795 & 54.8209 & 53.8358 & 53.1287 \\
\hline \multicolumn{13}{|c|}{${ }^{1} \mathrm{H}$} & 46.5577 \\
\hline 2 & 3.51 & 29.0222 & 28.5163 & 28.4530 & 28.9315 & 28.6358 & 28.6040 & 28.8373 & 28.3256 & 28.2553 & 28.7462 & 28.4423 & 28.4076 \\
\hline 3 & 4.14 & 28.2528 & 27.8193 & 27.6430 & 28.2070 & 28.0277 & 27.9512 & 28.2114 & 27.7770 & 27.5874 & 28.1574 & 27.9698 & 27.8851 \\
\hline 4 & 1.31 & 30.9715 & 30.5185 & 30.4103 & 30.8712 & 30.6101 & 30.5760 & 30.9482 & 30.4932 & 30.3759 & 30.8456 & 30.5832 & 30.5406 \\
\hline 5 & 1.26 & 30.7157 & 30.2574 & 30.2679 & 30.7252 & 30.4382 & 30.4512 & 30.6917 & 30.2325 & 30.2244 & 30.6931 & 30.4110 & 30.4124 \\
\hline 7 & 7.99 & 24.0126 & 23.3177 & 23.1373 & 23.9299 & 23.4237 & 23.3378 & 23.9545 & 23.2610 & 23.0725 & 23.8672 & 23.3664 & 23.2739 \\
\hline 8 & 7.55 & 24.6305 & 23.9956 & 23.8397 & 24.5266 & 24.0889 & 24.0543 & 24.4821 & 23.8474 & 23.6850 & 24.3724 & 23.9426 & 23.9033 \\
\hline 9 & 7.55 & 24.5530 & 23.9265 & 23.7348 & 24.4390 & 24.0097 & 23.9631 & 24.3710 & 23.7450 & 23.5423 & 24.2503 & 23.8286 & 23.7745 \\
\hline
\end{tabular}




\section{Compound 75a}<smiles>CC(C)C[C@H](C)[C@@H](O)[C@@H](C)C(=O)O</smiles>

$75 a$

\begin{tabular}{|c|c|c|c|c|c|c|c|c|c|c|c|c|c|}
\hline \multirow{3}{*}{\multicolumn{2}{|c|}{ Exp }} & \multicolumn{12}{|c|}{ B3LYP } \\
\hline & & \multicolumn{6}{|c|}{ Gas phase } & \multicolumn{6}{|c|}{ PCM } \\
\hline & & 6-31G* & 6-31G** & $6-31+G * *$ & 6-311G* & 6-311G** & $6-311+G^{* *}$ & 6-31G* & 6-31G** & $6-31+G * *$ & 6-311G* & 6-311 & $6-311+G$ \\
\hline \\
\hline 1 & 181.4 & 19.7001 & 19.4437 & 16.8059 & -3.1757 & -3.0152 & -5.0220 & 18.7511 & 18.4802 & 15.5864 & -4.3125 & -4.2211 & -6.4512 \\
\hline 2 & 39.4 & 148.8775 & 149.5699 & 147.9514 & 139.2281 & 139.2033 & 137.6482 & 148.9091 & 149.6015 & 148.0159 & 139.3023 & 139.2212 & 137.637 \\
\hline 3 & 78.2 & 111.6843 & 112.2301 & 111.7385 & 99.9207 & 99.4699 & 99.7992 & 111.7537 & 112.2972 & 111.7094 & 99.8741 & 99.4745 & 99.7414 \\
\hline 4 & 33.4 & 152.7692 & 153.5536 & 153.8334 & 143.9953 & 143.4747 & 143.6939 & 152.7214 & 153.4976 & 153.6585 & 143.7965 & 143.3263 & 143.472 \\
\hline 5 & 42.3 & 148.8816 & 149.9601 & 150.7875 & 140.1759 & 139.2789 & 139.9591 & 148.6524 & 149.7290 & 150.4570 & 139.7959 & 139.0050 & 139.607 \\
\hline 6 & 25.2 & 162.6711 & 163.6431 & 163.0035 & 152.9575 & 152.9421 & 152.3943 & 162.7677 & 163.7443 & 163.0803 & 153.1008 & 153.0880 & 152.524 \\
\hline 7 & 21.3 & 169.0790 & 70.8472 & 171.1574 & 162.2926 & 162.1012 & 161.9652 & 169.2871 & 171.0464 & 171.3819 & 162.5643 & 162.3515 & 162.232 \\
\hline 8 & 24.3 & 64.5918 & 166.3148 & 166.7191 & 157.3339 & 157.2327 & 156.8446 & 164.8277 & 166.5421 & 167.0215 & 157.6606 & 157.5300 & 157.141 \\
\hline 9 & 16.6 & 171.5045 & 173.2999 & 174.9167 & 165.3141 & 165.0367 & 165.4648 & 171.5956 & 173.3782 & 174.8537 & 165.2688 & 165.0648 & 165.381 \\
\hline 10 & 14.6 & 173.6060 & 175.4486 & 175.3109 & 167.2128 & 167.2612 & 166.4861 & 173.8971 & 175.7320 & 175.6508 & 167.6228 & 167.6071 & 166.868 \\
\hline \multicolumn{14}{|c|}{ 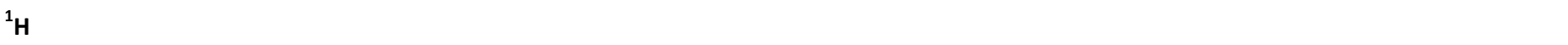 } \\
\hline 2 & 2.74 & 29.5214 & 29.0194 & 28.9036 & 29.4688 & 29.2228 & 29.1702 & 29.4358 & 28.9284 & 28.7928 & 29.3787 & 29.1237 & 29.0597 \\
\hline 3 & 3.48 & 29.0152 & 28.6478 & 28.4182 & 28.9721 & 28.8976 & 28.7699 & 28.9896 & 28.6229 & 28.3972 & 28.9588 & 28.8687 & 28.7407 \\
\hline 4 & 1.64 & 30.4772 & 30.0617 & 30.0203 & 30.6155 & 30.4161 & 30.3634 & 30.4525 & 30.0374 & 29.9781 & 30.5801 & 30.3844 & 30.3207 \\
\hline $5 a$ & 1.18 & 31.0369 & 30.6972 & 30.4487 & 30.9689 & 30.8988 & 30.7373 & 31.0282 & 30.6908 & 30.5116 & 31.0027 & 30.9074 & 30.7877 \\
\hline $5 b$ & 1.15 & 30.1093 & 29.6979 & 29.9797 & 30.3405 & 30.0542 & 30.2687 & 30.2079 & 29.7981 & 29.9719 & 30.3624 & 30.1136 & 30.2645 \\
\hline 6 & 1.69 & 30.3169 & 29.8783 & 29.9224 & 30.4629 & 30.2512 & 30.2473 & 30.2741 & 29.8340 & 29.8849 & 30.4208 & 30.2028 & 30.2049 \\
\hline 7 & 0.92 & 31.2728 & 30.8464 & 30.8191 & 31.3009 & 31.0653 & 31.0561 & 31.2699 & 30.8427 & 30.8125 & 31.2960 & 31.0617 & 31.0508 \\
\hline 8 & 0.95 & 31.1985 & 30.7872 & 30.7165 & 31.1707 & 30.9544 & 30.9259 & 31.2020 & 30.7896 & 30.7204 & 31.1750 & 30.9582 & 309291 \\
\hline 9 & 1.23 & 30.7824 & 30.3384 & 30.2779 & 30.7838 & 30.5227 & 30.5072 & 30.7609 & 30.3154 & 30.2354 & 30.7476 & 30.4922 & 30.4638 \\
\hline 19 & 0.84 & 31.3214 & 30.8881 & 30.7475 & 31.3000 & 31.0888 & 31.0081 & 31.3014 & 30.8668 & 30.7495 & 31.3000 & 31.0771 & 31.0089 \\
\hline
\end{tabular}

\begin{tabular}{|c|c|c|c|c|c|c|c|c|c|c|c|c|c|}
\hline \multirow{3}{*}{\multicolumn{2}{|c|}{ Exp }} & \multicolumn{12}{|c|}{ mPW1PW91 } \\
\hline & & \multicolumn{6}{|c|}{ Gas phase } & \multicolumn{6}{|c|}{ PCM } \\
\hline & & 6-31G* & $6-31 G^{* *}$ & $6-31+G^{* *}$ & 6-311G* & $6-311 \mathrm{G} * *$ & $6-311+G^{* *}$ & 6-31G* & $6-31 G^{* *}$ & $6-31+G^{* *}$ & 6-311G* & $6-311 \mathrm{G} * *$ & $6-311+G * *$ \\
\hline \\
\hline 1 & 181.4 & 21.9310 & 21.6542 & 19.6036 & 2.0251 & 2.1792 & 0.3962 & 20.9635 & 20.6711 & 18.4000 & 0.9014 & 0.9806 & -0.9938 \\
\hline 2 & 39.4 & 153.2423 & 153.8875 & 152.4224 & 144.6718 & 144.6766 & 143.2844 & 153.2879 & 153.9336 & 152.4463 & 144.7320 & 144.6854 & 143.2588 \\
\hline 3 & 78.2 & 117.0243 & 117.5085 & 117.2033 & 106.9008 & 106.4497 & 106.6384 & 117.0624 & 117.5459 & 117.1847 & 106.8202 & 106.4160 & 106.5657 \\
\hline 4 & 33.4 & 158.1356 & 158.8609 & 159.2061 & 150.3703 & 149.8418 & 149.8591 & 158.0572 & 158.7741 & 159.0956 & 150.1619 & 149.6784 & 149.6568 \\
\hline 5 & 42.3 & 154.6084 & 155.5618 & 156.4508 & 146.8117 & 145.8799 & 146.3194 & 154.3554 & 155.3071 & 156.2300 & 146.4326 & 145.6019 & 146.0100 \\
\hline 6 & 25.2 & 167.8770 & 168.7973 & 168.2939 & 159.0163 & 159.0001 & 158.4120 & 167.9779 & 168.9031 & 168.3775 & 159.1550 & 159.1429 & 158.5454 \\
\hline 7 & 21.3 & 173.4171 & 175.0506 & 175.2161 & 167.1479 & 166.9517 & 166.7898 & 173.6638 & 175.2879 & 175.4995 & 167.4348 & 167.2179 & 167.0722 \\
\hline 8 & 24.3 & 169.0409 & 170.6299 & 170.8214 & 162.2965 & 162.1967 & 161.8588 & 169.3220 & 170.9023 & 171.1676 & 162.6381 & 162.5105 & 162.1759 \\
\hline
\end{tabular}




\begin{tabular}{ccccccccccccccc}
\hline 9 & 16.6 & 176.2339 & 177.8967 & 179.3559 & 170.4151 & 170.1043 & 170.3487 & 176.3045 & 177.9554 & 179.3523 & 170.3534 & 170.1178 & 170.2906 \\
10 & 14.6 & 177.6904 & 179.4106 & 179.2978 & 171.7197 & 171.7991 & 171.1705 & 178.0286 & 179.7410 & 179.6472 & 172.1366 & 172.1563 & 171.5493 \\
${ }^{1} \mathbf{H}$ & & & & & & & & & & & & & & \\
2 & 2.74 & 29.4672 & 28.9791 & 28.8632 & 29.3758 & 29.1406 & 29.0843 & 29.3793 & 28.8846 & 28.7567 & 29.2859 & 29.0400 & 28.9753 \\
3 & 3.48 & 28.8879 & 28.5281 & 28.2998 & 28.8187 & 28.7473 & 28.6478 & 28.8658 & 28.5065 & 28.2690 & 28.8078 & 28.7210 & 28.6168 \\
4 & 1.64 & 30.4294 & 30.0153 & 29.9531 & 30.4865 & 30.2989 & 30.2507 & 30.4052 & 29.9913 & 29.9069 & 30.4512 & 30.2676 & 30.2087 \\
$5 a$ & 1.18 & 30.9392 & 30.6021 & 30.3605 & 30.8192 & 30.7592 & 30.6253 & 30.9468 & 30.6120 & 30.4258 & 30.8686 & 30.7807 & 30.6783 \\
$5 b$ & 1.15 & 30.1638 & 29.7564 & 30.0149 & 30.3402 & 30.0444 & 30.2119 & 30.2380 & 29.8320 & 30.0061 & 30.3418 & 30.0859 & 30.2053 \\
6 & 1.69 & 30.2907 & 29.8581 & 29.8716 & 30.3560 & 30.1586 & 30.1433 & 30.2515 & 29.8170 & 29.8382 & 30.3186 & 30.1141 & 30.1036 \\
7 & 0.92 & 31.2315 & 30.8065 & 30.7739 & 31.2200 & 30.9795 & 30.9656 & 31.2257 & 30.8000 & 30.7640 & 31.2120 & 30.9731 & 30.9578 \\
8 & 0.95 & 31.1480 & 30.7371 & 30.6721 & 31.0870 & 30.8661 & 30.8397 & 31.1485 & 30.7365 & 30.6730 & 31.0881 & 30.8667 & 30.8401 \\
9 & 1.23 & 30.7392 & 30.2916 & 30.2401 & 30.7270 & 30.4548 & 30.4325 & 30.7094 & 30.2604 & 30.1953 & 30.6840 & 30.4178 & 30.3859 \\
10 & 0.84 & 31.2598 & 30.8284 & 30.6873 & 31.1956 & 30.9857 & 30.9182 & 31.2437 & 30.8106 & 30.6874 & 31.1984 & 30.9759 & 30.9174 \\
\hline
\end{tabular}

\section{Compound 75b}<smiles>CC(C)C[C@H](C)[C@H](O)[C@@H](C)C(=O)O</smiles>

\begin{tabular}{|c|c|c|c|c|c|c|c|c|c|c|c|c|c|}
\hline \multirow{3}{*}{\multicolumn{2}{|c|}{ Exp }} & \multicolumn{12}{|c|}{ B3LYP } \\
\hline & & \multicolumn{6}{|c|}{ Gas phase } & \multicolumn{6}{|c|}{ PCM } \\
\hline & & 6-31G* & 6-31G** & $6-31+G * *$ & 6-311G* & 6-311G** & $6-311+G * *$ & 6-31G* & 6-31G** & $6-31+G * *$ & 6-311G* & 6-311G** & $6-311+G * *$ \\
\hline \multicolumn{14}{|c|}{ ( } \\
\hline 1 & 181.9 & 18.5053 & 18.2179 & 15.2117 & -4.3994 & -4.1892 & -6.5505 & 17.5913 & 17.2899 & 14.0469 & -5.4616 & -5.3329 & -7.9147 \\
\hline 2 & 41.6 & 148.9830 & 149.6719 & 148.8182 & 140.2999 & 140.0412 & 138.7562 & 148.9431 & 149.6293 & 148.7455 & 140.2059 & 139.9245 & 138.5782 \\
\hline 3 & 76.3 & 115.3343 & 115.8995 & 115.1705 & 103.7738 & 103.6356 & 103.4816 & 115.4144 & 115.9773 & 115.2755 & 103.8699 & 103.7118 & 103.5916 \\
\hline 4 & 33.5 & 155.7130 & 156.5098 & 156.3114 & 146.8387 & 146.6652 & 146.1216 & 155.6807 & 156.4784 & 156.2413 & 146.7618 & 146.5915 & 146.0359 \\
\hline 5 & 41.8 & 148.5326 & 149.5981 & 147.9968 & 138.8261 & 138.4346 & 137.7783 & 148.4183 & 149.4857 & 147.9490 & 138.7783 & 138.3731 & 137.7498 \\
\hline 6 & 25.3 & 162.5509 & 163.5291 & 162.6259 & 152.6645 & 152.6804 & 152.6692 & 162.6812 & 163.6635 & 162.7890 & 152.8610 & 152.8662 & 152.8757 \\
\hline 7 & 21.3 & 168.9673 & 170.7520 & 170.9246 & 162.1611 & 162.0194 & 161.7978 & 169.1695 & 170.9437 & 171.1328 & 162.4122 & 162.2451 & 162.0159 \\
\hline 8 & 24.3 & 164.4833 & 166.2096 & 166.5597 & 157.2392 & 157.1510 & 156.6503 & 164.7101 & 166.4267 & 166.8277 & 157.5523 & 157.4344 & 156.9296 \\
\hline 9 & 15.7 & 174.9671 & 176.8272 & 176.9906 & 168.9060 & 168.8125 & 168.4046 & 175.2854 & 177.1338 & 177.3437 & 169.2683 & 169.1535 & 168.7618 \\
\hline $\begin{array}{l}10 \\
{ }^{1} \mathbf{H}\end{array}$ & 9.9 & 177.6977 & 179.5611 & 180.5729 & 171.7142 & 171.8099 & 171.4797 & 177.8043 & 179.6570 & 180.6431 & 171.8149 & 171.8862 & 171.5437 \\
\hline 2 & 2.74 & 29.6446 & 29.1494 & 28.9569 & 29.5983 & 29.3555 & 29.2166 & 29.5462 & 29.0483 & 28.8324 & 29.4990 & 29.2495 & 29.0953 \\
\hline 3 & 3.67 & 28.5616 & 28.1758 & 28.1808 & 28.6262 & 28.4690 & 28.4772 & 28.5202 & 28.1339 & 28.1171 & 28.5692 & 28.4118 & 28.4074 \\
\hline 4 & 1.62 & 30.3054 & 29.9140 & 29.8591 & 30.4749 & 30.3302 & 30.2601 & 30.3155 & 29.9225 & 29.8570 & 30.4765 & 30.3273 & 30.2532 \\
\hline $5 a$ & 1.49 & 29.9403 & 29.5163 & 29.4105 & 29.9668 & 29.7638 & 29.7415 & 30.1150 & 29.6954 & 29.6022 & 30.1465 & 29.9412 & 29.9227 \\
\hline $5 b$ & 1.04 & 31.1772 & 30.8457 & 30.8374 & 31.2174 & 31.0837 & 31.0476 & 31.1226 & 30.7915 & 30.7726 & 31.1534 & 31.0234 & 30.9824 \\
\hline 6 & 1.67 & 30.2789 & 29.8436 & 29.8489 & 30.4176 & 30.2161 & 30.2125 & 30.2297 & 29.7923 & 29.7909 & 30.3634 & 30.1590 & 30.1518 \\
\hline 7 & 0.85 & 31.2205 & 30.8022 & 30.7608 & 31.2346 & 31.0208 & 31.0042 & 31.2243 & 30.8050 & 30.7650 & 31.2394 & 31.0245 & 31.0079 \\
\hline
\end{tabular}




\begin{tabular}{cccccccccccccc}
\hline 8 & 0.93 & 31.1964 & 30.7881 & 30.6989 & 31.1671 & 30.9554 & 30.9060 & 31.2005 & 30.7913 & 30.7064 & 31.1732 & 30.9611 & 30.9125 \\
9 & 1.22 & 30.8728 & 30.4196 & 30.3199 & 30.8912 & 30.6502 & 30.6029 & 30.8622 & 30.4075 & 30.3063 & 30.8819 & 30.6381 & 30.5881 \\
10 & 0.85 & 31.4151 & 30.9817 & 30.9445 & 31.4650 & 31.2292 & 31.2043 & 31.3747 & 30.9399 & 30.9007 & 31.4258 & 31.1891 & 31.1636 \\
\hline
\end{tabular}

\begin{tabular}{|c|c|c|c|c|c|c|c|c|c|c|c|c|c|}
\hline & & \multicolumn{12}{|c|}{ mPW1PW91 } \\
\hline & \multirow[b]{2}{*}{ Exp } & \multicolumn{6}{|c|}{ Gas phase } & \multicolumn{6}{|c|}{ PCM } \\
\hline & & 6-31G* & $6-31 G * *$ & $6-31+\mathrm{G} * *$ & 6-311G* & $6-311 G * *$ & $6-311+G * *$ & $6-31 G *$ & $6-31 G * *$ & $6-31+G * *$ & 6-311G* & $6-311 G * *$ & $6-311+G * *$ \\
\hline \multicolumn{14}{|l|}{${ }^{13} \mathrm{C}$} \\
\hline 1 & 181.9 & 20.7266 & 20.4230 & 18.0933 & 0.8019 & 1.0026 & -1.0835 & 19.7970 & 19.4781 & 16.9375 & -0.2493 & -0.1341 & -2.4146 \\
\hline 2 & 41.6 & 153.4429 & 154.0813 & 153.3760 & 145.7863 & 145.5404 & 144.3289 & 153.4000 & 154.0362 & 153.3084 & 145.6864 & 145.4178 & 144.1541 \\
\hline 3 & 76.3 & 120.4307 & 120.9462 & 120.4399 & 110.4285 & 110.3035 & 110.1132 & 120.5013 & 121.0153 & 120.5275 & 110.4930 & 110.3502 & 110.1883 \\
\hline 4 & 33.5 & 160.7446 & 161.4848 & 161.3040 & 152.8162 & 152.6678 & 152.1220 & 160.7166 & 161.4578 & 161.2396 & 152.7395 & 152.5948 & 152.0375 \\
\hline 5 & 41.8 & 153.9163 & 154.8610 & 153.5475 & 145.0977 & 144.7202 & 144.0929 & 153.8298 & 154.7760 & 153.5260 & 145.0663 & 144.6762 & 144.0811 \\
\hline 6 & 25.3 & 167.7624 & 168.6878 & 167.8875 & 158.7747 & 158.7856 & 158.6516 & 167.9031 & 168.8327 & 168.0596 & 158.9705 & 158.9713 & 158.8569 \\
\hline 7 & 21.3 & 173.2676 & 174.9162 & 174.9306 & 166.9633 & 166.8226 & 166.6042 & 173.5067 & 175.1448 & 175.1792 & 167.2267 & 167.0628 & 166.8401 \\
\hline 8 & 24.3 & 168.9384 & 170.5298 & 170.6694 & 162.1994 & 162.1114 & 161.6739 & 169.2078 & 170.7892 & 170.9794 & 162.5244 & 162.4091 & 161.9718 \\
\hline 9 & 15.7 & 179.1720 & 180.9067 & 181.0054 & 173.5704 & 173.4790 & 173.1248 & 179.5228 & 181.2463 & 181.3854 & 173.9371 & 173.8277 & 173.4894 \\
\hline 10 & 9.9 & 181.9612 & 183.7014 & 184.3968 & 176.3259 & 176.4298 & 176.0972 & 182.0964 & 183.8259 & 184.5003 & 176.4430 & 176.5240 & 176.1843 \\
\hline \multicolumn{14}{|l|}{${ }^{1} \mathrm{H}$} \\
\hline 2 & 2.74 & 29.5968 & 29.1085 & 28.9190 & 29.4961 & 29.2644 & 29.1299 & 29.4932 & 29.0018 & 28.7923 & 29.3941 & 29.1549 & 29.0065 \\
\hline 3 & 3.67 & 28.5010 & 28.1214 & 28.1041 & 28.5262 & 28.3729 & 28.3736 & 28.4559 & 28.0759 & 28.0390 & 28.4687 & 28.3150 & 28.3042 \\
\hline 4 & 1.62 & 30.2588 & 29.8699 & 29.8022 & 30.3519 & 30.2183 & 30.1439 & 30.2708 & 29.8801 & 29.8029 & 30.3559 & 30.2172 & 30.1389 \\
\hline $5 a$ & 1.49 & 29.9102 & 29.4885 & 29.3724 & 29.8833 & 29.6837 & 29.6583 & 30.0886 & 29.6715 & 29.5657 & 30.0646 & 29.8625 & 29.8397 \\
\hline $5 b$ & 1.04 & 31.1305 & 30.8032 & 30.7793 & 31.1155 & 30.9842 & 30.9460 & 31.0723 & 30.7453 & 30.7123 & 31.0502 & 30.9226 & 30.8801 \\
\hline 6 & 1.67 & 30.2544 & 29.8243 & 29.8083 & 30.3137 & 30.1235 & 30.1129 & 30.2052 & 29.7727 & 29.7509 & 30.2606 & 30.0673 & 30.0530 \\
\hline 7 & 0.85 & 31.1750 & 30.7581 & 30.7144 & 31.1514 & 30.9328 & 30.9150 & 31.1771 & 30.7591 & 30.7165 & 31.1543 & 30.9346 & 30.9166 \\
\hline 8 & 0.93 & 31.1445 & 30.7365 & 30.6569 & 31.0824 & 30.8657 & 30.8198 & 31.1458 & 30.7369 & 30.6610 & 31.0854 & 30.8683 & 30.8231 \\
\hline 9 & 1.22 & 30.8160 & 30.3616 & 30.2721 & 30.8114 & 30.5653 & 30.5207 & 30.8027 & 30.3468 & 30.2566 & 30.7994 & 30.5505 & 30.5035 \\
\hline 10 & 0.85 & 31.3754 & 30.9433 & 30.9026 & 31.3823 & 31.1430 & 31.1225 & 31.3312 & 30.8976 & 30.8556 & 31.3400 & 31.0998 & 31.0789 \\
\hline
\end{tabular}

\section{Compound 75c}<smiles>CC(C)C[C@H](O)[C@@H](O)[C@@H](C)C(=O)O</smiles>

\begin{tabular}{|c|c|c|c|c|c|c|c|c|c|c|c|c|c|}
\hline \multirow{3}{*}{\multicolumn{2}{|c|}{ Exp }} & \multicolumn{12}{|c|}{ B3LYP } \\
\hline & & \multicolumn{6}{|c|}{ Gas phase } & \multicolumn{6}{|c|}{ PCM } \\
\hline & & 6-31G* & $6-31 G * *$ & $6-31+\mathrm{G} * *$ & 6-311G* & $6-311 G * *$ & $6-311+G * *$ & 6-31G* & $6-31 G * *$ & $6-31+G * *$ & 6-311G* & $6-311 G * *$ & $6-311+\mathrm{G} * *$ \\
\hline \multicolumn{14}{|c|}{${ }^{13} \mathrm{C}$} \\
\hline 1 & 181.6 & 18.5409 & 18.2562 & 15.1583 & -4.3494 & -4.1362 & -6.4738 & 17.6315 & 17.3327 & 14.0169 & -5.4041 & -5.2726 & -7.8350 \\
\hline 2 & 42.1 & 149.4870 & 150.1736 & 149.2254 & 140.8540 & 140.5898 & 139.3795 & 149.4425 & 150.1270 & 149.1435 & 140.7498 & 140.4632 & 139.1963 \\
\hline 3 & 75.6 & 115.1100 & 115.6473 & 114.8112 & 103.5265 & 103.3920 & 103.2009 & 115.1708 & 115.7055 & 114.8638 & 103.5805 & 103.4252 & 103.2584 \\
\hline 4 & 33.3 & 155.2287 & 156.0384 & 155.2943 & 146.1239 & 146.0096 & 145.7380 & 155.2681 & 156.0770 & 155.3042 & 146.1468 & 146.0317 & 145.7169 \\
\hline
\end{tabular}




\begin{tabular}{cccccccccccccc}
\hline 5 & 42.6 & 149.5685 & 150.6743 & 149.4689 & 139.7148 & 139.3142 & 138.9626 & 149.9848 & 151.0816 & 150.0015 & 140.2588 & 139.8377 & 139.5299 \\
6 & 25.0 & 162.4470 & 163.4266 & 162.7614 & 152.6965 & 152.7032 & 152.4803 & 162.4614 & 163.4466 & 162.7800 & 152.7565 & 152.7645 & 152.5378 \\
7 & 23.6 & 164.6009 & 166.3246 & 166.5586 & 157.3911 & 157.2889 & 157.0201 & 164.9132 & 166.6289 & 166.9487 & 157.8304 & 157.6989 & 157.4384 \\
8 & 21.6 & 169.4721 & 171.2551 & 171.6691 & 162.6878 & 162.5203 & 162.3521 & 169.7324 & 171.5078 & 171.9957 & 163.0541 & 162.8622 & 162.7038 \\
9 & 14.5 & 173.4251 & 175.2830 & 176.0604 & 167.3356 & 167.3011 & 167.2687 & 173.5711 & 175.4293 & 176.3104 & 167.6020 & 167.5473 & 167.5332 \\
10 & 11.1 & 177.8173 & 179.6760 & 180.4223 & 171.8256 & 171.9318 & 171.5529 & 177.9172 & 179.7649 & 180.4837 & 171.9200 & 172.0004 & 171.6108 \\
${ }^{1} \mathrm{H}$ & & & & & & & & & & & & & \\
\hline 2 & 2.72 & 29.6395 & 29.1355 & 28.9206 & 29.5840 & 29.3433 & 29.1932 & 29.5439 & 29.0374 & 28.7990 & 29.4889 & 29.2413 & 29.0743 \\
3 & 3.68 & 28.5694 & 28.1831 & 28.1754 & 28.6359 & 28.4868 & 28.4893 & 28.5268 & 28.1395 & 28.1104 & 28.5779 & 28.4278 & 28.4173 \\
4 & 1.66 & 30.2985 & 29.9119 & 29.9117 & 30.4651 & 30.3299 & 30.2869 & 30.3062 & 29.9176 & 29.9060 & 30.4647 & 30.3250 & 30.2763 \\
$5 a$ & 1.07 & 31.1606 & 30.7971 & 30.7855 & 31.2513 & 31.0937 & 31.0720 & 31.1146 & 30.7506 & 30.7324 & 31.2007 & 31.0439 & 31.0199 \\
$5 b$ & 1.07 & 31.1456 & 30.7716 & 30.7764 & 31.2846 & 31.1115 & 31.1043 & 31.0714 & 30.6967 & 30.6954 & 31.2075 & 31.0351 & 31.0262 \\
6 & 1.66 & 30.1993 & 29.7688 & 29.8074 & 30.3417 & 30.1585 & 30.1402 & 30.1998 & 29.7719 & 29.8054 & 30.3389 & 30.1574 & 30.1387 \\
7 & 0.89 & 31.2221 & 30.8072 & 30.7486 & 31.1956 & 30.9777 & 30.9509 & 31.2136 & 30.7978 & 30.7425 & 31.1894 & 30.9712 & 30.9464 \\
8 & 0.85 & 31.2342 & 30.8103 & 30.7681 & 31.2636 & 31.0385 & 31.0201 & 31.2263 & 30.8020 & 30.7607 & 31.2566 & 31.0317 & 31.0146 \\
9 & 1.22 & 30.8942 & 30.4397 & 30.3358 & 30.9185 & 30.6754 & 30.6116 & 30.8839 & 30.4280 & 30.3223 & 30.9097 & 30.6639 & 30.5967 \\
10 & 0.95 & 31.0733 & 30.6391 & 30.5614 & 31.0684 & 30.8418 & 30.8023 & 31.1070 & 30.6724 & 30.5947 & 31.1029 & 30.8733 & 30.8331 \\
\hline
\end{tabular}

\begin{tabular}{|c|c|c|c|c|c|c|c|c|c|c|c|c|c|}
\hline \multirow{3}{*}{\multicolumn{2}{|c|}{ Exp }} & \multicolumn{12}{|c|}{ mPW1PW91 } \\
\hline & & \multicolumn{6}{|c|}{ Gas phase } & \multicolumn{6}{|c|}{ PCM } \\
\hline & & 6-31G* & 6-31G** & $6-31+G * *$ & 6-311G* & 6-311G** & $6-311+G * *$ & 6-31G* & 6-31G** & $6-31+G * *$ & 6-311G* & 6-311G** & $6-311+G * *$ \\
\hline \multicolumn{14}{|c|}{ 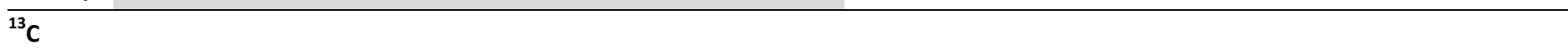 } \\
\hline 1 & 181.6 & 20.7544 & 20.4528 & 18.0852 & 0.8396 & 1.0445 & -1.0476 & 19.8309 & 19.5141 & 16.9556 & -0.2024 & -0.0835 & -2.3748 \\
\hline 2 & 42.1 & 153.9135 & 154.5531 & 153.7366 & 146.3185 & 146.0668 & 144.9377 & 153.8668 & 154.5047 & 153.6610 & 146.2099 & 145.9359 & 144.7597 \\
\hline 3 & 75.6 & 120.2123 & 120.7029 & 120.1162 & 110.1687 & 110.0494 & 109.8062 & 120.2650 & 120.7539 & 120.1550 & 110.1937 & 110.0558 & 109.8330 \\
\hline 4 & 33.3 & 160.3232 & 161.0789 & 160.4348 & 152.1608 & 152.0676 & 151.7346 & 160.3625 & 161.1175 & 160.4505 & 152.1829 & 152.0891 & 151.7146 \\
\hline 5 & 42.6 & 154.9177 & 155.8999 & 154.9272 & 146.0199 & 145.6317 & 145.3322 & 155.3455 & 156.3207 & 155.4595 & 146.5445 & 146.1386 & 145.8785 \\
\hline 6 & 25.0 & 167.6488 & 168.5794 & 168.0156 & 158.7248 & 158.7282 & 158.4331 & 167.6738 & 168.6102 & 168.0473 & 158.7899 & 158.7952 & 158.4962 \\
\hline 7 & 23.6 & 169.0500 & 170.6397 & 170.6850 & 162.3488 & 162.2495 & 162.0139 & 169.4036 & 170.9853 & 171.1126 & 162.7937 & 162.6676 & 162.4420 \\
\hline 8 & 21.6 & 173.7563 & 175.4039 & 175.6206 & 167.4733 & 167.3083 & 167.1611 & 174.0496 & 175.6897 & 175.9755 & 167.8421 & 167.6549 & 167.5177 \\
\hline 9 & 14.5 & 177.7535 & 179.4794 & 180.1391 & 172.0591 & 172.0294 & 172.0087 & 177.9364 & 179.6619 & 180.4148 & 172.3312 & 172.2821 & 172.2770 \\
\hline $\begin{array}{l}10 \\
{ }^{1} \mathbf{H}\end{array}$ & 11.1 & 182.0806 & 183.8169 & 184.3684 & 176.4382 & 176.5539 & 176.1653 & 182.2074 & 183.9329 & 184.4602 & 176.5478 & 176.6393 & 176.2461 \\
\hline 2 & 2.72 & 29.5946 & 29.0983 & 28.8864 & 29.4857 & 29.2555 & 29.1085 & 29.4944 & 28.9952 & 28.7634 & 29.3883 & 29.1504 & 28.9879 \\
\hline 3 & 3.68 & 28.5088 & 28.1293 & 28.1019 & 28.5357 & 28.3911 & 28.3884 & 28.4627 & 28.0823 & 28.0360 & 28.4773 & 28.3315 & 28.3169 \\
\hline 4 & 1.66 & 30.2516 & 29.8683 & 29.8511 & 30.3428 & 30.2195 & 30.1685 & 30.2612 & 29.8758 & 29.8488 & 30.3448 & 30.2165 & 30.1603 \\
\hline $5 a$ & 1.07 & 31.1350 & 30.7747 & 30.7467 & 31.1627 & 31.0041 & 30.9842 & 31.0855 & 30.7244 & 30.6915 & 31.1111 & 30.9529 & 30.9313 \\
\hline $5 b$ & 1.07 & 31.1282 & 30.7600 & 30.7489 & 31.1909 & 31.0232 & 31.0147 & 31.0520 & 30.6829 & 30.6672 & 31.1137 & 30.9463 & 30.9363 \\
\hline 6 & 1.66 & 30.1692 & 29.7433 & 29.7541 & 30.2347 & 30.0625 & 30.0338 & 30.1712 & 29.7477 & 29.7542 & 30.2339 & 30.0633 & 30.0338 \\
\hline 7 & 0.89 & 31.1714 & 30.7572 & 30.7017 & 31.1121 & 30.8893 & 30.8625 & 31.1601 & 30.7449 & 30.6927 & 31.1030 & 30.8799 & 30.8551 \\
\hline 8 & 0.85 & 31.1905 & 30.7677 & 30.7235 & 31.1815 & 30.9514 & 30.9310 & 31.1807 & 30.7574 & 30.7142 & 31.1724 & 30.9426 & 30.9235 \\
\hline 9 & 1.22 & 30.8376 & 30.3822 & 30.2856 & 30.8378 & 30.5906 & 30.5275 & 30.8249 & 30.3681 & 30.2705 & 30.8267 & 30.5766 & 30.5107 \\
\hline 10 & 0.95 & 31.0228 & 30.5892 & 30.5136 & 30.9838 & 30.7544 & 30.7176 & 31.0549 & 30.6211 & 30.5451 & 31.0163 & 30.7840 & 30.7465 \\
\hline
\end{tabular}




\section{Compound 75d}<smiles>CC(C)C[C@H]([18OH])[C@@H](O)[C@@H](C)C(=O)O</smiles>

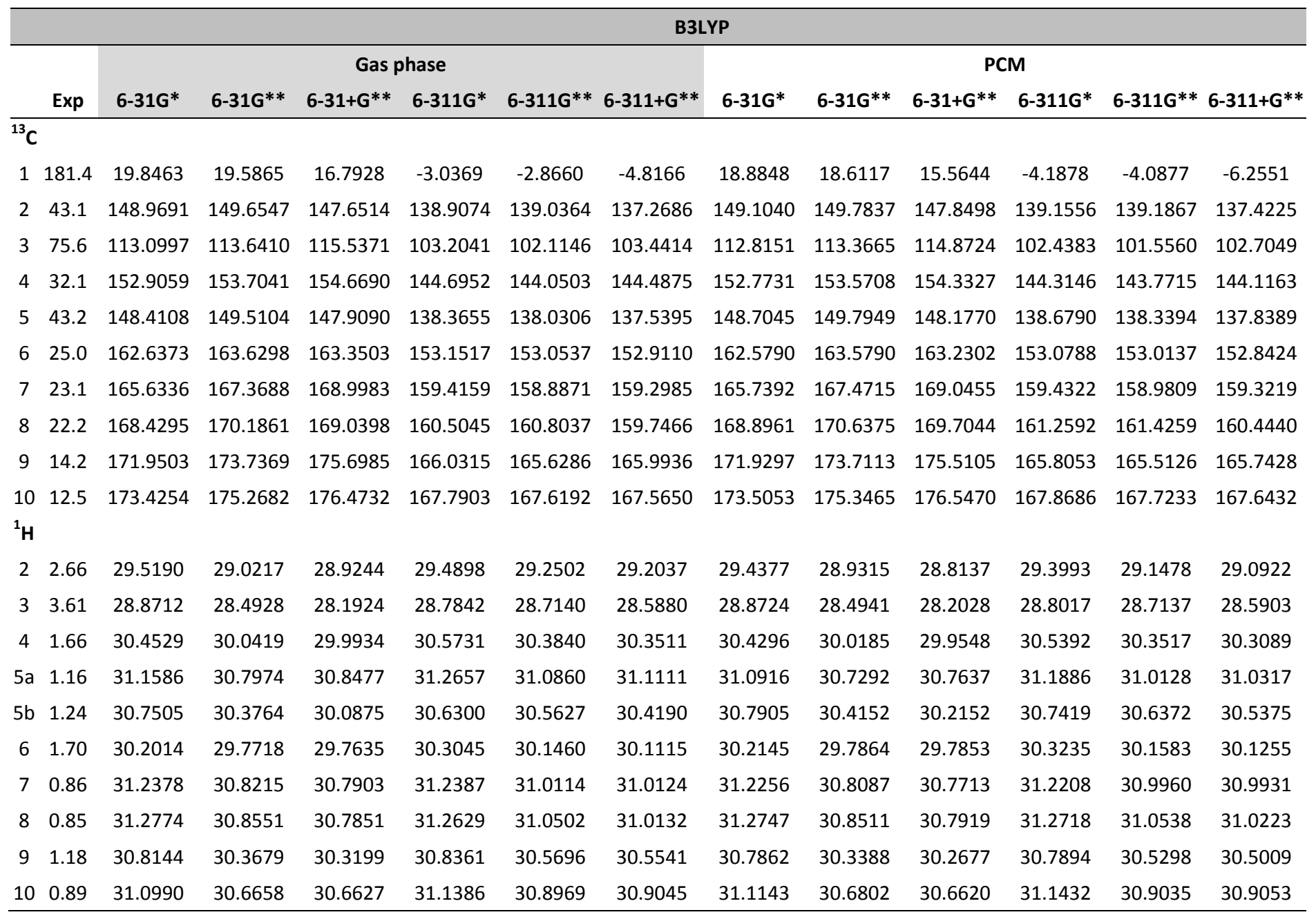

\begin{tabular}{|c|c|c|c|c|c|c|c|c|c|c|c|c|c|}
\hline & & \multicolumn{12}{|c|}{ mPW1PW91 } \\
\hline & \multirow[b]{2}{*}{ Exp } & \multicolumn{6}{|c|}{ Gas phase } & \multicolumn{6}{|c|}{ PCM } \\
\hline & & 6-31G* & $6-31 G^{* *}$ & $6-31+G * *$ & 6-311G* & 6-311G** & $6-311+G^{* *}$ & 6-31G* & $6-31 G^{* *}$ & $6-31+G^{* *}$ & 6-311G* & $6-311 \mathrm{G} * *$ & $6-311+G * *$ \\
\hline \\
\hline 1 & 181.4 & 22.0515 & 21.7724 & 19.5769 & 2.1223 & 2.2930 & 0.5575 & 21.0771 & 20.7833 & 18.3686 & 0.9913 & 1.0855 & -0.8380 \\
\hline 2 & 43.1 & 153.2830 & 153.9289 & 152.2862 & 144.4497 & 144.5632 & 143.0426 & 153.4216 & 154.0612 & 152.4259 & 144.6375 & 144.6670 & 143.1417 \\
\hline 3 & 75.6 & 118.6934 & 119.1648 & 120.8170 & 110.0428 & 109.0437 & 109.9103 & 118.3687 & 118.8530 & 120.2436 & 109.3530 & 108.5308 & 109.2802 \\
\hline 4 & 32.1 & 158.3030 & 159.0380 & 159.8193 & 150.8332 & 150.2616 & 150.4250 & 158.1501 & 158.8861 & 159.5545 & 150.5136 & 150.0242 & 150.1248 \\
\hline 5 & 43.2 & 153.7278 & 154.7091 & 153.4174 & 144.6441 & 144.3310 & 143.8937 & 154.0225 & 154.9964 & 153.6915 & 144.9430 & 144.6245 & 144.1927 \\
\hline 6 & 25.0 & 167.8945 & 168.8353 & 168.6164 & 159.1861 & 159.0908 & 158.8401 & 167.8407 & 168.7892 & 168.5272 & 159.1275 & 159.0616 & 158.7910 \\
\hline 7 & 23.1 & 170.2777 & 171.8728 & 173.2033 & 164.4103 & 163.9062 & 164.1604 & 170.4079 & 172.0009 & 173.3191 & 164.4716 & 164.0359 & 164.2479 \\
\hline 8 & 22.2 & 172.4924 & 174.1247 & 173.0897 & 165.2641 & 165.5332 & 164.7251 & 173.0086 & 174.6243 & 173.7355 & 165.9766 & 166.1283 & 165.3746 \\
\hline
\end{tabular}




\begin{tabular}{ccccccccccccccc}
\hline 9 & 14.2 & 176.6279 & 178.2832 & 179.9009 & 170.9215 & 170.5671 & 170.7407 & 176.6141 & 178.2655 & 179.7974 & 170.7527 & 170.4924 & 170.5697 \\
10 & 12.5 & 177.8028 & 179.5184 & 180.4769 & 172.5032 & 172.3492 & 172.2765 & 177.9114 & 179.6244 & 180.6068 & 172.6064 & 172.4727 & 172.3895 \\
${ }^{1} \mathbf{H}$ & & & & & & & & & & & & & & \\
2 & 2.66 & 29.4647 & 28.9816 & 28.8771 & 29.3913 & 29.1627 & 29.1111 & 29.3812 & 28.8889 & 28.7705 & 29.3022 & 29.0612 & 29.0033 \\
3 & 3.61 & 28.7456 & 28.3750 & 28.1142 & 28.6625 & 28.5890 & 28.4995 & 28.7471 & 28.3764 & 28.1146 & 28.6727 & 28.5823 & 28.4921 \\
4 & 1.66 & 30.4018 & 29.9931 & 29.9406 & 30.4511 & 30.2725 & 30.2423 & 30.3785 & 29.9695 & 29.9003 & 30.4166 & 30.2397 & 30.2003 \\
$5 a$ & 1.16 & 31.1315 & 30.7753 & 30.7937 & 31.1669 & 30.9895 & 31.0076 & 31.0613 & 30.7034 & 30.7105 & 31.0908 & 30.9166 & 30.9297 \\
$5 b$ & 1.24 & 30.6766 & 30.3087 & 30.0469 & 30.5212 & 30.4540 & 30.3464 & 30.7315 & 30.3617 & 30.1703 & 30.6315 & 30.5291 & 30.4543 \\
6 & 1.70 & 30.1607 & 29.7373 & 29.7147 & 30.1945 & 30.0479 & 30.0092 & 30.1780 & 29.7557 & 29.7382 & 30.2151 & 30.0621 & 30.0238 \\
7 & 0.86 & 31.1934 & 30.7774 & 30.7461 & 31.1583 & 30.9264 & 30.9230 & 31.1772 & 30.7605 & 30.7245 & 31.1377 & 30.9080 & 30.9022 \\
8 & 0.85 & 31.2299 & 30.8097 & 30.7419 & 31.1777 & 30.9601 & 30.9289 & 31.2273 & 30.8055 & 30.7459 & 31.1838 & 30.9615 & 30.9347 \\
9 & 1.18 & 30.7678 & 30.3181 & 30.2712 & 30.7646 & 30.4919 & 30.4680 & 30.7326 & 30.2821 & 30.2175 & 30.7156 & 30.4490 & 30.4147 \\
10 & 0.89 & 31.0608 & 30.6283 & 30.6180 & 31.0587 & 30.8151 & 30.8166 & 31.0724 & 30.6391 & 30.6174 & 31.0622 & 30.8203 & 30.8173 \\
\hline
\end{tabular}

\section{Compound 76a}

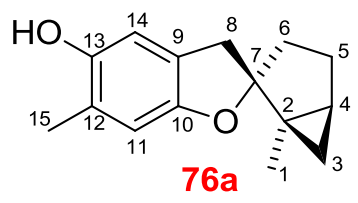

\begin{tabular}{|c|c|c|c|c|c|c|c|c|c|c|c|c|c|}
\hline & \multirow[b]{3}{*}{ Exp } & \multicolumn{12}{|c|}{ B3LYP } \\
\hline & & \multicolumn{6}{|c|}{ Gas phase } & \multicolumn{6}{|c|}{ PCM } \\
\hline & & 6-31G* & 6-31G** & $6-31+G * *$ & 6-311G* & 6-311G** & $6-311+G * *$ & 6-31G* & 6-31G** & $6-31+G * *$ & 6-311G* & 6-311G** & $6-311+G^{* *}$ \\
\hline \multicolumn{14}{|l|}{${ }^{13} \mathrm{C}$} \\
\hline 1 & 15.2 & 172.9461 & 174.8809 & 176.1017 & 166.8352 & 166.6409 & 166.3202 & 173.3019 & 175.2255 & 176.4808 & 167.2669 & 167.0481 & 166.7382 \\
\hline 2 & 31.6 & 158.1618 & 158.3541 & 158.2472 & 148.0375 & 147.8803 & 147.5531 & 158.2682 & 158.4610 & 158.3455 & 148.1697 & 148.0078 & 147.6736 \\
\hline 3 & 14.5 & 174.0847 & 175.6458 & 175.2726 & 167.0890 & 166.5140 & 166.3553 & 174.4780 & 176.0175 & 175.6844 & 167.5293 & 166.9282 & 166.7665 \\
\hline 4 & 25.6 & 163.3977 & 164.3437 & 163.7909 & 153.8288 & 153.3922 & 152.8080 & 163.6787 & 164.6211 & 164.0895 & 154.1220 & 153.6829 & 153.1183 \\
\hline 5 & 26.2 & 162.1871 & 163.6012 & 163.2754 & 153.7796 & 153.5628 & 153.3910 & 162.3150 & 163.7260 & 163.3789 & 153.9021 & 153.6757 & 153.5133 \\
\hline 6 & 36.6 & 150.8322 & 152.2230 & 152.8712 & 142.2933 & 142.0338 & 141.4854 & 151.0437 & 152.4269 & 153.0899 & 142.5137 & 142.2377 & 141.6929 \\
\hline 7 & 98.2 & 92.0253 & 92.3133 & 92.2250 & 78.9948 & 78.9690 & 78.5957 & 91.6607 & 91.9464 & 91.7681 & 78.5282 & 78.5020 & 78.0945 \\
\hline 8 & 36.7 & 152.1918 & 153.4119 & 152.4439 & 143.4217 & 142.8960 & 143.0344 & 152.4644 & 153.6767 & 152.7013 & 143.7096 & 143.1639 & 143.3033 \\
\hline 9 & 125.6 & 69.9341 & 70.0214 & 70.7398 & 52.7935 & 52.6994 & 51.6255 & 69.1560 & 69.2515 & 69.8338 & 51.9292 & 51.8585 & 50.6480 \\
\hline 10 & 153.5 & 44.3176 & 44.2618 & 43.1096 & 23.6795 & 23.5152 & 22.6611 & 44.5661 & 44.5177 & 43.3095 & 23.8897 & 23.7429 & 22.8829 \\
\hline 11 & 111.1 & 83.4591 & 84.2465 & 83.7585 & 68.0647 & 67.4836 & 66.8073 & 84.1020 & 84.8824 & 84.3243 & 68.6423 & 68.0648 & 67.4472 \\
\hline 12 & 123.8 & 73.2772 & 73.2677 & 72.5467 & 55.6968 & 55.5374 & 54.3061 & 73.0983 & 73.0937 & 72.3503 & 55.2676 & 55.1306 & 54.0382 \\
\hline 13 & 149.4 & 48.6592 & 48.4112 & 46.8795 & 26.7913 & 26.8416 & 26.5456 & 48.7815 & 48.5257 & 47.1013 & 26.9380 & 26.9740 & 26.7930 \\
\hline 14 & 112.1 & 86.0319 & 86.6792 & 86.7212 & 71.2213 & 70.4470 & 69.4992 & 85.7466 & 86.3879 & 86.3244 & 70.8104 & 70.0448 & 69.0239 \\
\hline $\begin{array}{l}15 \\
{ }^{1} \mathbf{H}\end{array}$ & 16.5 & 171.3581 & 173.0860 & 174.1617 & 165.1078 & 164.8286 & 164.7687 & 171.6930 & 173.4044 & 174.5202 & 165.3911 & 165.1096 & 165.1486 \\
\hline 1 & 1.06 & 30.9953 & 30.5384 & 30.4519 & 30.9843 & 30.7180 & 30.6961 & 30.9962 & 30.5380 & 30.4499 & 30.9843 & 30.7167 & 30.6929 \\
\hline $3 a$ & 0.45 & 31.6971 & 31.2660 & 31.1319 & 31.8052 & 31.6124 & 31.5369 & 31.6271 & 31.1917 & 31.0495 & 31.7287 & 31.5343 & 31.4549 \\
\hline $3 b$ & 0.29 & 31.8692 & 31.4650 & 31.3598 & 31.8337 & 31.6900 & 31.6642 & 31.8066 & 31.3989 & 31.2909 & 31.7695 & 31.6244 & 31.5973 \\
\hline 4 & 1.25 & 30.8826 & 30.4856 & 30.3897 & 30.8903 & 30.7266 & 30.6672 & 30.8374 & 30.4381 & 30.3352 & 30.8353 & 30.6717 & 30.6102 \\
\hline
\end{tabular}




\begin{tabular}{llllllllllllll}
\hline 5a & 1.98 & 30.0359 & 29.5794 & 29.4106 & 29.9956 & 29.7595 & 29.6667 & 30.0862 & 29.6317 & 29.4630 & 30.0418 & 29.8087 & 29.7175 \\
5b & 1.64 & 30.5797 & 30.1118 & 30.0437 & 30.5071 & 30.2534 & 30.2082 & 30.5380 & 30.0674 & 29.9986 & 30.4663 & 30.2102 & 30.1651 \\
6a & 1.85 & 30.3344 & 29.8699 & 29.7497 & 30.2969 & 30.0192 & 29.9954 & 30.3647 & 29.8996 & 29.7817 & 30.3285 & 30.0495 & 30.0240 \\
6b & 1.45 & 31.0408 & 30.5898 & 30.4930 & 31.0224 & 30.7997 & 30.7207 & 30.9238 & 30.4691 & 30.3592 & 30.9019 & 30.6784 & 30.5928 \\
8a & 3.27 & 28.7513 & 28.2206 & 28.0770 & 28.7413 & 28.4178 & 28.4010 & 28.7010 & 28.1703 & 28.0180 & 28.6907 & 28.3688 & 28.3458 \\
8b & 2.89 & 29.6095 & 29.1379 & 28.9493 & 29.5279 & 29.2681 & 29.2516 & 29.4865 & 29.0110 & 28.8101 & 29.4029 & 29.1427 & 29.1178 \\
11 & 6.41 & 25.9573 & 25.3073 & 25.1093 & 25.8039 & 25.3447 & 25.3471 & 25.9141 & 25.2626 & 25.0453 & 25.7367 & 25.2830 & 25.2755 \\
14 & 6.63 & 26.1149 & 25.4278 & 25.0830 & 25.9346 & 25.4552 & 25.3799 & 25.9489 & 25.2587 & 24.8920 & 25.7673 & 25.2915 & 25.1952 \\
15 & 2.12 & 30.1792 & 29.6981 & 29.5318 & 30.0592 & 29.7826 & 29.7388 & 30.1426 & 29.6606 & 29.4898 & 30.0170 & 29.7415 & 29.6953 \\
\hline
\end{tabular}

\begin{tabular}{|c|c|c|c|c|c|c|c|c|c|c|c|c|c|}
\hline & \multirow[b]{3}{*}{ Exp } & \multicolumn{12}{|c|}{ mPW1PW91 } \\
\hline & & \multicolumn{6}{|c|}{ Gas phase } & \multicolumn{6}{|c|}{ PCM } \\
\hline & & 6-31G* & 6-31G** & $6-31+G * *$ & 6-311G* & 6-311G** & $6-311+G * *$ & 6-31G* & 6-31G** & $6-31+G^{* *}$ & 6-311G* & 6-311G** & $6-311+G * *$ \\
\hline \\
\hline 1 & 15.2 & 176.9844 & 178.7978 & 180.0907 & 171.4952 & 171.3065 & 170.9656 & 177.3943 & 179.1963 & 177.3944 & 171.9454 & 171.7344 & 171.4027 \\
\hline 2 & 31.6 & 162.6843 & 162.8678 & 162.7673 & 153.3543 & 153.1727 & 152.8294 & 162.7982 & 162.9821 & 162.7981 & 153.4840 & 153.2988 & 152.9473 \\
\hline 3 & 14.5 & 178.1903 & 179.6665 & 179.2007 & 171.8210 & 171.2502 & 171.1083 & 178.6148 & 180.0699 & 178.6150 & 172.2680 & 171.6736 & 171.5284 \\
\hline 4 & 25.6 & 167.8009 & 168.6898 & 168.1155 & 159.0915 & 158.6453 & 158.0566 & 168.1154 & 169.0002 & 168.1154 & 159.3959 & 158.9482 & 158.3742 \\
\hline 5 & 26.2 & 166.5690 & 167.8861 & 167.7077 & 158.8322 & 158.6024 & 158.3404 & 166.7398 & 168.0534 & 166.7398 & 158.9826 & 158.7426 & 158.4882 \\
\hline 6 & 36.6 & 155.6840 & 156.9660 & 157.7109 & 147.9117 & 147.6549 & 147.1445 & 155.9135 & 157.1887 & 155.9132 & 148.1298 & 147.8587 & 147.3553 \\
\hline 7 & 98.2 & 96.5438 & 96.8330 & 97.0093 & 85.1835 & 85.1743 & 84.7752 & 96.1680 & 96.4544 & 96.1686 & 84.7071 & 84.6967 & 84.2685 \\
\hline 8 & 36.7 & 156.0756 & 157.2285 & 156.4026 & 148.4259 & 147.9066 & 148.0571 & 156.3690 & 157.5152 & 156.3691 & 148.7150 & 148.1772 & 148.3325 \\
\hline 9 & 125.6 & 72.7536 & 72.8237 & 73.6529 & 57.7375 & 57.6325 & 56.6095 & 72.0257 & 72.1080 & 72.0322 & 56.9578 & 56.8745 & 55.7259 \\
\hline 10 & 153.5 & 47.2643 & 47.2048 & 46.4593 & 29.3485 & 29.1779 & 28.3421 & 47.5177 & 47.4662 & 47.5166 & 29.5599 & 29.4077 & 28.5686 \\
\hline 11 & 111.1 & 85.8871 & 86.6369 & 86.4674 & 72.3829 & 71.7910 & 71.0477 & 86.5087 & 87.2474 & 86.5062 & 72.8829 & 72.2960 & 71.6104 \\
\hline 12 & 123.8 & 76.6655 & 76.6565 & 76.0031 & 61.4503 & 61.2664 & 60.0214 & 76.3325 & 76.3224 & 76.3179 & 60.8110 & 60.6578 & 59.5588 \\
\hline 13 & 149.4 & 51.7333 & 51.4623 & 50.6456 & 32.8546 & 32.8875 & 32.4091 & 51.8640 & 51.5860 & 51.8660 & 33.0013 & 33.0204 & 32.6467 \\
\hline 14 & 112.1 & 88.3320 & 88.9309 & 89.0021 & 75.1913 & 74.4111 & 73.4496 & 88.1011 & 88.6978 & 88.1055 & 74.8694 & 74.0936 & 73.0590 \\
\hline $\begin{array}{l}15 \\
{ }^{1} \mathbf{H}\end{array}$ & 16.5 & 175.2559 & 176.8755 & 178.2297 & 169.6055 & 169.3142 & 169.2516 & 175.6216 & 177.2230 & 175.6129 & 169.8752 & 169.5869 & 169.6195 \\
\hline 1 & 1.06 & 30.9427 & 30.4838 & 30.4097 & 30.8929 & 30.6192 & 30.6048 & 30.9414 & 30.4812 & 30.9414 & 30.8904 & 30.6155 & 30.5991 \\
\hline $3 a$ & 0.45 & 31.6250 & 31.1948 & 31.0494 & 31.6913 & 31.4924 & 31.4250 & 31.5497 & 31.1147 & 31.6844 & 31.6116 & 31.4112 & 31.3398 \\
\hline $3 b$ & 0.29 & 31.7555 & 31.3589 & 31.2697 & 31.7069 & 31.5601 & 31.5422 & 31.6843 & 31.2836 & 31.5498 & 31.6360 & 31.4873 & 31.4683 \\
\hline 4 & 1.25 & 30.7958 & 30.3976 & 30.3192 & 30.7854 & 30.6080 & 30.5522 & 30.7449 & 30.3436 & 30.7449 & 30.7263 & 30.5487 & 30.4904 \\
\hline $5 a$ & 1.98 & 29.9695 & 29.5137 & 29.3334 & 29.8941 & 29.6566 & 29.5571 & 30.0184 & 29.5645 & 30.0184 & 29.9380 & 29.7038 & 29.6053 \\
\hline $5 b$ & 1.64 & 30.5173 & 30.0487 & 29.9755 & 30.4102 & 30.1537 & 30.0996 & 30.4695 & 29.9978 & 30.4695 & 30.3639 & 30.1050 & 30.0518 \\
\hline $6 a$ & 1.85 & 30.2315 & 29.7644 & 29.6593 & 30.1778 & 29.8943 & 29.8673 & 30.2645 & 29.7967 & 30.2645 & 30.2111 & 29.9263 & 29.8977 \\
\hline $6 b$ & 1.45 & 30.9998 & 30.5536 & 30.4307 & 30.9304 & 30.7166 & 30.6254 & 30.8742 & 30.4236 & 30.8742 & 30.8040 & 30.5890 & 30.4923 \\
\hline $8 a$ & 3.27 & 28.6842 & 28.1515 & 28.0288 & 28.6369 & 28.3163 & 28.3102 & 28.6277 & 28.0951 & 28.6278 & 28.5813 & 28.2624 & 28.2511 \\
\hline $8 b$ & 2.89 & 29.5203 & 29.0450 & 28.8856 & 29.4133 & 29.1510 & 29.1376 & 29.3869 & 28.9070 & 29.3870 & 29.2807 & 29.0175 & 28.9972 \\
\hline 11 & 6.41 & 25.7628 & 25.1108 & 24.9490 & 25.6317 & 25.1463 & 25.1633 & 25.7030 & 25.0487 & 25.7029 & 25.5492 & 25.0697 & 25.0778 \\
\hline 14 & 6.63 & 25.8885 & 25.2030 & 24.9002 & 25.7337 & 25.2346 & 25.1694 & 25.7193 & 25.0309 & 25.7206 & 25.5698 & 25.0735 & 24.9878 \\
\hline 15 & 2.12 & 30.0956 & 29.6104 & 29.4656 & 29.9683 & 29.6798 & 29.6524 & 30.0460 & 29.5596 & 30.0458 & 29.9127 & 29.6260 & 29.5958 \\
\hline
\end{tabular}




\section{Compound 76b}

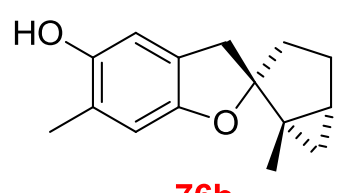

$76 b$

\begin{tabular}{|c|c|c|c|c|c|c|c|c|c|c|c|c|c|}
\hline & \multirow[b]{3}{*}{ Exp } & \multicolumn{12}{|c|}{ B3LYP } \\
\hline & & \multicolumn{6}{|c|}{ Gas phase } & \multicolumn{6}{|c|}{ PCM } \\
\hline & & 6-31G* & 6-31G** & $6-31+G * *$ & 6-311G* & 6-311G** & $6-311+G^{* *}$ & 6-31G* & 6-31G** & $6-31+G * *$ & 6-311G* & 6-311G** & $6-311+G * *$ \\
\hline \\
\hline 1 & 16.3 & 171.7543 & 173.6570 & 174.2158 & 165.4607 & 165.1730 & 164.9134 & 172.1403 & 174.0296 & 174.6006 & 165.8833 & 165.5712 & 165.3209 \\
\hline 2 & 28.0 & 159.4923 & 159.7320 & 159.5345 & 149.7116 & 149.5779 & 148.4465 & 159.4918 & 159.7328 & 159.4564 & 149.7143 & 149.5801 & 148.3798 \\
\hline 3 & 16.1 & 172.8608 & 174.4228 & 175.0584 & 165.9873 & 165.4052 & 164.9036 & 173.3984 & 174.9436 & 175.6739 & 166.6342 & 166.0406 & 165.5572 \\
\hline 4 & 24.2 & 164.6662 & 165.6316 & 164.7479 & 155.1822 & 154.8378 & 154.2744 & 164.7075 & 165.6672 & 164.7821 & 155.2397 & 154.8828 & 154.2900 \\
\hline 5 & 26.9 & 163.5341 & 164.9454 & 164.1112 & 155.0118 & 154.7805 & 154.3903 & 163.7062 & 165.1135 & 164.2808 & 155.2091 & 154.9685 & 154.5593 \\
\hline 6 & 34.1 & 152.8670 & 154.2968 & 154.1055 & 143.8234 & 143.4948 & 143.3513 & 153.0261 & 154.4464 & 154.2495 & 144.0070 & 143.6680 & 143.4702 \\
\hline 7 & 97.5 & 93.5519 & 93.8453 & 93.3238 & 80.3557 & 80.3018 & 79.9323 & 93.2085 & 93.4986 & 92.9178 & 79.8833 & 79.8280 & 79.4534 \\
\hline 8 & 38.5 & 149.5142 & 150.7153 & 150.2650 & 140.8401 & 140.2822 & 140.0200 & 149.7436 & 150.9338 & 150.4993 & 141.0963 & 140.5135 & 140.2419 \\
\hline 9 & 125.2 & 70.4001 & 70.4610 & 71.4824 & 53.2364 & 53.1258 & 51.9183 & 69.5332 & 69.6034 & 70.4937 & 52.2681 & 52.1832 & 50.8558 \\
\hline 10 & 153.9 & 43.4406 & 43.3833 & 41.6791 & 22.5779 & 22.4539 & 21.6722 & 43.7899 & 43.7388 & 41.9836 & 22.9030 & 22.7950 & 22.0051 \\
\hline 11 & 112.0 & 84.0186 & 84.8025 & 84.2309 & 68.7295 & 68.1525 & 67.7365 & 84.6916 & 85.4680 & 84.8352 & 69.3519 & 68.7786 & 68.3987 \\
\hline 12 & 122.7 & 73.4161 & 73.4072 & 72.4397 & 55.8827 & 55.7075 & 54.5757 & 73.2322 & 73.2291 & 72.2232 & 55.4446 & 55.2975 & 54.2839 \\
\hline 13 & 147.3 & 48.7460 & 48.4962 & 46.8372 & 26.8871 & 26.9409 & 26.5564 & 48.8191 & 48.5614 & 47.0048 & 26.9824 & 27.0209 & 26.7308 \\
\hline 14 & 110.5 & 85.8948 & 86.5385 & 86.2861 & 71.1460 & 70.3730 & 69.3142 & 85.5708 & 86.2086 & 85.8434 & 70.6936 & 69.9244 & 68.7720 \\
\hline $\begin{array}{l}15 \\
{ }^{1} \mathbf{H}\end{array}$ & 14.5 & 171.3885 & 173.1174 & 174.5078 & 165.1897 & 164.8954 & 164.8919 & 171.7175 & 173.4303 & 174.8659 & 165.4589 & 165.1652 & 165.2605 \\
\hline 1 & 1.11 & 31.0648 & 30.6054 & 30.5369 & 31.0567 & 30.7882 & 30.7808 & 31.0531 & 30.5918 & 30.5226 & 31.0439 & 30.7735 & 30.7657 \\
\hline $3 a$ & 1.00 & 31.3930 & 30.9541 & 30.8871 & 31.4334 & 31.2343 & 31.2599 & 31.4017 & 30.9617 & 30.8968 & 31.4358 & 31.2368 & 31.2647 \\
\hline $3 b$ & 0.39 & 31.5331 & 31.1220 & 30.9898 & 31.5468 & 31.3722 & 31.2646 & 31.5204 & 31.1053 & 30.9709 & 31.5298 & 31.3533 & 31.2471 \\
\hline 4 & 1.18 & 31.0865 & 30.6836 & 30.5472 & 31.0931 & 30.9216 & 30.8307 & 30.9924 & 30.5862 & 30.4430 & 30.9922 & 30.8212 & 30.7273 \\
\hline $5 a$ & 1.69 & 30.5263 & 30.0611 & 29.9985 & 30.4421 & 30.2013 & 30.1726 & 30.5025 & 30.0361 & 29.9753 & 30.4220 & 30.1788 & 30.1513 \\
\hline $5 b$ & 1.69 & 30.4015 & 29.9436 & 29.8387 & 30.3653 & 30.1322 & 30.0987 & 30.3302 & 29.8702 & 29.7646 & 30.2954 & 30.0621 & 30.0277 \\
\hline $6 a$ & 1.69 & 30.6755 & 30.2435 & 30.1894 & 30.6207 & 30.3812 & 30.4315 & 30.6125 & 30.1775 & 30.1105 & 30.5551 & 30.3128 & 30.3534 \\
\hline $6 b$ & 1.69 & 30.4721 & 30.0038 & 29.9257 & 30.4150 & 30.1869 & 30.1894 & 30.5034 & 30.0334 & 29.9565 & 30.4448 & 30.2156 & 30.2187 \\
\hline $8 a$ & 3.43 & 28.7139 & 28.1727 & 27.9805 & 28.7045 & 28.3636 & 28.3404 & 28.6436 & 28.1019 & 27.8999 & 28.6332 & 28.2934 & 28.2627 \\
\hline $8 b$ & 2.73 & 29.5461 & 29.0924 & 28.9779 & 29.5035 & 29.2385 & 29.2420 & 29.4290 & 28.9723 & 28.8416 & 29.3830 & 29.1187 & 29.1115 \\
\hline 11 & 6.64 & 25.9555 & 25.3080 & 25.1064 & 25.8023 & 25.3462 & 25.3390 & 25.9174 & 25.2682 & 25.0434 & 25.7401 & 25.2893 & 25.2701 \\
\hline 14 & 6.55 & 26.0969 & 25.4140 & 25.1345 & 25.9107 & 25.4448 & 25.3941 & 25.9293 & 25.2432 & 24.9433 & 25.7424 & 25.2795 & 25.2079 \\
\hline 15 & 2.19 & 30.1820 & 29.7008 & 29.5187 & 30.0614 & 29.7855 & 29.7409 & 30.1441 & 29.6622 & 29.4744 & 30.0178 & 29.7434 & 29.6957 \\
\hline
\end{tabular}




\begin{tabular}{|c|c|c|c|c|c|c|c|c|c|c|c|c|c|}
\hline & & \multicolumn{12}{|c|}{ mPW1PW91 } \\
\hline & \multirow[b]{2}{*}{ Exp } & \multicolumn{6}{|c|}{ Gas phase } & \multicolumn{6}{|c|}{ PCM } \\
\hline & & 6-31G* & $6-31 G * *$ & $6-31+G * *$ & 6-311G* & 6-311G** & $6-311+G * *$ & 6-31G* & $6-31 G * *$ & $6-31+\mathrm{G} * *$ & 6-311G* & 6-311G** & $6-311+G * *$ \\
\hline \multicolumn{14}{|l|}{${ }^{13} \mathrm{C}$} \\
\hline 1 & 16.3 & 175.8114 & 177.5942 & 178.2150 & 170.1872 & 169.9073 & 169.6105 & 176.2490 & 178.0186 & 176.2505 & 170.6274 & 170.3256 & 170.0373 \\
\hline 2 & 28.0 & 163.9674 & 164.1975 & 164.0835 & 154.9050 & 154.7489 & 153.7137 & 163.9781 & 164.2093 & 163.9720 & 154.9129 & 154.7569 & 153.6562 \\
\hline 3 & 16.1 & 177.0260 & 178.5059 & 179.0417 & 170.7749 & 170.1886 & 169.7273 & 177.5989 & 179.0614 & 177.6004 & 171.4192 & 170.8231 & 170.3759 \\
\hline 4 & 24.2 & 169.1487 & 170.0556 & 169.1971 & 160.4350 & 160.0807 & 159.4826 & 169.2101 & 170.1108 & 169.2087 & 160.5041 & 160.1381 & 159.5147 \\
\hline 5 & 26.9 & 167.9442 & 169.2568 & 168.6269 & 160.1020 & 159.8640 & 159.4198 & 168.1394 & 169.4483 & 168.1380 & 160.3090 & 160.0622 & 159.6017 \\
\hline 6 & 34.1 & 157.7178 & 159.0393 & 159.0141 & 149.4863 & 149.1582 & 148.9600 & 157.8963 & 159.2090 & 157.8924 & 149.6717 & 149.3345 & 149.0936 \\
\hline 7 & 97.5 & 98.0148 & 98.3091 & 98.3054 & 86.5779 & 86.5422 & 86.1084 & 97.6610 & 97.9514 & 97.6621 & 86.0924 & 86.0550 & 85.6198 \\
\hline 8 & 38.5 & 153.4293 & 154.5459 & 154.3452 & 145.8383 & 145.2748 & 144.9922 & 153.6736 & 154.7812 & 153.6686 & 146.0928 & 145.5064 & 145.2135 \\
\hline 9 & 125.2 & 73.2315 & 73.2780 & 74.3997 & 58.1875 & 58.0703 & 56.9937 & 72.4096 & 72.4693 & 72.4130 & 57.3036 & 57.2097 & 56.0211 \\
\hline 10 & 153.9 & 46.3972 & 46.3347 & 45.0052 & 28.2923 & 28.1617 & 27.3451 & 46.7558 & 46.7003 & 46.7550 & 28.6191 & 28.5051 & 27.6841 \\
\hline 11 & 112.0 & 86.4174 & 87.1643 & 86.9243 & 73.0050 & 72.4185 & 71.9133 & 87.0715 & 87.8071 & 87.0707 & 73.5519 & 72.9709 & 72.5005 \\
\hline 12 & 122.7 & 76.7942 & 76.7856 & 75.8870 & 61.6104 & 61.4091 & 60.2787 & 76.4567 & 76.4479 & 76.4484 & 60.9655 & 60.8012 & 59.7971 \\
\hline 13 & 147.3 & 51.8102 & 51.5381 & 50.6597 & 32.9434 & 32.9799 & 32.4028 & 51.8889 & 51.6093 & 51.8902 & 33.0363 & 33.0581 & 32.5680 \\
\hline 14 & 110.5 & 88.2064 & 88.8030 & 88.5807 & 75.1253 & 74.3491 & 73.3382 & 87.9371 & 88.5311 & 87.9407 & 74.7623 & 73.9850 & 72.8857 \\
\hline 15 & 14.5 & 175.2840 & 176.9045 & 178.5464 & 169.6812 & 169.3738 & 169.3640 & 175.6432 & 177.2458 & 175.6385 & 169.9361 & 169.6349 & 169.7186 \\
\hline \multicolumn{14}{|l|}{${ }^{1} \mathrm{H}$} \\
\hline 1 & 1.11 & 31.0159 & 30.5546 & 30.4924 & 30.9740 & 30.6983 & 30.6936 & 31.0020 & 30.5386 & 31.0023 & 30.9589 & 30.6813 & 30.6763 \\
\hline $3 a$ & 1.00 & 31.3013 & 30.8674 & 30.8182 & 31.3249 & 31.1240 & 31.1457 & 31.3045 & 30.8692 & 31.0286 & 31.3209 & 31.1201 & 31.1433 \\
\hline $3 b$ & 0.39 & 31.4217 & 31.0144 & 30.8848 & 31.4071 & 31.2289 & 31.1255 & 31.4027 & 30.9910 & 31.6789 & 31.3859 & 31.2059 & 31.1034 \\
\hline 4 & 1.18 & 31.0069 & 30.6037 & 30.4776 & 30.9979 & 30.8115 & 30.7247 & 30.9059 & 30.4988 & 30.9059 & 30.8928 & 30.7064 & 30.6169 \\
\hline $5 a$ & 1.69 & 30.4713 & 30.0057 & 29.9302 & 30.3520 & 30.1084 & 30.0721 & 30.4414 & 29.9743 & 30.4413 & 30.3265 & 30.0803 & 30.0453 \\
\hline $5 b$ & 1.69 & 30.3529 & 29.8968 & 29.7832 & 30.2768 & 30.0420 & 30.0053 & 30.2756 & 29.8172 & 30.2756 & 30.2025 & 29.9673 & 29.9305 \\
\hline $6 a$ & 1.69 & 30.5809 & 30.1497 & 30.1141 & 30.5126 & 30.2689 & 30.3106 & 30.5144 & 30.0800 & 30.5145 & 30.4448 & 30.1978 & 30.2306 \\
\hline $6 b$ & 1.69 & 30.3942 & 29.9313 & 29.8536 & 30.2949 & 30.0770 & 30.0689 & 30.4230 & 29.9582 & 30.4221 & 30.3221 & 30.1029 & 30.0945 \\
\hline $8 a$ & 3.43 & 28.6557 & 28.1120 & 27.9306 & 28.6129 & 28.2735 & 28.2572 & 28.5794 & 28.0350 & 28.5797 & 28.5358 & 28.1974 & 28.1747 \\
\hline $8 b$ & 2.73 & 29.4513 & 28.9937 & 28.8966 & 29.3795 & 29.1131 & 29.1182 & 29.3244 & 28.8632 & 29.3237 & 29.2528 & 28.9869 & 28.9829 \\
\hline 11 & 6.64 & 25.7606 & 25.1107 & 24.9450 & 25.6291 & 25.1470 & 25.1512 & 25.7060 & 25.0536 & 25.7060 & 25.5518 & 25.0752 & 25.0685 \\
\hline 14 & 6.55 & 25.8690 & 25.1870 & 24.9592 & 25.7104 & 25.2227 & 25.1931 & 25.6983 & 25.0133 & 25.6990 & 25.5454 & 25.0599 & 25.0101 \\
\hline 15 & 2.19 & 30.0982 & 29.6130 & 29.4524 & 29.9700 & 29.6823 & 29.6530 & 30.0472 & 29.5610 & 30.0471 & 29.9130 & 29.6274 & 29.5947 \\
\hline
\end{tabular}




\section{Compound 77a}<smiles>C=C(C)[C@@H](O)[C@@H](C)C(=O)[C@H](C)COCc1ccccc1</smiles>

\begin{tabular}{|c|c|c|c|c|c|c|c|c|c|c|c|c|c|}
\hline \multirow{3}{*}{\multicolumn{2}{|c|}{ Exp }} & \multicolumn{12}{|c|}{ B3LYP } \\
\hline & & \multicolumn{6}{|c|}{ Gas phase } & \multicolumn{6}{|c|}{ PCM } \\
\hline & & 6-31G* & $6-31 G * *$ & $6-31+G * *$ & 6-311G* & $6-311 G * *$ & $6-311+G * *$ & 6-31G* & $6-31 G * *$ & $6-31+G * *$ & 6-311G* & $6-311 G * *$ & $6-311+\mathrm{G} * *$ \\
\hline \multicolumn{14}{|l|}{${ }^{13} \mathrm{C}$} \\
\hline 1 & 111.4 & 81.0371 & 82.4077 & 84.5327 & 67.4289 & 66.2941 & 66.1856 & 81.7193 & 83.1516 & 85.3639 & 68.5151 & 67.6947 & 67.4559 \\
\hline 2 & 143.3 & 52.2586 & 52.0012 & 48.5900 & 30.6910 & 30.5011 & 28.2323 & 51.8805 & 51.5471 & 47.8098 & 29.7090 & 29.0239 & 27.1268 \\
\hline 3 & 73.1 & 117.4488 & 117.9990 & 117.5058 & 105.4685 & 105.3019 & 104.9954 & 117.8438 & 118.3730 & 117.6003 & 105.6694 & 105.5159 & 105.2653 \\
\hline 4 & 44.8 & 137.1637 & 138.0055 & 137.1866 & 126.1812 & 126.4029 & 125.4300 & 138.2784 & 139.0421 & 138.3614 & 126.9928 & 126.8035 & 125.9875 \\
\hline 5 & 218.1 & -24.9376 & -24.8997 & -26.5587 & -47.4912 & -46.5099 & -50.0014 & -24.3334 & -24.3759 & -26.1856 & -47.2568 & -46.7902 & -50.0419 \\
\hline 6 & 44.6 & 144.1356 & 144.9323 & 144.4675 & 135.1662 & 135.0217 & 133.8640 & 144.4600 & 145.2285 & 144.8438 & 135.4292 & 135.1654 & 134.0367 \\
\hline 7 & 73.5 & 115.2415 & 116.3690 & 115.4743 & 104.6632 & 104.1988 & 103.5449 & 116.3692 & 117.4975 & 116.8849 & 105.8784 & 105.2360 & 104.8293 \\
\hline 8 & 19.6 & 168.4454 & 170.1929 & 170.3861 & 161.0075 & 160.7076 & 160.1047 & 168.8210 & 170.5439 & 170.6965 & 161.3720 & 160.9609 & 160.3309 \\
\hline 9 & 8.3 & 179.5276 & 181.3281 & 182.8682 & 174.2994 & 174.4718 & 174.1755 & 179.8261 & 181.6466 & 183.5709 & 174.8726 & 174.9917 & 174.8677 \\
\hline 10 & 13.6 & 174.2937 & 176.0700 & 177.2924 & 167.8884 & 167.7713 & 167.4464 & 173.8544 & 175.6287 & 176.6114 & 167.4089 & 167.3498 & 166.9013 \\
\hline 11 & 72.6 & 116.5769 & 117.6720 & 117.4556 & 106.1112 & 105.4625 & 105.2698 & 117.1757 & 118.2669 & 117.8539 & 106.7178 & 106.0042 & 105.9878 \\
\hline 12 & 137.5 & 58.5863 & 58.3778 & 56.6413 & 38.9079 & 38.4810 & 36.6864 & 58.4376 & 58.2345 & 56.0924 & 38.4688 & 38.0667 & 36.3208 \\
\hline 13 & 128.4 & 68.6070 & 69.2186 & 68.3832 & 51.0866 & 50.2244 & 49.3535 & 68.2767 & 68.8850 & 68.0383 & 50.6529 & 49.8365 & 48.7462 \\
\hline 14 & 127.7 & 68.1954 & 68.7693 & 68.2099 & 50.7222 & 49.7357 & 48.6524 & 68.1278 & 68.6964 & 67.9360 & 50.5695 & 49.6093 & 48.6683 \\
\hline 15 & 127.8 & 68.5080 & 69.1082 & 68.9522 & 50.8495 & 49.9356 & 49.0827 & 68.3683 & 68.9692 & 68.8665 & 50.6463 & 49.7791 & 48.9843 \\
\hline \multicolumn{14}{|l|}{${ }^{1} \mathrm{H}$} \\
\hline 1 & 4.92 & 27.3394 & 26.7196 & 26.5616 & 27.1983 & 26.7519 & 26.7646 & 27.2453 & 26.6308 & 26.4497 & 27.1000 & 26.6889 & 26.6662 \\
\hline 1 & 5.08 & 27.0009 & 26.3280 & 26.2759 & 26.9185 & 26.4327 & 26.4676 & 26.9169 & 26.2470 & 26.1926 & 26.8734 & 26.4733 & 26.3998 \\
\hline 3 & 4.50 & 27.6255 & 27.1730 & 27.0432 & 27.4607 & 27.2319 & 27.2918 & 27.4296 & 26.9571 & 26.7592 & 27.2129 & 26.9706 & 26.9727 \\
\hline 4 & 2.85 & 29.4377 & 29.0027 & 28.8660 & 29.4511 & 29.2408 & 29.1251 & 29.2623 & 28.8154 & 28.6772 & 29.2815 & 29.0712 & 28.9095 \\
\hline 6 & 3.15 & 29.1315 & 28.6468 & 28.4955 & 29.0570 & 28.7752 & 28.7109 & 29.0079 & 28.5051 & 28.3407 & 28.9117 & 28.6164 & 28.5382 \\
\hline 7 & 3.65 & 28.6890 & 28.2313 & 28.2247 & 28.6659 & 28.4012 & 28.4307 & 28.6249 & 28.1605 & 28.1347 & 28.6112 & 28.3397 & 28.3542 \\
\hline 7 & 3.45 & 28.7491 & 28.2533 & 28.2090 & 28.7866 & 28.5037 & 28.4637 & 28.7553 & 28.2443 & 28.2328 & 28.8303 & 28.5146 & 28.5009 \\
\hline 8 & 1.68 & 30.7227 & 30.2562 & 30.1474 & 30.6101 & 30.3585 & 30.3248 & 30.6173 & 30.1377 & 29.9694 & 30.4842 & 30.2035 & 30.1431 \\
\hline 9 & 1.07 & 31.1521 & 30.7051 & 30.6407 & 31.2287 & 30.9879 & 30.8966 & 31.2217 & 30.7763 & 30.7215 & 31.3173 & 31.0670 & 30.9734 \\
\hline 10 & 1.06 & 31.2619 & 30.7992 & 30.7170 & 31.2600 & 30.9979 & 30.9437 & 31.2514 & 30.7907 & 30.7312 & 31.2530 & 30.9882 & 30.9477 \\
\hline 11 & 4.49 & 27.8982 & 27.4246 & 27.3442 & 27.9203 & 27.6961 & 27.5788 & 27.9175 & 27.4460 & 27.3980 & 27.9194 & 27.6817 & 27.6239 \\
\hline 11 & 4.46 & 27.7611 & 27.2728 & 27.0966 & 27.5397 & 27.2013 & 27.2703 & 27.5664 & 27.0618 & 26.7902 & 27.3706 & 27.0360 & 26.9869 \\
\hline 13 & 7.29 & 25.0478 & 24.4162 & 24.2350 & 24.9212 & 24.5103 & 24.4572 & 25.0008 & 24.3651 & 24.1466 & 24.8503 & 24.4254 & 24.3687 \\
\hline 14 & 7.29 & 24.9513 & 24.3280 & 24.1175 & 24.8088 & 24.4074 & 24.3525 & 24.8525 & 24.2271 & 24.0101 & 24.7004 & 24.3037 & 24.2410 \\
\hline 15 & 7.29 & 24.9949 & 24.3743 & 24.1833 & 24.8478 & 24.4573 & 24.4148 & 24.8928 & 24.2712 & 24.0579 & 24.7360 & 24.3525 & 24.3003 \\
\hline
\end{tabular}




\begin{tabular}{|c|c|c|c|c|c|c|c|c|c|c|c|c|c|}
\hline \multirow{3}{*}{\multicolumn{2}{|c|}{ Exp }} & \multicolumn{12}{|c|}{ mPW1PW91 } \\
\hline & & \multicolumn{6}{|c|}{ Gas phase } & \multicolumn{6}{|c|}{ PCM } \\
\hline & & $6-31 G *$ & $6-31 G * *$ & $6-31+\mathrm{G} * *$ & 6-311G* & $6-311 G * *$ & $6-311+G^{* *}$ & $6-31 G^{*}$ & $6-31 G * *$ & $6-31+\mathrm{G} * *$ & 6-311G* & $6-311 G * *$ & $6-311+G^{* *}$ \\
\hline \multicolumn{14}{|c|}{${ }^{13} \mathrm{C}$} \\
\hline 1 & 111.4 & 82.4792 & 83.7596 & 85.7838 & 70.1244 & 69.2314 & 69.0800 & 83.2063 & 84.5598 & 86.6928 & 71.4627 & 70.6237 & 70.4079 \\
\hline 2 & 143.3 & 55.7703 & 55.4812 & 52.4791 & 37.1899 & 36.5181 & 34.6050 & 55.2756 & 54.8914 & 51.5444 & 35.6800 & 34.9664 & 33.2741 \\
\hline 3 & 73.1 & 121.9776 & 122.4763 & 122.2747 & 111.6213 & 111.4707 & 111.1400 & 122.2932 & 122.7709 & 122.3250 & 111.7507 & 111.6090 & 111.3247 \\
\hline 4 & 44.8 & 141.8454 & 142.6535 & 141.8327 & 131.9854 & 131.9254 & 131.3008 & 142.7613 & 143.4861 & 142.7881 & 132.3262 & 132.1782 & 131.5563 \\
\hline 5 & 218.1 & -21.8277 & -21.7599 & -22.9174 & -40.8194 & -40.2493 & -43.6369 & -21.4397 & -21.4708 & -22.7345 & -41.2038 & -40.8058 & -43.8818 \\
\hline 6 & 44.6 & 148.6616 & 149.4144 & 149.1422 & 140.9902 & 140.7534 & 139.6013 & 148.8984 & 149.6220 & 149.4249 & 141.0621 & 140.8039 & 139.7179 \\
\hline 7 & 73.5 & 120.2179 & 121.2815 & 120.5550 & 111.1838 & 110.5479 & 109.8626 & 121.2054 & 122.2655 & 121.7788 & 112.0617 & 111.4186 & 110.9905 \\
\hline 8 & 19.6 & 172.3108 & 173.9448 & 174.1920 & 165.5743 & 165.2310 & 164.7090 & 172.7188 & 174.3262 & 174.5744 & 165.8873 & 165.4835 & 164.8848 \\
\hline 9 & 8.3 & 183.6645 & 185.3361 & 186.7734 & 178.7874 & 178.9199 & 178.5991 & 183.9963 & 185.6860 & 187.5259 & 179.3217 & 179.4368 & 179.2500 \\
\hline 10 & 13.6 & 178.4901 & 180.1380 & 180.9003 & 172.3050 & 172.2622 & 172.0883 & 178.1122 & 179.7612 & 180.3459 & 171.9369 & 171.8980 & 171.5895 \\
\hline 11 & 72.6 & 121.0120 & 122.0473 & 121.9667 & 112.0628 & 111.3795 & 111.1683 & 121.5945 & 122.6217 & 122.3029 & 112.5362 & 111.8407 & 111.7833 \\
\hline 12 & 137.5 & 61.7382 & 61.5046 & 60.2515 & 44.5734 & 44.1180 & 42.3864 & 61.5667 & 61.3408 & 59.7440 & 44.1387 & 43.7156 & 42.0367 \\
\hline 13 & 128.4 & 70.9466 & 71.4896 & 70.8038 & 55.4929 & 54.6397 & 53.7365 & 70.6192 & 71.1601 & 70.4598 & 55.0816 & 54.2485 & 53.1499 \\
\hline 14 & 127.7 & 70.6454 & 71.1316 & 70.8158 & 55.2946 & 54.2716 & 53.1697 & 70.5570 & 71.0386 & 70.4699 & 55.1099 & 54.1104 & 53.1500 \\
\hline 15 & 127.8 & 70.9065 & 71.4196 & 71.3347 & 55.3629 & 54.4257 & 53.5775 & 70.7493 & 71.2636 & 71.2180 & 55.1377 & 54.2344 & 53.4593 \\
\hline \multicolumn{14}{|l|}{${ }^{1} \mathrm{H}$} \\
\hline 1 & 4.92 & 27.1255 & 26.5022 & 26.3788 & 26.9734 & 26.5354 & 26.5576 & 27.0211 & 26.4037 & 26.2672 & 26.8910 & 26.4637 & 26.4618 \\
\hline 1 & 5.08 & 26.7854 & 26.1059 & 26.0738 & 26.6310 & 26.2090 & 26.2316 & 26.7183 & 26.0452 & 26.0086 & 26.6696 & 26.2520 & 26.1904 \\
\hline 3 & 4.50 & 27.5714 & 27.1200 & 26.9839 & 27.3401 & 27.1307 & 27.2030 & 27.3758 & 26.9051 & 26.7064 & 27.1002 & 26.8723 & 26.8889 \\
\hline 4 & 2.85 & 29.3790 & 28.9463 & 28.8073 & 29.3258 & 29.1308 & 29.0076 & 29.2011 & 28.7552 & 28.6146 & 29.1583 & 28.9582 & 28.7966 \\
\hline 6 & 3.15 & 29.0684 & 28.5887 & 28.4266 & 28.9311 & 28.6506 & 28.5850 & 28.9377 & 28.4408 & 28.2735 & 28.7812 & 28.4913 & 28.4140 \\
\hline 7 & 3.65 & 28.6344 & 28.1790 & 28.1528 & 28.5717 & 28.3058 & 28.3268 & 28.5648 & 28.1040 & 28.0589 & 28.5134 & 28.2449 & 28.2500 \\
\hline 7 & 3.45 & 28.7215 & 28.2273 & 28.1899 & 28.7795 & 28.4695 & 28.4098 & 28.7208 & 28.2122 & 28.2098 & 28.8007 & 28.4764 & 28.4392 \\
\hline 8 & 1.68 & 30.6542 & 30.1825 & 30.0823 & 30.5207 & 30.2485 & 30.2383 & 30.5393 & 30.0540 & 29.8926 & 30.3779 & 30.0920 & 30.0442 \\
\hline 9 & 1.07 & 31.1024 & 30.6536 & 30.5772 & 31.1489 & 30.8971 & 30.8055 & 31.1651 & 30.7180 & 30.6530 & 31.2260 & 30.9711 & 30.8721 \\
\hline 10 & 1.06 & 31.2028 & 30.7390 & 30.6683 & 31.1900 & 30.9145 & 30.8601 & 31.1867 & 30.7249 & 30.6759 & 31.1711 & 30.8993 & 30.8604 \\
\hline 11 & 4.49 & 27.8470 & 27.3744 & 27.3147 & 27.8674 & 27.6187 & 27.5056 & 27.8316 & 27.3620 & 27.3388 & 27.8245 & 27.5807 & 27.5207 \\
\hline 11 & 4.46 & 27.6646 & 27.1739 & 27.0150 & 27.4135 & 27.0963 & 27.1809 & 27.4876 & 26.9804 & 26.7366 & 27.2789 & 26.9404 & 26.9125 \\
\hline 13 & 7.29 & 24.8476 & 24.2138 & 24.0593 & 24.7572 & 24.2999 & 24.2590 & 24.7822 & 24.1435 & 23.9497 & 24.6596 & 24.2026 & 24.1525 \\
\hline 14 & 7.29 & 24.7480 & 24.1200 & 23.9358 & 24.6294 & 24.1939 & 24.1455 & 24.6357 & 24.0059 & 23.8165 & 24.5112 & 24.0805 & 24.0264 \\
\hline 15 & 7.29 & 24.7898 & 24.1647 & 24.0036 & 24.6673 & 24.2423 & 24.2047 & 24.6751 & 24.0493 & 23.8675 & 24.5460 & 24.1280 & 24.0827 \\
\hline
\end{tabular}




\section{Compound 77b}<smiles>C=C(C)[C@H](O)[C@@H](C)C(=O)[C@H](C)COCc1ccccc1</smiles>

\begin{tabular}{|c|c|c|c|c|c|c|c|c|c|c|c|c|c|}
\hline \multirow{3}{*}{\multicolumn{2}{|c|}{ Exp }} & \multicolumn{12}{|c|}{ B3LYP } \\
\hline & & \multicolumn{6}{|c|}{ Gas phase } & \multicolumn{6}{|c|}{ PCM } \\
\hline & & 6-31G* & 6-31G** & $6-31+G^{* *}$ & 6-311G* & 6-311G** & $6-311+G^{* *}$ & 6-31G* & 6-31G** & $6-31+G^{* *}$ & 6-311G* & 6-311G** & $6-311+G * *$ \\
\hline \multicolumn{14}{|c|}{${ }^{13} \mathrm{C}$} \\
\hline 1 & 114.0 & 81.4385 & 82.7631 & 83.8723 & 67.3795 & 66.6241 & 66.0503 & 81.5109 & 82.8723 & 84.0759 & 67.2191 & 66.4928 & 66.0394 \\
\hline 2 & 144.5 & 49.4888 & 49.0917 & 45.5237 & 27.4777 & 26.7256 & 24.4561 & 49.1465 & 48.7144 & 45.4466 & 27.2102 & 26.4362 & 24.1382 \\
\hline 3 & 78.3 & 111.4148 & 112.0390 & 110.4101 & 97.7354 & 97.8582 & 97.1172 & 111.8133 & 112.4269 & 111.0900 & 98.4062 & 98.3981 & 98.0717 \\
\hline 4 & 49.1 & 136.2895 & 137.1199 & 136.5454 & 125.3665 & 124.8872 & 125.3411 & 135.1452 & 136.0076 & 134.7133 & 123.3415 & 123.2037 & 122.9905 \\
\hline 5 & 217.3 & -22.7963 & -23.0539 & -24.8633 & -46.0306 & -45.5676 & -48.7370 & -24.1187 & -24.4066 & -26.3817 & -47.3158 & -47.3902 & -50.6687 \\
\hline 6 & 46.0 & 144.6055 & 145.2052 & 143.3680 & 134.5708 & 134.6923 & 132.7040 & 145.6732 & 146.2289 & 144.9866 & 136.1276 & 135.7615 & 134.6731 \\
\hline 7 & 73.3 & 117.6287 & 118.7245 & 117.2942 & 106.5993 & 105.6992 & 105.0864 & 117.2771 & 118.3772 & 116.5861 & 106.0341 & 105.3422 & 104.1359 \\
\hline 8 & 16.8 & 171.4957 & 173.1688 & 174.3882 & 165.4074 & 164.8163 & 164.7347 & 171.9886 & 173.6380 & 174.6513 & 165.7533 & 165.1385 & 165.0554 \\
\hline 9 & 13.7 & 174.5182 & 176.2167 & 177.1565 & 168.0845 & 167.8802 & 167.9333 & 174.7225 & 176.4158 & 177.1390 & 168.1075 & 167.9034 & 167.8672 \\
\hline 10 & 13.5 & 173.1458 & 174.9654 & 176.3001 & 167.1114 & 166.9704 & 166.9397 & 172.8591 & 174.6701 & 175.9880 & 166.5828 & 166.5594 & 165.9101 \\
\hline 11 & 72.2 & 117.3527 & 118.4184 & 117.6189 & 106.5186 & 105.7401 & 105.4122 & 117.7160 & 118.7579 & 117.8091 & 106.8082 & 106.0891 & 105.3877 \\
\hline 12 & 137.8 & 59.1719 & 58.8783 & 55.9436 & 39.0715 & 38.7924 & 36.9889 & 58.7911 & 58.5140 & 55.8859 & 38.8353 & 38.4313 & 37.1496 \\
\hline 13 & 128.4 & 69.1687 & 69.8068 & 69.2949 & 51.6523 & 50.8236 & 50.1155 & 68.9547 & 69.5989 & 68.9810 & 51.2892 & 50.5124 & 49.7824 \\
\hline 14 & 127.6 & 68.0901 & 68.6766 & 68.0982 & 50.7183 & 49.7503 & 48.9071 & 68.0849 & 68.6678 & 68.0644 & 50.6004 & 49.6607 & 48.8966 \\
\hline 15 & 127.7 & 68.7379 & 69.3576 & 69.2235 & 51.1764 & 50.2575 & 49.6668 & 68.6446 & 69.2736 & 68.9192 & 50.9315 & 50.1080 & 49.5175 \\
\hline 1 & 4.91 & 27.3818 & 26.7626 & 26.6695 & 27.2592 & 26.8288 & 26.8337 & 27.3135 & 26.6969 & 26.6072 & 27.1766 & 26.7530 & 26.7835 \\
\hline 1 & 4.94 & 27.3223 & 26.6790 & 26.6523 & 27.2920 & 26.8520 & 26.9060 & 27.2822 & 26.6462 & 26.5883 & 27.2179 & 26.7872 & 26.8457 \\
\hline 3 & 4.18 & 27.8534 & 27.4483 & 27.4014 & 27.8077 & 27.6233 & 27.6541 & 27.7421 & 27.3352 & 27.2629 & 27.6916 & 27.5055 & 27.5067 \\
\hline 4 & 2.92 & 29.3868 & 28.9433 & 28.8170 & 29.3581 & 29.1969 & 29.0542 & 29.4181 & 28.9530 & 28.8615 & 29.4136 & 29.2183 & 29.1059 \\
\hline 6 & 3.07 & 29.0697 & 28.5887 & 28.4449 & 28.9937 & 28.7426 & 28.6471 & 28.9204 & 28.4232 & 28.2387 & 28.8657 & 28.5912 & 28.4531 \\
\hline 7 & 3.43 & 28.4407 & 27.9412 & 28.0321 & 28.5756 & 28.2449 & 28.3029 & 28.4471 & 27.9510 & 27.9367 & 28.5527 & 28.2388 & 28.2022 \\
\hline 7 & 3.64 & 29.0977 & 28.6295 & 28.4063 & 28.9973 & 28.7139 & 28.6274 & 28.9045 & 28.4369 & 28.2188 & 28.7932 & 28.5268 & 28.4126 \\
\hline 8 & 1.65 & 30.4123 & 29.9320 & 29.8129 & 30.2978 & 30.0496 & 30.0124 & 30.4140 & 29.9284 & 29.8506 & 30.3214 & 30.0540 & 30.0586 \\
\hline 9 & 1.03 & 31.1222 & 30.6617 & 30.6459 & 31.2182 & 30.9492 & 30.8887 & 31.1199 & 30.6586 & 30.6286 & 31.2076 & 30.9352 & 30.8704 \\
\hline 10 & 0.97 & 31.2916 & 30.8403 & 30.7222 & 31.2641 & 31.0073 & 30.9256 & 31.2364 & 30.7835 & 30.6420 & 31.2149 & 30.9595 & 30.8488 \\
\hline 11 & 4.47 & 27.5839 & 27.0902 & 27.1282 & 27.6335 & 27.3344 & 27.3668 & 27.5338 & 27.0265 & 26.9319 & 27.5555 & 27.2383 & 27.2001 \\
\hline 11 & 4.49 & 27.9642 & 27.4974 & 27.1250 & 27.6972 & 27.4981 & 27.3970 & 27.8978 & 27.4302 & 27.2006 & 27.7060 & 27.4749 & 27.4347 \\
\hline 13 & 7.29 & 25.0117 & 24.3605 & 24.1248 & 24.8822 & 24.4393 & 24.3495 & 24.9411 & 24.2923 & 24.0496 & 24.8073 & 24.3697 & 24.2522 \\
\hline 14 & 7.29 & 24.9506 & 24.3316 & 24.1377 & 24.8199 & 24.4292 & 24.3637 & 24.8670 & 24.2489 & 24.0466 & 24.7274 & 24.3437 & 24.2872 \\
\hline 15 & 7.29 & 25.0201 & 24.4022 & 24.1958 & 24.8850 & 24.5001 & 24.4352 & 24.9234 & 24.3072 & 24.1143 & 24.7789 & 24.4045 & 24.3426 \\
\hline
\end{tabular}




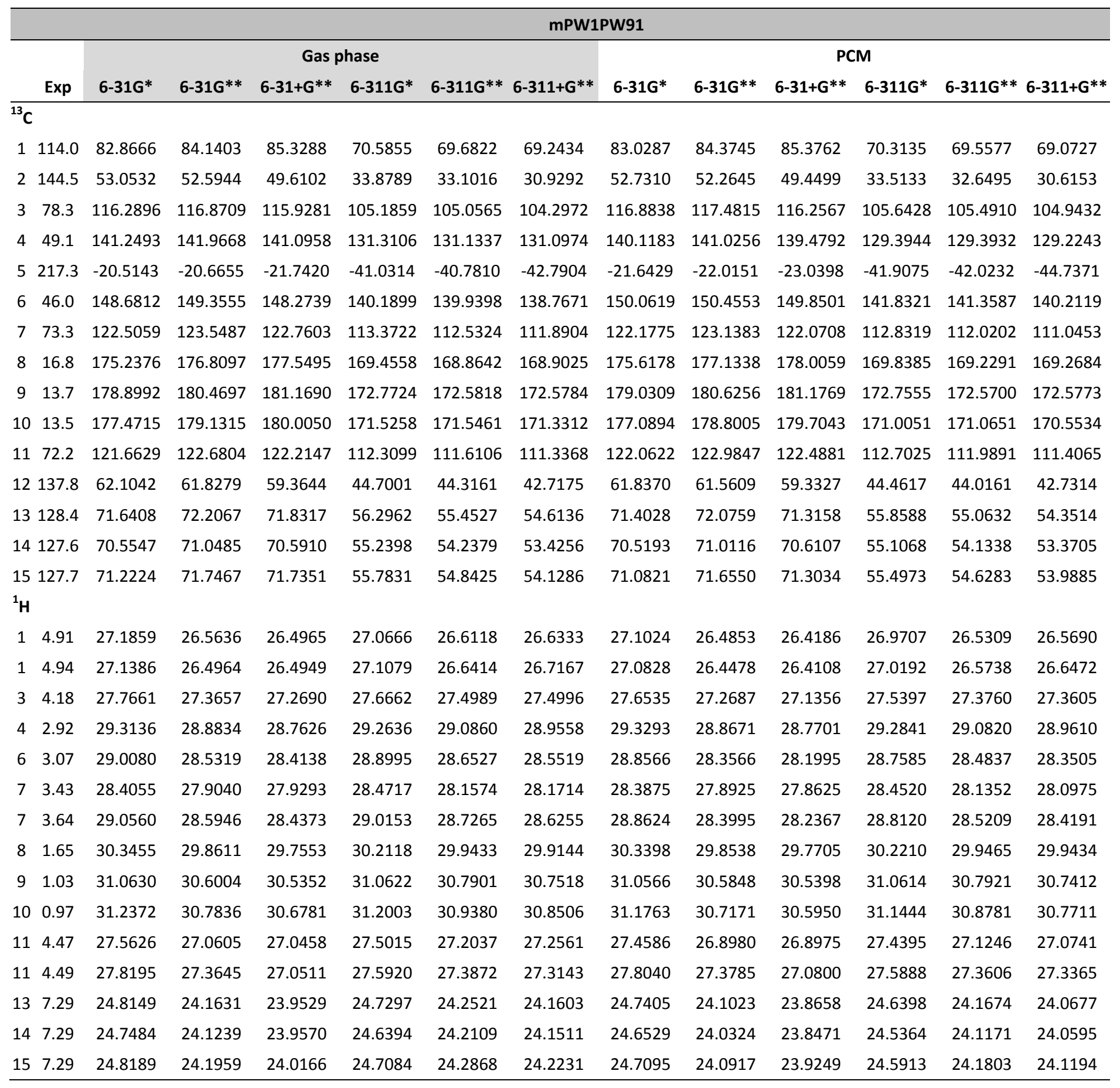




\section{Compound 77c}<smiles>C=C(C)[C@@H](O)[C@@H](C)C(=O)[C@H](C)COCc1ccccc1</smiles>

\begin{tabular}{|c|c|c|c|c|c|c|c|c|c|c|c|c|}
\hline \multirow[b]{3}{*}{ Exp } & \multicolumn{12}{|c|}{ B3LYP } \\
\hline & \multicolumn{6}{|c|}{ Gas phase } & \multicolumn{6}{|c|}{ PCM } \\
\hline & $6-31 G^{*}$ & 6-31G** & $6-31+G * *$ & 6-311G* & 6-311G** & $6-311+G^{* *}$ & 6-31G* & 6-31G** & $6-31+\mathrm{G} * *$ & 6-311G* & 6-311G** & $6-311+G * *$ \\
\hline \multicolumn{13}{|l|}{${ }^{13} \mathrm{C}$} \\
\hline 1 & 81.4702 & 82.7956 & 84.1669 & 67.6964 & 66.6748 & 66.4628 & 81.5145 & 82.8536 & 84.0035 & 67.4655 & 66.4742 & 66.2673 \\
\hline 2 & 49.3270 & 48.9602 & 45.7682 & 27.6256 & 26.9308 & 24.7320 & 49.0076 & 48.6283 & 45.4536 & 27.2838 & 26.6074 & 24.3024 \\
\hline 3 & 108.6672 & 109.3265 & 108.0985 & 95.5732 & 95.3695 & 95.2591 & 109.1133 & 109.7663 & 108.4719 & 95.9842 & 95.7498 & 95.6496 \\
\hline 4 & 137.1228 & 137.8862 & 137.4232 & 126.5431 & 126.4139 & 125.8520 & 137.3229 & 138.0804 & 137.5753 & 126.7433 & 126.5871 & 125.9436 \\
\hline 5 & -23.2111 & -23.2650 & -24.7840 & -46.6579 & -46.2715 & -48.4315 & -25.6292 & -25.6944 & -27.7871 & -49.4961 & -49.1409 & -51.8036 \\
\hline 6 & 142.0250 & 142.7398 & 141.7205 & 131.9533 & 131.5296 & 130.9689 & 141.2082 & 141.9373 & 140.7459 & 130.9405 & 130.5390 & 129.8773 \\
\hline 7 & 117.4526 & 118.5930 & 117.8355 & 106.7253 & 106.0760 & 105.7632 & 117.3527 & 118.4829 & 117.7808 & 106.6179 & 105.9490 & 105.6647 \\
\hline 8 & 172.4313 & 174.1785 & 175.1655 & 165.8604 & 165.5939 & 165.0049 & 172.6906 & 174.4089 & 175.4367 & 166.1318 & 165.8396 & 165.2723 \\
\hline 9 & 174.7098 & 176.5503 & 177.2756 & 168.5037 & 168.4945 & 168.1335 & 174.9111 & 176.7429 & 177.5460 & 168.8188 & 168.7950 & 168.4047 \\
\hline 10 & 174.3568 & 176.1156 & 176.7772 & 168.1227 & 168.1097 & 167.5974 & 174.4924 & 176.2354 & 176.8640 & 168.2339 & 168.2013 & 167.6561 \\
\hline 11 & 116.9835 & 118.0917 & 117.1290 & 106.2332 & 105.5090 & 105.4237 & 117.2010 & 118.2997 & 117.2939 & 106.4553 & 105.7141 & 105.6286 \\
\hline 12 & 59.5197 & 59.2863 & 56.0952 & 39.6239 & 39.1604 & 38.1327 & 58.8338 & 58.6070 & 55.3252 & 38.8111 & 38.3922 & 37.4812 \\
\hline 13 & 69.1174 & 69.7406 & 68.8945 & 51.6287 & 50.7934 & 49.7287 & 68.9749 & 69.5924 & 68.6769 & 51.3412 & 50.5195 & 49.4416 \\
\hline 14 & 67.9311 & 68.5074 & 67.8813 & 50.5184 & 49.5383 & 48.7574 & 67.9509 & 68.5243 & 67.7683 & 50.4288 & 49.4726 & 48.7502 \\
\hline 15 & 68.5125 & 69.1247 & 68.7324 & 50.9095 & 50.0302 & 49.2366 & 68.5509 & 69.1627 & 68.7417 & 50.8603 & 50.0066 & 49.2496 \\
\hline \multicolumn{13}{|l|}{${ }^{1} \mathrm{H}$} \\
\hline 1 & 27.4635 & 26.8489 & 26.7347 & 27.3454 & 26.9211 & 26.9432 & 27.3936 & 26.7801 & 26.6559 & 27.2626 & 26.8465 & 26.8620 \\
\hline 1 & 27.4161 & 26.7907 & 26.7484 & 27.3902 & 26.9711 & 27.0187 & 27.3604 & 26.7354 & 26.6831 & 27.3187 & 26.9079 & 26.9525 \\
\hline 3 & 28.1039 & 27.6996 & 27.6478 & 28.0948 & 27.9024 & 27.9073 & 28.0846 & 27.6791 & 27.6095 & 28.0555 & 27.8660 & 27.8633 \\
\hline 4 & 29.1521 & 28.7307 & 28.6585 & 29.2165 & 29.0444 & 28.9294 & 29.0243 & 28.5981 & 28.4945 & 29.0779 & 28.9003 & 28.7666 \\
\hline 6 & 28.6921 & 28.2160 & 28.0305 & 28.6158 & 28.3697 & 28.2536 & 28.6891 & 28.2132 & 28.0059 & 28.6052 & 28.3571 & 28.2299 \\
\hline 7 & 28.3065 & 27.8000 & 27.8218 & 28.3480 & 28.0361 & 28.0751 & 28.3557 & 27.8580 & 27.8515 & 28.3764 & 28.0700 & 28.0992 \\
\hline 7 & 29.0624 & 28.5813 & 28.4405 & 29.0353 & 28.7458 & 28.6611 & 28.9115 & 28.4243 & 28.2900 & 28.8878 & 28.5970 & 28.5204 \\
\hline 8 & 30.4079 & 29.9230 & 29.8002 & 30.3052 & 30.0368 & 30.0086 & 30.3878 & 29.9010 & 29.7769 & 30.2888 & 30.0188 & 29.9839 \\
\hline 9 & 31.3095 & 30.8584 & 30.8213 & 31.3803 & 31.1243 & 31.0647 & 31.3105 & 30.8572 & 30.8322 & 31.3923 & 31.1360 & 31.0751 \\
\hline 10 & 31.3407 & 30.8897 & 30.8110 & 31.3246 & 31.0691 & 31.0065 & 31.2865 & 30.8327 & 30.7451 & 31.2673 & 31.0103 & 30.9440 \\
\hline 11 & 27.7773 & 27.2746 & 27.2584 & 27.6944 & 27.4199 & 27.4574 & 27.7507 & 27.2536 & 27.2077 & 27.6493 & 27.3808 & 27.3966 \\
\hline 11 & 27.6833 & 27.1990 & 26.8622 & 27.5681 & 27.2720 & 27.1187 & 27.5748 & 27.0836 & 26.7699 & 27.4777 & 27.1774 & 27.0316 \\
\hline 13 & 24.9786 & 24.3214 & 24.1220 & 24.8858 & 24.4271 & 24.3544 & 24.9187 & 24.2623 & 24.0432 & 24.8117 & 24.3590 & 24.2743 \\
\hline 14 & 24.9138 & 24.2915 & 24.1425 & 24.7927 & 24.3956 & 24.3530 & 24.8365 & 24.2139 & 24.0579 & 24.7018 & 24.3115 & 24.2662 \\
\hline 15 & 24.9988 & 24.3774 & 24.1701 & 24.8688 & 24.4785 & 24.4064 & 24.9120 & 24.2910 & 24.0709 & 24.7709 & 24.3884 & 24.3119 \\
\hline
\end{tabular}




\begin{tabular}{|c|c|c|c|c|c|c|c|c|c|c|c|c|}
\hline \multirow[b]{3}{*}{$\operatorname{Exp}$} & \multicolumn{12}{|c|}{ mPW1PW91 } \\
\hline & \multicolumn{6}{|c|}{ Gas phase } & \multicolumn{6}{|c|}{ PCM } \\
\hline & $6-31 G^{*}$ & $6-31 G * *$ & $6-31+G * *$ & $6-311 G *$ & $6-311 G * *$ & $6-311+G * *$ & 6-31G* & $6-31 G * *$ & $6-31+G * *$ & $6-311 G *$ & $6-311 G * *$ & $6-311+G * *$ \\
\hline \multicolumn{13}{|l|}{${ }^{13} \mathrm{C}$} \\
\hline 1 & 82.9131 & 84.1664 & 85.5253 & 70.6236 & 69.5742 & 69.3615 & 82.9508 & 84.2280 & 85.2975 & 70.3235 & 69.4877 & 69.1101 \\
\hline 2 & 52.8657 & 52.4485 & 49.6563 & 33.7135 & 32.9939 & 31.0295 & 52.5189 & 52.0831 & 49.3937 & 33.3836 & 32.6019 & 30.6270 \\
\hline 3 & 113.5720 & 114.1709 & 113.0945 & 102.2669 & 102.0781 & 102.0025 & 114.0350 & 114.6300 & 113.4864 & 102.6497 & 102.6253 & 102.3690 \\
\hline 4 & 141.6831 & 142.4063 & 141.9853 & 132.2236 & 132.1195 & 131.6383 & 141.8907 & 142.6112 & 142.1481 & 132.4011 & 132.3769 & 131.7279 \\
\hline 5 & -20.3299 & -20.4030 & -21.3436 & -40.7541 & -40.3817 & -42.2511 & -22.8348 & -22.9214 & -24.3187 & -43.5987 & -43.4743 & -45.5638 \\
\hline 6 & 146.5845 & 147.2547 & 146.3431 & 137.6121 & 137.2159 & 136.7158 & 145.7877 & 146.4757 & 145.3994 & 136.6120 & 136.3539 & 135.6556 \\
\hline 7 & 122.2080 & 123.2681 & 122.5312 & 112.8691 & 112.2473 & 111.9222 & 122.1137 & 123.1647 & 122.3956 & 112.7831 & 112.0524 & 111.8270 \\
\hline 8 & 176.0209 & 177.6410 & 178.6277 & 170.0686 & 169.8048 & 169.2934 & 176.3028 & 177.8890 & 178.9542 & 170.3579 & 169.8497 & 169.5792 \\
\hline 9 & 178.9318 & 180.6375 & 181.3531 & 173.1178 & 173.1154 & 172.7690 & 179.1428 & 180.8369 & 181.6620 & 173.4339 & 173.2993 & 173.0469 \\
\hline 10 & 178.5129 & 180.1508 & 180.6763 & 172.6344 & 172.6250 & 172.1855 & 178.6708 & 180.2931 & 180.7782 & 172.7555 & 172.6921 & 172.2674 \\
\hline 11 & 121.3506 & 122.3966 & 121.5665 & 112.0512 & 111.3488 & 111.2647 & 121.5912 & 122.6298 & 121.6914 & 112.2749 & 111.5934 & 111.4739 \\
\hline 12 & 62.6474 & 62.3849 & 59.7671 & 45.3029 & 44.8151 & 43.7208 & 61.9259 & 61.6692 & 58.8256 & 44.4765 & 43.9588 & 43.0474 \\
\hline 13 & 71.5773 & 72.1464 & 71.3978 & 56.2394 & 55.3863 & 54.2828 & 71.4305 & 71.9933 & 71.1292 & 55.9569 & 55.0981 & 53.9926 \\
\hline 14 & 70.3875 & 70.8750 & 70.5224 & 55.0928 & 54.0673 & 53.2354 & 70.3866 & 70.8721 & 70.3448 & 54.9693 & 53.9756 & 53.2023 \\
\hline 15 & 70.9667 & 71.4962 & 71.1644 & 55.5014 & 54.5789 & 53.7722 & 70.9835 & 71.5132 & 71.1565 & 55.4171 & 54.5240 & 53.7515 \\
\hline \multicolumn{13}{|l|}{${ }^{1} \mathrm{H}$} \\
\hline 1 & 27.2565 & 26.6362 & 26.5459 & 27.1490 & 26.7046 & 26.7293 & 27.1742 & 26.5549 & 26.4650 & 27.0557 & 26.6146 & 26.6409 \\
\hline 1 & 27.2115 & 26.5776 & 26.5668 & 27.1925 & 26.7550 & 26.8005 & 27.1463 & 26.5124 & 26.5032 & 27.1134 & 26.6628 & 26.7291 \\
\hline 3 & 28.0168 & 27.6175 & 27.5557 & 27.9703 & 27.7786 & 27.7881 & 27.9958 & 27.5950 & 27.5299 & 27.9289 & 27.7518 & 27.7450 \\
\hline 4 & 29.0751 & 28.6567 & 28.5758 & 29.0695 & 28.9022 & 28.7974 & 28.9439 & 28.5200 & 28.4144 & 28.9299 & 28.7489 & 28.6362 \\
\hline 6 & 28.6283 & 28.1605 & 27.9790 & 28.4802 & 28.2458 & 28.1405 & 28.6249 & 28.1571 & 27.9512 & 28.4692 & 28.2629 & 28.1169 \\
\hline 7 & 28.2718 & 27.7697 & 27.7657 & 28.2544 & 27.9474 & 27.9812 & 28.3237 & 27.8322 & 27.7989 & 28.2865 & 28.0160 & 28.0070 \\
\hline 7 & 29.0304 & 28.5542 & 28.4248 & 28.9988 & 28.7046 & 28.6151 & 28.8801 & 28.3969 & 28.2497 & 28.8509 & 28.5530 & 28.4689 \\
\hline 8 & 30.3306 & 29.8418 & 29.7235 & 30.2033 & 29.9268 & 29.9071 & 30.3059 & 29.8148 & 29.7016 & 30.1829 & 29.9049 & 29.8791 \\
\hline 9 & 31.2484 & 30.7941 & 30.7606 & 31.2911 & 31.0304 & 30.9723 & 31.2415 & 30.7842 & 30.7707 & 31.3006 & 31.0031 & 30.9809 \\
\hline 10 & 31.2880 & 30.8350 & 30.7599 & 31.2449 & 30.9821 & 30.9198 & 31.2301 & 30.7743 & 30.6861 & 31.1841 & 30.9221 & 30.8540 \\
\hline 11 & 27.6811 & 27.1764 & 27.1528 & 27.5711 & 27.2840 & 27.3271 & 27.6505 & 27.1530 & 27.1237 & 27.5232 & 27.2706 & 27.2665 \\
\hline 11 & 27.6025 & 27.1198 & 26.8531 & 27.4907 & 27.1951 & 27.0782 & 27.4859 & 26.9943 & 26.7482 & 27.3898 & 27.0768 & 26.9810 \\
\hline 13 & 24.7749 & 24.1172 & 23.9452 & 24.7012 & 24.2149 & 24.1537 & 24.7036 & 24.0464 & 23.8523 & 24.6203 & 24.1369 & 24.0670 \\
\hline 14 & 24.7097 & 24.0830 & 23.9454 & 24.6113 & 24.1792 & 24.1378 & 24.6188 & 23.9919 & 23.8594 & 24.5103 & 24.0861 & 24.0423 \\
\hline 15 & 24.7964 & 24.1711 & 24.0034 & 24.6919 & 24.2658 & 24.1983 & 24.6958 & 24.0708 & 23.8877 & 24.5835 & 24.1654 & 24.0939 \\
\hline
\end{tabular}




\section{Compound 77d}<smiles>C=C(C)[C@@H](O)[C@@H](C)C(=O)[C@H](C)COCc1ccccc1</smiles>

77d

\begin{tabular}{|c|c|c|c|c|c|c|c|c|c|c|c|c|c|}
\hline \multirow{3}{*}{\multicolumn{2}{|c|}{ Exp }} & \multicolumn{12}{|c|}{ B3LYP } \\
\hline & & \multicolumn{6}{|c|}{ Gas phase } & \multicolumn{6}{|c|}{ PCM } \\
\hline & & 6-31G* & 6-31G** & $6-31+G^{* *}$ & 6-311G* & 6-311G** & $6-311+G^{* *}$ & 6-31G* & $6-31 G^{* *}$ & $6-31+G^{* *}$ & 6-311G* & 6-311G** & $6-311+G * *$ \\
\hline \multicolumn{14}{|c|}{ 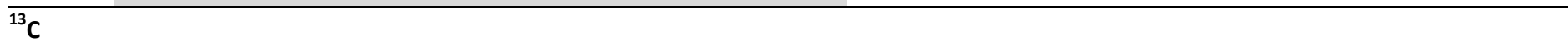 } \\
\hline 1 & 111.7 & 81.1495 & 82.5179 & 85.3563 & 67.9793 & 67.0465 & 67.0493 & 82.2010 & 83.5990 & 86.1640 & 69.0760 & 68.2125 & 67.9345 \\
\hline 2 & 143.5 & 51.7991 & 51.5175 & 47.2834 & 29.6563 & 29.1052 & 27.0347 & 50.9435 & 50.6307 & 46.8532 & 28.7485 & 28.1583 & 26.4159 \\
\hline 3 & 73.6 & 116.3576 & 116.8941 & 116.5474 & 104.2852 & 104.0431 & 103.6906 & 116.6259 & 117.1535 & 116.7450 & 104.5032 & 104.2406 & 104.0438 \\
\hline 4 & 47.6 & 138.5328 & 139.3598 & 137.6294 & 127.8027 & 127.8084 & 126.6600 & 139.5839 & 140.3878 & 139.6231 & 129.1366 & 129.0786 & 128.5200 \\
\hline 5 & 227.7 & -27.2752 & -27.2985 & -28.6902 & -51.7645 & -51.2419 & -52.7891 & -29.0445 & -29.0795 & -31.0714 & -53.8521 & -53.3895 & -55.4425 \\
\hline 6 & 45.0 & 141.8889 & 142.6734 & 141.9996 & 131.7824 & 131.5079 & 131.0491 & 141.4410 & 142.2250 & 141.2199 & 131.2387 & 130.9650 & 130.2311 \\
\hline 7 & 72.9 & 119.4292 & 120.6172 & 119.7503 & 108.7536 & 108.1395 & 107.9093 & 119.5460 & 120.7314 & 119.5291 & 108.9180 & 108.2925 & 107.7328 \\
\hline 8 & 19.2 & 168.4863 & 170.2347 & 170.4989 & 161.1648 & 160.8490 & 160.4320 & 168.9562 & 170.6826 & 170.9697 & 161.6337 & 161.2735 & 160.9696 \\
\hline 9 & 8.6 & 179.7625 & 181.5444 & 183.0329 & 174.3412 & 174.4428 & 174.3571 & 179.8922 & 181.6685 & 183.0335 & 174.5278 & 174.6190 & 174.4304 \\
\hline 10 & 13.6 & 173.9189 & 175.6564 & 176.2658 & 167.5923 & 167.5237 & 167.1625 & 174.0521 & 175.7750 & 176.4517 & 167.6961 & 167.6093 & 167.3246 \\
\hline 11 & 72.0 & 117.3056 & 118.4233 & 117.3690 & 106.4149 & 105.6998 & 105.8601 & 117.7413 & 118.8463 & 117.6059 & 106.8607 & 106.1312 & 106.2271 \\
\hline 12 & 137.4 & 58.6972 & 58.4667 & 55.8228 & 38.8085 & 38.3894 & 37.1464 & 57.9780 & 57.7499 & 55.1773 & 37.9858 & 37.5874 & 36.3865 \\
\hline 13 & 127.3 & 69.6148 & 70.2325 & 69.7430 & 52.2017 & 51.3841 & 50.7623 & 69.6327 & 70.2464 & 69.3120 & 52.0980 & 51.2960 & 50.4388 \\
\hline 14 & 128.3 & 68.0702 & 68.6422 & 68.0138 & 50.6754 & 49.6838 & 48.7445 & 68.0927 & 68.6621 & 68.0331 & 50.5985 & 49.6342 & 48.7122 \\
\hline 15 & 127.4 & 68.9570 & 69.5544 & 69.3514 & 51.4267 & 50.5226 & 49.6328 & 69.0845 & 69.6823 & 69.2632 & 51.4792 & 50.6062 & 49.7279 \\
\hline 1 & 4.93 & 27.2397 & 26.6170 & 26.5158 & 27.1263 & 26.6955 & 26.7321 & 27.2105 & 26.5911 & 26.4928 & 27.0811 & 26.6622 & 26.7068 \\
\hline 1 & 5.06 & 26.9492 & 26.2777 & 26.2278 & 26.9435 & 26.5217 & 26.5189 & 26.9806 & 26.3120 & 26.1995 & 26.9493 & 26.5403 & 26.4979 \\
\hline 3 & 4.37 & 27.6432 & 27.1988 & 27.0475 & 27.5225 & 27.3460 & 27.3108 & 27.6089 & 27.1605 & 27.0268 & 27.4799 & 27.2983 & 27.2807 \\
\hline 4 & 2.85 & 29.6280 & 29.1826 & 29.0433 & 29.6262 & 29.3977 & 29.2569 & 29.4194 & 28.9626 & 28.7746 & 29.4099 & 29.1706 & 29.0017 \\
\hline 6 & 3.12 & 29.2186 & 28.7548 & 28.6761 & 29.1801 & 28.9352 & 28.8567 & 29.0988 & 28.6317 & 28.5701 & 29.0518 & 28.8051 & 28.7482 \\
\hline 7 & 3.65 & 28.3488 & 27.8136 & 27.8106 & 28.4169 & 28.0804 & 28.0631 & 28.4072 & 27.8725 & 27.8475 & 28.4730 & 28.1328 & 28.0972 \\
\hline 7 & 3.05 & 29.3500 & 28.9046 & 28.7682 & 29.2648 & 29.0283 & 28.9807 & 29.2281 & 28.7818 & 28.5862 & 29.1413 & 28.9080 & 28.8131 \\
\hline 8 & 1.68 & 30.4977 & 30.0252 & 29.8526 & 30.3921 & 30.1325 & 30.0563 & 30.4672 & 29.9902 & 29.8955 & 30.3699 & 30.1042 & 30.0869 \\
\hline 9 & 1.07 & 30.9024 & 30.4402 & 30.3275 & 30.9623 & 30.6980 & 30.6014 & 30.9295 & 30.4675 & 30.3883 & 30.9937 & 30.7291 & 30.6566 \\
\hline 10 & 1.06 & 31.3052 & 30.8435 & 30.7763 & 31.2738 & 31.0023 & 30.9602 & 31.2476 & 30.7836 & 30.6744 & 31.2173 & 30.9452 & 30.8710 \\
\hline 11 & 4.46 & 27.4169 & 26.8970 & 26.7618 & 27.3348 & 27.0343 & 27.0389 & 27.3541 & 26.8304 & 26.6985 & 27.2563 & 26.9498 & 26.9671 \\
\hline 11 & 4.49 & 28.0108 & 27.5749 & 27.3048 & 27.7999 & 27.6073 & 27.5690 & 27.9337 & 27.4968 & 27.2532 & 27.7286 & 27.5407 & 27.5071 \\
\hline 13 & 7.32 & 25.0628 & 24.4084 & 24.1717 & 24.9575 & 24.5080 & 24.4140 & 25.0082 & 24.3535 & 24.0999 & 24.8912 & 24.4468 & 24.3349 \\
\hline 14 & 7.32 & 24.9412 & 24.3190 & 24.1289 & 24.8128 & 24.4158 & 24.3314 & 24.8606 & 24.2386 & 24.0307 & 24.7189 & 24.3295 & 24.2404 \\
\hline 15 & 7.32 & 25.0405 & 24.4157 & 24.2125 & 24.9133 & 24.5145 & 24.4366 & 24.9563 & 24.3316 & 24.1257 & 24.8195 & 24.4280 & 24.3472 \\
\hline
\end{tabular}




\begin{tabular}{|c|c|c|c|c|c|c|c|c|c|c|c|c|c|}
\hline & \multirow[b]{3}{*}{ Exp } & \multicolumn{12}{|c|}{ mPW1PW91 } \\
\hline & & \multicolumn{6}{|c|}{ Gas phase } & \multicolumn{6}{|c|}{ PCM } \\
\hline & & $6-31 G^{*}$ & $6-31 G * *$ & $6-31+\mathrm{G} * *$ & $6-311 G *$ & $6-311 G * *$ & $6-311+\mathrm{G} * *$ & 6-31G* & $6-31 G * *$ & $6-31+G * *$ & $6-311 G *$ & $6-311 G * *$ & $6-311+G * *$ \\
\hline \multicolumn{14}{|c|}{${ }^{13} \mathrm{C}$} \\
\hline 1 & 111.7 & 82.4635 & 83.7458 & 86.2261 & 70.6567 & 69.6991 & 69.6577 & 83.6098 & 84.9312 & 87.2350 & 71.8877 & 71.0055 & 70.7754 \\
\hline 2 & 143.5 & 55.5448 & 55.2319 & 51.5912 & 36.2405 & 35.6515 & 33.8804 & 54.5264 & 54.1694 & 50.8413 & 35.0702 & 34.4380 & 32.8743 \\
\hline 3 & 73.6 & 121.0108 & 121.4975 & 121.4164 & 110.6317 & 110.3937 & 110.0565 & 121.2468 & 121.7254 & 121.5802 & 110.7831 & 110.5268 & 110.3075 \\
\hline 4 & 47.6 & 143.3835 & 144.1699 & 142.7594 & 133.9233 & 133.9464 & 133.0509 & 144.3560 & 145.1154 & 144.4637 & 135.0980 & 135.0478 & 134.6366 \\
\hline 5 & 227.7 & -24.3548 & -24.3894 & -25.3967 & -45.7599 & -45.2486 & -46.6086 & -26.1657 & -26.2115 & -27.7176 & -47.8452 & -47.3962 & -49.1973 \\
\hline 6 & 45.0 & 146.5070 & 147.2427 & 146.6854 & 137.5496 & 137.2995 & 136.8691 & 146.0526 & 146.7893 & 145.9892 & 137.0041 & 136.7565 & 136.0956 \\
\hline 7 & 72.9 & 124.1008 & 125.2033 & 124.5411 & 114.8311 & 114.2392 & 114.0395 & 124.2305 & 125.3314 & 124.4260 & 114.9882 & 114.3862 & 113.9078 \\
\hline 8 & 19.2 & 172.3561 & 173.9933 & 174.1639 & 165.7176 & 165.4065 & 165.0565 & 172.8561 & 174.4697 & 174.6508 & 166.1724 & 165.8172 & 165.5511 \\
\hline 9 & 8.6 & 183.9431 & 185.5967 & 186.6898 & 178.7956 & 178.9027 & 178.8477 & 184.0994 & 185.7468 & 186.7282 & 178.9888 & 179.0852 & 178.9458 \\
\hline 10 & 13.6 & 178.1504 & 179.7679 & 180.2660 & 172.1617 & 172.1009 & 171.7515 & 178.3111 & 179.9149 & 180.4663 & 172.2768 & 172.2005 & 171.9248 \\
\hline 11 & 72.0 & 121.6622 & 122.7109 & 121.8118 & 112.2520 & 111.5591 & 111.7293 & 122.1219 & 123.1586 & 122.1201 & 112.6913 & 111.9856 & 112.1086 \\
\hline 12 & 137.4 & 61.8201 & 61.5634 & 59.3211 & 44.4532 & 44.0165 & 42.7814 & 61.0514 & 60.7975 & 58.6350 & 43.5909 & 43.1767 & 41.9733 \\
\hline 13 & 127.3 & 72.0006 & 72.5576 & 72.1175 & 56.6926 & 55.8538 & 55.2169 & 72.0261 & 72.5787 & 71.7608 & 56.5912 & 55.7696 & 54.9231 \\
\hline 14 & 128.3 & 70.5372 & 71.0210 & 70.6106 & 55.2531 & 54.2150 & 53.2801 & 70.5348 & 71.0164 & 70.5944 & 55.1350 & 54.1272 & 53.2167 \\
\hline 15 & 127.4 & 71.3827 & 71.8953 & 71.7095 & 55.9674 & 55.0198 & 54.1318 & 71.4923 & 72.0057 & 71.6222 & 55.9828 & 55.0691 & 54.1978 \\
\hline \multicolumn{14}{|c|}{${ }^{1} \mathrm{H}$} \\
\hline 1 & 4.93 & 27.0303 & 26.4055 & 26.3282 & 26.9266 & 26.4797 & 26.5159 & 26.9911 & 26.3699 & 26.2949 & 26.8760 & 26.4414 & 26.4855 \\
\hline 1 & 5.06 & 26.7241 & 26.0465 & 26.0029 & 26.6998 & 26.2620 & 26.2568 & 26.7633 & 26.0902 & 25.9969 & 26.7229 & 26.2978 & 26.2653 \\
\hline 3 & 4.37 & 27.6140 & 27.1742 & 27.0240 & 27.4698 & 27.2959 & 27.2597 & 27.5746 & 27.1300 & 26.9916 & 27.4213 & 27.2416 & 27.2188 \\
\hline 4 & 2.85 & 29.5653 & 29.1219 & 28.9533 & 29.4918 & 29.2668 & 29.1237 & 29.3502 & 28.8955 & 28.6889 & 29.2727 & 29.0374 & 28.8716 \\
\hline 6 & 3.12 & 29.1407 & 28.6813 & 28.5865 & 29.0462 & 28.8045 & 28.7196 & 29.0175 & 28.5545 & 28.4706 & 28.9175 & 28.6736 & 28.6091 \\
\hline 7 & 3.65 & 28.3130 & 27.7785 & 27.7409 & 28.3247 & 27.9951 & 27.9586 & 28.3808 & 27.8477 & 27.7933 & 28.3915 & 28.0593 & 28.0097 \\
\hline 7 & 3.05 & 29.2906 & 28.8521 & 28.7329 & 29.2065 & 28.9653 & 28.9223 & 29.1552 & 28.7150 & 28.5501 & 29.0699 & 28.8307 & 28.7453 \\
\hline 8 & 1.68 & 30.4410 & 29.9655 & 29.8079 & 30.3207 & 30.0515 & 29.9780 & 30.4004 & 29.9198 & 29.8214 & 30.2862 & 30.0108 & 29.9876 \\
\hline 9 & 1.07 & 30.8562 & 30.3918 & 30.2784 & 30.8798 & 30.6129 & 30.5236 & 30.8825 & 30.4184 & 30.3334 & 30.9093 & 30.6420 & 30.5741 \\
\hline 10 & 1.06 & 31.2479 & 30.7847 & 30.7130 & 31.1905 & 30.9124 & 30.8659 & 31.1860 & 30.7201 & 30.6175 & 31.1304 & 30.8516 & 30.7783 \\
\hline 11 & 4.46 & 27.3375 & 26.8096 & 26.6661 & 27.2403 & 26.9256 & 26.9209 & 27.2756 & 26.7443 & 26.6024 & 27.1709 & 26.8515 & 26.8617 \\
\hline 11 & 4.49 & 27.9317 & 27.5035 & 27.2873 & 27.7328 & 27.5407 & 27.5331 & 27.8362 & 27.4061 & 27.2088 & 27.6402 & 27.4515 & 27.4437 \\
\hline 13 & 7.32 & 24.8633 & 24.2087 & 24.0019 & 24.7782 & 24.3018 & 24.2188 & 24.7989 & 24.1439 & 23.9221 & 24.7041 & 24.2332 & 24.1326 \\
\hline 14 & 7.32 & 24.7397 & 24.1136 & 23.9454 & 24.6345 & 24.2025 & 24.1261 & 24.6456 & 24.0196 & 23.8361 & 24.5301 & 24.1061 & 24.0249 \\
\hline 15 & 7.32 & 24.8371 & 24.2089 & 24.0404 & 24.7340 & 24.3008 & 24.2269 & 24.7394 & 24.1112 & 23.9417 & 24.6294 & 24.2039 & 24.1271 \\
\hline
\end{tabular}




\section{Compound 78a}

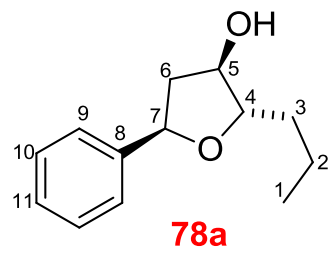

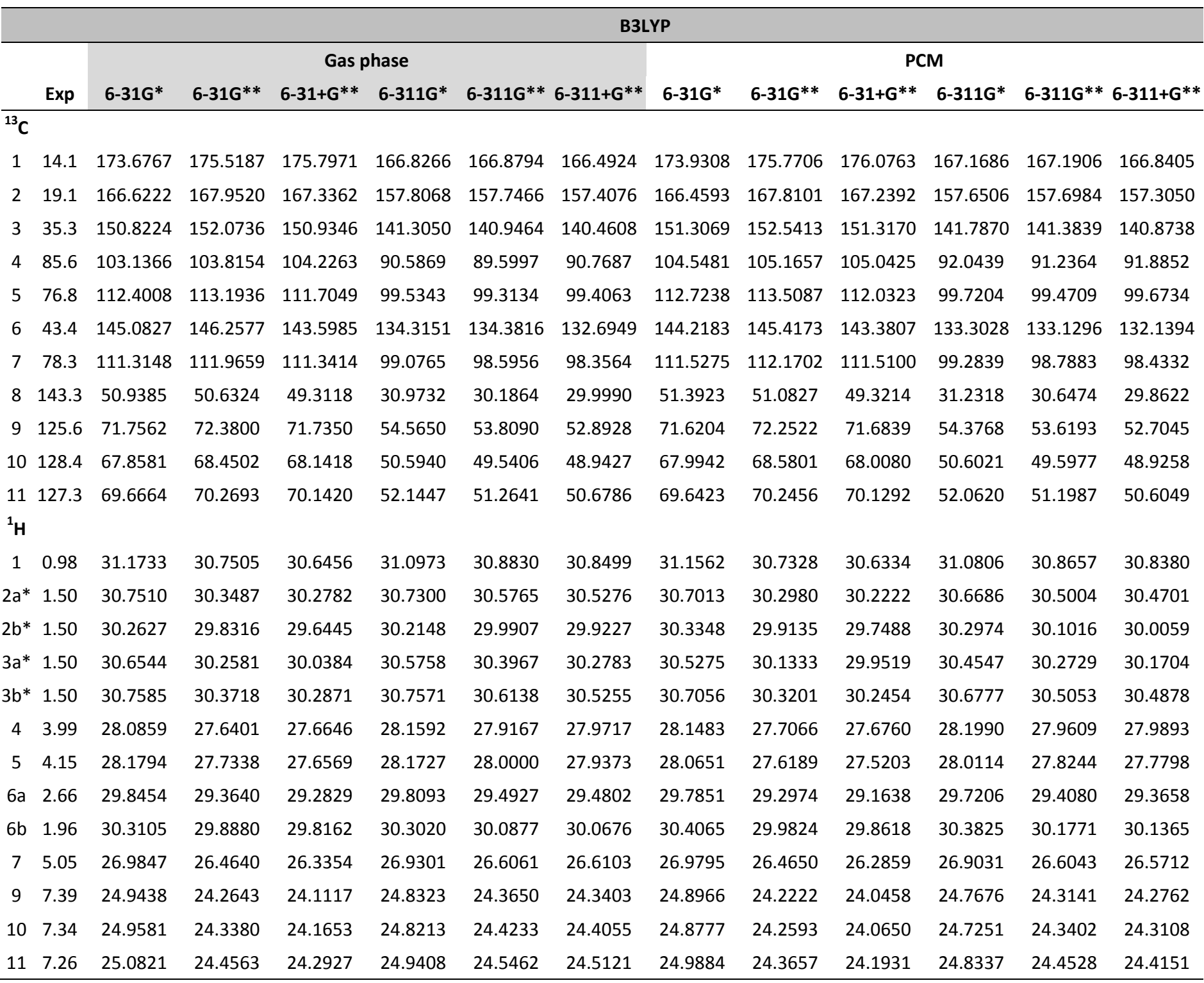

\begin{tabular}{|c|c|c|c|c|c|c|c|c|c|c|c|c|c|}
\hline & \multirow[b]{3}{*}{ Exp } & \multicolumn{12}{|c|}{ mPW1PW91 } \\
\hline & & \multicolumn{6}{|c|}{ Gas phase } & \multicolumn{6}{|c|}{ PCM } \\
\hline & & 6-31G* & $6-31 G * *$ & $6-31+\mathrm{G} * *$ & 6-311G* & $6-311 G * *$ & $6-311+G * *$ & 6-31G* & $6-31 G * *$ & $6-31+G * *$ & 6-311G* & $6-311 \mathrm{G} * *$ & $6-311+\mathrm{G} * *$ \\
\hline \multicolumn{14}{|c|}{${ }^{13} \mathrm{C}$} \\
\hline 1 & 14.1 & 177.8959 & 179.6245 & 179.8325 & 171.5676 & 171.5979 & 171.1960 & 178.1938 & 179.9196 & 180.1227 & 171.9163 & 171.9367 & 171.5567 \\
\hline 2 & 19.1 & 171.7465 & 173.0055 & 172.5527 & 163.7406 & 163.7698 & 163.1808 & 171.6157 & 172.8964 & 172.4026 & 163.6072 & 163.6771 & 163.1405 \\
\hline 3 & 35.3 & 155.9052 & 157.0455 & 156.0972 & 147.4023 & 147.0041 & 146.5691 & 156.4376 & 157.5646 & 156.6755 & 147.9357 & 147.5210 & 147.0213 \\
\hline
\end{tabular}




\begin{tabular}{|c|c|c|c|c|c|c|c|c|c|c|c|c|c|}
\hline 4 & 85.6 & 107.2395 & 107.8016 & 108.0915 & 95.9073 & 95.2300 & 95.9348 & 108.6362 & 109.1356 & 109.3989 & 97.7437 & 96.8016 & 97.4639 \\
\hline 5 & 76.8 & 116.9485 & 117.6918 & 116.6691 & 106.2044 & 105.8496 & 105.8186 & 117.3099 & 118.0431 & 116.9990 & 106.3159 & 106.0146 & 106.1154 \\
\hline 6 & 43.4 & 150.5500 & 151.6938 & 149.7128 & 141.5704 & 141.2931 & 140.2393 & 149.7336 & 150.8993 & 148.9426 & 140.2583 & 140.1700 & 139.2373 \\
\hline 7 & 78.3 & 115.5771 & 116.1772 & 115.7701 & 104.9615 & 104.4660 & 104.3500 & 115.8189 & 116.4113 & 116.0505 & 105.2439 & 104.7188 & 104.4849 \\
\hline 8 & 143.3 & 53.7638 & 53.3646 & 51.9588 & 35.9619 & 35.2663 & 35.0482 & 54.2288 & 53.8342 & 52.3922 & 36.5406 & 35.8169 & 35.2451 \\
\hline 9 & 125.6 & 74.3324 & 74.9104 & 74.2215 & 59.2172 & 58.4062 & 57.6120 & 74.1609 & 74.7449 & 74.1425 & 58.9593 & 58.1932 & 57.3098 \\
\hline 10 & 128.4 & 70.1794 & 70.6632 & 70.7167 & 54.9099 & 53.8533 & 53.2598 & 70.2895 & 70.7683 & 70.5820 & 54.9605 & 53.8869 & 53.2764 \\
\hline $\begin{array}{l}11 \\
{ }^{\mathbf{1}} \mathbf{H}\end{array}$ & 127.3 & 72.0768 & 72.5922 & 72.2541 & 56.6350 & 55.7203 & 55.1741 & 72.0117 & 72.5268 & 72.2864 & 56.5037 & 55.5994 & 55.0295 \\
\hline 1 & 0.98 & 31.1318 & 30.7088 & 30.6150 & 31.0346 & 30.8105 & 30.7790 & 31.1112 & 30.6875 & 30.5976 & 31.0124 & 30.7900 & 30.7619 \\
\hline $2 a *$ & 1.50 & 30.7146 & 30.3136 & 30.2455 & 30.6706 & 30.4941 & 30.4542 & 30.6661 & 30.2644 & 30.1875 & 30.5996 & 30.4314 & 30.3864 \\
\hline $2 b^{*}$ & 1.50 & 30.2602 & 29.8291 & 29.6707 & 30.1695 & 29.9664 & 29.8729 & 30.3238 & 29.9020 & 29.7486 & 30.2447 & 30.0499 & 29.9680 \\
\hline 3a* & 1.50 & 30.6893 & 30.2988 & 30.1152 & 30.6056 & 30.4240 & 30.3156 & 30.5492 & 30.1591 & 29.9702 & 30.4488 & 30.2749 & 30.1788 \\
\hline $3 b^{*}$ & 1.50 & 30.7106 & 30.3230 & 30.2235 & 30.7082 & 30.5149 & 30.4549 & 30.6532 & 30.2663 & 30.1765 & 30.6011 & 30.4207 & 30.3717 \\
\hline 4 & 3.99 & 27.9632 & 27.5041 & 27.5034 & 27.9843 & 27.7192 & 27.7837 & 28.0274 & 27.5730 & 27.5530 & 28.0424 & 27.7789 & 27.8243 \\
\hline 5 & 4.15 & 28.1399 & 27.6937 & 27.6425 & 28.1680 & 27.9621 & 27.9398 & 28.0269 & 27.5810 & 27.4894 & 27.9902 & 27.7991 & 27.7601 \\
\hline $6 a$ & 2.66 & 29.7466 & 29.2628 & 29.1781 & 29.6803 & 29.3843 & 29.3562 & 29.6681 & 29.1760 & 29.0678 & 29.5955 & 29.2801 & 29.2461 \\
\hline $6 b$ & 1.96 & 30.1269 & 29.6908 & 29.6304 & 30.0865 & 29.8526 & 29.8567 & 30.2143 & 29.7766 & 29.7048 & 30.1774 & 29.9385 & 29.9336 \\
\hline 7 & 5.05 & 26.8808 & 26.3417 & 26.2229 & 26.7913 & 26.4523 & 26.4546 & 26.8737 & 26.3421 & 26.2014 & 26.7787 & 26.4510 & 26.4446 \\
\hline 9 & 7.39 & 24.7264 & 24.0431 & 23.8855 & 24.6290 & 24.1282 & 24.0968 & 24.6683 & 23.9898 & 23.8270 & 24.5639 & 24.0732 & 24.0330 \\
\hline 10 & 7.34 & 24.7422 & 24.1150 & 23.9583 & 24.6279 & 24.1931 & 24.1718 & 24.6480 & 24.0226 & 23.8561 & 24.5257 & 24.1004 & 24.0734 \\
\hline 11 & 7.26 & 24.8738 & 24.2423 & 24.1157 & 24.7613 & 24.3268 & 24.2938 & 24.7655 & 24.1368 & 24.0031 & 24.6427 & 24.2221 & 24.1898 \\
\hline
\end{tabular}

\section{Compound 78b}<smiles>CCC[C@H]1O[C@@H](c2ccccc2)C[C@H]1O</smiles>

\begin{tabular}{|c|c|c|c|c|c|c|c|c|c|c|c|c|c|}
\hline & \multirow[b]{3}{*}{ Exp } & \multicolumn{12}{|c|}{ B3LYP } \\
\hline & & \multicolumn{6}{|c|}{ Gas phase } & \multicolumn{6}{|c|}{ PCM } \\
\hline & & 6-31G* & 6-31G** & $6-31+G * *$ & 6-311G* & $6-311 G * *$ & $6-311+G * *$ & 6-31G* & 6-31G** & $6-31+G * *$ & 6-311G* & 6-311G** & $6-311+G * *$ \\
\hline \multicolumn{14}{|l|}{${ }^{13} \mathrm{C}$} \\
\hline 1 & 14.1 & 173.8954 & 175.7283 & 176.2037 & 167.0666 & 167.1166 & 166.7838 & 174.1630 & 175.9918 & 176.5330 & 167.4183 & 167.4479 & 167.1388 \\
\hline 2 & 19.1 & 166.9869 & 168.3223 & 168.0596 & 158.3774 & 158.3725 & 157.8739 & 167.0512 & 168.3919 & 168.1622 & 158.5079 & 158.5038 & 158.0284 \\
\hline 3 & 36.5 & 150.8468 & 152.0904 & 151.1854 & 141.3323 & 141.0316 & 140.5874 & 151.1903 & 152.4266 & 151.6099 & 141.7666 & 141.4411 & 141.0487 \\
\hline 4 & 87.1 & 103.2982 & 103.8870 & 102.4552 & 90.0301 & 89.4629 & 89.3754 & 103.1832 & 103.7810 & 102.2272 & 89.7645 & 89.2073 & 89.1076 \\
\hline 5 & 76.8 & 111.6802 & 112.5603 & 111.0532 & 99.0452 & 98.8107 & 98.9351 & 111.9405 & 112.8207 & 111.2852 & 99.3200 & 99.0865 & 99.1382 \\
\hline 6 & 44.0 & 141.3917 & 142.7163 & 141.9874 & 131.5233 & 131.0163 & 131.0975 & 141.6939 & 143.0027 & 142.4242 & 131.8865 & 131.3706 & 131.5412 \\
\hline 7 & 79.5 & 109.5695 & 110.2435 & 109.4572 & 97.4365 & 96.8950 & 96.8281 & 109.6971 & 110.3696 & 109.5658 & 97.5362 & 96.9951 & 96.9501 \\
\hline 8 & 142.1 & 54.2580 & 53.9827 & 52.2602 & 34.0750 & 33.6844 & 32.2918 & 53.9375 & 53.6670 & 51.8564 & 33.6190 & 33.2510 & 31.9085 \\
\hline 9 & 125.9 & 70.5183 & 71.1579 & 70.3336 & 53.5374 & 52.7323 & 51.6446 & 70.6162 & 71.2495 & 70.3261 & 53.5074 & 52.7173 & 51.6457 \\
\hline 10 & 128.3 & 68.5117 & 69.0970 & 68.7444 & 51.2073 & 50.2285 & 49.1734 & 68.3749 & 68.9584 & 68.5047 & 50.9361 & 49.9869 & 49.0012 \\
\hline 11 & 127.4 & 69.5207 & 70.1235 & 69.4047 & 52.0324 & 51.1483 & 50.5439 & 69.4223 & 70.0263 & 69.2440 & 51.8304 & 50.9832 & 50.4276 \\
\hline
\end{tabular}




\begin{tabular}{ccccccccccccccc}
\hline${ }^{1} \mathbf{H}$ & & & & & & & & & & & & & \\
1 & 0.99 & 31.1397 & 30.7144 & 30.6119 & 31.0665 & 30.8471 & 30.8149 & 31.1309 & 30.7040 & 30.6039 & 31.0603 & 30.8391 & 30.8081 \\
$2 a^{*}$ & 1.57 & 30.7106 & 30.3041 & 30.2641 & 30.6805 & 30.4998 & 30.4700 & 30.6907 & 30.2815 & 30.2398 & 30.6644 & 30.4804 & 30.4496 \\
$2 b^{*}$ & 1.57 & 30.3390 & 29.9113 & 29.8075 & 30.3120 & 30.1110 & 30.0357 & 30.4099 & 29.9850 & 29.8917 & 30.3845 & 30.1851 & 30.1178 \\
$3 a^{*}$ & 1.57 & 30.6503 & 30.2662 & 30.1506 & 30.6226 & 30.4377 & 30.3769 & 30.5838 & 30.1968 & 30.0784 & 30.5611 & 30.3753 & 30.3088 \\
$3 b^{*}$ & 1.57 & 30.5374 & 30.1480 & 29.9923 & 30.5396 & 30.3574 & 30.2569 & 30.5115 & 30.1218 & 29.9542 & 30.5009 & 30.3190 & 30.2131 \\
4 & 3.91 & 28.1718 & 27.7255 & 27.6497 & 28.1312 & 27.9076 & 27.9616 & 28.1967 & 27.7473 & 27.6672 & 28.1559 & 27.9252 & 27.9731 \\
5 & 4.17 & 28.0739 & 27.5992 & 27.6166 & 28.0616 & 27.8031 & 27.8642 & 27.9609 & 27.4817 & 27.4793 & 27.9386 & 27.6773 & 27.7258 \\
$6 a$ & 2.21 & 30.4065 & 29.9518 & 29.9184 & 30.3930 & 30.1339 & 30.1175 & 30.2886 & 29.8309 & 29.7765 & 30.2566 & 30.0013 & 29.9854 \\
$6 \mathrm{~b}$ & 1.97 & 30.7030 & 30.2647 & 30.1280 & 30.6599 & 30.4535 & 30.4025 & 30.6456 & 30.2051 & 30.0665 & 30.6070 & 30.3993 & 30.3476 \\
7 & 5.12 & 26.9620 & 26.4954 & 26.4330 & 26.8841 & 26.6600 & 26.6851 & 26.9771 & 26.5073 & 26.4241 & 26.8851 & 26.6549 & 26.6723 \\
9 & 7.31 & 24.9251 & 24.2615 & 24.0287 & 24.8202 & 24.3735 & 24.2059 & 24.8621 & 24.1978 & 23.9447 & 24.7403 & 24.2990 & 24.1193 \\
10 & 7.31 & 25.0119 & 24.3961 & 24.1019 & 24.8743 & 24.4833 & 24.3842 & 24.9084 & 24.2927 & 23.9872 & 24.7586 & 24.3754 & 24.2669 \\
11 & 7.31 & 25.0753 & 24.4591 & 24.2732 & 24.9337 & 24.5535 & 24.5107 & 24.9716 & 24.3552 & 24.1600 & 24.8199 & 24.4470 & 24.4022 \\
\hline
\end{tabular}

\begin{tabular}{|c|c|c|c|c|c|c|c|c|c|c|c|c|c|}
\hline \multirow{3}{*}{\multicolumn{2}{|c|}{$\operatorname{Exp}$}} & \multicolumn{12}{|c|}{ mPW1PW91 } \\
\hline & & \multicolumn{6}{|c|}{ Gas phase } & \multicolumn{6}{|c|}{ PCM } \\
\hline & & 6-31G* & $6-31 G * *$ & $6-31+G^{* *}$ & 6-311G* & $6-311 G * *$ & $6-311+G * *$ & 6-31G* & $6-31 G * *$ & $6-31+G * *$ & 6-311G* & 6-311G** & $6-311+G * *$ \\
\hline \multicolumn{14}{|c|}{ ( } \\
\hline 1 & 14.1 & 178.1094 & 179.8315 & 180.1220 & 171.7975 & 171.8363 & 171.4859 & 178.4203 & 180.1373 & 180.4862 & 172.1593 & 172.1792 & 171.8515 \\
\hline 2 & 19.1 & 171.9840 & 173.2426 & 172.9778 & 164.0977 & 164.0821 & 163.4972 & 172.0878 & 173.3515 & 173.1160 & 164.2532 & 164.2385 & 163.6731 \\
\hline 3 & 36.5 & 156.1502 & 157.2967 & 156.6229 & 147.6753 & 147.3832 & 146.9067 & 156.5015 & 157.6417 & 157.0453 & 148.0888 & 147.7746 & 147.3418 \\
\hline 4 & 87.1 & 108.0979 & 108.6467 & 107.5884 & 96.6834 & 96.1364 & 96.0445 & 107.9861 & 108.5436 & 107.3834 & 96.4411 & 95.8995 & 95.7936 \\
\hline 5 & 76.8 & 116.2715 & 117.1006 & 116.0190 & 105.5920 & 105.3574 & 105.2528 & 116.4942 & 117.3266 & 116.2261 & 105.8212 & 105.5905 & 105.4271 \\
\hline 6 & 44.0 & 146.3355 & 147.5607 & 146.8366 & 137.6392 & 137.1442 & 137.1614 & 146.6272 & 147.8390 & 147.2380 & 137.9539 & 137.4563 & 137.5495 \\
\hline 7 & 79.5 & 113.9286 & 114.5622 & 114.0075 & 103.5441 & 103.0253 & 102.8686 & 114.0727 & 114.7047 & 114.1342 & 103.6461 & 103.1263 & 102.9894 \\
\hline 8 & 142.1 & 57.3526 & 57.0438 & 55.7494 & 39.7605 & 39.3382 & 37.9692 & 57.0250 & 56.7221 & 55.3383 & 39.3164 & 38.9179 & 37.5944 \\
\hline 9 & 125.9 & 72.9474 & 73.5288 & 72.7326 & 58.0399 & 57.2134 & 56.1196 & 73.0489 & 73.6240 & 72.7197 & 58.0060 & 57.1961 & 56.1295 \\
\hline 10 & 128.3 & 70.9852 & 71.4824 & 71.4118 & 55.7975 & 54.7737 & 53.6536 & 70.8118 & 71.3076 & 71.1277 & 55.4789 & 54.4881 & 53.4463 \\
\hline $\begin{array}{l}11 \\
{ }^{1} \mathbf{H}\end{array}$ & 127.4 & 71.9732 & 72.4915 & 71.8464 & 56.6007 & 55.6717 & 55.0376 & 71.8359 & 72.3561 & 71.6369 & 56.3451 & 55.4566 & 54.8800 \\
\hline 1 & 0.99 & 31.0924 & 30.6669 & 30.5756 & 30.9953 & 30.7681 & 30.7373 & 31.0810 & 30.6539 & 30.5646 & 30.9865 & 30.7577 & 30.7279 \\
\hline $2 a^{*}$ & 1.57 & 30.6733 & 30.2659 & 30.2212 & 30.6054 & 30.4198 & 30.3849 & 30.6521 & 30.2418 & 30.1960 & 30.5879 & 30.3992 & 30.3637 \\
\hline $2 b^{*}$ & 1.57 & 30.3244 & 29.8991 & 29.8056 & 30.2596 & 30.0594 & 29.9804 & 30.3911 & 29.9680 & 29.8822 & 30.3257 & 30.1272 & 30.0557 \\
\hline $3 a^{*}$ & 1.57 & 30.6222 & 30.2381 & 30.1138 & 30.5590 & 30.3704 & 30.3082 & 30.5501 & 30.1629 & 30.0354 & 30.4921 & 30.3025 & 30.2346 \\
\hline $3 b^{*}$ & 1.57 & 30.4709 & 30.0804 & 29.9386 & 30.4331 & 30.2455 & 30.1561 & 30.4457 & 30.0551 & 29.9029 & 30.3979 & 30.2107 & 30.1163 \\
\hline 4 & 3.91 & 28.1204 & 27.6713 & 27.6075 & 28.0517 & 27.8242 & 27.8800 & 28.1386 & 27.6859 & 27.6184 & 28.0677 & 27.8335 & 27.8843 \\
\hline 5 & 4.17 & 28.0358 & 27.5614 & 27.5758 & 27.9989 & 27.7356 & 27.7883 & 27.9123 & 27.4333 & 27.4310 & 27.8689 & 27.6029 & 27.6446 \\
\hline $6 a$ & 2.21 & 30.3085 & 29.8504 & 29.8144 & 30.2659 & 30.0018 & 29.9886 & 30.1854 & 29.7240 & 29.6716 & 30.1310 & 29.8698 & 29.8572 \\
\hline $6 b$ & 1.97 & 30.6187 & 30.1813 & 30.0541 & 30.5535 & 30.3429 & 30.2932 & 30.5588 & 30.1193 & 29.9915 & 30.4984 & 30.2864 & 30.2373 \\
\hline 7 & 5.12 & 26.8804 & 26.4141 & 26.3618 & 26.7813 & 26.5602 & 26.5905 & 26.8919 & 26.4220 & 26.3505 & 26.7812 & 26.5534 & 26.5754 \\
\hline 9 & 7.31 & 24.7166 & 24.0543 & 23.8484 & 24.6343 & 24.1602 & 24.0072 & 24.6429 & 23.9798 & 23.7555 & 24.5474 & 24.0788 & 23.9135 \\
\hline 10 & 7.31 & 24.8071 & 24.1873 & 23.9278 & 24.6962 & 24.2699 & 24.1787 & 24.6885 & 24.0685 & 23.7992 & 24.5687 & 24.1504 & 24.0504 \\
\hline 11 & 7.31 & 24.8714 & 24.2513 & 24.1161 & 24.7571 & 24.3410 & 24.3104 & 24.7523 & 24.1320 & 23.9901 & 24.6311 & 24.2227 & 24.1900 \\
\hline
\end{tabular}




\section{Compound 78c}<smiles>CCC[C@H]1O[C@@H](c2ccccc2)C[C@H]1O</smiles>

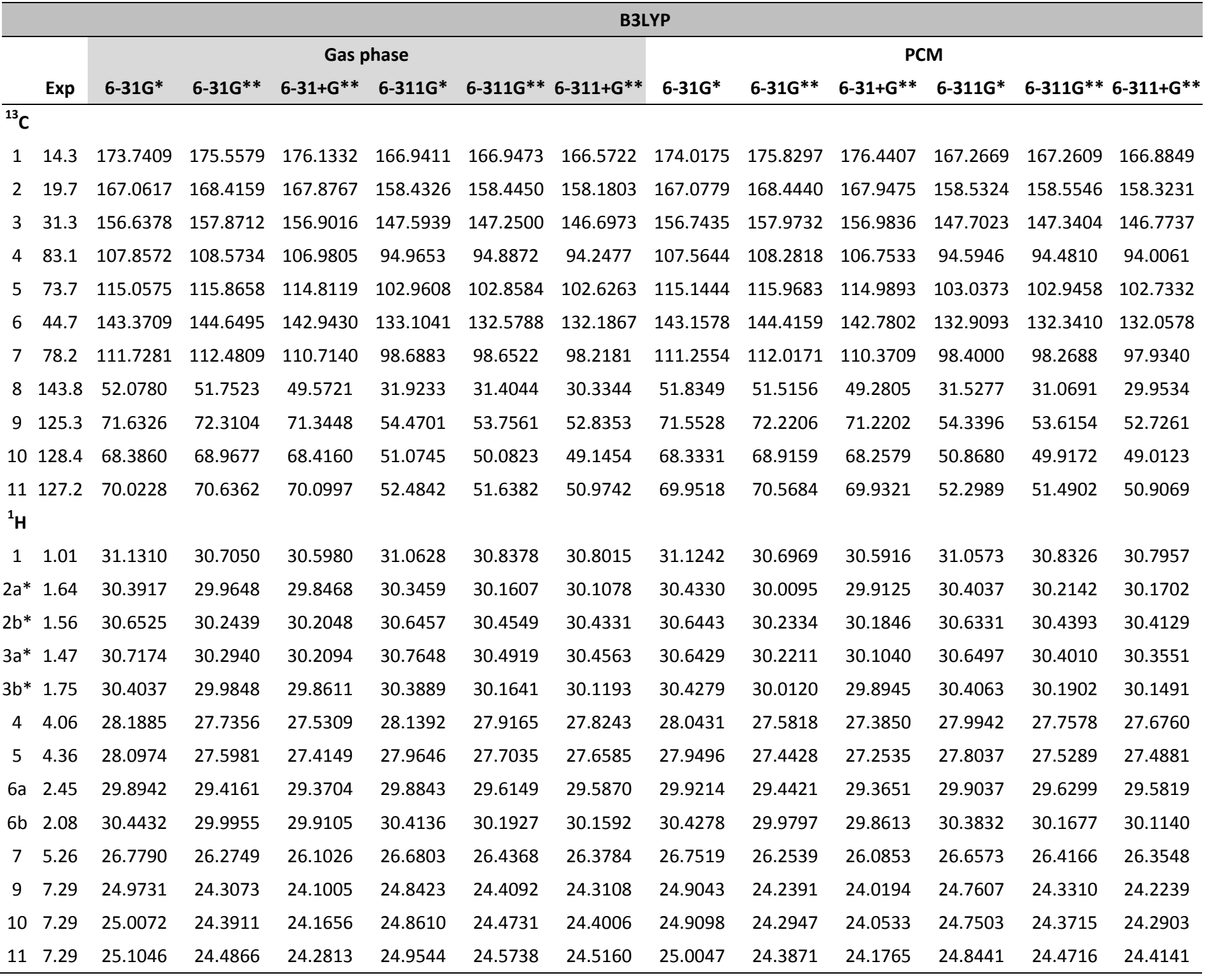

\begin{tabular}{|c|c|c|c|c|c|c|c|c|c|c|c|c|c|}
\hline & \multirow[b]{3}{*}{ Exp } & \multicolumn{12}{|c|}{ mPW1PW91 } \\
\hline & & \multicolumn{6}{|c|}{ Gas phase } & \multicolumn{6}{|c|}{ PCM } \\
\hline & & 6-31G* & $6-31 G * *$ & $6-31+G * *$ & 6-311G* & $6-311 G * *$ & $6-311+G * *$ & 6-31G* & $6-31 G * *$ & $6-31+\mathrm{G} * *$ & 6-311G* & $6-311 G * *$ & $6-311+G * *$ \\
\hline \multicolumn{14}{|c|}{${ }^{13} \mathrm{C}$} \\
\hline 1 & 14.3 & 177.8962 & 179.6038 & 180.0172 & 171.6453 & 171.6380 & 171.2879 & 178.2390 & 179.9399 & 180.3888 & 172.0115 & 171.9910 & 171.6342 \\
\hline 2 & 19.7 & 172.1219 & 173.4018 & 172.9280 & 164.2384 & 164.2338 & 163.8559 & 172.1619 & 173.4542 & 173.0013 & 164.3416 & 164.3514 & 164.0001 \\
\hline 3 & 31.3 & 161.7477 & 162.8965 & 162.2831 & 153.6995 & 153.3807 & 152.8422 & 161.8743 & 163.0213 & 162.3655 & 153.8150 & 153.4814 & 152.9221 \\
\hline
\end{tabular}




\begin{tabular}{|c|c|c|c|c|c|c|c|c|c|c|c|c|c|}
\hline 4 & 83.1 & 112.6508 & 113.3207 & 112.2515 & 101.7665 & 101.6587 & 100.9419 & 112.3126 & 112.9933 & 111.8964 & 101.2623 & 101.1573 & 100.5873 \\
\hline 5 & 73.7 & 119.6017 & 120.3471 & 119.4921 & 109.3560 & 109.1878 & 108.8300 & 119.6517 & 120.4208 & 119.6192 & 109.3280 & 109.2061 & 108.8740 \\
\hline 6 & 44.7 & 148.4369 & 149.6373 & 148.1692 & 139.2665 & 138.7804 & 138.3940 & 148.1943 & 149.3826 & 147.8905 & 139.0268 & 138.5106 & 138.1998 \\
\hline 7 & 78.2 & 116.5923 & 117.3064 & 115.9620 & 105.2521 & 105.2256 & 104.7369 & 115.9814 & 116.7205 & 115.3959 & 104.7615 & 104.6803 & 104.2582 \\
\hline 8 & 143.8 & 55.0320 & 54.6634 & 52.8681 & 37.4756 & 36.9103 & 35.8598 & 54.8004 & 54.4331 & 52.6434 & 37.1466 & 36.6302 & 35.5329 \\
\hline 9 & 125.3 & 74.1873 & 74.8123 & 74.0421 & 59.0809 & 58.3520 & 57.3634 & 74.0700 & 74.6877 & 73.8373 & 58.8989 & 58.1673 & 57.2159 \\
\hline 10 & 128.4 & 70.8310 & 71.3227 & 70.9590 & 55.6354 & 54.5972 & 53.6390 & 70.7630 & 71.2565 & 70.8036 & 55.4053 & 54.4109 & 53.4834 \\
\hline $\begin{array}{l}11 \\
{ }^{1} \mathrm{H}\end{array}$ & 127.2 & 72.5187 & 73.0487 & 72.7114 & 57.1012 & 56.2104 & 55.4847 & 72.4185 & 72.9547 & 72.4672 & 56.8622 & 56.0176 & 55.3810 \\
\hline 1 & 1.01 & 31.0838 & 30.6580 & 30.5657 & 30.9902 & 30.7583 & 30.7268 & 31.0749 & 30.6474 & 30.5552 & 30.9825 & 30.7503 & 30.7173 \\
\hline $2 a^{*}$ & 1.64 & 30.4008 & 29.9760 & 29.8711 & 30.3197 & 30.1292 & 30.0730 & 30.4237 & 30.0020 & 29.9113 & 30.3526 & 30.1614 & 30.1138 \\
\hline $2 b^{*}$ & 1.56 & 30.6048 & 30.1982 & 30.1550 & 30.5561 & 30.3687 & 30.3407 & 30.6017 & 30.1920 & 30.1425 & 30.5505 & 30.3571 & 30.3252 \\
\hline $3 a *$ & 1.47 & 30.6384 & 30.2151 & 30.1404 & 30.6492 & 30.3757 & 30.3511 & 30.5839 & 30.1614 & 30.0533 & 30.5577 & 30.3038 & 30.2659 \\
\hline $3 b^{*}$ & 1.75 & 30.3516 & 29.9327 & 29.8197 & 30.3032 & 30.0733 & 30.0380 & 30.3711 & 29.9549 & 29.8436 & 30.3159 & 30.0939 & 30.0602 \\
\hline 4 & 4.06 & 28.2247 & 27.7846 & 27.5778 & 28.1462 & 27.9314 & 27.8400 & 28.0580 & 27.6090 & 27.4000 & 27.9707 & 27.7453 & 27.6615 \\
\hline 5 & 4.36 & 28.0747 & 27.5784 & 27.4222 & 27.9559 & 27.6896 & 27.6502 & 27.9227 & 27.4200 & 27.2487 & 27.7805 & 27.5045 & 27.4640 \\
\hline $6 a$ & 2.45 & 29.7452 & 29.2594 & 29.2218 & 29.7202 & 29.4363 & 29.4113 & 29.7757 & 29.2871 & 29.2349 & 29.7506 & 29.4617 & 29.4226 \\
\hline $6 b$ & 2.08 & 30.3164 & 29.8655 & 29.7965 & 30.2635 & 30.0409 & 30.0157 & 30.3095 & 29.8576 & 29.7601 & 30.2496 & 30.0288 & 29.9832 \\
\hline 7 & 5.26 & 26.7422 & 26.2250 & 26.0713 & 26.6234 & 26.3621 & 26.3148 & 26.6916 & 26.1840 & 26.0369 & 26.5782 & 26.3276 & 26.2774 \\
\hline 9 & 7.29 & 24.7728 & 24.1070 & 23.9152 & 24.6649 & 24.2014 & 24.1136 & 24.6909 & 24.0261 & 23.8246 & 24.5737 & 24.1148 & 24.0177 \\
\hline 10 & 7.29 & 24.8015 & 24.1806 & 23.9858 & 24.6829 & 24.2578 & 24.1911 & 24.6907 & 24.0707 & 23.8608 & 24.5617 & 24.1465 & 24.0720 \\
\hline 11 & 7.29 & 24.9018 & 24.2797 & 24.1104 & 24.7788 & 24.3613 & 24.3087 & 24.7880 & 24.1664 & 23.9956 & 24.6571 & 24.2488 & 24.1982 \\
\hline
\end{tabular}

\section{Compound 78d}

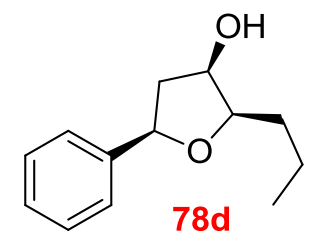

\begin{tabular}{|c|c|c|c|c|c|c|c|c|c|c|c|c|c|}
\hline & \multirow[b]{3}{*}{ Exp } & \multicolumn{12}{|c|}{ B3LYP } \\
\hline & & \multicolumn{6}{|c|}{ Gas phase } & \multicolumn{6}{|c|}{ PCM } \\
\hline & & 6-31G* & $6-31 G * *$ & $6-31+G * *$ & 6-311G* & $6-311 G * *$ & $6-311+G^{* *}$ & $6-31 G *$ & $6-31 G * *$ & $6-31+G * *$ & 6-311G* & $6-311 \mathrm{G} * *$ & $6-311+\mathrm{G} * *$ \\
\hline \multicolumn{14}{|l|}{${ }^{13} \mathrm{C}$} \\
\hline 1 & 14.3 & 173.8139 & 175.6612 & 176.1662 & 166.9922 & 167.0593 & 166.6350 & 173.9897 & 175.8274 & 176.3879 & 167.0985 & 167.2545 & 166.7577 \\
\hline 2 & 19.6 & 167.2000 & 168.5592 & 168.0933 & 158.5154 & 158.5733 & 158.0661 & 167.0589 & 168.4286 & 168.1020 & 158.3166 & 158.4869 & 158.0180 \\
\hline 3 & 30.9 & 156.4251 & 157.6837 & 157.4107 & 147.5524 & 147.2074 & 146.8400 & 156.9012 & 158.1389 & 157.7578 & 148.1535 & 147.7775 & 147.2495 \\
\hline 4 & 83.6 & 106.5443 & 107.2032 & 105.8851 & 93.7873 & 93.4674 & 92.8237 & 106.6348 & 107.2978 & 106.0112 & 93.8331 & 93.6831 & 92.9613 \\
\hline 5 & 73.1 & 115.5591 & 116.3534 & 115.2945 & 103.4318 & 103.2295 & 103.1627 & 115.5647 & 116.3928 & 115.4167 & 103.7530 & 103.5494 & 103.2657 \\
\hline 6 & 44.2 & 144.6116 & 145.8175 & 145.2051 & 134.7187 & 134.1486 & 133.9440 & 144.4989 & 145.7132 & 145.0472 & 134.6463 & 134.0517 & 133.9737 \\
\hline 7 & 78.7 & 111.7202 & 112.3964 & 110.4619 & 99.2684 & 99.0367 & 98.2384 & 110.8699 & 111.5807 & 109.6224 & 97.9641 & 97.9407 & 97.3788 \\
\hline 8 & 143.0 & 49.5146 & 49.1557 & 47.6012 & 29.4443 & 28.7623 & 28.3007 & 49.8888 & 49.5446 & 47.7177 & 30.0891 & 29.3756 & 28.5776 \\
\hline 9 & 125.9 & 71.5830 & 72.2117 & 70.2258 & 53.9779 & 53.3954 & 51.6064 & 70.5861 & 71.2458 & 69.0155 & 52.2422 & 51.9034 & 50.5722 \\
\hline 10 & 128.4 & 67.7020 & 68.2733 & 68.0685 & 50.4837 & 49.4409 & 48.7989 & 67.8807 & 68.4457 & 68.1971 & 50.7026 & 49.6391 & 48.8765 \\
\hline 11 & 127.3 & 69.6885 & 70.2910 & 69.7726 & 52.1527 & 51.3150 & 50.5669 & 69.5312 & 70.1407 & 69.3605 & 51.8155 & 51.0314 & 50.4084 \\
\hline
\end{tabular}




\begin{tabular}{ccccccccccccccc}
\hline${ }^{1} \mathbf{H}$ & & & & & & & & & & & & & \\
1 & 1.00 & 31.1245 & 30.7010 & 30.6091 & 31.0605 & 30.8388 & 30.8034 & 31.1249 & 30.6995 & 30.6058 & 31.0643 & 30.8382 & 30.8037 \\
$2 \mathrm{a}^{*}$ & 1.52 & 30.6087 & 30.2059 & 30.2004 & 30.6087 & 30.4304 & 30.4259 & 30.6429 & 30.2369 & 30.2091 & 30.6749 & 30.4629 & 30.4620 \\
$2 \mathrm{~b} *$ & 1.52 & 30.4646 & 30.0370 & 29.9099 & 30.4046 & 30.2070 & 30.1604 & 30.4756 & 30.0540 & 29.9440 & 30.4617 & 30.2648 & 30.2119 \\
$3 \mathrm{a}^{*}$ & 1.72 & 30.5295 & 30.1236 & 30.0387 & 30.5981 & 30.3689 & 30.2813 & 30.5123 & 30.0989 & 29.9866 & 30.5140 & 30.2862 & 30.2159 \\
$3 \mathrm{~b}^{*}$ & 1.79 & 30.2992 & 29.8799 & 29.7379 & 30.2813 & 30.0542 & 30.0041 & 30.3399 & 29.9226 & 29.7971 & 30.3364 & 30.1010 & 30.0765 \\
4 & 3.76 & 28.2402 & 27.7884 & 27.7376 & 28.2824 & 28.0225 & 28.0433 & 28.2382 & 27.7897 & 27.6949 & 28.2983 & 28.0311 & 28.0128 \\
5 & 4.27 & 28.2841 & 27.8004 & 27.6304 & 28.2451 & 28.0052 & 27.8990 & 28.1277 & 27.6388 & 27.4189 & 27.9919 & 27.7557 & 27.6565 \\
$6 a$ & 2.70 & 29.7341 & 29.2526 & 29.1306 & 29.6521 & 29.3753 & 29.3509 & 29.6194 & 29.1308 & 28.9867 & 29.5457 & 29.2576 & 29.2136 \\
$6 \mathrm{~b}$ & 1.92 & 30.2090 & 29.7708 & 29.6867 & 30.1907 & 29.9593 & 29.9320 & 30.2754 & 29.8368 & 29.7858 & 30.3310 & 30.0827 & 30.0391 \\
7 & 4.87 & 27.0958 & 26.5803 & 26.5540 & 27.0436 & 26.7128 & 26.8073 & 27.1624 & 26.6521 & 26.6061 & 27.1515 & 26.8149 & 26.8634 \\
9 & 7.40 & 24.9093 & 24.2115 & 23.9448 & 24.7841 & 24.3176 & 24.1763 & 24.8062 & 24.1280 & 23.8428 & 24.6359 & 24.2137 & 24.0716 \\
10 & 7.34 & 24.9637 & 24.3377 & 24.1388 & 24.8332 & 24.4290 & 24.3805 & 24.8797 & 24.2576 & 24.0477 & 24.7422 & 24.3540 & 24.2963 \\
11 & 7.26 & 25.0830 & 24.4588 & 24.2501 & 24.9465 & 24.5537 & 24.5100 & 24.9840 & 24.3636 & 24.1590 & 24.8324 & 24.4579 & 24.4097 \\
\hline
\end{tabular}

\begin{tabular}{|c|c|c|c|c|c|c|c|c|c|c|c|c|c|}
\hline \multirow{3}{*}{\multicolumn{2}{|c|}{$\operatorname{Exp}$}} & \multicolumn{12}{|c|}{ mPW1PW91 } \\
\hline & & \multicolumn{6}{|c|}{ Gas phase } & \multicolumn{6}{|c|}{ PCM } \\
\hline & & 6-31G* & $6-31 G * *$ & $6-31+G^{* *}$ & 6-311G* & $6-311 G * *$ & $6-311+G * *$ & 6-31G* & $6-31 G * *$ & $6-31+G * *$ & 6-311G* & 6-311G** & $6-311+G * *$ \\
\hline \multicolumn{14}{|c|}{ ( } \\
\hline 1 & 14.3 & 178.0559 & 179.7985 & 180.1232 & 171.7856 & 171.8432 & 171.4727 & 178.2835 & 180.0162 & 180.3628 & 172.0100 & 172.0454 & 171.6544 \\
\hline 2 & 19.6 & 172.3012 & 173.5918 & 173.1595 & 164.5233 & 164.5287 & 163.9082 & 172.2329 & 173.5358 & 173.1595 & 164.3873 & 164.4384 & 163.8860 \\
\hline 3 & 30.9 & 161.3841 & 162.5473 & 162.4093 & 153.4219 & 153.1365 & 152.5558 & 161.8044 & 162.9474 & 162.7920 & 154.0024 & 153.6307 & 153.1444 \\
\hline 4 & 83.6 & 111.0496 & 111.6606 & 110.8588 & 100.1213 & 99.8363 & 99.0189 & 111.1654 & 111.7789 & 110.9462 & 100.3487 & 100.0255 & 99.3319 \\
\hline 5 & 73.1 & 120.1287 & 120.8646 & 120.1740 & 109.9015 & 109.6212 & 109.3018 & 120.1447 & 120.9119 & 120.2038 & 109.9731 & 109.7653 & 109.4054 \\
\hline 6 & 44.2 & 149.3666 & 150.4637 & 149.7923 & 140.3965 & 139.8584 & 139.6858 & 149.2768 & 150.3874 & 149.6683 & 140.3203 & 139.7550 & 139.5637 \\
\hline 7 & 78.7 & 116.5360 & 117.1704 & 116.1704 & 106.0467 & 105.6653 & 105.4922 & 115.9531 & 116.6315 & 115.2801 & 104.9911 & 104.7899 & 104.3648 \\
\hline 8 & 143.0 & 52.5727 & 52.1602 & 50.6222 & 34.7372 & 34.1109 & 33.1991 & 52.8033 & 52.3925 & 50.8202 & 35.3039 & 34.6246 & 33.7855 \\
\hline 9 & 125.9 & 74.4714 & 75.0624 & 73.9158 & 59.3491 & 58.6038 & 57.3728 & 73.6771 & 74.3109 & 72.5906 & 57.8156 & 57.2969 & 55.9707 \\
\hline 10 & 128.4 & 70.0708 & 70.5445 & 70.3224 & 54.8351 & 53.7833 & 52.9471 & 70.1714 & 70.6357 & 70.5055 & 54.9723 & 53.9054 & 53.1286 \\
\hline $\begin{array}{l}11 \\
{ }^{1} \mathbf{H}\end{array}$ & 127.3 & 72.1709 & 72.6866 & 72.4792 & 56.8032 & 55.9060 & 55.1208 & 72.0073 & 72.5315 & 72.0105 & 56.4462 & 55.6071 & 54.9195 \\
\hline 1 & 1.00 & 31.0742 & 30.6506 & 30.5653 & 30.9788 & 30.7520 & 30.7176 & 31.0697 & 30.6440 & 30.5589 & 30.9797 & 30.7489 & 30.7150 \\
\hline $2 a^{*}$ & 1.52 & 30.5476 & 30.1442 & 30.1092 & 30.4832 & 30.3096 & 30.2651 & 30.5836 & 30.1779 & 30.1382 & 30.5439 & 30.3563 & 30.3227 \\
\hline $2 b^{*}$ & 1.52 & 30.4642 & 30.0386 & 29.9439 & 30.4002 & 30.1899 & 30.1035 & 30.4634 & 30.0426 & 29.9457 & 30.4058 & 30.2056 & 30.1419 \\
\hline $3 a^{*}$ & 1.72 & 30.4646 & 30.0601 & 29.9578 & 30.4443 & 30.2316 & 30.1930 & 30.4486 & 30.0376 & 29.9224 & 30.4097 & 30.1836 & 30.1256 \\
\hline $3 b^{*}$ & 1.79 & 30.2407 & 29.8212 & 29.6823 & 30.1984 & 29.9697 & 29.9100 & 30.2768 & 29.8596 & 29.7335 & 30.2230 & 29.9992 & 29.9563 \\
\hline 4 & 3.76 & 28.1893 & 27.7351 & 27.6547 & 28.1627 & 27.9074 & 27.9026 & 28.1618 & 27.7074 & 27.6027 & 28.1511 & 27.8941 & 27.8731 \\
\hline 5 & 4.27 & 28.2451 & 27.7608 & 27.6516 & 28.2279 & 27.9736 & 27.9525 & 28.1105 & 27.6226 & 27.4485 & 28.0136 & 27.7584 & 27.6859 \\
\hline $6 a$ & 2.70 & 29.6638 & 29.1853 & 29.0549 & 29.5513 & 29.2794 & 29.2390 & 29.5267 & 29.0403 & 28.8921 & 29.4211 & 29.1412 & 29.0936 \\
\hline $6 b$ & 1.92 & 30.0888 & 29.6455 & 29.5349 & 30.0212 & 29.7881 & 29.7029 & 30.1303 & 29.6836 & 29.6194 & 30.1323 & 29.8842 & 29.8363 \\
\hline 7 & 4.87 & 26.9802 & 26.4510 & 26.3734 & 26.8725 & 26.5426 & 26.5614 & 27.0048 & 26.4771 & 26.4175 & 26.9428 & 26.6070 & 26.6502 \\
\hline 9 & 7.40 & 24.7241 & 24.0221 & 23.7909 & 24.6408 & 24.1263 & 24.0148 & 24.6174 & 23.9303 & 23.6812 & 24.4911 & 24.0171 & 23.8869 \\
\hline 10 & 7.34 & 24.7521 & 24.1203 & 23.9390 & 24.6460 & 24.2029 & 24.1385 & 24.6528 & 24.0235 & 23.8369 & 24.5429 & 24.1148 & 24.0554 \\
\hline 11 & 7.26 & 24.8786 & 24.2488 & 24.0706 & 24.7709 & 24.3382 & 24.2934 & 24.7666 & 24.1398 & 23.9715 & 24.6475 & 24.2313 & 24.1883 \\
\hline
\end{tabular}




\section{Compound 79a}<smiles>CC[C@H](O)[C@H](C)C1=C(C)C(=O)[C@H](C)[C@H]([C@H](C)CC)O1</smiles>

\begin{tabular}{|c|c|c|c|c|c|c|c|c|c|c|c|c|c|}
\hline & \multirow[b]{3}{*}{ Exp } & \multicolumn{12}{|c|}{ B3LYP } \\
\hline & & \multicolumn{6}{|c|}{ Gas phase } & \multicolumn{6}{|c|}{ PCM } \\
\hline & & 6-31G* & 6-31G** & $6-31+G * *$ & 6-311G* & $6-311 G^{* *}$ & $6-311+G^{* *}$ & $6-31 G^{*}$ & $6-31 G^{* *}$ & $6-31+G * *$ & 6-311G* & 6-311G** & $6-311+G^{* *}$ \\
\hline \multicolumn{14}{|l|}{${ }^{13} \mathrm{C}$} \\
\hline 1 & 10.2 & 176.5725 & 178.4599 & 179.3462 & 170.3830 & 170.4902 & 170.1254 & 176.8225 & 178.6971 & 179.6123 & 170.6950 & 170.7841 & 170.4008 \\
\hline 2 & 27.9 & 160.1913 & 161.4744 & 160.4374 & 151.6674 & 151.4938 & 151.3665 & 160.5358 & 161.8039 & 160.7468 & 151.9670 & 151.7725 & 151.5857 \\
\hline 3 & 75.1 & 117.2306 & 117.8056 & 116.1744 & 104.5787 & 104.5462 & 104.0358 & 117.1776 & 117.7198 & 115.9591 & 104.5452 & 104.5314 & 103.9353 \\
\hline 4 & 41.6 & 146.7693 & 147.4691 & 146.1759 & 136.9208 & 136.7996 & 135.9596 & 146.7907 & 147.4202 & 145.8848 & 136.8510 & 136.7364 & 135.7480 \\
\hline 5 & 173.0 & 19.7704 & 19.8516 & 19.3717 & -2.2783 & -2.1910 & -2.7496 & 18.2974 & 18.4018 & 17.8493 & -3.9548 & -3.8860 & -4.6459 \\
\hline 6 & 108.6 & 83.5374 & 83.7209 & 84.3951 & 68.9880 & 69.0879 & 68.1026 & 83.7532 & 83.9248 & 84.4821 & 69.2440 & 69.3445 & 68.2411 \\
\hline 7 & 195.7 & 7.6455 & 7.6843 & 5.5404 & -13.1678 & -12.9949 & -16.6740 & 5.6627 & 5.7075 & 3.0412 & -15.5345 & -15.3539 & -19.4729 \\
\hline 8 & 40.6 & 149.1176 & 149.8010 & 148.7037 & 139.3049 & 138.9693 & 138.2566 & 149.0616 & 149.7474 & 148.7185 & 139.2425 & 138.9055 & 138.1643 \\
\hline 9 & 87.0 & 101.9355 & 102.4650 & 101.6404 & 89.2449 & 88.8733 & 88.5666 & 102.0957 & 102.6353 & 102.0090 & 89.5236 & 89.1426 & 88.8530 \\
\hline 10 & 35.1 & 152.0048 & 152.7971 & 152.0602 & 141.8126 & 141.6674 & 140.8404 & 152.1354 & 152.9335 & 152.2554 & 142.0091 & 141.8573 & 141.1074 \\
\hline 11 & 21.9 & 166.4479 & 167.6827 & 167.5212 & 157.8636 & 157.8122 & 157.2838 & 166.5833 & 167.8198 & 167.7487 & 158.0781 & 158.0022 & 157.5353 \\
\hline 12 & 11.7 & 175.8068 & 177.6782 & 178.0881 & 169.3948 & 169.3715 & 169.0971 & 176.0396 & 177.9124 & 178.4189 & 169.7021 & 169.6707 & 169.4165 \\
\hline 13 & 13.3 & 176.4379 & 178.2280 & 179.5586 & 170.6389 & 170.8323 & 169.9908 & 176.5997 & 178.3241 & 179.2591 & 170.6999 & 170.8867 & 169.9454 \\
\hline 14 & 9.3 & 180.0526 & 181.8912 & 181.7080 & 174.6493 & 174.4486 & 174.0103 & 180.3065 & 182.1345 & 181.9085 & 174.9964 & 174.7803 & 174.3184 \\
\hline 15 & 10.6 & 178.2662 & 180.1576 & 180.9710 & 172.8112 & 172.7192 & 172.4710 & 178.4939 & 180.3849 & 181.3263 & 173.1224 & 173.0183 & 172.8036 \\
\hline $\begin{array}{l}16 \\
{ }^{1} \mathrm{H}\end{array}$ & 16.1 & 171.8378 & 173.6317 & 174.4137 & 165.7664 & 165.7029 & 165.6540 & 172.0076 & 173.8043 & 174.6566 & 166.0183 & 165.9394 & 165.8788 \\
\hline 1 & 0.96 & 31.0939 & 30.6690 & 30.5774 & 31.0477 & 30.8336 & 30.8142 & 31.1127 & 30.6854 & 30.5998 & 31.0643 & 30.8469 & 30.8287 \\
\hline $2 a$ & 1.49 & 30.4228 & 30.0018 & 30.0089 & 30.4475 & 30.2602 & 30.2836 & 30.4733 & 30.0559 & 30.0785 & 30.4984 & 30.3059 & 30.3334 \\
\hline $2 b$ & 1.39 & 30.8983 & 30.4733 & 30.3789 & 30.8450 & 30.6199 & 30.5988 & 30.7833 & 30.3542 & 30.2627 & 30.7471 & 30.5230 & 30.5088 \\
\hline 3 & 3.65 & 28.2207 & 27.8043 & 27.8579 & 28.2899 & 28.1128 & 28.1996 & 28.1718 & 27.7575 & 27.8089 & 28.2319 & 28.0519 & 28.1350 \\
\hline 4 & 2.77 & 29.6894 & 29.2011 & 29.1279 & 29.6321 & 29.3770 & 29.3453 & 29.5891 & 29.1026 & 29.0471 & 29.5417 & 29.2880 & 29.2665 \\
\hline 8 & 2.49 & 29.7431 & 29.3122 & 29.2986 & 29.8816 & 29.6258 & 29.5583 & 29.6313 & 29.1982 & 29.1527 & 29.7644 & 29.5103 & 29.4275 \\
\hline 9 & 3.77 & 28.2264 & 27.8409 & 27.9020 & 28.3917 & 28.2106 & 28.2166 & 28.1127 & 27.7276 & 27.7835 & 28.2703 & 28.0927 & 28.0949 \\
\hline 10 & 1.73 & 30.4396 & 30.0160 & 30.0087 & 30.5865 & 30.3490 & 30.3103 & 30.3614 & 29.9394 & 29.9383 & 30.5093 & 30.2736 & 30.2417 \\
\hline $11 a$ & 1.57 & 30.5027 & 30.0322 & 29.9941 & 30.5919 & 30.3218 & 30.2990 & 30.4676 & 29.9992 & 29.9548 & 30.5518 & 30.2848 & 30.2583 \\
\hline $11 b$ & 1.25 & 30.7129 & 30.2897 & 30.2470 & 30.8338 & 30.6028 & 30.5712 & 30.7194 & 30.3000 & 30.2530 & 30.8374 & 30.6147 & 30.5854 \\
\hline 12 & 0.94 & 31.1564 & 30.7123 & 30.6455 & 31.1443 & 30.8892 & 30.8603 & 31.1482 & 30.7034 & 30.6404 & 31.1379 & 30.8827 & 30.8548 \\
\hline 13 & 1.20 & 30.9690 & 30.5184 & 30.4464 & 30.9966 & 30.7421 & 30.7187 & 30.9515 & 30.4982 & 30.4222 & 30.9755 & 30.7197 & 30.6917 \\
\hline 14 & 1.73 & 30.5918 & 30.0562 & 29.8687 & 30.4836 & 30.1370 & 30.0400 & 30.5907 & 30.0559 & 29.8713 & 30.4827 & 30.1387 & 30.0414 \\
\hline 15 & 1.08 & 31.2097 & 30.7258 & 30.6174 & 31.2175 & 30.9107 & 30.8513 & 31.2095 & 30.7261 & 30.6208 & 31.2161 & 30.9096 & 30.8531 \\
\hline 16 & 1.03 & 31.0130 & 30.5530 & 30.5099 & 31.0721 & 30.7747 & 30.7563 & 31.0114 & 30.5517 & 30.5080 & 31.0671 & 30.7732 & 30.7524 \\
\hline
\end{tabular}




\begin{tabular}{|c|c|c|c|c|c|c|c|c|c|c|c|c|c|}
\hline & \multirow[b]{3}{*}{ Exp } & \multicolumn{12}{|c|}{ mPW1PW91 } \\
\hline & & \multicolumn{6}{|c|}{ Gas phase } & \multicolumn{6}{|c|}{ PCM } \\
\hline & & 6-31G* & $6-31 G * *$ & $6-31+\mathrm{G} * *$ & 6-311G* & $6-311 G * *$ & $6-311+G * *$ & 6-31G* & $6-31 G * *$ & $6-31+G * *$ & 6-311G* & $6-311 G * *$ & $6-311+\mathrm{G} * *$ \\
\hline \multicolumn{14}{|l|}{${ }^{13} \mathrm{C}$} \\
\hline 1 & 10.2 & 180.7933 & 182.5640 & 183.2342 & 175.0165 & 175.1195 & 174.7498 & 181.0711 & 182.8279 & 183.5452 & 175.3302 & 175.4200 & 175.0337 \\
\hline 2 & 27.9 & 165.1697 & 166.3681 & 165.5912 & 157.4278 & 157.2634 & 157.0547 & 165.5026 & 166.6849 & 165.8289 & 157.6930 & 157.5140 & 157.2489 \\
\hline 3 & 75.1 & 122.2019 & 122.7197 & 121.3738 & 111.1532 & 111.1551 & 110.6281 & 122.0485 & 122.5397 & 121.1028 & 111.0471 & 111.0784 & 110.4776 \\
\hline 4 & 41.6 & 151.4354 & 152.0985 & 151.0741 & 142.6503 & 142.5913 & 141.7966 & 151.3327 & 151.9316 & 150.6916 & 142.4913 & 142.4561 & 141.5275 \\
\hline 5 & 173.0 & 22.9517 & 23.0385 & 22.8900 & 3.6999 & 3.7594 & 3.3060 & 21.4695 & 21.5793 & 21.3679 & 2.0009 & 2.0390 & 1.3998 \\
\hline 6 & 108.6 & 86.9642 & 87.1425 & 87.8263 & 74.2146 & 74.3286 & 73.2767 & 87.2071 & 87.3733 & 87.9443 & 74.4949 & 74.6107 & 73.4560 \\
\hline 7 & 195.7 & 9.9245 & 9.9561 & 8.5407 & -7.9684 & -7.8024 & -11.1360 & 7.8674 & 7.9052 & 6.0280 & -10.3668 & -10.1925 & -13.9286 \\
\hline 8 & 40.6 & 153.5139 & 154.1611 & 153.2850 & 144.8495 & 144.5308 & 143.8823 & 153.4379 & 154.0884 & 153.2709 & 144.7515 & 144.4309 & 143.7521 \\
\hline 9 & 87.0 & 107.0372 & 107.5237 & 106.9618 & 95.9069 & 95.5542 & 95.2449 & 107.1986 & 107.6965 & 107.3217 & 96.1477 & 95.7828 & 95.4871 \\
\hline 10 & 35.1 & 157.2079 & 157.9434 & 157.3308 & 147.9593 & 147.8309 & 147.0823 & 157.3432 & 158.0865 & 157.5131 & 148.1471 & 148.0121 & 147.3300 \\
\hline 11 & 21.9 & 171.3910 & 172.5404 & 172.3774 & 163.4844 & 163.4300 & 162.9132 & 171.5534 & 172.7063 & 172.6267 & 163.7129 & 163.6327 & 163.1626 \\
\hline 12 & 11.7 & 179.9729 & 181.7299 & 182.1063 & 174.0481 & 174.0193 & 173.7165 & 180.2565 & 182.0140 & 182.4810 & 174.3751 & 174.3385 & 174.0573 \\
\hline 13 & 13.3 & 180.5877 & 182.2464 & 183.0904 & 175.0299 & 175.2575 & 174.5183 & 180.6879 & 182.2830 & 182.7700 & 175.0417 & 175.2763 & 174.4561 \\
\hline 14 & 9.3 & 183.7523 & 185.4830 & 185.3552 & 178.8981 & 178.7135 & 178.2602 & 184.0308 & 185.7503 & 185.5888 & 179.2415 & 179.0445 & 178.5693 \\
\hline 15 & 10.6 & 182.4869 & 184.2561 & 185.0563 & 177.3450 & 177.2581 & 177.0509 & 182.7478 & 184.5165 & 185.4427 & 177.6598 & 177.5619 & 177.3843 \\
\hline 16 & 16.1 & 176.1613 & 177.8152 & 178.5807 & 170.4677 & 170.4041 & 170.2893 & 176.3678 & 178.0245 & 178.8513 & 170.7213 & 170.6447 & 170.5287 \\
\hline \multicolumn{14}{|c|}{${ }^{1} \mathrm{H}$} \\
\hline 1 & 0.96 & 31.0521 & 30.6263 & 30.5430 & 30.9788 & 30.7582 & 30.7361 & 31.0661 & 30.6380 & 30.5588 & 30.9905 & 30.7667 & 30.7458 \\
\hline $2 a$ & 1.49 & 30.3865 & 29.9670 & 29.9672 & 30.3669 & 30.1777 & 30.1953 & 30.4408 & 30.0251 & 30.0407 & 30.4187 & 30.2239 & 30.2439 \\
\hline $2 b$ & 1.39 & 30.8534 & 30.4271 & 30.3348 & 30.7581 & 30.5306 & 30.5116 & 30.7340 & 30.3040 & 30.2251 & 30.6617 & 30.4345 & 30.4251 \\
\hline 3 & 3.65 & 28.1908 & 27.7821 & 27.8252 & 28.2202 & 28.0425 & 28.1208 & 28.1422 & 27.7361 & 27.7760 & 28.1623 & 27.9816 & 28.0555 \\
\hline 4 & 2.77 & 29.6190 & 29.1365 & 29.0391 & 29.4953 & 29.2488 & 29.2121 & 29.5153 & 29.0347 & 28.9604 & 29.4046 & 29.1588 & 29.1324 \\
\hline 8 & 2.49 & 29.6850 & 29.2481 & 29.2240 & 29.7544 & 29.5036 & 29.4397 & 29.5760 & 29.1368 & 29.0822 & 29.6400 & 29.3906 & 29.3119 \\
\hline 9 & 3.77 & 28.1523 & 27.7751 & 27.8208 & 28.2612 & 28.0838 & 28.0872 & 28.0430 & 27.6663 & 27.7058 & 28.1453 & 27.9706 & 27.9690 \\
\hline 10 & 1.73 & 30.4046 & 29.9866 & 29.9638 & 30.4712 & 30.2442 & 30.2006 & 30.3246 & 29.9079 & 29.8925 & 30.3940 & 30.1682 & 30.1307 \\
\hline $11 a$ & 1.57 & 30.4850 & 30.0196 & 29.9820 & 30.5153 & 30.2479 & 30.2226 & 30.4500 & 29.9867 & 29.9429 & 30.4760 & 30.2117 & 30.1825 \\
\hline $11 b$ & 1.25 & 30.6858 & 30.2656 & 30.2242 & 30.7380 & 30.5126 & 30.4792 & 30.6927 & 30.2768 & 30.2310 & 30.7424 & 30.5258 & 30.4929 \\
\hline 12 & 0.94 & 31.1177 & 30.6732 & 30.6087 & 31.0697 & 30.8084 & 30.7806 & 31.1065 & 30.6613 & 30.6005 & 31.0600 & 30.7987 & 30.7721 \\
\hline 13 & 1.20 & 30.9165 & 30.4644 & 30.4045 & 30.9113 & 30.6516 & 30.6327 & 30.8937 & 30.4388 & 30.3749 & 30.8848 & 30.6241 & 30.6014 \\
\hline 14 & 1.73 & 30.5217 & 29.9844 & 29.8066 & 30.3955 & 30.0430 & 29.9512 & 30.5148 & 29.9786 & 29.8035 & 30.3899 & 30.0403 & 29.9484 \\
\hline 15 & 1.08 & 31.1474 & 30.6639 & 30.5698 & 31.1228 & 30.8103 & 30.7574 & 31.1442 & 30.6611 & 30.5695 & 31.1182 & 30.8059 & 30.7558 \\
\hline 16 & 1.03 & 30.9698 & 30.5098 & 30.4665 & 30.9868 & 30.6865 & 30.6684 & 30.9652 & 30.5059 & 30.4616 & 30.9780 & 30.6820 & 30.5059 \\
\hline
\end{tabular}




\section{Compound 79b}<smiles>CC[C@H](C)[C@H]1OC([C@H](C)CC)=C(C)C(=O)[C@H]1C</smiles>

\begin{tabular}{|c|c|c|c|c|c|c|c|c|c|c|c|c|c|}
\hline & \multirow[b]{3}{*}{ Exp } & \multicolumn{12}{|c|}{ B3LYP } \\
\hline & & \multicolumn{6}{|c|}{ Gas phase } & \multicolumn{6}{|c|}{ PCM } \\
\hline & & 6-31G* & $6-31 \mathrm{G} * *$ & $6-31+G * *$ & $6-311 G^{*}$ & $6-311 G * *$ & $6-311+G * *$ & 6-31G* & $6-31 G^{* *}$ & $6-31+G * *$ & 6-311G* & 6-311G** & $6-311+G * *$ \\
\hline${ }^{13} \mathrm{C}$ & & & & & & & & & & & & & \\
\hline 1 & 10.0 & 176.5969 & 178.4643 & 179.2144 & 170.5018 & 170.6012 & 170.2255 & 176.8104 & 178.6772 & 179.5098 & 170.8063 & 170.8882 & 170.5309 \\
\hline 2 & 28.1 & 158.3043 & 159.6667 & 159.2990 & 149.6073 & 149.2792 & 148.8914 & 158.6290 & 159.9673 & 159.4474 & 149.9052 & 149.5929 & 149.1600 \\
\hline 3 & 75.4 & 113.9782 & 114.6258 & 113.7463 & 101.5561 & 101.3307 & 101.1461 & 113.9288 & 114.5672 & 113.5409 & 101.3815 & 101.1859 & 100.9299 \\
\hline 4 & 41.4 & 146.1943 & 146.7804 & 145.0875 & 135.6513 & 135.6789 & 135.1513 & 146.2993 & 146.8713 & 145.2286 & 135.6977 & 135.6921 & 135.2169 \\
\hline 5 & 172.3 & 21.1447 & 21.2824 & 21.2056 & -0.2117 & -0.2563 & -0.9223 & 19.4415 & 19.5532 & 18.9540 & -2.2969 & -2.2975 & -3.2423 \\
\hline 6 & 109.7 & 81.5057 & 81.6311 & 82.0881 & 66.5716 & 66.7383 & 66.0537 & 81.8840 & 82.0305 & 82.6062 & 67.1034 & 67.2279 & 66.4314 \\
\hline 7 & 195.6 & 7.7156 & 7.7308 & 5.1687 & -13.1232 & -12.9223 & -16.3588 & 5.7490 & 5.7720 & 2.7325 & -15.4631 & -15.2618 & -19.0752 \\
\hline 8 & 40.6 & 149.1235 & 149.8113 & 148.8342 & 139.2860 & 138.9585 & 138.1316 & 149.0396 & 149.7280 & 148.7921 & 139.1730 & 138.8473 & 137.9421 \\
\hline 9 & 87.0 & 102.3163 & 102.8399 & 101.9226 & 89.7596 & 89.3994 & 89.0674 & 102.4452 & 102.9726 & 102.0908 & 89.9914 & 89.6202 & 89.2883 \\
\hline 10 & 35.1 & 152.0658 & 152.8478 & 152.1898 & 141.9400 & 141.7906 & 141.2739 & 152.1828 & 152.9649 & 152.3182 & 142.0986 & 141.9506 & 141.4567 \\
\hline 11 & 22.2 & 166.4122 & 167.6695 & 167.4402 & 157.7235 & 157.6078 & 157.0218 & 166.4121 & 167.6664 & 167.3354 & 157.7373 & 157.6227 & 156.9731 \\
\hline 12 & 11.7 & 175.8063 & 177.6715 & 178.2869 & 169.3477 & 169.3376 & 169.0952 & 176.0279 & 177.8927 & 178.5449 & 169.6557 & 169.6368 & 169.3946 \\
\hline 13 & 14.7 & 171.4929 & 173.4181 & 175.0684 & 165.8922 & 165.7748 & 165.6446 & 171.7061 & 173.6082 & 175.1307 & 166.0766 & 165.9741 & 165.7896 \\
\hline 14 & 9.4 & 179.7038 & 181.5374 & 181.7251 & 174.4129 & 174.1900 & 173.8599 & 179.9568 & 181.7812 & 182.0064 & 174.7458 & 174.5145 & 174.2009 \\
\hline 15 & 11.0 & 178.0530 & 179.9641 & 180.6761 & 172.1422 & 172.0461 & 171.5927 & 178.1947 & 180.1048 & 180.7237 & 172.3111 & 172.2042 & 171.6532 \\
\hline $\begin{array}{l}16 \\
{ }^{1} \mathrm{H}\end{array}$ & 16.3 & 171.4511 & 173.2648 & 174.2087 & 165.5932 & 165.4909 & 165.5832 & 171.6788 & 173.4924 & 174.5865 & 165.9257 & 165.8033 & 165.9118 \\
\hline 1 & 1.00 & 31.0654 & 30.6381 & 30.5468 & 31.0134 & 30.7976 & 30.7802 & 31.0857 & 30.6575 & 30.5701 & 31.0355 & 30.8162 & 30.7987 \\
\hline $2 a$ & 1.41 & 30.7566 & 30.3138 & 30.1991 & 30.6819 & 30.4546 & 30.4504 & 30.6729 & 30.2268 & 30.0975 & 30.5933 & 30.3636 & 30.3548 \\
\hline $2 b$ & 1.58 & 30.7482 & 30.3536 & 30.4048 & 30.8002 & 30.6105 & 30.6422 & 30.7179 & 30.3229 & 30.3714 & 30.7807 & 30.5845 & 30.6000 \\
\hline 3 & 3.57 & 28.6142 & 28.2161 & 28.1643 & 28.6568 & 28.4966 & 28.5194 & 28.5729 & 28.1746 & 28.1086 & 28.6052 & 28.4424 & 28.4604 \\
\hline 4 & 2.87 & 29.5617 & 29.0809 & 29.0815 & 29.5285 & 29.2901 & 29.2942 & 29.4768 & 28.9957 & 28.9931 & 29.4497 & 29.2045 & 29.2026 \\
\hline 8 & 2.50 & 29.7457 & 29.3156 & 29.2260 & 29.8762 & 29.6234 & 29.5200 & 29.6334 & 29.1985 & 29.1067 & 29.7598 & 29.5088 & 29.4022 \\
\hline 9 & 3.85 & 28.2513 & 27.8596 & 27.8836 & 28.4339 & 28.2320 & 28.2064 & 28.1176 & 27.7238 & 27.7305 & 28.2869 & 28.0948 & 28.0629 \\
\hline 10 & 1.76 & 30.4568 & 30.0279 & 30.0199 & 30.5941 & 30.3512 & 30.3158 & 30.3772 & 29.9496 & 29.9475 & 30.5194 & 30.2801 & 30.2478 \\
\hline 11a & 1.58 & 30.5148 & 30.0472 & 29.9656 & 30.5754 & 30.3045 & 30.2513 & 30.4706 & 30.0041 & 29.9205 & 30.5287 & 30.2616 & 30.2089 \\
\hline $11 \mathrm{~b}$ & 1.27 & 30.6844 & 30.2678 & 30.2243 & 30.8197 & 30.6126 & 30.5852 & 30.7076 & 30.2940 & 30.2587 & 30.8354 & 30.6329 & 30.6114 \\
\hline 12 & 0.94 & 31.1629 & 30.7188 & 30.6468 & 31.1469 & 30.8958 & 30.8590 & 31.1533 & 30.7083 & 30.6409 & 31.1397 & 30.8886 & 30.8550 \\
\hline 13 & 1.17 & 30.8421 & 30.4134 & 30.3888 & 30.8929 & 30.6389 & 30.6373 & 30.8229 & 30.3917 & 30.3501 & 30.8672 & 30.6160 & 30.6050 \\
\hline 14 & 1.75 & 30.5640 & 30.0242 & 29.8539 & 30.4645 & 30.1170 & 30.0456 & 30.5722 & 30.0339 & 29.8679 & 30.4766 & 30.1271 & 30.0544 \\
\hline 15 & 1.10 & 31.2003 & 30.7171 & 30.6101 & 31.1947 & 30.8908 & 30.8379 & 31.1943 & 30.7117 & 30.6020 & 31.1855 & 30.8828 & 30.8266 \\
\hline 16 & 1.06 & 31.0579 & 30.5915 & 30.5338 & 31.0979 & 30.8115 & 30.7938 & 31.0517 & 30.5851 & 30.5317 & 31.0898 & 30.8059 & 30.7942 \\
\hline
\end{tabular}




\begin{tabular}{|c|c|c|c|c|c|c|c|c|c|c|c|c|c|}
\hline & \multirow[b]{3}{*}{ Exp } & \multicolumn{12}{|c|}{ mPW1PW91 } \\
\hline & & \multicolumn{6}{|c|}{ Gas phase } & \multicolumn{6}{|c|}{ PCM } \\
\hline & & 6-31G* & 6-31G** & $6-31+\mathrm{G} * *$ & 6-311G* & 6-311G** & $6-311+G^{* *}$ & 6-31G* & $6-31 G * *$ & $6-31+G * *$ & 6-311G* & $6-311 G * *$ & $6-311+G * *$ \\
\hline \multicolumn{14}{|l|}{${ }^{13} \mathrm{C}$} \\
\hline 1 & 10.0 & 180.7806 & 182.5317 & 183.1881 & 175.0646 & 175.1548 & 174.7588 & 181.0377 & 182.7868 & 183.5191 & 175.3848 & 175.4580 & 175.0814 \\
\hline 2 & 28.1 & 163.3755 & 164.6287 & 164.4878 & 155.4560 & 155.1048 & 154.7152 & 163.6604 & 164.8917 & 164.5699 & 155.7102 & 155.3808 & 154.9463 \\
\hline 3 & 75.4 & 119.2164 & 119.7898 & 119.1467 & 108.4221 & 108.1842 & 107.9367 & 119.1471 & 119.7127 & 118.9333 & 108.2266 & 108.0212 & 107.7131 \\
\hline 4 & 41.4 & 150.7682 & 151.3467 & 149.9721 & 141.4164 & 141.5056 & 140.9838 & 150.8882 & 151.4599 & 150.1434 & 141.4506 & 141.5056 & 141.0364 \\
\hline 5 & 172.3 & 24.4223 & 24.5451 & 24.8945 & 5.6746 & 5.5900 & 4.9778 & 22.5993 & 22.7014 & 22.5659 & 3.5439 & 3.5108 & 2.6532 \\
\hline 6 & 109.7 & 84.8823 & 85.0134 & 85.4411 & 71.8190 & 72.0038 & 71.1955 & 85.3513 & 85.4994 & 86.0520 & 72.4016 & 72.5388 & 71.6352 \\
\hline 7 & 195.6 & 9.9831 & 9.9959 & 8.2697 & -7.9389 & -7.7402 & -10.8700 & 7.9466 & 7.9667 & 5.7979 & -10.3107 & -10.1125 & -13.5835 \\
\hline 8 & 40.6 & 153.5395 & 154.1892 & 153.4141 & 144.8427 & 144.5314 & 143.7546 & 153.4319 & 154.0823 & 153.3509 & 144.6928 & 144.3847 & 143.5432 \\
\hline 9 & 87.0 & 107.3796 & 107.8567 & 107.1937 & 96.3403 & 95.9989 & 95.6551 & 107.4835 & 107.9645 & 107.3299 & 96.5177 & 96.1680 & 95.8251 \\
\hline 10 & 35.1 & 157.2676 & 157.9955 & 157.4070 & 148.0825 & 147.9496 & 147.4403 & 157.3845 & 158.1133 & 157.5328 & 148.2278 & 148.0965 & 147.6040 \\
\hline 11 & 22.2 & 171.3629 & 172.5299 & 172.3198 & 163.3148 & 163.1870 & 162.6269 & 171.3721 & 172.5352 & 172.2294 & 163.3212 & 163.1969 & 162.5821 \\
\hline 12 & 11.7 & 179.9867 & 181.7384 & 182.3045 & 174.0241 & 174.0038 & 173.7250 & 180.2501 & 182.0004 & 182.5910 & 174.3460 & 174.3177 & 174.0393 \\
\hline 13 & 14.7 & 176.0643 & 177.8396 & 179.0169 & 170.6851 & 170.5406 & 170.4768 & 176.2564 & 178.0081 & 179.0454 & 170.8477 & 170.7271 & 170.6154 \\
\hline 14 & 9.4 & 183.4336 & 185.1586 & 185.3764 & 178.6946 & 178.4878 & 178.1388 & 183.7155 & 185.4319 & 185.6318 & 179.0281 & 178.8151 & 178.4782 \\
\hline 15 & 11.0 & 182.2919 & 184.0750 & 184.8023 & 176.6611 & 176.5567 & 176.1829 & 182.4545 & 184.2351 & 184.8775 & 176.8140 & 176.7013 & 176.2411 \\
\hline 16 & 16.3 & 175.8359 & 177.5085 & 178.3702 & 170.3616 & 170.2618 & 170.2806 & 176.0951 & 177.7662 & 178.7594 & 170.7040 & 170.5845 & 170.6225 \\
\hline \multicolumn{14}{|l|}{${ }^{1} \mathbf{H}$} \\
\hline 1 & 1.00 & 31.0218 & 30.5934 & 30.5092 & 30.9425 & 30.7208 & 30.7031 & 31.0393 & 30.6099 & 30.5295 & 30.9611 & 30.7357 & 30.7180 \\
\hline $2 a$ & 1.41 & 30.7320 & 30.2911 & 30.1804 & 30.6130 & 30.3881 & 30.3841 & 30.6449 & 30.2008 & 30.0802 & 30.5219 & 30.2947 & 30.2863 \\
\hline $2 b$ & 1.58 & 30.6970 & 30.2989 & 30.3376 & 30.6877 & 30.4950 & 30.5251 & 30.6746 & 30.2753 & 30.3140 & 30.6734 & 30.4737 & 30.4898 \\
\hline 3 & 3.57 & 28.5504 & 28.1604 & 28.1042 & 28.5698 & 28.4091 & 28.4239 & 28.5038 & 28.1136 & 28.0435 & 28.5152 & 28.3513 & 28.3620 \\
\hline 4 & 2.87 & 29.4905 & 29.0176 & 28.9900 & 29.3938 & 29.1625 & 29.1538 & 29.4059 & 28.9311 & 28.9020 & 29.3152 & 29.0755 & 29.0626 \\
\hline 8 & 2.50 & 29.6880 & 29.2516 & 29.1557 & 29.7464 & 29.4979 & 29.3978 & 29.5763 & 29.1349 & 29.0364 & 29.6318 & 29.3846 & 29.2809 \\
\hline 9 & 3.85 & 28.1836 & 27.7999 & 27.8057 & 28.3026 & 28.1040 & 28.0736 & 28.0444 & 27.6587 & 27.6488 & 28.1543 & 27.9657 & 27.9295 \\
\hline 10 & 1.76 & 30.4187 & 29.9944 & 29.9653 & 30.4758 & 30.2414 & 30.1992 & 30.3371 & 29.9137 & 29.8921 & 30.4014 & 30.1699 & 30.1305 \\
\hline $11 a$ & 1.58 & 30.4948 & 30.0315 & 29.9551 & 30.4953 & 30.2261 & 30.1763 & 30.4493 & 29.9869 & 29.9097 & 30.4486 & 30.1833 & 30.1339 \\
\hline $11 b$ & 1.27 & 30.6520 & 30.2386 & 30.1948 & 30.7224 & 30.5211 & 30.4892 & 30.6781 & 30.2678 & 30.2328 & 30.7404 & 30.5432 & 30.5168 \\
\hline 12 & 0.94 & 31.1230 & 30.6787 & 30.6082 & 31.0727 & 30.8148 & 30.7773 & 31.1101 & 30.6647 & 30.5990 & 31.0623 & 30.8046 & 30.7702 \\
\hline 13 & 1.17 & 30.8039 & 30.3706 & 30.3581 & 30.8133 & 30.5506 & 30.5506 & 30.7766 & 30.3409 & 30.3096 & 30.7819 & 30.5227 & 30.5139 \\
\hline 14 & 1.75 & 30.4890 & 29.9483 & 29.7867 & 30.3734 & 30.0203 & 29.9511 & 30.4961 & 29.9567 & 29.7997 & 30.3831 & 30.0277 & 29.9572 \\
\hline 15 & 1.10 & 31.1381 & 30.6550 & 30.5594 & 31.0997 & 30.7895 & 30.7438 & 31.1284 & 30.6458 & 30.5483 & 31.0863 & 30.7774 & 30.7293 \\
\hline 16 & 1.06 & 31.0083 & 30.5435 & 30.4842 & 31.0128 & 30.7240 & 30.7044 & 31.0004 & 30.5354 & 30.4801 & 31.0028 & 30.7164 & 30.5354 \\
\hline
\end{tabular}




\section{Compound 79c}

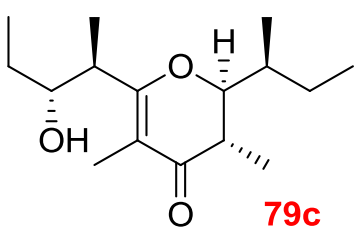

\begin{tabular}{|c|c|c|c|c|c|c|c|c|c|c|c|c|c|}
\hline & \multirow[b]{3}{*}{ Exp } & \multicolumn{12}{|c|}{ B3LYP } \\
\hline & & \multicolumn{6}{|c|}{ Gas phase } & \multicolumn{6}{|c|}{ PCM } \\
\hline & & 6-31G* & $6-31 \mathrm{G} * *$ & $6-31+G * *$ & 6-311G* & $6-311 G * *$ & $6-311+G * *$ & $6-31 G *$ & $6-31 G * *$ & $6-31+G * *$ & 6-311G* & $6-311 G * *$ & $6-311+G * *$ \\
\hline \multicolumn{14}{|l|}{${ }^{13} \mathrm{C}$} \\
\hline 1 & 12.0 & 176.5940 & 178.4506 & 179.1168 & 170.5235 & 170.6078 & 170.2586 & 176.8099 & 178.6653 & 179.4000 & 170.8221 & 170.8890 & 170.5473 \\
\hline 2 & 28.1 & 157.8457 & 159.1219 & 159.2328 & 149.1614 & 148.8480 & 148.8302 & 158.2148 & 159.4890 & 159.5725 & 149.5227 & 149.2077 & 149.1335 \\
\hline 3 & 75.5 & 113.2818 & 113.8855 & 113.2668 & 100.9181 & 100.6271 & 100.7362 & 113.3013 & 113.9083 & 113.2550 & 100.8966 & 100.6244 & 100.6919 \\
\hline 4 & 41.1 & 146.4835 & 147.2217 & 145.6732 & 136.3614 & 136.4611 & 135.7522 & 146.6107 & 147.3376 & 145.7670 & 136.4523 & 136.5282 & 135.8177 \\
\hline 5 & 172.4 & 21.4781 & 21.5183 & 21.2280 & -0.5760 & -0.4746 & -1.2319 & 19.8892 & 19.9311 & 19.2685 & -2.4122 & -2.3154 & -3.2879 \\
\hline 6 & 109.9 & 81.7055 & 81.8950 & 82.4870 & 66.6163 & 66.7520 & 65.6312 & 81.9798 & 82.1688 & 82.7469 & 66.9761 & 67.0933 & 65.8796 \\
\hline 7 & 195.6 & 7.7753 & 7.8069 & 5.4327 & -13.0077 & -12.8386 & -16.1660 & 5.7673 & 5.8028 & 2.9393 & -15.4276 & -15.2445 & -18.9945 \\
\hline 8 & 40.9 & 148.7641 & 149.4370 & 148.7022 & 139.1780 & 138.8469 & 138.1010 & 148.7291 & 149.3997 & 148.7185 & 139.1274 & 138.7960 & 138.0098 \\
\hline 9 & 87.8 & 102.1100 & 102.6132 & 101.6164 & 89.2010 & 88.8548 & 88.3762 & 102.1912 & 102.6917 & 101.7464 & 89.3618 & 89.0151 & 88.5293 \\
\hline 10 & 35.4 & 152.2151 & 153.0384 & 152.3503 & 142.3143 & 142.1575 & 141.5222 & 152.3578 & 153.1818 & 152.5292 & 142.4870 & 142.3286 & 141.6902 \\
\hline 11 & 22.0 & 166.5304 & 167.7771 & 167.5065 & 158.1687 & 158.1150 & 157.6866 & 166.5781 & 167.8171 & 167.5389 & 158.2296 & 158.1527 & 157.7075 \\
\hline 12 & 10.1 & 175.8500 & 177.7067 & 178.2144 & 169.4793 & 169.4489 & 169.1414 & 176.0649 & 177.9225 & 178.4728 & 169.7759 & 169.7357 & 169.4351 \\
\hline 13 & 14.7 & 171.5438 & 173.3372 & 174.6233 & 165.5132 & 165.4990 & 165.4824 & 171.8086 & 173.5933 & 174.8666 & 165.8054 & 165.7839 & 165.7547 \\
\hline 14 & 9.4 & 179.7648 & 181.6199 & 181.1544 & 174.5322 & 174.3013 & 174.2424 & 180.0295 & 181.8819 & 181.4854 & 174.8854 & 174.6387 & 174.5777 \\
\hline 15 & 10.0 & 178.4873 & 180.3741 & 181.1971 & 173.0233 & 172.9416 & 172.7510 & 178.7064 & 180.5899 & 181.4940 & 173.3192 & 173.2234 & 173.0576 \\
\hline 16 & 16.2 & 171.9057 & 173.6813 & 174.3488 & 165.4253 & 165.3759 & 165.0275 & 172.1074 & 173.8807 & 174.6154 & 165.7252 & 165.6543 & 165.3141 \\
\hline \multicolumn{14}{|l|}{${ }^{1} \mathrm{H}$} \\
\hline 1 & 1.00 & 31.0733 & 30.6437 & 30.5609 & 31.0447 & 30.8260 & 30.8098 & 31.0914 & 30.6610 & 30.5838 & 31.0628 & 30.8411 & 30.8259 \\
\hline $2 a$ & 1.58 & 30.7419 & 30.3104 & 30.2246 & 30.7280 & 30.4991 & 30.4888 & 30.6697 & 30.2361 & 30.1257 & 30.6468 & 30.4183 & 30.3943 \\
\hline $2 b$ & 1.41 & 30.6565 & 30.2478 & 30.2455 & 30.6798 & 30.4811 & 30.4800 & 30.6335 & 30.2235 & 30.2299 & 30.6738 & 30.4727 & 30.4728 \\
\hline 3 & 3.57 & 28.6022 & 28.2114 & 28.1549 & 28.6684 & 28.5000 & 28.4983 & 28.5596 & 28.1680 & 28.0838 & 28.6074 & 28.4356 & 28.4226 \\
\hline 4 & 2.87 & 29.6246 & 29.1467 & 29.0906 & 29.5574 & 29.3243 & 29.3171 & 29.5274 & 29.0472 & 28.9857 & 29.4688 & 29.2308 & 29.2169 \\
\hline 8 & 2.50 & 29.7213 & 29.2841 & 29.2922 & 29.8612 & 29.5964 & 29.5565 & 29.6011 & 29.1595 & 29.1489 & 29.7308 & 29.4674 & 29.4143 \\
\hline 9 & 3.85 & 28.3095 & 27.9160 & 27.9172 & 28.4432 & 28.2576 & 28.2232 & 28.1839 & 27.7882 & 27.7779 & 28.3095 & 28.1288 & 28.0862 \\
\hline 10 & 1.76 & 30.4741 & 30.0518 & 30.0497 & 30.6272 & 30.3881 & 30.3438 & 30.3919 & 29.9694 & 29.9675 & 30.5467 & 30.3083 & 30.2649 \\
\hline $11 a$ & 1.58 & 30.4902 & 30.0090 & 29.9690 & 30.5794 & 30.2898 & 30.2640 & 30.4475 & 29.9673 & 29.9260 & 30.5330 & 30.2457 & 30.2209 \\
\hline $11 b$ & 1.28 & 30.8582 & 30.4257 & 30.4035 & 31.0254 & 30.7537 & 30.7009 & 30.8743 & 30.4461 & 30.4211 & 31.0332 & 30.7711 & 30.7217 \\
\hline 12 & 0.94 & 31.1531 & 30.7080 & 30.6447 & 31.1487 & 30.8925 & 30.8625 & 31.1453 & 30.6991 & 30.6389 & 31.1431 & 30.8860 & 30.8578 \\
\hline 13 & 1.17 & 30.8354 & 30.4011 & 30.3755 & 30.8685 & 30.6172 & 30.6368 & 30.8187 & 30.3831 & 30.3469 & 30.8448 & 30.5957 & 30.6086 \\
\hline 14 & 1.75 & 30.5936 & 30.0618 & 29.8693 & 30.4915 & 30.1494 & 30.0491 & 30.5945 & 30.0631 & 29.8664 & 30.4932 & 30.1516 & 30.0466 \\
\hline 15 & 1.10 & 31.2227 & 30.7383 & 30.6535 & 31.2364 & 30.9294 & 30.8747 & 31.2216 & 30.7369 & 30.6518 & 31.2341 & 30.9269 & 30.8715 \\
\hline 16 & 1.06 & 30.9960 & 30.5413 & 30.4792 & 31.0307 & 30.7508 & 30.7266 & 30.9909 & 30.5356 & 30.4728 & 31.0240 & 30.7451 & 30.7215 \\
\hline
\end{tabular}




\begin{tabular}{|c|c|c|c|c|c|c|c|c|c|c|c|c|c|}
\hline \multicolumn{14}{|c|}{ mPW1PW91 } \\
\hline & \multirow[b]{2}{*}{ Exp } & \multicolumn{6}{|c|}{ Gas phase } & \multicolumn{6}{|c|}{ PCM } \\
\hline & & 6-31G* & $6-31 G * *$ & $6-31+G * *$ & 6-311G* & $6-311 G * *$ & $6-311+G * *$ & 6-31G* & $6-31 G * *$ & $6-31+\mathrm{G} * *$ & 6-311G* & $6-311 G * *$ & $6-311+\mathrm{G} * *$ \\
\hline \multicolumn{14}{|l|}{${ }^{13} \mathrm{C}$} \\
\hline 1 & 12.0 & 180.7701 & 182.5103 & 183.0975 & 175.0990 & 175.1706 & 174.8179 & 181.0301 & 182.7687 & 183.4112 & 175.4136 & 175.4688 & 175.1232 \\
\hline 2 & 28.1 & 162.8246 & 164.0050 & 164.0074 & 154.8925 & 154.6009 & 154.4942 & 163.1982 & 164.3771 & 164.3814 & 155.2501 & 154.9530 & 154.8108 \\
\hline 3 & 75.5 & 118.4527 & 118.9885 & 118.4623 & 107.6717 & 107.3959 & 107.3568 & 118.4724 & 119.0128 & 118.4636 & 107.6435 & 107.3853 & 107.3142 \\
\hline 4 & 41.1 & 151.2692 & 151.9755 & 150.8949 & 142.2770 & 142.3810 & 141.7833 & 151.3715 & 152.0688 & 150.9493 & 142.3250 & 142.4104 & 141.8043 \\
\hline 5 & 172.4 & 24.5783 & 24.6194 & 24.6103 & 5.2071 & 5.3070 & 4.5814 & 22.9260 & 22.9697 & 22.6401 & 3.3461 & 3.4379 & 2.5372 \\
\hline 6 & 109.9 & 85.1550 & 85.3439 & 85.9762 & 71.9165 & 72.0499 & 70.9881 & 85.4803 & 85.6682 & 86.2732 & 72.3025 & 72.4201 & 71.2692 \\
\hline 7 & 195.6 & 10.0669 & 10.0928 & 8.3783 & -7.7981 & -7.6359 & -10.6175 & 7.9821 & 8.0123 & 5.8738 & -10.2468 & -10.0706 & -13.4338 \\
\hline 8 & 40.9 & 153.1957 & 153.8320 & 153.3278 & 144.7258 & 144.4148 & 143.6875 & 153.1321 & 153.7668 & 153.3028 & 144.6328 & 144.3226 & 143.5605 \\
\hline 9 & 87.8 & 107.1777 & 107.6369 & 106.9208 & 95.8188 & 95.4989 & 95.0033 & 107.2259 & 107.6839 & 107.0130 & 95.9230 & 95.6048 & 95.1041 \\
\hline 10 & 35.4 & 157.4075 & 158.1687 & 157.5237 & 148.4117 & 148.2707 & 147.6236 & 157.5478 & 158.3107 & 157.7078 & 148.5719 & 148.4295 & 147.7808 \\
\hline 11 & 22.0 & 171.4642 & 172.6112 & 172.3476 & 163.7472 & 163.6892 & 163.2241 & 171.5278 & 172.6667 & 172.3954 & 163.8114 & 163.7322 & 163.2520 \\
\hline 12 & 10.1 & 180.0256 & 181.7706 & 182.2077 & 174.1495 & 174.1098 & 173.7902 & 180.2848 & 182.0298 & 182.5067 & 174.4626 & 174.4134 & 174.1018 \\
\hline 13 & 14.7 & 175.9322 & 177.5915 & 178.3866 & 170.1736 & 170.1872 & 170.1518 & 176.2260 & 177.8769 & 178.6668 & 170.4763 & 170.4808 & 170.4455 \\
\hline 14 & 9.4 & 183.4863 & 185.2316 & 184.8255 & 178.7793 & 178.5686 & 178.4517 & 183.7932 & 185.5343 & 185.1855 & 179.1436 & 178.9164 & 178.8018 \\
\hline 15 & 10.0 & 182.7026 & 184.4662 & 185.2594 & 177.5362 & 177.4573 & 177.2863 & 182.9511 & 184.7112 & 185.5747 & 177.8336 & 177.7421 & 177.5946 \\
\hline 16 & 16.2 & 176.2792 & 177.9206 & 178.5575 & 170.1910 & 170.1423 & 169.8100 & 176.5145 & 178.1528 & 178.8470 & 170.4939 & 170.4254 & 170.1045 \\
\hline \multicolumn{14}{|l|}{${ }^{1} \mathrm{H}$} \\
\hline 1 & 1.00 & 31.0344 & 30.6037 & 30.5309 & 30.9801 & 30.7538 & 30.7399 & 31.0490 & 30.6173 & 30.5485 & 30.9932 & 30.7642 & 30.7508 \\
\hline $2 a$ & 1.58 & 30.7308 & 30.3005 & 30.2284 & 30.6588 & 30.4292 & 30.4264 & 30.6548 & 30.2218 & 30.1269 & 30.5765 & 30.3473 & 30.3311 \\
\hline $2 b$ & 1.41 & 30.6078 & 30.1979 & 30.1956 & 30.5708 & 30.3706 & 30.3732 & 30.5902 & 30.1792 & 30.1812 & 30.5680 & 30.3657 & 30.3675 \\
\hline 3 & 3.57 & 28.5437 & 28.1592 & 28.1050 & 28.5893 & 28.4167 & 28.4138 & 28.4947 & 28.1092 & 28.0302 & 28.5245 & 28.3481 & 28.3350 \\
\hline 4 & 2.87 & 29.5597 & 29.0892 & 29.0114 & 29.4254 & 29.1985 & 29.1843 & 29.4578 & 28.9844 & 28.9010 & 29.3340 & 29.1020 & 29.0804 \\
\hline 8 & 2.50 & 29.6641 & 29.2209 & 29.2124 & 29.7313 & 29.4709 & 29.4271 & 29.5438 & 29.0960 & 29.0723 & 29.6025 & 29.3432 & 29.2873 \\
\hline 9 & 3.85 & 28.2290 & 27.8430 & 27.8260 & 28.3082 & 28.1260 & 28.0883 & 28.1031 & 27.7148 & 27.6875 & 28.1769 & 27.9994 & 27.9539 \\
\hline 10 & 1.76 & 30.4368 & 30.0206 & 29.9958 & 30.5102 & 30.2816 & 30.2249 & 30.3516 & 29.9346 & 29.9114 & 30.4284 & 30.2000 & 30.1444 \\
\hline $11 a$ & 1.58 & 30.4731 & 29.9972 & 29.9667 & 30.5025 & 30.2161 & 30.1924 & 30.4290 & 29.9537 & 29.9219 & 30.4559 & 30.1716 & 30.1485 \\
\hline $11 b$ & 1.28 & 30.8347 & 30.4045 & 30.3890 & 30.9311 & 30.6616 & 30.6082 & 30.8538 & 30.4282 & 30.4102 & 30.9416 & 30.6818 & 30.6314 \\
\hline 12 & 0.94 & 31.1146 & 30.6688 & 30.6095 & 31.0747 & 30.8116 & 30.7841 & 31.1037 & 30.6566 & 30.6003 & 31.0657 & 30.8018 & 30.7762 \\
\hline 13 & 1.17 & 30.7786 & 30.3419 & 30.3104 & 30.7750 & 30.5211 & 30.5341 & 30.7593 & 30.3210 & 30.2835 & 30.7504 & 30.4978 & 30.5061 \\
\hline 14 & 1.75 & 30.5245 & 29.9910 & 29.8119 & 30.4044 & 30.0547 & 29.9603 & 30.5208 & 29.9877 & 29.8035 & 30.4016 & 30.0526 & 29.9531 \\
\hline 15 & 1.10 & 31.1599 & 30.6754 & 30.6026 & 31.1402 & 30.8268 & 30.7763 & 31.1552 & 30.6703 & 30.5968 & 31.1341 & 30.8207 & 30.7697 \\
\hline 16 & 1.06 & 30.9508 & 30.4962 & 30.4396 & 30.9473 & 30.6632 & 30.6426 & 30.9433 & 30.4880 & 30.4309 & 30.9377 & 30.6548 & 30.4880 \\
\hline
\end{tabular}




\section{Compound 79d}<smiles>CC[C@H](C)[C@@H]1OC([C@@H](C)[C@@H](O)CC)=C(C)C(=O)[C@@H]1C</smiles>

\begin{tabular}{|c|c|c|c|c|c|c|c|c|c|c|c|c|c|}
\hline & \multirow[b]{3}{*}{ Exp } & \multicolumn{12}{|c|}{ B3LYP } \\
\hline & & \multicolumn{6}{|c|}{ Gas phase } & \multicolumn{6}{|c|}{ PCM } \\
\hline & & 6-31G* & $6-31 \mathrm{G} * *$ & $6-31+G * *$ & 6-311G* & $6-311 G * *$ & $6-311+G * *$ & 6-31G* & 6-31G** & $6-31+G * *$ & 6-311G* & 6-311G** & $6-311+G * *$ \\
\hline \\
\hline 1 & 10.2 & 177.2823 & 179.1554 & 180.3538 & 171.6583 & 171.9408 & 171.5185 & 177.3071 & 179.1812 & 180.3796 & 171.8043 & 172.0510 & 171.5994 \\
\hline 2 & 27.8 & 160.0318 & 161.3657 & 160.8842 & 151.3007 & 151.0795 & 150.7120 & 160.2318 & 161.5563 & 161.1279 & 151.5816 & 151.3585 & 150.9976 \\
\hline 3 & 74.8 & 114.9941 & 115.6672 & 114.9490 & 102.8102 & 102.8855 & 102.6111 & 115.0955 & 115.7636 & 115.0210 & 102.9687 & 103.0155 & 102.6512 \\
\hline 4 & 41.5 & 144.1848 & 144.9107 & 144.5910 & 135.2956 & 135.2327 & 134.6567 & 143.8554 & 144.5820 & 144.1979 & 134.9494 & 134.8858 & 134.2489 \\
\hline 5 & 173.1 & 22.7715 & 22.8542 & 22.2451 & 0.9200 & 1.1028 & 0.3345 & 20.7620 & 20.8481 & 19.9585 & -1.2942 & -1.0980 & -2.0817 \\
\hline 6 & 108.5 & 82.4331 & 82.5992 & 82.9788 & 67.3576 & 67.4140 & 66.6151 & 82.8899 & 83.0531 & 83.4241 & 67.9003 & 67.9431 & 67.0144 \\
\hline 7 & 195.7 & 7.3797 & 7.4191 & 5.1126 & -13.6188 & -13.4570 & -16.6495 & 5.4755 & 5.5171 & 2.7647 & -15.9518 & -15.7857 & -19.4092 \\
\hline 8 & 40.7 & 149.0498 & 149.7385 & 149.0742 & 139.4183 & 139.1053 & 138.4087 & 149.0471 & 149.7328 & 149.1042 & 139.3992 & 139.0828 & 138.3435 \\
\hline 9 & 87.4 & 102.7840 & 103.3089 & 102.2088 & 90.0738 & 89.7466 & 89.3117 & 102.9155 & 103.4369 & 102.3974 & 90.2763 & 89.9439 & 89.5045 \\
\hline 10 & 35.2 & 152.1059 & 152.9424 & 152.2990 & 142.2926 & 142.1796 & 141.4002 & 152.2404 & 153.0771 & 152.4445 & 142.4493 & 142.3340 & 141.5650 \\
\hline 11 & 21.9 & 166.9380 & 168.2094 & 168.1306 & 158.8077 & 158.7337 & 158.4464 & 166.9979 & 168.2662 & 168.2016 & 158.9084 & 158.8240 & 158.5352 \\
\hline 12 & 11.9 & 175.8872 & 177.7641 & 178.2875 & 169.4772 & 169.4892 & 169.1817 & 176.1213 & 177.9965 & 178.5738 & 169.7910 & 169.7908 & 169.4844 \\
\hline 13 & 12.9 & 173.3407 & 175.1248 & 176.3149 & 166.9579 & 166.8994 & 166.6800 & 173.5248 & 175.2951 & 176.5209 & 167.1057 & 167.0324 & 166.8272 \\
\hline 14 & 9.2 & 179.1367 & 180.9960 & 180.7205 & 173.5691 & 173.2826 & 172.8862 & 179.4570 & 181.3120 & 181.0015 & 173.9530 & 173.6561 & 173.2901 \\
\hline 15 & 10.1 & 178.4238 & 180.3304 & 181.0436 & 173.0691 & 173.0069 & 172.7591 & 178.6388 & 180.5419 & 181.3241 & 173.3629 & 173.2854 & 173.0695 \\
\hline $\begin{array}{l}16 \\
{ }^{1} \mathrm{H}\end{array}$ & 16.1 & 171.7892 & 173.6000 & 174.6122 & 165.4266 & 165.3904 & 164.9332 & 172.0766 & 173.8798 & 174.9577 & 165.7851 & 165.7296 & 165.2868 \\
\hline 1 & 0.95 & 31.0754 & 30.6509 & 30.5684 & 31.0587 & 30.8478 & 30.8341 & 31.0895 & 30.6640 & 30.5856 & 31.0772 & 30.8633 & 30.8500 \\
\hline $2 a$ & 1.43 & 30.6303 & 30.2163 & 30.2226 & 30.6510 & 30.4494 & 30.4515 & 30.6723 & 30.2593 & 30.2657 & 30.6897 & 30.4874 & 30.4875 \\
\hline $2 b$ & 1.55 & 30.5867 & 30.1503 & 30.1838 & 30.6711 & 30.4237 & 30.4610 & 30.5376 & 30.1017 & 30.1258 & 30.6117 & 30.3625 & 30.3946 \\
\hline 3 & 3.67 & 28.2463 & 27.8421 & 27.8317 & 28.3170 & 28.0932 & 28.1034 & 28.1918 & 27.7874 & 27.7699 & 28.2527 & 28.0316 & 28.0419 \\
\hline 4 & 2.76 & 29.5478 & 29.0919 & 29.0646 & 29.5067 & 29.2950 & 29.2842 & 29.5524 & 29.0967 & 29.0613 & 29.5007 & 29.2870 & 29.2731 \\
\hline 8 & 2.47 & 29.7753 & 29.3538 & 29.3634 & 29.9139 & 29.6721 & 29.6467 & 29.6667 & 29.2406 & 29.2345 & 29.7968 & 29.5539 & 29.5160 \\
\hline 9 & 3.77 & 28.3592 & 27.9823 & 27.9958 & 28.5000 & 28.3322 & 28.2733 & 28.2506 & 27.8707 & 27.8733 & 28.3848 & 28.2195 & 28.1513 \\
\hline 10 & 1.72 & 30.4825 & 30.0719 & 30.0655 & 30.6234 & 30.4050 & 30.3627 & 30.4125 & 30.0023 & 29.9926 & 30.5545 & 30.3369 & 30.2929 \\
\hline $11 a$ & 1.55 & 30.5341 & 30.0721 & 30.0439 & 30.6093 & 30.3502 & 30.3242 & 30.5026 & 30.0423 & 30.0131 & 30.5759 & 30.3197 & 30.2943 \\
\hline $11 b$ & 1.18 & 30.6960 & 30.2933 & 30.2763 & 30.8267 & 30.6312 & 30.5954 & 30.7142 & 30.3136 & 30.2928 & 30.8400 & 30.6475 & 30.6109 \\
\hline 12 & 0.94 & 31.1880 & 30.7489 & 30.6872 & 31.1694 & 30.9240 & 30.8979 & 31.1736 & 30.7336 & 30.6768 & 31.1591 & 30.9131 & 30.8898 \\
\hline 13 & 1.18 & 30.9827 & 30.5325 & 30.4390 & 30.9547 & 30.6909 & 30.6719 & 30.9427 & 30.4907 & 30.3967 & 30.9198 & 30.6569 & 30.6381 \\
\hline 14 & 1.72 & 30.5408 & 30.0213 & 29.8531 & 30.4522 & 30.1267 & 30.0549 & 30.5553 & 30.0350 & 29.8601 & 30.4622 & 30.1359 & 30.0573 \\
\hline 15 & 1.06 & 31.2267 & 30.7503 & 30.6841 & 31.2401 & 30.9438 & 30.9043 & 31.2311 & 30.7542 & 30.6860 & 31.2427 & 30.9456 & 30.9047 \\
\hline 16 & 1.06 & 31.0224 & 30.5761 & 30.5198 & 31.0373 & 30.7756 & 30.7560 & 31.0008 & 30.5540 & 30.5002 & 31.0187 & 30.7580 & 30.7402 \\
\hline
\end{tabular}




\begin{tabular}{|c|c|c|c|c|c|c|c|c|c|c|c|c|c|}
\hline \multicolumn{14}{|c|}{ mPW1PW91 } \\
\hline & \multirow[b]{2}{*}{ Exp } & \multicolumn{6}{|c|}{ Gas phase } & \multicolumn{6}{|c|}{ PCM } \\
\hline & & 6-31G* & $6-31 G * *$ & $6-31+G * *$ & 6-311G* & $6-311 G * *$ & $6-311+G * *$ & 6-31G* & $6-31 G * *$ & $6-31+\mathrm{G} * *$ & 6-311G* & $6-311 G * *$ & $6-311+\mathrm{G} * *$ \\
\hline \multicolumn{14}{|l|}{${ }^{13} \mathrm{C}$} \\
\hline 1 & 10.2 & 181.4594 & 183.2251 & 184.2975 & 176.3171 & 176.5955 & 176.1706 & 181.5288 & 183.2933 & 184.3227 & 176.4771 & 176.7195 & 176.2704 \\
\hline 2 & 27.8 & 164.9079 & 166.1426 & 165.7179 & 156.9102 & 156.6867 & 156.3042 & 165.1192 & 166.3468 & 165.9957 & 157.1943 & 156.9700 & 156.5922 \\
\hline 3 & 74.8 & 120.0867 & 120.7122 & 120.2601 & 109.5702 & 109.6511 & 109.3149 & 120.1735 & 120.7960 & 120.3026 & 109.7017 & 109.7571 & 109.3453 \\
\hline 4 & 41.5 & 149.0651 & 149.7586 & 149.7051 & 141.2585 & 141.2318 & 140.6474 & 148.7422 & 149.4351 & 149.2741 & 140.9108 & 140.8829 & 140.2476 \\
\hline 5 & 173.1 & 25.9461 & 26.0329 & 25.7196 & 6.7974 & 6.9802 & 6.2785 & 23.8742 & 23.9642 & 23.3875 & 4.5516 & 4.7469 & 3.8536 \\
\hline 6 & 108.5 & 85.8631 & 86.0303 & 86.4597 & 72.5968 & 72.6568 & 71.8632 & 86.3767 & 86.5408 & 86.9777 & 73.1757 & 73.2219 & 72.3183 \\
\hline 7 & 195.7 & 9.6674 & 9.7000 & 8.0777 & -8.4367 & -8.2815 & -11.1476 & 7.6819 & 7.7172 & 5.7098 & -10.8037 & -10.6440 & -13.8997 \\
\hline 8 & 40.7 & 153.4809 & 154.1324 & 153.6608 & 144.9620 & 144.6677 & 144.0060 & 153.4504 & 154.0997 & 153.6632 & 144.9008 & 144.6041 & 143.9030 \\
\hline 9 & 87.4 & 107.9016 & 108.3805 & 107.6279 & 96.7181 & 96.4171 & 95.9570 & 108.0037 & 108.4800 & 107.7827 & 96.8670 & 96.5622 & 96.0965 \\
\hline 10 & 35.2 & 157.2957 & 158.0702 & 157.4971 & 148.3936 & 148.2951 & 147.5217 & 157.4310 & 158.2065 & 157.6359 & 148.5389 & 148.4388 & 147.6763 \\
\hline 11 & 21.9 & 171.8552 & 173.0371 & 172.8971 & 164.3416 & 164.2709 & 163.9400 & 171.9339 & 173.1131 & 172.9848 & 164.4469 & 164.3672 & 164.0345 \\
\hline 12 & 11.9 & 180.0638 & 181.8280 & 182.2829 & 174.1462 & 174.1451 & 173.8340 & 180.3419 & 182.1037 & 182.6084 & 174.4761 & 174.4635 & 174.1558 \\
\hline 13 & 12.9 & 177.6261 & 179.2726 & 180.0566 & 171.5513 & 171.5043 & 171.3639 & 177.8338 & 179.4671 & 180.2832 & 171.7062 & 171.6464 & 171.5188 \\
\hline 14 & 9.2 & 182.8548 & 184.6015 & 184.5014 & 177.8008 & 177.5299 & 177.1383 & 183.2151 & 184.9568 & 184.8295 & 178.1959 & 177.9157 & 177.5496 \\
\hline 15 & 10.1 & 182.6464 & 184.4294 & 185.1834 & 177.5800 & 177.5203 & 177.2989 & 182.8927 & 184.6718 & 185.4856 & 177.8770 & 177.8032 & 177.6115 \\
\hline 16 & 16.1 & 176.1582 & 177.8393 & 178.7506 & 170.1990 & 170.1634 & 169.7609 & 176.4824 & 178.1558 & 179.1197 & 170.5610 & 170.5082 & 170.1222 \\
\hline \multicolumn{14}{|l|}{${ }^{1} \mathrm{H}$} \\
\hline 1 & 0.95 & 31.0343 & 30.6089 & 30.5301 & 30.9892 & 30.7724 & 30.7539 & 31.0458 & 30.6193 & 30.5427 & 31.0048 & 30.7850 & 30.7671 \\
\hline $2 a$ & 1.43 & 30.5883 & 30.1745 & 30.1612 & 30.5516 & 30.3516 & 30.3459 & 30.6276 & 30.2149 & 30.2049 & 30.5905 & 30.3894 & 30.3807 \\
\hline $2 b$ & 1.55 & 30.5729 & 30.1370 & 30.1700 & 30.6017 & 30.3530 & 30.3878 & 30.5277 & 30.0919 & 30.1133 & 30.5425 & 30.2913 & 30.3216 \\
\hline 3 & 3.67 & 28.2172 & 27.8186 & 27.7855 & 28.2193 & 28.0026 & 28.0083 & 28.1580 & 27.7589 & 27.7231 & 28.1536 & 27.9393 & 27.9443 \\
\hline 4 & 2.76 & 29.4563 & 29.0053 & 28.9660 & 29.3539 & 29.1479 & 29.1397 & 29.4583 & 29.0072 & 28.9644 & 29.3473 & 29.1387 & 29.1275 \\
\hline 8 & 2.47 & 29.7203 & 29.2928 & 29.2959 & 29.7871 & 29.5496 & 29.5205 & 29.6117 & 29.1791 & 29.1694 & 29.6713 & 29.4324 & 29.3915 \\
\hline 9 & 3.77 & 28.2791 & 27.9096 & 27.9007 & 28.3640 & 28.2010 & 28.1393 & 28.1701 & 27.7975 & 27.7788 & 28.2515 & 28.0907 & 28.0203 \\
\hline 10 & 1.72 & 30.4433 & 30.0387 & 30.0093 & 30.5051 & 30.2985 & 30.2462 & 30.3705 & 29.9658 & 29.9342 & 30.4353 & 30.2290 & 30.1749 \\
\hline $11 a$ & 1.55 & 30.5169 & 30.0600 & 30.0391 & 30.5324 & 30.2774 & 30.2526 & 30.4844 & 30.0290 & 30.0071 & 30.4990 & 30.2468 & 30.2221 \\
\hline $11 b$ & 1.18 & 30.6718 & 30.2707 & 30.2539 & 30.7310 & 30.5385 & 30.5010 & 30.6920 & 30.2929 & 30.2718 & 30.7467 & 30.5571 & 30.5180 \\
\hline 12 & 0.94 & 31.1504 & 30.7106 & 30.6506 & 31.0964 & 30.8442 & 30.8206 & 31.1328 & 30.6919 & 30.6366 & 31.0827 & 30.8300 & 30.8092 \\
\hline 13 & 1.18 & 30.9253 & 30.4738 & 30.3959 & 30.8645 & 30.5971 & 30.5851 & 30.8815 & 30.4280 & 30.3518 & 30.8272 & 30.5606 & 30.5487 \\
\hline 14 & 1.72 & 30.4687 & 29.9480 & 29.7875 & 30.3639 & 30.0322 & 29.9612 & 30.4797 & 29.9580 & 29.7902 & 30.3703 & 30.0378 & 29.9600 \\
\hline 15 & 1.06 & 31.1648 & 30.6881 & 30.6302 & 31.1442 & 30.8420 & 30.8062 & 31.1656 & 30.6882 & 30.6286 & 31.1431 & 30.8401 & 30.8029 \\
\hline 16 & 1.06 & 30.9772 & 30.5310 & 30.4800 & 30.9531 & 30.6879 & 30.6714 & 30.9524 & 30.5056 & 30.4573 & 30.9315 & 30.6673 & 30.5056 \\
\hline
\end{tabular}




\section{Compound 79e}

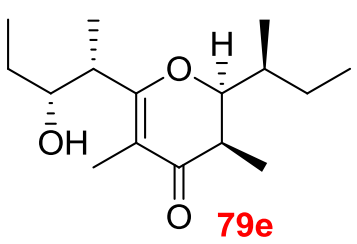

\begin{tabular}{|c|c|c|c|c|c|c|c|c|c|c|c|c|c|}
\hline & \multirow[b]{3}{*}{ Exp } & \multicolumn{12}{|c|}{ B3LYP } \\
\hline & & \multicolumn{6}{|c|}{ Gas phase } & \multicolumn{6}{|c|}{ PCM } \\
\hline & & 6-31G* & $6-31 G * *$ & $6-31+G * *$ & 6-311G* & $6-311 G * *$ & $6-311+G * *$ & 6-31G* & $6-31 G * *$ & $6-31+\mathrm{G} * *$ & 6-311G* & $6-311 G * *$ & $6-311+G * *$ \\
\hline \multicolumn{14}{|l|}{${ }^{13} \mathrm{C}$} \\
\hline 1 & 11.8 & 176.4484 & 178.3552 & 179.3458 & 170.4002 & 170.4839 & 170.3290 & 176.7080 & 178.6119 & 179.7062 & 170.7382 & 170.7967 & 170.6704 \\
\hline 2 & 27.8 & 159.6967 & 160.9889 & 160.5087 & 151.1072 & 150.9208 & 150.6524 & 160.0532 & 161.3305 & 160.7743 & 151.4062 & 151.2010 & 150.8486 \\
\hline 3 & 74.8 & 116.7449 & 117.3177 & 115.5165 & 104.0211 & 103.9455 & 103.3543 & 116.6962 & 117.2496 & 115.3866 & 104.0183 & 103.9578 & 103.3395 \\
\hline 4 & 41.5 & 146.0394 & 146.7720 & 145.7711 & 136.2369 & 136.0613 & 135.5471 & 146.0192 & 146.7128 & 145.5963 & 136.1782 & 136.0058 & 135.4041 \\
\hline 5 & 173.2 & 20.4608 & 20.5127 & 20.3693 & -1.4671 & -1.3426 & -1.8194 & 18.8227 & 18.8911 & 18.4154 & -3.3522 & -3.2392 & -3.9498 \\
\hline 6 & 108.6 & 83.8820 & 84.0575 & 84.3683 & 69.4092 & 69.4690 & 68.1404 & 84.0551 & 84.2167 & 84.3271 & 69.5946 & 69.6540 & 68.1103 \\
\hline 7 & 195.7 & 5.4909 & 5.5038 & 2.6463 & -15.2873 & -15.2457 & -18.2865 & 3.3854 & 3.4005 & 0.0618 & -17.8289 & -17.7834 & -21.2807 \\
\hline 8 & 40.7 & 147.3852 & 148.1933 & 147.2080 & 137.5743 & 137.2782 & 136.4250 & 147.2028 & 148.0055 & 146.8467 & 137.3074 & 137.0053 & 136.0438 \\
\hline 9 & 84.7 & 105.4958 & 106.0473 & 105.1383 & 93.3817 & 93.1572 & 92.9731 & 105.5627 & 106.1225 & 105.3024 & 93.5314 & 93.2939 & 93.1239 \\
\hline 10 & 35.5 & 153.0602 & 153.7762 & 152.7352 & 143.2219 & 143.0874 & 142.5399 & 153.0966 & 153.8105 & 152.7435 & 143.2640 & 143.1362 & 142.5563 \\
\hline 11 & 26.5 & 162.3989 & 163.6079 & 163.1252 & 153.7024 & 153.5859 & 153.3999 & 162.5118 & 163.7262 & 163.3579 & 153.8905 & 153.7595 & 153.6043 \\
\hline 12 & 10.2 & 177.0913 & 178.9072 & 179.3771 & 170.7483 & 170.8429 & 170.2430 & 177.1989 & 179.0188 & 179.4082 & 170.9137 & 171.0005 & 170.4023 \\
\hline 13 & 12.9 & 176.6450 & 178.4296 & 179.5284 & 170.8062 & 170.9607 & 170.0940 & 176.7408 & 178.4661 & 179.1020 & 170.7756 & 170.9262 & 169.9646 \\
\hline 14 & 9.2 & 180.2076 & 182.0613 & 182.3481 & 174.8587 & 174.6453 & 174.1900 & 180.4668 & 182.3139 & 182.6938 & 175.2296 & 174.9960 & 174.5241 \\
\hline 15 & 9.5 & 178.0282 & 179.8469 & 180.8110 & 172.1741 & 172.1548 & 171.8619 & 178.1181 & 179.9232 & 180.8143 & 172.1922 & 172.1554 & 171.8688 \\
\hline 16 & 12.4 & 175.2738 & 177.1399 & 177.8542 & 169.2504 & 169.1068 & 168.8930 & 175.6258 & 177.4817 & 178.2127 & 169.6586 & 169.4951 & 169.2847 \\
\hline \multicolumn{14}{|l|}{${ }^{1} \mathrm{H}$} \\
\hline 1 & 0.95 & 31.0636 & 30.6425 & 30.6001 & 31.0293 & 30.8132 & 30.8233 & 31.0904 & 30.6667 & 30.6214 & 31.0528 & 30.8322 & 30.8357 \\
\hline $2 a$ & 1.48 & 30.6860 & 30.2587 & 30.1944 & 30.6850 & 30.4737 & 30.4616 & 30.6463 & 30.2190 & 30.1695 & 30.6549 & 30.4412 & 30.4357 \\
\hline $2 b$ & 1.48 & 30.6860 & 30.2587 & 30.1944 & 30.6850 & 30.4737 & 30.4616 & 30.6463 & 30.2190 & 30.1695 & 30.6549 & 30.4412 & 30.4357 \\
\hline 3 & 3.68 & 28.2337 & 27.8128 & 27.8719 & 28.3051 & 28.1242 & 28.1783 & 28.1913 & 27.7722 & 27.8102 & 28.2505 & 28.0671 & 28.1097 \\
\hline 4 & 2.76 & 29.6737 & 29.1847 & 29.0719 & 29.6149 & 29.3633 & 29.3063 & 29.5743 & 29.0868 & 28.9786 & 29.5258 & 29.2762 & 29.2253 \\
\hline 8 & 2.44 & 30.0525 & 29.5900 & 29.4946 & 30.0198 & 29.7701 & 29.6976 & 30.0140 & 29.5471 & 29.4565 & 29.9755 & 29.7234 & 29.6570 \\
\hline 9 & 3.86 & 28.2236 & 27.8350 & 27.9361 & 28.3936 & 28.2088 & 28.2716 & 28.1250 & 27.7368 & 27.8368 & 28.2856 & 28.1056 & 28.1611 \\
\hline 10 & 1.65 & 30.1925 & 29.7850 & 29.8260 & 30.3497 & 30.1354 & 30.1259 & 30.1404 & 29.7353 & 29.7892 & 30.2991 & 30.0892 & 30.0862 \\
\hline $11 a$ & 1.48 & 30.5720 & 30.1219 & 30.1150 & 30.6556 & 30.3835 & 30.3645 & 30.5776 & 30.1289 & 30.1157 & 30.6500 & 30.3839 & 30.3651 \\
\hline $11 b$ & 1.48 & 30.5720 & 30.1219 & 30.1150 & 30.6556 & 30.3835 & 30.3645 & 30.5776 & 30.1289 & 30.1157 & 30.6500 & 30.3839 & 30.3651 \\
\hline 12 & 0.94 & 31.1367 & 30.6927 & 30.6305 & 31.1488 & 30.8956 & 30.8673 & 31.1311 & 30.6861 & 30.6251 & 31.1401 & 30.8869 & 30.8588 \\
\hline 13 & 1.17 & 30.9416 & 30.4868 & 30.3857 & 30.9581 & 30.7063 & 30.6546 & 30.9203 & 30.4632 & 30.3542 & 30.9347 & 30.6824 & 30.6278 \\
\hline 14 & 1.73 & 30.6260 & 30.0889 & 29.9048 & 30.5265 & 30.1753 & 30.1002 & 30.6231 & 30.0870 & 29.8991 & 30.5230 & 30.1746 & 30.0975 \\
\hline 15 & 1.04 & 31.1295 & 30.6744 & 30.6528 & 31.1963 & 30.9308 & 30.9381 & 31.1032 & 30.6476 & 30.6256 & 31.1688 & 30.9034 & 30.9086 \\
\hline 16 & 0.92 & 31.3087 & 30.8552 & 30.8347 & 31.3670 & 31.0902 & 31.0723 & 31.2813 & 30.8274 & 30.8099 & 31.3404 & 31.0649 & 31.0482 \\
\hline
\end{tabular}




\begin{tabular}{|c|c|c|c|c|c|c|c|c|c|c|c|c|c|}
\hline \multicolumn{14}{|c|}{ mPW1PW91 } \\
\hline & \multirow[b]{2}{*}{ Exp } & \multicolumn{6}{|c|}{ Gas phase } & \multicolumn{6}{|c|}{ PCM } \\
\hline & & 6-31G* & $6-31 G * *$ & $6-31+G * *$ & 6-311G* & $6-311 G * *$ & $6-311+G * *$ & 6-31G* & $6-31 G * *$ & $6-31+\mathrm{G} * *$ & 6-311G* & $6-311 G * *$ & $6-311+\mathrm{G} * *$ \\
\hline \multicolumn{14}{|l|}{${ }^{13} \mathrm{C}$} \\
\hline 1 & 11.8 & 180.6782 & 182.4637 & 183.2712 & 175.0331 & 175.1068 & 174.9132 & 180.9888 & 182.7687 & 183.6723 & 175.3881 & 175.4369 & 175.2683 \\
\hline 2 & 27.8 & 164.6587 & 165.8618 & 165.5651 & 156.8662 & 156.6836 & 156.3615 & 165.0147 & 166.2037 & 165.8032 & 157.1383 & 156.9428 & 156.5462 \\
\hline 3 & 74.8 & 121.7092 & 122.2331 & 120.8139 & 110.6279 & 110.5801 & 110.0089 & 121.5913 & 122.1013 & 120.6546 & 110.5719 & 110.5440 & 109.9490 \\
\hline 4 & 41.5 & 150.7442 & 151.4346 & 150.7497 & 142.0137 & 141.8775 & 141.4137 & 150.6699 & 151.3265 & 150.5391 & 141.9079 & 141.7836 & 141.2432 \\
\hline 5 & 173.2 & 23.7319 & 23.7824 & 23.8227 & 4.5315 & 4.6376 & 4.2283 & 22.0632 & 22.1289 & 21.8464 & 2.6184 & 2.7118 & 2.0985 \\
\hline 6 & 108.6 & 87.2722 & 87.4482 & 87.9547 & 74.5764 & 74.6495 & 73.2825 & 87.4765 & 87.6394 & 87.9752 & 74.7915 & 74.8663 & 73.3112 \\
\hline 7 & 195.7 & 7.9067 & 7.9144 & 5.9482 & -9.9978 & -9.9620 & -12.7479 & 5.7268 & 5.7369 & 3.3330 & -12.5693 & -12.5290 & -15.7231 \\
\hline 8 & 40.7 & 152.2470 & 153.0054 & 152.0897 & 143.4987 & 143.2152 & 142.4041 & 152.0578 & 152.8117 & 151.7477 & 143.2197 & 142.9308 & 142.0280 \\
\hline 9 & 84.7 & 110.5777 & 111.0825 & 110.4415 & 99.9126 & 99.6998 & 99.4636 & 110.6231 & 111.1363 & 110.5636 & 100.0100 & 99.7852 & 99.5612 \\
\hline 10 & 35.5 & 158.2631 & 158.9296 & 158.1651 & 149.3991 & 149.2808 & 148.7096 & 158.2741 & 158.9405 & 158.1510 & 149.4152 & 149.3081 & 148.7123 \\
\hline 11 & 26.5 & 167.5208 & 168.6345 & 168.2013 & 159.4957 & 159.3673 & 159.1564 & 167.6719 & 168.7901 & 168.4404 & 159.6974 & 159.5547 & 159.3724 \\
\hline 12 & 10.2 & 181.2723 & 182.9769 & 183.4181 & 175.4158 & 175.4992 & 174.9174 & 181.4035 & 183.1114 & 183.4707 & 175.5821 & 175.6615 & 175.0811 \\
\hline 13 & 12.9 & 180.8594 & 182.5162 & 183.2590 & 175.2663 & 175.4494 & 174.7663 & 180.8967 & 182.5000 & 182.8491 & 175.1912 & 175.3835 & 174.6140 \\
\hline 14 & 9.2 & 183.9222 & 185.6702 & 185.9256 & 179.1162 & 178.9188 & 178.4524 & 184.2110 & 185.9520 & 186.2863 & 179.4832 & 179.2676 & 178.7839 \\
\hline 15 & 9.5 & 182.2066 & 183.9042 & 184.5874 & 176.6647 & 176.6602 & 176.4198 & 182.3265 & 184.0107 & 184.6434 & 176.6999 & 176.6791 & 176.4472 \\
\hline 16 & 12.4 & 179.5069 & 181.2462 & 181.8481 & 173.9119 & 173.7705 & 173.5731 & 179.8931 & 181.6222 & 182.2529 & 174.3239 & 174.1649 & 173.9710 \\
\hline \multicolumn{14}{|l|}{${ }^{1} \mathrm{H}$} \\
\hline 1 & 0.95 & 31.0215 & 30.5987 & 30.5607 & 30.9608 & 30.7367 & 30.7438 & 31.0461 & 30.6204 & 30.5785 & 30.9805 & 30.7519 & 30.7531 \\
\hline $2 a$ & 1.48 & 30.6519 & 30.2252 & 30.1619 & 30.6042 & 30.3917 & 30.3767 & 30.6114 & 30.1846 & 30.1390 & 30.5741 & 30.3586 & 30.3506 \\
\hline $2 b$ & 1.48 & 30.6519 & 30.2252 & 30.1619 & 30.6042 & 30.3917 & 30.3767 & 30.6114 & 30.1846 & 30.1390 & 30.5741 & 30.3586 & 30.3506 \\
\hline 3 & 3.68 & 28.2060 & 27.7932 & 27.8331 & 28.2331 & 28.0547 & 28.0989 & 28.1627 & 27.7519 & 27.7722 & 28.1780 & 27.9973 & 28.0298 \\
\hline 4 & 2.76 & 29.6024 & 29.1207 & 28.9876 & 29.4786 & 29.2344 & 29.1761 & 29.4970 & 29.0167 & 28.8920 & 29.3873 & 29.1445 & 29.0917 \\
\hline 8 & 2.44 & 29.9573 & 29.4994 & 29.4078 & 29.8745 & 29.6280 & 29.5674 & 29.9165 & 29.4538 & 29.3683 & 29.8287 & 29.5796 & 29.5247 \\
\hline 9 & 3.86 & 28.1532 & 27.7692 & 27.8416 & 28.2561 & 28.0784 & 28.1349 & 28.0587 & 27.6752 & 27.7462 & 28.1533 & 27.9799 & 28.0289 \\
\hline 10 & 1.65 & 30.1386 & 29.7345 & 29.7558 & 30.2199 & 30.0129 & 29.9970 & 30.0900 & 29.6884 & 29.7233 & 30.1735 & 29.9703 & 29.9599 \\
\hline $11 a$ & 1.48 & 30.5436 & 30.0971 & 30.0839 & 30.5646 & 30.2972 & 30.2762 & 30.5490 & 30.1043 & 30.0863 & 30.5592 & 30.2982 & 30.2773 \\
\hline $11 b$ & 1.48 & 30.5436 & 30.0971 & 30.0839 & 30.5646 & 30.2972 & 30.2762 & 30.5490 & 30.1043 & 30.0863 & 30.5592 & 30.2982 & 30.2773 \\
\hline 12 & 0.94 & 31.0985 & 30.6536 & 30.5952 & 31.0745 & 30.8145 & 30.7872 & 31.0895 & 30.6435 & 30.5860 & 31.0623 & 30.8026 & 30.7756 \\
\hline 13 & 1.17 & 30.8865 & 30.4307 & 30.3446 & 30.8699 & 30.6142 & 30.5681 & 30.8595 & 30.4015 & 30.3077 & 30.8414 & 30.5855 & 30.5367 \\
\hline 14 & 1.73 & 30.5563 & 30.0179 & 29.8484 & 30.4365 & 30.0792 & 30.0086 & 30.5478 & 30.0106 & 29.8379 & 30.4283 & 30.0742 & 30.0015 \\
\hline 15 & 1.04 & 31.0805 & 30.6245 & 30.6075 & 31.1131 & 30.8432 & 30.8522 & 31.0526 & 30.5960 & 30.5787 & 31.0837 & 30.8139 & 30.8212 \\
\hline 16 & 0.92 & 31.2713 & 30.8185 & 30.7920 & 31.2857 & 31.0054 & 30.9886 & 31.2405 & 30.7872 & 30.7639 & 31.2559 & 30.9769 & 30.7872 \\
\hline
\end{tabular}




\section{Compound 79f}

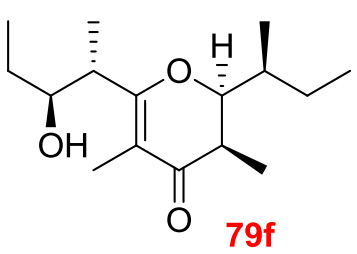

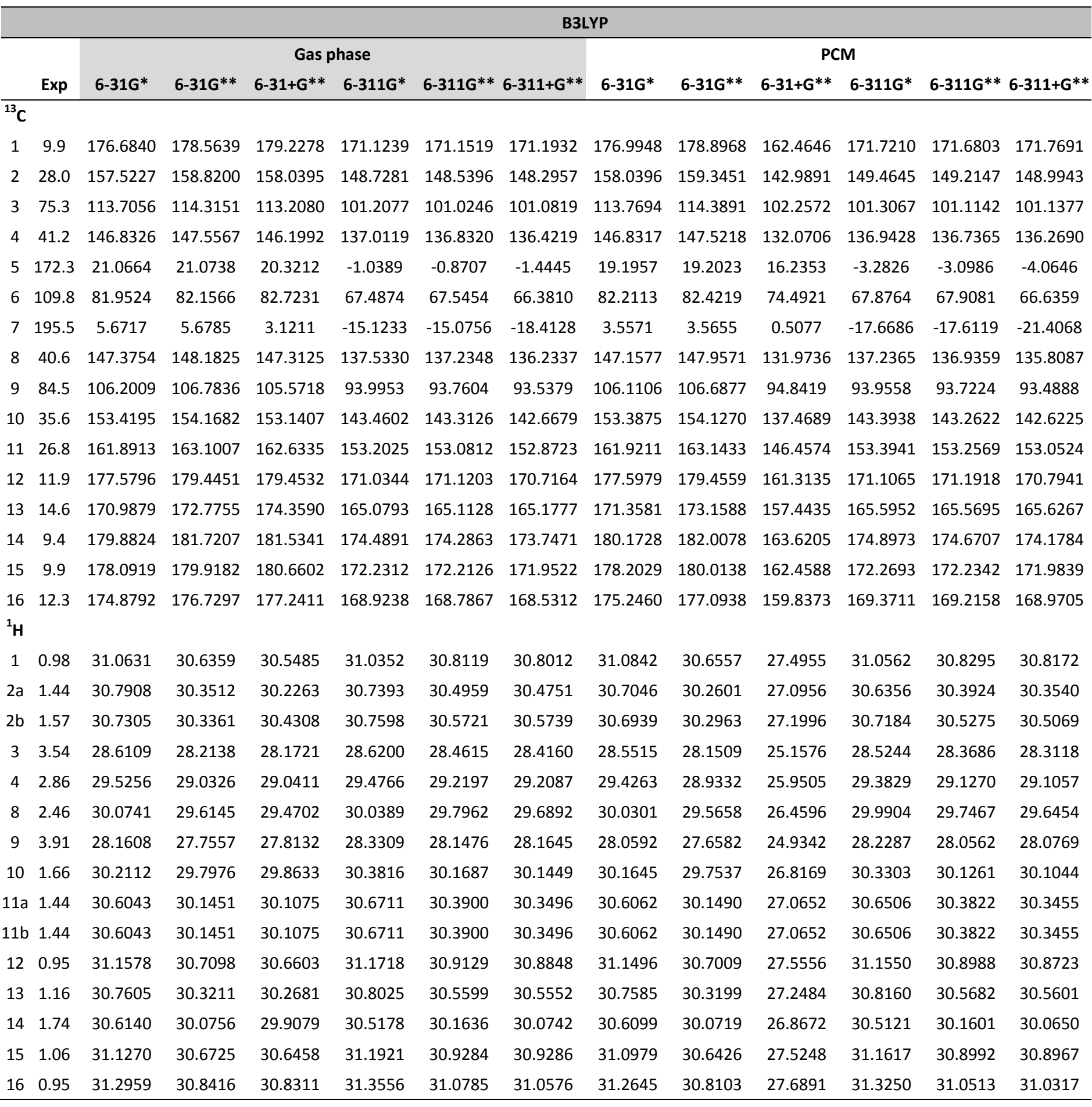




\begin{tabular}{|c|c|c|c|c|c|c|c|c|c|c|c|c|c|}
\hline & \multirow[b]{3}{*}{ Exp } & \multicolumn{12}{|c|}{ mPW1PW91 } \\
\hline & & \multicolumn{6}{|c|}{ Gas phase } & \multicolumn{6}{|c|}{ PCM } \\
\hline & & 6-31G* & 6-31G** & $6-31+\mathrm{G} * *$ & 6-311G* & 6-311G** & $6-311+G^{* *}$ & 6-31G* & $6-31 G * *$ & $6-31+G * *$ & 6-311G* & $6-311 G * *$ & $6-311+G * *$ \\
\hline \multicolumn{14}{|l|}{${ }^{13} \mathrm{C}$} \\
\hline 1 & 9.9 & 180.8453 & 182.6019 & 183.6994 & 175.5879 & 175.6126 & 175.4939 & 181.2203 & 182.9941 & 184.4645 & 176.2166 & 176.1671 & 176.1103 \\
\hline 2 & 28.0 & 162.4701 & 163.6648 & 163.1400 & 154.4713 & 154.2870 & 153.9841 & 163.0022 & 164.2016 & 163.9719 & 155.1874 & 154.9373 & 154.6539 \\
\hline 3 & 75.3 & 118.8817 & 119.4209 & 118.8234 & 108.0052 & 107.8190 & 107.7253 & 118.9487 & 119.4966 & 119.0250 & 108.0882 & 107.8938 & 107.7762 \\
\hline 4 & 41.2 & 151.5478 & 152.2461 & 151.6741 & 142.7658 & 142.6359 & 142.2531 & 151.5273 & 152.2036 & 151.5395 & 142.6954 & 142.5389 & 142.1103 \\
\hline 5 & 172.3 & 24.2758 & 24.2805 & 23.8799 & 4.8085 & 4.9617 & 4.4046 & 22.3344 & 22.3410 & 21.5397 & 2.5529 & 2.7226 & 1.8547 \\
\hline 6 & 109.8 & 85.3884 & 85.5891 & 86.1780 & 72.6763 & 72.7370 & 71.5590 & 85.7116 & 85.9153 & 86.4995 & 73.1027 & 73.1366 & 71.8529 \\
\hline 7 & 195.5 & 8.0983 & 8.1017 & 6.3855 & -9.8151 & -9.7744 & -12.8090 & 5.9080 & 5.9132 & 3.7647 & -12.3953 & -12.3444 & -15.7936 \\
\hline 8 & 40.6 & 152.2311 & 152.9890 & 152.0897 & 143.4322 & 143.1478 & 142.1907 & 152.0002 & 152.7517 & 151.6703 & 143.1205 & 142.8349 & 141.7754 \\
\hline 9 & 84.5 & 111.2477 & 111.7829 & 110.8984 & 100.4872 & 100.2631 & 100.0138 & 111.1229 & 111.6546 & 110.7005 & 100.3966 & 100.1794 & 99.9232 \\
\hline 10 & 35.6 & 158.6655 & 159.3662 & 158.5428 & 149.7209 & 149.5778 & 148.9281 & 158.6159 & 159.3112 & 158.3223 & 149.6313 & 149.5110 & 148.8546 \\
\hline 11 & 26.8 & 167.0475 & 168.1577 & 167.6452 & 159.0139 & 158.8762 & 158.6440 & 167.1043 & 168.2235 & 167.9272 & 159.2055 & 159.0530 & 158.8229 \\
\hline 12 & 11.9 & 181.8760 & 183.6298 & 183.6238 & 175.8143 & 175.8772 & 175.4946 & 181.9183 & 183.6644 & 183.5539 & 175.8774 & 175.9472 & 175.5602 \\
\hline 13 & 14.6 & 175.4074 & 177.0547 & 178.3596 & 169.7511 & 169.7911 & 169.8145 & 175.8170 & 177.4718 & 178.9973 & 170.2762 & 170.2551 & 170.2922 \\
\hline 14 & 9.4 & 183.6125 & 185.3446 & 185.3738 & 178.7796 & 178.5918 & 177.9921 & 183.9419 & 185.6699 & 185.7470 & 179.1889 & 178.9772 & 178.4275 \\
\hline 15 & 9.9 & 182.2693 & 183.9734 & 184.4360 & 176.7310 & 176.7251 & 176.4984 & 182.4081 & 184.0973 & 184.4785 & 176.7834 & 176.7627 & 176.5486 \\
\hline 16 & 12.3 & 179.0894 & 180.8140 & 181.2194 & 173.5573 & 173.4236 & 173.1693 & 179.4907 & 181.2112 & 181.7161 & 174.0129 & 173.8615 & 173.6218 \\
\hline \multicolumn{14}{|l|}{${ }^{1} \mathbf{H}$} \\
\hline 1 & 0.98 & 31.0204 & 30.5922 & 30.5181 & 30.9636 & 30.7342 & 30.7207 & 31.0383 & 30.6086 & 30.5352 & 30.9812 & 30.7482 & 30.7331 \\
\hline $2 a$ & 1.44 & 30.7790 & 30.3418 & 30.2522 & 30.6726 & 30.4330 & 30.4224 & 30.6862 & 30.2443 & 30.1046 & 30.5664 & 30.3271 & 30.2984 \\
\hline $2 b$ & 1.57 & 30.6888 & 30.2897 & 30.3182 & 30.6558 & 30.4644 & 30.4753 & 30.6571 & 30.2546 & 30.2508 & 30.6192 & 30.4249 & 30.4138 \\
\hline 3 & 3.54 & 28.5525 & 28.1625 & 28.0695 & 28.5449 & 28.3828 & 28.3487 & 28.4849 & 28.0918 & 27.9463 & 28.4425 & 28.2846 & 28.2388 \\
\hline 4 & 2.86 & 29.4661 & 28.9794 & 28.9194 & 29.3476 & 29.0948 & 29.0809 & 29.3613 & 28.8735 & 28.8045 & 29.2502 & 28.9980 & 28.9741 \\
\hline 8 & 2.46 & 29.9799 & 29.5232 & 29.3797 & 29.8959 & 29.6531 & 29.5540 & 29.9325 & 29.4707 & 29.3305 & 29.8446 & 29.6009 & 29.5073 \\
\hline 9 & 3.91 & 28.0831 & 27.6832 & 27.7226 & 28.1843 & 28.0093 & 28.0214 & 27.9815 & 27.5855 & 27.6328 & 28.0836 & 27.9189 & 27.9334 \\
\hline 10 & 1.66 & 30.1475 & 29.7358 & 29.7766 & 30.2418 & 30.0342 & 30.0035 & 30.1028 & 29.6941 & 29.7376 & 30.1944 & 29.9946 & 29.9653 \\
\hline $11 a$ & 1.44 & 30.5685 & 30.1139 & 30.0746 & 30.5801 & 30.3030 & 30.2567 & 30.5700 & 30.1180 & 30.0635 & 30.5610 & 30.2965 & 30.2535 \\
\hline $11 b$ & 1.44 & 30.5685 & 30.1139 & 30.0746 & 30.5801 & 30.3030 & 30.2567 & 30.5700 & 30.1180 & 30.0635 & 30.5610 & 30.2965 & 30.2535 \\
\hline 12 & 0.95 & 31.1220 & 30.6729 & 30.6260 & 31.1027 & 30.8352 & 30.8082 & 31.1105 & 30.6609 & 30.6101 & 31.0825 & 30.8184 & 30.7927 \\
\hline 13 & 1.16 & 30.7059 & 30.2636 & 30.2301 & 30.7094 & 30.4614 & 30.4464 & 30.7030 & 30.2607 & 30.2537 & 30.7210 & 30.4673 & 30.4512 \\
\hline 14 & 1.74 & 30.5476 & 30.0073 & 29.8424 & 30.4298 & 30.0683 & 29.9872 & 30.5390 & 29.9991 & 29.8249 & 30.4195 & 30.0608 & 29.9736 \\
\hline 15 & 1.06 & 31.0781 & 30.6225 & 30.5984 & 31.1093 & 30.8402 & 30.8420 & 31.0472 & 30.5905 & 30.5645 & 31.0767 & 30.8089 & 30.8083 \\
\hline 16 & 0.95 & 31.2579 & 30.8043 & 30.7841 & 31.2747 & 30.9931 & 30.9769 & 31.2227 & 30.7691 & 30.7516 & 31.2407 & 30.9626 & 30.7691 \\
\hline
\end{tabular}




\section{Compound 79g}

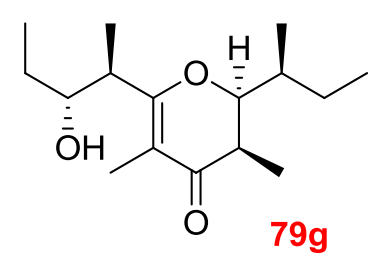

\begin{tabular}{|c|c|c|c|c|c|c|c|c|c|c|c|c|c|}
\hline & \multirow[b]{3}{*}{ Exp } & \multicolumn{12}{|c|}{ B3LYP } \\
\hline & & \multicolumn{6}{|c|}{ Gas phase } & \multicolumn{6}{|c|}{ PCM } \\
\hline & & 6-31G* & 6-31G** & $6-31+G * *$ & 6-311G* & $6-311 G * *$ & $6-311+\mathrm{G} * *$ & 6-31G* & $6-31 G * *$ & $6-31+G * *$ & 6-311G* & $6-311 G * *$ & $6-311+G * *$ \\
\hline \multicolumn{14}{|l|}{${ }^{13} \mathrm{C}$} \\
\hline 1 & 11.9 & 176.4854 & 178.3316 & 179.1035 & 170.3546 & 170.4355 & 170.1352 & 176.6920 & 178.5379 & 179.3641 & 170.6525 & 170.7147 & 170.4264 \\
\hline 2 & 28.0 & 157.8595 & 159.1059 & 158.6893 & 148.9705 & 148.6546 & 148.4522 & 158.1960 & 159.4401 & 159.0044 & 149.2805 & 148.9710 & 148.7229 \\
\hline 3 & 75.3 & 113.3648 & 113.9719 & 113.2797 & 100.9300 & 100.7015 & 100.3595 & 113.3911 & 113.9997 & 113.2408 & 100.9214 & 100.7100 & 100.3351 \\
\hline 4 & 41.2 & 146.2706 & 147.0246 & 145.9142 & 136.2297 & 136.2944 & 135.7823 & 146.3892 & 147.1323 & 146.0576 & 136.3150 & 136.3521 & 135.8555 \\
\hline 5 & 172.6 & 21.5441 & 21.5655 & 21.1438 & -0.4869 & -0.3791 & -1.3334 & 19.7007 & 19.7274 & 18.8521 & -2.6054 & -2.4927 & -3.6916 \\
\hline 6 & 109.8 & 81.6823 & 81.8758 & 81.9733 & 66.8867 & 66.9906 & 66.2282 & 81.9395 & 82.1306 & 82.1797 & 67.2188 & 67.3040 & 66.3779 \\
\hline 7 & 195.5 & 5.6630 & 5.6756 & 3.1801 & -15.1305 & -15.0875 & -18.4117 & 3.5514 & 3.5656 & 0.5589 & -17.6969 & -17.6451 & -21.4233 \\
\hline 8 & 40.5 & 147.1748 & 147.9746 & 146.9835 & 137.4538 & 137.1083 & 136.1779 & 146.9700 & 147.7650 & 146.6497 & 137.1671 & 136.8207 & 135.7513 \\
\hline 9 & 84.5 & 104.4931 & 105.0557 & 103.7129 & 92.0161 & 91.6950 & 91.4318 & 104.5209 & 105.0815 & 103.7760 & 92.0965 & 91.7817 & 91.5163 \\
\hline 10 & 35.6 & 151.9983 & 152.7423 & 151.4416 & 142.0518 & 141.8885 & 141.6366 & 152.1571 & 152.8996 & 151.6386 & 142.2557 & 142.0816 & 141.8197 \\
\hline 11 & 26.8 & 162.7897 & 163.9631 & 163.5002 & 153.8404 & 153.7254 & 153.4520 & 162.8515 & 164.0298 & 163.6146 & 154.0092 & 153.8799 & 153.6080 \\
\hline 12 & 9.9 & 176.3761 & 178.2498 & 178.6312 & 169.9431 & 169.9558 & 169.6006 & 176.6054 & 178.4783 & 178.9045 & 170.2455 & 170.2452 & 169.8963 \\
\hline 13 & 14.6 & 171.5119 & 173.2965 & 174.4868 & 165.4576 & 165.4588 & 165.1029 & 171.7721 & 173.5482 & 174.7399 & 165.7363 & 165.7348 & 165.3792 \\
\hline 14 & 9.4 & 180.1159 & 181.9761 & 182.0040 & 174.8747 & 174.6652 & 174.4508 & 180.3918 & 182.2482 & 182.3340 & 175.2404 & 175.0139 & 174.7971 \\
\hline 15 & 9.9 & 178.0357 & 179.7933 & 180.6808 & 172.0318 & 171.9957 & 171.7754 & 178.1245 & 179.8683 & 180.6075 & 172.0157 & 171.9680 & 171.7385 \\
\hline 16 & 12.3 & 175.5976 & 177.4323 & 177.9809 & 169.5281 & 169.4168 & 168.9820 & 175.8858 & 177.7124 & 178.3230 & 169.8747 & 169.7446 & 169.3276 \\
\hline \multicolumn{14}{|l|}{${ }^{1} \mathbf{H}$} \\
\hline 1 & 0.99 & 31.0520 & 30.6215 & 30.5389 & 31.0171 & 30.7977 & 30.7878 & 31.0699 & 30.6386 & 30.5616 & 31.0365 & 30.8140 & 30.8039 \\
\hline $2 a$ & 1.57 & 30.6980 & 30.2619 & 30.2098 & 30.6915 & 30.4541 & 30.4725 & 30.6242 & 30.1859 & 30.1111 & 30.6082 & 30.3711 & 30.3773 \\
\hline $2 b$ & 1.43 & 30.6104 & 30.1972 & 30.2372 & 30.6574 & 30.4482 & 30.4647 & 30.5885 & 30.1736 & 30.2167 & 30.6511 & 30.4396 & 30.4537 \\
\hline 3 & 3.55 & 28.5976 & 28.2084 & 28.2099 & 28.6491 & 28.4860 & 28.5419 & 28.5539 & 28.1642 & 28.1389 & 28.5872 & 28.4214 & 28.4650 \\
\hline 4 & 2.86 & 29.6125 & 29.1313 & 29.1113 & 29.5532 & 29.3129 & 29.3130 & 29.5167 & 29.0326 & 28.9977 & 29.4636 & 29.2181 & 29.2070 \\
\hline 8 & 2.46 & 30.0297 & 29.5674 & 29.4593 & 30.0090 & 29.7599 & 29.6544 & 29.9908 & 29.5231 & 29.4078 & 29.9628 & 29.7108 & 29.6056 \\
\hline 9 & 3.91 & 28.3579 & 27.9715 & 27.9483 & 28.4744 & 28.3079 & 28.2853 & 28.2427 & 27.8544 & 27.8204 & 28.3529 & 28.1908 & 28.1605 \\
\hline 10 & 1.66 & 30.3057 & 29.8957 & 29.9581 & 30.5012 & 30.2838 & 30.2431 & 30.2326 & 29.8227 & 29.8806 & 30.4210 & 30.2079 & 30.1678 \\
\hline $11 a$ & 1.43 & 30.5233 & 30.0849 & 30.0726 & 30.5910 & 30.3445 & 30.3415 & 30.5281 & 30.0909 & 30.0718 & 30.5863 & 30.3430 & 30.3397 \\
\hline $11 b$ & 1.43 & 30.5233 & 30.0849 & 30.0726 & 30.5910 & 30.3445 & 30.3415 & 30.5281 & 30.0909 & 30.0718 & 30.5863 & 30.3430 & 30.3397 \\
\hline 12 & 0.95 & 31.1358 & 30.6922 & 30.6273 & 31.1255 & 30.8702 & 30.8470 & 31.1329 & 30.6880 & 30.6243 & 31.1234 & 30.8672 & 30.8448 \\
\hline 13 & 1.16 & 30.8440 & 30.4087 & 30.3606 & 30.8844 & 30.6339 & 30.6134 & 30.8241 & 30.3875 & 30.3256 & 30.8541 & 30.6066 & 30.5804 \\
\hline 14 & 1.74 & 30.6267 & 30.0930 & 29.8931 & 30.5308 & 30.1828 & 30.0776 & 30.6257 & 30.0922 & 29.8894 & 30.5300 & 30.1824 & 30.0735 \\
\hline 15 & 1.06 & 31.0859 & 30.6202 & 30.6106 & 31.1824 & 30.8959 & 30.8781 & 31.0589 & 30.5921 & 30.5802 & 31.1546 & 30.8677 & 30.8472 \\
\hline 16 & 0.95 & 31.3321 & 30.8745 & 30.8215 & 31.3884 & 31.1093 & 31.0593 & 31.3038 & 30.8456 & 30.7947 & 31.3621 & 31.0840 & 31.0343 \\
\hline
\end{tabular}




\begin{tabular}{|c|c|c|c|c|c|c|c|c|c|c|c|c|c|}
\hline \multicolumn{14}{|c|}{ mPW1PW91 } \\
\hline & \multirow[b]{2}{*}{ Exp } & \multicolumn{6}{|c|}{ Gas phase } & \multicolumn{6}{|c|}{ PCM } \\
\hline & & 6-31G* & $6-31 G * *$ & $6-31+G * *$ & 6-311G* & $6-311 G * *$ & $6-311+G * *$ & 6-31G* & $6-31 G * *$ & $6-31+\mathrm{G} * *$ & 6-311G* & $6-311 G * *$ & $6-311+\mathrm{G} * *$ \\
\hline \multicolumn{14}{|l|}{${ }^{13} \mathrm{C}$} \\
\hline 1 & 11.9 & 180.6691 & 182.3991 & 183.0876 & 174.9474 & 175.0161 & 174.6777 & 180.9186 & 182.6482 & 183.3847 & 175.2592 & 175.3103 & 174.9820 \\
\hline 2 & 28.0 & 162.8328 & 163.9706 & 163.5653 & 154.6855 & 154.3863 & 154.1159 & 163.1795 & 164.3159 & 163.9109 & 155.0027 & 154.7062 & 154.4045 \\
\hline 3 & 75.3 & 118.5463 & 119.0870 & 118.5767 & 107.7244 & 107.5008 & 107.1349 & 118.5720 & 119.1158 & 118.5529 & 107.7047 & 107.4984 & 107.1022 \\
\hline 4 & 41.2 & 151.0472 & 151.7708 & 150.9880 & 142.1389 & 142.2146 & 141.7546 & 151.1332 & 151.8484 & 151.0850 & 142.1747 & 142.2271 & 141.7723 \\
\hline 5 & 172.6 & 24.6752 & 24.6970 & 24.4633 & 5.3130 & 5.4214 & 4.6125 & 22.7681 & 22.7960 & 22.1862 & 3.1721 & 3.2820 & 2.2582 \\
\hline 6 & 109.8 & 85.1196 & 85.3155 & 85.5509 & 72.1410 & 72.2434 & 71.3500 & 85.4366 & 85.6293 & 85.8133 & 72.5071 & 72.5932 & 71.5620 \\
\hline 7 & 195.5 & 8.0820 & 8.0895 & 6.4279 & -9.8308 & -9.7944 & -12.8003 & 5.8956 & 5.9051 & 3.7810 & -12.4247 & -12.3791 & -15.7924 \\
\hline 8 & 40.5 & 152.0018 & 152.7532 & 151.8308 & 143.3418 & 143.0102 & 142.1095 & 151.7882 & 152.5355 & 151.5046 & 143.0408 & 142.7097 & 141.6904 \\
\hline 9 & 84.5 & 109.4951 & 110.0106 & 108.9816 & 98.4826 & 98.1866 & 97.9204 & 109.4899 & 110.0045 & 109.0099 & 98.5141 & 98.2248 & 97.9574 \\
\hline 10 & 35.6 & 157.2315 & 157.9218 & 156.8909 & 148.2855 & 148.1338 & 147.8251 & 157.3806 & 158.0703 & 157.0745 & 148.4670 & 148.3064 & 147.9912 \\
\hline 11 & 26.8 & 167.9106 & 168.9895 & 168.4978 & 159.6344 & 159.5111 & 159.2086 & 167.9935 & 169.0772 & 168.6306 & 159.8050 & 159.6693 & 159.3692 \\
\hline 12 & 9.9 & 180.5135 & 182.2722 & 182.6354 & 174.5755 & 174.5745 & 174.2334 & 180.7897 & 182.5464 & 182.9481 & 174.8947 & 174.8813 & 174.5470 \\
\hline 13 & 14.6 & 175.8948 & 177.5423 & 178.3894 & 170.1063 & 170.1297 & 169.8014 & 176.1863 & 177.8256 & 178.6813 & 170.3983 & 170.4172 & 170.0951 \\
\hline 14 & 9.4 & 183.8396 & 185.5911 & 185.6706 & 179.1221 & 178.9283 & 178.6649 & 184.1578 & 185.9043 & 186.0356 & 179.4978 & 179.2867 & 179.0211 \\
\hline 15 & 9.9 & 182.2156 & 183.8430 & 184.5064 & 176.5300 & 176.5059 & 176.3023 & 182.3325 & 183.9462 & 184.4703 & 176.5324 & 176.4976 & 176.2899 \\
\hline 16 & 12.3 & 179.8125 & 181.5221 & 181.9903 & 174.1733 & 174.0631 & 173.6808 & 180.1333 & 181.8349 & 182.3591 & 174.5237 & 174.3973 & 174.0325 \\
\hline \multicolumn{14}{|l|}{${ }^{1} \mathrm{H}$} \\
\hline 1 & 0.99 & 31.0116 & 30.5799 & 30.5036 & 30.9519 & 30.7249 & 30.7123 & 31.0259 & 30.5934 & 30.5212 & 30.9665 & 30.7364 & 30.7235 \\
\hline $2 a$ & 1.57 & 30.6844 & 30.2487 & 30.1953 & 30.6216 & 30.3823 & 30.3970 & 30.6063 & 30.1680 & 30.0935 & 30.5365 & 30.2975 & 30.3005 \\
\hline $2 b$ & 1.43 & 30.5642 & 30.1495 & 30.1816 & 30.5487 & 30.3396 & 30.3534 & 30.5473 & 30.1314 & 30.1654 & 30.5451 & 30.3343 & 30.3453 \\
\hline 3 & 3.55 & 28.5386 & 28.1570 & 28.1519 & 28.5695 & 28.4041 & 28.4537 & 28.4880 & 28.1056 & 28.0760 & 28.5034 & 28.3348 & 28.3725 \\
\hline 4 & 2.86 & 29.5473 & 29.0737 & 29.0281 & 29.4200 & 29.1868 & 29.1813 & 29.4472 & 28.9703 & 28.9124 & 29.3284 & 29.0896 & 29.0733 \\
\hline 8 & 2.46 & 29.9361 & 29.4777 & 29.3693 & 29.8623 & 29.6164 & 29.5205 & 29.8942 & 29.4298 & 29.3151 & 29.8145 & 29.5653 & 29.4698 \\
\hline 9 & 3.91 & 28.2775 & 27.8966 & 27.8576 & 28.3340 & 28.1759 & 28.1542 & 28.1625 & 27.7797 & 27.7298 & 28.2153 & 28.0615 & 28.0319 \\
\hline 10 & 1.66 & 30.2564 & 29.8505 & 29.8923 & 30.3752 & 30.1654 & 30.1167 & 30.1839 & 29.7779 & 29.8155 & 30.2966 & 30.0909 & 30.0421 \\
\hline $11 a$ & 1.43 & 30.4928 & 30.0574 & 30.0426 & 30.5044 & 30.2605 & 30.2447 & 30.4979 & 30.0636 & 30.0423 & 30.5004 & 30.2598 & 30.2435 \\
\hline $11 b$ & 1.43 & 30.4928 & 30.0574 & 30.0426 & 30.5044 & 30.2605 & 30.2447 & 30.4979 & 30.0636 & 30.0423 & 30.5004 & 30.2598 & 30.2435 \\
\hline 12 & 0.95 & 31.0950 & 30.6506 & 30.5905 & 31.0514 & 30.7887 & 30.7665 & 31.0893 & 30.6434 & 30.5845 & 31.0461 & 30.7825 & 30.7610 \\
\hline 13 & 1.16 & 30.7904 & 30.3525 & 30.3031 & 30.7914 & 30.5385 & 30.5109 & 30.7679 & 30.3286 & 30.2690 & 30.7606 & 30.5099 & 30.4777 \\
\hline 14 & 1.74 & 30.5590 & 30.0233 & 29.8378 & 30.4422 & 30.0863 & 29.9896 & 30.5536 & 30.0180 & 29.8298 & 30.4370 & 30.0817 & 29.9815 \\
\hline 15 & 1.06 & 31.0375 & 30.5704 & 30.5629 & 31.0968 & 30.8057 & 30.7913 & 31.0088 & 30.5405 & 30.5310 & 31.0670 & 30.7755 & 30.7584 \\
\hline 16 & 0.95 & 31.2936 & 30.8369 & 30.7830 & 31.3073 & 31.0246 & 30.9812 & 31.2617 & 30.8043 & 30.7521 & 31.2775 & 30.9956 & 30.8043 \\
\hline
\end{tabular}




\section{Compound 79h}

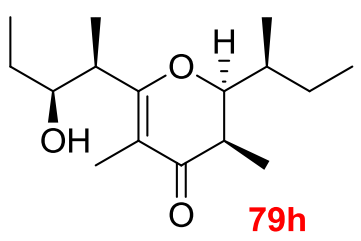

\begin{tabular}{|c|c|c|c|c|c|c|c|c|c|c|c|c|c|}
\hline & \multirow[b]{3}{*}{ Exp } & \multicolumn{12}{|c|}{ B3LYP } \\
\hline & & \multicolumn{6}{|c|}{ Gas phase } & \multicolumn{6}{|c|}{ PCM } \\
\hline & & 6-31G* & $6-31 G * *$ & $6-31+G * *$ & 6-311G* & $6-311 G * *$ & $6-311+G * *$ & 6-31G* & $6-31 G * *$ & $6-31+G * *$ & 6-311G* & $6-311 G * *$ & $6-311+\mathrm{G} * *$ \\
\hline \multicolumn{14}{|l|}{${ }^{13} \mathrm{C}$} \\
\hline 1 & 10.2 & 176.5698 & 178.4714 & 179.3519 & 170.6120 & 170.6813 & 170.4961 & 176.8983 & 178.7943 & 179.7961 & 171.0166 & 171.0551 & 170.9072 \\
\hline 2 & 28.0 & 159.9053 & 161.1898 & 160.4047 & 151.4250 & 151.2426 & 150.9824 & 160.2468 & 161.5229 & 160.6865 & 151.6838 & 151.4802 & 151.1763 \\
\hline 3 & 75.2 & 116.0729 & 116.7030 & 115.9372 & 103.7461 & 103.6896 & 103.5571 & 116.2554 & 116.8831 & 116.1199 & 103.9236 & 103.8559 & 103.7162 \\
\hline 4 & 41.8 & 146.6583 & 147.4534 & 147.0883 & 137.4194 & 137.3075 & 136.8492 & 146.8797 & 147.6587 & 147.2678 & 137.5370 & 137.3923 & 136.9496 \\
\hline 5 & 173.2 & 20.4467 & 20.4595 & 19.8707 & -1.6022 & -1.4405 & -2.2704 & 18.7073 & 18.7274 & 17.7683 & -3.5474 & -3.3924 & -4.4361 \\
\hline 6 & 108.7 & 83.8413 & 84.0264 & 84.1387 & 69.3309 & 69.3677 & 68.6653 & 84.0470 & 84.2278 & 84.2793 & 69.5601 & 69.5959 & 68.6491 \\
\hline 7 & 195.7 & 5.2604 & 5.2717 & 2.9498 & -15.5305 & -15.4912 & -18.9010 & 3.1359 & 3.1495 & 0.3698 & -18.0942 & -18.0489 & -21.9198 \\
\hline 8 & 40.5 & 147.1556 & 147.9595 & 146.5688 & 137.3224 & 136.9961 & 136.3070 & 146.9538 & 147.7521 & 146.2342 & 137.0342 & 136.7060 & 135.8952 \\
\hline 9 & 84.2 & 104.7661 & 105.3294 & 103.9740 & 92.3367 & 92.0470 & 91.7769 & 104.8078 & 105.3685 & 104.0335 & 92.4453 & 92.1548 & 91.9033 \\
\hline 10 & 35.6 & 151.9377 & 152.6832 & 151.6366 & 142.0311 & 141.8705 & 141.5859 & 152.0885 & 152.8338 & 151.8099 & 142.2057 & 142.0403 & 141.7734 \\
\hline 11 & 26.5 & 162.7923 & 163.9743 & 163.5217 & 153.8514 & 153.7532 & 153.4377 & 162.8705 & 164.0567 & 163.6661 & 154.0313 & 153.9205 & 153.6074 \\
\hline 12 & 11.8 & 176.3191 & 178.1997 & 178.6896 & 169.9148 & 169.9238 & 169.5690 & 176.5478 & 178.4279 & 178.9746 & 170.2121 & 170.2099 & 169.8598 \\
\hline 13 & 13.4 & 177.5016 & 179.3553 & 180.7064 & 171.6823 & 171.8242 & 171.3288 & 177.7549 & 179.5862 & 180.7954 & 171.8509 & 171.9756 & 171.4750 \\
\hline 14 & 9.3 & 180.1325 & 181.9974 & 182.5700 & 174.8685 & 174.6560 & 174.3823 & 180.4115 & 182.2728 & 182.8071 & 175.2504 & 175.0153 & 174.7607 \\
\hline 15 & 9.7 & 177.9873 & 179.8024 & 180.7669 & 172.1296 & 172.1119 & 171.9196 & 178.0947 & 179.8950 & 180.7518 & 172.1604 & 172.1246 & 171.9168 \\
\hline 16 & 12.3 & 175.5535 & 177.3864 & 177.9009 & 169.4781 & 169.3720 & 169.0339 & 175.8382 & 177.6633 & 178.2505 & 169.8166 & 169.6931 & 169.3737 \\
\hline \multicolumn{14}{|l|}{${ }^{1} \mathrm{H}$} \\
\hline 1 & 0.96 & 31.0949 & 30.6685 & 30.5930 & 31.0890 & 30.8648 & 30.8532 & 31.1157 & 30.6866 & 30.6170 & 31.1035 & 30.8744 & 30.8611 \\
\hline $2 a$ & 1.44 & 30.6405 & 30.2206 & 30.1502 & 30.6529 & 30.4470 & 30.3998 & 30.6103 & 30.1897 & 30.1149 & 30.6296 & 30.4221 & 30.3744 \\
\hline $2 b$ & 1.44 & 30.6405 & 30.2206 & 30.1502 & 30.6529 & 30.4470 & 30.3998 & 30.6103 & 30.1897 & 30.1149 & 30.6296 & 30.4221 & 30.3744 \\
\hline 3 & 3.61 & 28.1845 & 27.7572 & 27.7629 & 28.2471 & 28.0509 & 28.1067 & 28.1433 & 27.7154 & 27.7081 & 28.1962 & 27.9988 & 28.0447 \\
\hline 4 & 2.77 & 29.6854 & 29.2049 & 29.1514 & 29.6421 & 29.3955 & 29.3179 & 29.5831 & 29.1005 & 29.0205 & 29.5458 & 29.2999 & 29.2161 \\
\hline 8 & 2.45 & 30.0295 & 29.5606 & 29.4776 & 30.0023 & 29.7446 & 29.6948 & 29.9935 & 29.5192 & 29.4322 & 29.9601 & 29.6993 & 29.6494 \\
\hline 9 & 3.84 & 28.3565 & 27.9733 & 27.9597 & 28.4698 & 28.3109 & 28.3262 & 28.2425 & 27.8575 & 27.8397 & 28.3520 & 28.1968 & 28.2055 \\
\hline 10 & 1.64 & 30.2850 & 29.8823 & 29.9289 & 30.4645 & 30.2657 & 30.2346 & 30.2161 & 29.8139 & 29.8594 & 30.3920 & 30.1977 & 30.1717 \\
\hline $11 a$ & 1.44 & 30.5281 & 30.0908 & 30.0509 & 30.5822 & 30.3400 & 30.3450 & 30.5281 & 30.0922 & 30.0494 & 30.5761 & 30.3370 & 30.3434 \\
\hline $11 b$ & 1.44 & 30.5281 & 30.0908 & 30.0509 & 30.5822 & 30.3400 & 30.3450 & 30.5281 & 30.0922 & 30.0494 & 30.5761 & 30.3370 & 30.3434 \\
\hline 12 & 0.93 & 31.1320 & 30.6889 & 30.6344 & 31.1152 & 30.8627 & 30.8463 & 31.1297 & 30.6853 & 30.6327 & 31.1134 & 30.8599 & 30.8446 \\
\hline 13 & 1.21 & 30.9877 & 30.5365 & 30.4320 & 30.9916 & 30.7503 & 30.6962 & 30.9692 & 30.5165 & 30.4079 & 30.9730 & 30.7303 & 30.6747 \\
\hline 14 & 1.73 & 30.6347 & 30.1001 & 29.9208 & 30.5383 & 30.1896 & 30.1036 & 30.6328 & 30.0990 & 29.9173 & 30.5348 & 30.1884 & 30.0982 \\
\hline 15 & 1.05 & 31.0862 & 30.6262 & 30.6301 & 31.1690 & 30.8951 & 30.9081 & 31.0609 & 30.5997 & 30.5999 & 31.1414 & 30.8672 & 30.8771 \\
\hline 16 & 0.94 & 31.3145 & 30.8587 & 30.8130 & 31.3690 & 31.0932 & 31.0487 & 31.2872 & 30.8307 & 30.7881 & 31.3431 & 31.0685 & 31.0244 \\
\hline
\end{tabular}




\begin{tabular}{|c|c|c|c|c|c|c|c|c|c|c|c|c|c|}
\hline & & \multicolumn{12}{|c|}{ mPW1PW91 } \\
\hline & \multirow[b]{2}{*}{ Exp } & \multicolumn{6}{|c|}{ Gas phase } & \multicolumn{6}{|c|}{ PCM } \\
\hline & & 6-31G* & $6-31 G * *$ & $6-31+G * *$ & 6-311G* & $6-311 G * *$ & $6-311+\mathrm{G} * *$ & 6-31G* & $6-31 G * *$ & $6-31+G * *$ & $6-311 G *$ & $6-311 G * *$ & $6-311+G * *$ \\
\hline \multicolumn{14}{|c|}{${ }^{13} \mathrm{C}$} \\
\hline 1 & 10.2 & 180.8293 & 182.6157 & 183.3173 & 175.2743 & 175.3360 & 175.1145 & 181.2123 & 182.9933 & 183.7930 & 175.6994 & 175.7297 & 175.5380 \\
\hline 2 & 28.0 & 164.9160 & 166.1185 & 165.4913 & 157.2140 & 157.0401 & 156.7404 & 165.2602 & 166.4547 & 165.7521 & 157.4390 & 157.2487 & 156.9078 \\
\hline 3 & 75.2 & 121.1736 & 121.7471 & 121.2114 & 110.4393 & 110.3885 & 110.2314 & 121.3281 & 121.9009 & 121.3583 & 110.5694 & 110.5110 & 110.3498 \\
\hline 4 & 41.8 & 151.4939 & 152.2529 & 152.1101 & 143.2262 & 143.1521 & 142.7363 & 151.6948 & 152.4399 & 152.2597 & 143.2886 & 143.1869 & 142.7780 \\
\hline 5 & 173.2 & 23.6456 & 23.6610 & 23.2757 & 4.3510 & 4.5061 & 3.8165 & 21.8492 & 21.8723 & 21.1596 & 2.3772 & 2.5236 & 1.6523 \\
\hline 6 & 108.7 & 87.2445 & 87.4313 & 87.7050 & 74.5051 & 74.5525 & 73.6860 & 87.5013 & 87.6835 & 87.9032 & 74.7703 & 74.8177 & 73.7385 \\
\hline 7 & 195.7 & 7.6860 & 7.6916 & 6.1728 & -10.2180 & -10.1855 & -13.2723 & 5.4880 & 5.4961 & 3.5685 & -12.8081 & -12.7694 & -16.2729 \\
\hline 8 & 40.5 & 151.9952 & 152.7498 & 151.4456 & 143.2246 & 142.9128 & 142.2389 & 151.7817 & 152.5318 & 151.0942 & 142.9202 & 142.6078 & 141.8387 \\
\hline 9 & 84.2 & 109.7668 & 110.2848 & 109.2534 & 98.8196 & 98.5503 & 98.2863 & 109.7753 & 110.2918 & 109.2710 & 98.8783 & 98.6091 & 98.3662 \\
\hline 10 & 35.6 & 157.1787 & 157.8706 & 157.0744 & 148.2637 & 148.1166 & 147.7912 & 157.3223 & 158.0151 & 157.2405 & 148.4215 & 148.2709 & 147.9624 \\
\hline 11 & 26.5 & 167.9151 & 169.0026 & 168.5054 & 159.6445 & 159.5372 & 159.2023 & 168.0155 & 169.1075 & 168.6710 & 159.8298 & 159.7113 & 159.3797 \\
\hline 12 & 11.8 & 180.4572 & 182.2209 & 182.7311 & 174.5439 & 174.5401 & 174.1814 & 180.7335 & 182.4954 & 183.0534 & 174.8591 & 174.8446 & 174.4897 \\
\hline 13 & 13.4 & 181.7438 & 183.4660 & 184.4992 & 176.0916 & 176.2557 & 175.8541 & 181.9976 & 183.6984 & 184.6092 & 176.2196 & 176.3748 & 175.9629 \\
\hline 14 & 9.3 & 183.8665 & 185.6264 & 186.1501 & 179.1408 & 178.9412 & 178.6682 & 184.1835 & 185.9388 & 186.4214 & 179.5243 & 179.3031 & 179.0412 \\
\hline 15 & 9.7 & 182.1701 & 183.8610 & 184.6509 & 176.6213 & 176.6161 & 176.4240 & 182.3064 & 183.9820 & 184.6721 & 176.6653 & 176.6437 & 176.4400 \\
\hline 16 & 12.3 & 179.7825 & 181.4909 & 181.9351 & 174.1318 & 174.0274 & 173.7322 & 180.0989 & 181.7993 & 182.3123 & 174.4731 & 174.3542 & 174.0747 \\
\hline \multicolumn{14}{|l|}{${ }^{1} \mathrm{H}$} \\
\hline 1 & 0.96 & 31.0582 & 30.6308 & 30.5583 & 31.0254 & 30.7934 & 30.7776 & 31.0752 & 30.6448 & 30.5770 & 31.0349 & 30.7978 & 30.7805 \\
\hline $2 a$ & 1.44 & 30.5983 & 30.1787 & 30.1082 & 30.5638 & 30.3571 & 30.3104 & 30.5670 & 30.1466 & 30.0736 & 30.5414 & 30.3325 & 30.2859 \\
\hline $2 b$ & 1.44 & 30.5983 & 30.1787 & 30.1082 & 30.5638 & 30.3571 & 30.3104 & 30.5670 & 30.1466 & 30.0736 & 30.5414 & 30.3325 & 30.2859 \\
\hline 3 & 3.61 & 28.1501 & 27.7296 & 27.7169 & 28.1743 & 27.9784 & 28.0246 & 28.1066 & 27.6853 & 27.6616 & 28.1230 & 27.9257 & 27.9625 \\
\hline 4 & 2.77 & 29.6168 & 29.1416 & 29.0645 & 29.5054 & 29.2646 & 29.1919 & 29.5096 & 29.0318 & 28.9323 & 29.4074 & 29.1669 & 29.0892 \\
\hline 8 & 2.45 & 29.9350 & 29.4711 & 29.3910 & 29.8569 & 29.6031 & 29.5591 & 29.8963 & 29.4263 & 29.3418 & 29.8135 & 29.5559 & 29.5123 \\
\hline 9 & 3.84 & 28.2778 & 27.8994 & 27.8722 & 28.3304 & 28.1793 & 28.1951 & 28.1645 & 27.7842 & 27.7525 & 28.2161 & 28.0686 & 28.0776 \\
\hline 10 & 1.64 & 30.2374 & 29.8390 & 29.8659 & 30.3402 & 30.1496 & 30.1115 & 30.1694 & 29.7713 & 29.7974 & 30.2699 & 30.0836 & 30.0495 \\
\hline 11a & 1.44 & 30.4987 & 30.0642 & 30.0251 & 30.4952 & 30.2562 & 30.2515 & 30.4991 & 30.0659 & 30.0241 & 30.4895 & 30.2538 & 30.2503 \\
\hline $11 b$ & 1.44 & 30.4987 & 30.0642 & 30.0251 & 30.4952 & 30.2562 & 30.2515 & 30.4991 & 30.0659 & 30.0241 & 30.4895 & 30.2538 & 30.2503 \\
\hline 12 & 0.93 & 31.0917 & 30.6479 & 30.5937 & 31.0412 & 30.7817 & 30.7652 & 31.0866 & 30.6414 & 30.5890 & 31.0363 & 30.7758 & 30.7605 \\
\hline 13 & 1.21 & 30.9316 & 30.4797 & 30.3873 & 30.8997 & 30.6559 & 30.6066 & 30.9093 & 30.4558 & 30.3597 & 30.8766 & 30.6315 & 30.5806 \\
\hline 14 & 1.73 & 30.5652 & 30.0292 & 29.8698 & 30.4482 & 30.0930 & 30.0144 & 30.5587 & 30.0233 & 29.8611 & 30.4402 & 30.0876 & 30.0045 \\
\hline 15 & 1.05 & 31.0395 & 30.5786 & 30.5823 & 31.0868 & 30.8080 & 30.8206 & 31.0122 & 30.5500 & 30.5501 & 31.0573 & 30.7783 & 30.7882 \\
\hline 16 & 0.94 & 31.2768 & 30.8214 & 30.7721 & 31.2882 & 31.0087 & 30.9657 & 31.2460 & 30.7899 & 30.7427 & 31.2589 & 30.9807 & 30.7899 \\
\hline
\end{tabular}




\section{Compound 80a}<smiles>CC(C)(C)C(O)C[C@@H](O)C[C@H](O)C(C)(C)C</smiles>

\begin{tabular}{|c|c|c|c|c|c|c|c|c|c|c|c|c|c|}
\hline \multirow{3}{*}{\multicolumn{2}{|c|}{ Exp }} & \multicolumn{12}{|c|}{ B3LYP } \\
\hline & & \multicolumn{6}{|c|}{ Gas phase } & \multicolumn{6}{|c|}{ PCM } \\
\hline & & 6-31G* & 6-31G** & $6-31+G^{* *}$ & 6-311G* & 6-311G** & $6-311+\mathrm{G}^{* *}$ & $6-31 G^{*}$ & $6-31 G^{* *}$ & $6-31+G^{* *}$ & 6-311G* & 6-311G** & $6-311+G^{* *}$ \\
\hline \\
\hline 1 & 26.1 & 163.9388 & 165.6456 & 166.6608 & 157.2487 & 156.9910 & 156.5456 & 164.2049 & 165.9012 & 166.9955 & 157.6206 & 157.3372 & 156.9203 \\
\hline 2 & 35.8 & 152.6860 & 153.1955 & 153.3218 & 142.7707 & 142.7134 & 141.8836 & 152.6773 & 153.1915 & 153.3101 & 142.7912 & 142.7346 & 141.8760 \\
\hline 3 & 79.9 & 108.1292 & 108.7040 & 107.4402 & 95.6796 & 95.5170 & 95.4815 & 108.3691 & 108.9400 & 107.6756 & 95.9671 & 95.7800 & 95.7335 \\
\hline 4 & 38.8 & 152.0008 & 153.1898 & 152.4492 & 142.5607 & 142.0442 & 142.1534 & 152.1261 & 153.3095 & 152.5472 & 142.6377 & 142.1168 & 142.2128 \\
\hline 5 & 73.3 & 114.1823 & 114.8246 & 113.6184 & 101.5412 & 101.4819 & 101.1292 & 114.2253 & 114.8694 & 113.6250 & 101.5985 & 101.5273 & 101.1413 \\
\hline \\
\hline 1 & 0.90 & 31.2122 & 30.7858 & 30.7403 & 31.2434 & 31.0097 & 30.9602 & 31.2070 & 30.7801 & 30.7338 & 31.2387 & 31.0046 & 30.9544 \\
\hline 3 & 3.39 & 28.4112 & 28.0253 & 28.0135 & 28.4388 & 28.2561 & 28.3067 & 28.3423 & 27.9561 & 27.9263 & 28.3551 & 28.1729 & 28.2149 \\
\hline $4 b$ & 1.71 & 30.5118 & 30.1261 & 30.1456 & 30.5862 & 30.4283 & 30.4167 & 30.5689 & 30.1814 & 30.2000 & 30.6446 & 30.4804 & 30.4652 \\
\hline $4 a$ & 1.44 & 30.8281 & 30.3661 & 30.3065 & 30.8579 & 30.5578 & 30.5500 & 30.7112 & 30.2482 & 30.1596 & 30.7323 & 30.4332 & 30.4100 \\
\hline 5 & 4.03 & 28.0399 & 27.6159 & 27.5872 & 28.0323 & 27.8038 & 27.8635 & 27.9681 & 27.5442 & 27.4973 & 27.9501 & 27.7221 & 27.7693 \\
\hline
\end{tabular}

\begin{tabular}{|c|c|c|c|c|c|c|c|c|c|c|c|c|c|}
\hline \multirow{3}{*}{\multicolumn{2}{|c|}{ Exp }} & \multicolumn{12}{|c|}{ mPW1PW91 } \\
\hline & & \multicolumn{6}{|c|}{ Gas phase } & \multicolumn{6}{|c|}{ PCM } \\
\hline & & 6-31G* & $6-31 G^{* *}$ & $6-31+G^{* *}$ & 6-311G* & $6-311 G * *$ & $6-311+G * *$ & 6-31G* & $6-31 G^{* *}$ & $6-31+G * *$ & 6-311G* & $6-311 \mathrm{G} * *$ & $6-311+G * *$ \\
\hline \\
\hline 1 & 26.1 & 168.3476 & 169.9074 & 170.4966 & 162.0989 & 161.8529 & 161.4922 & 168.6499 & 170.1997 & 170.8646 & 162.4750 & 162.2063 & 161.8730 \\
\hline 2 & 35.8 & 157.7265 & 158.2413 & 158.4672 & 148.5556 & 148.5104 & 147.7850 & 157.7184 & 158.2376 & 158.4575 & 148.5751 & 148.5310 & 147.7796 \\
\hline 3 & 79.9 & 113.2851 & 113.7930 & 112.7937 & 102.4793 & 102.3276 & 102.1900 & 113.5151 & 114.0211 & 113.0224 & 102.7268 & 102.5528 & 102.4133 \\
\hline 4 & 38.8 & 156.8735 & 157.9622 & 157.3557 & 148.4811 & 147.9964 & 148.0524 & 157.0036 & 158.0875 & 157.4531 & 148.5512 & 148.0623 & 148.1012 \\
\hline 5 & 73.3 & 119.1418 & 119.7320 & 118.7409 & 108.3760 & 108.3118 & 107.9318 & 119.1773 & 119.7704 & 118.7421 & 108.3989 & 108.3238 & 107.9169 \\
\hline \\
\hline 1 & 0.90 & 31.1588 & 30.7349 & 30.6829 & 31.1483 & 30.9127 & 30.8619 & 31.1515 & 30.7271 & 30.6745 & 31.1416 & 30.9056 & 30.8542 \\
\hline 3 & 3.39 & 28.3761 & 27.9995 & 27.9551 & 28.3508 & 28.1751 & 28.2116 & 28.3017 & 27.9247 & 27.8648 & 28.2652 & 28.0899 & 28.1187 \\
\hline $4 b$ & 1.71 & 30.4300 & 30.0404 & 30.0413 & 30.4575 & 30.2975 & 30.2850 & 30.4889 & 30.0975 & 30.0979 & 30.5167 & 30.3499 & 30.3345 \\
\hline $4 a$ & 1.44 & 30.8152 & 30.3527 & 30.2703 & 30.7922 & 30.4877 & 30.4741 & 30.6908 & 30.2271 & 30.1196 & 30.6619 & 30.3580 & 30.3310 \\
\hline 5 & 4.03 & 27.9935 & 27.5731 & 27.5080 & 27.9535 & 27.7292 & 27.7759 & 27.9160 & 27.4956 & 27.4145 & 27.8685 & 27.6445 & 27.6801 \\
\hline
\end{tabular}




\section{Compound 80b}<smiles>CC(C)(C)[C@@H](O)CC(O)C[C@H](O)C(C)(C)C</smiles>

\begin{tabular}{|c|c|c|c|c|c|c|c|c|c|c|c|c|c|}
\hline \multirow{3}{*}{\multicolumn{2}{|c|}{ Exp }} & \multicolumn{12}{|c|}{ B3LYP } \\
\hline & & \multicolumn{6}{|c|}{ Gas phase } & \multicolumn{6}{|c|}{ PCM } \\
\hline & & 6-31G* & 6-31G** & $6-31+G * *$ & 6-311G* & 6-311G** & $6-311+G * *$ & 6-31G* & 6-31G** & $6-31+G^{* *}$ & 6-311G* & $6-311 G * *$ & $6-311+G * *$ \\
\hline \\
\hline 1 & 26.3 & 163.9858 & 165.6874 & 166.6424 & 157.2911 & 157.0467 & 156.6356 & 164.2684 & 165.9599 & 167.0142 & 157.6906 & 157.4174 & 157.0501 \\
\hline 2 & 40.7 & 152.7857 & 153.2887 & 153.7139 & 143.0528 & 142.9807 & 141.9808 & 152.7439 & 153.2599 & 153.6447 & 143.0249 & 142.9666 & 141.9300 \\
\hline 3 & 76.9 & 112.7693 & 113.4191 & 113.2397 & 101.4240 & 101.2713 & 101.4428 & 112.8621 & 113.4987 & 113.1153 & 101.4684 & 101.2818 & 101.2148 \\
\hline 4 & 35.7 & 154.6656 & 155.8549 & 155.2401 & 145.9466 & 145.1268 & 144.9506 & 154.5667 & 155.7370 & 154.9680 & 145.6120 & 144.8518 & 144.6272 \\
\hline 5 & 67.2 & 120.4332 & 121.1900 & 120.1858 & 108.6314 & 108.5103 & 108.5523 & 120.3912 & 121.1909 & 120.2924 & 108.6078 & 108.5805 & 108.8504 \\
\hline \multicolumn{14}{|c|}{${ }^{1} \mathrm{H}$} \\
\hline 1 & 0.80 & 31.2070 & 30.7775 & 30.7453 & 31.2410 & 31.0026 & 30.9656 & 31.2002 & 30.7708 & 30.7416 & 31.2355 & 30.9967 & 30.9625 \\
\hline 3 & 3.39 & 28.1893 & 27.7728 & 27.8079 & 28.2911 & 28.0798 & 28.1249 & 28.1304 & 27.7195 & 27.7497 & 28.2239 & 28.0223 & 28.0561 \\
\hline $4 b$ & 1.50 & 30.6191 & 30.1547 & 30.0754 & 30.6878 & 30.3594 & 30.3128 & 30.4920 & 30.0273 & 29.9088 & 30.5209 & 30.2164 & 30.1572 \\
\hline $4 a$ & 1.28 & 30.3019 & 29.8868 & 29.9887 & 30.3781 & 30.2098 & 30.2952 & 30.3539 & 29.9444 & 30.0673 & 30.4649 & 30.2832 & 30.3694 \\
\hline 5 & 3.94 & 27.6662 & 27.1942 & 27.0482 & 27.5917 & 27.3701 & 27.3596 & 27.6697 & 27.2055 & 27.1043 & 27.6264 & 27.4238 & 27.4564 \\
\hline
\end{tabular}

\begin{tabular}{|c|c|c|c|c|c|c|c|c|c|c|c|c|c|}
\hline & \multirow[b]{3}{*}{ Exp } & \multicolumn{12}{|c|}{ mPW1PW91 } \\
\hline & & \multicolumn{6}{|c|}{ Gas phase } & \multicolumn{6}{|c|}{ PCM } \\
\hline & & 6-31G* & $6-31 G * *$ & $6-31+\mathrm{G} * *$ & 6-311G* & $6-311 G * *$ & $6-311+G * *$ & $6-31 G *$ & $6-31 \mathrm{G} * *$ & $6-31+G * *$ & 6-311G* & $6-311 G * *$ & $6-311+G * *$ \\
\hline \multicolumn{14}{|c|}{${ }^{13} \mathrm{C}$} \\
\hline 1 & 26.3 & 168.3844 & 169.9369 & 170.4908 & 162.1218 & 161.8844 & 161.5391 & 168.7012 & 170.2441 & 170.8795 & 162.5239 & 162.2619 & 161.9625 \\
\hline 2 & 40.7 & 157.8132 & 158.3167 & 158.7746 & 148.7749 & 148.7134 & 147.8602 & 157.7691 & 158.2843 & 158.7079 & 148.7450 & 148.6995 & 147.8187 \\
\hline 3 & 76.9 & 117.7006 & 118.2627 & 118.3101 & 107.7994 & 107.6480 & 107.6764 & 117.7936 & 118.3506 & 118.2214 & 107.8320 & 107.6580 & 107.5132 \\
\hline 4 & 35.7 & 159.4487 & 160.5571 & 160.1498 & 151.6640 & 150.9489 & 150.7931 & 159.3919 & 160.4833 & 159.8844 & 151.3870 & 150.7036 & 150.4832 \\
\hline 5 & 67.2 & 125.2001 & 125.9042 & 125.0772 & 115.0163 & 114.9019 & 114.8084 & 125.1502 & 125.8895 & 125.1375 & 114.9895 & 114.9530 & 115.0274 \\
\hline \multicolumn{14}{|l|}{${ }^{1} \mathrm{H}$} \\
\hline 1 & 0.80 & 31.1554 & 30.7281 & 30.6855 & 31.1472 & 30.9062 & 30.8650 & 31.1463 & 30.7192 & 30.6790 & 31.1394 & 30.8982 & 30.8594 \\
\hline 3 & 3.39 & 28.1676 & 27.7601 & 27.7661 & 28.2134 & 28.0087 & 28.0484 & 28.1026 & 27.6996 & 27.7001 & 28.1398 & 27.9441 & 27.9724 \\
\hline $4 b$ & 1.50 & 30.5892 & 30.1285 & 30.0566 & 30.6046 & 30.2842 & 30.2442 & 30.4607 & 29.9996 & 29.8826 & 30.4399 & 30.1386 & 30.0837 \\
\hline $4 a$ & 1.28 & 30.2533 & 29.8309 & 29.8856 & 30.2610 & 30.0831 & 30.1414 & 30.2990 & 29.8817 & 29.9672 & 30.3397 & 30.1536 & 30.2186 \\
\hline 5 & 3.94 & 27.6305 & 27.1616 & 27.0308 & 27.5422 & 27.3182 & 27.3135 & 27.6250 & 27.1613 & 27.0621 & 27.5658 & 27.3558 & 27.3797 \\
\hline
\end{tabular}




\section{Compound 81a}

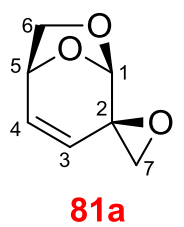

\begin{tabular}{|c|c|c|c|c|c|c|c|c|c|c|c|c|c|}
\hline & \multirow[b]{3}{*}{ Exp } & \multicolumn{12}{|c|}{ B3LYP } \\
\hline & & \multicolumn{6}{|c|}{ Gas phase } & \multicolumn{6}{|c|}{ PCM } \\
\hline & & 6-31G* & 6-31G** & $6-31+G * *$ & 6-311G* & 6-311G** & $6-311+G * *$ & 6-31G* & $6-31 G * *$ & $6-31+G * *$ & 6-311G* & $6-311 G * *$ & $6-311+G * *$ \\
\hline \multicolumn{14}{|l|}{${ }^{13} \mathrm{C}$} \\
\hline 1 & 102.7 & 86.8640 & 87.6171 & 86.3265 & 74.0651 & 73.3594 & 73.4894 & 87.1719 & 87.9229 & 86.5598 & 74.3039 & 73.5959 & 73.6800 \\
\hline 2 & 59.1 & 131.0282 & 131.1429 & 130.9907 & 121.4112 & 121.0947 & 119.6528 & 130.9795 & 131.0972 & 130.8992 & 121.3308 & 121.0214 & 119.5556 \\
\hline 3 & 127.3 & 63.5434 & 63.9948 & 63.4288 & 45.6198 & 44.6740 & 43.9468 & 64.8736 & 65.3179 & 64.9627 & 47.0244 & 46.0932 & 45.4845 \\
\hline 4 & 135.9 & 60.0296 & 60.5911 & 59.8573 & 42.2893 & 41.3436 & 40.1225 & 58.5646 & 59.1292 & 58.1579 & 40.5447 & 39.6340 & 38.2619 \\
\hline 5 & 71.6 & 116.8996 & 117.7435 & 116.6947 & 106.1537 & 105.5957 & 105.3316 & 116.9466 & 117.7874 & 116.6640 & 106.1369 & 105.5647 & 105.2800 \\
\hline 6 & 70.8 & 119.1137 & 120.4542 & 120.2652 & 108.8746 & 108.1998 & 108.0650 & 119.2214 & 120.5531 & 120.1834 & 108.8341 & 108.1346 & 108.0040 \\
\hline 7 & 52.4 & 139.4628 & 140.9987 & 139.6411 & 130.6293 & 129.6787 & 127.7230 & 139.3636 & 140.8769 & 139.1552 & 130.3677 & 129.3816 & 127.2142 \\
\hline \multicolumn{14}{|l|}{${ }^{1} \mathrm{H}$} \\
\hline 1 & 5.02 & 27.4706 & 26.9015 & 26.6568 & 27.3772 & 27.0304 & 26.9656 & 27.4048 & 26.8319 & 26.5634 & 27.2898 & 26.9447 & 26.8663 \\
\hline 3 & 5.33 & 26.8921 & 26.2449 & 26.0601 & 26.7792 & 26.3460 & 26.3357 & 26.8406 & 26.1933 & 26.0037 & 26.7207 & 26.2941 & 26.2777 \\
\hline 4 & 6.40 & 26.0454 & 25.3611 & 25.1551 & 25.9331 & 25.4437 & 25.4094 & 25.8441 & 25.1616 & 24.9403 & 25.7282 & 25.2473 & 25.1990 \\
\hline 5 & 4.80 & 27.9150 & 27.3408 & 27.1350 & 27.8084 & 27.4388 & 27.3967 & 27.7304 & 27.1492 & 26.9241 & 27.6184 & 27.2443 & 27.1890 \\
\hline $6 n$ & 3.78 & 28.3733 & 27.8471 & 27.7191 & 28.3531 & 28.0439 & 27.9755 & 28.2854 & 27.7562 & 27.6120 & 28.2638 & 27.9504 & 27.8700 \\
\hline $6 x$ & 4.00 & 28.4810 & 27.9440 & 27.7688 & 28.3646 & 28.0734 & 28.0158 & 28.4095 & 27.8746 & 27.6822 & 28.2873 & 28.0008 & 27.9311 \\
\hline $7^{*}$ & 2.92 & 29.7733 & 29.2972 & 29.0791 & 29.6796 & 29.4442 & 29.3374 & 29.6015 & 29.1209 & 28.8636 & 29.4987 & 29.2634 & 29.1265 \\
\hline $7^{*}$ & 2.92 & 29.6135 & 29.1093 & 28.8379 & 29.5038 & 29.2447 & 29.1172 & 29.4536 & 28.9449 & 28.6407 & 29.3334 & 29.0746 & 28.9232 \\
\hline \multicolumn{14}{|c|}{ mPW1PW91 } \\
\hline & & \multicolumn{6}{|c|}{ Gas phase } & \multicolumn{6}{|c|}{ PCM } \\
\hline & Exp & 6-31G* & $6-31 G * *$ & $6-31+G * *$ & 6-311G* & $6-311 G * *$ & $6-311+G * *$ & 6-31G* & $6-31 G * *$ & $6-31+G * *$ & 6-311G* & $6-311 G * *$ & $6-311+G * *$ \\
\hline \multicolumn{14}{|c|}{${ }^{13} \mathrm{C}$} \\
\hline 1 & 102.7 & 91.2768 & 91.9979 & 90.9928 & 80.6556 & 79.9756 & 80.0299 & 91.5844 & 92.3035 & 91.2275 & 80.8753 & 80.1938 & 80.2082 \\
\hline 2 & 59.1 & 135.0827 & 135.1910 & 135.2454 & 126.9323 & 126.6030 & 125.2421 & 135.0458 & 135.1573 & 135.1598 & 126.8508 & 126.5295 & 125.1393 \\
\hline 3 & 127.3 & 66.1913 & 66.5706 & 66.0506 & 50.5020 & 49.5266 & 48.8509 & 67.5512 & 67.9248 & 67.5824 & 51.8926 & 50.9359 & 50.3607 \\
\hline 4 & 135.9 & 62.7612 & 63.2334 & 62.6116 & 46.9775 & 45.9945 & 44.8297 & 61.2070 & 61.6828 & 60.8556 & 45.1827 & 44.2368 & 42.9466 \\
\hline 5 & 71.6 & 121.3073 & 122.0978 & 121.2966 & 112.0457 & 111.4929 & 111.2084 & 121.3316 & 122.1196 & 121.2473 & 111.9970 & 111.4319 & 111.1333 \\
\hline 6 & 70.8 & 123.0953 & 124.3608 & 124.4401 & 114.2975 & 113.6522 & 113.4799 & 123.1771 & 124.4352 & 124.3348 & 114.2237 & 113.5553 & 113.3867 \\
\hline 7 & 52.4 & 142.8825 & 144.3823 & 143.2532 & 135.4817 & 134.5484 & 132.7608 & 142.7485 & 144.2276 & 142.7613 & 135.1901 & 134.2252 & 132.2548 \\
\hline \multicolumn{14}{|l|}{${ }^{1} \mathrm{H}$} \\
\hline 1 & 5.02 & 27.3838 & 26.8178 & 26.6054 & 27.3372 & 26.9566 & 26.9078 & 27.3106 & 26.7404 & 26.5063 & 27.2437 & 26.8651 & 26.8043 \\
\hline 3 & 5.33 & 26.6993 & 26.0512 & 25.8946 & 26.6297 & 26.1608 & 26.1632 & 26.6397 & 25.9914 & 25.8312 & 26.5653 & 26.1033 & 26.0993 \\
\hline 4 & 6.40 & 25.8731 & 25.1863 & 25.0041 & 25.7892 & 25.2683 & 25.2379 & 25.6568 & 24.9716 & 24.7774 & 25.5734 & 25.0608 & 25.0184 \\
\hline 5 & 4.80 & 27.8168 & 27.2414 & 27.0639 & 27.7196 & 27.3307 & 27.3031 & 27.6203 & 27.0371 & 26.8446 & 27.5214 & 27.1277 & 27.0893 \\
\hline $6 n$ & 3.78 & 28.3062 & 27.7804 & 27.6756 & 28.2766 & 27.9551 & 27.8992 & 28.2107 & 27.6817 & 27.5640 & 28.1824 & 27.8565 & 27.7906 \\
\hline $6 x$ & 4.00 & 28.4297 & 27.8960 & 27.7442 & 28.3131 & 28.0127 & 27.9684 & 28.3470 & 27.8154 & 27.6488 & 28.2272 & 27.9315 & 27.8766 \\
\hline $7^{*}$ & 2.92 & 29.6799 & 29.2053 & 29.0092 & 29.5979 & 29.3455 & 29.2509 & 29.4946 & 29.0148 & 28.7850 & 29.4076 & 29.1551 & 29.0341 \\
\hline
\end{tabular}




\begin{tabular}{|c|}
\hline 2.92 \\
\hline
\end{tabular}

\section{Compound 81b}<smiles>Brc1ccc(C(Br)(Br)Br)cc1</smiles>

\begin{tabular}{|c|c|c|c|c|c|c|c|c|c|c|c|c|}
\hline \multirow[b]{3}{*}{ Exp } & \multicolumn{12}{|c|}{ B3LYP } \\
\hline & \multicolumn{6}{|c|}{ Gas phase } & \multicolumn{6}{|c|}{ PCM } \\
\hline & 6-31G* & 6-31G** & $6-31+\mathrm{G} * *$ & 6-311G* & $6-311 G * *$ & $6-311+G * *$ & 6-31G* & $6-31 G * *$ & $6-31+\mathrm{G} * *$ & 6-311G* & $6-311 G * *$ & $6-311+\mathrm{G} * *$ \\
\hline \multicolumn{13}{|l|}{${ }^{13} \mathrm{C}$} \\
\hline 1 & 86.0722 & 86.8160 & 85.3536 & 72.8735 & 72.2259 & 72.5798 & 86.3263 & 87.0681 & 85.5325 & 73.0668 & 72.4187 & 72.7440 \\
\hline 2 & 135.5039 & 135.6458 & 135.4781 & 125.8509 & 125.5819 & 124.2772 & 135.6023 & 135.7478 & 135.5252 & 125.9099 & 125.6480 & 124.3165 \\
\hline 3 & 65.3123 & 65.7746 & 65.4033 & 47.7436 & 46.7757 & 46.0106 & 66.6061 & 67.0612 & 66.9089 & 49.1143 & 48.1627 & 47.5127 \\
\hline 4 & 60.0397 & 60.6244 & 60.0120 & 42.4716 & 41.5200 & 40.2792 & 58.4981 & 59.0854 & 58.1639 & 40.6027 & 39.6861 & 38.2393 \\
\hline 5 & 117.9184 & 118.7648 & 117.8600 & 107.3844 & 106.8248 & 106.4739 & 117.9051 & 118.7503 & 117.7432 & 107.2805 & 106.7112 & 106.3173 \\
\hline 6 & 120.9332 & 122.2933 & 122.0150 & 110.6792 & 109.9939 & 109.8639 & 121.1375 & 122.4859 & 122.1183 & 110.7707 & 110.0623 & 109.9647 \\
\hline 7 & 139.7284 & 141.2752 & 140.2754 & 131.4013 & 130.4405 & 128.5525 & 139.8615 & 141.3861 & 140.0954 & 131.4317 & 130.4410 & 128.3599 \\
\hline \multicolumn{13}{|l|}{${ }^{1} \mathrm{H}$} \\
\hline 1 & 27.4132 & 26.8576 & 26.6585 & 27.3354 & 27.0153 & 26.9755 & 27.3748 & 26.8158 & 26.5956 & 27.2763 & 26.9555 & 26.9065 \\
\hline 3 & 26.8730 & 26.2318 & 26.0255 & 26.7724 & 26.3367 & 26.3126 & 26.8095 & 26.1679 & 25.9521 & 26.7024 & 26.2732 & 26.2410 \\
\hline 4 & 25.9710 & 25.2871 & 25.0710 & 25.8501 & 25.3589 & 25.3161 & 25.7641 & 25.0820 & 24.8469 & 25.6385 & 25.1561 & 25.0962 \\
\hline 5 & 27.8567 & 27.2901 & 27.0911 & 27.7526 & 27.3964 & 27.3618 & 27.6754 & 27.1015 & 26.8789 & 27.5629 & 27.2011 & 27.1514 \\
\hline $6 n$ & 28.6089 & 28.0842 & 27.9589 & 28.5779 & 28.2660 & 28.1958 & 28.4978 & 27.9698 & 27.8368 & 28.4704 & 28.1547 & 28.0766 \\
\hline $6 x$ & 28.5667 & 28.0295 & 27.8559 & 28.4556 & 28.1589 & 28.1006 & 28.4921 & 27.9570 & 27.7669 & 28.3758 & 28.0843 & 28.0144 \\
\hline $7^{*}$ & 29.4761 & 28.9782 & 28.7189 & 29.3701 & 29.1218 & 28.9981 & 29.3554 & 28.8532 & 28.5620 & 29.2394 & 28.9915 & 28.8457 \\
\hline $7^{*}$ & 29.7165 & 29.2390 & 28.9955 & 29.6087 & 29.3753 & 29.2676 & 29.5463 & 29.0633 & 28.7809 & 29.4276 & 29.1940 & 29.0580 \\
\hline & \multicolumn{12}{|c|}{ mPW1PW91 } \\
\hline & \multicolumn{6}{|c|}{ Gas phase } & \multicolumn{6}{|c|}{ PCM } \\
\hline Exp & 6-31G* & $6-31 G * *$ & $6-31+G * *$ & 6-311G* & $6-311 G * *$ & $6-311+G * *$ & 6-31G* & $6-31 G * *$ & $6-31+G * *$ & 6-311G* & $6-311 G * *$ & $6-311+G * *$ \\
\hline \multicolumn{13}{|l|}{${ }^{13} \mathrm{C}$} \\
\hline 1 & 90.5221 & 91.2379 & 90.1153 & 79.5599 & 78.9326 & 79.1812 & 90.7799 & 91.4937 & 90.2999 & 79.7419 & 79.1149 & 79.3364 \\
\hline 2 & 139.4965 & 139.6291 & 139.6551 & 131.2838 & 131.0015 & 129.7453 & 139.6140 & 139.7503 & 139.7112 & 131.3455 & 131.0709 & 129.7826 \\
\hline 3 & 67.8957 & 68.2853 & 67.9480 & 52.5062 & 51.5097 & 50.7992 & 69.2266 & 69.6105 & 69.4625 & 53.8735 & 52.8971 & 52.2833 \\
\hline 4 & 62.7543 & 63.2475 & 62.7019 & 47.1338 & 46.1420 & 44.9802 & 61.1220 & 61.6182 & 60.8048 & 45.2151 & 44.2605 & 42.9268 \\
\hline 5 & 122.3405 & 123.1332 & 122.4880 & 113.2703 & 112.7157 & 112.3357 & 122.3016 & 123.0938 & 122.3525 & 113.1330 & 112.5708 & 112.1571 \\
\hline 6 & 124.9108 & 126.1966 & 126.2474 & 116.1021 & 115.4458 & 115.2614 & 125.0981 & 126.3735 & 126.3288 & 116.1640 & 115.4864 & 115.3289 \\
\hline 7 & 143.2465 & 144.7626 & 144.1205 & 136.3365 & 135.3950 & 133.6742 & 143.3503 & 144.8462 & 143.9285 & 136.3336 & 135.3658 & 133.4745 \\
\hline \multicolumn{13}{|l|}{${ }^{1} \mathbf{H}$} \\
\hline 1 & 27.3209 & 26.7698 & 26.6091 & 27.2841 & 26.9344 & 26.9099 & 27.2765 & 26.7215 & 26.5417 & 27.2201 & 26.8700 & 26.8374 \\
\hline 3 & 26.6754 & 26.0331 & 25.8553 & 26.6199 & 26.1476 & 26.1379 & 26.6037 & 25.9610 & 25.7747 & 26.5442 & 26.0786 & 26.0604 \\
\hline 4 & 25.7935 & 25.1072 & 24.9175 & 25.7035 & 25.1800 & 25.1435 & 25.5716 & 24.8869 & 24.6821 & 25.4809 & 24.9660 & 24.9148 \\
\hline 5 & 27.7529 & 27.1846 & 27.0166 & 27.6585 & 27.2834 & 27.2628 & 27.5601 & 26.9836 & 26.7963 & 27.4606 & 27.0796 & 27.0462 \\
\hline
\end{tabular}




\begin{tabular}{lllllllllllll}
\hline $6 n$ & 28.5505 & 28.0265 & 27.9195 & 28.5097 & 28.1845 & 28.1215 & 28.4318 & 27.9041 & 27.7925 & 28.3978 & 28.0685 & 27.9993 \\
$6 \mathrm{x}$ & 28.5160 & 27.9818 & 27.8249 & 28.4052 & 28.0983 & 28.0496 & 28.4303 & 27.8982 & 27.7272 & 28.3171 & 28.0155 & 27.9565 \\
$7^{*}$ & 29.3884 & 28.8920 & 28.6680 & 29.2942 & 29.0288 & 28.9233 & 29.2549 & 28.7536 & 28.5021 & 29.1546 & 28.8894 & 28.7646 \\
$7^{*}$ & 29.6151 & 29.1402 & 28.9287 & 29.5243 & 29.2742 & 29.1834 & 29.4307 & 28.9496 & 28.7049 & 29.3332 & 29.0827 & 28.9674 \\
\hline
\end{tabular}

\section{Compound 82a}

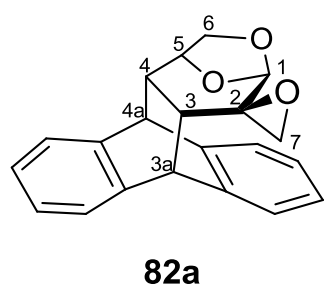

\begin{tabular}{|c|c|c|c|c|c|c|c|c|c|c|c|c|}
\hline \multirow[b]{3}{*}{ Exp } & \multicolumn{12}{|c|}{ B3LYP } \\
\hline & \multicolumn{6}{|c|}{ Gas phase } & \multicolumn{6}{|c|}{ PCM } \\
\hline & $6-31 G *$ & $6-31 G * *$ & $6-31+\mathrm{G} * *$ & 6-311G* & $6-311 G * *$ & $6-311+G * *$ & 6-31G* & $6-31 G * *$ & $6-31+G * *$ & 6-311G* & $6-311 G * *$ & $6-311+\mathrm{G} * *$ \\
\hline \multicolumn{13}{|l|}{${ }^{13} \mathrm{C}$} \\
\hline 1 & 87.4619 & 88.2062 & 86.7711 & 74.5662 & 73.9394 & 73.9496 & 87.7762 & 88.5193 & 86.9947 & 74.8124 & 74.1810 & 74.1544 \\
\hline 2 & 132.1543 & 132.3051 & 132.7680 & 122.7185 & 122.5136 & 120.7658 & 132.0339 & 132.1865 & 132.5636 & 122.5434 & 122.3456 & 120.5552 \\
\hline 3 & 149.0353 & 149.8075 & 149.2005 & 139.6885 & 139.6403 & 139.2062 & 149.2620 & 150.0291 & 149.4608 & 139.8609 & 139.8140 & 139.4042 \\
\hline 4 & 141.6180 & 142.4174 & 140.2576 & 130.9872 & 130.8007 & 130.2112 & 141.8602 & 142.6560 & 140.6520 & 131.2925 & 131.1025 & 130.5603 \\
\hline 5 & 112.1288 & 112.9045 & 112.2927 & 100.8540 & 100.3483 & 99.9987 & 112.2877 & 113.0620 & 112.4285 & 101.0307 & 100.5138 & 100.1489 \\
\hline 6 & 119.8092 & 121.1652 & 121.0898 & 109.4229 & 108.6537 & 108.3871 & 120.0056 & 121.3543 & 121.0874 & 109.5209 & 108.7279 & 108.4078 \\
\hline $3 a$ & 140.9538 & 141.5853 & 140.0567 & 129.7053 & 129.4207 & 129.4219 & 141.3411 & 141.9668 & 140.5230 & 130.2405 & 129.9422 & 129.9605 \\
\hline $4 a$ & 136.8437 & 137.4560 & 136.3408 & 124.3325 & 124.0096 & 123.3850 & 137.4380 & 138.0434 & 137.1088 & 125.1810 & 124.8397 & 124.2541 \\
\hline 7 & 136.5258 & 138.1178 & 137.9257 & 127.8264 & 126.8912 & 125.4568 & 136.5768 & 138.1467 & 137.6899 & 127.7724 & 126.8051 & 125.2189 \\
\hline \multicolumn{13}{|l|}{${ }^{1} \mathbf{H}$} \\
\hline 1 & 27.9724 & 27.4229 & 27.2325 & 27.9122 & 27.5901 & 27.5540 & 27.9252 & 27.3721 & 27.1618 & 27.8415 & 27.5207 & 27.4769 \\
\hline 3 & 29.6908 & 29.2631 & 29.0529 & 29.7412 & 29.4935 & 29.3345 & 29.6918 & 29.2614 & 29.0377 & 29.7397 & 29.4881 & 29.3194 \\
\hline 4 & 30.2209 & 29.8227 & 29.6169 & 30.2004 & 29.9904 & 29.8100 & 30.0863 & 29.6838 & 29.4568 & 30.0714 & 29.8580 & 29.6580 \\
\hline 5 & 27.8385 & 27.3157 & 27.1817 & 27.8402 & 27.5099 & 27.4894 & 27.6657 & 27.1359 & 26.9807 & 27.6613 & 27.3251 & 27.2922 \\
\hline $6 n$ & 28.5735 & 28.0674 & 27.9169 & 28.5482 & 28.2257 & 28.1378 & 28.4523 & 27.9435 & 27.7730 & 28.4284 & 28.1017 & 27.9999 \\
\hline $6 x$ & 28.5685 & 28.0363 & 27.8161 & 28.4287 & 28.1426 & 28.0741 & 28.5124 & 27.9821 & 27.7512 & 28.3707 & 28.0888 & 28.0107 \\
\hline $3 a$ & 28.3007 & 27.8493 & 27.7301 & 28.2372 & 28.0246 & 27.8959 & 28.1646 & 27.7073 & 27.5550 & 28.0810 & 27.8654 & 27.7218 \\
\hline $4 a$ & 28.3009 & 27.8792 & 27.6366 & 28.1639 & 27.9596 & 27.8451 & 28.0933 & 27.6612 & 27.3883 & 27.9403 & 27.7285 & 27.6021 \\
\hline $7 s$ & 29.9972 & 29.5280 & 29.3058 & 29.9079 & 29.6744 & 29.5368 & 29.8532 & 29.3789 & 29.1268 & 29.7543 & 29.5194 & 29.3631 \\
\hline $7 a$ & 29.1344 & 28.6143 & 28.4561 & 29.1110 & 28.8221 & 28.7996 & 28.9716 & 28.4478 & 28.2499 & 28.9388 & 28.6512 & 28.6020 \\
\hline
\end{tabular}

\begin{tabular}{|c|c|c|c|c|c|c|c|c|c|c|c|c|}
\hline \multirow[b]{3}{*}{ Exp } & \multicolumn{12}{|c|}{ mPW1PW91 } \\
\hline & \multicolumn{6}{|c|}{ Gas phase } & \multicolumn{6}{|c|}{ PCM } \\
\hline & 6-31G* & $6-31 G * *$ & $6-31+\mathrm{G} * *$ & 6-311G* & 6-311G** & $6-311+G * *$ & 6-31G* & $6-31 \mathrm{G} * *$ & $6-31+\mathrm{G} * *$ & 6-311G* & 6-311G** & $6-311+G * *$ \\
\hline \multicolumn{13}{|l|}{${ }^{13} \mathrm{C}$} \\
\hline 1 & 91.8929 & 92.6066 & 91.5115 & 81.1315 & 80.5279 & 80.5182 & 92.2159 & 92.9285 & 91.7492 & 81.3684 & 80.7609 & 80.7196 \\
\hline 2 & 136.2602 & 136.3959 & 136.9953 & 128.2233 & 128.0085 & 126.3383 & 136.1537 & 136.2910 & 136.8097 & 128.0581 & 127.8507 & 126.1337 \\
\hline
\end{tabular}




\begin{tabular}{|c|c|c|c|c|c|c|c|c|c|c|c|c|}
\hline 3 & 154.4765 & 155.1764 & 154.3627 & 145.9771 & 145.9264 & 145.5412 & 154.7060 & 155.4006 & 154.6271 & 146.1424 & 146.0923 & 145.7302 \\
\hline 4 & 147.1675 & 147.8746 & 145.7630 & 137.3371 & 137.1467 & 136.6145 & 147.3930 & 148.0973 & 146.1322 & 137.5979 & 137.4047 & 136.9124 \\
\hline 5 & 116.8393 & 117.5610 & 117.2311 & 107.1226 & 106.6195 & 106.2214 & 116.9811 & 117.7028 & 117.3487 & 107.2637 & 106.7506 & 106.3380 \\
\hline 6 & 123.9727 & 125.2549 & 125.3282 & 115.0282 & 114.2831 & 114.0233 & 124.1530 & 125.4296 & 125.3198 & 115.0926 & 114.3257 & 114.0195 \\
\hline $3 a$ & 145.6763 & 146.2346 & 144.7194 & 135.6167 & 135.3303 & 135.2453 & 146.0881 & 146.6410 & 145.1959 & 136.1513 & 135.8529 & 135.7776 \\
\hline $4 a$ & 141.7958 & 142.3367 & 141.1347 & 130.6235 & 130.3060 & 129.7838 & 142.4111 & 142.9463 & 141.8942 & 131.4434 & 131.1100 & 130.6211 \\
\hline 7 & 140.2021 & 141.7472 & 141.7391 & 133.0126 & 132.0934 & 130.8205 & 140.2345 & 141.7593 & 141.4975 & 132.9286 & 131.9813 & 130.5722 \\
\hline \multicolumn{13}{|l|}{${ }^{1} \mathbf{H}$} \\
\hline 1 & 27.9059 & 27.3597 & 27.1958 & 27.8830 & 27.5267 & 27.4995 & 27.8519 & 27.3018 & 27.1205 & 27.8067 & 27.4521 & 27.4190 \\
\hline 3 & 29.6237 & 29.1860 & 29.0185 & 29.6257 & 29.3752 & 29.2460 & 29.6242 & 29.1836 & 29.0026 & 29.6240 & 29.3695 & 29.2293 \\
\hline 4 & 30.1824 & 29.7836 & 29.5970 & 30.0993 & 29.8918 & 29.7334 & 30.0420 & 29.6386 & 29.4336 & 29.9677 & 29.7566 & 29.5788 \\
\hline 5 & 27.7614 & 27.2352 & 27.1128 & 27.7505 & 27.4059 & 27.3875 & 27.5760 & 27.0420 & 26.9028 & 27.5634 & 27.2125 & 27.1834 \\
\hline $6 n$ & 28.5260 & 28.0193 & 27.9017 & 28.4914 & 28.1542 & 28.0830 & 28.3935 & 27.8839 & 27.7501 & 28.3642 & 28.0226 & 27.9388 \\
\hline $6 x$ & 28.5231 & 27.9924 & 27.8029 & 28.3879 & 28.0905 & 28.0441 & 28.4557 & 27.9270 & 27.7282 & 28.3206 & 28.0275 & 27.9723 \\
\hline $3 a$ & 28.1316 & 27.6871 & 27.5667 & 28.0434 & 27.8339 & 27.7127 & 27.9814 & 27.5304 & 27.3806 & 27.8778 & 27.6648 & 27.5294 \\
\hline $4 a$ & 28.1194 & 27.7019 & 27.4957 & 27.9619 & 27.7496 & 27.6575 & 27.8929 & 27.4638 & 27.2321 & 27.7263 & 27.5058 & 27.4022 \\
\hline $7 s$ & 29.9157 & 29.4484 & 29.2701 & 29.8445 & 29.5916 & 29.4663 & 29.7583 & 29.2854 & 29.0813 & 29.6813 & 29.4268 & 29.2855 \\
\hline $7 a$ & 29.0703 & 28.5549 & 28.4117 & 29.0435 & 28.7433 & 28.7327 & 28.8947 & 28.3753 & 28.1995 & 28.8627 & 28.5639 & 28.5307 \\
\hline
\end{tabular}

\section{Compound 82b}

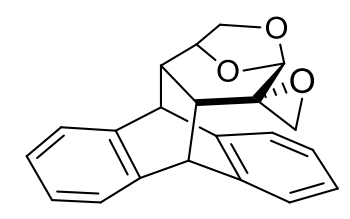

$82 b$

\begin{tabular}{|c|c|c|c|c|c|c|c|c|c|c|c|c|c|}
\hline & & \multicolumn{12}{|c|}{ B3LYP } \\
\hline & \multirow[b]{2}{*}{ Exp } & \multicolumn{6}{|c|}{ Gas phase } & \multicolumn{6}{|c|}{ PCM } \\
\hline & & 6-31G* & $6-31 G * *$ & $6-31+G * *$ & 6-311G* & 6-311G** & $6-311+G * *$ & 6-31G* & 6-31G** & $6-31+G * *$ & 6-311G* & $6-311 G * *$ & $6-311+\mathrm{G} * *$ \\
\hline \multicolumn{14}{|c|}{${ }^{13} \mathrm{C}$} \\
\hline 1 & 103.5 & 86.5680 & 87.3197 & 85.7341 & 73.0929 & 72.5124 & 72.7394 & 86.7780 & 87.5286 & 85.8132 & 73.2297 & 72.6447 & 72.8048 \\
\hline 2 & 56.0 & 134.4599 & 134.6221 & 134.4799 & 124.6386 & 124.4615 & 122.8724 & 134.4212 & 134.5859 & 134.3370 & 124.5629 & 124.3928 & 122.7645 \\
\hline 3 & 35.7 & 149.4070 & 150.2195 & 150.2279 & 140.3206 & 140.2010 & 140.0228 & 149.7747 & 150.5779 & 150.6601 & 140.6741 & 140.5462 & 140.4151 \\
\hline 4 & 42.3 & 144.0702 & 144.8794 & 142.3725 & 133.7498 & 133.6003 & 133.7974 & 144.4417 & 145.2442 & 142.9086 & 134.1834 & 134.0279 & 134.2988 \\
\hline 5 & 76.2 & 113.1571 & 113.9372 & 113.4404 & 101.9076 & 101.4171 & 100.9860 & 113.2389 & 114.0198 & 113.4533 & 101.9897 & 101.4934 & 101.0413 \\
\hline 6 & 69.8 & 120.6894 & 122.0495 & 121.5275 & 110.2152 & 109.4170 & 109.4332 & 120.9495 & 122.3011 & 121.6277 & 110.4125 & 109.5900 & 109.5682 \\
\hline $3 a$ & 45.1 & 142.6602 & 143.2895 & 141.8697 & 131.2307 & 131.0293 & 131.0766 & 142.7968 & 143.4193 & 142.0370 & 131.4634 & 131.2497 & 131.2982 \\
\hline $4 a$ & 51.0 & 136.7753 & 137.3896 & 136.4134 & 124.2573 & 123.9377 & 123.3613 & 137.3389 & 137.9449 & 137.1650 & 125.0647 & 124.7274 & 124.1781 \\
\hline 7 & 50.1 & 140.0629 & 141.6286 & 141.1633 & 131.9534 & 131.0535 & 129.8729 & 140.1490 & 141.6934 & 141.1254 & 131.9724 & 131.0417 & 129.8022 \\
\hline \multicolumn{14}{|c|}{${ }^{1} \mathrm{H}$} \\
\hline 1 & 4.33 & 27.9818 & 27.4443 & 27.3746 & 27.9558 & 27.6592 & 27.6571 & 27.9679 & 27.4273 & 27.3454 & 27.9219 & 27.6239 & 27.6190 \\
\hline 3 & 2.61 & 29.7635 & 29.3525 & 29.1878 & 29.8034 & 29.5602 & 29.4363 & 29.6532 & 29.2368 & 29.0631 & 29.6985 & 29.4514 & 29.3170 \\
\hline 4 & 2.09 & 30.4006 & 30.0223 & 29.9577 & 30.3986 & 30.2129 & 30.1389 & 30.2620 & 29.8776 & 29.7981 & 30.2642 & 30.0732 & 29.9847 \\
\hline 5 & 4.65 & 27.8524 & 27.3392 & 27.1906 & 27.8759 & 27.5566 & 27.4806 & 27.7047 & 27.1854 & 27.0139 & 27.7208 & 27.3957 & 27.3066 \\
\hline
\end{tabular}




\begin{tabular}{llllllllllllll}
\hline 6n & 3.68 & 28.6752 & 28.1714 & 28.0522 & 28.6642 & 28.3414 & 28.2502 & 28.5444 & 28.0376 & 27.9044 & 28.5372 & 28.2108 & 28.1080 \\
6x & 3.68 & 28.6205 & 28.0875 & 27.9386 & 28.5077 & 28.2130 & 28.1786 & 28.5645 & 28.0337 & 27.8757 & 28.4509 & 28.1611 & 28.1170 \\
3a & 4.25 & 28.1606 & 27.6983 & 27.5111 & 28.0913 & 27.8781 & 27.7311 & 28.0779 & 27.6107 & 27.3937 & 27.9926 & 27.7757 & 27.6154 \\
4a & 4.29 & 28.2906 & 27.8725 & 27.7431 & 28.1560 & 27.9592 & 27.9319 & 28.0873 & 27.6586 & 27.4962 & 27.9355 & 27.7305 & 27.6884 \\
7s & 2.76 & 29.6278 & 29.1404 & 28.9876 & 29.5561 & 29.3154 & 29.2457 & 29.5266 & 29.0361 & 28.8729 & 29.4459 & 29.2059 & 29.1333 \\
7a & 2.83 & 29.6777 & 29.2130 & 29.0097 & 29.6049 & 29.3856 & 29.2947 & 29.4986 & 29.0280 & 28.7974 & 29.4180 & 29.1973 & 29.0898 \\
\hline
\end{tabular}

\begin{tabular}{|c|c|c|c|c|c|c|c|c|c|c|c|c|c|}
\hline \multirow{3}{*}{\multicolumn{2}{|c|}{ Exp }} & \multicolumn{12}{|c|}{ mPW1PW91 } \\
\hline & & \multicolumn{6}{|c|}{ Gas phase } & \multicolumn{6}{|c|}{ PCM } \\
\hline & & 6-31G* & $6-31 \mathrm{G} * *$ & $6-31+G * *$ & 6-311G* & 6-311G** & $6-311+G^{* *}$ & 6-31G* & 6-31G** & $6-31+G * *$ & 6-311G* & 6-311G** & $6-311+G * *$ \\
\hline \multicolumn{14}{|c|}{ 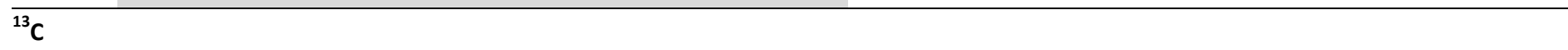 } \\
\hline 1 & 103.5 & 91.0317 & 91.7576 & 90.5631 & 79.7909 & 79.2337 & 79.4003 & 91.2483 & 91.9731 & 90.6588 & 79.9250 & 79.3636 & 79.4697 \\
\hline 2 & 56.0 & 138.5764 & 138.7257 & 138.8456 & 130.1463 & 129.9599 & 128.4793 & 138.5540 & 138.7058 & 138.7222 & 130.0834 & 129.9044 & 128.3824 \\
\hline 3 & 35.7 & 154.7906 & 155.5270 & 155.3907 & 146.4629 & 146.3416 & 146.2374 & 155.1536 & 155.8811 & 155.8160 & 146.7930 & 146.6639 & 146.6011 \\
\hline 4 & 42.3 & 149.6360 & 150.3547 & 147.8569 & 140.1109 & 139.9534 & 140.1479 & 149.9966 & 150.7095 & 148.3722 & 140.5008 & 140.3382 & 140.5962 \\
\hline 5 & 76.2 & 117.8700 & 118.5938 & 118.3988 & 108.1571 & 107.6699 & 107.2012 & 117.9342 & 118.6600 & 118.3963 & 108.2069 & 107.7154 & 107.2310 \\
\hline 6 & 69.8 & 124.8124 & 126.1009 & 125.8280 & 115.8149 & 115.0399 & 115.0061 & 125.0687 & 126.3505 & 125.9278 & 115.9874 & 115.1901 & 115.1213 \\
\hline $3 a$ & 45.1 & 147.4574 & 148.0240 & 146.5825 & 137.1527 & 136.9617 & 136.9139 & 147.6080 & 148.1682 & 146.7539 & 137.3834 & 137.1814 & 137.1353 \\
\hline $4 a$ & 51.0 & 141.7433 & 142.2870 & 141.2378 & 130.5561 & 130.2454 & 129.7492 & 142.3243 & 142.8610 & 141.9791 & 131.3312 & 131.0051 & 130.5346 \\
\hline 7 & 50.1 & 143.7170 & 145.2474 & 144.9171 & 136.9531 & 136.0712 & 135.0306 & 143.7717 & 145.2828 & 144.8504 & 136.9336 & 136.0241 & 134.9311 \\
\hline \multicolumn{14}{|c|}{${ }^{1} \mathrm{H}$} \\
\hline 1 & 4.33 & 27.9130 & 27.3797 & 27.3204 & 27.9158 & 27.5900 & 27.5996 & 27.8944 & 27.3579 & 27.2865 & 27.8781 & 27.5513 & 27.5581 \\
\hline 3 & 2.61 & 29.7182 & 29.2980 & 29.1537 & 29.7043 & 29.4611 & 29.3518 & 29.6039 & 29.1780 & 29.0255 & 29.5976 & 29.3502 & 29.2302 \\
\hline 4 & 2.09 & 30.3682 & 29.9881 & 29.9236 & 30.3030 & 30.1153 & 30.0533 & 30.2228 & 29.8363 & 29.7589 & 30.1651 & 29.9718 & 29.8957 \\
\hline 5 & 4.65 & 27.7780 & 27.2617 & 27.1251 & 27.7834 & 27.4523 & 27.3828 & 27.6190 & 27.0959 & 26.9404 & 27.6207 & 27.2836 & 27.2035 \\
\hline $6 n$ & 3.68 & 28.6339 & 28.1297 & 28.0328 & 28.6101 & 28.2721 & 28.1961 & 28.4921 & 27.9845 & 27.8772 & 28.4759 & 28.1340 & 28.0487 \\
\hline $6 x$ & 3.68 & 28.5780 & 28.0466 & 27.9129 & 28.4699 & 28.1624 & 28.1343 & 28.5115 & 27.9823 & 27.8411 & 28.4044 & 28.1021 & 28.0661 \\
\hline $3 a$ & 4.25 & 27.9982 & 27.5408 & 27.3607 & 27.9037 & 27.6874 & 27.5500 & 27.9028 & 27.4397 & 27.2334 & 27.7961 & 27.5755 & 27.4256 \\
\hline $4 a$ & 4.29 & 28.1064 & 27.6928 & 27.5836 & 27.9497 & 27.7447 & 27.7334 & 27.8845 & 27.4592 & 27.3216 & 27.7173 & 27.5034 & 27.4786 \\
\hline $7 \mathrm{~s}$ & 2.76 & 29.5479 & 29.0632 & 28.9366 & 29.4862 & 29.2285 & 29.1749 & 29.4351 & 28.9469 & 28.8121 & 29.3672 & 29.1101 & 29.0543 \\
\hline $7 a$ & 2.83 & 29.5844 & 29.1248 & 28.9534 & 29.5175 & 29.2844 & 29.2105 & 29.3909 & 28.9247 & 28.7315 & 29.3210 & 29.0863 & 28.9987 \\
\hline
\end{tabular}




\section{Compound 83a}

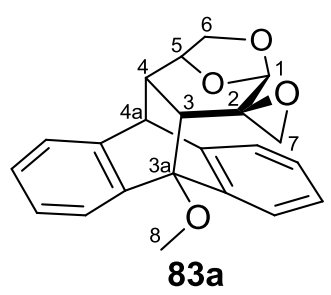

\begin{tabular}{|c|c|c|c|c|c|c|c|c|c|c|c|c|}
\hline \multirow[b]{3}{*}{ Exp } & \multicolumn{12}{|c|}{ B3LYP } \\
\hline & \multicolumn{6}{|c|}{ Gas phase } & \multicolumn{6}{|c|}{ PCM } \\
\hline & $6-31 G *$ & $6-31 \mathrm{G} * *$ & $6-31+G^{* *}$ & 6-311G* & $6-311 G * *$ & $6-311+G * *$ & 6-31G* & $6-31 G * *$ & $6-31+G * *$ & 6-311G* & $6-311 G * *$ & $6-311+G * *$ \\
\hline \multicolumn{13}{|l|}{${ }^{13} \mathrm{C}$} \\
\hline 1 & 86.0089 & 86.7565 & 85.5444 & 73.6173 & 72.9555 & 72.9851 & 86.3368 & 87.0832 & 85.8001 & 73.9048 & 73.2401 & 72.8020 \\
\hline 2 & 132.4072 & 132.5817 & 133.2300 & 123.5477 & 123.3640 & 122.0020 & 132.2296 & 132.4035 & 132.9752 & 123.3432 & 123.1624 & 120.4870 \\
\hline 3 & 150.1604 & 150.8797 & 152.2084 & 133.9127 & 133.8208 & 134.1279 & 150.4712 & 151.1839 & 152.4284 & 133.9620 & 133.8514 & 141.8622 \\
\hline 4 & 140.6967 & 141.4972 & 139.7617 & 128.8833 & 128.6423 & 128.9316 & 140.9967 & 141.7911 & 140.1881 & 129.1797 & 128.9324 & 129.7623 \\
\hline 5 & 111.6863 & 112.4487 & 111.7081 & 100.8831 & 100.3601 & 100.0825 & 111.8823 & 112.6426 & 111.8900 & 101.0980 & 100.5632 & 99.7392 \\
\hline 6 & 120.3907 & 121.7511 & 121.3490 & 109.9417 & 109.1753 & 108.7318 & 120.6179 & 121.9701 & 121.3855 & 110.0639 & 109.2721 & 109.2199 \\
\hline $3 a$ & 105.1212 & 105.1726 & 105.7809 & 86.5262 & 86.6114 & 85.9073 & 105.1094 & 105.1601 & 105.6597 & 86.6918 & 86.7585 & 91.0179 \\
\hline $4 a$ & 137.8116 & 138.4220 & 136.4961 & 124.5107 & 124.1743 & 123.8501 & 138.4277 & 139.0311 & 137.2919 & 125.4497 & 125.0933 & 125.8127 \\
\hline 7 & 137.5873 & 139.1522 & 139.4771 & 129.7992 & 128.9183 & 127.5140 & 137.6217 & 139.1691 & 139.2606 & 129.7391 & 128.8340 & 126.2392 \\
\hline 8 & 136.8946 & 138.7123 & 138.2620 & 125.6263 & 124.8570 & 124.3381 & 136.7418 & 138.5528 & 137.9262 & 125.5296 & 124.7275 & 127.5289 \\
\hline \multicolumn{13}{|l|}{${ }^{1} \mathrm{H}$} \\
\hline 1 & 28.0326 & 27.4904 & 27.3549 & 28.0797 & 27.7480 & 27.7090 & 27.9933 & 27.4476 & 27.2984 & 28.0147 & 27.6852 & 27.6149 \\
\hline 3 & 29.1551 & 28.7031 & 28.6093 & 29.6772 & 29.4396 & 29.3109 & 29.0746 & 28.6180 & 28.5166 & 29.7217 & 29.4812 & 28.7359 \\
\hline 4 & 30.1883 & 29.7796 & 29.5144 & 30.3895 & 30.1618 & 30.0863 & 30.0424 & 29.6287 & 29.3497 & 30.2473 & 30.0180 & 29.5666 \\
\hline 5 & 27.7850 & 27.2620 & 27.0350 & 27.9582 & 27.6255 & 27.6052 & 27.6040 & 27.0736 & 26.8322 & 27.7639 & 27.4249 & 27.1566 \\
\hline $6 n$ & 28.5567 & 28.0481 & 27.9158 & 28.6747 & 28.3525 & 28.2947 & 28.4279 & 27.9163 & 27.7648 & 28.5523 & 28.2261 & 27.9626 \\
\hline $6 x$ & 28.5369 & 27.9989 & 27.7665 & 28.5199 & 28.2310 & 28.1409 & 28.4786 & 27.9422 & 27.7028 & 28.4586 & 28.1741 & 27.9725 \\
\hline $4 a$ & 28.4539 & 28.0246 & 27.8172 & 28.2929 & 28.0812 & 28.0595 & 28.2319 & 27.7915 & 27.5539 & 28.0594 & 27.8401 & 27.8183 \\
\hline $7 s$ & 30.2083 & 29.7476 & 29.5712 & 30.1510 & 29.9078 & 29.7398 & 30.0711 & 29.6045 & 29.4030 & 29.9901 & 29.7463 & 29.6357 \\
\hline $7 a$ & 28.2411 & 27.6139 & 27.4758 & 28.1599 & 27.7348 & 27.5646 & 28.1948 & 27.5672 & 27.4014 & 28.0376 & 27.6134 & 27.7027 \\
\hline 8 & 28.3499 & 27.7941 & 27.6474 & 28.2834 & 27.9485 & 27.9553 & 28.3194 & 27.7654 & 27.6162 & 28.2205 & 27.8885 & 27.8599 \\
\hline
\end{tabular}

\begin{tabular}{|c|c|c|c|c|c|c|c|c|c|c|c|c|}
\hline \multirow[b]{3}{*}{ Exp } & \multicolumn{12}{|c|}{ mPW1PW91 } \\
\hline & \multicolumn{6}{|c|}{ Gas phase } & \multicolumn{6}{|c|}{ PCM } \\
\hline & 6-31G* & 6-31G** & $6-31+G * *$ & 6-311G* & $6-311 G * *$ & $6-311+G * *$ & 6-31G* & 6-31G** & $6-31+\mathrm{G} * *$ & 6-311G* & 6-311G** & $6-311+G * *$ \\
\hline \multicolumn{13}{|l|}{${ }^{13} \mathrm{C}$} \\
\hline 1 & 90.4651 & 91.1804 & 90.2676 & 80.1876 & 79.5522 & 79.4942 & 90.8046 & 91.5188 & 90.5219 & 80.4697 & 79.8331 & 79.4007 \\
\hline 2 & 136.6146 & 136.7783 & 137.5072 & 129.1149 & 128.9202 & 127.5703 & 136.4461 & 136.6088 & 137.2730 & 128.9076 & 128.7177 & 126.1384 \\
\hline 3 & 155.4687 & 156.1203 & 157.0803 & 140.3455 & 140.2545 & 140.5328 & 155.7737 & 156.4196 & 157.4884 & 140.4178 & 140.2926 & 147.9470 \\
\hline 4 & 146.2897 & 147.0021 & 145.2041 & 135.2728 & 135.0383 & 135.2523 & 146.5703 & 147.2777 & 145.6450 & 135.5248 & 135.2819 & 136.2182 \\
\hline 5 & 116.4179 & 117.1271 & 116.6167 & 107.1617 & 106.6428 & 106.3495 & 116.5967 & 117.3053 & 116.7697 & 107.3353 & 106.8070 & 105.9565 \\
\hline
\end{tabular}




\begin{tabular}{|c|c|c|c|c|c|c|c|c|c|c|c|c|}
\hline 6 & 124.5457 & 125.8320 & 125.6024 & 115.5505 & 114.8078 & 114.3770 & 124.7547 & 126.0346 & 125.6152 & 115.6346 & 114.8681 & 114.8270 \\
\hline 3a & 109.4791 & 109.5233 & 110.2681 & 92.7443 & 92.8298 & 91.9596 & 109.4675 & 109.5110 & 110.2653 & 92.9191 & 92.9760 & 97.0740 \\
\hline $4 a$ & 142.8215 & 143.3608 & 141.3854 & 130.8257 & 130.4945 & 130.2191 & 143.4586 & 143.9927 & 142.1869 & 131.7413 & 131.3906 & 132.1391 \\
\hline 7 & 141.2723 & 142.7852 & 143.1745 & 134.8599 & 133.9867 & 132.7060 & 141.3038 & 142.8000 & 142.9682 & 134.7754 & 133.8828 & 131.5545 \\
\hline 8 & 140.5242 & 142.2588 & 141.7336 & 130.5930 & 129.8547 & 129.3427 & 140.3617 & 142.0906 & 141.4594 & 130.4823 & 129.7088 & 132.3625 \\
\hline \multicolumn{13}{|l|}{${ }^{1} \mathrm{H}$} \\
\hline 1 & 27.9633 & 27.4248 & 27.3135 & 28.0479 & 27.6829 & 27.6454 & 27.9180 & 27.3757 & 27.2520 & 27.9770 & 27.6149 & 27.5570 \\
\hline 3 & 29.0932 & 28.6328 & 28.5455 & 29.5409 & 29.3039 & 29.1892 & 29.0102 & 28.5447 & 28.4387 & 29.5847 & 29.3462 & 28.6516 \\
\hline 4 & 30.1458 & 29.7391 & 29.4815 & 30.2876 & 30.0660 & 29.9951 & 29.9946 & 29.5826 & 29.3053 & 30.1426 & 29.9198 & 29.4935 \\
\hline 5 & 27.7069 & 27.1804 & 26.9604 & 27.8737 & 27.5259 & 27.5035 & 27.5123 & 26.9775 & 26.7414 & 27.6700 & 27.3159 & 27.0544 \\
\hline $6 n$ & 28.5093 & 27.9999 & 27.8930 & 28.6173 & 28.2810 & 28.2283 & 28.3692 & 27.8565 & 27.7320 & 28.4867 & 28.1466 & 27.8957 \\
\hline $6 x$ & 28.4888 & 27.9521 & 27.7505 & 28.4794 & 28.1788 & 28.1017 & 28.4187 & 27.8838 & 27.6737 & 28.4076 & 28.1119 & 27.9258 \\
\hline $4 a$ & 28.2733 & 27.8506 & 27.6617 & 28.0931 & 27.8740 & 27.8589 & 28.0322 & 27.5972 & 27.3818 & 27.8476 & 27.6202 & 27.6194 \\
\hline $7 \mathrm{~s}$ & 30.1270 & 29.6694 & 29.5308 & 30.0819 & 29.8232 & 29.6743 & 29.9787 & 29.5143 & 29.3552 & 29.9109 & 29.6512 & 29.5625 \\
\hline $7 a$ & 28.1960 & 27.5737 & 27.4412 & 28.0872 & 27.6553 & 27.4947 & 28.1452 & 27.5227 & 27.3732 & 27.9611 & 27.5305 & 27.6310 \\
\hline 8 & 28.2867 & 27.7333 & 27.6128 & 28.2122 & 27.8755 & 27.8879 & 28.2514 & 27.6997 & 27.5783 & 28.1445 & 27.8104 & 27.7840 \\
\hline
\end{tabular}

\section{Compound 83b}

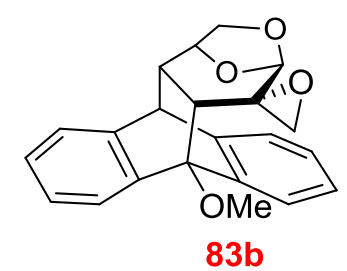

\begin{tabular}{|c|c|c|c|c|c|c|c|c|c|c|c|c|c|}
\hline \multirow{3}{*}{\multicolumn{2}{|c|}{ Exp }} & \multicolumn{12}{|c|}{ B3LYP } \\
\hline & & \multicolumn{6}{|c|}{ Gas phase } & \multicolumn{6}{|c|}{ PCM } \\
\hline & & 6-31G* & $6-31 G * *$ & $6-31+G * *$ & 6-311G* & $6-311 G * *$ & $6-311+G * *$ & $6-31 G *$ & $6-31 G * *$ & $6-31+G * *$ & 6-311G* & $6-311 G * *$ & $6-311+G * *$ \\
\hline \multicolumn{14}{|l|}{${ }^{13} \mathrm{C}$} \\
\hline 1 & 104.9 & 85.3390 & 86.0929 & 84.8569 & 71.9802 & 71.3683 & 71.2643 & 85.6117 & 86.3643 & 85.0194 & 72.1863 & 71.5700 & 71.4049 \\
\hline 2 & 55.8 & 134.5661 & 134.7551 & 133.9913 & 124.8478 & 124.6759 & 123.0831 & 134.4681 & 134.6539 & 133.8219 & 124.7355 & 124.5639 & 122.9276 \\
\hline 3 & 37.2 & 146.9596 & 147.6823 & 146.0690 & 137.2589 & 137.1873 & 137.6246 & 147.2050 & 147.9216 & 146.2938 & 137.4523 & 137.3759 & 137.8462 \\
\hline 4 & 45.3 & 141.5520 & 142.3473 & 139.2865 & 131.0128 & 130.7894 & 131.3761 & 141.8933 & 142.6832 & 139.8149 & 131.4127 & 131.1845 & 131.8557 \\
\hline 5 & 77.2 & 112.8264 & 113.5943 & 113.2843 & 101.5906 & 101.0646 & 100.6333 & 112.9633 & 113.7305 & 113.3517 & 101.7351 & 101.2000 & 100.7397 \\
\hline 6 & 69.9 & 120.3556 & 121.7089 & 121.7800 & 109.9217 & 109.1319 & 108.5707 & 120.6216 & 121.9672 & 121.9052 & 110.0981 & 109.2826 & 108.6969 \\
\hline $3 a$ & 87.0 & 100.8212 & 100.8768 & 100.9375 & 87.3423 & 87.4528 & 86.1433 & 100.8668 & 100.9203 & 100.9440 & 87.3768 & 87.4824 & 86.1337 \\
\hline $4 a$ & 50.5 & 137.2122 & 137.8129 & 136.2295 & 124.8006 & 124.4568 & 124.1257 & 137.8453 & 138.4388 & 137.0673 & 125.7009 & 125.3388 & 125.0316 \\
\hline 7 & 49.7 & 140.4750 & 142.0120 & 141.3054 & 132.6468 & 131.7401 & 130.2430 & 140.4853 & 142.0092 & 141.1679 & 132.5983 & 131.6684 & 130.1056 \\
\hline 8 & 56.4 & 133.4710 & 135.3754 & 134.8633 & 125.5905 & 124.8822 & 124.7021 & 133.4842 & 135.3790 & 134.7208 & 125.4610 & 124.7324 & 124.6035 \\
\hline \multicolumn{14}{|l|}{${ }^{1} \mathbf{H}$} \\
\hline 1 & 4.26 & 28.0911 & 27.5587 & 27.3869 & 28.0630 & 27.7582 & 27.6893 & 28.0831 & 27.5476 & 27.3644 & 28.0342 & 27.7290 & 27.6574 \\
\hline 3 & 2.89 & 29.5979 & 29.2003 & 28.9964 & 29.6483 & 29.4118 & 29.2196 & 29.5439 & 29.1412 & 28.9269 & 29.5953 & 29.3540 & 29.1507 \\
\hline 4 & 2.00 & 30.6323 & 30.2275 & 30.1370 & 30.6073 & 30.3949 & 30.2803 & 30.4927 & 30.0829 & 29.9800 & 30.4757 & 30.2595 & 30.1331 \\
\hline 5 & 4.75 & 27.9093 & 27.3903 & 27.2933 & 27.9142 & 27.5892 & 27.5557 & 27.7430 & 27.2168 & 27.0962 & 27.7401 & 27.4084 & 27.3605 \\
\hline $6 n$ & 3.70 & 28.7658 & 28.2602 & 28.1178 & 28.7572 & 28.4348 & 28.3658 & 28.6321 & 28.1233 & 27.9666 & 28.6277 & 28.3016 & 28.2199 \\
\hline
\end{tabular}




\begin{tabular}{|c|c|c|c|c|c|c|c|c|c|c|c|c|c|}
\hline $6 x$ & 3.70 & 28.6806 & 28.1464 & 27.9054 & 28.5620 & 28.2634 & 28.1698 & 28.6210 & 28.0888 & 27.8409 & 28.5021 & 28.2086 & 28.1069 \\
\hline $4 a$ & 4.21 & 28.4605 & 28.0292 & 27.8957 & 28.3082 & 28.1054 & 28.0539 & 28.2502 & 27.8077 & 27.6461 & 28.0799 & 27.8690 & 27.8053 \\
\hline $7 \mathrm{~s}$ & 2.52 & 29.9249 & 29.4440 & 29.1810 & 29.8645 & 29.6239 & 29.4241 & 29.8131 & 29.3282 & 29.0537 & 29.7409 & 29.5004 & 29.2979 \\
\hline $7 a$ & 3.18 & 29.2550 & 28.7493 & 28.4890 & 29.1783 & 28.9208 & 28.7958 & 29.2094 & 28.7033 & 28.4253 & 29.1282 & 28.8723 & 28.7319 \\
\hline 8 & 4.03 & 28.2201 & 27.7073 & 27.5656 & 28.2045 & 27.8764 & 27.8495 & 28.1736 & 27.6632 & 27.5181 & 28.1555 & 27.8314 & 27.8042 \\
\hline
\end{tabular}

\begin{tabular}{|c|c|c|c|c|c|c|c|c|c|c|c|c|c|}
\hline \multirow{3}{*}{\multicolumn{2}{|c|}{ Exp }} & \multicolumn{12}{|c|}{ mPW1PW91 } \\
\hline & & \multicolumn{6}{|c|}{ Gas phase } & \multicolumn{6}{|c|}{ PCM } \\
\hline & & 6-31G* & $6-31 G * *$ & $6-31+\mathrm{G} * *$ & 6-311G* & $6-311 G * *$ & $6-311+G * *$ & $6-31 G *$ & $6-31 G * *$ & $6-31+\mathrm{G} * *$ & 6-311G* & $6-311 G * *$ & $6-311+G * *$ \\
\hline \multicolumn{14}{|l|}{${ }^{13} \mathrm{C}$} \\
\hline 1 & 104.9 & 89.8268 & 90.5513 & 89.6785 & 78.6872 & 78.0985 & 77.9579 & 90.1107 & 90.8338 & 89.8557 & 78.8933 & 78.3007 & 78.1080 \\
\hline 2 & 55.8 & 138.7415 & 138.9181 & 138.5849 & 130.3943 & 130.2157 & 128.6601 & 138.6543 & 138.8271 & 138.4341 & 130.2887 & 130.1105 & 128.5093 \\
\hline 3 & 37.2 & 152.3866 & 153.0393 & 151.3578 & 143.5022 & 143.4322 & 143.9066 & 152.6318 & 153.2787 & 151.5593 & 143.6818 & 143.6073 & 144.1137 \\
\hline 4 & 45.3 & 147.1829 & 147.8916 & 144.8057 & 137.4712 & 137.2527 & 137.7585 & 147.5092 & 148.2135 & 145.2809 & 137.8260 & 137.6035 & 138.1843 \\
\hline 5 & 77.2 & 117.5519 & 118.2649 & 118.2945 & 107.8658 & 107.3440 & 106.8826 & 117.6706 & 118.3842 & 118.3421 & 107.9728 & 107.4436 & 106.9543 \\
\hline 6 & 69.9 & 124.5015 & 125.7804 & 125.9479 & 115.5222 & 114.7549 & 114.2145 & 124.7577 & 126.0310 & 126.0505 & 115.6696 & 114.8786 & 114.3149 \\
\hline $3 a$ & 87.0 & 105.2828 & 105.3248 & 105.7285 & 93.5549 & 93.6647 & 92.2882 & 105.3246 & 105.3645 & 105.7049 & 93.5908 & 93.6952 & 92.2783 \\
\hline $4 a$ & 50.5 & 142.2076 & 142.7377 & 141.0412 & 131.1310 & 130.7941 & 130.4973 & 142.8662 & 143.3904 & 141.8691 & 132.0036 & 131.6511 & 131.3674 \\
\hline 7 & 49.7 & 144.0062 & 145.5047 & 144.9965 & 137.5134 & 136.6225 & 135.3094 & 144.0024 & 145.4890 & 144.8566 & 137.4473 & 136.5353 & 135.1647 \\
\hline 8 & 56.4 & 137.2306 & 139.0468 & 138.4576 & 130.5522 & 129.8724 & 129.6369 & 137.2432 & 139.0515 & 138.2853 & 130.4155 & 129.7176 & 129.5290 \\
\hline \multicolumn{14}{|l|}{${ }^{1} \mathrm{H}$} \\
\hline 1 & 4.26 & 28.0228 & 27.4944 & 27.3523 & 28.0232 & 27.6898 & 27.6344 & 28.0101 & 27.4781 & 27.3256 & 27.9901 & 27.6566 & 27.5991 \\
\hline 3 & 2.89 & 29.5358 & 29.1274 & 28.9248 & 29.5276 & 29.2937 & 29.0896 & 29.4805 & 29.0669 & 28.8534 & 29.4748 & 29.2360 & 29.0209 \\
\hline 4 & 2.00 & 30.6075 & 30.2041 & 30.1048 & 30.5137 & 30.3056 & 30.2023 & 30.4629 & 30.0540 & 29.9481 & 30.3801 & 30.1677 & 30.0537 \\
\hline 5 & 4.75 & 27.8357 & 27.3141 & 27.2175 & 27.8263 & 27.4872 & 27.4582 & 27.6570 & 27.1273 & 27.0110 & 27.6438 & 27.2975 & 27.2563 \\
\hline $6 n$ & 3.70 & 28.7245 & 28.2181 & 28.0981 & 28.7005 & 28.3641 & 28.3011 & 28.5798 & 28.0698 & 27.9386 & 28.5637 & 28.2232 & 28.1499 \\
\hline $6 x$ & 3.70 & 28.6367 & 28.1047 & 27.8966 & 28.5214 & 28.2118 & 28.1396 & 28.5661 & 28.0361 & 27.8221 & 28.4523 & 28.1480 & 28.0688 \\
\hline $4 a$ & 4.21 & 28.2771 & 27.8524 & 27.7379 & 28.1054 & 27.8945 & 27.8572 & 28.0482 & 27.6112 & 27.4732 & 27.8653 & 27.6456 & 27.5980 \\
\hline $7 \mathrm{~s}$ & 2.52 & 29.8365 & 29.3589 & 29.1142 & 29.7833 & 29.5266 & 29.3333 & 29.7127 & 29.2304 & 28.9776 & 29.6507 & 29.3939 & 29.1991 \\
\hline $7 a$ & 3.18 & 29.1711 & 28.6706 & 28.4121 & 29.0830 & 28.8165 & 28.6771 & 29.1167 & 28.6156 & 28.3379 & 29.0261 & 28.7615 & 28.6080 \\
\hline 8 & 4.03 & 28.1582 & 27.6512 & 27.5364 & 28.1366 & 27.8066 & 27.7836 & 28.1060 & 27.6015 & 27.4810 & 28.0834 & 27.7573 & 27.7338 \\
\hline
\end{tabular}




\section{Compound 84a}

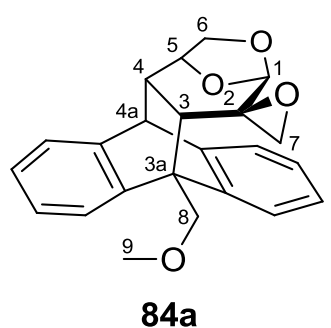

\begin{tabular}{|c|c|c|c|c|c|c|c|c|c|c|c|c|}
\hline \multirow[b]{3}{*}{ Exp } & \multicolumn{12}{|c|}{ B3LYP } \\
\hline & \multicolumn{6}{|c|}{ Gas phase } & \multicolumn{6}{|c|}{ PCM } \\
\hline & 6-31G* & 6-31G** & $6-31+\mathrm{G} * *$ & 6-311G* & 6-311G** & $6-311+\mathrm{G} * *$ & 6-31G* & 6-31G** & $6-31+\mathrm{G} * *$ & 6-311G* & $6-311 G * *$ & $6-311+G * *$ \\
\hline \multicolumn{13}{|l|}{${ }^{13} \mathrm{C}$} \\
\hline 1 & 84.9712 & 85.7044 & 84.1859 & 71.6926 & 71.0138 & 71.5530 & 85.3296 & 86.0608 & 84.4335 & 71.9622 & 71.2768 & 71.7526 \\
\hline 2 & 130.6100 & 130.7428 & 129.6194 & 120.0271 & 119.7800 & 118.7332 & 130.5955 & 130.7303 & 129.6022 & 119.9645 & 119.7259 & 118.6373 \\
\hline 3 & 151.5208 & 152.1992 & 153.0446 & 144.6168 & 144.6229 & 143.5203 & 151.3500 & 152.0227 & 152.5403 & 144.6832 & 144.6910 & 143.6150 \\
\hline 4 & 139.1846 & 140.0138 & 138.0360 & 128.4920 & 128.2502 & 128.1499 & 139.4325 & 140.2575 & 138.5116 & 128.8230 & 128.5788 & 128.5512 \\
\hline 5 & 111.6281 & 112.3893 & 111.5307 & 100.1416 & 99.5875 & 99.0881 & 111.8637 & 112.6218 & 111.7542 & 100.3811 & 99.8149 & 99.3017 \\
\hline 6 & 119.4307 & 120.7716 & 119.9548 & 108.8913 & 108.1102 & 107.9143 & 119.6184 & 120.9520 & 119.8647 & 108.9538 & 108.1485 & 107.8870 \\
\hline $3 a$ & 136.6669 & 136.8425 & 137.5756 & 125.9849 & 125.9849 & 124.9407 & 136.5827 & 136.7571 & 137.3091 & 125.9485 & 125.9480 & 124.8768 \\
\hline $4 a$ & 137.0309 & 137.6302 & 136.5404 & 124.6038 & 124.2448 & 123.6538 & 137.6728 & 138.2643 & 137.6178 & 125.4888 & 125.1118 & 124.5512 \\
\hline 7 & 138.0905 & 139.7532 & 139.6893 & 130.1260 & 129.1791 & 128.0103 & 138.1137 & 139.7550 & 139.4251 & 130.0647 & 129.0858 & 127.7830 \\
\hline 8 & 118.9825 & 120.1357 & 118.0657 & 110.0743 & 109.4594 & 109.3642 & 119.0539 & 120.1975 & 117.8325 & 110.2373 & 109.6053 & 109.5300 \\
\hline 9 & 132.5830 & 134.3170 & 134.9103 & 124.1805 & 123.5249 & 122.9539 & 132.6711 & 134.3851 & 134.9116 & 124.1102 & 123.4351 & 122.8829 \\
\hline \multicolumn{13}{|l|}{${ }^{1} \mathrm{H}$} \\
\hline 1 & 28.0063 & 27.4637 & 27.3206 & 27.9421 & 27.6169 & 27.6087 & 27.9619 & 27.4159 & 27.2515 & 27.8746 & 27.5509 & 27.5413 \\
\hline 3 & 29.2039 & 28.7775 & 28.6232 & 28.9251 & 28.6764 & 28.4742 & 29.3037 & 28.8783 & 28.7475 & 28.9986 & 28.7449 & 28.5291 \\
\hline 4 & 30.3906 & 29.9721 & 29.7979 & 30.3399 & 30.1143 & 29.9579 & 30.2844 & 29.8628 & 29.6793 & 30.2456 & 30.0167 & 29.8410 \\
\hline 5 & 27.7672 & 27.2429 & 27.0174 & 27.7504 & 27.4229 & 27.3531 & 27.5904 & 27.0584 & 26.8173 & 27.5681 & 27.2341 & 27.1526 \\
\hline $6 n$ & 28.6117 & 28.1041 & 28.0278 & 28.5698 & 28.2479 & 28.1414 & 28.4921 & 27.9815 & 27.8936 & 28.4535 & 28.1280 & 28.0035 \\
\hline $6 x$ & 28.5388 & 28.0112 & 27.8929 & 28.3896 & 28.1084 & 28.0800 & 28.4795 & 27.9536 & 27.8371 & 28.3275 & 28.0505 & 28.0122 \\
\hline $4 a$ & 28.4659 & 28.0332 & 27.7846 & 28.3041 & 28.1112 & 27.9433 & 28.2607 & 27.8160 & 27.5438 & 28.0796 & 27.8791 & 27.6986 \\
\hline $7 s$ & 30.3358 & 29.8638 & 29.6984 & 30.2777 & 30.0483 & 30.0011 & 30.1897 & 29.7117 & 29.5006 & 30.1241 & 29.8927 & 29.8341 \\
\hline $7 a$ & 29.1648 & 28.5418 & 28.4086 & 29.2751 & 28.8637 & 28.7239 & 29.0424 & 28.4180 & 28.2383 & 29.1597 & 28.7492 & 28.5912 \\
\hline $8 a$ & 27.8110 & 27.3196 & 27.1921 & 27.6342 & 27.3655 & 27.2409 & 27.8120 & 27.3189 & 27.1932 & 27.5375 & 27.2695 & 27.1318 \\
\hline $8 b$ & 28.2971 & 27.8448 & 27.8548 & 28.5331 & 28.2513 & 28.1485 & 28.2098 & 27.7586 & 27.7335 & 28.4870 & 28.2047 & 28.0942 \\
\hline 9 & 28.6239 & 28.1412 & 28.0323 & 28.5732 & 28.2948 & 28.2642 & 28.5799 & 28.0967 & 27.9802 & 28.5332 & 28.2558 & 28.2246 \\
\hline
\end{tabular}




\begin{tabular}{|c|c|c|c|c|c|c|c|c|c|c|c|c|}
\hline \multirow[b]{3}{*}{ Exp } & \multicolumn{12}{|c|}{ mPW1PW91 } \\
\hline & \multicolumn{6}{|c|}{ Gas phase } & \multicolumn{6}{|c|}{ PCM } \\
\hline & 6-31G* & 6-31G** & $6-31+\mathrm{G} * *$ & 6-311G* & $6-311 G * *$ & $6-311+G * *$ & 6-31G* & $6-31 G * *$ & $6-31+\mathrm{G} * *$ & 6-311G* & 6-311G** & $6-311+G * *$ \\
\hline \multicolumn{13}{|l|}{${ }^{13} \mathrm{C}$} \\
\hline 1 & 89.4827 & 90.1826 & 88.9994 & 78.3379 & 77.6870 & 78.0841 & 89.8554 & 90.5540 & 89.2654 & 78.5991 & 77.9424 & 78.2859 \\
\hline 2 & 134.8376 & 134.9574 & 134.3161 & 125.6632 & 125.4131 & 124.3394 & 134.8388 & 134.9607 & 134.2844 & 125.6034 & 125.3617 & 124.2414 \\
\hline 3 & 156.9357 & 157.5566 & 158.3068 & 150.5986 & 150.6124 & 149.4752 & 156.7505 & 157.3631 & 157.8451 & 150.6654 & 150.6802 & 149.5649 \\
\hline 4 & 144.7967 & 145.5375 & 143.4507 & 134.9846 & 134.7479 & 134.6494 & 145.0186 & 145.7558 & 143.9044 & 135.2677 & 135.0292 & 135.0033 \\
\hline 5 & 116.2985 & 117.0085 & 116.3842 & 106.4008 & 105.8477 & 105.3993 & 116.5224 & 117.2306 & 116.6003 & 106.6034 & 106.0396 & 105.5778 \\
\hline 6 & 123.6327 & 124.8954 & 124.2309 & 114.4993 & 113.7412 & 113.5620 & 123.8024 & 125.0599 & 124.1283 & 114.5283 & 113.7477 & 113.5089 \\
\hline $3 a$ & 141.3032 & 141.4763 & 142.3219 & 131.6781 & 131.6820 & 130.6580 & 141.2126 & 141.3837 & 142.0387 & 131.6398 & 131.6432 & 130.5907 \\
\hline $4 a$ & 141.9841 & 142.5135 & 141.4336 & 130.9069 & 130.5522 & 129.8776 & 142.6536 & 143.1767 & 142.4791 & 131.7659 & 131.3956 & 130.7474 \\
\hline 7 & 141.7892 & 143.4003 & 143.4367 & 135.2089 & 134.2725 & 133.1074 & 141.8155 & 143.4068 & 143.2137 & 135.1429 & 134.1788 & 132.8974 \\
\hline 8 & 123.7581 & 124.8246 & 122.7766 & 116.0860 & 115.4986 & 115.4310 & 123.8531 & 124.9082 & 122.5891 & 116.2490 & 115.6456 & 115.5908 \\
\hline 9 & 136.3314 & 137.9857 & 138.6745 & 129.0830 & 128.4622 & 127.8967 & 136.4462 & 138.0802 & 138.6811 & 129.0179 & 128.3788 & 127.8301 \\
\hline \multicolumn{13}{|l|}{${ }^{1} \mathrm{H}$} \\
\hline 1 & 27.9342 & 27.3960 & 27.2860 & 27.9073 & 27.5498 & 27.5553 & 27.8834 & 27.3415 & 27.2129 & 27.8346 & 27.4786 & 27.4827 \\
\hline 3 & 29.1330 & 28.6951 & 28.5622 & 28.7935 & 28.5494 & 28.3542 & 29.2424 & 28.8063 & 28.6830 & 28.8691 & 28.6197 & 28.4103 \\
\hline 4 & 30.3586 & 29.9429 & 29.7498 & 30.2326 & 30.0164 & 29.8650 & 30.2501 & 29.8310 & 29.6321 & 30.1380 & 29.9182 & 29.7490 \\
\hline 5 & 27.6847 & 27.1568 & 26.9450 & 27.6578 & 27.3129 & 27.2481 & 27.4946 & 26.9579 & 26.7352 & 27.4663 & 27.1146 & 27.0403 \\
\hline $6 n$ & 28.5624 & 28.0540 & 27.9916 & 28.5031 & 28.1701 & 28.0619 & 28.4332 & 27.9214 & 27.8519 & 28.3801 & 28.0433 & 27.9196 \\
\hline $6 x$ & 28.4886 & 27.9631 & 27.8657 & 28.3435 & 28.0514 & 28.0244 & 28.4184 & 27.8945 & 27.8017 & 28.2720 & 27.9842 & 27.9492 \\
\hline $4 a$ & 28.2822 & 27.8538 & 27.6269 & 28.1001 & 27.8983 & 27.7526 & 28.0584 & 27.6169 & 27.3674 & 27.8637 & 27.6535 & 27.4963 \\
\hline $7 \mathrm{~s}$ & 30.2507 & 29.7841 & 29.6291 & 30.2132 & 29.9689 & 29.9143 & 30.0923 & 29.6189 & 29.4248 & 30.0519 & 29.8053 & 29.7425 \\
\hline $7 a$ & 29.1243 & 28.5031 & 28.3727 & 29.2027 & 28.7842 & 28.6311 & 28.9964 & 28.3739 & 28.2091 & 29.0856 & 28.6684 & 28.5007 \\
\hline $8 a$ & 27.7255 & 27.2369 & 27.1370 & 27.5131 & 27.2535 & 27.1477 & 27.7266 & 27.2365 & 27.1339 & 27.4125 & 27.1534 & 27.0351 \\
\hline $8 b$ & 28.2165 & 27.7707 & 27.7851 & 28.4080 & 28.1334 & 28.0316 & 28.1212 & 27.6765 & 27.6645 & 28.3591 & 28.0840 & 27.9756 \\
\hline 9 & 28.5679 & 28.0867 & 28.0089 & 28.5145 & 28.2241 & 28.2033 & 28.5174 & 28.0357 & 27.9524 & 28.4699 & 28.1806 & 28.1595 \\
\hline
\end{tabular}

\section{Compound 84b}

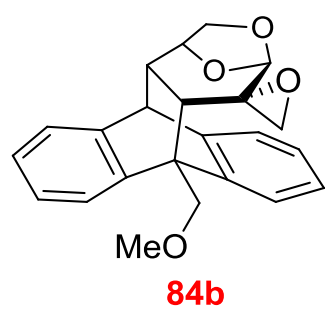

\begin{tabular}{|c|c|c|c|c|c|c|c|c|c|c|c|c|c|}
\hline \multirow{3}{*}{\multicolumn{2}{|c|}{ Exp }} & \multicolumn{12}{|c|}{ B3LYP } \\
\hline & & \multicolumn{6}{|c|}{ Gas phase } & \multicolumn{6}{|c|}{ PCM } \\
\hline & & 6-31G* & 6-31G** & $6-31+G * *$ & 6-311G* & $6-311 G * *$ & $6-311+\mathrm{G} * *$ & 6-31G* & 6-31G** & $6-31+G * *$ & 6-311G* & 6-311G** & $6-311+G * *$ \\
\hline \multicolumn{14}{|c|}{${ }^{13} \mathrm{C}$} \\
\hline 1 & 104.8 & 85.2777 & 86.0470 & 84.6308 & 71.7511 & 71.1601 & 71.1899 & 85.5137 & 86.2807 & 84.7506 & 71.9217 & 71.3246 & 71.3028 \\
\hline 2 & 57.0 & 133.0451 & 133.2166 & 133.3515 & 122.8499 & 122.6746 & 120.8214 & 133.0284 & 133.2013 & 133.2046 & 122.7987 & 122.6291 & 120.7267 \\
\hline 3 & 31.8 & 152.9070 & 153.6602 & 154.0630 & 144.1897 & 144.1868 & 144.0746 & 153.0650 & 153.8125 & 154.2191 & 144.3243 & 144.3171 & 144.2411 \\
\hline
\end{tabular}




\begin{tabular}{|c|c|c|c|c|c|c|c|c|c|c|c|c|c|}
\hline 4 & 4.6 & 2.2166 & 3.0477 & 0.0550 & 31.6942 & 131.5193 & 131.9005 & 142.5708 & 143.3952 & 140.5623 & 132.1057 & 131.9257 & 132.3 \\
\hline 5 & .1 & 2.0146 & 2.7867 & 2.1051 & 0.8054 & 0.2985 & 138 & 797 & 12.9509 & 2.2099 & 00.9902 & 744 & 9.96 \\
\hline 6 & 9.2 & & 2.3899 & 2.5472 & 0.6227 & & & 1.2974 & 122.6522 & 22.6990 & 110.8232 & & נש. \\
\hline $3 a$ & & & & & & & & & & & & & \\
\hline $4 a$ & & 6.1117 & 6.7179 & & 3.7378 & 3.4274 & & 36.7536 & 137.35 & 5.8726 & & & $124.32 \mathrm{~S}$ \\
\hline 7 & 8.6 & 1.9767 & 3.4935 & 142.7557 & 34.2116 & 133.3634 & 132.2140 & 141.8859 & 143.3869 & 142.5336 & 134.0340 & 133.1615 & 131.947 \\
\hline 8 & 1.4 & 8.3074 & 9.4531 & 8.3789 & 8.3742 & & 107.0225 & 118.6197 & 119.7588 & 118.6965 & .7086 & 3667 & 364 \\
\hline 9 & 8.3 & & & & & & & & & & & & \\
\hline \\
\hline 1 & & & & & & & & & & & & & \\
\hline 3 & 04 & & & & & & & & & & & & \\
\hline 4 & 2.04 & 30.4797 & 30.0893 & 29.9561 & 30.4368 & 30.2409 & 30.1284 & & 29.9661 & & & & 9955 \\
\hline 5 & .79 & .7868 & .2765 & 1676 & .7806 & 629 & .4846 & 267 & 1094 & & 6129 & & 2958 \\
\hline $\mathrm{n}$ & 68 & - & & & 824 & & & & & & & & $28.203 \mathrm{~s}$ \\
\hline on & & & & & & & & & & & & & 20.072 \\
\hline $4 d$ & 4.21 & & & & & & & & & & & & 27.8785 \\
\hline $7 \mathrm{~s}$ & 2.47 & 29.9266 & 29.4574 & 29.2609 & 29.8911 & 29.6662 & 29.5168 & 29.8106 & 29.3373 & 29.1271 & 29.7638 & 29.5387 & 29.3863 \\
\hline $7 a$ & 2.93 & .3736 & 8772 & 7060 & 163 & .0628 & 3.9860 & 29.2652 & 28.7655 & 8.5659 & 29.2004 & 28.9472 & 28.8525 \\
\hline $8 a$ & 4.7 & 2290 & 6566 & 5916 & 2878 & 9509 & 8070 & 2576 & 26.6873 & .6100 & 27.3091 & 6.9728 & 26.8236 \\
\hline $8 b$ & 4.38 & 27.9738 & 27.5381 & 27.4533 & 27.9997 & 27.7711 & 27.6244 & 27.9126 & 27.4751 & 27.3729 & 27.9319 & 27.7029 & 27.5469 \\
\hline 9 & 3.60 & 28.6627 & 28.1643 & 28.0379 & 28.6237 & 28.3163 & 28.2714 & 28.5922 & 28.0942 & 27.9622 & 28.5531 & 28.2477 & 20.15 \\
\hline
\end{tabular}

\begin{tabular}{|c|c|c|c|c|c|c|c|c|c|c|c|c|c|}
\hline \multicolumn{14}{|c|}{ mPW1PW91 } \\
\hline & \multirow[b]{2}{*}{ Exp } & \multicolumn{6}{|c|}{ Gas phase } & \multicolumn{6}{|c|}{ PCM } \\
\hline & & 6-31G* & $6-31 G * *$ & $6-31+G * *$ & 6-311G* & $6-311 G * *$ & $6-311+G * *$ & $6-31 G *$ & $6-31 G * *$ & $6-31+G * *$ & 6-311G* & $6-311 G * *$ & $6-311+\mathrm{G} * *$ \\
\hline \multicolumn{14}{|l|}{${ }^{13} \mathrm{C}$} \\
\hline 1 & 104.8 & 89.7638 & 90.5039 & 89.4469 & 78.4620 & 77.8944 & 77.8624 & 90.0112 & 90.7490 & 89.5877 & 78.6331 & 78.0596 & 77.9809 \\
\hline 2 & 57.0 & 137.2195 & 137.3761 & 137.7444 & 128.4398 & 128.2542 & 126.5338 & 137.2184 & 137.3760 & 137.6139 & 128.4018 & 128.2216 & 126.4519 \\
\hline 3 & 31.8 & 158.1533 & 158.8360 & 159.1674 & 150.1651 & 150.1646 & 150.0724 & 158.3190 & 158.9961 & 159.3252 & 150.2962 & 150.2912 & 150.2315 \\
\hline 4 & 44.6 & 147.8211 & 148.5612 & 145.7182 & 138.1288 & 137.9536 & 138.2737 & 148.1588 & 148.8931 & 146.2024 & 138.4928 & 138.3130 & 138.7043 \\
\hline 5 & 77.1 & 116.7591 & 117.4749 & 117.0471 & 107.1071 & 106.6035 & 106.1394 & 116.9078 & 117.6242 & 117.1321 & 107.2563 & 106.7452 & 106.2516 \\
\hline 6 & 69.2 & 125.1430 & 126.4335 & 126.7489 & 116.2015 & 115.4286 & 114.9530 & 125.4091 & 126.6920 & 126.8931 & 116.3763 & 115.5799 & 115.0898 \\
\hline $3 a$ & 49.7 & 141.8940 & 142.0588 & 141.2151 & 132.1955 & 132.2399 & 132.0309 & 141.8579 & 142.0185 & 141.1364 & 132.1119 & 132.1553 & 131.9256 \\
\hline $4 a$ & 51.3 & 141.1676 & 141.7010 & 139.8888 & 130.0742 & 129.7701 & 129.8484 & 141.8361 & 142.3623 & 140.7132 & 130.9483 & 130.6268 & 130.7183 \\
\hline 7 & 48.6 & 145.5106 & 146.9846 & 146.3904 & 139.0220 & 138.1876 & 137.1426 & 145.3963 & 146.8559 & 146.1525 & 138.8255 & 137.9691 & 136.8641 \\
\hline 8 & 71.4 & 123.0505 & 124.1090 & 123.0257 & 114.5154 & 113.8806 & 113.3712 & 123.3887 & 124.4413 & 123.3599 & 114.8623 & 114.2188 & 113.7180 \\
\hline 9 & 58.3 & 136.7110 & 138.3406 & 139.0177 & 129.7748 & 129.0669 & 128.6817 & 136.8123 & 138.4253 & 138.9785 & 129.7585 & 129.0291 & 128.6393 \\
\hline \multicolumn{14}{|l|}{${ }^{1} \mathrm{H}$} \\
\hline 1 & 4.21 & 28.0041 & 27.4815 & 27.4321 & 28.0121 & 27.6897 & 27.6679 & 27.9903 & 27.4641 & 27.4028 & 27.9791 & 27.6556 & 27.6316 \\
\hline 3 & 3.04 & 29.0912 & 28.6574 & 28.5901 & 29.1034 & 28.8609 & 28.7594 & 29.0840 & 28.6461 & 28.5665 & 29.0979 & 28.8498 & 28.7355 \\
\hline 4 & 2.04 & 30.4468 & 30.0564 & 29.9169 & 30.3365 & 30.1431 & 30.0536 & 30.3238 & 29.9282 & 29.7743 & 30.2214 & 30.0232 & 29.9175 \\
\hline 5 & 4.79 & 27.7127 & 27.1994 & 27.1007 & 27.6911 & 27.3594 & 27.3855 & 27.5400 & 27.0190 & 26.8999 & 27.5148 & 27.1762 & 27.1899 \\
\hline $6 n$ & 3.68 & 28.6478 & 28.1467 & 28.0618 & 28.6249 & 28.2963 & 28.2792 & 28.5136 & 28.0090 & 27.9129 & 28.4981 & 28.1653 & 28.1376 \\
\hline $6 x$ & 3.68 & 28.5806 & 28.0466 & 27.8381 & 28.4698 & 28.1583 & 28.0963 & 28.5101 & 27.9780 & 27.7629 & 28.4006 & 28.0941 & 28.0242 \\
\hline $4 a$ & 4.21 & 28.2276 & 27.8242 & 27.7900 & 28.0468 & 27.8542 & 27.9222 & 28.0073 & 27.5916 & 27.5279 & 27.8148 & 27.6131 & 27.6689 \\
\hline
\end{tabular}




\begin{tabular}{|c|c|c|c|c|c|c|c|c|c|c|c|c|c|}
\hline $7 s$ & 2.47 & 29.8375 & 29.3709 & 29.2066 & 29.8080 & 29.5665 & 29.4379 & 29.7098 & 29.2384 & 29.0633 & 29.6721 & 29.4301 & 29.2992 \\
\hline $7 a$ & 2.93 & 29.2824 & 28.7923 & 28.6189 & 29.2070 & 28.9489 & 28.8728 & 29.1658 & 28.6725 & 28.4744 & 29.0856 & 28.8279 & 28.7362 \\
\hline $8 a$ & 4.73 & 27.1645 & 26.6003 & 26.5415 & 27.1820 & 26.8585 & 26.7174 & 27.1898 & 26.6277 & 26.5574 & 27.2005 & 26.8775 & 26.7311 \\
\hline $8 b$ & 4.38 & 27.8777 & 27.4495 & 27.3625 & 27.8683 & 27.6493 & 27.5088 & 27.8128 & 27.3825 & 27.2782 & 27.7982 & 27.5783 & 27.4283 \\
\hline 9 & 3.60 & 28.6023 & 28.1065 & 28.0028 & 28.5656 & 28.2473 & 28.2130 & 28.5240 & 28.0285 & 27.9203 & 28.4890 & 28.1726 & 28.1352 \\
\hline
\end{tabular}

\section{Compound 85a}<smiles>CS(=O)(=O)[C@H](O)C[C@@H](O)/C=C/c1ccccc1</smiles>

\begin{tabular}{|c|c|c|c|c|c|c|c|c|c|c|c|c|c|}
\hline & \multirow[b]{3}{*}{ Exp } & \multicolumn{12}{|c|}{ B3LYP } \\
\hline & & \multicolumn{6}{|c|}{ Gas phase } & \multicolumn{6}{|c|}{ PCM } \\
\hline & & 6-31G* & 6-31G** & $6-31+\mathrm{G} * *$ & 6-311G* & $6-311 G * *$ & $6-311+G * *$ & 6-31G* & 6-31G** & $6-31+\mathrm{G} * *$ & 6-311G* & 6-311G** & $6-311+G^{* *}$ \\
\hline \multicolumn{14}{|l|}{${ }^{13} \mathrm{C}$} \\
\hline 5 & 29.8 & 158.5145 & 159.7089 & 158.8282 & 149.2000 & 148.8412 & 148.4138 & 158.7568 & 159.9344 & 159.0815 & 149.4038 & 149.0245 & 148.5743 \\
\hline $1^{\prime}$ & 42.8 & 145.6754 & 146.7080 & 146.1025 & 136.2284 & 135.7060 & 135.5453 & 146.1237 & 147.1445 & 146.5210 & 136.6939 & 136.1604 & 136.0083 \\
\hline $3^{\prime}$ & 43.5 & 146.1795 & 147.2933 & 146.5184 & 136.0434 & 135.5458 & 135.3701 & 146.4192 & 147.5353 & 146.6597 & 136.2195 & 135.7205 & 135.4906 \\
\hline $2^{\prime}$ & 67.4 & 121.6847 & 122.6096 & 120.4239 & 109.4516 & 109.3826 & 108.2139 & 121.7045 & 122.6129 & 120.6074 & 109.4133 & 109.3569 & 108.2775 \\
\hline $4^{\prime}$ & 73.4 & 114.2198 & 114.9486 & 112.7413 & 101.1579 & 101.0787 & 100.3665 & 114.6197 & 115.3314 & 113.1952 & 101.6397 & 101.5307 & 100.8473 \\
\hline 6 & 74.7 & 117.2961 & 118.1565 & 116.3817 & 105.6298 & 105.5022 & 104.1242 & 116.6183 & 117.4554 & 115.7672 & 104.9116 & 104.7807 & 103.4529 \\
\hline 3 & 121.1 & 71.7494 & 72.2058 & 72.2289 & 54.7274 & 53.9026 & 53.4398 & 73.4006 & 73.8655 & 74.1525 & 56.6377 & 55.8434 & 55.4936 \\
\hline 2"/6" & " 126.5 & 69.3144 & 69.9494 & 69.2603 & 52.5716 & 51.7172 & 50.7780 & 69.4183 & 70.0433 & 69.1847 & 52.5249 & 51.6822 & 50.7919 \\
\hline 4" & 127.8 & 68.7545 & 69.3545 & 68.5707 & 51.0535 & 50.1671 & 49.5986 & 68.7934 & 69.4026 & 68.4836 & 50.9494 & 50.1021 & 49.5950 \\
\hline 3"/5" & " 128.6 & 67.9674 & 68.5491 & 68.2029 & 50.6558 & 49.6777 & 48.7022 & 67.8677 & 68.4517 & 68.0813 & 50.3962 & 49.4522 & 48.5157 \\
\hline $5^{\prime}$ & 130.2 & 64.6684 & 64.9829 & 64.1005 & 46.7003 & 45.7131 & 44.6476 & 63.6267 & 63.9242 & 63.0607 & 45.4690 & 44.4955 & 43.5542 \\
\hline $6^{\prime}$ & 131.5 & 66.2815 & 66.7459 & 64.8076 & 49.0381 & 48.1122 & 46.5465 & 66.8933 & 67.3507 & 65.5529 & 49.6319 & 48.7287 & 47.1503 \\
\hline 1" & 136.4 & 61.0244 & 60.6883 & 58.9458 & 41.7824 & 41.4347 & 40.2710 & 60.8601 & 60.5231 & 58.7110 & 41.6082 & 41.2806 & 40.0838 \\
\hline 4 & 145.6 & 52.6994 & 53.4399 & 51.6650 & 33.5418 & 32.7848 & 31.2961 & 48.3037 & 49.0239 & 46.5154 & 28.5015 & 27.7197 & 25.7280 \\
\hline 2 & 164.8 & 36.9558 & 37.0029 & 34.9022 & 17.3768 & 17.4800 & 14.6380 & 34.7803 & 34.8208 & 32.2568 & 14.9465 & 15.0342 & 11.7131 \\
\hline \multicolumn{14}{|l|}{${ }^{1} \mathrm{H}$} \\
\hline $1^{\prime} / 3^{\prime}$ & 1.76 & 30.5951 & 30.2044 & 30.0230 & 30.5019 & 30.3249 & 30.1959 & 30.5530 & 30.1561 & 29.9882 & 30.4616 & 30.2791 & 30.1554 \\
\hline 5 & 2.37 & 30.1797 & 29.7242 & 29.5650 & 30.0842 & 29.8424 & 29.7513 & 30.0097 & 29.5460 & 29.3719 & 29.9171 & 29.6689 & 29.5680 \\
\hline $2^{\prime}$ & 4.34 & 27.6477 & 27.2053 & 27.1902 & 27.6565 & 27.4276 & 27.4911 & 27.6877 & 27.2508 & 27.1677 & 27.6699 & 27.4367 & 27.4572 \\
\hline $4^{\prime}$ & 4.61 & 27.4920 & 27.0017 & 26.9179 & 27.4481 & 27.1860 & 27.2197 & 27.3925 & 26.8993 & 26.7894 & 27.3367 & 27.0754 & 27.0995 \\
\hline 6 & 4.78 & 27.2382 & 26.7887 & 26.7776 & 27.3563 & 27.1635 & 27.1442 & 27.2264 & 26.7803 & 26.7160 & 27.3289 & 27.1311 & 27.0736 \\
\hline 3 & 6.03 & 26.5163 & 25.8400 & 25.6535 & 26.4350 & 25.9750 & 25.8862 & 26.5112 & 25.8265 & 25.6352 & 26.4248 & 25.9622 & 25.8720 \\
\hline $5^{\prime}$ & 6.23 & 25.9101 & 25.2532 & 24.9303 & 25.7208 & 25.2654 & 25.1780 & 25.7810 & 25.1212 & 24.7805 & 25.5840 & 25.1322 & 25.0456 \\
\hline $6^{\prime}$ & 6.60 & 25.8485 & 25.1743 & 24.9604 & 25.6753 & 25.1976 & 25.0789 & 25.7963 & 25.1216 & 24.8909 & 25.6099 & 25.1370 & 25.0076 \\
\hline 4 & 6.90 & 25.6011 & 24.9847 & 24.7503 & 25.4393 & 25.0278 & 24.9392 & 25.2645 & 24.6460 & 24.3793 & 25.0985 & 24.6912 & 24.5753 \\
\hline $\mathrm{Ar}$ & 7.32 & 25.0366 & 24.4013 & 24.1783 & 24.8682 & 24.4528 & 24.3795 & 24.9412 & 24.3064 & 24.0698 & 24.7576 & 24.3508 & 24.2675 \\
\hline
\end{tabular}




\begin{tabular}{|c|c|c|c|c|c|c|c|c|c|c|c|c|c|}
\hline & & \multicolumn{12}{|c|}{ mPW1PW91 } \\
\hline & \multirow[b]{2}{*}{ Exp } & \multicolumn{6}{|c|}{ Gas phase } & \multicolumn{6}{|c|}{ PCM } \\
\hline & & 6-31G* & $6-31 G * *$ & $6-31+\mathrm{G} * *$ & 6-311G* & $6-311 G * *$ & $6-311+G * *$ & 6-31G* & $6-31 G * *$ & $6-31+\mathrm{G} * *$ & 6-311G* & $6-311 G * *$ & $6-311+G * *$ \\
\hline \multicolumn{14}{|l|}{${ }^{13} \mathrm{C}$} \\
\hline 5 & 29.8 & 163.0050 & 164.1155 & 163.3368 & 154.6854 & 154.3269 & 153.8351 & 163.2185 & 164.3137 & 163.5542 & 154.8400 & 154.4632 & 153.9463 \\
\hline $1^{\prime}$ & 42.8 & 150.5845 & 151.5359 & 151.1213 & 142.3159 & 141.8220 & 141.5826 & 151.0652 & 152.0060 & 151.5699 & 142.7697 & 142.2663 & 142.0296 \\
\hline $3^{\prime}$ & 43.5 & 151.3104 & 152.3328 & 151.6095 & 142.3071 & 141.8397 & 141.5843 & 151.5943 & 152.6170 & 151.8092 & 142.5089 & 142.0395 & 141.7438 \\
\hline $2^{\prime}$ & 67.4 & 126.6397 & 127.4707 & 125.9660 & 116.1281 & 116.0484 & 115.0114 & 126.5972 & 127.4178 & 126.0465 & 116.0550 & 115.9886 & 115.0177 \\
\hline $4^{\prime}$ & 73.4 & 118.9633 & 119.6148 & 117.9961 & 107.7671 & 107.6669 & 106.9855 & 119.2964 & 119.9364 & 118.3881 & 108.1975 & 108.0682 & 107.4127 \\
\hline 6 & 74.7 & 122.0209 & 122.8094 & 121.4088 & 111.8634 & 111.7436 & 110.5510 & 121.2583 & 122.0293 & 120.6776 & 111.0943 & 110.9720 & 109.8062 \\
\hline 3 & 121.1 & 74.0952 & 74.5013 & 74.5211 & 59.1975 & 58.3505 & 57.8223 & 75.8354 & 76.2520 & 76.5177 & 61.1415 & 60.3302 & 59.9046 \\
\hline 2"/6" & " 126.5 & 71.8106 & 72.3792 & 71.5247 & 57.0933 & 56.2158 & 55.2549 & 71.9185 & 72.4773 & 71.4495 & 57.0395 & 56.1763 & 55.2661 \\
\hline 4" & 127.8 & 71.1109 & 71.6274 & 70.7278 & 55.5576 & 54.6297 & 54.0736 & 71.1143 & 71.6389 & 70.5769 & 55.3971 & 54.5109 & 54.0192 \\
\hline 3"/5" & " 128.6 & 70.4492 & 70.9434 & 71.0294 & 55.2723 & 54.2492 & 53.2926 & 70.3103 & 70.8068 & 70.8612 & 54.9615 & 53.9752 & 53.0649 \\
\hline $5^{\prime}$ & 130.2 & 67.2149 & 67.4496 & 66.8330 & 51.3849 & 50.3505 & 49.3597 & 66.0090 & 66.2304 & 65.6128 & 50.0363 & 49.0189 & 48.1440 \\
\hline $6^{\prime}$ & 131.5 & 69.0033 & 69.3863 & 68.1055 & 53.8988 & 52.9410 & 51.4059 & 69.6701 & 70.0462 & 68.8880 & 54.5442 & 53.6103 & 52.0630 \\
\hline $1^{\prime \prime}$ & 136.4 & 64.0058 & 63.6380 & 62.3026 & 47.4737 & 47.0858 & 45.7741 & 63.8616 & 63.4960 & 62.1062 & 47.3305 & 46.9648 & 45.6210 \\
\hline 4 & 145.6 & 54.9519 & 55.5863 & 54.0248 & 38.0900 & 37.2808 & 35.8939 & 50.3724 & 50.9864 & 48.7763 & 32.9774 & 32.1434 & 30.3233 \\
\hline 2 & 164.8 & 39.2821 & 39.3252 & 37.8737 & 22.3556 & 22.4525 & 19.8642 & 37.0617 & 37.0978 & 35.2239 & 19.9275 & 20.0077 & 16.9941 \\
\hline \multicolumn{14}{|l|}{${ }^{1} \mathrm{H}$} \\
\hline $1^{\prime} / 3^{\prime}$ & 1.76 & 30.5261 & 30.1313 & 29.9830 & 30.4019 & 30.2187 & 30.1031 & 30.4724 & 30.0716 & 29.9323 & 30.3547 & 30.1655 & 30.0532 \\
\hline 5 & 2.37 & 30.1150 & 29.6540 & 29.5011 & 29.9893 & 29.7395 & 29.6532 & 29.9329 & 29.4630 & 29.2975 & 29.8130 & 29.5562 & 29.4612 \\
\hline $2^{\prime}$ & 4.34 & 27.5919 & 27.1500 & 27.1026 & 27.5725 & 27.3457 & 27.3902 & 27.6370 & 27.2001 & 27.0953 & 27.5882 & 27.3573 & 27.3641 \\
\hline $4^{\prime}$ & 4.61 & 27.4225 & 26.9349 & 26.8215 & 27.3511 & 27.0892 & 27.1088 & 27.3152 & 26.8246 & 26.6924 & 27.2341 & 26.9729 & 26.9860 \\
\hline 6 & 4.78 & 27.1653 & 26.7133 & 26.6750 & 27.2497 & 27.0574 & 27.0275 & 27.1634 & 26.7141 & 26.6330 & 27.2279 & 27.0299 & 26.9672 \\
\hline 3 & 6.03 & 26.3035 & 25.6348 & 25.4705 & 26.2677 & 25.7771 & 25.7015 & 26.2927 & 25.6156 & 25.4485 & 26.2537 & 25.7611 & 25.6849 \\
\hline $5^{\prime}$ & 6.23 & 25.7374 & 25.0772 & 24.7855 & 25.5660 & 25.0935 & 25.0152 & 25.5873 & 24.9257 & 24.6200 & 25.4160 & 24.9479 & 24.8700 \\
\hline $6^{\prime}$ & 6.60 & 25.6153 & 24.9465 & 24.7584 & 25.4887 & 24.9884 & 24.8841 & 25.5609 & 24.8912 & 24.6899 & 25.4197 & 24.9242 & 24.8110 \\
\hline 4 & 6.90 & 25.4155 & 24.7924 & 24.5867 & 25.2865 & 24.8423 & 24.7628 & 25.0615 & 24.4363 & 24.2024 & 24.9332 & 24.4927 & 24.3891 \\
\hline $\mathrm{Ar}$ & 7.32 & 24.8183 & 24.1807 & 23.9962 & 24.6846 & 24.2358 & 24.1723 & 24.7083 & 24.0712 & 23.8752 & 24.5631 & 24.1231 & 24.0497 \\
\hline
\end{tabular}

\section{Compound 85b}

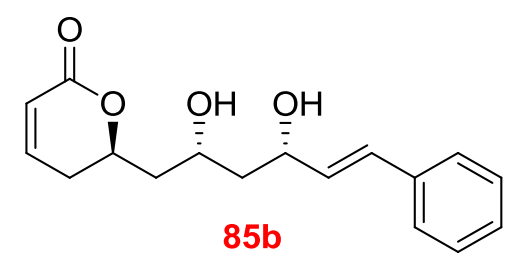

\begin{tabular}{|c|c|c|c|c|c|c|c|c|c|c|c|c|c|}
\hline & \multirow[b]{3}{*}{ Exp } & \multicolumn{12}{|c|}{ B3LYP } \\
\hline & & \multicolumn{6}{|c|}{ Gas phase } & \multicolumn{6}{|c|}{ PCM } \\
\hline & & 6-31G* & 6-31G** & $6-31+\mathrm{G} * *$ & 6-311G* & $6-311 G * *$ & $6-311+G * *$ & 6-31G* & $6-31 G * *$ & $6-31+G^{*} *$ & 6-311G* & $6-311 G * *$ & $6-311+G * *$ \\
\hline \multicolumn{14}{|l|}{${ }^{13} \mathrm{C}$} \\
\hline 5 & 29.9 & 158.3213 & 159.4845 & 158.6339 & 148.9414 & 148.5779 & 147.9565 & 158.5651 & 159.7143 & 158.8530 & 149.1532 & 148.7738 & 148.1217 \\
\hline $1^{\prime}$ & 42.4 & 148.1470 & 149.2219 & 146.1869 & 137.2074 & 136.8535 & 135.9020 & 148.7872 & 149.8451 & 147.0492 & 137.9874 & 137.5964 & 136.7424 \\
\hline
\end{tabular}




\begin{tabular}{|c|c|c|c|c|c|c|c|c|c|c|c|c|c|}
\hline $3^{\prime}$ & 43.2 & 150.7186 & 151.8320 & 148.2284 & 138.8493 & 138.5989 & 137.5655 & 151.1279 & 152.2099 & 148.6621 & 139.2555 & 138.9790 & 137.8913 \\
\hline $2^{\prime}$ & 64.8 & 122.3117 & 123.0612 & 125.5606 & 112.1955 & 111.8536 & 113.0525 & 121.7139 & 122.5005 & 124.8387 & 111.5348 & 111.2164 & 112.4184 \\
\hline $4^{\prime}$ & 70.6 & 117.4019 & 118.0393 & 112.4883 & 102.8745 & 102.9145 & 101.0588 & 117.7155 & 118.3441 & 113.3801 & 103.6088 & 103.5832 & 102.0732 \\
\hline 6 & 75.0 & 114.9864 & 115.7407 & 116.2504 & 104.2325 & 104.0163 & 103.9074 & 114.3606 & 115.1231 & 115.3816 & 103.5472 & 103.3251 & 103.1428 \\
\hline 3 & 121.3 & 72.4721 & 72.9340 & 72.3718 & 54.9184 & 54.1401 & 53.3276 & 74.1194 & 74.5781 & 74.2798 & 56.8237 & 56.0756 & 55.3348 \\
\hline 2"/6" & " 126.5 & 69.1463 & 69.7664 & 68.0575 & 52.1819 & 51.3676 & 50.7419 & 69.3249 & 69.9326 & 68.3161 & 52.2005 & 51.3935 & 50.6982 \\
\hline 4" & 127.8 & 69.2081 & 69.8015 & 68.0759 & 50.6819 & 49.8895 & 48.7046 & 69.1585 & 69.7480 & 68.2604 & 50.7076 & 49.9263 & 48.8477 \\
\hline 3"/5" & " 128.6 & 68.1051 & 68.6976 & 68.1909 & 50.5264 & 49.5772 & 48.4357 & 67.9487 & 68.5404 & 67.9278 & 50.3001 & 49.3754 & 48.3522 \\
\hline $5^{\prime}$ & 130.4 & 66.0160 & 66.3938 & 67.5751 & 49.2153 & 47.9875 & 47.6935 & 64.3293 & 64.7247 & 66.5728 & 47.5610 & 46.3619 & 46.3716 \\
\hline $6^{\prime}$ & 131.5 & 63.1532 & 63.5245 & 60.4495 & 44.9818 & 44.2616 & 42.3729 & 64.5325 & 64.8857 & 61.1122 & 46.1491 & 45.4227 & 43.3357 \\
\hline 1" & 136.5 & 60.6928 & 60.3755 & 58.8461 & 41.9465 & 41.5277 & 40.8018 & 60.7081 & 60.3989 & 58.6033 & 41.6878 & 41.3154 & 40.4521 \\
\hline 4 & 145.4 & 51.9476 & 52.6469 & 51.6909 & 33.1711 & 32.3751 & 30.9709 & 47.6528 & 48.3477 & 46.6196 & 28.2584 & 27.4240 & 25.5443 \\
\hline $\begin{array}{c}2 \\
{ }^{1} \mathrm{H}\end{array}$ & 164.5 & 38.1629 & 38.1811 & 34.8070 & 17.6937 & 17.8520 & 14.5542 & 36.1138 & 36.1156 & 32.2476 & 15.3433 & 15.4791 & 11.6863 \\
\hline $1^{\prime} / 3^{\prime}$ & 1.86 & 30.4473 & 30.0239 & 29.9891 & 30.3950 & 30.1881 & 30.1497 & 30.3361 & 29.9080 & 29.8796 & 30.3031 & 30.0900 & 30.0534 \\
\hline 5 & 2.40 & 30.1090 & 29.6300 & 29.4146 & 29.9552 & 29.7072 & 29.5866 & 29.9593 & 29.4735 & 29.2650 & 29.8256 & 29.5671 & 29.4498 \\
\hline $2^{\prime}$ & 4.41 & 27.7809 & 27.3057 & 26.8372 & 27.7186 & 27.4866 & 27.2960 & 27.8361 & 27.3629 & 26.9601 & 27.7878 & 27.5507 & 27.3772 \\
\hline $4^{\prime}$ & 4.68 & 27.4936 & 27.0018 & 27.0321 & 27.4441 & 27.1937 & 27.2386 & 27.4460 & 26.9497 & 26.9255 & 27.3551 & 27.1025 & 27.1254 \\
\hline 6 & 4.78 & 27.3203 & 26.8640 & 26.6870 & 27.3695 & 27.1793 & 27.1127 & 27.2095 & 26.7524 & 26.6529 & 27.3037 & 27.1068 & 27.0413 \\
\hline 3 & 6.04 & 26.4513 & 25.7695 & 25.5904 & 26.4036 & 25.9404 & 25.8397 & 26.4544 & 25.7652 & 25.5685 & 26.3931 & 25.9294 & 25.8229 \\
\hline $5^{\prime}$ & 6.32 & 25.8724 & 25.2046 & 24.6004 & 25.5887 & 25.1483 & 24.9827 & 25.7343 & 25.0681 & 24.6056 & 25.5026 & 25.0598 & 24.9447 \\
\hline $6^{\prime}$ & 6.66 & 25.6065 & 24.9636 & 25.1497 & 25.6492 & 25.1682 & 25.1910 & 25.6311 & 24.9871 & 25.0068 & 25.5776 & 25.1107 & 25.0518 \\
\hline 4 & 6.92 & 25.5571 & 24.9304 & 24.7133 & 25.4172 & 24.9975 & 24.9136 & 25.2332 & 24.6057 & 24.3473 & 25.0828 & 24.6666 & 24.5565 \\
\hline Ar & 7.34 & 25.0265 & 24.3940 & 24.1444 & 24.8346 & 24.4208 & 24.3346 & 24.9293 & 24.2969 & 24.0443 & 24.7315 & 24.3246 & 24.2377 \\
\hline
\end{tabular}

mPW1PW91

\begin{tabular}{|c|c|c|c|c|c|c|c|c|c|c|c|c|c|}
\hline & \multirow[b]{2}{*}{ Exp } & \multicolumn{6}{|c|}{ Gas phase } & \multicolumn{6}{|c|}{ PCM } \\
\hline & & 6-31G* & $6-31 G^{* *}$ & $6-31+G^{* *}$ & 6-311G* & 6-311G** & $6-311+G * *$ & 6-31G* & $6-31 G^{* *}$ & $6-31+G^{* *}$ & 6-311G* & $6-311 G^{* *}$ & $6-311+G^{* *}$ \\
\hline \multicolumn{14}{|l|}{${ }^{13} \mathrm{C}$} \\
\hline 5 & 29.9 & 162.7888 & 163.8660 & 163.2022 & 154.4291 & 154.0626 & 153.4180 & 163.0033 & 164.0682 & 163.3804 & 154.6028 & 154.2204 & 153.5328 \\
\hline $1^{\prime}$ & 42.4 & 153.2718 & 154.2765 & 151.5617 & 143.5088 & 143.1721 & 142.2522 & 153.8424 & 154.8292 & 152.4769 & 144.2128 & 143.8373 & 142.9786 \\
\hline $3^{\prime}$ & 43.2 & 156.0337 & 157.0894 & 153.4813 & 145.2871 & 145.0651 & 143.8941 & 156.3784 & 157.4021 & 154.0337 & 145.6571 & 145.4044 & 144.2481 \\
\hline $2^{\prime}$ & 64.8 & 126.7624 & 127.4117 & 130.0530 & 118.1287 & 117.7827 & 118.8717 & 126.1979 & 126.8798 & 129.2015 & 117.4817 & 117.1567 & 118.2005 \\
\hline $4^{\prime}$ & 70.6 & 122.3018 & 122.9387 & 117.8840 & 109.8378 & 109.8673 & 108.1295 & 122.4595 & 123.0776 & 118.8533 & 110.3976 & 110.3553 & 108.8757 \\
\hline 6 & 75.0 & 119.4787 & 120.1640 & 120.8200 & 110.2505 & 110.0530 & 110.0561 & 118.8241 & 119.5157 & 119.9319 & 109.5493 & 109.3407 & 109.1830 \\
\hline 3 & 121.3 & 74.9238 & 75.3412 & 74.7729 & 59.5015 & 58.7025 & 57.7965 & 76.6385 & 77.0562 & 76.7489 & 61.4302 & 60.6671 & 59.8559 \\
\hline 2"/6" & 126.5 & 71.6577 & 72.2197 & 70.5562 & 56.7667 & 55.9323 & 55.1620 & 71.8420 & 72.3921 & 70.9081 & 56.7823 & 55.9573 & 55.1406 \\
\hline $4 "$ & 127.8 & 71.7375 & 72.2669 & 70.4692 & 55.4096 & 54.5833 & 53.3518 & 71.5928 & 72.1133 & 70.6787 & 55.3344 & 54.5189 & 53.4070 \\
\hline 3"/5" & 128.6 & 70.6449 & 71.1562 & 70.9322 & 55.1972 & 54.2065 & 53.1125 & 70.4249 & 70.9330 & 70.5412 & 54.8968 & 53.9325 & 52.9565 \\
\hline $5^{\prime}$ & 130.4 & 68.3131 & 68.5902 & 70.0995 & 53.5690 & 52.3097 & 52.1613 & 66.4573 & 66.7446 & 68.7687 & 51.7076 & 50.4743 & 50.7662 \\
\hline $6^{\prime}$ & 131.5 & 66.0318 & 66.3688 & 63.4351 & 50.0811 & 49.3349 & 47.6039 & 67.5210 & 67.8458 & 64.4399 & 51.3975 & 50.6493 & 48.5124 \\
\hline $1 "$ & 136.5 & 63.5611 & 63.2076 & 62.0750 & 47.4561 & 47.0026 & 46.2405 & 63.6562 & 63.3173 & 61.8323 & 47.2731 & 46.8710 & 45.9445 \\
\hline 4 & 145.4 & 54.1233 & 54.7150 & 53.9301 & 37.6511 & 36.7987 & 35.5925 & 49.6621 & 50.2452 & 48.7691 & 32.6732 & 31.7817 & 30.1238 \\
\hline 2 & 164.5 & 40.6629 & 40.6960 & 37.9533 & 22.8805 & 23.0338 & 19.9396 & 38.5400 & 38.5565 & 35.4571 & 20.5238 & 20.6530 & 17.1330 \\
\hline
\end{tabular}




\begin{tabular}{|c|c|c|c|c|c|c|c|c|c|c|c|c|c|}
\hline $1^{\prime} / 3^{\prime}$ & 1.86 & 30.3794 & 29.9534 & 29.9177 & 30.2883 & 30.0737 & 30.0417 & 30.2573 & 29.8259 & 29.7987 & 30.1876 & 29.9664 & 29.9377 \\
\hline 5 & 2.40 & 30.0587 & 29.5750 & 29.3567 & 29.8779 & 29.6196 & 29.5066 & 29.8921 & 29.4002 & 29.1996 & 29.7366 & 29.4670 & 29.3533 \\
\hline $2^{\prime}$ & 4.41 & 27.7510 & 27.2775 & 26.8395 & 27.6782 & 27.4408 & 27.2699 & 27.7979 & 27.3259 & 26.9702 & 27.7403 & 27.4969 & 27.3382 \\
\hline $4^{\prime}$ & 4.68 & 27.4139 & 26.9199 & 26.9506 & 27.3507 & 27.0959 & 27.1354 & 27.3655 & 26.8675 & 26.8351 & 27.2603 & 27.0034 & 27.0253 \\
\hline 6 & 4.78 & 27.2603 & 26.8004 & 26.6304 & 27.2742 & 27.0795 & 27.0182 & 27.1411 & 26.6801 & 26.5881 & 27.1998 & 26.9990 & 26.9479 \\
\hline 3 & 6.04 & 26.2371 & 25.5605 & 25.4107 & 26.2350 & 25.7390 & 25.6561 & 26.2377 & 25.5539 & 25.3863 & 26.2230 & 25.7270 & 25.6380 \\
\hline $5^{\prime}$ & 6.32 & 25.7433 & 25.0815 & 24.5174 & 25.4812 & 25.0253 & 24.8751 & 25.5654 & 24.9021 & 24.5054 & 25.3635 & 24.9030 & 24.8061 \\
\hline $6^{\prime}$ & 6.66 & 25.3460 & 24.6963 & 24.9104 & 25.4148 & 24.9135 & 24.9459 & 25.3870 & 24.7390 & 24.7558 & 25.3582 & 24.8718 & 24.8241 \\
\hline 4 & 6.92 & 25.3683 & 24.7340 & 24.5484 & 25.2610 & 24.8079 & 24.7390 & 25.0298 & 24.3945 & 24.1725 & 24.9160 & 24.4661 & 24.3719 \\
\hline $\mathrm{Ar}$ & 7.34 & 24.8139 & 24.1807 & 23.9640 & 24.6559 & 24.2104 & 24.1327 & 24.6992 & 24.0655 & 23.8505 & 24.5397 & 24.1011 & 24.0225 \\
\hline
\end{tabular}

\section{Compound 85c}<smiles>CC(C)(C)[C@H](O)C[C@@H](O)/C=C/c1ccccc1</smiles>

\begin{tabular}{|c|c|c|c|c|c|c|c|c|c|c|c|c|c|}
\hline & \multirow[b]{3}{*}{ Exp } & \multicolumn{12}{|c|}{ B3LYP } \\
\hline & & \multicolumn{6}{|c|}{ Gas phase } & \multicolumn{6}{|c|}{ PCM } \\
\hline & & 6-31G* & $6-31 G^{* *}$ & $6-31+G^{* *}$ & 6-311G* & 6-311G** & $6-311+G * *$ & $6-31 G^{*}$ & $6-31 \mathrm{G} * *$ & $6-31+G^{* *}$ & 6-311G* & 6-311G** & $6-311+G^{* *}$ \\
\hline \multicolumn{14}{|l|}{${ }^{13} \mathrm{C}$} \\
\hline 5 & 29.5 & 159.1567 & 160.3750 & 159.8006 & 150.2055 & 149.8145 & 149.3567 & 159.2729 & 160.4822 & 159.9104 & 150.3334 & 149.9237 & 149.3893 \\
\hline $1^{\prime}$ & 41.9 & 147.4873 & 148.5937 & 147.7060 & 137.5816 & 137.1488 & 137.0346 & 147.7745 & 148.8500 & 147.8032 & 137.8348 & 137.3865 & 137.1849 \\
\hline $3^{\prime}$ & 42.8 & 146.4259 & 147.6818 & 147.2706 & 136.7088 & 136.3386 & 136.4690 & 146.4924 & 147.7410 & 147.0887 & 136.7446 & 136.3655 & 136.3671 \\
\hline $2^{\prime}$ & 66.8 & 121.5809 & 122.6431 & 123.0277 & 111.4064 & 111.3325 & 111.1975 & 120.9874 & 122.0247 & 121.9879 & 110.7912 & 110.7227 & 110.3043 \\
\hline $4^{\prime}$ & 70.4 & 115.4859 & 116.0856 & 113.2733 & 102.2310 & 101.9177 & 101.6321 & 116.0270 & 116.6762 & 113.9200 & 102.8114 & 102.4922 & 102.3892 \\
\hline 6 & 77.0 & 112.7639 & 113.6909 & 113.5002 & 102.3560 & 102.2076 & 102.1702 & 111.8460 & 112.7506 & 111.9583 & 101.3143 & 101.1590 & 100.8478 \\
\hline 3 & 121.2 & 72.7414 & 73.2010 & 73.1051 & 55.5396 & 54.7328 & 54.1273 & 74.2519 & 74.6935 & 74.8736 & 57.2286 & 56.4512 & 55.9662 \\
\hline 2"/6" & 126.5 & 69.3553 & 69.9468 & 69.1223 & 52.4290 & 51.5660 & 50.6990 & 69.4528 & 70.0504 & 69.1715 & 52.4145 & 51.5631 & 50.6831 \\
\hline $4 "$ & 127.8 & 69.0693 & 69.5949 & 68.7717 & 50.9513 & 50.0483 & 49.2065 & 68.9452 & 69.5063 & 68.7354 & 50.8342 & 49.9660 & 49.2592 \\
\hline 3"/5" & 128.6 & 68.0335 & 68.6048 & 68.1317 & 50.5763 & 49.6033 & 48.4830 & 67.8885 & 68.4691 & 67.9498 & 50.3266 & 49.3877 & 48.3101 \\
\hline $5^{\prime}$ & 130.3 & 65.3784 & 65.8666 & 65.1657 & 48.3880 & 47.3903 & 46.5571 & 64.6276 & 65.0385 & 64.0082 & 47.3080 & 46.3664 & 45.5326 \\
\hline $6^{\prime}$ & 131.5 & 64.2501 & 64.3939 & 63.3156 & 46.2996 & 45.3048 & 43.9450 & 64.9791 & 65.1996 & 64.4719 & 47.0985 & 46.0733 & 44.7763 \\
\hline $1 "$ & 136.5 & 60.4948 & 60.2221 & 58.9988 & 41.5976 & 41.2357 & 40.1373 & 60.5346 & 60.2439 & 59.0349 & 41.5169 & 41.1738 & 39.9234 \\
\hline 4 & 145.2 & 51.4834 & 52.1723 & 50.7652 & 32.4280 & 31.6404 & 30.2318 & 47.4577 & 48.1723 & 46.1311 & 27.8540 & 27.0356 & 25.1448 \\
\hline $\begin{array}{c}2 \\
{ }^{1} \mathrm{H}\end{array}$ & 163.80 & 37.8554 & 37.8077 & 35.2988 & 17.7477 & 17.8123 & 14.9710 & 35.9249 & 35.8798 & 33.0163 & 15.5271 & 15.5744 & 12.2872 \\
\hline $1^{\prime} / 3^{\prime}$ & 1.96 & 30.4892 & 30.0731 & 29.9175 & 30.1415 & 29.9403 & 29.9125 & 30.4292 & 30.0112 & 29.8688 & 29.9917 & 29.7870 & 29.7850 \\
\hline 5 & 2.44 & 29.9996 & 29.4989 & 29.2626 & 29.4172 & 29.1369 & 29.1178 & 29.9117 & 29.4185 & 29.2364 & 29.1974 & 28.9063 & 28.9299 \\
\hline $2^{\prime}$ & 4.28 & 27.5209 & 27.0230 & 26.8880 & 27.3011 & 26.9922 & 27.0426 & 27.5355 & 27.0560 & 26.9337 & 27.2162 & 26.9054 & 26.9752 \\
\hline $4^{\prime}$ & 4.71 & 27.8006 & 27.3177 & 27.2279 & 27.4022 & 27.1688 & 27.2239 & 27.7768 & 27.2907 & 27.2358 & 27.2373 & 27.0013 & 27.0951 \\
\hline 6 & 4.71 & 27.5246 & 27.0742 & 26.9079 & 27.2320 & 27.0098 & 26.9905 & 27.4043 & 26.9539 & 26.8070 & 27.0284 & 26.7976 & 26.8161 \\
\hline 3 & 6.04 & 26.4677 & 25.7823 & 25.5984 & 26.2588 & 25.7913 & 25.7414 & 26.4676 & 25.7728 & 25.5798 & 26.1764 & 25.7100 & 25.6761 \\
\hline
\end{tabular}




\begin{tabular}{|c|c|c|c|c|c|c|c|c|c|c|c|c|c|}
\hline $5^{\prime}$ & 6.30 & 25.3777 & 24.7321 & 24.5417 & 25.1872 & 24.7433 & 24.7683 & 25.3273 & 24.6899 & 24.4675 & 25.0671 & 24.6362 & 24.6648 \\
\hline $6^{\prime}$ & 6.65 & 26.1572 & 25.5182 & 25.2233 & 25.7581 & 25.3115 & 25.2397 & 26.0630 & 25.4241 & 25.1223 & 25.6134 & 25.1825 & 25.1103 \\
\hline 4 & 6.90 & 25.5446 & 24.9208 & 24.6989 & 25.3232 & 24.9082 & 24.8499 & 25.2297 & 24.6059 & 24.3515 & 25.0114 & 24.6031 & 24.5153 \\
\hline $\mathrm{Ar}$ & 7.40 & 25.0223 & 24.3853 & 24.1759 & 24.8432 & 24.4255 & 24.3679 & 24.9201 & 24.2855 & 24.0724 & 24.7310 & 24.3221 & 24.2612 \\
\hline
\end{tabular}

\begin{tabular}{|c|c|c|c|c|c|c|c|c|c|c|c|c|c|}
\hline & & \multicolumn{12}{|c|}{ mPW1PW91 } \\
\hline & \multirow[b]{2}{*}{ Exp } & \multicolumn{6}{|c|}{ Gas phase } & \multicolumn{6}{|c|}{ PCM } \\
\hline & & 6-31G* & $6-31 G * *$ & $6-31+\mathrm{G} * *$ & 6-311G* & $6-311 G * *$ & $6-311+G * *$ & 6-31G* & $6-31 G * *$ & $6-31+G * *$ & 6-311G* & $6-311 G * *$ & $6-311+G * *$ \\
\hline \multicolumn{14}{|l|}{${ }^{13} \mathrm{C}$} \\
\hline 5 & 29.5 & 163.6186 & 164.7483 & 164.3155 & 155.6505 & 155.2548 & 154.6896 & 163.7453 & 164.8742 & 164.4492 & 155.7572 & 155.3424 & 154.6970 \\
\hline $1^{\prime}$ & 41.9 & 152.4040 & 153.4164 & 152.6947 & 143.6570 & 143.2356 & 143.0504 & 152.7121 & 153.7035 & 152.8808 & 143.9316 & 143.4951 & 143.2291 \\
\hline $3^{\prime}$ & 42.8 & 151.6790 & 152.8162 & 152.1576 & 142.9304 & 142.5751 & 142.6032 & 151.8251 & 152.9668 & 152.0530 & 143.0280 & 142.6588 & 142.5636 \\
\hline $2^{\prime}$ & 66.8 & 126.4090 & 127.3820 & 127.8343 & 117.7912 & 117.7105 & 117.3608 & 125.9599 & 126.9296 & 126.9563 & 117.2815 & 117.1962 & 116.5762 \\
\hline $4^{\prime}$ & 70.4 & 120.2546 & 120.8244 & 118.4592 & 108.9163 & 108.6029 & 108.2346 & 120.7654 & 121.3742 & 119.0885 & 109.4413 & 109.1269 & 108.9178 \\
\hline 6 & 77.0 & 117.5441 & 118.3992 & 118.2109 & 108.5921 & 108.4465 & 108.2807 & 116.6663 & 117.5150 & 116.7462 & 107.5683 & 107.4117 & 106.9925 \\
\hline 3 & 121.2 & 75.0693 & 75.4752 & 75.4723 & 59.9716 & 59.1449 & 58.5482 & 76.6461 & 77.0378 & 77.2965 & 61.6965 & 60.9052 & 60.4239 \\
\hline 2"/6" & 126.5 & 71.8309 & 72.3641 & 71.5795 & 56.9656 & 56.0778 & 55.1003 & 71.9237 & 72.4609 & 71.6262 & 56.9334 & 56.0605 & 55.0727 \\
\hline $4 "$ & 127.8 & 71.4082 & 71.8589 & 71.0835 & 55.4675 & 54.5254 & 53.7033 & 71.2291 & 71.7117 & 70.9679 & 55.2824 & 54.3791 & 53.6771 \\
\hline 3"/5" & 128.6 & 70.5184 & 71.0036 & 70.9009 & 55.1966 & 54.1788 & 53.1052 & 70.3291 & 70.8225 & 70.6302 & 54.8928 & 53.9122 & 52.8856 \\
\hline $5^{\prime}$ & 130.3 & 67.8185 & 68.2002 & 67.6093 & 52.9750 & 51.9345 & 51.2041 & 66.9906 & 67.3014 & 66.4046 & 51.8247 & 50.8359 & 50.1059 \\
\hline $6^{\prime}$ & 131.5 & 67.0768 & 67.2027 & 66.5751 & 51.3274 & 50.3035 & 48.9116 & 67.8412 & 68.0391 & 67.7642 & 52.1361 & 51.0897 & 49.7498 \\
\hline 1" & 136.5 & 63.5338 & 63.2275 & 62.2999 & 47.2935 & 46.8912 & 45.6712 & 63.6140 & 63.2952 & 62.3915 & 47.2483 & 46.8659 & 45.4913 \\
\hline 4 & 145.2 & 53.7650 & 54.3614 & 53.1340 & 37.0459 & 36.2064 & 34.9030 & 49.5779 & 50.1961 & 48.3922 & 32.3921 & 31.5233 & 29.8102 \\
\hline 2 & 163.80 & 40.1698 & 40.1274 & 38.3556 & 22.7571 & 22.8183 & 20.2522 & 38.1484 & 38.1032 & 36.0447 & 20.5077 & 20.5548 & 17.6028 \\
\hline \multicolumn{14}{|l|}{${ }^{1} \mathrm{H}$} \\
\hline $1^{\prime} / 3^{\prime}$ & 1.96 & 30.4163 & 29.9989 & 29.8609 & 29.9625 & 29.7603 & 29.7485 & 30.3488 & 29.9289 & 29.8013 & 29.7859 & 29.5814 & 29.5963 \\
\hline 5 & 2.44 & 29.9432 & 29.4423 & 29.2231 & 29.1942 & 28.9043 & 28.9098 & 29.8394 & 29.3444 & 29.1744 & 28.9216 & 28.6217 & 28.6734 \\
\hline $2^{\prime}$ & 4.28 & 27.5105 & 27.0214 & 26.8637 & 27.1645 & 26.8526 & 26.9073 & 27.5235 & 27.0518 & 26.9019 & 27.0465 & 26.7317 & 26.8088 \\
\hline $4^{\prime}$ & 4.71 & 27.6829 & 27.2045 & 27.1467 & 27.2303 & 26.9831 & 27.0572 & 27.6344 & 27.1494 & 27.1224 & 27.0155 & 26.7653 & 26.8796 \\
\hline 6 & 4.71 & 27.4541 & 27.0047 & 26.8582 & 27.0313 & 26.8039 & 26.8125 & 27.3344 & 26.8852 & 26.7512 & 26.7945 & 26.5583 & 26.6064 \\
\hline 3 & 6.04 & 26.2662 & 25.5866 & 25.4256 & 26.0463 & 25.5495 & 25.5222 & 26.2625 & 25.5743 & 25.4054 & 25.9403 & 25.4474 & 25.4372 \\
\hline $5^{\prime}$ & 6.30 & 25.2226 & 24.5849 & 24.4196 & 24.9977 & 24.5436 & 24.5851 & 25.1639 & 24.5340 & 24.3406 & 24.8589 & 24.4172 & 24.4618 \\
\hline $6^{\prime}$ & 6.65 & 25.9310 & 25.2952 & 25.0358 & 25.5415 & 25.0789 & 25.0212 & 25.8190 & 25.1817 & 24.9180 & 25.3760 & 24.9290 & 24.8738 \\
\hline 4 & 6.90 & 25.3632 & 24.7335 & 24.5437 & 25.1458 & 24.7002 & 24.6538 & 25.0335 & 24.4038 & 24.1894 & 24.8259 & 24.3867 & 24.3142 \\
\hline $\mathrm{Ar}$ & 7.40 & 24.8062 & 24.1673 & 23.9925 & 24.6593 & 24.2085 & 24.1595 & 24.6886 & 24.0516 & 23.8727 & 24.5353 & 24.0935 & 24.0403 \\
\hline
\end{tabular}




\section{Compound 85d}

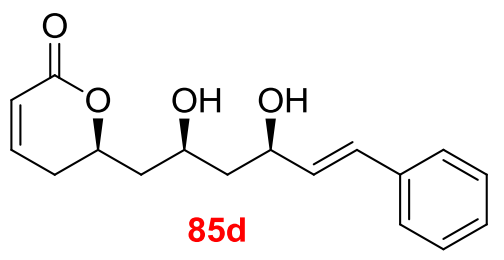

\begin{tabular}{|c|c|c|c|c|c|c|c|c|c|c|c|c|c|}
\hline & \multirow[b]{3}{*}{ Exp } & \multicolumn{12}{|c|}{ B3LYP } \\
\hline & & \multicolumn{6}{|c|}{ Gas phase } & \multicolumn{6}{|c|}{ PCM } \\
\hline & & 6-31G* & $6-31 \mathrm{G} * *$ & $6-31+\mathrm{G} * *$ & 6-311G* & $6-311 G * *$ & $6-311+G * *$ & 6-31G* & $6-31 G * *$ & $6-31+G * *$ & 6-311G* & $6-311 G * *$ & $6-311+G * *$ \\
\hline \multicolumn{14}{|l|}{${ }^{13} \mathrm{C}$} \\
\hline 5 & 29.4 & 159.4195 & 160.6514 & 159.7595 & 150.3395 & 149.9561 & 149.7520 & 159.3978 & 160.6135 & 159.7437 & 150.3593 & 149.9624 & 149.7095 \\
\hline $1^{\prime}$ & 42.2 & 147.3157 & 148.4649 & 147.4844 & 137.4463 & 137.0318 & 136.8800 & 147.5530 & 148.6654 & 147.6360 & 137.6728 & 137.2421 & 137.0755 \\
\hline $3^{\prime}$ & 43.2 & 145.8850 & 147.0909 & 146.0709 & 135.9017 & 135.4571 & 135.4384 & 145.9935 & 147.1859 & 146.1214 & 136.0182 & 135.5785 & 135.5051 \\
\hline $2^{\prime}$ & 69.3 & 118.8192 & 119.8543 & 119.4612 & 107.8725 & 107.8017 & 107.5494 & 118.0203 & 119.0227 & 118.3064 & 107.2701 & 107.2260 & 106.7362 \\
\hline $4^{\prime}$ & 73.6 & 112.7182 & 113.2997 & 111.1978 & 99.6928 & 99.4592 & 99.2899 & 113.4046 & 114.0321 & 111.9992 & 100.4641 & 100.1889 & 100.0991 \\
\hline 6 & 76.4 & 113.1796 & 114.1307 & 113.7882 & 102.4914 & 102.3547 & 101.7823 & 112.1640 & 113.1010 & 112.4017 & 101.4703 & 101.3597 & 100.4966 \\
\hline 3 & 121.2 & 72.7242 & 73.2043 & 73.0778 & 55.5709 & 54.7851 & 54.2295 & 74.2363 & 74.6883 & 74.8637 & 57.2595 & 56.4877 & 56.0813 \\
\hline 2"/6" & 126.5 & 69.1970 & 69.7963 & 69.2297 & 52.3724 & 51.5090 & 50.7142 & 69.2927 & 69.8921 & 69.2364 & 52.3235 & 51.4678 & 50.7348 \\
\hline $4 "$ & 127.8 & 68.8529 & 69.3803 & 68.9543 & 50.8447 & 49.9322 & 49.1943 & 68.7894 & 69.3542 & 68.9185 & 50.7710 & 49.8922 & 49.2447 \\
\hline 3"/5" & 128.6 & 68.0629 & 68.6348 & 67.6670 & 50.5930 & 49.6162 & 48.6843 & 67.9333 & 68.5159 & 67.4132 & 50.3512 & 49.4086 & 48.5159 \\
\hline $5^{\prime}$ & 130.4 & 64.1484 & 64.7072 & 64.1475 & 47.3281 & 46.3936 & 45.2818 & 63.4509 & 63.9254 & 63.2813 & 46.2822 & 45.4029 & 44.3598 \\
\hline $6^{\prime}$ & 131.4 & 64.5193 & 64.7052 & 63.4933 & 47.0567 & 46.0630 & 44.5727 & 65.1379 & 65.3778 & 64.2200 & 47.6571 & 46.6478 & 45.1248 \\
\hline $1 "$ & 136.4 & 60.7564 & 60.4973 & 58.2720 & 41.7124 & 41.3713 & 40.3947 & 60.6723 & 60.3877 & 57.7640 & 41.4919 & 41.1765 & 40.1352 \\
\hline 4 & 145.3 & 51.4429 & 52.0979 & 50.9021 & 32.3573 & 31.5578 & 30.0618 & 47.4504 & 48.1414 & 46.2797 & 27.7870 & 26.9726 & 24.9474 \\
\hline 2 & 164.1 & 37.6084 & 37.5380 & 35.2648 & 17.5500 & 17.6016 & 14.8937 & 35.7694 & 35.6985 & 33.0852 & 15.3379 & 15.3612 & 12.2113 \\
\hline \multicolumn{14}{|l|}{${ }^{1} \mathrm{H}$} \\
\hline $1^{\prime} / 3^{\prime}$ & 1.89 & 30.5690 & 30.1599 & 30.0337 & 30.4804 & 30.2902 & 30.2147 & 30.5190 & 30.1070 & 29.9933 & 30.4383 & 30.2426 & 30.1652 \\
\hline 5 & 2.44 & 29.8938 & 29.3789 & 29.1960 & 29.7339 & 29.4638 & 29.3858 & 29.8406 & 29.3342 & 29.1974 & 29.6831 & 29.4070 & 29.3428 \\
\hline $2^{\prime}$ & 4.23 & 27.7225 & 27.2571 & 27.2047 & 27.7448 & 27.4998 & 27.5122 & 27.6957 & 27.2463 & 27.1922 & 27.7139 & 27.4721 & 27.4689 \\
\hline $4^{\prime}$ & 4.60 & 27.6085 & 27.1623 & 27.1456 & 27.5624 & 27.3276 & 27.3749 & 27.5276 & 27.0781 & 27.0222 & 27.4452 & 27.2146 & 27.2482 \\
\hline 6 & 4.70 & 27.5371 & 27.0988 & 26.9946 & 27.5479 & 27.3608 & 27.3167 & 27.3957 & 26.9534 & 26.8638 & 27.4361 & 27.2406 & 27.1877 \\
\hline 3 & 6.03 & 26.4746 & 25.7963 & 25.6091 & 26.4161 & 25.9552 & 25.8465 & 26.4621 & 25.7718 & 25.5782 & 26.3966 & 25.9326 & 25.8232 \\
\hline $5^{\prime}$ & 6.23 & 25.8338 & 25.2224 & 24.9644 & 25.7288 & 25.3162 & 25.2460 & 25.7773 & 25.1614 & 24.8690 & 25.6399 & 25.2298 & 25.1542 \\
\hline $6^{\prime}$ & 6.61 & 26.1126 & 25.4832 & 25.1662 & 25.8567 & 25.4004 & 25.2560 & 26.0039 & 25.3770 & 25.0360 & 25.7377 & 25.2927 & 25.1445 \\
\hline 4 & 6.90 & 25.5312 & 24.9106 & 24.6804 & 25.3820 & 24.9732 & 24.9057 & 25.2115 & 24.5891 & 24.3311 & 25.0548 & 24.6511 & 24.5599 \\
\hline $\mathrm{Ar}$ & 7.32 & 25.0519 & 24.4132 & 24.1763 & 24.8648 & 24.4469 & 24.3715 & 24.9502 & 24.3144 & 24.0671 & 24.7539 & 24.3455 & 24.2599 \\
\hline
\end{tabular}

\begin{tabular}{|c|c|c|c|c|c|c|c|c|c|c|c|c|c|}
\hline & \multirow[b]{3}{*}{ Exp } & \multicolumn{12}{|c|}{ mPW1PW91 } \\
\hline & & \multicolumn{6}{|c|}{ Gas phase } & \multicolumn{6}{|c|}{ PCM } \\
\hline & & 6-31G* & $6-31 G * *$ & $6-31+G * *$ & 6-311G* & $6-311 G * *$ & $6-311+G * *$ & 6-31G* & $6-31 G * *$ & $6-31+G * *$ & 6-311G* & $6-311 \mathrm{G} * *$ & $6-311+G * *$ \\
\hline \multicolumn{14}{|c|}{${ }^{13} \mathrm{C}$} \\
\hline 5 & 29.4 & 163.9001 & 165.0274 & 164.1734 & 155.7626 & 155.3704 & 155.0778 & 163.8693 & 164.9897 & 164.1576 & 155.7598 & 155.3552 & 155.0009 \\
\hline $1^{\prime}$ & 42.2 & 152.2653 & 153.3101 & 152.5979 & 143.5133 & 143.1050 & 142.8927 & 152.5191 & 153.5364 & 152.7966 & 143.7591 & 143.3363 & 143.0949 \\
\hline
\end{tabular}




\begin{tabular}{|c|c|c|c|c|c|c|c|c|c|c|c|c|c|}
\hline 3' & 43.2 & 151.0967 & 152.1765 & 151.2323 & 142.1815 & 141.7492 & 141.6447 & 151.2321 & 152.3031 & 151.3082 & 142.3144 & 141.8857 & 141.7289 \\
\hline $2^{\prime}$ & 69.3 & 123.7367 & 124.6453 & 124.3925 & 114.4085 & 114.3107 & 113.9668 & 123.0003 & 123.8915 & 123.3046 & 113.8638 & 113.7855 & 113.2226 \\
\hline $4^{\prime}$ & 73.6 & 117.4789 & 118.0539 & 116.3804 & 106.4728 & 106.2514 & 105.9678 & 118.1637 & 118.7752 & 117.1679 & 107.2037 & 106.9429 & 106.7310 \\
\hline 6 & 76.4 & 118.0532 & 118.9078 & 118.6572 & 108.7706 & 108.6246 & 107.9961 & 116.9974 & 117.8479 & 117.2339 & 107.7195 & 107.5966 & 106.7083 \\
\hline 3 & 121.2 & 75.0402 & 75.4690 & 75.4620 & 60.0005 & 59.1924 & 58.6361 & 76.6278 & 77.0328 & 77.3030 & 61.7267 & 60.9393 & 60.5174 \\
\hline 2"/6" & 126.5 & 71.6401 & 72.1829 & 71.6827 & 56.8792 & 55.9958 & 55.1679 & 71.7402 & 72.2826 & 71.7320 & 56.8215 & 55.9489 & 55.1782 \\
\hline $4 "$ & 127.8 & 71.1639 & 71.6263 & 71.3118 & 55.3469 & 54.4022 & 53.6735 & 71.0701 & 71.5628 & 71.2297 & 55.2122 & 54.3021 & 53.6584 \\
\hline 3"/5" & 128.6 & 70.5471 & 71.0357 & 70.4130 & 55.2147 & 54.1956 & 53.2684 & 70.3793 & 70.8763 & 70.0789 & 54.9204 & 53.9376 & 53.0549 \\
\hline $5^{\prime}$ & 130.4 & 66.8498 & 67.2655 & 67.0139 & 52.0695 & 51.0660 & 50.0703 & 66.0170 & 66.3621 & 66.0582 & 50.9276 & 49.9816 & 49.0576 \\
\hline $6^{\prime}$ & 131.4 & 67.1713 & 67.3551 & 66.4241 & 51.9139 & 50.9189 & 49.4106 & 67.8663 & 68.0988 & 67.2259 & 52.5600 & 51.5519 & 49.9992 \\
\hline 1" & 136.4 & 63.8007 & 63.4951 & 61.4681 & 47.3899 & 47.0017 & 45.9332 & 63.7320 & 63.4100 & 61.0345 & 47.2045 & 46.8457 & 45.7263 \\
\hline 4 & 145.3 & 53.7437 & 54.3021 & 53.2729 & 36.9730 & 36.1202 & 34.7292 & 49.5832 & 50.1724 & 48.5528 & 32.3224 & 31.4539 & 29.6146 \\
\hline $\begin{array}{c}2 \\
{ }^{1} \mathrm{H}\end{array}$ & 164.1 & 39.9132 & 39.8614 & 38.3035 & 22.5700 & 22.6248 & 20.1599 & 38.0122 & 37.9560 & 36.1244 & 20.3451 & 20.3736 & 17.5250 \\
\hline $1^{\prime} / 3^{\prime}$ & 1.89 & 30.4851 & 30.0748 & 29.9623 & 30.3696 & 30.1744 & 30.1042 & 30.4313 & 30.0174 & 29.9160 & 30.3239 & 30.1227 & 30.0509 \\
\hline 5 & 2.44 & 29.8323 & 29.3196 & 29.1516 & 29.6538 & 29.3791 & 29.3034 & 29.7647 & 29.2580 & 29.1354 & 29.5895 & 29.3087 & 29.2457 \\
\hline $2^{\prime}$ & 4.23 & 27.6963 & 27.2377 & 27.1614 & 27.6905 & 27.4500 & 27.4497 & 27.6577 & 27.2133 & 27.1320 & 27.6494 & 27.4120 & 27.3961 \\
\hline $4^{\prime}$ & 4.60 & 27.5275 & 27.0822 & 27.0583 & 27.4652 & 27.2254 & 27.2634 & 27.4317 & 26.9831 & 26.9249 & 27.3407 & 27.1046 & 27.1311 \\
\hline 6 & 4.70 & 27.4574 & 27.0193 & 26.9317 & 27.4407 & 27.2558 & 27.2158 & 27.3199 & 26.8767 & 26.7976 & 27.3303 & 27.1367 & 27.0870 \\
\hline 3 & 6.03 & 26.2697 & 25.5977 & 25.4295 & 26.2525 & 25.7599 & 25.6655 & 26.2515 & 25.5678 & 25.3949 & 26.2293 & 25.7342 & 25.6391 \\
\hline $5^{\prime}$ & 6.23 & 25.6542 & 25.0386 & 24.8205 & 25.5674 & 25.1367 & 25.0833 & 25.5797 & 24.9600 & 24.7173 & 25.4661 & 25.0385 & 24.9817 \\
\hline $6^{\prime}$ & 6.61 & 25.8869 & 25.2576 & 24.9843 & 25.6724 & 25.1940 & 25.0636 & 25.7670 & 25.1395 & 24.8442 & 25.5469 & 25.0794 & 24.9442 \\
\hline 4 & 6.90 & 25.3468 & 24.7198 & 24.5202 & 25.2294 & 24.7884 & 24.7306 & 25.0101 & 24.3812 & 24.1608 & 24.8897 & 24.4536 & 24.3750 \\
\hline $\mathrm{Ar}$ & 7.32 & 24.8312 & 24.1907 & 23.9933 & 24.6805 & 24.2291 & 24.1652 & 24.7150 & 24.0770 & 23.8704 & 24.5583 & 24.1167 & 24.0430 \\
\hline
\end{tabular}

\section{Compound 86a}

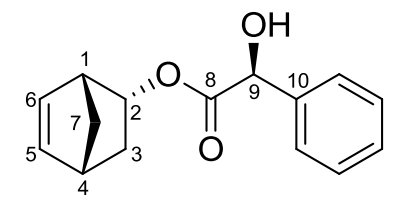

$86 a$

\begin{tabular}{|c|c|c|c|c|c|c|c|c|c|c|c|c|c|}
\hline & \multirow[b]{3}{*}{ Exp } & \multicolumn{12}{|c|}{ B3LYP } \\
\hline & & \multicolumn{6}{|c|}{ Gas phase } & \multicolumn{6}{|c|}{ PCM } \\
\hline & & 6-31G* & $6-31 G * *$ & $6-31+\mathrm{G} * *$ & 6-311G* & 6-311G** & $6-311+G * *$ & 6-31G* & $6-31 G * *$ & $6-31+G * *$ & 6-311G* & $6-311 G * *$ & $6-311+\mathrm{G} * *$ \\
\hline \multicolumn{14}{|c|}{${ }^{13} \mathrm{C}$} \\
\hline 1 & 45.6 & 141.7165 & 142.6020 & 142.5365 & 131.5814 & 131.2302 & 130.8514 & 141.6254 & 142.5131 & 142.4332 & 131.4779 & 131.1282 & 130.7297 \\
\hline 2 & 77.0 & 111.8191 & 112.6868 & 112.1858 & 100.6475 & 100.3808 & 100.2922 & 111.2149 & 112.0799 & 111.5541 & 99.9989 & 99.7356 & 99.5822 \\
\hline 3 & 34.2 & 153.8456 & 155.1645 & 155.2558 & 145.5369 & 145.1804 & 145.2900 & 153.9736 & 155.2853 & 155.3943 & 145.7011 & 145.3279 & 145.4329 \\
\hline 4 & 42.0 & 145.2117 & 146.1043 & 145.3079 & 134.8941 & 134.5521 & 134.3772 & 145.1642 & 146.0554 & 145.2446 & 134.8514 & 134.5037 & 134.3004 \\
\hline 5 & 138.5 & 55.9397 & 56.4301 & 55.8493 & 36.9448 & 36.0014 & 35.0964 & 55.2839 & 55.7766 & 55.0154 & 36.1096 & 35.1899 & 34.1898 \\
\hline 6 & 131.0 & 61.8331 & 62.3613 & 62.2714 & 43.4868 & 42.5943 & 41.8208 & 61.9983 & 62.5226 & 62.4236 & 43.6015 & 42.7359 & 41.9483 \\
\hline 7 & 47.4 & 141.0556 & 142.3394 & 141.4638 & 131.1347 & 130.7100 & 130.4597 & 141.0409 & 142.3164 & 141.3702 & 131.1077 & 130.6683 & 130.3655 \\
\hline 8 & 173.5 & 21.5630 & 21.6086 & 19.1945 & 0.0877 & 0.2553 & -1.6358 & 21.2107 & 21.2445 & 18.6910 & -0.3566 & -0.2410 & -2.2504 \\
\hline 9 & 72.5 & 118.8514 & 119.3646 & 118.2690 & 107.4972 & 106.8252 & 106.2675 & 119.0695 & 119.5826 & 118.4122 & 107.6835 & 107.0067 & 106.4259 \\
\hline
\end{tabular}




\begin{tabular}{lllllllllllllll}
\hline 10 & 138.1 & 57.0784 & 56.8187 & 54.5098 & 37.1145 & 36.7423 & 35.3693 & 57.1563 & 56.8972 & 54.5711 & 37.0861 & 36.7452 & 35.5090 \\
${ }^{1} \mathbf{H}$ & & & & & & & & & & & & & & \\
1 & 3.19 & 29.0932 & 28.5493 & 28.4152 & 29.0281 & 28.7042 & 28.6497 & 29.0571 & 28.5108 & 28.3818 & 28.9973 & 28.6730 & 28.6160 \\
2 & 5.34 & 26.8414 & 26.2870 & 26.2239 & 26.9081 & 26.5502 & 26.4787 & 26.8209 & 26.2684 & 26.1971 & 26.8861 & 26.5299 & 26.4559 \\
$3 \mathrm{n}$ & 0.68 & 31.1628 & 30.7466 & 30.7555 & 31.1777 & 30.9795 & 31.0145 & 31.1797 & 30.7611 & 30.7607 & 31.1876 & 30.9868 & 31.0117 \\
$3 \mathrm{x}$ & 2.04 & 30.1699 & 29.7055 & 29.6552 & 30.1848 & 29.9239 & 29.8853 & 30.1012 & 29.6348 & 29.5705 & 30.1080 & 29.8474 & 29.8066 \\
4 & 2.78 & 29.5089 & 28.9743 & 28.8715 & 29.4765 & 29.1821 & 29.1341 & 29.4297 & 28.8919 & 28.7820 & 29.3934 & 29.0965 & 29.0451 \\
5 & 6.22 & 25.7644 & 25.0678 & 24.8450 & 25.6837 & 25.2259 & 25.1645 & 25.6708 & 24.9745 & 24.7412 & 25.5792 & 25.1268 & 25.0611 \\
6 & 5.92 & 25.9751 & 25.2915 & 25.1698 & 25.9717 & 25.5223 & 25.4662 & 25.9374 & 25.2539 & 25.1284 & 25.9255 & 25.4819 & 25.4254 \\
$7 s$ & 1.47 & 30.5585 & 30.1475 & 30.0770 & 30.5594 & 30.3581 & 30.2895 & 30.5613 & 30.1491 & 30.0911 & 30.5721 & 30.3691 & 30.3027 \\
$7 \mathrm{a}$ & 1.28 & 30.8513 & 30.4034 & 30.3273 & 30.8406 & 30.6059 & 30.5473 & 30.7764 & 30.3264 & 30.2385 & 30.7606 & 30.5251 & 30.4620 \\
9 & 5.08 & 27.0884 & 26.6352 & 26.4622 & 26.9639 & 26.7763 & 26.6887 & 27.0007 & 26.5420 & 26.3393 & 26.8611 & 26.6670 & 26.5617 \\
\hline
\end{tabular}

\begin{tabular}{|c|c|c|c|c|c|c|c|c|c|c|c|c|c|}
\hline & \multirow[b]{3}{*}{ Exp } & \multicolumn{12}{|c|}{ mPW1PW91 } \\
\hline & & \multicolumn{6}{|c|}{ Gas phase } & \multicolumn{6}{|c|}{ PCM } \\
\hline & & 6-31G* & $6-31 G * *$ & $6-31+\mathrm{G} * *$ & 6-311G* & $6-311 G * *$ & $6-311+G * *$ & 6-31G* & $6-31 G * *$ & $6-31+G * *$ & 6-311G* & $6-311 G * *$ & $6-311+G * *$ \\
\hline \multicolumn{14}{|c|}{${ }^{13} \mathrm{C}$} \\
\hline 1 & 45.6 & 146.1875 & 146.9981 & 146.8333 & 136.9921 & 136.6299 & 136.2529 & 146.1071 & 146.9196 & 146.7489 & 136.8972 & 136.5363 & 136.1436 \\
\hline 2 & 77.0 & 116.3057 & 117.1103 & 116.7917 & 106.3398 & 106.0780 & 105.9929 & 115.7068 & 116.5088 & 116.1657 & 105.6985 & 105.4400 & 105.3026 \\
\hline 3 & 34.2 & 158.5248 & 159.7283 & 159.7289 & 150.7765 & 150.4257 & 150.4185 & 158.6806 & 159.8765 & 159.8937 & 150.9535 & 150.5844 & 150.5750 \\
\hline 4 & 42.0 & 149.8337 & 150.6509 & 149.8350 & 140.4024 & 140.0407 & 139.8262 & 149.7994 & 150.6148 & 149.7885 & 140.3650 & 139.9982 & 139.7602 \\
\hline 5 & 138.5 & 58.3679 & 58.7718 & 58.2768 & 41.3867 & 40.4046 & 39.5958 & 57.6843 & 58.0904 & 57.4334 & 40.5251 & 39.5654 & 38.6763 \\
\hline 6 & 131.0 & 64.3378 & 64.7809 & 64.7311 & 47.8648 & 46.9410 & 46.2368 & 64.4759 & 64.9156 & 64.8651 & 47.9484 & 47.0563 & 46.3350 \\
\hline 7 & 47.4 & 145.4197 & 146.5917 & 145.5780 & 136.3685 & 135.9410 & 135.7007 & 145.4124 & 146.5771 & 145.5056 & 136.3374 & 135.8960 & 135.6122 \\
\hline 8 & 173.5 & 24.1576 & 24.1906 & 22.3566 & 5.3834 & 5.5436 & 3.8561 & 23.7776 & 23.7989 & 21.8319 & 4.9262 & 5.0343 & 3.2336 \\
\hline 9 & 72.5 & 122.6018 & 123.0870 & 122.3820 & 113.1965 & 112.5134 & 111.9764 & 122.8030 & 123.2892 & 122.5082 & 113.3483 & 112.6625 & 112.1110 \\
\hline 10 & 138.1 & 60.3732 & 60.0849 & 58.3248 & 42.9279 & 42.5244 & 41.2001 & 60.4441 & 60.1576 & 58.3532 & 42.8985 & 42.5287 & 41.3113 \\
\hline \multicolumn{14}{|l|}{${ }^{1} \mathrm{H}$} \\
\hline 1 & 3.19 & 28.9870 & 28.4414 & 28.3235 & 28.9171 & 28.5733 & 28.5257 & 28.9439 & 28.3958 & 28.2820 & 28.8801 & 28.5366 & 28.4856 \\
\hline 2 & 5.34 & 26.7832 & 26.2290 & 26.1495 & 26.7989 & 26.4447 & 26.3686 & 26.7615 & 26.2089 & 26.1216 & 26.7773 & 26.4245 & 26.3450 \\
\hline $3 n$ & 0.68 & 31.1200 & 30.6999 & 30.6970 & 31.0887 & 30.8815 & 30.9175 & 31.1360 & 30.7136 & 30.7016 & 31.0959 & 30.8864 & 30.9130 \\
\hline $3 x$ & 2.04 & 30.1112 & 29.6449 & 29.5841 & 30.0821 & 29.8212 & 29.7724 & 30.0372 & 29.5683 & 29.4963 & 30.0022 & 29.7410 & 29.6910 \\
\hline 4 & 2.78 & 29.4228 & 28.8877 & 28.7908 & 29.3761 & 29.0635 & 29.0166 & 29.3351 & 28.7964 & 28.6939 & 29.2865 & 28.9712 & 28.9219 \\
\hline 5 & 6.22 & 25.5815 & 24.8814 & 24.6862 & 25.5406 & 25.0478 & 24.9918 & 25.4778 & 24.7779 & 24.5740 & 25.4285 & 24.9414 & 24.8816 \\
\hline 6 & 5.92 & 25.8040 & 25.1187 & 25.0075 & 25.8279 & 25.3471 & 25.2816 & 25.7595 & 25.0742 & 24.9605 & 25.7777 & 25.3029 & 25.2367 \\
\hline $7 \mathrm{~s}$ & 1.47 & 30.4844 & 30.0749 & 29.9925 & 30.4577 & 30.2514 & 30.1761 & 30.4868 & 30.0762 & 30.0042 & 30.4690 & 30.2613 & 30.1876 \\
\hline $7 a$ & 1.28 & 30.7930 & 30.3525 & 30.2800 & 30.7426 & 30.5104 & 30.4514 & 30.7123 & 30.2694 & 30.1864 & 30.6589 & 30.4257 & 30.3617 \\
\hline 9 & 5.08 & 27.0161 & 26.5592 & 26.3815 & 26.8762 & 26.6831 & 26.5911 & 26.9194 & 26.4568 & 26.2514 & 26.7666 & 26.5669 & 26.4588 \\
\hline
\end{tabular}




\section{Compound 86b}<smiles>O=C(O[C@H]1C[C@H]2C=C[C@@H]1C2)[C@H](O)c1ccccc1</smiles>

$86 b$

\begin{tabular}{|c|c|c|c|c|c|c|c|c|c|c|c|c|c|}
\hline \multirow{3}{*}{\multicolumn{2}{|c|}{ Exp }} & \multicolumn{12}{|c|}{ B3LYP } \\
\hline & & \multicolumn{6}{|c|}{ Gas phase } & \multicolumn{6}{|c|}{ PCM } \\
\hline & & 6-31G* & 6-31G** & $6-31+G * *$ & 6-311G* & 6-311G** & $6-311+G^{* *}$ & 6-31G* & 6-31G** & $6-31+\mathrm{G} * *$ & 6-311G* & 6-311G** & $6-311+G^{* *}$ \\
\hline \\
\hline 1 & 45.5 & 141.7945 & 142.6905 & 142.5016 & 131.8630 & 131.5207 & 131.3136 & 141.7659 & 142.6568 & 142.3825 & 131.7896 & 131.4451 & 131.2249 \\
\hline 2 & 76.8 & 111.8620 & 112.7266 & 112.1801 & 100.6875 & 100.4351 & 100.1026 & 111.2104 & 112.0750 & 111.4669 & 99.9144 & 99.6765 & 99.3176 \\
\hline 3 & 34.1 & 153.5422 & 154.9009 & 153.8473 & 144.5286 & 144.1837 & 143.8539 & 153.3732 & 154.7333 & 153.6811 & 144.2556 & 143.9280 & 143.5964 \\
\hline 4 & 42.0 & 145.1729 & 146.0712 & 145.2591 & 134.8843 & 134.5302 & 134.4979 & 145.1168 & 146.0117 & 145.1664 & 134.8004 & 134.4390 & 134.3940 \\
\hline 5 & 138.2 & 56.6959 & 57.2050 & 56.2073 & 37.8143 & 36.8763 & 35.8860 & 55.7301 & 56.2411 & 55.0675 & 36.6110 & 35.7102 & 34.7054 \\
\hline 6 & 130.9 & 61.3671 & 61.8951 & 61.6932 & 42.8357 & 41.9654 & 41.1575 & 61.8061 & 62.3363 & 62.0523 & 43.2300 & 42.3805 & 41.4977 \\
\hline 7 & 47.4 & 141.2843 & 142.5597 & 142.1369 & 131.4945 & 131.0672 & 130.8485 & 141.2772 & 142.5434 & 142.1647 & 131.5139 & 131.0688 & 130.8281 \\
\hline 8 & 173.7 & 21.3754 & 21.4275 & 19.1467 & -0.0861 & 0.0884 & -1.8287 & 20.9986 & 21.0402 & 18.5523 & -0.6011 & -0.4738 & -2.4793 \\
\hline 9 & 72.6 & 118.6924 & 119.2007 & 117.9320 & 107.3864 & 106.6931 & 106.1143 & 118.9314 & 119.4401 & 118.0959 & 107.5829 & 106.8885 & 106.2724 \\
\hline $\begin{array}{l}10 \\
{ }^{1} \mathbf{H}\end{array}$ & 138.3 & 56.8527 & 56.5982 & 54.2754 & 36.8461 & 36.4818 & 35.1023 & 57.0146 & 56.7597 & 54.3524 & 36.9009 & 36.5600 & 35.3000 \\
\hline 1 & 3.00 & 29.3691 & 28.8251 & 28.7322 & 29.3473 & 29.0241 & 28.9613 & 29.3613 & 28.8179 & 28.7146 & 29.3218 & 29.0023 & 28.9348 \\
\hline 2 & 5.34 & 26.8372 & 26.2856 & 26.2583 & 26.8996 & 26.5456 & 26.5033 & 26.8381 & 26.2876 & 26.2677 & 26.9034 & 26.5514 & 26.5139 \\
\hline $3 n$ & 0.99 & 30.9006 & 30.4691 & 30.5437 & 30.9605 & 30.7484 & 30.8130 & 30.9215 & 30.4883 & 30.5797 & 30.9939 & 30.7766 & 30.8523 \\
\hline $3 x$ & 2.13 & 29.9855 & 29.5289 & 29.3974 & 29.9389 & 29.6891 & 29.6217 & 29.8833 & 29.4237 & 29.2953 & 29.8424 & 29.5921 & 29.5284 \\
\hline 4 & 2.82 & 29.4766 & 28.9394 & 28.8350 & 29.4446 & 29.1460 & 29.0967 & 29.3950 & 28.8532 & 28.7420 & 29.3605 & 29.0587 & 29.0088 \\
\hline 5 & 6.22 & 25.8633 & 25.1692 & 24.9374 & 25.7860 & 25.3251 & 25.2583 & 25.7499 & 25.0556 & 24.8148 & 25.6586 & 25.2035 & 25.1342 \\
\hline 6 & 5.40 & 26.4914 & 25.8255 & 25.5695 & 26.4316 & 25.9960 & 25.8557 & 26.4098 & 25.7449 & 25.4395 & 26.2994 & 25.8733 & 25.7162 \\
\hline $7 \mathrm{~s}$ & 1.40 & 30.6711 & 30.2601 & 30.1840 & 30.6698 & 30.4631 & 30.3981 & 30.6633 & 30.2519 & 30.1820 & 30.6668 & 30.4602 & 30.3969 \\
\hline $7 a$ & 1.28 & 30.8875 & 30.4450 & 30.3941 & 30.8788 & 30.6571 & 30.6452 & 30.8058 & 30.3612 & 30.3086 & 30.7917 & 30.5695 & 30.5616 \\
\hline 9 & 5.06 & 27.0949 & 26.6437 & 26.4634 & 26.9576 & 26.7726 & 26.6887 & 27.0110 & 26.5535 & 26.3457 & 26.8617 & 26.6686 & 26.5650 \\
\hline
\end{tabular}

\begin{tabular}{|c|c|c|c|c|c|c|c|c|c|c|c|c|c|}
\hline \multirow{3}{*}{\multicolumn{2}{|c|}{ Exp }} & \multicolumn{12}{|c|}{ mPW1PW91 } \\
\hline & & \multicolumn{6}{|c|}{ Gas phase } & \multicolumn{6}{|c|}{ PCM } \\
\hline & & 6-31G* & 6-31G** & $6-31+G * *$ & 6-311G* & 6-311G** & $6-311+G * *$ & 6-31G* & 6-31G** & $6-31+G * *$ & 6-311G* & 6-311G** & $6-311+G^{* *}$ \\
\hline \multicolumn{14}{|c|}{${ }^{13} \mathrm{C}$} \\
\hline 1 & 45.5 & 146.2487 & 147.0678 & 146.8378 & 137.2384 & 136.8858 & 136.6500 & 146.2449 & 147.0576 & 146.7387 & 137.1800 & 136.8244 & 136.5720 \\
\hline 2 & 76.8 & 116.3633 & 117.1681 & 116.9280 & 106.4136 & 106.1668 & 105.8640 & 115.7100 & 116.5147 & 116.2174 & 105.6510 & 105.4182 & 105.0894 \\
\hline 3 & 34.1 & 158.3001 & 159.5478 & 158.5697 & 150.0137 & 149.6570 & 149.3854 & 158.1159 & 159.3661 & 158.4082 & 149.7367 & 149.3980 & 149.1074 \\
\hline 4 & 42.0 & 149.8040 & 150.6279 & 149.7758 & 140.3977 & 140.0257 & 139.9198 & 149.7521 & 150.5723 & 149.6912 & 140.3106 & 139.9321 & 139.8228 \\
\hline 5 & 138.2 & 59.2237 & 59.6528 & 58.7634 & 42.3314 & 41.3540 & 40.4489 & 58.1633 & 58.5934 & 57.5339 & 41.0481 & 40.1093 & 39.1952 \\
\hline 6 & 130.9 & 63.8302 & 64.2692 & 64.1422 & 47.2338 & 46.3242 & 45.5886 & 64.2977 & 64.7412 & 64.5190 & 47.6470 & 46.7605 & 45.9615 \\
\hline 7 & 47.4 & 145.6732 & 146.8369 & 146.1014 & 136.7320 & 136.3034 & 136.0650 & 145.6828 & 146.8378 & 146.1530 & 136.7531 & 136.3081 & 136.0585 \\
\hline
\end{tabular}




\begin{tabular}{cccccccccccccc}
\hline 8 & 173.7 & 23.9715 & 24.0114 & 22.3036 & 5.2277 & 5.3973 & 3.6798 & 23.5713 & 23.6008 & 21.7058 & 4.7071 & 4.8274 & 3.0294 \\
9 & 72.6 & 122.4545 & 122.9378 & 122.0393 & 113.0793 & 112.3828 & 111.8721 & 122.6807 & 123.1650 & 122.1778 & 113.2487 & 112.5515 & 112.0116 \\
10 & 138.3 & 60.1462 & 59.8652 & 58.0397 & 42.6226 & 42.2384 & 40.9289 & 60.3049 & 60.0250 & 58.1043 & 42.6862 & 42.3256 & 41.1030 \\
${ }^{1}$ H & & & & & & & & & & & & & \\
1 & 3.00 & 29.2665 & 28.7205 & 28.6242 & 29.2341 & 28.8909 & 28.8225 & 29.2560 & 28.7106 & 28.6060 & 29.2075 & 28.8682 & 28.7965 \\
2 & 5.34 & 26.7763 & 26.2235 & 26.1696 & 26.7861 & 26.4330 & 26.3774 & 26.7802 & 26.2284 & 26.1835 & 26.7927 & 26.4420 & 26.3922 \\
$3 n$ & 0.99 & 30.8451 & 30.4102 & 30.4596 & 30.8486 & 30.6314 & 30.6763 & 30.8697 & 30.4332 & 30.4988 & 30.8859 & 30.6631 & 30.7203 \\
$3 \mathrm{~B}$ & 2.13 & 29.9308 & 29.4724 & 29.3522 & 29.8514 & 29.5992 & 29.5309 & 29.8174 & 29.3552 & 29.2361 & 29.7451 & 29.4925 & 29.4256 \\
4 & 2.82 & 29.3893 & 28.8527 & 28.7531 & 29.3427 & 29.0266 & 28.9775 & 29.2988 & 28.7569 & 28.6532 & 29.2514 & 28.9321 & 28.8828 \\
5 & 6.22 & 25.6861 & 24.9893 & 24.7863 & 25.6481 & 25.1525 & 25.0930 & 25.5582 & 24.8610 & 24.6506 & 25.5094 & 25.0197 & 24.9571 \\
6 & 5.40 & 26.3530 & 25.6859 & 25.4598 & 26.3309 & 25.8636 & 25.7414 & 26.2486 & 25.5837 & 25.3119 & 26.1855 & 25.7279 & 25.5892 \\
$7 \mathrm{7s}$ & 1.40 & 30.6037 & 30.1945 & 30.1124 & 30.5768 & 30.3647 & 30.2941 & 30.5928 & 30.1834 & 30.1073 & 30.5708 & 30.3590 & 30.2900 \\
$7 \mathrm{7a}$ & 1.28 & 30.8349 & 30.3996 & 30.3476 & 30.7856 & 30.5656 & 30.5465 & 30.7466 & 30.3090 & 30.2575 & 30.6940 & 30.4735 & 30.4598 \\
9 & 5.06 & 27.0200 & 26.5657 & 26.3859 & 26.8683 & 26.6776 & 26.5981 & 26.9276 & 26.4666 & 26.2602 & 26.7656 & 26.5667 & 26.4692 \\
\hline
\end{tabular}

\section{Compound 86c}

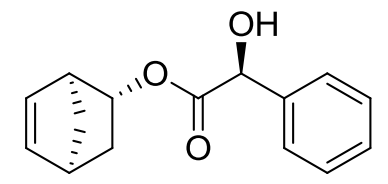

$86 c$

\begin{tabular}{|c|c|c|c|c|c|c|c|c|c|c|c|c|c|}
\hline \multirow{3}{*}{\multicolumn{2}{|c|}{ Exp }} & \multicolumn{12}{|c|}{ B3LYP } \\
\hline & & \multicolumn{6}{|c|}{ Gas phase } & \multicolumn{6}{|c|}{ PCM } \\
\hline & & 6-31G* & 6-31G** & $6-31+G^{* *}$ & 6-311G* & $6-311 G * *$ & $6-311+G * *$ & 6-31G* & 6-31G** & $6-31+G^{* *}$ & 6-311G* & 6-311G** & $6-311+G * *$ \\
\hline \multicolumn{14}{|c|}{${ }^{13} \mathrm{C}$} \\
\hline 1 & 47.1 & 139.8000 & 140.6328 & 140.2197 & 129.9816 & 129.5745 & 129.1617 & 139.7711 & 140.6054 & 140.1881 & 129.9284 & 129.5233 & 129.1027 \\
\hline 2 & 77.1 & 111.2693 & 112.1365 & 111.6266 & 100.1610 & 99.8377 & 99.5533 & 110.7571 & 111.6219 & 111.0935 & 99.6125 & 99.2910 & 98.9633 \\
\hline 3 & 34.4 & 153.4775 & 154.8651 & 155.0612 & 144.9324 & 144.5733 & 144.3899 & 153.5730 & 154.9532 & 155.1724 & 145.0595 & 144.6866 & 144.4960 \\
\hline 4 & 40.4 & 146.3516 & 147.2844 & 146.8197 & 136.6042 & 136.2783 & 136.1447 & 146.3246 & 147.2573 & 146.7603 & 136.5748 & 136.2423 & 136.0914 \\
\hline 5 & 141.4 & 52.9776 & 53.4760 & 52.5321 & 33.8166 & 32.8764 & 31.7815 & 52.4843 & 52.9831 & 51.9385 & 33.1887 & 32.2667 & 31.1195 \\
\hline 6 & 132.0 & 61.3363 & 61.8437 & 61.4997 & 43.0743 & 42.1151 & 41.0991 & 61.3627 & 61.8737 & 61.5199 & 43.0208 & 42.1015 & 41.0796 \\
\hline 7 & 46.1 & 141.7518 & 143.0498 & 143.4639 & 132.4733 & 132.0824 & 131.8557 & 141.7836 & 143.0758 & 143.4506 & 132.4853 & 132.0822 & 131.8204 \\
\hline 8 & 173.5 & 21.5466 & 21.5946 & 19.6249 & 0.1603 & 0.3226 & -1.5601 & 21.2023 & 21.2386 & 19.1164 & -0.3000 & -0.1905 & -2.1864 \\
\hline 9 & 72.8 & 118.0240 & 118.5425 & 117.4457 & 106.6903 & 105.9639 & 105.4763 & 118.3382 & 118.8552 & 117.6913 & 106.9832 & 106.2549 & 105.7451 \\
\hline $\begin{array}{l}10 \\
{ }^{1} \mathrm{H}\end{array}$ & 138.3 & 56.6064 & 56.3358 & 54.0751 & 36.4308 & 36.0699 & 35.0012 & 56.7786 & 56.5091 & 54.2921 & 36.5556 & 36.2168 & 35.2460 \\
\hline 1 & 2.94 & 29.3504 & 28.8168 & 28.7107 & 29.3093 & 28.9950 & 28.9579 & 29.3127 & 28.7781 & 28.6649 & 29.2664 & 28.9533 & 28.9120 \\
\hline 2 & 4.74 & 27.4915 & 26.9619 & 26.9225 & 27.5522 & 27.2411 & 27.1963 & 27.4773 & 26.9484 & 26.9098 & 27.5375 & 27.2282 & 27.1830 \\
\hline $3 n$ & 1.62 & 30.5681 & 30.1057 & 30.0905 & 30.6227 & 30.3842 & 30.3356 & 30.5280 & 30.0630 & 30.0487 & 30.5842 & 30.3439 & 30.2981 \\
\hline $3 x$ & 1.11 & 30.8565 & 30.4370 & 30.4426 & 30.8964 & 30.6776 & 30.7051 & 30.8507 & 30.4296 & 30.4272 & 30.8810 & 30.6623 & 30.6888 \\
\hline 4 & 2.77 & 29.5819 & 29.0501 & 28.9731 & 29.5471 & 29.2545 & 29.2306 & 29.5002 & 28.9652 & 28.8817 & 29.4597 & 29.1648 & 29.1399 \\
\hline 5 & 6.22 & 25.9417 & 25.2443 & 25.0081 & 25.8698 & 25.4083 & 25.3272 & 25.8497 & 25.1529 & 24.9103 & 25.7688 & 25.3136 & 25.2308 \\
\hline 6 & 5.95 & 26.1817 & 25.4833 & 25.2470 & 26.1251 & 25.6577 & 25.5739 & 26.1217 & 25.4238 & 25.1842 & 26.0562 & 25.5955 & 25.5093 \\
\hline
\end{tabular}




\begin{tabular}{cccccccccccccc}
\hline $7 s$ & 1.56 & 30.4401 & 30.0199 & 29.9871 & 30.4699 & 30.2611 & 30.2217 & 30.4335 & 30.0111 & 29.9832 & 30.4671 & 30.2568 & 30.2159 \\
$7 \mathrm{a}$ & 1.56 & 30.4509 & 30.0034 & 29.9148 & 30.4602 & 30.2096 & 30.1620 & 30.4636 & 30.0170 & 29.9208 & 30.4646 & 30.2155 & 30.1680 \\
9 & 5.15 & 27.0562 & 26.6092 & 26.3926 & 26.8957 & 26.7096 & 26.6184 & 26.9578 & 26.5036 & 26.2564 & 26.7829 & 26.5884 & 26.4779 \\
\hline
\end{tabular}

\begin{tabular}{|c|c|c|c|c|c|c|c|c|c|c|c|c|c|}
\hline & & \multicolumn{12}{|c|}{ mPW1PW91 } \\
\hline & \multirow[b]{2}{*}{ Exp } & \multicolumn{6}{|c|}{ Gas phase } & \multicolumn{6}{|c|}{ PCM } \\
\hline & & 6-31G* & $6-31 G * *$ & $6-31+G * *$ & 6-311G* & $6-311 G * *$ & $6-311+G * *$ & 6-31G* & $6-31 G * *$ & $6-31+G * *$ & 6-311G* & $6-311 \mathrm{G} * *$ & $6-311+G * *$ \\
\hline \multicolumn{14}{|l|}{${ }^{13} \mathrm{C}$} \\
\hline 1 & 47.1 & 144.4272 & 145.1828 & 144.8310 & 135.5317 & 135.1069 & 134.7065 & 144.4019 & 145.1593 & 144.8093 & 135.4806 & 135.0587 & 134.6494 \\
\hline 2 & 77.1 & 115.7557 & 116.5579 & 116.3028 & 105.8379 & 105.5182 & 105.2770 & 115.2623 & 116.0615 & 115.7932 & 105.3096 & 104.9904 & 104.7164 \\
\hline 3 & 34.4 & 158.1917 & 159.4608 & 159.6031 & 150.1944 & 149.8418 & 149.5959 & 158.3168 & 159.5780 & 159.7403 & 150.3353 & 149.9688 & 149.7148 \\
\hline 4 & 40.4 & 150.8841 & 151.7395 & 151.2099 & 141.9502 & 141.5991 & 141.4224 & 150.8675 & 151.7225 & 151.1636 & 141.9247 & 141.5676 & 141.3772 \\
\hline 5 & 141.4 & 55.3442 & 55.7531 & 54.8522 & 38.2404 & 37.2644 & 36.2777 & 54.8246 & 55.2328 & 54.2531 & 37.5908 & 36.6316 & 35.6045 \\
\hline 6 & 132.0 & 63.9846 & 64.4019 & 64.0143 & 47.6441 & 46.6323 & 45.7474 & 63.9705 & 64.3934 & 63.9997 & 47.5462 & 46.5801 & 45.6850 \\
\hline 7 & 46.1 & 146.0754 & 147.2586 & 147.4768 & 137.5245 & 137.1336 & 136.8685 & 146.1259 & 147.3032 & 147.4880 & 137.5467 & 137.1430 & 136.8497 \\
\hline 8 & 173.5 & 24.1498 & 24.1855 & 22.7075 & 5.4598 & 5.6150 & 3.9573 & 23.7771 & 23.8010 & 22.1812 & 4.9868 & 5.0880 & 3.3275 \\
\hline 9 & 72.8 & 121.8286 & 122.3181 & 121.6266 & 112.4128 & 111.6827 & 111.2065 & 122.1278 & 122.6164 & 121.8591 & 112.6719 & 111.9414 & 111.4485 \\
\hline 10 & 138.3 & 59.9262 & 59.6276 & 57.9376 & 42.2519 & 41.8695 & 40.8090 & 60.0989 & 59.8025 & 58.1256 & 42.3834 & 42.0239 & 41.0391 \\
\hline \multicolumn{14}{|l|}{${ }^{1} \mathbf{H}$} \\
\hline 1 & 2.94 & 29.2427 & 28.7085 & 28.6152 & 29.1900 & 28.8579 & 28.8264 & 29.1978 & 28.6625 & 28.5617 & 29.1419 & 28.8111 & 28.7745 \\
\hline 2 & 4.74 & 27.4564 & 26.9280 & 26.8851 & 27.4639 & 27.1549 & 27.1137 & 27.4414 & 26.9133 & 26.8715 & 27.4490 & 27.1420 & 27.1002 \\
\hline $3 n$ & 1.62 & 30.5248 & 30.0609 & 30.0346 & 30.5269 & 30.2865 & 30.2333 & 30.4845 & 30.0176 & 29.9930 & 30.4888 & 30.2461 & 30.1968 \\
\hline $3 x$ & 1.11 & 30.8070 & 30.3846 & 30.3831 & 30.8076 & 30.5823 & 30.6199 & 30.7970 & 30.3727 & 30.3642 & 30.7877 & 30.5627 & 30.6002 \\
\hline 4 & 2.77 & 29.4950 & 28.9623 & 28.8921 & 29.4466 & 29.1371 & 29.1201 & 29.4042 & 28.8678 & 28.7927 & 29.3521 & 29.0403 & 29.0228 \\
\hline 5 & 6.22 & 25.7677 & 25.0663 & 24.8571 & 25.7303 & 25.2325 & 25.1565 & 25.6653 & 24.9644 & 24.7507 & 25.6216 & 25.1302 & 25.0534 \\
\hline 6 & 5.95 & 26.0204 & 25.3180 & 25.1096 & 25.9967 & 25.4946 & 25.4170 & 25.9495 & 25.2478 & 25.0373 & 25.9196 & 25.4248 & 25.3443 \\
\hline $7 s$ & 1.56 & 30.3654 & 29.9472 & 29.9027 & 30.3686 & 30.1559 & 30.1190 & 30.3565 & 29.9362 & 29.8957 & 30.3634 & 30.1495 & 30.1108 \\
\hline $7 a$ & 1.56 & 30.3979 & 29.9541 & 29.8708 & 30.3694 & 30.1173 & 30.0626 & 30.4083 & 29.9654 & 29.8760 & 30.3725 & 30.1217 & 30.0681 \\
\hline 9 & 5.15 & 26.9731 & 26.5237 & 26.3142 & 26.8029 & 26.6109 & 26.5210 & 26.8643 & 26.4070 & 26.1690 & 26.6821 & 26.4815 & 26.3742 \\
\hline
\end{tabular}

\section{Compound 86d}<smiles>O=C(O[C@H]1C[C@H]2C=C[C@@H]1C2)[C@H](O)c1ccccc1</smiles>

$86 d$

\begin{tabular}{|c|c|c|c|c|c|c|c|c|c|c|c|c|c|}
\hline & \multirow[b]{3}{*}{ Exp } & \multicolumn{12}{|c|}{ B3LYP } \\
\hline & & \multicolumn{6}{|c|}{ Gas phase } & \multicolumn{6}{|c|}{ PCM } \\
\hline & & 6-31G* & $6-31 G * *$ & $6-31+\mathrm{G} * *$ & 6-311G* & $6-311 G * *$ & $6-311+G^{* *}$ & 6-31G* & 6-31G** & $6-31+G * *$ & 6-311G* & $6-311 G * *$ & $6-311+G^{* *}$ \\
\hline \multicolumn{14}{|c|}{${ }^{13} \mathrm{C}$} \\
\hline 1 & 47.0 & 139.9139 & 140.7659 & 140.6822 & 130.3723 & 129.9825 & 129.6962 & 139.9724 & 140.8214 & 140.6602 & 130.3829 & 129.9921 & 129.6923 \\
\hline 2 & 77.1 & 111.2966 & 112.1658 & 111.4792 & 100.3007 & 99.9706 & 99.8050 & 110.7992 & 111.6675 & 110.9364 & 99.7480 & 99.4229 & 99.2121 \\
\hline
\end{tabular}




\begin{tabular}{cccccccccccccc}
\hline 3 & 34.3 & 153.5554 & 154.9308 & 154.5767 & 144.5826 & 144.2077 & 144.0952 & 153.5565 & 154.9255 & 154.5577 & 144.5612 & 144.1820 & 144.0771 \\
4 & 40.4 & 146.3046 & 147.2374 & 146.7068 & 136.6109 & 136.3028 & 136.0694 & 146.2737 & 147.2064 & 146.6240 & 136.5565 & 136.2417 & 135.9984 \\
5 & 141.3 & 53.2108 & 53.7060 & 52.4713 & 34.0872 & 33.1442 & 31.9835 & 52.5869 & 53.0857 & 51.7819 & 33.3347 & 32.4167 & 31.2096 \\
6 & 132.1 & 60.9686 & 61.4866 & 61.0236 & 42.7944 & 41.8523 & 41.0923 & 61.2381 & 61.7550 & 61.2238 & 43.0026 & 42.0844 & 41.3046 \\
7 & 46.0 & 141.8862 & 143.1785 & 143.6390 & 132.5657 & 132.1776 & 131.7974 & 141.8822 & 143.1708 & 143.5852 & 132.5465 & 132.1510 & 131.7368 \\
8 & 173.7 & 21.3332 & 21.3823 & 19.4207 & -0.0765 & 0.0844 & -1.6950 & 20.9825 & 21.0197 & 18.8872 & -0.5411 & -0.4365 & -2.3461 \\
9 & 72.8 & 118.0824 & 118.5961 & 117.5573 & 106.7695 & 106.0484 & 105.6605 & 118.3926 & 118.9045 & 117.7871 & 107.0560 & 106.3323 & 105.9068 \\
10 & 138.5 & 56.4588 & 56.1922 & 54.0476 & 36.2280 & 35.8596 & 34.9325 & 56.6729 & 56.4071 & 54.2659 & 36.4017 & 36.0540 & 35.2067 \\
1 H & & & & & & & & & & & & & \\
1 & 2.68 & 29.6580 & 29.1296 & 29.0669 & 29.6417 & 29.3330 & 29.3068 & 29.6503 & 29.1222 & 29.0473 & 29.6163 & 29.3126 & 29.2809 \\
2 & 4.74 & 27.4411 & 26.9095 & 26.9217 & 27.5117 & 27.1985 & 27.1650 & 27.4413 & 26.9100 & 26.9264 & 27.5135 & 27.2013 & 27.1711 \\
$3 n$ & 1.72 & 30.4309 & 29.9644 & 29.9055 & 30.4325 & 30.1858 & 30.1453 & 30.3606 & 29.8915 & 29.8414 & 30.3715 & 30.1235 & 30.0856 \\
$3 \mathrm{x}$ & 1.44 & 30.4853 & 30.0550 & 30.0651 & 30.5356 & 30.3132 & 30.3282 & 30.4621 & 30.0312 & 30.0442 & 30.5141 & 30.2909 & 30.3085 \\
4 & 2.82 & 29.5190 & 28.9900 & 28.8901 & 29.4776 & 29.1881 & 29.1397 & 29.4311 & 28.8986 & 28.7904 & 29.3845 & 29.0930 & 29.0416 \\
5 & 6.22 & 25.9587 & 25.2628 & 25.0012 & 25.8894 & 25.4290 & 25.3311 & 25.8580 & 25.1629 & 24.8964 & 25.7811 & 25.3269 & 25.2278 \\
6 & 5.90 & 26.2566 & 25.5580 & 25.3635 & 26.2182 & 25.7488 & 25.6651 & 26.2100 & 25.5120 & 25.3130 & 26.1599 & 25.6972 & 25.6131 \\
$7 s$ & 1.44 & 30.6204 & 30.2110 & 30.1641 & 30.6393 & 30.4431 & 30.4184 & 30.6032 & 30.1918 & 30.1445 & 30.6188 & 30.4217 & 30.3953 \\
$7 a$ & 1.44 & 30.6213 & 30.1792 & 30.1067 & 30.6117 & 30.3726 & 30.3477 & 30.6164 & 30.1751 & 30.0844 & 30.5895 & 30.3527 & 30.3240 \\
9 & 5.14 & 27.0516 & 26.6026 & 26.4107 & 26.8973 & 26.7074 & 26.6297 & 26.9542 & 26.4981 & 26.2734 & 26.7852 & 26.5872 & 26.4886 \\
\hline
\end{tabular}

\begin{tabular}{|c|c|c|c|c|c|c|c|c|c|c|c|c|c|}
\hline & \multirow[b]{3}{*}{ Exp } & \multicolumn{12}{|c|}{ mPW1PW91 } \\
\hline & & \multicolumn{6}{|c|}{ Gas phase } & \multicolumn{6}{|c|}{ PCM } \\
\hline & & 6-31G* & 6-31G** & $6-31+G * *$ & 6-311G* & $6-311 G^{* *}$ & $6-311+G^{* *}$ & 6-31G* & 6-31G** & $6-31+G^{* *}$ & 6-311G* & 6-311G** & $6-311+G^{* *}$ \\
\hline \multicolumn{14}{|c|}{${ }^{13} \mathrm{C}$} \\
\hline 1 & 47.0 & 144.4916 & 145.2667 & 145.2466 & 135.8396 & 135.4395 & 135.1171 & 144.5779 & 145.3497 & 145.2530 & 135.8663 & 135.4647 & 135.1289 \\
\hline 2 & 77.1 & 115.7956 & 116.6002 & 116.1654 & 105.9670 & 105.6409 & 105.5159 & 115.3153 & 116.1186 & 115.6474 & 105.4376 & 105.1161 & 104.9519 \\
\hline 3 & 34.3 & 158.3212 & 159.5777 & 159.2534 & 150.0088 & 149.6286 & 149.5300 & 158.3359 & 159.5860 & 159.2546 & 149.9913 & 149.6076 & 149.5148 \\
\hline 4 & 40.4 & 150.8608 & 151.7153 & 151.1228 & 141.9697 & 141.6338 & 141.3878 & 150.8344 & 151.6887 & 151.0474 & 141.9149 & 141.5733 & 141.3227 \\
\hline 5 & 141.3 & 55.6491 & 56.0523 & 54.8106 & 38.5668 & 37.5831 & 36.5555 & 54.9725 & 55.3793 & 54.0859 & 37.7604 & 36.8034 & 35.7367 \\
\hline 6 & 132.1 & 63.5552 & 63.9841 & 63.4478 & 47.2806 & 46.2951 & 45.5859 & 63.8242 & 64.2532 & 63.6640 & 47.4839 & 46.5251 & 45.8040 \\
\hline 7 & 46.0 & 146.2272 & 147.4064 & 147.7167 & 137.6533 & 137.2633 & 136.9076 & 146.2403 & 147.4159 & 147.6823 & 137.6401 & 137.2428 & 136.8545 \\
\hline 8 & 173.7 & 23.9444 & 23.9818 & 22.4904 & 5.2381 & 5.3947 & 3.8218 & 23.5669 & 23.5924 & 21.9555 & 4.7645 & 4.8628 & 3.1717 \\
\hline 9 & 72.8 & 121.8805 & 122.3668 & 121.7611 & 112.4792 & 111.7559 & 111.3742 & 122.1780 & 122.6631 & 121.9794 & 112.7359 & 112.0110 & 111.6018 \\
\hline $\begin{array}{l}10 \\
{ }^{1} \mathbf{H}\end{array}$ & 138.5 & 59.7936 & 59.4988 & 57.8003 & 42.0640 & 41.6737 & 40.7629 & 60.0056 & 59.7130 & 57.9974 & 42.2406 & 41.8725 & 41.0182 \\
\hline 1 & 2.68 & 29.5592 & 29.0300 & 28.9691 & 29.5312 & 29.2039 & 29.1861 & 29.5482 & 29.0193 & 28.9487 & 29.5043 & 29.1822 & 29.1603 \\
\hline 2 & 4.74 & 27.4090 & 26.8772 & 26.8767 & 27.4233 & 27.1102 & 27.0717 & 27.4097 & 26.8781 & 26.8822 & 27.4263 & 27.1144 & 27.0790 \\
\hline $3 n$ & 1.72 & 30.3863 & 29.9194 & 29.8648 & 30.3462 & 30.0978 & 30.0601 & 30.3124 & 29.8424 & 29.7947 & 30.2816 & 30.0317 & 29.9951 \\
\hline $3 x$ & 1.44 & 30.4176 & 29.9854 & 29.9861 & 30.4186 & 30.1930 & 30.2053 & 30.3891 & 29.9561 & 29.9599 & 30.3935 & 30.1671 & 30.1817 \\
\hline 4 & 2.82 & 29.4270 & 28.8974 & 28.8065 & 29.3735 & 29.0675 & 29.0270 & 29.3299 & 28.7964 & 28.6995 & 29.2735 & 28.9654 & 28.9227 \\
\hline 5 & 6.22 & 25.7858 & 25.0862 & 24.8618 & 25.7510 & 25.2542 & 25.1653 & 25.6735 & 24.9744 & 24.7477 & 25.6336 & 25.1433 & 25.0537 \\
\hline 6 & 5.90 & 26.0959 & 25.3934 & 25.2204 & 26.0877 & 25.5837 & 25.4978 & 26.0405 & 25.3386 & 25.1631 & 26.0235 & 25.5267 & 25.4406 \\
\hline $7 \mathrm{~s}$ & 1.44 & 30.5488 & 30.1408 & 30.0925 & 30.5452 & 30.3456 & 30.3291 & 30.5285 & 30.1186 & 30.0691 & 30.5217 & 30.3216 & 30.3032 \\
\hline $7 a$ & 1.44 & 30.5752 & 30.1376 & 30.0756 & 30.5317 & 30.2921 & 30.2710 & 30.5648 & 30.1282 & 30.0504 & 30.5063 & 30.2692 & 30.2454 \\
\hline 9 & 5.14 & 26.9709 & 26.5192 & 26.3312 & 26.8069 & 26.6112 & 26.5339 & 26.8626 & 26.4033 & 26.1848 & 26.6863 & 26.4823 & 26.3870 \\
\hline
\end{tabular}




\section{Compound 87a}

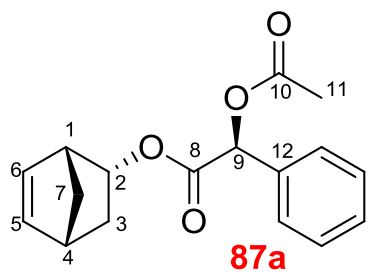

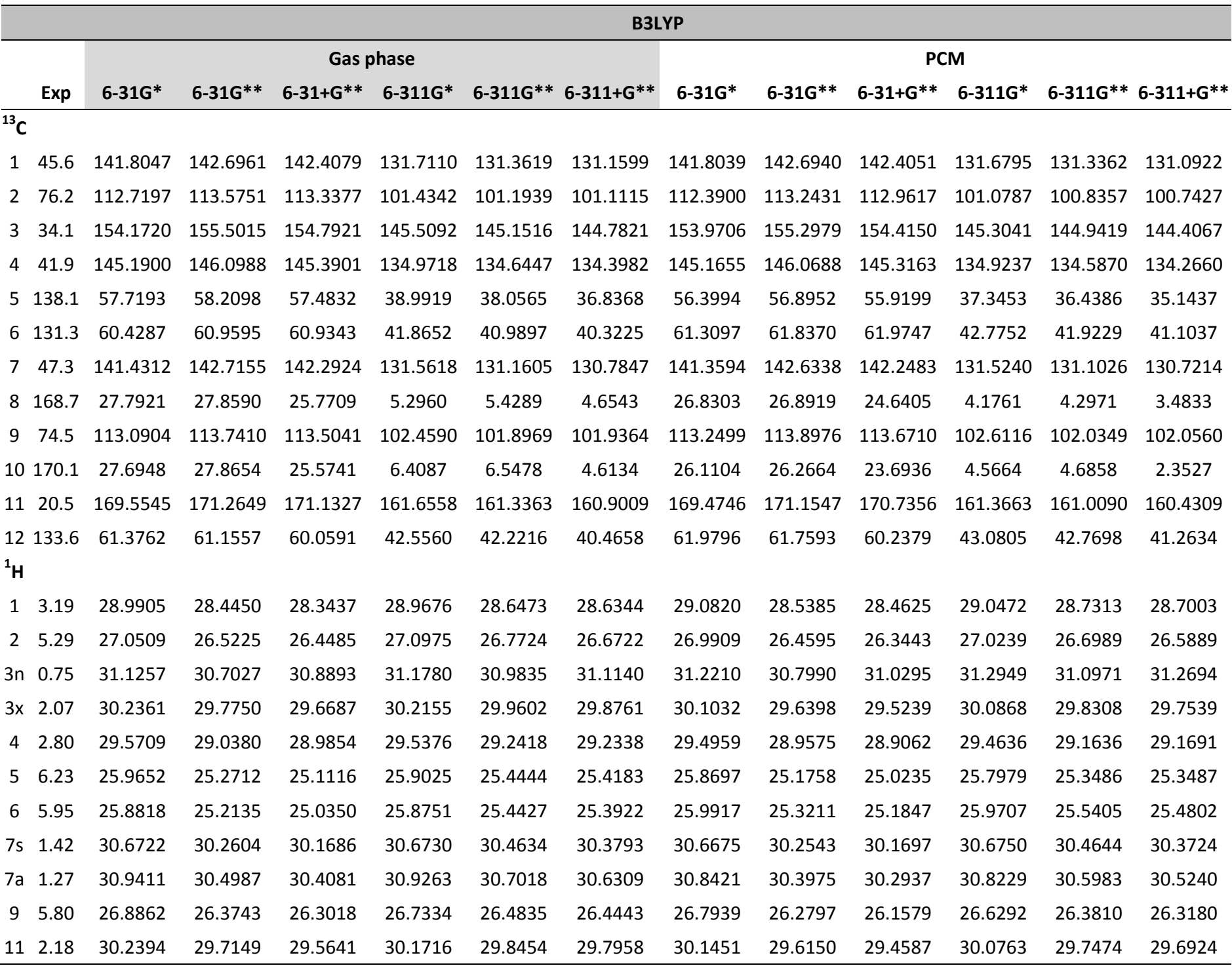

\begin{tabular}{|c|c|c|c|c|c|c|c|c|c|c|c|c|c|}
\hline & \multirow[b]{3}{*}{ Exp } & \multicolumn{12}{|c|}{ mPW1PW91 } \\
\hline & & \multicolumn{6}{|c|}{ Gas phase } & \multicolumn{6}{|c|}{ PCM } \\
\hline & & 6-31G* & 6-31G** & $6-31+\mathrm{G} * *$ & 6-311G* & $6-311 G * *$ & $6-311+G * *$ & $6-31 G *$ & $6-31 G * *$ & $6-31+G * *$ & 6-311G* & $6-311 G * *$ & $6-311+G * *$ \\
\hline \multicolumn{14}{|c|}{${ }^{13} \mathrm{C}$} \\
\hline 1 & 45.6 & 146.2983 & 147.1146 & 146.7940 & 137.1264 & 136.7678 & 136.5382 & 146.3207 & 147.1345 & 146.7863 & 137.1163 & 136.7613 & 136.4764 \\
\hline 2 & 76.2 & 117.2713 & 118.0655 & 118.0613 & 107.1964 & 106.9589 & 106.8810 & 116.9392 & 117.7306 & 117.6183 & 106.8408 & 106.6007 & 106.5199 \\
\hline 3 & 34.1 & 158.8039 & 160.0132 & 159.4749 & 150.7749 & 150.4044 & 150.0645 & 158.6222 & 159.8321 & 159.0704 & 150.5915 & 150.2197 & 149.7216 \\
\hline 4 & 41.9 & 149.8596 & 150.6920 & 149.8956 & 140.5031 & 140.1524 & 139.8864 & 149.8307 & 150.6571 & 149.8608 & 140.4426 & 140.0837 & 139.7598 \\
\hline
\end{tabular}




\begin{tabular}{|c|c|c|c|c|c|c|c|c|c|c|c|c|c|}
\hline 5 & 138.1 & 60.1197 & 60.5233 & 59.8233 & 43.3494 & 42.3741 & 41.2971 & 58.7585 & 59.1693 & 58.3539 & 41.6927 & 40.7465 & 39.5884 \\
\hline 6 & 131.3 & 63.0313 & 63.4778 & 63.5610 & 46.3888 & 45.4749 & 44.8724 & 63.8850 & 64.3261 & 64.4266 & 47.2419 & 46.3545 & 45.6324 \\
\hline 7 & 47.3 & 145.7998 & 146.9706 & 146.2961 & 136.7696 & 136.3623 & 136.0167 & 145.7484 & 146.9103 & 146.3306 & 136.7345 & 136.3090 & 135.9627 \\
\hline 8 & 168.7 & 30.5017 & 30.5657 & 29.1319 & 10.6862 & 10.8159 & 10.0953 & 29.5204 & 29.5784 & 27.8753 & 9.5645 & 9.6824 & 8.9392 \\
\hline 9 & 74.5 & 117.3206 & 117.9366 & 117.6718 & 108.3379 & 107.7929 & 107.7816 & 117.4981 & 118.1110 & 117.9528 & 108.4868 & 107.9285 & 107.9042 \\
\hline 10 & 170.1 & 30.0320 & 30.2029 & 28.4474 & 11.5055 & 11.6438 & 9.8922 & 28.4039 & 28.5589 & 26.5089 & 9.6383 & 9.7588 & 7.6851 \\
\hline 11 & 20.5 & 172.8738 & 174.4871 & 174.6477 & 165.7637 & 165.4660 & 165.0363 & 172.8051 & 174.3879 & 174.2580 & 165.4726 & 165.1411 & 164.5732 \\
\hline 12 & 133.6 & 64.7581 & 64.5095 & 63.6179 & 48.2936 & 47.9325 & 46.3374 & 65.3665 & 65.1181 & 63.9870 & 48.8321 & 48.4961 & 47.1021 \\
\hline \multicolumn{14}{|l|}{${ }^{1} \mathrm{H}$} \\
\hline 1 & 3.19 & 28.9206 & 28.3762 & 28.2888 & 28.8784 & 28.5434 & 28.5108 & 29.0022 & 28.4588 & 28.3680 & 28.9490 & 28.6172 & 28.5818 \\
\hline 2 & 5.29 & 26.9951 & 26.4645 & 26.3677 & 26.9914 & 26.6677 & 26.5688 & 26.9314 & 26.3982 & 26.2826 & 26.9175 & 26.5938 & 26.4885 \\
\hline $3 n$ & 0.75 & 31.0820 & 30.6544 & 30.7734 & 31.0772 & 30.8767 & 30.9896 & 31.1869 & 30.7602 & 30.9530 & 31.1990 & 30.9953 & 31.1318 \\
\hline $3 x$ & 2.07 & 30.1663 & 29.7031 & 29.5906 & 30.1073 & 29.8514 & 29.7678 & 30.0300 & 29.5641 & 29.4470 & 29.9767 & 29.7197 & 29.6368 \\
\hline 4 & 2.80 & 29.4834 & 28.9504 & 28.8898 & 29.4337 & 29.1215 & 29.1084 & 29.4016 & 28.8626 & 28.8124 & 29.3542 & 29.0375 & 29.0314 \\
\hline 5 & 6.23 & 25.7878 & 25.0897 & 24.9387 & 25.7596 & 25.2664 & 25.2415 & 25.6839 & 24.9857 & 24.8528 & 25.6480 & 25.1636 & 25.1530 \\
\hline 6 & 5.95 & 25.7474 & 25.0764 & 24.9400 & 25.7581 & 25.2922 & 25.2320 & 25.8464 & 25.1731 & 25.0042 & 25.8455 & 25.3824 & 25.3123 \\
\hline $7 s$ & 1.42 & 30.6022 & 30.1921 & 30.1011 & 30.5747 & 30.3603 & 30.2720 & 30.5970 & 30.1855 & 30.0889 & 30.5756 & 30.3600 & 30.2633 \\
\hline $7 a$ & 1.27 & 30.8837 & 30.4481 & 30.3588 & 30.8270 & 30.6049 & 30.5336 & 30.7796 & 30.3416 & 30.2409 & 30.7208 & 30.4985 & 30.4232 \\
\hline 9 & 5.80 & 26.7624 & 26.2599 & 26.1700 & 26.5995 & 26.3512 & 26.3253 & 26.6650 & 26.1598 & 26.0426 & 26.4926 & 26.2465 & 26.1865 \\
\hline 11 & 2.18 & 30.1496 & 29.6177 & 29.4938 & 30.0726 & 29.7308 & 29.6979 & 30.0472 & 29.5088 & 29.3765 & 29.9699 & 29.6250 & 29.5842 \\
\hline
\end{tabular}

\section{Compound 87b}

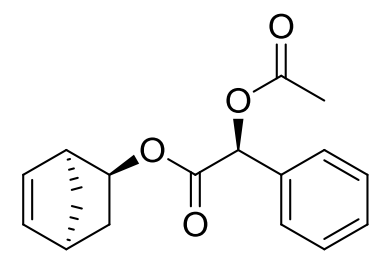

$87 b$

\begin{tabular}{|c|c|c|c|c|c|c|c|c|c|c|c|c|c|}
\hline \multirow{3}{*}{\multicolumn{2}{|c|}{ Exp }} & \multicolumn{12}{|c|}{ B3LYP } \\
\hline & & \multicolumn{6}{|c|}{ Gas phase } & \multicolumn{6}{|c|}{ PCM } \\
\hline & & 6-31G* & $6-31 \mathrm{G} * *$ & $6-31+\mathrm{G} * *$ & 6-311G* & 6-311G** & $6-311+G * *$ & 6-31G* & 6-31G** & $6-31+\mathrm{G} * *$ & 6-311G* & $6-311 G * *$ & $6-311+G * *$ \\
\hline \multicolumn{14}{|c|}{${ }^{13} \mathrm{C}$} \\
\hline 1 & 45.4 & 142.0220 & 142.9058 & 142.6654 & 132.0069 & 131.6674 & 131.5390 & 142.0182 & 142.9013 & 142.6156 & 131.9771 & 131.6397 & 131.4309 \\
\hline 2 & 76.2 & 112.8316 & 113.6978 & 113.1054 & 101.5730 & 101.3360 & 101.1209 & 112.3318 & 113.1902 & 112.5953 & 101.0094 & 100.7778 & 100.5847 \\
\hline 3 & 34.0 & 153.9208 & 155.3042 & 154.7624 & 144.9997 & 144.6811 & 144.4045 & 153.7194 & 155.0957 & 154.5522 & 144.7872 & 144.4779 & 144.1473 \\
\hline 4 & 41.9 & 145.2519 & 146.1628 & 145.2682 & 134.9962 & 134.6699 & 134.3983 & 145.2305 & 146.1380 & 145.2044 & 134.9438 & 134.6073 & 134.3326 \\
\hline 5 & 138.2 & 57.2401 & 57.7799 & 56.3912 & 38.3924 & 37.4691 & 36.2028 & 56.1713 & 56.6928 & 55.3530 & 37.0904 & 36.1927 & 34.9468 \\
\hline 6 & 131.0 & 61.3515 & 61.8417 & 62.3325 & 42.9354 & 42.0503 & 41.7218 & 61.9221 & 62.4420 & 62.6926 & 43.4791 & 42.6333 & 42.1136 \\
\hline 7 & 47.4 & 141.2349 & 142.5117 & 142.1392 & 131.4592 & 131.0507 & 130.8016 & 141.2727 & 142.5419 & 142.1130 & 131.5125 & 131.0801 & 130.8318 \\
\hline 8 & 168.7 & 27.9613 & 28.0351 & 25.7128 & 5.5265 & 5.6563 & 4.7935 & 26.8642 & 26.9307 & 24.5272 & 4.1522 & 4.2821 & 3.4562 \\
\hline 9 & 74.4 & 113.5673 & 114.2197 & 114.1261 & 102.9000 & 102.3327 & 101.9169 & 113.6106 & 114.2625 & 114.2017 & 102.9726 & 102.3978 & 101.8957 \\
\hline 10 & 170.1 & 28.0041 & 28.1602 & 25.8170 & 6.6386 & 6.7598 & 4.7059 & 26.3060 & 26.4569 & 23.7450 & 4.6528 & 4.7619 & 2.4065 \\
\hline 11 & 20.6 & 169.5167 & 171.2281 & 171.0892 & 161.6718 & 161.3480 & 160.9427 & 169.4335 & 171.1131 & 170.6700 & 161.3456 & 160.9916 & 160.4477 \\
\hline
\end{tabular}




\begin{tabular}{cccccccccccccc}
\hline $\begin{array}{c}12 \\
{ }^{1} \mathbf{H}\end{array}$ & 133.8 & 61.1243 & 60.9231 & 58.3831 & 42.0923 & 41.7785 & 40.5208 & 61.7874 & 61.5763 & 59.1296 & 42.7073 & 42.4078 & 41.3586 \\
1 & 3.02 & 29.2294 & 28.6756 & 28.6956 & 29.2239 & 28.9042 & 28.9195 & 29.2648 & 28.7158 & 28.6934 & 29.2454 & 28.9276 & 28.9155 \\
2 & 5.29 & 27.0086 & 26.4756 & 26.4412 & 27.0573 & 26.7281 & 26.6968 & 26.9910 & 26.4579 & 26.4095 & 27.0289 & 26.7004 & 26.6584 \\
$3 \mathrm{n}$ & 0.99 & 30.9215 & 30.4988 & 30.4708 & 31.0116 & 30.8099 & 30.7430 & 31.0095 & 30.5864 & 30.5927 & 31.0855 & 30.8798 & 30.8613 \\
$3 \mathrm{x}$ & 2.07 & 30.0977 & 29.6477 & 29.4906 & 30.0702 & 29.8237 & 29.7177 & 29.9909 & 29.5360 & 29.3888 & 29.9641 & 29.7166 & 29.6189 \\
4 & 2.80 & 29.5734 & 29.0397 & 28.9386 & 29.5397 & 29.2385 & 29.1733 & 29.4879 & 28.9496 & 28.8428 & 29.4499 & 29.1455 & 29.0827 \\
5 & 6.23 & 25.9904 & 25.2954 & 25.0636 & 25.9169 & 25.4557 & 25.3755 & 25.8843 & 25.1905 & 24.9581 & 25.7983 & 25.3459 & 25.2716 \\
6 & 5.47 & 26.4732 & 25.7932 & 25.7633 & 26.4349 & 26.0015 & 26.0521 & 26.4936 & 25.8212 & 25.7592 & 26.4465 & 26.0256 & 26.0354 \\
$7 s$ & 1.42 & 30.7202 & 30.3085 & 30.2515 & 30.7172 & 30.5094 & 30.4679 & 30.7121 & 30.3002 & 30.2489 & 30.7203 & 30.5120 & 30.4640 \\
$7 a$ & 1.27 & 30.9314 & 30.4912 & 30.4184 & 30.9172 & 30.6987 & 30.6602 & 30.8493 & 30.4068 & 30.3249 & 30.8313 & 30.6120 & 30.5680 \\
9 & 5.81 & 26.7646 & 26.2452 & 26.0785 & 26.6228 & 26.3673 & 26.3282 & 26.7324 & 26.2125 & 25.9994 & 26.5849 & 26.3295 & 26.2451 \\
11 & 2.18 & 30.2444 & 29.7200 & 29.5537 & 30.1739 & 29.8480 & 29.7870 & 30.1454 & 29.6149 & 29.4367 & 30.0765 & 29.7473 & 29.6736 \\
\hline
\end{tabular}

\section{mPW1PW91}

\section{Gas phase}

PCM

$\begin{array}{llllllllllll}\operatorname{Exp} & 6-31 \mathrm{G} * & 6-31 \mathrm{G} * & 6-31+\mathrm{G} * * & 6-311 \mathrm{G} * & 6-311 \mathrm{G} * & 6-311+\mathrm{G} * * & 6-31 \mathrm{G} * & 6-31 \mathrm{G} * * & 6-31+\mathrm{G} * * & 6-311 \mathrm{G} * & 6-311 \mathrm{G} * * 6-311+\mathrm{G} * * \\ \mathrm{C} & & & \end{array}$

\begin{tabular}{|c|c|c|c|c|c|c|c|c|c|c|c|c|c|}
\hline & 45.4 & 146.4808 & 147.2855 & 147.0350 & 137.4034 & 137.0507 & 136.9036 & 146.5067 & 147.3120 & 146.9935 & 137.3845 & 137.0339 & 136.8344 \\
\hline 2 & 76.2 & 117.3641 & 118.1692 & 117.8838 & 107.3419 & 107.1098 & 106.9091 & 116.8713 & 117.6680 & 117.3734 & 106.7758 & 106.5487 & 106.3671 \\
\hline & 34.0 & 158.6413 & 159.9084 & 159.3792 & 150.4529 & 150.1246 & 149.8147 & 158.5062 & 159.7694 & 159.2789 & 150.2467 & 149.9293 & 149.6061 \\
\hline & 41.9 & 149.9055 & 150.7396 & 149.7903 & 140.5126 & 140.1651 & 139.8864 & 149.8787 & 150.7097 & 149.7330 & 140.4535 & 140.0971 & 139.8146 \\
\hline & 138.2 & 59.7381 & 60.1983 & 58.9317 & 42.9011 & 41.9404 & 40.7720 & 58.6019 & 59.0417 & 57.8351 & 41.5133 & 40.5773 & 39.4630 \\
\hline & 131.0 & 63.8189 & 64.2124 & 64.7645 & 47.3023 & 46.3747 & 46.0489 & 64.4305 & 64.8594 & 65.1311 & 47.8835 & 47.0018 & 46.5186 \\
\hline & 47.4 & 145.6341 & 146.7977 & 146.1524 & 136.6774 & 136.2615 & 136.0259 & 145.6777 & 146.8342 & 146.1399 & 136.7296 & 136.2913 & 136.0550 \\
\hline & 168.7 & 30.6400 & 30.7102 & 29.0492 & 10.8781 & 11.0084 & 10.2579 & 29.5240 & 29.5865 & 27.8488 & 9.5072 & 9.6365 & 8.9019 \\
\hline & 74.4 & 117.7640 & 118.3821 & 118.5069 & 108.7400 & 108.1916 & 107.8112 & 117.8292 & 118.4468 & 118.6016 & 108.8336 & 108.2788 & 107.7972 \\
\hline & 170.1 & 30.3580 & 30.5151 & 28.6832 & 11.7166 & 11.8392 & 10.0534 & 28.5758 & 28.7262 & 26.5899 & 9.7193 & 9.8308 & 7.7159 \\
\hline & 20.6 & 172.8370 & 174.4500 & 174.6010 & 165.7745 & 165.4723 & 165.0623 & 172.7642 & 174.3462 & 174.2037 & 165.4525 & 165.1233 & 164.5970 \\
\hline & 133.8 & 64.5052 & 64.2749 & 62.0410 & 47.8692 & 47.5255 & 46.3221 & 65.1760 & 64.9353 & 62.7811 & 48.5039 & 48.1751 & 47.1243 \\
\hline & 3.02 & 29.1245 & 28.5676 & 28.5697 & 29.0975 & 28.7583 & 28.7654 & 29.1582 & 28.6062 & 28.5606 & 29.1226 & 28.7861 & 28.7698 \\
\hline & 5.29 & 26.9523 & 26.4186 & 26.3665 & 26.9516 & 26.6246 & 26.5896 & 26.9332 & 26.3993 & 26.3359 & 26.9224 & 26.5959 & 26.5516 \\
\hline & 0.99 & 30.9129 & 30.4888 & 30.4620 & 30.9202 & 30.7129 & 30.6829 & 30.9753 & 30.5490 & 30.5467 & 30.9925 & 30.7808 & 30.7585 \\
\hline & 2.07 & 30.0500 & 29.5988 & 29.4521 & 29.9843 & 29.7367 & 29.6348 & 29.9335 & 29.4766 & 29.3442 & 29.8687 & 29.6201 & 29.5217 \\
\hline & 2.80 & 29.4919 & 28.9584 & 28.8628 & 29.4389 & 29.1214 & 29.0614 & 29.3958 & 28.8573 & 28.7570 & 29.3426 & 29.0219 & 28.9593 \\
\hline & 6.23 & 25.8256 & 25.1277 & 24.9261 & 25.7864 & 25.2913 & 25.2241 & 25.7068 & 25.0101 & 24.8043 & 25.6578 & 25.1718 & 25.1083 \\
\hline & 5.47 & 26.3234 & 25.6377 & 25.6159 & 26.3233 & 25.8597 & 25.9007 & 26.3581 & 25.6828 & 25.6072 & 26.3362 & 25.8859 & 25.9114 \\
\hline & 1.42 & 30.6486 & 30.2383 & 30.1752 & 30.6195 & 30.4075 & 30.3598 & 30.6432 & 30.2328 & 30.1717 & 30.6216 & 30.4090 & 30.3589 \\
\hline & 1.27 & 30.8778 & 30.4442 & 30.3745 & 30.8233 & 30.6071 & 30.5674 & 30.7910 & 30.3551 & 30.2762 & 30.7327 & 30.5158 & 30.4735 \\
\hline & 5.81 & 26.6371 & 26.1267 & 25.9899 & 26.4897 & 26.2375 & 26.2074 & 26.6130 & 26.1009 & 25.9118 & 26.4506 & 26.1981 & 26.1316 \\
\hline & 2.18 & 30.1499 & 29.6175 & 29.4825 & 30.0715 & 29.7300 & 29.6850 & 30.0451 & 29.5060 & 29.3603 & 29.9675 & 29.6223 & 29.5699 \\
\hline
\end{tabular}




\section{Compound 87c}<smiles>CC(=O)O[C@H](C(=O)O[C@H]1C[C@H]2C=C[C@@H]1CC2)c1ccccc1</smiles>

$87 c$

\begin{tabular}{|c|c|c|c|c|c|c|c|c|c|c|c|c|c|}
\hline \multirow{3}{*}{\multicolumn{2}{|c|}{ Exp }} & \multicolumn{12}{|c|}{ B3LYP } \\
\hline & & \multicolumn{6}{|c|}{ Gas phase } & \multicolumn{6}{|c|}{ PCM } \\
\hline & & 6-31G* & 6-31G** & $6-31+G * *$ & 6-311G* & 6-311G** & $6-311+G * *$ & 6-31G* & 6-31G** & $6-31+G * *$ & 6-311G* & $6-311 \mathrm{G} * *$ & $6-311+G$ \\
\hline \\
\hline 1 & 47.0 & 39.8801 & 140.7323 & 140.0481 & 130.2313 & 129.8223 & 129.3044 & 139.9200 & 140.7718 & 140.1154 & 130.2143 & 129.8096 & 129.317 \\
\hline 2 & 76.5 & 12.6750 & 113.5375 & 113.1334 & 101.5790 & 101.2722 & 100.9749 & 112.2573 & 113.1180 & 112.6739 & 101.1289 & 100.8240 & 100.485 \\
\hline 3 & 34.2 & 153.8528 & 55.2404 & 155.1320 & 145.2412 & 144.8778 & 144.6031 & 153.8357 & 155.2158 & 155.2041 & 145.2475 & 144.8720 & 144.475 \\
\hline 4 & 40.3 & 146.2404 & 147.1806 & 146.6581 & 136.5725 & 136.2552 & 136.0509 & 146.2489 & 147.1882 & 146.6555 & 136.5722 & 136.2463 & 136.028 \\
\hline 5 & 141.2 & 53.6292 & 54.1210 & 53.5429 & 34.3725 & 33.4257 & 32.3066 & 52.9361 & 53.4321 & 52.6996 & 33.5233 & 32.6004 & 31.4286 \\
\hline 6 & 132.1 & 60.9223 & 61.4382 & 61.2043 & 42.4220 & 41.4767 & 40.4716 & 61.2471 & 61.7621 & 61.5451 & 42.7099 & 41.7976 & 40.8247 \\
\hline 7 & 46.1 & 141.7971 & 143.0984 & 143.5255 & 132.5817 & 132.1904 & 131.8350 & 141.8801 & 143.1747 & 143.5746 & 132.6458 & 132.2442 & $131.840 \mathrm{C}$ \\
\hline 8 & 168.7 & 27.9601 & 28.0179 & 25.7735 & 5.5983 & 5.6951 & 5.0877 & 26.8994 & 26.9540 & 24.6195 & 4.3387 & 4.4315 & 3.7460 \\
\hline 9 & 74.6 & 113.2798 & 113.9231 & 113.7510 & 102.6021 & 102.0194 & 101.9186 & 113.3783 & 114.0205 & 113.7913 & 102.7143 & 102.1223 & 102.028 \\
\hline 10 & 170.2 & 27.9948 & 28.1538 & 25.8535 & 6.6654 & 6.7789 & 4.6282 & 26.2607 & 26.4079 & 23.8204 & 4.6312 & 4.7351 & 2.3205 \\
\hline 11 & 20.5 & 169.5747 & 171.2838 & 171.0202 & 161.7112 & 161.3887 & 160.9335 & 169.4699 & 171.1481 & 170.6079 & 161.3749 & 161.0175 & 160.4135 \\
\hline 12 & 133.7 & 61.0913 & 60.8729 & 59.1499 & 42.0995 & 41.7746 & 40.3134 & 61.7535 & 61.5332 & 59.8919 & 42.7408 & 42.4362 & 41.060 \\
\hline \multicolumn{14}{|c|}{${ }^{1} \mathrm{H}$} \\
\hline 1 & 2.93 & 29.3277 & 28.8012 & 28.6629 & 29.3045 & 28.9959 & 28.9067 & 29.3737 & 28.8474 & 28.7319 & 29.3405 & 29.0361 & 28.9496 \\
\hline 2 & 4.69 & 27.5430 & 27.0181 & 26.9533 & 27.6049 & 27.3099 & 27.2567 & 27.5538 & 27.0303 & 26.9685 & 27.6091 & 27.3155 & 27.2682 \\
\hline $3 n$ & 1.61 & 30.6078 & 30.1497 & 30.1449 & 30.6481 & 30.4228 & 30.3857 & 30.5432 & 30.0821 & 30.0663 & 30.5877 & 30.3597 & 30.3170 \\
\hline $3 x$ & 1.17 & 30.7805 & 30.3590 & 30.4032 & 30.8175 & 30.6053 & 30.6425 & 30.8093 & 30.3878 & 30.4299 & 30.8469 & 30.6336 & 30.6857 \\
\hline 4 & 2.77 & 29.6146 & 29.0861 & 29.0076 & 29.5748 & 29.2860 & 29.2586 & 29.5339 & 29.0016 & 28.9244 & 29.4916 & 29.2000 & 29.1788 \\
\hline 5 & 6.22 & 25.9785 & 25.2849 & 25.0262 & 25.9068 & 25.4558 & 25.3642 & 25.8699 & 25.1770 & 24.9184 & 25.7899 & 25.3455 & 25.2517 \\
\hline 6 & 5.93 & 26.1817 & 25.4868 & 25.2623 & 26.1371 & 25.6784 & 25.5540 & 26.1322 & 25.4374 & 25.2178 & 26.0777 & 25.6249 & 25.5067 \\
\hline $7 \mathrm{~s}$ & 1.54 & 30.5175 & 30.1053 & 30.0204 & 30.5426 & 30.3366 & 30.2768 & 30.5200 & 30.1059 & 30.0430 & 30.5495 & 30.3426 & 30.2871 \\
\hline $7 a$ & 1.54 & 30.4621 & 30.0156 & 29.9075 & 30.4877 & 30.2451 & 30.1766 & 30.5010 & 30.0550 & 29.9765 & 30.5211 & 30.2804 & 30.2280 \\
\hline 9 & 5.89 & 26.7336 & 26.2178 & 26.1341 & 26.5849 & 26.3263 & 26.3232 & 26.6570 & 26.1386 & & 26.4920 & 26.2343 & 26.2102 \\
\hline 11 & 2.19 & 30.2194 & 29.6937 & 29.5386 & 30.1457 & 29.8176 & 29.7636 & 30.1200 & 29.5886 & 29.4240 & 30.0450 & 29.7142 & 29.6518 \\
\hline
\end{tabular}

\begin{tabular}{|c|c|c|c|c|c|c|c|c|c|c|c|c|c|}
\hline \multirow{3}{*}{\multicolumn{2}{|c|}{ Exp }} & \multicolumn{12}{|c|}{ mPW1PW91 } \\
\hline & & \multicolumn{6}{|c|}{ Gas phase } & \multicolumn{6}{|c|}{ PCM } \\
\hline & & 6-31G* & 6-31G** & $6-31+\mathrm{G} * *$ & 6-311G* & 6-311G** & $6-311+G * *$ & 6-31G* & $6-31 G * *$ & $6-31+G * *$ & 6-311G* & $6-311 \mathrm{G} * *$ & $6-311+G * *$ \\
\hline \multicolumn{14}{|c|}{${ }^{13} \mathrm{C}$} \\
\hline 1 & 47.0 & 144.4933 & 145.2682 & 144.8173 & 135.7379 & 135.3175 & 134.8180 & 144.5525 & 145.3263 & 144.8504 & 135.7363 & 135.3194 & 134.8074 \\
\hline
\end{tabular}




\begin{tabular}{|c|c|c|c|c|c|c|c|c|c|c|c|c|c|}
\hline 2 & 76.5 & 117.1761 & 117.9752 & 117.7226 & 107.2579 & 106.9541 & 106.7019 & 116.7737 & 117.5703 & 117.3053 & 106.8238 & 106.5214 & 106.2503 \\
\hline 3 & 34.2 & 158.5672 & 59.8350 & 159.7419 & 150.5208 & 150.1564 & 149.8568 & 158.5752 & 159.8360 & 159.7880 & 150.5335 & 150.1585 & 149.7878 \\
\hline 4 & 40.3 & 150.7991 & 151.6596 & 151.0875 & 141.9313 & 141.5861 & 141.3622 & 150.8107 & 151.6704 & 151.0688 & 141.9278 & 141.5751 & 141.3555 \\
\hline 5 & 141.2 & 56.0160 & 56.4175 & 55.7901 & 38.8367 & 37.8518 & 36.8300 & 55.2862 & 55.6915 & 54.9576 & 37.9586 & 36.9972 & 35.9398 \\
\hline 6 & 132.1 & 63.5578 & 63.9839 & 63.7251 & 47.0091 & 46.0135 & 45.1741 & 63.8460 & 64.2729 & 64.0278 & 47.2490 & 46.2922 & 45.3958 \\
\hline 7 & 46.1 & 146.1113 & 147.2977 & 147.5765 & 137.6205 & 137.2281 & 136.8617 & 146.2131 & 147.3929 & 147.6151 & 137.6908 & 137.2883 & 136.8975 \\
\hline 8 & 168.7 & 30.6580 & 30.7114 & 29.1030 & 10.9532 & 11.0442 & 10.5278 & 29.5754 & 29.6252 & 27.9145 & 9.6870 & 9.7750 & 9.1882 \\
\hline 9 & 74.6 & 117.4793 & 118.0878 & 118.0360 & 108.4565 & 107.8922 & 107.7937 & 117.5983 & 118.2061 & 118.1108 & 108.5755 & 108.0025 & 107.8934 \\
\hline 10 & 170.2 & 30.3074 & 30.4659 & 28.7013 & 11.7364 & 11.8507 & 9.9368 & 28.5272 & 28.6728 & 26.6306 & 9.6844 & 9.7903 & 7.6359 \\
\hline 11 & 20.5 & 172.8937 & 174.5051 & 174.5521 & 165.8142 & 165.5137 & 165.0543 & 172.7986 & 174.3794 & 174.1539 & 165.4789 & 165.1469 & 164.5588 \\
\hline $\begin{array}{l}12 \\
{ }^{1} \mathrm{H}\end{array}$ & 133.7 & 64.4879 & 64.2415 & 62.8227 & 47.8915 & 47.5380 & 46.1813 & 65.1566 & 64.9087 & 63.5320 & 48.5405 & 48.2087 & 46.8878 \\
\hline 1 & 2.93 & 29.2385 & 28.7118 & 28.6031 & 29.1989 & 28.8733 & 28.7969 & 29.2733 & 28.7467 & 28.6365 & 29.2249 & 28.9040 & 28.8181 \\
\hline 2 & 4.69 & 27.5180 & 26.9935 & 26.9247 & 27.5226 & 27.2282 & 27.1774 & 27.5279 & 27.0045 & 26.9356 & 27.5270 & 27.2339 & 27.1783 \\
\hline $3 n$ & 1.61 & 30.5592 & 30.0992 & 30.0720 & 30.5470 & 30.3190 & 30.2702 & 30.4962 & 30.0328 & 30.0014 & 30.4885 & 30.2572 & 30.2138 \\
\hline $3 x$ & 1.17 & 30.7227 & 30.2972 & 30.3076 & 30.7126 & 30.4966 & 30.5291 & 30.7532 & 30.3276 & 30.3494 & 30.7430 & 30.5256 & 30.5799 \\
\hline 4 & 2.77 & 29.5244 & 28.9949 & 28.9184 & 29.4694 & 29.1650 & 29.1403 & 29.4362 & 28.9023 & 28.8297 & 29.3802 & 29.0728 & 29.0580 \\
\hline 5 & 6.22 & 25.8080 & 25.1103 & 24.8765 & 25.7685 & 25.2805 & 25.1880 & 25.6891 & 24.9919 & 24.7590 & 25.6439 & 25.1625 & 25.0751 \\
\hline 6 & 5.93 & 26.0275 & 25.3289 & 25.1301 & 26.0112 & 25.5175 & 25.4013 & 25.9668 & 25.2682 & 25.0697 & 25.9433 & 25.4563 & 25.3374 \\
\hline $7 \mathrm{~s}$ & 1.54 & 30.4449 & 30.0338 & 29.9464 & 30.4429 & 30.2331 & 30.1658 & 30.4453 & 30.0322 & 29.9536 & 30.4471 & 30.2367 & 30.1767 \\
\hline $7 a$ & 1.54 & 30.4226 & 29.9796 & 29.8785 & 30.4036 & 30.1576 & 30.0790 & 30.4581 & 30.0156 & 29.9269 & 30.4335 & 30.1898 & 30.1295 \\
\hline 9 & 5.89 & 26.6072 & 26.1012 & 26.0113 & 26.4471 & 26.1924 & 26.1878 & 26.5268 & 26.0175 & 25.9026 & 26.3525 & 26.0984 & 26.0787 \\
\hline 11 & 2.19 & 30.1254 & 29.5922 & 29.4620 & 30.0434 & 29.6997 & 29.6581 & 30.0181 & 29.4782 & 29.3404 & 29.9359 & 29.5892 & 29.5444 \\
\hline
\end{tabular}

\section{Compound 87d}<smiles>CC(=O)O[C@H](C(=O)O[C@H]1C[C@@H]2C=C[C@@H]1C2)c1ccccc1</smiles>

87d

\begin{tabular}{|c|c|c|c|c|c|c|c|c|c|c|c|c|c|}
\hline & \multirow[b]{3}{*}{ Exp } & \multicolumn{12}{|c|}{ B3LYP } \\
\hline & & \multicolumn{6}{|c|}{ Gas phase } & \multicolumn{6}{|c|}{ PCM } \\
\hline & & 6-31G* & $6-31 G * *$ & $6-31+G * *$ & 6-311G* & $6-311 G * *$ & $6-311+G * *$ & 6-31G* & $6-31 G * *$ & $6-31+G * *$ & 6-311G* & $6-311 G * *$ & $6-311+G * *$ \\
\hline \multicolumn{14}{|c|}{${ }^{13} \mathrm{C}$} \\
\hline 1 & 46.9 & 140.5160 & 141.3461 & 140.5092 & 130.7909 & 130.3972 & 130.2009 & 140.4812 & 141.3174 & 140.4642 & 130.7549 & 130.3578 & 130.1001 \\
\hline 2 & 76.5 & 112.8199 & 113.6979 & 112.7327 & 101.7005 & 101.4073 & 101.4113 & 112.4323 & 113.3016 & 112.2965 & 101.2988 & 101.0054 & 100.9909 \\
\hline 3 & 34.2 & 153.6073 & 154.9561 & 154.7736 & 144.7166 & 144.3662 & 144.4270 & 153.6124 & 155.0164 & 154.9463 & 144.7783 & 144.4300 & 144.4872 \\
\hline 4 & 40.4 & 146.2970 & 147.2338 & 146.6797 & 136.5763 & 136.2709 & 136.0331 & 146.2917 & 147.2304 & 146.6891 & 136.5725 & 136.2611 & 136.0208 \\
\hline 5 & 141.1 & 53.2824 & 53.7841 & 52.7476 & 34.1299 & 33.1915 & 32.0464 & 52.7545 & 53.2564 & 52.1744 & 33.4687 & 32.5525 & 31.4191 \\
\hline 6 & 132.0 & 61.3701 & 61.8937 & 61.7063 & 43.1006 & 42.1547 & 41.3507 & 61.4424 & 61.9502 & 61.6920 & 43.0736 & 42.1594 & 41.3025 \\
\hline 7 & 46.0 & 141.8692 & 143.1421 & 143.8545 & 132.5739 & 132.2028 & 131.9010 & 141.9343 & 143.2332 & 143.9388 & 132.6607 & 132.2782 & 131.9267 \\
\hline 8 & 168.6 & 27.8066 & 27.8942 & 25.5452 & 5.4522 & 5.5525 & 4.9443 & 26.7628 & 26.8239 & 24.3411 & 4.1851 & 4.2843 & 3.6355 \\
\hline
\end{tabular}




\begin{tabular}{|c|c|c|c|c|c|c|c|c|c|c|c|c|c|}
\hline 9 & 74.6 & 113.2357 & 113.9080 & 113.7453 & 102.6249 & 102.0525 & 101.8483 & 113.3363 & 113.9842 & 113.8260 & 102.7151 & 102.1342 & 101.9293 \\
\hline 10 & 170.2 & 27.8930 & 28.1056 & 25.7795 & 6.6175 & 6.7418 & 4.5616 & 26.1870 & 26.3426 & 23.7080 & 4.5701 & 4.6827 & 2.2969 \\
\hline 11 & 20.5 & 169.5817 & 171.2971 & 171.0503 & 161.7277 & 161.4062 & 160.9345 & 169.4793 & 171.1593 & 170.6094 & 161.3885 & 161.0321 & 160.4195 \\
\hline 12 & 133.7 & 61.0487 & 60.8397 & 58.7433 & 42.0549 & 41.7137 & 40.5028 & 61.7343 & 61.5081 & 59.5045 & 42.7029 & 42.3800 & 41.3015 \\
\hline 1 & 2.71 & 29.6466 & 29.1143 & 29.1422 & 29.6160 & 29.3208 & 29.3617 & 29.6330 & 29.1080 & 29.1275 & 29.6044 & 29.3104 & 29.3310 \\
\hline 2 & 4.69 & 27.5327 & 27.0087 & 26.9778 & 27.5954 & 27.2984 & 27.2428 & 27.5403 & 27.0133 & 26.9745 & 27.5934 & 27.2962 & 27.2422 \\
\hline $3 n$ & 1.70 & 30.4556 & 29.9995 & 29.9285 & 30.4943 & 30.2613 & 30.1910 & 30.4172 & 29.9573 & 29.8864 & 30.4552 & 30.2199 & 30.1579 \\
\hline $3 x$ & 1.47 & 30.4174 & 30.0343 & 29.9598 & 30.5176 & 30.3036 & 30.2165 & 30.5041 & 30.0821 & 29.9968 & 30.5622 & 30.3456 & 30.3031 \\
\hline 4 & 2.82 & 29.5528 & 29.0301 & 28.9130 & 29.5253 & 29.2405 & 29.1795 & 29.4732 & 28.9431 & 28.8260 & 29.4384 & 29.1512 & 29.1003 \\
\hline 5 & 6.22 & 25.9833 & 25.2896 & 25.0156 & 25.9103 & 25.4578 & 25.3602 & 25.8824 & 25.1904 & 24.9176 & 25.8030 & 25.3572 & 25.2600 \\
\hline 6 & 5.88 & 26.2908 & 25.5891 & 25.4109 & 26.2422 & 25.7774 & 25.7013 & 26.2226 & 25.5253 & 25.3436 & 26.1692 & 25.7109 & 25.6287 \\
\hline $7 \mathrm{~s}$ & 1.47 & 30.6455 & 30.2315 & 30.2084 & 30.6591 & 30.4675 & 30.4705 & 30.6340 & 30.2257 & 30.2221 & 30.6622 & 30.4694 & 30.4653 \\
\hline $7 a$ & 1.47 & 30.5467 & 30.0999 & 30.0563 & 30.5665 & 30.3319 & 30.3019 & 30.5843 & 30.1431 & 30.1264 & 30.6030 & 30.3727 & 30.3572 \\
\hline 9 & 5.89 & 26.7551 & 26.2214 & 26.1084 & 26.5822 & 26.3169 & 26.3109 & 26.6687 & 26.1465 & 26.0077 & 26.4951 & 26.2313 & 26.2053 \\
\hline 11 & 2.19 & 30.2291 & 29.7010 & 29.5450 & 30.1529 & 29.8248 & 29.7675 & 30.1261 & 29.5946 & 29.4293 & 30.0504 & 29.7193 & 29.6509 \\
\hline
\end{tabular}

\section{mPW1PW91}

\section{Gas phase PCM}

$\operatorname{Exp} 6-31 G^{*} \quad 6-31 G * * 6-31+G * * 6-311 G * 6-311 G * * 6-311+G * * \quad 6-31 G * \quad 6-31 G * * \quad 6-31+G * * \quad 6-311 G * 6-311 G * * 6-311+G * *$

\section{${ }^{13} \mathrm{C}$}

$\begin{array}{llllllll}1 & 46.9 & 145.0192 & 145.7777 & 145.1470 & 136.2249 & 135.8196 & 135\end{array}$

$\begin{array}{llllllll}2 & 76.5 & 117.3112 & 118.1183 & 117.4647 & 107.3778 & 107.0859 & 107\end{array}$

$\begin{array}{lllllll}3 & 34.2 & 158.3687 & 159.6565 & 159.4155 & 150.1083 & 149.7559\end{array}$

$\begin{array}{llllllll}4 & 40.4 & 150.8358 & 151.6975 & 151.0505 & 141.9308 & 141.5980\end{array}$

$\begin{array}{llll}5 & 141.155 .7306 \quad 56.1469\end{array}$

$\begin{array}{llll}6 & 132.0 & 63.9085 & 64.3180\end{array}$

$\begin{array}{llll}7 & 46.0 & 146.1710 & 147.3636\end{array}$

$\begin{array}{llll}8 & 168.6 & 30.5407 & 30.6027\end{array}$

$9 \quad 74.6 \quad 117.4717 \quad 118.0877$

$10 \quad 170.2 \quad 30.2647 \quad 30.4349$

$\begin{array}{llll}11 & 20.5 & 172.9014 & 174.5145\end{array}$

$\begin{array}{llll}12 & 133.7 & 64.4583 & 64.2053\end{array}$

$\begin{array}{llll}1 & 2.71 & 29.5282 & 29.0007\end{array}$

$\begin{array}{llll}2 & 4.69 & 27.5019 & 26.9744\end{array}$

3n $\quad 1.70 \quad 30.4255 \quad 29.9685$

$3 x \quad 1.47 \quad 30.4047 \quad 29.9830$

$\begin{array}{llll}4 & 2.82 & 29.4718 & 28.9448\end{array}$

$\begin{array}{llll}5 & 6.22 & 25.8148 & 25.1182\end{array}$

$\begin{array}{llll}6 & 5.88 & 26.1214 & 25.4191\end{array}$

$\begin{array}{llll}7 s & 1.47 & 30.5638 & 30.1589\end{array}$

$\begin{array}{llll}7 a & 1.47 & 30.5087 & 30.0714\end{array}$

$\begin{array}{llll}9 & 5.89 & 26.6146 \quad 26.1046\end{array}$

$\begin{array}{llll}11 & 2.19 & 30.1321 & 29.5985\end{array}$ $\begin{array}{llll}55.1601 & 38.6301 & 37.6513 & 36.6541\end{array}$

$\begin{array}{llll}64.0008 & 47.5451 & 46.5528 & 45.7846\end{array}$

147.8858

28.8739

118.0910

28.6638

174.6052

62.3600

$28.9913 \quad 29.4875$

$26.9421 \quad 27.5075$

$29.9009 \quad 30.4098$

29.9350

30.4162

28.8405

29.4219

24.8760

25.2519

25.7737

26.1051

30.1086

30.5550

30.4833

26.0018

26.4472

29.4670

$\begin{array}{lll}30.0497 & 29.7059 & 29.6597\end{array}$
29.1850

27.1666

30.1130

30.1748

30.1988

29.1207

25.2842

25.6066

30.3611

30.2488

26.1851

25.5226

30.3431

26.1803 $\begin{array}{lllllll}35.5204 & 145.0114 & 145.7708 & 145.1271 & 136.1989 & 135.7912 & 135.4836\end{array}$

$\begin{array}{lllllll}158.4547 & 159.7387 & 159.5630 & 150.1797 & 149.8290 & 149.8505\end{array}$

$\begin{array}{llllll}150.8385 & 151.6999 & 151.0618 & 141.9255 & 141.5878 & 141.3324\end{array}$

$\begin{array}{llllll}55.1373 & 55.5489 & 54.5195 & 37.9055 & 36.9496 & 35.9082\end{array}$

$\begin{array}{llllll}63.9769 & 64.3966 & 64.0395 & 47.5296 & 46.5730 & 45.8108\end{array}$

$\begin{array}{llllll}146.2837 & 147.4691 & 147.9781 & 137.7283 & 137.3441 & 136.9847\end{array}$

$\begin{array}{llllll}29.4534 & 29.5113 & 27.6451 & 9.5589 & 9.6570 & 9.1059\end{array}$

$\begin{array}{llllll}117.5713 & 118.1862 & 118.1887 & 108.5741 & 108.0131 & 107.8168\end{array}$

$\begin{array}{lllllll}8.4676 & 28.6232 & 26.5712 & 9.6313 & 9.7471 & 7.6104\end{array}$

$\begin{array}{llllll}172.8047 & 174.3867 & 174.1929 & 165.4862 & 165.1549 & 164.5630\end{array}$

$\begin{array}{llllll}65.1342 & 64.8794 & 63.0685 & 48.5090 & 48.1580 & 47.0746\end{array}$

$\begin{array}{llllll}29.5242 & 28.9981 & 28.9791 & 29.4785 & 29.1679 & 29.1954\end{array}$

$\begin{array}{llllll}27.5064 & 26.9794 & 26.9423 & 27.5068 & 27.2098 & 27.1533\end{array}$

$\begin{array}{llllll}30.3814 & 29.9203 & 29.8548 & 30.3670 & 30.1293 & 30.0587\end{array}$

$\begin{array}{lllllll}30.1564 & 30.4458 & 30.0213 & 29.9701 & 30.4539 & 30.2335 & 30.1914\end{array}$

$\begin{array}{lllllll}29.0712 & 29.3793 & 28.8481 & 28.7445 & 29.3283 & 29.0243 & 28.9794\end{array}$

$\begin{array}{lllllll}25.1911 & 25.7029 & 25.0065 & 24.7658 & 25.6568 & 25.1742 & 25.0829\end{array}$

26.0498

25.3484

25.1786

26.0270

25.5355

25.4561

30.5602

30.1531

30.1156

30.5570

30.3621

30.3576

$30.5468 \quad 30.1109$

$26.5372 \quad 26.0244$

30.0723

30.5165

30.2862

30.2649

30.0233

$26.3574 \quad 26.0968$

26.0813

29.5422 


\section{Compound 88a}

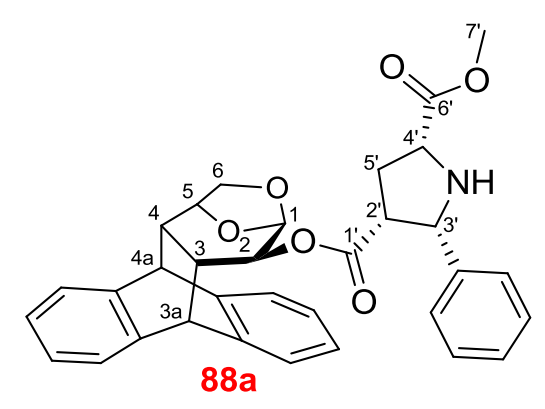

\begin{tabular}{|c|c|c|c|c|c|c|c|c|c|c|c|c|c|}
\hline & \multirow[b]{3}{*}{ Exp } & \multicolumn{12}{|c|}{ B3LYP } \\
\hline & & \multicolumn{6}{|c|}{ Gas phase } & \multicolumn{6}{|c|}{ PCM } \\
\hline & & 6-31G* & 6-31G** & $6-31+\mathrm{G} * *$ & 6-311G* & $6-311 G * *$ & $6-311+\mathrm{G} * *$ & 6-31G* & $6-31 G * *$ & $6-31+G * *$ & $6-311 G *$ & $6-311 G * *$ & $6-311+G * *$ \\
\hline \multicolumn{14}{|c|}{${ }^{13} \mathrm{C}$} \\
\hline 1 & 97.0 & 93.0086 & 93.7678 & 92.8804 & 80.7229 & 80.2649 & 79.9687 & 92.8878 & 93.6388 & 92.6433 & 80.5208 & 80.0500 & 79.6803 \\
\hline 2 & 72.1 & 117.5409 & 118.3544 & 117.7414 & 107.3023 & 107.1210 & 106.1093 & 117.4595 & 118.2675 & 117.5473 & 107.0774 & 106.8957 & 105.7849 \\
\hline 3 & 36.9 & 148.8582 & 149.6698 & 147.7053 & 139.4162 & 139.3769 & 138.6241 & 148.8543 & 149.6556 & 147.9903 & 139.4506 & 139.3933 & 138.5922 \\
\hline 4 & 46.4 & 140.4874 & 141.2796 & 139.1120 & 129.9101 & 129.7165 & 129.6185 & 140.6789 & 141.4661 & 139.4101 & 130.1622 & 129.9600 & 129.9786 \\
\hline 5 & 76.3 & 112.9195 & 113.6847 & 112.8625 & 101.7733 & 101.2521 & 100.8405 & 113.0219 & 113.7817 & 113.0105 & 101.8847 & 101.3460 & 100.8823 \\
\hline 6 & 70.6 & 119.7512 & 121.0940 & 120.7201 & 109.4721 & 108.7083 & 108.3966 & 119.9119 & 121.2484 & 120.6953 & 109.5288 & 108.7375 & 108.4401 \\
\hline $3 a$ & 47.0 & 141.3939 & 142.0287 & 141.9843 & 129.7845 & 129.5466 & 129.2806 & 141.4389 & 142.0683 & 141.8936 & 129.8951 & 129.6496 & 129.4621 \\
\hline $4 a$ & 50.2 & 137.5137 & 138.1268 & 136.7435 & 124.9738 & 124.6662 & 123.9798 & 138.0996 & 138.7043 & 137.4958 & 125.8032 & 125.4770 & 124.8412 \\
\hline $1^{\prime}$ & 172.7 & 24.3825 & 24.3998 & 22.7657 & 2.6728 & 2.7156 & 1.3937 & 23.2704 & 23.2779 & 21.3206 & 1.3264 & 1.3655 & 0.0629 \\
\hline $2^{\prime}$ & 49.1 & 138.6207 & 139.3720 & 138.4972 & 127.7720 & 127.6158 & 126.1775 & 138.9883 & 139.7490 & 139.0281 & 128.1295 & 127.9826 & 126.6013 \\
\hline $3^{\prime}$ & 65.9 & 121.0719 & 121.6663 & 120.6947 & 109.2714 & 108.8485 & 109.7131 & 121.6353 & 122.2095 & 121.4009 & 109.8182 & 109.4137 & 110.3231 \\
\hline $4^{\prime}$ & 59.8 & 128.8504 & 129.4962 & 128.1205 & 118.2217 & 117.7372 & 117.1700 & 129.2952 & 129.9277 & 128.4256 & 118.5731 & 118.1038 & 117.5863 \\
\hline $5^{\prime}$ & 34.5 & 153.4015 & 154.7703 & 156.3246 & 144.5352 & 144.1888 & 144.7681 & 153.2592 & 154.6094 & 155.9101 & 144.4121 & 144.0511 & 144.8258 \\
\hline $6^{\prime}$ & 173.4 & 22.1325 & 22.0796 & 19.5289 & -1.0404 & -0.9173 & -2.0414 & 21.4868 & 21.4341 & 18.6931 & -1.8808 & -1.7812 & -3.0163 \\
\hline $7^{\prime}$ & 52.3 & 138.6430 & 140.3584 & 140.0202 & 129.7821 & 129.2031 & 128.8285 & 138.3881 & 140.0900 & 139.5624 & 129.4119 & 128.8122 & 128.4645 \\
\hline \multicolumn{14}{|l|}{${ }^{1} \mathrm{H}$} \\
\hline 1 & 4.83 & 27.7050 & 27.1420 & 27.1970 & 27.8841 & 27.5485 & 27.5135 & 27.7116 & 27.1446 & 27.1218 & 27.8457 & 27.5118 & 27.4376 \\
\hline 2 & 3.92 & 28.2870 & 27.7299 & 27.7056 & 28.4009 & 28.0273 & 27.9783 & 28.2860 & 27.7179 & 27.6700 & 28.4055 & 28.0237 & 27.9558 \\
\hline 3 & 1.62 & 30.2147 & 29.8015 & 29.6654 & 30.2362 & 30.0157 & 29.9033 & 30.1737 & 29.7565 & 29.5895 & 30.1981 & 29.9778 & 29.8424 \\
\hline 4 & 1.91 & 30.4476 & 30.0551 & 29.9549 & 30.4466 & 30.2434 & 30.1111 & 30.3198 & 29.9231 & 29.8432 & 30.3301 & 30.1249 & 29.9935 \\
\hline 5 & 4.52 & 28.0004 & 27.4830 & 27.3688 & 28.0266 & 27.7044 & 27.6476 & 27.8154 & 27.2904 & 27.1506 & 27.8362 & 27.5086 & 27.4460 \\
\hline $6 n$ & 3.60 & 28.6248 & 28.1221 & 28.0715 & 28.6289 & 28.2997 & 28.2546 & 28.5002 & 27.9944 & 27.9178 & 28.5165 & 28.1848 & 28.1068 \\
\hline $6 x$ & 3.67 & 28.6062 & 28.0749 & 27.9636 & 28.5197 & 28.2374 & 28.1979 & 28.5198 & 27.9900 & 27.8727 & 28.4292 & 28.1519 & 28.1055 \\
\hline $3 a$ & 3.55 & 28.7390 & 28.2747 & 27.7595 & 28.5289 & 28.3511 & 28.0272 & 28.6448 & 28.1758 & 27.7013 & 28.4485 & 28.2583 & 27.9520 \\
\hline $4 a$ & 4.10 & 28.4628 & 28.0380 & 27.9084 & 28.3423 & 28.1350 & 28.0700 & 28.2522 & 27.8173 & 27.6654 & 28.1161 & 27.9024 & 27.8472 \\
\hline $2^{\prime}$ & 3.44 & 29.2135 & 28.6890 & 28.3500 & 29.0311 & 28.7501 & 28.5868 & 29.0603 & 28.5265 & 28.1746 & 28.8858 & 28.5951 & 28.4088 \\
\hline $3^{\prime}$ & 4.56 & 27.6379 & 27.1760 & 26.9326 & 27.4958 & 27.2483 & 27.1639 & 27.5920 & 27.1297 & 26.8908 & 27.4502 & 27.1975 & 27.1239 \\
\hline $4^{\prime}$ & 3.99 & 28.4087 & 27.9189 & 27.6440 & 28.2176 & 27.9327 & 27.8499 & 28.3100 & 27.8129 & 27.5294 & 28.1123 & 27.8213 & 27.7309 \\
\hline 5'a & 2.47 & 29.9493 & 29.4602 & 29.2182 & 29.8821 & 29.6219 & 29.4483 & 29.8424 & 29.3492 & 29.1438 & 29.7869 & 29.5231 & 29.3808 \\
\hline 5 'b & 2.47 & 29.8705 & 29.3952 & 29.3253 & 29.8688 & 29.5924 & 29.5582 & 29.8634 & 29.3867 & 29.3101 & 29.8344 & 29.5615 & 29.5327 \\
\hline $7^{\prime}$ & 3.81 & 28.5206 & 28.0064 & 27.8325 & 28.4950 & 28.1606 & 28.1328 & 28.4515 & 27.9366 & 27.7468 & 28.4180 & 28.0844 & 28.0498 \\
\hline
\end{tabular}




\begin{tabular}{|c|c|c|c|c|c|c|c|c|c|c|c|c|c|}
\hline & \multirow[b]{3}{*}{ Exp } & \multicolumn{12}{|c|}{ mPW1PW91 } \\
\hline & & \multicolumn{6}{|c|}{ Gas phase } & \multicolumn{6}{|c|}{ PCM } \\
\hline & & 6-31G* & $6-31 G * *$ & $6-31+G * *$ & 6-311G* & $6-311 G * *$ & $6-311+G^{* *}$ & 6-31G* & $6-31 G * *$ & $6-31+\mathrm{G} * *$ & 6-311G* & $6-311 G * *$ & $6-311+G * *$ \\
\hline \multicolumn{14}{|l|}{${ }^{13} \mathrm{C}$} \\
\hline 1 & 97.0 & 97.1377 & 97.8723 & 97.4398 & 86.8664 & 86.4561 & 86.2103 & 96.9584 & 97.6853 & 97.2443 & 86.5983 & 86.1723 & 85.8722 \\
\hline 2 & 72.1 & 121.9305 & 122.6811 & 122.3101 & 113.0300 & 112.8383 & 111.9905 & 121.8634 & 122.6120 & 122.1301 & 112.8066 & 112.6239 & 111.7044 \\
\hline 3 & 36.9 & 154.1734 & 154.9043 & 153.0367 & 145.4787 & 145.4611 & 144.9613 & 154.1089 & 154.8309 & 153.3018 & 145.4517 & 145.4097 & 144.8724 \\
\hline 4 & 46.4 & 145.9979 & 146.7024 & 144.2653 & 136.2420 & 136.0564 & 136.0632 & 146.1638 & 146.8638 & 144.5374 & 136.4416 & 136.2456 & 136.3617 \\
\hline 5 & 76.3 & 117.5850 & 118.2965 & 117.7693 & 108.0105 & 107.4950 & 107.1158 & 117.6364 & 118.3436 & 117.9070 & 108.0508 & 107.5170 & 107.0759 \\
\hline 6 & 70.6 & 123.9059 & 125.1741 & 124.9977 & 115.0103 & 114.2631 & 114.0597 & 124.0393 & 125.3045 & 124.9466 & 115.0260 & 114.2560 & 114.0717 \\
\hline 3a & 47.0 & 146.1164 & 146.6870 & 146.6152 & 135.6963 & 135.4882 & 135.1430 & 146.2226 & 146.7838 & 146.6471 & 135.8376 & 135.6128 & 135.3615 \\
\hline $4 a$ & 50.2 & 142.4162 & 142.9576 & 141.5906 & 131.2547 & 130.9477 & 130.3653 & 143.0210 & 143.5556 & 142.3061 & 132.0517 & 131.7294 & 131.1986 \\
\hline $1^{\prime}$ & 172.7 & 26.9881 & 26.9913 & 25.7913 & 7.9195 & 7.9259 & 6.7922 & 25.5488 & 25.5467 & 24.2190 & 6.3200 & 6.3545 & 5.2230 \\
\hline $2^{\prime}$ & 49.1 & 143.2953 & 143.9648 & 143.3105 & 133.5910 & 133.4109 & 132.2391 & 143.9891 & 144.6746 & 143.9376 & 134.2706 & 134.0909 & 133.0335 \\
\hline $3^{\prime}$ & 65.9 & 125.9206 & 126.4479 & 125.6385 & 115.4972 & 115.0377 & 115.5626 & 126.4286 & 126.9224 & 126.2385 & 115.9365 & 115.5140 & 116.0255 \\
\hline $4^{\prime}$ & 59.8 & 133.1796 & 133.7645 & 132.7636 & 124.0436 & 123.5227 & 122.8625 & 133.5633 & 134.1350 & 133.0457 & 124.2930 & 123.8069 & 123.1779 \\
\hline $5^{\prime}$ & 34.5 & 158.1120 & 159.3971 & 160.5684 & 150.0189 & 149.6690 & 149.9739 & 157.6328 & 158.8950 & 159.8607 & 149.5658 & 149.2208 & 149.6872 \\
\hline $6^{\prime}$ & 173.4 & 24.7769 & 24.6831 & 22.6785 & 4.4083 & 4.4920 & 3.5219 & 24.3071 & 24.2206 & 21.8676 & 3.7675 & 3.8181 & 2.6932 \\
\hline $7^{\prime}$ & 52.3 & 142.0667 & 143.7075 & 143.5182 & 134.2679 & 133.7259 & 133.4617 & 141.8124 & 143.4429 & 143.0571 & 133.9139 & 133.3548 & 133.1214 \\
\hline \multicolumn{14}{|l|}{${ }^{1} \mathrm{H}$} \\
\hline 1 & 4.83 & 27.6803 & 27.1141 & 27.1687 & 27.8217 & 27.4484 & 27.4416 & 27.6225 & 27.0540 & 27.0625 & 27.7209 & 27.3569 & 27.3178 \\
\hline 2 & 3.92 & 28.2582 & 27.7017 & 27.6436 & 28.3151 & 27.9595 & 27.8776 & 28.2392 & 27.6669 & 27.5988 & 28.3033 & 27.9350 & 27.8309 \\
\hline 3 & 1.62 & 30.1564 & 29.7376 & 29.6156 & 30.1385 & 29.9233 & 29.7991 & 30.1272 & 29.7033 & 29.5449 & 30.1110 & 29.8951 & 29.7485 \\
\hline 4 & 1.91 & 30.4139 & 30.0219 & 29.9331 & 30.3547 & 30.1557 & 30.0409 & 30.2829 & 29.8860 & 29.8188 & 30.2377 & 30.0356 & 29.9339 \\
\hline 5 & 4.52 & 27.9227 & 27.4021 & 27.2905 & 27.9382 & 27.6001 & 27.5573 & 27.7191 & 27.1893 & 27.0563 & 27.7342 & 27.3897 & 27.3427 \\
\hline $6 n$ & 3.60 & 28.5837 & 28.0825 & 28.0375 & 28.5834 & 28.2444 & 28.1856 & 28.4407 & 27.9345 & 27.8722 & 28.4501 & 28.1070 & 28.0205 \\
\hline $6 x$ & 3.67 & 28.5603 & 28.0315 & 27.9483 & 28.4739 & 28.1834 & 28.1611 & 28.4520 & 27.9248 & 27.8444 & 28.3629 & 28.0785 & 28.0536 \\
\hline 3a & 3.55 & 28.5280 & 28.0677 & 27.5890 & 28.3273 & 28.1605 & 27.8539 & 28.4562 & 27.9902 & 27.5416 & 28.2805 & 28.0929 & 27.7971 \\
\hline $4 a$ & 4.10 & 28.2856 & 27.8651 & 27.7611 & 28.1438 & 27.9297 & 27.8949 & 28.0589 & 27.6265 & 27.5023 & 27.9087 & 27.6864 & 27.6690 \\
\hline $2^{\prime}$ & 3.44 & 29.0941 & 28.5730 & 28.2797 & 28.8987 & 28.6222 & 28.4887 & 28.9344 & 28.3991 & 28.1075 & 28.7498 & 28.4578 & 28.3027 \\
\hline $3^{\prime}$ & 4.56 & 27.5670 & 27.0980 & 26.8918 & 27.3956 & 27.1431 & 27.0909 & 27.5258 & 27.0572 & 26.8420 & 27.3603 & 27.1039 & 27.0502 \\
\hline $4^{\prime}$ & 3.99 & 28.3451 & 27.8483 & 27.5981 & 28.1353 & 27.8520 & 27.7768 & 28.2212 & 27.7149 & 27.4740 & 28.0118 & 27.7185 & 27.6348 \\
\hline $5^{\prime} \mathrm{a}$ & 2.47 & 29.8521 & 29.3623 & 29.1429 & 29.7550 & 29.4946 & 29.3300 & 29.7312 & 29.2366 & 29.0547 & 29.6481 & 29.3817 & 29.2441 \\
\hline 5 'b & 2.47 & 29.8239 & 29.3433 & 29.2527 & 29.7764 & 29.4884 & 29.4374 & 29.7957 & 29.3128 & 29.2199 & 29.7287 & 29.4495 & 29.3940 \\
\hline $7^{\prime}$ & 3.81 & 28.4517 & 27.9351 & 27.7938 & 28.4172 & 28.0668 & 28.0644 & 28.3657 & 27.8486 & 27.7023 & 28.3291 & 27.9797 & 27.9701 \\
\hline
\end{tabular}




\section{Compound 88b}

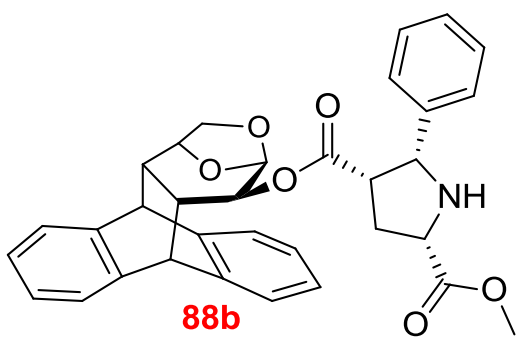

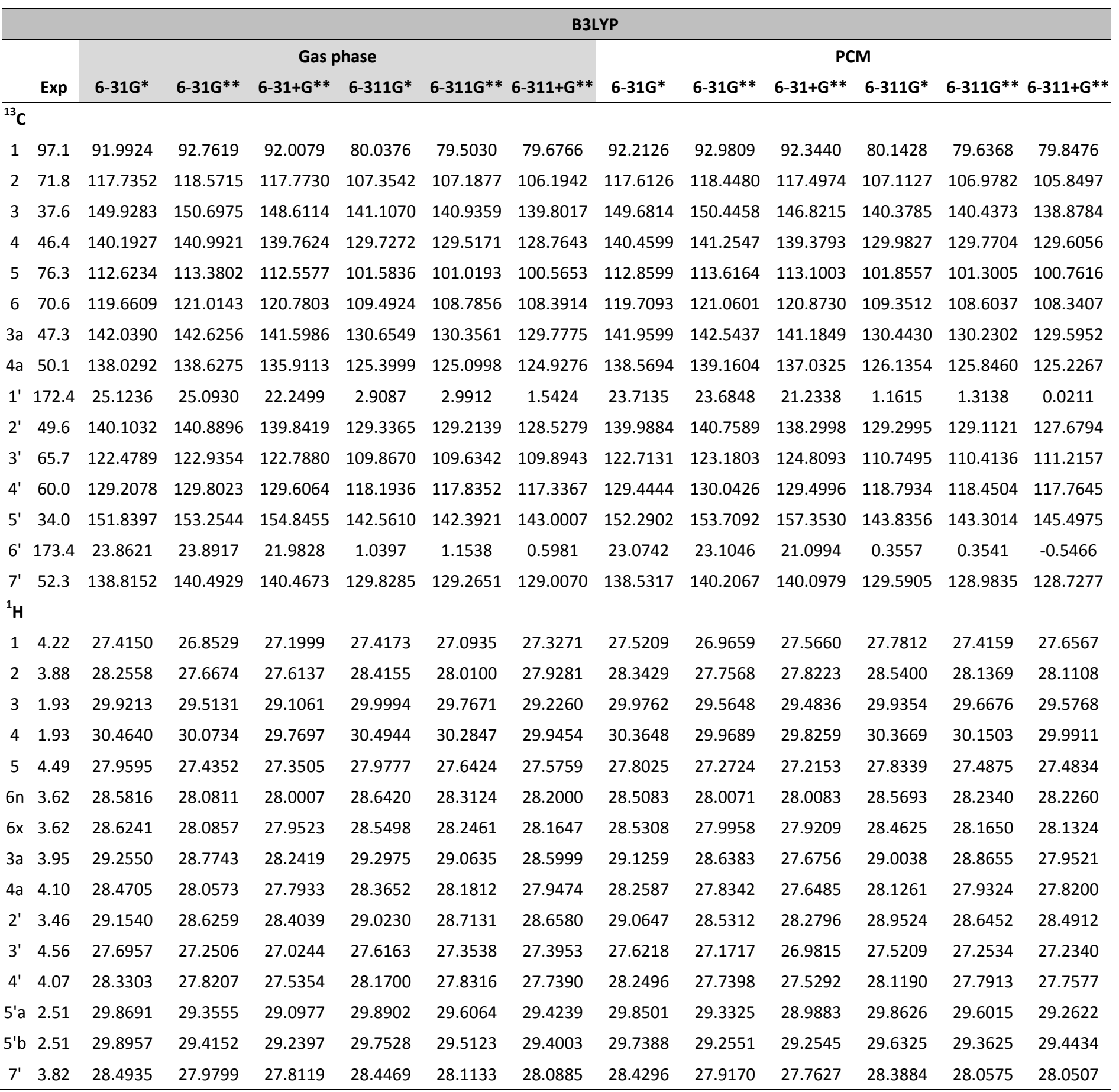




\begin{tabular}{|c|c|c|c|c|c|c|c|c|c|c|c|c|c|}
\hline & & \multicolumn{12}{|c|}{ mPW1PW91 } \\
\hline & \multirow[b]{2}{*}{ Exp } & \multicolumn{6}{|c|}{ Gas phase } & \multicolumn{6}{|c|}{ PCM } \\
\hline & & 6-31G* & $6-31 G * *$ & $6-31+\mathrm{G} * *$ & 6-311G* & $6-311 G * *$ & $6-311+G * *$ & 6-31G* & $6-31 G * *$ & $6-31+\mathrm{G} * *$ & 6-311G* & $6-311 G * *$ & $6-311+\mathrm{G} * *$ \\
\hline \multicolumn{14}{|l|}{${ }^{13} \mathrm{C}$} \\
\hline 1 & 97.1 & 96.3073 & 97.0458 & 96.4884 & 86.3519 & 85.8371 & 85.9247 & 96.5879 & 97.2851 & 96.8080 & 86.5329 & 85.9781 & 86.1950 \\
\hline 2 & 71.8 & 122.0887 & 122.8789 & 122.3305 & 113.1601 & 113.0180 & 112.0714 & 121.9989 & 122.7874 & 122.0562 & 112.9108 & 112.8561 & 111.6903 \\
\hline 3 & 37.6 & 155.4443 & 156.1443 & 154.8207 & 147.6149 & 147.5148 & 146.3495 & 155.4738 & 155.9233 & 152.9688 & 146.8363 & 146.8287 & 145.7801 \\
\hline 4 & 46.4 & 145.7505 & 146.4573 & 145.2773 & 136.0319 & 135.8177 & 135.1983 & 145.9722 & 146.7062 & 145.0237 & 136.3015 & 136.0849 & 135.7919 \\
\hline 5 & 76.3 & 117.3227 & 118.0249 & 117.3051 & 107.9116 & 107.3520 & 106.8154 & 117.5792 & 118.2453 & 117.8148 & 108.1014 & 107.5332 & 107.0091 \\
\hline 6 & 70.6 & 123.8808 & 125.1609 & 124.9474 & 115.0976 & 114.4010 & 114.1011 & 123.8882 & 125.1938 & 125.0002 & 114.9637 & 114.2633 & 113.9702 \\
\hline $3 a$ & 47.3 & 146.8186 & 147.3365 & 146.4578 & 136.6069 & 136.3455 & 135.7542 & 146.8638 & 147.2720 & 146.1753 & 136.3903 & 136.1667 & 135.6099 \\
\hline $4 a$ & 50.1 & 142.9433 & 143.4744 & 140.6397 & 131.7158 & 131.4277 & 131.2625 & 143.5512 & 144.0296 & 141.6518 & 132.4060 & 132.1247 & 131.6952 \\
\hline $1^{\prime}$ & 172.4 & 27.8252 & 27.7988 & 25.6267 & 8.4680 & 8.5669 & 7.2279 & 26.5386 & 26.3739 & 23.8534 & 6.6006 & 6.7617 & 5.4529 \\
\hline $2^{\prime}$ & 49.6 & 144.8572 & 145.5859 & 145.0521 & 135.2474 & 135.1214 & 134.3550 & 144.7700 & 145.4394 & 143.8566 & 135.0629 & 134.9702 & 133.7509 \\
\hline $3^{\prime}$ & 65.7 & 127.0268 & 127.4248 & 127.0158 & 115.8094 & 115.5826 & 115.5845 & 127.2173 & 127.6733 & 128.7873 & 116.5344 & 116.2316 & 116.5926 \\
\hline $4^{\prime}$ & 60.0 & 133.5729 & 134.1369 & 134.4916 & 124.2801 & 123.9375 & 123.0389 & 133.9809 & 134.3649 & 134.0611 & 124.4182 & 124.2063 & 123.2592 \\
\hline $5^{\prime}$ & 34.0 & 156.3892 & 157.6860 & 157.9841 & 147.5634 & 147.3209 & 147.8116 & 156.5343 & 158.0986 & 160.1841 & 148.6606 & 148.3268 & 149.3904 \\
\hline $6^{\prime}$ & 173.4 & 26.3963 & 26.3966 & 24.6543 & 6.2681 & 6.3471 & 5.8456 & 25.3541 & 25.5400 & 24.2670 & 5.7465 & 5.5631 & 4.9537 \\
\hline $7^{\prime}$ & 52.3 & 142.2722 & 143.8749 & 143.8669 & 134.4241 & 133.9137 & 133.6438 & 142.0425 & 143.5888 & 143.5495 & 134.1107 & 133.5515 & 133.3404 \\
\hline \multicolumn{14}{|l|}{${ }^{1} \mathrm{H}$} \\
\hline 1 & 4.22 & 27.3582 & 26.7974 & 26.8778 & 27.3886 & 27.0376 & 27.0962 & 27.4689 & 26.8973 & 27.3982 & 27.6001 & 27.2547 & 27.3824 \\
\hline 2 & 3.88 & 28.2490 & 27.6607 & 27.4928 & 28.3386 & 27.9433 & 27.8083 & 28.3556 & 27.7557 & 27.7598 & 28.4600 & 28.0527 & 28.0179 \\
\hline 3 & 1.93 & 29.8179 & 29.3916 & 28.9219 & 29.7028 & 29.4369 & 29.0648 & 29.7374 & 29.4466 & 29.2711 & 29.8077 & 29.5332 & 29.3270 \\
\hline 4 & 1.93 & 30.4178 & 30.0263 & 29.6733 & 30.3531 & 30.1431 & 29.8451 & 30.2873 & 29.9197 & 29.7235 & 30.2631 & 30.0514 & 29.8563 \\
\hline 5 & 4.49 & 27.8829 & 27.3544 & 27.2348 & 27.8869 & 27.5322 & 27.4686 & 27.7075 & 27.1776 & 27.1004 & 27.7285 & 27.3679 & 27.3491 \\
\hline $6 n$ & 3.62 & 28.5377 & 28.0360 & 27.9143 & 28.5525 & 28.2111 & 28.0993 & 28.4327 & 27.9529 & 27.9322 & 28.4989 & 28.1525 & 28.1084 \\
\hline $6 x$ & 3.62 & 28.5845 & 28.0457 & 27.9073 & 28.5073 & 28.1861 & 28.1194 & 28.4752 & 27.9430 & 27.8771 & 28.4050 & 28.0985 & 28.0720 \\
\hline $3 a$ & 3.95 & 29.1742 & 28.7070 & 28.3776 & 29.3378 & 29.1369 & 28.6238 & 29.2051 & 28.5741 & 27.7397 & 28.9558 & 28.7992 & 28.0952 \\
\hline $4 a$ & 4.10 & 28.2934 & 27.8830 & 27.6199 & 28.1601 & 27.9662 & 27.7648 & 28.0543 & 27.6398 & 27.4413 & 27.9192 & 27.7139 & 27.6065 \\
\hline $2^{\prime}$ & 3.46 & 29.0528 & 28.5282 & 28.3216 & 28.8991 & 28.5923 & 28.5470 & 28.9590 & 28.4205 & 28.2154 & 28.7960 & 28.4954 & 28.3804 \\
\hline $3^{\prime}$ & 4.56 & 27.6324 & 27.1726 & 26.9269 & 27.4931 & 27.2319 & 27.3033 & 27.5288 & 27.0867 & 26.9161 & 27.4225 & 27.1473 & 27.1488 \\
\hline $4^{\prime}$ & 4.07 & 28.2797 & 27.7604 & 27.4574 & 28.0896 & 27.7508 & 27.6320 & 28.2049 & 27.6720 & 27.4495 & 28.0287 & 27.6849 & 27.6381 \\
\hline 5'a & 2.51 & 29.8148 & 29.3039 & 29.0862 & 29.8493 & 29.5810 & 29.3715 & 29.8433 & 29.2758 & 29.0043 & 29.7783 & 29.5022 & 29.2567 \\
\hline 5 'b & 2.51 & 29.7926 & 29.3086 & 29.1147 & 29.5971 & 29.3463 & 29.2610 & 29.5927 & 29.1336 & 29.0485 & 29.4779 & 29.2154 & 29.2122 \\
\hline $7^{\prime}$ & 3.82 & 28.4361 & 27.9209 & 27.7688 & 28.3888 & 28.0449 & 28.0192 & 28.3671 & 27.8516 & 27.7190 & 28.3347 & 27.9375 & 27.9806 \\
\hline
\end{tabular}




\section{Compound 89a}<smiles>CC(C)(C)OC12C[C@H]3CC4(C[C@H]3CC1(C)O4)OCCO2</smiles>

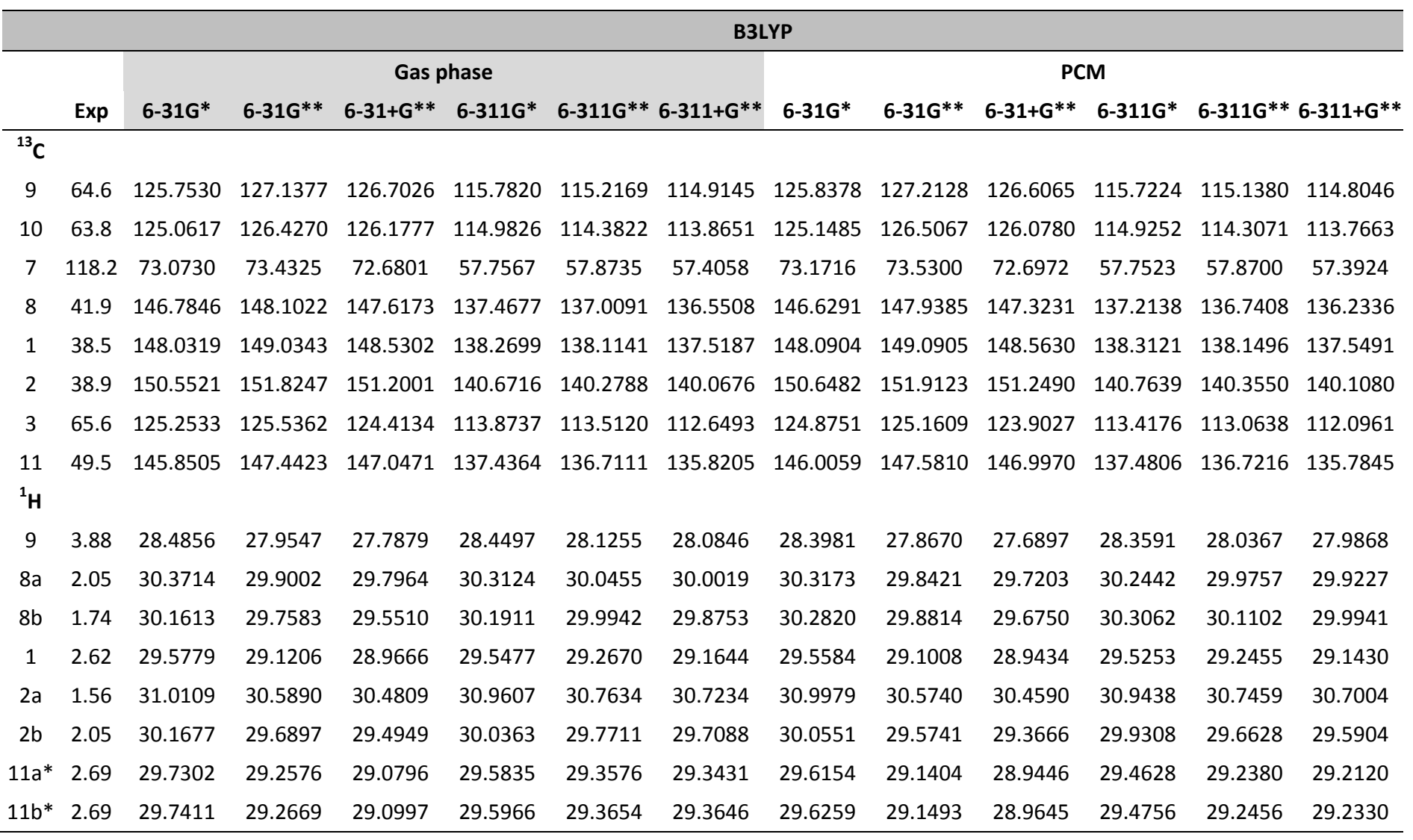

\begin{tabular}{|c|c|c|c|c|c|c|c|c|c|c|c|c|c|}
\hline & \multirow[b]{3}{*}{ Exp } & \multicolumn{12}{|c|}{ mPW1PW91 } \\
\hline & & \multicolumn{6}{|c|}{ Gas phase } & \multicolumn{6}{|c|}{ PCM } \\
\hline & & 6-31G* & $6-31 \mathrm{G} * *$ & $6-31+G * *$ & 6-311G* & $6-311 \mathrm{G} * *$ & $6-311+G * *$ & 6-31G* & $6-31 G * *$ & $6-31+\mathrm{G} * *$ & 6-311G* & $6-311 G * *$ & $6-311+G * *$ \\
\hline \multicolumn{14}{|l|}{${ }^{13} \mathrm{C}$} \\
\hline 9 & 64.6 & 129.7493 & 131.0691 & 130.8563 & 121.2158 & 120.6631 & 120.3395 & 129.8286 & 131.1401 & 130.7619 & 121.1429 & 120.5729 & 120.2256 \\
\hline 10 & 63.8 & 129.0105 & 130.3118 & 130.2584 & 120.4072 & 119.8202 & 119.3303 & 129.0867 & 130.3823 & 130.1536 & 120.3292 & 119.7259 & 119.2174 \\
\hline 7 & 118.2 & 77.3418 & 77.6977 & 77.2869 & 64.2984 & 64.4168 & 63.9755 & 77.4390 & 77.7935 & 77.3049 & 64.2862 & 64.4052 & 63.9564 \\
\hline 8 & 41.9 & 151.4046 & 152.6200 & 152.0948 & 143.0263 & 142.5916 & 142.1561 & 151.2800 & 152.4865 & 151.8462 & 142.8074 & 142.3582 & 141.8816 \\
\hline 1 & 38.5 & 153.0043 & 153.9274 & 153.5165 & 144.1012 & 143.9365 & 143.3336 & 153.0825 & 154.0033 & 153.5726 & 144.1591 & 143.9876 & 143.3809 \\
\hline 2 & 38.9 & 155.0072 & 156.1778 & 155.4702 & 146.1734 & 145.7788 & 145.5243 & 155.1139 & 156.2767 & 155.5370 & 146.2672 & 145.8577 & 145.5708 \\
\hline 3 & 65.6 & 129.3768 & 129.6688 & 128.8682 & 119.4403 & 119.0837 & 118.2307 & 128.9979 & 129.2926 & 128.3686 & 118.9823 & 118.6339 & 117.6878 \\
\hline 11 & 49.5 & 149.1848 & 150.7453 & 150.6871 & 142.2048 & 141.4932 & 140.6785 & 149.3316 & 150.8768 & 150.6381 & 142.2378 & 141.4949 & 140.6421 \\
\hline
\end{tabular}




\begin{tabular}{cccccccccccccc}
\hline 9 & 3.88 & 28.4398 & 27.9101 & 27.7579 & 28.3963 & 28.0616 & 28.0336 & 28.3435 & 27.8133 & 27.6529 & 28.2993 & 27.9662 & 27.9302 \\
$8 \mathrm{a}$ & 2.05 & 30.2939 & 29.8199 & 29.7238 & 30.2116 & 29.9361 & 29.8992 & 30.2371 & 29.7587 & 29.6123 & 30.1416 & 29.8644 & 29.8195 \\
$8 \mathrm{~b}$ & 1.74 & 30.0876 & 29.6831 & 29.4880 & 30.0761 & 29.8820 & 29.7696 & 30.2097 & 29.8078 & 29.6469 & 30.1910 & 29.9978 & 29.8872 \\
1 & 2.62 & 29.5328 & 29.0684 & 28.9181 & 29.4490 & 29.1676 & 29.0699 & 29.5110 & 29.0463 & 28.8934 & 29.4248 & 29.1446 & 29.0466 \\
$2 \mathrm{a}$ & 1.56 & 30.9256 & 30.5031 & 30.4132 & 30.8535 & 30.6484 & 30.6119 & 30.9083 & 30.4836 & 30.3881 & 30.8327 & 30.6267 & 30.5856 \\
$2 \mathrm{~b}$ & 2.05 & 30.1388 & 29.6604 & 29.4672 & 29.9624 & 29.6959 & 29.6354 & 30.0209 & 29.5391 & 29.3347 & 29.8528 & 29.5833 & 29.5138 \\
$11 \mathrm{a} *$ & 2.69 & 29.6534 & 29.1830 & 29.0250 & 29.5189 & 29.2754 & 29.2697 & 29.5282 & 29.0550 & 28.8822 & 29.3911 & 29.1487 & 29.1333 \\
$11 \mathrm{~b} *$ & 2.69 & 29.6636 & 29.1917 & 29.0489 & 29.5306 & 29.2821 & 29.2898 & 29.5379 & 29.0633 & 28.9060 & 29.4026 & 29.1551 & 29.1531 \\
\hline
\end{tabular}

\section{Compound 89b}<smiles>C1COC2(C1)C[C@@H]1CC3(CO3)C[C@H]1C2</smiles>

$89 b$

\begin{tabular}{|c|c|c|c|c|c|c|c|c|c|c|c|c|c|}
\hline & \multirow[b]{3}{*}{ Exp } & \multicolumn{12}{|c|}{ B3LYP } \\
\hline & & \multicolumn{6}{|c|}{ Gas phase } & \multicolumn{6}{|c|}{ PCM } \\
\hline & & 6-31G* & $6-31 G * *$ & $6-31+G * *$ & 6-311G* & $6-311 G * *$ & $6-311+G * *$ & 6-31G* & $6-31 \mathrm{G} * *$ & $6-31+G * *$ & 6-311G* & $6-311 G * *$ & $6-311+G * *$ \\
\hline \multicolumn{14}{|l|}{${ }^{13} \mathrm{C}$} \\
\hline 9 & & 125.4861 & 126.8654 & 126.7766 & 115.6051 & 115.0277 & 114.7944 & 125.5055 & 126.8787 & 126.6551 & 115.5064 & 114.9114 & 114.6617 \\
\hline 10 & & 125.4556 & 126.8111 & 126.5179 & 115.3527 & 114.7230 & 114.2901 & 125.5191 & 126.8687 & 126.4111 & 115.2767 & 114.6319 & 114.1884 \\
\hline 7 & & 71.7041 & 72.0017 & 70.6087 & 56.6491 & 56.6677 & 56.0827 & 71.7543 & 72.0521 & 70.6086 & 56.6257 & 56.6488 & 56.0336 \\
\hline 8 & & 144.6642 & 145.9253 & 145.1971 & 134.6663 & 134.1729 & 133.7554 & 144.7457 & 146.0007 & 145.2178 & 134.7594 & 134.2554 & 133.7887 \\
\hline 1 & & 148.4001 & 149.3638 & 148.3945 & 138.2552 & 138.0177 & 137.4069 & 148.4270 & 149.3914 & 148.4111 & 138.3240 & 138.0809 & 137.4323 \\
\hline 2 & & 150.0665 & 151.3508 & 150.2844 & 140.3766 & 139.8815 & 139.3333 & 150.2549 & 151.5313 & 150.4325 & 140.5701 & 140.0667 & 139.4597 \\
\hline 3 & & 126.7197 & 127.0292 & 126.5649 & 115.8890 & 115.5965 & 114.6830 & 126.3097 & 126.6188 & 126.0601 & 115.3791 & 115.0933 & 114.1037 \\
\hline 11 & & 132.2505 & 133.8335 & 132.4783 & 121.9038 & 121.0160 & 120.1975 & 132.4829 & 134.0438 & 132.3905 & 122.0587 & 121.1379 & 120.1575 \\
\hline \multicolumn{14}{|l|}{${ }^{1} \mathrm{H}$} \\
\hline 9 & & 28.4870 & 27.9529 & 27.7980 & 28.4632 & 28.1327 & 28.1091 & 28.4071 & 27.8733 & 27.7104 & 28.3811 & 28.0530 & 28.0232 \\
\hline $8 a$ & & 30.1099 & 29.6542 & 29.4773 & 30.0363 & 29.7943 & 29.7048 & 30.1193 & 29.6628 & 29.4893 & 30.0499 & 29.8069 & 29.7183 \\
\hline $8 b$ & & 30.5613 & 30.1349 & 30.0621 & 30.5718 & 30.3383 & 30.3039 & 30.5207 & 30.0914 & 30.0025 & 30.5222 & 30.2869 & 30.2414 \\
\hline 1 & & 29.6067 & 29.1235 & 28.9784 & 29.5068 & 29.2046 & 29.1482 & 29.5214 & 29.0368 & 28.8814 & 29.4245 & 29.1220 & 29.0573 \\
\hline $2 a$ & & 30.7962 & 30.3737 & 30.2572 & 30.7619 & 30.5308 & 30.4895 & 30.7607 & 30.3347 & 30.2055 & 30.7209 & 30.4871 & 30.4359 \\
\hline $2 b$ & & 30.0859 & 29.6110 & 29.4377 & 29.9929 & 29.7409 & 29.6451 & 30.0866 & 29.6097 & 29.4293 & 29.9935 & 29.7387 & 29.6365 \\
\hline $11 a^{*}$ & & 29.4595 & 29.0282 & 28.8459 & 29.3741 & 29.1768 & 29.1309 & 29.3923 & 28.9590 & 28.7465 & 29.2978 & 29.1017 & 29.0337 \\
\hline $11 b^{*}$ & & 29.4901 & 29.0485 & 28.8666 & 29.4076 & 29.1964 & 29.1315 & 29.3741 & 28.9285 & 28.7143 & 29.2813 & 29.0701 & 28.9804 \\
\hline
\end{tabular}




\begin{tabular}{|c|c|c|c|c|c|c|c|c|c|c|c|c|c|}
\hline & \multirow[b]{3}{*}{ Exp } & \multicolumn{12}{|c|}{ mPW1PW91 } \\
\hline & & \multicolumn{6}{|c|}{ Gas phase } & \multicolumn{6}{|c|}{ PCM } \\
\hline & & 6-31G* & $6-31 G * *$ & $6-31+G * *$ & 6-311G* & 6-311G** & $6-311+G^{* *}$ & 6-31G* & $6-31 G * *$ & $6-31+G * *$ & 6-311G* & $6-311 G * *$ & $6-311+G * *$ \\
\hline \multicolumn{14}{|l|}{${ }^{13} \mathrm{C}$} \\
\hline 9 & & 129.4732 & 130.7879 & 130.9028 & 120.9902 & 120.4260 & 120.1864 & 129.4934 & 130.8031 & 130.7877 & 120.8885 & 120.3081 & 120.0552 \\
\hline 10 & & 129.3958 & 130.6892 & 130.6629 & 120.7594 & 120.1429 & 119.6922 & 129.4546 & 130.7430 & 130.5559 & 120.6687 & 120.0382 & 119.5808 \\
\hline 7 & & 76.0014 & 76.2972 & 75.4029 & 63.2469 & 63.2692 & 62.6806 & 76.0485 & 76.3439 & 75.4021 & 63.2170 & 63.2436 & 62.6278 \\
\hline 8 & & 149.3441 & 150.4965 & 149.7720 & 140.3385 & 139.8592 & 139.4479 & 149.4532 & 150.5996 & 149.8246 & 140.4451 & 139.9556 & 139.5006 \\
\hline 1 & & 153.3437 & 154.2289 & 153.3191 & 144.0765 & 143.8296 & 143.2165 & 153.3788 & 154.2648 & 153.3504 & 144.1498 & 143.8982 & 143.2547 \\
\hline 2 & & 154.6684 & 155.8422 & 154.8640 & 146.0089 & 145.5142 & 144.9746 & 154.8850 & 156.0514 & 155.0497 & 146.2145 & 145.7118 & 145.1233 \\
\hline 3 & & 130.8732 & 131.1880 & 131.0626 & 121.5280 & 121.2376 & 120.2999 & 130.4708 & 130.7844 & 130.5713 & 121.0190 & 120.7356 & 119.7262 \\
\hline 11 & & 136.1957 & 137.7313 & 136.7617 & 127.4917 & 126.6086 & 125.8085 & 136.4192 & 137.9342 & 136.6828 & 127.6244 & 126.7108 & 125.7678 \\
\hline \multicolumn{14}{|l|}{${ }^{1} \mathrm{H}$} \\
\hline 9 & & 28.4463 & 27.9128 & 27.7694 & 28.4138 & 28.0717 & 28.0566 & 28.3585 & 27.8251 & 27.6756 & 28.3262 & 27.9864 & 27.9658 \\
\hline $8 a$ & & 30.0600 & 29.6032 & 29.4290 & 29.9420 & 29.7007 & 29.6189 & 30.0680 & 29.6102 & 29.4386 & 29.9533 & 29.7109 & 29.6297 \\
\hline $8 b$ & & 30.4940 & 30.0638 & 29.9901 & 30.4718 & 30.2317 & 30.1952 & 30.4505 & 30.0172 & 29.9294 & 30.4206 & 30.1785 & 30.1321 \\
\hline 1 & & 29.5619 & 29.0736 & 28.9311 & 29.4203 & 29.1129 & 29.0557 & 29.4707 & 28.9806 & 28.8301 & 29.3337 & 29.0257 & 28.9614 \\
\hline $2 a$ & & 30.7311 & 30.3059 & 30.2008 & 30.6694 & 30.4318 & 30.3920 & 30.6912 & 30.2622 & 30.1465 & 30.6255 & 30.3849 & 30.3363 \\
\hline $2 b$ & & 30.0127 & 29.5357 & 29.3745 & 29.8811 & 29.6284 & 29.5456 & 30.0129 & 29.5337 & 29.3656 & 29.8798 & 29.6245 & 29.5354 \\
\hline $11 a^{*}$ & & 29.4075 & 28.9764 & 28.8129 & 29.3244 & 29.1084 & 29.0747 & 29.3319 & 28.8985 & 28.7089 & 29.2424 & 29.0274 & 28.9744 \\
\hline $11 b^{*}$ & & 29.4406 & 28.9988 & 28.8308 & 29.3639 & 29.1332 & 29.0697 & 29.3140 & 28.8678 & 28.6720 & 29.2304 & 28.9996 & 28.9146 \\
\hline
\end{tabular}

\section{Compound 90a}

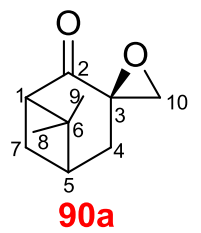

\begin{tabular}{|c|c|c|c|c|c|c|c|c|c|c|c|c|c|}
\hline & \multirow[b]{3}{*}{ Exp } & \multicolumn{12}{|c|}{ B3LYP } \\
\hline & & \multicolumn{6}{|c|}{ Gas phase } & \multicolumn{6}{|c|}{ PCM } \\
\hline & & 6-31G* & $6-31 G * *$ & $6-31+\mathrm{G} * *$ & 6-311G* & $6-311 G * *$ & $6-311+G * *$ & 6-31G* & $6-31 G * *$ & $6-31+\mathrm{G} * *$ & 6-311G* & $6-311 G * *$ & $6-311+G * *$ \\
\hline \multicolumn{14}{|l|}{${ }^{13} \mathrm{C}$} \\
\hline 1 & 57.3 & 133.4696 & 134.3185 & 132.3384 & 122.5217 & 122.2125 & 121.3700 & 132.6439 & 133.4893 & 131.2639 & 121.5568 & 121.2500 & 120.1307 \\
\hline 2 & 207.8 & -11.2467 & -11.1066 & -13.1171 & -32.1522 & -31.9964 & -35.7488 & -14.8769 & -14.7403 & -17.4880 & -36.2907 & -36.1318 & -40.6232 \\
\hline 3 & 54.3 & 136.2753 & 136.4099 & 136.2757 & 126.5274 & 126.2567 & 125.4664 & 135.8442 & 135.9750 & 135.8259 & 126.0319 & 125.7660 & 124.9319 \\
\hline 4 & 27.0 & 156.7757 & 158.0248 & 157.0727 & 147.1778 & 146.7265 & 146.6027 & 156.9266 & 158.1647 & 157.1564 & 147.2873 & 146.8222 & 146.6457 \\
\hline 5 & 40.3 & 148.2002 & 149.1176 & 147.5453 & 138.6015 & 138.3490 & 137.9699 & 148.1984 & 149.1189 & 147.5655 & 138.6302 & 138.3766 & 137.9698 \\
\hline 6 & 40.8 & 145.4445 & 145.9667 & 145.5537 & 134.8666 & 135.0344 & 134.4545 & 144.9133 & 145.4378 & 144.9159 & 134.3108 & 134.4820 & 133.8124 \\
\hline 7 & 31.6 & 161.7361 & 163.3055 & 162.9285 & 154.5077 & 154.2242 & 154.0359 & 161.7801 & 163.3376 & 162.8544 & 154.5352 & 154.2315 & 153.9757 \\
\hline 8 & 25.8 & 163.6016 & 165.2659 & 165.4083 & 156.3014 & 156.0751 & 155.7613 & 163.8652 & 165.5135 & 165.7077 & 156.6323 & 156.3790 & 156.0726 \\
\hline 9 & 22.3 & 167.5656 & 169.3182 & 169.0704 & 160.4099 & 160.1461 & 159.7657 & 167.6074 & 169.3467 & 169.1023 & 160.4646 & 160.1736 & 159.7737 \\
\hline 10 & 52.4 & 139.0066 & 140.5059 & 139.9309 & 130.3074 & 129.4204 & 128.5865 & 138.9552 & 140.4495 & 139.6629 & 130.1581 & 129.2468 & 128.3090 \\
\hline${ }^{1} H$ & & & & & & & & & & & & & \\
\hline 1 & 2.76 & 29.6578 & 29.1443 & 29.0690 & 29.6751 & 29.3935 & 29.3330 & 29.6286 & 29.1080 & 29.0222 & 29.6416 & 29.3525 & 29.2832 \\
\hline
\end{tabular}




\begin{tabular}{cccccccccccccc}
\hline 4a & 2.75 & 29.6740 & 29.1969 & 29.1342 & 29.7244 & 29.4494 & 29.4326 & 29.5330 & 29.0550 & 28.9792 & 29.5889 & 29.3171 & 29.2855 \\
4b & 1.75 & 30.2669 & 29.8057 & 29.6637 & 30.2714 & 30.0285 & 29.9090 & 30.2531 & 29.7921 & 29.6435 & 30.2560 & 30.0145 & 29.8863 \\
5 & 2.36 & 29.9057 & 29.4652 & 29.3958 & 29.9607 & 29.7259 & 29.6794 & 29.8047 & 29.3619 & 29.2781 & 29.8578 & 29.6219 & 29.5641 \\
$7 \mathrm{a}$ & 2.50 & 29.6588 & 29.1743 & 29.0506 & 29.6525 & 29.4068 & 29.3660 & 29.5335 & 29.0451 & 28.9075 & 29.5248 & 29.2783 & 29.2264 \\
$7 \mathrm{~b}$ & 2.09 & 30.2885 & 29.8731 & 29.7819 & 30.3674 & 30.1638 & 30.1205 & 30.3324 & 29.9185 & 29.8287 & 30.4069 & 30.2056 & 30.1625 \\
8 & 1.40 & 30.8912 & 30.4432 & 30.3333 & 30.8570 & 30.6034 & 30.5345 & 30.8487 & 30.3989 & 30.2868 & 30.8165 & 30.5617 & 30.4905 \\
9 & 1.00 & 31.1856 & 30.7456 & 30.7158 & 31.2689 & 31.0095 & 30.9747 & 31.1578 & 30.7172 & 30.6872 & 31.2427 & 30.9828 & 30.9463 \\
$10 a$ & 2.82 & 29.6482 & 29.1765 & 29.0713 & 29.5520 & 29.3448 & 29.3249 & 29.4487 & 28.9714 & 28.8323 & 29.3455 & 29.1366 & 29.0920 \\
$10 \mathrm{~b}$ & 3.39 & 28.7414 & 28.1894 & 28.0583 & 28.6621 & 28.3460 & 28.3040 & 28.7712 & 28.2246 & 28.0749 & 28.6849 & 28.3754 & 28.3148 \\
\hline
\end{tabular}

\begin{tabular}{|c|c|c|c|c|c|c|c|c|c|c|c|c|c|}
\hline & & \multicolumn{12}{|c|}{ mPW1PW91 } \\
\hline & \multirow[b]{2}{*}{$\operatorname{Exp}$} & \multicolumn{6}{|c|}{ Gas phase } & \multicolumn{6}{|c|}{ PCM } \\
\hline & & 6-31G* & 6-31G** & $6-31+G * *$ & 6-311G* & 6-311G** & $6-311+G^{* *}$ & 6-31G* & $6-31 G^{* *}$ & $6-31+G * *$ & 6-311G* & 6-311G** & $6-311+G^{* *}$ \\
\hline \multicolumn{14}{|l|}{${ }^{13} \mathrm{C}$} \\
\hline 1 & 57.3 & 138.2790 & 139.0709 & 137.2800 & 128.3410 & 128.0332 & 127.2236 & 137.4565 & 138.2444 & 136.2339 & 127.3770 & 127.0710 & 126.0205 \\
\hline 2 & 207.8 & -8.6756 & -8.5481 & -9.9187 & -26.7140 & -26.5692 & -29.8547 & -12.4225 & -12.2988 & -14.3265 & -30.8967 & -30.7491 & -34.6896 \\
\hline 3 & 54.3 & 140.4827 & 140.6148 & 140.7341 & 131.9971 & 131.7289 & 130.9576 & 140.0474 & 140.1756 & 140.2790 & 131.4935 & 131.2302 & 130.4174 \\
\hline 4 & 27.0 & 161.6556 & 162.8116 & 161.9248 & 152.9679 & 152.5255 & 152.3374 & 161.8077 & 162.9539 & 162.0158 & 153.0744 & 152.6193 & 152.3870 \\
\hline 5 & 40.3 & 153.2399 & 154.0860 & 152.6762 & 144.4811 & 144.2194 & 143.7729 & 153.2495 & 154.0988 & 152.7074 & 144.5134 & 144.2506 & 143.7783 \\
\hline 6 & 40.8 & 151.0347 & 151.5699 & 151.3060 & 140.9748 & 141.1562 & 140.6302 & 150.5174 & 151.0547 & 150.7005 & 140.4375 & 140.6229 & 140.0252 \\
\hline 7 & 31.6 & 166.5205 & 167.9779 & 167.4033 & 159.6071 & 159.3318 & 159.1765 & 166.5977 & 168.0435 & 167.3781 & 159.6568 & 159.3626 & 159.1501 \\
\hline 8 & 25.8 & 168.0023 & 169.5369 & 169.6092 & 161.2617 & 161.0513 & 160.7361 & 168.2934 & 169.8129 & 169.9332 & 161.5870 & 161.3535 & 161.0462 \\
\hline 9 & 22.3 & 171.6101 & 173.2383 & 173.0980 & 165.0748 & 164.8174 & 164.4679 & 171.6708 & 173.2865 & 173.1465 & 165.1234 & 164.8420 & 164.4766 \\
\hline 10 & 52.4 & 142.6196 & 144.0878 & 143.6804 & 135.3114 & 134.4476 & 133.7258 & 142.5506 & 144.0153 & 143.4153 & 135.1496 & 134.2631 & 133.4567 \\
\hline \multicolumn{14}{|c|}{ P } \\
\hline 1 & 2.76 & 29.5450 & 29.0332 & 28.9685 & 29.5467 & 29.2437 & 29.1981 & 29.5144 & 28.9951 & 28.9214 & 29.5127 & 29.2021 & 29.1489 \\
\hline $4 a$ & 2.75 & 29.6442 & 29.1677 & 29.1055 & 29.6468 & 29.3801 & 29.3657 & 29.4969 & 29.0191 & 28.9461 & 29.5064 & 29.2426 & 29.2153 \\
\hline $4 b$ & 1.75 & 30.1775 & 29.7170 & 29.5907 & 30.1507 & 29.9087 & 29.8039 & 30.1592 & 29.6985 & 29.5667 & 30.1308 & 29.8898 & 29.7771 \\
\hline 5 & 2.36 & 29.8486 & 29.4055 & 29.3343 & 29.8686 & 29.6187 & 29.5702 & 29.7414 & 29.2957 & 29.2126 & 29.7629 & 29.5114 & 29.4530 \\
\hline $7 a$ & 2.50 & 29.5698 & 29.0939 & 28.9895 & 29.5335 & 29.2973 & 29.2609 & 29.4374 & 28.9572 & 28.8409 & 29.4000 & 29.1627 & 29.1164 \\
\hline $7 \mathrm{~b}$ & 2.09 & 30.2217 & 29.8034 & 29.7380 & 30.2572 & 30.0519 & 30.0279 & 30.2670 & 29.8504 & 29.7851 & 30.2972 & 30.0942 & 30.0698 \\
\hline 8 & 1.40 & 30.8227 & 30.3755 & 30.2748 & 30.7585 & 30.5015 & 30.4291 & 30.7763 & 30.3270 & 30.2248 & 30.7145 & 30.4562 & 30.3819 \\
\hline 9 & 1.00 & 31.1499 & 30.7098 & 30.6701 & 31.1798 & 30.9160 & 30.8794 & 31.1203 & 30.6795 & 30.6402 & 31.1522 & 30.8879 & 30.8500 \\
\hline $10 a$ & 2.82 & 29.5527 & 29.0868 & 29.0007 & 29.4763 & 29.2536 & 29.2508 & 29.3399 & 28.8675 & 28.7524 & 29.2606 & 29.0359 & 29.0117 \\
\hline $10 \mathrm{~b}$ & 3.39 & 28.6643 & 28.1160 & 28.0232 & 28.5943 & 28.2628 & 28.2413 & 28.6858 & 28.1432 & 28.0346 & 28.6114 & 28.2869 & 28.2487 \\
\hline
\end{tabular}




\section{Compound 90b}

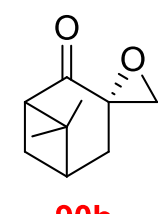

90b

\begin{tabular}{|c|c|c|c|c|c|c|c|c|c|c|c|c|c|}
\hline & \multirow[b]{3}{*}{ Exp } & \multicolumn{12}{|c|}{ B3LYP } \\
\hline & & \multicolumn{6}{|c|}{ Gas phase } & \multicolumn{6}{|c|}{ PCM } \\
\hline & & 6-31G* & $-31 G * *$ & $6-31+G * *$ & 6-311G* & 6-311G** & $6-311+G * *$ & 6-31G* & 6-31G** & $6-31+G^{* *}$ & 6-311G* & 6-311G** & 6 \\
\hline \multicolumn{14}{|c|}{ 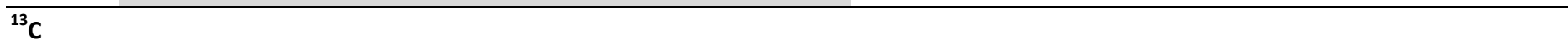 } \\
\hline 1 & 56.6 & 133.8278 & 134.6764 & 132.5991 & 122.7313 & 122.4395 & 121.5827 & 133.0917 & 133.9362 & 131.6427 & 121.8796 & 121.5881 & 120.489 \\
\hline 2 & 208.8 & -11.2406 & -11.1140 & -13.2776 & -32.6669 & -32.5422 & -35.7483 & -14.9831 & -14.8613 & -17.7351 & -36.9523 & -36.8285 & -40.7257 \\
\hline 3 & 55.2 & 135.5306 & 135.7229 & 135.9287 & 125.7243 & 125.5080 & 124.9572 & 134.9797 & 135.1696 & 135.3721 & 125.0924 & 124.8800 & 124.2926 \\
\hline 4 & 27.0 & 156.8710 & 158.1126 & 157.2318 & 147.4660 & 147.0044 & 146.8373 & 157.1505 & 158.3833 & 157.5246 & 147.7655 & 147.2953 & 147.090 \\
\hline 5 & 40.1 & 148.2803 & 149.2025 & 147.4003 & 138.3955 & 138.1901 & 137.6135 & 148.3122 & 149.2385 & 147.4672 & 138.4788 & 138.2750 & 137.671 \\
\hline 6 & 41.8 & 145.6050 & 146.1140 & 144.9283 & 134.9892 & 135.1597 & 134.7474 & 145.3137 & 145.8276 & 144.5671 & 134.7298 & 134.9052 & 134.432 \\
\hline 7 & 30.9 & 161.1294 & 162.6825 & 162.2527 & 153.7348 & 153.4749 & 153.1791 & 161.3850 & 162.9231 & 162.4575 & 154.0056 & 153.7240 & 153.3973 \\
\hline 8 & 25.8 & 163.5265 & 165.1856 & 165.2900 & 156.2271 & 155.9987 & 155.5556 & 163.8874 & 165.5313 & 165.6966 & 156.6651 & 156.4107 & 155.986 \\
\hline 9 & 21.2 & 168.4720 & 170.2036 & 170.3619 & 161.2213 & 160.9420 & 160.6440 & 168.4509 & 170.1821 & 170.4000 & 161.3093 & 161.0074 & 160.709 \\
\hline 10 & 56.7 & 132.6576 & 134.1399 & 133.4446 & 123.2594 & 122.2904 & 121.4214 & 132.5560 & 134.0295 & 133.0444 & 123.0147 & 122.0146 & 121.0298 \\
\hline \multicolumn{14}{|l|}{${ }^{1} \mathrm{H}$} \\
\hline 1 & 2.70 & 29.6990 & 29.1825 & 29.0422 & 29.6937 & 29.4119 & 29.3003 & 29.6761 & 29.1525 & 29.0010 & 29.6679 & 29.3790 & 29.2574 \\
\hline $4 a$ & 2.66 & 29.9665 & 29.4906 & 29.3595 & 29.9008 & 29.6224 & 29.5779 & 29.8148 & 29.3374 & 29.1906 & 29.7526 & 29.4769 & 29.4176 \\
\hline $4 \mathrm{~b}$ & 1.76 & 29.9493 & 29.4890 & 29.3687 & 30.0223 & 29.7804 & 29.6836 & 29.9495 & 29.4889 & 29.3634 & 30.0224 & 29.7805 & 29.6764 \\
\hline 5 & 2.36 & 29.9375 & 29.4954 & 29.4138 & 29.9802 & 29.7397 & 29.6854 & 29.8383 & 29.3940 & 29.2996 & 29.8799 & 29.6386 & 29.5749 \\
\hline $7 a$ & 2.30 & 29.6840 & 29.1999 & 29.0808 & 29.6635 & 29.4156 & 29.3903 & 29.5741 & 29.0880 & 28.9602 & 29.5556 & 29.3092 & 29.2735 \\
\hline $7 b$ & 2.28 & 30.5902 & 30.1773 & 30.0974 & 30.6358 & 30.4333 & 30.4254 & 30.5090 & 30.0928 & 30.0104 & 30.5574 & 30.3531 & 30.3406 \\
\hline 8 & 1.40 & 30.8943 & 30.4472 & 30.3578 & 30.8592 & 30.6093 & 30.5433 & 30.8502 & 30.4011 & 30.3086 & 30.8171 & 30.5656 & 30.4973 \\
\hline 9 & 1.01 & 31.0689 & 30.6259 & 30.5721 & 31.1518 & 30.8975 & 30.8508 & 31.0822 & 30.6389 & 30.5864 & 31.1617 & 30.9071 & 30.8607 \\
\hline $10 a$ & 2.83 & 29.5920 & 29.1341 & 29.0422 & 29.5027 & 29.2926 & 29.2962 & 29.3827 & 28.9194 & 28.7964 & 29.2838 & 29.0726 & 29.0568 \\
\hline $10 \mathrm{~b}$ & 3.12 & 28.9215 & 28.3998 & 28.3332 & 28.8659 & 28.5710 & 28.5799 & 28.9247 & 28.4078 & 28.3230 & 28.8590 & 28.5709 & 28.5681 \\
\hline
\end{tabular}

mPW1PW91

Gas phase

PCM

Exp 6-31G* 6-31G** 6-31+G** 6-311G* 6-311G**6-311+G** 6-31G* 6-31G** 6-31+G** 6-311G* 6-311G** 6-311+G** ${ }^{13} \mathrm{C}$

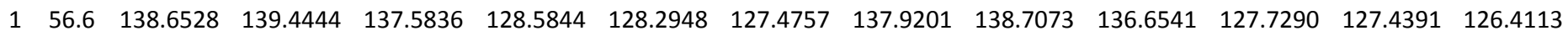

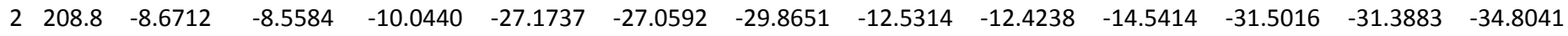

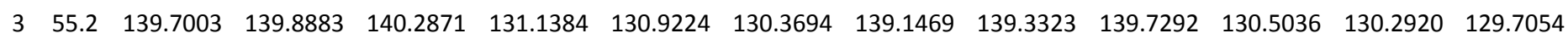

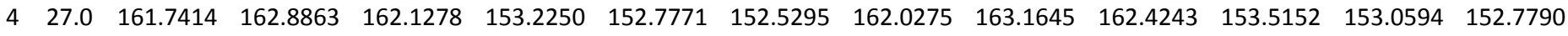

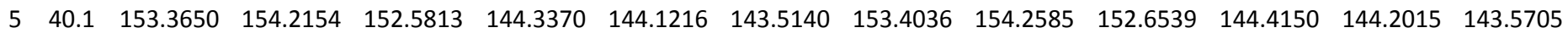

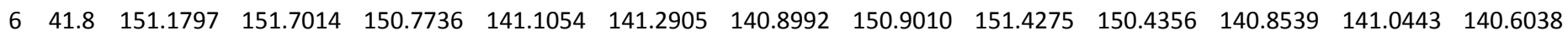

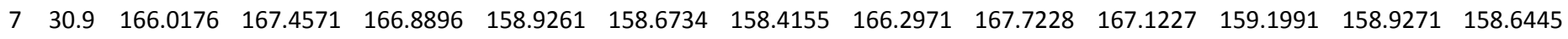

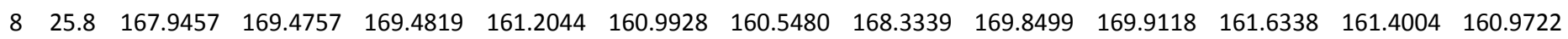




\begin{tabular}{ccccccccccccccc}
\hline 9 & 21.2 & 172.4851 & 174.0925 & 174.3553 & 165.8480 & 165.5759 & 165.3084 & 172.4875 & 174.0941 & 174.4036 & 165.9343 & 165.6410 & 165.3763 \\
10 & 56.7 & 136.4303 & 137.8785 & 137.5117 & 128.5397 & 127.5933 & 126.7713 & 136.3092 & 137.7502 & 137.1091 & 128.2860 & 127.3106 & 126.3930 \\
${ }^{1}$ H & & & & & & & & & & & & & & \\
1 & 2.70 & 29.5851 & 29.0715 & 28.9445 & 29.5682 & 29.2647 & 29.1705 & 29.5604 & 29.0395 & 28.9023 & 29.5414 & 29.2306 & 29.1274 \\
4a & 2.66 & 29.9061 & 29.4311 & 29.3127 & 29.8104 & 29.5365 & 29.4959 & 29.7455 & 29.2683 & 29.1370 & 29.6556 & 29.3839 & 29.3302 \\
4b & 1.76 & 29.8843 & 29.4234 & 29.3110 & 29.9140 & 29.6760 & 29.5841 & 29.8835 & 29.4222 & 29.3045 & 29.9118 & 29.6737 & 29.5747 \\
5 & 2.36 & 29.8814 & 29.4383 & 29.3546 & 29.8913 & 29.6363 & 29.5835 & 29.7758 & 29.3301 & 29.2356 & 29.7877 & 29.5316 & 29.4703 \\
$7 \mathrm{a}$ & 2.30 & 29.6018 & 29.1259 & 29.0273 & 29.5500 & 29.3112 & 29.2920 & 29.4836 & 29.0055 & 28.8995 & 29.4350 & 29.1975 & 29.1689 \\
$7 \mathrm{~b}$ & 2.28 & 30.5385 & 30.1234 & 30.0656 & 30.5313 & 30.3259 & 30.3324 & 30.4568 & 30.0384 & 29.9794 & 30.4540 & 30.2468 & 30.2493 \\
8 & 1.40 & 30.8259 & 30.3796 & 30.2983 & 30.7603 & 30.5070 & 30.4424 & 30.7778 & 30.3293 & 30.2455 & 30.7146 & 30.4596 & 30.3930 \\
9 & 1.01 & 31.0210 & 30.5775 & 30.5205 & 31.0566 & 30.7976 & 30.7521 & 31.0339 & 30.5900 & 30.5346 & 31.0656 & 30.8063 & 30.7611 \\
$10 a$ & 2.83 & 29.5100 & 29.0565 & 28.9833 & 29.4375 & 29.2110 & 29.2268 & 29.2867 & 28.8267 & 28.7271 & 29.2093 & 28.9812 & 28.9803 \\
$10 \mathrm{~b}$ & 3.12 & 28.8520 & 28.3341 & 28.2858 & 28.8061 & 28.4978 & 28.5118 & 28.8470 & 28.3340 & 28.2703 & 28.7939 & 28.4925 & 28.4965 \\
\hline
\end{tabular}


sDP4+, $u$ DP4+ and DP4+ probabilities (\%) for compound 73a<smiles>CCOC(=O)[C@H](C)[C@H](O)c1ccccc1</smiles>

correct isomer<smiles>CCOC(=O)[C@H](C)[C@@H](O)c1ccccc1</smiles>

Level of theory: B3LYP/6-31G* (gas phase)

\begin{tabular}{|c|c|c|c|c|c|c|c|c|c|}
\hline & & \multicolumn{8}{|c|}{ Level of theory: B3LYP/6-31G* (gas phase) } \\
\hline & & $73 a$ & $73 b$ & - & - & - & - & - & - \\
\hline \multirow{3}{*}{ sDP4+ } & ${ }^{1} \mathrm{H}$ data & 93.26 & 6.74 & - & - & - & - & - & - \\
\hline & ${ }^{13} \mathrm{C}$ data & 99.72 & 0.28 & - & - & - & - & - & - \\
\hline & All data & 99.98 & 0.02 & - & - & - & - & - & - \\
\hline \multirow{3}{*}{ uDP4+ } & ${ }^{1} \mathrm{H}$ data & 94.53 & 5.47 & - & - & - & - & - & - \\
\hline & ${ }^{13} \mathrm{C}$ data & 96.58 & 3.42 & - & - & - & - & - & - \\
\hline & All data & 99.80 & 0.20 & - & - & - & - & - & - \\
\hline \multirow{3}{*}{ DP4+ } & ${ }^{1} \mathrm{H}$ data & 99.58 & 0.42 & - & - & - & - & - & - \\
\hline & ${ }^{13} \mathrm{C}$ data & 99.99 & 0.01 & - & - & - & - & - & - \\
\hline & All data & 100.00 & 0.00 & - & - & - & - & - & - \\
\hline & & \multicolumn{8}{|c|}{ Level of theory: B3LYP/6-31G** (gas phase) } \\
\hline & & $73 a$ & $73 b$ & - & - & - & - & - & - \\
\hline \multirow{3}{*}{ sDP4+ } & ${ }^{1} \mathrm{H}$ data & 98.19 & 1.81 & - & - & - & - & - & - \\
\hline & ${ }^{13} \mathrm{C}$ data & 99.73 & 0.27 & - & - & - & - & - & - \\
\hline & All data & 100.00 & 0.00 & - & - & - & - & - & - \\
\hline \multirow{3}{*}{ uDP4+ } & ${ }^{1} \mathrm{H}$ data & 84.08 & 15.92 & - & - & - & - & - & - \\
\hline & ${ }^{13} \mathrm{C}$ data & 97.88 & 2.12 & - & - & - & - & - & - \\
\hline & All data & 99.59 & 0.41 & - & - & - & - & - & - \\
\hline \multirow{5}{*}{ DP4+ } & ${ }^{1} \mathrm{H}$ data & 99.65 & 0.35 & - & - & - & - & - & - \\
\hline & ${ }^{13}$ C data & 99.99 & 0.01 & - & - & - & - & - & - \\
\hline & All data & 100.00 & 0.00 & - & - & - & - & - & - \\
\hline & & \multicolumn{8}{|c|}{ Level of theory: B3LYP/6-31+G** (gas phase) } \\
\hline & & $73 a$ & 73b & - & - & - & - & - & - \\
\hline \multirow{3}{*}{ sDP4+ } & ${ }^{1} \mathrm{H}$ data & 99.60 & 0.40 & - & - & - & - & - & - \\
\hline & ${ }^{13} \mathrm{C}$ data & 99.57 & 0.43 & - & - & - & - & - & - \\
\hline & All data & 100.00 & 0.00 & - & - & - & - & - & - \\
\hline \multirow{3}{*}{ uDP4+ } & ${ }^{1} \mathrm{H}$ data & 77.44 & 22.56 & - & - & - & - & - & - \\
\hline & ${ }^{13} \mathrm{C}$ data & 97.12 & 2.88 & - & - & - & - & - & - \\
\hline & All data & 99.14 & 0.86 & - & - & - & - & - & - \\
\hline \multirow{3}{*}{ DP4+ } & ${ }^{1} \mathrm{H}$ data & 99.88 & 0.12 & - & - & - & - & - & - \\
\hline & ${ }^{13} \mathrm{C}$ data & 99.99 & 0.01 & - & - & - & - & - & - \\
\hline & All data & 100.00 & 0.00 & - & - & - & - & - & - \\
\hline & & \multicolumn{8}{|c|}{ Level of theory: B3LYP/6-311G* (gas phase) } \\
\hline & & $73 a$ & $73 b$ & - & - & - & - & - & - \\
\hline \multirow{3}{*}{ sDP4+ } & ${ }^{1}$ H data & 96.92 & 3.08 & - & - & - & - & - & - \\
\hline & ${ }^{13} \mathrm{C}$ data & 99.74 & 0.26 & - & - & - & - & - & - \\
\hline & All data & 99.99 & 0.01 & - & - & - & - & - & - \\
\hline \multirow{3}{*}{ uDP4+ } & ${ }^{1} \mathrm{H}$ data & 56.30 & 43.70 & - & - & - & - & - & - \\
\hline & ${ }^{13} \mathrm{C}$ data & 93.06 & 6.94 & - & - & - & - & - & - \\
\hline & All data & 94.53 & 5.47 & - & - & - & - & - & - \\
\hline \multirow{3}{*}{ DP4+ } & ${ }^{1} \mathrm{H}$ data & 97.59 & 2.41 & - & - & - & - & - & - \\
\hline & ${ }^{13} \mathrm{C}$ data & 99.98 & 0.02 & - & - & - & - & - & - \\
\hline & All data & 100.00 & 0.00 & - & - & - & - & - & - \\
\hline & & \multicolumn{8}{|c|}{ Level of theory: B3LYP/6-311G** (gas phase) } \\
\hline & & $73 a$ & $73 b$ & - & - & - & - & - & - \\
\hline \multirow{2}{*}{ sDP4+ } & ${ }_{12}^{1} \mathrm{H}$ data & 99.26 & 0.74 & - & - & - & - & - & - \\
\hline & ${ }^{13} \mathrm{C}$ data & 9966 & 034 & _- & - & - & _. & - & \\
\hline
\end{tabular}




\begin{tabular}{|c|c|c|c|c|c|c|c|c|c|}
\hline & All data & 100.00 & 0.00 & - & - & - & - & - & - \\
\hline & ${ }^{1}$ H data & 52.13 & 47.87 & - & - & - & - & - & - \\
\hline \multirow[t]{3}{*}{ uDP4+ } & ${ }^{13} \mathrm{C}$ data & 89.92 & 10.08 & - & - & - & - & - & - \\
\hline & All data & 90.67 & 9.33 & - & - & - & - & - & - \\
\hline & ${ }^{1} \mathrm{H}$ data & 99.32 & 0.68 & - & - & - & - & - & - \\
\hline \multirow[t]{5}{*}{ DP4+ } & ${ }^{13} \mathrm{C}$ data & 99.96 & 0.04 & - & - & - & - & - & - \\
\hline & All data & 100.00 & 0.00 & - & - & - & - & - & - \\
\hline & & \multicolumn{8}{|c|}{ Level of theory: B3LYP/6-311+G** (gas phase) } \\
\hline & & $73 a$ & $73 b$ & - & - & - & - & - & - \\
\hline & ${ }^{1} \mathrm{H}$ data & 99.90 & 0.10 & - & - & - & - & - & - \\
\hline \multirow[t]{3}{*}{ sDP4+ } & ${ }^{13} \mathrm{C}$ data & 99.58 & 0.42 & - & - & - & - & - & - \\
\hline & All data & 100.00 & 0.00 & - & - & - & - & - & - \\
\hline & ${ }^{1} \mathrm{H}$ data & 83.07 & 16.93 & - & - & - & - & - & - \\
\hline \multirow[t]{3}{*}{ uDP4+ } & ${ }^{13} \mathrm{C}$ data & 85.29 & 14.71 & - & - & - & - & - & - \\
\hline & All data & 96.61 & 3.39 & - & - & - & - & - & - \\
\hline & ${ }^{1} \mathrm{H}$ data & 99.98 & 0.02 & - & - & - & - & - & - \\
\hline \multirow[t]{5}{*}{ DP4+ } & ${ }^{13} \mathrm{C}$ data & 99.93 & 0.07 & - & - & - & - & - & - \\
\hline & All data & 100.00 & 0.00 & - & - & - & - & - & - \\
\hline & & \multicolumn{8}{|c|}{ Level of theory: B3LYP/6-31G* (solution, PCM) } \\
\hline & & $73 a$ & $73 b$ & - & - & - & - & - & - \\
\hline & ${ }^{1}$ H data & 98.26 & 1.74 & - & - & - & - & - & - \\
\hline \multirow[t]{3}{*}{ sDP4+ } & ${ }^{13}$ C data & 99.44 & 0.56 & - & - & - & - & - & - \\
\hline & All data & 99.99 & 0.01 & - & - & - & - & - & - \\
\hline & ${ }^{1} \mathrm{H}$ data & 94.34 & 5.66 & - & - & - & - & - & - \\
\hline \multirow[t]{3}{*}{$u$ DP4+ } & ${ }^{13} \mathrm{C}$ data & 93.33 & 6.67 & - & - & - & - & - & - \\
\hline & All data & 99.57 & 0.43 & - & - & - & - & - & - \\
\hline & ${ }^{1}$ H data & 99.89 & 0.11 & - & - & - & - & - & - \\
\hline \multirow[t]{5}{*}{ DP4+ } & ${ }^{13} \mathrm{C}$ data & 99.96 & 0.04 & - & - & - & - & - & - \\
\hline & All data & 100.00 & 0.00 & - & - & - & - & - & - \\
\hline & & \multicolumn{8}{|c|}{ Level of theory: B3LYP/6-31G** (solution, PCM) } \\
\hline & & $73 a$ & $73 b$ & - & - & - & - & - & - \\
\hline & ${ }^{1} \mathrm{H}$ data & 99.55 & 0.45 & - & - & - & - & - & - \\
\hline \multirow[t]{3}{*}{ sDP4+ } & ${ }^{13} \mathrm{C}$ data & 99.49 & 0.51 & - & - & - & - & - & - \\
\hline & All data & 100.00 & 0.00 & - & - & - & - & - & - \\
\hline & ${ }^{1} \mathrm{H}$ data & 85.70 & 14.30 & - & - & - & - & - & - \\
\hline \multirow[t]{3}{*}{ uDP4+ } & ${ }^{13} \mathrm{C}$ data & 95.49 & 4.51 & - & - & - & - & - & - \\
\hline & All data & 99.22 & 0.78 & - & - & - & - & - & - \\
\hline & ${ }^{1} \mathrm{H}$ data & 99.92 & 0.08 & - & - & - & - & - & - \\
\hline \multirow[t]{5}{*}{ DP4+ } & ${ }^{13} \mathrm{C}$ data & 99.98 & 0.02 & - & - & - & - & - & - \\
\hline & All data & 100.00 & 0.00 & - & - & - & - & - & - \\
\hline & & \multicolumn{8}{|c|}{ Level of theory: B3LYP/6-31+G** (solution, PCM) } \\
\hline & & $73 a$ & $73 b$ & - & - & - & - & - & - \\
\hline & ${ }^{1} \mathrm{H}$ data & 99.95 & 0.05 & - & - & - & - & - & - \\
\hline \multirow[t]{3}{*}{ sDP4+ } & ${ }^{13} \mathrm{C}$ data & 99.16 & 0.84 & - & - & - & - & - & - \\
\hline & All data & 100.00 & 0.00 & - & - & - & - & - & - \\
\hline & ${ }^{1} \mathrm{H}$ data & 76.83 & 23.17 & - & - & - & - & - & - \\
\hline uDP4+ & ${ }^{13} \mathrm{C}$ data & 95.95 & 4.05 & - & - & - & - & - & - \\
\hline & All data & 98.74 & 1.26 & - & - & - & - & - & - \\
\hline & ${ }^{1} \mathrm{H}$ data & 99.99 & 0.01 & - & - & - & - & - & - \\
\hline DP4+ & ${ }^{13} \mathrm{C}$ data & 99.96 & 0.04 & - & - & - & - & - & - \\
\hline & All data & 100.00 & 0.00 & - & - & - & - & - & - \\
\hline & & & & ! & B3 & j* & $\overline{P C}$ & & \\
\hline & & $73 a$ & $73 b$ & - & - & - & - & - & - \\
\hline & ${ }^{1} \mathrm{H}$ data & 99.37 & 0.63 & - & - & - & - & - & - \\
\hline sDP4+ & ${ }^{13} \mathrm{C}$ data & 99.49 & 0.51 & - & - & - & - & - & - \\
\hline & All data & 100.00 & 0.00 & - & - & - & - & - & - \\
\hline
\end{tabular}




\begin{tabular}{|c|c|c|c|c|c|c|c|c|c|}
\hline & ${ }^{1} \mathrm{H}$ data & 59.24 & 40.76 & - & - & - & - & - & - \\
\hline \multirow[t]{3}{*}{ uDP4+ } & ${ }^{13} \mathrm{C}$ data & 78.11 & 21.89 & - & - & - & - & - & - \\
\hline & All data & 83.83 & 16.17 & - & - & - & - & - & - \\
\hline & ${ }^{1} \mathrm{H}$ data & 99.57 & 0.43 & - & - & - & - & - & - \\
\hline \multirow[t]{5}{*}{ DP4+ } & ${ }^{13} \mathrm{C}$ data & 99.86 & 0.14 & - & - & - & - & - & - \\
\hline & All data & 100.00 & 0.00 & - & - & - & - & - & - \\
\hline & & \multicolumn{8}{|c|}{ Level of theory: B3LYP/6-311G** (solution, PCM) } \\
\hline & & $73 a$ & 73b & - & - & - & - & - & - \\
\hline & ${ }^{1} \mathrm{H}$ data & 99.38 & 0.62 & - & - & - & - & - & - \\
\hline \multirow[t]{3}{*}{ sDP4+ } & ${ }^{13} \mathrm{C}$ data & 99.33 & 0.67 & - & - & - & - & - & - \\
\hline & All data & 100.00 & 0.00 & - & - & - & - & - & - \\
\hline & ${ }^{1} \mathrm{H}$ data & 44.11 & 55.89 & - & - & - & - & - & - \\
\hline \multirow[t]{3}{*}{ uDP4+ } & ${ }^{13} \mathrm{C}$ data & 73.07 & 26.93 & - & - & - & - & - & - \\
\hline & All data & 68.17 & 31.83 & - & - & - & - & - & - \\
\hline & ${ }^{1} \mathrm{H}$ data & 99.22 & 0.78 & - & - & - & - & - & - \\
\hline \multirow[t]{5}{*}{ DP4+ } & ${ }^{13} \mathrm{C}$ data & 99.75 & 0.25 & - & - & - & - & - & - \\
\hline & All data & 100.00 & 0.00 & - & - & - & - & - & - \\
\hline & & \multicolumn{8}{|c|}{ Level of theory: B3LYP/6-311+G** (solution, PCM) } \\
\hline & & $73 a$ & $73 b$ & - & - & - & - & - & - \\
\hline & ${ }^{1} \mathrm{H}$ data & 99.93 & 0.07 & - & - & - & - & - & - \\
\hline \multirow[t]{3}{*}{ sDP4+ } & ${ }^{13} \mathrm{C}$ data & 98.76 & 1.24 & - & - & - & - & - & - \\
\hline & All data & 100.00 & 0.00 & - & - & - & - & - & - \\
\hline & ${ }^{1} \mathrm{H}$ data & 89.75 & 10.25 & - & - & - & - & - & - \\
\hline \multirow[t]{3}{*}{ uDP4+ } & ${ }^{13} \mathrm{C}$ data & 48.34 & 51.66 & - & - & - & - & - & - \\
\hline & All data & 89.12 & 10.88 & - & - & - & - & - & - \\
\hline & ${ }^{1} \mathrm{H}$ data & 99.99 & 0.01 & - & - & - & - & - & - \\
\hline \multirow[t]{5}{*}{ DP4+ } & ${ }^{13} \mathrm{C}$ data & 98.68 & 1.32 & - & - & - & - & - & - \\
\hline & All data & 100.00 & 0.00 & - & - & - & - & - & - \\
\hline & & \multicolumn{8}{|c|}{ Level of theory: mPW1PW91/6-31G* (gas phase) } \\
\hline & & $73 a$ & $73 b$ & - & - & - & - & - & - \\
\hline & ${ }^{1} \mathrm{H}$ data & 99.46 & 0.54 & - & - & - & - & - & - \\
\hline \multirow[t]{3}{*}{ sDP4+ } & ${ }^{13}$ C data & 99.77 & 0.23 & - & - & - & - & - & - \\
\hline & All data & 100.00 & 0.00 & - & - & - & - & - & - \\
\hline & ${ }^{1} \mathrm{H}$ data & 94.93 & 5.07 & - & - & - & - & - & - \\
\hline \multirow[t]{3}{*}{ uDP4+ } & ${ }^{13} \mathrm{C}$ data & 94.14 & 5.86 & - & - & - & - & - & - \\
\hline & All data & 99.67 & 0.33 & - & - & - & - & - & - \\
\hline & ${ }^{1} \mathrm{H}$ data & 99.97 & 0.03 & - & - & - & - & - & - \\
\hline \multirow[t]{5}{*}{ DP4+ } & ${ }^{13} \mathrm{C}$ data & 99.99 & 0.01 & - & - & - & - & - & - \\
\hline & All data & 100.00 & 0.00 & - & - & - & - & - & - \\
\hline & & \multicolumn{8}{|c|}{ Level of theory: mPW1PW91/6-31G** (gas phase) } \\
\hline & & $73 a$ & $73 b$ & - & - & - & - & - & - \\
\hline & ${ }^{1} \mathrm{H}$ data & 99.83 & 0.17 & - & - & - & - & - & - \\
\hline \multirow[t]{2}{*}{ sDP4+ } & ${ }^{13} \mathrm{C}$ data & 99.90 & 0.10 & - & - & - & - & - & - \\
\hline & All data & 100.00 & 0.00 & - & - & - & - & - & - \\
\hline & ${ }^{1}$ H data & 85.23 & 14.77 & - & - & - & - & - & - \\
\hline uDP4+ & ${ }^{13}$ C data & 98.61 & 1.39 & - & - & - & - & - & - \\
\hline & All data & 99.76 & 0.24 & - & - & - & - & - & - \\
\hline & ${ }^{1} \mathrm{H}$ data & 99.97 & 0.03 & - & - & - & - & - & - \\
\hline DP4+ & ${ }^{13} \mathrm{C}$ data & 100.00 & 0.00 & - & - & - & - & - & - \\
\hline & All data & 100.00 & 0.00 & - & - & - & - & - & - \\
\hline & & & & 0 & $|\mathbf{P}|$ & -3 & pr & & \\
\hline & & $73 a$ & $73 b$ & - & - & - & - & - & - \\
\hline & ${ }^{1} \mathrm{H}$ data & 99.94 & 0.06 & - & - & - & - & - & - \\
\hline sDP4+ & ${ }^{13} \mathrm{C}$ data & 99.90 & 0.10 & - & - & - & - & - & - \\
\hline & All data & 100.00 & 0.00 & - & - & - & - & - & - \\
\hline uDP4+ & ${ }^{1} \mathrm{H}$ data & 72.66 & 27.34 & - & - & - & - & - & - \\
\hline
\end{tabular}




\begin{tabular}{|c|c|c|c|c|c|c|c|c|c|}
\hline & ${ }^{13} \mathrm{C}$ data & 98.40 & 1.60 & - & - & - & - & - & - \\
\hline & All data & 99.39 & 0.61 & - & - & - & - & - & - \\
\hline & ${ }^{1} \mathrm{H}$ data & 99.98 & 0.02 & - & - & - & - & - & - \\
\hline \multirow[t]{5}{*}{ DP4+ } & ${ }^{13} \mathrm{C}$ data & 100.00 & 0.00 & - & - & - & - & - & - \\
\hline & All data & 100.00 & 0.00 & - & - & - & - & - & - \\
\hline & & \multicolumn{8}{|c|}{ Level of theory: mPW1PW91/6-311G* (gas phase) } \\
\hline & & $73 a$ & $73 b$ & - & - & - & - & - & - \\
\hline & ${ }^{1}$ H data & 99.67 & 0.33 & - & - & - & - & - & - \\
\hline \multirow[t]{3}{*}{ sDP4+ } & ${ }^{13}$ C data & 98.72 & 1.28 & - & - & - & - & - & - \\
\hline & All data & 100.00 & 0.00 & - & - & - & - & - & - \\
\hline & ${ }^{1} \mathrm{H}$ data & 68.81 & 31.19 & - & - & - & - & - & - \\
\hline \multirow[t]{3}{*}{ uDP4+ } & ${ }^{13} \mathrm{C}$ data & 97.39 & 2.61 & - & - & - & - & - & - \\
\hline & All data & 98.80 & 1.20 & - & - & - & - & - & - \\
\hline & ${ }^{1} \mathrm{H}$ data & 99.85 & 0.15 & - & - & - & - & - & - \\
\hline \multirow[t]{5}{*}{ DP4+ } & ${ }^{13} \mathrm{C}$ data & 99.97 & 0.03 & - & - & - & - & - & - \\
\hline & All data & 100.00 & 0.00 & - & - & - & - & - & - \\
\hline & & \multicolumn{8}{|c|}{ Level of theory: mPW1PW91/6-311G** (gas phase) } \\
\hline & & $73 a$ & $73 b$ & - & - & - & - & - & - \\
\hline & ${ }^{1} \mathrm{H}$ data & 99.91 & 0.09 & - & - & - & - & - & - \\
\hline \multirow[t]{3}{*}{ sDP4+ } & ${ }^{13} \mathrm{C}$ data & 98.74 & 1.26 & - & - & - & - & - & - \\
\hline & All data & 100.00 & 0.00 & - & - & - & - & - & - \\
\hline & ${ }^{1} \mathrm{H}$ data & 65.13 & 34.87 & - & - & - & - & - & - \\
\hline \multirow[t]{3}{*}{ uDP4+ } & ${ }^{13} \mathrm{C}$ data & 95.03 & 4.97 & - & - & - & - & - & - \\
\hline & All data & 97.27 & 2.73 & - & - & - & - & - & - \\
\hline & ${ }^{1} \mathrm{H}$ data & 99.95 & 0.05 & - & - & - & - & - & - \\
\hline \multirow[t]{5}{*}{ DP4+ } & ${ }^{13} \mathrm{C}$ data & 99.93 & 0.07 & - & - & - & - & - & - \\
\hline & All data & 100.00 & 0.00 & - & - & - & - & - & - \\
\hline & & \multicolumn{8}{|c|}{ Level of theory: mPW1PW91/6-311+G** (gas phase) } \\
\hline & & $73 a$ & $73 b$ & - & - & - & - & - & - \\
\hline & ${ }^{1}$ H data & 99.98 & 0.02 & - & - & - & - & - & - \\
\hline \multirow[t]{2}{*}{ sDP4+ } & ${ }^{13} \mathrm{C}$ data & 98.49 & 1.51 & - & - & - & - & - & - \\
\hline & All data & 100.00 & 0.00 & - & - & - & - & - & - \\
\hline \multirow{3}{*}{ uDP4+ } & ${ }^{1} \mathrm{H}$ data & 90.05 & 9.95 & - & - & - & - & - & - \\
\hline & ${ }^{13}$ C data & 94.71 & 5.29 & - & - & - & - & - & - \\
\hline & All data & 99.39 & 0.61 & - & - & - & - & - & - \\
\hline \multirow{5}{*}{ DP4+ } & ${ }^{1} \mathrm{H}$ data & 100.00 & 0.00 & - & - & - & - & - & - \\
\hline & ${ }^{13} \mathrm{C}$ data & 99.91 & 0.09 & - & - & - & - & - & - \\
\hline & All data & 100.00 & 0.00 & - & - & - & - & - & - \\
\hline & & \multicolumn{8}{|c|}{ Level of theory: mPW1PW91/6-31G* (solution, PCM) } \\
\hline & & $73 a$ & $73 b$ & - & - & - & - & - & - \\
\hline \multirow{3}{*}{ sDP4+ } & ${ }^{1} \mathrm{H}$ data & 99.93 & 0.07 & - & - & - & - & - & - \\
\hline & ${ }^{13} \mathrm{C}$ data & 98.98 & 1.02 & - & - & - & - & - & - \\
\hline & All data & 100.00 & 0.00 & - & - & - & - & - & - \\
\hline \multirow{3}{*}{ uDP4+ } & ${ }^{1} \mathrm{H}$ data & 94.14 & 5.86 & - & - & - & - & - & - \\
\hline & ${ }^{13} \mathrm{C}$ data & 90.21 & 9.79 & - & - & - & - & - & - \\
\hline & All data & 99.33 & 0.67 & - & - & - & - & - & - \\
\hline & ${ }^{1} \mathrm{H}$ data & 100.00 & 0.00 & - & - & - & - & - & - \\
\hline DP4+ & ${ }^{13} \mathrm{C}$ data & 99.89 & 0.11 & - & - & - & - & - & - \\
\hline & All data & 100.00 & 0.00 & - & - & - & - & - & - \\
\hline & & & & of & $\mathbf{W}$ & 1 & on, & & \\
\hline & & $73 a$ & $73 b$ & - & - & - & - & - & - \\
\hline & ${ }^{1} \mathrm{H}$ data & 99.98 & 0.02 & - & - & - & - & - & - \\
\hline sDP4+ & ${ }^{13} \mathrm{C}$ data & 99.72 & 0.28 & - & - & - & - & - & - \\
\hline & All data & 100.00 & 0.00 & - & - & - & - & - & - \\
\hline uDP4+ & ${ }_{12}^{1} \mathrm{H}$ data & 87.26 & 12.74 & - & - & - & - & - & - \\
\hline UDP4+ & ${ }^{13} \mathrm{C}$ data & 96.42 & 3.58 & - & - & - & - & - & - \\
\hline
\end{tabular}




\begin{tabular}{|c|c|c|c|c|c|c|c|c|c|}
\hline & All data & 99.46 & 0.54 & - & - & - & - & - & - \\
\hline & ${ }^{1} \mathrm{H}$ data & 100.00 & 0.00 & - & - & - & - & - & - \\
\hline \multirow[t]{5}{*}{ DP4+ } & ${ }^{13} \mathrm{C}$ data & 99.99 & 0.01 & - & - & - & - & - & - \\
\hline & All data & 100.00 & 0.00 & - & - & - & - & - & - \\
\hline & & \multicolumn{8}{|c|}{ Level of theory: mPW1PW91/6-31+G** (solution, PCM) } \\
\hline & & $73 a$ & $73 b$ & - & - & - & - & - & - \\
\hline & ${ }^{1} \mathrm{H}$ data & 99.83 & 0.17 & - & - & - & - & - & - \\
\hline \multirow[t]{3}{*}{ sDP4+ } & ${ }^{13} \mathrm{C}$ data & 99.66 & 0.34 & - & - & - & - & - & - \\
\hline & All data & 100.00 & 0.00 & - & - & - & - & - & - \\
\hline & ${ }^{1} \mathrm{H}$ data & 18.96 & 81.04 & - & - & - & - & - & - \\
\hline \multirow[t]{3}{*}{ uDP4+ } & ${ }^{13} \mathrm{C}$ data & 97.34 & 2.66 & - & - & - & - & - & - \\
\hline & All data & 89.55 & 10.45 & - & - & - & - & - & - \\
\hline & ${ }^{1} \mathrm{H}$ data & 99.29 & 0.71 & - & - & - & - & - & - \\
\hline \multirow[t]{5}{*}{ DP4+ } & ${ }^{13} \mathrm{C}$ data & 99.99 & 0.01 & - & - & - & - & - & - \\
\hline & All data & 100.00 & 0.00 & - & - & - & - & - & - \\
\hline & & \multicolumn{8}{|c|}{ Level of theory: mPW1PW91/6-311G* (solution, PCM) } \\
\hline & & $73 a$ & $73 b$ & - & - & - & - & - & - \\
\hline & ${ }^{1} \mathrm{H}$ data & 99.96 & 0.04 & - & - & - & - & - & - \\
\hline \multirow[t]{3}{*}{ sDP4+ } & ${ }^{13} \mathrm{C}$ data & 97.26 & 2.74 & - & - & - & - & - & - \\
\hline & All data & 100.00 & 0.00 & - & - & - & - & - & - \\
\hline & ${ }^{1}$ H data & 78.02 & 21.98 & - & - & - & - & - & - \\
\hline \multirow[t]{3}{*}{ uDP4+ } & ${ }^{13}$ C data & 84.13 & 15.87 & - & - & - & - & - & - \\
\hline & All data & 94.95 & 5.05 & - & - & - & - & - & - \\
\hline & ${ }^{1} \mathrm{H}$ data & 99.99 & 0.01 & - & - & - & - & - & - \\
\hline \multirow[t]{5}{*}{ DP4+ } & ${ }^{13} \mathrm{C}$ data & 99.47 & 0.53 & - & - & - & - & - & - \\
\hline & All data & 100.00 & 0.00 & - & - & - & - & - & - \\
\hline & & \multicolumn{8}{|c|}{ Level of theory: mPW1PW91/6-311G** (solution, PCM) } \\
\hline & & $73 a$ & 73b & - & - & - & - & - & - \\
\hline & ${ }^{1} \mathrm{H}$ data & 99.94 & 0.06 & - & - & - & - & - & - \\
\hline \multirow[t]{2}{*}{ sDP4+ } & ${ }^{13} \mathrm{C}$ data & 97.12 & 2.88 & - & - & - & - & - & - \\
\hline & All data & 100.00 & 0.00 & - & - & - & - & - & - \\
\hline \multirow{3}{*}{ uDP4+ } & ${ }^{1} \mathrm{H}$ data & 51.96 & 48.04 & - & - & - & - & - & - \\
\hline & ${ }^{13} \mathrm{C}$ data & 79.22 & 20.78 & - & - & - & - & - & - \\
\hline & All data & 80.48 & 19.52 & - & - & - & - & - & - \\
\hline \multirow{5}{*}{ DP4+ } & ${ }^{1} \mathrm{H}$ data & 99.94 & 0.06 & - & - & - & - & - & - \\
\hline & ${ }^{13} \mathrm{C}$ data & 99.23 & 0.77 & - & - & - & - & - & - \\
\hline & All data & 100.00 & 0.00 & - & - & - & - & - & - \\
\hline & & \multicolumn{8}{|c|}{ Level of theory: mPW1PW91/6-311+G** (solution, PCM) } \\
\hline & & $73 a$ & $73 b$ & - & - & - & - & - & - \\
\hline \multirow{3}{*}{ sDP4+ } & ${ }^{1} \mathrm{H}$ data & 99.98 & 0.02 & - & - & - & - & - & - \\
\hline & ${ }^{13} \mathrm{C}$ data & 95.07 & 4.93 & - & - & - & - & - & - \\
\hline & All data & 100.00 & 0.00 & - & - & - & - & - & - \\
\hline \multirow{3}{*}{ uDP4+ } & ${ }^{1} \mathrm{H}$ data & 87.23 & 12.77 & - & - & - & - & - & - \\
\hline & ${ }^{13} \mathrm{C}$ data & 55.31 & 44.69 & - & - & - & - & - & - \\
\hline & All data & 89.42 & 10.58 & - & - & - & - & - & - \\
\hline \multirow{3}{*}{ DP4+ } & ${ }^{1} \mathrm{H}$ data & 100.00 & 0.00 & - & - & - & - & - & - \\
\hline & ${ }^{13} \mathrm{C}$ data & 95.98 & 4.02 & - & - & - & - & - & - \\
\hline & All data & 100.00 & 0.00 & - & - & - & - & - & - \\
\hline
\end{tabular}


sDP4+, $u$ DP4+ and DP4+ probabilities (\%) for compound 73b<smiles>CCOC(=O)[C@H](C)[C@@H](O)c1ccccc1</smiles><smiles>CCOC(=O)[C@H](C)[C@H](O)c1ccccc1</smiles>

correct isomer

\begin{tabular}{|c|c|c|c|c|c|c|c|c|c|}
\hline & & \multicolumn{8}{|c|}{ Level of theory: B3LYP/6-31G* (gas phase) } \\
\hline & & $73 a$ & 73b & - & - & - & - & - & - \\
\hline \multirow{3}{*}{ sDP4+ } & ${ }^{1} \mathrm{H}$ data & 79.56 & 20.44 & - & - & - & - & - & - \\
\hline & ${ }^{13} \mathrm{C}$ data & 22.73 & 77.27 & - & - & - & - & - & - \\
\hline & All data & 53.39 & 46.61 & - & - & - & - & - & - \\
\hline \multirow{3}{*}{ uDP4+ } & ${ }^{1} \mathrm{H}$ data & 63.29 & 36.71 & - & - & - & - & - & - \\
\hline & ${ }^{13} \mathrm{C}$ data & 12.23 & 87.77 & - & - & - & - & - & - \\
\hline & All data & 19.37 & 80.63 & - & - & - & - & - & - \\
\hline \multirow{5}{*}{ DP4+ } & ${ }^{1} \mathrm{H}$ data & 87.03 & 12.97 & - & - & - & - & - & - \\
\hline & ${ }^{13} \mathrm{C}$ data & 3.94 & 96.06 & - & - & - & - & - & - \\
\hline & All data & 21.58 & 78.42 & - & - & - & - & - & - \\
\hline & & \multicolumn{8}{|c|}{ Level of theory: B3LYP/6-31G** (gas phase) } \\
\hline & & $73 a$ & $73 b$ & - & - & - & - & - & - \\
\hline \multirow{3}{*}{ sDP4+ } & ${ }^{1} \mathrm{H}$ data & 74.54 & 25.46 & - & - & - & - & - & - \\
\hline & ${ }^{13} \mathrm{C}$ data & 56.32 & 43.68 & - & - & - & - & - & - \\
\hline & All data & 79.05 & 20.95 & - & - & - & - & - & - \\
\hline \multirow{3}{*}{ uDP4+ } & ${ }^{1} \mathrm{H}$ data & 31.14 & 68.86 & - & - & - & - & - & - \\
\hline & ${ }^{13} \mathrm{C}$ data & 7.15 & 92.85 & - & - & - & - & - & - \\
\hline & All data & 3.37 & 96.63 & - & - & - & - & - & - \\
\hline \multirow{5}{*}{ DP4+ } & ${ }^{1} \mathrm{H}$ data & 56.97 & 43.03 & - & - & - & - & - & - \\
\hline & ${ }^{13} \mathrm{C}$ data & 9.04 & 90.96 & - & - & - & - & - & - \\
\hline & All data & 11.62 & 88.38 & - & - & - & - & - & - \\
\hline & & \multicolumn{8}{|c|}{ Level of theory: B3LYP/6-31+G** (gas phase) } \\
\hline & & $73 a$ & $73 b$ & - & - & - & - & - & - \\
\hline \multirow{3}{*}{ sDP4+ } & ${ }^{1} \mathrm{H}$ data & 91.92 & 8.08 & - & - & - & - & - & - \\
\hline & ${ }^{13} \mathrm{C}$ data & 68.64 & 31.36 & - & - & - & - & - & - \\
\hline & All data & 96.14 & 3.86 & - & - & - & - & - & - \\
\hline \multirow{3}{*}{ uDP4+ } & ${ }^{1} \mathrm{H}$ data & 43.81 & 56.19 & - & - & - & - & - & - \\
\hline & ${ }^{13} \mathrm{C}$ data & 5.50 & 94.50 & - & - & - & - & - & - \\
\hline & All data & 4.34 & 95.66 & - & - & - & - & - & - \\
\hline \multirow{5}{*}{ DP4+ } & ${ }^{1} \mathrm{H}$ data & 89.87 & 10.13 & - & - & - & - & - & - \\
\hline & ${ }^{13} \mathrm{C}$ data & 11.30 & 88.70 & - & - & - & - & - & - \\
\hline & All data & 53.06 & 46.94 & - & - & - & - & - & - \\
\hline & & \multicolumn{8}{|c|}{ Level of theory: B3LYP/6-311G* (gas phase) } \\
\hline & & $73 a$ & $73 b$ & - & - & - & - & - & - \\
\hline \multirow{3}{*}{ sDP4+ } & ${ }^{1} \mathrm{H}$ data & 84.62 & 15.38 & - & - & - & - & - & - \\
\hline & ${ }^{13} \mathrm{C}$ data & 33.19 & 66.81 & - & - & - & - & - & - \\
\hline & All data & 73.22 & 26.78 & - & - & - & - & - & - \\
\hline \multirow{3}{*}{ uDP4+ } & ${ }^{1} \mathrm{H}$ data & 44.43 & 55.57 & - & - & - & - & - & - \\
\hline & ${ }^{13} \mathrm{C}$ data & 0.77 & 99.23 & - & - & - & - & - & - \\
\hline & All data & 0.62 & 99.38 & - & - & - & - & - & - \\
\hline \multirow{3}{*}{ DP4+ } & ${ }^{1} \mathrm{H}$ data & 81.48 & 18.52 & - & - & - & - & - & - \\
\hline & ${ }^{13} \mathrm{C}$ data & 0.39 & 99.61 & - & - & - & - & - & - \\
\hline & All data & 1.67 & 98.33 & - & - & - & - & - & - \\
\hline & & \multicolumn{8}{|c|}{ Level of theory: B3LYP/6-311G** (gas phase) } \\
\hline & & $73 a$ & $73 b$ & - & - & - & - & - & - \\
\hline \multirow{2}{*}{ sDP4+ } & ${ }^{1} \mathrm{H}$ data & 92.10 & 7.90 & - & - & - & - & - & - \\
\hline & ${ }^{13} \mathrm{C}$ data & 22.18 & 77.82 & - & - & - & - & - & - \\
\hline
\end{tabular}




\begin{tabular}{|c|c|c|c|c|c|c|c|c|c|}
\hline & All data & 76.87 & 23.13 & - & - & - & - & - & - \\
\hline & ${ }^{1} \mathrm{H}$ data & 16.11 & 83.89 & - & - & - & - & - & - \\
\hline \multirow[t]{3}{*}{ uDP4+ } & ${ }^{13} \mathrm{C}$ data & 0.43 & 99.57 & - & - & - & - & - & - \\
\hline & All data & 0.08 & 99.92 & - & - & - & - & - & - \\
\hline & ${ }^{1} \mathrm{H}$ data & 69.14 & 30.86 & - & - & - & - & - & - \\
\hline \multirow[t]{5}{*}{ DP4+ } & ${ }^{13} \mathrm{C}$ data & 0.12 & 99.88 & - & - & - & - & - & - \\
\hline & All data & 0.27 & 99.73 & - & - & - & - & - & - \\
\hline & & \multicolumn{8}{|c|}{ Level of theory: B3LYP/6-311+G** (gas phase) } \\
\hline & & $73 a$ & $73 b$ & - & - & - & - & - & - \\
\hline & ${ }^{1} \mathrm{H}$ data & 87.91 & 12.09 & - & - & - & - & - & - \\
\hline \multirow[t]{3}{*}{ sDP4+ } & ${ }^{13} \mathrm{C}$ data & 14.87 & 85.13 & - & - & - & - & - & - \\
\hline & All data & 55.96 & 44.04 & - & - & - & - & - & - \\
\hline & ${ }^{1} \mathrm{H}$ data & 34.80 & 65.20 & - & - & - & - & - & - \\
\hline \multirow[t]{3}{*}{ uDP4+ } & ${ }^{13} \mathrm{C}$ data & 0.36 & 99.64 & - & - & - & - & - & - \\
\hline & All data & 0.19 & 99.81 & - & - & - & - & - & - \\
\hline & ${ }^{1} \mathrm{H}$ data & 79.51 & 20.49 & - & - & - & - & - & - \\
\hline \multirow[t]{5}{*}{ DP4+ } & ${ }^{13} \mathrm{C}$ data & 0.06 & 99.94 & - & - & - & - & - & - \\
\hline & All data & 0.25 & 99.75 & - & - & - & - & - & - \\
\hline & & \multicolumn{8}{|c|}{ Level of theory: B3LYP/6-31G* (solution, PCM) } \\
\hline & & $73 a$ & $73 b$ & - & - & - & - & - & - \\
\hline & ${ }^{1} \mathrm{H}$ data & 86.25 & 13.75 & - & - & - & - & - & - \\
\hline \multirow[t]{3}{*}{ sDP4+ } & ${ }^{13} \mathrm{C}$ data & 10.53 & 89.47 & - & - & - & - & - & - \\
\hline & All data & 42.46 & 57.54 & - & - & - & - & - & - \\
\hline & ${ }^{1} \mathrm{H}$ data & 69.95 & 30.05 & - & - & - & - & - & - \\
\hline \multirow[t]{3}{*}{ uDP4+ } & ${ }^{13} \mathrm{C}$ data & 5.32 & 94.68 & - & - & - & - & - & - \\
\hline & All data & 11.57 & 88.43 & - & - & - & - & - & - \\
\hline & ${ }^{1} \mathrm{H}$ data & 93.59 & 6.41 & - & - & - & - & - & - \\
\hline \multirow[t]{5}{*}{ DP4+ } & ${ }^{13} \mathrm{C}$ data & 0.66 & 99.34 & - & - & - & - & - & - \\
\hline & All data & 8.80 & 91.20 & - & - & - & - & - & - \\
\hline & & \multicolumn{8}{|c|}{ Level of theory: B3LYP/6-31G** (solution, PCM) } \\
\hline & & $73 a$ & $73 b$ & - & - & - & - & - & - \\
\hline & ${ }^{1} \mathrm{H}$ data & 88.87 & 11.13 & - & - & - & - & - & - \\
\hline \multirow[t]{3}{*}{ sDP4+ } & ${ }^{13} \mathrm{C}$ data & 33.92 & 66.08 & - & - & - & - & - & - \\
\hline & All data & 80.39 & 19.61 & - & - & - & - & - & - \\
\hline & ${ }^{1} \mathrm{H}$ data & 40.17 & 59.83 & - & - & - & - & - & - \\
\hline \multirow[t]{3}{*}{ uDP4+ } & ${ }^{13} \mathrm{C}$ data & 3.77 & 96.23 & - & - & - & - & - & - \\
\hline & All data & 2.56 & 97.44 & - & - & - & - & - & - \\
\hline & ${ }^{1} \mathrm{H}$ data & 84.28 & 15.72 & - & - & - & - & - & - \\
\hline \multirow[t]{5}{*}{ DP4+ } & ${ }^{13} \mathrm{C}$ data & 1.97 & 98.03 & - & - & - & - & - & - \\
\hline & All data & 9.73 & 90.27 & - & - & - & - & - & - \\
\hline & & \multicolumn{8}{|c|}{ Level of theory: B3LYP/6-31+G** (solution, PCM) } \\
\hline & & 73a & $73 b$ & - & - & - & - & - & - \\
\hline & ${ }^{1} \mathrm{H}$ data & 94.20 & 5.80 & - & - & - & - & - & - \\
\hline \multirow[t]{3}{*}{ sDP4+ } & ${ }^{13} \mathrm{C}$ data & 55.31 & 44.69 & - & - & - & - & - & - \\
\hline & All data & 95.26 & 4.74 & - & - & - & - & - & - \\
\hline & ${ }^{1} \mathrm{H}$ data & 75.88 & 24.12 & - & - & - & - & - & - \\
\hline uDP4+ & ${ }^{13} \mathrm{C}$ data & 3.89 & 96.11 & - & - & - & - & - & - \\
\hline & All data & 11.29 & 88.71 & - & - & - & - & - & - \\
\hline & ${ }^{1} \mathrm{H}$ data & 98.08 & 1.92 & - & - & - & - & - & - \\
\hline DP4+ & ${ }^{13} \mathrm{C}$ data & 4.77 & 95.23 & - & - & - & - & - & - \\
\hline & All data & 71.90 & 28.10 & - & - & - & - & - & - \\
\hline & & & & el & $\mathrm{B} 3$ & $\mathbf{G}^{*}$ & $\mathrm{PC}$ & & \\
\hline & & $73 a$ & $73 b$ & - & - & - & - & - & - \\
\hline & ${ }^{1} \mathrm{H}$ data & 80.68 & 19.32 & - & - & - & - & - & - \\
\hline sDP4+ & ${ }^{13} \mathrm{C}$ data & 17.90 & 82.10 & - & - & - & - & - & - \\
\hline & All data & 47.66 & 52.34 & - & - & - & - & - & - \\
\hline
\end{tabular}




\begin{tabular}{|c|c|c|c|c|c|c|c|c|c|}
\hline & ${ }^{1} \mathrm{H}$ data & 55.44 & 44.56 & - & - & - & - & - & - \\
\hline \multirow[t]{3}{*}{ uDP4+ } & ${ }^{13} \mathrm{C}$ data & 0.22 & 99.78 & - & - & - & - & - & - \\
\hline & All data & 0.27 & 99.73 & - & - & - & - & - & - \\
\hline & ${ }^{1} \mathrm{H}$ data & 83.86 & 16.14 & - & - & - & - & - & - \\
\hline \multirow[t]{5}{*}{ DP4+ } & ${ }^{13} \mathrm{C}$ data & 0.05 & 99.95 & - & - & - & - & - & - \\
\hline & All data & 0.24 & 99.76 & - & - & - & - & - & - \\
\hline & & \multicolumn{8}{|c|}{ Level of theory: B3LYP/6-311G** (solution, PCM) } \\
\hline & & $73 a$ & $73 b$ & - & - & - & - & - & - \\
\hline & ${ }^{1} \mathrm{H}$ data & 90.01 & 9.99 & - & - & - & - & - & - \\
\hline \multirow[t]{3}{*}{ sDP4+ } & ${ }^{13} \mathrm{C}$ data & 10.24 & 89.76 & - & - & - & - & - & - \\
\hline & All data & 50.69 & 49.31 & - & - & - & - & - & - \\
\hline & ${ }^{1} \mathrm{H}$ data & 24.69 & 75.31 & - & - & - & - & - & - \\
\hline \multirow[t]{3}{*}{ uDP4+ } & ${ }^{13} \mathrm{C}$ data & 0.15 & 99.85 & - & - & - & - & - & - \\
\hline & All data & 0.05 & 99.95 & - & - & - & - & - & - \\
\hline & ${ }^{1} \mathrm{H}$ data & 74.70 & 25.30 & - & - & - & - & - & - \\
\hline \multirow[t]{5}{*}{ DP4+ } & ${ }^{13} \mathrm{C}$ data & 0.02 & 99.98 & - & - & - & - & - & - \\
\hline & All data & 0.05 & 99.95 & - & - & - & - & - & - \\
\hline & & \multicolumn{8}{|c|}{ Level of theory: B3LYP/6-311+G** (solution, PCM) } \\
\hline & & $73 a$ & $73 b$ & - & - & - & - & - & - \\
\hline & ${ }^{1} \mathrm{H}$ data & 89.60 & 10.40 & - & - & - & - & - & - \\
\hline \multirow[t]{3}{*}{ sDP4+ } & ${ }^{13} \mathrm{C}$ data & 6.11 & 93.89 & - & - & - & - & - & - \\
\hline & All data & 35.93 & 64.07 & - & - & - & - & - & - \\
\hline & ${ }^{1} \mathrm{H}$ data & 77.11 & 22.89 & - & - & - & - & - & - \\
\hline \multirow[t]{3}{*}{ uDP4+ } & ${ }^{13} \mathrm{C}$ data & 0.09 & 99.91 & - & - & - & - & - & - \\
\hline & All data & 0.31 & 99.69 & - & - & - & - & - & - \\
\hline & ${ }^{1} \mathrm{H}$ data & 96.67 & 3.33 & - & - & - & - & - & - \\
\hline \multirow[t]{5}{*}{ DP4+ } & ${ }^{13} \mathrm{C}$ data & 0.01 & 99.99 & - & - & - & - & - & - \\
\hline & All data & 0.17 & 99.83 & - & - & - & - & - & - \\
\hline & & \multicolumn{8}{|c|}{ Level of theory: mPW1PW91/6-31G* (gas phase) } \\
\hline & & $73 a$ & $73 b$ & - & - & - & - & - & - \\
\hline & ${ }_{12}^{1} \mathrm{H}$ data & 92.94 & 7.06 & - & - & - & - & - & - \\
\hline \multirow[t]{3}{*}{ sDP4+ } & ${ }^{13} \mathrm{C}$ data & 2.95 & 97.05 & - & - & - & - & - & - \\
\hline & All data & 28.60 & 71.40 & - & - & - & - & - & - \\
\hline & ${ }^{1} \mathrm{H}$ data & 74.13 & 25.87 & - & - & - & - & - & - \\
\hline \multirow[t]{3}{*}{ uDP4+ } & ${ }^{13} \mathrm{C}$ data & 16.50 & 83.50 & - & - & - & - & - & - \\
\hline & All data & 36.15 & 63.85 & - & - & - & - & - & - \\
\hline & ${ }^{1} \mathrm{H}$ data & 97.42 & 2.58 & - & - & - & - & - & - \\
\hline \multirow[t]{5}{*}{ DP4+ } & ${ }^{13} \mathrm{C}$ data & 0.60 & 99.40 & - & - & - & - & - & - \\
\hline & All data & 18.49 & 81.51 & - & - & - & - & - & - \\
\hline & & \multicolumn{8}{|c|}{ Level of theory: mPW1PW91/6-31G** (gas phase) } \\
\hline & & $73 a$ & $73 b$ & - & - & - & - & - & - \\
\hline & ${ }^{1} \mathrm{H}$ data & 93.19 & 6.81 & - & - & - & - & - & - \\
\hline \multirow[t]{3}{*}{ sDP4+ } & ${ }^{13} \mathrm{C}$ data & 12.12 & 87.88 & - & - & - & - & - & - \\
\hline & All data & 65.36 & 34.64 & - & - & - & - & - & - \\
\hline & ${ }^{1} \mathrm{H}$ data & 40.60 & 59.40 & - & - & - & - & - & - \\
\hline uDP4+ & ${ }^{13} \mathrm{C}$ data & 8.08 & 91.92 & - & - & - & - & - & - \\
\hline & All data & 5.67 & 94.33 & - & - & - & - & - & - \\
\hline & ${ }^{1} \mathrm{H}$ data & 90.34 & 9.66 & - & - & - & - & - & - \\
\hline DP4+ & ${ }^{13} \mathrm{C}$ data & 1.20 & 98.80 & - & - & - & - & - & - \\
\hline & All data & 10.18 & 89.82 & - & - & - & - & - & - \\
\hline & & & & 10 & PV & -31 & ph & & \\
\hline & & $73 a$ & $73 b$ & - & - & - & - & - & - \\
\hline & ${ }^{1} \mathrm{H}$ data & 94.05 & 5.95 & - & - & - & - & - & - \\
\hline sDP4+ & ${ }^{13} \mathrm{C}$ data & 29.58 & 70.42 & - & - & - & - & - & - \\
\hline & All data & 86.91 & 13.09 & - & - & - & - & - & - \\
\hline uDP4+ & ${ }^{1} \mathrm{H}$ data & 49.03 & 50.97 & - & - & - & - & - & - \\
\hline
\end{tabular}




\begin{tabular}{|c|c|c|c|c|c|c|c|c|c|}
\hline & ${ }^{13} \mathrm{C}$ data & 10.21 & 89.79 & - & - & - & - & - & - \\
\hline & All data & 9.86 & 90.14 & - & - & - & - & - & - \\
\hline & ${ }^{1} \mathrm{H}$ data & 93.83 & 6.17 & - & - & - & - & - & - \\
\hline \multirow[t]{5}{*}{ DP4+ } & ${ }^{13} \mathrm{C}$ data & 4.56 & 95.44 & - & - & - & - & - & - \\
\hline & All data & 42.07 & 57.93 & - & - & - & - & - & - \\
\hline & & \multicolumn{8}{|c|}{ Level of theory: mPW1PW91/6-311G* (gas phase) } \\
\hline & & $73 a$ & $73 b$ & - & - & - & - & - & - \\
\hline & ${ }^{1}$ H data & 89.15 & 10.85 & - & - & - & - & - & - \\
\hline \multirow[t]{3}{*}{ sDP4+ } & ${ }^{13}$ C data & 15.39 & 84.61 & - & - & - & - & - & - \\
\hline & All data & 59.90 & 40.10 & - & - & - & - & - & - \\
\hline & ${ }^{1} \mathrm{H}$ data & 54.89 & 45.11 & - & - & - & - & - & - \\
\hline \multirow[t]{3}{*}{ uDP4+ } & ${ }^{13} \mathrm{C}$ data & 1.50 & 98.50 & - & - & - & - & - & - \\
\hline & All data & 1.83 & 98.17 & - & - & - & - & - & - \\
\hline & ${ }^{1} \mathrm{H}$ data & 90.91 & 9.09 & - & - & - & - & - & - \\
\hline \multirow[t]{5}{*}{ DP4+ } & ${ }^{13} \mathrm{C}$ data & 0.28 & 99.72 & - & - & - & - & - & - \\
\hline & All data & 2.70 & 97.30 & - & - & - & - & - & - \\
\hline & & \multicolumn{8}{|c|}{ Level of theory: mPW1PW91/6-311G** (gas phase) } \\
\hline & & $73 a$ & $73 b$ & - & - & - & - & - & - \\
\hline & ${ }^{1} \mathrm{H}$ data & 97.40 & 2.60 & - & - & - & - & - & - \\
\hline \multirow[t]{3}{*}{ sDP4+ } & ${ }^{13} \mathrm{C}$ data & 8.52 & 91.48 & - & - & - & - & - & - \\
\hline & All data & 77.74 & 22.26 & - & - & - & - & - & - \\
\hline & ${ }^{1} \mathrm{H}$ data & 23.09 & 76.91 & - & - & - & - & - & - \\
\hline \multirow[t]{3}{*}{ uDP4+ } & ${ }^{13} \mathrm{C}$ data & 0.62 & 99.38 & - & - & - & - & - & - \\
\hline & All data & 0.19 & 99.81 & - & - & - & - & - & - \\
\hline & ${ }^{1} \mathrm{H}$ data & 91.84 & 8.16 & - & - & - & - & - & - \\
\hline \multirow[t]{5}{*}{ DP4+ } & ${ }^{13} \mathrm{C}$ data & 0.06 & 99.94 & - & - & - & - & - & - \\
\hline & All data & 0.65 & 99.35 & - & - & - & - & - & - \\
\hline & & \multicolumn{8}{|c|}{ Level of theory: mPW1PW91/6-311+G** (gas phase) } \\
\hline & & $73 a$ & $73 b$ & - & - & - & - & - & - \\
\hline & ${ }^{1} \mathrm{H}$ data & 95.15 & 4.85 & - & - & - & - & - & - \\
\hline \multirow[t]{2}{*}{ sDP4+ } & ${ }^{13} \mathrm{C}$ data & 5.98 & 94.02 & - & - & - & - & - & - \\
\hline & All data & 55.50 & 44.50 & - & - & - & - & - & - \\
\hline \multirow{3}{*}{ uDP4+ } & ${ }^{1} \mathrm{H}$ data & 50.15 & 49.85 & - & - & - & - & - & - \\
\hline & ${ }^{13}$ C data & 0.46 & 99.54 & - & - & - & - & - & - \\
\hline & All data & 0.46 & 99.54 & - & - & - & - & - & - \\
\hline \multirow{5}{*}{ DP4+ } & ${ }^{1} \mathrm{H}$ data & 95.18 & 4.82 & - & - & - & - & - & - \\
\hline & ${ }^{13} \mathrm{C}$ data & 0.03 & 99.97 & - & - & - & - & - & - \\
\hline & All data & 0.57 & 99.43 & - & - & - & - & - & - \\
\hline & & \multicolumn{8}{|c|}{ Level of theory: mPW1PW91/6-31G* (solution, PCM) } \\
\hline & & $73 a$ & $73 b$ & - & - & - & - & - & - \\
\hline \multirow{3}{*}{ sDP4+ } & ${ }^{1} \mathrm{H}$ data & 96.93 & 3.07 & - & - & - & - & - & - \\
\hline & ${ }^{13} \mathrm{C}$ data & 1.29 & 98.71 & - & - & - & - & - & - \\
\hline & All data & 29.27 & 70.73 & - & - & - & - & - & - \\
\hline \multirow{3}{*}{ uDP4+ } & ${ }^{1} \mathrm{H}$ data & 79.88 & 20.12 & - & - & - & - & - & - \\
\hline & ${ }^{13} \mathrm{C}$ data & 7.21 & 92.79 & - & - & - & - & - & - \\
\hline & All data & 23.56 & 76.44 & - & - & - & - & - & - \\
\hline & ${ }^{1} \mathrm{H}$ data & 99.21 & 0.79 & - & - & - & - & - & - \\
\hline DP4+ & ${ }^{13} \mathrm{C}$ data & 0.10 & 99.90 & - & - & - & - & - & - \\
\hline & All data & 11.31 & 88.69 & - & - & - & - & - & - \\
\hline & & & & of & $\mathbf{W}$ & 10 & on, & & \\
\hline & & $73 a$ & $73 b$ & - & - & - & - & - & - \\
\hline & ${ }^{1} \mathrm{H}$ data & 97.77 & 2.23 & - & - & - & - & - & - \\
\hline sDP4+ & ${ }^{13} \mathrm{C}$ data & 3.72 & 96.28 & - & - & - & - & - & - \\
\hline & All data & 62.91 & 37.09 & - & - & - & - & - & - \\
\hline & ${ }_{13}^{1}$ data & 57.56 & 42.44 & - & - & - & - & - & - \\
\hline uDP4+ & ${ }^{13} \mathrm{C}$ data & 3.56 & 96.44 & - & - & - & - & - & - \\
\hline
\end{tabular}




\begin{tabular}{|c|c|c|c|c|c|c|c|c|c|}
\hline & All data & 4.77 & 95.23 & - & - & - & - & - & - \\
\hline & ${ }^{1} \mathrm{H}$ data & 98.35 & 1.65 & - & - & - & - & - & - \\
\hline \multirow[t]{5}{*}{ DP4+ } & ${ }^{13} \mathrm{C}$ data & 0.14 & 99.86 & - & - & - & - & - & - \\
\hline & All data & 7.83 & 92.17 & - & - & - & - & - & - \\
\hline & & \multicolumn{8}{|c|}{ Level of theory: mPW1PW91/6-31+G** (solution, PCM) } \\
\hline & & $73 a$ & 73b & - & - & - & - & - & - \\
\hline & ${ }^{1} \mathrm{H}$ data & 89.17 & 10.83 & - & - & - & - & - & - \\
\hline \multirow[t]{3}{*}{ sDP4+ } & ${ }^{13} \mathrm{C}$ data & 14.91 & 85.09 & - & - & - & - & - & - \\
\hline & All data & 59.07 & 40.93 & - & - & - & - & - & - \\
\hline & ${ }^{1} \mathrm{H}$ data & 32.59 & 67.41 & - & - & - & - & - & - \\
\hline \multirow[t]{3}{*}{ uDP4+ } & ${ }^{13} \mathrm{C}$ data & 3.88 & 96.12 & - & - & - & - & - & - \\
\hline & All data & 1.91 & 98.09 & - & - & - & - & - & - \\
\hline & ${ }^{1} \mathrm{H}$ data & 79.93 & 20.07 & - & - & - & - & - & - \\
\hline \multirow[t]{5}{*}{ DP4+ } & ${ }^{13} \mathrm{C}$ data & 0.70 & 99.30 & - & - & - & - & - & - \\
\hline & All data & 2.74 & 97.26 & - & - & - & - & - & - \\
\hline & & \multicolumn{8}{|c|}{ Level of theory: mPW1PW91/6-311G* (solution, PCM) } \\
\hline & & $73 a$ & $73 b$ & - & - & - & - & - & - \\
\hline & ${ }^{1} \mathrm{H}$ data & 90.05 & 9.95 & - & - & - & - & - & - \\
\hline \multirow[t]{3}{*}{ sDP4+ } & ${ }^{13} \mathrm{C}$ data & 7.09 & 92.91 & - & - & - & - & - & - \\
\hline & All data & 40.87 & 59.13 & - & - & - & - & - & - \\
\hline & ${ }^{1}$ H data & 74.43 & 25.57 & - & - & - & - & - & - \\
\hline \multirow[t]{3}{*}{ uDP4+ } & ${ }^{13}$ C data & 0.15 & 99.85 & - & - & - & - & - & - \\
\hline & All data & 0.44 & 99.56 & - & - & - & - & - & - \\
\hline & ${ }^{1} \mathrm{H}$ data & 96.34 & 3.66 & - & - & - & - & - & - \\
\hline \multirow[t]{5}{*}{ DP4+ } & ${ }^{13} \mathrm{C}$ data & 0.01 & 99.99 & - & - & - & - & - & - \\
\hline & All data & 0.31 & 99.69 & - & - & - & - & - & - \\
\hline & & \multicolumn{8}{|c|}{ Level of theory: mPW1PW91/6-311G** (solution, PCM) } \\
\hline & & $73 a$ & 73b & - & - & - & - & - & - \\
\hline & ${ }^{1} \mathrm{H}$ data & 97.16 & 2.84 & - & - & - & - & - & - \\
\hline \multirow[t]{3}{*}{ sDP4+ } & ${ }^{13} \mathrm{C}$ data & 3.38 & 96.62 & - & - & - & - & - & - \\
\hline & All data & 54.48 & 45.52 & - & - & - & - & - & - \\
\hline & ${ }^{1} \mathrm{H}$ data & 37.44 & 62.56 & - & - & - & - & - & - \\
\hline \multirow[t]{3}{*}{ uDP4+ } & ${ }^{13} \mathrm{C}$ data & 0.10 & 99.90 & - & - & - & - & - & - \\
\hline & All data & 0.06 & 99.94 & - & - & - & - & - & - \\
\hline & ${ }^{1}$ H data & 95.34 & 4.66 & - & - & - & - & - & - \\
\hline \multirow[t]{5}{*}{ DP4+ } & ${ }^{13} \mathrm{C}$ data & 0.00 & 100.00 & - & - & - & - & - & - \\
\hline & All data & 0.07 & 99.93 & - & - & - & - & - & - \\
\hline & & \multicolumn{8}{|c|}{ Level of theory: mPW1PW91/6-311+G** (solution, PCM) } \\
\hline & & $73 a$ & $73 b$ & - & - & - & - & - & - \\
\hline & ${ }^{1} \mathrm{H}$ data & 97.13 & 2.87 & - & - & - & - & - & - \\
\hline \multirow[t]{2}{*}{ sDP4+ } & ${ }^{13} \mathrm{C}$ data & 2.23 & 97.77 & - & - & - & - & - & - \\
\hline & All data & 43.57 & 56.43 & - & - & - & - & - & - \\
\hline \multirow{3}{*}{ uDP4+ } & ${ }^{1} \mathrm{H}$ data & 82.43 & 17.57 & - & - & - & - & - & - \\
\hline & ${ }^{13} \mathrm{C}$ data & 0.03 & 99.97 & - & - & - & - & - & - \\
\hline & All data & 0.14 & 99.86 & - & - & - & - & - & - \\
\hline \multirow{3}{*}{ DP4+ } & ${ }^{1} \mathrm{H}$ data & 99.37 & 0.63 & - & - & - & - & - & - \\
\hline & ${ }^{13} \mathrm{C}$ data & 0.00 & 100.00 & - & - & - & - & - & - \\
\hline & All data & 0.11 & 99.89 & - & - & - & - & - & - \\
\hline
\end{tabular}


$s \mathrm{DP4}+, u \mathrm{DP} 4+$ and DP4+ probabilities (\%) for compound 74a<smiles>[Z16]C(C(=O)c1ccccc1)[C@@H](C)O</smiles>

correct isomer<smiles>[R4][14C](C(=O)c1ccccc1)[C@@H](C)O</smiles>

$74 b$

\begin{tabular}{|c|c|c|c|c|c|c|c|c|c|}
\hline & & \multicolumn{8}{|c|}{ Level of theory: B3LYP/6-31G* (gas phase) } \\
\hline & & $74 a$ & 74b & - & - & - & - & - & - \\
\hline \multirow{3}{*}{ sDP4+ } & ${ }^{1} \mathrm{H}$ data & 68.58 & 31.42 & - & - & - & - & - & - \\
\hline & ${ }^{13}$ C data & 99.99 & 0.01 & - & - & - & - & - & - \\
\hline & All data & 99.99 & 0.01 & - & - & - & - & - & - \\
\hline \multirow{3}{*}{ uDP4+ } & ${ }^{1} \mathrm{H}$ data & 77.66 & 22.34 & - & - & - & - & - & - \\
\hline & ${ }^{13} \mathrm{C}$ data & 99.04 & 0.96 & - & - & - & - & - & - \\
\hline & All data & 99.72 & 0.28 & - & - & - & - & - & - \\
\hline \multirow{3}{*}{ DP4+ } & ${ }^{1} \mathrm{H}$ data & 88.36 & 11.64 & - & - & - & - & - & - \\
\hline & ${ }^{13} \mathrm{C}$ data & 100.00 & 0.00 & - & - & - & - & - & - \\
\hline & All data & 100.00 & 0.00 & - & - & - & - & - & - \\
\hline & & \multicolumn{8}{|c|}{ Level of theory: B3LYP/6-31G** (gas phase) } \\
\hline & & $74 a$ & 74b & - & - & - & - & - & - \\
\hline \multirow{3}{*}{ sDP4+ } & ${ }^{1} \mathrm{H}$ data & 85.11 & 14.89 & - & - & - & - & - & - \\
\hline & ${ }^{13} \mathrm{C}$ data & 99.97 & 0.03 & - & - & - & - & - & - \\
\hline & All data & 99.99 & 0.01 & - & - & - & - & - & - \\
\hline \multirow{3}{*}{ uDP4+ } & ${ }^{1} \mathrm{H}$ data & 74.95 & 25.05 & - & - & - & - & - & - \\
\hline & ${ }^{13} \mathrm{C}$ data & 98.03 & 1.97 & - & - & - & - & - & - \\
\hline & All data & 99.33 & 0.67 & - & - & - & - & - & - \\
\hline \multirow{3}{*}{ DP4+ } & ${ }^{1} \mathrm{H}$ data & 94.48 & 5.52 & - & - & - & - & - & - \\
\hline & ${ }^{13} \mathrm{C}$ data & 100.00 & 0.00 & - & - & - & - & - & - \\
\hline & All data & 100.00 & 0.00 & - & - & - & - & - & - \\
\hline & & \multicolumn{8}{|c|}{ Level of theory: B3LYP/6-31+G** (gas phase) } \\
\hline & & $74 a$ & $74 b$ & - & - & - & - & - & - \\
\hline \multirow{3}{*}{$s \mathrm{DP} 4+$} & ${ }^{1}$ H data & 96.58 & 3.42 & - & - & - & - & - & - \\
\hline & ${ }^{13} \mathrm{C}$ data & 99.69 & 0.31 & - & - & - & - & - & - \\
\hline & All data & 99.99 & 0.01 & - & - & - & - & - & - \\
\hline \multirow{3}{*}{ uDP4+ } & ${ }^{1} \mathrm{H}$ data & 81.35 & 18.65 & - & - & - & - & - & - \\
\hline & ${ }^{13}$ C data & 86.74 & 13.26 & - & - & - & - & - & - \\
\hline & All data & 96.61 & 3.39 & - & - & - & - & - & - \\
\hline \multirow{3}{*}{ DP4+ } & ${ }^{1} \mathrm{H}$ data & 99.19 & 0.81 & - & - & - & - & - & - \\
\hline & ${ }^{13} \mathrm{C}$ data & 99.95 & 0.05 & - & - & - & - & - & - \\
\hline & All data & 100.00 & 0.00 & - & - & - & - & - & - \\
\hline & & \multicolumn{8}{|c|}{ Level of theory: B3LYP/6-311G* (gas phase) } \\
\hline & & $74 a$ & 74b & - & - & - & - & - & - \\
\hline \multirow{3}{*}{ sDP4+ } & ${ }^{1} \mathrm{H}$ data & 86.55 & 13.45 & - & - & - & - & - & - \\
\hline & ${ }^{13} \mathrm{C}$ data & 99.88 & 0.12 & - & - & - & - & - & - \\
\hline & All data & 99.98 & 0.02 & - & - & - & - & - & - \\
\hline \multirow{3}{*}{ uDP4+ } & ${ }^{1} \mathrm{H}$ data & 73.79 & 26.21 & - & - & - & - & - & - \\
\hline & ${ }^{13} \mathrm{C}$ data & 55.71 & 44.29 & - & - & - & - & - & - \\
\hline & All data & 77.98 & 22.02 & - & - & - & - & - & - \\
\hline \multirow{3}{*}{ DP4+ } & ${ }^{1} \mathrm{H}$ data & 94.77 & 5.23 & - & - & - & - & - & - \\
\hline & ${ }^{13} \mathrm{C}$ data & 99.90 & 0.10 & - & - & - & - & - & - \\
\hline & All data & 99.99 & 0.01 & - & - & - & - & - & - \\
\hline & & \multicolumn{8}{|c|}{ Level of theory: B3LYP/6-311G** (gas phase) } \\
\hline & & $74 a$ & 74b & - & - & - & - & - & - \\
\hline \multirow{2}{*}{ sDP4+ } & ${ }^{1} \mathrm{H}$ data & 97.65 & 2.35 & - & - & - & - & - & - \\
\hline & ${ }^{13} \mathrm{C}$ data & 99.89 & 0.11 & - & - & - & - & - & - \\
\hline
\end{tabular}




\begin{tabular}{|c|c|c|c|c|c|c|c|c|c|}
\hline & All data & 100.00 & 0.00 & - & - & - & - & - & - \\
\hline & ${ }^{1}$ H data & 75.98 & 24.02 & - & - & - & - & - & - \\
\hline \multirow[t]{3}{*}{ uDP4+ } & ${ }^{13} \mathrm{C}$ data & 53.44 & 46.56 & - & - & - & - & - & - \\
\hline & All data & 78.41 & 21.59 & - & - & - & - & - & - \\
\hline & ${ }^{1} \mathrm{H}$ data & 99.24 & 0.76 & - & - & - & - & - & - \\
\hline \multirow[t]{5}{*}{ DP4+ } & ${ }^{13} \mathrm{C}$ data & 99.91 & 0.09 & - & - & - & - & - & - \\
\hline & All data & 100.00 & 0.00 & - & - & - & - & - & - \\
\hline & & \multicolumn{8}{|c|}{ Level of theory: B3LYP/6-311+G** (gas phase) } \\
\hline & & $74 a$ & $74 b$ & - & - & - & - & - & - \\
\hline & ${ }^{1} \mathrm{H}$ data & 98.12 & 1.88 & - & - & - & - & - & - \\
\hline \multirow[t]{3}{*}{ sDP4+ } & ${ }^{13} \mathrm{C}$ data & 98.70 & 1.30 & - & - & - & - & - & - \\
\hline & All data & 99.97 & 0.03 & - & - & - & - & - & - \\
\hline & ${ }^{1} \mathrm{H}$ data & 82.68 & 17.32 & - & - & - & - & - & - \\
\hline \multirow[t]{3}{*}{ uDP4+ } & ${ }^{13} \mathrm{C}$ data & 26.52 & 73.48 & - & - & - & - & - & - \\
\hline & All data & 63.27 & 36.73 & - & - & - & - & - & - \\
\hline & ${ }^{1} \mathrm{H}$ data & 99.60 & 0.40 & - & - & - & - & - & - \\
\hline \multirow[t]{5}{*}{ DP4+ } & ${ }^{13} \mathrm{C}$ data & 96.47 & 3.53 & - & - & - & - & - & - \\
\hline & All data & 99.99 & 0.01 & - & - & - & - & - & - \\
\hline & & \multicolumn{8}{|c|}{ Level of theory: B3LYP/6-31G* (solution, PCM) } \\
\hline & & $74 a$ & $74 b$ & - & - & - & - & - & - \\
\hline & ${ }^{1}$ H data & 90.66 & 9.34 & - & - & - & - & - & - \\
\hline \multirow[t]{3}{*}{ sDP4+ } & ${ }^{13}$ C data & 99.99 & 0.01 & - & - & - & - & - & - \\
\hline & All data & 100.00 & 0.00 & - & - & - & - & - & - \\
\hline & ${ }^{1} \mathrm{H}$ data & 88.57 & 11.43 & - & - & - & - & - & - \\
\hline \multirow[t]{3}{*}{$u$ DP4+ } & ${ }^{13} \mathrm{C}$ data & 98.85 & 1.15 & - & - & - & - & - & - \\
\hline & All data & 99.85 & 0.15 & - & - & - & - & - & - \\
\hline & ${ }^{1}$ H data & 98.69 & 1.31 & - & - & - & - & - & - \\
\hline \multirow[t]{5}{*}{ DP4+ } & ${ }^{13} \mathrm{C}$ data & 100.00 & 0.00 & - & - & - & - & - & - \\
\hline & All data & 100.00 & 0.00 & - & - & - & - & - & - \\
\hline & & \multicolumn{8}{|c|}{ Level of theory: B3LYP/6-31G** (solution, PCM) } \\
\hline & & $74 a$ & 74b & - & - & - & - & - & - \\
\hline & ${ }^{1} \mathrm{H}$ data & 96.25 & 3.75 & - & - & - & - & - & - \\
\hline \multirow[t]{3}{*}{ sDP4+ } & ${ }^{13} \mathrm{C}$ data & 99.97 & 0.03 & - & - & - & - & - & - \\
\hline & All data & 100.00 & 0.00 & - & - & - & - & - & - \\
\hline & ${ }^{1} \mathrm{H}$ data & 85.24 & 14.76 & - & - & - & - & - & - \\
\hline \multirow[t]{3}{*}{ uDP4+ } & ${ }^{13} \mathrm{C}$ data & 97.38 & 2.62 & - & - & - & - & - & - \\
\hline & All data & 99.54 & 0.46 & - & - & - & - & - & - \\
\hline & ${ }^{1} \mathrm{H}$ data & 99.33 & 0.67 & - & - & - & - & - & - \\
\hline \multirow[t]{5}{*}{ DP4+ } & ${ }^{13} \mathrm{C}$ data & 100.00 & 0.00 & - & - & - & - & - & - \\
\hline & All data & 100.00 & 0.00 & - & - & - & - & - & - \\
\hline & & \multicolumn{8}{|c|}{ Level of theory: B3LYP/6-31+G** (solution, PCM) } \\
\hline & & $74 a$ & 74b & - & - & - & - & - & - \\
\hline & ${ }^{1} \mathrm{H}$ data & 99.17 & 0.83 & - & - & - & - & - & - \\
\hline \multirow[t]{3}{*}{ sDP4+ } & ${ }^{13} \mathrm{C}$ data & 99.80 & 0.20 & - & - & - & - & - & - \\
\hline & All data & 100.00 & 0.00 & - & - & - & - & - & - \\
\hline & ${ }^{1} \mathrm{H}$ data & 90.95 & 9.05 & - & - & - & - & - & - \\
\hline uDP4+ & ${ }^{13} \mathrm{C}$ data & 87.70 & 12.30 & - & - & - & - & - & - \\
\hline & All data & 98.62 & 1.38 & - & - & - & - & - & - \\
\hline & ${ }^{1} \mathrm{H}$ data & 99.92 & 0.08 & - & - & - & - & - & - \\
\hline DP4+ & ${ }^{13} \mathrm{C}$ data & 99.97 & 0.03 & - & - & - & - & - & - \\
\hline & All data & 100.00 & 0.00 & - & - & - & - & - & - \\
\hline & & & & ! & B3 & $\mathrm{G}^{*}$ & $\overline{P C}$ & & \\
\hline & & $74 a$ & $74 b$ & - & - & - & - & - & - \\
\hline & ${ }^{1} \mathrm{H}$ data & 93.26 & 6.74 & - & - & - & - & - & - \\
\hline sDP4+ & ${ }^{13} \mathrm{C}$ data & 99.90 & 0.10 & - & - & - & - & - & - \\
\hline & All data & 99.99 & 0.01 & - & - & - & - & - & - \\
\hline
\end{tabular}




\begin{tabular}{|c|c|c|c|c|c|c|c|c|c|}
\hline & ${ }^{1} \mathrm{H}$ data & 76.10 & 23.90 & - & - & - & - & - & - \\
\hline \multirow[t]{3}{*}{ uDP4+ } & ${ }^{13} \mathrm{C}$ data & 51.72 & 48.28 & - & - & - & - & - & - \\
\hline & All data & 77.33 & 22.67 & - & - & - & - & - & - \\
\hline & ${ }^{1} \mathrm{H}$ data & 97.78 & 2.22 & - & - & - & - & - & - \\
\hline \multirow[t]{5}{*}{ DP4+ } & ${ }^{13} \mathrm{C}$ data & 99.91 & 0.09 & - & - & - & - & - & - \\
\hline & All data & 100.00 & 0.00 & - & - & - & - & - & - \\
\hline & & \multicolumn{8}{|c|}{ Level of theory: B3LYP/6-311G** (solution, PCM) } \\
\hline & & $74 a$ & 74b & - & - & - & - & - & - \\
\hline & ${ }^{1} \mathrm{H}$ data & 97.55 & 2.45 & - & - & - & - & - & - \\
\hline \multirow[t]{3}{*}{ sDP4+ } & ${ }^{13} \mathrm{C}$ data & 99.94 & 0.06 & - & - & - & - & - & - \\
\hline & All data & 100.00 & 0.00 & - & - & - & - & - & - \\
\hline & ${ }^{1} \mathrm{H}$ data & 36.61 & 63.39 & - & - & - & - & - & - \\
\hline \multirow[t]{3}{*}{ uDP4+ } & ${ }^{13} \mathrm{C}$ data & 54.07 & 45.93 & - & - & - & - & - & - \\
\hline & All data & 40.47 & 59.53 & - & - & - & - & - & - \\
\hline & ${ }^{1} \mathrm{H}$ data & 95.84 & 4.16 & - & - & - & - & - & - \\
\hline \multirow[t]{5}{*}{ DP4+ } & ${ }^{13} \mathrm{C}$ data & 99.95 & 0.05 & - & - & - & - & - & - \\
\hline & All data & 100.00 & 0.00 & - & - & - & - & - & - \\
\hline & & \multicolumn{8}{|c|}{ Level of theory: B3LYP/6-311+G** (solution, PCM) } \\
\hline & & $74 a$ & 74b & - & - & - & - & - & - \\
\hline & ${ }^{1}$ H data & 97.03 & 2.97 & - & - & - & - & - & - \\
\hline \multirow[t]{3}{*}{ sDP4+ } & ${ }^{13} \mathrm{C}$ data & 98.93 & 1.07 & - & - & - & - & - & - \\
\hline & All data & 99.97 & 0.03 & - & - & - & - & - & - \\
\hline & ${ }^{1} \mathrm{H}$ data & 75.24 & 24.76 & - & - & - & - & - & - \\
\hline \multirow[t]{3}{*}{ uDP4+ } & ${ }^{13} \mathrm{C}$ data & 27.72 & 72.28 & - & - & - & - & - & - \\
\hline & All data & 53.82 & 46.18 & - & - & - & - & - & - \\
\hline & ${ }^{1} \mathrm{H}$ data & 99.00 & 1.00 & - & - & - & - & - & - \\
\hline \multirow[t]{5}{*}{ DP4+ } & ${ }^{13} \mathrm{C}$ data & 97.26 & 2.74 & - & - & - & - & - & - \\
\hline & All data & 99.97 & 0.03 & - & - & - & - & - & - \\
\hline & & \multicolumn{8}{|c|}{ Level of theory: mPW1PW91/6-31G* (gas phase) } \\
\hline & & $74 a$ & 74b & - & - & - & - & - & - \\
\hline & ${ }^{1} \mathrm{H}$ data & 86.32 & 13.68 & - & - & - & - & - & - \\
\hline \multirow[t]{2}{*}{ sDP4+ } & ${ }^{13} \mathrm{C}$ data & 99.99 & 0.01 & - & - & - & - & - & - \\
\hline & All data & 100.00 & 0.00 & - & - & - & - & - & - \\
\hline \multirow{3}{*}{ uDP4+ } & ${ }^{1} \mathrm{H}$ data & 75.56 & 24.44 & - & - & - & - & - & - \\
\hline & ${ }^{13} \mathrm{C}$ data & 99.05 & 0.95 & - & - & - & - & - & - \\
\hline & All data & 99.69 & 0.31 & - & - & - & - & - & - \\
\hline \multirow{5}{*}{ DP4+ } & ${ }^{1} \mathrm{H}$ data & 95.12 & 4.88 & - & - & - & - & - & - \\
\hline & ${ }^{13} \mathrm{C}$ data & 100.00 & 0.00 & - & - & - & - & - & - \\
\hline & All data & 100.00 & 0.00 & - & - & - & - & - & - \\
\hline & & \multicolumn{8}{|c|}{ Level of theory: mPW1PW91/6-31G** (gas phase) } \\
\hline & & $74 a$ & 74b & - & - & - & - & - & - \\
\hline \multirow{3}{*}{ sDP4+ } & ${ }^{1} \mathrm{H}$ data & 96.12 & 3.88 & - & - & - & - & - & - \\
\hline & ${ }^{13} \mathrm{C}$ data & 99.99 & 0.01 & - & - & - & - & - & - \\
\hline & All data & 100.00 & 0.00 & - & - & - & - & - & - \\
\hline & ${ }^{1} \mathrm{H}$ data & 75.40 & 24.60 & - & - & - & - & - & - \\
\hline uDP4+ & ${ }^{13} \mathrm{C}$ data & 99.16 & 0.84 & - & - & - & - & - & - \\
\hline & All data & 99.72 & 0.28 & - & - & - & - & - & - \\
\hline & ${ }^{1} \mathrm{H}$ data & 98.70 & 1.30 & - & - & - & - & - & - \\
\hline DP4+ & ${ }^{13} \mathrm{C}$ data & 100.00 & 0.00 & - & - & - & - & - & - \\
\hline & All data & 100.00 & 0.00 & - & - & - & - & - & - \\
\hline & & & & 10 & $|P|$ & -3 & pr & & \\
\hline & & $74 a$ & $74 b$ & - & - & - & - & - & - \\
\hline & ${ }^{1} \mathrm{H}$ data & 98.23 & 1.77 & - & - & - & - & - & - \\
\hline sDP4+ & ${ }^{13} \mathrm{C}$ data & 99.84 & 0.16 & - & - & - & - & - & - \\
\hline & All data & 100.00 & 0.00 & - & - & - & - & - & - \\
\hline uDP4+ & ${ }^{1} \mathrm{H}$ data & 77.09 & 22.91 & - & - & - & - & - & - \\
\hline
\end{tabular}




\begin{tabular}{|c|c|c|c|c|c|c|c|c|c|}
\hline & ${ }^{13} \mathrm{C}$ data & 89.85 & 10.15 & - & - & - & - & - & - \\
\hline & All data & 96.75 & 3.25 & - & - & - & - & - & - \\
\hline & ${ }^{1} \mathrm{H}$ data & 99.47 & 0.53 & - & - & - & - & - & - \\
\hline \multirow[t]{5}{*}{ DP4+ } & ${ }^{13} \mathrm{C}$ data & 99.98 & 0.02 & - & - & - & - & - & - \\
\hline & All data & 100.00 & 0.00 & - & - & - & - & - & - \\
\hline & & \multicolumn{8}{|c|}{ Level of theory: mPW1PW91/6-311G* (gas phase) } \\
\hline & & $74 a$ & $74 b$ & - & - & - & - & - & - \\
\hline & ${ }^{1}$ H data & 91.66 & 8.34 & - & - & - & - & - & - \\
\hline \multirow[t]{3}{*}{ sDP4+ } & ${ }^{13}$ C data & 99.81 & 0.19 & - & - & - & - & - & - \\
\hline & All data & 99.98 & 0.02 & - & - & - & - & - & - \\
\hline & ${ }^{1} \mathrm{H}$ data & 70.20 & 29.80 & - & - & - & - & - & - \\
\hline \multirow[t]{3}{*}{ uDP4+ } & ${ }^{13} \mathrm{C}$ data & 67.87 & 32.13 & - & - & - & - & - & - \\
\hline & All data & 83.26 & 16.74 & - & - & - & - & - & - \\
\hline & ${ }^{1} \mathrm{H}$ data & 96.28 & 3.72 & - & - & - & - & - & - \\
\hline \multirow[t]{5}{*}{ DP4+ } & ${ }^{13} \mathrm{C}$ data & 99.91 & 0.09 & - & - & - & - & - & - \\
\hline & All data & 100.00 & 0.00 & - & - & - & - & - & - \\
\hline & & \multicolumn{8}{|c|}{ Level of theory: mPW1PW91/6-311G** (gas phase) } \\
\hline & & $74 a$ & 74b & - & - & - & - & - & - \\
\hline & ${ }^{1} \mathrm{H}$ data & 95.23 & 4.77 & - & - & - & - & - & - \\
\hline \multirow[t]{3}{*}{ sDP4+ } & ${ }^{13} \mathrm{C}$ data & 100.00 & 0.00 & - & - & - & - & - & - \\
\hline & All data & 100.00 & 0.00 & - & - & - & - & - & - \\
\hline & ${ }^{1} \mathrm{H}$ data & 23.78 & 76.22 & - & - & - & - & - & - \\
\hline \multirow[t]{3}{*}{ uDP4+ } & ${ }^{13} \mathrm{C}$ data & 3.16 & 96.84 & - & - & - & - & - & - \\
\hline & All data & 1.01 & 98.99 & - & - & - & - & - & - \\
\hline & ${ }^{1} \mathrm{H}$ data & 86.16 & 13.84 & - & - & - & - & - & - \\
\hline \multirow[t]{5}{*}{ DP4+ } & ${ }^{13} \mathrm{C}$ data & 100.00 & 0.00 & - & - & - & - & - & - \\
\hline & All data & 100.00 & 0.00 & - & - & - & - & - & - \\
\hline & & \multicolumn{8}{|c|}{ Level of theory: mPW1PW91/6-311+G** (gas phase) } \\
\hline & & $74 a$ & 74b & - & - & - & - & - & - \\
\hline & ${ }^{1} \mathrm{H}$ data & 98.14 & 1.86 & - & - & - & - & - & - \\
\hline \multirow[t]{2}{*}{ sDP4+ } & ${ }^{13} \mathrm{C}$ data & 98.07 & 1.93 & - & - & - & - & - & - \\
\hline & All data & 99.96 & 0.04 & - & - & - & - & - & - \\
\hline \multirow{3}{*}{ uDP4+ } & ${ }^{1} \mathrm{H}$ data & 78.92 & 21.08 & - & - & - & - & - & - \\
\hline & ${ }^{13}$ C data & 44.51 & 55.49 & - & - & - & - & - & - \\
\hline & All data & 75.02 & 24.98 & - & - & - & - & - & - \\
\hline \multirow{5}{*}{ DP4+ } & ${ }^{1} \mathrm{H}$ data & 99.50 & 0.50 & - & - & - & - & - & - \\
\hline & ${ }^{13} \mathrm{C}$ data & 97.61 & 2.39 & - & - & - & - & - & - \\
\hline & All data & 99.99 & 0.01 & - & - & - & - & - & - \\
\hline & & \multicolumn{8}{|c|}{ Level of theory: mPW1PW91/6-31G* (solution, PCM) } \\
\hline & & $74 a$ & $74 b$ & - & - & - & - & - & - \\
\hline \multirow{3}{*}{ sDP4+ } & ${ }^{1} \mathrm{H}$ data & 95.96 & 4.04 & - & - & - & - & - & - \\
\hline & ${ }^{13} \mathrm{C}$ data & 99.99 & 0.01 & - & - & - & - & - & - \\
\hline & All data & 100.00 & 0.00 & - & - & - & - & - & - \\
\hline \multirow{3}{*}{ uDP4+ } & ${ }^{1} \mathrm{H}$ data & 87.15 & 12.85 & - & - & - & - & - & - \\
\hline & ${ }^{13} \mathrm{C}$ data & 98.76 & 1.24 & - & - & - & - & - & - \\
\hline & All data & 99.82 & 0.18 & - & - & - & - & - & - \\
\hline & ${ }^{1} \mathrm{H}$ data & 99.38 & 0.62 & - & - & - & - & - & - \\
\hline DP4+ & ${ }^{13} \mathrm{C}$ data & 100.00 & 0.00 & - & - & - & - & - & - \\
\hline & All data & 100.00 & 0.00 & - & - & - & - & - & - \\
\hline & & & & of & $\mathbf{W}$ & 10 & on, & & \\
\hline & & $74 a$ & $74 b$ & - & - & - & - & - & - \\
\hline & ${ }^{1} \mathrm{H}$ data & 98.52 & 1.48 & - & - & - & - & - & - \\
\hline sDP4+ & ${ }^{13} \mathrm{C}$ data & 99.99 & 0.01 & - & - & - & - & - & - \\
\hline & All data & 100.00 & 0.00 & - & - & - & - & - & - \\
\hline & ${ }_{12}^{1} \mathrm{H}$ data & 86.33 & 13.67 & - & - & - & - & - & - \\
\hline uDP4+ & ${ }^{13} \mathrm{C}$ data & 98.70 & 1.30 & - & - & - & - & - & - \\
\hline
\end{tabular}




\begin{tabular}{|c|c|c|c|c|c|c|c|c|c|}
\hline & All data & 99.79 & 0.21 & - & - & - & - & - & - \\
\hline & ${ }^{1} \mathrm{H}$ data & 99.76 & 0.24 & - & - & - & - & - & - \\
\hline \multirow[t]{5}{*}{ DP4+ } & ${ }^{13} \mathrm{C}$ data & 100.00 & 0.00 & - & - & - & - & - & - \\
\hline & All data & 100.00 & 0.00 & - & - & - & - & - & - \\
\hline & & \multicolumn{8}{|c|}{ Level of theory: mPW1PW91/6-31+G** (solution, PCM) } \\
\hline & & $74 a$ & 74b & - & - & - & - & - & - \\
\hline & ${ }^{1} \mathrm{H}$ data & 98.68 & 1.32 & - & - & - & - & - & - \\
\hline \multirow[t]{3}{*}{ sDP4+ } & ${ }^{13} \mathrm{C}$ data & 99.91 & 0.09 & - & - & - & - & - & - \\
\hline & All data & 100.00 & 0.00 & - & - & - & - & - & - \\
\hline & ${ }^{1} \mathrm{H}$ data & 87.50 & 12.50 & - & - & - & - & - & - \\
\hline \multirow[t]{3}{*}{ uDP4+ } & ${ }^{13} \mathrm{C}$ data & 86.81 & 13.19 & - & - & - & - & - & - \\
\hline & All data & 97.88 & 2.12 & - & - & - & - & - & - \\
\hline & ${ }^{1} \mathrm{H}$ data & 99.81 & 0.19 & - & - & - & - & - & - \\
\hline \multirow[t]{5}{*}{ DP4+ } & ${ }^{13} \mathrm{C}$ data & 99.99 & 0.01 & - & - & - & - & - & - \\
\hline & All data & 100.00 & 0.00 & - & - & - & - & - & - \\
\hline & & \multicolumn{8}{|c|}{ Level of theory: mPW1PW91/6-311G* (solution, PCM) } \\
\hline & & $74 a$ & $74 b$ & - & - & - & - & - & - \\
\hline & ${ }^{1} \mathrm{H}$ data & 96.69 & 3.31 & - & - & - & - & - & - \\
\hline \multirow[t]{3}{*}{ sDP4+ } & ${ }^{13} \mathrm{C}$ data & 99.88 & 0.12 & - & - & - & - & - & - \\
\hline & All data & 100.00 & 0.00 & - & - & - & - & - & - \\
\hline & ${ }^{1}$ H data & 66.88 & 33.12 & - & - & - & - & - & - \\
\hline \multirow[t]{3}{*}{ uDP4+ } & ${ }^{13}$ C data & 70.55 & 29.45 & - & - & - & - & - & - \\
\hline & All data & 82.87 & 17.13 & - & - & - & - & - & - \\
\hline & ${ }^{1} \mathrm{H}$ data & 98.33 & 1.67 & - & - & - & - & - & - \\
\hline \multirow[t]{5}{*}{ DP4+ } & ${ }^{13} \mathrm{C}$ data & 99.95 & 0.05 & - & - & - & - & - & - \\
\hline & All data & 100.00 & 0.00 & - & - & - & - & - & - \\
\hline & & \multicolumn{8}{|c|}{ Level of theory: mPW1PW91/6-311G** (solution, PCM) } \\
\hline & & $74 a$ & 74b & - & - & - & - & - & - \\
\hline & ${ }^{1} \mathrm{H}$ data & 97.93 & 2.07 & - & - & - & - & - & - \\
\hline \multirow[t]{3}{*}{ sDP4+ } & ${ }^{13} \mathrm{C}$ data & 99.92 & 0.08 & - & - & - & - & - & - \\
\hline & All data & 100.00 & 0.00 & - & - & - & - & - & - \\
\hline & ${ }^{1} \mathrm{H}$ data & 26.35 & 73.65 & - & - & - & - & - & - \\
\hline \multirow[t]{3}{*}{ uDP4+ } & ${ }^{13} \mathrm{C}$ data & 67.63 & 32.37 & - & - & - & - & - & - \\
\hline & All data & 42.77 & 57.23 & - & - & - & - & - & - \\
\hline & ${ }^{1} \mathrm{H}$ data & 94.42 & 5.58 & - & - & - & - & - & - \\
\hline \multirow[t]{5}{*}{ DP4+ } & ${ }^{13}$ C data & 99.96 & 0.04 & - & - & - & - & - & - \\
\hline & All data & 100.00 & 0.00 & - & - & - & - & - & - \\
\hline & & \multicolumn{8}{|c|}{ Level of theory: mPW1PW91/6-311+G** (solution, PCM) } \\
\hline & & $74 a$ & 74b & - & - & - & - & - & - \\
\hline & ${ }^{1} \mathrm{H}$ data & 96.68 & 3.32 & - & - & - & - & - & - \\
\hline \multirow[t]{2}{*}{ sDP4+ } & ${ }^{13} \mathrm{C}$ data & 97.77 & 2.23 & - & - & - & - & - & - \\
\hline & All data & 99.92 & 0.08 & - & - & - & - & - & - \\
\hline \multirow{3}{*}{ uDP4+ } & ${ }^{1} \mathrm{H}$ data & 68.83 & 31.17 & - & - & - & - & - & - \\
\hline & ${ }^{13} \mathrm{C}$ data & 42.70 & 57.30 & - & - & - & - & - & - \\
\hline & All data & 62.20 & 37.80 & - & - & - & - & - & - \\
\hline \multirow{3}{*}{ DP4+ } & ${ }^{1} \mathrm{H}$ data & 98.47 & 1.53 & - & - & - & - & - & - \\
\hline & ${ }^{13} \mathrm{C}$ data & 97.04 & 2.96 & - & - & - & - & - & - \\
\hline & All data & 99.95 & 0.05 & - & - & - & - & - & - \\
\hline
\end{tabular}


sDP4+, $u$ DP4+ and DP4+ probabilities (\%) for compound 74b<smiles>C[C@@H](O)[C@@H](C)C(=O)c1ccccc1</smiles>

$74 a$<smiles>C[C@H](O)[C@@H](C)C(=O)c1ccccc1</smiles>

correct isomer

\begin{tabular}{|c|c|c|c|c|c|c|c|c|c|}
\hline & & \multicolumn{8}{|c|}{ Level of theory: B3LYP/6-31G* (gas phase) } \\
\hline & & $74 a$ & $74 b$ & - & - & - & - & - & - \\
\hline & ${ }^{1}$ H data & 15.58 & 84.42 & - & - & - & - & - & - \\
\hline \multirow[t]{2}{*}{ sDP4+ } & ${ }^{13}$ C data & 99.69 & 0.31 & - & - & - & - & - & - \\
\hline & All data & 98.37 & 1.63 & - & - & - & - & - & - \\
\hline \multirow{3}{*}{ uDP4+ } & ${ }^{1} \mathrm{H}$ data & 39.23 & 60.77 & - & - & - & - & - & - \\
\hline & ${ }^{13} \mathrm{C}$ data & 0.76 & 99.24 & - & - & - & - & - & - \\
\hline & All data & 0.49 & 99.51 & - & - & - & - & - & - \\
\hline \multirow{3}{*}{ DP4+ } & ${ }^{1} \mathrm{H}$ data & 10.65 & 89.35 & - & - & - & - & - & - \\
\hline & ${ }^{13} \mathrm{C}$ data & 71.45 & 28.55 & - & - & - & - & - & - \\
\hline & All data & 22.97 & 77.03 & - & - & - & - & - & - \\
\hline & & \multicolumn{8}{|c|}{ Level of theory: B3LYP/6-31G** (gas phase) } \\
\hline & & $74 a$ & 74b & - & - & - & - & - & - \\
\hline \multirow{3}{*}{ sDP4+ } & ${ }^{1}$ H data & 35.41 & 64.59 & - & - & - & - & - & - \\
\hline & ${ }^{13} \mathrm{C}$ data & 98.98 & 1.02 & - & - & - & - & - & - \\
\hline & All data & 98.15 & 1.85 & - & - & - & - & - & - \\
\hline \multirow{3}{*}{ uDP4+ } & ${ }^{1} \mathrm{H}$ data & 39.03 & 60.97 & - & - & - & - & - & - \\
\hline & ${ }^{13} \mathrm{C}$ data & 0.24 & 99.76 & - & - & - & - & - & - \\
\hline & All data & 0.15 & 99.85 & - & - & - & - & - & - \\
\hline \multirow{3}{*}{ DP4+ } & ${ }^{1} \mathrm{H}$ data & 25.97 & 74.03 & - & - & - & - & - & - \\
\hline & ${ }^{13} \mathrm{C}$ data & 19.05 & 80.95 & - & - & - & - & - & - \\
\hline & All data & 7.63 & 92.37 & - & - & - & - & - & - \\
\hline & & \multicolumn{8}{|c|}{ Level of theory: B3LYP/6-31+G** (gas phase) } \\
\hline & & $74 a$ & 74b & - & - & - & - & - & - \\
\hline \multirow{3}{*}{ sDP4+ } & ${ }^{1}$ H data & 70.90 & 29.10 & - & - & - & - & - & - \\
\hline & ${ }^{13} \mathrm{C}$ data & 88.20 & 11.80 & - & - & - & - & - & - \\
\hline & All data & 94.79 & 5.21 & - & - & - & - & - & - \\
\hline \multirow{3}{*}{ uDP4+ } & ${ }^{1}$ H data & 41.27 & 58.73 & - & - & - & - & - & - \\
\hline & ${ }^{13} \mathrm{C}$ data & 0.02 & 99.98 & - & - & - & - & - & - \\
\hline & All data & 0.01 & 99.99 & - & - & - & - & - & - \\
\hline \multirow{3}{*}{ DP4+ } & ${ }^{1} \mathrm{H}$ data & 63.12 & 36.88 & - & - & - & - & - & - \\
\hline & ${ }^{13} \mathrm{C}$ data & 0.15 & 99.85 & - & - & - & - & - & - \\
\hline & All data & 0.26 & 99.74 & - & - & - & - & - & - \\
\hline & & \multicolumn{8}{|c|}{ Level of theory: B3LYP/6-311G* (gas phase) } \\
\hline & & $74 a$ & 74b & - & - & - & - & - & - \\
\hline \multirow{3}{*}{ sDP4+ } & ${ }^{1} \mathrm{H}$ data & 43.51 & 56.49 & - & - & - & - & - & - \\
\hline & ${ }^{13}$ C data & 91.96 & 8.04 & - & - & - & - & - & - \\
\hline & All data & 89.80 & 10.20 & - & - & - & - & - & - \\
\hline \multirow{3}{*}{ uDP4+ } & ${ }^{1} \mathrm{H}$ data & 35.43 & 64.57 & - & - & - & - & - & - \\
\hline & ${ }^{13} \mathrm{C}$ data & 0.03 & 99.97 & - & - & - & - & - & - \\
\hline & All data & 0.02 & 99.98 & - & - & - & - & - & - \\
\hline \multirow{3}{*}{ DP4+ } & ${ }^{1} \mathrm{H}$ data & 29.71 & 70.29 & - & - & - & - & - & - \\
\hline & ${ }^{13} \mathrm{C}$ data & 0.33 & 99.67 & - & - & - & - & - & - \\
\hline & All data & 0.14 & 99.86 & - & - & - & - & - & - \\
\hline & & \multicolumn{8}{|c|}{ Level of theory: B3LYP/6-311G** (gas phase) } \\
\hline & & $74 a$ & $74 b$ & - & - & - & - & - & - \\
\hline \multirow{2}{*}{ sDP4+ } & ${ }^{1} \mathrm{H}$ data & 85.03 & 14.97 & - & - & - & - & - & - \\
\hline & ${ }^{13}$ C data & 87.31 & 12.69 & - & - & - & - & - & - \\
\hline
\end{tabular}




\begin{tabular}{|c|c|c|c|c|c|c|c|c|c|}
\hline & All data & 97.50 & 2.50 & - & - & - & - & - & - \\
\hline & ${ }^{1} \mathrm{H}$ data & 16.15 & 83.85 & - & - & - & - & - & - \\
\hline \multirow[t]{3}{*}{ uDP4+ } & ${ }^{13} \mathrm{C}$ data & 0.02 & 99.98 & - & - & - & - & - & - \\
\hline & All data & 0.00 & 100.00 & - & - & - & - & - & - \\
\hline & ${ }^{1} \mathrm{H}$ data & 52.25 & 47.75 & - & - & - & - & - & - \\
\hline \multirow[t]{5}{*}{ DP4+ } & ${ }^{13} \mathrm{C}$ data & 0.15 & 99.85 & - & - & - & - & - & - \\
\hline & All data & 0.16 & 99.84 & - & - & - & - & - & - \\
\hline & & \multicolumn{8}{|c|}{ Level of theory: B3LYP/6-311+G** (gas phase) } \\
\hline & & $74 a$ & $74 b$ & - & - & - & - & - & - \\
\hline & ${ }^{1}$ H data & 88.10 & 11.90 & - & - & - & - & - & - \\
\hline \multirow[t]{3}{*}{ sDP4+ } & ${ }^{13} \mathrm{C}$ data & 58.02 & 41.98 & - & - & - & - & - & - \\
\hline & All data & 91.09 & 8.91 & - & - & - & - & - & - \\
\hline & ${ }^{1} \mathrm{H}$ data & 36.84 & 63.16 & - & - & - & - & - & - \\
\hline \multirow[t]{3}{*}{ uDP4+ } & ${ }^{13} \mathrm{C}$ data & 0.00 & 100.00 & - & - & - & - & - & - \\
\hline & All data & 0.00 & 100.00 & - & - & - & - & - & - \\
\hline & ${ }^{1} \mathrm{H}$ data & 81.19 & 18.81 & - & - & - & - & - & - \\
\hline \multirow[t]{5}{*}{ DP4+ } & ${ }^{13} \mathrm{C}$ data & 0.00 & 100.00 & - & - & - & - & - & - \\
\hline & All data & 0.02 & 99.98 & - & - & - & - & - & - \\
\hline & & \multicolumn{8}{|c|}{ Level of theory: B3LYP/6-31G* (solution, PCM) } \\
\hline & & $74 a$ & $74 b$ & - & - & - & - & - & - \\
\hline & ${ }^{1} \mathrm{H}$ data & 32.48 & 67.52 & - & - & - & - & - & - \\
\hline \multirow[t]{3}{*}{ sDP4+ } & ${ }^{13} \mathrm{C}$ data & 99.86 & 0.14 & - & - & - & - & - & - \\
\hline & All data & 99.71 & 0.29 & - & - & - & - & - & - \\
\hline & ${ }^{1} \mathrm{H}$ data & 48.87 & 51.13 & - & - & - & - & - & - \\
\hline \multirow[t]{3}{*}{ uDP4+ } & ${ }^{13}$ C data & 0.44 & 99.56 & - & - & - & - & - & - \\
\hline & All data & 0.42 & 99.58 & - & - & - & - & - & - \\
\hline & ${ }^{1} \mathrm{H}$ data & 31.49 & 68.51 & - & - & - & - & - & - \\
\hline \multirow[t]{5}{*}{ DP4+ } & ${ }^{13} \mathrm{C}$ data & 75.69 & 24.31 & - & - & - & - & - & - \\
\hline & All data & 58.87 & 41.13 & - & - & - & - & - & - \\
\hline & & \multicolumn{8}{|c|}{ Level of theory: B3LYP/6-31G** (solution, PCM) } \\
\hline & & $74 a$ & $74 b$ & - & - & - & - & - & - \\
\hline & ${ }^{1} \mathrm{H}$ data & 66.47 & 33.53 & - & - & - & - & - & - \\
\hline \multirow[t]{3}{*}{ sDP4+ } & ${ }^{13} \mathrm{C}$ data & 99.29 & 0.71 & - & - & - & - & - & - \\
\hline & All data & 99.64 & 0.36 & - & - & - & - & - & - \\
\hline & ${ }^{1} \mathrm{H}$ data & 47.76 & 52.24 & - & - & - & - & - & - \\
\hline \multirow[t]{3}{*}{ uDP4+ } & ${ }^{13} \mathrm{C}$ data & 0.19 & 99.81 & - & - & - & - & - & - \\
\hline & All data & 0.17 & 99.83 & - & - & - & - & - & - \\
\hline & ${ }^{1} \mathrm{H}$ data & 64.45 & 35.55 & - & - & - & - & - & - \\
\hline \multirow[t]{5}{*}{ DP4+ } & ${ }^{13} \mathrm{C}$ data & 20.96 & 79.04 & - & - & - & - & - & - \\
\hline & All data & 32.46 & 67.54 & - & - & - & - & - & - \\
\hline & & \multicolumn{8}{|c|}{ Level of theory: B3LYP/6-31+G** (solution, PCM) } \\
\hline & & $74 a$ & $74 b$ & - & - & - & - & - & - \\
\hline & ${ }^{1} \mathrm{H}$ data & 88.90 & 11.10 & - & - & - & - & - & - \\
\hline \multirow[t]{3}{*}{ sDP4+ } & ${ }^{13} \mathrm{C}$ data & 90.76 & 9.24 & - & - & - & - & - & - \\
\hline & All data & 98.74 & 1.26 & - & - & - & - & - & - \\
\hline & ${ }^{1} \mathrm{H}$ data & 64.27 & 35.73 & - & - & - & - & - & - \\
\hline uDP4+ & ${ }^{13} \mathrm{C}$ data & 0.02 & 99.98 & - & - & - & - & - & - \\
\hline & All data & 0.03 & 99.97 & - & - & - & - & - & - \\
\hline & ${ }^{1} \mathrm{H}$ data & 93.51 & 6.49 & - & - & - & - & - & - \\
\hline DP4+ & ${ }^{13} \mathrm{C}$ data & 0.19 & 99.81 & - & - & - & - & - & - \\
\hline & All data & 2.67 & 97.33 & - & - & - & - & - & - \\
\hline & & & & el & B3 & $\mathrm{G}^{*}$ & $\overrightarrow{P C}$ & & \\
\hline & & $74 a$ & $74 b$ & - & - & - & - & - & - \\
\hline & ${ }^{1} \mathrm{H}$ data & 50.62 & 49.38 & - & - & - & - & - & - \\
\hline sDP4+ & ${ }^{13} \mathrm{C}$ data & 94.07 & 5.93 & - & - & - & - & - & - \\
\hline & All data & 94.20 & 5.80 & - & - & - & - & - & - \\
\hline
\end{tabular}




\begin{tabular}{|c|c|c|c|c|c|c|c|c|c|}
\hline & ${ }^{1} \mathrm{H}$ data & 30.51 & 69.49 & - & - & - & - & - & - \\
\hline \multirow[t]{3}{*}{ uDP4+ } & ${ }^{13} \mathrm{C}$ data & 0.03 & 99.97 & - & - & - & - & - & - \\
\hline & All data & 0.01 & 99.99 & - & - & - & - & - & - \\
\hline & ${ }^{1} \mathrm{H}$ data & 31.04 & 68.96 & - & - & - & - & - & - \\
\hline \multirow[t]{5}{*}{ DP4+ } & ${ }^{13} \mathrm{C}$ data & 0.44 & 99.56 & - & - & - & - & - & - \\
\hline & All data & 0.20 & 99.80 & - & - & - & - & - & - \\
\hline & & \multicolumn{8}{|c|}{ Level of theory: B3LYP/6-311G** (solution, PCM) } \\
\hline & & $74 a$ & 74b & - & - & - & - & - & - \\
\hline & ${ }^{1} \mathrm{H}$ data & 87.68 & 12.32 & - & - & - & - & - & - \\
\hline \multirow[t]{3}{*}{ sDP4+ } & ${ }^{13} \mathrm{C}$ data & 93.47 & 6.53 & - & - & - & - & - & - \\
\hline & All data & 99.03 & 0.97 & - & - & - & - & - & - \\
\hline & ${ }^{1} \mathrm{H}$ data & 8.52 & 91.48 & - & - & - & - & - & - \\
\hline \multirow[t]{3}{*}{ uDP4+ } & ${ }^{13} \mathrm{C}$ data & 0.02 & 99.98 & - & - & - & - & - & - \\
\hline & All data & 0.00 & 100.00 & - & - & - & - & - & - \\
\hline & ${ }^{1} \mathrm{H}$ data & 39.85 & 60.15 & - & - & - & - & - & - \\
\hline \multirow[t]{5}{*}{ DP4+ } & ${ }^{13} \mathrm{C}$ data & 0.32 & 99.68 & - & - & - & - & - & - \\
\hline & All data & 0.21 & 99.79 & - & - & - & - & - & - \\
\hline & & \multicolumn{8}{|c|}{ Level of theory: B3LYP/6-311+G** (solution, PCM) } \\
\hline & & $74 a$ & 74b & - & - & - & - & - & - \\
\hline & ${ }^{1}$ H data & 92.08 & 7.92 & - & - & - & - & - & - \\
\hline \multirow[t]{3}{*}{ sDP4+ } & ${ }^{13} \mathrm{C}$ data & 59.57 & 40.43 & - & - & - & - & - & - \\
\hline & All data & 94.49 & 5.51 & - & - & - & - & - & - \\
\hline & ${ }^{1} \mathrm{H}$ data & 33.56 & 66.44 & - & - & - & - & - & - \\
\hline \multirow[t]{3}{*}{ uDP4+ } & ${ }^{13} \mathrm{C}$ data & 0.00 & 100.00 & - & - & - & - & - & - \\
\hline & All data & 0.00 & 100.00 & - & - & - & - & - & - \\
\hline & ${ }^{1} \mathrm{H}$ data & 85.46 & 14.54 & - & - & - & - & - & - \\
\hline \multirow[t]{5}{*}{ DP4+ } & ${ }^{13} \mathrm{C}$ data & 0.01 & 99.99 & - & - & - & - & - & - \\
\hline & All data & 0.03 & 99.97 & - & - & - & - & - & - \\
\hline & & \multicolumn{8}{|c|}{ Level of theory: mPW1PW91/6-31G* (gas phase) } \\
\hline & & $74 a$ & 74b & - & - & - & - & - & - \\
\hline & ${ }^{1} \mathrm{H}$ data & 30.64 & 69.36 & - & - & - & - & - & - \\
\hline \multirow[t]{3}{*}{ sDP4+ } & ${ }^{13} \mathrm{C}$ data & 99.62 & 0.38 & - & - & - & - & - & - \\
\hline & All data & 99.14 & 0.86 & - & - & - & - & - & - \\
\hline & ${ }^{1} \mathrm{H}$ data & 33.94 & 66.06 & - & - & - & - & - & - \\
\hline \multirow[t]{3}{*}{ uDP4+ } & ${ }^{13} \mathrm{C}$ data & 1.63 & 98.37 & - & - & - & - & - & - \\
\hline & All data & 0.85 & 99.15 & - & - & - & - & - & - \\
\hline & ${ }^{1} \mathrm{H}$ data & 18.50 & 81.50 & - & - & - & - & - & - \\
\hline \multirow[t]{5}{*}{ DP4+ } & ${ }^{13} \mathrm{C}$ data & 81.29 & 18.71 & - & - & - & - & - & - \\
\hline & All data & 49.65 & 50.35 & - & - & - & - & - & - \\
\hline & & \multicolumn{8}{|c|}{ Level of theory: mPW1PW91/6-31G** (gas phase) } \\
\hline & & $74 a$ & $74 b$ & - & - & - & - & - & - \\
\hline & ${ }^{1} \mathrm{H}$ data & 69.76 & 30.24 & - & - & - & - & - & - \\
\hline \multirow[t]{2}{*}{ sDP4+ } & ${ }^{13} \mathrm{C}$ data & 99.18 & 0.82 & - & - & - & - & - & - \\
\hline & All data & 99.64 & 0.36 & - & - & - & - & - & - \\
\hline & ${ }^{1} \mathrm{H}$ data & 37.27 & 62.73 & - & - & - & - & - & - \\
\hline uDP4+ & ${ }^{13} \mathrm{C}$ data & 0.24 & 99.76 & - & - & - & - & - & - \\
\hline & All data & 0.14 & 99.86 & - & - & - & - & - & - \\
\hline & ${ }^{1} \mathrm{H}$ data & 57.82 & 42.18 & - & - & - & - & - & - \\
\hline DP4+ & ${ }^{13} \mathrm{C}$ data & 22.35 & 77.65 & - & - & - & - & - & - \\
\hline & All data & 28.29 & 71.71 & - & - & - & - & - & - \\
\hline & & & & 10 & $\mathrm{PI}$ & -3 & $\mathrm{pr}$ & & \\
\hline & & $74 a$ & $74 b$ & - & - & - & - & - & - \\
\hline & ${ }^{1} \mathrm{H}$ data & 83.26 & 16.74 & - & - & - & - & - & - \\
\hline sDP4+ & ${ }^{13} \mathrm{C}$ data & 72.21 & 27.79 & - & - & - & - & - & - \\
\hline & All data & 92.82 & 7.18 & - & - & - & - & - & - \\
\hline uDP4+ & ${ }^{1} \mathrm{H}$ data & 37.90 & 62.10 & - & - & - & - & - & - \\
\hline
\end{tabular}




\begin{tabular}{|c|c|c|c|c|c|c|c|c|c|}
\hline & ${ }^{13} \mathrm{C}$ data & 0.01 & 99.99 & - & - & - & - & - & - \\
\hline & All data & 0.01 & 99.99 & - & - & - & - & - & - \\
\hline & ${ }^{1} \mathrm{H}$ data & 75.22 & 24.78 & - & - & - & - & - & - \\
\hline \multirow[t]{5}{*}{ DP4+ } & ${ }^{13} \mathrm{C}$ data & 0.04 & 99.96 & - & - & - & - & - & - \\
\hline & All data & 0.11 & 99.89 & - & - & - & - & - & - \\
\hline & & \multicolumn{8}{|c|}{ Level of theory: mPW1PW91/6-311G* (gas phase) } \\
\hline & & $74 a$ & 74b & - & - & - & - & - & - \\
\hline & ${ }^{1} \mathrm{H}$ data & 55.87 & 44.13 & - & - & - & - & - & - \\
\hline \multirow[t]{2}{*}{$s D P 4+$} & ${ }^{13} \mathrm{C}$ data & 77.82 & 22.18 & - & - & - & - & - & - \\
\hline & All data & 81.62 & 18.38 & - & - & - & - & - & - \\
\hline \multirow{3}{*}{ uDP4+ } & ${ }^{1} \mathrm{H}$ data & 28.77 & 71.23 & - & - & - & - & - & - \\
\hline & ${ }^{13} \mathrm{C}$ data & 0.02 & 99.98 & - & - & - & - & - & - \\
\hline & All data & 0.01 & 99.99 & - & - & - & - & - & - \\
\hline \multirow{5}{*}{ DP4+ } & ${ }^{1} \mathrm{H}$ data & 33.83 & 66.17 & - & - & - & - & - & - \\
\hline & ${ }^{13} \mathrm{C}$ data & 0.06 & 99.94 & - & - & - & - & - & - \\
\hline & All data & 0.03 & 99.97 & - & - & - & - & - & - \\
\hline & & \multicolumn{8}{|c|}{ Level of theory: mPW1PW91/6-311G** (gas phase) } \\
\hline & & $74 a$ & 74b & - & - & - & - & - & - \\
\hline \multirow{3}{*}{ sDP4+ } & ${ }^{1} \mathrm{H}$ data & 94.19 & 5.81 & - & - & - & - & - & - \\
\hline & ${ }^{13} \mathrm{C}$ data & 100.00 & 0.00 & - & - & - & - & - & - \\
\hline & All data & 100.00 & 0.00 & - & - & - & - & - & - \\
\hline \multirow{3}{*}{ uDP4+ } & ${ }^{1} \mathrm{H}$ data & 18.37 & 81.63 & - & - & - & - & - & - \\
\hline & ${ }^{13} \mathrm{C}$ data & 3.95 & 96.05 & - & - & - & - & - & - \\
\hline & All data & 0.92 & 99.08 & - & - & - & - & - & - \\
\hline \multirow{3}{*}{ DP4+ } & ${ }^{1} \mathrm{H}$ data & 78.49 & 21.51 & - & - & - & - & - & - \\
\hline & ${ }^{13} \mathrm{C}$ data & 100.00 & 0.00 & - & - & - & - & - & - \\
\hline & All data & 100.00 & 0.00 & - & - & - & - & - & - \\
\hline & & \multicolumn{8}{|c|}{ Level of theory: mPW1PW91/6-311+G** (gas phase) } \\
\hline & & $74 a$ & 74b & - & - & - & - & - & - \\
\hline \multirow{3}{*}{ sDP4+ } & ${ }^{1} \mathrm{H}$ data & 94.70 & 5.30 & - & - & - & - & - & - \\
\hline & ${ }^{13} \mathrm{C}$ data & 21.88 & 78.12 & - & - & - & - & - & - \\
\hline & All data & 83.36 & 16.64 & - & - & - & - & - & - \\
\hline \multirow{3}{*}{ uDP4+ } & ${ }^{1} \mathrm{H}$ data & 37.87 & 62.13 & - & - & - & - & - & - \\
\hline & ${ }^{13} \mathrm{C}$ data & 0.00 & 100.00 & - & - & - & - & - & - \\
\hline & All data & 0.00 & 100.00 & - & - & - & - & - & - \\
\hline \multirow{5}{*}{ DP4+ } & ${ }^{1} \mathrm{H}$ data & 91.60 & 8.40 & - & - & - & - & - & - \\
\hline & ${ }^{13} \mathrm{C}$ data & 0.00 & 100.00 & - & - & - & - & - & - \\
\hline & All data & 0.00 & 100.00 & - & - & - & - & - & - \\
\hline & & \multicolumn{8}{|c|}{ Level of theory: mPW1PW91/6-31G* (solution, PCM) } \\
\hline & & $74 a$ & 74b & - & - & - & - & - & - \\
\hline \multirow{3}{*}{ sDP4+ } & ${ }^{1} \mathrm{H}$ data & 56.46 & 43.54 & - & - & - & - & - & - \\
\hline & ${ }^{13} \mathrm{C}$ data & 99.78 & 0.22 & - & - & - & - & - & - \\
\hline & All data & 99.83 & 0.17 & - & - & - & - & - & - \\
\hline \multirow{3}{*}{ uDP4+ } & ${ }^{1} \mathrm{H}$ data & 43.77 & 56.23 & - & - & - & - & - & - \\
\hline & ${ }^{13} \mathrm{C}$ data & 0.79 & 99.21 & - & - & - & - & - & - \\
\hline & All data & 0.61 & 99.39 & - & - & - & - & - & - \\
\hline & ${ }^{1} \mathrm{H}$ data & 50.23 & 49.77 & - & - & - & - & - & - \\
\hline DP4+ & ${ }^{13} \mathrm{C}$ data & 78.62 & 21.38 & - & - & - & - & - & - \\
\hline & All data & 78.77 & 21.23 & - & - & - & - & - & - \\
\hline & & & & of $t$ & $\mathbf{W}$ & 1( & on, & & \\
\hline & & $74 a$ & $74 b$ & - & - & - & - & - & - \\
\hline & ${ }^{1} \mathrm{H}$ data & 86.00 & 14.00 & - & - & - & - & - & - \\
\hline sDP4+ & ${ }^{13} \mathrm{C}$ data & 99.58 & 0.42 & - & - & - & - & - & - \\
\hline & All data & 99.93 & 0.07 & - & - & - & - & - & - \\
\hline & ${ }^{1} \mathrm{H}$ data & 47.18 & 52.82 & - & - & - & - & - & - \\
\hline UDP4+ & ${ }^{13} \mathrm{C}$ data & 0.13 & 99.87 & - & - & - & - & - & - \\
\hline
\end{tabular}




\begin{tabular}{|c|c|c|c|c|c|c|c|c|c|}
\hline & All data & 0.12 & 99.88 & - & - & - & - & - & - \\
\hline & ${ }^{1} \mathrm{H}$ data & 84.59 & 15.41 & - & - & - & - & - & - \\
\hline \multirow[t]{5}{*}{ DP4+ } & ${ }^{13} \mathrm{C}$ data & 23.75 & 76.25 & - & - & - & - & - & - \\
\hline & All data & 63.09 & 36.91 & - & - & - & - & - & - \\
\hline & & \multicolumn{8}{|c|}{ Level of theory: mPW1PW91/6-31+G** (solution, PCM) } \\
\hline & & $74 a$ & 74b & - & - & - & - & - & - \\
\hline & ${ }^{1} \mathrm{H}$ data & 93.37 & 6.63 & - & - & - & - & - & - \\
\hline \multirow[t]{3}{*}{ sDP4+ } & ${ }^{13} \mathrm{C}$ data & 84.23 & 15.77 & - & - & - & - & - & - \\
\hline & All data & 98.69 & 1.31 & - & - & - & - & - & - \\
\hline & ${ }^{1} \mathrm{H}$ data & 58.27 & 41.73 & - & - & - & - & - & - \\
\hline \multirow[t]{3}{*}{ uDP4+ } & ${ }^{13} \mathrm{C}$ data & 0.01 & 99.99 & - & - & - & - & - & - \\
\hline & All data & 0.01 & 99.99 & - & - & - & - & - & - \\
\hline & ${ }^{1} \mathrm{H}$ data & 95.16 & 4.84 & - & - & - & - & - & - \\
\hline \multirow[t]{5}{*}{ DP4+ } & ${ }^{13} \mathrm{C}$ data & 0.03 & 99.97 & - & - & - & - & - & - \\
\hline & All data & 0.63 & 99.37 & - & - & - & - & - & - \\
\hline & & \multicolumn{8}{|c|}{ Level of theory: mPW1PW91/6-311G* (solution, PCM) } \\
\hline & & $74 a$ & $74 b$ & - & - & - & - & - & - \\
\hline & ${ }^{1} \mathrm{H}$ data & 65.60 & 34.40 & - & - & - & - & - & - \\
\hline \multirow[t]{3}{*}{ sDP4+ } & ${ }^{13} \mathrm{C}$ data & 87.41 & 12.59 & - & - & - & - & - & - \\
\hline & All data & 92.98 & 7.02 & - & - & - & - & - & - \\
\hline & ${ }^{1}$ H data & 12.31 & 87.69 & - & - & - & - & - & - \\
\hline \multirow[t]{3}{*}{ uDP4+ } & ${ }^{13}$ C data & 0.01 & 99.99 & - & - & - & - & - & - \\
\hline & All data & 0.00 & 100.00 & - & - & - & - & - & - \\
\hline & ${ }^{1} \mathrm{H}$ data & 21.11 & 78.89 & - & - & - & - & - & - \\
\hline \multirow[t]{5}{*}{ DP4+ } & ${ }^{13} \mathrm{C}$ data & 0.07 & 99.93 & - & - & - & - & - & - \\
\hline & All data & 0.02 & 99.98 & - & - & - & - & - & - \\
\hline & & \multicolumn{8}{|c|}{ Level of theory: mPW1PW91/6-311G** (solution, PCM) } \\
\hline & & $74 a$ & 74b & - & - & - & - & - & - \\
\hline & ${ }^{1} \mathrm{H}$ data & 94.89 & 5.11 & - & - & - & - & - & - \\
\hline \multirow[t]{2}{*}{ sDP4+ } & ${ }^{13} \mathrm{C}$ data & 75.13 & 24.87 & - & - & - & - & - & - \\
\hline & All data & 98.25 & 1.75 & - & - & - & - & - & - \\
\hline \multirow{3}{*}{ uDP4+ } & ${ }^{1} \mathrm{H}$ data & 9.08 & 90.92 & - & - & - & - & - & - \\
\hline & ${ }^{13} \mathrm{C}$ data & 0.01 & 99.99 & - & - & - & - & - & - \\
\hline & All data & 0.00 & 100.00 & - & - & - & - & - & - \\
\hline \multirow{5}{*}{ DP4+ } & ${ }^{1} \mathrm{H}$ data & 64.94 & 35.06 & - & - & - & - & - & - \\
\hline & ${ }^{13} \mathrm{C}$ data & 0.03 & 99.97 & - & - & - & - & - & - \\
\hline & All data & 0.05 & 99.95 & - & - & - & - & - & - \\
\hline & & \multicolumn{8}{|c|}{ Level of theory: mPW1PW91/6-311+G** (solution, PCM) } \\
\hline & & $74 a$ & $74 b$ & - & - & - & - & - & - \\
\hline \multirow{3}{*}{ sDP4+ } & ${ }^{1} \mathrm{H}$ data & 96.31 & 3.69 & - & - & - & - & - & - \\
\hline & ${ }^{13} \mathrm{C}$ data & 25.09 & 74.91 & - & - & - & - & - & - \\
\hline & All data & 89.75 & 10.25 & - & - & - & - & - & - \\
\hline \multirow{3}{*}{ uDP4+ } & ${ }^{1} \mathrm{H}$ data & 37.01 & 62.99 & - & - & - & - & - & - \\
\hline & ${ }^{13} \mathrm{C}$ data & 0.00 & 100.00 & - & - & - & - & - & - \\
\hline & All data & 0.00 & 100.00 & - & - & - & - & - & - \\
\hline \multirow{3}{*}{ DP4+ } & ${ }^{1} \mathrm{H}$ data & 93.89 & 6.11 & - & - & - & - & - & - \\
\hline & ${ }^{13} \mathrm{C}$ data & 0.00 & 100.00 & - & - & - & - & - & - \\
\hline & All data & 0.00 & 100.00 & - & - & - & - & - & - \\
\hline
\end{tabular}


$s \mathrm{DP} 4+, u \mathrm{DP} 4+$ and DP4+ probabilities (\%) for compound 75a<smiles>CC(C)C[C@H](C)[C@@H](O)[C@H](C)C(=O)O</smiles>

$75 a$<smiles>CC(C)C[C@H](C)[C@@H](O)[C@H](C)C(=O)O</smiles>

$75 b$<smiles>CC(C)C[C@H](C)[C@@H](O)[C@H](C)C(=O)O</smiles>

$75 c$<smiles>CC(C)C[C@H]1CCC[C@H]1C(=O)O</smiles>

$75 d$

\begin{tabular}{|c|c|c|c|c|c|c|c|c|c|}
\hline & & \multicolumn{8}{|c|}{ Level of theory: B3LYP/6-31G* (gas phase) } \\
\hline & & $75 a$ & $75 \mathrm{~b}$ & $75 \mathrm{c}$ & 75d & - & - & - & - \\
\hline & ${ }^{1} \mathrm{H}$ data & 0.19 & 0.02 & 24.01 & 75.78 & - & - & - & - \\
\hline \multirow[t]{2}{*}{ sDP4+ } & ${ }^{13} \mathrm{C}$ data & 41.23 & 35.46 & 0.50 & 22.81 & - & - & - & - \\
\hline & All data & 0.45 & 0.03 & 0.69 & 98.82 & - & - & - & - \\
\hline & ${ }^{1} \mathrm{H}$ data & 3.68 & 0.37 & 38.84 & 57.12 & - & - & - & - \\
\hline \multirow[t]{2}{*}{ uDP4+ } & ${ }^{13} \mathrm{C}$ data & 96.13 & 0.34 & 0.01 & 3.52 & - & - & - & - \\
\hline & All data & 63.68 & 0.02 & 0.05 & 36.25 & - & - & - & - \\
\hline \multirow{6}{*}{ DP4+ } & ${ }^{1} \mathrm{H}$ data & 0.01 & 0.00 & 17.73 & 82.26 & - & - & - & - \\
\hline & ${ }^{13} \mathrm{C}$ data & 97.72 & 0.30 & 0.00 & 1.98 & - & - & - & - \\
\hline & All data & 0.80 & 0.00 & 0.00 & 99.20 & - & - & - & - \\
\hline & & \multicolumn{8}{|c|}{ Level of theory: B3LYP/6-31G** (gas phase) } \\
\hline & & $75 a$ & $75 b$ & $75 c$ & 75d & - & - & - & - \\
\hline & ${ }^{1} \mathrm{H}$ data & 0.06 & 0.14 & 49.28 & 50.52 & - & - & - & - \\
\hline \multirow[t]{3}{*}{ sDP4+ } & ${ }^{13} \mathrm{C}$ data & 33.93 & 41.74 & 0.75 & 23.58 & - & - & - & - \\
\hline & All data & 0.16 & 0.47 & 2.98 & 96.39 & - & - & - & - \\
\hline & ${ }^{1} \mathrm{H}$ data & 5.80 & 1.72 & 51.93 & 40.54 & - & - & - & - \\
\hline \multirow[t]{3}{*}{ uDP4+ } & ${ }^{13} \mathrm{C}$ data & 92.01 & 0.22 & 0.02 & 7.75 & - & - & - & - \\
\hline & All data & 62.87 & 0.05 & 0.09 & 37.00 & - & - & - & - \\
\hline & ${ }^{1} \mathrm{H}$ data & 0.01 & 0.01 & 55.54 & 44.44 & - & - & - & - \\
\hline \multirow[t]{5}{*}{ DP4+ } & ${ }^{13} \mathrm{C}$ data & 94.21 & 0.28 & 0.00 & 5.51 & - & - & - & - \\
\hline & All data & 0.28 & 0.00 & 0.01 & 99.72 & - & - & - & - \\
\hline & & \multicolumn{8}{|c|}{ Level of theory: B3LYP/6-31+G** (gas phase) } \\
\hline & & $75 a$ & $75 b$ & $75 c$ & 75d & - & - & - & - \\
\hline & ${ }^{1} \mathrm{H}$ data & 44.45 & 0.13 & 42.92 & 12.50 & - & - & - & - \\
\hline \multirow[t]{2}{*}{ sDP4+ } & ${ }^{13} \mathrm{C}$ data & 15.75 & 19.14 & 0.78 & 64.33 & - & - & - & - \\
\hline & All data & 45.45 & 0.17 & 2.17 & 52.22 & - & - & - & - \\
\hline \multirow{3}{*}{ uDP4+ } & ${ }^{1} \mathrm{H}$ data & 56.50 & 1.08 & 32.49 & 9.94 & - & - & - & - \\
\hline & ${ }^{13} \mathrm{C}$ data & 82.55 & 1.98 & 0.34 & 15.13 & - & - & - & - \\
\hline & All data & 96.61 & 0.04 & 0.23 & 3.11 & - & - & - & - \\
\hline \multirow{6}{*}{ DP4+ } & ${ }^{1} \mathrm{H}$ data & 62.31 & 0.00 & 34.61 & 3.08 & - & - & - & - \\
\hline & ${ }^{13} \mathrm{C}$ data & 56.25 & 1.64 & 0.01 & 42.10 & - & - & - & - \\
\hline & All data & 96.42 & 0.00 & 0.01 & 3.57 & - & - & - & - \\
\hline & & \multicolumn{8}{|c|}{ Level of theory: B3LYP/6-311G* (gas phase) } \\
\hline & & $75 a$ & $75 \mathrm{~b}$ & $75 c$ & $75 d$ & - & - & - & - \\
\hline & ${ }^{1} \mathrm{H}$ data & 10.66 & 0.86 & 36.41 & 52.07 & - & - & - & - \\
\hline \multirow[t]{2}{*}{ sDP4+ } & ${ }^{13} \mathrm{C}$ data & 34.82 & 11.06 & 0.41 & 53.70 & - & - & - & - \\
\hline & All data & 11.63 & 0.30 & 0.47 & 87.60 & - & - & - & - \\
\hline \multirow{3}{*}{ uDP4+ } & ${ }^{1} \mathrm{H}$ data & 29.34 & 5.18 & 32.32 & 33.16 & - & - & - & - \\
\hline & ${ }^{13} \mathrm{C}$ data & 71.42 & 0.05 & 0.02 & 28.51 & - & - & - & - \\
\hline & All data & 68.89 & 0.01 & 0.02 & 31.08 & - & - & - & - \\
\hline \multirow{3}{*}{ DP4+ } & ${ }^{1} \mathrm{H}$ data & 9.71 & 0.14 & 36.54 & 53.61 & - & - & - & - \\
\hline & ${ }^{13} \mathrm{C}$ data & 61.89 & 0.01 & 0.00 & 38.09 & - & - & - & - \\
\hline & All data & 22.74 & 0.00 & 0.00 & 77.26 & - & - & - & - \\
\hline & & \multicolumn{8}{|c|}{ Level of theory: B3LYP/6-311G** (gas phase) } \\
\hline & & $75 a$ & $75 b$ & $75 c$ & $75 d$ & - & - & - & - \\
\hline \multirow{2}{*}{ sDP4+ } & ${ }^{1} \mathrm{H}$ data & 0.17 & 1.92 & 62.16 & 35.75 & - & - & - & - \\
\hline & ${ }^{13} \mathrm{C}$ data & 35.16 & 8.60 & 0.42 & 55.82 & - & - & - & - \\
\hline
\end{tabular}




\begin{tabular}{|c|c|c|c|c|c|c|c|c|c|}
\hline & All data & 0.29 & 0.81 & 1.29 & 97.61 & - & - & - & - \\
\hline & ${ }^{1} \mathrm{H}$ data & 6.95 & 12.82 & 62.06 & 18.16 & - & - & - & - \\
\hline \multirow[t]{3}{*}{ uDP4+ } & ${ }^{13} \mathrm{C}$ data & 66.73 & 0.03 & 0.02 & 33.22 & - & - & - & - \\
\hline & All data & 43.42 & 0.04 & 0.09 & 56.45 & - & - & - & - \\
\hline & ${ }^{1} \mathrm{H}$ data & 0.03 & 0.54 & 85.10 & 14.33 & - & - & - & - \\
\hline \multirow[t]{5}{*}{ DP4+ } & ${ }^{13} \mathrm{C}$ data & 55.85 & 0.01 & 0.00 & 44.14 & - & - & - & - \\
\hline & All data & 0.23 & 0.00 & 0.00 & 99.77 & - & - & - & - \\
\hline & & \multicolumn{8}{|c|}{ Level of theory: B3LYP/6-311+G** (gas phase) } \\
\hline & & $75 a$ & 75b & $75 c$ & 75d & - & - & - & - \\
\hline & ${ }^{1} \mathrm{H}$ data & 15.33 & 4.32 & 58.63 & 21.72 & - & - & - & - \\
\hline \multirow[t]{3}{*}{ sDP4+ } & ${ }^{13} \mathrm{C}$ data & 51.33 & 8.76 & 0.35 & 39.55 & - & - & - & - \\
\hline & All data & 46.17 & 2.22 & 1.20 & 50.41 & - & - & - & - \\
\hline & ${ }^{1} \mathrm{H}$ data & 29.81 & 16.38 & 39.10 & 14.71 & - & - & - & - \\
\hline \multirow[t]{3}{*}{ uDP4+ } & ${ }^{13} \mathrm{C}$ data & 80.03 & 0.10 & 0.02 & 19.86 & - & - & - & - \\
\hline & All data & 89.02 & 0.06 & 0.03 & 10.90 & - & - & - & - \\
\hline & ${ }^{1} \mathrm{H}$ data & 14.55 & 2.25 & 73.02 & 10.17 & - & - & - & - \\
\hline \multirow[t]{5}{*}{ DP4+ } & ${ }^{13} \mathrm{C}$ data & 83.94 & 0.02 & 0.00 & 16.05 & - & - & - & - \\
\hline & All data & 88.21 & 0.00 & 0.00 & 11.79 & - & - & - & - \\
\hline & & \multicolumn{8}{|c|}{ Level of theory: B3LYP/6-31G* (solution, PCM) } \\
\hline & & $75 a$ & 75b & $75 c$ & 75d & - & - & - & - \\
\hline & ${ }^{1}$ H data & 0.02 & 0.03 & 54.51 & 45.45 & - & - & - & - \\
\hline \multirow[t]{3}{*}{ sDP4+ } & ${ }^{13} \mathrm{C}$ data & 61.10 & 25.95 & 0.22 & 12.72 & - & - & - & - \\
\hline & All data & 0.18 & 0.13 & 2.07 & 97.61 & - & - & - & - \\
\hline & ${ }^{1} \mathrm{H}$ data & 1.22 & 0.30 & 59.15 & 39.33 & - & - & - & - \\
\hline \multirow[t]{3}{*}{ uDP4+ } & ${ }^{13} \mathrm{C}$ data & 97.69 & 0.21 & 0.00 & 2.10 & - & - & - & - \\
\hline & All data & 58.95 & 0.03 & 0.10 & 40.92 & - & - & - & - \\
\hline & ${ }^{1} \mathrm{H}$ data & 0.00 & 0.00 & 64.34 & 35.66 & - & - & - & - \\
\hline \multirow[t]{5}{*}{ DP4+ } & ${ }^{13} \mathrm{C}$ data & 99.47 & 0.09 & 0.00 & 0.45 & - & - & - & - \\
\hline & All data & 0.27 & 0.00 & 0.01 & 99.72 & - & - & - & - \\
\hline & & \multicolumn{8}{|c|}{ Level of theory: B3LYP/6-31G** (solution, PCM) } \\
\hline & & $75 a$ & 75b & $75 c$ & 75d & - & - & - & - \\
\hline & ${ }^{1} \mathrm{H}$ data & 0.00 & 0.27 & 90.61 & 9.12 & - & - & - & - \\
\hline \multirow[t]{3}{*}{ sDP4+ } & ${ }^{13} \mathrm{C}$ data & 49.00 & 34.44 & 0.54 & 16.02 & - & - & - & - \\
\hline & All data & 0.09 & 4.49 & 23.83 & 71.58 & - & - & - & - \\
\hline & ${ }^{1} \mathrm{H}$ data & 1.04 & 2.16 & 80.09 & 16.72 & - & - & - & - \\
\hline \multirow[t]{3}{*}{ uDP4+ } & ${ }^{13} \mathrm{C}$ data & 93.04 & 0.21 & 0.01 & 6.75 & - & - & - & - \\
\hline & All data & 45.89 & 0.21 & 0.48 & 53.42 & - & - & - & - \\
\hline & ${ }^{1} \mathrm{H}$ data & 0.00 & 0.01 & 97.93 & 2.06 & - & - & - & - \\
\hline \multirow[t]{5}{*}{ DP4+ } & ${ }^{13} \mathrm{C}$ data & 97.54 & 0.15 & 0.00 & 2.31 & - & - & - & - \\
\hline & All data & 0.11 & 0.02 & 0.30 & 99.57 & - & - & - & - \\
\hline & & \multicolumn{8}{|c|}{ Level of theory: B3LYP/6-31+G** (solution, PCM) } \\
\hline & & $75 a$ & $75 b$ & $75 c$ & 75d & - & - & - & - \\
\hline & ${ }^{1} \mathrm{H}$ data & 1.76 & 0.40 & 84.20 & 13.64 & - & - & - & - \\
\hline \multirow[t]{3}{*}{ sDP4+ } & ${ }^{13} \mathrm{C}$ data & 20.15 & 14.32 & 0.48 & 65.04 & - & - & - & - \\
\hline & All data & 3.66 & 0.59 & 4.17 & 91.58 & - & - & - & - \\
\hline & ${ }^{1} \mathrm{H}$ data & 32.65 & 2.73 & 44.13 & 20.49 & - & - & - & - \\
\hline uDP4+ & ${ }^{13} \mathrm{C}$ data & 81.90 & 1.50 & 0.22 & 16.38 & - & - & - & - \\
\hline & All data & 88.44 & 0.14 & 0.32 & 11.10 & - & - & - & - \\
\hline & ${ }^{1} \mathrm{H}$ data & 1.42 & 0.03 & 91.66 & 6.89 & - & - & - & - \\
\hline DP4+ & ${ }^{13} \mathrm{C}$ data & 60.29 & 0.78 & 0.00 & 38.92 & - & - & - & - \\
\hline & All data & 24.11 & 0.01 & 0.10 & 75.79 & - & - & - & - \\
\hline & & & & Level of & $y: B 3 L Y$ & $\mathbf{G}^{*}$ & PC & & \\
\hline & & $75 a$ & $75 b$ & $75 c$ & $75 d$ & - & - & - & - \\
\hline & ${ }^{1} \mathrm{H}$ data & 0.43 & 1.70 & 71.32 & 26.55 & - & - & - & - \\
\hline sDP4+ & ${ }^{13} \mathrm{C}$ data & 47.12 & 8.93 & 0.25 & 43.69 & - & - & - & - \\
\hline & All data & 1.66 & 1.25 & 1.50 & 95.60 & - & - & - & - \\
\hline
\end{tabular}




\begin{tabular}{|c|c|c|c|c|c|c|c|c|c|}
\hline & ${ }^{1} \mathrm{H}$ data & 13.26 & 9.62 & 50.99 & 26.14 & - & - & - & - \\
\hline \multirow[t]{3}{*}{ uDP4+ } & ${ }^{13} \mathrm{C}$ data & 70.11 & 0.03 & 0.01 & 29.85 & - & - & - & - \\
\hline & All data & 54.34 & 0.02 & 0.03 & 45.61 & - & - & - & - \\
\hline & ${ }^{1} \mathrm{H}$ data & 0.13 & 0.37 & 83.55 & 15.94 & - & - & - & - \\
\hline \multirow[t]{5}{*}{ DP4+ } & ${ }^{13} \mathrm{C}$ data & 71.70 & 0.01 & 0.00 & 28.30 & - & - & - & - \\
\hline & All data & 2.02 & 0.00 & 0.00 & 97.97 & - & - & - & - \\
\hline & & \multicolumn{8}{|c|}{ Level of theory: B3LYP/6-311G** (solution, PCM) } \\
\hline & & $75 a$ & 75b & $75 c$ & 75d & - & - & - & - \\
\hline & ${ }^{1} \mathrm{H}$ data & 0.03 & 7.38 & 85.87 & 6.72 & - & - & - & - \\
\hline \multirow[t]{3}{*}{ sDP4+ } & ${ }^{13} \mathrm{C}$ data & 46.83 & 6.53 & 0.26 & 46.38 & - & - & - & - \\
\hline & All data & 0.36 & 12.58 & 5.71 & 81.34 & - & - & - & - \\
\hline & ${ }^{1} \mathrm{H}$ data & 2.02 & 27.78 & 61.99 & 8.21 & - & - & - & - \\
\hline \multirow[t]{3}{*}{ uDP4+ } & ${ }^{13} \mathrm{C}$ data & 68.70 & 0.02 & 0.01 & 31.27 & - & - & - & - \\
\hline & All data & 34.96 & 0.16 & 0.10 & 64.78 & - & - & - & - \\
\hline & ${ }^{1} \mathrm{H}$ data & 0.00 & 3.67 & 95.34 & 0.99 & - & - & - & - \\
\hline \multirow[t]{5}{*}{ DP4+ } & ${ }^{13} \mathrm{C}$ data & 68.93 & 0.00 & 0.00 & 31.07 & - & - & - & - \\
\hline & All data & 0.24 & 0.04 & 0.01 & 99.71 & - & - & - & - \\
\hline & & \multicolumn{8}{|c|}{ Level of theory: B3LYP/6-311+G** (solution, PCM) } \\
\hline & & $75 a$ & 75b & $75 c$ & 75d & - & - & - & - \\
\hline & ${ }^{1}$ H data & 1.29 & 26.67 & 61.58 & 10.46 & - & - & - & - \\
\hline \multirow[t]{3}{*}{ sDP4+ } & ${ }^{13} \mathrm{C}$ data & 58.33 & 5.58 & 0.16 & 35.92 & - & - & - & - \\
\hline & All data & 12.32 & 24.42 & 1.63 & 61.63 & - & - & - & - \\
\hline & ${ }^{1} \mathrm{H}$ data & 10.02 & 28.94 & 48.93 & 12.11 & - & - & - & - \\
\hline \multirow[t]{3}{*}{ uDP4+ } & ${ }^{13} \mathrm{C}$ data & 75.89 & 0.06 & 0.01 & 24.04 & - & - & - & - \\
\hline & All data & 72.15 & 0.18 & 0.04 & 27.64 & - & - & - & - \\
\hline & ${ }^{1} \mathrm{H}$ data & 0.33 & 19.67 & 76.78 & 3.23 & - & - & - & - \\
\hline \multirow[t]{5}{*}{ DP4+ } & ${ }^{13} \mathrm{C}$ data & 83.67 & 0.01 & 0.00 & 16.32 & - & - & - & - \\
\hline & All data & 34.23 & 0.17 & 0.00 & 65.60 & - & - & - & - \\
\hline & & \multicolumn{8}{|c|}{ Level of theory: mPW1PW91/6-31G* (gas phase) } \\
\hline & & $75 a$ & 75b & $75 c$ & 75d & - & - & - & - \\
\hline & ${ }^{1} \mathrm{H}$ data & 2.81 & 0.02 & 13.47 & 83.70 & - & - & - & - \\
\hline \multirow[t]{3}{*}{ sDP4+ } & ${ }^{13} \mathrm{C}$ data & 64.69 & 10.03 & 0.05 & 25.23 & - & - & - & - \\
\hline & All data & 7.93 & 0.01 & 0.03 & 92.03 & - & - & - & - \\
\hline & ${ }^{1} \mathrm{H}$ data & 10.40 & 0.27 & 20.92 & 68.41 & - & - & - & - \\
\hline \multirow[t]{3}{*}{ uDP4+ } & ${ }^{13} \mathrm{C}$ data & 95.02 & 0.77 & 0.01 & 4.20 & - & - & - & - \\
\hline & All data & 77.48 & 0.02 & 0.02 & 22.49 & - & - & - & - \\
\hline & ${ }^{1} \mathrm{H}$ data & 0.48 & 0.00 & 4.67 & 94.85 & - & - & - & - \\
\hline \multirow[t]{5}{*}{ DP4+ } & ${ }^{13} \mathrm{C}$ data & 98.19 & 0.12 & 0.00 & 1.69 & - & - & - & - \\
\hline & All data & 22.89 & 0.00 & 0.00 & 77.11 & - & - & - & - \\
\hline & & \multicolumn{8}{|c|}{ Level of theory: mPW1PW91/6-31G** (gas phase) } \\
\hline & & $75 a$ & $75 b$ & $75 c$ & 75d & - & - & - & - \\
\hline & ${ }^{1} \mathrm{H}$ data & 0.76 & 0.14 & 22.27 & 76.83 & - & - & - & - \\
\hline \multirow[t]{3}{*}{ sDP4+ } & ${ }^{13} \mathrm{C}$ data & 49.00 & 13.99 & 0.10 & 36.92 & - & - & - & - \\
\hline & All data & 1.29 & 0.07 & 0.07 & 98.56 & - & - & - & - \\
\hline & ${ }^{1}$ H data & 26.80 & 1.29 & 23.78 & 48.13 & - & - & - & - \\
\hline uDP4+ & ${ }^{13} \mathrm{C}$ data & 93.80 & 0.16 & 0.01 & 6.03 & - & - & - & - \\
\hline & All data & 89.64 & 0.01 & 0.00 & 10.35 & - & - & - & - \\
\hline & ${ }^{1} \mathrm{H}$ data & 0.48 & 0.00 & 12.47 & 87.05 & - & - & - & - \\
\hline DP4+ & ${ }^{13} \mathrm{C}$ data & 95.34 & 0.05 & 0.00 & 4.62 & - & - & - & - \\
\hline & All data & 10.21 & 0.00 & 0.00 & 89.79 & - & - & - & - \\
\hline & & & & evel of $t$ & : mPW1 & -3 & pr & & \\
\hline & & $75 a$ & $75 b$ & $75 c$ & 75d & - & - & - & - \\
\hline & ${ }^{1} \mathrm{H}$ data & 92.37 & 0.03 & 4.93 & 2.68 & - & - & - & - \\
\hline sDP4+ & ${ }^{13} \mathrm{C}$ data & 14.47 & 20.91 & 0.12 & 64.50 & - & - & - & - \\
\hline & All data & 88.49 & 0.03 & 0.04 & 11.43 & - & - & - & - \\
\hline uDP4+ & ${ }^{1} \mathrm{H}$ data & 80.85 & 1.23 & 11.53 & 6.38 & - & - & - & - \\
\hline
\end{tabular}




\begin{tabular}{|c|c|c|c|c|c|c|c|c|c|}
\hline & ${ }^{13} \mathrm{C}$ data & 83.14 & 1.56 & 0.11 & 15.18 & - & - & - & - \\
\hline & All data & 98.53 & 0.03 & 0.02 & 1.42 & - & - & - & - \\
\hline & ${ }^{1} \mathrm{H}$ data & 99.02 & 0.00 & 0.75 & 0.23 & - & - & - & - \\
\hline \multirow[t]{5}{*}{ DP4+ } & ${ }^{13} \mathrm{C}$ data & 54.31 & 1.47 & 0.00 & 44.22 & - & - & - & - \\
\hline & All data & 99.81 & 0.00 & 0.00 & 0.19 & - & - & - & - \\
\hline & & \multicolumn{8}{|c|}{ Level of theory: mPW1PW91/6-311G* (gas phase) } \\
\hline & & $75 a$ & 75b & $75 c$ & 75d & - & - & - & - \\
\hline & ${ }^{1} \mathrm{H}$ data & 63.77 & 0.39 & 11.59 & 24.25 & - & - & - & - \\
\hline \multirow[t]{3}{*}{ sDP4+ } & ${ }^{13}$ C data & 79.72 & 6.48 & 0.11 & 13.70 & - & - & - & - \\
\hline & All data & 93.80 & 0.05 & 0.02 & 6.13 & - & - & - & - \\
\hline & ${ }^{1} \mathrm{H}$ data & 44.26 & 3.69 & 21.90 & 30.15 & - & - & - & - \\
\hline \multirow[t]{3}{*}{ uDP4+ } & ${ }^{13} \mathrm{C}$ data & 83.59 & 0.04 & 0.01 & 16.36 & - & - & - & - \\
\hline & All data & 88.23 & 0.00 & 0.01 & 11.77 & - & - & - & - \\
\hline & ${ }^{1} \mathrm{H}$ data & 74.10 & 0.04 & 6.67 & 19.19 & - & - & - & - \\
\hline \multirow[t]{5}{*}{ DP4+ } & ${ }^{13} \mathrm{C}$ data & 96.74 & 0.00 & 0.00 & 3.26 & - & - & - & - \\
\hline & All data & 99.14 & 0.00 & 0.00 & 0.86 & - & - & - & - \\
\hline & & \multicolumn{8}{|c|}{ Level of theory: mPW1PW91/6-311G** (gas phase) } \\
\hline & & $75 a$ & $75 b$ & $75 c$ & 75d & - & - & - & - \\
\hline & ${ }^{1} \mathrm{H}$ data & 1.77 & 1.88 & 42.82 & 53.53 & - & - & - & - \\
\hline \multirow[t]{3}{*}{ sDP4+ } & ${ }^{13} \mathrm{C}$ data & 79.88 & 4.86 & 0.07 & 15.19 & - & - & - & - \\
\hline & All data & 14.65 & 0.94 & 0.32 & 84.08 & - & - & - & - \\
\hline & ${ }^{1} \mathrm{H}$ data & 24.28 & 11.19 & 43.22 & 21.31 & - & - & - & - \\
\hline \multirow[t]{3}{*}{ uDP4+ } & ${ }^{13} \mathrm{C}$ data & 82.02 & 0.02 & 0.01 & 17.95 & - & - & - & - \\
\hline & All data & 83.87 & 0.01 & 0.01 & 16.11 & - & - & - & - \\
\hline & ${ }^{1} \mathrm{H}$ data & 1.41 & 0.69 & 60.57 & 37.34 & - & - & - & - \\
\hline \multirow[t]{5}{*}{ DP4+ } & ${ }^{13} \mathrm{C}$ data & 96.00 & 0.00 & 0.00 & 4.00 & - & - & - & - \\
\hline & All data & 47.56 & 0.00 & 0.00 & 52.44 & - & - & - & - \\
\hline & & \multicolumn{8}{|c|}{ Level of theory: mPW1PW91/6-311+G** (gas phase) } \\
\hline & & $75 a$ & $75 b$ & $75 c$ & 75d & - & - & - & - \\
\hline & ${ }^{1}$ H data & 40.99 & 3.37 & 36.68 & 18.96 & - & - & - & - \\
\hline \multirow[t]{2}{*}{ sDP4+ } & ${ }^{13} \mathrm{C}$ data & 77.04 & 6.77 & 0.08 & 16.11 & - & - & - & - \\
\hline & All data & 90.51 & 0.65 & 0.08 & 8.76 & - & - & - & - \\
\hline \multirow{3}{*}{ uDP4+ } & ${ }^{1} \mathrm{H}$ data & 44.43 & 12.65 & 28.24 & 14.69 & - & - & - & - \\
\hline & ${ }^{13} \mathrm{C}$ data & 86.24 & 0.08 & 0.01 & 13.66 & - & - & - & - \\
\hline & All data & 94.99 & 0.03 & 0.01 & 4.97 & - & - & - & - \\
\hline \multirow{5}{*}{ DP4+ } & ${ }^{1} \mathrm{H}$ data & 57.31 & 1.34 & 32.59 & 8.76 & - & - & - & - \\
\hline & ${ }^{13} \mathrm{C}$ data & 96.78 & 0.01 & 0.00 & 3.21 & - & - & - & - \\
\hline & All data & 99.50 & 0.00 & 0.00 & 0.50 & - & - & - & - \\
\hline & & \multicolumn{8}{|c|}{ Level of theory: mPW1PW91/6-31G* (solution, PCM) } \\
\hline & & $75 a$ & 75b & 75c & 75d & - & - & - & - \\
\hline \multirow{3}{*}{ sDP4+ } & ${ }^{1} \mathrm{H}$ data & 0.05 & 0.53 & 74.06 & 25.37 & - & - & - & - \\
\hline & ${ }^{13} \mathrm{C}$ data & 67.52 & 10.87 & 0.04 & 21.57 & - & - & - & - \\
\hline & All data & 0.56 & 1.03 & 0.56 & 97.86 & - & - & - & - \\
\hline \multirow{3}{*}{ uDP4+ } & ${ }^{1} \mathrm{H}$ data & 4.90 & 2.64 & 56.98 & 35.48 & - & - & - & - \\
\hline & ${ }^{13} \mathrm{C}$ data & 94.97 & 0.13 & 0.00 & 4.90 & - & - & - & - \\
\hline & All data & 72.75 & 0.05 & 0.04 & 27.16 & - & - & - & - \\
\hline & ${ }^{1} \mathrm{H}$ data & 0.00 & 0.03 & 82.39 & 17.57 & - & - & - & - \\
\hline DP4+ & ${ }^{13} \mathrm{C}$ data & 98.36 & 0.02 & 0.00 & 1.62 & - & - & - & - \\
\hline & All data & 1.51 & 0.00 & 0.00 & 98.49 & - & - & - & - \\
\hline & & & & el of th & nPW1PI & 10 & on, & & \\
\hline & & $75 a$ & $75 b$ & $75 c$ & 75d & - & - & - & - \\
\hline & ${ }^{1} \mathrm{H}$ data & 0.27 & 0.05 & 30.54 & 69.14 & - & - & - & - \\
\hline sDP4+ & ${ }^{13} \mathrm{C}$ data & 83.57 & 5.60 & 0.02 & 10.82 & - & - & - & - \\
\hline & All data & 2.93 & 0.04 & 0.07 & 96.97 & - & - & - & - \\
\hline & ${ }^{1} \mathrm{H}$ data & 6.76 & 0.40 & 35.11 & 57.73 & - & - & - & - \\
\hline UDP4+ & ${ }^{13} \mathrm{C}$ data & 97.00 & 0.42 & 0.01 & 2.57 & - & - & - & - \\
\hline
\end{tabular}




\begin{tabular}{|c|c|c|c|c|c|c|c|c|c|}
\hline & All data & 81.51 & 0.02 & 0.03 & 18.45 & - & - & - & - \\
\hline & ${ }^{1} \mathrm{H}$ data & 0.04 & 0.00 & 21.17 & 78.79 & - & - & - & - \\
\hline \multirow[t]{5}{*}{ DP4+ } & ${ }^{13} \mathrm{C}$ data & 99.63 & 0.03 & 0.00 & 0.34 & - & - & - & - \\
\hline & All data & 11.77 & 0.00 & 0.00 & 88.23 & - & - & - & - \\
\hline & & \multicolumn{8}{|c|}{ Level of theory: mPW1PW91/6-31+G** (solution, PCM) } \\
\hline & & $75 a$ & $75 b$ & $75 c$ & $75 d$ & - & - & - & - \\
\hline & ${ }^{1} \mathrm{H}$ data & 26.80 & 0.91 & 54.43 & 17.86 & - & - & - & - \\
\hline \multirow[t]{3}{*}{ sDP4+ } & ${ }^{13} \mathrm{C}$ data & 25.39 & 18.05 & 0.07 & 56.49 & - & - & - & - \\
\hline & All data & 39.81 & 0.96 & 0.22 & 59.01 & - & - & - & - \\
\hline & ${ }^{1} \mathrm{H}$ data & 69.07 & 1.23 & 14.51 & 15.18 & - & - & - & - \\
\hline \multirow[t]{3}{*}{ uDP4+ } & ${ }^{13} \mathrm{C}$ data & 80.98 & 1.34 & 0.10 & 17.58 & - & - & - & - \\
\hline & All data & 95.40 & 0.03 & 0.02 & 4.55 & - & - & - & - \\
\hline & ${ }^{1} \mathrm{H}$ data & 63.54 & 0.04 & 27.11 & 9.31 & - & - & - & - \\
\hline \multirow[t]{5}{*}{ DP4+ } & ${ }^{13} \mathrm{C}$ data & 66.90 & 0.79 & 0.00 & 32.31 & - & - & - & - \\
\hline & All data & 93.39 & 0.00 & 0.00 & 6.61 & - & - & - & - \\
\hline & & \multicolumn{8}{|c|}{ Level of theory: mPW1PW91/6-311G* (solution, PCM) } \\
\hline & & $75 a$ & $75 b$ & $75 c$ & 75d & - & - & - & - \\
\hline & ${ }^{1} \mathrm{H}$ data & 4.73 & 1.80 & 53.42 & 40.05 & - & - & - & - \\
\hline \multirow[t]{3}{*}{ sDP4+ } & ${ }^{13} \mathrm{C}$ data & 88.03 & 3.60 & 0.03 & 8.34 & - & - & - & - \\
\hline & All data & 54.89 & 0.85 & 0.20 & 44.06 & - & - & - & - \\
\hline & ${ }^{1}$ H data & 29.15 & 8.34 & 34.50 & 28.01 & - & - & - & - \\
\hline \multirow[t]{3}{*}{ uDP4+ } & ${ }^{13}$ C data & 82.93 & 0.02 & 0.00 & 17.06 & - & - & - & - \\
\hline & All data & 83.50 & 0.00 & 0.00 & 16.50 & - & - & - & - \\
\hline & ${ }^{1} \mathrm{H}$ data & 4.42 & 0.48 & 59.12 & 35.98 & - & - & - & - \\
\hline \multirow[t]{5}{*}{ DP4+ } & ${ }^{13} \mathrm{C}$ data & 98.09 & 0.00 & 0.00 & 1.91 & - & - & - & - \\
\hline & All data & 86.31 & 0.00 & 0.00 & 13.69 & - & - & - & - \\
\hline & & \multicolumn{8}{|c|}{ Level of theory: mPW1PW91/6-311G** (solution, PCM) } \\
\hline & & $75 a$ & 75b & $75 c$ & $75 d$ & - & - & - & - \\
\hline & ${ }^{1} \mathrm{H}$ data & 0.18 & 8.82 & 78.86 & 12.14 & - & - & - & - \\
\hline \multirow[t]{2}{*}{ sDP4+ } & ${ }^{13} \mathrm{C}$ data & 89.35 & 2.31 & 0.02 & 8.32 & - & - & - & - \\
\hline & All data & 11.60 & 14.67 & 0.97 & 72.76 & - & - & - & - \\
\hline \multirow{3}{*}{ uDP4+ } & ${ }^{1} \mathrm{H}$ data & 5.10 & 27.63 & 55.61 & 11.67 & - & - & - & - \\
\hline & ${ }^{13} \mathrm{C}$ data & 82.80 & 0.01 & 0.00 & 17.19 & - & - & - & - \\
\hline & All data & 67.75 & 0.04 & 0.02 & 32.19 & - & - & - & - \\
\hline \multirow{5}{*}{ DP4+ } & ${ }^{1} \mathrm{H}$ data & 0.02 & 5.11 & 91.91 & 2.97 & - & - & - & - \\
\hline & ${ }^{13} \mathrm{C}$ data & 98.10 & 0.00 & 0.00 & 1.90 & - & - & - & - \\
\hline & All data & 25.12 & 0.02 & 0.00 & 74.86 & - & - & - & - \\
\hline & & \multicolumn{8}{|c|}{ Level of theory: mPW1PW91/6-311+G** (solution, PCM) } \\
\hline & & $75 a$ & $75 b$ & $75 c$ & $75 d$ & - & - & - & - \\
\hline \multirow{3}{*}{ sDP4+ } & ${ }^{1} \mathrm{H}$ data & 3.58 & 33.64 & 50.36 & 12.41 & - & - & - & - \\
\hline & ${ }^{13} \mathrm{C}$ data & 83.97 & 4.40 & 0.03 & 11.60 & - & - & - & - \\
\hline & All data & 50.63 & 24.91 & 0.24 & 24.23 & - & - & - & - \\
\hline \multirow{3}{*}{ uDP4+ } & ${ }^{1} \mathrm{H}$ data & 20.93 & 30.27 & 38.94 & 9.86 & - & - & - & - \\
\hline & ${ }^{13} \mathrm{C}$ data & 83.92 & 0.03 & 0.00 & 16.04 & - & - & - & - \\
\hline & All data & 91.68 & 0.05 & 0.01 & 8.26 & - & - & - & - \\
\hline \multirow{3}{*}{ DP4+ } & ${ }^{1} \mathrm{H}$ data & 2.36 & 32.05 & 61.73 & 3.85 & - & - & - & - \\
\hline & ${ }^{13} \mathrm{C}$ data & 97.42 & 0.00 & 0.00 & 2.57 & - & - & - & - \\
\hline & All data & 95.84 & 0.03 & 0.00 & 4.13 & - & - & - & - \\
\hline
\end{tabular}


$s \mathrm{DP} 4+, u \mathrm{DP} 4+$ and DP4+ probabilities (\%) for compound 75b<smiles>CC(C)C[C@H](C)[C@@H](O)[C@@H](C)C(=O)O</smiles><smiles>CC(C)C[C@H](C)[C@@H](O)[C@@H](C)C(=O)O</smiles>

correct isomer<smiles>CC(C)C[C@H](C)[C@@H](O)[C@@H](CC(C)C)C(=O)O</smiles><smiles>CC(C)C[C@H](C)[C@@H](O)[C@H](C)C(=O)O</smiles>

\begin{tabular}{|c|c|c|c|c|c|c|c|c|c|}
\hline & & \multicolumn{8}{|c|}{ Level of theory: B3LYP/6-31G* (gas phase) } \\
\hline & & $75 a$ & $75 \mathrm{~b}$ & $75 \mathrm{c}$ & 75d & - & - & - & - \\
\hline & ${ }^{1} \mathrm{H}$ data & 0.01 & 17.46 & 71.03 & 11.50 & - & - & - & - \\
\hline \multirow[t]{2}{*}{ sDP4+ } & ${ }^{13} \mathrm{C}$ data & 0.03 & 99.30 & 0.61 & 0.05 & - & - & - & - \\
\hline & All data & 0.00 & 97.52 & 2.45 & 0.03 & - & - & - & - \\
\hline & ${ }^{1} \mathrm{H}$ data & 0.35 & 40.63 & 56.53 & 2.48 & - & - & - & - \\
\hline \multirow[t]{2}{*}{ uDP4+ } & ${ }^{13} \mathrm{C}$ data & 9.12 & 88.60 & 1.51 & 0.76 & - & - & - & - \\
\hline & All data & 0.09 & 97.54 & 2.32 & 0.05 & - & - & - & - \\
\hline \multirow{6}{*}{ DP4+ } & ${ }^{1} \mathrm{H}$ data & 0.00 & 14.93 & 84.47 & 0.60 & - & - & - & - \\
\hline & ${ }^{13} \mathrm{C}$ data & 0.00 & 99.99 & 0.01 & 0.00 & - & - & - & - \\
\hline & All data & 0.00 & 99.94 & 0.06 & 0.00 & - & - & - & - \\
\hline & & \multicolumn{8}{|c|}{ Level of theory: B3LYP/6-31G** (gas phase) } \\
\hline & & $75 a$ & $75 \mathrm{~b}$ & $75 c$ & 75d & - & - & - & - \\
\hline & ${ }^{1} \mathrm{H}$ data & 0.00 & 19.05 & 78.66 & 2.30 & - & - & - & - \\
\hline \multirow[t]{2}{*}{ sDP4+ } & ${ }^{13} \mathrm{C}$ data & 0.09 & 98.62 & 1.16 & 0.13 & - & - & - & - \\
\hline & All data & 0.00 & 95.37 & 4.62 & 0.02 & - & - & - & - \\
\hline & ${ }^{1} \mathrm{H}$ data & 0.28 & 52.21 & 46.04 & 1.46 & - & - & - & - \\
\hline \multirow[t]{2}{*}{ uDP4+ } & ${ }^{13} \mathrm{C}$ data & 9.24 & 84.06 & 3.58 & 3.12 & - & - & - & - \\
\hline & All data & 0.06 & 96.23 & 3.62 & 0.10 & - & - & - & - \\
\hline \multirow{5}{*}{ DP4+ } & ${ }^{1} \mathrm{H}$ data & 0.00 & 21.53 & 78.40 & 0.07 & - & - & - & - \\
\hline & ${ }^{13} \mathrm{C}$ data & 0.01 & 99.93 & 0.05 & 0.00 & - & - & - & - \\
\hline & All data & 0.00 & 99.82 & 0.18 & 0.00 & - & - & - & - \\
\hline & & \multicolumn{8}{|c|}{ Level of theory: B3LYP/6-31+G** (gas phase) } \\
\hline & & $75 a$ & $75 b$ & $75 c$ & $75 d$ & - & - & - & - \\
\hline & ${ }^{1} \mathrm{H}$ data & 10.10 & 29.34 & 49.70 & 10.86 & - & - & - & - \\
\hline \multirow[t]{2}{*}{ sDP4+ } & ${ }^{13} \mathrm{C}$ data & 0.16 & 84.93 & 3.01 & 11.89 & - & - & - & - \\
\hline & All data & 0.06 & 89.89 & 5.40 & 4.66 & - & - & - & - \\
\hline \multirow{3}{*}{ uDP4+ } & ${ }^{1} \mathrm{H}$ data & 24.47 & 28.26 & 44.25 & 3.02 & - & - & - & - \\
\hline & ${ }^{13} \mathrm{C}$ data & 6.18 & 54.44 & 6.61 & 32.77 & - & - & - & - \\
\hline & All data & 7.26 & 73.92 & 14.06 & 4.76 & - & - & - & - \\
\hline \multirow{6}{*}{ DP4+ } & ${ }^{1} \mathrm{H}$ data & 7.47 & 25.07 & 66.47 & 0.99 & - & - & - & - \\
\hline & ${ }^{13} \mathrm{C}$ data & 0.02 & 91.84 & 0.40 & 7.74 & - & - & - & - \\
\hline & All data & 0.01 & 98.54 & 1.12 & 0.33 & - & - & - & - \\
\hline & & \multicolumn{8}{|c|}{ Level of theory: B3LYP/6-311G* (gas phase) } \\
\hline & & $75 a$ & $75 b$ & $75 c$ & $75 d$ & - & - & - & - \\
\hline & ${ }^{1} \mathrm{H}$ data & 0.22 & 80.83 & 13.99 & 4.96 & - & - & - & - \\
\hline \multirow[t]{2}{*}{ sDP4+ } & ${ }^{13} \mathrm{C}$ data & 0.36 & 83.31 & 1.61 & 14.73 & - & - & - & - \\
\hline & All data & 0.00 & 98.60 & 0.33 & 1.07 & - & - & - & - \\
\hline \multirow{3}{*}{ uDP4+ } & ${ }^{1} \mathrm{H}$ data & 5.55 & 66.23 & 26.53 & 1.70 & - & - & - & - \\
\hline & ${ }^{13} \mathrm{C}$ data & 10.68 & 1.49 & 0.53 & 87.29 & - & - & - & - \\
\hline & All data & 18.51 & 30.85 & 4.43 & 46.20 & - & - & - & - \\
\hline \multirow{3}{*}{ DP4+ } & ${ }^{1} \mathrm{H}$ data & 0.02 & 93.36 & 6.47 & 0.15 & - & - & - & - \\
\hline & ${ }^{13} \mathrm{C}$ data & 0.27 & 8.79 & 0.06 & 90.88 & - & - & - & - \\
\hline & All data & 0.00 & 98.35 & 0.05 & 1.60 & - & - & - & - \\
\hline & & \multicolumn{8}{|c|}{ Level of theory: B3LYP/6-311G** (gas phase) } \\
\hline & & $75 a$ & $75 b$ & $75 c$ & 75d & - & - & - & - \\
\hline \multirow{2}{*}{ sDP4+ } & ${ }^{1} \mathrm{H}$ data & 0.00 & 87.71 & 11.03 & 1.25 & - & - & - & - \\
\hline & ${ }^{13} \mathrm{C}$ data & 0.34 & 91.99 & 2.20 & 5.47 & - & - & - & - \\
\hline
\end{tabular}




\begin{tabular}{|c|c|c|c|c|c|c|c|c|c|}
\hline & All data & 0.00 & 99.62 & 0.30 & 0.08 & - & - & - & - \\
\hline & ${ }^{1} \mathrm{H}$ data & 0.65 & 77.16 & 20.69 & 1.51 & - & - & - & - \\
\hline \multirow[t]{3}{*}{ uDP4+ } & ${ }^{13} \mathrm{C}$ data & 14.92 & 1.24 & 0.51 & 83.33 & - & - & - & - \\
\hline & All data & 3.99 & 39.64 & 4.33 & 52.04 & - & - & - & - \\
\hline & ${ }^{1} \mathrm{H}$ data & 0.00 & 96.71 & 3.26 & 0.03 & - & - & - & - \\
\hline \multirow[t]{5}{*}{ DP4+ } & ${ }^{13} \mathrm{C}$ data & 0.89 & 19.81 & 0.19 & 79.11 & - & - & - & - \\
\hline & All data & 0.00 & 99.86 & 0.03 & 0.11 & - & - & - & - \\
\hline & & \multicolumn{8}{|c|}{ Level of theory: B3LYP/6-311+G** (gas phase) } \\
\hline & & $75 a$ & 75b & $75 c$ & 75d & - & - & - & - \\
\hline & ${ }^{1} \mathrm{H}$ data & 0.35 & 96.23 & 2.27 & 1.15 & - & - & - & - \\
\hline \multirow[t]{3}{*}{ sDP4+ } & ${ }^{13} \mathrm{C}$ data & 0.29 & 89.14 & 2.08 & 8.48 & - & - & - & - \\
\hline & All data & 0.00 & 99.83 & 0.05 & 0.11 & - & - & - & - \\
\hline & ${ }^{1}$ H data & 6.07 & 76.48 & 15.55 & 1.91 & - & - & - & - \\
\hline \multirow[t]{3}{*}{ uDP4+ } & ${ }^{13} \mathrm{C}$ data & 8.74 & 1.55 & 0.25 & 89.46 & - & - & - & - \\
\hline & All data & 15.32 & 34.31 & 1.14 & 49.22 & - & - & - & - \\
\hline & ${ }^{1} \mathrm{H}$ data & 0.03 & 99.47 & 0.48 & 0.03 & - & - & - & - \\
\hline \multirow[t]{5}{*}{ DP4+ } & ${ }^{13} \mathrm{C}$ data & 0.29 & 15.38 & 0.06 & 84.27 & - & - & - & - \\
\hline & All data & 0.00 & 99.83 & 0.00 & 0.16 & - & - & - & - \\
\hline & & \multicolumn{8}{|c|}{ Level of theory: B3LYP/6-31G* (solution, PCM) } \\
\hline & & $75 a$ & 75b & $75 c$ & 75d & - & - & - & - \\
\hline & ${ }^{1}$ H data & 0.00 & 28.75 & 70.32 & 0.92 & - & - & - & - \\
\hline \multirow[t]{3}{*}{ sDP4+ } & ${ }^{13}$ C data & 0.03 & 99.63 & 0.33 & 0.01 & - & - & - & - \\
\hline & All data & 0.00 & 99.19 & 0.81 & 0.00 & - & - & - & - \\
\hline & ${ }^{1} \mathrm{H}$ data & 0.03 & 62.63 & 36.64 & 0.70 & - & - & - & - \\
\hline \multirow[t]{3}{*}{ uDP4+ } & ${ }^{13} \mathrm{C}$ data & 11.15 & 87.30 & 1.15 & 0.40 & - & - & - & - \\
\hline & All data & 0.01 & 99.22 & 0.77 & 0.01 & - & - & - & - \\
\hline & ${ }^{1} \mathrm{H}$ data & 0.00 & 41.13 & 58.85 & 0.01 & - & - & - & - \\
\hline \multirow[t]{5}{*}{ DP4+ } & ${ }^{13} \mathrm{C}$ data & 0.00 & 99.99 & 0.00 & 0.00 & - & - & - & - \\
\hline & All data & 0.00 & 99.99 & 0.01 & 0.00 & - & - & - & - \\
\hline & & \multicolumn{8}{|c|}{ Level of theory: B3LYP/6-31G** (solution, PCM) } \\
\hline & & $75 a$ & $75 b$ & $75 c$ & $75 d$ & - & - & - & - \\
\hline & ${ }^{1} \mathrm{H}$ data & 0.00 & 21.19 & 78.69 & 0.12 & - & - & - & - \\
\hline \multirow[t]{3}{*}{ sDP4+ } & ${ }^{13} \mathrm{C}$ data & 0.12 & 98.84 & 0.98 & 0.05 & - & - & - & - \\
\hline & All data & 0.00 & 96.43 & 3.57 & 0.00 & - & - & - & - \\
\hline & ${ }^{1} \mathrm{H}$ data & 0.03 & 59.93 & 39.82 & 0.22 & - & - & - & - \\
\hline \multirow[t]{3}{*}{ uDP4+ } & ${ }^{13} \mathrm{C}$ data & 11.86 & 83.23 & 3.00 & 1.91 & - & - & - & - \\
\hline & All data & 0.01 & 97.65 & 2.34 & 0.01 & - & - & - & - \\
\hline & ${ }^{1} \mathrm{H}$ data & 0.00 & 28.84 & 71.16 & 0.00 & - & - & - & - \\
\hline \multirow[t]{5}{*}{ DP4+ } & ${ }^{13} \mathrm{C}$ data & 0.02 & 99.95 & 0.04 & 0.00 & - & - & - & - \\
\hline & All data & 0.00 & 99.91 & 0.09 & 0.00 & - & - & - & - \\
\hline & & \multicolumn{8}{|c|}{ Level of theory: B3LYP/6-31+G** (solution, PCM) } \\
\hline & & $75 a$ & $75 b$ & $75 c$ & 75d & - & - & - & - \\
\hline & ${ }^{1} \mathrm{H}$ data & 0.10 & 60.52 & 37.51 & 1.86 & - & - & - & - \\
\hline \multirow[t]{3}{*}{ sDP4+ } & ${ }^{13} \mathrm{C}$ data & 0.23 & 89.77 & 2.55 & 7.45 & - & - & - & - \\
\hline & All data & 0.00 & 98.03 & 1.72 & 0.25 & - & - & - & - \\
\hline & ${ }^{1} \mathrm{H}$ data & 4.33 & 52.63 & 40.55 & 2.49 & - & - & - & - \\
\hline uDP4+ & ${ }^{13} \mathrm{C}$ data & 9.21 & 51.25 & 5.57 & 33.98 & - & - & - & - \\
\hline & All data & 1.31 & 88.51 & 7.41 & 2.78 & - & - & - & - \\
\hline & ${ }^{1} \mathrm{H}$ data & 0.01 & 67.61 & 32.28 & 0.10 & - & - & - & - \\
\hline DP4+ & ${ }^{13} \mathrm{C}$ data & 0.04 & 94.46 & 0.29 & 5.20 & - & - & - & - \\
\hline & All data & 0.00 & 99.85 & 0.15 & 0.01 & - & - & - & - \\
\hline & & & & Level of & y: B3LY & G* & $\overline{P C}$ & & \\
\hline & & $75 a$ & $75 b$ & $75 c$ & $75 d$ & - & - & - & - \\
\hline & ${ }^{1} \mathrm{H}$ data & 0.00 & 89.04 & 10.57 & 0.39 & - & - & - & - \\
\hline sDP4+ & ${ }^{13} \mathrm{C}$ data & 0.56 & 92.13 & 1.54 & 5.78 & - & - & - & - \\
\hline & All data & 0.00 & 99.77 & 0.20 & 0.03 & - & - & - & - \\
\hline
\end{tabular}




\begin{tabular}{|c|c|c|c|c|c|c|c|c|c|}
\hline & ${ }^{1} \mathrm{H}$ data & 0.34 & 78.26 & 21.10 & 0.30 & - & - & - & - \\
\hline \multirow[t]{3}{*}{ uDP4+ } & ${ }^{13} \mathrm{C}$ data & 21.65 & 1.71 & 0.38 & 76.26 & - & - & - & - \\
\hline & All data & 4.23 & 77.92 & 4.64 & 13.21 & - & - & - & - \\
\hline & ${ }^{1} \mathrm{H}$ data & 0.00 & 96.90 & 3.10 & 0.00 & - & - & - & - \\
\hline \multirow[t]{5}{*}{ DP4+ } & ${ }^{13} \mathrm{C}$ data & 1.98 & 25.79 & 0.10 & 72.14 & - & - & - & - \\
\hline & All data & 0.00 & 99.98 & 0.01 & 0.00 & - & - & - & - \\
\hline & & \multicolumn{8}{|c|}{ Level of theory: B3LYP/6-311G** (solution, PCM) } \\
\hline & & $75 a$ & 75b & $75 c$ & 75d & - & - & - & - \\
\hline & ${ }^{1}$ H data & 0.00 & 90.54 & 9.12 & 0.34 & - & - & - & - \\
\hline \multirow[t]{3}{*}{ sDP4+ } & ${ }^{13} \mathrm{C}$ data & 0.43 & 95.13 & 2.15 & 2.29 & - & - & - & - \\
\hline & All data & 0.00 & 99.76 & 0.23 & 0.01 & - & - & - & - \\
\hline & ${ }^{1} \mathrm{H}$ data & 0.34 & 80.09 & 18.98 & 0.59 & - & - & - & - \\
\hline \multirow[t]{3}{*}{ uDP4+ } & ${ }^{13} \mathrm{C}$ data & 34.14 & 1.98 & 0.47 & 63.40 & - & - & - & - \\
\hline & All data & 5.32 & 73.28 & 4.12 & 17.28 & - & - & - & - \\
\hline & ${ }^{1} \mathrm{H}$ data & 0.00 & 97.67 & 2.33 & 0.00 & - & - & - & - \\
\hline \multirow[t]{5}{*}{ DP4+ } & ${ }^{13} \mathrm{C}$ data & 4.19 & 54.02 & 0.29 & 41.50 & - & - & - & - \\
\hline & All data & 0.00 & 99.99 & 0.01 & 0.00 & - & - & - & - \\
\hline & & \multicolumn{8}{|c|}{ Level of theory: B3LYP/6-311+G** (solution, PCM) } \\
\hline & & $75 a$ & 75b & $75 c$ & 75d & - & - & - & - \\
\hline & ${ }^{1}$ H data & 0.05 & 96.51 & 2.67 & 0.76 & - & - & - & - \\
\hline \multirow[t]{3}{*}{ sDP4+ } & ${ }^{13} \mathrm{C}$ data & 0.65 & 87.53 & 1.70 & 10.12 & - & - & - & - \\
\hline & All data & 0.00 & 99.85 & 0.05 & 0.09 & - & - & - & - \\
\hline & ${ }^{1} \mathrm{H}$ data & 0.59 & 92.10 & 6.84 & 0.47 & - & - & - & - \\
\hline \multirow[t]{3}{*}{ uDP4+ } & ${ }^{13} \mathrm{C}$ data & 14.40 & 1.32 & 0.13 & 84.16 & - & - & - & - \\
\hline & All data & 4.94 & 71.16 & 0.50 & 23.40 & - & - & - & - \\
\hline & ${ }^{1} \mathrm{H}$ data & 0.00 & 99.79 & 0.21 & 0.00 & - & - & - & - \\
\hline \multirow[t]{5}{*}{ DP4+ } & ${ }^{13} \mathrm{C}$ data & 0.96 & 11.83 & 0.02 & 87.20 & - & - & - & - \\
\hline & All data & 0.00 & 99.97 & 0.00 & 0.03 & - & - & - & - \\
\hline & & \multicolumn{8}{|c|}{ Level of theory: mPW1PW91/6-31G* (gas phase) } \\
\hline & & $75 a$ & 75b & $75 c$ & $75 d$ & - & - & - & - \\
\hline & ${ }_{12}^{1} \mathrm{H}$ data & 0.19 & 32.85 & 44.42 & 22.54 & - & - & - & - \\
\hline \multirow[t]{3}{*}{ sDP4+ } & ${ }^{13} \mathrm{C}$ data & 0.01 & 99.78 & 0.18 & 0.03 & - & - & - & - \\
\hline & All data & 0.00 & 99.73 & 0.25 & 0.02 & - & - & - & - \\
\hline & ${ }^{1} \mathrm{H}$ data & 1.41 & 46.58 & 49.51 & 2.50 & - & - & - & - \\
\hline \multirow[t]{3}{*}{ uDP4+ } & ${ }^{13} \mathrm{C}$ data & 12.82 & 85.56 & 0.83 & 0.79 & - & - & - & - \\
\hline & All data & 0.45 & 98.49 & 1.02 & 0.05 & - & - & - & - \\
\hline & ${ }^{1} \mathrm{H}$ data & 0.01 & 40.42 & 58.08 & 1.49 & - & - & - & - \\
\hline \multirow[t]{5}{*}{ DP4+ } & ${ }^{13} \mathrm{C}$ data & 0.00 & 100.00 & 0.00 & 0.00 & - & - & - & - \\
\hline & All data & 0.00 & 100.00 & 0.00 & 0.00 & - & - & - & - \\
\hline & & \multicolumn{8}{|c|}{ Level of theory: mPW1PW91/6-31G** (gas phase) } \\
\hline & & $75 a$ & $75 b$ & $75 c$ & $75 d$ & - & - & - & - \\
\hline & ${ }^{1} \mathrm{H}$ data & 0.03 & 40.47 & 53.46 & 6.04 & - & - & - & - \\
\hline \multirow[t]{3}{*}{ sDP4+ } & ${ }^{13} \mathrm{C}$ data & 0.02 & 99.48 & 0.42 & 0.09 & - & - & - & - \\
\hline & All data & 0.00 & 99.44 & 0.55 & 0.01 & - & - & - & - \\
\hline & ${ }^{1} \mathrm{H}$ data & 1.28 & 63.74 & 32.95 & 2.03 & - & - & - & - \\
\hline uDP4+ & ${ }^{13} \mathrm{C}$ data & 12.91 & 82.64 & 1.68 & 2.77 & - & - & - & - \\
\hline & All data & 0.31 & 98.55 & 1.04 & 0.11 & - & - & - & - \\
\hline & ${ }^{1} \mathrm{H}$ data & 0.00 & 59.26 & 40.46 & 0.28 & - & - & - & - \\
\hline DP4+ & ${ }^{13} \mathrm{C}$ data & 0.00 & 99.99 & 0.01 & 0.00 & - & - & - & - \\
\hline & All data & 0.00 & 99.99 & 0.01 & 0.00 & - & - & - & - \\
\hline & & & & evel of $\mathrm{tl}$ & mPW1 & -5 & pr & & \\
\hline & & $75 a$ & $75 b$ & $75 c$ & $75 d$ & - & - & - & - \\
\hline & ${ }^{1} \mathrm{H}$ data & 54.57 & 17.51 & 20.43 & 7.49 & - & - & - & - \\
\hline sDP4+ & ${ }^{13} \mathrm{C}$ data & 0.01 & 98.09 & 0.36 & 1.54 & - & - & - & - \\
\hline & All data & 0.02 & 98.89 & 0.42 & 0.67 & - & - & - & - \\
\hline uDP4+ & ${ }^{1} \mathrm{H}$ data & 55.56 & 20.92 & 19.90 & 3.61 & - & - & - & - \\
\hline
\end{tabular}




\begin{tabular}{|c|c|c|c|c|c|c|c|c|c|}
\hline & ${ }^{13} \mathrm{C}$ data & 7.37 & 74.65 & 3.49 & 14.49 & - & - & - & - \\
\hline & All data & 19.57 & 74.61 & 3.32 & 2.50 & - & - & - & - \\
\hline & ${ }^{1} \mathrm{H}$ data & 79.12 & 9.56 & 10.61 & 0.71 & - & - & - & - \\
\hline \multirow[t]{5}{*}{ DP4+ } & ${ }^{13} \mathrm{C}$ data & 0.00 & 99.68 & 0.02 & 0.30 & - & - & - & - \\
\hline & All data & 0.01 & 99.95 & 0.02 & 0.02 & - & - & - & - \\
\hline & & \multicolumn{8}{|c|}{ Level of theory: mPW1PW91/6-311G* (gas phase) } \\
\hline & & $75 a$ & 75b & 75c & 75d & - & - & - & - \\
\hline & ${ }^{1} \mathrm{H}$ data & 3.78 & 71.35 & 15.10 & 9.77 & - & - & - & - \\
\hline \multirow[t]{3}{*}{ sDP4+ } & ${ }^{13} \mathrm{C}$ data & 0.84 & 87.48 & 0.88 & 10.80 & - & - & - & - \\
\hline & All data & 0.05 & 98.08 & 0.21 & 1.66 & - & - & - & - \\
\hline & ${ }^{1} \mathrm{H}$ data & 15.76 & 59.49 & 22.92 & 1.83 & - & - & - & - \\
\hline \multirow[t]{3}{*}{ uDP4+ } & ${ }^{13} \mathrm{C}$ data & 13.33 & 2.55 & 0.72 & 83.40 & - & - & - & - \\
\hline & All data & 39.53 & 28.59 & 3.10 & 28.78 & - & - & - & - \\
\hline & ${ }^{1} \mathrm{H}$ data & 1.28 & 90.93 & 7.41 & 0.38 & - & - & - & - \\
\hline \multirow[t]{5}{*}{ DP4+ } & ${ }^{13} \mathrm{C}$ data & 0.99 & 19.66 & 0.06 & 79.29 & - & - & - & - \\
\hline & All data & 0.07 & 98.24 & 0.02 & 1.67 & - & - & - & - \\
\hline & & \multicolumn{8}{|c|}{ Level of theory: mPW1PW91/6-311G** (gas phase) } \\
\hline & & $75 a$ & $75 b$ & $75 c$ & $75 d$ & - & - & - & - \\
\hline & ${ }^{1} \mathrm{H}$ data & 0.02 & 90.88 & 7.39 & 1.71 & - & - & - & - \\
\hline \multirow[t]{2}{*}{ sDP4+ } & ${ }^{13} \mathrm{C}$ data & 0.50 & 92.68 & 0.86 & 5.96 & - & - & - & - \\
\hline & All data & 0.00 & 99.80 & 0.08 & 0.12 & - & - & - & - \\
\hline \multirow{3}{*}{ uDP4+ } & ${ }^{1} \mathrm{H}$ data & 2.24 & 78.10 & 17.64 & 2.02 & - & - & - & - \\
\hline & ${ }^{13} \mathrm{C}$ data & 16.03 & 1.30 & 0.42 & 82.26 & - & - & - & - \\
\hline & All data & 11.54 & 32.68 & 2.38 & 53.41 & - & - & - & - \\
\hline \multirow{3}{*}{ DP4+ } & ${ }^{1} \mathrm{H}$ data & 0.00 & 98.15 & 1.80 & 0.05 & - & - & - & - \\
\hline & ${ }^{13} \mathrm{C}$ data & 1.29 & 19.46 & 0.06 & 79.18 & - & - & - & - \\
\hline & All data & 0.00 & 99.80 & 0.01 & 0.20 & - & - & - & - \\
\hline & & \multicolumn{8}{|c|}{ Level of theory: mPW1PW91/6-311+G** (gas phase) } \\
\hline & & $75 a$ & 75b & 75c & 75d & - & - & - & - \\
\hline \multirow{3}{*}{ sDP4+ } & ${ }^{1} \mathrm{H}$ data & 1.12 & 95.45 & 2.05 & 1.38 & - & - & - & - \\
\hline & ${ }^{13} \mathrm{C}$ data & 0.53 & 94.03 & 0.52 & 4.93 & - & - & - & - \\
\hline & All data & 0.01 & 99.91 & 0.01 & 0.08 & - & - & - & - \\
\hline \multirow{3}{*}{ uDP4+ } & ${ }^{1} \mathrm{H}$ data & 12.41 & 72.08 & 13.21 & 2.31 & - & - & - & - \\
\hline & ${ }^{13} \mathrm{C}$ data & 15.28 & 3.23 & 0.39 & 81.10 & - & - & - & - \\
\hline & All data & 30.88 & 37.84 & 0.84 & 30.44 & - & - & - & - \\
\hline \multirow{5}{*}{ DP4+ } & ${ }^{1} \mathrm{H}$ data & 0.20 & 99.36 & 0.39 & 0.05 & - & - & - & - \\
\hline & ${ }^{13} \mathrm{C}$ data & 1.13 & 42.66 & 0.03 & 56.18 & - & - & - & - \\
\hline & All data & 0.01 & 99.93 & 0.00 & 0.06 & - & - & - & - \\
\hline & & \multicolumn{8}{|c|}{ Level of theory: mPW1PW91/6-31G* (solution, PCM) } \\
\hline & & $75 a$ & $75 b$ & 75c & 75d & - & - & - & - \\
\hline \multirow{3}{*}{ sDP4+ } & ${ }^{1} \mathrm{H}$ data & 0.00 & 55.10 & 43.09 & 1.80 & - & - & - & - \\
\hline & ${ }^{13} \mathrm{C}$ data & 0.01 & 99.86 & 0.12 & 0.01 & - & - & - & - \\
\hline & All data & 0.00 & 99.91 & 0.09 & 0.00 & - & - & - & - \\
\hline \multirow{3}{*}{ uDP4+ } & ${ }^{1} \mathrm{H}$ data & 0.12 & 72.04 & 27.30 & 0.53 & - & - & - & - \\
\hline & ${ }^{13} \mathrm{C}$ data & 15.56 & 83.23 & 0.70 & 0.51 & - & - & - & - \\
\hline & All data & 0.03 & 99.65 & 0.32 & 0.00 & - & - & - & - \\
\hline & ${ }^{1} \mathrm{H}$ data & 0.00 & 77.12 & 22.86 & 0.02 & - & - & - & - \\
\hline DP4+ & ${ }^{13} \mathrm{C}$ data & 0.00 & 100.00 & 0.00 & 0.00 & - & - & - & - \\
\hline & All data & 0.00 & 100.00 & 0.00 & 0.00 & - & - & - & - \\
\hline & & & & el of the & nPW1P & 1( & on, & & \\
\hline & & $75 a$ & $75 b$ & $75 c$ & $75 d$ & - & - & - & - \\
\hline & ${ }^{1} \mathrm{H}$ data & 0.00 & 44.53 & 55.21 & 0.27 & - & - & - & - \\
\hline sDP4+ & ${ }^{13} \mathrm{C}$ data & 0.02 & 99.70 & 0.24 & 0.03 & - & - & - & - \\
\hline & All data & 0.00 & 99.70 & 0.30 & 0.00 & - & - & - & - \\
\hline & ${ }^{1} \mathrm{H}$ data & 0.10 & 75.88 & 23.77 & 0.25 & - & - & - & - \\
\hline UDP4+ & ${ }^{13} \mathrm{C}$ data & 15.54 & 81.08 & 1.58 & 1.79 & - & - & - & - \\
\hline
\end{tabular}




\begin{tabular}{|c|c|c|c|c|c|c|c|c|c|}
\hline & All data & 0.02 & 99.36 & 0.61 & 0.01 & - & - & - & - \\
\hline & ${ }^{1} \mathrm{H}$ data & 0.00 & 72.02 & 27.97 & 0.00 & - & - & - & - \\
\hline \multirow[t]{5}{*}{ DP4+ } & ${ }^{13} \mathrm{C}$ data & 0.00 & 99.99 & 0.00 & 0.00 & - & - & - & - \\
\hline & All data & 0.00 & 100.00 & 0.00 & 0.00 & - & - & - & - \\
\hline & & \multicolumn{8}{|c|}{ Level of theory: mPW1PW91/6-31+G** (solution, PCM) } \\
\hline & & $75 a$ & $75 b$ & $75 c$ & $75 d$ & - & - & - & - \\
\hline & ${ }^{1} \mathrm{H}$ data & 1.48 & 72.17 & 23.03 & 3.32 & - & - & - & - \\
\hline \multirow[t]{3}{*}{ sDP4+ } & ${ }^{13} \mathrm{C}$ data & 0.01 & 98.22 & 0.29 & 1.47 & - & - & - & - \\
\hline & All data & 0.00 & 99.84 & 0.10 & 0.07 & - & - & - & - \\
\hline & ${ }^{1} \mathrm{H}$ data & 8.91 & 59.39 & 28.76 & 2.95 & - & - & - & - \\
\hline \multirow[t]{3}{*}{ uDP4+ } & ${ }^{13} \mathrm{C}$ data & 9.76 & 70.29 & 3.23 & 16.71 & - & - & - & - \\
\hline & All data & 1.98 & 94.80 & 2.11 & 1.12 & - & - & - & - \\
\hline & ${ }^{1} \mathrm{H}$ data & 0.27 & 86.21 & 13.33 & 0.20 & - & - & - & - \\
\hline \multirow[t]{5}{*}{ DP4+ } & ${ }^{13} \mathrm{C}$ data & 0.00 & 99.63 & 0.01 & 0.36 & - & - & - & - \\
\hline & All data & 0.00 & 100.00 & 0.00 & 0.00 & - & - & - & - \\
\hline & & \multicolumn{8}{|c|}{ Level of theory: mPW1PW91/6-311G* (solution, PCM) } \\
\hline & & $75 a$ & $75 b$ & $75 c$ & 75d & - & - & - & - \\
\hline & ${ }^{1} \mathrm{H}$ data & 0.03 & 87.45 & 11.86 & 0.66 & - & - & - & - \\
\hline \multirow[t]{3}{*}{ sDP4+ } & ${ }^{13} \mathrm{C}$ data & 1.03 & 93.05 & 0.43 & 5.49 & - & - & - & - \\
\hline & All data & 0.00 & 99.89 & 0.06 & 0.04 & - & - & - & - \\
\hline & ${ }^{1}$ H data & 1.17 & 81.24 & 17.23 & 0.35 & - & - & - & - \\
\hline \multirow[t]{3}{*}{ uDP4+ } & ${ }^{13}$ C data & 21.91 & 1.87 & 0.31 & 75.92 & - & - & - & - \\
\hline & All data & 12.28 & 72.37 & 2.51 & 12.84 & - & - & - & - \\
\hline & ${ }^{1} \mathrm{H}$ data & 0.00 & 97.20 & 2.80 & 0.00 & - & - & - & - \\
\hline \multirow[t]{5}{*}{ DP4+ } & ${ }^{13} \mathrm{C}$ data & 3.67 & 28.33 & 0.02 & 67.98 & - & - & - & - \\
\hline & All data & 0.00 & 99.99 & 0.00 & 0.01 & - & - & - & - \\
\hline & & \multicolumn{8}{|c|}{ Level of theory: mPW1PW91/6-311G** (solution, PCM) } \\
\hline & & $75 a$ & 75b & $75 c$ & $75 d$ & - & - & - & - \\
\hline & ${ }^{1} \mathrm{H}$ data & 0.00 & 93.69 & 5.88 & 0.43 & - & - & - & - \\
\hline \multirow[t]{2}{*}{ sDP4+ } & ${ }^{13} \mathrm{C}$ data & 0.60 & 96.26 & 0.40 & 2.73 & - & - & - & - \\
\hline & All data & 0.00 & 99.96 & 0.03 & 0.01 & - & - & - & - \\
\hline \multirow{3}{*}{ uDP4+ } & ${ }^{1} \mathrm{H}$ data & 0.82 & 84.25 & 14.19 & 0.74 & - & - & - & - \\
\hline & ${ }^{13} \mathrm{C}$ data & 24.43 & 1.25 & 0.26 & 74.06 & - & - & - & - \\
\hline & All data & 10.91 & 57.29 & 2.03 & 29.78 & - & - & - & - \\
\hline \multirow{5}{*}{ DP4+ } & ${ }^{1} \mathrm{H}$ data & 0.00 & 98.95 & 1.05 & 0.00 & - & - & - & - \\
\hline & ${ }^{13} \mathrm{C}$ data & 4.37 & 35.64 & 0.03 & 59.96 & - & - & - & - \\
\hline & All data & 0.00 & 99.99 & 0.00 & 0.01 & - & - & - & - \\
\hline & & \multicolumn{8}{|c|}{ Level of theory: mPW1PW91/6-311+G** (solution, PCM) } \\
\hline & & $75 a$ & $75 b$ & $75 c$ & $75 d$ & - & - & - & - \\
\hline \multirow{3}{*}{ sDP4+ } & ${ }^{1} \mathrm{H}$ data & 0.09 & 97.55 & 1.71 & 0.64 & - & - & - & - \\
\hline & ${ }^{13} \mathrm{C}$ data & 1.04 & 94.01 & 0.34 & 4.61 & - & - & - & - \\
\hline & All data & 0.00 & 99.96 & 0.01 & 0.03 & - & - & - & - \\
\hline \multirow{3}{*}{ uDP4+ } & ${ }^{1} \mathrm{H}$ data & 3.42 & 86.49 & 8.64 & 1.46 & - & - & - & - \\
\hline & ${ }^{13} \mathrm{C}$ data & 22.23 & 1.72 & 0.16 & 75.89 & - & - & - & - \\
\hline & All data & 22.54 & 44.16 & 0.41 & 32.90 & - & - & - & - \\
\hline \multirow{3}{*}{ DP4+ } & ${ }^{1} \mathrm{H}$ data & 0.00 & 99.81 & 0.17 & 0.01 & - & - & - & - \\
\hline & ${ }^{13} \mathrm{C}$ data & 4.32 & 30.24 & 0.01 & 65.43 & - & - & - & - \\
\hline & All data & 0.00 & 99.98 & 0.00 & 0.02 & - & - & - & - \\
\hline
\end{tabular}


$s \mathrm{DP} 4+, u \mathrm{DP} 4+$ and DP4+ probabilities (\%) for compound $75 \mathrm{c}$<smiles>CC(C)C[C@H](C)[C@@H](O)[C@@H](C)C(=O)O</smiles><smiles>CC(C)C[C@H](C)[C@@H](O)[C@@H](C)C(=O)O</smiles><smiles>CC(C)C[C@H](C)[C@@H](O)[C@@H](CC(C)(C)C)C(=O)O</smiles><smiles>CC(C)C[C@H](C)[C@@H](O)[C@@H](C)C(=O)O</smiles>
correct isomer

\begin{tabular}{|c|c|c|c|c|c|c|c|c|c|}
\hline & & \multicolumn{8}{|c|}{ Level of theory: B3LYP/6-31G* (gas phase) } \\
\hline & & $75 a$ & $75 \mathrm{~b}$ & $75 c$ & 75d & - & - & - & - \\
\hline \multirow{3}{*}{ sDP4+ } & ${ }^{1} \mathrm{H}$ data & 0.00 & 0.00 & 91.65 & 8.35 & - & - & - & - \\
\hline & ${ }^{13} \mathrm{C}$ data & 0.05 & 22.08 & 76.17 & 1.69 & - & - & - & - \\
\hline & All data & 0.00 & 0.00 & 99.80 & 0.20 & - & - & - & - \\
\hline & ${ }^{1} \mathrm{H}$ data & 0.05 & 0.09 & 95.78 & 4.09 & - & - & - & - \\
\hline \multirow[t]{2}{*}{ uDP4+ } & ${ }^{13} \mathrm{C}$ data & 0.11 & 10.31 & 81.88 & 7.71 & - & - & - & - \\
\hline & All data & 0.00 & 0.01 & 99.59 & 0.40 & - & - & - & - \\
\hline \multirow{6}{*}{ DP4+ } & ${ }^{1} \mathrm{H}$ data & 0.00 & 0.00 & 99.61 & 0.39 & - & - & - & - \\
\hline & ${ }^{13} \mathrm{C}$ data & 0.00 & 3.51 & 96.29 & 0.20 & - & - & - & - \\
\hline & All data & 0.00 & 0.00 & 100.00 & 0.00 & - & - & - & - \\
\hline & & \multicolumn{8}{|c|}{ Level of theory: B3LYP/6-31G** (gas phase) } \\
\hline & & $75 a$ & $75 \mathrm{~b}$ & $75 c$ & $75 d$ & - & - & - & - \\
\hline & ${ }^{1} \mathrm{H}$ data & 0.00 & 0.00 & 97.34 & 2.66 & - & - & - & - \\
\hline \multirow[t]{2}{*}{ sDP4+ } & ${ }^{13} \mathrm{C}$ data & 0.10 & 15.67 & 81.72 & 2.50 & - & - & - & - \\
\hline & All data & 0.00 & 0.00 & 99.92 & 0.08 & - & - & - & - \\
\hline & ${ }^{1} \mathrm{H}$ data & 0.19 & 0.21 & 95.96 & 3.64 & - & - & - & - \\
\hline \multirow[t]{2}{*}{ uDP4+ } & ${ }^{13} \mathrm{C}$ data & 0.38 & 12.04 & 73.22 & 14.35 & - & - & - & - \\
\hline & All data & 0.00 & 0.04 & 99.22 & 0.74 & - & - & - & - \\
\hline \multirow{6}{*}{ DP4+ } & ${ }^{1} \mathrm{H}$ data & 0.00 & 0.00 & 99.90 & 0.10 & - & - & - & - \\
\hline & ${ }^{13} \mathrm{C}$ data & 0.00 & 3.04 & 96.38 & 0.58 & - & - & - & - \\
\hline & All data & 0.00 & 0.00 & 100.00 & 0.00 & - & - & - & - \\
\hline & & \multicolumn{8}{|c|}{ Level of theory: B3LYP/6-31+G** (gas phase) } \\
\hline & & $75 a$ & $75 b$ & $75 c$ & 75d & - & - & - & - \\
\hline & ${ }^{1} \mathrm{H}$ data & 0.88 & 0.01 & 95.37 & 3.73 & - & - & - & - \\
\hline \multirow[t]{2}{*}{ sDP4+ } & ${ }^{13} \mathrm{C}$ data & 0.06 & 5.74 & 27.51 & 66.69 & - & - & - & - \\
\hline & All data & 0.00 & 0.00 & 91.33 & 8.67 & - & - & - & - \\
\hline \multirow{3}{*}{ uDP4+ } & ${ }^{1} \mathrm{H}$ data & 3.08 & 0.08 & 88.15 & 8.69 & - & - & - & - \\
\hline & ${ }^{13} \mathrm{C}$ data & 1.23 & 6.47 & 12.30 & 79.99 & - & - & - & - \\
\hline & All data & 0.21 & 0.03 & 60.79 & 38.97 & - & - & - & - \\
\hline & ${ }^{1} \mathrm{H}$ data & 0.03 & 0.00 & 99.58 & 0.38 & - & - & - & - \\
\hline \multirow[t]{5}{*}{ DP4+ } & ${ }^{13} \mathrm{C}$ data & 0.00 & 0.65 & 5.93 & 93.42 & - & - & - & - \\
\hline & All data & 0.00 & 0.00 & 94.26 & 5.74 & - & - & - & - \\
\hline & & \multicolumn{8}{|c|}{ Level of theory: B3LYP/6-311G* (gas phase) } \\
\hline & & $75 a$ & $75 b$ & $75 c$ & 75d & - & - & - & - \\
\hline & ${ }^{1} \mathrm{H}$ data & 0.05 & 0.03 & 97.70 & 2.22 & - & - & - & - \\
\hline \multirow[t]{2}{*}{ sDP4+ } & ${ }^{13} \mathrm{C}$ data & 0.12 & 5.07 & 32.29 & 62.52 & - & - & - & - \\
\hline & All data & 0.00 & 0.00 & 95.78 & 4.22 & - & - & - & - \\
\hline \multirow{3}{*}{ uDP4+ } & ${ }^{1} \mathrm{H}$ data & 0.78 & 1.19 & 92.08 & 5.95 & - & - & - & - \\
\hline & ${ }^{13} \mathrm{C}$ data & 1.46 & 0.15 & 0.34 & 98.04 & - & - & - & - \\
\hline & All data & 0.19 & 0.03 & 5.14 & 94.65 & - & - & - & - \\
\hline \multirow{5}{*}{ DP4+ } & ${ }^{1} \mathrm{H}$ data & 0.00 & 0.00 & 99.85 & 0.15 & - & - & - & - \\
\hline & ${ }^{13} \mathrm{C}$ data & 0.00 & 0.01 & 0.18 & 99.80 & - & - & - & - \\
\hline & All data & 0.00 & 0.00 & 55.21 & 44.79 & - & - & - & - \\
\hline & & \multicolumn{8}{|c|}{ Level of theory: B3LYP/6-311G** (gas phase) } \\
\hline & & $75 a$ & $75 b$ & $75 c$ & $75 d$ & - & - & - & - \\
\hline \multirow{2}{*}{ sDP4+ } & ${ }^{1} \mathrm{H}$ data & 0.00 & 0.08 & 97.80 & 2.11 & - & - & - & - \\
\hline & ${ }^{13} \mathrm{C}$ data & 0.23 & 8.50 & 58.44 & 32.83 & - & - & - & - \\
\hline
\end{tabular}




\begin{tabular}{|c|c|c|c|c|c|c|c|c|c|}
\hline & All data & 0.00 & 0.01 & 98.79 & 1.20 & - & - & - & - \\
\hline & ${ }^{1} \mathrm{H}$ data & 0.93 & 1.32 & 92.18 & 5.57 & - & - & - & - \\
\hline \multirow[t]{3}{*}{ uDP4+ } & ${ }^{13} \mathrm{C}$ data & 3.48 & 0.27 & 0.55 & 95.70 & - & - & - & - \\
\hline & All data & 0.55 & 0.06 & 8.62 & 90.77 & - & - & - & - \\
\hline & ${ }^{1} \mathrm{H}$ data & 0.00 & 0.00 & 99.87 & 0.13 & - & - & - & - \\
\hline \multirow[t]{5}{*}{ DP4+ } & ${ }^{13} \mathrm{C}$ data & 0.03 & 0.07 & 1.01 & 98.89 & - & - & - & - \\
\hline & All data & 0.00 & 0.00 & 88.67 & 11.33 & - & - & - & - \\
\hline & & \multicolumn{8}{|c|}{ Level of theory: B3LYP/6-311+G** (gas phase) } \\
\hline & & $75 a$ & $75 b$ & $75 c$ & 75d & - & - & - & - \\
\hline & ${ }^{1} \mathrm{H}$ data & 0.84 & 0.40 & 91.71 & 7.05 & - & - & - & - \\
\hline \multirow[t]{2}{*}{ sDP4+ } & ${ }^{13} \mathrm{C}$ data & 0.05 & 4.29 & 30.67 & 64.99 & - & - & - & - \\
\hline & All data & 0.00 & 0.05 & 85.94 & 14.01 & - & - & - & - \\
\hline \multirow{3}{*}{ uDP4+ } & ${ }^{1} \mathrm{H}$ data & 4.61 & 1.92 & 85.14 & 8.32 & - & - & - & - \\
\hline & ${ }^{13} \mathrm{C}$ data & 1.43 & 0.17 & 0.19 & 98.21 & - & - & - & - \\
\hline & All data & 0.78 & 0.04 & 1.88 & 97.30 & - & - & - & - \\
\hline \multirow{3}{*}{ DP4+ } & ${ }^{1} \mathrm{H}$ data & 0.05 & 0.01 & 99.20 & 0.75 & - & - & - & - \\
\hline & ${ }^{13} \mathrm{C}$ data & 0.00 & 0.01 & 0.09 & 99.90 & - & - & - & - \\
\hline & All data & 0.00 & 0.00 & 10.58 & 89.42 & - & - & - & - \\
\hline & & \multicolumn{8}{|c|}{ Level of theory: B3LYP/6-31G* (solution, PCM) } \\
\hline & & $75 a$ & 75b & $75 c$ & 75d & - & - & - & - \\
\hline \multirow{3}{*}{ sDP4+ } & ${ }^{1}$ H data & 0.00 & 0.00 & 99.21 & 0.79 & - & - & - & - \\
\hline & ${ }^{13}$ C data & 0.04 & 27.75 & 71.87 & 0.34 & - & - & - & - \\
\hline & All data & 0.00 & 0.00 & 100.00 & 0.00 & - & - & - & - \\
\hline \multirow{3}{*}{ uDP4+ } & ${ }^{1} \mathrm{H}$ data & 0.00 & 0.04 & 98.68 & 1.28 & - & - & - & - \\
\hline & ${ }^{13} \mathrm{C}$ data & 0.18 & 14.41 & 81.19 & 4.22 & - & - & - & - \\
\hline & All data & 0.00 & 0.01 & 99.93 & 0.07 & - & - & - & - \\
\hline \multirow{3}{*}{ DP4+ } & ${ }^{1} \mathrm{H}$ data & 0.00 & 0.00 & 99.99 & 0.01 & - & - & - & - \\
\hline & ${ }^{13} \mathrm{C}$ data & 0.00 & 6.41 & 93.56 & 0.02 & - & - & - & - \\
\hline & All data & 0.00 & 0.00 & 100.00 & 0.00 & - & - & - & - \\
\hline & & \multicolumn{8}{|c|}{ Level of theory: B3LYP/6-31G** (solution, PCM) } \\
\hline & & $75 a$ & $75 b$ & $75 c$ & $75 d$ & - & - & - & - \\
\hline \multirow{3}{*}{ sDP4+ } & ${ }^{1} \mathrm{H}$ data & 0.00 & 0.00 & 99.85 & 0.15 & - & - & - & - \\
\hline & ${ }^{13} \mathrm{C}$ data & 0.12 & 20.67 & 78.44 & 0.77 & - & - & - & - \\
\hline & All data & 0.00 & 0.00 & 100.00 & 0.00 & - & - & - & - \\
\hline \multirow{3}{*}{ uDP4+ } & ${ }^{1} \mathrm{H}$ data & 0.02 & 0.10 & 98.70 & 1.18 & - & - & - & - \\
\hline & ${ }^{13} \mathrm{C}$ data & 0.92 & 22.18 & 68.32 & 8.57 & - & - & - & - \\
\hline & All data & 0.00 & 0.03 & 99.82 & 0.15 & - & - & - & - \\
\hline \multirow{3}{*}{ DP4+ } & ${ }^{1} \mathrm{H}$ data & 0.00 & 0.00 & 100.00 & 0.00 & - & - & - & - \\
\hline & ${ }^{13} \mathrm{C}$ data & 0.00 & 7.87 & 92.01 & 0.11 & - & - & - & - \\
\hline & All data & 0.00 & 0.00 & 100.00 & 0.00 & - & - & - & - \\
\hline & & \multicolumn{8}{|c|}{ Level of theory: B3LYP/6-31+G** (solution, PCM) } \\
\hline & & $75 a$ & $75 b$ & $75 c$ & 75d & - & - & - & - \\
\hline \multirow{3}{*}{ sDP4+ } & ${ }^{1} \mathrm{H}$ data & 0.01 & 0.00 & 98.79 & 1.20 & - & - & - & - \\
\hline & ${ }^{13} \mathrm{C}$ data & 0.14 & 10.21 & 40.71 & 48.93 & - & - & - & - \\
\hline & All data & 0.00 & 0.00 & 98.56 & 1.43 & - & - & - & - \\
\hline & ${ }^{1} \mathrm{H}$ data & 1.09 & 0.09 & 90.87 & 7.94 & - & - & - & - \\
\hline uDP4+ & ${ }^{13} \mathrm{C}$ data & 2.65 & 8.97 & 7.01 & 81.38 & - & - & - & - \\
\hline & All data & 0.22 & 0.07 & 49.49 & 50.22 & - & - & - & - \\
\hline & ${ }^{1} \mathrm{H}$ data & 0.00 & 0.00 & 99.89 & 0.11 & - & - & - & - \\
\hline DP4+ & ${ }^{13} \mathrm{C}$ data & 0.01 & 2.10 & 6.55 & 91.34 & - & - & - & - \\
\hline & All data & 0.00 & 0.00 & 98.55 & 1.45 & - & - & - & - \\
\hline & & & & Level of & y: B3LY & G* & $\overline{P C}$ & & \\
\hline & & $75 a$ & $75 b$ & $75 c$ & $75 d$ & - & - & - & - \\
\hline & ${ }^{1} \mathrm{H}$ data & 0.00 & 0.02 & 99.80 & 0.18 & - & - & - & - \\
\hline sDP4+ & ${ }^{13} \mathrm{C}$ data & 0.24 & 12.28 & 60.78 & 26.69 & - & - & - & - \\
\hline & All data & 0.00 & 0.00 & 99.92 & 0.08 & - & - & - & - \\
\hline
\end{tabular}




\begin{tabular}{|c|c|c|c|c|c|c|c|c|c|}
\hline & ${ }^{1} \mathrm{H}$ data & 0.06 & 0.59 & 97.86 & 1.50 & - & - & - & - \\
\hline \multirow[t]{3}{*}{ uDP4+ } & ${ }^{13} \mathrm{C}$ data & 4.39 & 0.31 & 0.40 & 94.91 & - & - & - & - \\
\hline & All data & 0.14 & 0.10 & 21.43 & 78.33 & - & - & - & - \\
\hline & ${ }^{1} \mathrm{H}$ data & 0.00 & 0.00 & 100.00 & 0.00 & - & - & - & - \\
\hline \multirow[t]{5}{*}{ DP4+ } & ${ }^{13} \mathrm{C}$ data & 0.04 & 0.15 & 0.94 & 98.87 & - & - & - & - \\
\hline & All data & 0.00 & 0.00 & 99.71 & 0.29 & - & - & - & - \\
\hline & & \multicolumn{8}{|c|}{ Level of theory: B3LYP/6-311G** (solution, PCM) } \\
\hline & & $75 a$ & $75 b$ & 75c & 75d & - & - & - & - \\
\hline & ${ }^{1} \mathrm{H}$ data & 0.00 & 0.68 & 98.19 & 1.13 & - & - & - & - \\
\hline \multirow[t]{3}{*}{ sDP4+ } & ${ }^{13} \mathrm{C}$ data & 0.29 & 13.25 & 76.47 & 9.99 & - & - & - & - \\
\hline & All data & 0.00 & 0.12 & 99.73 & 0.15 & - & - & - & - \\
\hline & ${ }^{1} \mathrm{H}$ data & 0.33 & 1.90 & 92.21 & 5.57 & - & - & - & - \\
\hline \multirow[t]{3}{*}{ uDP4+ } & ${ }^{13} \mathrm{C}$ data & 9.27 & 0.48 & 0.58 & 89.68 & - & - & - & - \\
\hline & All data & 0.55 & 0.16 & 9.56 & 89.73 & - & - & - & - \\
\hline & ${ }^{1}$ H data & 0.00 & 0.01 & 99.92 & 0.07 & - & - & - & - \\
\hline \multirow[t]{5}{*}{ DP4+ } & ${ }^{13} \mathrm{C}$ data & 0.28 & 0.66 & 4.65 & 94.41 & - & - & - & - \\
\hline & All data & 0.00 & 0.00 & 98.61 & 1.39 & - & - & - & - \\
\hline & & \multicolumn{8}{|c|}{ Level of theory: B3LYP/6-311+G** (solution, PCM) } \\
\hline & & $75 a$ & $75 b$ & 75c & $75 d$ & - & - & - & - \\
\hline & ${ }^{1} \mathrm{H}$ data & 0.16 & 1.58 & 93.53 & 4.73 & - & - & - & - \\
\hline \multirow[t]{3}{*}{ sDP4+ } & ${ }^{13} \mathrm{C}$ data & 0.13 & 6.51 & 46.27 & 47.09 & - & - & - & - \\
\hline & All data & 0.00 & 0.23 & 94.89 & 4.88 & - & - & - & - \\
\hline & ${ }^{1} \mathrm{H}$ data & 0.94 & 1.14 & 88.08 & 9.84 & - & - & - & - \\
\hline \multirow[t]{3}{*}{ uDP4+ } & ${ }^{13} \mathrm{C}$ data & 2.44 & 0.19 & 0.12 & 97.26 & - & - & - & - \\
\hline & All data & 0.24 & 0.02 & 1.05 & 98.69 & - & - & - & - \\
\hline & ${ }^{1} \mathrm{H}$ data & 0.00 & 0.02 & 99.42 & 0.56 & - & - & - & - \\
\hline \multirow[t]{5}{*}{ DP4+ } & ${ }^{13} \mathrm{C}$ data & 0.01 & 0.03 & 0.12 & 99.85 & - & - & - & - \\
\hline & All data & 0.00 & 0.00 & 17.14 & 82.86 & - & - & - & - \\
\hline & & \multicolumn{8}{|c|}{ Level of theory: mPW1PW91/6-31G* (gas phase) } \\
\hline & & $75 a$ & 75b & $75 c$ & 75d & - & - & - & - \\
\hline & ${ }^{1} \mathrm{H}$ data & 0.01 & 0.00 & 86.17 & 13.82 & - & - & - & - \\
\hline \multirow[t]{3}{*}{ sDP4+ } & ${ }^{13} \mathrm{C}$ data & 0.00 & 36.73 & 61.63 & 1.63 & - & - & - & - \\
\hline & All data & 0.00 & 0.00 & 99.58 & 0.42 & - & - & - & - \\
\hline & ${ }^{1} \mathrm{H}$ data & 0.09 & 0.06 & 94.34 & 5.51 & - & - & - & - \\
\hline \multirow[t]{3}{*}{ uDP4+ } & ${ }^{13} \mathrm{C}$ data & 0.12 & 11.27 & 77.35 & 11.26 & - & - & - & - \\
\hline & All data & 0.00 & 0.01 & 99.15 & 0.84 & - & - & - & - \\
\hline & ${ }^{1} \mathrm{H}$ data & 0.00 & 0.00 & 99.07 & 0.93 & - & - & - & - \\
\hline \multirow[t]{5}{*}{ DP4+ } & ${ }^{13} \mathrm{C}$ data & 0.00 & 7.96 & 91.69 & 0.35 & - & - & - & - \\
\hline & All data & 0.00 & 0.00 & 100.00 & 0.00 & - & - & - & - \\
\hline & & \multicolumn{8}{|c|}{ Level of theory: mPW1PW91/6-31G** (gas phase) } \\
\hline & & $75 a$ & 75b & $75 c$ & 75d & - & - & - & - \\
\hline & ${ }^{1}$ H data & 0.00 & 0.01 & 92.84 & 7.15 & - & - & - & - \\
\hline \multirow[t]{3}{*}{ sDP4+ } & ${ }^{13} \mathrm{C}$ data & 0.02 & 24.63 & 72.75 & 2.61 & - & - & - & - \\
\hline & All data & 0.00 & 0.00 & 99.72 & 0.28 & - & - & - & - \\
\hline & ${ }^{1} \mathrm{H}$ data & 0.69 & 0.23 & 91.09 & 8.00 & - & - & - & - \\
\hline uDP4+ & ${ }^{13} \mathrm{C}$ data & 0.14 & 6.76 & 79.39 & 13.71 & - & - & - & - \\
\hline & All data & 0.00 & 0.02 & 98.48 & 1.49 & - & - & - & - \\
\hline & ${ }^{1} \mathrm{H}$ data & 0.00 & 0.00 & 99.33 & 0.67 & - & - & - & - \\
\hline DP4+ & ${ }^{13} \mathrm{C}$ data & 0.00 & 2.79 & 96.62 & 0.60 & - & - & - & - \\
\hline & All data & 0.00 & 0.00 & 100.00 & 0.00 & - & - & - & - \\
\hline & & & & evel of $\mathrm{th}$ & : mPW1 & $5-3$ & $\mathrm{pr}$ & & \\
\hline & & $75 a$ & $75 b$ & $75 c$ & 75d & - & - & - & - \\
\hline & ${ }^{1} \mathrm{H}$ data & 5.13 & 0.01 & 89.29 & 5.57 & - & - & - & - \\
\hline sDP4+ & ${ }^{13} \mathrm{C}$ data & 0.01 & 9.90 & 31.97 & 58.12 & - & - & - & - \\
\hline & All data & 0.00 & 0.00 & 89.81 & 10.19 & - & - & - & - \\
\hline uDP4+ & ${ }^{1} \mathrm{H}$ data & 10.06 & 0.28 & 70.04 & 19.61 & - & - & - & - \\
\hline
\end{tabular}




\begin{tabular}{|c|c|c|c|c|c|c|c|c|c|}
\hline & ${ }^{13} \mathrm{C}$ data & 0.99 & 4.71 & 25.35 & 68.95 & - & - & - & - \\
\hline & All data & 0.32 & 0.04 & 56.56 & 43.08 & - & - & - & - \\
\hline & ${ }^{1} \mathrm{H}$ data & 0.80 & 0.00 & 97.49 & 1.70 & - & - & - & - \\
\hline \multirow[t]{5}{*}{ DP4+ } & ${ }^{13} \mathrm{C}$ data & 0.00 & 0.96 & 16.66 & 82.38 & - & - & - & - \\
\hline & All data & 0.00 & 0.00 & 92.05 & 7.95 & - & - & - & - \\
\hline & & \multicolumn{8}{|c|}{ Level of theory: mPW1PW91/6-311G* (gas phase) } \\
\hline & & $75 a$ & $75 b$ & $75 c$ & $75 d$ & - & - & - & - \\
\hline & ${ }^{1}$ H data & 0.31 & 0.04 & 96.54 & 3.11 & - & - & - & - \\
\hline \multirow[t]{3}{*}{ sDP4+ } & ${ }^{13}$ C data & 0.10 & 10.34 & 38.29 & 51.26 & - & - & - & - \\
\hline & All data & 0.00 & 0.01 & 95.85 & 4.14 & - & - & - & - \\
\hline & ${ }^{1} \mathrm{H}$ data & 1.70 & 1.13 & 89.56 & 7.62 & - & - & - & - \\
\hline \multirow[t]{3}{*}{ uDP4+ } & ${ }^{13} \mathrm{C}$ data & 1.69 & 0.14 & 0.49 & 97.67 & - & - & - & - \\
\hline & All data & 0.36 & 0.02 & 5.58 & 94.04 & - & - & - & - \\
\hline & ${ }^{1} \mathrm{H}$ data & 0.01 & 0.00 & 99.72 & 0.27 & - & - & - & - \\
\hline \multirow[t]{5}{*}{ DP4+ } & ${ }^{13} \mathrm{C}$ data & 0.00 & 0.03 & 0.38 & 99.59 & - & - & - & - \\
\hline & All data & 0.00 & 0.00 & 57.90 & 42.10 & - & - & - & - \\
\hline & & \multicolumn{8}{|c|}{ Level of theory: mPW1PW91/6-311G** (gas phase) } \\
\hline & & $75 a$ & 75b & 75c & 75d & - & - & - & - \\
\hline & ${ }^{1} \mathrm{H}$ data & 0.01 & 0.10 & 96.47 & 3.42 & - & - & - & - \\
\hline \multirow[t]{3}{*}{ sDP4+ } & ${ }^{13} \mathrm{C}$ data & 0.11 & 12.33 & 55.16 & 32.40 & - & - & - & - \\
\hline & All data & 0.00 & 0.02 & 97.94 & 2.04 & - & - & - & - \\
\hline & ${ }^{1} \mathrm{H}$ data & 2.13 & 1.24 & 87.63 & 9.00 & - & - & - & - \\
\hline \multirow[t]{3}{*}{$u$ DP4+ } & ${ }^{13} \mathrm{C}$ data & 2.38 & 0.11 & 0.30 & 97.20 & - & - & - & - \\
\hline & All data & 0.56 & 0.02 & 2.93 & 96.50 & - & - & - & - \\
\hline & ${ }^{1} \mathrm{H}$ data & 0.00 & 0.00 & 99.64 & 0.36 & - & - & - & - \\
\hline \multirow[t]{5}{*}{ DP4+ } & ${ }^{13} \mathrm{C}$ data & 0.01 & 0.04 & 0.53 & 99.42 & - & - & - & - \\
\hline & All data & 0.00 & 0.00 & 59.25 & 40.75 & - & - & - & - \\
\hline & & \multicolumn{8}{|c|}{ Level of theory: mPW1PW91/6-311+G** (gas phase) } \\
\hline & & $75 a$ & $75 b$ & $75 c$ & $75 d$ & - & - & - & - \\
\hline & ${ }^{1} \mathrm{H}$ data & 3.04 & 0.56 & 88.03 & 8.37 & - & - & - & - \\
\hline \multirow[t]{2}{*}{ sDP4+ } & ${ }^{13} \mathrm{C}$ data & 0.07 & 7.25 & 42.67 & 50.00 & - & - & - & - \\
\hline & All data & 0.01 & 0.10 & 89.89 & 10.01 & - & - & - & - \\
\hline \multirow{3}{*}{ uDP4+ } & ${ }^{1} \mathrm{H}$ data & 6.96 & 1.90 & 79.92 & 11.22 & - & - & - & - \\
\hline & ${ }^{13} \mathrm{C}$ data & 1.65 & 0.13 & 0.18 & 98.04 & - & - & - & - \\
\hline & All data & 1.02 & 0.02 & 1.25 & 97.71 & - & - & - & - \\
\hline \multirow{5}{*}{ DP4+ } & ${ }^{1} \mathrm{H}$ data & 0.30 & 0.01 & 98.38 & 1.31 & - & - & - & - \\
\hline & ${ }^{13} \mathrm{C}$ data & 0.00 & 0.02 & 0.15 & 99.83 & - & - & - & - \\
\hline & All data & 0.00 & 0.00 & 10.27 & 89.73 & - & - & - & - \\
\hline & & \multicolumn{8}{|c|}{ Level of theory: mPW1PW91/6-31G* (solution, PCM) } \\
\hline & & $75 a$ & $75 b$ & $75 c$ & $75 d$ & - & - & - & - \\
\hline \multirow{3}{*}{ sDP4+ } & ${ }^{1} \mathrm{H}$ data & 0.00 & 0.00 & 98.70 & 1.30 & - & - & - & - \\
\hline & ${ }^{13} \mathrm{C}$ data & 0.01 & 51.07 & 48.42 & 0.51 & - & - & - & - \\
\hline & All data & 0.00 & 0.00 & 99.99 & 0.01 & - & - & - & - \\
\hline \multirow{3}{*}{ uDP4+ } & ${ }^{1} \mathrm{H}$ data & 0.01 & 0.04 & 97.95 & 2.00 & - & - & - & - \\
\hline & ${ }^{13} \mathrm{C}$ data & 0.17 & 15.04 & 78.39 & 6.40 & - & - & - & - \\
\hline & All data & 0.00 & 0.01 & 99.83 & 0.17 & - & - & - & - \\
\hline & ${ }^{1} \mathrm{H}$ data & 0.00 & 0.00 & 99.97 & 0.03 & - & - & - & - \\
\hline DP4+ & ${ }^{13} \mathrm{C}$ data & 0.00 & 16.82 & 83.11 & 0.07 & - & - & - & - \\
\hline & All data & 0.00 & 0.00 & 100.00 & 0.00 & - & - & - & - \\
\hline & & & & el of the & nPW1P & & on, & & \\
\hline & & $75 a$ & $75 b$ & $75 c$ & $75 d$ & - & - & - & - \\
\hline & ${ }^{1} \mathrm{H}$ data & 0.00 & 0.00 & 99.65 & 0.35 & - & - & - & - \\
\hline sDP4+ & ${ }^{13} \mathrm{C}$ data & 0.02 & 34.69 & 64.67 & 0.63 & - & - & - & - \\
\hline & All data & 0.00 & 0.00 & 99.99 & 0.00 & - & - & - & - \\
\hline & ${ }^{1} \mathrm{H}$ data & 0.05 & 0.09 & 97.14 & 2.71 & - & - & - & - \\
\hline uDP4+ & ${ }^{13} \mathrm{C}$ data & 0.33 & 12.34 & 77.20 & 10.13 & - & - & - & - \\
\hline
\end{tabular}




\begin{tabular}{|c|c|c|c|c|c|c|c|c|c|}
\hline & All data & 0.00 & 0.02 & 99.62 & 0.37 & - & - & - & - \\
\hline & ${ }^{1} \mathrm{H}$ data & 0.00 & 0.00 & 99.99 & 0.01 & - & - & - & - \\
\hline \multirow[t]{5}{*}{ DP4+ } & ${ }^{13} \mathrm{C}$ data & 0.00 & 7.89 & 92.00 & 0.12 & - & - & - & - \\
\hline & All data & 0.00 & 0.00 & 100.00 & 0.00 & - & - & - & - \\
\hline & & \multicolumn{8}{|c|}{ Level of theory: mPW1PW91/6-31+G** (solution, PCM) } \\
\hline & & $75 a$ & $75 b$ & $75 c$ & $75 d$ & - & - & - & - \\
\hline & ${ }^{1} \mathrm{H}$ data & 0.55 & 0.04 & 96.24 & 3.17 & - & - & - & - \\
\hline \multirow[t]{3}{*}{ sDP4+ } & ${ }^{13} \mathrm{C}$ data & 0.02 & 18.77 & 42.08 & 39.14 & - & - & - & - \\
\hline & All data & 0.00 & 0.02 & 97.01 & 2.97 & - & - & - & - \\
\hline & ${ }^{1} \mathrm{H}$ data & 2.27 & 0.10 & 87.92 & 9.70 & - & - & - & - \\
\hline \multirow[t]{3}{*}{ uDP4+ } & ${ }^{13} \mathrm{C}$ data & 2.30 & 7.53 & 14.81 & 75.37 & - & - & - & - \\
\hline & All data & 0.26 & 0.04 & 63.85 & 35.86 & - & - & - & - \\
\hline & ${ }^{1} \mathrm{H}$ data & 0.01 & 0.00 & 99.62 & 0.36 & - & - & - & - \\
\hline \multirow[t]{5}{*}{ DP4+ } & ${ }^{13} \mathrm{C}$ data & 0.00 & 3.80 & 16.78 & 79.42 & - & - & - & - \\
\hline & All data & 0.00 & 0.00 & 98.31 & 1.69 & - & - & - & - \\
\hline & & \multicolumn{8}{|c|}{ Level of theory: mPW1PW91/6-311G* (solution, PCM) } \\
\hline & & $75 a$ & $75 b$ & 75c & 75d & - & - & - & - \\
\hline & ${ }^{1} \mathrm{H}$ data & 0.00 & 0.02 & 99.66 & 0.31 & - & - & - & - \\
\hline \multirow[t]{2}{*}{ sDP4+ } & ${ }^{13} \mathrm{C}$ data & 0.14 & 15.29 & 52.79 & 31.77 & - & - & - & - \\
\hline & All data & 0.00 & 0.01 & 99.81 & 0.19 & - & - & - & - \\
\hline \multirow{3}{*}{ uDP4+ } & ${ }^{1}$ H data & 0.11 & 0.59 & 96.66 & 2.64 & - & - & - & - \\
\hline & ${ }^{13} \mathrm{C}$ data & 2.79 & 0.14 & 0.20 & 96.87 & - & - & - & - \\
\hline & All data & 0.12 & 0.03 & 6.94 & 92.91 & - & - & - & - \\
\hline \multirow{3}{*}{ DP4+ } & ${ }^{1} \mathrm{H}$ data & 0.00 & 0.00 & 99.99 & 0.01 & - & - & - & - \\
\hline & ${ }^{13} \mathrm{C}$ data & 0.01 & 0.07 & 0.34 & 99.58 & - & - & - & - \\
\hline & All data & 0.00 & 0.00 & 97.57 & 2.43 & - & - & - & - \\
\hline & & \multicolumn{8}{|c|}{ Level of theory: mPW1PW91/6-311G** (solution, PCM) } \\
\hline & & $75 a$ & 75b & $75 c$ & $75 d$ & - & - & - & - \\
\hline \multirow{3}{*}{ sDP4+ } & ${ }^{1} \mathrm{H}$ data & 0.00 & 0.67 & 97.93 & 1.40 & - & - & - & - \\
\hline & ${ }^{13} \mathrm{C}$ data & 0.13 & 16.96 & 69.03 & 13.89 & - & - & - & - \\
\hline & All data & 0.00 & 0.17 & 99.55 & 0.29 & - & - & - & - \\
\hline \multirow{3}{*}{ uDP4+ } & ${ }^{1} \mathrm{H}$ data & 0.70 & 1.78 & 88.74 & 8.79 & - & - & - & - \\
\hline & ${ }^{13} \mathrm{C}$ data & 5.63 & 0.17 & 0.19 & 94.02 & - & - & - & - \\
\hline & All data & 0.46 & 0.03 & 1.98 & 97.52 & - & - & - & - \\
\hline \multirow{5}{*}{ DP4+ } & ${ }^{1} \mathrm{H}$ data & 0.00 & 0.01 & 99.85 & 0.14 & - & - & - & - \\
\hline & ${ }^{13} \mathrm{C}$ data & 0.05 & 0.21 & 0.99 & 98.74 & - & - & - & - \\
\hline & All data & 0.00 & 0.00 & 87.63 & 12.37 & - & - & - & - \\
\hline & & \multicolumn{8}{|c|}{ Level of theory: mPW1PW91/6-311+G** (solution, PCM) } \\
\hline & & $75 a$ & $75 b$ & 75c & $75 d$ & - & - & - & - \\
\hline \multirow{3}{*}{ sDP4+ } & ${ }^{1} \mathrm{H}$ data & 0.35 & 1.57 & 93.52 & 4.56 & - & - & - & - \\
\hline & ${ }^{13} \mathrm{C}$ data & 0.14 & 12.00 & 53.29 & 34.57 & - & - & - & - \\
\hline & All data & 0.00 & 0.36 & 96.58 & 3.06 & - & - & - & - \\
\hline \multirow{3}{*}{ uDP4+ } & ${ }^{1} \mathrm{H}$ data & 3.07 & 2.93 & 75.71 & 18.29 & - & - & - & - \\
\hline & ${ }^{13} \mathrm{C}$ data & 2.85 & 0.14 & 0.08 & 96.93 & - & - & - & - \\
\hline & All data & 0.49 & 0.02 & 0.32 & 99.16 & - & - & - & - \\
\hline \multirow{3}{*}{ DP4+ } & ${ }^{1} \mathrm{H}$ data & 0.01 & 0.06 & 98.76 & 1.16 & - & - & - & - \\
\hline & ${ }^{13} \mathrm{C}$ data & 0.01 & 0.05 & 0.12 & 99.82 & - & - & - & - \\
\hline & All data & 0.00 & 0.00 & 9.34 & 90.66 & - & - & - & - \\
\hline
\end{tabular}


sDP4+, $u \mathrm{DP} 4+$ and DP4+ probabilities (\%) for compound 75d<smiles>CC(C)C[C@H](C)[C@@H](O)[C@@H](C)C(=O)O</smiles><smiles>CC(C)C[C@H](C)[C@@H](O)[C@@H](C)C(=O)O</smiles><smiles>CC(C)C[C@H](C)[C@@H](O)[C@@H](CC(C)(C)C)C(=O)O</smiles><smiles>CC(C)C[C@H](C)[C@@H](O)[C@@H](C)C(=O)O</smiles>

\begin{tabular}{|c|c|c|c|c|c|c|c|c|c|}
\hline & \multicolumn{8}{|c|}{ Level of theory: B3LYP/6-31G* (gas phase) } \\
\hline & & $75 a$ & $75 \mathrm{~b}$ & $75 c$ & $75 d$ & - & - & - & - \\
\hline \multirow{3}{*}{$s \mathrm{DP} 4+$} & ${ }^{1} \mathrm{H}$ data & 0.02 & 0.01 & 27.45 & 72.52 & - & - & - & - \\
\hline & ${ }^{13} \mathrm{C}$ data & 0.37 & 73.26 & 19.35 & 7.02 & - & - & - & - \\
\hline & All data & 0.00 & 0.07 & 51.02 & 48.91 & - & - & - & - \\
\hline \multirow{3}{*}{ uDP4+ } & ${ }^{1} \mathrm{H}$ data & 0.87 & 0.24 & 69.40 & 29.49 & - & - & - & - \\
\hline & ${ }^{13} \mathrm{C}$ data & 0.97 & 47.70 & 27.27 & 24.06 & - & - & - & - \\
\hline & All data & 0.03 & 0.44 & 72.39 & 27.14 & - & - & - & - \\
\hline \multirow{3}{*}{ DP4+ } & ${ }^{1} \mathrm{H}$ data & 0.00 & 0.00 & 47.12 & 52.88 & - & - & - & - \\
\hline & ${ }^{13} \mathrm{C}$ data & 0.01 & 83.37 & 12.59 & 4.03 & - & - & - & - \\
\hline & All data & 0.00 & 0.00 & 73.56 & 26.44 & - & - & - & - \\
\hline & & \multicolumn{8}{|c|}{ Level of theory: B3LYP/6-31G** (gas phase) } \\
\hline & & $75 a$ & $75 \mathrm{~b}$ & $75 c$ & $75 d$ & - & - & - & - \\
\hline \multirow{3}{*}{$s \mathrm{DP} 4+$} & ${ }^{1} \mathrm{H}$ data & 0.01 & 0.15 & 58.68 & 41.15 & - & - & - & - \\
\hline & ${ }^{13} \mathrm{C}$ data & 0.60 & 61.80 & 28.80 & 8.80 & - & - & - & - \\
\hline & All data & 0.00 & 0.46 & 81.97 & 17.57 & - & - & - & - \\
\hline \multirow{3}{*}{ uDP4+ } & ${ }^{1} \mathrm{H}$ data & 1.51 & 1.07 & 77.34 & 20.08 & - & - & - & - \\
\hline & ${ }^{13} \mathrm{C}$ data & 2.44 & 28.38 & 32.74 & 36.44 & - & - & - & - \\
\hline & All data & 0.11 & 0.92 & 76.78 & 22.18 & - & - & - & - \\
\hline \multirow{3}{*}{ DP4+ } & ${ }^{1} \mathrm{H}$ data & 0.00 & 0.00 & 84.60 & 15.40 & - & - & - & - \\
\hline & ${ }^{13} \mathrm{C}$ data & 0.05 & 58.10 & 31.23 & 10.62 & - & - & - & - \\
\hline & All data & 0.00 & 0.01 & 94.16 & 5.83 & - & - & - & - \\
\hline & & \multicolumn{8}{|c|}{ Level of theory: B3LYP/6-31+G** (gas phase) } \\
\hline & & 75a & $75 \mathrm{~b}$ & $75 c$ & $75 d$ & - & - & - & - \\
\hline \multirow{3}{*}{ sDP4+ } & ${ }^{1} \mathrm{H}$ data & 27.48 & 0.11 & 14.38 & 58.04 & - & - & - & - \\
\hline & ${ }^{13} \mathrm{C}$ data & 0.07 & 3.47 & 1.82 & 94.64 & - & - & - & - \\
\hline & All data & 0.03 & 0.01 & 0.47 & 99.49 & - & - & - & - \\
\hline \multirow{3}{*}{ uDP4+ } & ${ }^{1} \mathrm{H}$ data & 49.61 & 0.16 & 20.14 & 30.08 & - & - & - & - \\
\hline & ${ }^{13} \mathrm{C}$ data & 2.71 & 4.77 & 1.74 & 90.78 & - & - & - & - \\
\hline & All data & 4.64 & 0.03 & 1.21 & 94.13 & - & - & - & - \\
\hline \multirow{3}{*}{ DP4+ } & ${ }^{1} \mathrm{H}$ data & 40.11 & 0.00 & 8.52 & 51.37 & - & - & - & - \\
\hline & ${ }^{13} \mathrm{C}$ data & 0.00 & 0.19 & 0.04 & 99.77 & - & - & - & - \\
\hline & All data & 0.00 & 0.00 & 0.01 & 99.99 & - & - & - & - \\
\hline & & \multicolumn{8}{|c|}{ Level of theory: B3LYP/6-311G* (gas phase) } \\
\hline & & $75 a$ & $75 b$ & $75 c$ & $75 d$ & - & - & - & - \\
\hline \multirow{3}{*}{ sDP4+ } & ${ }^{1} \mathrm{H}$ data & 1.18 & 0.99 & 41.38 & 56.45 & - & - & - & - \\
\hline & ${ }^{13} \mathrm{C}$ data & 0.21 & 5.68 & 4.00 & 90.11 & - & - & - & - \\
\hline & All data & 0.00 & 0.11 & 3.15 & 96.74 & - & - & - & - \\
\hline \multirow{3}{*}{ uDP4+ } & ${ }^{1} \mathrm{H}$ data & 8.15 & 4.84 & 68.61 & 18.40 & - & - & - & - \\
\hline & ${ }^{13} \mathrm{C}$ data & 2.41 & 0.29 & 0.21 & 97.09 & - & - & - & - \\
\hline & All data & 1.08 & 0.08 & 0.79 & 98.06 & - & - & - & - \\
\hline \multirow{3}{*}{ DP4+ } & ${ }^{1} \mathrm{H}$ data & 0.25 & 0.12 & 72.95 & 26.68 & - & - & - & - \\
\hline & ${ }^{13} \mathrm{C}$ data & 0.01 & 0.02 & 0.01 & 99.97 & - & - & - & - \\
\hline & All data & 0.00 & 0.00 & 0.03 & 99.97 & - & - & - & - \\
\hline & & \multicolumn{8}{|c|}{ Level of theory: B3LYP/6-311G** (gas phase) } \\
\hline & & $75 a$ & $75 \mathrm{~b}$ & $75 c$ & $75 d$ & - & - & - & - \\
\hline \multirow{2}{*}{ sDP4+ } & ${ }^{1} \mathrm{H}$ data & 0.04 & 2.91 & 44.83 & 52.23 & - & - & - & - \\
\hline & ${ }^{13} \mathrm{C}$ data & 0.70 & 15.97 & 13.10 & 70.22 & - & - & - & - \\
\hline
\end{tabular}




\begin{tabular}{|c|c|c|c|c|c|c|c|c|c|}
\hline & All data & 0.00 & 1.08 & 13.66 & 85.26 & - & - & - & - \\
\hline & ${ }^{1} \mathrm{H}$ data & 4.50 & 6.37 & 70.11 & 19.03 & - & - & - & - \\
\hline \multirow[t]{3}{*}{ uDP4+ } & ${ }^{13} \mathrm{C}$ data & 4.87 & 0.62 & 0.48 & 94.03 & - & - & - & - \\
\hline & All data & 1.18 & 0.21 & 1.81 & 96.80 & - & - & - & - \\
\hline & ${ }^{1} \mathrm{H}$ data & 0.00 & 0.45 & 75.63 & 23.92 & - & - & - & - \\
\hline \multirow[t]{5}{*}{ DP4+ } & ${ }^{13} \mathrm{C}$ data & 0.05 & 0.15 & 0.09 & 99.70 & - & - & - & - \\
\hline & All data & 0.00 & 0.00 & 0.30 & 99.70 & - & - & - & - \\
\hline & & \multicolumn{8}{|c|}{ Level of theory: B3LYP/6-311+G** (gas phase) } \\
\hline & & $75 a$ & 75b & $75 c$ & 75d & - & - & - & - \\
\hline & ${ }^{1} \mathrm{H}$ data & 10.01 & 1.44 & 15.84 & 72.71 & - & - & - & - \\
\hline \multirow[t]{3}{*}{ sDP4+ } & ${ }^{13} \mathrm{C}$ data & 0.07 & 2.79 & 2.35 & 94.78 & - & - & - & - \\
\hline & All data & 0.01 & 0.06 & 0.54 & 99.39 & - & - & - & - \\
\hline & ${ }^{1} \mathrm{H}$ data & 42.15 & 3.75 & 28.64 & 25.45 & - & - & - & - \\
\hline \multirow[t]{3}{*}{ uDP4+ } & ${ }^{13} \mathrm{C}$ data & 2.56 & 0.27 & 0.11 & 97.06 & - & - & - & - \\
\hline & All data & 4.18 & 0.04 & 0.12 & 95.66 & - & - & - & - \\
\hline & ${ }^{1} \mathrm{H}$ data & 15.44 & 0.20 & 16.61 & 67.74 & - & - & - & - \\
\hline \multirow[t]{5}{*}{ DP4+ } & ${ }^{13} \mathrm{C}$ data & 0.00 & 0.01 & 0.00 & 99.99 & - & - & - & - \\
\hline & All data & 0.00 & 0.00 & 0.00 & 100.00 & - & - & - & - \\
\hline & & \multicolumn{8}{|c|}{ Level of theory: B3LYP/6-31G* (solution, PCM) } \\
\hline & & $75 a$ & $75 b$ & $75 c$ & $75 d$ & - & - & - & - \\
\hline & ${ }^{1} \mathrm{H}$ data & 0.00 & 0.02 & 77.63 & 22.35 & - & - & - & - \\
\hline \multirow[t]{3}{*}{ sDP4+ } & ${ }^{13} \mathrm{C}$ data & 0.22 & 85.88 & 12.76 & 1.14 & - & - & - & - \\
\hline & All data & 0.00 & 0.13 & 97.37 & 2.49 & - & - & - & - \\
\hline & ${ }^{1} \mathrm{H}$ data & 0.16 & 0.13 & 86.23 & 13.48 & - & - & - & - \\
\hline \multirow[t]{3}{*}{ uDP4+ } & ${ }^{13} \mathrm{C}$ data & 1.59 & 59.68 & 25.94 & 12.78 & - & - & - & - \\
\hline & All data & 0.01 & 0.32 & 92.54 & 7.13 & - & - & - & - \\
\hline & ${ }^{1} \mathrm{H}$ data & 0.00 & 0.00 & 95.69 & 4.31 & - & - & - & - \\
\hline \multirow[t]{5}{*}{ DP4+ } & ${ }^{13} \mathrm{C}$ data & 0.01 & 93.68 & 6.05 & 0.27 & - & - & - & - \\
\hline & All data & 0.00 & 0.00 & 99.80 & 0.20 & - & - & - & - \\
\hline & & \multicolumn{8}{|c|}{ Level of theory: B3LYP/6-31G** (solution, PCM) } \\
\hline & & $75 a$ & 75b & $75 c$ & 75d & - & - & - & - \\
\hline & ${ }^{1} \mathrm{H}$ data & 0.00 & 0.20 & 93.21 & 6.59 & - & - & - & - \\
\hline \multirow[t]{3}{*}{ sDP4+ } & ${ }^{13} \mathrm{C}$ data & 0.62 & 74.66 & 22.41 & 2.31 & - & - & - & - \\
\hline & All data & 0.00 & 0.69 & 98.59 & 0.72 & - & - & - & - \\
\hline & ${ }^{1} \mathrm{H}$ data & 0.27 & 0.84 & 85.68 & 13.22 & - & - & - & - \\
\hline \multirow[t]{3}{*}{ uDP4+ } & ${ }^{13} \mathrm{C}$ data & 5.22 & 43.95 & 31.37 & 19.46 & - & - & - & - \\
\hline & All data & 0.05 & 1.23 & 90.10 & 8.62 & - & - & - & - \\
\hline & ${ }^{1} \mathrm{H}$ data & 0.00 & 0.00 & 98.92 & 1.08 & - & - & - & - \\
\hline \multirow[t]{5}{*}{ DP4+ } & ${ }^{13} \mathrm{C}$ data & 0.08 & 81.37 & 17.44 & 1.11 & - & - & - & - \\
\hline & All data & 0.00 & 0.01 & 99.92 & 0.07 & - & - & - & - \\
\hline & & \multicolumn{8}{|c|}{ Level of theory: B3LYP/6-31+G** (solution, PCM) } \\
\hline & & $75 a$ & $75 b$ & $75 c$ & 75d & - & - & - & - \\
\hline & ${ }^{1} \mathrm{H}$ data & 1.29 & 0.13 & 25.69 & 72.88 & - & - & - & - \\
\hline \multirow[t]{3}{*}{ sDP4+ } & ${ }^{13} \mathrm{C}$ data & 0.25 & 11.61 & 4.23 & 83.91 & - & - & - & - \\
\hline & All data & 0.01 & 0.03 & 1.74 & 98.23 & - & - & - & - \\
\hline & ${ }^{1} \mathrm{H}$ data & 26.11 & 0.30 & 24.94 & 48.65 & - & - & - & - \\
\hline uDP4+ & ${ }^{13} \mathrm{C}$ data & 4.92 & 6.55 & 1.12 & 87.41 & - & - & - & - \\
\hline & All data & 2.92 & 0.04 & 0.63 & 96.41 & - & - & - & - \\
\hline & ${ }^{1} \mathrm{H}$ data & 0.80 & 0.00 & 15.18 & 84.02 & - & - & - & - \\
\hline DP4+ & ${ }^{13} \mathrm{C}$ data & 0.02 & 1.02 & 0.06 & 98.89 & - & - & - & - \\
\hline & All data & 0.00 & 0.00 & 0.01 & 99.99 & - & - & - & - \\
\hline & & & & Level of & $y: B 3 L Y$ & G* & PC & & \\
\hline & & $75 a$ & $75 b$ & $75 c$ & 75d & - & - & - & - \\
\hline & ${ }^{1} \mathrm{H}$ data & 0.05 & 2.45 & 72.48 & 25.02 & - & - & - & - \\
\hline sDP4+ & ${ }^{13} \mathrm{C}$ data & 0.80 & 27.09 & 13.06 & 59.04 & - & - & - & - \\
\hline & All data & 0.00 & 2.67 & 38.01 & 59.32 & - & - & - & - \\
\hline
\end{tabular}




\begin{tabular}{|c|c|c|c|c|c|c|c|c|c|}
\hline & ${ }^{1} \mathrm{H}$ data & 2.57 & 4.30 & 81.87 & 11.25 & - & - & - & - \\
\hline \multirow[t]{3}{*}{ uDP4+ } & ${ }^{13} \mathrm{C}$ data & 6.04 & 0.61 & 0.25 & 93.10 & - & - & - & - \\
\hline & All data & 1.43 & 0.24 & 1.85 & 96.48 & - & - & - & - \\
\hline & ${ }^{1} \mathrm{H}$ data & 0.00 & 0.17 & 95.31 & 4.52 & - & - & - & - \\
\hline \multirow[t]{5}{*}{ DP4+ } & ${ }^{13} \mathrm{C}$ data & 0.09 & 0.30 & 0.06 & 99.55 & - & - & - & - \\
\hline & All data & 0.00 & 0.01 & 1.21 & 98.78 & - & - & - & - \\
\hline & & \multicolumn{8}{|c|}{ Level of theory: B3LYP/6-311G** (solution, PCM) } \\
\hline & & $75 a$ & 75b & $75 c$ & 75d & - & - & - & - \\
\hline & ${ }^{1} \mathrm{H}$ data & 0.03 & 14.30 & 45.22 & 40.45 & - & - & - & - \\
\hline \multirow[t]{3}{*}{ sDP4+ } & ${ }^{13} \mathrm{C}$ data & 1.42 & 42.04 & 24.04 & 32.49 & - & - & - & - \\
\hline & All data & 0.00 & 20.02 & 36.21 & 43.77 & - & - & - & - \\
\hline & ${ }^{1} \mathrm{H}$ data & 1.74 & 9.23 & 66.73 & 22.30 & - & - & - & - \\
\hline \multirow[t]{3}{*}{ uDP4+ } & ${ }^{13} \mathrm{C}$ data & 11.29 & 1.12 & 0.46 & 87.12 & - & - & - & - \\
\hline & All data & 0.98 & 0.52 & 1.54 & 96.96 & - & - & - & - \\
\hline & ${ }^{1} \mathrm{H}$ data & 0.00 & 3.26 & 74.48 & 22.26 & - & - & - & - \\
\hline \multirow[t]{5}{*}{ DP4+ } & ${ }^{13} \mathrm{C}$ data & 0.55 & 1.62 & 0.38 & 97.44 & - & - & - & - \\
\hline & All data & 0.00 & 0.24 & 1.30 & 98.46 & - & - & - & - \\
\hline & & \multicolumn{8}{|c|}{ Level of theory: B3LYP/6-311+G** (solution, PCM) } \\
\hline & & $75 a$ & 75b & $75 c$ & 75d & - & - & - & - \\
\hline & ${ }^{1} \mathrm{H}$ data & 1.63 & 5.48 & 19.43 & 73.46 & - & - & - & - \\
\hline \multirow[t]{3}{*}{ sDP4+ } & ${ }^{13} \mathrm{C}$ data & 0.33 & 10.57 & 7.60 & 81.50 & - & - & - & - \\
\hline & All data & 0.01 & 0.94 & 2.38 & 96.67 & - & - & - & - \\
\hline & ${ }^{1} \mathrm{H}$ data & 15.86 & 3.25 & 29.50 & 51.39 & - & - & - & - \\
\hline \multirow[t]{3}{*}{ uDP4+ } & ${ }^{13} \mathrm{C}$ data & 3.57 & 0.32 & 0.07 & 96.04 & - & - & - & - \\
\hline & All data & 1.13 & 0.02 & 0.04 & 98.80 & - & - & - & - \\
\hline & ${ }^{1} \mathrm{H}$ data & 0.59 & 0.40 & 13.05 & 85.96 & - & - & - & - \\
\hline \multirow[t]{5}{*}{ DP4+ } & ${ }^{13} \mathrm{C}$ data & 0.01 & 0.04 & 0.01 & 99.93 & - & - & - & - \\
\hline & All data & 0.00 & 0.00 & 0.00 & 100.00 & - & - & - & - \\
\hline & & \multicolumn{8}{|c|}{ Level of theory: mPW1PW91/6-31G* (gas phase) } \\
\hline & & $75 a$ & 75b & $75 c$ & 75d & - & - & - & - \\
\hline & ${ }^{1} \mathrm{H}$ data & 0.18 & 0.01 & 8.18 & 91.63 & - & - & - & - \\
\hline \multirow[t]{3}{*}{ sDP4+ } & ${ }^{13} \mathrm{C}$ data & 0.05 & 83.79 & 8.28 & 7.88 & - & - & - & - \\
\hline & All data & 0.00 & 0.06 & 8.57 & 91.36 & - & - & - & - \\
\hline & ${ }^{1} \mathrm{H}$ data & 2.89 & 0.21 & 47.79 & 49.11 & - & - & - & - \\
\hline \multirow[t]{3}{*}{ uDP4+ } & ${ }^{13} \mathrm{C}$ data & 0.67 & 55.09 & 23.14 & 21.11 & - & - & - & - \\
\hline & All data & 0.09 & 0.53 & 51.29 & 48.09 & - & - & - & - \\
\hline & ${ }^{1} \mathrm{H}$ data & 0.01 & 0.00 & 7.99 & 92.00 & - & - & - & - \\
\hline \multirow[t]{5}{*}{ DP4+ } & ${ }^{13} \mathrm{C}$ data & 0.00 & 92.80 & 3.85 & 3.34 & - & - & - & - \\
\hline & All data & 0.00 & 0.00 & 9.10 & 90.90 & - & - & - & - \\
\hline & & \multicolumn{8}{|c|}{ Level of theory: mPW1PW91/6-31G** (gas phase) } \\
\hline & & $75 a$ & $75 b$ & $75 c$ & 75d & - & - & - & - \\
\hline & ${ }^{1} \mathrm{H}$ data & 0.08 & 0.16 & 25.14 & 74.61 & - & - & - & - \\
\hline \multirow[t]{3}{*}{ sDP4+ } & ${ }^{13} \mathrm{C}$ data & 0.14 & 75.43 & 13.88 & 10.55 & - & - & - & - \\
\hline & All data & 0.00 & 1.06 & 30.39 & 68.55 & - & - & - & - \\
\hline & ${ }^{1} \mathrm{H}$ data & 8.85 & 1.07 & 51.94 & 38.13 & - & - & - & - \\
\hline uDP4+ & ${ }^{13} \mathrm{C}$ data & 1.30 & 29.42 & 26.30 & 42.99 & - & - & - & - \\
\hline & All data & 0.38 & 1.03 & 44.81 & 53.78 & - & - & - & - \\
\hline & ${ }^{1} \mathrm{H}$ data & 0.02 & 0.00 & 31.45 & 68.53 & - & - & - & - \\
\hline DP4+ & ${ }^{13} \mathrm{C}$ data & 0.01 & 73.05 & 12.01 & 14.93 & - & - & - & - \\
\hline & All data & 0.00 & 0.02 & 26.97 & 73.01 & - & - & - & - \\
\hline & & & & evel of $\mathrm{tl}$ & : mPW1 & -3 & pr & & \\
\hline & & $75 a$ & $75 b$ & $75 c$ & $75 d$ & - & - & - & - \\
\hline & ${ }^{1} \mathrm{H}$ data & 77.87 & 0.03 & 2.54 & 19.56 & - & - & - & - \\
\hline sDP4+ & ${ }^{13} \mathrm{C}$ data & 0.01 & 2.91 & 0.64 & 96.44 & - & - & - & - \\
\hline & All data & 0.04 & 0.01 & 0.09 & 99.86 & - & - & - & - \\
\hline uDP4+ & ${ }^{1} \mathrm{H}$ data & 66.67 & 0.27 & 8.64 & 24.41 & - & - & - & - \\
\hline
\end{tabular}




\begin{tabular}{|c|c|c|c|c|c|c|c|c|c|}
\hline & ${ }^{13} \mathrm{C}$ data & 2.76 & 4.95 & 3.09 & 89.21 & - & - & - & - \\
\hline & All data & 7.70 & 0.06 & 1.12 & 91.12 & - & - & - & - \\
\hline & ${ }^{1} \mathrm{H}$ data & 91.23 & 0.00 & 0.39 & 8.39 & - & - & - & - \\
\hline \multirow[t]{4}{*}{ DP4+ } & ${ }^{13} \mathrm{C}$ data & 0.00 & 0.17 & 0.02 & 99.81 & - & - & - & - \\
\hline & All data & 0.00 & 0.00 & 0.00 & 100.00 & - & - & - & - \\
\hline & & \multicolumn{8}{|c|}{ Level of theory: mPW1PW91/6-311G* (gas phase) } \\
\hline & & $75 a$ & $75 b$ & $75 c$ & $75 d$ & - & - & - & - \\
\hline & ${ }^{1} \mathrm{H}$ data & 13.43 & 0.94 & 26.43 & 59.19 & - & - & - & - \\
\hline \multirow[t]{2}{*}{ sDP4+ } & ${ }^{13} \mathrm{C}$ data & 0.25 & 12.38 & 4.42 & 82.95 & - & - & - & - \\
\hline & All data & 0.07 & 0.23 & 2.32 & 97.38 & - & - & - & - \\
\hline & ${ }^{1} \mathrm{H}$ data & 16.23 & 4.17 & 58.82 & 20.78 & - & - & - & - \\
\hline \multirow[t]{2}{*}{ uDP4+ } & ${ }^{13} \mathrm{C}$ data & 5.52 & 0.28 & 0.28 & 93.92 & - & - & - & - \\
\hline & All data & 4.35 & 0.06 & 0.81 & 94.78 & - & - & - & - \\
\hline \multirow{5}{*}{ DP4+ } & ${ }^{1} \mathrm{H}$ data & 7.25 & 0.13 & 51.71 & 40.91 & - & - & - & - \\
\hline & ${ }^{13} \mathrm{C}$ data & 0.02 & 0.04 & 0.02 & 99.92 & - & - & - & - \\
\hline & All data & 0.00 & 0.00 & 0.02 & 99.98 & - & - & - & - \\
\hline & & \multicolumn{8}{|c|}{ Level of theory: mPW1PW91/6-311G** (gas phase) } \\
\hline & & $75 a$ & $75 b$ & $75 c$ & 75d & - & - & - & - \\
\hline & ${ }^{1} \mathrm{H}$ data & 0.30 & 2.77 & 30.23 & 66.71 & - & - & - & - \\
\hline \multirow[t]{2}{*}{$s \mathrm{DP} 4+$} & ${ }^{13} \mathrm{C}$ data & 0.39 & 21.37 & 8.46 & 69.79 & - & - & - & - \\
\hline & All data & 0.00 & 1.19 & 5.14 & 93.66 & - & - & - & - \\
\hline \multirow{3}{*}{ uDP4+ } & ${ }^{1} \mathrm{H}$ data & 15.79 & 5.15 & 54.43 & 24.63 & - & - & - & - \\
\hline & ${ }^{13} \mathrm{C}$ data & 7.57 & 0.32 & 0.31 & 91.80 & - & - & - & - \\
\hline & All data & 4.98 & 0.07 & 0.70 & 94.25 & - & - & - & - \\
\hline \multirow{3}{*}{ DP4+ } & ${ }^{1} \mathrm{H}$ data & 0.14 & 0.43 & 49.75 & 49.67 & - & - & - & - \\
\hline & ${ }^{13} \mathrm{C}$ data & 0.05 & 0.11 & 0.04 & 99.81 & - & - & - & - \\
\hline & All data & 0.00 & 0.00 & 0.04 & 99.96 & - & - & - & - \\
\hline & & \multicolumn{8}{|c|}{ Level of theory: mPW1PW91/6-311+G** (gas phase) } \\
\hline & & $75 a$ & $75 b$ & $75 c$ & 75d & - & - & - & - \\
\hline \multirow{3}{*}{ sDP4+ } & ${ }^{1} \mathrm{H}$ data & 26.50 & 1.60 & 9.86 & 62.04 & - & - & - & - \\
\hline & ${ }^{13} \mathrm{C}$ data & 0.19 & 8.66 & 4.02 & 87.13 & - & - & - & - \\
\hline & All data & 0.09 & 0.25 & 0.73 & 98.93 & - & - & - & - \\
\hline \multirow{3}{*}{ uDP4+ } & ${ }^{1} \mathrm{H}$ data & 54.02 & 2.63 & 18.68 & 24.67 & - & - & - & - \\
\hline & ${ }^{13} \mathrm{C}$ data & 6.65 & 0.30 & 0.13 & 92.92 & - & - & - & - \\
\hline & All data & 13.54 & 0.03 & 0.09 & 86.34 & - & - & - & - \\
\hline \multirow{5}{*}{ DP4+ } & ${ }^{1} \mathrm{H}$ data & 45.44 & 0.13 & 5.85 & 48.58 & - & - & - & - \\
\hline & ${ }^{13} \mathrm{C}$ data & 0.02 & 0.03 & 0.01 & 99.95 & - & - & - & - \\
\hline & All data & 0.01 & 0.00 & 0.00 & 99.98 & - & - & - & - \\
\hline & & \multicolumn{8}{|c|}{ Level of theory: mPW1PW91/6-31G* (solution, PCM) } \\
\hline & & $75 a$ & $75 b$ & $75 c$ & 75d & - & - & - & - \\
\hline \multirow{3}{*}{ sDP4+ } & ${ }^{1} \mathrm{H}$ data & 0.02 & 0.04 & 45.12 & 54.81 & - & - & - & - \\
\hline & ${ }^{13} \mathrm{C}$ data & 0.04 & 94.22 & 4.48 & 1.26 & - & - & - & - \\
\hline & All data & 0.00 & 1.53 & 73.40 & 25.07 & - & - & - & - \\
\hline \multirow{3}{*}{ uDP4+ } & ${ }^{1} \mathrm{H}$ data & 0.91 & 0.21 & 68.08 & 30.81 & - & - & - & - \\
\hline & ${ }^{13} \mathrm{C}$ data & 1.01 & 64.35 & 22.74 & 11.90 & - & - & - & - \\
\hline & All data & 0.05 & 0.69 & 80.25 & 19.01 & - & - & - & - \\
\hline & ${ }^{1} \mathrm{H}$ data & 0.00 & 0.00 & 64.53 & 35.47 & - & - & - & - \\
\hline DP4+ & ${ }^{13} \mathrm{C}$ data & 0.00 & 98.11 & 1.65 & 0.24 & - & - & - & - \\
\hline & All data & 0.00 & 0.02 & 92.50 & 7.48 & - & - & - & - \\
\hline & & & & el of th & mPW1PV & $31 C$ & n, & & \\
\hline & & $75 a$ & $75 b$ & $75 c$ & 75d & - & - & - & - \\
\hline & ${ }^{1} \mathrm{H}$ data & 0.00 & 0.40 & 75.48 & 24.11 & - & - & - & - \\
\hline sDP4+ & ${ }^{13} \mathrm{C}$ data & 0.13 & 88.84 & 8.97 & 2.06 & - & - & - & - \\
\hline & All data & 0.00 & 4.70 & 88.78 & 6.52 & - & - & - & - \\
\hline & ${ }^{1} \mathrm{H}$ data & 1.02 & 0.95 & 70.61 & 27.42 & - & - & - & - \\
\hline uDP4+ & ${ }^{13} \mathrm{C}$ data & 2.55 & 40.86 & 29.99 & 26.59 & - & - & - & - \\
\hline
\end{tabular}




\begin{tabular}{|c|c|c|c|c|c|c|c|c|c|}
\hline & All data & 0.09 & 1.34 & 73.33 & 25.24 & - & - & - & - \\
\hline & ${ }^{1} \mathrm{H}$ data & 0.00 & 0.01 & 88.96 & 11.04 & - & - & - & - \\
\hline \multirow[t]{5}{*}{ DP4+ } & ${ }^{13} \mathrm{C}$ data & 0.01 & 91.80 & 6.80 & 1.39 & - & - & - & - \\
\hline & All data & 0.00 & 0.09 & 97.44 & 2.46 & - & - & - & - \\
\hline & & \multicolumn{8}{|c|}{ Level of theory: mPW1PW91/6-31+G** (solution, PCM) } \\
\hline & & $75 a$ & $75 b$ & $75 c$ & $75 d$ & - & - & - & - \\
\hline & ${ }^{1} \mathrm{H}$ data & 16.53 & 0.42 & 12.62 & 70.42 & - & - & - & - \\
\hline \multirow[t]{3}{*}{ sDP4+ } & ${ }^{13} \mathrm{C}$ data & 0.04 & 13.23 & 1.76 & 84.97 & - & - & - & - \\
\hline & All data & 0.01 & 0.09 & 0.37 & 99.53 & - & - & - & - \\
\hline & ${ }^{1}$ H data & 51.93 & 0.20 & 12.14 & 35.73 & - & - & - & - \\
\hline \multirow[t]{3}{*}{ uDP4+ } & ${ }^{13} \mathrm{C}$ data & 5.72 & 6.91 & 1.91 & 85.47 & - & - & - & - \\
\hline & All data & 8.79 & 0.04 & 0.69 & 90.48 & - & - & - & - \\
\hline & ${ }^{1} \mathrm{H}$ data & 24.33 & 0.00 & 4.34 & 71.32 & - & - & - & - \\
\hline \multirow[t]{5}{*}{ DP4+ } & ${ }^{13} \mathrm{C}$ data & 0.00 & 1.24 & 0.05 & 98.71 & - & - & - & - \\
\hline & All data & 0.00 & 0.00 & 0.00 & 100.00 & - & - & - & - \\
\hline & & \multicolumn{8}{|c|}{ Level of theory: mPW1PW91/6-311G* (solution, PCM) } \\
\hline & & $75 a$ & $75 b$ & $75 c$ & 75d & - & - & - & - \\
\hline & ${ }^{1} \mathrm{H}$ data & 0.62 & 2.52 & 57.40 & 39.46 & - & - & - & - \\
\hline \multirow[t]{3}{*}{ sDP4+ } & ${ }^{13} \mathrm{C}$ data & 0.49 & 29.68 & 7.16 & 62.67 & - & - & - & - \\
\hline & All data & 0.01 & 2.53 & 13.89 & 83.57 & - & - & - & - \\
\hline & ${ }^{1}$ H data & 6.72 & 3.58 & 71.64 & 18.05 & - & - & - & - \\
\hline \multirow[t]{3}{*}{ uDP4+ } & ${ }^{13}$ C data & 6.76 & 0.24 & 0.10 & 92.90 & - & - & - & - \\
\hline & All data & 2.63 & 0.05 & 0.42 & 96.91 & - & - & - & - \\
\hline & ${ }^{1} \mathrm{H}$ data & 0.09 & 0.19 & 85.00 & 14.72 & - & - & - & - \\
\hline \multirow[t]{5}{*}{ DP4+ } & ${ }^{13} \mathrm{C}$ data & 0.06 & 0.12 & 0.01 & 99.81 & - & - & - & - \\
\hline & All data & 0.00 & 0.00 & 0.07 & 99.93 & - & - & - & - \\
\hline & & \multicolumn{8}{|c|}{ Level of theory: mPW1PW91/6-311G** (solution, PCM) } \\
\hline & & $75 a$ & $75 b$ & $75 c$ & $75 d$ & - & - & - & - \\
\hline & ${ }^{1} \mathrm{H}$ data & 0.12 & 12.78 & 36.97 & 50.14 & - & - & - & - \\
\hline \multirow[t]{3}{*}{ sDP4+ } & ${ }^{13} \mathrm{C}$ data & 0.67 & 46.39 & 12.41 & 40.53 & - & - & - & - \\
\hline & All data & 0.00 & 19.22 & 14.88 & 65.90 & - & - & - & - \\
\hline & ${ }^{1} \mathrm{H}$ data & 3.74 & 7.90 & 60.70 & 27.66 & - & - & - & - \\
\hline \multirow[t]{3}{*}{ uDP4+ } & ${ }^{13} \mathrm{C}$ data & 10.56 & 0.32 & 0.16 & 88.96 & - & - & - & - \\
\hline & All data & 1.57 & 0.10 & 0.38 & 97.95 & - & - & - & - \\
\hline & ${ }^{1} \mathrm{H}$ data & 0.01 & 2.70 & 60.12 & 37.16 & - & - & - & - \\
\hline \multirow[t]{5}{*}{ DP4+ } & ${ }^{13} \mathrm{C}$ data & 0.19 & 0.41 & 0.05 & 99.34 & - & - & - & - \\
\hline & All data & 0.00 & 0.03 & 0.09 & 99.88 & - & - & - & - \\
\hline & & \multicolumn{8}{|c|}{ Level of theory: mPW1PW91/6-311+G** (solution, PCM) } \\
\hline & & $75 a$ & $75 b$ & $75 c$ & 75d & - & - & - & - \\
\hline & ${ }^{1} \mathrm{H}$ data & 3.66 & 6.29 & 17.21 & 72.83 & - & - & - & - \\
\hline \multirow[t]{2}{*}{ sDP4+ } & ${ }^{13} \mathrm{C}$ data & 0.47 & 21.37 & 6.65 & 71.50 & - & - & - & - \\
\hline & All data & 0.03 & 2.46 & 2.10 & 95.41 & - & - & - & - \\
\hline \multirow{3}{*}{ uDP4+ } & ${ }^{1} \mathrm{H}$ data & 25.69 & 5.24 & 23.38 & 45.70 & - & - & - & - \\
\hline & ${ }^{13} \mathrm{C}$ data & 8.23 & 0.28 & 0.06 & 91.43 & - & - & - & - \\
\hline & All data & 4.81 & 0.03 & 0.03 & 95.12 & - & - & - & - \\
\hline \multirow{3}{*}{ DP4+ } & ${ }^{1} \mathrm{H}$ data & 2.44 & 0.85 & 10.43 & 86.28 & - & - & - & - \\
\hline & ${ }^{13} \mathrm{C}$ data & 0.06 & 0.09 & 0.01 & 99.84 & - & - & - & - \\
\hline & All data & 0.00 & 0.00 & 0.00 & 100.00 & - & - & - & - \\
\hline
\end{tabular}


$s \mathrm{DP4}+, u \mathrm{DP} 4+$ and DP4+ probabilities (\%) for compound 76a

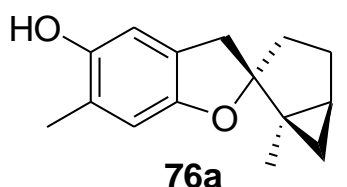

correct isomer

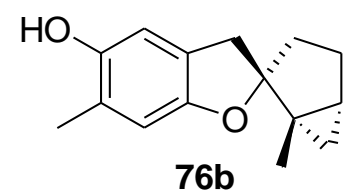

$76 b$

\begin{tabular}{|c|c|c|c|c|c|c|c|c|c|}
\hline & & \multicolumn{8}{|c|}{ Level of theory: B3LYP/6-31G* (gas phase) } \\
\hline & & $76 a$ & $76 b$ & - & - & - & - & - & - \\
\hline & ${ }^{1}$ H data & 99.98 & 0.02 & - & - & - & - & - & - \\
\hline \multirow[t]{2}{*}{ sDP4+ } & ${ }^{13} \mathrm{C}$ data & 90.43 & 9.57 & - & - & - & - & - & - \\
\hline & All data & 100.00 & 0.00 & - & - & - & - & - & - \\
\hline \multirow{3}{*}{ uDP4+ } & ${ }^{1} \mathrm{H}$ data & 99.96 & 0.04 & - & - & - & - & - & - \\
\hline & ${ }^{13} \mathrm{C}$ data & 99.71 & 0.29 & - & - & - & - & - & - \\
\hline & All data & 100.00 & 0.00 & - & - & - & - & - & - \\
\hline \multirow{5}{*}{ DP4+ } & ${ }^{1}$ H data & 100.00 & 0.00 & - & - & - & - & - & - \\
\hline & ${ }^{13}$ C data & 99.97 & 0.03 & - & - & - & - & - & - \\
\hline & All data & 100.00 & 0.00 & - & - & - & - & - & - \\
\hline & & \multicolumn{8}{|c|}{ Level of theory: B3LYP/6-31G** (gas phase) } \\
\hline & & $76 a$ & $76 b$ & - & - & - & - & - & - \\
\hline \multirow{3}{*}{ sDP4+ } & ${ }^{1} \mathrm{H}$ data & 100.00 & 0.00 & - & - & - & - & - & - \\
\hline & ${ }^{13} \mathrm{C}$ data & 77.89 & 22.11 & - & - & - & - & - & - \\
\hline & All data & 100.00 & 0.00 & - & - & - & - & - & - \\
\hline \multirow{3}{*}{ uDP4+ } & ${ }^{1} \mathrm{H}$ data & 99.99 & 0.01 & - & - & - & - & - & - \\
\hline & ${ }^{13} \mathrm{C}$ data & 98.75 & 1.25 & - & - & - & - & - & - \\
\hline & All data & 100.00 & 0.00 & - & - & - & - & - & - \\
\hline \multirow{3}{*}{ DP4+ } & ${ }^{1} \mathrm{H}$ data & 100.00 & 0.00 & - & - & - & - & - & - \\
\hline & ${ }^{13} \mathrm{C}$ data & 99.64 & 0.36 & - & - & - & - & - & - \\
\hline & All data & 100.00 & 0.00 & - & - & - & - & - & - \\
\hline & & \multicolumn{8}{|c|}{ Level of theory: B3LYP/6-31+G** (gas phase) } \\
\hline & & $76 a$ & $76 b$ & - & - & - & - & - & - \\
\hline \multirow{3}{*}{ sDP4+ } & ${ }^{1} \mathrm{H}$ data & 100.00 & 0.00 & - & - & - & - & - & - \\
\hline & ${ }^{13} \mathrm{C}$ data & 53.46 & 46.54 & - & - & - & - & - & - \\
\hline & All data & 100.00 & 0.00 & - & - & - & - & - & - \\
\hline \multirow{3}{*}{ uDP4+ } & ${ }^{1} \mathrm{H}$ data & 100.00 & 0.00 & - & - & - & - & - & - \\
\hline & ${ }^{13} \mathrm{C}$ data & 93.59 & 6.41 & - & - & - & - & - & - \\
\hline & All data & 100.00 & 0.00 & - & - & - & - & - & - \\
\hline \multirow{5}{*}{ DP4+ } & ${ }^{1} \mathrm{H}$ data & 100.00 & 0.00 & - & - & - & - & - & - \\
\hline & ${ }^{13} \mathrm{C}$ data & 94.37 & 5.63 & - & - & - & - & - & - \\
\hline & All data & 100.00 & 0.00 & - & - & - & - & - & - \\
\hline & & \multicolumn{8}{|c|}{ Level of theory: B3LYP/6-311G* (gas phase) } \\
\hline & & $76 a$ & $76 b$ & - & - & - & - & - & - \\
\hline \multirow{3}{*}{ sDP4+ } & ${ }^{1}$ H data & 99.94 & 0.06 & - & - & - & - & - & - \\
\hline & ${ }^{13}$ C data & 46.45 & 53.55 & - & - & - & - & - & - \\
\hline & All data & 99.93 & 0.07 & - & - & - & - & - & - \\
\hline \multirow{3}{*}{ uDP4+ } & ${ }^{1} \mathrm{H}$ data & 99.75 & 0.25 & - & - & - & - & - & - \\
\hline & ${ }^{13} \mathrm{C}$ data & 86.18 & 13.82 & - & - & - & - & - & - \\
\hline & All data & 99.96 & 0.04 & - & - & - & - & - & - \\
\hline \multirow{3}{*}{ DP4+ } & ${ }^{1} \mathrm{H}$ data & 100.00 & 0.00 & - & - & - & - & - & - \\
\hline & ${ }^{13} \mathrm{C}$ data & 84.39 & 15.61 & - & - & - & - & - & - \\
\hline & All data & 100.00 & 0.00 & - & - & - & - & - & - \\
\hline & & \multicolumn{8}{|c|}{ Level of theory: B3LYP/6-311G** (gas phase) } \\
\hline & & $76 a$ & $76 b$ & - & - & - & - & - & - \\
\hline \multirow{2}{*}{ sDP4+ } & ${ }^{1} \mathrm{H}$ data & 99.90 & 0.10 & - & - & - & - & - & - \\
\hline & ${ }^{13} \mathrm{C}$ data & 59.65 & 40.35 & - & - & - & - & - & - \\
\hline
\end{tabular}




\begin{tabular}{|c|c|c|c|c|c|c|c|c|c|}
\hline & All data & 99.94 & 0.06 & - & - & - & - & - & - \\
\hline & ${ }^{1} \mathrm{H}$ data & 99.92 & 0.08 & - & - & - & - & - & - \\
\hline \multirow[t]{3}{*}{ uDP4+ } & ${ }^{13} \mathrm{C}$ data & 90.04 & 9.96 & - & - & - & - & - & - \\
\hline & All data & 99.99 & 0.01 & - & - & - & - & - & - \\
\hline & ${ }^{1} \mathrm{H}$ data & 100.00 & 0.00 & - & - & - & - & - & - \\
\hline \multirow[t]{5}{*}{ DP4+ } & ${ }^{13} \mathrm{C}$ data & 93.04 & 6.96 & - & - & - & - & - & - \\
\hline & All data & 100.00 & 0.00 & - & - & - & - & - & - \\
\hline & & \multicolumn{8}{|c|}{ Level of theory: B3LYP/6-311+G** (gas phase) } \\
\hline & & $76 a$ & $76 b$ & - & - & - & - & - & - \\
\hline & ${ }^{1} \mathrm{H}$ data & 99.94 & 0.06 & - & - & - & - & - & - \\
\hline \multirow[t]{3}{*}{ sDP4+ } & ${ }^{13} \mathrm{C}$ data & 51.98 & 48.02 & - & - & - & - & - & - \\
\hline & All data & 99.94 & 0.06 & - & - & - & - & - & - \\
\hline & ${ }^{1} \mathrm{H}$ data & 99.92 & 0.08 & - & - & - & - & - & - \\
\hline \multirow[t]{3}{*}{ uDP4+ } & ${ }^{13} \mathrm{C}$ data & 76.20 & 23.80 & - & - & - & - & - & - \\
\hline & All data & 99.98 & 0.02 & - & - & - & - & - & - \\
\hline & ${ }^{1} \mathrm{H}$ data & 100.00 & 0.00 & - & - & - & - & - & - \\
\hline \multirow[t]{5}{*}{ DP4+ } & ${ }^{13} \mathrm{C}$ data & 77.61 & 22.39 & - & - & - & - & - & - \\
\hline & All data & 100.00 & 0.00 & - & - & - & - & - & - \\
\hline & & \multicolumn{8}{|c|}{ Level of theory: B3LYP/6-31G* (solution, PCM) } \\
\hline & & $76 a$ & $76 b$ & - & - & - & - & - & - \\
\hline & ${ }^{1}$ H data & 100.00 & 0.00 & - & - & - & - & - & - \\
\hline \multirow[t]{3}{*}{ sDP4+ } & ${ }^{13} \mathrm{C}$ data & 83.32 & 16.68 & - & - & - & - & - & - \\
\hline & All data & 100.00 & 0.00 & - & - & - & - & - & - \\
\hline & ${ }^{1} \mathrm{H}$ data & 99.99 & 0.01 & - & - & - & - & - & - \\
\hline \multirow[t]{3}{*}{ uDP4+ } & ${ }^{13} \mathrm{C}$ data & 99.54 & 0.46 & - & - & - & - & - & - \\
\hline & All data & 100.00 & 0.00 & - & - & - & - & - & - \\
\hline & ${ }^{1} \mathrm{H}$ data & 100.00 & 0.00 & - & - & - & - & - & - \\
\hline \multirow[t]{5}{*}{ DP4+ } & ${ }^{13} \mathrm{C}$ data & 99.91 & 0.09 & - & - & - & - & - & - \\
\hline & All data & 100.00 & 0.00 & - & - & - & - & - & - \\
\hline & & \multicolumn{8}{|c|}{ Level of theory: B3LYP/6-31G** (solution, PCM) } \\
\hline & & $76 a$ & $76 b$ & - & - & - & - & - & - \\
\hline & ${ }^{1} \mathrm{H}$ data & 100.00 & 0.00 & - & - & - & - & - & - \\
\hline \multirow[t]{3}{*}{ sDP4+ } & ${ }^{13} \mathrm{C}$ data & 56.30 & 43.70 & - & - & - & - & - & - \\
\hline & All data & 100.00 & 0.00 & - & - & - & - & - & - \\
\hline & ${ }^{1} \mathrm{H}$ data & 100.00 & 0.00 & - & - & - & - & - & - \\
\hline \multirow[t]{3}{*}{ uDP4+ } & ${ }^{13} \mathrm{C}$ data & 97.49 & 2.51 & - & - & - & - & - & - \\
\hline & All data & 100.00 & 0.00 & - & - & - & - & - & - \\
\hline & ${ }^{1} \mathrm{H}$ data & 100.00 & 0.00 & - & - & - & - & - & - \\
\hline \multirow[t]{5}{*}{ DP4+ } & ${ }^{13} \mathrm{C}$ data & 98.04 & 1.96 & - & - & - & - & - & - \\
\hline & All data & 100.00 & 0.00 & - & - & - & - & - & - \\
\hline & & \multicolumn{8}{|c|}{ Level of theory: B3LYP/6-31+G** (solution, PCM) } \\
\hline & & $76 a$ & $76 b$ & - & - & - & - & - & - \\
\hline & ${ }^{1} \mathrm{H}$ data & 100.00 & 0.00 & - & - & - & - & - & - \\
\hline \multirow[t]{3}{*}{ sDP4+ } & ${ }^{13} \mathrm{C}$ data & 37.48 & 62.52 & - & - & - & - & - & - \\
\hline & All data & 100.00 & 0.00 & - & - & - & - & - & - \\
\hline & ${ }^{1} \mathrm{H}$ data & 100.00 & 0.00 & - & - & - & - & - & - \\
\hline uDP4+ & ${ }^{13} \mathrm{C}$ data & 90.56 & 9.44 & - & - & - & - & - & - \\
\hline & All data & 100.00 & 0.00 & - & - & - & - & - & - \\
\hline & ${ }^{1} \mathrm{H}$ data & 100.00 & 0.00 & - & - & - & - & - & - \\
\hline DP4+ & ${ }^{13} \mathrm{C}$ data & 85.19 & 14.81 & - & - & - & - & - & - \\
\hline & All data & 100.00 & 0.00 & - & - & - & - & - & - \\
\hline & & & & el & B3 & G* & PC & & \\
\hline & & $76 a$ & $76 \mathrm{~b}$ & - & - & - & - & - & - \\
\hline & ${ }^{1} \mathrm{H}$ data & 99.99 & 0.01 & - & - & - & - & - & - \\
\hline sDP4+ & ${ }^{13} \mathrm{C}$ data & 26.82 & 73.18 & - & - & - & - & - & - \\
\hline & All data & 99.98 & 0.02 & - & - & - & - & - & - \\
\hline
\end{tabular}




\begin{tabular}{|c|c|c|c|c|c|c|c|c|c|}
\hline & ${ }^{1} \mathrm{H}$ data & 99.97 & 0.03 & - & - & - & - & - & - \\
\hline \multirow[t]{3}{*}{ uDP4+ } & ${ }^{13} \mathrm{C}$ data & 71.31 & 28.69 & - & - & - & - & - & - \\
\hline & All data & 99.99 & 0.01 & - & - & - & - & - & - \\
\hline & ${ }^{1} \mathrm{H}$ data & 100.00 & 0.00 & - & - & - & - & - & - \\
\hline \multirow[t]{5}{*}{ DP4+ } & ${ }^{13} \mathrm{C}$ data & 47.68 & 52.32 & - & - & - & - & - & - \\
\hline & All data & 100.00 & 0.00 & - & - & - & - & - & - \\
\hline & & \multicolumn{8}{|c|}{ Level of theory: B3LYP/6-311G** (solution, PCM) } \\
\hline & & $76 a$ & $76 b$ & - & - & - & - & - & - \\
\hline & ${ }^{1} \mathrm{H}$ data & 99.97 & 0.03 & - & - & - & - & - & - \\
\hline \multirow[t]{3}{*}{ sDP4+ } & ${ }^{13} \mathrm{C}$ data & 36.70 & 63.30 & - & - & - & - & - & - \\
\hline & All data & 99.96 & 0.04 & - & - & - & - & - & - \\
\hline & ${ }^{1} \mathrm{H}$ data & 99.97 & 0.03 & - & - & - & - & - & - \\
\hline \multirow[t]{3}{*}{ uDP4+ } & ${ }^{13} \mathrm{C}$ data & 76.53 & 23.47 & - & - & - & - & - & - \\
\hline & All data & 99.99 & 0.01 & - & - & - & - & - & - \\
\hline & ${ }^{1} \mathrm{H}$ data & 100.00 & 0.00 & - & - & - & - & - & - \\
\hline \multirow[t]{5}{*}{ DP4+ } & ${ }^{13} \mathrm{C}$ data & 65.41 & 34.59 & - & - & - & - & - & - \\
\hline & All data & 100.00 & 0.00 & - & - & - & - & - & - \\
\hline & & \multicolumn{8}{|c|}{ Level of theory: B3LYP/6-311+G** (solution, PCM) } \\
\hline & & $76 a$ & $76 b$ & - & - & - & - & - & - \\
\hline & ${ }^{1}$ H data & 99.98 & 0.02 & - & - & - & - & - & - \\
\hline \multirow[t]{3}{*}{ sDP4+ } & ${ }^{13} \mathrm{C}$ data & 35.53 & 64.47 & - & - & - & - & - & - \\
\hline & All data & 99.96 & 0.04 & - & - & - & - & - & - \\
\hline & ${ }^{1} \mathrm{H}$ data & 99.99 & 0.01 & - & - & - & - & - & - \\
\hline \multirow[t]{3}{*}{ uDP4+ } & ${ }^{13} \mathrm{C}$ data & 50.63 & 49.37 & - & - & - & - & - & - \\
\hline & All data & 99.99 & 0.01 & - & - & - & - & - & - \\
\hline & ${ }^{1} \mathrm{H}$ data & 100.00 & 0.00 & - & - & - & - & - & - \\
\hline \multirow[t]{5}{*}{ DP4+ } & ${ }^{13} \mathrm{C}$ data & 36.11 & 63.89 & - & - & - & - & - & - \\
\hline & All data & 100.00 & 0.00 & - & - & - & - & - & - \\
\hline & & \multicolumn{8}{|c|}{ Level of theory: mPW1PW91/6-31G* (gas phase) } \\
\hline & & $76 a$ & $76 b$ & - & - & - & - & - & - \\
\hline & ${ }^{1} \mathrm{H}$ data & 100.00 & 0.00 & - & - & - & - & - & - \\
\hline \multirow[t]{2}{*}{ sDP4+ } & ${ }^{13} \mathrm{C}$ data & 99.42 & 0.58 & - & - & - & - & - & - \\
\hline & All data & 100.00 & 0.00 & - & - & - & - & - & - \\
\hline \multirow{3}{*}{ uDP4+ } & ${ }^{1} \mathrm{H}$ data & 99.94 & 0.06 & - & - & - & - & - & - \\
\hline & ${ }^{13} \mathrm{C}$ data & 99.93 & 0.07 & - & - & - & - & - & - \\
\hline & All data & 100.00 & 0.00 & - & - & - & - & - & - \\
\hline \multirow{5}{*}{ DP4+ } & ${ }^{1} \mathrm{H}$ data & 100.00 & 0.00 & - & - & - & - & - & - \\
\hline & ${ }^{13} \mathrm{C}$ data & 100.00 & 0.00 & - & - & - & - & - & - \\
\hline & All data & 100.00 & 0.00 & - & - & - & - & - & - \\
\hline & & \multicolumn{8}{|c|}{ Level of theory: mPW1PW91/6-31G** (gas phase) } \\
\hline & & $76 a$ & $76 b$ & - & - & - & - & - & - \\
\hline \multirow{3}{*}{ sDP4+ } & ${ }^{1} \mathrm{H}$ data & 100.00 & 0.00 & - & - & - & - & - & - \\
\hline & ${ }^{13} \mathrm{C}$ data & 98.13 & 1.87 & - & - & - & - & - & - \\
\hline & All data & 100.00 & 0.00 & - & - & - & - & - & - \\
\hline & ${ }^{1} \mathrm{H}$ data & 99.98 & 0.02 & - & - & - & - & - & - \\
\hline uDP4+ & ${ }^{13} \mathrm{C}$ data & 99.95 & 0.05 & - & - & - & - & - & - \\
\hline & All data & 100.00 & 0.00 & - & - & - & - & - & - \\
\hline & ${ }^{1} \mathrm{H}$ data & 100.00 & 0.00 & - & - & - & - & - & - \\
\hline DP4+ & ${ }^{13} \mathrm{C}$ data & 100.00 & 0.00 & - & - & - & - & - & - \\
\hline & All data & 100.00 & 0.00 & - & - & - & - & - & - \\
\hline & & & & 10 & $\mathrm{PI}$ & -3 & $\mathrm{pr}$ & & \\
\hline & & $76 a$ & $76 b$ & - & - & - & - & - & - \\
\hline & ${ }^{1} \mathrm{H}$ data & 100.00 & 0.00 & - & - & - & - & - & - \\
\hline sDP4+ & ${ }^{13} \mathrm{C}$ data & 87.24 & 12.76 & - & - & - & - & - & - \\
\hline & All data & 100.00 & 0.00 & - & - & - & - & - & - \\
\hline uDP4+ & ${ }^{1} \mathrm{H}$ data & 99.98 & 0.02 & - & - & - & - & - & - \\
\hline
\end{tabular}




\begin{tabular}{|c|c|c|c|c|c|c|c|c|c|}
\hline & ${ }^{13} \mathrm{C}$ data & 98.71 & 1.29 & - & - & - & - & - & - \\
\hline & All data & 100.00 & 0.00 & - & - & - & - & - & - \\
\hline & ${ }^{1} \mathrm{H}$ data & 100.00 & 0.00 & - & - & - & - & - & - \\
\hline \multirow[t]{5}{*}{ DP4+ } & ${ }^{13} \mathrm{C}$ data & 99.81 & 0.19 & - & - & - & - & - & - \\
\hline & All data & 100.00 & 0.00 & - & - & - & - & - & - \\
\hline & & \multicolumn{8}{|c|}{ Level of theory: mPW1PW91/6-311G* (gas phase) } \\
\hline & & $76 a$ & $76 b$ & - & - & - & - & - & - \\
\hline & ${ }^{1}$ H data & 99.98 & 0.02 & - & - & - & - & - & - \\
\hline \multirow[t]{3}{*}{ sDP4+ } & ${ }^{13}$ C data & 78.39 & 21.61 & - & - & - & - & - & - \\
\hline & All data & 99.99 & 0.01 & - & - & - & - & - & - \\
\hline & ${ }^{1} \mathrm{H}$ data & 99.70 & 0.30 & - & - & - & - & - & - \\
\hline \multirow[t]{3}{*}{ uDP4+ } & ${ }^{13} \mathrm{C}$ data & 96.26 & 3.74 & - & - & - & - & - & - \\
\hline & All data & 99.99 & 0.01 & - & - & - & - & - & - \\
\hline & ${ }^{1} \mathrm{H}$ data & 100.00 & 0.00 & - & - & - & - & - & - \\
\hline \multirow[t]{5}{*}{ DP4+ } & ${ }^{13} \mathrm{C}$ data & 98.94 & 1.06 & - & - & - & - & - & - \\
\hline & All data & 100.00 & 0.00 & - & - & - & - & - & - \\
\hline & & \multicolumn{8}{|c|}{ Level of theory: mPW1PW91/6-311G** (gas phase) } \\
\hline & & $76 a$ & $76 b$ & - & - & - & - & - & - \\
\hline & ${ }^{1} \mathrm{H}$ data & 99.97 & 0.03 & - & - & - & - & - & - \\
\hline \multirow[t]{3}{*}{ sDP4+ } & ${ }^{13} \mathrm{C}$ data & 89.09 & 10.91 & - & - & - & - & - & - \\
\hline & All data & 100.00 & 0.00 & - & - & - & - & - & - \\
\hline & ${ }^{1} \mathrm{H}$ data & 99.89 & 0.11 & - & - & - & - & - & - \\
\hline \multirow[t]{3}{*}{ uDP4+ } & ${ }^{13} \mathrm{C}$ data & 97.56 & 2.44 & - & - & - & - & - & - \\
\hline & All data & 100.00 & 0.00 & - & - & - & - & - & - \\
\hline & ${ }^{1} \mathrm{H}$ data & 100.00 & 0.00 & - & - & - & - & - & - \\
\hline \multirow[t]{5}{*}{ DP4+ } & ${ }^{13} \mathrm{C}$ data & 99.69 & 0.31 & - & - & - & - & - & - \\
\hline & All data & 100.00 & 0.00 & - & - & - & - & - & - \\
\hline & & \multicolumn{8}{|c|}{ Level of theory: mPW1PW91/6-311+G** (gas phase) } \\
\hline & & $76 a$ & $76 b$ & - & - & - & - & - & - \\
\hline & ${ }^{1} \mathrm{H}$ data & 99.98 & 0.02 & - & - & - & - & - & - \\
\hline \multirow[t]{2}{*}{ sDP4+ } & ${ }^{13} \mathrm{C}$ data & 88.35 & 11.65 & - & - & - & - & - & - \\
\hline & All data & 100.00 & 0.00 & - & - & - & - & - & - \\
\hline \multirow{3}{*}{ uDP4+ } & ${ }^{1} \mathrm{H}$ data & 99.90 & 0.10 & - & - & - & - & - & - \\
\hline & ${ }^{13}$ C data & 89.20 & 10.80 & - & - & - & - & - & - \\
\hline & All data & 99.99 & 0.01 & - & - & - & - & - & - \\
\hline \multirow{5}{*}{ DP4+ } & ${ }^{1} \mathrm{H}$ data & 100.00 & 0.00 & - & - & - & - & - & - \\
\hline & ${ }^{13} \mathrm{C}$ data & 98.43 & 1.57 & - & - & - & - & - & - \\
\hline & All data & 100.00 & 0.00 & - & - & - & - & - & - \\
\hline & & \multicolumn{8}{|c|}{ Level of theory: mPW1PW91/6-31G* (solution, PCM) } \\
\hline & & $76 a$ & $76 b$ & - & - & - & - & - & - \\
\hline \multirow{3}{*}{ sDP4+ } & ${ }^{1} \mathrm{H}$ data & 100.00 & 0.00 & - & - & - & - & - & - \\
\hline & ${ }^{13} \mathrm{C}$ data & 97.41 & 2.59 & - & - & - & - & - & - \\
\hline & All data & 100.00 & 0.00 & - & - & - & - & - & - \\
\hline \multirow{3}{*}{ uDP4+ } & ${ }^{1} \mathrm{H}$ data & 99.99 & 0.01 & - & - & - & - & - & - \\
\hline & ${ }^{13} \mathrm{C}$ data & 99.93 & 0.07 & - & - & - & - & - & - \\
\hline & All data & 100.00 & 0.00 & - & - & - & - & - & - \\
\hline & ${ }^{1} \mathrm{H}$ data & 100.00 & 0.00 & - & - & - & - & - & - \\
\hline DP4+ & ${ }^{13} \mathrm{C}$ data & 100.00 & 0.00 & - & - & - & - & - & - \\
\hline & All data & 100.00 & 0.00 & - & - & - & - & - & - \\
\hline & & & & of & $\mathbf{W}$ & 1 & on, & & \\
\hline & & $76 a$ & $76 b$ & - & - & - & - & - & - \\
\hline & ${ }^{1} \mathrm{H}$ data & 100.00 & 0.00 & - & - & - & - & - & - \\
\hline sDP4+ & ${ }^{13} \mathrm{C}$ data & 95.03 & 4.97 & - & - & - & - & - & - \\
\hline & All data & 100.00 & 0.00 & - & - & - & - & - & - \\
\hline uDP4+ & ${ }_{13}^{1}$ data & 100.00 & 0.00 & - & - & - & - & - & - \\
\hline UDP4+ & ${ }^{13} \mathrm{C}$ data & 99.91 & 0.09 & - & - & - & - & - & - \\
\hline
\end{tabular}




\begin{tabular}{|c|c|c|c|c|c|c|c|c|c|}
\hline & All data & 100.00 & 0.00 & - & - & - & - & - & - \\
\hline & ${ }^{1} \mathrm{H}$ data & 100.00 & 0.00 & - & - & - & - & - & - \\
\hline \multirow[t]{5}{*}{ DP4+ } & ${ }^{13} \mathrm{C}$ data & 100.00 & 0.00 & - & - & - & - & - & - \\
\hline & All data & 100.00 & 0.00 & - & - & - & - & - & - \\
\hline & & \multicolumn{8}{|c|}{ Level of theory: mPW1PW91/6-31+G** (solution, PCM) } \\
\hline & & $76 a$ & $76 b$ & - & - & - & - & - & - \\
\hline & ${ }^{1} \mathrm{H}$ data & 99.95 & 0.05 & - & - & - & - & - & - \\
\hline \multirow[t]{3}{*}{ sDP4+ } & ${ }^{13} \mathrm{C}$ data & 95.42 & 4.58 & - & - & - & - & - & - \\
\hline & All data & 100.00 & 0.00 & - & - & - & - & - & - \\
\hline & ${ }^{1}$ H data & 7.73 & 92.27 & - & - & - & - & - & - \\
\hline \multirow[t]{3}{*}{ uDP4+ } & ${ }^{13} \mathrm{C}$ data & 99.74 & 0.26 & - & - & - & - & - & - \\
\hline & All data & 96.93 & 3.07 & - & - & - & - & - & - \\
\hline & ${ }^{1} \mathrm{H}$ data & 99.42 & 0.58 & - & - & - & - & - & - \\
\hline \multirow[t]{5}{*}{ DP4+ } & ${ }^{13} \mathrm{C}$ data & 99.99 & 0.01 & - & - & - & - & - & - \\
\hline & All data & 100.00 & 0.00 & - & - & - & - & - & - \\
\hline & & \multicolumn{8}{|c|}{ Level of theory: mPW1PW91/6-311G* (solution, PCM) } \\
\hline & & $76 a$ & $76 b$ & - & - & - & - & - & - \\
\hline & ${ }^{1} \mathrm{H}$ data & 100.00 & 0.00 & - & - & - & - & - & - \\
\hline \multirow[t]{3}{*}{ sDP4+ } & ${ }^{13} \mathrm{C}$ data & 64.22 & 35.78 & - & - & - & - & - & - \\
\hline & All data & 100.00 & 0.00 & - & - & - & - & - & - \\
\hline & ${ }^{1}$ H data & 99.95 & 0.05 & - & - & - & - & - & - \\
\hline \multirow[t]{3}{*}{ uDP4+ } & ${ }^{13}$ C data & 85.15 & 14.85 & - & - & - & - & - & - \\
\hline & All data & 99.99 & 0.01 & - & - & - & - & - & - \\
\hline & ${ }^{1} \mathrm{H}$ data & 100.00 & 0.00 & - & - & - & - & - & - \\
\hline \multirow[t]{5}{*}{ DP4+ } & ${ }^{13} \mathrm{C}$ data & 91.15 & 8.85 & - & - & - & - & - & - \\
\hline & All data & 100.00 & 0.00 & - & - & - & - & - & - \\
\hline & & \multicolumn{8}{|c|}{ Level of theory: mPW1PW91/6-311G** (solution, PCM) } \\
\hline & & $76 a$ & $76 b$ & - & - & - & - & - & - \\
\hline & ${ }^{1} \mathrm{H}$ data & 99.99 & 0.01 & - & - & - & - & - & - \\
\hline \multirow[t]{2}{*}{ sDP4+ } & ${ }^{13} \mathrm{C}$ data & 72.82 & 27.18 & - & - & - & - & - & - \\
\hline & All data & 99.99 & 0.01 & - & - & - & - & - & - \\
\hline \multirow{3}{*}{ uDP4+ } & ${ }^{1} \mathrm{H}$ data & 99.96 & 0.04 & - & - & - & - & - & - \\
\hline & ${ }^{13} \mathrm{C}$ data & 90.48 & 9.52 & - & - & - & - & - & - \\
\hline & All data & 100.00 & 0.00 & - & - & - & - & - & - \\
\hline \multirow{5}{*}{ DP4+ } & ${ }^{1} \mathrm{H}$ data & 100.00 & 0.00 & - & - & - & - & - & - \\
\hline & ${ }^{13} \mathrm{C}$ data & 96.22 & 3.78 & - & - & - & - & - & - \\
\hline & All data & 100.00 & 0.00 & - & - & - & - & - & - \\
\hline & & \multicolumn{8}{|c|}{ Level of theory: mPW1PW91/6-311+G** (solution, PCM) } \\
\hline & & $76 a$ & $76 b$ & - & - & - & - & - & - \\
\hline \multirow{3}{*}{ sDP4+ } & ${ }^{1} \mathrm{H}$ data & 99.99 & 0.01 & - & - & - & - & - & - \\
\hline & ${ }^{13} \mathrm{C}$ data & 70.54 & 29.46 & - & - & - & - & - & - \\
\hline & All data & 100.00 & 0.00 & - & - & - & - & - & - \\
\hline \multirow{3}{*}{ uDP4+ } & ${ }^{1} \mathrm{H}$ data & 99.95 & 0.05 & - & - & - & - & - & - \\
\hline & ${ }^{13} \mathrm{C}$ data & 74.96 & 25.04 & - & - & - & - & - & - \\
\hline & All data & 99.98 & 0.02 & - & - & - & - & - & - \\
\hline \multirow{3}{*}{ DP4+ } & ${ }^{1} \mathrm{H}$ data & 100.00 & 0.00 & - & - & - & - & - & - \\
\hline & ${ }^{13} \mathrm{C}$ data & 87.75 & 12.25 & - & - & - & - & - & - \\
\hline & All data & 100.00 & 0.00 & - & - & - & - & - & - \\
\hline
\end{tabular}


$s \mathrm{DP4}+, u \mathrm{DP} 4+$ and DP4+ probabilities (\%) for compound 76b

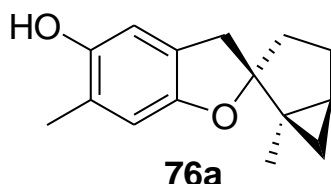

$76 a$<smiles>Cc1cc2c(cc1O)C[C@]1(CCCC1(C)C)O2</smiles>

correct isomer

\begin{tabular}{|c|c|c|c|c|c|c|c|c|c|}
\hline & & \multicolumn{8}{|c|}{ Level of theory: B3LYP/6-31G* (gas phase) } \\
\hline & & $76 a$ & 76b & - & - & - & - & - & - \\
\hline & ${ }^{1} \mathrm{H}$ data & 0.00 & 100.00 & - & - & - & - & - & - \\
\hline \multirow[t]{2}{*}{ sDP4+ } & ${ }^{13} \mathrm{C}$ data & 0.45 & 99.55 & - & - & - & - & - & - \\
\hline & All data & 0.00 & 100.00 & - & - & - & - & - & - \\
\hline \multirow{3}{*}{ uDP4+ } & ${ }^{1} \mathrm{H}$ data & 0.02 & 99.98 & - & - & - & - & - & - \\
\hline & ${ }^{13} \mathrm{C}$ data & 2.12 & 97.88 & - & - & - & - & - & - \\
\hline & All data & 0.00 & 100.00 & - & - & - & - & - & - \\
\hline \multirow{5}{*}{ DP4+ } & ${ }^{1} \mathrm{H}$ data & 0.00 & 100.00 & - & - & - & - & - & - \\
\hline & ${ }^{13} \mathrm{C}$ data & 0.01 & 99.99 & - & - & - & - & - & - \\
\hline & All data & 0.00 & 100.00 & - & - & - & - & - & - \\
\hline & & \multicolumn{8}{|c|}{ Level of theory: B3LYP/6-31G** (gas phase) } \\
\hline & & $76 a$ & $76 \mathrm{~b}$ & - & - & - & - & - & - \\
\hline & ${ }^{1} \mathrm{H}$ data & 0.01 & 99.99 & - & - & - & - & - & - \\
\hline \multirow[t]{2}{*}{ sDP4+ } & ${ }^{13} \mathrm{C}$ data & 0.22 & 99.78 & - & - & - & - & - & - \\
\hline & All data & 0.00 & 100.00 & - & - & - & - & - & - \\
\hline \multirow{3}{*}{ uDP4+ } & ${ }^{1} \mathrm{H}$ data & 0.05 & 99.95 & - & - & - & - & - & - \\
\hline & ${ }^{13} \mathrm{C}$ data & 0.94 & 99.06 & - & - & - & - & - & - \\
\hline & All data & 0.00 & 100.00 & - & - & - & - & - & - \\
\hline \multirow{5}{*}{ DP4+ } & ${ }^{1} \mathrm{H}$ data & 0.00 & 100.00 & - & - & - & - & - & - \\
\hline & ${ }^{13} \mathrm{C}$ data & 0.00 & 100.00 & - & - & - & - & - & - \\
\hline & All data & 0.00 & 100.00 & - & - & - & - & - & - \\
\hline & & \multicolumn{8}{|c|}{ Level of theory: B3LYP/6-31+G** (gas phase) } \\
\hline & & $76 a$ & $76 b$ & - & - & - & - & - & - \\
\hline \multirow{3}{*}{$s \mathrm{DP4+}$} & ${ }^{1} \mathrm{H}$ data & 0.04 & 99.96 & - & - & - & - & - & - \\
\hline & ${ }^{13} \mathrm{C}$ data & 3.13 & 96.87 & - & - & - & - & - & - \\
\hline & All data & 0.00 & 100.00 & - & - & - & - & - & - \\
\hline \multirow{3}{*}{ uDP4+ } & ${ }^{1} \mathrm{H}$ data & 0.07 & 99.93 & - & - & - & - & - & - \\
\hline & ${ }^{13} \mathrm{C}$ data & 2.10 & 97.90 & - & - & - & - & - & - \\
\hline & All data & 0.00 & 100.00 & - & - & - & - & - & - \\
\hline \multirow{3}{*}{ DP4+ } & ${ }^{1} \mathrm{H}$ data & 0.00 & 100.00 & - & - & - & - & - & - \\
\hline & ${ }^{13} \mathrm{C}$ data & 0.07 & 99.93 & - & - & - & - & - & - \\
\hline & All data & 0.00 & 100.00 & - & - & - & - & - & - \\
\hline & & \multicolumn{8}{|c|}{ Level of theory: B3LYP/6-311G* (gas phase) } \\
\hline & & $76 a$ & $76 \mathrm{~b}$ & - & - & - & - & - & - \\
\hline \multirow{3}{*}{ sDP4+ } & ${ }^{1} \mathrm{H}$ data & 0.02 & 99.98 & - & - & - & - & - & - \\
\hline & ${ }^{13} \mathrm{C}$ data & 0.62 & 99.38 & - & - & - & - & - & - \\
\hline & All data & 0.00 & 100.00 & - & - & - & - & - & - \\
\hline \multirow{3}{*}{ uDP4+ } & ${ }^{1} \mathrm{H}$ data & 0.11 & 99.89 & - & - & - & - & - & - \\
\hline & ${ }^{13} \mathrm{C}$ data & 1.42 & 98.58 & - & - & - & - & - & - \\
\hline & All data & 0.00 & 100.00 & - & - & - & - & - & - \\
\hline \multirow{5}{*}{ DP4+ } & ${ }^{1} \mathrm{H}$ data & 0.00 & 100.00 & - & - & - & - & - & - \\
\hline & ${ }^{13} \mathrm{C}$ data & 0.01 & 99.99 & - & - & - & - & - & - \\
\hline & All data & 0.00 & 100.00 & - & - & - & - & - & - \\
\hline & & \multicolumn{8}{|c|}{ Level of theory: B3LYP/6-311G** (gas phase) } \\
\hline & & $76 a$ & $76 b$ & - & - & - & - & - & - \\
\hline \multirow{2}{*}{ sDP4+ } & ${ }^{1} \mathrm{H}$ data & 0.01 & 99.99 & - & - & - & - & - & - \\
\hline & ${ }^{13} \mathrm{C}$ data & 0.65 & 99.35 & - & - & - & - & - & - \\
\hline
\end{tabular}




\begin{tabular}{|c|c|c|c|c|c|c|c|c|c|}
\hline & All data & 0.00 & 100.00 & - & - & - & - & - & - \\
\hline & ${ }^{1}$ H data & 0.07 & 99.93 & - & - & - & - & - & - \\
\hline \multirow[t]{3}{*}{ uDP4+ } & ${ }^{13} \mathrm{C}$ data & 1.11 & 98.89 & - & - & - & - & - & - \\
\hline & All data & 0.00 & 100.00 & - & - & - & - & - & - \\
\hline & ${ }^{1} \mathrm{H}$ data & 0.00 & 100.00 & - & - & - & - & - & - \\
\hline \multirow[t]{5}{*}{ DP4+ } & ${ }^{13} \mathrm{C}$ data & 0.01 & 99.99 & - & - & - & - & - & - \\
\hline & All data & 0.00 & 100.00 & - & - & - & - & - & - \\
\hline & & \multicolumn{8}{|c|}{ Level of theory: B3LYP/6-311+G** (gas phase) } \\
\hline & & $76 a$ & $76 b$ & - & - & - & - & - & - \\
\hline & ${ }^{1} \mathrm{H}$ data & 0.05 & 99.95 & - & - & - & - & - & - \\
\hline \multirow[t]{2}{*}{ sDP4+ } & ${ }^{13} \mathrm{C}$ data & 0.36 & 99.64 & - & - & - & - & - & - \\
\hline & All data & 0.00 & 100.00 & - & - & - & - & - & - \\
\hline \multirow{3}{*}{ uDP4+ } & ${ }^{1} \mathrm{H}$ data & 0.16 & 99.84 & - & - & - & - & - & - \\
\hline & ${ }^{13} \mathrm{C}$ data & 0.56 & 99.44 & - & - & - & - & - & - \\
\hline & All data & 0.00 & 100.00 & - & - & - & - & - & - \\
\hline \multirow{5}{*}{ DP4+ } & ${ }^{1} \mathrm{H}$ data & 0.00 & 100.00 & - & - & - & - & - & - \\
\hline & ${ }^{13} \mathrm{C}$ data & 0.00 & 100.00 & - & - & - & - & - & - \\
\hline & All data & 0.00 & 100.00 & - & - & - & - & - & - \\
\hline & & \multicolumn{8}{|c|}{ Level of theory: B3LYP/6-31G* (solution, PCM) } \\
\hline & & $76 a$ & $76 b$ & - & - & - & - & - & - \\
\hline \multirow{3}{*}{ sDP4+ } & ${ }^{1}$ H data & 0.01 & 99.99 & - & - & - & - & - & - \\
\hline & ${ }^{13}$ C data & 0.19 & 99.81 & - & - & - & - & - & - \\
\hline & All data & 0.00 & 100.00 & - & - & - & - & - & - \\
\hline \multirow{3}{*}{ uDP4+ } & ${ }^{1} \mathrm{H}$ data & 0.06 & 99.94 & - & - & - & - & - & - \\
\hline & ${ }^{13} \mathrm{C}$ data & 1.73 & 98.27 & - & - & - & - & - & - \\
\hline & All data & 0.00 & 100.00 & - & - & - & - & - & - \\
\hline \multirow{3}{*}{ DP4+ } & ${ }^{1}$ H data & 0.00 & 100.00 & - & - & - & - & - & - \\
\hline & ${ }^{13} \mathrm{C}$ data & 0.00 & 100.00 & - & - & - & - & - & - \\
\hline & All data & 0.00 & 100.00 & - & - & - & - & - & - \\
\hline & & \multicolumn{8}{|c|}{ Level of theory: B3LYP/6-31G** (solution, PCM) } \\
\hline & & $76 a$ & $76 b$ & - & - & - & - & - & - \\
\hline \multirow{3}{*}{ sDP4+ } & ${ }^{1} \mathrm{H}$ data & 0.08 & 99.92 & - & - & - & - & - & - \\
\hline & ${ }^{13} \mathrm{C}$ data & 0.16 & 99.84 & - & - & - & - & - & - \\
\hline & All data & 0.00 & 100.00 & - & - & - & - & - & - \\
\hline \multirow{3}{*}{ uDP4+ } & ${ }^{1} \mathrm{H}$ data & 0.22 & 99.78 & - & - & - & - & - & - \\
\hline & ${ }^{13} \mathrm{C}$ data & 0.94 & 99.06 & - & - & - & - & - & - \\
\hline & All data & 0.00 & 100.00 & - & - & - & - & - & - \\
\hline \multirow{3}{*}{ DP4+ } & ${ }^{1} \mathrm{H}$ data & 0.00 & 100.00 & - & - & - & - & - & - \\
\hline & ${ }^{13} \mathrm{C}$ data & 0.00 & 100.00 & - & - & - & - & - & - \\
\hline & All data & 0.00 & 100.00 & - & - & - & - & - & - \\
\hline & & \multicolumn{8}{|c|}{ Level of theory: B3LYP/6-31+G** (solution, PCM) } \\
\hline & & $76 a$ & $76 b$ & - & - & - & - & - & - \\
\hline \multirow{3}{*}{ sDP4+ } & ${ }^{1} \mathrm{H}$ data & 0.23 & 99.77 & - & - & - & - & - & - \\
\hline & ${ }^{13} \mathrm{C}$ data & 2.55 & 97.45 & - & - & - & - & - & - \\
\hline & All data & 0.01 & 99.99 & - & - & - & - & - & - \\
\hline & ${ }^{1} \mathrm{H}$ data & 0.61 & 99.39 & - & - & - & - & - & - \\
\hline uDP4+ & ${ }^{13} \mathrm{C}$ data & 2.71 & 97.29 & - & - & - & - & - & - \\
\hline & All data & 0.02 & 99.98 & - & - & - & - & - & - \\
\hline & ${ }^{1} \mathrm{H}$ data & 0.00 & 100.00 & - & - & - & - & - & - \\
\hline DP4+ & ${ }^{13} \mathrm{C}$ data & 0.07 & 99.93 & - & - & - & - & - & - \\
\hline & All data & 0.00 & 100.00 & - & - & - & - & - & - \\
\hline & & & & el & B3 & G* & $\overline{P C}$ & & \\
\hline & & $76 a$ & $76 b$ & - & - & - & - & - & - \\
\hline & ${ }^{1} \mathrm{H}$ data & 0.06 & 99.94 & - & - & - & - & - & - \\
\hline sDP4+ & ${ }^{13} \mathrm{C}$ data & 0.30 & 99.70 & - & - & - & - & - & - \\
\hline & All data & 0.00 & 100.00 & - & - & - & - & - & - \\
\hline
\end{tabular}




\begin{tabular}{|c|c|c|c|c|c|c|c|c|c|}
\hline & ${ }^{1} \mathrm{H}$ data & 0.21 & 99.79 & - & - & - & - & - & - \\
\hline \multirow[t]{3}{*}{ uDP4+ } & ${ }^{13} \mathrm{C}$ data & 0.92 & 99.08 & - & - & - & - & - & - \\
\hline & All data & 0.00 & 100.00 & - & - & - & - & - & - \\
\hline & ${ }^{1} \mathrm{H}$ data & 0.00 & 100.00 & - & - & - & - & - & - \\
\hline \multirow[t]{5}{*}{ DP4+ } & ${ }^{13} \mathrm{C}$ data & 0.00 & 100.00 & - & - & - & - & - & - \\
\hline & All data & 0.00 & 100.00 & - & - & - & - & - & - \\
\hline & & \multicolumn{8}{|c|}{ Level of theory: B3LYP/6-311G** (solution, PCM) } \\
\hline & & $76 a$ & $76 b$ & - & - & - & - & - & - \\
\hline & ${ }^{1} \mathrm{H}$ data & 0.24 & 99.76 & - & - & - & - & - & - \\
\hline \multirow[t]{3}{*}{ sDP4+ } & ${ }^{13} \mathrm{C}$ data & 0.29 & 99.71 & - & - & - & - & - & - \\
\hline & All data & 0.00 & 100.00 & - & - & - & - & - & - \\
\hline & ${ }^{1} \mathrm{H}$ data & 0.56 & 99.44 & - & - & - & - & - & - \\
\hline \multirow[t]{3}{*}{ uDP4+ } & ${ }^{13} \mathrm{C}$ data & 0.73 & 99.27 & - & - & - & - & - & - \\
\hline & All data & 0.00 & 100.00 & - & - & - & - & - & - \\
\hline & ${ }^{1} \mathrm{H}$ data & 0.00 & 100.00 & - & - & - & - & - & - \\
\hline \multirow[t]{5}{*}{ DP4+ } & ${ }^{13} \mathrm{C}$ data & 0.00 & 100.00 & - & - & - & - & - & - \\
\hline & All data & 0.00 & 100.00 & - & - & - & - & - & - \\
\hline & & \multicolumn{8}{|c|}{ Level of theory: B3LYP/6-311+G** (solution, PCM) } \\
\hline & & $76 a$ & $76 b$ & - & - & - & - & - & - \\
\hline & ${ }^{1}$ H data & 0.85 & 99.15 & - & - & - & - & - & - \\
\hline \multirow[t]{3}{*}{ sDP4+ } & ${ }^{13} \mathrm{C}$ data & 0.25 & 99.75 & - & - & - & - & - & - \\
\hline & All data & 0.00 & 100.00 & - & - & - & - & - & - \\
\hline & ${ }^{1}$ H data & 0.36 & 99.64 & - & - & - & - & - & - \\
\hline \multirow[t]{3}{*}{ uDP4+ } & ${ }^{13} \mathrm{C}$ data & 0.63 & 99.37 & - & - & - & - & - & - \\
\hline & All data & 0.00 & 100.00 & - & - & - & - & - & - \\
\hline & ${ }^{1} \mathrm{H}$ data & 0.00 & 100.00 & - & - & - & - & - & - \\
\hline \multirow[t]{4}{*}{ DP4+ } & ${ }^{13} \mathrm{C}$ data & 0.00 & 100.00 & - & - & - & - & - & - \\
\hline & All data & 0.00 & 100.00 & - & - & - & - & - & - \\
\hline & & \multicolumn{8}{|c|}{ Level of theory: mPW1PW91/6-31G* (gas phase) } \\
\hline & & $76 a$ & $76 b$ & - & - & - & - & - & - \\
\hline \multirow{3}{*}{ sDP4+ } & ${ }^{1} \mathrm{H}$ data & 0.00 & 100.00 & - & - & - & - & - & - \\
\hline & ${ }^{13} \mathrm{C}$ data & 0.31 & 99.69 & - & - & - & - & - & - \\
\hline & All data & 0.00 & 100.00 & - & - & - & - & - & - \\
\hline \multirow{3}{*}{ uDP4+ } & ${ }^{1} \mathrm{H}$ data & 0.00 & 100.00 & - & - & - & - & - & - \\
\hline & ${ }^{13} \mathrm{C}$ data & 8.30 & 91.70 & - & - & - & - & - & - \\
\hline & All data & 0.00 & 100.00 & - & - & - & - & - & - \\
\hline \multirow{5}{*}{ DP4+ } & ${ }^{1} \mathrm{H}$ data & 0.00 & 100.00 & - & - & - & - & - & - \\
\hline & ${ }^{13} \mathrm{C}$ data & 0.03 & 99.97 & - & - & - & - & - & - \\
\hline & All data & 0.00 & 100.00 & - & - & - & - & - & - \\
\hline & & \multicolumn{8}{|c|}{ Level of theory: mPW1PW91/6-31G** (gas phase) } \\
\hline & & $76 a$ & $76 b$ & - & - & - & - & - & - \\
\hline \multirow{3}{*}{ sDP4+ } & ${ }^{1} \mathrm{H}$ data & 0.00 & 100.00 & - & - & - & - & - & - \\
\hline & ${ }^{13} \mathrm{C}$ data & 0.19 & 99.81 & - & - & - & - & - & - \\
\hline & All data & 0.00 & 100.00 & - & - & - & - & - & - \\
\hline & ${ }^{1} \mathrm{H}$ data & 0.01 & 99.99 & - & - & - & - & - & - \\
\hline uDP4+ & ${ }^{13} \mathrm{C}$ data & 1.78 & 98.22 & - & - & - & - & - & - \\
\hline & All data & 0.00 & 100.00 & - & - & - & - & - & - \\
\hline & ${ }^{1} \mathrm{H}$ data & 0.00 & 100.00 & - & - & - & - & - & - \\
\hline DP4+ & ${ }^{13} \mathrm{C}$ data & 0.00 & 100.00 & - & - & - & - & - & - \\
\hline & All data & 0.00 & 100.00 & - & - & - & - & - & - \\
\hline & & & & 10 & IPV & -3 & $\mathrm{pr}$ & & \\
\hline & & $76 a$ & $76 b$ & - & - & - & - & - & - \\
\hline & ${ }^{1} \mathrm{H}$ data & 0.05 & 99.95 & - & - & - & - & - & - \\
\hline sDP4+ & ${ }^{13} \mathrm{C}$ data & 1.48 & 98.52 & - & - & - & - & - & - \\
\hline & All data & 0.00 & 100.00 & - & - & - & - & - & - \\
\hline uDP4+ & ${ }^{1} \mathrm{H}$ data & 0.23 & 99.77 & - & - & - & - & - & - \\
\hline
\end{tabular}




\begin{tabular}{|c|c|c|c|c|c|c|c|c|c|}
\hline & ${ }^{13} \mathrm{C}$ data & 2.59 & 97.41 & - & - & - & - & - & - \\
\hline & All data & 0.01 & 99.99 & - & - & - & - & - & - \\
\hline & ${ }^{1} \mathrm{H}$ data & 0.00 & 100.00 & - & - & - & - & - & - \\
\hline \multirow[t]{5}{*}{ DP4+ } & ${ }^{13} \mathrm{C}$ data & 0.04 & 99.96 & - & - & - & - & - & - \\
\hline & All data & 0.00 & 100.00 & - & - & - & - & - & - \\
\hline & & \multicolumn{8}{|c|}{ Level of theory: mPW1PW91/6-311G* (gas phase) } \\
\hline & & $76 a$ & $76 \mathrm{~b}$ & - & - & - & - & - & - \\
\hline & ${ }^{1} \mathrm{H}$ data & 0.03 & 99.97 & - & - & - & - & - & - \\
\hline \multirow[t]{2}{*}{ sDP4+ } & ${ }^{13} \mathrm{C}$ data & 0.53 & 99.47 & - & - & - & - & - & - \\
\hline & All data & 0.00 & 100.00 & - & - & - & - & - & - \\
\hline & ${ }^{1} \mathrm{H}$ data & 0.10 & 99.90 & - & - & - & - & - & - \\
\hline \multirow[t]{2}{*}{ uDP4+ } & ${ }^{13} \mathrm{C}$ data & 0.34 & 99.66 & - & - & - & - & - & - \\
\hline & All data & 0.00 & 100.00 & - & - & - & - & - & - \\
\hline \multirow{5}{*}{ DP4+ } & ${ }^{1} \mathrm{H}$ data & 0.00 & 100.00 & - & - & - & - & - & - \\
\hline & ${ }^{13} \mathrm{C}$ data & 0.00 & 100.00 & - & - & - & - & - & - \\
\hline & All data & 0.00 & 100.00 & - & - & - & - & - & - \\
\hline & & \multicolumn{8}{|c|}{ Level of theory: mPW1PW91/6-311G** (gas phase) } \\
\hline & & $76 a$ & $76 \mathrm{~b}$ & - & - & - & - & - & - \\
\hline \multirow{3}{*}{$s \mathrm{DP} 4+$} & ${ }^{1} \mathrm{H}$ data & 0.01 & 99.99 & - & - & - & - & - & - \\
\hline & ${ }^{13} \mathrm{C}$ data & 0.72 & 99.28 & - & - & - & - & - & - \\
\hline & All data & 0.00 & 100.00 & - & - & - & - & - & - \\
\hline \multirow{3}{*}{ uDP4+ } & ${ }^{1} \mathrm{H}$ data & 0.05 & 99.95 & - & - & - & - & - & - \\
\hline & ${ }^{13} \mathrm{C}$ data & 0.28 & 99.72 & - & - & - & - & - & - \\
\hline & All data & 0.00 & 100.00 & - & - & - & - & - & - \\
\hline \multirow{5}{*}{ DP4+ } & ${ }^{1} \mathrm{H}$ data & 0.00 & 100.00 & - & - & - & - & - & - \\
\hline & ${ }^{13} \mathrm{C}$ data & 0.00 & 100.00 & - & - & - & - & - & - \\
\hline & All data & 0.00 & 100.00 & - & - & - & - & - & - \\
\hline & & \multicolumn{8}{|c|}{ Level of theory: mPW1PW91/6-311+G** (gas phase) } \\
\hline & & $76 a$ & $76 \mathrm{~b}$ & - & - & - & - & - & - \\
\hline \multirow{3}{*}{$s \mathrm{DP} 4+$} & ${ }^{1} \mathrm{H}$ data & 0.06 & 99.94 & - & - & - & - & - & - \\
\hline & ${ }^{13} \mathrm{C}$ data & 0.42 & 99.58 & - & - & - & - & - & - \\
\hline & All data & 0.00 & 100.00 & - & - & - & - & - & - \\
\hline \multirow{3}{*}{ uDP4+ } & ${ }^{1} \mathrm{H}$ data & 0.13 & 99.87 & - & - & - & - & - & - \\
\hline & ${ }^{13} \mathrm{C}$ data & 0.19 & 99.81 & - & - & - & - & - & - \\
\hline & All data & 0.00 & 100.00 & - & - & - & - & - & - \\
\hline \multirow{5}{*}{ DP4+ } & ${ }^{1} \mathrm{H}$ data & 0.00 & 100.00 & - & - & - & - & - & - \\
\hline & ${ }^{13} \mathrm{C}$ data & 0.00 & 100.00 & - & - & - & - & - & - \\
\hline & All data & 0.00 & 100.00 & - & - & - & - & - & - \\
\hline & & \multicolumn{8}{|c|}{ Level of theory: mPW1PW91/6-31G* (solution, PCM) } \\
\hline & & $76 a$ & $76 \mathrm{~b}$ & - & - & - & - & - & - \\
\hline \multirow{3}{*}{ sDP4+ } & ${ }^{1} \mathrm{H}$ data & 0.02 & 99.98 & - & - & - & - & - & - \\
\hline & ${ }^{13} \mathrm{C}$ data & 0.29 & 99.71 & - & - & - & - & - & - \\
\hline & All data & 0.00 & 100.00 & - & - & - & - & - & - \\
\hline \multirow{3}{*}{ uDP4+ } & ${ }^{1} \mathrm{H}$ data & 0.02 & 99.98 & - & - & - & - & - & - \\
\hline & ${ }^{13} \mathrm{C}$ data & 6.98 & 93.02 & - & - & - & - & - & - \\
\hline & All data & 0.00 & 100.00 & - & - & - & - & - & - \\
\hline & ${ }^{1} \mathrm{H}$ data & 0.00 & 100.00 & - & - & - & - & - & - \\
\hline DP4+ & ${ }^{13} \mathrm{C}$ data & 0.02 & 99.98 & - & - & - & - & - & - \\
\hline & All data & 0.00 & 100.00 & - & - & - & - & - & - \\
\hline & & & & of & $\mathbf{W}$ & $31 C$ & on, & & \\
\hline & & $76 a$ & $76 b$ & - & - & - & - & - & - \\
\hline & ${ }^{1} \mathrm{H}$ data & 0.05 & 99.95 & - & - & - & - & - & - \\
\hline sDP4+ & ${ }^{13} \mathrm{C}$ data & 0.18 & 99.82 & - & - & - & - & - & - \\
\hline & All data & 0.00 & 100.00 & - & - & - & - & - & - \\
\hline & ${ }^{1} \mathrm{H}$ data & 0.07 & 99.93 & - & - & - & - & - & - \\
\hline uDP4+ & ${ }^{13} \mathrm{C}$ data & 1.27 & 98.73 & - & - & - & - & - & - \\
\hline
\end{tabular}




\begin{tabular}{|c|c|c|c|c|c|c|c|c|c|}
\hline & All data & 0.00 & 100.00 & - & - & - & - & - & - \\
\hline & ${ }^{1} \mathrm{H}$ data & 0.00 & 100.00 & - & - & - & - & - & - \\
\hline \multirow[t]{5}{*}{ DP4+ } & ${ }^{13} \mathrm{C}$ data & 0.00 & 100.00 & - & - & - & - & - & - \\
\hline & All data & 0.00 & 100.00 & - & - & - & - & - & - \\
\hline & & \multicolumn{8}{|c|}{ Level of theory: mPW1PW91/6-31+G** (solution, PCM) } \\
\hline & & $76 a$ & $76 b$ & - & - & - & - & - & - \\
\hline & ${ }^{1} \mathrm{H}$ data & 0.00 & 100.00 & - & - & - & - & - & - \\
\hline \multirow[t]{3}{*}{ sDP4+ } & ${ }^{13} \mathrm{C}$ data & 0.56 & 99.44 & - & - & - & - & - & - \\
\hline & All data & 0.00 & 100.00 & - & - & - & - & - & - \\
\hline & ${ }^{1} \mathrm{H}$ data & 61.64 & 38.36 & - & - & - & - & - & - \\
\hline \multirow[t]{3}{*}{ uDP4+ } & ${ }^{13} \mathrm{C}$ data & 16.46 & 83.54 & - & - & - & - & - & - \\
\hline & All data & 24.04 & 75.96 & - & - & - & - & - & - \\
\hline & ${ }^{1} \mathrm{H}$ data & 0.00 & 100.00 & - & - & - & - & - & - \\
\hline \multirow[t]{5}{*}{ DP4+ } & ${ }^{13} \mathrm{C}$ data & 0.11 & 99.89 & - & - & - & - & - & - \\
\hline & All data & 0.00 & 100.00 & - & - & - & - & - & - \\
\hline & & \multicolumn{8}{|c|}{ Level of theory: mPW1PW91/6-311G* (solution, PCM) } \\
\hline & & $76 a$ & $76 b$ & - & - & - & - & - & - \\
\hline & ${ }^{1} \mathrm{H}$ data & 0.09 & 99.91 & - & - & - & - & - & - \\
\hline \multirow[t]{3}{*}{ sDP4+ } & ${ }^{13} \mathrm{C}$ data & 0.17 & 99.83 & - & - & - & - & - & - \\
\hline & All data & 0.00 & 100.00 & - & - & - & - & - & - \\
\hline & ${ }^{1}$ H data & 0.16 & 99.84 & - & - & - & - & - & - \\
\hline \multirow[t]{3}{*}{ uDP4+ } & ${ }^{13}$ C data & 0.25 & 99.75 & - & - & - & - & - & - \\
\hline & All data & 0.00 & 100.00 & - & - & - & - & - & - \\
\hline & ${ }^{1} \mathrm{H}$ data & 0.00 & 100.00 & - & - & - & - & - & - \\
\hline \multirow[t]{5}{*}{ DP4+ } & ${ }^{13} \mathrm{C}$ data & 0.00 & 100.00 & - & - & - & - & - & - \\
\hline & All data & 0.00 & 100.00 & - & - & - & - & - & - \\
\hline & & \multicolumn{8}{|c|}{ Level of theory: mPW1PW91/6-311G** (solution, PCM) } \\
\hline & & $76 a$ & $76 b$ & - & - & - & - & - & - \\
\hline & ${ }^{1} \mathrm{H}$ data & 0.30 & 99.70 & - & - & - & - & - & - \\
\hline \multirow[t]{2}{*}{ sDP4+ } & ${ }^{13} \mathrm{C}$ data & 0.18 & 99.82 & - & - & - & - & - & - \\
\hline & All data & 0.00 & 100.00 & - & - & - & - & - & - \\
\hline \multirow{3}{*}{ uDP4+ } & ${ }^{1} \mathrm{H}$ data & 0.41 & 99.59 & - & - & - & - & - & - \\
\hline & ${ }^{13} \mathrm{C}$ data & 0.27 & 99.73 & - & - & - & - & - & - \\
\hline & All data & 0.00 & 100.00 & - & - & - & - & - & - \\
\hline \multirow{5}{*}{ DP4+ } & ${ }^{1} \mathrm{H}$ data & 0.00 & 100.00 & - & - & - & - & - & - \\
\hline & ${ }^{13} \mathrm{C}$ data & 0.00 & 100.00 & - & - & - & - & - & - \\
\hline & All data & 0.00 & 100.00 & - & - & - & - & - & - \\
\hline & & \multicolumn{8}{|c|}{ Level of theory: mPW1PW91/6-311+G** (solution, PCM) } \\
\hline & & $76 a$ & $76 b$ & - & - & - & - & - & - \\
\hline \multirow{3}{*}{ sDP4+ } & ${ }^{1} \mathrm{H}$ data & 0.81 & 99.19 & - & - & - & - & - & - \\
\hline & ${ }^{13} \mathrm{C}$ data & 0.21 & 99.79 & - & - & - & - & - & - \\
\hline & All data & 0.00 & 100.00 & - & - & - & - & - & - \\
\hline \multirow{3}{*}{ uDP4+ } & ${ }^{1} \mathrm{H}$ data & 0.96 & 99.04 & - & - & - & - & - & - \\
\hline & ${ }^{13} \mathrm{C}$ data & 0.30 & 99.70 & - & - & - & - & - & - \\
\hline & All data & 0.00 & 100.00 & - & - & - & - & - & - \\
\hline \multirow{3}{*}{ DP4+ } & ${ }^{1} \mathrm{H}$ data & 0.01 & 99.99 & - & - & - & - & - & - \\
\hline & ${ }^{13} \mathrm{C}$ data & 0.00 & 100.00 & - & - & - & - & - & - \\
\hline & All data & 0.00 & 100.00 & - & - & - & - & - & - \\
\hline
\end{tabular}


$s \mathrm{DP4}+, u \mathrm{DP} 4+$ and DP4+ probabilities (\%) for compound 77a<smiles>C=C(C)[C@H](O)[C@@H](C)C(=O)[C@@H](C)C(=O)OCc1ccccc1</smiles>

\begin{tabular}{|c|c|c|c|c|c|c|c|c|c|}
\hline & & \multicolumn{8}{|c|}{ Level of theory: B3LYP/6-31G* (gas phase) } \\
\hline & & $77 a$ & 77b & 77c & 77d & - & - & - & - \\
\hline \multirow{3}{*}{ sDP4+ } & ${ }^{1} \mathrm{H}$ data & 98.48 & 1.52 & 0.00 & 0.00 & - & - & - & - \\
\hline & ${ }^{13} \mathrm{C}$ data & 97.22 & 0.01 & 0.00 & 2.77 & - & - & - & - \\
\hline & All data & 100.00 & 0.00 & 0.00 & 0.00 & - & - & - & - \\
\hline & ${ }^{1} \mathrm{H}$ data & 84.16 & 15.83 & 0.01 & 0.00 & - & - & - & - \\
\hline \multirow[t]{2}{*}{ uDP4+ } & ${ }^{13} \mathrm{C}$ data & 99.40 & 0.00 & 0.00 & 0.60 & - & - & - & - \\
\hline & All data & 100.00 & 0.00 & 0.00 & 0.00 & - & - & - & - \\
\hline \multirow{5}{*}{ DP4+ } & ${ }^{1} \mathrm{H}$ data & 99.71 & 0.29 & 0.00 & 0.00 & - & - & - & - \\
\hline & ${ }^{13} \mathrm{C}$ data & 99.98 & 0.00 & 0.00 & 0.02 & - & - & - & - \\
\hline & All data & 100.00 & 0.00 & 0.00 & 0.00 & - & - & - & - \\
\hline & & \multicolumn{8}{|c|}{ Level of theory: B3LYP/6-31G** (gas phase) } \\
\hline & & $77 a$ & $77 \mathrm{~b}$ & 77c & 77d & - & - & - & - \\
\hline & ${ }^{1} \mathrm{H}$ data & 98.57 & 1.43 & 0.00 & 0.00 & - & - & - & - \\
\hline \multirow[t]{2}{*}{ sDP4+ } & ${ }^{13} \mathrm{C}$ data & 98.65 & 0.00 & 0.00 & 1.34 & - & - & - & - \\
\hline & All data & 100.00 & 0.00 & 0.00 & 0.00 & - & - & - & - \\
\hline & ${ }^{1} \mathrm{H}$ data & 94.99 & 5.00 & 0.00 & 0.00 & - & - & - & - \\
\hline \multirow[t]{2}{*}{ uDP4+ } & ${ }^{13} \mathrm{C}$ data & 99.62 & 0.00 & 0.00 & 0.38 & - & - & - & - \\
\hline & All data & 100.00 & 0.00 & 0.00 & 0.00 & - & - & - & - \\
\hline & ${ }^{1} \mathrm{H}$ data & 99.92 & 0.08 & 0.00 & 0.00 & - & - & - & - \\
\hline \multirow[t]{5}{*}{ DP4+ } & ${ }^{13} \mathrm{C}$ data & 99.99 & 0.00 & 0.00 & 0.01 & - & - & - & - \\
\hline & All data & 100.00 & 0.00 & 0.00 & 0.00 & - & - & - & - \\
\hline & & \multicolumn{8}{|c|}{ Level of theory: B3LYP/6-31+G** (gas phase) } \\
\hline & & $77 a$ & $77 \mathrm{~b}$ & 77c & 77d & - & - & - & - \\
\hline & ${ }^{1} \mathrm{H}$ data & 69.51 & 30.49 & 0.00 & 0.00 & - & - & - & - \\
\hline \multirow[t]{2}{*}{ sDP4+ } & ${ }^{13} \mathrm{C}$ data & 99.60 & 0.00 & 0.00 & 0.40 & - & - & - & - \\
\hline & All data & 100.00 & 0.00 & 0.00 & 0.00 & - & - & - & - \\
\hline \multirow{3}{*}{ uDP4+ } & ${ }^{1} \mathrm{H}$ data & 86.91 & 13.09 & 0.00 & 0.00 & - & - & - & - \\
\hline & ${ }^{13} \mathrm{C}$ data & 99.88 & 0.00 & 0.00 & 0.11 & - & - & - & - \\
\hline & All data & 100.00 & 0.00 & 0.00 & 0.00 & - & - & - & - \\
\hline \multirow{5}{*}{ DP4+ } & ${ }^{1} \mathrm{H}$ data & 93.80 & 6.20 & 0.00 & 0.00 & - & - & - & - \\
\hline & ${ }^{13} \mathrm{C}$ data & 100.00 & 0.00 & 0.00 & 0.00 & - & - & - & - \\
\hline & All data & 100.00 & 0.00 & 0.00 & 0.00 & - & - & - & - \\
\hline & & \multicolumn{8}{|c|}{ Level of theory: B3LYP/6-311G* (gas phase) } \\
\hline & & $77 a$ & 77b & 77c & 77d & - & - & - & - \\
\hline & ${ }^{1} \mathrm{H}$ data & 46.51 & 53.49 & 0.00 & 0.00 & - & - & - & - \\
\hline \multirow[t]{2}{*}{ sDP4+ } & ${ }^{13} \mathrm{C}$ data & 99.97 & 0.00 & 0.00 & 0.03 & - & - & - & - \\
\hline & All data & 100.00 & 0.00 & 0.00 & 0.00 & - & - & - & - \\
\hline & ${ }^{1} \mathrm{H}$ data & 12.02 & 87.95 & 0.00 & 0.02 & - & - & - & - \\
\hline \multirow[t]{2}{*}{ uDP4+ } & ${ }^{13} \mathrm{C}$ data & 99.29 & 0.01 & 0.00 & 0.71 & - & - & - & - \\
\hline & All data & 99.95 & 0.05 & 0.00 & 0.00 & - & - & - & - \\
\hline \multirow{5}{*}{ DP4+ } & ${ }^{1} \mathrm{H}$ data & 10.62 & 89.38 & 0.00 & 0.00 & - & - & - & - \\
\hline & ${ }^{13} \mathrm{C}$ data & 100.00 & 0.00 & 0.00 & 0.00 & - & - & - & - \\
\hline & All data & 100.00 & 0.00 & 0.00 & 0.00 & - & - & - & - \\
\hline & & \multicolumn{8}{|c|}{ Level of theory: B3LYP/6-311G** (gas phase) } \\
\hline & & $77 a$ & $77 \mathrm{~b}$ & 77c & 77d & - & - & - & - \\
\hline \multirow{3}{*}{ sDP4+ } & ${ }^{1} \mathrm{H}$ data & 12.54 & 87.45 & 0.01 & 0.00 & - & - & - & - \\
\hline & ${ }^{13} \mathrm{C}$ data & 99.98 & 0.00 & 0.00 & 0.02 & - & - & - & - \\
\hline & All data & 100.00 & 0.00 & 0.00 & 0.00 & - & - & - & - \\
\hline
\end{tabular}




\begin{tabular}{|c|c|c|c|c|c|c|c|c|c|}
\hline & ${ }^{1} \mathrm{H}$ data & 5.15 & 94.84 & 0.00 & 0.01 & - & - & - & - \\
\hline \multirow[t]{3}{*}{ uDP4+ } & ${ }^{13} \mathrm{C}$ data & 99.57 & 0.01 & 0.00 & 0.43 & - & - & - & - \\
\hline & All data & 99.89 & 0.11 & 0.00 & 0.00 & - & - & - & - \\
\hline & ${ }^{1} \mathrm{H}$ data & 0.77 & 99.23 & 0.00 & 0.00 & - & - & - & - \\
\hline \multirow[t]{5}{*}{ DP4+ } & ${ }^{13} \mathrm{C}$ data & 100.00 & 0.00 & 0.00 & 0.00 & - & - & - & - \\
\hline & All data & 100.00 & 0.00 & 0.00 & 0.00 & - & - & - & - \\
\hline & & \multicolumn{8}{|c|}{ Level of theory: B3LYP/6-311+G** (gas phase) } \\
\hline & & $77 a$ & 77b & 77c & 77d & - & - & - & - \\
\hline & ${ }^{1}$ H data & 81.80 & 18.20 & 0.00 & 0.00 & - & - & - & - \\
\hline \multirow[t]{3}{*}{ sDP4+ } & ${ }^{13} \mathrm{C}$ data & 99.97 & 0.00 & 0.00 & 0.03 & - & - & - & - \\
\hline & All data & 100.00 & 0.00 & 0.00 & 0.00 & - & - & - & - \\
\hline & ${ }^{1} \mathrm{H}$ data & 83.78 & 16.21 & 0.00 & 0.01 & - & - & - & - \\
\hline \multirow[t]{3}{*}{ uDP4+ } & ${ }^{13} \mathrm{C}$ data & 99.49 & 0.01 & 0.00 & 0.50 & - & - & - & - \\
\hline & All data & 100.00 & 0.00 & 0.00 & 0.00 & - & - & - & - \\
\hline & ${ }^{1} \mathrm{H}$ data & 95.87 & 4.13 & 0.00 & 0.00 & - & - & - & - \\
\hline \multirow[t]{5}{*}{ DP4+ } & ${ }^{13} \mathrm{C}$ data & 100.00 & 0.00 & 0.00 & 0.00 & - & - & - & - \\
\hline & All data & 100.00 & 0.00 & 0.00 & 0.00 & - & - & - & - \\
\hline & & \multicolumn{8}{|c|}{ Level of theory: B3LYP/6-31G* (solution, PCM) } \\
\hline & & $77 a$ & 77b & 77c & 77d & - & - & - & - \\
\hline & ${ }^{1}$ H data & 18.37 & 81.60 & 0.00 & 0.02 & - & - & - & - \\
\hline \multirow[t]{3}{*}{ sDP4+ } & ${ }^{13} \mathrm{C}$ data & 99.99 & 0.00 & 0.00 & 0.01 & - & - & - & - \\
\hline & All data & 100.00 & 0.00 & 0.00 & 0.00 & - & - & - & - \\
\hline & ${ }^{1} \mathrm{H}$ data & 3.55 & 96.40 & 0.00 & 0.05 & - & - & - & - \\
\hline \multirow[t]{3}{*}{ uDP4+ } & ${ }^{13} \mathrm{C}$ data & 99.82 & 0.00 & 0.00 & 0.18 & - & - & - & - \\
\hline & All data & 100.00 & 0.00 & 0.00 & 0.00 & - & - & - & - \\
\hline & ${ }^{1} \mathrm{H}$ data & 0.82 & 99.18 & 0.00 & 0.00 & - & - & - & - \\
\hline \multirow[t]{5}{*}{ DP4+ } & ${ }^{13} \mathrm{C}$ data & 100.00 & 0.00 & 0.00 & 0.00 & - & - & - & - \\
\hline & All data & 100.00 & 0.00 & 0.00 & 0.00 & - & - & - & - \\
\hline & & \multicolumn{8}{|c|}{ Level of theory: B3LYP/6-31G** (solution, PCM) } \\
\hline & & $77 a$ & 77b & 77c & 77d & - & - & - & - \\
\hline & ${ }^{1} \mathrm{H}$ data & 46.86 & 53.12 & 0.01 & 0.01 & - & - & - & - \\
\hline \multirow[t]{3}{*}{ sDP4+ } & ${ }^{13} \mathrm{C}$ data & 99.99 & 0.00 & 0.00 & 0.01 & - & - & - & - \\
\hline & All data & 100.00 & 0.00 & 0.00 & 0.00 & - & - & - & - \\
\hline & ${ }^{1} \mathrm{H}$ data & 6.52 & 93.41 & 0.00 & 0.06 & - & - & - & - \\
\hline \multirow[t]{3}{*}{ uDP4+ } & ${ }^{13} \mathrm{C}$ data & 99.93 & 0.00 & 0.00 & 0.07 & - & - & - & - \\
\hline & All data & 100.00 & 0.00 & 0.00 & 0.00 & - & - & - & - \\
\hline & ${ }^{1} \mathrm{H}$ data & 5.80 & 94.20 & 0.00 & 0.00 & - & - & - & - \\
\hline \multirow[t]{5}{*}{ DP4+ } & ${ }^{13} \mathrm{C}$ data & 100.00 & 0.00 & 0.00 & 0.00 & - & - & - & - \\
\hline & All data & 100.00 & 0.00 & 0.00 & 0.00 & - & - & - & - \\
\hline & & \multicolumn{8}{|c|}{ Level of theory: B3LYP/6-31+G** (solution, PCM) } \\
\hline & & $77 a$ & 77b & 77c & 77d & - & - & - & - \\
\hline & ${ }^{1} \mathrm{H}$ data & 0.24 & 99.47 & 0.00 & 0.28 & - & - & - & - \\
\hline \multirow[t]{3}{*}{ sDP4+ } & ${ }^{13} \mathrm{C}$ data & 99.98 & 0.00 & 0.00 & 0.02 & - & - & - & - \\
\hline & All data & 99.95 & 0.03 & 0.00 & 0.02 & - & - & - & - \\
\hline & ${ }^{1} \mathrm{H}$ data & 0.89 & 88.62 & 0.00 & 10.49 & - & - & - & - \\
\hline uDP4+ & ${ }^{13}$ C data & 99.96 & 0.01 & 0.00 & 0.04 & - & - & - & - \\
\hline & All data & 98.93 & 0.65 & 0.00 & 0.42 & - & - & - & - \\
\hline & ${ }^{1} \mathrm{H}$ data & 0.00 & 99.96 & 0.00 & 0.03 & - & - & - & - \\
\hline DP4+ & ${ }^{13} \mathrm{C}$ data & 100.00 & 0.00 & 0.00 & 0.00 & - & - & - & - \\
\hline & All data & 100.00 & 0.00 & 0.00 & 0.00 & - & - & - & - \\
\hline & & & & Level o & y: B3LY & & PC & & \\
\hline & & $77 a$ & $77 b$ & 77c & $77 d$ & - & - & - & - \\
\hline & ${ }^{1} \mathrm{H}$ data & 0.05 & 99.85 & 0.00 & 0.11 & - & - & - & - \\
\hline sDP4+ & ${ }^{13} \mathrm{C}$ data & 100.00 & 0.00 & 0.00 & 0.00 & - & - & - & - \\
\hline & All data & 99.99 & 0.00 & 0.00 & 0.00 & - & - & - & - \\
\hline uDP4+ & ${ }^{1} \mathrm{H}$ data & 0.04 & 99.42 & 0.00 & 0.54 & - & - & - & - \\
\hline
\end{tabular}




\begin{tabular}{|c|c|c|c|c|c|c|c|c|c|}
\hline & ${ }^{13} \mathrm{C}$ data & 99.98 & 0.00 & 0.00 & 0.02 & - & - & - & - \\
\hline & All data & 98.84 & 0.95 & 0.00 & 0.22 & - & - & - & - \\
\hline & ${ }^{1} \mathrm{H}$ data & 0.00 & 100.00 & 0.00 & 0.00 & - & - & - & - \\
\hline \multirow[t]{5}{*}{ DP4+ } & ${ }^{13} \mathrm{C}$ data & 100.00 & 0.00 & 0.00 & 0.00 & - & - & - & - \\
\hline & All data & 100.00 & 0.00 & 0.00 & 0.00 & - & - & - & - \\
\hline & & \multicolumn{8}{|c|}{ Level of theory: B3LYP/6-311G** (solution, PCM) } \\
\hline & & $77 a$ & 77b & 77c & 77d & - & - & - & - \\
\hline & ${ }^{1} \mathrm{H}$ data & 0.41 & 99.25 & 0.03 & 0.31 & - & - & - & - \\
\hline \multirow[t]{3}{*}{ sDP4+ } & ${ }^{13} \mathrm{C}$ data & 100.00 & 0.00 & 0.00 & 0.00 & - & - & - & - \\
\hline & All data & 100.00 & 0.00 & 0.00 & 0.00 & - & - & - & - \\
\hline & ${ }^{1} \mathrm{H}$ data & 0.42 & 98.28 & 0.00 & 1.30 & - & - & - & - \\
\hline \multirow[t]{3}{*}{ uDP4+ } & ${ }^{13} \mathrm{C}$ data & 99.95 & 0.00 & 0.00 & 0.05 & - & - & - & - \\
\hline & All data & 99.68 & 0.16 & 0.00 & 0.16 & - & - & - & - \\
\hline & ${ }^{1} \mathrm{H}$ data & 0.00 & 99.99 & 0.00 & 0.00 & - & - & - & - \\
\hline \multirow[t]{5}{*}{ DP4+ } & ${ }^{13} \mathrm{C}$ data & 100.00 & 0.00 & 0.00 & 0.00 & - & - & - & - \\
\hline & All data & 100.00 & 0.00 & 0.00 & 0.00 & - & - & - & - \\
\hline & & \multicolumn{8}{|c|}{ Level of theory: B3LYP/6-311+G** (solution, PCM) } \\
\hline & & $77 a$ & $77 b$ & 77c & 77d & - & - & - & - \\
\hline & ${ }^{1} \mathrm{H}$ data & 0.47 & 87.94 & 0.00 & 11.58 & - & - & - & - \\
\hline \multirow[t]{2}{*}{ sDP4+ } & ${ }^{13} \mathrm{C}$ data & 99.99 & 0.00 & 0.00 & 0.01 & - & - & - & - \\
\hline & All data & 99.67 & 0.00 & 0.00 & 0.33 & - & - & - & - \\
\hline \multirow{3}{*}{ uDP4+ } & ${ }^{1} \mathrm{H}$ data & 0.14 & 77.36 & 0.00 & 22.50 & - & - & - & - \\
\hline & ${ }^{13} \mathrm{C}$ data & 99.98 & 0.00 & 0.00 & 0.02 & - & - & - & - \\
\hline & All data & 97.07 & 0.30 & 0.00 & 2.63 & - & - & - & - \\
\hline \multirow{5}{*}{ DP4+ } & ${ }^{1} \mathrm{H}$ data & 0.00 & 96.31 & 0.00 & 3.69 & - & - & - & - \\
\hline & ${ }^{13} \mathrm{C}$ data & 100.00 & 0.00 & 0.00 & 0.00 & - & - & - & - \\
\hline & All data & 99.99 & 0.00 & 0.00 & 0.01 & - & - & - & - \\
\hline & & \multicolumn{8}{|c|}{ Level of theory: mPW1PW91/6-31G* (gas phase) } \\
\hline & & $77 a$ & 77b & 77c & 77d & - & - & - & - \\
\hline \multirow{3}{*}{ sDP4+ } & ${ }^{1} \mathrm{H}$ data & 91.84 & 8.16 & 0.00 & 0.00 & - & - & - & - \\
\hline & ${ }^{13} \mathrm{C}$ data & 99.39 & 0.00 & 0.00 & 0.61 & - & - & - & - \\
\hline & All data & 100.00 & 0.00 & 0.00 & 0.00 & - & - & - & - \\
\hline \multirow{3}{*}{ uDP4+ } & ${ }^{1}$ H data & 65.53 & 34.47 & 0.00 & 0.00 & - & - & - & - \\
\hline & ${ }^{13} \mathrm{C}$ data & 99.50 & 0.01 & 0.00 & 0.50 & - & - & - & - \\
\hline & All data & 100.00 & 0.00 & 0.00 & 0.00 & - & - & - & - \\
\hline \multirow{5}{*}{ DP4+ } & ${ }^{1} \mathrm{H}$ data & 95.54 & 4.46 & 0.00 & 0.00 & - & - & - & - \\
\hline & ${ }^{13} \mathrm{C}$ data & 100.00 & 0.00 & 0.00 & 0.00 & - & - & - & - \\
\hline & All data & 100.00 & 0.00 & 0.00 & 0.00 & - & - & - & - \\
\hline & & \multicolumn{8}{|c|}{ Level of theory: mPW1PW91/6-31G** (gas phase) } \\
\hline & & $77 a$ & 77b & 77c & 77d & - & - & - & - \\
\hline \multirow{3}{*}{ sDP4+ } & ${ }^{1} \mathrm{H}$ data & 93.43 & 6.55 & 0.02 & 0.00 & - & - & - & - \\
\hline & ${ }^{13} \mathrm{C}$ data & 99.78 & 0.00 & 0.00 & 0.22 & - & - & - & - \\
\hline & All data & 100.00 & 0.00 & 0.00 & 0.00 & - & - & - & - \\
\hline \multirow{3}{*}{ uDP4+ } & ${ }^{1} \mathrm{H}$ data & 89.40 & 10.60 & 0.00 & 0.00 & - & - & - & - \\
\hline & ${ }^{13} \mathrm{C}$ data & 99.77 & 0.00 & 0.00 & 0.23 & - & - & - & - \\
\hline & All data & 100.00 & 0.00 & 0.00 & 0.00 & - & - & - & - \\
\hline & ${ }^{1} \mathrm{H}$ data & 99.18 & 0.82 & 0.00 & 0.00 & - & - & - & - \\
\hline DP4+ & ${ }^{13} \mathrm{C}$ data & 100.00 & 0.00 & 0.00 & 0.00 & - & - & - & - \\
\hline & All data & 100.00 & 0.00 & 0.00 & 0.00 & - & - & - & - \\
\hline & & & & vel of $t$ & mPW1 & -3 & pr & & \\
\hline & & $77 a$ & $77 b$ & 77c & 77d & - & - & - & - \\
\hline & ${ }^{1} \mathrm{H}$ data & 42.35 & 57.65 & 0.00 & 0.00 & - & - & - & - \\
\hline sDP4+ & ${ }^{13} \mathrm{C}$ data & 99.82 & 0.00 & 0.00 & 0.18 & - & - & - & - \\
\hline & All data & 100.00 & 0.00 & 0.00 & 0.00 & - & - & - & - \\
\hline & ${ }^{1} \mathrm{H}$ data & 91.74 & 8.26 & 0.00 & 0.00 & - & - & - & - \\
\hline UDP4+ & ${ }^{13} \mathrm{C}$ data & 99.80 & 0.00 & 0.00 & 0.20 & - & - & - & - \\
\hline
\end{tabular}




\begin{tabular}{|c|c|c|c|c|c|c|c|c|c|}
\hline & All data & 100.00 & 0.00 & 0.00 & 0.00 & - & - & - & - \\
\hline & ${ }^{1} \mathrm{H}$ data & 89.08 & 10.92 & 0.00 & 0.00 & - & - & - & - \\
\hline \multirow[t]{5}{*}{ DP4+ } & ${ }^{13} \mathrm{C}$ data & 100.00 & 0.00 & 0.00 & 0.00 & - & - & - & - \\
\hline & All data & 100.00 & 0.00 & 0.00 & 0.00 & - & - & - & - \\
\hline & & \multicolumn{8}{|c|}{ Level of theory: mPW1PW91/6-311G* (gas phase) } \\
\hline & & $77 a$ & $77 \mathrm{~b}$ & $77 c$ & $77 d$ & - & - & - & - \\
\hline & ${ }^{1} \mathrm{H}$ data & 4.93 & 95.07 & 0.00 & 0.00 & - & - & - & - \\
\hline \multirow[t]{2}{*}{ sDP4+ } & ${ }^{13} \mathrm{C}$ data & 99.87 & 0.00 & 0.00 & 0.13 & - & - & - & - \\
\hline & All data & 100.00 & 0.00 & 0.00 & 0.00 & - & - & - & - \\
\hline & ${ }^{1} \mathrm{H}$ data & 1.08 & 98.88 & 0.00 & 0.03 & - & - & - & - \\
\hline \multirow[t]{2}{*}{ uDP4+ } & ${ }^{13} \mathrm{C}$ data & 99.76 & 0.00 & 0.00 & 0.24 & - & - & - & - \\
\hline & All data & 99.88 & 0.11 & 0.00 & 0.01 & - & - & - & - \\
\hline \multirow{5}{*}{ DP4+ } & ${ }^{1} \mathrm{H}$ data & 0.06 & 99.94 & 0.00 & 0.00 & - & - & - & - \\
\hline & ${ }^{13} \mathrm{C}$ data & 100.00 & 0.00 & 0.00 & 0.00 & - & - & - & - \\
\hline & All data & 100.00 & 0.00 & 0.00 & 0.00 & - & - & - & - \\
\hline & & \multicolumn{8}{|c|}{ Level of theory: mPW1PW91/6-311G** (gas phase) } \\
\hline & & $77 a$ & $77 \mathrm{~b}$ & 77c & 77d & - & - & - & - \\
\hline \multirow{3}{*}{ sDP4+ } & ${ }^{1} \mathrm{H}$ data & 14.34 & 85.61 & 0.04 & 0.00 & - & - & - & - \\
\hline & ${ }^{13} \mathrm{C}$ data & 99.76 & 0.00 & 0.00 & 0.24 & - & - & - & - \\
\hline & All data & 100.00 & 0.00 & 0.00 & 0.00 & - & - & - & - \\
\hline \multirow{3}{*}{ uDP4+ } & ${ }^{1} \mathrm{H}$ data & 5.58 & 94.40 & 0.00 & 0.01 & - & - & - & - \\
\hline & ${ }^{13} \mathrm{C}$ data & 99.56 & 0.00 & 0.00 & 0.44 & - & - & - & - \\
\hline & All data & 99.94 & 0.06 & 0.00 & 0.00 & - & - & - & - \\
\hline \multirow{3}{*}{ DP4+ } & ${ }^{1} \mathrm{H}$ data & 0.98 & 99.02 & 0.00 & 0.00 & - & - & - & - \\
\hline & ${ }^{13} \mathrm{C}$ data & 100.00 & 0.00 & 0.00 & 0.00 & - & - & - & - \\
\hline & All data & 100.00 & 0.00 & 0.00 & 0.00 & - & - & - & - \\
\hline & & \multicolumn{8}{|c|}{ Level of theory: mPW1PW91/6-311+G** (gas phase) } \\
\hline & & $77 a$ & $77 \mathrm{~b}$ & $77 c$ & 77d & - & - & - & - \\
\hline \multirow{3}{*}{ sDP4+ } & ${ }^{1} \mathrm{H}$ data & 83.20 & 16.79 & 0.00 & 0.00 & - & - & - & - \\
\hline & ${ }^{13} \mathrm{C}$ data & 99.90 & 0.00 & 0.00 & 0.10 & - & - & - & - \\
\hline & All data & 100.00 & 0.00 & 0.00 & 0.00 & - & - & - & - \\
\hline \multirow{3}{*}{ uDP4+ } & ${ }^{1} \mathrm{H}$ data & 87.50 & 12.49 & 0.00 & 0.01 & - & - & - & - \\
\hline & ${ }^{13} \mathrm{C}$ data & 99.35 & 0.00 & 0.00 & 0.64 & - & - & - & - \\
\hline & All data & 100.00 & 0.00 & 0.00 & 0.00 & - & - & - & - \\
\hline \multirow{5}{*}{ DP4+ } & ${ }^{1} \mathrm{H}$ data & 97.20 & 2.80 & 0.00 & 0.00 & - & - & - & - \\
\hline & ${ }^{13} \mathrm{C}$ data & 100.00 & 0.00 & 0.00 & 0.00 & - & - & - & - \\
\hline & All data & 100.00 & 0.00 & 0.00 & 0.00 & - & - & - & - \\
\hline & & \multicolumn{8}{|c|}{ Level of theory: mPW1PW91/6-31G* (solution, PCM) } \\
\hline & & $77 a$ & 77b & $77 c$ & $77 d$ & - & - & - & - \\
\hline \multirow{3}{*}{ sDP4+ } & ${ }^{1} \mathrm{H}$ data & 20.03 & 79.95 & 0.00 & 0.02 & - & - & - & - \\
\hline & ${ }^{13} \mathrm{C}$ data & 99.98 & 0.00 & 0.00 & 0.02 & - & - & - & - \\
\hline & All data & 100.00 & 0.00 & 0.00 & 0.00 & - & - & - & - \\
\hline \multirow{3}{*}{ uDP4+ } & ${ }^{1} \mathrm{H}$ data & 2.27 & 97.67 & 0.00 & 0.06 & - & - & - & - \\
\hline & ${ }^{13} \mathrm{C}$ data & 99.75 & 0.00 & 0.00 & 0.25 & - & - & - & - \\
\hline & All data & 99.98 & 0.01 & 0.00 & 0.01 & - & - & - & - \\
\hline & ${ }^{1} \mathrm{H}$ data & 0.58 & 99.42 & 0.00 & 0.00 & - & - & - & - \\
\hline DP4+ & ${ }^{13} \mathrm{C}$ data & 100.00 & 0.00 & 0.00 & 0.00 & - & - & - & - \\
\hline & All data & 100.00 & 0.00 & 0.00 & 0.00 & - & - & - & - \\
\hline & & & & of th & PPW1F & $1 \mathrm{G}$ & n, & & \\
\hline & & $77 a$ & $77 \mathrm{~b}$ & $77 c$ & $77 d$ & - & - & - & - \\
\hline & ${ }^{1} \mathrm{H}$ data & 88.37 & 11.61 & 0.02 & 0.00 & - & - & - & - \\
\hline$s D P 4+$ & ${ }^{13} \mathrm{C}$ data & 99.99 & 0.00 & 0.00 & 0.01 & - & - & - & - \\
\hline & All data & 100.00 & 0.00 & 0.00 & 0.00 & - & - & - & - \\
\hline & ${ }^{1} \mathrm{H}$ data & 17.86 & 81.96 & 0.00 & 0.18 & - & - & - & - \\
\hline uDP4+ & ${ }^{13} \mathrm{C}$ data & 99.93 & 0.00 & 0.00 & 0.07 & - & - & - & - \\
\hline & All data & 100.00 & 0.00 & 0.00 & 0.00 & - & - & - & - \\
\hline
\end{tabular}




\begin{tabular}{|c|c|c|c|c|c|c|c|c|c|}
\hline & ${ }^{1}$ H data & 62.39 & 37.61 & 0.00 & 0.00 & - & - & - & - \\
\hline \multirow[t]{5}{*}{ DP4+ } & ${ }^{13} \mathrm{C}$ data & 100.00 & 0.00 & 0.00 & 0.00 & - & - & - & - \\
\hline & All data & 100.00 & 0.00 & 0.00 & 0.00 & - & - & - & - \\
\hline & & \multicolumn{8}{|c|}{ Level of theory: mPW1PW91/6-31+G** (solution, PCM) } \\
\hline & & $77 a$ & 77b & 77c & 77d & - & - & - & - \\
\hline & ${ }^{1} \mathrm{H}$ data & 0.49 & 99.42 & 0.00 & 0.09 & - & - & - & - \\
\hline \multirow[t]{3}{*}{ sDP4+ } & ${ }^{13} \mathrm{C}$ data & 99.99 & 0.00 & 0.00 & 0.01 & - & - & - & - \\
\hline & All data & 100.00 & 0.00 & 0.00 & 0.00 & - & - & - & - \\
\hline & ${ }^{1} \mathrm{H}$ data & 0.53 & 92.92 & 0.00 & 6.55 & - & - & - & - \\
\hline \multirow[t]{3}{*}{ uDP4+ } & ${ }^{13} \mathrm{C}$ data & 99.96 & 0.00 & 0.00 & 0.04 & - & - & - & - \\
\hline & All data & 99.49 & 0.08 & 0.00 & 0.43 & - & - & - & - \\
\hline & ${ }^{1} \mathrm{H}$ data & 0.00 & 99.99 & 0.00 & 0.01 & - & - & - & - \\
\hline \multirow[t]{5}{*}{ DP4+ } & ${ }^{13} \mathrm{C}$ data & 100.00 & 0.00 & 0.00 & 0.00 & - & - & - & - \\
\hline & All data & 100.00 & 0.00 & 0.00 & 0.00 & - & - & - & - \\
\hline & & \multicolumn{8}{|c|}{ Level of theory: mPW1PW91/6-311G* (solution, PCM) } \\
\hline & & $77 a$ & $77 b$ & 77c & 77d & - & - & - & - \\
\hline & ${ }^{1} \mathrm{H}$ data & 0.02 & 99.80 & 0.00 & 0.18 & - & - & - & - \\
\hline \multirow[t]{2}{*}{ sDP4+ } & ${ }^{13} \mathrm{C}$ data & 99.99 & 0.00 & 0.00 & 0.01 & - & - & - & - \\
\hline & All data & 99.88 & 0.04 & 0.00 & 0.08 & - & - & - & - \\
\hline \multirow{3}{*}{ uDP4+ } & ${ }^{1} \mathrm{H}$ data & 0.02 & 98.06 & 0.00 & 1.92 & - & - & - & - \\
\hline & ${ }^{13} \mathrm{C}$ data & 99.97 & 0.00 & 0.00 & 0.03 & - & - & - & - \\
\hline & All data & 95.67 & 1.41 & 0.00 & 2.93 & - & - & - & - \\
\hline \multirow{5}{*}{ DP4+ } & ${ }^{1} \mathrm{H}$ data & 0.00 & 100.00 & 0.00 & 0.00 & - & - & - & - \\
\hline & ${ }^{13} \mathrm{C}$ data & 100.00 & 0.00 & 0.00 & 0.00 & - & - & - & - \\
\hline & All data & 100.00 & 0.00 & 0.00 & 0.00 & - & - & - & - \\
\hline & & \multicolumn{8}{|c|}{ Level of theory: mPW1PW91/6-311G** (solution, PCM) } \\
\hline & & $77 a$ & $77 b$ & 77c & 77d & - & - & - & - \\
\hline \multirow{3}{*}{ sDP4+ } & ${ }^{1} \mathrm{H}$ data & 0.78 & 98.63 & 0.14 & 0.46 & - & - & - & - \\
\hline & ${ }^{13} \mathrm{C}$ data & 99.99 & 0.00 & 0.00 & 0.01 & - & - & - & - \\
\hline & All data & 99.99 & 0.00 & 0.00 & 0.01 & - & - & - & - \\
\hline \multirow{3}{*}{ uDP4+ } & ${ }^{1} \mathrm{H}$ data & 0.12 & 96.57 & 0.00 & 3.30 & - & - & - & - \\
\hline & ${ }^{13} \mathrm{C}$ data & 99.93 & 0.00 & 0.00 & 0.07 & - & - & - & - \\
\hline & All data & 97.86 & 0.33 & 0.00 & 1.80 & - & - & - & - \\
\hline \multirow{3}{*}{ DP4+ } & ${ }^{1} \mathrm{H}$ data & 0.00 & 99.98 & 0.00 & 0.02 & - & - & - & - \\
\hline & ${ }^{13} \mathrm{C}$ data & 100.00 & 0.00 & 0.00 & 0.00 & - & - & - & - \\
\hline & All data & 100.00 & 0.00 & 0.00 & 0.00 & - & - & - & - \\
\hline & & \multicolumn{8}{|c|}{ Level of theory: mPW1PW91/6-311+G** (solution, PCM) } \\
\hline & & $77 a$ & 77b & 77c & 77d & - & - & - & - \\
\hline \multirow{3}{*}{ sDP4+ } & ${ }^{1} \mathrm{H}$ data & 2.17 & 84.22 & 0.05 & 13.57 & - & - & - & - \\
\hline & ${ }^{13}$ C data & 99.98 & 0.00 & 0.00 & 0.02 & - & - & - & - \\
\hline & All data & 99.86 & 0.00 & 0.00 & 0.14 & - & - & - & - \\
\hline \multirow{3}{*}{ uDP4+ } & ${ }^{1} \mathrm{H}$ data & 0.22 & 83.47 & 0.00 & 16.31 & - & - & - & - \\
\hline & ${ }^{13} \mathrm{C}$ data & 99.99 & 0.00 & 0.00 & 0.01 & - & - & - & - \\
\hline & All data & 98.70 & 0.30 & 0.00 & 1.01 & - & - & - & - \\
\hline \multirow{3}{*}{ DP4+ } & ${ }^{1} \mathrm{H}$ data & 0.01 & 96.94 & 0.00 & 3.05 & - & - & - & - \\
\hline & ${ }^{13} \mathrm{C}$ data & 100.00 & 0.00 & 0.00 & 0.00 & - & - & - & - \\
\hline & All data & 100.00 & 0.00 & 0.00 & 0.00 & - & - & - & - \\
\hline
\end{tabular}


sDP4+, $u \mathrm{DP} 4+$ and DP4+ probabilities (\%) for compound 77b<smiles>C=C(C)[C@H](O)[C@@H](C)C(=O)[C@@H](C)C(=O)[C@@H](C)[C@@H](O)COCc1ccccc1</smiles>

\begin{tabular}{|c|c|c|c|c|c|c|c|c|c|}
\hline & & \multicolumn{8}{|c|}{ Level of theory: B3LYP/6-31G* (gas phase) } \\
\hline & & 77a & $77 \mathrm{~b}$ & 77c & 77d & - & - & - & - \\
\hline \multirow{3}{*}{ sDP4+ } & ${ }^{1} \mathrm{H}$ data & 93.98 & 5.90 & 0.13 & 0.00 & - & - & - & - \\
\hline & ${ }^{13} \mathrm{C}$ data & 0.00 & 95.30 & 4.69 & 0.00 & - & - & - & - \\
\hline & All data & 0.05 & 99.85 & 0.10 & 0.00 & - & - & - & - \\
\hline & ${ }^{1} \mathrm{H}$ data & 91.11 & 7.16 & 1.73 & 0.00 & - & - & - & - \\
\hline \multirow[t]{2}{*}{ uDP4+ } & ${ }^{13} \mathrm{C}$ data & 0.01 & 8.14 & 91.85 & 0.00 & - & - & - & - \\
\hline & All data & 0.47 & 26.75 & 72.78 & 0.00 & - & - & - & - \\
\hline \multirow{6}{*}{ DP4+ } & ${ }^{1} \mathrm{H}$ data & 99.51 & 0.49 & 0.00 & 0.00 & - & - & - & - \\
\hline & ${ }^{13} \mathrm{C}$ data & 0.00 & 64.27 & 35.73 & 0.00 & - & - & - & - \\
\hline & All data & 0.00 & 99.71 & 0.28 & 0.00 & - & - & - & - \\
\hline & & \multicolumn{8}{|c|}{ Level of theory: B3LYP/6-31G** (gas phase) } \\
\hline & & $77 a$ & 77b & 77c & 77d & - & - & - & - \\
\hline & ${ }^{1} \mathrm{H}$ data & 81.22 & 17.38 & 1.40 & 0.00 & - & - & - & - \\
\hline \multirow[t]{2}{*}{ sDP4+ } & ${ }^{13} \mathrm{C}$ data & 0.02 & 97.74 & 2.24 & 0.00 & - & - & - & - \\
\hline & All data & 0.09 & 99.72 & 0.18 & 0.00 & - & - & - & - \\
\hline & ${ }^{1} \mathrm{H}$ data & 95.06 & 4.38 & 0.56 & 0.00 & - & - & - & - \\
\hline \multirow[t]{2}{*}{ uDP4+ } & ${ }^{13} \mathrm{C}$ data & 0.12 & 41.30 & 58.58 & 0.00 & - & - & - & - \\
\hline & All data & 4.94 & 80.57 & 14.49 & 0.00 & - & - & - & - \\
\hline & ${ }^{1} \mathrm{H}$ data & 99.01 & 0.98 & 0.01 & 0.00 & - & - & - & - \\
\hline \multirow[t]{4}{*}{ DP4+ } & ${ }^{13} \mathrm{C}$ data & 0.00 & 96.86 & 3.14 & 0.00 & - & - & - & - \\
\hline & All data & 0.01 & 99.96 & 0.03 & 0.00 & - & - & - & - \\
\hline & & \multicolumn{8}{|c|}{ Level of theory: B3LYP/6-31+G** (gas phase) } \\
\hline & & $77 a$ & $77 \mathrm{~b}$ & 77c & 77d & - & - & - & - \\
\hline & ${ }^{1} \mathrm{H}$ data & 0.98 & 99.02 & 0.00 & 0.00 & - & - & - & - \\
\hline \multirow[t]{2}{*}{ sDP4+ } & ${ }^{13} \mathrm{C}$ data & 0.34 & 90.17 & 9.48 & 0.01 & - & - & - & - \\
\hline & All data & 0.00 & 100.00 & 0.00 & 0.00 & - & - & - & - \\
\hline \multirow{3}{*}{ uDP4+ } & ${ }^{1} \mathrm{H}$ data & 55.72 & 44.28 & 0.00 & 0.00 & - & - & - & - \\
\hline & ${ }^{13} \mathrm{C}$ data & 0.07 & 83.68 & 16.24 & 0.00 & - & - & - & - \\
\hline & All data & 0.11 & 99.89 & 0.00 & 0.00 & - & - & - & - \\
\hline & ${ }^{1} \mathrm{H}$ data & 1.23 & 98.77 & 0.00 & 0.00 & - & - & - & - \\
\hline \multirow[t]{5}{*}{ DP4+ } & ${ }^{13} \mathrm{C}$ data & 0.00 & 98.00 & 2.00 & 0.00 & - & - & - & - \\
\hline & All data & 0.00 & 100.00 & 0.00 & 0.00 & - & - & - & - \\
\hline & & \multicolumn{8}{|c|}{ Level of theory: B3LYP/6-311G* (gas phase) } \\
\hline & & $77 a$ & 77b & 77c & 77d & - & - & - & - \\
\hline & ${ }^{1} \mathrm{H}$ data & 3.65 & 96.30 & 0.05 & 0.00 & - & - & - & - \\
\hline \multirow[t]{2}{*}{$s \mathrm{DP4+}$} & ${ }^{13} \mathrm{C}$ data & 0.79 & 92.37 & 6.84 & 0.00 & - & - & - & - \\
\hline & All data & 0.03 & 99.96 & 0.00 & 0.00 & - & - & - & - \\
\hline \multirow{3}{*}{ uDP4+ } & ${ }^{1} \mathrm{H}$ data & 3.69 & 95.76 & 0.55 & 0.00 & - & - & - & - \\
\hline & ${ }^{13} \mathrm{C}$ data & 1.07 & 84.54 & 14.21 & 0.18 & - & - & - & - \\
\hline & All data & 0.05 & 99.86 & 0.10 & 0.00 & - & - & - & - \\
\hline \multirow{5}{*}{ DP4+ } & ${ }^{1} \mathrm{H}$ data & 0.15 & 99.85 & 0.00 & 0.00 & - & - & - & - \\
\hline & ${ }^{13} \mathrm{C}$ data & 0.01 & 98.76 & 1.23 & 0.00 & - & - & - & - \\
\hline & All data & 0.00 & 100.00 & 0.00 & 0.00 & - & - & - & - \\
\hline & & \multicolumn{8}{|c|}{ Level of theory: B3LYP/6-311G** (gas phase) } \\
\hline & & $77 a$ & $77 \mathrm{~b}$ & 77c & 77d & - & - & - & - \\
\hline \multirow{3}{*}{ sDP4+ } & ${ }^{1} \mathrm{H}$ data & 0.65 & 99.12 & 0.23 & 0.00 & - & - & - & - \\
\hline & ${ }^{13} \mathrm{C}$ data & 1.50 & 92.48 & 6.02 & 0.00 & - & - & - & - \\
\hline & All data & 0.01 & 99.97 & 0.02 & 0.00 & - & - & - & - \\
\hline
\end{tabular}




\begin{tabular}{|c|c|c|c|c|c|c|c|c|c|}
\hline & ${ }^{1} \mathrm{H}$ data & 1.17 & 98.52 & 0.31 & 0.00 & - & - & - & - \\
\hline \multirow[t]{3}{*}{ uDP4+ } & ${ }^{13} \mathrm{C}$ data & 1.16 & 90.02 & 8.68 & 0.13 & - & - & - & - \\
\hline & All data & 0.02 & 99.95 & 0.03 & 0.00 & - & - & - & - \\
\hline & ${ }^{1} \mathrm{H}$ data & 0.01 & 99.99 & 0.00 & 0.00 & - & - & - & - \\
\hline \multirow[t]{5}{*}{ DP4+ } & ${ }^{13} \mathrm{C}$ data & 0.02 & 99.36 & 0.62 & 0.00 & - & - & - & - \\
\hline & All data & 0.00 & 100.00 & 0.00 & 0.00 & - & - & - & - \\
\hline & & \multicolumn{8}{|c|}{ Level of theory: B3LYP/6-311+G** (gas phase) } \\
\hline & & $77 a$ & 77b & $77 c$ & 77d & - & - & - & - \\
\hline & ${ }^{1}$ H data & 2.79 & 97.21 & 0.00 & 0.00 & - & - & - & - \\
\hline \multirow[t]{3}{*}{ sDP4+ } & ${ }^{13} \mathrm{C}$ data & 0.14 & 92.76 & 7.09 & 0.00 & - & - & - & - \\
\hline & All data & 0.00 & 100.00 & 0.00 & 0.00 & - & - & - & - \\
\hline & ${ }^{1} \mathrm{H}$ data & 31.63 & 68.36 & 0.01 & 0.00 & - & - & - & - \\
\hline \multirow[t]{3}{*}{ uDP4+ } & ${ }^{13} \mathrm{C}$ data & 0.34 & 80.65 & 18.96 & 0.06 & - & - & - & - \\
\hline & All data & 0.19 & 99.80 & 0.00 & 0.00 & - & - & - & - \\
\hline & ${ }^{1} \mathrm{H}$ data & 1.31 & 98.69 & 0.00 & 0.00 & - & - & - & - \\
\hline \multirow[t]{5}{*}{ DP4+ } & ${ }^{13} \mathrm{C}$ data & 0.00 & 98.23 & 1.77 & 0.00 & - & - & - & - \\
\hline & All data & 0.00 & 100.00 & 0.00 & 0.00 & - & - & - & - \\
\hline & & \multicolumn{8}{|c|}{ Level of theory: B3LYP/6-31G* (solution, PCM) } \\
\hline & & $77 a$ & 77b & 77c & 77d & - & - & - & - \\
\hline & ${ }^{1}$ H data & 34.98 & 63.97 & 1.05 & 0.00 & - & - & - & - \\
\hline \multirow[t]{3}{*}{ sDP4+ } & ${ }^{13} \mathrm{C}$ data & 0.43 & 89.55 & 10.02 & 0.00 & - & - & - & - \\
\hline & All data & 0.26 & 99.56 & 0.18 & 0.00 & - & - & - & - \\
\hline & ${ }^{1} \mathrm{H}$ data & 22.19 & 70.13 & 7.68 & 0.01 & - & - & - & - \\
\hline \multirow[t]{3}{*}{ uDP4+ } & ${ }^{13} \mathrm{C}$ data & 0.03 & 1.87 & 98.10 & 0.00 & - & - & - & - \\
\hline & All data & 0.07 & 14.80 & 85.13 & 0.00 & - & - & - & - \\
\hline & ${ }^{1} \mathrm{H}$ data & 14.72 & 85.12 & 0.15 & 0.00 & - & - & - & - \\
\hline \multirow[t]{5}{*}{ DP4+ } & ${ }^{13} \mathrm{C}$ data & 0.00 & 14.53 & 85.47 & 0.00 & - & - & - & - \\
\hline & All data & 0.00 & 98.96 & 1.04 & 0.00 & - & - & - & - \\
\hline & & \multicolumn{8}{|c|}{ Level of theory: B3LYP/6-31G** (solution, PCM) } \\
\hline & & $77 a$ & 77b & 77c & 77d & - & - & - & - \\
\hline & ${ }^{1} \mathrm{H}$ data & 20.74 & 76.35 & 2.91 & 0.00 & - & - & - & - \\
\hline \multirow[t]{3}{*}{ sDP4+ } & ${ }^{13}$ C data & 2.75 & 92.28 & 4.96 & 0.01 & - & - & - & - \\
\hline & All data & 0.80 & 99.00 & 0.20 & 0.00 & - & - & - & - \\
\hline & ${ }^{1} \mathrm{H}$ data & 31.88 & 64.06 & 4.06 & 0.00 & - & - & - & - \\
\hline \multirow[t]{3}{*}{ uDP4+ } & ${ }^{13} \mathrm{C}$ data & 0.58 & 14.37 & 85.05 & 0.00 & - & - & - & - \\
\hline & All data & 1.44 & 71.67 & 26.89 & 0.00 & - & - & - & - \\
\hline & ${ }^{1} \mathrm{H}$ data & 11.88 & 87.91 & 0.21 & 0.00 & - & - & - & - \\
\hline \multirow[t]{5}{*}{ DP4+ } & ${ }^{13} \mathrm{C}$ data & 0.09 & 75.79 & 24.12 & 0.00 & - & - & - & - \\
\hline & All data & 0.02 & 99.91 & 0.08 & 0.00 & - & - & - & - \\
\hline & & \multicolumn{8}{|c|}{ Level of theory: B3LYP/6-31+G** (solution, PCM) } \\
\hline & & $77 a$ & 77b & 77c & 77d & - & - & - & - \\
\hline & ${ }^{1} \mathrm{H}$ data & 0.24 & 99.75 & 0.00 & 0.01 & - & - & - & - \\
\hline \multirow[t]{3}{*}{ sDP4+ } & ${ }^{13} \mathrm{C}$ data & 6.67 & 86.04 & 7.28 & 0.01 & - & - & - & - \\
\hline & All data & 0.02 & 99.98 & 0.00 & 0.00 & - & - & - & - \\
\hline & ${ }^{1} \mathrm{H}$ data & 3.42 & 95.62 & 0.03 & 0.93 & - & - & - & - \\
\hline uDP4+ & ${ }^{13} \mathrm{C}$ data & 0.75 & 84.51 & 14.74 & 0.00 & - & - & - & - \\
\hline & All data & 0.03 & 99.96 & 0.01 & 0.00 & - & - & - & - \\
\hline & ${ }^{1} \mathrm{H}$ data & 0.01 & 99.99 & 0.00 & 0.00 & - & - & - & - \\
\hline DP4+ & ${ }^{13} \mathrm{C}$ data & 0.07 & 98.48 & 1.45 & 0.00 & - & - & - & - \\
\hline & All data & 0.00 & 100.00 & 0.00 & 0.00 & - & - & - & - \\
\hline & & & & Level of & $y: B 3 L Y$ & G* & $P C$ & & \\
\hline & & $77 a$ & $77 b$ & 77c & 77d & - & - & - & - \\
\hline & ${ }^{1} \mathrm{H}$ data & 0.14 & 99.74 & 0.10 & 0.02 & - & - & - & - \\
\hline sDP4+ & ${ }^{13} \mathrm{C}$ data & 6.91 & 77.27 & 15.81 & 0.00 & - & - & - & - \\
\hline & All data & 0.01 & 99.97 & 0.02 & 0.00 & - & - & - & - \\
\hline uDP4+ & ${ }^{1} \mathrm{H}$ data & 0.09 & 99.52 & 0.34 & 0.05 & - & - & - & - \\
\hline
\end{tabular}




\begin{tabular}{|c|c|c|c|c|c|c|c|c|c|}
\hline & ${ }^{13} \mathrm{C}$ data & 8.17 & 79.59 & 12.21 & 0.02 & - & - & - & - \\
\hline & All data & 0.01 & 99.94 & 0.05 & 0.00 & - & - & - & - \\
\hline & ${ }^{1} \mathrm{H}$ data & 0.00 & 100.00 & 0.00 & 0.00 & - & - & - & - \\
\hline \multirow[t]{5}{*}{ DP4+ } & ${ }^{13} \mathrm{C}$ data & 0.88 & 96.10 & 3.02 & 0.00 & - & - & - & - \\
\hline & All data & 0.00 & 100.00 & 0.00 & 0.00 & - & - & - & - \\
\hline & & \multicolumn{8}{|c|}{ Level of theory: B3LYP/6-311G** (solution, PCM) } \\
\hline & & $77 a$ & 77b & 77c & 77d & - & - & - & - \\
\hline & ${ }^{1}$ H data & 0.20 & 96.58 & 3.21 & 0.02 & - & - & - & - \\
\hline \multirow[t]{3}{*}{ sDP4+ } & ${ }^{13} \mathrm{C}$ data & 2.98 & 85.75 & 11.27 & 0.00 & - & - & - & - \\
\hline & All data & 0.01 & 99.56 & 0.43 & 0.00 & - & - & - & - \\
\hline & ${ }^{1} \mathrm{H}$ data & 0.22 & 99.08 & 0.60 & 0.10 & - & - & - & - \\
\hline \multirow[t]{3}{*}{ uDP4+ } & ${ }^{13} \mathrm{C}$ data & 4.99 & 82.76 & 12.19 & 0.06 & - & - & - & - \\
\hline & All data & 0.01 & 99.90 & 0.09 & 0.00 & - & - & - & - \\
\hline & ${ }^{1} \mathrm{H}$ data & 0.00 & 99.98 & 0.02 & 0.00 & - & - & - & - \\
\hline \multirow[t]{5}{*}{ DP4+ } & ${ }^{13} \mathrm{C}$ data & 0.21 & 97.90 & 1.89 & 0.00 & - & - & - & - \\
\hline & All data & 0.00 & 100.00 & 0.00 & 0.00 & - & - & - & - \\
\hline & & \multicolumn{8}{|c|}{ Level of theory: B3LYP/6-311+G** (solution, PCM) } \\
\hline & & $77 a$ & 77b & 77c & 77d & - & - & - & - \\
\hline & ${ }^{1} \mathrm{H}$ data & 0.34 & 99.31 & 0.06 & 0.30 & - & - & - & - \\
\hline \multirow[t]{3}{*}{ sDP4+ } & ${ }^{13} \mathrm{C}$ data & 1.54 & 78.35 & 20.11 & 0.00 & - & - & - & - \\
\hline & All data & 0.01 & 99.98 & 0.01 & 0.00 & - & - & - & - \\
\hline & ${ }^{1} \mathrm{H}$ data & 0.41 & 99.05 & 0.03 & 0.52 & - & - & - & - \\
\hline \multirow[t]{3}{*}{ uDP4+ } & ${ }^{13} \mathrm{C}$ data & 5.01 & 76.50 & 18.47 & 0.01 & - & - & - & - \\
\hline & All data & 0.03 & 99.97 & 0.01 & 0.00 & - & - & - & - \\
\hline & ${ }^{1} \mathrm{H}$ data & 0.00 & 100.00 & 0.00 & 0.00 & - & - & - & - \\
\hline \multirow[t]{5}{*}{ DP4+ } & ${ }^{13} \mathrm{C}$ data & 0.12 & 94.05 & 5.83 & 0.00 & - & - & - & - \\
\hline & All data & 0.00 & 100.00 & 0.00 & 0.00 & - & - & - & - \\
\hline & & \multicolumn{8}{|c|}{ Level of theory: mPW1PW91/6-31G* (gas phase) } \\
\hline & & $77 a$ & 77b & 77c & 77d & - & - & - & - \\
\hline & ${ }^{1} \mathrm{H}$ data & 45.91 & 53.12 & 0.97 & 0.00 & - & - & - & - \\
\hline \multirow[t]{2}{*}{ sDP4+ } & ${ }^{13} \mathrm{C}$ data & 0.00 & 83.75 & 16.25 & 0.00 & - & - & - & - \\
\hline & All data & 0.00 & 99.65 & 0.35 & 0.00 & - & - & - & - \\
\hline \multirow{3}{*}{ uDP4+ } & ${ }^{1}$ H data & 78.94 & 19.94 & 1.12 & 0.00 & - & - & - & - \\
\hline & ${ }^{13} \mathrm{C}$ data & 0.00 & 4.58 & 95.41 & 0.00 & - & - & - & - \\
\hline & All data & 0.20 & 46.12 & 53.69 & 0.00 & - & - & - & - \\
\hline \multirow{5}{*}{ DP4+ } & ${ }^{1} \mathrm{H}$ data & 77.36 & 22.62 & 0.02 & 0.00 & - & - & - & - \\
\hline & ${ }^{13} \mathrm{C}$ data & 0.00 & 19.85 & 80.15 & 0.00 & - & - & - & - \\
\hline & All data & 0.00 & 99.59 & 0.41 & 0.00 & - & - & - & - \\
\hline & & \multicolumn{8}{|c|}{ Level of theory: mPW1PW91/6-31G** (gas phase) } \\
\hline & & $77 a$ & 77b & 77c & 77d & - & - & - & - \\
\hline \multirow{3}{*}{ sDP4+ } & ${ }^{1} \mathrm{H}$ data & 35.90 & 56.34 & 7.75 & 0.00 & - & - & - & - \\
\hline & ${ }^{13} \mathrm{C}$ data & 0.00 & 94.13 & 5.86 & 0.00 & - & - & - & - \\
\hline & All data & 0.00 & 99.15 & 0.85 & 0.00 & - & - & - & - \\
\hline \multirow{3}{*}{ uDP4+ } & ${ }^{1} \mathrm{H}$ data & 88.60 & 10.97 & 0.43 & 0.00 & - & - & - & - \\
\hline & ${ }^{13} \mathrm{C}$ data & 0.01 & 21.50 & 78.48 & 0.00 & - & - & - & - \\
\hline & All data & 0.42 & 87.09 & 12.49 & 0.00 & - & - & - & - \\
\hline & ${ }^{1} \mathrm{H}$ data & 83.66 & 16.25 & 0.09 & 0.00 & - & - & - & - \\
\hline DP4+ & ${ }^{13} \mathrm{C}$ data & 0.00 & 81.48 & 18.52 & 0.00 & - & - & - & - \\
\hline & All data & 0.00 & 99.88 & 0.12 & 0.00 & - & - & - & - \\
\hline & & & & vel of $t$ & mPW & -31 & ph & & \\
\hline & & $77 a$ & $77 b$ & 77c & 77d & - & - & - & - \\
\hline & ${ }^{1} \mathrm{H}$ data & 0.62 & 99.37 & 0.01 & 0.00 & - & - & - & - \\
\hline sDP4+ & ${ }^{13} \mathrm{C}$ data & 0.01 & 97.44 & 2.54 & 0.00 & - & - & - & - \\
\hline & All data & 0.00 & 100.00 & 0.00 & 0.00 & - & - & - & - \\
\hline uDP4+ & ${ }^{1} \mathrm{H}$ data & 76.68 & 23.30 & 0.02 & 0.00 & - & - & - & - \\
\hline UDP4+ & ${ }^{13} \mathrm{C}$ data & 0.03 & 66.17 & 33.80 & 0.00 & - & - & - & - \\
\hline
\end{tabular}




\begin{tabular}{|c|c|c|c|c|c|c|c|c|c|}
\hline & All data & 0.15 & 99.81 & 0.04 & 0.00 & - & - & - & - \\
\hline & ${ }^{1} \mathrm{H}$ data & 2.01 & 97.99 & 0.00 & 0.00 & - & - & - & - \\
\hline \multirow[t]{5}{*}{ DP4+ } & ${ }^{13} \mathrm{C}$ data & 0.00 & 98.68 & 1.32 & 0.00 & - & - & - & - \\
\hline & All data & 0.00 & 100.00 & 0.00 & 0.00 & - & - & - & - \\
\hline & & \multicolumn{8}{|c|}{ Level of theory: mPW1PW91/6-311G* (gas phase) } \\
\hline & & $77 a$ & 77b & 77c & 77d & - & - & - & - \\
\hline & ${ }^{1} \mathrm{H}$ data & 0.42 & 99.38 & 0.20 & 0.00 & - & - & - & - \\
\hline \multirow[t]{3}{*}{ sDP4+ } & ${ }^{13} \mathrm{C}$ data & 1.94 & 78.92 & 19.14 & 0.00 & - & - & - & - \\
\hline & All data & 0.01 & 99.94 & 0.05 & 0.00 & - & - & - & - \\
\hline & ${ }^{1} \mathrm{H}$ data & 0.83 & 97.88 & 1.28 & 0.00 & - & - & - & - \\
\hline \multirow[t]{3}{*}{ uDP4+ } & ${ }^{13} \mathrm{C}$ data & 0.84 & 87.75 & 11.34 & 0.06 & - & - & - & - \\
\hline & All data & 0.01 & 99.82 & 0.17 & 0.00 & - & - & - & - \\
\hline & ${ }^{1} \mathrm{H}$ data & 0.00 & 99.99 & 0.00 & 0.00 & - & - & - & - \\
\hline \multirow[t]{5}{*}{ DP4+ } & ${ }^{13} \mathrm{C}$ data & 0.02 & 96.94 & 3.04 & 0.00 & - & - & - & - \\
\hline & All data & 0.00 & 100.00 & 0.00 & 0.00 & - & - & - & - \\
\hline & & \multicolumn{8}{|c|}{ Level of theory: mPW1PW91/6-311G** (gas phase) } \\
\hline & & $77 a$ & 77b & 77c & 77d & - & - & - & - \\
\hline & ${ }^{1} \mathrm{H}$ data & 1.61 & 95.78 & 2.61 & 0.00 & - & - & - & - \\
\hline \multirow[t]{3}{*}{$s D P 4+$} & ${ }^{13} \mathrm{C}$ data & 0.67 & 82.44 & 16.89 & 0.00 & - & - & - & - \\
\hline & All data & 0.01 & 99.43 & 0.56 & 0.00 & - & - & - & - \\
\hline & ${ }^{1} \mathrm{H}$ data & 1.86 & 97.51 & 0.63 & 0.00 & - & - & - & - \\
\hline \multirow[t]{3}{*}{ uDP4+ } & ${ }^{13} \mathrm{C}$ data & 0.21 & 93.79 & 5.96 & 0.04 & - & - & - & - \\
\hline & All data & 0.00 & 99.95 & 0.04 & 0.00 & - & - & - & - \\
\hline & ${ }^{1} \mathrm{H}$ data & 0.03 & 99.95 & 0.02 & 0.00 & - & - & - & - \\
\hline \multirow[t]{5}{*}{ DP4+ } & ${ }^{13} \mathrm{C}$ data & 0.00 & 98.71 & 1.28 & 0.00 & - & - & - & - \\
\hline & All data & 0.00 & 100.00 & 0.00 & 0.00 & - & - & - & - \\
\hline & & \multicolumn{8}{|c|}{ Level of theory: mPW1PW91/6-311+G** (gas phase) } \\
\hline & & $77 a$ & 77b & 77c & 77d & - & - & - & - \\
\hline & ${ }^{1} \mathrm{H}$ data & 3.46 & 96.45 & 0.10 & 0.00 & - & - & - & - \\
\hline \multirow[t]{3}{*}{ sDP4+ } & ${ }^{13} \mathrm{C}$ data & 0.18 & 82.44 & 17.38 & 0.00 & - & - & - & - \\
\hline & All data & 0.01 & 99.97 & 0.02 & 0.00 & - & - & - & - \\
\hline & ${ }^{1} \mathrm{H}$ data & 41.82 & 58.14 & 0.03 & 0.00 & - & - & - & - \\
\hline \multirow[t]{3}{*}{ uDP4+ } & ${ }^{13} \mathrm{C}$ data & 0.07 & 87.78 & 12.14 & 0.02 & - & - & - & - \\
\hline & All data & 0.06 & 99.94 & 0.01 & 0.00 & - & - & - & - \\
\hline & ${ }^{1} \mathrm{H}$ data & 2.51 & 97.49 & 0.00 & 0.00 & - & - & - & - \\
\hline \multirow[t]{5}{*}{ DP4+ } & ${ }^{13} \mathrm{C}$ data & 0.00 & 97.17 & 2.83 & 0.00 & - & - & - & - \\
\hline & All data & 0.00 & 100.00 & 0.00 & 0.00 & - & - & - & - \\
\hline & & \multicolumn{8}{|c|}{ Level of theory: mPW1PW91/6-31G* (solution, PCM) } \\
\hline & & $77 a$ & 77b & 77c & 77d & - & - & - & - \\
\hline & ${ }^{1} \mathrm{H}$ data & 24.09 & 72.89 & 3.02 & 0.00 & - & - & - & - \\
\hline \multirow[t]{3}{*}{ sDP4+ } & ${ }^{13} \mathrm{C}$ data & 0.06 & 77.07 & 22.87 & 0.00 & - & - & - & - \\
\hline & All data & 0.03 & 98.76 & 1.21 & 0.00 & - & - & - & - \\
\hline & ${ }^{1} \mathrm{H}$ data & 25.85 & 67.95 & 6.19 & 0.01 & - & - & - & - \\
\hline \multirow[t]{3}{*}{ uDP4+ } & ${ }^{13} \mathrm{C}$ data & 0.01 & 0.59 & 99.40 & 0.00 & - & - & - & - \\
\hline & All data & 0.04 & 6.13 & 93.83 & 0.00 & - & - & - & - \\
\hline & ${ }^{1} \mathrm{H}$ data & 11.13 & 88.53 & 0.33 & 0.00 & - & - & - & - \\
\hline DP4+ & ${ }^{13} \mathrm{C}$ data & 0.00 & 1.97 & 98.03 & 0.00 & - & - & - & - \\
\hline & All data & 0.00 & 84.17 & 15.83 & 0.00 & - & - & - & - \\
\hline & & & & el of th & IPW1P & & on, & & \\
\hline & & $77 a$ & $77 b$ & 77c & 77d & - & - & - & - \\
\hline & ${ }^{1} \mathrm{H}$ data & 44.27 & 34.36 & 21.38 & 0.00 & - & - & - & - \\
\hline sDP4+ & ${ }^{13} \mathrm{C}$ data & 0.35 & 91.65 & 8.00 & 0.00 & - & - & - & - \\
\hline & All data & 0.47 & 94.40 & 5.12 & 0.00 & - & - & - & - \\
\hline & ${ }^{1} \mathrm{H}$ data & 54.80 & 42.50 & 2.70 & 0.01 & - & - & - & - \\
\hline uDP4+ & ${ }^{13} \mathrm{C}$ data & 0.06 & 6.16 & 93.78 & 0.00 & - & - & - & - \\
\hline & All data & 0.61 & 50.56 & 48.83 & 0.00 & - & - & - & - \\
\hline
\end{tabular}




\begin{tabular}{|c|c|c|c|c|c|c|c|c|c|}
\hline & ${ }^{1}$ H data & 61.51 & 37.03 & 1.46 & 0.00 & - & - & - & - \\
\hline \multirow[t]{5}{*}{ DP4+ } & ${ }^{13} \mathrm{C}$ data & 0.00 & 42.95 & 57.04 & 0.00 & - & - & - & - \\
\hline & All data & 0.01 & 95.01 & 4.98 & 0.00 & - & - & - & - \\
\hline & & \multicolumn{8}{|c|}{ Level of theory: mPW1PW91/6-31+G** (solution, PCM) } \\
\hline & & $77 a$ & 77b & 77c & 77d & - & - & - & - \\
\hline & ${ }^{1} \mathrm{H}$ data & 0.30 & 99.67 & 0.03 & 0.01 & - & - & - & - \\
\hline \multirow[t]{3}{*}{ sDP4+ } & ${ }^{13} \mathrm{C}$ data & 0.17 & 97.29 & 2.54 & 0.00 & - & - & - & - \\
\hline & All data & 0.00 & 100.00 & 0.00 & 0.00 & - & - & - & - \\
\hline & ${ }^{1} \mathrm{H}$ data & 3.01 & 96.17 & 0.03 & 0.79 & - & - & - & - \\
\hline \multirow[t]{3}{*}{ uDP4+ } & ${ }^{13} \mathrm{C}$ data & 0.26 & 60.05 & 39.68 & 0.00 & - & - & - & - \\
\hline & All data & 0.01 & 99.96 & 0.02 & 0.00 & - & - & - & - \\
\hline & ${ }^{1} \mathrm{H}$ data & 0.01 & 99.99 & 0.00 & 0.00 & - & - & - & - \\
\hline \multirow[t]{5}{*}{ DP4+ } & ${ }^{13} \mathrm{C}$ data & 0.00 & 98.30 & 1.70 & 0.00 & - & - & - & - \\
\hline & All data & 0.00 & 100.00 & 0.00 & 0.00 & - & - & - & - \\
\hline & & \multicolumn{8}{|c|}{ Level of theory: mPW1PW91/6-311G* (solution, PCM) } \\
\hline & & $77 a$ & 77b & 77c & 77d & - & - & - & - \\
\hline & ${ }^{1} \mathrm{H}$ data & 0.07 & 99.56 & 0.34 & 0.03 & - & - & - & - \\
\hline \multirow[t]{2}{*}{ sDP4+ } & ${ }^{13} \mathrm{C}$ data & 3.54 & 62.16 & 34.30 & 0.00 & - & - & - & - \\
\hline & All data & 0.00 & 99.81 & 0.19 & 0.00 & - & - & - & - \\
\hline \multirow{3}{*}{ uDP4+ } & ${ }^{1} \mathrm{H}$ data & 0.05 & 99.46 & 0.39 & 0.11 & - & - & - & - \\
\hline & ${ }^{13} \mathrm{C}$ data & 1.46 & 80.61 & 17.92 & 0.01 & - & - & - & - \\
\hline & All data & 0.00 & 99.91 & 0.09 & 0.00 & - & - & - & - \\
\hline \multirow{5}{*}{ DP4+ } & ${ }^{1} \mathrm{H}$ data & 0.00 & 100.00 & 0.00 & 0.00 & - & - & - & - \\
\hline & ${ }^{13} \mathrm{C}$ data & 0.09 & 88.99 & 10.92 & 0.00 & - & - & - & - \\
\hline & All data & 0.00 & 100.00 & 0.00 & 0.00 & - & - & - & - \\
\hline & & \multicolumn{8}{|c|}{ Level of theory: mPW1PW91/6-311G** (solution, PCM) } \\
\hline & & $77 a$ & 77b & 77c & 77d & - & - & - & - \\
\hline \multirow{3}{*}{ sDP4+ } & ${ }^{1} \mathrm{H}$ data & 0.27 & 89.85 & 9.84 & 0.04 & - & - & - & - \\
\hline & ${ }^{13} \mathrm{C}$ data & 0.99 & 43.47 & 55.54 & 0.00 & - & - & - & - \\
\hline & All data & 0.01 & 87.72 & 12.27 & 0.00 & - & - & - & - \\
\hline \multirow{3}{*}{ uDP4+ } & ${ }^{1} \mathrm{H}$ data & 0.10 & 98.98 & 0.79 & 0.13 & - & - & - & - \\
\hline & ${ }^{13} \mathrm{C}$ data & 0.59 & 84.32 & 15.07 & 0.01 & - & - & - & - \\
\hline & All data & 0.00 & 99.86 & 0.14 & 0.00 & - & - & - & - \\
\hline \multirow{3}{*}{ DP4+ } & ${ }^{1} \mathrm{H}$ data & 0.00 & 99.91 & 0.09 & 0.00 & - & - & - & - \\
\hline & ${ }^{13} \mathrm{C}$ data & 0.01 & 81.40 & 18.59 & 0.00 & - & - & - & - \\
\hline & All data & 0.00 & 99.98 & 0.02 & 0.00 & - & - & - & - \\
\hline & & \multicolumn{8}{|c|}{ Level of theory: mPW1PW91/6-311+G** (solution, PCM) } \\
\hline & & $77 a$ & 77b & 77c & 77d & - & - & - & - \\
\hline \multirow{3}{*}{ sDP4+ } & ${ }^{1} \mathrm{H}$ data & 0.69 & 98.56 & 0.41 & 0.33 & - & - & - & - \\
\hline & ${ }^{13}$ C data & 0.71 & 48.69 & 50.60 & 0.00 & - & - & - & - \\
\hline & All data & 0.01 & 99.56 & 0.43 & 0.00 & - & - & - & - \\
\hline \multirow{3}{*}{ uDP4+ } & ${ }^{1} \mathrm{H}$ data & 1.66 & 95.87 & 0.18 & 2.28 & - & - & - & - \\
\hline & ${ }^{13} \mathrm{C}$ data & 0.45 & 88.94 & 10.61 & 0.00 & - & - & - & - \\
\hline & All data & 0.01 & 99.97 & 0.02 & 0.00 & - & - & - & - \\
\hline \multirow{3}{*}{ DP4+ } & ${ }^{1} \mathrm{H}$ data & 0.01 & 99.98 & 0.00 & 0.01 & - & - & - & - \\
\hline & ${ }^{13} \mathrm{C}$ data & 0.01 & 88.96 & 11.03 & 0.00 & - & - & - & - \\
\hline & All data & 0.00 & 100.00 & 0.00 & 0.00 & - & - & - & - \\
\hline
\end{tabular}


$s \mathrm{DP4}+, u \mathrm{DP} 4+$ and DP4+ probabilities (\%) for compound $77 \mathrm{~d}$<smiles>C=C(C)[C@@H](O)[C@@H](C)C(=O)[C@H](C)COCc1ccccc1</smiles><smiles>C=C(C)[C@@H](O)[C@@H](C)C(=O)[C@@H](C)COc1ccccc1</smiles><smiles>C=C(C)[C@H](O)[C@@H](C)C(=O)C(C)COCc1ccccc1</smiles>

\begin{tabular}{|c|c|c|c|c|c|c|c|c|c|}
\hline & & \multicolumn{8}{|c|}{ Level of theory: B3LYP/6-31G* (gas phase) } \\
\hline & & $77 a$ & $77 \mathrm{~b}$ & 77c & 77d & - & - & - & - \\
\hline & ${ }^{1} \mathrm{H}$ data & 2.43 & 0.05 & 0.00 & 97.51 & - & - & - & - \\
\hline \multirow{2}{*}{ sDP4+ } & ${ }^{13} \mathrm{C}$ data & 58.07 & 0.01 & 0.00 & 41.92 & - & - & - & - \\
\hline & All data & 3.34 & 0.00 & 0.00 & 96.66 & - & - & - & - \\
\hline & ${ }^{1} \mathrm{H}$ data & 2.92 & 96.73 & 0.16 & 0.19 & - & - & - & - \\
\hline \multirow[t]{2}{*}{ uDP4+ } & ${ }^{13} \mathrm{C}$ data & 75.35 & 0.00 & 0.00 & 24.64 & - & - & - & - \\
\hline & All data & 97.91 & 0.03 & 0.00 & 2.06 & - & - & - & - \\
\hline \multirow{6}{*}{ DP4+ } & ${ }^{1} \mathrm{H}$ data & 23.34 & 16.58 & 0.00 & 60.08 & - & - & - & - \\
\hline & ${ }^{13} \mathrm{C}$ data & 80.90 & 0.00 & 0.00 & 19.10 & - & - & - & - \\
\hline & All data & 62.21 & 0.00 & 0.00 & 37.79 & - & - & - & - \\
\hline & & \multicolumn{8}{|c|}{ Level of theory: B3LYP/6-31G** (gas phase) } \\
\hline & & $77 a$ & 77b & 77c & 77d & - & - & - & - \\
\hline & ${ }^{1} \mathrm{H}$ data & 5.62 & 0.27 & 0.00 & 94.11 & - & - & - & - \\
\hline \multirow[t]{2}{*}{ sDP4+ } & ${ }^{13} \mathrm{C}$ data & 61.04 & 0.01 & 0.00 & 38.95 & - & - & - & - \\
\hline & All data & 8.56 & 0.00 & 0.00 & 91.44 & - & - & - & - \\
\hline & ${ }^{1} \mathrm{H}$ data & 2.33 & 97.42 & 0.08 & 0.17 & - & - & - & - \\
\hline \multirow[t]{2}{*}{ uDP4+ } & ${ }^{13} \mathrm{C}$ data & 82.88 & 0.00 & 0.00 & 17.12 & - & - & - & - \\
\hline & All data & 98.50 & 0.03 & 0.00 & 1.47 & - & - & - & - \\
\hline \multirow{5}{*}{ DP4+ } & ${ }^{1} \mathrm{H}$ data & 23.65 & 47.80 & 0.00 & 28.55 & - & - & - & - \\
\hline & ${ }^{13} \mathrm{C}$ data & 88.35 & 0.00 & 0.00 & 11.65 & - & - & - & - \\
\hline & All data & 86.27 & 0.00 & 0.00 & 13.73 & - & - & - & - \\
\hline & & \multicolumn{8}{|c|}{ Level of theory: B3LYP/6-31+G** (gas phase) } \\
\hline & & $77 a$ & 77b & 77c & 77d & - & - & - & - \\
\hline & ${ }^{1} \mathrm{H}$ data & 4.25 & 0.14 & 0.00 & 95.62 & - & - & - & - \\
\hline \multirow[t]{2}{*}{ sDP4+ } & ${ }^{13} \mathrm{C}$ data & 91.86 & 0.00 & 0.00 & 8.14 & - & - & - & - \\
\hline & All data & 33.38 & 0.00 & 0.00 & 66.62 & - & - & - & - \\
\hline \multirow{3}{*}{ uDP4+ } & ${ }^{1} \mathrm{H}$ data & 39.01 & 55.94 & 0.02 & 5.03 & - & - & - & - \\
\hline & ${ }^{13} \mathrm{C}$ data & 92.25 & 0.01 & 0.00 & 7.75 & - & - & - & - \\
\hline & All data & 98.92 & 0.01 & 0.00 & 1.07 & - & - & - & - \\
\hline & ${ }^{1} \mathrm{H}$ data & 25.31 & 1.16 & 0.00 & 73.53 & - & - & - & - \\
\hline \multirow[t]{5}{*}{ DP4+ } & ${ }^{13} \mathrm{C}$ data & 99.26 & 0.00 & 0.00 & 0.74 & - & - & - & - \\
\hline & All data & 97.88 & 0.00 & 0.00 & 2.12 & - & - & - & - \\
\hline & & \multicolumn{8}{|c|}{ Level of theory: B3LYP/6-311G* (gas phase) } \\
\hline & & $77 a$ & 77b & 77c & 77d & - & - & - & - \\
\hline & ${ }^{1} \mathrm{H}$ data & 0.10 & 99.89 & 0.00 & 0.00 & - & - & - & - \\
\hline \multirow[t]{2}{*}{ sDP4+ } & ${ }^{13} \mathrm{C}$ data & 57.88 & 0.00 & 0.00 & 42.12 & - & - & - & - \\
\hline & All data & 96.89 & 0.65 & 0.00 & 2.46 & - & - & - & - \\
\hline \multirow{3}{*}{ uDP4+ } & ${ }^{1} \mathrm{H}$ data & 0.39 & 99.54 & 0.02 & 0.05 & - & - & - & - \\
\hline & ${ }^{13} \mathrm{C}$ data & 79.50 & 0.01 & 0.00 & 20.49 & - & - & - & - \\
\hline & All data & 94.66 & 2.03 & 0.00 & 3.30 & - & - & - & - \\
\hline \multirow{5}{*}{ DP4+ } & ${ }^{1} \mathrm{H}$ data & 0.00 & 100.00 & 0.00 & 0.00 & - & - & - & - \\
\hline & ${ }^{13} \mathrm{C}$ data & 84.20 & 0.00 & 0.00 & 15.80 & - & - & - & - \\
\hline & All data & 99.90 & 0.01 & 0.00 & 0.09 & - & - & - & - \\
\hline & & \multicolumn{8}{|c|}{ Level of theory: B3LYP/6-311G** (gas phase) } \\
\hline & & $77 a$ & 77b & 77c & 77d & - & - & - & - \\
\hline \multirow{3}{*}{ sDP4+ } & ${ }^{1} \mathrm{H}$ data & 0.03 & 99.92 & 0.05 & 0.00 & - & - & - & - \\
\hline & ${ }^{13} \mathrm{C}$ data & 27.66 & 0.00 & 0.00 & 72.34 & - & - & - & - \\
\hline & All data & 78.72 & 11.27 & 0.00 & 10.01 & - & - & - & - \\
\hline
\end{tabular}




\begin{tabular}{|c|c|c|c|c|c|c|c|c|c|}
\hline & ${ }^{1} \mathrm{H}$ data & 0.07 & 99.91 & 0.00 & 0.01 & - & - & - & - \\
\hline \multirow[t]{3}{*}{ uDP4+ } & ${ }^{13} \mathrm{C}$ data & 55.10 & 0.01 & 0.00 & 44.89 & - & - & - & - \\
\hline & All data & 76.76 & 11.68 & 0.00 & 11.56 & - & - & - & - \\
\hline & ${ }^{1} \mathrm{H}$ data & 0.00 & 100.00 & 0.00 & 0.00 & - & - & - & - \\
\hline \multirow[t]{5}{*}{ DP4+ } & ${ }^{13} \mathrm{C}$ data & 31.94 & 0.00 & 0.00 & 68.06 & - & - & - & - \\
\hline & All data & 96.07 & 2.09 & 0.00 & 1.84 & - & - & - & - \\
\hline & & \multicolumn{8}{|c|}{ Level of theory: B3LYP/6-311+G** (gas phase) } \\
\hline & & $77 a$ & 77b & 77c & 77d & - & - & - & - \\
\hline & ${ }^{1} \mathrm{H}$ data & 4.55 & 95.39 & 0.00 & 0.06 & - & - & - & - \\
\hline \multirow[t]{3}{*}{ sDP4+ } & ${ }^{13} \mathrm{C}$ data & 75.71 & 0.00 & 0.00 & 24.29 & - & - & - & - \\
\hline & All data & 99.55 & 0.03 & 0.00 & 0.42 & - & - & - & - \\
\hline & ${ }^{1} \mathrm{H}$ data & 12.48 & 87.11 & 0.00 & 0.41 & - & - & - & - \\
\hline \multirow[t]{3}{*}{ uDP4+ } & ${ }^{13} \mathrm{C}$ data & 88.69 & 0.01 & 0.00 & 11.30 & - & - & - & - \\
\hline & All data & 99.50 & 0.08 & 0.00 & 0.42 & - & - & - & - \\
\hline & ${ }^{1} \mathrm{H}$ data & 0.68 & 99.32 & 0.00 & 0.00 & - & - & - & - \\
\hline \multirow[t]{5}{*}{ DP4+ } & ${ }^{13} \mathrm{C}$ data & 96.07 & 0.00 & 0.00 & 3.93 & - & - & - & - \\
\hline & All data & 100.00 & 0.00 & 0.00 & 0.00 & - & - & - & - \\
\hline & & \multicolumn{8}{|c|}{ Level of theory: B3LYP/6-31G* (solution, PCM) } \\
\hline & & $77 a$ & 77b & 77c & 77d & - & - & - & - \\
\hline & ${ }^{1}$ H data & 5.98 & 0.47 & 0.00 & 93.55 & - & - & - & - \\
\hline \multirow[t]{3}{*}{ sDP4+ } & ${ }^{13} \mathrm{C}$ data & 97.59 & 0.00 & 0.00 & 2.41 & - & - & - & - \\
\hline & All data & 72.12 & 0.00 & 0.00 & 27.88 & - & - & - & - \\
\hline & ${ }^{1} \mathrm{H}$ data & 1.21 & 86.31 & 0.12 & 12.37 & - & - & - & - \\
\hline \multirow[t]{3}{*}{ uDP4+ } & ${ }^{13} \mathrm{C}$ data & 48.86 & 0.00 & 0.00 & 51.14 & - & - & - & - \\
\hline & All data & 8.52 & 0.00 & 0.00 & 91.48 & - & - & - & - \\
\hline & ${ }^{1} \mathrm{H}$ data & 0.60 & 3.39 & 0.00 & 96.01 & - & - & - & - \\
\hline \multirow[t]{5}{*}{ DP4+ } & ${ }^{13} \mathrm{C}$ data & 97.48 & 0.00 & 0.00 & 2.52 & - & - & - & - \\
\hline & All data & 19.42 & 0.00 & 0.00 & 80.58 & - & - & - & - \\
\hline & & \multicolumn{8}{|c|}{ Level of theory: B3LYP/6-31G** (solution, PCM) } \\
\hline & & $77 a$ & 77b & 77c & 77d & - & - & - & - \\
\hline & ${ }^{1} \mathrm{H}$ data & 12.77 & 2.02 & 0.00 & 85.21 & - & - & - & - \\
\hline \multirow[t]{3}{*}{ sDP4+ } & ${ }^{13} \mathrm{C}$ data & 98.31 & 0.00 & 0.00 & 1.69 & - & - & - & - \\
\hline & All data & 89.73 & 0.00 & 0.00 & 10.27 & - & - & - & - \\
\hline & ${ }^{1} \mathrm{H}$ data & 0.89 & 84.74 & 0.05 & 14.32 & - & - & - & - \\
\hline \multirow[t]{3}{*}{ uDP4+ } & ${ }^{13} \mathrm{C}$ data & 76.18 & 0.00 & 0.00 & 23.82 & - & - & - & - \\
\hline & All data & 16.52 & 0.00 & 0.00 & 83.48 & - & - & - & - \\
\hline & ${ }^{1} \mathrm{H}$ data & 0.81 & 12.19 & 0.00 & 87.00 & - & - & - & - \\
\hline \multirow[t]{5}{*}{ DP4+ } & ${ }^{13} \mathrm{C}$ data & 99.47 & 0.00 & 0.00 & 0.53 & - & - & - & - \\
\hline & All data & 63.36 & 0.00 & 0.00 & 36.64 & - & - & - & - \\
\hline & & \multicolumn{8}{|c|}{ Level of theory: B3LYP/6-31+G** (solution, PCM) } \\
\hline & & $77 a$ & 77b & 77c & 77d & - & - & - & - \\
\hline & ${ }^{1} \mathrm{H}$ data & 10.84 & 1.33 & 0.00 & 87.82 & - & - & - & - \\
\hline \multirow[t]{2}{*}{ sDP4+ } & ${ }^{13} \mathrm{C}$ data & 97.09 & 0.00 & 0.00 & 2.91 & - & - & - & - \\
\hline & All data & 80.49 & 0.00 & 0.00 & 19.51 & - & - & - & - \\
\hline & ${ }^{1}$ H data & 2.18 & 7.32 & 0.00 & 90.49 & - & - & - & - \\
\hline uDP4+ & ${ }^{13} \mathrm{C}$ data & 66.63 & 0.02 & 0.00 & 33.35 & - & - & - & - \\
\hline & All data & 4.60 & 0.00 & 0.00 & 95.40 & - & - & - & - \\
\hline & ${ }^{1} \mathrm{H}$ data & 0.30 & 0.12 & 0.00 & 99.58 & - & - & - & - \\
\hline DP4+ & ${ }^{13} \mathrm{C}$ data & 98.52 & 0.00 & 0.00 & 1.48 & - & - & - & - \\
\hline & All data & 16.60 & 0.00 & 0.00 & 83.40 & - & - & - & - \\
\hline & & & & Level o & $y: B 3 L Y$ & & PC & & \\
\hline & & $77 a$ & $77 b$ & 77c & $77 d$ & - & - & - & - \\
\hline & ${ }^{1} \mathrm{H}$ data & 0.16 & 85.49 & 0.02 & 14.34 & - & - & - & - \\
\hline sDP4+ & ${ }^{13} \mathrm{C}$ data & 70.09 & 0.00 & 0.00 & 29.91 & - & - & - & - \\
\hline & All data & 2.59 & 0.00 & 0.00 & 97.41 & - & - & - & - \\
\hline uDP4+ & ${ }^{1} \mathrm{H}$ data & 0.06 & 65.69 & 0.01 & 34.25 & - & - & - & - \\
\hline
\end{tabular}




\begin{tabular}{|c|c|c|c|c|c|c|c|c|c|}
\hline & ${ }^{13} \mathrm{C}$ data & 93.00 & 0.00 & 0.00 & 7.00 & - & - & - & - \\
\hline & All data & 2.28 & 0.05 & 0.00 & 97.66 & - & - & - & - \\
\hline & ${ }^{1} \mathrm{H}$ data & 0.00 & 91.96 & 0.00 & 8.04 & - & - & - & - \\
\hline \multirow[t]{5}{*}{ DP4+ } & ${ }^{13} \mathrm{C}$ data & 96.89 & 0.00 & 0.00 & 3.11 & - & - & - & - \\
\hline & All data & 0.06 & 0.00 & 0.00 & 99.94 & - & - & - & - \\
\hline & & \multicolumn{8}{|c|}{ Level of theory: B3LYP/6-311G** (solution, PCM) } \\
\hline & & $77 a$ & 77b & 77c & 77d & - & - & - & - \\
\hline & ${ }^{1} \mathrm{H}$ data & 0.13 & 93.38 & 0.80 & 5.70 & - & - & - & - \\
\hline \multirow[t]{2}{*}{$s$ DP4+ } & ${ }^{13} \mathrm{C}$ data & 20.80 & 0.00 & 0.00 & 79.19 & - & - & - & - \\
\hline & All data & 0.58 & 0.01 & 0.00 & 99.41 & - & - & - & - \\
\hline \multirow{3}{*}{ uDP4+ } & ${ }^{1} \mathrm{H}$ data & 0.19 & 72.03 & 0.01 & 27.78 & - & - & - & - \\
\hline & ${ }^{13} \mathrm{C}$ data & 75.69 & 0.01 & 0.00 & 24.30 & - & - & - & - \\
\hline & All data & 2.04 & 0.05 & 0.00 & 97.91 & - & - & - & - \\
\hline \multirow{5}{*}{ DP4+ } & ${ }^{1} \mathrm{H}$ data & 0.00 & 97.70 & 0.00 & 2.30 & - & - & - & - \\
\hline & ${ }^{13} \mathrm{C}$ data & 45.00 & 0.00 & 0.00 & 55.00 & - & - & - & - \\
\hline & All data & 0.01 & 0.00 & 0.00 & 99.99 & - & - & - & - \\
\hline & & \multicolumn{8}{|c|}{ Level of theory: B3LYP/6-311+G** (solution, PCM) } \\
\hline & & $77 a$ & $77 b$ & 77c & 77d & - & - & - & - \\
\hline \multirow{3}{*}{ sDP4+ } & ${ }^{1} \mathrm{H}$ data & 0.11 & 10.55 & 0.01 & 89.33 & - & - & - & - \\
\hline & ${ }^{13} \mathrm{C}$ data & 50.19 & 0.00 & 0.00 & 49.81 & - & - & - & - \\
\hline & All data & 0.13 & 0.00 & 0.00 & 99.87 & - & - & - & - \\
\hline \multirow{3}{*}{ uDP4+ } & ${ }^{1} \mathrm{H}$ data & 0.01 & 8.50 & 0.00 & 91.48 & - & - & - & - \\
\hline & ${ }^{13} \mathrm{C}$ data & 90.01 & 0.00 & 0.00 & 9.99 & - & - & - & - \\
\hline & All data & 0.12 & 0.00 & 0.00 & 99.87 & - & - & - & - \\
\hline \multirow{3}{*}{ DP4+ } & ${ }^{1} \mathrm{H}$ data & 0.00 & 1.09 & 0.00 & 98.91 & - & - & - & - \\
\hline & ${ }^{13} \mathrm{C}$ data & 90.08 & 0.00 & 0.00 & 9.92 & - & - & - & - \\
\hline & All data & 0.00 & 0.00 & 0.00 & 100.00 & - & - & - & - \\
\hline & & \multicolumn{8}{|c|}{ Level of theory: mPW1PW91/6-31G* (gas phase) } \\
\hline & & $77 a$ & 77b & 77c & 77d & - & - & - & - \\
\hline \multirow{3}{*}{ sDP4+ } & ${ }^{1} \mathrm{H}$ data & 3.38 & 0.06 & 0.00 & 96.55 & - & - & - & - \\
\hline & ${ }^{13} \mathrm{C}$ data & 20.48 & 0.00 & 0.00 & 79.52 & - & - & - & - \\
\hline & All data & 0.89 & 0.00 & 0.00 & 99.11 & - & - & - & - \\
\hline \multirow{3}{*}{ uDP4+ } & ${ }^{1} \mathrm{H}$ data & 2.40 & 97.29 & 0.15 & 0.16 & - & - & - & - \\
\hline & ${ }^{13} \mathrm{C}$ data & 60.37 & 0.01 & 0.00 & 39.62 & - & - & - & - \\
\hline & All data & 95.24 & 0.65 & 0.00 & 4.11 & - & - & - & - \\
\hline \multirow{3}{*}{ DP4+ } & ${ }^{1} \mathrm{H}$ data & 27.57 & 20.78 & 0.00 & 51.65 & - & - & - & - \\
\hline & ${ }^{13} \mathrm{C}$ data & 28.18 & 0.00 & 0.00 & 71.82 & - & - & - & - \\
\hline & All data & 17.32 & 0.00 & 0.00 & 82.68 & - & - & - & - \\
\hline & & \multicolumn{8}{|c|}{ Level of theory: mPW1PW91/6-31G** (gas phase) } \\
\hline & & $77 a$ & 77b & 77c & 77d & - & - & - & - \\
\hline \multirow{3}{*}{ sDP4+ } & ${ }^{1} \mathrm{H}$ data & 7.16 & 0.33 & 0.00 & 92.51 & - & - & - & - \\
\hline & ${ }^{13} \mathrm{C}$ data & 33.46 & 0.00 & 0.00 & 66.54 & - & - & - & - \\
\hline & All data & 3.75 & 0.00 & 0.00 & 96.25 & - & - & - & - \\
\hline \multirow{3}{*}{ uDP4+ } & ${ }^{1} \mathrm{H}$ data & 1.14 & 98.71 & 0.05 & 0.09 & - & - & - & - \\
\hline & ${ }^{13} \mathrm{C}$ data & 83.12 & 0.00 & 0.00 & 16.88 & - & - & - & - \\
\hline & All data & 98.35 & 0.08 & 0.00 & 1.57 & - & - & - & - \\
\hline & ${ }^{1} \mathrm{H}$ data & 16.57 & 66.60 & 0.00 & 16.82 & - & - & - & - \\
\hline DP4+ & ${ }^{13} \mathrm{C}$ data & 71.24 & 0.00 & 0.00 & 28.76 & - & - & - & - \\
\hline & All data & 70.93 & 0.00 & 0.00 & 29.07 & - & - & - & - \\
\hline & & & & vel of $t$ & : mPW1 & -3 & pr & & \\
\hline & & $77 a$ & $77 b$ & 77c & 77d & - & - & - & - \\
\hline & ${ }^{1} \mathrm{H}$ data & 6.32 & 0.28 & 0.00 & 93.40 & - & - & - & - \\
\hline sDP4+ & ${ }^{13} \mathrm{C}$ data & 84.10 & 0.00 & 0.00 & 15.89 & - & - & - & - \\
\hline & All data & 26.36 & 0.00 & 0.00 & 73.64 & - & - & - & - \\
\hline & ${ }^{1} \mathrm{H}$ data & 21.78 & 73.12 & 0.03 & 5.06 & - & - & - & - \\
\hline UDP4+ & ${ }^{13} \mathrm{C}$ data & 83.36 & 0.00 & 0.00 & 16.64 & - & - & - & - \\
\hline
\end{tabular}




\begin{tabular}{|c|c|c|c|c|c|c|c|c|c|}
\hline & All data & 95.56 & 0.01 & 0.00 & 4.43 & - & - & - & - \\
\hline & ${ }^{1} \mathrm{H}$ data & 21.80 & 3.27 & 0.00 & 74.93 & - & - & - & - \\
\hline \multirow[t]{5}{*}{ DP4+ } & ${ }^{13} \mathrm{C}$ data & 96.37 & 0.00 & 0.00 & 3.63 & - & - & - & - \\
\hline & All data & 88.53 & 0.00 & 0.00 & 11.47 & - & - & - & - \\
\hline & & \multicolumn{8}{|c|}{ Level of theory: mPW1PW91/6-311G* (gas phase) } \\
\hline & & $77 a$ & $77 b$ & 77c & 77d & - & - & - & - \\
\hline & ${ }^{1} \mathrm{H}$ data & 0.00 & 99.99 & 0.00 & 0.00 & - & - & - & - \\
\hline \multirow[t]{3}{*}{ sDP4+ } & ${ }^{13} \mathrm{C}$ data & 4.00 & 0.00 & 0.00 & 96.00 & - & - & - & - \\
\hline & All data & 10.51 & 64.72 & 0.00 & 24.77 & - & - & - & - \\
\hline & ${ }^{1} \mathrm{H}$ data & 0.03 & 99.93 & 0.01 & 0.02 & - & - & - & - \\
\hline \multirow[t]{3}{*}{ uDP4+ } & ${ }^{13} \mathrm{C}$ data & 45.14 & 0.01 & 0.00 & 54.85 & - & - & - & - \\
\hline & All data & 43.79 & 16.84 & 0.00 & 39.38 & - & - & - & - \\
\hline & ${ }^{1} \mathrm{H}$ data & 0.00 & 100.00 & 0.00 & 0.00 & - & - & - & - \\
\hline \multirow[t]{5}{*}{ DP4+ } & ${ }^{13} \mathrm{C}$ data & 3.31 & 0.00 & 0.00 & 96.69 & - & - & - & - \\
\hline & All data & 18.22 & 43.16 & 0.00 & 38.63 & - & - & - & - \\
\hline & & \multicolumn{8}{|c|}{ Level of theory: mPW1PW91/6-311G** (gas phase) } \\
\hline & & $77 a$ & $77 b$ & 77c & 77d & - & - & - & - \\
\hline & ${ }^{1} \mathrm{H}$ data & 0.02 & 99.85 & 0.13 & 0.00 & - & - & - & - \\
\hline \multirow[t]{3}{*}{ sDP4+ } & ${ }^{13} \mathrm{C}$ data & 0.36 & 0.00 & 0.00 & 99.64 & - & - & - & - \\
\hline & All data & 3.68 & 72.38 & 0.05 & 23.89 & - & - & - & - \\
\hline & ${ }^{1} \mathrm{H}$ data & 0.02 & 99.97 & 0.00 & 0.01 & - & - & - & - \\
\hline \multirow[t]{3}{*}{ uDP4+ } & ${ }^{13} \mathrm{C}$ data & 7.08 & 0.01 & 0.00 & 92.91 & - & - & - & - \\
\hline & All data & 11.91 & 50.07 & 0.00 & 38.02 & - & - & - & - \\
\hline & ${ }^{1} \mathrm{H}$ data & 0.00 & 100.00 & 0.00 & 0.00 & - & - & - & - \\
\hline \multirow[t]{5}{*}{ DP4+ } & ${ }^{13} \mathrm{C}$ data & 0.03 & 0.00 & 0.00 & 99.97 & - & - & - & - \\
\hline & All data & 0.96 & 79.19 & 0.00 & 19.85 & - & - & - & - \\
\hline & & \multicolumn{8}{|c|}{ Level of theory: mPW1PW91/6-311+G** (gas phase) } \\
\hline & & $77 a$ & 77b & 77c & 77d & - & - & - & - \\
\hline & ${ }^{1} \mathrm{H}$ data & 1.80 & 98.15 & 0.04 & 0.02 & - & - & - & - \\
\hline \multirow[t]{3}{*}{ sDP4+ } & ${ }^{13} \mathrm{C}$ data & 12.94 & 0.00 & 0.00 & 87.06 & - & - & - & - \\
\hline & All data & 93.49 & 0.97 & 0.00 & 5.54 & - & - & - & - \\
\hline & ${ }^{1} \mathrm{H}$ data & 3.78 & 96.14 & 0.00 & 0.08 & - & - & - & - \\
\hline \multirow[t]{3}{*}{ uDP4+ } & ${ }^{13} \mathrm{C}$ data & 42.09 & 0.01 & 0.00 & 57.90 & - & - & - & - \\
\hline & All data & 96.55 & 0.47 & 0.00 & 2.98 & - & - & - & - \\
\hline & ${ }^{1} \mathrm{H}$ data & 0.07 & 99.93 & 0.00 & 0.00 & - & - & - & - \\
\hline \multirow[t]{5}{*}{ DP4+ } & ${ }^{13} \mathrm{C}$ data & 9.75 & 0.00 & 0.00 & 90.25 & - & - & - & - \\
\hline & All data & 99.81 & 0.01 & 0.00 & 0.18 & - & - & - & - \\
\hline & & \multicolumn{8}{|c|}{ Level of theory: mPW1PW91/6-31G* (solution, PCM) } \\
\hline & & $77 a$ & 77b & 77c & 77d & - & - & - & - \\
\hline & ${ }^{1} \mathrm{H}$ data & 10.97 & 1.20 & 0.00 & 87.83 & - & - & - & - \\
\hline \multirow[t]{3}{*}{ sDP4+ } & ${ }^{13} \mathrm{C}$ data & 60.51 & 0.00 & 0.00 & 39.49 & - & - & - & - \\
\hline & All data & 16.06 & 0.00 & 0.00 & 83.94 & - & - & - & - \\
\hline & ${ }^{1} \mathrm{H}$ data & 1.00 & 83.77 & 0.09 & 15.14 & - & - & - & - \\
\hline \multirow[t]{3}{*}{ uDP4+ } & ${ }^{13} \mathrm{C}$ data & 27.43 & 0.00 & 0.00 & 72.57 & - & - & - & - \\
\hline & All data & 2.43 & 0.00 & 0.00 & 97.57 & - & - & - & - \\
\hline & ${ }^{1} \mathrm{H}$ data & 0.76 & 6.96 & 0.00 & 92.28 & - & - & - & - \\
\hline DP4+ & ${ }^{13} \mathrm{C}$ data & 36.67 & 0.00 & 0.00 & 63.33 & - & - & - & - \\
\hline & All data & 0.47 & 0.00 & 0.00 & 99.53 & - & - & - & - \\
\hline & & & & of th & nPW1P & 1 & on, & & \\
\hline & & $77 a$ & $77 b$ & 77c & 77d & - & - & - & - \\
\hline & ${ }^{1} \mathrm{H}$ data & 14.65 & 1.99 & 0.00 & 83.35 & - & - & - & - \\
\hline sDP4+ & ${ }^{13} \mathrm{C}$ data & 75.07 & 0.00 & 0.00 & 24.93 & - & - & - & - \\
\hline & All data & 34.61 & 0.00 & 0.00 & 65.39 & - & - & - & - \\
\hline & ${ }^{1} \mathrm{H}$ data & 0.60 & 84.95 & 0.03 & 14.41 & - & - & - & - \\
\hline uDP4+ & ${ }^{13} \mathrm{C}$ data & 63.78 & 0.00 & 0.00 & 36.22 & - & - & - & - \\
\hline & All data & 6.86 & 0.00 & 0.00 & 93.14 & - & - & - & - \\
\hline
\end{tabular}




\begin{tabular}{|c|c|c|c|c|c|c|c|c|c|}
\hline & ${ }^{1}$ H data & 0.64 & 12.25 & 0.00 & 87.11 & - & - & - & - \\
\hline \multirow[t]{5}{*}{ DP4+ } & ${ }^{13} \mathrm{C}$ data & 84.13 & 0.00 & 0.00 & 15.87 & - & - & - & - \\
\hline & All data & 3.75 & 0.00 & 0.00 & 96.25 & - & - & - & - \\
\hline & & \multicolumn{8}{|c|}{ Level of theory: mPW1PW91/6-31+G** (solution, PCM) } \\
\hline & & $77 a$ & 77b & 77c & 77d & - & - & - & - \\
\hline & ${ }^{1} \mathrm{H}$ data & 0.05 & 99.19 & 0.00 & 0.76 & - & - & - & - \\
\hline \multirow[t]{3}{*}{ sDP4+ } & ${ }^{13} \mathrm{C}$ data & 89.89 & 0.00 & 0.00 & 10.11 & - & - & - & - \\
\hline & All data & 38.08 & 0.52 & 0.00 & 61.40 & - & - & - & - \\
\hline & ${ }^{1} \mathrm{H}$ data & 0.09 & 35.81 & 0.00 & 64.10 & - & - & - & - \\
\hline \multirow[t]{3}{*}{ uDP4+ } & ${ }^{13} \mathrm{C}$ data & 58.86 & 0.00 & 0.00 & 41.14 & - & - & - & - \\
\hline & All data & 0.19 & 0.00 & 0.00 & 99.81 & - & - & - & - \\
\hline & ${ }^{1} \mathrm{H}$ data & 0.00 & 98.65 & 0.00 & 1.35 & - & - & - & - \\
\hline \multirow[t]{5}{*}{ DP4+ } & ${ }^{13} \mathrm{C}$ data & 92.71 & 0.00 & 0.00 & 7.29 & - & - & - & - \\
\hline & All data & 0.12 & 0.00 & 0.00 & 99.88 & - & - & - & - \\
\hline & & \multicolumn{8}{|c|}{ Level of theory: mPW1PW91/6-311G* (solution, PCM) } \\
\hline & & $77 a$ & 77b & 77c & 77d & - & - & - & - \\
\hline & ${ }^{1} \mathrm{H}$ data & 0.03 & 98.30 & 0.03 & 1.63 & - & - & - & - \\
\hline \multirow[t]{2}{*}{ sDP4+ } & ${ }^{13} \mathrm{C}$ data & 2.43 & 0.00 & 0.00 & 97.57 & - & - & - & - \\
\hline & All data & 0.05 & 0.00 & 0.00 & 99.95 & - & - & - & - \\
\hline \multirow{3}{*}{ uDP4+ } & ${ }^{1} \mathrm{H}$ data & 0.02 & 82.41 & 0.01 & 17.57 & - & - & - & - \\
\hline & ${ }^{13} \mathrm{C}$ data & 66.74 & 0.00 & 0.00 & 33.26 & - & - & - & - \\
\hline & All data & 0.20 & 0.03 & 0.00 & 99.77 & - & - & - & - \\
\hline \multirow{5}{*}{ DP4+ } & ${ }^{1} \mathrm{H}$ data & 0.00 & 99.65 & 0.00 & 0.35 & - & - & - & - \\
\hline & ${ }^{13} \mathrm{C}$ data & 4.75 & 0.00 & 0.00 & 95.25 & - & - & - & - \\
\hline & All data & 0.00 & 0.00 & 0.00 & 100.00 & - & - & - & - \\
\hline & & \multicolumn{8}{|c|}{ Level of theory: mPW1PW91/6-311G** (solution, PCM) } \\
\hline & & $77 a$ & 77b & 77c & 77d & - & - & - & - \\
\hline \multirow{3}{*}{ sDP4+ } & ${ }^{1} \mathrm{H}$ data & 0.05 & 97.63 & 1.16 & 1.16 & - & - & - & - \\
\hline & ${ }^{13} \mathrm{C}$ data & 0.12 & 0.00 & 0.00 & 99.88 & - & - & - & - \\
\hline & All data & 0.00 & 0.00 & 0.00 & 99.99 & - & - & - & - \\
\hline \multirow{3}{*}{ uDP4+ } & ${ }^{1} \mathrm{H}$ data & 0.05 & 83.98 & 0.01 & 15.97 & - & - & - & - \\
\hline & ${ }^{13} \mathrm{C}$ data & 34.71 & 0.00 & 0.00 & 65.29 & - & - & - & - \\
\hline & All data & 0.16 & 0.03 & 0.00 & 99.81 & - & - & - & - \\
\hline \multirow{3}{*}{ DP4+ } & ${ }^{1} \mathrm{H}$ data & 0.00 & 99.77 & 0.00 & 0.23 & - & - & - & - \\
\hline & ${ }^{13} \mathrm{C}$ data & 0.06 & 0.00 & 0.00 & 99.94 & - & - & - & - \\
\hline & All data & 0.00 & 0.00 & 0.00 & 100.00 & - & - & - & - \\
\hline & & \multicolumn{8}{|c|}{ Level of theory: mPW1PW91/6-311+G** (solution, PCM) } \\
\hline & & $77 a$ & 77b & 77c & 77d & - & - & - & - \\
\hline \multirow{3}{*}{ sDP4+ } & ${ }^{1} \mathrm{H}$ data & 0.39 & 48.67 & 0.13 & 50.81 & - & - & - & - \\
\hline & ${ }^{13}$ C data & 1.81 & 0.00 & 0.00 & 98.19 & - & - & - & - \\
\hline & All data & 0.01 & 0.00 & 0.00 & 99.99 & - & - & - & - \\
\hline \multirow{3}{*}{ uDP4+ } & ${ }^{1} \mathrm{H}$ data & 0.06 & 23.76 & 0.00 & 76.18 & - & - & - & - \\
\hline & ${ }^{13} \mathrm{C}$ data & 68.78 & 0.01 & 0.00 & 31.21 & - & - & - & - \\
\hline & All data & 0.16 & 0.01 & 0.00 & 99.83 & - & - & - & - \\
\hline \multirow{3}{*}{ DP4+ } & ${ }^{1} \mathrm{H}$ data & 0.00 & 23.00 & 0.00 & 77.00 & - & - & - & - \\
\hline & ${ }^{13} \mathrm{C}$ data & 3.90 & 0.00 & 0.00 & 96.10 & - & - & - & - \\
\hline & All data & 0.00 & 0.00 & 0.00 & 100.00 & - & - & - & - \\
\hline
\end{tabular}


$s \mathrm{DP4}+, u \mathrm{DP} 4+$ and DP4+ probabilities (\%) for compound 78a<smiles>CCC[C@H]1O[C@@H](c2ccccc2)C[C@H]1O</smiles><smiles>CCC[C@H]1O[C@@H](c2ccccc2)C[C@H]1O</smiles>
$78 \mathrm{~b}$<smiles>CCC[C@H]1O[C@@H](c2ccccc2)C[C@H]1O</smiles><smiles>CCC[C@H]1O[C@@H](c2ccccc2)C[C@H]1O</smiles>
correct isomer

\begin{tabular}{|c|c|c|c|c|c|c|c|c|c|}
\hline & & \multicolumn{8}{|c|}{ Level of theory: B3LYP/6-31G* (gas phase) } \\
\hline & & 78a & $78 \mathrm{~b}$ & $78 \mathrm{c}$ & 78d & - & - & - & - \\
\hline & ${ }^{1} \mathrm{H}$ data & 14.55 & 0.01 & 0.81 & 84.63 & - & - & - & - \\
\hline \multirow[t]{2}{*}{ sDP4+ } & ${ }^{13} \mathrm{C}$ data & 17.19 & 3.05 & 53.54 & 26.22 & - & - & - & - \\
\hline & All data & 9.95 & 0.00 & 1.73 & 88.31 & - & - & - & - \\
\hline & ${ }^{1} \mathrm{H}$ data & 83.15 & 0.08 & 3.88 & 12.90 & - & - & - & - \\
\hline \multirow[t]{2}{*}{ uDP4+ } & ${ }^{13} \mathrm{C}$ data & 76.20 & 23.63 & 0.04 & 0.12 & - & - & - & - \\
\hline & All data & 99.94 & 0.03 & 0.00 & 0.03 & - & - & - & - \\
\hline \multirow{3}{*}{ DP4+ } & ${ }^{1} \mathrm{H}$ data & 52.49 & 0.00 & 0.14 & 47.37 & - & - & - & - \\
\hline & ${ }^{13} \mathrm{C}$ data & 94.41 & 5.19 & 0.17 & 0.23 & - & - & - & - \\
\hline & All data & 99.78 & 0.00 & 0.00 & 0.22 & - & - & - & - \\
\hline & & \multicolumn{8}{|c|}{ Level of theory: B3LYP/6-31G** (gas phase) } \\
\hline & & 78a & $78 \mathrm{~b}$ & $78 \mathrm{c}$ & 78d & - & - & - & - \\
\hline \multirow{3}{*}{ sDP4+ } & ${ }^{1} \mathrm{H}$ data & 24.31 & 0.04 & 2.83 & 72.83 & - & - & - & - \\
\hline & ${ }^{13} \mathrm{C}$ data & 14.02 & 6.30 & 56.64 & 23.04 & - & - & - & - \\
\hline & All data & 15.64 & 0.01 & 7.35 & 77.00 & - & - & - & - \\
\hline \multirow{3}{*}{ uDP4+ } & ${ }^{1} \mathrm{H}$ data & 54.57 & 0.36 & 5.56 & 39.51 & - & - & - & - \\
\hline & ${ }^{13} \mathrm{C}$ data & 75.76 & 24.03 & 0.06 & 0.15 & - & - & - & - \\
\hline & All data & 99.64 & 0.21 & 0.01 & 0.14 & - & - & - & - \\
\hline \multirow{6}{*}{ DP4+ } & ${ }^{1} \mathrm{H}$ data & 31.43 & 0.00 & 0.37 & 68.19 & - & - & - & - \\
\hline & ${ }^{13} \mathrm{C}$ data & 87.01 & 12.41 & 0.29 & 0.28 & - & - & - & - \\
\hline & All data & 99.30 & 0.00 & 0.00 & 0.70 & - & - & - & - \\
\hline & & \multicolumn{8}{|c|}{ Level of theory: B3LYP/6-31+G** (gas phase) } \\
\hline & & $78 a$ & $78 \mathrm{~b}$ & $78 \mathrm{c}$ & 78d & - & - & - & - \\
\hline & ${ }^{1} \mathrm{H}$ data & 16.47 & 0.09 & 5.15 & 78.29 & - & - & - & - \\
\hline \multirow[t]{2}{*}{ sDP4+ } & ${ }^{13} \mathrm{C}$ data & 26.89 & 5.48 & 30.99 & 36.64 & - & - & - & - \\
\hline & All data & 12.76 & 0.01 & 4.60 & 82.63 & - & - & - & - \\
\hline \multirow{3}{*}{ uDP4+ } & ${ }^{1} \mathrm{H}$ data & 12.35 & 0.07 & 0.26 & 87.32 & - & - & - & - \\
\hline & ${ }^{13} \mathrm{C}$ data & 36.93 & 57.39 & 1.39 & 4.29 & - & - & - & - \\
\hline & All data & 54.65 & 0.46 & 0.04 & 44.85 & - & - & - & - \\
\hline & ${ }^{1} \mathrm{H}$ data & 2.89 & 0.00 & 0.02 & 97.09 & - & - & - & - \\
\hline \multirow[t]{5}{*}{ DP4+ } & ${ }^{13} \mathrm{C}$ data & 65.88 & 20.85 & 2.85 & 10.42 & - & - & - & - \\
\hline & All data & 15.84 & 0.00 & 0.00 & 84.16 & - & - & - & - \\
\hline & & \multicolumn{8}{|c|}{ Level of theory: B3LYP/6-311G* (gas phase) } \\
\hline & & 78a & $78 \mathrm{~b}$ & $78 \mathrm{c}$ & 78d & - & - & - & - \\
\hline & ${ }^{1} \mathrm{H}$ data & 24.88 & 0.10 & 0.94 & 74.08 & - & - & - & - \\
\hline \multirow[t]{2}{*}{ sDP4+ } & ${ }^{13} \mathrm{C}$ data & 43.84 & 38.39 & 7.67 & 10.10 & - & - & - & - \\
\hline & All data & 58.97 & 0.20 & 0.39 & 40.44 & - & - & - & - \\
\hline \multirow{3}{*}{ uDP4+ } & ${ }^{1} \mathrm{H}$ data & 77.69 & 0.23 & 1.44 & 20.63 & - & - & - & - \\
\hline & ${ }^{13} \mathrm{C}$ data & 23.82 & 4.94 & 20.75 & 50.48 & - & - & - & - \\
\hline & All data & 63.31 & 0.04 & 1.02 & 35.63 & - & - & - & - \\
\hline \multirow{5}{*}{ DP4+ } & ${ }^{1} \mathrm{H}$ data & 55.82 & 0.00 & 0.04 & 44.14 & - & - & - & - \\
\hline & ${ }^{13} \mathrm{C}$ data & 54.88 & 9.97 & 8.37 & 26.78 & - & - & - & - \\
\hline & All data & 72.15 & 0.00 & 0.01 & 27.84 & - & - & - & - \\
\hline & & \multicolumn{8}{|c|}{ Level of theory: B3LYP/6-311G** (gas phase) } \\
\hline & & 78a & $78 \mathrm{~b}$ & $78 \mathrm{c}$ & 78d & - & - & - & - \\
\hline$s$ DP4+ & ${ }^{1} \mathrm{H}$ data & 16.70 & 0.44 & 9.90 & 72.95 & - & - & - & - \\
\hline
\end{tabular}




\begin{tabular}{|c|c|c|c|c|c|c|c|c|c|}
\hline & ${ }^{13} \mathrm{C}$ data & 37.44 & 42.54 & 9.37 & 10.66 & - & - & - & - \\
\hline & All data & 41.29 & 1.24 & 6.13 & 51.35 & - & - & - & - \\
\hline & ${ }^{1} \mathrm{H}$ data & 36.72 & 1.04 & 4.44 & 57.80 & - & - & - & - \\
\hline \multirow[t]{3}{*}{ uDP4+ } & ${ }^{13} \mathrm{C}$ data & 16.26 & 3.68 & 24.91 & 55.15 & - & - & - & - \\
\hline & All data & 15.31 & 0.10 & 2.84 & 81.75 & - & - & - & - \\
\hline & ${ }^{1} \mathrm{H}$ data & 12.58 & 0.01 & 0.90 & 86.50 & - & - & - & - \\
\hline \multirow[t]{5}{*}{ DP4+ } & ${ }^{13} \mathrm{C}$ data & 38.37 & 9.88 & 14.71 & 37.05 & - & - & - & - \\
\hline & All data & 13.05 & 0.00 & 0.36 & 86.59 & - & - & - & - \\
\hline & & \multicolumn{8}{|c|}{ Level of theory: B3LYP/6-311+G** (gas phase) } \\
\hline & & $78 a$ & $78 b$ & $78 \mathrm{c}$ & 78d & - & - & - & - \\
\hline & ${ }^{1} \mathrm{H}$ data & 17.88 & 0.15 & 24.89 & 57.07 & - & - & - & - \\
\hline \multirow[t]{3}{*}{ sDP4+ } & ${ }^{13}$ C data & 46.19 & 47.31 & 2.52 & 3.98 & - & - & - & - \\
\hline & All data & 73.54 & 0.65 & 5.58 & 20.23 & - & - & - & - \\
\hline & ${ }^{1} \mathrm{H}$ data & 51.80 & 0.32 & 1.29 & 46.60 & - & - & - & - \\
\hline \multirow[t]{3}{*}{ uDP4+ } & ${ }^{13} \mathrm{C}$ data & 25.29 & 6.44 & 18.92 & 49.35 & - & - & - & - \\
\hline & All data & 36.03 & 0.06 & 0.67 & 63.25 & - & - & - & - \\
\hline & ${ }^{1} \mathrm{H}$ data & 25.60 & 0.00 & 0.89 & 73.51 & - & - & - & - \\
\hline \multirow[t]{5}{*}{ DP4+ } & ${ }^{13} \mathrm{C}$ data & 68.04 & 17.74 & 2.78 & 11.44 & - & - & - & - \\
\hline & All data & 67.37 & 0.00 & 0.10 & 32.53 & - & - & - & - \\
\hline & & \multicolumn{8}{|c|}{ Level of theory: B3LYP/6-31G* (solution, PCM) } \\
\hline & & $78 a$ & $78 b$ & 78c & 78d & - & - & - & - \\
\hline & ${ }^{1}$ H data & 6.83 & 0.00 & 0.04 & 93.13 & - & - & - & - \\
\hline \multirow[t]{3}{*}{ sDP4+ } & ${ }^{13} \mathrm{C}$ data & 80.53 & 1.88 & 10.11 & 7.48 & - & - & - & - \\
\hline & All data & 44.11 & 0.00 & 0.04 & 55.85 & - & - & - & - \\
\hline & ${ }^{1} \mathrm{H}$ data & 31.11 & 0.03 & 0.16 & 68.69 & - & - & - & - \\
\hline \multirow[t]{3}{*}{ uDP4+ } & ${ }^{13} \mathrm{C}$ data & 43.78 & 55.73 & 0.09 & 0.41 & - & - & - & - \\
\hline & All data & 97.87 & 0.13 & 0.00 & 2.01 & - & - & - & - \\
\hline & ${ }^{1} \mathrm{H}$ data & 3.21 & 0.00 & 0.00 & 96.79 & - & - & - & - \\
\hline \multirow[t]{5}{*}{ DP4+ } & ${ }^{13} \mathrm{C}$ data & 97.01 & 2.89 & 0.03 & 0.08 & - & - & - & - \\
\hline & All data & 97.47 & 0.00 & 0.00 & 2.53 & - & - & - & - \\
\hline & & \multicolumn{8}{|c|}{ Level of theory: B3LYP/6-31G** (solution, PCM) } \\
\hline & & $78 a$ & $78 \mathrm{~b}$ & 78c & 78d & - & - & - & - \\
\hline & ${ }^{1}$ H data & 28.54 & 0.03 & 0.65 & 70.78 & - & - & - & - \\
\hline \multirow[t]{3}{*}{ sDP4+ } & ${ }^{13}$ C data & 65.49 & 6.02 & 17.30 & 11.19 & - & - & - & - \\
\hline & All data & 69.94 & 0.01 & 0.42 & 29.64 & - & - & - & - \\
\hline & ${ }^{1} \mathrm{H}$ data & 6.69 & 0.06 & 0.06 & 93.19 & - & - & - & - \\
\hline \multirow[t]{3}{*}{ uDP4+ } & ${ }^{13} \mathrm{C}$ data & 53.23 & 46.24 & 0.11 & 0.42 & - & - & - & - \\
\hline & All data & 89.37 & 0.70 & 0.00 & 9.93 & - & - & - & - \\
\hline & ${ }^{1} \mathrm{H}$ data & 2.82 & 0.00 & 0.00 & 97.18 & - & - & - & - \\
\hline \multirow[t]{5}{*}{ DP4+ } & ${ }^{13} \mathrm{C}$ data & 92.45 & 7.38 & 0.05 & 0.13 & - & - & - & - \\
\hline & All data & 95.50 & 0.00 & 0.00 & 4.50 & - & - & - & - \\
\hline & & \multicolumn{8}{|c|}{ Level of theory: B3LYP/6-31+G** (solution, PCM) } \\
\hline & & $78 a$ & $78 b$ & $78 \mathrm{c}$ & 78d & - & - & - & - \\
\hline & ${ }^{1} \mathrm{H}$ data & 20.46 & 0.14 & 3.20 & 76.20 & - & - & - & - \\
\hline \multirow[t]{3}{*}{ sDP4+ } & ${ }^{13} \mathrm{C}$ data & 47.45 & 7.22 & 17.97 & 27.36 & - & - & - & - \\
\hline & All data & 31.17 & 0.03 & 1.85 & 66.95 & - & - & - & - \\
\hline & ${ }^{1} \mathrm{H}$ data & 4.88 & 0.24 & 0.04 & 94.84 & - & - & - & - \\
\hline uDP4+ & ${ }^{13} \mathrm{C}$ data & 19.94 & 75.61 & 0.86 & 3.59 & - & - & - & - \\
\hline & All data & 21.30 & 4.06 & 0.01 & 74.64 & - & - & - & - \\
\hline & ${ }^{1} \mathrm{H}$ data & 1.36 & 0.00 & 0.00 & 98.64 & - & - & - & - \\
\hline DP4+ & ${ }^{13} \mathrm{C}$ data & 58.92 & 33.99 & 0.97 & 6.12 & - & - & - & - \\
\hline & All data & 11.73 & 0.00 & 0.00 & 88.27 & - & - & - & - \\
\hline & & & & Level of & y: B3LY & 3* & C & & \\
\hline & & $78 a$ & $78 b$ & $78 c$ & $78 d$ & - & - & - & - \\
\hline sDP4+ & ${ }_{12}^{1} \mathrm{H}$ data & 21.32 & 0.14 & 0.40 & 78.14 & - & - & - & - \\
\hline SDP4+ & ${ }^{13} \mathrm{C}$ data & 60.52 & 32.90 & 2.71 & 3.86 & - & - & - & - \\
\hline
\end{tabular}




\begin{tabular}{|c|c|c|c|c|c|c|c|c|c|}
\hline & All data & 80.76 & 0.28 & 0.07 & 18.89 & - & - & - & - \\
\hline & ${ }^{1} \mathrm{H}$ data & 25.79 & 0.11 & 0.06 & 74.04 & - & - & - & - \\
\hline \multirow[t]{3}{*}{ uDP4+ } & ${ }^{13} \mathrm{C}$ data & 43.57 & 5.61 & 15.10 & 35.72 & - & - & - & - \\
\hline & All data & 29.80 & 0.02 & 0.02 & 70.15 & - & - & - & - \\
\hline & ${ }^{1} \mathrm{H}$ data & 8.68 & 0.00 & 0.00 & 91.32 & - & - & - & - \\
\hline \multirow[t]{5}{*}{ DP4+ } & ${ }^{13} \mathrm{C}$ data & 87.89 & 6.15 & 1.36 & 4.60 & - & - & - & - \\
\hline & All data & 64.49 & 0.00 & 0.00 & 35.51 & - & - & - & - \\
\hline & & \multicolumn{8}{|c|}{ Level of theory: B3LYP/6-311G** (solution, PCM) } \\
\hline & & $78 a$ & $78 b$ & $78 c$ & 78d & - & - & - & - \\
\hline & ${ }^{1} \mathrm{H}$ data & 21.62 & 1.61 & 9.28 & 67.50 & - & - & - & - \\
\hline \multirow[t]{3}{*}{ sDP4+ } & ${ }^{13} \mathrm{C}$ data & 65.20 & 29.00 & 2.48 & 3.32 & - & - & - & - \\
\hline & All data & 82.76 & 2.74 & 1.35 & 13.15 & - & - & - & - \\
\hline & ${ }^{1} \mathrm{H}$ data & 19.22 & 0.65 & 0.15 & 79.98 & - & - & - & - \\
\hline \multirow[t]{3}{*}{ uDP4+ } & ${ }^{13} \mathrm{C}$ data & 41.98 & 4.64 & 17.22 & 36.15 & - & - & - & - \\
\hline & All data & 21.78 & 0.08 & 0.07 & 78.07 & - & - & - & - \\
\hline & ${ }^{1} \mathrm{H}$ data & 7.14 & 0.02 & 0.02 & 92.82 & - & - & - & - \\
\hline \multirow[t]{5}{*}{ DP4+ } & ${ }^{13} \mathrm{C}$ data & 90.20 & 4.44 & 1.41 & 3.95 & - & - & - & - \\
\hline & All data & 63.72 & 0.01 & 0.00 & 36.27 & - & - & - & - \\
\hline & & \multicolumn{8}{|c|}{ Level of theory: B3LYP/6-311+G** (solution, PCM) } \\
\hline & & $78 a$ & $78 b$ & $78 \mathrm{c}$ & 78d & - & - & - & - \\
\hline & ${ }^{1}$ H data & 15.61 & 0.70 & 34.43 & 49.26 & - & - & - & - \\
\hline \multirow[t]{3}{*}{ sDP4+ } & ${ }^{13} \mathrm{C}$ data & 37.42 & 58.01 & 1.64 & 2.93 & - & - & - & - \\
\hline & All data & 70.73 & 4.93 & 6.83 & 17.51 & - & - & - & - \\
\hline & ${ }^{1} \mathrm{H}$ data & 11.87 & 0.23 & 0.14 & 87.76 & - & - & - & - \\
\hline \multirow[t]{3}{*}{ uDP4+ } & ${ }^{13} \mathrm{C}$ data & 39.72 & 6.22 & 13.72 & 40.34 & - & - & - & - \\
\hline & All data & 11.75 & 0.04 & 0.05 & 88.17 & - & - & - & - \\
\hline & ${ }^{1} \mathrm{H}$ data & 4.11 & 0.00 & 0.10 & 95.79 & - & - & - & - \\
\hline \multirow[t]{5}{*}{ DP4+ } & ${ }^{13} \mathrm{C}$ data & 74.77 & 18.14 & 1.13 & 5.96 & - & - & - & - \\
\hline & All data & 34.98 & 0.01 & 0.01 & 65.00 & - & - & - & - \\
\hline & & \multicolumn{8}{|c|}{ Level of theory: mPW1PW91/6-31G* (gas phase) } \\
\hline & & $78 a$ & $78 b$ & $78 c$ & 78d & - & - & - & - \\
\hline & ${ }^{1} \mathrm{H}$ data & 25.05 & 0.02 & 10.64 & 64.29 & - & - & - & - \\
\hline \multirow[t]{3}{*}{ sDP4+ } & ${ }^{13} \mathrm{C}$ data & 33.89 & 17.97 & 37.10 & 11.04 & - & - & - & - \\
\hline & All data & 43.44 & 0.02 & 20.20 & 36.34 & - & - & - & - \\
\hline & ${ }^{1} \mathrm{H}$ data & 60.32 & 0.14 & 31.53 & 8.01 & - & - & - & - \\
\hline \multirow[t]{3}{*}{ uDP4+ } & ${ }^{13} \mathrm{C}$ data & 80.50 & 19.42 & 0.02 & 0.06 & - & - & - & - \\
\hline & All data & 99.92 & 0.06 & 0.01 & 0.01 & - & - & - & - \\
\hline & ${ }^{1} \mathrm{H}$ data & 63.99 & 0.00 & 14.21 & 21.81 & - & - & - & - \\
\hline \multirow[t]{5}{*}{ DP4+ } & ${ }^{13} \mathrm{C}$ data & 88.62 & 11.34 & 0.02 & 0.02 & - & - & - & - \\
\hline & All data & 99.99 & 0.00 & 0.01 & 0.01 & - & - & - & - \\
\hline & & \multicolumn{8}{|c|}{ Level of theory: mPW1PW91/6-31G** (gas phase) } \\
\hline & & $78 a$ & $78 b$ & $78 \mathrm{c}$ & 78d & - & - & - & - \\
\hline & ${ }^{1} \mathrm{H}$ data & 25.45 & 0.12 & 14.63 & 59.79 & - & - & - & - \\
\hline \multirow[t]{3}{*}{ sDP4+ } & ${ }^{13} \mathrm{C}$ data & 15.64 & 32.64 & 40.01 & 11.71 & - & - & - & - \\
\hline & All data & 23.58 & 0.24 & 34.68 & 41.50 & - & - & - & - \\
\hline & ${ }^{1} \mathrm{H}$ data & 13.82 & 0.54 & 67.64 & 17.99 & - & - & - & - \\
\hline uDP4+ & ${ }^{13} \mathrm{C}$ data & 69.85 & 30.05 & 0.02 & 0.08 & - & - & - & - \\
\hline & All data & 98.06 & 1.66 & 0.15 & 0.14 & - & - & - & - \\
\hline & ${ }^{1} \mathrm{H}$ data & 14.56 & 0.00 & 40.93 & 44.51 & - & - & - & - \\
\hline DP4+ & ${ }^{13} \mathrm{C}$ data & 52.64 & 47.27 & 0.04 & 0.04 & - & - & - & - \\
\hline & All data & 99.52 & 0.02 & 0.22 & 0.25 & - & - & - & - \\
\hline & & & & evel of $t$ & mPW1 & -3 & ph & & \\
\hline & & $78 a$ & $78 b$ & $78 c$ & $78 d$ & - & - & - & - \\
\hline & ${ }^{1} \mathrm{H}$ data & 50.42 & 0.03 & 15.64 & 33.91 & - & - & - & - \\
\hline sDP4+ & ${ }^{13} \mathrm{C}$ data & 51.79 & 25.50 & 11.13 & 11.57 & - & - & - & - \\
\hline & All data & 82.15 & 0.02 & 5.48 & 12.34 & - & - & - & - \\
\hline
\end{tabular}




\begin{tabular}{|c|c|c|c|c|c|c|c|c|c|}
\hline & ${ }^{1} \mathrm{H}$ data & 37.62 & 0.54 & 29.95 & 31.89 & - & - & - & - \\
\hline \multirow[t]{3}{*}{ uDP4+ } & ${ }^{13} \mathrm{C}$ data & 74.79 & 25.03 & 0.06 & 0.12 & - & - & - & - \\
\hline & All data & 99.33 & 0.48 & 0.06 & 0.13 & - & - & - & - \\
\hline & ${ }^{1} \mathrm{H}$ data & 55.03 & 0.00 & 13.59 & 31.37 & - & - & - & - \\
\hline \multirow[t]{5}{*}{ DP4+ } & ${ }^{13} \mathrm{C}$ data & 85.81 & 14.14 & 0.01 & 0.03 & - & - & - & - \\
\hline & All data & 99.98 & 0.00 & 0.00 & 0.02 & - & - & - & - \\
\hline & & \multicolumn{8}{|c|}{ Level of theory: mPW1PW91/6-311G* (gas phase) } \\
\hline & & $78 a$ & $78 b$ & 78c & 78d & - & - & - & - \\
\hline & ${ }^{1} \mathrm{H}$ data & 18.31 & 0.09 & 4.71 & 76.90 & - & - & - & - \\
\hline \multirow[t]{3}{*}{ sDP4+ } & ${ }^{13} \mathrm{C}$ data & 6.93 & 92.34 & 0.44 & 0.29 & - & - & - & - \\
\hline & All data & 79.55 & 4.95 & 1.30 & 14.20 & - & - & - & - \\
\hline & ${ }^{1} \mathrm{H}$ data & 44.32 & 0.85 & 44.80 & 10.04 & - & - & - & - \\
\hline \multirow[t]{3}{*}{ uDP4+ } & ${ }^{13} \mathrm{C}$ data & 50.28 & 20.44 & 7.08 & 22.20 & - & - & - & - \\
\hline & All data & 79.99 & 0.62 & 11.39 & 8.00 & - & - & - & - \\
\hline & ${ }^{1} \mathrm{H}$ data & 45.22 & 0.00 & 11.75 & 43.02 & - & - & - & - \\
\hline \multirow[t]{5}{*}{ DP4+ } & ${ }^{13} \mathrm{C}$ data & 15.51 & 84.06 & 0.14 & 0.29 & - & - & - & - \\
\hline & All data & 97.98 & 0.05 & 0.23 & 1.75 & - & - & - & - \\
\hline & & \multicolumn{8}{|c|}{ Level of theory: mPW1PW91/6-311G** (gas phase) } \\
\hline & & $78 a$ & $78 b$ & 78c & 78d & - & - & - & - \\
\hline & ${ }^{1}$ H data & 27.02 & 0.43 & 17.15 & 55.40 & - & - & - & - \\
\hline \multirow[t]{3}{*}{ sDP4+ } & ${ }^{13} \mathrm{C}$ data & 8.73 & 90.53 & 0.45 & 0.29 & - & - & - & - \\
\hline & All data & 79.11 & 12.94 & 2.59 & 5.37 & - & - & - & - \\
\hline & ${ }^{1} \mathrm{H}$ data & 8.29 & 2.27 & 77.13 & 12.31 & - & - & - & - \\
\hline \multirow[t]{3}{*}{ uDP4+ } & ${ }^{13} \mathrm{C}$ data & 41.64 & 20.02 & 10.79 & 27.54 & - & - & - & - \\
\hline & All data & 22.11 & 2.90 & 53.28 & 21.70 & - & - & - & - \\
\hline & ${ }^{1} \mathrm{H}$ data & 10.05 & 0.04 & 59.33 & 30.58 & - & - & - & - \\
\hline \multirow[t]{5}{*}{ DP4+ } & ${ }^{13} \mathrm{C}$ data & 16.61 & 82.80 & 0.22 & 0.36 & - & - & - & - \\
\hline & All data & 85.70 & 1.84 & 6.76 & 5.71 & - & - & - & - \\
\hline & & \multicolumn{8}{|c|}{ Level of theory: mPW1PW91/6-311+G** (gas phase) } \\
\hline & & $78 a$ & $78 b$ & $78 \mathrm{c}$ & 78d & - & - & - & - \\
\hline & ${ }^{1} \mathrm{H}$ data & 48.59 & 0.05 & 23.70 & 27.66 & - & - & - & - \\
\hline \multirow[t]{3}{*}{ sDP4+ } & ${ }^{13} \mathrm{C}$ data & 9.76 & 90.09 & 0.08 & 0.07 & - & - & - & - \\
\hline & All data & 98.28 & 0.93 & 0.41 & 0.38 & - & - & - & - \\
\hline & ${ }^{1} \mathrm{H}$ data & 47.50 & 0.60 & 48.45 & 3.45 & - & - & - & - \\
\hline \multirow[t]{3}{*}{ uDP4+ } & ${ }^{13} \mathrm{C}$ data & 58.38 & 18.01 & 4.86 & 18.74 & - & - & - & - \\
\hline & All data & 89.92 & 0.35 & 7.64 & 2.10 & - & - & - & - \\
\hline & ${ }^{1} \mathrm{H}$ data & 64.98 & 0.00 & 32.33 & 2.69 & - & - & - & - \\
\hline \multirow[t]{5}{*}{ DP4+ } & ${ }^{13} \mathrm{C}$ data & 25.97 & 73.95 & 0.02 & 0.06 & - & - & - & - \\
\hline & All data & 99.95 & 0.00 & 0.04 & 0.01 & - & - & - & - \\
\hline & & \multicolumn{8}{|c|}{ Level of theory: mPW1PW91/6-31G* (solution, PCM) } \\
\hline & & $78 a$ & $78 b$ & 78c & 78d & - & - & - & - \\
\hline & ${ }^{1} \mathrm{H}$ data & 56.75 & 0.01 & 2.69 & 40.55 & - & - & - & - \\
\hline \multirow[t]{3}{*}{ sDP4+ } & ${ }^{13} \mathrm{C}$ data & 95.02 & 2.52 & 1.72 & 0.74 & - & - & - & - \\
\hline & All data & 99.36 & 0.00 & 0.09 & 0.55 & - & - & - & - \\
\hline & ${ }^{1}$ H data & 64.30 & 0.03 & 0.97 & 34.70 & - & - & - & - \\
\hline uDP4+ & ${ }^{13} \mathrm{C}$ data & 79.77 & 20.15 & 0.02 & 0.07 & - & - & - & - \\
\hline & All data & 99.94 & 0.01 & 0.00 & 0.04 & - & - & - & - \\
\hline & ${ }^{1} \mathrm{H}$ data & 72.13 & 0.00 & 0.05 & 27.82 & - & - & - & - \\
\hline DP4+ & ${ }^{13} \mathrm{C}$ data & 99.33 & 0.66 & 0.00 & 0.00 & - & - & - & - \\
\hline & All data & 100.00 & 0.00 & 0.00 & 0.00 & - & - & - & - \\
\hline & & & & el of th & nPW1PI & 31 & on, & & \\
\hline & & $78 a$ & $78 b$ & $78 c$ & 78d & - & - & - & - \\
\hline & ${ }^{1} \mathrm{H}$ data & 76.78 & 0.01 & 6.47 & 16.74 & - & - & - & - \\
\hline sDP4+ & ${ }^{13} \mathrm{C}$ data & 81.96 & 11.90 & 3.92 & 2.21 & - & - & - & - \\
\hline & All data & 99.02 & 0.00 & 0.40 & 0.58 & - & - & - & - \\
\hline uDP4+ & ${ }^{1} \mathrm{H}$ data & 19.16 & 0.08 & 0.88 & 79.88 & - & - & - & - \\
\hline
\end{tabular}




\begin{tabular}{|c|c|c|c|c|c|c|c|c|c|}
\hline & ${ }^{13} \mathrm{C}$ data & 80.35 & 19.58 & 0.01 & 0.05 & - & - & - & - \\
\hline & All data & 99.65 & 0.10 & 0.00 & 0.24 & - & - & - & - \\
\hline & ${ }^{1} \mathrm{H}$ data & 52.28 & 0.00 & 0.20 & 47.52 & - & - & - & - \\
\hline \multirow[t]{5}{*}{ DP4+ } & ${ }^{13} \mathrm{C}$ data & 96.58 & 3.42 & 0.00 & 0.00 & - & - & - & - \\
\hline & All data & 100.00 & 0.00 & 0.00 & 0.00 & - & - & - & - \\
\hline & & \multicolumn{8}{|c|}{ Level of theory: mPW1PW91/6-31+G** (solution, PCM) } \\
\hline & & 78a & 78b & 78c & 78d & - & - & - & - \\
\hline & ${ }^{1} \mathrm{H}$ data & 28.57 & 0.04 & 48.24 & 23.15 & - & - & - & - \\
\hline \multirow[t]{2}{*}{ sDP4+ } & ${ }^{13} \mathrm{C}$ data & 79.67 & 15.91 & 1.63 & 2.80 & - & - & - & - \\
\hline & All data & 94.05 & 0.03 & 3.24 & 2.68 & - & - & - & - \\
\hline \multirow{3}{*}{ uDP4+ } & ${ }^{1} \mathrm{H}$ data & 8.62 & 0.09 & 0.10 & 91.20 & - & - & - & - \\
\hline & ${ }^{13} \mathrm{C}$ data & 40.65 & 58.83 & 0.10 & 0.42 & - & - & - & - \\
\hline & All data & 89.06 & 1.29 & 0.00 & 9.65 & - & - & - & - \\
\hline & ${ }^{1} \mathrm{H}$ data & 10.42 & 0.00 & 0.21 & 89.37 & - & - & - & - \\
\hline \multirow[t]{5}{*}{ DP4+ } & ${ }^{13} \mathrm{C}$ data & 77.56 & 22.41 & 0.00 & 0.03 & - & - & - & - \\
\hline & All data & 99.69 & 0.00 & 0.00 & 0.31 & - & - & - & - \\
\hline & & \multicolumn{8}{|c|}{ Level of theory: mPW1PW91/6-311G* (solution, PCM) } \\
\hline & & 78a & $78 \mathrm{~b}$ & $78 \mathrm{c}$ & 78d & - & - & - & - \\
\hline & ${ }^{1} \mathrm{H}$ data & 39.30 & 0.09 & 6.12 & 54.49 & - & - & - & - \\
\hline \multirow[t]{2}{*}{ sDP4+ } & ${ }^{13} \mathrm{C}$ data & 42.69 & 57.13 & 0.10 & 0.08 & - & - & - & - \\
\hline & All data & 99.38 & 0.31 & 0.04 & 0.27 & - & - & - & - \\
\hline \multirow{3}{*}{ uDP4+ } & ${ }^{1} \mathrm{H}$ data & 41.45 & 0.12 & 0.36 & 58.07 & - & - & - & - \\
\hline & ${ }^{13} \mathrm{C}$ data & 88.39 & 5.81 & 1.50 & 4.30 & - & - & - & - \\
\hline & All data & 93.60 & 0.02 & 0.01 & 6.37 & - & - & - & - \\
\hline \multirow{5}{*}{ DP4+ } & ${ }^{1} \mathrm{H}$ data & 33.97 & 0.00 & 0.05 & 65.98 & - & - & - & - \\
\hline & ${ }^{13} \mathrm{C}$ data & 91.90 & 8.08 & 0.00 & 0.01 & - & - & - & - \\
\hline & All data & 99.98 & 0.00 & 0.00 & 0.02 & - & - & - & - \\
\hline & & \multicolumn{8}{|c|}{ Level of theory: mPW1PW91/6-311G** (solution, PCM) } \\
\hline & & 78a & 78b & $78 \mathrm{c}$ & 78d & - & - & - & - \\
\hline \multirow{3}{*}{ sDP4+ } & ${ }^{1} \mathrm{H}$ data & 33.30 & 0.50 & 45.75 & 20.45 & - & - & - & - \\
\hline & ${ }^{13} \mathrm{C}$ data & 46.25 & 53.61 & 0.08 & 0.06 & - & - & - & - \\
\hline & All data & 98.00 & 1.69 & 0.22 & 0.08 & - & - & - & - \\
\hline & ${ }^{1} \mathrm{H}$ data & 23.06 & 1.01 & 1.24 & 74.68 & - & - & - & - \\
\hline \multirow[t]{2}{*}{ uDP4+ } & ${ }^{13} \mathrm{C}$ data & 85.73 & 6.29 & 2.15 & 5.83 & - & - & - & - \\
\hline & All data & 81.65 & 0.26 & 0.11 & 17.98 & - & - & - & - \\
\hline \multirow{5}{*}{ DP4+ } & ${ }^{1} \mathrm{H}$ data & 32.65 & 0.02 & 2.42 & 64.91 & - & - & - & - \\
\hline & ${ }^{13} \mathrm{C}$ data & 92.15 & 7.84 & 0.00 & 0.01 & - & - & - & - \\
\hline & All data & 99.98 & 0.01 & 0.00 & 0.02 & - & - & - & - \\
\hline & & \multicolumn{8}{|c|}{ Level of theory: mPW1PW91/6-311+G** (solution, PCM) } \\
\hline & & $78 \mathrm{a}$ & 78b & $78 c$ & 78d & - & - & - & - \\
\hline \multirow{3}{*}{ sDP4+ } & ${ }^{1} \mathrm{H}$ data & 9.92 & 0.05 & 83.29 & 6.75 & - & - & - & - \\
\hline & ${ }^{13} \mathrm{C}$ data & 14.64 & 85.27 & 0.04 & 0.05 & - & - & - & - \\
\hline & All data & 94.73 & 2.67 & 2.36 & 0.23 & - & - & - & - \\
\hline \multirow{3}{*}{ uDP4+ } & ${ }^{1} \mathrm{H}$ data & 36.07 & 0.31 & 1.06 & 62.57 & - & - & - & - \\
\hline & ${ }^{13} \mathrm{C}$ data & 85.89 & 6.44 & 1.67 & 6.00 & - & - & - & - \\
\hline & All data & 89.10 & 0.06 & 0.05 & 10.79 & - & - & - & - \\
\hline \multirow{3}{*}{ DP4+ } & ${ }^{1} \mathrm{H}$ data & 41.21 & 0.00 & 10.14 & 48.65 & - & - & - & - \\
\hline & ${ }^{13} \mathrm{C}$ data & 69.58 & 30.40 & 0.00 & 0.02 & - & - & - & - \\
\hline & All data & 99.97 & 0.00 & 0.00 & 0.03 & - & - & - & - \\
\hline
\end{tabular}


$s \mathrm{DP4}+, u \mathrm{DP} 4+$ and DP4+ probabilities (\%) for compound 78b<smiles>CCC[C@H]1O[C@@H](c2ccccc2)C[C@H]1O</smiles><smiles>CCC[C@H]1O[C@@H](c2ccccc2)C[C@H]1O</smiles>

correct isomer<smiles>CCC[C@H]1O[C@@H](c2ccccc2)C[C@H]1O</smiles>

$78 \mathrm{c}$<smiles>CCC[C@H]1O[C@@H](c2ccccc2)C[C@H]1O</smiles>

78d

\begin{tabular}{|c|c|c|c|c|c|c|c|c|c|}
\hline & & \multicolumn{8}{|c|}{ Level of theory: B3LYP/6-31G* (gas phase) } \\
\hline & & 78a & $78 \mathrm{~b}$ & $78 \mathrm{c}$ & 78d & - & - & - & - \\
\hline & ${ }^{1} \mathrm{H}$ data & 5.31 & 0.14 & 5.00 & 89.55 & - & - & - & - \\
\hline \multirow[t]{2}{*}{ sDP4+ } & ${ }^{13} \mathrm{C}$ data & 41.79 & 42.94 & 8.37 & 6.90 & - & - & - & - \\
\hline & All data & 24.99 & 0.70 & 4.72 & 69.60 & - & - & - & - \\
\hline & ${ }^{1} \mathrm{H}$ data & 62.08 & 3.22 & 23.71 & 10.99 & - & - & - & - \\
\hline \multirow[t]{2}{*}{ uDP4+ } & ${ }^{13} \mathrm{C}$ data & 16.95 & 83.04 & 0.00 & 0.00 & - & - & - & - \\
\hline & All data & 79.75 & 20.24 & 0.01 & 0.00 & - & - & - & - \\
\hline \multirow{6}{*}{ DP4+ } & ${ }^{1} \mathrm{H}$ data & 23.00 & 0.03 & 8.27 & 68.70 & - & - & - & - \\
\hline & ${ }^{13} \mathrm{C}$ data & 16.58 & 83.42 & 0.00 & 0.00 & - & - & - & - \\
\hline & All data & 99.28 & 0.70 & 0.00 & 0.01 & - & - & - & - \\
\hline & & \multicolumn{8}{|c|}{ Level of theory: B3LYP/6-31G** (gas phase) } \\
\hline & & 78a & $78 \mathrm{~b}$ & $78 \mathrm{c}$ & 78d & - & - & - & - \\
\hline & ${ }^{1} \mathrm{H}$ data & 5.22 & 1.31 & 17.92 & 75.55 & - & - & - & - \\
\hline \multirow[t]{3}{*}{ sDP4+ } & ${ }^{13} \mathrm{C}$ data & 30.57 & 51.77 & 9.70 & 7.96 & - & - & - & - \\
\hline & All data & 15.91 & 6.77 & 17.33 & 60.00 & - & - & - & - \\
\hline & ${ }^{1} \mathrm{H}$ data & 22.08 & 7.55 & 33.75 & 36.62 & - & - & - & - \\
\hline \multirow[t]{2}{*}{ uDP4+ } & ${ }^{13} \mathrm{C}$ data & 13.85 & 86.15 & 0.00 & 0.00 & - & - & - & - \\
\hline & All data & 31.98 & 68.00 & 0.01 & 0.01 & - & - & - & - \\
\hline & ${ }^{1} \mathrm{H}$ data & 3.30 & 0.28 & 17.29 & 79.13 & - & - & - & - \\
\hline \multirow[t]{5}{*}{ DP4+ } & ${ }^{13} \mathrm{C}$ data & 8.67 & 91.33 & 0.00 & 0.00 & - & - & - & - \\
\hline & All data & 52.46 & 47.45 & 0.02 & 0.08 & - & - & - & - \\
\hline & & \multicolumn{8}{|c|}{ Level of theory: B3LYP/6-31+G** (gas phase) } \\
\hline & & $78 a$ & $78 \mathrm{~b}$ & $78 \mathrm{c}$ & 78d & - & - & - & - \\
\hline & ${ }^{1} \mathrm{H}$ data & 27.18 & 1.00 & 53.31 & 18.50 & - & - & - & - \\
\hline \multirow[t]{2}{*}{$s \mathrm{DP} 4+$} & ${ }^{13} \mathrm{C}$ data & 41.00 & 33.26 & 6.83 & 18.91 & - & - & - & - \\
\hline & All data & 59.86 & 1.79 & 19.56 & 18.79 & - & - & - & - \\
\hline \multirow{3}{*}{ uDP4+ } & ${ }^{1} \mathrm{H}$ data & 26.95 & 2.27 & 3.65 & 67.13 & - & - & - & - \\
\hline & ${ }^{13} \mathrm{C}$ data & 2.84 & 97.09 & 0.02 & 0.05 & - & - & - & - \\
\hline & All data & 25.52 & 73.37 & 0.02 & 1.09 & - & - & - & - \\
\hline & ${ }^{1} \mathrm{H}$ data & 33.73 & 0.10 & 8.96 & 57.20 & - & - & - & - \\
\hline \multirow[t]{5}{*}{ DP4+ } & ${ }^{13} \mathrm{C}$ data & 3.48 & 96.48 & 0.00 & 0.03 & - & - & - & - \\
\hline & All data & 90.93 & 7.82 & 0.03 & 1.22 & - & - & - & - \\
\hline & & \multicolumn{8}{|c|}{ Level of theory: B3LYP/6-311G* (gas phase) } \\
\hline & & 78a & $78 \mathrm{~b}$ & $78 \mathrm{c}$ & 78d & - & - & - & - \\
\hline & ${ }^{1} \mathrm{H}$ data & 30.40 & 1.22 & 14.04 & 54.35 & - & - & - & - \\
\hline \multirow[t]{2}{*}{ sDP4+ } & ${ }^{13} \mathrm{C}$ data & 23.16 & 74.71 & 0.97 & 1.16 & - & - & - & - \\
\hline & All data & 80.77 & 10.43 & 1.56 & 7.24 & - & - & - & - \\
\hline & ${ }^{1} \mathrm{H}$ data & 66.64 & 6.76 & 7.83 & 18.77 & - & - & - & - \\
\hline \multirow[t]{2}{*}{ uDP4+ } & ${ }^{13} \mathrm{C}$ data & 65.70 & 26.79 & 2.16 & 5.35 & - & - & - & - \\
\hline & All data & 93.62 & 3.87 & 0.36 & 2.15 & - & - & - & - \\
\hline \multirow{5}{*}{ DP4+ } & ${ }^{1} \mathrm{H}$ data & 64.02 & 0.26 & 3.47 & 32.24 & - & - & - & - \\
\hline & ${ }^{13} \mathrm{C}$ data & 43.08 & 56.68 & 0.06 & 0.18 & - & - & - & - \\
\hline & All data & 99.26 & 0.53 & 0.01 & 0.20 & - & - & - & - \\
\hline & & \multicolumn{8}{|c|}{ Level of theory: B3LYP/6-311G** (gas phase) } \\
\hline & & 78a & 78b & $78 \mathrm{c}$ & 78d & - & - & - & - \\
\hline sDP4+ & ${ }^{1} \mathrm{H}$ data & 2.60 & 4.96 & 72.87 & 19.57 & - & - & - & - \\
\hline
\end{tabular}




\begin{tabular}{|c|c|c|c|c|c|c|c|c|c|}
\hline & ${ }^{13} \mathrm{C}$ data & 16.53 & 81.51 & 0.91 & 1.05 & - & - & - & - \\
\hline & All data & 8.05 & 75.66 & 12.43 & 3.85 & - & - & - & - \\
\hline & ${ }^{1} \mathrm{H}$ data & 10.22 & 28.75 & 25.07 & 35.97 & - & - & - & - \\
\hline \multirow[t]{3}{*}{ uDP4+ } & ${ }^{13} \mathrm{C}$ data & 63.26 & 27.43 & 2.67 & 6.65 & - & - & - & - \\
\hline & All data & 37.13 & 45.30 & 3.84 & 13.74 & - & - & - & - \\
\hline & ${ }^{1} \mathrm{H}$ data & 0.99 & 5.28 & 67.66 & 26.08 & - & - & - & - \\
\hline \multirow[t]{5}{*}{ DP4+ } & ${ }^{13} \mathrm{C}$ data & 31.77 & 67.94 & 0.07 & 0.21 & - & - & - & - \\
\hline & All data & 7.81 & 89.56 & 1.25 & 1.38 & - & - & - & - \\
\hline & & \multicolumn{8}{|c|}{ Level of theory: B3LYP/6-311+G** (gas phase) } \\
\hline & & $78 a$ & $78 b$ & $78 c$ & 78d & - & - & - & - \\
\hline & ${ }^{1} \mathrm{H}$ data & 13.67 & 2.09 & 77.17 & 7.07 & - & - & - & - \\
\hline \multirow[t]{3}{*}{$s D P 4+$} & ${ }^{13} \mathrm{C}$ data & 11.76 & 87.37 & 0.35 & 0.53 & - & - & - & - \\
\hline & All data & 42.99 & 48.86 & 7.14 & 1.00 & - & - & - & - \\
\hline & ${ }^{1} \mathrm{H}$ data & 38.35 & 6.62 & 6.26 & 48.77 & - & - & - & - \\
\hline \multirow[t]{3}{*}{ uDP4+ } & ${ }^{13} \mathrm{C}$ data & 60.40 & 32.78 & 1.73 & 5.09 & - & - & - & - \\
\hline & All data & 82.95 & 7.77 & 0.39 & 8.89 & - & - & - & - \\
\hline & ${ }^{1} \mathrm{H}$ data & 38.37 & 1.01 & 35.38 & 25.23 & - & - & - & - \\
\hline \multirow[t]{5}{*}{ DP4+ } & ${ }^{13} \mathrm{C}$ data & 19.85 & 80.05 & 0.02 & 0.08 & - & - & - & - \\
\hline & All data & 90.11 & 9.59 & 0.07 & 0.22 & - & - & - & - \\
\hline & & \multicolumn{8}{|c|}{ Level of theory: B3LYP/6-31G* (solution, PCM) } \\
\hline & & $78 a$ & $78 b$ & $78 c$ & $78 d$ & - & - & - & - \\
\hline & ${ }^{1} \mathrm{H}$ data & 46.92 & 1.61 & 12.58 & 38.89 & - & - & - & - \\
\hline \multirow[t]{2}{*}{ sDP4+ } & ${ }^{13} \mathrm{C}$ data & 86.73 & 11.68 & 0.76 & 0.83 & - & - & - & - \\
\hline & All data & 98.53 & 0.46 & 0.23 & 0.78 & - & - & - & - \\
\hline \multirow{3}{*}{ uDP4+ } & ${ }^{1} \mathrm{H}$ data & 63.11 & 6.37 & 14.66 & 15.86 & - & - & - & - \\
\hline & ${ }^{13} \mathrm{C}$ data & 5.23 & 94.76 & 0.00 & 0.01 & - & - & - & - \\
\hline & All data & 35.32 & 64.66 & 0.00 & 0.01 & - & - & - & - \\
\hline \multirow{5}{*}{ DP4+ } & ${ }^{1} \mathrm{H}$ data & 78.49 & 0.27 & 4.88 & 16.35 & - & - & - & - \\
\hline & ${ }^{13} \mathrm{C}$ data & 29.05 & 70.95 & 0.00 & 0.00 & - & - & - & - \\
\hline & All data & 99.16 & 0.84 & 0.00 & 0.00 & - & - & - & - \\
\hline & & \multicolumn{8}{|c|}{ Level of theory: B3LYP/6-31G** (solution, PCM) } \\
\hline & & $78 a$ & $78 b$ & $78 c$ & $78 d$ & - & - & - & - \\
\hline \multirow{3}{*}{ sDP4+ } & ${ }^{1} \mathrm{H}$ data & 23.12 & 9.47 & 58.09 & 9.32 & - & - & - & - \\
\hline & ${ }^{13} \mathrm{C}$ data & 73.32 & 22.88 & 1.82 & 1.97 & - & - & - & - \\
\hline & All data & 83.26 & 10.64 & 5.20 & 0.90 & - & - & - & - \\
\hline \multirow{3}{*}{ uDP4+ } & ${ }^{1} \mathrm{H}$ data & 21.73 & 14.26 & 7.62 & 56.39 & - & - & - & - \\
\hline & ${ }^{13} \mathrm{C}$ data & 4.43 & 95.56 & 0.00 & 0.00 & - & - & - & - \\
\hline & All data & 6.60 & 93.38 & 0.00 & 0.02 & - & - & - & - \\
\hline \multirow{5}{*}{ DP4+ } & ${ }^{1} \mathrm{H}$ data & 31.29 & 8.41 & 27.57 & 32.73 & - & - & - & - \\
\hline & ${ }^{13} \mathrm{C}$ data & 12.94 & 87.06 & 0.00 & 0.00 & - & - & - & - \\
\hline & All data & 35.62 & 64.38 & 0.00 & 0.00 & - & - & - & - \\
\hline & & \multicolumn{8}{|c|}{ Level of theory: B3LYP/6-31+G** (solution, PCM) } \\
\hline & & $78 a$ & $78 \mathrm{~b}$ & $78 c$ & 78d & - & - & - & - \\
\hline \multirow{3}{*}{ sDP4+ } & ${ }^{1} \mathrm{H}$ data & 5.71 & 2.42 & 90.79 & 1.08 & - & - & - & - \\
\hline & ${ }^{13} \mathrm{C}$ data & 47.56 & 38.47 & 3.59 & 10.38 & - & - & - & - \\
\hline & All data & 38.66 & 13.28 & 46.47 & 1.59 & - & - & - & - \\
\hline & ${ }^{1} \mathrm{H}$ data & 26.96 & 35.58 & 5.52 & 31.94 & - & - & - & - \\
\hline uDP4+ & ${ }^{13} \mathrm{C}$ data & 0.62 & 99.32 & 0.01 & 0.06 & - & - & - & - \\
\hline & All data & 0.47 & 99.48 & 0.00 & 0.05 & - & - & - & - \\
\hline & ${ }_{12}^{1} \mathrm{H}$ data & 19.83 & 11.12 & 64.63 & 4.43 & - & - & - & - \\
\hline DP4+ & ${ }^{13} \mathrm{C}$ data & 0.76 & 99.22 & 0.00 & 0.02 & - & - & - & - \\
\hline & All data & 1.35 & 98.64 & 0.00 & 0.01 & - & - & - & - \\
\hline & & & & Level of & $y: B 3 L Y$ & J & $\bar{C}$ & & \\
\hline & & $78 a$ & $78 b$ & $78 c$ & $78 d$ & - & - & - & - \\
\hline & ${ }^{1} \mathrm{H}$ data & 71.41 & 11.67 & 10.85 & 6.07 & - & - & - & - \\
\hline SUP4+ & ${ }^{13} \mathrm{C}$ data & 16.55 & 81.97 & 0.60 & 0.88 & - & - & - & - \\
\hline
\end{tabular}




\begin{tabular}{|c|c|c|c|c|c|c|c|c|c|}
\hline & All data & 54.96 & 44.49 & 0.30 & 0.25 & - & - & - & - \\
\hline & ${ }^{1} \mathrm{H}$ data & 65.28 & 9.49 & 3.83 & 21.41 & - & - & - & - \\
\hline \multirow[t]{3}{*}{ uDP4+ } & ${ }^{13} \mathrm{C}$ data & 66.65 & 28.25 & 1.55 & 3.54 & - & - & - & - \\
\hline & All data & 92.56 & 5.70 & 0.13 & 1.61 & - & - & - & - \\
\hline & ${ }^{1} \mathrm{H}$ data & 94.29 & 2.24 & 0.84 & 2.63 & - & - & - & - \\
\hline \multirow[t]{5}{*}{ DP4+ } & ${ }^{13} \mathrm{C}$ data & 32.23 & 67.65 & 0.03 & 0.09 & - & - & - & - \\
\hline & All data & 95.24 & 4.75 & 0.00 & 0.01 & - & - & - & - \\
\hline & & \multicolumn{8}{|c|}{ Level of theory: B3LYP/6-311G** (solution, PCM) } \\
\hline & & $78 a$ & $78 b$ & $78 c$ & 78d & - & - & - & - \\
\hline & ${ }^{1} \mathrm{H}$ data & 3.79 & 30.19 & 63.75 & 2.28 & - & - & - & - \\
\hline \multirow[t]{3}{*}{ sDP4+ } & ${ }^{13} \mathrm{C}$ data & 19.62 & 79.36 & 0.42 & 0.59 & - & - & - & - \\
\hline & All data & 2.97 & 95.90 & 1.08 & 0.05 & - & - & - & - \\
\hline & ${ }^{1} \mathrm{H}$ data & 7.91 & 21.68 & 3.17 & 67.24 & - & - & - & - \\
\hline \multirow[t]{3}{*}{ uDP4+ } & ${ }^{13} \mathrm{C}$ data & 73.79 & 22.01 & 1.36 & 2.84 & - & - & - & - \\
\hline & All data & 46.45 & 37.99 & 0.34 & 15.22 & - & - & - & - \\
\hline & ${ }^{1} \mathrm{H}$ data & 2.88 & 62.95 & 19.46 & 14.71 & - & - & - & - \\
\hline \multirow[t]{5}{*}{ DP4+ } & ${ }^{13} \mathrm{C}$ data & 45.29 & 54.64 & 0.02 & 0.05 & - & - & - & - \\
\hline & All data & 3.65 & 96.32 & 0.01 & 0.02 & - & - & - & - \\
\hline & & \multicolumn{8}{|c|}{ Level of theory: B3LYP/6-311+G** (solution, PCM) } \\
\hline & & $78 a$ & $78 b$ & $78 \mathrm{c}$ & 78d & - & - & - & - \\
\hline & ${ }^{1} \mathrm{H}$ data & 1.84 & 1.89 & 95.26 & 1.02 & - & - & - & - \\
\hline \multirow[t]{3}{*}{ sDP4+ } & ${ }^{13} \mathrm{C}$ data & 4.97 & 94.42 & 0.22 & 0.39 & - & - & - & - \\
\hline & All data & 4.38 & 85.58 & 9.84 & 0.19 & - & - & - & - \\
\hline & ${ }^{1} \mathrm{H}$ data & 29.10 & 46.30 & 4.44 & 20.16 & - & - & - & - \\
\hline \multirow[t]{3}{*}{ uDP4+ } & ${ }^{13} \mathrm{C}$ data & 55.04 & 38.02 & 1.76 & 5.18 & - & - & - & - \\
\hline & All data & 46.10 & 50.67 & 0.23 & 3.01 & - & - & - & - \\
\hline & ${ }^{1} \mathrm{H}$ data & 9.15 & 14.96 & 72.38 & 3.51 & - & - & - & - \\
\hline \multirow[t]{5}{*}{ DP4+ } & ${ }^{13} \mathrm{C}$ data & 7.08 & 92.86 & 0.01 & 0.05 & - & - & - & - \\
\hline & All data & 4.45 & 95.49 & 0.05 & 0.01 & - & - & - & - \\
\hline & & \multicolumn{8}{|c|}{ Level of theory: mPW1PW91/6-31G* (gas phase) } \\
\hline & & $78 a$ & $78 b$ & $78 c$ & 78d & - & - & - & - \\
\hline & ${ }^{1}$ H data & 2.08 & 0.25 & 16.06 & 81.61 & - & - & - & - \\
\hline \multirow[t]{3}{*}{ sDP4+ } & ${ }^{13} \mathrm{C}$ data & 15.88 & 83.59 & 0.38 & 0.15 & - & - & - & - \\
\hline & All data & 45.62 & 28.78 & 8.48 & 17.12 & - & - & - & - \\
\hline & ${ }^{1} \mathrm{H}$ data & 10.30 & 4.77 & 79.20 & 5.74 & - & - & - & - \\
\hline \multirow[t]{3}{*}{ uDP4+ } & ${ }^{13} \mathrm{C}$ data & 16.01 & 83.99 & 0.00 & 0.00 & - & - & - & - \\
\hline & All data & 29.15 & 70.84 & 0.02 & 0.00 & - & - & - & - \\
\hline & ${ }^{1} \mathrm{H}$ data & 1.22 & 0.07 & 72.16 & 26.56 & - & - & - & - \\
\hline \multirow[t]{5}{*}{ DP4+ } & ${ }^{13} \mathrm{C}$ data & 3.49 & 96.51 & 0.00 & 0.00 & - & - & - & - \\
\hline & All data & 39.47 & 60.52 & 0.00 & 0.00 & - & - & - & - \\
\hline & & \multicolumn{8}{|c|}{ Level of theory: mPW1PW91/6-31G** (gas phase) } \\
\hline & & $78 a$ & $78 b$ & $78 \mathrm{c}$ & 78d & - & - & - & - \\
\hline & ${ }^{1} \mathrm{H}$ data & 1.75 & 2.53 & 20.73 & 74.99 & - & - & - & - \\
\hline \multirow[t]{3}{*}{ sDP4+ } & ${ }^{13} \mathrm{C}$ data & 9.51 & 89.58 & 0.65 & 0.26 & - & - & - & - \\
\hline & All data & 6.01 & 82.02 & 4.87 & 7.10 & - & - & - & - \\
\hline & ${ }^{1} \mathrm{H}$ data & 1.09 & 6.17 & 82.37 & 10.37 & - & - & - & - \\
\hline uDP4+ & ${ }^{13} \mathrm{C}$ data & 11.88 & 88.12 & 0.00 & 0.00 & - & - & - & - \\
\hline & All data & 2.33 & 97.66 & 0.01 & 0.00 & - & - & - & - \\
\hline & ${ }^{1} \mathrm{H}$ data & 0.08 & 0.62 & 68.23 & 31.07 & - & - & - & - \\
\hline DP4+ & ${ }^{13} \mathrm{C}$ data & 1.41 & 98.59 & 0.00 & 0.00 & - & - & - & - \\
\hline & All data & 0.17 & 99.82 & 0.00 & 0.00 & - & - & - & - \\
\hline & & & & vel of $t$ l & mPW1 & 3 & ph & & \\
\hline & & $78 a$ & $78 b$ & $78 c$ & $78 d$ & - & - & - & - \\
\hline & ${ }^{1} \mathrm{H}$ data & 7.02 & 0.49 & 74.29 & 18.20 & - & - & - & - \\
\hline sDP4+ & ${ }^{13} \mathrm{C}$ data & 35.41 & 63.46 & 0.59 & 0.55 & - & - & - & - \\
\hline & All data & 74.52 & 9.38 & 13.08 & 3.01 & - & - & - & - \\
\hline
\end{tabular}




\begin{tabular}{|c|c|c|c|c|c|c|c|c|c|}
\hline & ${ }^{1} \mathrm{H}$ data & 13.32 & 7.17 & 59.30 & 20.21 & - & - & - & - \\
\hline \multirow[t]{3}{*}{ uDP4+ } & ${ }^{13} \mathrm{C}$ data & 9.50 & 90.50 & 0.00 & 0.00 & - & - & - & - \\
\hline & All data & 16.32 & 83.67 & 0.01 & 0.00 & - & - & - & - \\
\hline & ${ }^{1} \mathrm{H}$ data & 1.92 & 0.07 & 90.45 & 7.55 & - & - & - & - \\
\hline \multirow[t]{5}{*}{ DP4+ } & ${ }^{13} \mathrm{C}$ data & 5.53 & 94.47 & 0.00 & 0.00 & - & - & - & - \\
\hline & All data & 60.76 & 39.23 & 0.01 & 0.00 & - & - & - & - \\
\hline & & \multicolumn{8}{|c|}{ Level of theory: mPW1PW91/6-311G* (gas phase) } \\
\hline & & $78 a$ & $78 \mathrm{~b}$ & $78 \mathrm{c}$ & 78d & - & - & - & - \\
\hline & ${ }^{1}$ H data & 1.72 & 0.73 & 16.63 & 80.92 & - & - & - & - \\
\hline \multirow[t]{3}{*}{ sDP4+ } & ${ }^{13} \mathrm{C}$ data & 4.72 & 95.21 & 0.05 & 0.02 & - & - & - & - \\
\hline & All data & 10.25 & 87.01 & 1.12 & 1.62 & - & - & - & - \\
\hline & ${ }^{1} \mathrm{H}$ data & 9.52 & 11.12 & 74.29 & 5.06 & - & - & - & - \\
\hline \multirow[t]{3}{*}{ uDP4+ } & ${ }^{13} \mathrm{C}$ data & 39.53 & 60.08 & 0.17 & 0.23 & - & - & - & - \\
\hline & All data & 35.57 & 63.14 & 1.19 & 0.11 & - & - & - & - \\
\hline & ${ }^{1} \mathrm{H}$ data & 0.98 & 0.48 & 74.01 & 24.52 & - & - & - & - \\
\hline \multirow[t]{5}{*}{ DP4+ } & ${ }^{13} \mathrm{C}$ data & 3.16 & 96.84 & 0.00 & 0.00 & - & - & - & - \\
\hline & All data & 6.22 & 93.75 & 0.02 & 0.00 & - & - & - & - \\
\hline & & \multicolumn{8}{|c|}{ Level of theory: mPW1PW91/6-311G** (gas phase) } \\
\hline & & $78 a$ & $78 b$ & 78c & 78d & - & - & - & - \\
\hline & ${ }^{1}$ H data & 1.14 & 7.87 & 31.09 & 59.91 & - & - & - & - \\
\hline \multirow[t]{3}{*}{ sDP4+ } & ${ }^{13} \mathrm{C}$ data & 3.21 & 96.76 & 0.02 & 0.01 & - & - & - & - \\
\hline & All data & 0.48 & 99.40 & 0.08 & 0.04 & - & - & - & - \\
\hline & ${ }^{1} \mathrm{H}$ data & 0.36 & 14.40 & 82.14 & 3.11 & - & - & - & - \\
\hline \multirow[t]{3}{*}{ uDP4+ } & ${ }^{13} \mathrm{C}$ data & 34.70 & 64.84 & 0.18 & 0.28 & - & - & - & - \\
\hline & All data & 1.28 & 97.06 & 1.57 & 0.09 & - & - & - & - \\
\hline & ${ }^{1} \mathrm{H}$ data & 0.01 & 3.97 & 89.49 & 6.52 & - & - & - & - \\
\hline \multirow[t]{5}{*}{ DP4+ } & ${ }^{13} \mathrm{C}$ data & 1.74 & 98.26 & 0.00 & 0.00 & - & - & - & - \\
\hline & All data & 0.01 & 99.99 & 0.00 & 0.00 & - & - & - & - \\
\hline & & \multicolumn{8}{|c|}{ Level of theory: mPW1PW91/6-311+G** (gas phase) } \\
\hline & & $78 a$ & $78 b$ & $78 c$ & 78d & - & - & - & - \\
\hline & ${ }^{1} \mathrm{H}$ data & 11.41 & 1.37 & 70.77 & 16.45 & - & - & - & - \\
\hline \multirow[t]{3}{*}{ sDP4+ } & ${ }^{13} \mathrm{C}$ data & 4.97 & 95.02 & 0.01 & 0.00 & - & - & - & - \\
\hline & All data & 30.23 & 69.49 & 0.25 & 0.02 & - & - & - & - \\
\hline & ${ }^{1} \mathrm{H}$ data & 10.46 & 12.73 & 73.94 & 2.88 & - & - & - & - \\
\hline \multirow[t]{3}{*}{ uDP4+ } & ${ }^{13} \mathrm{C}$ data & 54.89 & 44.86 & 0.08 & 0.17 & - & - & - & - \\
\hline & All data & 49.86 & 49.58 & 0.52 & 0.04 & - & - & - & - \\
\hline & ${ }^{1} \mathrm{H}$ data & 2.20 & 0.32 & 96.60 & 0.87 & - & - & - & - \\
\hline \multirow[t]{5}{*}{ DP4+ } & ${ }^{13} \mathrm{C}$ data & 6.01 & 93.99 & 0.00 & 0.00 & - & - & - & - \\
\hline & All data & 30.43 & 69.56 & 0.00 & 0.00 & - & - & - & - \\
\hline & & \multicolumn{8}{|c|}{ Level of theory: mPW1PW91/6-31G* (solution, PCM) } \\
\hline & & $78 a$ & $78 \mathrm{~b}$ & $78 \mathrm{c}$ & 78d & - & - & - & - \\
\hline & ${ }^{1} \mathrm{H}$ data & 27.55 & 3.56 & 62.43 & 6.47 & - & - & - & - \\
\hline \multirow[t]{3}{*}{ sDP4+ } & ${ }^{13} \mathrm{C}$ data & 75.30 & 24.62 & 0.06 & 0.03 & - & - & - & - \\
\hline & All data & 95.78 & 4.05 & 0.16 & 0.01 & - & - & - & - \\
\hline & ${ }^{1}$ H data & 46.12 & 9.04 & 29.29 & 15.55 & - & - & - & - \\
\hline uDP4+ & ${ }^{13} \mathrm{C}$ data & 8.07 & 91.92 & 0.00 & 0.00 & - & - & - & - \\
\hline & All data & 30.93 & 69.07 & 0.00 & 0.00 & - & - & - & - \\
\hline & ${ }^{1} \mathrm{H}$ data & 39.31 & 1.00 & 56.58 & 3.11 & - & - & - & - \\
\hline DP4+ & ${ }^{13} \mathrm{C}$ data & 21.17 & 78.83 & 0.00 & 0.00 & - & - & - & - \\
\hline & All data & 91.37 & 8.63 & 0.00 & 0.00 & - & - & - & - \\
\hline & & & & el of the & mPW1P & 31 & on, & & \\
\hline & & $78 a$ & $78 b$ & $78 c$ & $78 d$ & - & - & - & - \\
\hline & ${ }^{1} \mathrm{H}$ data & 5.53 & 8.77 & 84.08 & 1.62 & - & - & - & - \\
\hline sDP4+ & ${ }^{13} \mathrm{C}$ data & 58.32 & 41.47 & 0.14 & 0.07 & - & - & - & - \\
\hline & All data & 46.20 & 52.09 & 1.70 & 0.02 & - & - & - & - \\
\hline uDP4+ & ${ }^{1} \mathrm{H}$ data & 7.01 & 20.36 & 22.45 & 50.17 & - & - & - & - \\
\hline
\end{tabular}




\begin{tabular}{|c|c|c|c|c|c|c|c|c|c|}
\hline & ${ }^{13} \mathrm{C}$ data & 4.82 & 95.18 & 0.00 & 0.00 & - & - & - & - \\
\hline & All data & 1.71 & 98.28 & 0.00 & 0.00 & - & - & - & - \\
\hline & ${ }^{1} \mathrm{H}$ data & 1.77 & 8.17 & 86.34 & 3.72 & - & - & - & - \\
\hline \multirow[t]{5}{*}{ DP4+ } & ${ }^{13} \mathrm{C}$ data & 6.65 & 93.35 & 0.00 & 0.00 & - & - & - & - \\
\hline & All data & 1.52 & 98.48 & 0.00 & 0.00 & - & - & - & - \\
\hline & & \multicolumn{8}{|c|}{ Level of theory: mPW1PW91/6-31+G** (solution, PCM) } \\
\hline & & 78a & 78b & 78c & 78d & - & - & - & - \\
\hline & ${ }^{1} \mathrm{H}$ data & 2.69 & 0.18 & 96.87 & 0.26 & - & - & - & - \\
\hline \multirow[t]{2}{*}{ sDP4+ } & ${ }^{13} \mathrm{C}$ data & 39.30 & 60.17 & 0.22 & 0.31 & - & - & - & - \\
\hline & All data & 76.27 & 7.99 & 15.68 & 0.06 & - & - & - & - \\
\hline \multirow{3}{*}{ uDP4+ } & ${ }^{1} \mathrm{H}$ data & 11.19 & 20.86 & 6.31 & 61.64 & - & - & - & - \\
\hline & ${ }^{13} \mathrm{C}$ data & 0.93 & 99.07 & 0.00 & 0.00 & - & - & - & - \\
\hline & All data & 0.50 & 99.49 & 0.00 & 0.01 & - & - & - & - \\
\hline & ${ }^{1} \mathrm{H}$ data & 4.55 & 0.58 & 92.48 & 2.39 & - & - & - & - \\
\hline \multirow[t]{5}{*}{ DP4+ } & ${ }^{13} \mathrm{C}$ data & 0.61 & 99.39 & 0.00 & 0.00 & - & - & - & - \\
\hline & All data & 4.60 & 95.40 & 0.00 & 0.00 & - & - & - & - \\
\hline & & \multicolumn{8}{|c|}{ Level of theory: mPW1PW91/6-311G* (solution, PCM) } \\
\hline & & $78 \mathrm{a}$ & 78b & $78 c$ & $78 d$ & - & - & - & - \\
\hline & ${ }^{1} \mathrm{H}$ data & 22.38 & 5.21 & 69.86 & 2.55 & - & - & - & - \\
\hline \multirow[t]{2}{*}{ sDP4+ } & ${ }^{13} \mathrm{C}$ data & 8.17 & 91.80 & 0.02 & 0.01 & - & - & - & - \\
\hline & All data & 27.62 & 72.20 & 0.18 & 0.00 & - & - & - & - \\
\hline \multirow{3}{*}{ uDP4+ } & ${ }^{1} \mathrm{H}$ data & 35.72 & 11.61 & 10.46 & 42.21 & - & - & - & - \\
\hline & ${ }^{13} \mathrm{C}$ data & 69.18 & 30.63 & 0.05 & 0.13 & - & - & - & - \\
\hline & All data & 87.23 & 12.56 & 0.02 & 0.19 & - & - & - & - \\
\hline \multirow{5}{*}{ DP4+ } & ${ }^{1} \mathrm{H}$ data & 47.07 & 3.56 & 43.03 & 6.34 & - & - & - & - \\
\hline & ${ }^{13} \mathrm{C}$ data & 16.74 & 83.26 & 0.00 & 0.00 & - & - & - & - \\
\hline & All data & 72.66 & 27.34 & 0.00 & 0.00 & - & - & - & - \\
\hline & & \multicolumn{8}{|c|}{ Level of theory: mPW1PW91/6-311G** (solution, PCM) } \\
\hline & & 78a & $78 \mathrm{~b}$ & $78 c$ & 78d & - & - & - & - \\
\hline \multirow{3}{*}{ sDP4+ } & ${ }^{1} \mathrm{H}$ data & 2.45 & 6.78 & 90.31 & 0.46 & - & - & - & - \\
\hline & ${ }^{13} \mathrm{C}$ data & 7.97 & 92.02 & 0.01 & 0.00 & - & - & - & - \\
\hline & All data & 3.03 & 96.88 & 0.08 & 0.00 & - & - & - & - \\
\hline & ${ }^{1} \mathrm{H}$ data & 5.93 & 38.25 & 11.20 & 44.63 & - & - & - & - \\
\hline \multirow[t]{2}{*}{ uDP4+ } & ${ }^{13} \mathrm{C}$ data & 76.91 & 22.93 & 0.04 & 0.12 & - & - & - & - \\
\hline & All data & 34.05 & 65.53 & 0.04 & 0.38 & - & - & - & - \\
\hline \multirow{5}{*}{ DP4+ } & ${ }^{1} \mathrm{H}$ data & 1.11 & 19.85 & 77.45 & 1.59 & - & - & - & - \\
\hline & ${ }^{13} \mathrm{C}$ data & 22.50 & 77.50 & 0.00 & 0.00 & - & - & - & - \\
\hline & All data & 1.60 & 98.40 & 0.00 & 0.00 & - & - & - & - \\
\hline & & \multicolumn{8}{|c|}{ Level of theory: mPW1PW91/6-311+G** (solution, PCM) } \\
\hline & & $78 a$ & $78 \mathrm{~b}$ & $78 c$ & 78d & - & - & - & - \\
\hline \multirow{3}{*}{ sDP4+ } & ${ }^{1} \mathrm{H}$ data & 1.65 & 0.16 & 97.94 & 0.25 & - & - & - & - \\
\hline & ${ }^{13} \mathrm{C}$ data & 2.96 & 97.03 & 0.00 & 0.00 & - & - & - & - \\
\hline & All data & 23.92 & 74.27 & 1.81 & 0.00 & - & - & - & - \\
\hline \multirow{3}{*}{ uDP4+ } & ${ }^{1} \mathrm{H}$ data & 16.85 & 25.86 & 7.56 & 49.72 & - & - & - & - \\
\hline & ${ }^{13} \mathrm{C}$ data & 63.60 & 36.12 & 0.06 & 0.21 & - & - & - & - \\
\hline & All data & 53.14 & 46.32 & 0.02 & 0.52 & - & - & - & - \\
\hline \multirow{3}{*}{ DP4+ } & ${ }^{1} \mathrm{H}$ data & 3.55 & 0.52 & 94.33 & 1.60 & - & - & - & - \\
\hline & ${ }^{13} \mathrm{C}$ data & 5.09 & 94.91 & 0.00 & 0.00 & - & - & - & - \\
\hline & All data & 26.98 & 73.02 & 0.00 & 0.00 & - & - & - & - \\
\hline
\end{tabular}


$s \mathrm{DP4}+, u \mathrm{DP} 4+$ and DP4+ probabilities (\%) for compound 78c<smiles>CCC[C@H]1O[C@@H](c2ccccc2)C[C@H]1O</smiles>

$78 a$<smiles>CCC[C@H]1O[C@@H](c2ccccc2)C[C@H]1O</smiles>

$78 b$<smiles>CCC[C@H]1O[C@@H](c2ccccc2)C[C@H]1O</smiles>

correct isomer<smiles>CCC[C@H]1O[C@@H](c2ccccc2)C[C@H]1O</smiles>

78d

\begin{tabular}{|c|c|c|c|c|c|c|c|c|c|}
\hline & & \multicolumn{8}{|c|}{ Level of theory: B3LYP/6-31G* (gas phase) } \\
\hline & & 78a & $78 \mathrm{~b}$ & $78 \mathrm{c}$ & 78d & - & - & - & - \\
\hline & ${ }^{1} \mathrm{H}$ data & 1.75 & 0.02 & 61.38 & 36.84 & - & - & - & - \\
\hline \multirow[t]{2}{*}{ sDP4+ } & ${ }^{13} \mathrm{C}$ data & 0.00 & 0.00 & 85.13 & 14.87 & - & - & - & - \\
\hline & All data & 0.00 & 0.00 & 90.51 & 9.49 & - & - & - & - \\
\hline & ${ }^{1} \mathrm{H}$ data & 4.63 & 0.12 & 81.56 & 13.68 & - & - & - & - \\
\hline \multirow[t]{2}{*}{ uDP4+ } & ${ }^{13} \mathrm{C}$ data & 0.09 & 0.04 & 55.86 & 44.00 & - & - & - & - \\
\hline & All data & 0.01 & 0.00 & 88.32 & 11.67 & - & - & - & - \\
\hline \multirow{3}{*}{ DP4+ } & ${ }^{1} \mathrm{H}$ data & 0.15 & 0.00 & 90.72 & 9.14 & - & - & - & - \\
\hline & ${ }^{13} \mathrm{C}$ data & 0.00 & 0.00 & 87.91 & 12.09 & - & - & - & - \\
\hline & All data & 0.00 & 0.00 & 98.63 & 1.37 & - & - & - & - \\
\hline & & \multicolumn{8}{|c|}{ Level of theory: B3LYP/6-31G** (gas phase) } \\
\hline & & 78a & $78 \mathrm{~b}$ & $78 \mathrm{c}$ & 78d & - & - & - & - \\
\hline \multirow{3}{*}{ sDP4+ } & ${ }^{1} \mathrm{H}$ data & 1.54 & 0.08 & 90.39 & 7.99 & - & - & - & - \\
\hline & ${ }^{13} \mathrm{C}$ data & 0.00 & 0.00 & 88.41 & 11.58 & - & - & - & - \\
\hline & All data & 0.00 & 0.00 & 98.85 & 1.15 & - & - & - & - \\
\hline \multirow{3}{*}{ uDP4+ } & ${ }^{1} \mathrm{H}$ data & 1.09 & 0.12 & 84.97 & 13.83 & - & - & - & - \\
\hline & ${ }^{13} \mathrm{C}$ data & 0.04 & 0.02 & 62.66 & 37.28 & - & - & - & - \\
\hline & All data & 0.00 & 0.00 & 91.17 & 8.83 & - & - & - & - \\
\hline \multirow{6}{*}{ DP4+ } & ${ }^{1} \mathrm{H}$ data & 0.02 & 0.00 & 98.56 & 1.42 & - & - & - & - \\
\hline & ${ }^{13} \mathrm{C}$ data & 0.00 & 0.00 & 92.77 & 7.23 & - & - & - & - \\
\hline & All data & 0.00 & 0.00 & 99.89 & 0.11 & - & - & - & - \\
\hline & & \multicolumn{8}{|c|}{ Level of theory: B3LYP/6-31+G** (gas phase) } \\
\hline & & $78 a$ & $78 \mathrm{~b}$ & $78 \mathrm{c}$ & 78d & - & - & - & - \\
\hline & ${ }^{1} \mathrm{H}$ data & 0.21 & 0.01 & 99.35 & 0.43 & - & - & - & - \\
\hline \multirow[t]{2}{*}{ sDP4+ } & ${ }^{13} \mathrm{C}$ data & 0.02 & 0.00 & 56.78 & 43.19 & - & - & - & - \\
\hline & All data & 0.00 & 0.00 & 99.67 & 0.33 & - & - & - & - \\
\hline \multirow{3}{*}{ uDP4+ } & ${ }^{1} \mathrm{H}$ data & 0.88 & 0.08 & 89.63 & 9.41 & - & - & - & - \\
\hline & ${ }^{13} \mathrm{C}$ data & 0.03 & 0.01 & 29.23 & 70.74 & - & - & - & - \\
\hline & All data & 0.00 & 0.00 & 79.73 & 20.26 & - & - & - & - \\
\hline & ${ }^{1} \mathrm{H}$ data & 0.00 & 0.00 & 99.95 & 0.05 & - & - & - & - \\
\hline \multirow[t]{5}{*}{ DP4+ } & ${ }^{13} \mathrm{C}$ data & 0.00 & 0.00 & 35.20 & 64.80 & - & - & - & - \\
\hline & All data & 0.00 & 0.00 & 99.92 & 0.08 & - & - & - & - \\
\hline & & \multicolumn{8}{|c|}{ Level of theory: B3LYP/6-311G* (gas phase) } \\
\hline & & 78a & $78 \mathrm{~b}$ & $78 \mathrm{c}$ & 78d & - & - & - & - \\
\hline & ${ }^{1} \mathrm{H}$ data & 1.37 & 0.07 & 91.68 & 6.88 & - & - & - & - \\
\hline \multirow[t]{2}{*}{ sDP4+ } & ${ }^{13} \mathrm{C}$ data & 0.01 & 0.02 & 74.18 & 25.80 & - & - & - & - \\
\hline & All data & 0.00 & 0.00 & 97.45 & 2.55 & - & - & - & - \\
\hline \multirow{3}{*}{ uDP4+ } & ${ }^{1} \mathrm{H}$ data & 4.20 & 0.30 & 84.86 & 10.65 & - & - & - & - \\
\hline & ${ }^{13} \mathrm{C}$ data & 0.00 & 0.00 & 60.58 & 39.41 & - & - & - & - \\
\hline & All data & 0.00 & 0.00 & 92.45 & 7.55 & - & - & - & - \\
\hline \multirow{5}{*}{ DP4+ } & ${ }^{1} \mathrm{H}$ data & 0.07 & 0.00 & 98.99 & 0.93 & - & - & - & - \\
\hline & ${ }^{13} \mathrm{C}$ data & 0.00 & 0.00 & 81.55 & 18.45 & - & - & - & - \\
\hline & All data & 0.00 & 0.00 & 99.79 & 0.21 & - & - & - & - \\
\hline & & \multicolumn{8}{|c|}{ Level of theory: B3LYP/6-311G** (gas phase) } \\
\hline & & 78a & $78 \mathrm{~b}$ & $78 \mathrm{c}$ & 78d & - & - & - & - \\
\hline$s$ DP4+ & ${ }^{1} \mathrm{H}$ data & 0.15 & 0.13 & 98.17 & 1.55 & - & - & - & - \\
\hline
\end{tabular}




\begin{tabular}{|c|c|c|c|c|c|c|c|c|c|}
\hline & ${ }^{13} \mathrm{C}$ data & 0.00 & 0.01 & 78.56 & 21.43 & - & - & - & - \\
\hline & All data & 0.00 & 0.00 & 99.57 & 0.43 & - & - & - & - \\
\hline & ${ }^{1} \mathrm{H}$ data & 0.17 & 0.11 & 95.21 & 4.50 & - & - & - & - \\
\hline \multirow[t]{3}{*}{ uDP4+ } & ${ }^{13} \mathrm{C}$ data & 0.00 & 0.00 & 67.50 & 32.49 & - & - & - & - \\
\hline & All data & 0.00 & 0.00 & 97.77 & 2.23 & - & - & - & - \\
\hline & ${ }^{1} \mathrm{H}$ data & 0.00 & 0.00 & 99.92 & 0.07 & - & - & - & - \\
\hline \multirow[t]{5}{*}{ DP4+ } & ${ }^{13} \mathrm{C}$ data & 0.00 & 0.00 & 88.39 & 11.61 & - & - & - & - \\
\hline & All data & 0.00 & 0.00 & 99.99 & 0.01 & - & - & - & - \\
\hline & & \multicolumn{8}{|c|}{ Level of theory: B3LYP/6-311+G** (gas phase) } \\
\hline & & $78 a$ & $78 b$ & $78 \mathrm{c}$ & 78d & - & - & - & - \\
\hline & ${ }^{1} \mathrm{H}$ data & 0.13 & 0.01 & 99.70 & 0.16 & - & - & - & - \\
\hline \multirow[t]{3}{*}{ sDP4+ } & ${ }^{13} \mathrm{C}$ data & 0.02 & 0.02 & 67.69 & 32.27 & - & - & - & - \\
\hline & All data & 0.00 & 0.00 & 99.92 & 0.08 & - & - & - & - \\
\hline & ${ }^{1} \mathrm{H}$ data & 0.81 & 0.06 & 97.21 & 1.92 & - & - & - & - \\
\hline \multirow[t]{3}{*}{ uDP4+ } & ${ }^{13} \mathrm{C}$ data & 0.01 & 0.00 & 56.14 & 43.85 & - & - & - & - \\
\hline & All data & 0.00 & 0.00 & 98.48 & 1.52 & - & - & - & - \\
\hline & ${ }^{1} \mathrm{H}$ data & 0.00 & 0.00 & 100.00 & 0.00 & - & - & - & - \\
\hline \multirow[t]{5}{*}{ DP4+ } & ${ }^{13} \mathrm{C}$ data & 0.00 & 0.00 & 72.87 & 27.13 & - & - & - & - \\
\hline & All data & 0.00 & 0.00 & 100.00 & 0.00 & - & - & - & - \\
\hline & & \multicolumn{8}{|c|}{ Level of theory: B3LYP/6-31G* (solution, PCM) } \\
\hline & & $78 a$ & $78 b$ & $78 \mathrm{c}$ & 78d & - & - & - & - \\
\hline & ${ }^{1} \mathrm{H}$ data & 3.46 & 0.14 & 93.19 & 3.21 & - & - & - & - \\
\hline \multirow[t]{2}{*}{ sDP4+ } & ${ }^{13} \mathrm{C}$ data & 0.01 & 0.00 & 63.46 & 36.53 & - & - & - & - \\
\hline & All data & 0.00 & 0.00 & 98.05 & 1.95 & - & - & - & - \\
\hline \multirow{3}{*}{ uDP4+ } & ${ }^{1} \mathrm{H}$ data & 2.00 & 0.07 & 95.28 & 2.65 & - & - & - & - \\
\hline & ${ }^{13} \mathrm{C}$ data & 0.23 & 0.01 & 25.02 & 74.74 & - & - & - & - \\
\hline & All data & 0.02 & 0.00 & 92.30 & 7.68 & - & - & - & - \\
\hline \multirow{5}{*}{ DP4+ } & ${ }^{1} \mathrm{H}$ data & 0.08 & 0.00 & 99.83 & 0.10 & - & - & - & - \\
\hline & ${ }^{13} \mathrm{C}$ data & 0.00 & 0.00 & 36.77 & 63.23 & - & - & - & - \\
\hline & All data & 0.00 & 0.00 & 99.84 & 0.16 & - & - & - & - \\
\hline & & \multicolumn{8}{|c|}{ Level of theory: B3LYP/6-31G** (solution, PCM) } \\
\hline & & $78 a$ & $78 b$ & $78 \mathrm{c}$ & 78d & - & - & - & - \\
\hline \multirow{3}{*}{ sDP4+ } & ${ }^{1}$ H data & 0.28 & 0.09 & 99.48 & 0.15 & - & - & - & - \\
\hline & ${ }^{13} \mathrm{C}$ data & 0.01 & 0.00 & 68.09 & 31.90 & - & - & - & - \\
\hline & All data & 0.00 & 0.00 & 99.93 & 0.07 & - & - & - & - \\
\hline \multirow{3}{*}{ uDP4+ } & ${ }^{1} \mathrm{H}$ data & 1.16 & 0.17 & 95.80 & 2.88 & - & - & - & - \\
\hline & ${ }^{13} \mathrm{C}$ data & 0.13 & 0.01 & 37.06 & 62.79 & - & - & - & - \\
\hline & All data & 0.00 & 0.00 & 95.15 & 4.85 & - & - & - & - \\
\hline \multirow{5}{*}{ DP4+ } & ${ }^{1} \mathrm{H}$ data & 0.00 & 0.00 & 99.99 & 0.00 & - & - & - & - \\
\hline & ${ }^{13} \mathrm{C}$ data & 0.00 & 0.00 & 55.75 & 44.25 & - & - & - & - \\
\hline & All data & 0.00 & 0.00 & 100.00 & 0.00 & - & - & - & - \\
\hline & & \multicolumn{8}{|c|}{ Level of theory: B3LYP/6-31+G** (solution, PCM) } \\
\hline & & $78 a$ & $78 b$ & $78 \mathrm{c}$ & 78d & - & - & - & - \\
\hline \multirow{3}{*}{ sDP4+ } & ${ }^{1} \mathrm{H}$ data & 0.03 & 0.00 & 99.97 & 0.01 & - & - & - & - \\
\hline & ${ }^{13} \mathrm{C}$ data & 0.08 & 0.01 & 34.13 & 65.79 & - & - & - & - \\
\hline & All data & 0.00 & 0.00 & 99.98 & 0.02 & - & - & - & - \\
\hline & ${ }^{1} \mathrm{H}$ data & 0.80 & 0.20 & 97.03 & 1.97 & - & - & - & - \\
\hline uDP4+ & ${ }^{13} \mathrm{C}$ data & 0.04 & 0.01 & 19.54 & 80.42 & - & - & - & - \\
\hline & All data & 0.00 & 0.00 & 92.30 & 7.70 & - & - & - & - \\
\hline & ${ }_{12}^{1} \mathrm{H}$ data & 0.00 & 0.00 & 100.00 & 0.00 & - & - & - & - \\
\hline DP4+ & ${ }^{13} \mathrm{C}$ data & 0.00 & 0.00 & 11.19 & 88.81 & - & - & - & - \\
\hline & All data & 0.00 & 0.00 & 100.00 & 0.00 & - & - & - & - \\
\hline & & & & Level of & y: B3LY & $\mathbf{G}^{*}$ & PC & & \\
\hline & & $78 a$ & $78 b$ & $78 c$ & 78d & - & - & - & - \\
\hline & ${ }^{1} \mathrm{H}$ data & 0.25 & 0.15 & 99.59 & 0.02 & - & - & - & - \\
\hline SUP4+ & ${ }^{13} \mathrm{C}$ data & 0.06 & 0.02 & 40.60 & 59.32 & - & - & - & - \\
\hline
\end{tabular}




\begin{tabular}{|c|c|c|c|c|c|c|c|c|c|}
\hline & All data & 0.00 & 0.00 & 99.97 & 0.03 & - & - & - & - \\
\hline & ${ }^{1} \mathrm{H}$ data & 2.84 & 0.69 & 95.87 & 0.60 & - & - & - & - \\
\hline \multirow[t]{3}{*}{ uDP4+ } & ${ }^{13} \mathrm{C}$ data & 0.02 & 0.00 & 36.77 & 63.21 & - & - & - & - \\
\hline & All data & 0.00 & 0.00 & 98.94 & 1.06 & - & - & - & - \\
\hline & ${ }^{1} \mathrm{H}$ data & 0.01 & 0.00 & 99.99 & 0.00 & - & - & - & - \\
\hline \multirow[t]{5}{*}{ DP4+ } & ${ }^{13} \mathrm{C}$ data & 0.00 & 0.00 & 28.47 & 71.53 & - & - & - & - \\
\hline & All data & 0.00 & 0.00 & 100.00 & 0.00 & - & - & - & - \\
\hline & & \multicolumn{8}{|c|}{ Level of theory: B3LYP/6-311G** (solution, PCM) } \\
\hline & & $78 a$ & $78 b$ & $78 \mathrm{c}$ & 78d & - & - & - & - \\
\hline & ${ }^{1} \mathrm{H}$ data & 0.03 & 0.14 & 99.82 & 0.01 & - & - & - & - \\
\hline \multirow[t]{3}{*}{ sDP4+ } & ${ }^{13} \mathrm{C}$ data & 0.02 & 0.01 & 37.87 & 62.11 & - & - & - & - \\
\hline & All data & 0.00 & 0.00 & 99.98 & 0.02 & - & - & - & - \\
\hline & ${ }^{1} \mathrm{H}$ data & 0.45 & 1.28 & 97.29 & 0.98 & - & - & - & - \\
\hline \multirow[t]{3}{*}{ uDP4+ } & ${ }^{13} \mathrm{C}$ data & 0.01 & 0.00 & 38.07 & 61.93 & - & - & - & - \\
\hline & All data & 0.00 & 0.00 & 98.39 & 1.61 & - & - & - & - \\
\hline & ${ }^{1} \mathrm{H}$ data & 0.00 & 0.00 & 100.00 & 0.00 & - & - & - & - \\
\hline \multirow[t]{5}{*}{ DP4+ } & ${ }^{13} \mathrm{C}$ data & 0.00 & 0.00 & 27.26 & 72.74 & - & - & - & - \\
\hline & All data & 0.00 & 0.00 & 100.00 & 0.00 & - & - & - & - \\
\hline & & \multicolumn{8}{|c|}{ Level of theory: B3LYP/6-311+G** (solution, PCM) } \\
\hline & & $78 a$ & $78 b$ & $78 \mathrm{c}$ & 78d & - & - & - & - \\
\hline & ${ }^{1}$ H data & 0.02 & 0.00 & 99.97 & 0.01 & - & - & - & - \\
\hline \multirow[t]{3}{*}{ sDP4+ } & ${ }^{13} \mathrm{C}$ data & 0.11 & 0.03 & 42.04 & 57.83 & - & - & - & - \\
\hline & All data & 0.00 & 0.00 & 99.98 & 0.02 & - & - & - & - \\
\hline & ${ }^{1} \mathrm{H}$ data & 0.20 & 0.05 & 99.32 & 0.44 & - & - & - & - \\
\hline \multirow[t]{3}{*}{ uDP4+ } & ${ }^{13} \mathrm{C}$ data & 0.03 & 0.00 & 38.65 & 61.33 & - & - & - & - \\
\hline & All data & 0.00 & 0.00 & 99.30 & 0.70 & - & - & - & - \\
\hline & ${ }^{1} \mathrm{H}$ data & 0.00 & 0.00 & 100.00 & 0.00 & - & - & - & - \\
\hline \multirow[t]{5}{*}{ DP4+ } & ${ }^{13} \mathrm{C}$ data & 0.00 & 0.00 & 31.42 & 68.58 & - & - & - & - \\
\hline & All data & 0.00 & 0.00 & 100.00 & 0.00 & - & - & - & - \\
\hline & & \multicolumn{8}{|c|}{ Level of theory: mPW1PW91/6-31G* (gas phase) } \\
\hline & & $78 a$ & $78 b$ & $78 \mathrm{c}$ & 78d & - & - & - & - \\
\hline & ${ }^{1} \mathrm{H}$ data & 4.88 & 0.02 & 81.76 & 13.35 & - & - & - & - \\
\hline \multirow[t]{3}{*}{ sDP4+ } & ${ }^{13} \mathrm{C}$ data & 0.00 & 0.00 & 94.20 & 5.80 & - & - & - & - \\
\hline & All data & 0.00 & 0.00 & 99.00 & 1.00 & - & - & - & - \\
\hline & ${ }^{1} \mathrm{H}$ data & 3.62 & 0.06 & 85.80 & 10.53 & - & - & - & - \\
\hline \multirow[t]{3}{*}{ uDP4+ } & ${ }^{13} \mathrm{C}$ data & 0.03 & 0.03 & 55.84 & 44.10 & - & - & - & - \\
\hline & All data & 0.00 & 0.00 & 91.16 & 8.83 & - & - & - & - \\
\hline & ${ }^{1} \mathrm{H}$ data & 0.25 & 0.00 & 97.79 & 1.96 & - & - & - & - \\
\hline \multirow[t]{5}{*}{ DP4+ } & ${ }^{13} \mathrm{C}$ data & 0.00 & 0.00 & 95.36 & 4.64 & - & - & - & - \\
\hline & All data & 0.00 & 0.00 & 99.90 & 0.10 & - & - & - & - \\
\hline & & \multicolumn{8}{|c|}{ Level of theory: mPW1PW91/6-31G** (gas phase) } \\
\hline & & $78 a$ & $78 b$ & $78 \mathrm{c}$ & 78d & - & - & - & - \\
\hline & ${ }^{1} \mathrm{H}$ data & 4.16 & 0.09 & 90.07 & 5.68 & - & - & - & - \\
\hline \multirow[t]{2}{*}{ sDP4+ } & ${ }^{13} \mathrm{C}$ data & 0.00 & 0.00 & 94.94 & 5.06 & - & - & - & - \\
\hline & All data & 0.00 & 0.00 & 99.66 & 0.34 & - & - & - & - \\
\hline & ${ }^{1} \mathrm{H}$ data & 0.44 & 0.08 & 81.13 & 18.35 & - & - & - & - \\
\hline uDP4+ & ${ }^{13} \mathrm{C}$ data & 0.01 & 0.01 & 51.50 & 48.49 & - & - & - & - \\
\hline & All data & 0.00 & 0.00 & 82.45 & 17.55 & - & - & - & - \\
\hline & ${ }^{1} \mathrm{H}$ data & 0.02 & 0.00 & 98.57 & 1.41 & - & - & - & - \\
\hline DP4+ & ${ }^{13} \mathrm{C}$ data & 0.00 & 0.00 & 95.22 & 4.78 & - & - & - & - \\
\hline & All data & 0.00 & 0.00 & 99.93 & 0.07 & - & - & - & - \\
\hline & & & & evel of th & mPW1 & -3 & ph & & \\
\hline & & $78 a$ & $78 b$ & $78 c$ & $78 d$ & - & - & - & - \\
\hline & ${ }^{1} \mathrm{H}$ data & 0.02 & 0.06 & 2.46 & 97.46 & - & - & - & - \\
\hline sDP4+ & ${ }^{13} \mathrm{C}$ data & 0.00 & 0.00 & 75.61 & 24.39 & - & - & - & - \\
\hline & All data & 0.00 & 0.00 & 7.25 & 92.75 & - & - & - & - \\
\hline
\end{tabular}




\begin{tabular}{|c|c|c|c|c|c|c|c|c|c|}
\hline & ${ }^{1} \mathrm{H}$ data & 0.45 & 0.03 & 95.19 & 4.33 & - & - & - & - \\
\hline \multirow[t]{3}{*}{ uDP4+ } & ${ }^{13} \mathrm{C}$ data & 0.02 & 0.00 & 46.69 & 53.29 & - & - & - & - \\
\hline & All data & 0.00 & 0.00 & 95.06 & 4.94 & - & - & - & - \\
\hline & ${ }^{1} \mathrm{H}$ data & 0.00 & 0.00 & 35.65 & 64.35 & - & - & - & - \\
\hline \multirow[t]{5}{*}{ DP4+ } & ${ }^{13} \mathrm{C}$ data & 0.00 & 0.00 & 73.09 & 26.91 & - & - & - & - \\
\hline & All data & 0.00 & 0.00 & 60.08 & 39.92 & - & - & - & - \\
\hline & & \multicolumn{8}{|c|}{ Level of theory: mPW1PW91/6-311G* (gas phase) } \\
\hline & & $78 a$ & $78 b$ & $78 \mathrm{c}$ & 78d & - & - & - & - \\
\hline & ${ }^{1} \mathrm{H}$ data & 2.36 & 0.06 & 85.90 & 11.69 & - & - & - & - \\
\hline \multirow[t]{3}{*}{ sDP4+ } & ${ }^{13} \mathrm{C}$ data & 0.00 & 0.07 & 78.64 & 21.29 & - & - & - & - \\
\hline & All data & 0.00 & 0.00 & 96.45 & 3.55 & - & - & - & - \\
\hline & ${ }^{1} \mathrm{H}$ data & 1.81 & 0.20 & 88.78 & 9.21 & - & - & - & - \\
\hline \multirow[t]{3}{*}{ uDP4+ } & ${ }^{13} \mathrm{C}$ data & 0.00 & 0.00 & 67.64 & 32.36 & - & - & - & - \\
\hline & All data & 0.00 & 0.00 & 95.27 & 4.73 & - & - & - & - \\
\hline & ${ }^{1} \mathrm{H}$ data & 0.06 & 0.00 & 98.55 & 1.39 & - & - & - & - \\
\hline \multirow[t]{5}{*}{ DP4+ } & ${ }^{13} \mathrm{C}$ data & 0.00 & 0.00 & 88.53 & 11.47 & - & - & - & - \\
\hline & All data & 0.00 & 0.00 & 99.82 & 0.18 & - & - & - & - \\
\hline & & \multicolumn{8}{|c|}{ Level of theory: mPW1PW91/6-311G** (gas phase) } \\
\hline & & $78 a$ & $78 b$ & 78c & 78d & - & - & - & - \\
\hline & ${ }^{1} \mathrm{H}$ data & 1.95 & 0.11 & 94.36 & 3.58 & - & - & - & - \\
\hline \multirow[t]{3}{*}{ sDP4+ } & ${ }^{13} \mathrm{C}$ data & 0.00 & 0.03 & 78.58 & 21.39 & - & - & - & - \\
\hline & All data & 0.00 & 0.00 & 98.98 & 1.02 & - & - & - & - \\
\hline & ${ }^{1} \mathrm{H}$ data & 0.16 & 0.13 & 96.17 & 3.54 & - & - & - & - \\
\hline \multirow[t]{3}{*}{ uDP4+ } & ${ }^{13} \mathrm{C}$ data & 0.00 & 0.00 & 72.70 & 27.29 & - & - & - & - \\
\hline & All data & 0.00 & 0.00 & 98.64 & 1.36 & - & - & - & - \\
\hline & ${ }^{1} \mathrm{H}$ data & 0.00 & 0.00 & 99.86 & 0.14 & - & - & - & - \\
\hline \multirow[t]{5}{*}{ DP4+ } & ${ }^{13} \mathrm{C}$ data & 0.00 & 0.00 & 90.73 & 9.27 & - & - & - & - \\
\hline & All data & 0.00 & 0.00 & 99.99 & 0.01 & - & - & - & - \\
\hline & & \multicolumn{8}{|c|}{ Level of theory: mPW1PW91/6-311+G** (gas phase) } \\
\hline & & $78 a$ & $78 b$ & 78c & 78d & - & - & - & - \\
\hline & ${ }^{1} \mathrm{H}$ data & 0.58 & 0.00 & 98.42 & 0.99 & - & - & - & - \\
\hline \multirow[t]{3}{*}{ sDP4+ } & ${ }^{13} \mathrm{C}$ data & 0.00 & 0.08 & 89.90 & 10.02 & - & - & - & - \\
\hline & All data & 0.00 & 0.00 & 99.89 & 0.11 & - & - & - & - \\
\hline & ${ }^{1}$ H data & 0.43 & 0.02 & 98.51 & 1.04 & - & - & - & - \\
\hline \multirow[t]{3}{*}{ uDP4+ } & ${ }^{13} \mathrm{C}$ data & 0.00 & 0.00 & 76.93 & 23.07 & - & - & - & - \\
\hline & All data & 0.00 & 0.00 & 99.68 & 0.32 & - & - & - & - \\
\hline & ${ }^{1} \mathrm{H}$ data & 0.00 & 0.00 & 99.99 & 0.01 & - & - & - & - \\
\hline \multirow[t]{5}{*}{ DP4+ } & ${ }^{13} \mathrm{C}$ data & 0.00 & 0.00 & 96.77 & 3.23 & - & - & - & - \\
\hline & All data & 0.00 & 0.00 & 100.00 & 0.00 & - & - & - & - \\
\hline & & \multicolumn{8}{|c|}{ Level of theory: mPW1PW91/6-31G* (solution, PCM) } \\
\hline & & $78 a$ & $78 b$ & $78 \mathrm{c}$ & 78d & - & - & - & - \\
\hline & ${ }^{1} \mathrm{H}$ data & 0.87 & 0.01 & 98.94 & 0.17 & - & - & - & - \\
\hline \multirow[t]{3}{*}{ sDP4+ } & ${ }^{13} \mathrm{C}$ data & 0.00 & 0.00 & 77.49 & 22.50 & - & - & - & - \\
\hline & All data & 0.00 & 0.00 & 99.95 & 0.05 & - & - & - & - \\
\hline & ${ }^{1} \mathrm{H}$ data & 1.74 & 0.02 & 97.09 & 1.15 & - & - & - & - \\
\hline uDP4+ & ${ }^{13} \mathrm{C}$ data & 0.12 & 0.01 & 33.08 & 66.78 & - & - & - & - \\
\hline & All data & 0.01 & 0.00 & 97.66 & 2.34 & - & - & - & - \\
\hline & ${ }^{1} \mathrm{H}$ data & 0.02 & 0.00 & 99.98 & 0.00 & - & - & - & - \\
\hline DP4+ & ${ }^{13} \mathrm{C}$ data & 0.00 & 0.00 & 63.04 & 36.96 & - & - & - & - \\
\hline & All data & 0.00 & 0.00 & 100.00 & 0.00 & - & - & - & - \\
\hline & & & & el of the & nPW1P & $31 C$ & on, & & \\
\hline & & $78 a$ & $78 b$ & $78 c$ & $78 d$ & - & - & - & - \\
\hline & ${ }^{1} \mathrm{H}$ data & 0.15 & 0.01 & 99.81 & 0.03 & - & - & - & - \\
\hline sDP4+ & ${ }^{13} \mathrm{C}$ data & 0.00 & 0.00 & 80.40 & 19.59 & - & - & - & - \\
\hline & All data & 0.00 & 0.00 & 99.99 & 0.01 & - & - & - & - \\
\hline uDP4+ & ${ }^{1} \mathrm{H}$ data & 0.21 & 0.03 & 98.04 & 1.72 & - & - & - & - \\
\hline
\end{tabular}




\begin{tabular}{|c|c|c|c|c|c|c|c|c|c|}
\hline & ${ }^{13} \mathrm{C}$ data & 0.04 & 0.00 & 48.71 & 51.24 & - & - & - & - \\
\hline & All data & 0.00 & 0.00 & 98.19 & 1.81 & - & - & - & - \\
\hline & ${ }^{1} \mathrm{H}$ data & 0.00 & 0.00 & 100.00 & 0.00 & - & - & - & - \\
\hline \multirow[t]{5}{*}{ DP4+ } & ${ }^{13} \mathrm{C}$ data & 0.00 & 0.00 & 79.59 & 20.41 & - & - & - & - \\
\hline & All data & 0.00 & 0.00 & 100.00 & 0.00 & - & - & - & - \\
\hline & & \multicolumn{8}{|c|}{ Level of theory: mPW1PW91/6-31+G** (solution, PCM) } \\
\hline & & 78a & 78b & 78c & 78d & - & - & - & - \\
\hline & ${ }^{1} \mathrm{H}$ data & 0.02 & 0.00 & 99.97 & 0.01 & - & - & - & - \\
\hline \multirow[t]{2}{*}{ sDP4+ } & ${ }^{13} \mathrm{C}$ data & 0.02 & 0.00 & 43.81 & 56.16 & - & - & - & - \\
\hline & All data & 0.00 & 0.00 & 99.99 & 0.01 & - & - & - & - \\
\hline & ${ }^{1} \mathrm{H}$ data & 0.21 & 0.03 & 98.24 & 1.52 & - & - & - & - \\
\hline \multirow[t]{2}{*}{ uDP4+ } & ${ }^{13} \mathrm{C}$ data & 0.05 & 0.00 & 29.14 & 70.81 & - & - & - & - \\
\hline & All data & 0.00 & 0.00 & 96.38 & 3.62 & - & - & - & - \\
\hline & ${ }^{1} \mathrm{H}$ data & 0.00 & 0.00 & 100.00 & 0.00 & - & - & - & - \\
\hline \multirow[t]{5}{*}{ DP4+ } & ${ }^{13} \mathrm{C}$ data & 0.00 & 0.00 & 24.30 & 75.70 & - & - & - & - \\
\hline & All data & 0.00 & 0.00 & 100.00 & 0.00 & - & - & - & - \\
\hline & & \multicolumn{8}{|c|}{ Level of theory: mPW1PW91/6-311G* (solution, PCM) } \\
\hline & & 78a & $78 \mathrm{~b}$ & $78 \mathrm{c}$ & 78d & - & - & - & - \\
\hline & ${ }^{1} \mathrm{H}$ data & 0.15 & 0.03 & 99.80 & 0.02 & - & - & - & - \\
\hline \multirow[t]{2}{*}{ sDP4+ } & ${ }^{13} \mathrm{C}$ data & 0.02 & 0.03 & 37.98 & 61.98 & - & - & - & - \\
\hline & All data & 0.00 & 0.00 & 99.97 & 0.03 & - & - & - & - \\
\hline \multirow{3}{*}{ uDP4+ } & ${ }^{1} \mathrm{H}$ data & 1.26 & 0.17 & 97.90 & 0.67 & - & - & - & - \\
\hline & ${ }^{13} \mathrm{C}$ data & 0.01 & 0.00 & 43.50 & 56.49 & - & - & - & - \\
\hline & All data & 0.00 & 0.00 & 99.12 & 0.88 & - & - & - & - \\
\hline \multirow{5}{*}{ DP4+ } & ${ }^{1} \mathrm{H}$ data & 0.00 & 0.00 & 100.00 & 0.00 & - & - & - & - \\
\hline & ${ }^{13} \mathrm{C}$ data & 0.00 & 0.00 & 32.05 & 67.95 & - & - & - & - \\
\hline & All data & 0.00 & 0.00 & 100.00 & 0.00 & - & - & - & - \\
\hline & & \multicolumn{8}{|c|}{ Level of theory: mPW1PW91/6-311G** (solution, PCM) } \\
\hline & & 78a & 78b & 78c & 78d & - & - & - & - \\
\hline \multirow{3}{*}{ sDP4+ } & ${ }^{1} \mathrm{H}$ data & 0.08 & 0.03 & 99.87 & 0.02 & - & - & - & - \\
\hline & ${ }^{13} \mathrm{C}$ data & 0.00 & 0.01 & 33.23 & 66.75 & - & - & - & - \\
\hline & All data & 0.00 & 0.00 & 99.95 & 0.05 & - & - & - & - \\
\hline & ${ }^{1} \mathrm{H}$ data & 0.12 & 0.18 & 99.23 & 0.47 & - & - & - & - \\
\hline \multirow[t]{2}{*}{ uDP4+ } & ${ }^{13} \mathrm{C}$ data & 0.00 & 0.00 & 49.38 & 50.62 & - & - & - & - \\
\hline & All data & 0.00 & 0.00 & 99.52 & 0.48 & - & - & - & - \\
\hline \multirow{5}{*}{ DP4+ } & ${ }^{1} \mathrm{H}$ data & 0.00 & 0.00 & 100.00 & 0.00 & - & - & - & - \\
\hline & ${ }^{13} \mathrm{C}$ data & 0.00 & 0.00 & 32.69 & 67.31 & - & - & - & - \\
\hline & All data & 0.00 & 0.00 & 100.00 & 0.00 & - & - & - & - \\
\hline & & \multicolumn{8}{|c|}{ Level of theory: mPW1PW91/6-311+G** (solution, PCM) } \\
\hline & & $78 \mathrm{a}$ & $78 \mathrm{~b}$ & 78c & 78d & - & - & - & - \\
\hline \multirow{3}{*}{ sDP4+ } & ${ }^{1} \mathrm{H}$ data & 0.02 & 0.00 & 99.97 & 0.01 & - & - & - & - \\
\hline & ${ }^{13} \mathrm{C}$ data & 0.03 & 0.05 & 42.78 & 57.14 & - & - & - & - \\
\hline & All data & 0.00 & 0.00 & 99.99 & 0.01 & - & - & - & - \\
\hline \multirow{3}{*}{ uDP4+ } & ${ }^{1} \mathrm{H}$ data & 0.25 & 0.03 & 99.36 & 0.36 & - & - & - & - \\
\hline & ${ }^{13} \mathrm{C}$ data & 0.02 & 0.00 & 48.05 & 51.93 & - & - & - & - \\
\hline & All data & 0.00 & 0.00 & 99.61 & 0.39 & - & - & - & - \\
\hline \multirow{3}{*}{ DP4+ } & ${ }^{1} \mathrm{H}$ data & 0.00 & 0.00 & 100.00 & 0.00 & - & - & - & - \\
\hline & ${ }^{13} \mathrm{C}$ data & 0.00 & 0.00 & 40.93 & 59.07 & - & - & - & - \\
\hline & All data & 0.00 & 0.00 & 100.00 & 0.00 & - & - & - & - \\
\hline
\end{tabular}


$s \mathrm{DP} 4+, u \mathrm{DP} 4+$ and DP4+ probabilities (\%) for compound 78d<smiles>CCC[C@H]1O[C@@H](c2ccccc2)C[C@H]1O</smiles>

$78 a$<smiles>CCC[C@H]1O[C@@H](c2ccccc2)C[C@H]1O</smiles>

$78 b$<smiles>CCC[C@H]1O[C@@H](c2ccccc2)C[C@H]1O</smiles>

$78 c$

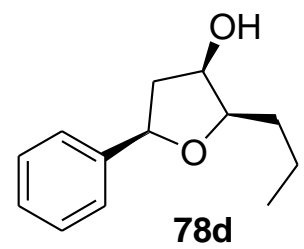

correct isomer

\begin{tabular}{|c|c|c|c|c|c|c|c|c|c|}
\hline & & \multicolumn{8}{|c|}{ Level of theory: B3LYP/6-31G* (gas phase) } \\
\hline & & $78 a$ & $78 b$ & $78 \mathrm{c}$ & 78d & - & - & - & - \\
\hline \multirow{3}{*}{ sDP4+ } & ${ }^{1} \mathrm{H}$ data & 0.02 & 0.00 & 0.05 & 99.93 & - & - & - & - \\
\hline & ${ }^{13} \mathrm{C}$ data & 0.00 & 0.00 & 81.62 & 18.38 & - & - & - & - \\
\hline & All data & 0.00 & 0.00 & 0.24 & 99.76 & - & - & - & - \\
\hline \multirow{3}{*}{ uDP4+ } & ${ }^{1} \mathrm{H}$ data & 1.18 & 0.05 & 3.32 & 95.44 & - & - & - & - \\
\hline & ${ }^{13} \mathrm{C}$ data & 0.13 & 0.12 & 36.53 & 63.22 & - & - & - & - \\
\hline & All data & 0.00 & 0.00 & 1.97 & 98.02 & - & - & - & - \\
\hline \multirow{3}{*}{ DP4+ } & ${ }^{1} \mathrm{H}$ data & 0.00 & 0.00 & 0.00 & 100.00 & - & - & - & - \\
\hline & ${ }^{13} \mathrm{C}$ data & 0.00 & 0.00 & 71.96 & 28.04 & - & - & - & - \\
\hline & All data & 0.00 & 0.00 & 0.00 & 100.00 & - & - & - & - \\
\hline & & \multicolumn{8}{|c|}{ Level of theory: B3LYP/6-31G** (gas phase) } \\
\hline & & $78 a$ & $78 b$ & $78 \mathrm{c}$ & 78d & - & - & - & - \\
\hline \multirow{3}{*}{ sDP4+ } & ${ }^{1} \mathrm{H}$ data & 0.03 & 0.00 & 0.21 & 99.76 & - & - & - & - \\
\hline & ${ }^{13} \mathrm{C}$ data & 0.00 & 0.00 & 86.40 & 13.60 & - & - & - & - \\
\hline & All data & 0.00 & 0.00 & 1.30 & 98.70 & - & - & - & - \\
\hline \multirow{3}{*}{ uDP4+ } & ${ }^{1} \mathrm{H}$ data & 0.27 & 0.03 & 4.67 & 95.02 & - & - & - & - \\
\hline & ${ }^{13} \mathrm{C}$ data & 0.07 & 0.07 & 38.31 & 61.55 & - & - & - & - \\
\hline & All data & 0.00 & 0.00 & 2.97 & 97.03 & - & - & - & - \\
\hline \multirow{3}{*}{ DP4+ } & ${ }^{1} \mathrm{H}$ data & 0.00 & 0.00 & 0.01 & 99.99 & - & - & - & - \\
\hline & ${ }^{13} \mathrm{C}$ data & 0.00 & 0.00 & 79.81 & 20.19 & - & - & - & - \\
\hline & All data & 0.00 & 0.00 & 0.04 & 99.96 & - & - & - & - \\
\hline & & \multicolumn{8}{|c|}{ Level of theory: B3LYP/6-31+G** (gas phase) } \\
\hline & & $78 a$ & $78 b$ & $78 c$ & 78d & - & - & - & - \\
\hline \multirow{3}{*}{ sDP4+ } & ${ }^{1} \mathrm{H}$ data & 0.05 & 0.00 & 0.03 & 99.92 & - & - & - & - \\
\hline & ${ }^{13} \mathrm{C}$ data & 0.02 & 0.01 & 44.53 & 55.45 & - & - & - & - \\
\hline & All data & 0.00 & 0.00 & 0.02 & 99.98 & - & - & - & - \\
\hline \multirow{3}{*}{ uDP4+ } & ${ }^{1} \mathrm{H}$ data & 0.12 & 0.00 & 0.32 & 99.55 & - & - & - & - \\
\hline & ${ }^{13} \mathrm{C}$ data & 0.02 & 0.02 & 13.43 & 86.54 & - & - & - & - \\
\hline & All data & 0.00 & 0.00 & 0.05 & 99.95 & - & - & - & - \\
\hline \multirow{3}{*}{ DP4+ } & ${ }^{1} \mathrm{H}$ data & 0.00 & 0.00 & 0.00 & 100.00 & - & - & - & - \\
\hline & ${ }^{13} \mathrm{C}$ data & 0.00 & 0.00 & 11.08 & 88.92 & - & - & - & - \\
\hline & All data & 0.00 & 0.00 & 0.00 & 100.00 & - & - & - & - \\
\hline & & \multicolumn{8}{|c|}{ Level of theory: B3LYP/6-311G* (gas phase) } \\
\hline & & $78 a$ & $78 b$ & $78 \mathrm{c}$ & 78d & - & - & - & - \\
\hline \multirow{3}{*}{ sDP4+ } & ${ }^{1} \mathrm{H}$ data & 0.11 & 0.01 & 0.06 & 99.83 & - & - & - & - \\
\hline & ${ }^{13} \mathrm{C}$ data & 0.01 & 0.03 & 64.64 & 35.32 & - & - & - & - \\
\hline & All data & 0.00 & 0.00 & 0.11 & 99.89 & - & - & - & - \\
\hline \multirow{3}{*}{ uDP4+ } & ${ }^{1} \mathrm{H}$ data & 6.82 & 0.35 & 7.02 & 85.81 & - & - & - & - \\
\hline & ${ }^{13} \mathrm{C}$ data & 0.00 & 0.00 & 48.36 & 51.63 & - & - & - & - \\
\hline & All data & 0.00 & 0.00 & 7.12 & 92.88 & - & - & - & - \\
\hline \multirow{3}{*}{ DP4+ } & ${ }^{1} \mathrm{H}$ data & 0.01 & 0.00 & 0.00 & 99.99 & - & - & - & - \\
\hline & ${ }^{13} \mathrm{C}$ data & 0.00 & 0.00 & 63.15 & 36.85 & - & - & - & - \\
\hline & All data & 0.00 & 0.00 & 0.01 & 99.99 & - & - & - & - \\
\hline & & \multicolumn{8}{|c|}{ Level of theory: B3LYP/6-311G** (gas phase) } \\
\hline & & $78 a$ & $78 b$ & $78 c$ & 78d & - & - & - & - \\
\hline sDP4+ & ${ }^{1} \mathrm{H}$ data & 0.11 & 0.02 & 0.63 & 99.24 & - & - & - & - \\
\hline
\end{tabular}




\begin{tabular}{|c|c|c|c|c|c|c|c|c|c|}
\hline & ${ }^{13} \mathrm{C}$ data & 0.00 & 0.02 & 64.95 & 35.02 & - & - & - & - \\
\hline & All data & 0.00 & 0.00 & 1.17 & 98.83 & - & - & - & - \\
\hline & ${ }^{1} \mathrm{H}$ data & 0.54 & 0.10 & 13.63 & 85.73 & - & - & - & - \\
\hline \multirow[t]{3}{*}{ uDP4+ } & ${ }^{13} \mathrm{C}$ data & 0.00 & 0.00 & 59.09 & 40.91 & - & - & - & - \\
\hline & All data & 0.00 & 0.00 & 18.68 & 81.32 & - & - & - & - \\
\hline & ${ }^{1} \mathrm{H}$ data & 0.00 & 0.00 & 0.10 & 99.90 & - & - & - & - \\
\hline \multirow[t]{5}{*}{ DP4+ } & ${ }^{13} \mathrm{C}$ data & 0.00 & 0.00 & 72.81 & 27.19 & - & - & - & - \\
\hline & All data & 0.00 & 0.00 & 0.27 & 99.73 & - & - & - & - \\
\hline & & \multicolumn{8}{|c|}{ Level of theory: B3LYP/6-311+G** (gas phase) } \\
\hline & & $78 a$ & $78 b$ & $78 c$ & 78d & - & - & - & - \\
\hline & ${ }^{1} \mathrm{H}$ data & 0.22 & 0.01 & 0.10 & 99.66 & - & - & - & - \\
\hline \multirow[t]{3}{*}{ sDP4+ } & ${ }^{13}$ C data & 0.02 & 0.04 & 43.35 & 56.59 & - & - & - & - \\
\hline & All data & 0.00 & 0.00 & 0.08 & 99.92 & - & - & - & - \\
\hline & ${ }^{1} \mathrm{H}$ data & 1.08 & 0.03 & 1.57 & 97.32 & - & - & - & - \\
\hline \multirow[t]{3}{*}{ uDP4+ } & ${ }^{13} \mathrm{C}$ data & 0.00 & 0.00 & 43.43 & 56.57 & - & - & - & - \\
\hline & All data & 0.00 & 0.00 & 1.22 & 98.78 & - & - & - & - \\
\hline & ${ }^{1} \mathrm{H}$ data & 0.00 & 0.00 & 0.00 & 100.00 & - & - & - & - \\
\hline \multirow[t]{5}{*}{ DP4+ } & ${ }^{13} \mathrm{C}$ data & 0.00 & 0.00 & 37.03 & 62.97 & - & - & - & - \\
\hline & All data & 0.00 & 0.00 & 0.00 & 100.00 & - & - & - & - \\
\hline & & \multicolumn{8}{|c|}{ Level of theory: B3LYP/6-31G* (solution, PCM) } \\
\hline & & $78 a$ & $78 b$ & $78 c$ & 78d & - & - & - & - \\
\hline & ${ }^{1}$ H data & 0.01 & 0.00 & 0.00 & 99.99 & - & - & - & - \\
\hline \multirow[t]{3}{*}{ sDP4+ } & ${ }^{13} \mathrm{C}$ data & 0.01 & 0.00 & 48.96 & 51.04 & - & - & - & - \\
\hline & All data & 0.00 & 0.00 & 0.00 & 100.00 & - & - & - & - \\
\hline & ${ }^{1} \mathrm{H}$ data & 0.05 & 0.00 & 0.01 & 99.94 & - & - & - & - \\
\hline \multirow[t]{3}{*}{ uDP4+ } & ${ }^{13} \mathrm{C}$ data & 0.19 & 0.03 & 15.43 & 84.35 & - & - & - & - \\
\hline & All data & 0.00 & 0.00 & 0.00 & 100.00 & - & - & - & - \\
\hline & ${ }^{1} \mathrm{H}$ data & 0.00 & 0.00 & 0.00 & 100.00 & - & - & - & - \\
\hline \multirow[t]{5}{*}{ DP4+ } & ${ }^{13} \mathrm{C}$ data & 0.00 & 0.00 & 14.93 & 85.07 & - & - & - & - \\
\hline & All data & 0.00 & 0.00 & 0.00 & 100.00 & - & - & - & - \\
\hline & & \multicolumn{8}{|c|}{ Level of theory: B3LYP/6-31G** (solution, PCM) } \\
\hline & & $78 a$ & $78 b$ & $78 \mathrm{c}$ & 78d & - & - & - & - \\
\hline & ${ }^{1}$ H data & 0.01 & 0.00 & 0.00 & 99.99 & - & - & - & - \\
\hline \multirow[t]{3}{*}{ sDP4+ } & ${ }^{13}$ C data & 0.01 & 0.00 & 56.15 & 43.84 & - & - & - & - \\
\hline & All data & 0.00 & 0.00 & 0.00 & 100.00 & - & - & - & - \\
\hline & ${ }^{1} \mathrm{H}$ data & 0.01 & 0.00 & 0.01 & 99.97 & - & - & - & - \\
\hline \multirow[t]{3}{*}{ uDP4+ } & ${ }^{13} \mathrm{C}$ data & 0.13 & 0.03 & 17.33 & 82.50 & - & - & - & - \\
\hline & All data & 0.00 & 0.00 & 0.00 & 100.00 & - & - & - & - \\
\hline & ${ }^{1} \mathrm{H}$ data & 0.00 & 0.00 & 0.00 & 100.00 & - & - & - & - \\
\hline \multirow[t]{5}{*}{ DP4+ } & ${ }^{13} \mathrm{C}$ data & 0.00 & 0.00 & 21.20 & 78.80 & - & - & - & - \\
\hline & All data & 0.00 & 0.00 & 0.00 & 100.00 & - & - & - & - \\
\hline & & \multicolumn{8}{|c|}{ Level of theory: B3LYP/6-31+G** (solution, PCM) } \\
\hline & & $78 a$ & $78 b$ & $78 \mathrm{c}$ & 78d & - & - & - & - \\
\hline & ${ }^{1} \mathrm{H}$ data & 0.01 & 0.00 & 0.00 & 99.99 & - & - & - & - \\
\hline \multirow[t]{2}{*}{ sDP4+ } & ${ }^{13} \mathrm{C}$ data & 0.05 & 0.01 & 19.76 & 80.18 & - & - & - & - \\
\hline & All data & 0.00 & 0.00 & 0.00 & 100.00 & - & - & - & - \\
\hline & ${ }^{1} \mathrm{H}$ data & 0.02 & 0.00 & 0.01 & 99.98 & - & - & - & - \\
\hline uDP4+ & ${ }^{13} \mathrm{C}$ data & 0.01 & 0.01 & 7.21 & 92.76 & - & - & - & - \\
\hline & All data & 0.00 & 0.00 & 0.00 & 100.00 & - & - & - & - \\
\hline & ${ }^{1} \mathrm{H}$ data & 0.00 & 0.00 & 0.00 & 100.00 & - & - & - & - \\
\hline DP4+ & ${ }^{13} \mathrm{C}$ data & 0.00 & 0.00 & 1.88 & 98.12 & - & - & - & - \\
\hline & All data & 0.00 & 0.00 & 0.00 & 100.00 & - & - & - & - \\
\hline & & & & Level of & ry: B3LY & G* & C & & \\
\hline & & $78 a$ & $78 b$ & $78 c$ & $78 d$ & - & - & - & - \\
\hline sDP4+ & ${ }_{12}^{1} \mathrm{H}$ data & 0.01 & 0.00 & 0.00 & 99.99 & - & - & - & - \\
\hline SDP4+ & ${ }^{13} \mathrm{C}$ data & 0.02 & 0.02 & 25.47 & 74.49 & - & - & - & - \\
\hline
\end{tabular}




\begin{tabular}{|c|c|c|c|c|c|c|c|c|c|}
\hline & All data & 0.00 & 0.00 & 0.00 & 100.00 & - & - & - & - \\
\hline & ${ }^{1} \mathrm{H}$ data & 0.10 & 0.00 & 0.01 & 99.89 & - & - & - & - \\
\hline \multirow[t]{3}{*}{ uDP4+ } & ${ }^{13} \mathrm{C}$ data & 0.01 & 0.00 & 21.48 & 78.51 & - & - & - & - \\
\hline & All data & 0.00 & 0.00 & 0.00 & 100.00 & - & - & - & - \\
\hline & ${ }^{1} \mathrm{H}$ data & 0.00 & 0.00 & 0.00 & 100.00 & - & - & - & - \\
\hline \multirow[t]{5}{*}{ DP4+ } & ${ }^{13} \mathrm{C}$ data & 0.00 & 0.00 & 8.55 & 91.45 & - & - & - & - \\
\hline & All data & 0.00 & 0.00 & 0.00 & 100.00 & - & - & - & - \\
\hline & & \multicolumn{8}{|c|}{ Level of theory: B3LYP/6-311G** (solution, PCM) } \\
\hline & & $78 a$ & $78 b$ & $78 \mathrm{c}$ & 78d & - & - & - & - \\
\hline & ${ }^{1}$ H data & 0.10 & 0.02 & 0.02 & 99.86 & - & - & - & - \\
\hline \multirow[t]{2}{*}{ sDP4+ } & ${ }^{13} \mathrm{C}$ data & 0.01 & 0.01 & 25.33 & 74.64 & - & - & - & - \\
\hline & All data & 0.00 & 0.00 & 0.01 & 99.99 & - & - & - & - \\
\hline \multirow{3}{*}{ uDP4+ } & ${ }^{1} \mathrm{H}$ data & 0.10 & 0.01 & 0.04 & 99.85 & - & - & - & - \\
\hline & ${ }^{13} \mathrm{C}$ data & 0.00 & 0.00 & 25.58 & 74.42 & - & - & - & - \\
\hline & All data & 0.00 & 0.00 & 0.01 & 99.99 & - & - & - & - \\
\hline \multirow{3}{*}{ DP4+ } & ${ }^{1} \mathrm{H}$ data & 0.00 & 0.00 & 0.00 & 100.00 & - & - & - & - \\
\hline & ${ }^{13} \mathrm{C}$ data & 0.00 & 0.00 & 10.45 & 89.55 & - & - & - & - \\
\hline & All data & 0.00 & 0.00 & 0.00 & 100.00 & - & - & - & - \\
\hline & & \multicolumn{8}{|c|}{ Level of theory: B3LYP/6-311+G** (solution, PCM) } \\
\hline & & $78 a$ & $78 b$ & $78 c$ & 78d & - & - & - & - \\
\hline \multirow{3}{*}{ sDP4+ } & ${ }^{1}$ H data & 0.07 & 0.01 & 0.01 & 99.91 & - & - & - & - \\
\hline & ${ }^{13}$ C data & 0.05 & 0.03 & 20.44 & 79.48 & - & - & - & - \\
\hline & All data & 0.00 & 0.00 & 0.00 & 100.00 & - & - & - & - \\
\hline \multirow{3}{*}{ uDP4+ } & ${ }^{1} \mathrm{H}$ data & 0.02 & 0.00 & 0.00 & 99.98 & - & - & - & - \\
\hline & ${ }^{13} \mathrm{C}$ data & 0.01 & 0.00 & 23.86 & 76.13 & - & - & - & - \\
\hline & All data & 0.00 & 0.00 & 0.00 & 100.00 & - & - & - & - \\
\hline \multirow{3}{*}{ DP4+ } & ${ }^{1} \mathrm{H}$ data & 0.00 & 0.00 & 0.00 & 100.00 & - & - & - & - \\
\hline & ${ }^{13} \mathrm{C}$ data & 0.00 & 0.00 & 7.46 & 92.54 & - & - & - & - \\
\hline & All data & 0.00 & 0.00 & 0.00 & 100.00 & - & - & - & - \\
\hline & & \multicolumn{8}{|c|}{ Level of theory: mPW1PW91/6-31G* (gas phase) } \\
\hline & & $78 a$ & $78 b$ & $78 \mathrm{c}$ & 78d & - & - & - & - \\
\hline \multirow{3}{*}{ sDP4+ } & ${ }^{1} \mathrm{H}$ data & 0.00 & 0.00 & 2.58 & 97.41 & - & - & - & - \\
\hline & ${ }^{13} \mathrm{C}$ data & 0.00 & 0.00 & 86.50 & 13.50 & - & - & - & - \\
\hline & All data & 0.00 & 0.00 & 14.52 & 85.48 & - & - & - & - \\
\hline \multirow{3}{*}{ uDP4+ } & ${ }^{1} \mathrm{H}$ data & 0.08 & 0.04 & 47.23 & 52.64 & - & - & - & - \\
\hline & ${ }^{13} \mathrm{C}$ data & 0.05 & 0.11 & 35.81 & 64.03 & - & - & - & - \\
\hline & All data & 0.00 & 0.00 & 33.41 & 66.59 & - & - & - & - \\
\hline \multirow{3}{*}{ DP4+ } & ${ }^{1} \mathrm{H}$ data & 0.00 & 0.00 & 2.32 & 97.68 & - & - & - & - \\
\hline & ${ }^{13} \mathrm{C}$ data & 0.00 & 0.00 & 78.18 & 21.82 & - & - & - & - \\
\hline & All data & 0.00 & 0.00 & 7.85 & 92.15 & - & - & - & - \\
\hline & & \multicolumn{8}{|c|}{ Level of theory: mPW1PW91/6-31G** (gas phase) } \\
\hline & & $78 a$ & $78 b$ & $78 \mathrm{c}$ & 78d & - & - & - & - \\
\hline \multirow{3}{*}{ sDP4+ } & ${ }^{1} \mathrm{H}$ data & 0.00 & 0.01 & 11.06 & 88.93 & - & - & - & - \\
\hline & ${ }^{13} \mathrm{C}$ data & 0.00 & 0.00 & 90.08 & 9.92 & - & - & - & - \\
\hline & All data & 0.00 & 0.00 & 53.03 & 46.97 & - & - & - & - \\
\hline & ${ }^{1} \mathrm{H}$ data & 0.01 & 0.02 & 63.57 & 36.40 & - & - & - & - \\
\hline uDP4+ & ${ }^{13} \mathrm{C}$ data & 0.01 & 0.04 & 32.89 & 67.05 & - & - & - & - \\
\hline & All data & 0.00 & 0.00 & 46.14 & 53.86 & - & - & - & - \\
\hline & ${ }^{1} \mathrm{H}$ data & 0.00 & 0.00 & 17.84 & 82.16 & - & - & - & - \\
\hline DP4+ & ${ }^{13} \mathrm{C}$ data & 0.00 & 0.00 & 81.66 & 18.34 & - & - & - & - \\
\hline & All data & 0.00 & 0.00 & 49.17 & 50.83 & - & - & - & - \\
\hline & & & & vel of $t$ & : mPW1F & 3 & $\overline{p h}$ & & \\
\hline & & $78 a$ & $78 b$ & $78 c$ & $78 d$ & - & - & - & - \\
\hline & ${ }^{1} \mathrm{H}$ data & 0.00 & 0.00 & 0.05 & 99.95 & - & - & - & - \\
\hline sDP4+ & ${ }^{13} \mathrm{C}$ data & 0.00 & 0.01 & 67.22 & 32.78 & - & - & - & - \\
\hline & All data & 0.00 & 0.00 & 0.09 & 99.91 & - & - & - & - \\
\hline
\end{tabular}




\begin{tabular}{|c|c|c|c|c|c|c|c|c|c|}
\hline & ${ }^{1} \mathrm{H}$ data & 0.09 & 0.05 & 29.39 & 70.48 & - & - & - & - \\
\hline \multirow[t]{3}{*}{ uDP4+ } & ${ }^{13} \mathrm{C}$ data & 0.03 & 0.03 & 23.62 & 76.32 & - & - & - & - \\
\hline & All data & 0.00 & 0.00 & 11.43 & 88.57 & - & - & - & - \\
\hline & ${ }^{1} \mathrm{H}$ data & 0.00 & 0.00 & 0.02 & 99.98 & - & - & - & - \\
\hline \multirow[t]{5}{*}{ DP4+ } & ${ }^{13} \mathrm{C}$ data & 0.00 & 0.00 & 38.83 & 61.17 & - & - & - & - \\
\hline & All data & 0.00 & 0.00 & 0.01 & 99.99 & - & - & - & - \\
\hline & & \multicolumn{8}{|c|}{ Level of theory: mPW1PW91/6-311G* (gas phase) } \\
\hline & & $78 a$ & $78 b$ & 78c & 78d & - & - & - & - \\
\hline & ${ }^{1} \mathrm{H}$ data & 0.01 & 0.02 & 1.56 & 98.41 & - & - & - & - \\
\hline \multirow[t]{3}{*}{ sDP4+ } & ${ }^{13} \mathrm{C}$ data & 0.00 & 0.30 & 75.97 & 23.73 & - & - & - & - \\
\hline & All data & 0.00 & 0.00 & 4.84 & 95.16 & - & - & - & - \\
\hline & ${ }^{1} \mathrm{H}$ data & 0.33 & 0.46 & 50.61 & 48.60 & - & - & - & - \\
\hline \multirow[t]{3}{*}{ uDP4+ } & ${ }^{13} \mathrm{C}$ data & 0.00 & 0.00 & 50.47 & 49.53 & - & - & - & - \\
\hline & All data & 0.00 & 0.00 & 51.49 & 48.51 & - & - & - & - \\
\hline & ${ }^{1} \mathrm{H}$ data & 0.00 & 0.00 & 1.63 & 98.37 & - & - & - & - \\
\hline \multirow[t]{5}{*}{ DP4+ } & ${ }^{13} \mathrm{C}$ data & 0.00 & 0.00 & 76.54 & 23.46 & - & - & - & - \\
\hline & All data & 0.00 & 0.00 & 5.12 & 94.88 & - & - & - & - \\
\hline & & \multicolumn{8}{|c|}{ Level of theory: mPW1PW91/6-311G** (gas phase) } \\
\hline & & $78 a$ & $78 b$ & 78c & 78d & - & - & - & - \\
\hline & ${ }^{1}$ H data & 0.01 & 0.04 & 14.06 & 85.89 & - & - & - & - \\
\hline \multirow[t]{3}{*}{ sDP4+ } & ${ }^{13} \mathrm{C}$ data & 0.00 & 0.22 & 75.00 & 24.78 & - & - & - & - \\
\hline & All data & 0.00 & 0.00 & 33.14 & 66.86 & - & - & - & - \\
\hline & ${ }^{1} \mathrm{H}$ data & 0.01 & 0.05 & 83.13 & 16.81 & - & - & - & - \\
\hline \multirow[t]{3}{*}{ uDP4+ } & ${ }^{13} \mathrm{C}$ data & 0.00 & 0.00 & 57.51 & 42.49 & - & - & - & - \\
\hline & All data & 0.00 & 0.00 & 87.00 & 13.00 & - & - & - & - \\
\hline & ${ }^{1} \mathrm{H}$ data & 0.00 & 0.00 & 44.74 & 55.26 & - & - & - & - \\
\hline \multirow[t]{5}{*}{ DP4+ } & ${ }^{13} \mathrm{C}$ data & 0.00 & 0.00 & 80.38 & 19.62 & - & - & - & - \\
\hline & All data & 0.00 & 0.00 & 76.83 & 23.17 & - & - & - & - \\
\hline & & \multicolumn{8}{|c|}{ Level of theory: mPW1PW91/6-311+G** (gas phase) } \\
\hline & & $78 a$ & $78 b$ & $78 \mathrm{c}$ & 78d & - & - & - & - \\
\hline & ${ }^{1} \mathrm{H}$ data & 0.12 & 0.09 & 13.65 & 86.14 & - & - & - & - \\
\hline \multirow[t]{3}{*}{ sDP4+ } & ${ }^{13} \mathrm{C}$ data & 0.01 & 0.72 & 77.21 & 22.06 & - & - & - & - \\
\hline & All data & 0.00 & 0.00 & 35.67 & 64.32 & - & - & - & - \\
\hline & ${ }^{1} \mathrm{H}$ data & 0.26 & 0.15 & 81.64 & 17.94 & - & - & - & - \\
\hline \multirow[t]{3}{*}{ uDP4+ } & ${ }^{13} \mathrm{C}$ data & 0.00 & 0.00 & 63.21 & 36.79 & - & - & - & - \\
\hline & All data & 0.00 & 0.00 & 88.66 & 11.34 & - & - & - & - \\
\hline & ${ }^{1} \mathrm{H}$ data & 0.00 & 0.00 & 41.90 & 58.10 & - & - & - & - \\
\hline \multirow[t]{5}{*}{ DP4+ } & ${ }^{13} \mathrm{C}$ data & 0.00 & 0.00 & 85.74 & 14.26 & - & - & - & - \\
\hline & All data & 0.00 & 0.00 & 81.26 & 18.74 & - & - & - & - \\
\hline & & \multicolumn{8}{|c|}{ Level of theory: mPW1PW91/6-31G* (solution, PCM) } \\
\hline & & $78 a$ & $78 b$ & 78c & 78d & - & - & - & - \\
\hline & ${ }^{1} \mathrm{H}$ data & 0.01 & 0.00 & 0.02 & 99.97 & - & - & - & - \\
\hline \multirow[t]{3}{*}{ sDP4+ } & ${ }^{13} \mathrm{C}$ data & 0.00 & 0.00 & 54.72 & 45.28 & - & - & - & - \\
\hline & All data & 0.00 & 0.00 & 0.02 & 99.98 & - & - & - & - \\
\hline & ${ }^{1}$ H data & 0.08 & 0.01 & 0.35 & 99.56 & - & - & - & - \\
\hline uDP4+ & ${ }^{13} \mathrm{C}$ data & 0.15 & 0.03 & 21.69 & 78.13 & - & - & - & - \\
\hline & All data & 0.00 & 0.00 & 0.10 & 99.90 & - & - & - & - \\
\hline & ${ }^{1} \mathrm{H}$ data & 0.00 & 0.00 & 0.00 & 100.00 & - & - & - & - \\
\hline DP4+ & ${ }^{13} \mathrm{C}$ data & 0.00 & 0.00 & 25.12 & 74.88 & - & - & - & - \\
\hline & All data & 0.00 & 0.00 & 0.00 & 100.00 & - & - & - & - \\
\hline & & & & el of th & nPW1PI & 31 & on, & & \\
\hline & & $78 a$ & $78 b$ & $78 c$ & 78d & - & - & - & - \\
\hline & ${ }^{1} \mathrm{H}$ data & 0.01 & 0.01 & 0.07 & 99.91 & - & - & - & - \\
\hline sDP4+ & ${ }^{13} \mathrm{C}$ data & 0.00 & 0.00 & 66.57 & 33.42 & - & - & - & - \\
\hline & All data & 0.00 & 0.00 & 0.14 & 99.86 & - & - & - & - \\
\hline uDP4+ & ${ }^{1} \mathrm{H}$ data & 0.01 & 0.00 & 0.35 & 99.64 & - & - & - & - \\
\hline
\end{tabular}




\begin{tabular}{|c|c|c|c|c|c|c|c|c|c|}
\hline & ${ }^{13} \mathrm{C}$ data & 0.06 & 0.02 & 25.35 & 74.56 & - & - & - & - \\
\hline & All data & 0.00 & 0.00 & 0.12 & 99.88 & - & - & - & - \\
\hline & ${ }^{1} \mathrm{H}$ data & 0.00 & 0.00 & 0.00 & 100.00 & - & - & - & - \\
\hline \multirow[t]{5}{*}{ DP4+ } & ${ }^{13} \mathrm{C}$ data & 0.00 & 0.00 & 40.38 & 59.62 & - & - & - & - \\
\hline & All data & 0.00 & 0.00 & 0.00 & 100.00 & - & - & - & - \\
\hline & & \multicolumn{8}{|c|}{ Level of theory: mPW1PW91/6-31+G** (solution, PCM) } \\
\hline & & 78a & 78b & 78c & 78d & - & - & - & - \\
\hline & ${ }^{1} \mathrm{H}$ data & 0.04 & 0.01 & 0.03 & 99.92 & - & - & - & - \\
\hline \multirow[t]{2}{*}{ sDP4+ } & ${ }^{13} \mathrm{C}$ data & 0.01 & 0.01 & 31.64 & 68.34 & - & - & - & - \\
\hline & All data & 0.00 & 0.00 & 0.01 & 99.99 & - & - & - & - \\
\hline \multirow{3}{*}{ uDP4+ } & ${ }^{1} \mathrm{H}$ data & 0.02 & 0.00 & 0.09 & 99.88 & - & - & - & - \\
\hline & ${ }^{13} \mathrm{C}$ data & 0.02 & 0.01 & 7.72 & 92.25 & - & - & - & - \\
\hline & All data & 0.00 & 0.00 & 0.01 & 99.99 & - & - & - & - \\
\hline & ${ }^{1} \mathrm{H}$ data & 0.00 & 0.00 & 0.00 & 100.00 & - & - & - & - \\
\hline \multirow[t]{5}{*}{ DP4+ } & ${ }^{13} \mathrm{C}$ data & 0.00 & 0.00 & 3.73 & 96.27 & - & - & - & - \\
\hline & All data & 0.00 & 0.00 & 0.00 & 100.00 & - & - & - & - \\
\hline & & \multicolumn{8}{|c|}{ Level of theory: mPW1PW91/6-311G* (solution, PCM) } \\
\hline & & 78a & $78 \mathrm{~b}$ & $78 \mathrm{c}$ & 78d & - & - & - & - \\
\hline & ${ }^{1} \mathrm{H}$ data & 0.01 & 0.00 & 0.01 & 99.98 & - & - & - & - \\
\hline \multirow[t]{2}{*}{ sDP4+ } & ${ }^{13} \mathrm{C}$ data & 0.01 & 0.10 & 26.85 & 73.03 & - & - & - & - \\
\hline & All data & 0.00 & 0.00 & 0.00 & 100.00 & - & - & - & - \\
\hline \multirow{3}{*}{ uDP4+ } & ${ }^{1} \mathrm{H}$ data & 0.19 & 0.02 & 0.29 & 99.51 & - & - & - & - \\
\hline & ${ }^{13} \mathrm{C}$ data & 0.01 & 0.00 & 26.50 & 73.50 & - & - & - & - \\
\hline & All data & 0.00 & 0.00 & 0.10 & 99.90 & - & - & - & - \\
\hline \multirow{5}{*}{ DP4+ } & ${ }^{1} \mathrm{H}$ data & 0.00 & 0.00 & 0.00 & 100.00 & - & - & - & - \\
\hline & ${ }^{13} \mathrm{C}$ data & 0.00 & 0.00 & 11.70 & 88.30 & - & - & - & - \\
\hline & All data & 0.00 & 0.00 & 0.00 & 100.00 & - & - & - & - \\
\hline & & \multicolumn{8}{|c|}{ Level of theory: mPW1PW91/6-311G** (solution, PCM) } \\
\hline & & 78a & 78b & $78 \mathrm{c}$ & 78d & - & - & - & - \\
\hline \multirow{3}{*}{ sDP4+ } & ${ }^{1} \mathrm{H}$ data & 0.07 & 0.04 & 0.19 & 99.70 & - & - & - & - \\
\hline & ${ }^{13} \mathrm{C}$ data & 0.01 & 0.07 & 39.00 & 60.92 & - & - & - & - \\
\hline & All data & 0.00 & 0.00 & 0.12 & 99.88 & - & - & - & - \\
\hline & ${ }^{1} \mathrm{H}$ data & 0.06 & 0.03 & 0.76 & 99.16 & - & - & - & - \\
\hline \multirow[t]{2}{*}{ uDP4+ } & ${ }^{13} \mathrm{C}$ data & 0.00 & 0.00 & 32.53 & 67.46 & - & - & - & - \\
\hline & All data & 0.00 & 0.00 & 0.37 & 99.63 & - & - & - & - \\
\hline \multirow{5}{*}{ DP4+ } & ${ }^{1} \mathrm{H}$ data & 0.00 & 0.00 & 0.00 & 100.00 & - & - & - & - \\
\hline & ${ }^{13} \mathrm{C}$ data & 0.00 & 0.00 & 23.59 & 76.41 & - & - & - & - \\
\hline & All data & 0.00 & 0.00 & 0.00 & 100.00 & - & - & - & - \\
\hline & & \multicolumn{8}{|c|}{ Level of theory: mPW1PW91/6-311+G** (solution, PCM) } \\
\hline & & $78 \mathrm{a}$ & $78 \mathrm{~b}$ & $78 c$ & 78d & - & - & - & - \\
\hline \multirow{3}{*}{ sDP4+ } & ${ }^{1} \mathrm{H}$ data & 0.24 & 0.03 & 0.12 & 99.62 & - & - & - & - \\
\hline & ${ }^{13} \mathrm{C}$ data & 0.04 & 0.23 & 32.18 & 67.56 & - & - & - & - \\
\hline & All data & 0.00 & 0.00 & 0.06 & 99.94 & - & - & - & - \\
\hline \multirow{3}{*}{ uDP4+ } & ${ }^{1} \mathrm{H}$ data & 0.33 & 0.03 & 0.64 & 99.00 & - & - & - & - \\
\hline & ${ }^{13} \mathrm{C}$ data & 0.01 & 0.00 & 30.28 & 69.71 & - & - & - & - \\
\hline & All data & 0.00 & 0.00 & 0.28 & 99.72 & - & - & - & - \\
\hline \multirow{3}{*}{ DP4+ } & ${ }^{1} \mathrm{H}$ data & 0.00 & 0.00 & 0.00 & 100.00 & - & - & - & - \\
\hline & ${ }^{13} \mathrm{C}$ data & 0.00 & 0.00 & 17.14 & 82.86 & - & - & - & - \\
\hline & All data & 0.00 & 0.00 & 0.00 & 100.00 & - & - & - & - \\
\hline
\end{tabular}


sDP4+, $u \mathrm{DP} 4+$ and DP4+ probabilities (\%) for compound 79a<smiles>CC[C@H](O)[C@H](C)C1=C(C)C(=O)[C@H](C)[C@H]([C@H](C)C(=O)O[Na])O1</smiles><smiles>CC[C@H](O)[C@@H](C)C1=C(C)C(=O)[C@@H](C)[C@@H]([C@@H](C)CC)O1</smiles><smiles>CC[C@H](O)[C@@H](C)C1=C(C)C(=O)[C@@H](C)[C@@H]([C@@H](C)CC)O1</smiles><smiles>CC[C@H](O)[C@@H](C)C1=C(C)C(=O)[C@@H](C)[C@@H]([C@@H](C)CC)O1</smiles><smiles>CC[C@H](O)[C@@H](C)C1=C(C)C(=O)[C@@H](C)[C@@H]([C@@H](C)CC)O1</smiles><smiles>CC[C@H](O)[C@@H](C)C1=C(C)C(=O)[C@@H](C)[C@@H]([C@@H](C)CC)O1</smiles><smiles>CC[C@H](O)[C@@H](C)C1=C(C)C(=O)[C@@H](C)[C@@H]([C@@H](C)CC)O1</smiles><smiles>CC[C@H](O)[C@@H](C)C1=C(C)C(=O)[C@@H](C)[C@@H]([C@@H](C)CC)O1</smiles>

\begin{tabular}{|c|c|c|c|c|c|c|c|c|c|}
\hline & & \multicolumn{8}{|c|}{ Level of theory: B3LYP/6-31G* (gas phase) } \\
\hline & & $79 a$ & $79 b$ & $79 c$ & 79d & $79 e$ & $79 f$ & $79 \mathrm{~g}$ & $79 h$ \\
\hline & ${ }^{1} \mathrm{H}$ data & 0.42 & 43.52 & 41.66 & 14.32 & 0.01 & 0.02 & 0.04 & 0.01 \\
\hline \multirow[t]{2}{*}{ sDP4+ } & ${ }^{13}$ C data & 28.33 & 2.38 & 0.86 & 59.42 & 2.81 & 0.04 & 0.41 & 5.74 \\
\hline & All data & 1.20 & 10.32 & 3.58 & 84.89 & 0.00 & 0.00 & 0.00 & 0.01 \\
\hline \multirow{3}{*}{ uDP4+ } & ${ }^{1} \mathrm{H}$ data & 0.34 & 52.39 & 40.35 & 6.46 & 0.01 & 0.23 & 0.20 & 0.02 \\
\hline & ${ }^{13} \mathrm{C}$ data & 24.82 & 5.48 & 13.96 & 55.19 & 0.18 & 0.02 & 0.06 & 0.29 \\
\hline & All data & 0.69 & 23.63 & 46.35 & 29.33 & 0.00 & 0.00 & 0.00 & 0.00 \\
\hline \multirow{5}{*}{ DP4+ } & ${ }^{1} \mathrm{H}$ data & 0.00 & 56.24 & 41.47 & 2.28 & 0.00 & 0.00 & 0.00 & 0.00 \\
\hline & ${ }^{13} \mathrm{C}$ data & 17.54 & 0.32 & 0.30 & 81.78 & 0.01 & 0.00 & 0.00 & 0.04 \\
\hline & All data & 0.03 & 8.41 & 5.72 & 85.84 & 0.00 & 0.00 & 0.00 & 0.00 \\
\hline & & \multicolumn{8}{|c|}{ Level of theory: B3LYP/6-31G** (gas phase) } \\
\hline & & $79 a$ & $79 b$ & $79 c$ & $79 d$ & $79 e$ & $79 f$ & $79 g$ & $79 h$ \\
\hline \multirow{3}{*}{ sDP4+ } & ${ }^{1} \mathrm{H}$ data & 0.48 & 40.46 & 49.84 & 8.92 & 0.02 & 0.02 & 0.21 & 0.04 \\
\hline & ${ }^{13} \mathrm{C}$ data & 32.24 & 3.71 & 1.30 & 56.10 & 2.92 & 0.07 & 0.33 & 3.34 \\
\hline & All data & 2.13 & 20.53 & 8.86 & 68.44 & 0.01 & 0.00 & 0.01 & 0.02 \\
\hline \multirow{3}{*}{ uDP4+ } & ${ }^{1} \mathrm{H}$ data & 0.56 & 48.00 & 41.73 & 9.04 & 0.04 & 0.26 & 0.31 & 0.06 \\
\hline & ${ }^{13} \mathrm{C}$ data & 11.80 & 11.58 & 10.11 & 66.42 & 0.02 & 0.00 & 0.02 & 0.05 \\
\hline & All data & 0.42 & 35.07 & 26.62 & 37.89 & 0.00 & 0.00 & 0.00 & 0.00 \\
\hline \multirow{3}{*}{ DP4+ } & ${ }^{1} \mathrm{H}$ data & 0.01 & 47.34 & 50.69 & 1.96 & 0.00 & 0.00 & 0.00 & 0.00 \\
\hline & ${ }^{13} \mathrm{C}$ data & 9.14 & 1.03 & 0.32 & 89.51 & 0.00 & 0.00 & 0.00 & 0.00 \\
\hline & All data & 0.03 & 20.29 & 6.64 & 73.05 & 0.00 & 0.00 & 0.00 & 0.00 \\
\hline & & \multicolumn{8}{|c|}{ Level of theory: B3LYP/6-31+G** (gas phase) } \\
\hline & & $79 a$ & $79 b$ & $79 c$ & $79 d$ & $79 e$ & $79 f$ & $79 g$ & $79 h$ \\
\hline \multirow{3}{*}{ sDP4+ } & ${ }^{1} \mathrm{H}$ data & 3.43 & 23.29 & 66.56 & 4.86 & 0.29 & 0.05 & 1.34 & 0.16 \\
\hline & ${ }^{13} \mathrm{C}$ data & 11.10 & 15.55 & 9.82 & 61.14 & 1.31 & 0.37 & 0.35 & 0.35 \\
\hline & All data & 2.82 & 26.78 & 48.35 & 21.98 & 0.03 & 0.00 & 0.03 & 0.00 \\
\hline \multirow{3}{*}{ uDP4+ } & ${ }^{1} \mathrm{H}$ data & 5.59 & 26.28 & 54.46 & 9.09 & 1.60 & 0.66 & 1.99 & 0.31 \\
\hline & ${ }^{13} \mathrm{C}$ data & 3.06 & 30.62 & 30.14 & 36.08 & 0.02 & 0.02 & 0.05 & 0.01 \\
\hline & All data & 0.61 & 28.83 & 58.80 & 11.75 & 0.00 & 0.00 & 0.00 & 0.00 \\
\hline \multirow{3}{*}{ DP4+ } & ${ }^{1} \mathrm{H}$ data & 0.45 & 14.22 & 84.23 & 1.03 & 0.01 & 0.00 & 0.06 & 0.00 \\
\hline & ${ }^{13} \mathrm{C}$ data & 1.13 & 15.81 & 9.83 & 73.23 & 0.00 & 0.00 & 0.00 & 0.00 \\
\hline & All data & 0.04 & 19.93 & 73.36 & 6.67 & 0.00 & 0.00 & 0.00 & 0.00 \\
\hline & & \multicolumn{8}{|c|}{ Level of theory: B3LYP/6-311G* (gas phase) } \\
\hline & & $79 a$ & $79 b$ & $79 c$ & 79d & $79 e$ & $79 f$ & $79 g$ & $79 h$ \\
\hline \multirow{3}{*}{ sDP4+ } & ${ }^{1} \mathrm{H}$ data & 0.27 & 90.42 & 5.87 & 3.07 & 0.03 & 0.19 & 0.14 & 0.02 \\
\hline & ${ }^{13} \mathrm{C}$ data & 6.64 & 4.10 & 1.11 & 87.45 & 0.24 & 0.01 & 0.05 & 0.39 \\
\hline & All data & 0.28 & 57.25 & 1.00 & 41.47 & 0.00 & 0.00 & 0.00 & 0.00 \\
\hline \multirow{2}{*}{ uDP4+ } & ${ }^{1}$ H data & 2.87 & 55.07 & 29.96 & 5.03 & 0.44 & 4.49 & 1.79 & 0.36 \\
\hline & ${ }^{13} \mathrm{C}$ data & 2.06 & 28.45 & 6.21 & 61.28 & 0.34 & 0.36 & 1.01 & 0.29 \\
\hline
\end{tabular}




\begin{tabular}{|c|c|c|c|c|c|c|c|c|c|}
\hline & All data & 0.29 & 75.67 & 8.98 & 14.88 & 0.01 & 0.08 & 0.09 & 0.00 \\
\hline & ${ }^{1} \mathrm{H}$ data & 0.02 & 96.27 & 3.40 & 0.30 & 0.00 & 0.02 & 0.00 & 0.00 \\
\hline \multirow[t]{5}{*}{ DP4+ } & ${ }^{13} \mathrm{C}$ data & 0.25 & 2.12 & 0.12 & 97.50 & 0.00 & 0.00 & 0.00 & 0.00 \\
\hline & All data & 0.00 & 87.37 & 0.18 & 12.45 & 0.00 & 0.00 & 0.00 & 0.00 \\
\hline & & \multicolumn{8}{|c|}{ Level of theory: B3LYP/6-311G** (gas phase) } \\
\hline & & $79 a$ & $79 b$ & $79 c$ & $79 d$ & $79 e$ & $79 f$ & $79 \mathrm{~g}$ & $79 \mathrm{~h}$ \\
\hline & ${ }^{1} \mathrm{H}$ data & 1.42 & 76.84 & 16.29 & 3.47 & 0.24 & 0.36 & 1.29 & 0.09 \\
\hline \multirow[t]{2}{*}{ sDP4+ } & ${ }^{13} \mathrm{C}$ data & 6.36 & 3.82 & 1.11 & 87.88 & 0.26 & 0.01 & 0.06 & 0.50 \\
\hline & All data & 1.45 & 46.89 & 2.88 & 48.76 & 0.01 & 0.00 & 0.01 & 0.01 \\
\hline & ${ }^{1} \mathrm{H}$ data & 8.40 & 46.98 & 25.95 & 4.68 & 2.37 & 9.29 & 1.23 & 1.11 \\
\hline \multirow[t]{2}{*}{ uDP4+ } & ${ }^{13} \mathrm{C}$ data & 0.80 & 30.06 & 6.16 & 60.97 & 0.15 & 0.44 & 1.28 & 0.14 \\
\hline & All data & 0.36 & 75.51 & 8.54 & 15.26 & 0.02 & 0.22 & 0.08 & 0.01 \\
\hline \multirow{5}{*}{ DP4+ } & ${ }^{1} \mathrm{H}$ data & 0.29 & 88.77 & 10.40 & 0.40 & 0.01 & 0.08 & 0.04 & 0.00 \\
\hline & ${ }^{13} \mathrm{C}$ data & 0.09 & 2.09 & 0.12 & 97.69 & 0.00 & 0.00 & 0.00 & 0.00 \\
\hline & All data & 0.01 & 82.16 & 0.57 & 17.26 & 0.00 & 0.00 & 0.00 & 0.00 \\
\hline & & \multicolumn{8}{|c|}{ Level of theory: B3LYP/6-311+G** (gas phase) } \\
\hline & & 79a & $79 b$ & 79c & 79d & $79 e$ & $79 f$ & $79 \mathrm{~g}$ & $79 \mathrm{~h}$ \\
\hline \multirow{3}{*}{$s \mathrm{DP4+}$} & ${ }^{1} \mathrm{H}$ data & 10.73 & 46.18 & 29.18 & 9.47 & 0.67 & 1.02 & 2.40 & 0.35 \\
\hline & ${ }^{13} \mathrm{C}$ data & 4.95 & 6.21 & 1.60 & 86.91 & 0.12 & 0.01 & 0.03 & 0.18 \\
\hline & All data & 4.39 & 23.71 & 3.86 & 68.03 & 0.01 & 0.00 & 0.01 & 0.01 \\
\hline \multirow{3}{*}{ uDP4+ } & ${ }^{1} \mathrm{H}$ data & 22.87 & 20.12 & 22.95 & 14.45 & 4.32 & 11.69 & 1.67 & 1.93 \\
\hline & ${ }^{13} \mathrm{C}$ data & 0.78 & 37.33 & 5.06 & 55.33 & 0.15 & 0.35 & 0.94 & 0.05 \\
\hline & All data & 1.06 & 44.42 & 6.87 & 47.27 & 0.04 & 0.24 & 0.09 & 0.01 \\
\hline \multirow{5}{*}{ DP4+ } & ${ }^{1} \mathrm{H}$ data & 12.26 & 46.45 & 33.48 & 6.84 & 0.15 & 0.59 & 0.20 & 0.03 \\
\hline & ${ }^{13} \mathrm{C}$ data & 0.08 & 4.59 & 0.16 & 95.17 & 0.00 & 0.00 & 0.00 & 0.00 \\
\hline & All data & 0.11 & 24.49 & 0.62 & 74.78 & 0.00 & 0.00 & 0.00 & 0.00 \\
\hline & & \multicolumn{8}{|c|}{ Level of theory: B3LYP/6-31G* (solution, PCM) } \\
\hline & & $79 a$ & $79 b$ & 79c & 79d & $79 e$ & $79 f$ & $79 \mathrm{~g}$ & $79 \mathrm{~h}$ \\
\hline & ${ }^{1} \mathrm{H}$ data & 4.22 & 54.91 & 34.07 & 6.75 & 0.00 & 0.01 & 0.04 & 0.01 \\
\hline \multirow[t]{2}{*}{$s \mathrm{DP} 4+$} & ${ }^{13} \mathrm{C}$ data & 22.79 & 4.66 & 1.73 & 69.31 & 0.49 & 0.02 & 0.17 & 0.81 \\
\hline & All data & 10.94 & 29.12 & 6.72 & 53.22 & 0.00 & 0.00 & 0.00 & 0.00 \\
\hline \multirow{3}{*}{ uDP4+ } & ${ }^{1} \mathrm{H}$ data & 0.59 & 51.67 & 41.92 & 5.21 & 0.01 & 0.39 & 0.19 & 0.02 \\
\hline & ${ }^{13} \mathrm{C}$ data & 25.87 & 7.74 & 21.55 & 44.53 & 0.09 & 0.01 & 0.05 & 0.15 \\
\hline & All data & 0.98 & 25.79 & 58.26 & 14.97 & 0.00 & 0.00 & 0.00 & 0.00 \\
\hline \multirow{5}{*}{ DP4+ } & ${ }^{1} \mathrm{H}$ data & 0.06 & 65.94 & 33.19 & 0.82 & 0.00 & 0.00 & 0.00 & 0.00 \\
\hline & ${ }^{13} \mathrm{C}$ data & 15.72 & 0.96 & 1.00 & 82.31 & 0.00 & 0.00 & 0.00 & 0.00 \\
\hline & All data & 0.55 & 38.52 & 20.07 & 40.86 & 0.00 & 0.00 & 0.00 & 0.00 \\
\hline & & \multicolumn{8}{|c|}{ Level of theory: B3LYP/6-31G** (solution, PCM) } \\
\hline & & $79 a$ & $79 b$ & 79c & $79 d$ & $79 e$ & $79 f$ & $79 \mathrm{~g}$ & $79 \mathrm{~h}$ \\
\hline & ${ }^{1} \mathrm{H}$ data & 7.17 & 60.40 & 26.08 & 6.05 & 0.02 & 0.02 & 0.23 & 0.04 \\
\hline \multirow[t]{2}{*}{ sDP4+ } & ${ }^{13} \mathrm{C}$ data & 29.82 & 6.77 & 2.69 & 58.33 & 0.91 & 0.04 & 0.20 & 1.23 \\
\hline & All data & 20.44 & 39.12 & 6.70 & 33.73 & 0.00 & 0.00 & 0.00 & 0.00 \\
\hline \multirow{3}{*}{ uDP4+ } & ${ }^{1} \mathrm{H}$ data & 1.27 & 56.55 & 29.93 & 11.67 & 0.03 & 0.29 & 0.21 & 0.05 \\
\hline & ${ }^{13} \mathrm{C}$ data & 12.14 & 20.96 & 16.81 & 50.01 & 0.02 & 0.01 & 0.02 & 0.04 \\
\hline & All data & 0.67 & 51.83 & 21.99 & 25.51 & 0.00 & 0.00 & 0.00 & 0.00 \\
\hline & ${ }^{1} \mathrm{H}$ data & 0.21 & 79.88 & 18.25 & 1.65 & 0.00 & 0.00 & 0.00 & 0.00 \\
\hline DP4+ & ${ }^{13} \mathrm{C}$ data & 10.44 & 4.09 & 1.30 & 84.16 & 0.00 & 0.00 & 0.00 & 0.00 \\
\hline & All data & 0.45 & 66.49 & 4.83 & 28.23 & 0.00 & 0.00 & 0.00 & 0.00 \\
\hline & & & & Level of & V: B3LYF & $+\mathrm{G}^{* *}($ & on, PCN & & \\
\hline & & $79 a$ & $79 b$ & 79c & $79 d$ & $79 e$ & $79 f$ & $79 \mathrm{~g}$ & $79 \mathrm{~h}$ \\
\hline & ${ }^{1} \mathrm{H}$ data & 36.94 & 11.08 & 49.33 & 1.07 & 0.07 & 0.82 & 0.61 & 0.08 \\
\hline sDP4+ & ${ }^{13} \mathrm{C}$ data & 8.03 & 18.54 & 14.10 & 58.52 & 0.24 & 0.20 & 0.28 & 0.10 \\
\hline & All data & 23.52 & 16.30 & 55.18 & 4.97 & 0.00 & 0.01 & 0.01 & 0.00 \\
\hline & ${ }^{1} \mathrm{H}$ data & 29.73 & 8.41 & 56.07 & 3.69 & 0.49 & 0.00 & 1.28 & 0.32 \\
\hline uDP4+ & ${ }^{13} \mathrm{C}$ data & 4.00 & 33.24 & 39.65 & 23.04 & 0.01 & 0.00 & 0.05 & 0.00 \\
\hline & All data & 4.39 & 10.33 & 82.14 & 3.14 & 0.00 & 0.00 & 0.00 & 0.00 \\
\hline
\end{tabular}




\begin{tabular}{|c|c|c|c|c|c|c|c|c|c|}
\hline & ${ }^{1} \mathrm{H}$ data & 27.71 & 2.35 & 69.81 & 0.10 & 0.00 & 0.00 & 0.02 & 0.00 \\
\hline \multirow[t]{5}{*}{ DP4+ } & ${ }^{13} \mathrm{C}$ data & 1.26 & 24.12 & 21.88 & 52.75 & 0.00 & 0.00 & 0.00 & 0.00 \\
\hline & All data & 2.14 & 3.49 & 94.04 & 0.32 & 0.00 & 0.00 & 0.00 & 0.00 \\
\hline & & \multicolumn{8}{|c|}{ Level of theory: B3LYP/6-311G* (solution, PCM) } \\
\hline & & 79a & $79 b$ & 79c & 79d & $79 e$ & $79 f$ & $79 \mathrm{~g}$ & $79 \mathrm{~h}$ \\
\hline & ${ }^{1} \mathrm{H}$ data & 3.19 & 84.76 & 8.72 & 2.84 & 0.02 & 0.23 & 0.21 & 0.03 \\
\hline \multirow[t]{2}{*}{$s \mathrm{DP} 4+$} & ${ }^{13} \mathrm{C}$ data & 3.73 & 4.44 & 1.82 & 89.89 & 0.03 & 0.01 & 0.02 & 0.06 \\
\hline & All data & 1.80 & 57.09 & 2.42 & 38.69 & 0.00 & 0.00 & 0.00 & 0.00 \\
\hline \multirow{3}{*}{ uDP4+ } & ${ }^{1} \mathrm{H}$ data & 3.84 & 69.14 & 17.34 & 8.27 & 0.06 & 0.68 & 0.55 & 0.11 \\
\hline & ${ }^{13} \mathrm{C}$ data & 0.70 & 37.25 & 8.68 & 51.91 & 0.09 & 0.33 & 0.99 & 0.05 \\
\hline & All data & 0.09 & 81.53 & 4.76 & 13.60 & 0.00 & 0.01 & 0.02 & 0.00 \\
\hline \multirow{3}{*}{ DP4+ } & ${ }^{1} \mathrm{H}$ data & 0.20 & 96.90 & 2.50 & 0.39 & 0.00 & 0.00 & 0.00 & 0.00 \\
\hline & ${ }^{13} \mathrm{C}$ data & 0.05 & 3.41 & 0.33 & 96.21 & 0.00 & 0.00 & 0.00 & 0.00 \\
\hline & All data & 0.00 & 89.64 & 0.22 & 10.13 & 0.00 & 0.00 & 0.00 & 0.00 \\
\hline & & \multicolumn{8}{|c|}{ Level of theory: B3LYP/6-311G** (solution, PCM) } \\
\hline & & $79 a$ & $79 b$ & 79c & 79d & $79 e$ & $79 f$ & $79 \mathrm{~g}$ & $79 \mathrm{~h}$ \\
\hline \multirow{3}{*}{ sDP4+ } & ${ }^{1} \mathrm{H}$ data & 15.84 & 60.38 & 17.52 & 1.60 & 0.26 & 0.78 & 3.27 & 0.34 \\
\hline & ${ }^{13} \mathrm{C}$ data & 3.01 & 4.19 & 1.79 & 90.89 & 0.03 & 0.01 & 0.03 & 0.06 \\
\hline & All data & 9.99 & 52.90 & 6.57 & 30.52 & 0.00 & 0.00 & 0.02 & 0.00 \\
\hline \multirow{3}{*}{ uDP4+ } & ${ }^{1} \mathrm{H}$ data & 18.49 & 44.85 & 22.36 & 4.55 & 1.17 & 3.05 & 2.67 & 2.86 \\
\hline & ${ }^{13} \mathrm{C}$ data & 0.32 & 39.91 & 8.62 & 49.27 & 0.05 & 0.43 & 1.37 & 0.03 \\
\hline & All data & 0.26 & 80.72 & 8.69 & 10.10 & 0.00 & 0.06 & 0.16 & 0.00 \\
\hline & ${ }^{1} \mathrm{H}$ data & 8.59 & 79.36 & 11.48 & 0.21 & 0.01 & 0.07 & 0.26 & 0.03 \\
\hline \multirow[t]{4}{*}{ DP4+ } & ${ }^{13} \mathrm{C}$ data & 0.02 & 3.58 & 0.33 & 96.06 & 0.00 & 0.00 & 0.00 & 0.00 \\
\hline & All data & 0.06 & 92.07 & 1.23 & 6.65 & 0.00 & 0.00 & 0.00 & 0.00 \\
\hline & & \multicolumn{8}{|c|}{ Level of theory: B3LYP/6-311+G** (solution, PCM) } \\
\hline & & $79 a$ & $79 b$ & $79 c$ & $79 d$ & $79 e$ & $79 f$ & $79 \mathrm{~g}$ & $79 \mathrm{~h}$ \\
\hline & ${ }_{12}^{1} \mathrm{H}$ data & 54.56 & 22.58 & 15.27 & 2.69 & 0.37 & 1.26 & 2.75 & 0.52 \\
\hline \multirow[t]{2}{*}{ sDP4+ } & ${ }^{13} \mathrm{C}$ data & 2.38 & 6.41 & 2.66 & 88.50 & 0.02 & 0.00 & 0.01 & 0.02 \\
\hline & All data & 23.46 & 26.15 & 7.34 & 43.04 & 0.00 & 0.00 & 0.01 & 0.00 \\
\hline \multirow{3}{*}{ uDP4+ } & ${ }^{1} \mathrm{H}$ data & 66.51 & 13.24 & 13.20 & 3.67 & 0.52 & 1.62 & 0.63 & 0.62 \\
\hline & ${ }^{13} \mathrm{C}$ data & 0.47 & 60.09 & 9.66 & 29.16 & 0.04 & 0.11 & 0.46 & 0.01 \\
\hline & All data & 2.94 & 74.93 & 12.01 & 10.08 & 0.00 & 0.02 & 0.03 & 0.00 \\
\hline \multirow{5}{*}{ DP4+ } & ${ }^{1} \mathrm{H}$ data & 87.58 & 7.21 & 4.87 & 0.24 & 0.00 & 0.05 & 0.04 & 0.01 \\
\hline & ${ }^{13} \mathrm{C}$ data & 0.04 & 12.86 & 0.86 & 86.24 & 0.00 & 0.00 & 0.00 & 0.00 \\
\hline & All data & 2.70 & 76.82 & 3.46 & 17.02 & 0.00 & 0.00 & 0.00 & 0.00 \\
\hline & & \multicolumn{8}{|c|}{ Level of theory: mPW1PW91/6-31G* (gas phase) } \\
\hline & & $79 a$ & $79 b$ & 79c & 79d & $79 e$ & $79 f$ & $79 \mathrm{~g}$ & $79 \mathrm{~h}$ \\
\hline \multirow{3}{*}{ sDP4+ } & ${ }^{1} \mathrm{H}$ data & 0.45 & 39.46 & 23.43 & 36.61 & 0.01 & 0.00 & 0.02 & 0.01 \\
\hline & ${ }^{13} \mathrm{C}$ data & 5.73 & 3.47 & 0.88 & 88.38 & 0.40 & 0.00 & 0.17 & 0.96 \\
\hline & All data & 0.08 & 4.03 & 0.61 & 95.28 & 0.00 & 0.00 & 0.00 & 0.00 \\
\hline \multirow{3}{*}{ uDP4+ } & ${ }^{1} \mathrm{H}$ data & 0.34 & 53.03 & 33.89 & 12.48 & 0.01 & 0.08 & 0.16 & 0.02 \\
\hline & ${ }^{13} \mathrm{C}$ data & 67.42 & 3.50 & 12.82 & 14.09 & 0.89 & 0.03 & 0.12 & 1.13 \\
\hline & All data & 2.78 & 22.65 & 53.08 & 21.49 & 0.00 & 0.00 & 0.00 & 0.00 \\
\hline & ${ }^{1} \mathrm{H}$ data & 0.00 & 62.58 & 23.75 & 13.66 & 0.00 & 0.00 & 0.00 & 0.00 \\
\hline DP4+ & ${ }^{13} \mathrm{C}$ data & 23.31 & 0.73 & 0.68 & 75.18 & 0.02 & 0.00 & 0.00 & 0.07 \\
\hline & All data & 0.01 & 4.21 & 1.49 & 94.29 & 0.00 & 0.00 & 0.00 & 0.00 \\
\hline & & & & evel of $t$ & : $\mathrm{mPW}$ & $/ 6-31$ & s phas & & \\
\hline & & $79 a$ & $79 b$ & $79 c$ & $79 d$ & $79 e$ & $79 f$ & $79 \mathrm{~g}$ & $79 \mathrm{~h}$ \\
\hline & ${ }^{1} \mathrm{H}$ data & 0.75 & 43.24 & 24.84 & 30.94 & 0.02 & 0.01 & 0.15 & 0.06 \\
\hline sDP4+ & ${ }^{13} \mathrm{C}$ data & 15.08 & 5.44 & 1.51 & 76.24 & 0.43 & 0.01 & 0.16 & 1.12 \\
\hline & All data & 0.43 & 8.90 & 1.42 & 89.24 & 0.00 & 0.00 & 0.00 & 0.00 \\
\hline & ${ }^{1} \mathrm{H}$ data & 0.79 & 51.23 & 28.93 & 18.58 & 0.04 & 0.10 & 0.25 & 0.07 \\
\hline uDP4+ & ${ }^{13} \mathrm{C}$ data & 26.47 & 9.96 & 19.53 & 43.78 & 0.07 & 0.00 & 0.04 & 0.15 \\
\hline & All data & 1.10 & 26.71 & 29.59 & 42.60 & 0.00 & 0.00 & 0.00 & 0.00 \\
\hline DP4+ & ${ }^{1} \mathrm{H}$ data & 0.02 & 63.13 & 20.47 & 16.38 & 0.00 & 0.00 & 0.00 & 0.00 \\
\hline
\end{tabular}




\begin{tabular}{|c|c|c|c|c|c|c|c|c|c|}
\hline & ${ }^{13} \mathrm{C}$ data & 10.45 & 1.42 & 0.77 & 87.36 & 0.00 & 0.00 & 0.00 & 0.00 \\
\hline & All data & 0.01 & 5.83 & 1.03 & 93.13 & 0.00 & 0.00 & 0.00 & 0.00 \\
\hline & & \multicolumn{8}{|c|}{ Level of theory: mPW1PW91/6-31+G** (gas phase) } \\
\hline & & $79 a$ & $79 b$ & 79c & 79d & $79 e$ & $79 f$ & $79 \mathrm{~g}$ & $79 \mathrm{~h}$ \\
\hline & ${ }^{1} \mathrm{H}$ data & 7.83 & 35.17 & 36.42 & 18.75 & 0.34 & 0.07 & 1.04 & 0.39 \\
\hline \multirow[t]{2}{*}{ sDP4+ } & ${ }^{13} \mathrm{C}$ data & 8.96 & 6.99 & 3.82 & 79.38 & 0.54 & 0.04 & 0.11 & 0.16 \\
\hline & All data & 3.61 & 12.65 & 7.16 & 76.57 & 0.01 & 0.00 & 0.01 & 0.00 \\
\hline \multirow{3}{*}{ uDP4+ } & ${ }^{1} \mathrm{H}$ data & 7.40 & 28.17 & 34.75 & 25.10 & 1.56 & 0.68 & 1.69 & 0.66 \\
\hline & ${ }^{13} \mathrm{C}$ data & 8.44 & 25.47 & 38.14 & 27.88 & 0.02 & 0.02 & 0.03 & 0.01 \\
\hline & All data & 2.23 & 25.58 & 47.24 & 24.95 & 0.00 & 0.00 & 0.00 & 0.00 \\
\hline \multirow{5}{*}{ DP4+ } & ${ }^{1} \mathrm{H}$ data & 2.08 & 35.54 & 45.40 & 16.88 & 0.02 & 0.00 & 0.06 & 0.01 \\
\hline & ${ }^{13} \mathrm{C}$ data & 2.89 & 6.81 & 5.58 & 84.72 & 0.00 & 0.00 & 0.00 & 0.00 \\
\hline & All data & 0.31 & 12.54 & 13.11 & 74.05 & 0.00 & 0.00 & 0.00 & 0.00 \\
\hline & & \multicolumn{8}{|c|}{ Level of theory: mPW1PW91/6-311G* (gas phase) } \\
\hline & & $79 a$ & $79 b$ & $79 c$ & 79d & $79 e$ & $79 f$ & $79 \mathrm{~g}$ & $79 \mathrm{~h}$ \\
\hline & ${ }^{1} \mathrm{H}$ data & 0.34 & 91.49 & 3.85 & 4.11 & 0.02 & 0.03 & 0.13 & 0.03 \\
\hline \multirow[t]{2}{*}{ sDP4+ } & ${ }^{13} \mathrm{C}$ data & 5.48 & 6.83 & 1.47 & 85.90 & 0.08 & 0.01 & 0.04 & 0.19 \\
\hline & All data & 0.19 & 63.43 & 0.58 & 35.80 & 0.00 & 0.00 & 0.00 & 0.00 \\
\hline \multirow{3}{*}{ uDP4+ } & ${ }^{1} \mathrm{H}$ data & 3.75 & 58.90 & 25.75 & 8.79 & 0.32 & 0.98 & 1.21 & 0.31 \\
\hline & ${ }^{13} \mathrm{C}$ data & 1.12 & 20.06 & 2.70 & 75.74 & 0.06 & 0.03 & 0.21 & 0.08 \\
\hline & All data & 0.22 & 61.50 & 3.62 & 34.65 & 0.00 & 0.00 & 0.01 & 0.00 \\
\hline \multirow{6}{*}{ DP4+ } & ${ }^{1} \mathrm{H}$ data & 0.02 & 97.53 & 1.79 & 0.65 & 0.00 & 0.00 & 0.00 & 0.00 \\
\hline & ${ }^{13} \mathrm{C}$ data & 0.09 & 2.06 & 0.06 & 97.79 & 0.00 & 0.00 & 0.00 & 0.00 \\
\hline & All data & 0.00 & 75.84 & 0.04 & 24.12 & 0.00 & 0.00 & 0.00 & 0.00 \\
\hline & & \multicolumn{8}{|c|}{ Level of theory: mPW1PW91/6-311G** (gas phase) } \\
\hline & & $79 a$ & $79 b$ & 79c & $79 d$ & $79 e$ & $79 f$ & $79 \mathrm{~g}$ & $79 \mathrm{~h}$ \\
\hline & ${ }^{1} \mathrm{H}$ data & 2.70 & 78.31 & 10.38 & 6.85 & 0.24 & 0.08 & 1.24 & 0.20 \\
\hline \multirow[t]{2}{*}{ sDP4+ } & ${ }^{13} \mathrm{C}$ data & 4.59 & 6.17 & 1.40 & 87.49 & 0.07 & 0.01 & 0.04 & 0.21 \\
\hline & All data & 1.11 & 43.55 & 1.31 & 54.01 & 0.00 & 0.00 & 0.01 & 0.00 \\
\hline \multirow{3}{*}{ uDP4+ } & ${ }^{1} \mathrm{H}$ data & 8.88 & 55.77 & 20.97 & 8.44 & 1.60 & 1.45 & 1.30 & 1.58 \\
\hline & ${ }^{13} \mathrm{C}$ data & 0.40 & 30.55 & 4.17 & 64.42 & 0.02 & 0.04 & 0.37 & 0.03 \\
\hline & All data & 0.15 & 72.83 & 3.74 & 23.25 & 0.00 & 0.00 & 0.02 & 0.00 \\
\hline \multirow{5}{*}{ DP4+ } & ${ }^{1} \mathrm{H}$ data & 0.51 & 93.54 & 4.66 & 1.24 & 0.01 & 0.00 & 0.03 & 0.01 \\
\hline & ${ }^{13} \mathrm{C}$ data & 0.03 & 3.23 & 0.10 & 96.63 & 0.00 & 0.00 & 0.00 & 0.00 \\
\hline & All data & 0.00 & 71.55 & 0.11 & 28.33 & 0.00 & 0.00 & 0.00 & 0.00 \\
\hline & & \multicolumn{8}{|c|}{ Level of theory: mPW1PW91/6-311+G** (gas phase) } \\
\hline & & $79 a$ & $79 b$ & 79c & 79d & $79 e$ & $79 f$ & $79 \mathrm{~g}$ & $79 \mathrm{~h}$ \\
\hline \multirow{3}{*}{ sDP4+ } & ${ }^{1} \mathrm{H}$ data & 16.31 & 41.74 & 20.24 & 19.03 & 0.69 & 0.14 & 1.22 & 0.62 \\
\hline & ${ }^{13} \mathrm{C}$ data & 3.53 & 14.44 & 2.42 & 79.50 & 0.03 & 0.00 & 0.02 & 0.06 \\
\hline & All data & 2.59 & 27.11 & 2.21 & 68.09 & 0.00 & 0.00 & 0.00 & 0.00 \\
\hline \multirow{3}{*}{ uDP4+ } & ${ }^{1} \mathrm{H}$ data & 28.27 & 21.96 & 20.04 & 22.69 & 2.98 & 1.58 & 0.81 & 1.67 \\
\hline & ${ }^{13} \mathrm{C}$ data & 0.44 & 46.00 & 4.77 & 48.42 & 0.02 & 0.05 & 0.27 & 0.01 \\
\hline & All data & 0.56 & 45.56 & 4.31 & 49.55 & 0.00 & 0.00 & 0.01 & 0.00 \\
\hline \multirow{3}{*}{ DP4+ } & ${ }^{1} \mathrm{H}$ data & 20.77 & 41.30 & 18.27 & 19.46 & 0.09 & 0.01 & 0.04 & 0.05 \\
\hline & ${ }^{13} \mathrm{C}$ data & 0.03 & 14.67 & 0.26 & 85.04 & 0.00 & 0.00 & 0.00 & 0.00 \\
\hline & All data & 0.03 & 26.74 & 0.21 & 73.02 & 0.00 & 0.00 & 0.00 & 0.00 \\
\hline & & & & vel of th & mPW1P & 6-31G & ion, $\mathrm{PC}$ & & \\
\hline & & $79 a$ & $79 b$ & 79c & $79 d$ & $79 e$ & $79 f$ & $79 \mathrm{~g}$ & $79 \mathrm{~h}$ \\
\hline & ${ }^{1} \mathrm{H}$ data & 7.82 & 55.42 & 16.75 & 19.96 & 0.00 & 0.00 & 0.03 & 0.01 \\
\hline sDP4+ & ${ }^{13} \mathrm{C}$ data & 9.53 & 11.81 & 3.45 & 74.57 & 0.21 & 0.00 & 0.13 & 0.29 \\
\hline & All data & 3.27 & 28.76 & 2.54 & 65.42 & 0.00 & 0.00 & 0.00 & 0.00 \\
\hline & ${ }^{1} \mathrm{H}$ data & 0.70 & 54.76 & 35.08 & 9.21 & 0.01 & 0.11 & 0.12 & 0.01 \\
\hline uDP4+ & ${ }^{13} \mathrm{C}$ data & 51.13 & 6.37 & 23.88 & 17.59 & 0.46 & 0.02 & 0.07 & 0.46 \\
\hline & All data & 2.59 & 25.19 & 60.51 & 11.70 & 0.00 & 0.00 & 0.00 & 0.00 \\
\hline & ${ }^{1} \mathrm{H}$ data & 0.14 & 79.62 & 15.41 & 4.82 & 0.00 & 0.00 & 0.00 & 0.00 \\
\hline DP4+ & ${ }^{13} \mathrm{C}$ data & 24.90 & 3.84 & 4.21 & 67.04 & 0.01 & 0.00 & 0.00 & 0.01 \\
\hline
\end{tabular}




\begin{tabular}{|c|c|c|c|c|c|c|c|c|c|}
\hline & All data & 0.51 & 43.85 & 9.30 & 46.34 & 0.00 & 0.00 & 0.00 & 0.00 \\
\hline & & \multicolumn{8}{|c|}{ Level of theory: mPW1PW91/6-31G** (solution, PCM) } \\
\hline & & $79 a$ & $79 b$ & 79c & 79d & $79 e$ & $79 f$ & $79 \mathrm{~g}$ & $79 \mathrm{~h}$ \\
\hline & ${ }^{1} \mathrm{H}$ data & 15.05 & 56.79 & 9.13 & 18.87 & 0.01 & 0.01 & 0.10 & 0.04 \\
\hline \multirow[t]{2}{*}{ sDP4+ } & ${ }^{13} \mathrm{C}$ data & 16.10 & 11.25 & 3.60 & 68.52 & 0.15 & 0.01 & 0.08 & 0.29 \\
\hline & All data & 10.98 & 28.96 & 1.49 & 58.58 & 0.00 & 0.00 & 0.00 & 0.00 \\
\hline & ${ }^{1} \mathrm{H}$ data & 1.73 & 62.05 & 19.52 & 16.48 & 0.02 & 0.05 & 0.11 & 0.03 \\
\hline \multirow[t]{2}{*}{ uDP4+ } & ${ }^{13} \mathrm{C}$ data & 18.54 & 13.46 & 25.74 & 42.11 & 0.04 & 0.00 & 0.03 & 0.07 \\
\hline & All data & 1.56 & 40.48 & 24.34 & 33.62 & 0.00 & 0.00 & 0.00 & 0.00 \\
\hline & ${ }^{1} \mathrm{H}$ data & 0.65 & 87.25 & 4.41 & 7.70 & 0.00 & 0.00 & 0.00 & 0.00 \\
\hline \multirow[t]{4}{*}{ DP4+ } & ${ }^{13} \mathrm{C}$ data & 8.70 & 4.42 & 2.71 & 84.17 & 0.00 & 0.00 & 0.00 & 0.00 \\
\hline & All data & 0.53 & 36.69 & 1.14 & 61.64 & 0.00 & 0.00 & 0.00 & 0.00 \\
\hline & & \multicolumn{8}{|c|}{ Level of theory: mPW1PW91/6-31+G** (solution, PCM) } \\
\hline & & 79a & $79 b$ & 79c & 79d & $79 e$ & $79 f$ & $79 \mathrm{~g}$ & $79 \mathrm{~h}$ \\
\hline & ${ }^{1} \mathrm{H}$ data & 66.65 & 12.86 & 15.49 & 3.95 & 0.10 & 0.21 & 0.54 & 0.20 \\
\hline \multirow[t]{2}{*}{$s \mathrm{DP} 4+$} & ${ }^{13} \mathrm{C}$ data & 7.02 & 10.62 & 6.80 & 75.41 & 0.06 & 0.02 & 0.05 & 0.02 \\
\hline & All data & 46.46 & 13.55 & 10.45 & 29.54 & 0.00 & 0.00 & 0.00 & 0.00 \\
\hline \multirow{3}{*}{ uDP4+ } & ${ }^{1} \mathrm{H}$ data & 47.42 & 13.63 & 28.30 & 8.68 & 0.25 & 0.62 & 0.81 & 0.30 \\
\hline & ${ }^{13} \mathrm{C}$ data & 6.05 & 31.17 & 45.01 & 17.71 & 0.02 & 0.01 & 0.03 & 0.00 \\
\hline & All data & 13.41 & 19.87 & 59.54 & 7.18 & 0.00 & 0.00 & 0.00 & 0.00 \\
\hline \multirow{6}{*}{ DP4+ } & ${ }_{12}^{1} \mathrm{H}$ data & 82.97 & 4.60 & 11.51 & 0.90 & 0.00 & 0.00 & 0.01 & 0.00 \\
\hline & ${ }^{13} \mathrm{C}$ data & 2.11 & 16.43 & 15.18 & 66.28 & 0.00 & 0.00 & 0.00 & 0.00 \\
\hline & All data & 36.08 & 15.59 & 36.04 & 12.29 & 0.00 & 0.00 & 0.00 & 0.00 \\
\hline & & \multicolumn{8}{|c|}{ Level of theory: mPW1PW91/6-311G* (solution, PCM) } \\
\hline & & $79 a$ & $79 b$ & 79c & 79d & $79 e$ & $79 f$ & $79 \mathrm{~g}$ & $79 \mathrm{~h}$ \\
\hline & ${ }^{1} \mathrm{H}$ data & 5.03 & 85.16 & 5.42 & 4.21 & 0.01 & 0.04 & 0.10 & 0.03 \\
\hline \multirow[t]{2}{*}{$s \mathrm{DP} 4+$} & ${ }^{13} \mathrm{C}$ data & 4.88 & 10.49 & 2.91 & 81.65 & 0.01 & 0.00 & 0.02 & 0.02 \\
\hline & All data & 1.92 & 69.94 & 1.24 & 26.90 & 0.00 & 0.00 & 0.00 & 0.00 \\
\hline & ${ }^{1} \mathrm{H}$ data & 5.38 & 68.15 & 14.33 & 11.55 & 0.05 & 0.16 & 0.30 & 0.09 \\
\hline \multirow[t]{2}{*}{ uDP4+ } & ${ }^{13} \mathrm{C}$ data & 0.37 & 35.91 & 5.35 & 58.01 & 0.02 & 0.05 & 0.29 & 0.01 \\
\hline & All data & 0.06 & 76.57 & 2.40 & 20.96 & 0.00 & 0.00 & 0.00 & 0.00 \\
\hline \multirow{6}{*}{ DP4+ } & ${ }^{1} \mathrm{H}$ data & 0.45 & 97.42 & 1.30 & 0.82 & 0.00 & 0.00 & 0.00 & 0.00 \\
\hline & ${ }^{13} \mathrm{C}$ data & 0.04 & 7.34 & 0.30 & 92.32 & 0.00 & 0.00 & 0.00 & 0.00 \\
\hline & All data & 0.00 & 90.43 & 0.05 & 9.52 & 0.00 & 0.00 & 0.00 & 0.00 \\
\hline & & \multicolumn{8}{|c|}{ Level of theory: mPW1PW91/6-311G** (solution, PCM) } \\
\hline & & $79 a$ & $79 b$ & 79c & 79d & $79 e$ & $79 f$ & $79 \mathrm{~g}$ & $79 \mathrm{~h}$ \\
\hline & ${ }^{1} \mathrm{H}$ data & 22.69 & 65.69 & 7.04 & 2.32 & 0.18 & 0.15 & 1.49 & 0.44 \\
\hline \multirow[t]{2}{*}{$s \mathrm{DP} 4+$} & ${ }^{13} \mathrm{C}$ data & 2.82 & 10.83 & 2.78 & 83.52 & 0.01 & 0.00 & 0.02 & 0.02 \\
\hline & All data & 6.47 & 71.94 & 1.98 & 19.61 & 0.00 & 0.00 & 0.00 & 0.00 \\
\hline \multirow{3}{*}{ uDP4+ } & ${ }^{1} \mathrm{H}$ data & 23.76 & 47.82 & 13.88 & 8.41 & 0.48 & 0.52 & 2.04 & 3.09 \\
\hline & ${ }^{13} \mathrm{C}$ data & 0.15 & 52.06 & 7.99 & 39.13 & 0.01 & 0.06 & 0.60 & 0.01 \\
\hline & All data & 0.12 & 84.84 & 3.78 & 11.22 & 0.00 & 0.00 & 0.04 & 0.00 \\
\hline \multirow{5}{*}{ DP4+ } & ${ }^{1} \mathrm{H}$ data & 14.18 & 82.62 & 2.57 & 0.51 & 0.00 & 0.00 & 0.08 & 0.04 \\
\hline & ${ }^{13} \mathrm{C}$ data & 0.01 & 14.63 & 0.58 & 84.78 & 0.00 & 0.00 & 0.00 & 0.00 \\
\hline & All data & 0.01 & 96.40 & 0.12 & 3.47 & 0.00 & 0.00 & 0.00 & 0.00 \\
\hline & & & & I of the & PW1PV & $311+G$ & ution, & & \\
\hline & & $79 a$ & $79 b$ & 79c & $79 d$ & $79 e$ & $79 f$ & $79 \mathrm{~g}$ & $79 h$ \\
\hline & ${ }^{1} \mathrm{H}$ data & 51.98 & 12.86 & 7.15 & 5.71 & 3.87 & 2.45 & 10.12 & 5.85 \\
\hline sDP4+ & ${ }^{13} \mathrm{C}$ data & 3.70 & 18.35 & 7.55 & 70.37 & 0.01 & 0.00 & 0.01 & 0.01 \\
\hline & All data & 21.75 & 26.68 & 6.10 & 45.43 & 0.00 & 0.00 & 0.01 & 0.00 \\
\hline & ${ }^{1} \mathrm{H}$ data & 45.83 & 9.25 & 7.33 & 8.68 & 6.78 & 5.31 & 6.97 & 9.86 \\
\hline uDP4+ & ${ }^{13} \mathrm{C}$ data & 0.19 & 71.59 & 9.48 & 18.50 & 0.01 & 0.03 & 0.19 & 0.00 \\
\hline & All data & 0.95 & 73.38 & 7.70 & 17.79 & 0.00 & 0.02 & 0.15 & 0.00 \\
\hline & ${ }^{1} \mathrm{H}$ data & 85.98 & 4.29 & 1.89 & 1.79 & 0.95 & 0.47 & 2.54 & 2.08 \\
\hline DP4+ & ${ }^{13} \mathrm{C}$ data & 0.03 & 48.87 & 2.66 & 48.44 & 0.00 & 0.00 & 0.00 & 0.00 \\
\hline & All data & 0.73 & 69.09 & 1.66 & 28.52 & 0.00 & 0.00 & 0.00 & 0.00 \\
\hline
\end{tabular}


$s \mathrm{DP4}+, u \mathrm{DP} 4+$ and DP4+ probabilities (\%) for compound 79b<smiles>CC[C@H](C)[C@H]1OC([C@H](C)CC)=C(C)C(=O)[C@H]1C</smiles><smiles>CC[C@H](O)[C@@H](C)C1=C(C)C(=O)[C@@H](C)[C@@H]([C@@H](C)CC)O1</smiles><smiles>CC[C@H](O)[C@@H](C)C1=C(C)C(=O)[C@@H](C)[C@@H]([C@@H](C)CC)O1</smiles><smiles>CC[C@H](O)[C@@H](C)C1=C(C)C(=O)[C@@H](C)[C@@H]([C@@H](C)CC)O1</smiles><smiles>CC[C@H](O)[C@@H](C)C1=C(C)C(=O)[C@@H](C)[C@@H]([C@@H](C)CC)O1</smiles><smiles>CC[C@H](O)[C@@H](C)C1=C(C)C(=O)[C@@H](C)[C@@H]([C@@H](C)CC)O1</smiles><smiles>CC[C@H](O)[C@@H](C)C1=C(C)C(=O)[C@@H](C)[C@@H]([C@@H](C)CC)O1</smiles><smiles>CC[C@H](O)[C@@H](C)C1=C(C)C(=O)[C@@H](C)[C@@H]([C@@H](C)CC)O1</smiles>

\begin{tabular}{|c|c|c|c|c|c|c|c|c|c|}
\hline & & \multicolumn{8}{|c|}{ Level of theory: B3LYP/6-31G* (gas phase) } \\
\hline & & $79 a$ & $79 b$ & $79 c$ & 79d & $79 \mathrm{e}$ & $79 f$ & $79 g$ & $79 \mathrm{~h}$ \\
\hline & ${ }^{1} \mathrm{H}$ data & 0.02 & 30.37 & 59.12 & 10.40 & 0.00 & 0.02 & 0.06 & 0.01 \\
\hline \multirow[t]{3}{*}{ sDP4+ } & ${ }^{13} \mathrm{C}$ data & 4.70 & 7.81 & 2.76 & 82.68 & 0.35 & 0.16 & 0.97 & 0.56 \\
\hline & All data & 0.01 & 18.82 & 12.96 & 68.20 & 0.00 & 0.00 & 0.00 & 0.00 \\
\hline & ${ }^{1} \mathrm{H}$ data & 0.02 & 34.05 & 58.87 & 6.58 & 0.01 & 0.23 & 0.22 & 0.01 \\
\hline \multirow[t]{3}{*}{ uDP4+ } & ${ }^{13} \mathrm{C}$ data & 3.52 & 9.57 & 23.14 & 63.56 & 0.03 & 0.03 & 0.12 & 0.04 \\
\hline & All data & 0.00 & 15.46 & 64.67 & 19.86 & 0.00 & 0.00 & 0.00 & 0.00 \\
\hline & ${ }^{1} \mathrm{H}$ data & 0.00 & 22.56 & 75.94 & 1.49 & 0.00 & 0.00 & 0.00 & 0.00 \\
\hline \multirow[t]{5}{*}{ DP4+ } & ${ }^{13} \mathrm{C}$ data & 0.31 & 1.38 & 1.18 & 97.13 & 0.00 & 0.00 & 0.00 & 0.00 \\
\hline & All data & 0.00 & 11.72 & 33.75 & 54.54 & 0.00 & 0.00 & 0.00 & 0.00 \\
\hline & & \multicolumn{8}{|c|}{ Level of theory: B3LYP/6-31G** (gas phase) } \\
\hline & & $79 a$ & $79 b$ & 79c & $79 d$ & $79 \mathrm{e}$ & $79 f$ & $79 \mathrm{~g}$ & $79 \mathrm{~h}$ \\
\hline & ${ }^{1} \mathrm{H}$ data & 0.03 & 31.45 & 60.22 & 8.06 & 0.01 & 0.02 & 0.19 & 0.01 \\
\hline \multirow[t]{3}{*}{ sDP4+ } & ${ }^{13} \mathrm{C}$ data & 7.40 & 14.62 & 5.30 & 69.80 & 0.71 & 0.31 & 1.18 & 0.70 \\
\hline & All data & 0.02 & 34.26 & 23.78 & 41.93 & 0.00 & 0.00 & 0.02 & 0.00 \\
\hline & ${ }^{1} \mathrm{H}$ data & 0.04 & 30.72 & 63.85 & 4.71 & 0.02 & 0.24 & 0.40 & 0.03 \\
\hline \multirow[t]{3}{*}{ uDP4+ } & ${ }^{13} \mathrm{C}$ data & 1.65 & 21.65 & 22.76 & 53.85 & 0.00 & 0.01 & 0.06 & 0.01 \\
\hline & All data & 0.00 & 28.04 & 61.25 & 10.70 & 0.00 & 0.00 & 0.00 & 0.00 \\
\hline & ${ }^{1} \mathrm{H}$ data & 0.00 & 19.92 & 79.29 & 0.78 & 0.00 & 0.00 & 0.00 & 0.00 \\
\hline \multirow[t]{5}{*}{ DP4+ } & ${ }^{13} \mathrm{C}$ data & 0.29 & 7.52 & 2.87 & 89.32 & 0.00 & 0.00 & 0.00 & 0.00 \\
\hline & All data & 0.00 & 33.52 & 50.82 & 15.66 & 0.00 & 0.00 & 0.00 & 0.00 \\
\hline & & \multicolumn{8}{|c|}{ Level of theory: B3LYP/6-31+G** (gas phase) } \\
\hline & & $79 a$ & $79 b$ & $79 c$ & 79d & $79 \mathrm{e}$ & $79 f$ & $79 \mathrm{~g}$ & $79 \mathrm{~h}$ \\
\hline & ${ }^{1} \mathrm{H}$ data & 0.88 & 24.96 & 66.24 & 6.45 & 0.24 & 0.08 & 1.04 & 0.12 \\
\hline \multirow[t]{3}{*}{ sDP4+ } & ${ }^{13} \mathrm{C}$ data & 2.07 & 19.88 & 17.45 & 58.45 & 0.26 & 1.00 & 0.83 & 0.06 \\
\hline & All data & 0.09 & 24.41 & 56.88 & 18.57 & 0.00 & 0.00 & 0.04 & 0.00 \\
\hline & ${ }^{1} \mathrm{H}$ data & 0.65 & 17.19 & 71.65 & 6.91 & 0.82 & 0.78 & 1.72 & 0.29 \\
\hline \multirow[t]{3}{*}{ uDP4+ } & ${ }^{13} \mathrm{C}$ data & 0.68 & 30.93 & 54.51 & 13.64 & 0.01 & 0.08 & 0.15 & 0.00 \\
\hline & All data & 0.01 & 11.73 & 86.17 & 2.08 & 0.00 & 0.00 & 0.01 & 0.00 \\
\hline & ${ }^{1} \mathrm{H}$ data & 0.01 & 8.22 & 90.88 & 0.85 & 0.00 & 0.00 & 0.03 & 0.00 \\
\hline \multirow[t]{5}{*}{ DP4+ } & ${ }^{13} \mathrm{C}$ data & 0.06 & 25.99 & 40.22 & 33.72 & 0.00 & 0.00 & 0.01 & 0.00 \\
\hline & All data & 0.00 & 5.48 & 93.78 & 0.74 & 0.00 & 0.00 & 0.00 & 0.00 \\
\hline & & \multicolumn{8}{|c|}{ Level of theory: B3LYP/6-311G* (gas phase) } \\
\hline & & $79 a$ & $79 b$ & $79 c$ & $79 d$ & $79 \mathrm{e}$ & $79 f$ & $79 \mathrm{~g}$ & $79 h$ \\
\hline & ${ }^{1} \mathrm{H}$ data & 0.27 & 50.28 & 42.16 & 6.01 & 0.09 & 0.48 & 0.64 & 0.07 \\
\hline \multirow[t]{2}{*}{ sDP4+ } & ${ }^{13} \mathrm{C}$ data & 0.81 & 8.01 & 3.13 & 87.79 & 0.03 & 0.04 & 0.13 & 0.05 \\
\hline & All data & 0.02 & 37.92 & 12.43 & 49.62 & 0.00 & 0.00 & 0.01 & 0.00 \\
\hline \multirow{2}{*}{ uDP4+ } & ${ }^{1} \mathrm{H}$ data & 0.65 & 31.40 & 55.76 & 4.34 & 0.54 & 4.63 & 2.36 & 0.31 \\
\hline & ${ }^{13} \mathrm{C}$ data & 0.41 & 37.75 & 9.35 & 49.06 & 0.08 & 1.09 & 2.18 & 0.08 \\
\hline
\end{tabular}




\begin{tabular}{|c|c|c|c|c|c|c|c|c|c|}
\hline & All data & 0.01 & 61.42 & 27.00 & 11.03 & 0.00 & 0.26 & 0.27 & 0.00 \\
\hline & ${ }^{1} \mathrm{H}$ data & 0.00 & 39.87 & 59.37 & 0.66 & 0.00 & 0.06 & 0.04 & 0.00 \\
\hline \multirow[t]{5}{*}{ DP4+ } & ${ }^{13} \mathrm{C}$ data & 0.01 & 6.52 & 0.63 & 92.83 & 0.00 & 0.00 & 0.01 & 0.00 \\
\hline & All data & 0.00 & 72.50 & 10.45 & 17.05 & 0.00 & 0.00 & 0.00 & 0.00 \\
\hline & & \multicolumn{8}{|c|}{ Level of theory: B3LYP/6-311G** (gas phase) } \\
\hline & & $79 a$ & $79 \mathrm{~b}$ & $79 c$ & 79d & $79 e$ & $79 f$ & $79 \mathrm{~g}$ & $79 \mathrm{~h}$ \\
\hline & ${ }^{1} \mathrm{H}$ data & 0.45 & 52.21 & 40.70 & 3.84 & 0.25 & 0.90 & 1.54 & 0.10 \\
\hline \multirow[t]{2}{*}{ sDP4+ } & ${ }^{13} \mathrm{C}$ data & 0.84 & 8.56 & 3.41 & 86.86 & 0.04 & 0.05 & 0.18 & 0.06 \\
\hline & All data & 0.04 & 48.57 & 15.08 & 36.27 & 0.00 & 0.00 & 0.03 & 0.00 \\
\hline & ${ }^{1} \mathrm{H}$ data & 1.16 & 22.75 & 47.63 & 6.97 & 2.04 & 15.47 & 3.04 & 0.94 \\
\hline \multirow[t]{2}{*}{ uDP4+ } & ${ }^{13} \mathrm{C}$ data & 0.14 & 40.25 & 8.83 & 47.06 & 0.04 & 1.07 & 2.58 & 0.04 \\
\hline & All data & 0.01 & 54.22 & 24.91 & 19.42 & 0.00 & 0.98 & 0.46 & 0.00 \\
\hline \multirow{6}{*}{ DP4+ } & ${ }^{1} \mathrm{H}$ data & 0.02 & 37.43 & 61.10 & 0.84 & 0.02 & 0.44 & 0.15 & 0.00 \\
\hline & ${ }^{13} \mathrm{C}$ data & 0.00 & 7.72 & 0.67 & 91.59 & 0.00 & 0.00 & 0.01 & 0.00 \\
\hline & All data & 0.00 & 70.91 & 10.12 & 18.97 & 0.00 & 0.00 & 0.00 & 0.00 \\
\hline & & \multicolumn{8}{|c|}{ Level of theory: B3LYP/6-311+G** (gas phase) } \\
\hline & & 79a & $79 b$ & 79c & 79d & $79 e$ & $79 f$ & $79 \mathrm{~g}$ & $79 \mathrm{~h}$ \\
\hline & ${ }^{1} \mathrm{H}$ data & 1.68 & 49.60 & 41.77 & 2.43 & 0.20 & 0.72 & 3.43 & 0.16 \\
\hline \multirow[t]{2}{*}{$s \mathrm{DP} 4+$} & ${ }^{13} \mathrm{C}$ data & 0.50 & 11.01 & 3.87 & 84.46 & 0.02 & 0.03 & 0.10 & 0.02 \\
\hline & All data & 0.09 & 59.69 & 17.69 & 22.49 & 0.00 & 0.00 & 0.04 & 0.00 \\
\hline \multirow{3}{*}{ uDP4+ } & ${ }^{1} \mathrm{H}$ data & 4.75 & 14.77 & 51.38 & 10.70 & 2.95 & 10.45 & 2.89 & 2.11 \\
\hline & ${ }^{13} \mathrm{C}$ data & 0.14 & 51.42 & 7.40 & 38.36 & 0.04 & 0.72 & 1.92 & 0.01 \\
\hline & All data & 0.04 & 48.56 & 24.31 & 26.25 & 0.01 & 0.48 & 0.35 & 0.00 \\
\hline \multirow{5}{*}{ DP4+ } & ${ }^{1} \mathrm{H}$ data & 0.27 & 24.99 & 73.22 & 0.89 & 0.02 & 0.26 & 0.34 & 0.01 \\
\hline & ${ }^{13} \mathrm{C}$ data & 0.00 & 14.76 & 0.75 & 84.49 & 0.00 & 0.00 & 0.00 & 0.00 \\
\hline & All data & 0.00 & 73.96 & 10.97 & 15.06 & 0.00 & 0.00 & 0.00 & 0.00 \\
\hline & & \multicolumn{8}{|c|}{ Level of theory: B3LYP/6-316* (solution, PCM) } \\
\hline & & 79a & $79 b$ & $79 c$ & 79d & $79 e$ & $79 f$ & $79 \mathrm{~g}$ & $79 \mathrm{~h}$ \\
\hline & ${ }^{1} \mathrm{H}$ data & 0.16 & 41.58 & 47.63 & 10.59 & 0.00 & 0.01 & 0.02 & 0.00 \\
\hline \multirow[t]{2}{*}{ sDP4+ } & ${ }^{13} \mathrm{C}$ data & 1.09 & 14.23 & 4.98 & 79.17 & 0.02 & 0.07 & 0.40 & 0.03 \\
\hline & All data & 0.01 & 35.49 & 14.23 & 50.27 & 0.00 & 0.00 & 0.00 & 0.00 \\
\hline \multirow{3}{*}{ uDP4+ } & ${ }^{1} \mathrm{H}$ data & 0.05 & 30.71 & 60.85 & 7.90 & 0.01 & 0.21 & 0.26 & 0.01 \\
\hline & ${ }^{13} \mathrm{C}$ data & 3.01 & 14.44 & 36.35 & 46.05 & 0.01 & 0.02 & 0.09 & 0.02 \\
\hline & All data & 0.00 & 14.68 & 73.26 & 12.05 & 0.00 & 0.00 & 0.00 & 0.00 \\
\hline \multirow{6}{*}{ DP4+ } & ${ }^{1} \mathrm{H}$ data & 0.00 & 29.98 & 68.05 & 1.96 & 0.00 & 0.00 & 0.00 & 0.00 \\
\hline & ${ }^{13} \mathrm{C}$ data & 0.08 & 5.09 & 4.49 & 90.34 & 0.00 & 0.00 & 0.00 & 0.00 \\
\hline & All data & 0.00 & 24.02 & 48.06 & 27.92 & 0.00 & 0.00 & 0.00 & 0.00 \\
\hline & & \multicolumn{8}{|c|}{ Level of theory: B3LYP/6-31G** (solution, PCM) } \\
\hline & & $79 a$ & $79 b$ & 79c & $79 d$ & $79 e$ & $79 f$ & $79 \mathrm{~g}$ & $79 h$ \\
\hline & ${ }^{1} \mathrm{H}$ data & 0.33 & 43.03 & 42.52 & 13.94 & 0.01 & 0.03 & 0.14 & 0.01 \\
\hline \multirow[t]{2}{*}{ sDP4+ } & ${ }^{13} \mathrm{C}$ data & 3.15 & 23.44 & 9.13 & 63.42 & 0.08 & 0.15 & 0.54 & 0.09 \\
\hline & All data & 0.05 & 44.21 & 17.00 & 38.74 & 0.00 & 0.00 & 0.00 & 0.00 \\
\hline & ${ }^{1} \mathrm{H}$ data & 0.10 & 24.98 & 57.12 & 17.00 & 0.01 & 0.20 & 0.55 & 0.03 \\
\hline \multirow[t]{2}{*}{ uDP4+ } & ${ }^{13} \mathrm{C}$ data & 1.63 & 33.40 & 35.45 & 29.44 & 0.00 & 0.02 & 0.06 & 0.01 \\
\hline & All data & 0.00 & 24.83 & 60.26 & 14.90 & 0.00 & 0.00 & 0.00 & 0.00 \\
\hline & ${ }^{1} \mathrm{H}$ data & 0.00 & 28.74 & 64.92 & 6.34 & 0.00 & 0.00 & 0.00 & 0.00 \\
\hline DP4+ & ${ }^{13} \mathrm{C}$ data & 0.17 & 26.29 & 10.86 & 62.68 & 0.00 & 0.00 & 0.00 & 0.00 \\
\hline & All data & 0.00 & 40.67 & 37.96 & 21.38 & 0.00 & 0.00 & 0.00 & 0.00 \\
\hline & & & & Level of & : B3LYI & $+\mathrm{G} * *($ & n, PCN & & \\
\hline & & $79 a$ & $79 b$ & 79c & $79 d$ & $79 e$ & $79 f$ & $79 \mathrm{~g}$ & $79 h$ \\
\hline & ${ }^{1} \mathrm{H}$ data & 14.21 & 19.13 & 54.53 & 10.70 & 0.12 & 0.50 & 0.67 & 0.13 \\
\hline sDP4+ & ${ }^{13} \mathrm{C}$ data & 2.14 & 28.62 & 27.55 & 40.51 & 0.08 & 0.36 & 0.72 & 0.02 \\
\hline & All data & 1.21 & 21.78 & 59.75 & 17.24 & 0.00 & 0.01 & 0.02 & 0.00 \\
\hline & ${ }^{1} \mathrm{H}$ data & 9.81 & 12.69 & 64.70 & 10.97 & 0.27 & 0.00 & 1.23 & 0.34 \\
\hline uDP4+ & ${ }^{13} \mathrm{C}$ data & 0.90 & 36.01 & 56.49 & 6.47 & 0.00 & 0.00 & 0.12 & 0.00 \\
\hline & All data & 0.21 & 10.90 & 87.20 & 1.69 & 0.00 & 0.00 & 0.00 & 0.00 \\
\hline
\end{tabular}




\begin{tabular}{|c|c|c|c|c|c|c|c|c|c|}
\hline & ${ }^{1} \mathrm{H}$ data & 3.46 & 6.03 & 87.58 & 2.91 & 0.00 & 0.00 & 0.02 & 0.00 \\
\hline \multirow[t]{5}{*}{ DP4+ } & ${ }^{13} \mathrm{C}$ data & 0.07 & 36.15 & 54.59 & 9.19 & 0.00 & 0.00 & 0.00 & 0.00 \\
\hline & All data & 0.00 & 4.33 & 95.13 & 0.53 & 0.00 & 0.00 & 0.00 & 0.00 \\
\hline & & \multicolumn{8}{|c|}{ Level of theory: B3LYP/6-311G* (solution, PCM) } \\
\hline & & 79a & $79 b$ & 79c & 79d & $79 e$ & $79 f$ & $79 \mathrm{~g}$ & $79 \mathrm{~h}$ \\
\hline & ${ }^{1} \mathrm{H}$ data & 1.76 & 52.21 & 32.30 & 12.98 & 0.05 & 0.19 & 0.40 & 0.10 \\
\hline \multirow[t]{2}{*}{$s \mathrm{DP} 4+$} & ${ }^{13} \mathrm{C}$ data & 0.22 & 9.55 & 4.84 & 85.34 & 0.00 & 0.01 & 0.04 & 0.00 \\
\hline & All data & 0.02 & 28.28 & 8.86 & 62.83 & 0.00 & 0.00 & 0.00 & 0.00 \\
\hline \multirow{3}{*}{ uDP4+ } & ${ }^{1} \mathrm{H}$ data & 1.51 & 42.07 & 42.55 & 11.81 & 0.09 & 0.54 & 1.24 & 0.18 \\
\hline & ${ }^{13} \mathrm{C}$ data & 0.14 & 54.96 & 13.52 & 28.93 & 0.02 & 0.45 & 1.95 & 0.02 \\
\hline & All data & 0.01 & 71.54 & 17.80 & 10.57 & 0.00 & 0.01 & 0.08 & 0.00 \\
\hline \multirow{3}{*}{ DP4+ } & ${ }^{1} \mathrm{H}$ data & 0.07 & 58.93 & 36.87 & 4.11 & 0.00 & 0.00 & 0.01 & 0.00 \\
\hline & ${ }^{13} \mathrm{C}$ data & 0.00 & 17.15 & 2.14 & 80.70 & 0.00 & 0.00 & 0.00 & 0.00 \\
\hline & All data & 0.00 & 71.11 & 5.54 & 23.34 & 0.00 & 0.00 & 0.00 & 0.00 \\
\hline & & \multicolumn{8}{|c|}{ Level of theory: B3LYP/6-311G** (solution, PCM) } \\
\hline & & 79a & $79 b$ & 79c & 79d & $79 e$ & $79 f$ & $79 \mathrm{~g}$ & $79 \mathrm{~h}$ \\
\hline \multirow{3}{*}{ sDP4+ } & ${ }^{1} \mathrm{H}$ data & 4.54 & 60.72 & 20.80 & 10.48 & 0.34 & 0.74 & 1.94 & 0.43 \\
\hline & ${ }^{13} \mathrm{C}$ data & 0.18 & 9.53 & 4.77 & 85.46 & 0.00 & 0.01 & 0.05 & 0.00 \\
\hline & All data & 0.05 & 36.75 & 6.30 & 56.89 & 0.00 & 0.00 & 0.01 & 0.00 \\
\hline \multirow{3}{*}{ uDP4+ } & ${ }^{1} \mathrm{H}$ data & 8.31 & 38.64 & 24.88 & 16.65 & 1.29 & 6.44 & 2.40 & 1.39 \\
\hline & ${ }^{13} \mathrm{C}$ data & 0.08 & 57.14 & 14.21 & 25.28 & 0.02 & 0.58 & 2.69 & 0.01 \\
\hline & All data & 0.02 & 73.76 & 11.81 & 14.06 & 0.00 & 0.12 & 0.22 & 0.00 \\
\hline & ${ }^{1} \mathrm{H}$ data & 1.22 & 76.02 & 16.76 & 5.65 & 0.01 & 0.16 & 0.15 & 0.02 \\
\hline \multirow[t]{4}{*}{ DP4+ } & ${ }^{13} \mathrm{C}$ data & 0.00 & 19.64 & 2.44 & 77.91 & 0.00 & 0.00 & 0.00 & 0.00 \\
\hline & All data & 0.00 & 75.61 & 2.08 & 22.31 & 0.00 & 0.00 & 0.00 & 0.00 \\
\hline & & \multicolumn{8}{|c|}{ Level of theory: B3LYP/6-311+G** (solution, PCM) } \\
\hline & & $79 a$ & $79 b$ & $79 c$ & $79 d$ & $79 e$ & $79 f$ & $79 \mathrm{~g}$ & $79 \mathrm{~h}$ \\
\hline \multirow{3}{*}{ sDP4+ } & ${ }_{12}^{1} \mathrm{H}$ data & 23.35 & 36.06 & 23.26 & 11.79 & 0.30 & 0.93 & 3.73 & 0.58 \\
\hline & ${ }^{13} \mathrm{C}$ data & 0.23 & 13.19 & 9.32 & 77.23 & 0.00 & 0.01 & 0.03 & 0.00 \\
\hline & All data & 0.33 & 29.57 & 13.47 & 56.62 & 0.00 & 0.00 & 0.01 & 0.00 \\
\hline \multirow{3}{*}{ uDP4+ } & ${ }^{1} \mathrm{H}$ data & 22.28 & 29.30 & 25.95 & 18.26 & 0.45 & 2.57 & 0.61 & 0.58 \\
\hline & ${ }^{13} \mathrm{C}$ data & 0.14 & 65.36 & 13.66 & 20.02 & 0.02 & 0.15 & 0.66 & 0.00 \\
\hline & All data & 0.11 & 72.57 & 13.44 & 13.85 & 0.00 & 0.01 & 0.02 & 0.00 \\
\hline \multirow{5}{*}{ DP4+ } & ${ }^{1} \mathrm{H}$ data & 21.67 & 44.01 & 25.14 & 8.97 & 0.01 & 0.10 & 0.09 & 0.01 \\
\hline & ${ }^{13} \mathrm{C}$ data & 0.00 & 34.00 & 5.02 & 60.98 & 0.00 & 0.00 & 0.00 & 0.00 \\
\hline & All data & 0.00 & 68.98 & 5.82 & 25.20 & 0.00 & 0.00 & 0.00 & 0.00 \\
\hline & & \multicolumn{8}{|c|}{ Level of theory: mPW1PW91/6-31G* (gas phase) } \\
\hline & & $79 a$ & $79 b$ & 79c & 79d & $79 e$ & $79 f$ & $79 \mathrm{~g}$ & $79 \mathrm{~h}$ \\
\hline \multirow{3}{*}{ sDP4+ } & ${ }^{1} \mathrm{H}$ data & 0.02 & 30.99 & 53.73 & 15.19 & 0.00 & 0.01 & 0.05 & 0.01 \\
\hline & ${ }^{13} \mathrm{C}$ data & 0.72 & 8.28 & 2.37 & 88.07 & 0.07 & 0.01 & 0.40 & 0.09 \\
\hline & All data & 0.00 & 14.91 & 7.38 & 77.71 & 0.00 & 0.00 & 0.00 & 0.00 \\
\hline \multirow{3}{*}{ uDP4+ } & ${ }^{1} \mathrm{H}$ data & 0.02 & 36.07 & 54.77 & 8.89 & 0.00 & 0.08 & 0.16 & 0.01 \\
\hline & ${ }^{13} \mathrm{C}$ data & 12.25 & 11.94 & 34.88 & 39.78 & 0.26 & 0.10 & 0.46 & 0.34 \\
\hline & All data & 0.01 & 15.98 & 70.88 & 13.12 & 0.00 & 0.00 & 0.00 & 0.00 \\
\hline & ${ }^{1} \mathrm{H}$ data & 0.00 & 26.65 & 70.13 & 3.22 & 0.00 & 0.00 & 0.00 & 0.00 \\
\hline DP4+ & ${ }^{13} \mathrm{C}$ data & 0.24 & 2.68 & 2.23 & 94.85 & 0.00 & 0.00 & 0.00 & 0.00 \\
\hline & All data & 0.00 & 13.37 & 29.38 & 57.25 & 0.00 & 0.00 & 0.00 & 0.00 \\
\hline & & & & evel of $t$ & : $\mathrm{mPW}$ & $/ 6-31$ & s phas & & \\
\hline & & $79 a$ & $79 b$ & $79 c$ & $79 d$ & $79 e$ & $79 f$ & $79 \mathrm{~g}$ & $79 \mathrm{~h}$ \\
\hline & ${ }^{1} \mathrm{H}$ data & 0.04 & 41.90 & 43.17 & 14.59 & 0.01 & 0.01 & 0.28 & 0.02 \\
\hline sDP4+ & ${ }^{13} \mathrm{C}$ data & 1.28 & 9.64 & 2.94 & 85.65 & 0.06 & 0.02 & 0.29 & 0.11 \\
\hline & All data & 0.00 & 22.69 & 7.13 & 70.17 & 0.00 & 0.00 & 0.00 & 0.00 \\
\hline & ${ }^{1} \mathrm{H}$ data & 0.03 & 27.07 & 64.72 & 7.67 & 0.01 & 0.08 & 0.40 & 0.02 \\
\hline uDP4+ & ${ }^{13} \mathrm{C}$ data & 2.22 & 15.48 & 30.24 & 51.94 & 0.01 & 0.01 & 0.08 & 0.02 \\
\hline & All data & 0.00 & 15.10 & 70.54 & 14.35 & 0.00 & 0.00 & 0.00 & 0.00 \\
\hline DP4+ & ${ }^{1} \mathrm{H}$ data & 0.00 & 28.07 & 69.16 & 2.77 & 0.00 & 0.00 & 0.00 & 0.00 \\
\hline
\end{tabular}




\begin{tabular}{|c|c|c|c|c|c|c|c|c|c|}
\hline & ${ }^{13} \mathrm{C}$ data & 0.06 & 3.18 & 1.90 & 94.86 & 0.00 & 0.00 & 0.00 & 0.00 \\
\hline & All data & 0.00 & 18.49 & 27.16 & 54.35 & 0.00 & 0.00 & 0.00 & 0.00 \\
\hline & & \multicolumn{8}{|c|}{ Level of theory: mPW1PW91/6-31+G** (gas phase) } \\
\hline & & $79 a$ & $79 \mathrm{~b}$ & $79 c$ & 79d & $79 e$ & $79 f$ & $79 \mathrm{~g}$ & $79 \mathrm{~h}$ \\
\hline & ${ }^{1} \mathrm{H}$ data & 1.01 & 37.32 & 53.84 & 5.93 & 0.18 & 0.12 & 1.45 & 0.14 \\
\hline \multirow[t]{2}{*}{ sDP4+ } & ${ }^{13} \mathrm{C}$ data & 0.76 & 14.60 & 12.89 & 71.06 & 0.06 & 0.18 & 0.45 & 0.02 \\
\hline & All data & 0.05 & 32.79 & 41.75 & 25.37 & 0.00 & 0.00 & 0.04 & 0.00 \\
\hline \multirow{3}{*}{ uDP4+ } & ${ }^{1} \mathrm{H}$ data & 0.80 & 21.50 & 68.49 & 5.47 & 0.50 & 1.09 & 1.81 & 0.34 \\
\hline & ${ }^{13} \mathrm{C}$ data & 0.84 & 28.30 & 59.23 & 11.51 & 0.00 & 0.04 & 0.07 & 0.00 \\
\hline & All data & 0.01 & 12.87 & 85.78 & 1.33 & 0.00 & 0.00 & 0.00 & 0.00 \\
\hline & ${ }^{1} \mathrm{H}$ data & 0.02 & 17.73 & 81.47 & 0.72 & 0.00 & 0.00 & 0.06 & 0.00 \\
\hline \multirow[t]{4}{*}{ DP4+ } & ${ }^{13} \mathrm{C}$ data & 0.03 & 20.71 & 38.26 & 40.99 & 0.00 & 0.00 & 0.00 & 0.00 \\
\hline & All data & 0.00 & 10.45 & 88.71 & 0.84 & 0.00 & 0.00 & 0.00 & 0.00 \\
\hline & & \multicolumn{8}{|c|}{ Level of theory: mPW1PW91/6-311G* (gas phase) } \\
\hline & & $79 a$ & $79 \mathrm{~b}$ & $79 c$ & 79d & $79 e$ & $79 f$ & $79 \mathrm{~g}$ & $79 \mathrm{~h}$ \\
\hline & ${ }^{1} \mathrm{H}$ data & 0.16 & 52.89 & 44.03 & 2.08 & 0.03 & 0.16 & 0.61 & 0.04 \\
\hline \multirow[t]{2}{*}{ sDP4+ } & ${ }^{13} \mathrm{C}$ data & 0.62 & 9.67 & 2.35 & 87.27 & 0.01 & 0.02 & 0.06 & 0.02 \\
\hline & All data & 0.01 & 64.20 & 12.97 & 22.81 & 0.00 & 0.00 & 0.00 & 0.00 \\
\hline \multirow{3}{*}{ uDP4+ } & ${ }^{1} \mathrm{H}$ data & 0.46 & 37.30 & 54.65 & 3.65 & 0.19 & 1.34 & 2.22 & 0.19 \\
\hline & ${ }^{13} \mathrm{C}$ data & 0.18 & 44.21 & 11.32 & 42.54 & 0.01 & 0.26 & 1.45 & 0.02 \\
\hline & All data & 0.00 & 67.96 & 25.50 & 6.39 & 0.00 & 0.01 & 0.13 & 0.00 \\
\hline \multirow{6}{*}{ DP4+ } & ${ }^{1} \mathrm{H}$ data & 0.00 & 44.95 & 54.84 & 0.17 & 0.00 & 0.00 & 0.03 & 0.00 \\
\hline & ${ }^{13} \mathrm{C}$ data & 0.00 & 10.26 & 0.64 & 89.10 & 0.00 & 0.00 & 0.00 & 0.00 \\
\hline & All data & 0.00 & 90.15 & 6.84 & 3.01 & 0.00 & 0.00 & 0.00 & 0.00 \\
\hline & & \multicolumn{8}{|c|}{ Level of theory: mPW1PW91/6-311G** (gas phase) } \\
\hline & & 79a & $79 b$ & $79 c$ & $79 d$ & $79 e$ & $79 f$ & $79 \mathrm{~g}$ & $79 \mathrm{~h}$ \\
\hline & ${ }^{1} \mathrm{H}$ data & 0.32 & 56.00 & 39.62 & 1.81 & 0.09 & 0.38 & 1.71 & 0.07 \\
\hline \multirow[t]{2}{*}{ sDP4+ } & ${ }^{13} \mathrm{C}$ data & 0.41 & 11.01 & 2.63 & 85.85 & 0.01 & 0.01 & 0.06 & 0.01 \\
\hline & All data & 0.01 & 70.37 & 11.91 & 17.70 & 0.00 & 0.00 & 0.01 & 0.00 \\
\hline \multirow{3}{*}{ uDP4+ } & ${ }^{1} \mathrm{H}$ data & 1.10 & 28.56 & 53.46 & 6.17 & 1.18 & 5.90 & 2.82 & 0.82 \\
\hline & ${ }^{13} \mathrm{C}$ data & 0.06 & 55.52 & 12.27 & 30.08 & 0.01 & 0.24 & 1.82 & 0.01 \\
\hline & All data & 0.00 & 65.16 & 26.94 & 7.63 & 0.00 & 0.06 & 0.21 & 0.00 \\
\hline \multirow{5}{*}{ DP4+ } & ${ }^{1} \mathrm{H}$ data & 0.01 & 42.81 & 56.69 & 0.30 & 0.00 & 0.06 & 0.13 & 0.00 \\
\hline & ${ }^{13} \mathrm{C}$ data & 0.00 & 18.95 & 1.00 & 80.04 & 0.00 & 0.00 & 0.00 & 0.00 \\
\hline & All data & 0.00 & 90.96 & 6.37 & 2.68 & 0.00 & 0.00 & 0.00 & 0.00 \\
\hline & & \multicolumn{8}{|c|}{ Level of theory: mPW1PW91/6-311+G** (gas phase) } \\
\hline & & 79a & $79 b$ & 79c & 79d & $79 e$ & $79 f$ & $79 \mathrm{~g}$ & $79 \mathrm{~h}$ \\
\hline \multirow{3}{*}{ sDP4+ } & ${ }^{1} \mathrm{H}$ data & 1.32 & 51.35 & 42.26 & 1.51 & 0.09 & 0.26 & 3.09 & 0.12 \\
\hline & ${ }^{13} \mathrm{C}$ data & 0.33 & 23.47 & 4.52 & 71.62 & 0.00 & 0.01 & 0.04 & 0.00 \\
\hline & All data & 0.03 & 80.08 & 12.70 & 7.18 & 0.00 & 0.00 & 0.01 & 0.00 \\
\hline \multirow{3}{*}{ uDP4+ } & ${ }^{1} \mathrm{H}$ data & 3.96 & 17.94 & 61.32 & 8.27 & 1.38 & 3.89 & 1.87 & 1.36 \\
\hline & ${ }^{13} \mathrm{C}$ data & 0.06 & 68.25 & 10.79 & 19.30 & 0.01 & 0.23 & 1.35 & 0.00 \\
\hline & All data & 0.01 & 59.73 & 32.29 & 7.79 & 0.00 & 0.04 & 0.12 & 0.00 \\
\hline \multirow{3}{*}{ DP4+ } & ${ }^{1} \mathrm{H}$ data & 0.15 & 26.04 & 73.26 & 0.35 & 0.00 & 0.03 & 0.16 & 0.00 \\
\hline & ${ }^{13} \mathrm{C}$ data & 0.00 & 52.82 & 1.61 & 45.57 & 0.00 & 0.00 & 0.00 & 0.00 \\
\hline & All data & 0.00 & 91.12 & 7.81 & 1.07 & 0.00 & 0.00 & 0.00 & 0.00 \\
\hline & & & & rel of th & mPW1P & 6-31G & ion, $\mathrm{PC}$ & & \\
\hline & & $79 a$ & $79 b$ & $79 c$ & $79 d$ & $79 e$ & $79 f$ & $79 \mathrm{~g}$ & $79 \mathrm{~h}$ \\
\hline & ${ }^{1} \mathrm{H}$ data & 0.19 & 43.06 & 37.01 & 19.69 & 0.00 & 0.01 & 0.03 & 0.00 \\
\hline sDP4+ & ${ }^{13} \mathrm{C}$ data & 0.41 & 29.04 & 8.97 & 61.28 & 0.01 & 0.01 & 0.28 & 0.01 \\
\hline & All data & 0.00 & 44.84 & 11.90 & 43.26 & 0.00 & 0.00 & 0.00 & 0.00 \\
\hline & ${ }^{1} \mathrm{H}$ data & 0.05 & 26.31 & 57.41 & 15.97 & 0.00 & 0.05 & 0.19 & 0.01 \\
\hline uDP4+ & ${ }^{13} \mathrm{C}$ data & 6.42 & 15.47 & 49.46 & 28.18 & 0.10 & 0.06 & 0.22 & 0.10 \\
\hline & All data & 0.01 & 11.01 & 76.81 & 12.17 & 0.00 & 0.00 & 0.00 & 0.00 \\
\hline & ${ }^{1} \mathrm{H}$ data & 0.00 & 31.72 & 59.48 & 8.80 & 0.00 & 0.00 & 0.00 & 0.00 \\
\hline DP4+ & ${ }^{13} \mathrm{C}$ data & 0.10 & 17.13 & 16.91 & 65.85 & 0.00 & 0.00 & 0.00 & 0.00 \\
\hline
\end{tabular}




\begin{tabular}{|c|c|c|c|c|c|c|c|c|c|}
\hline & All data & 0.00 & 25.52 & 47.25 & 27.23 & 0.00 & 0.00 & 0.00 & 0.00 \\
\hline & & \multicolumn{8}{|c|}{ Level of theory: mPW1PW91/6-31G** (solution, PCM) } \\
\hline & & 79a & $79 b$ & $79 c$ & 79d & $79 e$ & $79 f$ & $79 \mathrm{~g}$ & $79 \mathrm{~h}$ \\
\hline & ${ }^{1} \mathrm{H}$ data & 0.40 & 47.26 & 28.24 & 23.93 & 0.00 & 0.01 & 0.14 & 0.01 \\
\hline \multirow[t]{2}{*}{ sDP4+ } & ${ }^{13} \mathrm{C}$ data & 1.03 & 25.96 & 8.83 & 63.95 & 0.01 & 0.02 & 0.18 & 0.01 \\
\hline & All data & 0.01 & 40.81 & 8.29 & 50.89 & 0.00 & 0.00 & 0.00 & 0.00 \\
\hline \multirow{3}{*}{ uDP4+ } & ${ }^{1} \mathrm{H}$ data & 0.10 & 29.39 & 36.28 & 33.84 & 0.00 & 0.04 & 0.32 & 0.02 \\
\hline & ${ }^{13} \mathrm{C}$ data & 1.74 & 24.13 & 42.10 & 31.94 & 0.01 & 0.01 & 0.08 & 0.01 \\
\hline & All data & 0.01 & 21.38 & 46.04 & 32.58 & 0.00 & 0.00 & 0.00 & 0.00 \\
\hline \multirow{5}{*}{ DP4+ } & ${ }^{1} \mathrm{H}$ data & 0.00 & 43.10 & 31.78 & 25.12 & 0.00 & 0.00 & 0.00 & 0.00 \\
\hline & ${ }^{13} \mathrm{C}$ data & 0.06 & 20.59 & 12.22 & 67.13 & 0.00 & 0.00 & 0.00 & 0.00 \\
\hline & All data & 0.00 & 29.96 & 13.11 & 56.93 & 0.00 & 0.00 & 0.00 & 0.00 \\
\hline & & \multicolumn{8}{|c|}{ Level of theory: mPW1PW91/6-31+G** (solution, PCM) } \\
\hline & & $79 a$ & $79 b$ & 79c & $79 d$ & $79 e$ & $79 f$ & $79 \mathrm{~g}$ & $79 \mathrm{~h}$ \\
\hline \multirow{3}{*}{ sDP4+ } & ${ }^{1} \mathrm{H}$ data & 18.60 & 28.04 & 37.49 & 14.40 & 0.15 & 0.21 & 0.85 & 0.25 \\
\hline & ${ }^{13} \mathrm{C}$ data & 0.39 & 33.88 & 19.87 & 45.61 & 0.01 & 0.06 & 0.18 & 0.00 \\
\hline & All data & 0.31 & 40.27 & 31.58 & 27.84 & 0.00 & 0.00 & 0.01 & 0.00 \\
\hline & ${ }^{1} \mathrm{H}$ data & 12.97 & 19.71 & 52.54 & 13.01 & 0.17 & 0.30 & 1.05 & 0.25 \\
\hline \multirow[t]{2}{*}{ uDP4+ } & ${ }^{13} \mathrm{C}$ data & 0.43 & 33.09 & 63.13 & 3.24 & 0.00 & 0.03 & 0.07 & 0.00 \\
\hline & All data & 0.14 & 16.24 & 82.57 & 1.05 & 0.00 & 0.00 & 0.00 & 0.00 \\
\hline \multirow{5}{*}{ DP4+ } & ${ }^{1} \mathrm{H}$ data & 8.17 & 18.72 & 66.72 & 6.35 & 0.00 & 0.00 & 0.03 & 0.00 \\
\hline & ${ }^{13} \mathrm{C}$ data & 0.01 & 44.43 & 49.72 & 5.85 & 0.00 & 0.00 & 0.00 & 0.00 \\
\hline & All data & 0.00 & 19.87 & 79.24 & 0.89 & 0.00 & 0.00 & 0.00 & 0.00 \\
\hline & & \multicolumn{8}{|c|}{ Level of theory: mPW1PW91/6-311G* (solution, PCM) } \\
\hline & & 79a & $79 b$ & 79c & 79d & $79 e$ & $79 f$ & $79 \mathrm{~g}$ & $79 \mathrm{~h}$ \\
\hline \multirow{3}{*}{$s \mathrm{DP} 4+$} & ${ }^{1} \mathrm{H}$ data & 1.26 & 55.73 & 36.51 & 6.07 & 0.02 & 0.08 & 0.29 & 0.05 \\
\hline & ${ }^{13} \mathrm{C}$ data & 0.13 & 14.31 & 3.95 & 81.59 & 0.00 & 0.01 & 0.02 & 0.00 \\
\hline & All data & 0.01 & 55.50 & 10.04 & 34.45 & 0.00 & 0.00 & 0.00 & 0.00 \\
\hline \multirow{3}{*}{ uDP4+ } & ${ }^{1} \mathrm{H}$ data & 1.38 & 44.72 & 38.22 & 14.12 & 0.04 & 0.21 & 1.19 & 0.13 \\
\hline & ${ }^{13} \mathrm{C}$ data & 0.06 & 64.79 & 16.13 & 17.67 & 0.00 & 0.14 & 1.21 & 0.00 \\
\hline & All data & 0.00 & 76.96 & 16.37 & 6.63 & 0.00 & 0.00 & 0.04 & 0.00 \\
\hline \multirow{5}{*}{ DP4+ } & ${ }^{1} \mathrm{H}$ data & 0.04 & 62.69 & 35.10 & 2.15 & 0.00 & 0.00 & 0.01 & 0.00 \\
\hline & ${ }^{13} \mathrm{C}$ data & 0.00 & 38.11 & 2.62 & 59.27 & 0.00 & 0.00 & 0.00 & 0.00 \\
\hline & All data & 0.00 & 91.58 & 3.52 & 4.89 & 0.00 & 0.00 & 0.00 & 0.00 \\
\hline & & \multicolumn{8}{|c|}{ Level of theory: mPW1PW91/6-311G** (solution, PCM) } \\
\hline & & 79a & $79 b$ & $79 c$ & 79d & $79 e$ & $79 f$ & $79 \mathrm{~g}$ & $79 \mathrm{~h}$ \\
\hline & ${ }^{1} \mathrm{H}$ data & 4.63 & 65.25 & 19.17 & 7.56 & 0.17 & 0.48 & 2.37 & 0.35 \\
\hline \multirow[t]{2}{*}{$s \mathrm{DP} 4+$} & ${ }^{13} \mathrm{C}$ data & 0.06 & 17.80 & 4.45 & 77.67 & 0.00 & 0.00 & 0.02 & 0.00 \\
\hline & All data & 0.02 & 63.31 & 4.65 & 32.03 & 0.00 & 0.00 & 0.00 & 0.00 \\
\hline \multirow{3}{*}{ uDP4+ } & ${ }^{1} \mathrm{H}$ data & 7.83 & 42.56 & 26.67 & 17.45 & 0.56 & 1.78 & 1.99 & 1.16 \\
\hline & ${ }^{13} \mathrm{C}$ data & 0.03 & 70.34 & 16.47 & 11.23 & 0.00 & 0.16 & 1.78 & 0.00 \\
\hline & All data & 0.01 & 82.41 & 12.09 & 5.39 & 0.00 & 0.01 & 0.10 & 0.00 \\
\hline \multirow{5}{*}{ DP4+ } & ${ }^{1} \mathrm{H}$ data & 1.05 & 80.20 & 14.76 & 3.81 & 0.00 & 0.02 & 0.14 & 0.01 \\
\hline & ${ }^{13} \mathrm{C}$ data & 0.00 & 56.97 & 3.33 & 39.69 & 0.00 & 0.00 & 0.00 & 0.00 \\
\hline & All data & 0.00 & 95.80 & 1.03 & 3.17 & 0.00 & 0.00 & 0.00 & 0.00 \\
\hline & & & & of the & PW1PV & $311+G$ & tion, & & \\
\hline & & $79 a$ & $79 b$ & $79 c$ & $79 d$ & $79 e$ & $79 f$ & $79 \mathrm{~g}$ & $79 h$ \\
\hline & ${ }^{1} \mathrm{H}$ data & 17.30 & 19.32 & 15.33 & 8.28 & 2.00 & 6.47 & 28.14 & 3.15 \\
\hline sDP4+ & ${ }^{13} \mathrm{C}$ data & 0.12 & 24.10 & 10.85 & 64.91 & 0.00 & 0.01 & 0.02 & 0.00 \\
\hline & All data & 0.18 & 39.73 & 14.19 & 45.86 & 0.00 & 0.00 & 0.04 & 0.00 \\
\hline & ${ }^{1} \mathrm{H}$ data & 15.80 & 17.92 & 20.60 & 13.69 & 3.59 & 17.21 & 5.45 & 5.74 \\
\hline uDP4+ & ${ }^{13} \mathrm{C}$ data & 0.04 & 76.45 & 14.25 & 8.70 & 0.00 & 0.06 & 0.50 & 0.00 \\
\hline & All data & 0.04 & 76.66 & 16.42 & 6.66 & 0.00 & 0.06 & 0.15 & 0.00 \\
\hline & ${ }^{1} \mathrm{H}$ data & 20.42 & 25.86 & 23.58 & 8.47 & 0.54 & 8.32 & 11.46 & 1.35 \\
\hline DP4+ & ${ }^{13} \mathrm{C}$ data & 0.00 & 71.93 & 6.03 & 22.04 & 0.00 & 0.00 & 0.00 & 0.00 \\
\hline & All data & 0.00 & 84.97 & 6.50 & 8.53 & 0.00 & 0.00 & 0.00 & 0.00 \\
\hline
\end{tabular}


$s \mathrm{DP} 4+, u \mathrm{DP} 4+$ and DP4+ probabilities (\%) for compound 79c<smiles>CC[C@H](C)[C@@H]1OC([C@@H](C)[C@@H](O)CC)=C(C)C(=O)[C@@H]1C</smiles><smiles>CC[C@H](O)C1=C(C)C(=O)C(C)[C@H]([C@H](C)CC)O1</smiles><smiles>CC[C@H](C)[C@@H]1OC([C@@H](C)[C@@H](O)CC)=C(C)C(=O)[C@@H]1C</smiles><smiles>CC[C@H](C)[C@@H]1OC([C@@H](C)[C@@H](O)CC)=C(C)C(=O)[C@@H]1C</smiles><smiles>CC[C@H](O)[C@H](C)C1=C(C)C(=O)[C@H](C)[C@H]([C@H](C)CC)O1</smiles><smiles>CC[C@H](C)[C@@H]1OC([C@@H](C)[C@@H](O)CC)=C(C)C(=O)[C@@H]1C</smiles><smiles>CC[C@H](C)[C@@H]1OC([C@@H](C)[C@@H](O)CC)=C(C)C(=O)[C@@H]1C</smiles>

$79 d$<smiles>CC[C@H](C)[C@@H]1OC([C@@H](C)[C@@H](O)CC)=C(C)C(=O)[C@@H]1C</smiles>

\begin{tabular}{|c|c|c|c|c|c|c|c|c|c|}
\hline & & \multicolumn{8}{|c|}{ Level of theory: B3LYP/6-31G* (gas phase) } \\
\hline & & $79 a$ & $79 b$ & $79 c$ & 79d & $79 e$ & $79 f$ & $79 \mathrm{~g}$ & $79 h$ \\
\hline \multirow{3}{*}{ sDP4+ } & ${ }^{1} \mathrm{H}$ data & 1.16 & 58.66 & 29.13 & 10.98 & 0.01 & 0.02 & 0.03 & 0.01 \\
\hline & ${ }^{13}$ C data & 9.84 & 12.73 & 5.13 & 68.18 & 1.32 & 0.34 & 1.71 & 0.75 \\
\hline & All data & 0.69 & 45.07 & 9.02 & 45.22 & 0.00 & 0.00 & 0.00 & 0.00 \\
\hline \multirow{3}{*}{ uDP4+ } & ${ }^{1}$ H data & 0.56 & 52.93 & 35.82 & 10.25 & 0.01 & 0.19 & 0.21 & 0.02 \\
\hline & ${ }^{13} \mathrm{C}$ data & 5.58 & 10.69 & 43.18 & 40.11 & 0.07 & 0.10 & 0.20 & 0.06 \\
\hline & All data & 0.12 & 22.38 & 61.22 & 16.28 & 0.00 & 0.00 & 0.00 & 0.00 \\
\hline \multirow{3}{*}{ DP4+ } & ${ }^{1} \mathrm{H}$ data & 0.02 & 72.85 & 24.49 & 2.64 & 0.00 & 0.00 & 0.00 & 0.00 \\
\hline & ${ }^{13} \mathrm{C}$ data & 1.75 & 4.32 & 7.03 & 86.89 & 0.00 & 0.00 & 0.01 & 0.00 \\
\hline & All data & 0.00 & 43.92 & 24.03 & 32.04 & 0.00 & 0.00 & 0.00 & 0.00 \\
\hline & & \multicolumn{8}{|c|}{ Level of theory: B3LYP/6-31G** (gas phase) } \\
\hline & & $79 a$ & $79 b$ & $79 c$ & $79 d$ & $79 e$ & $79 f$ & $79 \mathrm{~g}$ & $79 h$ \\
\hline \multirow{3}{*}{ sDP4+ } & ${ }^{1} \mathrm{H}$ data & 1.09 & 58.04 & 36.15 & 4.56 & 0.01 & 0.04 & 0.10 & 0.02 \\
\hline & ${ }^{13} \mathrm{C}$ data & 9.46 & 19.06 & 7.84 & 59.74 & 1.35 & 0.38 & 1.44 & 0.73 \\
\hline & All data & 0.62 & 66.16 & 16.94 & 16.28 & 0.00 & 0.00 & 0.01 & 0.00 \\
\hline \multirow{3}{*}{ uDP4+ } & ${ }^{1} \mathrm{H}$ data & 0.59 & 62.78 & 31.76 & 4.36 & 0.02 & 0.23 & 0.22 & 0.03 \\
\hline & ${ }^{13}$ C data & 2.51 & 27.89 & 39.51 & 29.79 & 0.02 & 0.09 & 0.17 & 0.02 \\
\hline & All data & 0.05 & 55.81 & 40.00 & 4.14 & 0.00 & 0.00 & 0.00 & 0.00 \\
\hline \multirow{3}{*}{ DP4+ } & ${ }^{1} \mathrm{H}$ data & 0.01 & 75.71 & 23.86 & 0.41 & 0.00 & 0.00 & 0.00 & 0.00 \\
\hline & ${ }^{13} \mathrm{C}$ data & 0.90 & 20.10 & 11.71 & 67.28 & 0.00 & 0.00 & 0.01 & 0.00 \\
\hline & All data & 0.00 & 83.21 & 15.27 & 1.52 & 0.00 & 0.00 & 0.00 & 0.00 \\
\hline & & \multicolumn{8}{|c|}{ Level of theory: B3LYP/6-31+G** (gas phase) } \\
\hline & & $79 a$ & $79 b$ & $79 c$ & 79d & $79 e$ & $79 f$ & $79 \mathrm{~g}$ & $79 h$ \\
\hline \multirow{3}{*}{ sDP4+ } & ${ }^{1} \mathrm{H}$ data & 10.31 & 35.43 & 50.76 & 2.08 & 0.15 & 0.12 & 1.07 & 0.07 \\
\hline & ${ }^{13} \mathrm{C}$ data & 1.05 & 27.10 & 25.63 & 44.31 & 0.19 & 0.64 & 1.03 & 0.04 \\
\hline & All data & 0.46 & 40.58 & 55.00 & 3.90 & 0.00 & 0.00 & 0.05 & 0.00 \\
\hline \multirow{3}{*}{ uDP4+ } & ${ }^{1} \mathrm{H}$ data & 6.17 & 40.07 & 48.81 & 1.91 & 0.41 & 1.17 & 1.32 & 0.15 \\
\hline & ${ }^{13} \mathrm{C}$ data & 0.58 & 25.54 & 65.66 & 7.90 & 0.01 & 0.12 & 0.18 & 0.00 \\
\hline & All data & 0.08 & 24.09 & 75.46 & 0.35 & 0.00 & 0.00 & 0.01 & 0.00 \\
\hline \multirow{3}{*}{ DP4+ } & ${ }^{1} \mathrm{H}$ data & 1.60 & 35.78 & 62.47 & 0.10 & 0.00 & 0.00 & 0.04 & 0.00 \\
\hline & ${ }^{13} \mathrm{C}$ data & 0.02 & 25.39 & 61.73 & 12.84 & 0.00 & 0.00 & 0.01 & 0.00 \\
\hline & All data & 0.00 & 19.06 & 80.91 & 0.03 & 0.00 & 0.00 & 0.00 & 0.00 \\
\hline & & \multicolumn{8}{|c|}{ Level of theory: B3LYP/6-311G* (gas phase) } \\
\hline & & $79 a$ & $79 b$ & $79 c$ & $79 d$ & $79 \mathrm{e}$ & $79 f$ & $79 \mathrm{~g}$ & $79 h$ \\
\hline \multirow{3}{*}{ sDP4+ } & ${ }^{1} \mathrm{H}$ data & 1.29 & 91.18 & 4.48 & 2.68 & 0.03 & 0.22 & 0.10 & 0.02 \\
\hline & ${ }^{13} \mathrm{C}$ data & 1.61 & 10.30 & 5.64 & 82.11 & 0.07 & 0.06 & 0.17 & 0.04 \\
\hline & All data & 0.17 & 79.15 & 2.13 & 18.55 & 0.00 & 0.00 & 0.00 & 0.00 \\
\hline \multirow{2}{*}{ uDP4+ } & ${ }^{1} \mathrm{H}$ data & 3.21 & 75.89 & 13.61 & 2.90 & 0.28 & 3.14 & 0.80 & 0.16 \\
\hline & ${ }^{13} \mathrm{C}$ data & 0.40 & 41.15 & 11.97 & 44.47 & 0.04 & 0.56 & 1.37 & 0.04 \\
\hline
\end{tabular}




\begin{tabular}{|c|c|c|c|c|c|c|c|c|c|}
\hline & All data & 0.04 & 91.34 & 4.77 & 3.77 & 0.00 & 0.05 & 0.03 & 0.00 \\
\hline & ${ }^{1} \mathrm{H}$ data & 0.06 & 98.95 & 0.87 & 0.11 & 0.00 & 0.01 & 0.00 & 0.00 \\
\hline \multirow[t]{5}{*}{ DP4+ } & ${ }^{13} \mathrm{C}$ data & 0.02 & 10.23 & 1.63 & 88.12 & 0.00 & 0.00 & 0.01 & 0.00 \\
\hline & All data & 0.00 & 98.90 & 0.14 & 0.96 & 0.00 & 0.00 & 0.00 & 0.00 \\
\hline & & \multicolumn{8}{|c|}{ Level of theory: B3LYP/6-311G** (gas phase) } \\
\hline & & $79 a$ & $79 \mathrm{~b}$ & $79 c$ & 79d & $79 e$ & $79 f$ & $79 \mathrm{~g}$ & $79 \mathrm{~h}$ \\
\hline & ${ }^{1} \mathrm{H}$ data & 3.48 & 83.31 & 10.64 & 1.03 & 0.11 & 0.78 & 0.60 & 0.05 \\
\hline \multirow[t]{2}{*}{ sDP4+ } & ${ }^{13} \mathrm{C}$ data & 1.08 & 9.68 & 5.26 & 83.62 & 0.07 & 0.06 & 0.20 & 0.04 \\
\hline & All data & 0.39 & 84.67 & 5.88 & 9.05 & 0.00 & 0.00 & 0.01 & 0.00 \\
\hline & ${ }^{1} \mathrm{H}$ data & 6.27 & 59.11 & 12.09 & 1.85 & 0.78 & 18.46 & 1.06 & 0.36 \\
\hline \multirow[t]{2}{*}{ uDP4+ } & ${ }^{13} \mathrm{C}$ data & 0.13 & 44.14 & 11.40 & 42.22 & 0.02 & 0.48 & 1.60 & 0.02 \\
\hline & All data & 0.03 & 91.98 & 4.86 & 2.76 & 0.00 & 0.31 & 0.06 & 0.00 \\
\hline \multirow{6}{*}{ DP4+ } & ${ }^{1} \mathrm{H}$ data & 0.43 & 96.71 & 2.53 & 0.04 & 0.00 & 0.28 & 0.01 & 0.00 \\
\hline & ${ }^{13} \mathrm{C}$ data & 0.00 & 10.63 & 1.49 & 87.86 & 0.00 & 0.00 & 0.01 & 0.00 \\
\hline & All data & 0.00 & 99.32 & 0.36 & 0.32 & 0.00 & 0.00 & 0.00 & 0.00 \\
\hline & & \multicolumn{8}{|c|}{ Level of theory: B3LYP/6-311+G** (gas phase) } \\
\hline & & 79a & $79 b$ & 79c & 79d & $79 e$ & $79 f$ & $79 \mathrm{~g}$ & $79 \mathrm{~h}$ \\
\hline & ${ }^{1} \mathrm{H}$ data & 17.02 & 60.24 & 18.28 & 1.70 & 0.12 & 0.75 & 1.79 & 0.10 \\
\hline \multirow[t]{2}{*}{$s \mathrm{DP} 4+$} & ${ }^{13} \mathrm{C}$ data & 0.71 & 14.51 & 6.59 & 78.03 & 0.02 & 0.03 & 0.09 & 0.01 \\
\hline & All data & 1.06 & 76.72 & 10.58 & 11.62 & 0.00 & 0.00 & 0.01 & 0.00 \\
\hline \multirow{3}{*}{ uDP4+ } & ${ }^{1} \mathrm{H}$ data & 30.14 & 28.42 & 18.61 & 5.80 & 1.38 & 13.44 & 1.20 & 0.99 \\
\hline & ${ }^{13} \mathrm{C}$ data & 0.14 & 57.46 & 10.02 & 30.88 & 0.02 & 0.31 & 1.16 & 0.01 \\
\hline & All data & 0.21 & 81.30 & 9.29 & 8.92 & 0.00 & 0.21 & 0.07 & 0.00 \\
\hline \multirow{5}{*}{ DP4+ } & ${ }^{1} \mathrm{H}$ data & 19.82 & 66.16 & 13.15 & 0.38 & 0.01 & 0.39 & 0.08 & 0.00 \\
\hline & ${ }^{13} \mathrm{C}$ data & 0.00 & 25.19 & 2.00 & 72.80 & 0.00 & 0.00 & 0.00 & 0.00 \\
\hline & All data & 0.00 & 96.86 & 1.53 & 1.61 & 0.00 & 0.00 & 0.00 & 0.00 \\
\hline & & \multicolumn{8}{|c|}{ Level of theory: B3LYP/6-31G* (solution, PCM) } \\
\hline & & $79 a$ & $79 b$ & $79 \mathrm{c}$ & 79d & $79 e$ & $79 f$ & $79 \mathrm{~g}$ & $79 \mathrm{~h}$ \\
\hline & ${ }^{1} \mathrm{H}$ data & 4.06 & 76.25 & 18.44 & 1.22 & 0.00 & 0.01 & 0.01 & 4.06 \\
\hline \multirow[t]{2}{*}{ sDP4+ } & ${ }^{13} \mathrm{C}$ data & 2.20 & 21.77 & 9.11 & 66.03 & 0.06 & 0.16 & 0.64 & 2.20 \\
\hline & All data & 0.47 & 86.57 & 8.77 & 4.20 & 0.00 & 0.00 & 0.00 & 0.47 \\
\hline \multirow{3}{*}{ uDP4+ } & ${ }^{1} \mathrm{H}$ data & 0.83 & 60.07 & 36.04 & 2.66 & 0.01 & 0.19 & 0.20 & 0.83 \\
\hline & ${ }^{13} \mathrm{C}$ data & 4.11 & 12.52 & 54.03 & 29.10 & 0.03 & 0.05 & 0.12 & 4.11 \\
\hline & All data & 0.12 & 27.06 & 70.04 & 2.78 & 0.00 & 0.00 & 0.00 & 0.12 \\
\hline \multirow{6}{*}{ DP4+ } & ${ }^{1} \mathrm{H}$ data & 0.06 & 87.22 & 12.66 & 0.06 & 0.00 & 0.00 & 0.00 & 0.06 \\
\hline & ${ }^{13} \mathrm{C}$ data & 0.34 & 10.11 & 18.27 & 71.28 & 0.00 & 0.00 & 0.00 & 0.34 \\
\hline & All data & 0.00 & 78.92 & 20.69 & 0.39 & 0.00 & 0.00 & 0.00 & 0.00 \\
\hline & & \multicolumn{8}{|c|}{ Level of theory: B3LYP/6-31G** (solution, PCM) } \\
\hline & & $79 a$ & $79 b$ & $79 c$ & $79 d$ & $79 e$ & $79 f$ & $79 \mathrm{~g}$ & $79 \mathrm{~h}$ \\
\hline & ${ }^{1} \mathrm{H}$ data & 6.20 & 75.17 & 17.88 & 0.63 & 0.00 & 0.03 & 0.07 & 0.01 \\
\hline \multirow[t]{2}{*}{ sDP4+ } & ${ }^{13} \mathrm{C}$ data & 4.31 & 28.59 & 13.25 & 52.79 & 0.13 & 0.18 & 0.68 & 0.07 \\
\hline & All data & 1.09 & 87.85 & 9.68 & 1.37 & 0.00 & 0.00 & 0.00 & 0.00 \\
\hline & ${ }^{1} \mathrm{H}$ data & 1.09 & 69.26 & 27.39 & 1.74 & 0.01 & 0.22 & 0.27 & 0.02 \\
\hline \multirow[t]{2}{*}{ uDP4+ } & ${ }^{13} \mathrm{C}$ data & 1.78 & 33.08 & 47.80 & 17.10 & 0.02 & 0.07 & 0.14 & 0.01 \\
\hline & All data & 0.05 & 63.08 & 36.05 & 0.82 & 0.00 & 0.00 & 0.00 & 0.00 \\
\hline & ${ }^{1} \mathrm{H}$ data & 0.12 & 91.27 & 8.59 & 0.02 & 0.00 & 0.00 & 0.00 & 0.00 \\
\hline DP4+ & ${ }^{13} \mathrm{C}$ data & 0.31 & 37.99 & 25.44 & 36.26 & 0.00 & 0.00 & 0.00 & 0.00 \\
\hline & All data & 0.00 & 94.05 & 5.93 & 0.02 & 0.00 & 0.00 & 0.00 & 0.00 \\
\hline & & & & Level of & : B3LYI & $+\mathrm{G} * *($ & n, PCM & & \\
\hline & & 79a & $79 \mathrm{~b}$ & 79c & 79d & $79 e$ & $79 f$ & $79 \mathrm{~g}$ & $79 \mathrm{~h}$ \\
\hline & ${ }^{1} \mathrm{H}$ data & 27.69 & 28.45 & 42.33 & 0.16 & 0.02 & 0.49 & 0.85 & 0.02 \\
\hline sDP4+ & ${ }^{13} \mathrm{C}$ data & 1.24 & 33.87 & 35.03 & 29.11 & 0.04 & 0.17 & 0.52 & 0.01 \\
\hline & All data & 1.38 & 38.76 & 59.65 & 0.19 & 0.00 & 0.00 & 0.02 & 0.00 \\
\hline & ${ }^{1} \mathrm{H}$ data & 22.85 & 20.44 & 54.96 & 0.34 & 0.05 & 0.00 & 1.30 & 0.06 \\
\hline uDP4+ & ${ }^{13} \mathrm{C}$ data & 0.62 & 30.44 & 64.55 & 4.23 & 0.01 & 0.00 & 0.16 & 0.00 \\
\hline & All data & 0.34 & 14.86 & 84.76 & 0.03 & 0.00 & 0.00 & 0.00 & 0.00 \\
\hline
\end{tabular}




\begin{tabular}{|c|c|c|c|c|c|c|c|c|c|}
\hline & ${ }^{1} \mathrm{H}$ data & 17.86 & 16.42 & 65.69 & 0.00 & 0.00 & 0.00 & 0.03 & 0.00 \\
\hline \multirow[t]{5}{*}{ DP4+ } & ${ }^{13} \mathrm{C}$ data & 0.02 & 30.18 & 66.20 & 3.60 & 0.00 & 0.00 & 0.00 & 0.00 \\
\hline & All data & 0.01 & 10.23 & 89.77 & 0.00 & 0.00 & 0.00 & 0.00 & 0.00 \\
\hline & & \multicolumn{8}{|c|}{ Level of theory: B3LYP/6-311G* (solution, PCM) } \\
\hline & & 79a & $79 b$ & 79c & 79d & $79 e$ & $79 f$ & $79 \mathrm{~g}$ & $79 \mathrm{~h}$ \\
\hline & ${ }^{1} \mathrm{H}$ data & 2.83 & 93.30 & 3.49 & 0.21 & 0.00 & 0.08 & 0.07 & 0.01 \\
\hline \multirow[t]{2}{*}{$s \mathrm{DP4+}$} & ${ }^{13} \mathrm{C}$ data & 0.54 & 11.84 & 7.76 & 79.79 & 0.01 & 0.01 & 0.05 & 0.00 \\
\hline & All data & 0.13 & 96.03 & 2.35 & 1.48 & 0.00 & 0.00 & 0.00 & 0.00 \\
\hline \multirow{3}{*}{ uDP4+ } & ${ }^{1} \mathrm{H}$ data & 2.35 & 88.37 & 8.10 & 0.51 & 0.01 & 0.30 & 0.34 & 0.03 \\
\hline & ${ }^{13} \mathrm{C}$ data & 0.15 & 55.94 & 17.96 & 24.57 & 0.01 & 0.21 & 1.16 & 0.01 \\
\hline & All data & 0.01 & 96.89 & 2.85 & 0.24 & 0.00 & 0.00 & 0.01 & 0.00 \\
\hline \multirow{3}{*}{ DP4+ } & ${ }^{1} \mathrm{H}$ data & 0.08 & 99.58 & 0.34 & 0.00 & 0.00 & 0.00 & 0.00 & 0.00 \\
\hline & ${ }^{13} \mathrm{C}$ data & 0.00 & 23.99 & 5.04 & 70.96 & 0.00 & 0.00 & 0.00 & 0.00 \\
\hline & All data & 0.00 & 99.92 & 0.07 & 0.00 & 0.00 & 0.00 & 0.00 & 0.00 \\
\hline & & \multicolumn{8}{|c|}{ Level of theory: B3LYP/6-311G** (solution, PCM) } \\
\hline & & $79 a$ & $79 b$ & 79c & $79 d$ & $79 e$ & $79 f$ & $79 \mathrm{~g}$ & $79 h$ \\
\hline \multirow{3}{*}{ sDP4+ } & ${ }^{1} \mathrm{H}$ data & 11.57 & 79.04 & 7.18 & 0.12 & 0.05 & 0.86 & 1.12 & 0.06 \\
\hline & ${ }^{13} \mathrm{C}$ data & 0.30 & 11.29 & 7.91 & 80.43 & 0.00 & 0.01 & 0.05 & 0.00 \\
\hline & All data & 0.36 & 92.71 & 5.90 & 1.03 & 0.00 & 0.00 & 0.01 & 0.00 \\
\hline \multirow{3}{*}{ uDP4+ } & ${ }^{1} \mathrm{H}$ data & 16.46 & 67.89 & 6.81 & 0.25 & 0.16 & 7.32 & 0.93 & 0.17 \\
\hline & ${ }^{13} \mathrm{C}$ data & 0.08 & 58.82 & 17.63 & 21.58 & 0.01 & 0.27 & 1.61 & 0.01 \\
\hline & All data & 0.03 & 96.84 & 2.91 & 0.13 & 0.00 & 0.05 & 0.04 & 0.00 \\
\hline \multirow{5}{*}{ DP4+ } & ${ }^{1} \mathrm{H}$ data & 3.39 & 95.60 & 0.87 & 0.00 & 0.00 & 0.11 & 0.02 & 0.00 \\
\hline & ${ }^{13} \mathrm{C}$ data & 0.00 & 26.14 & 5.49 & 68.36 & 0.00 & 0.00 & 0.00 & 0.00 \\
\hline & All data & 0.00 & 99.81 & 0.19 & 0.00 & 0.00 & 0.00 & 0.00 & 0.00 \\
\hline & & \multicolumn{8}{|c|}{ Level of theory: B3LYP/6-311+G** (solution, PCM) } \\
\hline & & $79 a$ & $79 b$ & $79 c$ & 79d & $79 e$ & $79 f$ & $79 \mathrm{~g}$ & $79 \mathrm{~h}$ \\
\hline \multirow{3}{*}{ sDP4+ } & ${ }_{12}^{1} \mathrm{H}$ data & 44.28 & 34.53 & 15.75 & 0.45 & 0.06 & 1.59 & 3.24 & 0.11 \\
\hline & ${ }^{13} \mathrm{C}$ data & 0.45 & 14.61 & 15.09 & 69.82 & 0.00 & 0.01 & 0.03 & 0.00 \\
\hline & All data & 2.50 & 63.59 & 29.94 & 3.95 & 0.00 & 0.00 & 0.01 & 0.00 \\
\hline \multirow{3}{*}{ uDP4+ } & ${ }^{1} \mathrm{H}$ data & 44.13 & 39.20 & 12.04 & 0.55 & 0.06 & 3.60 & 0.33 & 0.08 \\
\hline & ${ }^{13} \mathrm{C}$ data & 0.15 & 65.14 & 17.03 & 17.21 & 0.01 & 0.07 & 0.40 & 0.00 \\
\hline & All data & 0.24 & 92.02 & 7.39 & 0.34 & 0.00 & 0.01 & 0.00 & 0.00 \\
\hline \multirow{5}{*}{ DP4+ } & ${ }^{1} \mathrm{H}$ data & 55.76 & 38.63 & 5.41 & 0.01 & 0.00 & 0.16 & 0.03 & 0.00 \\
\hline & ${ }^{13} \mathrm{C}$ data & 0.00 & 39.48 & 10.66 & 49.86 & 0.00 & 0.00 & 0.00 & 0.00 \\
\hline & All data & 0.01 & 96.33 & 3.64 & 0.02 & 0.00 & 0.00 & 0.00 & 0.00 \\
\hline & & \multicolumn{8}{|c|}{ Level of theory: mPW1PW91/6-31G* (gas phase) } \\
\hline & & $79 a$ & $79 b$ & 79c & $79 d$ & $79 e$ & $79 f$ & $79 \mathrm{~g}$ & $79 \mathrm{~h}$ \\
\hline \multirow{3}{*}{ sDP4+ } & ${ }^{1} \mathrm{H}$ data & 1.26 & 55.25 & 17.32 & 26.14 & 0.00 & 0.01 & 0.01 & 0.01 \\
\hline & ${ }^{13} \mathrm{C}$ data & 4.34 & 16.49 & 5.67 & 72.27 & 0.22 & 0.08 & 0.80 & 0.13 \\
\hline & All data & 0.19 & 31.37 & 3.38 & 65.06 & 0.00 & 0.00 & 0.00 & 0.00 \\
\hline \multirow{3}{*}{ uDP4+ } & ${ }^{1} \mathrm{H}$ data & 0.66 & 54.12 & 24.54 & 20.46 & 0.01 & 0.07 & 0.11 & 0.02 \\
\hline & ${ }^{13} \mathrm{C}$ data & 13.27 & 9.36 & 50.18 & 25.98 & 0.21 & 0.18 & 0.49 & 0.33 \\
\hline & All data & 0.39 & 22.24 & 54.04 & 23.33 & 0.00 & 0.00 & 0.00 & 0.00 \\
\hline & ${ }^{1} \mathrm{H}$ data & 0.02 & 75.68 & 10.76 & 13.54 & 0.00 & 0.00 & 0.00 & 0.00 \\
\hline DP4+ & ${ }^{13} \mathrm{C}$ data & 2.43 & 6.50 & 11.99 & 79.06 & 0.00 & 0.00 & 0.02 & 0.00 \\
\hline & All data & 0.00 & 29.09 & 7.62 & 63.28 & 0.00 & 0.00 & 0.00 & 0.00 \\
\hline & & & & evel of $t$ & V: $\mathrm{mPW}$ & $/ 6-31$ & s phas & & \\
\hline & & $79 a$ & $79 b$ & $79 c$ & $79 d$ & $79 e$ & $79 f$ & $79 \mathrm{~g}$ & $79 \mathrm{~h}$ \\
\hline & ${ }^{1} \mathrm{H}$ data & 1.62 & 70.03 & 16.11 & 12.12 & 0.01 & 0.01 & 0.07 & 0.03 \\
\hline sDP4+ & ${ }^{13} \mathrm{C}$ data & 5.31 & 18.87 & 6.94 & 67.90 & 0.21 & 0.08 & 0.56 & 0.14 \\
\hline & All data & 0.38 & 58.33 & 4.94 & 36.35 & 0.00 & 0.00 & 0.00 & 0.00 \\
\hline & ${ }^{1} \mathrm{H}$ data & 0.97 & 61.48 & 26.98 & 10.23 & 0.02 & 0.10 & 0.18 & 0.04 \\
\hline uDP4+ & ${ }^{13} \mathrm{C}$ data & 3.05 & 18.69 & 53.60 & 24.44 & 0.02 & 0.05 & 0.12 & 0.02 \\
\hline & All data & 0.10 & 40.34 & 50.78 & 8.77 & 0.00 & 0.00 & 0.00 & 0.00 \\
\hline DP4+ & ${ }^{1} \mathrm{H}$ data & 0.03 & 88.49 & 8.93 & 2.55 & 0.00 & 0.00 & 0.00 & 0.00 \\
\hline
\end{tabular}




\begin{tabular}{|c|c|c|c|c|c|c|c|c|c|}
\hline & ${ }^{13} \mathrm{C}$ data & 0.67 & 14.69 & 15.50 & 69.13 & 0.00 & 0.00 & 0.00 & 0.00 \\
\hline & All data & 0.00 & 80.51 & 8.58 & 10.91 & 0.00 & 0.00 & 0.00 & 0.00 \\
\hline & & \multicolumn{8}{|c|}{ Level of theory: mPW1PW91/6-31+G** (gas phase) } \\
\hline & & $79 a$ & $79 b$ & $79 c$ & 79d & $79 e$ & $79 f$ & $79 \mathrm{~g}$ & $79 \mathrm{~h}$ \\
\hline & ${ }^{1} \mathrm{H}$ data & 19.04 & 45.55 & 28.44 & 5.65 & 0.15 & 0.12 & 0.94 & 0.11 \\
\hline \multirow[t]{2}{*}{ sDP4+ } & ${ }^{13} \mathrm{C}$ data & 0.97 & 22.78 & 21.71 & 53.88 & 0.08 & 0.19 & 0.39 & 0.01 \\
\hline & All data & 0.93 & 52.45 & 31.20 & 15.39 & 0.00 & 0.00 & 0.02 & 0.00 \\
\hline \multirow{3}{*}{ uDP4+ } & ${ }^{1} \mathrm{H}$ data & 11.71 & 45.59 & 33.70 & 5.84 & 0.46 & 1.04 & 1.34 & 0.31 \\
\hline & ${ }^{13} \mathrm{C}$ data & 0.65 & 27.99 & 66.51 & 4.63 & 0.01 & 0.08 & 0.12 & 0.00 \\
\hline & All data & 0.22 & 35.92 & 63.09 & 0.76 & 0.00 & 0.00 & 0.00 & 0.00 \\
\hline \multirow{5}{*}{ DP4+ } & ${ }^{1} \mathrm{H}$ data & 6.77 & 63.07 & 29.11 & 1.00 & 0.00 & 0.00 & 0.04 & 0.00 \\
\hline & ${ }^{13} \mathrm{C}$ data & 0.03 & 27.35 & 61.92 & 10.71 & 0.00 & 0.00 & 0.00 & 0.00 \\
\hline & All data & 0.01 & 48.75 & 50.94 & 0.30 & 0.00 & 0.00 & 0.00 & 0.00 \\
\hline & & \multicolumn{8}{|c|}{ Level of theory: mPW1PW91/6-311G* (gas phase) } \\
\hline & & $79 a$ & $79 b$ & $79 c$ & 79d & $79 e$ & $79 f$ & $79 \mathrm{~g}$ & $79 \mathrm{~h}$ \\
\hline \multirow{3}{*}{ sDP4+ } & ${ }^{1} \mathrm{H}$ data & 1.38 & 92.78 & 3.27 & 2.41 & 0.01 & 0.06 & 0.07 & 0.02 \\
\hline & ${ }^{13} \mathrm{C}$ data & 3.05 & 18.08 & 6.14 & 72.47 & 0.04 & 0.06 & 0.12 & 0.03 \\
\hline & All data & 0.23 & 89.39 & 1.07 & 9.32 & 0.00 & 0.00 & 0.00 & 0.00 \\
\hline \multirow{3}{*}{ uDP4+ } & ${ }^{1} \mathrm{H}$ data & 3.52 & 77.79 & 11.70 & 5.15 & 0.15 & 0.83 & 0.72 & 0.15 \\
\hline & ${ }^{13} \mathrm{C}$ data & 0.16 & 46.73 & 15.51 & 36.37 & 0.01 & 0.14 & 1.07 & 0.01 \\
\hline & All data & 0.01 & 90.76 & 4.53 & 4.68 & 0.00 & 0.00 & 0.02 & 0.00 \\
\hline \multirow{6}{*}{ DP4+ } & ${ }^{1} \mathrm{H}$ data & 0.07 & 99.24 & 0.53 & 0.17 & 0.00 & 0.00 & 0.00 & 0.00 \\
\hline & ${ }^{13} \mathrm{C}$ data & 0.01 & 23.63 & 2.66 & 73.69 & 0.00 & 0.00 & 0.00 & 0.00 \\
\hline & All data & 0.00 & 99.41 & 0.06 & 0.53 & 0.00 & 0.00 & 0.00 & 0.00 \\
\hline & & \multicolumn{8}{|c|}{ Level of theory: mPW1PW91/6-311G** (gas phase) } \\
\hline & & 79a & $79 b$ & $79 c$ & $79 d$ & $79 e$ & $79 f$ & $79 \mathrm{~g}$ & $79 \mathrm{~h}$ \\
\hline & ${ }^{1} \mathrm{H}$ data & 4.23 & 86.35 & 7.26 & 1.27 & 0.07 & 0.29 & 0.49 & 0.05 \\
\hline \multirow[t]{2}{*}{ sDP4+ } & ${ }^{13} \mathrm{C}$ data & 1.77 & 20.13 & 6.16 & 71.70 & 0.03 & 0.06 & 0.13 & 0.02 \\
\hline & All data & 0.40 & 92.37 & 2.38 & 4.85 & 0.00 & 0.00 & 0.00 & 0.00 \\
\hline \multirow{3}{*}{ uDP4+ } & ${ }^{1} \mathrm{H}$ data & 9.94 & 69.96 & 9.58 & 3.45 & 0.64 & 5.22 & 0.77 & 0.44 \\
\hline & ${ }^{13} \mathrm{C}$ data & 0.05 & 58.64 & 16.55 & 23.60 & 0.00 & 0.09 & 1.06 & 0.00 \\
\hline & All data & 0.01 & 94.43 & 3.65 & 1.88 & 0.00 & 0.01 & 0.02 & 0.00 \\
\hline \multirow{5}{*}{ DP4+ } & ${ }^{1} \mathrm{H}$ data & 0.68 & 98.08 & 1.13 & 0.07 & 0.00 & 0.02 & 0.01 & 0.00 \\
\hline & ${ }^{13} \mathrm{C}$ data & 0.00 & 39.68 & 3.43 & 56.88 & 0.00 & 0.00 & 0.00 & 0.00 \\
\hline & All data & 0.00 & 99.80 & 0.10 & 0.10 & 0.00 & 0.00 & 0.00 & 0.00 \\
\hline & & \multicolumn{8}{|c|}{ Level of theory: mPW1PW91/6-311+G** (gas phase) } \\
\hline & & 79a & $79 b$ & $79 c$ & 79d & $79 e$ & $79 f$ & $79 \mathrm{~g}$ & $79 \mathrm{~h}$ \\
\hline \multirow{3}{*}{ sDP4+ } & ${ }^{1} \mathrm{H}$ data & 20.44 & 58.05 & 17.07 & 2.75 & 0.08 & 0.26 & 1.25 & 0.10 \\
\hline & ${ }^{13} \mathrm{C}$ data & 1.48 & 28.14 & 11.99 & 58.26 & 0.01 & 0.04 & 0.08 & 0.01 \\
\hline & All data & 1.49 & 80.51 & 10.09 & 7.91 & 0.00 & 0.00 & 0.00 & 0.00 \\
\hline \multirow{3}{*}{ uDP4+ } & ${ }^{1} \mathrm{H}$ data & 37.24 & 31.27 & 15.43 & 10.03 & 0.87 & 3.68 & 0.61 & 0.86 \\
\hline & ${ }^{13} \mathrm{C}$ data & 0.06 & 71.15 & 14.38 & 13.64 & 0.00 & 0.08 & 0.69 & 0.00 \\
\hline & All data & 0.08 & 86.02 & 8.58 & 5.29 & 0.00 & 0.01 & 0.02 & 0.00 \\
\hline \multirow{3}{*}{ DP4+ } & ${ }^{1} \mathrm{H}$ data & 26.53 & 63.26 & 9.18 & 0.96 & 0.00 & 0.03 & 0.03 & 0.00 \\
\hline & ${ }^{13} \mathrm{C}$ data & 0.00 & 67.42 & 5.81 & 26.77 & 0.00 & 0.00 & 0.00 & 0.00 \\
\hline & All data & 0.00 & 98.18 & 1.23 & 0.59 & 0.00 & 0.00 & 0.00 & 0.00 \\
\hline & & & & rel of th & mPW1P & 6-31G & ion, $\mathrm{PC}$ & & \\
\hline & & $79 a$ & $79 b$ & $79 c$ & $79 d$ & $79 e$ & $79 f$ & $79 \mathrm{~g}$ & $79 \mathrm{~h}$ \\
\hline & ${ }^{1} \mathrm{H}$ data & 7.55 & 79.24 & 9.66 & 3.53 & 0.00 & 0.00 & 0.01 & 0.00 \\
\hline sDP4+ & ${ }^{13} \mathrm{C}$ data & 1.98 & 36.33 & 16.91 & 44.21 & 0.01 & 0.08 & 0.48 & 0.00 \\
\hline & All data & 0.47 & 89.60 & 5.08 & 4.85 & 0.00 & 0.00 & 0.00 & 0.00 \\
\hline & ${ }^{1} \mathrm{H}$ data & 1.50 & 64.39 & 27.59 & 6.33 & 0.00 & 0.05 & 0.12 & 0.01 \\
\hline uDP4+ & ${ }^{13} \mathrm{C}$ data & 6.21 & 9.69 & 65.92 & 17.79 & 0.07 & 0.08 & 0.19 & 0.07 \\
\hline & All data & 0.36 & 24.32 & 70.92 & 4.39 & 0.00 & 0.00 & 0.00 & 0.00 \\
\hline & ${ }^{1} \mathrm{H}$ data & 0.21 & 94.44 & 4.93 & 0.41 & 0.00 & 0.00 & 0.00 & 0.00 \\
\hline DP4+ & ${ }^{13} \mathrm{C}$ data & 0.54 & 15.53 & 49.21 & 34.71 & 0.00 & 0.00 & 0.00 & 0.00 \\
\hline
\end{tabular}




\begin{tabular}{|c|c|c|c|c|c|c|c|c|c|}
\hline & All data & 0.01 & 85.08 & 14.08 & 0.83 & 0.00 & 0.00 & 0.00 & 0.00 \\
\hline & & \multicolumn{8}{|c|}{ Level of theory: mPW1PW91/6-31G** (solution, PCM) } \\
\hline & & $79 a$ & $79 b$ & $79 c$ & 79d & $79 e$ & $79 f$ & $79 \mathrm{~g}$ & $79 h$ \\
\hline & ${ }^{1} \mathrm{H}$ data & 13.13 & 77.14 & 7.93 & 1.72 & 0.00 & 0.01 & 0.05 & 0.01 \\
\hline \multirow[t]{2}{*}{ sDP4+ } & ${ }^{13} \mathrm{C}$ data & 3.28 & 35.40 & 15.10 & 45.88 & 0.01 & 0.06 & 0.26 & 0.01 \\
\hline & All data & 1.45 & 91.87 & 4.03 & 2.65 & 0.00 & 0.00 & 0.00 & 0.00 \\
\hline \multirow{3}{*}{ uDP4+ } & ${ }^{1} \mathrm{H}$ data & 2.57 & 81.72 & 12.29 & 3.21 & 0.00 & 0.05 & 0.14 & 0.02 \\
\hline & ${ }^{13} \mathrm{C}$ data & 1.95 & 23.17 & 57.87 & 16.86 & 0.01 & 0.04 & 0.10 & 0.01 \\
\hline & All data & 0.19 & 71.08 & 26.70 & 2.03 & 0.00 & 0.00 & 0.00 & 0.00 \\
\hline \multirow{5}{*}{ DP4+ } & ${ }^{1} \mathrm{H}$ data & 0.52 & 97.88 & 1.51 & 0.09 & 0.00 & 0.00 & 0.00 & 0.00 \\
\hline & ${ }^{13} \mathrm{C}$ data & 0.26 & 33.15 & 35.33 & 31.27 & 0.00 & 0.00 & 0.00 & 0.00 \\
\hline & All data & 0.00 & 98.29 & 1.62 & 0.08 & 0.00 & 0.00 & 0.00 & 0.00 \\
\hline & & \multicolumn{8}{|c|}{ Level of theory: mPW1PW91/6-31+G** (solution, PCM) } \\
\hline & & $79 a$ & $79 b$ & 79c & 79d & $79 e$ & $79 f$ & $79 \mathrm{~g}$ & $79 \mathrm{~h}$ \\
\hline \multirow{3}{*}{ sDP4+ } & ${ }^{1} \mathrm{H}$ data & 51.23 & 28.39 & 18.48 & 0.75 & 0.03 & 0.24 & 0.82 & 0.06 \\
\hline & ${ }^{13} \mathrm{C}$ data & 0.49 & 44.00 & 28.39 & 26.96 & 0.00 & 0.03 & 0.12 & 0.00 \\
\hline & All data & 1.39 & 68.66 & 28.83 & 1.11 & 0.00 & 0.00 & 0.01 & 0.00 \\
\hline & ${ }^{1} \mathrm{H}$ data & 41.47 & 27.50 & 28.01 & 1.47 & 0.05 & 0.29 & 1.13 & 0.08 \\
\hline \multirow[t]{2}{*}{ uDP4+ } & ${ }^{13} \mathrm{C}$ data & 0.28 & 29.95 & 67.73 & 1.86 & 0.00 & 0.06 & 0.12 & 0.00 \\
\hline & All data & 0.42 & 30.11 & 69.36 & 0.10 & 0.00 & 0.00 & 0.01 & 0.00 \\
\hline \multirow{5}{*}{ DP4+ } & ${ }^{1} \mathrm{H}$ data & 62.04 & 22.79 & 15.11 & 0.03 & 0.00 & 0.00 & 0.03 & 0.00 \\
\hline & ${ }^{13} \mathrm{C}$ data & 0.00 & 40.04 & 58.43 & 1.53 & 0.00 & 0.00 & 0.00 & 0.00 \\
\hline & All data & 0.01 & 50.82 & 49.16 & 0.00 & 0.00 & 0.00 & 0.00 & 0.00 \\
\hline & & \multicolumn{8}{|c|}{ Level of theory: mPW1PW91/6-311G* (solution, PCM) } \\
\hline & & $79 a$ & $79 b$ & 79c & 79d & $79 e$ & $79 f$ & $79 \mathrm{~g}$ & $79 h$ \\
\hline \multirow{3}{*}{$s \mathrm{DP} 4+$} & ${ }^{1} \mathrm{H}$ data & 3.75 & 93.32 & 2.62 & 0.25 & 0.00 & 0.02 & 0.04 & 0.00 \\
\hline & ${ }^{13} \mathrm{C}$ data & 1.33 & 22.12 & 15.48 & 60.96 & 0.00 & 0.02 & 0.08 & 0.00 \\
\hline & All data & 0.23 & 97.15 & 1.91 & 0.71 & 0.00 & 0.00 & 0.00 & 0.00 \\
\hline \multirow{3}{*}{ uDP4+ } & ${ }^{1} \mathrm{H}$ data & 3.51 & 89.40 & 5.44 & 1.26 & 0.01 & 0.09 & 0.26 & 0.02 \\
\hline & ${ }^{13} \mathrm{C}$ data & 0.06 & 64.12 & 21.72 & 13.37 & 0.00 & 0.05 & 0.68 & 0.00 \\
\hline & All data & 0.00 & 97.69 & 2.01 & 0.29 & 0.00 & 0.00 & 0.00 & 0.00 \\
\hline \multirow{5}{*}{ DP4+ } & ${ }^{1} \mathrm{H}$ data & 0.16 & 99.67 & 0.17 & 0.00 & 0.00 & 0.00 & 0.00 & 0.00 \\
\hline & ${ }^{13} \mathrm{C}$ data & 0.00 & 55.19 & 13.08 & 31.72 & 0.00 & 0.00 & 0.00 & 0.00 \\
\hline & All data & 0.00 & 99.96 & 0.04 & 0.00 & 0.00 & 0.00 & 0.00 & 0.00 \\
\hline & & \multicolumn{8}{|c|}{ Level of theory: mPW1PW91/6-311G** (solution, PCM) } \\
\hline & & $79 a$ & $79 b$ & $79 c$ & $79 d$ & $79 e$ & $79 f$ & $79 \mathrm{~g}$ & $79 h$ \\
\hline & ${ }^{1} \mathrm{H}$ data & 18.52 & 75.00 & 4.66 & 0.20 & 0.03 & 0.46 & 1.04 & 0.07 \\
\hline \multirow[t]{2}{*}{$s \mathrm{DP} 4+$} & ${ }^{13} \mathrm{C}$ data & 0.44 & 22.64 & 15.24 & 61.61 & 0.00 & 0.01 & 0.05 & 0.00 \\
\hline & All data & 0.46 & 94.87 & 3.97 & 0.70 & 0.00 & 0.00 & 0.00 & 0.00 \\
\hline \multirow{3}{*}{ uDP4+ } & ${ }^{1} \mathrm{H}$ data & 25.35 & 66.58 & 5.16 & 0.44 & 0.09 & 1.57 & 0.62 & 0.19 \\
\hline & ${ }^{13} \mathrm{C}$ data & 0.03 & 70.85 & 20.13 & 8.08 & 0.00 & 0.05 & 0.87 & 0.00 \\
\hline & All data & 0.01 & 97.75 & 2.15 & 0.07 & 0.00 & 0.00 & 0.01 & 0.00 \\
\hline \multirow{5}{*}{ DP4+ } & ${ }^{1} \mathrm{H}$ data & 8.55 & 90.98 & 0.44 & 0.00 & 0.00 & 0.01 & 0.01 & 0.00 \\
\hline & ${ }^{13} \mathrm{C}$ data & 0.00 & 66.58 & 12.74 & 20.68 & 0.00 & 0.00 & 0.00 & 0.00 \\
\hline & All data & 0.00 & 99.91 & 0.09 & 0.00 & 0.00 & 0.00 & 0.00 & 0.00 \\
\hline & & & & of the & PW1PV & $311+G$ & ation, & & \\
\hline & & $79 a$ & $79 b$ & $79 c$ & $79 d$ & $79 e$ & $79 f$ & 79g & $79 h$ \\
\hline & ${ }^{1} \mathrm{H}$ data & 44.72 & 14.81 & 7.99 & 0.93 & 0.67 & 6.73 & 23.05 & 1.09 \\
\hline sDP4+ & ${ }^{13} \mathrm{C}$ data & 0.58 & 28.60 & 31.53 & 39.23 & 0.00 & 0.01 & 0.04 & 0.00 \\
\hline & All data & 3.51 & 57.32 & 34.11 & 4.93 & 0.00 & 0.01 & 0.13 & 0.00 \\
\hline & ${ }^{1} \mathrm{H}$ data & 43.06 & 20.93 & 9.17 & 1.53 & 1.05 & 19.29 & 3.30 & 1.67 \\
\hline uDP4+ & ${ }^{13} \mathrm{C}$ data & 0.05 & 75.35 & 17.96 & 6.38 & 0.00 & 0.02 & 0.25 & 0.00 \\
\hline & All data & 0.11 & 89.88 & 9.39 & 0.55 & 0.00 & 0.02 & 0.05 & 0.00 \\
\hline & ${ }^{1} \mathrm{H}$ data & 76.46 & 12.30 & 2.91 & 0.06 & 0.03 & 5.15 & 3.01 & 0.07 \\
\hline DP4+ & ${ }^{13} \mathrm{C}$ data & 0.00 & 72.53 & 19.05 & 8.42 & 0.00 & 0.00 & 0.00 & 0.00 \\
\hline & All data & 0.01 & 94.09 & 5.85 & 0.05 & 0.00 & 0.00 & 0.00 & 0.00 \\
\hline
\end{tabular}


$s \mathrm{DP4}+, u \mathrm{DP} 4+$ and DP4+ probabilities (\%) for compound 79d<smiles>CC[C@H](C)[C@@H]1OC([C@@H](C)[C@@H](O)CC)=C(C)C(=O)[C@@H]1C</smiles><smiles>CC[C@H](O)[C@H](C)C1=C(C)C(=O)C(C)[C@H]([C@H](C)CC)O1</smiles><smiles>CC[C@H](C)[C@@H]1OC([C@@H](C)[C@@H](O)CC)=C(C)C(=O)[C@@H]1C</smiles><smiles>CC[C@H](C)[C@@H]1OC([C@@H](C)[C@@H](O)CC)=C(C)C(=O)[C@@H]1C</smiles><smiles>CC[C@H](O)[C@H](C)C1=C(C)C(=O)[C@H](C)[C@H]([C@H](C)CC)O1</smiles><smiles>CC[C@H](C)[C@@H]1OC([C@@H](C)[C@@H](O)CC)=C(C)C(=O)C1C</smiles><smiles>CCC(C)[C@H]1OC([C@H](C)[C@H](O)CC)=C(C)C(=O)[C@H]1C</smiles><smiles>CC[C@H](C)[C@@H]1OC([C@@H](C)[C@@H](O)CC)=C(C)C(=O)[C@@H]1C</smiles>

\begin{tabular}{|c|c|c|c|c|c|c|c|c|c|}
\hline & & \multicolumn{8}{|c|}{ Level of theory: B3LYP/6-31G* (gas phase) } \\
\hline & & $79 a$ & $79 b$ & $79 c$ & 79d & $79 \mathrm{e}$ & $79 f$ & $79 \mathrm{~g}$ & $79 \mathrm{~h}$ \\
\hline & ${ }^{1}$ H data & 0.05 & 11.58 & 56.90 & 31.40 & 0.00 & 0.00 & 0.06 & 0.01 \\
\hline \multirow[t]{2}{*}{ sDP4+ } & ${ }^{13} \mathrm{C}$ data & 53.90 & 1.27 & 0.46 & 35.74 & 2.96 & 0.01 & 0.17 & 5.49 \\
\hline & All data & 0.24 & 1.26 & 2.25 & 96.24 & 0.00 & 0.00 & 0.00 & 0.00 \\
\hline \multirow{3}{*}{ uDP4+ } & ${ }^{1} \mathrm{H}$ data & 0.06 & 31.40 & 54.33 & 13.82 & 0.02 & 0.17 & 0.18 & 0.02 \\
\hline & ${ }^{13} \mathrm{C}$ data & 42.80 & 3.52 & 8.81 & 44.23 & 0.20 & 0.01 & 0.03 & 0.40 \\
\hline & All data & 0.20 & 9.19 & 39.79 & 50.81 & 0.00 & 0.00 & 0.00 & 0.00 \\
\hline \multirow{5}{*}{ DP4+ } & ${ }^{1}$ H data & 0.00 & 9.35 & 79.49 & 11.16 & 0.00 & 0.00 & 0.00 & 0.00 \\
\hline & ${ }^{13} \mathrm{C}$ data & 59.16 & 0.11 & 0.10 & 40.55 & 0.01 & 0.00 & 0.00 & 0.06 \\
\hline & All data & 0.00 & 0.23 & 1.79 & 97.97 & 0.00 & 0.00 & 0.00 & 0.00 \\
\hline & & \multicolumn{8}{|c|}{ Level of theory: B3LYP/6-31G** (gas phase) } \\
\hline & & $79 a$ & $79 b$ & $79 c$ & 79d & $79 e$ & $79 f$ & $79 \mathrm{~g}$ & $79 \mathrm{~h}$ \\
\hline \multirow{3}{*}{ sDP4+ } & ${ }^{1} \mathrm{H}$ data & 0.10 & 13.35 & 50.99 & 35.26 & 0.01 & 0.00 & 0.27 & 0.03 \\
\hline & ${ }^{13} \mathrm{C}$ data & 55.87 & 2.05 & 0.72 & 35.01 & 2.90 & 0.02 & 0.14 & 3.30 \\
\hline & All data & 0.41 & 2.10 & 2.81 & 94.67 & 0.00 & 0.00 & 0.00 & 0.01 \\
\hline \multirow{3}{*}{ uDP4+ } & ${ }^{1} \mathrm{H}$ data & 0.11 & 17.03 & 58.24 & 24.06 & 0.04 & 0.13 & 0.32 & 0.09 \\
\hline & ${ }^{13} \mathrm{C}$ data & 24.46 & 9.48 & 6.69 & 59.24 & 0.04 & 0.00 & 0.01 & 0.08 \\
\hline & All data & 0.13 & 8.16 & 19.69 & 72.01 & 0.00 & 0.00 & 0.00 & 0.00 \\
\hline \multirow{3}{*}{ DP4+ } & ${ }^{1} \mathrm{H}$ data & 0.00 & 5.62 & 73.41 & 20.97 & 0.00 & 0.00 & 0.00 & 0.00 \\
\hline & ${ }^{13} \mathrm{C}$ data & 39.44 & 0.56 & 0.14 & 59.85 & 0.00 & 0.00 & 0.00 & 0.01 \\
\hline & All data & 0.00 & 0.25 & 0.80 & 98.95 & 0.00 & 0.00 & 0.00 & 0.00 \\
\hline & & \multicolumn{8}{|c|}{ Level of theory: B3LYP/6-31+G** (gas phase) } \\
\hline & & $79 a$ & $79 b$ & 79c & 79d & $79 e$ & $79 f$ & $79 \mathrm{~g}$ & $79 h$ \\
\hline \multirow{3}{*}{ sDP4+ } & ${ }^{1} \mathrm{H}$ data & 1.07 & 10.46 & 74.34 & 13.02 & 0.29 & 0.02 & 0.59 & 0.21 \\
\hline & ${ }^{13} \mathrm{C}$ data & 16.79 & 11.48 & 7.28 & 62.43 & 1.13 & 0.17 & 0.25 & 0.48 \\
\hline & All data & 1.21 & 8.04 & 36.26 & 54.45 & 0.02 & 0.00 & 0.01 & 0.01 \\
\hline \multirow{3}{*}{ uDP4+ } & ${ }^{1} \mathrm{H}$ data & 1.45 & 4.88 & 56.70 & 33.09 & 2.20 & 0.12 & 0.84 & 0.72 \\
\hline & ${ }^{13} \mathrm{C}$ data & 7.05 & 21.32 & 26.73 & 44.82 & 0.03 & 0.01 & 0.03 & 0.01 \\
\hline & All data & 0.33 & 3.34 & 48.69 & 47.63 & 0.00 & 0.00 & 0.00 & 0.00 \\
\hline \multirow{3}{*}{ DP4+ } & ${ }^{1} \mathrm{H}$ data & 0.03 & 1.09 & 89.69 & 9.17 & 0.01 & 0.00 & 0.01 & 0.00 \\
\hline & ${ }^{13} \mathrm{C}$ data & 3.52 & 7.30 & 5.80 & 83.38 & 0.00 & 0.00 & 0.00 & 0.00 \\
\hline & All data & 0.01 & 0.61 & 40.25 & 59.13 & 0.00 & 0.00 & 0.00 & 0.00 \\
\hline & & \multicolumn{8}{|c|}{ Level of theory: B3LYP/6-311G* (gas phase) } \\
\hline & & $79 a$ & $79 b$ & $79 c$ & 79d & $79 e$ & $79 f$ & $79 \mathrm{~g}$ & $79 \mathrm{~h}$ \\
\hline \multirow{3}{*}{ sDP4+ } & ${ }^{1} \mathrm{H}$ data & 0.11 & 19.82 & 71.76 & 7.81 & 0.03 & 0.11 & 0.31 & 0.05 \\
\hline & ${ }^{13} \mathrm{C}$ data & 16.83 & 3.52 & 0.90 & 77.99 & 0.26 & 0.01 & 0.03 & 0.47 \\
\hline & All data & 0.24 & 9.36 & 8.65 & 81.74 & 0.00 & 0.00 & 0.00 & 0.00 \\
\hline \multirow{2}{*}{ uDP4+ } & ${ }^{1} \mathrm{H}$ data & 0.63 & 14.36 & 72.44 & 8.31 & 0.47 & 1.66 & 1.69 & 0.42 \\
\hline & ${ }^{13} \mathrm{C}$ data & 3.64 & 24.08 & 4.31 & 66.62 & 0.38 & 0.15 & 0.49 & 0.33 \\
\hline
\end{tabular}




\begin{tabular}{|c|c|c|c|c|c|c|c|c|c|}
\hline & All data & 0.19 & 28.45 & 25.69 & 45.56 & 0.01 & 0.02 & 0.07 & 0.01 \\
\hline & ${ }^{1} \mathrm{H}$ data & 0.00 & 5.13 & 93.68 & 1.17 & 0.00 & 0.00 & 0.01 & 0.00 \\
\hline \multirow[t]{5}{*}{ DP4+ } & ${ }^{13} \mathrm{C}$ data & 1.14 & 1.59 & 0.07 & 97.19 & 0.00 & 0.00 & 0.00 & 0.00 \\
\hline & All data & 0.00 & 6.32 & 5.28 & 88.40 & 0.00 & 0.00 & 0.00 & 0.00 \\
\hline & & \multicolumn{8}{|c|}{ Level of theory: B3LYP/6-311G** (gas phase) } \\
\hline & & $79 a$ & $79 b$ & $79 c$ & $79 d$ & $79 e$ & $79 f$ & $79 \mathrm{~g}$ & $79 \mathrm{~h}$ \\
\hline & ${ }^{1} \mathrm{H}$ data & 0.47 & 26.11 & 61.87 & 9.73 & 0.24 & 0.10 & 1.36 & 0.12 \\
\hline \multirow[t]{2}{*}{ sDP4+ } & ${ }^{13} \mathrm{C}$ data & 15.64 & 3.09 & 0.84 & 79.56 & 0.27 & 0.01 & 0.03 & 0.56 \\
\hline & All data & 0.81 & 8.82 & 5.71 & 84.64 & 0.01 & 0.00 & 0.00 & 0.01 \\
\hline & ${ }^{1} \mathrm{H}$ data & 2.53 & 9.60 & 59.09 & 19.37 & 3.52 & 2.09 & 1.69 & 2.11 \\
\hline \multirow[t]{2}{*}{ uDP4+ } & ${ }^{13} \mathrm{C}$ data & 1.53 & 26.50 & 4.70 & 66.08 & 0.18 & 0.18 & 0.66 & 0.17 \\
\hline & All data & 0.21 & 13.99 & 15.27 & 70.40 & 0.03 & 0.02 & 0.06 & 0.02 \\
\hline \multirow{6}{*}{ DP4+ } & ${ }^{1} \mathrm{H}$ data & 0.03 & 6.11 & 89.17 & 4.60 & 0.02 & 0.00 & 0.06 & 0.01 \\
\hline & ${ }^{13} \mathrm{C}$ data & 0.45 & 1.53 & 0.07 & 97.95 & 0.00 & 0.00 & 0.00 & 0.00 \\
\hline & All data & 0.00 & 2.00 & 1.41 & 96.58 & 0.00 & 0.00 & 0.00 & 0.00 \\
\hline & & \multicolumn{8}{|c|}{ Level of theory: B3LYP/6-311+G** (gas phase) } \\
\hline & & 79a & $79 b$ & 79c & 79d & $79 e$ & $79 f$ & $79 \mathrm{~g}$ & $79 \mathrm{~h}$ \\
\hline & ${ }^{1} \mathrm{H}$ data & 3.54 & 20.18 & 60.62 & 12.48 & 0.71 & 0.28 & 1.56 & 0.63 \\
\hline \multirow[t]{2}{*}{$s \mathrm{DP4+}$} & ${ }^{13} \mathrm{C}$ data & 11.96 & 4.90 & 1.35 & 81.39 & 0.12 & 0.00 & 0.02 & 0.26 \\
\hline & All data & 3.42 & 7.98 & 6.58 & 82.00 & 0.01 & 0.00 & 0.00 & 0.01 \\
\hline \multirow{3}{*}{ uDP4+ } & ${ }^{1} \mathrm{H}$ data & 8.32 & 5.32 & 35.62 & 32.92 & 6.10 & 3.23 & 1.80 & 6.68 \\
\hline & ${ }^{13} \mathrm{C}$ data & 1.46 & 32.83 & 4.71 & 60.10 & 0.16 & 0.18 & 0.48 & 0.08 \\
\hline & All data & 0.52 & 7.48 & 7.19 & 84.69 & 0.04 & 0.02 & 0.04 & 0.02 \\
\hline \multirow{5}{*}{ DP4+ } & ${ }^{1} \mathrm{H}$ data & 1.08 & 3.95 & 79.41 & 15.11 & 0.16 & 0.03 & 0.10 & 0.15 \\
\hline & ${ }^{13} \mathrm{C}$ data & 0.35 & 3.17 & 0.12 & 96.36 & 0.00 & 0.00 & 0.00 & 0.00 \\
\hline & All data & 0.03 & 0.85 & 0.67 & 98.46 & 0.00 & 0.00 & 0.00 & 0.00 \\
\hline & & \multicolumn{8}{|c|}{ Level of theory: B3LYP/6-31G* (solution, PCM) } \\
\hline & & 79a & $79 b$ & 79c & 79d & $79 e$ & $79 f$ & $79 \mathrm{~g}$ & $79 \mathrm{~h}$ \\
\hline & ${ }^{1} \mathrm{H}$ data & 0.53 & 10.10 & 37.27 & 52.06 & 0.00 & 0.00 & 0.03 & 0.00 \\
\hline \multirow[t]{2}{*}{$s \mathrm{DP} 4+$} & ${ }^{13} \mathrm{C}$ data & 55.79 & 2.27 & 0.86 & 39.44 & 0.55 & 0.01 & 0.06 & 1.03 \\
\hline & All data & 1.40 & 1.07 & 1.50 & 96.03 & 0.00 & 0.00 & 0.00 & 0.00 \\
\hline \multirow{3}{*}{ uDP4+ } & ${ }^{1} \mathrm{H}$ data & 0.12 & 21.43 & 64.38 & 13.68 & 0.01 & 0.17 & 0.18 & 0.02 \\
\hline & ${ }^{13} \mathrm{C}$ data & 45.01 & 4.97 & 12.81 & 36.87 & 0.10 & 0.01 & 0.02 & 0.21 \\
\hline & All data & 0.39 & 7.39 & 57.22 & 35.00 & 0.00 & 0.00 & 0.00 & 0.00 \\
\hline & ${ }^{1} \mathrm{H}$ data & 0.00 & 6.50 & 72.10 & 21.40 & 0.00 & 0.00 & 0.00 & 0.00 \\
\hline \multirow[t]{4}{*}{ DP4+ } & ${ }^{13} \mathrm{C}$ data & 62.97 & 0.28 & 0.28 & 36.47 & 0.00 & 0.00 & 0.00 & 0.01 \\
\hline & All data & 0.02 & 0.23 & 2.48 & 97.27 & 0.00 & 0.00 & 0.00 & 0.00 \\
\hline & & \multicolumn{8}{|c|}{ Level of theory: B3LYP/6-31G** (solution, PCM) } \\
\hline & & $79 a$ & $79 b$ & 79c & $79 d$ & $79 e$ & $79 f$ & $79 \mathrm{~g}$ & $79 \mathrm{~h}$ \\
\hline & ${ }^{1} \mathrm{H}$ data & 0.99 & 10.82 & 25.17 & 62.89 & 0.01 & 0.00 & 0.09 & 0.02 \\
\hline \multirow[t]{2}{*}{ sDP4+ } & ${ }^{13} \mathrm{C}$ data & 57.16 & 3.57 & 1.44 & 35.41 & 0.88 & 0.01 & 0.08 & 1.45 \\
\hline & All data & 2.40 & 1.64 & 1.53 & 94.43 & 0.00 & 0.00 & 0.00 & 0.00 \\
\hline \multirow{3}{*}{ uDP4+ } & ${ }^{1} \mathrm{H}$ data & 0.26 & 12.26 & 35.84 & 51.16 & 0.04 & 0.07 & 0.27 & 0.09 \\
\hline & ${ }^{13} \mathrm{C}$ data & 25.74 & 15.00 & 11.23 & 47.92 & 0.03 & 0.00 & 0.01 & 0.07 \\
\hline & All data & 0.22 & 6.04 & 13.22 & 80.52 & 0.00 & 0.00 & 0.00 & 0.00 \\
\hline & ${ }^{1} \mathrm{H}$ data & 0.01 & 3.12 & 21.22 & 75.66 & 0.00 & 0.00 & 0.00 & 0.00 \\
\hline DP4+ & ${ }^{13} \mathrm{C}$ data & 45.44 & 1.65 & 0.50 & 52.40 & 0.00 & 0.00 & 0.00 & 0.00 \\
\hline & All data & 0.01 & 0.13 & 0.27 & 99.60 & 0.00 & 0.00 & 0.00 & 0.00 \\
\hline & & & & Level of & V: B3LYF & $+\mathrm{G} * *$ & $n, P C N$ & & \\
\hline & & $79 a$ & $79 \mathrm{~b}$ & $79 c$ & 79d & $79 e$ & $79 f$ & $79 \mathrm{~g}$ & $79 \mathrm{~h}$ \\
\hline & ${ }^{1} \mathrm{H}$ data & 22.65 & 2.42 & 57.95 & 16.20 & 0.15 & 0.22 & 0.19 & 0.23 \\
\hline sDP4+ & ${ }^{13} \mathrm{C}$ data & 12.44 & 13.78 & 10.79 & 62.41 & 0.20 & 0.09 & 0.15 & 0.13 \\
\hline & All data & 14.44 & 1.71 & 32.03 & 51.82 & 0.00 & 0.00 & 0.00 & 0.00 \\
\hline & ${ }^{1} \mathrm{H}$ data & 12.23 & 2.12 & 39.73 & 43.23 & 1.09 & 0.00 & 0.51 & 1.10 \\
\hline uDP4+ & ${ }^{13} \mathrm{C}$ data & 7.34 & 23.33 & 35.74 & 33.52 & 0.02 & 0.00 & 0.04 & 0.01 \\
\hline & All data & 2.99 & 1.64 & 47.20 & 48.17 & 0.00 & 0.00 & 0.00 & 0.00 \\
\hline
\end{tabular}




\begin{tabular}{|c|c|c|c|c|c|c|c|c|c|}
\hline & ${ }^{1} \mathrm{H}$ data & 8.43 & 0.16 & 70.07 & 21.32 & 0.00 & 0.00 & 0.00 & 0.01 \\
\hline \multirow[t]{5}{*}{ DP4+ } & ${ }^{13} \mathrm{C}$ data & 3.16 & 11.12 & 13.34 & 72.38 & 0.00 & 0.00 & 0.00 & 0.00 \\
\hline & All data & 1.06 & 0.07 & 37.29 & 61.57 & 0.00 & 0.00 & 0.00 & 0.00 \\
\hline & & \multicolumn{8}{|c|}{ Level of theory: B3LYP/6-311G* (solution, PCM) } \\
\hline & & 79a & $79 b$ & 79c & 79d & $79 e$ & $79 f$ & $79 \mathrm{~g}$ & $79 \mathrm{~h}$ \\
\hline & ${ }^{1} \mathrm{H}$ data & 1.63 & 12.89 & 48.13 & 36.81 & 0.03 & 0.07 & 0.34 & 0.10 \\
\hline \multirow[t]{2}{*}{$s \mathrm{DP} 4+$} & ${ }^{13} \mathrm{C}$ data & 11.76 & 3.83 & 1.54 & 82.70 & 0.05 & 0.00 & 0.01 & 0.09 \\
\hline & All data & 0.60 & 1.55 & 2.33 & 95.52 & 0.00 & 0.00 & 0.00 & 0.00 \\
\hline \multirow{3}{*}{ uDP4+ } & ${ }^{1} \mathrm{H}$ data & 1.81 & 12.86 & 57.00 & 27.28 & 0.10 & 0.26 & 0.50 & 0.19 \\
\hline & ${ }^{13} \mathrm{C}$ data & 1.34 & 33.64 & 6.98 & 57.21 & 0.10 & 0.15 & 0.52 & 0.07 \\
\hline & All data & 0.10 & 18.07 & 16.62 & 65.20 & 0.00 & 0.00 & 0.01 & 0.00 \\
\hline \multirow{3}{*}{ DP4+ } & ${ }^{1} \mathrm{H}$ data & 0.07 & 4.23 & 70.05 & 25.64 & 0.00 & 0.00 & 0.00 & 0.00 \\
\hline & ${ }^{13} \mathrm{C}$ data & 0.32 & 2.64 & 0.22 & 96.82 & 0.00 & 0.00 & 0.00 & 0.00 \\
\hline & All data & 0.00 & 0.44 & 0.62 & 98.94 & 0.00 & 0.00 & 0.00 & 0.00 \\
\hline & & \multicolumn{8}{|c|}{ Level of theory: B3LYP/6-311G** (solution, PCM) } \\
\hline & & 79a & $79 b$ & 79c & 79d & $79 e$ & $79 f$ & $79 \mathrm{~g}$ & $79 \mathrm{~h}$ \\
\hline \multirow{3}{*}{ sDP4+ } & ${ }^{1} \mathrm{H}$ data & 8.89 & 24.51 & 32.43 & 29.97 & 0.54 & 0.16 & 2.40 & 1.10 \\
\hline & ${ }^{13} \mathrm{C}$ data & 8.76 & 3.27 & 1.34 & 86.49 & 0.04 & 0.00 & 0.01 & 0.09 \\
\hline & All data & 2.79 & 2.87 & 1.56 & 92.78 & 0.00 & 0.00 & 0.00 & 0.00 \\
\hline \multirow{3}{*}{ uDP4+ } & ${ }^{1} \mathrm{H}$ data & 6.92 & 8.26 & 44.64 & 33.00 & 1.49 & 0.51 & 1.81 & 3.38 \\
\hline & ${ }^{13} \mathrm{C}$ data & 0.61 & 35.66 & 7.99 & 54.55 & 0.06 & 0.22 & 0.85 & 0.05 \\
\hline & All data & 0.17 & 11.99 & 14.51 & 73.25 & 0.00 & 0.00 & 0.06 & 0.01 \\
\hline \multirow{5}{*}{ DP4+ } & ${ }^{1} \mathrm{H}$ data & 2.27 & 7.47 & 53.43 & 36.50 & 0.03 & 0.00 & 0.16 & 0.14 \\
\hline & ${ }^{13} \mathrm{C}$ data & 0.11 & 2.40 & 0.22 & 97.27 & 0.00 & 0.00 & 0.00 & 0.00 \\
\hline & All data & 0.01 & 0.50 & 0.33 & 99.16 & 0.00 & 0.00 & 0.00 & 0.00 \\
\hline & & \multicolumn{8}{|c|}{ Level of theory: B3LYP/6-311+G** (solution, PCM) } \\
\hline & & $79 a$ & $79 b$ & $79 c$ & $79 d$ & $79 e$ & $79 f$ & $79 \mathrm{~g}$ & $79 \mathrm{~h}$ \\
\hline \multirow{3}{*}{ sDP4+ } & ${ }_{12}^{1} \mathrm{H}$ data & 41.08 & 8.49 & 23.11 & 22.27 & 0.96 & 0.36 & 1.42 & 2.31 \\
\hline & ${ }^{13} \mathrm{C}$ data & 6.53 & 5.64 & 2.51 & 85.26 & 0.02 & 0.00 & 0.01 & 0.03 \\
\hline & All data & 11.80 & 2.11 & 2.55 & 83.54 & 0.00 & 0.00 & 0.00 & 0.00 \\
\hline \multirow{3}{*}{ uDP4+ } & ${ }^{1} \mathrm{H}$ data & 32.86 & 2.85 & 22.69 & 37.06 & 1.14 & 0.37 & 0.41 & 2.61 \\
\hline & ${ }^{13} \mathrm{C}$ data & 0.86 & 55.38 & 9.40 & 33.94 & 0.04 & 0.07 & 0.30 & 0.01 \\
\hline & All data & 1.71 & 9.53 & 12.86 & 75.88 & 0.00 & 0.00 & 0.01 & 0.00 \\
\hline \multirow{5}{*}{ DP4+ } & ${ }^{1} \mathrm{H}$ data & 49.42 & 0.89 & 19.19 & 30.21 & 0.04 & 0.00 & 0.02 & 0.22 \\
\hline & ${ }^{13} \mathrm{C}$ data & 0.17 & 9.65 & 0.73 & 89.44 & 0.00 & 0.00 & 0.00 & 0.00 \\
\hline & All data & 0.31 & 0.31 & 0.51 & 98.86 & 0.00 & 0.00 & 0.00 & 0.00 \\
\hline & & \multicolumn{8}{|c|}{ Level of theory: mPW1PW91/6-31G* (gas phase) } \\
\hline & & $79 a$ & $79 b$ & 79c & 79d & $79 e$ & $79 f$ & $79 \mathrm{~g}$ & $79 \mathrm{~h}$ \\
\hline \multirow{3}{*}{ sDP4+ } & ${ }^{1} \mathrm{H}$ data & 0.05 & 10.28 & 38.07 & 51.56 & 0.00 & 0.00 & 0.03 & 0.01 \\
\hline & ${ }^{13} \mathrm{C}$ data & 23.73 & 2.59 & 0.59 & 70.38 & 0.55 & 0.00 & 0.09 & 2.08 \\
\hline & All data & 0.03 & 0.72 & 0.61 & 98.64 & 0.00 & 0.00 & 0.00 & 0.00 \\
\hline \multirow{3}{*}{ uDP4+ } & ${ }^{1} \mathrm{H}$ data & 0.03 & 28.62 & 52.54 & 18.65 & 0.01 & 0.04 & 0.10 & 0.01 \\
\hline & ${ }^{13} \mathrm{C}$ data & 77.73 & 1.97 & 9.00 & 8.63 & 0.99 & 0.01 & 0.07 & 1.60 \\
\hline & All data & 0.37 & 8.14 & 68.24 & 23.24 & 0.00 & 0.00 & 0.00 & 0.00 \\
\hline & ${ }^{1} \mathrm{H}$ data & 0.00 & 9.03 & 61.43 & 29.54 & 0.00 & 0.00 & 0.00 & 0.00 \\
\hline DP4+ & ${ }^{13} \mathrm{C}$ data & 74.79 & 0.21 & 0.21 & 24.63 & 0.02 & 0.00 & 0.00 & 0.14 \\
\hline & All data & 0.00 & 0.25 & 1.77 & 97.98 & 0.00 & 0.00 & 0.00 & 0.00 \\
\hline & & & & evel of $t$ & : $\mathrm{mPW}$ & $/ 6-31$ & s phas & & \\
\hline & & $79 a$ & $79 b$ & $79 c$ & $79 d$ & $79 e$ & $79 f$ & $79 \mathrm{~g}$ & $79 \mathrm{~h}$ \\
\hline & ${ }^{1} \mathrm{H}$ data & 0.11 & 10.49 & 24.46 & 64.75 & 0.01 & 0.00 & 0.16 & 0.03 \\
\hline sDP4+ & ${ }^{13} \mathrm{C}$ data & 41.36 & 3.21 & 0.87 & 52.55 & 0.49 & 0.00 & 0.07 & 1.45 \\
\hline & All data & 0.13 & 0.97 & 0.62 & 98.28 & 0.00 & 0.00 & 0.00 & 0.00 \\
\hline & ${ }^{1} \mathrm{H}$ data & 0.08 & 14.93 & 49.31 & 35.35 & 0.02 & 0.03 & 0.21 & 0.07 \\
\hline uDP4+ & ${ }^{13} \mathrm{C}$ data & 52.12 & 7.39 & 10.92 & 29.25 & 0.08 & 0.00 & 0.02 & 0.22 \\
\hline & All data & 0.24 & 6.54 & 31.92 & 61.30 & 0.00 & 0.00 & 0.00 & 0.00 \\
\hline DP4+ & ${ }^{1} \mathrm{H}$ data & 0.00 & 4.29 & 33.03 & 62.68 & 0.00 & 0.00 & 0.00 & 0.00 \\
\hline
\end{tabular}




\begin{tabular}{|c|c|c|c|c|c|c|c|c|c|}
\hline & ${ }^{13} \mathrm{C}$ data & 57.85 & 0.64 & 0.26 & 41.25 & 0.00 & 0.00 & 0.00 & 0.01 \\
\hline & All data & 0.00 & 0.10 & 0.33 & 99.57 & 0.00 & 0.00 & 0.00 & 0.00 \\
\hline & & \multicolumn{8}{|c|}{ Level of theory: mPW1PW91/6-31+G** (gas phase) } \\
\hline & & $79 a$ & $79 b$ & $79 c$ & 79d & $79 e$ & $79 f$ & $79 \mathrm{~g}$ & $79 \mathrm{~h}$ \\
\hline & ${ }^{1} \mathrm{H}$ data & 2.04 & 12.06 & 59.09 & 25.66 & 0.29 & 0.02 & 0.47 & 0.37 \\
\hline \multirow[t]{2}{*}{ sDP4+ } & ${ }^{13} \mathrm{C}$ data & 21.65 & 4.87 & 2.64 & 70.00 & 0.54 & 0.02 & 0.05 & 0.23 \\
\hline & All data & 2.15 & 2.86 & 7.59 & 87.39 & 0.01 & 0.00 & 0.00 & 0.00 \\
\hline \multirow{3}{*}{ uDP4+ } & ${ }^{1} \mathrm{H}$ data & 1.95 & 6.13 & 50.62 & 36.97 & 1.78 & 0.24 & 1.10 & 1.21 \\
\hline & ${ }^{13} \mathrm{C}$ data & 19.24 & 24.90 & 27.42 & 28.34 & 0.04 & 0.01 & 0.02 & 0.02 \\
\hline & All data & 1.43 & 5.81 & 52.85 & 39.90 & 0.00 & 0.00 & 0.00 & 0.00 \\
\hline & ${ }^{1} \mathrm{H}$ data & 0.10 & 1.84 & 74.42 & 23.60 & 0.01 & 0.00 & 0.01 & 0.01 \\
\hline \multirow[t]{4}{*}{ DP4+ } & ${ }^{13} \mathrm{C}$ data & 16.05 & 4.67 & 2.79 & 76.48 & 0.00 & 0.00 & 0.00 & 0.00 \\
\hline & All data & 0.08 & 0.42 & 10.27 & 89.23 & 0.00 & 0.00 & 0.00 & 0.00 \\
\hline & & \multicolumn{8}{|c|}{ Level of theory: mPW1PW91/6-311G* (gas phase) } \\
\hline & & $79 a$ & $79 \mathrm{~b}$ & 79c & 79d & $79 e$ & $79 f$ & $79 \mathrm{~g}$ & $79 \mathrm{~h}$ \\
\hline & ${ }^{1} \mathrm{H}$ data & 0.14 & 22.29 & 70.11 & 6.89 & 0.02 & 0.02 & 0.47 & 0.06 \\
\hline \multirow[t]{2}{*}{ sDP4+ } & ${ }^{13} \mathrm{C}$ data & 16.53 & 4.96 & 1.06 & 77.01 & 0.11 & 0.00 & 0.02 & 0.31 \\
\hline & All data & 0.33 & 15.38 & 10.34 & 73.94 & 0.00 & 0.00 & 0.00 & 0.00 \\
\hline \multirow{3}{*}{ uDP4+ } & ${ }^{1} \mathrm{H}$ data & 0.73 & 17.46 & 70.09 & 9.56 & 0.27 & 0.48 & 1.08 & 0.34 \\
\hline & ${ }^{13} \mathrm{C}$ data & 2.30 & 12.51 & 1.65 & 83.28 & 0.07 & 0.01 & 0.08 & 0.11 \\
\hline & All data & 0.15 & 19.30 & 10.21 & 70.33 & 0.00 & 0.00 & 0.01 & 0.00 \\
\hline \multirow{6}{*}{ DP4+ } & ${ }^{1} \mathrm{H}$ data & 0.00 & 7.25 & 91.51 & 1.23 & 0.00 & 0.00 & 0.01 & 0.00 \\
\hline & ${ }^{13} \mathrm{C}$ data & 0.58 & 0.95 & 0.03 & 98.44 & 0.00 & 0.00 & 0.00 & 0.00 \\
\hline & All data & 0.00 & 5.30 & 1.88 & 92.82 & 0.00 & 0.00 & 0.00 & 0.00 \\
\hline & & \multicolumn{8}{|c|}{ Level of theory: mPW1PW91/6-311G** (gas phase) } \\
\hline & & 79a & $79 b$ & $79 c$ & $79 d$ & $79 e$ & $79 f$ & $79 \mathrm{~g}$ & $79 \mathrm{~h}$ \\
\hline & ${ }^{1} \mathrm{H}$ data & 0.80 & 29.87 & 53.16 & 13.80 & 0.21 & 0.02 & 1.88 & 0.25 \\
\hline \multirow[t]{2}{*}{ sDP4+ } & ${ }^{13} \mathrm{C}$ data & 15.26 & 4.01 & 0.91 & 79.42 & 0.09 & 0.00 & 0.02 & 0.30 \\
\hline & All data & 0.95 & 9.38 & 3.77 & 85.88 & 0.00 & 0.00 & 0.00 & 0.01 \\
\hline \multirow{3}{*}{ uDP4+ } & ${ }^{1} \mathrm{H}$ data & 1.64 & 8.23 & 63.87 & 21.16 & 1.41 & 0.38 & 1.49 & 1.82 \\
\hline & ${ }^{13} \mathrm{C}$ data & 0.73 & 15.94 & 2.14 & 81.01 & 0.02 & 0.01 & 0.12 & 0.04 \\
\hline & All data & 0.06 & 6.61 & 6.88 & 86.43 & 0.00 & 0.00 & 0.01 & 0.00 \\
\hline \multirow{5}{*}{ DP4+ } & ${ }^{1} \mathrm{H}$ data & 0.03 & 6.24 & 86.22 & 7.42 & 0.01 & 0.00 & 0.07 & 0.01 \\
\hline & ${ }^{13} \mathrm{C}$ data & 0.17 & 0.98 & 0.03 & 98.82 & 0.00 & 0.00 & 0.00 & 0.00 \\
\hline & All data & 0.00 & 0.83 & 0.35 & 98.83 & 0.00 & 0.00 & 0.00 & 0.00 \\
\hline & & \multicolumn{8}{|c|}{ Level of theory: mPW1PW91/6-311+G** (gas phase) } \\
\hline & & 79a & $79 b$ & $79 c$ & 79d & $79 e$ & $79 f$ & $79 \mathrm{~g}$ & $79 \mathrm{~h}$ \\
\hline \multirow{3}{*}{$s \mathrm{DP} 4+$} & ${ }^{1} \mathrm{H}$ data & 5.52 & 16.34 & 55.91 & 19.33 & 0.67 & 0.04 & 1.11 & 1.08 \\
\hline & ${ }^{13} \mathrm{C}$ data & 11.91 & 8.43 & 1.64 & 77.88 & 0.03 & 0.00 & 0.01 & 0.10 \\
\hline & All data & 3.65 & 7.65 & 5.08 & 83.61 & 0.00 & 0.00 & 0.00 & 0.01 \\
\hline \multirow{3}{*}{ uDP4+ } & ${ }^{1} \mathrm{H}$ data & 8.04 & 5.74 & 46.32 & 30.55 & 3.40 & 0.54 & 1.17 & 4.23 \\
\hline & ${ }^{13} \mathrm{C}$ data & 0.90 & 27.93 & 2.96 & 68.05 & 0.03 & 0.02 & 0.10 & 0.02 \\
\hline & All data & 0.30 & 6.73 & 5.75 & 87.21 & 0.00 & 0.00 & 0.01 & 0.00 \\
\hline \multirow{3}{*}{ DP4+ } & ${ }^{1} \mathrm{H}$ data & 1.33 & 2.82 & 77.85 & 17.75 & 0.07 & 0.00 & 0.04 & 0.14 \\
\hline & ${ }^{13} \mathrm{C}$ data & 0.19 & 4.24 & 0.09 & 95.48 & 0.00 & 0.00 & 0.00 & 0.00 \\
\hline & All data & 0.02 & 0.70 & 0.40 & 98.89 & 0.00 & 0.00 & 0.00 & 0.00 \\
\hline & & & & vel of th & mPW1P & 6-31G & ion, $\mathrm{PC}$ & & \\
\hline & & $79 a$ & $79 b$ & $79 c$ & $79 d$ & $79 e$ & $79 f$ & $79 \mathrm{~g}$ & $79 \mathrm{~h}$ \\
\hline & ${ }^{1} \mathrm{H}$ data & 0.59 & 7.05 & 20.89 & 71.44 & 0.00 & 0.00 & 0.02 & 0.00 \\
\hline sDP4+ & ${ }^{13} \mathrm{C}$ data & 30.27 & 6.17 & 1.63 & 61.33 & 0.18 & 0.00 & 0.05 & 0.36 \\
\hline & All data & 0.40 & 0.97 & 0.76 & 97.87 & 0.00 & 0.00 & 0.00 & 0.00 \\
\hline & ${ }^{1} \mathrm{H}$ data & 0.10 & 17.01 & 59.97 & 22.73 & 0.01 & 0.06 & 0.11 & 0.01 \\
\hline uDP4+ & ${ }^{13} \mathrm{C}$ data & 68.94 & 2.78 & 16.13 & 11.11 & 0.38 & 0.01 & 0.04 & 0.62 \\
\hline & All data & 0.54 & 3.71 & 75.92 & 19.83 & 0.00 & 0.00 & 0.00 & 0.00 \\
\hline & ${ }^{1} \mathrm{H}$ data & 0.00 & 4.00 & 41.80 & 54.19 & 0.00 & 0.00 & 0.00 & 0.00 \\
\hline DP4+ & ${ }^{13} \mathrm{C}$ data & 74.21 & 0.61 & 0.94 & 24.23 & 0.00 & 0.00 & 0.00 & 0.01 \\
\hline
\end{tabular}




\begin{tabular}{|c|c|c|c|c|c|c|c|c|c|}
\hline & All data & 0.01 & 0.18 & 2.89 & 96.92 & 0.00 & 0.00 & 0.00 & 0.00 \\
\hline & & \multicolumn{8}{|c|}{ Level of theory: mPW1PW91/6-31G** (solution, PCM) } \\
\hline & & $79 a$ & $79 \mathrm{~b}$ & $79 c$ & 79d & $79 \mathrm{e}$ & $79 f$ & $79 \mathrm{~g}$ & $79 \mathrm{~h}$ \\
\hline & ${ }^{1} \mathrm{H}$ data & 1.09 & 5.39 & 9.20 & 84.25 & 0.00 & 0.00 & 0.05 & 0.02 \\
\hline \multirow[t]{2}{*}{ sDP4+ } & ${ }^{13} \mathrm{C}$ data & 44.84 & 5.29 & 1.76 & 47.63 & 0.14 & 0.00 & 0.03 & 0.30 \\
\hline & All data & 1.19 & 0.69 & 0.39 & 97.72 & 0.00 & 0.00 & 0.00 & 0.00 \\
\hline \multirow{3}{*}{ uDP4+ } & ${ }^{1} \mathrm{H}$ data & 0.16 & 6.45 & 21.64 & 71.62 & 0.01 & 0.01 & 0.08 & 0.03 \\
\hline & ${ }^{13} \mathrm{C}$ data & 37.58 & 10.35 & 15.14 & 36.75 & 0.06 & 0.00 & 0.01 & 0.11 \\
\hline & All data & 0.19 & 2.20 & 10.81 & 86.80 & 0.00 & 0.00 & 0.00 & 0.00 \\
\hline \multirow{5}{*}{ DP4+ } & ${ }^{1} \mathrm{H}$ data & 0.00 & 0.56 & 3.18 & 96.27 & 0.00 & 0.00 & 0.00 & 0.00 \\
\hline & ${ }^{13} \mathrm{C}$ data & 47.92 & 1.56 & 0.76 & 49.77 & 0.00 & 0.00 & 0.00 & 0.00 \\
\hline & All data & 0.00 & 0.02 & 0.05 & 99.93 & 0.00 & 0.00 & 0.00 & 0.00 \\
\hline & & \multicolumn{8}{|c|}{ Level of theory: mPW1PW91/6-31+G** (solution, PCM) } \\
\hline & & $79 a$ & $79 b$ & 79c & $79 d$ & $79 e$ & $79 f$ & $79 \mathrm{~g}$ & $79 \mathrm{~h}$ \\
\hline \multirow{3}{*}{ sDP4+ } & ${ }^{1} \mathrm{H}$ data & 36.48 & 4.66 & 33.15 & 24.68 & 0.20 & 0.07 & 0.22 & 0.54 \\
\hline & ${ }^{13} \mathrm{C}$ data & 19.07 & 7.72 & 5.24 & 67.83 & 0.08 & 0.01 & 0.03 & 0.02 \\
\hline & All data & 26.97 & 1.40 & 6.73 & 64.89 & 0.00 & 0.00 & 0.00 & 0.00 \\
\hline & ${ }^{1} \mathrm{H}$ data & 13.07 & 2.58 & 42.07 & 40.61 & 0.43 & 0.15 & 0.29 & 0.81 \\
\hline \multirow[t]{2}{*}{ uDP4+ } & ${ }^{13} \mathrm{C}$ data & 13.87 & 30.40 & 33.32 & 22.33 & 0.03 & 0.01 & 0.02 & 0.01 \\
\hline & All data & 7.06 & 3.05 & 54.58 & 35.31 & 0.00 & 0.00 & 0.00 & 0.00 \\
\hline \multirow{5}{*}{ DP4+ } & ${ }^{1} \mathrm{H}$ data & 16.51 & 0.42 & 48.32 & 34.72 & 0.00 & 0.00 & 0.00 & 0.02 \\
\hline & ${ }^{13} \mathrm{C}$ data & 12.09 & 10.72 & 7.98 & 69.21 & 0.00 & 0.00 & 0.00 & 0.00 \\
\hline & All data & 6.67 & 0.15 & 12.88 & 80.30 & 0.00 & 0.00 & 0.00 & 0.00 \\
\hline & & \multicolumn{8}{|c|}{ Level of theory: mPW1PW91/6-311G* (solution, PCM) } \\
\hline & & 79a & $79 b$ & 79c & 79d & $79 e$ & $79 f$ & $79 \mathrm{~g}$ & $79 \mathrm{~h}$ \\
\hline \multirow{3}{*}{$s \mathrm{DP} 4+$} & ${ }^{1} \mathrm{H}$ data & 2.44 & 12.02 & 45.12 & 40.12 & 0.02 & 0.01 & 0.16 & 0.10 \\
\hline & ${ }^{13} \mathrm{C}$ data & 22.52 & 6.12 & 2.62 & 68.63 & 0.03 & 0.00 & 0.01 & 0.06 \\
\hline & All data & 1.83 & 2.45 & 3.94 & 91.78 & 0.00 & 0.00 & 0.00 & 0.00 \\
\hline \multirow{3}{*}{ uDP4+ } & ${ }^{1} \mathrm{H}$ data & 2.23 & 13.68 & 50.98 & 32.52 & 0.06 & 0.06 & 0.33 & 0.14 \\
\hline & ${ }^{13} \mathrm{C}$ data & 0.76 & 21.78 & 3.31 & 73.98 & 0.02 & 0.01 & 0.11 & 0.02 \\
\hline & All data & 0.06 & 10.37 & 5.88 & 83.70 & 0.00 & 0.00 & 0.00 & 0.00 \\
\hline \multirow{5}{*}{ DP4+ } & ${ }^{1} \mathrm{H}$ data & 0.14 & 4.36 & 60.93 & 34.57 & 0.00 & 0.00 & 0.00 & 0.00 \\
\hline & ${ }^{13} \mathrm{C}$ data & 0.33 & 2.55 & 0.17 & 96.96 & 0.00 & 0.00 & 0.00 & 0.00 \\
\hline & All data & 0.00 & 0.33 & 0.30 & 99.37 & 0.00 & 0.00 & 0.00 & 0.00 \\
\hline & & \multicolumn{8}{|c|}{ Level of theory: mPW1PW91/6-311G** (solution, PCM) } \\
\hline & & $79 a$ & $79 b$ & $79 c$ & 79d & $79 e$ & $79 f$ & $79 \mathrm{~g}$ & $79 \mathrm{~h}$ \\
\hline & ${ }^{1} \mathrm{H}$ data & 13.15 & 21.69 & 22.79 & 38.86 & 0.38 & 0.05 & 1.35 & 1.74 \\
\hline \multirow[t]{2}{*}{$s \mathrm{DP} 4+$} & ${ }^{13} \mathrm{C}$ data & 14.02 & 5.83 & 2.07 & 78.02 & 0.01 & 0.00 & 0.01 & 0.04 \\
\hline & All data & 5.44 & 3.73 & 1.39 & 89.44 & 0.00 & 0.00 & 0.00 & 0.00 \\
\hline \multirow{3}{*}{ uDP4+ } & ${ }^{1} \mathrm{H}$ data & 5.94 & 6.84 & 23.54 & 58.45 & 0.54 & 0.08 & 1.44 & 3.17 \\
\hline & ${ }^{13} \mathrm{C}$ data & 0.32 & 32.41 & 5.05 & 61.94 & 0.01 & 0.02 & 0.24 & 0.01 \\
\hline & All data & 0.05 & 5.60 & 3.00 & 91.35 & 0.00 & 0.00 & 0.01 & 0.00 \\
\hline \multirow{5}{*}{ DP4+ } & ${ }^{1} \mathrm{H}$ data & 2.57 & 4.88 & 17.63 & 74.67 & 0.01 & 0.00 & 0.06 & 0.18 \\
\hline & ${ }^{13} \mathrm{C}$ data & 0.09 & 3.75 & 0.21 & 95.95 & 0.00 & 0.00 & 0.00 & 0.00 \\
\hline & All data & 0.00 & 0.25 & 0.05 & 99.69 & 0.00 & 0.00 & 0.00 & 0.00 \\
\hline & & & & of the & PW1PV & $311+G$ & ution, & & \\
\hline & & $79 a$ & $79 b$ & $79 c$ & $79 d$ & $79 e$ & $79 f$ & $79 \mathrm{~g}$ & $79 h$ \\
\hline & ${ }^{1} \mathrm{H}$ data & 28.98 & 11.25 & 12.12 & 22.13 & 4.42 & 0.75 & 4.64 & 15.72 \\
\hline sDP4+ & ${ }^{13} \mathrm{C}$ data & 15.96 & 11.25 & 6.06 & 66.68 & 0.02 & 0.00 & 0.01 & 0.02 \\
\hline & All data & 21.63 & 5.91 & 3.44 & 69.00 & 0.00 & 0.00 & 0.00 & 0.01 \\
\hline & ${ }^{1} \mathrm{H}$ data & 15.70 & 1.88 & 12.03 & 31.50 & 10.75 & 1.16 & 4.48 & 22.50 \\
\hline uDP4+ & ${ }^{13} \mathrm{C}$ data & 0.47 & 57.94 & 7.77 & 33.69 & 0.01 & 0.01 & 0.10 & 0.00 \\
\hline & All data & 0.58 & 8.56 & 7.35 & 83.46 & 0.01 & 0.00 & 0.04 & 0.00 \\
\hline & ${ }^{1} \mathrm{H}$ data & 26.12 & 1.21 & 8.37 & 40.02 & 2.73 & 0.05 & 1.19 & 20.30 \\
\hline DP4+ & ${ }^{13} \mathrm{C}$ data & 0.25 & 22.07 & 1.59 & 76.09 & 0.00 & 0.00 & 0.00 & 0.00 \\
\hline & All data & 0.21 & 0.87 & 0.43 & 98.49 & 0.00 & 0.00 & 0.00 & 0.00 \\
\hline
\end{tabular}


sDP4+, $u$ DP4+ and DP4+ probabilities (\%) for compound 79e<smiles>CC[C@H](C)[C@H]1OC([C@H](C)CC)=C(C)C(=O)[C@H]1C</smiles><smiles>CC[C@H](C)[C@@H]1OC([C@@H](C)[C@@H](O)CC)=C(C)C(=O)[C@@H]1C</smiles>
correct isomer<smiles>CC[C@H](O)[C@@H](C)C1=C(C)C(=O)[C@@H](C)[C@@H]([C@@H](C)CC)O1</smiles><smiles>CC[C@H](O)[C@@H](C)C1=C(C)C(=O)[C@@H](C)[C@@H]([C@@H](C)CC)O1</smiles><smiles>CC[C@H](C)[C@H]1OC([C@@H](C)[C@H](O)CC)=C(C)C(=O)[C@H]1C</smiles><smiles>CC[C@H](C)[C@H]1OC([C@H](C)[C@H](O)CC)=C(C)C(=O)[C@H]1C</smiles><smiles>CC[C@H](O)[C@@H](C)C1=C(C)C(=O)[C@@H](C)[C@@H]([C@@H](C)CC)O1</smiles><smiles>CC[C@H](O)[C@@H](C)C1=C(C)C(=O)[C@@H](C)[C@@H]([C@@H](C)CC)O1</smiles>

\begin{tabular}{|c|c|c|c|c|c|c|c|c|c|}
\hline & & \multicolumn{8}{|c|}{ Level of theory: B3LYP/6-31G* (gas phase) } \\
\hline & & $79 a$ & $79 b$ & 79c & 79d & $79 e$ & $79 f$ & $79 \mathrm{~g}$ & $79 \mathrm{~h}$ \\
\hline \multirow{3}{*}{ sDP4+ } & ${ }^{1} \mathrm{H}$ data & 0.21 & 50.31 & 5.27 & 42.52 & 0.15 & 0.18 & 1.15 & 0.22 \\
\hline & ${ }^{13} \mathrm{C}$ data & 0.00 & 0.00 & 0.00 & 0.00 & 85.75 & 0.75 & 0.24 & 13.26 \\
\hline & All data & 0.00 & 0.01 & 0.00 & 0.19 & 79.01 & 0.86 & 1.76 & 18.17 \\
\hline \multirow{3}{*}{ uDP4+ } & ${ }^{1}$ H data & 0.19 & 68.12 & 11.27 & 17.11 & 0.16 & 1.50 & 1.43 & 0.20 \\
\hline & ${ }^{13} \mathrm{C}$ data & 0.01 & 0.00 & 0.00 & 0.01 & 49.73 & 2.89 & 3.61 & 43.75 \\
\hline & All data & 0.00 & 0.22 & 0.08 & 0.55 & 30.37 & 16.22 & 19.35 & 33.20 \\
\hline \multirow{3}{*}{ DP4+ } & ${ }^{1} \mathrm{H}$ data & 0.00 & 81.29 & 1.41 & 17.26 & 0.00 & 0.01 & 0.04 & 0.00 \\
\hline & ${ }^{13} \mathrm{C}$ data & 0.00 & 0.00 & 0.00 & 0.00 & 87.97 & 0.04 & 0.02 & 11.97 \\
\hline & All data & 0.00 & 0.00 & 0.00 & 0.00 & 78.65 & 0.46 & 1.11 & 19.77 \\
\hline & & \multicolumn{8}{|c|}{ Level of theory: B3LYP/6-31G** (gas phase) } \\
\hline & & $79 a$ & $79 b$ & $79 c$ & 79d & $79 e$ & $79 f$ & $79 \mathrm{~g}$ & $79 \mathrm{~h}$ \\
\hline \multirow{3}{*}{ sDP4+ } & ${ }^{1} \mathrm{H}$ data & 0.42 & 52.68 & 6.72 & 28.69 & 0.52 & 0.25 & 10.01 & 0.71 \\
\hline & ${ }^{13} \mathrm{C}$ data & 0.00 & 0.00 & 0.00 & 0.00 & 84.72 & 1.86 & 0.37 & 13.05 \\
\hline & All data & 0.00 & 0.02 & 0.00 & 0.12 & 76.62 & 0.82 & 6.44 & 15.98 \\
\hline \multirow{3}{*}{ uDP4+ } & ${ }^{1} \mathrm{H}$ data & 0.45 & 50.08 & 14.18 & 30.74 & 0.60 & 1.69 & 1.74 & 0.51 \\
\hline & ${ }^{13} \mathrm{C}$ data & 0.01 & 0.00 & 0.00 & 0.02 & 60.41 & 6.65 & 4.28 & 28.63 \\
\hline & All data & 0.00 & 0.20 & 0.05 & 0.87 & 51.47 & 16.01 & 10.57 & 20.83 \\
\hline \multirow{3}{*}{ DP4+ } & ${ }^{1} \mathrm{H}$ data & 0.01 & 72.60 & 2.62 & 24.27 & 0.01 & 0.01 & 0.48 & 0.01 \\
\hline & ${ }^{13} \mathrm{C}$ data & 0.00 & 0.00 & 0.00 & 0.00 & 92.96 & 0.22 & 0.03 & 6.79 \\
\hline & All data & 0.00 & 0.00 & 0.00 & 0.00 & 90.50 & 0.30 & 1.56 & 7.64 \\
\hline & & \multicolumn{8}{|c|}{ Level of theory: B3LYP/6-31+G** (gas phase) } \\
\hline & & $79 a$ & $79 b$ & 79c & 79d & $79 e$ & $79 f$ & $79 \mathrm{~g}$ & $79 \mathrm{~h}$ \\
\hline \multirow{3}{*}{ sDP4+ } & ${ }^{1} \mathrm{H}$ data & 3.23 & 20.39 & 8.45 & 6.80 & 11.29 & 0.98 & 43.21 & 5.64 \\
\hline & ${ }^{13} \mathrm{C}$ data & 0.01 & 0.03 & 0.02 & 0.09 & 60.66 & 31.79 & 2.27 & 5.13 \\
\hline & All data & 0.00 & 0.07 & 0.02 & 0.07 & 81.11 & 3.71 & 11.60 & 3.43 \\
\hline \multirow{3}{*}{ uDP4+ } & ${ }^{1} \mathrm{H}$ data & 3.14 & 11.27 & 9.26 & 30.98 & 26.64 & 5.78 & 9.37 & 3.57 \\
\hline & ${ }^{13} \mathrm{C}$ data & 0.03 & 0.11 & 0.11 & 0.36 & 42.75 & 41.13 & 10.09 & 5.43 \\
\hline & All data & 0.01 & 0.08 & 0.07 & 0.73 & 75.73 & 15.81 & 6.29 & 1.29 \\
\hline \multirow{5}{*}{ DP4+ } & ${ }^{1} \mathrm{H}$ data & 0.81 & 18.23 & 6.21 & 16.73 & 23.87 & 0.45 & 32.11 & 1.60 \\
\hline & ${ }^{13} \mathrm{C}$ data & 0.00 & 0.00 & 0.00 & 0.00 & 65.62 & 33.09 & 0.58 & 0.70 \\
\hline & All data & 0.00 & 0.00 & 0.00 & 0.00 & 97.83 & 0.93 & 1.16 & 0.07 \\
\hline & & \multicolumn{8}{|c|}{ Level of theory: B3LYP/6-311G* (gas phase) } \\
\hline & & $79 a$ & $79 b$ & $79 c$ & 79d & $79 e$ & $79 f$ & $79 \mathrm{~g}$ & $79 \mathrm{~h}$ \\
\hline \multirow{3}{*}{ sDP4+ } & ${ }^{1} \mathrm{H}$ data & 0.12 & 62.28 & 4.30 & 4.54 & 2.39 & 7.92 & 17.75 & 0.70 \\
\hline & ${ }^{13} \mathrm{C}$ data & 0.01 & 0.01 & 0.00 & 0.03 & 78.63 & 3.86 & 1.57 & 15.89 \\
\hline & All data & 0.00 & 0.16 & 0.00 & 0.05 & 72.77 & 11.88 & 10.83 & 4.31 \\
\hline \multirow{2}{*}{ uDP4+ } & ${ }^{1} \mathrm{H}$ data & 2.08 & 29.96 & 3.50 & 5.77 & 5.24 & 46.23 & 5.82 & 1.39 \\
\hline & ${ }^{13} \mathrm{C}$ data & 0.06 & 0.37 & 0.04 & 0.55 & 36.10 & 44.33 & 10.31 & 8.24 \\
\hline
\end{tabular}




\begin{tabular}{|c|c|c|c|c|c|c|c|c|c|}
\hline & All data & 0.01 & 0.47 & 0.01 & 0.14 & 8.13 & 88.17 & 2.58 & 0.49 \\
\hline & ${ }^{1} \mathrm{H}$ data & 0.01 & 78.05 & 0.63 & 1.09 & 0.52 & 15.32 & 4.32 & 0.04 \\
\hline \multirow[t]{4}{*}{ DP4+ } & ${ }^{13} \mathrm{C}$ data & 0.00 & 0.00 & 0.00 & 0.00 & 89.91 & 5.43 & 0.51 & 4.15 \\
\hline & All data & 0.00 & 0.00 & 0.00 & 0.00 & 35.45 & 62.74 & 1.68 & 0.13 \\
\hline & & \multicolumn{8}{|c|}{ Level of theory: B3LYP/6-311G** (gas phase) } \\
\hline & & $79 a$ & $79 \mathrm{~b}$ & $79 c$ & 79d & $79 e$ & $79 f$ & $79 \mathrm{~g}$ & $79 \mathrm{~h}$ \\
\hline & ${ }^{1} \mathrm{H}$ data & 0.11 & 7.42 & 2.25 & 0.66 & 2.82 & 2.02 & 82.27 & 2.45 \\
\hline \multirow[t]{2}{*}{ sDP4+ } & ${ }^{13} \mathrm{C}$ data & 0.01 & 0.01 & 0.00 & 0.03 & 78.18 & 4.97 & 1.52 & 15.27 \\
\hline & All data & 0.00 & 0.01 & 0.00 & 0.01 & 56.07 & 2.55 & 31.86 & 9.50 \\
\hline & ${ }^{1} \mathrm{H}$ data & 2.54 & 6.04 & 2.66 & 4.73 & 24.16 & 45.48 & 6.79 & 7.60 \\
\hline \multirow[t]{2}{*}{ uDP4+ } & ${ }^{13} \mathrm{C}$ data & 0.03 & 0.45 & 0.05 & 0.66 & 19.89 & 60.37 & 14.03 & 4.52 \\
\hline & All data & 0.00 & 0.08 & 0.00 & 0.09 & 14.30 & 81.67 & 2.83 & 1.02 \\
\hline \multirow{6}{*}{ DP4+ } & ${ }^{1} \mathrm{H}$ data & 0.04 & 5.67 & 0.75 & 0.39 & 8.61 & 11.60 & 70.59 & 2.35 \\
\hline & ${ }^{13} \mathrm{C}$ data & 0.00 & 0.00 & 0.00 & 0.00 & 79.93 & 15.42 & 1.10 & 3.55 \\
\hline & All data & 0.00 & 0.00 & 0.00 & 0.00 & 72.23 & 18.76 & 8.14 & 0.88 \\
\hline & & \multicolumn{8}{|c|}{ Level of theory: B3LYP/6-311+G** (gas phase) } \\
\hline & & $79 a$ & $79 \mathrm{~b}$ & 79c & 79d & $79 e$ & $79 f$ & $79 \mathrm{~g}$ & $79 \mathrm{~h}$ \\
\hline & ${ }^{1} \mathrm{H}$ data & 1.24 & 3.38 & 2.68 & 1.27 & 5.17 & 5.21 & 66.41 & 14.63 \\
\hline \multirow[t]{2}{*}{ sDP4+ } & ${ }^{13} \mathrm{C}$ data & 0.01 & 0.02 & 0.00 & 0.02 & 80.81 & 4.14 & 1.89 & 13.10 \\
\hline & All data & 0.00 & 0.01 & 0.00 & 0.00 & 55.24 & 2.85 & 16.56 & 25.33 \\
\hline \multirow{3}{*}{ uDP4+ } & ${ }^{1} \mathrm{H}$ data & 4.02 & 1.64 & 1.49 & 9.99 & 33.24 & 24.21 & 7.81 & 17.60 \\
\hline & ${ }^{13} \mathrm{C}$ data & 0.04 & 1.20 & 0.06 & 0.56 & 18.67 & 65.48 & 11.68 & 2.31 \\
\hline & All data & 0.01 & 0.08 & 0.00 & 0.24 & 26.46 & 67.58 & 3.89 & 1.73 \\
\hline \multirow{5}{*}{ DP4+ } & ${ }^{1} \mathrm{H}$ data & 0.45 & 0.50 & 0.36 & 1.15 & 15.61 & 11.45 & 47.11 & 23.37 \\
\hline & ${ }^{13} \mathrm{C}$ data & 0.00 & 0.00 & 0.00 & 0.00 & 82.34 & 14.80 & 1.20 & 1.65 \\
\hline & All data & 0.00 & 0.00 & 0.00 & 0.00 & 82.92 & 10.93 & 3.66 & 2.49 \\
\hline & & \multicolumn{8}{|c|}{ Level of theory: B3LYP/6-31G* (solution, PCM) } \\
\hline & & 79a & $79 b$ & $79 \mathrm{c}$ & 79d & $79 e$ & $79 f$ & $79 \mathrm{~g}$ & $79 \mathrm{~h}$ \\
\hline & ${ }^{1} \mathrm{H}$ data & 3.23 & 35.37 & 3.15 & 47.87 & 0.93 & 1.19 & 7.32 & 0.94 \\
\hline \multirow[t]{2}{*}{ sDP4+ } & ${ }^{13} \mathrm{C}$ data & 0.00 & 0.00 & 0.00 & 0.00 & 85.84 & 3.14 & 1.05 & 9.97 \\
\hline & All data & 0.00 & 0.00 & 0.00 & 0.04 & 79.32 & 3.71 & 7.65 & 9.28 \\
\hline \multirow{3}{*}{ uDP4+ } & ${ }^{1} \mathrm{H}$ data & 0.48 & 68.09 & 5.02 & 15.74 & 0.44 & 6.30 & 3.44 & 0.50 \\
\hline & ${ }^{13} \mathrm{C}$ data & 0.01 & 0.00 & 0.00 & 0.01 & 55.21 & 3.78 & 5.08 & 35.91 \\
\hline & All data & 0.00 & 0.10 & 0.02 & 0.20 & 28.80 & 28.42 & 20.85 & 21.61 \\
\hline \multirow{6}{*}{ DP4+ } & ${ }^{1} \mathrm{H}$ data & 0.05 & 74.96 & 0.49 & 23.46 & 0.01 & 0.23 & 0.78 & 0.01 \\
\hline & ${ }^{13} \mathrm{C}$ data & 0.00 & 0.00 & 0.00 & 0.00 & 92.67 & 0.23 & 0.10 & 7.00 \\
\hline & All data & 0.00 & 0.00 & 0.00 & 0.00 & 83.07 & 3.83 & 5.80 & 7.29 \\
\hline & & \multicolumn{8}{|c|}{ Level of theory: B3LYP/6-31G** (solution, PCM) } \\
\hline & & $79 a$ & $79 b$ & $79 c$ & $79 d$ & $79 e$ & $79 f$ & $79 \mathrm{~g}$ & $79 \mathrm{~h}$ \\
\hline & ${ }^{1} \mathrm{H}$ data & 4.86 & 18.35 & 2.04 & 23.92 & 3.49 & 1.60 & 41.87 & 3.86 \\
\hline \multirow[t]{2}{*}{ sDP4+ } & ${ }^{13} \mathrm{C}$ data & 0.00 & 0.00 & 0.00 & 0.00 & 81.91 & 4.53 & 0.89 & 12.67 \\
\hline & All data & 0.00 & 0.00 & 0.00 & 0.02 & 75.36 & 1.91 & 9.80 & 12.91 \\
\hline \multirow{3}{*}{ uDP4+ } & ${ }^{1} \mathrm{H}$ data & 1.01 & 39.75 & 4.72 & 38.70 & 2.05 & 4.94 & 6.16 & 2.67 \\
\hline & ${ }^{13} \mathrm{C}$ data & 0.01 & 0.01 & 0.01 & 0.03 & 57.01 & 12.63 & 5.95 & 24.36 \\
\hline & All data & 0.00 & 0.08 & 0.01 & 0.42 & 41.36 & 22.09 & 12.99 & 23.06 \\
\hline & ${ }^{1} \mathrm{H}$ data & 0.25 & 37.35 & 0.49 & 47.41 & 0.37 & 0.40 & 13.20 & 0.53 \\
\hline DP4+ & ${ }^{13} \mathrm{C}$ data & 0.00 & 0.00 & 0.00 & 0.00 & 92.64 & 1.14 & 0.10 & 6.12 \\
\hline & All data & 0.00 & 0.00 & 0.00 & 0.00 & 86.96 & 1.18 & 3.55 & 8.31 \\
\hline & & & & evel of & : B3LY & $1+G * *(s$ & on, PCM & & \\
\hline & & $79 a$ & $79 \mathrm{~b}$ & $79 c$ & $79 d$ & $79 e$ & $79 f$ & $79 \mathrm{~g}$ & $79 \mathrm{~h}$ \\
\hline & ${ }^{1} \mathrm{H}$ data & 14.38 & 1.62 & 1.69 & 1.24 & 9.13 & 39.96 & 27.43 & 4.55 \\
\hline sDP4+ & ${ }^{13} \mathrm{C}$ data & 0.01 & 0.07 & 0.05 & 0.14 & 47.91 & 43.24 & 5.27 & 3.30 \\
\hline & All data & 0.01 & 0.00 & 0.00 & 0.01 & 18.80 & 74.31 & 6.22 & 0.65 \\
\hline & ${ }^{1} \mathrm{H}$ data & 17.14 & 2.75 & 4.24 & 6.52 & 42.28 & 0.00 & 14.09 & 12.99 \\
\hline uDP4+ & ${ }^{13} \mathrm{C}$ data & 0.14 & 0.57 & 0.55 & 1.43 & 64.39 & 0.00 & 26.07 & 6.85 \\
\hline & All data & 0.07 & 0.05 & 0.07 & 0.29 & 85.23 & 0.00 & 11.50 & 2.78 \\
\hline
\end{tabular}




\begin{tabular}{|c|c|c|c|c|c|c|c|c|c|}
\hline & ${ }^{1} \mathrm{H}$ data & 22.45 & 0.41 & 0.65 & 0.74 & 35.15 & 0.00 & 35.21 & 5.38 \\
\hline \multirow[t]{5}{*}{ DP4+ } & ${ }^{13} \mathrm{C}$ data & 0.00 & 0.00 & 0.00 & 0.01 & 95.06 & 0.00 & 4.23 & 0.70 \\
\hline & All data & 0.00 & 0.00 & 0.00 & 0.00 & 95.63 & 0.00 & 4.27 & 0.11 \\
\hline & & \multicolumn{8}{|c|}{ Level of theory: B3LYP/6-311G* (solution, PCM) } \\
\hline & & 79a & $79 b$ & 79c & 79d & $79 e$ & $79 f$ & $79 \mathrm{~g}$ & $79 \mathrm{~h}$ \\
\hline & ${ }^{1} \mathrm{H}$ data & 1.43 & 30.46 & 5.88 & 3.44 & 6.54 & 20.72 & 29.49 & 2.04 \\
\hline \multirow[t]{2}{*}{ sDP4+ } & ${ }^{13} \mathrm{C}$ data & 0.01 & 0.02 & 0.00 & 0.06 & 71.23 & 7.59 & 5.87 & 15.21 \\
\hline & All data & 0.00 & 0.08 & 0.00 & 0.03 & 56.26 & 18.99 & 20.90 & 3.74 \\
\hline \multirow{3}{*}{ uDP4+ } & ${ }^{1} \mathrm{H}$ data & 4.22 & 31.02 & 6.16 & 10.30 & 6.36 & 28.60 & 10.10 & 3.22 \\
\hline & ${ }^{13} \mathrm{C}$ data & 0.04 & 1.26 & 0.15 & 1.10 & 15.59 & 60.04 & 19.01 & 2.80 \\
\hline & All data & 0.01 & 1.89 & 0.05 & 0.55 & 4.80 & 83.00 & 9.28 & 0.44 \\
\hline \multirow{5}{*}{ DP4+ } & ${ }^{1} \mathrm{H}$ data & 0.31 & 48.18 & 1.84 & 1.81 & 2.12 & 30.21 & 15.19 & 0.33 \\
\hline & ${ }^{13} \mathrm{C}$ data & 0.00 & 0.00 & 0.00 & 0.00 & 64.53 & 26.50 & 6.48 & 2.48 \\
\hline & All data & 0.00 & 0.01 & 0.00 & 0.00 & 13.21 & 77.20 & 9.50 & 0.08 \\
\hline & & \multicolumn{8}{|c|}{ Level of theory: B3LYP/6-311G** (solution, PCM) } \\
\hline & & $79 a$ & $79 b$ & 79c & 79d & $79 e$ & $79 f$ & $79 \mathrm{~g}$ & $79 \mathrm{~h}$ \\
\hline & ${ }^{1} \mathrm{H}$ data & 1.51 & 4.80 & 2.08 & 0.38 & 10.01 & 7.10 & 66.77 & 7.36 \\
\hline \multirow[t]{2}{*}{ sDP4+ } & ${ }^{13} \mathrm{C}$ data & 0.01 & 0.02 & 0.00 & 0.07 & 71.72 & 9.04 & 5.57 & 13.57 \\
\hline & All data & 0.00 & 0.01 & 0.00 & 0.00 & 57.27 & 5.11 & 29.64 & 7.97 \\
\hline \multirow{3}{*}{ uDP4+ } & ${ }^{1} \mathrm{H}$ data & 8.12 & 5.26 & 2.89 & 1.61 & 37.24 & 23.62 & 8.23 & 13.02 \\
\hline & ${ }^{13} \mathrm{C}$ data & 0.01 & 0.98 & 0.13 & 0.86 & 7.62 & 68.67 & 20.34 & 1.38 \\
\hline & All data & 0.01 & 0.24 & 0.02 & 0.07 & 13.52 & 77.30 & 7.98 & 0.86 \\
\hline \multirow{5}{*}{ DP4+ } & ${ }^{1} \mathrm{H}$ data & 0.99 & 2.05 & 0.49 & 0.05 & 30.31 & 13.63 & 44.68 & 7.79 \\
\hline & ${ }^{13} \mathrm{C}$ data & 0.00 & 0.00 & 0.00 & 0.00 & 42.06 & 47.77 & 8.72 & 1.45 \\
\hline & All data & 0.00 & 0.00 & 0.00 & 0.00 & 54.80 & 27.98 & 16.74 & 0.48 \\
\hline & & \multicolumn{8}{|c|}{ Level of theory: B3LYP/6-311+G** (solution, PCM) } \\
\hline & & $79 a$ & $79 b$ & $79 c$ & $79 d$ & $79 e$ & $79 f$ & $79 \mathrm{~g}$ & $79 \mathrm{~h}$ \\
\hline \multirow{3}{*}{ sDP4+ } & ${ }^{1} \mathrm{H}$ data & 5.11 & 1.06 & 1.03 & 0.85 & 6.19 & 12.05 & 48.09 & 25.61 \\
\hline & ${ }^{13} \mathrm{C}$ data & 0.03 & 0.18 & 0.01 & 0.12 & 61.38 & 11.20 & 12.76 & 14.32 \\
\hline & All data & 0.01 & 0.01 & 0.00 & 0.01 & 25.41 & 9.03 & 41.03 & 24.51 \\
\hline \multirow{3}{*}{ uDP4+ } & ${ }^{1} \mathrm{H}$ data & 11.34 & 0.61 & 0.86 & 2.47 & 25.76 & 18.24 & 7.76 & 32.96 \\
\hline & ${ }^{13} \mathrm{C}$ data & 0.11 & 9.83 & 0.58 & 1.53 & 13.05 & 53.38 & 20.79 & 0.74 \\
\hline & All data & 0.08 & 0.40 & 0.03 & 0.25 & 22.30 & 64.62 & 10.70 & 1.61 \\
\hline \multirow{5}{*}{ DP4+ } & ${ }^{1} \mathrm{H}$ data & 3.49 & 0.04 & 0.05 & 0.13 & 9.62 & 13.26 & 22.50 & 50.90 \\
\hline & ${ }^{13} \mathrm{C}$ data & 0.00 & 0.11 & 0.00 & 0.01 & 47.76 & 35.67 & 15.82 & 0.63 \\
\hline & All data & 0.00 & 0.00 & 0.00 & 0.00 & 34.80 & 35.81 & 26.97 & 2.42 \\
\hline & & \multicolumn{8}{|c|}{ Level of theory: mPW1PW91/6-31G* (gas phase) } \\
\hline & & $79 a$ & $79 b$ & 79c & 79d & $79 e$ & $79 f$ & $79 \mathrm{~g}$ & $79 \mathrm{~h}$ \\
\hline \multirow{3}{*}{ sDP4+ } & ${ }^{1} \mathrm{H}$ data & 0.13 & 29.42 & 1.38 & 68.18 & 0.09 & 0.04 & 0.56 & 0.22 \\
\hline & ${ }^{13} \mathrm{C}$ data & 0.00 & 0.00 & 0.00 & 0.00 & 76.99 & 0.62 & 0.94 & 21.45 \\
\hline & All data & 0.00 & 0.01 & 0.00 & 0.48 & 55.87 & 0.19 & 4.40 & 39.05 \\
\hline \multirow{3}{*}{ uDP4+ } & ${ }^{1} \mathrm{H}$ data & 0.15 & 60.88 & 5.33 & 31.38 & 0.17 & 0.67 & 1.24 & 0.19 \\
\hline & ${ }^{13} \mathrm{C}$ data & 0.01 & 0.00 & 0.00 & 0.00 & 37.76 & 0.88 & 2.92 & 58.42 \\
\hline & All data & 0.01 & 0.16 & 0.05 & 0.52 & 29.79 & 2.70 & 16.48 & 50.31 \\
\hline & ${ }^{1} \mathrm{H}$ data & 0.00 & 45.48 & 0.19 & 54.32 & 0.00 & 0.00 & 0.02 & 0.00 \\
\hline DP4+ & ${ }^{13} \mathrm{C}$ data & 0.00 & 0.00 & 0.00 & 0.00 & 69.82 & 0.01 & 0.07 & 30.10 \\
\hline & All data & 0.00 & 0.00 & 0.00 & 0.01 & 44.95 & 0.01 & 1.96 & 53.07 \\
\hline & & & & vel of & : $\mathrm{mPW}$ & $1 / 6-31 G$ & as phas & & \\
\hline & & 79a & $79 b$ & $79 c$ & $79 d$ & $79 e$ & $79 f$ & $79 \mathrm{~g}$ & $79 \mathrm{~h}$ \\
\hline & ${ }^{1} \mathrm{H}$ data & 0.43 & 33.34 & 1.84 & 55.84 & 0.44 & 0.07 & 7.07 & 0.97 \\
\hline sDP4+ & ${ }^{13} \mathrm{C}$ data & 0.00 & 0.00 & 0.00 & 0.00 & 85.92 & 0.65 & 0.66 & 12.77 \\
\hline & All data & 0.00 & 0.01 & 0.00 & 0.11 & 69.09 & 0.08 & 8.42 & 22.29 \\
\hline & ${ }^{1} \mathrm{H}$ data & 0.30 & 32.10 & 4.50 & 59.15 & 0.82 & 0.65 & 1.76 & 0.71 \\
\hline uDP4+ & ${ }^{13} \mathrm{C}$ data & 0.01 & 0.00 & 0.00 & 0.01 & 51.54 & 3.21 & 4.70 & 40.53 \\
\hline & All data & 0.00 & 0.05 & 0.01 & 0.43 & 51.52 & 2.54 & 10.14 & 35.31 \\
\hline DP4+ & ${ }^{1} \mathrm{H}$ data & 0.00 & 24.35 & 0.19 & 75.15 & 0.01 & 0.00 & 0.28 & 0.02 \\
\hline
\end{tabular}




\begin{tabular}{|c|c|c|c|c|c|c|c|c|c|}
\hline & ${ }^{13} \mathrm{C}$ data & 0.00 & 0.00 & 0.00 & 0.00 & 89.44 & 0.04 & 0.06 & 10.45 \\
\hline & All data & 0.00 & 0.00 & 0.00 & 0.00 & 80.31 & 0.00 & 1.93 & 17.76 \\
\hline & & \multicolumn{8}{|c|}{ Level of theory: mPW1PW91/6-31+G** (gas phase) } \\
\hline & & $79 a$ & $79 b$ & $79 c$ & 79d & $79 e$ & $79 f$ & $79 \mathrm{~g}$ & $79 \mathrm{~h}$ \\
\hline & ${ }^{1} \mathrm{H}$ data & 5.12 & 16.42 & 3.55 & 10.77 & 16.82 & 1.53 & 33.19 & 12.61 \\
\hline \multirow[t]{2}{*}{ sDP4+ } & ${ }^{13} \mathrm{C}$ data & 0.00 & 0.00 & 0.00 & 0.01 & 93.12 & 4.76 & 0.37 & 1.74 \\
\hline & All data & 0.00 & 0.00 & 0.00 & 0.00 & 97.42 & 0.45 & 0.76 & 1.36 \\
\hline \multirow{3}{*}{ uDP4+ } & ${ }^{1} \mathrm{H}$ data & 3.37 & 8.27 & 4.03 & 33.46 & 33.95 & 5.15 & 6.64 & 5.14 \\
\hline & ${ }^{13} \mathrm{C}$ data & 0.01 & 0.02 & 0.02 & 0.04 & 62.30 & 23.02 & 8.94 & 5.65 \\
\hline & All data & 0.00 & 0.01 & 0.00 & 0.05 & 91.03 & 5.10 & 2.56 & 1.25 \\
\hline & ${ }^{1} \mathrm{H}$ data & 1.24 & 9.75 & 1.03 & 25.90 & 41.02 & 0.56 & 15.83 & 4.66 \\
\hline \multirow[t]{5}{*}{ DP4+ } & ${ }^{13} \mathrm{C}$ data & 0.00 & 0.00 & 0.00 & 0.00 & 97.93 & 1.85 & 0.06 & 0.17 \\
\hline & All data & 0.00 & 0.00 & 0.00 & 0.00 & 99.93 & 0.03 & 0.02 & 0.02 \\
\hline & & \multicolumn{8}{|c|}{ Level of theory: mPW1PW91/6-311G* (gas phase) } \\
\hline & & 79a & $79 b$ & $79 c$ & 79d & $79 e$ & $79 f$ & $79 \mathrm{~g}$ & $79 \mathrm{~h}$ \\
\hline & ${ }^{1} \mathrm{H}$ data & 0.26 & 73.13 & 3.50 & 6.96 & 2.35 & 1.44 & 11.42 & 0.94 \\
\hline \multirow[t]{2}{*}{$s \mathrm{DP} 4+$} & ${ }^{13} \mathrm{C}$ data & 0.00 & 0.00 & 0.00 & 0.01 & 77.39 & 1.51 & 1.67 & 19.41 \\
\hline & All data & 0.00 & 0.12 & 0.00 & 0.04 & 82.03 & 0.98 & 8.61 & 8.22 \\
\hline \multirow{3}{*}{ uDP4+ } & ${ }^{1} \mathrm{H}$ data & 3.07 & 54.95 & 5.11 & 9.52 & 5.61 & 13.21 & 6.33 & 2.20 \\
\hline & ${ }^{13} \mathrm{C}$ data & 0.02 & 0.10 & 0.01 & 0.52 & 52.94 & 17.23 & 10.67 & 18.52 \\
\hline & All data & 0.01 & 0.83 & 0.01 & 0.77 & 46.16 & 35.38 & 10.49 & 6.35 \\
\hline \multirow{6}{*}{ DP4+ } & ${ }^{1} \mathrm{H}$ data & 0.02 & 95.45 & 0.42 & 1.57 & 0.31 & 0.45 & 1.72 & 0.05 \\
\hline & ${ }^{13} \mathrm{C}$ data & 0.00 & 0.00 & 0.00 & 0.00 & 91.04 & 0.58 & 0.40 & 7.99 \\
\hline & All data & 0.00 & 0.00 & 0.00 & 0.00 & 95.53 & 0.88 & 2.28 & 1.32 \\
\hline & & \multicolumn{8}{|c|}{ Level of theory: mPW1PW91/6-311G** (gas phase) } \\
\hline & & $79 a$ & $79 b$ & $79 c$ & 79d & $79 e$ & $79 f$ & $79 \mathrm{~g}$ & $79 \mathrm{~h}$ \\
\hline & ${ }^{1} \mathrm{H}$ data & 0.32 & 6.42 & 1.86 & 1.76 & 4.46 & 0.68 & 78.85 & 5.64 \\
\hline \multirow[t]{2}{*}{$s \mathrm{DP} 4+$} & ${ }^{13} \mathrm{C}$ data & 0.00 & 0.00 & 0.00 & 0.01 & 82.10 & 1.44 & 1.33 & 15.12 \\
\hline & All data & 0.00 & 0.00 & 0.00 & 0.00 & 65.72 & 0.18 & 18.79 & 15.31 \\
\hline \multirow{3}{*}{ uDP4+ } & ${ }^{1} \mathrm{H}$ data & 3.52 & 8.84 & 2.24 & 8.12 & 39.68 & 18.40 & 6.92 & 12.29 \\
\hline & ${ }^{13} \mathrm{C}$ data & 0.01 & 0.28 & 0.03 & 1.37 & 28.59 & 34.55 & 24.57 & 10.58 \\
\hline & All data & 0.00 & 0.12 & 0.00 & 0.53 & 54.44 & 30.51 & 8.16 & 6.24 \\
\hline \multirow{5}{*}{ DP4+ } & ${ }^{1} \mathrm{H}$ data & 0.13 & 6.45 & 0.47 & 1.62 & 20.10 & 1.43 & 61.94 & 7.87 \\
\hline & ${ }^{13} \mathrm{C}$ data & 0.00 & 0.00 & 0.00 & 0.00 & 90.64 & 1.92 & 1.26 & 6.18 \\
\hline & All data & 0.00 & 0.00 & 0.00 & 0.00 & 93.37 & 0.14 & 4.00 & 2.49 \\
\hline & & \multicolumn{8}{|c|}{ Level of theory: mPW1PW91/6-311+G** (gas phase) } \\
\hline & & $79 a$ & $79 b$ & $79 c$ & 79d & $79 e$ & $79 f$ & $79 \mathrm{~g}$ & $79 \mathrm{~h}$ \\
\hline \multirow{3}{*}{$s \mathrm{DP} 4+$} & ${ }^{1} \mathrm{H}$ data & 3.98 & 3.84 & 2.65 & 4.34 & 9.05 & 1.24 & 44.28 & 30.64 \\
\hline & ${ }^{13} \mathrm{C}$ data & 0.01 & 0.02 & 0.00 & 0.01 & 63.24 & 3.61 & 3.76 & 29.36 \\
\hline & All data & 0.00 & 0.00 & 0.00 & 0.00 & 34.83 & 0.27 & 10.13 & 54.76 \\
\hline \multirow{3}{*}{ uDP4+ } & ${ }^{1} \mathrm{H}$ data & 5.72 & 2.22 & 1.74 & 17.45 & 39.69 & 8.13 & 4.87 & 20.19 \\
\hline & ${ }^{13} \mathrm{C}$ data & 0.02 & 0.84 & 0.04 & 0.74 & 28.10 & 44.09 & 21.36 & 4.81 \\
\hline & All data & 0.01 & 0.11 & 0.00 & 0.76 & 66.01 & 21.20 & 6.15 & 5.74 \\
\hline \multirow{5}{*}{ DP4+ } & ${ }^{1} \mathrm{H}$ data & 1.73 & 0.65 & 0.35 & 5.75 & 27.31 & 0.76 & 16.40 & 47.05 \\
\hline & ${ }^{13} \mathrm{C}$ data & 0.00 & 0.00 & 0.00 & 0.00 & 82.36 & 7.38 & 3.72 & 6.54 \\
\hline & All data & 0.00 & 0.00 & 0.00 & 0.00 & 85.73 & 0.21 & 2.33 & 11.73 \\
\hline & & & & el of $t$ & mPW1F & /6-31G* & tion, $\mathrm{PC}$ & & \\
\hline & & $79 a$ & $79 b$ & $79 c$ & 79d & $79 e$ & $79 f$ & $79 \mathrm{~g}$ & $79 \mathrm{~h}$ \\
\hline & ${ }^{1} \mathrm{H}$ data & 2.61 & 15.36 & 0.84 & 72.92 & 1.01 & 0.43 & 5.30 & 1.51 \\
\hline sDP4+ & ${ }^{13} \mathrm{C}$ data & 0.00 & 0.00 & 0.00 & 0.00 & 80.41 & 2.42 & 3.43 & 13.74 \\
\hline & All data & 0.00 & 0.00 & 0.00 & 0.06 & 67.05 & 0.86 & 14.94 & 17.09 \\
\hline & ${ }^{1} \mathrm{H}$ data & 0.41 & 60.60 & 3.06 & 26.23 & 0.73 & 3.80 & 4.48 & 0.69 \\
\hline uDP4+ & ${ }^{13} \mathrm{C}$ data & 0.02 & 0.00 & 0.00 & 0.01 & 39.71 & 1.80 & 4.09 & 54.37 \\
\hline & All data & 0.01 & 0.07 & 0.01 & 0.20 & 31.57 & 7.43 & 19.99 & 40.73 \\
\hline & ${ }^{1} \mathrm{H}$ data & 0.04 & 32.39 & 0.09 & 66.54 & 0.03 & 0.06 & 0.83 & 0.04 \\
\hline DP4+ & ${ }^{13} \mathrm{C}$ data & 0.00 & 0.00 & 0.00 & 0.00 & 80.67 & 0.11 & 0.35 & 18.87 \\
\hline
\end{tabular}




\begin{tabular}{|c|c|c|c|c|c|c|c|c|c|}
\hline & All data & 0.00 & 0.00 & 0.00 & 0.00 & 67.89 & 0.20 & 9.58 & 22.33 \\
\hline & & \multicolumn{8}{|c|}{ Level of theory: mPW1PW91/6-31G** (solution, PCM) } \\
\hline & & 79a & $79 \mathrm{~b}$ & $79 \mathrm{c}$ & 79d & $79 e$ & $79 f$ & $79 \mathrm{~g}$ & $79 \mathrm{~h}$ \\
\hline & ${ }^{1} \mathrm{H}$ data & 4.95 & 8.15 & 0.54 & 44.70 & 3.70 & 0.54 & 30.95 & 6.47 \\
\hline \multirow[t]{2}{*}{ sDP4+ } & ${ }^{13} \mathrm{C}$ data & 0.00 & 0.00 & 0.00 & 0.00 & 86.94 & 1.52 & 1.71 & 9.82 \\
\hline & All data & 0.00 & 0.00 & 0.00 & 0.01 & 73.28 & 0.19 & 12.04 & 14.47 \\
\hline \multirow{3}{*}{ uDP4+ } & ${ }^{1} \mathrm{H}$ data & 0.79 & 24.12 & 1.78 & 55.97 & 3.73 & 1.83 & 7.62 & 4.15 \\
\hline & ${ }^{13} \mathrm{C}$ data & 0.01 & 0.00 & 0.00 & 0.02 & 56.88 & 5.09 & 6.37 & 31.64 \\
\hline & All data & 0.00 & 0.01 & 0.00 & 0.24 & 52.75 & 2.32 & 12.04 & 32.64 \\
\hline \multirow{5}{*}{ DP4+ } & ${ }^{1} \mathrm{H}$ data & 0.13 & 6.59 & 0.03 & 83.93 & 0.46 & 0.03 & 7.91 & 0.90 \\
\hline & ${ }^{13} \mathrm{C}$ data & 0.00 & 0.00 & 0.00 & 0.00 & 93.76 & 0.15 & 0.21 & 5.89 \\
\hline & All data & 0.00 & 0.00 & 0.00 & 0.00 & 86.22 & 0.01 & 3.24 & 10.53 \\
\hline & & \multicolumn{8}{|c|}{ Level of theory: mPW1PW91/6-31+G** (solution, PCM) } \\
\hline & & $79 a$ & $79 \mathrm{~b}$ & $79 c$ & $79 d$ & $79 e$ & $79 f$ & $79 \mathrm{~g}$ & $79 \mathrm{~h}$ \\
\hline \multirow{3}{*}{$s \mathrm{DP4+}$} & ${ }^{1} \mathrm{H}$ data & 18.84 & 1.64 & 0.83 & 2.98 & 17.25 & 10.87 & 31.74 & 15.86 \\
\hline & ${ }^{13} \mathrm{C}$ data & 0.00 & 0.01 & 0.00 & 0.03 & 75.70 & 20.27 & 2.74 & 1.25 \\
\hline & All data & 0.00 & 0.00 & 0.00 & 0.00 & 79.95 & 13.49 & 5.33 & 1.22 \\
\hline & ${ }^{1} \mathrm{H}$ data & 9.55 & 1.37 & 1.14 & 5.83 & 28.17 & 17.08 & 17.82 & 19.03 \\
\hline \multirow[t]{2}{*}{ uDP4+ } & ${ }^{13} \mathrm{C}$ data & 0.03 & 0.07 & 0.06 & 0.14 & 50.50 & 32.53 & 12.98 & 3.69 \\
\hline & All data & 0.01 & 0.00 & 0.00 & 0.04 & 62.37 & 24.36 & 10.14 & 3.08 \\
\hline \multirow{5}{*}{ DP4+ } & ${ }^{1} \mathrm{H}$ data & 10.34 & 0.13 & 0.05 & 1.00 & 27.93 & 10.67 & 32.51 & 17.35 \\
\hline & ${ }^{13} \mathrm{C}$ data & 0.00 & 0.00 & 0.00 & 0.00 & 84.53 & 14.58 & 0.79 & 0.10 \\
\hline & All data & 0.00 & 0.00 & 0.00 & 0.00 & 92.81 & 6.12 & 1.01 & 0.07 \\
\hline & & \multicolumn{8}{|c|}{ Level of theory: mPW1PW91/6-311G* (solution, PCM) } \\
\hline & & 79a & $79 b$ & $79 c$ & 79d & $79 e$ & $79 f$ & $79 \mathrm{~g}$ & $79 \mathrm{~h}$ \\
\hline & ${ }^{1} \mathrm{H}$ data & 4.23 & 38.85 & 4.06 & 10.82 & 10.69 & 7.66 & 19.48 & 4.21 \\
\hline \multirow[t]{2}{*}{$s \mathrm{DP} 4+$} & ${ }^{13} \mathrm{C}$ data & 0.01 & 0.02 & 0.00 & 0.02 & 59.98 & 8.77 & 9.21 & 21.99 \\
\hline & All data & 0.00 & 0.07 & 0.00 & 0.03 & 65.34 & 6.84 & 18.28 & 9.44 \\
\hline \multirow{3}{*}{ uDP4+ } & ${ }^{1} \mathrm{H}$ data & 5.07 & 27.36 & 4.55 & 27.20 & 7.33 & 10.30 & 12.47 & 5.73 \\
\hline & ${ }^{13} \mathrm{C}$ data & 0.01 & 0.49 & 0.04 & 1.07 & 22.61 & 43.77 & 26.67 & 5.33 \\
\hline & All data & 0.01 & 1.31 & 0.02 & 2.86 & 16.20 & 44.10 & 32.52 & 2.99 \\
\hline \multirow{5}{*}{ DP4+ } & ${ }^{1} \mathrm{H}$ data & 1.18 & 58.36 & 1.01 & 16.16 & 4.30 & 4.33 & 13.33 & 1.33 \\
\hline & ${ }^{13} \mathrm{C}$ data & 0.00 & 0.00 & 0.00 & 0.00 & 64.49 & 18.25 & 11.68 & 5.58 \\
\hline & All data & 0.00 & 0.00 & 0.00 & 0.00 & 53.38 & 15.22 & 29.97 & 1.42 \\
\hline & & \multicolumn{8}{|c|}{ Level of theory: mPW1PW91/6-311G** (solution, PCM) } \\
\hline & & 79a & $79 b$ & 79c & 79d & $79 e$ & $79 f$ & $79 \mathrm{~g}$ & $79 \mathrm{~h}$ \\
\hline & ${ }^{1} \mathrm{H}$ data & 3.91 & 3.66 & 0.97 & 1.10 & 14.39 & 3.14 & 58.45 & 14.38 \\
\hline \multirow[t]{2}{*}{$s \mathrm{DP} 4+$} & ${ }^{13} \mathrm{C}$ data & 0.00 & 0.01 & 0.00 & 0.02 & 63.04 & 8.88 & 10.94 & 17.11 \\
\hline & All data & 0.00 & 0.00 & 0.00 & 0.00 & 49.84 & 1.53 & 35.11 & 13.51 \\
\hline \multirow{3}{*}{ uDP4+ } & ${ }^{1} \mathrm{H}$ data & 7.99 & 4.09 & 2.37 & 2.57 & 45.96 & 7.29 & 8.69 & 21.05 \\
\hline & ${ }^{13} \mathrm{C}$ data & 0.01 & 0.67 & 0.07 & 0.92 & 8.31 & 49.65 & 38.09 & 2.29 \\
\hline & All data & 0.00 & 0.24 & 0.01 & 0.21 & 33.85 & 32.08 & 29.33 & 4.27 \\
\hline \multirow{5}{*}{ DP4+ } & ${ }^{1} \mathrm{H}$ data & 2.02 & 0.97 & 0.15 & 0.18 & 42.79 & 1.48 & 32.84 & 19.58 \\
\hline & ${ }^{13} \mathrm{C}$ data & 0.00 & 0.00 & 0.00 & 0.00 & 36.87 & 31.04 & 29.33 & 2.76 \\
\hline & All data & 0.00 & 0.00 & 0.00 & 0.00 & 59.74 & 1.74 & 36.48 & 2.04 \\
\hline & & & & of the & PW1PV & $-311+G$ & Iution, & & \\
\hline & & $79 a$ & $79 b$ & $79 c$ & $79 d$ & $79 e$ & $79 f$ & $79 g$ & $79 h$ \\
\hline & ${ }^{1} \mathrm{H}$ data & 1.09 & 0.09 & 0.09 & 0.31 & 9.93 & 3.90 & 37.84 & 46.76 \\
\hline sDP4+ & ${ }^{13} \mathrm{C}$ data & 0.01 & 0.15 & 0.01 & 0.04 & 51.32 & 15.78 & 15.47 & 17.22 \\
\hline & All data & 0.00 & 0.00 & 0.00 & 0.00 & 25.97 & 3.14 & 29.85 & 41.04 \\
\hline & ${ }^{1} \mathrm{H}$ data & 6.65 & 0.54 & 0.96 & 3.75 & 27.74 & 8.65 & 12.23 & 39.48 \\
\hline uDP4+ & ${ }^{13} \mathrm{C}$ data & 0.03 & 4.83 & 0.23 & 0.89 & 12.35 & 47.32 & 33.42 & 0.92 \\
\hline & All data & 0.01 & 0.22 & 0.02 & 0.28 & 28.48 & 34.01 & 33.95 & 3.03 \\
\hline & ${ }^{1} \mathrm{H}$ data & 0.28 & 0.00 & 0.00 & 0.04 & 10.48 & 1.29 & 17.62 & 70.29 \\
\hline DP4+ & ${ }^{13} \mathrm{C}$ data & 0.00 & 0.04 & 0.00 & 0.00 & 33.12 & 39.01 & 27.01 & 0.83 \\
\hline & All data & 0.00 & 0.00 & 0.00 & 0.00 & 37.27 & 5.38 & 51.08 & 6.27 \\
\hline
\end{tabular}


$s \mathrm{DP4}+, u \mathrm{DP} 4+$ and DP4+ probabilities (\%) for compound $79 \mathrm{f}$<smiles>CC[C@H](C)[C@@H]1OC([C@@H](C)[C@@H](O)CC)=C(C)C(=O)[C@@H]1C</smiles><smiles>CC[C@H](O)[C@H](C)C1=C(C)C(=O)C(C)[C@H]([C@H](C)CC)O1</smiles><smiles>CC[C@H](C)[C@@H]1OC([C@@H](C)[C@@H](O)CC)=C(C)C(=O)[C@@H]1C</smiles><smiles>CC[C@H](C)[C@H]1OC([C@H](C)CC)=C(C)C(=O)[C@H]1C</smiles>
correct isomer<smiles>CC[C@H](O)[C@H](C)C1=C(C)C(=O)[C@H](C)[C@H]([C@H](C)CC)O1</smiles><smiles>CC[C@H](C)[C@@H]1OC([C@@H](C)[C@@H](O)CC)=C(C)C(=O)[C@@H]1C</smiles><smiles>CC[C@H](C)[C@@H]1OC([C@@H](C)[C@@H](O)CC)=C(C)C(=O)[C@@H]1C</smiles><smiles>CC[C@H](C)[C@@H]1OC([C@@H](C)[C@@H](O)CC)=C(C)C(=O)[C@@H]1C</smiles>

\begin{tabular}{|c|c|c|c|c|c|c|c|c|c|}
\hline & & \multicolumn{8}{|c|}{ Level of theory: B3LYP/6-31G* (gas phase) } \\
\hline & & $79 a$ & $79 b$ & $79 c$ & 79d & $79 e$ & $79 f$ & $79 \mathrm{~g}$ & $79 \mathrm{~h}$ \\
\hline & ${ }^{1} \mathrm{H}$ data & 0.06 & 69.79 & 7.82 & 21.56 & 0.03 & 0.14 & 0.58 & 0.03 \\
\hline \multirow[t]{2}{*}{ sDP4+ } & ${ }^{13} \mathrm{C}$ data & 0.00 & 0.01 & 0.00 & 0.05 & 18.48 & 52.88 & 15.92 & 12.66 \\
\hline & All data & 0.00 & 1.99 & 0.16 & 5.44 & 2.65 & 39.82 & 48.11 & 1.82 \\
\hline & ${ }^{1} \mathrm{H}$ data & 0.04 & 58.40 & 28.94 & 9.69 & 0.06 & 1.81 & 1.00 & 0.06 \\
\hline \multirow[t]{2}{*}{ uDP4+ } & ${ }^{13} \mathrm{C}$ data & 0.00 & 0.01 & 0.03 & 0.19 & 11.63 & 9.41 & 54.76 & 23.97 \\
\hline & All data & 0.00 & 0.71 & 1.13 & 2.33 & 0.95 & 22.06 & 70.85 & 1.97 \\
\hline & ${ }^{1} \mathrm{H}$ data & 0.00 & 90.34 & 5.01 & 4.63 & 0.00 & 0.01 & 0.01 & 0.00 \\
\hline \multirow[t]{5}{*}{ DP4+ } & ${ }^{13} \mathrm{C}$ data & 0.00 & 0.00 & 0.00 & 0.00 & 11.38 & 26.36 & 46.18 & 16.07 \\
\hline & All data & 0.00 & 0.03 & 0.00 & 0.29 & 0.06 & 20.39 & 79.14 & 0.08 \\
\hline & & \multicolumn{8}{|c|}{ Level of theory: B3LYP/6-31G** (gas phase) } \\
\hline & & 79a & $79 b$ & 79c & 79d & $79 e$ & $79 f$ & $79 \mathrm{~g}$ & $79 \mathrm{~h}$ \\
\hline & ${ }^{1} \mathrm{H}$ data & 0.07 & 78.84 & 7.62 & 11.18 & 0.07 & 0.20 & 1.96 & 0.06 \\
\hline \multirow[t]{3}{*}{ sDP4+ } & ${ }^{13} \mathrm{C}$ data & 0.00 & 0.01 & 0.00 & 0.03 & 22.59 & 57.12 & 10.45 & 9.79 \\
\hline & All data & 0.00 & 1.44 & 0.09 & 0.83 & 4.60 & 32.21 & 59.23 & 1.60 \\
\hline & ${ }^{1} \mathrm{H}$ data & 0.08 & 60.59 & 27.70 & 8.14 & 0.13 & 2.01 & 1.27 & 0.09 \\
\hline \multirow[t]{2}{*}{ uDP4+ } & ${ }^{13} \mathrm{C}$ data & 0.00 & 0.03 & 0.04 & 0.22 & 9.82 & 22.89 & 53.72 & 13.29 \\
\hline & All data & 0.00 & 1.56 & 0.89 & 1.46 & 1.02 & 37.92 & 56.17 & 0.98 \\
\hline \multirow{5}{*}{ DP4+ } & ${ }^{1} \mathrm{H}$ data & 0.00 & 94.00 & 4.16 & 1.79 & 0.00 & 0.01 & 0.05 & 0.00 \\
\hline & ${ }^{13} \mathrm{C}$ data & 0.00 & 0.00 & 0.00 & 0.00 & 9.99 & 58.87 & 25.29 & 5.86 \\
\hline & All data & 0.00 & 0.05 & 0.00 & 0.03 & 0.10 & 26.79 & 72.99 & 0.03 \\
\hline & & \multicolumn{8}{|c|}{ Level of theory: B3LYP/6-31+G** (gas phase) } \\
\hline & & $79 a$ & $79 b$ & $79 c$ & 79d & $79 e$ & $79 f$ & $79 \mathrm{~g}$ & $79 \mathrm{~h}$ \\
\hline & ${ }^{1} \mathrm{H}$ data & 1.23 & 56.44 & 12.80 & 4.44 & 1.88 & 1.09 & 21.36 & 0.77 \\
\hline \multirow[t]{2}{*}{ sDP4+ } & ${ }^{13} \mathrm{C}$ data & 0.00 & 0.04 & 0.05 & 0.11 & 9.32 & 81.02 & 8.37 & 1.09 \\
\hline & All data & 0.00 & 0.82 & 0.20 & 0.16 & 6.07 & 30.63 & 61.83 & 0.29 \\
\hline \multirow{3}{*}{ uDP4+ } & ${ }^{1} \mathrm{H}$ data & 0.84 & 31.70 & 27.02 & 13.53 & 3.84 & 9.71 & 12.17 & 1.18 \\
\hline & ${ }^{13} \mathrm{C}$ data & 0.00 & 0.13 & 0.24 & 0.13 & 3.81 & 75.81 & 19.33 & 0.54 \\
\hline & All data & 0.00 & 0.42 & 0.65 & 0.17 & 1.47 & 73.69 & 23.54 & 0.06 \\
\hline \multirow{6}{*}{ DP4+ } & ${ }^{1} \mathrm{H}$ data & 0.04 & 72.30 & 13.97 & 2.43 & 0.29 & 0.43 & 10.50 & 0.04 \\
\hline & ${ }^{13} \mathrm{C}$ data & 0.00 & 0.00 & 0.00 & 0.00 & 0.56 & 96.88 & 2.55 & 0.01 \\
\hline & All data & 0.00 & 0.01 & 0.00 & 0.00 & 0.24 & 60.65 & 39.10 & 0.00 \\
\hline & & \multicolumn{8}{|c|}{ Level of theory: B3LYP/6-311G* (gas phase) } \\
\hline & & 79a & $79 \mathrm{~b}$ & 79c & 79d & $79 e$ & $79 f$ & $79 \mathrm{~g}$ & $79 \mathrm{~h}$ \\
\hline & ${ }^{1} \mathrm{H}$ data & 0.11 & 67.68 & 9.98 & 3.06 & 0.56 & 8.16 & 10.16 & 0.30 \\
\hline \multirow[t]{2}{*}{ sDP4+ } & ${ }^{13} \mathrm{C}$ data & 0.00 & 0.06 & 0.01 & 0.20 & 10.50 & 64.35 & 17.97 & 6.91 \\
\hline & All data & 0.00 & 0.52 & 0.02 & 0.09 & 0.81 & 72.92 & 25.36 & 0.28 \\
\hline \multirow{2}{*}{ uDP4+ } & ${ }^{1} \mathrm{H}$ data & 0.72 & 39.46 & 12.93 & 5.07 & 1.68 & 30.49 & 8.90 & 0.75 \\
\hline & ${ }^{13} \mathrm{C}$ data & 0.00 & 0.34 & 0.05 & 0.31 & 2.58 & 81.07 & 14.75 & 0.89 \\
\hline
\end{tabular}




\begin{tabular}{|c|c|c|c|c|c|c|c|c|c|}
\hline & All data & 0.00 & 0.51 & 0.03 & 0.06 & 0.17 & 94.21 & 5.00 & 0.03 \\
\hline & ${ }^{1} \mathrm{H}$ data & 0.00 & 84.63 & 4.09 & 0.49 & 0.03 & 7.88 & 2.86 & 0.01 \\
\hline \multirow[t]{5}{*}{ DP4+ } & ${ }^{13} \mathrm{C}$ data & 0.00 & 0.00 & 0.00 & 0.00 & 0.49 & 94.59 & 4.81 & 0.11 \\
\hline & All data & 0.00 & 0.00 & 0.00 & 0.00 & 0.00 & 98.18 & 1.81 & 0.00 \\
\hline & & \multicolumn{8}{|c|}{ Level of theory: B3LYP/6-311G** (gas phase) } \\
\hline & & $79 a$ & $79 \mathrm{~b}$ & $79 c$ & 79d & $79 e$ & $79 f$ & $79 \mathrm{~g}$ & $79 \mathrm{~h}$ \\
\hline & ${ }^{1} \mathrm{H}$ data & 0.13 & 32.06 & 8.29 & 0.91 & 1.29 & 11.15 & 45.06 & 1.11 \\
\hline \multirow[t]{2}{*}{ sDP4+ } & ${ }^{13} \mathrm{C}$ data & 0.00 & 0.05 & 0.01 & 0.19 & 8.62 & 70.66 & 14.40 & 6.07 \\
\hline & All data & 0.00 & 0.11 & 0.01 & 0.01 & 0.76 & 54.10 & 44.55 & 0.46 \\
\hline & ${ }^{1} \mathrm{H}$ data & 0.67 & 13.69 & 9.37 & 3.62 & 4.19 & 51.66 & 14.53 & 2.26 \\
\hline \multirow[t]{2}{*}{ uDP4+ } & ${ }^{13} \mathrm{C}$ data & 0.00 & 0.37 & 0.06 & 0.34 & 1.13 & 81.92 & 15.77 & 0.42 \\
\hline & All data & 0.00 & 0.11 & 0.01 & 0.03 & 0.11 & 94.60 & 5.12 & 0.02 \\
\hline \multirow{6}{*}{ DP4+ } & ${ }^{1} \mathrm{H}$ data & 0.00 & 24.96 & 4.42 & 0.19 & 0.31 & 32.76 & 37.22 & 0.14 \\
\hline & ${ }^{13} \mathrm{C}$ data & 0.00 & 0.00 & 0.00 & 0.00 & 0.16 & 96.03 & 3.77 & 0.04 \\
\hline & All data & 0.00 & 0.00 & 0.00 & 0.00 & 0.00 & 95.73 & 4.27 & 0.00 \\
\hline & & \multicolumn{8}{|c|}{ Level of theory: B3LYP/6-311+G** (gas phase) } \\
\hline & & 79a & $79 b$ & 79c & 79d & $79 e$ & $79 f$ & $79 \mathrm{~g}$ & $79 \mathrm{~h}$ \\
\hline & ${ }^{1} \mathrm{H}$ data & 0.48 & 20.60 & 6.38 & 0.73 & 0.92 & 5.64 & 63.31 & 1.95 \\
\hline \multirow[t]{2}{*}{$s \mathrm{DP} 4+$} & ${ }^{13} \mathrm{C}$ data & 0.00 & 0.13 & 0.01 & 0.11 & 12.82 & 63.68 & 19.60 & 3.65 \\
\hline & All data & 0.00 & 0.16 & 0.00 & 0.00 & 0.73 & 22.14 & 76.52 & 0.44 \\
\hline \multirow{3}{*}{ uDP4+ } & ${ }^{1} \mathrm{H}$ data & 2.16 & 8.31 & 9.65 & 6.29 & 7.40 & 37.99 & 18.52 & 9.68 \\
\hline & ${ }^{13} \mathrm{C}$ data & 0.00 & 1.18 & 0.06 & 0.29 & 1.66 & 80.31 & 16.28 & 0.20 \\
\hline & All data & 0.00 & 0.29 & 0.02 & 0.05 & 0.36 & 90.29 & 8.92 & 0.06 \\
\hline \multirow{5}{*}{ DP4+ } & ${ }^{1} \mathrm{H}$ data & 0.06 & 10.37 & 3.73 & 0.28 & 0.41 & 12.97 & 71.03 & 1.14 \\
\hline & ${ }^{13} \mathrm{C}$ data & 0.00 & 0.00 & 0.00 & 0.00 & 0.39 & 93.74 & 5.85 & 0.01 \\
\hline & All data & 0.00 & 0.00 & 0.00 & 0.00 & 0.01 & 74.53 & 25.46 & 0.00 \\
\hline & & \multicolumn{8}{|c|}{ Level of theory: B3LYP/6-31G* (solution, PCM) } \\
\hline & & 79a & $79 b$ & $79 \mathrm{c}$ & 79d & $79 e$ & $79 f$ & $79 \mathrm{~g}$ & $79 \mathrm{~h}$ \\
\hline & ${ }^{1} \mathrm{H}$ data & 0.27 & 76.29 & 4.05 & 18.01 & 0.04 & 0.57 & 0.73 & 0.04 \\
\hline \multirow[t]{2}{*}{ sDP4+ } & ${ }^{13} \mathrm{C}$ data & 0.00 & 0.00 & 0.00 & 0.01 & 3.92 & 74.50 & 19.75 & 1.80 \\
\hline & All data & 0.00 & 0.44 & 0.02 & 0.46 & 0.27 & 73.77 & 24.93 & 0.12 \\
\hline \multirow{3}{*}{ uDP4+ } & ${ }^{1} \mathrm{H}$ data & 0.10 & 63.87 & 15.62 & 12.61 & 0.12 & 4.10 & 3.42 & 0.17 \\
\hline & ${ }^{13} \mathrm{C}$ data & 0.00 & 0.01 & 0.03 & 0.12 & 9.65 & 13.49 & 57.29 & 19.41 \\
\hline & All data & 0.00 & 0.28 & 0.20 & 0.58 & 0.45 & 21.40 & 75.84 & 1.26 \\
\hline \multirow{6}{*}{ DP4+ } & ${ }^{1} \mathrm{H}$ data & 0.00 & 94.29 & 1.23 & 4.39 & 0.00 & 0.05 & 0.05 & 0.00 \\
\hline & ${ }^{13} \mathrm{C}$ data & 0.00 & 0.00 & 0.00 & 0.00 & 1.71 & 45.49 & 51.22 & 1.58 \\
\hline & All data & 0.00 & 0.00 & 0.00 & 0.01 & 0.00 & 45.49 & 54.49 & 0.00 \\
\hline & & \multicolumn{8}{|c|}{ Level of theory: B3LYP/6-31G** (solution, PCM) } \\
\hline & & $79 a$ & $79 b$ & $79 c$ & $79 d$ & $79 e$ & $79 f$ & $79 \mathrm{~g}$ & $79 \mathrm{~h}$ \\
\hline & ${ }^{1} \mathrm{H}$ data & 0.40 & 75.98 & 4.72 & 13.16 & 0.20 & 1.46 & 3.95 & 0.13 \\
\hline \multirow[t]{2}{*}{ sDP4+ } & ${ }^{13} \mathrm{C}$ data & 0.00 & 0.01 & 0.01 & 0.02 & 6.32 & 79.02 & 12.22 & 2.41 \\
\hline & All data & 0.00 & 0.35 & 0.01 & 0.15 & 0.77 & 69.44 & 29.10 & 0.19 \\
\hline & ${ }^{1} \mathrm{H}$ data & 0.19 & 60.04 & 11.26 & 18.87 & 0.25 & 4.05 & 5.03 & 0.31 \\
\hline \multirow[t]{2}{*}{ uDP4+ } & ${ }^{13} \mathrm{C}$ data & 0.00 & 0.04 & 0.05 & 0.14 & 8.26 & 37.69 & 44.81 & 9.02 \\
\hline & All data & 0.00 & 0.66 & 0.15 & 0.66 & 0.53 & 39.24 & 58.04 & 0.72 \\
\hline & ${ }^{1} \mathrm{H}$ data & 0.00 & 93.30 & 1.09 & 5.08 & 0.00 & 0.12 & 0.41 & 0.00 \\
\hline DP4+ & ${ }^{13} \mathrm{C}$ data & 0.00 & 0.00 & 0.00 & 0.00 & 1.45 & 82.73 & 15.21 & 0.60 \\
\hline & All data & 0.00 & 0.01 & 0.00 & 0.00 & 0.01 & 61.72 & 38.26 & 0.00 \\
\hline & & & & Level of & : B3LY & $1+\mathrm{G} * *(\mathrm{~s}$ & on, PCM & & \\
\hline & & 79a & $79 \mathrm{~b}$ & 79c & 79d & $79 e$ & $79 f$ & $79 \mathrm{~g}$ & $79 \mathrm{~h}$ \\
\hline & ${ }^{1} \mathrm{H}$ data & 10.68 & 21.20 & 8.63 & 2.59 & 2.31 & 28.36 & 25.18 & 1.05 \\
\hline sDP4+ & ${ }^{13} \mathrm{C}$ data & 0.00 & 0.07 & 0.06 & 0.07 & 9.26 & 79.31 & 10.62 & 0.62 \\
\hline & All data & 0.00 & 0.05 & 0.02 & 0.01 & 0.84 & 88.53 & 10.52 & 0.03 \\
\hline & ${ }^{1} \mathrm{H}$ data & 9.36 & 33.68 & 17.77 & 8.00 & 6.21 & 0.00 & 21.77 & 3.21 \\
\hline uDP4+ & ${ }^{13} \mathrm{C}$ data & 0.04 & 1.36 & 1.88 & 0.64 & 14.71 & 0.00 & 79.81 & 1.55 \\
\hline & All data & 0.02 & 2.39 & 1.74 & 0.27 & 4.76 & 0.00 & 90.56 & 0.26 \\
\hline
\end{tabular}




\begin{tabular}{|c|c|c|c|c|c|c|c|c|c|}
\hline & ${ }^{1} \mathrm{H}$ data & 6.43 & 45.95 & 9.86 & 1.33 & 0.92 & 0.00 & 35.28 & 0.22 \\
\hline \multirow[t]{5}{*}{ DP4+ } & ${ }^{13} \mathrm{C}$ data & 0.00 & 0.01 & 0.01 & 0.00 & 13.84 & 0.00 & 86.04 & 0.10 \\
\hline & All data & 0.00 & 0.01 & 0.00 & 0.00 & 0.42 & 0.00 & 99.56 & 0.00 \\
\hline & & \multicolumn{8}{|c|}{ Level of theory: B3LYP/6-311G* (solution, PCM) } \\
\hline & & 79a & $79 b$ & $79 c$ & 79d & $79 e$ & $79 f$ & $79 \mathrm{~g}$ & $79 \mathrm{~h}$ \\
\hline & ${ }^{1} \mathrm{H}$ data & 0.76 & 60.20 & 11.73 & 5.93 & 1.14 & 12.03 & 7.68 & 0.54 \\
\hline \multirow[t]{2}{*}{ sDP4+ } & ${ }^{13} \mathrm{C}$ data & 0.00 & 0.21 & 0.05 & 0.40 & 7.50 & 54.41 & 33.10 & 4.32 \\
\hline & All data & 0.00 & 1.37 & 0.06 & 0.25 & 0.92 & 69.97 & 27.18 & 0.25 \\
\hline \multirow{3}{*}{ uDP4+ } & ${ }^{1} \mathrm{H}$ data & 2.12 & 56.09 & 12.56 & 8.12 & 1.73 & 12.79 & 5.80 & 0.80 \\
\hline & ${ }^{13} \mathrm{C}$ data & 0.00 & 1.49 & 0.23 & 0.53 & 1.73 & 67.12 & 28.50 & 0.40 \\
\hline & All data & 0.00 & 7.49 & 0.26 & 0.38 & 0.27 & 76.80 & 14.78 & 0.03 \\
\hline \multirow{5}{*}{ DP4+ } & ${ }^{1} \mathrm{H}$ data & 0.04 & 89.46 & 3.90 & 1.27 & 0.05 & 4.07 & 1.18 & 0.01 \\
\hline & ${ }^{13} \mathrm{C}$ data & 0.00 & 0.01 & 0.00 & 0.00 & 0.28 & 79.21 & 20.46 & 0.04 \\
\hline & All data & 0.00 & 0.18 & 0.00 & 0.00 & 0.00 & 92.87 & 6.94 & 0.00 \\
\hline & & \multicolumn{8}{|c|}{ Level of theory: B3LYP/6-311G** (solution, PCM) } \\
\hline & & $79 a$ & $79 b$ & 79c & 79d & $79 e$ & $79 f$ & $79 \mathrm{~g}$ & $79 \mathrm{~h}$ \\
\hline & ${ }^{1} \mathrm{H}$ data & 1.23 & 30.71 & 8.57 & 1.36 & 2.68 & 19.05 & 34.10 & 2.30 \\
\hline \multirow[t]{2}{*}{ sDP4+ } & ${ }^{13} \mathrm{C}$ data & 0.00 & 0.16 & 0.04 & 0.36 & 6.28 & 58.05 & 31.26 & 3.86 \\
\hline & All data & 0.00 & 0.22 & 0.02 & 0.02 & 0.76 & 50.19 & 48.38 & 0.40 \\
\hline \multirow{3}{*}{ uDP4+ } & ${ }^{1} \mathrm{H}$ data & 3.32 & 18.02 & 8.51 & 4.08 & 5.61 & 42.95 & 13.01 & 4.50 \\
\hline & ${ }^{13} \mathrm{C}$ data & 0.00 & 1.27 & 0.20 & 0.43 & 0.99 & 68.55 & 28.30 & 0.26 \\
\hline & All data & 0.00 & 0.69 & 0.05 & 0.05 & 0.17 & 88.00 & 11.00 & 0.04 \\
\hline & ${ }^{1} \mathrm{H}$ data & 0.21 & 28.77 & 3.79 & 0.29 & 0.78 & 42.55 & 23.07 & 0.54 \\
\hline \multirow[t]{5}{*}{ DP4+ } & ${ }^{13} \mathrm{C}$ data & 0.00 & 0.00 & 0.00 & 0.00 & 0.13 & 81.69 & 18.16 & 0.02 \\
\hline & All data & 0.00 & 0.00 & 0.00 & 0.00 & 0.00 & 89.24 & 10.76 & 0.00 \\
\hline & & \multicolumn{8}{|c|}{ Level of theory: B3LYP/6-311+G** (solution, PCM) } \\
\hline & & $79 a$ & $79 b$ & $79 c$ & $79 d$ & $79 e$ & $79 f$ & $79 \mathrm{~g}$ & $79 \mathrm{~h}$ \\
\hline & ${ }^{1} \mathrm{H}$ data & 3.42 & 12.28 & 5.79 & 0.85 & 1.53 & 9.86 & 61.69 & 4.57 \\
\hline \multirow[t]{2}{*}{ sDP4+ } & ${ }^{13} \mathrm{C}$ data & 0.00 & 0.49 & 0.06 & 0.21 & 9.47 & 52.59 & 33.88 & 3.29 \\
\hline & All data & 0.00 & 0.23 & 0.01 & 0.01 & 0.55 & 19.60 & 79.03 & 0.57 \\
\hline \multirow{3}{*}{ uDP4+ } & ${ }^{1} \mathrm{H}$ data & 8.72 & 8.34 & 7.73 & 4.05 & 6.91 & 31.92 & 18.96 & 13.37 \\
\hline & ${ }^{13} \mathrm{C}$ data & 0.02 & 11.12 & 0.82 & 1.18 & 3.07 & 60.17 & 23.46 & 0.16 \\
\hline & All data & 0.01 & 3.72 & 0.26 & 0.19 & 0.85 & 77.05 & 17.84 & 0.08 \\
\hline \multirow{5}{*}{ DP4+ } & ${ }^{1} \mathrm{H}$ data & 1.72 & 5.90 & 2.58 & 0.20 & 0.61 & 18.12 & 67.35 & 3.52 \\
\hline & ${ }^{13} \mathrm{C}$ data & 0.00 & 0.14 & 0.00 & 0.01 & 0.73 & 79.22 & 19.90 & 0.01 \\
\hline & All data & 0.00 & 0.03 & 0.00 & 0.00 & 0.02 & 51.69 & 48.26 & 0.00 \\
\hline & & \multicolumn{8}{|c|}{ Level of theory: mPW1PW91/6-31G* (gas phase) } \\
\hline & & $79 a$ & $79 b$ & 79c & 79d & $79 e$ & $79 f$ & $79 \mathrm{~g}$ & $79 \mathrm{~h}$ \\
\hline \multirow{3}{*}{ sDP4+ } & ${ }^{1} \mathrm{H}$ data & 0.04 & 70.07 & 3.27 & 26.17 & 0.02 & 0.04 & 0.35 & 0.03 \\
\hline & ${ }^{13} \mathrm{C}$ data & 0.00 & 0.01 & 0.00 & 0.10 & 13.93 & 16.01 & 59.01 & 10.93 \\
\hline & All data & 0.00 & 2.04 & 0.05 & 10.68 & 1.10 & 2.84 & 82.12 & 1.17 \\
\hline \multirow{3}{*}{ uDP4+ } & ${ }^{1} \mathrm{H}$ data & 0.02 & 59.33 & 19.15 & 19.90 & 0.04 & 0.56 & 0.96 & 0.06 \\
\hline & ${ }^{13} \mathrm{C}$ data & 0.01 & 0.01 & 0.03 & 0.10 & 16.17 & 4.40 & 42.90 & 36.38 \\
\hline & All data & 0.00 & 0.99 & 1.16 & 4.03 & 1.28 & 4.99 & 83.34 & 4.20 \\
\hline & ${ }^{1} \mathrm{H}$ data & 0.00 & 87.69 & 1.32 & 10.98 & 0.00 & 0.00 & 0.01 & 0.00 \\
\hline DP4+ & ${ }^{13} \mathrm{C}$ data & 0.00 & 0.00 & 0.00 & 0.00 & 6.99 & 2.19 & 78.49 & 12.34 \\
\hline & All data & 0.00 & 0.03 & 0.00 & 0.62 & 0.02 & 0.21 & 99.05 & 0.07 \\
\hline & & & & evel of & : $\mathrm{mPW}$ & $1 / 6-31 G$ & as phas & & \\
\hline & & 79a & $79 b$ & $79 c$ & $79 d$ & $79 e$ & $79 f$ & $79 \mathrm{~g}$ & $79 \mathrm{~h}$ \\
\hline & ${ }^{1} \mathrm{H}$ data & 0.08 & 80.85 & 3.00 & 14.15 & 0.07 & 0.09 & 1.69 & 0.08 \\
\hline sDP4+ & ${ }^{13} \mathrm{C}$ data & 0.00 & 0.01 & 0.01 & 0.07 & 26.46 & 29.31 & 28.70 & 15.44 \\
\hline & All data & 0.00 & 1.44 & 0.03 & 1.76 & 3.12 & 4.57 & 86.99 & 2.09 \\
\hline & ${ }^{1} \mathrm{H}$ data & 0.06 & 60.88 & 19.48 & 17.36 & 0.09 & 0.66 & 1.38 & 0.08 \\
\hline uDP4+ & ${ }^{13} \mathrm{C}$ data & 0.00 & 0.01 & 0.03 & 0.17 & 6.85 & 6.39 & 66.07 & 20.47 \\
\hline & All data & 0.00 & 0.83 & 0.58 & 2.88 & 0.61 & 4.15 & 89.42 & 1.53 \\
\hline DP4+ & ${ }^{1} \mathrm{H}$ data & 0.00 & 94.14 & 1.12 & 4.70 & 0.00 & 0.00 & 0.04 & 0.00 \\
\hline
\end{tabular}




\begin{tabular}{|c|c|c|c|c|c|c|c|c|c|}
\hline & ${ }^{13} \mathrm{C}$ data & 0.00 & 0.00 & 0.00 & 0.00 & 7.03 & 7.26 & 73.46 & 12.25 \\
\hline & All data & 0.00 & 0.02 & 0.00 & 0.06 & 0.02 & 0.24 & 99.61 & 0.04 \\
\hline & & \multicolumn{8}{|c|}{ Level of theory: mPW1PW91/6-31+G** (gas phase) } \\
\hline & & $79 a$ & $79 \mathrm{~b}$ & $79 c$ & 79d & $79 e$ & $79 f$ & $79 \mathrm{~g}$ & $79 \mathrm{~h}$ \\
\hline & ${ }^{1} \mathrm{H}$ data & 1.43 & 57.95 & 7.90 & 5.17 & 2.44 & 1.76 & 22.22 & 1.13 \\
\hline \multirow[t]{2}{*}{ sDP4+ } & ${ }^{13} \mathrm{C}$ data & 0.00 & 0.01 & 0.01 & 0.03 & 6.34 & 87.73 & 5.55 & 0.33 \\
\hline & All data & 0.00 & 0.15 & 0.03 & 0.06 & 5.25 & 52.51 & 41.88 & 0.13 \\
\hline \multirow{3}{*}{ uDP4+ } & ${ }^{1} \mathrm{H}$ data & 1.23 & 44.10 & 16.99 & 9.04 & 3.54 & 10.75 & 12.75 & 1.59 \\
\hline & ${ }^{13} \mathrm{C}$ data & 0.00 & 0.08 & 0.14 & 0.15 & 4.02 & 56.74 & 37.73 & 1.13 \\
\hline & All data & 0.00 & 0.30 & 0.22 & 0.12 & 1.28 & 54.75 & 43.17 & 0.16 \\
\hline & ${ }^{1} \mathrm{H}$ data & 0.06 & 83.76 & 4.40 & 1.53 & 0.28 & 0.62 & 9.28 & 0.06 \\
\hline \multirow[t]{4}{*}{ DP4+ } & ${ }^{13} \mathrm{C}$ data & 0.00 & 0.00 & 0.00 & 0.00 & 0.49 & 95.49 & 4.01 & 0.01 \\
\hline & All data & 0.00 & 0.00 & 0.00 & 0.00 & 0.14 & 61.30 & 38.56 & 0.00 \\
\hline & & \multicolumn{8}{|c|}{ Level of theory: mPW1PW91/6-311G* (gas phase) } \\
\hline & & $79 a$ & $79 \mathrm{~b}$ & $79 c$ & 79d & $79 e$ & $79 f$ & $79 \mathrm{~g}$ & $79 \mathrm{~h}$ \\
\hline & ${ }^{1} \mathrm{H}$ data & 0.08 & 81.26 & 8.49 & 1.52 & 0.19 & 2.06 & 6.28 & 0.12 \\
\hline \multirow[t]{2}{*}{ sDP4+ } & ${ }^{13} \mathrm{C}$ data & 0.00 & 0.09 & 0.02 & 0.33 & 15.96 & 41.68 & 29.88 & 12.05 \\
\hline & All data & 0.00 & 2.68 & 0.05 & 0.17 & 1.07 & 30.03 & 65.49 & 0.49 \\
\hline \multirow{3}{*}{ uDP4+ } & ${ }^{1} \mathrm{H}$ data & 0.76 & 56.74 & 16.47 & 4.88 & 1.05 & 12.77 & 6.76 & 0.57 \\
\hline & ${ }^{13} \mathrm{C}$ data & 0.00 & 0.20 & 0.04 & 0.20 & 1.84 & 65.36 & 31.25 & 1.11 \\
\hline & All data & 0.00 & 1.08 & 0.06 & 0.09 & 0.18 & 78.62 & 19.91 & 0.06 \\
\hline \multirow{6}{*}{ DP4+ } & ${ }^{1} \mathrm{H}$ data & 0.00 & 95.52 & 2.90 & 0.15 & 0.00 & 0.55 & 0.88 & 0.00 \\
\hline & ${ }^{13} \mathrm{C}$ data & 0.00 & 0.00 & 0.00 & 0.00 & 0.79 & 73.61 & 25.23 & 0.36 \\
\hline & All data & 0.00 & 0.08 & 0.00 & 0.00 & 0.01 & 64.36 & 35.55 & 0.00 \\
\hline & & \multicolumn{8}{|c|}{ Level of theory: mPW1PW91/6-311G** (gas phase) } \\
\hline & & 79a & $79 b$ & $79 c$ & $79 d$ & $79 e$ & $79 f$ & $79 \mathrm{~g}$ & $79 \mathrm{~h}$ \\
\hline & ${ }^{1} \mathrm{H}$ data & 0.11 & 35.55 & 6.53 & 0.60 & 0.67 & 4.29 & 51.51 & 0.74 \\
\hline \multirow[t]{2}{*}{ sDP4+ } & ${ }^{13} \mathrm{C}$ data & 0.00 & 0.08 & 0.02 & 0.23 & 10.72 & 50.82 & 28.37 & 9.74 \\
\hline & All data & 0.00 & 0.17 & 0.01 & 0.01 & 0.42 & 12.85 & 86.12 & 0.43 \\
\hline \multirow{3}{*}{ uDP4+ } & ${ }^{1} \mathrm{H}$ data & 0.70 & 18.67 & 11.45 & 3.83 & 3.53 & 44.09 & 15.37 & 2.36 \\
\hline & ${ }^{13} \mathrm{C}$ data & 0.00 & 0.27 & 0.05 & 0.17 & 0.63 & 64.74 & 33.71 & 0.44 \\
\hline & All data & 0.00 & 0.15 & 0.02 & 0.02 & 0.07 & 84.40 & 15.32 & 0.03 \\
\hline \multirow{5}{*}{ DP4+ } & ${ }^{1} \mathrm{H}$ data & 0.00 & 38.46 & 4.33 & 0.13 & 0.14 & 10.96 & 45.88 & 0.10 \\
\hline & ${ }^{13} \mathrm{C}$ data & 0.00 & 0.00 & 0.00 & 0.00 & 0.16 & 77.28 & 22.47 & 0.10 \\
\hline & All data & 0.00 & 0.00 & 0.00 & 0.00 & 0.00 & 45.11 & 54.89 & 0.00 \\
\hline & & \multicolumn{8}{|c|}{ Level of theory: mPW1PW91/6-311+G** (gas phase) } \\
\hline & & $79 a$ & $79 b$ & $79 c$ & 79d & $79 e$ & $79 f$ & $79 \mathrm{~g}$ & $79 \mathrm{~h}$ \\
\hline \multirow{3}{*}{ sDP4+ } & ${ }^{1} \mathrm{H}$ data & 0.82 & 28.05 & 9.38 & 1.12 & 0.85 & 2.95 & 54.88 & 1.95 \\
\hline & ${ }^{13} \mathrm{C}$ data & 0.00 & 0.13 & 0.01 & 0.09 & 8.84 & 52.34 & 33.91 & 4.68 \\
\hline & All data & 0.00 & 0.18 & 0.01 & 0.01 & 0.37 & 7.60 & 91.39 & 0.45 \\
\hline \multirow{3}{*}{ uDP4+ } & ${ }^{1} \mathrm{H}$ data & 2.93 & 16.57 & 15.67 & 8.49 & 6.41 & 25.48 & 16.29 & 8.16 \\
\hline & ${ }^{13} \mathrm{C}$ data & 0.00 & 0.62 & 0.04 & 0.09 & 0.85 & 63.44 & 34.74 & 0.22 \\
\hline & All data & 0.00 & 0.47 & 0.03 & 0.03 & 0.25 & 73.44 & 25.70 & 0.08 \\
\hline \multirow{3}{*}{ DP4+ } & ${ }^{1} \mathrm{H}$ data & 0.15 & 28.80 & 9.11 & 0.59 & 0.34 & 4.66 & 55.37 & 0.98 \\
\hline & ${ }^{13} \mathrm{C}$ data & 0.00 & 0.00 & 0.00 & 0.00 & 0.17 & 73.68 & 26.13 & 0.02 \\
\hline & All data & 0.00 & 0.00 & 0.00 & 0.00 & 0.00 & 19.19 & 80.80 & 0.00 \\
\hline & & & & rel of th & mPW1F & $6-31 G^{*}$ & tion, $\mathrm{PC}$ & & \\
\hline & & $79 a$ & $79 b$ & $79 c$ & $79 d$ & $79 e$ & $79 f$ & $79 \mathrm{~g}$ & $79 \mathrm{~h}$ \\
\hline & ${ }^{1} \mathrm{H}$ data & 0.37 & 65.72 & 2.33 & 29.88 & 0.08 & 0.38 & 1.16 & 0.08 \\
\hline sDP4+ & ${ }^{13} \mathrm{C}$ data & 0.00 & 0.01 & 0.01 & 0.03 & 2.12 & 21.06 & 76.03 & 0.74 \\
\hline & All data & 0.00 & 0.61 & 0.01 & 1.05 & 0.16 & 8.24 & 89.86 & 0.06 \\
\hline & ${ }^{1} \mathrm{H}$ data & 0.09 & 58.10 & 7.48 & 28.47 & 0.11 & 2.09 & 3.48 & 0.17 \\
\hline uDP4+ & ${ }^{13} \mathrm{C}$ data & 0.01 & 0.01 & 0.05 & 0.11 & 14.45 & 7.62 & 48.76 & 28.99 \\
\hline & All data & 0.00 & 0.39 & 0.21 & 1.60 & 0.84 & 8.12 & 86.30 & 2.55 \\
\hline & ${ }^{1} \mathrm{H}$ data & 0.00 & 81.40 & 0.37 & 18.13 & 0.00 & 0.02 & 0.09 & 0.00 \\
\hline DP4+ & ${ }^{13} \mathrm{C}$ data & 0.00 & 0.00 & 0.00 & 0.00 & 0.78 & 4.09 & 94.58 & 0.55 \\
\hline
\end{tabular}




\begin{tabular}{|c|c|c|c|c|c|c|c|c|c|}
\hline & All data & 0.00 & 0.00 & 0.00 & 0.02 & 0.00 & 0.86 & 99.12 & 0.00 \\
\hline & & \multicolumn{8}{|c|}{ Level of theory: mPW1PW91/6-31G** (solution, PCM) } \\
\hline & & 79a & $79 \mathrm{~b}$ & $79 c$ & 79d & $79 e$ & $79 f$ & $79 \mathrm{~g}$ & $79 \mathrm{~h}$ \\
\hline & ${ }^{1} \mathrm{H}$ data & 0.62 & 69.07 & 2.37 & 18.85 & 0.31 & 1.01 & 7.57 & 0.21 \\
\hline \multirow[t]{2}{*}{ sDP4+ } & ${ }^{13} \mathrm{C}$ data & 0.00 & 0.01 & 0.01 & 0.03 & 6.08 & 46.30 & 45.55 & 2.01 \\
\hline & All data & 0.00 & 0.26 & 0.01 & 0.16 & 0.47 & 11.80 & 87.20 & 0.11 \\
\hline \multirow{3}{*}{ uDP4+ } & ${ }^{1} \mathrm{H}$ data & 0.13 & 49.35 & 5.92 & 36.15 & 0.22 & 2.22 & 5.70 & 0.31 \\
\hline & ${ }^{13} \mathrm{C}$ data & 0.00 & 0.02 & 0.05 & 0.14 & 7.52 & 12.60 & 66.89 & 12.78 \\
\hline & All data & 0.00 & 0.27 & 0.06 & 1.19 & 0.39 & 6.64 & 90.52 & 0.93 \\
\hline \multirow{5}{*}{ DP4+ } & ${ }^{1} \mathrm{H}$ data & 0.00 & 82.14 & 0.34 & 16.42 & 0.00 & 0.05 & 1.04 & 0.00 \\
\hline & ${ }^{13} \mathrm{C}$ data & 0.00 & 0.00 & 0.00 & 0.00 & 1.24 & 15.76 & 82.31 & 0.70 \\
\hline & All data & 0.00 & 0.00 & 0.00 & 0.00 & 0.00 & 0.98 & 99.01 & 0.00 \\
\hline & & \multicolumn{8}{|c|}{ Level of theory: mPW1PW91/6-31+G** (solution, PCM) } \\
\hline & & $79 a$ & $79 b$ & 79c & $79 d$ & $79 e$ & $79 f$ & $79 g$ & $79 \mathrm{~h}$ \\
\hline \multirow{3}{*}{ sDP4+ } & ${ }^{1} \mathrm{H}$ data & 10.82 & 23.37 & 8.55 & 3.07 & 3.99 & 12.48 & 35.39 & 2.33 \\
\hline & ${ }^{13} \mathrm{C}$ data & 0.00 & 0.03 & 0.02 & 0.03 & 4.14 & 83.91 & 11.72 & 0.14 \\
\hline & All data & 0.00 & 0.05 & 0.01 & 0.01 & 1.12 & 70.77 & 28.02 & 0.02 \\
\hline \multirow{3}{*}{ uDP4+ } & ${ }^{1} \mathrm{H}$ data & 6.21 & 22.95 & 14.38 & 3.72 & 5.79 & 16.47 & 27.21 & 3.28 \\
\hline & ${ }^{13} \mathrm{C}$ data & 0.00 & 0.18 & 0.25 & 0.09 & 2.39 & 61.97 & 34.62 & 0.50 \\
\hline & All data & 0.00 & 0.21 & 0.18 & 0.02 & 0.70 & 51.38 & 47.43 & 0.08 \\
\hline \multirow{5}{*}{ DP4+ } & ${ }^{1} \mathrm{H}$ data & 3.47 & 27.70 & 6.34 & 0.59 & 1.19 & 10.61 & 49.70 & 0.39 \\
\hline & ${ }^{13} \mathrm{C}$ data & 0.00 & 0.00 & 0.00 & 0.00 & 0.18 & 92.60 & 7.22 & 0.00 \\
\hline & All data & 0.00 & 0.00 & 0.00 & 0.00 & 0.02 & 73.23 & 26.76 & 0.00 \\
\hline & & \multicolumn{8}{|c|}{ Level of theory: mPW1PW91/6-311G* (solution, PCM) } \\
\hline & & $79 a$ & $79 b$ & $79 c$ & $79 d$ & $79 e$ & $79 f$ & $79 \mathrm{~g}$ & $79 \mathrm{~h}$ \\
\hline \multirow{3}{*}{ sDP4+ } & ${ }^{1} \mathrm{H}$ data & 0.68 & 72.65 & 9.68 & 3.47 & 0.53 & 6.23 & 6.48 & 0.27 \\
\hline & ${ }^{13} \mathrm{C}$ data & 0.00 & 0.15 & 0.02 & 0.26 & 2.08 & 48.05 & 47.62 & 1.83 \\
\hline & All data & 0.00 & 1.71 & 0.03 & 0.14 & 0.18 & 48.16 & 49.69 & 0.08 \\
\hline \multirow{3}{*}{ uDP4+ } & ${ }^{1} \mathrm{H}$ data & 1.53 & 58.04 & 15.95 & 9.32 & 1.12 & 6.08 & 7.20 & 0.76 \\
\hline & ${ }^{13} \mathrm{C}$ data & 0.00 & 0.52 & 0.08 & 0.13 & 0.78 & 56.21 & 41.99 & 0.28 \\
\hline & All data & 0.00 & 4.49 & 0.18 & 0.18 & 0.13 & 50.38 & 44.60 & 0.03 \\
\hline \multirow{5}{*}{ DP4+ } & ${ }^{1} \mathrm{H}$ data & 0.02 & 93.92 & 3.44 & 0.72 & 0.01 & 0.84 & 1.04 & 0.00 \\
\hline & ${ }^{13} \mathrm{C}$ data & 0.00 & 0.00 & 0.00 & 0.00 & 0.03 & 57.44 & 42.52 & 0.01 \\
\hline & All data & 0.00 & 0.17 & 0.00 & 0.00 & 0.00 & 52.18 & 47.66 & 0.00 \\
\hline & & \multicolumn{8}{|c|}{ Level of theory: mPW1PW91/6-311G** (solution, PCM) } \\
\hline & & 79a & $79 b$ & 79c & 79d & $79 e$ & $79 f$ & $79 \mathrm{~g}$ & $79 \mathrm{~h}$ \\
\hline \multirow{3}{*}{$s \mathrm{DP} 4+$} & ${ }^{1} \mathrm{H}$ data & 1.07 & 33.62 & 7.13 & 0.94 & 1.67 & 15.75 & 38.30 & 1.52 \\
\hline & ${ }^{13} \mathrm{C}$ data & 0.00 & 0.12 & 0.02 & 0.19 & 1.59 & 51.14 & 45.48 & 1.46 \\
\hline & All data & 0.00 & 0.16 & 0.00 & 0.01 & 0.10 & 31.51 & 68.13 & 0.09 \\
\hline \multirow{3}{*}{ uDP4+ } & ${ }^{1} \mathrm{H}$ data & 2.87 & 20.50 & 6.74 & 3.71 & 4.33 & 45.29 & 12.45 & 4.10 \\
\hline & ${ }^{13} \mathrm{C}$ data & 0.00 & 0.58 & 0.09 & 0.10 & 0.39 & 54.06 & 44.61 & 0.18 \\
\hline & All data & 0.00 & 0.39 & 0.02 & 0.01 & 0.06 & 81.10 & 18.40 & 0.02 \\
\hline \multirow{3}{*}{ DP4+ } & ${ }^{1} \mathrm{H}$ data & 0.16 & 35.39 & 2.47 & 0.18 & 0.37 & 36.63 & 24.49 & 0.32 \\
\hline & ${ }^{13} \mathrm{C}$ data & 0.00 & 0.00 & 0.00 & 0.00 & 0.01 & 57.67 & 42.31 & 0.01 \\
\hline & All data & 0.00 & 0.00 & 0.00 & 0.00 & 0.00 & 67.09 & 32.91 & 0.00 \\
\hline & & & & of the & IPW1PV & $311+G$ & lution, $\mathrm{P}$ & & \\
\hline & & 79a & $79 b$ & 79c & $79 d$ & $79 e$ & $79 f$ & $79 \mathrm{~g}$ & $79 \mathrm{~h}$ \\
\hline & ${ }^{1} \mathrm{H}$ data & 0.28 & 0.90 & 0.51 & 0.09 & 1.38 & 12.96 & 80.44 & 3.44 \\
\hline sDP4+ & ${ }^{13} \mathrm{C}$ data & 0.00 & 0.51 & 0.04 & 0.13 & 2.29 & 48.01 & 47.75 & 1.27 \\
\hline & All data & 0.00 & 0.01 & 0.00 & 0.00 & 0.07 & 13.91 & 85.91 & 0.10 \\
\hline & ${ }^{1} \mathrm{H}$ data & 3.53 & 3.90 & 4.61 & 2.68 & 10.44 & 37.53 & 21.56 & 15.75 \\
\hline uDP4+ & ${ }^{13} \mathrm{C}$ data & 0.00 & 3.96 & 0.27 & 0.22 & 1.08 & 51.82 & 42.55 & 0.10 \\
\hline & All data & 0.00 & 0.53 & 0.04 & 0.02 & 0.39 & 67.24 & 31.72 & 0.06 \\
\hline & ${ }^{1} \mathrm{H}$ data & 0.04 & 0.15 & 0.10 & 0.01 & 0.63 & 21.18 & 75.52 & 2.36 \\
\hline DP4+ & ${ }^{13} \mathrm{C}$ data & 0.00 & 0.04 & 0.00 & 0.00 & 0.05 & 54.98 & 44.91 & 0.00 \\
\hline & All data & 0.00 & 0.00 & 0.00 & 0.00 & 0.00 & 25.56 & 74.44 & 0.00 \\
\hline
\end{tabular}


sDP4+, $u$ DP4+ and DP4+ probabilities (\%) for compound 79g<smiles>CC[C@H](C)[C@H]1OC([C@H](C)CC)=C(C)C(=O)[C@H]1C</smiles><smiles>CC[C@H](C)[C@H]1OC([C@H](C)[C@H](O)CC)=C(C)C(=O)[C@H]1C</smiles><smiles>CC[C@H](O)[C@@H](C)C1=C(C)C(=O)[C@@H](C)[C@@H]([C@@H](C)CC)O1</smiles><smiles>CC[C@H](O)[C@@H](C)C1=C(C)C(=O)[C@@H](C)[C@@H]([C@@H](C)CC)O1</smiles><smiles>CC[C@H](O)[C@H](C)C1=C(C)C(=O)[C@@H](C)[C@@H]([C@@H](C)CC)O1</smiles><smiles>CC[C@H](O)[C@H](C)C1=C(C)C(=O)[C@H](C)[C@H]([C@H](C)C=O)O1</smiles><smiles>CC[C@H](O)[C@@H](C)C1=C(C)C(=O)[C@@H](C)[C@@H]([C@@H](C)CC)O1</smiles><smiles>CC[C@H](O)[C@@H](C)C1=C(C)C(=O)[C@@H](C)[C@@H]([C@@H](C)CC)O1</smiles>

\begin{tabular}{|c|c|c|c|c|c|c|c|c|c|}
\hline & & \multicolumn{8}{|c|}{ Level of theory: B3LYP/6-31G* (gas phase) } \\
\hline & & 79a & $79 b$ & $79 c$ & 79d & $79 e$ & $79 f$ & $79 \mathrm{~g}$ & $79 \mathrm{~h}$ \\
\hline & ${ }^{1}$ H data & 1.01 & 82.06 & 4.00 & 12.59 & 0.03 & 0.14 & 0.15 & 0.03 \\
\hline \multirow[t]{3}{*}{ sDP4+ } & ${ }^{13}$ C data & 0.00 & 0.00 & 0.00 & 0.01 & 29.46 & 57.23 & 7.72 & 5.58 \\
\hline & All data & 0.00 & 0.92 & 0.03 & 0.66 & 8.07 & 77.75 & 11.06 & 1.51 \\
\hline & ${ }^{1}$ H data & 0.43 & 68.56 & 17.38 & 11.52 & 0.08 & 1.16 & 0.79 & 0.08 \\
\hline \multirow[t]{3}{*}{ uDP4+ } & ${ }^{13} \mathrm{C}$ data & 0.00 & 0.01 & 0.02 & 0.05 & 19.57 & 23.66 & 40.39 & 16.30 \\
\hline & All data & 0.00 & 0.66 & 0.53 & 0.88 & 2.51 & 43.10 & 50.24 & 2.07 \\
\hline & ${ }^{1} \mathrm{H}$ data & 0.01 & 96.31 & 1.19 & 2.48 & 0.00 & 0.00 & 0.00 & 0.00 \\
\hline \multirow[t]{5}{*}{ DP4+ } & ${ }^{13} \mathrm{C}$ data & 0.00 & 0.00 & 0.00 & 0.00 & 24.70 & 58.04 & 13.36 & 3.90 \\
\hline & All data & 0.00 & 0.02 & 0.00 & 0.01 & 0.52 & 85.24 & 14.13 & 0.08 \\
\hline & & \multicolumn{8}{|c|}{ Level of theory: B3LYP/6-31G** (gas phase) } \\
\hline & & $79 a$ & $79 b$ & $79 c$ & 79d & $79 e$ & $79 f$ & $79 \mathrm{~g}$ & $79 \mathrm{~h}$ \\
\hline & ${ }^{1} \mathrm{H}$ data & 0.78 & 89.15 & 5.55 & 3.64 & 0.06 & 0.18 & 0.61 & 0.05 \\
\hline \multirow[t]{3}{*}{ sDP4+ } & ${ }^{13} \mathrm{C}$ data & 0.00 & 0.00 & 0.00 & 0.01 & 34.44 & 53.55 & 6.62 & 5.38 \\
\hline & All data & 0.00 & 1.29 & 0.05 & 0.13 & 12.95 & 59.10 & 24.93 & 1.54 \\
\hline & ${ }^{1}$ H data & 0.56 & 80.69 & 11.02 & 5.66 & 0.10 & 1.38 & 0.52 & 0.07 \\
\hline \multirow[t]{3}{*}{ uDP4+ } & ${ }^{13} \mathrm{C}$ data & 0.00 & 0.01 & 0.01 & 0.03 & 13.52 & 46.66 & 33.18 & 6.59 \\
\hline & All data & 0.00 & 0.88 & 0.16 & 0.20 & 1.58 & 76.09 & 20.55 & 0.54 \\
\hline & ${ }^{1} \mathrm{H}$ data & 0.01 & 98.86 & 0.84 & 0.28 & 0.00 & 0.00 & 0.00 & 0.00 \\
\hline \multirow[t]{5}{*}{ DP4+ } & ${ }^{13} \mathrm{C}$ data & 0.00 & 0.00 & 0.00 & 0.00 & 14.47 & 77.61 & 6.82 & 1.10 \\
\hline & All data & 0.00 & 0.02 & 0.00 & 0.00 & 0.41 & 89.37 & 10.18 & 0.02 \\
\hline & & \multicolumn{8}{|c|}{ Level of theory: B3LYP/6-31+G** (gas phase) } \\
\hline & & $79 a$ & $79 b$ & 79c & $79 d$ & $79 e$ & $79 f$ & $79 \mathrm{~g}$ & $79 \mathrm{~h}$ \\
\hline & ${ }^{1} \mathrm{H}$ data & 10.18 & 56.11 & 11.62 & 2.33 & 1.26 & 0.95 & 17.05 & 0.49 \\
\hline \multirow[t]{2}{*}{ sDP4+ } & ${ }^{13} \mathrm{C}$ data & 0.00 & 0.03 & 0.03 & 0.03 & 9.52 & 83.27 & 6.59 & 0.53 \\
\hline & All data & 0.00 & 0.70 & 0.16 & 0.04 & 5.84 & 38.44 & 54.68 & 0.13 \\
\hline \multirow{3}{*}{ uDP4+ } & ${ }^{1}$ H data & 5.11 & 48.91 & 20.80 & 4.62 & 2.01 & 9.70 & 8.24 & 0.61 \\
\hline & ${ }^{13} \mathrm{C}$ data & 0.00 & 0.06 & 0.10 & 0.04 & 4.00 & 83.83 & 11.64 & 0.33 \\
\hline & All data & 0.00 & 0.31 & 0.22 & 0.02 & 0.87 & 88.15 & 10.40 & 0.02 \\
\hline \multirow{5}{*}{ DP4+ } & ${ }^{1} \mathrm{H}$ data & 1.62 & 85.73 & 7.55 & 0.34 & 0.08 & 0.29 & 4.39 & 0.01 \\
\hline & ${ }^{13} \mathrm{C}$ data & 0.00 & 0.00 & 0.00 & 0.00 & 0.54 & 98.38 & 1.08 & 0.00 \\
\hline & All data & 0.00 & 0.01 & 0.00 & 0.00 & 0.13 & 85.51 & 14.35 & 0.00 \\
\hline & & \multicolumn{8}{|c|}{ Level of theory: B3LYP/6-311G* (gas phase) } \\
\hline & & $79 a$ & $79 b$ & 79c & 79d & $79 e$ & $79 f$ & $79 \mathrm{~g}$ & $79 \mathrm{~h}$ \\
\hline \multirow{3}{*}{ sDP4+ } & ${ }^{1} \mathrm{H}$ data & 0.51 & 89.17 & 1.93 & 2.05 & 0.22 & 3.89 & 2.11 & 0.12 \\
\hline & ${ }^{13} \mathrm{C}$ data & 0.00 & 0.02 & 0.01 & 0.05 & 17.06 & 67.29 & 12.00 & 3.57 \\
\hline & All data & 0.00 & 0.70 & 0.00 & 0.03 & 1.30 & 89.21 & 8.61 & 0.14 \\
\hline \multirow{2}{*}{ uDP4+ } & ${ }^{1} \mathrm{H}$ data & 2.02 & 74.06 & 3.07 & 2.53 & 0.64 & 15.13 & 2.27 & 0.28 \\
\hline & ${ }^{13} \mathrm{C}$ data & 0.00 & 0.29 & 0.05 & 0.23 & 2.84 & 81.91 & 13.89 & 0.78 \\
\hline
\end{tabular}




\begin{tabular}{|c|c|c|c|c|c|c|c|c|c|}
\hline & All data & 0.00 & 1.68 & 0.01 & 0.04 & 0.14 & 95.67 & 2.44 & 0.02 \\
\hline & ${ }^{1} \mathrm{H}$ data & 0.02 & 98.86 & 0.09 & 0.08 & 0.00 & 0.88 & 0.07 & 0.00 \\
\hline \multirow[t]{5}{*}{ DP4+ } & ${ }^{13} \mathrm{C}$ data & 0.00 & 0.00 & 0.00 & 0.00 & 0.85 & 96.19 & 2.91 & 0.05 \\
\hline & All data & 0.00 & 0.01 & 0.00 & 0.00 & 0.00 & 99.74 & 0.25 & 0.00 \\
\hline & & \multicolumn{8}{|c|}{ Level of theory: B3LYP/6-311G** (gas phase) } \\
\hline & & $79 a$ & $79 \mathrm{~b}$ & $79 \mathrm{c}$ & 79d & $79 \mathrm{e}$ & $79 f$ & $79 \mathrm{~g}$ & $79 \mathrm{~h}$ \\
\hline & ${ }^{1} \mathrm{H}$ data & 1.21 & 48.80 & 7.06 & 0.50 & 0.97 & 10.18 & 30.48 & 0.79 \\
\hline \multirow[t]{2}{*}{ sDP4+ } & ${ }^{13} \mathrm{C}$ data & 0.00 & 0.02 & 0.01 & 0.04 & 14.11 & 73.06 & 10.00 & 2.77 \\
\hline & All data & 0.00 & 0.09 & 0.00 & 0.00 & 1.29 & 69.81 & 28.60 & 0.21 \\
\hline & ${ }^{1} \mathrm{H}$ data & 3.24 & 31.33 & 3.39 & 1.34 & 1.92 & 52.42 & 5.33 & 1.03 \\
\hline \multirow[t]{2}{*}{ uDP4+ } & ${ }^{13} \mathrm{C}$ data & 0.00 & 0.32 & 0.05 & 0.25 & 1.23 & 83.23 & 14.57 & 0.36 \\
\hline & All data & 0.00 & 0.22 & 0.00 & 0.01 & 0.05 & 97.96 & 1.74 & 0.01 \\
\hline \multirow{6}{*}{ DP4+ } & ${ }^{1} \mathrm{H}$ data & 0.17 & 67.77 & 1.06 & 0.03 & 0.08 & 23.65 & 7.19 & 0.04 \\
\hline & ${ }^{13} \mathrm{C}$ data & 0.00 & 0.00 & 0.00 & 0.00 & 0.28 & 97.37 & 2.33 & 0.02 \\
\hline & All data & 0.00 & 0.00 & 0.00 & 0.00 & 0.00 & 99.28 & 0.72 & 0.00 \\
\hline & & \multicolumn{8}{|c|}{ Level of theory: B3LYP/6-311+G** (gas phase) } \\
\hline & & 79a & $79 b$ & 79c & 79d & $79 e$ & $79 f$ & $79 \mathrm{~g}$ & $79 \mathrm{~h}$ \\
\hline & ${ }^{1} \mathrm{H}$ data & 4.53 & 22.63 & 6.86 & 0.84 & 0.80 & 5.55 & 57.21 & 1.58 \\
\hline \multirow[t]{2}{*}{$s \mathrm{DP} 4+$} & ${ }^{13} \mathrm{C}$ data & 0.00 & 0.06 & 0.00 & 0.02 & 15.17 & 69.60 & 13.46 & 1.68 \\
\hline & All data & 0.00 & 0.11 & 0.00 & 0.00 & 1.04 & 32.95 & 65.67 & 0.23 \\
\hline \multirow{3}{*}{ uDP4+ } & ${ }^{1} \mathrm{H}$ data & 13.07 & 15.23 & 5.26 & 5.04 & 4.48 & 42.48 & 8.65 & 5.77 \\
\hline & ${ }^{13} \mathrm{C}$ data & 0.00 & 1.10 & 0.06 & 0.22 & 1.79 & 80.76 & 15.87 & 0.18 \\
\hline & All data & 0.00 & 0.47 & 0.01 & 0.03 & 0.22 & 95.42 & 3.82 & 0.03 \\
\hline \multirow{5}{*}{ DP4+ } & ${ }^{1} \mathrm{H}$ data & 4.98 & 29.03 & 3.04 & 0.36 & 0.30 & 19.86 & 41.65 & 0.77 \\
\hline & ${ }^{13} \mathrm{C}$ data & 0.00 & 0.00 & 0.00 & 0.00 & 0.46 & 95.88 & 3.65 & 0.01 \\
\hline & All data & 0.00 & 0.00 & 0.00 & 0.00 & 0.01 & 92.61 & 7.38 & 0.00 \\
\hline & & \multicolumn{8}{|c|}{ Level of theory: B3LYP/6-31G* (solution, PCM) } \\
\hline & & $79 a$ & $79 b$ & $79 \mathrm{c}$ & 79d & $79 e$ & $79 f$ & $79 \mathrm{~g}$ & $79 \mathrm{~h}$ \\
\hline & ${ }^{1} \mathrm{H}$ data & 3.94 & 91.14 & 2.02 & 2.09 & 0.03 & 0.47 & 0.28 & 0.03 \\
\hline \multirow[t]{2}{*}{ sDP4+ } & ${ }^{13} \mathrm{C}$ data & 0.00 & 0.00 & 0.00 & 0.00 & 5.04 & 84.50 & 9.81 & 0.65 \\
\hline & All data & 0.00 & 0.13 & 0.00 & 0.01 & 0.36 & 92.95 & 6.49 & 0.05 \\
\hline \multirow{3}{*}{ uDP4+ } & ${ }^{1} \mathrm{H}$ data & 0.68 & 83.29 & 7.75 & 3.57 & 0.08 & 2.50 & 2.01 & 0.11 \\
\hline & ${ }^{13} \mathrm{C}$ data & 0.00 & 0.01 & 0.02 & 0.04 & 16.50 & 27.36 & 45.13 & 10.93 \\
\hline & All data & 0.00 & 0.36 & 0.10 & 0.09 & 0.86 & 42.10 & 55.72 & 0.77 \\
\hline \multirow{6}{*}{ DP4+ } & ${ }^{1} \mathrm{H}$ data & 0.04 & 99.64 & 0.21 & 0.10 & 0.00 & 0.02 & 0.01 & 0.00 \\
\hline & ${ }^{13} \mathrm{C}$ data & 0.00 & 0.00 & 0.00 & 0.00 & 2.92 & 81.26 & 15.56 & 0.25 \\
\hline & All data & 0.00 & 0.00 & 0.00 & 0.00 & 0.01 & 91.53 & 8.46 & 0.00 \\
\hline & & \multicolumn{8}{|c|}{ Level of theory: B3LYP/6-31G** (solution, PCM) } \\
\hline & & $79 a$ & $79 b$ & 79c & $79 d$ & $79 e$ & $79 f$ & $79 \mathrm{~g}$ & $79 \mathrm{~h}$ \\
\hline & ${ }^{1} \mathrm{H}$ data & 4.77 & 87.37 & 3.29 & 1.05 & 0.14 & 1.25 & 2.04 & 0.08 \\
\hline \multirow[t]{2}{*}{ sDP4+ } & ${ }^{13} \mathrm{C}$ data & 0.00 & 0.00 & 0.00 & 0.00 & 9.18 & 80.57 & 8.94 & 1.30 \\
\hline & All data & 0.00 & 0.19 & 0.00 & 0.00 & 1.07 & 83.55 & 15.09 & 0.09 \\
\hline & ${ }^{1} \mathrm{H}$ data & 0.97 & 87.88 & 4.52 & 1.85 & 0.10 & 2.87 & 1.69 & 0.12 \\
\hline \multirow[t]{2}{*}{ uDP4+ } & ${ }^{13} \mathrm{C}$ data & 0.00 & 0.01 & 0.02 & 0.03 & 10.03 & 55.17 & 30.60 & 4.14 \\
\hline & All data & 0.00 & 0.56 & 0.04 & 0.02 & 0.47 & 74.45 & 24.22 & 0.24 \\
\hline & ${ }^{1} \mathrm{H}$ data & 0.06 & 99.63 & 0.19 & 0.03 & 0.00 & 0.05 & 0.04 & 0.00 \\
\hline DP4+ & ${ }^{13} \mathrm{C}$ data & 0.00 & 0.00 & 0.00 & 0.00 & 1.91 & 92.30 & 5.68 & 0.11 \\
\hline & All data & 0.00 & 0.00 & 0.00 & 0.00 & 0.01 & 94.44 & 5.55 & 0.00 \\
\hline & & & & Level of & : B3LYI & $+\mathrm{G}^{* *}($ & on, PCM & & \\
\hline & & 79a & $79 \mathrm{~b}$ & 79c & 79d & $79 \mathrm{e}$ & $79 f$ & $79 \mathrm{~g}$ & $79 \mathrm{~h}$ \\
\hline & ${ }^{1} \mathrm{H}$ data & 18.78 & 25.48 & 7.09 & 0.19 & 0.52 & 20.46 & 27.26 & 0.23 \\
\hline sDP4+ & ${ }^{13} \mathrm{C}$ data & 0.00 & 0.05 & 0.04 & 0.03 & 9.24 & 78.46 & 11.81 & 0.36 \\
\hline & All data & 0.00 & 0.07 & 0.02 & 0.00 & 0.25 & 83.01 & 16.65 & 0.00 \\
\hline & ${ }^{1} \mathrm{H}$ data & 19.63 & 42.74 & 16.84 & 0.53 & 1.24 & 0.00 & 18.40 & 0.62 \\
\hline uDP4+ & ${ }^{13} \mathrm{C}$ data & 0.02 & 0.96 & 1.25 & 0.38 & 18.23 & 0.00 & 77.70 & 1.46 \\
\hline & All data & 0.03 & 2.70 & 1.38 & 0.01 & 1.49 & 0.00 & 94.32 & 0.06 \\
\hline
\end{tabular}




\begin{tabular}{|c|c|c|c|c|c|c|c|c|c|}
\hline & ${ }^{1} \mathrm{H}$ data & 17.73 & 52.37 & 5.74 & 0.00 & 0.03 & 0.00 & 24.12 & 0.01 \\
\hline \multirow[t]{5}{*}{ DP4+ } & ${ }^{13} \mathrm{C}$ data & 0.00 & 0.00 & 0.01 & 0.00 & 15.49 & 0.00 & 84.45 & 0.05 \\
\hline & All data & 0.00 & 0.01 & 0.00 & 0.00 & 0.02 & 0.00 & 99.96 & 0.00 \\
\hline & & \multicolumn{8}{|c|}{ Level of theory: B3LYP/6-311G* (solution, PCM) } \\
\hline & & $79 a$ & $79 \mathrm{~b}$ & $79 c$ & 79d & $79 e$ & $79 f$ & $79 \mathrm{~g}$ & $79 \mathrm{~h}$ \\
\hline & ${ }^{1} \mathrm{H}$ data & 1.60 & 86.77 & 2.74 & 0.18 & 0.13 & 6.42 & 2.09 & 0.06 \\
\hline \multirow[t]{2}{*}{ sDP4+ } & ${ }^{13} \mathrm{C}$ data & 0.00 & 0.09 & 0.02 & 0.09 & 11.95 & 58.88 & 26.68 & 2.29 \\
\hline & All data & 0.00 & 1.77 & 0.01 & 0.00 & 0.35 & 85.25 & 12.58 & 0.03 \\
\hline & ${ }^{1} \mathrm{H}$ data & 3.04 & 85.09 & 3.11 & 0.41 & 0.24 & 6.50 & 1.50 & 0.11 \\
\hline \multirow[t]{2}{*}{ uDP4+ } & ${ }^{13} \mathrm{C}$ data & 0.00 & 1.40 & 0.22 & 0.42 & 2.00 & 66.56 & 29.02 & 0.37 \\
\hline & All data & 0.00 & 19.99 & 0.12 & 0.03 & 0.08 & 72.50 & 7.28 & 0.01 \\
\hline \multirow{5}{*}{ DP4+ } & ${ }^{1} \mathrm{H}$ data & 0.07 & 99.22 & 0.11 & 0.00 & 0.00 & 0.56 & 0.04 & 0.00 \\
\hline & ${ }^{13} \mathrm{C}$ data & 0.00 & 0.00 & 0.00 & 0.00 & 0.51 & 83.06 & 16.41 & 0.02 \\
\hline & All data & 0.00 & 0.56 & 0.00 & 0.00 & 0.00 & 97.99 & 1.45 & 0.00 \\
\hline & & \multicolumn{8}{|c|}{ Level of theory: B3LYP/6-311G** (solution, PCM) } \\
\hline & & $79 a$ & $79 b$ & $79 c$ & 79d & $79 e$ & $79 f$ & $79 \mathrm{~g}$ & $79 \mathrm{~h}$ \\
\hline & ${ }^{1} \mathrm{H}$ data & 3.84 & 41.30 & 5.30 & 0.06 & 0.61 & 16.38 & 31.99 & 0.52 \\
\hline \multirow[t]{2}{*}{ sDP4+ } & ${ }^{13} \mathrm{C}$ data & 0.00 & 0.07 & 0.02 & 0.08 & 10.12 & 64.92 & 23.01 & 1.79 \\
\hline & All data & 0.00 & 0.16 & 0.01 & 0.00 & 0.34 & 58.76 & 40.68 & 0.05 \\
\hline \multirow{3}{*}{ uDP4+ } & ${ }^{1} \mathrm{H}$ data & 7.06 & 31.43 & 3.77 & 0.11 & 0.94 & 49.83 & 6.11 & 0.76 \\
\hline & ${ }^{13} \mathrm{C}$ data & 0.00 & 1.17 & 0.19 & 0.33 & 1.13 & 68.88 & 28.06 & 0.24 \\
\hline & All data & 0.00 & 1.01 & 0.02 & 0.00 & 0.03 & 94.23 & 4.71 & 0.01 \\
\hline & ${ }^{1} \mathrm{H}$ data & 1.15 & 55.05 & 0.85 & 0.00 & 0.02 & 34.62 & 8.29 & 0.02 \\
\hline \multirow[t]{4}{*}{ DP4+ } & ${ }^{13} \mathrm{C}$ data & 0.00 & 0.00 & 0.00 & 0.00 & 0.22 & 87.18 & 12.59 & 0.01 \\
\hline & All data & 0.00 & 0.00 & 0.00 & 0.00 & 0.00 & 96.66 & 3.34 & 0.00 \\
\hline & & \multicolumn{8}{|c|}{ Level of theory: B3LYP/6-311+G** (solution, PCM) } \\
\hline & & $79 a$ & $79 b$ & $79 c$ & $79 d$ & $79 e$ & $79 f$ & $79 \mathrm{~g}$ & $79 \mathrm{~h}$ \\
\hline & ${ }^{1} \mathrm{H}$ data & 6.61 & 13.00 & 4.40 & 0.17 & 0.53 & 13.43 & 60.36 & 1.52 \\
\hline \multirow[t]{2}{*}{ sDP4+ } & ${ }^{13} \mathrm{C}$ data & 0.00 & 0.24 & 0.03 & 0.06 & 11.24 & 58.71 & 28.14 & 1.58 \\
\hline & All data & 0.00 & 0.13 & 0.00 & 0.00 & 0.24 & 31.55 & 67.98 & 0.10 \\
\hline \multirow{3}{*}{ uDP4+ } & ${ }^{1} \mathrm{H}$ data & 21.18 & 11.55 & 5.31 & 0.34 & 1.45 & 45.45 & 12.02 & 2.70 \\
\hline & ${ }^{13} \mathrm{C}$ data & 0.02 & 11.00 & 0.82 & 0.95 & 3.42 & 59.17 & 24.47 & 0.15 \\
\hline & All data & 0.02 & 4.07 & 0.14 & 0.01 & 0.16 & 86.17 & 9.42 & 0.01 \\
\hline \multirow{6}{*}{ DP4+ } & ${ }^{1} \mathrm{H}$ data & 8.46 & 9.08 & 1.41 & 0.00 & 0.05 & 36.90 & 43.85 & 0.25 \\
\hline & ${ }^{13} \mathrm{C}$ data & 0.00 & 0.06 & 0.00 & 0.00 & 0.92 & 82.63 & 16.38 & 0.01 \\
\hline & All data & 0.00 & 0.02 & 0.00 & 0.00 & 0.00 & 80.92 & 19.07 & 0.00 \\
\hline & & \multicolumn{8}{|c|}{ Level of theory: mPW1PW91/6-31G* (gas phase) } \\
\hline & & 79a & $79 b$ & $79 c$ & 79d & $79 e$ & $79 f$ & $79 \mathrm{~g}$ & $79 \mathrm{~h}$ \\
\hline & ${ }^{1} \mathrm{H}$ data & 0.80 & 74.70 & 1.23 & 23.12 & 0.02 & 0.04 & 0.07 & 0.03 \\
\hline \multirow[t]{2}{*}{$s \mathrm{DP} 4+$} & ${ }^{13} \mathrm{C}$ data & 0.00 & 0.00 & 0.00 & 0.01 & 24.10 & 46.51 & 24.49 & 4.88 \\
\hline & All data & 0.00 & 1.99 & 0.02 & 3.04 & 11.03 & 43.39 & 37.57 & 2.96 \\
\hline & ${ }^{1} \mathrm{H}$ data & 0.41 & 60.59 & 7.43 & 30.49 & 0.06 & 0.41 & 0.51 & 0.09 \\
\hline \multirow[t]{2}{*}{ uDP4+ } & ${ }^{13} \mathrm{C}$ data & 0.01 & 0.01 & 0.03 & 0.06 & 19.07 & 11.70 & 37.85 & 31.28 \\
\hline & All data & 0.01 & 1.52 & 0.67 & 5.58 & 3.87 & 15.83 & 63.49 & 9.02 \\
\hline & ${ }^{1} \mathrm{H}$ data & 0.01 & 86.37 & 0.17 & 13.45 & 0.00 & 0.00 & 0.00 & 0.00 \\
\hline DP4+ & ${ }^{13} \mathrm{C}$ data & 0.00 & 0.00 & 0.00 & 0.00 & 22.06 & 26.12 & 44.49 & 7.33 \\
\hline & All data & 0.00 & 0.10 & 0.00 & 0.54 & 1.35 & 21.72 & 75.45 & 0.84 \\
\hline & & & & vel of & : $\mathrm{mPW}$ & $1 / 6-31 G$ & as phas & & \\
\hline & & 79a & $79 b$ & $79 c$ & $79 d$ & $79 e$ & $79 f$ & $79 \mathrm{~g}$ & $79 \mathrm{~h}$ \\
\hline & ${ }^{1} \mathrm{H}$ data & 1.03 & 89.36 & 1.79 & 7.21 & 0.06 & 0.08 & 0.42 & 0.06 \\
\hline sDP4+ & ${ }^{13} \mathrm{C}$ data & 0.00 & 0.00 & 0.00 & 0.01 & 34.77 & 47.28 & 12.72 & 5.23 \\
\hline & All data & 0.00 & 1.38 & 0.02 & 0.32 & 18.09 & 31.66 & 45.68 & 2.85 \\
\hline & ${ }^{1} \mathrm{H}$ data & 0.76 & 77.23 & 6.49 & 14.30 & 0.10 & 0.65 & 0.39 & 0.08 \\
\hline uDP4+ & ${ }^{13} \mathrm{C}$ data & 0.00 & 0.01 & 0.02 & 0.03 & 13.25 & 26.35 & 49.59 & 10.75 \\
\hline & All data & 0.00 & 1.53 & 0.29 & 1.24 & 3.28 & 43.28 & 48.19 & 2.19 \\
\hline DP4+ & ${ }^{1} \mathrm{H}$ data & 0.01 & 98.35 & 0.17 & 1.47 & 0.00 & 0.00 & 0.00 & 0.00 \\
\hline
\end{tabular}




\begin{tabular}{|c|c|c|c|c|c|c|c|c|c|}
\hline & ${ }^{13} \mathrm{C}$ data & 0.00 & 0.00 & 0.00 & 0.00 & 19.25 & 52.05 & 26.35 & 2.35 \\
\hline & All data & 0.00 & 0.06 & 0.00 & 0.01 & 1.63 & 37.65 & 60.49 & 0.17 \\
\hline & & \multicolumn{8}{|c|}{ Level of theory: mPW1PW91/6-31+G** (gas phase) } \\
\hline & & $79 a$ & $79 b$ & $79 c$ & 79d & $79 \mathrm{e}$ & $79 f$ & $79 \mathrm{~g}$ & $79 \mathrm{~h}$ \\
\hline \multirow{3}{*}{ sDP4+ } & ${ }^{1} \mathrm{H}$ data & 13.62 & 55.20 & 6.65 & 4.78 & 1.69 & 1.37 & 15.94 & 0.75 \\
\hline & ${ }^{13} \mathrm{C}$ data & 0.00 & 0.00 & 0.00 & 0.01 & 8.80 & 88.01 & 3.02 & 0.16 \\
\hline & All data & 0.00 & 0.07 & 0.01 & 0.01 & 8.06 & 65.60 & 26.17 & 0.07 \\
\hline \multirow{3}{*}{ uDP4+ } & ${ }^{1} \mathrm{H}$ data & 10.28 & 49.74 & 11.45 & 8.87 & 2.60 & 9.23 & 6.67 & 1.15 \\
\hline & ${ }^{13} \mathrm{C}$ data & 0.00 & 0.03 & 0.06 & 0.02 & 6.40 & 66.95 & 25.87 & 0.66 \\
\hline & All data & 0.00 & 0.20 & 0.09 & 0.03 & 2.05 & 76.23 & 21.31 & 0.09 \\
\hline \multirow{3}{*}{ DP4+ } & ${ }^{1} \mathrm{H}$ data & 4.48 & 87.76 & 2.44 & 1.35 & 0.14 & 0.40 & 3.40 & 0.03 \\
\hline & ${ }^{13} \mathrm{C}$ data & 0.00 & 0.00 & 0.00 & 0.00 & 0.93 & 97.77 & 1.30 & 0.00 \\
\hline & All data & 0.00 & 0.00 & 0.00 & 0.00 & 0.30 & 89.70 & 10.00 & 0.00 \\
\hline & & \multicolumn{8}{|c|}{ Level of theory: mPW1PW91/6-311G* (gas phase) } \\
\hline & & 79a & $79 b$ & $79 c$ & 79d & $79 e$ & $79 f$ & $79 \mathrm{~g}$ & $79 \mathrm{~h}$ \\
\hline \multirow{3}{*}{ sDP4+ } & ${ }^{1} \mathrm{H}$ data & 0.54 & 93.79 & 1.21 & 2.31 & 0.10 & 1.17 & 0.82 & 0.06 \\
\hline & ${ }^{13} \mathrm{C}$ data & 0.00 & 0.03 & 0.01 & 0.03 & 26.31 & 51.26 & 16.99 & 5.36 \\
\hline & All data & 0.00 & 3.01 & 0.01 & 0.10 & 3.38 & 75.53 & 17.55 & 0.41 \\
\hline \multirow{3}{*}{ uDP4+ } & ${ }^{1} \mathrm{H}$ data & 2.90 & 82.18 & 2.93 & 4.43 & 0.52 & 5.17 & 1.58 & 0.28 \\
\hline & ${ }^{13} \mathrm{C}$ data & 0.00 & 0.10 & 0.02 & 0.09 & 2.07 & 69.99 & 26.90 & 0.83 \\
\hline & All data & 0.00 & 1.92 & 0.01 & 0.10 & 0.26 & 87.35 & 10.29 & 0.06 \\
\hline \multirow{3}{*}{ DP4+ } & ${ }^{1} \mathrm{H}$ data & 0.02 & 99.71 & 0.05 & 0.13 & 0.00 & 0.08 & 0.02 & 0.00 \\
\hline & ${ }^{13} \mathrm{C}$ data & 0.00 & 0.00 & 0.00 & 0.00 & 1.33 & 87.43 & 11.14 & 0.11 \\
\hline & All data & 0.00 & 0.09 & 0.00 & 0.00 & 0.01 & 97.24 & 2.66 & 0.00 \\
\hline & & \multicolumn{8}{|c|}{ Level of theory: mPW1PW91/6-311G** (gas phase) } \\
\hline & & $79 a$ & $79 b$ & $79 c$ & 79d & $79 \mathrm{e}$ & $79 f$ & $79 \mathrm{~g}$ & 79h \\
\hline \multirow{3}{*}{ sDP4+ } & ${ }^{1} \mathrm{H}$ data & 1.78 & 58.38 & 5.68 & 0.81 & 0.79 & 5.52 & 26.22 & 0.82 \\
\hline & ${ }^{13} \mathrm{C}$ data & 0.00 & 0.02 & 0.00 & 0.03 & 19.71 & 60.64 & 15.76 & 3.84 \\
\hline & All data & 0.00 & 0.15 & 0.00 & 0.00 & 2.03 & 43.61 & 53.79 & 0.41 \\
\hline \multirow{3}{*}{ uDP4+ } & ${ }^{1} \mathrm{H}$ data & 5.61 & 41.21 & 3.08 & 3.02 & 2.33 & 38.74 & 4.47 & 1.54 \\
\hline & ${ }^{13} \mathrm{C}$ data & 0.00 & 0.16 & 0.03 & 0.10 & 0.69 & 68.09 & 30.58 & 0.36 \\
\hline & All data & 0.00 & 0.23 & 0.00 & 0.01 & 0.06 & 94.77 & 4.91 & 0.02 \\
\hline \multirow{5}{*}{ DP4+ } & ${ }^{1} \mathrm{H}$ data & 0.36 & 86.85 & 0.63 & 0.09 & 0.07 & 7.72 & 4.23 & 0.05 \\
\hline & ${ }^{13} \mathrm{C}$ data & 0.00 & 0.00 & 0.00 & 0.00 & 0.29 & 89.26 & 10.42 & 0.03 \\
\hline & All data & 0.00 & 0.00 & 0.00 & 0.00 & 0.00 & 93.99 & 6.01 & 0.00 \\
\hline & & \multicolumn{8}{|c|}{ Level of theory: mPW1PW91/6-311+G** (gas phase) } \\
\hline & & $79 a$ & $79 b$ & $79 c$ & 79d & $79 \mathrm{e}$ & $79 f$ & $79 \mathrm{~g}$ & $79 \mathrm{~h}$ \\
\hline \multirow{3}{*}{ sDP4+ } & ${ }^{1} \mathrm{H}$ data & 8.62 & 32.19 & 10.12 & 1.89 & 0.88 & 2.97 & 41.60 & 1.72 \\
\hline & ${ }^{13} \mathrm{C}$ data & 0.00 & 0.05 & 0.00 & 0.01 & 11.85 & 63.92 & 21.99 & 2.18 \\
\hline & All data & 0.00 & 0.13 & 0.00 & 0.00 & 0.93 & 16.93 & 81.66 & 0.33 \\
\hline \multirow{3}{*}{ uDP4+ } & ${ }^{1} \mathrm{H}$ data & 22.38 & 23.96 & 5.90 & 12.86 & 4.54 & 19.49 & 5.21 & 5.67 \\
\hline & ${ }^{13} \mathrm{C}$ data & 0.00 & 0.41 & 0.03 & 0.05 & 0.89 & 66.38 & 32.05 & 0.18 \\
\hline & All data & 0.00 & 0.67 & 0.01 & 0.05 & 0.27 & 87.61 & 11.32 & 0.07 \\
\hline \multirow{3}{*}{ DP4+ } & ${ }^{1} \mathrm{H}$ data & 14.44 & 57.69 & 4.47 & 1.82 & 0.30 & 4.33 & 16.22 & 0.73 \\
\hline & ${ }^{13} \mathrm{C}$ data & 0.00 & 0.00 & 0.00 & 0.00 & 0.21 & 85.57 & 14.21 & 0.01 \\
\hline & All data & 0.00 & 0.00 & 0.00 & 0.00 & 0.01 & 61.61 & 38.38 & 0.00 \\
\hline & & & & el of th & nPW1F & 6-31G* & tion, $\mathrm{PC}$ & & \\
\hline & & $79 a$ & $79 b$ & $79 c$ & $79 d$ & $79 e$ & $79 f$ & $79 \mathrm{~g}$ & $79 \mathrm{~h}$ \\
\hline & ${ }^{1} \mathrm{H}$ data & 7.41 & 85.22 & 1.02 & 5.56 & 0.07 & 0.34 & 0.31 & 0.07 \\
\hline sDP4+ & ${ }^{13} \mathrm{C}$ data & 0.00 & 0.00 & 0.00 & 0.00 & 3.37 & 55.30 & 40.91 & 0.41 \\
\hline & All data & 0.00 & 0.46 & 0.00 & 0.06 & 0.69 & 58.78 & 39.92 & 0.10 \\
\hline & ${ }^{1} \mathrm{H}$ data & 1.13 & 84.26 & 2.99 & 8.69 & 0.10 & 1.24 & 1.42 & 0.15 \\
\hline uDP4+ & ${ }^{13} \mathrm{C}$ data & 0.01 & 0.01 & 0.05 & 0.06 & 17.00 & 18.02 & 42.36 & 22.49 \\
\hline & All data & 0.01 & 1.10 & 0.16 & 0.62 & 1.95 & 24.98 & 67.40 & 3.78 \\
\hline DP4+ & ${ }^{1} \mathrm{H}$ data & 0.12 & 99.16 & 0.04 & 0.67 & 0.00 & 0.01 & 0.01 & 0.00 \\
\hline DP4+ & ${ }^{13} \mathrm{C}$ data & 0.00 & 0.00 & 0.00 & 0.00 & 2.05 & 35.64 & 61.98 & 0.33 \\
\hline
\end{tabular}




\begin{tabular}{|c|c|c|c|c|c|c|c|c|c|}
\hline & All data & 0.00 & 0.01 & 0.00 & 0.00 & 0.03 & 35.29 & 64.66 & 0.01 \\
\hline & & \multicolumn{8}{|c|}{ Level of theory: mPW1PW91/6-31G** (solution, PCM) } \\
\hline & & $79 a$ & $79 b$ & $79 \mathrm{c}$ & 79d & $79 e$ & $79 f$ & $79 \mathrm{~g}$ & $79 \mathrm{~h}$ \\
\hline & ${ }^{1} \mathrm{H}$ data & 10.87 & 81.03 & 1.75 & 2.55 & 0.24 & 0.90 & 2.52 & 0.15 \\
\hline \multirow[t]{2}{*}{ sDP4+ } & ${ }^{13} \mathrm{C}$ data & 0.00 & 0.00 & 0.00 & 0.00 & 6.68 & 71.47 & 21.25 & 0.59 \\
\hline & All data & 0.00 & 0.18 & 0.00 & 0.01 & 1.32 & 53.72 & 44.70 & 0.07 \\
\hline \multirow{3}{*}{ uDP4+ } & ${ }^{1} \mathrm{H}$ data & 1.59 & 88.21 & 2.28 & 3.85 & 0.15 & 1.78 & 1.94 & 0.20 \\
\hline & ${ }^{13} \mathrm{C}$ data & 0.00 & 0.01 & 0.02 & 0.03 & 11.26 & 30.85 & 52.30 & 5.53 \\
\hline & All data & 0.00 & 0.58 & 0.03 & 0.07 & 1.02 & 34.19 & 63.41 & 0.68 \\
\hline \multirow{5}{*}{ DP4+ } & ${ }^{1} \mathrm{H}$ data & 0.24 & 99.48 & 0.06 & 0.14 & 0.00 & 0.02 & 0.07 & 0.00 \\
\hline & ${ }^{13} \mathrm{C}$ data & 0.00 & 0.00 & 0.00 & 0.00 & 2.22 & 64.95 & 32.74 & 0.10 \\
\hline & All data & 0.00 & 0.00 & 0.00 & 0.00 & 0.03 & 39.30 & 60.66 & 0.00 \\
\hline & & \multicolumn{8}{|c|}{ Level of theory: mPW1PW91/6-31+G** (solution, PCM) } \\
\hline & & $79 a$ & $79 b$ & 79c & $79 d$ & $79 e$ & $79 f$ & $79 \mathrm{~g}$ & $79 \mathrm{~h}$ \\
\hline \multirow{3}{*}{ sDP4+ } & ${ }^{1} \mathrm{H}$ data & 25.73 & 22.53 & 4.89 & 0.80 & 1.55 & 10.17 & 33.47 & 0.86 \\
\hline & ${ }^{13} \mathrm{C}$ data & 0.00 & 0.02 & 0.01 & 0.01 & 5.46 & 83.78 & 10.63 & 0.08 \\
\hline & All data & 0.00 & 0.03 & 0.00 & 0.00 & 0.70 & 70.02 & 29.24 & 0.01 \\
\hline & ${ }^{1} \mathrm{H}$ data & 19.81 & 28.57 & 9.58 & 1.02 & 2.27 & 14.28 & 23.26 & 1.20 \\
\hline \multirow[t]{2}{*}{ uDP4+ } & ${ }^{13} \mathrm{C}$ data & 0.00 & 0.10 & 0.14 & 0.03 & 3.15 & 63.45 & 32.82 & 0.31 \\
\hline & All data & 0.00 & 0.17 & 0.08 & 0.00 & 0.42 & 53.89 & 45.40 & 0.02 \\
\hline \multirow{5}{*}{ DP4+ } & ${ }^{1} \mathrm{H}$ data & 23.93 & 30.23 & 2.20 & 0.04 & 0.17 & 6.82 & 36.56 & 0.05 \\
\hline & ${ }^{13} \mathrm{C}$ data & 0.00 & 0.00 & 0.00 & 0.00 & 0.30 & 93.55 & 6.14 & 0.00 \\
\hline & All data & 0.00 & 0.00 & 0.00 & 0.00 & 0.01 & 73.97 & 26.02 & 0.00 \\
\hline & & \multicolumn{8}{|c|}{ Level of theory: mPW1PW91/6-311G* (solution, PCM) } \\
\hline & & 79a & $79 b$ & $79 c$ & 79d & $79 e$ & $79 f$ & $79 \mathrm{~g}$ & $79 \mathrm{~h}$ \\
\hline \multirow{3}{*}{$s \mathrm{DP} 4+$} & ${ }^{1} \mathrm{H}$ data & 2.07 & 92.36 & 1.44 & 0.25 & 0.08 & 2.61 & 1.15 & 0.04 \\
\hline & ${ }^{13} \mathrm{C}$ data & 0.00 & 0.06 & 0.01 & 0.04 & 4.50 & 49.37 & 44.83 & 1.19 \\
\hline & All data & 0.00 & 2.96 & 0.01 & 0.00 & 0.20 & 69.15 & 27.65 & 0.03 \\
\hline \multirow{3}{*}{ uDP4+ } & ${ }^{1} \mathrm{H}$ data & 2.95 & 89.18 & 2.80 & 0.90 & 0.19 & 2.43 & 1.41 & 0.13 \\
\hline & ${ }^{13} \mathrm{C}$ data & 0.00 & 0.35 & 0.06 & 0.08 & 0.89 & 57.19 & 41.18 & 0.24 \\
\hline & All data & 0.00 & 13.58 & 0.07 & 0.03 & 0.08 & 60.85 & 25.37 & 0.01 \\
\hline \multirow{5}{*}{ DP4+ } & ${ }^{1} \mathrm{H}$ data & 0.07 & 99.78 & 0.05 & 0.00 & 0.00 & 0.08 & 0.02 & 0.00 \\
\hline & ${ }^{13} \mathrm{C}$ data & 0.00 & 0.00 & 0.00 & 0.00 & 0.09 & 60.41 & 39.50 & 0.01 \\
\hline & All data & 0.00 & 0.81 & 0.00 & 0.00 & 0.00 & 85.01 & 14.17 & 0.00 \\
\hline & & \multicolumn{8}{|c|}{ Level of theory: mPW1PW91/6-311G** (solution, PCM) } \\
\hline & & 79a & $79 b$ & 79c & 79d & $79 e$ & $79 f$ & $79 \mathrm{~g}$ & $79 \mathrm{~h}$ \\
\hline & ${ }^{1} \mathrm{H}$ data & 5.61 & 45.92 & 4.38 & 0.10 & 0.63 & 12.19 & 30.60 & 0.56 \\
\hline \multirow[t]{2}{*}{$s \mathrm{DP} 4+$} & ${ }^{13} \mathrm{C}$ data & 0.00 & 0.04 & 0.01 & 0.02 & 3.57 & 58.85 & 36.77 & 0.74 \\
\hline & All data & 0.00 & 0.10 & 0.00 & 0.00 & 0.12 & 38.84 & 60.92 & 0.02 \\
\hline \multirow{3}{*}{ uDP4+ } & ${ }^{1} \mathrm{H}$ data & 10.57 & 35.66 & 2.38 & 0.19 & 1.07 & 43.90 & 5.23 & 1.01 \\
\hline & ${ }^{13} \mathrm{C}$ data & 0.00 & 0.45 & 0.08 & 0.08 & 0.43 & 56.02 & 42.78 & 0.15 \\
\hline & All data & 0.00 & 0.60 & 0.01 & 0.00 & 0.02 & 91.09 & 8.28 & 0.01 \\
\hline \multirow{5}{*}{ DP4+ } & ${ }^{1} \mathrm{H}$ data & 2.47 & 68.13 & 0.43 & 0.00 & 0.03 & 22.27 & 6.65 & 0.02 \\
\hline & ${ }^{13} \mathrm{C}$ data & 0.00 & 0.00 & 0.00 & 0.00 & 0.03 & 67.68 & 32.29 & 0.00 \\
\hline & All data & 0.00 & 0.00 & 0.00 & 0.00 & 0.00 & 87.52 & 12.48 & 0.00 \\
\hline & & & & of the & PW1PV & $-311+G$ & lution, $\mathrm{P}$ & & \\
\hline & & 79a & $79 b$ & $79 c$ & $79 d$ & $79 e$ & $79 f$ & $79 g$ & $79 h$ \\
\hline & ${ }^{1} \mathrm{H}$ data & 0.74 & 0.80 & 0.40 & 0.07 & 1.21 & 12.76 & 81.92 & 2.11 \\
\hline sDP4+ & ${ }^{13} \mathrm{C}$ data & 0.00 & 0.23 & 0.02 & 0.02 & 3.46 & 47.05 & 48.40 & 0.83 \\
\hline & All data & 0.00 & 0.00 & 0.00 & 0.00 & 0.09 & 13.13 & 86.74 & 0.04 \\
\hline & ${ }^{1} \mathrm{H}$ data & 12.83 & 5.55 & 3.49 & 0.85 & 4.79 & 48.19 & 17.29 & 7.02 \\
\hline uDP4+ & ${ }^{13} \mathrm{C}$ data & 0.00 & 3.60 & 0.25 & 0.16 & 1.16 & 52.60 & 42.14 & 0.09 \\
\hline & All data & 0.00 & 0.61 & 0.03 & 0.00 & 0.17 & 77.03 & 22.15 & 0.02 \\
\hline & ${ }^{1} \mathrm{H}$ data & 0.46 & 0.21 & 0.07 & 0.00 & 0.28 & 29.74 & 68.52 & 0.72 \\
\hline DP4+ & ${ }^{13} \mathrm{C}$ data & 0.00 & 0.02 & 0.00 & 0.00 & 0.09 & 54.76 & 45.13 & 0.00 \\
\hline & All data & 0.00 & 0.00 & 0.00 & 0.00 & 0.00 & 34.49 & 65.51 & 0.00 \\
\hline
\end{tabular}


$s \mathrm{DP4}+, u \mathrm{DP} 4+$ and DP4+ probabilities (\%) for compound 79h<smiles>CC[C@H](C)[C@H]1OC([C@H](C)CC)=C(C)C(=O)[C@H]1C</smiles><smiles>CC[C@H](C)[C@H]1OC([C@H](C)CC)=C(C)C(=O)[C@H]1C</smiles><smiles>CC[C@H](O)[C@@H](C)C1=C(C)C(=O)[C@@H](C)[C@@H]([C@@H](C)CC)O1</smiles><smiles>CC[C@H](O)[C@@H](C)C1=C(C)C(=O)[C@@H](Br)[C@@H]([C@@H](C)CC)O1</smiles><smiles>CC[C@H](C)[C@H]1OC([C@H](C)[C@H](O)CC)=C(C)C(=O)[C@H]1C</smiles><smiles>CC[C@H](O)[C@@H](C)C1=C(C)C(=O)[C@@H](C)[C@@H]([C@@H](C)CC)O1</smiles><smiles>CC[C@H](O)[C@@H](C)C1=C(C)C(=O)[C@@H](C)[C@@H]([C@@H](C)CC)O1</smiles><smiles>CC[C@H](O)[C@@H](C)C1=C(C)C(=O)[C@@H](C)[C@@H]([C@@H](C)CC)O1</smiles>

\begin{tabular}{|c|c|c|c|c|c|c|c|c|c|}
\hline & & \multicolumn{8}{|c|}{ Level of theory: B3LYP/6-31G* (gas phase) } \\
\hline & & 79a & $79 b$ & $79 c$ & 79d & $79 e$ & $79 f$ & $79 \mathrm{~g}$ & $79 \mathrm{~h}$ \\
\hline & ${ }^{1}$ H data & 0.07 & 86.32 & 5.92 & 7.20 & 0.03 & 0.16 & 0.28 & 0.02 \\
\hline \multirow[t]{3}{*}{ sDP4+ } & ${ }^{13}$ C data & 0.00 & 0.00 & 0.00 & 0.01 & 55.96 & 4.69 & 1.81 & 37.53 \\
\hline & All data & 0.00 & 1.29 & 0.07 & 2.32 & 47.93 & 19.75 & 13.12 & 15.52 \\
\hline & ${ }^{1}$ H data & 0.13 & 73.72 & 16.56 & 6.78 & 0.05 & 1.88 & 0.81 & 0.06 \\
\hline \multirow[t]{3}{*}{ uDP4+ } & ${ }^{13} \mathrm{C}$ data & 0.01 & 0.00 & 0.01 & 0.05 & 31.11 & 2.27 & 14.95 & 51.61 \\
\hline & All data & 0.00 & 0.72 & 0.48 & 1.51 & 7.58 & 19.67 & 56.02 & 14.00 \\
\hline & ${ }^{1} \mathrm{H}$ data & 0.00 & 97.74 & 1.51 & 0.75 & 0.00 & 0.00 & 0.00 & 0.00 \\
\hline \multirow[t]{5}{*}{ DP4+ } & ${ }^{13} \mathrm{C}$ data & 0.00 & 0.00 & 0.00 & 0.00 & 46.86 & 0.29 & 0.73 & 52.13 \\
\hline & All data & 0.00 & 0.05 & 0.00 & 0.20 & 21.27 & 22.73 & 43.02 & 12.72 \\
\hline & & \multicolumn{8}{|c|}{ Level of theory: B3LYP/6-31G** (gas phase) } \\
\hline & & $79 a$ & $79 b$ & $79 c$ & 79d & $79 e$ & $79 f$ & $79 \mathrm{~g}$ & $79 \mathrm{~h}$ \\
\hline & ${ }^{1} \mathrm{H}$ data & 0.09 & 82.49 & 11.34 & 3.95 & 0.10 & 0.18 & 1.79 & 0.05 \\
\hline \multirow[t]{3}{*}{ sDP4+ } & ${ }^{13} \mathrm{C}$ data & 0.00 & 0.00 & 0.00 & 0.02 & 61.30 & 7.50 & 1.67 & 29.51 \\
\hline & All data & 0.00 & 0.83 & 0.07 & 0.50 & 51.70 & 10.68 & 24.35 & 11.87 \\
\hline & ${ }^{1}$ H data & 0.20 & 68.94 & 19.54 & 7.78 & 0.11 & 2.31 & 1.00 & 0.11 \\
\hline \multirow[t]{3}{*}{ uDP4+ } & ${ }^{13} \mathrm{C}$ data & 0.01 & 0.01 & 0.01 & 0.13 & 33.42 & 9.25 & 16.12 & 41.05 \\
\hline & All data & 0.00 & 1.59 & 0.49 & 2.07 & 7.83 & 44.60 & 33.70 & 9.72 \\
\hline & ${ }^{1} \mathrm{H}$ data & 0.00 & 95.71 & 3.73 & 0.52 & 0.00 & 0.01 & 0.03 & 0.00 \\
\hline \multirow[t]{5}{*}{ DP4+ } & ${ }^{13} \mathrm{C}$ data & 0.00 & 0.00 & 0.00 & 0.00 & 61.05 & 2.07 & 0.80 & 36.09 \\
\hline & All data & 0.00 & 0.07 & 0.00 & 0.06 & 22.26 & 26.17 & 45.10 & 6.34 \\
\hline & & \multicolumn{8}{|c|}{ Level of theory: B3LYP/6-31+G** (gas phase) } \\
\hline & & $79 a$ & $79 b$ & 79c & 79d & $79 e$ & $79 f$ & $79 \mathrm{~g}$ & $79 \mathrm{~h}$ \\
\hline & ${ }^{1} \mathrm{H}$ data & 0.91 & 57.88 & 11.32 & 3.68 & 3.59 & 0.90 & 21.25 & 0.47 \\
\hline \multirow[t]{2}{*}{$s \mathrm{DP} 4+$} & ${ }^{13} \mathrm{C}$ data & 0.01 & 0.04 & 0.03 & 0.11 & 42.06 & 48.81 & 3.59 & 5.34 \\
\hline & All data & 0.00 & 0.81 & 0.13 & 0.15 & 54.51 & 15.93 & 27.56 & 0.90 \\
\hline \multirow{3}{*}{ uDP4+ } & ${ }^{1}$ H data & 2.46 & 28.64 & 28.65 & 18.28 & 5.42 & 6.67 & 9.18 & 0.70 \\
\hline & ${ }^{13} \mathrm{C}$ data & 0.02 & 0.18 & 0.20 & 0.29 & 20.18 & 64.77 & 12.49 & 1.86 \\
\hline & All data & 0.01 & 0.77 & 0.85 & 0.79 & 16.22 & 64.14 & 17.03 & 0.19 \\
\hline \multirow{5}{*}{ DP4+ } & ${ }^{1} \mathrm{H}$ data & 0.10 & 72.95 & 14.27 & 2.96 & 0.85 & 0.27 & 8.58 & 0.01 \\
\hline & ${ }^{13} \mathrm{C}$ data & 0.00 & 0.00 & 0.00 & 0.00 & 20.88 & 77.78 & 1.10 & 0.25 \\
\hline & All data & 0.00 & 0.03 & 0.00 & 0.00 & 37.21 & 42.99 & 19.75 & 0.01 \\
\hline & & \multicolumn{8}{|c|}{ Level of theory: B3LYP/6-311G* (gas phase) } \\
\hline & & $79 a$ & $79 b$ & 79c & 79d & $79 e$ & $79 f$ & $79 \mathrm{~g}$ & $79 \mathrm{~h}$ \\
\hline \multirow{3}{*}{ sDP4+ } & ${ }^{1} \mathrm{H}$ data & 0.06 & 79.20 & 4.86 & 1.74 & 0.45 & 8.42 & 5.19 & 0.08 \\
\hline & ${ }^{13} \mathrm{C}$ data & 0.02 & 0.02 & 0.00 & 0.15 & 50.95 & 15.15 & 4.30 & 29.40 \\
\hline & All data & 0.00 & 1.10 & 0.01 & 0.15 & 12.97 & 71.90 & 12.61 & 1.27 \\
\hline \multirow{2}{*}{ uDP4+ } & ${ }^{1} \mathrm{H}$ data & 1.68 & 63.28 & 4.85 & 2.93 & 1.07 & 22.64 & 3.24 & 0.31 \\
\hline & ${ }^{13} \mathrm{C}$ data & 0.03 & 0.45 & 0.07 & 0.55 & 14.74 & 64.79 & 15.82 & 3.55 \\
\hline
\end{tabular}




\begin{tabular}{|c|c|c|c|c|c|c|c|c|c|}
\hline & All data & 0.00 & 1.83 & 0.02 & 0.10 & 1.00 & 93.69 & 3.27 & 0.07 \\
\hline & ${ }^{1} \mathrm{H}$ data & 0.00 & 95.49 & 0.45 & 0.10 & 0.01 & 3.63 & 0.32 & 0.00 \\
\hline \multirow[t]{5}{*}{ DP4+ } & ${ }^{13} \mathrm{C}$ data & 0.00 & 0.00 & 0.00 & 0.00 & 39.42 & 51.52 & 3.57 & 5.48 \\
\hline & All data & 0.00 & 0.03 & 0.00 & 0.00 & 0.19 & 99.17 & 0.61 & 0.00 \\
\hline & & \multicolumn{8}{|c|}{ Level of theory: B3LYP/6-311G** (gas phase) } \\
\hline & & 79a & $79 \mathrm{~b}$ & $79 c$ & $79 d$ & $79 e$ & $79 f$ & $79 \mathrm{~g}$ & $79 \mathrm{~h}$ \\
\hline & ${ }^{1} \mathrm{H}$ data & 0.07 & 30.10 & 7.19 & 0.54 & 1.54 & 7.06 & 53.06 & 0.45 \\
\hline \multirow[t]{2}{*}{ sDP4+ } & ${ }^{13} \mathrm{C}$ data & 0.02 & 0.02 & 0.00 & 0.17 & 49.47 & 16.28 & 4.03 & 30.00 \\
\hline & All data & 0.00 & 0.18 & 0.01 & 0.02 & 18.16 & 27.42 & 51.01 & 3.20 \\
\hline & ${ }^{1} \mathrm{H}$ data & 2.74 & 21.08 & 5.37 & 2.69 & 5.12 & 56.95 & 4.42 & 1.63 \\
\hline \multirow[t]{2}{*}{ uDP4+ } & ${ }^{13} \mathrm{C}$ data & 0.01 & 0.46 & 0.06 & 0.55 & 6.23 & 75.45 & 15.82 & 1.41 \\
\hline & All data & 0.00 & 0.22 & 0.01 & 0.03 & 0.72 & 97.38 & 1.58 & 0.05 \\
\hline \multirow{6}{*}{ DP4+ } & ${ }^{1} \mathrm{H}$ data & 0.01 & 48.08 & 2.93 & 0.11 & 0.60 & 30.46 & 17.76 & 0.06 \\
\hline & ${ }^{13} \mathrm{C}$ data & 0.00 & 0.00 & 0.00 & 0.01 & 18.77 & 74.76 & 3.88 & 2.58 \\
\hline & All data & 0.00 & 0.00 & 0.00 & 0.00 & 0.48 & 96.59 & 2.92 & 0.01 \\
\hline & & \multicolumn{8}{|c|}{ Level of theory: B3LYP/6-311+G** (gas phase) } \\
\hline & & 79a & $79 b$ & 79c & 79d & $79 e$ & $79 f$ & $79 \mathrm{~g}$ & $79 \mathrm{~h}$ \\
\hline & ${ }^{1} \mathrm{H}$ data & 0.41 & 13.82 & 7.15 & 1.13 & 2.13 & 7.80 & 66.24 & 1.31 \\
\hline \multirow[t]{2}{*}{$s \mathrm{DP} 4+$} & ${ }^{13} \mathrm{C}$ data & 0.02 & 0.06 & 0.00 & 0.08 & 61.21 & 15.56 & 4.45 & 18.61 \\
\hline & All data & 0.00 & 0.15 & 0.00 & 0.02 & 22.84 & 21.23 & 51.49 & 4.27 \\
\hline \multirow{3}{*}{ uDP4+ } & ${ }^{1} \mathrm{H}$ data & 6.56 & 9.15 & 6.45 & 10.21 & 11.00 & 42.35 & 8.74 & 5.55 \\
\hline & ${ }^{13} \mathrm{C}$ data & 0.02 & 1.23 & 0.06 & 0.50 & 7.77 & 75.88 & 13.84 & 0.71 \\
\hline & All data & 0.00 & 0.33 & 0.01 & 0.15 & 2.49 & 93.40 & 3.51 & 0.11 \\
\hline \multirow{5}{*}{ DP4+ } & ${ }^{1} \mathrm{H}$ data & 0.24 & 11.22 & 4.09 & 1.02 & 2.08 & 29.33 & 51.37 & 0.65 \\
\hline & ${ }^{13} \mathrm{C}$ data & 0.00 & 0.00 & 0.00 & 0.00 & 27.47 & 68.20 & 3.55 & 0.76 \\
\hline & All data & 0.00 & 0.00 & 0.00 & 0.00 & 2.56 & 89.27 & 8.15 & 0.02 \\
\hline & & \multicolumn{8}{|c|}{ Level of theory: B3LYP/6-31G* (solution, PCM) } \\
\hline & & 79a & $79 b$ & $79 \mathrm{c}$ & 79d & $79 e$ & $79 f$ & $79 \mathrm{~g}$ & $79 \mathrm{~h}$ \\
\hline & ${ }^{1} \mathrm{H}$ data & 0.72 & 83.80 & 5.24 & 8.35 & 0.10 & 0.88 & 0.86 & 0.05 \\
\hline \multirow[t]{2}{*}{ sDP4+ } & ${ }^{13} \mathrm{C}$ data & 0.00 & 0.00 & 0.00 & 0.01 & 51.38 & 20.95 & 6.22 & 21.43 \\
\hline & All data & 0.00 & 0.23 & 0.01 & 0.32 & 16.72 & 61.48 & 17.81 & 3.42 \\
\hline \multirow{3}{*}{ uDP4+ } & ${ }^{1} \mathrm{H}$ data & 0.26 & 80.78 & 7.62 & 5.34 & 0.09 & 4.18 & 1.62 & 0.10 \\
\hline & ${ }^{13} \mathrm{C}$ data & 0.01 & 0.00 & 0.01 & 0.05 & 32.26 & 4.17 & 21.21 & 42.29 \\
\hline & All data & 0.00 & 0.45 & 0.14 & 0.45 & 5.07 & 29.30 & 57.76 & 6.83 \\
\hline \multirow{6}{*}{ DP4+ } & ${ }^{1} \mathrm{H}$ data & 0.00 & 98.69 & 0.58 & 0.65 & 0.00 & 0.05 & 0.02 & 0.00 \\
\hline & ${ }^{13} \mathrm{C}$ data & 0.00 & 0.00 & 0.00 & 0.00 & 59.55 & 3.14 & 4.74 & 32.57 \\
\hline & All data & 0.00 & 0.00 & 0.00 & 0.00 & 2.88 & 61.30 & 35.01 & 0.80 \\
\hline & & \multicolumn{8}{|c|}{ Level of theory: B3LYP/6-31G** (solution, PCM) } \\
\hline & & 79a & $79 b$ & 79c & 79d & $79 e$ & $79 f$ & $79 \mathrm{~g}$ & $79 \mathrm{~h}$ \\
\hline & ${ }^{1} \mathrm{H}$ data & 1.29 & 72.59 & 7.18 & 5.85 & 0.84 & 1.85 & 10.17 & 0.23 \\
\hline \multirow[t]{2}{*}{ sDP4+ } & ${ }^{13} \mathrm{C}$ data & 0.00 & 0.00 & 0.00 & 0.02 & 56.78 & 18.31 & 3.70 & 21.18 \\
\hline & All data & 0.00 & 0.13 & 0.01 & 0.08 & 38.27 & 27.22 & 30.31 & 3.97 \\
\hline & ${ }^{1} \mathrm{H}$ data & 0.53 & 73.74 & 5.58 & 12.72 & 0.25 & 4.58 & 2.30 & 0.31 \\
\hline \multirow[t]{2}{*}{ uDP4+ } & ${ }^{13} \mathrm{C}$ data & 0.01 & 0.02 & 0.02 & 0.13 & 32.36 & 19.20 & 17.48 & 30.77 \\
\hline & All data & 0.00 & 1.01 & 0.08 & 1.10 & 5.35 & 59.04 & 27.04 & 6.37 \\
\hline & ${ }^{1} \mathrm{H}$ data & 0.01 & 97.32 & 0.73 & 1.35 & 0.00 & 0.15 & 0.43 & 0.00 \\
\hline DP4+ & ${ }^{13} \mathrm{C}$ data & 0.00 & 0.00 & 0.00 & 0.00 & 63.24 & 12.10 & 2.23 & 22.43 \\
\hline & All data & 0.00 & 0.01 & 0.00 & 0.00 & 7.70 & 60.49 & 30.85 & 0.95 \\
\hline & & & & Level of & : B3LYI & $+\mathrm{G}^{* *}($ & on, PCM & & \\
\hline & & 79a & $79 \mathrm{~b}$ & 79c & 79d & $79 \mathrm{e}$ & $79 f$ & $79 \mathrm{~g}$ & $79 \mathrm{~h}$ \\
\hline & ${ }^{1} \mathrm{H}$ data & 9.22 & 9.96 & 9.96 & 1.63 & 4.34 & 36.36 & 27.91 & 0.61 \\
\hline sDP4+ & ${ }^{13} \mathrm{C}$ data & 0.01 & 0.08 & 0.05 & 0.15 & 29.57 & 61.05 & 6.72 & 2.38 \\
\hline & All data & 0.00 & 0.03 & 0.02 & 0.01 & 5.05 & 87.44 & 7.39 & 0.06 \\
\hline & ${ }^{1} \mathrm{H}$ data & 19.70 & 11.04 & 16.36 & 9.54 & 15.76 & 0.00 & 24.40 & 3.19 \\
\hline uDP4+ & ${ }^{13} \mathrm{C}$ data & 0.11 & 1.23 & 1.26 & 1.24 & 50.95 & 0.00 & 42.02 & 3.18 \\
\hline & All data & 0.11 & 0.72 & 1.09 & 0.63 & 42.57 & 0.00 & 54.33 & 0.54 \\
\hline
\end{tabular}




\begin{tabular}{|c|c|c|c|c|c|c|c|c|c|}
\hline & ${ }^{1} \mathrm{H}$ data & 14.88 & 9.01 & 13.34 & 1.27 & 5.60 & 0.00 & 55.74 & 0.16 \\
\hline \multirow[t]{5}{*}{ DP4+ } & ${ }^{13} \mathrm{C}$ data & 0.00 & 0.01 & 0.00 & 0.01 & 83.85 & 0.00 & 15.71 & 0.42 \\
\hline & All data & 0.00 & 0.00 & 0.00 & 0.00 & 34.88 & 0.00 & 65.10 & 0.01 \\
\hline & & \multicolumn{8}{|c|}{ Level of theory: B3LYP/6-311G* (solution, PCM) } \\
\hline & & $79 a$ & $79 \mathrm{~b}$ & $79 c$ & 79d & $79 e$ & $79 f$ & $79 \mathrm{~g}$ & $79 \mathrm{~h}$ \\
\hline & ${ }^{1} \mathrm{H}$ data & 0.51 & 62.26 & 10.33 & 2.31 & 1.01 & 17.31 & 6.13 & 0.15 \\
\hline \multirow[t]{2}{*}{ sDP4+ } & ${ }^{13} \mathrm{C}$ data & 0.02 & 0.07 & 0.01 & 0.32 & 36.98 & 29.76 & 13.71 & 19.12 \\
\hline & All data & 0.00 & 0.72 & 0.02 & 0.11 & 5.81 & 79.88 & 13.03 & 0.43 \\
\hline & ${ }^{1} \mathrm{H}$ data & 2.58 & 66.05 & 8.90 & 6.12 & 0.94 & 9.56 & 5.29 & 0.55 \\
\hline \multirow[t]{2}{*}{ uDP4+ } & ${ }^{13} \mathrm{C}$ data & 0.01 & 1.15 & 0.15 & 0.86 & 5.52 & 71.53 & 19.88 & 0.89 \\
\hline & All data & 0.00 & 8.65 & 0.16 & 0.60 & 0.59 & 77.95 & 11.98 & 0.06 \\
\hline \multirow{5}{*}{ DP4+ } & ${ }^{1} \mathrm{H}$ data & 0.03 & 93.07 & 2.08 & 0.32 & 0.02 & 3.75 & 0.73 & 0.00 \\
\hline & ${ }^{13} \mathrm{C}$ data & 0.00 & 0.00 & 0.00 & 0.01 & 7.78 & 81.16 & 10.39 & 0.65 \\
\hline & All data & 0.00 & 0.10 & 0.00 & 0.00 & 0.05 & 97.40 & 2.44 & 0.00 \\
\hline & & \multicolumn{8}{|c|}{ Level of theory: B3LYP/6-311G** (solution, PCM) } \\
\hline & & 79a & $79 b$ & 79c & 79d & $79 e$ & $79 f$ & $79 \mathrm{~g}$ & $79 \mathrm{~h}$ \\
\hline & ${ }^{1} \mathrm{H}$ data & 0.66 & 15.05 & 10.08 & 0.37 & 4.22 & 12.84 & 55.88 & 0.89 \\
\hline \multirow[t]{2}{*}{$s \mathrm{DP} 4+$} & ${ }^{13} \mathrm{C}$ data & 0.01 & 0.07 & 0.01 & 0.31 & 33.44 & 35.18 & 12.95 & 18.02 \\
\hline & All data & 0.00 & 0.07 & 0.01 & 0.01 & 10.59 & 33.86 & 54.25 & 1.21 \\
\hline \multirow{3}{*}{ uDP4+ } & ${ }^{1} \mathrm{H}$ data & 11.29 & 24.96 & 10.04 & 2.30 & 10.10 & 29.89 & 7.46 & 3.96 \\
\hline & ${ }^{13} \mathrm{C}$ data & 0.01 & 1.06 & 0.14 & 0.72 & 2.69 & 74.15 & 20.80 & 0.43 \\
\hline & All data & 0.00 & 1.09 & 0.06 & 0.07 & 1.12 & 91.21 & 6.38 & 0.07 \\
\hline & ${ }^{1} \mathrm{H}$ data & 0.56 & 28.19 & 7.60 & 0.06 & 3.20 & 28.82 & 31.30 & 0.27 \\
\hline \multirow[t]{5}{*}{ DP4+ } & ${ }^{13} \mathrm{C}$ data & 0.00 & 0.00 & 0.00 & 0.01 & 3.02 & 87.65 & 9.05 & 0.26 \\
\hline & All data & 0.00 & 0.00 & 0.00 & 0.00 & 0.34 & 89.60 & 10.05 & 0.00 \\
\hline & & \multicolumn{8}{|c|}{ Level of theory: B3LYP/6-311+G** (solution, PCM) } \\
\hline & & $79 a$ & $79 b$ & 79c & $79 d$ & $79 e$ & $79 f$ & $79 \mathrm{~g}$ & $79 \mathrm{~h}$ \\
\hline & ${ }^{1} \mathrm{H}$ data & 3.03 & 4.00 & 5.33 & 0.91 & 4.22 & 17.06 & 62.07 & 3.37 \\
\hline \multirow[t]{2}{*}{ sDP4+ } & ${ }^{13} \mathrm{C}$ data & 0.02 & 0.26 & 0.02 & 0.21 & 45.44 & 25.10 & 16.68 & 12.27 \\
\hline & All data & 0.00 & 0.06 & 0.01 & 0.01 & 11.30 & 25.21 & 60.98 & 2.44 \\
\hline \multirow{3}{*}{ uDP4+ } & ${ }^{1} \mathrm{H}$ data & 15.90 & 4.47 & 5.64 & 4.19 & 13.20 & 34.81 & 13.84 & 7.96 \\
\hline & ${ }^{13} \mathrm{C}$ data & 0.05 & 10.63 & 0.60 & 1.40 & 6.75 & 59.01 & 21.30 & 0.26 \\
\hline & All data & 0.03 & 1.90 & 0.14 & 0.24 & 3.56 & 82.25 & 11.80 & 0.08 \\
\hline \multirow{6}{*}{ DP4+ } & ${ }^{1} \mathrm{H}$ data & 2.95 & 1.09 & 1.84 & 0.23 & 3.41 & 36.32 & 52.52 & 1.64 \\
\hline & ${ }^{13} \mathrm{C}$ data & 0.00 & 0.13 & 0.00 & 0.01 & 14.26 & 68.90 & 16.54 & 0.15 \\
\hline & All data & 0.00 & 0.00 & 0.00 & 0.00 & 1.42 & 73.17 & 25.40 & 0.01 \\
\hline & & \multicolumn{8}{|c|}{ Level of theory: mPW1PW91/6-31G* (gas phase) } \\
\hline & & 79a & $79 b$ & 79c & 79d & $79 e$ & $79 f$ & $79 \mathrm{~g}$ & $79 \mathrm{~h}$ \\
\hline & ${ }^{1} \mathrm{H}$ data & 0.05 & 82.49 & 2.51 & 14.67 & 0.03 & 0.05 & 0.18 & 0.02 \\
\hline \multirow[t]{2}{*}{$s \mathrm{DP} 4+$} & ${ }^{13} \mathrm{C}$ data & 0.00 & 0.00 & 0.00 & 0.03 & 41.20 & 2.93 & 8.47 & 47.36 \\
\hline & All data & 0.00 & 2.52 & 0.03 & 10.13 & 30.85 & 3.12 & 33.94 & 19.41 \\
\hline \multirow{3}{*}{ uDP4+ } & ${ }^{1} \mathrm{H}$ data & 0.08 & 78.19 & 9.92 & 10.44 & 0.04 & 0.70 & 0.58 & 0.04 \\
\hline & ${ }^{13} \mathrm{C}$ data & 0.01 & 0.00 & 0.00 & 0.01 & 34.89 & 0.74 & 8.79 & 55.56 \\
\hline & All data & 0.01 & 0.94 & 0.40 & 1.16 & 14.96 & 5.47 & 53.44 & 23.62 \\
\hline & ${ }^{1} \mathrm{H}$ data & 0.00 & 97.31 & 0.38 & 2.31 & 0.00 & 0.00 & 0.00 & 0.00 \\
\hline DP4+ & ${ }^{13} \mathrm{C}$ data & 0.00 & 0.00 & 0.00 & 0.00 & 34.68 & 0.05 & 1.80 & 63.47 \\
\hline & All data & 0.00 & 0.09 & 0.00 & 0.42 & 16.69 & 0.62 & 65.60 & 16.59 \\
\hline & & & & evel of $t$ & : $\mathrm{mPW}$ & $1 / 6-31 G$ & as phas & & \\
\hline & & 79a & $79 b$ & 79c & $79 d$ & $79 e$ & $79 f$ & $79 \mathrm{~g}$ & $79 \mathrm{~h}$ \\
\hline & ${ }^{1} \mathrm{H}$ data & 0.11 & 83.86 & 4.96 & 9.30 & 0.12 & 0.06 & 1.51 & 0.08 \\
\hline sDP4+ & ${ }^{13} \mathrm{C}$ data & 0.00 & 0.00 & 0.00 & 0.02 & 54.53 & 5.95 & 4.63 & 34.86 \\
\hline & All data & 0.00 & 0.91 & 0.03 & 1.09 & 39.05 & 2.18 & 40.34 & 16.41 \\
\hline & ${ }^{1} \mathrm{H}$ data & 0.16 & 69.73 & 10.43 & 17.56 & 0.12 & 0.89 & 0.99 & 0.12 \\
\hline uDP4+ & ${ }^{13} \mathrm{C}$ data & 0.00 & 0.00 & 0.01 & 0.04 & 23.89 & 2.02 & 18.86 & 55.17 \\
\hline & All data & 0.00 & 0.69 & 0.25 & 2.19 & 8.93 & 5.83 & 60.30 & 21.82 \\
\hline DP4+ & ${ }^{1} \mathrm{H}$ data & 0.00 & 96.43 & 0.85 & 2.69 & 0.00 & 0.00 & 0.02 & 0.00 \\
\hline
\end{tabular}




\begin{tabular}{|c|c|c|c|c|c|c|c|c|c|}
\hline & ${ }^{13} \mathrm{C}$ data & 0.00 & 0.00 & 0.00 & 0.00 & 39.18 & 0.36 & 2.63 & 57.83 \\
\hline & All data & 0.00 & 0.02 & 0.00 & 0.08 & 11.05 & 0.40 & 77.10 & 11.35 \\
\hline & & \multicolumn{8}{|c|}{ Level of theory: mPW1PW91/6-31+G** (gas phase) } \\
\hline & & $79 a$ & $79 \mathrm{~b}$ & $79 c$ & 79d & $79 e$ & $79 f$ & $79 \mathrm{~g}$ & $79 \mathrm{~h}$ \\
\hline & ${ }^{1} \mathrm{H}$ data & 1.36 & 51.26 & 8.34 & 6.71 & 5.24 & 1.56 & 24.58 & 0.94 \\
\hline \multirow[t]{2}{*}{ sDP4+ } & ${ }^{13} \mathrm{C}$ data & 0.00 & 0.00 & 0.00 & 0.04 & 74.94 & 20.56 & 1.36 & 3.10 \\
\hline & All data & 0.00 & 0.05 & 0.01 & 0.06 & 85.04 & 6.96 & 7.25 & 0.63 \\
\hline \multirow{3}{*}{ uDP4+ } & ${ }^{1} \mathrm{H}$ data & 2.96 & 33.98 & 18.43 & 20.90 & 6.49 & 7.15 & 9.08 & 1.01 \\
\hline & ${ }^{13} \mathrm{C}$ data & 0.02 & 0.06 & 0.09 & 0.16 & 32.65 & 39.00 & 23.59 & 4.43 \\
\hline & All data & 0.01 & 0.28 & 0.24 & 0.48 & 29.56 & 38.92 & 29.88 & 0.62 \\
\hline & ${ }^{1} \mathrm{H}$ data & 0.17 & 75.44 & 6.65 & 6.07 & 1.47 & 0.48 & 9.66 & 0.04 \\
\hline \multirow[t]{4}{*}{ DP4+ } & ${ }^{13} \mathrm{C}$ data & 0.00 & 0.00 & 0.00 & 0.00 & 74.28 & 24.33 & 0.97 & 0.42 \\
\hline & All data & 0.00 & 0.00 & 0.00 & 0.00 & 83.74 & 9.03 & 7.22 & 0.01 \\
\hline & & \multicolumn{8}{|c|}{ Level of theory: mPW1PW91/6-311G* (gas phase) } \\
\hline & & $79 a$ & $79 \mathrm{~b}$ & $79 c$ & 79d & $79 e$ & $79 f$ & $79 \mathrm{~g}$ & $79 \mathrm{~h}$ \\
\hline & ${ }^{1} \mathrm{H}$ data & 0.05 & 92.00 & 2.49 & 1.48 & 0.22 & 1.41 & 2.31 & 0.04 \\
\hline \multirow[t]{2}{*}{ sDP4+ } & ${ }^{13} \mathrm{C}$ data & 0.01 & 0.02 & 0.00 & 0.09 & 51.62 & 6.89 & 6.01 & 35.35 \\
\hline & All data & 0.00 & 5.52 & 0.02 & 0.35 & 29.68 & 25.00 & 35.84 & 3.59 \\
\hline \multirow{3}{*}{ uDP4+ } & ${ }^{1} \mathrm{H}$ data & 1.46 & 78.16 & 6.01 & 4.30 & 0.72 & 6.38 & 2.61 & 0.37 \\
\hline & ${ }^{13} \mathrm{C}$ data & 0.01 & 0.32 & 0.03 & 0.68 & 19.90 & 50.73 & 22.23 & 6.10 \\
\hline & All data & 0.00 & 5.83 & 0.04 & 0.68 & 3.35 & 75.97 & 13.60 & 0.53 \\
\hline \multirow{6}{*}{ DP4+ } & ${ }^{1} \mathrm{H}$ data & 0.00 & 99.49 & 0.21 & 0.09 & 0.00 & 0.12 & 0.08 & 0.00 \\
\hline & ${ }^{13} \mathrm{C}$ data & 0.00 & 0.00 & 0.00 & 0.00 & 59.50 & 20.26 & 7.74 & 12.49 \\
\hline & All data & 0.00 & 1.28 & 0.00 & 0.01 & 3.95 & 75.35 & 19.34 & 0.08 \\
\hline & & \multicolumn{8}{|c|}{ Level of theory: mPW1PW91/6-311G** (gas phase) } \\
\hline & & 79a & $79 b$ & $79 c$ & $79 d$ & $79 e$ & $79 f$ & $79 \mathrm{~g}$ & $79 \mathrm{~h}$ \\
\hline & ${ }^{1} \mathrm{H}$ data & 0.12 & 32.33 & 7.37 & 1.10 & 1.55 & 1.75 & 55.14 & 0.64 \\
\hline \multirow[t]{2}{*}{ sDP4+ } & ${ }^{13} \mathrm{C}$ data & 0.01 & 0.02 & 0.00 & 0.08 & 48.07 & 8.61 & 5.94 & 37.27 \\
\hline & All data & 0.00 & 0.13 & 0.00 & 0.02 & 16.86 & 3.42 & 74.13 & 5.43 \\
\hline \multirow{3}{*}{ uDP4+ } & ${ }^{1} \mathrm{H}$ data & 4.19 & 36.14 & 7.09 & 5.84 & 8.82 & 29.87 & 5.22 & 2.82 \\
\hline & ${ }^{13} \mathrm{C}$ data & 0.00 & 0.48 & 0.05 & 0.63 & 6.85 & 58.21 & 31.72 & 2.05 \\
\hline & All data & 0.00 & 0.87 & 0.02 & 0.18 & 3.03 & 87.28 & 8.32 & 0.29 \\
\hline \multirow{5}{*}{ DP4+ } & ${ }^{1} \mathrm{H}$ data & 0.03 & 73.79 & 3.30 & 0.40 & 0.86 & 3.31 & 18.19 & 0.11 \\
\hline & ${ }^{13} \mathrm{C}$ data & 0.00 & 0.00 & 0.00 & 0.00 & 30.06 & 45.74 & 17.21 & 6.98 \\
\hline & All data & 0.00 & 0.01 & 0.00 & 0.00 & 5.29 & 30.81 & 63.73 & 0.16 \\
\hline & & \multicolumn{8}{|c|}{ Level of theory: mPW1PW91/6-311+G** (gas phase) } \\
\hline & & 79a & $79 b$ & 79c & 79d & $79 e$ & $79 f$ & $79 \mathrm{~g}$ & $79 \mathrm{~h}$ \\
\hline \multirow{3}{*}{ sDP4+ } & ${ }^{1} \mathrm{H}$ data & 1.41 & 19.38 & 12.00 & 3.87 & 4.00 & 2.67 & 53.87 & 2.80 \\
\hline & ${ }^{13} \mathrm{C}$ data & 0.00 & 0.05 & 0.00 & 0.04 & 46.78 & 10.08 & 9.07 & 33.97 \\
\hline & All data & 0.00 & 0.13 & 0.00 & 0.02 & 23.42 & 3.36 & 61.16 & 11.90 \\
\hline \multirow{3}{*}{ uDP4+ } & ${ }^{1} \mathrm{H}$ data & 8.32 & 13.84 & 9.15 & 20.03 & 12.35 & 24.37 & 6.46 & 5.48 \\
\hline & ${ }^{13} \mathrm{C}$ data & 0.01 & 1.07 & 0.04 & 0.34 & 7.65 & 64.97 & 25.01 & 0.91 \\
\hline & All data & 0.00 & 0.79 & 0.02 & 0.36 & 5.06 & 84.84 & 8.65 & 0.27 \\
\hline \multirow{5}{*}{ DP4+ } & ${ }^{1} \mathrm{H}$ data & 1.25 & 28.39 & 11.62 & 8.21 & 5.23 & 6.88 & 36.81 & 1.62 \\
\hline & ${ }^{13} \mathrm{C}$ data & 0.00 & 0.00 & 0.00 & 0.00 & 28.17 & 51.54 & 17.86 & 2.43 \\
\hline & All data & 0.00 & 0.01 & 0.00 & 0.00 & 12.67 & 30.48 & 56.50 & 0.34 \\
\hline & & & & rel of th & mPW1F & /6-31G* & tion, $\mathrm{PC}$ & & \\
\hline & & $79 a$ & $79 b$ & $79 c$ & 79d & $79 e$ & $79 f$ & $79 \mathrm{~g}$ & $79 \mathrm{~h}$ \\
\hline & ${ }^{1} \mathrm{H}$ data & 1.02 & 69.74 & 2.61 & 24.16 & 0.27 & 0.55 & 1.55 & 0.11 \\
\hline sDP4+ & ${ }^{13} \mathrm{C}$ data & 0.00 & 0.00 & 0.00 & 0.02 & 49.55 & 8.85 & 21.79 & 19.78 \\
\hline & All data & 0.00 & 0.39 & 0.01 & 0.97 & 24.28 & 8.83 & 61.44 & 4.07 \\
\hline & ${ }^{1} \mathrm{H}$ data & 0.23 & 83.08 & 3.56 & 8.96 & 0.10 & 2.71 & 1.28 & 0.08 \\
\hline uDP4+ & ${ }^{13} \mathrm{C}$ data & 0.01 & 0.00 & 0.01 & 0.02 & 40.77 & 1.90 & 12.95 & 44.34 \\
\hline & All data & 0.01 & 0.65 & 0.11 & 0.67 & 13.63 & 17.36 & 55.75 & 11.81 \\
\hline & ${ }^{1} \mathrm{H}$ data & 0.00 & 96.19 & 0.15 & 3.59 & 0.00 & 0.02 & 0.03 & 0.00 \\
\hline DP4+ & ${ }^{13} \mathrm{C}$ data & 0.00 & 0.00 & 0.00 & 0.00 & 63.20 & 0.53 & 8.82 & 27.44 \\
\hline
\end{tabular}




\begin{tabular}{|c|c|c|c|c|c|c|c|c|c|}
\hline & All data & 0.00 & 0.01 & 0.00 & 0.02 & 8.36 & 3.87 & 86.53 & 1.21 \\
\hline & & \multicolumn{8}{|c|}{ Level of theory: mPW1PW91/6-31G** (solution, PCM) } \\
\hline & & 79a & $79 b$ & $79 c$ & 79d & $79 e$ & $79 f$ & $79 \mathrm{~g}$ & $79 \mathrm{~h}$ \\
\hline & ${ }^{1} \mathrm{H}$ data & 2.14 & 55.14 & 3.06 & 18.58 & 1.44 & 0.93 & 18.22 & 0.49 \\
\hline \multirow[t]{2}{*}{ sDP4+ } & ${ }^{13} \mathrm{C}$ data & 0.00 & 0.00 & 0.00 & 0.01 & 56.59 & 12.24 & 9.55 & 21.60 \\
\hline & All data & 0.00 & 0.07 & 0.00 & 0.10 & 29.34 & 4.08 & 62.64 & 3.78 \\
\hline \multirow{3}{*}{ uDP4+ } & ${ }^{1} \mathrm{H}$ data & 0.43 & 66.29 & 3.58 & 23.23 & 0.38 & 2.97 & 2.74 & 0.38 \\
\hline & ${ }^{13} \mathrm{C}$ data & 0.01 & 0.01 & 0.01 & 0.06 & 30.14 & 4.81 & 25.93 & 39.03 \\
\hline & All data & 0.00 & 0.38 & 0.05 & 1.31 & 10.08 & 12.59 & 62.53 & 13.05 \\
\hline \multirow{5}{*}{ DP4+ } & ${ }^{1} \mathrm{H}$ data & 0.02 & 88.03 & 0.26 & 10.40 & 0.01 & 0.07 & 1.20 & 0.00 \\
\hline & ${ }^{13} \mathrm{C}$ data & 0.00 & 0.00 & 0.00 & 0.00 & 59.74 & 2.06 & 8.67 & 29.52 \\
\hline & All data & 0.00 & 0.00 & 0.00 & 0.00 & 6.86 & 1.19 & 90.80 & 1.14 \\
\hline & & \multicolumn{8}{|c|}{ Level of theory: mPW1PW91/6-31+G** (solution, PCM) } \\
\hline & & $79 a$ & $79 b$ & 79c & $79 d$ & $79 e$ & $79 f$ & $79 \mathrm{~g}$ & $79 \mathrm{~h}$ \\
\hline \multirow{3}{*}{ sDP4+ } & ${ }^{1} \mathrm{H}$ data & 14.37 & 10.09 & 6.11 & 4.45 & 10.04 & 18.28 & 34.65 & 2.00 \\
\hline & ${ }^{13} \mathrm{C}$ data & 0.00 & 0.01 & 0.01 & 0.06 & 35.67 & 59.21 & 3.99 & 1.05 \\
\hline & All data & 0.00 & 0.01 & 0.00 & 0.02 & 22.66 & 68.44 & 8.74 & 0.13 \\
\hline & ${ }^{1} \mathrm{H}$ data & 8.48 & 5.55 & 6.66 & 10.36 & 21.69 & 16.97 & 26.40 & 3.88 \\
\hline \multirow[t]{2}{*}{ uDP4+ } & ${ }^{13} \mathrm{C}$ data & 0.03 & 0.16 & 0.19 & 0.26 & 24.28 & 48.47 & 24.99 & 1.63 \\
\hline & All data & 0.01 & 0.04 & 0.06 & 0.13 & 26.07 & 40.71 & 32.66 & 0.31 \\
\hline \multirow{5}{*}{ DP4+ } & ${ }^{1} \mathrm{H}$ data & 7.11 & 3.27 & 2.37 & 2.69 & 12.70 & 18.09 & 53.32 & 0.45 \\
\hline & ${ }^{13} \mathrm{C}$ data & 0.00 & 0.00 & 0.00 & 0.00 & 22.57 & 74.79 & 2.60 & 0.04 \\
\hline & All data & 0.00 & 0.00 & 0.00 & 0.00 & 16.13 & 76.08 & 7.79 & 0.00 \\
\hline & & \multicolumn{8}{|c|}{ Level of theory: mPW1PW91/6-311G* (solution, PCM) } \\
\hline & & 79a & $79 b$ & 79c & 79d & $79 e$ & $79 f$ & $79 \mathrm{~g}$ & $79 \mathrm{~h}$ \\
\hline \multirow{3}{*}{ sDP4+ } & ${ }^{1} \mathrm{H}$ data & 0.97 & 71.71 & 6.60 & 4.49 & 1.32 & 8.21 & 6.51 & 0.19 \\
\hline & ${ }^{13} \mathrm{C}$ data & 0.01 & 0.06 & 0.01 & 0.11 & 32.38 & 24.57 & 23.33 & 19.54 \\
\hline & All data & 0.00 & 1.10 & 0.01 & 0.12 & 10.54 & 49.81 & 37.49 & 0.92 \\
\hline \multirow{3}{*}{ uDP4+ } & ${ }^{1} \mathrm{H}$ data & 2.62 & 66.54 & 5.72 & 14.32 & 1.02 & 4.21 & 4.89 & 0.68 \\
\hline & ${ }^{13} \mathrm{C}$ data & 0.00 & 0.58 & 0.05 & 0.49 & 4.78 & 65.77 & 27.42 & 0.91 \\
\hline & All data & 0.00 & 8.37 & 0.06 & 1.52 & 1.05 & 59.86 & 29.01 & 0.13 \\
\hline \multirow{5}{*}{ DP4+ } & ${ }^{1} \mathrm{H}$ data & 0.05 & 96.51 & 0.76 & 1.30 & 0.03 & 0.70 & 0.64 & 0.00 \\
\hline & ${ }^{13} \mathrm{C}$ data & 0.00 & 0.00 & 0.00 & 0.00 & 6.37 & 66.55 & 26.34 & 0.73 \\
\hline & All data & 0.00 & 0.23 & 0.00 & 0.00 & 0.27 & 72.91 & 26.59 & 0.00 \\
\hline & & \multicolumn{8}{|c|}{ Level of theory: mPW1PW91/6-311G** (solution, PCM) } \\
\hline & & 79a & $79 b$ & $79 c$ & 79d & $79 e$ & $79 f$ & $79 \mathrm{~g}$ & $79 \mathrm{~h}$ \\
\hline & ${ }^{1} \mathrm{H}$ data & 1.25 & 18.57 & 7.53 & 0.81 & 5.85 & 6.41 & 58.12 & 1.47 \\
\hline \multirow[t]{2}{*}{ sDP4+ } & ${ }^{13} \mathrm{C}$ data & 0.00 & 0.04 & 0.01 & 0.08 & 29.89 & 26.42 & 25.52 & 18.03 \\
\hline & All data & 0.00 & 0.04 & 0.00 & 0.00 & 9.43 & 9.13 & 79.97 & 1.43 \\
\hline \multirow{3}{*}{ uDP4+ } & ${ }^{1} \mathrm{H}$ data & 12.03 & 24.49 & 11.16 & 5.52 & 15.32 & 13.08 & 10.54 & 7.86 \\
\hline & ${ }^{13} \mathrm{C}$ data & 0.00 & 0.71 & 0.07 & 0.30 & 1.59 & 62.03 & 34.97 & 0.33 \\
\hline & All data & 0.00 & 1.41 & 0.06 & 0.13 & 1.98 & 66.14 & 30.05 & 0.21 \\
\hline \multirow{5}{*}{ DP4+ } & ${ }^{1} \mathrm{H}$ data & 1.11 & 33.54 & 6.19 & 0.33 & 6.61 & 6.18 & 45.18 & 0.85 \\
\hline & ${ }^{13} \mathrm{C}$ data & 0.00 & 0.00 & 0.00 & 0.00 & 1.84 & 63.40 & 34.53 & 0.23 \\
\hline & All data & 0.00 & 0.00 & 0.00 & 0.00 & 0.62 & 19.96 & 79.41 & 0.01 \\
\hline & & & & of the & PW1PV & $-311+G$ & Iution, $\mathrm{P}$ & & \\
\hline & & $79 a$ & $79 b$ & $79 c$ & $79 d$ & $79 e$ & $79 f$ & $79 g$ & $79 \mathrm{~h}$ \\
\hline & ${ }^{1} \mathrm{H}$ data & 0.49 & 0.42 & 0.45 & 0.27 & 6.57 & 13.05 & 72.77 & 5.98 \\
\hline sDP4+ & ${ }^{13} \mathrm{C}$ data & 0.01 & 0.24 & 0.01 & 0.08 & 28.83 & 22.08 & 34.56 & 14.19 \\
\hline & All data & 0.00 & 0.00 & 0.00 & 0.00 & 6.16 & 9.37 & 81.72 & 2.76 \\
\hline & ${ }^{1} \mathrm{H}$ data & 6.83 & 1.90 & 4.82 & 6.25 & 30.42 & 15.88 & 19.82 & 14.08 \\
\hline uDP4+ & ${ }^{13} \mathrm{C}$ data & 0.01 & 4.40 & 0.22 & 0.33 & 3.39 & 61.39 & 30.09 & 0.16 \\
\hline & All data & 0.00 & 0.49 & 0.06 & 0.12 & 6.11 & 57.75 & 35.32 & 0.13 \\
\hline & ${ }^{1} \mathrm{H}$ data & 0.17 & 0.04 & 0.11 & 0.09 & 10.29 & 10.67 & 74.28 & 4.34 \\
\hline DP4+ & ${ }^{13} \mathrm{C}$ data & 0.00 & 0.04 & 0.00 & 0.00 & 3.92 & 54.31 & 41.64 & 0.09 \\
\hline & All data & 0.00 & 0.00 & 0.00 & 0.00 & 1.09 & 15.61 & 83.30 & 0.01 \\
\hline
\end{tabular}


sDP4+, $u$ DP4+ and DP4+ probabilities (\%) for compound 80a<smiles>CC(C)(C)[C@H](O)CC(O)C[C@@H](O)C(C)(C)C</smiles>

correct isomer

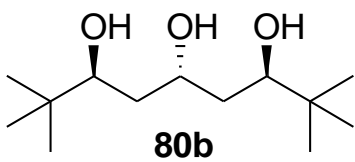

$80 b$

\begin{tabular}{|c|c|c|c|c|c|c|c|c|c|}
\hline & & \multicolumn{8}{|c|}{ Level of theory: B3LYP/6-31G* (gas phase) } \\
\hline & & $80 a$ & $80 \mathrm{~b}$ & - & - & - & - & - & - \\
\hline & ${ }^{1} \mathrm{H}$ data & 85.93 & 14.07 & - & - & - & - & - & - \\
\hline \multirow[t]{2}{*}{ sDP4+ } & ${ }^{13} \mathrm{C}$ data & 93.10 & 6.90 & - & - & - & - & - & - \\
\hline & All data & 98.80 & 1.20 & - & - & - & - & - & - \\
\hline \multirow{3}{*}{ uDP4+ } & ${ }^{1} \mathrm{H}$ data & 99.98 & 0.02 & - & - & - & - & - & - \\
\hline & ${ }^{13} \mathrm{C}$ data & 99.98 & 0.02 & - & - & - & - & - & - \\
\hline & All data & 100.00 & 0.00 & - & - & - & - & - & - \\
\hline \multirow{5}{*}{ DP4+ } & ${ }^{1} \mathrm{H}$ data & 100.00 & 0.00 & - & - & - & - & - & - \\
\hline & ${ }^{13} \mathrm{C}$ data & 100.00 & 0.00 & - & - & - & - & - & - \\
\hline & All data & 100.00 & 0.00 & - & - & - & - & - & - \\
\hline & & \multicolumn{8}{|c|}{ Level of theory: B3LYP/6-31G** (gas phase) } \\
\hline & & $80 a$ & $80 \mathrm{~b}$ & - & - & - & - & - & - \\
\hline \multirow{3}{*}{ sDP4+ } & ${ }^{1} \mathrm{H}$ data & 79.44 & 20.56 & - & - & - & - & - & - \\
\hline & ${ }^{13} \mathrm{C}$ data & 91.23 & 8.77 & - & - & - & - & - & - \\
\hline & All data & 97.57 & 2.43 & - & - & - & - & - & - \\
\hline \multirow{3}{*}{ uDP4+ } & ${ }^{1} \mathrm{H}$ data & 99.97 & 0.03 & - & - & - & - & - & - \\
\hline & ${ }^{13} \mathrm{C}$ data & 99.93 & 0.07 & - & - & - & - & - & - \\
\hline & All data & 100.00 & 0.00 & - & - & - & - & - & - \\
\hline \multirow{5}{*}{ DP4+ } & ${ }^{1} \mathrm{H}$ data & 99.99 & 0.01 & - & - & - & - & - & - \\
\hline & ${ }^{13} \mathrm{C}$ data & 99.99 & 0.01 & - & - & - & - & - & - \\
\hline & All data & 100.00 & 0.00 & - & - & - & - & - & - \\
\hline & & \multicolumn{8}{|c|}{ Level of theory: B3LYP/6-31+G** (gas phase) } \\
\hline & & $80 a$ & $80 \mathrm{~b}$ & - & - & - & - & - & - \\
\hline \multirow{3}{*}{ sDP4+ } & ${ }^{1} \mathrm{H}$ data & 31.01 & 68.99 & - & - & - & - & - & - \\
\hline & ${ }^{13} \mathrm{C}$ data & 76.76 & 23.24 & - & - & - & - & - & - \\
\hline & All data & 59.75 & 40.25 & - & - & - & - & - & - \\
\hline \multirow{3}{*}{ uDP4+ } & ${ }^{1}$ H data & 99.89 & 0.11 & - & - & - & - & - & - \\
\hline & ${ }^{13} \mathrm{C}$ data & 99.73 & 0.27 & - & - & - & - & - & - \\
\hline & All data & 100.00 & 0.00 & - & - & - & - & - & - \\
\hline \multirow{3}{*}{ DP4+ } & ${ }^{1} \mathrm{H}$ data & 99.75 & 0.25 & - & - & - & - & - & - \\
\hline & ${ }^{13} \mathrm{C}$ data & 99.92 & 0.08 & - & - & - & - & - & - \\
\hline & All data & 100.00 & 0.00 & - & - & - & - & - & - \\
\hline & & \multicolumn{8}{|c|}{ Level of theory: B3LYP/6-311G* (gas phase) } \\
\hline & & $80 a$ & $80 \mathrm{~b}$ & - & - & - & - & - & - \\
\hline \multirow{3}{*}{ sDP4+ } & ${ }^{1} \mathrm{H}$ data & 63.05 & 36.95 & - & - & - & - & - & - \\
\hline & ${ }^{13} \mathrm{C}$ data & 81.78 & 18.22 & - & - & - & - & - & - \\
\hline & All data & 88.45 & 11.55 & - & - & - & - & - & - \\
\hline \multirow{3}{*}{ uDP4+ } & ${ }^{1} \mathrm{H}$ data & 99.82 & 0.18 & - & - & - & - & - & - \\
\hline & ${ }^{13} \mathrm{C}$ data & 77.64 & 22.36 & - & - & - & - & - & - \\
\hline & All data & 99.95 & 0.05 & - & - & - & - & - & - \\
\hline \multirow{3}{*}{ DP4+ } & ${ }^{1} \mathrm{H}$ data & 99.89 & 0.11 & - & - & - & - & - & - \\
\hline & ${ }^{13} \mathrm{C}$ data & 93.97 & 6.03 & - & - & - & - & - & - \\
\hline & All data & 99.99 & 0.01 & - & - & - & - & - & - \\
\hline & & \multicolumn{8}{|c|}{ Level of theory: B3LYP/6-311G** (gas phase) } \\
\hline & & $80 a$ & $80 \mathrm{~b}$ & - & - & - & - & - & - \\
\hline \multirow{3}{*}{ sDP4+ } & ${ }^{1}$ H data & 40.46 & 59.54 & - & - & - & - & - & - \\
\hline & ${ }^{13} \mathrm{C}$ data & 80.01 & 19.99 & - & - & - & - & - & - \\
\hline & All data & 73.12 & 26.88 & - & - & - & - & - & - \\
\hline
\end{tabular}




\begin{tabular}{|c|c|c|c|c|c|c|c|c|c|}
\hline & ${ }^{1} \mathrm{H}$ data & 99.68 & 0.32 & - & - & - & - & - & - \\
\hline \multirow[t]{3}{*}{ uDP4+ } & ${ }^{13} \mathrm{C}$ data & 81.27 & 18.73 & - & - & - & - & - & - \\
\hline & All data & 99.93 & 0.07 & - & - & - & - & - & - \\
\hline & ${ }^{1} \mathrm{H}$ data & 99.53 & 0.47 & - & - & - & - & - & - \\
\hline \multirow[t]{5}{*}{ DP4+ } & ${ }^{13} \mathrm{C}$ data & 94.56 & 5.44 & - & - & - & - & - & - \\
\hline & All data & 99.97 & 0.03 & - & - & - & - & - & - \\
\hline & & \multicolumn{8}{|c|}{ Level of theory: B3LYP/6-311+G** (gas phase) } \\
\hline & & $80 a$ & $80 \mathrm{~b}$ & - & - & - & - & - & - \\
\hline & ${ }^{1} \mathrm{H}$ data & 20.58 & 79.42 & - & - & - & - & - & - \\
\hline \multirow[t]{3}{*}{ sDP4+ } & ${ }^{13} \mathrm{C}$ data & 82.65 & 17.35 & - & - & - & - & - & - \\
\hline & All data & 55.24 & 44.76 & - & - & - & - & - & - \\
\hline & ${ }^{1} \mathrm{H}$ data & 99.33 & 0.67 & - & - & - & - & - & - \\
\hline \multirow[t]{3}{*}{ uDP4+ } & ${ }^{13} \mathrm{C}$ data & 89.90 & 10.10 & - & - & - & - & - & - \\
\hline & All data & 99.92 & 0.08 & - & - & - & - & - & - \\
\hline & ${ }^{1} \mathrm{H}$ data & 97.46 & 2.54 & - & - & - & - & - & - \\
\hline \multirow[t]{5}{*}{ DP4+ } & ${ }^{13} \mathrm{C}$ data & 97.70 & 2.30 & - & - & - & - & - & - \\
\hline & All data & 99.94 & 0.06 & - & - & - & - & - & - \\
\hline & & \multicolumn{8}{|c|}{ Level of theory: B3LYP/6-31G* (solution, PCM) } \\
\hline & & $80 a$ & $80 b$ & - & - & - & - & - & - \\
\hline & ${ }^{1} \mathrm{H}$ data & 73.78 & 26.22 & - & - & - & - & - & - \\
\hline \multirow[t]{3}{*}{ sDP4+ } & ${ }^{13} \mathrm{C}$ data & 91.18 & 8.82 & - & - & - & - & - & - \\
\hline & All data & 96.68 & 3.32 & - & - & - & - & - & - \\
\hline & ${ }^{1} \mathrm{H}$ data & 99.94 & 0.06 & - & - & - & - & - & - \\
\hline \multirow[t]{3}{*}{ uDP4+ } & ${ }^{13} \mathrm{C}$ data & 99.99 & 0.01 & - & - & - & - & - & - \\
\hline & All data & 100.00 & 0.00 & - & - & - & - & - & - \\
\hline & ${ }^{1} \mathrm{H}$ data & 99.98 & 0.02 & - & - & - & - & - & - \\
\hline \multirow[t]{4}{*}{ DP4+ } & ${ }^{13} \mathrm{C}$ data & 100.00 & 0.00 & - & - & - & - & - & - \\
\hline & All data & 100.00 & 0.00 & - & - & - & - & - & - \\
\hline & & \multicolumn{8}{|c|}{ Level of theory: B3LYP/6-31G** (solution, PCM) } \\
\hline & & $80 a$ & $80 \mathrm{~b}$ & - & - & - & - & - & - \\
\hline \multirow{3}{*}{ sDP4+ } & ${ }^{1} \mathrm{H}$ data & 51.23 & 48.77 & - & - & - & - & - & - \\
\hline & ${ }^{13} \mathrm{C}$ data & 89.99 & 10.01 & - & - & - & - & - & - \\
\hline & All data & 90.43 & 9.57 & - & - & - & - & - & - \\
\hline \multirow{3}{*}{ uDP4+ } & ${ }^{1} \mathrm{H}$ data & 99.68 & 0.32 & - & - & - & - & - & - \\
\hline & ${ }^{13} \mathrm{C}$ data & 99.93 & 0.07 & - & - & - & - & - & - \\
\hline & All data & 100.00 & 0.00 & - & - & - & - & - & - \\
\hline \multirow{5}{*}{ DP4+ } & ${ }^{1} \mathrm{H}$ data & 99.70 & 0.30 & - & - & - & - & - & - \\
\hline & ${ }^{13} \mathrm{C}$ data & 99.99 & 0.01 & - & - & - & - & - & - \\
\hline & All data & 100.00 & 0.00 & - & - & - & - & - & - \\
\hline & & \multicolumn{8}{|c|}{ Level of theory: B3LYP/6-31+G** (solution, PCM) } \\
\hline & & $80 a$ & $80 \mathrm{~b}$ & - & - & - & - & - & - \\
\hline \multirow{3}{*}{ sDP4+ } & ${ }^{1} \mathrm{H}$ data & 1.98 & 98.02 & - & - & - & - & - & - \\
\hline & ${ }^{13} \mathrm{C}$ data & 75.47 & 24.53 & - & - & - & - & - & - \\
\hline & All data & 5.85 & 94.15 & - & - & - & - & - & - \\
\hline & ${ }^{1} \mathrm{H}$ data & 93.11 & 6.89 & - & - & - & - & - & - \\
\hline uDP4+ & ${ }^{13} \mathrm{C}$ data & 99.68 & 0.32 & - & - & - & - & - & - \\
\hline & All data & 99.98 & 0.02 & - & - & - & - & - & - \\
\hline & ${ }^{1} \mathrm{H}$ data & 21.43 & 78.57 & - & - & - & - & - & - \\
\hline DP4+ & ${ }^{13} \mathrm{C}$ data & 99.90 & 0.10 & - & - & - & - & - & - \\
\hline & All data & 99.62 & 0.38 & - & - & - & - & - & - \\
\hline & & & & rel & B3 & G* & $P$ & & \\
\hline & & $80 a$ & $80 \mathrm{~b}$ & - & - & - & - & - & - \\
\hline & ${ }^{1} \mathrm{H}$ data & 26.45 & 73.55 & - & - & - & - & - & - \\
\hline sDP4+ & ${ }^{13} \mathrm{C}$ data & 77.79 & 22.21 & - & - & - & - & - & - \\
\hline & All data & 55.76 & 44.24 & - & - & - & - & - & - \\
\hline uDP4+ & ${ }^{1} \mathrm{H}$ data & 98.07 & 1.93 & - & - & - & - & - & - \\
\hline
\end{tabular}




\begin{tabular}{|c|c|c|c|c|c|c|c|c|c|}
\hline & ${ }^{13} \mathrm{C}$ data & 74.10 & 25.90 & - & - & - & - & - & - \\
\hline & All data & 99.32 & 0.68 & - & - & - & - & - & - \\
\hline & ${ }^{1} \mathrm{H}$ data & 94.80 & 5.20 & - & - & - & - & - & - \\
\hline \multirow[t]{5}{*}{ DP4+ } & ${ }^{13} \mathrm{C}$ data & 90.93 & 9.07 & - & - & - & - & - & - \\
\hline & All data & 99.46 & 0.54 & - & - & - & - & - & - \\
\hline & & \multicolumn{8}{|c|}{ Level of theory: B3LYP/6-311G** (solution, PCM) } \\
\hline & & $80 a$ & $80 b$ & - & - & - & - & - & - \\
\hline & ${ }^{1} \mathrm{H}$ data & 7.44 & 92.56 & - & - & - & - & - & - \\
\hline \multirow[t]{2}{*}{ sDP4+ } & ${ }^{13} \mathrm{C}$ data & 77.73 & 22.27 & - & - & - & - & - & - \\
\hline & All data & 21.90 & 78.10 & - & - & - & - & - & - \\
\hline & ${ }^{1} \mathrm{H}$ data & 83.06 & 16.94 & - & - & - & - & - & - \\
\hline \multirow[t]{2}{*}{ uDP4+ } & ${ }^{13} \mathrm{C}$ data & 78.81 & 21.19 & - & - & - & - & - & - \\
\hline & All data & 94.80 & 5.20 & - & - & - & - & - & - \\
\hline \multirow{5}{*}{ DP4+ } & ${ }^{1} \mathrm{H}$ data & 28.27 & 71.73 & - & - & - & - & - & - \\
\hline & ${ }^{13} \mathrm{C}$ data & 92.85 & 7.15 & - & - & - & - & - & - \\
\hline & All data & 83.65 & 16.35 & - & - & - & - & - & - \\
\hline & & \multicolumn{8}{|c|}{ Level of theory: B3LYP/6-311+G** (solution, PCM) } \\
\hline & & $80 a$ & $80 b$ & - & - & - & - & - & - \\
\hline \multirow{3}{*}{ sDP4+ } & ${ }^{1} \mathrm{H}$ data & 2.84 & 97.16 & - & - & - & - & - & - \\
\hline & ${ }^{13} \mathrm{C}$ data & 82.74 & 17.26 & - & - & - & - & - & - \\
\hline & All data & 12.29 & 87.71 & - & - & - & - & - & - \\
\hline \multirow{3}{*}{ uDP4+ } & ${ }^{1} \mathrm{H}$ data & 83.93 & 16.07 & - & - & - & - & - & - \\
\hline & ${ }^{13} \mathrm{C}$ data & 88.47 & 11.53 & - & - & - & - & - & - \\
\hline & All data & 97.57 & 2.43 & - & - & - & - & - & - \\
\hline \multirow{5}{*}{ DP4+ } & ${ }^{1} \mathrm{H}$ data & 13.24 & 86.76 & - & - & - & - & - & - \\
\hline & ${ }^{13} \mathrm{C}$ data & 97.36 & 2.64 & - & - & - & - & - & - \\
\hline & All data & 84.89 & 15.11 & - & - & - & - & - & - \\
\hline & & \multicolumn{8}{|c|}{ Level of theory: mPW1PW91/6-31G* (gas phase) } \\
\hline & & $80 a$ & $80 \mathrm{~b}$ & - & - & - & - & - & - \\
\hline \multirow{3}{*}{ sDP4+ } & ${ }^{1} \mathrm{H}$ data & 88.62 & 11.38 & - & - & - & - & - & - \\
\hline & ${ }^{13} \mathrm{C}$ data & 94.50 & 5.50 & - & - & - & - & - & - \\
\hline & All data & 99.26 & 0.74 & - & - & - & - & - & - \\
\hline \multirow{3}{*}{ uDP4+ } & ${ }^{1} \mathrm{H}$ data & 99.98 & 0.02 & - & - & - & - & - & - \\
\hline & ${ }^{13} \mathrm{C}$ data & 99.99 & 0.01 & - & - & - & - & - & - \\
\hline & All data & 100.00 & 0.00 & - & - & - & - & - & - \\
\hline \multirow{5}{*}{ DP4+ } & ${ }^{1} \mathrm{H}$ data & 100.00 & 0.00 & - & - & - & - & - & - \\
\hline & ${ }^{13} \mathrm{C}$ data & 100.00 & 0.00 & - & - & - & - & - & - \\
\hline & All data & 100.00 & 0.00 & - & - & - & - & - & - \\
\hline & & \multicolumn{8}{|c|}{ Level of theory: mPW1PW91/6-31G** (gas phase) } \\
\hline & & $80 a$ & $80 b$ & - & - & - & - & - & - \\
\hline \multirow{3}{*}{ sDP4+ } & ${ }^{1} \mathrm{H}$ data & 84.56 & 15.44 & - & - & - & - & - & - \\
\hline & ${ }^{13} \mathrm{C}$ data & 93.31 & 6.69 & - & - & - & - & - & - \\
\hline & All data & 98.71 & 1.29 & - & - & - & - & - & - \\
\hline \multirow{3}{*}{ uDP4+ } & ${ }^{1} \mathrm{H}$ data & 99.98 & 0.02 & - & - & - & - & - & - \\
\hline & ${ }^{13} \mathrm{C}$ data & 99.98 & 0.02 & - & - & - & - & - & - \\
\hline & All data & 100.00 & 0.00 & - & - & - & - & - & - \\
\hline & ${ }^{1} \mathrm{H}$ data & 100.00 & 0.00 & - & - & - & - & - & - \\
\hline DP4+ & ${ }^{13} \mathrm{C}$ data & 100.00 & 0.00 & - & - & - & - & - & - \\
\hline & All data & 100.00 & 0.00 & - & - & - & - & - & - \\
\hline & & & & 10 & $\mathrm{nPV}$ & -3 & ph & & \\
\hline & & $80 a$ & $80 \mathrm{~b}$ & - & - & - & - & - & - \\
\hline & ${ }^{1} \mathrm{H}$ data & 54.82 & 45.18 & - & - & - & - & - & - \\
\hline$s \mathrm{DP} 4+$ & ${ }^{13} \mathrm{C}$ data & 81.37 & 18.63 & - & - & - & - & - & - \\
\hline & All data & 84.12 & 15.88 & - & - & - & - & - & - \\
\hline & ${ }^{1} \mathrm{H}$ data & 99.84 & 0.16 & - & - & - & - & - & - \\
\hline uDP4+ & ${ }^{13} \mathrm{C}$ data & 99.97 & 0.03 & - & - & - & - & - & - \\
\hline
\end{tabular}




\begin{tabular}{|c|c|c|c|c|c|c|c|c|c|}
\hline & All data & 100.00 & 0.00 & - & - & - & - & - & - \\
\hline & ${ }^{1} \mathrm{H}$ data & 99.87 & 0.13 & - & - & - & - & - & - \\
\hline \multirow[t]{5}{*}{ DP4+ } & ${ }^{13} \mathrm{C}$ data & 99.99 & 0.01 & - & - & - & - & - & - \\
\hline & All data & 100.00 & 0.00 & - & - & - & - & - & - \\
\hline & & \multicolumn{8}{|c|}{ Level of theory: mPW1PW91/6-311G* (gas phase) } \\
\hline & & $80 a$ & $80 b$ & - & - & - & - & - & - \\
\hline & ${ }^{1} \mathrm{H}$ data & 69.02 & 30.98 & - & - & - & - & - & - \\
\hline \multirow[t]{3}{*}{ sDP4+ } & ${ }^{13} \mathrm{C}$ data & 84.51 & 15.49 & - & - & - & - & - & - \\
\hline & All data & 92.40 & 7.60 & - & - & - & - & - & - \\
\hline & ${ }^{1} \mathrm{H}$ data & 99.84 & 0.16 & - & - & - & - & - & - \\
\hline \multirow[t]{3}{*}{ uDP4+ } & ${ }^{13} \mathrm{C}$ data & 97.85 & 2.15 & - & - & - & - & - & - \\
\hline & All data & 100.00 & 0.00 & - & - & - & - & - & - \\
\hline & ${ }^{1} \mathrm{H}$ data & 99.93 & 0.07 & - & - & - & - & - & - \\
\hline \multirow[t]{5}{*}{ DP4+ } & ${ }^{13} \mathrm{C}$ data & 99.60 & 0.40 & - & - & - & - & - & - \\
\hline & All data & 100.00 & 0.00 & - & - & - & - & - & - \\
\hline & & \multicolumn{8}{|c|}{ Level of theory: mPW1PW91/6-311G** (gas phase) } \\
\hline & & $80 a$ & $80 \mathrm{~b}$ & - & - & - & - & - & - \\
\hline & ${ }^{1} \mathrm{H}$ data & 51.82 & 48.18 & - & - & - & - & - & - \\
\hline \multirow[t]{3}{*}{ sDP4+ } & ${ }^{13} \mathrm{C}$ data & 84.52 & 15.48 & - & - & - & - & - & - \\
\hline & All data & 85.45 & 14.55 & - & - & - & - & - & - \\
\hline & ${ }^{1}$ H data & 99.83 & 0.17 & - & - & - & - & - & - \\
\hline \multirow[t]{3}{*}{ uDP4+ } & ${ }^{13}$ C data & 98.78 & 1.22 & - & - & - & - & - & - \\
\hline & All data & 100.00 & 0.00 & - & - & - & - & - & - \\
\hline & ${ }^{1} \mathrm{H}$ data & 99.84 & 0.16 & - & - & - & - & - & - \\
\hline \multirow[t]{5}{*}{ DP4+ } & ${ }^{13} \mathrm{C}$ data & 99.77 & 0.23 & - & - & - & - & - & - \\
\hline & All data & 100.00 & 0.00 & - & - & - & - & - & - \\
\hline & & \multicolumn{8}{|c|}{ Level of theory: mPW1PW91/6-311+G** (gas phase) } \\
\hline & & $80 a$ & $80 b$ & - & - & - & - & - & - \\
\hline & ${ }^{1} \mathrm{H}$ data & 32.46 & 67.54 & - & - & - & - & - & - \\
\hline \multirow[t]{3}{*}{ sDP4+ } & ${ }^{13} \mathrm{C}$ data & 85.85 & 14.15 & - & - & - & - & - & - \\
\hline & All data & 74.46 & 25.54 & - & - & - & - & - & - \\
\hline & ${ }^{1} \mathrm{H}$ data & 99.64 & 0.36 & - & - & - & - & - & - \\
\hline \multirow[t]{3}{*}{ uDP4+ } & ${ }^{13} \mathrm{C}$ data & 99.36 & 0.64 & - & - & - & - & - & - \\
\hline & All data & 100.00 & 0.00 & - & - & - & - & - & - \\
\hline & ${ }^{1} \mathrm{H}$ data & 99.26 & 0.74 & - & - & - & - & - & - \\
\hline \multirow[t]{5}{*}{ DP4+ } & ${ }^{13} \mathrm{C}$ data & 99.89 & 0.11 & - & - & - & - & - & - \\
\hline & All data & 100.00 & 0.00 & - & - & - & - & - & - \\
\hline & & \multicolumn{8}{|c|}{ Level of theory: mPW1PW91/6-31G* (solution, PCM) } \\
\hline & & $80 a$ & $80 b$ & - & - & - & - & - & - \\
\hline & ${ }^{1} \mathrm{H}$ data & 78.59 & 21.41 & - & - & - & - & - & - \\
\hline \multirow[t]{2}{*}{ sDP4+ } & ${ }^{13} \mathrm{C}$ data & 92.06 & 7.94 & - & - & - & - & - & - \\
\hline & All data & 97.71 & 2.29 & - & - & - & - & - & - \\
\hline \multirow{3}{*}{ uDP4+ } & ${ }^{1} \mathrm{H}$ data & 99.96 & 0.04 & - & - & - & - & - & - \\
\hline & ${ }^{13} \mathrm{C}$ data & 99.99 & 0.01 & - & - & - & - & - & - \\
\hline & All data & 100.00 & 0.00 & - & - & - & - & - & - \\
\hline & ${ }^{1} \mathrm{H}$ data & 99.99 & 0.01 & - & - & - & - & - & - \\
\hline DP4+ & ${ }^{13} \mathrm{C}$ data & 100.00 & 0.00 & - & - & - & - & - & - \\
\hline & All data & 100.00 & 0.00 & - & - & - & - & - & - \\
\hline & & & & of & $\mathbf{W}$ & $1 C$ & on, & & \\
\hline & & $80 a$ & $80 \mathrm{~b}$ & - & - & - & - & - & - \\
\hline & ${ }^{1} \mathrm{H}$ data & 70.07 & 29.93 & - & - & - & - & - & - \\
\hline sDP4+ & ${ }^{13} \mathrm{C}$ data & 91.47 & 8.53 & - & - & - & - & - & - \\
\hline & All data & 96.17 & 3.83 & - & - & - & - & - & - \\
\hline & ${ }^{1} \mathrm{H}$ data & 99.90 & 0.10 & - & - & - & - & - & - \\
\hline uDP4+ & ${ }^{13} \mathrm{C}$ data & 99.98 & 0.02 & - & - & - & - & - & - \\
\hline & All data & 100.00 & 0.00 & - & - & - & - & - & - \\
\hline
\end{tabular}




\begin{tabular}{|c|c|c|c|c|c|c|c|c|c|}
\hline & ${ }^{1} \mathrm{H}$ data & 99.96 & 0.04 & - & - & - & - & - & - \\
\hline \multirow[t]{5}{*}{ DP4+ } & ${ }^{13} \mathrm{C}$ data & 100.00 & 0.00 & - & - & - & - & - & - \\
\hline & All data & 100.00 & 0.00 & - & - & - & - & - & - \\
\hline & & \multicolumn{8}{|c|}{ Level of theory: mPW1PW91/6-31+G** (solution, PCM) } \\
\hline & & $80 a$ & $80 b$ & - & - & - & - & - & - \\
\hline & ${ }^{1} \mathrm{H}$ data & 7.86 & 92.14 & - & - & - & - & - & - \\
\hline \multirow[t]{3}{*}{ sDP4+ } & ${ }^{13} \mathrm{C}$ data & 78.29 & 21.71 & - & - & - & - & - & - \\
\hline & All data & 23.53 & 76.47 & - & - & - & - & - & - \\
\hline & ${ }^{1} \mathrm{H}$ data & 95.76 & 4.24 & - & - & - & - & - & - \\
\hline \multirow[t]{3}{*}{ uDP4+ } & ${ }^{13} \mathrm{C}$ data & 99.96 & 0.04 & - & - & - & - & - & - \\
\hline & All data & 100.00 & 0.00 & - & - & - & - & - & - \\
\hline & ${ }^{1} \mathrm{H}$ data & 65.83 & 34.17 & - & - & - & - & - & - \\
\hline \multirow[t]{5}{*}{ DP4+ } & ${ }^{13} \mathrm{C}$ data & 99.99 & 0.01 & - & - & - & - & - & - \\
\hline & All data & 99.99 & 0.01 & - & - & - & - & - & - \\
\hline & & \multicolumn{8}{|c|}{ Level of theory: mPW1PW91/6-311G* (solution, PCM) } \\
\hline & & $80 a$ & $80 \mathrm{~b}$ & - & - & - & - & - & - \\
\hline & ${ }^{1} \mathrm{H}$ data & 39.58 & 60.42 & - & - & - & - & - & - \\
\hline \multirow[t]{3}{*}{ sDP4+ } & ${ }^{13} \mathrm{C}$ data & 82.68 & 17.32 & - & - & - & - & - & - \\
\hline & All data & 75.77 & 24.23 & - & - & - & - & - & - \\
\hline & ${ }^{1} \mathrm{H}$ data & 98.72 & 1.28 & - & - & - & - & - & - \\
\hline \multirow[t]{3}{*}{ uDP4+ } & ${ }^{13} \mathrm{C}$ data & 98.25 & 1.75 & - & - & - & - & - & - \\
\hline & All data & 99.98 & 0.02 & - & - & - & - & - & - \\
\hline & ${ }^{1} \mathrm{H}$ data & 98.06 & 1.94 & - & - & - & - & - & - \\
\hline \multirow[t]{5}{*}{ DP4+ } & ${ }^{13} \mathrm{C}$ data & 99.63 & 0.37 & - & - & - & - & - & - \\
\hline & All data & 99.99 & 0.01 & - & - & - & - & - & - \\
\hline & & \multicolumn{8}{|c|}{ Level of theory: mPW1PW91/6-311G** (solution, PCM) } \\
\hline & & $80 a$ & $80 \mathrm{~b}$ & - & - & - & - & - & - \\
\hline & ${ }^{1} \mathrm{H}$ data & 14.63 & 85.37 & - & - & - & - & - & - \\
\hline \multirow[t]{3}{*}{ sDP4+ } & ${ }^{13} \mathrm{C}$ data & 83.65 & 16.35 & - & - & - & - & - & - \\
\hline & All data & 46.71 & 53.29 & - & - & - & - & - & - \\
\hline & ${ }^{1} \mathrm{H}$ data & 91.12 & 8.88 & - & - & - & - & - & - \\
\hline \multirow[t]{3}{*}{ uDP4+ } & ${ }^{13}$ C data & 98.92 & 1.08 & - & - & - & - & - & - \\
\hline & All data & 99.89 & 0.11 & - & - & - & - & - & - \\
\hline & ${ }^{1} \mathrm{H}$ data & 63.75 & 36.25 & - & - & - & - & - & - \\
\hline \multirow[t]{5}{*}{ DP4+ } & ${ }^{13} \mathrm{C}$ data & 99.79 & 0.21 & - & - & - & - & - & - \\
\hline & All data & 99.88 & 0.12 & - & - & - & - & - & - \\
\hline & & \multicolumn{8}{|c|}{ Level of theory: mPW1PW91/6-311+G** (solution, PCM) } \\
\hline & & $80 a$ & $80 \mathrm{~b}$ & - & - & - & - & - & - \\
\hline & ${ }^{1} \mathrm{H}$ data & 6.26 & 93.74 & - & - & - & - & - & - \\
\hline \multirow[t]{2}{*}{ sDP4+ } & ${ }^{13} \mathrm{C}$ data & 86.11 & 13.89 & - & - & - & - & - & - \\
\hline & All data & 29.28 & 70.72 & - & - & - & - & - & - \\
\hline \multirow{3}{*}{ uDP4+ } & ${ }^{1}$ H data & 84.13 & 15.87 & - & - & - & - & - & - \\
\hline & ${ }^{13} \mathrm{C}$ data & 99.35 & 0.65 & - & - & - & - & - & - \\
\hline & All data & 99.88 & 0.12 & - & - & - & - & - & - \\
\hline \multirow{3}{*}{ DP4+ } & ${ }^{1} \mathrm{H}$ data & 26.16 & 73.84 & - & - & - & - & - & - \\
\hline & ${ }^{13} \mathrm{C}$ data & 99.89 & 0.11 & - & - & - & - & - & - \\
\hline & All data & 99.70 & 0.30 & - & - & - & - & - & - \\
\hline
\end{tabular}


$s \mathrm{DP4}+, u \mathrm{DP} 4+$ and DP4+ probabilities (\%) for compound 80b
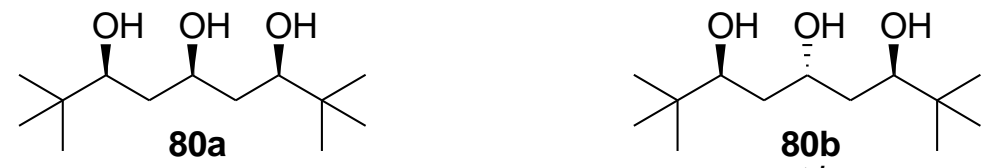

correct isomer

\begin{tabular}{|c|c|c|c|c|c|c|c|c|c|}
\hline & & \multicolumn{8}{|c|}{ Level of theory: B3LYP/6-31G* (gas phase) } \\
\hline & & $80 a$ & $80 \mathrm{~b}$ & - & - & - & - & - & - \\
\hline & ${ }^{1}$ H data & 94.39 & 5.61 & - & - & - & - & - & - \\
\hline \multirow[t]{3}{*}{ sDP4+ } & ${ }^{13} \mathrm{C}$ data & 3.97 & 96.03 & - & - & - & - & - & - \\
\hline & All data & 40.97 & 59.03 & - & - & - & - & - & - \\
\hline & ${ }^{1} \mathrm{H}$ data & 99.96 & 0.04 & - & - & - & - & - & - \\
\hline \multirow[t]{3}{*}{ uDP4+ } & ${ }^{13} \mathrm{C}$ data & 0.40 & 99.60 & - & - & - & - & - & - \\
\hline & All data & 91.26 & 8.74 & - & - & - & - & - & - \\
\hline & ${ }^{1} \mathrm{H}$ data & 100.00 & 0.00 & - & - & - & - & - & - \\
\hline \multirow[t]{5}{*}{ DP4+ } & ${ }^{13} \mathrm{C}$ data & 0.02 & 99.98 & - & - & - & - & - & - \\
\hline & All data & 87.87 & 12.13 & - & - & - & - & - & - \\
\hline & & \multicolumn{8}{|c|}{ Level of theory: B3LYP/6-31G** (gas phase) } \\
\hline & & $80 a$ & $80 b$ & - & - & - & - & - & - \\
\hline & ${ }^{1} \mathrm{H}$ data & 93.17 & 6.83 & - & - & - & - & - & - \\
\hline \multirow[t]{3}{*}{ sDP4+ } & ${ }^{13} \mathrm{C}$ data & 4.66 & 95.34 & - & - & - & - & - & - \\
\hline & All data & 40.04 & 59.96 & - & - & - & - & - & - \\
\hline & ${ }^{1} \mathrm{H}$ data & 99.95 & 0.05 & - & - & - & - & - & - \\
\hline \multirow[t]{3}{*}{ uDP4+ } & ${ }^{13} \mathrm{C}$ data & 0.20 & 99.80 & - & - & - & - & - & - \\
\hline & All data & 79.36 & 20.64 & - & - & - & - & - & - \\
\hline & ${ }^{1} \mathrm{H}$ data & 100.00 & 0.00 & - & - & - & - & - & - \\
\hline \multirow[t]{5}{*}{ DP4+ } & ${ }^{13} \mathrm{C}$ data & 0.01 & 99.99 & - & - & - & - & - & - \\
\hline & All data & 71.97 & 28.03 & - & - & - & - & - & - \\
\hline & & \multicolumn{8}{|c|}{ Level of theory: B3LYP/6-31+G** (gas phase) } \\
\hline & & $80 a$ & $80 \mathrm{~b}$ & - & - & - & - & - & - \\
\hline & ${ }^{1} \mathrm{H}$ data & 77.96 & 22.04 & - & - & - & - & - & - \\
\hline \multirow[t]{2}{*}{ sDP4+ } & ${ }^{13} \mathrm{C}$ data & 10.18 & 89.82 & - & - & - & - & - & - \\
\hline & All data & 28.62 & 71.38 & - & - & - & - & - & - \\
\hline \multirow{3}{*}{ uDP4+ } & ${ }^{1} \mathrm{H}$ data & 99.98 & 0.02 & - & - & - & - & - & - \\
\hline & ${ }^{13} \mathrm{C}$ data & 0.17 & 99.83 & - & - & - & - & - & - \\
\hline & All data & 88.36 & 11.64 & - & - & - & - & - & - \\
\hline \multirow{5}{*}{ DP4+ } & ${ }^{1} \mathrm{H}$ data & 99.99 & 0.01 & - & - & - & - & - & - \\
\hline & ${ }^{13} \mathrm{C}$ data & 0.02 & 99.98 & - & - & - & - & - & - \\
\hline & All data & 75.27 & 24.73 & - & - & - & - & - & - \\
\hline & & \multicolumn{8}{|c|}{ Level of theory: B3LYP/6-311G* (gas phase) } \\
\hline & & $80 a$ & $80 \mathrm{~b}$ & - & - & - & - & - & - \\
\hline \multirow{3}{*}{ sDP4+ } & ${ }^{1} \mathrm{H}$ data & 91.70 & 8.30 & - & - & - & - & - & - \\
\hline & ${ }^{13} \mathrm{C}$ data & 5.13 & 94.87 & - & - & - & - & - & - \\
\hline & All data & 37.38 & 62.62 & - & - & - & - & - & - \\
\hline \multirow{3}{*}{ uDP4+ } & ${ }^{1} \mathrm{H}$ data & 99.51 & 0.49 & - & - & - & - & - & - \\
\hline & ${ }^{13} \mathrm{C}$ data & 0.02 & 99.98 & - & - & - & - & - & - \\
\hline & All data & 3.55 & 96.45 & - & - & - & - & - & - \\
\hline \multirow{3}{*}{ DP4+ } & ${ }^{1} \mathrm{H}$ data & 99.96 & 0.04 & - & - & - & - & - & - \\
\hline & ${ }^{13} \mathrm{C}$ data & 0.00 & 100.00 & - & - & - & - & - & - \\
\hline & All data & 2.15 & 97.85 & - & - & - & - & - & - \\
\hline & & \multicolumn{8}{|c|}{ Level of theory: B3LYP/6-311G** (gas phase) } \\
\hline & & $80 a$ & $80 \mathrm{~b}$ & - & - & - & - & - & - \\
\hline \multirow{3}{*}{ sDP4+ } & ${ }^{1} \mathrm{H}$ data & 84.34 & 15.66 & - & - & - & - & - & - \\
\hline & ${ }^{13} \mathrm{C}$ data & 5.15 & 94.85 & - & - & - & - & - & - \\
\hline & All data & 22.61 & 77.39 & - & - & - & - & - & - \\
\hline
\end{tabular}




\begin{tabular}{|c|c|c|c|c|c|c|c|c|c|}
\hline & ${ }^{1} \mathrm{H}$ data & 99.84 & 0.16 & - & - & - & - & - & - \\
\hline \multirow[t]{3}{*}{ uDP4+ } & ${ }^{13} \mathrm{C}$ data & 0.01 & 99.99 & - & - & - & - & - & - \\
\hline & All data & 6.22 & 93.78 & - & - & - & - & - & - \\
\hline & ${ }^{1} \mathrm{H}$ data & 99.97 & 0.03 & - & - & - & - & - & - \\
\hline \multirow[t]{5}{*}{ DP4+ } & ${ }^{13} \mathrm{C}$ data & 0.00 & 100.00 & - & - & - & - & - & - \\
\hline & All data & 1.90 & 98.10 & - & - & - & - & - & - \\
\hline & & \multicolumn{8}{|c|}{ Level of theory: B3LYP/6-311+G** (gas phase) } \\
\hline & & $80 a$ & $80 \mathrm{~b}$ & - & - & - & - & - & - \\
\hline & ${ }^{1} \mathrm{H}$ data & 65.72 & 34.28 & - & - & - & - & - & - \\
\hline \multirow[t]{3}{*}{ sDP4+ } & ${ }^{13} \mathrm{C}$ data & 4.45 & 95.55 & - & - & - & - & - & - \\
\hline & All data & 8.19 & 91.81 & - & - & - & - & - & - \\
\hline & ${ }^{1} \mathrm{H}$ data & 99.88 & 0.12 & - & - & - & - & - & - \\
\hline \multirow[t]{3}{*}{ uDP4+ } & ${ }^{13} \mathrm{C}$ data & 0.01 & 99.99 & - & - & - & - & - & - \\
\hline & All data & 7.15 & 92.85 & - & - & - & - & - & - \\
\hline & ${ }^{1} \mathrm{H}$ data & 99.94 & 0.06 & - & - & - & - & - & - \\
\hline \multirow[t]{5}{*}{ DP4+ } & ${ }^{13} \mathrm{C}$ data & 0.00 & 100.00 & - & - & - & - & - & - \\
\hline & All data & 0.68 & 99.32 & - & - & - & - & - & - \\
\hline & & \multicolumn{8}{|c|}{ Level of theory: B3LYP/6-31G* (solution, PCM) } \\
\hline & & $80 a$ & $80 \mathrm{~b}$ & - & - & - & - & - & - \\
\hline & ${ }^{1}$ H data & 89.40 & 10.60 & - & - & - & - & - & - \\
\hline \multirow[t]{3}{*}{ sDP4+ } & ${ }^{13} \mathrm{C}$ data & 2.94 & 97.06 & - & - & - & - & - & - \\
\hline & All data & 20.32 & 79.68 & - & - & - & - & - & - \\
\hline & ${ }^{1} \mathrm{H}$ data & 99.98 & 0.02 & - & - & - & - & - & - \\
\hline \multirow[t]{3}{*}{ uDP4+ } & ${ }^{13} \mathrm{C}$ data & 0.38 & 99.62 & - & - & - & - & - & - \\
\hline & All data & 95.92 & 4.08 & - & - & - & - & - & - \\
\hline & ${ }^{1} \mathrm{H}$ data & 100.00 & 0.00 & - & - & - & - & - & - \\
\hline \multirow[t]{5}{*}{ DP4+ } & ${ }^{13} \mathrm{C}$ data & 0.01 & 99.99 & - & - & - & - & - & - \\
\hline & All data & 85.72 & 14.28 & - & - & - & - & - & - \\
\hline & & \multicolumn{8}{|c|}{ Level of theory: B3LYP/6-31G** (solution, PCM) } \\
\hline & & $80 a$ & $80 \mathrm{~b}$ & - & - & - & - & - & - \\
\hline & ${ }^{1} \mathrm{H}$ data & 75.32 & 24.68 & - & - & - & - & - & - \\
\hline \multirow[t]{3}{*}{ sDP4+ } & ${ }^{13} \mathrm{C}$ data & 4.03 & 95.97 & - & - & - & - & - & - \\
\hline & All data & 11.35 & 88.65 & - & - & - & - & - & - \\
\hline & ${ }^{1} \mathrm{H}$ data & 99.96 & 0.04 & - & - & - & - & - & - \\
\hline \multirow[t]{3}{*}{ uDP4+ } & ${ }^{13} \mathrm{C}$ data & 0.21 & 99.79 & - & - & - & - & - & - \\
\hline & All data & 83.62 & 16.38 & - & - & - & - & - & - \\
\hline & ${ }^{1} \mathrm{H}$ data & 99.99 & 0.01 & - & - & - & - & - & - \\
\hline \multirow[t]{5}{*}{ DP4+ } & ${ }^{13} \mathrm{C}$ data & 0.01 & 99.99 & - & - & - & - & - & - \\
\hline & All data & 39.52 & 60.48 & - & - & - & - & - & - \\
\hline & & \multicolumn{8}{|c|}{ Level of theory: B3LYP/6-31+G** (solution, PCM) } \\
\hline & & $80 a$ & $80 \mathrm{~b}$ & - & - & - & - & - & - \\
\hline & ${ }^{1} \mathrm{H}$ data & 6.12 & 93.88 & - & - & - & - & - & - \\
\hline \multirow[t]{3}{*}{ sDP4+ } & ${ }^{13} \mathrm{C}$ data & 8.03 & 91.97 & - & - & - & - & - & - \\
\hline & All data & 0.57 & 99.43 & - & - & - & - & - & - \\
\hline & ${ }^{1} \mathrm{H}$ data & 99.67 & 0.33 & - & - & - & - & - & - \\
\hline uDP4+ & ${ }^{13} \mathrm{C}$ data & 0.14 & 99.86 & - & - & - & - & - & - \\
\hline & All data & 29.98 & 70.02 & - & - & - & - & - & - \\
\hline & ${ }^{1} \mathrm{H}$ data & 95.23 & 4.77 & - & - & - & - & - & - \\
\hline DP4+ & ${ }^{13} \mathrm{C}$ data & 0.01 & 99.99 & - & - & - & - & - & - \\
\hline & All data & 0.24 & 99.76 & - & - & - & - & - & - \\
\hline & & & & el & B3 & G* & $P C$ & & \\
\hline & & $80 a$ & $80 \mathrm{~b}$ & - & - & - & - & - & - \\
\hline & ${ }^{1} \mathrm{H}$ data & 47.32 & 52.68 & - & - & - & - & - & - \\
\hline sDP4+ & ${ }^{13} \mathrm{C}$ data & 4.35 & 95.65 & - & - & - & - & - & - \\
\hline & All data & 3.92 & 96.08 & - & - & - & - & - & - \\
\hline uDP4+ & ${ }^{1} \mathrm{H}$ data & 99.58 & 0.42 & - & - & - & - & - & - \\
\hline
\end{tabular}




\begin{tabular}{|c|c|c|c|c|c|c|c|c|c|}
\hline & ${ }^{13} \mathrm{C}$ data & 0.01 & 99.99 & - & - & - & - & - & - \\
\hline & All data & 2.80 & 97.20 & - & - & - & - & - & - \\
\hline & ${ }^{1} \mathrm{H}$ data & 99.53 & 0.47 & - & - & - & - & - & - \\
\hline \multirow[t]{5}{*}{ DP4+ } & ${ }^{13} \mathrm{C}$ data & 0.00 & 100.00 & - & - & - & - & - & - \\
\hline & All data & 0.12 & 99.88 & - & - & - & - & - & - \\
\hline & & \multicolumn{8}{|c|}{ Level of theory: B3LYP/6-311G** (solution, PCM) } \\
\hline & & $80 a$ & $80 \mathrm{~b}$ & - & - & - & - & - & - \\
\hline & ${ }^{1} \mathrm{H}$ data & 13.24 & 86.76 & - & - & - & - & - & - \\
\hline \multirow[t]{3}{*}{ sDP4+ } & ${ }^{13}$ C data & 4.38 & 95.62 & - & - & - & - & - & - \\
\hline & All data & 0.69 & 99.31 & - & - & - & - & - & - \\
\hline & ${ }^{1}$ H data & 98.66 & 1.34 & - & - & - & - & - & - \\
\hline \multirow[t]{3}{*}{ uDP4+ } & ${ }^{13} \mathrm{C}$ data & 0.01 & 99.99 & - & - & - & - & - & - \\
\hline & All data & 0.62 & 99.38 & - & - & - & - & - & - \\
\hline & ${ }^{1} \mathrm{H}$ data & 91.84 & 8.16 & - & - & - & - & - & - \\
\hline \multirow[t]{5}{*}{ DP4+ } & ${ }^{13} \mathrm{C}$ data & 0.00 & 100.00 & - & - & - & - & - & - \\
\hline & All data & 0.00 & 100.00 & - & - & - & - & - & - \\
\hline & & \multicolumn{8}{|c|}{ Level of theory: B3LYP/6-311+G** (solution, PCM) } \\
\hline & & $80 a$ & $80 \mathrm{~b}$ & - & - & - & - & - & - \\
\hline & ${ }^{1} \mathrm{H}$ data & 3.53 & 96.47 & - & - & - & - & - & - \\
\hline \multirow[t]{3}{*}{ sDP4+ } & ${ }^{13} \mathrm{C}$ data & 3.43 & 96.57 & - & - & - & - & - & - \\
\hline & All data & 0.13 & 99.87 & - & - & - & - & - & - \\
\hline & ${ }^{1} \mathrm{H}$ data & 99.35 & 0.65 & - & - & - & - & - & - \\
\hline \multirow[t]{3}{*}{ uDP4+ } & ${ }^{13} \mathrm{C}$ data & 0.01 & 99.99 & - & - & - & - & - & - \\
\hline & All data & 1.17 & 98.83 & - & - & - & - & - & - \\
\hline & ${ }^{1} \mathrm{H}$ data & 84.79 & 15.21 & - & - & - & - & - & - \\
\hline \multirow[t]{5}{*}{ DP4+ } & ${ }^{13} \mathrm{C}$ data & 0.00 & 100.00 & - & - & - & - & - & - \\
\hline & All data & 0.00 & 100.00 & - & - & - & - & - & - \\
\hline & & \multicolumn{8}{|c|}{ Level of theory: mPW1PW91/6-31G* (gas phase) } \\
\hline & & $80 a$ & $80 \mathrm{~b}$ & - & - & - & - & - & - \\
\hline & ${ }^{1} \mathrm{H}$ data & 93.74 & 6.26 & - & - & - & - & - & - \\
\hline \multirow[t]{3}{*}{ sDP4+ } & ${ }^{13} \mathrm{C}$ data & 2.88 & 97.12 & - & - & - & - & - & - \\
\hline & All data & 30.77 & 69.23 & - & - & - & - & - & - \\
\hline & ${ }^{1} \mathrm{H}$ data & 99.97 & 0.03 & - & - & - & - & - & - \\
\hline \multirow[t]{3}{*}{ uDP4+ } & ${ }^{13}$ C data & 0.93 & 99.07 & - & - & - & - & - & - \\
\hline & All data & 96.64 & 3.36 & - & - & - & - & - & - \\
\hline & ${ }^{1} \mathrm{H}$ data & 100.00 & 0.00 & - & - & - & - & - & - \\
\hline \multirow[t]{5}{*}{ DP4+ } & ${ }^{13} \mathrm{C}$ data & 0.03 & 99.97 & - & - & - & - & - & - \\
\hline & All data & 92.74 & 7.26 & - & - & - & - & - & - \\
\hline & & \multicolumn{8}{|c|}{ Level of theory: mPW1PW91/6-31G** (gas phase) } \\
\hline & & $80 a$ & $80 \mathrm{~b}$ & - & - & - & - & - & - \\
\hline & ${ }^{1} \mathrm{H}$ data & 94.97 & 5.03 & - & - & - & - & - & - \\
\hline \multirow[t]{2}{*}{ sDP4+ } & ${ }^{13} \mathrm{C}$ data & 2.85 & 97.15 & - & - & - & - & - & - \\
\hline & All data & 35.64 & 64.36 & - & - & - & - & - & - \\
\hline \multirow{3}{*}{ uDP4+ } & ${ }^{1} \mathrm{H}$ data & 99.96 & 0.04 & - & - & - & - & - & - \\
\hline & ${ }^{13} \mathrm{C}$ data & 0.30 & 99.70 & - & - & - & - & - & - \\
\hline & All data & 88.01 & 11.99 & - & - & - & - & - & - \\
\hline & ${ }^{1} \mathrm{H}$ data & 100.00 & 0.00 & - & - & - & - & - & - \\
\hline DP4+ & ${ }^{13} \mathrm{C}$ data & 0.01 & 99.99 & - & - & - & - & - & - \\
\hline & All data & 80.25 & 19.75 & - & - & - & - & - & - \\
\hline & & & & 10 & $|P|$ & -3 & $\mathrm{pr}$ & & \\
\hline & & $80 a$ & $80 \mathrm{~b}$ & - & - & - & - & - & - \\
\hline & ${ }^{1} \mathrm{H}$ data & 91.02 & 8.98 & - & - & - & - & - & - \\
\hline sDP4+ & ${ }^{13} \mathrm{C}$ data & 6.10 & 93.90 & - & - & - & - & - & - \\
\hline & All data & 39.71 & 60.29 & - & - & - & - & - & - \\
\hline & ${ }_{12}^{1} \mathrm{H}$ data & 99.87 & 0.13 & - & - & - & - & - & - \\
\hline uDP4+ & ${ }^{13} \mathrm{C}$ data & 0.37 & 99.63 & - & - & - & - & - & - \\
\hline
\end{tabular}




\begin{tabular}{|c|c|c|c|c|c|c|c|c|c|}
\hline & All data & 73.77 & 26.23 & - & - & - & - & - & - \\
\hline & ${ }^{1} \mathrm{H}$ data & 99.99 & 0.01 & - & - & - & - & - & - \\
\hline \multirow[t]{5}{*}{ DP4+ } & ${ }^{13} \mathrm{C}$ data & 0.02 & 99.98 & - & - & - & - & - & - \\
\hline & All data & 64.94 & 35.06 & - & - & - & - & - & - \\
\hline & & \multicolumn{8}{|c|}{ Level of theory: mPW1PW91/6-311G* (gas phase) } \\
\hline & & $80 a$ & $80 \mathrm{~b}$ & - & - & - & - & - & - \\
\hline & ${ }^{1} \mathrm{H}$ data & 92.63 & 7.37 & - & - & - & - & - & - \\
\hline \multirow[t]{2}{*}{ sDP4+ } & ${ }^{13} \mathrm{C}$ data & 5.18 & 94.82 & - & - & - & - & - & - \\
\hline & All data & 40.70 & 59.30 & - & - & - & - & - & - \\
\hline \multirow{3}{*}{ uDP4+ } & ${ }^{1} \mathrm{H}$ data & 99.45 & 0.55 & - & - & - & - & - & - \\
\hline & ${ }^{13} \mathrm{C}$ data & 0.15 & 99.85 & - & - & - & - & - & - \\
\hline & All data & 21.82 & 78.18 & - & - & - & - & - & - \\
\hline \multirow{5}{*}{ DP4+ } & ${ }^{1} \mathrm{H}$ data & 99.96 & 0.04 & - & - & - & - & - & - \\
\hline & ${ }^{13} \mathrm{C}$ data & 0.01 & 99.99 & - & - & - & - & - & - \\
\hline & All data & 16.08 & 83.92 & - & - & - & - & - & - \\
\hline & & \multicolumn{8}{|c|}{ Level of theory: mPW1PW91/6-311G** (gas phase) } \\
\hline & & $80 a$ & $80 \mathrm{~b}$ & - & - & - & - & - & - \\
\hline \multirow{3}{*}{ sDP4+ } & ${ }^{1} \mathrm{H}$ data & 91.36 & 8.64 & - & - & - & - & - & - \\
\hline & ${ }^{13} \mathrm{C}$ data & 5.10 & 94.90 & - & - & - & - & - & - \\
\hline & All data & 36.23 & 63.77 & - & - & - & - & - & - \\
\hline \multirow{3}{*}{ uDP4+ } & ${ }^{1} \mathrm{H}$ data & 99.85 & 0.15 & - & - & - & - & - & - \\
\hline & ${ }^{13} \mathrm{C}$ data & 0.09 & 99.91 & - & - & - & - & - & - \\
\hline & All data & 37.15 & 62.85 & - & - & - & - & - & - \\
\hline \multirow{5}{*}{ DP4+ } & ${ }^{1} \mathrm{H}$ data & 99.99 & 0.01 & - & - & - & - & - & - \\
\hline & ${ }^{13} \mathrm{C}$ data & 0.00 & 100.00 & - & - & - & - & - & - \\
\hline & All data & 25.14 & 74.86 & - & - & - & - & - & - \\
\hline & & \multicolumn{8}{|c|}{ Level of theory: mPW1PW91/6-311+G** (gas phase) } \\
\hline & & $80 a$ & $80 \mathrm{~b}$ & - & - & - & - & - & - \\
\hline \multirow{3}{*}{ sDP4+ } & ${ }^{1} \mathrm{H}$ data & 79.52 & 20.48 & - & - & - & - & - & - \\
\hline & ${ }^{13} \mathrm{C}$ data & 3.75 & 96.25 & - & - & - & - & - & - \\
\hline & All data & 13.14 & 86.86 & - & - & - & - & - & - \\
\hline \multirow{3}{*}{ uDP4+ } & ${ }^{1} \mathrm{H}$ data & 99.88 & 0.12 & - & - & - & - & - & - \\
\hline & ${ }^{13} \mathrm{C}$ data & 0.08 & 99.92 & - & - & - & - & - & - \\
\hline & All data & 39.57 & 60.43 & - & - & - & - & - & - \\
\hline \multirow{5}{*}{ DP4+ } & ${ }^{1} \mathrm{H}$ data & 99.97 & 0.03 & - & - & - & - & - & - \\
\hline & ${ }^{13} \mathrm{C}$ data & 0.00 & 100.00 & - & - & - & - & - & - \\
\hline & All data & 9.01 & 90.99 & - & - & - & - & - & - \\
\hline & & \multicolumn{8}{|c|}{ Level of theory: mPW1PW91/6-31G* (solution, PCM) } \\
\hline & & $80 a$ & $80 \mathrm{~b}$ & - & - & - & - & - & - \\
\hline \multirow{3}{*}{ sDP4+ } & ${ }^{1} \mathrm{H}$ data & 93.45 & 6.55 & - & - & - & - & - & - \\
\hline & ${ }^{13} \mathrm{C}$ data & 2.59 & 97.41 & - & - & - & - & - & - \\
\hline & All data & 27.47 & 72.53 & - & - & - & - & - & - \\
\hline \multirow{3}{*}{ uDP4+ } & ${ }^{1} \mathrm{H}$ data & 99.98 & 0.02 & - & - & - & - & - & - \\
\hline & ${ }^{13} \mathrm{C}$ data & 0.96 & 99.04 & - & - & - & - & - & - \\
\hline & All data & 97.87 & 2.13 & - & - & - & - & - & - \\
\hline & ${ }^{1} \mathrm{H}$ data & 100.00 & 0.00 & - & - & - & - & - & - \\
\hline DP4+ & ${ }^{13} \mathrm{C}$ data & 0.03 & 99.97 & - & - & - & - & - & - \\
\hline & All data & 94.55 & 5.45 & - & - & - & - & - & - \\
\hline & & & & of & W & 11 & n, & & \\
\hline & & $80 a$ & $80 \mathrm{~b}$ & - & - & - & - & - & - \\
\hline & ${ }^{1} \mathrm{H}$ data & 89.13 & 10.87 & - & - & - & - & - & - \\
\hline sDP4+ & ${ }^{13} \mathrm{C}$ data & 2.41 & 97.59 & - & - & - & - & - & - \\
\hline & All data & 16.83 & 83.17 & - & - & - & - & - & - \\
\hline & ${ }^{1} \mathrm{H}$ data & 99.97 & 0.03 & - & - & - & - & - & - \\
\hline uDP4+ & ${ }^{13} \mathrm{C}$ data & 0.33 & 99.67 & - & - & - & - & - & - \\
\hline & All data & 92.88 & 7.12 & - & - & - & - & - & - \\
\hline
\end{tabular}




\begin{tabular}{|c|c|c|c|c|c|c|c|c|c|}
\hline & ${ }^{1} \mathrm{H}$ data & 100.00 & 0.00 & - & - & - & - & - & - \\
\hline \multirow[t]{5}{*}{ DP4+ } & ${ }^{13} \mathrm{C}$ data & 0.01 & 99.99 & - & - & - & - & - & - \\
\hline & All data & 72.54 & 27.46 & - & - & - & - & - & - \\
\hline & & \multicolumn{8}{|c|}{ Level of theory: mPW1PW91/6-31+G** (solution, PCM) } \\
\hline & & $80 a$ & $80 \mathrm{~b}$ & - & - & - & - & - & - \\
\hline & ${ }^{1} \mathrm{H}$ data & 20.78 & 79.22 & - & - & - & - & - & - \\
\hline \multirow[t]{3}{*}{ sDP4+ } & ${ }^{13} \mathrm{C}$ data & 4.88 & 95.12 & - & - & - & - & - & - \\
\hline & All data & 1.33 & 98.67 & - & - & - & - & - & - \\
\hline & ${ }^{1} \mathrm{H}$ data & 99.78 & 0.22 & - & - & - & - & - & - \\
\hline \multirow[t]{3}{*}{ uDP4+ } & ${ }^{13} \mathrm{C}$ data & 0.29 & 99.71 & - & - & - & - & - & - \\
\hline & All data & 57.25 & 42.75 & - & - & - & - & - & - \\
\hline & ${ }^{1} \mathrm{H}$ data & 99.17 & 0.83 & - & - & - & - & - & - \\
\hline \multirow[t]{5}{*}{ DP4+ } & ${ }^{13} \mathrm{C}$ data & 0.02 & 99.98 & - & - & - & - & - & - \\
\hline & All data & 1.77 & 98.23 & - & - & - & - & - & - \\
\hline & & \multicolumn{8}{|c|}{ Level of theory: mPW1PW91/6-311G* (solution, PCM) } \\
\hline & & $80 a$ & $80 b$ & - & - & - & - & - & - \\
\hline & ${ }^{1} \mathrm{H}$ data & 72.41 & 27.59 & - & - & - & - & - & - \\
\hline \multirow[t]{3}{*}{ sDP4+ } & ${ }^{13} \mathrm{C}$ data & 3.78 & 96.22 & - & - & - & - & - & - \\
\hline & All data & 9.36 & 90.64 & - & - & - & - & - & - \\
\hline & ${ }^{1} \mathrm{H}$ data & 99.56 & 0.44 & - & - & - & - & - & - \\
\hline \multirow[t]{3}{*}{ uDP4+ } & ${ }^{13} \mathrm{C}$ data & 0.05 & 99.95 & - & - & - & - & - & - \\
\hline & All data & 10.80 & 89.20 & - & - & - & - & - & - \\
\hline & ${ }^{1} \mathrm{H}$ data & 99.83 & 0.17 & - & - & - & - & - & - \\
\hline \multirow[t]{5}{*}{ DP4+ } & ${ }^{13} \mathrm{C}$ data & 0.00 & 100.00 & - & - & - & - & - & - \\
\hline & All data & 1.23 & 98.77 & - & - & - & - & - & - \\
\hline & & \multicolumn{8}{|c|}{ Level of theory: mPW1PW91/6-311G** (solution, PCM) } \\
\hline & & $80 a$ & $80 \mathrm{~b}$ & - & - & - & - & - & - \\
\hline & ${ }^{1} \mathrm{H}$ data & 29.03 & 70.97 & - & - & - & - & - & - \\
\hline \multirow[t]{2}{*}{ sDP4+ } & ${ }^{13} \mathrm{C}$ data & 3.37 & 96.63 & - & - & - & - & - & - \\
\hline & All data & 1.41 & 98.59 & - & - & - & - & - & - \\
\hline \multirow{3}{*}{ uDP4+ } & ${ }^{1} \mathrm{H}$ data & 99.23 & 0.77 & - & - & - & - & - & - \\
\hline & ${ }^{13} \mathrm{C}$ data & 0.04 & 99.96 & - & - & - & - & - & - \\
\hline & All data & 5.10 & 94.90 & - & - & - & - & - & - \\
\hline \multirow{3}{*}{ DP4+ } & ${ }^{1} \mathrm{H}$ data & 98.13 & 1.87 & - & - & - & - & - & - \\
\hline & ${ }^{13} \mathrm{C}$ data & 0.00 & 100.00 & - & - & - & - & - & - \\
\hline & All data & 0.08 & 99.92 & - & - & - & - & - & - \\
\hline & & \multicolumn{8}{|c|}{ Level of theory: mPW1PW91/6-311+G** (solution, PCM) } \\
\hline & & $80 a$ & $80 \mathrm{~b}$ & - & - & - & - & - & - \\
\hline \multirow{3}{*}{$s \mathrm{DP} 4+$} & ${ }^{1} \mathrm{H}$ data & 9.49 & 90.51 & - & - & - & - & - & - \\
\hline & ${ }^{13} \mathrm{C}$ data & 2.37 & 97.63 & - & - & - & - & - & - \\
\hline & All data & 0.25 & 99.75 & - & - & - & - & - & - \\
\hline \multirow{3}{*}{ uDP4+ } & ${ }^{1} \mathrm{H}$ data & 98.74 & 1.26 & - & - & - & - & - & - \\
\hline & ${ }^{13} \mathrm{C}$ data & 0.04 & 99.96 & - & - & - & - & - & - \\
\hline & All data & 2.68 & 97.32 & - & - & - & - & - & - \\
\hline \multirow{3}{*}{ DP4+ } & ${ }^{1} \mathrm{H}$ data & 89.17 & 10.83 & - & - & - & - & - & - \\
\hline & ${ }^{13} \mathrm{C}$ data & 0.00 & 100.00 & - & - & - & - & - & - \\
\hline & All data & 0.01 & 99.99 & - & - & - & - & - & - \\
\hline
\end{tabular}


$s \mathrm{DP4}+, u \mathrm{DP} 4+$ and DP4+ probabilities (\%) for compound 81a<smiles>C1=CC2(CO2)C2OCC1O2</smiles>

$81 a$

correct isomer<smiles>C1=CC23COC2OC3O1</smiles>

81b

\begin{tabular}{|c|c|c|c|c|c|c|c|c|c|}
\hline & & \multicolumn{8}{|c|}{ Level of theory: B3LYP/6-31G* (gas phase) } \\
\hline & & $81 a$ & 81b & - & - & - & - & - & - \\
\hline & ${ }^{1} \mathrm{H}$ data & 10.91 & 89.09 & - & - & - & - & - & - \\
\hline \multirow[t]{2}{*}{ sDP4+ } & ${ }^{13} \mathrm{C}$ data & 76.15 & 23.85 & - & - & - & - & - & - \\
\hline & All data & 28.11 & 71.89 & - & - & - & - & - & - \\
\hline & ${ }^{1} \mathrm{H}$ data & 17.36 & 82.64 & - & - & - & - & - & - \\
\hline \multirow[t]{2}{*}{ uDP4+ } & ${ }^{13} \mathrm{C}$ data & 98.81 & 1.19 & - & - & - & - & - & - \\
\hline & All data & 94.56 & 5.44 & - & - & - & - & - & - \\
\hline \multirow{5}{*}{ DP4+ } & ${ }^{1} \mathrm{H}$ data & 2.51 & 97.49 & - & - & - & - & - & - \\
\hline & ${ }^{13} \mathrm{C}$ data & 99.62 & 0.38 & - & - & - & - & - & - \\
\hline & All data & 87.17 & 12.83 & - & - & - & - & - & - \\
\hline & & \multicolumn{8}{|c|}{ Level of theory: B3LYP/6-31G** (gas phase) } \\
\hline & & $81 a$ & $81 \mathrm{~b}$ & - & - & - & - & - & - \\
\hline & ${ }^{1} \mathrm{H}$ data & 13.93 & 86.07 & - & - & - & - & - & - \\
\hline \multirow[t]{2}{*}{ sDP4+ } & ${ }^{13} \mathrm{C}$ data & 62.94 & 37.06 & - & - & - & - & - & - \\
\hline & All data & 21.56 & 78.44 & - & - & - & - & - & - \\
\hline & ${ }^{1} \mathrm{H}$ data & 9.72 & 90.28 & - & - & - & - & - & - \\
\hline \multirow[t]{2}{*}{ uDP4+ } & ${ }^{13} \mathrm{C}$ data & 98.50 & 1.50 & - & - & - & - & - & - \\
\hline & All data & 87.58 & 12.42 & - & - & - & - & - & - \\
\hline & ${ }^{1} \mathrm{H}$ data & 1.71 & 98.29 & - & - & - & - & - & - \\
\hline \multirow[t]{5}{*}{ DP4+ } & ${ }^{13} \mathrm{C}$ data & 99.11 & 0.89 & - & - & - & - & - & - \\
\hline & All data & 65.97 & 34.03 & - & - & - & - & - & - \\
\hline & & \multicolumn{8}{|c|}{ Level of theory: B3LYP/6-31+G** (gas phase) } \\
\hline & & $81 a$ & $81 \mathrm{~b}$ & - & - & - & - & - & - \\
\hline & ${ }^{1} \mathrm{H}$ data & 24.44 & 75.56 & - & - & - & - & - & - \\
\hline \multirow[t]{2}{*}{ sDP4+ } & ${ }^{13} \mathrm{C}$ data & 81.66 & 18.34 & - & - & - & - & - & - \\
\hline & All data & 59.02 & 40.98 & - & - & - & - & - & - \\
\hline \multirow{3}{*}{ uDP4+ } & ${ }^{1} \mathrm{H}$ data & 16.86 & 83.14 & - & - & - & - & - & - \\
\hline & ${ }^{13} \mathrm{C}$ data & 98.52 & 1.48 & - & - & - & - & - & - \\
\hline & All data & 93.10 & 6.90 & - & - & - & - & - & - \\
\hline \multirow{6}{*}{ DP4+ } & ${ }^{1} \mathrm{H}$ data & 6.16 & 93.84 & - & - & - & - & - & - \\
\hline & ${ }^{13} \mathrm{C}$ data & 99.66 & 0.34 & - & - & - & - & - & - \\
\hline & All data & 95.10 & 4.90 & - & - & - & - & - & - \\
\hline & & \multicolumn{8}{|c|}{ Level of theory: B3LYP/6-311G* (gas phase) } \\
\hline & & $81 a$ & $81 \mathrm{~b}$ & - & - & - & - & - & - \\
\hline & ${ }^{1} \mathrm{H}$ data & 10.66 & 89.34 & - & - & - & - & - & - \\
\hline \multirow[t]{2}{*}{$s \mathrm{DP4+}$} & ${ }^{13} \mathrm{C}$ data & 72.58 & 27.42 & - & - & - & - & - & - \\
\hline & All data & 23.99 & 76.01 & - & - & - & - & - & - \\
\hline & ${ }^{1} \mathrm{H}$ data & 17.26 & 82.74 & - & - & - & - & - & - \\
\hline \multirow[t]{2}{*}{ uDP4+ } & ${ }^{13} \mathrm{C}$ data & 98.50 & 1.50 & - & - & - & - & - & - \\
\hline & All data & 93.18 & 6.82 & - & - & - & - & - & - \\
\hline \multirow{3}{*}{ DP4+ } & ${ }^{1} \mathrm{H}$ data & 2.43 & 97.57 & - & - & - & - & - & - \\
\hline & ${ }^{13} \mathrm{C}$ data & 99.43 & 0.57 & - & - & - & - & - & - \\
\hline & All data & 81.19 & 18.81 & - & - & - & - & - & - \\
\hline & & \multicolumn{8}{|c|}{ Level of theory: B3LYP/6-311G** (gas phase) } \\
\hline & & $81 a$ & $81 b$ & - & - & - & - & - & - \\
\hline \multirow{2}{*}{ sDP4+ } & ${ }^{1} \mathrm{H}$ data & 20.29 & 79.71 & - & - & - & - & - & - \\
\hline & ${ }^{13} \mathrm{C}$ data & 75.23 & 24.77 & - & - & - & - & - & - \\
\hline
\end{tabular}




\begin{tabular}{|c|c|c|c|c|c|c|c|c|c|}
\hline & All data & 43.60 & 56.40 & - & - & - & - & - & - \\
\hline & ${ }^{1} \mathrm{H}$ data & 13.07 & 86.93 & - & - & - & - & - & - \\
\hline \multirow[t]{3}{*}{ uDP4+ } & ${ }^{13} \mathrm{C}$ data & 98.19 & 1.81 & - & - & - & - & - & - \\
\hline & All data & 89.10 & 10.90 & - & - & - & - & - & - \\
\hline & ${ }^{1} \mathrm{H}$ data & 3.69 & 96.31 & - & - & - & - & - & - \\
\hline \multirow[t]{5}{*}{ DP4+ } & ${ }^{13} \mathrm{C}$ data & 99.40 & 0.60 & - & - & - & - & - & - \\
\hline & All data & 86.34 & 13.66 & - & - & - & - & - & - \\
\hline & & \multicolumn{8}{|c|}{ Level of theory: B3LYP/6-311+G** (gas phase) } \\
\hline & & $81 a$ & 81b & - & - & - & - & - & - \\
\hline & ${ }^{1} \mathrm{H}$ data & 35.70 & 64.30 & - & - & - & - & - & - \\
\hline \multirow[t]{3}{*}{ sDP4+ } & ${ }^{13} \mathrm{C}$ data & 74.27 & 25.73 & - & - & - & - & - & - \\
\hline & All data & 61.57 & 38.43 & - & - & - & - & - & - \\
\hline & ${ }^{1} \mathrm{H}$ data & 13.82 & 86.18 & - & - & - & - & - & - \\
\hline \multirow[t]{3}{*}{ uDP4+ } & ${ }^{13} \mathrm{C}$ data & 96.74 & 3.26 & - & - & - & - & - & - \\
\hline & All data & 82.65 & 17.35 & - & - & - & - & - & - \\
\hline & ${ }^{1} \mathrm{H}$ data & 8.18 & 91.82 & - & - & - & - & - & - \\
\hline \multirow[t]{5}{*}{ DP4+ } & ${ }^{13} \mathrm{C}$ data & 98.85 & 1.15 & - & - & - & - & - & - \\
\hline & All data & 88.42 & 11.58 & - & - & - & - & - & - \\
\hline & & \multicolumn{8}{|c|}{ Level of theory: B3LYP/6-31G* (solution, PCM) } \\
\hline & & $81 a$ & $81 b$ & - & - & - & - & - & - \\
\hline & ${ }^{1}$ H data & 21.44 & 78.56 & - & - & - & - & - & - \\
\hline \multirow[t]{3}{*}{ sDP4+ } & ${ }^{13} \mathrm{C}$ data & 96.01 & 3.99 & - & - & - & - & - & - \\
\hline & All data & 86.78 & 13.22 & - & - & - & - & - & - \\
\hline & ${ }^{1} \mathrm{H}$ data & 27.58 & 72.42 & - & - & - & - & - & - \\
\hline \multirow[t]{3}{*}{ uDP4+ } & ${ }^{13} \mathrm{C}$ data & 99.17 & 0.83 & - & - & - & - & - & - \\
\hline & All data & 97.86 & 2.14 & - & - & - & - & - & - \\
\hline & ${ }^{1} \mathrm{H}$ data & 9.41 & 90.59 & - & - & - & - & - & - \\
\hline \multirow[t]{5}{*}{ DP4+ } & ${ }^{13} \mathrm{C}$ data & 99.97 & 0.03 & - & - & - & - & - & - \\
\hline & All data & 99.67 & 0.33 & - & - & - & - & - & - \\
\hline & & \multicolumn{8}{|c|}{ Level of theory: B3LYP/6-31G** (solution, PCM) } \\
\hline & & $81 a$ & $81 b$ & - & - & - & - & - & - \\
\hline & ${ }^{1} \mathrm{H}$ data & 27.33 & 72.67 & - & - & - & - & - & - \\
\hline \multirow[t]{3}{*}{ sDP4+ } & ${ }^{13} \mathrm{C}$ data & 87.08 & 12.92 & - & - & - & - & - & - \\
\hline & All data & 71.70 & 28.30 & - & - & - & - & - & - \\
\hline & ${ }^{1} \mathrm{H}$ data & 14.31 & 85.69 & - & - & - & - & - & - \\
\hline \multirow[t]{3}{*}{ uDP4+ } & ${ }^{13} \mathrm{C}$ data & 98.78 & 1.22 & - & - & - & - & - & - \\
\hline & All data & 93.14 & 6.86 & - & - & - & - & - & - \\
\hline & ${ }^{1} \mathrm{H}$ data & 5.91 & 94.09 & - & - & - & - & - & - \\
\hline \multirow[t]{5}{*}{ DP4+ } & ${ }^{13} \mathrm{C}$ data & 99.82 & 0.18 & - & - & - & - & - & - \\
\hline & All data & 97.17 & 2.83 & - & - & - & - & - & - \\
\hline & & \multicolumn{8}{|c|}{ Level of theory: B3LYP/6-31+G** (solution, PCM) } \\
\hline & & $81 a$ & 81b & - & - & - & - & - & - \\
\hline & ${ }^{1} \mathrm{H}$ data & 46.41 & 53.59 & - & - & - & - & - & - \\
\hline \multirow[t]{3}{*}{ sDP4+ } & ${ }^{13} \mathrm{C}$ data & 96.79 & 3.21 & - & - & - & - & - & - \\
\hline & All data & 96.32 & 3.68 & - & - & - & - & - & - \\
\hline & ${ }^{1} \mathrm{H}$ data & 35.94 & 64.06 & - & - & - & - & - & - \\
\hline uDP4+ & ${ }^{13} \mathrm{C}$ data & 98.92 & 1.08 & - & - & - & - & - & - \\
\hline & All data & 98.09 & 1.91 & - & - & - & - & - & - \\
\hline & ${ }^{1} \mathrm{H}$ data & 32.70 & 67.30 & - & - & - & - & - & - \\
\hline DP4+ & ${ }^{13} \mathrm{C}$ data & 99.96 & 0.04 & - & - & - & - & - & - \\
\hline & All data & 99.93 & 0.07 & - & - & - & - & - & - \\
\hline & & & & el & B3 & G* & PC & & \\
\hline & & $81 a$ & $81 b$ & - & - & - & - & - & - \\
\hline & ${ }^{1} \mathrm{H}$ data & 29.92 & 70.08 & - & - & - & - & - & - \\
\hline sDP4+ & ${ }^{13} \mathrm{C}$ data & 86.55 & 13.45 & - & - & - & - & - & - \\
\hline & All data & 73.31 & 26.69 & - & - & - & - & - & - \\
\hline
\end{tabular}




\begin{tabular}{|c|c|c|c|c|c|c|c|c|c|}
\hline & ${ }^{1} \mathrm{H}$ data & 35.72 & 64.28 & - & - & - & - & - & - \\
\hline \multirow[t]{3}{*}{ uDP4+ } & ${ }^{13} \mathrm{C}$ data & 99.07 & 0.93 & - & - & - & - & - & - \\
\hline & All data & 98.35 & 1.65 & - & - & - & - & - & - \\
\hline & ${ }^{1} \mathrm{H}$ data & 19.18 & 80.82 & - & - & - & - & - & - \\
\hline \multirow[t]{5}{*}{ DP4+ } & ${ }^{13} \mathrm{C}$ data & 99.86 & 0.14 & - & - & - & - & - & - \\
\hline & All data & 99.39 & 0.61 & - & - & - & - & - & - \\
\hline & & \multicolumn{8}{|c|}{ Level of theory: B3LYP/6-311G** (solution, PCM) } \\
\hline & & $81 a$ & 81b & - & - & - & - & - & - \\
\hline & ${ }^{1} \mathrm{H}$ data & 39.52 & 60.48 & - & - & - & - & - & - \\
\hline \multirow[t]{3}{*}{ sDP4+ } & ${ }^{13} \mathrm{C}$ data & 88.22 & 11.78 & - & - & - & - & - & - \\
\hline & All data & 83.03 & 16.97 & - & - & - & - & - & - \\
\hline & ${ }^{1} \mathrm{H}$ data & 27.42 & 72.58 & - & - & - & - & - & - \\
\hline \multirow[t]{3}{*}{ uDP4+ } & ${ }^{13} \mathrm{C}$ data & 98.87 & 1.13 & - & - & - & - & - & - \\
\hline & All data & 97.07 & 2.93 & - & - & - & - & - & - \\
\hline & ${ }^{1} \mathrm{H}$ data & 19.80 & 80.20 & - & - & - & - & - & - \\
\hline \multirow[t]{5}{*}{ DP4+ } & ${ }^{13} \mathrm{C}$ data & 99.85 & 0.15 & - & - & - & - & - & - \\
\hline & All data & 99.39 & 0.61 & - & - & - & - & - & - \\
\hline & & \multicolumn{8}{|c|}{ Level of theory: B3LYP/6-311+G** (solution, PCM) } \\
\hline & & $81 a$ & $81 b$ & - & - & - & - & - & - \\
\hline & ${ }^{1} \mathrm{H}$ data & 48.46 & 51.54 & - & - & - & - & - & - \\
\hline \multirow[t]{3}{*}{ sDP4+ } & ${ }^{13} \mathrm{C}$ data & 86.65 & 13.35 & - & - & - & - & - & - \\
\hline & All data & 85.92 & 14.08 & - & - & - & - & - & - \\
\hline & ${ }^{1} \mathrm{H}$ data & 51.84 & 48.16 & - & - & - & - & - & - \\
\hline \multirow[t]{3}{*}{ uDP4+ } & ${ }^{13} \mathrm{C}$ data & 98.29 & 1.71 & - & - & - & - & - & - \\
\hline & All data & 98.41 & 1.59 & - & - & - & - & - & - \\
\hline & ${ }^{1} \mathrm{H}$ data & 50.30 & 49.70 & - & - & - & - & - & - \\
\hline \multirow[t]{5}{*}{ DP4+ } & ${ }^{13} \mathrm{C}$ data & 99.73 & 0.27 & - & - & - & - & - & - \\
\hline & All data & 99.74 & 0.26 & - & - & - & - & - & - \\
\hline & & \multicolumn{8}{|c|}{ Level of theory: mPW1PW91/6-31G* (gas phase) } \\
\hline & & $81 a$ & $81 b$ & - & - & - & - & - & - \\
\hline & ${ }^{1} \mathrm{H}$ data & 11.42 & 88.58 & - & - & - & - & - & - \\
\hline \multirow[t]{3}{*}{ sDP4+ } & ${ }^{13} \mathrm{C}$ data & 79.75 & 20.25 & - & - & - & - & - & - \\
\hline & All data & 33.66 & 66.34 & - & - & - & - & - & - \\
\hline & ${ }^{1} \mathrm{H}$ data & 12.72 & 87.28 & - & - & - & - & - & - \\
\hline \multirow[t]{3}{*}{ uDP4+ } & ${ }^{13} \mathrm{C}$ data & 98.61 & 1.39 & - & - & - & - & - & - \\
\hline & All data & 91.20 & 8.80 & - & - & - & - & - & - \\
\hline & ${ }^{1} \mathrm{H}$ data & 1.84 & 98.16 & - & - & - & - & - & - \\
\hline \multirow[t]{5}{*}{ DP4+ } & ${ }^{13} \mathrm{C}$ data & 99.64 & 0.36 & - & - & - & - & - & - \\
\hline & All data & 84.03 & 15.97 & - & - & - & - & - & - \\
\hline & & \multicolumn{8}{|c|}{ Level of theory: mPW1PW91/6-31G** (gas phase) } \\
\hline & & $81 a$ & $81 b$ & - & - & - & - & - & - \\
\hline & ${ }^{1} \mathrm{H}$ data & 14.88 & 85.12 & - & - & - & - & - & - \\
\hline \multirow[t]{3}{*}{ sDP4+ } & ${ }^{13} \mathrm{C}$ data & 62.78 & 37.22 & - & - & - & - & - & - \\
\hline & All data & 22.77 & 77.23 & - & - & - & - & - & - \\
\hline & ${ }^{1}$ H data & 5.81 & 94.19 & - & - & - & - & - & - \\
\hline uDP4+ & ${ }^{13} \mathrm{C}$ data & 98.88 & 1.12 & - & - & - & - & - & - \\
\hline & All data & 84.44 & 15.56 & - & - & - & - & - & - \\
\hline & ${ }^{1} \mathrm{H}$ data & 1.07 & 98.93 & - & - & - & - & - & - \\
\hline DP4+ & ${ }^{13} \mathrm{C}$ data & 99.33 & 0.67 & - & - & - & - & - & - \\
\hline & All data & 61.55 & 38.45 & - & - & - & - & - & - \\
\hline & & & & 10 & $\mathbf{P} \mid$ & -3 & pr & & \\
\hline & & $81 a$ & $81 b$ & - & - & - & - & - & - \\
\hline & ${ }^{1} \mathrm{H}$ data & 32.14 & 67.86 & - & - & - & - & - & - \\
\hline sDP4+ & ${ }^{13} \mathrm{C}$ data & 82.22 & 17.78 & - & - & - & - & - & - \\
\hline & All data & 68.66 & 31.34 & - & - & - & - & - & - \\
\hline uDP4+ & ${ }^{1} \mathrm{H}$ data & 16.48 & 83.52 & - & - & - & - & - & - \\
\hline
\end{tabular}




\begin{tabular}{|c|c|c|c|c|c|c|c|c|c|}
\hline & ${ }^{13} \mathrm{C}$ data & 99.25 & 0.75 & - & - & - & - & - & - \\
\hline & All data & 96.30 & 3.70 & - & - & - & - & - & - \\
\hline & ${ }^{1} \mathrm{H}$ data & 8.55 & 91.45 & - & - & - & - & - & - \\
\hline \multirow[t]{4}{*}{ DP4+ } & ${ }^{13} \mathrm{C}$ data & 99.84 & 0.16 & - & - & - & - & - & - \\
\hline & All data & 98.28 & 1.72 & - & - & - & - & - & - \\
\hline & & \multicolumn{8}{|c|}{ Level of theory: mPW1PW91/6-311G* (gas phase) } \\
\hline & & $81 a$ & $81 \mathrm{~b}$ & - & - & - & - & - & - \\
\hline & ${ }^{1} \mathrm{H}$ data & 16.53 & 83.47 & - & - & - & - & - & - \\
\hline \multirow[t]{2}{*}{ sDP4+ } & ${ }^{13} \mathrm{C}$ data & 56.49 & 43.51 & - & - & - & - & - & - \\
\hline & All data & 20.45 & 79.55 & - & - & - & - & - & - \\
\hline \multirow{3}{*}{ uDP4+ } & ${ }^{1} \mathrm{H}$ data & 18.20 & 81.80 & - & - & - & - & - & - \\
\hline & ${ }^{13} \mathrm{C}$ data & 99.01 & 0.99 & - & - & - & - & - & - \\
\hline & All data & 95.71 & 4.29 & - & - & - & - & - & - \\
\hline \multirow{5}{*}{ DP4+ } & ${ }^{1} \mathrm{H}$ data & 4.22 & 95.78 & - & - & - & - & - & - \\
\hline & ${ }^{13} \mathrm{C}$ data & 99.24 & 0.76 & - & - & - & - & - & - \\
\hline & All data & 85.17 & 14.83 & - & - & - & - & - & - \\
\hline & & \multicolumn{8}{|c|}{ Level of theory: mPW1PW91/6-311G** (gas phase) } \\
\hline & & $81 a$ & $81 \mathrm{~b}$ & - & - & - & - & - & - \\
\hline \multirow{3}{*}{ sDP4+ } & ${ }^{1} \mathrm{H}$ data & 25.00 & 75.00 & - & - & - & - & - & - \\
\hline & ${ }^{13} \mathrm{C}$ data & 64.64 & 35.36 & - & - & - & - & - & - \\
\hline & All data & 37.85 & 62.15 & - & - & - & - & - & - \\
\hline \multirow{3}{*}{ uDP4+ } & ${ }^{1} \mathrm{H}$ data & 9.44 & 90.56 & - & - & - & - & - & - \\
\hline & ${ }^{13} \mathrm{C}$ data & 98.64 & 1.36 & - & - & - & - & - & - \\
\hline & All data & 88.35 & 11.65 & - & - & - & - & - & - \\
\hline \multirow{5}{*}{ DP4+ } & ${ }^{1} \mathrm{H}$ data & 3.36 & 96.64 & - & - & - & - & - & - \\
\hline & ${ }^{13} \mathrm{C}$ data & 99.25 & 0.75 & - & - & - & - & - & - \\
\hline & All data & 82.20 & 17.80 & - & - & - & - & - & - \\
\hline & & \multicolumn{8}{|c|}{ Level of theory: mPW1PW91/6-311+G** (gas phase) } \\
\hline & & $81 a$ & $81 \mathrm{~b}$ & - & - & - & - & - & - \\
\hline \multirow{3}{*}{ sDP4+ } & ${ }^{1} \mathrm{H}$ data & 42.57 & 57.43 & - & - & - & - & - & - \\
\hline & ${ }^{13} \mathrm{C}$ data & 58.26 & 41.74 & - & - & - & - & - & - \\
\hline & All data & 50.86 & 49.14 & - & - & - & - & - & - \\
\hline \multirow{3}{*}{ uDP4+ } & ${ }^{1} \mathrm{H}$ data & 9.58 & 90.42 & - & - & - & - & - & - \\
\hline & ${ }^{13} \mathrm{C}$ data & 98.25 & 1.75 & - & - & - & - & - & - \\
\hline & All data & 85.61 & 14.39 & - & - & - & - & - & - \\
\hline \multirow{5}{*}{ DP4+ } & ${ }^{1} \mathrm{H}$ data & 7.28 & 92.72 & - & - & - & - & - & - \\
\hline & ${ }^{13} \mathrm{C}$ data & 98.74 & 1.26 & - & - & - & - & - & - \\
\hline & All data & 86.03 & 13.97 & - & - & - & - & - & - \\
\hline & & \multicolumn{8}{|c|}{ Level of theory: mPW1PW91/6-31G* (solution, PCM) } \\
\hline & & $81 a$ & 81b & - & - & - & - & - & - \\
\hline \multirow{3}{*}{$s \mathrm{DP} 4+$} & ${ }^{1} \mathrm{H}$ data & 23.64 & 76.36 & - & - & - & - & - & - \\
\hline & ${ }^{13} \mathrm{C}$ data & 95.56 & 4.44 & - & - & - & - & - & - \\
\hline & All data & 86.96 & 13.04 & - & - & - & - & - & - \\
\hline \multirow{3}{*}{ uDP4+ } & ${ }^{1} \mathrm{H}$ data & 21.66 & 78.34 & - & - & - & - & - & - \\
\hline & ${ }^{13} \mathrm{C}$ data & 99.04 & 0.96 & - & - & - & - & - & - \\
\hline & All data & 96.63 & 3.37 & - & - & - & - & - & - \\
\hline & ${ }^{1} \mathrm{H}$ data & 7.89 & 92.11 & - & - & - & - & - & - \\
\hline DP4+ & ${ }^{13} \mathrm{C}$ data & 99.96 & 0.04 & - & - & - & - & - & - \\
\hline & All data & 99.48 & 0.52 & - & - & - & - & - & - \\
\hline & & & & of & W & $31 C$ & on, & & \\
\hline & & $81 a$ & 81b & - & - & - & - & - & - \\
\hline & ${ }^{1} \mathrm{H}$ data & 27.26 & 72.74 & - & - & - & - & - & - \\
\hline sDP4+ & ${ }^{13} \mathrm{C}$ data & 84.30 & 15.70 & - & - & - & - & - & - \\
\hline & All data & 66.80 & 33.20 & - & - & - & - & - & - \\
\hline & ${ }^{1} \mathrm{H}$ data & 9.53 & 90.47 & - & - & - & - & - & - \\
\hline uDP4+ & ${ }^{13} \mathrm{C}$ data & 98.86 & 1.14 & - & - & - & - & - & - \\
\hline
\end{tabular}




\begin{tabular}{|c|c|c|c|c|c|c|c|c|c|}
\hline & All data & 90.12 & 9.88 & - & - & - & - & - & - \\
\hline & ${ }^{1} \mathrm{H}$ data & 3.80 & 96.20 & - & - & - & - & - & - \\
\hline \multirow[t]{5}{*}{ DP4+ } & ${ }^{13} \mathrm{C}$ data & 99.79 & 0.21 & - & - & - & - & - & - \\
\hline & All data & 94.84 & 5.16 & - & - & - & - & - & - \\
\hline & & \multicolumn{8}{|c|}{ Level of theory: mPW1PW91/6-31+G** (solution, PCM) } \\
\hline & & $81 a$ & $81 b$ & - & - & - & - & - & - \\
\hline & ${ }^{1} \mathrm{H}$ data & 64.27 & 35.73 & - & - & - & - & - & - \\
\hline \multirow[t]{3}{*}{ sDP4+ } & ${ }^{13} \mathrm{C}$ data & 97.16 & 2.84 & - & - & - & - & - & - \\
\hline & All data & 98.40 & 1.60 & - & - & - & - & - & - \\
\hline & ${ }^{1} \mathrm{H}$ data & 36.15 & 63.85 & - & - & - & - & - & - \\
\hline \multirow[t]{3}{*}{ uDP4+ } & ${ }^{13} \mathrm{C}$ data & 99.32 & 0.68 & - & - & - & - & - & - \\
\hline & All data & 98.80 & 1.20 & - & - & - & - & - & - \\
\hline & ${ }^{1} \mathrm{H}$ data & 50.46 & 49.54 & - & - & - & - & - & - \\
\hline \multirow[t]{5}{*}{ DP4+ } & ${ }^{13} \mathrm{C}$ data & 99.98 & 0.02 & - & - & - & - & - & - \\
\hline & All data & 99.98 & 0.02 & - & - & - & - & - & - \\
\hline & & \multicolumn{8}{|c|}{ Level of theory: mPW1PW91/6-311G* (solution, PCM) } \\
\hline & & $81 a$ & 81b & - & - & - & - & - & - \\
\hline & ${ }^{1} \mathrm{H}$ data & 29.96 & 70.04 & - & - & - & - & - & - \\
\hline \multirow[t]{3}{*}{ sDP4+ } & ${ }^{13} \mathrm{C}$ data & 61.42 & 38.58 & - & - & - & - & - & - \\
\hline & All data & 40.51 & 59.49 & - & - & - & - & - & - \\
\hline & ${ }^{1}$ H data & 30.06 & 69.94 & - & - & - & - & - & - \\
\hline \multirow[t]{3}{*}{ uDP4+ } & ${ }^{13}$ C data & 99.47 & 0.53 & - & - & - & - & - & - \\
\hline & All data & 98.77 & 1.23 & - & - & - & - & - & - \\
\hline & ${ }^{1} \mathrm{H}$ data & 15.53 & 84.47 & - & - & - & - & - & - \\
\hline \multirow[t]{5}{*}{ DP4+ } & ${ }^{13} \mathrm{C}$ data & 99.67 & 0.33 & - & - & - & - & - & - \\
\hline & All data & 98.21 & 1.79 & - & - & - & - & - & - \\
\hline & & \multicolumn{8}{|c|}{ Level of theory: mPW1PW91/6-311G** (solution, PCM) } \\
\hline & & $81 a$ & 81b & - & - & - & - & - & - \\
\hline & ${ }^{1} \mathrm{H}$ data & 37.52 & 62.48 & - & - & - & - & - & - \\
\hline \multirow[t]{2}{*}{ sDP4+ } & ${ }^{13} \mathrm{C}$ data & 69.85 & 30.15 & - & - & - & - & - & - \\
\hline & All data & 58.18 & 41.82 & - & - & - & - & - & - \\
\hline \multirow{3}{*}{ uDP4+ } & ${ }^{1} \mathrm{H}$ data & 26.02 & 73.98 & - & - & - & - & - & - \\
\hline & ${ }^{13} \mathrm{C}$ data & 99.24 & 0.76 & - & - & - & - & - & - \\
\hline & All data & 97.87 & 2.13 & - & - & - & - & - & - \\
\hline \multirow{5}{*}{ DP4+ } & ${ }^{1} \mathrm{H}$ data & 17.44 & 82.56 & - & - & - & - & - & - \\
\hline & ${ }^{13} \mathrm{C}$ data & 99.67 & 0.33 & - & - & - & - & - & - \\
\hline & All data & 98.46 & 1.54 & - & - & - & - & - & - \\
\hline & & \multicolumn{8}{|c|}{ Level of theory: mPW1PW91/6-311+G** (solution, PCM) } \\
\hline & & $81 a$ & $81 b$ & - & - & - & - & - & - \\
\hline \multirow{3}{*}{ sDP4+ } & ${ }^{1} \mathrm{H}$ data & 49.75 & 50.25 & - & - & - & - & - & - \\
\hline & ${ }^{13} \mathrm{C}$ data & 61.37 & 38.63 & - & - & - & - & - & - \\
\hline & All data & 61.14 & 38.86 & - & - & - & - & - & - \\
\hline \multirow{3}{*}{ uDP4+ } & ${ }^{1} \mathrm{H}$ data & 58.14 & 41.86 & - & - & - & - & - & - \\
\hline & ${ }^{13} \mathrm{C}$ data & 99.01 & 0.99 & - & - & - & - & - & - \\
\hline & All data & 99.28 & 0.72 & - & - & - & - & - & - \\
\hline \multirow{3}{*}{ DP4+ } & ${ }^{1} \mathrm{H}$ data & 57.90 & 42.10 & - & - & - & - & - & - \\
\hline & ${ }^{13} \mathrm{C}$ data & 99.37 & 0.63 & - & - & - & - & - & - \\
\hline & All data & 99.54 & 0.46 & - & - & - & - & - & - \\
\hline
\end{tabular}


sDP4+, $u$ DP4+ and DP4+ probabilities (\%) for compound 82b

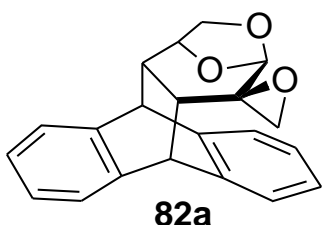

$82 a$

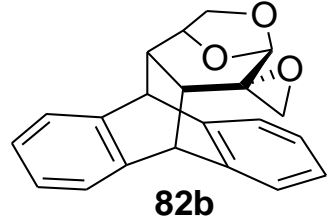

correct isomer

\begin{tabular}{|c|c|c|c|c|c|c|c|c|c|}
\hline & & \multicolumn{8}{|c|}{ Level of theory: B3LYP/6-31G* (gas phase) } \\
\hline & & $82 a$ & $82 b$ & - & - & - & - & - & - \\
\hline \multirow{3}{*}{ sDP4+ } & ${ }^{1} \mathrm{H}$ data & 0.04 & 99.96 & - & - & - & - & - & - \\
\hline & ${ }^{13} \mathrm{C}$ data & 91.90 & 8.10 & - & - & - & - & - & - \\
\hline & All data & 0.49 & 99.51 & - & - & - & - & - & - \\
\hline \multirow{3}{*}{ uDP4+ } & ${ }^{1} \mathrm{H}$ data & 20.17 & 79.83 & - & - & - & - & - & - \\
\hline & ${ }^{13} \mathrm{C}$ data & 12.76 & 87.24 & - & - & - & - & - & - \\
\hline & All data & 3.56 & 96.44 & - & - & - & - & - & - \\
\hline \multirow{5}{*}{ DP4+ } & ${ }^{1} \mathrm{H}$ data & 0.01 & 99.99 & - & - & - & - & - & - \\
\hline & ${ }^{13} \mathrm{C}$ data & 62.39 & 37.61 & - & - & - & - & - & - \\
\hline & All data & 0.02 & 99.98 & - & - & - & - & - & - \\
\hline & & \multicolumn{8}{|c|}{ Level of theory: B3LYP/6-31G** (gas phase) } \\
\hline & & $82 a$ & $82 b$ & - & - & - & - & - & - \\
\hline \multirow{3}{*}{ sDP4+ } & ${ }^{1} \mathrm{H}$ data & 0.02 & 99.98 & - & - & - & - & - & - \\
\hline & ${ }^{13} \mathrm{C}$ data & 92.12 & 7.88 & - & - & - & - & - & - \\
\hline & All data & 0.28 & 99.72 & - & - & - & - & - & - \\
\hline \multirow{3}{*}{ uDP4+ } & ${ }^{1} \mathrm{H}$ data & 6.93 & 93.07 & - & - & - & - & - & - \\
\hline & ${ }^{13} \mathrm{C}$ data & 5.58 & 94.42 & - & - & - & - & - & - \\
\hline & All data & 0.44 & 99.56 & - & - & - & - & - & - \\
\hline \multirow{3}{*}{ DP4+ } & ${ }^{1} \mathrm{H}$ data & 0.00 & 100.00 & - & - & - & - & - & - \\
\hline & ${ }^{13} \mathrm{C}$ data & 40.89 & 59.11 & - & - & - & - & - & - \\
\hline & All data & 0.00 & 100.00 & - & - & - & - & - & - \\
\hline & & \multicolumn{8}{|c|}{ Level of theory: B3LYP/6-31+G** (gas phase) } \\
\hline & & $82 a$ & $82 b$ & - & - & - & - & - & - \\
\hline \multirow{3}{*}{ sDP4+ } & ${ }^{1} \mathrm{H}$ data & 0.02 & 99.98 & - & - & - & - & - & - \\
\hline & ${ }^{13} \mathrm{C}$ data & 77.92 & 22.08 & - & - & - & - & - & - \\
\hline & All data & 0.06 & 99.94 & - & - & - & - & - & - \\
\hline \multirow{3}{*}{ uDP4+ } & ${ }^{1} \mathrm{H}$ data & 0.68 & 99.32 & - & - & - & - & - & - \\
\hline & ${ }^{13} \mathrm{C}$ data & 7.77 & 92.23 & - & - & - & - & - & - \\
\hline & All data & 0.06 & 99.94 & - & - & - & - & - & - \\
\hline \multirow{3}{*}{ DP4+ } & ${ }^{1} \mathrm{H}$ data & 0.00 & 100.00 & - & - & - & - & - & - \\
\hline & ${ }^{13} \mathrm{C}$ data & 22.93 & 77.07 & - & - & - & - & - & - \\
\hline & All data & 0.00 & 100.00 & - & - & - & - & - & - \\
\hline & & \multicolumn{8}{|c|}{ Level of theory: B3LYP/6-311G* (gas phase) } \\
\hline & & $82 a$ & $82 b$ & - & - & - & - & - & - \\
\hline \multirow{3}{*}{ sDP4+ } & ${ }^{1}$ H data & 0.06 & 99.94 & - & - & - & - & - & - \\
\hline & ${ }^{13} \mathrm{C}$ data & 97.55 & 2.45 & - & - & - & - & - & - \\
\hline & All data & 2.45 & 97.55 & - & - & - & - & - & - \\
\hline \multirow{3}{*}{ uDP4+ } & ${ }^{1} \mathrm{H}$ data & 18.42 & 81.58 & - & - & - & - & - & - \\
\hline & ${ }^{13} \mathrm{C}$ data & 26.83 & 73.17 & - & - & - & - & - & - \\
\hline & All data & 7.65 & 92.35 & - & - & - & - & - & - \\
\hline \multirow{5}{*}{ DP4+ } & ${ }^{1} \mathrm{H}$ data & 0.01 & 99.99 & - & - & - & - & - & - \\
\hline & ${ }^{13} \mathrm{C}$ data & 93.58 & 6.42 & - & - & - & - & - & - \\
\hline & All data & 0.21 & 99.79 & - & - & - & - & - & - \\
\hline & & \multicolumn{8}{|c|}{ Level of theory: B3LYP/6-311G** (gas phase) } \\
\hline & & $82 a$ & $82 b$ & - & - & - & - & - & - \\
\hline
\end{tabular}




\begin{tabular}{|c|c|c|c|c|c|c|c|c|c|}
\hline & ${ }^{13} \mathrm{C}$ data & 97.00 & 3.00 & - & - & - & - & - & - \\
\hline & All data & 0.85 & 99.15 & - & - & - & - & - & - \\
\hline & ${ }^{1} \mathrm{H}$ data & 3.92 & 96.08 & - & - & - & - & - & - \\
\hline \multirow[t]{3}{*}{ uDP4+ } & ${ }^{13} \mathrm{C}$ data & 12.32 & 87.68 & - & - & - & - & - & - \\
\hline & All data & 0.57 & 99.43 & - & - & - & - & - & - \\
\hline & ${ }^{1} \mathrm{H}$ data & 0.00 & 100.00 & - & - & - & - & - & - \\
\hline \multirow[t]{5}{*}{ DP4+ } & ${ }^{13} \mathrm{C}$ data & 81.97 & 18.03 & - & - & - & - & - & - \\
\hline & All data & 0.00 & 100.00 & - & - & - & - & - & - \\
\hline & & \multicolumn{8}{|c|}{ Level of theory: B3LYP/6-311+G** (gas phase) } \\
\hline & & $82 a$ & $82 \mathrm{~b}$ & - & - & - & - & - & - \\
\hline & ${ }^{1} \mathrm{H}$ data & 0.22 & 99.78 & - & - & - & - & - & - \\
\hline \multirow[t]{3}{*}{$s D P 4+$} & ${ }^{13} \mathrm{C}$ data & 95.02 & 4.98 & - & - & - & - & - & - \\
\hline & All data & 3.96 & 96.04 & - & - & - & - & - & - \\
\hline & ${ }^{1} \mathrm{H}$ data & 2.99 & 97.01 & - & - & - & - & - & - \\
\hline \multirow[t]{3}{*}{ uDP4+ } & ${ }^{13} \mathrm{C}$ data & 0.63 & 99.37 & - & - & - & - & - & - \\
\hline & All data & 0.02 & 99.98 & - & - & - & - & - & - \\
\hline & ${ }^{1} \mathrm{H}$ data & 0.01 & 99.99 & - & - & - & - & - & - \\
\hline \multirow[t]{5}{*}{ DP4+ } & ${ }^{13} \mathrm{C}$ data & 10.82 & 89.18 & - & - & - & - & - & - \\
\hline & All data & 0.00 & 100.00 & - & - & - & - & - & - \\
\hline & & \multicolumn{8}{|c|}{ Level of theory: B3LYP/6-31G* (solution, PCM) } \\
\hline & & $82 a$ & $82 b$ & - & - & - & - & - & - \\
\hline & ${ }^{1} \mathrm{H}$ data & 0.01 & 99.99 & - & - & - & - & - & - \\
\hline \multirow[t]{3}{*}{ sDP4+ } & ${ }^{13} \mathrm{C}$ data & 86.68 & 13.32 & - & - & - & - & - & - \\
\hline & All data & 0.03 & 99.97 & - & - & - & - & - & - \\
\hline & ${ }^{1} \mathrm{H}$ data & 0.98 & 99.02 & - & - & - & - & - & - \\
\hline \multirow[t]{3}{*}{ uDP4+ } & ${ }^{13} \mathrm{C}$ data & 10.59 & 89.41 & - & - & - & - & - & - \\
\hline & All data & 0.12 & 99.88 & - & - & - & - & - & - \\
\hline & ${ }^{1} \mathrm{H}$ data & 0.00 & 100.00 & - & - & - & - & - & - \\
\hline \multirow[t]{5}{*}{ DP4+ } & ${ }^{13} \mathrm{C}$ data & 43.53 & 56.47 & - & - & - & - & - & - \\
\hline & All data & 0.00 & 100.00 & - & - & - & - & - & - \\
\hline & & \multicolumn{8}{|c|}{ Level of theory: B3LYP/6-31G** (solution, PCM) } \\
\hline & & $82 a$ & $82 b$ & - & - & - & - & - & - \\
\hline & ${ }^{1} \mathrm{H}$ data & 0.00 & 100.00 & - & - & - & - & - & - \\
\hline \multirow[t]{3}{*}{ sDP4+ } & ${ }^{13} \mathrm{C}$ data & 89.73 & 10.27 & - & - & - & - & - & - \\
\hline & All data & 0.03 & 99.97 & - & - & - & - & - & - \\
\hline & ${ }^{1} \mathrm{H}$ data & 0.27 & 99.73 & - & - & - & - & - & - \\
\hline \multirow[t]{3}{*}{ uDP4+ } & ${ }^{13} \mathrm{C}$ data & 4.84 & 95.16 & - & - & - & - & - & - \\
\hline & All data & 0.01 & 99.99 & - & - & - & - & - & - \\
\hline & ${ }^{1} \mathrm{H}$ data & 0.00 & 100.00 & - & - & - & - & - & - \\
\hline \multirow[t]{5}{*}{ DP4+ } & ${ }^{13} \mathrm{C}$ data & 30.77 & 69.23 & - & - & - & - & - & - \\
\hline & All data & 0.00 & 100.00 & - & - & - & - & - & - \\
\hline & & \multicolumn{8}{|c|}{ Level of theory: B3LYP/6-31+G** (solution, PCM) } \\
\hline & & $82 a$ & $82 b$ & - & - & - & - & - & - \\
\hline & ${ }^{1} \mathrm{H}$ data & 0.01 & 99.99 & - & - & - & - & - & - \\
\hline \multirow[t]{2}{*}{ sDP4+ } & ${ }^{13} \mathrm{C}$ data & 71.78 & 28.22 & - & - & - & - & - & - \\
\hline & All data & 0.02 & 99.98 & - & - & - & - & - & - \\
\hline & ${ }^{1} \mathrm{H}$ data & 0.04 & 99.96 & - & - & - & - & - & - \\
\hline uDP4+ & ${ }^{13} \mathrm{C}$ data & 5.49 & 94.51 & - & - & - & - & - & - \\
\hline & All data & 0.00 & 100.00 & - & - & - & - & - & - \\
\hline & ${ }_{12}^{1} \mathrm{H}$ data & 0.00 & 100.00 & - & - & - & - & - & - \\
\hline DP4+ & ${ }^{13} \mathrm{C}$ data & 12.86 & 87.14 & - & - & - & - & - & - \\
\hline & All data & 0.00 & 100.00 & - & - & - & - & - & - \\
\hline & & & & vel & 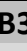 & $\mathrm{J}^{\mathrm{N}}$ & $\overline{P C}$ & & \\
\hline & & $82 a$ & $82 b$ & - & - & - & - & - & - \\
\hline & ${ }^{1} \mathrm{H}$ data & 0.02 & 99.98 & - & - & - & - & - & - \\
\hline SDP4+ & ${ }^{13} \mathrm{C}$ data & 98.38 & 1.62 & - & - & - & - & - & - \\
\hline
\end{tabular}




\begin{tabular}{|c|c|c|c|c|c|c|c|c|c|}
\hline & All data & 1.03 & 98.97 & - & - & - & - & - & - \\
\hline & ${ }^{1}$ H data & 0.79 & 99.21 & - & - & - & - & - & - \\
\hline \multirow[t]{3}{*}{ uDP4+ } & ${ }^{13} \mathrm{C}$ data & 26.24 & 73.76 & - & - & - & - & - & - \\
\hline & All data & 0.28 & 99.72 & - & - & - & - & - & - \\
\hline & ${ }^{1} \mathrm{H}$ data & 0.00 & 100.00 & - & - & - & - & - & - \\
\hline \multirow[t]{5}{*}{ DP4+ } & ${ }^{13} \mathrm{C}$ data & 95.57 & 4.43 & - & - & - & - & - & - \\
\hline & All data & 0.00 & 100.00 & - & - & - & - & - & - \\
\hline & & \multicolumn{8}{|c|}{ Level of theory: B3LYP/6-311G** (solution, PCM) } \\
\hline & & $82 a$ & $82 b$ & - & - & - & - & - & - \\
\hline & ${ }^{1} \mathrm{H}$ data & 0.03 & 99.97 & - & - & - & - & - & - \\
\hline \multirow[t]{3}{*}{ sDP4+ } & ${ }^{13} \mathrm{C}$ data & 98.12 & 1.88 & - & - & - & - & - & - \\
\hline & All data & 1.44 & 98.56 & - & - & - & - & - & - \\
\hline & ${ }^{1} \mathrm{H}$ data & 0.49 & 99.51 & - & - & - & - & - & - \\
\hline \multirow[t]{3}{*}{ uDP4+ } & ${ }^{13} \mathrm{C}$ data & 12.59 & 87.41 & - & - & - & - & - & - \\
\hline & All data & 0.07 & 99.93 & - & - & - & - & - & - \\
\hline & ${ }^{1} \mathrm{H}$ data & 0.00 & 100.00 & - & - & - & - & - & - \\
\hline \multirow[t]{5}{*}{ DP4+ } & ${ }^{13} \mathrm{C}$ data & 88.29 & 11.71 & - & - & - & - & - & - \\
\hline & All data & 0.00 & 100.00 & - & - & - & - & - & - \\
\hline & & \multicolumn{8}{|c|}{ Level of theory: B3LYP/6-311+G** (solution, PCM) } \\
\hline & & $82 a$ & $82 b$ & - & - & - & - & - & - \\
\hline & ${ }^{1}$ H data & 0.16 & 99.84 & - & - & - & - & - & - \\
\hline \multirow[t]{3}{*}{ sDP4+ } & ${ }^{13}$ C data & 96.05 & 3.95 & - & - & - & - & - & - \\
\hline & All data & 3.83 & 96.17 & - & - & - & - & - & - \\
\hline & ${ }^{1} \mathrm{H}$ data & 0.10 & 99.90 & - & - & - & - & - & - \\
\hline \multirow[t]{3}{*}{$u$ DP4+ } & ${ }^{13} \mathrm{C}$ data & 0.74 & 99.26 & - & - & - & - & - & - \\
\hline & All data & 0.00 & 100.00 & - & - & - & - & - & - \\
\hline & ${ }^{1}$ H data & 0.00 & 100.00 & - & - & - & - & - & - \\
\hline \multirow[t]{5}{*}{ DP4+ } & ${ }^{13} \mathrm{C}$ data & 15.43 & 84.57 & - & - & - & - & - & - \\
\hline & All data & 0.00 & 100.00 & - & - & - & - & - & - \\
\hline & & \multicolumn{8}{|c|}{ Level of theory: mPW1PW91/6-31G* (gas phase) } \\
\hline & & $82 a$ & $82 b$ & - & - & - & - & - & - \\
\hline & ${ }^{1} \mathrm{H}$ data & 0.09 & 99.91 & - & - & - & - & - & - \\
\hline \multirow[t]{3}{*}{ sDP4+ } & ${ }^{13} \mathrm{C}$ data & 61.69 & 38.31 & - & - & - & - & - & - \\
\hline & All data & 0.15 & 99.85 & - & - & - & - & - & - \\
\hline & ${ }^{1} \mathrm{H}$ data & 22.36 & 77.64 & - & - & - & - & - & - \\
\hline \multirow[t]{3}{*}{ uDP4+ } & ${ }^{13} \mathrm{C}$ data & 1.06 & 98.94 & - & - & - & - & - & - \\
\hline & All data & 0.31 & 99.69 & - & - & - & - & - & - \\
\hline & ${ }^{1} \mathrm{H}$ data & 0.03 & 99.97 & - & - & - & - & - & - \\
\hline \multirow[t]{5}{*}{ DP4+ } & ${ }^{13} \mathrm{C}$ data & 1.70 & 98.30 & - & - & - & - & - & - \\
\hline & All data & 0.00 & 100.00 & - & - & - & - & - & - \\
\hline & & \multicolumn{8}{|c|}{ Level of theory: mPW1PW91/6-31G** (gas phase) } \\
\hline & & $82 a$ & $82 b$ & - & - & - & - & - & - \\
\hline & ${ }^{1} \mathrm{H}$ data & 0.05 & 99.95 & - & - & - & - & - & - \\
\hline \multirow[t]{3}{*}{ sDP4+ } & ${ }^{13} \mathrm{C}$ data & 85.15 & 14.85 & - & - & - & - & - & - \\
\hline & All data & 0.27 & 99.73 & - & - & - & - & - & - \\
\hline & ${ }^{1} \mathrm{H}$ data & 7.22 & 92.78 & - & - & - & - & - & - \\
\hline uDP4+ & ${ }^{13} \mathrm{C}$ data & 0.36 & 99.64 & - & - & - & - & - & - \\
\hline & All data & 0.03 & 99.97 & - & - & - & - & - & - \\
\hline & ${ }^{1} \mathrm{H}$ data & 0.00 & 100.00 & - & - & - & - & - & - \\
\hline DP4+ & ${ }^{13} \mathrm{C}$ data & 2.02 & 97.98 & - & - & - & - & - & - \\
\hline & All data & 0.00 & 100.00 & - & - & - & - & - & - \\
\hline & & & & 10 & $\mathbf{P I}$ & -3 & $\overline{p h}$ & & \\
\hline & & $82 a$ & $82 b$ & - & - & - & - & - & - \\
\hline & ${ }^{1} \mathrm{H}$ data & 0.09 & 99.91 & - & - & - & - & - & - \\
\hline sDP4+ & ${ }^{13} \mathrm{C}$ data & 83.50 & 16.50 & - & - & - & - & - & - \\
\hline & All data & 0.44 & 99.56 & - & - & - & - & - & - \\
\hline
\end{tabular}




\begin{tabular}{|c|c|c|c|c|c|c|c|c|c|}
\hline & ${ }^{1} \mathrm{H}$ data & 4.68 & 95.32 & - & - & - & - & - & - \\
\hline \multirow[t]{3}{*}{ uDP4+ } & ${ }^{13} \mathrm{C}$ data & 1.65 & 98.35 & - & - & - & - & - & - \\
\hline & All data & 0.08 & 99.92 & - & - & - & - & - & - \\
\hline & ${ }^{1} \mathrm{H}$ data & 0.00 & 100.00 & - & - & - & - & - & - \\
\hline \multirow[t]{5}{*}{ DP4+ } & ${ }^{13} \mathrm{C}$ data & 7.81 & 92.19 & - & - & - & - & - & - \\
\hline & All data & 0.00 & 100.00 & - & - & - & - & - & - \\
\hline & & \multicolumn{8}{|c|}{ Level of theory: mPW1PW91/6-311G* (gas phase) } \\
\hline & & $82 a$ & $82 b$ & - & - & - & - & - & - \\
\hline & ${ }^{1} \mathrm{H}$ data & 0.49 & 99.51 & - & - & - & - & - & - \\
\hline \multirow[t]{3}{*}{ sDP4+ } & ${ }^{13} \mathrm{C}$ data & 96.76 & 3.24 & - & - & - & - & - & - \\
\hline & All data & 12.80 & 87.20 & - & - & - & - & - & - \\
\hline & ${ }^{1} \mathrm{H}$ data & 32.01 & 67.99 & - & - & - & - & - & - \\
\hline \multirow[t]{3}{*}{ uDP4+ } & ${ }^{13} \mathrm{C}$ data & 16.08 & 83.92 & - & - & - & - & - & - \\
\hline & All data & 8.28 & 91.72 & - & - & - & - & - & - \\
\hline & ${ }^{1} \mathrm{H}$ data & 0.23 & 99.77 & - & - & - & - & - & - \\
\hline \multirow[t]{5}{*}{ DP4+ } & ${ }^{13} \mathrm{C}$ data & 85.11 & 14.89 & - & - & - & - & - & - \\
\hline & All data & 1.31 & 98.69 & - & - & - & - & - & - \\
\hline & & \multicolumn{8}{|c|}{ Level of theory: mPW1PW91/6-311G** (gas phase) } \\
\hline & & $82 a$ & $82 b$ & - & - & - & - & - & - \\
\hline & ${ }^{1} \mathrm{H}$ data & 0.06 & 99.94 & - & - & - & - & - & - \\
\hline \multirow[t]{3}{*}{ sDP4+ } & ${ }^{13} \mathrm{C}$ data & 96.04 & 3.96 & - & - & - & - & - & - \\
\hline & All data & 1.51 & 98.49 & - & - & - & - & - & - \\
\hline & ${ }^{1} \mathrm{H}$ data & 9.23 & 90.77 & - & - & - & - & - & - \\
\hline \multirow[t]{3}{*}{ uDP4+ } & ${ }^{13} \mathrm{C}$ data & 5.24 & 94.76 & - & - & - & - & - & - \\
\hline & All data & 0.56 & 99.44 & - & - & - & - & - & - \\
\hline & ${ }^{1} \mathrm{H}$ data & 0.01 & 99.99 & - & - & - & - & - & - \\
\hline \multirow[t]{4}{*}{ DP4+ } & ${ }^{13} \mathrm{C}$ data & 57.31 & 42.69 & - & - & - & - & - & - \\
\hline & All data & 0.01 & 99.99 & - & - & - & - & - & - \\
\hline & & \multicolumn{8}{|c|}{ Level of theory: mPW1PW91/6-311+G** (gas phase) } \\
\hline & & $82 a$ & $82 b$ & - & - & - & - & - & - \\
\hline \multirow{3}{*}{ sDP4+ } & ${ }^{1} \mathrm{H}$ data & 1.45 & 98.55 & - & - & - & - & - & - \\
\hline & ${ }^{13} \mathrm{C}$ data & 90.61 & 9.39 & - & - & - & - & - & - \\
\hline & All data & 12.46 & 87.54 & - & - & - & - & - & - \\
\hline \multirow{3}{*}{ uDP4+ } & ${ }^{1} \mathrm{H}$ data & 25.98 & 74.02 & - & - & - & - & - & - \\
\hline & ${ }^{13} \mathrm{C}$ data & 0.16 & 99.84 & - & - & - & - & - & - \\
\hline & All data & 0.06 & 99.94 & - & - & - & - & - & - \\
\hline \multirow{5}{*}{ DP4+ } & ${ }^{1} \mathrm{H}$ data & 0.51 & 99.49 & - & - & - & - & - & - \\
\hline & ${ }^{13} \mathrm{C}$ data & 1.52 & 98.48 & - & - & - & - & - & - \\
\hline & All data & 0.01 & 99.99 & - & - & - & - & - & - \\
\hline & & \multicolumn{8}{|c|}{ Level of theory: mPW1PW91/6-31G* (solution, PCM) } \\
\hline & & $82 a$ & $82 b$ & - & - & - & - & - & - \\
\hline \multirow{3}{*}{ sDP4+ } & ${ }^{1} \mathrm{H}$ data & 0.04 & 99.96 & - & - & - & - & - & - \\
\hline & ${ }^{13} \mathrm{C}$ data & 43.89 & 56.11 & - & - & - & - & - & - \\
\hline & All data & 0.03 & 99.97 & - & - & - & - & - & - \\
\hline & ${ }^{1} \mathrm{H}$ data & 1.14 & 98.86 & - & - & - & - & - & - \\
\hline uDP4+ & ${ }^{13} \mathrm{C}$ data & 0.69 & 99.31 & - & - & - & - & - & - \\
\hline & All data & 0.01 & 99.99 & - & - & - & - & - & - \\
\hline & ${ }^{1} \mathrm{H}$ data & 0.00 & 100.00 & - & - & - & - & - & - \\
\hline DP4+ & ${ }^{13} \mathrm{C}$ data & 0.54 & 99.46 & - & - & - & - & - & - \\
\hline & All data & 0.00 & 100.00 & - & - & - & - & - & - \\
\hline & & & & of & $\mathbf{W}$ & $11 C$ & n, & & \\
\hline & & $82 a$ & $82 b$ & - & - & - & - & - & - \\
\hline & ${ }^{1} \mathrm{H}$ data & 0.01 & 99.99 & - & - & - & - & - & - \\
\hline sDP4+ & ${ }^{13} \mathrm{C}$ data & 64.60 & 35.40 & - & - & - & - & - & - \\
\hline & All data & 0.01 & 99.99 & - & - & - & - & - & - \\
\hline uDP4+ & ${ }^{1} \mathrm{H}$ data & 0.13 & 99.87 & - & - & - & - & - & - \\
\hline
\end{tabular}




\begin{tabular}{|c|c|c|c|c|c|c|c|c|c|}
\hline & ${ }^{13} \mathrm{C}$ data & 0.29 & 99.71 & - & - & - & - & - & - \\
\hline & All data & 0.00 & 100.00 & - & - & - & - & - & - \\
\hline & ${ }^{1} \mathrm{H}$ data & 0.00 & 100.00 & - & - & - & - & - & - \\
\hline \multirow[t]{5}{*}{ DP4+ } & ${ }^{13} \mathrm{C}$ data & 0.52 & 99.48 & - & - & - & - & - & - \\
\hline & All data & 0.00 & 100.00 & - & - & - & - & - & - \\
\hline & & \multicolumn{8}{|c|}{ Level of theory: mPW1PW91/6-31+G** (solution, PCM) } \\
\hline & & $82 a$ & $82 \mathrm{~b}$ & - & - & - & - & - & - \\
\hline & ${ }^{1} \mathrm{H}$ data & 0.19 & 99.81 & - & - & - & - & - & - \\
\hline \multirow[t]{2}{*}{ sDP4+ } & ${ }^{13} \mathrm{C}$ data & 75.04 & 24.96 & - & - & - & - & - & - \\
\hline & All data & 0.58 & 99.42 & - & - & - & - & - & - \\
\hline \multirow{3}{*}{ uDP4+ } & ${ }^{1} \mathrm{H}$ data & 0.09 & 99.91 & - & - & - & - & - & - \\
\hline & ${ }^{13} \mathrm{C}$ data & 0.92 & 99.08 & - & - & - & - & - & - \\
\hline & All data & 0.00 & 100.00 & - & - & - & - & - & - \\
\hline & ${ }^{1} \mathrm{H}$ data & 0.00 & 100.00 & - & - & - & - & - & - \\
\hline \multirow[t]{5}{*}{ DP4+ } & ${ }^{13} \mathrm{C}$ data & 2.71 & 97.29 & - & - & - & - & - & - \\
\hline & All data & 0.00 & 100.00 & - & - & - & - & - & - \\
\hline & & \multicolumn{8}{|c|}{ Level of theory: mPW1PW91/6-311G* (solution, PCM) } \\
\hline & & $82 a$ & $82 \mathrm{~b}$ & - & - & - & - & - & - \\
\hline & ${ }^{1} \mathrm{H}$ data & 0.20 & 99.80 & - & - & - & - & - & - \\
\hline \multirow[t]{2}{*}{ sDP4+ } & ${ }^{13} \mathrm{C}$ data & 97.99 & 2.01 & - & - & - & - & - & - \\
\hline & All data & 8.81 & 91.19 & - & - & - & - & - & - \\
\hline & ${ }^{1} \mathrm{H}$ data & 4.15 & 95.85 & - & - & - & - & - & - \\
\hline \multirow[t]{2}{*}{ uDP4+ } & ${ }^{13} \mathrm{C}$ data & 10.42 & 89.58 & - & - & - & - & - & - \\
\hline & All data & 0.50 & 99.50 & - & - & - & - & - & - \\
\hline \multirow{6}{*}{ DP4+ } & ${ }^{1} \mathrm{H}$ data & 0.01 & 99.99 & - & - & - & - & - & - \\
\hline & ${ }^{13} \mathrm{C}$ data & 85.01 & 14.99 & - & - & - & - & - & - \\
\hline & All data & 0.05 & 99.95 & - & - & - & - & - & - \\
\hline & & \multicolumn{8}{|c|}{ Level of theory: mPW1PW91/6-311G** (solution, PCM) } \\
\hline & & $82 a$ & $82 b$ & - & - & - & - & - & - \\
\hline & ${ }^{1} \mathrm{H}$ data & 0.19 & 99.81 & - & - & - & - & - & - \\
\hline \multirow[t]{2}{*}{ sDP4+ } & ${ }^{13} \mathrm{C}$ data & 97.30 & 2.70 & - & - & - & - & - & - \\
\hline & All data & 6.27 & 93.73 & - & - & - & - & - & - \\
\hline \multirow{3}{*}{ uDP4+ } & ${ }^{1} \mathrm{H}$ data & 0.84 & 99.16 & - & - & - & - & - & - \\
\hline & ${ }^{13} \mathrm{C}$ data & 4.19 & 95.81 & - & - & - & - & - & - \\
\hline & All data & 0.04 & 99.96 & - & - & - & - & - & - \\
\hline \multirow{5}{*}{ DP4+ } & ${ }^{1} \mathrm{H}$ data & 0.00 & 100.00 & - & - & - & - & - & - \\
\hline & ${ }^{13} \mathrm{C}$ data & 61.20 & 38.80 & - & - & - & - & - & - \\
\hline & All data & 0.00 & 100.00 & - & - & - & - & - & - \\
\hline & & \multicolumn{8}{|c|}{ Level of theory: mPW1PW91/6-311+G** (solution, PCM) } \\
\hline & & $82 a$ & $82 b$ & - & - & - & - & - & - \\
\hline \multirow{3}{*}{ sDP4+ } & ${ }^{1} \mathrm{H}$ data & 0.79 & 99.21 & - & - & - & - & - & - \\
\hline & ${ }^{13} \mathrm{C}$ data & 84.94 & 15.06 & - & - & - & - & - & - \\
\hline & All data & 4.32 & 95.68 & - & - & - & - & - & - \\
\hline \multirow{3}{*}{ uDP4+ } & ${ }^{1} \mathrm{H}$ data & 0.92 & 99.08 & - & - & - & - & - & - \\
\hline & ${ }^{13} \mathrm{C}$ data & 0.13 & 99.87 & - & - & - & - & - & - \\
\hline & All data & 0.00 & 100.00 & - & - & - & - & - & - \\
\hline \multirow{3}{*}{ DP4+ } & ${ }^{1} \mathrm{H}$ data & 0.01 & 99.99 & - & - & - & - & - & - \\
\hline & ${ }^{13} \mathrm{C}$ data & 0.73 & 99.27 & - & - & - & - & - & - \\
\hline & All data & 0.00 & 100.00 & - & - & - & - & - & - \\
\hline
\end{tabular}


sDP4+, $u$ DP4+ and DP4+ probabilities (\%) for compound 83b
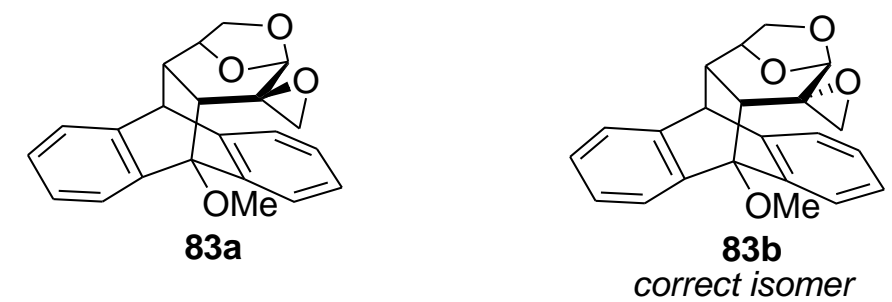

\begin{tabular}{|c|c|c|c|c|c|c|c|c|c|}
\hline & & \multicolumn{8}{|c|}{ Level of theory: B3LYP/6-31G* (gas phase) } \\
\hline & & $83 a$ & $83 b$ & - & - & - & - & - & - \\
\hline & ${ }^{1}$ H data & 0.00 & 100.00 & - & - & - & - & - & - \\
\hline \multirow[t]{3}{*}{ sDP4+ } & ${ }^{13} \mathrm{C}$ data & 76.69 & 23.31 & - & - & - & - & - & - \\
\hline & All data & 0.00 & 100.00 & - & - & - & - & - & - \\
\hline & ${ }^{1} \mathrm{H}$ data & 26.89 & 73.11 & - & - & - & - & - & - \\
\hline \multirow[t]{3}{*}{ uDP4+ } & ${ }^{13} \mathrm{C}$ data & 24.08 & 75.92 & - & - & - & - & - & - \\
\hline & All data & 10.45 & 89.55 & - & - & - & - & - & - \\
\hline & ${ }^{1} \mathrm{H}$ data & 0.00 & 100.00 & - & - & - & - & - & - \\
\hline \multirow[t]{5}{*}{ DP4+ } & ${ }^{13} \mathrm{C}$ data & 51.06 & 48.94 & - & - & - & - & - & - \\
\hline & All data & 0.00 & 100.00 & - & - & - & - & - & - \\
\hline & & \multicolumn{8}{|c|}{ Level of theory: B3LYP/6-31G** (gas phase) } \\
\hline & & $83 a$ & $83 b$ & - & - & - & - & - & - \\
\hline & ${ }^{1} \mathrm{H}$ data & 0.01 & 99.99 & - & - & - & - & - & - \\
\hline \multirow[t]{3}{*}{ sDP4+ } & ${ }^{13} \mathrm{C}$ data & 84.93 & 15.07 & - & - & - & - & - & - \\
\hline & All data & 0.04 & 99.96 & - & - & - & - & - & - \\
\hline & ${ }^{1} \mathrm{H}$ data & 20.20 & 79.80 & - & - & - & - & - & - \\
\hline \multirow[t]{3}{*}{ uDP4+ } & ${ }^{13} \mathrm{C}$ data & 46.93 & 53.07 & - & - & - & - & - & - \\
\hline & All data & 18.29 & 81.71 & - & - & - & - & - & - \\
\hline & ${ }^{1} \mathrm{H}$ data & 0.00 & 100.00 & - & - & - & - & - & - \\
\hline \multirow[t]{5}{*}{ DP4+ } & ${ }^{13} \mathrm{C}$ data & 83.29 & 16.71 & - & - & - & - & - & - \\
\hline & All data & 0.01 & 99.99 & - & - & - & - & - & - \\
\hline & & \multicolumn{8}{|c|}{ Level of theory: B3LYP/6-31+G** (gas phase) } \\
\hline & & $83 a$ & $83 b$ & - & - & - & - & - & - \\
\hline & ${ }^{1} \mathrm{H}$ data & 2.03 & 97.97 & - & - & - & - & - & - \\
\hline \multirow[t]{3}{*}{ sDP4+ } & ${ }^{13} \mathrm{C}$ data & 92.96 & 7.04 & - & - & - & - & - & - \\
\hline & All data & 21.50 & 78.50 & - & - & - & - & - & - \\
\hline & ${ }^{1} \mathrm{H}$ data & 0.54 & 99.46 & - & - & - & - & - & - \\
\hline \multirow[t]{3}{*}{ uDP4+ } & ${ }^{13} \mathrm{C}$ data & 86.69 & 13.31 & - & - & - & - & - & - \\
\hline & All data & 3.40 & 96.60 & - & - & - & - & - & - \\
\hline & ${ }^{1} \mathrm{H}$ data & 0.01 & 99.99 & - & - & - & - & - & - \\
\hline \multirow[t]{5}{*}{ DP4+ } & ${ }^{13} \mathrm{C}$ data & 98.85 & 1.15 & - & - & - & - & - & - \\
\hline & All data & 0.96 & 99.04 & - & - & - & - & - & - \\
\hline & & \multicolumn{8}{|c|}{ Level of theory: B3LYP/6-311G* (gas phase) } \\
\hline & & $83 a$ & $83 b$ & - & - & - & - & - & - \\
\hline & ${ }^{1} \mathrm{H}$ data & 0.02 & 99.98 & - & - & - & - & - & - \\
\hline \multirow[t]{2}{*}{ sDP4+ } & ${ }^{13} \mathrm{C}$ data & 10.58 & 89.42 & - & - & - & - & - & - \\
\hline & All data & 0.00 & 100.00 & - & - & - & - & - & - \\
\hline \multirow{3}{*}{ uDP4+ } & ${ }^{1} \mathrm{H}$ data & 1.15 & 98.85 & - & - & - & - & - & - \\
\hline & ${ }^{13} \mathrm{C}$ data & 6.86 & 93.14 & - & - & - & - & - & - \\
\hline & All data & 0.09 & 99.91 & - & - & - & - & - & - \\
\hline \multirow{5}{*}{ DP4+ } & ${ }^{1} \mathrm{H}$ data & 0.00 & 100.00 & - & - & - & - & - & - \\
\hline & ${ }^{13} \mathrm{C}$ data & 0.86 & 99.14 & - & - & - & - & - & - \\
\hline & All data & 0.00 & 100.00 & - & - & - & - & - & - \\
\hline & & \multicolumn{8}{|c|}{ Level of theory: B3LYP/6-311G** (gas phase) } \\
\hline & & $83 a$ & $83 b$ & - & - & - & - & - & - \\
\hline sDP4+ & ${ }^{1}$ H data & 0.01 & 99.99 & - & - & - & - & - & - \\
\hline
\end{tabular}




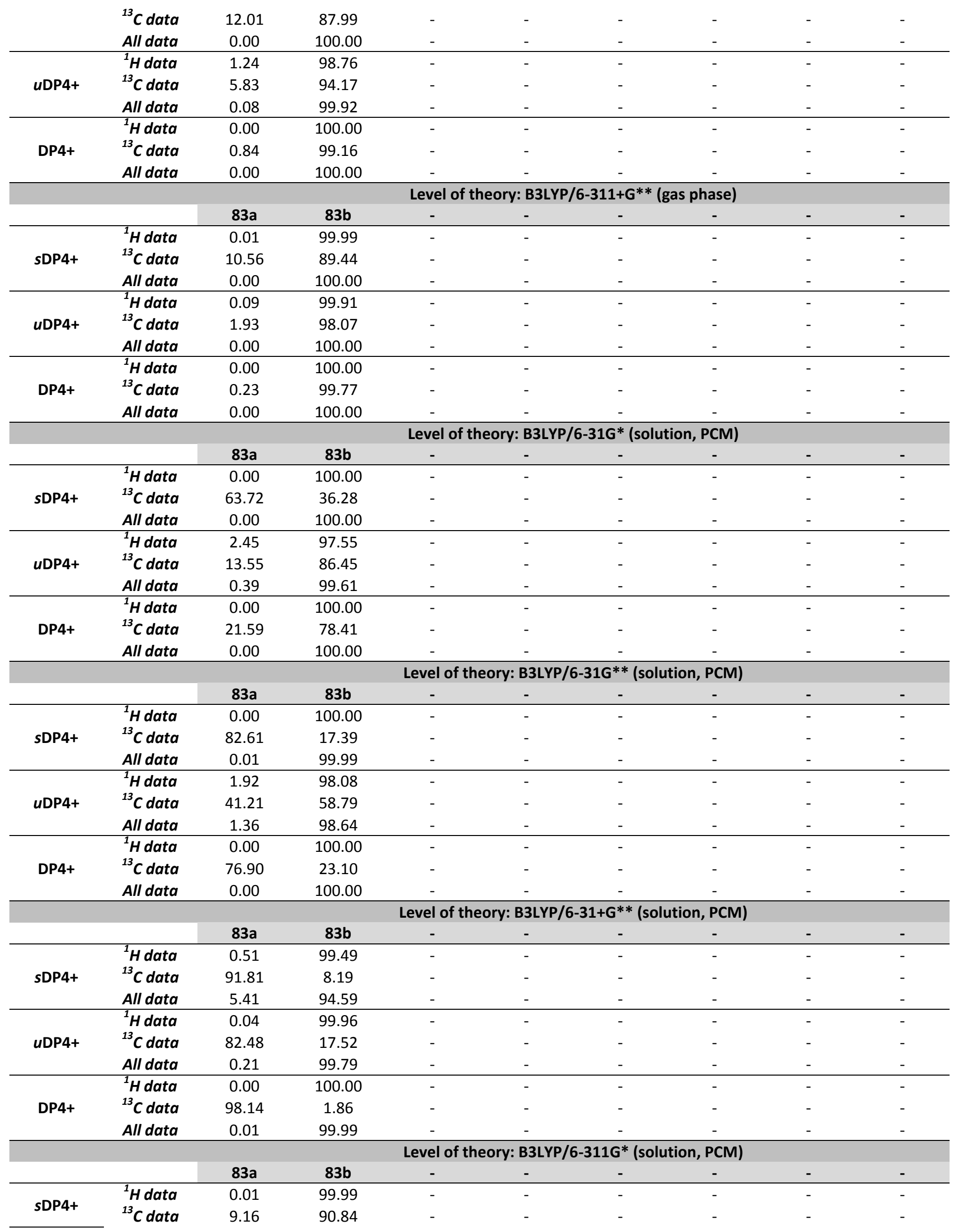




\begin{tabular}{|c|c|c|c|c|c|c|c|c|c|}
\hline & All data & 0.00 & 100.00 & - & - & - & - & - & - \\
\hline & ${ }^{1} \mathrm{H}$ data & 0.65 & 99.35 & - & - & - & - & - & - \\
\hline \multirow[t]{3}{*}{ uDP4+ } & ${ }^{13} \mathrm{C}$ data & 5.85 & 94.15 & - & - & - & - & - & - \\
\hline & All data & 0.04 & 99.96 & - & - & - & - & - & - \\
\hline & ${ }^{1} \mathrm{H}$ data & 0.00 & 100.00 & - & - & - & - & - & - \\
\hline \multirow[t]{5}{*}{ DP4+ } & ${ }^{13} \mathrm{C}$ data & 0.62 & 99.38 & - & - & - & - & - & - \\
\hline & All data & 0.00 & 100.00 & - & - & - & - & - & - \\
\hline & & \multicolumn{8}{|c|}{ Level of theory: B3LYP/6-311G** (solution, PCM) } \\
\hline & & $83 a$ & $83 b$ & - & - & - & - & - & - \\
\hline & ${ }^{1} \mathrm{H}$ data & 0.08 & 99.92 & - & - & - & - & - & - \\
\hline \multirow[t]{3}{*}{ sDP4+ } & ${ }^{13} \mathrm{C}$ data & 10.25 & 89.75 & - & - & - & - & - & - \\
\hline & All data & 0.01 & 99.99 & - & - & - & - & - & - \\
\hline & ${ }^{1} \mathrm{H}$ data & 3.49 & 96.51 & - & - & - & - & - & - \\
\hline \multirow[t]{3}{*}{ uDP4+ } & ${ }^{13} \mathrm{C}$ data & 4.73 & 95.27 & - & - & - & - & - & - \\
\hline & All data & 0.18 & 99.82 & - & - & - & - & - & - \\
\hline & ${ }^{1} \mathrm{H}$ data & 0.00 & 100.00 & - & - & - & - & - & - \\
\hline \multirow[t]{5}{*}{ DP4+ } & ${ }^{13} \mathrm{C}$ data & 0.56 & 99.44 & - & - & - & - & - & - \\
\hline & All data & 0.00 & 100.00 & - & - & - & - & - & - \\
\hline & & \multicolumn{8}{|c|}{ Level of theory: B3LYP/6-311+G** (solution, PCM) } \\
\hline & & $83 a$ & $83 b$ & - & - & - & - & - & - \\
\hline & ${ }^{1}$ H data & 0.33 & 99.67 & - & - & - & - & - & - \\
\hline \multirow[t]{3}{*}{ sDP4+ } & ${ }^{13} \mathrm{C}$ data & 54.19 & 45.81 & - & - & - & - & - & - \\
\hline & All data & 0.39 & 99.61 & - & - & - & - & - & - \\
\hline & ${ }^{1} \mathrm{H}$ data & 0.00 & 100.00 & - & - & - & - & - & - \\
\hline \multirow[t]{3}{*}{ uDP4+ } & ${ }^{13} \mathrm{C}$ data & 56.78 & 43.22 & - & - & - & - & - & - \\
\hline & All data & 0.01 & 99.99 & - & - & - & - & - & - \\
\hline & ${ }^{1} \mathrm{H}$ data & 0.00 & 100.00 & - & - & - & - & - & - \\
\hline \multirow[t]{5}{*}{ DP4+ } & ${ }^{13} \mathrm{C}$ data & 60.85 & 39.15 & - & - & - & - & - & - \\
\hline & All data & 0.00 & 100.00 & - & - & - & - & - & - \\
\hline & & \multicolumn{8}{|c|}{ Level of theory: mPW1PW91/6-31G* (gas phase) } \\
\hline & & $83 a$ & $83 b$ & - & - & - & - & - & - \\
\hline & ${ }^{1} \mathrm{H}$ data & 0.00 & 100.00 & - & - & - & - & - & - \\
\hline \multirow[t]{3}{*}{ sDP4+ } & ${ }^{13} \mathrm{C}$ data & 15.81 & 84.19 & - & - & - & - & - & - \\
\hline & All data & 0.00 & 100.00 & - & - & - & - & - & - \\
\hline & ${ }^{1} \mathrm{H}$ data & 35.52 & 64.48 & - & - & - & - & - & - \\
\hline \multirow[t]{3}{*}{ uDP4+ } & ${ }^{13} \mathrm{C}$ data & 3.71 & 96.29 & - & - & - & - & - & - \\
\hline & All data & 2.08 & 97.92 & - & - & - & - & - & - \\
\hline & ${ }^{1} \mathrm{H}$ data & 0.00 & 100.00 & - & - & - & - & - & - \\
\hline \multirow[t]{5}{*}{ DP4+ } & ${ }^{13} \mathrm{C}$ data & 0.72 & 99.28 & - & - & - & - & - & - \\
\hline & All data & 0.00 & 100.00 & - & - & - & - & - & - \\
\hline & & \multicolumn{8}{|c|}{ Level of theory: mPW1PW91/6-31G** (gas phase) } \\
\hline & & $83 a$ & $83 b$ & - & - & - & - & - & - \\
\hline & ${ }^{1} \mathrm{H}$ data & 0.01 & 99.99 & - & - & - & - & - & - \\
\hline \multirow[t]{3}{*}{ sDP4+ } & ${ }^{13} \mathrm{C}$ data & 57.50 & 42.50 & - & - & - & - & - & - \\
\hline & All data & 0.01 & 99.99 & - & - & - & - & - & - \\
\hline & ${ }^{1} \mathrm{H}$ data & 25.29 & 74.71 & - & - & - & - & - & - \\
\hline uDP4+ & ${ }^{13} \mathrm{C}$ data & 12.22 & 87.78 & - & - & - & - & - & - \\
\hline & All data & 4.50 & 95.50 & - & - & - & - & - & - \\
\hline & ${ }^{1} \mathrm{H}$ data & 0.00 & 100.00 & - & - & - & - & - & - \\
\hline DP4+ & ${ }^{13} \mathrm{C}$ data & 15.85 & 84.15 & - & - & - & - & - & - \\
\hline & All data & 0.00 & 100.00 & - & - & - & - & - & - \\
\hline & & & & 0 & $|P|$ & -3 & ph & & \\
\hline & & $83 a$ & $83 b$ & - & - & - & - & - & - \\
\hline & ${ }^{1} \mathrm{H}$ data & 1.55 & 98.45 & - & - & - & - & - & - \\
\hline sDP4+ & ${ }^{13} \mathrm{C}$ data & 86.72 & 13.28 & - & - & - & - & - & - \\
\hline & All data & 9.30 & 90.70 & - & - & - & - & - & - \\
\hline
\end{tabular}




\begin{tabular}{|c|c|c|c|c|c|c|c|c|c|}
\hline & ${ }^{1} \mathrm{H}$ data & 2.18 & 97.82 & - & - & - & - & - & - \\
\hline \multirow[t]{3}{*}{ uDP4+ } & ${ }^{13} \mathrm{C}$ data & 67.30 & 32.70 & - & - & - & - & - & - \\
\hline & All data & 4.38 & 95.62 & - & - & - & - & - & - \\
\hline & ${ }^{1} \mathrm{H}$ data & 0.03 & 99.97 & - & - & - & - & - & - \\
\hline \multirow[t]{5}{*}{ DP4+ } & ${ }^{13} \mathrm{C}$ data & 93.07 & 6.93 & - & - & - & - & - & - \\
\hline & All data & 0.47 & 99.53 & - & - & - & - & - & - \\
\hline & & \multicolumn{8}{|c|}{ Level of theory: mPW1PW91/6-311G* (gas phase) } \\
\hline & & $83 a$ & $83 b$ & - & - & - & - & - & - \\
\hline & ${ }^{1} \mathrm{H}$ data & 0.03 & 99.97 & - & - & - & - & - & - \\
\hline \multirow[t]{3}{*}{ sDP4+ } & ${ }^{13} \mathrm{C}$ data & 14.88 & 85.12 & - & - & - & - & - & - \\
\hline & All data & 0.00 & 100.00 & - & - & - & - & - & - \\
\hline & ${ }^{1} \mathrm{H}$ data & 1.40 & 98.60 & - & - & - & - & - & - \\
\hline \multirow[t]{3}{*}{ uDP4+ } & ${ }^{13} \mathrm{C}$ data & 11.85 & 88.15 & - & - & - & - & - & - \\
\hline & All data & 0.19 & 99.81 & - & - & - & - & - & - \\
\hline & ${ }^{1} \mathrm{H}$ data & 0.00 & 100.00 & - & - & - & - & - & - \\
\hline \multirow[t]{5}{*}{ DP4+ } & ${ }^{13} \mathrm{C}$ data & 2.29 & 97.71 & - & - & - & - & - & - \\
\hline & All data & 0.00 & 100.00 & - & - & - & - & - & - \\
\hline & & \multicolumn{8}{|c|}{ Level of theory: mPW1PW91/6-311G** (gas phase) } \\
\hline & & $83 a$ & $83 b$ & - & - & - & - & - & - \\
\hline & ${ }^{1} \mathrm{H}$ data & 0.01 & 99.99 & - & - & - & - & - & - \\
\hline \multirow[t]{3}{*}{ sDP4+ } & ${ }^{13} \mathrm{C}$ data & 14.92 & 85.08 & - & - & - & - & - & - \\
\hline & All data & 0.00 & 100.00 & - & - & - & - & - & - \\
\hline & ${ }^{1} \mathrm{H}$ data & 1.28 & 98.72 & - & - & - & - & - & - \\
\hline \multirow[t]{3}{*}{ uDP4+ } & ${ }^{13} \mathrm{C}$ data & 6.15 & 93.85 & - & - & - & - & - & - \\
\hline & All data & 0.08 & 99.92 & - & - & - & - & - & - \\
\hline & ${ }^{1} \mathrm{H}$ data & 0.00 & 100.00 & - & - & - & - & - & - \\
\hline \multirow[t]{5}{*}{ DP4+ } & ${ }^{13} \mathrm{C}$ data & 1.14 & 98.86 & - & - & - & - & - & - \\
\hline & All data & 0.00 & 100.00 & - & - & - & - & - & - \\
\hline & & \multicolumn{8}{|c|}{ Level of theory: mPW1PW91/6-311+G** (gas phase) } \\
\hline & & $83 a$ & $83 b$ & - & - & - & - & - & - \\
\hline & ${ }^{1} \mathrm{H}$ data & 0.01 & 99.99 & - & - & - & - & - & - \\
\hline \multirow[t]{3}{*}{ sDP4+ } & ${ }^{13} \mathrm{C}$ data & 10.80 & 89.20 & - & - & - & - & - & - \\
\hline & All data & 0.00 & 100.00 & - & - & - & - & - & - \\
\hline & ${ }^{1} \mathrm{H}$ data & 0.04 & 99.96 & - & - & - & - & - & - \\
\hline \multirow[t]{3}{*}{ uDP4+ } & ${ }^{13} \mathrm{C}$ data & 0.57 & 99.43 & - & - & - & - & - & - \\
\hline & All data & 0.00 & 100.00 & - & - & - & - & - & - \\
\hline & ${ }^{1} \mathrm{H}$ data & 0.00 & 100.00 & - & - & - & - & - & - \\
\hline \multirow[t]{5}{*}{ DP4+ } & ${ }^{13} \mathrm{C}$ data & 0.07 & 99.93 & - & - & - & - & - & - \\
\hline & All data & 0.00 & 100.00 & - & - & - & - & - & - \\
\hline & & \multicolumn{8}{|c|}{ Level of theory: mPW1PW91/6-31G* (solution, PCM) } \\
\hline & & $83 a$ & $83 b$ & - & - & - & - & - & - \\
\hline & ${ }^{1} \mathrm{H}$ data & 0.00 & 100.00 & - & - & - & - & - & - \\
\hline \multirow[t]{3}{*}{ sDP4+ } & ${ }^{13} \mathrm{C}$ data & 9.07 & 90.93 & - & - & - & - & - & - \\
\hline & All data & 0.00 & 100.00 & - & - & - & - & - & - \\
\hline & ${ }^{1}$ H data & 4.83 & 95.17 & - & - & - & - & - & - \\
\hline uDP4+ & ${ }^{13} \mathrm{C}$ data & 1.67 & 98.33 & - & - & - & - & - & - \\
\hline & All data & 0.09 & 99.91 & - & - & - & - & - & - \\
\hline & ${ }^{1} \mathrm{H}$ data & 0.00 & 100.00 & - & - & - & - & - & - \\
\hline DP4+ & ${ }^{13} \mathrm{C}$ data & 0.17 & 99.83 & - & - & - & - & - & - \\
\hline & All data & 0.00 & 100.00 & - & - & - & - & - & - \\
\hline & & & & of $t$ & W & 31 & on, & & \\
\hline & & $83 a$ & $83 b$ & - & - & - & - & - & - \\
\hline & ${ }^{1} \mathrm{H}$ data & 0.00 & 100.00 & - & - & - & - & - & - \\
\hline sDP4+ & ${ }^{13} \mathrm{C}$ data & 34.18 & 65.82 & - & - & - & - & - & - \\
\hline & All data & 0.00 & 100.00 & - & - & - & - & - & - \\
\hline uDP4+ & ${ }^{1} \mathrm{H}$ data & 1.95 & 98.05 & - & - & - & - & - & - \\
\hline
\end{tabular}




\begin{tabular}{|c|c|c|c|c|c|c|c|c|c|}
\hline & ${ }^{13} \mathrm{C}$ data & 6.97 & 93.03 & - & - & - & - & - & - \\
\hline & All data & 0.15 & 99.85 & - & - & - & - & - & - \\
\hline & ${ }^{1} \mathrm{H}$ data & 0.00 & 100.00 & - & - & - & - & - & - \\
\hline \multirow[t]{5}{*}{ DP4+ } & ${ }^{13} \mathrm{C}$ data & 3.74 & 96.26 & - & - & - & - & - & - \\
\hline & All data & 0.00 & 100.00 & - & - & - & - & - & - \\
\hline & & \multicolumn{8}{|c|}{ Level of theory: mPW1PW91/6-31+G** (solution, PCM) } \\
\hline & & $83 a$ & $83 b$ & - & - & - & - & - & - \\
\hline & ${ }^{1} \mathrm{H}$ data & 0.96 & 99.04 & - & - & - & - & - & - \\
\hline \multirow[t]{2}{*}{ sDP4+ } & ${ }^{13} \mathrm{C}$ data & 74.49 & 25.51 & - & - & - & - & - & - \\
\hline & All data & 2.74 & 97.26 & - & - & - & - & - & - \\
\hline \multirow{3}{*}{ uDP4+ } & ${ }^{1} \mathrm{H}$ data & 0.03 & 99.97 & - & - & - & - & - & - \\
\hline & ${ }^{13} \mathrm{C}$ data & 46.08 & 53.92 & - & - & - & - & - & - \\
\hline & All data & 0.03 & 99.97 & - & - & - & - & - & - \\
\hline & ${ }^{1} \mathrm{H}$ data & 0.00 & 100.00 & - & - & - & - & - & - \\
\hline \multirow[t]{5}{*}{ DP4+ } & ${ }^{13} \mathrm{C}$ data & 71.39 & 28.61 & - & - & - & - & - & - \\
\hline & All data & 0.00 & 100.00 & - & - & - & - & - & - \\
\hline & & \multicolumn{8}{|c|}{ Level of theory: mPW1PW91/6-311G* (solution, PCM) } \\
\hline & & $83 a$ & 83b & - & - & - & - & - & - \\
\hline & ${ }^{1} \mathrm{H}$ data & 0.01 & 99.99 & - & - & - & - & - & - \\
\hline \multirow[t]{2}{*}{ sDP4+ } & ${ }^{13} \mathrm{C}$ data & 10.07 & 89.93 & - & - & - & - & - & - \\
\hline & All data & 0.00 & 100.00 & - & - & - & - & - & - \\
\hline & ${ }^{1} \mathrm{H}$ data & 0.72 & 99.28 & - & - & - & - & - & - \\
\hline \multirow[t]{2}{*}{ uDP4+ } & ${ }^{13} \mathrm{C}$ data & 5.21 & 94.79 & - & - & - & - & - & - \\
\hline & All data & 0.04 & 99.96 & - & - & - & - & - & - \\
\hline \multirow{5}{*}{ DP4+ } & ${ }^{1} \mathrm{H}$ data & 0.00 & 100.00 & - & - & - & - & - & - \\
\hline & ${ }^{13} \mathrm{C}$ data & 0.61 & 99.39 & - & - & - & - & - & - \\
\hline & All data & 0.00 & 100.00 & - & - & - & - & - & - \\
\hline & & \multicolumn{8}{|c|}{ Level of theory: mPW1PW91/6-311G** (solution, PCM) } \\
\hline & & $83 a$ & 83b & - & - & - & - & - & - \\
\hline \multirow{3}{*}{ sDP4+ } & ${ }^{1} \mathrm{H}$ data & 0.10 & 99.90 & - & - & - & - & - & - \\
\hline & ${ }^{13} \mathrm{C}$ data & 10.18 & 89.82 & - & - & - & - & - & - \\
\hline & All data & 0.01 & 99.99 & - & - & - & - & - & - \\
\hline & ${ }^{1} \mathrm{H}$ data & 3.83 & 96.17 & - & - & - & - & - & - \\
\hline \multirow[t]{2}{*}{ uDP4+ } & ${ }^{13} \mathrm{C}$ data & 2.94 & 97.06 & - & - & - & - & - & - \\
\hline & All data & 0.12 & 99.88 & - & - & - & - & - & - \\
\hline \multirow{5}{*}{ DP4+ } & ${ }^{1} \mathrm{H}$ data & 0.00 & 100.00 & - & - & - & - & - & - \\
\hline & ${ }^{13} \mathrm{C}$ data & 0.34 & 99.66 & - & - & - & - & - & - \\
\hline & All data & 0.00 & 100.00 & - & - & - & - & - & - \\
\hline & & \multicolumn{8}{|c|}{ Level of theory: mPW1PW91/6-311+G** (solution, PCM) } \\
\hline & & $83 a$ & $83 b$ & - & - & - & - & - & - \\
\hline \multirow{3}{*}{ sDP4+ } & ${ }^{1} \mathrm{H}$ data & 0.25 & 99.75 & - & - & - & - & - & - \\
\hline & ${ }^{13} \mathrm{C}$ data & 29.71 & 70.29 & - & - & - & - & - & - \\
\hline & All data & 0.11 & 99.89 & - & - & - & - & - & - \\
\hline \multirow{3}{*}{ uDP4+ } & ${ }^{1} \mathrm{H}$ data & 0.05 & 99.95 & - & - & - & - & - & - \\
\hline & ${ }^{13} \mathrm{C}$ data & 22.83 & 77.17 & - & - & - & - & - & - \\
\hline & All data & 0.02 & 99.98 & - & - & - & - & - & - \\
\hline \multirow{3}{*}{ DP4+ } & ${ }^{1} \mathrm{H}$ data & 0.00 & 100.00 & - & - & - & - & - & - \\
\hline & ${ }^{13} \mathrm{C}$ data & 11.12 & 88.88 & - & - & - & - & - & - \\
\hline & All data & 0.00 & 100.00 & - & - & - & - & - & - \\
\hline
\end{tabular}


sDP4+, $u$ DP4+ and DP4+ probabilities (\%) for compound 84b
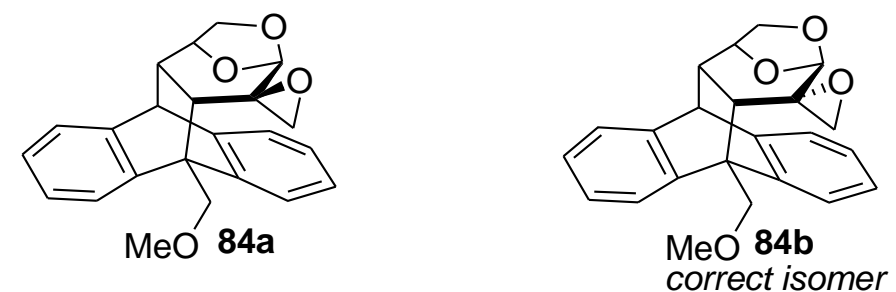

\begin{tabular}{|c|c|c|c|c|c|c|c|c|c|}
\hline & & \multicolumn{8}{|c|}{ Level of theory: B3LYP/6-31G* (gas phase) } \\
\hline & & $84 a$ & $84 b$ & - & - & - & - & - & - \\
\hline & ${ }^{1}$ H data & 0.05 & 99.95 & - & - & - & - & - & - \\
\hline \multirow[t]{3}{*}{ sDP4+ } & ${ }^{13} \mathrm{C}$ data & 52.61 & 47.39 & - & - & - & - & - & - \\
\hline & All data & 0.05 & 99.95 & - & - & - & - & - & - \\
\hline & ${ }^{1} \mathrm{H}$ data & 0.76 & 99.24 & - & - & - & - & - & - \\
\hline \multirow[t]{3}{*}{ uDP4+ } & ${ }^{13} \mathrm{C}$ data & 20.27 & 79.73 & - & - & - & - & - & - \\
\hline & All data & 0.19 & 99.81 & - & - & - & - & - & - \\
\hline & ${ }^{1} \mathrm{H}$ data & 0.00 & 100.00 & - & - & - & - & - & - \\
\hline \multirow[t]{5}{*}{ DP4+ } & ${ }^{13} \mathrm{C}$ data & 22.01 & 77.99 & - & - & - & - & - & - \\
\hline & All data & 0.00 & 100.00 & - & - & - & - & - & - \\
\hline & & \multicolumn{8}{|c|}{ Level of theory: B3LYP/6-31G** (gas phase) } \\
\hline & & $84 a$ & $84 b$ & - & - & - & - & - & - \\
\hline & ${ }^{1} \mathrm{H}$ data & 0.02 & 99.98 & - & - & - & - & - & - \\
\hline \multirow[t]{3}{*}{ sDP4+ } & ${ }^{13} \mathrm{C}$ data & 42.70 & 57.30 & - & - & - & - & - & - \\
\hline & All data & 0.02 & 99.98 & - & - & - & - & - & - \\
\hline & ${ }^{1}$ H data & 0.23 & 99.77 & - & - & - & - & - & - \\
\hline \multirow[t]{3}{*}{ uDP4+ } & ${ }^{13} \mathrm{C}$ data & 11.90 & 88.10 & - & - & - & - & - & - \\
\hline & All data & 0.03 & 99.97 & - & - & - & - & - & - \\
\hline & ${ }^{1} \mathrm{H}$ data & 0.00 & 100.00 & - & - & - & - & - & - \\
\hline \multirow[t]{5}{*}{ DP4+ } & ${ }^{13} \mathrm{C}$ data & 9.14 & 90.86 & - & - & - & - & - & - \\
\hline & All data & 0.00 & 100.00 & - & - & - & - & - & - \\
\hline & & \multicolumn{8}{|c|}{ Level of theory: B3LYP/6-31+G** (gas phase) } \\
\hline & & $84 a$ & $84 b$ & - & - & - & - & - & - \\
\hline & ${ }^{1} \mathrm{H}$ data & 0.47 & 99.53 & - & - & - & - & - & - \\
\hline \multirow[t]{3}{*}{ sDP4+ } & ${ }^{13} \mathrm{C}$ data & 96.84 & 3.16 & - & - & - & - & - & - \\
\hline & All data & 12.58 & 87.42 & - & - & - & - & - & - \\
\hline & ${ }^{1} \mathrm{H}$ data & 1.02 & 98.98 & - & - & - & - & - & - \\
\hline \multirow[t]{3}{*}{ uDP4+ } & ${ }^{13} \mathrm{C}$ data & 86.42 & 13.58 & - & - & - & - & - & - \\
\hline & All data & 6.14 & 93.86 & - & - & - & - & - & - \\
\hline & ${ }^{1} \mathrm{H}$ data & 0.00 & 100.00 & - & - & - & - & - & - \\
\hline \multirow[t]{5}{*}{ DP4+ } & ${ }^{13} \mathrm{C}$ data & 99.49 & 0.51 & - & - & - & - & - & - \\
\hline & All data & 0.93 & 99.07 & - & - & - & - & - & - \\
\hline & & \multicolumn{8}{|c|}{ Level of theory: B3LYP/6-311G* (gas phase) } \\
\hline & & $84 a$ & $84 b$ & - & - & - & - & - & - \\
\hline & ${ }^{1} \mathrm{H}$ data & 0.00 & 100.00 & - & - & - & - & - & - \\
\hline \multirow[t]{2}{*}{ sDP4+ } & ${ }^{13} \mathrm{C}$ data & 91.82 & 8.18 & - & - & - & - & - & - \\
\hline & All data & 0.02 & 99.98 & - & - & - & - & - & - \\
\hline \multirow{3}{*}{ uDP4+ } & ${ }^{1} \mathrm{H}$ data & 0.14 & 99.86 & - & - & - & - & - & - \\
\hline & ${ }^{13} \mathrm{C}$ data & 27.80 & 72.20 & - & - & - & - & - & - \\
\hline & All data & 0.05 & 99.95 & - & - & - & - & - & - \\
\hline \multirow{5}{*}{ DP4+ } & ${ }^{1} \mathrm{H}$ data & 0.00 & 100.00 & - & - & - & - & - & - \\
\hline & ${ }^{13} \mathrm{C}$ data & 81.22 & 18.78 & - & - & - & - & - & - \\
\hline & All data & 0.00 & 100.00 & - & - & - & - & - & - \\
\hline & & \multicolumn{8}{|c|}{ Level of theory: B3LYP/6-311G** (gas phase) } \\
\hline & & $84 a$ & $84 b$ & - & - & - & - & - & - \\
\hline sDP4+ & ${ }^{1} \mathrm{H}$ data & 0.00 & 100.00 & - & - & - & - & - & - \\
\hline
\end{tabular}




\begin{tabular}{|c|c|c|c|c|c|c|c|c|c|}
\hline & ${ }^{13} \mathrm{C}$ data & 91.80 & 8.20 & - & - & - & - & - & - \\
\hline & All data & 0.01 & 99.99 & - & - & - & - & - & - \\
\hline & ${ }^{1} \mathrm{H}$ data & 0.02 & 99.98 & - & - & - & - & - & - \\
\hline \multirow[t]{3}{*}{ uDP4+ } & ${ }^{13} \mathrm{C}$ data & 8.26 & 91.74 & - & - & - & - & - & - \\
\hline & All data & 0.00 & 100.00 & - & - & - & - & - & - \\
\hline & ${ }^{1} \mathrm{H}$ data & 0.00 & 100.00 & - & - & - & - & - & - \\
\hline \multirow[t]{5}{*}{ DP4+ } & ${ }^{13} \mathrm{C}$ data & 50.22 & 49.78 & - & - & - & - & - & - \\
\hline & All data & 0.00 & 100.00 & - & - & - & - & - & - \\
\hline & & \multicolumn{8}{|c|}{ Level of theory: B3LYP/6-311+G** (gas phase) } \\
\hline & & $84 a$ & $84 b$ & - & - & - & - & - & - \\
\hline & ${ }^{1} \mathrm{H}$ data & 0.01 & 99.99 & - & - & - & - & - & - \\
\hline \multirow[t]{3}{*}{$s D P 4+$} & ${ }^{13} \mathrm{C}$ data & 32.67 & 67.33 & - & - & - & - & - & - \\
\hline & All data & 0.00 & 100.00 & - & - & - & - & - & - \\
\hline & ${ }^{1} \mathrm{H}$ data & 0.10 & 99.90 & - & - & - & - & - & - \\
\hline \multirow[t]{3}{*}{ uDP4+ } & ${ }^{13} \mathrm{C}$ data & 0.33 & 99.67 & - & - & - & - & - & - \\
\hline & All data & 0.00 & 100.00 & - & - & - & - & - & - \\
\hline & ${ }^{1} \mathrm{H}$ data & 0.00 & 100.00 & - & - & - & - & - & - \\
\hline \multirow[t]{5}{*}{ DP4+ } & ${ }^{13} \mathrm{C}$ data & 0.16 & 99.84 & - & - & - & - & - & - \\
\hline & All data & 0.00 & 100.00 & - & - & - & - & - & - \\
\hline & & \multicolumn{8}{|c|}{ Level of theory: B3LYP/6-31G* (solution, PCM) } \\
\hline & & $84 a$ & $84 b$ & - & - & - & - & - & - \\
\hline & ${ }^{1} \mathrm{H}$ data & 0.01 & 99.99 & - & - & - & - & - & - \\
\hline \multirow[t]{2}{*}{ sDP4+ } & ${ }^{13} \mathrm{C}$ data & 26.94 & 73.06 & - & - & - & - & - & - \\
\hline & All data & 0.00 & 100.00 & - & - & - & - & - & - \\
\hline \multirow{3}{*}{ uDP4+ } & ${ }^{1} \mathrm{H}$ data & 0.01 & 99.99 & - & - & - & - & - & - \\
\hline & ${ }^{13} \mathrm{C}$ data & 7.75 & 92.25 & - & - & - & - & - & - \\
\hline & All data & 0.00 & 100.00 & - & - & - & - & - & - \\
\hline \multirow{5}{*}{ DP4+ } & ${ }^{1} \mathrm{H}$ data & 0.00 & 100.00 & - & - & - & - & - & - \\
\hline & ${ }^{13} \mathrm{C}$ data & 3.00 & 97.00 & - & - & - & - & - & - \\
\hline & All data & 0.00 & 100.00 & - & - & - & - & - & - \\
\hline & & \multicolumn{8}{|c|}{ Level of theory: B3LYP/6-31G** (solution, PCM) } \\
\hline & & $84 a$ & $84 b$ & - & - & - & - & - & - \\
\hline \multirow{3}{*}{ sDP4+ } & ${ }^{1} \mathrm{H}$ data & 0.00 & 100.00 & - & - & - & - & - & - \\
\hline & ${ }^{13} \mathrm{C}$ data & 21.00 & 79.00 & - & - & - & - & - & - \\
\hline & All data & 0.00 & 100.00 & - & - & - & - & - & - \\
\hline \multirow{3}{*}{ uDP4+ } & ${ }^{1} \mathrm{H}$ data & 0.00 & 100.00 & - & - & - & - & - & - \\
\hline & ${ }^{13} \mathrm{C}$ data & 7.25 & 92.75 & - & - & - & - & - & - \\
\hline & All data & 0.00 & 100.00 & - & - & - & - & - & - \\
\hline \multirow{5}{*}{ DP4+ } & ${ }^{1} \mathrm{H}$ data & 0.00 & 100.00 & - & - & - & - & - & - \\
\hline & ${ }^{13} \mathrm{C}$ data & 2.03 & 97.97 & - & - & - & - & - & - \\
\hline & All data & 0.00 & 100.00 & - & - & - & - & - & - \\
\hline & & \multicolumn{8}{|c|}{ Level of theory: B3LYP/6-31+G** (solution, PCM) } \\
\hline & & $84 a$ & $84 b$ & - & - & - & - & - & - \\
\hline \multirow{3}{*}{ sDP4+ } & ${ }^{1} \mathrm{H}$ data & 0.01 & 99.99 & - & - & - & - & - & - \\
\hline & ${ }^{13} \mathrm{C}$ data & 90.76 & 9.24 & - & - & - & - & - & - \\
\hline & All data & 0.09 & 99.91 & - & - & - & - & - & - \\
\hline & ${ }^{1} \mathrm{H}$ data & 0.21 & 99.79 & - & - & - & - & - & - \\
\hline uDP4+ & ${ }^{13} \mathrm{C}$ data & 76.14 & 23.86 & - & - & - & - & - & - \\
\hline & All data & 0.66 & 99.34 & - & - & - & - & - & - \\
\hline & ${ }_{12}^{1} \mathrm{H}$ data & 0.00 & 100.00 & - & - & - & - & - & - \\
\hline DP4+ & ${ }^{13} \mathrm{C}$ data & 96.91 & 3.09 & - & - & - & - & - & - \\
\hline & All data & 0.00 & 100.00 & - & - & - & - & - & - \\
\hline & & & & vel & 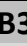 & $\mathrm{J}^{*}$ & PC & & \\
\hline & & $84 a$ & $84 b$ & - & - & - & - & - & - \\
\hline & ${ }^{1} \mathrm{H}$ data & 0.00 & 100.00 & - & - & - & - & - & - \\
\hline SDP4+ & ${ }^{13} \mathrm{C}$ data & 91.35 & 8.65 & - & - & - & - & - & - \\
\hline
\end{tabular}




\begin{tabular}{|c|c|c|c|c|c|c|c|c|c|}
\hline & All data & 0.01 & 99.99 & - & - & - & - & - & - \\
\hline & ${ }^{1} \mathrm{H}$ data & 0.07 & 99.93 & - & - & - & - & - & - \\
\hline \multirow[t]{3}{*}{ uDP4+ } & ${ }^{13} \mathrm{C}$ data & 24.31 & 75.69 & - & - & - & - & - & - \\
\hline & All data & 0.02 & 99.98 & - & - & - & - & - & - \\
\hline & ${ }^{1} \mathrm{H}$ data & 0.00 & 100.00 & - & - & - & - & - & - \\
\hline \multirow[t]{5}{*}{ DP4+ } & ${ }^{13} \mathrm{C}$ data & 77.23 & 22.77 & - & - & - & - & - & - \\
\hline & All data & 0.00 & 100.00 & - & - & - & - & - & - \\
\hline & & \multicolumn{8}{|c|}{ Level of theory: B3LYP/6-311G** (solution, PCM) } \\
\hline & & $84 a$ & $84 b$ & - & - & - & - & - & - \\
\hline & ${ }^{1} \mathrm{H}$ data & 0.01 & 99.99 & - & - & - & - & - & - \\
\hline \multirow[t]{3}{*}{ sDP4+ } & ${ }^{13} \mathrm{C}$ data & 91.57 & 8.43 & - & - & - & - & - & - \\
\hline & All data & 0.07 & 99.93 & - & - & - & - & - & - \\
\hline & ${ }^{1} \mathrm{H}$ data & 0.03 & 99.97 & - & - & - & - & - & - \\
\hline \multirow[t]{3}{*}{ uDP4+ } & ${ }^{13} \mathrm{C}$ data & 7.14 & 92.86 & - & - & - & - & - & - \\
\hline & All data & 0.00 & 100.00 & - & - & - & - & - & - \\
\hline & ${ }^{1} \mathrm{H}$ data & 0.00 & 100.00 & - & - & - & - & - & - \\
\hline \multirow[t]{5}{*}{ DP4+ } & ${ }^{13} \mathrm{C}$ data & 45.52 & 54.48 & - & - & - & - & - & - \\
\hline & All data & 0.00 & 100.00 & - & - & - & - & - & - \\
\hline & & \multicolumn{8}{|c|}{ Level of theory: B3LYP/6-311+G** (solution, PCM) } \\
\hline & & $84 a$ & $84 b$ & - & - & - & - & - & - \\
\hline & ${ }^{1}$ H data & 0.08 & 99.92 & - & - & - & - & - & - \\
\hline \multirow[t]{3}{*}{ sDP4+ } & ${ }^{13} \mathrm{C}$ data & 29.49 & 70.51 & - & - & - & - & - & - \\
\hline & All data & 0.03 & 99.97 & - & - & - & - & - & - \\
\hline & ${ }^{1} \mathrm{H}$ data & 0.02 & 99.98 & - & - & - & - & - & - \\
\hline \multirow[t]{3}{*}{ uDP4+ } & ${ }^{13} \mathrm{C}$ data & 0.36 & 99.64 & - & - & - & - & - & - \\
\hline & All data & 0.00 & 100.00 & - & - & - & - & - & - \\
\hline & ${ }^{1} \mathrm{H}$ data & 0.00 & 100.00 & - & - & - & - & - & - \\
\hline \multirow[t]{5}{*}{ DP4+ } & ${ }^{13} \mathrm{C}$ data & 0.15 & 99.85 & - & - & - & - & - & - \\
\hline & All data & 0.00 & 100.00 & - & - & - & - & - & - \\
\hline & & \multicolumn{8}{|c|}{ Level of theory: mPW1PW91/6-31G* (gas phase) } \\
\hline & & $84 a$ & $84 b$ & - & - & - & - & - & - \\
\hline & ${ }^{1} \mathrm{H}$ data & 0.05 & 99.95 & - & - & - & - & - & - \\
\hline \multirow[t]{3}{*}{ sDP4+ } & ${ }^{13} \mathrm{C}$ data & 7.20 & 92.80 & - & - & - & - & - & - \\
\hline & All data & 0.00 & 100.00 & - & - & - & - & - & - \\
\hline & ${ }^{1} \mathrm{H}$ data & 0.64 & 99.36 & - & - & - & - & - & - \\
\hline \multirow[t]{3}{*}{ uDP4+ } & ${ }^{13} \mathrm{C}$ data & 1.24 & 98.76 & - & - & - & - & - & - \\
\hline & All data & 0.01 & 99.99 & - & - & - & - & - & - \\
\hline & ${ }^{1} \mathrm{H}$ data & 0.00 & 100.00 & - & - & - & - & - & - \\
\hline \multirow[t]{5}{*}{ DP4+ } & ${ }^{13} \mathrm{C}$ data & 0.10 & 99.90 & - & - & - & - & - & - \\
\hline & All data & 0.00 & 100.00 & - & - & - & - & - & - \\
\hline & & \multicolumn{8}{|c|}{ Level of theory: mPW1PW91/6-31G** (gas phase) } \\
\hline & & $84 a$ & $84 b$ & - & - & - & - & - & - \\
\hline & ${ }^{1} \mathrm{H}$ data & 0.03 & 99.97 & - & - & - & - & - & - \\
\hline \multirow[t]{3}{*}{ sDP4+ } & ${ }^{13} \mathrm{C}$ data & 16.72 & 83.28 & - & - & - & - & - & - \\
\hline & All data & 0.01 & 99.99 & - & - & - & - & - & - \\
\hline & ${ }^{1} \mathrm{H}$ data & 0.17 & 99.83 & - & - & - & - & - & - \\
\hline uDP4+ & ${ }^{13} \mathrm{C}$ data & 1.03 & 98.97 & - & - & - & - & - & - \\
\hline & All data & 0.00 & 100.00 & - & - & - & - & - & - \\
\hline & ${ }^{1} \mathrm{H}$ data & 0.00 & 100.00 & - & - & - & - & - & - \\
\hline DP4+ & ${ }^{13} \mathrm{C}$ data & 0.21 & 99.79 & - & - & - & - & - & - \\
\hline & All data & 0.00 & 100.00 & - & - & - & - & - & - \\
\hline & & & & 0 & $|P|$ & -3 & ph & & \\
\hline & & $84 a$ & $84 b$ & - & - & - & - & - & - \\
\hline & ${ }^{1} \mathrm{H}$ data & 1.07 & 98.93 & - & - & - & - & - & - \\
\hline sDP4+ & ${ }^{13} \mathrm{C}$ data & 97.37 & 2.63 & - & - & - & - & - & - \\
\hline & All data & 28.53 & 71.47 & - & - & - & - & - & - \\
\hline
\end{tabular}




\begin{tabular}{|c|c|c|c|c|c|c|c|c|c|}
\hline & ${ }^{1} \mathrm{H}$ data & 2.64 & 97.36 & - & - & - & - & - & - \\
\hline \multirow[t]{3}{*}{ uDP4+ } & ${ }^{13} \mathrm{C}$ data & 65.50 & 34.50 & - & - & - & - & - & - \\
\hline & All data & 4.90 & 95.10 & - & - & - & - & - & - \\
\hline & ${ }^{1} \mathrm{H}$ data & 0.03 & 99.97 & - & - & - & - & - & - \\
\hline \multirow[t]{5}{*}{ DP4+ } & ${ }^{13} \mathrm{C}$ data & 98.60 & 1.40 & - & - & - & - & - & - \\
\hline & All data & 2.01 & 97.99 & - & - & - & - & - & - \\
\hline & & \multicolumn{8}{|c|}{ Level of theory: mPW1PW91/6-311G* (gas phase) } \\
\hline & & $84 a$ & $84 b$ & - & - & - & - & - & - \\
\hline & ${ }^{1} \mathrm{H}$ data & 0.00 & 100.00 & - & - & - & - & - & - \\
\hline \multirow[t]{3}{*}{ sDP4+ } & ${ }^{13} \mathrm{C}$ data & 90.12 & 9.88 & - & - & - & - & - & - \\
\hline & All data & 0.03 & 99.97 & - & - & - & - & - & - \\
\hline & ${ }^{1} \mathrm{H}$ data & 0.41 & 99.59 & - & - & - & - & - & - \\
\hline \multirow[t]{3}{*}{ uDP4+ } & ${ }^{13} \mathrm{C}$ data & 11.70 & 88.30 & - & - & - & - & - & - \\
\hline & All data & 0.05 & 99.95 & - & - & - & - & - & - \\
\hline & ${ }^{1} \mathrm{H}$ data & 0.00 & 100.00 & - & - & - & - & - & - \\
\hline \multirow[t]{5}{*}{ DP4+ } & ${ }^{13} \mathrm{C}$ data & 54.74 & 45.26 & - & - & - & - & - & - \\
\hline & All data & 0.00 & 100.00 & - & - & - & - & - & - \\
\hline & & \multicolumn{8}{|c|}{ Level of theory: mPW1PW91/6-311G** (gas phase) } \\
\hline & & $84 a$ & $84 b$ & - & - & - & - & - & - \\
\hline & ${ }^{1} \mathrm{H}$ data & 0.00 & 100.00 & - & - & - & - & - & - \\
\hline \multirow[t]{3}{*}{ sDP4+ } & ${ }^{13} \mathrm{C}$ data & 84.85 & 15.15 & - & - & - & - & - & - \\
\hline & All data & 0.00 & 100.00 & - & - & - & - & - & - \\
\hline & ${ }^{1}$ H data & 0.02 & 99.98 & - & - & - & - & - & - \\
\hline \multirow[t]{3}{*}{ uDP4+ } & ${ }^{13} \mathrm{C}$ data & 2.00 & 98.00 & - & - & - & - & - & - \\
\hline & All data & 0.00 & 100.00 & - & - & - & - & - & - \\
\hline & ${ }^{1}$ H data & 0.00 & 100.00 & - & - & - & - & - & - \\
\hline \multirow[t]{5}{*}{ DP4+ } & ${ }^{13} \mathrm{C}$ data & 10.25 & 89.75 & - & - & - & - & - & - \\
\hline & All data & 0.00 & 100.00 & - & - & - & - & - & - \\
\hline & & \multicolumn{8}{|c|}{ Level of theory: mPW1PW91/6-311+G** (gas phase) } \\
\hline & & $84 a$ & 84b & - & - & - & - & - & - \\
\hline & ${ }^{1} \mathrm{H}$ data & 0.02 & 99.98 & - & - & - & - & - & - \\
\hline \multirow[t]{2}{*}{ sDP4+ } & ${ }^{13} \mathrm{C}$ data & 17.03 & 82.97 & - & - & - & - & - & - \\
\hline & All data & 0.00 & 100.00 & - & - & - & - & - & - \\
\hline \multirow{3}{*}{ uDP4+ } & ${ }^{1} \mathrm{H}$ data & 0.22 & 99.78 & - & - & - & - & - & - \\
\hline & ${ }^{13} \mathrm{C}$ data & 0.04 & 99.96 & - & - & - & - & - & - \\
\hline & All data & 0.00 & 100.00 & - & - & - & - & - & - \\
\hline \multirow{5}{*}{ DP4+ } & ${ }^{1} \mathrm{H}$ data & 0.00 & 100.00 & - & - & - & - & - & - \\
\hline & ${ }^{13} \mathrm{C}$ data & 0.01 & 99.99 & - & - & - & - & - & - \\
\hline & All data & 0.00 & 100.00 & - & - & - & - & - & - \\
\hline & & \multicolumn{8}{|c|}{ Level of theory: mPW1PW91/6-31G* (solution, PCM) } \\
\hline & & $84 a$ & 84b & - & - & - & - & - & - \\
\hline \multirow{3}{*}{ sDP4+ } & ${ }^{1} \mathrm{H}$ data & 0.01 & 99.99 & - & - & - & - & - & - \\
\hline & ${ }^{13} \mathrm{C}$ data & 1.91 & 98.09 & - & - & - & - & - & - \\
\hline & All data & 0.00 & 100.00 & - & - & - & - & - & - \\
\hline & ${ }^{1} \mathrm{H}$ data & 0.01 & 99.99 & - & - & - & - & - & - \\
\hline uDP4+ & ${ }^{13} \mathrm{C}$ data & 0.34 & 99.66 & - & - & - & - & - & - \\
\hline & All data & 0.00 & 100.00 & - & - & - & - & - & - \\
\hline & ${ }^{1} \mathrm{H}$ data & 0.00 & 100.00 & - & - & - & - & - & - \\
\hline DP4+ & ${ }^{13} \mathrm{C}$ data & 0.01 & 99.99 & - & - & - & - & - & - \\
\hline & All data & 0.00 & 100.00 & - & - & - & - & - & - \\
\hline & & & & of & $\mathbf{W}$ & $11 C$ & n, & & \\
\hline & & $84 a$ & $84 b$ & - & - & - & - & - & - \\
\hline & ${ }^{1} \mathrm{H}$ data & 0.00 & 100.00 & - & - & - & - & - & - \\
\hline sDP4+ & ${ }^{13} \mathrm{C}$ data & 4.58 & 95.42 & - & - & - & - & - & - \\
\hline & All data & 0.00 & 100.00 & - & - & - & - & - & - \\
\hline uDP4+ & ${ }^{1} \mathrm{H}$ data & 0.00 & 100.00 & - & - & - & - & - & - \\
\hline
\end{tabular}




\begin{tabular}{|c|c|c|c|c|c|c|c|c|c|}
\hline & ${ }^{13} \mathrm{C}$ data & 0.43 & 99.57 & - & - & - & - & - & - \\
\hline & All data & 0.00 & 100.00 & - & - & - & - & - & - \\
\hline & ${ }^{1} \mathrm{H}$ data & 0.00 & 100.00 & - & - & - & - & - & - \\
\hline \multirow[t]{5}{*}{ DP4+ } & ${ }^{13} \mathrm{C}$ data & 0.02 & 99.98 & - & - & - & - & - & - \\
\hline & All data & 0.00 & 100.00 & - & - & - & - & - & - \\
\hline & & \multicolumn{8}{|c|}{ Level of theory: mPW1PW91/6-31+G** (solution, PCM) } \\
\hline & & $84 a$ & $84 b$ & - & - & - & - & - & - \\
\hline & ${ }^{1} \mathrm{H}$ data & 0.03 & 99.97 & - & - & - & - & - & - \\
\hline \multirow[t]{2}{*}{ sDP4+ } & ${ }^{13} \mathrm{C}$ data & 89.61 & 10.39 & - & - & - & - & - & - \\
\hline & All data & 0.28 & 99.72 & - & - & - & - & - & - \\
\hline \multirow{3}{*}{ uDP4+ } & ${ }^{1} \mathrm{H}$ data & 0.27 & 99.73 & - & - & - & - & - & - \\
\hline & ${ }^{13} \mathrm{C}$ data & 38.37 & 61.63 & - & - & - & - & - & - \\
\hline & All data & 0.17 & 99.83 & - & - & - & - & - & - \\
\hline & ${ }^{1} \mathrm{H}$ data & 0.00 & 100.00 & - & - & - & - & - & - \\
\hline \multirow[t]{5}{*}{ DP4+ } & ${ }^{13} \mathrm{C}$ data & 84.30 & 15.70 & - & - & - & - & - & - \\
\hline & All data & 0.00 & 100.00 & - & - & - & - & - & - \\
\hline & & \multicolumn{8}{|c|}{ Level of theory: mPW1PW91/6-311G* (solution, PCM) } \\
\hline & & $84 a$ & $84 b$ & - & - & - & - & - & - \\
\hline & ${ }^{1} \mathrm{H}$ data & 0.00 & 100.00 & - & - & - & - & - & - \\
\hline \multirow[t]{2}{*}{ sDP4+ } & ${ }^{13} \mathrm{C}$ data & 87.89 & 12.11 & - & - & - & - & - & - \\
\hline & All data & 0.02 & 99.98 & - & - & - & - & - & - \\
\hline & ${ }^{1} \mathrm{H}$ data & 0.62 & 99.38 & - & - & - & - & - & - \\
\hline \multirow[t]{2}{*}{ uDP4+ } & ${ }^{13} \mathrm{C}$ data & 9.36 & 90.64 & - & - & - & - & - & - \\
\hline & All data & 0.06 & 99.94 & - & - & - & - & - & - \\
\hline \multirow{5}{*}{ DP4+ } & ${ }^{1} \mathrm{H}$ data & 0.00 & 100.00 & - & - & - & - & - & - \\
\hline & ${ }^{13} \mathrm{C}$ data & 42.85 & 57.15 & - & - & - & - & - & - \\
\hline & All data & 0.00 & 100.00 & - & - & - & - & - & - \\
\hline & & \multicolumn{8}{|c|}{ Level of theory: mPW1PW91/6-311G** (solution, PCM) } \\
\hline & & $84 a$ & 84b & - & - & - & - & - & - \\
\hline \multirow{3}{*}{ sDP4+ } & ${ }^{1} \mathrm{H}$ data & 0.01 & 99.99 & - & - & - & - & - & - \\
\hline & ${ }^{13} \mathrm{C}$ data & 80.25 & 19.75 & - & - & - & - & - & - \\
\hline & All data & 0.03 & 99.97 & - & - & - & - & - & - \\
\hline \multirow{3}{*}{ uDP4+ } & ${ }^{1} \mathrm{H}$ data & 0.05 & 99.95 & - & - & - & - & - & - \\
\hline & ${ }^{13} \mathrm{C}$ data & 1.66 & 98.34 & - & - & - & - & - & - \\
\hline & All data & 0.00 & 100.00 & - & - & - & - & - & - \\
\hline \multirow{5}{*}{ DP4+ } & ${ }^{1} \mathrm{H}$ data & 0.00 & 100.00 & - & - & - & - & - & - \\
\hline & ${ }^{13} \mathrm{C}$ data & 6.44 & 93.56 & - & - & - & - & - & - \\
\hline & All data & 0.00 & 100.00 & - & - & - & - & - & - \\
\hline & & \multicolumn{8}{|c|}{ Level of theory: mPW1PW91/6-311+G** (solution, PCM) } \\
\hline & & $84 a$ & $84 b$ & - & - & - & - & - & - \\
\hline \multirow{3}{*}{ sDP4+ } & ${ }^{1} \mathrm{H}$ data & 0.07 & 99.93 & - & - & - & - & - & - \\
\hline & ${ }^{13} \mathrm{C}$ data & 9.06 & 90.94 & - & - & - & - & - & - \\
\hline & All data & 0.01 & 99.99 & - & - & - & - & - & - \\
\hline \multirow{3}{*}{ uDP4+ } & ${ }^{1} \mathrm{H}$ data & 0.07 & 99.93 & - & - & - & - & - & - \\
\hline & ${ }^{13} \mathrm{C}$ data & 0.04 & 99.96 & - & - & - & - & - & - \\
\hline & All data & 0.00 & 100.00 & - & - & - & - & - & - \\
\hline \multirow{3}{*}{ DP4+ } & ${ }^{1} \mathrm{H}$ data & 0.00 & 100.00 & - & - & - & - & - & - \\
\hline & ${ }^{13} \mathrm{C}$ data & 0.00 & 100.00 & - & - & - & - & - & - \\
\hline & All data & 0.00 & 100.00 & - & - & - & - & - & - \\
\hline
\end{tabular}


$s \mathrm{DP} 4+, u \mathrm{DP} 4+$ and DP4+ probabilities (\%) for compound 85a<smiles>O=C1C=CCC(CC(O)CC(O)C=Cc2ccccc2)O1</smiles>

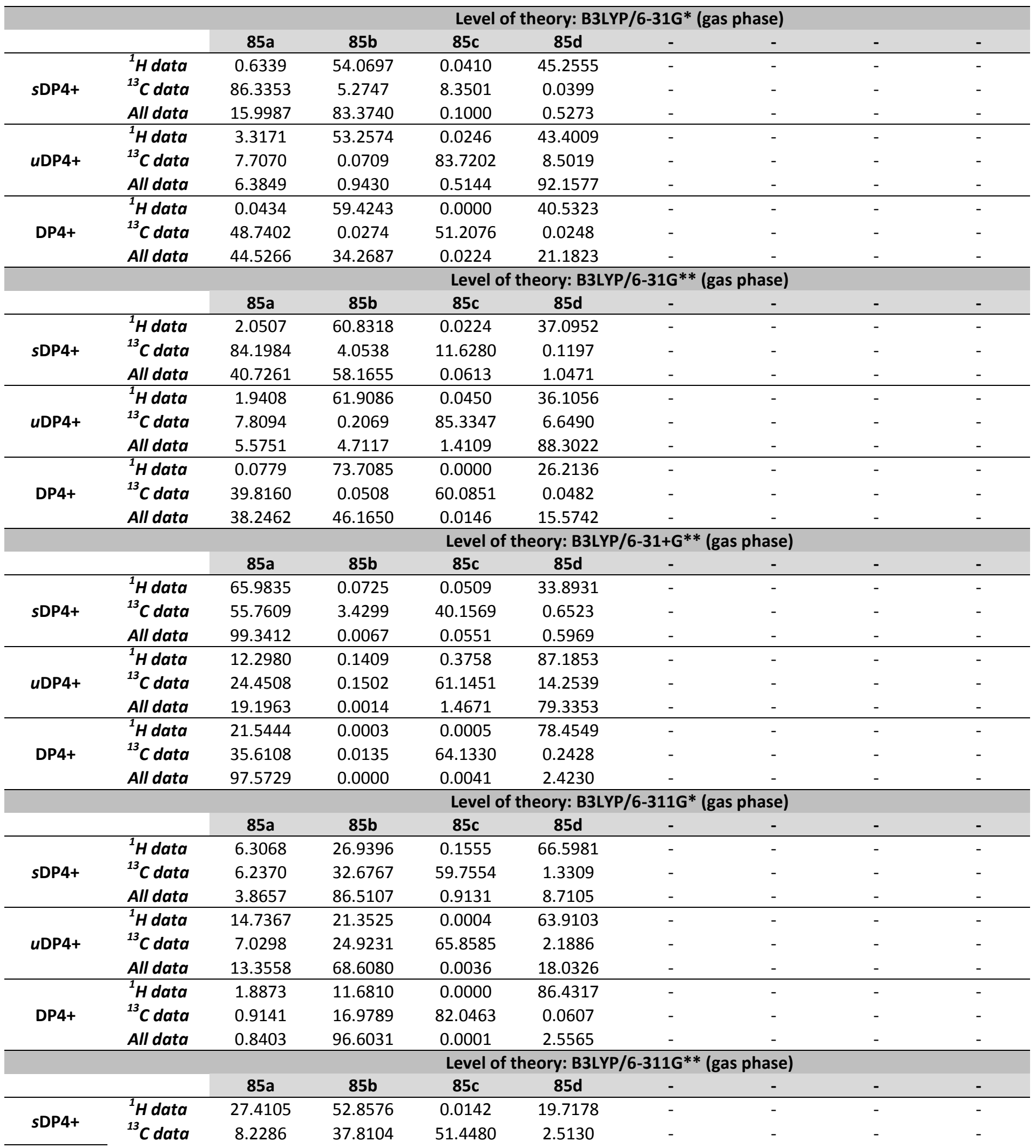




\begin{tabular}{|c|c|c|c|c|c|c|c|c|c|}
\hline & All data & 9.9169 & 87.8724 & 0.0321 & 2.1786 & - & - & - & - \\
\hline & ${ }^{1} \mathrm{H}$ data & 12.5497 & 41.3716 & 0.0005 & 46.0782 & - & - & - & - \\
\hline \multirow[t]{3}{*}{ uDP4+ } & ${ }^{13} \mathrm{C}$ data & 6.7441 & 25.9056 & 65.1850 & 2.1653 & - & - & - & - \\
\hline & All data & 6.7375 & 85.3176 & 0.0025 & 7.9423 & - & - & - & - \\
\hline & ${ }^{1} \mathrm{H}$ data & 10.0017 & 63.5817 & 0.0000 & 26.4166 & - & - & - & - \\
\hline \multirow[t]{5}{*}{ DP4+ } & ${ }^{13} \mathrm{C}$ data & 1.2629 & 22.2914 & 76.3218 & 0.1238 & - & - & - & - \\
\hline & All data & 0.8813 & 98.8904 & 0.0000 & 0.2282 & - & - & - & - \\
\hline & & \multicolumn{8}{|c|}{ Level of theory: B3LYP/6-311+G** (gas phase) } \\
\hline & & $85 a$ & $85 b$ & $85 c$ & $85 d$ & - & - & - & - \\
\hline & ${ }^{1} \mathrm{H}$ data & 69.2333 & 8.3052 & 0.0496 & 22.4119 & - & - & - & - \\
\hline \multirow[t]{2}{*}{ sDP4+ } & ${ }^{13} \mathrm{C}$ data & 8.9619 & 2.5071 & 86.8500 & 1.6810 & - & - & - & - \\
\hline & All data & 90.8078 & 3.0474 & 0.6309 & 5.5139 & - & - & - & - \\
\hline \multirow{3}{*}{ uDP4+ } & ${ }^{1} \mathrm{H}$ data & 38.7749 & 6.3385 & 0.0026 & 54.8839 & - & - & - & - \\
\hline & ${ }^{13} \mathrm{C}$ data & 11.5497 & 6.6022 & 78.9687 & 2.8794 & - & - & - & - \\
\hline & All data & 69.1189 & 6.4588 & 0.0317 & 24.3906 & - & - & - & - \\
\hline \multirow{5}{*}{ DP4+ } & ${ }^{1} \mathrm{H}$ data & 67.6676 & 1.3269 & 0.0000 & 31.0054 & - & - & - & - \\
\hline & ${ }^{13} \mathrm{C}$ data & 1.4822 & 0.2370 & 98.2115 & 0.0693 & - & - & - & - \\
\hline & All data & 97.6023 & 0.3061 & 0.0003 & 2.0913 & - & - & - & - \\
\hline & & \multicolumn{8}{|c|}{ Level of theory: B3LYP/6-31G* (solution. PCM) } \\
\hline & & $85 a$ & $85 b$ & $85 c$ & $85 d$ & - & - & - & - \\
\hline \multirow{3}{*}{ sDP4+ } & ${ }^{1}$ H data & 1.4962 & 90.3824 & 0.0090 & 8.1124 & - & - & - & - \\
\hline & ${ }^{13}$ C data & 97.8560 & 1.3249 & 0.8186 & 0.0005 & - & - & - & - \\
\hline & All data & 55.0073 & 44.9884 & 0.0028 & 0.0015 & - & - & - & - \\
\hline \multirow{3}{*}{ uDP4+ } & ${ }^{1} \mathrm{H}$ data & 4.7785 & 52.8266 & 0.0233 & 42.3716 & - & - & - & - \\
\hline & ${ }^{13} \mathrm{C}$ data & 42.5871 & 0.2990 & 53.2702 & 3.8437 & - & - & - & - \\
\hline & All data & 53.0781 & 4.1193 & 0.3239 & 42.4787 & - & - & - & - \\
\hline \multirow{3}{*}{ DP4+ } & ${ }^{1} \mathrm{H}$ data & 0.1395 & 93.1541 & 0.0000 & 6.7064 & - & - & - & - \\
\hline & ${ }^{13} \mathrm{C}$ data & 98.9551 & 0.0094 & 1.0354 & 0.0000 & - & - & - & - \\
\hline & All data & 94.0297 & 5.9683 & 0.0000 & 0.0020 & - & - & - & - \\
\hline & & \multicolumn{8}{|c|}{ Level of theory: B3LYP/6-31G** (solution. PCM) } \\
\hline & & $85 a$ & $85 b$ & $85 c$ & $85 d$ & - & - & - & - \\
\hline \multirow{3}{*}{ sDP4+ } & ${ }^{1} \mathrm{H}$ data & 8.4410 & 75.4692 & 0.0175 & 16.0723 & - & - & - & - \\
\hline & ${ }^{13} \mathrm{C}$ data & 96.3137 & 1.8098 & 1.8737 & 0.0028 & - & - & - & - \\
\hline & All data & 85.6092 & 14.3825 & 0.0034 & 0.0048 & - & - & - & - \\
\hline \multirow{3}{*}{ uDP4+ } & ${ }^{1} \mathrm{H}$ data & 2.6704 & 61.7584 & 0.0472 & 35.5240 & - & - & - & - \\
\hline & ${ }^{13} \mathrm{C}$ data & 41.6158 & 0.7582 & 54.9069 & 2.7191 & - & - & - & - \\
\hline & All data & 43.2173 & 18.2103 & 1.0085 & 37.5640 & - & - & - & - \\
\hline \multirow{3}{*}{ DP4+ } & ${ }^{1} \mathrm{H}$ data & 0.4290 & 88.7047 & 0.0000 & 10.8663 & - & - & - & - \\
\hline & ${ }^{13} \mathrm{C}$ data & 97.4647 & 0.0334 & 2.5017 & 0.0002 & - & - & - & - \\
\hline & All data & 93.3847 & 6.6107 & 0.0001 & 0.0045 & - & - & - & - \\
\hline & & \multicolumn{8}{|c|}{ Level of theory: B3LYP/6-31+G** (solution. PCM) } \\
\hline & & $85 a$ & $85 b$ & $85 c$ & $85 d$ & - & - & - & - \\
\hline \multirow{3}{*}{ sDP4+ } & ${ }^{1} \mathrm{H}$ data & 80.1909 & 2.7227 & 0.0106 & 17.0757 & - & - & - & - \\
\hline & ${ }^{13} \mathrm{C}$ data & 36.4444 & 60.4904 & 3.0425 & 0.0227 & - & - & - & - \\
\hline & All data & 94.6522 & 5.3342 & 0.0010 & 0.0125 & - & - & - & - \\
\hline & ${ }^{1} \mathrm{H}$ data & 4.5309 & 0.8162 & 0.1175 & 94.5355 & - & - & - & - \\
\hline uDP4+ & ${ }^{13} \mathrm{C}$ data & 70.5263 & 6.5646 & 20.0876 & 2.8215 & - & - & - & - \\
\hline & All data & 53.7959 & 0.9021 & 0.3972 & 44.9048 & - & - & - & - \\
\hline & ${ }^{1} \mathrm{H}$ data & 18.3518 & 0.1122 & 0.0001 & 81.5359 & - & - & - & - \\
\hline DP4+ & ${ }^{13} \mathrm{C}$ data & 84.8681 & 13.1118 & 2.0180 & 0.0021 & - & - & - & - \\
\hline & All data & 99.8945 & 0.0944 & 0.0000 & 0.0110 & - & - & - & - \\
\hline & & & & Level of $t$ & ry: B3LYP & G* & $\overline{P C}$ & & \\
\hline & & $85 a$ & $85 b$ & $85 c$ & $85 d$ & - & - & - & - \\
\hline & ${ }^{1} \mathrm{H}$ data & 22.4213 & 67.9723 & 0.1160 & 9.4904 & - & - & - & - \\
\hline sDP4+ & ${ }^{13} \mathrm{C}$ data & 3.3913 & 78.7413 & 17.7936 & 0.0738 & - & - & - & - \\
\hline & All data & 1.4001 & 98.5490 & 0.0380 & 0.0129 & - & - & - & - \\
\hline
\end{tabular}




\begin{tabular}{|c|c|c|c|c|c|c|c|c|c|}
\hline & ${ }^{1} \mathrm{H}$ data & 20.5596 & 29.9441 & 0.0000 & 49.4963 & - & - & - & - \\
\hline \multirow[t]{3}{*}{ uDP4+ } & ${ }^{13} \mathrm{C}$ data & 13.6602 & 72.6955 & 13.2442 & 0.4001 & - & - & - & - \\
\hline & All data & 11.3362 & 87.8645 & 0.0000 & 0.7993 & - & - & - & - \\
\hline & ${ }^{1} \mathrm{H}$ data & 15.5415 & 68.6215 & 0.0000 & 15.8370 & - & - & - & - \\
\hline \multirow[t]{5}{*}{ DP4+ } & ${ }^{13} \mathrm{C}$ data & 0.7713 & 95.3045 & 3.9237 & 0.0005 & - & - & - & - \\
\hline & All data & 0.1830 & 99.8169 & 0.0000 & 0.0001 & - & - & - & - \\
\hline & & \multicolumn{8}{|c|}{ Level of theory: B3LYP/6-311G** (solution. PCM) } \\
\hline & & $85 a$ & $85 b$ & $85 c$ & $85 d$ & - & - & - & - \\
\hline & ${ }^{1} \mathrm{H}$ data & 57.3278 & 31.0249 & 0.0401 & 11.6073 & - & - & - & - \\
\hline \multirow[t]{3}{*}{ sDP4+ } & ${ }^{13} \mathrm{C}$ data & 4.1441 & 62.1406 & 33.5569 & 0.1584 & - & - & - & - \\
\hline & All data & 10.9549 & 88.8984 & 0.0620 & 0.0848 & - & - & - & - \\
\hline & ${ }^{1} \mathrm{H}$ data & 16.6951 & 65.0159 & 0.0001 & 18.2890 & - & - & - & - \\
\hline \multirow[t]{3}{*}{ uDP4+ } & ${ }^{13} \mathrm{C}$ data & 11.5526 & 70.9259 & 17.1725 & 0.3491 & - & - & - & - \\
\hline & All data & 4.0093 & 95.8579 & 0.0000 & 0.1327 & - & - & - & - \\
\hline & ${ }^{1} \mathrm{H}$ data & 30.0360 & 63.3020 & 0.0000 & 6.6621 & - & - & - & - \\
\hline \multirow[t]{5}{*}{ DP4+ } & ${ }^{13} \mathrm{C}$ data & 0.9515 & 87.5946 & 11.4528 & 0.0011 & - & - & - & - \\
\hline & All data & 0.5128 & 99.4871 & 0.0000 & 0.0001 & - & - & - & - \\
\hline & & \multicolumn{8}{|c|}{ Level of theory: B3LYP/6-311+G** (solution. PCM) } \\
\hline & & $85 a$ & $85 b$ & $85 c$ & $85 d$ & - & - & - & - \\
\hline & ${ }^{1}$ H data & 65.7135 & 28.4928 & 0.0341 & 5.7596 & - & - & - & - \\
\hline \multirow[t]{3}{*}{ sDP4+ } & ${ }^{13} \mathrm{C}$ data & 4.5217 & 33.0279 & 62.3323 & 0.1180 & - & - & - & - \\
\hline & All data & 23.9434 & 75.8307 & 0.1711 & 0.0548 & - & - & - & - \\
\hline & ${ }^{1} \mathrm{H}$ data & 35.9940 & 3.6482 & 0.0000 & 60.3578 & - & - & - & - \\
\hline \multirow[t]{3}{*}{ uDP4+ } & ${ }^{13} \mathrm{C}$ data & 17.5344 & 56.5507 & 25.4761 & 0.4387 & - & - & - & - \\
\hline & All data & 73.0547 & 23.8804 & 0.0001 & 3.0649 & - & - & - & - \\
\hline & ${ }^{1} \mathrm{H}$ data & 83.9686 & 3.6901 & 0.0000 & 12.3413 & - & - & - & - \\
\hline \multirow[t]{5}{*}{ DP4+ } & ${ }^{13} \mathrm{C}$ data & 2.2428 & 52.8349 & 44.9208 & 0.0015 & - & - & - & - \\
\hline & All data & 49.1313 & 50.8640 & 0.0000 & 0.0047 & - & - & - & - \\
\hline & & \multicolumn{8}{|c|}{ Level of theory: mPW1PW91/6-31G* (gas phase) } \\
\hline & & $85 a$ & $85 b$ & $85 c$ & $85 d$ & - & - & - & - \\
\hline & ${ }^{1} \mathrm{H}$ data & 1.5288 & 39.6898 & 0.0285 & 58.7529 & - & - & - & - \\
\hline \multirow[t]{3}{*}{ sDP4+ } & ${ }^{13} \mathrm{C}$ data & 79.6898 & 1.2805 & 18.9497 & 0.0800 & - & - & - & - \\
\hline & All data & 68.4857 & 28.5706 & 0.3031 & 2.6406 & - & - & - & - \\
\hline & ${ }^{1} \mathrm{H}$ data & 2.0919 & 29.5970 & 0.0432 & 68.2679 & - & - & - & - \\
\hline \multirow[t]{3}{*}{ uDP4+ } & ${ }^{13} \mathrm{C}$ data & 6.5135 & 0.0550 & 81.7142 & 11.7174 & - & - & - & - \\
\hline & All data & 1.6643 & 0.1987 & 0.4310 & 97.7060 & - & - & - & - \\
\hline & ${ }^{1} \mathrm{H}$ data & 0.0616 & 22.6390 & 0.0000 & 77.2994 & - & - & - & - \\
\hline \multirow[t]{5}{*}{ DP4+ } & ${ }^{13} \mathrm{C}$ data & 25.0931 & 0.0034 & 74.8582 & 0.0453 & - & - & - & - \\
\hline & All data & 30.1702 & 1.5026 & 0.0346 & 68.2926 & - & - & - & - \\
\hline & & \multicolumn{8}{|c|}{ Level of theory: mPW1PW91/6-31G** (gas phase) } \\
\hline & & $85 a$ & $85 b$ & $85 c$ & $85 d$ & - & - & - & - \\
\hline & ${ }^{1} \mathrm{H}$ data & 7.9655 & 57.2265 & 0.0255 & 34.7824 & - & - & - & - \\
\hline \multirow[t]{3}{*}{ sDP4+ } & ${ }^{13} \mathrm{C}$ data & 68.6563 & 0.9491 & 30.1981 & 0.1965 & - & - & - & - \\
\hline & All data & 89.8288 & 8.9217 & 0.1267 & 1.1229 & - & - & - & - \\
\hline & ${ }^{1} \mathrm{H}$ data & 1.2622 & 37.0143 & 0.1084 & 61.6150 & - & - & - & - \\
\hline uDP4+ & ${ }^{13} \mathrm{C}$ data & 5.9388 & 0.0916 & 87.3651 & 6.6045 & - & - & - & - \\
\hline & All data & 1.7543 & 0.7934 & 2.2164 & 95.2359 & - & - & - & - \\
\hline & ${ }^{1} \mathrm{H}$ data & 0.2354 & 49.5906 & 0.0001 & 50.1740 & - & - & - & - \\
\hline DP4+ & ${ }^{13} \mathrm{C}$ data & 13.3800 & 0.0029 & 86.5746 & 0.0426 & - & - & - & - \\
\hline & All data & 57.9614 & 2.6034 & 0.1033 & 39.3319 & - & - & - & - \\
\hline & & & & Level of th & V: mPW1P & -3 & pr & & \\
\hline & & $85 a$ & $85 b$ & $85 c$ & $85 d$ & - & - & - & - \\
\hline & ${ }^{1} \mathrm{H}$ data & 72.1038 & 0.6127 & 0.0540 & 27.2294 & - & - & - & - \\
\hline sDP4+ & ${ }^{13} \mathrm{C}$ data & 60.9936 & 0.7468 & 37.5276 & 0.7320 & - & - & - & - \\
\hline & All data & 99.4928 & 0.0104 & 0.0459 & 0.4509 & - & - & - & - \\
\hline uDP4+ & ${ }^{1} \mathrm{H}$ data & 3.3581 & 0.4563 & 0.6838 & 95.5018 & - & - & - & - \\
\hline
\end{tabular}




\begin{tabular}{|c|c|c|c|c|c|c|c|c|c|}
\hline & ${ }^{13} \mathrm{C}$ data & 23.8932 & 0.0630 & 56.5433 & 19.5006 & - & - & - & - \\
\hline & All data & 4.0497 & 0.0015 & 1.9515 & 93.9973 & - & - & - & - \\
\hline & ${ }^{1} \mathrm{H}$ data & 8.5171 & 0.0098 & 0.0013 & 91.4718 & - & - & - & - \\
\hline \multirow[t]{4}{*}{ DP4+ } & ${ }^{13}$ C data & 40.5537 & 0.0013 & 59.0478 & 0.3972 & - & - & - & - \\
\hline & All data & 90.4630 & 0.0000 & 0.0201 & 9.5169 & - & - & - & - \\
\hline & & \multicolumn{8}{|c|}{ Level of theory: mPW1PW91/6-311G* (gas phase) } \\
\hline & & $85 a$ & $85 b$ & $85 c$ & $85 d$ & - & - & - & - \\
\hline & ${ }^{1} \mathrm{H}$ data & 7.3022 & 54.1405 & 0.0585 & 38.4988 & - & - & - & - \\
\hline \multirow[t]{2}{*}{ sDP4+ } & ${ }^{13} \mathrm{C}$ data & 7.3253 & 14.6090 & 73.7700 & 4.2958 & - & - & - & - \\
\hline & All data & 5.2745 & 77.9920 & 0.4255 & 16.3079 & - & - & - & - \\
\hline & ${ }^{1} \mathrm{H}$ data & 10.4920 & 19.3449 & 0.0000 & 70.1630 & - & - & - & - \\
\hline \multirow[t]{2}{*}{ uDP4+ } & ${ }^{13} \mathrm{C}$ data & 6.9412 & 14.2810 & 74.6429 & 4.1348 & - & - & - & - \\
\hline & All data & 11.3934 & 43.2201 & 0.0003 & 45.3862 & - & - & - & - \\
\hline \multirow{5}{*}{ DP4+ } & ${ }^{1} \mathrm{H}$ data & 2.0029 & 27.3805 & 0.0000 & 70.6166 & - & - & - & - \\
\hline & ${ }^{13} \mathrm{C}$ data & 0.8791 & 3.6073 & 95.2065 & 0.3071 & - & - & - & - \\
\hline & All data & 1.4408 & 80.8143 & 0.0000 & 17.7450 & - & - & - & - \\
\hline & & \multicolumn{8}{|c|}{ Level of theory: mPW1PW91/6-311G** (gas phase) } \\
\hline & & $85 a$ & $85 b$ & $85 c$ & $85 d$ & - & - & - & - \\
\hline \multirow{3}{*}{ sDP4+ } & ${ }^{1} \mathrm{H}$ data & 38.6710 & 35.9448 & 0.0099 & 25.3743 & - & - & - & - \\
\hline & ${ }^{13} \mathrm{C}$ data & 9.7762 & 11.3715 & 70.7231 & 8.1292 & - & - & - & - \\
\hline & All data & 38.0424 & 41.1308 & 0.0705 & 20.7564 & - & - & - & - \\
\hline \multirow{3}{*}{ uDP4+ } & ${ }^{1} \mathrm{H}$ data & 7.9699 & 39.1451 & 0.0000 & 52.8849 & - & - & - & - \\
\hline & ${ }^{13} \mathrm{C}$ data & 11.1783 & 22.2819 & 62.1850 & 4.3548 & - & - & - & - \\
\hline & All data & 7.4764 & 73.1967 & 0.0001 & 19.3268 & - & - & - & - \\
\hline \multirow{3}{*}{ DP4+ } & ${ }^{1} \mathrm{H}$ data & 10.0813 & 46.0248 & 0.0000 & 43.8938 & - & - & - & - \\
\hline & ${ }^{13} \mathrm{C}$ data & 2.2786 & 5.2831 & 91.7001 & 0.7381 & - & - & - & - \\
\hline & All data & 7.6949 & 81.4520 & 0.0000 & 10.8531 & - & - & - & - \\
\hline & & \multicolumn{8}{|c|}{ Level of theory: mPW1PW91/6-311+G** (gas phase) } \\
\hline & & $85 a$ & $85 b$ & $85 c$ & $85 d$ & - & - & - & - \\
\hline \multirow{3}{*}{ sDP4+ } & ${ }^{1} \mathrm{H}$ data & 67.1954 & 21.9404 & 0.0474 & 10.8168 & - & - & - & - \\
\hline & ${ }^{13} \mathrm{C}$ data & 17.2602 & 0.8447 & 74.5326 & 7.3625 & - & - & - & - \\
\hline & All data & 91.9378 & 1.4691 & 0.2801 & 6.3130 & - & - & - & - \\
\hline \multirow{3}{*}{ uDP4+ } & ${ }^{1} \mathrm{H}$ data & 18.8837 & 11.2321 & 0.0002 & 69.8840 & - & - & - & - \\
\hline & ${ }^{13} \mathrm{C}$ data & 16.1705 & 3.8361 & 76.2120 & 3.7814 & - & - & - & - \\
\hline & All data & 49.8365 & 7.0322 & 0.0024 & 43.1289 & - & - & - & - \\
\hline \multirow{5}{*}{ DP4+ } & ${ }^{1} \mathrm{H}$ data & 55.8677 & 10.8502 & 0.0000 & 33.2821 & - & - & - & - \\
\hline & ${ }^{13} \mathrm{C}$ data & 4.6592 & 0.0541 & 94.8220 & 0.4647 & - & - & - & - \\
\hline & All data & 94.1905 & 0.2124 & 0.0000 & 5.5971 & - & - & - & - \\
\hline & & \multicolumn{8}{|c|}{ Level of theory: mPW1PW91/6-31G* (solution. PCM) } \\
\hline & & $85 a$ & $85 b$ & $85 c$ & 85d & - & - & - & - \\
\hline \multirow{3}{*}{ sDP4+ } & ${ }^{1} \mathrm{H}$ data & 9.2596 & 64.9372 & 0.0227 & 25.7805 & - & - & - & - \\
\hline & ${ }^{13} \mathrm{C}$ data & 94.3715 & 0.2712 & 5.3537 & 0.0036 & - & - & - & - \\
\hline & All data & 98.0007 & 1.9752 & 0.0136 & 0.0105 & - & - & - & - \\
\hline \multirow{3}{*}{ uDP4+ } & ${ }^{1} \mathrm{H}$ data & 5.2538 & 36.0554 & 0.0797 & 58.6111 & - & - & - & - \\
\hline & ${ }^{13} \mathrm{C}$ data & 37.6478 & 0.2019 & 56.0150 & 6.1353 & - & - & - & - \\
\hline & All data & 34.7535 & 1.2790 & 0.7845 & 63.1830 & - & - & - & - \\
\hline & ${ }^{1} \mathrm{H}$ data & 1.2471 & 60.0187 & 0.0000 & 38.7342 & - & - & - & - \\
\hline DP4+ & ${ }^{13} \mathrm{C}$ data & 92.2145 & 0.0014 & 7.7835 & 0.0006 & - & - & - & - \\
\hline & All data & 99.9062 & 0.0741 & 0.0003 & 0.0194 & - & - & - & - \\
\hline & & & & vel of the & mPW1PV & $31 C$ & on. & & \\
\hline & & $85 a$ & $85 b$ & $85 c$ & $85 d$ & - & - & - & - \\
\hline & ${ }^{1} \mathrm{H}$ data & 52.2779 & 36.5458 & 0.0147 & 11.1616 & - & - & - & - \\
\hline sDP4+ & ${ }^{13} \mathrm{C}$ data & 91.9474 & 0.2344 & 7.8099 & 0.0083 & - & - & - & - \\
\hline & All data & 99.8178 & 0.1779 & 0.0024 & 0.0019 & - & - & - & - \\
\hline & ${ }^{1} \mathrm{H}$ data & 2.8772 & 45.7494 & 0.2828 & 51.0906 & - & - & - & - \\
\hline uDP4+ & ${ }^{13} \mathrm{C}$ data & 37.0429 & 0.2767 & 59.2945 & 3.3858 & - & - & - & - \\
\hline
\end{tabular}




\begin{tabular}{|c|c|c|c|c|c|c|c|c|c|}
\hline & All data & 34.4927 & 4.0973 & 5.4267 & 55.9833 & - & - & - & - \\
\hline & ${ }^{1} \mathrm{H}$ data & 6.2866 & 69.8795 & 0.0002 & 23.8338 & - & - & - & - \\
\hline \multirow[t]{5}{*}{ DP4+ } & ${ }^{13} \mathrm{C}$ data & 88.0290 & 0.0017 & 11.9686 & 0.0007 & - & - & - & - \\
\hline & All data & 99.9753 & 0.0212 & 0.0004 & 0.0031 & - & - & - & - \\
\hline & & \multicolumn{8}{|c|}{ Level of theory: mPW1PW91/6-31+G** (solution. PCM) } \\
\hline & & $85 a$ & $85 b$ & $85 c$ & $85 d$ & - & - & - & - \\
\hline & ${ }^{1} \mathrm{H}$ data & 77.9142 & 13.4547 & 0.0246 & 8.6065 & - & - & - & - \\
\hline \multirow[t]{3}{*}{ sDP4+ } & ${ }^{13} \mathrm{C}$ data & 64.1548 & 33.5691 & 2.2532 & 0.0229 & - & - & - & - \\
\hline & All data & 91.7087 & 8.2866 & 0.0010 & 0.0036 & - & - & - & - \\
\hline & ${ }^{1} \mathrm{H}$ data & 2.5972 & 2.2741 & 0.3571 & 94.7715 & - & - & - & - \\
\hline \multirow[t]{3}{*}{ uDP4+ } & ${ }^{13} \mathrm{C}$ data & 76.3331 & 6.7560 & 13.1191 & 3.7919 & - & - & - & - \\
\hline & All data & 34.3201 & 2.6596 & 0.8111 & 62.2092 & - & - & - & - \\
\hline & ${ }^{1} \mathrm{H}$ data & 19.2980 & 2.9179 & 0.0008 & 77.7833 & - & - & - & - \\
\hline \multirow[t]{5}{*}{ DP4+ } & ${ }^{13} \mathrm{C}$ data & 95.0240 & 4.4007 & 0.5736 & 0.0017 & - & - & - & - \\
\hline & All data & 99.2976 & 0.6953 & 0.0000 & 0.0071 & - & - & - & - \\
\hline & & \multicolumn{8}{|c|}{ Level of theory: mPW1PW91/6-311G* (solution. PCM) } \\
\hline & & $85 a$ & $85 b$ & $85 c$ & $85 d$ & - & - & - & - \\
\hline & ${ }^{1} \mathrm{H}$ data & 52.2483 & 33.6949 & 0.0658 & 13.9909 & - & - & - & - \\
\hline \multirow[t]{2}{*}{ sDP4+ } & ${ }^{13} \mathrm{C}$ data & 2.8010 & 32.9831 & 63.7470 & 0.4688 & - & - & - & - \\
\hline & All data & 11.5374 & 87.6147 & 0.3308 & 0.5171 & - & - & - & - \\
\hline \multirow{3}{*}{ uDP4+ } & ${ }^{1}$ H data & 16.7232 & 35.7185 & 0.0000 & 47.5583 & - & - & - & - \\
\hline & ${ }^{13}$ C data & 18.6524 & 49.9740 & 30.5757 & 0.7979 & - & - & - & - \\
\hline & All data & 14.6111 & 83.6114 & 0.0000 & 1.7776 & - & - & - & - \\
\hline \multirow{3}{*}{ DP4+ } & ${ }^{1} \mathrm{H}$ data & 31.8578 & 43.8817 & 0.0000 & 24.2604 & - & - & - & - \\
\hline & ${ }^{13} \mathrm{C}$ data & 1.4314 & 45.1585 & 53.3999 & 0.0102 & - & - & - & - \\
\hline & All data & 2.2491 & 97.7386 & 0.0000 & 0.0123 & - & - & - & - \\
\hline & & \multicolumn{8}{|c|}{ Level of theory: mPW1PW91/6-311G** (solution. PCM) } \\
\hline & & $85 a$ & $85 b$ & $85 c$ & $85 d$ & - & - & - & - \\
\hline \multirow{3}{*}{ sDP4+ } & ${ }^{1} \mathrm{H}$ data & 79.5049 & 15.9877 & 0.0269 & 4.4805 & - & - & - & - \\
\hline & ${ }^{13} \mathrm{C}$ data & 2.9522 & 24.0038 & 71.9704 & 1.0736 & - & - & - & - \\
\hline & All data & 37.5406 & 61.3802 & 0.3098 & 0.7694 & - & - & - & - \\
\hline \multirow{3}{*}{ uDP4+ } & ${ }^{1} \mathrm{H}$ data & 18.4442 & 61.4147 & 0.0000 & 20.1412 & - & - & - & - \\
\hline & ${ }^{13} \mathrm{C}$ data & 19.8031 & 39.0493 & 40.0040 & 1.1436 & - & - & - & - \\
\hline & All data & 13.1079 & 86.0654 & 0.0000 & 0.8266 & - & - & - & - \\
\hline \multirow{5}{*}{ DP4+ } & ${ }^{1} \mathrm{H}$ data & 57.7660 & 38.6791 & 0.0000 & 3.5549 & - & - & - & - \\
\hline & ${ }^{13} \mathrm{C}$ data & 1.5083 & 24.1822 & 74.2779 & 0.0317 & - & - & - & - \\
\hline & All data & 8.5202 & 91.4688 & 0.0000 & 0.0110 & - & - & - & - \\
\hline & & \multicolumn{8}{|c|}{ Level of theory: mPW1PW91/6-311+G** (solution. PCM) } \\
\hline & & $85 a$ & $85 b$ & $85 c$ & $85 d$ & - & - & - & - \\
\hline \multirow{3}{*}{ sDP4+ } & ${ }^{1} \mathrm{H}$ data & 79.9752 & 17.0863 & 0.0626 & 2.8759 & - & - & - & - \\
\hline & ${ }^{13} \mathrm{C}$ data & 3.1969 & 16.3722 & 79.8615 & 0.5693 & - & - & - & - \\
\hline & All data & 47.1679 & 51.6073 & 0.9227 & 0.3021 & - & - & - & - \\
\hline \multirow{3}{*}{ uDP4+ } & ${ }^{1} \mathrm{H}$ data & 32.0992 & 8.1485 & 0.0000 & 59.7523 & - & - & - & - \\
\hline & ${ }^{13} \mathrm{C}$ data & 21.7141 & 35.1583 & 42.5487 & 0.5788 & - & - & - & - \\
\hline & All data & 68.4627 & 28.1399 & 0.0002 & 3.3972 & - & - & - & - \\
\hline \multirow{3}{*}{ DP4+ } & ${ }^{1} \mathrm{H}$ data & 89.1922 & 4.8373 & 0.0000 & 5.9705 & - & - & - & - \\
\hline & ${ }^{13} \mathrm{C}$ data & 1.7169 & 14.2361 & 84.0389 & 0.0082 & - & - & - & - \\
\hline & All data & 68.9642 & 31.0139 & 0.0000 & 0.0219 & - & - & - & - \\
\hline
\end{tabular}


$s \mathrm{DP4}+, u \mathrm{DP} 4+$ and DP4+ probabilities (\%) for compound $85 \mathrm{~b}$<smiles>O=C1C=CCC(C[C@@H](O)C[C@@H](O)/C=C/c2ccccc2)O1</smiles>

\begin{tabular}{|c|c|c|c|c|c|c|c|c|c|}
\hline & & \multicolumn{8}{|c|}{ Level of theory: B3LYP/6-31G* (gas phase) } \\
\hline & & $85 a$ & $85 b$ & $85 c$ & $85 d$ & - & - & - & - \\
\hline & ${ }^{1} \mathrm{H}$ data & 0.4560 & 76.5490 & 0.0277 & 22.9673 & - & - & - & - \\
\hline \multirow[t]{2}{*}{ sDP4+ } & ${ }^{13} \mathrm{C}$ data & 60.5405 & 25.3430 & 14.0262 & 0.0903 & - & - & - & - \\
\hline & All data & 1.4014 & 98.4736 & 0.0197 & 0.1053 & - & - & - & - \\
\hline & ${ }^{1} \mathrm{H}$ data & 1.5305 & 87.8098 & 0.0063 & 10.6534 & - & - & - & - \\
\hline \multirow[t]{2}{*}{ uDP4+ } & ${ }^{13} \mathrm{C}$ data & 1.9971 & 2.3438 & 94.5184 & 1.1408 & - & - & - & - \\
\hline & All data & 1.3792 & 92.8679 & 0.2689 & 5.4840 & - & - & - & - \\
\hline \multirow{6}{*}{ DP4+ } & ${ }^{1} \mathrm{H}$ data & 0.0100 & 96.4781 & 0.0000 & 3.5119 & - & - & - & - \\
\hline & ${ }^{13} \mathrm{C}$ data & 8.0275 & 3.9437 & 88.0219 & 0.0068 & - & - & - & - \\
\hline & All data & 0.0211 & 99.9725 & 0.0001 & 0.0063 & - & - & - & - \\
\hline & & \multicolumn{8}{|c|}{ Level of theory: B3LYP/6-31G** (gas phase) } \\
\hline & & $85 a$ & $85 \mathrm{~b}$ & $85 c$ & $85 d$ & - & - & - & - \\
\hline & ${ }^{1} \mathrm{H}$ data & 1.2367 & 84.1553 & 0.0165 & 14.5915 & - & - & - & - \\
\hline \multirow[t]{2}{*}{ sDP4+ } & ${ }^{13} \mathrm{C}$ data & 56.3627 & 21.6187 & 21.8822 & 0.1364 & - & - & - & - \\
\hline & All data & 3.6852 & 96.1905 & 0.0191 & 0.1052 & - & - & - & - \\
\hline & ${ }^{1} \mathrm{H}$ data & 1.0281 & 88.1705 & 0.0080 & 10.7934 & - & - & - & - \\
\hline \multirow[t]{2}{*}{ uDP4+ } & ${ }^{13} \mathrm{C}$ data & 2.6013 & 3.8750 & 92.0168 & 1.5069 & - & - & - & - \\
\hline & All data & 0.7401 & 94.5541 & 0.2046 & 4.5011 & - & - & - & - \\
\hline \multirow{6}{*}{ DP4+ } & ${ }^{1} \mathrm{H}$ data & 0.0168 & 97.9052 & 0.0000 & 2.0781 & - & - & - & - \\
\hline & ${ }^{13} \mathrm{C}$ data & 6.5335 & 3.7329 & 89.7245 & 0.0092 & - & - & - & - \\
\hline & All data & 0.0300 & 99.9648 & 0.0000 & 0.0052 & - & - & - & - \\
\hline & & \multicolumn{8}{|c|}{ Level of theory: B3LYP/6-31+G** (gas phase) } \\
\hline & & $85 a$ & $85 b$ & $85 c$ & $85 d$ & - & - & - & - \\
\hline & ${ }^{1} \mathrm{H}$ data & 82.7084 & 0.0438 & 0.0386 & 17.2092 & - & - & - & - \\
\hline \multirow[t]{2}{*}{ sDP4+ } & ${ }^{13} \mathrm{C}$ data & 19.6149 & 29.8503 & 50.1455 & 0.3894 & - & - & - & - \\
\hline & All data & 99.3908 & 0.0801 & 0.1186 & 0.4105 & - & - & - & - \\
\hline \multirow{3}{*}{ uDP4+ } & ${ }^{1} \mathrm{H}$ data & 24.2598 & 0.1739 & 0.3665 & 75.1998 & - & - & - & - \\
\hline & ${ }^{13} \mathrm{C}$ data & 2.9282 & 0.6712 & 93.1221 & 3.2785 & - & - & - & - \\
\hline & All data & 20.1910 & 0.0332 & 9.7008 & 70.0750 & - & - & - & - \\
\hline \multirow{5}{*}{ DP4+ } & ${ }^{1} \mathrm{H}$ data & 60.7909 & 0.0002 & 0.0004 & 39.2085 & - & - & - & - \\
\hline & ${ }^{13} \mathrm{C}$ data & 1.2096 & 0.4219 & 98.3416 & 0.0269 & - & - & - & - \\
\hline & All data & 98.5310 & 0.0001 & 0.0565 & 1.4124 & - & - & - & - \\
\hline & & \multicolumn{8}{|c|}{ Level of theory: B3LYP/6-311G* (gas phase) } \\
\hline & & $85 a$ & $85 b$ & $85 c$ & $85 d$ & - & - & - & - \\
\hline \multirow{3}{*}{ sDP4+ } & ${ }^{1} \mathrm{H}$ data & 6.9103 & 37.3155 & 0.1096 & 55.6646 & - & - & - & - \\
\hline & ${ }^{13} \mathrm{C}$ data & 1.4612 & 48.1642 & 49.8576 & 0.5170 & - & - & - & - \\
\hline & All data & 0.5483 & 97.5923 & 0.2967 & 1.5628 & - & - & - & - \\
\hline \multirow{3}{*}{ uDP4+ } & ${ }^{1} \mathrm{H}$ data & 10.5943 & 45.2825 & 0.0015 & 44.1217 & - & - & - & - \\
\hline & ${ }^{13} \mathrm{C}$ data & 2.0392 & 42.6013 & 54.6679 & 0.6917 & - & - & - & - \\
\hline & All data & 1.0904 & 97.3653 & 0.0040 & 1.5403 & - & - & - & - \\
\hline \multirow{5}{*}{ DP4+ } & ${ }^{1} \mathrm{H}$ data & 1.7353 & 40.0510 & 0.0000 & 58.2138 & - & - & - & - \\
\hline & ${ }^{13} \mathrm{C}$ data & 0.0623 & 42.9187 & 57.0115 & 0.0075 & - & - & - & - \\
\hline & All data & 0.0063 & 99.9684 & 0.0000 & 0.0253 & - & - & - & - \\
\hline & & \multicolumn{8}{|c|}{ Level of theory: B3LYP/6-311G** (gas phase) } \\
\hline & & $85 a$ & $85 b$ & $85 c$ & $85 d$ & - & - & - & - \\
\hline \multirow{2}{*}{ sDP4+ } & ${ }^{1} \mathrm{H}$ data & 26.1844 & 69.5061 & 0.0035 & 4.3060 & - & - & - & - \\
\hline & ${ }^{13} \mathrm{C}$ data & 1.4575 & 37.7533 & 60.2334 & 0.5558 & - & - & - & - \\
\hline
\end{tabular}




\begin{tabular}{|c|c|c|c|c|c|c|c|c|c|}
\hline & All data & 1.4321 & 98.4702 & 0.0079 & 0.0898 & - & - & - & - \\
\hline & ${ }^{1} \mathrm{H}$ data & 7.6761 & 70.6416 & 0.0011 & 21.6812 & - & - & - & - \\
\hline \multirow[t]{2}{*}{ uDP4+ } & ${ }^{13} \mathrm{C}$ data & 1.8302 & 44.0443 & 53.4704 & 0.6551 & - & - & - & - \\
\hline & All data & 0.4475 & 99.0983 & 0.0018 & 0.4524 & - & - & - & - \\
\hline & ${ }^{1} \mathrm{H}$ data & 3.8621 & 94.3441 & 0.0000 & 1.7939 & - & - & - & - \\
\hline \multirow[t]{5}{*}{ DP4+ } & ${ }^{13}$ C data & 0.0546 & 34.0284 & 65.9095 & 0.0075 & - & - & - & - \\
\hline & All data & 0.0066 & 99.9930 & 0.0000 & 0.0004 & - & - & - & - \\
\hline & & \multicolumn{8}{|c|}{ Level of theory: B3LYP/6-311+G** (gas phase) } \\
\hline & & $85 a$ & $85 b$ & $85 c$ & $85 d$ & - & - & - & - \\
\hline & ${ }^{1} \mathrm{H}$ data & 92.8686 & 3.9541 & 0.0092 & 3.1681 & - & - & - & - \\
\hline \multirow[t]{2}{*}{ sDP4+ } & ${ }^{13} \mathrm{C}$ data & 1.1231 & 13.2780 & 85.2651 & 0.3339 & - & - & - & - \\
\hline & All data & 65.7427 & 33.0945 & 0.4961 & 0.6667 & - & - & - & - \\
\hline \multirow{3}{*}{ uDP4+ } & ${ }^{1} \mathrm{H}$ data & 69.9767 & 8.4738 & 0.0080 & 21.5415 & - & - & - & - \\
\hline & ${ }^{13} \mathrm{C}$ data & 2.2254 & 30.3546 & 66.8108 & 0.6092 & - & - & - & - \\
\hline & All data & 36.5032 & 60.2945 & 0.1260 & 3.0764 & - & - & - & - \\
\hline & ${ }^{1} \mathrm{H}$ data & 98.4584 & 0.5076 & 0.0000 & 1.0340 & - & - & - & - \\
\hline \multirow[t]{5}{*}{ DP4+ } & ${ }^{13} \mathrm{C}$ data & 0.0410 & 6.6048 & 93.3509 & 0.0033 & - & - & - & - \\
\hline & All data & 54.5743 & 45.3777 & 0.0014 & 0.0466 & - & - & - & - \\
\hline & & \multicolumn{8}{|c|}{ Level of theory: B3LYP/6-31G* (solution. PCM) } \\
\hline & & $85 a$ & $85 b$ & $85 c$ & $85 d$ & - & - & - & - \\
\hline & ${ }^{1} \mathrm{H}$ data & 1.2771 & 94.5753 & 0.0055 & 4.1422 & - & - & - & - \\
\hline \multirow[t]{2}{*}{ sDP4+ } & ${ }^{13} \mathrm{C}$ data & 86.8286 & 11.9504 & 1.2200 & 0.0010 & - & - & - & - \\
\hline & All data & 8.9343 & 91.0648 & 0.0005 & 0.0003 & - & - & - & - \\
\hline & ${ }^{1} \mathrm{H}$ data & 2.2252 & 84.9896 & 0.0049 & 12.7803 & - & - & - & - \\
\hline \multirow[t]{2}{*}{ uDP4+ } & ${ }^{13} \mathrm{C}$ data & 20.7934 & 9.4781 & 69.0310 & 0.6976 & - & - & - & - \\
\hline & All data & 5.3736 & 93.5520 & 0.0390 & 1.0354 & - & - & - & - \\
\hline \multirow{6}{*}{ DP4+ } & ${ }^{1} \mathrm{H}$ data & 0.0351 & 99.3108 & 0.0000 & 0.6541 & - & - & - & - \\
\hline & ${ }^{13} \mathrm{C}$ data & 90.1402 & 5.6550 & 4.2047 & 0.0000 & - & - & - & - \\
\hline & All data & 0.5604 & 99.4396 & 0.0000 & 0.0000 & - & - & - & - \\
\hline & & \multicolumn{8}{|c|}{ Level of theory: B3LYP/6-31G** (solution. PCM) } \\
\hline & & $85 a$ & $85 b$ & $85 c$ & 85d & - & - & - & - \\
\hline & ${ }^{1} \mathrm{H}$ data & 7.6503 & 83.1946 & 0.0204 & 9.1346 & - & - & - & - \\
\hline \multirow[t]{2}{*}{ sDP4+ } & ${ }^{13} \mathrm{C}$ data & 85.2407 & 11.7019 & 3.0539 & 0.0035 & - & - & - & - \\
\hline & All data & 40.1120 & 59.8822 & 0.0038 & 0.0020 & - & - & - & - \\
\hline \multirow{3}{*}{ uDP4+ } & ${ }^{1} \mathrm{H}$ data & 1.3327 & 83.8303 & 0.0068 & 14.8302 & - & - & - & - \\
\hline & ${ }^{13} \mathrm{C}$ data & 25.1047 & 10.5500 & 63.6625 & 0.6829 & - & - & - & - \\
\hline & All data & 3.6036 & 95.2590 & 0.0467 & 1.0908 & - & - & - & - \\
\hline \multirow{3}{*}{ DP4+ } & ${ }^{1} \mathrm{H}$ data & 0.1432 & 97.9541 & 0.0000 & 1.9027 & - & - & - & - \\
\hline & ${ }^{13} \mathrm{C}$ data & 87.0667 & 5.0229 & 7.9103 & 0.0001 & - & - & - & - \\
\hline & All data & 2.4713 & 97.5286 & 0.0000 & 0.0000 & - & - & - & - \\
\hline & & \multicolumn{8}{|c|}{ Level of theory: B3LYP/6-31+G** (solution. PCM) } \\
\hline & & $85 a$ & $85 b$ & $85 c$ & 85d & - & - & - & - \\
\hline \multirow{3}{*}{ sDP4+ } & ${ }^{1} \mathrm{H}$ data & 85.4578 & 1.8945 & 0.0136 & 12.6341 & - & - & - & - \\
\hline & ${ }^{13} \mathrm{C}$ data & 3.0292 & 96.1492 & 0.8176 & 0.0041 & - & - & - & - \\
\hline & All data & 58.6887 & 41.2972 & 0.0025 & 0.0116 & - & - & - & - \\
\hline & ${ }^{1} \mathrm{H}$ data & 7.6754 & 1.4077 & 0.0816 & 90.8353 & - & - & - & - \\
\hline uDP4+ & ${ }^{13} \mathrm{C}$ data & 19.3794 & 61.1498 & 18.3614 & 1.1094 & - & - & - & - \\
\hline & All data & 44.1256 & 25.5360 & 0.4446 & 29.8938 & - & - & - & - \\
\hline & ${ }^{1} \mathrm{H}$ data & 36.3146 & 0.1476 & 0.0001 & 63.5377 & - & - & - & - \\
\hline DP4+ & ${ }^{13} \mathrm{C}$ data & 0.9861 & 98.7617 & 0.2522 & 0.0001 & - & - & - & - \\
\hline & All data & 71.0554 & 28.9350 & 0.0000 & 0.0095 & - & - & - & - \\
\hline & & & & evel of thec & 3 LYP/6-31 & sol & M) & & \\
\hline & & $85 a$ & $85 \mathrm{~b}$ & $85 c$ & $85 d$ & - & - & - & - \\
\hline & ${ }^{1} \mathrm{H}$ data & 24.6509 & 66.6347 & 0.0377 & 8.6767 & - & - & - & - \\
\hline$s \mathrm{DP} 4+$ & ${ }^{13} \mathrm{C}$ data & 0.3903 & 93.8801 & 5.7166 & 0.0130 & - & - & - & - \\
\hline & All data & 0.1536 & 99.8412 & 0.0034 & 0.0018 & - & - & - & - \\
\hline
\end{tabular}




\begin{tabular}{|c|c|c|c|c|c|c|c|c|c|}
\hline & ${ }^{1} \mathrm{H}$ data & 10.2752 & 57.2591 & 0.0000 & 32.4658 & - & - & - & - \\
\hline \multirow[t]{3}{*}{ uDP4+ } & ${ }^{13} \mathrm{C}$ data & 4.3657 & 85.6370 & 9.9011 & 0.0962 & - & - & - & - \\
\hline & All data & 0.9060 & 99.0310 & 0.0000 & 0.0631 & - & - & - & - \\
\hline & ${ }^{1} \mathrm{H}$ data & 5.8222 & 87.7026 & 0.0000 & 6.4751 & - & - & - & - \\
\hline \multirow[t]{5}{*}{ DP4+ } & ${ }^{13} \mathrm{C}$ data & 0.0210 & 99.2800 & 0.6989 & 0.0000 & - & - & - & - \\
\hline & All data & 0.0014 & 99.9986 & 0.0000 & 0.0000 & - & - & - & - \\
\hline & & \multicolumn{8}{|c|}{ Level of theory: B3LYP/6-311G** (solution. PCM) } \\
\hline & & $85 a$ & $85 b$ & $85 c$ & $85 d$ & - & - & - & - \\
\hline & ${ }^{1} \mathrm{H}$ data & 65.5955 & 30.8623 & 0.0149 & 3.5273 & - & - & - & - \\
\hline \multirow[t]{3}{*}{ sDP4+ } & ${ }^{13} \mathrm{C}$ data & 0.5330 & 87.9752 & 11.4612 & 0.0306 & - & - & - & - \\
\hline & All data & 1.2711 & 98.7188 & 0.0062 & 0.0039 & - & - & - & - \\
\hline & ${ }^{1} \mathrm{H}$ data & 7.4544 & 80.1148 & 0.0000 & 12.4307 & - & - & - & - \\
\hline \multirow[t]{3}{*}{ uDP4+ } & ${ }^{13} \mathrm{C}$ data & 3.3451 & 84.5613 & 12.0071 & 0.0865 & - & - & - & - \\
\hline & All data & 0.3667 & 99.6175 & 0.0000 & 0.0158 & - & - & - & - \\
\hline & ${ }^{1} \mathrm{H}$ data & 16.2702 & 82.2708 & 0.0000 & 1.4590 & - & - & - & - \\
\hline \multirow[t]{5}{*}{ DP4+ } & ${ }^{13} \mathrm{C}$ data & 0.0235 & 98.1606 & 1.8158 & 0.0000 & - & - & - & - \\
\hline & All data & 0.0047 & 99.9953 & 0.0000 & 0.0000 & - & - & - & - \\
\hline & & \multicolumn{8}{|c|}{ Level of theory: B3LYP/6-311+G** (solution. PCM) } \\
\hline & & $85 a$ & $85 b$ & $85 c$ & $85 d$ & - & - & - & - \\
\hline & ${ }^{1} \mathrm{H}$ data & 79.4109 & 18.5353 & 0.0201 & 2.0337 & - & - & - & - \\
\hline \multirow[t]{3}{*}{ sDP4+ } & ${ }^{13} \mathrm{C}$ data & 0.3330 & 79.9877 & 19.6609 & 0.0184 & - & - & - & - \\
\hline & All data & 1.7519 & 98.2195 & 0.0262 & 0.0025 & - & - & - & - \\
\hline & ${ }^{1} \mathrm{H}$ data & 46.2487 & 17.1666 & 0.0001 & 36.5847 & - & - & - & - \\
\hline \multirow[t]{3}{*}{ uDP4+ } & ${ }^{13} \mathrm{C}$ data & 2.0355 & 89.5408 & 8.3833 & 0.0403 & - & - & - & - \\
\hline & All data & 5.7659 & 94.1437 & 0.0000 & 0.0904 & - & - & - & - \\
\hline & ${ }^{1} \mathrm{H}$ data & 90.3428 & 7.8270 & 0.0000 & 1.8302 & - & - & - & - \\
\hline \multirow[t]{5}{*}{ DP4+ } & ${ }^{13} \mathrm{C}$ data & 0.0093 & 97.7414 & 2.2493 & 0.0000 & - & - & - & - \\
\hline & All data & 0.1091 & 99.8909 & 0.0000 & 0.0000 & - & - & - & - \\
\hline & & \multicolumn{8}{|c|}{ Level of theory: mPW1PW91/6-31G* (gas phase) } \\
\hline & & $85 a$ & $85 b$ & $85 c$ & $85 d$ & - & - & - & - \\
\hline & ${ }^{1} \mathrm{H}$ data & 1.0098 & 61.4725 & 0.0247 & 37.4929 & - & - & - & - \\
\hline \multirow[t]{3}{*}{ sDP4+ } & ${ }^{13} \mathrm{C}$ data & 47.1963 & 18.3359 & 34.1531 & 0.3147 & - & - & - & - \\
\hline & All data & 4.0136 & 94.9217 & 0.0712 & 0.9936 & - & - & - & - \\
\hline & ${ }^{1} \mathrm{H}$ data & 1.3328 & 72.5116 & 0.0139 & 26.1417 & - & - & - & - \\
\hline \multirow[t]{3}{*}{ uDP4+ } & ${ }^{13} \mathrm{C}$ data & 2.3308 & 1.7702 & 94.4112 & 1.4878 & - & - & - & - \\
\hline & All data & 1.8096 & 74.7726 & 0.7621 & 22.6557 & - & - & - & - \\
\hline & ${ }^{1} \mathrm{H}$ data & 0.0247 & 81.9547 & 0.0000 & 18.0206 & - & - & - & - \\
\hline \multirow[t]{5}{*}{ DP4+ } & ${ }^{13} \mathrm{C}$ data & 3.2668 & 0.9639 & 95.7554 & 0.0139 & - & - & - & - \\
\hline & All data & 0.1019 & 99.5815 & 0.0008 & 0.3158 & - & - & - & - \\
\hline & & \multicolumn{8}{|c|}{ Level of theory: mPW1PW91/6-31G** (gas phase) } \\
\hline & & $85 a$ & $85 b$ & $85 c$ & $85 d$ & - & - & - & - \\
\hline & ${ }^{1} \mathrm{H}$ data & 5.3778 & 84.4668 & 0.0252 & 10.1302 & - & - & - & - \\
\hline \multirow[t]{2}{*}{ sDP4+ } & ${ }^{13} \mathrm{C}$ data & 34.7169 & 13.3510 & 51.5541 & 0.3779 & - & - & - & - \\
\hline & All data & 14.1488 & 85.4625 & 0.0986 & 0.2901 & - & - & - & - \\
\hline & ${ }^{1} \mathrm{H}$ data & 0.7839 & 73.3195 & 0.0228 & 25.8738 & - & - & - & - \\
\hline uDP4+ & ${ }^{13} \mathrm{C}$ data & 2.4225 & 1.9118 & 94.4501 & 1.2156 & - & - & - & - \\
\hline & All data & 1.0809 & 79.7896 & 1.2269 & 17.9026 & - & - & - & - \\
\hline & ${ }^{1} \mathrm{H}$ data & 0.0653 & 95.8770 & 0.0000 & 4.0578 & - & - & - & - \\
\hline DP4+ & ${ }^{13} \mathrm{C}$ data & 1.6890 & 0.5126 & 97.7892 & 0.0092 & - & - & - & - \\
\hline & All data & 0.2236 & 99.6987 & 0.0018 & 0.0759 & - & - & - & - \\
\hline & & & & of theor & W1PW91 & $\mathbf{G}^{*}$ & las & & \\
\hline & & $85 a$ & $85 b$ & $85 c$ & $85 d$ & - & - & - & - \\
\hline & ${ }^{1} \mathrm{H}$ data & 89.1172 & 0.3458 & 0.0383 & 10.4987 & - & - & - & - \\
\hline sDP4+ & ${ }^{13} \mathrm{C}$ data & 19.6457 & 11.8345 & 67.9245 & 0.5954 & - & - & - & - \\
\hline & All data & 99.2660 & 0.2320 & 0.1475 & 0.3544 & - & - & - & - \\
\hline uDP4+ & ${ }^{1} \mathrm{H}$ data & 7.0856 & 0.6355 & 0.6389 & 91.6400 & - & - & - & - \\
\hline
\end{tabular}




\begin{tabular}{|c|c|c|c|c|c|c|c|c|c|}
\hline & ${ }^{13} \mathrm{C}$ data & 2.2172 & 0.3626 & 94.3943 & 3.0260 & - & - & - & - \\
\hline & All data & 4.4435 & 0.0652 & 17.0576 & 78.4337 & - & - & - & - \\
\hline & ${ }^{1} \mathrm{H}$ data & 39.6193 & 0.0138 & 0.0015 & 60.3654 & - & - & - & - \\
\hline \multirow[t]{4}{*}{ DP4+ } & ${ }^{13} \mathrm{C}$ data & 0.6741 & 0.0664 & 99.2316 & 0.0279 & - & - & - & - \\
\hline & All data & 93.5666 & 0.0032 & 0.5339 & 5.8963 & - & - & - & - \\
\hline & & \multicolumn{8}{|c|}{ Level of theory: mPW1PW91/6-311G* (gas phase) } \\
\hline & & $85 a$ & $85 b$ & $85 c$ & $85 d$ & - & - & - & - \\
\hline & ${ }^{1} \mathrm{H}$ data & 6.5912 & 67.2997 & 0.0269 & 26.0822 & - & - & - & - \\
\hline \multirow[t]{2}{*}{ sDP4+ } & ${ }^{13} \mathrm{C}$ data & 1.0066 & 14.0804 & 84.2295 & 0.6835 & - & - & - & - \\
\hline & All data & 0.6810 & 97.2572 & 0.2323 & 1.8296 & - & - & - & - \\
\hline \multirow{3}{*}{ uDP4+ } & ${ }^{1} \mathrm{H}$ data & 5.4709 & 57.4912 & 0.0001 & 37.0379 & - & - & - & - \\
\hline & ${ }^{13} \mathrm{C}$ data & 2.3936 & 21.3799 & 74.3385 & 1.8880 & - & - & - & - \\
\hline & All data & 0.9980 & 93.6726 & 0.0003 & 5.3291 & - & - & - & - \\
\hline \multirow{5}{*}{ DP4+ } & ${ }^{1} \mathrm{H}$ data & 0.7403 & 79.4284 & 0.0000 & 19.8314 & - & - & - & - \\
\hline & ${ }^{13} \mathrm{C}$ data & 0.0367 & 4.5846 & 95.3590 & 0.0197 & - & - & - & - \\
\hline & All data & 0.0075 & 99.8856 & 0.0000 & 0.1069 & - & - & - & - \\
\hline & & \multicolumn{8}{|c|}{ Level of theory: mPW1PW91/6-311G** (gas phase) } \\
\hline & & $85 a$ & $85 b$ & $85 c$ & $85 d$ & - & - & - & - \\
\hline \multirow{3}{*}{ sDP4+ } & ${ }^{1} \mathrm{H}$ data & 54.8316 & 41.8993 & 0.0020 & 3.2671 & - & - & - & - \\
\hline & ${ }^{13} \mathrm{C}$ data & 0.9531 & 12.3556 & 85.9895 & 0.7018 & - & - & - & - \\
\hline & All data & 9.1295 & 90.4405 & 0.0294 & 0.4005 & - & - & - & - \\
\hline \multirow{3}{*}{ uDP4+ } & ${ }^{1}$ H data & 3.5363 & 79.6420 & 0.0000 & 16.8217 & - & - & - & - \\
\hline & ${ }^{13} \mathrm{C}$ data & 2.8162 & 21.8160 & 73.7795 & 1.5883 & - & - & - & - \\
\hline & All data & 0.5613 & 97.9326 & 0.0001 & 1.5059 & - & - & - & - \\
\hline \multirow{3}{*}{ DP4+ } & ${ }^{1} \mathrm{H}$ data & 5.4074 & 93.0599 & 0.0000 & 1.5327 & - & - & - & - \\
\hline & ${ }^{13} \mathrm{C}$ data & 0.0406 & 4.0732 & 95.8694 & 0.0168 & - & - & - & - \\
\hline & All data & 0.0578 & 99.9354 & 0.0000 & 0.0068 & - & - & - & - \\
\hline & & \multicolumn{8}{|c|}{ Level of theory: mPW1PW91/6-311+G** (gas phase) } \\
\hline & & $85 a$ & $85 b$ & $85 c$ & 85d & - & - & - & - \\
\hline \multirow{3}{*}{ sDP4+ } & ${ }^{1} \mathrm{H}$ data & 82.7513 & 14.9612 & 0.0116 & 2.2758 & - & - & - & - \\
\hline & ${ }^{13} \mathrm{C}$ data & 1.2492 & 3.6424 & 94.6652 & 0.4433 & - & - & - & - \\
\hline & All data & 64.6161 & 34.0648 & 0.6885 & 0.6306 & - & - & - & - \\
\hline \multirow{3}{*}{ uDP4+ } & ${ }^{1} \mathrm{H}$ data & 43.4292 & 26.2737 & 0.0007 & 30.2964 & - & - & - & - \\
\hline & ${ }^{13} \mathrm{C}$ data & 3.4954 & 20.7481 & 74.8348 & 0.9217 & - & - & - & - \\
\hline & All data & 20.9411 & 75.1996 & 0.0072 & 3.8521 & - & - & - & - \\
\hline \multirow{3}{*}{ DP4+ } & ${ }^{1} \mathrm{H}$ data & 88.6082 & 9.6918 & 0.0000 & 1.7000 & - & - & - & - \\
\hline & ${ }^{13} \mathrm{C}$ data & 0.0609 & 1.0548 & 98.8785 & 0.0057 & - & - & - & - \\
\hline & All data & 34.5431 & 65.3948 & 0.0001 & 0.0620 & - & - & - & - \\
\hline & & \multicolumn{8}{|c|}{ Level of theory: mPW1PW91/6-31G* (solution. PCM) } \\
\hline & & $85 a$ & $85 b$ & $85 c$ & 85d & - & - & - & - \\
\hline \multirow{3}{*}{ sDP4+ } & ${ }^{1} \mathrm{H}$ data & 8.7783 & 79.3924 & 0.0403 & 11.7890 & - & - & - & - \\
\hline & ${ }^{13} \mathrm{C}$ data & 84.5075 & 7.5907 & 7.8894 & 0.0123 & - & - & - & - \\
\hline & All data & 55.1573 & 44.8083 & 0.0236 & 0.0108 & - & - & - & - \\
\hline \multirow{3}{*}{ uDP4+ } & ${ }^{1} \mathrm{H}$ data & 2.6193 & 72.6681 & 0.0189 & 24.6937 & - & - & - & - \\
\hline & ${ }^{13} \mathrm{C}$ data & 17.0242 & 4.1381 & 77.7707 & 1.0670 & - & - & - & - \\
\hline & All data & 11.9513 & 80.5934 & 0.3936 & 7.0617 & - & - & - & - \\
\hline & ${ }^{1} \mathrm{H}$ data & 0.3780 & 94.8366 & 0.0000 & 4.7854 & - & - & - & - \\
\hline DP4+ & ${ }^{13} \mathrm{C}$ data & 69.0455 & 1.5075 & 29.4464 & 0.0006 & - & - & - & - \\
\hline & All data & 15.4360 & 84.5620 & 0.0002 & 0.0018 & - & - & - & - \\
\hline & & & & of theory & N1PW91/E & $*$ & PC & & \\
\hline & & $85 a$ & $85 b$ & $85 c$ & $85 d$ & - & - & - & - \\
\hline & ${ }^{1} \mathrm{H}$ data & 42.0162 & 53.0167 & 0.0188 & 4.9483 & - & - & - & - \\
\hline sDP4+ & ${ }^{13} \mathrm{C}$ data & 81.9543 & 4.9417 & 13.0857 & 0.0183 & - & - & - & - \\
\hline & All data & 92.9210 & 7.0700 & 0.0066 & 0.0024 & - & - & - & - \\
\hline & ${ }_{12}^{1} \mathrm{H}$ data & 1.0605 & 76.9120 & 0.0267 & 22.0007 & - & 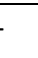 & - & - \\
\hline uDP4+ & ${ }^{13} \mathrm{C}$ data & 19.9957 & 3.5185 & 75.6822 & 0.8036 & - & - & - & - \\
\hline
\end{tabular}




\begin{tabular}{|c|c|c|c|c|c|c|c|c|c|}
\hline & All data & 6.8071 & 86.8683 & 0.6497 & 5.6749 & - & - & - & - \\
\hline & ${ }^{1} \mathrm{H}$ data & 1.0531 & 96.3738 & 0.0000 & 2.5730 & - & - & - & - \\
\hline \multirow[t]{5}{*}{ DP4+ } & ${ }^{13} \mathrm{C}$ data & 61.9210 & 0.6570 & 37.4214 & 0.0006 & - & - & - & - \\
\hline & All data & 50.7360 & 49.2625 & 0.0003 & 0.0011 & - & - & - & - \\
\hline & & \multicolumn{8}{|c|}{ Level of theory: mPW1PW91/6-31+G** (solution. PCM) } \\
\hline & & $85 a$ & $85 b$ & $85 c$ & $85 d$ & - & - & - & - \\
\hline & ${ }^{1} \mathrm{H}$ data & 78.8878 & 16.9047 & 0.0280 & 4.1795 & - & - & - & - \\
\hline \multirow[t]{3}{*}{ sDP4+ } & ${ }^{13} \mathrm{C}$ data & 2.6677 & 97.0204 & 0.3084 & 0.0035 & - & - & - & - \\
\hline & All data & 11.3719 & 88.6268 & 0.0005 & 0.0008 & - & - & - & - \\
\hline & ${ }^{1} \mathrm{H}$ data & 2.4522 & 3.3895 & 0.1582 & 94.0001 & - & - & - & - \\
\hline \multirow[t]{3}{*}{ uDP4+ } & ${ }^{13} \mathrm{C}$ data & 17.4006 & 68.3152 & 13.0036 & 1.2805 & - & - & - & - \\
\hline & All data & 10.7575 & 58.3774 & 0.5186 & 30.3465 & - & - & - & - \\
\hline & ${ }^{1} \mathrm{H}$ data & 30.0561 & 8.9025 & 0.0007 & 61.0408 & - & - & - & - \\
\hline \multirow[t]{5}{*}{ DP4+ } & ${ }^{13} \mathrm{C}$ data & 0.6951 & 99.2448 & 0.0601 & 0.0001 & - & - & - & - \\
\hline & All data & 2.3099 & 97.6897 & 0.0000 & 0.0005 & - & - & - & - \\
\hline & & \multicolumn{8}{|c|}{ Level of theory: mPW1PW91/6-311G* (solution. PCM) } \\
\hline & & $85 a$ & $85 b$ & $85 c$ & $85 d$ & - & - & - & - \\
\hline & ${ }^{1} \mathrm{H}$ data & 57.2923 & 34.7349 & 0.0131 & 7.9597 & - & - & - & - \\
\hline \multirow[t]{3}{*}{ sDP4+ } & ${ }^{13} \mathrm{C}$ data & 0.6821 & 54.8665 & 44.3343 & 0.1172 & - & - & - & - \\
\hline & All data & 2.0077 & 97.9145 & 0.0299 & 0.0479 & - & - & - & - \\
\hline & ${ }^{1} \mathrm{H}$ data & 11.1104 & 48.0784 & 0.0000 & 40.8112 & - & - & - & - \\
\hline \multirow[t]{3}{*}{ uDP4+ } & ${ }^{13} \mathrm{C}$ data & 5.8556 & 69.7862 & 24.0656 & 0.2926 & - & - & - & - \\
\hline & All data & 1.8955 & 97.7566 & 0.0000 & 0.3479 & - & - & - & - \\
\hline & ${ }^{1} \mathrm{H}$ data & 24.1904 & 63.4647 & 0.0000 & 12.3450 & - & - & - & - \\
\hline \multirow[t]{5}{*}{ DP4+ } & ${ }^{13} \mathrm{C}$ data & 0.0815 & 78.1432 & 21.7746 & 0.0007 & - & - & - & - \\
\hline & All data & 0.0397 & 99.9601 & 0.0000 & 0.0002 & - & - & - & - \\
\hline & & \multicolumn{8}{|c|}{ Level of theory: mPW1PW91/6-311G** (solution. PCM) } \\
\hline & & $85 a$ & $85 b$ & $85 c$ & $85 d$ & - & - & - & - \\
\hline & ${ }^{1} \mathrm{H}$ data & 85.9793 & 12.9645 & 0.0115 & 1.0447 & - & - & - & - \\
\hline \multirow[t]{3}{*}{$s D P 4+$} & ${ }^{13} \mathrm{C}$ data & 0.5386 & 26.2019 & 73.0927 & 0.1668 & - & - & - & - \\
\hline & All data & 11.9651 & 87.7730 & 0.2168 & 0.0450 & - & - & - & - \\
\hline & ${ }^{1} \mathrm{H}$ data & 8.4372 & 77.5355 & 0.0000 & 14.0273 & - & - & - & - \\
\hline \multirow[t]{3}{*}{ uDP4+ } & ${ }^{13} \mathrm{C}$ data & 7.1387 & 52.8054 & 39.6143 & 0.4416 & - & - & - & - \\
\hline & All data & 1.4476 & 98.4035 & 0.0000 & 0.1489 & - & - & - & - \\
\hline & ${ }^{1} \mathrm{H}$ data & 41.5648 & 57.5955 & 0.0000 & 0.8396 & - & - & - & - \\
\hline \multirow[t]{5}{*}{ DP4+ } & ${ }^{13} \mathrm{C}$ data & 0.0898 & 32.3043 & 67.6043 & 0.0017 & - & - & - & - \\
\hline & All data & 0.2001 & 99.7998 & 0.0000 & 0.0001 & - & - & - & - \\
\hline & & \multicolumn{8}{|c|}{ Level of theory: mPW1PW91/6-311+G** (solution. PCM) } \\
\hline & & $85 a$ & $85 b$ & $85 c$ & $85 d$ & - & - & - & - \\
\hline & ${ }^{1} \mathrm{H}$ data & 84.3048 & 13.6260 & 0.0491 & 2.0200 & - & - & - & - \\
\hline \multirow[t]{3}{*}{ sDP4+ } & ${ }^{13} \mathrm{C}$ data & 0.4073 & 42.0670 & 57.4737 & 0.0520 & - & - & - & - \\
\hline & All data & 5.6251 & 93.8956 & 0.4621 & 0.0172 & - & - & - & - \\
\hline & ${ }^{1} \mathrm{H}$ data & 28.8781 & 39.7923 & 0.0001 & 31.3296 & - & - & - & - \\
\hline \multirow[t]{3}{*}{ uDP4+ } & ${ }^{13} \mathrm{C}$ data & 3.0126 & 80.7118 & 16.1951 & 0.0806 & - & - & - & - \\
\hline & All data & 2.6353 & 97.2882 & 0.0000 & 0.0765 & - & - & - & - \\
\hline & ${ }^{1} \mathrm{H}$ data & 80.0827 & 17.8355 & 0.0000 & 2.0818 & - & - & - & - \\
\hline \multirow[t]{2}{*}{ DP4+ } & ${ }^{13} \mathrm{C}$ data & 0.0284 & 78.4619 & 21.5096 & 0.0001 & - & - & - & - \\
\hline & All data & 0.1620 & 99.8380 & 0.0000 & 0.0000 & - & - & - & - \\
\hline
\end{tabular}


$s \mathrm{DP} 4+, u \mathrm{DP} 4+$ and DP4+ probabilities (\%) for compound $85 \mathrm{c}$<smiles>O=C1C=CCC(C[C@@H](O)C[C@@H](O)/C=C/c2ccccc2)O1</smiles>

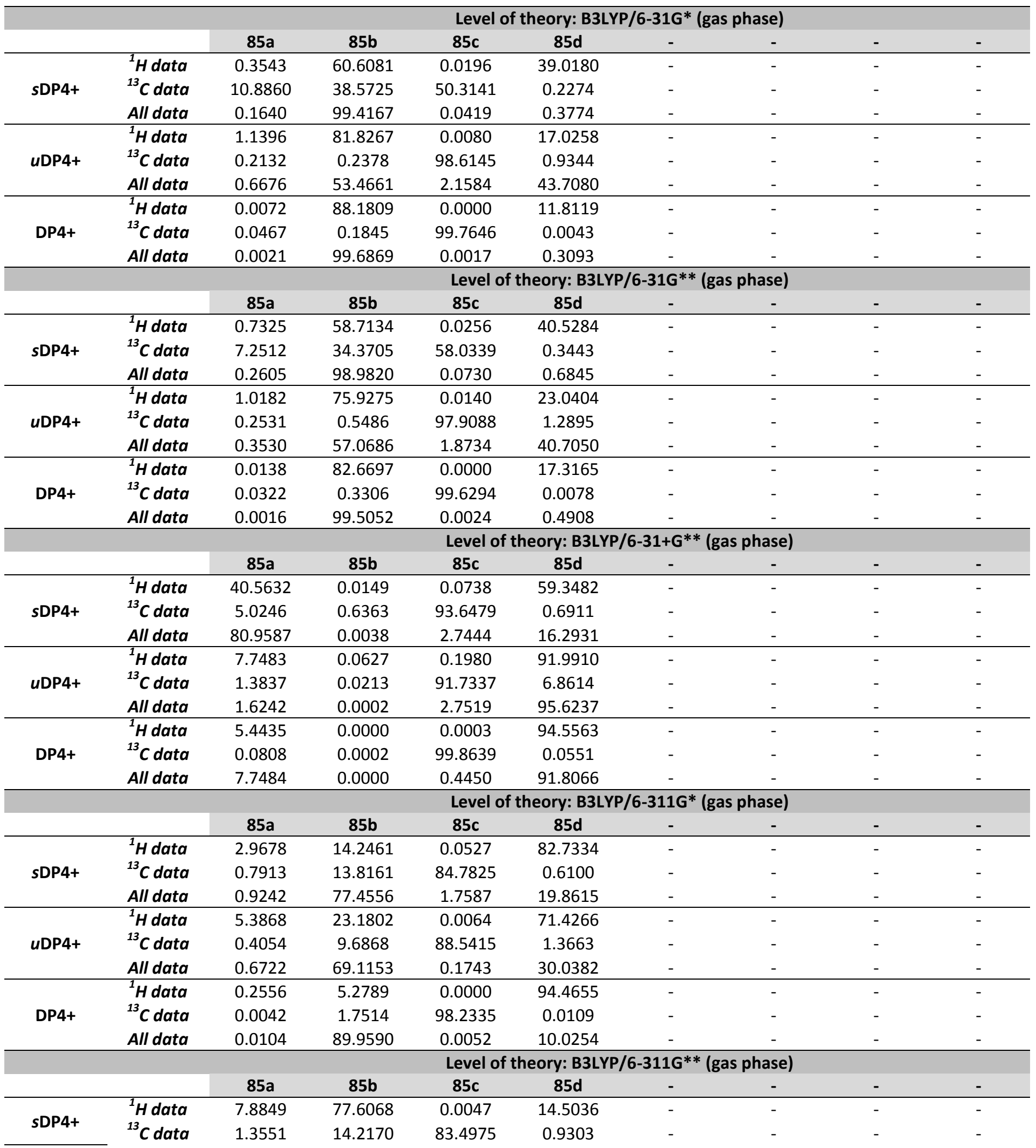




\begin{tabular}{|c|c|c|c|c|c|c|c|c|c|}
\hline & All data & 0.9473 & 97.8217 & 0.0346 & 1.1963 & - & - & - & - \\
\hline & ${ }^{1} \mathrm{H}$ data & 4.1095 & 49.7854 & 0.0112 & 46.0939 & - & - & - & - \\
\hline \multirow[t]{3}{*}{ uDP4+ } & ${ }^{13} \mathrm{C}$ data & 0.3081 & 12.7013 & 85.8434 & 1.1472 & - & - & - & - \\
\hline & All data & 0.1842 & 91.9842 & 0.1395 & 7.6921 & - & - & - & - \\
\hline & ${ }^{1} \mathrm{H}$ data & 0.7099 & 84.6442 & 0.0000 & 14.6459 & - & - & - & - \\
\hline \multirow[t]{5}{*}{ DP4+ } & ${ }^{13} \mathrm{C}$ data & 0.0057 & 2.4569 & 97.5229 & 0.0145 & - & - & - & - \\
\hline & All data & 0.0019 & 99.8958 & 0.0001 & 0.1022 & - & - & - & - \\
\hline & & \multicolumn{8}{|c|}{ Level of theory: B3LYP/6-311+G** (gas phase) } \\
\hline & & $85 a$ & $85 b$ & $85 c$ & $85 d$ & - & - & - & - \\
\hline & ${ }^{1} \mathrm{H}$ data & 83.3486 & 2.7201 & 0.0119 & 13.9194 & - & - & - & - \\
\hline \multirow[t]{2}{*}{ sDP4+ } & ${ }^{13} \mathrm{C}$ data & 1.5985 & 0.7066 & 96.7819 & 0.9130 & - & - & - & - \\
\hline & All data & 89.4080 & 1.2899 & 0.7737 & 8.5284 & - & - & - & - \\
\hline \multirow{3}{*}{ uDP4+ } & ${ }^{1} \mathrm{H}$ data & 37.0844 & 9.1024 & 0.0688 & 53.7444 & - & - & - & - \\
\hline & ${ }^{13} \mathrm{C}$ data & 0.7847 & 1.6772 & 95.3525 & 2.1857 & - & - & - & - \\
\hline & All data & 17.2803 & 9.0661 & 3.8944 & 69.7592 & - & - & - & - \\
\hline \multirow{5}{*}{ DP4+ } & ${ }^{1} \mathrm{H}$ data & 79.9976 & 0.6408 & 0.0000 & 19.3615 & - & - & - & - \\
\hline & ${ }^{13} \mathrm{C}$ data & 0.0136 & 0.0128 & 99.9520 & 0.0216 & - & - & - & - \\
\hline & All data & 71.7056 & 0.5428 & 0.1398 & 27.6118 & - & - & - & - \\
\hline & & \multicolumn{8}{|c|}{ Level of theory: B3LYP/6-31G* (solution. PCM) } \\
\hline & & $85 a$ & $85 b$ & $85 c$ & $85 d$ & - & - & - & - \\
\hline \multirow{3}{*}{ sDP4+ } & ${ }^{1}$ H data & 0.3525 & 97.6337 & 0.0013 & 2.0126 & - & - & - & - \\
\hline & ${ }^{13}$ C data & 31.7054 & 49.2972 & 18.9847 & 0.0128 & - & - & - & - \\
\hline & All data & 0.2316 & 99.7673 & 0.0005 & 0.0005 & - & - & - & - \\
\hline \multirow{3}{*}{ uDP4+ } & ${ }^{1} \mathrm{H}$ data & 0.7007 & 95.4741 & 0.0013 & 3.8239 & - & - & - & - \\
\hline & ${ }^{13} \mathrm{C}$ data & 0.7143 & 0.6319 & 98.0061 & 0.6477 & - & - & - & - \\
\hline & All data & 0.7890 & 95.1040 & 0.2030 & 3.9040 & - & - & - & - \\
\hline \multirow{3}{*}{ DP4+ } & ${ }^{1} \mathrm{H}$ data & 0.0026 & 99.9149 & 0.0000 & 0.0825 & - & - & - & - \\
\hline & ${ }^{13} \mathrm{C}$ data & 1.1831 & 1.6272 & 97.1893 & 0.0004 & - & - & - & - \\
\hline & All data & 0.0019 & 99.9981 & 0.0000 & 0.0000 & - & - & - & - \\
\hline & & \multicolumn{8}{|c|}{ Level of theory: B3LYP/6-31G** (solution. PCM) } \\
\hline & & $85 a$ & $85 b$ & $85 c$ & $85 d$ & - & - & - & - \\
\hline \multirow{3}{*}{ sDP4+ } & ${ }^{1} \mathrm{H}$ data & 0.9283 & 97.3765 & 0.0040 & 1.6912 & - & - & - & - \\
\hline & ${ }^{13} \mathrm{C}$ data & 26.0091 & 42.1276 & 31.8272 & 0.0361 & - & - & - & - \\
\hline & All data & 0.5851 & 99.4103 & 0.0031 & 0.0015 & - & - & - & - \\
\hline \multirow{3}{*}{ uDP4+ } & ${ }^{1} \mathrm{H}$ data & 0.7442 & 92.4348 & 0.0027 & 6.8183 & - & - & - & - \\
\hline & ${ }^{13} \mathrm{C}$ data & 0.9772 & 1.0293 & 97.2084 & 0.7850 & - & - & - & - \\
\hline & All data & 0.7166 & 93.7462 & 0.2632 & 5.2740 & - & - & - & - \\
\hline \multirow{3}{*}{ DP4+ } & ${ }^{1} \mathrm{H}$ data & 0.0077 & 99.8644 & 0.0000 & 0.1279 & - & - & - & - \\
\hline & ${ }^{13} \mathrm{C}$ data & 0.8037 & 1.3711 & 97.8244 & 0.0009 & - & - & - & - \\
\hline & All data & 0.0045 & 99.9954 & 0.0000 & 0.0001 & - & - & - & - \\
\hline & & \multicolumn{8}{|c|}{ Level of theory: B3LYP/6-31+G** (solution. PCM) } \\
\hline & & $85 a$ & $85 b$ & $85 c$ & $85 d$ & - & - & - & - \\
\hline \multirow{3}{*}{ sDP4+ } & ${ }^{1} \mathrm{H}$ data & 67.9248 & 0.7560 & 0.0314 & 31.2878 & - & - & - & - \\
\hline & ${ }^{13} \mathrm{C}$ data & 5.7749 & 69.2266 & 24.8095 & 0.1891 & - & - & - & - \\
\hline & All data & 86.9198 & 11.5970 & 0.1725 & 1.3107 & - & - & - & - \\
\hline & ${ }^{1} \mathrm{H}$ data & 5.3436 & 1.5396 & 0.0523 & 93.0646 & - & - & - & - \\
\hline uDP4+ & ${ }^{13} \mathrm{C}$ data & 3.5222 & 1.3571 & 90.8697 & 4.2509 & - & - & - & - \\
\hline & All data & 4.4678 & 0.4960 & 1.1281 & 93.9081 & - & - & - & - \\
\hline & ${ }^{1} \mathrm{H}$ data & 11.0797 & 0.0355 & 0.0001 & 88.8847 & - & - & - & - \\
\hline DP4+ & ${ }^{13} \mathrm{C}$ data & 0.8584 & 3.9649 & 95.1428 & 0.0339 & - & - & - & - \\
\hline & All data & 75.0597 & 1.1117 & 0.0376 & 23.7909 & - & - & - & - \\
\hline & & & & Level of $t$ & ry: B3LYP & G* & $\overline{P C}$ & & \\
\hline & & $85 a$ & $85 b$ & $85 c$ & $85 d$ & - & - & - & - \\
\hline & ${ }^{1} \mathrm{H}$ data & 4.0214 & 81.6148 & 0.0082 & 14.3556 & - & - & - & - \\
\hline sDP4+ & ${ }^{13} \mathrm{C}$ data & 0.3105 & 50.9762 & 48.6493 & 0.0641 & - & - & - & - \\
\hline & All data & 0.0300 & 99.9383 & 0.0096 & 0.0221 & - & - & - & - \\
\hline
\end{tabular}




\begin{tabular}{|c|c|c|c|c|c|c|c|c|c|}
\hline & ${ }^{1} \mathrm{H}$ data & 3.6667 & 51.6549 & 0.0000 & 44.6784 & - & - & - & - \\
\hline \multirow[t]{3}{*}{ uDP4+ } & ${ }^{13} \mathrm{C}$ data & 1.6897 & 55.4831 & 42.2530 & 0.5742 & - & - & - & - \\
\hline & All data & 0.2138 & 98.9009 & 0.0001 & 0.8852 & - & - & - & - \\
\hline & ${ }^{1} \mathrm{H}$ data & 0.3027 & 86.5325 & 0.0000 & 13.1649 & - & - & - & - \\
\hline \multirow[t]{5}{*}{ DP4+ } & ${ }^{13} \mathrm{C}$ data & 0.0107 & 57.9044 & 42.0841 & 0.0008 & - & - & - & - \\
\hline & All data & 0.0001 & 99.9997 & 0.0000 & 0.0002 & - & - & - & - \\
\hline & & \multicolumn{8}{|c|}{ Level of theory: B3LYP/6-311G** (solution. PCM) } \\
\hline & & $85 a$ & $85 b$ & $85 c$ & $85 d$ & - & - & - & - \\
\hline & ${ }^{1}$ H data & 4.7900 & 94.4693 & 0.0024 & 0.7383 & - & - & - & - \\
\hline \multirow[t]{3}{*}{ sDP4+ } & ${ }^{13} \mathrm{C}$ data & 0.3718 & 33.4134 & 66.1220 & 0.0927 & - & - & - & - \\
\hline & All data & 0.0564 & 99.9363 & 0.0051 & 0.0022 & - & - & - & - \\
\hline & ${ }^{1} \mathrm{H}$ data & 3.3101 & 60.0117 & 0.0005 & 36.6777 & - & - & - & - \\
\hline \multirow[t]{3}{*}{ uDP4+ } & ${ }^{13} \mathrm{C}$ data & 0.8450 & 48.4931 & 50.2266 & 0.4353 & - & - & - & - \\
\hline & All data & 0.0955 & 99.3585 & 0.0009 & 0.5451 & - & - & - & - \\
\hline & ${ }^{1} \mathrm{H}$ data & 0.2776 & 99.2484 & 0.0000 & 0.4740 & - & - & - & - \\
\hline \multirow[t]{5}{*}{ DP4+ } & ${ }^{13} \mathrm{C}$ data & 0.0064 & 32.7883 & 67.2045 & 0.0008 & - & - & - & - \\
\hline & All data & 0.0001 & 99.9999 & 0.0000 & 0.0000 & - & - & - & - \\
\hline & & \multicolumn{8}{|c|}{ Level of theory: B3LYP/6-311+G** (solution. PCM) } \\
\hline & & $85 a$ & $85 b$ & $85 c$ & $85 d$ & - & - & - & - \\
\hline & ${ }^{1} \mathrm{H}$ data & 83.4990 & 13.9237 & 0.0083 & 2.5690 & - & - & - & - \\
\hline \multirow[t]{3}{*}{ sDP4+ } & ${ }^{13} \mathrm{C}$ data & 0.2852 & 5.9446 & 93.6748 & 0.0955 & - & - & - & - \\
\hline & All data & 22.1304 & 76.9171 & 0.7247 & 0.2279 & - & - & - & - \\
\hline & ${ }^{1} \mathrm{H}$ data & 16.0305 & 19.4710 & 0.0005 & 64.4979 & - & - & - & - \\
\hline \multirow[t]{3}{*}{ uDP4+ } & ${ }^{13} \mathrm{C}$ data & 1.9054 & 30.8025 & 66.7013 & 0.5908 & - & - & - & - \\
\hline & All data & 4.5695 & 89.7247 & 0.0053 & 5.7005 & - & - & - & - \\
\hline & ${ }^{1} \mathrm{H}$ data & 75.3960 & 15.2709 & 0.0000 & 9.3330 & - & - & - & - \\
\hline \multirow[t]{5}{*}{ DP4+ } & ${ }^{13} \mathrm{C}$ data & 0.0084 & 2.8468 & 97.1438 & 0.0009 & - & - & - & - \\
\hline & All data & 1.4438 & 98.5375 & 0.0001 & 0.0185 & - & - & - & - \\
\hline & & \multicolumn{8}{|c|}{ Level of theory: mPW1PW91/6-31G* (gas phase) } \\
\hline & & $85 a$ & $85 b$ & $85 c$ & $85 d$ & - & - & - & - \\
\hline & ${ }^{1} \mathrm{H}$ data & 0.3586 & 49.1976 & 0.0153 & 50.4285 & - & - & - & - \\
\hline \multirow[t]{3}{*}{ sDP4+ } & ${ }^{13}$ C data & 6.1738 & 3.5577 & 90.0863 & 0.1822 & - & - & - & - \\
\hline & All data & 1.1789 & 93.1960 & 0.7336 & 4.8914 & - & - & - & - \\
\hline & ${ }^{1} \mathrm{H}$ data & 0.8612 & 66.2292 & 0.0154 & 32.8942 & - & - & - & - \\
\hline \multirow[t]{3}{*}{ uDP4+ } & ${ }^{13} \mathrm{C}$ data & 0.2312 & 0.2519 & 98.4589 & 1.0581 & - & - & - & - \\
\hline & All data & 0.3742 & 31.3569 & 2.8495 & 65.4193 & - & - & - & - \\
\hline & ${ }^{1} \mathrm{H}$ data & 0.0063 & 66.2605 & 0.0000 & 33.7332 & - & - & - & - \\
\hline \multirow[t]{5}{*}{ DP4+ } & ${ }^{13} \mathrm{C}$ data & 0.0161 & 0.0101 & 99.9716 & 0.0022 & - & - & - & - \\
\hline & All data & 0.0136 & 90.0605 & 0.0644 & 9.8615 & - & - & - & - \\
\hline & & \multicolumn{8}{|c|}{ Level of theory: mPW1PW91/6-31G** (gas phase) } \\
\hline & & $85 a$ & $85 b$ & $85 c$ & $85 d$ & - & - & - & - \\
\hline & ${ }^{1} \mathrm{H}$ data & 1.2305 & 72.9641 & 0.0208 & 25.7846 & - & - & - & - \\
\hline \multirow[t]{3}{*}{ sDP4+ } & ${ }^{13} \mathrm{C}$ data & 2.8403 & 2.7766 & 94.1769 & 0.2062 & - & - & - & - \\
\hline & All data & 1.6381 & 94.9518 & 0.9180 & 2.4921 & - & - & - & - \\
\hline & ${ }^{1} \mathrm{H}$ data & 0.6413 & 51.9612 & 0.0282 & 47.3693 & - & - & - & - \\
\hline uDP4+ & ${ }^{13} \mathrm{C}$ data & 0.1894 & 0.3515 & 98.5454 & 0.9137 & - & - & - & - \\
\hline & All data & 0.1885 & 28.3374 & 4.3170 & 67.1571 & - & - & - & - \\
\hline & ${ }^{1} \mathrm{H}$ data & 0.0157 & 75.6220 & 0.0000 & 24.3623 & - & - & - & - \\
\hline DP4+ & ${ }^{13} \mathrm{C}$ data & 0.0058 & 0.0105 & 99.9817 & 0.0020 & - & - & - & - \\
\hline & All data & 0.0108 & 94.0036 & 0.1385 & 5.8472 & - & - & - & - \\
\hline & & & & Level of th & $y:$ mPW1P & -31 & ph & & \\
\hline & & $85 a$ & $85 b$ & $85 c$ & $85 d$ & - & - & - & - \\
\hline & ${ }^{1} \mathrm{H}$ data & 42.5101 & 0.1103 & 0.0840 & 57.2955 & - & - & - & - \\
\hline sDP4+ & ${ }^{13} \mathrm{C}$ data & 2.0670 & 0.0794 & 97.5689 & 0.2847 & - & - & - & - \\
\hline & All data & 78.1859 & 0.0078 & 7.2910 & 14.5153 & - & - & - & - \\
\hline uDP4+ & ${ }^{1} \mathrm{H}$ data & 2.2745 & 0.2829 & 0.2513 & 97.1913 & - & - & - & - \\
\hline
\end{tabular}




\begin{tabular}{|c|c|c|c|c|c|c|c|c|c|}
\hline & ${ }^{13} \mathrm{C}$ data & 1.2593 & 0.0132 & 90.7804 & 7.9471 & - & - & - & - \\
\hline & All data & 0.3589 & 0.0005 & 2.8581 & 96.7825 & - & - & - & - \\
\hline & ${ }^{1} \mathrm{H}$ data & 1.7067 & 0.0006 & 0.0004 & 98.2924 & - & - & - & - \\
\hline \multirow[t]{5}{*}{ DP4+ } & ${ }^{13} \mathrm{C}$ data & 0.0294 & 0.0000 & 99.9451 & 0.0255 & - & - & - & - \\
\hline & All data & 1.9302 & 0.0000 & 1.4335 & 96.6363 & - & - & - & - \\
\hline & & \multicolumn{8}{|c|}{ Level of theory: mPW1PW91/6-311G* (gas phase) } \\
\hline & & $85 a$ & $85 b$ & $85 c$ & $85 d$ & - & - & - & - \\
\hline & ${ }^{1} \mathrm{H}$ data & 1.9207 & 40.9462 & 0.0067 & 57.1264 & - & - & - & - \\
\hline \multirow[t]{2}{*}{$s$ DP4+ } & ${ }^{13} \mathrm{C}$ data & 1.5469 & 4.1103 & 93.1380 & 1.2049 & - & - & - & - \\
\hline & All data & 1.2342 & 69.9135 & 0.2596 & 28.5926 & - & - & - & - \\
\hline \multirow{3}{*}{ uDP4+ } & ${ }^{1} \mathrm{H}$ data & 2.7133 & 33.6295 & 0.0003 & 63.6569 & - & - & - & - \\
\hline & ${ }^{13} \mathrm{C}$ data & 0.4285 & 3.5546 & 93.5737 & 2.4431 & - & - & - & - \\
\hline & All data & 0.4209 & 43.2725 & 0.0093 & 56.2974 & - & - & - & - \\
\hline \multirow{5}{*}{ DP4+ } & ${ }^{1} \mathrm{H}$ data & 0.1038 & 27.4374 & 0.0000 & 72.4588 & - & - & - & - \\
\hline & ${ }^{13} \mathrm{C}$ data & 0.0076 & 0.1673 & 99.7914 & 0.0337 & - & - & - & - \\
\hline & All data & 0.0112 & 65.2638 & 0.0001 & 34.7249 & - & - & - & - \\
\hline & & \multicolumn{8}{|c|}{ Level of theory: mPW1PW91/6-311G** (gas phase) } \\
\hline & & $85 a$ & $85 b$ & $85 c$ & $85 d$ & - & - & - & - \\
\hline \multirow{3}{*}{ sDP4+ } & ${ }^{1} \mathrm{H}$ data & 8.9195 & 85.6964 & 0.0008 & 5.3834 & - & - & - & - \\
\hline & ${ }^{13} \mathrm{C}$ data & 3.0627 & 5.8424 & 88.5077 & 2.5872 & - & - & - & - \\
\hline & All data & 5.0402 & 92.3777 & 0.0123 & 2.5698 & - & - & - & - \\
\hline \multirow{3}{*}{ uDP4+ } & ${ }^{1} \mathrm{H}$ data & 2.0837 & 53.2070 & 0.0003 & 44.7089 & - & - & - & - \\
\hline & ${ }^{13} \mathrm{C}$ data & 0.5885 & 4.8754 & 91.8512 & 2.6849 & - & - & - & - \\
\hline & All data & 0.3221 & 68.1389 & 0.0077 & 31.5313 & - & - & - & - \\
\hline \multirow{3}{*}{ DP4+ } & ${ }^{1} \mathrm{H}$ data & 0.3857 & 94.6197 & 0.0000 & 4.9946 & - & - & - & - \\
\hline & ${ }^{13} \mathrm{C}$ data & 0.0221 & 0.3488 & 99.5441 & 0.0851 & - & - & - & - \\
\hline & All data & 0.0255 & 98.7039 & 0.0000 & 1.2706 & - & - & - & - \\
\hline & & \multicolumn{8}{|c|}{ Level of theory: mPW1PW91/6-311+G** (gas phase) } \\
\hline & & $85 a$ & $85 b$ & $85 c$ & $85 d$ & - & - & - & - \\
\hline \multirow{3}{*}{ sDP4+ } & ${ }^{1} \mathrm{H}$ data & 72.8576 & 13.3233 & 0.0069 & 13.8122 & - & - & - & - \\
\hline & ${ }^{13} \mathrm{C}$ data & 4.3815 & 0.3412 & 92.9912 & 2.2860 & - & - & - & - \\
\hline & All data & 89.6720 & 1.2771 & 0.1813 & 8.8696 & - & - & - & - \\
\hline \multirow{3}{*}{ uDP4+ } & ${ }^{1} \mathrm{H}$ data & 17.0753 & 16.4093 & 0.0055 & 66.5099 & - & - & - & - \\
\hline & ${ }^{13} \mathrm{C}$ data & 1.1109 & 0.9527 & 94.7440 & 3.1924 & - & - & - & - \\
\hline & All data & 7.6660 & 6.3179 & 0.2117 & 85.8044 & - & - & - & - \\
\hline \multirow{3}{*}{ DP4+ } & ${ }^{1} \mathrm{H}$ data & 52.2423 & 9.1808 & 0.0000 & 38.5770 & - & - & - & - \\
\hline & ${ }^{13} \mathrm{C}$ data & 0.0552 & 0.0037 & 99.8584 & 0.0827 & - & - & - & - \\
\hline & All data & 47.1941 & 0.5540 & 0.0026 & 52.2493 & - & - & - & - \\
\hline & & \multicolumn{8}{|c|}{ Level of theory: mPW1PW91/6-31G* (solution. PCM) } \\
\hline & & $85 a$ & $85 b$ & $85 c$ & $85 d$ & - & - & - & - \\
\hline \multirow{3}{*}{ sDP4+ } & ${ }^{1} \mathrm{H}$ data & 0.3830 & 98.1472 & 0.0021 & 1.4678 & - & - & - & - \\
\hline & ${ }^{13} \mathrm{C}$ data & 19.7309 & 8.2693 & 71.9276 & 0.0721 & - & - & - & - \\
\hline & All data & 0.9222 & 99.0466 & 0.0183 & 0.0129 & - & - & - & - \\
\hline \multirow{3}{*}{ uDP4+ } & ${ }^{1} \mathrm{H}$ data & 0.5610 & 93.9065 & 0.0036 & 5.5289 & - & - & - & - \\
\hline & ${ }^{13} \mathrm{C}$ data & 0.5114 & 0.2393 & 98.4853 & 0.7641 & - & - & - & - \\
\hline & All data & 1.0497 & 82.2021 & 1.2930 & 15.4552 & - & - & - & - \\
\hline & ${ }^{1} \mathrm{H}$ data & 0.0023 & 99.9097 & 0.0000 & 0.0880 & - & - & - & - \\
\hline DP4+ & ${ }^{13} \mathrm{C}$ data & 0.1422 & 0.0279 & 99.8291 & 0.0008 & - & - & - & - \\
\hline & All data & 0.0119 & 99.9854 & 0.0003 & 0.0025 & - & - & - & - \\
\hline & & & & vel of the & mPW1PV & 10 & on. & & \\
\hline & & $85 a$ & $85 b$ & $85 c$ & $85 d$ & - & - & - & - \\
\hline & ${ }^{1} \mathrm{H}$ data & 1.6932 & 97.4343 & 0.0048 & 0.8677 & - & - & - & - \\
\hline sDP4+ & ${ }^{13} \mathrm{C}$ data & 10.9872 & 4.8381 & 84.0887 & 0.0860 & - & - & - & - \\
\hline & All data & 3.7931 & 96.1096 & 0.0821 & 0.0152 & - & - & - & - \\
\hline & ${ }^{1} \mathrm{H}$ data & 0.4007 & 90.6862 & 0.0076 & 8.9056 & - & - & - & - \\
\hline 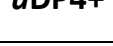 & ${ }^{13} \mathrm{C}$ data & 0.5219 & 0.2259 & 98.5648 & 0.6874 & - & - & - & - \\
\hline
\end{tabular}




\begin{tabular}{|c|c|c|c|c|c|c|c|c|c|}
\hline & All data & 0.7587 & 74.3229 & 2.7078 & 22.2106 & - & - & - & - \\
\hline & ${ }^{1} \mathrm{H}$ data & 0.0077 & 99.9050 & 0.0000 & 0.0874 & - & - & - & - \\
\hline \multirow[t]{5}{*}{ DP4+ } & ${ }^{13} \mathrm{C}$ data & 0.0691 & 0.0132 & 99.9170 & 0.0007 & - & - & - & - \\
\hline & All data & 0.0403 & 99.9519 & 0.0031 & 0.0047 & - & - & - & - \\
\hline & & \multicolumn{8}{|c|}{ Level of theory: mPW1PW91/6-31+G** (solution. PCM) } \\
\hline & & $85 a$ & $85 b$ & $85 c$ & $85 d$ & - & - & - & - \\
\hline & ${ }^{1} \mathrm{H}$ data & 74.7262 & 6.7197 & 0.1064 & 18.4477 & - & - & - & - \\
\hline \multirow[t]{3}{*}{ sDP4+ } & ${ }^{13} \mathrm{C}$ data & 8.1965 & 61.3538 & 30.1202 & 0.3294 & - & - & - & - \\
\hline & All data & 59.2321 & 39.8702 & 0.3100 & 0.5877 & - & - & - & - \\
\hline & ${ }^{1} \mathrm{H}$ data & 1.7083 & 3.8980 & 0.1004 & 94.2933 & - & - & - & - \\
\hline \multirow[t]{3}{*}{ uDP4+ } & ${ }^{13} \mathrm{C}$ data & 3.3647 & 1.1092 & 89.7145 & 5.8116 & - & - & - & - \\
\hline & All data & 1.0136 & 0.7624 & 1.5880 & 96.6360 & - & - & - & - \\
\hline & ${ }^{1} \mathrm{H}$ data & 6.7424 & 1.3834 & 0.0006 & 91.8736 & - & - & - & - \\
\hline \multirow[t]{5}{*}{ DP4+ } & ${ }^{13} \mathrm{C}$ data & 0.9850 & 2.4306 & 96.5160 & 0.0684 & - & - & - & - \\
\hline & All data & 40.6435 & 20.5779 & 0.3332 & 38.4453 & - & - & - & - \\
\hline & & \multicolumn{8}{|c|}{ Level of theory: mPW1PW91/6-311G* (solution. PCM) } \\
\hline & & $85 a$ & $85 b$ & $85 c$ & $85 d$ & - & - & - & - \\
\hline & ${ }^{1} \mathrm{H}$ data & 2.1635 & 92.0219 & 0.0005 & 5.8140 & - & - & - & - \\
\hline \multirow[t]{2}{*}{ sDP4+ } & ${ }^{13} \mathrm{C}$ data & 0.2026 & 7.3600 & 92.3131 & 0.1243 & - & - & - & - \\
\hline & All data & 0.0646 & 99.8220 & 0.0069 & 0.1065 & - & - & - & - \\
\hline \multirow{3}{*}{ uDP4+ } & ${ }^{1}$ H data & 2.5140 & 64.1918 & 0.0000 & 33.2941 & - & - & - & - \\
\hline & ${ }^{13} \mathrm{C}$ data & 0.9961 & 16.4540 & 81.4095 & 1.1404 & - & - & - & - \\
\hline & All data & 0.2284 & 96.3095 & 0.0000 & 3.4622 & - & - & - & - \\
\hline \multirow{3}{*}{ DP4+ } & ${ }^{1} \mathrm{H}$ data & 0.0891 & 96.7408 & 0.0000 & 3.1702 & - & - & - & - \\
\hline & ${ }^{13} \mathrm{C}$ data & 0.0026 & 1.5858 & 98.4097 & 0.0019 & - & - & - & - \\
\hline & All data & 0.0002 & 99.9960 & 0.0000 & 0.0038 & - & - & - & - \\
\hline & & \multicolumn{8}{|c|}{ Level of theory: mPW1PW91/6-311G** (solution. PCM) } \\
\hline & & $85 a$ & $85 b$ & $85 c$ & $85 d$ & - & - & - & - \\
\hline \multirow{3}{*}{ sDP4+ } & ${ }^{1} \mathrm{H}$ data & 20.9732 & 77.9148 & 0.0016 & 1.1105 & - & - & - & - \\
\hline & ${ }^{13} \mathrm{C}$ data & 0.2329 & 4.7315 & 94.8427 & 0.1929 & - & - & - & - \\
\hline & All data & 1.3063 & 98.5961 & 0.0402 & 0.0573 & - & - & - & - \\
\hline \multirow{3}{*}{ uDP4+ } & ${ }^{1} \mathrm{H}$ data & 1.8314 & 74.1562 & 0.0000 & 24.0123 & - & - & - & - \\
\hline & ${ }^{13} \mathrm{C}$ data & 0.6375 & 8.3561 & 89.8807 & 1.1257 & - & - & - & - \\
\hline & All data & 0.1802 & 95.6474 & 0.0002 & 4.1722 & - & - & - & - \\
\hline \multirow{5}{*}{ DP4+ } & ${ }^{1} \mathrm{H}$ data & 0.6574 & 98.8862 & 0.0000 & 0.4564 & - & - & - & - \\
\hline & ${ }^{13} \mathrm{C}$ data & 0.0017 & 0.4616 & 99.5341 & 0.0025 & - & - & - & - \\
\hline & All data & 0.0025 & 99.9950 & 0.0000 & 0.0025 & - & - & - & - \\
\hline & & \multicolumn{8}{|c|}{ Level of theory: mPW1PW91/6-311+G** (solution. PCM) } \\
\hline & & $85 a$ & $85 b$ & $85 c$ & $85 d$ & - & - & - & - \\
\hline \multirow{3}{*}{ sDP4+ } & ${ }^{1} \mathrm{H}$ data & 69.9676 & 27.0815 & 0.0051 & 2.9457 & - & - & - & - \\
\hline & ${ }^{13} \mathrm{C}$ data & 0.2547 & 2.3872 & 97.1634 & 0.1946 & - & - & - & - \\
\hline & All data & 21.3342 & 77.3807 & 0.5988 & 0.6863 & - & - & - & - \\
\hline \multirow{3}{*}{ uDP4+ } & ${ }^{1} \mathrm{H}$ data & 9.8455 & 32.2927 & 0.0004 & 57.8615 & - & - & - & - \\
\hline & ${ }^{13} \mathrm{C}$ data & 1.0737 & 8.1585 & 90.0011 & 0.7666 & - & - & - & - \\
\hline & All data & 3.3198 & 82.7392 & 0.0100 & 13.9310 & - & - & - & - \\
\hline \multirow{3}{*}{ DP4+ } & ${ }^{1} \mathrm{H}$ data & 39.7305 & 50.4391 & 0.0000 & 9.8304 & - & - & - & - \\
\hline & ${ }^{13} \mathrm{C}$ data & 0.0031 & 0.2222 & 99.7730 & 0.0017 & - & - & - & - \\
\hline & All data & 1.0925 & 98.7599 & 0.0001 & 0.1475 & - & - & - & - \\
\hline
\end{tabular}


$s \mathrm{DP4}+, u \mathrm{DP} 4+$ and DP4+ probabilities (\%) for compound 85d<smiles>O=C1C=CCC(C[C@@H](O)CC(O)/C=C/c2ccccc2)O1</smiles>

\begin{tabular}{|c|c|c|c|c|c|c|c|c|c|}
\hline & & \multicolumn{8}{|c|}{ Level of theory: B3LYP/6-31G* (gas phase) } \\
\hline & & $85 a$ & $85 \mathrm{~b}$ & $85 c$ & $85 d$ & - & - & - & - \\
\hline & ${ }^{1} \mathrm{H}$ data & 0.3208 & 58.6340 & 0.0605 & 40.9846 & - & - & - & - \\
\hline \multirow[t]{2}{*}{ sDP4+ } & ${ }^{13} \mathrm{C}$ data & 33.3450 & 10.9482 & 55.2742 & 0.4325 & - & - & - & - \\
\hline & All data & 1.5880 & 95.2840 & 0.4967 & 2.6313 & - & - & - & - \\
\hline & ${ }^{1} \mathrm{H}$ data & 1.1723 & 43.5025 & 0.0322 & 55.2930 & - & - & - & - \\
\hline \multirow[t]{2}{*}{ uDP4+ } & ${ }^{13} \mathrm{C}$ data & 0.4101 & 0.0041 & 43.9242 & 55.6616 & - & - & - & - \\
\hline & All data & 0.0156 & 0.0058 & 0.0460 & 99.9326 & - & - & - & - \\
\hline \multirow{6}{*}{ DP4+ } & ${ }^{1} \mathrm{H}$ data & 0.0078 & 52.9496 & 0.0000 & 47.0425 & - & - & - & - \\
\hline & ${ }^{13} \mathrm{C}$ data & 0.5546 & 0.0018 & 98.4671 & 0.9764 & - & - & - & - \\
\hline & All data & 0.0094 & 0.2110 & 0.0087 & 99.7709 & - & - & - & - \\
\hline & & \multicolumn{8}{|c|}{ Level of theory: B3LYP/6-31G** (gas phase) } \\
\hline & & $85 a$ & $85 b$ & $85 c$ & $85 d$ & - & - & - & - \\
\hline & ${ }^{1} \mathrm{H}$ data & 0.3679 & 31.8629 & 0.0308 & 67.7384 & - & - & - & - \\
\hline \multirow[t]{2}{*}{$s \mathrm{DP} 4+$} & ${ }^{13}$ C data & 28.2009 & 11.2890 & 59.2055 & 1.3046 & - & - & - & - \\
\hline & All data & 2.2543 & 78.1494 & 0.3961 & 19.2002 & - & - & - & - \\
\hline & ${ }^{1} \mathrm{H}$ data & 0.7173 & 30.2456 & 0.0613 & 68.9758 & - & - & - & - \\
\hline \multirow[t]{2}{*}{ uDP4+ } & ${ }^{13} \mathrm{C}$ data & 0.4359 & 0.0156 & 54.5065 & 45.0420 & - & - & - & - \\
\hline & All data & 0.0101 & 0.0152 & 0.1074 & 99.8673 & - & - & - & - \\
\hline \multirow{6}{*}{ DP4+ } & ${ }^{1} \mathrm{H}$ data & 0.0047 & 17.0984 & 0.0000 & 82.8969 & - & - & - & - \\
\hline & ${ }^{13} \mathrm{C}$ data & 0.3727 & 0.0053 & 97.8404 & 1.7816 & - & - & - & - \\
\hline & All data & 0.0012 & 0.0618 & 0.0022 & 99.9348 & - & - & - & - \\
\hline & & \multicolumn{8}{|c|}{ Level of theory: B3LYP/6-31+G** (gas phase) } \\
\hline & & $85 a$ & $85 b$ & $85 c$ & $85 d$ & - & - & - & - \\
\hline & ${ }^{1} \mathrm{H}$ data & 14.3221 & 0.0179 & 0.0626 & 85.5974 & - & - & - & - \\
\hline \multirow[t]{2}{*}{ sDP4+ } & ${ }^{13} \mathrm{C}$ data & 54.0627 & 0.1266 & 41.8889 & 3.9218 & - & - & - & - \\
\hline & All data & 69.5925 & 0.0002 & 0.2356 & 30.1717 & - & - & - & - \\
\hline \multirow{3}{*}{ uDP4+ } & ${ }^{1} \mathrm{H}$ data & 1.1737 & 0.0195 & 0.2638 & 98.5430 & - & - & - & - \\
\hline & ${ }^{13} \mathrm{C}$ data & 7.7448 & 0.0067 & 28.5681 & 63.6805 & - & - & - & - \\
\hline & All data & 0.1445 & 0.0000 & 0.1198 & 99.7358 & - & - & - & - \\
\hline & ${ }^{1} \mathrm{H}$ data & 0.1989 & 0.0000 & 0.0002 & 99.8009 & - & - & - & - \\
\hline \multirow[t]{4}{*}{ DP4+ } & ${ }^{13} \mathrm{C}$ data & 22.4491 & 0.0000 & 64.1610 & 13.3899 & - & - & - & - \\
\hline & All data & 0.3330 & 0.0000 & 0.0009 & 99.6661 & - & - & - & - \\
\hline & & \multicolumn{8}{|c|}{ Level of theory: B3LYP/6-311G* (gas phase) } \\
\hline & & $85 a$ & $85 b$ & $85 c$ & $85 d$ & - & - & - & - \\
\hline \multirow{3}{*}{ sDP4+ } & ${ }^{1} \mathrm{H}$ data & 1.4470 & 7.1692 & 0.1916 & 91.1922 & - & - & - & - \\
\hline & ${ }^{13} \mathrm{C}$ data & 7.3945 & 3.2862 & 79.6053 & 9.7141 & - & - & - & - \\
\hline & All data & 1.1440 & 2.5187 & 1.6303 & 94.7070 & - & - & - & - \\
\hline \multirow{3}{*}{ uDP4+ } & ${ }^{1} \mathrm{H}$ data & 2.8515 & 10.5960 & 0.0015 & 86.5510 & - & - & - & - \\
\hline & ${ }^{13} \mathrm{C}$ data & 4.4116 & 3.1873 & 72.4313 & 19.9698 & - & - & - & - \\
\hline & All data & 0.7088 & 1.9029 & 0.0059 & 97.3824 & - & - & - & - \\
\hline \multirow{5}{*}{ DP4+ } & ${ }^{1} \mathrm{H}$ data & 0.0518 & 0.9528 & 0.0000 & 98.9955 & - & - & - & - \\
\hline & ${ }^{13} \mathrm{C}$ data & 0.5434 & 0.1745 & 96.0506 & 3.2315 & - & - & - & - \\
\hline & All data & 0.0088 & 0.0519 & 0.0001 & 99.9392 & - & - & - & - \\
\hline & & \multicolumn{8}{|c|}{ Level of theory: B3LYP/6-311G** (gas phase) } \\
\hline & & $85 a$ & $85 b$ & $85 c$ & $85 d$ & - & - & - & - \\
\hline \multirow{2}{*}{ sDP4+ } & ${ }^{1} \mathrm{H}$ data & 7.2606 & 59.5874 & 0.0202 & 33.1318 & - & - & - & - \\
\hline & ${ }^{13} \mathrm{C}$ data & 7.5211 & 3.5905 & 73.3377 & 15.5506 & - & - & - & - \\
\hline
\end{tabular}




\begin{tabular}{|c|c|c|c|c|c|c|c|c|c|}
\hline & All data & 6.9541 & 27.2455 & 0.1890 & 65.6113 & - & - & - & - \\
\hline & ${ }^{1} \mathrm{H}$ data & 2.5270 & 15.7007 & 0.0025 & 81.7698 & - & - & - & - \\
\hline \multirow[t]{2}{*}{ uDP4+ } & ${ }^{13} \mathrm{C}$ data & 4.3963 & 4.3969 & 68.7952 & 22.4116 & - & - & - & - \\
\hline & All data & 0.5808 & 3.6089 & 0.0091 & 95.8013 & - & - & - & - \\
\hline & ${ }^{1} \mathrm{H}$ data & 0.5009 & 25.5403 & 0.0000 & 73.9588 & - & - & - & - \\
\hline \multirow[t]{5}{*}{ DP4+ } & ${ }^{13} \mathrm{C}$ data & 0.6075 & 0.2901 & 92.6990 & 6.4034 & - & - & - & - \\
\hline & All data & 0.0632 & 1.5392 & 0.0000 & 98.3975 & - & - & - & - \\
\hline & & \multicolumn{8}{|c|}{ Level of theory: B3LYP/6-311+G** (gas phase) } \\
\hline & & $85 a$ & $85 \mathrm{~b}$ & $85 c$ & $85 d$ & - & - & - & - \\
\hline & ${ }^{1} \mathrm{H}$ data & 74.6720 & 3.5322 & 0.0298 & 21.7660 & - & - & - & - \\
\hline \multirow[t]{2}{*}{ sDP4+ } & ${ }^{13} \mathrm{C}$ data & 20.0175 & 0.1074 & 65.4255 & 14.4496 & - & - & - & - \\
\hline & All data & 82.5104 & 0.0209 & 0.1077 & 17.3610 & - & - & - & - \\
\hline \multirow{3}{*}{ uDP4+ } & ${ }^{1} \mathrm{H}$ data & 7.0694 & 2.2798 & 0.0097 & 90.6412 & - & - & - & - \\
\hline & ${ }^{13} \mathrm{C}$ data & 11.9786 & 0.6515 & 58.4220 & 28.9479 & - & - & - & - \\
\hline & All data & 3.1241 & 0.0548 & 0.0208 & 96.8003 & - & - & - & - \\
\hline & ${ }^{1} \mathrm{H}$ data & 21.0410 & 0.3210 & 0.0000 & 78.6380 & - & - & - & - \\
\hline \multirow[t]{4}{*}{ DP4+ } & ${ }^{13} \mathrm{C}$ data & 5.3518 & 0.0016 & 85.3108 & 9.3358 & - & - & - & - \\
\hline & All data & 13.2985 & 0.0001 & 0.0001 & 86.7013 & - & - & - & - \\
\hline & & \multicolumn{8}{|c|}{ Level of theory: B3LYP/6-31G* (solution. PCM) } \\
\hline & & $85 a$ & $85 \mathrm{~b}$ & $85 c$ & $85 d$ & - & - & - & - \\
\hline \multirow{3}{*}{ sDP4+ } & ${ }^{1} \mathrm{H}$ data & 0.3325 & 96.9827 & 0.0055 & 2.6793 & - & - & - & - \\
\hline & ${ }^{13} \mathrm{C}$ data & 68.1284 & 10.7835 & 21.0695 & 0.0186 & - & - & - & - \\
\hline & All data & 2.1200 & 97.8646 & 0.0108 & 0.0047 & - & - & - & - \\
\hline \multirow{3}{*}{ uDP4+ } & ${ }^{1} \mathrm{H}$ data & 1.0409 & 83.6870 & 0.0108 & 15.2613 & - & - & - & - \\
\hline & ${ }^{13} \mathrm{C}$ data & 1.4442 & 0.0105 & 43.9179 & 54.6274 & - & - & - & - \\
\hline & All data & 0.1797 & 0.1050 & 0.0567 & 99.6586 & - & - & - & - \\
\hline \multirow{5}{*}{ DP4+ } & ${ }^{1} \mathrm{H}$ data & 0.0042 & 99.4945 & 0.0000 & 0.5013 & - & - & - & - \\
\hline & ${ }^{13} \mathrm{C}$ data & 9.6005 & 0.0110 & 90.2890 & 0.0994 & - & - & - & - \\
\hline & All data & 3.4257 & 92.3786 & 0.0055 & 4.1902 & - & - & - & - \\
\hline & & \multicolumn{8}{|c|}{ Level of theory: B3LYP/6-31G** (solution. PCM) } \\
\hline & & $85 a$ & $85 \mathrm{~b}$ & $85 c$ & $85 d$ & - & - & - & - \\
\hline \multirow{3}{*}{ sDP4+ } & ${ }^{1} \mathrm{H}$ data & 0.7126 & 92.0351 & 0.0153 & 7.2370 & - & - & - & - \\
\hline & ${ }^{13} \mathrm{C}$ data & 56.4771 & 12.2135 & 31.2253 & 0.0841 & - & - & - & - \\
\hline & All data & 3.4533 & 96.4534 & 0.0411 & 0.0522 & - & - & - & - \\
\hline \multirow{3}{*}{ uDP4+ } & ${ }^{1} \mathrm{H}$ data & 0.7965 & 77.5771 & 0.0260 & 21.6004 & - & - & - & - \\
\hline & ${ }^{13} \mathrm{C}$ data & 2.1214 & 0.0461 & 57.8056 & 40.0269 & - & - & - & - \\
\hline & All data & 0.1939 & 0.4107 & 0.1724 & 99.2230 & - & - & - & - \\
\hline \multirow{3}{*}{ DP4+ } & ${ }^{1} \mathrm{H}$ data & 0.0078 & 97.8499 & 0.0000 & 2.1424 & - & - & - & - \\
\hline & ${ }^{13} \mathrm{C}$ data & 6.2119 & 0.0292 & 93.5844 & 0.1745 & - & - & - & - \\
\hline & All data & 1.4727 & 87.1190 & 0.0156 & 11.3927 & - & - & - & - \\
\hline & & \multicolumn{8}{|c|}{ Level of theory: B3LYP/6-31+G** (solution. PCM) } \\
\hline & & $85 a$ & $85 \mathrm{~b}$ & $85 c$ & $85 d$ & - & - & - & - \\
\hline \multirow{3}{*}{ sDP4+ } & ${ }^{1} \mathrm{H}$ data & 25.7038 & 1.1670 & 0.0575 & 73.0718 & - & - & - & - \\
\hline & ${ }^{13} \mathrm{C}$ data & 59.8057 & 10.3618 & 29.2201 & 0.6123 & - & - & - & - \\
\hline & All data & 96.3331 & 0.7578 & 0.1052 & 2.8040 & - & - & - & - \\
\hline & ${ }_{12}^{1} \mathrm{H}$ data & 1.2807 & 0.3937 & 0.1187 & 98.2068 & - & - & - & - \\
\hline uDP4+ & ${ }^{13} \mathrm{C}$ data & 18.9236 & 0.3865 & 32.0420 & 48.6478 & - & - & - & - \\
\hline & All data & 0.5043 & 0.0032 & 0.0791 & 99.4134 & - & - & - & - \\
\hline & ${ }^{1} \mathrm{H}$ data & 0.4566 & 0.0064 & 0.0001 & 99.5369 & - & - & - & - \\
\hline DP4+ & ${ }^{13} \mathrm{C}$ data & 53.8461 & 0.1906 & 44.5461 & 1.4173 & - & - & - & - \\
\hline & All data & 14.8413 & 0.0007 & 0.0025 & 85.1554 & - & - & - & - \\
\hline & & & & Level of & गry: B3LYP & $\mathrm{G}^{*}$ & $\overline{P C}$ & & \\
\hline & & $85 a$ & $85 b$ & $85 c$ & $85 d$ & - & - & - & - \\
\hline & ${ }^{1} \mathrm{H}$ data & 3.7095 & 73.2044 & 0.0741 & 23.0119 & - & - & - & - \\
\hline sDP4+ & ${ }^{13} \mathrm{C}$ data & 3.5945 & 34.5953 & 60.4152 & 1.3950 & - & - & - & - \\
\hline & All data & 0.5163 & 98.0671 & 0.1735 & 1.2431 & - & - & - & - \\
\hline
\end{tabular}




\begin{tabular}{|c|c|c|c|c|c|c|c|c|c|}
\hline & ${ }^{1} \mathrm{H}$ data & 4.1156 & 39.7032 & 0.0000 & 56.1812 & - & - & - & - \\
\hline \multirow[t]{3}{*}{ uDP4+ } & ${ }^{13} \mathrm{C}$ data & 18.8805 & 31.2570 & 43.1915 & 6.6710 & - & - & - & - \\
\hline & All data & 4.5884 & 73.2806 & 0.0000 & 22.1309 & - & - & - & - \\
\hline & ${ }^{1} \mathrm{H}$ data & 0.3622 & 68.9622 & 0.0000 & 30.6755 & - & - & - & - \\
\hline \multirow[t]{5}{*}{ DP4+ } & ${ }^{13} \mathrm{C}$ data & 1.8011 & 28.6986 & 69.2533 & 0.2470 & - & - & - & - \\
\hline & All data & 0.0328 & 99.5859 & 0.0000 & 0.3812 & - & - & - & - \\
\hline & & \multicolumn{8}{|c|}{ Level of theory: B3LYP/6-311G** (solution. PCM) } \\
\hline & & $85 a$ & $85 b$ & $85 c$ & $85 d$ & - & - & - & - \\
\hline & ${ }^{1} \mathrm{H}$ data & 6.7428 & 89.5607 & 0.0202 & 3.6763 & - & - & - & - \\
\hline \multirow[t]{3}{*}{ sDP4+ } & ${ }^{13} \mathrm{C}$ data & 2.3701 & 18.7992 & 76.0637 & 2.7670 & - & - & - & - \\
\hline & All data & 0.9338 & 98.3819 & 0.0899 & 0.5944 & - & - & - & - \\
\hline & ${ }^{1} \mathrm{H}$ data & 3.9852 & 51.4105 & 0.0003 & 44.6039 & - & - & - & - \\
\hline \multirow[t]{3}{*}{ uDP4+ } & ${ }^{13} \mathrm{C}$ data & 12.1088 & 31.1894 & 50.3322 & 6.3697 & - & - & - & - \\
\hline & All data & 2.4928 & 82.8300 & 0.0009 & 14.6764 & - & - & - & - \\
\hline & ${ }^{1} \mathrm{H}$ data & 0.5604 & 96.0200 & 0.0000 & 3.4196 & - & - & - & - \\
\hline \multirow[t]{5}{*}{ DP4+ } & ${ }^{13} \mathrm{C}$ data & 0.6433 & 13.1433 & 85.8183 & 0.3951 & - & - & - & - \\
\hline & All data & 0.0285 & 99.8646 & 0.0000 & 0.1069 & - & - & - & - \\
\hline & & \multicolumn{8}{|c|}{ Level of theory: B3LYP/6-311+G** (solution. PCM) } \\
\hline & & $85 a$ & $85 b$ & $85 c$ & $85 d$ & - & - & - & - \\
\hline & ${ }^{1}$ H data & 60.4010 & 32.9659 & 0.0224 & 6.6107 & - & - & - & - \\
\hline \multirow[t]{3}{*}{$s \mathrm{DP4+}$} & ${ }^{13} \mathrm{C}$ data & 4.7489 & 1.7619 & 90.6940 & 2.7952 & - & - & - & - \\
\hline & All data & 78.4938 & 15.8948 & 0.5548 & 5.0566 & - & - & - & - \\
\hline & ${ }^{1} \mathrm{H}$ data & 5.2802 & 6.2645 & 0.0001 & 88.4552 & - & - & - & - \\
\hline \multirow[t]{3}{*}{ uDP4+ } & ${ }^{13} \mathrm{C}$ data & 25.0240 & 10.6096 & 58.1210 & 6.2454 & - & - & - & - \\
\hline & All data & 17.5932 & 8.8495 & 0.0008 & 73.5565 & - & - & - & - \\
\hline & ${ }^{1} \mathrm{H}$ data & 28.7273 & 18.6016 & 0.0000 & 52.6711 & - & - & - & - \\
\hline \multirow[t]{5}{*}{ DP4+ } & ${ }^{13} \mathrm{C}$ data & 2.1900 & 0.3445 & 97.1437 & 0.3217 & - & - & - & - \\
\hline & All data & 72.9290 & 7.4284 & 0.0000 & 19.6426 & - & - & - & - \\
\hline & & \multicolumn{8}{|c|}{ Level of theory: mPW1PW91/6-31G* (gas phase) } \\
\hline & & $85 a$ & $85 b$ & $85 c$ & $85 d$ & - & - & - & - \\
\hline & ${ }_{12}^{1} \mathrm{H}$ data & 0.3397 & 50.0873 & 0.0505 & 49.5226 & - & - & - & - \\
\hline \multirow[t]{3}{*}{ sDP4+ } & ${ }^{13} \mathrm{C}$ data & 9.1856 & 3.1999 & 86.3255 & 1.2890 & - & - & - & - \\
\hline & All data & 1.3473 & 69.2065 & 1.8817 & 27.5645 & - & - & - & - \\
\hline & ${ }^{1} \mathrm{H}$ data & 0.6797 & 27.1704 & 0.0526 & 72.0973 & - & - & - & - \\
\hline \multirow[t]{3}{*}{ uDP4+ } & ${ }^{13} \mathrm{C}$ data & 0.3912 & 0.0029 & 32.8310 & 66.7750 & - & - & - & - \\
\hline & All data & 0.0055 & 0.0016 & 0.0359 & 99.9570 & - & - & - & - \\
\hline & ${ }^{1} \mathrm{H}$ data & 0.0047 & 27.5955 & 0.0001 & 72.3998 & - & - & - & - \\
\hline \multirow[t]{5}{*}{ DP4+ } & ${ }^{13} \mathrm{C}$ data & 0.1229 & 0.0003 & 96.9329 & 2.9439 & - & - & - & - \\
\hline & All data & 0.0003 & 0.0041 & 0.0024 & 99.9932 & - & - & - & - \\
\hline & & \multicolumn{8}{|c|}{ Level of theory: mPW1PW91/6-31G** (gas phase) } \\
\hline & & $85 a$ & $85 b$ & $85 c$ & $85 d$ & - & - & - & - \\
\hline & ${ }^{1} \mathrm{H}$ data & 0.7341 & 44.0048 & 0.0276 & 55.2334 & - & - & - & - \\
\hline \multirow[t]{2}{*}{ sDP4+ } & ${ }^{13} \mathrm{C}$ data & 6.7945 & 2.1743 & 88.5898 & 2.4415 & - & - & - & - \\
\hline & All data & 2.0961 & 40.2078 & 1.0270 & 56.6690 & - & - & - & - \\
\hline & ${ }^{1} \mathrm{H}$ data & 0.3395 & 15.2338 & 0.0988 & 84.3279 & - & - & - & - \\
\hline uDP4+ & ${ }^{13} \mathrm{C}$ data & 0.2958 & 0.0052 & 49.9778 & 49.7212 & - & - & - & - \\
\hline & All data & 0.0024 & 0.0019 & 0.1176 & 99.8781 & - & - & - & - \\
\hline & ${ }^{1} \mathrm{H}$ data & 0.0047 & 12.5811 & 0.0001 & 87.4142 & - & - & - & - \\
\hline DP4+ & ${ }^{13} \mathrm{C}$ data & 0.0442 & 0.0002 & 97.2882 & 2.6674 & - & - & - & - \\
\hline & All data & 0.0001 & 0.0013 & 0.0021 & 99.9964 & - & - & - & - \\
\hline & & & & Level of th & $y:$ mPW1P & -31 & pn & & \\
\hline & & $85 a$ & $85 b$ & $85 c$ & $85 d$ & - & - & - & - \\
\hline & ${ }^{1} \mathrm{H}$ data & 15.8050 & 0.1426 & 0.0808 & 83.9716 & - & - & - & - \\
\hline sDP4+ & ${ }^{13} \mathrm{C}$ data & 43.8972 & 0.0149 & 45.6053 & 10.4827 & - & - & - & - \\
\hline & All data & 43.9742 & 0.0001 & 0.2336 & 55.7920 & - & - & - & - \\
\hline uDP4+ & ${ }^{1} \mathrm{H}$ data & 0.3838 & 0.0682 & 0.3722 & 99.1757 & - & - & - & - \\
\hline
\end{tabular}




\begin{tabular}{|c|c|c|c|c|c|c|c|c|c|}
\hline & ${ }^{13} \mathrm{C}$ data & 2.4942 & 0.0037 & 25.0090 & 72.4931 & - & - & - & - \\
\hline & All data & 0.0133 & 0.0000 & 0.1293 & 99.8574 & - & - & - & - \\
\hline & ${ }^{1} \mathrm{H}$ data & 0.0728 & 0.0001 & 0.0004 & 99.9267 & - & - & - & - \\
\hline \multirow[t]{5}{*}{ DP4+ } & ${ }^{13} \mathrm{C}$ data & 5.4474 & 0.0000 & 56.7447 & 37.8079 & - & - & - & - \\
\hline & All data & 0.0105 & 0.0000 & 0.0005 & 99.9890 & - & - & - & - \\
\hline & & \multicolumn{8}{|c|}{ Level of theory: mPW1PW91/6-311G* (gas phase) } \\
\hline & & $85 a$ & $85 b$ & $85 c$ & $85 d$ & - & - & - & - \\
\hline & ${ }^{1} \mathrm{H}$ data & 1.6091 & 33.4679 & 0.0468 & 64.8763 & - & - & - & - \\
\hline \multirow[t]{2}{*}{ sDP4+ } & ${ }^{13} \mathrm{C}$ data & 3.0809 & 1.4559 & 68.8617 & 26.6015 & - & - & - & - \\
\hline & All data & 0.2781 & 2.7333 & 0.1808 & 96.8078 & - & - & - & - \\
\hline \multirow{3}{*}{ uDP4+ } & ${ }^{1} \mathrm{H}$ data & 1.7425 & 10.5087 & 0.0001 & 87.7487 & - & - & - & - \\
\hline & ${ }^{13} \mathrm{C}$ data & 2.9740 & 1.4553 & 53.9270 & 41.6438 & - & - & - & - \\
\hline & All data & 0.1410 & 0.4162 & 0.0001 & 99.4427 & - & - & - & - \\
\hline \multirow{5}{*}{ DP4+ } & ${ }^{1} \mathrm{H}$ data & 0.0464 & 5.8159 & 0.0000 & 94.1378 & - & - & - & - \\
\hline & ${ }^{13} \mathrm{C}$ data & 0.1896 & 0.0438 & 76.8432 & 22.9233 & - & - & - & - \\
\hline & All data & 0.0004 & 0.0118 & 0.0000 & 99.9878 & - & - & - & - \\
\hline & & \multicolumn{8}{|c|}{ Level of theory: mPW1PW91/6-311G** (gas phase) } \\
\hline & & $85 a$ & $85 b$ & $85 c$ & $85 d$ & - & - & - & - \\
\hline \multirow{3}{*}{ sDP4+ } & ${ }^{1} \mathrm{H}$ data & 11.2832 & 70.4350 & 0.0048 & 18.2770 & - & - & - & - \\
\hline & ${ }^{13} \mathrm{C}$ data & 2.7558 & 1.5317 & 50.7007 & 45.0118 & - & - & - & - \\
\hline & All data & 3.2326 & 11.2156 & 0.0251 & 85.5267 & - & - & - & - \\
\hline \multirow{3}{*}{ uDP4+ } & ${ }^{1} \mathrm{H}$ data & 1.3116 & 15.5023 & 0.0001 & 83.1860 & - & - & - & - \\
\hline & ${ }^{13} \mathrm{C}$ data & 3.4134 & 2.0247 & 49.1988 & 45.3632 & - & - & - & - \\
\hline & All data & 0.1175 & 0.8239 & 0.0001 & 99.0584 & - & - & - & - \\
\hline \multirow{3}{*}{ DP4+ } & ${ }^{1} \mathrm{H}$ data & 0.5633 & 41.5631 & 0.0000 & 57.8735 & - & - & - & - \\
\hline & ${ }^{13} \mathrm{C}$ data & 0.2068 & 0.0682 & 54.8367 & 44.8883 & - & - & - & - \\
\hline & All data & 0.0045 & 0.1089 & 0.0000 & 99.8866 & - & - & - & - \\
\hline & & \multicolumn{8}{|c|}{ Level of theory: mPW1PW91/6-311+G** (gas phase) } \\
\hline & & $85 a$ & $85 b$ & $85 c$ & $85 d$ & - & - & - & - \\
\hline \multirow{3}{*}{ sDP4+ } & ${ }^{1} \mathrm{H}$ data & 63.4889 & 14.9429 & 0.0175 & 21.5507 & - & - & - & - \\
\hline & ${ }^{13} \mathrm{C}$ data & 10.3718 & 0.0584 & 32.4727 & 57.0972 & - & - & - & - \\
\hline & All data & 34.8332 & 0.0462 & 0.0300 & 65.0907 & - & - & - & - \\
\hline \multirow{3}{*}{ uDP4+ } & ${ }^{1} \mathrm{H}$ data & 2.4570 & 3.4861 & 0.0006 & 94.0564 & - & - & - & - \\
\hline & ${ }^{13} \mathrm{C}$ data & 13.8389 & 0.2709 & 39.4339 & 46.4563 & - & - & - & - \\
\hline & All data & 0.7720 & 0.0214 & 0.0005 & 99.2061 & - & - & - & - \\
\hline \multirow{5}{*}{ DP4+ } & ${ }^{1} \mathrm{H}$ data & 6.9794 & 2.3307 & 0.0000 & 90.6900 & - & - & - & - \\
\hline & ${ }^{13} \mathrm{C}$ data & 3.5209 & 0.0004 & 31.4116 & 65.0671 & - & - & - & - \\
\hline & All data & 0.4147 & 0.0000 & 0.0000 & 99.5853 & - & - & - & - \\
\hline & & \multicolumn{8}{|c|}{ Level of theory: mPW1PW91/6-31G* (solution. PCM) } \\
\hline & & $85 a$ & $85 b$ & $85 c$ & $85 d$ & - & - & - & - \\
\hline \multirow{3}{*}{ sDP4+ } & ${ }^{1} \mathrm{H}$ data & 0.4845 & 96.4125 & 0.0117 & 3.0913 & - & - & - & - \\
\hline & ${ }^{13} \mathrm{C}$ data & 6.4467 & 1.4844 & 91.9750 & 0.0939 & - & - & - & - \\
\hline & All data & 2.1163 & 96.9600 & 0.7271 & 0.1966 & - & - & - & - \\
\hline \multirow{3}{*}{ uDP4+ } & ${ }^{1} \mathrm{H}$ data & 0.9467 & 81.6050 & 0.0395 & 17.4088 & - & - & - & - \\
\hline & ${ }^{13} \mathrm{C}$ data & 0.8293 & 0.0038 & 27.7951 & 71.3718 & - & - & - & - \\
\hline & All data & 0.0631 & 0.0249 & 0.0882 & 99.8238 & - & - & - & - \\
\hline & ${ }^{1} \mathrm{H}$ data & 0.0058 & 99.3149 & 0.0000 & 0.6793 & - & - & - & - \\
\hline DP4+ & ${ }^{13} \mathrm{C}$ data & 0.2082 & 0.0002 & 99.5307 & 0.2609 & - & - & - & - \\
\hline & All data & 0.6002 & 10.8550 & 0.2883 & 88.2565 & - & - & - & - \\
\hline & & & & vel of the & mPW1PV & 10 & on. & & \\
\hline & & $85 a$ & $85 b$ & $85 c$ & $85 d$ & - & - & - & - \\
\hline & ${ }^{1} \mathrm{H}$ data & 1.2387 & 93.0234 & 0.0104 & 5.7276 & - & - & - & - \\
\hline sDP4+ & ${ }^{13} \mathrm{C}$ data & 6.7044 & 1.3283 & 91.7406 & 0.2268 & - & - & - & - \\
\hline & All data & 6.1920 & 92.1288 & 0.7108 & 0.9684 & - & - & - & - \\
\hline & ${ }^{1} \mathrm{H}$ data & 0.6097 & 71.9463 & 0.1136 & 27.3303 & - & - & - & - \\
\hline UDP4+ & ${ }^{13} \mathrm{C}$ data & 0.9770 & 0.0091 & 40.9983 & 58.0155 & - & - & - & - \\
\hline
\end{tabular}




\begin{tabular}{|c|c|c|c|c|c|c|c|c|c|}
\hline & All data & 0.0374 & 0.0411 & 0.2928 & 99.6287 & - & - & - & - \\
\hline & ${ }^{1} \mathrm{H}$ data & 0.0110 & 97.7037 & 0.0000 & 2.2852 & - & - & - & - \\
\hline \multirow[t]{5}{*}{ DP4+ } & ${ }^{13} \mathrm{C}$ data & 0.1732 & 0.0003 & 99.4785 & 0.3480 & - & - & - & - \\
\hline & All data & 0.2301 & 3.7592 & 0.2066 & 95.8040 & - & - & - & - \\
\hline & & \multicolumn{8}{|c|}{ Level of theory: mPW1PW91/6-31+G** (solution. PCM) } \\
\hline & & $85 a$ & $85 b$ & $85 c$ & $85 d$ & - & - & - & - \\
\hline & ${ }^{1} \mathrm{H}$ data & 40.6281 & 14.0809 & 0.2172 & 45.0739 & - & - & - & - \\
\hline \multirow[t]{3}{*}{ sDP4+ } & ${ }^{13} \mathrm{C}$ data & 65.1772 & 6.9894 & 26.0096 & 1.8238 & - & - & - & - \\
\hline & All data & 93.4279 & 3.4724 & 0.1993 & 2.9004 & - & - & - & - \\
\hline & ${ }^{1} \mathrm{H}$ data & 0.7821 & 1.5735 & 0.4432 & 97.2013 & - & - & - & - \\
\hline \multirow[t]{3}{*}{ uDP4+ } & ${ }^{13} \mathrm{C}$ data & 6.4802 & 0.2383 & 17.5111 & 75.7703 & - & - & - & - \\
\hline & All data & 0.0687 & 0.0051 & 0.1052 & 99.8211 & - & - & - & - \\
\hline & ${ }^{1} \mathrm{H}$ data & 0.7164 & 0.4995 & 0.0022 & 98.7819 & - & - & - & - \\
\hline \multirow[t]{5}{*}{ DP4+ } & ${ }^{13} \mathrm{C}$ data & 41.5028 & 0.1637 & 44.7546 & 13.5790 & - & - & - & - \\
\hline & All data & 2.1682 & 0.0060 & 0.0071 & 97.8187 & - & - & - & - \\
\hline & & \multicolumn{8}{|c|}{ Level of theory: mPW1PW91/6-311G* (solution. PCM) } \\
\hline & & $85 a$ & $85 b$ & $85 c$ & $85 d$ & - & - & - & - \\
\hline & ${ }^{1} \mathrm{H}$ data & 2.9955 & 87.8436 & 0.0058 & 9.1551 & - & - & - & - \\
\hline \multirow[t]{2}{*}{ sDP4+ } & ${ }^{13} \mathrm{C}$ data & 0.7679 & 10.5319 & 77.7654 & 10.9348 & - & - & - & - \\
\hline & All data & 0.2238 & 89.9943 & 0.0439 & 9.7380 & - & - & - & - \\
\hline \multirow{3}{*}{ uDP4+ } & ${ }^{1}$ H data & 2.8558 & 54.1940 & 0.0000 & 42.9501 & - & - & - & - \\
\hline & ${ }^{13} \mathrm{C}$ data & 9.3680 & 11.2919 & 57.2562 & 22.0840 & - & - & - & - \\
\hline & All data & 1.6856 & 38.5551 & 0.0000 & 59.7593 & - & - & - & - \\
\hline \multirow{3}{*}{ DP4+ } & ${ }^{1} \mathrm{H}$ data & 0.1657 & 92.2174 & 0.0000 & 7.6169 & - & - & - & - \\
\hline & ${ }^{13} \mathrm{C}$ data & 0.1492 & 2.4672 & 92.3736 & 5.0099 & - & - & - & - \\
\hline & All data & 0.0093 & 85.6291 & 0.0000 & 14.3616 & - & - & - & - \\
\hline & & \multicolumn{8}{|c|}{ Level of theory: mPW1PW91/6-311G** (solution. PCM) } \\
\hline & & $85 a$ & $85 b$ & $85 c$ & $85 d$ & - & - & - & - \\
\hline \multirow{3}{*}{ sDP4+ } & ${ }^{1} \mathrm{H}$ data & 15.3028 & 79.7774 & 0.0082 & 4.9116 & - & - & - & - \\
\hline & ${ }^{13} \mathrm{C}$ data & 0.3946 & 4.7453 & 75.6952 & 19.1650 & - & - & - & - \\
\hline & All data & 1.2595 & 78.9739 & 0.1299 & 19.6367 & - & - & - & - \\
\hline \multirow{3}{*}{ uDP4+ } & ${ }^{1} \mathrm{H}$ data & 2.7539 & 63.0639 & 0.0000 & 34.1822 & - & - & - & - \\
\hline & ${ }^{13} \mathrm{C}$ data & 5.8545 & 6.8971 & 62.1730 & 25.0753 & - & - & - & - \\
\hline & All data & 1.2324 & 33.2484 & 0.0001 & 65.5191 & - & - & - & - \\
\hline \multirow{5}{*}{ DP4+ } & ${ }^{1} \mathrm{H}$ data & 0.8041 & 95.9926 & 0.0000 & 3.2033 & - & - & - & - \\
\hline & ${ }^{13} \mathrm{C}$ data & 0.0442 & 0.6268 & 90.1259 & 9.2031 & - & - & - & - \\
\hline & All data & 0.0397 & 67.0882 & 0.0000 & 32.8721 & - & - & - & - \\
\hline & & \multicolumn{8}{|c|}{ Level of theory: mPW1PW91/6-311+G** (solution. PCM) } \\
\hline & & $85 a$ & $85 b$ & $85 c$ & $85 d$ & - & - & - & - \\
\hline \multirow{3}{*}{ sDP4+ } & ${ }^{1} \mathrm{H}$ data & 57.7968 & 37.2768 & 0.0091 & 4.9174 & - & - & - & - \\
\hline & ${ }^{13} \mathrm{C}$ data & 0.8997 & 0.8226 & 86.0208 & 12.2569 & - & - & - & - \\
\hline & All data & 36.1823 & 21.3362 & 0.5440 & 41.9375 & - & - & - & - \\
\hline \multirow{3}{*}{ uDP4+ } & ${ }^{1} \mathrm{H}$ data & 5.1541 & 18.8294 & 0.0002 & 76.0164 & - & - & - & - \\
\hline & ${ }^{13} \mathrm{C}$ data & 16.1258 & 3.3506 & 66.8092 & 13.7144 & - & - & - & - \\
\hline & All data & 6.9918 & 5.3072 & 0.0010 & 87.7000 & - & - & - & - \\
\hline \multirow{3}{*}{ DP4+ } & ${ }^{1} \mathrm{H}$ data & 21.6869 & 51.0995 & 0.0000 & 27.2136 & - & - & - & - \\
\hline & ${ }^{13} \mathrm{C}$ data & 0.2446 & 0.0465 & 96.8754 & 2.8336 & - & - & - & - \\
\hline & All data & 6.2554 & 2.8000 & 0.0000 & 90.9445 & - & - & - & - \\
\hline
\end{tabular}


$s \mathrm{DP} 4+, u \mathrm{DP} 4+$ and DP4+ probabilities (\%) for compound 86a<smiles>O=C(O[C@@H]1C[C@H]2C=C[C@H]1C2)[C@H](O)c1ccccc1</smiles>

correct isomer<smiles>CC(C)(C)OC(=O)C(O)[C@H]1CC2C=CC1C2</smiles>

86b<smiles>CC(C)(C)[O+]=O</smiles>

$86 c$<smiles>O=C(OC1CC2C=CC1C2)C(O)c1ccccc1</smiles>

86d

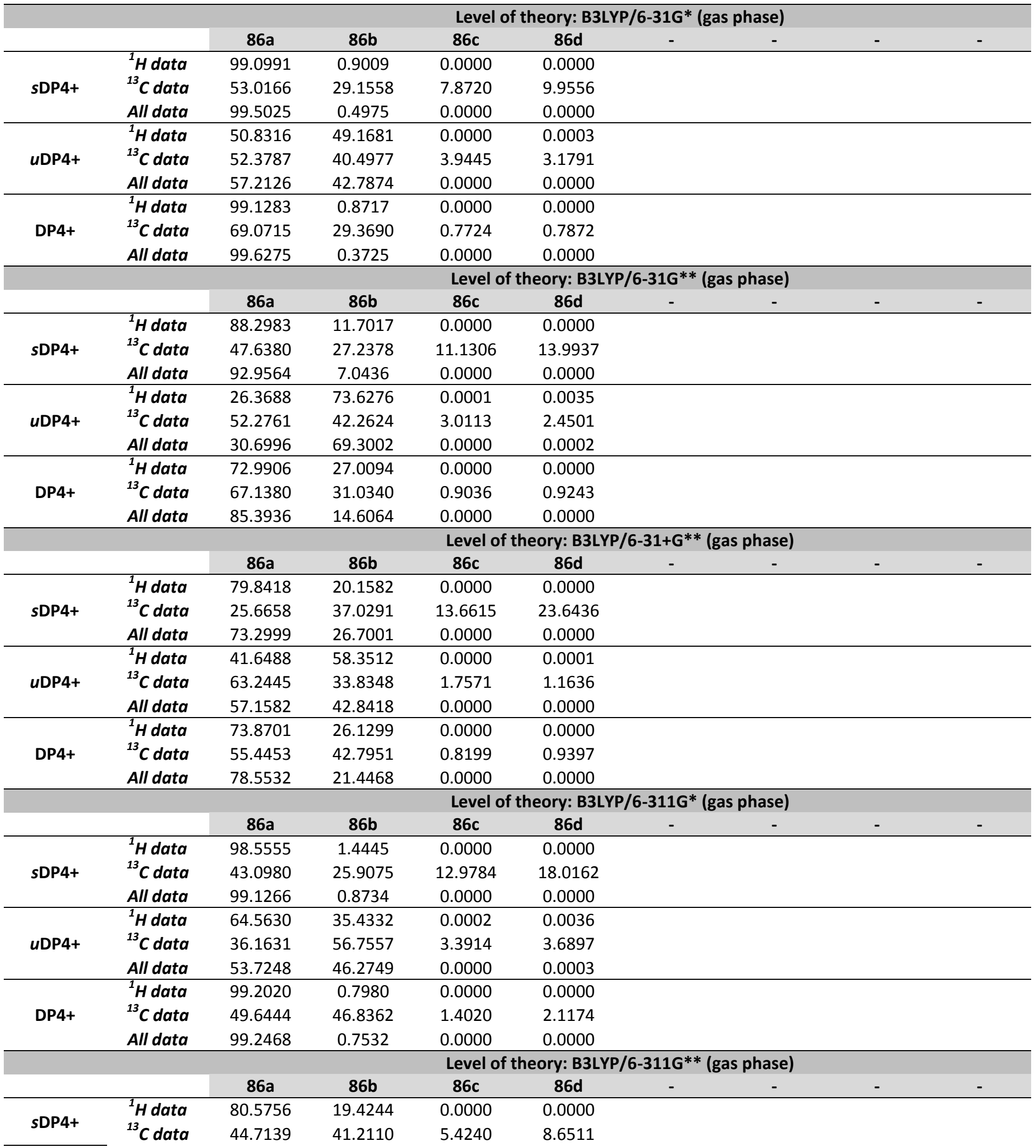




\begin{tabular}{|c|c|c|c|c|c|c|c|c|c|}
\hline & All data & 81.8207 & 18.1793 & 0.0000 & 0.0000 & & & & \\
\hline & ${ }^{1} \mathrm{H}$ data & 49.6934 & 50.3023 & 0.0002 & 0.0041 & & & & \\
\hline \multirow[t]{3}{*}{ uDP4+ } & ${ }^{13} \mathrm{C}$ data & 36.3091 & 58.0186 & 2.7175 & 2.9548 & & & & \\
\hline & All data & 38.2045 & 61.7952 & 0.0000 & 0.0003 & & & & \\
\hline & ${ }^{1} \mathrm{H}$ data & 80.3843 & 19.6157 & 0.0000 & 0.0000 & & & & \\
\hline \multirow[t]{5}{*}{ DP4+ } & ${ }^{13} \mathrm{C}$ data & 40.0392 & 58.9668 & 0.3635 & 0.6304 & & & & \\
\hline & All data & 73.5630 & 26.4370 & 0.0000 & 0.0000 & & & & \\
\hline & & \multicolumn{8}{|c|}{ Level of theory: B3LYP/6-311+G** (gas phase) } \\
\hline & & $86 a$ & $86 b$ & $86 c$ & 86d & - & - & - & - \\
\hline & ${ }^{1} \mathrm{H}$ data & 74.5201 & 25.4799 & 0.0000 & 0.0000 & & & & \\
\hline \multirow[t]{3}{*}{ sDP4+ } & ${ }^{13} \mathrm{C}$ data & 46.2186 & 39.5948 & 5.2119 & 8.9748 & & & & \\
\hline & All data & 77.3444 & 22.6556 & 0.0000 & 0.0000 & & & & \\
\hline & ${ }^{1} \mathrm{H}$ data & 26.5565 & 73.4418 & 0.0001 & 0.0016 & & & & \\
\hline \multirow[t]{3}{*}{ uDP4+ } & ${ }^{13} \mathrm{C}$ data & 33.4422 & 61.8347 & 1.5792 & 3.1438 & & & & \\
\hline & All data & 16.3575 & 83.6424 & 0.0000 & 0.0001 & & & & \\
\hline & ${ }^{1} \mathrm{H}$ data & 51.3986 & 48.6014 & 0.0000 & 0.0000 & & & & \\
\hline \multirow[t]{5}{*}{ DP4+ } & ${ }^{13} \mathrm{C}$ data & 38.3495 & 60.7462 & 0.2042 & 0.7001 & & & & \\
\hline & All data & 40.0351 & 59.9649 & 0.0000 & 0.0000 & & & & \\
\hline & & \multicolumn{8}{|c|}{ Level of theory: B3LYP/6-31G* (solution. PCM) } \\
\hline & & $86 a$ & $86 b$ & $86 c$ & $86 d$ & - & - & - & - \\
\hline & ${ }^{1}$ H data & 99.8510 & 0.1490 & 0.0000 & 0.0000 & & & & \\
\hline \multirow[t]{3}{*}{ sDP4+ } & ${ }^{13} \mathrm{C}$ data & 46.1461 & 45.3492 & 3.4453 & 5.0595 & & & & \\
\hline & All data & 99.8536 & 0.1464 & 0.0000 & 0.0000 & & & & \\
\hline & ${ }^{1} \mathrm{H}$ data & 87.8690 & 12.1310 & 0.0000 & 0.0000 & & & & \\
\hline \multirow[t]{3}{*}{ uDP4+ } & ${ }^{13} \mathrm{C}$ data & 55.4640 & 41.0228 & 1.8697 & 1.6435 & & & & \\
\hline & All data & 90.7349 & 9.2651 & 0.0000 & 0.0000 & & & & \\
\hline & ${ }^{1} \mathrm{H}$ data & 99.9794 & 0.0206 & 0.0000 & 0.0000 & & & & \\
\hline \multirow[t]{5}{*}{ DP4+ } & ${ }^{13} \mathrm{C}$ data & 57.7159 & 41.9513 & 0.1453 & 0.1875 & & & & \\
\hline & All data & 99.9850 & 0.0150 & 0.0000 & 0.0000 & & & & \\
\hline & & \multicolumn{8}{|c|}{ Level of theory: B3LYP/6-31G** (solution. PCM) } \\
\hline & & $86 a$ & $86 b$ & $86 c$ & $86 d$ & - & - & - & - \\
\hline & ${ }^{1} \mathrm{H}$ data & 95.6660 & 4.3340 & 0.0000 & 0.0000 & & & & \\
\hline \multirow[t]{3}{*}{ sDP4+ } & ${ }^{13} \mathrm{C}$ data & 43.8178 & 41.9529 & 5.4411 & 8.7882 & & & & \\
\hline & All data & 95.8428 & 4.1572 & 0.0000 & 0.0000 & & & & \\
\hline & ${ }^{1} \mathrm{H}$ data & 62.7075 & 37.2918 & 0.0000 & 0.0007 & & & & \\
\hline \multirow[t]{3}{*}{ uDP4+ } & ${ }^{13} \mathrm{C}$ data & 54.3687 & 42.2790 & 1.7752 & 1.5771 & & & & \\
\hline & All data & 68.3782 & 31.6218 & 0.0000 & 0.0000 & & & & \\
\hline & ${ }^{1} \mathrm{H}$ data & 97.3765 & 2.6235 & 0.0000 & 0.0000 & & & & \\
\hline \multirow[t]{5}{*}{ DP4+ } & ${ }^{13} \mathrm{C}$ data & 56.9992 & 42.4381 & 0.2311 & 0.3316 & & & & \\
\hline & All data & 98.0335 & 1.9665 & 0.0000 & 0.0000 & & & & \\
\hline & & \multicolumn{8}{|c|}{ Level of theory: B3LYP/6-31+G** (solution. PCM) } \\
\hline & & $86 a$ & $86 b$ & $86 c$ & $86 d$ & - & - & - & - \\
\hline & ${ }^{1} \mathrm{H}$ data & 96.8449 & 3.1551 & 0.0000 & 0.0000 & & & & \\
\hline \multirow[t]{3}{*}{ sDP4+ } & ${ }^{13} \mathrm{C}$ data & 27.7813 & 47.0300 & 9.6538 & 15.5349 & & & & \\
\hline & All data & 94.7731 & 5.2269 & 0.0000 & 0.0000 & & & & \\
\hline & ${ }^{1} \mathrm{H}$ data & 62.2861 & 37.7137 & 0.0000 & 0.0001 & & & & \\
\hline uDP4+ & ${ }^{13} \mathrm{C}$ data & 66.3624 & 30.7645 & 1.6971 & 1.1760 & & & & \\
\hline & All data & 78.0825 & 21.9175 & 0.0000 & 0.0000 & & & & \\
\hline & ${ }^{1} \mathrm{H}$ data & 98.0655 & 1.9345 & 0.0000 & 0.0000 & & & & \\
\hline DP4+ & ${ }^{13} \mathrm{C}$ data & 55.4453 & 43.5126 & 0.4927 & 0.5494 & & & & \\
\hline & All data & 98.4755 & 1.5245 & 0.0000 & 0.0000 & & & & \\
\hline & & & & Level of & ry: B3LYP & & PC & & \\
\hline & & $86 a$ & $86 b$ & $86 c$ & $86 d$ & - & - & - & - \\
\hline & ${ }^{1} \mathrm{H}$ data & 99.6376 & 0.3624 & 0.0000 & 0.0000 & & & & \\
\hline sDP4+ & ${ }^{13} \mathrm{C}$ data & 37.2003 & 45.0254 & 6.3761 & 11.3982 & & & & \\
\hline & All data & 99.5617 & 0.4383 & 0.0000 & 0.0000 & & & & \\
\hline
\end{tabular}




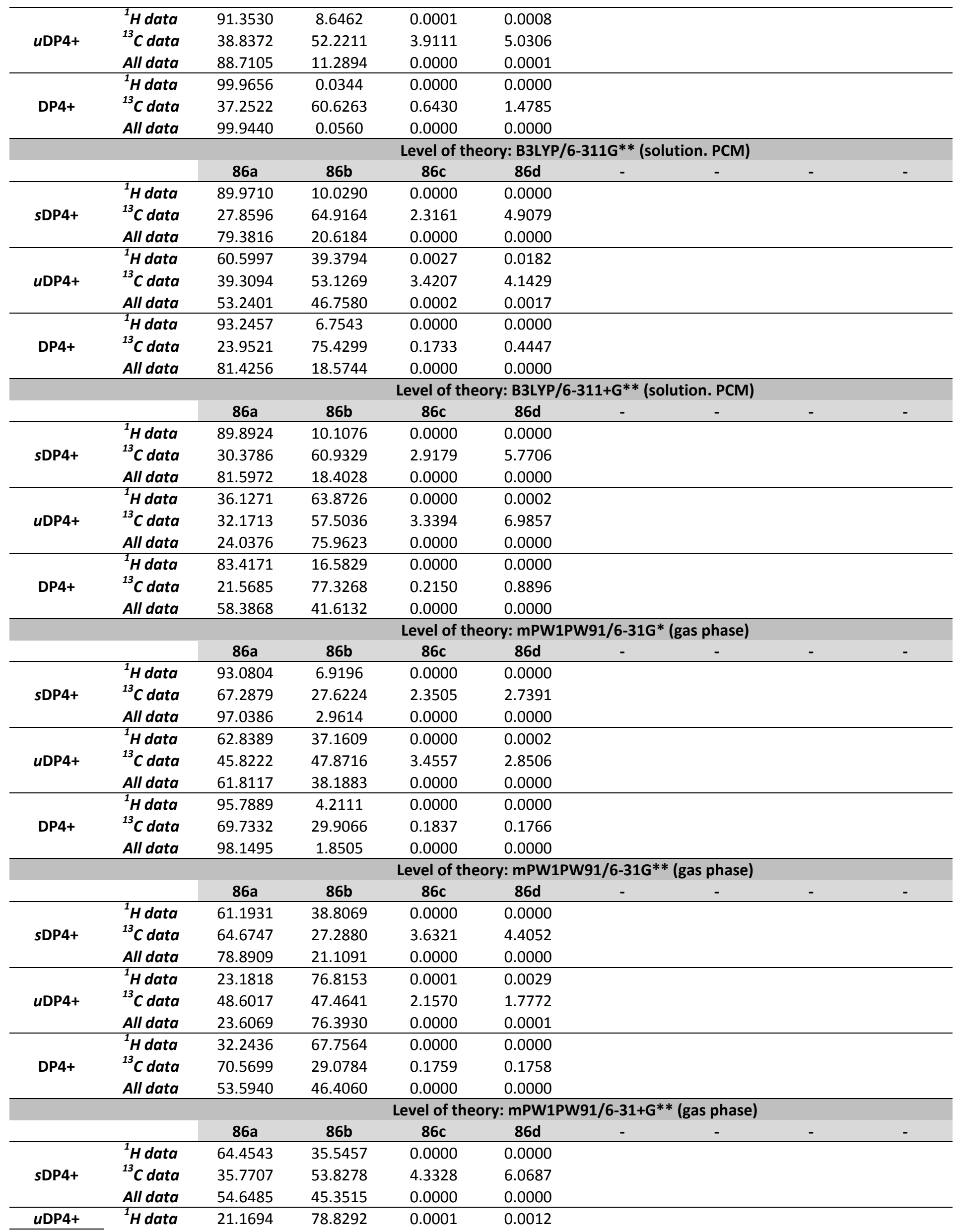




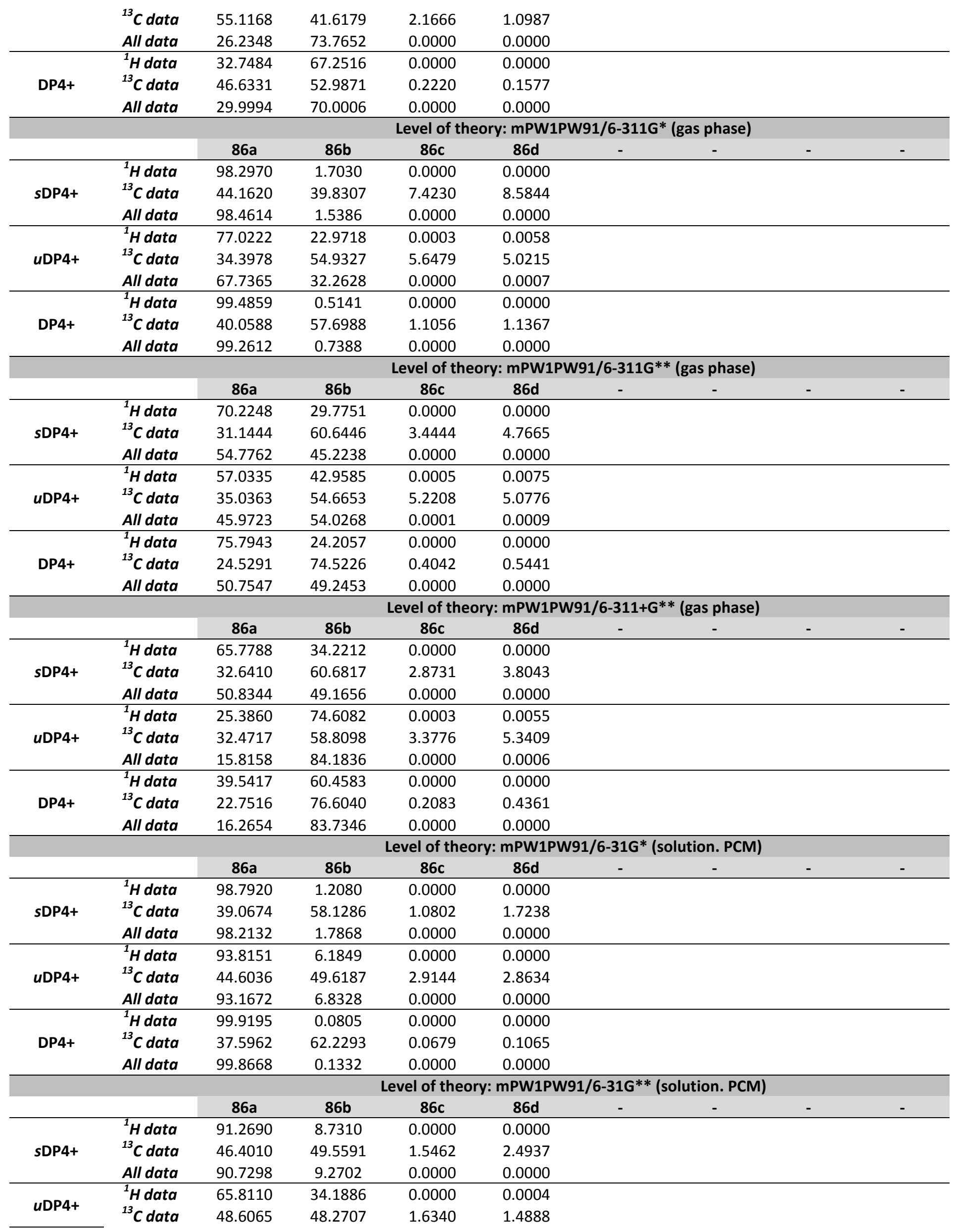




\begin{tabular}{|c|c|c|c|c|c|c|c|c|c|}
\hline & All data & 65.9671 & 34.0329 & 0.0000 & 0.0000 & & & & \\
\hline & ${ }^{1}$ H data & 95.2656 & 4.7344 & 0.0000 & 0.0000 & & & & \\
\hline \multirow[t]{5}{*}{ DP4+ } & ${ }^{13} \mathrm{C}$ data & 48.4626 & 51.4033 & 0.0543 & 0.0798 & & & & \\
\hline & All data & 94.9927 & 5.0073 & 0.0000 & 0.0000 & & & & \\
\hline & & \multicolumn{8}{|c|}{ Level of theory: mPW1PW91/6-31+G** (solution. PCM) } \\
\hline & & $86 a$ & $86 b$ & $86 c$ & $86 d$ & - & - & - & - \\
\hline & ${ }^{1} \mathrm{H}$ data & 90.9589 & 9.0411 & 0.0000 & 0.0000 & & & & \\
\hline \multirow[t]{3}{*}{ sDP4+ } & ${ }^{13} \mathrm{C}$ data & 22.8559 & 71.9952 & 1.9555 & 3.1934 & & & & \\
\hline & All data & 76.1558 & 23.8442 & 0.0000 & 0.0000 & & & & \\
\hline & ${ }^{1} \mathrm{H}$ data & 40.2552 & 59.7445 & 0.0000 & 0.0002 & & & & \\
\hline \multirow[t]{3}{*}{ uDP4+ } & ${ }^{13} \mathrm{C}$ data & 56.1087 & 40.9931 & 1.8810 & 1.0173 & & & & \\
\hline & All data & 47.9773 & 52.0227 & 0.0000 & 0.0000 & & & & \\
\hline & ${ }^{1} \mathrm{H}$ data & 87.1445 & 12.8555 & 0.0000 & 0.0000 & & & & \\
\hline \multirow[t]{5}{*}{ DP4+ } & ${ }^{13} \mathrm{C}$ data & 30.2410 & 69.5957 & 0.0867 & 0.0766 & & & & \\
\hline & All data & 74.6549 & 25.3451 & 0.0000 & 0.0000 & & & & \\
\hline & & \multicolumn{8}{|c|}{ Level of theory: mPW1PW91/6-311G* (solution. PCM) } \\
\hline & & $86 a$ & $86 b$ & $86 c$ & $86 d$ & - & - & - & - \\
\hline & ${ }^{1} \mathrm{H}$ data & 99.6602 & 0.3398 & 0.0000 & 0.0000 & & & & \\
\hline \multirow[t]{3}{*}{ sDP4+ } & ${ }^{13} \mathrm{C}$ data & 32.9841 & 57.8717 & 3.7370 & 5.4073 & & & & \\
\hline & All data & 99.4053 & 0.5947 & 0.0000 & 0.0000 & & & & \\
\hline & ${ }^{1} \mathrm{H}$ data & 95.4405 & 4.5585 & 0.0001 & 0.0009 & & & & \\
\hline \multirow[t]{3}{*}{ uDP4+ } & ${ }^{13} \mathrm{C}$ data & 39.1481 & 49.8851 & 5.3601 & 5.6067 & & & & \\
\hline & All data & 94.2629 & 5.7370 & 0.0000 & 0.0001 & & & & \\
\hline & ${ }^{1} \mathrm{H}$ data & 99.9837 & 0.0163 & 0.0000 & 0.0000 & & & & \\
\hline \multirow[t]{5}{*}{ DP4+ } & ${ }^{13} \mathrm{C}$ data & 30.5368 & 68.2725 & 0.4737 & 0.7170 & & & & \\
\hline & All data & 99.9636 & 0.0364 & 0.0000 & 0.0000 & & & & \\
\hline & & \multicolumn{8}{|c|}{ Level of theory: mPW1PW91/6-311G** (solution. PCM) } \\
\hline & & $86 a$ & $86 b$ & $86 c$ & 86d & - & - & - & - \\
\hline & ${ }^{1} \mathrm{H}$ data & 84.8883 & 15.1116 & 0.0000 & 0.0000 & & & & \\
\hline \multirow[t]{3}{*}{ sDP4+ } & ${ }^{13} \mathrm{C}$ data & 26.2458 & 68.2469 & 2.0127 & 3.4946 & & & & \\
\hline & All data & 68.3574 & 31.6426 & 0.0000 & 0.0000 & & & & \\
\hline & ${ }^{1} \mathrm{H}$ data & 67.1238 & 32.8292 & 0.0068 & 0.0402 & & & & \\
\hline \multirow[t]{3}{*}{ uDP4+ } & ${ }^{13} \mathrm{C}$ data & 39.0622 & 49.0646 & 5.8239 & 6.0493 & & & & \\
\hline & All data & 61.9414 & 38.0519 & 0.0009 & 0.0057 & & & & \\
\hline & ${ }^{1}$ H data & 91.9908 & 8.0092 & 0.0000 & 0.0000 & & & & \\
\hline \multirow[t]{5}{*}{ DP4+ } & ${ }^{13} \mathrm{C}$ data & 23.2656 & 75.9887 & 0.2660 & 0.4797 & & & & \\
\hline & All data & 77.8593 & 22.1407 & 0.0000 & 0.0000 & & & & \\
\hline & & \multicolumn{8}{|c|}{ Level of theory: mPW1PW91/6-311+G** (solution. PCM) } \\
\hline & & $86 a$ & $86 b$ & $86 c$ & 86d & - & - & - & - \\
\hline & ${ }^{1} \mathrm{H}$ data & 80.3584 & 19.6416 & 0.0000 & 0.0000 & & & & \\
\hline \multirow[t]{3}{*}{ sDP4+ } & ${ }^{13} \mathrm{C}$ data & 23.9856 & 71.5709 & 1.7119 & 2.7316 & & & & \\
\hline & All data & 57.8255 & 42.1745 & 0.0000 & 0.0000 & & & & \\
\hline & ${ }^{1} \mathrm{H}$ data & 26.2490 & 73.7217 & 0.0041 & 0.0251 & & & & \\
\hline \multirow[t]{3}{*}{ uDP4+ } & ${ }^{13} \mathrm{C}$ data & 35.1249 & 55.2107 & 3.6760 & 5.9885 & & & & \\
\hline & All data & 18.4680 & 81.5287 & 0.0003 & 0.0030 & & & & \\
\hline & ${ }^{1} \mathrm{H}$ data & 59.2951 & 40.7049 & 0.0000 & 0.0000 & & & & \\
\hline \multirow[t]{2}{*}{ DP4+ } & ${ }^{13} \mathrm{C}$ data & 17.4914 & 82.0384 & 0.1306 & 0.3396 & & & & \\
\hline & All data & 23.6981 & 76.3019 & 0.0000 & 0.0000 & & & & \\
\hline
\end{tabular}


$s \mathrm{DP4}+, u \mathrm{DP} 4+$ and DP4+ probabilities (\%) for compound 86b<smiles>CC(C)(C)O[C@H](OC(=O)[C@@H](O)c1ccccc1)C1CC2C=CC1C2</smiles><smiles>O=C(O[C@H]1C[C@H]2C=C[C@@H]1C2)[C@@H](O)c1ccccc1</smiles><smiles>O=C(O[C@@H]1C[C@H]2C=C[C@@H]1C2)[C@@H](O)c1ccccc1</smiles><smiles>CC(C)(C)OC(=O)C1CC2C=CC1C2</smiles>
correct isomer

\begin{tabular}{|c|c|c|c|c|c|c|c|c|c|}
\hline & \multicolumn{8}{|c|}{ Level of theory: B3LYP/6-31G* (gas phase) } \\
\hline & & $86 a$ & $86 b$ & $86 c$ & 86d & - & - & - & - \\
\hline & ${ }^{1} \mathrm{H}$ data & 1.1260 & 98.8740 & 0.0000 & 0.0000 & & & & \\
\hline \multirow[t]{2}{*}{$s \mathrm{DP} 4+$} & ${ }^{13} \mathrm{C}$ data & 57.4858 & 33.0869 & 4.1433 & 5.2841 & & & & \\
\hline & All data & 1.9403 & 98.0597 & 0.0000 & 0.0000 & & & & \\
\hline \multirow{3}{*}{ uDP4+ } & ${ }^{1} \mathrm{H}$ data & 0.7592 & 99.2408 & 0.0000 & 0.0000 & & & & \\
\hline & ${ }^{13} \mathrm{C}$ data & 52.7376 & 41.6803 & 3.0794 & 2.5026 & & & & \\
\hline & All data & 0.9586 & 99.0414 & 0.0000 & 0.0000 & & & & \\
\hline \multirow{5}{*}{ DP4+ } & ${ }^{1} \mathrm{H}$ data & 0.0087 & 99.9913 & 0.0000 & 0.0000 & & & & \\
\hline & ${ }^{13} \mathrm{C}$ data & 68.3313 & 31.0831 & 0.2876 & 0.2981 & & & & \\
\hline & All data & 0.0191 & 99.9809 & 0.0000 & 0.0000 & & & & \\
\hline & & \multicolumn{8}{|c|}{ Level of theory: B3LYP/6-31G** (gas phase) } \\
\hline & & $86 a$ & $86 b$ & $86 c$ & 86d & - & - & - & - \\
\hline \multirow{3}{*}{ sDP4+ } & ${ }^{1} \mathrm{H}$ data & 1.8864 & 98.1136 & 0.0000 & 0.0000 & & & & \\
\hline & ${ }^{13} \mathrm{C}$ data & 53.2045 & 31.8013 & 6.5880 & 8.4063 & & & & \\
\hline & All data & 3.1165 & 96.8835 & 0.0000 & 0.0000 & & & & \\
\hline \multirow{3}{*}{ uDP4+ } & ${ }^{1} \mathrm{H}$ data & 0.4933 & 99.5066 & 0.0000 & 0.0001 & & & & \\
\hline & ${ }^{13} \mathrm{C}$ data & 51.9787 & 43.6744 & 2.3887 & 1.9581 & & & & \\
\hline & All data & 0.5865 & 99.4135 & 0.0000 & 0.0000 & & & & \\
\hline \multirow{5}{*}{ DP4+ } & ${ }^{1} \mathrm{H}$ data & 0.0095 & 99.9905 & 0.0000 & 0.0000 & & & & \\
\hline & ${ }^{13} \mathrm{C}$ data & 66.0560 & 33.1749 & 0.3759 & 0.3932 & & & & \\
\hline & All data & 0.0190 & 99.9810 & 0.0000 & 0.0000 & & & & \\
\hline & & \multicolumn{8}{|c|}{ Level of theory: B3LYP/6-31+G** (gas phase) } \\
\hline & & $86 a$ & $86 \mathrm{~b}$ & $86 c$ & $86 d$ & - & - & - & - \\
\hline \multirow{3}{*}{$s \mathrm{DP} 4+$} & ${ }^{1} \mathrm{H}$ data & 2.8444 & 97.1556 & 0.0000 & 0.0000 & & & & \\
\hline & ${ }^{13} \mathrm{C}$ data & 30.6199 & 47.0998 & 8.2088 & 14.0715 & & & & \\
\hline & All data & 1.8678 & 98.1322 & 0.0000 & 0.0000 & & & & \\
\hline \multirow{3}{*}{ uDP4+ } & ${ }^{1} \mathrm{H}$ data & 0.4998 & 99.5002 & 0.0000 & 0.0000 & & & & \\
\hline & ${ }^{13} \mathrm{C}$ data & 65.7140 & 32.0052 & 1.4223 & 0.8585 & & & & \\
\hline & All data & 1.0208 & 98.9792 & 0.0000 & 0.0000 & & & & \\
\hline \multirow{3}{*}{ DP4+ } & ${ }^{1} \mathrm{H}$ data & 0.0147 & 99.9853 & 0.0000 & 0.0000 & & & & \\
\hline & ${ }^{13} \mathrm{C}$ data & 56.7868 & 42.5428 & 0.3295 & 0.3409 & & & & \\
\hline & All data & 0.0196 & 99.9804 & 0.0000 & 0.0000 & & & & \\
\hline & & \multicolumn{8}{|c|}{ Level of theory: B3LYP/6-311G* (gas phase) } \\
\hline & & $86 a$ & $86 b$ & $86 c$ & $86 d$ & - & - & - & - \\
\hline \multirow{3}{*}{$s \mathrm{DP} 4+$} & ${ }^{1} \mathrm{H}$ data & 4.5845 & 95.4155 & 0.0000 & 0.0000 & & & & \\
\hline & ${ }^{13} \mathrm{C}$ data & 47.7172 & 33.1181 & 7.8962 & 11.2685 & & & & \\
\hline & All data & 6.4745 & 93.5255 & 0.0000 & 0.0000 & & & & \\
\hline \multirow{3}{*}{ uDP4+ } & ${ }^{1} \mathrm{H}$ data & 0.5666 & 99.4333 & 0.0000 & 0.0001 & & & & \\
\hline & ${ }^{13} \mathrm{C}$ data & 37.5226 & 55.5568 & 3.4321 & 3.4885 & & & & \\
\hline & All data & 0.3834 & 99.6166 & 0.0000 & 0.0000 & & & & \\
\hline \multirow{3}{*}{ DP4+ } & ${ }^{1} \mathrm{H}$ data & 0.0274 & 99.9726 & 0.0000 & 0.0000 & & & & \\
\hline & ${ }^{13} \mathrm{C}$ data & 48.4329 & 49.7707 & 0.7331 & 1.0633 & & & & \\
\hline & All data & 0.0266 & 99.9734 & 0.0000 & 0.0000 & & & & \\
\hline & & \multicolumn{8}{|c|}{ Level of theory: B3LYP/6-311G** (gas phase) } \\
\hline & & $86 a$ & $86 b$ & $86 c$ & $86 d$ & - & - & - & - \\
\hline \multirow{2}{*}{ sDP4+ } & ${ }^{1} \mathrm{H}$ data & 2.4852 & 97.5148 & 0.0000 & 0.0000 & & & & \\
\hline & ${ }^{13} \mathrm{C}$ data & 38.5527 & 51.6401 & 3.7920 & 6.0152 & & & & \\
\hline
\end{tabular}




\begin{tabular}{|c|c|c|c|c|c|c|c|c|c|}
\hline & All data & 1.8671 & 98.1329 & 0.0000 & 0.0000 & & & & \\
\hline & ${ }^{1} \mathrm{H}$ data & 0.4868 & 99.5131 & 0.0000 & 0.0001 & & & & \\
\hline \multirow[t]{3}{*}{ uDP4+ } & ${ }^{13}$ C data & 37.6372 & 56.3969 & 2.9877 & 2.9781 & & & & \\
\hline & All data & 0.3254 & 99.6746 & 0.0000 & 0.0000 & & & & \\
\hline & ${ }^{1} \mathrm{H}$ data & 0.0125 & 99.9875 & 0.0000 & 0.0000 & & & & \\
\hline \multirow[t]{5}{*}{ DP4+ } & ${ }^{13} \mathrm{C}$ data & 33.0332 & 66.3011 & 0.2579 & 0.4078 & & & & \\
\hline & All data & 0.0062 & 99.9938 & 0.0000 & 0.0000 & & & & \\
\hline & & \multicolumn{8}{|c|}{ Level of theory: B3LYP/6-311+G** (gas phase) } \\
\hline & & $86 a$ & $86 b$ & $86 c$ & 86d & - & - & - & - \\
\hline & ${ }^{1} \mathrm{H}$ data & 2.0331 & 97.9669 & 0.0000 & 0.0000 & & & & \\
\hline \multirow[t]{3}{*}{ sDP4+ } & ${ }^{13} \mathrm{C}$ data & 39.5698 & 52.0717 & 3.0743 & 5.2842 & & & & \\
\hline & All data & 1.5525 & 98.4475 & 0.0000 & 0.0000 & & & & \\
\hline & ${ }^{1} \mathrm{H}$ data & 0.5913 & 99.4086 & 0.0000 & 0.0001 & & & & \\
\hline \multirow[t]{3}{*}{ uDP4+ } & ${ }^{13} \mathrm{C}$ data & 34.8299 & 60.2615 & 1.6909 & 3.2177 & & & & \\
\hline & All data & 0.3426 & 99.6574 & 0.0000 & 0.0000 & & & & \\
\hline & ${ }^{1} \mathrm{H}$ data & 0.0123 & 99.9877 & 0.0000 & 0.0000 & & & & \\
\hline \multirow[t]{5}{*}{ DP4+ } & ${ }^{13} \mathrm{C}$ data & 30.3683 & 69.1426 & 0.1145 & 0.3747 & & & & \\
\hline & All data & 0.0054 & 99.9946 & 0.0000 & 0.0000 & & & & \\
\hline & & \multicolumn{8}{|c|}{ Level of theory: B3LYP/6-31G* (solution. PCM) } \\
\hline & & $86 a$ & $86 b$ & $86 c$ & $86 d$ & - & - & - & - \\
\hline & ${ }^{1}$ H data & 2.2880 & 97.7120 & 0.0000 & 0.0000 & & & & \\
\hline \multirow[t]{3}{*}{ sDP4+ } & ${ }^{13}$ C data & 42.9557 & 52.3285 & 1.8060 & 2.9098 & & & & \\
\hline & All data & 1.8859 & 98.1141 & 0.0000 & 0.0000 & & & & \\
\hline & ${ }^{1} \mathrm{H}$ data & 3.9133 & 96.0867 & 0.0000 & 0.0000 & & & & \\
\hline \multirow[t]{3}{*}{ uDP4+ } & ${ }^{13} \mathrm{C}$ data & 52.3382 & 43.9418 & 1.9710 & 1.7490 & & & & \\
\hline & All data & 4.6265 & 95.3735 & 0.0000 & 0.0000 & & & & \\
\hline & ${ }^{1} \mathrm{H}$ data & 0.0953 & 99.9047 & 0.0000 & 0.0000 & & & & \\
\hline \multirow[t]{5}{*}{ DP4+ } & ${ }^{13} \mathrm{C}$ data & 49.3434 & 50.4668 & 0.0781 & 0.1117 & & & & \\
\hline & All data & 0.0932 & 99.9068 & 0.0000 & 0.0000 & & & & \\
\hline & & \multicolumn{8}{|c|}{ Level of theory: B3LYP/6-31G** (solution. PCM) } \\
\hline & & $86 a$ & $86 b$ & $86 c$ & $86 d$ & - & - & - & - \\
\hline & ${ }^{1}$ H data & 5.4487 & 94.5513 & 0.0000 & 0.0000 & & & & \\
\hline \multirow[t]{3}{*}{ sDP4+ } & ${ }^{13} \mathrm{C}$ data & 46.1114 & 45.3816 & 3.2347 & 5.2723 & & & & \\
\hline & All data & 5.5315 & 94.4685 & 0.0000 & 0.0000 & & & & \\
\hline & ${ }^{1} \mathrm{H}$ data & 2.2139 & 97.7860 & 0.0000 & 0.0000 & & & & \\
\hline \multirow[t]{3}{*}{ uDP4+ } & ${ }^{13}$ C data & 54.0540 & 42.6642 & 1.7316 & 1.5502 & & & & \\
\hline & All data & 2.7885 & 97.2115 & 0.0000 & 0.0000 & & & & \\
\hline & ${ }^{1} \mathrm{H}$ data & 0.1303 & 99.8697 & 0.0000 & 0.0000 & & & & \\
\hline \multirow[t]{5}{*}{ DP4+ } & ${ }^{13}$ C data & 56.1065 & 43.5834 & 0.1261 & 0.1840 & & & & \\
\hline & All data & 0.1677 & 99.8323 & 0.0000 & 0.0000 & & & & \\
\hline & & \multicolumn{8}{|c|}{ Level of theory: B3LYP/6-31+G** (solution. PCM) } \\
\hline & & $86 a$ & $86 b$ & $86 c$ & $86 d$ & - & - & - & - \\
\hline & ${ }^{1} \mathrm{H}$ data & 9.0624 & 90.9376 & 0.0000 & 0.0000 & & & & \\
\hline \multirow[t]{3}{*}{ sDP4+ } & ${ }^{13} \mathrm{C}$ data & 31.5470 & 51.5142 & 6.4247 & 10.5142 & & & & \\
\hline & All data & 5.7518 & 94.2482 & 0.0000 & 0.0000 & & & & \\
\hline & ${ }^{1} \mathrm{H}$ data & 0.7703 & 99.2297 & 0.0000 & 0.0000 & & & & \\
\hline uDP4+ & ${ }^{13} \mathrm{C}$ data & 68.7007 & 28.6686 & 1.6121 & 1.0185 & & & & \\
\hline & All data & 1.8262 & 98.1738 & 0.0000 & 0.0000 & & & & \\
\hline & ${ }^{1} \mathrm{H}$ data & 0.0773 & 99.9227 & 0.0000 & 0.0000 & & & & \\
\hline DP4+ & ${ }^{13} \mathrm{C}$ data & 59.1317 & 40.2935 & 0.2826 & 0.2922 & & & & \\
\hline & All data & 0.1134 & 99.8866 & 0.0000 & 0.0000 & & & & \\
\hline & & & & Level of & ry: B3LYP & C* & PC & & \\
\hline & & $86 a$ & $86 b$ & $86 c$ & $86 d$ & - & - & - & - \\
\hline & ${ }^{1} \mathrm{H}$ data & 25.0093 & 74.9907 & 0.0000 & 0.0000 & & & & \\
\hline sDP4+ & ${ }^{13} \mathrm{C}$ data & 34.1285 & 53.8985 & 4.1948 & 7.7782 & & & & \\
\hline & All data & 17.4353 & 82.5647 & 0.0000 & 0.0000 & & & & \\
\hline
\end{tabular}




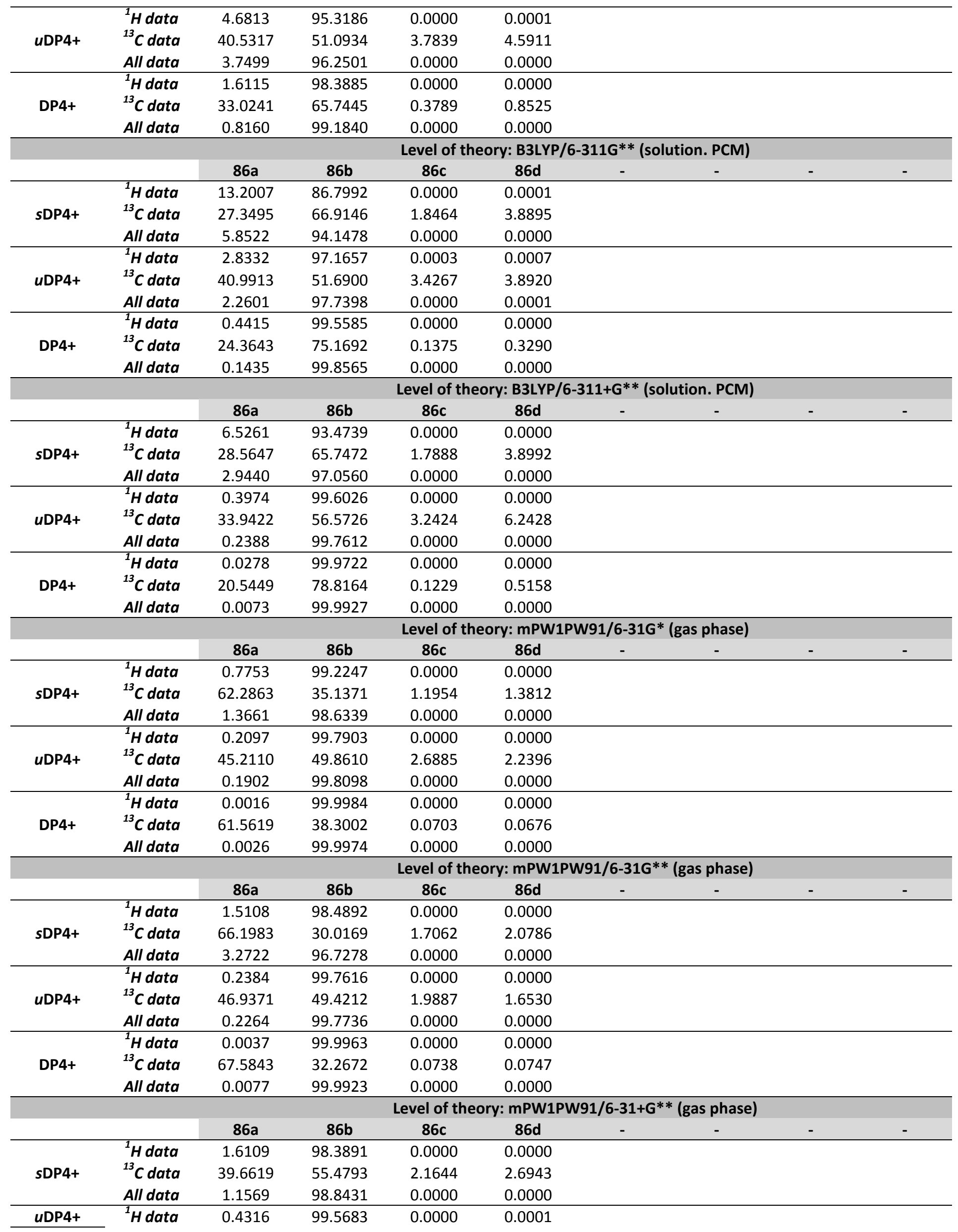




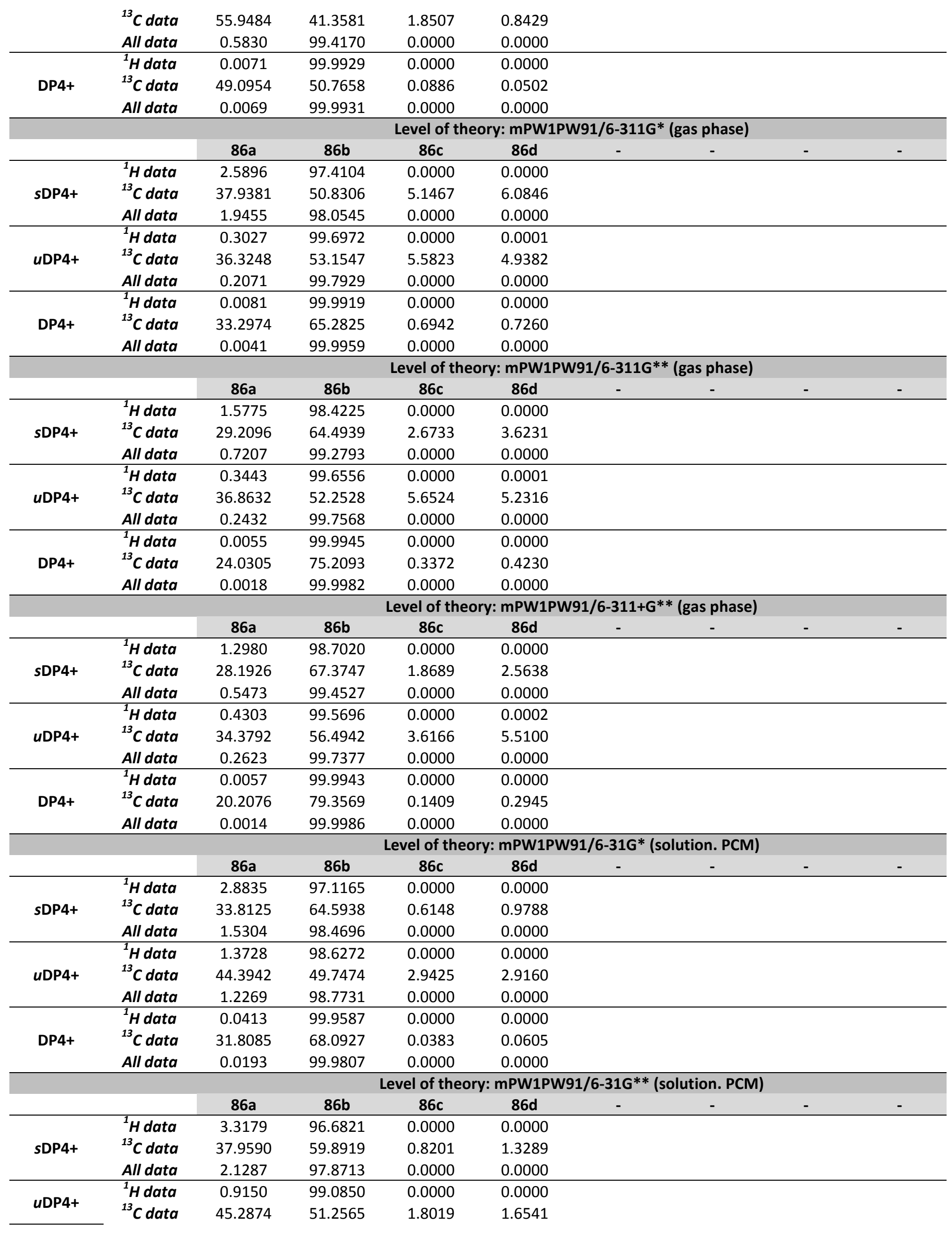




\begin{tabular}{|c|c|c|c|c|c|c|c|c|c|}
\hline & All data & 0.8093 & 99.1907 & 0.0000 & 0.0000 & & & & \\
\hline & ${ }^{1} \mathrm{H}$ data & 0.0317 & 99.9683 & 0.0000 & 0.0000 & & & & \\
\hline \multirow[t]{5}{*}{ DP4+ } & ${ }^{13} \mathrm{C}$ data & 35.8692 & 64.0541 & 0.0308 & 0.0459 & & & & \\
\hline & All data & 0.0177 & 99.9823 & 0.0000 & 0.0000 & & & & \\
\hline & & \multicolumn{8}{|c|}{ Level of theory: mPW1PW91/6-31+G** (solution. PCM) } \\
\hline & & $86 a$ & $86 b$ & $86 \mathrm{c}$ & $86 d$ & - & - & - & - \\
\hline & ${ }^{1} \mathrm{H}$ data & 7.1720 & 92.8280 & 0.0000 & 0.0000 & & & & \\
\hline \multirow[t]{3}{*}{ sDP4+ } & ${ }^{13} \mathrm{C}$ data & 24.4943 & 73.1145 & 0.8945 & 1.4967 & & & & \\
\hline & All data & 2.5230 & 97.4770 & 0.0000 & 0.0000 & & & & \\
\hline & ${ }^{1} \mathrm{H}$ data & 0.4122 & 99.5878 & 0.0000 & 0.0000 & & & & \\
\hline \multirow[t]{3}{*}{ uDP4+ } & ${ }^{13} \mathrm{C}$ data & 58.7347 & 38.3813 & 1.9400 & 0.9439 & & & & \\
\hline & All data & 0.6294 & 99.3706 & 0.0000 & 0.0000 & & & & \\
\hline & ${ }^{1} \mathrm{H}$ data & 0.0320 & 99.9680 & 0.0000 & 0.0000 & & & & \\
\hline \multirow[t]{5}{*}{ DP4+ } & ${ }^{13} \mathrm{C}$ data & 33.8666 & 66.0593 & 0.0408 & 0.0333 & & & & \\
\hline & All data & 0.0164 & 99.9836 & 0.0000 & 0.0000 & & & & \\
\hline & & \multicolumn{8}{|c|}{ Level of theory: mPW1PW91/6-311G* (solution. PCM) } \\
\hline & & $86 a$ & $86 b$ & $86 c$ & $86 d$ & - & - & - & - \\
\hline & ${ }^{1}$ H data & 15.6896 & 84.3104 & 0.0000 & 0.0000 & & & & \\
\hline \multirow[t]{2}{*}{ sDP4+ } & ${ }^{13} \mathrm{C}$ data & 31.2235 & 61.7331 & 2.7801 & 4.2633 & & & & \\
\hline & All data & 8.6026 & 91.3974 & 0.0000 & 0.0000 & & & & \\
\hline \multirow{3}{*}{ uDP4+ } & ${ }^{1} \mathrm{H}$ data & 2.5923 & 97.4077 & 0.0000 & 0.0001 & & & & \\
\hline & ${ }^{13} \mathrm{C}$ data & 41.3247 & 48.3765 & 5.0275 & 5.2713 & & & & \\
\hline & All data & 2.2228 & 97.7772 & 0.0000 & 0.0000 & & & & \\
\hline \multirow{3}{*}{ DP4+ } & ${ }^{1} \mathrm{H}$ data & 0.4928 & 99.5072 & 0.0000 & 0.0000 & & & & \\
\hline & ${ }^{13} \mathrm{C}$ data & 29.9153 & 69.2396 & 0.3240 & 0.5210 & & & & \\
\hline & All data & 0.2135 & 99.7865 & 0.0000 & 0.0000 & & & & \\
\hline & & \multicolumn{8}{|c|}{ Level of theory: mPW1PW91/6-311G** (solution. PCM) } \\
\hline & & $86 a$ & $86 b$ & $86 \mathrm{c}$ & $86 d$ & - & - & - & - \\
\hline \multirow{3}{*}{$s D P 4+$} & ${ }^{1} \mathrm{H}$ data & 9.2792 & 90.7206 & 0.0001 & 0.0002 & & & & \\
\hline & ${ }^{13} \mathrm{C}$ data & 27.0967 & 68.2610 & 1.6894 & 2.9529 & & & & \\
\hline & All data & 3.9018 & 96.0982 & 0.0000 & 0.0000 & & & & \\
\hline \multirow{3}{*}{ uDP4+ } & ${ }^{1} \mathrm{H}$ data & 1.1531 & 98.8462 & 0.0002 & 0.0005 & & & & \\
\hline & ${ }^{13} \mathrm{C}$ data & 41.2702 & 47.1326 & 5.6939 & 5.9034 & & & & \\
\hline & All data & 1.0112 & 98.9888 & 0.0000 & 0.0001 & & & & \\
\hline \multirow{5}{*}{ DP4+ } & ${ }^{1} \mathrm{H}$ data & 0.1192 & 99.8808 & 0.0000 & 0.0000 & & & & \\
\hline & ${ }^{13} \mathrm{C}$ data & 25.6331 & 73.7468 & 0.2205 & 0.3996 & & & & \\
\hline & All data & 0.0415 & 99.9585 & 0.0000 & 0.0000 & & & & \\
\hline & & \multicolumn{8}{|c|}{ Level of theory: mPW1PW91/6-311+G** (solution. PCM) } \\
\hline & & $86 a$ & $86 b$ & $86 c$ & $86 d$ & - & - & - & - \\
\hline \multirow{3}{*}{ sDP4+ } & ${ }^{1} \mathrm{H}$ data & 4.6697 & 95.3302 & 0.0000 & 0.0001 & & & & \\
\hline & ${ }^{13} \mathrm{C}$ data & 25.3342 & 70.9730 & 1.3655 & 2.3273 & & & & \\
\hline & All data & 1.7185 & 98.2815 & 0.0000 & 0.0000 & & & & \\
\hline \multirow{3}{*}{ uDP4+ } & ${ }^{1} \mathrm{H}$ data & 0.4940 & 99.5051 & 0.0002 & 0.0007 & & & & \\
\hline & ${ }^{13} \mathrm{C}$ data & 37.4239 & 54.0880 & 3.2064 & 5.2816 & & & & \\
\hline & All data & 0.3424 & 99.6576 & 0.0000 & 0.0001 & & & & \\
\hline \multirow{3}{*}{ DP4+ } & ${ }^{1} \mathrm{H}$ data & 0.0243 & 99.9757 & 0.0000 & 0.0000 & & & & \\
\hline & ${ }^{13} \mathrm{C}$ data & 19.7375 & 79.9155 & 0.0911 & 0.2559 & & & & \\
\hline & All data & 0.0060 & 99.9940 & 0.0000 & 0.0000 & & & & \\
\hline
\end{tabular}


$s \mathrm{DP} 4+, u \mathrm{DP} 4+$ and DP4+ probabilities (\%) for compound 86c<smiles>O=C(O[C@H]1C[C@H]2C=C[C@H]1C2)[C@@H](O)c1ccccc1</smiles>

$86 a$<smiles>O=C(O[14C]1C[C@H]2C=C[C@@H]1C2)[C@@H](O)c1ccccc1</smiles><smiles>CC(C)(C)[O+]=O</smiles>

correct isomer<smiles>CC(C)(C)OC(=O)C1CC2C=CC1C2</smiles>
86d

\begin{tabular}{|c|c|c|c|c|c|c|c|c|c|}
\hline & \multicolumn{8}{|c|}{ Level of theory: B3LYP/6-31G* (gas phase) } \\
\hline & & $86 a$ & $86 b$ & $86 c$ & 86d & - & - & - & - \\
\hline \multirow{3}{*}{ sDP4+ } & ${ }^{1} \mathrm{H}$ data & 0.0001 & 0.0000 & 99.4435 & 0.5564 & & & & \\
\hline & ${ }^{13} \mathrm{C}$ data & 0.8073 & 0.4814 & 60.6225 & 38.0888 & & & & \\
\hline & All data & 0.0000 & 0.0000 & 99.6497 & 0.3503 & & & & \\
\hline \multirow{3}{*}{ uDP4+ } & ${ }^{1} \mathrm{H}$ data & 0.0000 & 0.0062 & 94.7333 & 5.2604 & & & & \\
\hline & ${ }^{13} \mathrm{C}$ data & 15.6768 & 7.7278 & 40.1906 & 36.4048 & & & & \\
\hline & All data & 0.0000 & 0.0012 & 95.2099 & 4.7889 & & & & \\
\hline \multirow{5}{*}{ DP4+ } & ${ }^{1} \mathrm{H}$ data & 0.0000 & 0.0000 & 99.9689 & 0.0311 & & & & \\
\hline & ${ }^{13} \mathrm{C}$ data & 0.3296 & 0.0969 & 63.4585 & 36.1150 & & & & \\
\hline & All data & 0.0000 & 0.0000 & 99.9823 & 0.0177 & & & & \\
\hline & & \multicolumn{8}{|c|}{ Level of theory: B3LYP/6-31G** (gas phase) } \\
\hline & & $86 a$ & $86 b$ & $86 c$ & $86 d$ & - & - & - & - \\
\hline \multirow{3}{*}{ sDP4+ } & ${ }^{1} \mathrm{H}$ data & 0.0003 & 0.0000 & 98.8656 & 1.1342 & & & & \\
\hline & ${ }^{13} \mathrm{C}$ data & 2.1310 & 1.1614 & 53.3439 & 43.3637 & & & & \\
\hline & All data & 0.0000 & 0.0000 & 99.0761 & 0.9239 & & & & \\
\hline \multirow{3}{*}{ uDP4+ } & ${ }^{1} \mathrm{H}$ data & 0.0000 & 0.0094 & 92.1542 & 7.8364 & & & & \\
\hline & ${ }^{13} \mathrm{C}$ data & 19.5851 & 10.1258 & 37.0937 & 33.1955 & & & & \\
\hline & All data & 0.0000 & 0.0026 & 92.9258 & 7.0716 & & & & \\
\hline \multirow{5}{*}{ DP4+ } & ${ }^{1} \mathrm{H}$ data & 0.0000 & 0.0000 & 99.9025 & 0.0975 & & & & \\
\hline & ${ }^{13} \mathrm{C}$ data & 1.2022 & 0.3387 & 56.9958 & 41.4633 & & & & \\
\hline & All data & 0.0000 & 0.0000 & 99.9291 & 0.0709 & & & & \\
\hline & & \multicolumn{8}{|c|}{ Level of theory: B3LYP/6-31+G** (gas phase) } \\
\hline & & $86 a$ & $86 b$ & $86 c$ & $86 d$ & - & - & - & - \\
\hline \multirow{3}{*}{ sDP4+ } & ${ }^{1} \mathrm{H}$ data & 0.0015 & 0.0000 & 98.4819 & 1.5166 & & & & \\
\hline & ${ }^{13} \mathrm{C}$ data & 0.5705 & 1.6733 & 38.5058 & 59.2504 & & & & \\
\hline & All data & 0.0000 & 0.0000 & 97.6852 & 2.3147 & & & & \\
\hline \multirow{3}{*}{ uDP4+ } & ${ }^{1} \mathrm{H}$ data & 0.0000 & 0.0006 & 93.9491 & 6.0503 & & & & \\
\hline & ${ }^{13} \mathrm{C}$ data & 9.7083 & 8.7692 & 45.7426 & 35.7800 & & & & \\
\hline & All data & 0.0000 & 0.0001 & 95.2041 & 4.7958 & & & & \\
\hline \multirow{5}{*}{ DP4+ } & ${ }^{1} \mathrm{H}$ data & 0.0000 & 0.0000 & 99.9009 & 0.0991 & & & & \\
\hline & ${ }^{13} \mathrm{C}$ data & 0.1420 & 0.3761 & 45.1451 & 54.3369 & & & & \\
\hline & All data & 0.0000 & 0.0000 & 99.8808 & 0.1192 & & & & \\
\hline & & \multicolumn{8}{|c|}{ Level of theory: B3LYP/6-311G* (gas phase) } \\
\hline & & $86 a$ & $86 b$ & $86 c$ & $86 d$ & - & - & - & - \\
\hline \multirow{3}{*}{ sDP4+ } & ${ }^{1} \mathrm{H}$ data & 0.0148 & 0.0001 & 97.2281 & 2.7570 & & & & \\
\hline & ${ }^{13} \mathrm{C}$ data & 0.7700 & 0.4026 & 52.5199 & 46.3076 & & & & \\
\hline & All data & 0.0002 & 0.0000 & 97.5606 & 2.4392 & & & & \\
\hline \multirow{3}{*}{ uDP4+ } & ${ }^{1} \mathrm{H}$ data & 0.0003 & 0.0271 & 84.4034 & 15.5693 & & & & \\
\hline & ${ }^{13} \mathrm{C}$ data & 24.9713 & 19.7259 & 25.2760 & 30.0268 & & & & \\
\hline & All data & 0.0002 & 0.0205 & 82.0084 & 17.9708 & & & & \\
\hline \multirow{5}{*}{ DP4+ } & ${ }^{1} \mathrm{H}$ data & 0.0000 & 0.0000 & 99.4797 & 0.5203 & & & & \\
\hline & ${ }^{13} \mathrm{C}$ data & 0.7004 & 0.2893 & 48.3581 & 50.6522 & & & & \\
\hline & All data & 0.0000 & 0.0000 & 99.4551 & 0.5449 & & & & \\
\hline & & \multicolumn{8}{|c|}{ Level of theory: B3LYP/6-311G** (gas phase) } \\
\hline & & $86 a$ & $86 b$ & $86 c$ & $86 d$ & - & - & - & - \\
\hline \multirow{2}{*}{ sDP4+ } & ${ }^{1} \mathrm{H}$ data & 0.0021 & 0.0000 & 96.2750 & 3.7229 & & & & \\
\hline & ${ }^{13} \mathrm{C}$ data & 1.1344 & 0.5888 & 43.9269 & 54.3500 & & & & \\
\hline
\end{tabular}




\begin{tabular}{|c|c|c|c|c|c|c|c|c|c|}
\hline & All data & 0.0001 & 0.0000 & 95.4339 & 4.5660 & & & & \\
\hline & ${ }^{1} \mathrm{H}$ data & 0.0000 & 0.0028 & 90.0530 & 9.9442 & & & & \\
\hline \multirow[t]{3}{*}{ uDP4+ } & ${ }^{13} \mathrm{C}$ data & 33.5703 & 28.1603 & 17.2254 & 21.0441 & & & & \\
\hline & All data & 0.0000 & 0.0045 & 88.1090 & 11.8864 & & & & \\
\hline & ${ }^{1} \mathrm{H}$ data & 0.0000 & 0.0000 & 99.5748 & 0.4252 & & & & \\
\hline \multirow[t]{5}{*}{ DP4+ } & ${ }^{13} \mathrm{C}$ data & 1.9478 & 0.8481 & 38.7024 & 58.5017 & & & & \\
\hline & All data & 0.0000 & 0.0000 & 99.3587 & 0.6413 & & & & \\
\hline & & \multicolumn{8}{|c|}{ Level of theory: B3LYP/6-311+G** (gas phase) } \\
\hline & & $86 a$ & $86 b$ & $86 c$ & 86d & - & - & - & - \\
\hline & ${ }^{1} \mathrm{H}$ data & 0.0030 & 0.0001 & 96.9006 & 3.0963 & & & & \\
\hline \multirow[t]{3}{*}{ sDP4+ } & ${ }^{13} \mathrm{C}$ data & 0.5570 & 0.4355 & 44.3527 & 54.6547 & & & & \\
\hline & All data & 0.0000 & 0.0000 & 96.2116 & 3.7883 & & & & \\
\hline & ${ }^{1} \mathrm{H}$ data & 0.0000 & 0.0027 & 91.6841 & 8.3131 & & & & \\
\hline \multirow[t]{3}{*}{ uDP4+ } & ${ }^{13} \mathrm{C}$ data & 28.3709 & 26.9190 & 17.8989 & 26.8113 & & & & \\
\hline & All data & 0.0000 & 0.0039 & 88.0387 & 11.9574 & & & & \\
\hline & ${ }^{1} \mathrm{H}$ data & 0.0000 & 0.0000 & 99.7111 & 0.2889 & & & & \\
\hline \multirow[t]{5}{*}{ DP4+ } & ${ }^{13} \mathrm{C}$ data & 0.6911 & 0.5127 & 34.7157 & 64.0805 & & & & \\
\hline & All data & 0.0000 & 0.0000 & 99.4681 & 0.5319 & & & & \\
\hline & & \multicolumn{8}{|c|}{ Level of theory: B3LYP/6-31G* (solution. PCM) } \\
\hline & & $86 a$ & $86 b$ & $86 c$ & $86 d$ & - & - & - & - \\
\hline & ${ }^{1} \mathrm{H}$ data & 0.0000 & 0.0000 & 99.9454 & 0.0546 & & & & \\
\hline \multirow[t]{3}{*}{ sDP4+ } & ${ }^{13} \mathrm{C}$ data & 0.9168 & 0.8898 & 50.1009 & 48.0925 & & & & \\
\hline & All data & 0.0000 & 0.0000 & 99.9476 & 0.0524 & & & & \\
\hline & ${ }^{1} \mathrm{H}$ data & 0.0000 & 0.0001 & 98.6987 & 1.3012 & & & & \\
\hline \multirow[t]{3}{*}{ uDP4+ } & ${ }^{13} \mathrm{C}$ data & 23.8767 & 13.0057 & 32.1322 & 30.9853 & & & & \\
\hline & All data & 0.0000 & 0.0000 & 98.7446 & 1.2553 & & & & \\
\hline & ${ }^{1} \mathrm{H}$ data & 0.0000 & 0.0000 & 99.9993 & 0.0007 & & & & \\
\hline \multirow[t]{5}{*}{ DP4+ } & ${ }^{13} \mathrm{C}$ data & 0.6986 & 0.3693 & 51.3759 & 47.5562 & & & & \\
\hline & All data & 0.0000 & 0.0000 & 99.9993 & 0.0007 & & & & \\
\hline & & \multicolumn{8}{|c|}{ Level of theory: B3LYP/6-31G** (solution. PCM) } \\
\hline & & $86 a$ & $86 b$ & $86 c$ & $86 d$ & - & - & - & - \\
\hline & ${ }^{1} \mathrm{H}$ data & 0.0001 & 0.0000 & 99.8649 & 0.1350 & & & & \\
\hline \multirow[t]{3}{*}{ sDP4+ } & ${ }^{13} \mathrm{C}$ data & 2.3127 & 2.4155 & 48.0444 & 47.2274 & & & & \\
\hline & All data & 0.0000 & 0.0000 & 99.8673 & 0.1327 & & & & \\
\hline & ${ }^{1} \mathrm{H}$ data & 0.0000 & 0.0003 & 98.9559 & 1.0438 & & & & \\
\hline \multirow[t]{3}{*}{ uDP4+ } & ${ }^{13} \mathrm{C}$ data & 24.6291 & 13.9748 & 31.4130 & 29.9832 & & & & \\
\hline & All data & 0.0000 & 0.0001 & 99.0031 & 0.9968 & & & & \\
\hline & ${ }^{1} \mathrm{H}$ data & 0.0000 & 0.0000 & 99.9986 & 0.0014 & & & & \\
\hline \multirow[t]{5}{*}{ DP4+ } & ${ }^{13} \mathrm{C}$ data & 1.8886 & 1.1192 & 50.0410 & 46.9511 & & & & \\
\hline & All data & 0.0000 & 0.0000 & 99.9987 & 0.0013 & & & & \\
\hline & & \multicolumn{8}{|c|}{ Level of theory: B3LYP/6-31+G** (solution. PCM) } \\
\hline & & $86 a$ & $86 b$ & $86 c$ & $86 d$ & - & - & - & - \\
\hline & ${ }^{1} \mathrm{H}$ data & 0.0004 & 0.0000 & 99.8075 & 0.1921 & & & & \\
\hline \multirow[t]{3}{*}{ sDP4+ } & ${ }^{13} \mathrm{C}$ data & 0.6535 & 2.6769 & 39.6839 & 56.9858 & & & & \\
\hline & All data & 0.0000 & 0.0000 & 99.7244 & 0.2756 & & & & \\
\hline & ${ }^{1} \mathrm{H}$ data & 0.0000 & 0.0002 & 98.9256 & 1.0742 & & & & \\
\hline uDP4+ & ${ }^{13} \mathrm{C}$ data & 9.8081 & 10.8246 & 43.6139 & 35.7534 & & & & \\
\hline & All data & 0.0000 & 0.0001 & 99.1177 & 0.8823 & & & & \\
\hline & ${ }^{1} \mathrm{H}$ data & 0.0000 & 0.0000 & 99.9979 & 0.0021 & & & & \\
\hline DP4+ & ${ }^{13} \mathrm{C}$ data & 0.1685 & 0.7618 & 45.5036 & 53.5661 & & & & \\
\hline & All data & 0.0000 & 0.0000 & 99.9975 & 0.0025 & & & & \\
\hline & & & & Level of $t$ & ry: B3LYP & & PC & & \\
\hline & & $86 a$ & $86 b$ & $86 c$ & $86 d$ & - & - & - & - \\
\hline & ${ }^{1} \mathrm{H}$ data & 0.0118 & 0.0000 & 99.5153 & 0.4729 & & & & \\
\hline sDP4+ & ${ }^{13} \mathrm{C}$ data & 0.8764 & 0.9428 & 39.6003 & 58.5805 & & & & \\
\hline & All data & 0.0003 & 0.0000 & 99.3017 & 0.6980 & & & & \\
\hline
\end{tabular}




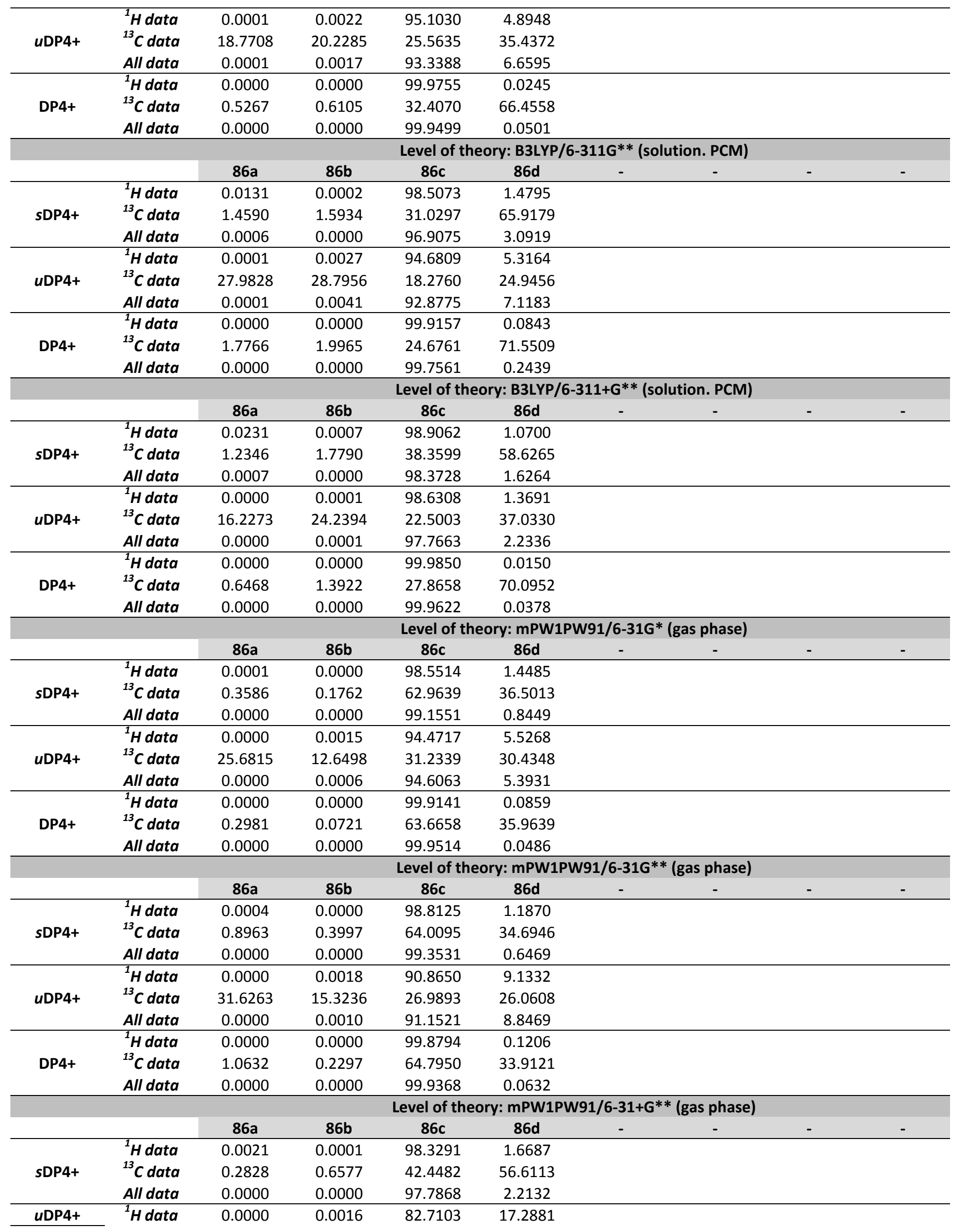




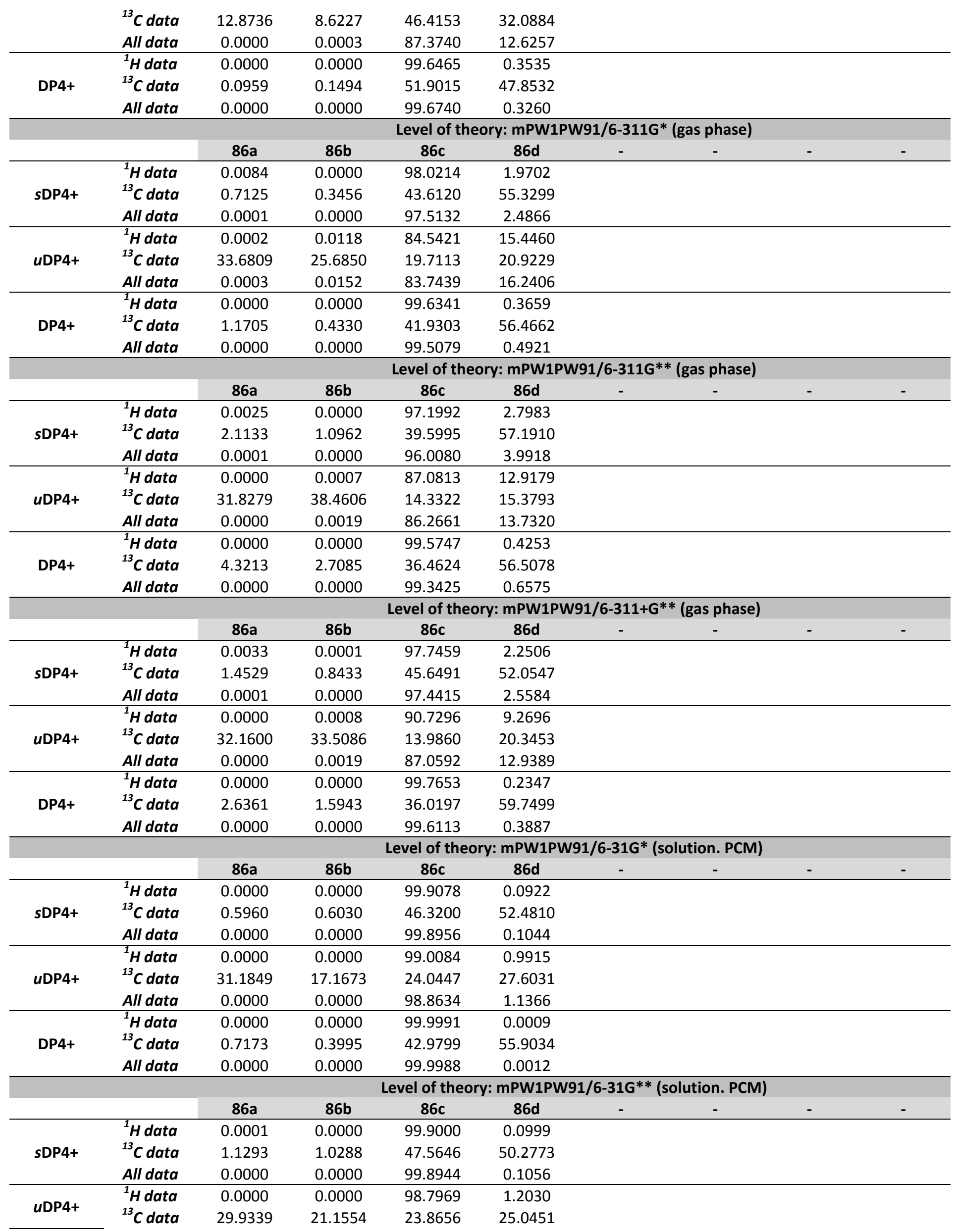




\begin{tabular}{|c|c|c|c|c|c|c|c|c|c|}
\hline & All data & 0.0000 & 0.0000 & 98.7382 & 1.2617 & & & & \\
\hline & ${ }^{1} \mathrm{H}$ data & 0.0000 & 0.0000 & 99.9988 & 0.0012 & & & & \\
\hline \multirow[t]{5}{*}{ DP4+ } & ${ }^{13}$ C data & 1.3798 & 0.8884 & 46.3344 & 51.3974 & & & & \\
\hline & All data & 0.0000 & 0.0000 & 99.9986 & 0.0014 & & & & \\
\hline & & \multicolumn{8}{|c|}{ Level of theory: mPW1PW91/6-31+G** (solution. PCM) } \\
\hline & & $86 a$ & $86 b$ & $86 \mathrm{c}$ & $86 d$ & - & - & - & - \\
\hline & ${ }^{1} \mathrm{H}$ data & 0.0060 & 0.0001 & 99.4634 & 0.5304 & & & & \\
\hline \multirow[t]{3}{*}{ sDP4+ } & ${ }^{13} \mathrm{C}$ data & 0.3361 & 2.0256 & 39.2799 & 58.3584 & & & & \\
\hline & All data & 0.0001 & 0.0000 & 99.2138 & 0.7861 & & & & \\
\hline & ${ }^{1} \mathrm{H}$ data & 0.0000 & 0.0001 & 98.4990 & 1.5009 & & & & \\
\hline \multirow[t]{3}{*}{ uDP4+ } & ${ }^{13} \mathrm{C}$ data & 15.0288 & 15.3389 & 39.9470 & 29.6853 & & & & \\
\hline & All data & 0.0000 & 0.0000 & 98.8803 & 1.1197 & & & & \\
\hline & ${ }^{1} \mathrm{H}$ data & 0.0000 & 0.0000 & 99.9919 & 0.0081 & & & & \\
\hline \multirow[t]{5}{*}{ DP4+ } & ${ }^{13} \mathrm{C}$ data & 0.1513 & 0.9309 & 47.0130 & 51.9048 & & & & \\
\hline & All data & 0.0000 & 0.0000 & 99.9910 & 0.0090 & & & & \\
\hline & & \multicolumn{8}{|c|}{ Level of theory: mPW1PW91/6-311G* (solution. PCM) } \\
\hline & & $86 a$ & $86 b$ & $86 c$ & 86d & - & - & - & - \\
\hline & ${ }^{1}$ H data & 0.0097 & 0.0000 & 99.6883 & 0.3019 & & & & \\
\hline \multirow[t]{2}{*}{ sDP4+ } & ${ }^{13} \mathrm{C}$ data & 1.2320 & 1.4340 & 38.4971 & 58.8369 & & & & \\
\hline & All data & 0.0003 & 0.0000 & 99.5389 & 0.4608 & & & & \\
\hline \multirow{3}{*}{ uDP4+ } & ${ }^{1}$ H data & 0.0000 & 0.0008 & 96.9066 & 3.0926 & & & & \\
\hline & ${ }^{13} \mathrm{C}$ data & 26.2812 & 25.9780 & 21.4424 & 26.2984 & & & & \\
\hline & All data & 0.0001 & 0.0009 & 96.2324 & 3.7666 & & & & \\
\hline \multirow{3}{*}{ DP4+ } & ${ }^{1} \mathrm{H}$ data & 0.0000 & 0.0000 & 99.9903 & 0.0097 & & & & \\
\hline & ${ }^{13} \mathrm{C}$ data & 1.3256 & 1.5253 & 33.7973 & 63.3518 & & & & \\
\hline & All data & 0.0000 & 0.0000 & 99.9819 & 0.0181 & & & & \\
\hline & & \multicolumn{8}{|c|}{ Level of theory: mPW1PW91/6-311G** (solution. PCM) } \\
\hline & & $86 a$ & $86 b$ & $86 \mathrm{c}$ & $86 d$ & - & - & - & - \\
\hline \multirow{3}{*}{$s D P 4+$} & ${ }^{1} \mathrm{H}$ data & 0.0149 & 0.0003 & 98.4126 & 1.5722 & & & & \\
\hline & ${ }^{13} \mathrm{C}$ data & 3.0387 & 3.6119 & 32.0241 & 61.3254 & & & & \\
\hline & All data & 0.0014 & 0.0000 & 97.0302 & 2.9684 & & & & \\
\hline \multirow{3}{*}{ uDP4+ } & ${ }^{1} \mathrm{H}$ data & 0.0000 & 0.0009 & 91.5500 & 8.4490 & & & & \\
\hline & ${ }^{13} \mathrm{C}$ data & 27.4940 & 38.7820 & 15.5530 & 18.1710 & & & & \\
\hline & All data & 0.0001 & 0.0022 & 90.2650 & 9.7327 & & & & \\
\hline \multirow{5}{*}{ DP4+ } & ${ }^{1}$ H data & 0.0000 & 0.0000 & 99.8528 & 0.1472 & & & & \\
\hline & ${ }^{13} \mathrm{C}$ data & 4.5503 & 7.6293 & 27.1274 & 60.6929 & & & & \\
\hline & All data & 0.0000 & 0.0000 & 99.6712 & 0.3288 & & & & \\
\hline & & \multicolumn{8}{|c|}{ Level of theory: mPW1PW91/6-311+G** (solution. PCM) } \\
\hline & & $86 a$ & $86 b$ & $86 c$ & $86 d$ & - & - & - & - \\
\hline \multirow{3}{*}{ sDP4+ } & ${ }^{1} \mathrm{H}$ data & 0.0234 & 0.0008 & 98.9253 & 1.0505 & & & & \\
\hline & ${ }^{13} \mathrm{C}$ data & 2.5100 & 3.7664 & 38.6642 & 55.0593 & & & & \\
\hline & All data & 0.0015 & 0.0001 & 98.5087 & 1.4897 & & & & \\
\hline \multirow{3}{*}{ uDP4+ } & ${ }^{1} \mathrm{H}$ data & 0.0000 & 0.0011 & 96.2225 & 3.7764 & & & & \\
\hline & ${ }^{13} \mathrm{C}$ data & 21.8357 & 28.8950 & 18.6969 & 30.5724 & & & & \\
\hline & All data & 0.0000 & 0.0017 & 93.9679 & 6.0304 & & & & \\
\hline \multirow{3}{*}{ DP4+ } & ${ }^{1} \mathrm{H}$ data & 0.0000 & 0.0000 & 99.9583 & 0.0417 & & & & \\
\hline & ${ }^{13} \mathrm{C}$ data & 2.1328 & 4.2349 & 28.1302 & 65.5021 & & & & \\
\hline & All data & 0.0000 & 0.0000 & 99.9030 & 0.0970 & & & & \\
\hline
\end{tabular}


sDP4+, $u \mathrm{DP} 4+$ and DP4+ probabilities (\%) for compound 86d<smiles>O=C(O[C@H]1C[C@H]2C=C[C@H]1C2)[C@@H](O)c1ccccc1</smiles>

$86 a$<smiles>O=C(O[C@H]1C[C@H]2C=C[C@@H]1C2)[C@@H](O)c1ccccc1</smiles><smiles>CC(C)(C)[O+]=O</smiles><smiles>CC(C)(C)OC(=O)C1CC2C=CC1C2</smiles>

correct isomer

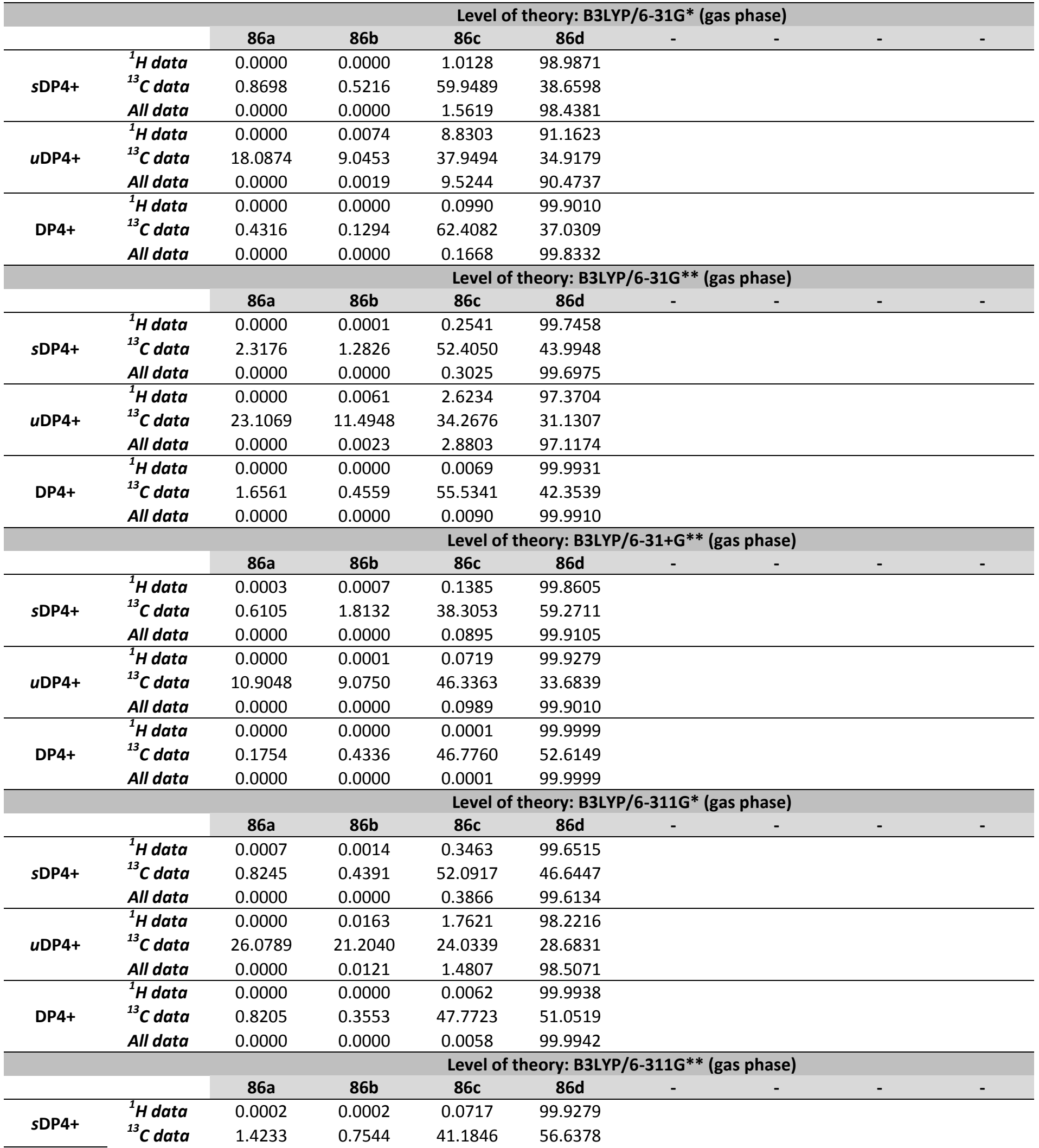




\begin{tabular}{|c|c|c|c|c|c|c|c|c|c|}
\hline & All data & 0.0000 & 0.0000 & 0.0522 & 99.9478 & & & & \\
\hline & ${ }^{1} \mathrm{H}$ data & 0.0000 & 0.0012 & 0.4120 & 99.5867 & & & & \\
\hline \multirow[t]{2}{*}{ uDP4+ } & ${ }^{13} \mathrm{C}$ data & 35.0975 & 28.6321 & 16.9306 & 19.3398 & & & & \\
\hline & All data & 0.0000 & 0.0018 & 0.3609 & 99.6373 & & & & \\
\hline & ${ }^{1} \mathrm{H}$ data & 0.0000 & 0.0000 & 0.0003 & 99.9997 & & & & \\
\hline \multirow[t]{5}{*}{ DP4+ } & ${ }^{13} \mathrm{C}$ data & 2.6796 & 1.1586 & 37.4038 & 58.7580 & & & & \\
\hline & All data & 0.0000 & 0.0000 & 0.0002 & 99.9998 & & & & \\
\hline & & \multicolumn{8}{|c|}{ Level of theory: B3LYP/6-311+G** (gas phase) } \\
\hline & & $86 a$ & $86 b$ & $86 c$ & 86d & - & - & - & - \\
\hline & ${ }^{1} \mathrm{H}$ data & 0.0003 & 0.0005 & 0.0640 & 99.9352 & & & & \\
\hline \multirow[t]{2}{*}{ sDP4+ } & ${ }^{13}$ C data & 0.7107 & 0.5677 & 41.7605 & 56.9611 & & & & \\
\hline & All data & 0.0000 & 0.0000 & 0.0470 & 99.9530 & & & & \\
\hline \multirow{3}{*}{ uDP4+ } & ${ }^{1} \mathrm{H}$ data & 0.0000 & 0.0006 & 0.1613 & 99.8381 & & & & \\
\hline & ${ }^{13} \mathrm{C}$ data & 29.5926 & 28.3001 & 16.8183 & 25.2891 & & & & \\
\hline & All data & 0.0000 & 0.0007 & 0.1073 & 99.8920 & & & & \\
\hline \multirow{5}{*}{ DP4+ } & ${ }^{1} \mathrm{H}$ data & 0.0000 & 0.0000 & 0.0001 & 99.9999 & & & & \\
\hline & ${ }^{13} \mathrm{C}$ data & 0.9648 & 0.7370 & 32.2185 & 66.0797 & & & & \\
\hline & All data & 0.0000 & 0.0000 & 0.0001 & 99.9999 & & & & \\
\hline & & \multicolumn{8}{|c|}{ Level of theory: B3LYP/6-31G* (solution. PCM) } \\
\hline & & $86 a$ & $86 b$ & $86 c$ & $86 d$ & - & - & - & - \\
\hline \multirow{3}{*}{ sDP4+ } & ${ }^{1} \mathrm{H}$ data & 0.0000 & 0.0000 & 1.2944 & 98.7056 & & & & \\
\hline & ${ }^{13} \mathrm{C}$ data & 1.0593 & 1.0558 & 47.4421 & 50.4428 & & & & \\
\hline & All data & 0.0000 & 0.0000 & 1.2184 & 98.7816 & & & & \\
\hline \multirow{3}{*}{ uDP4+ } & ${ }^{1} \mathrm{H}$ data & 0.0000 & 0.0006 & 5.5691 & 94.4303 & & & & \\
\hline & ${ }^{13} \mathrm{C}$ data & 24.7336 & 13.6000 & 31.1550 & 30.5115 & & & & \\
\hline & All data & 0.0000 & 0.0003 & 5.6799 & 94.3198 & & & & \\
\hline \multirow{5}{*}{ DP4+ } & ${ }^{1} \mathrm{H}$ data & 0.0000 & 0.0000 & 0.0773 & 99.9227 & & & & \\
\hline & ${ }^{13} \mathrm{C}$ data & 0.8568 & 0.4696 & 48.3389 & 50.3346 & & & & \\
\hline & All data & 0.0000 & 0.0000 & 0.0742 & 99.9258 & & & & \\
\hline & & \multicolumn{8}{|c|}{ Level of theory: B3LYP/6-31G** (solution. PCM) } \\
\hline & & $86 a$ & $86 b$ & $86 c$ & $86 d$ & - & - & - & - \\
\hline \multirow{3}{*}{ sDP4+ } & ${ }^{1} \mathrm{H}$ data & 0.0000 & 0.0000 & 0.2847 & 99.7152 & & & & \\
\hline & ${ }^{13} \mathrm{C}$ data & 2.5134 & 2.6632 & 47.5569 & 47.2665 & & & & \\
\hline & All data & 0.0000 & 0.0000 & 0.2865 & 99.7135 & & & & \\
\hline \multirow{3}{*}{ uDP4+ } & ${ }^{1} \mathrm{H}$ data & 0.0000 & 0.0011 & 1.9657 & 98.0333 & & & & \\
\hline & ${ }^{13} \mathrm{C}$ data & 28.7628 & 15.7009 & 28.2223 & 27.3140 & & & & \\
\hline & All data & 0.0000 & 0.0006 & 2.0297 & 97.9697 & & & & \\
\hline \multirow{3}{*}{ DP4+ } & ${ }^{1} \mathrm{H}$ data & 0.0000 & 0.0000 & 0.0057 & 99.9943 & & & & \\
\hline & ${ }^{13} \mathrm{C}$ data & 2.6314 & 1.5220 & 48.8538 & 46.9927 & & & & \\
\hline & All data & 0.0000 & 0.0000 & 0.0060 & 99.9940 & & & & \\
\hline & & \multicolumn{8}{|c|}{ Level of theory: B3LYP/6-31+G** (solution. PCM) } \\
\hline & & $86 a$ & $86 \mathrm{~b}$ & $86 c$ & $86 d$ & - & - & - & - \\
\hline \multirow{3}{*}{ sDP4+ } & ${ }^{1} \mathrm{H}$ data & 0.0004 & 0.0008 & 0.1378 & 99.8610 & & & & \\
\hline & ${ }^{13} \mathrm{C}$ data & 0.7629 & 3.1582 & 39.3865 & 56.6924 & & & & \\
\hline & All data & 0.0000 & 0.0000 & 0.0958 & 99.9042 & & & & \\
\hline & ${ }^{1} \mathrm{H}$ data & 0.0000 & 0.0004 & 0.0890 & 99.9106 & & & & \\
\hline uDP4+ & ${ }^{13} \mathrm{C}$ data & 10.8284 & 11.2617 & 44.2954 & 33.6145 & & & & \\
\hline & All data & 0.0000 & 0.0001 & 0.1173 & 99.8826 & & & & \\
\hline & ${ }^{1} \mathrm{H}$ data & 0.0000 & 0.0000 & 0.0001 & 99.9999 & & & & \\
\hline DP4+ & ${ }^{13} \mathrm{C}$ data & 0.2236 & 0.9628 & 47.2271 & 51.5865 & & & & \\
\hline & All data & 0.0000 & 0.0000 & 0.0001 & 99.9999 & & & & \\
\hline & & & & Level of $t$ & ory: B3LYP & $\mathrm{G}^{*}$ & $\overline{P C}$ & & \\
\hline & & $86 a$ & $86 b$ & $86 c$ & $86 d$ & - & - & - & - \\
\hline & ${ }^{1} \mathrm{H}$ data & 0.0023 & 0.0030 & 0.3369 & 99.6577 & & & & \\
\hline$s \mathrm{DP4+}$ & ${ }^{13} \mathrm{C}$ data & 1.1285 & 1.2369 & 36.2785 & 61.3560 & & & & \\
\hline & All data & 0.0000 & 0.0001 & 0.1995 & 99.8004 & & & & \\
\hline
\end{tabular}




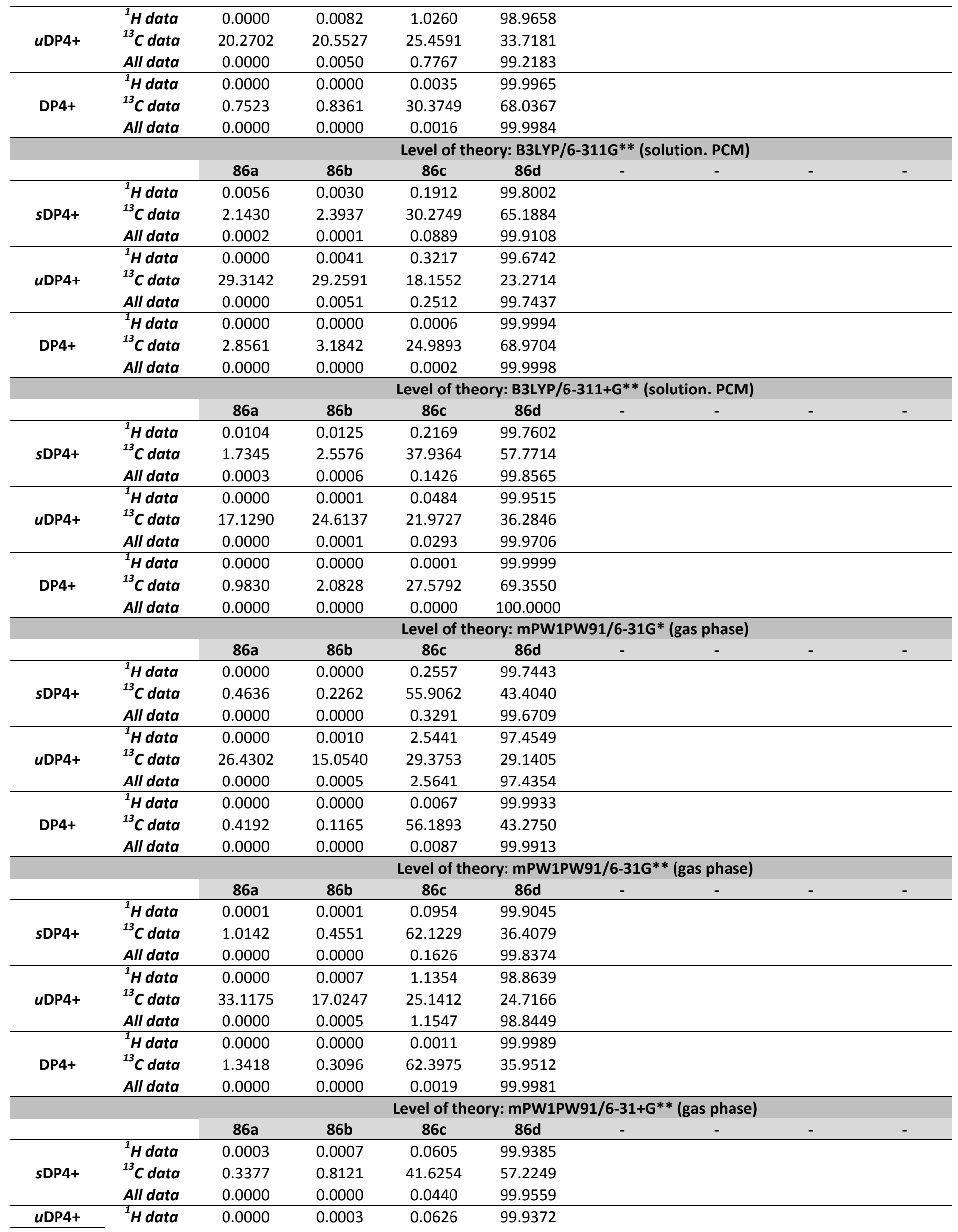




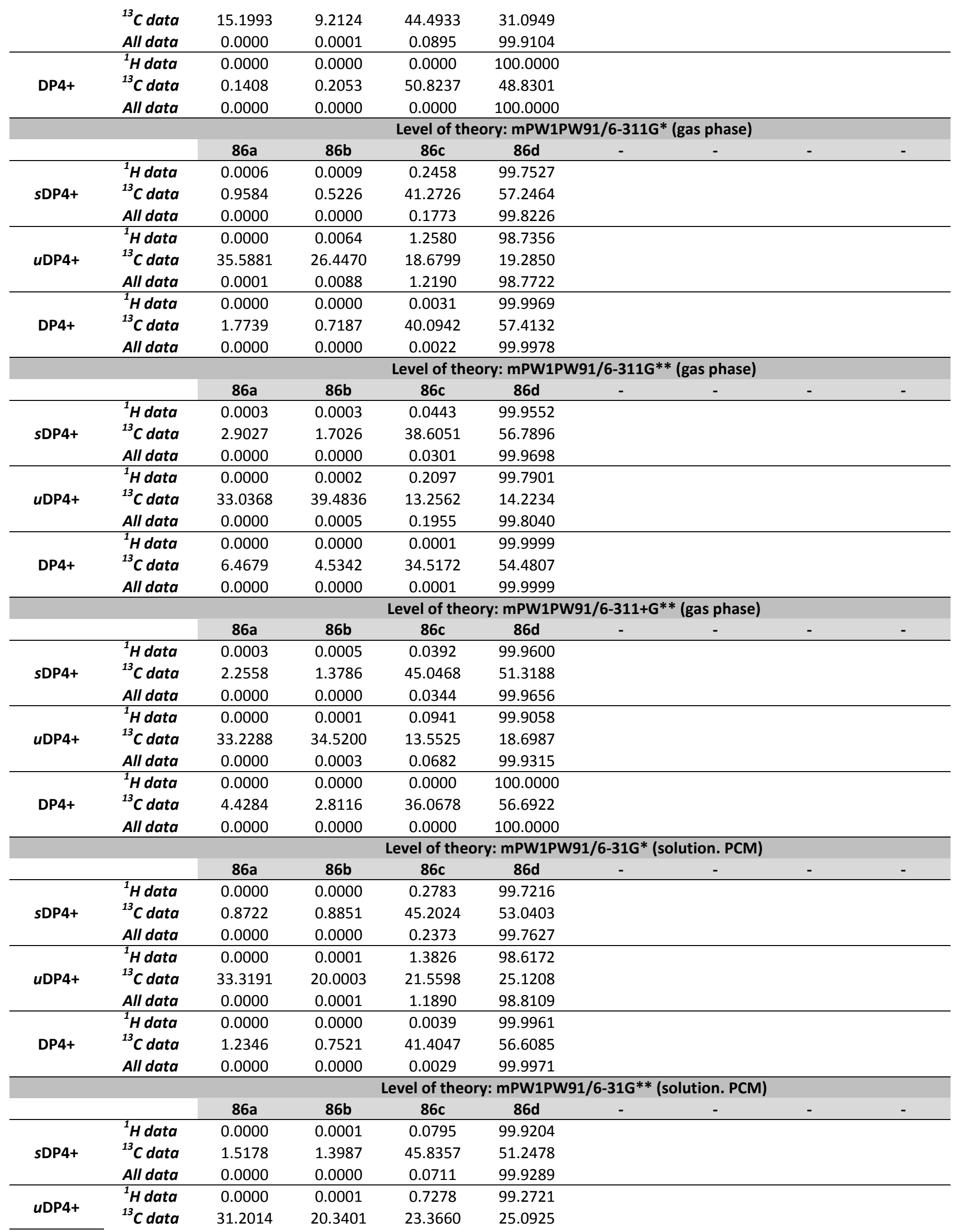




\begin{tabular}{|c|c|c|c|c|c|c|c|c|c|}
\hline & All data & 0.0000 & 0.0001 & 0.6781 & 99.3218 & & & & \\
\hline & ${ }^{1}$ H data & 0.0000 & 0.0000 & 0.0006 & 99.9994 & & & & \\
\hline \multirow[t]{5}{*}{ DP4+ } & ${ }^{13} \mathrm{C}$ data & 1.9467 & 1.1694 & 44.0242 & 52.8596 & & & & \\
\hline & All data & 0.0000 & 0.0000 & 0.0005 & 99.9995 & & & & \\
\hline & & \multicolumn{8}{|c|}{ Level of theory: mPW1PW91/6-31+G** (solution. PCM) } \\
\hline & & $86 a$ & $86 b$ & $86 c$ & $86 d$ & - & - & - & - \\
\hline & ${ }^{1} \mathrm{H}$ data & 0.0028 & 0.0059 & 0.0958 & 99.8954 & & & & \\
\hline \multirow[t]{3}{*}{ sDP4+ } & ${ }^{13} \mathrm{C}$ data & 0.4473 & 2.7347 & 38.8201 & 57.9979 & & & & \\
\hline & All data & 0.0000 & 0.0003 & 0.0642 & 99.9355 & & & & \\
\hline & ${ }^{1} \mathrm{H}$ data & 0.0000 & 0.0001 & 0.0413 & 99.9586 & & & & \\
\hline \multirow[t]{3}{*}{ uDP4+ } & ${ }^{13} \mathrm{C}$ data & 17.8255 & 16.3604 & 37.9183 & 27.8959 & & & & \\
\hline & All data & 0.0000 & 0.0001 & 0.0561 & 99.9439 & & & & \\
\hline & ${ }^{1} \mathrm{H}$ data & 0.0000 & 0.0000 & 0.0000 & 100.0000 & & & & \\
\hline \multirow[t]{5}{*}{ DP4+ } & ${ }^{13} \mathrm{C}$ data & 0.2537 & 1.4237 & 46.8399 & 51.4828 & & & & \\
\hline & All data & 0.0000 & 0.0000 & 0.0000 & 100.0000 & & & & \\
\hline & & \multicolumn{8}{|c|}{ Level of theory: mPW1PW91/6-311G* (solution. PCM) } \\
\hline & & $86 a$ & $86 b$ & $86 c$ & $86 d$ & - & - & - & - \\
\hline & ${ }^{1} \mathrm{H}$ data & 0.0029 & 0.0024 & 0.2486 & 99.7462 & & & & \\
\hline \multirow[t]{3}{*}{ sDP4+ } & ${ }^{13} \mathrm{C}$ data & 1.6576 & 1.9762 & 37.4532 & 58.9130 & & & & \\
\hline & All data & 0.0001 & 0.0001 & 0.1582 & 99.8417 & & & & \\
\hline & ${ }^{1} \mathrm{H}$ data & 0.0000 & 0.0028 & 0.7291 & 99.2680 & & & & \\
\hline \multirow[t]{3}{*}{ uDP4+ } & ${ }^{13} \mathrm{C}$ data & 27.3622 & 26.5760 & 21.5578 & 24.5040 & & & & \\
\hline & All data & 0.0000 & 0.0031 & 0.6420 & 99.3549 & & & & \\
\hline & ${ }^{1} \mathrm{H}$ data & 0.0000 & 0.0000 & 0.0018 & 99.9982 & & & & \\
\hline \multirow[t]{5}{*}{ DP4+ } & ${ }^{13} \mathrm{C}$ data & 1.9309 & 2.2360 & 34.3741 & 61.4591 & & & & \\
\hline & All data & 0.0000 & 0.0000 & 0.0010 & 99.9990 & & & & \\
\hline & & \multicolumn{8}{|c|}{ Level of theory: mPW1PW91/6-311G** (solution. PCM) } \\
\hline & & $86 a$ & $86 b$ & $86 c$ & $86 d$ & - & - & - & - \\
\hline & ${ }^{1} \mathrm{H}$ data & 0.0067 & 0.0035 & 0.1146 & 99.8753 & & & & \\
\hline \multirow[t]{3}{*}{$s D P 4+$} & ${ }^{13} \mathrm{C}$ data & 4.2168 & 5.1774 & 30.8172 & 59.7887 & & & & \\
\hline & All data & 0.0005 & 0.0003 & 0.0591 & 99.9401 & & & & \\
\hline & ${ }^{1} \mathrm{H}$ data & 0.0000 & 0.0007 & 0.2025 & 99.7967 & & & & \\
\hline \multirow[t]{3}{*}{ uDP4+ } & ${ }^{13} \mathrm{C}$ data & 29.2990 & 37.9660 & 15.3085 & 17.4265 & & & & \\
\hline & All data & 0.0000 & 0.0015 & 0.1780 & 99.8205 & & & & \\
\hline & ${ }^{1} \mathrm{H}$ data & 0.0000 & 0.0000 & 0.0002 & 99.9998 & & & & \\
\hline \multirow[t]{5}{*}{ DP4+ } & ${ }^{13} \mathrm{C}$ data & 6.7374 & 10.7190 & 25.7263 & 56.8174 & & & & \\
\hline & All data & 0.0000 & 0.0000 & 0.0001 & 99.9999 & & & & \\
\hline & & \multicolumn{8}{|c|}{ Level of theory: mPW1PW91/6-311+G** (solution. PCM) } \\
\hline & & $86 a$ & $86 b$ & $86 c$ & $86 d$ & - & - & - & - \\
\hline & ${ }^{1} \mathrm{H}$ data & 0.0096 & 0.0096 & 0.1127 & 99.8681 & & & & \\
\hline \multirow[t]{3}{*}{ sDP4+ } & ${ }^{13}$ C data & 3.5977 & 5.5677 & 37.2865 & 53.5481 & & & & \\
\hline & All data & 0.0006 & 0.0010 & 0.0785 & 99.9198 & & & & \\
\hline & ${ }^{1} \mathrm{H}$ data & 0.0000 & 0.0008 & 0.1016 & 99.8976 & & & & \\
\hline \multirow[t]{3}{*}{ uDP4+ } & ${ }^{13} \mathrm{C}$ data & 22.8502 & 29.7221 & 18.8312 & 28.5965 & & & & \\
\hline & All data & 0.0000 & 0.0008 & 0.0670 & 99.9322 & & & & \\
\hline & ${ }^{1} \mathrm{H}$ data & 0.0000 & 0.0000 & 0.0001 & 99.9999 & & & & \\
\hline \multirow[t]{2}{*}{ DP4+ } & ${ }^{13} \mathrm{C}$ data & 3.3133 & 6.6697 & 28.2996 & 61.7173 & & & & \\
\hline & All data & 0.0000 & 0.0000 & 0.0001 & 99.9999 & & & & \\
\hline
\end{tabular}


$s \mathrm{DP} 4+, u \mathrm{DP} 4+$ and DP4+ probabilities (\%) for compound 87a<smiles>CC(=O)O[C@@H](C(=O)O[C@H]1C[C@H]2C=C[C@H]1C2)c1ccccc1</smiles>

correct isomer<smiles>O=C(OC1CC2C=CC1C2)[C@@H](O)c1ccccc1</smiles>

87b<smiles>O=C(O[C@@H]1C[C@H]2C=C[C@@H]1C2)[C@@H](O)c1ccccc1</smiles>

87c<smiles>O=C(OC1CC2C=CC1C2)[C@H](O)c1ccccc1</smiles>

87d

\begin{tabular}{|c|c|c|c|c|c|c|c|c|c|}
\hline & & \multicolumn{8}{|c|}{ Level of theory: B3LYP/6-31G* (gas phase) } \\
\hline & & $87 a$ & $87 b$ & $87 c$ & 87d & - & - & - & - \\
\hline & ${ }^{1} \mathrm{H}$ data & 5.0687 & 94.9313 & 0.0000 & 0.0000 & & & & \\
\hline \multirow[t]{2}{*}{ sDP4+ } & ${ }^{13} \mathrm{C}$ data & 9.3182 & 84.9972 & 1.5312 & 4.1533 & & & & \\
\hline & All data & 0.5819 & 99.4181 & 0.0000 & 0.0000 & & & & \\
\hline & ${ }^{1} \mathrm{H}$ data & 5.4309 & 94.5690 & 0.0000 & 0.0000 & & & & \\
\hline \multirow[t]{2}{*}{ uDP4+ } & ${ }^{13} \mathrm{C}$ data & 37.7070 & 57.8439 & 1.7937 & 2.6555 & & & & \\
\hline & All data & 3.6085 & 96.3915 & 0.0000 & 0.0000 & & & & \\
\hline \multirow{6}{*}{ DP4+ } & ${ }^{1} \mathrm{H}$ data & 0.3057 & 99.6943 & 0.0000 & 0.0000 & & & & \\
\hline & ${ }^{13} \mathrm{C}$ data & 6.6524 & 93.0867 & 0.0520 & 0.2088 & & & & \\
\hline & All data & 0.0219 & 99.9781 & 0.0000 & 0.0000 & & & & \\
\hline & & \multicolumn{8}{|c|}{ Level of theory: B3LYP/6-31G** (gas phase) } \\
\hline & & $87 a$ & 87b & $87 c$ & 87d & - & - & - & - \\
\hline & ${ }^{1} \mathrm{H}$ data & 2.4030 & 97.5970 & 0.0000 & 0.0000 & & & & \\
\hline \multirow[t]{2}{*}{$s \mathrm{DP} 4+$} & ${ }^{13} \mathrm{C}$ data & 12.2170 & 75.4077 & 3.6836 & 8.6917 & & & & \\
\hline & All data & 0.3973 & 99.6027 & 0.0000 & 0.0000 & & & & \\
\hline \multirow{3}{*}{ uDP4+ } & ${ }^{1} \mathrm{H}$ data & 1.0595 & 98.9404 & 0.0000 & 0.0000 & & & & \\
\hline & ${ }^{13} \mathrm{C}$ data & 32.1207 & 63.1428 & 1.9321 & 2.8044 & & & & \\
\hline & All data & 0.5418 & 99.4582 & 0.0000 & 0.0000 & & & & \\
\hline \multirow{5}{*}{ DP4+ } & ${ }^{1} \mathrm{H}$ data & 0.0264 & 99.9736 & 0.0000 & 0.0000 & & & & \\
\hline & ${ }^{13} \mathrm{C}$ data & 7.5678 & 91.8249 & 0.1372 & 0.4701 & & & & \\
\hline & All data & 0.0022 & 99.9978 & 0.0000 & 0.0000 & & & & \\
\hline & & \multicolumn{8}{|c|}{ Level of theory: B3LYP/6-31+G** (gas phase) } \\
\hline & & $87 a$ & $87 \mathrm{~b}$ & $87 \mathrm{c}$ & 87d & - & - & - & - \\
\hline \multirow{3}{*}{ sDP4+ } & ${ }^{1} \mathrm{H}$ data & 14.7506 & 85.2494 & 0.0000 & 0.0000 & & & & \\
\hline & ${ }^{13} \mathrm{C}$ data & 24.9255 & 55.1335 & 12.6535 & 7.2874 & & & & \\
\hline & All data & 7.2550 & 92.7450 & 0.0000 & 0.0000 & & & & \\
\hline \multirow{3}{*}{ uDP4+ } & ${ }^{1} \mathrm{H}$ data & 31.8320 & 68.1679 & 0.0000 & 0.0000 & & & & \\
\hline & ${ }^{13} \mathrm{C}$ data & 46.3181 & 51.4310 & 1.2839 & 0.9670 & & & & \\
\hline & All data & 29.6044 & 70.3956 & 0.0000 & 0.0000 & & & & \\
\hline \multirow{5}{*}{ DP4+ } & ${ }^{1} \mathrm{H}$ data & 7.4758 & 92.5242 & 0.0000 & 0.0000 & & & & \\
\hline & ${ }^{13} \mathrm{C}$ data & 28.7664 & 70.6532 & 0.4048 & 0.1756 & & & & \\
\hline & All data & 3.1849 & 96.8151 & 0.0000 & 0.0000 & & & & \\
\hline & & \multicolumn{8}{|c|}{ Level of theory: B3LYP/6-311G* (gas phase) } \\
\hline & & $87 a$ & $87 b$ & $87 c$ & $87 d$ & - & - & - & - \\
\hline \multirow{3}{*}{ sDP4+ } & ${ }^{1} \mathrm{H}$ data & 1.8829 & 98.1171 & 0.0000 & 0.0000 & & & & \\
\hline & ${ }^{13} \mathrm{C}$ data & 13.6109 & 71.1485 & 5.3629 & 9.8777 & & & & \\
\hline & All data & 0.3658 & 99.6342 & 0.0000 & 0.0000 & & & & \\
\hline \multirow{3}{*}{ uDP4+ } & ${ }^{1} \mathrm{H}$ data & 13.8721 & 86.1256 & 0.0004 & 0.0018 & & & & \\
\hline & ${ }^{13} \mathrm{C}$ data & 25.3268 & 66.9678 & 2.5498 & 5.1557 & & & & \\
\hline & All data & 5.7417 & 94.2581 & 0.0000 & 0.0002 & & & & \\
\hline \multirow{3}{*}{ DP4+ } & ${ }^{1} \mathrm{H}$ data & 0.3081 & 99.6919 & 0.0000 & 0.0000 & & & & \\
\hline & ${ }^{13} \mathrm{C}$ data & 6.6626 & 92.0888 & 0.2643 & 0.9843 & & & & \\
\hline & All data & 0.0224 & 99.9776 & 0.0000 & 0.0000 & & & & \\
\hline & & \multicolumn{8}{|c|}{ Level of theory: B3LYP/6-311G** (gas phase) } \\
\hline & & $87 a$ & $87 b$ & $87 c$ & $87 d$ & - & - & - & - \\
\hline \multirow{2}{*}{$s \mathrm{DP} 4+$} & ${ }^{1} \mathrm{H}$ data & 1.8636 & 98.1364 & 0.0000 & 0.0000 & & & & \\
\hline & ${ }^{13} \mathrm{C}$ data & 14.9657 & 79.9222 & 1.6417 & 3.4704 & & & & \\
\hline
\end{tabular}




\begin{tabular}{|c|c|c|c|c|c|c|c|c|c|}
\hline & All data & 0.3543 & 99.6457 & 0.0000 & 0.0000 & & & & \\
\hline & ${ }^{1} \mathrm{H}$ data & 4.8358 & 95.1640 & 0.0000 & 0.0002 & & & & \\
\hline \multirow[t]{2}{*}{ uDP4+ } & ${ }^{13} \mathrm{C}$ data & 25.9094 & 67.9218 & 2.0602 & 4.1086 & & & & \\
\hline & All data & 1.9015 & 98.0985 & 0.0000 & 0.0000 & & & & \\
\hline & ${ }^{1} \mathrm{H}$ data & 0.0964 & 99.9036 & 0.0000 & 0.0000 & & & & \\
\hline \multirow[t]{5}{*}{ DP4+ } & ${ }^{13} \mathrm{C}$ data & 6.6466 & 93.0510 & 0.0580 & 0.2444 & & & & \\
\hline & All data & 0.0069 & 99.9931 & 0.0000 & 0.0000 & & & & \\
\hline & & \multicolumn{8}{|c|}{ Level of theory: B3LYP/6-311+G** (gas phase) } \\
\hline & & $87 a$ & $87 \mathrm{~b}$ & $87 c$ & 87d & - & - & - & - \\
\hline & ${ }^{1} \mathrm{H}$ data & 41.9260 & 58.0740 & 0.0000 & 0.0000 & & & & \\
\hline \multirow[t]{2}{*}{ sDP4+ } & ${ }^{13} \mathrm{C}$ data & 17.1814 & 79.6120 & 0.9395 & 2.2672 & & & & \\
\hline & All data & 13.4802 & 86.5198 & 0.0000 & 0.0000 & & & & \\
\hline \multirow{3}{*}{ uDP4+ } & ${ }^{1} \mathrm{H}$ data & 74.7064 & 25.2930 & 0.0000 & 0.0006 & & & & \\
\hline & ${ }^{13} \mathrm{C}$ data & 25.4412 & 69.4376 & 1.2075 & 3.9137 & & & & \\
\hline & All data & 51.9734 & 48.0265 & 0.0000 & 0.0001 & & & & \\
\hline & ${ }^{1} \mathrm{H}$ data & 68.0751 & 31.9249 & 0.0000 & 0.0000 & & & & \\
\hline \multirow[t]{4}{*}{ DP4+ } & ${ }^{13} \mathrm{C}$ data & 7.3155 & 92.5170 & 0.0190 & 0.1485 & & & & \\
\hline & All data & 14.4282 & 85.5718 & 0.0000 & 0.0000 & & & & \\
\hline & & \multicolumn{8}{|c|}{ Level of theory: B3LYP/6-31G* (solution. PCM) } \\
\hline & & $87 a$ & $87 b$ & $87 c$ & $87 d$ & - & - & - & - \\
\hline \multirow{3}{*}{ sDP4+ } & ${ }^{1} \mathrm{H}$ data & 71.9842 & 28.0158 & 0.0000 & 0.0000 & & & & \\
\hline & ${ }^{13} \mathrm{C}$ data & 19.3553 & 77.7711 & 0.8457 & 2.0280 & & & & \\
\hline & All data & 39.0044 & 60.9956 & 0.0000 & 0.0000 & & & & \\
\hline \multirow{3}{*}{ uDP4+ } & ${ }^{1} \mathrm{H}$ data & 87.2509 & 12.7491 & 0.0000 & 0.0000 & & & & \\
\hline & ${ }^{13} \mathrm{C}$ data & 34.3529 & 62.4252 & 1.3244 & 1.8975 & & & & \\
\hline & All data & 79.0185 & 20.9815 & 0.0000 & 0.0000 & & & & \\
\hline \multirow{5}{*}{ DP4+ } & ${ }^{1} \mathrm{H}$ data & 94.6191 & 5.3809 & 0.0000 & 0.0000 & & & & \\
\hline & ${ }^{13} \mathrm{C}$ data & 12.0351 & 87.8750 & 0.0203 & 0.0697 & & & & \\
\hline & All data & 70.6597 & 29.3403 & 0.0000 & 0.0000 & & & & \\
\hline & & \multicolumn{8}{|c|}{ Level of theory: B3LYP/6-31G** (solution. PCM) } \\
\hline & & $87 a$ & $87 b$ & $87 c$ & $87 d$ & - & - & - & - \\
\hline \multirow{3}{*}{$s \mathrm{DP} 4+$} & ${ }^{1} \mathrm{H}$ data & 36.0202 & 63.9798 & 0.0000 & 0.0000 & & & & \\
\hline & ${ }^{13} \mathrm{C}$ data & 22.6291 & 70.8673 & 2.1382 & 4.3654 & & & & \\
\hline & All data & 15.2379 & 84.7621 & 0.0000 & 0.0000 & & & & \\
\hline \multirow{3}{*}{ uDP4+ } & ${ }^{1} \mathrm{H}$ data & 73.7940 & 26.2060 & 0.0000 & 0.0000 & & & & \\
\hline & ${ }^{13} \mathrm{C}$ data & 37.9451 & 59.0745 & 1.1760 & 1.8045 & & & & \\
\hline & All data & 64.3968 & 35.6032 & 0.0000 & 0.0000 & & & & \\
\hline \multirow{3}{*}{ DP4+ } & ${ }^{1} \mathrm{H}$ data & 61.3204 & 38.6796 & 0.0000 & 0.0000 & & & & \\
\hline & ${ }^{13} \mathrm{C}$ data & 16.9847 & 82.8098 & 0.0497 & 0.1558 & & & & \\
\hline & All data & 24.5375 & 75.4625 & 0.0000 & 0.0000 & & & & \\
\hline & & \multicolumn{8}{|c|}{ Level of theory: B3LYP/6-31+G** (solution. PCM) } \\
\hline & & $87 a$ & $87 \mathrm{~b}$ & $87 c$ & $87 d$ & - & - & - & - \\
\hline \multirow{3}{*}{$s \mathrm{DP} 4+$} & ${ }^{1} \mathrm{H}$ data & 64.8205 & 35.1795 & 0.0000 & 0.0000 & & & & \\
\hline & ${ }^{13} \mathrm{C}$ data & 28.4942 & 61.6800 & 5.4900 & 4.3359 & & & & \\
\hline & All data & 45.9812 & 54.0188 & 0.0000 & 0.0000 & & & & \\
\hline & ${ }_{12}^{1} \mathrm{H}$ data & 98.0219 & 1.9781 & 0.0000 & 0.0000 & & & & \\
\hline uDP4+ & ${ }^{13} \mathrm{C}$ data & 51.9343 & 46.1139 & 1.3111 & 0.6407 & & & & \\
\hline & All data & 98.2397 & 1.7603 & 0.0000 & 0.0000 & & & & \\
\hline & ${ }^{1} \mathrm{H}$ data & 98.9167 & 1.0833 & 0.0000 & 0.0000 & & & & \\
\hline DP4+ & ${ }^{13} \mathrm{C}$ data & 34.1437 & 65.6261 & 0.1661 & 0.0641 & & & & \\
\hline & All data & 97.9383 & 2.0617 & 0.0000 & 0.0000 & & & & \\
\hline & & & & Level of & ry: B3LYF & G* & PC & & \\
\hline & & $87 a$ & $87 \mathrm{~b}$ & $87 c$ & $87 d$ & - & - & - & - \\
\hline & ${ }^{1} \mathrm{H}$ data & 61.2129 & 38.7871 & 0.0000 & 0.0000 & & & & \\
\hline sDP4+ & ${ }^{13} \mathrm{C}$ data & 22.5334 & 67.5764 & 4.1248 & 5.7653 & & & & \\
\hline & All data & 34.4797 & 65.5203 & 0.0000 & 0.0000 & & & & \\
\hline
\end{tabular}




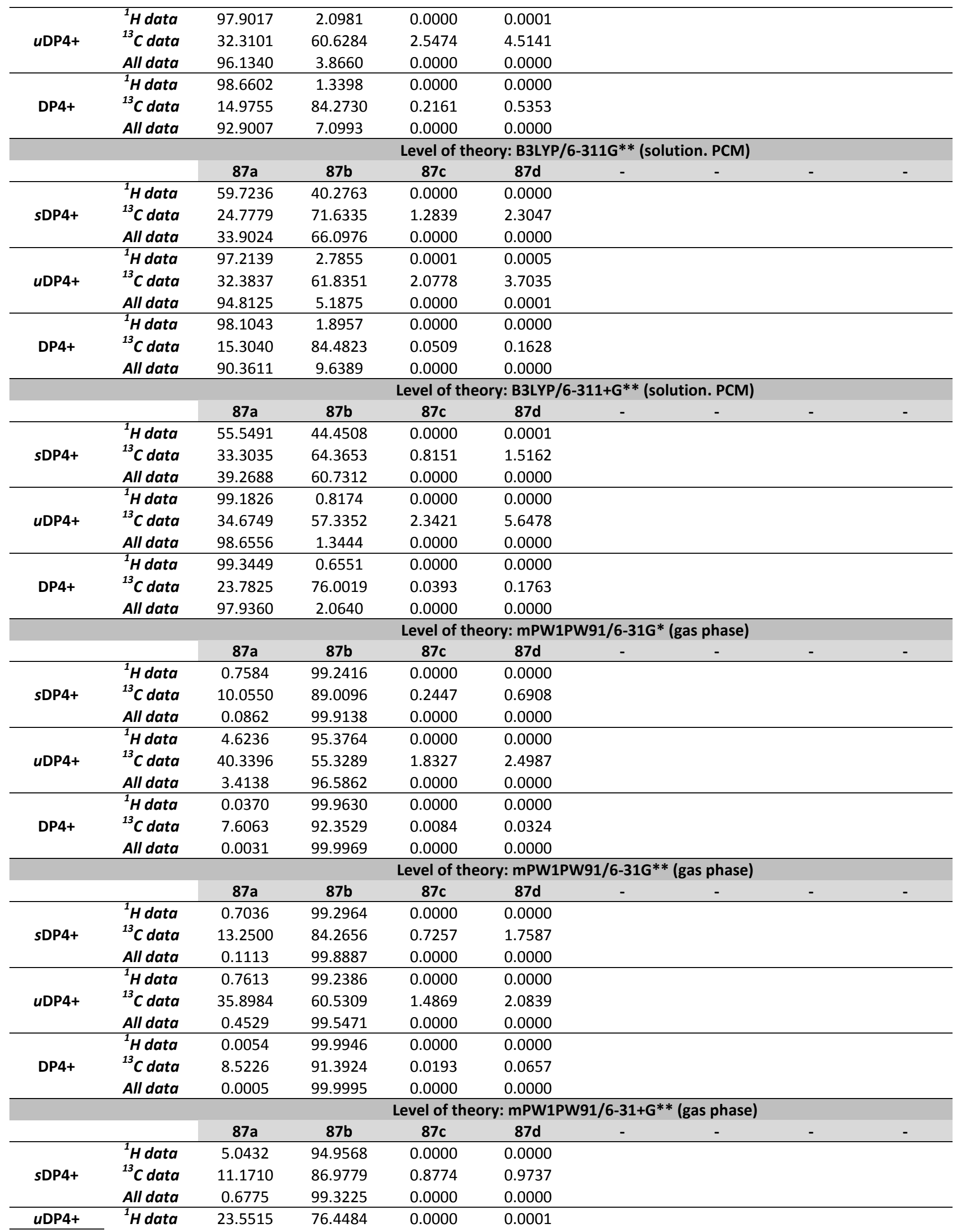




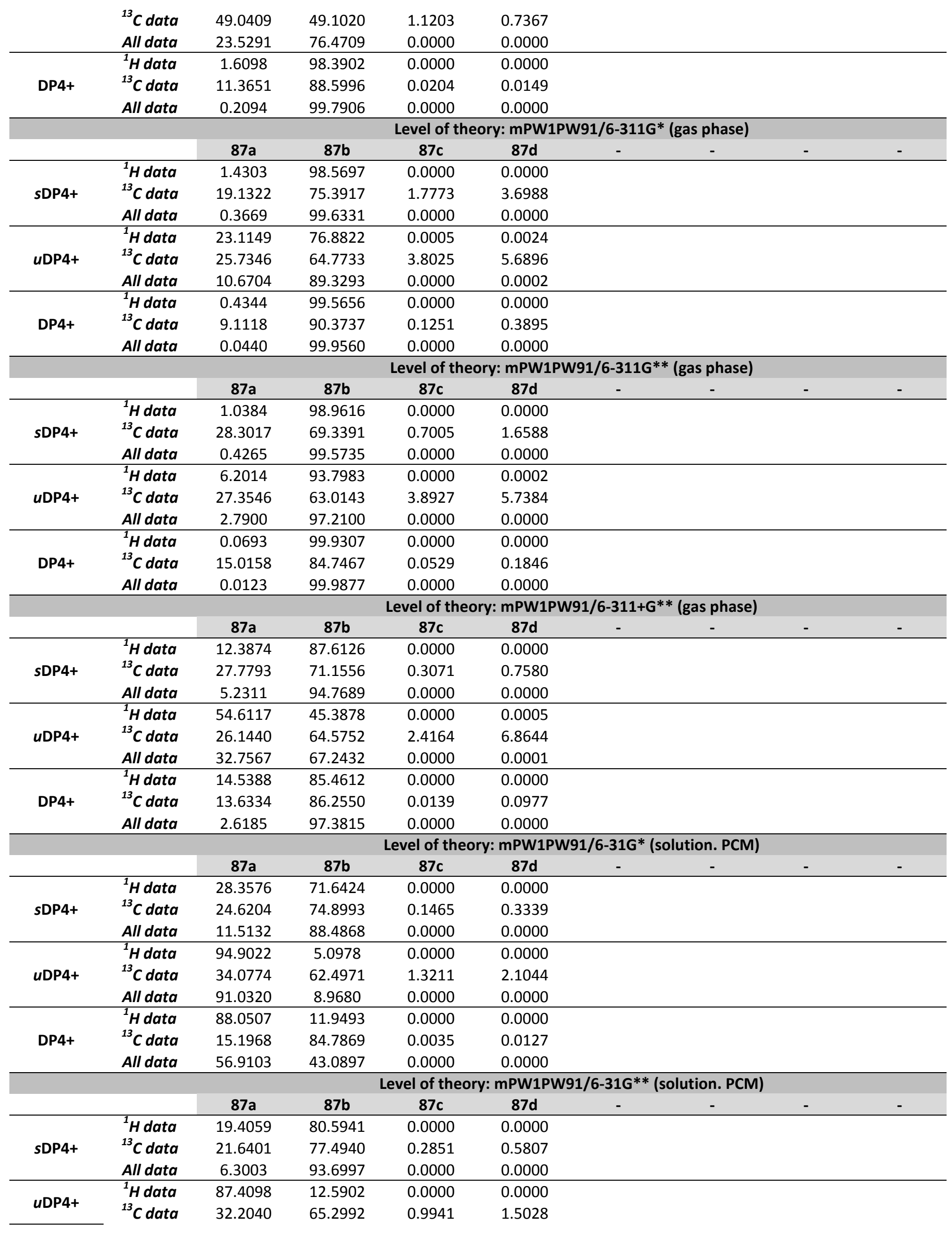




\begin{tabular}{|c|c|c|c|c|c|c|c|c|c|}
\hline & All data & 77.3958 & 22.6042 & 0.0000 & 0.0000 & & & & \\
\hline & ${ }^{1}$ H data & 62.5706 & 37.4294 & 0.0000 & 0.0000 & & & & \\
\hline \multirow[t]{5}{*}{ DP4+ } & ${ }^{13} \mathrm{C}$ data & 12.1024 & 87.8775 & 0.0049 & 0.0152 & & & & \\
\hline & All data & 18.7140 & 81.2860 & 0.0000 & 0.0000 & & & & \\
\hline & & \multicolumn{8}{|c|}{ Level of theory: mPW1PW91/6-31+G** (solution. PCM) } \\
\hline & & $87 a$ & $87 b$ & 87c & 87d & - & - & - & - \\
\hline & ${ }^{1} \mathrm{H}$ data & 34.5058 & 65.4942 & 0.0000 & 0.0000 & & & & \\
\hline \multirow[t]{3}{*}{ sDP4+ } & ${ }^{13} \mathrm{C}$ data & 19.4790 & 79.4858 & 0.5462 & 0.4890 & & & & \\
\hline & All data & 11.4348 & 88.5652 & 0.0000 & 0.0000 & & & & \\
\hline & ${ }^{1} \mathrm{H}$ data & 96.8045 & 3.1955 & 0.0000 & 0.0000 & & & & \\
\hline \multirow[t]{3}{*}{ uDP4+ } & ${ }^{13} \mathrm{C}$ data & 46.6182 & 51.9462 & 0.9983 & 0.4373 & & & & \\
\hline & All data & 96.4522 & 3.5478 & 0.0000 & 0.0000 & & & & \\
\hline & ${ }^{1} \mathrm{H}$ data & 94.1039 & 5.8961 & 0.0000 & 0.0000 & & & & \\
\hline \multirow[t]{5}{*}{ DP4+ } & ${ }^{13} \mathrm{C}$ data & 18.0252 & 81.9597 & 0.0108 & 0.0042 & & & & \\
\hline & All data & 77.8278 & 22.1722 & 0.0000 & 0.0000 & & & & \\
\hline & & \multicolumn{8}{|c|}{ Level of theory: mPW1PW91/6-311G* (solution. PCM) } \\
\hline & & $87 a$ & $87 b$ & 87c & 87d & - & - & - & - \\
\hline & ${ }^{1}$ H data & 50.4531 & 49.5469 & 0.0000 & 0.0000 & & & & \\
\hline \multirow[t]{3}{*}{ sDP4+ } & ${ }^{13} \mathrm{C}$ data & 30.4808 & 65.3461 & 1.4895 & 2.6836 & & & & \\
\hline & All data & 32.2026 & 67.7974 & 0.0000 & 0.0000 & & & & \\
\hline & ${ }^{1} \mathrm{H}$ data & 99.0766 & 0.9233 & 0.0000 & 0.0001 & & & & \\
\hline \multirow[t]{3}{*}{ uDP4+ } & ${ }^{13} \mathrm{C}$ data & 29.4049 & 62.6647 & 3.2238 & 4.7067 & & & & \\
\hline & All data & 98.0527 & 1.9473 & 0.0000 & 0.0000 & & & & \\
\hline & ${ }^{1} \mathrm{H}$ data & 99.0932 & 0.9068 & 0.0000 & 0.0000 & & & & \\
\hline \multirow[t]{5}{*}{ DP4+ } & ${ }^{13} \mathrm{C}$ data & 17.8949 & 81.7571 & 0.0959 & 0.2522 & & & & \\
\hline & All data & 95.9867 & 4.0133 & 0.0000 & 0.0000 & & & & \\
\hline & & \multicolumn{8}{|c|}{ Level of theory: mPW1PW91/6-311G** (solution. PCM) } \\
\hline & & $87 a$ & $87 b$ & 87c & 87d & - & - & - & - \\
\hline & ${ }^{1} \mathrm{H}$ data & 50.8326 & 49.1672 & 0.0000 & 0.0001 & & & & \\
\hline \multirow[t]{3}{*}{ sDP4+ } & ${ }^{13} \mathrm{C}$ data & 29.7713 & 68.3403 & 0.6099 & 1.2785 & & & & \\
\hline & All data & 31.0530 & 68.9470 & 0.0000 & 0.0000 & & & & \\
\hline & ${ }^{1} \mathrm{H}$ data & 98.9089 & 1.0907 & 0.0000 & 0.0004 & & & & \\
\hline \multirow[t]{3}{*}{ uDP4+ } & ${ }^{13} \mathrm{C}$ data & 29.3327 & 61.9474 & 3.4630 & 5.2569 & & & & \\
\hline & All data & 97.7241 & 2.2758 & 0.0000 & 0.0001 & & & & \\
\hline & ${ }^{1} \mathrm{H}$ data & 98.9447 & 1.0553 & 0.0000 & 0.0000 & & & & \\
\hline \multirow[t]{5}{*}{ DP4+ } & ${ }^{13} \mathrm{C}$ data & 17.0708 & 82.7566 & 0.0413 & 0.1314 & & & & \\
\hline & All data & 95.0835 & 4.9165 & 0.0000 & 0.0000 & & & & \\
\hline & & \multicolumn{8}{|c|}{ Level of theory: mPW1PW91/6-311+G** (solution. PCM) } \\
\hline & & $87 a$ & $87 b$ & 87c & 87d & - & - & - & - \\
\hline & ${ }^{1} \mathrm{H}$ data & 50.5884 & 49.4114 & 0.0000 & 0.0001 & & & & \\
\hline \multirow[t]{3}{*}{ sDP4+ } & ${ }^{13} \mathrm{C}$ data & 23.7789 & 75.2889 & 0.3069 & 0.6253 & & & & \\
\hline & All data & 24.4348 & 75.5652 & 0.0000 & 0.0000 & & & & \\
\hline & ${ }^{1} \mathrm{H}$ data & 99.3089 & 0.6903 & 0.0000 & 0.0008 & & & & \\
\hline \multirow[t]{3}{*}{ uDP4+ } & ${ }^{13} \mathrm{C}$ data & 34.6320 & 57.7650 & 2.2331 & 5.3699 & & & & \\
\hline & All data & 98.8538 & 1.1461 & 0.0000 & 0.0001 & & & & \\
\hline & ${ }^{1} \mathrm{H}$ data & 99.3257 & 0.6743 & 0.0000 & 0.0000 & & & & \\
\hline \multirow[t]{2}{*}{ DP4+ } & ${ }^{13} \mathrm{C}$ data & 15.9083 & 84.0136 & 0.0132 & 0.0649 & & & & \\
\hline & All data & 96.5386 & 3.4614 & 0.0000 & 0.0000 & & & & \\
\hline
\end{tabular}


$s \mathrm{DP4}+, u \mathrm{DP} 4+$ and DP4+ probabilities (\%) for compound 87b<smiles>CC(=O)O[C@H](C(=O)O[C@H]1C[C@H]2C=C[C@H]1C2)c1ccccc1</smiles>
$87 a$<smiles>O=C(O[C@H]1C[C@H]2C=C[C@@H]1C2)[C@@H](O)c1ccccc1</smiles>

correct isomer<smiles>O=C(O[C@@H]1C[C@H]2C=C[C@@H]1C2)[C@@H](O)c1ccccc1</smiles>

$87 c$<smiles>O=C(OC1CC2C=CC1C2)[C@H](O)c1ccccc1</smiles>

87d

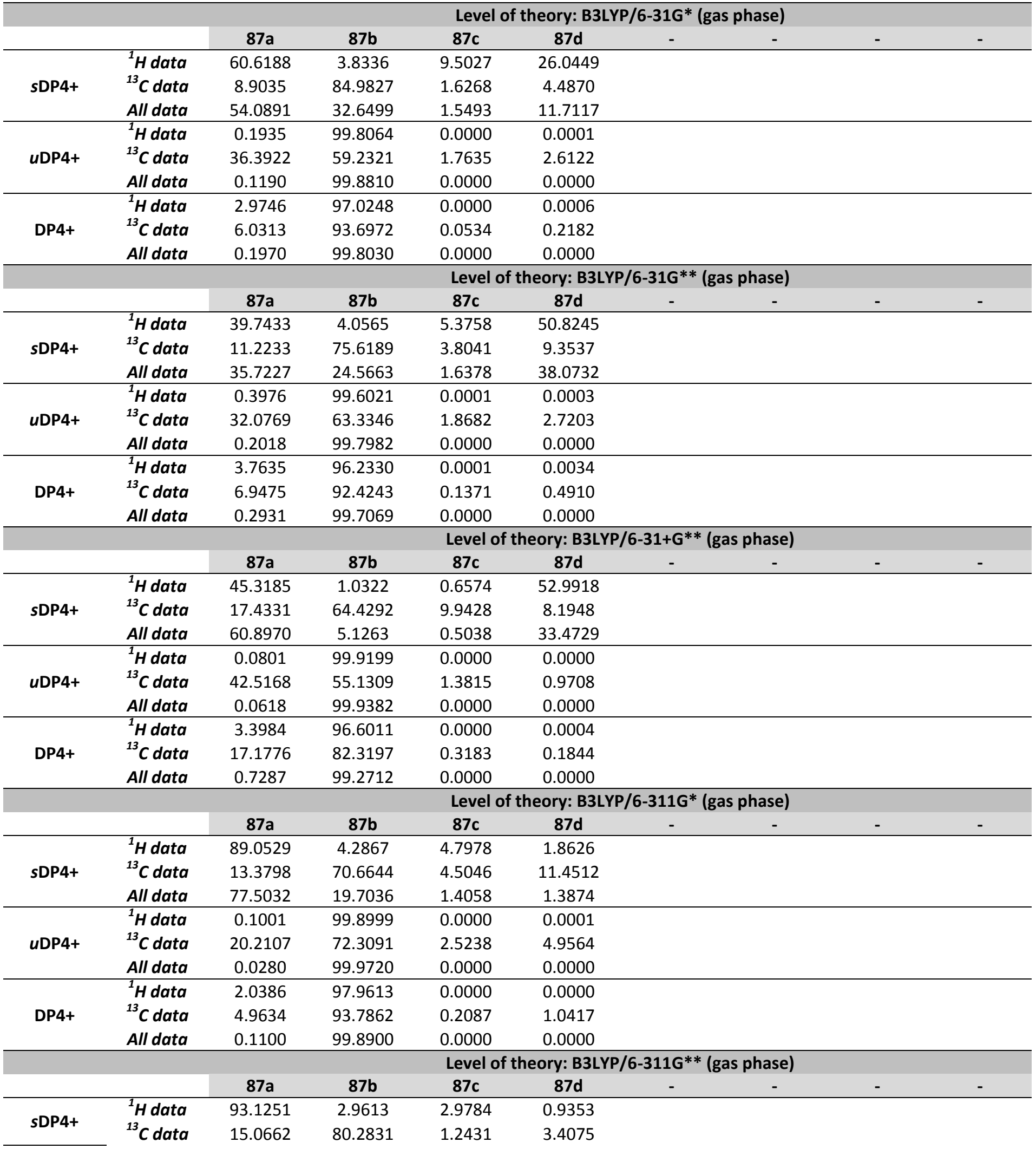




\begin{tabular}{|c|c|c|c|c|c|c|c|c|c|}
\hline & All data & 85.1531 & 14.4288 & 0.2247 & 0.1934 & & & & \\
\hline & ${ }^{1} \mathrm{H}$ data & 0.1320 & 99.8680 & 0.0000 & 0.0000 & & & & \\
\hline \multirow[t]{3}{*}{ uDP4+ } & ${ }^{13} \mathrm{C}$ data & 21.6825 & 72.4002 & 2.0084 & 3.9089 & & & & \\
\hline & All data & 0.0396 & 99.9604 & 0.0000 & 0.0000 & & & & \\
\hline & ${ }^{1} \mathrm{H}$ data & 3.9901 & 96.0098 & 0.0000 & 0.0000 & & & & \\
\hline \multirow[t]{5}{*}{ DP4+ } & ${ }^{13} \mathrm{C}$ data & 5.3074 & 94.4356 & 0.0406 & 0.2164 & & & & \\
\hline & All data & 0.2330 & 99.7670 & 0.0000 & 0.0000 & & & & \\
\hline & & \multicolumn{8}{|c|}{ Level of theory: B3LYP/6-311+G** (gas phase) } \\
\hline & & $87 a$ & $87 b$ & 87c & 87d & - & - & - & - \\
\hline & ${ }^{1} \mathrm{H}$ data & 70.1590 & 15.5360 & 9.8334 & 4.4716 & & & & \\
\hline \multirow[t]{3}{*}{ sDP4+ } & ${ }^{13} \mathrm{C}$ data & 15.5695 & 82.7442 & 0.3811 & 1.3052 & & & & \\
\hline & All data & 45.7536 & 53.8450 & 0.1570 & 0.2445 & & & & \\
\hline & ${ }^{1} \mathrm{H}$ data & 0.1095 & 99.8905 & 0.0000 & 0.0000 & & & & \\
\hline \multirow[t]{3}{*}{ uDP4+ } & ${ }^{13} \mathrm{C}$ data & 25.2611 & 69.9942 & 1.1938 & 3.5508 & & & & \\
\hline & All data & 0.0396 & 99.9604 & 0.0000 & 0.0000 & & & & \\
\hline & ${ }^{1} \mathrm{H}$ data & 0.4927 & 99.5073 & 0.0000 & 0.0000 & & & & \\
\hline \multirow[t]{5}{*}{ DP4+ } & ${ }^{13} \mathrm{C}$ data & 6.3538 & 93.5639 & 0.0074 & 0.0749 & & & & \\
\hline & All data & 0.0336 & 99.9664 & 0.0000 & 0.0000 & & & & \\
\hline & & \multicolumn{8}{|c|}{ Level of theory: B3LYP/6-31G* (solution. PCM) } \\
\hline & & $87 a$ & $87 b$ & 87c & 87d & - & - & - & - \\
\hline & ${ }^{1}$ H data & 39.1630 & 2.1408 & 3.0453 & 55.6509 & & & & \\
\hline \multirow[t]{3}{*}{ sDP4+ } & ${ }^{13} \mathrm{C}$ data & 18.4652 & 78.4537 & 0.9014 & 2.1797 & & & & \\
\hline & All data & 71.2359 & 16.5445 & 0.2704 & 11.9492 & & & & \\
\hline & ${ }^{1} \mathrm{H}$ data & 3.8364 & 96.1636 & 0.0000 & 0.0000 & & & & \\
\hline \multirow[t]{3}{*}{ uDP4+ } & ${ }^{13} \mathrm{C}$ data & 33.9035 & 63.0066 & 1.2741 & 1.8158 & & & & \\
\hline & All data & 2.1016 & 97.8984 & 0.0000 & 0.0000 & & & & \\
\hline & ${ }^{1} \mathrm{H}$ data & 42.1907 & 57.8093 & 0.0000 & 0.0000 & & & & \\
\hline \multirow[t]{5}{*}{ DP4+ } & ${ }^{13} \mathrm{C}$ data & 11.2308 & 88.6775 & 0.0206 & 0.0710 & & & & \\
\hline & All data & 8.4611 & 91.5389 & 0.0000 & 0.0000 & & & & \\
\hline & & \multicolumn{8}{|c|}{ Level of theory: B3LYP/6-31G** (solution. PCM) } \\
\hline & & $87 a$ & $87 b$ & 87c & 87d & - & - & - & - \\
\hline & ${ }^{1} \mathrm{H}$ data & 19.4724 & 2.2987 & 1.9912 & 76.2377 & & & & \\
\hline \multirow[t]{3}{*}{ sDP4+ } & ${ }^{13} \mathrm{C}$ data & 21.6328 & 71.3501 & 2.2847 & 4.7324 & & & & \\
\hline & All data & 44.3138 & 17.2536 & 0.4786 & 37.9541 & & & & \\
\hline & ${ }^{1} \mathrm{H}$ data & 1.0507 & 98.9493 & 0.0000 & 0.0000 & & & & \\
\hline \multirow[t]{3}{*}{ uDP4+ } & ${ }^{13} \mathrm{C}$ data & 37.4573 & 59.6668 & 1.1357 & 1.7402 & & & & \\
\hline & All data & 0.6622 & 99.3378 & 0.0000 & 0.0000 & & & & \\
\hline & ${ }^{1} \mathrm{H}$ data & 8.2526 & 91.7473 & 0.0000 & 0.0001 & & & & \\
\hline \multirow[t]{5}{*}{ DP4+ } & ${ }^{13} \mathrm{C}$ data & 15.9560 & 83.8307 & 0.0511 & 0.1622 & & & & \\
\hline & All data & 1.6832 & 98.3168 & 0.0000 & 0.0000 & & & & \\
\hline & & \multicolumn{8}{|c|}{ Level of theory: B3LYP/6-31+G** (solution. PCM) } \\
\hline & & $87 a$ & $87 b$ & 87c & 87d & - & - & - & - \\
\hline & ${ }^{1} \mathrm{H}$ data & 31.4516 & 0.6161 & 0.2222 & 67.7102 & & & & \\
\hline \multirow[t]{3}{*}{ sDP4+ } & ${ }^{13} \mathrm{C}$ data & 25.8071 & 64.0502 & 5.6116 & 4.5311 & & & & \\
\hline & All data & 70.0212 & 3.4041 & 0.1075 & 26.4671 & & & & \\
\hline & ${ }^{1} \mathrm{H}$ data & 0.1690 & 99.8310 & 0.0000 & 0.0000 & & & & \\
\hline uDP4+ & ${ }^{13} \mathrm{C}$ data & 49.9336 & 48.1048 & 1.3424 & 0.6192 & & & & \\
\hline & All data & 0.1754 & 99.8246 & 0.0000 & 0.0000 & & & & \\
\hline & ${ }^{1} \mathrm{H}$ data & 7.9557 & 92.0440 & 0.0000 & 0.0003 & & & & \\
\hline DP4+ & ${ }^{13} \mathrm{C}$ data & 29.4204 & 70.3436 & 0.1720 & 0.0641 & & & & \\
\hline & All data & 3.4889 & 96.5111 & 0.0000 & 0.0000 & & & & \\
\hline & & & & Level of $t$ & ry: B3LYP & & PC & & \\
\hline & & $87 a$ & $87 b$ & $87 c$ & 87d & - & - & - & - \\
\hline & ${ }^{1} \mathrm{H}$ data & 59.3727 & 17.7942 & 14.7842 & 8.0489 & & & & \\
\hline sDP4+ & ${ }^{13} \mathrm{C}$ data & 27.5411 & 61.1653 & 3.4688 & 7.8248 & & & & \\
\hline & All data & 57.6209 & 38.3527 & 1.8071 & 2.2193 & & & & \\
\hline
\end{tabular}




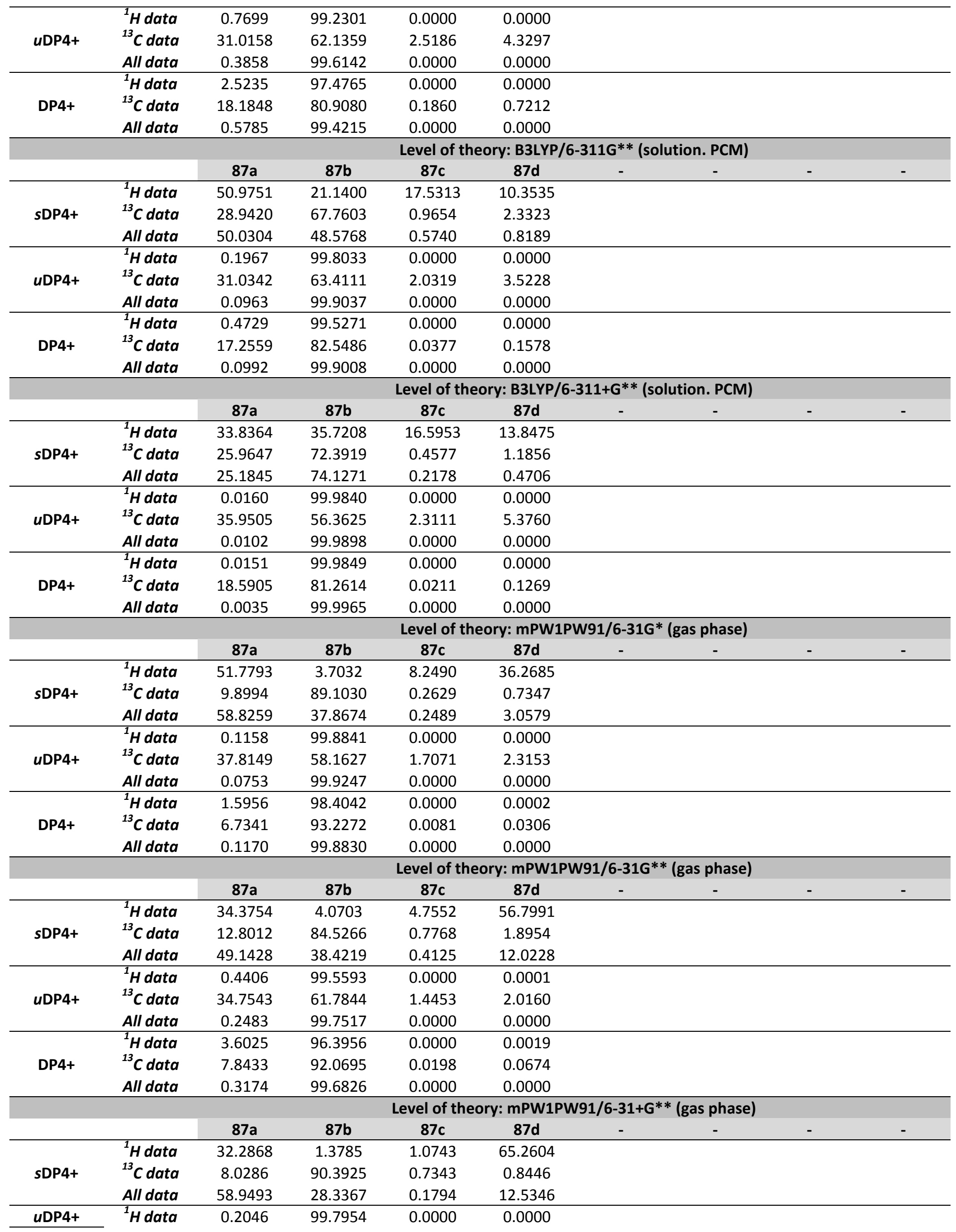




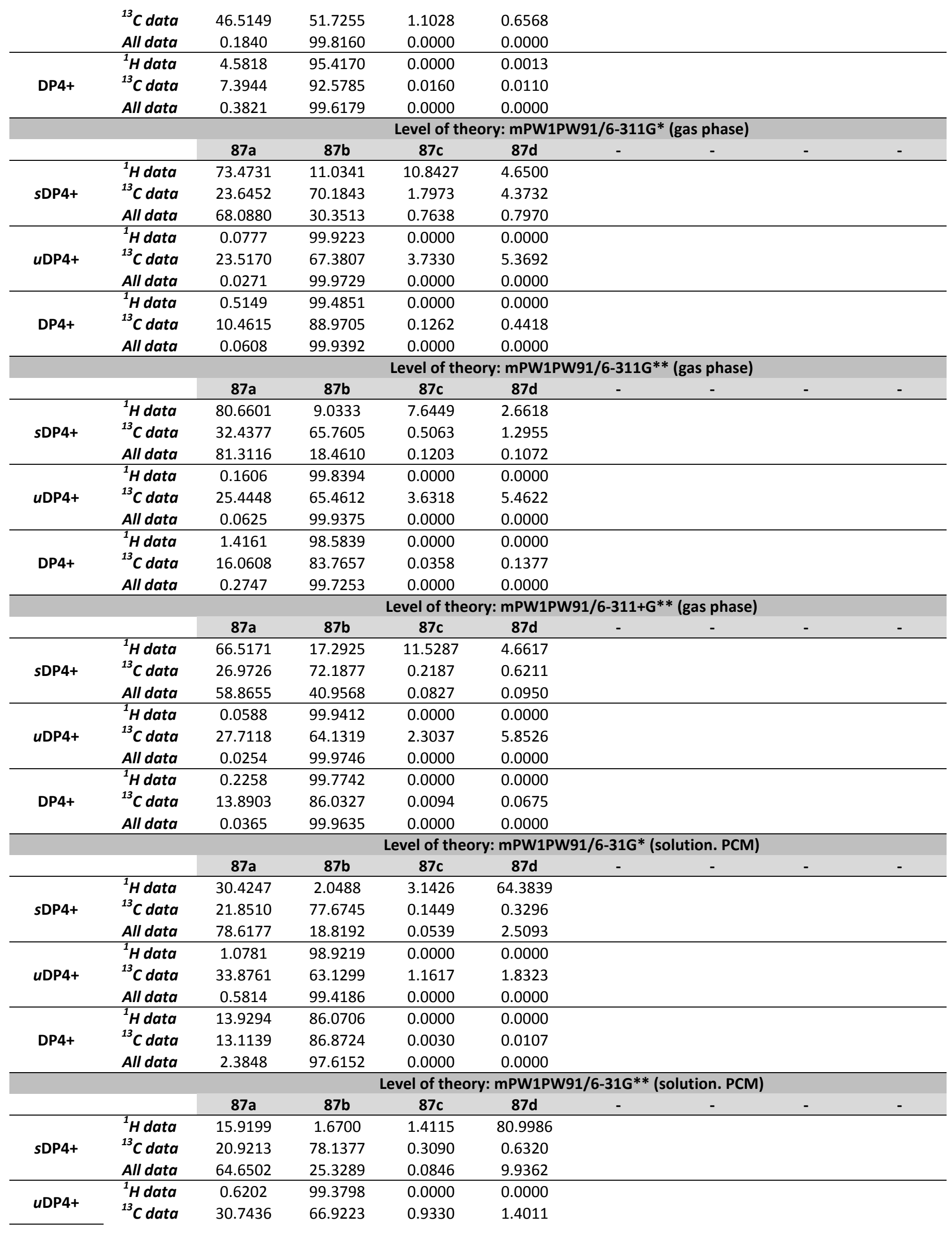




\begin{tabular}{|c|c|c|c|c|c|c|c|c|c|}
\hline & All data & 0.2859 & 99.7141 & 0.0000 & 0.0000 & & & & \\
\hline & ${ }^{1}$ H data & 5.6149 & 94.3851 & 0.0000 & 0.0000 & & & & \\
\hline \multirow[t]{5}{*}{ DP4+ } & ${ }^{13} \mathrm{C}$ data & 10.9508 & 89.0292 & 0.0049 & 0.0151 & & & & \\
\hline & All data & 0.7264 & 99.2736 & 0.0000 & 0.0000 & & & & \\
\hline & & \multicolumn{8}{|c|}{ Level of theory: mPW1PW91/6-31+G** (solution. PCM) } \\
\hline & & $87 a$ & $87 b$ & 87c & 87d & - & - & - & - \\
\hline & ${ }^{1} \mathrm{H}$ data & 0.3116 & 99.6884 & 0.0000 & 0.0000 & & & & \\
\hline \multirow[t]{3}{*}{ sDP4+ } & ${ }^{13} \mathrm{C}$ data & 13.7363 & 85.2576 & 0.4802 & 0.5259 & & & & \\
\hline & All data & 0.0503 & 99.9497 & 0.0000 & 0.0000 & & & & \\
\hline & ${ }^{1} \mathrm{H}$ data & 0.0115 & 99.9885 & 0.0000 & 0.0000 & & & & \\
\hline \multirow[t]{3}{*}{ uDP4+ } & ${ }^{13} \mathrm{C}$ data & 39.4019 & 59.1630 & 1.0253 & 0.4098 & & & & \\
\hline & All data & 0.0076 & 99.9924 & 0.0000 & 0.0000 & & & & \\
\hline & ${ }^{1} \mathrm{H}$ data & 0.0000 & 100.0000 & 0.0000 & 0.0000 & & & & \\
\hline \multirow[t]{5}{*}{ DP4+ } & ${ }^{13} \mathrm{C}$ data & 9.6890 & 90.2983 & 0.0088 & 0.0039 & & & & \\
\hline & All data & 0.0000 & 100.0000 & 0.0000 & 0.0000 & & & & \\
\hline & & \multicolumn{8}{|c|}{ Level of theory: mPW1PW91/6-311G* (solution. PCM) } \\
\hline & & $87 a$ & $87 b$ & $87 c$ & 87d & - & - & - & - \\
\hline & ${ }^{1} \mathrm{H}$ data & 44.3705 & 25.1798 & 19.4778 & 10.9719 & & & & \\
\hline \multirow[t]{2}{*}{ sDP4+ } & ${ }^{13} \mathrm{C}$ data & 35.2428 & 61.3696 & 1.0720 & 2.3157 & & & & \\
\hline & All data & 49.5592 & 48.9738 & 0.6617 & 0.8052 & & & & \\
\hline \multirow{3}{*}{ uDP4+ } & ${ }^{1} \mathrm{H}$ data & 0.3999 & 99.6001 & 0.0000 & 0.0000 & & & & \\
\hline & ${ }^{13} \mathrm{C}$ data & 33.8600 & 59.2605 & 2.8536 & 4.0259 & & & & \\
\hline & All data & 0.2289 & 99.7711 & 0.0000 & 0.0000 & & & & \\
\hline \multirow{3}{*}{ DP4+ } & ${ }^{1} \mathrm{H}$ data & 0.7025 & 99.2974 & 0.0000 & 0.0000 & & & & \\
\hline & ${ }^{13} \mathrm{C}$ data & 24.6427 & 75.1016 & 0.0632 & 0.1925 & & & & \\
\hline & All data & 0.2316 & 99.7684 & 0.0000 & 0.0000 & & & & \\
\hline & & \multicolumn{8}{|c|}{ Level of theory: mPW1PW91/6-311G** (solution. PCM) } \\
\hline & & $87 a$ & $87 b$ & 87c & 87d & - & - & - & - \\
\hline \multirow{3}{*}{ sDP4+ } & ${ }^{1} \mathrm{H}$ data & 40.8240 & 26.3078 & 20.4372 & 12.4310 & & & & \\
\hline & ${ }^{13} \mathrm{C}$ data & 35.3940 & 63.3973 & 0.3676 & 0.8411 & & & & \\
\hline & All data & 46.1529 & 53.2732 & 0.2400 & 0.3340 & & & & \\
\hline \multirow{3}{*}{$u$ DP4+ } & ${ }^{1} \mathrm{H}$ data & 0.0954 & 99.9046 & 0.0000 & 0.0000 & & & & \\
\hline & ${ }^{13} \mathrm{C}$ data & 32.4302 & 60.0451 & 3.0196 & 4.5052 & & & & \\
\hline & All data & 0.0515 & 99.9485 & 0.0000 & 0.0000 & & & & \\
\hline \multirow{5}{*}{ DP4+ } & ${ }^{1} \mathrm{H}$ data & 0.1479 & 99.8521 & 0.0000 & 0.0000 & & & & \\
\hline & ${ }^{13} \mathrm{C}$ data & 23.1445 & 76.7567 & 0.0224 & 0.0764 & & & & \\
\hline & All data & 0.0447 & 99.9553 & 0.0000 & 0.0000 & & & & \\
\hline & & \multicolumn{8}{|c|}{ Level of theory: mPW1PW91/6-311+G** (solution. PCM) } \\
\hline & & $87 a$ & $87 b$ & 87c & 87d & - & - & - & - \\
\hline \multirow{3}{*}{ sDP4+ } & ${ }^{1} \mathrm{H}$ data & 31.3182 & 36.6334 & 19.0974 & 12.9509 & & & & \\
\hline & ${ }^{13} \mathrm{C}$ data & 24.0155 & 75.1764 & 0.2302 & 0.5779 & & & & \\
\hline & All data & 21.3795 & 78.2828 & 0.1249 & 0.2127 & & & & \\
\hline \multirow{3}{*}{ uDP4+ } & ${ }^{1} \mathrm{H}$ data & 0.0218 & 99.9781 & 0.0000 & 0.0000 & & & & \\
\hline & ${ }^{13} \mathrm{C}$ data & 33.8301 & 59.5628 & 1.8205 & 4.7866 & & & & \\
\hline & All data & 0.0124 & 99.9876 & 0.0000 & 0.0000 & & & & \\
\hline \multirow{3}{*}{ DP4+ } & ${ }^{1} \mathrm{H}$ data & 0.0187 & 99.9813 & 0.0000 & 0.0000 & & & & \\
\hline & ${ }^{13} \mathrm{C}$ data & 15.3485 & 84.5914 & 0.0079 & 0.0523 & & & & \\
\hline & All data & 0.0034 & 99.9966 & 0.0000 & 0.0000 & & & & \\
\hline
\end{tabular}


$s \mathrm{DP} 4+, u \mathrm{DP} 4+$ and DP4+ probabilities (\%) for compound 87c<smiles>CC(=O)O[C@H](C(=O)O[C@H]1C[C@H]2C=C[C@H]1C2)c1ccccc1</smiles>

$87 a$<smiles>O=C(OC1CC2C=CC1C2)[C@@H](O)c1ccccc1</smiles>

87b<smiles>O=C(O[C@@H]1C[C@H]2C=C[C@@H]1C2)[C@@H](O)c1ccccc1</smiles>

correct isomer<smiles>O=C(OC1CC2C=CC1C2)[C@H](O)c1ccccc1</smiles>

87d

\begin{tabular}{|c|c|c|c|c|c|c|c|c|c|}
\hline & & \multicolumn{8}{|c|}{ Level of theory: B3LYP/6-31G* (gas phase) } \\
\hline & & $87 a$ & $87 \mathrm{~b}$ & $87 c$ & 87d & - & - & - & - \\
\hline \multirow{3}{*}{ sDP4+ } & ${ }^{1} \mathrm{H}$ data & 14.9606 & 69.4965 & 9.7333 & 5.8096 & & & & \\
\hline & ${ }^{13} \mathrm{C}$ data & 0.2334 & 1.2359 & 24.0758 & 74.4549 & & & & \\
\hline & All data & 0.4616 & 11.3571 & 30.9858 & 57.1954 & & & & \\
\hline & ${ }^{1} \mathrm{H}$ data & 0.0000 & 0.0235 & 98.2614 & 1.7150 & & & & \\
\hline \multirow[t]{2}{*}{ uDP4+ } & ${ }^{13} \mathrm{C}$ data & 9.2770 & 12.7324 & 30.5176 & 47.4729 & & & & \\
\hline & All data & 0.0000 & 0.0097 & 97.3472 & 2.6430 & & & & \\
\hline \multirow{6}{*}{ DP4+ } & ${ }^{1} \mathrm{H}$ data & 0.0001 & 0.1691 & 98.8016 & 1.0293 & & & & \\
\hline & ${ }^{13} \mathrm{C}$ data & 0.0505 & 0.3670 & 17.1378 & 82.4447 & & & & \\
\hline & All data & 0.0000 & 0.0035 & 95.2243 & 4.7722 & & & & \\
\hline & & \multicolumn{8}{|c|}{ Level of theory: B3LYP/6-31G** (gas phase) } \\
\hline & & $87 a$ & $87 \mathrm{~b}$ & $87 c$ & 87d & - & - & - & - \\
\hline & ${ }^{1} \mathrm{H}$ data & 11.3943 & 69.5806 & 10.8484 & 8.1767 & & & & \\
\hline \multirow[t]{2}{*}{ sDP4+ } & ${ }^{13} \mathrm{C}$ data & 0.3971 & 1.2192 & 23.9768 & 74.4068 & & & & \\
\hline & All data & 0.4724 & 8.8563 & 27.1551 & 63.5162 & & & & \\
\hline \multirow{3}{*}{ uDP4+ } & ${ }^{1} \mathrm{H}$ data & 0.0000 & 0.0259 & 94.3179 & 5.6562 & & & & \\
\hline & ${ }^{13} \mathrm{C}$ data & 8.0201 & 13.9139 & 31.5432 & 46.5228 & & & & \\
\hline & All data & 0.0000 & 0.0111 & 91.8637 & 8.1252 & & & & \\
\hline \multirow{5}{*}{ DP4+ } & ${ }^{1} \mathrm{H}$ data & 0.0000 & 0.1681 & 95.5146 & 4.3173 & & & & \\
\hline & ${ }^{13} \mathrm{C}$ data & 0.0752 & 0.4003 & 17.8455 & 81.6791 & & & & \\
\hline & All data & 0.0000 & 0.0033 & 82.8554 & 17.1413 & & & & \\
\hline & & \multicolumn{8}{|c|}{ Level of theory: B3LYP/6-31+G** (gas phase) } \\
\hline & & $87 a$ & $87 \mathrm{~b}$ & $87 \mathrm{c}$ & 87d & - & - & - & - \\
\hline & ${ }^{1} \mathrm{H}$ data & 0.7919 & 44.3490 & 29.2722 & 25.5869 & & & & \\
\hline \multirow[t]{2}{*}{ sDP4+ } & ${ }^{13} \mathrm{C}$ data & 2.3156 & 2.4684 & 47.1194 & 48.0965 & & & & \\
\hline & All data & 0.0674 & 4.0228 & 50.6862 & 45.2236 & & & & \\
\hline \multirow{3}{*}{ uDP4+ } & ${ }^{1} \mathrm{H}$ data & 0.0000 & 0.0049 & 98.3204 & 1.6746 & & & & \\
\hline & ${ }^{13} \mathrm{C}$ data & 10.0167 & 15.5397 & 36.5407 & 37.9029 & & & & \\
\hline & All data & 0.0000 & 0.0021 & 98.2619 & 1.7360 & & & & \\
\hline \multirow{5}{*}{ DP4+ } & ${ }^{1} \mathrm{H}$ data & 0.0000 & 0.0075 & 98.5257 & 1.4669 & & & & \\
\hline & ${ }^{13} \mathrm{C}$ data & 0.6432 & 1.0636 & 47.7432 & 50.5500 & & & & \\
\hline & All data & 0.0000 & 0.0002 & 98.4480 & 1.5519 & & & & \\
\hline & & \multicolumn{8}{|c|}{ Level of theory: B3LYP/6-311G* (gas phase) } \\
\hline & & $87 a$ & 87b & $87 c$ & 87d & - & - & - & - \\
\hline \multirow{3}{*}{ sDP4+ } & ${ }^{1} \mathrm{H}$ data & 87.5431 & 4.7827 & 5.4937 & 2.1805 & & & & \\
\hline & ${ }^{13} \mathrm{C}$ data & 0.5030 & 2.0291 & 32.9118 & 64.5561 & & & & \\
\hline & All data & 11.7318 & 2.5858 & 48.1754 & 37.5070 & & & & \\
\hline \multirow{3}{*}{ uDP4+ } & ${ }^{1} \mathrm{H}$ data & 0.0001 & 0.0276 & 87.8010 & 12.1713 & & & & \\
\hline & ${ }^{13} \mathrm{C}$ data & 3.9742 & 19.6304 & 25.5743 & 50.8211 & & & & \\
\hline & All data & 0.0000 & 0.0189 & 78.3875 & 21.5935 & & & & \\
\hline \multirow{5}{*}{ DP4+ } & ${ }^{1} \mathrm{H}$ data & 0.0019 & 0.0260 & 94.7583 & 5.2138 & & & & \\
\hline & ${ }^{13} \mathrm{C}$ data & 0.0480 & 0.9565 & 20.2120 & 78.7835 & & & & \\
\hline & All data & 0.0000 & 0.0011 & 82.3397 & 17.6593 & & & & \\
\hline & & \multicolumn{8}{|c|}{ Level of theory: B3LYP/6-311G** (gas phase) } \\
\hline & & $87 a$ & $87 \mathrm{~b}$ & $87 \mathrm{c}$ & 87d & - & - & - & - \\
\hline \multirow{2}{*}{ sDP4+ } & ${ }^{1} \mathrm{H}$ data & 91.8762 & 3.4410 & 3.5428 & 1.1400 & & & & \\
\hline & ${ }^{13} \mathrm{C}$ data & 0.9441 & 3.9174 & 31.0120 & 64.1264 & & & & \\
\hline
\end{tabular}




\begin{tabular}{|c|c|c|c|c|c|c|c|c|c|}
\hline & All data & 30.6300 & 4.7599 & 38.7967 & 25.8134 & & & & \\
\hline & ${ }^{1} \mathrm{H}$ data & 0.0000 & 0.0019 & 80.7149 & 19.2832 & & & & \\
\hline \multirow[t]{3}{*}{ uDP4+ } & ${ }^{13}$ C data & 6.1869 & 33.2343 & 19.7660 & 40.8127 & & & & \\
\hline & All data & 0.0000 & 0.0027 & 66.9645 & 33.0328 & & & & \\
\hline & ${ }^{1} \mathrm{H}$ data & 0.0002 & 0.0022 & 92.8593 & 7.1383 & & & & \\
\hline \multirow[t]{5}{*}{ DP4+ } & ${ }^{13} \mathrm{C}$ data & 0.1735 & 3.8676 & 18.2100 & 77.7488 & & & & \\
\hline & All data & 0.0000 & 0.0004 & 75.2890 & 24.7106 & & & & \\
\hline & & \multicolumn{8}{|c|}{ Level of theory: B3LYP/6-311+G** (gas phase) } \\
\hline & & $87 a$ & $87 b$ & 87c & 87d & - & - & - & - \\
\hline & ${ }^{1} \mathrm{H}$ data & 67.5961 & 16.8615 & 10.5652 & 4.9771 & & & & \\
\hline \multirow[t]{3}{*}{ sDP4+ } & ${ }^{13} \mathrm{C}$ data & 1.4231 & 6.3744 & 27.9816 & 64.2209 & & & & \\
\hline & All data & 11.7465 & 13.1244 & 36.0990 & 39.0301 & & & & \\
\hline & ${ }^{1} \mathrm{H}$ data & 0.0000 & 0.0058 & 87.3978 & 12.5964 & & & & \\
\hline \multirow[t]{3}{*}{ uDP4+ } & ${ }^{13} \mathrm{C}$ data & 8.0031 & 31.4453 & 16.7999 & 43.7517 & & & & \\
\hline & All data & 0.0000 & 0.0090 & 72.7023 & 27.2887 & & & & \\
\hline & ${ }^{1} \mathrm{H}$ data & 0.0002 & 0.0099 & 93.6326 & 6.3573 & & & & \\
\hline \multirow[t]{5}{*}{ DP4+ } & ${ }^{13} \mathrm{C}$ data & 0.3262 & 5.7406 & 13.4630 & 80.4702 & & & & \\
\hline & All data & 0.0000 & 0.0032 & 71.1303 & 28.8665 & & & & \\
\hline & & \multicolumn{8}{|c|}{ Level of theory: B3LYP/6-31G* (solution. PCM) } \\
\hline & & $87 a$ & $87 b$ & 87c & 87d & - & - & - & - \\
\hline & ${ }^{1}$ H data & 4.4669 & 44.2645 & 31.9051 & 19.3634 & & & & \\
\hline \multirow[t]{3}{*}{ sDP4+ } & ${ }^{13}$ C data & 0.4297 & 1.3409 & 32.9326 & 65.2968 & & & & \\
\hline & All data & 0.0808 & 2.4978 & 44.2154 & 53.2061 & & & & \\
\hline & ${ }^{1} \mathrm{H}$ data & 0.0000 & 0.0005 & 98.4463 & 1.5532 & & & & \\
\hline \multirow[t]{3}{*}{ uDP4+ } & ${ }^{13} \mathrm{C}$ data & 9.7349 & 15.7227 & 30.9274 & 43.6150 & & & & \\
\hline & All data & 0.0000 & 0.0002 & 97.8232 & 2.1766 & & & & \\
\hline & ${ }^{1} \mathrm{H}$ data & 0.0000 & 0.0007 & 99.0509 & 0.9484 & & & & \\
\hline \multirow[t]{5}{*}{ DP4+ } & ${ }^{13} \mathrm{C}$ data & 0.1075 & 0.5417 & 26.1715 & 73.1792 & & & & \\
\hline & All data & 0.0000 & 0.0000 & 97.3924 & 2.6076 & & & & \\
\hline & & \multicolumn{8}{|c|}{ Level of theory: B3LYP/6-31G** (solution. PCM) } \\
\hline & & $87 a$ & $87 b$ & 87c & 87d & - & - & - & - \\
\hline & ${ }^{1} \mathrm{H}$ data & 5.2328 & 45.1898 & 26.7630 & 22.8144 & & & & \\
\hline \multirow[t]{3}{*}{ sDP4+ } & ${ }^{13} \mathrm{C}$ data & 1.1837 & 2.8781 & 34.9615 & 60.9767 & & & & \\
\hline & All data & 0.2515 & 5.2803 & 37.9881 & 56.4801 & & & & \\
\hline & ${ }^{1} \mathrm{H}$ data & 0.0000 & 0.0004 & 98.7703 & 1.2292 & & & & \\
\hline \multirow[t]{3}{*}{ uDP4+ } & ${ }^{13}$ C data & 11.5569 & 16.7016 & 31.0361 & 40.7053 & & & & \\
\hline & All data & 0.0000 & 0.0002 & 98.3937 & 1.6060 & & & & \\
\hline & ${ }^{1} \mathrm{H}$ data & 0.0000 & 0.0007 & 98.9495 & 1.0498 & & & & \\
\hline \multirow[t]{5}{*}{ DP4+ } & ${ }^{13}$ C data & 0.3770 & 1.3246 & 29.9008 & 68.3976 & & & & \\
\hline & All data & 0.0000 & 0.0000 & 97.6307 & 2.3693 & & & & \\
\hline & & \multicolumn{8}{|c|}{ Level of theory: B3LYP/6-31+G** (solution. PCM) } \\
\hline & & $87 a$ & $87 b$ & 87c & 87d & - & - & - & - \\
\hline & ${ }^{1} \mathrm{H}$ data & 0.1938 & 10.7091 & 41.3749 & 47.7222 & & & & \\
\hline \multirow[t]{3}{*}{ sDP4+ } & ${ }^{13} \mathrm{C}$ data & 2.4673 & 3.1553 & 41.6999 & 52.6775 & & & & \\
\hline & All data & 0.0112 & 0.7907 & 40.3729 & 58.8252 & & & & \\
\hline & ${ }^{1} \mathrm{H}$ data & 0.0000 & 0.0001 & 99.8616 & 0.1383 & & & & \\
\hline uDP4+ & ${ }^{13} \mathrm{C}$ data & 12.5282 & 13.2760 & 45.4136 & 28.7822 & & & & \\
\hline & All data & 0.0000 & 0.0000 & 99.9123 & 0.0877 & & & & \\
\hline & ${ }^{1} \mathrm{H}$ data & 0.0000 & 0.0000 & 99.8405 & 0.1595 & & & & \\
\hline DP4+ & ${ }^{13} \mathrm{C}$ data & 0.8876 & 1.2028 & 54.3755 & 43.5342 & & & & \\
\hline & All data & 0.0000 & 0.0000 & 99.8723 & 0.1277 & & & & \\
\hline & & & & Level of $t$ & ry: B3LYP & C* & PC & & \\
\hline & & $87 a$ & $87 b$ & $87 c$ & 87d & - & - & - & - \\
\hline & ${ }^{1} \mathrm{H}$ data & 57.8259 & 18.0467 & 15.5337 & 8.5937 & & & & \\
\hline sDP4+ & ${ }^{13} \mathrm{C}$ data & 1.4957 & 2.4076 & 36.1789 & 59.9177 & & & & \\
\hline & All data & 7.1668 & 3.6003 & 46.5668 & 42.6661 & & & & \\
\hline
\end{tabular}




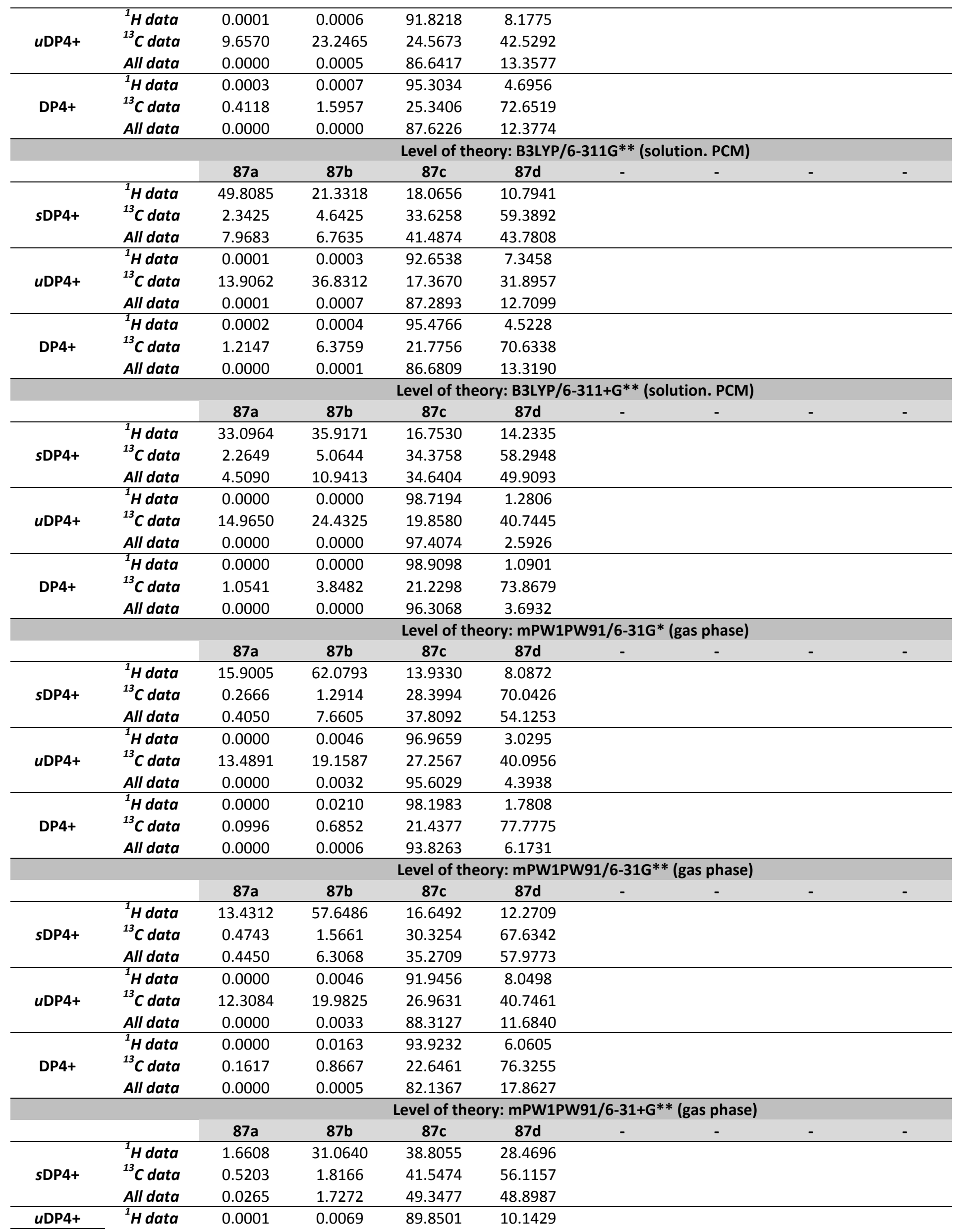




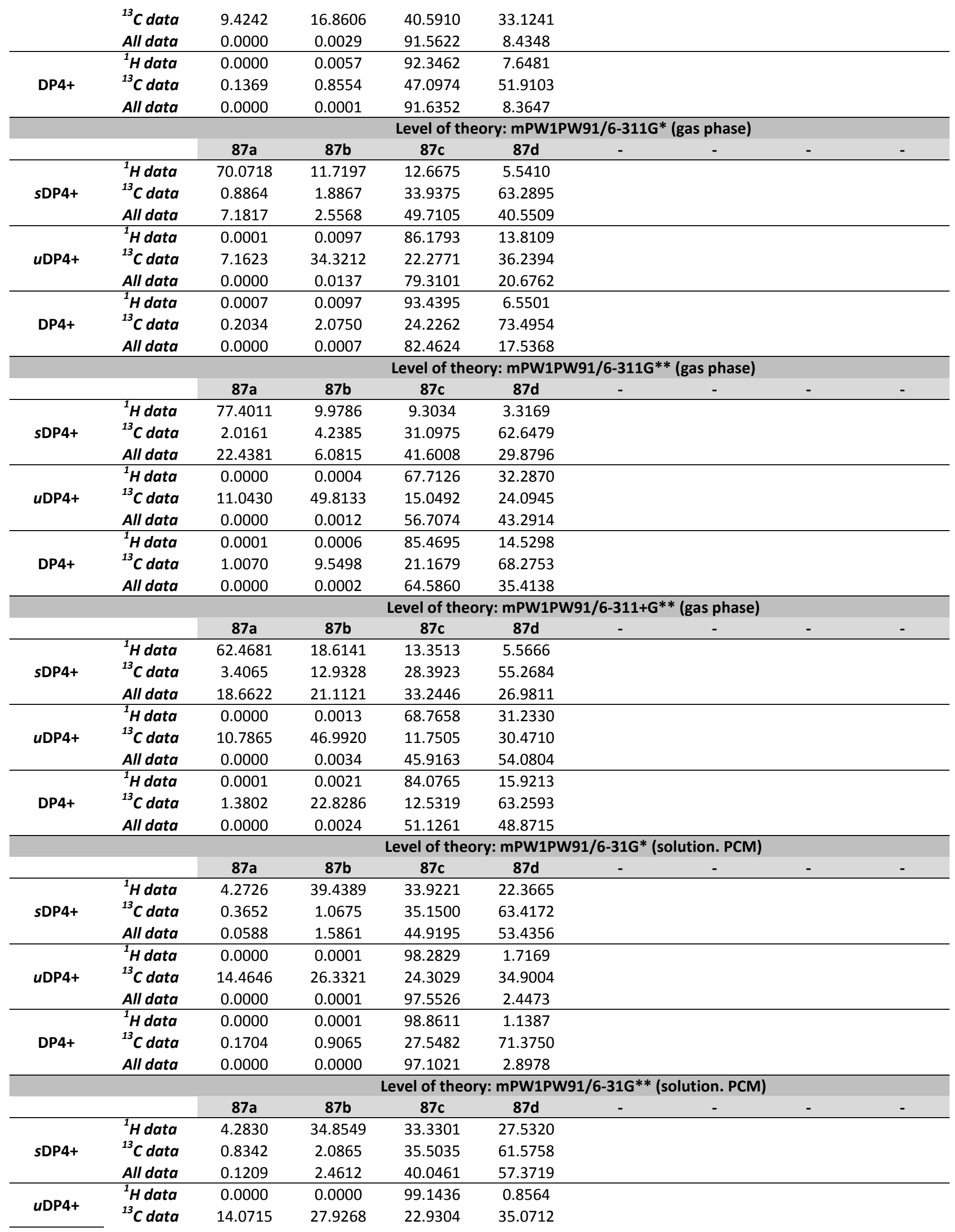




\begin{tabular}{|c|c|c|c|c|c|c|c|c|c|}
\hline & All data & 0.0000 & 0.0000 & 98.6961 & 1.3039 & & & & \\
\hline & ${ }^{1} \mathrm{H}$ data & 0.0000 & 0.0000 & 99.2915 & 0.7085 & & & & \\
\hline \multirow[t]{5}{*}{ DP4+ } & ${ }^{13} \mathrm{C}$ data & 0.3857 & 1.9145 & 26.7477 & 70.9521 & & & & \\
\hline & All data & 0.0000 & 0.0000 & 98.1424 & 1.8576 & & & & \\
\hline & & \multicolumn{8}{|c|}{ Level of theory: mPW1PW91/6-31+G** (solution. PCM) } \\
\hline & & $87 a$ & $87 b$ & 87c & 87d & - & - & - & - \\
\hline & ${ }^{1} \mathrm{H}$ data & 0.0003 & 0.0008 & 99.2315 & 0.7675 & & & & \\
\hline \multirow[t]{2}{*}{ sDP4+ } & ${ }^{13} \mathrm{C}$ data & 0.8223 & 1.9837 & 43.1757 & 54.0183 & & & & \\
\hline & All data & 0.0000 & 0.0000 & 99.0416 & 0.9584 & & & & \\
\hline \multirow{3}{*}{ uDP4+ } & ${ }^{1} \mathrm{H}$ data & 0.0000 & 0.0000 & 99.3240 & 0.6760 & & & & \\
\hline & ${ }^{13} \mathrm{C}$ data & 11.7895 & 22.4932 & 42.3208 & 23.3966 & & & & \\
\hline & All data & 0.0000 & 0.0000 & 99.6252 & 0.3748 & & & & \\
\hline \multirow{5}{*}{ DP4+ } & ${ }^{1}$ H data & 0.0000 & 0.0000 & 99.9947 & 0.0053 & & & & \\
\hline & ${ }^{13} \mathrm{C}$ data & 0.3082 & 1.4186 & 58.0924 & 40.1808 & & & & \\
\hline & All data & 0.0000 & 0.0000 & 99.9964 & 0.0036 & & & & \\
\hline & & \multicolumn{8}{|c|}{ Level of theory: mPW1PW91/6-311G* (solution. PCM) } \\
\hline & & $87 a$ & $87 b$ & 87c & 87d & - & - & - & - \\
\hline \multirow{3}{*}{ sDP4+ } & ${ }^{1} \mathrm{H}$ data & 41.9678 & 24.6576 & 21.2505 & 12.1241 & & & & \\
\hline & ${ }^{13} \mathrm{C}$ data & 1.8407 & 2.8401 & 36.4801 & 58.8391 & & & & \\
\hline & All data & 4.7222 & 4.2809 & 47.3888 & 43.6081 & & & & \\
\hline \multirow{3}{*}{ uDP4+ } & ${ }^{1} \mathrm{H}$ data & 0.0001 & 0.0001 & 91.5733 & 8.4265 & & & & \\
\hline & ${ }^{13} \mathrm{C}$ data & 15.7269 & 34.6201 & 19.7391 & 29.9138 & & & & \\
\hline & All data & 0.0001 & 0.0003 & 87.7613 & 12.2384 & & & & \\
\hline \multirow{3}{*}{ DP4+ } & ${ }^{1} \mathrm{H}$ data & 0.0001 & 0.0002 & 95.0116 & 4.9881 & & & & \\
\hline & ${ }^{13} \mathrm{C}$ data & 1.1102 & 3.7709 & 27.6163 & 67.5026 & & & & \\
\hline & All data & 0.0000 & 0.0000 & 88.6269 & 11.3731 & & & & \\
\hline & & \multicolumn{8}{|c|}{ Level of theory: mPW1PW91/6-311G** (solution. PCM) } \\
\hline & & $87 a$ & $87 b$ & 87c & 87d & - & - & - & - \\
\hline \multirow{3}{*}{ sDP4+ } & ${ }^{1} \mathrm{H}$ data & 39.1079 & 25.9526 & 21.6416 & 13.2979 & & & & \\
\hline & ${ }^{13} \mathrm{C}$ data & 3.8845 & 6.2658 & 33.2864 & 56.5633 & & & & \\
\hline & All data & 8.5008 & 9.0995 & 40.3102 & 42.0895 & & & & \\
\hline \multirow{3}{*}{ uDP4+ } & ${ }^{1} \mathrm{H}$ data & 0.0000 & 0.0000 & 94.8285 & 5.1714 & & & & \\
\hline & ${ }^{13} \mathrm{C}$ data & 22.1085 & 48.1407 & 11.7622 & 17.9886 & & & & \\
\hline & All data & 0.0001 & 0.0001 & 92.3016 & 7.6982 & & & & \\
\hline \multirow{5}{*}{ DP4+ } & ${ }^{1} \mathrm{H}$ data & 0.0001 & 0.0000 & 96.7576 & 3.2423 & & & & \\
\hline & ${ }^{13} \mathrm{C}$ data & 4.7804 & 16.7901 & 21.7931 & 56.6364 & & & & \\
\hline & All data & 0.0000 & 0.0000 & 91.9891 & 8.0108 & & & & \\
\hline & & \multicolumn{8}{|c|}{ Level of theory: mPW1PW91/6-311+G** (solution. PCM) } \\
\hline & & $87 a$ & $87 b$ & $87 c$ & $87 d$ & - & - & - & - \\
\hline \multirow{3}{*}{ sDP4+ } & ${ }^{1} \mathrm{H}$ data & 30.0704 & 36.1469 & 20.0001 & 13.7827 & & & & \\
\hline & ${ }^{13} \mathrm{C}$ data & 6.1933 & 13.7729 & 30.8567 & 49.1771 & & & & \\
\hline & All data & 9.4105 & 25.1564 & 31.1841 & 34.2490 & & & & \\
\hline \multirow{3}{*}{ uDP4+ } & ${ }^{1} \mathrm{H}$ data & 0.0004 & 0.0001 & 96.3233 & 3.6762 & & & & \\
\hline & ${ }^{13} \mathrm{C}$ data & 20.0546 & 34.0513 & 13.9578 & 31.9362 & & & & \\
\hline & All data & 0.0005 & 0.0002 & 91.9683 & 8.0309 & & & & \\
\hline \multirow{3}{*}{ DP4+ } & ${ }^{1} \mathrm{H}$ data & 0.0006 & 0.0002 & 97.4366 & 2.5626 & & & & \\
\hline & ${ }^{13} \mathrm{C}$ data & 4.7874 & 18.0768 & 16.6008 & 60.5351 & & & & \\
\hline & All data & 0.0002 & 0.0002 & 91.2484 & 8.7512 & & & & \\
\hline
\end{tabular}


sDP4+, $u \mathrm{DP} 4+$ and DP4+ probabilities (\%) for compound 87d<smiles>CC(=O)O[C@H](C(=O)O[C@H]1C[C@H]2C=C[C@H]1C2)c1ccccc1</smiles>

$87 a$<smiles>O=C(OC1C[C@H]2C=C[C@@H]1C2)[C@@H](O)c1ccccc1</smiles>

87b<smiles>O=C(O[C@@H]1C[C@H]2C=C[C@@H]1C2)[C@@H](O)c1ccccc1</smiles>

87c<smiles>O=C(OC1CC2C=CC1C2)[C@@H](O)c1ccccc1</smiles>

correct isomer

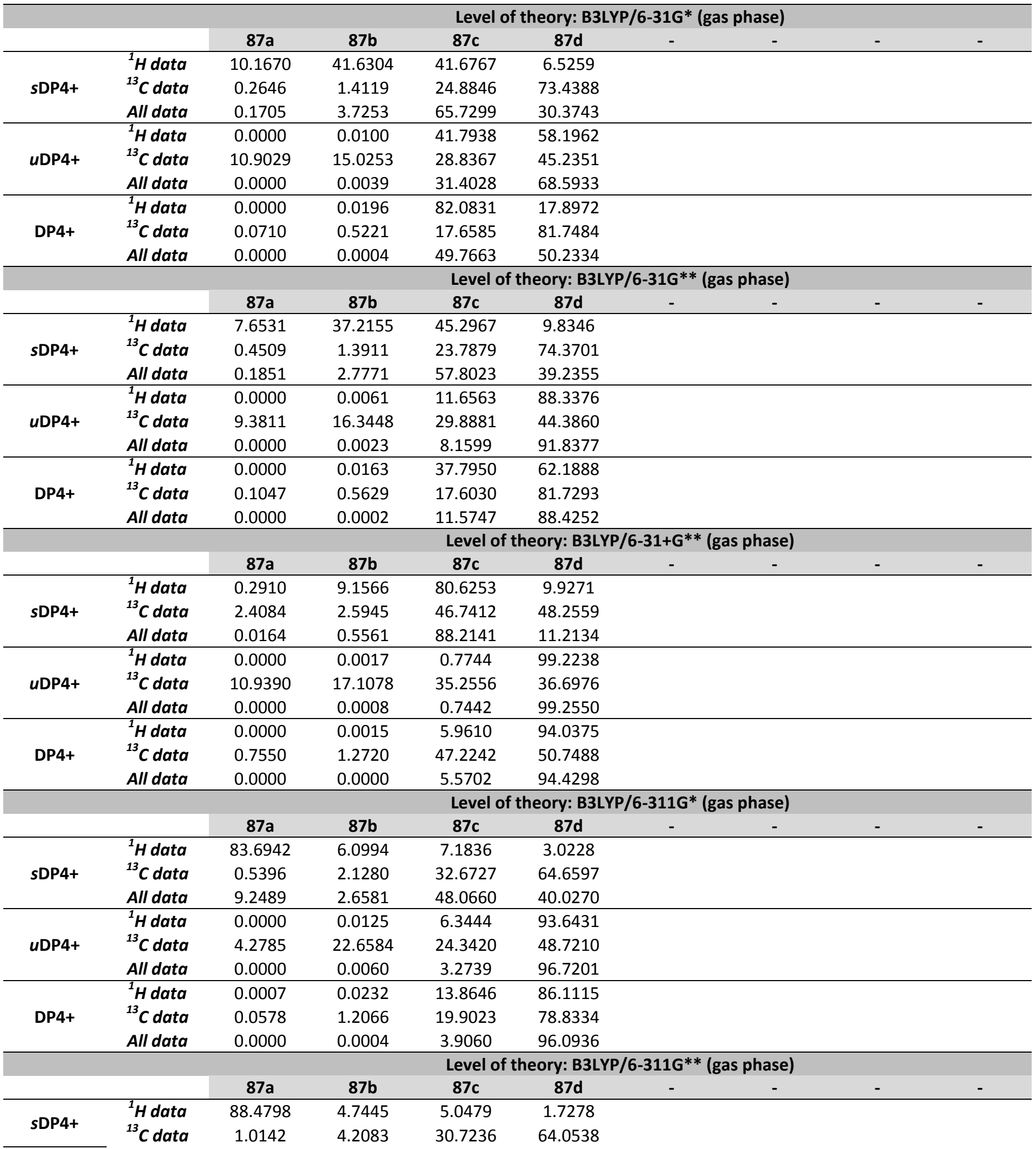




\begin{tabular}{|c|c|c|c|c|c|c|c|c|c|}
\hline & All data & 23.9001 & 5.3178 & 41.3061 & 29.4760 & & & & \\
\hline & ${ }^{1} \mathrm{H}$ data & 0.0000 & 0.0005 & 0.8555 & 99.1440 & & & & \\
\hline \multirow[t]{3}{*}{ uDP4+ } & ${ }^{13} \mathrm{C}$ data & 6.5963 & 36.4317 & 18.5050 & 38.4670 & & & & \\
\hline & All data & 0.0000 & 0.0005 & 0.4134 & 99.5861 & & & & \\
\hline & ${ }^{1} \mathrm{H}$ data & 0.0001 & 0.0014 & 2.4590 & 97.5395 & & & & \\
\hline \multirow[t]{5}{*}{ DP4+ } & ${ }^{13} \mathrm{C}$ data & 0.2096 & 4.8024 & 17.8086 & 77.1794 & & & & \\
\hline & All data & 0.0000 & 0.0001 & 0.5783 & 99.4216 & & & & \\
\hline & & \multicolumn{8}{|c|}{ Level of theory: B3LYP/6-311+G** (gas phase) } \\
\hline & & $87 a$ & $87 b$ & 87c & 87d & - & - & - & - \\
\hline & ${ }^{1} \mathrm{H}$ data & 61.0766 & 20.0694 & 12.5107 & 6.3433 & & & & \\
\hline \multirow[t]{3}{*}{ sDP4+ } & ${ }^{13} \mathrm{C}$ data & 1.5087 & 6.8183 & 27.0449 & 64.6281 & & & & \\
\hline & All data & 9.4288 & 14.0019 & 34.6213 & 41.9480 & & & & \\
\hline & ${ }^{1} \mathrm{H}$ data & 0.0000 & 0.0017 & 0.4060 & 99.5923 & & & & \\
\hline \multirow[t]{3}{*}{ uDP4+ } & ${ }^{13} \mathrm{C}$ data & 8.4072 & 35.5631 & 15.4016 & 40.6280 & & & & \\
\hline & All data & 0.0000 & 0.0015 & 0.1543 & 99.8442 & & & & \\
\hline & ${ }^{1} \mathrm{H}$ data & 0.0000 & 0.0054 & 0.7976 & 99.1970 & & & & \\
\hline \multirow[t]{5}{*}{ DP4+ } & ${ }^{13} \mathrm{C}$ data & 0.3847 & 7.3536 & 12.6321 & 79.6296 & & & & \\
\hline & All data & 0.0000 & 0.0005 & 0.1274 & 99.8721 & & & & \\
\hline & & \multicolumn{8}{|c|}{ Level of theory: B3LYP/6-31G* (solution. PCM) } \\
\hline & & $87 a$ & $87 b$ & 87c & 87d & - & - & - & - \\
\hline & ${ }^{1}$ H data & 1.2052 & 8.9785 & 78.1408 & 11.6755 & & & & \\
\hline \multirow[t]{3}{*}{ sDP4+ } & ${ }^{13} \mathrm{C}$ data & 0.5373 & 1.6919 & 32.7922 & 64.9787 & & & & \\
\hline & All data & 0.0194 & 0.4552 & 76.7900 & 22.7354 & & & & \\
\hline & ${ }^{1} \mathrm{H}$ data & 0.0000 & 0.0002 & 30.7950 & 69.2047 & & & & \\
\hline \multirow[t]{3}{*}{ uDP4+ } & ${ }^{13} \mathrm{C}$ data & 11.6026 & 18.8744 & 28.7388 & 40.7843 & & & & \\
\hline & All data & 0.0000 & 0.0001 & 23.8710 & 76.1289 & & & & \\
\hline & ${ }^{1} \mathrm{H}$ data & 0.0000 & 0.0001 & 74.8627 & 25.1372 & & & & \\
\hline \multirow[t]{5}{*}{ DP4+ } & ${ }^{13} \mathrm{C}$ data & 0.1717 & 0.8795 & 25.9568 & 72.9920 & & & & \\
\hline & All data & 0.0000 & 0.0000 & 51.4343 & 48.5657 & & & & \\
\hline & & \multicolumn{8}{|c|}{ Level of theory: B3LYP/6-31G** (solution. PCM) } \\
\hline & & $87 a$ & $87 b$ & 87c & 87d & - & - & - & - \\
\hline & ${ }^{1} \mathrm{H}$ data & 1.9366 & 11.5336 & 70.2673 & 16.2626 & & & & \\
\hline \multirow[t]{3}{*}{ sDP4+ } & ${ }^{13} \mathrm{C}$ data & 1.3704 & 3.4842 & 33.5751 & 61.5703 & & & & \\
\hline & All data & 0.0780 & 1.1808 & 69.3206 & 29.4207 & & & & \\
\hline & ${ }^{1} \mathrm{H}$ data & 0.0000 & 0.0002 & 8.7827 & 91.2171 & & & & \\
\hline \multirow[t]{3}{*}{ uDP4+ } & ${ }^{13} \mathrm{C}$ data & 13.2923 & 19.9819 & 29.6459 & 37.0799 & & & & \\
\hline & All data & 0.0000 & 0.0001 & 7.1478 & 92.8521 & & & & \\
\hline & ${ }^{1} \mathrm{H}$ data & 0.0000 & 0.0001 & 29.3795 & 70.6204 & & & & \\
\hline \multirow[t]{5}{*}{ DP4+ } & ${ }^{13} \mathrm{C}$ data & 0.5411 & 2.0682 & 29.5692 & 67.8214 & & & & \\
\hline & All data & 0.0000 & 0.0000 & 15.3532 & 84.6468 & & & & \\
\hline & & \multicolumn{8}{|c|}{ Level of theory: B3LYP/6-31+G** (solution. PCM) } \\
\hline & & $87 a$ & $87 b$ & 87c & 87d & - & - & - & - \\
\hline & ${ }^{1} \mathrm{H}$ data & 0.0459 & 1.3602 & 86.7623 & 11.8315 & & & & \\
\hline \multirow[t]{3}{*}{ sDP4+ } & ${ }^{13} \mathrm{C}$ data & 2.6342 & 3.5435 & 42.4031 & 51.4192 & & & & \\
\hline & All data & 0.0028 & 0.1123 & 85.7115 & 14.1734 & & & & \\
\hline & ${ }^{1} \mathrm{H}$ data & 0.0000 & 0.0005 & 1.9114 & 98.0881 & & & & \\
\hline uDP4+ & ${ }^{13} \mathrm{C}$ data & 13.6940 & 15.7815 & 43.1484 & 27.3762 & & & & \\
\hline & All data & 0.0000 & 0.0003 & 2.9798 & 97.0199 & & & & \\
\hline & ${ }^{1} \mathrm{H}$ data & 0.0000 & 0.0000 & 12.5031 & 87.4968 & & & & \\
\hline DP4+ & ${ }^{13} \mathrm{C}$ data & 1.0835 & 1.6797 & 54.9555 & 42.2812 & & & & \\
\hline & All data & 0.0000 & 0.0000 & 15.6640 & 84.3360 & & & & \\
\hline & & & & Level of $t$ & ory: B3LYP & G* & PC & & \\
\hline & & $87 a$ & $87 b$ & $87 c$ & 87d & - & - & - & - \\
\hline & ${ }^{1} \mathrm{H}$ data & 54.6204 & 19.0581 & 16.6839 & 9.6376 & & & & \\
\hline sDP4+ & ${ }^{13} \mathrm{C}$ data & 1.5961 & 2.7139 & 35.6442 & 60.0458 & & & & \\
\hline & All data & 6.6434 & 3.9414 & 45.3166 & 44.0985 & & & & \\
\hline
\end{tabular}




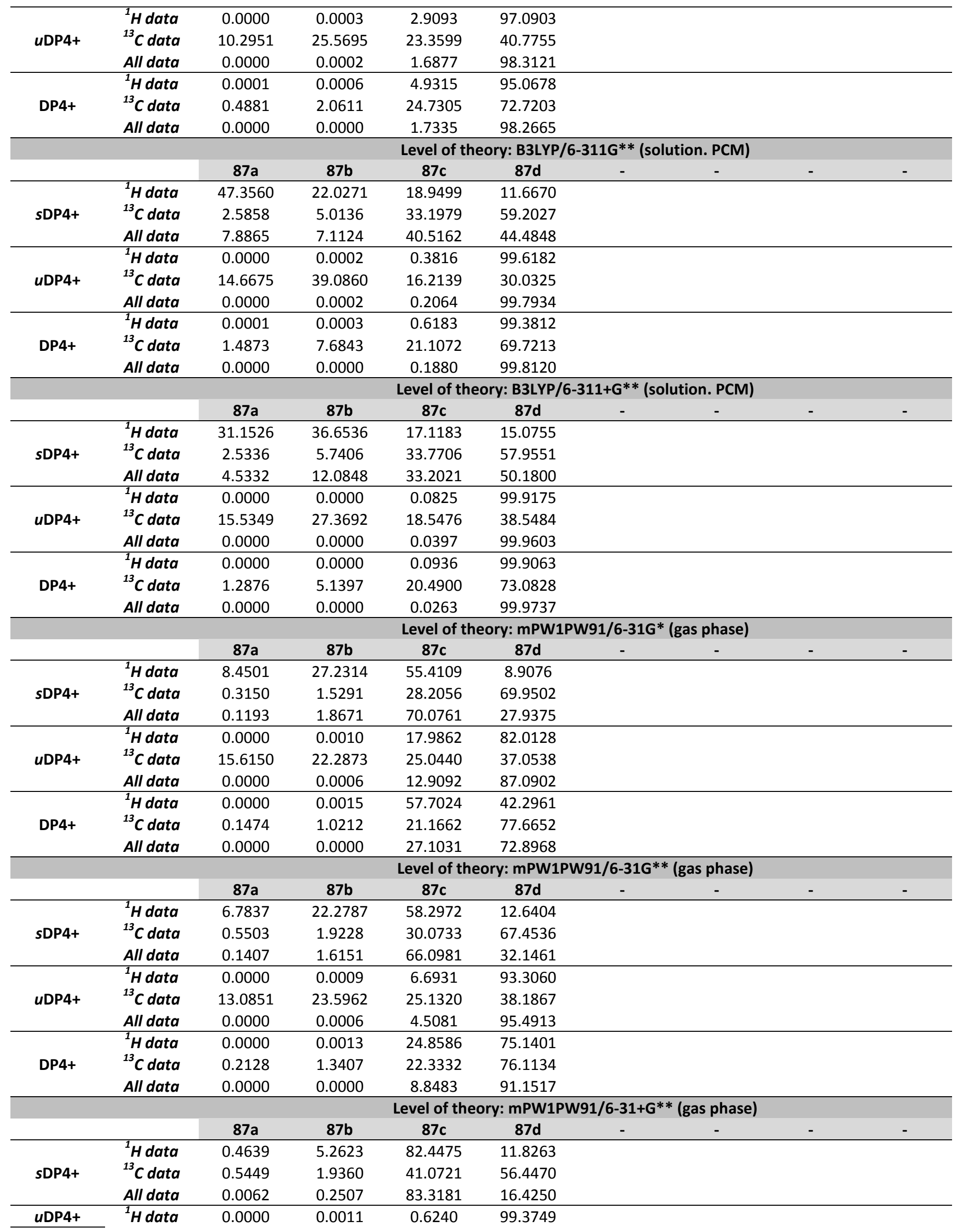




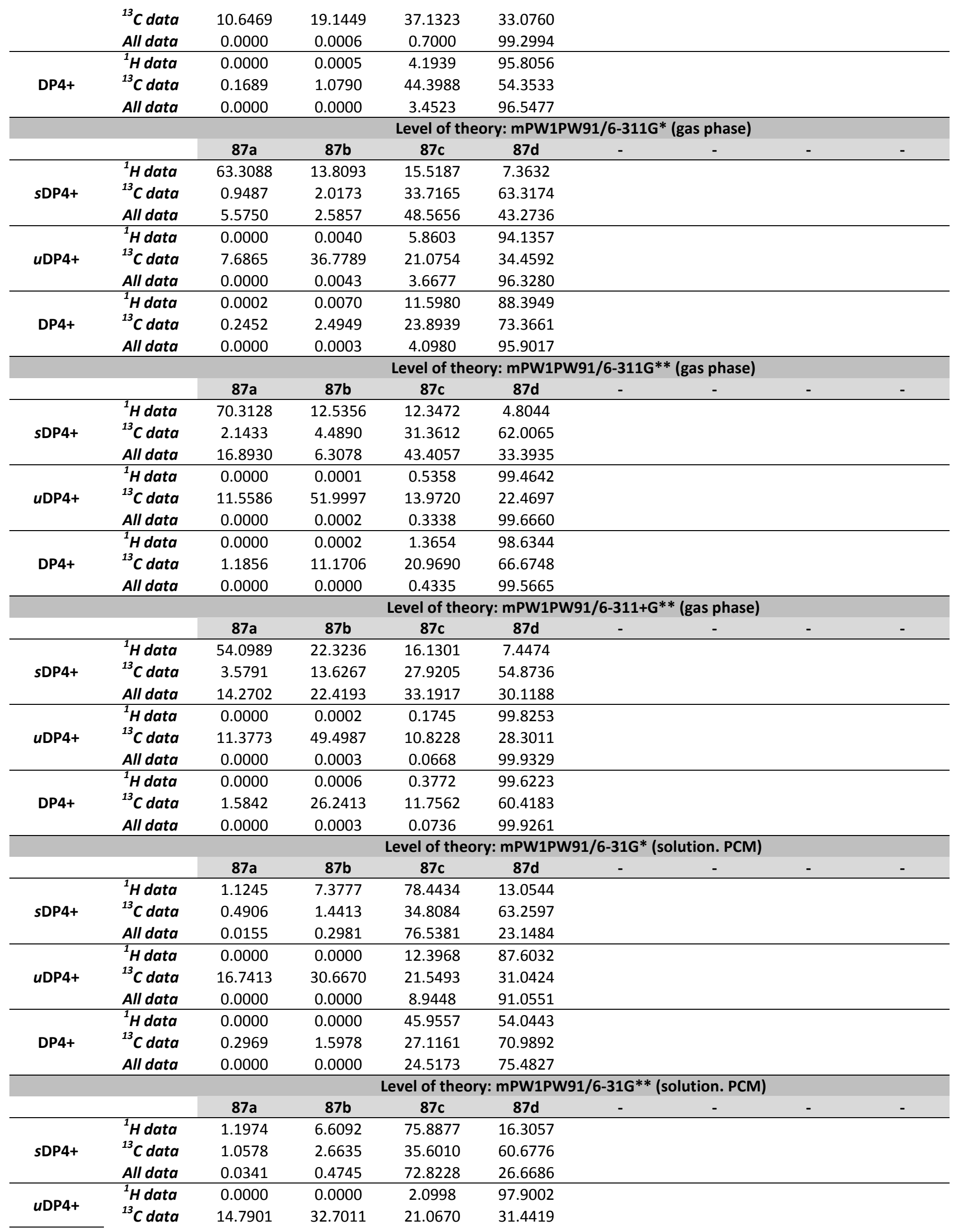




\begin{tabular}{|c|c|c|c|c|c|c|c|c|c|}
\hline & All data & 0.0000 & 0.0000 & 1.4167 & 98.5832 & & & & \\
\hline & ${ }^{1}$ H data & 0.0000 & 0.0000 & 9.0762 & 90.9238 & & & & \\
\hline \multirow[t]{5}{*}{ DP4+ } & ${ }^{13} \mathrm{C}$ data & 0.5667 & 3.1551 & 27.1685 & 69.1096 & & & & \\
\hline & All data & 0.0000 & 0.0000 & 3.7761 & 96.2239 & & & & \\
\hline & & \multicolumn{8}{|c|}{ Level of theory: mPW1PW91/6-31+G** (solution. PCM) } \\
\hline & & $87 a$ & $87 b$ & 87c & 87d & - & - & - & - \\
\hline & ${ }^{1} \mathrm{H}$ data & 0.0002 & 0.0017 & 0.3088 & 99.6892 & & & & \\
\hline \multirow[t]{3}{*}{ sDP4+ } & ${ }^{13} \mathrm{C}$ data & 0.9070 & 2.2245 & 45.4236 & 51.4449 & & & & \\
\hline & All data & 0.0000 & 0.0001 & 0.2728 & 99.7271 & & & & \\
\hline & ${ }^{1} \mathrm{H}$ data & 0.0000 & 0.0000 & 0.0789 & 99.9211 & & & & \\
\hline \multirow[t]{3}{*}{ uDP4+ } & ${ }^{13} \mathrm{C}$ data & 13.2126 & 25.7799 & 38.0728 & 22.9347 & & & & \\
\hline & All data & 0.0000 & 0.0000 & 0.1309 & 99.8691 & & & & \\
\hline & ${ }^{1} \mathrm{H}$ data & 0.0000 & 0.0000 & 0.0002 & 99.9998 & & & & \\
\hline \multirow[t]{5}{*}{ DP4+ } & ${ }^{13} \mathrm{C}$ data & 0.4023 & 1.9253 & 58.0608 & 39.6115 & & & & \\
\hline & All data & 0.0000 & 0.0000 & 0.0004 & 99.9996 & & & & \\
\hline & & \multicolumn{8}{|c|}{ Level of theory: mPW1PW91/6-311G* (solution. PCM) } \\
\hline & & $87 a$ & $87 b$ & 87c & 87d & - & - & - & - \\
\hline & ${ }^{1} \mathrm{H}$ data & 38.5402 & 25.4722 & 22.3856 & 13.6020 & & & & \\
\hline \multirow[t]{3}{*}{ sDP4+ } & ${ }^{13} \mathrm{C}$ data & 1.9829 & 3.0765 & 36.1198 & 58.8208 & & & & \\
\hline & All data & 4.3336 & 4.4440 & 45.8517 & 45.3707 & & & & \\
\hline & ${ }^{1} \mathrm{H}$ data & 0.0000 & 0.0001 & 2.4381 & 97.5619 & & & & \\
\hline \multirow[t]{3}{*}{ uDP4+ } & ${ }^{13} \mathrm{C}$ data & 16.5900 & 36.8032 & 18.4357 & 28.1712 & & & & \\
\hline & All data & 0.0000 & 0.0001 & 1.6091 & 98.3908 & & & & \\
\hline & ${ }^{1} \mathrm{H}$ data & 0.0000 & 0.0001 & 3.9502 & 96.0496 & & & & \\
\hline \multirow[t]{5}{*}{ DP4+ } & ${ }^{13} \mathrm{C}$ data & 1.3323 & 4.5858 & 26.9694 & 67.1125 & & & & \\
\hline & All data & 0.0000 & 0.0000 & 1.6258 & 98.3741 & & & & \\
\hline & & \multicolumn{8}{|c|}{ Level of theory: mPW1PW91/6-311G** (solution. PCM) } \\
\hline & & $87 a$ & $87 b$ & 87c & 87d & - & - & - & - \\
\hline & ${ }^{1} \mathrm{H}$ data & 36.5431 & 26.4567 & 22.5299 & 14.4704 & & & & \\
\hline \multirow[t]{3}{*}{$s D P 4+$} & ${ }^{13} \mathrm{C}$ data & 4.1823 & 6.7629 & 32.8643 & 56.1905 & & & & \\
\hline & All data & 8.1067 & 9.4905 & 39.2741 & 43.1287 & & & & \\
\hline & ${ }^{1} \mathrm{H}$ data & 0.0000 & 0.0000 & 0.1593 & 99.8407 & & & & \\
\hline \multirow[t]{3}{*}{ uDP4+ } & ${ }^{13} \mathrm{C}$ data & 22.7800 & 49.8581 & 10.7703 & 16.5916 & & & & \\
\hline & All data & 0.0000 & 0.0001 & 0.1035 & 99.8965 & & & & \\
\hline & ${ }^{1} \mathrm{H}$ data & 0.0000 & 0.0000 & 0.2478 & 99.7521 & & & & \\
\hline \multirow[t]{5}{*}{ DP4+ } & ${ }^{13} \mathrm{C}$ data & 5.5433 & 19.6185 & 20.5945 & 54.2437 & & & & \\
\hline & All data & 0.0000 & 0.0000 & 0.0942 & 99.9058 & & & & \\
\hline & & \multicolumn{8}{|c|}{ Level of theory: mPW1PW91/6-311+G** (solution. PCM) } \\
\hline & & $87 a$ & $87 b$ & 87c & 87d & - & - & - & - \\
\hline & ${ }^{1} \mathrm{H}$ data & 27.9890 & 36.8022 & 20.3518 & 14.8570 & & & & \\
\hline \multirow[t]{3}{*}{ sDP4+ } & ${ }^{13} \mathrm{C}$ data & 6.8910 & 15.5077 & 29.7006 & 47.9007 & & & & \\
\hline & All data & 9.2740 & 27.4421 & 29.0647 & 34.2192 & & & & \\
\hline & ${ }^{1} \mathrm{H}$ data & 0.0001 & 0.0001 & 0.1153 & 99.8846 & & & & \\
\hline \multirow[t]{3}{*}{ uDP4+ } & ${ }^{13} \mathrm{C}$ data & 20.9398 & 36.0560 & 12.9531 & 30.0511 & & & & \\
\hline & All data & 0.0000 & 0.0001 & 0.0497 & 99.9501 & & & & \\
\hline & ${ }^{1} \mathrm{H}$ data & 0.0001 & 0.0002 & 0.1578 & 99.8418 & & & & \\
\hline \multirow[t]{2}{*}{ DP4+ } & ${ }^{13} \mathrm{C}$ data & 5.7088 & 22.1213 & 15.2205 & 56.9495 & & & & \\
\hline & All data & 0.0000 & 0.0001 & 0.0422 & 99.9577 & & & & \\
\hline
\end{tabular}


$s \mathrm{DP4}+, u \mathrm{DP} 4+$ and DP4+ probabilities (\%) for compound 88a

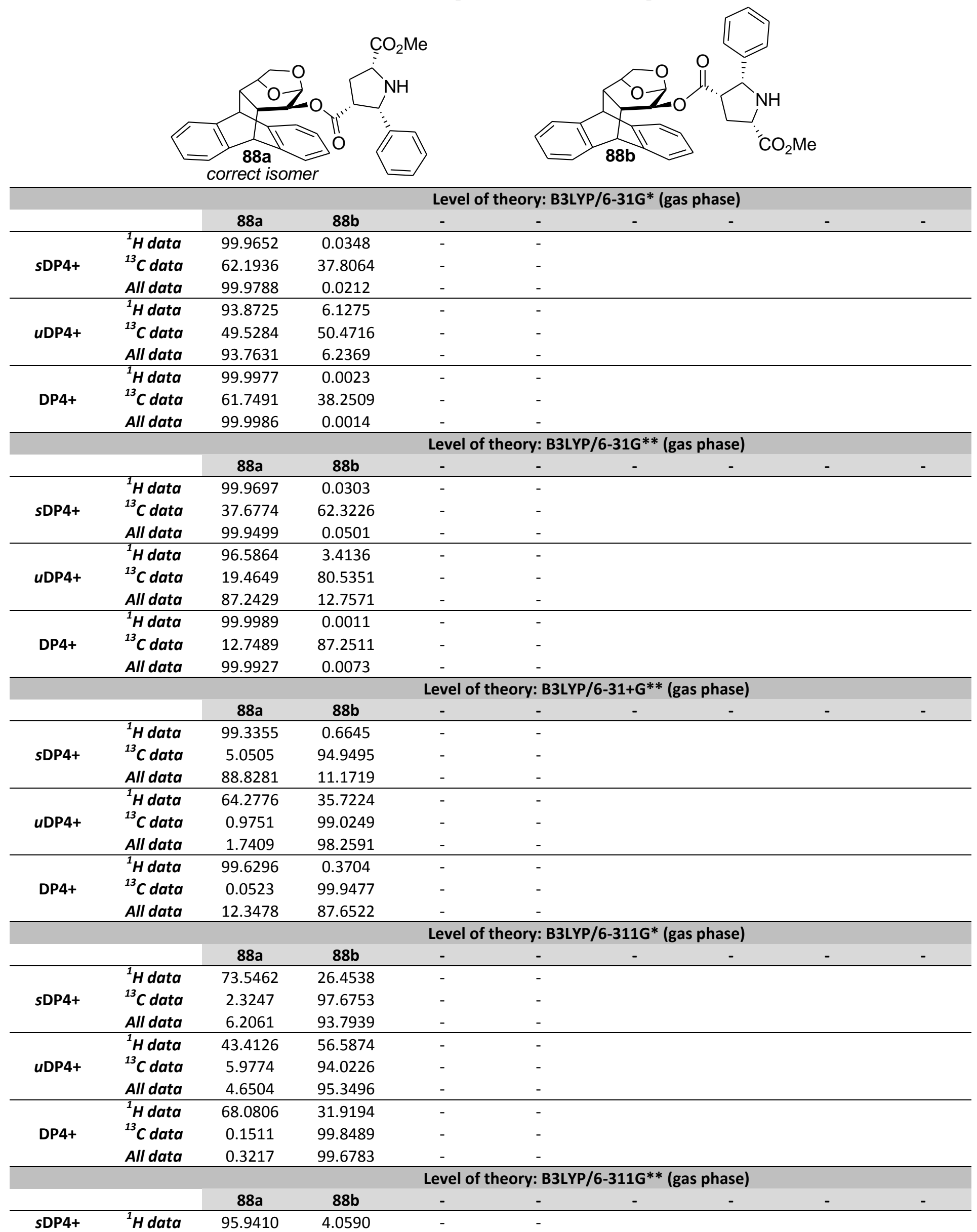




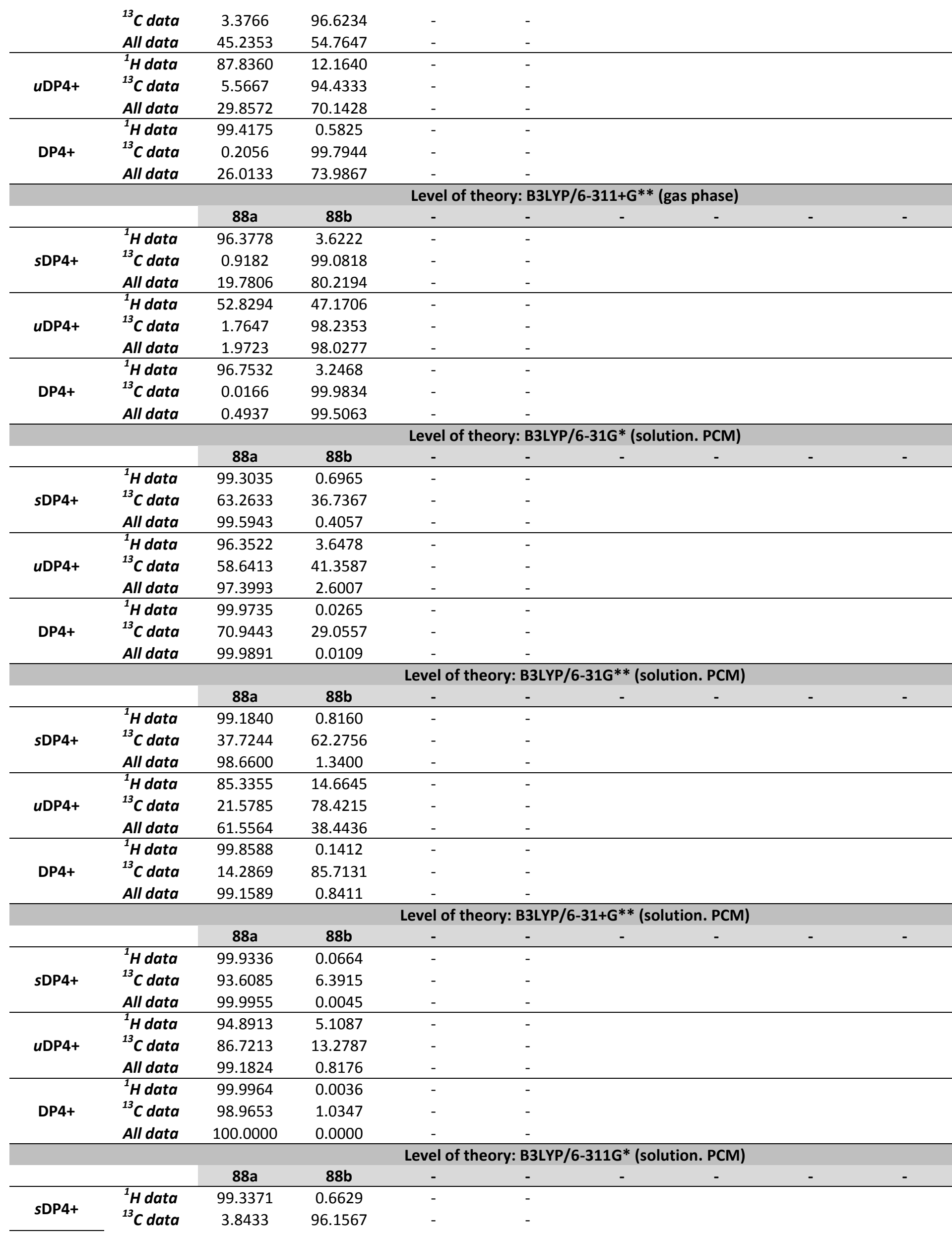




\begin{tabular}{|c|c|c|c|c|c|c|c|c|c|}
\hline & All data & 85.6919 & 14.3081 & - & - & & & & \\
\hline & ${ }^{1}$ H data & 96.4236 & 3.5764 & - & - & & & & \\
\hline \multirow[t]{3}{*}{ uDP4+ } & ${ }^{13}$ C data & 4.6085 & 95.3915 & - & - & & & & \\
\hline & All data & 56.5695 & 43.4305 & - & - & & & & \\
\hline & ${ }^{1} \mathrm{H}$ data & 99.9753 & 0.0247 & - & - & & & & \\
\hline \multirow[t]{5}{*}{ DP4+ } & ${ }^{13} \mathrm{C}$ data & 0.1927 & 99.8073 & - & - & & & & \\
\hline & All data & 88.6375 & 11.3625 & - & - & & & & \\
\hline & & \multicolumn{8}{|c|}{ Level of theory: B3LYP/6-311G** (solution. PCM) } \\
\hline & & $88 a$ & $88 b$ & - & - & - & - & - & - \\
\hline & ${ }^{1} \mathrm{H}$ data & 99.5160 & 0.4840 & - & - & & & & \\
\hline \multirow[t]{3}{*}{ sDP4+ } & ${ }^{13} \mathrm{C}$ data & 3.9413 & 96.0587 & - & - & & & & \\
\hline & All data & 89.4030 & 10.5970 & - & - & & & & \\
\hline & ${ }^{1} \mathrm{H}$ data & 99.1666 & 0.8334 & - & - & & & & \\
\hline \multirow[t]{3}{*}{ uDP4+ } & ${ }^{13} \mathrm{C}$ data & 4.4689 & 95.5311 & - & - & & & & \\
\hline & All data & 84.7701 & 15.2299 & - & - & & & & \\
\hline & ${ }^{1} \mathrm{H}$ data & 99.9959 & 0.0041 & - & - & & & & \\
\hline \multirow[t]{5}{*}{ DP4+ } & ${ }^{13} \mathrm{C}$ data & 0.1916 & 99.8084 & - & - & & & & \\
\hline & All data & 97.9149 & 2.0851 & - & - & & & & \\
\hline & & \multicolumn{8}{|c|}{ Level of theory: B3LYP/6-311+G** (solution. PCM) } \\
\hline & & $88 a$ & $88 b$ & - & - & - & - & - & - \\
\hline & ${ }^{1} \mathrm{H}$ data & 97.5737 & 2.4263 & - & - & & & & \\
\hline \multirow[t]{3}{*}{ sDP4+ } & ${ }^{13} \mathrm{C}$ data & 12.4722 & 87.5278 & - & - & & & & \\
\hline & All data & 85.1419 & 14.8581 & - & - & & & & \\
\hline & ${ }^{1} \mathrm{H}$ data & 78.8238 & 21.1762 & - & - & & & & \\
\hline \multirow[t]{3}{*}{ uDP4+ } & ${ }^{13} \mathrm{C}$ data & 9.1726 & 90.8274 & - & - & & & & \\
\hline & All data & 27.3209 & 72.6791 & - & - & & & & \\
\hline & ${ }^{1} \mathrm{H}$ data & 99.3364 & 0.6636 & - & - & & & & \\
\hline \multirow[t]{5}{*}{ DP4+ } & ${ }^{13} \mathrm{C}$ data & 1.4186 & 98.5814 & - & - & & & & \\
\hline & All data & 68.2952 & 31.7048 & - & - & & & & \\
\hline & & \multicolumn{8}{|c|}{ Level of theory: mPW1PW91/6-31G* (gas phase) } \\
\hline & & $88 a$ & $88 b$ & - & - & - & - & - & - \\
\hline & ${ }^{1} \mathrm{H}$ data & 99.8273 & 0.1727 & - & - & & & & \\
\hline \multirow[t]{2}{*}{ sDP4+ } & ${ }^{13} \mathrm{C}$ data & 80.3347 & 19.6653 & - & - & & & & \\
\hline & All data & 99.9577 & 0.0423 & - & - & & & & \\
\hline \multirow{3}{*}{ uDP4+ } & ${ }^{1} \mathrm{H}$ data & 90.5512 & 9.4488 & - & - & & & & \\
\hline & ${ }^{13} \mathrm{C}$ data & 56.3392 & 43.6608 & - & - & & & & \\
\hline & All data & 92.5185 & 7.4815 & - & - & & & & \\
\hline \multirow{5}{*}{ DP4+ } & ${ }^{1} \mathrm{H}$ data & 99.9820 & 0.0180 & - & - & & & & \\
\hline & ${ }^{13} \mathrm{C}$ data & 84.0544 & 15.9456 & - & - & & & & \\
\hline & All data & 99.9966 & 0.0034 & - & - & & & & \\
\hline & & \multicolumn{8}{|c|}{ Level of theory: mPW1PW91/6-31G** (gas phase) } \\
\hline & & $88 a$ & $88 b$ & - & - & - & - & - & - \\
\hline \multirow{3}{*}{ sDP4+ } & ${ }^{1}$ H data & 99.9089 & 0.0911 & - & - & & & & \\
\hline & ${ }^{13} \mathrm{C}$ data & 44.4753 & 55.5247 & - & - & & & & \\
\hline & All data & 99.8863 & 0.1137 & - & - & & & & \\
\hline & ${ }^{1} \mathrm{H}$ data & 92.9651 & 7.0349 & - & - & & & & \\
\hline uDP4+ & ${ }^{13} \mathrm{C}$ data & 21.6565 & 78.3435 & - & - & & & & \\
\hline & All data & 78.5085 & 21.4915 & - & - & & & & \\
\hline & ${ }^{1} \mathrm{H}$ data & 99.9931 & 0.0069 & - & - & & & & \\
\hline DP4+ & ${ }^{13} \mathrm{C}$ data & 18.1281 & 81.8719 & - & - & & & & \\
\hline & All data & 99.9688 & 0.0312 & - & - & & & & \\
\hline & & & & 10 & PI & -3 & ph & & \\
\hline & & $88 a$ & $88 b$ & - & - & - & - & - & - \\
\hline & ${ }^{1} \mathrm{H}$ data & 99.7297 & 0.2703 & - & - & & & & \\
\hline sDP4+ & ${ }^{13} \mathrm{C}$ data & 1.3477 & 98.6523 & - & - & & & & \\
\hline & All data & 83.4424 & 16.5576 & - & - & & & & \\
\hline
\end{tabular}




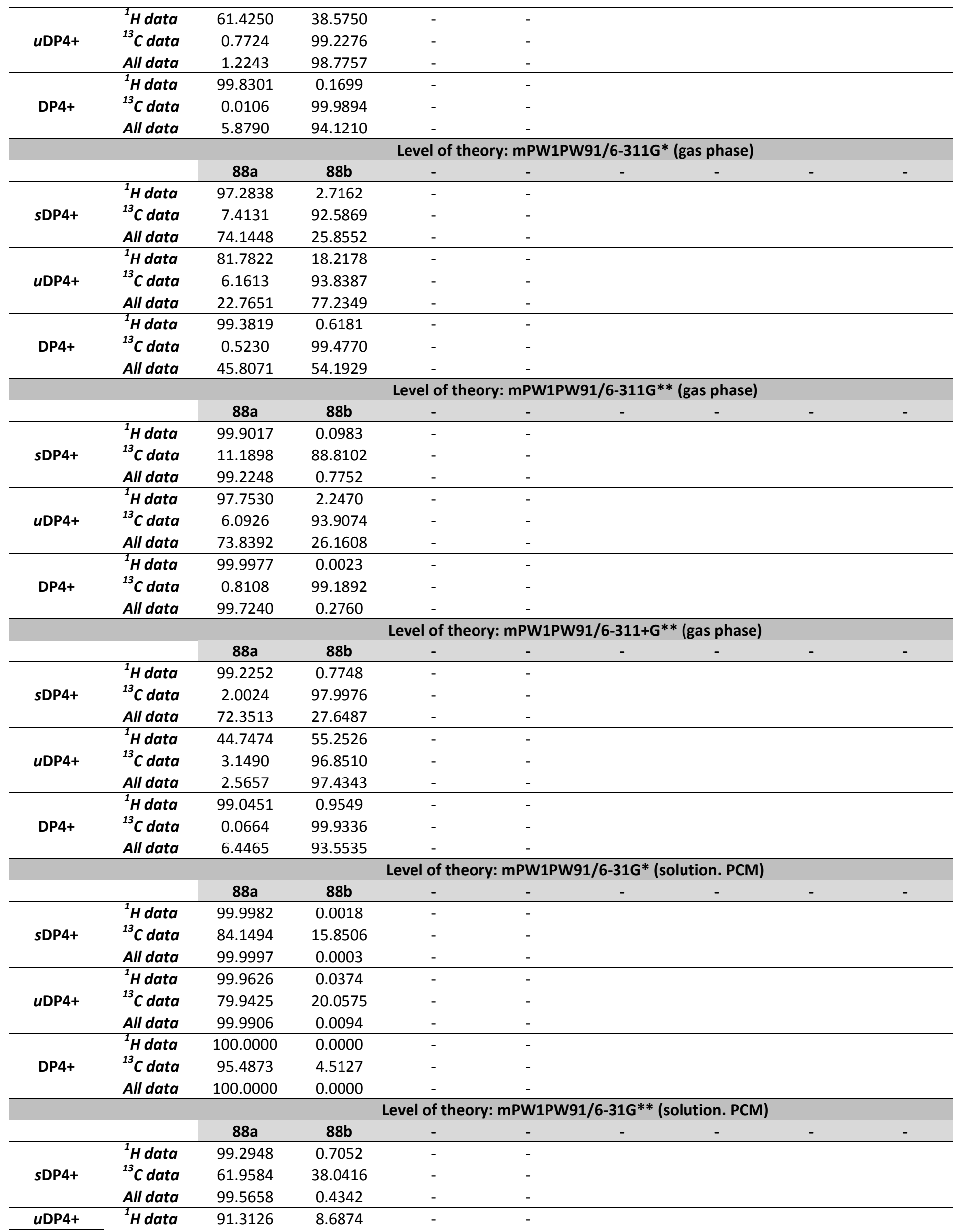




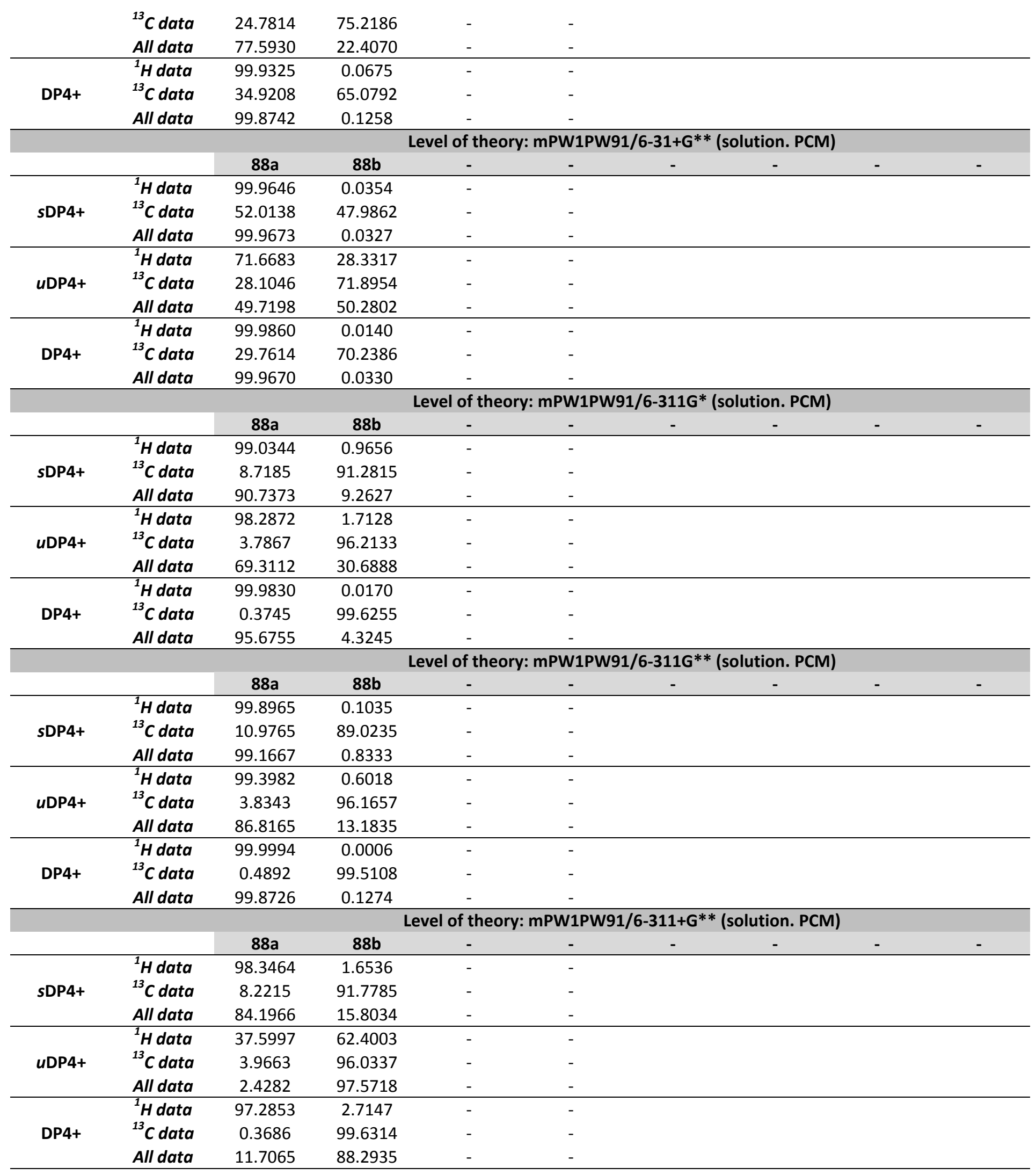


$s \mathrm{DP4}+, u \mathrm{DP} 4+$ and DP4+ probabilities (\%) for compound $88 \mathrm{~b}$

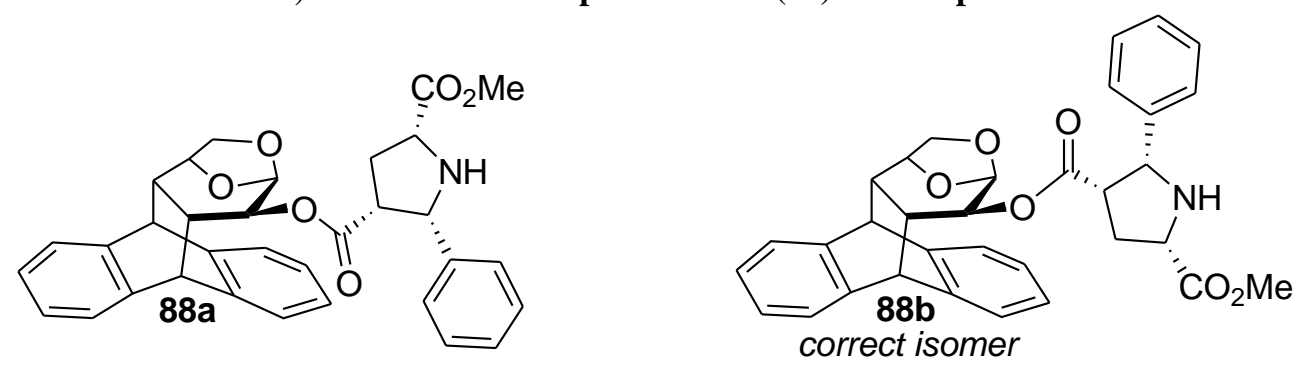

\begin{tabular}{|c|c|c|c|c|c|c|c|c|c|}
\hline & & \multicolumn{8}{|c|}{ Level of theory: B3LYP/6-31G* (gas phase) } \\
\hline & & $88 a$ & $88 \mathrm{~b}$ & - & - & - & - & - & - \\
\hline \multirow{3}{*}{ sDP4+ } & ${ }^{1} \mathrm{H}$ data & 99.9831 & 0.0169 & - & - & & & & \\
\hline & ${ }^{13} \mathrm{C}$ data & 89.6809 & 10.3191 & - & - & & & & \\
\hline & All data & 99.9980 & 0.0020 & - & - & & & & \\
\hline & ${ }^{1} \mathrm{H}$ data & 99.8204 & 0.1796 & - & - & & & & \\
\hline \multirow[t]{2}{*}{ uDP4+ } & ${ }^{13} \mathrm{C}$ data & 67.7387 & 32.2613 & - & - & & & & \\
\hline & All data & 99.9144 & 0.0856 & - & - & & & & \\
\hline & ${ }^{1} \mathrm{H}$ data & 100.0000 & 0.0000 & - & - & & & & \\
\hline \multirow[t]{5}{*}{ DP4+ } & ${ }^{13} \mathrm{C}$ data & 94.8046 & 5.1954 & - & - & & & & \\
\hline & All data & 100.0000 & 0.0000 & - & - & & & & \\
\hline & & \multicolumn{8}{|c|}{ Level of theory: B3LYP/6-31G** (gas phase) } \\
\hline & & $88 a$ & $88 \mathrm{~b}$ & - & - & - & - & - & - \\
\hline & ${ }^{1} \mathrm{H}$ data & 99.9301 & 0.0699 & - & - & & & & \\
\hline \multirow[t]{2}{*}{ sDP4+ } & ${ }^{13} \mathrm{C}$ data & 72.3881 & 27.6119 & - & - & & & & \\
\hline & All data & 99.9733 & 0.0267 & - & - & & & & \\
\hline \multirow{3}{*}{ uDP4+ } & ${ }^{1} \mathrm{H}$ data & 99.2299 & 0.7701 & - & - & & & & \\
\hline & ${ }^{13} \mathrm{C}$ data & 43.3529 & 56.6471 & - & - & & & & \\
\hline & All data & 98.9962 & 1.0038 & - & - & & & & \\
\hline \multirow{5}{*}{ DP4+ } & ${ }^{1} \mathrm{H}$ data & 99.9995 & 0.0005 & - & - & & & & \\
\hline & ${ }^{13} \mathrm{C}$ data & 66.7374 & 33.2626 & - & - & & & & \\
\hline & All data & 99.9997 & 0.0003 & - & - & & & & \\
\hline & & \multicolumn{8}{|c|}{ Level of theory: B3LYP/6-31+G** (gas phase) } \\
\hline & & $88 \mathrm{a}$ & $88 \mathrm{~b}$ & - & - & - & - & - & - \\
\hline \multirow{3}{*}{ sDP4+ } & ${ }^{1} \mathrm{H}$ data & 99.9995 & 0.0005 & - & - & & & & \\
\hline & ${ }^{13} \mathrm{C}$ data & 11.5789 & 88.4211 & - & - & & & & \\
\hline & All data & 99.9962 & 0.0038 & - & - & & & & \\
\hline \multirow{3}{*}{ uDP4+ } & ${ }^{1} \mathrm{H}$ data & 99.8862 & 0.1138 & - & - & & & & \\
\hline & ${ }^{13} \mathrm{C}$ data & 2.0673 & 97.9327 & - & - & & & & \\
\hline & All data & 94.8781 & 5.1219 & - & - & & & & \\
\hline \multirow{5}{*}{ DP4+ } & ${ }^{1} \mathrm{H}$ data & 100.0000 & 0.0000 & - & - & & & & \\
\hline & ${ }^{13} \mathrm{C}$ data & 0.2757 & 99.7243 & - & - & & & & \\
\hline & All data & 99.9998 & 0.0002 & - & - & & & & \\
\hline & & \multicolumn{8}{|c|}{ Level of theory: B3LYP/6-311G* (gas phase) } \\
\hline & & $88 \mathrm{a}$ & $88 \mathrm{~b}$ & - & - & - & - & - & - \\
\hline \multirow{3}{*}{ sDP4+ } & ${ }^{1} \mathrm{H}$ data & 99.9932 & 0.0068 & - & - & & & & \\
\hline & ${ }^{13} \mathrm{C}$ data & 9.7630 & 90.2370 & - & - & & & & \\
\hline & All data & 99.9367 & 0.0633 & - & - & & & & \\
\hline \multirow{3}{*}{ uDP4+ } & ${ }^{1} \mathrm{H}$ data & 99.6977 & 0.3023 & - & - & & & & \\
\hline & ${ }^{13} \mathrm{C}$ data & 9.4935 & 90.5065 & - & - & & & & \\
\hline & All data & 97.1905 & 2.8095 & - & - & & & & \\
\hline \multirow{5}{*}{ DP4+ } & ${ }^{1} \mathrm{H}$ data & 100.0000 & 0.0000 & - & - & & & & \\
\hline & ${ }^{13} \mathrm{C}$ data & 1.1221 & 98.8779 & - & - & & & & \\
\hline & All data & 99.9982 & 0.0018 & - & - & & & & \\
\hline & & \multicolumn{8}{|c|}{ Level of theory: B3LYP/6-311G** (gas phase) } \\
\hline & & $88 \mathrm{a}$ & $88 \mathrm{~b}$ & - & - & - & - & - & - \\
\hline \multirow{2}{*}{ sDP4+ } & ${ }^{1} \mathrm{H}$ data & 99.9901 & 0.0099 & - & - & & & & \\
\hline & ${ }^{13} \mathrm{C}$ data & 15.0320 & 84.9680 & - & - & & & & \\
\hline
\end{tabular}




\begin{tabular}{|c|c|c|c|c|c|c|c|c|c|}
\hline & All data & 99.9439 & 0.0561 & - & - & & & & \\
\hline & ${ }^{1} \mathrm{H}$ data & 99.8794 & 0.1206 & - & - & & & & \\
\hline \multirow[t]{2}{*}{ uDP4+ } & ${ }^{13} \mathrm{C}$ data & 9.1105 & 90.8895 & - & - & & & & \\
\hline & All data & 98.8100 & 1.1900 & - & - & & & & \\
\hline \multirow{5}{*}{ DP4+ } & ${ }^{1} \mathrm{H}$ data & 100.0000 & 0.0000 & - & - & & & & \\
\hline & ${ }^{13} \mathrm{C}$ data & 1.7424 & 98.2576 & - & - & & & & \\
\hline & All data & 99.9993 & 0.0007 & - & - & & & & \\
\hline & & \multicolumn{8}{|c|}{ Level of theory: B3LYP/6-311+G** (gas phase) } \\
\hline & & $88 a$ & $88 b$ & - & - & - & - & - & - \\
\hline \multirow{3}{*}{ sDP4+ } & ${ }^{1} \mathrm{H}$ data & 99.9999 & 0.0001 & - & - & & & & \\
\hline & ${ }^{13} \mathrm{C}$ data & 3.8991 & 96.1009 & - & - & & & & \\
\hline & All data & 99.9971 & 0.0029 & - & - & & & & \\
\hline \multirow{3}{*}{ uDP4+ } & ${ }^{1} \mathrm{H}$ data & 99.9573 & 0.0427 & - & - & & & & \\
\hline & ${ }^{13} \mathrm{C}$ data & 3.3948 & 96.6052 & - & - & & & & \\
\hline & All data & 98.7994 & 1.2006 & - & - & & & & \\
\hline \multirow{5}{*}{ DP4+ } & ${ }^{1} \mathrm{H}$ data & 100.0000 & 0.0000 & - & - & & & & \\
\hline & ${ }^{13} \mathrm{C}$ data & 0.1424 & 99.8576 & - & - & & & & \\
\hline & All data & 100.0000 & 0.0000 & - & - & & & & \\
\hline & & \multicolumn{8}{|c|}{ Level of theory: B3LYP/6-31G* (solution. PCM) } \\
\hline & & $88 a$ & $88 b$ & - & - & - & - & - & - \\
\hline \multirow{3}{*}{ sDP4+ } & ${ }^{1} \mathrm{H}$ data & 99.9460 & 0.0540 & - & - & & & & \\
\hline & ${ }^{13} \mathrm{C}$ data & 84.6498 & 15.3502 & - & - & & & & \\
\hline & All data & 99.9902 & 0.0098 & - & - & & & & \\
\hline \multirow{3}{*}{ uDP4+ } & ${ }^{1} \mathrm{H}$ data & 99.7516 & 0.2484 & - & - & & & & \\
\hline & ${ }^{13} \mathrm{C}$ data & 66.8963 & 33.1037 & - & - & & & & \\
\hline & All data & 99.8769 & 0.1231 & - & - & & & & \\
\hline \multirow{3}{*}{ DP4+ } & ${ }^{1} \mathrm{H}$ data & 99.9999 & 0.0001 & - & - & & & & \\
\hline & ${ }^{13} \mathrm{C}$ data & 91.7654 & 8.2346 & - & - & & & & \\
\hline & All data & 100.0000 & 0.0000 & - & - & & & & \\
\hline & & \multicolumn{8}{|c|}{ Level of theory: B3LYP/6-31G** (solution. PCM) } \\
\hline & & $88 a$ & $88 b$ & - & - & - & - & - & - \\
\hline \multirow{3}{*}{ sDP4+ } & ${ }^{1} \mathrm{H}$ data & 99.9460 & 0.0540 & - & - & & & & \\
\hline & ${ }^{13} \mathrm{C}$ data & 84.6498 & 15.3502 & - & - & & & & \\
\hline & All data & 99.9902 & 0.0098 & - & - & & & & \\
\hline \multirow{3}{*}{ uDP4+ } & ${ }^{1} \mathrm{H}$ data & 99.7516 & 0.2484 & - & - & & & & \\
\hline & ${ }^{13} \mathrm{C}$ data & 66.8963 & 33.1037 & - & - & & & & \\
\hline & All data & 99.8769 & 0.1231 & - & - & & & & \\
\hline \multirow{5}{*}{ DP4+ } & ${ }^{1} \mathrm{H}$ data & 99.9999 & 0.0001 & - & - & & & & \\
\hline & ${ }^{13}$ C data & 91.7654 & 8.2346 & - & - & & & & \\
\hline & All data & 100.0000 & 0.0000 & - & - & & & & \\
\hline & & \multicolumn{8}{|c|}{ Level of theory: B3LYP/6-31+G** (solution. PCM) } \\
\hline & & $88 a$ & $88 b$ & - & - & - & - & - & - \\
\hline \multirow{3}{*}{ sDP4+ } & ${ }^{1}$ H data & 99.9460 & 0.0540 & - & - & & & & \\
\hline & ${ }^{13} \mathrm{C}$ data & 84.6498 & 15.3502 & - & - & & & & \\
\hline & All data & 99.9902 & 0.0098 & - & - & & & & \\
\hline & ${ }^{1} \mathrm{H}$ data & 99.7516 & 0.2484 & - & - & & & & \\
\hline uDP4+ & ${ }^{13} \mathrm{C}$ data & 66.8963 & 33.1037 & - & - & & & & \\
\hline & All data & 99.8769 & 0.1231 & - & - & & & & \\
\hline & ${ }^{1} \mathrm{H}$ data & 99.9999 & 0.0001 & - & - & & & & \\
\hline DP4+ & ${ }^{13} \mathrm{C}$ data & 91.7654 & 8.2346 & - & - & & & & \\
\hline & All data & 100.0000 & 0.0000 & - & - & & & & \\
\hline & & & & el & B3 & 6* & PC & & \\
\hline & & $88 a$ & $88 b$ & - & - & - & - & - & - \\
\hline & ${ }^{1} \mathrm{H}$ data & 99.9460 & 0.0540 & - & - & & & & \\
\hline sDP4+ & ${ }^{13} \mathrm{C}$ data & 84.6498 & 15.3502 & - & - & & & & \\
\hline & All data & 99.9902 & 0.0098 & - & - & & & & \\
\hline uDP4+ & ${ }^{1} \mathrm{H}$ data & 99.7516 & 0.2484 & - & - & & & & \\
\hline
\end{tabular}




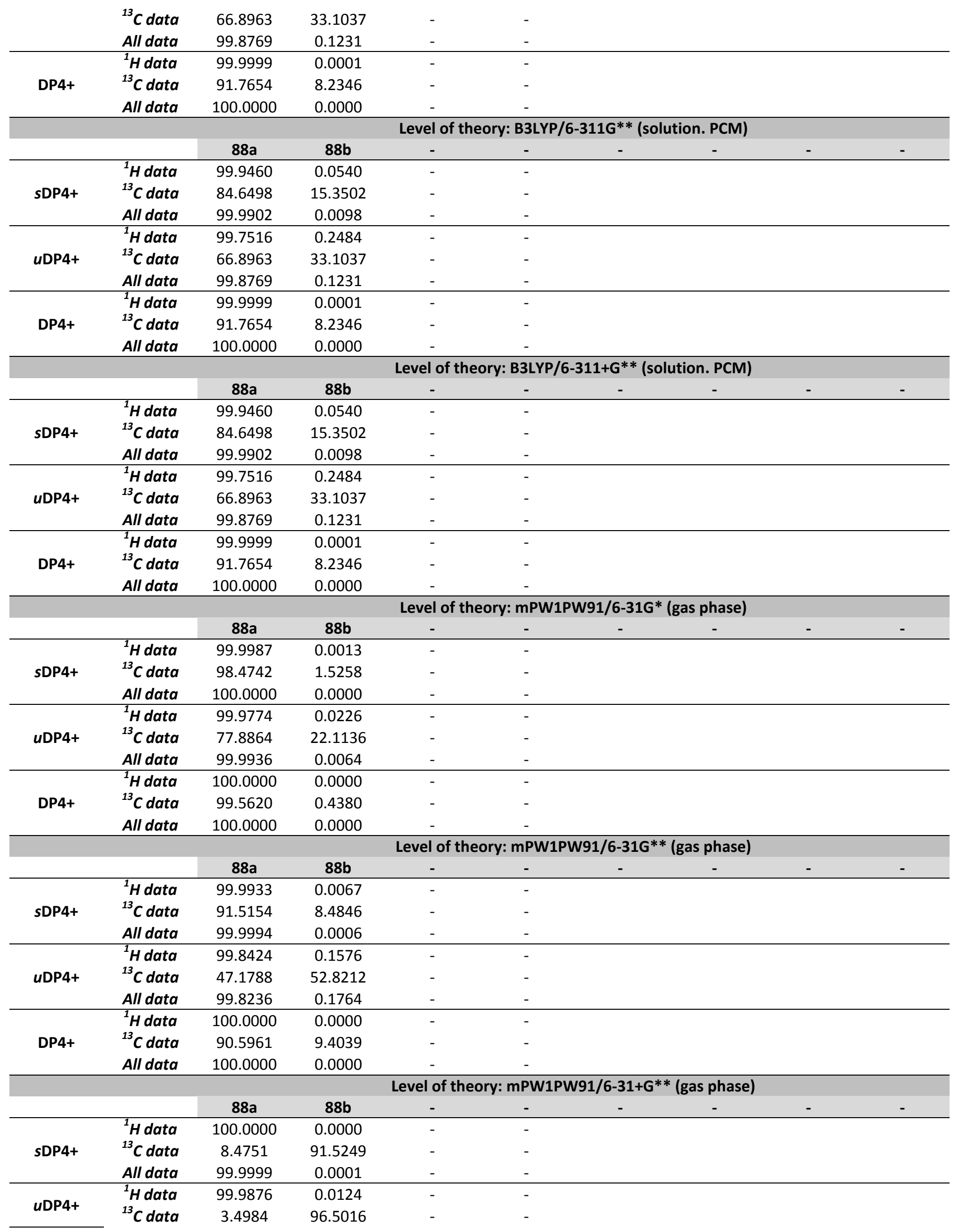




\begin{tabular}{|c|c|c|c|c|c|c|c|c|c|}
\hline & All data & 99.6580 & 0.3420 & - & - & & & & \\
\hline & ${ }^{1} \mathrm{H}$ data & 100.0000 & 0.0000 & - & - & & & & \\
\hline \multirow[t]{4}{*}{ DP4+ } & ${ }^{13} \mathrm{C}$ data & 0.3346 & 99.6654 & - & - & & & & \\
\hline & All data & 100.0000 & 0.0000 & - & - & & & & \\
\hline & & \multicolumn{8}{|c|}{ Level of theory: mPW1PW91/6-311G* (gas phase) } \\
\hline & & $88 a$ & $88 \mathrm{~b}$ & - & - & - & - & - & - \\
\hline & ${ }^{1} \mathrm{H}$ data & 99.9999 & 0.0001 & - & - & & & & \\
\hline \multirow[t]{2}{*}{ sDP4+ } & ${ }^{13} \mathrm{C}$ data & 32.5703 & 67.4297 & - & - & & & & \\
\hline & All data & 99.9999 & 0.0001 & - & - & & & & \\
\hline & ${ }^{1} \mathrm{H}$ data & 99.9743 & 0.0257 & - & - & & & & \\
\hline \multirow[t]{2}{*}{ uDP4+ } & ${ }^{13} \mathrm{C}$ data & 20.6068 & 79.3932 & - & - & & & & \\
\hline & All data & 99.9011 & 0.0989 & - & - & & & & \\
\hline \multirow{5}{*}{ DP4+ } & ${ }^{1} \mathrm{H}$ data & 100.0000 & 0.0000 & - & - & & & & \\
\hline & ${ }^{13} \mathrm{C}$ data & 11.1404 & 88.8596 & - & - & & & & \\
\hline & All data & 100.0000 & 0.0000 & - & - & & & & \\
\hline & & \multicolumn{8}{|c|}{ Level of theory: mPW1PW91/6-311G** (gas phase) } \\
\hline & & $88 \mathrm{a}$ & 88b & - & - & - & - & - & - \\
\hline \multirow{3}{*}{ sDP4+ } & ${ }^{1} \mathrm{H}$ data & 100.0000 & 0.0000 & - & - & & & & \\
\hline & ${ }^{13} \mathrm{C}$ data & 45.6151 & 54.3849 & - & - & & & & \\
\hline & All data & 100.0000 & 0.0000 & - & - & & & & \\
\hline \multirow{3}{*}{ uDP4+ } & ${ }^{1} \mathrm{H}$ data & 99.9945 & 0.0055 & - & - & & & & \\
\hline & ${ }^{13} \mathrm{C}$ data & 23.9617 & 76.0383 & - & - & & & & \\
\hline & All data & 99.9827 & 0.0173 & - & - & & & & \\
\hline \multirow{5}{*}{ DP4+ } & ${ }^{1} \mathrm{H}$ data & 100.0000 & 0.0000 & - & - & & & & \\
\hline & ${ }^{13} \mathrm{C}$ data & 20.9055 & 79.0945 & - & - & & & & \\
\hline & All data & 100.0000 & 0.0000 & - & - & & & & \\
\hline & & \multicolumn{8}{|c|}{ Level of theory: mPW1PW91/6-311+G** (gas phase) } \\
\hline & & $88 a$ & $88 \mathrm{~b}$ & - & - & - & - & - & - \\
\hline \multirow{3}{*}{ sDP4+ } & ${ }^{1} \mathrm{H}$ data & 100.0000 & 0.0000 & - & - & & & & \\
\hline & ${ }^{13} \mathrm{C}$ data & 14.0356 & 85.9644 & - & - & & & & \\
\hline & All data & 99.9999 & 0.0001 & - & - & & & & \\
\hline \multirow{3}{*}{ uDP4+ } & ${ }^{1} \mathrm{H}$ data & 99.9951 & 0.0049 & - & - & & & & \\
\hline & ${ }^{13}$ C data & 7.3813 & 92.6187 & - & - & & & & \\
\hline & All data & 99.9386 & 0.0614 & - & - & & & & \\
\hline \multirow{5}{*}{ DP4+ } & ${ }^{1} \mathrm{H}$ data & 100.0000 & 0.0000 & - & - & & & & \\
\hline & ${ }^{13} \mathrm{C}$ data & 1.2845 & 98.7155 & - & - & & & & \\
\hline & All data & 100.0000 & 0.0000 & - & - & & & & \\
\hline & & \multicolumn{8}{|c|}{ Level of theory: mPW1PW91/6-31G* (solution. PCM) } \\
\hline & & $88 a$ & $88 \mathrm{~b}$ & - & - & - & - & - & - \\
\hline \multirow{3}{*}{$s \mathrm{DP} 4+$} & ${ }^{1} \mathrm{H}$ data & 100.0000 & 0.0000 & - & - & & & & \\
\hline & ${ }^{13} \mathrm{C}$ data & 95.8604 & 4.1396 & - & - & & & & \\
\hline & All data & 100.0000 & 0.0000 & - & - & & & & \\
\hline \multirow{3}{*}{ uDP4+ } & ${ }^{1} \mathrm{H}$ data & 99.9989 & 0.0011 & - & - & & & & \\
\hline & ${ }^{13} \mathrm{C}$ data & 82.7056 & 17.2944 & - & - & & & & \\
\hline & All data & 99.9998 & 0.0002 & - & - & & & & \\
\hline & ${ }^{1} \mathrm{H}$ data & 100.0000 & 0.0000 & - & - & & & & \\
\hline DP4+ & ${ }^{13} \mathrm{C}$ data & 99.1051 & 0.8949 & - & - & & & & \\
\hline & All data & 100.0000 & 0.0000 & - & - & & & & \\
\hline & & & & f & $\mathbf{W}$ & 16 & on. & & \\
\hline & & $88 \mathrm{a}$ & $88 \mathrm{~b}$ & - & - & - & - & - & - \\
\hline & ${ }^{1} \mathrm{H}$ data & 99.9809 & 0.0191 & - & - & & & & \\
\hline sDP4+ & ${ }^{13} \mathrm{C}$ data & 78.0139 & 21.9861 & - & - & & & & \\
\hline & All data & 99.9946 & 0.0054 & - & - & & & & \\
\hline & ${ }^{1} \mathrm{H}$ data & 99.5731 & 0.4269 & - & - & & & & \\
\hline uDP4+ & ${ }^{13} \mathrm{C}$ data & 42.0021 & 57.9979 & - & - & & & & \\
\hline & All data & 99.4114 & 0.5886 & - & - & & & & \\
\hline
\end{tabular}




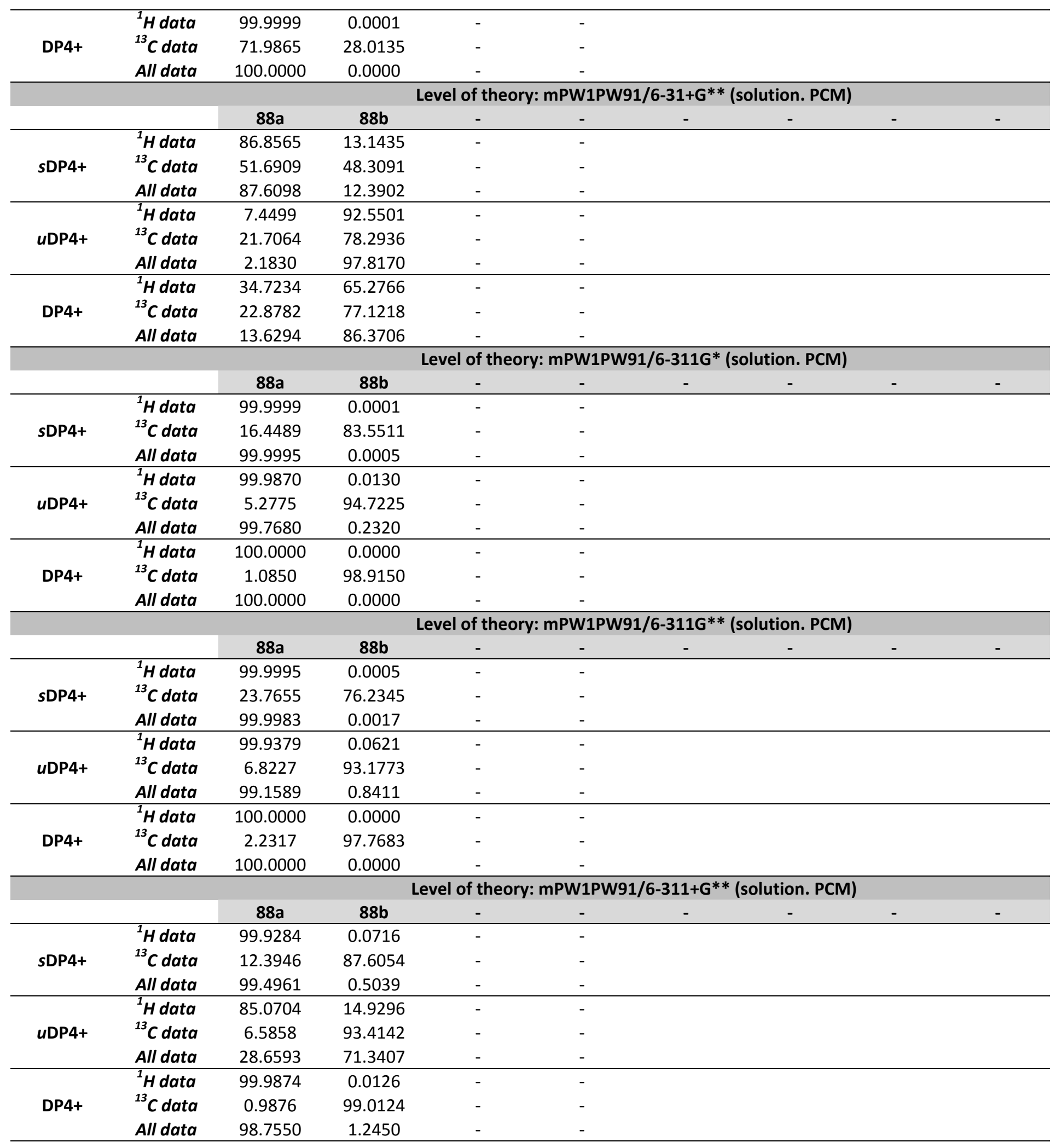


sDP4+, $u$ DP4+ and DP4+ probabilities (\%) for compound 89a<smiles>C1COC2(C1)CC1C[C@@]3(CO3)C[C@H]1C2</smiles>

$89 a$

correct isomer<smiles>C1COC2(C[C@@H]3C[C@@]4(CO4)C[C@@H]3C2)O1</smiles>

89b

\begin{tabular}{|c|c|c|c|c|c|c|c|c|c|}
\hline & & \multicolumn{8}{|c|}{ Level of theory: B3LYP/6-31G* (gas phase) } \\
\hline & & $89 a$ & $89 b$ & - & - & - & - & - & - \\
\hline & ${ }^{1} \mathrm{H}$ data & 0.4572 & 99.5428 & - & - & - & - & - & - \\
\hline \multirow[t]{3}{*}{ sDP4+ } & ${ }^{13} \mathrm{C}$ data & 96.2403 & 3.7597 & - & - & - & - & - & - \\
\hline & All data & 10.5209 & 89.4791 & - & - & - & - & - & - \\
\hline & ${ }^{1} \mathrm{H}$ data & 0.3455 & 99.6545 & - & - & - & - & - & - \\
\hline \multirow[t]{3}{*}{ uDP4+ } & ${ }^{13} \mathrm{C}$ data & 60.7304 & 39.2696 & - & - & - & - & - & - \\
\hline & All data & 0.5332 & 99.4668 & - & - & - & - & - & - \\
\hline & ${ }^{1} \mathrm{H}$ data & 0.0016 & 99.9984 & - & - & - & - & - & - \\
\hline \multirow[t]{5}{*}{ DP4+ } & ${ }^{13} \mathrm{C}$ data & 97.5362 & 2.4638 & - & - & - & - & - & - \\
\hline & All data & 0.0630 & 99.9370 & - & - & - & - & - & - \\
\hline & & \multicolumn{8}{|c|}{ Level of theory: B3LYP/6-31G** (gas phase) } \\
\hline & & $89 a$ & $89 b$ & - & - & - & - & - & - \\
\hline & ${ }^{1} \mathrm{H}$ data & 0.6666 & 99.3334 & - & - & - & - & - & - \\
\hline \multirow[t]{3}{*}{ sDP4+ } & ${ }^{13} \mathrm{C}$ data & 91.7727 & 8.2273 & - & - & - & - & - & - \\
\hline & All data & 6.9644 & 93.0356 & - & - & - & - & - & - \\
\hline & ${ }^{1} \mathrm{H}$ data & 0.7628 & 99.2372 & - & - & - & - & - & - \\
\hline \multirow[t]{3}{*}{ uDP4+ } & ${ }^{13} \mathrm{C}$ data & 37.7468 & 62.2532 & - & - & - & - & - & - \\
\hline & All data & 0.4639 & 99.5361 & - & - & - & - & - & - \\
\hline & ${ }^{1} \mathrm{H}$ data & 0.0052 & 99.9948 & - & - & - & - & - & - \\
\hline \multirow[t]{5}{*}{ DP4+ } & ${ }^{13} \mathrm{C}$ data & 87.1193 & 12.8807 & - & - & - & - & - & - \\
\hline & All data & 0.0349 & 99.9651 & - & - & - & - & - & - \\
\hline & & \multicolumn{8}{|c|}{ Level of theory: B3LYP/6-31+G** (gas phase) } \\
\hline & & $89 a$ & $89 \mathrm{~b}$ & - & - & - & - & - & - \\
\hline & ${ }^{1} \mathrm{H}$ data & 0.9630 & 99.0370 & - & - & - & - & - & - \\
\hline \multirow[t]{3}{*}{ sDP4+ } & ${ }^{13} \mathrm{C}$ data & 97.1995 & 2.8005 & - & - & - & - & - & - \\
\hline & All data & 25.2335 & 74.7665 & - & - & - & - & - & - \\
\hline & ${ }^{1} \mathrm{H}$ data & 6.0837 & 93.9163 & - & - & - & - & - & - \\
\hline \multirow[t]{3}{*}{ uDP4+ } & ${ }^{13} \mathrm{C}$ data & 70.6823 & 29.3177 & - & - & - & - & - & - \\
\hline & All data & 13.5077 & 86.4923 & - & - & - & - & - & - \\
\hline & ${ }^{1} \mathrm{H}$ data & 0.0629 & 99.9371 & - & - & - & - & - & - \\
\hline \multirow[t]{5}{*}{ DP4+ } & ${ }^{13} \mathrm{C}$ data & 98.8190 & 1.1810 & - & - & - & - & - & - \\
\hline & All data & 5.0069 & 94.9931 & - & - & - & - & - & - \\
\hline & & \multicolumn{8}{|c|}{ Level of theory: B3LYP/6-311G* (gas phase) } \\
\hline & & $89 a$ & $89 \mathrm{~b}$ & - & - & - & - & - & - \\
\hline & ${ }^{1} \mathrm{H}$ data & 20.7573 & 79.2427 & - & - & - & - & - & - \\
\hline \multirow[t]{3}{*}{ sDP4+ } & ${ }^{13} \mathrm{C}$ data & 95.9014 & 4.0986 & - & - & - & - & - & - \\
\hline & All data & 85.9730 & 14.0270 & - & - & - & - & - & - \\
\hline & ${ }^{1} \mathrm{H}$ data & 31.0254 & 68.9746 & - & - & - & - & - & - \\
\hline \multirow[t]{3}{*}{ uDP4+ } & ${ }^{13} \mathrm{C}$ data & 89.7062 & 10.2938 & - & - & - & - & - & - \\
\hline & All data & 79.6743 & 20.3257 & - & - & - & - & - & - \\
\hline & ${ }^{1} \mathrm{H}$ data & 10.5406 & 89.4594 & - & - & - & - & - & - \\
\hline \multirow[t]{4}{*}{ DP4+ } & ${ }^{13} \mathrm{C}$ data & 99.5120 & 0.4880 & - & - & - & - & - & - \\
\hline & All data & 96.0041 & 3.9959 & - & - & - & - & - & - \\
\hline & & \multicolumn{8}{|c|}{ Level of theory: B3LYP/6-311G** (gas phase) } \\
\hline & & $89 a$ & $89 b$ & - & - & - & - & - & - \\
\hline
\end{tabular}




\begin{tabular}{|c|c|c|c|c|c|c|c|c|c|}
\hline & ${ }^{13} \mathrm{C}$ data & 97.7124 & 2.2876 & - & - & - & - & - & - \\
\hline & All data & 75.5737 & 24.4263 & - & - & - & - & - & - \\
\hline & ${ }^{1} \mathrm{H}$ data & 21.7994 & 78.2006 & - & - & - & - & - & - \\
\hline \multirow[t]{3}{*}{ uDP4+ } & ${ }^{13} \mathrm{C}$ data & 96.6978 & 3.3022 & - & - & - & - & - & - \\
\hline & All data & 89.0867 & 10.9133 & - & - & - & - & - & - \\
\hline & ${ }^{1} \mathrm{H}$ data & 1.9792 & 98.0208 & - & - & - & - & - & - \\
\hline \multirow[t]{5}{*}{ DP4+ } & ${ }^{13} \mathrm{C}$ data & 99.9201 & 0.0799 & - & - & - & - & - & - \\
\hline & All data & 96.1914 & 3.8086 & - & - & - & - & - & - \\
\hline & & \multicolumn{8}{|c|}{ Level of theory: B3LYP/6-311+G** (gas phase) } \\
\hline & & $89 a$ & $89 \mathrm{~b}$ & - & - & - & - & - & - \\
\hline & ${ }^{1} \mathrm{H}$ data & 3.8768 & 96.1232 & - & - & - & - & - & - \\
\hline \multirow[t]{3}{*}{ sDP4+ } & ${ }^{13} \mathrm{C}$ data & 98.7504 & 1.2496 & - & - & - & - & - & - \\
\hline & All data & 76.1170 & 23.8830 & - & - & - & - & - & - \\
\hline & ${ }^{1} \mathrm{H}$ data & 19.3063 & 80.6937 & - & - & - & - & - & - \\
\hline \multirow[t]{3}{*}{ uDP4+ } & ${ }^{13} \mathrm{C}$ data & 98.5138 & 1.4862 & - & - & - & - & - & - \\
\hline & All data & 94.0684 & 5.9316 & - & - & - & - & - & - \\
\hline & ${ }^{1} \mathrm{H}$ data & 0.9557 & 99.0443 & - & - & - & - & - & - \\
\hline \multirow[t]{5}{*}{ DP4+ } & ${ }^{13} \mathrm{C}$ data & 99.9809 & 0.0191 & - & - & - & - & - & - \\
\hline & All data & 98.0599 & 1.9401 & - & - & - & - & - & - \\
\hline & & \multicolumn{8}{|c|}{ Level of theory: B3LYP/6-31G* (solution. PCM) } \\
\hline & & $89 a$ & $89 \mathrm{~b}$ & - & - & - & - & - & - \\
\hline & ${ }^{1} \mathrm{H}$ data & 2.4318 & 97.5682 & - & - & - & - & - & - \\
\hline \multirow[t]{3}{*}{ sDP4+ } & ${ }^{13} \mathrm{C}$ data & 96.0853 & 3.9147 & - & - & - & - & - & - \\
\hline & All data & 37.9565 & 62.0435 & - & - & - & - & - & - \\
\hline & ${ }^{1} \mathrm{H}$ data & 1.5692 & 98.4308 & - & - & - & - & - & - \\
\hline \multirow[t]{3}{*}{ uDP4+ } & ${ }^{13} \mathrm{C}$ data & 57.5362 & 42.4638 & - & - & - & - & - & - \\
\hline & All data & 2.1144 & 97.8856 & - & - & - & - & - & - \\
\hline & ${ }^{1} \mathrm{H}$ data & 0.0397 & 99.9603 & - & - & - & - & - & - \\
\hline \multirow[t]{5}{*}{ DP4+ } & ${ }^{13} \mathrm{C}$ data & 97.0809 & 2.9191 & - & - & - & - & - & - \\
\hline & All data & 1.3042 & 98.6958 & - & - & - & - & - & - \\
\hline & & \multicolumn{8}{|c|}{ Level of theory: B3LYP/6-31G** (solution. PCM) } \\
\hline & & $89 a$ & $89 b$ & - & - & - & - & - & - \\
\hline & ${ }^{1} \mathrm{H}$ data & 3.4635 & 96.5365 & - & - & - & - & - & - \\
\hline \multirow[t]{3}{*}{ sDP4+ } & ${ }^{13} \mathrm{C}$ data & 85.3921 & 14.6079 & - & - & - & - & - & - \\
\hline & All data & 17.3369 & 82.6631 & - & - & - & - & - & - \\
\hline & ${ }^{1} \mathrm{H}$ data & 4.6373 & 95.3627 & - & - & - & - & - & - \\
\hline \multirow[t]{3}{*}{ uDP4+ } & ${ }^{13} \mathrm{C}$ data & 33.3901 & 66.6099 & - & - & - & - & - & - \\
\hline & All data & 2.3796 & 97.6204 & - & - & - & - & - & - \\
\hline & ${ }^{1} \mathrm{H}$ data & 0.1742 & 99.8258 & - & - & - & - & - & - \\
\hline \multirow[t]{5}{*}{ DP4+ } & ${ }^{13} \mathrm{C}$ data & 74.5566 & 25.4434 & - & - & - & - & - & - \\
\hline & All data & 0.5086 & 99.4914 & - & - & - & - & - & - \\
\hline & & \multicolumn{8}{|c|}{ Level of theory: B3LYP/6-31+G** (solution. PCM) } \\
\hline & & $89 a$ & $89 b$ & - & - & - & - & - & - \\
\hline & ${ }^{1} \mathrm{H}$ data & 5.9614 & 94.0386 & - & - & - & - & - & - \\
\hline \multirow[t]{2}{*}{ sDP4+ } & ${ }^{13} \mathrm{C}$ data & 95.7727 & 4.2273 & - & - & - & - & - & - \\
\hline & All data & 58.9529 & 41.0471 & - & - & - & - & - & - \\
\hline & ${ }^{1} \mathrm{H}$ data & 49.7039 & 50.2961 & - & - & - & - & - & - \\
\hline uDP4+ & ${ }^{13} \mathrm{C}$ data & 74.5766 & 25.4234 & - & - & - & - & - & - \\
\hline & All data & 74.3514 & 25.6486 & - & - & - & - & - & - \\
\hline & ${ }^{1} \mathrm{H}$ data & 5.8953 & 94.1047 & - & - & - & - & - & - \\
\hline DP4+ & ${ }^{13} \mathrm{C}$ data & 98.5176 & 1.4824 & - & - & - & - & - & - \\
\hline & All data & 80.6329 & 19.3671 & - & - & - & - & - & - \\
\hline & & & & vel & B3 & $\mathbf{G}^{*}$ & PC & & \\
\hline & & $89 a$ & $89 b$ & - & - & - & - & - & - \\
\hline & ${ }^{1} \mathrm{H}$ data & 40.6891 & 59.3109 & - & - & - & - & - & - \\
\hline SDP4+ & ${ }^{13} \mathrm{C}$ data & 93.2032 & 6.7968 & - & - & - & - & - & - \\
\hline
\end{tabular}




\begin{tabular}{|c|c|c|c|c|c|c|c|c|c|}
\hline & All data & 90.3914 & 9.6086 & - & - & - & - & - & - \\
\hline & ${ }^{1} \mathrm{H}$ data & 69.9236 & 30.0764 & - & - & - & - & - & - \\
\hline \multirow[t]{3}{*}{ uDP4+ } & ${ }^{13} \mathrm{C}$ data & 86.6663 & 13.3337 & - & - & - & - & - & - \\
\hline & All data & 93.7931 & 6.2069 & - & - & - & - & - & - \\
\hline & ${ }^{1} \mathrm{H}$ data & 61.4633 & 38.5367 & - & - & - & - & - & - \\
\hline \multirow[t]{5}{*}{ DP4+ } & ${ }^{13} \mathrm{C}$ data & 98.8905 & 1.1095 & - & - & - & - & - & - \\
\hline & All data & 99.3015 & 0.6985 & - & - & - & - & - & - \\
\hline & & \multicolumn{8}{|c|}{ Level of theory: B3LYP/6-311G** (solution. PCM) } \\
\hline & & $89 a$ & $89 b$ & - & - & - & - & - & - \\
\hline & ${ }^{1} \mathrm{H}$ data & 38.8911 & 61.1089 & - & - & - & - & - & - \\
\hline \multirow[t]{3}{*}{ sDP4+ } & ${ }^{13} \mathrm{C}$ data & 96.6055 & 3.3945 & - & - & - & - & - & - \\
\hline & All data & 94.7678 & 5.2322 & - & - & - & - & - & - \\
\hline & ${ }^{1} \mathrm{H}$ data & 75.9282 & 24.0718 & - & - & - & - & - & - \\
\hline \multirow[t]{3}{*}{ uDP4+ } & ${ }^{13} \mathrm{C}$ data & 96.2333 & 3.7667 & - & - & - & - & - & - \\
\hline & All data & 98.7743 & 1.2257 & - & - & - & - & - & - \\
\hline & ${ }^{1}$ H data & 66.7490 & 33.2510 & - & - & - & - & - & - \\
\hline \multirow[t]{5}{*}{ DP4+ } & ${ }^{13}$ C data & 99.8627 & 0.1373 & - & - & - & - & - & - \\
\hline & All data & 99.9315 & 0.0685 & - & - & - & - & - & - \\
\hline & & \multicolumn{8}{|c|}{ Level of theory: B3LYP/6-311+G** (solution. PCM) } \\
\hline & & $89 a$ & $89 b$ & - & - & - & - & - & - \\
\hline & ${ }^{1} \mathrm{H}$ data & 32.3685 & 67.6315 & - & - & - & - & - & - \\
\hline \multirow[t]{3}{*}{ sDP4+ } & ${ }^{13} \mathrm{C}$ data & 97.8129 & 2.1871 & - & - & - & - & - & - \\
\hline & All data & 95.5367 & 4.4633 & - & - & - & - & - & - \\
\hline & ${ }^{1} \mathrm{H}$ data & 72.1333 & 27.8667 & - & - & - & - & - & - \\
\hline \multirow[t]{3}{*}{ uDP4+ } & ${ }^{13} \mathrm{C}$ data & 97.7101 & 2.2899 & - & - & - & - & - & - \\
\hline & All data & 99.1028 & 0.8972 & - & - & - & - & - & - \\
\hline & ${ }^{1} \mathrm{H}$ data & 55.3345 & 44.6655 & - & - & - & - & - & - \\
\hline \multirow[t]{5}{*}{ DP4+ } & ${ }^{13} \mathrm{C}$ data & 99.9476 & 0.0524 & - & - & - & - & - & - \\
\hline & All data & 99.9577 & 0.0423 & - & - & - & - & - & - \\
\hline & & \multicolumn{8}{|c|}{ Level of theory: mPW1PW91/6-31G* (gas phase) } \\
\hline & & $89 a$ & $89 b$ & - & - & - & - & - & - \\
\hline & ${ }^{1} \mathrm{H}$ data & 0.6585 & 99.3415 & - & - & - & - & - & - \\
\hline \multirow[t]{2}{*}{ sDP4+ } & ${ }^{13} \mathrm{C}$ data & 98.9097 & 1.0903 & - & - & - & - & - & - \\
\hline & All data & 37.5522 & 62.4478 & - & - & - & - & - & - \\
\hline \multirow{3}{*}{ uDP4+ } & ${ }^{1} \mathrm{H}$ data & 0.3567 & 99.6433 & - & - & - & - & - & - \\
\hline & ${ }^{13} \mathrm{C}$ data & 85.1016 & 14.8984 & - & - & - & - & - & - \\
\hline & All data & 2.0038 & 97.9962 & - & - & - & - & - & - \\
\hline \multirow{5}{*}{ DP4+ } & ${ }^{1} \mathrm{H}$ data & 0.0024 & 99.9976 & - & - & - & - & - & - \\
\hline & ${ }^{13} \mathrm{C}$ data & 99.8074 & 0.1926 & - & - & - & - & - & - \\
\hline & All data & 1.2146 & 98.7854 & - & - & - & - & - & - \\
\hline & & \multicolumn{8}{|c|}{ Level of theory: mPW1PW91/6-31G** (gas phase) } \\
\hline & & $89 a$ & $89 b$ & - & - & - & - & - & - \\
\hline \multirow{3}{*}{ sDP4+ } & ${ }^{1} \mathrm{H}$ data & 1.0216 & 98.9784 & - & - & - & - & - & - \\
\hline & ${ }^{13} \mathrm{C}$ data & 97.8304 & 2.1696 & - & - & - & - & - & - \\
\hline & All data & 31.7607 & 68.2393 & - & - & - & - & - & - \\
\hline & ${ }^{1} \mathrm{H}$ data & 0.8316 & 99.1684 & - & - & - & - & - & - \\
\hline uDP4+ & ${ }^{13} \mathrm{C}$ data & 78.5641 & 21.4359 & - & - & - & - & - & - \\
\hline & All data & 2.9816 & 97.0184 & - & - & - & - & - & - \\
\hline & ${ }^{1} \mathrm{H}$ data & 0.0087 & 99.9913 & - & - & - & - & - & - \\
\hline DP4+ & ${ }^{13} \mathrm{C}$ data & 99.3986 & 0.6014 & - & - & - & - & - & - \\
\hline & All data & 1.4102 & 98.5898 & - & - & - & - & - & - \\
\hline & & & & 10 & $\mathbf{P} \mid$ & -3 & ph & & \\
\hline & & $89 a$ & $89 b$ & - & - & - & - & - & - \\
\hline & ${ }^{1} \mathrm{H}$ data & 1.1078 & 98.8922 & - & - & - & - & - & - \\
\hline sDP4+ & ${ }^{13} \mathrm{C}$ data & 99.3014 & 0.6986 & - & - & - & - & - & - \\
\hline & All data & 61.4218 & 38.5782 & - & - & - & - & - & - \\
\hline
\end{tabular}




\begin{tabular}{|c|c|c|c|c|c|c|c|c|c|}
\hline & ${ }^{1} \mathrm{H}$ data & 8.0187 & 91.9813 & - & - & - & - & - & - \\
\hline \multirow[t]{3}{*}{ uDP4+ } & ${ }^{13} \mathrm{C}$ data & 91.9461 & 8.0539 & - & - & - & - & - & - \\
\hline & All data & 49.8809 & 50.1191 & - & - & - & - & - & - \\
\hline & ${ }^{1} \mathrm{H}$ data & 0.0976 & 99.9024 & - & - & - & - & - & - \\
\hline \multirow[t]{5}{*}{ DP4+ } & ${ }^{13} \mathrm{C}$ data & 99.9384 & 0.0616 & - & - & - & - & - & - \\
\hline & All data & 61.3088 & 38.6912 & - & - & - & - & - & - \\
\hline & & \multicolumn{8}{|c|}{ Level of theory: mPW1PW91/6-311G* (gas phase) } \\
\hline & & $89 a$ & $89 b$ & - & - & - & - & - & - \\
\hline & ${ }^{1} \mathrm{H}$ data & 16.5171 & 83.4829 & - & - & - & - & - & - \\
\hline \multirow[t]{3}{*}{ sDP4+ } & ${ }^{13} \mathrm{C}$ data & 97.7260 & 2.2740 & - & - & - & - & - & - \\
\hline & All data & 89.4767 & 10.5233 & - & - & - & - & - & - \\
\hline & ${ }^{1} \mathrm{H}$ data & 15.8450 & 84.1550 & - & - & - & - & - & - \\
\hline \multirow[t]{3}{*}{ uDP4+ } & ${ }^{13} \mathrm{C}$ data & 96.8644 & 3.1356 & - & - & - & - & - & - \\
\hline & All data & 85.3296 & 14.6704 & - & - & - & - & - & - \\
\hline & ${ }^{1} \mathrm{H}$ data & 3.5914 & 96.4086 & - & - & - & - & - & - \\
\hline \multirow[t]{5}{*}{ DP4+ } & ${ }^{13} \mathrm{C}$ data & 99.9247 & 0.0753 & - & - & - & - & - & - \\
\hline & All data & 98.0181 & 1.9819 & - & - & - & - & - & - \\
\hline & & \multicolumn{8}{|c|}{ Level of theory: mPW1PW91/6-311G** (gas phase) } \\
\hline & & $89 a$ & $89 \mathrm{~b}$ & - & - & - & - & - & - \\
\hline & ${ }^{1}$ H data & 6.9146 & 93.0854 & - & - & - & - & - & - \\
\hline \multirow[t]{3}{*}{ sDP4+ } & ${ }^{13} \mathrm{C}$ data & 98.6063 & 1.3937 & - & - & - & - & - & - \\
\hline & All data & 84.0140 & 15.9860 & - & - & - & - & - & - \\
\hline & ${ }^{1} \mathrm{H}$ data & 14.2875 & 85.7125 & - & - & - & - & - & - \\
\hline \multirow[t]{3}{*}{ uDP4+ } & ${ }^{13} \mathrm{C}$ data & 99.0178 & 0.9822 & - & - & - & - & - & - \\
\hline & All data & 94.3837 & 5.6163 & - & - & - & - & - & - \\
\hline & ${ }^{1} \mathrm{H}$ data & 1.2231 & 98.7769 & - & - & - & - & - & - \\
\hline \multirow[t]{5}{*}{ DP4+ } & ${ }^{13} \mathrm{C}$ data & 99.9860 & 0.0140 & - & - & - & - & - & - \\
\hline & All data & 98.8804 & 1.1196 & - & - & - & - & - & - \\
\hline & & \multicolumn{8}{|c|}{ Level of theory: mPW1PW91/6-311+G** (gas phase) } \\
\hline & & $89 a$ & $89 b$ & - & - & - & - & - & - \\
\hline & ${ }^{1} \mathrm{H}$ data & 3.2335 & 96.7665 & - & - & - & - & - & - \\
\hline \multirow[t]{3}{*}{ sDP4+ } & ${ }^{13} \mathrm{C}$ data & 99.1697 & 0.8303 & - & - & - & - & - & - \\
\hline & All data & 79.9637 & 20.0363 & - & - & - & - & - & - \\
\hline & ${ }^{1} \mathrm{H}$ data & 9.3007 & 90.6993 & - & - & - & - & - & - \\
\hline \multirow[t]{3}{*}{ uDP4+ } & ${ }^{13} \mathrm{C}$ data & 99.4163 & 0.5837 & - & - & - & - & - & - \\
\hline & All data & 94.5848 & 5.4152 & - & - & - & - & - & - \\
\hline & ${ }^{1} \mathrm{H}$ data & 0.3415 & 99.6585 & - & - & - & - & - & - \\
\hline \multirow[t]{5}{*}{ DP4+ } & ${ }^{13} \mathrm{C}$ data & 99.9951 & 0.0049 & - & - & - & - & - & - \\
\hline & All data & 98.5857 & 1.4143 & - & - & - & - & - & - \\
\hline & & \multicolumn{8}{|c|}{ Level of theory: mPW1PW91/6-31G* (solution. PCM) } \\
\hline & & $89 a$ & $89 \mathrm{~b}$ & - & - & - & - & - & - \\
\hline & ${ }^{1} \mathrm{H}$ data & 5.4672 & 94.5328 & - & - & - & - & - & - \\
\hline \multirow[t]{3}{*}{ sDP4+ } & ${ }^{13} \mathrm{C}$ data & 98.7296 & 1.2704 & - & - & - & - & - & - \\
\hline & All data & 81.8008 & 18.1992 & - & - & - & - & - & - \\
\hline & ${ }^{1} \mathrm{H}$ data & 2.6344 & 97.3656 & - & - & - & - & - & - \\
\hline uDP4+ & ${ }^{13} \mathrm{C}$ data & 84.3396 & 15.6604 & - & - & - & - & - & - \\
\hline & All data & 12.7183 & 87.2817 & - & - & - & - & - & - \\
\hline & ${ }^{1} \mathrm{H}$ data & 0.1562 & 99.8438 & - & - & - & - & - & - \\
\hline DP4+ & ${ }^{13} \mathrm{C}$ data & 99.7617 & 0.2383 & - & - & - & - & - & - \\
\hline & All data & 39.5754 & 60.4246 & - & - & - & - & - & - \\
\hline & & & & of $t$ & $\mathbf{W}$ & $31 C$ & on. & & \\
\hline & & $89 a$ & $89 b$ & - & - & - & - & - & - \\
\hline & ${ }^{1} \mathrm{H}$ data & 6.4010 & 93.5990 & - & - & - & - & - & - \\
\hline sDP4+ & ${ }^{13} \mathrm{C}$ data & 95.9185 & 4.0815 & - & - & - & - & - & - \\
\hline & All data & 61.6443 & 38.3557 & - & - & - & - & - & - \\
\hline uDP4+ & ${ }^{1} \mathrm{H}$ data & 7.5580 & 92.4420 & - & - & - & - & - & - \\
\hline
\end{tabular}




\begin{tabular}{|c|c|c|c|c|c|c|c|c|c|}
\hline & ${ }^{13} \mathrm{C}$ data & 75.9084 & 24.0916 & - & - & - & - & - & - \\
\hline & All data & 20.4839 & 79.5161 & - & - & - & - & - & - \\
\hline & ${ }^{1} \mathrm{H}$ data & 0.5560 & 99.4440 & - & - & - & - & - & - \\
\hline \multirow[t]{5}{*}{ DP4+ } & ${ }^{13} \mathrm{C}$ data & 98.6675 & 1.3325 & - & - & - & - & - & - \\
\hline & All data & 29.2797 & 70.7203 & - & - & - & - & - & - \\
\hline & & \multicolumn{8}{|c|}{ Level of theory: mPW1PW91/6-31+G** (solution. PCM) } \\
\hline & & $89 a$ & $89 \mathrm{~b}$ & - & - & - & - & - & - \\
\hline & ${ }^{1} \mathrm{H}$ data & 19.8948 & 80.1052 & - & - & - & - & - & - \\
\hline \multirow[t]{2}{*}{ sDP4+ } & ${ }^{13} \mathrm{C}$ data & 98.8955 & 1.1045 & - & - & - & - & - & - \\
\hline & All data & 95.6966 & 4.3034 & - & - & - & - & - & - \\
\hline \multirow{3}{*}{ uDP4+ } & ${ }^{1} \mathrm{H}$ data & 63.4652 & 36.5348 & - & - & - & - & - & - \\
\hline & ${ }^{13} \mathrm{C}$ data & 93.4645 & 6.5355 & - & - & - & - & - & - \\
\hline & All data & 96.1304 & 3.8696 & - & - & - & - & - & - \\
\hline & ${ }^{1} \mathrm{H}$ data & 30.1397 & 69.8603 & - & - & - & - & - & - \\
\hline \multirow[t]{5}{*}{ DP4+ } & ${ }^{13} \mathrm{C}$ data & 99.9220 & 0.0780 & - & - & - & - & - & - \\
\hline & All data & 99.8193 & 0.1807 & - & - & - & - & - & - \\
\hline & & \multicolumn{8}{|c|}{ Level of theory: mPW1PW91/6-311G* (solution. PCM) } \\
\hline & & $89 a$ & $89 \mathrm{~b}$ & - & - & - & - & - & - \\
\hline & ${ }^{1} \mathrm{H}$ data & 44.7882 & 55.2118 & - & - & - & - & - & - \\
\hline \multirow[t]{2}{*}{ sDP4+ } & ${ }^{13} \mathrm{C}$ data & 96.6500 & 3.3500 & - & - & - & - & - & - \\
\hline & All data & 95.9023 & 4.0977 & - & - & - & - & - & - \\
\hline & ${ }^{1} \mathrm{H}$ data & 62.6157 & 37.3843 & - & - & - & - & - & - \\
\hline \multirow[t]{2}{*}{ uDP4+ } & ${ }^{13} \mathrm{C}$ data & 96.4161 & 3.5839 & - & - & - & - & - & - \\
\hline & All data & 97.8289 & 2.1711 & - & - & - & - & - & - \\
\hline \multirow{5}{*}{ DP4+ } & ${ }^{1} \mathrm{H}$ data & 57.6038 & 42.3962 & - & - & - & - & - & - \\
\hline & ${ }^{13} \mathrm{C}$ data & 99.8713 & 0.1287 & - & - & - & - & - & - \\
\hline & All data & 99.9053 & 0.0947 & - & - & - & - & - & - \\
\hline & & \multicolumn{8}{|c|}{ Level of theory: mPW1PW91/6-311G** (solution. PCM) } \\
\hline & & $89 a$ & $89 \mathrm{~b}$ & - & - & - & - & - & - \\
\hline \multirow{3}{*}{ sDP4+ } & ${ }^{1} \mathrm{H}$ data & 42.1376 & 57.8624 & - & - & - & - & - & - \\
\hline & ${ }^{13} \mathrm{C}$ data & 98.2678 & 1.7322 & - & - & - & - & - & - \\
\hline & All data & 97.6367 & 2.3633 & - & - & - & - & - & - \\
\hline & ${ }^{1} \mathrm{H}$ data & 76.7782 & 23.2218 & - & - & - & - & - & - \\
\hline \multirow[t]{2}{*}{ uDP4+ } & ${ }^{13} \mathrm{C}$ data & 98.8478 & 1.1522 & - & - & - & - & - & - \\
\hline & All data & 99.6487 & 0.3513 & - & - & - & - & - & - \\
\hline \multirow{5}{*}{ DP4+ } & ${ }^{1} \mathrm{H}$ data & 70.6553 & 29.3447 & - & - & - & - & - & - \\
\hline & ${ }^{13} \mathrm{C}$ data & 99.9795 & 0.0205 & - & - & - & - & - & - \\
\hline & All data & 99.9915 & 0.0085 & - & - & - & - & - & - \\
\hline & & \multicolumn{8}{|c|}{ Level of theory: mPW1PW91/6-311+G** (solution. PCM) } \\
\hline & & $89 a$ & $89 b$ & - & - & - & - & - & - \\
\hline \multirow{3}{*}{ sDP4+ } & ${ }^{1} \mathrm{H}$ data & 29.3046 & 70.6954 & - & - & - & - & - & - \\
\hline & ${ }^{13} \mathrm{C}$ data & 98.7886 & 1.2114 & - & - & - & - & - & - \\
\hline & All data & 97.1267 & 2.8733 & - & - & - & - & - & - \\
\hline \multirow{3}{*}{ uDP4+ } & ${ }^{1} \mathrm{H}$ data & 65.3242 & 34.6758 & - & - & - & - & - & - \\
\hline & ${ }^{13} \mathrm{C}$ data & 99.1129 & 0.8871 & - & - & - & - & - & - \\
\hline & All data & 99.5271 & 0.4729 & - & - & - & - & - & - \\
\hline \multirow{3}{*}{ DP4+ } & ${ }^{1} \mathrm{H}$ data & 43.8484 & 56.1516 & - & - & - & - & - & - \\
\hline & ${ }^{13} \mathrm{C}$ data & 99.9890 & 0.0110 & - & - & - & - & - & - \\
\hline & All data & 99.9859 & 0.0141 & - & - & - & - & - & - \\
\hline
\end{tabular}


sDP4+, $u$ DP4+ and DP4+ probabilities (\%) for compound 90a<smiles></smiles>

$90 a$

correct isomer<smiles>O=C1[C@H]2C[C@@H]3C[C@@]1(CO3)C21CO1</smiles>

90b

\begin{tabular}{|c|c|c|c|c|c|c|c|c|c|}
\hline & & \multicolumn{8}{|c|}{ Level of theory: B3LYP/6-31G* (gas phase) } \\
\hline & & $90 a$ & $90 \mathrm{~b}$ & - & - & - & - & - & - \\
\hline & ${ }^{1} \mathrm{H}$ data & 99.9855 & 0.0145 & - & - & - & - & - & - \\
\hline \multirow[t]{3}{*}{ sDP4+ } & ${ }^{13} \mathrm{C}$ data & 95.8128 & 4.1872 & - & - & - & - & - & - \\
\hline & All data & 99.9994 & 0.0006 & - & - & - & - & - & - \\
\hline & ${ }^{1} \mathrm{H}$ data & 99.9580 & 0.0420 & - & - & - & - & - & - \\
\hline \multirow[t]{3}{*}{ uDP4+ } & ${ }^{13} \mathrm{C}$ data & 52.8592 & 47.1408 & - & - & - & - & - & - \\
\hline & All data & 99.9625 & 0.0375 & - & - & - & - & - & - \\
\hline & ${ }^{1} \mathrm{H}$ data & 100.0000 & 0.0000 & - & - & - & - & - & - \\
\hline \multirow[t]{5}{*}{ DP4+ } & ${ }^{13} \mathrm{C}$ data & 96.2488 & 3.7512 & - & - & - & - & - & - \\
\hline & All data & 100.0000 & 0.0000 & - & - & - & - & - & - \\
\hline & & \multicolumn{8}{|c|}{ Level of theory: B3LYP/6-31G** (gas phase) } \\
\hline & & $90 a$ & $90 \mathrm{~b}$ & - & - & - & - & - & - \\
\hline & ${ }^{1} \mathrm{H}$ data & 99.7253 & 0.2747 & - & - & - & - & - & - \\
\hline \multirow[t]{3}{*}{ sDP4+ } & ${ }^{13} \mathrm{C}$ data & 89.7799 & 10.2201 & - & - & - & - & - & - \\
\hline & All data & 99.9687 & 0.0313 & - & - & - & - & - & - \\
\hline & ${ }^{1} \mathrm{H}$ data & 99.3090 & 0.6910 & - & - & - & - & - & - \\
\hline \multirow[t]{3}{*}{ uDP4+ } & ${ }^{13} \mathrm{C}$ data & 67.7360 & 32.2640 & - & - & - & - & - & - \\
\hline & All data & 99.6697 & 0.3303 & - & - & - & - & - & - \\
\hline & ${ }^{1} \mathrm{H}$ data & 99.9981 & 0.0019 & - & - & - & - & - & - \\
\hline \multirow[t]{5}{*}{ DP4+ } & ${ }^{13} \mathrm{C}$ data & 94.8567 & 5.1433 & - & - & - & - & - & - \\
\hline & All data & 99.9999 & 0.0001 & - & - & - & - & - & - \\
\hline & & \multicolumn{8}{|c|}{ Level of theory: B3LYP/6-31+G** (gas phase) } \\
\hline & & $90 a$ & $90 \mathrm{~b}$ & - & - & - & - & - & - \\
\hline & ${ }^{1} \mathrm{H}$ data & 99.9457 & 0.0543 & - & - & - & - & - & - \\
\hline \multirow[t]{3}{*}{ sDP4+ } & ${ }^{13} \mathrm{C}$ data & 91.7591 & 8.2409 & - & - & - & - & - & - \\
\hline & All data & 99.9951 & 0.0049 & - & - & - & - & - & - \\
\hline & ${ }^{1} \mathrm{H}$ data & 99.7717 & 0.2283 & - & - & - & - & - & - \\
\hline \multirow[t]{3}{*}{ uDP4+ } & ${ }^{13} \mathrm{C}$ data & 37.6742 & 62.3258 & - & - & - & - & - & - \\
\hline & All data & 99.6228 & 0.3772 & - & - & - & - & - & - \\
\hline & ${ }^{1} \mathrm{H}$ data & 99.9999 & 0.0001 & - & - & - & - & - & - \\
\hline \multirow[t]{5}{*}{ DP4+ } & ${ }^{13} \mathrm{C}$ data & 87.0643 & 12.9357 & - & - & - & - & - & - \\
\hline & All data & 100.0000 & 0.0000 & - & - & - & - & - & - \\
\hline & & \multicolumn{8}{|c|}{ Level of theory: B3LYP/6-311G* (gas phase) } \\
\hline & & $90 a$ & $90 \mathrm{~b}$ & - & - & - & - & - & - \\
\hline & ${ }^{1} \mathrm{H}$ data & 99.4032 & 0.5968 & - & - & - & - & - & - \\
\hline \multirow[t]{2}{*}{ sDP4+ } & ${ }^{13} \mathrm{C}$ data & 72.3111 & 27.6889 & - & - & - & - & - & - \\
\hline & All data & 99.7706 & 0.2294 & - & - & - & - & - & - \\
\hline \multirow{3}{*}{ uDP4+ } & ${ }^{1} \mathrm{H}$ data & 96.6140 & 3.3860 & - & - & - & - & - & - \\
\hline & ${ }^{13} \mathrm{C}$ data & 30.9029 & 69.0971 & - & - & - & - & - & - \\
\hline & All data & 92.7333 & 7.2667 & - & - & - & - & - & - \\
\hline \multirow{3}{*}{ DP4+ } & ${ }^{1} \mathrm{H}$ data & 99.9790 & 0.0210 & - & - & - & - & - & - \\
\hline & ${ }^{13} \mathrm{C}$ data & 53.8743 & 46.1257 & - & - & - & - & - & - \\
\hline & All data & 99.9820 & 0.0180 & - & - & - & - & - & - \\
\hline
\end{tabular}

Level of theory: B3LYP/6-311G** (gas phase)

sDP4+ $\quad{ }^{1}$ H data $\quad 97.6290 \quad 2.3710$

${ }^{13}$ C data $\quad 88.5556 \quad 11.4444$ 


\begin{tabular}{|c|c|c|c|c|c|c|c|c|c|}
\hline & All data & 99.6871 & 0.3129 & - & - & - & - & - & - \\
\hline & ${ }^{1} \mathrm{H}$ data & 95.5224 & 4.4776 & - & - & - & - & - & - \\
\hline \multirow[t]{3}{*}{ uDP4+ } & ${ }^{13} \mathrm{C}$ data & 56.1469 & 43.8531 & - & - & - & - & - & - \\
\hline & All data & 96.4682 & 3.5318 & - & - & - & - & - & - \\
\hline & ${ }^{1} \mathrm{H}$ data & 99.8863 & 0.1137 & - & - & - & - & - & - \\
\hline \multirow[t]{5}{*}{ DP4+ } & ${ }^{13} \mathrm{C}$ data & 90.8317 & 9.1683 & - & - & - & - & - & - \\
\hline & All data & 99.9885 & 0.0115 & - & - & - & - & - & - \\
\hline & & \multicolumn{8}{|c|}{ Level of theory: B3LYP/6-311+G** (gas phase) } \\
\hline & & $90 a$ & $90 \mathrm{~b}$ & - & - & - & - & - & - \\
\hline & ${ }^{1}$ H data & 92.2781 & 7.7219 & - & - & - & - & - & - \\
\hline \multirow[t]{3}{*}{ sDP4+ } & ${ }^{13} \mathrm{C}$ data & 90.8563 & 9.1437 & - & - & - & - & - & - \\
\hline & All data & 99.1649 & 0.8351 & - & - & - & - & - & - \\
\hline & ${ }^{1} \mathrm{H}$ data & 90.3355 & 9.6645 & - & - & - & - & - & - \\
\hline \multirow[t]{3}{*}{ uDP4+ } & ${ }^{13} \mathrm{C}$ data & 66.4850 & 33.5150 & - & - & - & - & - & - \\
\hline & All data & 94.8829 & 5.1171 & - & - & - & - & - & - \\
\hline & ${ }^{1} \mathrm{H}$ data & 99.1127 & 0.8873 & - & - & - & - & - & - \\
\hline \multirow[t]{5}{*}{ DP4+ } & ${ }^{13} \mathrm{C}$ data & 95.1718 & 4.8282 & - & - & - & - & - & - \\
\hline & All data & 99.9546 & 0.0454 & - & - & - & - & - & - \\
\hline & & \multicolumn{8}{|c|}{ Level of theory: B3LYP/6-31G* (solution. PCM) } \\
\hline & & $90 a$ & $90 b$ & - & - & - & - & - & - \\
\hline & ${ }^{1}$ H data & 99.9968 & 0.0032 & - & - & - & - & - & - \\
\hline \multirow[t]{3}{*}{ sDP4+ } & ${ }^{13}$ C data & 92.2579 & 7.7421 & - & - & - & - & - & - \\
\hline & All data & 99.9997 & 0.0003 & - & - & - & - & - & - \\
\hline & ${ }^{1} \mathrm{H}$ data & 99.9656 & 0.0344 & - & - & - & - & - & - \\
\hline \multirow[t]{3}{*}{ uDP4+ } & ${ }^{13} \mathrm{C}$ data & 58.3034 & 41.6966 & - & - & - & - & - & - \\
\hline & All data & 99.9754 & 0.0246 & - & - & - & - & - & - \\
\hline & ${ }^{1} \mathrm{H}$ data & 100.0000 & 0.0000 & - & - & - & - & - & - \\
\hline \multirow[t]{5}{*}{ DP4+ } & ${ }^{13} \mathrm{C}$ data & 94.3382 & 5.6618 & - & - & - & - & - & - \\
\hline & All data & 100.0000 & 0.0000 & - & - & - & - & - & - \\
\hline & & \multicolumn{8}{|c|}{ Level of theory: B3LYP/6-31G** (solution. PCM) } \\
\hline & & $90 a$ & $90 \mathrm{~b}$ & - & - & - & - & - & - \\
\hline & ${ }^{1} \mathrm{H}$ data & 99.9724 & 0.0276 & - & - & - & - & - & - \\
\hline \multirow[t]{3}{*}{ sDP4+ } & ${ }^{13} \mathrm{C}$ data & 85.9994 & 14.0006 & - & - & - & - & - & - \\
\hline & All data & 99.9955 & 0.0045 & - & - & - & - & - & - \\
\hline & ${ }^{1} \mathrm{H}$ data & 99.7630 & 0.2370 & - & - & - & - & - & - \\
\hline \multirow[t]{3}{*}{ uDP4+ } & ${ }^{13} \mathrm{C}$ data & 45.8472 & 54.1528 & - & - & - & - & - & - \\
\hline & All data & 99.7202 & 0.2798 & - & - & - & - & - & - \\
\hline & ${ }^{1} \mathrm{H}$ data & 99.9999 & 0.0001 & - & - & - & - & - & - \\
\hline \multirow[t]{5}{*}{ DP4+ } & ${ }^{13} \mathrm{C}$ data & 83.8721 & 16.1279 & - & - & - & - & - & - \\
\hline & All data & 100.0000 & 0.0000 & - & - & - & - & - & - \\
\hline & & \multicolumn{8}{|c|}{ Level of theory: B3LYP/6-31+G** (solution. PCM) } \\
\hline & & $90 a$ & $90 \mathrm{~b}$ & - & - & - & - & - & - \\
\hline & ${ }^{1} \mathrm{H}$ data & 99.8652 & 0.1348 & - & - & - & - & - & - \\
\hline \multirow[t]{3}{*}{ sDP4+ } & ${ }^{13} \mathrm{C}$ data & 80.1646 & 19.8354 & - & - & - & - & - & - \\
\hline & All data & 99.9666 & 0.0334 & - & - & - & - & - & - \\
\hline & ${ }^{1} \mathrm{H}$ data & 97.4982 & 2.5018 & - & - & - & - & - & - \\
\hline uDP4+ & ${ }^{13} \mathrm{C}$ data & 72.2267 & 27.7733 & - & - & - & - & - & - \\
\hline & All data & 99.0229 & 0.9771 & - & - & - & - & - & - \\
\hline & ${ }^{1} \mathrm{H}$ data & 99.9965 & 0.0035 & - & - & - & - & - & - \\
\hline DP4+ & ${ }^{13} \mathrm{C}$ data & 91.3121 & 8.6879 & - & - & - & - & - & - \\
\hline & All data & 99.9997 & 0.0003 & - & - & - & - & - & - \\
\hline & & & & el & B3 & $\mathrm{G}^{*}$ & $\overline{P C}$ & & \\
\hline & & $90 a$ & $90 \mathrm{~b}$ & - & - & - & - & - & - \\
\hline & ${ }^{1} \mathrm{H}$ data & 99.7747 & 0.2253 & - & - & - & - & - & - \\
\hline sDP4+ & ${ }^{13} \mathrm{C}$ data & 50.8878 & 49.1122 & - & - & - & - & - & - \\
\hline & All data & 99.7825 & 0.2175 & - & - & - & - & - & - \\
\hline
\end{tabular}




\begin{tabular}{|c|c|c|c|c|c|c|c|c|c|}
\hline & ${ }^{1} \mathrm{H}$ data & 97.9300 & 2.0700 & - & - & - & - & - & - \\
\hline \multirow[t]{3}{*}{ uDP4+ } & ${ }^{13} \mathrm{C}$ data & 41.2270 & 58.7730 & - & - & - & - & - & - \\
\hline & All data & 97.0747 & 2.9253 & - & - & - & - & - & - \\
\hline & ${ }^{1} \mathrm{H}$ data & 99.9952 & 0.0048 & - & - & - & - & - & - \\
\hline \multirow[t]{5}{*}{ DP4+ } & ${ }^{13} \mathrm{C}$ data & 42.0902 & 57.9098 & - & - & - & - & - & - \\
\hline & All data & 99.9934 & 0.0066 & - & - & - & - & - & - \\
\hline & & \multicolumn{8}{|c|}{ Level of theory: B3LYP/6-311G** (solution. PCM) } \\
\hline & & $90 a$ & $90 b$ & - & - & - & - & - & - \\
\hline & ${ }^{1} \mathrm{H}$ data & 98.1578 & 1.8422 & - & - & - & - & - & - \\
\hline \multirow[t]{3}{*}{ sDP4+ } & ${ }^{13} \mathrm{C}$ data & 76.6735 & 23.3265 & - & - & - & - & - & - \\
\hline & All data & 99.4323 & 0.5677 & - & - & - & - & - & - \\
\hline & ${ }^{1} \mathrm{H}$ data & 88.6670 & 11.3330 & - & - & - & - & - & - \\
\hline \multirow[t]{3}{*}{ uDP4+ } & ${ }^{13} \mathrm{C}$ data & 66.1206 & 33.8794 & - & - & - & - & - & - \\
\hline & All data & 93.8534 & 6.1466 & - & - & - & - & - & - \\
\hline & ${ }^{1} \mathrm{H}$ data & 99.7607 & 0.2393 & - & - & - & - & - & - \\
\hline \multirow[t]{5}{*}{ DP4+ } & ${ }^{13} \mathrm{C}$ data & 86.5139 & 13.4861 & - & - & - & - & - & - \\
\hline & All data & 99.9626 & 0.0374 & - & - & - & - & - & - \\
\hline & & \multicolumn{8}{|c|}{ Level of theory: B3LYP/6-311+G** (solution. PCM) } \\
\hline & & $90 a$ & 90b & - & - & - & - & - & - \\
\hline & ${ }^{1} \mathrm{H}$ data & 90.0459 & 9.9541 & - & - & - & - & - & - \\
\hline \multirow[t]{3}{*}{$s \mathrm{DP4+}$} & ${ }^{13} \mathrm{C}$ data & 77.2750 & 22.7250 & - & - & - & - & - & - \\
\hline & All data & 96.8515 & 3.1485 & - & - & - & - & - & - \\
\hline & ${ }^{1} \mathrm{H}$ data & 84.8478 & 15.1522 & - & - & - & - & - & - \\
\hline \multirow[t]{3}{*}{ uDP4+ } & ${ }^{13} \mathrm{C}$ data & 72.3526 & 27.6474 & - & - & - & - & - & - \\
\hline & All data & 93.6120 & 6.3880 & - & - & - & - & - & - \\
\hline & ${ }^{1} \mathrm{H}$ data & 98.0641 & 1.9359 & - & - & - & - & - & - \\
\hline \multirow[t]{5}{*}{ DP4+ } & ${ }^{13} \mathrm{C}$ data & 89.8979 & 10.1021 & - & - & - & - & - & - \\
\hline & All data & 99.7787 & 0.2213 & - & - & - & - & - & - \\
\hline & & \multicolumn{8}{|c|}{ Level of theory: mPW1PW91/6-31G* (gas phase) } \\
\hline & & $90 a$ & 90b & - & - & - & - & - & - \\
\hline & ${ }^{1} \mathrm{H}$ data & 99.9856 & 0.0144 & - & - & - & - & - & - \\
\hline \multirow[t]{3}{*}{ sDP4+ } & ${ }^{13} \mathrm{C}$ data & 99.4295 & 0.5705 & - & - & - & - & - & - \\
\hline & All data & 99.9999 & 0.0001 & - & - & - & - & - & - \\
\hline & ${ }^{1} \mathrm{H}$ data & 99.9620 & 0.0380 & - & - & - & - & - & - \\
\hline \multirow[t]{3}{*}{ uDP4+ } & ${ }^{13} \mathrm{C}$ data & 87.6802 & 12.3198 & - & - & - & - & - & - \\
\hline & All data & 99.9947 & 0.0053 & - & - & - & - & - & - \\
\hline & ${ }^{1} \mathrm{H}$ data & 100.0000 & 0.0000 & - & - & - & - & - & - \\
\hline \multirow[t]{5}{*}{ DP4+ } & ${ }^{13} \mathrm{C}$ data & 99.9194 & 0.0806 & - & - & - & - & - & - \\
\hline & All data & 100.0000 & 0.0000 & - & - & - & - & - & - \\
\hline & & \multicolumn{8}{|c|}{ Level of theory: mPW1PW91/6-31G** (gas phase) } \\
\hline & & $90 a$ & 90b & - & - & - & - & - & - \\
\hline & ${ }^{1} \mathrm{H}$ data & 99.9481 & 0.0519 & - & - & - & - & - & - \\
\hline \multirow[t]{2}{*}{ sDP4+ } & ${ }^{13} \mathrm{C}$ data & 98.7054 & 1.2946 & - & - & - & - & - & - \\
\hline & All data & 99.9993 & 0.0007 & - & - & - & - & - & - \\
\hline & ${ }^{1} \mathrm{H}$ data & 99.7704 & 0.2296 & - & - & - & - & - & - \\
\hline$u \mathrm{DP} 4+$ & ${ }^{13} \mathrm{C}$ data & 81.6159 & 18.3841 & - & - & - & - & - & - \\
\hline & All data & 99.9482 & 0.0518 & - & - & - & - & - & - \\
\hline & ${ }^{1} \mathrm{H}$ data & 99.9999 & 0.0001 & - & - & - & - & - & - \\
\hline DP4+ & ${ }^{13} \mathrm{C}$ data & 99.7054 & 0.2946 & - & - & - & - & - & - \\
\hline & All data & 100.0000 & 0.0000 & - & - & - & - & - & - \\
\hline & & & & 10 & PV & -31 & ph & & \\
\hline & & $90 a$ & $90 b$ & - & - & - & - & - & - \\
\hline & ${ }^{1} \mathrm{H}$ data & 99.8403 & 0.1597 & - & - & - & - & - & - \\
\hline sDP4+ & ${ }^{13} \mathrm{C}$ data & 98.3439 & 1.6561 & - & - & - & - & - & - \\
\hline & All data & 99.9973 & 0.0027 & - & - & - & - & - & - \\
\hline uDP4+ & ${ }^{1} \mathrm{H}$ data & 98.3026 & 1.6974 & - & - & - & - & - & - \\
\hline
\end{tabular}




\begin{tabular}{|c|c|c|c|c|c|c|c|c|c|}
\hline & ${ }^{13} \mathrm{C}$ data & 88.5315 & 11.4685 & - & - & - & - & - & - \\
\hline & All data & 99.7768 & 0.2232 & - & - & - & - & - & - \\
\hline & ${ }^{1} \mathrm{H}$ data & 99.9972 & 0.0028 & - & - & - & - & - & - \\
\hline \multirow[t]{5}{*}{ DP4+ } & ${ }^{13} \mathrm{C}$ data & 99.7823 & 0.2177 & - & - & - & - & - & - \\
\hline & All data & 100.0000 & 0.0000 & - & - & - & - & - & - \\
\hline & & \multicolumn{8}{|c|}{ Level of theory: mPW1PW91/6-311G* (gas phase) } \\
\hline & & $90 a$ & 90b & - & - & - & - & - & - \\
\hline & ${ }^{1} \mathrm{H}$ data & 99.0340 & 0.9660 & - & - & - & - & - & - \\
\hline \multirow[t]{2}{*}{$s D P 4+$} & ${ }^{13}$ C data & 83.3665 & 16.6335 & - & - & - & - & - & - \\
\hline & All data & 99.8058 & 0.1942 & - & - & - & - & - & - \\
\hline \multirow{3}{*}{ uDP4+ } & ${ }^{1} \mathrm{H}$ data & 97.4950 & 2.5050 & - & - & - & - & - & - \\
\hline & ${ }^{13} \mathrm{C}$ data & 50.5667 & 49.4333 & - & - & - & - & - & - \\
\hline & All data & 97.5497 & 2.4503 & - & - & - & - & - & - \\
\hline \multirow{5}{*}{ DP4+ } & ${ }^{1} \mathrm{H}$ data & 99.9749 & 0.0251 & - & - & - & - & - & - \\
\hline & ${ }^{13} \mathrm{C}$ data & 83.6785 & 16.3215 & - & - & - & - & - & - \\
\hline & All data & 99.9951 & 0.0049 & - & - & - & - & - & - \\
\hline & & \multicolumn{8}{|c|}{ Level of theory: mPW1PW91/6-311G** (gas phase) } \\
\hline & & $90 a$ & $90 \mathrm{~b}$ & - & - & - & - & - & - \\
\hline \multirow{3}{*}{ sDP4+ } & ${ }^{1} \mathrm{H}$ data & 96.9176 & 3.0824 & - & - & - & - & - & - \\
\hline & ${ }^{13} \mathrm{C}$ data & 93.4731 & 6.5269 & - & - & - & - & - & - \\
\hline & All data & 99.7784 & 0.2216 & - & - & - & - & - & - \\
\hline \multirow{3}{*}{ uDP4+ } & ${ }^{1} \mathrm{H}$ data & 94.8790 & 5.1210 & - & - & - & - & - & - \\
\hline & ${ }^{13} \mathrm{C}$ data & 75.9607 & 24.0393 & - & - & - & - & - & - \\
\hline & All data & 98.3206 & 1.6794 & - & - & - & - & - & - \\
\hline \multirow{3}{*}{ DP4+ } & ${ }^{1} \mathrm{H}$ data & 99.8286 & 0.1714 & - & - & - & - & - & - \\
\hline & ${ }^{13} \mathrm{C}$ data & 97.8380 & 2.1620 & - & - & - & - & - & - \\
\hline & All data & 99.9962 & 0.0038 & - & - & - & - & - & - \\
\hline & & \multicolumn{8}{|c|}{ Level of theory: mPW1PW91/6-311+G** (gas phase) } \\
\hline & & $90 a$ & 90b & - & - & - & - & - & - \\
\hline \multirow{3}{*}{ sDP4+ } & ${ }^{1} \mathrm{H}$ data & 95.4963 & 4.5037 & - & - & - & - & - & - \\
\hline & ${ }^{13} \mathrm{C}$ data & 94.9100 & 5.0900 & - & - & - & - & - & - \\
\hline & All data & 99.7477 & 0.2523 & - & - & - & - & - & - \\
\hline \multirow{3}{*}{ uDP4+ } & ${ }^{1} \mathrm{H}$ data & 92.8162 & 7.1838 & - & - & - & - & - & - \\
\hline & ${ }^{13} \mathrm{C}$ data & 82.7042 & 17.2958 & - & - & - & - & - & - \\
\hline & All data & 98.4072 & 1.5928 & - & - & - & - & - & - \\
\hline \multirow{5}{*}{ DP4+ } & ${ }^{1} \mathrm{H}$ data & 99.6363 & 0.3637 & - & - & - & - & - & - \\
\hline & ${ }^{13} \mathrm{C}$ data & 98.8909 & 1.1091 & - & - & - & - & - & - \\
\hline & All data & 99.9959 & 0.0041 & - & - & - & - & - & - \\
\hline & & \multicolumn{8}{|c|}{ Level of theory: mPW1PW91/6-31G* (solution. PCM) } \\
\hline & & $90 a$ & 90b & - & - & - & - & - & - \\
\hline \multirow{3}{*}{ sDP4+ } & ${ }^{1} \mathrm{H}$ data & 99.9951 & 0.0049 & - & - & - & - & - & - \\
\hline & ${ }^{13} \mathrm{C}$ data & 99.0256 & 0.9744 & - & - & - & - & - & - \\
\hline & All data & 100.0000 & 0.0000 & - & - & - & - & - & - \\
\hline \multirow{3}{*}{ uDP4+ } & ${ }^{1} \mathrm{H}$ data & 99.9632 & 0.0368 & - & - & - & - & - & - \\
\hline & ${ }^{13} \mathrm{C}$ data & 90.9986 & 9.0014 & - & - & - & - & - & - \\
\hline & All data & 99.9964 & 0.0036 & - & - & - & - & - & - \\
\hline & ${ }^{1} \mathrm{H}$ data & 100.0000 & 0.0000 & - & - & - & - & - & - \\
\hline DP4+ & ${ }^{13} \mathrm{C}$ data & 99.9028 & 0.0972 & - & - & - & - & - & - \\
\hline & All data & 100.0000 & 0.0000 & - & - & - & - & - & - \\
\hline & & & & of $t$ & $\mathbf{W}$ & 10 & on. & & \\
\hline & & $90 a$ & $90 \mathrm{~b}$ & - & - & - & - & - & - \\
\hline & ${ }^{1} \mathrm{H}$ data & 99.9767 & 0.0233 & - & - & - & - & - & - \\
\hline sDP4+ & ${ }^{13} \mathrm{C}$ data & 98.2000 & 1.8000 & - & - & - & - & - & - \\
\hline & All data & 99.9996 & 0.0004 & - & - & - & - & - & - \\
\hline & ${ }^{1} \mathrm{H}$ data & 99.7808 & 0.2192 & - & - & - & - & - & - \\
\hline UDP4+ & ${ }^{13} \mathrm{C}$ data & 92.1605 & 7.8395 & - & - & - & - & - & - \\
\hline
\end{tabular}




\begin{tabular}{|c|c|c|c|c|c|c|c|c|c|}
\hline & All data & 99.9813 & 0.0187 & - & - & - & - & - & - \\
\hline & ${ }^{1} \mathrm{H}$ data & 99.9999 & 0.0001 & - & - & - & - & - & - \\
\hline \multirow[t]{5}{*}{ DP4+ } & ${ }^{13} \mathrm{C}$ data & 99.8443 & 0.1557 & - & - & - & - & - & - \\
\hline & All data & 100.0000 & 0.0000 & - & - & - & - & - & - \\
\hline & & \multicolumn{8}{|c|}{ Level of theory: mPW1PW91/6-31+G** (solution. PCM) } \\
\hline & & $90 a$ & $90 \mathrm{~b}$ & - & - & - & - & - & - \\
\hline & ${ }^{1} \mathrm{H}$ data & 99.5849 & 0.4151 & - & - & - & - & - & - \\
\hline \multirow[t]{3}{*}{ sDP4+ } & ${ }^{13} \mathrm{C}$ data & 95.7619 & 4.2381 & - & - & - & - & - & - \\
\hline & All data & 99.9816 & 0.0184 & - & - & - & - & - & - \\
\hline & ${ }^{1} \mathrm{H}$ data & 97.1999 & 2.8001 & - & - & - & - & - & - \\
\hline \multirow[t]{3}{*}{ uDP4+ } & ${ }^{13} \mathrm{C}$ data & 92.2005 & 7.7995 & - & - & - & - & - & - \\
\hline & All data & 99.7569 & 0.2431 & - & - & - & - & - & - \\
\hline & ${ }^{1} \mathrm{H}$ data & 99.9880 & 0.0120 & - & - & - & - & - & - \\
\hline \multirow[t]{5}{*}{ DP4+ } & ${ }^{13} \mathrm{C}$ data & 99.6270 & 0.3730 & - & - & - & - & - & - \\
\hline & All data & 100.0000 & 0.0000 & - & - & - & - & - & - \\
\hline & & \multicolumn{8}{|c|}{ Level of theory: mPW1PW91/6-311G* (solution. PCM) } \\
\hline & & $90 a$ & 90b & - & - & - & - & - & - \\
\hline & ${ }^{1} \mathrm{H}$ data & 99.6825 & 0.3175 & - & - & - & - & - & - \\
\hline \multirow[t]{2}{*}{ sDP4+ } & ${ }^{13} \mathrm{C}$ data & 72.7044 & 27.2956 & - & - & - & - & - & - \\
\hline & All data & 99.8805 & 0.1195 & - & - & - & - & - & - \\
\hline \multirow{3}{*}{ uDP4+ } & ${ }^{1}$ H data & 98.1122 & 1.8878 & - & - & - & - & - & - \\
\hline & ${ }^{13}$ C data & 62.3884 & 37.6116 & - & - & - & - & - & - \\
\hline & All data & 98.8533 & 1.1467 & - & - & - & - & - & - \\
\hline \multirow{5}{*}{ DP4+ } & ${ }^{1} \mathrm{H}$ data & 99.9939 & 0.0061 & - & - & - & - & - & - \\
\hline & ${ }^{13} \mathrm{C}$ data & 81.5439 & 18.4561 & - & - & - & - & - & - \\
\hline & All data & 99.9986 & 0.0014 & - & - & - & - & - & - \\
\hline & & \multicolumn{8}{|c|}{ Level of theory: mPW1PW91/6-311G** (solution. PCM) } \\
\hline & & $90 a$ & $90 \mathrm{~b}$ & - & - & - & - & - & - \\
\hline \multirow{3}{*}{ sDP4+ } & ${ }^{1} \mathrm{H}$ data & 97.4850 & 2.5150 & - & - & - & - & - & - \\
\hline & ${ }^{13} \mathrm{C}$ data & 89.6088 & 10.3912 & - & - & - & - & - & - \\
\hline & All data & 99.7017 & 0.2983 & - & - & - & - & - & - \\
\hline \multirow{3}{*}{ uDP4+ } & ${ }^{1} \mathrm{H}$ data & 87.5196 & 12.4804 & - & - & - & - & - & - \\
\hline & ${ }^{13} \mathrm{C}$ data & 84.5438 & 15.4562 & - & - & - & - & - & - \\
\hline & All data & 97.4592 & 2.5408 & - & - & - & - & - & - \\
\hline \multirow{5}{*}{ DP4+ } & ${ }^{1} \mathrm{H}$ data & 99.6335 & 0.3665 & - & - & - & - & - & - \\
\hline & ${ }^{13} \mathrm{C}$ data & 97.9240 & 2.0760 & - & - & - & - & - & - \\
\hline & All data & 99.9922 & 0.0078 & - & - & - & - & - & - \\
\hline & & \multicolumn{8}{|c|}{ Level of theory: mPW1PW91/6-311+G** (solution. PCM) } \\
\hline & & $90 a$ & $90 \mathrm{~b}$ & - & - & - & - & - & - \\
\hline \multirow{3}{*}{ sDP4+ } & ${ }^{1} \mathrm{H}$ data & 86.2696 & 13.7304 & - & - & - & - & - & - \\
\hline & ${ }^{13} \mathrm{C}$ data & 88.9678 & 11.0322 & - & - & - & - & - & - \\
\hline & All data & 98.0646 & 1.9354 & - & - & - & - & - & - \\
\hline \multirow{3}{*}{ uDP4+ } & ${ }^{1} \mathrm{H}$ data & 73.6135 & 26.3865 & - & - & - & - & - & - \\
\hline & ${ }^{13} \mathrm{C}$ data & 85.8734 & 14.1266 & - & - & - & - & - & - \\
\hline & All data & 94.4317 & 5.5683 & - & - & - & - & - & - \\
\hline \multirow{3}{*}{ DP4+ } & ${ }^{1} \mathrm{H}$ data & 94.6030 & 5.3970 & - & - & - & - & - & - \\
\hline & ${ }^{13} \mathrm{C}$ data & 98.0009 & 1.9991 & - & - & - & - & - & - \\
\hline & All data & 99.8838 & 0.1162 & - & - & - & - & - & - \\
\hline
\end{tabular}


$s \mathrm{DP4}+, u \mathrm{DP} 4+$ and DP4+ probabilities (\%) for compound 90b
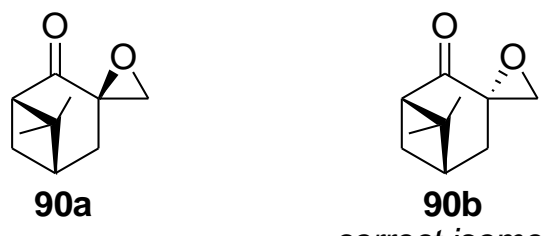

correct isomer

\begin{tabular}{|c|c|c|c|c|c|c|c|c|c|}
\hline & & \multicolumn{8}{|c|}{ Level of theory: B3LYP/6-31G* (gas phase) } \\
\hline & & $90 a$ & $90 \mathrm{~b}$ & - & - & - & - & - & - \\
\hline & ${ }^{1}$ H data & 99.9573 & 0.0427 & - & - & - & - & - & - \\
\hline \multirow[t]{3}{*}{ sDP4+ } & ${ }^{13} \mathrm{C}$ data & 1.1877 & 98.8123 & - & - & - & - & - & - \\
\hline & All data & 96.5717 & 3.4283 & - & - & - & - & - & - \\
\hline & ${ }^{1} \mathrm{H}$ data & 99.8118 & 0.1882 & - & - & - & - & - & - \\
\hline \multirow[t]{3}{*}{ uDP4+ } & ${ }^{13} \mathrm{C}$ data & 0.4418 & 99.5582 & - & - & - & - & - & - \\
\hline & All data & 70.1842 & 29.8158 & - & - & - & - & - & - \\
\hline & ${ }^{1} \mathrm{H}$ data & 99.9999 & 0.0001 & - & - & - & - & - & - \\
\hline \multirow[t]{5}{*}{ DP4+ } & ${ }^{13} \mathrm{C}$ data & 0.0053 & 99.9947 & - & - & - & - & - & - \\
\hline & All data & 98.5143 & 1.4857 & - & - & - & - & - & - \\
\hline & & \multicolumn{8}{|c|}{ Level of theory: B3LYP/6-31G** (gas phase) } \\
\hline & & $90 a$ & $90 \mathrm{~b}$ & - & - & - & - & - & - \\
\hline & ${ }^{1} \mathrm{H}$ data & 99.8661 & 0.1339 & - & - & - & - & - & - \\
\hline \multirow[t]{3}{*}{ sDP4+ } & ${ }^{13} \mathrm{C}$ data & 0.6304 & 99.3696 & - & - & - & - & - & - \\
\hline & All data & 82.5553 & 17.4447 & - & - & - & - & - & - \\
\hline & ${ }^{1} \mathrm{H}$ data & 99.6361 & 0.3639 & - & - & - & - & - & - \\
\hline \multirow[t]{3}{*}{ uDP4+ } & ${ }^{13} \mathrm{C}$ data & 0.4535 & 99.5465 & - & - & - & - & - & - \\
\hline & All data & 55.5041 & 44.4959 & - & - & - & - & - & - \\
\hline & ${ }^{1} \mathrm{H}$ data & 99.9995 & 0.0005 & - & - & - & - & - & - \\
\hline \multirow[t]{5}{*}{ DP4+ } & ${ }^{13} \mathrm{C}$ data & 0.0029 & 99.9971 & - & - & - & - & - & - \\
\hline & All data & 85.5139 & 14.4861 & - & - & - & - & - & - \\
\hline & & \multicolumn{8}{|c|}{ Level of theory: B3LYP/6-31+G** (gas phase) } \\
\hline & & $90 a$ & $90 \mathrm{~b}$ & - & - & - & - & - & - \\
\hline & ${ }^{1} \mathrm{H}$ data & 99.5443 & 0.4557 & - & - & - & - & - & - \\
\hline \multirow[t]{2}{*}{ sDP4+ } & ${ }^{13} \mathrm{C}$ data & 1.5919 & 98.4081 & - & - & - & - & - & - \\
\hline & All data & 77.9416 & 22.0584 & - & - & - & - & - & - \\
\hline \multirow{3}{*}{ uDP4+ } & ${ }^{1} \mathrm{H}$ data & 98.5957 & 1.4043 & - & - & - & - & - & - \\
\hline & ${ }^{13} \mathrm{C}$ data & 1.3441 & 98.6559 & - & - & - & - & - & - \\
\hline & All data & 48.8891 & 51.1109 & - & - & - & - & - & - \\
\hline \multirow{3}{*}{ DP4+ } & ${ }^{1} \mathrm{H}$ data & 99.9935 & 0.0065 & - & - & - & - & - & - \\
\hline & ${ }^{13} \mathrm{C}$ data & 0.0220 & 99.9780 & - & - & - & - & - & - \\
\hline & All data & 77.1680 & 22.8320 & - & - & - & - & - & - \\
\hline & & \multicolumn{8}{|c|}{ Level of theory: B3LYP/6-311G* (gas phase) } \\
\hline & & $90 a$ & $90 \mathrm{~b}$ & - & - & - & - & - & - \\
\hline \multirow{3}{*}{ sDP4+ } & ${ }^{1} \mathrm{H}$ data & 96.8790 & 3.1210 & - & - & - & - & - & - \\
\hline & ${ }^{13} \mathrm{C}$ data & 0.3604 & 99.6396 & - & - & - & - & - & - \\
\hline & All data & 10.0953 & 89.9047 & - & - & - & - & - & - \\
\hline \multirow{3}{*}{ uDP4+ } & ${ }^{1} \mathrm{H}$ data & 94.0050 & 5.9950 & - & - & - & - & - & - \\
\hline & ${ }^{13} \mathrm{C}$ data & 0.2475 & 99.7525 & - & - & - & - & - & - \\
\hline & All data & 3.7453 & 96.2547 & - & - & - & - & - & - \\
\hline \multirow{3}{*}{ DP4+ } & ${ }^{1} \mathrm{H}$ data & 99.7950 & 0.2050 & - & - & - & - & - & - \\
\hline & ${ }^{13} \mathrm{C}$ data & 0.0009 & 99.9991 & - & - & - & - & - & - \\
\hline & All data & 0.4350 & 99.5650 & - & - & - & - & - & - \\
\hline & & \multicolumn{8}{|c|}{ Level of theory: B3LYP/6-311G** (gas phase) } \\
\hline & & $90 a$ & $90 b$ & - & - & - & - & - & - \\
\hline \multirow{2}{*}{ sDP4+ } & ${ }^{1} \mathrm{H}$ data & 94.8514 & 5.1486 & - & - & - & - & - & - \\
\hline & ${ }^{13} \mathrm{C}$ data & 0.6082 & 99.3918 & - & - & - & - & - & - \\
\hline
\end{tabular}




\begin{tabular}{|c|c|c|c|c|c|c|c|c|c|}
\hline & All data & 10.1305 & 89.8695 & - & - & - & - & - & - \\
\hline & ${ }^{1} \mathrm{H}$ data & 95.5915 & 4.4085 & - & - & - & - & - & - \\
\hline \multirow[t]{3}{*}{ uDP4+ } & ${ }^{13} \mathrm{C}$ data & 0.2884 & 99.7116 & - & - & - & - & - & - \\
\hline & All data & 5.9010 & 94.0990 & - & - & - & - & - & - \\
\hline & ${ }^{1} \mathrm{H}$ data & 99.7503 & 0.2497 & - & - & - & - & - & - \\
\hline \multirow[t]{5}{*}{ DP4+ } & ${ }^{13} \mathrm{C}$ data & 0.0018 & 99.9982 & - & - & - & - & - & - \\
\hline & All data & 0.7019 & 99.2981 & - & - & - & - & - & - \\
\hline & & \multicolumn{8}{|c|}{ Level of theory: B3LYP/6-311+G** (gas phase) } \\
\hline & & $90 a$ & 90b & - & - & - & - & - & - \\
\hline & ${ }^{1} \mathrm{H}$ data & 90.6868 & 9.3132 & - & - & - & - & - & - \\
\hline \multirow[t]{3}{*}{ sDP4+ } & ${ }^{13} \mathrm{C}$ data & 0.5778 & 99.4222 & - & - & - & - & - & - \\
\hline & All data & 5.3557 & 94.6443 & - & - & - & - & - & - \\
\hline & ${ }^{1} \mathrm{H}$ data & 87.4261 & 12.5739 & - & - & - & - & - & - \\
\hline \multirow[t]{3}{*}{ uDP4+ } & ${ }^{13} \mathrm{C}$ data & 0.2790 & 99.7210 & - & - & - & - & - & - \\
\hline & All data & 1.9081 & 98.0919 & - & - & - & - & - & - \\
\hline & ${ }^{1} \mathrm{H}$ data & 98.5445 & 1.4555 & - & - & - & - & - & - \\
\hline \multirow[t]{5}{*}{ DP4+ } & ${ }^{13} \mathrm{C}$ data & 0.0016 & 99.9984 & - & - & - & - & - & - \\
\hline & All data & 0.1100 & 99.8900 & - & - & - & - & - & - \\
\hline & & \multicolumn{8}{|c|}{ Level of theory: B3LYP/6-31G* (solution. PCM) } \\
\hline & & $90 a$ & 90b & - & - & - & - & - & - \\
\hline & ${ }^{1} \mathrm{H}$ data & 99.9663 & 0.0337 & - & - & - & - & - & - \\
\hline \multirow[t]{3}{*}{ sDP4+ } & ${ }^{13} \mathrm{C}$ data & 0.1856 & 99.8144 & - & - & - & - & - & - \\
\hline & All data & 84.6526 & 15.3474 & - & - & - & - & - & - \\
\hline & ${ }^{1} \mathrm{H}$ data & 99.5162 & 0.4838 & - & - & - & - & - & - \\
\hline \multirow[t]{3}{*}{ uDP4+ } & ${ }^{13} \mathrm{C}$ data & 0.3252 & 99.6748 & - & - & - & - & - & - \\
\hline & All data & 40.1557 & 59.8443 & - & - & - & - & - & - \\
\hline & ${ }^{1} \mathrm{H}$ data & 99.9998 & 0.0002 & - & - & - & - & - & - \\
\hline \multirow[t]{5}{*}{ DP4+ } & ${ }^{13} \mathrm{C}$ data & 0.0006 & 99.9994 & - & - & - & - & - & - \\
\hline & All data & 78.7283 & 21.2717 & - & - & - & - & - & - \\
\hline & & \multicolumn{8}{|c|}{ Level of theory: B3LYP/6-31G** (solution. PCM) } \\
\hline & & $90 a$ & 90b & - & - & - & - & - & - \\
\hline & ${ }^{1} \mathrm{H}$ data & 99.6921 & 0.3079 & - & - & - & - & - & - \\
\hline \multirow[t]{3}{*}{ sDP4+ } & ${ }^{13} \mathrm{C}$ data & 0.2569 & 99.7431 & - & - & - & - & - & - \\
\hline & All data & 45.4741 & 54.5259 & - & - & - & - & - & - \\
\hline & ${ }^{1} \mathrm{H}$ data & 98.6599 & 1.3401 & - & - & - & - & - & - \\
\hline \multirow[t]{3}{*}{ uDP4+ } & ${ }^{13} \mathrm{C}$ data & 0.3994 & 99.6006 & - & - & - & - & - & - \\
\hline & All data & 22.7937 & 77.2063 & - & - & - & - & - & - \\
\hline & ${ }^{1} \mathrm{H}$ data & 99.9958 & 0.0042 & - & - & - & - & - & - \\
\hline \multirow[t]{5}{*}{ DP4+ } & ${ }^{13} \mathrm{C}$ data & 0.0010 & 99.9990 & - & - & - & - & - & - \\
\hline & All data & 19.7573 & 80.2427 & - & - & - & - & - & - \\
\hline & & \multicolumn{8}{|c|}{ Level of theory: B3LYP/6-31+G** (solution. PCM) } \\
\hline & & $90 a$ & $90 b$ & - & - & - & - & - & - \\
\hline & ${ }^{1} \mathrm{H}$ data & 98.6789 & 1.3211 & - & - & - & - & - & - \\
\hline \multirow[t]{2}{*}{ sDP4+ } & ${ }^{13} \mathrm{C}$ data & 0.8826 & 99.1174 & - & - & - & - & - & - \\
\hline & All data & 39.9423 & 60.0577 & - & - & - & - & - & - \\
\hline & ${ }^{1} \mathrm{H}$ data & 90.8349 & 9.1651 & - & - & - & - & - & - \\
\hline uDP4+ & ${ }^{13} \mathrm{C}$ data & 1.0928 & 98.9072 & - & - & - & - & - & - \\
\hline & All data & 9.8693 & 90.1307 & - & - & - & - & - & - \\
\hline & ${ }^{1} \mathrm{H}$ data & 99.8651 & 0.1349 & - & - & - & - & - & - \\
\hline DP4+ & ${ }^{13} \mathrm{C}$ data & 0.0098 & 99.9902 & - & - & - & - & - & - \\
\hline & All data & 6.7881 & 93.2119 & - & - & - & - & - & - \\
\hline & & & & el & B3 & $5^{*}$ & PC & & \\
\hline & & $90 a$ & $90 \mathrm{~b}$ & - & - & - & - & - & - \\
\hline & ${ }^{1} \mathrm{H}$ data & 99.1273 & 0.8727 & - & - & - & - & - & - \\
\hline sDP4+ & ${ }^{13} \mathrm{C}$ data & 0.1862 & 99.8138 & - & - & - & - & - & - \\
\hline & All data & 17.4817 & 82.5183 & - & - & - & - & - & - \\
\hline
\end{tabular}




\begin{tabular}{|c|c|c|c|c|c|c|c|c|c|}
\hline & ${ }^{1} \mathrm{H}$ data & 93.4018 & 6.5982 & - & - & - & - & - & - \\
\hline \multirow[t]{3}{*}{ uDP4+ } & ${ }^{13} \mathrm{C}$ data & 0.2093 & 99.7907 & - & - & - & - & - & - \\
\hline & All data & 2.8838 & 97.1162 & - & - & - & - & - & - \\
\hline & ${ }^{1} \mathrm{H}$ data & 99.9378 & 0.0622 & - & - & - & - & - & - \\
\hline \multirow[t]{5}{*}{ DP4+ } & ${ }^{13} \mathrm{C}$ data & 0.0004 & 99.9996 & - & - & - & - & - & - \\
\hline & All data & 0.6251 & 99.3749 & - & - & - & - & - & - \\
\hline & & \multicolumn{8}{|c|}{ Level of theory: B3LYP/6-311G** (solution. PCM) } \\
\hline & & $90 a$ & $90 \mathrm{~b}$ & - & - & - & - & - & - \\
\hline & ${ }^{1} \mathrm{H}$ data & 91.0789 & 8.9211 & - & - & - & - & - & - \\
\hline \multirow[t]{3}{*}{ sDP4+ } & ${ }^{13} \mathrm{C}$ data & 0.2333 & 99.7667 & - & - & - & - & - & - \\
\hline & All data & 2.3322 & 97.6678 & - & - & - & - & - & - \\
\hline & ${ }^{1} \mathrm{H}$ data & 89.1084 & 10.8916 & - & - & - & - & - & - \\
\hline \multirow[t]{3}{*}{ uDP4+ } & ${ }^{13} \mathrm{C}$ data & 0.2676 & 99.7324 & - & - & - & - & - & - \\
\hline & All data & 2.1483 & 97.8517 & - & - & - & - & - & - \\
\hline & ${ }^{1} \mathrm{H}$ data & 98.8169 & 1.1831 & - & - & - & - & - & - \\
\hline \multirow[t]{5}{*}{ DP4+ } & ${ }^{13} \mathrm{C}$ data & 0.0006 & 99.9994 & - & - & - & - & - & - \\
\hline & All data & 0.0524 & 99.9476 & - & - & - & - & - & - \\
\hline & & \multicolumn{8}{|c|}{ Level of theory: B3LYP/6-311+G** (solution. PCM) } \\
\hline & & $90 a$ & 90b & - & - & - & - & - & - \\
\hline & ${ }^{1}$ H data & 69.4216 & 30.5784 & - & - & - & - & - & - \\
\hline \multirow[t]{3}{*}{ sDP4+ } & ${ }^{13} \mathrm{C}$ data & 0.2568 & 99.7432 & - & - & - & - & - & - \\
\hline & All data & 0.5812 & 99.4188 & - & - & - & - & - & - \\
\hline & ${ }^{1} \mathrm{H}$ data & 66.3899 & 33.6101 & - & - & - & - & - & - \\
\hline \multirow[t]{3}{*}{ uDP4+ } & ${ }^{13} \mathrm{C}$ data & 0.4481 & 99.5519 & - & - & - & - & - & - \\
\hline & All data & 0.8812 & 99.1188 & - & - & - & - & - & - \\
\hline & ${ }^{1} \mathrm{H}$ data & 81.7667 & 18.2333 & - & - & - & - & - & - \\
\hline \multirow[t]{5}{*}{ DP4+ } & ${ }^{13} \mathrm{C}$ data & 0.0012 & 99.9988 & - & - & - & - & - & - \\
\hline & All data & 0.0052 & 99.9948 & - & - & - & - & - & - \\
\hline & & \multicolumn{8}{|c|}{ Level of theory: mPW1PW91/6-31G* (gas phase) } \\
\hline & & $90 a$ & $90 \mathrm{~b}$ & - & - & - & - & - & - \\
\hline & ${ }^{1} \mathrm{H}$ data & 99.9550 & 0.0450 & - & - & - & - & - & - \\
\hline \multirow[t]{2}{*}{ sDP4+ } & ${ }^{13} \mathrm{C}$ data & 1.7839 & 98.2161 & - & - & - & - & - & - \\
\hline & All data & 97.5792 & 2.4208 & - & - & - & - & - & - \\
\hline \multirow{3}{*}{ uDP4+ } & ${ }^{1} \mathrm{H}$ data & 99.8337 & 0.1663 & - & - & - & - & - & - \\
\hline & ${ }^{13} \mathrm{C}$ data & 0.4519 & 99.5481 & - & - & - & - & - & - \\
\hline & All data & 73.1525 & 26.8475 & - & - & - & - & - & - \\
\hline \multirow{5}{*}{ DP4+ } & ${ }^{1} \mathrm{H}$ data & 99.9999 & 0.0001 & - & - & - & - & - & - \\
\hline & ${ }^{13} \mathrm{C}$ data & 0.0082 & 99.9918 & - & - & - & - & - & - \\
\hline & All data & 99.0977 & 0.9023 & - & - & - & - & - & - \\
\hline & & \multicolumn{8}{|c|}{ Level of theory: mPW1PW91/6-31G** (gas phase) } \\
\hline & & $90 a$ & $90 \mathrm{~b}$ & - & - & - & - & - & - \\
\hline \multirow{3}{*}{ sDP4+ } & ${ }^{1} \mathrm{H}$ data & 99.8736 & 0.1264 & - & - & - & - & - & - \\
\hline & ${ }^{13} \mathrm{C}$ data & 1.7370 & 98.2630 & - & - & - & - & - & - \\
\hline & All data & 93.3200 & 6.6800 & - & - & - & - & - & - \\
\hline & ${ }^{1} \mathrm{H}$ data & 99.6761 & 0.3239 & - & - & - & - & - & - \\
\hline uDP4+ & ${ }^{13} \mathrm{C}$ data & 0.4627 & 99.5373 & - & - & - & - & - & - \\
\hline & All data & 58.8538 & 41.1462 & - & - & - & - & - & - \\
\hline & ${ }^{1} \mathrm{H}$ data & 99.9996 & 0.0004 & - & - & - & - & - & - \\
\hline DP4+ & ${ }^{13} \mathrm{C}$ data & 0.0082 & 99.9918 & - & - & - & - & - & - \\
\hline & All data & 95.2340 & 4.7660 & - & - & - & - & - & - \\
\hline & & & & 10 & $\mid \mathbf{P}$ & -3 & $\mathrm{pr}$ & & \\
\hline & & $90 a$ & $90 \mathrm{~b}$ & - & - & - & - & - & - \\
\hline & ${ }^{1} \mathrm{H}$ data & 99.7403 & 0.2597 & - & - & - & - & - & - \\
\hline sDP4+ & ${ }^{13} \mathrm{C}$ data & 1.6521 & 98.3479 & - & - & - & - & - & - \\
\hline & All data & 86.5804 & 13.4196 & - & - & - & - & - & - \\
\hline uDP4+ & ${ }^{1} \mathrm{H}$ data & 97.1132 & 2.8868 & - & - & - & - & - & - \\
\hline
\end{tabular}




\begin{tabular}{|c|c|c|c|c|c|c|c|c|c|}
\hline & ${ }^{13} \mathrm{C}$ data & 1.1300 & 98.8700 & - & - & - & - & - & - \\
\hline & All data & 27.7713 & 72.2287 & - & - & - & - & - & - \\
\hline & ${ }^{1} \mathrm{H}$ data & 99.9923 & 0.0077 & - & - & - & - & - & - \\
\hline \multirow[t]{5}{*}{ DP4+ } & ${ }^{13} \mathrm{C}$ data & 0.0192 & 99.9808 & - & - & - & - & - & - \\
\hline & All data & 71.2699 & 28.7301 & - & - & - & - & - & - \\
\hline & & \multicolumn{8}{|c|}{ Level of theory: mPW1PW91/6-311G* (gas phase) } \\
\hline & & $90 a$ & 90b & - & - & - & - & - & - \\
\hline & ${ }^{1} \mathrm{H}$ data & 96.8825 & 3.1175 & - & - & - & - & - & - \\
\hline \multirow[t]{2}{*}{$s D P 4+$} & ${ }^{13} \mathrm{C}$ data & 0.6107 & 99.3893 & - & - & - & - & - & - \\
\hline & All data & 16.0328 & 83.9672 & - & - & - & - & - & - \\
\hline \multirow{3}{*}{ uDP4+ } & ${ }^{1} \mathrm{H}$ data & 92.7458 & 7.2542 & - & - & - & - & - & - \\
\hline & ${ }^{13} \mathrm{C}$ data & 0.4305 & 99.5695 & - & - & - & - & - & - \\
\hline & All data & 5.2382 & 94.7618 & - & - & - & - & - & - \\
\hline \multirow{5}{*}{ DP4+ } & ${ }^{1} \mathrm{H}$ data & 99.7489 & 0.2511 & - & - & - & - & - & - \\
\hline & ${ }^{13} \mathrm{C}$ data & 0.0027 & 99.9973 & - & - & - & - & - & - \\
\hline & All data & 1.0444 & 98.9556 & - & - & - & - & - & - \\
\hline & & \multicolumn{8}{|c|}{ Level of theory: mPW1PW91/6-311G** (gas phase) } \\
\hline & & $90 a$ & $90 \mathrm{~b}$ & - & - & - & - & - & - \\
\hline \multirow{3}{*}{ sDP4+ } & ${ }^{1} \mathrm{H}$ data & 94.1900 & 5.8100 & - & - & - & - & - & - \\
\hline & ${ }^{13} \mathrm{C}$ data & 0.8711 & 99.1289 & - & - & - & - & - & - \\
\hline & All data & 12.4691 & 87.5309 & - & - & - & - & - & - \\
\hline \multirow{3}{*}{ uDP4+ } & ${ }^{1} \mathrm{H}$ data & 94.8396 & 5.1604 & - & - & - & - & - & - \\
\hline & ${ }^{13} \mathrm{C}$ data & 0.4008 & 99.5992 & - & - & - & - & - & - \\
\hline & All data & 6.8865 & 93.1135 & - & - & - & - & - & - \\
\hline \multirow{3}{*}{ DP4+ } & ${ }^{1} \mathrm{H}$ data & 99.6655 & 0.3345 & - & - & - & - & - & - \\
\hline & ${ }^{13} \mathrm{C}$ data & 0.0035 & 99.9965 & - & - & - & - & - & - \\
\hline & All data & 1.0426 & 98.9574 & - & - & - & - & - & - \\
\hline & & \multicolumn{8}{|c|}{ Level of theory: mPW1PW91/6-311+G** (gas phase) } \\
\hline & & $90 a$ & 90b & - & - & - & - & - & - \\
\hline \multirow{3}{*}{ sDP4+ } & ${ }^{1} \mathrm{H}$ data & 94.7698 & 5.2302 & - & - & - & - & - & - \\
\hline & ${ }^{13} \mathrm{C}$ data & 0.5558 & 99.4442 & - & - & - & - & - & - \\
\hline & All data & 9.1963 & 90.8037 & - & - & - & - & - & - \\
\hline \multirow{3}{*}{ uDP4+ } & ${ }^{1} \mathrm{H}$ data & 86.2097 & 13.7903 & - & - & - & - & - & - \\
\hline & ${ }^{13} \mathrm{C}$ data & 0.4097 & 99.5903 & - & - & - & - & - & - \\
\hline & All data & 2.5075 & 97.4925 & - & - & - & - & - & - \\
\hline \multirow{5}{*}{ DP4+ } & ${ }^{1} \mathrm{H}$ data & 99.1249 & 0.8751 & - & - & - & - & - & - \\
\hline & ${ }^{13} \mathrm{C}$ data & 0.0023 & 99.9977 & - & - & - & - & - & - \\
\hline & All data & 0.2598 & 99.7402 & - & - & - & - & - & - \\
\hline & & \multicolumn{8}{|c|}{ Level of theory: mPW1PW91/6-31G* (solution. PCM) } \\
\hline & & $90 a$ & 90b & - & - & - & - & - & - \\
\hline \multirow{3}{*}{ sDP4+ } & ${ }^{1} \mathrm{H}$ data & 99.9387 & 0.0613 & - & - & - & - & - & - \\
\hline & ${ }^{13} \mathrm{C}$ data & 0.3167 & 99.6833 & - & - & - & - & - & - \\
\hline & All data & 83.8215 & 16.1785 & - & - & - & - & - & - \\
\hline \multirow{3}{*}{ uDP4+ } & ${ }^{1} \mathrm{H}$ data & 99.4349 & 0.5651 & - & - & - & - & - & - \\
\hline & ${ }^{13} \mathrm{C}$ data & 0.4336 & 99.5664 & - & - & - & - & - & - \\
\hline & All data & 43.3817 & 56.6183 & - & - & - & - & - & - \\
\hline & ${ }^{1} \mathrm{H}$ data & 99.9997 & 0.0003 & - & - & - & - & - & - \\
\hline DP4+ & ${ }^{13} \mathrm{C}$ data & 0.0014 & 99.9986 & - & - & - & - & - & - \\
\hline & All data & 79.8784 & 20.1216 & - & - & - & - & - & - \\
\hline & & & & of $t$ & $\mathbf{W}$ & 10 & n. & & \\
\hline & & $90 a$ & $90 \mathrm{~b}$ & - & - & - & - & - & - \\
\hline & ${ }^{1} \mathrm{H}$ data & 99.6991 & 0.3009 & - & - & - & - & - & - \\
\hline sDP4+ & ${ }^{13} \mathrm{C}$ data & 0.4392 & 99.5608 & - & - & - & - & - & - \\
\hline & All data & 59.3826 & 40.6174 & - & - & - & - & - & - \\
\hline & ${ }^{1} \mathrm{H}$ data & 98.9731 & 1.0269 & - & - & - & - & - & - \\
\hline UDP4+ & ${ }^{13} \mathrm{C}$ data & 0.4508 & 99.5492 & - & - & - & - & - & - \\
\hline
\end{tabular}




\begin{tabular}{|c|c|c|c|c|c|c|c|c|c|}
\hline & All data & 30.3833 & 69.6167 & - & - & - & - & - & - \\
\hline & ${ }^{1} \mathrm{H}$ data & 99.9969 & 0.0031 & - & - & - & - & - & - \\
\hline \multirow[t]{5}{*}{ DP4+ } & ${ }^{13} \mathrm{C}$ data & 0.0020 & 99.9980 & - & - & - & - & - & - \\
\hline & All data & 38.9526 & 61.0474 & - & - & - & - & - & - \\
\hline & & \multicolumn{8}{|c|}{ Level of theory: mPW1PW91/6-31+G** (solution. PCM) } \\
\hline & & $90 a$ & $90 b$ & - & - & - & - & - & - \\
\hline & ${ }^{1} \mathrm{H}$ data & 59.0935 & 40.9065 & - & - & - & - & - & - \\
\hline \multirow[t]{3}{*}{ sDP4+ } & ${ }^{13} \mathrm{C}$ data & 0.1231 & 99.8769 & - & - & - & - & - & - \\
\hline & All data & 0.1777 & 99.8223 & - & - & - & - & - & - \\
\hline & ${ }^{1} \mathrm{H}$ data & 52.2416 & 47.7584 & - & - & - & - & - & - \\
\hline \multirow[t]{3}{*}{ uDP4+ } & ${ }^{13} \mathrm{C}$ data & 0.4118 & 99.5882 & - & - & - & - & - & - \\
\hline & All data & 0.4503 & 99.5497 & - & - & - & - & - & - \\
\hline & ${ }^{1} \mathrm{H}$ data & 61.2434 & 38.7566 & - & - & - & - & - & - \\
\hline \multirow[t]{5}{*}{ DP4+ } & ${ }^{13} \mathrm{C}$ data & 0.0005 & 99.9995 & - & - & - & - & - & - \\
\hline & All data & 0.0008 & 99.9992 & - & - & - & - & - & - \\
\hline & & \multicolumn{8}{|c|}{ Level of theory: mPW1PW91/6-311G* (solution. PCM) } \\
\hline & & $90 a$ & 90b & - & - & - & - & - & - \\
\hline & ${ }^{1} \mathrm{H}$ data & 98.7017 & 1.2983 & - & - & - & - & - & - \\
\hline \multirow[t]{3}{*}{ sDP4+ } & ${ }^{13} \mathrm{C}$ data & 0.1345 & 99.8655 & - & - & - & - & - & - \\
\hline & All data & 9.2895 & 90.7105 & - & - & - & - & - & - \\
\hline & ${ }^{1}$ H data & 88.5463 & 11.4537 & - & - & - & - & - & - \\
\hline \multirow[t]{3}{*}{ uDP4+ } & ${ }^{13}$ C data & 0.1783 & 99.8217 & - & - & - & - & - & - \\
\hline & All data & 1.3618 & 98.6382 & - & - & - & - & - & - \\
\hline & ${ }^{1} \mathrm{H}$ data & 99.8301 & 0.1699 & - & - & - & - & - & - \\
\hline \multirow[t]{5}{*}{ DP4+ } & ${ }^{13} \mathrm{C}$ data & 0.0002 & 99.9998 & - & - & - & - & - & - \\
\hline & All data & 0.1412 & 99.8588 & - & - & - & - & - & - \\
\hline & & \multicolumn{8}{|c|}{ Level of theory: mPW1PW91/6-311G** (solution. PCM) } \\
\hline & & $90 a$ & 90b & - & - & - & - & - & - \\
\hline & ${ }^{1} \mathrm{H}$ data & 87.5719 & 12.4281 & - & - & - & - & - & - \\
\hline \multirow[t]{2}{*}{ sDP4+ } & ${ }^{13} \mathrm{C}$ data & 0.1415 & 99.8585 & - & - & - & - & - & - \\
\hline & All data & 0.9886 & 99.0114 & - & - & - & - & - & - \\
\hline \multirow{3}{*}{ uDP4+ } & ${ }^{1} \mathrm{H}$ data & 83.8675 & 16.1325 & - & - & - & - & - & - \\
\hline & ${ }^{13} \mathrm{C}$ data & 0.2898 & 99.7102 & - & - & - & - & - & - \\
\hline & All data & 1.4884 & 98.5116 & - & - & - & - & - & - \\
\hline \multirow{5}{*}{ DP4+ } & ${ }^{1} \mathrm{H}$ data & 97.3426 & 2.6574 & - & - & - & - & - & - \\
\hline & ${ }^{13} \mathrm{C}$ data & 0.0004 & 99.9996 & - & - & - & - & - & - \\
\hline & All data & 0.0151 & 99.9849 & - & - & - & - & - & - \\
\hline & & \multicolumn{8}{|c|}{ Level of theory: mPW1PW91/6-311+G** (solution. PCM) } \\
\hline & & $90 a$ & 90b & - & - & - & - & - & - \\
\hline \multirow{3}{*}{ sDP4+ } & ${ }^{1} \mathrm{H}$ data & 59.0935 & 40.9065 & - & - & - & - & - & - \\
\hline & ${ }^{13} \mathrm{C}$ data & 0.1231 & 99.8769 & - & - & - & - & - & - \\
\hline & All data & 0.1777 & 99.8223 & - & - & - & - & - & - \\
\hline \multirow{3}{*}{ uDP4+ } & ${ }^{1} \mathrm{H}$ data & 52.2416 & 47.7584 & - & - & - & - & - & - \\
\hline & ${ }^{13} \mathrm{C}$ data & 0.4118 & 99.5882 & - & - & - & - & - & - \\
\hline & All data & 0.4503 & 99.5497 & - & - & - & - & - & - \\
\hline \multirow{3}{*}{ DP4+ } & ${ }^{1} \mathrm{H}$ data & 61.2434 & 38.7566 & - & - & - & - & - & - \\
\hline & ${ }^{13} \mathrm{C}$ data & 0.0005 & 99.9995 & - & - & - & - & - & - \\
\hline & All data & 0.0008 & 99.9992 & - & - & - & - & - & - \\
\hline
\end{tabular}




\section{Performance Evaluation}

The performance of the different probabilities developed in this study at the 24 levels of theory, using scaled data (sDP4+), unscaled data (uDP4+) and the combination of both (DP4+), using only proton data, carbon data or the combination of both (all data) are shown in the following figures. Each column represents the number of examples used in the validation test (total number $=48$ ) that a) were correctly assigned in high high confidence ( $>95 \%$, green column), b) were correctly assigned in modest confidence ( $>50 \%$, yellow column) or c) were incorrectly assigned ( $<50 \%$, red column). At the right is displayed the performance evaluation of the original DP4 (using all data) for comparison.

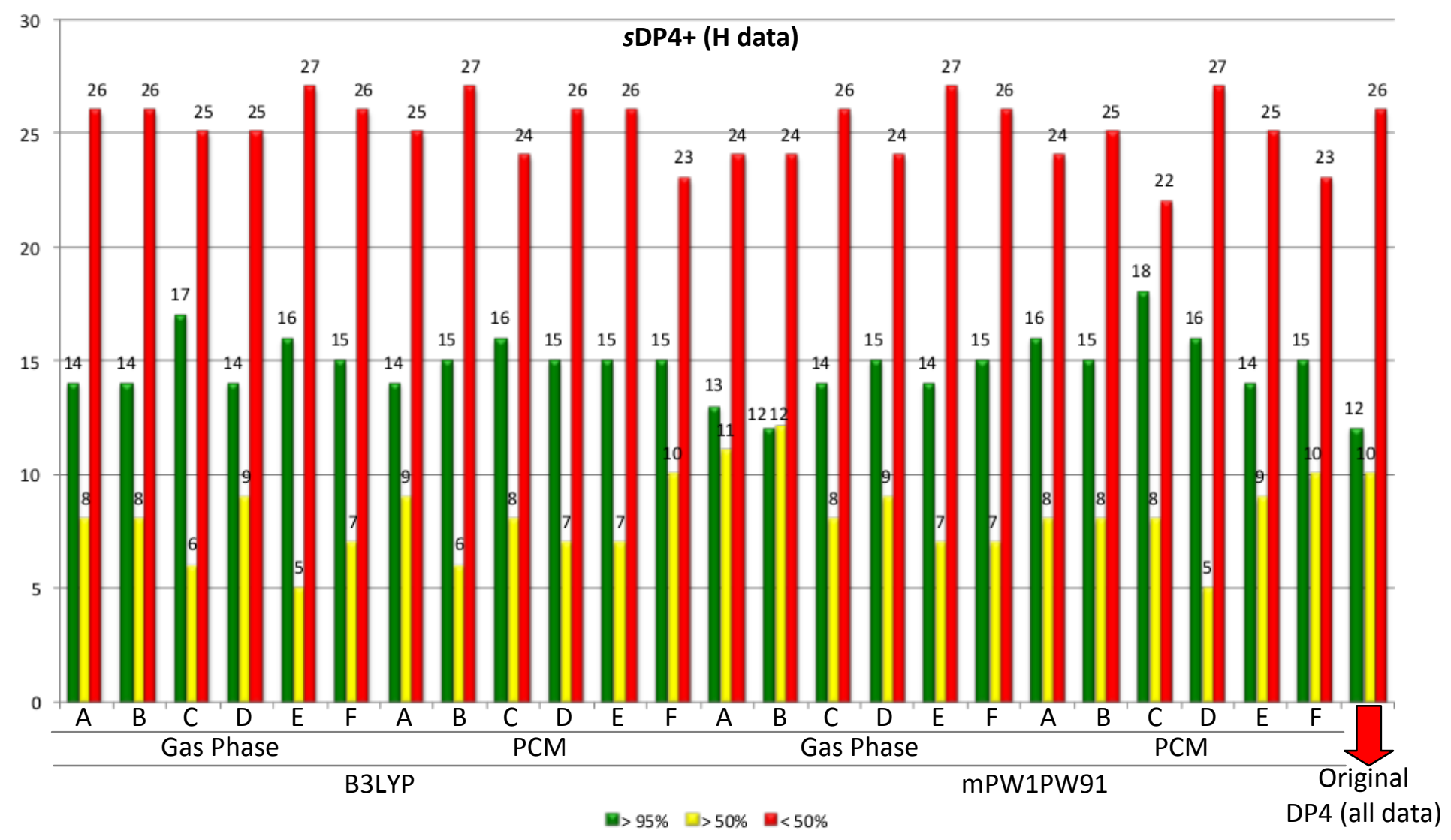



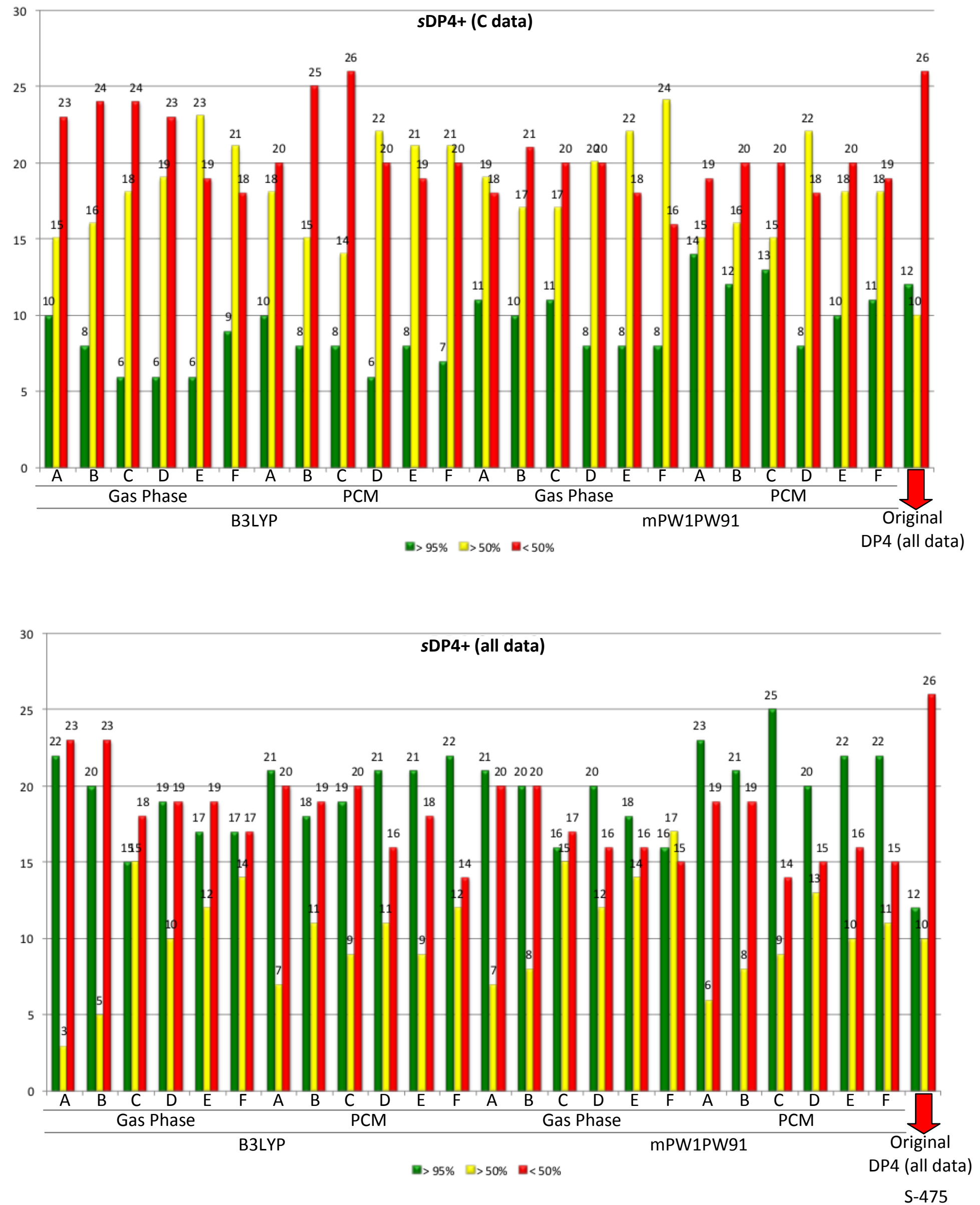

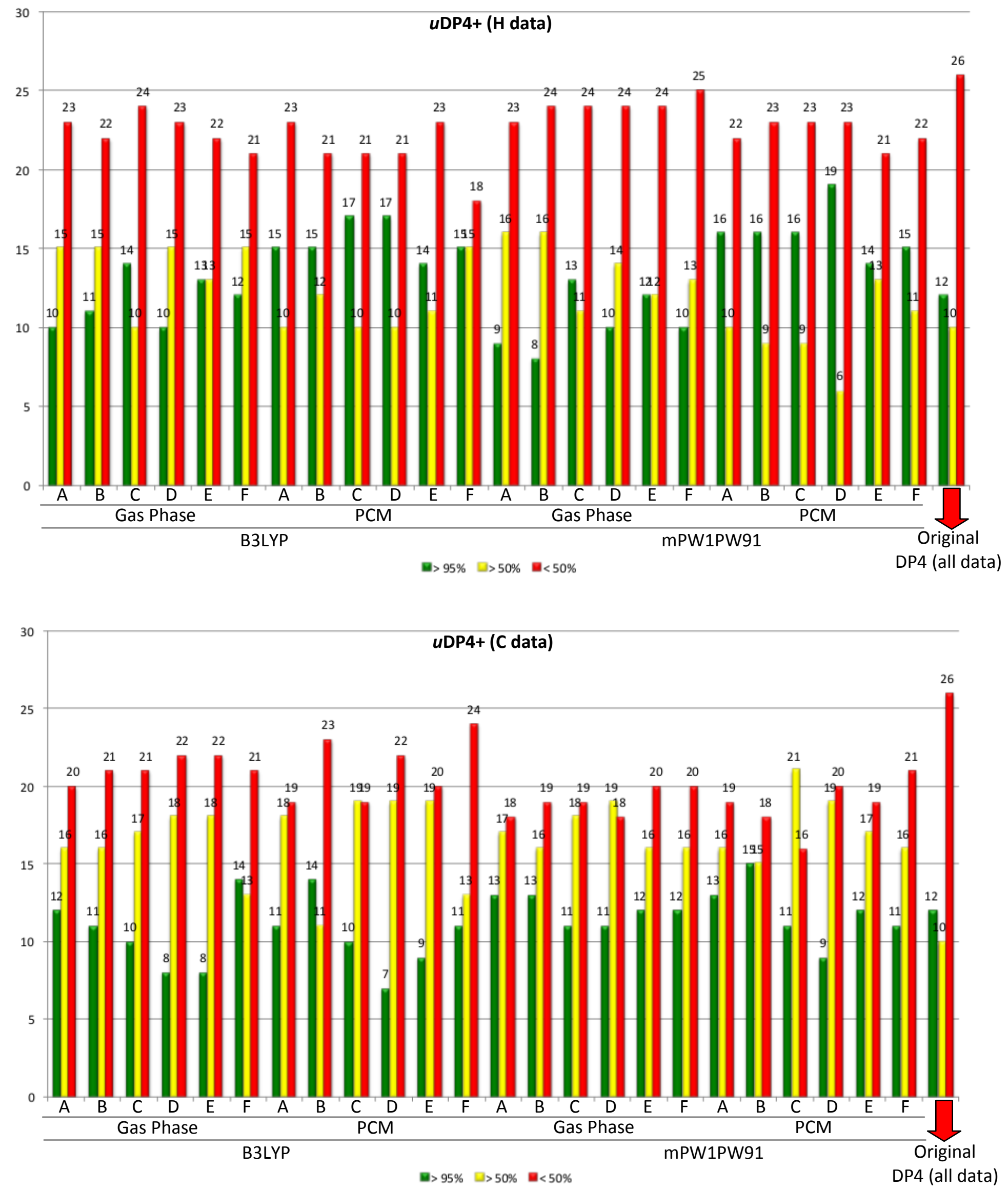

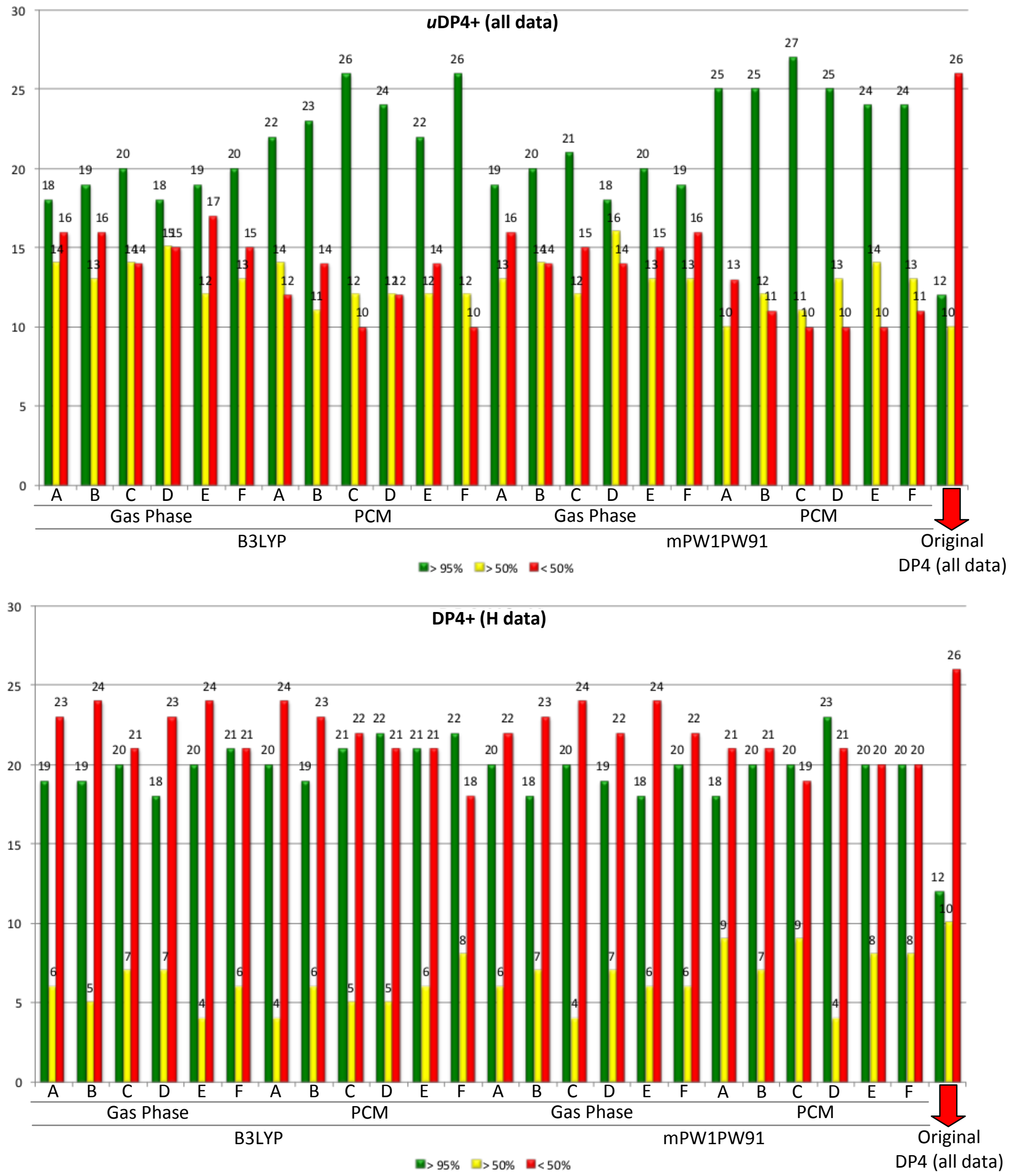

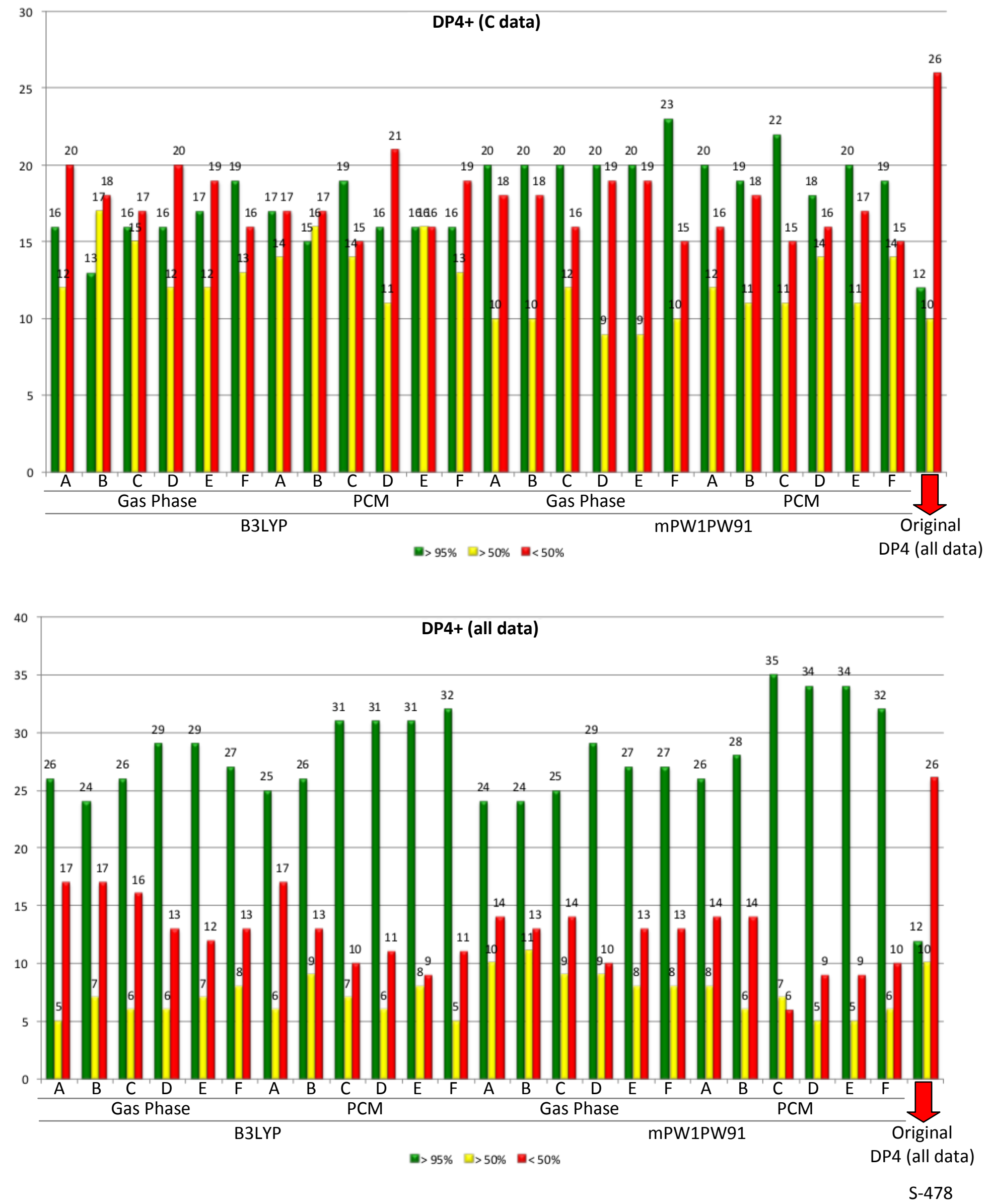